36401

\title{
Global Burden of Disease and Risk Factors
}

Editors

Alan D. Lopez, Colin D. Mathers, Majid Ezzati,

Dean T. Jamison, and Christopher J. L. Murray

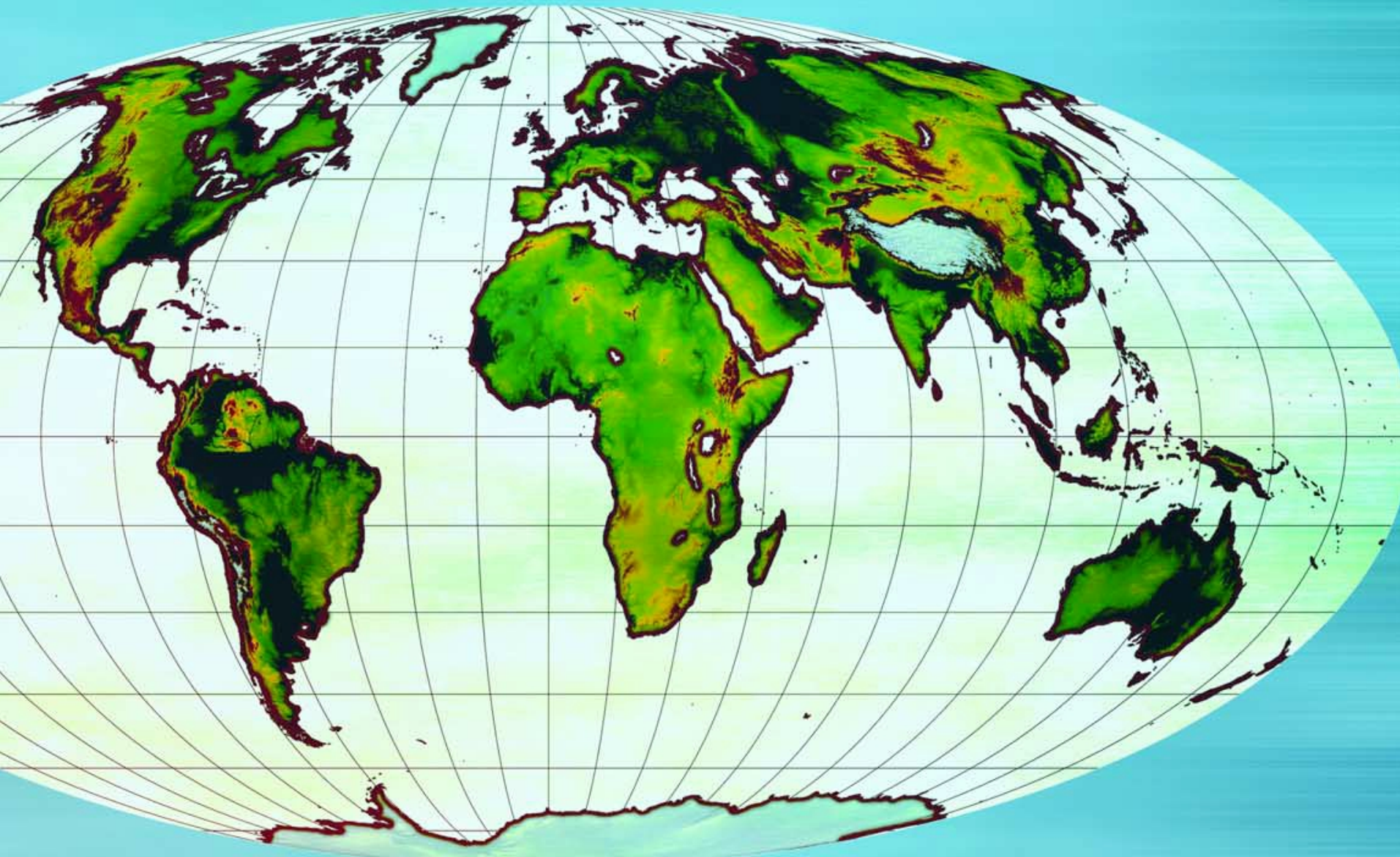




\section{The Developing World by Region}

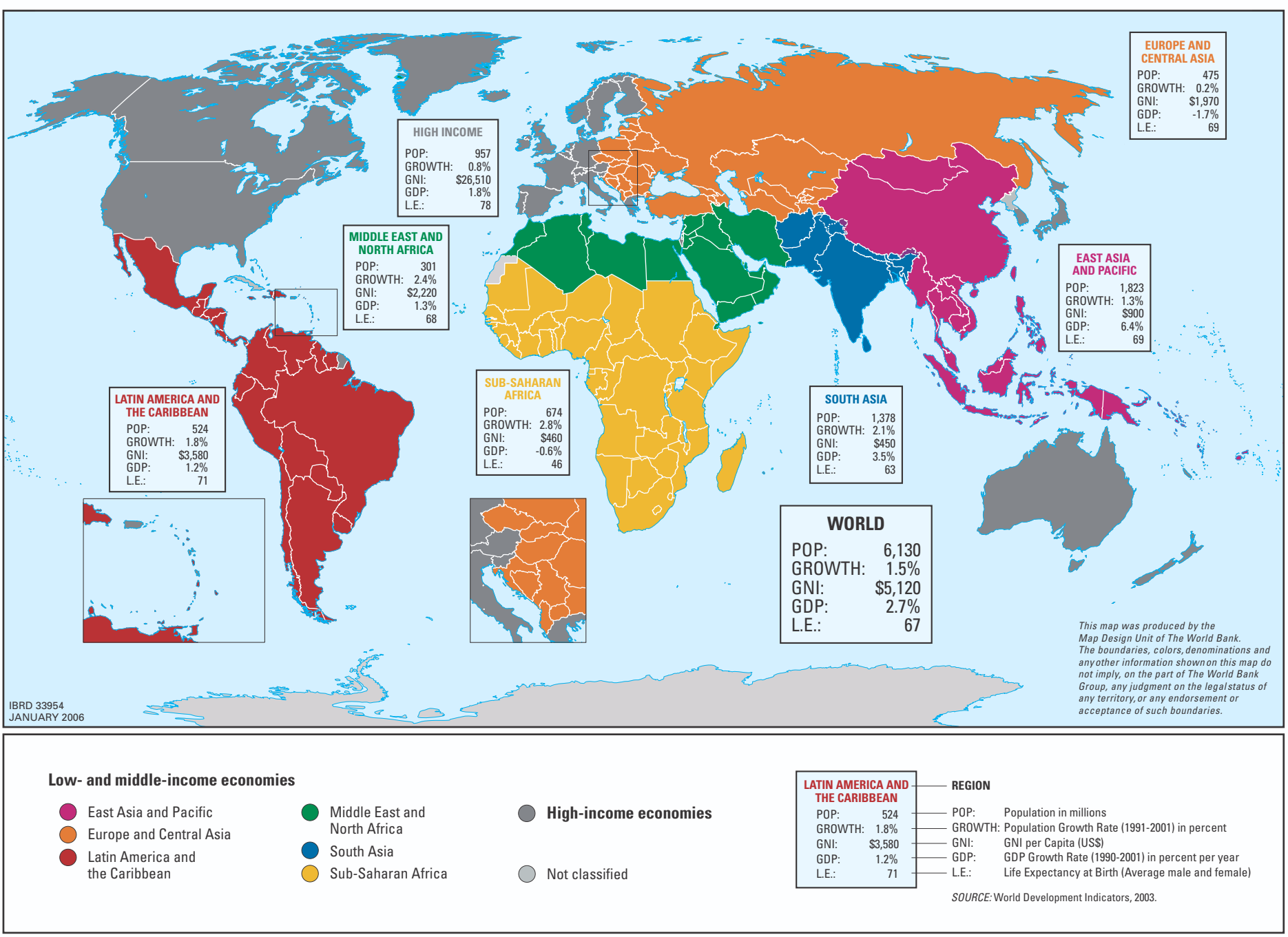




\section{Global Burden of Disease and Risk Factors}





\section{Global Burden \\ of Disease and \\ Risk Factors}

Editors

Alan D. Lopez

Colin D. Mathers

Majid Ezzati

Dean T. Jamison

Christopher J. L. Murray 
(C)2006 The International Bank for Reconstruction and Development / The World Bank

1818 H Street NW

Washington DC 20433

Telephone: 202-473-1000

Internet: www.worldbank.org

E-mail: feedback@worldbank.org

All rights reserved

$\begin{array}{llllllll}1 & 2 & 3 & 4 & 09 & 08 & 07 & 06\end{array}$

A copublication of The World Bank and Oxford University Press.

Oxford University Press

165 Madison Avenue

New York NY 10016

This volume was funded in part by a grant from the Bill \& Melinda Gates Foundation and is a product of the staff of the International Bank for Reconstruction and Development / The World Bank, the World Health Organization, and the Fogarty International Center of the National Institutes of Health. The findings, interpretations, and conclusions expressed in this volume do not necessarily reflect the views of the executive directors of The World Bank or the governments they represent, the World Health Organization, or the Fogarty International Center of the National Institutes of Health.

The World Bank, the World Health Organization, and the Fogarty International Center of the National Institutes of Health do not guarantee the accuracy of the data included in this work. The boundaries, colors, denominations, and other information shown on any map in this work do not imply any judgement on the part of The World Bank, the World Health Organization, or the Fogarty International Center of the National Institutes of Health concerning the legal status of any territory or the endorsement or acceptance of such boundaries.

\section{Rights and Permissions}

The material in this publication is copyrighted. Copying and/or transmitting portions or all of this work without permission may be a violation of applicable law. The International Bank for Reconstruction and Development / The World Bank encourages dissemination of its work and will normally grant permission to reproduce portions of the work promptly.

For permission to photocopy or reprint any part of this work, please send a request with complete information to the Copyright Clearance Center Inc., 222 Rosewood Drive, Danvers, MA 01923, USA; telephone: 978-750-8400; fax: 978-750-4470; Internet: www.copyright.com.

All other queries on rights and licenses, including subsidiary rights, should be addressed to the Office of the Publisher, The World Bank, 1818 H Street NW, Washington, DC 20433, USA; fax: 202-522-2422; e-mail: pubrights@worldbank.org.

ISBN-10: $0-8213-6262-3$

ISBN-13: 978-0-8213-6262-4

eISBN: $\quad 0-8213-6263-1$

DOI: $\quad 10.1596 / 978-0-8213-6262-4$

Library of Congress Cataloguing-in-Publication Data has been applied for. 
This book is dedicated to the memory of Sir Richard Doll, Fellow of the Royal Society (born Hampton, United Kingdom, October 28, 1912; died Oxford, United Kingdom, July 24, 2005). It is entirely fitting that an assessment of world health at the end of the 20th century should be dedicated to the memory of a man whose work did so much to improve it. 



\section{Contents}

Foreword by Samuel H. Preston $\quad$ xv

Preface xvii

Editors $\quad$ xix

Advisory Committee to the Editors $\quad$ xxi

Contributors $\quad$ xxiii

Disease Control Priorities Project Partners $\quad$ xxv

Acknowledgments $\quad$ xxvii

Abbreviations and Acronyms $\quad$ xxix

Chapter 1 Measuring the Global Burden of Disease and Risk Factors, 1990-2001 1

Alan D. Lopez, Colin D. Mathers, Majid Ezzati, Dean T. Jamison, and Christopher J. L. Murray History of Burden of Disease Studies

Applications of Burden of Disease Analysis 4

Improving the Comparative Quantification of Diseases,

Injuries, and Risk Factors: The 2001 GBD Study 5

Major Findings of the 2001 GBD Study $\quad 7$

$\begin{array}{lr}\text { Conclusions } & 10\end{array}$

$\begin{array}{ll}\text { References } & 11\end{array}$

Part I: Global Burden of Disease and Risk Factors 15

Chapter 2 Demographic and Epidemiological Characteristics of

Major Regions, 1990-2001

Alan D. Lopez, Stephen Begg, and Ed Bos

$\begin{array}{lr}\text { Regional Demographic Characteristics } & 18\end{array}$

Changes in Mortality, 1990-2001 21

Trends in Causes of Child Death, 1990-2001 28

Discussion $\quad 32$

$\begin{array}{ll}\text { Conclusions } & 35\end{array}$

Annex 2A: Key Demographic Indicators, by Country/Territory, 1990 and 2001

Acknowledgments $\quad 43$

Notes $\quad 43$

$\begin{array}{ll}\text { References } & 43\end{array}$ 
Chapter 3 The Burden of Disease and Mortality by Condition: Data, Methods, and Results for 2001

Colin D. Mathers, Alan D. Lopez, and Christopher J. L. Murray

Quantifying the Global Burden of Disease

Estimating Deaths by Cause: Methods and Data

Global and Regional Mortality in 2001

Estimating Incidence, Prevalence, and YLD: Methods and Data

Burden of Disability and Poor Health in 2001

Global Burden of Disease in 2001

Discussion and Conclusions

Annex 3A: Definitions, Mortality Data Sources, and Disability Weights

Annex 3B: Deaths by Cause, Sex, Age, and Region, 2001

Annex 3C: DALYs $(3,0)$ by Cause, Sex, Age, and Region, 2001

Acknowledgments

References

Chapter 4 Comparative Quantification of Mortality and Burden of Disease Attributable to Selected Risk Factors

Majid Ezzati, Stephen Vander Hoorn, Alan D. Lopez, Goodarz Danaei, Anthony Rodgers, Colin D. Mathers, and Christopher J. L. Murray

Burden of Disease Attributable to Risk Factors 242

Risk Factor Selection

Burden of Disease Attributable to Individual Risk Factors

Burden of Disease Attributable to Multiple Risk Factors

Directions for Future Research

Discussion

Annex 4A: Population Attributable Fractions, Attributable Deaths, Years of Life Lost Because of Premature Mortality (YLL), and Disability-Adjusted Life Years (DALYs) by Risk Factor, Disease Outcome, Age, Sex, and Region

\section{Part II: Sensitivity Analyses}

Chapter 5 Sensitivity and Uncertainty Analyses for Burden of Disease and Risk Factor Estimates

Colin D. Mathers, Joshua A. Salomon, Majid Ezzati, Stephen Begg, Stephen Vander Hoorn, and Alan D. Lopez

Discounting and Age Weighting in the DALY Measure

Sensitivity of Burden of Disease and Injury Results to Variations in Key Parameter Values

Sensitivity of Risk Factor Estimates to Variations in Key Parameter Values 
Chapter 6 Incorporating Deaths Near the Time of Birth into Estimates of the Global Burden of Disease

Dean T. Jamison, Sonbol A. Shahid-Salles, Julian Jamison, Joy E. Lawn, and Jelka Zupan

Stillbirths and Neonatal Mortality in the Context of the Global Burden of Disease

428

The Burden of Disease Resulting from Events Near the Time of Birth 431

Conclusions

442

Annex 6A: Flexible Functional Forms for the Acquisition of Life Potential Annex 6B: Supplementary Tables

Annex 6C: Causes of Neonatal Mortality: Comparison of Numbers from the Global Burden of Disease with those from the Child Health Epidemiology Reference Group

Acknowledgments

References

462

\section{List of Boxes}

Box $1.1 \quad$ Disability-Adjusted Life Years

\section{List of Figures}

Figure 1.1 Overview of Burden of Disease Framework 2

Figure 2.1 Changes in Population Age Distribution, 1990-2001 22

Figure 2.2 Population Sex Ratios at Different Ages, $2001 \quad 23$

Figure 2.3 UN's versus Authors' Life Table Parameters, $1990 \quad 24$

Figure 2.4 Change in Risk of Death for Children Under Five by Cause (probability of mortality per 1,000 live births), 1990-2001 31

Figure 3.1 Relationship between Health Expectancies and Health Gaps in a Stationary Population

Figure 3.4 CodMod Estimation of Major Cause Group Proportional Mortality for Islamic Republic of Iran, 2001

Figure 3.5 Proportional Distribution of Total Deaths by Broad Cause Group, 2001

Figure 3.6 Death Rates by Broad Cause Group, Region, and Broad Age Group, 2001

Figure 3.7 Leading Causes of Premature Death (YLL) and of Deaths, Worldwide, 2001

Figure 3.8 Disease Model Underlying DisMod

Figure 3.9 Input Prevalences and Incidence Rates Estimated Using DisMod II, for Diabetes Mellitus Cases in Males, Sub-Saharan Africa

Figure 3.10 Life Expectancy, HALE, and Lost Healthy Years by Region and Sex, 2001

Figure 3.11 YLL, YLD, and DALYs by Region, 2001

Figure 3.12 Burden of Disease by Broad Cause Group and Region, 2001

Figure 3.13 Age Distribution of Burden of Disease by Income Group, 2001

Figure 4.1 Mortality and the Burden of Disease Attributable to Leading Global Risk Factors, by World Bank Region

Figure 5.1 Age-Weighting Function Incorporated into the DALY

Figure 5.2 Effect of Age Weighting and Discounting on the YLL per Death at Various Ages for Females 
Figure 5.3 Effect of Age Weighting and Discounting on the Male-Female Gap in YLL per Death

Figure 5.4 Effect on YLL per Death of Varying the Parameter $\beta$ in the DALY Age-Weighting Function

Figure 5.5 Effects of Changing the Discount Rate and Age Weighting on DALYs' Broad Cause and Age Composition, 2001

Figure 5.6 Relationship between the Rank Order of Causes of the Global Burden Using DALYs(3,1) and DALYs(3,0) in 2001

Figure 5.7 Relationship between the Rank Order of Causes of the Global Burden of Disease in 2001, Using Uniform Age Weights and 3 Percent Discounting and No Discounting

Figure 5.8 Effects of Changing the Discount Rate and Age Weighting on Global Rankings for the Top 20 Causes of the Burden of Disease, 2001

Figure 5.9 Effects of Changes in Key DALY Parameters on Proportion of the Global Disease Burden Attributable to Risk Factors

Figure 5.10 Effects of Changes in Key DALY Parameters on Proportion of the Regional Disease Burden Attributable to Risk Factors for Low- and Middle-Income Countries

Figure 5.11 Effects of Changes in Key DALY Parameters on Proportion of the Regional Disease Burden Attributable to Risk Factors for High-Income Countries

Figure 5.12 Uncertainty Ranges for Child and Adult Mortality for WHO Member States, 2001

Figure 5.13 Uncertainty in Average Life Expectancy at Birth, by Sex and DCPP Region, 2001

Figure 5.14 Sensitivity of Uncertainty Ranges to Changes in Between-Country Correlation Assumptions

Figure 5.15 Assumed 95 Percent Uncertainty Ranges for Disability Weights Based on Constant Variance Distribution for Logit of Disability Weight

Figure 5.16 Relative 95 Percent Uncertainty Ranges for Disability Weights Based on the Assumption of a Constant Variance Distribution for Logit of Disability Weight across All Disability Weights

Figure 5.17 Estimated 95 Percent Uncertainty in Total DALYs $(3,0)$ Due to Uncertainty in Estimation of Disability Weights, Top 20 Causes, Low- and Middle-Income Countries

Figure 5.18 PAF Sensitivity to Exposure and Relative Risks

Figure 6.1 Age Distribution of Deaths of Children under Five in Low- and Middle-Income Countries, 2001

Figure 6.2 ALP, Traditional DALYs, and DALYs $(3,0, .54)$

Figure 6.3 Ratio of DALYs Lost at Age 20 to Age 0 as a Function of Age Weighting

Figure 6A.1 Relationship between Time to Complete ALP and Life Potential at Age 0 for Several Values of $A$

Figure 6A.2 Ratio of DALYs Lost at Age 20 to Age 0 as a Function of $A$

\section{List of Tables}

Table 1.1 Deaths and Burden of Disease by Cause-Low- and Middle-Income Countries, High-Income Countries, and World, 2001

Table 1.2 Deaths and Burden of Disease Attributable to Risk Factors-Low- and Middle-Income Countries, High-Income Countries, and World, 2001

Table 2.1

Percentage of Regional Population Covered by Censuses, 
Table 2.2 Population Size and Composition, Fertility, and GNP, by

World Bank Region, 1990 and 2001

Table 2.3 Selected Mortality Characteristics by Sex and World Bank Region, 1990 and 2001

26

$\begin{array}{lll}\text { Table 2.4 } & \text { Mortality in Children Under Five by Cause, } 1990 \text { and } 2001 & 29 \\ \text { Annex 2A } & \text { Key Demographic Indicators, by Country/Territory, } 1990 \text { and } 2001 & 36\end{array}$

Table 3.1 Availability of Data for Estimation of All-Cause Mortality Rates by Age and Sex

36

52

Table 3.2 Availability of Data for Estimation of Causes of Death by Age and Sex

55

Table 3.3 Distribution of Percentage of Total Deaths Assigned to Ill-Defined

Codes for 105 WHO Member States, Most Recent Available Year 57

Table 3.4 Correction Factors Giving Proportion of Ill-Defined CVD

Deaths to Be Reassigned to IHD, by Age and Sex 58

Table 3.5 Numbers of Data Sets Contributing to Epidemiologically Based Estimates of Deaths Due to Specific Causes

Table 3.6 The 10 Leading Causes of Death, by Broad Income Group, 2001

Table 3.7 The 10 Leading Causes of Death, by Sex, in Low- and Middle-Income Countries, 2001

Table 3.8 The 10 Leading Causes of Death in Children Ages 0-14, by Broad Income Group, 2001

Regional Reporting Categories for the Disease Control Priorities Project

GBD Cause Categories and ICD Codes

100 Age, and Sex

107

108

(Except Cancers and Injuries)

Table 3A.7 Disability Weights for Malignant Neoplasms and

Their Long-Term Sequelae

124

125

Table 3A.8 Disability Weights for Injuries

126

Countries, 2001

Table 3B.2 Deaths by Cause, Sex, and Age in the East Asia and Pacific Region, 2001

Table 3B.3 Deaths by Cause, Sex, and Age in the Europe and Central Asia Region, 2001

Table 3B.4 Deaths by Cause, Sex, and Age in the Latin America and the Caribbean Region, 2001 
Table 3B.5 Deaths by Cause, Sex, and Age in the Middle East and North Africa Region, 2001

Table 3B.6 Deaths by Cause, Sex, and Age in the South Asia Region, 2001

Table 3B.7 Deaths by Cause, Sex, and Age in the Sub-Saharan Africa Region, 2001

Table 3B.8 Deaths by Cause, Sex, and Age in High-Income Countries, 2001

Table 3B.9 Deaths by Cause, Sex, and Age in the World, 2001

Table 3C.1 DALYs $(3,0)$ by Cause, Sex, and Age in Low- and Middle-Income Countries, 2001

Table 3C.2 DALYs $(3,0)$ by Cause, Sex, and Age in the East Asia and Pacific Region, 2001

Table 3C.3 DALYs $(3,0)$ by Cause, Sex, and Age in the Europe and Central Asia Region, 2001

Table 3C.5 DALYs $(3,0)$ by Cause, Sex, and Age in the Middle East and North Africa Region, 2001

Table 3C.6 DALYs $(3,0)$ by Cause, Sex, and Age in the South Asia Region, 2001

Table 3C.7 DALYs $(3,0)$ by Cause, Sex, and Age in the Sub-Saharan Africa Region, 2001

Table 3C.9 DALYs $(3,0)$ by Cause, Sex, and Age in the World, 2001

Table 4.1 CRA Risk Factors, Exposure Variables, Theoretical-Minimum-Risk Exposure Distributions, and Disease Outcomes

Table 4.2 Distribution of Risk Factor-Attributable Mortality and Burden of Disease, by Age and Sex

Table 4.3 Joint Contributions (PAFs) of the Leading Risk Factors to Mortality and Burden of Disease, by Region

Table 4.4 Individual and Joint Contributions of Risk Factors to 10 Leading Diseases and Total Burden of Disease

Table 4.5 Individual and Joint Contributions of Risk Factors to Mortality and Burden of Disease from Site-Specific Cancers

Table 4.6 Individual and Joint Contributions of Risk Factors to Mortality and Burden of Disease from Cardiovascular Diseases

Table 4.7 Individual and Joint Contributions of Risk Factors to Mortality and Burden of Disease from Major Diseases of Children

Annex 4A Population Attributable Fractions, Attributable Deaths, Years of Life Lost Because of Premature Mortality (YLL), and Disability-Adjusted Life Years (DALYs) by Risk Factor, Disease Outcome, Age, Sex, and Region

Table 5.1 Standard Life Expectancies at Selected Exact Ages and Discounted YLL Due to a Death at Selected Ages

Table 5.2 Implications of Variation in Choice of Age-Weight Parameter $\beta$ on the Age-Weighting Function

Table 5.3 Comparison of the Effects of Changing the Discount Rate $(r)$ and the Age-Weighting Factor $(K)$ on the Composition of DALYs $(r, K), 2001$

Table 5.4 Effects of Changing the Discount Rate $(r)$ and the Age-Weighting Factor $(K)$ on the Second-Level Cause Group Composition of DALYs $(r, K), 2001$ (percentages of total DALYs)

Table 5.5 Estimated Total Deaths and 95 Percent Uncertainty Ranges for Selected Causes, by Region, 2001

Table 5.6 Estimated 95 Percent Uncertainty Ranges for YLD and DALYs Arising from Uncertainty in Disability Weights for Selected Causes for Low- and Middle-Income Countries, 2001

Table 5.7 Estimated 95 Percent Uncertainty Ranges Arising from Uncertainty in Disability Weights for the Top 40 Causes of the Burden of Disease in Low- and Middle-Income Countries, 2001 
Table 6.1 Population Totals and Numbers of Births, $2001 \quad 429$

Table 6.2 Age Distribution of Deaths under Age 5, 2001

Table 6.3 Estimated Death Rates under Age 5, by Country Income Level, 2001

Table 6.4 Deaths by Age and Cause, 2001

Table 6.5 Values of Selected ALP Functions $\quad 439$

Table 6.6 Discounted YLL at Different Ages of Death for Several DALY Formulations 440

Table 6.7 Disease Burden at Different Ages Using Different Measures,

Low- and Middle-Income Countries, 2001

Table 6.8 Disease Burden from Selected Groups of Causes Using Different Measures, Low- and Middle-Income Countries, 2001

Table 6B.1 Deaths (Excluding Stillbirths) from Selected Causes, by Age, 2001

Table 6B.2 YLL(3,0) from Selected Causes, by Age, 2001

Table 6B.3 YLD from Selected Causes, by Age, 2001 447

Table 6B.4 The Burden of Disease-DALYs(3,0) from Selected Causes, by Age, 2001 (Excluding Stillbirths)

Table 6B.5 $\operatorname{YLL}_{\mathrm{SB}}(3,0,1)$ Calculated to Include Stillbirths (Valued the Same as Newborn Deaths)

Table 6B.6 The Burden of Disease-DALYs $\mathrm{SB}_{\mathrm{SB}}(3,0,1)$. Calculated to Include Stillbirths (Valued the Same as Newborn Deaths)

Table 6B.7 $\mathrm{YLL}_{\mathrm{SB}}(3,0, .54)$ Calculated to Include Stillbirths and Gradual ALP

Table 6C.1 Causes of Neonatal Mortality, Worldwide in 2001 



\section{Foreword}

"Every observer of human misery among the poor reports that disease plays the leading role.” Irving Fisher (1909, p. 124) ${ }^{1}$

Before 1990, the global disease landscape was perceived "through a glass darkly." Mortality conditions by cause of death were known with some precision only for the relatively small minority of the world's population residing in countries with adequate vital statistics. Nowhere were estimates of disease incidence, prevalence, survival, and disabling sequelae consistently combined into population-level profiles of morbidity and mortality.

Publication of the Global Burden of Disease (1990) was a watershed event in the assessment of health and disease. Through careful synthesis of disease conditions revealed in thousands of piecemeal studies and data systems, it constructed a comprehensive portrait of diseases, injuries, and causes of death. It dealt creatively and carefully with the hundreds of issues that had to be addressed to develop useful, broadly gauged indicators of health. These included establishing terms of trade among disabling conditions, among age groups and generations, and between the living and the dead. At all points that offered tempting shortcuts, the authors decided in favor of comprehensiveness.

Like the microscope, the Global Burden of Disease (1990) brought diseases into much sharper focus. Like national income accounts, it connected parts to a whole and measured the whole with unprecedented precision. As a sophisticated measuring device, it could not be ignored by any serious student of epidemiology or development. One might have experimented with its calibrations, but the device itself was irreplaceable.

However, the value of a measuring device lies in its measurements, not in its abstract qualities on the shelf. The world has changed dramatically since 1990, and we must be grateful for the fresh assessment of disease conditions presented in this volume. The picture that it paints is not only updated; it is also more precise. Better data have become available through expanded vital statistics systems, improved surveys, and more extensive population surveillance systems. The measurement instrument has also been improved. Most notably, a critical new layer of physical risk factors and their distribution has been added, providing valuable new tools for policy makers.

This second application of the global burden of disease framework permits an analysis of trends observed since the first application. The intervening period was clearly one of slow progress, impeded by the HIV/AIDS epidemic and setbacks in Eastern Europe. The volume is appropriately cautious in drawing inferences about disease-specific trends because of changes in data sources and, in some instances, improvements in approaches to measurement.

The volume also contains a valuable and admirably frank chapter on the sensitivity of estimates to various sources of uncertainty in methods and data. Some estimates are found to have wide bands of uncertainty. While this outcome is disappointing, uncertainty about the burden of disease in all its dimensions-including the degree of uncertainty itselfwould be much greater without the heroic efforts reflected in this volume.

My congratulations to the authors and the sponsoring agencies.

\section{Samuel H. Preston, Fredrick J. Warren Professor of} Demography, University of Pennsylvania 



\section{Preface}

This book emerges from two separate, but intersecting, strands of work that began in the late 1980s, when the World Bank initiated a review of priorities for the control of specific diseases. The review generated findings about the comparative costeffectiveness of interventions for most diseases important in developing countries. The purpose of the cost-effectiveness analysis (CEA) was to inform decision making within the health sectors of highly resource-constrained countries. This process resulted in the publication of the first edition of Disease Control Priorities in Developing Countries (Jamison and others 1993). Also important for informing policy is a consistent, quantitative assessment of the relative magnitudes of diseases, injuries, and their risk factors. The first edition of Disease Control Priorities in Developing Countries included an initial assessment of health status for low- and middle-income countries as measured by deaths from specific causes; importantly, the numbers of cause-specific deaths for each age-sex group were constrained by the total number of deaths as estimated by demographers. This consistency constraint led to downward revision of the estimates of deaths from many diseases.

These two strands of work-CEA and burden of diseasewere further developed during preparation of the World Development Report 1993: Investing in Health (World Bank 1993). This report drew on both the CEA work in the first edition of Disease Control Priorities in Developing Countries and on a growing academic literature on CEA. In addition, the World Bank invested in generating improved estimates of deaths and the disease burden by age, cause, and region for 1990. Results of this initial assessment of the global burden of disease appeared both in the World Development Report 1993 and widely in the academic literature (see, for example, Murray and Lopez 1996a, 1996b; Murray, Lopez, and Jamison 1994). Over the past six years, the World Health Organization has undertaken a new assessment of the global burden of disease for 2000-2, with consecutive revisions and updates published annually in its World Health Reports. The World Health Organization has also invested in improving the conceptual, methodological, and empirical basis of burden of disease assessments and the assessment of the disease and injury burden from major risk factors (Ezzati and others 2004; Murray and others 2002; World Health Organization 2002).

In 2002, a number of organizations-the Fogarty International Center of the U.S. National Institutes of Health, the World Bank, the World Health Organization, and the Bill \& Melinda Gates Foundation-initiated the Disease Control Priorities Project (DCPP), located at the Fogarty International Center. The DCPP's purpose has been to review, generate, and disseminate information that contributes to the scientific evidence base for improving population health in developing countries. A major product is the second edition of Disease Control Priorities in Developing Countries (DCP2) (Jamison and others 2006), which updates and extends available CEA relevant to developing countries and explores the institutional, organizational, financial, and research capabilities essential for health systems to be able to select and deliver the appropriate interventions.

DCP2 was to have included two major chapters on burden, one dealing with deaths and the disease burden by cause and the other with the burden from major risk factors. Two points quickly became clear. First, even though DCP2 had allocated substantial space for these chapters, much valuable background, methodology, and results still had to be relegated to a separate document on the Web. Second, this material would generate substantial interest independently of its tie to DCP2, because health system activities, including the choice of interventions, depend partly on the magnitude of health problems, and because assessment of the burden of diseases, injuries, and risk factors includes important methodological and empirical dimensions. The sponsors of the DCPP therefore decided to publish this volume, which includes a full account of methods, the complete results of recent work, and an assessment of trends for total mortality and for major causes of death among children under five along with two chapters that cover sensitivity and uncertainty analyses in relation to a broad range of potentially important parameters.

During 1999-2004, the authors of this volume and many collaborators from around the world worked intensively to assemble an updated, comprehensive assessment of the global 
burden of disease and its causes. This book provides the definitive, scientific account of that effort and of the health conditions of the world's population at the beginning of the 21 st century.

Both DCP2 and this book are available on the DCPP Web site (http://www.dcp2.org), as well as through the National Library of Medicine's PubMedCentral. From the DCPP Web site, users can download individual chapters or create an ad hoc group of chapters formatted for printing booklets or course packets. We encourage users to construct variants of the book most suited to their work or their teaching. The DCPP Web site also allows access to Excel versions of all global burden of disease tables so that users can freely reanalyze the data to meet their own needs.

\section{REFERENCES}

Ezzati, M., A. D. Lopez, A. Rodgers, and C. J. L. Murray. 2004. Comparative Quantification of Health Risks: The Global and Regional Burden of Disease Attributable to Selected Major Risk Factors. Geneva: World Health Organization.
Jamison, D. T., J. G. Breman, A. R. Measham, G. Alleyne, M. Claeson, D. B. Evans, P. Jha, A. Mills, and P. Musgrove, eds. 2006. Disease Control Priorities in Developing Countries, 2nd ed. New York: Oxford University Press.

Jamison, D. T., W. H. Mosley, A. R. Measham and J. L. Bobadilla, eds. 1993. Disease Control Priorities in Developing Countries. New York: Oxford University Press.

Murray, C. J. L., and A. D. Lopez, eds. 1996a. The Global Burden of Disease. Cambridge, MA: Harvard University Press.

- 1996b. Global Health Statistics: A Compendium of Incidence. Prevalence, and Mortality Estimates for over 200 Conditions Cambridge, MA: Harvard University Press.

Murray, C. J. L., A. D. Lopez, and D. T. Jamison. 1994. The Global Burden of Disease in 1990: Summary Results, Sensitivity Analysis, and Future Directions." In Global Comparative Assessments in the Health Sector: Disease Burden, Expenditures, and Intervention Packages, eds. C. J. L. Murray and A. D. Lopez, 97-138. Geneva: World Health Organization.

Murray, C. J. L, J. A. Salomon, C. D. Mathers, and A. D. Lopez. 2002. Summary Measures of Population Health: Concepts, Ethics, Measurement, and Applications. Geneva: World Health Organization.

World Bank. 1993. World Development Report 1993: Investing in Health. New York: Oxford University Press.

World Health Organization. 2002. Reducing Risks: Promoting Healthy Life. World Health Report 2002. Geneva: World Health Organization. 


\section{Editors}

Alan D. Lopez is professor of medical statistics and population health and Head of the School of Population Health at the University of Queensland, Australia. Prior to joining the university in January 2003, he worked for 22 years at the World Health Organization in Geneva, where he held a series of technical and senior managerial posts, including chief epidemiologist in the Tobacco Control Program (1992-5), manager of the Program on Substance Abuse (1996-8), director of the Epidemiology and Burden of Disease Unit (1999-2001), and senior science adviser to the director-general (2002).

Professor Lopez has published widely on mortality analysis and causes of death, including the impact of the global tobacco epidemic, and on the global descriptive epidemiology of major diseases, injuries, and risk factors. He is the coauthor of the seminal Global Burden of Disease Study (1996), which has greatly influenced debates about priority setting and resource allocation in health. He has been awarded major research grants in epidemiology, health services research, and population health; chairs the Health and Medical Research Council of Queensland; and is a member of Australia's Medical Services Advisory Committee.

Professor Lopez graduated with an honors degree in mathematics from the University of Western Australia in 1973 and a master of science degree in statistics from Purdue University in the United States. He was awarded a Ph.D. in medical demography from the Australian National University in 1979. His principal research interests are analysis of mortality data; burden of disease methods and applications; and quantification of the health effects of tobacco, particularly in developing countries. He has collaborated extensively with leading researchers throughout the world on these issues, particularly at Harvard and Oxford universities, and he holds an adjunct appointment at Harvard University as professor of population and international health.

Colin D. Mathers is a senior scientist in the Evidence and Information for Policy Cluster at the World Health Organization in Geneva. From 2002 to 2005, he managed the World Health Organization's Epidemiology and Burden of
Disease Unit. Prior to joining the World Health Organization in 2000, he worked for the Australian Institute of Health and Welfare for 13 years in technical and senior managerial posts.

Dr. Mathers has published widely on population health and mortality analysis; on inequalities in health, health expectancies, and burden of disease; and on health system costs and performance. He developed the first set of Australian health accounts mapping health expenditures by age, sex, and disease and injury causes (1998) and carried out an influential national burden of disease and risk factors study (1999). At the World Health Organization, he played a key role in the development of comparable estimates of healthy life expectancy for 192 countries, in the reassessment of the global burden of disease for the years 2000-2, and in the development of software tools to support burden of disease analysis at the country level. He recently completed new projections of global, regional, and country mortality and burden of disease from 2002 to 2030.

Dr. Mathers graduated with an honors degree and university medal in physics from the University of Sydney in 1975 and was awarded a Ph.D. in theoretical physics from the University of Sydney in 1979. His principal research interests are the measurement and reporting of population health and its determinants, burden of disease methods and applications, measurement of health state prevalences, and cross-population comparability. He has collaborated with leading researchers throughout the world on issues relating to the development and applications of summary measures of population health.

Majid Ezzati is an assistant professor of international health at the Harvard School of Public Health. He holds bachelor's and master's degrees in engineering from McMaster and McGill Universities and a Ph.D. in science, technology, and environmental policy from Princeton University. Dr. Ezzati's research interests center around understanding the causal determinants of health and disease, especially as they change in the process of social and economic development and as a result of technological innovation and technology management. 
His current research focuses on two main areas. The first area is the relationship among energy, air pollution, and health in developing countries, on which he conducts field research projects in Asia and sub-Saharan Africa. This research has led to the identification and design of technological interventions for reducing exposure to indoor air pollution from household energy use. His second area of research is major health risk factors and their role in the current and future disease burden globally and in specific countries and regions. His research on risk factors focuses on environmental risks, smoking, and nutritional risks. He was the lead scientist for the World Health Organization's Comparative Risk Assessment Project, which was reported in the World Health Report 2002: Reducing Health, Promoting Healthy Life. He is currently studying the role of major risk factors in health inequalities.

Dean T. Jamison is a professor of health economics in the School of Medicine at the University of California, San Francisco (UCSF), and an affiliate of UCSF Global Health Sciences. Dr. Jamison concurrently serves as an Adjunct Professor in both the Peking University Guanghua School of Management and in the University of Queensland School of Population Health.

Before joining UCSF, Dr. Jamison was on the faculty of the University of California, Los Angeles, and also spent a number of years at the World Bank, where he was a senior economist in the research department, division chief for education policy, and division chief for population, health, and nutrition. In 1992-93 he temporarily rejoined the World Bank to serve as Director of the World Development Report Office and as lead author for the Bank's 1993 World Development Report: Investing in Health. His publications are in the areas of economic theory, public health and education. Dr. Jamison studied at Stanford (B.A., Philosophy; M.S., Engineering Sciences) and at Harvard (Ph.D., Economics, under K.J. Arrow). In 1994 he was elected to membership in the Institute of Medicine of the U.S. National Academy of Sciences.
Christopher J. L. Murray is the Richard Saltonstall professor of public policy, professor of social medicine, and director of the Harvard Initiative for Global Health. Prior to his return to the university, for five years he led the World Health Organization's Evidence and Information for Policy Cluster, which was dedicated to building the evidence base and fostering a culture of evidence to inform health decision making. The cluster was responsible for work on epidemiology and the burden of disease, the World Health Survey, cost-effectiveness analysis, national health accounts, catastrophic health spending, responsiveness, health financing policy, human resources for health systems, coverage of health interventions, quality of care and patient safety, stewardship of health systems, assessment of health system performance, health research policy, and a range of efforts to manage and disseminate information through print and the Web.

A physician and health economist, Dr. Murray's early work focused on tuberculosis control and the development with Alan D. Lopez of global burden of disease methods and applications. During the course of this work, they developed a new metric for comparing deaths and disabilities caused by various diseases and the contribution of risk factors to the overall burden of disease in developing and developed countries. This pioneering effort has been hailed as a major landmark in public health and an important foundation for policy formulation and priority setting. Recently, Dr. Murray has contributed to the development of a range of new methods and empirical studies for strengthening the basis for population health measurement and cost-effectiveness analysis. A main thrust of his work has been the conceptualization, measurement, and application of approaches to understanding the inputs, organization, outputs, and outcomes of health systems. He has authored or edited eight books, many book chapters, and more than 90 journal articles in internationally peer-reviewed publications.

Dr. Murray holds a B.A. from Harvard College, a D. Phil. from Oxford University, and an M.D. from Harvard Medical School. 


\section{Advisory Committee to the Editors}

\section{J. R. Aluoch}

Professor, Nairobi Women's Hospital, Nairobi, Kenya

\section{Jacques Baudouy}

Director, Health, Nutrition, and Population, World Bank, Washington, DC, United States

\section{Fred Binka}

Executive Director, INDEPTH Network, Accra, Ghana

\section{Mayra Buvinić}

Director, Gender and Development, World Bank, Washington,

DC, United States

\section{David Challoner, Co-chair}

Foreign Secretary, Institute of Medicine, U.S. National Academies, Gainesville, Florida, United States

\section{Guy de Thé, Co-chair}

Research Director and Professor Emeritus, Institut Pasteur, Paris, France

\section{Timothy Evans}

Assistant Director General, Evidence and Information for Policy, World Health Organization, Geneva, Switzerland

\section{Richard Horton}

Editor, The Lancet, London, United Kingdom

\section{Sharon Hrynkow}

Acting Director, Fogarty International Center, National Institutes of Health, Bethesda, Maryland, United States

\section{Gerald Keusch}

Provost and Dean for Global Health, Boston University School of Public Health, Boston, Massachusetts, United States

\section{Kiyoshi Kurokawa}

President, Science Council of Japan, Kanawaga, Japan

\section{Peter Lachmann}

Past President, U.K. Academy of Medical Sciences, Cambridge, United Kingdom

\section{Mary Ann Lansang}

Executive Director, INCLEN Trust International, Inc., Manila, Philippines

\section{Christopher Lovelace}

Director, Kyrgyz Republic Country Office and Central Asia Human Development, World Bank, Bishkek, Kyrgyz Republic

\section{Anthony Mbewu}

Executive Director, Medical Research Council of South Africa, Tygerberg, South Africa

\section{Rajiv Misra}

Former Secretary of Health, Government of India, Haryana, India

\section{Perla Santos Ocampo}

President, National Academy of Science and Technology, San Juan, Philippines

\section{G. B. A. Okelo}

Secretary General and Executive Director, African Academy of Sciences, Nairobi, Kenya

\section{Sevket Ruacan}

General Director, MESA Hospital, Ankara, Turkey

\section{Pramilla Senanayake}

Chairman, Foundation Council of the Global Forum for Health Research, Colombo, Sri Lanka

\section{Jaime Sepúlveda, Chair}

Director, National Institutes of Health of Mexico, Mexico City, Mexico

\section{Chitr Sitthi-amorn}

Director, Institute of Health Research, Dean, Chulalongkorn University, College of Public Health, Bangkok, Thailand

\section{Sally Stansfield}

Associate Director, Global Health Strategies, Bill \& Melinda

Gates Foundation, Seattle, Washington, United States 
Misael Uribe

President, National Academy of Medicine of Mexico, Mexico City, Mexico

\section{Zhengguo Wang}

Professor, Chinese Academy of Engineering, Daping, China
Witold Zatonski

Professor, Health Promotion Foundation, Warsaw, Poland 


\section{Contributors}

Stephen J. Begg

University of Queensland

Eduard R. Bos

World Bank

Goodarz Danaei

Harvard School of Public Health; Harvard University

Initiative for Global Health

\section{Majid Ezzati}

Harvard School of Public Health; Harvard University

Initiative for Global Health

\section{Dean T. Jamison}

University of California, San Francisco; Disease Control

Priorities Project

\section{Julian Jamison}

University of California, Berkeley

Joy E. Lawn

Save the Children-USA, Institute of Child Health, London

\section{Alan D. Lopez}

University of Queensland; Harvard School of Public Health

\section{Colin D. Mathers}

World Health Organization

\section{Christopher J. L. Murray}

Harvard University Initiative for Global Health; Harvard School of Public Health

Anthony Rodgers

University of Auckland

Joshua Salomon

Harvard School of Public Health

\section{Sonbol A. Shahid-Salles}

Population Reference Bureau; Disease Control Priorities Project

\author{
Stephen Robert Vander Hoorn \\ University of Auckland \\ Jelka Zupan \\ World Health Organization
}





\section{Disease Control Priorities Project Partners}

The Disease Control Priorities Project is a joint enterprise of the Fogarty International Center of the National Institutes of Health, the World Health Organization, the World Bank, and the Population Reference Bureau.

The Fogarty International Center is the international component of the National Institutes of Health. It addresses global health challenges through innovative and collaborative research and training programs and supports and advances the mission of the National Institutes of Health through international partnerships.

The World Health Organization is the United Nations' specialized agency for health. Its objective, as set out in its constitution, is the attainment by all peoples of the highest possible level of health, with health defined as a state of complete physical, mental, and social well-being and not merely the absence of disease or infirmity.

The World Bank Group is one of the world's largest sources of development assistance. The Bank, which provides US $\$ 18$ billion to $\$ 22$ billion each year in loans to its client countries, provided $\$ 1.27$ billion for health, nutrition, and population in 2004. The World Bank is working in more than 100 developing economies, bringing a mix of analytical work, policy dialogue, and lending to improve living standards—including health and education - and reduce poverty.

The Population Reference Bureau informs people around the world about health, population, and the environment and empowers them to use that information to advance the wellbeing of current and future generations. For 75 years, the bureau has analyzed complex data and research results to provide objective and timely information in a format easily understood by advocates, journalists, and decision makers; conducted workshops around the world to give key audiences the tools they need to understand and communicate effectively about relevant issues; and worked to ensure that developing country policy makers base policy decisions on sound evidence. 



\section{Acknowledgments}

This volume brings together results of the work of many institutions and individuals spanning a period of more than 15 years. Several contributions to this process should be acknowledged. The Disease Control Priorities Project (DCPP) provided sponsorship for the specific work leading to this volume and for its publication. The DCPP itself resulted from collaboration among the following four institutions and benefited from the contributions of those institutions and the efforts of the responsible individuals within them:

- The Fogarty International Center of the U.S. National Institutes of Health. The FIC supported both the senior editor and one of the co-managing editors of this project, as well as support staff. FIC also provided office space for the secretariat and other administrative support. Gerald Keusch, former director of the FIC, initiated and facilitated this effort, and FIC's acting director, Sharon Hrynkow, continued to provide support and counsel.

- The World Bank. Successive directors of the World Bank's Health, Nutrition, and Population Department, Christopher Lovelace and Jacques Baudouy, provided support, guidance, and critical reactions and facilitated the involvement of Bank staff as coauthors and reviewers.

- The World Health Organization. Successive leaders of the Evidence and Information for Policy Cluster, Christopher Murray and Timothy Evans, coordinated the involvement of the World Health Organization. For much of the past eight years, the Evidence and Information for Policy Cluster has sponsored research and analysis central to this volume and we are particularly grateful for that support.

- The Bill \& Melinda Gates Foundation. Richard Klausner, Sally Stansfield, and Beth Peterman arranged for the foundation to provide major financial support to the DCPP.

The DCPP was guided by a group of editors for its publication of the second edition of Disease Control Priorities in Developing Countries. The editors of this volume wish to thank the following for their inclusion of this book within the DCPP effort: George A. O. Alleyne, Joel G. Breman, Mariam Claeson, David B. Evans, Prabhat Jha, Anne Mills, Philip Musgrove, and, in particular, Anthony R. Measham.

We are grateful to the National Institute on Aging of the U.S. National Institutes of Health, which provided grant support for much of the research reported in this book under PO1-AG17625. Richard Suzman of the National Institute on Aging provided invaluable support and critical reactions.

We benefited from the strong collaboration with the InterAcademy Medical Panel (IAMP), an association of the medical academies or medical divisions of the scientific academies of 44 countries. David Challoner and Guy de Thé co-chaired the Steering Committee of the IAMP and invested much time and effort into facilitating the collaboration. The IAMP sponsored the peer review process for the chapters in this volume. The IAMP's second global meeting in Beijing in April 2006 included the launch of this volume, which was hosted by the Chinese Academies of Engineering and Science. The Institute of Medicine of the U.S. National Academy of Sciences, the U.S. member of the IAMP, played a critical role in facilitating all aspects of the IAMP's collaboration. Patrick Kelley and Dianne Stare of the Institute of Medicine managed this effort and provided critical, substantive inputs.

The Office of the Publisher at the World Bank provided outstanding assistance, enthusiastic advice, and support during every phase of production of this volume and helped coordinate publicity and initial distribution. We particularly wish to thank Dirk H. Koehler, the publisher, and Carlos Rossel, Mary Fisk, Randi Park, Santiago Pombo-Bejarano, Nancy Lammers, Alice Faintich, Nita Congress, Valentina Kalk, and Andrés Meneses for their timely, high-quality professionalism.

Donald Lindberg, director, and Julia Royall, chief, International Programs, of the National Library of Medicine of the U.S. National Institutes of Health, graciously offered the competent services of the Information Engineering Branch of the National Center for Biotechnology Information to convert 
the text into an electronic product available to all visitors to the National Library of Medicine's PubMed Web site. We would like to extend our gratitude to the National Center for Biotechnology Information team members-David Lipman, Jo McEntyre, Mohammad Al-Ubaydli, and Belinda Beckfor their technical expertise and commitment.

The Harvard Initiative for Global Health and the University of Queensland School of Population Health assisted with the production of chapters and hosted meetings in support of this effort, and we are grateful for their contributions and hospitality. We would like to thank Teri McGuane at Harvard University and Trish Sharkey and Kim Wicks at the University of Queensland for their valuable assistance.
Finally, we would like to acknowledge the critical role that Sonbol A. Shahid-Salles played in the creation of this book. She participated in all the meetings of the editors; she coordinated the peer review process, reacted critically to chapter content and presentation, and reviewed drafts and proofs; she interacted with the World Bank's Office of the Publisher on all aspects of production; and she kept the effort on its extremely tight time line in an environment where editors and authors were dispersed worldwide. The book simply could not have been completed without her efforts and we are very grateful indeed.

The Editors 


\section{Abbreviations and Acronyms}

$\begin{array}{llll}\text { ALP } & \text { acquisition of life potential } & \text { HALE } & \text { health-adjusted life expectancy } \\ \text { CHERG } & \text { Child Health Epidemiology Reference Group } & \text { ICD } & \text { international classification of diseases } \\ \text { CRA } & \text { comparative risk assessment } & \text { IHD } & \text { ischemic heart disease } \\ \text { CVD } & \text { cardiovascular disease } & \text { PAF } & \text { population attributable fraction } \\ \text { DALY } & \text { disability-adjusted life year } & \text { TB } & \text { tuberculosis } \\ \text { DCP2 } & \text { Disease Control Priorities in Developing } & \text { UN } & \text { United Nations } \\ & \text { Countries, second edition } & \text { WHO } & \text { World Health Organization } \\ \text { DCPP } & \text { Disease Control Priorities Project } & \text { YLD } & \text { years of life lost due to disability } \\ \text { GBD } & \text { global burden of disease } & \text { YLL } & \text { years of life lost due to premature mortality } \\ \text { GDP } & \text { gross domestic product } & & \end{array}$

All dollar amounts are U.S. dollars unless otherwise indicated. 



\section{Chapter 1}

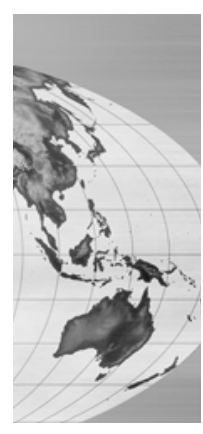

In an era when most societies must cope with increasing demand for health resources, they will inevitably have to make choices about the provision of health services, even if those choices are, by default, to continue current practices. Strategic health planning can accelerate health development and the attainment of health goals or reduce the cost of reaching such goals. Such planning must take into account the needs that the health system must address; that is, policy makers must be aware of the comparative burden of diseases and injuries and the risk factors that cause them, and how this burden is likely to change with the adoption of various policies and interventions. Needs are, of course, not the only factors determining service provision, but should be a critical component of the decision-making and planning processes.

The issue then becomes how to assess the comparative importance of risks to health and their outcomes in different demographic groups of the population. What is needed is a framework for integrating, validating, analyzing, and disseminating the fragmentary, and at times contradictory, information that is available on a population's health, along with some understanding of how that population's health is

\section{Measuring the Global Burden of Disease and Risk Factors, 1990-2001}

Alan D. Lopez, Colin D. Mathers, Majid Ezzati, Dean T. Jamison, and Christopher J. L. Murray

changing, so that the information is more relevant for health policy and planning purposes. The Global Burden of Disease (GBD) framework is the principal, if not the only, attempt to do so. Features of the GBD framework include the incorporation of data on nonfatal health outcomes into summary measures of population health, the development of methods for assessing the reliability of data and imputing missing data, and the use of a common metric to summarize the disease burden from diagnostic categories of the International Classification of Diseases and the major risk factors that cause those health outcomes. Figure 1.1 presents a simplified version of this framework and indicates the causal chain of events that matter for health outcomes, identifying the key components and determinants of health status that require quantification.

Many countries and health development agencies have adopted the GBD approach as the standard for health accounting and for guiding the determination of health research priorities, for example, Australia (Mathers, Vos, and Stevenson 1999); the state of Andra Pradesh, India (Mahapatra 2002); Mauritius (Vos and others 1995); Mexico (Lozano and others 1995); South Africa (Bradshaw and 


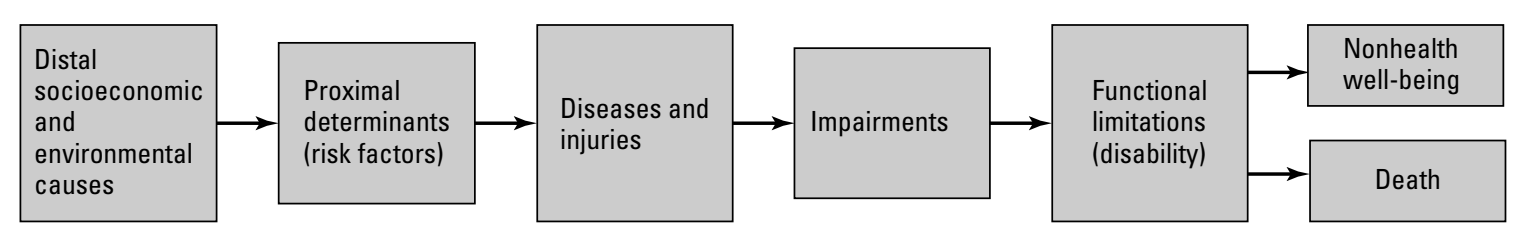

Source: Mathers and others 2002.

Note: This presentation is intended as a broad schema: for example, some exposures, such as environmental factors, can be proximate causes of disease, and injuries can lead directly to death.

Figure 1.1 Overview of Burden of Disease Framework

others 2003); Thailand (Bundhamcharoen and others 2002); Turkey (Baskent University 2005); the United States (McKenna and others 2005); and the World Health Organization (WHO 1996).

This chapter begins with a brief history of the work on burden of disease, including a discussion of the nature and origins of the disability-adjusted life year (DALY) as a measure of disease burden. Next it discusses applications of burden of disease analysis to the formulation of health policy. The chapter then summarizes the methods and findings of the 2001 GBD study, reported in more detail in chapters 3 and 4 of this volume. A concluding section takes stock of the work on disease burden since the early 1990s and suggests some key areas for further work.

Following this introductory and summarizing chapter, chapter 2 describes the demographic underpinnings for the epidemiological assessments that follow and provides context by briefly reviewing recent changes (from 1990 to 2001) in key demographic parameters. The chapter also assesses changes in the cause distribution of mortality among children under five between 1990 and 2001 and the difficulties of reliably assessing trends in mortality. Chapters 3 and 4 provide the definitive methods and results of the $2001 \mathrm{GBD}$ study. Chapter 3 reports on deaths and the disease and injury burden by age, sex, and 136 disease and injury categories. Chapter 4 reports on the disease and injury burden resulting from 19 risk factors, specifically for a number of important conditions. Both chapters present results using the World Bank's classification of low- and middle-income countries into six regional groups. Chapter 5 then explores the robustness of the major findings to uncertainties in the data and to alternative assumptions concerning construction of the DALY. Chapter 6 examines the implications of including stillbirths in a global burden of disease assessment. Their inclusion is potentially significant, both because the numbers are large (3.3 million in 2001), and because including stillbirths raises major questions about how to assess the DALY loss associated with deaths near the time of birth.

\section{HISTORY OF BURDEN OF DISEASE STUDIES}

In 1992, the World Bank commissioned the initial GBD study to provide a comprehensive assessment of the disease burden in 1990. The study was undertaken for the world as a whole and for 8 regions (Lopez and Murray 1998; Murray and Lopez 1996a,d; Murray, Lopez, and Jamison 1994; World Bank 1993). In order to recommend intervention packages for countries at different stages of development, the estimates were combined with analyses of the costeffectiveness of interventions in different populations (World Bank 1993; Jamison and Jardel 1994). Whereas earlier attempts to quantify global cause of death patterns (Hakulinen and others 1986; Lopez 1993) were valuable initial contributions to building the evidence base for policy, they were largely restricted to broad cause of death groups, for example, all infections and parasitic diseases combined, and did not address nonfatal health outcomes.

The methods and findings of the 1990 GBD study have been widely published and, as noted earlier, have spawned multiple disease burden exercises (Murray and Lopez $1996 c, d ; 1997 a, b, c)$. One of the basic principles guiding a burden of disease assessment is that almost all sources of health data are likely to contain useful information provided they are carefully screened for validity and completeness. With appropriate methods, investigator commitment, and expert judgment, obtaining internally consistent estimates of the global descriptive epidemiology of major conditions is possible. To prepare internally consistent estimates of incidence, prevalence, duration, and mortality for almost 500 sequelae of the diseases and injuries under consideration, a mathematical model, DisMod, was developed for the 1990 GBD study to convert partial, often nonspecific, data on disease and injury occurrence into a consistent description of the basic epidemiological parameters in each region by age group (Barendregt and others 2003; Murray and Lopez 1996b).

Many diseases, for example, neuropsychiatric conditions and hearing loss, and injuries may cause considerable ill health but no or few direct deaths. Therefore separate 


\section{Box 1.1}

\section{Disability-Adjusted Life Years}

The DALY is a health gap measure that extends the concept of potential years of life lost due to premature death to include equivalent years of healthy life lost by virtue of individuals being in states of poor health or disability (Murray 1996). One DALY can be thought of as one lost year of healthy life and the burden of disease as a measure of the gap between current health status and an ideal situation where everyone lives into old age free from disease and disability. This conceptualization of DALYs as a measure of health, and not of lost utility, is analogous to the principles of measuring gross domestic product as summarized by Eisner (1989, p. 7): "Our focus . . is on measures of all economic activity related to welfare [for example, gross domestic product], but not of welfare itself." Information on calculating DALYs, on time discounting, and on age weights is provided in chapter 3.

DALYs for a disease or health condition are calculated as the sum of YLL in the population and YLD for incident cases of the health condition. YLL is calculated from the number of deaths at each age multiplied by a global stan- dard life expectancy for the age at which death occurs. To estimate YLD for a particular cause for a particular time period, the number of incident cases in that period is multiplied by the average duration of the disease and a weight factor that reflects the severity of the disease on a scale from 0 (perfect health) to 1 (dead). The weights used in the 2001 GBD study are listed in detail elsewhere (see annex tables 3A.6 to 3A.8 in chapter 3).

In addition, in calculating DALYs, the GBD study used 3 percent time discounting and non-uniform age weights which give less weight to years lived at young and older ages. For the results reported in this volume and used in the Disease Control Priorities in Developing Countries, second edition (DCP2) 3 percent time discounting was applied but not non-uniform age weights. A death in infancy then corresponds to 30 DALYs, and deaths at age 20 to around 28 DALYs. Thus a disease burden of 3,000 DALYs in a population would be the equivalent of around 100 infant deaths or to approximately 5,000 persons aged 50 years living one year with blindness (disability weight 0.6 ). measures of survival and of health status among survivors, while useful inputs when formulating health policy, need to be combined in some fashion to provide a single, holistic measure of overall population health. To assess the burden of disease, the 1990 GBD study used a time-based metric that measures both premature mortality (years of life lost because of premature mortality or YLL) and disability (years of healthy life lost as a result of disability or YLD, weighted by the severity of the disability). The sum of the two components, namely, DALYs, provides a measure of the future stream of healthy life (years expected to be lived in full health) lost as a result of the incidence of specific diseases and injuries in 1990 (box 1.1). The effect of fatal cases (of disease or injury) is captured by years of life lost, while YLD captures the future health consequences in terms of sequelae of diseases or injuries of incident cases in 1990 that were not fatal. (For a more complete account of the DALY measure and the philosophy underlying parameter choices, see Murray 1996; Murray, Salomon, and others 2002).

DALYs are not unique to the GBD study. The World Bank used a variant of DALYs in its seminal review of health sector priorities (Jamison and others 1993), and they are derived from earlier work to develop time-based measures that better reflect the public health impact of death or illness at young ages (Dempsey 1947; Ghana Health Assessment Project Team 1981).

Much of the comment on, and criticism of, the GBD study focused on the construction of DALYs (Anand and Hanson 1998; Hyder, Rotllant, and Morrow 1998; Williams 1999), particularly the social choices pertaining to age weights and severity scores for disabilities. Relatively little criticism was directed at the vast uncertainty of the basic descriptive epidemiology for some populations, especially in Sub-Saharan Africa (see chapter 5 in this volume), which is likely to be far more consequential for setting health priorities (Cooper and others 1998).

The results of the 1990 GBD study confirmed what many health workers had suspected for some time, namely, that noncommunicable diseases and injuries were a significant cause of health burden in all regions, and in some rapidly industrializing regions such as East Asia and Pacific, were already by far the leading cause of death and disability. Neuropsychiatric disorders and injuries in particular were major causes of lost years of healthy life as measured by DALYs, and were vastly underappreciated when measured by mortality alone. The original GBD study estimated that noncommunicable diseases, including neuropsychiatric disorders, caused 41 percent of the global burden of disease in 1990, only slightly less than 
communicable, maternal, perinatal, and nutritional conditions combined (44 percent), and that 15 percent of the burden was due to injuries. Earlier assessments of global health priorities based on mortality data attributed no deaths to mental health disorders and less than half (7 percent) of that suggested by DALYs to injuries (Lopez 1993).

Estimates of the disease and injury burden caused by exposure to major risk factors are likely to be a much more useful guide to policies and priorities for prevention than a "league table" of the disease and injury burden. In recent decades, researchers have attempted to quantify the effects of specific exposures, for instance, tobacco smoking, on mortality from major diseases such as cancers (Doll and Peto 1981; Parkin and others 1994) or from multiple diseases (Peto and others 1992; United States Department of Health and Human Services 1992), either in individual countries or across groups of countries using comparable methods.

Specific country studies have examined the impact of several leading risk factors (Holman and others 1988; McGinnis and Foege 1993), but prior to the 1990 GBD study, no global assessments of the fatal and nonfatal burden of disease and injury resulting from exposure to multiple major health risks had been attempted. The 1990 study quantified 10 risk factors based on information about causation, prevalence, exposure, and disease and injury outcomes available at the time. The study attributed almost 16 percent of the entire global burden of disease and injury to malnutrition; another 7 percent to poor water and sanitation; and 2 to 3 percent to such risks as unsafe sex, tobacco, alcohol, and occupational exposures (Lopez and Murray 1998; Murray and Lopez 1996a; Murray and Lopez 1997a; Murray, Lopez, and Jamison 1994; World Bank 1993).

\section{APPLICATIONS OF BURDEN OF DISEASE ANALYSIS}

Burden of disease analyses are useful for informing health policy in at least five major ways as outlined in this section. Estimates of deaths by cause or years of life lost serve these same purposes, but for some uses, less well.

\section{Assessing Performance}

The burden of disease provides an indicator that can be used to judge progress over time within a single country or region or relative performance across countries and regions. In this application, burden of disease may be considered analogous to national income and product accounts, developed by
Simon Kuznets and others in the 1930s and culminating in 1939 with a complete national income and product account for the United Kingdom prepared at the request of the treasury. In subsequent decades, national income and product accounts have transformed the empirical underpinnings of economic policy analysis. As one leading scholar put it, "The national income and product accounts for the United States ..., and kindred accounts in other nations, have been among the major contributions to economic knowledge over the past half century ... Several generations of economists and practitioners have now been able to tie theoretical constructs of income, output, investment, consumption, and savings to the actual numbers of these remarkable accounts with all their fine detail and soundly meshed interrelations" (Eisner 1989, p. 1).

\section{Generating Forums for Informed Debate of Values and Priorities}

In practice, assessing the disease burden involves participation by a broad range of disease specialists, epidemiologists, and often, policy makers. Debating the appropriate values for, say, disability weights or for years of life lost at different ages helps clarify values and objectives for national health policy. Discussing the relationships between diseases and their risk factors in the light of local conditions sharpens consideration of priorities and of programs to address them.

\section{Identifying National Control Priorities}

Many countries now identify a relatively short list of interventions whose full implementation becomes an explicit priority for national political and administrative attention. Examples include interventions to control tuberculosis, poliomyelitis, HIV/AIDS, smoking, and specific micronutrient deficiencies. Because political attention and high-level administrative capacity are in relatively fixed and short supply, the benefits from using those resources will be maximized if they are directed toward interventions that are both cost-effective and aimed at problems associated with a high disease burden. National assessments of disease burden are one input into the process of establishing a shortlist of disease control priorities.

\section{Creating Knowledge}

Medical schools offer a fixed number of instructional hours, and training programs for other levels and types of health workers are similarly limited. A major instrument for 
implementing health policy priorities is to allocate this fixed time resource well. This implies allocating time to training for interventions where the disease burden is high and costeffective interventions exist.

Information on the disease or risk factor burden is also a vital input for informing resource allocation for research and development. In particular, whenever a fixed effort will have a benefit proportional not only to the size of that effort, but also to the size of the problem being addressed, estimates of the disease burden become essential for formulating and implementing research and development priorities. For example, developing a vaccine for a broad range of viral pneumonias would have perhaps hundreds of times the impact of a vaccine against hantavirus infection.

\section{Allocating Resources across Health Interventions}

A key task for priority-setting analyses in health is to create the evidence base to stimulate the reallocation of resources to interventions that, at the margin, will generate the greatest reduction in health loss. When there are major fixed costs in mounting an intervention, as is the case with political and managerial attention for national control priorities, burden estimates are required to improve resource allocation. Similarly, major fixed costs may be associated with the universalization (or major expansion) of an intervention and, if so, the cost-effectiveness of the expansion will depend in part on the size of the burden.

\section{IMPROVING THE COMPARATIVE QUANTIFICATION OF DISEASES, INJURIES, AND RISK FACTORS: THE 2001 GBD STUDY}

The 1990 GBD study represented a major advance in the quantification of the impact of diseases, injuries, and risk factors on population health globally and by region. Government and nongovernmental agencies alike have used its results to argue for more strategic allocations of health resources to disease prevention and control programs that are likely to yield the greatest gains in terms of population health. The results have also greatly increased understanding of the basic descriptive epidemiology of diseases and injuries worldwide.

Following publication of the initial results of the GBD study, several national applications of the methods it used have led to substantially more data on the descriptive epidemiology of diseases and injuries becoming available, as well as to improvements in analytical methods and mortality data in a number of countries. By emphasizing substan- tially more sophisticated approaches than in the past to the interpretation and presentation of population health data to policy makers, national burden of disease studies have stimulated efforts to improve and extend the collection of the health information data that are the basis for such analyses. A good example is the Islamic Republic of Iran where, over the last five years, the government has implemented a system of death registration with medical information on the cause of death that has been extended from four provinces initially to include 26 , or almost all of the country's provinces. Another example is the government of Thailand's extensive verbal autopsy study aimed at addressing major coding deficiencies in Thailand's national mortality data (Choprapawon and others 2005).

Critiques of the original study's approach, particularly of the methods used to assess the severity weightings for disabling health states, have led to fundamental changes in the way that investigators incorporate health state valuations, that is, the use of population-based rather than expert opinion as used in the 1990 study, and to substantially better methods for improving the cross-national comparability of survey data on health status (Murray, Tandon, and others 2002; Salomon and Murray 2004). Better methods for modeling the relationship between the level of mortality and the broad cause of death structure in populations that are based on proportions rather than rates have led to greater confidence in cause of death estimates for developing countries (Salomon and Murray 2002). In addition, improved population surveillance for some major diseases such as HIV/AIDS, and the wider availability of data from verbal autopsy methods, particularly in Sub-Saharan Africa, have lessened the dependence on models for cause of death estimates, although substantial uncertainty in the use of such data persists. For more details on these and other methodological advances, see chapter 3 in this volume.

Perhaps the major methodological progress since the 1990 GBD study has been with respect to the quantification of the disease burden from risk factors. The initial study quantified the population health effects of 10 risk factors, but serious concerns exist about the comparability of the methods and estimates used. Different risk factors have different epidemiological traditions, particularly with regard to the definitions of hazardous exposure, the strength of the evidence on causality, and the availability of epidemiological research on exposure and hazard. As a result, comparability across estimates of the disease burden caused by different risk factors has been difficult to establish. In particular, much of classical risk factor research has treated exposures as dichotomous, with individuals either exposed 
or not exposed, with exposure defined according to an often arbitrary threshold value, for example, systolic blood pressure of 140 millimeters of mercury as the threshold for hypertension. Recent evidence for such continuous exposures as cholesterol, blood pressure, and body mass index suggests that such arbitrarily defined thresholds are inappropriate, because the hazards for these risks decline continuously across the entire range of measured exposure levels, with no obvious threshold (Eastern Stroke and Coronary Heart Disease Collaborative Research Group 1998; Ezzati and others 2004; Rose 1985; WHO 2002).

For the 2001 GBD study, a new framework for risk factor assessment was defined that examines changes in the disease burden that would be expected under alternative population distributions of exposure to a risk factor or groups of risk factors (Murray and Lopez 1999). Attributable fractions of disease due to a risk factor were then calculated based on a comparison of the disease burden expected under the current estimated distribution of exposure by age, sex, and region with that expected under a counterfactual distribution of exposure. One such counterfactual distribution was defined for each risk factor as the population distribution of exposure that would lead to the lowest levels of disease burden. Thus, for example, in the case of tobacco, this theoreticalminimum-risk counterfactual exposure would be 100 percent of the population being never-smokers, for overweight and obesity it would be a narrow distribution of body mass index centered around an optimal level of $21 \mathrm{~kg} / \mathrm{m}^{2}$ and so on. The distributions of the theoretical-minimum-risk exposure for the risk factors quantified in the World Health Organization's study of comparative risk assessment (the methodological and empirical basis for the 2001 GBD study) were developed by expert groups for each risk factor based on available scientific knowledge of risk factor hazard. The study also used systematic reviews and analyses of extant sources on risk factor exposure and hazard in an iterative process that increased comparability across risk factors (Ezzati and others $2002,2004)$. These methods and results are described in more detail in chapter 4 in this volume.

Risk factors may affect disease and injury outcomes through other intermediate factors. For instance, some of the effects of diet and physical activity on cardiovascular diseases are mediated through changes in such intermediate factors as weight, blood pressure, and cholesterol. Risk factors may also affect disease and injury outcomes in combination with one another. For example, people who smoke and have elevated blood pressure and cholesterol have substantially higher probabilities of cardiovascular events. Finally, some risks have common social and behavioral determinants. For instance, members of poor households in rural areas are the most likely to be undernourished, use unsafe water sources, and be exposed to indoor smoke from solid fuels. Because of these epidemiological and social characteristics of risk factor exposure and hazard, policy-relevant analysis should include an assessment of the health benefits of simultaneous reductions in multiple risks. Multicausality also means that a range of interventions can be used for disease prevention, with the specific choices determined by such factors as costs, technology availability, infrastructure, and preferences. A novel aspect of the analysis of risk factors in the 2001 GBD study is the development and application of methods for estimating the disease burden attributable to the combined hazards of multiple risk factors (Ezzati and others 2003).

The basic units of analysis in the 1990 GBD study were the eight World Bank regions defined for the World Bank's (1993) World Development Report 1993. Designed to be geographically contiguous, these regions were nonetheless extremely heterogeneous with respect to health development, for example, the region referred to as Other Asia and Islands included countries with such diverse epidemiological profiles as Myanmar and Singapore. This seriously limited the applicability of these regions to comparative epidemiological assessments. Thus the 2001 GBD study followed a more refined approach. Estimates of overall mortality were first developed for World Health Organization member states using different methods for countries at different stages of health development. The choice of methods was largely determined by the availability of data (Lopez and others 2002). Age- and sex-specific death rates for countries were essentially determined using one of three standard approaches: the use of routine life table methods for countries with complete vital registration; the application of standard demographic methods to correct for underregistration of deaths; or the application of model life tables where no vital registration or survey data on adult mortality were available (Lopez and others 2002; Murray and others 2003).

The detailed methodological approaches adopted for estimating cause-specific mortality for countries and the descriptive epidemiology of nonfatal conditions for countries or subregions are described elsewhere (Mathers and others 2002; chapter 3 in this volume). This focus on individual countries as the unit of analysis, as well as the systematic application of standardized approaches for all countries in any given category of data availability, has vastly improved the cross-population comparability of disease and injury quantification.

A final major advance of the 2001 GBD study has been the systematic attempts to quantify some of the uncertainty 
in both national and global assessments of the disease burden (see chapter 5 in this volume). This uncertainty must be taken into account when making cross-national comparisons and needs to be carefully communicated to and interpreted by epidemiologists and policy makers alike.

\section{MAJOR FINDINGS OF THE 2001 GBD STUDY}

This section, and tables 1.1 and 1.2, summarize the principle findings of the 2001 GBD study. More detailed findings are reported in chapters 3 and 4 .

\section{Global and Regional Mortality}

Slightly more than 56 million people died in 2001, 10.5 million (or nearly 20 percent) of whom were children younger than five years of age. Almost 4 million children died before 1 month of age, with an additional 3.3 million stillbirths (see chapter 6). Of these child deaths, 99 percent occurred in low- and middle-income countries. Low- and middleincome countries also account for a comparatively large number of deaths at young and middle adult ages: 30 percent of all deaths occur at ages 15 to 59, compared with 15 percent in high-income countries. The causes of death at these ages, as well as in childhood, are thus important for assessing public health priorities.

Worldwide, one death in every three is from what the GBD study terms Group I causes (communicable diseases, maternal and perinatal conditions, and nutritional deficiencies) (see table 1.1). This proportion remains almost unchanged from 1990, with one major difference. Whereas HIV/AIDS accounted for only 2 percent of Group I deaths in 1990, it accounted for 14 percent in 2001. Excluding HIV/AIDS, Group I deaths fell from one-third of total deaths in 1990 to less than one-fifth in 2001. Virtually all Group I deaths are in low- and middle-income countries.

In low- and middle-countries, Group II causes (noncommunicable diseases) are now responsible for more than 50 percent of deaths in adults ages 15 to 59 in all regions except South Asia and Sub-Saharan Africa, where Group I causes, including HIV/AIDS, remain responsible for onethird and two-thirds of deaths, respectively. Outside these two regions, developing countries are now facing a triple burden of disease from communicable diseases, noncommunicable diseases, and injuries (Group III causes). Among low- and middle-income countries as a group, the three leading causes of death in 2001 included ischemic heart disease and cerebrovascular disease, which together accounted for almost one-fifth of all deaths. In other words, the epidemiological transition from infectious to chronic noncommunicable diseases in this group of countries is already well established and is of major relevance to health planning.

\section{Leading Causes of Disability}

The 1990 GBD study brought the previously largely ignored burden of nonfatal illnesses, particularly neuropsychiatric disorders, to the attention of health policy makers. The findings of the 2001 GBD study, based on updated data and analyses, confirm that disability and states of less than full health caused by diseases and injuries play a central role in determining the overall health status of populations in all regions of the world. Neuropsychiatric conditions, vision disorders, hearing loss, and alcohol use disorders dominate the overall burden of nonfatal disabling conditions.

In all regions, neuropsychiatric conditions are the most important causes of disability, accounting for more than 37 percent of YLD among adults aged 15 years and older worldwide. The disabling burden of neuropsychiatric conditions is almost the same for males and females, but the major contributing causes are different. While depression is the leading cause of disability for both males and females, the burden of depression is 50 percent higher for females than males, and females also have higher burdens from anxiety disorders, migraine, and senile dementia. In contrast, the male burden for alcohol and drug use disorders is nearly six times higher than that for females and accounts for a quarter of the male neuropsychiatric burden.

More than 85 percent of disease burden from nonfatal health outcomes occurs in low- and middle-income countries, and South Asia and Sub-Saharan Africa account for 40 percent of all YLD. Even though the prevalence of disabling conditions such as dementia and musculoskeletal disease is higher in countries with long life expectancies, this is offset by lower contributions to disability from conditions such as cardiovascular disease, chronic respiratory diseases, and long-term sequelae of communicable diseases and nutritional deficiencies. In other words, people living in developing countries not only face shorter life expectancies than those in developed countries, but also live a higher proportion of their lives in poor health.

\section{Burden of Disease and Injuries}

The results of the 2001 GBD study reinforce some of the conclusions of the $1990 \mathrm{GBD}$ study about the importance of including nonfatal outcomes in a comprehensive assessment 
Table 1.1 Deaths and Burden of Disease by Cause-Low- and Middle-Income Countries, High-Income Countries, and World, 2001

\begin{tabular}{|c|c|c|c|c|c|c|}
\hline & \multicolumn{2}{|c|}{ Low- and middle-income } & \multicolumn{2}{|c|}{ High-income } & \multicolumn{2}{|c|}{ World } \\
\hline & Deaths & $\operatorname{DALYs}(3,0)^{\mathrm{a}}$ & Deaths & $\operatorname{DALYs}(3,0)^{\mathrm{a}}$ & Deaths & $\operatorname{DALYs}(3,0)^{\mathrm{a}}$ \\
\hline \multicolumn{7}{|l|}{ All causes } \\
\hline Total number (thousands) & 48,351 & $1,386,709$ & 7,891 & 149,161 & 56,242 & 1,535 871 \\
\hline Rate per 1,000 population & 9.3 & 265.7 & 8.5 & 160.6 & 9.1 & 249.8 \\
\hline Age-standardized rate per $1,000^{b}$ & 11.4 & 281.7 & 5.0 & 128.2 & 10.0 & 256.5 \\
\hline Selected cause groups: & \multicolumn{6}{|c|}{ Number in thousands (percent) } \\
\hline $\begin{array}{l}\text { I. COMMUNICABLE DISEASES, } \\
\text { MATERNAL AND PERINATAL } \\
\text { CONDITIONS AND }\end{array}$ & $17,613(36.4)$ & $552,376(39.8)$ & $552(7.0)$ & $8,561(5.7)$ & $18,166(32.3)$ & $560,937(36.5)$ \\
\hline NUTRITIONAL DEFICIENCIES & & & & & & \\
\hline Tuberculosis & $1,590(3.3)$ & $35,874(2.6)$ & $16(0.2)$ & $219(0.1)$ & $1,606(2.9)$ & $36,093(2.3)$ \\
\hline HIV/AIDS & $2,552(5.3)$ & $70,796(5.1)$ & $22(0.3)$ & $665(0.4)$ & $2,574(4.6)$ & $71,461(4.7)$ \\
\hline Diarrheal diseases & $1,777(3.7)$ & $58,697(4.2)$ & $6(<.1)$ & $444(0.3)$ & $1,783(3.2)$ & $59,141(3.9)$ \\
\hline Measles & $762(1.6)$ & $23,091(1.7)$ & $1(<.1)$ & $23(<.1)$ & $763(1.4)$ & $23,113(1.5)$ \\
\hline Malaria & $1,207(2.5)$ & $39,961(2.9)$ & $0(0.0)$ & $9(<.1)$ & $1,208(2.1)$ & $39,970(2.6)$ \\
\hline Lower respiratory infections & $3,408(7.0)$ & $83,606(6.0)$ & $345(4.4)$ & $2,314(1.6)$ & $3,753(6.7)$ & $85,920(5.6)$ \\
\hline Perinatal conditions & $2,489(5.1)$ & $89,068(6.4)$ & $32(0.4)$ & $1,408(0.9)$ & $2,522(4.5)$ & $90,477(5.9)$ \\
\hline Protein-energy malnutrition & $241(0.5)$ & $15,449(1.1)$ & $9(0.1)$ & $130(<.1)$ & $250(0.4)$ & $15,578(1.0)$ \\
\hline II. NONCOMMUNICABLE CONDITIONS & $26,023(53.8)$ & $678,483(48.9)$ & $6,868(87.0)$ & $129,356(86.7)$ & $32,891(58.5)$ & $807,839(52.6)$ \\
\hline Stomach cancers & $696(1.4)$ & $9,616(0.7)$ & $146(1.9)$ & $1,628(1.1)$ & $842(1.5)$ & $11,244(0.7)$ \\
\hline Colon and rectum cancers & $357(0.7)$ & $5,060(0.4)$ & $257(3.3)$ & $3,175(2.1)$ & $614(1.1)$ & $8,236(0.5)$ \\
\hline Liver cancer & $505(1.0)$ & $7,945(0.6)$ & $102(1.3)$ & $1,223(0.8)$ & $607(1.1)$ & $9,169(0.6)$ \\
\hline Trachea, bronchus, and lung cancers & $771(1.6)$ & $10,701(0.8)$ & $456(5.8)$ & $5,397(3.6)$ & $1,227(2.2)$ & $16,099(1.0)$ \\
\hline Diabetes mellitus & $757(1.6)$ & $15,804(1.1)$ & $202(2.6)$ & $4,192(2.8)$ & $960(1.7)$ & $19,997(1.3)$ \\
\hline Unipolar depressive disorders & $10(<.1)$ & $43,427(3.1)$ & $3(<.1)$ & $8,408(5.6)$ & $13(<.1)$ & $51,835(3.4)$ \\
\hline Alcohol use disorders & $62(0.1)$ & $11,007(0.8)$ & $23(0.3)$ & $4,171(2.8)$ & $84(0.2)$ & $15,178(1.0)$ \\
\hline Cataracts & $0(0.0)$ & $28,150(2.0)$ & $0(0.0)$ & $493(0.3)$ & $0(0.0)$ & $28,643(1.9)$ \\
\hline Vision disorders, age-related & $0(0.0)$ & $15,364(1.1)$ & $0(0.0)$ & $1,525(1.0)$ & $0(0.0)$ & $16,889(1.1)$ \\
\hline Hearing loss, adult onset & $0(0.0)$ & $24,607(1.8)$ & $0(0.0)$ & $5,387(3.6)$ & $0(0.0)$ & $29,994(2.0)$ \\
\hline Hypertensive heart disease & $760(1.6)$ & $9,969(0.7)$ & $129(1.6)$ & $1,209(0.8)$ & $889(1.6)$ & $11,178(0.7)$ \\
\hline Ischemic heart disease & $5,699(11.8)$ & $71,882(5.2)$ & $1,364(17.3)$ & $12,390(8.3)$ & $7,063(12.6)$ & $84,273(5.5)$ \\
\hline Cerebrovascular disease & $4,608(9.5)$ & $62,669(4.5)$ & $781(9.9)$ & $9,354(6.3)$ & $5,390(9.6)$ & $72,024(4.7)$ \\
\hline Chronic obstructive pulmonary disease & $2,378(4.9)$ & $33,453(2.4)$ & $297(3.8)$ & $5,282(3.5)$ & $2,676(4.8)$ & $38,736(2.5)$ \\
\hline Cirrhosis of the liver & $654(1.4)$ & $13,633(1.0)$ & $118(1.5)$ & $2,146(1.4)$ & $771(1.4)$ & $15,778(1.0)$ \\
\hline Nephritis and nephrosis & $552(1.1)$ & $9,076(0.7)$ & $111(1.4)$ & $929(0.6)$ & $663(1.2)$ & $10,005(0.7)$ \\
\hline Osteoarthritis & $2(<.1)$ & $13,666(1.0)$ & $3(<.1)$ & $3,786(2.5)$ & $5(<.1)$ & $17,452(1.1)$ \\
\hline Congenital anomalies & $477(1.0)$ & $23,533(1.7)$ & $30(0.4)$ & $1,420(1.0)$ & $507(0.9)$ & $24,952(1.6)$ \\
\hline Alzheimer and other dementias & $173(0.4)$ & $9,640(0.7)$ & 207 (2.6) & $7,468(5.0)$ & $380(0.7)$ & $17,108(1.1)$ \\
\hline III. INJURIES & $4,715(9.8)$ & $155,850(11.2)$ & $471(6.0)$ & $11,244(7.5)$ & $5,186(9.2)$ & $167,094(10.9)$ \\
\hline Road traffic accidents & $1,069(2.2)$ & $32,017(2.3)$ & $121(1.5)$ & $3,045(2.0)$ & $1,189(2.1)$ & $35,063(2.3)$ \\
\hline Falls & $316(0.7)$ & $13,582(1.0)$ & $71(0.9)$ & $1,459(1.0)$ & $387(0.7)$ & $15,041(1.0)$ \\
\hline Self-inflicted injuries & $749(1.5)$ & $17,674(1.3)$ & $126(1.6)$ & $2,581(1.7)$ & $875(1.6)$ & $20,255(1.3)$ \\
\hline Violence & $532(1.1)$ & $18,132(1.3)$ & $24(0.3)$ & $765(0.5)$ & $556(1.0)$ & $18,897(1.2)$ \\
\hline
\end{tabular}

Source: Chapter 3.

Notes: Numbers in parentheses indicate percentage of column total.

Broad group totals in bold are additive but should not be summed with all other conditions listed in table.

a. DALYS $(3,0)$ refer to the version of the DALY based on a $3 \%$ annual discount rate and uniform age weights.

b. Age-standardized using the WHO World Standard Population.

c. Includes only causes responsible for more than $1 \%$ of global deaths or DALYs in 2001.

of global population health. They also confirm the growing importance of noncommunicable diseases in low- and middle-income countries and highlight important changes in population health in some regions since 1990.
HIV/AIDS is now the fourth leading cause of the burden of disease globally and the leading cause in Sub-Saharan Africa, where it is followed by malaria in second place. Seven other Group I causes also appear in the top 10 causes for this 
region. The epidemiological transition in low- and middleincome countries has resulted in a 20 percent reduction in the per capita disease burden due to Group I causes since 1990. Without the HIV/AIDS epidemic and the associated lack of decline in the burden of tuberculosis, this reduction would have been closer to 30 percent.

The per capita disease burden in Europe and Central Asia has increased by nearly 40 percent since 1990, and population health in this region is now worse than all other regions except South Asia and Sub-Saharan Africa. This reflects the sharp increase in adult male mortality and disability in the 1990s, leading to the highest male-female differential in the disease burden in the world. A significant factor in this increase is probably the high level of harmful alcohol consumption among men, which has led to high rates of accidents, violence, and cardiovascular disease. From 1991 to 1994, the risk of premature adult (15 to 59 years) death increased by 50 percent for Russian males. It improved somewhat between 1994 and 1998, but subsequently increased.

The burden of noncommunicable diseases is increasing, accounting for nearly half the total global burden of disease, a 10 percent increase from estimated levels in 1990. Almost 50 percent of the adult disease burden in low- and middle income countries is now attributable to noncommunicable diseases. The implementation of effective interventions for Group I diseases, coupled with population aging and the spread of risks for noncommunicable disease in many lowand middle-income countries, are the likely causes of this shift. Ischemic heart disease and stroke dominate the burden of disease in Europe and Central Asia and together account for more than a quarter of the total disease burden. In contrast, in Latin America and the Caribbean these diseases account for 8 percent of the disease burden, but this region also has high levels of diabetes and endocrine disorders compared with other regions. Violence is the fourth leading cause of the disease and injury burden in Latin America and the Caribbean. Violence does not appear among the top 10 causes of burden in any other region, but is nonetheless significant.

Injuries primarily affect young adults and often result in severe, disabling sequelae. All forms of injury accounted for 16 percent of the adult burden in 2001. In parts of Europe and Central Asia, Latin America and the Caribbean, and the Middle East and North Africa, more than 30 percent of the entire disease and injury burden among male adults aged 15 to 44 is attributable to injuries. Road traffic accidents, violence, and self-inflicted injuries are all among the top 10 leading causes of burden in these regions. The former Soviet Union and other high-mortality (among adults) countries of
Eastern Europe have rates of injury death and disability among males that are similar to those in Sub-Saharan Africa.

\section{Burden of Disease Attributable to Risk Factors}

As described earlier, a major advance of the 2001 GBD study has been in creating a unified framework for quantifying the burden of disease and injury attributable to major risk factors and in applying this framework to exposure and hazard data for selected major risk factors based on comprehensive and systematic reviews of published literature and other sources. Notwithstanding the inherent uncertainties in assessing the population-level health effects of risk factors, the quantification of the burden of disease attributable to the individual and joint hazards of selected risks suggests that the leading causes of mortality and disease burden include risk factors for Group I conditions (for example, undernutrition; indoor smoke from household use of solid fuels; poor water, sanitation, and hygiene; and unsafe sex), whose burden is primarily concentrated in South Asia and Sub-Saharan Africa, and risk factors for Group II conditions (especially, smoking, alcohol, high blood pressure and cholesterol, and overweight and obesity), which are widespread globally (see table 1.2). In low- and middle-income countries, the leading causes of disease burden included risk factors prevalent among the poor and associated with Group I conditions (for example, childhood underweight [ 8.7 percent of the disease burden in these regions]; unsafe water, sanitation, and hygiene [3.7 percent]; and indoor smoke from household use of solid fuels [3.0 percent]), unsafe sex (5.8 percent), and risk factors for noncommunicable diseases (for example, high blood pressure [5.6 percent], smoking [3.9 percent], and alcohol use [3.6 percent]). Across high-income countries, risk factors associated with Group II and Group III conditions were the leading causes of loss of healthy life (smoking [12.7 percent], high blood pressure [9.3 percent], overweight and obesity [7.2 percent], high cholesterol $[6.3$ percent], and alcohol use [4.4 percent]).

An estimated 45 percent of global mortality and 36 percent of the global burden of disease were attributable to the joint hazards of the 19 selected global risk factors. The joint hazards were even larger in regions where a relatively small number of diseases and their risk factors were responsible for large losses of life (HIV/AIDS and risk factors for child mortality in Sub-Saharan Africa; cardiovascular risks, including smoking and alcohol use in Europe and Central Asia). Globally, large fractions of major diseases such as diarrhea, lower respiratory infections, HIV/AIDS, lung cancer, 
Table 1.2 Deaths and Burden of Disease Attributable to Risk Factors-Low- and Middle-Income Countries, High-Income Countries, and World, 2001

\begin{tabular}{|c|c|c|c|c|c|c|}
\hline & \multicolumn{2}{|c|}{ Low- and middle-income } & \multicolumn{2}{|c|}{ High-income } & \multicolumn{2}{|c|}{ World } \\
\hline & Deaths & $\overline{D A L Y s(3,0)^{a}}$ & Deaths & $\operatorname{DALYs}(3,0)^{a}$ & Deaths & $\operatorname{DALYs}(3,0)^{\mathrm{a}}$ \\
\hline Total number (thousands) & 48,351 & 1,386709 & 7,891 & 149,161 & 56,242 & 1,535871 \\
\hline Rate per 1,000 population & 9.3 & 265.7 & 8.5 & 160.6 & 9.1 & 249.8 \\
\hline Age-standardized rate per $1,000^{b}$ & 11.4 & 281.7 & 5.0 & 128.2 & 10.0 & 256.5 \\
\hline Risk factor & \multicolumn{6}{|c|}{ Number in thousands (percent) } \\
\hline \multicolumn{7}{|l|}{ Childhood and maternal undernutrition } \\
\hline Childhood underweight & $3,630(7.5)$ & $120,579(8.7)$ & $0(0.0)$ & $67(<0.1)$ & $3,630(6.5)$ & $120,647(7.9)$ \\
\hline Iron-deficiency anemia & $613(1.3)$ & $23,933(1.7)$ & $8(0.1)$ & $789(0.5)$ & $621(1.1)$ & $24,722(1.6)$ \\
\hline Vitamin A deficiency & $800(1.7)$ & $24,686(1.8)$ & $0(0.0)$ & $0(0.0)$ & $800(1.4)$ & $24,686(1.6)$ \\
\hline Zinc deficiency & $849(1.8)$ & $27,631(2.0)$ & $0(0.0)$ & $5(<0.1)$ & $849(1.5)$ & $27,636(1.8)$ \\
\hline \multicolumn{7}{|l|}{ Other nutrition-related risk factors and physical activity } \\
\hline High blood pressure & $6,223(12.9)$ & $78,063(5.6)$ & $1,392(17.6)$ & $13,887(9.3)$ & $7,615(13.5)$ & $91,950(6.0)$ \\
\hline High cholesterol & $3,038(6.3)$ & $42,815(3.1)$ & $842(10.7)$ & $9,431(6.3)$ & $3,880(6.9)$ & $52,246(3.4)$ \\
\hline Overweight and obesity & $1,747(3.6)$ & $31,515(2.3)$ & $614(7.8)$ & $10,733(7.2)$ & $2,361(4.2)$ & $42,248(2.8)$ \\
\hline Low fruit and vegetable intake & $2,308(4.8)$ & $32,836(2.4)$ & $333(4.2)$ & $3,982(2.7)$ & $2,641(4.7)$ & $36,819(2.4)$ \\
\hline Physical inactivity & $1,559(3.2)$ & $22,679(1.6)$ & $376(4.8)$ & $4,732(3.2)$ & $1,935(3.4)$ & $27,411(1.8)$ \\
\hline \multicolumn{7}{|l|}{ Addictive substances } \\
\hline Smoking & $3,340(6.9)$ & $54,019(3.9)$ & $1,462(18.5)$ & $18,900(12.7)$ & $4,802(8.5)$ & $72,919(4.7)$ \\
\hline Alcohol use & $1,869(3.9)$ & $49,449(3.6)$ & $24(0.3)$ & $6,580(4.4)$ & $1,893(3.4)$ & $56,029(3.6)$ \\
\hline Illicit drug use & $189(0.4)$ & $7,890(0.6)$ & $37(0.5)$ & $2,024(1.4)$ & $226(0.4)$ & $9,914(0.6)$ \\
\hline \multicolumn{7}{|l|}{ Sexual and reproductive health } \\
\hline Unsafe sex & $2,819(5.8)$ & $80,270(5.8)$ & $32(0.4)$ & $909(0.6)$ & $2,851(5.1)$ & $81,179(5.3)$ \\
\hline Non-use and use of ineffective methods of contraception & $162(0.3)$ & $7,411(0.5)$ & $0(0.0)$ & $23(<0.1)$ & $162(0.3)$ & $7,434(0.5)$ \\
\hline \multicolumn{7}{|l|}{ Environmental risks } \\
\hline Unsafe water, sanitation, and hygiene & $1,563(3.2)$ & $51,622(3.7)$ & $4(<0.1)$ & $289(0.2)$ & $1,567(2.8)$ & $51,911(3.4)$ \\
\hline Urban air pollution & $735(1.5)$ & $8,707(0.6)$ & $76(1.0)$ & $664(0.4)$ & $811(1.4)$ & $9,371(0.6)$ \\
\hline Indoor smoke from household use of solid fuels & $1,791(3.7)$ & $41,731(3.0)$ & $0(0.0)$ & $2(<0.1)$ & $1,791(3.2)$ & $41,734(2.7)$ \\
\hline \multicolumn{7}{|l|}{ Other selected risks } \\
\hline Contaminated injections in health care setting & $407(0.8)$ & $8,974(0.6)$ & $4(<0.1)$ & $76(<0.1)$ & $412(0.7)$ & $9,050(0.6)$ \\
\hline Child sexual abuse & $65(0.1)$ & $5,381(0.4)$ & $6(<0.1)$ & $699(0.5)$ & $71(0.1)$ & $6,079(0.4)$ \\
\hline All selected risk factors together & $22,014(45.6)$ & $500,066(36.1)$ & $3,473(44.0)$ & $51,092(34.3)$ & $25,488(45.3)$ & $551,158(35.9)$ \\
\hline
\end{tabular}

Source: Chapter 4. Note that mortality and disease burden attributable to individual risk factors cannot be added due to multi-causality. See Chapter 4 for details. a. (some footnote as Table 1.1)

b. Age-standardized using the WHO World Standard Population

chronic obstructive pulmonary disease, ischemic heart disease, and stroke were attributable to the joint effects of the risk factors considered in this volume. The joint hazards of these 19 risks for a number of other important diseases and injuries, such as perinatal and maternal conditions, selected other cancers, and intentional and unintentional injuries, which have more diverse risk factors, were smaller, but nonnegligible. The relatively small number of risk factors that account for a large fraction of the disease burden underscores the need for policies, programs, and scientific research to take advantage of interventions for multiple major risks to health (Ezzati and others 2003).

\section{CONCLUSIONS}

The substantial scientific and policy interest in the methods and findings of the 1990 GBD study, the widespread application of the methods by countries at all levels of health development, and the adoption of the framework as the preferred method for health accounting by international health agencies such as the World Health Organization attest to the critical need for objective and systematic assessments of the disease burden for priority setting in health. The vast and comprehensive effort to quantify the disease burden worldwide dramatically changed views about the 
importance of some conditions, particularly psychiatric disorders, and drew global public health attention to the unrecognized burden of injuries. The methodological developments over the past decade, a more systematic approach to collecting key data and research findings on the health of populations, and the results of numerous national and subnational burden of disease studies have dramatically improved the methodological armamentarium and the empirical base for disease burden assessment, in particular, the comparability of the estimated contributions of diseases, injuries, and risk factors to this burden.

As reported in this volume, the 2001 GBD study provides a comprehensive update of the comparative importance of diseases, injuries, and risk factors for global health. The study incorporates a range of new data sources to develop internally consistent estimates of incidence, prevalence, severity and duration, and mortality for 136 major causes by sex and by eight age groups. Estimates of deaths by cause, age, and sex were carried out separately for 226 countries and territories, drawing on a total of 770 country-years of death registration data, 535 additional sources of information on levels of child and adult mortality, and more than 2,600 data sets providing information on specific causes of death in regions not well covered by death registration systems. Together with the more than 8,500 data sources (epidemiological studies, disease registers, notifications systems, and so on) used to estimate incidence, prevalence, and YLD by cause, the 2001 GBD study has incorporated information from more than 10,000 datasets relating to population health and mortality (see chapter 3 ). This represents one of the largest syntheses of global information on population health carried out to date.

Much of the research on the burden of disease undertaken over the past decade or so has relied on the methodological and empirical efforts that defined the 1990 GBD study as a major advance in global public health statistics. Progress in updating the epidemiological basis for assessing the disease burden from the various diseases and injuries of interest has been uneven, although improvements in the data and methods available for assessing global and regional mortality by cause have been substantial, and some advances have been made in the data for, and epidemiological understanding of some major causes of ill health such as HIV/AIDS and diabetes mellitus. Nevertheless, making more reliable estimates of global, regional, and national disease burdens still faces many methodological and empirical challenges. The substantive agenda, mapped out over a decade ago (Murray, Lopez and Jamison, 1994) remains equally valid today and needs to be addressed more systematically if the burden of disease framework is to gain greater acceptance as the international tool for health accounting.

Assessing and documenting in detail the state of the world's health at the beginning of the millennium is a useful undertaking. This volume will provide scholars today and in the future with a definitive historical record of the leading causes of the burden of disease for major regions of the world at the start of the 21st century. An account of global health at the beginning of the 20th century, or earlier, would no doubt have been of more than just historical interest, but given the methods of scientific interchange and the state of scientific and methodological knowledge at the time, this was impossible.

In presenting the comprehensive findings of the 2001 GBD study, this volume is, in many respects, a culmination of the effort launched in 1990 and represents the end of the beginning of global disease burden assessments. The widespread use of disease burden concepts by national and international bodies since the first results were published and the heightened interest in improving the basic descriptive epidemiology of diseases, injuries, and risk factors by both countries and agencies has laid the foundations for future population health assessments. As programs and policies to improve health worldwide become more widespread, so too will the need for more comprehensible, credible, and comparable assessments to periodically monitor world health and the success, or otherwise, of measures to promote health and reduce the burden of disease. New initiatives, and perhaps new global institutions, are required to measure the burden of disease worldwide and how it is changing, more reliably than hitherto. This book provides the baseline against which such progress with global health development will be measured.

\section{REFERENCES}

Anand, S., and K. Hanson. 1998. “DALYs: Efficiency Versus Equity.” World Development 26 (2): 307-10.

Barendregt, J. J., G. J. van Oortmarssen, T. Vos, and C. J. L. Murray. 2003. "A Generic Model for the Assessment of Disease Epidemiology: The Computational Basis of DisMod II." Population Health Metrics 1 (1): e4.

Baskent University. 2005. Burden of Disease Final Report. Ankara, Turkey: Baskent University and the School of Public Health, Refik Saydam Hygiene Center, Ministry of Health.

Bradshaw, D., P. Groenewald, R. Laubscher, N. Nannan, B. Nojilana, R. Norman, D. Pieterse, M. Schneider, D. E. Bourne, I. M. Timaeus, R. Dorrington, and L. Johnson. 2003. Initial Burden of Disease Estimates for South Africa, 2000. Cape Town: South African Medical Research Council. http://www.mrc.ac.za/bod/bod.htm. 
Bundhamcharoen, K., Y. Teerawatananon, T. Vos, and S. Begg. 2002. Burden of Disease and Injuries in Thailand: Priority Setting for Policy. Bangkok: Ministry of Public Health.

Choprapawon, C., Y. Porapakkham, O. Sablon, R. Panjajaru and B. Jhantharatat. 2005. "Thailand's National Death Registration Reform: Verifying the Causes of Death between July 1997 and December 1999." Asia-Pacific Journal of Public Health 17 (2): 110-116.

Cooper, R. S., B. Osotimehin, J. S. Kaufman, and T. Forrester. 1998. "Disease Burden in Sub-Saharan Africa: What Should We Conclude in the Absence of Data?" Lancet 351 (9087): 208-10.

Dempsey, M. 1947. "Decline in Tuberculosis: The Death Rate Fails to Tell the Entire Story." American Review of Tuberculosis 56 (2): 157-64.

Doll, R., and R. Peto. 1981. The Causes of Cancer. Oxford, U.K.: Oxford University Press.

Eastern Stroke and Coronary Heart Disease Collaborative Research Group. 1998. "Blood Pressure, Cholesterol, and Stroke in Eastern Asia." Lancet 352 (9143): 1801-7.

Eisner, R. 1989. The Total Incomes System of Account. Chicago and London: University of Chicago Press.

Ezzati, M., A. D. Lopez, A. Rodgers, and C. J. L. Murray. 2004. Comparative Quantification of Health Risks: The Global and Regional Burden of Disease Attributable to Selected Major Risk Factors. Geneva: World Health Organization.

Ezzati, M., A. D. Lopez, A. Rodgers, S. Vander Hoorn, C. J. L. Murray, and the Comparative Risk Assessment Collaborating Group. 2002. "Selected Major Risk Factors and Global and Regional Burden of Disease." Lancet 360 (9343): 1347-60.

Ezzati, M., S. Vander Hoorn, A. Rodgers, A. D. Lopez, C. D. Mathers, C. J. L. Murray, and the Comparative Risk Assessment Collaborating Group. 2003. "Estimates of Global and Regional Potential Health Gains from Reducing Multiple Major Risk Factors.” Lancet 362 (9380): 271-80.

Ghana Health Assessment Project Team. 1981. "Quantitative Method of Assessing the Health Impact of Different Diseases in Less Developed Countries." International Journal of Epidemiology 10 (1): 73-80.

Hakulinen, T., H. Hansluwka, A. D. Lopez, and T. Nakada. 1986. "Global and Regional Mortality Patterns by Cause of Death in 1980." International Journal of Epidemiology 15 (2): 226-33.

Holman, C. D. J., B. K. Armstrong, L. N. Arias, C. A. Martin, W. M. Hatton, L. D. Hayward, M. A. Salmon, R. E. Shean, V. P. Waddell. 1990. The Quantification of Drug Caused Morbidity and Mortality in Australia 1988. Canberra: Commonwealth Department of Community Services and Health.

Hyder, A. A., G. Rotllant, and R. Morrow. 1998. "Measuring the Burden of Disease: Healthy Life Years.” American Journal of Public Health 88 (2): 196-202.

Jamison, D. T., and J.-P. Jardel. 1994. "Comparative Health Data and Analyses." In Global Comparative Assessments in the Health Sector: Disease Burden, Expenditures, and Intervention Packages, ed. C. J. L. Murray and A. D. Lopez, v-vii. Geneva: World Health Organization.

Jamison, D. T., W. H. Mosely, A. R. Measham, and J. L. Bobadilla, eds. 1993. Disease Control Priorities in Developing Countries. New York: Oxford University.

Lopez, A. D. 1993. "Causes of Death in the Industrialized and Developing Countries: Estimates for 1985-1990.” In Disease Control Priorities in Developing Countries, ed. Dean Jamison, W. Henry Mosely, A. R. Measham, and J. L. Bobadilla, 15-30. New York: Oxford University Press.

Lopez, A. D., and C. J. L. Murray. 1998. "The Global Burden of Disease, 1990-2020." Nature Medicine 4 (11): 1241-43.

Lopez, A. D., O. B. Ahmad, M. Guillot, B. D. Ferguson, J. A. Salomon, C. J. L. Murray, and K. Hill. 2002. World Mortality in 2000: Life Tables for 191 Countries. Geneva: World Health Organization.
Lozano, R., C. J. L. Murray, J. Frenk, and J. Bobadilla. 1995. "Burden of Disease Assessment and Health System Reform: Results of a Study in Mexico." Journal for International Development 7 (3): 555-64.

Mahapatra, P. 2002. Estimating National Burden of Disease: The Burden of Disease in Andhra Pradesh, 1990s. Hyderabad, India: Institute of Health Systems.

Mathers, C. D., T. Vos, and C. Stevenson. 1999. The Burden of Disease and Injury in Australia. Canberra: Australian Institute of Health and Welfare. http://www.aihw.gov.au/publications/index.cfm/title/5180.

Mathers, C. D., C. Stein, M. Ma Fat, C. Rao, M. Inoue, K. Shibuya, N. Tomijima, C. Bernard, and H. Xu. 2002. The Global Burden of Disease 2000 Study (version 2): Methods and Results. Discussion Paper 50. Geneva: Global Program on Evidence for Health Policy, World Health Organization. http://www.who.int/evidence.

McGinnis, J. M., and W. H. Foege. 1993. "Actual Causes of Death in the United States." Journal of the American Medical Association 270 (18): 2207-12.

McKenna, M. T., C. M. Michaud, C. J. L. Murray, and J. S. Marks. 2005. "Assessing the Burden of Disease in the United States Using DisabilityAdjusted Life Years." American Journal of Preventive Medicine 28 (5): 415-23.

Murray, C. J. L. 1996. “Rethinking DALYs.” In The Global Burden of Disease, ed. C. J. L. Murray and A. D. Lopez, 1-89. Cambridge, MA: Harvard University Press

Murray, C. J. L., and A. D. Lopez. 1996a. "Evidence-Based Health Policy: Lessons from the Global Burden of Disease Study." Science 274 (5288): $740-43$.

1996b. "Global and Regional Descriptive Epidemiology of Disability: Incidence, Prevalence, Health Expectancies, and Years Lived with Disability." In The Global Burden of Disease, ed. C. J. L. Murray and A. D. Lopez, 201-46. Cambridge, MA: Harvard University Press.

1996c. Global Health Statistics: A Compendium of Incidence, Prevalence, and Mortality Estimates for over 200 Conditions. Cambridge, MA: Harvard University Press.

eds. 1996d. The Global Burden of Disease, vol. 1. Cambridge, MA: Harvard University Press.

1997a. "Global Mortality, Disability, and the Contribution of Risk Factors: Global Burden of Disease Study." Lancet 349 (9063): 1436-42.

1997b. "Mortality by Cause for Eight Regions of the World: Global Burden of Disease Study." Lancet 349 (9061): 1269-76.

1997c. "Regional Patterns of Disability-Free Life Expectancy and Disability-Adjusted Life Expectancy: Global Burden of Disease Study." Lancet 349 (9062): 1347-52.

1999. "On the Comparable Quantification of Health Risks: Lessons from the Global Burden of Disease Study." Epidemiology 10 (5): 594-605.

Murray, C. J. L., A. D. Lopez, and D. T. Jamison. 1994. “The Global Burden of Disease in 1990: Summary Results, Sensitivity Analyses, and Future Directions." Bulletin of the World Health Organization 72 (3): 495-508.

Murray, C. J. L., J. A. Salomon, C. D. Mathers, and A. D. Lopez. 2002. Summary Measures of Population Health: Concepts, Ethics, Measurement, and Applications. Geneva: World Health Organization.

Murray, C. J. L., A. Tandon, J. A. Salomon, C. D. Mathers, and R. Sadana. 2002. "New Approaches to Enhance Cross-Population Comparability of Survey Results". In Summary Measures of Population Health: Concepts, Ethics, Measurement, and Applications, ed. C. J. L. Murray, J. A. Salomon, C. D. Mathers, and A. D. Lopez, 421-32. Geneva: World Health Organization.

Murray, C. J. L., B. D. Ferguson, A. D. Lopez, M. Guillot, J. A. Salomon, and O.B. Ahmad. 2003. "Modified Logit Life Table System: Principles, Empirical Validation, and Application." Population Studies 57 (2): 165-82. 
Parkin, D. M., P. Pisani, A. D. Lopez, and E. Masuyer. 1994. "At Least One in Seven Cases of Cancer Is Caused by Smoking: Global Estimates for 1985." International Journal of Cancer 59 (4): 494-504.

Peto, R., A. D. Lopez, J. Boreham, M. Thun, and C. Heath. 1992. "Mortality from Tobacco in Developed Countries: Indirect Estimates from National Vital Statistics." Lancet 339 (8804): 1268-78.

Rose, G. 1985. "Sick Individuals and Sick Populations." International Journal of Epidemiology 14 (1): 32-38.

Salomon, J. A., and C. J. L. Murray. 2002. "The Epidemiologic Transition Revisited: Compositional Models for Causes of Death by Age and Sex.” Population and Development Review 28 (2): 205-28.

. 2004. "A Multimethod Approach to Measuring Health State Valuations." Health Economics 13: 281-90.

United States Department of Health and Human Services. 1992. Smoking and Health in the Americas. Report of the Surgeon General, in Collaboration with the Pan-American Health Organization, Department of Health and Human Services publication (CDC) 92-8419. Washington, DC: Office on Smoking and Health.

Vos, T., M. Tobias, H. Gareeboo, F. Roussety, S. Huttley, and C. J. L. Murray. 1995. Mauritius Health Sector Reform, National Burden of Disease Study, Final Report of Consultancy. Port Louis, Mauritius: Ministry of Health and Ministry of Economic Planning and Development.

Williams, A. 1999. "Calculating the Global Burden of Disease: Time for a Strategic Appraisal?” Health Economics 8 (1): 1-8.

World Bank. 1993. Investing in Health: World Development Report 1993. New York: Oxford University Press.

WHO (World Health Organization). 1996. Investing in Health Research and Development. Report of the Ad Hoc Committee on Health Research Relating to Future Intervention Options. Geneva: WHO.

2002. Reducing Risks: Promoting Healthy Life. World Health Report 2002. Geneva: WHO. 

Part I

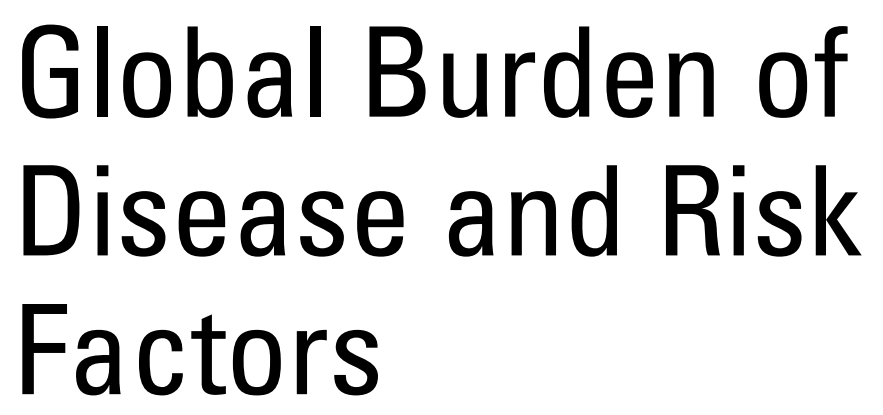





\section{Chapter 2}

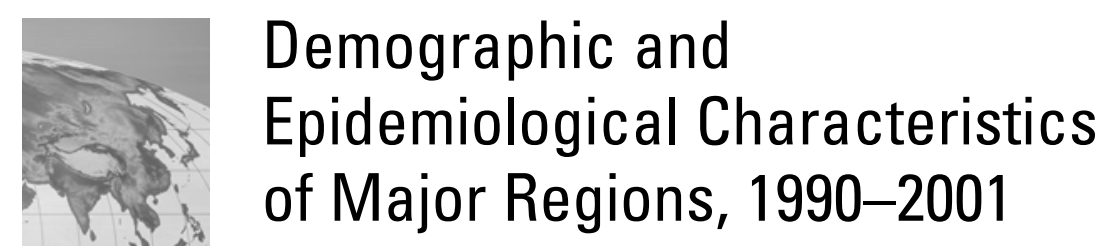

Health status is both a determinant of population change, between age and mortality and morbidity. Second, each of the dynamic processes influencing population size and growth, structure, and distribution, namely, fertility, mortality, and migration, will also affect health status. Thus, any discussion of disease control priorities and of the health system for delivering interventions requires an understanding of the demographic context and how it is changing.

This chapter begins by providing an overview of global population trends in each major region of the world and the current size and composition of the population. Given this volume's focus on the descriptive epidemiology of diseases, injuries, and risk factors, we then examine trends in mortality over the past decade in more detail as background against which the current assessment of the disease burden might be more usefully interpreted. This includes both an assessment of trends in age-specific mortality and summary measures of the age schedule of mortality, such as life expectancy and the probability of dying within certain age ranges, as well as a specific discussion of trends in the main causes of child mortality. The focus on child mortality is entirely appropriate because (a) the fact that at the end of 
the 20th century, we remained woefully ignorant of its levels, let alone its causes, is highlighted; (b) the reduction of child mortality should remain a priority for global health development efforts, and the moral imperative to do so remains as relevant today as it was 30 years ago, when efforts to improve child survival became increasingly organized and focused; and (c) the resulting emphasis by the global public health community on reducing child mortality has yielded vastly more epidemiological information that can be used to assess trends in levels and causes. Nevertheless, we argue later in the chapter that large and unacceptable uncertainties about trends in cause-specific child mortality rates persist, with important implications for program planning and evaluation.

\section{REGIONAL DEMOGRAPHIC CHARACTERISTICS}

The key characteristics of regional demography of concern for health services provision include the size, age structure, and sex structure of the population and its rate of growth and comparative measures of fertility and mortality.

\section{Sources of Population Data and Methodology}

The population and mortality estimates for various regions summarized here are based on different data sources and methods, and thus are not strictly comparable. This primarily concerns the impact of different estimates of deaths by age and sex on population size and structure. Because the effect of mortality on population size and structure is generally modest, such differences have little impact on the findings reported in this chapter. The population estimates are based on data the United Nations (UN) Population Division compiled and analyzed for its biennial assessment of global population trends and regional demographic patterns (United Nations 2003). The UN Population Division estimates population size and vital rates (births and deaths) from censuses, vital registration, and demographic and health surveys and evaluates the data for completeness, accuracy, and consistency. Where necessary, it adjusts the data to achieve internal consistency and cross-country comparability. The baseline from which the UN projections are made is mid-2003. Because the 2002 revision was produced without complete data for 2001 for all countries, the baseline estimates are also projections, and the population figures in this chapter are therefore a mixture of both observed and projected data. ${ }^{1}$
The UN Population Division assesses a number of demographic parameters to produce country projections. In addition to total population, the baseline assessment includes a breakdown of population by sex and age (in five-year aggregates). Fertility is specified as age-specific fertility rates for females and mortality rates are based on survival probabilities from life tables. Age-specific patterns of migration are also incorporated for countries in which migration flows are observed or are thought to occur. When these inputs are not available from any of the sources listed earlier, the UN uses demographic models, such as model life tables or indirect mortality estimation techniques, to generate the information. Additional modeling is applied to estimate mortality patterns in countries with significant HIV/AIDS prevalence levels.

The UN Population Division provides a limited amount of information about the data in its reports, including the dates of censuses, the adjustment factors applied to total census populations, and the type and year of the latest surveys that contained mortality and fertility estimates. It does not provide information about the adjustments made to reported fertility rates, age and sex structures, or mortality rates. Basic information on population size and composition is available for most countries for 1990, and with the exception of Sub-Saharan Africa, for 2000 (or thereabouts) as well (table 2.1). Around both dates, censuses covered more than 90 percent of populations in all the regions except Sub-Saharan Africa. Thus, the basic population estimates developed by the UN Population Division and summarized in this chapter have a reasonable evidence base.

The UN projections of population size and vital rates are based on assumptions about levels and trends in vital rates. Fertility is assumed to follow a path modeled on the experience of countries with declining fertility, except when a country's recent fertility trend deviates considerably from

Table 2.1 Percentage of Regional Population Covered by Censuses, circa 1990 and 2000

\begin{tabular}{lrc}
\hline Region & $\mathbf{1 9 9 0}$ & $\mathbf{2 0 0 0}$ \\
\hline East Asia and Pacific & 95.7 & 96.2 \\
Europe and Central Asia & 100.0 & 93.9 \\
Latin America and the Caribbean & 95.2 & 91.9 \\
Middle East and North Africa & 96.9 & 98.6 \\
South Asia & 87.0 & 98.1 \\
Sub-Saharan Africa & 81.6 & 53.4 \\
High-income countries & 90.2 & 99.0 \\
\hline
\end{tabular}

Source: U.S. Census Bureau, Population Division, International Programs Center (July 7, 2004). 
the model pattern, in which case the country-specific pattern is followed (United Nations 2003).

Our 2001 estimates and future projections are generated on the basis of the cohort component methodology. This approach applies estimated trends in birth and death rates and migration by age and sex to a baseline age and sex structure. Population growth rates are determined by the levels of age-specific fertility and mortality rates and migration and the size of the initial age groups (base year population) against which these levels are applied. We constructed demographic estimates for the aggregate regional and income groupings used for the second edition of Disease Control Priorities in Developing Countries (Jamison and others 2006) from the UN Population Division countrylevel estimates by aggregating populations in specific age and sex groups and age-specific fertility rates. The aggregates are thus weighted by the different population sizes of individual countries.

The mortality estimates presented in this chapter are developed from other sources using methods different than those the UN employed, as described later. As a result, the age and sex structures reported here, as well as any indicators derived from them (such as crude birth and death rates) are not strictly internally consistent. In particular, the mortality rates estimated for this chapter would, in some cases, have produced different age and sex population structures than those estimated by the UN, as well as different numbers of births and deaths. These differences are unlikely to be large, however, as the estimated age-specific mortality rates reported later in this chapter agree quite closely with those of the UN, except for Sub-Saharan Africa.

\section{Population Size and Growth}

Between 1990 and 2001, global population increased from about 5.3 billion to 6.1 billion people, an average rate of increase of 1.4 percent per year, equivalent to about 220,000 people per day (table 2.2). During the decade, the growth rate in developing regions ranged from 0.2 percent in Europe and Central Asia to 2.6 percent in Sub-Saharan Africa.

Estimates at the global level conceal large differences in population growth among regions, which in turn consist of countries that may have quite different demographic trends. For example, Europe and Central Asia added just 1 million people per year between 1990 and 2001, whereas South Asia added 25 million people each year.

The World Bank regions (see map 1 inside the front cover of this volume) vary substantially in terms of population size, with East Asia and the Pacific accounting for about 30 percent of the global population and South Asia for roughly another 20 percent. Thus, about half the world's population live in the low- and middle-income countries of these two regions. The smallest region in terms of population size is the Middle East and North Africa, with just 5 percent of the world's population. Just over 10 percent of the world's population live in Sub-Saharan Africa. Another 15 percent live in high-income countries, a proportion that is declining.

\section{Distribution by Age, Sex, and Location}

How populations are distributed by age matters a great deal for public health, because many aspects of risk behavior, as well as disease and injury outcomes, are strongly associated with age. While many other factors contribute to mortality and fertility levels, the age distribution of a population is an important factor in explaining differences in demographic and epidemiological indicators. Regions differ significantly in how their populations are distributed across age groups, with almost 45 percent of the population of Sub-Saharan Africa being younger than 15, compared with 20 percent of the population in high-income countries, where fertility has been low for decades. Nevertheless, the trends during 1990-2001 show a great deal of similarity: in all regions the proportion of the population in the youngest age groups was lower in 2001 than in 1990, with most of the increase occurring in the 15 through 69 age group. As a result, the median age of the population has increased in all regions. At the same time, the population aged 70 and older has been increasing in most regions as mortality has declined, and this age group now represents more than 10 percent of the population in the high-income countries.

These changes in the relative age distribution of populations since 1990 reflect changes in the growth rates of different age groups (figure 2.1). In three of the six regions (East Asia and Pacific, Europe and Central Asia, and the Middle East and North Africa), as well as the world as a whole, the number of children under five was smaller in absolute terms in 2001 than in 1990. The highest growth rates during this period were in the 40- through 55-year-old age group and among those over 70 . The irregularities in growth rates of different age groups reflect past trends in the initial size of each cohort and its subsequent mortality and migration experiences. This is particularly evident for Europe and Central Asia, where the impact of the regional conflicts in the early 1990s on demographic structure is particularly evident. 
Table 2.2 Population Size and Composition, Fertility, and GNP, by World Bank Region, 1990 and 2001

\begin{tabular}{|c|c|c|c|c|c|c|c|c|}
\hline \multirow[b]{2}{*}{ Population Characteristic } & \multicolumn{2}{|c|}{$\begin{array}{l}\text { Low- and middle- } \\
\text { income countries }\end{array}$} & \multicolumn{2}{|c|}{$\begin{array}{l}\text { East Asia and } \\
\text { Pacific }\end{array}$} & \multicolumn{2}{|c|}{$\begin{array}{l}\text { Europe and } \\
\text { Central Asia }\end{array}$} & \multicolumn{2}{|c|}{$\begin{array}{l}\text { Latin America } \\
\text { and the Caribbean }\end{array}$} \\
\hline & 1990 & 2001 & 1990 & 2001 & 1990 & 2001 & 1990 & 2001 \\
\hline \multicolumn{9}{|l|}{ Size } \\
\hline Total population (thousands) & $4,398,401$ & $5,216,587$ & $1,625,868$ & $1,848,388$ & 467,797 & 477,116 & 439,709 & 525,864 \\
\hline Proportion of world population (\%) & 83.6 & 84.9 & 30.9 & 30.1 & 8.9 & 7.8 & 8.4 & 8.6 \\
\hline $\begin{array}{l}\text { Annual average growth rate, } \\
\text { 1990-2001 (\%) }\end{array}$ & \multicolumn{2}{|c|}{1.6} & \multicolumn{2}{|c|}{1.2} & 0.2 & & 1.6 & \\
\hline \multicolumn{9}{|l|}{ Composition (\%) } \\
\hline \multicolumn{9}{|l|}{ Age } \\
\hline $0-14$ & 34.8 & 31.8 & 30.2 & 26.5 & 26.5 & 21.8 & 36.2 & 31.5 \\
\hline $15-59$ & 57.6 & 59.8 & 61.8 & 64.1 & 59.5 & 62.6 & 56.8 & 60.4 \\
\hline $60-69$ & 4.7 & 5.0 & 4.9 & 5.6 & 8.3 & 8.5 & 4.2 & 4.5 \\
\hline $70+$ & 2.9 & 3.4 & 3.0 & 3.8 & 5.6 & 7.2 & 2.9 & 3.6 \\
\hline Urban & 36.9 & 41.6 & 28.8 & 37.0 & 63.2 & 63.5 & 71.1 & 75.4 \\
\hline Female & 49.4 & 49.5 & 48.9 & 49.0 & 51.9 & 51.9 & 50.3 & 50.5 \\
\hline \multicolumn{9}{|l|}{ Fertility } \\
\hline Total fertility rate & 3.5 & 2.9 & 2.6 & 2.1 & 2.3 & 1.6 & 3.2 & 2.6 \\
\hline Total no. of births (thousands) & 123,400 & 122,400 & 36,200 & 31,500 & 8,300 & 6,300 & 11,700 & 11,600 \\
\hline Crude birth rate per 1,000 & 28.2 & 23.4 & 22.3 & 17.0 & 16.7 & 12.7 & 26.6 & 22.0 \\
\hline \multicolumn{9}{|l|}{ GNP (exchange rate dollars) } \\
\hline GNP per capita & 870 & 1,170 & 420 & 890 & 39,737 & 54,933 & 2,260 & 3,570 \\
\hline
\end{tabular}

Source: UN Population Division 2002 revision estimates.

Note: GNP = gross national product.

Along with the progressive aging of the population, the relentless trend toward increasing urbanization has continued, with consequences for health in terms of both health service provision, which, in principle, is better with urbanization, and risk of exposure to chronic disease, which is, on balance, worse (Ezzati and others 2005). Almost half the world's population lived in urban areas in 2001, up 4 percentage points from 1990. The increase in urbanization was particularly marked in East Asia and the Pacific (increase from 29 to 37 percent of the population) and in SubSaharan Africa (from 28 to 34 percent). Overall, 42 percent of the population in low- and middle-income countries now live in urban areas.

In general, more boys than girls are born, with sex ratios at birth of between 1.03 and 1.06 in most countries, though in some Asian countries, sex-selective abortions have skewed this ratio to more than 1.10. Differential mortality and, to a limited extent, migration, shape the sex ratio at other ages (figure 2.2). In South Asia, higher mortality for girls and for women during their childbearing years leads at first to an increasing and then to a constant sex ratio to about age 45, after which male mortality is higher. Excess mortality of adult males in Europe and Central Asia explains the particularly low sex ratio observed there (Lopez and others 2002). In all regions, the higher mortality of males relative to females accounts for the sharp decline in the population sex ratio after age 50 or thereabouts.

The overall effects of the age-specific mortality differences between the sexes are relatively minor in terms of total population sex ratios. All regions have roughly equal numbers of males and females in the population, with the proportion of males being slightly higher in Europe and Central Asia and in the high-income regions (51 to 52 percent) than in East Asia and the Pacific and South Asia (49 percent).

\section{Fertility}

Table 2.2 shows recent trends in fertility, as indicated by the total fertility rate for the period, that is, the average number of children a woman could expect to have if she were subject indefinitely to current age-specific fertility rates. Even though fertility levels vary a good deal among regions, all low- and middle-income regions witnessed large declines in fertility levels during the 1990s. Overall fertility levels in low- and middle-income countries fell by almost 20 percent over the decade, a remarkable decline, with levels falling by as much as 33 percent in the Middle East and North Africa, and even by 10 percent in Sub-Saharan Africa. However, fertility rates in Sub-Saharan Africa remain high, with the total fertility rate of 5.6 being about twice as high as that for any other region. 
Table 2.2 Continued

\begin{tabular}{|c|c|c|c|c|c|c|c|c|c|}
\hline \multicolumn{2}{|c|}{$\begin{array}{l}\text { Middle East and North } \\
\text { Africa }\end{array}$} & \multicolumn{2}{|c|}{ South Asia } & \multicolumn{2}{|c|}{$\begin{array}{c}\text { Sub-Saharan } \\
\text { Africa }\end{array}$} & \multicolumn{2}{|c|}{ High-income } & \multicolumn{2}{|c|}{ World } \\
\hline 1990 & 2001 & 1990 & 2001 & 1990 & 2001 & 1990 & 2001 & 1990 & 2001 \\
\hline 243,973 & 309,762 & $1,117,887$ & $1,387,873$ & 503,166 & 667,583 & 862,342 & 928,110 & $5,260,742$ & $6,144,696$ \\
\hline 4.6 & 5.0 & 21.2 & 22.6 & 9.6 & 10.9 & 16.4 & 15.1 & 100.0 & 100.0 \\
\hline \multicolumn{2}{|c|}{2.2} & \multicolumn{2}{|c|}{2.0} & \multicolumn{2}{|c|}{2.6} & \multicolumn{2}{|c|}{0.7} & \multicolumn{2}{|c|}{1.4} \\
\hline 43.1 & 36.4 & 37.8 & 35.3 & 45.7 & 44.3 & 20.0 & 18.5 & 32.4 & 29.8 \\
\hline 51.5 & 57.7 & 55.7 & 57.6 & 49.7 & 51.0 & 62.5 & 62.2 & 58.4 & 60.2 \\
\hline 3.5 & 3.6 & 4.2 & 4.4 & 3.0 & 3.0 & 9.0 & 9.1 & 5.4 & 5.6 \\
\hline 1.9 & 2.4 & 2.3 & 2.7 & 1.6 & 1.7 & 8.5 & 10.2 & 3.8 & 4.5 \\
\hline 53.5 & 57.5 & 25.0 & 27.4 & 27.9 & 34.0 & 74.4 & 77.1 & 43.0 & 46.9 \\
\hline 49.0 & 49.3 & 48.4 & 48.5 & 50.4 & 50.4 & 51.0 & 50.8 & 49.6 & 49.7 \\
\hline 5.0 & 3.6 & 4.3 & 3.4 & 6.3 & 5.6 & 1.7 & 1.7 & 3.2 & 2.7 \\
\hline 9,300 & 9,400 & 36,500 & 37,300 & 21,400 & 26,300 & 11,300 & 10,800 & 134,700 & 133,200 \\
\hline 34.8 & 27.3 & 32.7 & 26.7 & 44.6 & 40.8 & 13.4 & 11.9 & 25.7 & 21.6 \\
\hline 1,770 & 3,570 & 380 & 450 & 470 & 550 & 19,760 & 26,760 & 4,060 & 5,180 \\
\hline
\end{tabular}

Few low- and middle-income countries experienced increasing fertility during 1990-2001, ${ }^{2}$ though a few high-income countries have seen small upturns from previously low levels. Fertility is below replacement levels (about two children) in all but five high-income countries (Brunei Darussalam, Israel, Kuwait, Qatar, and the United Arab Emirates), as well as in most countries in Europe and Central Asia. When fertility drops to below replacement levels, population growth often continues for several decades, as the number of births exceeds the number of deaths because of the high proportion of women of childbearing age.

\section{CHANGES IN MORTALITY, 1990-2001}

Change in patterns of mortality is a major determinant of the demography of populations and underlies important population differentials. For example, the differences in mortality by sex across regions contribute to the variable pattern of population sex ratios described earlier. The theory of demographic transition suggests that the rapid declines in fertility observed during the 1990s in most regions would be preceded, and perhaps accompanied, by a similarly rapid decline in child mortality. To help interpret the broad regional demographic patterns described earlier, a review of trends in mortality and the causes underlying such trends is useful.

\section{Estimating Mortality}

Various methods are available to estimate age patterns and levels of mortality in populations. These fall into three broad categories depending on the available data: direct estimation from complete vital registration, estimates from vital registration corrected for undercounting, and estimates derived from models based on child mortality levels. Mathers and others (2005) review the availability and quality of mortality data and group the 192 member states of the World Health Organization into broad categories according to criteria pertaining to the coverage, completeness, and quality of cause of death data. Their findings indicate that only about 33 percent (64) of World Health Organization member states, mostly high-income countries, have complete mortality data and that another 26 percent (50 countries) have data that can be used for mortality estimation purposes. The approximately 40 percent of remaining countries either have no recent data or no data at all that can be used to estimate causes of death or the level of adult mortality directly.

The situation is somewhat different for levels of child mortality, where decades of interest in monitoring child survival by the global public health community have yielded either direct or indirect estimates of child mortality for all but a handful of countries (Hill and others 1999; Lopez and others 2002). Based on a careful review of the time trend of 

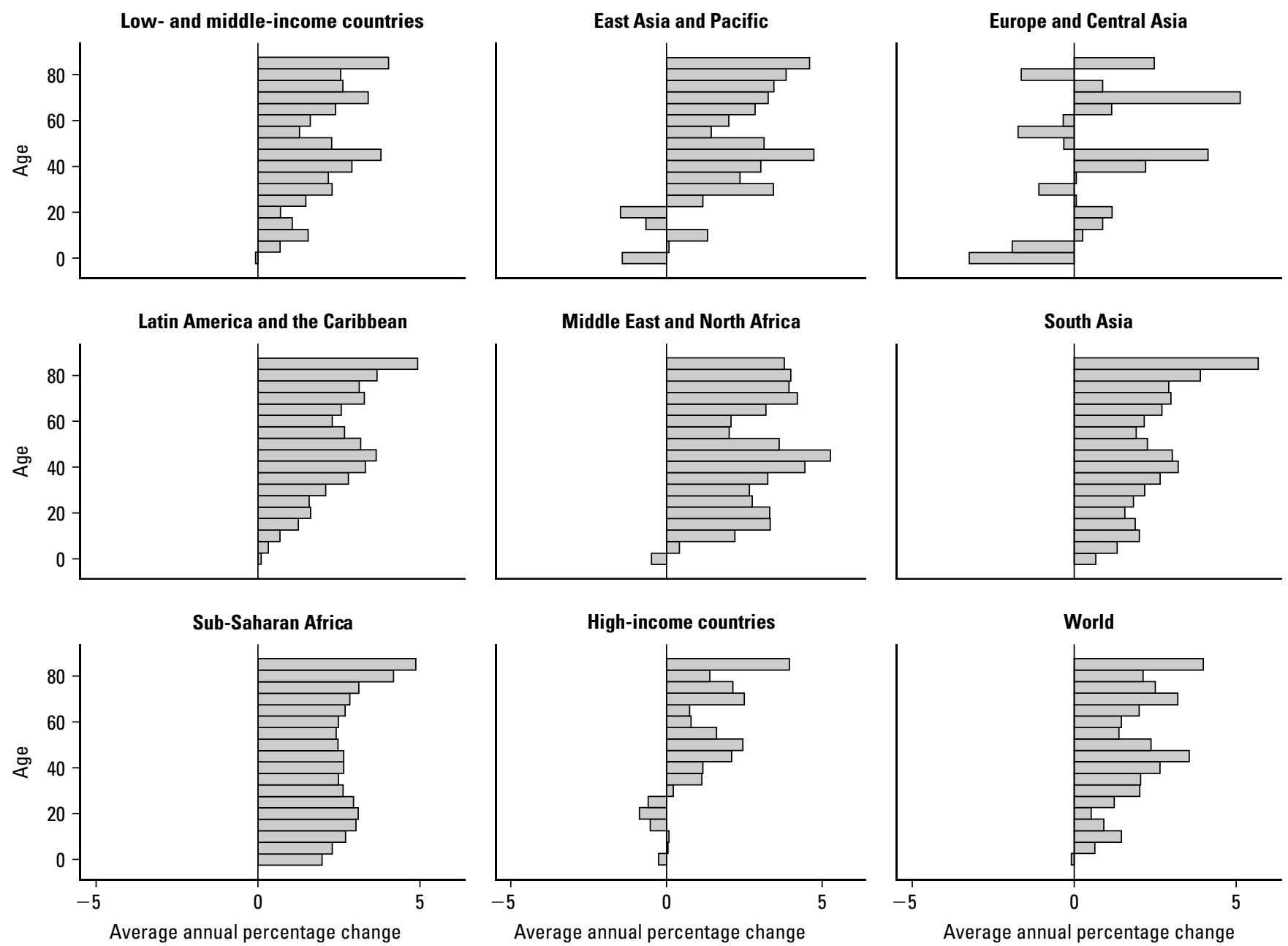

Source: Calculated from United Nations 2003.

Figure 2.1 Changes in Population Age Distribution, 1990-2001

these estimates of child deaths, which come primarily from censuses and surveys, estimating child mortality levels in 1990 and 2001 is possible for virtually all countries with an acceptable level of uncertainty. Levels of child mortality are unavailable for only about 10 countries that together account for about 2 percent of child deaths (Lopez and others 2002). Formal curve-fitting procedures to estimate time trends in child mortality can be applied to all the data, but given the subjective assessments that are required to judge which data points are plausible and which are not, simple averaging of all plausible observations at any given point in time is likely to be sufficient, and this was the procedure used to estimate child mortality levels for this chapter.

For those countries with complete vital registration data, age-specific and cause-specific death rates are easily derived directly from the registration data and from population censuses. For those countries where registration data are incomplete, demographers have developed indirect demographic methods to correct for underreporting of deaths before estimating age-specific mortality (Bennett and Horiuchi 1984; Hill 1987). These countries include China and India, where application of such methods suggest that data from the disease surveillance points system in China and the sample registration system in India are 85 to 90 percent complete (Mari Bhat 2002; Rao and others 2005).

For countries with no usable data on adult mortality levels, age-specific death rates were predicted from the modified logit life table system (Murray and others 2003). The median level of adult mortality was predicted based on a modeled relationship between adult and child mortality as determined from a historical data set of more than 1,800 life tables judged to be reasonably complete. Uncertainty about these predicted mean values of adult mortality is considerable given the few observations with comparatively 


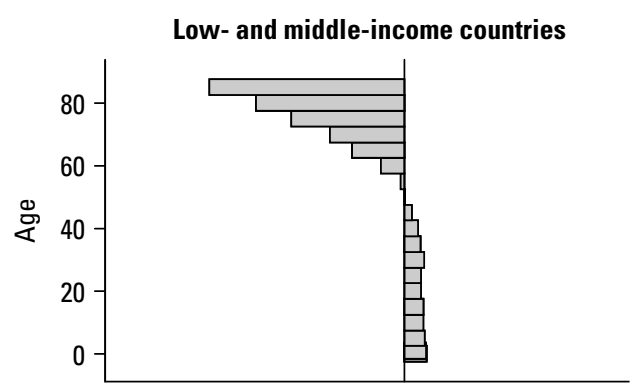

Latin America and the Caribbean

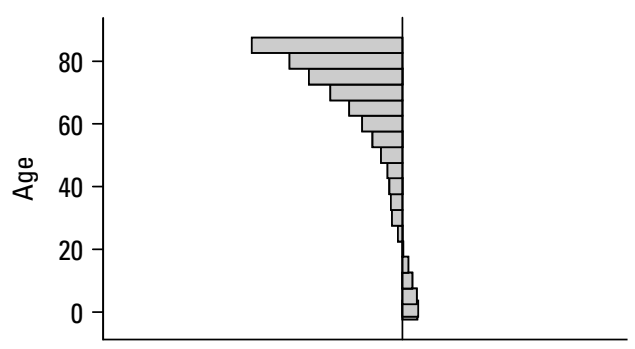

Sub-Saharan Africa

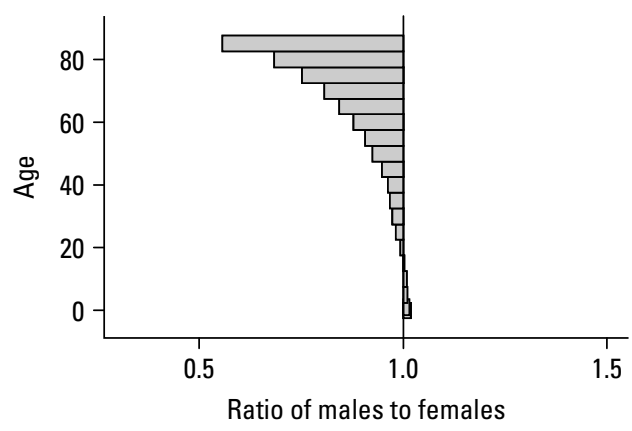

Source: Calculated from United Nations 2003

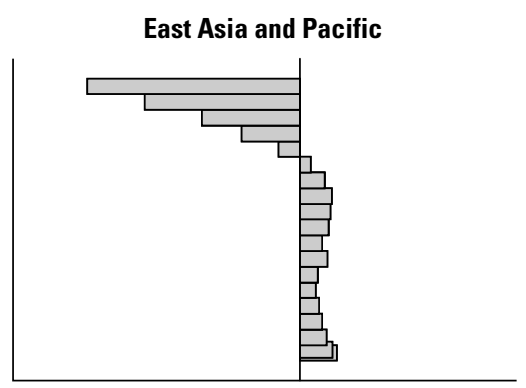

Middle East and North Africa
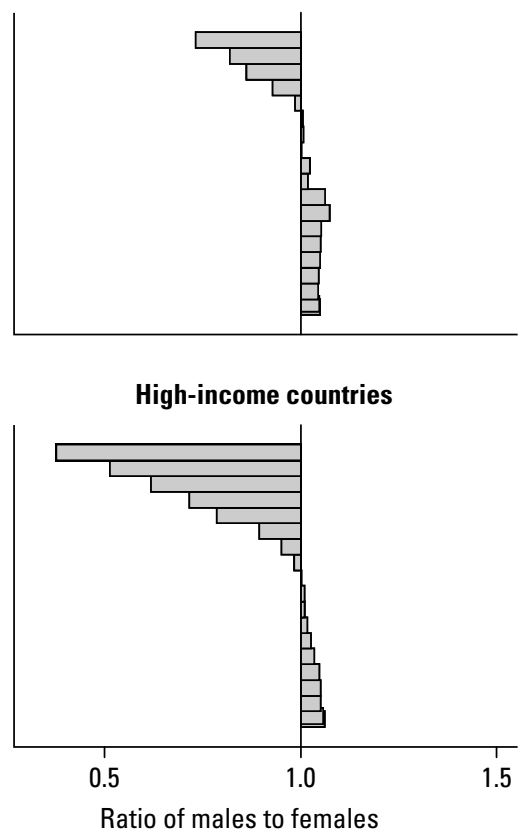

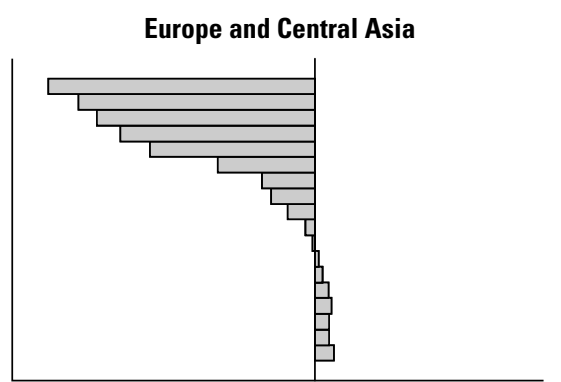

South Asia
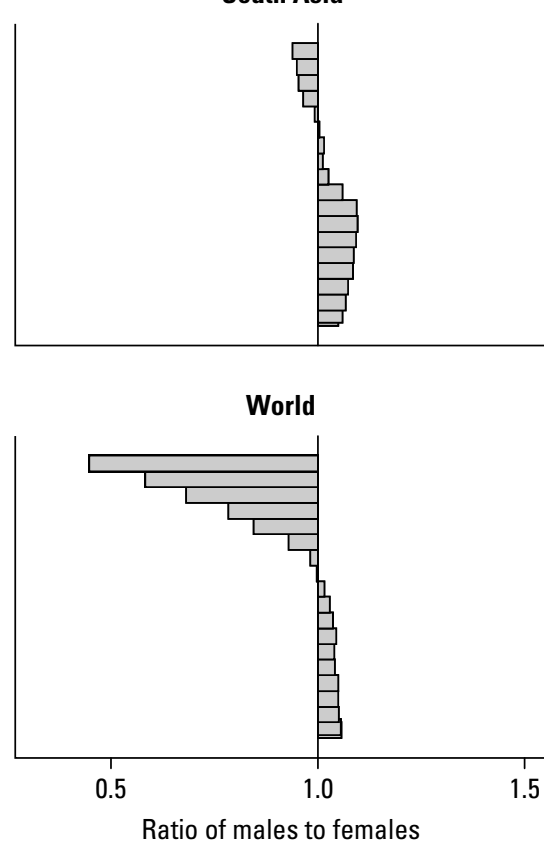

Figure 2.2 Population Sex Ratios at Different Ages, 2001

high levels of child and adult mortality. The estimated and predicted levels of child and adult mortality, respectively, were then applied to the modified life table system by selecting the best match from among 50,000 life tables to estimate a complete, smoothed set of age-specific death rates (Murray and others 2003). This method was applied for all but about 70 countries.

Obvious uncertainties are associated with this procedure. Hence, the life tables for East Asia and the Pacific, the Middle East and North Africa, and Sub-Saharan Africa (where HIV/AIDS mortality was added to the predicted adult mortality rates) in particular need to be viewed with caution, because the rates for many countries in these regions have been modeled using these methods.

Identical methods were applied to estimate national agespecific mortality rates for both 1990 and 2001; thus, the two sets of estimates are, in principle at least, comparable.
Annex 2A provides detailed estimates of summary measures of mortality by country for the two years based on these methods. The annex also shows the percentage decline in child mortality during the period.

Whether these methods correctly describe levels and patterns of mortality is difficult to ascertain given the substantial uncertainties in the data, particularly for adult mortality. The only other systematic attempt to estimate national and global death rates in 1990 is that of the UN Population Division (United Nations 2003). Figure 2.3 presents estimated mortality parameters for 1990 by region. For a comparison of mortality estimates for 2001, see Lopez and others (2002).

Despite the UN's different model life table approach for estimating age-specific death rates based on child mortality, the two sets of estimates shown in figure 2.3 are remarkably congruent. Regional estimates of child mortality ${ }_{5} q_{0}$ (the 


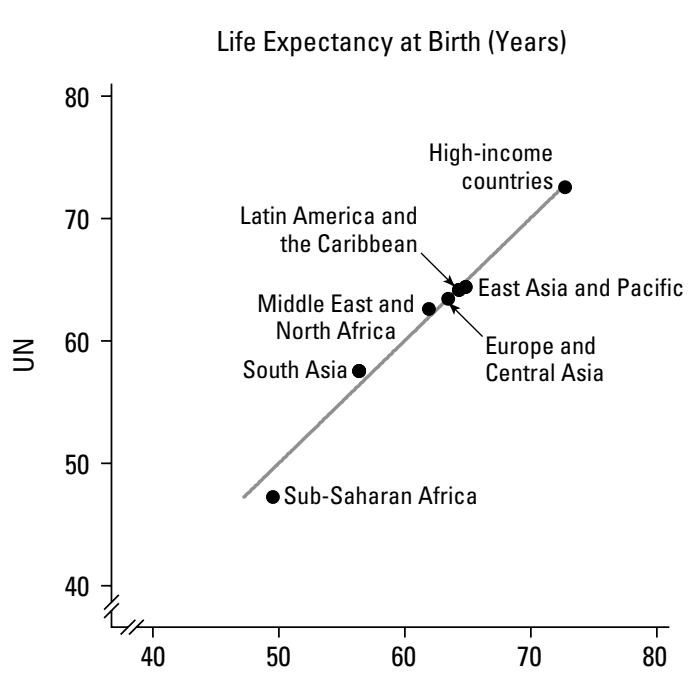

Probability of Mortality: 15 - to 59 -Year-Olds (per 1,000)

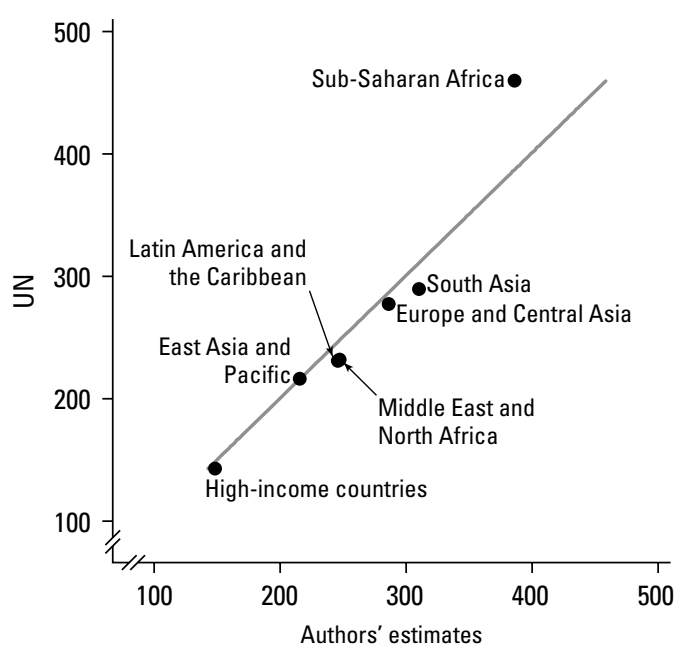

Probability of Mortality: Children under Five (per 1,000)

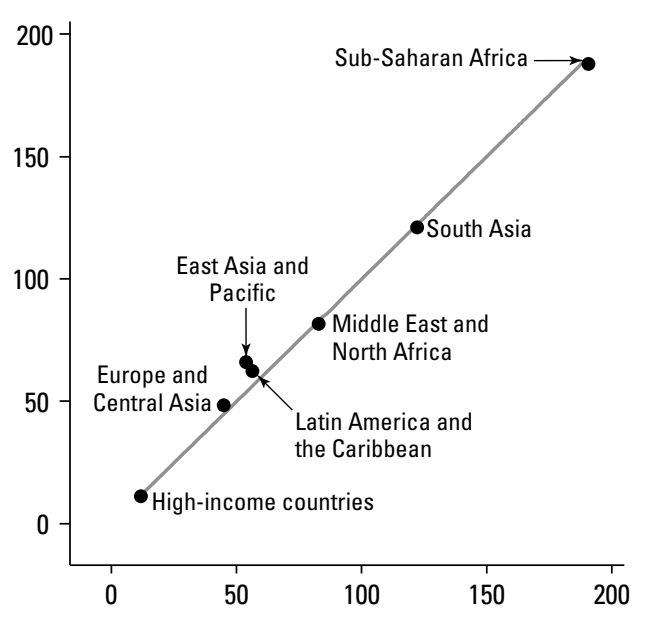

Probability of Mortality: 60- to 79-Year-Olds (per 1,000)

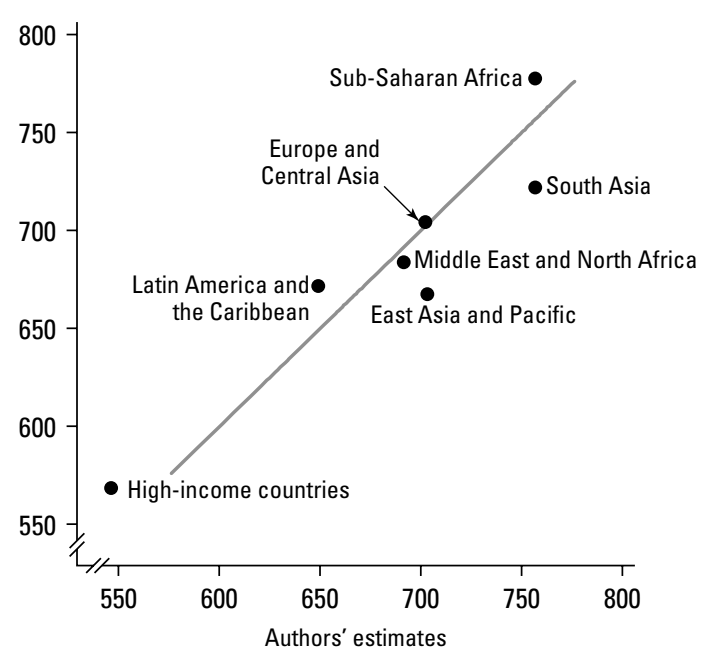

Figure 2.3 UN's versus Authors' Life Table Parameters, 1990

mortality risk for children under five years of age) are virtually identical, with a possible exception being the UN's slightly higher levels of child mortality for East Asia and the Pacific (which is dominated by China). This congruence is not unexpected given the intense collaborative efforts of the past five years or so by the World Health Organization, the United Nations Children's Fund, the United Nations, and the World Bank to agree upon a common interpretation of the extensive data available on trends in child mortality in low- and middle-income countries.

Somewhat surprisingly given the quite different methodological approaches, regional estimates of adult mortality ${ }_{45} q_{15}$ (the mortality risk for adults between the ages of 15 and $60)$ are remarkably similar, with our estimates tending to be slightly higher in the Middle East and North Africa and South Asia for males and slightly lower in the same regions for females. That is, we have estimated larger sex mortality differentials among adults than the UN on the basis of observed patterns of mortality where data were available (as in the Arab Republic of Egypt and India), and where not, on the basis of observed differences in child mortality for boys and girls. Some investigators expect male excess mortality to increase with social development and economic growth (Bhatia 1983), but whether this is better reflected in our estimates or those of the UN is not clear. In any case, the differences are minor. Significantly more disagreement is apparent for Sub-Saharan Africa, with the UN estimates of adult mortality in 1990 being one-quarter to one-third higher than ours. This is obviously 


\section{b. Females}

Life Expectancy at Birth (Years)

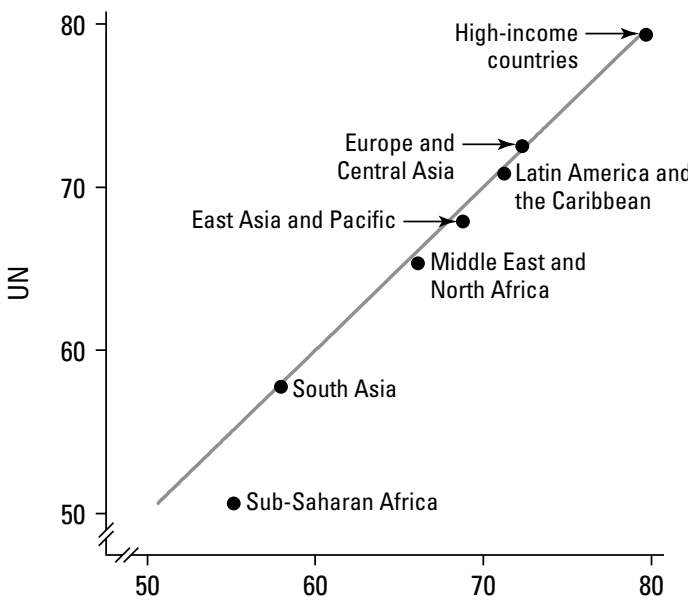

Probability of Mortality: 15- to 59-Year-Olds (per 1,000)

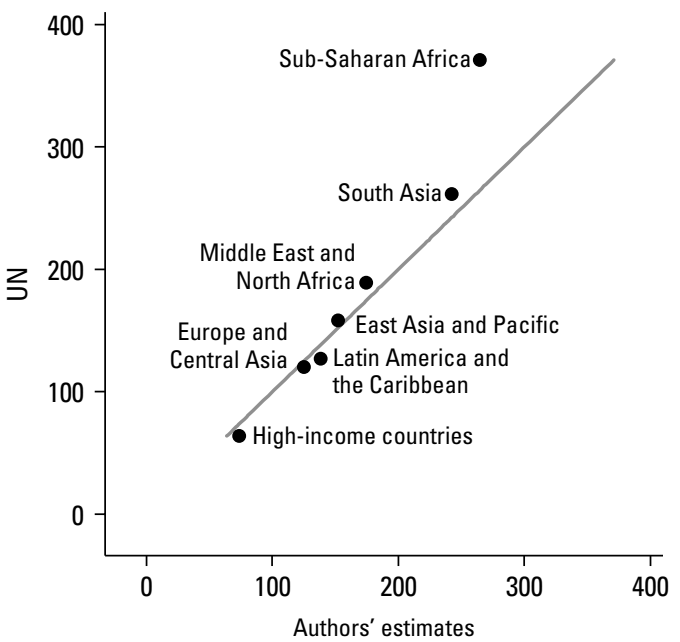

Probability of Mortality: Children under Five (per 1,000)

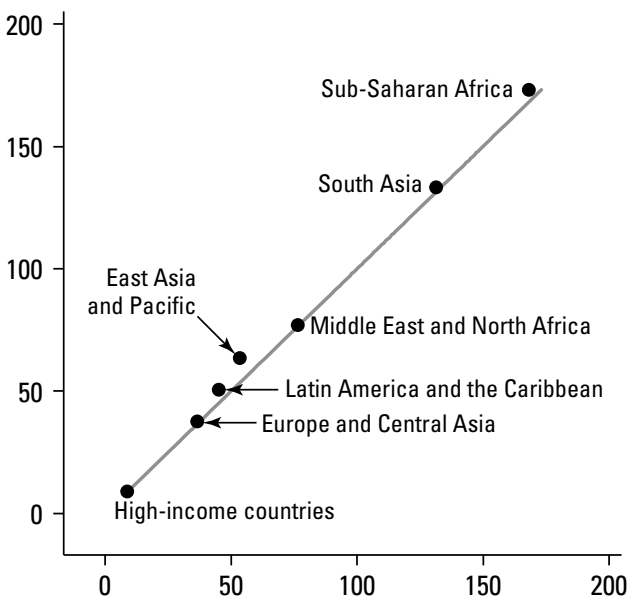

Probability of Mortality: 60- to 79-Year-Olds (per 1,000)

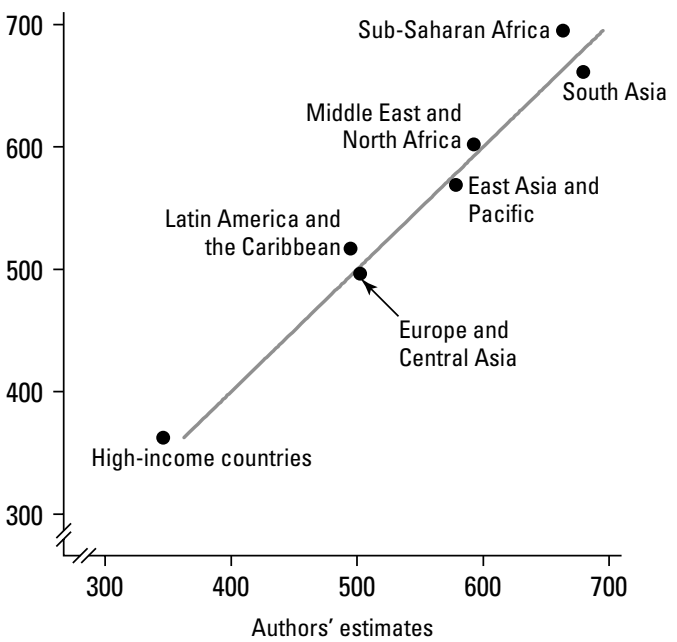

Sources: UN parameters are from United Nations 2005b; authors' estimates are from this chapter.

Figure 2.3 Continued

uncertain given the sparse data available on adult mortality in the region and the fact that the HIV epidemic in Sub-Saharan Africa was well established by then, and hence a higher estimate may be justified. Recent evidence, however, has suggested that basing mortality estimates on prenatal clinic data may well lead to an overestimation of death rates due to HIV.

Differences in methodology and adjustment criteria appear to have the greatest effect at older ages, especially for males. The UN estimates indicate significantly higher mortality in high-income countries at ages 60 to 79 even though complete vital registration data are available for virtually all the countries except some of the small Gulf states. Differences in estimated mortality for the Sub-Saharan
Africa region are not unexpected given the differences reported for younger adults, and are less extreme than at ages 15 to 59, as one might expect given that HIV/AIDS mortality is not of major consequence for older ages. Otherwise, estimates for females at older ages agree quite closely, but the UN's are significantly higher than ours for Latin America and the Caribbean and significantly lower for East Asia and the Pacific and South Asia. These differences arise because the model life table methods used by the UN tend to shift deaths from younger to older adult ages at lower levels of child mortality (Latin America and the Caribbean) and the converse at higher levels of child death rates (East Asia and the Pacific and South Asia). 
Table 2.3 Selected Mortality Characteristics by Sex and World Bank Region, 1990 and 2001

\begin{tabular}{|c|c|c|c|c|c|c|c|c|c|c|}
\hline \multirow[b]{3}{*}{ Sex and region } & \multirow[b]{3}{*}{ Year } & \multicolumn{9}{|c|}{ Male } \\
\hline & & \multirow{2}{*}{$\begin{array}{c}\text { Deaths } \\
\text { (millions) }\end{array}$} & \multirow{2}{*}{$\begin{array}{l}\text { Crude death } \\
\text { rate per } 1,000\end{array}$} & \multicolumn{2}{|c|}{$\%$ of deaths } & \multicolumn{4}{|c|}{ Probability of dying per 1,000} & \multirow{2}{*}{$\begin{array}{c}\text { Expectation } \\
\text { of life at } \\
\text { birth (years) }\end{array}$} \\
\hline & & & & Under age 5 & Over age 60 & Ages 0-5a & Ages 15-60 & Ages 0-60 & Ages $60-80$ & \\
\hline \multirow{2}{*}{$\begin{array}{l}\text { Low- and middle- } \\
\text { income countries }\end{array}$} & 1990 & 22.5 & 10.1 & 27.9 & 39.5 & 98 & 269 & 351 & 712 & 59.9 \\
\hline & 2001 & 25.5 & 9.7 & 21.2 & 42.2 & 86 & 269 & 341 & 667 & 61.2 \\
\hline \multirow[t]{2}{*}{ East Asia and Pacific } & 1990 & 6.6 & 8.0 & 16.3 & 50.6 & 54 & 215 & 265 & 699 & 64.9 \\
\hline & 2001 & 6.9 & 7.4 & 10.2 & 56.2 & 41 & 189 & 228 & 623 & 67.8 \\
\hline \multirow[t]{2}{*}{ Europe and Central Asia } & 1990 & 2.5 & 11.1 & 7.9 & 55.9 & 45 & 286 & 323 & 696 & 63.6 \\
\hline & 2001 & 3.0 & 13.0 & 3.2 & 59.6 & 32 & 328 & 353 & 711 & 63.0 \\
\hline \multirow{2}{*}{$\begin{array}{l}\text { Latin America and } \\
\text { the Caribbean }\end{array}$} & 1990 & 1.7 & 7.8 & 19.5 & 44.7 & 56 & 245 & 294 & 640 & 64.5 \\
\hline & 2001 & 1.8 & 7.0 & 12.4 & 49.1 & 38 & 218 & 252 & 572 & 67.6 \\
\hline \multirow{2}{*}{$\begin{array}{c}\text { Middle East and } \\
\text { North Africa }\end{array}$} & 1990 & 1.0 & 8.2 & 34.9 & 34.2 & 83 & 247 & 318 & 688 & 62.0 \\
\hline & 2001 & 1.1 & 6.8 & 21.6 & 45.2 & 56 & 216 & 267 & 674 & 65.2 \\
\hline \multirow[t]{2}{*}{ South Asia } & 1990 & 6.8 & 11.7 & 32.4 & 35.0 & 122 & 310 & 407 & 754 & 56.4 \\
\hline & 2001 & 7.1 & 9.9 & 25.1 & 39.2 & 94 & 285 & 362 & 710 & 59.9 \\
\hline \multirow[t]{2}{*}{ Sub-Saharan Africa } & 1990 & 3.9 & 15.6 & 54.1 & 17.0 & 191 & 386 & 517 & 758 & 49.6 \\
\hline & 2001 & 5.6 & 16.9 & 42.2 & 16.9 & 178 & 518 & 616 & 760 & 46.0 \\
\hline \multirow[t]{2}{*}{ High-income countries } & 1990 & 3.9 & 9.1 & 1.7 & 76.2 & 12 & 148 & 160 & 542 & 72.9 \\
\hline & 2001 & 4.0 & 8.8 & 1.0 & 78.7 & 7 & 124 & 132 & 469 & 75.5 \\
\hline \multirow[t]{2}{*}{ World } & 1990 & 26.4 & 10.0 & 24.0 & 44.8 & 91 & 245 & 323 & 667 & 61.7 \\
\hline & 2001 & 29.5 & 9.6 & 18.5 & 47.2 & 80 & 243 & 312 & 618 & 63.1 \\
\hline
\end{tabular}

Sources: Estimates for 1990 are authors' calculations, based on country-level life tables (see annex 2A). Estimates for 2001 are derived from Lopez and others 2002.

a. Estimates of child mortality are rounded to the nearest whole number.

Overall, as figure 2.3 demonstrates, the age patterns are largely compensatory, with the result that estimates of life expectancy at birth for the two series are remarkably similar for both males and females, with the notable exception being Sub-Saharan Africa, where the higher adult mortality assumptions favored by the UN result in life expectancies at birth that are about 2.5 years lower than ours for males and 5.0 years lower for females.

\section{Trends in Mortality Levels}

The 1990s were characterized by significant economic gains in most regions, with growth in gross national product per capita ranging from 18 percent in South Asia and SubSaharan Africa to more than 100 percent in East Asia and the Pacific and the Middle East and North Africa (table 2.2). Overall, gross national product per capita grew by about 35 percent in low- and middle-income countries during the decade. One would expect this to have led to a significant improvement in life expectancy, and this indeed occurred in most regions with the notable exception of Europe and Central Asia and, in particular, Sub-Saharan Africa (table 2.3). In the former region, life expectancy was largely unchanged over the decade, primarily because of the massive rise in adult mortality in countries such as the Russian Federation and its neighbors during the first part of the decade, which negated the declines in child mortality. Much of this extraordinary increase in adult mortality, which rose by about 50 percent between 1987 and 1994, has been attributed to alcohol abuse, particularly among men (Leon and others 1997; Shkolnikov, McKee, and Leon 2001).

Economic development and better coverage of the population with essential child health services have ensured continued declines in levels of child mortality, as measured by the risk of death from birth to age five, in all regions. The notable exception is Sub-Saharan Africa, where child mortality among girls remained unchanged at around 165 per 1,000 , with only a modest decline (5 percent) in the risk of death for boys. The absence of significant declines in child mortality in the 1990s in Sub-Saharan Africa is most likely largely due to the impact of HIV/AIDS. Overall, the risk of child death declined from 90 per 1,000 in 1990 to 80 per 1,000 in 2001, with the risk being remarkably similar for males and females (table 2.3); however, the differential in child mortality between the world's richest and poorest populations is stark, with a newborn in Sub-Saharan Africa 
Table 2.3 Continued

\begin{tabular}{|c|c|c|c|c|c|c|c|c|}
\hline \multicolumn{9}{|c|}{ Female } \\
\hline \multirow{2}{*}{$\begin{array}{r}\text { Deaths } \\
\text { (millions) }\end{array}$} & \multirow{2}{*}{$\begin{array}{c}\text { Crude death } \\
\text { rate per } 1,000\end{array}$} & \multicolumn{2}{|c|}{$\%$ of deaths } & \multicolumn{4}{|c|}{ Probability of dying per 1,000} & \multirow{2}{*}{$\begin{array}{c}\text { Expectation } \\
\text { of life at } \\
\text { birth (years) }\end{array}$} \\
\hline & & Under age 5 & Over age 60 & Ages 0-5a & Ages 15-60 & Ages 0-60 & Ages 60-80 & \\
\hline 19.4 & 8.9 & 29.7 & 44.6 & 95 & 182 & 270 & 585 & 64.2 \\
\hline 22.8 & 8.8 & 22.5 & 48.3 & 86 & 191 & 271 & 554 & 64.9 \\
\hline 5.5 & 7.0 & 17.8 & 56.2 & 53 & 152 & 204 & 577 & 68.8 \\
\hline 6.1 & 6.8 & 11.4 & 64.6 & 44 & 127 & 171 & 519 & 71.3 \\
\hline 2.4 & 9.9 & 6.4 & 77.5 & 37 & 125 & 162 & 503 & 72.3 \\
\hline 2.7 & 10.8 & 2.9 & 81.5 & 26 & 133 & 159 & 511 & 72.8 \\
\hline 1.3 & 5.7 & 20.1 & 53.9 & 45 & 138 & 182 & 493 & 71.3 \\
\hline 1.4 & 5.4 & 12.5 & 61.3 & 32 & 124 & 155 & 434 & 73.9 \\
\hline 0.8 & 6.9 & 37.7 & 36.1 & 76 & 174 & 245 & 593 & 66.1 \\
\hline 0.8 & 5.5 & 23.5 & 49.7 & 51 & 144 & 193 & 562 & 69.5 \\
\hline 6.1 & 11.2 & 37.0 & 33.7 & 131 & 243 & 357 & 680 & 57.9 \\
\hline 6.5 & 9.6 & 28.3 & 40.2 & 101 & 226 & 317 & 645 & 61.5 \\
\hline 3.3 & 12.9 & 54.8 & 19.6 & 168 & 265 & 403 & 664 & 55.1 \\
\hline 5.2 & 15.5 & 40.9 & 18.3 & 166 & 437 & 545 & 680 & 48.9 \\
\hline 3.6 & 8.2 & 1.3 & 87.3 & 9 & 74 & 83 & 346 & 79.7 \\
\hline 3.9 & 8.2 & 0.8 & 88.3 & 6 & 65 & 73 & 297 & 81.6 \\
\hline 23.0 & 8.8 & 25.2 & 51.3 & 88 & 161 & 244 & 516 & 66.6 \\
\hline 26.7 & 8.7 & 19.3 & 54.1 & 80 & 168 & 244 & 487 & 67.3 \\
\hline
\end{tabular}

facing 25 times the risk of death before the age of five than a newborn in a high-income country.

Despite the much greater uncertainty in relation to levels of adult mortality compared with those for children, the estimates shown in table 2.3 nonetheless indicate substantially different trends in adult mortality across different regions between 1990 and 2001. For most regions, the risk of death between ages 15 and 60 fell by about 10 to 17 percent over the decade. This was not the case in Europe and Central Asia, where policy shifts, particularly in relation to alcohol, together with broader social change, have largely been responsible for the 15 percent rise in adult male mortality and the 6 percent increase in the risk of death for women. Note that these estimates mask the large cyclical fluctuations in adult mortality in Russia, in particular, that characterized the region's mortality trends in the 1990s.

Table 2.3 also reveals the large increase in adult mortality in Sub-Saharan Africa, which was due primarily to the unfolding of the HIV/AIDS epidemic in southern Africa. Notwithstanding the substantial uncertainty surrounding these estimates, the epidemic appears to have been of proportionately greater consequence for women, with the rise in their risk of death (67 percent) being twice that of males, among whom other causes of death such as violence were more common. If these estimates are correct, then
52.0 percent of African males reaching age 15 and 44.0 percent of females will die before their 60th birthdays, compared with, for instance, 6.5 percent of women in highincome countries, who despite their already low risk enjoyed a further 11 percent decline in mortality during the 1990s. These reversals in mortality decline have effectively negated gains elsewhere, with the result that the global risk of adult death has remained essentially unchanged for males, and may even have risen slightly for females.

Taken together, the probability of death up to the age of five and between the ages of 15 and 60 are a better reflection of the risk of premature death than either alone, although both have particular public health implications. One might argue that health policy should be equally concerned with keeping adults alive into old age as it is with keeping children alive into adulthood. A convenient metric in this regard is the risk of death between birth and age 60 (table 2.3). In highincome countries, given 2001 mortality rates, only about 7 percent of females and 13 percent of males would be dead by age 60 , compared with 55 percent of females and 62 percent of males in Sub-Saharan Africa. Significant improvements in this summary measure of premature death can be observed in all regions except Europe and Central Asia and Sub-Saharan Africa. Worldwide, the index appears to have improved slightly for males and not at all for females. 
Other features of global mortality summarized in table 2.3 are worth highlighting. First is the impressive evidence of a continued decline in mortality among older age groups in high-income countries that began in the early 1970s. The risk of a 60 -year-old dying before age 80 declined by about 15 percent for both men and women in highincome countries so that at 2001 rates, less than 30 percent of women who reach age 60 will be dead by age 80 , as will less than 50 percent of men. Second, crude death rates in East Asia and the Pacific, Latin America and the Caribbean, and the Middle East and North Africa are lower than in high-income countries, reflecting the impact of the older age structure of rich countries, and are particularly low in Latin America and the Caribbean. Third, the proportion of deaths that occur below age five, while declining in all regions, varies enormously across them, from just over 1 percent in high-income countries to just over 40 percent in Sub-Saharan Africa. In some low- and middle-income regions, particularly East Asia and the Pacific, Europe and Central Asia, and Latin America and the Caribbean, the proportion is well below 20 percent. The net effect of these changes in age-specific mortality since 1990 has been to increase global life expectancy at birth by 0.7 years for females and by about twice this for males: a modest scorecard.

\section{TRENDS IN CAUSES OF CHILD DEATH, 1990-2001}

The estimation of cause of death patterns for world regions will, for the foreseeable future, be substantially uncertain given the paucity of data on medically certified deaths in many low- and middle-income countries (Mathers and others 2005; Sibai 2004). Verbal autopsies, that is, structured interviews with relatives of the deceased about symptoms experienced prior to death, will not yield the diagnostic accuracy achievable with medical certification based on good clinical case histories and medical records. This is not to deny that verbal autopsies can meet broad policy needs for information about causes of death, particularly with clinical input into the coding of interviewees' responses, but their reliability for diagnosing leading causes of child death is questionable (Snow and others 1992). Thus, estimates of child mortality derived from proportionate mortality models that are based largely on verbal autopsies need to be viewed with caution (Lopez 2003; Morris, Black, and Tomaskovic 2003).

Yet, despite these concerns about the quality of cause of death data, investigators can more confidently assess the comparative magnitude of causes of death for children than for adults. The fact that the demographic "envelope" of child deaths is reasonably well understood in all regions limits excessive claims about deaths due to individual causes, a constraint that is not a feature of adult mortality given the relative ignorance of age-specific death rates in many countries. In addition, the need for data on cause-specific outcomes to assess and monitor the impact of various child survival programs in recent decades has led to a reasonably substantial epidemiological literature that might permit cause-specific estimation, but under an unacceptably large number of assumptions (Black, Morris, and Bryce 2003).

A critical feature of any estimation exercise is a rigorous assessment of data sets for biases, study methods, and generalizability of results. Investigators have undertaken a number of efforts to estimate the causes of child mortality over the past decade or so (Bryce and others 2005; Lopez 1993; Morris, Black, and Tomaskovic 2004; Williams and others 2002), but undoubtedly the most comprehensive was the study by Murray and Lopez (1996) and its 2001 revision (chapter 3 in this volume). Both the latter Global Burden of Disease (GBD) studies apply methods to force epidemiological consistency according to the evidence available for each region, and inevitably the constraint of demography has meant that the GBD estimates of cause-specific mortality will differ from those developed largely independently of other causes. That is, the GBD estimates of specific causes of death are constrained to sum to the number of deaths derived from demographic analyses, whereas cause-specific estimates that are derived in the absence of such demographic constraints are unbounded and tend to be inclusive at the margin rather than exclusive. Differences in regional estimates between 1990 and 2001 arise in part because the countries included in the regions differed and, more important, because of better information for more recent periods. Yet, despite improved information, the true level of child death rates from major causes such as malaria and perinatal conditions (birth trauma, birth asphyxia, sepsis, and prematurity) remains largely unknown.

Notwithstanding methodological differences and uncertainties, deriving implied estimates of trends in the leading causes of child mortality is possible by comparing results from the two GBD studies, and these are summarized in table 2.4. These estimates have been simply obtained as the difference between the regional estimates for 1990 and 2001, but the implied pattern of change is interesting nonetheless. The conversion of the 1990 regional GBD estimates (Murray and Lopez 1996) to the regions used for the 2001 assessment 
Table 2.4 Mortality in Children Under Five by Cause, 1990 and 2001

\begin{tabular}{|c|c|c|c|c|c|c|c|c|c|c|c|c|c|c|c|c|c|c|}
\hline \multirow[b]{2}{*}{ Disease and indicator } & \multicolumn{2}{|c|}{$\begin{array}{l}\text { Low- and middle- } \\
\text { income countries }\end{array}$} & \multicolumn{2}{|c|}{$\begin{array}{l}\text { East Asia } \\
\text { and Pacific }\end{array}$} & \multicolumn{2}{|c|}{$\begin{array}{l}\text { Europe and } \\
\text { Central Asia }\end{array}$} & \multicolumn{2}{|c|}{$\begin{array}{l}\text { Latin America } \\
\text { and the Caribbean }\end{array}$} & \multicolumn{2}{|c|}{$\begin{array}{l}\text { Middle East and } \\
\text { North Africa }\end{array}$} & \multicolumn{2}{|c|}{ South Asia } & \multicolumn{2}{|c|}{$\begin{array}{c}\text { Sub-Saharan } \\
\text { Africa }\end{array}$} & \multicolumn{2}{|c|}{$\begin{array}{l}\text { High-income } \\
\text { countries }\end{array}$} & \multicolumn{2}{|c|}{ World } \\
\hline & 1990 & 2001 & 1990 & 2001 & 1990 & 2001 & 1990 & 2001 & 1990 & 2001 & 1990 & 2001 & 1990 & 2001 & 1990 & 2001 & 1990 & 2001 \\
\hline \multicolumn{19}{|l|}{ Acute respiratory infections } \\
\hline Deaths (thousands) & 2,521 & 1,943 & 492 & 197 & 68 & 36 & 83 & 44 & 138 & 76 & 1,027 & 833 & 713 & 757 & 13 & 2 & 2,533 & 1,944 \\
\hline$\%$ of childhood deaths & 21.0 & 18.4 & 23.8 & 14.0 & 19.5 & 20.6 & 14.1 & 10.9 & 20.6 & 17.7 & 23.2 & 23.1 & 18.3 & 16.8 & 11.1 & 2.3 & 20.9 & 18.3 \\
\hline $\begin{array}{l}\text { Probability of dying before } \\
\text { age } 5 \text { per } 1,000 \text { live births }\end{array}$ & 20 & 16 & 13 & 6 & 8 & 6 & 7 & 4 & 16 & 9 & 29 & 22 & 33 & 29 & 1 & 0 & 19 & 15 \\
\hline \multicolumn{19}{|l|}{ Congenital anomalies } \\
\hline Deaths (thousands) & 421 & 421 & 118 & 115 & 25 & 24 & 30 & 41 & 22 & 41 & 186 & 142 & 41 & 58 & 19 & 18 & 440 & 439 \\
\hline$\%$ of childhood deaths & 3.5 & 4.0 & 5.7 & 8.2 & 7.1 & 13.5 & 5.1 & 10.1 & 3.3 & 9.5 & 4.2 & 3.9 & 1.0 & 1.3 & 16.3 & 24.6 & 3.6 & 4.1 \\
\hline $\begin{array}{l}\text { Probability of dying before } \\
\text { age } 5 \text { per } 1,000 \text { live births }\end{array}$ & 3 & 3 & 3 & 4 & 3 & 4 & 3 & 4 & 3 & 5 & 5 & 4 & 2 & 2 & 2 & 2 & 3 & 3 \\
\hline \multicolumn{19}{|l|}{ Diarrheal diseases } \\
\hline Deaths (thousands) & 2,362 & 1,599 & 274 & 201 & 61 & 12 & 108 & 46 & 144 & 66 & 991 & 631 & 784 & 643 & 11 & 0 & 2,374 & 1,600 \\
\hline$\%$ of childhood deaths & 19.7 & 15.2 & 13.2 & 14.3 & 17.4 & 6.9 & 18.3 & 11.4 & 21.6 & 15.3 & 22.4 & 17.5 & 20.1 & 14.3 & 9.9 & 0.6 & 19.6 & 15.1 \\
\hline $\begin{array}{l}\text { Probability of dying before } \\
\text { age } 5 \text { per } 1,000 \text { live births }\end{array}$ & 19 & 13 & 7 & 6 & 7 & 2 & 9 & 4 & 17 & 8 & 28 & 17 & 36 & 25 & 1 & 0 & 17 & 12 \\
\hline \multicolumn{19}{|l|}{ HIV/AIDS } \\
\hline Deaths (thousands) & 62 & 340 & - & 5 & - & 0 & 2 & 6 & 0 & 1 & - & 14 & 60 & 313 & 0 & 0 & 62 & 340 \\
\hline$\%$ of childhood deaths & 0.5 & 3.2 & 0.0 & 0.4 & 0.0 & 0.2 & 0.3 & 1.4 & 0.0 & 0.1 & 0.0 & 0.4 & 1.5 & 7.0 & 0.0 & 0.1 & 0.5 & 3.2 \\
\hline $\begin{array}{l}\text { Probability of dying before } \\
\text { age } 5 \text { per 1,000 live births }\end{array}$ & 0 & 3 & 0 & 0 & 0 & 0 & 0 & 1 & 0 & 0 & 0 & 0 & 3 & 12 & 0 & 0 & 0 & 3 \\
\hline \multicolumn{19}{|l|}{ Injuries } \\
\hline Deaths (thousands) & 647 & 302 & 206 & 82 & 25 & 11 & 28 & 19 & 32 & 24 & 188 & 79 & 169 & 87 & 9 & 7 & 656 & 309 \\
\hline$\%$ of childhood deaths & 5.4 & 2.9 & 9.9 & 5.8 & 7.0 & 6.6 & 4.7 & 4.6 & 4.8 & 5.6 & 4.2 & 2.2 & 4.3 & 1.9 & 7.8 & 9.8 & 5.4 & 2.9 \\
\hline $\begin{array}{l}\text { Probability of dying before } \\
\text { age } 5 \text { per } 1,000 \text { live births }\end{array}$ & 5 & 2 & 5 & 2 & 3 & 2 & 2 & 2 & 4 & 3 & 5 & 2 & 8 & 3 & 1 & 1 & 5 & 2 \\
\hline \multicolumn{19}{|l|}{ Malaria } \\
\hline Deaths (thousands) & 588 & 1,086 & 7 & 27 & 0 & 0 & 2 & 1 & 1 & 17 & 9 & 57 & 570 & 984 & 0 & 0 & 588 & 1,086 \\
\hline$\%$ of childhood deaths & 4.9 & 10.3 & 0.3 & 1.9 & 0.1 & 0.0 & 0.3 & 0.3 & 0.1 & 3.9 & 0.2 & 1.6 & 14.6 & 21.8 & 0.2 & 0.1 & 4.8 & 10.2 \\
\hline $\begin{array}{l}\text { Probability of dying before } \\
\text { age } 5 \text { per } 1,000 \text { live births }\end{array}$ & 5 & 9 & 0 & 1 & 0 & 0 & 0 & 0 & 0 & 2 & 0 & 2 & 26 & 38 & 0 & 0 & 4 & 8 \\
\hline
\end{tabular}




\begin{tabular}{|c|c|c|c|c|c|c|c|c|c|c|c|c|c|c|c|c|c|c|}
\hline \multirow[b]{2}{*}{ Disease and indicator } & \multicolumn{2}{|c|}{$\begin{array}{l}\text { Low- and middle- } \\
\text { income countries }\end{array}$} & \multicolumn{2}{|c|}{$\begin{array}{c}\text { East Asia } \\
\text { and Pacific }\end{array}$} & \multicolumn{2}{|c|}{$\begin{array}{l}\text { Europe and } \\
\text { Central Asia }\end{array}$} & \multicolumn{2}{|c|}{$\begin{array}{l}\text { Latin America } \\
\text { and the Caribbean }\end{array}$} & \multicolumn{2}{|c|}{$\begin{array}{l}\text { Middle East and } \\
\text { North Africa }\end{array}$} & \multicolumn{2}{|c|}{ South Asia } & \multicolumn{2}{|c|}{$\begin{array}{l}\text { Sub-Saharan } \\
\text { Africa }\end{array}$} & \multicolumn{2}{|c|}{$\begin{array}{l}\text { High-income } \\
\text { countries }\end{array}$} & \multicolumn{2}{|c|}{ World } \\
\hline & 1990 & 2001 & 1990 & 2001 & 1990 & 2001 & 1990 & 2001 & 1990 & 2001 & 1990 & 2001 & 1990 & 2001 & 1990 & 2001 & 1990 & 200 \\
\hline \multicolumn{19}{|l|}{ Measles } \\
\hline Deaths (thousands) & 869 & 556 & 75 & 45 & 12 & 5 & 38 & - & 30 & 10 & 239 & 145 & 474 & 351 & 3 & 0 & 872 & 556 \\
\hline$\%$ of childhood deaths & 7.2 & 5.3 & 3.6 & 3.2 & 3.5 & 2.9 & 6.5 & 0.0 & 4.5 & 2.3 & 5.4 & 4.0 & 12.2 & 7.8 & 2.5 & 0.1 & 7.2 & 5.2 \\
\hline $\begin{array}{l}\text { Probability of dying before } \\
\text { age } 5 \text { per } 1,000 \text { live births }\end{array}$ & 7 & 5 & 2 & 1 & 1 & 1 & 3 & 0 & 4 & 1 & 7 & 4 & 22 & 13 & 0 & 0 & 6 & 4 \\
\hline \multicolumn{19}{|l|}{ Perinatal conditions } \\
\hline Deaths (thousands) & 2,261 & 2,492 & 480 & 506 & 83 & 57 & 162 & 164 & 141 & 106 & 906 & 1,086 & 487 & 573 & 38 & 32 & 2,298 & 2,524 \\
\hline$\%$ of childhood deaths & 18.8 & 23.7 & 23.2 & 36.0 & 23.6 & 32.9 & 27.6 & 40.3 & 21.2 & 24.7 & 20.4 & 30.1 & 12.5 & 12.7 & 33.0 & 44.5 & 18.9 & 23.8 \\
\hline $\begin{array}{l}\text { Probability of dying before } \\
\text { age } 5 \text { per } 1,000 \text { live births }\end{array}$ & 18 & 20 & 12 & 15 & 10 & 10 & 14 & 14 & 17 & 13 & 26 & 29 & 22 & 22 & 3 & 3 & 17 & 19 \\
\hline \multicolumn{19}{|l|}{ Other causes } \\
\hline Deaths (thousands) & 2,288 & 1,792 & 420 & 228 & 77 & 28 & 137 & 85 & 159 & 90 & 888 & 625 & 607 & 737 & 22 & 13 & 2,309 & 1,805 \\
\hline$\%$ of childhood deaths & 19.0 & 17.0 & 20.3 & 16.2 & 21.9 & 16.3 & 23.2 & 21.0 & 23.8 & 20.9 & 20.0 & 17.3 & 15.5 & 16.4 & 19.1 & 17.9 & 19.0 & 17.0 \\
\hline $\begin{array}{l}\text { Probability of dying before } \\
\text { age } 5 \text { per } 1,000 \text { live births }\end{array}$ & 18 & 15 & 11 & 7 & 9 & 5 & 12 & 7 & 19 & 11 & 25 & 17 & 28 & 28 & 2 & 1 & 17 & 14 \\
\hline \multicolumn{19}{|l|}{ Total } \\
\hline Deaths (thousands) & 12,019 & 10,532 & 2,072 & 1,407 & 352 & 174 & 588 & 407 & 668 & 429 & 4,434 & 3,612 & 3,904 & 4,504 & 115 & 73 & 12,134 & 10,605 \\
\hline$\%$ of childhood deaths & 100.0 & 100.0 & 100.0 & 100.0 & 100.0 & 100.0 & 100.0 & 100.0 & 100.0 & 100.0 & 100.0 & 100.0 & 100.0 & 100.0 & 100.0 & 100.0 & 100.0 & 100.0 \\
\hline $\begin{array}{l}\text { Probability of dying before } \\
\text { age } 5 \text { per } 1,000 \text { live births }\end{array}$ & 97 & 86 & 54 & 43 & 41 & 29 & 51 & 35 & 80 & 53 & 127 & 97 & 180 & 172 & 10 & 7 & 89 & 80 \\
\hline
\end{tabular}

Sources: Estimates for 1990 are based on Murray and Lopez 1996, weighted to World Bank regions using population under five years old. Estimates for 2001 are from chapter 3 in this volume. Note: $-=$ not available or not applicable. Estimates of child mortality are rounded to the nearest whole number 
Acute Respiratory Infections

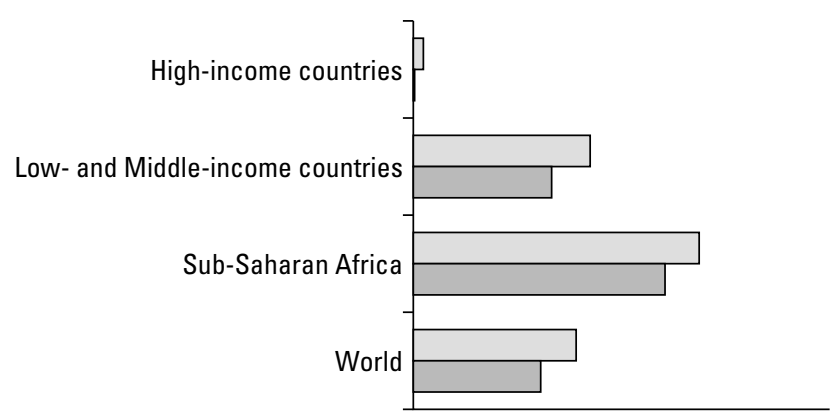

HIV/AIDS
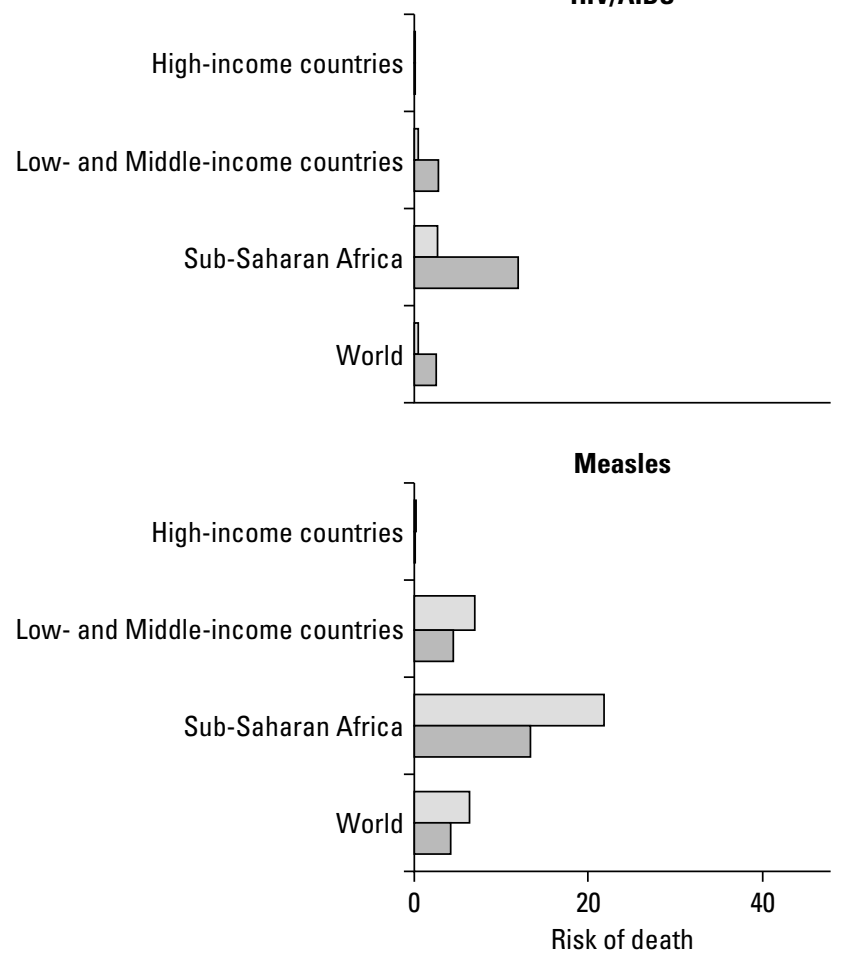

Congenital Anomalies

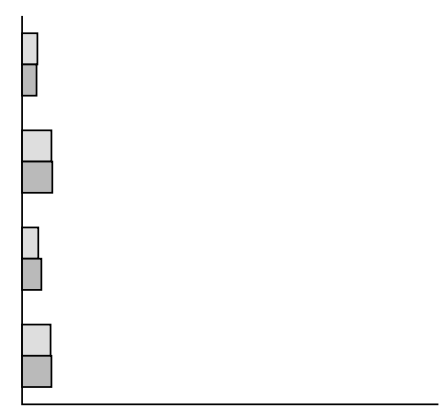

Injuries

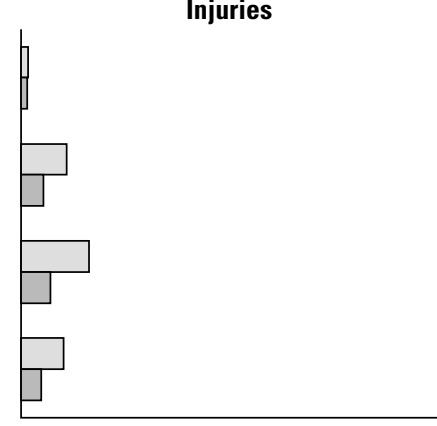

Perinatal Conditions

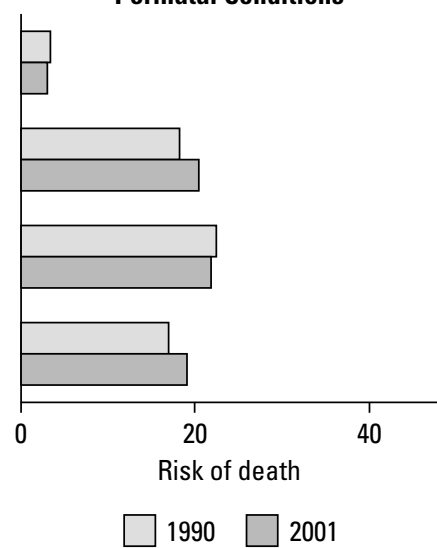

Diarrheal Diseases

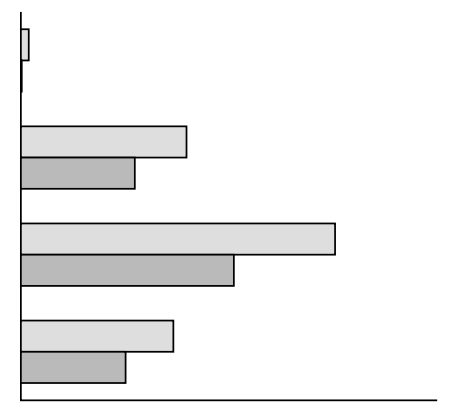

Malaria

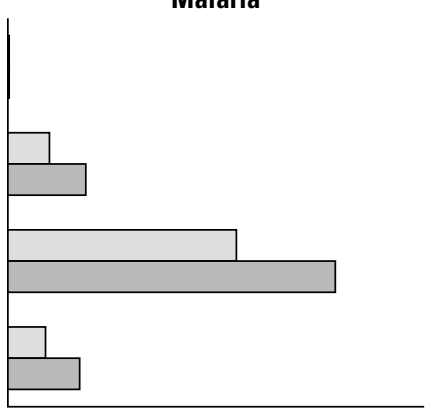

Other

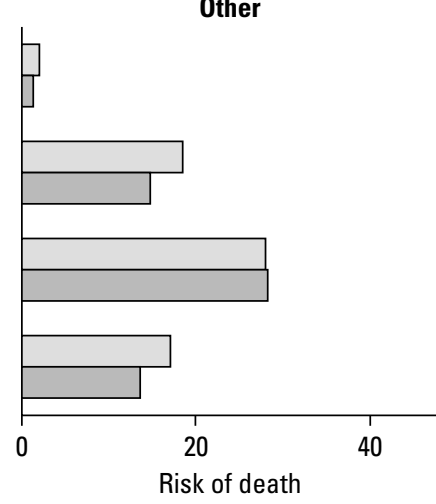

Sources: Estimates for 1990 are from Murray and Lopez 1996; estimates for 2001 are from chapter 3 in this volume.

Figure 2.4 Change in Risk of Death for Children Under Five by Cause (probability of mortality per 1,000 live births), 1990-2001

was done simply by population weighting, a very approximate procedure. By contrast, the 2001 estimates were prepared as regional aggregates of country-specific estimates (see chapter 3,) and this has undoubtedly affected comparisons further.

Global mortality from malaria increased by 0.5 million during the 1990s, with 80 percent of the deaths occurring in Sub-Saharan Africa. The proportion of all child deaths due to malaria doubled from 5 percent in 1990 to 10 percent in 2001 worldwide and increased from 15 percent in 1990 to
22 percent in 2001 in Sub-Saharan Africa. The only other causes that appear to have increased are HIV/AIDS in Africa, a reasonable conclusion given female prevalence levels, and the category of perinatal conditions, which are strongly dependent on the quality and availability of prenatal services. Causes that appear to have declined substantially include acute respiratory infections ( 2.5 million to 1.9 million deaths or 15 percent of all child deaths), diarrheal diseases (2.4 million to 1.6 million deaths or 13 percent of child deaths), measles ( 0.8 million to 0.5 million 
deaths or 5 percent of child deaths), and injuries ( 0.6 million to 0.3 million deaths or 2 percent of child deaths).

The implied pattern of change in the risk of child death varies across regions for all major conditions listed in table 2.4, particularly with regard to the magnitude of change. This can be seen more clearly from figure 2.4, which summarizes these trends for broad regional aggregates and for Sub-Saharan Africa. In general, the absolute change in risk of death has been greater in Sub-Saharan Africa than elsewhere, both for causes with increased risk (HIV/AIDS, malaria) and where risk has declined (diarrheal diseases, measles).

While these changes may be in accord with what is known about regional health development and economic growth, they need to be confirmed. Some of the suggested changes warrant further investigation, for example, death rates from perinatal causes appear to have risen in both East Asia and the Pacific and South Asia and remained unchanged in Latin America and the Caribbean, which may or may not be in line with what is known about developments in prenatal care and safe motherhood initiatives. Similarly, measles appears to have disappeared as a cause of child death in Latin America and the Caribbean. The risk of child death from congenital anomalies appears to have risen in both Latin America and the Caribbean and the Middle East and North Africa, but why is unclear. Similarly, the large suggested declines in the risk of child deaths because of injury in South Asia and Sub-Saharan Africa appear unlikely and may largely reflect better data and methods for measuring injury deaths.

\section{DISCUSSION}

Understanding the demographic context of health status assessments such as the GBD studies is essential if policy directions and program delivery are to be focused appropriately. Knowledge about the size and composition of populations and how they are changing is critical for health planning and priority setting. Demographers and demographic institutions such as the UN Population Division have applied the demographic ethos that available data permit making estimates and reasonable predictions of population change provided the data are interpreted and used appropriately.

Such estimates and projections have been useful for social and economic development for countries, regions, and the world as a whole. They suggest that health and social policies need to pay increasing attention to the key demographic trends observed in the 1990s, namely, rapidly falling fertility virtually everywhere, rapidly aging populations, and unprecedented reversals of the long-term path of mortality decline in Europe and Central Asia and Sub-Saharan Africa The causes of these so-called mortality shocks are reasonably well understood, but the lessons for health policy cannot be overemphasized. Globally, the mortality reversals caused by inadequate preventive programs, social disintegration, and failure to understand the gravity of rapidly expanding epidemics have meant that the 1990s were a lost decade for further improvements in adults' survival prospects. Thus, despite the substantial and continued declines in mortality from major vascular diseases in high-income countries, worldwide the risk of death in adulthood did not change in the 1990s, although some gains in reducing mortality in the elderly were achieved, particularly in rich countries.

The trend in child mortality during the 1990s was only marginally more satisfactory. While most regions achieved significant gains in child survival, progress was modest in Sub-Saharan Africa, and as a result, the global decline in child mortality slowed to an annual average of about 1 percent over the decade.

Decades of intensive data collection on child mortality in many low- and middle-income countries by dedicated international survey programs and the efforts of agencies such as the United Nations Children's Fund mean that trends in overall child mortality, and the numbers of child deaths they imply, can be established with reasonable certainty. The trends in the leading causes of child mortality are, however, much more difficult to establish (Rudan and others 2005). Much debate in the literature has centered on whether the risk of malaria infection in Sub-Saharan Africa increased in the 1990s, and thus whether the massive increase in malaria deaths suggested in table 2.4 is real (Korenromp and others 2003; Snow, Trape, and Marsh 2001; Trape 2001). Most malaria mortality in Sub-Saharan Africa is diagnosed via verbal autopsies, which, where studied, have been shown to be a poor diagnostic tool for malaria (Snow and others 1992). While some evidence from demographic surveillance sites using verbal autopsies indicates that malaria mortality rates have increased in eastern and southern Africa (summarized in Korenromp and others 2003) and that the spread of chloroquine resistance may have been the primary reason (Snow and others 1999; Trape 2001), whether this is sufficiently widespread to account for the implied rise of almost 50 percent in malaria mortality rates over the decade (figure 2.4) is unclear. Other factors, such as 
a general deterioration in clinical care and a decline in the efficacy of chloroquine therapy, may also have contributed (Snow and others 2001), but how much of the rise is real and how much is due to different interpretations of available data in 1990 and 2001 remains unknown.

Similarly, the substantial implied declines in the risk of child death from acute respiratory infections and diarrheal diseases need to be understood in the context of likely contributing factors. One of these is no doubt malnutrition, because it is a major risk factor for both conditions (Black, Morris, and Bryce 2003; Pelletier, Frongillo, and Habicht 1993; Rice and others 2000; Tupasi and others 1988). In the 1990s, malnutrition, as assessed by childhood stunting, declined in all regions except Sub-Saharan Africa (de Onis, Frongillo, and Blossner 2000), which is consistent with the modest declines in mortality from respiratory infections among children in the region. Increased use of oral rehydration therapy and improved access to safe water and sanitation in the 1990s would suggest some decline in mortality from diarrheal disease, but whether they were sufficient to account for the one-third decline in risk, including in Sub-Saharan Africa, is also unclear (Victora and others 2000). The large absolute decline in childhood diarrheal deaths from 2.4 million in 1990 to 1.6 million in 2001 is surprising, and suggests that the 2001 estimate may be an undercount. Some other studies (Morris, Black, and Tomaskovic 2003; UNICEF 2003) suggest a figure about 20 percent higher for 2001.

Malnutrition is also a leading risk factor for measles mortality, and hence changes in the proportion and distribution of underweight children should be broadly consistent with mortality trends from the disease (Fishman and others 2004). Effective vaccination coverage is a primary determinant of mortality from measles, and further increases in vaccination coverage in the 1990s should have led to lower mortality. This is certainly apparent from the estimates reported here, but the extent of that decline is subject to some controversy, depending on the methods used to estimate current mortality. Using proportionate mortality models largely derived from verbal autopsy data, Morris, Black, and Tomaskovic (2003) estimate that measles deaths account for only about 2.2 percent of all child deaths in South Asia and Sub-Saharan Africa, significantly less than Stein and others' (2003) estimates of 4 to 8 percent for the same period using data on vaccination coverage and assumptions about efficacy and case fatality rates. This implies a global estimate of measles deaths that is about half the 556,000 estimated for 2001 in chapter 3, and thus a much faster rate of decline in the measles mortality rate during the 1990s than the one-third reduction suggested by the GBD estimates. The truth may well lie somewhere in between and requires urgent resolution if measles control efforts are to be appropriately guided.

While the confirmation of mother to child transmission of HIV infection implies that mortality from the disease will increase with increasing prevalence among women, the extent of the impact on child mortality continues to be debated. The GBD estimates suggest that HIV/AIDS led to an increase in child mortality of, on average, 10 per 1,000 in Sub-Saharan Africa between 1990 and 2001. Recent research suggesting a potential overestimation of HIV/AIDS mortality may lead to lower estimates of child mortality from the disease, which may attenuate this trend estimate. What is clear is that HIV/AIDS has not been the only cause of recent reversals in the decline in child mortality in SubSaharan Africa (Walker, Schwartzlander, and Bryce 2002) and that its effect on child survival in the 1990s may not have been as great as initially thought (Adetunji 2000).

Perinatal conditions that cover specific risks for the newborn, primarily birth asphyxia, birth trauma, prematurity, and sepsis, are undoubtedly a major cause of death among children, but until recently did not receive sufficient attention in the epidemiological literature, perhaps because interventions are largely related to the delivery of prenatal care and the intrapartum period. Virtually all children born alive who die from these causes do so in the first few days of life (Lawn, Cousens, and Zupan 2005). Hence some constraint on the probable demographic envelope of mortality from these causes can be derived by estimating the neonatal mortality rate in different regions as was done for the 1990 GBD study (Murray and Lopez 1996) and repeated for the 2001 estimates (chapter 3 in this volume). This has undoubtedly removed a major source of uncertainty about mortality from these conditions, but substantial uncertainty remains about their relative importance as a cause of neonatal death when considering other conditions such as tetanus (classified under infectious diseases in the 1990 and 2001 GBD studies), neonatal diarrhea, congenital anomalies, and injuries. As a result, the global estimate of deaths from perinatal causes is influenced by the availability and reliability of data on the causes of neonatal mortality, particularly in countries with the largest number of neonatal deaths: China, India, Nigeria, and Pakistan account for half of all neonatal deaths, and with the possible exception of China, none has reliable, nationally representative systems for cause of death reporting. 
Given this context, judging whether mortality from perinatal causes indeed rose by 10 percent during the 1990s as suggested by figure 2.4 is difficult. If improvements in survival from these causes are largely related to better and more comprehensive service provision for pregnant women, which in turn is dependent on substantial infrastructure investments to improve health services, then modest declines in risk should be expected given economic growth in the 1990s. This was indeed the case in all regions except East Asia and the Pacific and South Asia, where the risk appears to have risen. Without compelling evidence that health service provision deteriorated in these regions during the 1990s, this increase in the risk of death from perinatal causes is probably a statistical artifact of data availability and different interpretation criteria used for 1990 and 2001. Privatization of the rural health care system in China during the 1990s may have led to a deterioration of prenatal care, but this remains to be established.

Finally, note the 50 percent reduction in the risk of child death from injuries implied by the GBD studies, which is primarily due to large reductions suggested for East Asia and the Pacific, South Asia, and Sub-Saharan Africa. Some decline in injury mortality is to be expected with economic and social development and the introduction of injury control programs and legislation, but the massive declines estimated for these regions may well be attributable to methodological differences in estimation procedures between the two dates (see chapter 3 in this volume). The descriptive epidemiology of injuries remains a major neglected area of the information base for policy to improve child health. For example, Rudan and others' (2005) review of information gaps in relation to assessing the burden of illness in children fails to even mention childhood injuries, even though burns, falls, and drownings are likely to be significant causes of child death (Etebu and Ekere 2004; Gali, Madziga, and Naaya 2004; Istre and others 2003; Mock and others 2004; Shen, Sanno-Duanda, and Bickler 2003). Thus, establishing the extent of changes in these risks, whose levels are based on essentially anecdotal evidence, remains difficult. Evidence of major declines in injury death rates therefore need to be viewed with great caution and may well be largely artifactual.

The global public health community's increasing interest in gaining a better understanding of the successes of, and challenges facing programs and policies to improve child survival has led to alternative assessments of the leading causes of child death. With the substantial data gaps and data quality issues pertaining to the estimation of child mortality, varying estimates of the leading causes of child death because of different estimation principles and variable interpretation of the data are hardly surprising. Scientific debate is to be encouraged insofar as it will guide data collection strategies to reduce unacceptable uncertainty, but the existence of alternative estimates of child mortality for 2001 makes the interpretation of changes over the past decade even more complex. The World Health Organization's Child Health Epidemiology Reference Group (CHERG), for example, working within the same total number of child deaths (10.5 million), has recently published quite different estimates of the causes of child mortality (Bryce and others 2005). According to CHERG's estimates, in 2001 perinatal causes were responsible for 3.9 million child deaths, that is, 37 percent of all child deaths and 55 percent more than the GBD figure (see chapter 3). Conversely, CHERG estimated lower levels of malaria mortality in children (853,000 deaths compared with 1,110,000 in the GBD study) and much lower measles mortality (395,000 versus 562,000 deaths). CHERG's implied rise in perinatal causes of child death is even more extreme than that suggested by the GBD study, whereas the rise in malaria deaths is less extreme. CHERG's estimates also imply far greater success of vaccination programs to reduce measles mortality than does the GBD figure. Note also that CHERG, which does not include any experts in noncommunicable diseases, including congenital anomalies, estimated only about half the number of noncommunicable disease deaths among children than estimated in the GBD study.

Public policy to accelerate the decline in child mortality would be well served through greater scientific collaboration to better understand the descriptive epidemiology of the leading causes of child death over the past decade or so and how this has changed. Notwithstanding the legitimate role of scientific discourse and the issue of comorbidity among the leading causes of child death, particularly diarrhea and pneumonia (Fenn, Morris, and Black 2005), the lack of clarity about the extent of the decline (or rise) in child deaths from specific causes or groups of causes, particularly those that have been the focus of massive programmatic efforts, hinders policy making. Having said this, it should also be borne in mind that for the GBD estimates at least, which have followed a consistent methodology and estimation framework, uncertainty in the rate of change of mortality for any given condition may well be less than period uncertainty around estimates for 1990 and 2001 because of the high likelihood of correlation of uncertainty of estimates for the two periods. 


\section{CONCLUSIONS}

Priority setting in health, as in other sectors concerned with social development, will increasingly depend on the availability of reliable, timely, representative, and relevant information on the comparative importance of diseases, injuries, and risk factors for the health of populations and how these are changing. Population scientists, particularly epidemiologists, have provided important insights into the descriptive epidemiology of some segments of some populations and on the causes of disease and injuries in those populations. Administrative requirements have resulted in most countries undertaking routine data collection efforts, but these are highly variable in terms of both quality and of what is being measured. As a result, we have substantial partial data collections on many aspects of population health status, but no country has complete data on all aspects of health relevant for policy, and in many parts of the world, health status is largely unknown. Efforts to bring these fragmentary pieces of data together to develop comprehensive estimates of the disease and injury burden and its causes are likely to be extremely valuable for policy making, particularly if the analytical methods and frameworks employed are understandable, transparent, and rigorously argued. Demographers were the first to attempt global, regional, and national efforts to estimate population size, structure, and determinants of change in a coherent fashion, and despite scientific differences of opinion about some of the methods and assumptions, the results have been enormously influential for guiding social development policies and programs.

The two GBD studies for 1990 (Murray and Lopez 1996) and 2001 (chapter 3 in this volume) attempted something similar for mortality and the burden of disease. Scholars and global health development agencies alike have repeatedly emphasized the interrelationship between demographic change and the health conditions of populations. This chapter has summarized the key quantitative findings about global demography and epidemiology that are relevant for disease control and public health development, leading to the following three broad conclusions:

- Despite significant investments in disease control in lowand middle-income countries over the past 50 years and the considerable success in reducing mortality, commensurate investments have not been made in the health intelligence base needed to monitor and evaluate changes in population health. As a result, uncertainty about the causes of child mortality in many countries and how these have changed over the last decade or so because of intervention programs is considerable. Moreover, data collection pertaining to health conditions among adults has been almost totally neglected, with the result that virtually nothing is known reliably about levels, let alone causes, of adult death in much of the developing world. HIV/AIDS has highlighted this neglect, but continued ignorance of the leading causes of adult mortality will continue to hinder policy action to reduce the large, avoidable causes of adult mortality that can be addressed through targeted prevention and treatment programs.

- Demographic change is often poorly understood, and thus potentially underappreciated in relation to health and social development policies. The evidence summarized in this chapter suggests that population aging is likely to become rapidly more pronounced in low- and middle-income countries than is currently appreciated, in part because swift fertility declines are under way in much of the developing world. The little evidence that is available about mortality trends among adults in developing countries suggests different paths of mortality change among regions, but indicates that globally, little progress was achieved in the 1990s. At older ages, the impressive and widely unappreciated declines in mortality that began in the high-income countries in the 1970s continued through the 1990s and show little sign of deceleration. In large part, these declines reflect progress in the control of major vascular diseases and point to continued steady gains in life expectancy in high-income countries.

- Despite at least two decades of intensive efforts by the global public health community to implement intervention programs and reorganize health services to reduce child mortality, knowledge about the major causes of death among children is insufficiently precise to resolve uncertainties about global progress with specific disease control strategies, and thus to be of maximum benefit for global policy action to reduce the more than 10 million child deaths that still occur each year. Results from the two GBD studies, while suggesting trends that are broadly consistent with public health knowledge, are equivocal about trends in specific conditions in some regions. Policy action to rapidly and substantially reduce this enormous burden of premature mortality will be better served if policy makers can be more appropriately informed about the causes of child death, including hitherto neglected areas such as perinatal conditions and injuries. 
Annex 2A Key Demographic Indicators, by Country/Territory, 1990 and 2001

\begin{tabular}{|c|c|c|c|c|c|c|c|c|c|c|c|c|c|c|c|c|c|}
\hline \multirow[b]{4}{*}{ Country/Territory } & \multirow[b]{4}{*}{ World Bank region } & \multicolumn{7}{|c|}{1990} & \multicolumn{7}{|c|}{2001} & \multirow{3}{*}{\multicolumn{2}{|c|}{$\begin{array}{c}\text { Annual change } \\
\text { in probability } \\
\text { of dying } \\
\text { under age 5, } \\
\text { 1990-2001 (\%) }\end{array}$}} \\
\hline & & \multirow{3}{*}{$\begin{array}{l}\text { Population } \\
\text { (thousands) }\end{array}$} & \multirow{2}{*}{\multicolumn{2}{|c|}{$\begin{array}{l}\text { Life expectancy } \\
\text { at birth (years) }\end{array}$}} & \multicolumn{4}{|c|}{$\begin{array}{l}\text { Probability of } \\
\text { dying per 1,000 }\end{array}$} & \multirow{3}{*}{$\begin{array}{c}\text { Population } \\
\text { (thousands) }\end{array}$} & \multirow{2}{*}{\multicolumn{2}{|c|}{$\begin{array}{l}\text { Life expectancy } \\
\text { at birth (years) }\end{array}$}} & \multicolumn{4}{|c|}{$\begin{array}{r}\text { Probability of } \\
\text { dying per } 1,000\end{array}$} & & \\
\hline & & & & & \multicolumn{2}{|c|}{ Under age 5} & \multicolumn{2}{|c|}{ Ages 15-59 } & & & & \multicolumn{2}{|c|}{ Under age 5} & \multicolumn{2}{|c|}{ Ages 15-59 } & & \\
\hline & & & Males & Females & Males & Females & Males & Females & & Males & Females & Males & Females & Males & Females & Males & Females \\
\hline Afghanistan & South Asia & 13,799 & 43.8 & 47.8 & 267 & 253 & 421 & 295 & 22,083 & 41.4 & 43.3 & 257 & 255 & 510 & 419 & -0.3 & 0.1 \\
\hline Albania & Europe and Central Asia & 3,289 & 64.1 & 71.2 & 52 & 38 & 253 & 140 & 3,122 & 67.0 & 73.4 & 28 & 23 & 171 & 97 & -5.6 & -4.4 \\
\hline Algeria & Middle East and North Africa & 25,017 & 65.4 & 67.8 & 70 & 68 & 188 & 153 & 30,746 & 67.4 & 71.0 & 55 & 44 & 172 & 129 & -2.3 & -3.9 \\
\hline American Samoa & East Asia and Pacific & - & - & - & - & - & - & - & 59 & 59.9 & 36.4 & 86 & 79 & 295 & 229 & - & - \\
\hline Andorra & High-income countries & - & - & - & - & - & - & - & 68 & 76.6 & 83.7 & 5 & 4 & 113 & 45 & - & - \\
\hline Angola & Sub-Saharan Africa & 9,340 & 42.7 & 49.0 & 280 & 239 & 427 & 288 & 12,768 & 37.5 & 42.3 & 277 & 246 & 605 & 475 & -0.1 & 0.2 \\
\hline Anguilla & Not included & - & - & - & - & - & - & - & 11 & 68.0 & 72.8 & 34 & 27 & 216 & 116 & - & - \\
\hline Antigua and Barbuda & Latin America and the Caribbean & - & - & - & - & - & - & - & 72 & 68.9 & 73.7 & 22 & 18 & 197 & 128 & - & - \\
\hline Argentina & Latin America and the Caribbean & 32,527 & 68.9 & 75.8 & 32 & 26 & 196 & 103 & 37,529 & 70.9 & 78.5 & 20 & 17 & 177 & 91 & -4.2 & -4.0 \\
\hline Armenia & Europe and Central Asia & 3,545 & 61.9 & 69.0 & 68 & 52 & 276 & 158 & 3,088 & 67.0 & 72.9 & 38 & 34 & 208 & 99 & -5.3 & -3.8 \\
\hline Aruba & High-income countries & - & - & - & - & - & - & - & 92 & 66.9 & 72.8 & 36 & 28 & 230 & 116 & - & - \\
\hline Australia & High-income countries & 16,888 & 74.1 & 80.4 & 10 & 8 & 124 & 66 & 19,352 & 77.6 & 83.0 & 7 & 5 & 94 & 54 & -4.2 & -4.1 \\
\hline Austria & High-income countries & 7,729 & 72.4 & 79.0 & 10 & 9 & 153 & 74 & 8,106 & 76.1 & 82.2 & 6 & 5 & 121 & 61 & -5.1 & -5.6 \\
\hline Azerbaijan & Europe and Central Asia & 7,192 & 56.4 & 63.6 & 119 & 93 & 327 & 199 & 8,226 & 62.5 & 68.2 & 77 & 68 & 247 & 129 & -3.9 & -2.8 \\
\hline Bahamas, The & High-income countries & 255 & 66.6 & 74.2 & 35 & 23 & 224 & 115 & 307 & 68.8 & 75.2 & 14 & 11 & 249 & 152 & -8.5 & -6.4 \\
\hline Bahrain & High-income countries & 490 & 71.7 & 73.6 & 25 & 26 & 124 & 104 & 693 & 72.4 & 74.3 & 14 & 10 & 112 & 82 & -5.6 & -8.6 \\
\hline Bangladesh & South Asia & 109,403 & 53.8 & 58.1 & 145 & 143 & 348 & 236 & 140,880 & 62.3 & 62.2 & 76 & 78 & 251 & 258 & -5.9 & -5.5 \\
\hline Barbados & Latin America and the Caribbean & 257 & 69.2 & 76.4 & 21 & 14 & 193 & 96 & 268 & 70.5 & 77.7 & 18 & 17 & 187 & 103 & -1.6 & 1.7 \\
\hline Belarus & Europe and Central Asia & 10,266 & 66.2 & 75.8 & 17 & 12 & 282 & 107 & 9,986 & 63.0 & 74.5 & 14 & 11 & 361 & 129 & -1.5 & -1.0 \\
\hline Belgium & High-income countries & 9,967 & 72.7 & 79.5 & 11 & 8 & 139 & 75 & 10,273 & 74.9 & 81.4 & 6 & 5 & 128 & 67 & -5.2 & -4.5 \\
\hline Belize & Latin America and the Caribbean & 186 & 64.4 & 69.5 & 50 & 48 & 250 & 154 & 245 & 67.2 & 72.2 & 45 & 34 & 191 & 124 & -0.9 & -3.2 \\
\hline Benin & Sub-Saharan Africa & 4,650 & 49.0 & 55.5 & 201 & 168 & 385 & 251 & 6,387 & 50.4 & 52.8 & 165 & 157 & 415 & 351 & -1.8 & -0.6 \\
\hline Bermuda & High-income countries & - & - & - & - & - & - & - & 80 & 68.0 & 72.8 & 34 & 27 & 216 & 116 & - & - \\
\hline Bhutan & South Asia & 1,696 & 53.8 & 59.5 & 145 & 129 & 349 & 227 & 2,125 & 60.0 & 62.1 & 95 & 94 & 275 & 228 & -3.8 & -2.9 \\
\hline Bolivia & Latin America and the Caribbean & 6,669 & 55.3 & 61.1 & 129 & 114 & 336 & 216 & 8,481 & 61.6 & 64.4 & 80 & 75 & 261 & 211 & -4.4 & -3.8 \\
\hline $\begin{array}{l}\text { Bosnia and } \\
\text { Herzegovina }\end{array}$ & Europe and Central Asia & 4,308 & 68.3 & 75.3 & 26 & 18 & 204 & 105 & 4,067 & 69.1 & 76.3 & 21 & 16 & 193 & 91 & -2.0 & -1.3 \\
\hline Botswana & Sub-Saharan Africa & 1,354 & 62.4 & 69.0 & 64 & 52 & 271 & 158 & 1,750 & 41.5 & 42.2 & 99 & 97 & 762 & 718 & 4.0 & 5.7 \\
\hline Brazil & Latin America and the Caribbean & 148,809 & 61.9 & 69.0 & 68 & 51 & 276 & 158 & 174,029 & 65.5 & 72.1 & 44 & 36 & 247 & 135 & -4.0 & -3.3 \\
\hline British Virgin Islands & Not included & - & - & - & - & - & - & - & 20 & 68.0 & 72.8 & 34 & 27 & 215 & 116 & - & - \\
\hline Brunei Darussalam & High-income countries & 257 & 70.0 & 77.7 & 18 & 11 & 182 & 85 & 342 & 74.7 & 77.4 & 15 & 13 & 115 & 87 & -1.9 & 1.6 \\
\hline Bulgaria & Europe and Central Asia & 8,718 & 68.2 & 74.9 & 20 & 15 & 216 & 97 & 8,033 & 68.6 & 75.2 & 18 & 15 & 221 & 97 & -1.2 & -0.1 \\
\hline Burkina Faso & Sub-Saharan Africa & 8,921 & 47.1 & 52.9 & 224 & 196 & 398 & 267 & 12,259 & 40.6 & 42.7 & 232 & 217 & 596 & 520 & 0.3 & 0.9 \\
\hline Burundi & Sub-Saharan Africa & 5,609 & 49.2 & 55.4 & 198 & 169 & 384 & 252 & 6,412 & 39.0 & 42.7 & 192 & 180 & 680 & 565 & -0.3 & 0.5 \\
\hline Cambodia & East Asia and Pacific & 9,744 & 55.7 & 62.4 & 126 & 103 & 334 & 207 & 13,478 & 52.3 & 57.5 & 147 & 123 & 392 & 290 & 1.4 & 1.6 \\
\hline Cameroon & Sub-Saharan Africa & 11,661 & 54.1 & 60.2 & 142 & 123 & 346 & 222 & 15,429 & 48.0 & 50.0 & 160 & 155 & 498 & 434 & 1.1 & 2.1 \\
\hline
\end{tabular}




\begin{tabular}{|c|c|c|c|c|c|c|c|c|c|c|c|c|c|c|c|c|c|}
\hline Canada & High-income countries & 27,701 & 74.0 & 80.7 & 9 & 7 & 132 & 71 & 31,025 & 77.0 & 82.5 & 6 & 5 & 98 & 59 & -3.5 & -3. \\
\hline Cape Verde & Sub-Saharan Africa & 349 & 61.6 & 69.4 & 71 & 49 & 279 & 155 & 445 & 66.4 & 72.7 & 44 & 32 & 209 & 121 & -4.3 & -4.1 \\
\hline Cayman Islands & High-income countries & - & - & - & - & - & - & - & 38 & 68.0 & 72.8 & 34 & 27 & 216 & 116 & - & - \\
\hline $\begin{array}{l}\text { Central African } \\
\text { Republic }\end{array}$ & Sub-Saharan Africa & 2,943 & 49.0 & 56.5 & 200 & 159 & 385 & 246 & 3,770 & 42.6 & 44.1 & 187 & 173 & 607 & 556 & -0.6 & 0.1 \\
\hline Chad & Sub-Saharan Africa & 5,822 & 47.5 & 53.7 & 218 & 187 & 395 & 262 & 8,103 & 45.7 & 49.2 & 203 & 181 & 485 & 401 & -0.7 & $-0 .:$ \\
\hline Channel Islands & High-income countries & 142 & - & - & - & - & - & - & 145 & 75.5 & 81.6 & 6 & 5 & 120 & 60 & - & - \\
\hline Chile & $\begin{array}{l}\text { Latin America and the } \\
\text { Caribbean }\end{array}$ & 13,100 & 69.8 & 76.6 & 21 & 17 & 191 & 97 & 15,419 & 73.1 & 79.9 & 17 & 14 & 137 & 68 & -1.9 & -2.1 \\
\hline Colombia & $\begin{array}{l}\text { Latin America and the } \\
\text { Caribbean }\end{array}$ & 34,970 & 65.8 & 72.4 & 40 & 31 & 234 & 130 & 42,826 & 67.5 & 76.3 & 27 & 19 & 235 & 102 & -3.7 & -4 \\
\hline Comoros & Sub-Saharan Africa & 527 & 55.0 & 61.9 & 132 & 107 & 339 & 210 & 726 & 60.9 & 64.5 & 84 & 75 & 273 & 210 & -4.2 & $-3:$ \\
\hline $\begin{array}{l}\text { Congo, Democratic } \\
\text { Republic of }\end{array}$ & Sub-Saharan Africa & 37,370 & 47.4 & 53.6 & 221 & 189 & 397 & 263 & 49,785 & 41.1 & 46.1 & 222 & 198 & 583 & 449 & 0.0 & 0. \\
\hline Congo, Republic of & Sub-Saharan Africa & 2,494 & 55.9 & 63.2 & 123 & 96 & 331 & 202 & 3,542 & 52.2 & 54.5 & 109 & 101 & 460 & 409 & -1.1 & $0 . !$ \\
\hline Cook Islands & Not included & - & - & - & - & - & - & - & 18 & 68.9 & 74.1 & 23 & 19 & 176 & 112 & - & - \\
\hline Costa Rica & Latin America and the Caribbean & 3,076 & 73.0 & 77.7 & 19 & 15 & 129 & 79 & 4,013 & 74.4 & 79.3 & 12 & 10 & 128 & 75 & -3.6 & -3.1 \\
\hline Côte d'Ivoire & Sub-Saharan Africa & 12,505 & 51.7 & 58.0 & 169 & 144 & 365 & 237 & 16,098 & 43.5 & 48.1 & 192 & 143 & 567 & 498 & 1.2 & -0 \\
\hline Cuba & Latin America and the Caribbean & 10,628 & 72.8 & 76.7 & 15 & 11 & 155 & 111 & 11,238 & 75.2 & 80.0 & 9 & 7 & 139 & 90 & -4.4 & $-4 .:$ \\
\hline Cyprus & High-income countries & 681 & 71.7 & 77.3 & 12 & 12 & 161 & 88 & 789 & 75.0 & 78.8 & 8 & 8 & 105 & 53 & -3.8 & $-3 . i$ \\
\hline Czech Republic & Europe and Central Asia & 10,306 & 68.0 & 75.5 & 14 & 10 & 220 & 95 & 10,257 & 72.1 & 79.0 & 5 & 4 & 168 & 74 & -8.7 & -8.1 \\
\hline Denmark & High-income countries & 5,140 & 72.1 & 77.9 & 10 & 8 & 152 & 99 & 5,338 & 74.7 & 79.6 & 6 & 5 & 124 & 77 & -4.5 & $-3 . !$ \\
\hline Djibouti & Middle East and North Africa & 528 & 46.4 & 49.8 & 188 & 161 & 487 & 417 & 681 & 49.5 & 51.9 & 157 & 144 & 455 & 400 & -1.7 & -1.1 \\
\hline Dominica & Latin America and the Caribbean & - & - & - & - & - & - & - & 78 & 71.1 & 75.7 & 13 & 14 & 204 & 121 & - & - \\
\hline Dominican Republic & Latin America and the Caribbean & 7,058 & 61.2 & 68.5 & 74 & 55 & 283 & 162 & 8,485 & 64.8 & 71.4 & 38 & 32 & 256 & 151 & -6.0 & -5.1 \\
\hline Ecuador & Latin America and the Caribbean & 10,264 & 62.5 & 69.2 & 63 & 50 & 270 & 156 & 12,616 & 67.5 & 73.1 & 36 & 32 & 219 & 135 & -5.2 & -4. \\
\hline $\begin{array}{l}\text { Egypt, Arab } \\
\text { Republic of }\end{array}$ & Middle East and North Africa & 55,768 & 57.8 & 62.4 & 109 & 99 & 307 & 215 & 69,124 & 65.0 & 68.7 & 42 & 42 & 240 & 158 & -8.7 & -7.1 \\
\hline El Salvador & Latin America and the Caribbean & 5,110 & 62.2 & 68.7 & 66 & 54 & 273 & 160 & 6,313 & 66.2 & 72.6 & 37 & 34 & 265 & 145 & -5.2 & -4.1 \\
\hline Equatorial Guinea & Sub-Saharan Africa & 354 & 47.3 & 53.5 & 222 & 190 & 397 & 263 & 468 & 51.8 & 54.7 & 160 & 147 & 382 & 317 & -3.0 & -2 \\
\hline Eritrea & Sub-Saharan Africa & 3,103 & 52.9 & 58.6 & 156 & 138 & 356 & 232 & 3,847 & 42.9 & 57.3 & 118 & 103 & 659 & 334 & -2.5 & -2 \\
\hline Estonia & Europe and Central Asia & 1,584 & 64.8 & 74.9 & 18 & 14 & 298 & 107 & 1,353 & 65.1 & 76.7 & 12 & 8 & 319 & 116 & -4.3 & $-5 !$ \\
\hline Ethiopia & Sub-Saharan Africa & 48,856 & 47.5 & 53.6 & 219 & 189 & 395 & 263 & 67,266 & 46.2 & 49.5 & 186 & 169 & 500 & 417 & -1.5 & -1.1 \\
\hline Faeroe Islands & High-income countries & - & - & - & - & - & - & - & 46 & 75.5 & 81.6 & 6 & 5 & 120 & 60 & - & - \\
\hline Falkland Islands & Not included & - & - & - & - & - & - & - & 3 & 68.0 & 72.7 & 34 & 27 & 216 & 117 & - & - \\
\hline France & High-income countries & 56,735 & 73.3 & 81.7 & 10 & 8 & 162 & 67 & 59,564 & 75.7 & 83.7 & 6 & 5 & 136 & 60 & -5.0 & $-4 !$ \\
\hline French Guiana & Not included & - & - & - & - & - & - & - & 169 & 68.1 & 72.8 & 33 & 27 & 216 & 116 & - & - \\
\hline French Polynesia & High-income countries & 195 & - & - & - & - & - & - & 237 & 59.8 & 36.6 & 88 & 81 & 295 & 230 & - & - \\
\hline Gabon & Sub-Saharan Africa & 953 & 58.0 & 64.1 & 103 & 89 & 313 & 196 & 1,283 & 57.2 & 61.3 & 101 & 80 & 342 & 281 & -0.2 & -1.1 \\
\hline
\end{tabular}

(Continues on the following page. 
Annex 2A Continued

\begin{tabular}{|c|c|c|c|c|c|c|c|c|c|c|c|c|c|c|c|c|c|}
\hline \multirow[b]{4}{*}{ Country/Territory } & \multirow[b]{4}{*}{ World Bank region } & \multicolumn{7}{|c|}{1990} & \multicolumn{7}{|c|}{2001} & \multirow{3}{*}{\multicolumn{2}{|c|}{$\begin{array}{c}\text { Annual change } \\
\text { in probability } \\
\text { of dying } \\
\text { under age } 5 \text {, } \\
\text { 1990-2001 (\%) }\end{array}$}} \\
\hline & & \multirow{3}{*}{$\begin{array}{l}\text { Population } \\
\text { (thousands) }\end{array}$} & \multirow{2}{*}{\multicolumn{2}{|c|}{$\begin{array}{l}\text { Life expectancy } \\
\text { at birth (years) }\end{array}$}} & \multicolumn{4}{|c|}{$\begin{array}{l}\text { Probability of } \\
\text { dying per } 1,000\end{array}$} & \multirow{3}{*}{$\begin{array}{l}\text { Population } \\
\text { (thousands) }\end{array}$} & \multirow{2}{*}{\multicolumn{2}{|c|}{$\begin{array}{l}\text { Life expectancy } \\
\text { at birth (years) }\end{array}$}} & \multicolumn{4}{|c|}{$\begin{array}{r}\text { Probability of } \\
\text { dying per } 1,000\end{array}$} & & \\
\hline & & & & & \multicolumn{2}{|c|}{ Under age 5} & \multicolumn{2}{|c|}{ Ages 15-59 } & & & & \multicolumn{2}{|c|}{ Under age 5} & \multicolumn{2}{|c|}{ Ages 15-59 } & & \\
\hline & & & Males & Females & Males & Females & Males & Females & & Males & Females & Males & Females & Males & Females & Males & Females \\
\hline Gambia, The & Sub-Saharan Africa & 936 & 52.0 & 58.1 & 165 & 143 & 362 & 235 & 1,351 & 55.3 & 58.8 & 134 & 119 & 330 & 264 & -1.9 & -1.6 \\
\hline Georgia & Europe and Central Asia & 5,460 & 67.9 & 74.9 & 28 & 20 & 210 & 109 & 5,224 & 68.3 & 74.3 & 26 & 20 & 216 & 89 & -0.7 & -0.1 \\
\hline Germany & High-income countries & 79,433 & 72.0 & 78.5 & 10 & 8 & 157 & 77 & 82,349 & 75.4 & 81.6 & 6 & 4 & 121 & 62 & -5.3 & -4.9 \\
\hline Ghana & Sub-Saharan Africa & 15,277 & 54.9 & 61.0 & 134 & 115 & 340 & 217 & 20,028 & 56.3 & 58.8 & 107 & 100 & 355 & 303 & -2.1 & -1.3 \\
\hline Gibraltar & Not included & - & - & - & - & - & - & - & 27 & 75.5 & 81.6 & 6 & 5 & 120 & 60 & - & - \\
\hline Greece & High-income countries & 10,161 & 74.7 & 79.5 & 11 & 10 & 117 & 56 & 10,947 & 75.5 & 80.9 & 7 & 6 & 118 & 49 & -3.8 & -5.7 \\
\hline Greenland & High-income countries & - & - & - & - & - & - & - & 56 & 75.5 & 81.6 & 6 & 5 & 120 & 60 & - & - \\
\hline Grenada & Latin America and the Caribbean & - & - & - & - & - & - & - & 81 & 65.8 & 68.7 & 25 & 21 & 263 & 224 & - & - \\
\hline Guadeloupe & Not included & - & - & - & - & - & - & - & 432 & 68.0 & 72.8 & 34 & 27 & 216 & 116 & - & - \\
\hline Guam & High-income countries & 134 & - & - & - & - & - & - & 158 & 59.9 & 36.5 & 86 & 80 & 295 & 229 & - & - \\
\hline Guatemala & Latin America and the Caribbean & 8,749 & 59.5 & 65.8 & 89 & 75 & 299 & 183 & 11,728 & 62.9 & 68.7 & 58 & 51 & 285 & 167 & -3.8 & -3.5 \\
\hline Guinea & Sub-Saharan Africa & 6,122 & 45.2 & 49.7 & 248 & 232 & 411 & 285 & 8,242 & 50.5 & 53.5 & 166 & 155 & 408 & 333 & -3.7 & -3.6 \\
\hline Guinea-Bissau & Sub-Saharan Africa & 1,016 & 43.3 & 49.6 & 273 & 232 & 424 & 285 & 1,407 & 45.4 & 48.4 & 219 & 201 & 464 & 384 & -2.0 & -1.3 \\
\hline Guyana & Latin America and the Caribbean & 731 & 57.9 & 65.7 & 104 & 76 & 314 & 184 & 762 & 61.3 & 66.7 & 62 & 51 & 302 & 206 & -4.6 & -3.5 \\
\hline Haiti & Latin America and the Caribbean & 6,914 & 52.7 & 58.2 & 157 & 142 & 357 & 235 & 8,111 & 48.8 & 50.8 & 140 & 130 & 497 & 444 & -1.0 & -0.8 \\
\hline $\begin{array}{l}\text { Holy See } \\
\quad \text { (Vatican City) }\end{array}$ & Not included & - & - & - & - & - & - & - & 1 & 75.5 & 81.6 & 6 & 5 & 120 & 60 & - & - \\
\hline Honduras & Latin America and the Caribbean & 4,868 & 61.9 & 68.7 & 68 & 54 & 276 & 160 & 6,619 & 64.4 & 70.4 & 45 & 42 & 263 & 148 & -3.8 & -2.1 \\
\hline Hungary & Europe and Central Asia & 10,365 & 65.1 & 73.8 & 19 & 15 & 305 & 133 & 9,968 & 68.0 & 76.7 & 11 & 9 & 264 & 113 & -5.0 & -4.6 \\
\hline Iceland & High-income countries & 255 & 75.5 & 80.7 & 9 & 5 & 116 & 77 & 285 & 78.1 & 81.7 & 4 & 3 & 88 & 56 & -8.0 & -5.5 \\
\hline India & South Asia & 846,418 & 57.3 & 58.0 & 113 & 126 & 301 & 246 & $1,033,395$ & 60.0 & 61.8 & 89 & 98 & 291 & 222 & -2.2 & -2.3 \\
\hline Indonesia & East Asia and Pacific & 182,117 & 58.3 & 64.9 & 100 & 82 & 310 & 189 & 214,356 & 64.4 & 67.4 & 50 & 40 & 246 & 213 & -6.2 & -6.4 \\
\hline $\begin{array}{l}\text { Iran, Islamic } \\
\text { Republic of }\end{array}$ & $\begin{array}{l}\text { Middle East and } \\
\text { North Africa }\end{array}$ & 56,703 & 62.5 & 66.5 & 73 & 71 & 253 & 173 & 67,245 & 65.8 & 71.1 & 45 & 38 & 225 & 140 & -4.4 & -5.8 \\
\hline Iraq & Middle East and North Africa & 17,341 & 63.5 & 68.3 & 79 & 71 & 215 & 138 & 23,860 & 58.7 & 62.8 & 122 & 112 & 258 & 180 & 3.9 & 4.2 \\
\hline Ireland & High-income countries & 3,515 & 71.9 & 77.6 & 11 & 9 & 133 & 81 & 3,865 & 74.1 & 79.5 & 8 & 6 & 117 & 68 & -3.0 & -3.6 \\
\hline Isle of Man & Europe and Central Asia & - & - & - & - & - & - & - & 74 & 75.5 & 81.6 & 6 & 5 & 120 & 60 & - & - \\
\hline Israel & High-income countries & 4,514 & 75.0 & 78.4 & 13 & 11 & 107 & 71 & 6,174 & 77.1 & 81.2 & 7 & 6 & 100 & 54 & -5.0 & -5.7 \\
\hline Italy & High-income countries & 56,719 & 73.7 & 80.4 & 10 & 8 & 129 & 60 & 57,521 & 76.6 & 82.6 & 6 & 5 & 99 & 50 & -5.5 & -4.6 \\
\hline Jamaica & Latin America and the Caribbean & in $\quad 2,369$ & 69.0 & 75.5 & 22 & 17 & 195 & 103 & 2,603 & 71.0 & 74.4 & 16 & 14 & 164 & 123 & -3.0 & -1.7 \\
\hline Japan & High-income countries & 123,537 & 76.1 & 82.4 & 7 & 6 & 109 & 53 & 127,271 & 78.2 & 85.8 & 5 & 4 & 97 & 47 & -3.9 & -3.3 \\
\hline Jordan & Middle East and North Africa & 3,254 & 66.5 & 71.0 & 44 & 42 & 209 & 137 & 5,183 & 68.5 & 73.1 & 29 & 27 & 193 & 122 & -3.9 & -4.0 \\
\hline Kazakhstan & Europe and Central Asia & 16,809 & 63.2 & 69.9 & 58 & 46 & 262 & 151 & 15,533 & 58.4 & 68.9 & 40 & 30 & 420 & 192 & -3.4 & -3.9 \\
\hline Kenya & Sub-Saharan Africa & 23,585 & 57.6 & 64.2 & 106 & 87 & 316 & 194 & 31,065 & 50.4 & 52.6 & 117 & 112 & 496 & 434 & 0.9 & 2.3 \\
\hline Kiribati & East Asia and Pacific & - & - & - & - & - & - & - & 85 & 61.8 & 66.5 & 82 & 69 & 288 & 194 & - & - \\
\hline
\end{tabular}




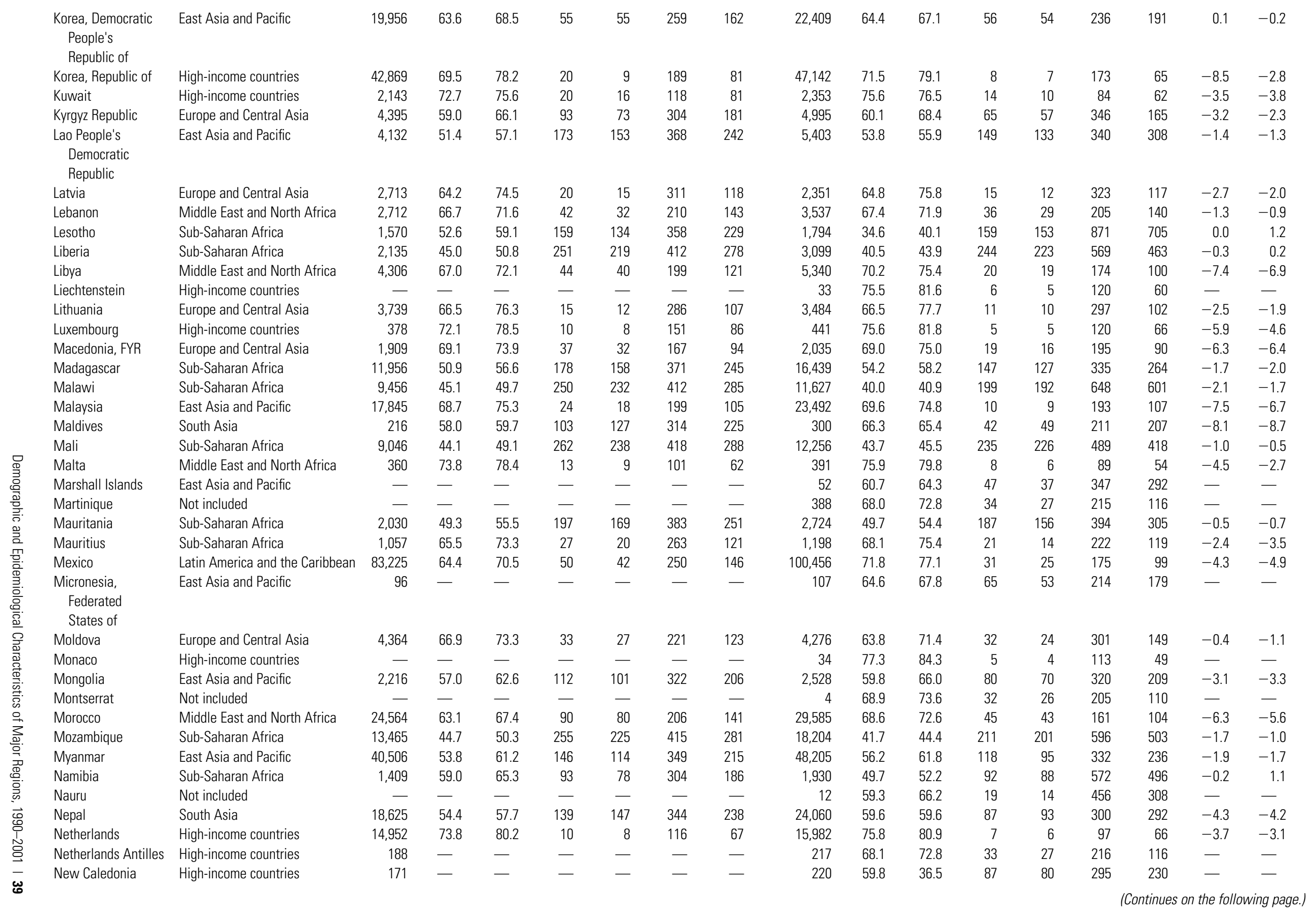


Annex 2A Continued

\begin{tabular}{|c|c|c|c|c|c|c|c|c|c|c|c|c|c|c|c|c|c|}
\hline \multirow[b]{4}{*}{ Country/ Territory } & \multirow[b]{4}{*}{ World Bank region } & \multicolumn{7}{|c|}{1990} & \multicolumn{7}{|c|}{2001} & \multirow{3}{*}{\multicolumn{2}{|c|}{$\begin{array}{c}\text { Annual change } \\
\text { in probability } \\
\text { of dying } \\
\text { under age } 5 \\
1990-2001(\%)\end{array}$}} \\
\hline & & \multirow{3}{*}{$\begin{array}{l}\text { Population } \\
\text { (thousands) }\end{array}$} & \multirow{2}{*}{\multicolumn{2}{|c|}{$\begin{array}{l}\text { Life expectancy } \\
\text { at birth (years) }\end{array}$}} & \multicolumn{4}{|c|}{$\begin{array}{l}\text { Probability of } \\
\text { dying per } 1,000\end{array}$} & \multirow{3}{*}{$\begin{array}{c}\text { Population } \\
\text { (thousands) }\end{array}$} & \multirow{2}{*}{\multicolumn{2}{|c|}{$\begin{array}{l}\text { Life expectancy } \\
\text { at birth (years) }\end{array}$}} & \multicolumn{4}{|c|}{$\begin{array}{r}\text { Probability of } \\
\text { dying per } 1,000\end{array}$} & & \\
\hline & & & & & \multicolumn{2}{|c|}{ Under age 5} & \multicolumn{2}{|c|}{ Ages 15-59 } & & & & \multicolumn{2}{|c|}{ Under age 5} & \multicolumn{2}{|c|}{ Ages 15-59 } & & \\
\hline & & & Males & Females & Males & Females & Males & Females & & Males & Females & Males & Females & Males & Females & Males & Females \\
\hline New Zealand & High-income countries & 3,360 & 72.5 & 78.5 & 13 & 9 & 143 & 93 & 3,815 & 76.5 & 81.6 & 8 & 6 & 101 & 65 & -4.8 & -3.1 \\
\hline Nicaragua & Latin America and the Caribbean & n $\quad 3,824$ & 61.3 & 68.0 & 73 & 58 & 282 & 166 & 5,204 & 67.7 & 72.3 & 39 & 34 & 213 & 146 & -5.7 & -5.0 \\
\hline Niger & Sub-Saharan Africa & 7,650 & 39.2 & 42.9 & 329 & 311 & 449 & 320 & 11,134 & 42.6 & 42.6 & 251 & 257 & 496 & 442 & -2.5 & 1.7 \\
\hline Nigeria & Sub-Saharan Africa & 86,018 & 49.8 & 54.7 & 191 & 177 & 379 & 256 & 117,823 & 48.1 & 49.8 & 184 & 181 & 448 & 387 & -0.3 & 0.2 \\
\hline Niue & Not included & - & - & - & - & - & - & - & 2 & 67.9 & 73.1 & 34 & 26 & 193 & 132 & - & - \\
\hline $\begin{array}{l}\text { Northern } \\
\quad \text { Mariana Islands }\end{array}$ & High-income countries & - & - & - & - & - & - & - & 73 & 65.3 & 36.4 & 86 & 80 & 295 & 229 & - & - \\
\hline Norway & High-income countries & 4,241 & 73.4 & 79.9 & 10 & 7 & 128 & 65 & 4,494 & 76.1 & 81.7 & 5 & 4 & 103 & 61 & -6.1 & -5.3 \\
\hline $\begin{array}{l}\text { West Bank and } \\
\text { Gaza }\end{array}$ & Middle East and North Africa & 2,154 & - & - & - & - & - & - & 3,310 & 68.4 & 72.0 & 27 & 29 & 200 & 140 & - & - \\
\hline Oman & Middle East and North Africa & 1,845 & 68.6 & 74.3 & 33 & 27 & 184 & 106 & 2,688 & 70.8 & 76.1 & 17 & 16 & 168 & 95 & -6.1 & -4.8 \\
\hline Pakistan & South Asia & 110,901 & 54.6 & 58.4 & 137 & 140 & 342 & 233 & 146,277 & 61.1 & 61.5 & 105 & 115 & 228 & 203 & -2.4 & -1.7 \\
\hline Palau & East Asia and Pacific & - & - & - & - & - & - & - & 20 & 66.3 & 71.4 & 24 & 22 & 241 & 194 & - & - \\
\hline Panama & Latin America and the Caribbean & n $\quad 2,411$ & 66.0 & 72.9 & 39 & 29 & 232 & 126 & 3,007 & 73.1 & 78.4 & 26 & 22 & 143 & 85 & -3.6 & -2.4 \\
\hline Papua New Guinea & East Asia and Pacific & 4,114 & 58.1 & 62.8 & 102 & 100 & 312 & 205 & 5,460 & 58.3 & 61.4 & 99 & 92 & 310 & 250 & -0.2 & -0.7 \\
\hline Paraguay & Latin America and the Caribbean & n $\quad 4,219$ & 65.6 & 72.2 & 42 & 32 & 237 & 131 & 5,604 & 68.7 & 74.2 & 37 & 27 & 170 & 123 & -1.0 & -1.6 \\
\hline Peru & Latin America and the Caribbean & n 21,753 & 59.5 & 66.3 & 88 & 71 & 299 & 179 & 26,362 & 67.1 & 71.6 & 41 & 37 & 206 & 145 & -6.9 & -6.0 \\
\hline Philippines & East Asia and Pacific & 61,104 & 62.2 & 69.3 & 65 & 50 & 272 & 156 & 77,151 & 64.9 & 71.4 & 41 & 35 & 260 & 136 & -4.2 & -3.3 \\
\hline Pitcairn & Not included & - & - & - & - & - & - & - & 0 & 59.9 & 36.4 & 86 & 80 & 295 & 230 & - & - \\
\hline Poland & Europe and Central Asia & 38,111 & 66.5 & 75.6 & 20 & 16 & 263 & 102 & 38,651 & 70.2 & 78.5 & 9 & 8 & 209 & 84 & -6.9 & -6.2 \\
\hline Portugal & High-income countries & 9,899 & 70.4 & 77.3 & 16 & 12 & 178 & 80 & 10,033 & 73.2 & 80.5 & 7 & 6 & 155 & 66 & -7.2 & -6.7 \\
\hline Puerto Rico & Latin America and the Caribbean & n $\quad 3,528$ & 68.7 & 77.6 & 17 & 13 & 237 & 90 & 3,838 & 70.5 & 78.4 & 13 & 11 & 217 & 93 & -2.1 & -1.2 \\
\hline Qatar & High-income countries & 467 & 67.7 & 74.6 & 29 & 21 & 211 & 111 & 591 & 75.2 & 74.3 & 15 & 13 & 93 & 82 & -5.7 & -4.3 \\
\hline Romania & Europe and Central Asia & 23,207 & 66.8 & 73.2 & 34 & 27 & 239 & 114 & 22,437 & 67.9 & 74.9 & 24 & 20 & 235 & 107 & -3.1 & -3.0 \\
\hline Russian Federation & Europe and Central Asia & 148,292 & 63.8 & 74.4 & 24 & 18 & 318 & 117 & 144,877 & 58.6 & 72.1 & 22 & 17 & 453 & 163 & -0.8 & -0.6 \\
\hline Rwanda & Sub-Saharan Africa & 6,775 & 50.6 & 55.9 & 181 & 165 & 373 & 249 & 8,066 & 41.6 & 46.3 & 189 & 173 & 608 & 483 & 0.4 & 0.5 \\
\hline Réunion & Not included & - & - & - & - & - & - & - & 734 & 49.4 & 52.0 & 176 & 168 & 422 & 352 & - & - \\
\hline Samoa & East Asia and Pacific & 160 & 65.0 & 71.1 & 45 & 39 & 243 & 141 & 175 & 66.7 & 69.6 & 28 & 22 & 235 & 203 & -4.4 & -5.1 \\
\hline San Marino & High-income countries & - & - & - & - & - & - & - & 27 & 77.2 & 84.0 & 6 & 3 & 85 & 32 & - & - \\
\hline $\begin{array}{l}\text { São Tomé } \\
\text { and Principe }\end{array}$ & Sub-Saharan Africa & 116 & - & - & - & - & - & - & 153 & 61.1 & 63.1 & 85 & 86 & 262 & 220 & - & - \\
\hline Saudi Arabia & Middle East and North Africa & 16,554 & 66.3 & 71.6 & 47 & 41 & 208 & 128 & 22,829 & 68.4 & 73.8 & 31 & 26 & 192 & 113 & -3.9 & -4.2 \\
\hline Senegal & Sub-Saharan Africa & 7,345 & 52.6 & 58.7 & 158 & 138 & 358 & 232 & 9,621 & 54.2 & 57.1 & 140 & 131 & 350 & 285 & -1.1 & -0.5 \\
\hline Serbia and & Europe and Central Asia & 10,156 & 66.8 & 74.0 & 34 & 24 & 223 & 116 & 10,545 & 69.7 & 74.8 & 17 & 13 & 187 & 98 & -6.2 & -5.1 \\
\hline
\end{tabular}




\begin{tabular}{|c|c|c|c|c|c|c|c|c|c|c|c|c|c|c|c|c|c|}
\hline Seychelles & Sub-Saharan Africa & - & - & - & - & - & - & - & 80 & 66.7 & 77.7 & 15 & 10 & 248 & 113 & - & - \\
\hline Sierra Leone & Sub-Saharan Africa & 4,054 & 39.5 & 45.6 & 324 & 279 & 447 & 306 & 4,573 & 31.2 & 35.8 & 330 & 301 & 718 & 567 & 0.2 & 0.7 \\
\hline Singapore & High-income countries & 3,016 & 72.8 & 77.8 & 10 & 8 & 152 & 93 & 4,105 & 76.8 & 81.7 & 4 & 3 & 93 & 54 & -8.3 & -7.4 \\
\hline Slovak Republic & Europe and Central Asia & 5,256 & 70.3 & 76.9 & 17 & 13 & 179 & 92 & 5,394 & 69.5 & 78.0 & 10 & 8 & 210 & 79 & -4.4 & -4.8 \\
\hline Slovenia & High-income countries & 1,918 & 69.9 & 77.9 & 12 & 8 & 207 & 81 & 1,988 & 72.5 & 80.2 & 6 & 4 & 165 & 72 & -6.6 & -5.2 \\
\hline Solomon Islands & East Asia and Pacific & 319 & - & - & - & - & - & - & 450 & 63.5 & 67.3 & 86 & 75 & 202 & 149 & - & - \\
\hline Somalia & Sub-Saharan Africa & 7,163 & 45.8 & 51.6 & 240 & 210 & 407 & 274 & 9,088 & 43.7 & 46.1 & 217 & 223 & 516 & 407 & -0.9 & 0.6 \\
\hline South Africa & Sub-Saharan Africa & 36,848 & 61.9 & 69.0 & 68 & 52 & 275 & 158 & 44,416 & 50.4 & 54.5 & 80 & 75 & 566 & 448 & 1.5 & 3.4 \\
\hline Spain & High-income countries & 39,303 & 73.3 & 80.4 & 10 & 8 & 146 & 60 & 40,875 & 76.1 & 83.2 & 6 & 5 & 121 & 48 & -5.3 & -5.1 \\
\hline Sri Lanka & South Asia & 16,830 & 67.2 & 74.9 & 32 & 20 & 218 & 109 & 18,752 & 66.1 & 74.2 & 21 & 17 & 260 & 125 & -3.8 & -1.6 \\
\hline St. Helena & Not included & - & - & - & - & - & - & - & 5 & 49.5 & 52.0 & 176 & 168 & 422 & 352 & - & - \\
\hline St. Kitts and Nevis & Latin America and the Caribbean & - & - & - & - & - & - & - & 42 & 68.4 & 72.1 & 21 & 24 & 210 & 150 & - & - \\
\hline St. Lucia & Latin America and the Caribbean & 131 & 66.9 & 72.3 & 22 & 17 & 246 & 158 & 147 & 69.5 & 74.9 & 14 & 14 & 217 & 138 & -4.2 & -1.7 \\
\hline $\begin{array}{l}\text { St. Pierre et } \\
\text { Miquelon }\end{array}$ & Not included & - & - & - & - & - & - & - & 6 & 74.7 & 80.0 & 9 & 7 & 139 & 81 & - & - \\
\hline $\begin{array}{l}\text { St. Vincent and } \\
\text { the Grenadines }\end{array}$ & High-income countries & 110 & - & - & - & - & - & - & 118 & 68.0 & 72.2 & 24 & 20 & 235 & 180 & - & - \\
\hline Sudan & Sub-Saharan Africa & 24,927 & 55.5 & 61.4 & 128 & 112 & 335 & 214 & 32,151 & 55.4 & 59.6 & 112 & 106 & 364 & 269 & -1.2 & -0.4 \\
\hline Suriname & Latin America and the Caribbean & 402 & 65.8 & 72.9 & 40 & 29 & 233 & 126 & 429 & 64.2 & 70.6 & 34 & 28 & 282 & 166 & -1.4 & -0.3 \\
\hline Swaziland & Sub-Saharan Africa & 847 & 56.1 & 63.0 & 122 & 98 & 330 & 203 & 1,058 & 38.5 & 42.1 & 145 & 137 & 784 & 673 & 1.6 & 3.0 \\
\hline Sweden & High-income countries & 8,559 & 74.8 & 80.5 & 8 & 6 & 114 & 66 & 8,860 & 77.8 & 82.5 & 4 & 3 & 85 & 54 & -5.9 & -6.3 \\
\hline Switzerland & High-income countries & 6,834 & 73.9 & 80.9 & 9 & 8 & 126 & 62 & 7,173 & 77.4 & 83.2 & 6 & 5 & 95 & 53 & -3.7 & -4.0 \\
\hline $\begin{array}{l}\text { Syrian } \\
\text { Arab Republic }\end{array}$ & Middle East and North Africa & 12,717 & 66.1 & 70.7 & 48 & 40 & 211 & 147 & 16,968 & 68.6 & 73.4 & 28 & 21 & 192 & 128 & -4.8 & -5.6 \\
\hline Tajikistan & Europe and Central Asia & 5,303 & 60.1 & 66.8 & 83 & 67 & 293 & 175 & 6,144 & 60.7 & 66.0 & 69 & 58 & 290 & 182 & -1.7 & -1.4 \\
\hline Tanzania & Sub-Saharan Africa & 26,068 & 51.6 & 56.8 & 170 & 156 & 366 & 244 & 35,565 & 45.4 & 47.5 & 164 & 145 & 559 & 510 & -0.3 & -0.7 \\
\hline Thailand & East Asia and Pacific & 54,389 & 64.6 & 72.4 & 48 & 31 & 248 & 130 & 61,555 & 66.1 & 72.6 & 33 & 27 & 276 & 156 & -3.4 & -1.5 \\
\hline Timor-Leste & East Asia and Pacific & 740 & - & - & - & - & - & - & 711 & 55.2 & 60.9 & 139 & 106 & 323 & 237 & - & - \\
\hline Togo & Sub-Saharan Africa & 3,455 & 52.1 & 58.5 & 165 & 139 & 362 & 233 & 4,686 & 50.1 & 53.5 & 150 & 128 & 454 & 388 & -0.8 & -0.7 \\
\hline Tokelau & Not included & - & - & - & - & - & - & - & 2 & 59.9 & 36.4 & 86 & 80 & 295 & 230 & - & - \\
\hline Tonga & East Asia and Pacific & 99 & - & - & - & - & - & - & 102 & 69.2 & 71.7 & 23 & 15 & 190 & 175 & - & - \\
\hline Trinidad and Tobago & Latin America and the Caribbean & 1,215 & 67.5 & 75.4 & 30 & 18 & 214 & 105 & 1,294 & 67.3 & 72.8 & 24 & 18 & 243 & 153 & -2.0 & -0.1 \\
\hline Tunisia & Middle East and North Africa & 8,207 & 66.4 & 70.1 & 55 & 49 & 194 & 141 & 9,624 & 69.3 & 73.8 & 32 & 25 & 171 & 116 & -5.0 & -6.3 \\
\hline Turkey & Europe and Central Asia & 57,593 & 60.3 & 65.9 & 82 & 74 & 292 & 182 & 69,303 & 67.8 & 72.1 & 44 & 42 & 180 & 113 & -5.7 & -5.2 \\
\hline Turkmenistan & Europe and Central Asia & 3,668 & 57.4 & 64.3 & 108 & 87 & 318 & 194 & 4,720 & 58.7 & 66.8 & 65 & 49 & 365 & 192 & -4.7 & -5.3 \\
\hline $\begin{array}{l}\text { Turks and } \\
\text { Caicos Islands }\end{array}$ & Not included & - & - & - & - & - & - & - & 19 & 68.0 & 72.8 & 34 & 27 & 216 & 116 & - & - \\
\hline Tuvalu & Not included & - & - & - & - & - & - & - & 10 & 60.3 & 61.4 & 73 & 57 & 293 & 279 & - & - \\
\hline Uganda & Sub-Saharan Africa & 17,359 & 51.4 & 57.6 & 172 & 148 & 367 & 239 & 24,225 & 47.2 & 50.0 & 150 & 138 & 518 & 449 & -1.2 & -0.6 \\
\hline Ukraine & Europe and Central Asia & 51,891 & 65.6 & 74.9 & 19 & 14 & 287 & 112 & 49,290 & 61.8 & 73.1 & 23 & 17 & 376 & 137 & 1.8 & 1.9 \\
\hline United Arab Emirates & High-income countries & 2,035 & 68.5 & 75.7 & 25 & 17 & 201 & 102 & 2,879 & 71.3 & 75.1 & 10 & 10 & 170 & 123 & -7.9 & -5.0 \\
\hline United Kingdom & High-income countries & 56,761 & 72.9 & 78.6 & 11 & 8 & 129 & 78 & 58,881 & 75.2 & 80.0 & 7 & 6 & 111 & 70 & -3.6 & -3.0 \\
\hline United States & High-income countries & 255,712 & 71.9 & 79.0 & 13 & 10 & 172 & 91 & 288,025 & 74.5 & 79.7 & 9 & 7 & 144 & 83 & -3.2 & -2.9 \\
\hline U.S. Virgin Islands & High-income countries & 101 & - & - & - & - & - & - & 110 & 68.0 & 72.8 & 34 & 27 & 216 & 116 & - & - \\
\hline
\end{tabular}


A Annex 2A Continued

\begin{tabular}{|c|c|c|c|c|c|c|c|c|c|c|c|c|c|c|c|c|c|}
\hline \multirow[b]{4}{*}{ Country/Territory } & \multirow[b]{4}{*}{ World Bank region } & \multicolumn{7}{|c|}{1990} & \multicolumn{7}{|c|}{2001} & \multirow{3}{*}{\multicolumn{2}{|c|}{$\begin{array}{c}\text { Annual change } \\
\text { in probability } \\
\text { of dying } \\
\text { under age 5, } \\
\text { 1990-2001 (\%) }\end{array}$}} \\
\hline & & \multirow{3}{*}{$\begin{array}{l}\text { Population } \\
\text { (thousands) }\end{array}$} & \multirow{2}{*}{\multicolumn{2}{|c|}{$\begin{array}{l}\text { Life expectancy } \\
\text { at birth (years) }\end{array}$}} & \multicolumn{4}{|c|}{$\begin{array}{l}\text { Probability of } \\
\text { dying per } 1,000\end{array}$} & \multirow{3}{*}{$\begin{array}{l}\text { Population } \\
\text { (thousands) }\end{array}$} & \multirow{2}{*}{\multicolumn{2}{|c|}{$\begin{array}{l}\text { Life expectancy } \\
\text { at birth (years) }\end{array}$}} & \multicolumn{4}{|c|}{$\begin{array}{r}\text { Probability of } \\
\text { dying per } 1,000\end{array}$} & & \\
\hline & & & & & \multicolumn{2}{|c|}{ Under age 5} & \multicolumn{2}{|c|}{ Ages 15-59 } & & & & \multicolumn{2}{|c|}{ Under age 5} & \multicolumn{2}{|c|}{ Ages 15-59 } & & \\
\hline & & & Males & Females & Males & Females & Males & Females & & Males & Females & Males & Females & Males & Females & Males & Females \\
\hline Uruguay & Latin America and the Caribbean & 3,106 & 69.0 & 76.5 & 27 & 23 & 196 & 98 & 3,366 & 71.1 & 79.5 & 18 & 13 & 181 & 89 & -3.6 & -5.2 \\
\hline Uzbekistan & Europe and Central Asia & 20,515 & 62.3 & 69.0 & 65 & 52 & 272 & 158 & 25,313 & 65.2 & 70.7 & 39 & 28 & 246 & 150 & -4.5 & -5.7 \\
\hline Vanuatu & East Asia and Pacific & 149 & 61.9 & 66.1 & 68 & 72 & 276 & 180 & 202 & 66.2 & 68.9 & 43 & 42 & 219 & 177 & -4.3 & -5.0 \\
\hline $\begin{array}{l}\text { Venezuela, } \\
\text { Républica } \\
\text { Bolivariana de }\end{array}$ & Latin America and the Caribbean & in 19,502 & 69.1 & 74.4 & 35 & 29 & 181 & 107 & 24,752 & 70.9 & 77.0 & 24 & 20 & 185 & 98 & -3.4 & -3.5 \\
\hline Vietnam & East Asia and Pacific & 66,074 & 63.1 & 69.8 & 59 & 47 & 264 & 152 & 79,197 & 67.1 & 72.0 & 42 & 33 & 200 & 132 & -3.2 & -3.1 \\
\hline $\begin{array}{l}\text { Wallis and } \\
\text { Futuna Islands }\end{array}$ & Not included & - & - & - & - & - & - & - & 15 & 59.9 & 36.4 & 86 & 80 & 295 & 230 & - & - \\
\hline Western Sahara & Not included & - & - & - & - & - & - & - & 293 & 63.2 & 49.7 & 72 & 67 & 233 & 159 & - & - \\
\hline Yemen, Republic of & Middle East and North Africa & 11,944 & 54.5 & 57.1 & 147 & 137 & 327 & 271 & 18,651 & 58.2 & 61.7 & 111 & 98 & 292 & 232 & -2.5 & -3.0 \\
\hline Zambia & Sub-Saharan Africa & 8,200 & 48.7 & 54.9 & 204 & 175 & 387 & 255 & 10,570 & 39.3 & 40.5 & 192 & 177 & 692 & 646 & -0.5 & 0.1 \\
\hline Zimbabwe & Sub-Saharan Africa & 10,467 & 59.7 & 66.0 & 87 & 73 & 297 & 181 & 12,756 & 38.5 & 38.8 & 114 & 105 & 805 & 775 & 2.5 & 3.3 \\
\hline
\end{tabular}

Sources: Population data are from United Nations 2003. Mortality estimates for 1990 are authors' calculations; estimates for 2001 are from chapter 3 in this volume. Note: $-=$ not available or not applicable. Estimates of child mortality are rounded to the nearest whole number. 


\section{ACKNOWLEDGMENTS}

We are grateful to Colin Mathers for his input into the estimation of child mortality levels in 1990 reported in this chapter.

\section{NOTES}

1. While it would have been much more informative to base this assessment of demographic change on the 2004 Revision of World Population Prospects (United Nations 2005a), the results were released too late to be incorporated into the estimates reported in this and subsequent chapters. The differences between the two revisions, at least for regional aggregates, are unlikely to be substantial.

2. An exception is Timor-Leste, where fertility increased following independence in 2002 and is currently higher than in any other country.

\section{REFERENCES}

Adetunji, J. 2000. "Trends in under-5 Mortality Rates and the HIV/AIDS Epidemic." Bulletin of the World Health Organization 78 (10): 1200-6.

Bennett, N. G., and S. Horiuchi. 1984. "Mortality Estimation from Registered Deaths in Less Developed Countries." Demography 21 (2): 217-33.

Bhatia, S. 1983. "Traditional Practices Affecting Female Health and Survival: Evidence from Countries of South Asia." In Sex Differentials in Mortality, ed. A. D. Lopez and L. T. Ruzicka, 165-77. Canberra: Australian National University Press.

Black, R. E., S. S. Morris, and J. Bryce. 2003. "Where and Why Are 10 Million Children Dying Every Year?” Lancet 361 (9376): 2226-34.

Bryce, J., C. Boschi-Pinto, K. Shibuya, R. E. Black, and the WHO Child Health Epidemiology Reference Group. 2005. "WHO Estimates of the Causes of Death in Children." Lancet 365 (9465): 1147-52.

de Onis, M., E. A. Frongillo, and M. Blossner. 2000. "Is Malnutrition Declining? An Analysis of Changes in Levels of Child Malnutrition since 1980." Bulletin of the World Health Organization 78 (10): 1222-33.

Etebu, E. N., and A. U. Ekere. 2004. "Paediatric Accidental Deaths in Port Harcourt, Nigeria: A 10-Year Retrospective Study." Nigerian Journal of Medicine 13 (2): 140-3.

Ezzati, M., S. Vander Hoorn, C. M. M. Lawes, R. Leach, W. P. T. James, A. D. Lopez, A. Rodgers, and C. J. L. Murray. 2005. "Rethinking the 'Diseases of Affluence' Paradigm: Global Patterns of Nutritional Risks in Relation to Economic Development." PLoS Medicine 2: e133.

Fenn, B., S. S. Morris, and R. F. Black. 2005. "Comorbidity in Childhood in Northern Ghana: Magnitude, Associated Factors, and Impact on Mortality." International Journal of Epidemiology 34 (2): 368-74.

Fishman, S., L. E. Caulfield, M. de Onis M, M. Blossner, A. A. Hyder, L. Mullany, and R. E. Black. 2004. "Childhood and Maternal Underweight." In Comparative Quantification of Health Risks: Global and Regional Burden of Disease Attributable to Selected Major Risk Factors, ed. M. Ezzati, A. D. Lopez, A. Rodgers, and C. J. L. Murray, 39-61. Geneva: World Health Organization.

Gali, B. M., A. G. Madziga, and H. U. Naaya. 2004. "Epidemiology of Childhood Burns in Maiduguri, Northeastern Nigeria." Nigerian Journal of Medicine 13 (2): 144-7.

Hill, K. 1987. "Estimating Census and Death Registration Completeness." Asian and Pacific Population Forum 1 (3): 8-24.
Hill, K., R. Pande, M. Mahy, and G. Jones. 1999. Trends in Child Mortality in the Developing World: 1960-1996. New York: United Nations Children's Fund.

Istre, G. R., M. A. McCoy, M. Stowe, K. Davies, D. Zane, R. J. Anderson, and R. Wieber. 2003. "Childhood Injuries Due to Falls from Apartment Balconies and Windows." Injury Prevention 9 (4): 349-52.

Jamison, D. T., G. Alleyne, J. G. Breman, M. Claeson, D. B. Evans, P. Jha, and others. 2006. Disease Control Priorities in Developing Countries, 2nd ed. New York: Oxford University Press.

Korenromp, E. L., B. G. Williams, E. Gouws, C. Dye, and R. W. Snow. 2003. "Measurement of Trends in Childhood Malaria Mortality in Africa: An Assessment of Progress toward Targets Based on Verbal Autopsy." Lancet Journal of Infectious Diseases 3 (6): 349-58.

Lawn, J. E., S. Cousens, and J. Zupan. 2005. “Four Million Neonatal Deaths: When? Where? Why?” Lancet 363 (9462): 9-18.

Leon, D., A. Chenet, V. Shkolnikov, S. Zakharov, J. Shapiro, G. Rakhmanova, S. Vassin, and M. McKee. 1997. "Huge Variation in Russian Mortality Rates, 1984-1994: Artefact, Alcohol, or What?" Lancet 350 (9075):383-8.

Lopez, A. D. 1993. "Causes of Death in Industrial and Developing Countries: Estimates for 1985.” In Disease Control Priorities in Developing Countries, ed. D. Jamison, H. Mosely, A. Measham, and J. L. Bobodilla, 35-50. New York: Oxford University Press.

Lopez, A. D. 2003. "Estimating the Causes of Child Deaths." International Journal of Epidemiology 32 (6): 1052-3.

Lopez, A. D, O. B. Ahmad, M. Guillot, M. Inoue, B. Fergusson, J. Salomon, C. J. L. Murray, and K. Hill. 2002. World Mortality in 2000: Life Tables for 191 Countries. Geneva: World Health Organization.

Mari Bhat, P. N. 2002. "Completeness of India's Sample Registration System: An Assessment Using the General Growth Balance Method." Population Studies 56 (2): 119-34.

Mathers, C. D., D. Mafat, M. Inoue, C. Rao, and A. D. Lopez. 2005. "Counting the Dead and What They Died from: An Assessment of the Global Status of Cause of Death Data." Bulletin of the World Health Organization 83 (3): 171-7.

Mock, C., R. Quansah, R. Krishnan, C. Arreola-Risa, and F. Rivara. 2004. "Strengthening the Prevention and Care of Injuries Worldwide." Lancet 363 (9427): 2172-9.

Morris, S. S., R. E. Black, and L. Tomaskovic. 2003. "Predicting the Distribution of Under-Five Deaths by Cause in Countries without Adequate Vital Registration Systems." International Journal of Epidemiology 32 (6): 1041-51.

Murray, C. J. L., B. D. Ferguson, A. D. Lopez, M. Guillot, J. A. Salomon, and O. B. Ahmad. 2003. "Modified Logit Life Table System: Principles, Empirical Validation, and Application." Population Studies 57 (2): 165-82.

Murray, C. J. L., and A. D. Lopez, eds. 1996. The Global Burden of Disease, vol. 1. Cambridge, MA: Harvard University Press.

Pelletier, D. L., E. A. Frongillo, and J. P. Habicht. 1993. "Epidemiologic Evidence for a Potentiating Effect of Malnutrition on Child Mortality." American Journal of Public Health 83 (8): 1130-3.

Rao, C., A. D. Lopez, G. Yang, S. Begg, and J. Ma. 2005. “Evaluating National Cause of Death Statistics: Principles and Application to the Case of China." Bulletin of the World Health Organization 83 (8): 618-25.

Rice, A. L., L. Sacco, A. Hyder, and R. E. Black. 2000. "Malnutrition as an Underlying Cause of Childhood Deaths Associated with Infectious Diseases in Developing Countries." Bulletin of the World Health Organization 78 (10): 1207-21.

Rudan, I., J. Lawn, S. Cousens, A. K. Rowe, C. Boschi-Pinto, L. Tomaskovic, W. Mendoza, C. F. Lanata, A. Roca-Feltrer, I. Carneira, J. A. Schellenberg, O. Polasek, M. Weber, J. Bryce, S. S. Morris, R. E. Black, and H. Campbell. 2005. "Gaps in Policy-Relevant Information on Burden of Disease in Children: A Systematic Review." Lancet 365 (9476): 2031-40. 
Shen, C., B. Sanno-Duanda, and S. W. Bickler. 2003. "Paediatric Trauma at a Government Referral Hospital in The Gambia." West African Journal of Medicine 22 (4): 287-90.

Shkolnikov, V., M. McKee, and D. A. Leon. 2001. "Changes in Life Expectancy in Russia in the Mid-1990s." Lancet 357 (9260): 917-21.

Sibai, A. M. 2004. "Mortality Certification and Cause of Death Reporting in Developing Countries." Bulletin of the World Health Organization 82 (2): 83.

Snow, R. W., M. T. Winstanley, V. M. Marsh, C. R. C. J. Newton, C. Waruiru, I. Mwangi, P. A. Winstanley, and K. Marsh. 1992. "Childhood Deaths in Africa: Uses and Limitations of Verbal Autopsies." Lancet 340 (8815): $351-5$.

Snow, R. W., M. Craig, U. Deichmann, and K. W. Marsh. 1999. "Estimating Mortality, Morbidity, and Disability Due to Malaria among Africa's Non-pregnant Population." Bulletin of the World Health Organization 77 (8): 624-40.

Snow, R. W., J.-F. Trape, and K. Marsh. 2001. “The Past, Present, and Future of Childhood Malaria Mortality in Africa." Trends in Parasitology 17 (12): 593-7.

Stein, C. E., M. Birmingham, M. Kurian, P. Duclos, and P. Strebel. 2003. "The Global Burden of Measles in the Year 2000: A Model That Uses Country-Specific Indicators.” Journal of Infectious Diseases 187 (Suppl1): S8-S14.

Trape, J.-F. 2001. "The Public Health Impact of Chloroquine Resistance in Africa." American Journal of Tropical Medicine and Hygiene 64 (1-2): $12-17$.
Tupasi, T. E., M. A. Velmonte, M. E. G. Sanvitores, L. Abraham, L. E. De Leon, S. A. Tan, C. A. Miquel, and M. C. Saniel. 1988. "Determinants of Morbidity and Mortality Due to Acute Respiratory Infections: Implications for Intervention." Journal of Infectious Diseases 157 (4): 615-23.

UNICEF (United Nations Children's Fund). 2003. State of the World's Children 2003. New York: UNICEF.

United Nations. 2003. World Population Prospects: The 2002 Assessment. ST/ESA/SER.A/222. New York: United Nations.

2005a. World Population Prospects: The 2004 Assessment. New York: United Nations.

2005b. World Population Prospects: The 2002 Revision and World Urbanization Prospects: The 2001 Revision. New York: United Nations, Department of Economic and Social Affairs, Population Division. http://esa.un.org/unpp.

Victora, C. G., J. Bryce, O. Fontaine, and R. Monash. 2000. "Reducing Deaths from Diarrhea through Oral Rehydration Therapy." Bulletin of the World Health Organization 78 (10): 1246-55.

Walker, N., B. Schwartlander, and J. Bryce. 2002. "Meeting International Goals in Child Survival and HIV/AIDS." Lancet 360 (9329): 284-9.

Williams, B. G., E. Gouws, C. Boshi-Pinto, C. Bryce, and C. Dye. 2002. "Estimates of Worldwide Distribution of Child Deaths from Acute Respiratory Infections." Lancet Journal of Infectious Diseases 2 (1): $25-32$. 


\section{Chapter 3}

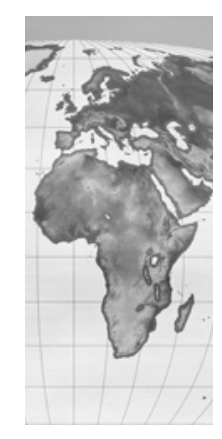

In 1993 the World Bank, in collaboration with the World Health Organization (WHO) and the Harvard School of Public Health, sponsored a study to assess the global burden of disease in 1990 (Murray, Lopez, and Jamison 1994; World Bank 1993). As well as generating the first comprehensive and consistent set of estimates of mortality and morbidity by age, sex, and region for the world, the Global Burden of Disease (GBD) study also introduced a new metric, the disability-adjusted life year (DALY), to quantify the burden of disease (Murray and Lopez 1996c, 1996d). The DALY is a summary measure of population health that combines years of life lost from premature death and years of life lived in less than full health and is described in more detail later. Thus, burden of disease analysis provides a unique perspective on health that integrates fatal and nonfatal outcomes, yet allows the two classes of outcomes to be examined separately as well.

The original (1990) GBD study analyzed and synthesized a large volume of data on population health to produce comprehensive and comparable information on the causes of loss of health globally and regionally, including low- and

\section{The Burden of Disease and Mortality by Condition: Data, Methods, and Results for 2001}

\author{
Colin D. Mathers, Alan D. Lopez, and Christopher J. L. Murray
}

middle-income countries with considerable limitations in data availability and comparability. In addition, the GBD study made estimates even for diseases and conditions for which data were limited and involved considerable uncertainty to ensure that causes of the disease burden for which information was sparse were not implicitly considered to impose no burden and hence be ignored by health policy makers (Murray, Mathers, and Salomon 2003).

Under the leadership of Chris Murray, WHO's executive director of the Evidence and Information for Policy Cluster from 1998 to 2003, WHO undertook a new assessment of the global burden of disease for 2000 to 2002, with consecutive revisions and updates published annually in WHO's world health reports. Version 1 estimates for 2000 were published in the World Health Report 2001 (WHO 2001d), and Version 3 estimates for 2002, with consistent back revision of the 2000 estimates, were published in the World Health Report 2003 (WHO 2003b).

The editors of the second edition of Disease Control Priorities in Developing Countries (DCP2) (Jamison and others 2006) decided to use the Version 3 GBD estimates for 
2001 to provide a common framework for assessing the causes of burden of disease in low- and middle-income countries and in analyzing priorities for interventions. We refer to these estimates as the GBD 2001. DCP2 measured the burden of disease in DALYs using a 3 percent discount rate, but without the nonuniform age weights used in the 1990 GBD study and in the results WHO published for 2000 to 2002 .

This chapter documents the data sources and methods used to prepare the GBD 2001 estimates for DCP2 and provides an overview of the global and regional results for causes of disease and injury. The results presented here are those DCP2 used as a starting point for disease-specific economic and intervention analyses. The GBD 2001 incorporates a range of new data sources for developing internally consistent estimates of incidence, health state prevalence, severity, duration, and mortality for 136 major causes by sex and by eight age groups. It estimates deaths by cause, age, and sex for 226 countries and territories drawing on a total of 770 country-years of death registration data, as well as 535 additional sources of information on levels of child and adult mortality and in excess of 2,700 data sets providing information on specific causes of death in regions not well covered by death registration systems. Estimates of incidence, prevalence, severity, duration, and DALYs by cause, age, and sex drew on more than 8,500 data sources, including epidemiological studies, disease registers, and notification systems.

The results are presented here in terms of the World Bank's income and regional groupings of countries, which DCP2 used to facilitate matching causes of death and disease burden data with the economic and social data compilation in the World Development Report 2003 (World Bank 2003). Countries are divided into seven groups: the high-income countries constitute one group and the low- and middle-income countries are divided into six geographical regions: East Asia and the Pacific, Europe and Central Asia, Latin America and the Caribbean, the Middle East and North Africa, South Asia, and SubSaharan Africa (see annex table 3A.1 and map 1 inside the front cover). Annex $3 \mathrm{~A}$ includes tables documenting definitions of cause and sequela categories and regional categories and summarizing country-specific sources of information on mortality and causes of death and the disability weights used for each cause-sequela category. The tables in annexes $3 \mathrm{~B}$ and $3 \mathrm{C}$ include results for the low- and middle-income countries as a whole as well as for the six regional groups.

\section{QUANTIFYING THE GLOBAL BURDEN OF DISEASE}

We first give an overview of the GBD approach toward summarizing the health of populations and the disease and injury causes of loss of health through the use of a particular form of summary measure, the DALY, and discuss the value choices incorporated in the DALY.

\section{The GBD Study}

The simplest and most widely used method for producing population health statistics is to aggregate data on individuals to generate estimates of quantities, for example, the proportion of the population (or of a particular grouping by age or sex) suffering from a particular health problem, being in a particular health state, or dying from a specific cause in a defined time period. This approach rapidly becomes unwieldy when a number of problems are being monitored and the intent is to make comparisons over time, across population groups, or before and after specific health interventions, as in cost-effectiveness analyses. Policy makers then face an explosion in the number of statistics they must compare and difficulties in comparing indicators relating to different health states, mortality risks, or disease events. Such statistics on the health status of populations also suffer from several other limitations that reduce their practical value for policy makers:

- Health statistics are partial and fragmented. In many countries, basic information on causes of death is not available for all important causes, and even where mortality data are available, they fail to capture the impact of nonfatal outcomes of disease and injury, such as mental disorders, musculoskeletal disorders, blindness, or deafness, on population health.

- Analyses of incidence, prevalence, or mortality for single causes often result in overestimates, even when carried out by well-intentioned epidemiologists, if not constrained to fit within demographically plausible limits and to be internally consistent and consistent with information on other causes. These problems are compounded when estimates are carried out by groups in competition for scarce resources that are acting as advocates for affected populations or by groups carrying out program evaluation that are also responsible for program implementation (Murray, Lopez, and Wibulpolprasert 2004).

- Health statistics based on a compilation of separate measures of mortality and of incidence and prevalence 
rates for a large number of conditions do not allow analysts or policy makers to evaluate outcomes of policies or to compare the relative cost-effectiveness of different interventions.

The 1990 GBD study developed a comprehensive framework for integrating, validating, analyzing, and disseminating fragmented information on the health of populations so that it is truly useful for health policy and planning (Murray and Lopez 1996b, 1996c, 1997a, 1997b). Features of this framework included the incorporation of data on nonfatal health outcomes into summary measures of population health (described in the next subsection), the development of methods and approaches to estimate missing data and to assess the reliability of data, and the use of a common metric to summarize the disease burden both from diagnostic categories of the International Classification of Diseases (ICD) and the major risk factors that cause those disease and injury outcomes.

The basic philosophy guiding the burden of disease approach is that almost all sources of health data are likely to have information content provided that they are carefully screened for plausibility and completeness and that internally consistent estimates of the global descriptive epidemiology of major conditions are possible with appropriate tools, investigator commitment, and expert opinion. This philosophy remains central to the $2001 \mathrm{GBD}$ study, which has expanded the framework of the 1990 GBD study to

- quantify the burden of premature mortality and disability by age, sex, and region for 136 causes;

- develop internally consistent estimates of incidence, prevalence, duration, and case fatality rates for more than 500 sequelae resulting from the foregoing causes;

- analyze the contribution to this burden of major physiological, behavioral, and social risk factors by age, sex, and region.

\section{Summary Measures of Population Health and the DALY}

To address the problems described above and to provide an outcome measure for cost-effectiveness analyses and priority-setting exercises, a common metric is required for mortality and for loss of health that can be disaggregated into disease and injury causes and risk factors. Since the mid-20th century, analysts have generally agreed that time is the most appropriate metric: time in years lived or lost because of mortality and years lived in various health states.
Investigators have developed a wide range of such timebased summary measures of population health, many of them generalizations of life expectancy, such as disabilityfree life expectancy or variants of the so-called qualityadjusted life year (QALY). For assessing the health of populations, summary measures of population health provide a simple and useful digest of the vast array of components of population health (Murray, Salomon, and Mathers 2000; Wolfson 1999). Summary measures of population health do not replace the more detailed reporting of data for specific aspects of health and mortality or for specific causes of health problems; rather, they supplement these data by providing a metric that can be used to monitor trends and compare health across populations or for measuring health outcomes in cost-effectiveness analyses. The last two decades have seen a marked increase in interest in the development, calculation, and use of summary measures (Field and Gold 1998; Murray, Salomon, and others 2002a; Robine and others 2003).

Two classes of summary measures of population health have been developed: health expectancies, for example, disability-free life expectancy; active life expectancy, and healthy life expectancy; and health gaps, such as DALYs and healthy life years (figure 3.1). Health expectancies extend the concept of life expectancy to refer to expectations of various states of health or of the overall expectation of years of equivalent full health, not just of life per se. Health gaps are a complementary class of indicators that measure lost years

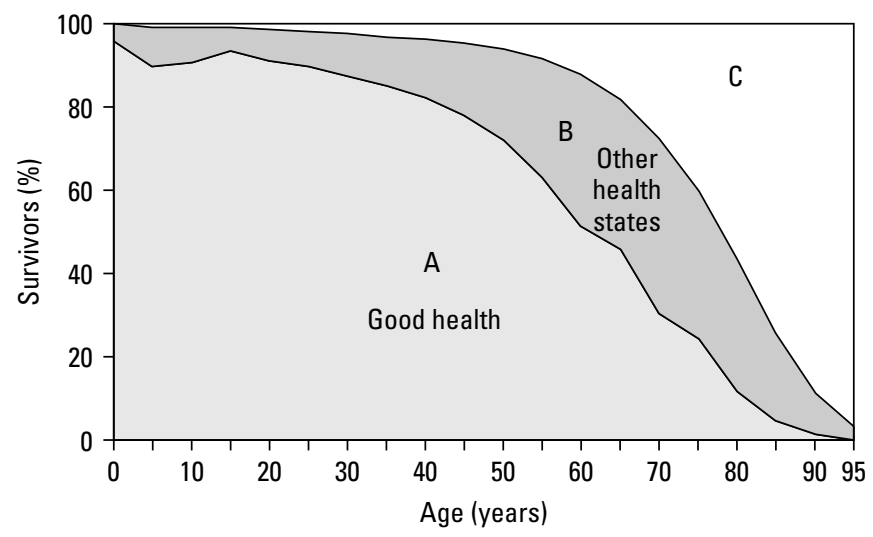

Source: Murray, Salomon, and Mathers 2000.

Note: The health gap is area $C+f(B)$ where $f(B)$ is a function of $B$ in the range 0 to area $B$ representing the lost equivalent years of full health lived in states $B$. The health expectancy is the area $A+g(B)$, where $g(B)=B-f(B)$ represents the equivalent years of full health lived in states $B$.

Figure 3.1 Relationship between Health Expectancies and Health Gaps in a Stationary Population 
of full health against some normative ideal. Measures of potential years of life lost due to premature mortality have been used for many years to measure the mortality burden of various causes of death. These all measure the gap in years between age at death and some arbitrary standard age before which death is considered premature (typically 65 or 75 ). The DALY, developed for the GBD study, is an example of a health gap indicator that extends the notion of mortality gaps to include time lived in states other than excellent health.

One of the fundamental goals in choosing a summary measure of population health for quantifying the global burden of disease was to be able to identify the relative magnitude of different health problems, including diseases, injuries, and risk factors. A health gap measure was chosen because it permits categorical attribution of the fatal and nonfatal burden of diseases and injuries to an exhaustive and mutually exclusive set of disease and injury causes (Mathers, Ezzati, and others 2002; Murray, Salomon, and Mathers 2000). The lost years of health (or DALYs) are additive across such a set of disease or injury categories. By contrast, health expectancy measures do not naturally lend themselves to disaggregation by categorically defined causes. Instead, counterfactual methods such as disease elimination are required to quantify the contribution of disease causes to overall health expectancy measures, as well as for dealing with risk factors. Health gap measures also generally require counterfactual analysis to attribute the burden of disease to health determinants and risk factors, as discussed in chapter 4 .

DALYs for a specific cause are calculated as the sum of the years of life lost due to premature mortality (YLL) from that cause and the years of healthy life lost as a result of disability (YLD) for incident cases of the health condition as follows:

$$
D A L Y=Y L L+Y L D .
$$

The YLL are essentially calculated as the number of cause-specific deaths multiplied by a loss function specifying the years lost as a function of the age at which death occurs. Ignoring for the moment other social preferences discussed later, the basic formula for YLL for a given cause $c$, age $a$, and sex $s$ is as follows:

$$
Y L L(c, a, s)=N(c, a, s) \times L(a, s),
$$

where $N(c, a, s)$ is the number of deaths due to cause $c$ for given age $a$ and sex $s$ and $L(a, s)$ is the standard loss function in years for age $a$ and sex $s$.

The 1990 GBD study did not use an arbitrary age cutoff such as 70 for the loss function used to calculate YLL, but instead specified the loss function in terms of the life expectancies at various ages in standard life tables, with life expectancy at birth fixed at 82.5 years for females and 80.0 years for males (Coale and Demeny West Model Levels 26 and 25, respectively, see Murray 1996), the highest observed life expectancies in the mid-1990s. The sex difference in the loss function was based on evidence of an intrinsic biological difference in life expectancy for males and females, but one that it is much less than the approximately five to seven years observed in developed countries (Murray 1996). Chapter 5 presents a more detailed specification of the loss function used in the standard DALY calculation.

Because YLL measure the incident stream of lost years of life due to deaths, an incidence perspective is also taken for the calculation of YLD. To estimate YLD for a particular cause during a particular time period, the number of incident cases in that period is multiplied by the average duration of the disease and a weight factor that reflects the severity of the resulting health states on a scale from 0 (perfect health) to 1 (dead). Again without yet considering other social preferences, the basic formula for YLD is as follows:

$$
Y L D(c, a, s)=I(c, a, s) \times D W(c, a, s) \times L(c, a, s),
$$

where $I(c, a, s)$ is the number of incident cases for cause $c$, age $a$, and sex $s$, DW $(c, a, s)$ is the disability weight for cause $c$, age $a$, and sex $s$; and $L(c, a, s)$ is the average duration in years of the case until remission or death.

The valuation of time lived in nonfatal health states formalizes and quantifies social preferences for different states of health as disability weights. Depending on how these weights are derived, they are variously referred to as disability weights, QALY weights, health state valuations, or health state preferences. Because the DALY is measuring loss of health (unlike the QALY, which measures equivalent healthy years lived), the disability weights for DALYs are inverted, running from 0 (ideal health) to 1 (state comparable to death). Health state valuations are discussed in more detail later.

DALYs are not unique to the GBD study. The World Bank used a variant of DALYs in its seminal study of health sector priorities (Jamison and others 1993), which was derived from earlier work to develop time-based measures that reflected the public health impact of death or illness at different ages better than mortality or prevalence counts or rates (Dempsey 1947; Ghana Health Assessment Project Team 1981). As noted, DALYs are an inverse form of the more general concept of QALYs, proposed by Zeckhauser and Shepard (1976) and widely used in economic evaluations. DCP2 (Jamison and others 2006) and WHO's generalized cost-effectiveness 
analyses for more than 170 health interventions (Tan-Torres Edejer and others 2003) use DALYs as the health outcome measure for their economic analyses.

Countries and health development agencies alike have widely adopted the burden of disease approach as the standard for health accounting, as well as for guiding the determination of health research priorities (Baskent University 2005; Bradshaw and others 2003; Bundhamcharoen and others 2002; Lozano and others 1995; Mahapatra 2002; Mathers and de Francisco 2004; Mathers, Vos, and Stevenson 1999; McKenna and others 2005; Vos and others 1995; WHO 1996).

\section{Making Social Value Choices Explicit}

In developing the DALY indicator, Murray (1996) identified three additional value choices that he argued should be made explicit in the formulation of the summary measure:

- How long "should" people in good health expect to live? This choice determines the loss function $L(a, s)$ for age $a$ and sex $s$. Should the loss function be determined at the national level or globally? The DALY uses a global loss function that is the same for all people of a given age and sex, irrespective of other characteristics such as race, socioeconomic status, or occupation.

- Is a year of healthy life gained now worth more to society than a year of healthy life gained in 20 years' time? In other words, should time discounting be applied to the stream of incident lost healthy years represented by the DALY?

- Are lost years of healthy life valued more at some ages than others? Is a year of life at young adult ages valued more than in old age or infancy? In other words, should unequal age weights be applied to years of healthy life lost at different ages?

Much of the comment on and criticism of the GBD study focused on the explicit social value choices incorporated in the DALY (Anand and Hanson 1997, 1998; Hyder, Rotllanat, and Morrow 1998; Williams 1997, 1999), particularly the social choices pertaining to age weights and severity scores for disabilities, rather than on the uncertainty of the basic descriptive epidemiology. The latter, particularly in the least developed regions, is likely to be far more consequential for setting health priorities (see chapter 5). See Murray and Acharya (1997) and Murray and Lopez (2000) for responses to the criticisms of the value choices made for the 1990 GBD study.
Murray (1996) argues on equity grounds for use of the same life expectancy "ideal" standard for specifying years of life lost for a death in all population subgroups, whether or not their current life expectancy was lower than that of other groups. In addition, he argues that the same disability weight should be used for people of the same age in the same health state.

The DALY measures the future stream of healthy years of life lost due to each incident case of disease or injury and for each death. It is thus an incidence-based rather than a prevalence-based measure. The GBD study applied a 3 percent time discount rate to years of life lost in the future to estimate the net present value of years of life lost. With this discount rate, a year of healthy life gained in 10 years' time is worth 24 percent less than one gained now. Discounting future benefits is standard practice in economic analysis and the following specific arguments can be made for applying discounting to the DALY when measuring population health (Murray and Acharya 1997):

- to be consistent with the measurement of health outcomes in cost-effectiveness analyses;

- to prevent giving excessive weight to deaths at younger ages;

- to address the disease eradication and research paradox, that is, assuming that investment in research or disease eradication has a nonzero chance of succeeding, then without discounting, all current expenditure should be shifted to such investment because the future stream of benefits is infinite.

Chapter 5 examines the sensitivity of the burden of disease results to the choice of discount rate.

In addition to 3 percent time discounting, the 1990 GBD study (Murray 1996) and the GBD results reported in recent world health reports (WHO 2000, 2002d, 2004b) used nonuniform age weights that give less weight to years lived at younger and older ages in calculating DALYs. The inclusion of nonuniform age weights was based on human capital arguments and on a number of studies that suggest the existence of a broad social preference to value a year lived by a young adult more highly than a year lived by a young child or an older person (Murray 1996). At its extreme, age preference manifests as a lack of policy interest in any deaths at ages where the death is not considered premature.

The particular age weights used in the GBD study result in greater weight being given to all deaths below age 39 compared with deaths at older ages. Age weights have perhaps 
been the most contentious social value incorporated into the DALY (Anand and Hanson 1997; Murray and Acharya 1997), and some national burden of disease studies have chosen not to use them (Mathers, Vos, and Stevenson 1999). The editors of DCP2 decided that uniform age weights should be used. Chapter 5 examines the sensitivity of the burden of disease results to different age weighting choices.

To denote different choices for discounting and age weights, we use the notation DALYs $(r, K)$, where $r$ is the discount rate in percent (not a fraction as in the GBD 1990) and $K$ is the age-weighting modulation factor. The age weights used in the GBD are denoted by $K=1$ and the nonuse of age weights (that is, uniform age weighting) is denoted by $K=0$. Thus DALYs $(3,0)$ denotes the DALY with 3 percent discounting and uniform age weights as used in DCP2 and DALYs $(3,1)$ denotes the 3 percent discounting and varying age weights as used in the GBD study. Using $\operatorname{DALYs}(3,0)$, an infant death represents the loss of 30.3 $\operatorname{DALYs}(3,0)$ for males and $30.5 \operatorname{DALYs}(3,0)$ for females, compared with 26.0 and $26.6 \operatorname{DALYs}(3,0)$ at age 30 for males and females, respectively. A death at age 60 represents 16.0 $\operatorname{DALYs}(3,0)$ for males and 17.5 for females.

\section{Comparing Time Lived in Different States of Health}

To use time as a common currency for nonfatal health states and for years of life lost due to mortality, we must define, measure, and numerically value time lived in nonfatal health states. The valuation of time lived in nonfatal health states formalizes and quantifies social preferences for different states of health as health state weights. While death is not difficult to define, nonfatal health states are. They involve multiple domains of health that relate to different functions, capacities, or aspects of living. During the last three decades, there has been general acceptance of an approach to describing individuals' health states in terms of multiple domains of health and to developing self-reporting instruments that seek information on a core set of these domains, typically no more than five to eight, that capture most of the important variations in health states across individuals (McDowell and Newell 1996; Sadana 2002).

One common approach is to describe health as a profile of levels on a series of domains. The Medical Outcomes Study (MOS) Short Form 36 is an example of such an instrument, with eight domains covering self-perceived health, vitality, bodily pain, mental health, physical functioning, social functioning, physical role limitations, and social role limitations (Ware and Sherbourne 1992). MOS Short Form 36 domains are scored on continuous scales from 0 to 100 , resulting in a large number of potential health states. Health state profiles intended for use with health state valuations tend to use a more limited number of levels in each domain.

Murray and colleagues argue that health state valuations should be conceptualized and operationalized as judgments about the overall level of health associated with a multidimensional description of an individual's health state, not about overall levels of well-being, quality of life, or utility (Murray, Salomon, and others. 2002b; Salomon, Mathers, and others 2003). In this conceptualization, health state valuations formalize the intuitive notion that health levels lie on a continuum and that we may characterize one individual as being more or less healthy than another individual at a particular moment in time. Health state valuations quantify departures from perfect health, that is, the reductions in health associated with particular health states. Note that these weights do not measure the quality of life of people with disabilities and do not measure the value of people to society.

By assigning a single number to an individual's health state with reference to ideal health, health state valuations permit aggregating individual health levels over time and comparing health across individuals, and thereby provide the critical link that allows individuals' nonfatal health experience to be combined with information about mortality in summary measures of population health. Researchers have developed a number of choice-based methods to measure preferences for health states (Salomon and Murray 2004).

The 1990 GBD used two forms of the person trade-off method and asked participants in weighting exercises to make a composite judgment about the severity distribution of the condition and the preference for time spent in each severity level (Murray 1996). This was largely necessitated by the lack of population information on the severity distribution of most conditions at the global and regional levels. The disability weights used in the GBD 2001 are still based in large part on the GBD 1990 disability weights (Murray 1996). Disability weights may vary by age, sex, and region, reflecting variations in the severity distributions of health states and the proportions of cases treated. A common global valuation function is assumed for the underlying health state valuations for specific health states. Despite the assertion by some commentators that valuations for certain health states are likely to be extremely heterogeneous across individuals and populations, empirical evidence suggests otherwise. Valuation studies carried out with deliberative small groups from a wide range of countries have found surprising consistency in valuations across cultures (Salomon and Murray 2002b). More recently, valuation studies carried out as part 
of the WHO multicountry survey study have also found reasonable consistency in health state valuations for most health states (Salomon, Murray, and others 2003).

Following the GBD terminology, the term disability is used here broadly to refer to departures from optimal health in any of the important domains of health, including mobility, self-care, participation in usual activities, pain and discomfort, anxiety and depression, and cognition and social participation. We thus refer to disability weights and healthy years lost due to disability as shorthand terms for health state preferences and years of healthy life lost because of time lived in states other than the reference state of optimal health, respectively. Note that with this usage, disability, that is, states other than ideal health, may be short term or long term: a day with a common cold is a day with disability.

\section{ESTIMATING DEATHS BY CAUSE: METHODS AND DATA}

Complete death registration data cover only one-third of the world's population. Some information on another third is available through the urban death registration systems and national sample registration systems of China and India. For the remaining one-third of the world's population, including most countries in Sub-Saharan Africa, only partial information is available from epidemiological studies, disease registers, and surveillance systems.

The original (1990) GBD study was the first attempt to estimate the global and regional numbers of deaths resulting from a comprehensive set of causes while ensuring consistency with death totals provided by death registration and demographic methods (Murray and Lopez 1996c). Ensuring this consistency was a major advance and is an essential first step in measuring the disease burden. Estimates of numbers of deaths carried out separately for individual causes that are not constrained to sum to a demographically derived total often result in substantial overestimates of deaths from each cause (Jamison 1996). In part, this occurs because in carrying out analysis for a single cause, researchers may easily be overinclusive in counting the deaths attributable to the cause of interest, even without any intent to maximize the size of the specific problem.

Thus, the first analytical step in estimating deaths by cause was to estimate age-specific total death rates by sex. The importance of this step cannot be overemphasized. The number of deaths by age and sex provided an essential "envelope" that constrained individual disease and injury estimates of deaths. Competing claims for the magnitude of deaths from various causes must then be reconciled within this envelope.

Next, to estimate the number of deaths by cause we drew on the following four broad sources of data:

- Death registration systems. Complete or incomplete death registration systems provide information about causes of death for almost all high-income countries and for many countries in Europe (Eastern) and Central Asia and in Latin America and the Caribbean. Some vital registration (VR) information is also available in all other regions.

- Sample death registration systems. In China and India, sample registration systems for rural areas supplement urban death registration systems. Information systems now provide data on causes of death for several other large countries for which information was not available at the time of the original GBD study.

- Epidemiological assessments. Epidemiologists have estimated deaths for specific causes, such as HIV/AIDS, malaria, and tuberculosis (TB), for most countries in the regions most affected. These estimates usually combine information from surveys on the incidence or prevalence of the disease with data on case fatality rates.

- Cause of death models. The cause of death models used in the original GBD study (Murray and Lopez 1996a) were substantially revised and enhanced for estimating deaths by broad cause group in regions with limited information on mortality. The CodMod software developed for this study and described later drew on a data set of 1,613 country-years of observation of cause of death distributions from 58 countries between 1950 and 2001.

\section{All-Cause Mortality for 192 Countries}

According to data provided by 112 WHO member states, only about one-third of the estimated 56 million deaths occurring annually are recorded in death registration systems. If the sample registration systems of China and India are considered to provide information on their entire populations, then information is available for around 72 percent of the global population. In recent years, considerable priority has also been given to obtaining data on child and maternal mortality through such instruments as the Demographic and Health Survey (DHS) program funded by the U.S. Agency for International Development and the Multiple Indicator Cluster Survey program carried out by the United Nations Children's Fund. Table 3.1 summarizes sources of information on levels of child and adult all-cause 
Table 3.1 Availability of Data for Estimation of All-Cause Mortality Rates by Age and Sex

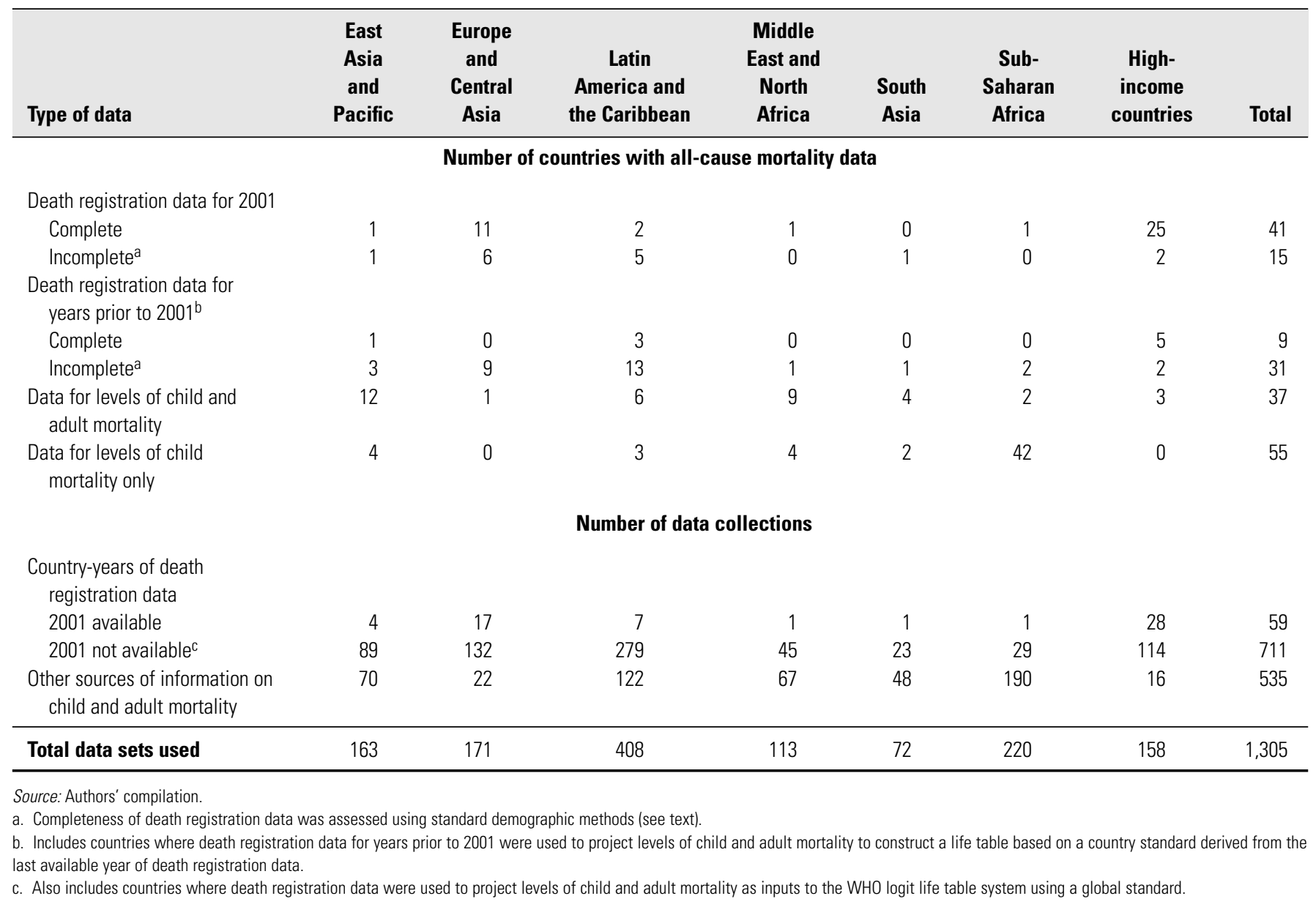

mortality used to construct life tables for 192 WHO member states by region and by type of data.

For countries with death registration data, demographic techniques (Preston-Coale, Brass growth-balance, generalized growth-balance, and Bennett-Horiuchi methods) were first applied, as appropriate, to assess the extent of completeness of the recorded mortality data for adults. If the data coverage estimates were high enough to be meaningful, death rates for those aged five years and over were then adjusted accordingly. The completeness of death registration for children was assessed separately using other available sources of information on child mortality. For countries without usable VR data, other available sources of adult mortality such as surveys and censuses were used to estimate the level of adult mortality as measured by ${ }_{45} q_{15}$ (the probability of dying between exact ages 15 and 60 ). For child mortality under five, again, all available survey, census, and VR data were assessed, adjusted, and averaged to estimate the probable trend in child mortality $\left({ }_{5} q_{0}\right)$ in recent decades.
The population estimates used for all countries were those prepared by the United Nations Population Division (2003). Note that these estimates refer to de facto populations, that is, they include residents such as guest workers and refugees, rather than de jure populations, meaning citizens, and in some countries, permanent residents. Member states that report death registration data to $\mathrm{WHO}$ also routinely report population data for the population the death registration system covers, which in some cases is a subset of the national population. Death registration data may cover less than 100 percent of the population not only because some geographical areas may be excluded, but also because registration may be restricted to a subset of the resident population, such as citizens, and may thus exclude deaths among groups such as guest workers or refugees.

For the GBD 2001 study, age- and sex-specific death rates were calculated from the death and population data provided by countries, with adjustments made for completeness of the registration data where needed, and then total deaths by age and sex were calculated for each country by applying 
these rates to the United Nations Population Division estimates of de facto populations for 2001.

Four methods were used to construct life tables for each country depending on the type of data available (Lopez and others 2002):

- Countries with death registration data for 2001. Such data were used directly to construct life tables for 56 countries after adjusting for incomplete registration if necessary.

- Countries with a time series of death registration data. Where the latest year of death registration data available was prior to 2001, a time series of annual life tables (adjusted if the registration level was incomplete) between 1985 and the latest available year was used to project levels of child and adult mortality for 2001. For small countries with populations of less than 500,000, moving averages were used to smooth the time series. Projected values of child and adult mortality were then applied to a modified logit life table model (Murray, Ferguson, and others 2003), using the most recent national data as the standard, to predict the full life table for 2001, and HIV/AIDS and war deaths were added to total mortality rates for 2001 where necessary. This method was applied for 40 countries using a total of 711 country-years of death registration data.

- Countries with other information on levels of child and adult mortality. For 37 countries, estimated levels of child and adult mortality were applied to a modified logit life table model (Murray, Ferguson, and others 2003), using a global standard, to estimate the full life table for 2001, and HIV/AIDS deaths and war deaths were added to total mortality rates as necessary. For most of these countries, data on levels of adult mortality were obtained from death registration data, official life tables, or mortality information derived from other sources such as censuses and surveys. The all-cause mortality envelope for China was derived from a time series analysis of deaths for every household in China reported in the 1982, 1990, and 2000 censuses. The extent of underreporting of deaths in the 2000 census was estimated at about 11.3 percent for males and 18.1 percent for females (Bannister and Hill 2004). The all-cause mortality envelope for India was derived from a time series analysis of age-specific death rates from the Sample Registration System after correction for underregistration (88 percent completeness) (Mari Bhat 2002).

- Countries with information on levels of child mortality only. For 55 countries, 42 of them in Sub-Saharan Africa, no information was available on levels of adult mortality.
Based on the predicted level of child mortality in 2001, the most likely corresponding level of adult mortality (excluding HIV/AIDS deaths where necessary) was selected, along with uncertainty ranges, based on regression models of child versus adult mortality as observed in a set of almost 2,000 life tables judged to be of good quality (Lopez and others 2002; Murray, Ferguson, and others 2003). These estimated levels of child and adult mortality were then applied to a modified logit life table model, using a global standard, to estimate the full life table in 2001, and HIV/AIDS deaths and war deaths were added to total mortality rates as necessary. Evidence on adult mortality in Sub-Saharan African countries remains limited, even in areas with successful child and maternal mortality surveys.

\section{Classification of Causes of Disease and Injury}

Disease and injury causes of death and of burden of disease were classified using the same tree structure as in the original GBD study (Murray and Lopez 1996c). The first level of disaggregation comprises the following three broad cause groups:

- Group I: communicable, maternal, perinatal, and nutritional conditions

- Group II: noncommunicable diseases

- Group III: injuries.

Each group was then divided into major cause subcategories, for example, cardiovascular disease (CVD) and malignant neoplasms (cancers) are two major cause subcategories of Group II. Beyond this level, two further disaggregation levels were used, resulting in a complete cause list of 136 categories of specific diseases and injuries. Annex table 3A.2 lists the GBD 2001 cause categories and their ICD codes in terms of the ICD 9th revision (ICD-9) and 10th revision (ICD-10) (WHO 1977, 1992).

Group I causes of death consist of the cluster of conditions that typically decline at a faster pace than all-cause mortality during the epidemiological transition. In highmortality populations, Group I dominates the cause of death pattern, whereas in low-mortality populations, Group I accounts for only a small proportion of deaths. The major cause subcategories are closely based on the ICD chapters with a few significant differences. Whereas the ICD classifies chronic respiratory diseases and acute respiratory infections into the same chapter, the GBD cause classification includes acute respiratory infections in Group I and 
chronic respiratory diseases in Group II. Note also that the Group I subcategory of "causes arising in the perinatal period" relates to the causes included in the corresponding ICD chapter, principally low birthweight, prematurity, birth asphyxia, and birth trauma, but does not include all causes of deaths occurring during the perinatal period, such as infections, congenital malformations, and injuries. In addition, the GBD includes only deaths among children born alive and does not estimate stillbirths (see chapter 6).

The development and successive revisions of the ICD have facilitated the comparability of cause of death data within and across countries. Although each revision has produced some discontinuities in cause of death data, the revision from ICD-9 to ICD-10 resulted in more substantial changes than previous revisions. ICD-10 is considerably more detailed than ICD-9, with almost twice the number of codes, and includes both conceptual and classification revisions as well as changes in the coding rules used to select the underlying cause of death. Additional problems in comparing data on causes of death across countries arise from variations in the accuracy of diagnoses of causes of death.

In most developed countries, medical practitioners certify the underlying cause of death even though they may not always have had prior contact with the deceased or access to relevant medical records. In developing countries, a significant proportion of deaths may occur without medical attention and such deaths may be registered without a medical opinion about the cause of death. At the same time, selecting a single underlying cause of death is often problematic for the elderly, who have often had several chronic diseases that concurrently led to their death. This results in higher levels of uncertainty about cause of death distributions in the oldest age group. Finally, in both developing and developed countries, legal, societal, and other reasons may lead to the underreporting of causes of death of a sensitive nature, such as suicide or HIV/AIDS. For this reason, other sources of information for specific causes such as HIV/AIDS, illicit drug use, and war have been used where necessary to modify cause-specific estimates based on death registration data.

The GBD classification system does not include the ICD category "symptoms, signs, and ill-defined conditions" as one of the major causes of deaths. The GBD classification scheme has reassigned deaths assigned to this ICD category, as well as some other codes used for ill-defined conditions, to specific causes of death. This is important from the perspective of generating useful information to compare cause of death patterns or to inform health policy making, because it allows unbiased comparisons of cause of death patterns across countries or regions.
Deaths are categorically attributed to one underlying cause using ICD rules and conventions. In some cases where the ICD rules are ambiguous, the GBD 2001 follows the conventions used by the GBD 1990 study (Murray and Lopez 1996a). Note also that a number of causes of death act as risk factors for other diseases. Total mortality attributable to such causes may be substantially larger than the mortality estimates for the cause in terms of ICD rules for underlying causes. For example, the GBD 2001 estimates that 960,000 deaths were due to diabetes mellitus as an underlying cause, but when deaths from CVD and renal failure attributable to diabetes are included, the global total of attributable deaths rises to almost 3 million (Roglic and others 2005). Other causes for which important components of attributable mortality are included elsewhere in the GBD cause list include hepatitis $\mathrm{B}$ or $\mathrm{C}$ (mortality attributable to liver cancer and renal failure), unipolar or bipolar depressive disorders and schizophrenia (mortality attributable to suicide), and blindness (mortality attributable to blindness whether from infectious or noninfectious causes).

\section{Countries with Complete or Incomplete Death Registration Data}

In the last decade, computerization of death registration data at the country level and electronic transmission to WHO have considerably improved the timeliness of information. In addition, the number of countries submitting their underlying cause of death data to WHO using ICD-10 increased from 4 in 1995 to 75 in 2003. Some 50 countries are still reporting data using ICD-9 and only 1 country is still using ICD-8 (Mathers and others 2005).

Several new features and changes from ICD-9 to ICD-10 have a major impact on the interpretation of statistical data, and the implications of these changes have been taken into account to a limited extent when making trend comparisons and estimations for causes of death. ICD-10 is more detailed, with about 10,000 codes compared with around 5,100 in ICD-9, and the rules for selecting the underlying cause of death have been reevaluated and sometimes changed. For example, ICD-10 considers pneumonia to be a consequence of a much wider range of conditions than ICD-9, and it therefore would be less likely to be selected as the underlying cause. Modification of the death certificate with the inclusion of an additional line in part 1 of the certificate (for diseases related to the chain of events leading directly to death) as recommended by WHO may also have had an impact on the selection of the underlying cause of death. 
Accuracy in diagnosing causes of death still varies substantially across countries with death registration systems. In addition, even in countries where medically qualified staff assign causes of death, some degree of misattribution or miscoding occurs during the process of coding underlying causes of death, mainly because of incorrect or systematic biases in diagnoses, incorrect or incomplete death certificates, misinterpretation of ICD rules for selecting underlying causes, and variations in the use of categories for unknown and ill-defined causes (Mathers and others 2005).

Death registration data containing usable information on cause of death distributions were available for 107 countries, mostly in the high-income group, Europe and Central Asia, and Latin America and the Caribbean (table 3.2, annex table
3A.3). Where the latest available year was earlier than 2001, death registration data from 1980 through the latest available year were analyzed as a basis for projecting recent trends for specific causes, and these trend estimates were used to project the cause distribution for 2001. When estimating cause of death distributions for very small countries, an average of the three last years of data was used to minimize stochastic variation.

In the case of the few countries still reporting data using the condensed ICD-9 Basic Tabulation List, algorithms based on data from countries with more detailed coding were applied to estimate deaths due to asthma as no Basic Tabulation List code for asthma is available. Also, China and some of the newly independent states of the former Soviet

Table 3.2 Availability of Data for Estimation of Causes of Death by Age and Sex

\begin{tabular}{|c|c|c|c|c|c|c|c|c|}
\hline Type of data & $\begin{array}{c}\text { East } \\
\text { Asia } \\
\text { and } \\
\text { Pacific }\end{array}$ & $\begin{array}{c}\text { Europe } \\
\text { and } \\
\text { Central } \\
\text { Asia }\end{array}$ & $\begin{array}{l}\text { Latin } \\
\text { America and } \\
\text { the Caribbean }\end{array}$ & $\begin{array}{c}\text { Middle } \\
\text { East and } \\
\text { North } \\
\text { Africa }\end{array}$ & $\begin{array}{l}\text { South } \\
\text { Asia }\end{array}$ & $\begin{array}{l}\text { Sub- } \\
\text { Saharan } \\
\text { Africa }\end{array}$ & $\begin{array}{l}\text { High- } \\
\text { income } \\
\text { countries }\end{array}$ & Total \\
\hline \multicolumn{9}{|c|}{ Number of countries } \\
\hline $\begin{array}{l}\text { Death registration data } \\
\text { (coverage of } 85 \% \text { or more) }^{\text {a }}\end{array}$ & 1 & 11 & 5 & 1 & 0 & 1 & 29 & 48 \\
\hline $\begin{array}{l}\text { Death registration data } \\
\text { (coverage }<85 \% \text { ) - adjusted } \\
\text { using cause-of-death models }\end{array}$ & 5 & 16 & 25 & 3 & 1 & 3 & 5 & 58 \\
\hline $\begin{array}{l}\text { Sample registration and } \\
\text { surveillance }^{\mathrm{b}}\end{array}$ & 2 & 0 & 0 & 1 & 1 & 1 & 0 & 5 \\
\hline $\begin{array}{l}\text { No data-cause-of-death models } \\
\text { used with detailed cause patterns } \\
\text { based on regional data }\end{array}$ & 14 & 0 & 2 & 10 & 6 & 42 & 3 & 77 \\
\hline $\begin{array}{l}\text { Epidemiological estimates for } \\
\text { mortality due to specific causes } \\
\text { used where applicable }\end{array}$ & c & $d$ & c & c & c & c & e & c \\
\hline \multicolumn{9}{|c|}{ Percent of population } \\
\hline 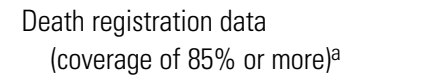 & 0.0 & 52.7 & 13.0 & 0.1 & 0.0 & 0.2 & 94.4 & 19.5 \\
\hline $\begin{array}{l}\text { Death registration data } \\
\text { (coverage }<85 \%) \text { - adjusted } \\
\text { using cause-of-death models }\end{array}$ & 5.6 & 47.3 & 84.2 & 50.0 & 1.4 & 8.6 & 5.3 & 17.1 \\
\hline $\begin{array}{l}\text { Sample registration and } \\
\text { surveillance }{ }^{b}\end{array}$ & 73.3 & 0.0 & 0.0 & 1.7 & 74.5 & 1.4 & 0.0 & 39.1 \\
\hline $\begin{array}{l}\text { No data-cause-of-death models } \\
\text { used with detailed cause patterns } \\
\text { based on regional data }\end{array}$ & 21.1 & 0.0 & 2.9 & 48.1 & 24.2 & 89.8 & 0.3 & 24.3 \\
\hline Total & 100.0 & 100.0 & 100.0 & 100.0 & 100.0 & 100.0 & 100.0 & 100.0 \\
\hline \multicolumn{9}{|c|}{$\begin{array}{l}\text { Source: Authors' compilation. } \\
\text { a. The threshold of coverage of } 85 \text { percent used for causes of death differs from that used for registration of deaths ( } 95 \text { percent) because the biases from underreporting of the fact of death are more } \\
\text { serious for assessing levels of all-cause mortality than for assessing the distribution of causes. } \\
\text { b. Includes countries with death registration or surveillance systems relying heavily on verbal autopsy methods for ascertaining causes of death. } \\
\text { c. HIV/AIDS, tuberculosis, measles, pertussis, poliomyelitis, tetanus, acute lower respiratory infections, Chagas' disease, maternal conditions, perinatal conditions, cancers, drug use disorders, } \\
\text { rheumathoid arthritis, and war. See table } 3.5 \text { for details. } \\
\text { d. AIDS, drug use disorders, and war. See table } 3.5 \text { for details. } \\
\text { e. Drug use disorders and war. See table } 3.5 \text { for details. }\end{array}$} \\
\hline
\end{tabular}


Union still use some special condensed ICD-9 cause of death classifications, which were then mapped to the GBD cause list. Missing values for some GBD conditions were estimated with the use of algorithms. Similarly, algorithms were also applied for countries reporting data using the condensed ICD-10 Mortality Tabulation List 1.

Deaths resulting from war are not systematically included in the cause of death data. For example, in the United States, the Department of Defense records deaths resulting from war, and for security reasons they are not included in the death registration system. Some death registration data undercount deaths due to HIV/AIDS and drug use partly because of miscoding and partly because of reluctance to record these diagnoses. In some cases, adjustments for deaths due to war, HIV/AIDS, and drug use have been made using other sources of information as described later.

Cause of death data were carefully analyzed to take incomplete coverage of VR into account and the likely differences in cause of death patterns among the uncovered and often poorer subpopulations. When the coverage of death registration data was assessed as less than 85 percent, cause of death modeling was used to adjust the proportions of deaths occurring in Groups I, II, and III by age and sex. Table 3.2 shows the regional distribution of the 58 countries for which such adjustments were carried out. In total, useful information on cause of death distributions was available for 37 percent of the world's population, or 76 percent if China and India's sample registration and mortality surveillance systems were included. Usable death registration information was available for only four Sub-Saharan African countries: Mauritius, the Seychelles, South Africa, and Zimbabwe. Death registration data are available for several other SubSaharan African countries, but are largely restricted to deaths in urban hospitals, with overall coverage being too low to provide useful population-level information on cause of death distributions (Rao, Bradshaw, and Mathers 2004).

Annex table 3A.3 summarizes the years of death registration data with information on underlying cause available for each country, together with information on the methods used to estimate cause of death distributions. As shown in table 3.1, a total of 770 country-years of death registration data were used in the analysis of causes of death for the GBD 2001.

\section{Redistribution of III-Defined Causes and "Garbage Codes"}

Even in countries where medically qualified staff assign causes there is substantial use of coding categories for unknown and ill-defined causes. In addition to the ICD codes for "symptoms, signs, and ill-defined conditions" (ICD-9 codes 780-799 and ICD-10 codes R00-R99), a number of other ICD codes do not represent useful underlying causes from a policy perspective and their inappropriate overuse compromises the usefulness of information on causes of death. These garbage codes or ill-defined codes include deaths from injuries where the intent was not determined (ICD-9 codes E980-989 and ICD-10 codes Y10-Y34 and Y872); CVD categories lacking diagnostic meaning, such as cardiac arrest and heart failure (ICD-9 codes 427.1, 427.4, 427.5, 428, 429.0, 429.1, 429.2, 429.9, and 440.9; and ICD-10 codes I47.2, I49.0, I46, I50, I51.4, I51.5, I51.6, I51.9, and I70.9); and cancer deaths coded to categories for secondary or unspecified sites (ICD-9 codes 195 and 199 and ICD-10 codes C76, C80, and C97). The percentage of deaths coded as ill-defined causes varies from 4 percent in New Zealand to more than 40 percent in Sri Lanka and Thailand.

Table 3.3 shows the distribution of deaths assigned to ill-defined codes for the 105 WHO member states reporting data on death registrations since 1990 with at least 50 percent completeness or coverage. The median percentage of deaths coded to ill-defined causes was 12 percent; the median percentage of symptoms, signs, and ill-defined conditions was 4.0 percent; and the median of ill-defined cardiovascular causes was 5.3 percent. In more than 15 high-income countries, more than 10 percent of deaths were coded to these ill-defined conditions, not so much because of overuse of codes for symptoms, signs, and illdefined conditions, but because of excessive use of garbage codes for CVD, cancers, and injuries (Mathers and others 2005).

To produce unbiased estimates of cause-specific death rates, and to maximize comparability across member states, deaths coded to general ill-defined categories (ICD- 9, chapter XVI; ICD-10, chapter XVIII) were redistributed pro rata across all Group I and Group II causes, that is, all causes excluding injuries. Correction algorithms were also applied to resolve problems of miscoding for the cardiovascular, cancer, and injury garbage codes.

Ill-Defined Cardiovascular Codes. Physicians may use a number of cardiovascular codes in ICD-9 and ICD-10 to assign deaths that are actually due to ischemic heart disease (IHD). They may assign IHD deaths to ill-defined cardiovascular codes because of insufficient clinical information at the time of death, local medical diagnostic practices, or simply by error. These include codes for heart failure, ventricular dysrhythmias, generalized atherosclerosis, and illdefined descriptions and complications of heart disease. 
Table 3.3 Distribution of Percentage of Total Deaths Assigned to III-Defined Codes for 105 WHO Member States, Most Recent Available Year

\begin{tabular}{lcccc}
\hline & \multicolumn{2}{c}{ Percentage of deaths assigned to ill-defined codes } & \\
\cline { 2 - 4 } III-defined code group & Median & 25th percentile & 75th percentile & Maximum \\
\hline Symptoms & 4.0 & 2.1 & 8.7 & 44.0 \\
Injury & 0.5 & 0.2 & 1.3 & $5.1^{\mathrm{a}}$ \\
Cancer & 1.0 & 0.5 & 1.5 & 2.7 \\
Cardiovascular disease & 5.3 & 2.7 & 7.7 & 23.4 \\
\hline Total ill-defined & 12.0 & 7.0 & 17.2 & 48.8 \\
\hline
\end{tabular}

Source: Mathers and others 2005

Note: Table includes those countries supplying data on death registration for most recent year since 1990 and with at least 50 percent completeness or coverage.

a. These data exclude South Africa, where 93 percent of deaths from external causes were coded to ill-defined injuries.

Figure 3.2 illustrates the enormous variation across countries in coding practice with respect to these ill-defined cardiovascular codes. For each country, the fraction of cardiovascular deaths (excluding stroke) assigned to the illdefined cardiovascular codes is plotted against the fraction of cardiovascular deaths (excluding stroke) assigned to IHD (ICD-9 codes $410-414$ or ICD-10 codes I20-I25). The strong negative relationship between IHD mortality and that from the ill-defined CVD codes $\left(\mathrm{r}^{2}=0.90\right)$ strongly supports the hypothesis that the quality of CVD death certification varies substantially across countries. The upper left portion of figure 3.2 shows countries where doctors certified, on average, more ill-defined CVD than IHD deaths, and these include France, Japan, Portugal, and Spain. The bottom right corner of the figure shows those countries where doctors assign, on average, a small proportion of illdefined CVD deaths. This second group includes Australia, Canada, Finland, New Zealand, Norway, and the United Kingdom (Scotland). We refer to these two groups of countries as the high ill-defined coding and low ill-defined coding groups.

To correct for the likely underregistration of IHD in countries such as France, Japan, and Spain in the original GBD study, Murray and Lopez (1996a) developed an algorithm based on the assumption that the cluster of countries comprising Canada, Finland, New Zealand, and Norway, where ill-defined coding was low, would define the standard coding practice. For all other countries, the percentage of cardiovascular deaths (excluding stroke) assigned to these codes in excess of this standard percentage was then assumed to be largely miscertified IHD. For the GBD 2001, Lozano and others (2001) developed a revised method to estimate the fraction of IHD deaths assigned to ill-defined cardiovascular codes. This involved estimating age- and sexspecific regression equations predicting observed IHD death

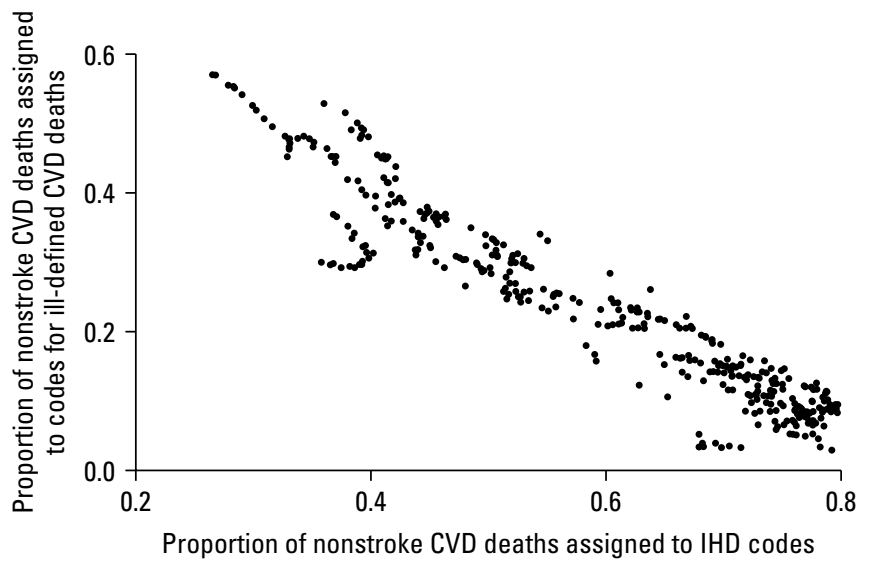

Source: Lozano and others 2001.

Figure 3.2 Variation across Selected Countries in Coding for IIIDefined CVD Causes, 1979-98

rates in terms of the ill-defined CVD death rates and the smoking impact ratio for a cross-national data set of 372 country-years of death registration data for 26 countries between 1979 and 1998. The smoking impact ratio, estimated from lung cancer mortality rates using the Peto-Lopez method (Peto and others 1992), is a measure of the cumulative effects of tobacco exposure as a risk factor for IHD.

Table 3.4 shows the resulting correction factors, that is, the proportion of ill-defined CVD deaths reassigned to IHD. As expected, the extent of miscoding at every age, for both males and females, was systematically higher in high ill-defined coding countries, where the results suggest that 50 to 95 percent of ill-defined CVD codes should be reassigned to IHD.

With correction, the age standardized death rates increased in all countries, but most notably in Japan (26 percent for males and 24 percent for females), France (27 percent 
Table 3.4 Correction Factors Giving Proportion of III-Defined CVD Deaths to Be Reassigned to IHD, by Age and Sex

\begin{tabular}{cccccc}
\hline & \multicolumn{2}{c}{ Males } & & \multicolumn{2}{c}{ Females } \\
\cline { 2 - 3 } Age group & $\begin{array}{c}\text { Low ill-defined } \\
\text { coding countries }\end{array}$ & $\begin{array}{c}\text { High ill-defined } \\
\text { coding countries }\end{array}$ & & $\begin{array}{c}\text { Low ill-defined } \\
\text { coding countries }\end{array}$ & $\begin{array}{c}\text { High ill-defined } \\
\text { coding countries }\end{array}$ \\
\hline $35-39$ & 0.000 & 0.000 & 0.000 & 0.000 \\
$40-44$ & 0.107 & 0.107 & 0.000 & 0.000 \\
$45-49$ & 0.039 & 0.273 & 0.000 & 0.041 \\
$50-54$ & 0.040 & 0.696 & 0.101 & 0.446 \\
$55-59$ & 0.203 & 0.941 & 0.139 & 0.689 \\
$60-64$ & 0.160 & 0.754 & 0.119 & 0.660 \\
$65-69$ & 0.253 & 0.827 & 0.251 & 0.615 \\
$70-74$ & 0.264 & 0.732 & 0.202 & 0.469 \\
$75-79$ & 0.233 & 0.576 & 0.170 & 0.358 \\
$80+$ & 0.030 & 0.242 & 0.060 & 0.198 \\
\hline
\end{tabular}

Source: Lozano and others 2001.

for males and 35 percent for females), and Greece (32 percent for males and 47 percent for females). Smaller increases were apparent for Belgium, the Czech Republic, Hungary, Italy, Portugal, and Spain (12 to 25 percent on average for males and females), and only small changes were observed for Austria, Germany, the Netherlands, and the United States (about 5 percent). In other countries, including Australia, Canada, Finland, Ireland, New Zealand, Norway, and the United Kingdom (Northern Ireland and Scotland), no corrections were suggested by this analysis.

Corrections for miscertification narrow the range in death rates across countries from a fivefold to a fourfold variation and also change the relative rankings of countries. The analysis of IHD miscertification is supported by the dramatic increase of more than 25 percent in recorded IHD mortality rates in Japan between 1994 and 1995 with the change from ICD-9 to ICD-10, whereby physicians were encouraged not to use heart failure as an underlying cause of death. Prior to the introduction of ICD-10, corrected rates were more than 80 percent higher in males and around 70 percent greater in females compared with what was recorded in vital statistics.

Lozano and others (2001) compare the miscertification levels estimated using their regression approach with those observed in the WHO Monitoring Cardiovascular Disease (MONICA) study sites. They find general agreement in relation to the existence of significant miscertification in each country, but less clear agreement on specific levels of miscertification. This latter finding is difficult to interpret given some difficulties in mapping the MONICA "possible IHD category" to ICD categories and the fact that the study sites may not be representative of national populations.
While the empirical results of applying the recoding model are encouraging, and the GBD 2001 has used it to reassign ill-defined CVD codes, two points are noteworthy. First, the fraction of ill-defined cardiovascular deaths that are due to IHD is assumed to be constant across countries within each of the low and high ill-defined code groups. Statistical models can only go so far in extracting truth from poorly coded deaths data, and more precise country-specific analyses really require recoding studies for samples of relevant deaths, ideally involving autopsy or other clinical diagnostic information. Second, due to the nonstandard disease classification used in Russia and other newly independent states (175 categories based on ICD-9), the method cannot be applied without further evidence from autopsies as to the true cause of cardiovascular deaths. The single most important cause of cardiovascular death in these countries is "coronary atherosclerosis" (093 in the Soviet classification of diseases), which in part reflects a disease process different than what the term implies elsewhere (Chenet and others 1998; Zatonski 1998). The use of the code "sudden death" to describe mortality often associated with binge drinking in Russia and neighboring countries may also conceal cases of IHD (Kauhanen and others 1997).

Ill-Defined Cancer Codes. In the GBD 1990 study, deaths coded to ICD-9 195-199 (malignant neoplasm of other and unspecified sites, including those whose point of origin cannot be determined, secondary and unspecified neoplasm) were redistributed pro rata across all malignant neoplasm categories within each age and sex group, so that the category "other malignant neoplasms" included only malignant neoplasms of other specified sites (Murray and Lopez 1996a). 
For the GBD 2001, the survival model developed for estimating cancer deaths by site from cancer incidence data (Mathers, Shibuya, and others 2002) was used to compare predicted deaths from the survival model for the United States with those reported in U.S. vital statistics. This comparison identified four sites that did not appear to have any significant coding of cancer deaths to the garbage codes ICD-9 195-199. The redistribution algorithm for cancer garbage codes was therefore revised for the GBD 2001 to redistribute cancer garbage code deaths pro rata across all cancer sites except liver; pancreas; ovary; and trachea, bronchus, and lung.

Intent of Injuries Undetermined. Deaths assigned to codes for injuries undetermined whether accidentally or purposefully inflicted (ICD-9 codes E980-989 and ICD-10 codes Y10-Y34 and Y872) are those where the person certifying the cause of death has not determined whether the injuries were unintentional or intentional, for example, an outcome of self-inflicted injury or assault. While there will remain a residue of deaths for which insufficient information is available to determine intent, this should be a small fraction of injury deaths if appropriate forensic and coronial investigations are carried out. Excluding South Africa, the proportion of injury deaths assigned to these codes varies from less than 0.5 percent in most developed countries to just over 5 percent (table 3.3). To reduce bias in estimating deaths due to unintentional and intentional injuries, deaths coded as undetermined intent were redistributed pro rata by age and sex to the GBD categories for intentional and unintentional injury.

\section{Data Sources and Methods for Some Specific Countries}

In some cases, either because of large population size, and hence implications for global mortality estimates, or because of recent national burden of disease research involving one or more of the authors, more detailed methods to estimate mortality patterns were applied, as summarized in the following subsections.

China. Cause-specific mortality data for China are available from two sources: the sample VR system administered by the Ministry of Health and the Disease Surveillance Point (DSP) system established by the Chinese Center for Disease Control (see Yang and others 2005 for an overview of the design and operational characteristics of these systems). The VR system covers a population of 120 million people at 137 sample sites and captures around 700,000 deaths per year. The DSP system has 145 surveillance points, covers a population of around 11 million, and collects information on around 50,000 deaths per year.

The Ministry of Health classifies sample sites for the DSP system into an urban stratum and four socioeconomic strata for rural areas, based on an analysis of nine indicators for rural counties from the 1990 national census. These indicators include birth and mortality rates, dependency ratios, literacy rates, and proportions of agricultural versus industrial occupations in the overall workforce. The VR system's sample sites are classified into one urban and three rural socioeconomic strata. Because the sample sites for the DSP system are considered to be nationally representative, the fraction of the national population in each socioeconomic stratum was assumed to follow the same population distribution as the DSP sites.

Data from the VR system for 2000 and a three-year average for the DSP system from 1997-9 were separately appraised for their usability in estimating national-level, cause-specific mortality for China. From the two systems, a comparison of age-standardized mortality rates for specific conditions was carried out for each socioeconomic stratum, as shown in figure 3.3.

We found that the mortality rates of the DSP system reflected the broad cause, group-specific mortality distribution more accurately, especially in rural areas. Also, the sampling distribution of sites in the DSP system was more nationally representative than that of theVR system. Thus, the proportional distribution of broad cause group mortality

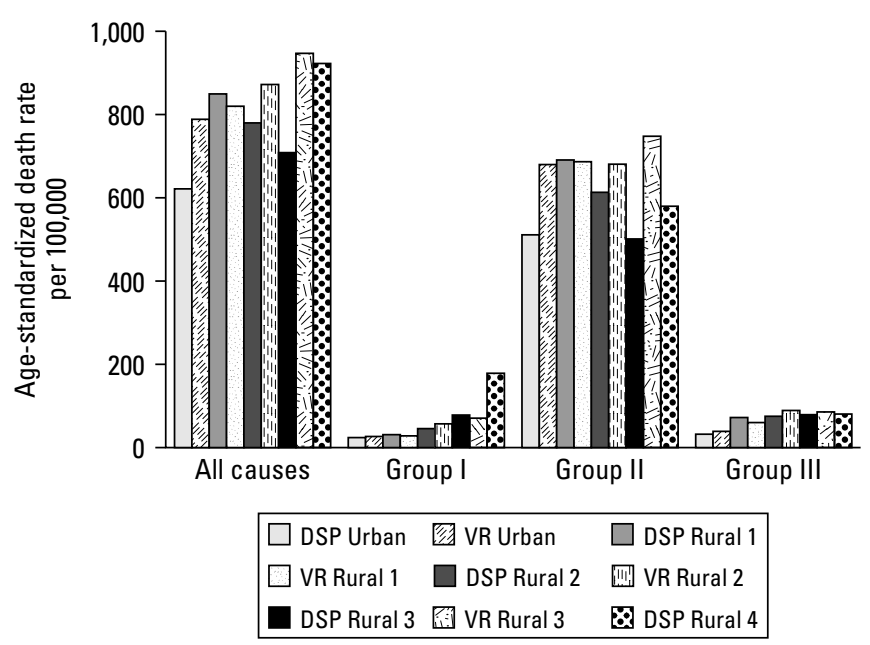

Source: Authors' calculations.

Note: Mortality rates are age standardized using the WHO world standard population.

Figure 3.3 Mortality Rates for Socioeconomic Strata, by Cause Group, from China's Two Mortality Data Systems 
for each stratum from the DSP data was applied to each stratum-specific mortality envelope to derive the broad cause group mortality in absolute numbers of deaths by age and sex.

The VR system's data captured mortality at the level of subgroup and specific cause more accurately, and because it was based on a significantly larger sample of deaths, it showed more plausible age patterns for specific causes. Hence, the specific cause-proportionate mortality distributions from the VR system's data were used for distributions within broad cause groups.

Finally, we summed the mortality estimates by cause, age, and sex from each stratum to obtain a national estimate of cause-specific mortality that had not been corrected for underregistration. We then inflated this cause-specific mortality to the national all-cause mortality envelope from the life table analysis to obtain the final national estimate of cause-specific mortality for 2001. We adjusted these estimates with information from WHO technical programs on maternal, perinatal, and childhood-cluster conditions and from epidemiological estimates for TB, HIV/AIDS, illicit drug dependence and problem use, rheumatoid arthritis, and war deaths.

India. For India, separate mortality recording systems for rural and urban areas were used to estimate all-cause death rates by age and sex for rural and urban areas and these were added to obtain national all-cause death rates to construct a national life table. The all-cause mortality envelope was derived from a time series analysis of age-specific death rates from the Sample Registration System after correcting them for underregistration (88 percent completeness) (Mari Bhat 2002).

Cause patterns of mortality were based on the Medical Certification of Cause of Death Database for urban areas of India and the Annual Survey of Causes of Death for rural areas of India. The all-cause mortality envelope was split into separate envelopes for urban and rural populations using a 70:30 ratio. Data on cause-specific mortality from separate sources for rural and urban areas were used with these mortality envelopes to build up independent estimates for urban and rural areas, which were summed to obtain national cause-specific mortality estimates.

For rural areas, the Andhra Pradesh burden of disease study (Mahapatra 2002) analyzed data from the Annual Survey of Causes of Death for 1996-8. The analysis included the redistribution of ill-defined deaths to specific causes based on a verbal autopsy retest survey conducted as part of the field studies for the project. For urban areas, data from the Medical Certification of Cause of Death system for 1996 were used. This system provides data on about 400,000 deaths annually coded to a national list of ICD-9 causes groups that approximates the ICD-9 Basic Tabulation List. These data were mapped onto the GBD classification and inflated to the urban mortality envelope. The proportion of urban deaths due to injuries was adjusted based on results from a large-scale verbal autopsy study in the city of Chennai, which detected that about 2.5 percent of deaths certified as due to ill-defined medical causes were actually due to injuries (Gajalakshmi and others 2002).

The summed national-level, cause-specific mortality estimates were adjusted with information from WHO technical programs on maternal, perinatal, and childhoodcluster conditions, as well as epidemiological estimates for TB, HIV/AIDS, illicit drug dependence and problem use, rheumatoid arthritis, and war deaths.

Egypt. Even though Lopez and others (2002) assessed Egyptian death registration data for 2000 to be almost complete, these data contained high proportions of deaths coded to symptoms and ill-defined conditions, as well as to conditions such as heart failure and cardiac arrest that were not underlying causes of death. Hence, a model-based prediction of the broad cause proportionate distribution by age and sex was used and applied to the cause-specific mortality structure from the country data after excluding a major proportion of the ill-defined deaths.

Turkey. The national life table for Turkey was estimated from separate urban and rural life tables. To estimate the urban life table, reported deaths during 1991-9 in the 81 provincial and distinct urban centers were evaluated for completeness using established demographic methods. These methods suggested that for more recent years, adult deaths were about 80 percent complete for males and 78 percent complete for females. These correction factors were used to estimate the level of adult mortality $\left.{ }_{(45} q_{15}\right)$ in 1999 and the rate was then projected forward to 2000 . The resulting estimates ( 0.190 for males and 0.106 for females) were similar to the levels estimated from the 2002-3 nationally representative mortality survey carried out by the Ministry of Health and Başkent University (Baskent University 2005). Together with estimated child mortality values from the 1998 DHS projected to 2000, a full life table was estimated for urban Turkey, which is equivalent to about two-thirds of the national population. Death rates were projected to 2001 assuming an annual rate of mortality decline of 1.25 percent. For rural 
areas, child mortality was first estimated from the DHS in the same way as for urban areas. Adult mortality $\left({ }_{45} q_{15}\right)$ was estimated from the WHO modified logit life table system $(0.235$ for males, 0.189 for females), values that were broadly similar to national mortality survey data, although the relatively small number of rural deaths in the survey, about 300 , gave rise to substantial uncertainty about the true levels of adult mortality in rural areas. The urban and rural death rates were then weighted by population size to obtain estimated national death rates, and hence the life table.

Data on causes of death were only available for urban areas of Turkey. These data were systematically reviewed for cause miscoding and adjusted based on clinical opinion and evidence on a sample of deaths from urban hospitals in Ismir and Ankara. In particular, most of the large proportion of deaths coded to "other heart disease" were reassigned to specific vascular pathologies based on this clinical evidence. For rural areas, causes of death were estimated using CodMod as described later. Adjusted proportions of Group I, II, and III deaths by age and sex were first estimated, and then the same proportionate distribution of deaths by cause as observed for urban areas was applied, after adjustment, to estimate the detailed pattern of causes of death.

Islamic Republic of Iran. Data from the VR system in Iran were compiled for 18 of the country's 26 provinces for 2001 . The data were coded to a condensed list of 150 cause categories using ICD-10. Because the registration system only covered part of the national population, a model-based prediction was used to estimate the broad cause proportionate mortality for the whole country. The results are shown in figure 3.4. The model predicted a higher proportion of Group I causes for both males and females in childhood and a higher proportion of Group I causes for females ages 15 to 44 , reflecting higher maternal mortality among the nonregistered population than among the registered population. The predicted distributions for the broad cause groups were then applied to the specific-cause proportionate mortality from the reported data and adjusted to the national mortality envelope derived from the life table analysis.

Thailand. VR data were available for 2000 with an estimated coverage of about 80 percent (Lopez and others 2002). However, the proportion of ill-defined conditions was nearly 50 percent, because many deaths in Thailand occur at home and the cause of death is often reported by lay persons. To improve the usability of data from the VR system, the Ministry of Public Health conducted a retest survey on a
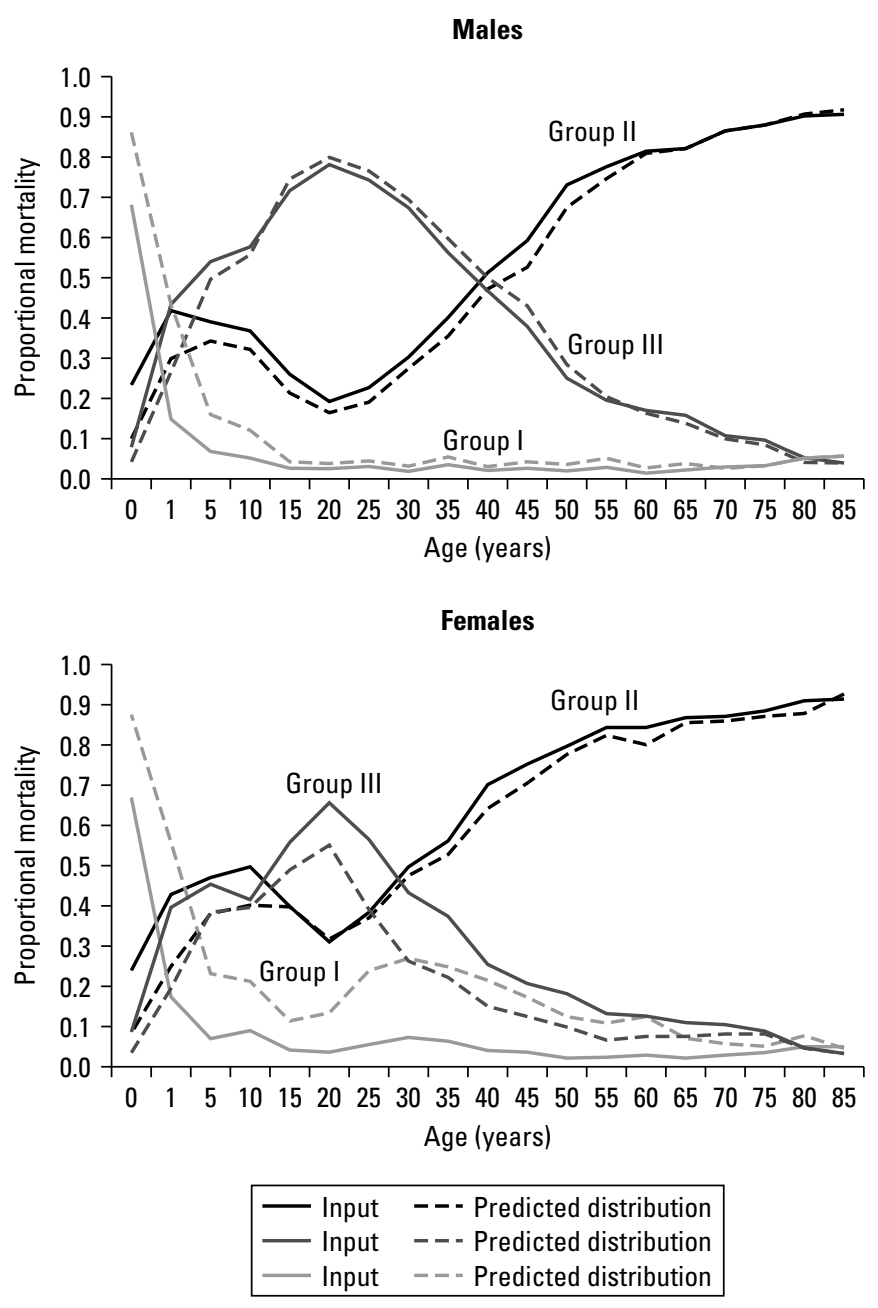

Source: Authors' calculations.

Figure 3.4 CodMod Estimation of Major Cause Group Proportional Mortality for Islamic Republic of Iran, 2001

sample of about 33,000 deaths, using verbal autopsy methods, to ascertain the true cause of death (Ministry of Public Health 2002). This included a sample of 12,000 deaths with illdefined causes. The study reallocated about 66 percent of deaths with ill-defined causes to specific causes, including reclassifying many deaths as caused by HIV/AIDS. The reallocation algorithm for ill-defined causes from the verbal autopsy study was used to correct the high proportion of ill-defined deaths from the VR data, and then the resultant cause-specific proportionate mortality was inflated to the national mortality envelope derived from the life table analysis.

\section{Epidemiological Estimates of Mortality for Specific Causes}

As outlined in table 3.2, specific epidemiological estimates for some causes were also taken into account in analyzing 
Table 3.5 Numbers of Data Sets Contributing to Epidemiologically Based Estimates of Deaths Due to Specific Causes

\begin{tabular}{|c|c|c|c|c|c|c|c|c|}
\hline Cause & $\begin{array}{c}\text { East } \\
\text { Asia } \\
\text { and } \\
\text { Pacific }\end{array}$ & $\begin{array}{l}\text { Europe } \\
\text { and } \\
\text { Central } \\
\text { Asia }\end{array}$ & $\begin{array}{l}\text { Latin } \\
\text { America and } \\
\text { the Caribbean }\end{array}$ & $\begin{array}{l}\text { Middle } \\
\text { East and } \\
\text { North } \\
\text { Africa }\end{array}$ & $\begin{array}{l}\text { South } \\
\text { Asia }\end{array}$ & $\begin{array}{c}\text { Sub- } \\
\text { Saharan } \\
\text { Africa }\end{array}$ & $\begin{array}{l}\text { High- } \\
\text { income } \\
\text { countries }\end{array}$ & Total \\
\hline Tuberculosis $^{\mathrm{a}}$ & 24 & 27 & 34 & 16 & 8 & 39 & 31 & 179 \\
\hline HIV/AIDS ${ }^{a}$ & 14 & 26 & 27 & 13 & 5 & 37 & 29 & 150 \\
\hline Diarrheal diseases & & 0 & 15 & 8 & 21 & 24 & 0 & 73 \\
\hline \multicolumn{9}{|l|}{ Childhood-cluster diseases } \\
\hline Pertussis & 14 & 33 & 64 & 14 & 8 & 45 & 124 & 302 \\
\hline Poliomyelitis & 22 & 27 & 32 & 15 & 8 & 47 & 37 & 192 \\
\hline Diphtheria & 12 & 25 & 2 & 14 & 8 & 46 & 8 & 115 \\
\hline Measles & 22 & 18 & 32 & 12 & 8 & 47 & 22 & 127 \\
\hline Tetanus & 48 & 23 & 27 & 40 & 32 & 79 & 34 & 289 \\
\hline Meningitis & 23 & 18 & 30 & 12 & 4 & 27 & 43 & 157 \\
\hline Hepatitis B and C & 40 & 27 & 47 & 43 & 18 & 67 & 113 & 355 \\
\hline Malaria & 9 & 0 & 2 & 1 & 7 & 142 & 0 & 161 \\
\hline \multicolumn{9}{|l|}{ Tropical-cluster diseases } \\
\hline Trypanosomiasis & 0 & 0 & 0 & 0 & 0 & 36 & 0 & 36 \\
\hline Chagas' disease & 0 & 0 & 31 & 0 & 0 & 0 & 0 & 31 \\
\hline Schistosomiasis & 6 & 0 & 3 & 8 & 0 & 37 & 1 & 55 \\
\hline Lower respiratory infections & 2 & 0 & 18 & 0 & 9 & 18 & 2 & 49 \\
\hline Maternal conditions (all causes) & 6 & 0 & 9 & 5 & 4 & 20 & 1 & 45 \\
\hline Unsafe abortion & 14 & 32 & 27 & 11 & 13 & 49 & 10 & 156 \\
\hline Perinatal causes & 7 & 0 & 7 & 11 & 19 & 12 & 0 & 56 \\
\hline Malignant neoplasms & 14 & 12 & 12 & 10 & 3 & 14 & 40 & 105 \\
\hline Drug use disorders & 11 & 11 & 18 & 10 & 6 & 15 & 43 & 114 \\
\hline War & 3 & 1 & 1 & 0 & 0 & 6 & 7 & 18 \\
\hline Total & 296 & 280 & 438 & 243 & 181 & 807 & 545 & 2,765 \\
\hline
\end{tabular}

Source: Authors' calculations.

Note: The data sources include population-based epidemiological studies, disease registers, and surveillance and notification systems. Where possible, regional and global totals refer to numbers of separate studies, or country-years of reported data from surveillance or notification systems. Refer to text for more information on data sources for specific causes.

a. Totals refer to numbers of countries for which data were available, not to total data sets or country-years.

causes of death for countries. Table 3.5 summarizes the numbers of studies (population-based epidemiological studies, disease registers, and notification systems) that contributed to the estimation of mortality due to 21 specific causes of death, including HIV/AIDS, malaria, and TB. As the table shows, more than 2,700 data sets contributed to the estimates for these 21 causes of death, with almost one-third of these relating to Sub-Saharan Africa.

Tuberculosis. In 1997, WHO began a study to develop country estimates of incidence, prevalence, and mortality from TB (for a detailed description of data sources and methods see Dye and others 1999). The study derived estimates of incidence from case notifications adjusted by estimated case detection rates, prevalence data on active disease combined with estimates of average case durations, or estimates of infection risk multiplied by a scalar factor relating the incidence of smear-positive pulmonary TB to annual risks of infection.
Since the original estimates for 1997 were completed, revised and updated estimates have been prepared. Most countries reporting to WHO have provided notification data with interpretable trends and with no other evidence for any significant change in the case detection rate. Trends in notification rates were assumed to represent trends in incidence rates for most countries except those with evidence of changes in case detection rates. China carried out a countrywide disease prevalence survey during 2000, and the results were used to reevaluate incidence for 1999. For other countries with evidence of changes in case detection rates, the trend for one of eight groups of epidemiologically similar countries was assumed to apply (Corbett and others 2003). Annual reports on TB control have included further details on surveillance methods, case notifications, and incidence estimates by country (WHO 2003a).

Deaths due to all forms of TB (excluding HIV-infected persons) were estimated for 2001. For countries with VR data, these estimates were based on the most recently 
available VR data. For other countries, estimates were based on the estimated TB incidence rates (excluding HIV-infected persons) multiplied by the estimated case fatality rates and weighted for the proportion of cases treated and the proportion smear-positive.

HIV/AIDS. The Joint United Nations Programme on HIVAIDS and WHO have developed country-specific estimates of HIV/AIDS mortality and revise them periodically to account for new data and improved methods (Schwartlander and others 1999; Walker and others 2003). For the most recent round of estimates, they used two different types of models depending on the nature of the epidemic in a particular country. For generalized epidemics, in which infection is spread primarily through heterosexual contact, they used a simple epidemiological model to estimate epidemic curves based on sentinel surveillance data on HIV seroprevalence (UNAIDS Reference Group on Estimates Model and Projections 2002). For countries with epidemics concentrated in high-risk groups, they used prevalence estimates derived from the estimated population size and prevalence surveillance data in each high-risk category, and then employed simple models to back-calculate incidence and mortality based on these estimated prevalence trends (Stover and others 2002).

For countries with death registration data, HIV/AIDS mortality estimates were generally based on the most recently available VR data except where miscoding of HIV/AIDS deaths was evident. In such cases, a time series analysis of causes was carried out to identify and reassign miscoded HIV/AIDS deaths. For other countries, estimates were based on the Joint United Nations Programme on HIV/AIDS and WHO estimates of HIV/AIDS mortality for 2001, or in some cases where these were not available, on the estimated prevalence of HIV/AIDS in 2001 multiplied by the average subregional mortality to prevalence ratio.

Diarrheal Diseases. For countries with usable death registration data, deaths due to diarrheal diseases were estimated directly from that data. For other countries, a regression model was used to estimate proportional mortality from diarrhea for children under five (Boschi-Pinto and others forthcoming). The regression model included the logit of the proportional mortality from diarrheal diseases in children from birth through four as a dependent variable and gross domestic product (GDP) per capita in international dollars, time, and region indicator variables as explanatory variables. The regression data were drawn from more than 60 community-based studies carried out since 1980 with study durations of multiples of 12 months. This model was validated and supplemented with vital statistics from developing countries where coverage was high.

Vaccine-Preventable Childhood Diseases. Mortality for measles was estimated using two approaches. In countries where routine vaccine coverage was low (less than 80 percent), incidence data were derived from a natural history model using country-specific vaccine coverage and attack rates from population-based studies (Crowcroft and others 2003). For countries with higher routine coverage and in the elimination phase, case notification and country-specific correction factors were used to estimate incidence. To obtain mortality in countries where VR data were not available, age- and region-specific case fatality rates from communitybased and outbreak studies were applied to incidence estimates derived from both approaches.

Pertussis cases and deaths were based on a natural history model using vaccine coverage and age-specific case fatality rates from community based studies, where available (Crowcroft and others 2003). The model is a revision of Galezka and Robertson's (2004) approach.

The incidence estimates for polio and diphtheria (Stein 2002b; Stein and Robertson 2002) were based on countryspecific reported cases of acute flaccid paralysis with adjustments for underreporting and on country-specific notifications of diphtheria cases with an assumed notification efficiency of 20 percent, respectively. A case fatality rate of 10 percent was assumed for diphtheria in countries without high death registration coverage.

Acute Respiratory Infections. Community-based studies with durations of one year or longer, published since 1980, were used to estimate the proportional mortality from acute respiratory infections in children under five in developing countries (Williams and others 2002). The results confirmed earlier findings that the proportion of deaths attributable to acute respiratory infections diminishes as general mortality diminishes. Much of the variability across studies in the proportion of child deaths attributed to acute respiratory infections was due to the use of verbal autopsies to determine the cause of death. Data from seven studies that compared verbal autopsies with hospital-based diagnoses indicated that the percentage of deaths due to acute respiratory infections could be underestimated by up to 4 percent. The modeled estimates were supplemented with vital statistics from developing countries where coverage was high to develop regional and global estimates. 
Malaria. Malaria mortality estimates for all regions except Sub-Saharan Africa were derived from the cause of death data sources described earlier. For Sub-Saharan Africa, country-specific estimates of malaria mortality were based on analyses by Snow and others (1999) and updated using the most recent geographical distributions of risks from the Mapping Malaria Risks in Africa International Collaboration. Subsequent adjustments were made to the estimated country-specific malaria deaths to ensure that total mortality for Group I causes, particularly in the $0-4$ year age group, and including estimates for other specific causes such as TB, HIV/AIDS, and measles, added to the total all-cause mortality envelopes for the relevant countries. Work is currently under way to refine and revise these country-specific estimates of malaria mortality in collaboration with other WHO programs and external expert groups (Korenromp and others 2003; Rowe and others 2004).

Chagas' Disease. Chagas' disease estimates were obtained from recent intensive surveillance activities in the Southern Cone American countries and community-based studies (Moncayo 2003; Moncayo, Guhl, and Stein 2002). These estimates were supplemented with and validated against vital statistics from Latin American countries where coverage was high.

Maternal Mortality. Mortality from maternal conditions was estimated following a similar approach to earlier analyses (Abdallah and Zehani 2000; Hill, AbouZahr, and Wardlaw 2001), using the most recently available mortality data for developing countries, together with improved estimates of the impact of HIV/AIDS as a competing cause of mortality (WHO, UNICEF, and UNFPA 2003). Depending on the availability and quality of data on detailed causes of maternal deaths, the methods used to estimate the proportion of deaths of women of reproductive age that is due to maternal causes (PMDF) varied and included vital records, DHSs and other surveys, Reproductive Age Mortality Study surveys, and epidemiological models. For countries without death registration data, both nationally reported data and specific criteria for a regression model were used to estimate maternal mortality. The dependent variable in this model was the logit of the PMDF after subtracting HIV/AIDS deaths and the explanatory variables were the proportion of deliveries with skilled birth attendance, the GDP per capita in international dollars, and the general fertility rate plus region dummy variables. The total number of deaths from maternal causes for each country was estimated by multi- plying the PMDF by the overall mortality envelope for women aged 15 to 49 after subtracting HIV/AIDS deaths.

Abortion-related mortality occurs mainly as a result of unsafe induced abortion. It has been estimated using published and unpublished reports for 131 countries together with other information on legal and social contexts and summed to give regional totals (WHO 2004a).

Perinatal Causes. The cause category "perinatal causes" refers to the ICD cause group "conditions arising in the perinatal period" (ICD chapter 16, P-codes). Deaths from these causes, primarily low birthweight, prematurity, and birth trauma or asphyxia, may occur at any age, and can include some maternal or placental causes, such as multiple pregnancy. Deaths from these causes should not be confused with deaths that occur during the perinatal period, which include stillbirths and neonatal deaths from other causes such as tetanus and congenital malformations. However, acknowledging that nearly all deaths due to perinatal causes occur during the neonatal period, we first estimated the envelope of neonatal mortality for every country (for details of the method see Murray and Lopez 1998). The analysis has been updated using recent death registration data and DHS data. Work is currently under way in collaboration with other WHO programs and external expert groups to refine and revise these country-specific estimates of mortality due to perinatal causes (Lawn, Cousens, and Zupan 2005).

Cancer. For countries without good VR data to estimate the site-specific distribution of cancer mortality, a site-specific model for relative interval survival was developed and applied to cancer incidence estimates by site (Mathers, Shibuya, and others 2002; Shibuya and others 2002). This age-period-cohort model of cancer survival was based on data from the Surveillance, Epidemiology, and End Results program of the National Cancer Institute (Ries and others 2002). The model was further adjusted by site for each country based on observed correlations in regional and country survival probabilities and level of economic development (GDP per capita in international dollars) (Mathers, Shibuya, and others 2002). Combined with available incidence data from the International Agency for Research on Cancer (Ferlay and others 2001), cancer death distributions were estimated and the model estimates were validated against available VR data from countries other than the United States.

Drug Use Disorders. This category includes dependence on and nondependent problem use of both licit and illicit 
drugs, excluding tobacco and alcohol (see table 3A.2). Estimating mortality directly attributable to drug use disorders, such as death from overdose, is difficult because of variations in the quality and quantity of mortality data. For some regions with a substantial prevalence of illicit drug use, available data sources do not record any deaths as due to drug dependence. As a result, it is necessary to make indirect estimates based on estimates of the prevalence of illicit drug use and of case fatality rates, on the assumption that almost all mortality directly attributable to drug use disorders is associated with illicit drugs. However, making even indirect estimates is difficult because the use of these drugs is illegal, stigmatized, and hidden.

The comparative risk assessment work carried out for the World Health Report 2002 (WHO 2002d) included estimating the prevalence of illicit drug dependence and direct mortality based on available data (Degenhardt and others 2003; Ezzati and others 2002). Data on the prevalence of problematic illicit drug use were derived from a range of sources, including a formal literature search of all studies that estimated the prevalence of problematic drug use, the United Nations Drug Control Program, and the European Monitoring Centre for Drugs and Drug Addiction (2002).

A search was also conducted for cohort studies of drug users that had estimated mortality due to individual causes of death (overdose, suicide, and trauma) and to all causes of death (updating previous systematic reviews). Data on the number of years of follow up were extracted from each study and a weighted average annual mortality rate was calculated for each cause of death and for their sum.

The total regional deaths due directly to illicit drug use were then distributed among countries in each region in proportion to estimated prevalences of drug dependence and problem use. For developed countries with good VR data, evidence suggests that deaths due to drug use disorders are underrecorded (European Monitoring Centre for Drugs and Drug Addiction 2002; Single and others 2002). For these countries, mortality figures were adjusted for age groups in which the estimated deaths derived from the comparative risk assessment analysis exceeded the number of deaths recorded on the assumption that these additional deaths were originally miscoded as due to accidental poisoning or ill-defined causes.

War Deaths. Country-specific estimates of war deaths and corresponding uncertainty ranges were obtained from a variety of published and unpublished databases. The Armed Conflict Report (Project Ploughshares 2001, 2002), a report that supplies several databases with mortality estimates (see, for example, Center for Research on the Epidemiology of Disasters 2001), was the primary source used for time trend and mortality estimates. This report was a preferred source of information, because it includes war deaths by country and year, a departure from the typical practice of supplying estimates by conflict and across years. The report's data were checked against historical and current estimates by other research groups, such as those of the Uppsala Conflict Data Project (Gleditsch and others 2002) and the Center for International Development and Conflict Management at the University of Maryland (Marshall and Gurr 2003).

These data sets rely on press reports of eyewitness accounts and official announcements of combatants, which are, unfortunately, the main and often only possible method of estimating casualties in armed conflicts. Murray, King, and others (2002) summarize the issues involved in estimating war deaths and emphasize the considerable uncertainty in the GBD 2000 and GBD 2001 estimates. Many of the available data sources on conflict deaths only count deaths in conflicts that involve the armed forces of at least one state or one or more armed factions seeking to gain control of all or part of the state, and in which more than a certain number of people have been killed, for instance, more than 1,000 total or more than 25 per year. Some sources count only battlefield deaths and deaths that occur concurrently with conflict.

In contrast, the GBD 2001 estimated deaths occurring in 2001 in which the underlying cause (following ICD conventions) was an injury due to operations of war or civil insurrection, whether or not that injury occurred during the time of war or following the cessation of hostilities, which in some cases occurred many years earlier than 2001. The GBD 2001 estimates included injury deaths resulting from all civil insurrection, whether or not the state was involved. They also included deaths due to terrorism carried out by organized groups. The GBD 2001 estimates of war deaths did not include deaths from other causes, such as starvation, infectious disease epidemics, or lack of medical intervention for chronic diseases, that may be counterfactually attributable to war or civil conflict.

Deaths due to landmines and unexploded ordnance were estimated separately by country. The primary sources for these data were the Landmine Monitor Report of the International Campaign to Ban Landmines (Human Rights Watch 2001) and Handicap International's annual report on landmine victims (Handicap International 2001).

Whereas total injury deaths for most countries were derived either from death registration data or from cause of 
death models, war deaths were treated as "outside the envelope," and for countries for which life tables were estimated from data for earlier years not affected by war, war deaths were added to the total deaths estimated from the life tables.

\section{Cause of Death Modeling for Countries with Poor Data}

Although epidemiological studies and other data sources described in the previous section allow the estimation of deaths due to certain causes in populations without death registration data, they do not cover many important causes of death in these populations, such as CVD or injuries. To address these information gaps, models for estimating broad cause of death patterns can serve as the starting point for indirect methods of estimating attributable mortality for a comprehensive list of causes.

Preston (1976) was the first to develop indirect methods for estimating cause of death structure. Preston modeled the relationship between total mortality and cause-specific mortality for 12 broad groups of causes using historical VR data for the industrial countries and a few developing countries. In particular, Preston postulated that cause-specific mortality was a linear function of total mortality. The GBD 1990 study (Murray and Lopez 1996a) used cause of death models to estimate mortality for the three major cause groups (Groups I, II, III) as a function of mortality from all causes, based on regression analysis of observations on recent mortality patterns from 67 countries. The log of cause-specific mortality was postulated to be a linear function of the log of total mortality, and poorly coded deaths were redistributed before estimating the regression equations.

The cause of death model used in the GBD 1990 has been substantially revised and enhanced for estimating deaths by broad cause group in regions with limited information on mortality. The statistical model has been improved by adapting models for compositional data that were previously developed in other areas, and a substantially larger data set of 1,613 country-years of observations was used for analysis. Income per capita has been added to the model as an explanatory variable in addition to the level of all-cause mortality (Salomon and Murray 2002a).

This section provides an overview of the new model, CodMod, developed by Salomon and Murray for the GBD 2001, and describes its application for estimating (a) broad cause patterns for populations where no cause of death information is available, and (b) broad cause of death patterns when incomplete death registration data are available. The estimation of broad cause of death patterns is critical to avoid overemphasizing or underemphasizing specific causes due to biases in the data sets available to estimate national mortality patterns, for example, if data are derived from urban hospital statistics.

Statistical Methods and Data. The statistical basis for cause of death models has also been enhanced by the adaptation of models for compositional data that were previously developed in other areas (Katz and King 1999). These models take account of the key features of this type of data, namely, that the fraction of deaths attributable to each cause is bounded by 0 and 1 and that all the fractions must sum to unity. Violations of both constraints were possible with the regression models used in the GBD 1990; an additional normalization step was undertaken to impose these constraints. The new model explicitly ensures both these constraints using a seemingly unrelated regression model (for a full description of this model and its application to analysis of the epidemiological transition, see Salomon and Murray 2002a).

In addition to revising the statistical model used in the previous study, Salomon and Murray also considered additional covariates beyond all-cause mortality. The objective was to identify variables likely to have a strong relationship to cause-specific mortality, but also variables for which estimates would be available in all countries, because one of the goals of the exercise was to use the model to predict broad patterns of mortality for countries without VR data. The variables that were selected based on these criteria were allcause mortality, as before, plus income per capita in international dollars. Both variables were included in logged form, because this formulation tended to provide a better fit than the linear form.

Perhaps most important, the new cause of death model incorporated a more extensive database on mortality by age, sex, and cause than previous efforts, with substantially more representation of middle-income countries. Increasing the range of observed cause of death patterns should improve the validity of extrapolations from countries with registration systems to data-poor settings.

Separate models were estimated for each sex and the following age groups: younger than 1 month, 1-11 months, 1-4 years, 5-9 years, 10-14 years, and so on by five-year age groups up to $80-84$ years and 85 years and older. For the two youngest age groups, a smaller number of observations were available because some countries for some periods reported only on the age range from birth to 11 months. A total of 586 country-years of observations were available for the first two age groups and 1,613 country-years of observations for each of the other 18 age groups. The regression 
results provided insights into the relationships between cause of death patterns, all-cause mortality levels, and increases in income per capita (Salomon and Murray 2002a).

Salomon and Murray also used Monte Carlo simulation techniques to estimate the probability distributions of the predicted cause of death components given a particular set of values for all-cause mortality and GDP per capita (Salomon and Murray 2001a). The results from this approach were useful in estimating cause of death patterns for residual areas in countries where VR covers only part of the population and in defining regional cause of death patterns.

\section{Application of CodMod for Countries without Good} Registration Data. As with the GBD 1990, one of the useful applications of cause of death models is to examine patterns of deviation from the expected cause composition across countries or regions based on the probability distribution for a predicted cause of death pattern. In other words, the models permit comparison of the observed pattern with the pattern that would be predicted conditional on the levels of all-cause mortality and income per capita associated with that observation.

Given some assumptions about the stability of this pattern of deviation over short time intervals within a country or across countries in the same mortality stratum, it is possible to use the observed cause of death pattern in a reference population to estimate the cause of death pattern for some other population while taking into account differences in the explanatory variables. Some examples of applications would be

- estimating the cause of death pattern in nonregistration areas for a country in which part of the population is covered by a VR system,

- forecasting the cause of death pattern for a country where the most recent VR data are for several years in the past, and

- estimating the cause of death pattern for a country for which information is not available but is available for other countries in the same region.

All these applications are based on the assumption that patterns of deviation from the cause compositions predicted by the model will have some stability across time and place, for example, if young adults in Canada tend to have a low proportion of Group I deaths and a high proportion of Group II deaths in one year given the levels of all-cause mortality and income in that year, a reasonable assumption would be that the next year's composition will be similarly low in Group I and high in Group II given that year's total income and mortality. This hypothesis builds on the notion that all-cause mortality and income per capita explain only some of the variation in cause of death patterns, while the other sources of this variation are unmeasured but are assumed to be relatively stable. In other words, the cause of death pattern in Canada differs from what we would predict based only on total mortality and income because other factors influence the pattern. We assume that these other factors will change gradually over time, which would imply that the deviation from the prediction should also move gradually.

Using similar arguments, Salomon and Murray (2001a) suggested that it may be possible to use patterns of deviation from one country to predict cause of death patterns in another country in the same demographic region. They demonstrated an example of this for mortality data from Chile and Mexico for women aged 35 to 39 for 1965-94. They estimated the percentiles at which the observed cause fractions for the two countries fell in the probability distribution of predicted fractions produced by the Monte Carlo simulations conditional on the mortality and income levels in those years for each country and found similarities in the deviation patterns. Overall, this example suggested that deviation patterns in groups of similar countries may be similar, allowing predictions of cause of death patterns in countries where registration data are not available but for which neighboring countries do have data.

The application of this method has been formalized in a simple spreadsheet program called CodMod (Salomon and Murray 2001a). The program incorporates the regression models described earlier and uses Monte Carlo simulation methods to generate probability distributions around predicted cause of death patterns conditional on values for allcause mortality and income per capita. CodMod allows two main operations: (a) analysis of deviations in observed cause of death patterns given levels of mortality and income, and (b) predictions of cause of death patterns conditional on a reference pattern of deviation and levels of mortality and income.

Thus, for example, if the VR system covers only one region in a country, CodMod may be used to examine the pattern of deviation in that region from the predicted cause of death pattern at local income and total mortality levels. We assume that a similar pattern of deviation will hold in the nonregistration areas of the country, then we can use information on total mortality levels and income in the nonregistration areas to predict cause of death patterns in these areas. The GBD 2001 used CodMod for countries with incomplete death registration data to adjust for biases in 
cause composition. Annex table 3A.3 lists countries for which such adjustments were carried out.

CodMod was also used to develop regional patterns of deviation from predicted cause compositions, which were then used to estimate mortality by broad causes for countries for which no registration data were available. Annex table 3A. 3 summarizes details of these regional models. In the case of the Sub-Saharan Africa region, where good VR data were available for only three countries, a regional pattern of specific causes of deaths was based on VR data from urban and rural South Africa. For the Middle East and North Africa, a similar pattern was built for the Gulf states based on the four latest years of data from Bahrain and Kuwait. For other countries in that region, regional models were based on weighted death rates using Egyptian and Iranian VR data. The weights used were determined by the income levels of the individual countries and overall death rates. For the Pacific islands, a regional pattern was based on data available from islands reporting death registration data.

Whereas the original GBD study used a more detailed cause of death model for 12 causes of death to estimate deaths below the broad group level for countries without death registration data, the increased availability of death registration data in most regions has enabled us to use detailed proportional cause distributions within Groups I, II, and III based on death registration data from within each region (see annex table 3A.3 for more details). Specific causes were further adjusted on the basis of epidemiological evidence from registries, verbal autopsy studies, disease surveillance systems, and analyses from WHO technical programs as described earlier.

\section{GLOBAL AND REGIONAL MORTALITY IN 2001}

Slightly more than 56 million people died in 2001, 10.5 million, or nearly 20 percent, of whom were children younger than five. Of these child deaths, 99 percent occurred in low- and middle-income countries. Those age 70 and over accounted for 70 percent of deaths in high-income countries, compared with 30 percent in other countries. Thus, a key point is the comparatively large number of deaths among the young and the middle-aged in low- and middle-income countries. In these countries, 30 percent of all deaths occur at ages 15 to 59, compared with 15 percent in high-income countries. The causes of death at these ages, as well as in childhood, are thus important in assessing public health priorities.
This section provides an overview of global and regional causes of death in 2001. Note that as described earlier, the results reported here are tabulated by underlying disease cause or external cause of injury. Total attributable deaths for some diseases that increase the risk of other diseases or injuries will be substantially larger than the estimates of direct deaths given here. Chapter 4 estimates deaths attributable to 26 global risk factors. The tables in annex $3 \mathrm{~B}$ provide detailed tabulations of deaths by cause and sex for regions, for low- and middle-income countries, for highincome countries, and for the world.

\section{Distribution of Deaths by Major Cause Group}

Worldwide, one death in every three is from a Group I cause. This proportion remains almost unchanged from 1990 with one big difference: whereas HIV/AIDS accounted for only 2 percent of Group I deaths in 1990, it accounted for 44 percent of Group I deaths in 2001. Excluding HIV/AIDS, Group I deaths fell from 33 percent of total deaths in 1990 to less than 20 percent in 2001. Virtually all the Group I deaths are in low- and middle-income countries. Just under 10 percent are from Group III causes (injuries) and almost 60 percent of deaths are from Group II causes (noncommunicable diseases). Figure 3.5 shows the proportional distribution of these major cause groups for low- and middleincome countries and high-income countries.

Group I causes remain the leading cause of child deaths in all regions, although they are now responsible for fewer child deaths than Group II and Group III combined in highincome countries (figure 3.6). In contrast, Group II causes are now responsible for more than 50 percent of deaths in adults ages 15 to 59 in all regions except South Asia and
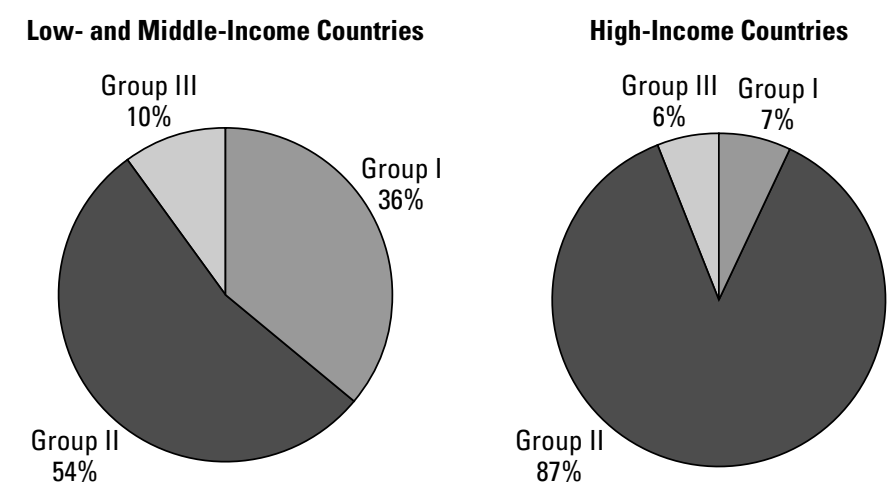

Source: Authors' calculations.

Figure 3.5 Proportional Distribution of Total Deaths by Broad Cause Group, 2001 


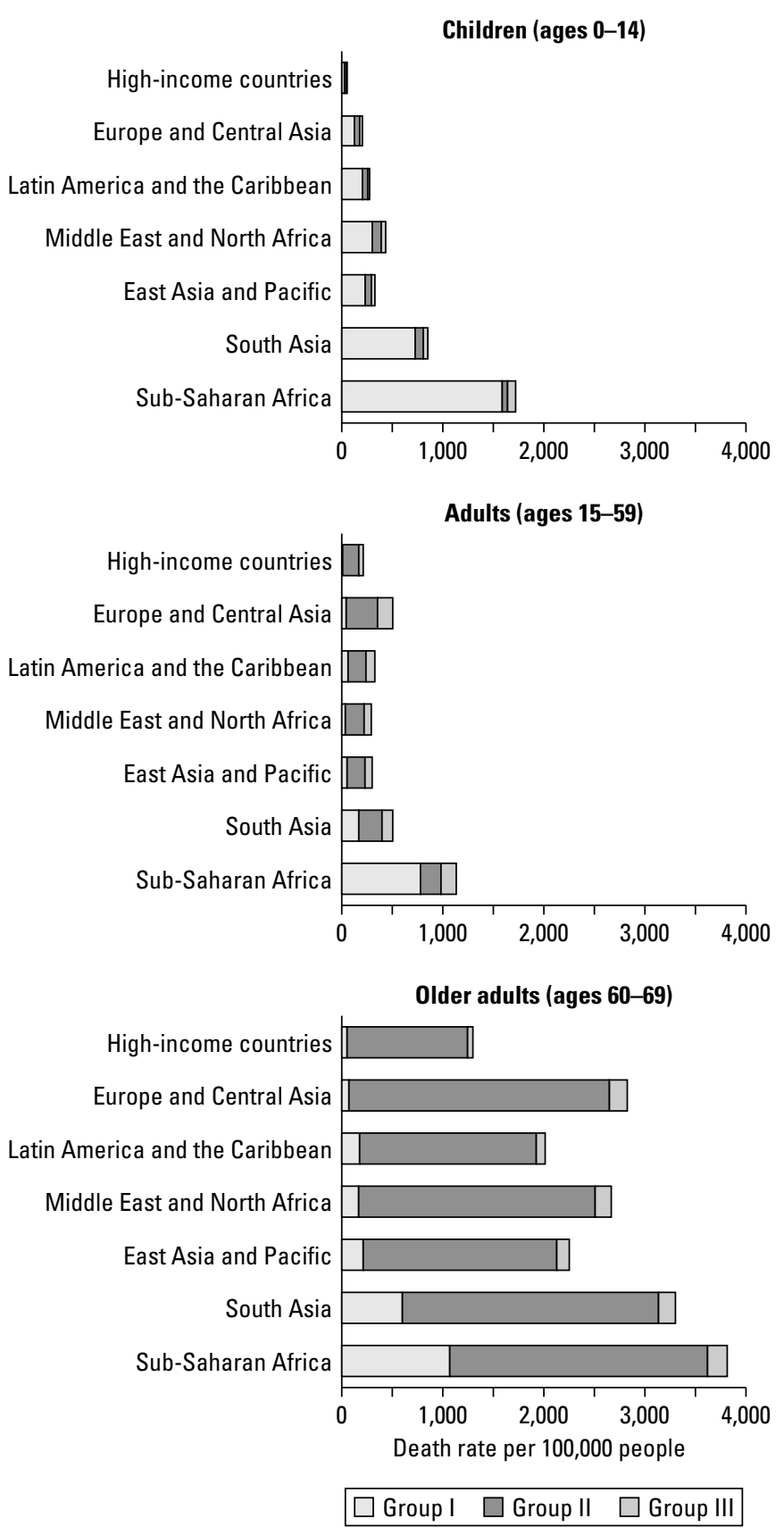

Source: Authors' calculations.

Figure 3.6 Death Rates by Broad Cause Group, Region, and Broad Age Group, 2001

Sub-Saharan Africa, where Group I causes, including HIV/AIDS, remain responsible for 33 and 67 percent of deaths, respectively. For adults ages 15 to 59, death rates from Group II causes are higher for all low- and middleincome regions than for high-income countries, and in Europe and Central Asia are almost double the rate for the high-income countries. These results show that premature mortality from noncommunicable diseases is higher in pop- ulations with high mortality and low incomes than in the high-income countries.

\section{Leading Causes of Death}

Table 3.6 shows the top 10 disease and injury causes of death in 2001 for low- and middle-income countries and for highincome countries. IHD and cerebrovascular disease (stroke) were the leading causes of death in both groups of countries in 2001, responsible for 12 million deaths globally, or almost one-quarter of the global total. Only 1.4 million of the 7.1 million who died of IHD were in the high-income countries. Stroke killed 5.4 million, of whom less than 1.0 million were in high-income countries.

Whereas lung cancer, predominantly due to tobacco smoking, remains the third leading cause of death in highincome countries, reflecting high levels of smoking in previous years, the increasing prevalence of smoking in low- and middle-income countries has not yet driven lung cancer into the top 10 causes of death for these countries. HIV/AIDS is the fourth leading cause of death in low- and middle-income countries, and HIV/AIDS death rates are projected to continue to rise, albeit at a slower pace, despite recent increased efforts to improve access to antiretroviral drugs.

Lower respiratory infections, conditions arising during the perinatal period, and diarrheal diseases remain among the top 10 causes of death in low- and middle-income countries. In 2001, these three causes of death together accounted for nearly 60 percent of child deaths globally.

Table 3.7 shows the 10 leading causes of death in lowand middle-income countries by sex in 2001. Leading causes of death are generally similar for males and females, although road traffic accidents appear in the top 10 only for males and diabetes appears only for females.

Leading Causes of Death in Children. Infectious and parasitic diseases remain the major killers of children in the developing world. Although notable success has been achieved in certain areas, for example, polio, communicable diseases still account for 7 out of the top 10 causes and are responsible for about 60 percent of all child deaths. Overall, the 10 leading causes in low- and middle-income countries represent 80 percent of all child deaths in those countries, and also worldwide (table 3.8).

Many Latin American and some Asian and Middle Eastern countries have shifted somewhat toward the cause of death pattern observed in developed countries. In these countries, conditions arising during the perinatal period, including birth asphyxia, birth trauma, and low birthweight, 
Table 3.6 The 10 Leading Causes of Death, by Broad Income Group, 2001

\begin{tabular}{|c|c|c|c|c|c|}
\hline \multicolumn{3}{|c|}{ Low- and middle-income countries } & \multicolumn{3}{|c|}{ High-income countries } \\
\hline Cause & $\begin{array}{c}\text { Deaths } \\
\text { (millions) }\end{array}$ & $\begin{array}{c}\text { Percentage } \\
\text { of total } \\
\text { deaths }\end{array}$ & Cause & $\begin{array}{c}\text { Deaths } \\
\text { (millions) }\end{array}$ & $\begin{array}{c}\text { Percentage } \\
\text { of total } \\
\text { deaths }\end{array}$ \\
\hline 1 Ischemic heart disease & 5.70 & 11.8 & 1 Ischemic heart disease & 1.36 & 17.3 \\
\hline 2 Cerebrovascular disease & 4.61 & 9.5 & 2 Cerebrovascular disease & 0.78 & 9.9 \\
\hline 3 Lower respiratory infections & 3.41 & 7.0 & 3 Trachea, bronchus, and lung cancers & 0.46 & 5.8 \\
\hline 4 HIV/AIDS & 2.55 & 5.3 & 4 Lower respiratory infections & 0.34 & 4.4 \\
\hline 5 Perinatal conditions & 2.49 & 5.1 & 5 Chronic obstructive pulmonary disease & 0.30 & 3.8 \\
\hline 6 Chronic obstructive pulmonary disease & 2.38 & 4.9 & 6 Colon and rectal cancers & 0.26 & 3.3 \\
\hline 7 Diarrheal diseases & 1.78 & 3.7 & 7 Alzheimer's and other dementias & 0.21 & 2.6 \\
\hline 8 Tuberculosis & 1.59 & 3.3 & 8 Diabetes mellitus & 0.20 & 2.6 \\
\hline 9 Malaria & 1.21 & 2.5 & 9 Breast cancer & 0.16 & 2.0 \\
\hline 10 Road traffic accidents & 1.07 & 2.2 & 10 Stomach cancer & 0.15 & 1.9 \\
\hline
\end{tabular}

Source: Authors' calculations.

Table 3.7 The 10 Leading Causes of Death, by Sex, in Low- and Middle-Income Countries, 2001

\begin{tabular}{|c|c|c|c|c|c|}
\hline \multicolumn{3}{|l|}{ Males } & \multicolumn{3}{|l|}{ Females } \\
\hline Cause & $\begin{array}{c}\text { Deaths } \\
\text { (millions) }\end{array}$ & $\begin{array}{c}\text { Percentage } \\
\text { of total } \\
\text { deaths }\end{array}$ & Cause & $\begin{array}{c}\text { Deaths } \\
\text { (millions) }\end{array}$ & $\begin{array}{c}\text { Percentage } \\
\text { of total } \\
\text { deaths }\end{array}$ \\
\hline 1 Ischemic heart disease & 3.01 & 11.8 & 1 Ischemic heart disease & 2.69 & 11.8 \\
\hline 2 Cerebrovascular disease & 2.17 & 8.5 & 2 Cerebrovascular disease & 2.44 & 10.7 \\
\hline 3 Lower respiratory infections & 1.72 & 6.7 & 3 Lower respiratory infections & 1.68 & 7.4 \\
\hline 4 Perinatal conditions & 1.38 & 5.4 & 4 HIV/AIDS & 1.18 & 5.2 \\
\hline 5 HIV/AIDS & 1.38 & 5.4 & 5 Chronic obstructive pulmonary disease & 1.17 & 5.1 \\
\hline 6 Chronic obstructive pulmonary disease & 1.21 & 4.7 & 6 Perinatal conditions & 1.11 & 4.9 \\
\hline 7 Tuberculosis & 1.04 & 4.1 & 7 Diarrheal diseases & 0.85 & 3.7 \\
\hline 8 Diarrheal diseases & 0.93 & 3.6 & 8 Malaria & 0.63 & 2.8 \\
\hline 9 Road traffic accidents & 0.78 & 3.1 & 9 Tuberculosis & 0.55 & 2.4 \\
\hline 10 Malaria & 0.58 & 2.3 & 10 Diabetes mellitus & 0.42 & 1.8 \\
\hline
\end{tabular}

Source: Authors' calculations.

Table 3.8 The 10 Leading Causes of Death in Children Ages 0-14, by Broad Income Group, 2001

\begin{tabular}{|c|c|c|c|c|c|}
\hline \multicolumn{3}{|c|}{ Low- and middle-income countries } & \multicolumn{3}{|c|}{ High-income countries } \\
\hline Cause & $\begin{array}{l}\text { Deaths } \\
\text { (millions) }\end{array}$ & $\begin{array}{l}\text { Percentage of } \\
\text { total deaths }\end{array}$ & Cause & $\begin{array}{l}\text { Deaths } \\
\text { (millions) }\end{array}$ & $\begin{array}{l}\text { Percentage of } \\
\text { total deaths }\end{array}$ \\
\hline 1 Perinatal conditions & 2.49 & 20.7 & 1 Perinatal conditions & 0.03 & 33.9 \\
\hline 2 Lower respiratory infections & 2.04 & 17.0 & 2 Congenital anomalies & 0.02 & 20.0 \\
\hline 3 Diarrheal diseases & 1.61 & 13.4 & 3 Road traffic accidents & 0.01 & 5.9 \\
\hline 4 Malaria & 1.10 & 9.2 & 4 Lower respiratory infections & 0.00 & 2.5 \\
\hline 5 Measles & 0.74 & 6.2 & 5 Endocrine disorders & 0.00 & 2.4 \\
\hline 6 HIV/AIDS & 0.44 & 3.7 & 6 Drownings & 0.00 & 2.4 \\
\hline 7 Congenital anomalies & 0.44 & 3.7 & 7 Leukemia & 0.00 & 1.9 \\
\hline 8 Whooping cough & 0.30 & 2.5 & 8 Violence & 0.00 & 1.8 \\
\hline 9 Tetanus & 0.22 & 1.9 & 9 Fires & 0.00 & 1.2 \\
\hline 10 Road traffic accidents & 0.18 & 1.5 & 10 Meningitis & 0.00 & 1.2 \\
\hline
\end{tabular}

Source: Authors' calculations. 
have replaced infectious diseases as the leading cause of death and are now responsible for 21 to 34 percent of deaths. Such a shift in the cause of death pattern has not occurred in Sub-Saharan Africa, where perinatal conditions rank in fourth place and malaria, lower respiratory infections, and diarrheal diseases continue to be the leading causes of death in children, accounting for 53 percent of all deaths.

About 90 percent of all HIV/AIDS and malaria deaths in children in developing countries occur in Sub-Saharan Africa, which accounts for 23 percent of the world's births and 42 percent of the world's child deaths. The immense surge of HIV/AIDS mortality in children in recent years means that HIV/AIDS is now responsible for 332,000 child deaths annually in Sub-Saharan Africa and nearly 8 percent of all child deaths in the region.

Some progress has been made against diarrheal diseases and measles in low- and middle-income countries. While the incidence of diarrheal diseases is thought to have remained stable, mortality from diarrheal diseases has fallen from 2.5 million deaths in 1990 to about 1.6 million deaths in 2001, and now accounts for 13 percent of all deaths of children under age 15. Deaths from measles have declined modestly, although more than half a million children under five still died from this disease in 2001. Malaria causes more than a million child deaths per year or nearly 11 percent of all deaths of children under five.

\section{Leading Causes of Death in Adults}

Table 3.9 shows the leading causes of deaths among adults ages 15 to 59 worldwide in 2001. Despite a global trend of declining communicable disease burden in adults, HIV/AIDS has become the leading cause of mortality and the single most important contributor to the burden of disease among adults in this age group.

Nearly 80 percent of the 2.1 million adult deaths from HIV/AIDS in 2001 occurred in Sub-Saharan Africa. In this region, HIV/AIDS is the leading cause of death, resulting in more than 6,000 deaths every day and accounting for almost one in five deaths for all ages and one in two deaths of adults ages 15 to 59. HIV/AIDS has reversed mortality trends among adults in the region, and in many countries, life expectancies have declined since 1990.

The 4.5 million adult injury deaths in 2001 were heavily concentrated among young adults, particularly men. In the 15 to 59 age group, road traffic accidents and suicide were among the 10 leading causes of death in high-income and low- and middle-income countries, and violence (homicide) was also among the 10 leading causes in low- and middle-income countries. Among adults ages 15 to 44 worldwide, road traffic accidents were the leading cause of death for men after HIV/AIDS, followed by TB and violence. Suicide was the third leading cause of death for women in this age group, after HIV/AIDS and TB, with road traffic accidents in fifth place.

The risk of death rises rapidly with age among adults age 60 and over in all regions. Globally, 60-year-olds have a 55 percent chance of dying before their 70th birthday. Regional variations in the risk of death are smaller at older ages than at younger ages, ranging from around 40 percent in the developed countries of Western Europe to 60 percent in most developing regions and 70 percent in Sub-Saharan Africa. Historical data from countries such as Australia and Sweden show that life expectancy at age 60 changed slowly during the first six to seven decades of the 20th century, but started to increase substantially since around 1970. Life

Table 3.9 The 10 Leading Causes of Death in Adults Ages 15-59, by Broad Income Group, 2001

\begin{tabular}{|c|c|c|c|c|c|}
\hline \multicolumn{3}{|c|}{ Low- and middle-income countries } & \multicolumn{3}{|c|}{ High-income countries } \\
\hline Cause & $\begin{array}{l}\text { Deaths } \\
\text { (millions) }\end{array}$ & $\begin{array}{l}\text { Percentage of } \\
\text { total deaths }\end{array}$ & Cause & $\begin{array}{l}\text { Deaths } \\
\text { (millions) }\end{array}$ & $\begin{array}{c}\text { Percentage of } \\
\text { total deaths }\end{array}$ \\
\hline 1 HIV/AIDS & 2.05 & 14.1 & 1 Ischemic heart disease & 0.13 & 10.8 \\
\hline 2 Ischemic heart disease & 1.18 & 8.1 & 2 Self-inflicted injuries & 0.09 & 7.2 \\
\hline 3 Tuberculosis & 1.03 & 7.1 & 3 Road traffic accidents & 0.08 & 6.9 \\
\hline 4 Road traffic accidents & 0.73 & 5.0 & 4 Trachea, bronchus, and lung cancers & 0.08 & 6.8 \\
\hline 5 Cerebrovascular disease & 0.71 & 4.9 & 5 Cerebrovascular disease & 0.05 & 4.4 \\
\hline 6 Self-inflicted injuries & 0.58 & 4.0 & 6 Cirrhosis of the liver & 0.05 & 4.4 \\
\hline 7 Violence & 0.45 & 3.1 & 7 Breast cancer & 0.05 & 4.0 \\
\hline 8 Lower respiratory infections & 0.33 & 2.3 & 8 Colon and rectal cancers & 0.04 & 3.1 \\
\hline 9 Cirrhosis of the liver & 0.32 & 2.2 & 9 Diabetes mellitus & 0.03 & 2.1 \\
\hline 10 Chronic obstructive pulmonary disease & 0.32 & 2.2 & 10 Stomach cancer & 0.02 & 2.0 \\
\hline
\end{tabular}

Source: Authors' calculations. 
Table 3.10 The 10 Leading Causes of Death in Low- and Middle-Income Countries, by Region, 2001

\begin{tabular}{|c|c|c|c|}
\hline East Asia and Pacific & $\begin{array}{l}\text { Percentage of } \\
\text { total deaths }\end{array}$ & Europe and Central Asia & $\begin{array}{l}\text { Percentage of } \\
\text { total deaths }\end{array}$ \\
\hline 1 Cerebrovascular disease & 14.6 & 1 Ischemic heart disease & 29.7 \\
\hline 2 Chronic obstructive pulmonary disease & 10.8 & 2 Cerebrovascular disease & 18.2 \\
\hline 3 Ischemic heart disease & 8.8 & 3 Trachea, bronchus, and lung cancers & 2.9 \\
\hline 4 Lower respiratory infections & 4.2 & 4 Chronic obstructive pulmonary disease & 2.3 \\
\hline 5 Tuberculosis & 4.1 & 5 Self-inflicted injuries & 2.1 \\
\hline 6 Perinatal conditions & 3.8 & 6 Hypertensive heart disease & 1.9 \\
\hline 7 Stomach cancer & 3.4 & 7 Poisonings & 1.9 \\
\hline 8 Trachea, bronchus, and lung cancers & 3.0 & 8 Lower respiratory infections & 1.8 \\
\hline 9 Liver cancer & 2.9 & 9 Cirrhosis of the liver & 1.8 \\
\hline 10 Road traffic accidents & 2.8 & 10 Stomach cancer & 1.8 \\
\hline Latin America and the Caribbean & $\begin{array}{l}\text { Percentage of } \\
\text { total deaths }\end{array}$ & Middle East and North Africa & $\begin{array}{l}\text { Percentage of } \\
\text { total deaths }\end{array}$ \\
\hline 1 Ischemic heart disease & 10.9 & 1 Ischemic heart disease & 16.9 \\
\hline 2 Cerebrovascular disease & 8.2 & 2 Cerebrovascular disease & 6.8 \\
\hline 3 Perinatal conditions & 5.0 & 3 Lower respiratory infections & 5.6 \\
\hline 4 Diabetes mellitus & 5.0 & 4 Perinatal conditions & 5.5 \\
\hline 5 Lower respiratory infections & 4.8 & 5 Road traffic accidents & 5.1 \\
\hline 6 Violence & 4.0 & 6 Hypertensive heart disease & 3.9 \\
\hline 7 Chronic obstructive pulmonary disease & 3.0 & 7 Diarrheal diseases & 3.9 \\
\hline 8 Road traffic accidents & 2.7 & 8 Congenital anomalies & 2.4 \\
\hline 9 Hypertensive heart disease & 2.7 & 9 Nephritis and nephrosis & 2.2 \\
\hline $10 \mathrm{HIV} / \mathrm{AIDS}$ & 2.5 & 10 Chronic obstructive pulmonary disease & 2.1 \\
\hline South Asia & $\begin{array}{l}\text { Percentage of } \\
\text { total deaths }\end{array}$ & Sub-Saharan Africa & $\begin{array}{l}\text { Percentage of } \\
\text { total deaths }\end{array}$ \\
\hline 1 Ischemic heart disease & 13.6 & 1 HIV/AIDS & 19.0 \\
\hline 2 Lower respiratory infections & 10.4 & 2 Malaria & 10.1 \\
\hline 3 Perinatal conditions & 8.0 & 3 Lower respiratory infections & 10.0 \\
\hline 4 Cerebrovascular disease & 6.8 & 4 Diarrheal diseases & 6.6 \\
\hline 5 Diarrheal diseases & 5.1 & 5 Perinatal conditions & 5.3 \\
\hline 6 Tuberculosis & 4.5 & 6 Measles & 4.1 \\
\hline 7 Chronic obstructive pulmonary disease & 4.3 & 7 Cerebrovascular disease & 3.3 \\
\hline 8 HIV/AIDS & 2.0 & 8 Ischemic heart disease & 3.2 \\
\hline 9 Road traffic accidents & 1.8 & 9 Tuberculosis & 2.9 \\
\hline 10 Self-inflicted injuries & 1.7 & 10 Road traffic accidents & 1.8 \\
\hline
\end{tabular}

Source: Authors' calculations.

expectancy at age 60 has now reached 25 years in Japan. In Eastern Europe from 1990 onward, Hungary and Poland started to experience similar improvements in mortality for older people, but Russia has not, and is actually experiencing a worsening trend.

\section{Regional Variations in Causes of Death}

The tables in annex 3B show total deaths by age, sex, and cause for each of the regions and the world. The 10 leading causes of mortality differ greatly among low-income and middle-income countries (table 3.10) and between them and high-income countries (table 3.6). IHD and cerebrovascular disease are among the top four causes of death in all low- and middle-income regions except Sub-Saharan Africa, where they are eighth and seventh, respectively. Cerebrovascular disease is the leading cause of death in East Asia and the Pacific, unlike in most other regions, where IHD causes more deaths than cerebrovascular disease. In Sub-Saharan Africa, 6 of the top 10 causes are communicable diseases, with HIV/AIDS being the leading cause of death, followed by malaria and lower respiratory infections.

South Asia (mainly India) and Latin America and the Caribbean are the only two other low- and middle-income regions where HIV/AIDS is one of the top 10 causes of death. Lower respiratory infections, primarily pneumonia, are the third leading cause of death, especially among children under five, who account for 60 percent of these 


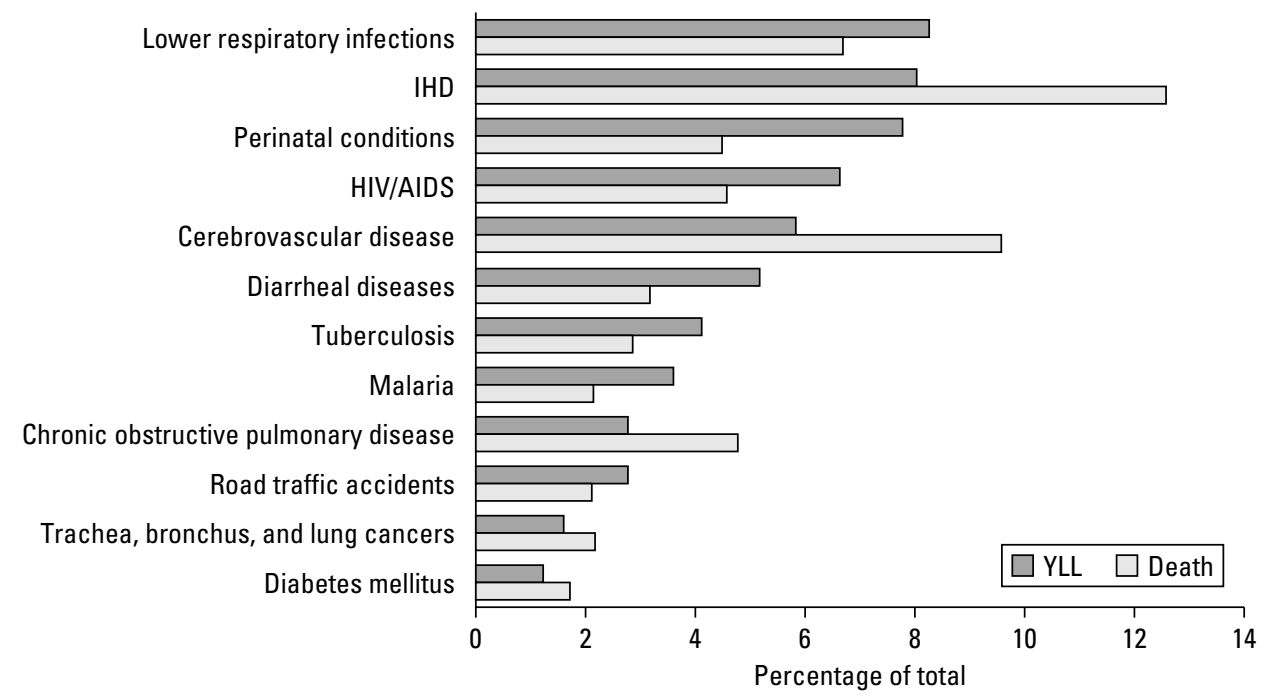

Source: Authors' calculations.

Figure 3.7 Leading Causes of Premature Death (YLL) and of Deaths, Worldwide, 2001

deaths. Chronic obstructive pulmonary disease kills more people (1.4 million) in the East Asia and Pacific region, primarily China, than anywhere else in the world, with 50 percent of global mortality from the disease occurring there.

Europe and Central Asia differs from all other low- and middle-income regions in the size of the CVD epidemic (with almost 50 percent of deaths due to CVD), followed by trachea, bronchus, and lung cancers in third place. Selfinflicted injuries (suicide) are the fifth leading cause of death in this region. South Asia is the only other region where suicide is in the top 10 causes of death. Latin America and the Caribbean is distinguished as the only region where violence falls in the top 10 causes of death, responsible for 1 in 25 deaths. In all low- and middle-income regions apart from Europe and Central Asia, road traffic accidents are included among the top 10 causes of death, reaching fifth position in the Middle East and North Africa, where they are responsible for 1 in 20 deaths.

\section{Years of Life Lost Due to Premature Death}

In contrast to crude numbers of deaths, a time-based measure such as YLL allows us to identify those causes that account for premature deaths by giving greater weight to deaths at younger ages. Thus, while noncommunicable diseases accounted for nearly 60 percent of deaths globally in 2001, they accounted for only 40 percent of YLL, whereas injuries accounted for 12 percent of YLL and 9 percent of deaths.

Figure 3.7 compares the 10 leading causes of YLL and 10 leading causes of death for 2001. YLL give relatively greater importance to HIV/AIDS, perinatal conditions, and diarrheal diseases, whereas counts of deaths give relatively greater importance to IHD, stroke, and chronic obstructive pulmonary disease.

\section{ESTIMATING INCIDENCE, PREVALENCE, AND YLD: METHODS AND DATA}

This section provides an overview of the methods, software tools, and data sources used to calculate YLD for the GBD 2001 together with a short description of the disease models, assumptions, and data sources for important cause groups. Estimating YLD is the most complex and time-consuming component of burden of disease analysis, because it requires systematic assessments of the available evidence on incidence, prevalence, duration, and severity of a wide range of conditions. The GBD study has developed various methods to reconcile often fragmented and partial estimates available from different studies. A specific software tool, DisMod, described later, has been developed to assist in the analysis of epidemiological data and the preparation of internally consistent estimates.

\section{Assessing YLD}

YLD are essentially calculated as follows (ignoring the complications of discounting):

$$
\mathrm{YLD}=I \times D \times L,
$$


where $I$ is the number of incident cases in the reference period, $D$ is the disability weight (in the range 0 to 1 ), and $L$ is the average duration of disability measured in years. With discounting at rate $r$, the formula for calculating YLD becomes

$$
\mathrm{YLD}=I \times D \times[1-\exp (-r L)] / r .
$$

To prepare consistent and unbiased estimates of YLD by cause, it is important to ensure that the disability weight and the population incidence and prevalence data relate to the same case definitions. The data required to estimate YLD are incidence, disability duration, age of onset, and distribution by severity class, all of which must be disaggregated by age and sex. These in turn require estimates of incidence, remission, and case fatality rates or relative risks by age and sex.

For some conditions, numbers of incident cases were available directly from disease registers or epidemiological studies, but for most conditions, only prevalence data were available. In these cases, the DisMod II software program was used to model incidence and duration from estimates of prevalence, remission, case fatality rates, and background mortality.

The sources of data and methods used for each of the major disease and injury groups are summarized in later subsections. Given the large number of categories analyzed and the paucity of epidemiological information for many of them, many of the disease models were necessarily simple and approximate. For most disease and injury groups, relevant experts were consulted during the development and revision of YLD estimates.

The disability weights used for the GBD 2001 are still largely based on the GBD 1990 disability weights and are summarized in annex tables 3A.6 to 3A.8. For certain conditions for which weights were not available from the original GBD study, provisional weights were used from Mathers, Vos, and Stevenson (1999) and Stouthard and others (1997).

As discussed earlier, the disability weights used in DALY calculations quantify societal preferences for different health states. These weights do not represent the lived experience of any disability or health state or imply any societal value of the person in a disability or health state. Thus, for example, disability weights of 0.57 for paraplegia and 0.43 for blindness quantify a social judgment that a year with blindness represents less loss of health than a year with paraplegia. It also means that, on average, a person who lives three years with paraplegia followed by death is considered to experience more equivalent healthy years than a person who has one year of good health followed by death ( 3 years $X$ $[1-0.57]=1.3$ "healthy" years is greater than 1 year of good health).

\section{Ensuring Internal Consistency Using DisMod}

Estimating prevalence and incidence is usually much harder than estimating mortality. Data collection, when done, is often limited in terms of both time and geographical area and problems of case definition abound. Not surprisingly, data are frequently incomplete, and when available, their validity may be in doubt. In particular, given differences in the way the data for incidence, prevalence, and mortality are collected, it is almost inevitable that observations are internally inconsistent. For example, when a cohort study misses more incident cases than deaths, the observed incidence will be too small to account for the observed mortality.

To address such issues, the GBD studies have exploited two kinds of knowledge. First, disease characteristics, such as remission, case fatality rates, and duration, may be relatively constant across countries and known from studies in some populations, from clinical studies, or from expert knowledge. Supplementing observed data with expert knowledge may help to overcome a lack of data. Second, because the various epidemiological variables are causally linked by a disease process, a disease model that explicitly describes these causal pathways allows us to infer missing data if existing data are sufficient to do so.

DisMod was developed for the original GBD study to help model the parameters needed for YLD calculations, to incorporate expert knowledge, and to check the consistency of different epidemiological estimates and ensure that the estimates used were internally consistent. Figure 3.8 shows the underlying model used by DisMod.

Based on experience with the DisMod software tool in the original GBD study, a new version, DisMod II, was developed with a number of additional features (Barendregt and others 2003). Unlike DisMod I, which used finite difference methods to "solve" the disease model, DisMod II implements an exact solution to the underlying differential equations. As well as calculating solutions when the three

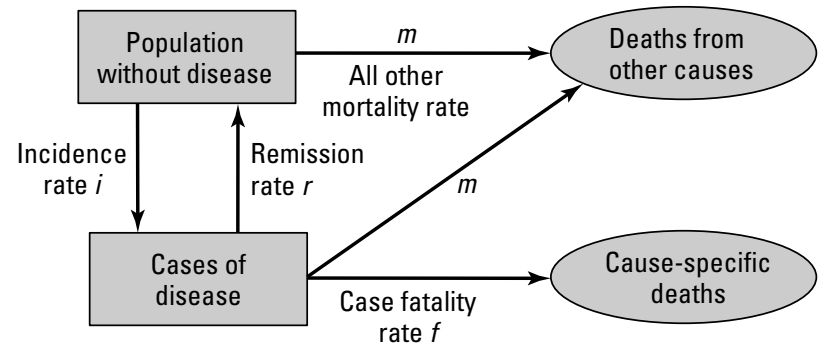

Source: Barendregt and others 2003.

Figure 3.8 Disease Model Underlying DisMod 
hazard rates (for incidence, remission, and mortality) are provided as inputs, DisMod II allows other combinations of inputs, such as prevalence, remission, and case fatality rates. In these cases, DisMod uses a goal-seeking algorithm to fit hazards such that the model reproduces the available input variables. DisMod II also has a range of advanced features, including the ability to undertake sensitivity analysis and uncertainty analysis, to give different weights to the various inputs, and to smooth inputs and specify age patterns for outputs. (The software may be downloaded from the WHO Web site at http://www.who.int/evidence/dismod.)

DisMod II was extensively used in the analyses for the GBD 2001 for four main purposes:

- to estimate a set of incidence rates by age from observed prevalences for a condition, given estimates of remission rates and cause-specific mortality risk derived from population data or epidemiological studies;

- to check whether available data for a condition are consistent with each other, for example, when separate estimates of incidence and prevalence were available for a condition;

- to calculate the average duration of incident cases, needed to calculate YLD for a condition;

- to extrapolate estimates in GBD age categories from epidemiological data for different age categories.

Whereas different assumptions regarding remission and case fatality rates affect the age distribution of incident cases and YLD estimates, total YLD are relatively insensitive to these assumptions if matched to a fixed prevalence distribution. This is because YLD estimates are proportional to incidence multiplied by duration, which approximately equals the prevalence of the condition. In other words, for most conditions the combination of incidence, case fatality, and remission rates (and thus derived durations) used in the YLD calculations makes relatively little difference to total YLD across age groups assuming the same prevalence figures are used as the basis. The effect of discounting complicates this, however, with low incidence and long duration conditions being more discounted than high incidence but short duration conditions.

Figure 3.9 illustrates the use of DisMod II to calculate the incidence of diabetes mellitus in males in Sub-Saharan Africa given estimates of the age-specific prevalence of cases, the relative risk of mortality for those with diabetes compared with those without diabetes (Roglic and others 2005), and the assumption that remission rates are zero.

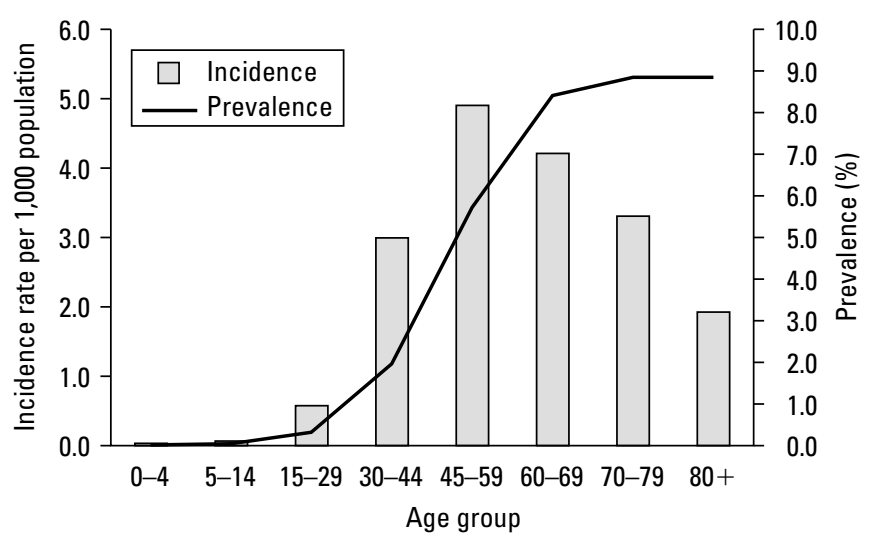

Source: Authors' calculations.

Figure 3.9 Input Prevalences and Incidence Rates Estimated Using DisMod II, for Diabetes Mellitus Cases in Males, Sub-Saharan Africa

\section{YLD Estimates for Regions in 2001}

The GBD 2001 estimated incidence, prevalence, and YLD for 17 epidemiological regions based on the 6 WHO regions subdivided by 5 mortality strata. The five mortality strata were defined in terms of quintiles of the distribution of child and adult mortality for males in 1999 (WHO 2002d, pp. 233-5). These regions are defined in annex table 3A.4.

The Disease Control Priorities Project followed the World Bank approach in treating all high-income countries as one region even though they are not geographically contiguous, and then dividing the rest of the world into six geographic regions that together are referred to as low- and middle-income countries. These regions are defined in annex table 3A.1.

To estimate YLD by cause, age, sex, and region for 2001, incidence and prevalence rates were imputed from the $17 \mathrm{epi}$ demiological subregions to the country level using cause-specific methods documented by Mathers, Murray, and Salomon (2003). Absolute incidence and prevalence numbers by age and sex were then added for all countries in each region to provide regional estimates for 2001. Because Version 3 estimates for 2000 had been prepared so that they were consistent with those for 2002, estimates for 2001 were imputed by averaging the Version 3 estimated cause-, age-, sex-, and countryspecific rates for mortality, incidence, and prevalence for 2000 and 2002 and applying them to population data for 2001.

\section{Overview of Data Sources}

A wide range of data sources were used to analyze incidence, prevalence, and YLD for the GBD 2001. These included

- Disease registers. Disease registers record new cases of disease based on reports by physicians and laboratories. 
Registers are common for infectious diseases, for instance, TB; cancer; congenital anomalies; for some relatively rare diseases, such as cystic fibrosis or thallassaemia; and sometimes for conditions such as diabetes, schizophrenia, and epilepsy.

- Population surveys. Interview surveys, such as the National Health Interview Survey in the United States, can provide self-reported information on disabilities, impairments, and diseases; however, self-reported data are generally not comparable across countries (Murray, Tandon, and others 2002; Sadana and others 2002). In addition, attributing impairment to the underlying causes is often difficult and frequently considerable differences are apparent between lay self-reporting of disease causes and actual underlying disease causes in terms of defined GBD disease categories. In general, the results of health examination surveys have contributed more to YLD calculations than self-reported interview surveys. The Composite International Diagnostic Interview (CIDI) and Diagnostic Interview Schedule (DIS) questionnaires used for mental health surveys are examples of standard questionnaires based on self-reporting that have undergone validity testing and have been used in assessing YLD for mental disorders for the GBD 2001.

- Epidemiological studies. Some of the most useful sources of information for the GBD 2001 were population-based epidemiological studies. In particular, longitudinal studies of the natural history of a disease have provided a wealth of information about incidence, average duration, levels of severity, remission, and case fatality rates. Such studies are rare because they are costly to undertake. In addition, as they are often conducted in a particular region or town, judgment is needed when extrapolating results to the entire population.

- Health facility data. In most cases, routine data on consultations by diagnosis were not found to be a great deal of use in estimating YLD. Unless coverage of the health system is virtually total, facilities-based data will be based on biased samples that do not reflect the prevalence or severity distributions of conditions in the community. Likewise, hospital deaths are unlikely to be useful because of the same problems of selection bias. Examples of conditions that were estimated from hospital data with national or quasi-national population coverage include perinatal and maternal conditions, meningitis, stroke, myocardial infarction, some sequelae identifiable from data on surgical interventions, and injuries.
The following sections provide an overview of data sources and methods for various specific causes and references to more detailed documentation. For some conditions, WHO programs maintain up-to-date databases based on diseases registers, population surveys, and epidemiological studies. These have been used where available. Many of the epidemiological reviews underlying the GBD 2001 estimates of YLD have been documented and published in draft form on the WHO Web site (http://www.who.int/ evidence/bod) and in peer-reviewed publications.

While it is difficult to quantify the exact numbers of data sources used for the YLD estimates for the GBD 2001, table 3.11 provides an approximate count by region. This table counts the number of data sources (registers, notifications, health facility and other official data sets, and epidemiological studies) for each of the causes included in the GBD 2001. For some causes, the only counts available were of the number of countries in each region for which countryspecific data were used. In some cases, an exact recount of studies by region was not feasible, and an approximate regional breakdown was estimated from prior counts according to 17 subdivisions of the $6 \mathrm{WHO}$ regions used in WHO documentation of GBD analyses and data sources (Mathers, Lopez, and others 2003). In addition, it was not always possible to be consistent in the counting of studies carried out across multiple countries or multiple years. Finally, note that there is huge variability in the information content across studies or data sets, and that small epidemiological studies are counted equally in table 3.11 with national hospital inpatient data on injuries for an entire population-year. Thus the counts in table 3.11 should be treated as reasonably indicative of the empirical bases underlying the GBD 2001 without overinterpreting differences between causes or regions.

That said, it is striking that of the more than 8,000 data sets estimated to have been used for the GBD 2001 estimation of YLD, nearly 6,600 relate to Group I causes and only 18 to Group III causes. Furthermore, one-quarter of the data sets relate to populations in Sub-Saharan Africa and around one-fifth to populations in high-income countries. While this predominance of data relating to Group I conditions and to Sub-Saharan Africa is not entirely surprising, the paucity of data for some of the leading noncommunicable diseases is more surprising. For example, for several of the leading causes of burden among mental disorders, one or no usable population-based studies were found for some regions, and for IHD, few studies of the incidence or prevalence of angina pectoris or acute myocardial infarction were found outside high-income countries. 
Table 3.11 Numbers of Country Data Sources Contributing to the Estimation of YLD, by Region and Cause

\begin{tabular}{|c|c|c|c|c|c|c|c|c|}
\hline GBD cause category & $\begin{array}{l}\text { East } \\
\text { Asia } \\
\text { and } \\
\text { Pacific }\end{array}$ & $\begin{array}{l}\text { Europe } \\
\text { and } \\
\text { Central } \\
\text { Asia }\end{array}$ & $\begin{array}{l}\text { Latin } \\
\text { America } \\
\text { and the } \\
\text { Caribbean }\end{array}$ & $\begin{array}{l}\text { Middle } \\
\text { East and } \\
\text { North } \\
\text { Africa }\end{array}$ & $\begin{array}{l}\text { South } \\
\text { Asia }\end{array}$ & $\begin{array}{l}\text { Sub- } \\
\text { Saharan } \\
\text { Africa }\end{array}$ & $\begin{array}{l}\text { High- } \\
\text { income } \\
\text { countries }\end{array}$ & Total $^{\mathbf{a}}$ \\
\hline \multicolumn{9}{|l|}{$\begin{array}{l}\text { I. Communicable, maternal, } \\
\text { perinatal, and nutritional } \\
\text { conditions }\end{array}$} \\
\hline Tuberculosis ${ }^{b}$ & 24 & 27 & 34 & 16 & 8 & 39 & 31 & 179 \\
\hline $\begin{array}{l}\text { Sexually transmitted diseases } \\
\text { excluding HIV/AIDSC }\end{array}$ & 143 & 318 & 148 & 45 & 99 & 406 & 297 & 1,456 \\
\hline HIV/AIDSb & 14 & 26 & 27 & 13 & 5 & 37 & 29 & 150 \\
\hline Diarrheal diseases & 155 & 0 & 27 & 55 & 29 & 91 & 0 & 357 \\
\hline \multicolumn{9}{|l|}{ Childhood-cluster diseases $^{d}$} \\
\hline Pertussis & 14 & 33 & 64 & 14 & 8 & 45 & 124 & 302 \\
\hline Poliomyelitis & 22 & 27 & 32 & 15 & 8 & 47 & 37 & 192 \\
\hline Diphtheria & 12 & 25 & 2 & 14 & 8 & 46 & 8 & 115 \\
\hline Measles & 22 & 18 & 32 & 12 & 8 & 47 & 22 & 127 \\
\hline Tetanus & 48 & 23 & 27 & 40 & 32 & 79 & 34 & 289 \\
\hline Meningitis & 23 & 18 & 30 & 12 & 4 & 27 & 43 & 157 \\
\hline Hepatitis B & 4 & 4 & 6 & 6 & 10 & 11 & 28 & 69 \\
\hline Hepatitis Ce & 36 & 23 & 41 & 37 & 8 & 56 & 85 & 286 \\
\hline Malaria & 9 & 0 & 2 & 1 & 7 & 98 & 0 & 117 \\
\hline \multicolumn{9}{|l|}{ Tropical-cluster diseases } \\
\hline Trypanosomiasis ${ }^{b}$ & 0 & 0 & 0 & 0 & 0 & 36 & 0 & 36 \\
\hline Chagas' disease & 0 & 0 & 31 & 0 & 0 & 0 & 0 & 31 \\
\hline Schistosomiasis & 6 & 0 & 3 & 8 & 0 & 37 & 1 & 55 \\
\hline Leishmaniasis $^{\dagger}$ & 3 & 7 & 15 & 13 & 4 & 20 & 4 & 66 \\
\hline Lymphatic filariasis $^{b}$ & 29 & 0 & 8 & 5 & 5 & 40 & 2 & 89 \\
\hline Onchocerciasis & 0 & 0 & 6 & 0 & 0 & 26 & 0 & 32 \\
\hline Leprosy ${ }^{b}$ & 32 & 10 & 8 & 14 & 8 & 45 & 3 & 120 \\
\hline Dengue $^{g}$ & 91 & 0 & 170 & 0 & 4 & 2 & 15 & 282 \\
\hline Japanese encephalitis $^{b}$ & 10 & 1 & 0 & 0 & 3 & 0 & 4 & 18 \\
\hline Trachoma & 11 & 0 & 4 & 5 & 4 & 19 & 0 & 43 \\
\hline Intestinal nematode infections & 29 & 0 & 23 & 13 & 10 & 53 & 6 & 134 \\
\hline Lower respiratory infections & 15 & 0 & 15 & 12 & 30 & 18 & 5 & 95 \\
\hline Otitis media & 4 & 0 & 0 & 2 & 2 & 7 & 9 & 24 \\
\hline \multicolumn{9}{|l|}{ Maternal conditions } \\
\hline Maternal hemorrhage & 3 & 0 & 2 & 0 & 1 & 13 & 9 & 28 \\
\hline Maternal sepsis & 2 & 0 & 3 & 0 & 1 & 14 & 11 & 31 \\
\hline $\begin{array}{l}\text { Hypertensive disorders of } \\
\text { pregnancy }\end{array}$ & 1 & 0 & 1 & 0 & 2 & 12 & 2 & 18 \\
\hline Obstructed labor & 2 & 0 & 2 & 0 & 1 & 14 & 2 & 21 \\
\hline Abortion & 32 & 10 & 11 & 13 & 49 & 156 & 27 & 32 \\
\hline \multicolumn{9}{|l|}{ Perinatal conditions } \\
\hline Low birthweighth & 28 & 27 & 33 & 15 & 7 & 41 & 33 & 184 \\
\hline Birth asphyxia and birth trauma & 7 & 0 & 7 & 11 & 19 & 12 & 0 & 56 \\
\hline \multicolumn{9}{|l|}{ Nutritional deficiencies } \\
\hline Protein-energy malnutrition ${ }^{i}$ & 61 & 28 & 116 & 37 & 30 & 132 & 15 & 419 \\
\hline lodine deficiency & 17 & 13 & 13 & 17 & 12 & 44 & 20 & 136 \\
\hline Vitamin A deficiency & 10 & 2 & 12 & 4 & 4 & 29 & 8 & 67 \\
\hline Iron-deficiency anemia & 14 & 1 & 1 & 15 & 5 & 33 & 0 & 69 \\
\hline \multicolumn{9}{|l|}{ II. Noncommunicable diseases } \\
\hline \multicolumn{9}{|l|}{ Malignant neoplasms } \\
\hline Incidence & 11 & 8 & 11 & 10 & 2 & 14 & 25 & 81 \\
\hline Survival & 3 & 4 & 1 & 0 & 1 & 0 & 15 & 24 \\
\hline
\end{tabular}




\begin{tabular}{|c|c|c|c|c|c|c|c|c|}
\hline GBD cause category & $\begin{array}{l}\text { East } \\
\text { Asia } \\
\text { and } \\
\text { Pacific }\end{array}$ & $\begin{array}{l}\text { Europe } \\
\text { and } \\
\text { Central } \\
\text { Asia }\end{array}$ & $\begin{array}{l}\text { Latin } \\
\text { America } \\
\text { and the } \\
\text { Caribbean }\end{array}$ & $\begin{array}{l}\text { Middle } \\
\text { East and } \\
\text { North } \\
\text { Africa }\end{array}$ & $\begin{array}{l}\text { South } \\
\text { Asia }\end{array}$ & $\begin{array}{l}\text { Sub- } \\
\text { Saharan } \\
\text { Africa }\end{array}$ & $\begin{array}{l}\text { High- } \\
\text { income } \\
\text { countries }\end{array}$ & Total $^{2}$ \\
\hline Diabetes mellitus - type $1^{j}$ & 22 & 12 & 17 & 5 & 1 & 2 & 41 & 100 \\
\hline Diabetes mellitus - type 2 & 6 & 4 & 5 & 8 & 3 & 6 & 8 & 40 \\
\hline \multicolumn{9}{|l|}{ Neuropsychiatric conditions } \\
\hline Unipolar depressive disorders & 5 & 5 & 6 & 3 & 4 & 6 & 27 & 56 \\
\hline Bipolar affective disorder & 2 & 1 & 1 & 1 & 2 & 0 & 14 & 21 \\
\hline Schizophrenia & 4 & 3 & 3 & 1 & 3 & 6 & 25 & 45 \\
\hline Epilepsyk & 1 & 1 & 6 & 1 & 4 & 8 & 7 & 28 \\
\hline Alcohol use disorders & 24 & 43 & 39 & 13 & 5 & 34 & 56 & 214 \\
\hline Alzheimer's and other dementias & 10 & 3 & 3 & 0 & 4 & 3 & 87 & 110 \\
\hline Parkinson's disease ${ }^{k}$ & 2 & 1 & 1 & 0 & 1 & 1 & 7 & 13 \\
\hline Multiple sclerosis & 4 & 24 & 3 & 5 & 1 & 1 & 116 & 154 \\
\hline Drug use disorders & 11 & 11 & 18 & 10 & 6 & 15 & 43 & 114 \\
\hline Post-traumatic stress disorder & 1 & 0 & 1 & 0 & 0 & 0 & 6 & 6 \\
\hline Obsessive-compulsive disorder & 2 & 0 & 3 & 0 & 1 & 0 & 14 & 20 \\
\hline Panic disorder & 2 & 0 & 3 & 1 & 0 & 2 & 22 & 30 \\
\hline Insomnia (primary) & 2 & 2 & 5 & 1 & 1 & 1 & 9 & 21 \\
\hline Migraine & 6 & 2 & 5 & 2 & 0 & 1 & 11 & 43 \\
\hline $\begin{array}{l}\text { Mental retardation attributable } \\
\text { to lead exposure }\end{array}$ & 10 & 12 & 21 & 4 & 14 & 9 & 23 & 93 \\
\hline \multicolumn{9}{|l|}{ Sense organ diseases } \\
\hline Vision disorders ${ }^{b}$ & 11 & 3 & 4 & 5 & 4 & 19 & 9 & 55 \\
\hline Hearing loss, adult onset & 5 & 0 & 1 & 1 & 5 & 1 & 12 & 25 \\
\hline Cardiovascular diseases & 0 & 0 & 0 & 0 & 0 & 0 & 0 & 0 \\
\hline Rheumatic heart disease & 15 & 0 & 12 & 9 & 15 & 26 & 7 & 84 \\
\hline Ischemic heart disease & 3 & 11 & 0 & 2 & 4 & 1 & 58 & 79 \\
\hline Cerebrovascular disease & 4 & 8 & 1 & 5 & 0 & 6 & 28 & 52 \\
\hline Other cardiovascular diseases & 0 & 0 & 0 & 0 & 0 & 0 & 5 & 5 \\
\hline \multicolumn{9}{|l|}{ Respiratory diseases } \\
\hline Chronic obstructive pulmonary disease & 24 & 10 & 10 & 4 & 16 & 8 & 32 & 104 \\
\hline Asthma & 17 & 14 & 20 & 12 & 6 & 7 & 74 & 149 \\
\hline \multicolumn{9}{|l|}{ Musculoskeletal diseases } \\
\hline Rheumatoid arthritis & 4 & 1 & 4 & 4 & 2 & 5 & 9 & 29 \\
\hline Osteoarthritis & 1 & 1 & 1 & 0 & 2 & 1 & 9 & 15 \\
\hline Congenital malformations & 3 & 42 & 29 & 6 & 9 & 6 & 5 & 100 \\
\hline Oral conditions $s^{\dagger}$ & 22 & 24 & 32 & 15 & 7 & 35 & 27 & 162 \\
\hline III. Injuries & 3 & 1 & 1 & 0 & 0 & 6 & 7 & 18 \\
\hline Total' & 1,155 & 914 & 1,239 & 590 & 522 & 1,955 & 1,735 & 8,096 \\
\hline
\end{tabular}

Source: Authors' compilation.

Note: The data sources include population-based epidemiological studies, disease registers, and surveillance and notification systems, but exclude death registration data (see tables 3.1 and 3.2).

Where possible, regional and global totals refer to numbers of separate studies, or country-years of reported data from surveillance or notification systems. For some causes, regional subtotals for the Disease Control Priorities Project regions were estimated from subtotals for WHO regions and subregions. See text for more information on data sources for specific causes.

a. Global totals may include global review studies not counted in regional subtotals.

b. Totals refer to numbers of countries for which data were available, not to total data sets or country-years.

c. Regional subtotals were estimated from the current distribution of studies in the WHO sexually transmitted infection (STI) surveillance database.

d. Regional subtotals were estimated from numbers of studies by WHO region, rather than by re-accessing original databases.

e. Country-years of data available for 133 countries

f. Approximate estimate from current WHO database; original extraction from surveillance data sources is not available.

g. Country-years of surveillance reports (approximate, minimum estimate for Latin America and the Caribbean).

h. Estimate based on final published literature review.

i. Regional distribution of the 419 national studies used is assumed to be similar to that of the current 442 national studies in the WH0 malnutrition database.

j. Total of 100 population-based registries in 50 countries.

k. Approximate minimum estimate. Several global reviews were used; studies were not separately counted.

I. Actual numbers of studies used exceed the minimums shown here, based on summed table entries for specific causes regardless of whether counts were of data sets or of countries 
Assuming that for causes in table 3.11 where the counts relate to countries rather than to data sets there are, on average, two data sets per country; then overall, approximately 8,700 data sets contributed to the estimation of YLD. Not counting again studies that also contributed to the estimation of cause-specific mortality rates, an additional 1,370 data sets were used to estimate YLL. In total, the GBD 2001 has drawn on more than 10,000 data sets or studies, making it almost certainly the largest synthesis and analysis of global population health data carried out to date.

\section{Communicable Diseases and Maternal, Perinatal, and Nutritional Conditions}

This section gives an overview of data sources and methods for specific Group I causes and references to more detailed documentation.

Tuberculosis. Estimates of incidence and deaths due to TB (excluding HIV-infected persons) for countries in 2001 formed the basis of estimates of TB prevalence in 2001. The methods and data used to estimate incidence and mortality for each country were described earlier. For countries with VR data for TB deaths, incidence estimates have been revised to be consistent with estimated deaths, estimated case fatality rates for treated and untreated cases, and proportion of incident cases treated.

Estimated prevalence of all forms of TB (excluding HIVinfected persons) for 2001 was calculated by multiplying estimated incidence by estimated duration. Country-specific estimates of duration were weighted for the proportion of cases treated and that were smear-positive.

\section{Sexually Transmitted Infections Other Than HIV/AIDS.} More than 300 community-based and prenatal care-based prevalence and incidence studies of pregnant women were used to generate region-specific estimates of the prevalence of syphilis, chlamydia, and gonorrhea. The methodology is described in detail elsewhere (Gerbase and others 1998; WHO 2001c) and was used to update estimates to 2001.

HIV/AIDS. The Joint United Nations Programme on HIV/AIDS and WHO have developed country-specific estimates of HIV/AIDS for most countries and revise them periodically to account for new data and improved methods (Salomon and Murray 2001b; Schwartlander and others 1999; Walker and others 2003). For the most recent round of estimates, they used two different types of models, one for generalized epidemics and one for epidemics concentrated in high-risk groups.

For a few countries where prevalence estimates for HIV seropositive cases were not directly available, they were derived by scaling regional prevalence estimates according to the ratio of country-specific HIV/AIDS mortality to regional HIV/AIDS mortality. Because different countries may be in different phases of the epidemic, the relationship between prevalence and mortality may vary across countries.

Diarrheal Diseases. To estimate the incidence of diarrheal diseases in children under five in developing and developed countries, 357 community-based studies and population surveys were used (Bern 2004; Murray and Lopez 1996d). Point prevalences were estimated assuming an average duration of six days per episode. Work is currently in progress to update these estimates with more recent evidence from community-based studies.

\section{Vaccine-Preventable Childhood Diseases and Meningitis.} The methods used to estimate incidence for childhood-cluster diseases were summarized earlier. The incidence of meningitis due to Haemophilus influenzae type b together with the incidence of meningitis due to Streptococcus pneumoniae and Neisseria meningitides, was updated from the 1990 estimates using information from the WHO Vaccines and Biologicals Program derived from country notifications of cases and deaths, from WHO surveillance centers and, where relevant, from immunization coverage data (WHO 2001b).

Hepatitis B and C. Available data on the prevalence of chronic hepatitis B and hepatitis C infection were used together with disease models to estimate regional incidence and mortality rates (Global Burden of Hepatitis C Working Group 2004; Lavanchy 2004; WHO 2002a, 2002b).

Malaria. Malaria prevalence was based on regional prevalence rates for acute symptomatic episodes estimated by Murray and Lopez (1996d). Country-specific estimates of malaria prevalence were derived by adjusting subregional prevalence by the ratio of country to subregional malaria mortality. Work is currently under way in collaboration with other WHO programs and external expert groups to refine and revise these country-specific estimates of malaria prevalence (Korenromp 2005).

Schistosomiasis. The CEGET/WHO Atlas of the Global Distribution of Schistosomiasis (Doumenge and others 1987) 
and population-based prevalence studies were used to estimate country-specific prevalence rates. Prevalence estimates were based on regional prevalence rates for schistosomiasis infection (Murray and Lopez 1996d) applied to updated estimates of country-specific populations at risk in 2001 (van der Werf and de Vlas 2001).

Lymphatic Filariasis. Estimates for lymphatic filariasis were developed for six of the eight regions defined for the GBD 1990 study (Murray and Lopez 1996d). The established market economies and formerly socialist economies of Europe were excluded, because infection was not considered to be endemic in these countries. The prevalence data were obtained from community-based surveys and complemented with reports by the Information and Reference Service of the Parasitic Diseases Program, WHO. Prevalence estimates were based on regional prevalence rates for cases of hydrocele or lymphodaema caused by infection with filariae. These estimates were updated using estimates of country-specific populations at risk in 2001 provided by the WHO Lymphatic Filiariasis Elimination Program.

Onchocerciasis. In the early 1990s, WHO estimated the prevalence of blindness due to onchocerciasis from surveys and national reports (WHO 1995). Following the continued success of the Onchocerciasis Control Program in western African countries and the introduction of population-wide administration of ivermectin in other endemic areas, the prevalence of onchocerciasis and its disabling sequelae has been dramatically reduced in all 36 endemic countries in Latin America and the Caribbean and Sub-Saharan Africa (Richards and others 2001). Therefore, the prevalence of blindness from onchocerciasis was reestimated by taking into account the declining trends in prevalence and the coverage and duration of onchocerciasis control programs (Alley and others 2001).

Reliable sources of information on the prevalence of blindness due to onchocerciasis are available from several population-based studies, usually as part of an overall blindness survey. However, prevalence studies of onchocerciasis-specific blindness are often carried out in hyperendemic areas and/or in local communities, and thus the estimated prevalence may not be generalizable to the country as a whole. For this reason, the current prevalence of blindness due to onchocerciasis was estimated by nationally reported data, if available, and extrapolation from 1993 estimates using trend analysis of onchocerciasis control programs in each endemic country (Shibuya and Ezzati 2003).
Leprosy. Regional incidence and prevalence rates for leprosy were based on case reporting and surveillance by 120 WHO member states (Stein 2002a; WHO 2002c).

Dengue and Dengue Hemorrhagic Fever. Regional incidence and prevalence rates for dengue and dengue hemorrhagic fever were based on a review of nearly 300 population-based studies, but data were sparse for regions apart from East Asia and the Pacific and Latin America and the Caribbean (LeDuc, Esteves, and Gratz 2004).

Trachoma. The baseline regional and subregional prevalence of blinding trachoma was first estimated as described elsewhere (Frick and others 2003; Ranson and Evans 1995) and then updated using several recent population-based studies in the Middle East and North Africa and Sub-Saharan Africa. As the prevalence of blinding trachoma declines with socioeconomic development even in the absence of a specific trachoma control program (Dolin and others 1997), the extrapolation from regional prevalence estimates made in the 1980s would overestimate current prevalence. For this reason, both nationally reported data and specific criteria for a regression model of time-series data were used to estimate the prevalence of blinding trachoma. The model estimates were then applied to countries that have reported cases of blinding trachoma (Shibuya and Mathers 2003).

Intestinal Nematode Infections. Updated estimates of the prevalence of intestinal nematode infections were based on WHO's new global databank on schistosomiasis and soiltransmitted helminths, which contains data derived from community-based, cross-sectional surveys for subnational administrative regions (Brooker and others 2000; de Silva and others 2003). In areas without comprehensive data, predictions of the distribution of soil-transmitted helminths were developed using environmental data derived from satellite remote sensing (Brooker and others 2002). Incidence rates and YLD for disabling sequelae of helminth infections were modeled using a mathematical model developed by Chan and others (Bundy and others 2004; Chan 1997).

Lower Respiratory Infections. Prevalence and incidence estimates for lower respiratory infections were based on an analysis of published data on the incidence of clinical pneumonia from 95 community-based studies published since 1961 (Rudan and others 2004). Most of the studies were longitudinal and conducted over long enough periods to account for seasonal variation. Studies over short periods of time were excluded. 
Maternal Conditions. Incidence rates for maternal conditions and disabling sequelae were derived from reviews of published population-based studies supplemented by studies of hospital-based deliveries adjusted for the proportion of deliveries occurring in hospitals (Dolea and AbouZahr 2003a, 2003b; Dolea, AbouZahr, and Stein 2003; Dolea and Stein 2003). The incidence of unsafe induced abortion was estimated at the country level using 156 published and unpublished reports for 131 countries together with information on legal and social contexts (Ahman, Dolea, and Shah 2003; WHO 2004a).

Perinatal Conditions. Incidence rates for low birthweight, birth asphyxia and trauma, and disabling sequelae were derived from health service-based data and national birth registration systems in high-income countries and from mothers participating in nationally representative household surveys (such as the U.S. Agency for International Development-funded DHSs and the Multiple Indicator Cluster Surveys carried out by the United Nations Children's Fund), supplemented by reviews of published populationbased and hospital-based studies (UNICEF and WHO 2005).

Protein-Energy Malnutrition. More than 400 recent nationally representative studies from WHO's global database on child growth and malnutrition (http://www. who.int/nutgrowthdb/) were used to estimate the prevalence of child stunting and wasting in every country (de Onis and Blossner 2003; de Onis, Frongillo, and Blossner 2000; de Onis and others 2004). Where country estimates were not available from the database, the regional average calculated from the available studies or data from other countries with similar epidemiological characteristics were used (Stein 2002c).

Iodine Deficiency and Vitamin A Deficiency. Countryspecific estimates for goiter rates were obtained and used to calculate regional estimates for total goiter rates. The primary data source was the WHO Nutrition and Health for Development Program, which is developing and refining a comprehensive database of country-specific estimates of both clinical and subclinical iodine deficiency disorders from national level and subnational nutrition surveys (Rastogi and Mathers 2002a; WHO 2001a; WHO Nutrition Program 2005).

Country-specific estimates were obtained and used to calculate regional estimates for both xerophthalmia and corneal scars resulting from vitamin A deficiency (Rastogi and Mathers 2002c). Again, the primary data source was the WHO Nutrition and Health for Development Program, which is also developing and refining a comprehensive database of country-specific estimates of both clinical and subclinical vitamin A deficiency from national-level and subnational nutrition surveys (WHO Nutrition Program 2002b). The database compiles information for all population groups, especially preschool-age children and women of childbearing age, and includes information on the prevalence of xerophthalmia, including night blindness and serum retinol distributions.

Iron Deficiency Anemia. Country-specific prevalence estimates of iron deficiency anemia were obtained from 69 studies and used to estimate regional age- and sex-specific prevalence rates for mild, moderate, and severe anemia. The primary data source was the WHO Nutrition and Health for Development Program. The program is currently preparing a comprehensive database of country-specific prevalence estimates of both clinical and subclinical iron deficiency anemia from national-level and subnational nutrition surveys (WHO Nutrition Program 2002a).

All prevalence estimates were reviewed, with priority being given to the most recent national-level estimates (most were obtained from studies conducted in the last 10 years). For countries for which no studies were available, the regional average was applied (Rastogi and Mathers 2002b).

\section{Noncommunicable Diseases}

This section gives an overview of data sources and methods for specific Group II causes and references to more detailed documentation.

Malignant Neoplasms. Regional survival models were developed for each cancer site and used to estimate numbers of incident cases from estimated deaths by site for each country (Mathers, Shibuya, and others 2002; Shibuya and others 2002). The same models were used to estimate numbers of prevalent cases, defined as cases of malignant neoplasms causing death within 15 years, and cases of nonfatal malignant neoplasms (where the person is likely to survive 15 years or more) diagnosed within the last five years.

Diabetes Mellitus. Diabetes prevalence estimates for those age 20 and older were based on an analysis of 41 representative population-based studies that used oral glucose tolerance tests and either 1980 WHO criteria to define diabetes cases or similar criteria that produced comparable prevalences (Wild and others 2004). For countries for which eligible data were not available, data from a proxy country believed to have similar diabetes prevalence were used. Most 
studies of diabetes prevalence did not indicate the type of diabetes, and consequently the estimates refer to all diabetes. The prevalence of diabetes among people under 20 years of age was estimated from incidence data derived from 100 published studies (Karvonen and others 2000).

Depressive Disorders. Point prevalence estimates for episodes of unipolar major depression were derived from a systematic review of available published and nonpublished population studies on depressive disorders, which identified 56 studies from all World Bank regions (Ustun and others 2005). Variations in the prevalence of unipolar depressive disorders in some European countries, Australia, Japan, and New Zealand were estimated directly from relevant population studies (Ayuso-Mateos and others 2001). For other highincome European countries, country-specific prevalences were estimated using a regression model of available prevalence data on suicide rates (for ages 15 to 59, both sexes combined). For other regions, prevalence estimates were based on regional prevalence rates applied to country-specific population estimates for 2002. Unlike the original GBD study, survey data on the severity of unipolar depressive disorders (mild, moderate, or severe) were used together with disability weights for these three severity classes from Stouthard and others (1997). This resulted in an overall disability weight for unipolar depressive disorders across regions from 0.30 to 0.46 . This compares reasonably well with a more recent analysis of the distribution of depression by severity and disability weights for a Dutch community, which resulted in an overall disability weight of 0.41 (Kruijshaar and others 2005). YLD due to dysthymia not associated with major depressive episodes were estimated separately using the disability weight for mild depressive disorders.

Subregional prevalence rates for bipolar disorder were derived from a systematic review of all available published and unpublished population studies using case definitions that met the diagnostic criteria of the fourth edition of the Diagnostic and Statistical Manual of Mental Disorders (DSM-IV) of the American Psychiatric Association (1994) or of ICD-10 (Ayuso-Mateos 2002a).

Anxiety Disorders and Schizophrenia. Subregional prevalence rates for panic disorder, obsessive-compulsive disorder, and post-traumatic stress disorder were also derived from systematic reviews of all available published and unpublished population studies using case definitions that met ICD-10 or DSM-IV criteria (Ayuso-Mateos 2002b, 2002c, 2002d; Ustun and Chisholm 2001). Those with comorbid depressive disorder or alcohol or drug use disorders were excluded from prevalence estimates. For data sources and methods for schizophrenia see Ayuso-Mateos (2002d).

Alcohol and Drug Use Disorders. The case definition for alcohol use disorders is based on ICD-10 criteria for alcohol dependence and harmful use, excluding cases with comorbid depressive episode. DSM-IV alcohol abuse is included in the case definition. All available population-based surveys using diagnostic criteria that could be mapped to this case definition were identified. Population estimates of the point prevalence of alcohol use disorders were obtained from 55 studies (Mathers and Ayuso-Mateos 2003).

Published data on alcohol production, trade, and sales, adjusted for estimates of illegally produced alcohol, were used to estimate country averages of the volume of alcohol consumed. These preliminary estimates were then further adjusted on the basis of survey data on alcohol consumption to estimate the prevalence of alcohol use disorders for countries where recent population-based survey data were not available (Rehm and others 2004).

Estimating the prevalence of illicit drug use is difficult, because the use of these drugs is illegal, stigmatized, and hidden. In addition, definitions differ from country to country, as does the quality of data collected. The definition used for the GBD 2001 was based on ICD-10 criteria for opioid dependence and harmful use or cocaine dependence and harmful use, excluding cases with comorbid depressive episodes. Data on the prevalence of problematic illicit drug use were derived from a range of sources (Degenhardt and others 2003). A literature search was conducted of all studies that estimated the prevalence of problematic drug use and more than 100 studies were identified. Other data sources included the United Nations Drug Control Program and the European Monitoring Centre for Drugs and Drug Addiction.

Insomnia (Primary). Subregional prevalence rates for primary insomnia were derived from systematic reviews of all available published and unpublished population studies using case definitions that met ICD-10 or DSM-IV criteria, where the insomnia causes problems with usual activity and is not secondary to other diseases. Persons with comorbid depressive disorder or alcohol or drug use disorders were excluded from the prevalence estimates.

Epilepsy and Multiple Sclerosis. Subregional prevalence rates for epilepsy, excluding epilepsy or seizure disorder secondary to other diseases or injury, were derived from systematic reviews of available published and unpublished 
population studies. Subregional prevalence rates for multiple sclerosis, derived for the GBD 1990, were updated using recent epidemiological studies (Warren and Warren 2001).

Alzheimer's Disease and Other Dementias. Subregional prevalence rates, incidence rates, and durations for Alzheimer's disease and other dementias were estimated based on 110 available population studies and assumed to apply to countries within each subregion (Mathers and Leonardi 2003).

Parkinson's Disease. Regional incidence to mortality rates for Parkinson's disease estimated by Murray and Lopez (1996d) were used to derive country-specific estimates for incidence from the estimated country-specific mortality rates.

Migraine. Regional prevalence rates for people who experience migraine were estimated from 43 available population studies and assumed to apply to countries within each subregion (Leonardi and Mathers 2003). Migraine has been treated as a chronic disease lasting from 15 years to around 45 years with sporadic episodes. The case definition was taken from the International Headache Society's definition of migraine. Available population studies using this definition provided prevalence estimates that were quite similar across most regions.

Mental Retardation. An attempt was made to assess the prevalence of all forms of mental retardation, but due to difficulties with data comparability, we decided to assess only the burden resulting from childhood exposure to environmental lead, plus mental retardation estimated as sequelae to diseases or injuries or associated with specific congenital malformations. The YLD associated with mental retardation as a sequela of diseases and injuries or as a component of a syndrome are included in the estimation of total YLD for such causes in the tables presented in annex 3C. In addition, YLD were estimated separately for mental retardation as a consequence of environmental lead exposure, because this was required for the assessment of the total attributable burden of environmental lead exposure. For details of methods and data sources see Fewtrell and others (2004) and PrussUstun and others (2004).

Low Vision and Blindness. Both regional and subregional prevalences for blindness and low vision were updated using all available data gathered since 1980 (Resnikoff and others 2004; Thylefors and others 1995). Subregional prevalences were estimated from more than 50 cross-sectional, population-based surveys of blindness and low vision, both published and unpublished. For countries for which no data were available, prevalences were extrapolated from available data for neighboring subregions or countries with a similar epidemiological and socioeconomic environment. The DisMod software was then used to obtain internally consistent age- and sex-specific estimates of incidence, prevalence, remission, and relative risks of mortality. Ratios of blindness to low vision for each region were used to estimate the prevalence of low vision and DisMod analyses were then carried out to ensure internal consistency among parameters.

Hearing Loss. Despite the number of published studies on hearing loss, many of them use different criteria and relate to subnational or nonrepresentative populations. Data from 25 representative population surveys of measured hearing loss (19 surveys for adults and 14 surveys for children) were used to estimate subregional prevalences of moderate or greater hearing loss according to the WHO definition (hearing threshold level in the better ear is 41 decibels or greater averaged over $0.5,1.0,2.0$, and 4.0 kilohertz) and of severe or greater hearing loss (hearing threshold level in the better ear is 61 decibels or greater averaged over 0.5, 1.0, 2.0, and 4.0 kilohertz) (Mathers, Smith, and Concha 2003). Regional estimates of the prevalence of hearing aid use were used in the calculation of average disability weights for moderate, severe, and profound hearing loss in each region, and thus to calculate YLD associated with hearing loss.

Congestive Heart Failure. The incidence of congestive heart failure following acute myocardial infarction was estimated using a model for IHD based on available population data on incidence and case fatality rates for acute myocardial infarction and on the proportion of acute myocardial infarction patients who go on to develop congestive heart failure (Mathers, Truelson, and others 2004). The incidence of congestive heart failure as a sequela to rheumatic heart disease, hypertensive heart disease, and inflammatory heart diseases was estimated using incidence to mortality ratios from the GBD 1990 (Murray and Lopez 1996d).

Angina Pectoris. The GBD 2001 study developed a model for IHD based on available population data on the incidence and case fatality rates for acute myocardial infarction and on the prevalence and case fatality rates for angina pectoris (Mathers, Truelson, and others 2004). Observed correlations between the prevalence of acute myocardial infarction survivors and the prevalence of angina pectoris (whether incident before or after acute myocardial infarction) were used 
to estimate the prevalence of angina pectoris from the modeled prevalences of acute myocardial infarction survivors. The latter were estimated from country-specific IHD mortality estimates together with estimated regional case fatality rates for acute myocardial infarction.

Stroke. The GBD 2001 study developed a model for stroke based on available population data on case fatality rates within 28 days for incident cases of first-ever stroke and on long-term survival in cases surviving this initial period, in which the risk of mortality is highest (Truelsen and others 2002). A consistent relationship between incidence, prevalence, and mortality was established using U.S. data. The resulting age- and sex-specific 28-day and long-term case fatality rates were used as the basis for estimating subregional case fatality rates after adjusting for the observed relationship between GDP per capita and overall 28-day case fatality rates in published studies from various countries. Consistent epidemiological models for the prevalence of stroke survivors in each subregion were then estimated using these case fatality rates and observed mortality after adjustment to account for the fact that deaths recorded as resulting from stroke in vital statistics do not fully reflect the true excess risk of mortality among survivors.

Chronic Obstructive Pulmonary Disease. Chronic obstructive pulmonary disease is characterized by airway obstruction with lung function levels of forced expiratory volume in one second $\left(\mathrm{FEV}_{1}\right)$ to forced vital capacity ratio of less than 70 percent and the presence of a postbronchodilator $\mathrm{FEV}_{1}$ of less than 80 percent of the predicted value that is not fully reversible. Because accurate prevalence data based on spirometry are not available in many regions, an alternative approach was used to infer disease occurrence from regional estimates of mortality due to chronic obstructive pulmonary disease that made use of the constraints imposed by the consistent epidemiological relationships among prevalence to incidence, remission, case fatality, and mortality rates. The relative risk of mortality due to chronic obstructive pulmonary disease across subregions was estimated as a function of its two leading risk factors- tobacco smoking and indoor air pollution from solid fuel used for cooking - along with regional fixed effects (Lopez and others forthcoming). Data on risk factors were derived from the comparative risk assessment carried out for the World Health Report 2002 (Ezzati and others 2002; WHO 2002d). The estimated relative risks were validated by comparing estimated regional prevalence with data from available population studies. For regions where surveys of representative populations based on spirometry were available, both direct estimation and model estimation were used.

Asthma. Asthma prevalence estimates were based on a case definition requiring a positive airway hyper-responsiveness test in addition to symptoms in the last 12 months. Specifically, the prevalence estimates related to cases defined in terms of reported wheeze in the last 12 months plus current bronchial hyper-responsiveness, defined as a mean provocation concentration of histamine required to produce a 20 percent fall in $\mathrm{FEV}_{1}$ of 8 milligrams per milliliter or less.

While epidemiological studies commonly use a broader definition of asthma based on symptom reporting, the 2001 GBD study used a narrower definition in order to identify cases experiencing a significant loss of health. The disability threshold for inclusion in the prevalence estimates is mild asthma, defined as occasional wheeze that does not affect usual activities, but which, if untreated, may result in occasional episodes that cause sleep disturbance and/or speech limitations.

A review of published literature identified studies using the foregoing definition, but also many studies using selfreported symptoms only, self-reported current asthma (asthma attack in the last 12 months or currently in treatment), or physician diagnosis of current asthma in the last 12 months. Based on study populations for which prevalence data were available according to one of these alternative definitions, as well as the foregoing stricter definition, we calculated adjustment factors to estimate asthma prevalence from community surveys using other definitions of asthma.

A total of 149 population-based studies were used to derive estimates of asthma prevalence for a wide range of countries for children, teenagers, and adults. In particular, extensive use was made of two multicountry studies: the International Study of Asthma and Allergies in Childhood using self-reported symptoms in children ages 6 to 7 and 13 to 14 (ISAAC Steering Committee 1998a, 1998b), and the European Community Respiratory Health Survey of adults ages 20 to 44 using self-reported symptoms and bronchial hyper-responsiveness (Chinn and others 1997; Pearce and others 2000). Estimates from the population-based studies were then used to derive subregional average prevalence rates, which were assumed to apply in countries without specific population studies.

Rheumatoid Arthritis. Subregional prevalence rates for rheumatoid arthritis were derived from available published population studies using case definitions for definite or 
classical rheumatoid arthritis (Symmons, Mathers, and Pfleger 2002b).

Osteoarthritis. Subregional prevalence rates for osteoarthritis were derived from available published population studies that provided prevalence data for symptomatic osteoarthritis of the hip or knee, radiologically confirmed as Kellgren-Lawrence grade 2 or greater (Symmons, Mathers, and Pfleger 2002a).

Edentulism. Prevalence numbers were based on regional prevalence rates for edentulism estimated by Murray and Lopez (1996d). New data from the 2002-4 WHO World Health Survey will enable revision of these estimates in the future.

\section{Injuries}

An incident episode of a nonfatal injury is defined as an episode that is severe enough for the person to be hospitalized or that requires emergency room care (if such care is available). Begg and others (2002) describe methods used to estimate injury-related prevalences and prevalence YLD. In brief, the incidence of nonfatal injuries by external cause category, age, and sex was estimated by applying regional and country-specific death to incidence ratios to the injury deaths estimated for each country in 2002.

Age- and sex-specific ratios were based on new analyses of health facility data provided by 18 countries in five World Bank regions. For most cause categories, extrapolations from observed death to incidence ratios were derived for all countries at a regional level, with final adjustments using mortality and per capita GDP as predictors of expected variability in case fatality rates.

Prevalences for disabling injuries were estimated from the proportions of cases by injury type estimated to result in long-term disability, together with estimates of short- and long-term disability durations. The latter were based on analyses of excess mortality risks from epidemiological studies (Begg and others 2002).

\section{BURDEN OF DISABILITY AND POOR HEALTH IN 2001}

As defined earlier, YLD measure the equivalent years of healthy life lost through time spent in states of less than full health. The original GBD study brought the previously largely ignored burden of nonfatal illnesses, particularly mental disorders, to the attention of health policy makers.
The findings of the GBD 2001, based on updated data and analyses, confirm that disability and states of less than full health caused by diseases and injuries play an important role in determining the overall health status of populations in all regions of the world.

\section{Leading Causes of YLD in 2001}

Tables 3.12 and 3.13 show the 10 leading causes of $\operatorname{YLD}(3,0)$ by broad income group and by sex. A relatively short list of causes dominates the overall burden of nonfatal disabling conditions. In both income regions, neuropsychiatric conditions are the most important causes of disability, accounting for more than 37 percent of $Y \operatorname{LDs}(3,0)$ among adults ages 15 and over. The disabling burden of neuropsychiatric conditions is almost the same for males and females, but the major contributing causes are different. While depression is the leading cause for both males and females, the burden of depression is 50 percent higher for females than for males, and females also have a higher burden from anxiety disorders, migraine, and senile dementias. In contrast, the male burden for alcohol and drug use disorders is nearly six times higher than that for females and accounts for one-quarter of the male neuropsychiatric burden.

Globally, cataracts and age-related vision disorders together account for more than 9 percent of total $\operatorname{YLD}(3,0)$, and adult-onset hearing loss accounts for another 5.2 percent. Adult-onset hearing loss is extremely prevalent, with more than 27 percent of men and 24 percent of women aged 45 and over experiencing mild hearing loss or greater. The GBD 2001 has estimated only the burden of moderate or greater hearing loss. Childhood-onset hearing loss is not included in this cause category, as most childhood hearing loss is due to congenital causes, infectious diseases, or other diseases or injuries, and is included as sequelae for such causes in the estimation of the burden of disease.

In both low- and middle-income countries and highincome countries, alcohol use disorders are among the 10 leading causes of $\operatorname{YLD}(3,0)$. This includes only the direct burden of alcohol dependence and problem use. The total attributable burden of disability due to alcohol use is much larger (see chapter 4 ).

More than 80 percent of global nonfatal health outcomes occur in developing countries, and high-mortality developing countries account for nearly half of all YLD. Although the prevalences of disabling conditions such as dementia and musculoskeletal disease are higher in countries with long life expectancies, this is offset by lower contributions to disability from conditions such as CVD, chronic respiratory 
Table 3.12 The 10 Leading Causes of YLD by Broad Income Group, 2001

\begin{tabular}{|c|c|c|c|c|c|}
\hline \multicolumn{3}{|c|}{ Low- and middle-income countries } & \multicolumn{3}{|c|}{ High-income countries } \\
\hline Cause & $\begin{array}{c}\text { YLD (millions } \\
\text { of years) }\end{array}$ & $\begin{array}{c}\text { Percentage of } \\
\text { total YLD }\end{array}$ & Cause & $\begin{array}{l}\text { YLD (millions of } \\
\text { years) }\end{array}$ & $\begin{array}{l}\text { Percentage } \\
\text { of total YLD }\end{array}$ \\
\hline 1 Unipolar depressive disorders & 43.22 & 9.1 & 1 Unipolar depressive disorders & 8.39 & 11.8 \\
\hline 2 Cataracts & 28.15 & 5.9 & 2 Alzheimer's and other dementias & 6.33 & 8.9 \\
\hline 3 Hearing loss, adult onset & 24.61 & 5.2 & 3 Hearing loss, adult onset & 5.39 & 7.6 \\
\hline 4 Vision disorders, age-related & 15.36 & 3.2 & 4 Alcohol use disorders & 3.77 & 5.3 \\
\hline 5 Osteoarthritis & 13.65 & 2.9 & 5 Osteoarthritis & 3.77 & 5.3 \\
\hline 6 Perinatal conditions & 13.52 & 2.8 & 6 Cerebrovascular disease & 3.46 & 4.9 \\
\hline 7 Cerebrovascular disease & 11.10 & 2.3 & 7 Chronic obstructive pulmonary disease & 2.86 & 4.0 \\
\hline 8 Schizophrenia & 10.15 & 2.1 & 8 Diabetes mellitus & 2.25 & 3.2 \\
\hline 9 Alcohol use disorders & 9.81 & 2.1 & 9 Endocrine disorders & 1.68 & 2.4 \\
\hline 10 Protein-energy malnutrition & 9.34 & 2.0 & 10 Vision disorders, age-related & 1.53 & 2.1 \\
\hline
\end{tabular}

Source: Authors' calculations.

Table 3.13 The 10 Leading Causes of YLD by Sex, Worldwide, 2001

\begin{tabular}{|c|c|c|c|c|c|}
\hline \multicolumn{3}{|l|}{ Males } & \multicolumn{3}{|c|}{ Females } \\
\hline Cause & $\begin{array}{l}\text { YLD (millions } \\
\text { of years) }\end{array}$ & $\begin{array}{c}\text { Percentage of } \\
\text { total YLD }\end{array}$ & Cause & $\begin{array}{l}\text { YLD (millions } \\
\text { of years) }\end{array}$ & $\begin{array}{l}\text { Percentage } \\
\text { of total YLD }\end{array}$ \\
\hline 1 Unipolar depressive disorders & 20.35 & 7.7 & 1 Unipolar depressive disorders & 31.26 & 11.0 \\
\hline 2 Hearing loss, adult onset & 14.96 & 5.6 & 2 Cataracts & 16.49 & 5.8 \\
\hline 3 Cataracts & 12.16 & 4.6 & 3 Hearing loss, adult onset & 15.03 & 5.3 \\
\hline 4 Alcohol use disorders & 11.50 & 4.3 & 4 Osteoarthritis & 10.83 & 3.8 \\
\hline 5 Cerebrovascular disease & 7.58 & 2.9 & 5 Vision disorders, age-related & 9.66 & 3.4 \\
\hline 6 Vision disorders, age-related & 7.23 & 2.7 & 6 Alzheimer's and other dementias & 9.46 & 3.3 \\
\hline 7 Perinatal conditions & 7.03 & 2.7 & 7 Cerebrovascular disease & 6.98 & 2.5 \\
\hline 8 Osteoarthritis & 6.59 & 2.5 & 8 Perinatal conditions & 6.91 & 2.4 \\
\hline 9 Chronic obstructive pulmonary disease & 6.55 & 2.5 & 9 Schizophrenia & 5.58 & 2.0 \\
\hline 10 Schizophrenia & 5.66 & 2.1 & 10 Bipolar disorder & 4.82 & 1.7 \\
\hline
\end{tabular}

Source: Authors' calculations.

diseases, and long-term sequelae of communicable diseases and nutritional deficiencies. In other words, people living in developing countries not only face shorter life expectancies than those in developed countries, but also live a larger proportion of their lives in poor health.

\section{Regional Variations in Healthy Life Expectancy}

In the original GBD study, Murray and Lopez (1996c) computed a form of health expectancy referred to as disabilityadjusted life expectancy using age- and sex-specific YLD rates and regional life tables to compute the expected equivalent years of healthy life in each region. Their results clearly demonstrated that populations with higher mortality also had higher prevalences of disability and lower health expectancies.
WHO has used a similar indicator, referred to as healthy life expectancy (HALE), to report on the average levels of population health for its 192 member countries (WHO 2004b). We calculated HALE at birth for regions in 2001 (figure 3.10) using the GBD 2001 estimates for YLD by region, age, and sex, together with information on health state prevalences and valuations from the WHO Multicountry Survey Study on Health and Responsiveness carried out in 2000 and 2001 (Ustun, Chatterji, Villanueva, and others 2003). For a description of the methods used to calculate HALE see Mathers, Salomon, and others (2003). Regional variations in HALE have also been discussed in more detail elsewhere, as have estimates of regional variations in increases in HALE associated with the elimination of selected health risks (Ezzati and others 2003; Mathers, Murray, and others 2003). 


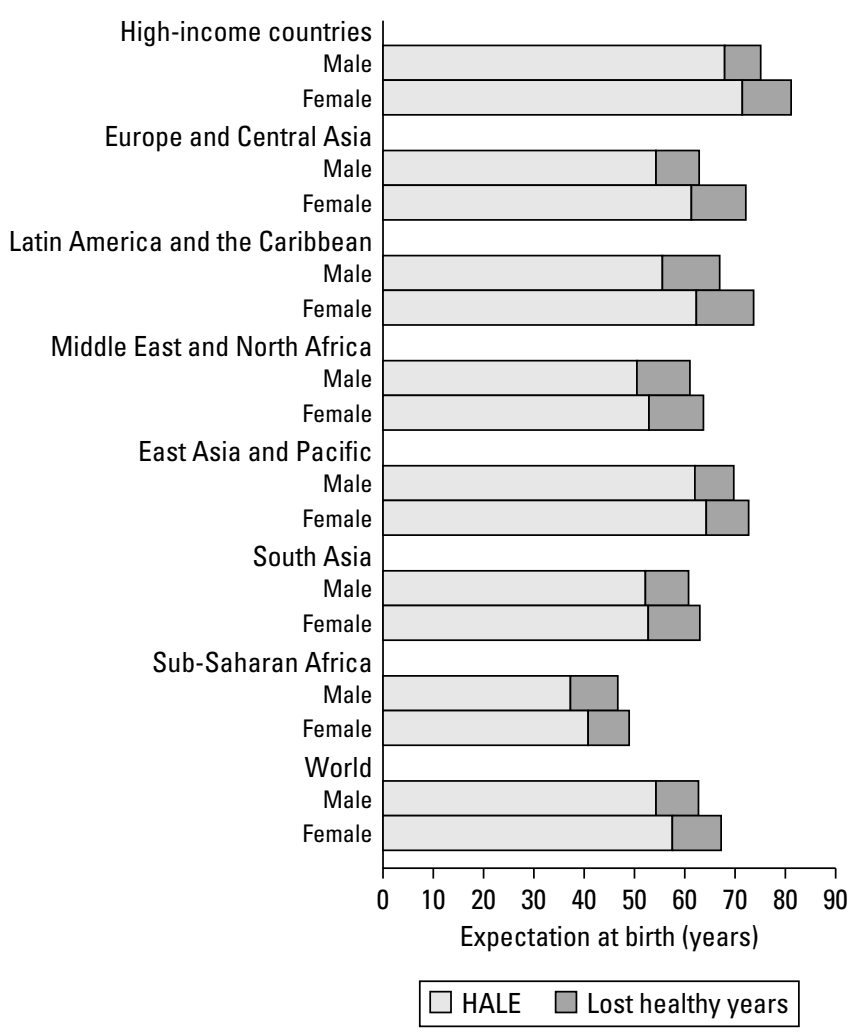

Source: Authors' calculations.

Figure 3.10 Life Expectancy, HALE, and Lost Healthy Years by Region and Sex, 2001

Overall, global HALE at birth in 2001 for males and females combined was 57.4 years, 7.5 years lower than total life expectancy at birth (figure 3.10). In other words, on average, poor health resulted in a loss of nearly eight years of healthy life globally. Global HALE at birth for females was only 2.7 years greater than that for males. In comparison, female life expectancy at birth was 4.2 years higher than that for males. Global HALE at age 60 was 12.7 years and 14.7 years for males and females, respectively, 4.3 years lower than total life expectancy at age 60 for males and 5.3 years lower for females.

HALE at birth ranged from a low of 40 years for males in Sub-Saharan Africa to more than 70 years for females in high-income countries. This reflects an almost twofold difference in HALE between major regional populations (figure 3.10). The equivalent "lost" healthy years (total life expectancy minus HALE) ranged from 15 percent of total life expectancy at birth in Sub-Saharan Africa to 8 percent in high-income countries. The sex gap was highest for Europe and Central Asia and lowest in the Middle East and North Africa.

\section{GLOBAL BURDEN OF DISEASE IN 2001}

This section provides an overview of the global and regional burden of disease in 2001 as measured in DALYs, more specifically, in DALYs $(3,0)$. As defined earlier, DALYs $(3,0)$ do not apply nonuniform age weights, but incorporate a 3 percent discount rate and should be distinguished from the DALYs $(3,1)$ used in the GBD results reported by WHO in recent world health reports. In 2001, the global average burden of disease across all regions was $250 \operatorname{DALYs}(3,0)$ per 1,000 population, of which almost two-thirds were due to premature death.

YLL varied dramatically across regions, with YLL rates nearly five times higher in Sub-Saharan Africa than in highincome countries (figure 3.11). In contrast, YLD rates were less varied, with Sub-Saharan Africa having 50 percent higher rates than high-income countries. South Asia and SubSaharan Africa together bore 45 percent of the total GBD in 2001, even though they account for only one-third of the world's population. East Asia and the Pacific is the "healthiest" of the low- and middle-income regions, with countries such as China now having life expectancies similar to those of many Latin American countries and higher than those in some European countries (see chapter 2).

Europe and Central Asia now experiences a higher burden of disease than all other low- and middle-income regions except South Asia and Sub-Saharan Africa. This reflects the sharp increase in adult male mortality and disability in the 1990s. A significant factor in this increase was the high incidence of male alcohol abuse, which led to high rates of accidents, violence, and CVD. From 1991 to 1994, the risk of premature death increased by 50 percent for Russian males (Gavrilova and others 2000; Semenova and

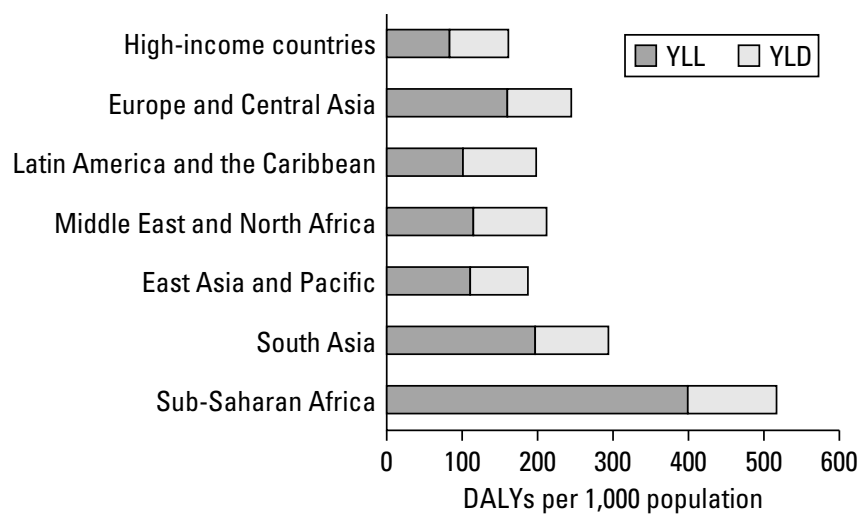

Source: Authors' calculations.

Figure 3.11 YLL, YLD, and DALYs by Region, 2001 


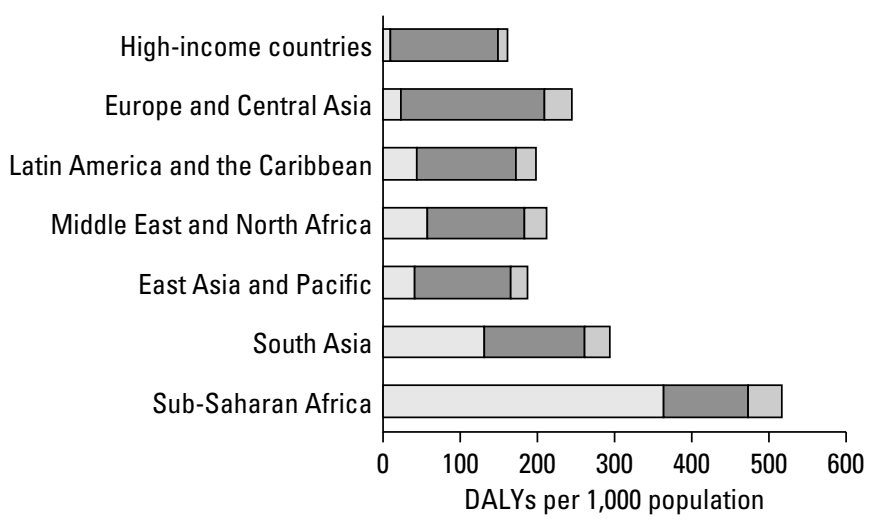

$\square$ Group I $\square$ Group II $\square$ Group III

Source: Authors' calculations.

Figure 3.12 Burden of Disease by Broad Cause Group and Region, 2001

others 2000; Shkolnikov, McKee, and Leon 2001). Between 1994 and 1998, life expectancy for males improved, but declined again significantly between 1998 and 2001 (Men and others 2003).

While countries in Europe and Central Asia have a substantially higher burden of noncommunicable disease than high-income countries (figure 3.12), they also have a higher burden due to Group I causes and Group III causes. Indeed, countries in Europe and Central Asia have the highest proportion of the burden due to injuries of all the regions, 14 percent, followed by the Middle East and North Africa.

\section{Leading Causes of the Burden of Disease in 2001}

The 20 leading causes of burden of disease for both sexes together are shown in table 3.14. While the two leading causes of death, IHD and cerebrovascular disease, remain among the top four causes of the burden of disease, four nonfatal condi- tions are also among the top 20 causes of burden: unipolar depressive disorders, adult-onset hearing loss, cataracts, and osteoarthritis. This once again illustrates the importance of taking nonfatal conditions into account, as well as deaths, when assessing the causes of loss of health in populations.

In 2001, the leading causes of the burden of disease in low- and middle-income countries were broadly similar to those for the world as a whole (table 3.15), and included six Group I causes among the top 10, but the leading causes in high- income countries consisted entirely of Group II conditions, including three (unipolar depressive disorders, adult-onset hearing loss, and alcohol use disorders) for which direct mortality is low.

\section{Age and Sex Differences in the Burden of Disease}

Measured in DALYs $(3,0)$, children younger than 15 accounted for 36 percent of the world's total burden of disease and injury in 2001 and adults ages 15 to 59 accounted for almost 50 percent. Low- and middle-income countries accounted for the vast majority of the disease burden for children (figure 3.13). While the proportion of the total burden of disease borne by adults ages 15 to 59 was the same in both groups of countries, adults older than 60 accounted for a significantly larger share of the disease burden in high-income countries.

Although injuries become more important for boys beyond infancy, the causes of the burden of disease are broadly similar for boys and girls. However, striking gender differences emerge in adulthood. In low- and middleincome countries, 5 of the 10 leading causes of DALYs $(3,0)$ for men ages 15 to 44 are injuries. Indeed, after HIV/AIDS, road traffic accidents were the second leading cause of the burden of disease for men in this age group. Other unintentional injuries and violence were the third and fourth

Table 3.14 The 20 Leading Causes of Global Burden of Disease, DALYs(3,0), 2001

\begin{tabular}{lccccc}
\hline Cause & $\begin{array}{c}\text { DALYs (millions } \\
\text { of years) }\end{array}$ & $\begin{array}{c}\text { Percentage of } \\
\text { total DALYs (3,0) }\end{array}$ & Cause & $\begin{array}{c}\text { DALYs (millions } \\
\text { of years) }\end{array}$ & $\begin{array}{c}\text { Percentage } \\
\text { of total DALYs (3,0) }\end{array}$ \\
\hline 1 Perinatal conditions & 90.48 & 5.9 & 11 Road traffic accidents & 35.06 & 2.3 \\
2 Lower respiratory infections & 85.92 & 5.6 & 12 Hearing loss, adult onset & 29.99 & 2.0 \\
3 Ischemic heart disease & 84.27 & 5.5 & 13 Cataracts & 28.64 & 1.9 \\
4 Cerebrovascular disease & 72.02 & 4.7 & 14 Congenital anomalies & 24.95 & 1.6 \\
5 HIV/AIDS & 71.46 & 4.7 & 15 Measles & 23.11 & 1.5 \\
6 Diarrheal diseases & 59.14 & 3.9 & 16 Self-inflicted injuries & 20.26 \\
7 Unipolar depressive disorders & 51.84 & 3.4 & 17 Diabetes mellitus & 1.3 \\
8 Malaria & 39.97 & 2.6 & 18 Violence & 18.90 \\
9 Chronic obstructive pulmonary disease & 38.74 & 2.5 & 19 Osteoarthritis & 17.45 \\
10 Tuberculosis & 36.09 & 2.3 & 20 Alzheimer's and other dementias & 17.11 \\
\hline
\end{tabular}

Source: Authors' calculations. 
Table 3.15 The 10 Leading Causes of Burden of Disease, by Broad Income Group, 2001

\begin{tabular}{|c|c|c|c|c|c|}
\hline \multicolumn{3}{|c|}{ Low- and middle-income countries } & \multicolumn{3}{|c|}{ High-income countries } \\
\hline Cause & $\begin{array}{c}\text { DALYs (millions } \\
\text { of years) }\end{array}$ & $\begin{array}{l}\text { Percentage of } \\
\text { total DALYs }(3,0)\end{array}$ & Cause & $\begin{array}{c}\text { DALYs (millions } \\
\text { of years) }\end{array}$ & $\begin{array}{c}\text { Percentage of } \\
\text { total DALYs }(3,0)\end{array}$ \\
\hline 1 Perinatal conditions & 89.07 & 6.4 & 1 Ischemic heart disease & 12.39 & 8.3 \\
\hline 2 Lower respiratory infections & 83.61 & 6.0 & 2 Cerebrovascular disease & 9.35 & 6.3 \\
\hline 3 Ischemic heart disease & 71.88 & 5.2 & 3 Unipolar depressive disorders & 8.41 & 5.6 \\
\hline 4 HIV/AIDS & 70.80 & 5.1 & 4 Alzheimer's and other dementias & 7.47 & 5.0 \\
\hline 5 Cerebrovascular disease & 62.67 & 4.5 & 5 Trachea, bronchus, and lung cancers & 5.40 & 3.6 \\
\hline 6 Diarrheal diseases & 58.70 & 4.2 & 6 Hearing loss, adult onset & 5.39 & 3.6 \\
\hline 7 Unipolar depressive disorders & 43.43 & 3.1 & 7 Chronic obstructive pulmonary disease & 5.28 & 3.5 \\
\hline 8 Malaria & 39.96 & 2.9 & 8 Diabetes mellitus & 4.19 & 2.8 \\
\hline 9 Tuberculosis & 35.87 & 2.6 & 9 Alcohol use disorders & 4.17 & 2.8 \\
\hline 10 Chronic obstructive pulmonary disease & 33.45 & 2.4 & 10 Osteoarthritis & 3.79 & 2.5 \\
\hline
\end{tabular}

Source: Authors' calculations
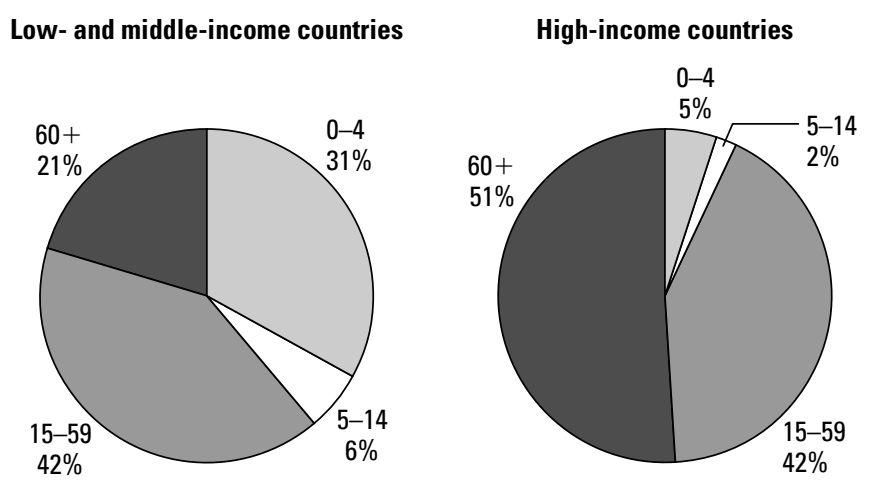

Source: Authors' calculations.

Note: The disease burden is measured in DALYs.

Figure 3.13 Age Distribution of Burden of Disease by Income Group, 2001

leading causes, with self-inflicted injuries and war also appearing in the top 10 causes. Injuries were also important for women ages 15 to 44, although road traffic accidents were the 10th leading cause, preceded by other unintentional injuries in 4th place and self-inflicted injuries in 6th place. Unipolar depressive disorders were the second leading cause of the burden for women in this age group, after HIV/AIDS.

\section{The Growing Burden of Noncommunicable Diseases}

The burden of noncommunicable diseases is increasing, accounting for nearly half the global burden of disease for all ages, a 10 percent increase from estimated levels in 1990. While the proportion of the burden from noncommunicable disease in high-income countries has remained stable at around 85 percent in adults ages 15 and older, the proportion in middle-income countries has already exceeded 70 percent. Surprisingly, almost 50 percent of the adult disease burden in low- and middle-income countries is now attributable to noncommunicable disease. Population aging and changes in the distribution of risk factors have accelerated the epidemic of noncommunicable disease in many developing countries.

CVD accounted for 13 percent of the disease burden among adults ages 15 and older in 2001. IHD and cerebrovascular disease (stroke) were the two leading causes of mortality and the disease burden among adults ages 60 and older and were also among the top 10 causes of the disease burden in adults ages 15 to 59. In low- and middle income countries, IHD and cerebrovascular disease (stroke) were together responsible for 15 percent of the disease burden in those ages 15 and older, and DALYs $(3,0)$ rates were higher for men than for women.

The proportion of the burden among adults ages 15 and older attributable to cancer was 6 percent in low- and middle income countries and 14 percent in high-income countries in 2001. Of the 7.1 million cancer deaths estimated to have occurred in that year, 17 percent, or 1.2 million, were attributable to lung cancer alone, and of these, three-quarters occurred among men. The number of cases of lung cancer increased nearly 30 percent since 1990, largely reflecting the emergence of the tobacco epidemic in low- and middleincome countries.

Stomach cancer, which until recently was the leading site of cancer mortality worldwide, has been declining in all parts of the world where trends can be reliably assessed, and in 2001 caused 842,000 deaths, or about two-thirds as many as lung cancer. Liver cancer was the third leading site, with 
607,000 deaths in 2001, more than 60 percent of them in the East Asia and Pacific region. Among women, the leading cause of cancer deaths was breast cancer. Breast cancer survival rates have been improving during the past decade, but the chance of survival varies according to the coverage of and access to secondary prevention. Globally, neuropsychiatric conditions accounted for 19 percent of the disease burden among adults, primarily from nonfatal health outcomes.

\section{Injuries: The Hidden Epidemic}

Injuries, both unintentional and intentional, primarily affect young adults, and often result in severe, disabling sequelae. In 2001, injuries accounted for 16 percent of the adult burden of ill-health and premature death worldwide. In parts of Europe and Central Asia, Latin America and the Caribbean, and the Middle East and North Africa, more than 30 percent of the entire disease and injury burden among male adults ages 15 to 44 was attributable to injuries, and road traffic accidents, violence, and self-inflicted injuries were all among the top 10 leading causes of the burden of disease. Globally, road traffic accidents were the third leading cause of burden in the same age and sex group, preceded only by HIV/AIDS and unipolar depression. The burden of road traffic accidents has been increasing, especially in the developing countries of Sub-Saharan Africa and South and Southeast Asia, and particularly affects males.

Intentional injuries, which include self-inflicted injuries and suicide, violence, and war, accounted for an increasing share of the burden, especially among economically productive young adults. In developed countries, suicides accounted for the largest share of the intentional injury burden, whereas in developing regions, violence and war were the major sources. The former Soviet Union and other high-mortality countries of Eastern Europe have rates of death and disability resulting from injury among males that are similar to those in Sub-Saharan Africa.

\section{Regional Variations in the Burden of Disease}

The tables in annex 3C show estimated total DALYs $(3,0)$ by age, sex, and cause in 2001 for each region and for the world as a whole. Table 3.16 summarizes the 10 leading causes of burden for each of the low- and middle-income regions.

In 2001, IHD and stroke dominated the burden of disease in Europe and Central Asia, and together accounted for more than a quarter of the total disease burden. In contrast, in Latin America and the Caribbean, these diseases accounted for 8 percent of disease burden. However, this region also had high levels of diabetes and endocrine disorders compared with other regions. Violence was the third leading cause of burden in Latin America and Caribbean countries, but did not reach the top 10 in any other region.

HIV/AIDS was the leading cause of the burden of disease in Sub-Saharan Africa, followed by malaria. Seven other Group I causes also appear in the top 10 causes for this region, with road traffic accidents being the only nonGroup I cause.

Group I, II, and III causes all appear among the top 10 causes of the disease burden for the Middle East and North Africa. Of particular note, road traffic accidents were the third leading cause and congenital anomalies were the seventh leading cause.

Group I causes of the disease burden remained dominant in South Asia, and this burden fell particularly on children, but noncommunicable diseases such as IHD, stroke, and chronic obstructive pulmonary disease also featured in the list of top 10 causes.

In East Asia and the Pacific, stroke was the leading cause of disease burden in 2001, with IHD in fourth place, although Group I causes such as conditions arising during the perinatal period, TB, lower respiratory infections, and diarrheal diseases remained important.

\section{DISCUSSION AND CONCLUSIONS}

The analysis presented in this chapter has confirmed some of the conclusions of the original GBD study about the importance of including nonfatal outcomes in a comprehensive assessment of global population health, and has also confirmed the growing importance of noncommunicable diseases in low- and middle-income countries. However, it has also documented dramatic changes in population health in some regions since 1990. The key findings include the following:

- HIV/AIDS is now the fourth leading cause of the burden of disease globally and the leading cause in Sub-Saharan Africa.

- In low- and middle-income countries, the epidemiological transition has resulted in a 20 percent reduction in the per capita disease burden due to Group I causes since 1990. Without the HIV/AIDS epidemic, this reduction would have been closer to 30 percent. Several of the "traditional" infectious diseases, such as TB and malaria, have not declined, in part because of weak public health services and the increased numbers of people with immune systems weakened by HIV/AIDS. 
Table 3.16 The 10 Leading Causes of the Burden of Disease in Low- and Middle-Income Countries, by Region, 2001

\begin{tabular}{|c|c|c|c|}
\hline East Asia and Pacific & $\begin{array}{l}\text { Percentage of } \\
\text { total DALYs }(3,0)\end{array}$ & Europe and Central Asia & $\begin{array}{c}\text { Percentage of } \\
\text { total DALYs }(3,0)\end{array}$ \\
\hline 1 Cerebrovascular disease & 7.5 & 1 Ischemic heart disease & 15.9 \\
\hline 2 Perinatal conditions & 5.4 & 2 Cerebrovascular disease & 10.8 \\
\hline 3 Chronic obstructive pulmonary disease & 5.0 & 3 Unipolar depressive disorders & 3.7 \\
\hline 4 Ischemic heart disease & 4.1 & 4 Self-inflicted injuries & 2.3 \\
\hline 5 Unipolar depressive disorders & 4.1 & 5 Hearing loss, adult onset & 2.2 \\
\hline 6 Tuberculosis & 3.1 & 6 Chronic obstructive pulmonary disease & 2.0 \\
\hline 7 Lower respiratory infections & 3.1 & 7 Trachea, bronchus, and lung cancers & 2.0 \\
\hline 8 Road traffic accidents & 3.0 & 8 Osteoarthritis & 2.0 \\
\hline 9 Cataracts & 2.8 & 9 Road traffic accidents & 1.9 \\
\hline 10 Diarrheal diseases & 2.5 & 10 Poisonings & 1.9 \\
\hline Latin America and the Caribbean & $\begin{array}{l}\text { Percentage of } \\
\text { total DALYs }(3,0)\end{array}$ & Middle East and North Africa & $\begin{array}{l}\text { Percentage of } \\
\text { total DALYs }(3,0)\end{array}$ \\
\hline 1 Perinatal conditions & 6.0 & 1 Ischemic heart disease & 6.6 \\
\hline 2 Unipolar depressive disorders & 5.0 & 2 Perinatal conditions & 6.3 \\
\hline 3 Violence & 4.9 & 3 Road traffic accidents & 4.6 \\
\hline 4 Ischemic heart disease & 4.2 & 4 Lower respiratory infections & 4.5 \\
\hline 5 Cerebrovascular disease & 3.8 & 5 Diarrheal diseases & 3.9 \\
\hline 6 Endocrine disorders & 3.0 & 6 Unipolar depressive disorders & 3.1 \\
\hline 7 Lower respiratory infections & 2.9 & 7 Congenital anomalies & 3.1 \\
\hline 8 Alcohol use disorders & 2.8 & 8 Cerebrovascular disease & 3.0 \\
\hline 9 Diabetes mellitus & 2.7 & 9 Vision disorders, age-related & 2.7 \\
\hline 10 Road traffic accidents & 2.6 & 10 Cataracts & 2.3 \\
\hline South Asia & $\begin{array}{l}\text { Percentage of } \\
\text { total DALYs }(3,0)\end{array}$ & Sub-Saharan Africa & $\begin{array}{c}\text { Percentage of } \\
\text { total DALYs }(3,0)\end{array}$ \\
\hline 1 Perinatal conditions & 9.2 & $1 \mathrm{HIV} / \mathrm{AIDS}$ & 16.5 \\
\hline 2 Lower respiratory infections & 8.4 & 2 Malaria & 10.3 \\
\hline 3 Ischemic heart disease & 6.3 & 3 Lower respiratory infections & 8.8 \\
\hline 4 Diarrheal diseases & 5.4 & 4 Diarrheal diseases & 6.4 \\
\hline 5 Unipolar depressive disorders & 3.6 & 5 Perinatal conditions & 5.8 \\
\hline 6 Tuberculosis & 3.4 & 6 Measles & 3.9 \\
\hline 7 Cerebrovascular disease & 3.2 & 7 Tuberculosis & 2.3 \\
\hline 8 Cataracts & 2.3 & 8 Road traffic accidents & 1.8 \\
\hline 9 Chronic obstructive pulmonary disease & 2.3 & 9 Pertussis & 1.8 \\
\hline 10 Hearing loss, adult onset & 2.0 & 10 Protein-energy malnutrition & 1.5 \\
\hline
\end{tabular}

Source: Authors' calculations.

- The per capita disease burden in Europe and Central Asia increased by nearly 40 percent during 1990-2001, meaning that this region now has worse health than all other regions except South Asia and Sub-Saharan Africa. The unexpected increase in the disease burden, and the concomitant reduction in life expectancy, in countries of this region appear to be related to such factors as alcohol abuse, suicide, and violence, which seem to be associated with societies facing dramatic social and economic changes. The rapidity of these declines has dramatically changed our perceptions of the time frames within which substantial changes in the burden of chronic disease can occur and of the potential for such adverse health trends to occur elsewhere.
- Adults under the age of 70 in low- and middle-income countries face a greater risk of death from noncommunicable diseases than adults of the same age in high-income countries.

- In Europe and Central Asia, Latin America and the Caribbean, and the Middle East and North Africa, more than 30 percent of the entire disease burden among male adults ages 15 to 44 is attributable to injuries, including road traffic accidents, violence, and self-inflicted injuries. In addition, injury deaths are noticeably higher for women in some parts of Asia and the Middle East and North Africa than in other regions, partly because of high levels of suicide and violence. Combined with higher rates of infant and child mortality for girls, 
this results in a narrower differential between male and female healthy life expectancy than in any other region.

- Sense organ disorders, principally hearing and sight loss, contribute significantly to disability in all regions of the world.

- Levels of nonfatal health loss are proportionately greater in low- and middle-income countries than in highincome countries, contrary to the perception that disability is associated with older populations. The gap between healthy life expectancy and total life expectancy is proportionately highest for the low-income countries.

The analysis presented in this chapter has aimed to produce a comprehensive and detailed assessment of the global burden of disease, based on all available relevant data. It has attempted to maximize the use of high-quality, populationbased data, and for regions and causes for which data are sparse has used the available evidence and the best available methods to make inferences and to assess the uncertainty in resulting estimates (see chapter 5). The need for internal consistency between estimates of incidence, prevalence, case fatality rates, and mortality rates for a given disease and for consistency across diseases and injuries with known total levels of mortality are crucial strategies for making the best use of multiple sources of uncertain and potentially biased data.

The data inputs used for the GBD 2001 estimation of global and regional causes of death have been summarized in tables 3.1, 3.2, and 3.5. In excess of 770 country-years of death registration data and more than 3,000 additional sources of information on levels of child and adult mortality and on specific causes of death were used to estimate global and regional patterns of mortality. Together with the more than 8,500 data sources used for the estimation of YLD, the GBD 2001 has incorporated information from more than 10,000 data sets relating to population health and mortality. This represents the largest synthesis of global information on population health carried out to date.

Despite the perceptions of some critics that the GBD study is inadequately empirically based for some regions, particularly Sub-Saharan Africa (Cooper and others 1998), it is notable that fully one-third of the more than 10,000 data sources used relate to Sub-Saharan African populations, albeit with the serious limitations on the information available on mortality noted earlier. We believe that the GBD studies have demonstrated the importance of including assessments of all causes of the disease and injury burden, even in the face of limited or missing data, to ensure that a comprehensive overview is provided to gain a better understanding of the importance of specific diseases and risk factors in causing loss of health. Otherwise, limitations in the evidence base for certain causes or regions might lead to their omission, and hence to the conclusion that they cause no burden, thereby presenting health decision makers with a misleading picture.

Nevertheless, the fact that estimates are possible does not obviate the need to put a higher priority on addressing the serious lack of information on levels of adult mortality and causes of death in some regions, particularly Sub-Saharan Africa. The key need for countries is to establish a system that registers the most common causes of death for the entire population without serious biases (such as an emphasis on urban mortality), in which there is reasonable confidence, and which yields timely data. Complete VR with annual population updates is the ideal system to generate this information, but it is not essential. Recent experience in countries such as China, India, and Tanzania suggests that sample registration based on a representative set of surveillance sites, and with appropriate controls and reporting procedures, can yield extremely useful information about levels, patterns, and causes of mortality for large populations (Setel and others 2005; Yang and others 2005). Low- and middleincome countries can benefit from the advantages of death registration without implementing a system of complete population coverage and medical certification (Rao, Bradshaw, and Mathers 2004). To support such systems, priority needs to be given to developing a standardized reporting form for verbal autopsies and to implementing validation studies to assess the reliability and accuracy of verbal autopsy methods.

Improved verbal autopsy methods will also contribute to improving the accuracy of estimates of the causes of child deaths under five, the majority of which occur in countries without useable death registration data. As discussed in chapters 5 and 6 , new data and syntheses for major causes of child death may result in future revisions to the estimates of child deaths for certain causes.

There is also a lack of good population-based epidemiological data for developing regions, particularly for noncommunicable diseases. For example, even though IHD and stroke are among the leading causes of the burden of disease in most regions, few recent and reliable sources of information on the prevalence and severity distribution of chronic cardiovascular conditions and long-term disability following stroke are available outside the high-income countries. Similarly, even in high-income countries, few population-based studies of the prevalence of chronic lung disease or musculoskeletal conditions have been carried out. 
Cross-national surveys, such as WHO's World Health Survey conducted during 2002 and 2003 in 73 countries, will fill some information gaps for some chronic diseases and mental disorders (Ustun, Chatterji, Mechbal, and others 2003). However, there remain significant issues that will need to be addressed relating to the comparability of prevalence data derived from self-reported survey data on symptoms of mental disorders, angina, and other chronic diseases.

Lack of information has resulted in limitations in the disease models used to estimate the burden of disease for some causes. Future iterations of burden of disease analysis will need to review disease models and sequelae chosen for estimating YLD to ensure that the best available estimates of the disease burden for each cause continue to remain based on current knowledge and data.

A particular difficulty is how to measure and characterize the average health states associated with sequelae. This is partly an issue of valuation of health states for the construction of disability weights, and partly an issue of lack of information on the population-level distribution of outcomes and the severity of health states. To a large extent, the disability weights used here derive from the original GBD study (Murray 1996), where typically an average disability weight was estimated for disease sequelae averaged across the distribution of outcomes, in some cases separately for treated and untreated cases. Stouthard and others (1997) have gone further in assessing disability weights for a range of severity levels of outcomes for a particular sequela, thereby allowing the overall final disability weight for a sequela to take account of regional variations in the severity distribution of outcomes.

The 2001 WHO Multicountry Survey Study collected health state valuation data on more than 500,000 health states from respondents in 71 countries, which Salomon and Murray (2004) used to construct a health state valuation function. The World Health Survey also included a health state valuation module, and analysis of the resulting data is under way (Salomon, Murray, and others 2003). In the next iteration of burden of disease analysis, it should be feasible to use health state valuations based on such survey data, together with descriptions of outcomes associated with disease sequelae, to produce updated disability weights that take into account not only the available information on health state distributions for disease sequelae, but also the health state preferences of people from all regions. A particular issue is the measurement of disability weights for low severity but highly prevalent conditions, such as anemia and hearing loss, where the current disability weights are small but quite uncertain and are multiplied by large prevalences (see chapter 5).

Burden of disease analysis provides a comprehensive, comparative overview of the state of population health and the factors affecting the health of populations. The 2001 GBD study is an expanded effort compared with the original 1990 study, with the incorporation of much new data and a greater understanding of the limitations of routinely available data sets. Nevertheless, substantial uncertainty remains about the comparative burden of diseases and injuries in many parts of the world that has significantly greater consequences for policy than the inclusion or otherwise of social choices such as age weighting in the basic burden of disease metric. We can conclude with some certainty that major causes of death and disability, such as tobacco and HIV/AIDS, are global pandemics and are likely to become more widespread unless control programs are more widely implemented. However, we remain substantially uncertain about the true levels of the disease burden from chronic lung disease, heart disease, stroke, mental disorders, various forms of injury, and a number of other key health concerns. International health agencies such as WHO and public health and epidemiological researchers need to make a concerted effort to improve data collection, and hence knowledge, about the true extent of the disease burden worldwide. Even efforts that substantially reduce uncertainty will be a major advance toward this goal.

With rising pressure on resources for health in all countries, priority setting in the health sector will increasingly depend on comprehensive, comparative information about the impact of diseases, injuries, and risk factors on population health. The burden of disease framework, with 15 years of development and application in numerous countries across the globe, offers the best, indeed the only, approach to comprehensively assess the impact of conditions and exposures that health systems need to deal with if population health is to improve rapidly. Yet to be even more useful for setting and monitoring global health priorities, a more concerted effort is needed to obtain and critically assess data sets on the health of populations in all countries. This must be a key focus of future efforts to assess the burden of disease. With WHO now giving greater emphasis to working with countries on capacity building and on specific organizational intervention priorities, new global partners such as the Ellison Institute for Global Health (Horton 2005) are urgently required to provide stewardship and guarantee that the evidence base for health policy and priority setting will develop at a pace commensurate with need. 


\section{ANNEX 3A: Definitions, Mortality Data Sources, and Disability Weights}

\section{Table 3A.1 Regional Reporting Categories for the Disease Control Priorities Project}

\begin{tabular}{|c|c|}
\hline Region & Countries included \\
\hline East Asia and Pacific & $\begin{array}{l}\text { American Samoa, Cambodia, China, Fiji, Indonesia, Kiribati, Democratic People's Republic of Korea, Lao People's Democratic } \\
\text { Republic, Malaysia, Marshall Islands, Federated States of Micronesia, Mongolia, Myanmar, Palau, Papua New Guinea, } \\
\text { Philippines, Samoa, Solomon Islands, Thailand, Timor-Leste, Tonga, Vanuatu, Vietnam }\end{array}$ \\
\hline Europe and Central Asia & $\begin{array}{l}\text { Albania, Armenia, Azerbaijan, Belarus, Bosnia and Herzegovina, Bulgaria, Croatia, Czech Republic, Estonia, Georgia, Hungary, } \\
\text { Isle of Man, Kazakhstan, Kyrgyz Republic, Latvia, Lithuania, the former Yugoslav Republic of Macedonia, Moldova, Poland, } \\
\text { Romania, Russian Federation, Serbia and Montenegro, Slovak Republic, Tajikistan, Turkey, Turkmenistan, Ukraine, Uzbekistan }\end{array}$ \\
\hline High-income countries & $\begin{array}{l}\text { Andorra, Aruba, Australia, Austria, The Bahamas, Bahrain, Belgium, Bermuda, Brunei Darussalam, Canada, Cayman Islands, } \\
\text { Channel Islands, Cyprus, Denmark, Faeroe Islands, Finland, France, French Polynesia, Germany, Greece, Greenland, Guam, } \\
\text { Iceland, Ireland, Israel, Italy, Japan, Kuwait, Liechtenstein, Luxembourg, Monaco, the Netherlands, Netherlands Antilles, New } \\
\text { Caledonia, New Zealand, Northern Mariana Islands, Norway, Portugal, Qatar, Republic of Korea, San Marino, Singapore, } \\
\text { Slovenia, Spain, Sweden, Switzerland, United Arab Emirates, United Kingdom, United States, U.S. Virgin Islands }\end{array}$ \\
\hline $\begin{array}{l}\text { Latin America and } \\
\text { the Caribbean }\end{array}$ & $\begin{array}{l}\text { Antigua and Barbuda, Argentina, Barbados, Belize, Bolivia, Brazil, Chile, Colombia, Costa Rica, Cuba, Dominica, Dominican } \\
\text { Republic, Ecuador, El Salvador, Grenada, Guatemala, Guyana, Haiti, Honduras, Jamaica, Mexico, Nicaragua, Panama, Paraguay, } \\
\text { Peru, Puerto Rico, St. Kitts and Nevis, St. Lucia, St. Vincent and the Grenadines, Suriname, Trinidad and Tobago, Uruguay, } \\
\text { República Bolivariana de Venezuela }\end{array}$ \\
\hline $\begin{array}{l}\text { Middle East and } \\
\text { North Africa }\end{array}$ & $\begin{array}{l}\text { Algeria, Djibouti, Arab Republic of Egypt, Islamic Republic of Iran, Iraq, Jordan, Lebanon, Libya, Malta, Morocco, Oman, Saudi } \\
\text { Arabia, Syrian Arab Republic, Tunisia, West Bank and Gaza, Republic of Yemen }\end{array}$ \\
\hline South Asia & Afghanistan, Bangladesh, Bhutan, India, Maldives, Nepal, Pakistan, Sri Lanka \\
\hline Sub-Saharan Africa & $\begin{array}{l}\text { Angola, Benin, Botswana, Burkina Faso, Burundi, Cameroon, Cape Verde, Central African Republic, Chad, Comoros, Democratic } \\
\text { Republic of Congo, Republic of Congo, Côte d'Ivoire, Equatorial Guinea, Eritrea, Ethiopia, Gabon, Gambia, Ghana, Guinea, } \\
\text { Guinea-Bissau, Kenya, Lesotho, Liberia, Madagascar, Malawi, Mali, Mauritania, Mauritius, Mozambique, Namibia, Niger, } \\
\text { Nigeria, Rwanda, São Tomé and Principe, Senegal, Seychelles, Sierra Leone, Somalia, South Africa, Sudan, Swaziland, } \\
\text { Tanzania, Togo, Uganda, Zambia, Zimbabwe }\end{array}$ \\
\hline Other & $\begin{array}{l}\text { Anguilla, British Virgin Islands, Cook Islands, Falkland Islands, French Guiana, Gibraltar, Guadeloupe, Holy See (Vatican City), } \\
\text { Martinique, Montserrat, Nauru, Niue, Pitcairn, Réunion, St. Helena, St. Pierre et Miquelon, Tokelau, Turks and Caicos Islands, } \\
\text { Tuvalu, Wallis and Futuna Islands, Western Sahara }\end{array}$ \\
\hline
\end{tabular}

Source: Jamison and others 2006 
Table 3A.2 GBD Cause Categories and ICD Codes

\begin{tabular}{|c|c|c|c|}
\hline Code & GBD cause name & ICD-9 code & ICD-10 code \\
\hline U000 & All causes & & \\
\hline U001 & $\begin{array}{l}\text { I. Communicable, maternal, perinatal, and } \\
\text { nutritional conditions }{ }^{a}\end{array}$ & $\begin{array}{l}001-139,243,260-269,279.5 \\
280-281,285.9,320-323 \\
381-382,460-465,466,480-487, \\
614-616,630-676,760-779\end{array}$ & $\begin{array}{l}\text { A00-B99, G00-G04, N70-N73, J00-J06, } \\
\text { J10-J18, J20-J22, H65-H66, 000-099, } \\
\text { P00-P96, E00-E02, E40-E46, E50, } \\
\text { D50-D53, D64.9, E51-64 }\end{array}$ \\
\hline U002 & A. Infectious and parasitic diseases & $\begin{array}{l}001-139,279.5,320-323,614-616, \\
771.3\end{array}$ & A00-B99, G00, G03-G04, N70-N73 \\
\hline U003 & 1. Tuberculosis & 010-018, 137 & A15-A19, B90 \\
\hline U004 & $\begin{array}{l}\text { 2. Sexually transmitted diseases } \\
\text { excluding HIV/AIDS }\end{array}$ & 090-099, 614-616 & A50-A64, N70-N73 \\
\hline U005 & a. Syphilis & 090-097 & A50-A53 \\
\hline U006 & b. Chlamydia & - & A55-A56 \\
\hline U007 & c. Gonorrhea & 098 & A54 \\
\hline U008 & d. Other sexually transmitted diseases & $099,614-616$ & A57-A64, N70-N73 \\
\hline U009 & 3. HIV/AIDS & $279.5(=042-044)$ & B20-B24 \\
\hline U010 & 4. Diarrheal diseases & $001,002,004,006-009$ & $\mathrm{~A} 00, \mathrm{~A} 01, \mathrm{~A} 03, \mathrm{~A} 04, \mathrm{~A} 06-\mathrm{A} 09$ \\
\hline U011 & 5. Childhood-cluster diseases & $032,033,037,045,055,138,771.3$ & A33-A37, A80, B05, B91 \\
\hline U012 & a. Pertussis & 033 & A37 \\
\hline U013 & b. Poliomyelitis & 045,138 & A80, B91 \\
\hline U014 & c. Diphtheria & 032 & A36 \\
\hline U015 & d. Measles & 055 & B05 \\
\hline U016 & e. Tetanus & $037,771.3$ & A33-A35 \\
\hline U017 & 6. Meningitis & $036,320-322$ & A39, G00, G03 \\
\hline U018 & 7. Hepatitis B & $070.2-070.9$ & B16-B19 (minus B17.1, B18.2) \\
\hline U019 & Hepatitis C & - & B17.1, B18.2 \\
\hline U020 & 8. Malaria & 084 & B50-B54 \\
\hline U021 & 9. Tropical-cluster diseases & $085,086,120,125.0,125.1,125.3$ & B55-B57, B65, B73, B74.0-B74.2 \\
\hline U022 & a. Trypanosomiasis & 086.3, 086.4, 086.5, & B56 \\
\hline U023 & b. Chagas' disease & $086.0,086.1,086.2,086.9$ & B57 \\
\hline U024 & c. Schistosomiasis & 120 & B65 \\
\hline U025 & d. Leishmaniasis & 085 & B55 \\
\hline U026 & e. Lymphatic filariasis & $125.0,125.1$ & B74.0-B74.2 \\
\hline U027 & f. Onchocerciasis & 125.3 & $\mathrm{~B} 73$ \\
\hline U028 & 10. Leprosy & 030 & A30 \\
\hline U029 & 11. Dengue & 061 & A90-A91 \\
\hline U030 & 12. Japanese encephalitis & 062.0 & A83.0 \\
\hline U031 & 13. Trachoma & 076 & A71 \\
\hline U032 & 14. Intestinal nematode infections & $126-129$ & B76-B81 \\
\hline U033 & a. Ascariasis & 127.0 & B77 \\
\hline U034 & b. Trichuriasis & 127.3 & B79 \\
\hline U035 & $\begin{array}{l}\text { c. Hookworm disease } \\
\text { (Ancylostomiasis and necatoriasis) }\end{array}$ & 126 & B76 \\
\hline U036 & Other intestinal infections & $127.1,127.2,127.4-127.9,128,129$ & B78, B80, B81 \\
\hline
\end{tabular}


Table 3A.2 Continued

\begin{tabular}{|c|c|c|c|}
\hline Code & GBD cause name & ICD-9 code & ICD-10 code \\
\hline U037 & Other infectious diseases & $\begin{array}{l}003,005,020-027,031,034,035, \\
038-041,046-049,050-054, \\
056-057,060,062.1-066, \\
070.0-070.1,071-075,077-079, \\
080-083,087-088,100-104, \\
110-118,121-124,125.2,125.4 \\
125.5,125.6,125.7,125.9,130-136, \\
139,323\end{array}$ & $\begin{array}{l}\text { A02, A05, A20-A28, A31, A32, A38, } \\
\text { A40-A49, A65-A70, A74-A79, A81, A82, } \\
\text { A83.1-A83.9, A84-A89, A92-A99, } \\
\text { B00-B04, B06-B15, B25-B49, B58-B60, } \\
\text { B64, B66-B72, B74.3-B74.9, B75, } \\
\text { B82-B89, B92-B99, G04 }\end{array}$ \\
\hline U038 & B. Respiratory infections & 460-466, 480-487, 381-382 & J00-J06, J10-J18, J20-J22, H65-H66 \\
\hline U039 & 1. Lower respiratory infections & $466,480-487$ & J10-J18, J20-J22 \\
\hline U040 & 2. Upper respiratory infections & 460-465 & J00-J06 \\
\hline U041 & 3. Otitis media & $381-382$ & H65-H66 \\
\hline U042 & C. Maternal conditions & $630-676$ & 000-099 \\
\hline U043 & 1. Maternal hemorrhage & $640,641,666$ & 044-046, 067, 072 \\
\hline U044 & 2. Maternal sepsis & 670 & 085-086 \\
\hline U045 & 3. Hypertensive disorders of pregnancy & 642 & 010-016 \\
\hline U046 & 4. Obstructed labor & 660 & 064-066 \\
\hline U047 & 5. Abortion & $630-639$ & 000-007 \\
\hline U048 & Other maternal conditions & $\begin{array}{l}643-659,661-665,667-669 \\
671-676\end{array}$ & $\begin{array}{l}\text { 020-043, 047-063, 068-071, 073-075, } \\
087-099\end{array}$ \\
\hline U049 & $\begin{array}{l}\text { D. Conditions arising during the perinatal } \\
\text { period }\end{array}$ & 760-779 (minus 771.3) & P00-P96 \\
\hline U050 & 1. Low birthweight & $764-765$ & P05-P07 \\
\hline U051 & 2. Birth asphyxia and birth trauma & $767-770$ & P03, P10-P15, P20-P29 \\
\hline U052 & Other perinatal conditions & $\begin{array}{l}\text { 760-763, 766, } 771 \text { (minus 771.3), } \\
\text { 772-779 }\end{array}$ & P00-P02, P04, P08, P35-P96 \\
\hline U053 & E. Nutritional deficiencies & $243,260-269,280-281,285.9$ & $\begin{array}{l}\text { E00-E02, E40-E46, E50, D50-D53, D64.9, } \\
\text { E51-E64 }\end{array}$ \\
\hline U054 & 1. Protein-energy malnutrition & $260-263$ & E40-E46 \\
\hline U055 & 2. Iodine deficiency & 243 & E00-E02 \\
\hline U056 & 3. Vitamin A deficiency & 264 & E50 \\
\hline U057 & 4. Iron-deficiency anemia & $280,285.9$ & D50, D64.9 \\
\hline U058 & Other nutritional disorders & 265-269, 281 & D51-D53, E51-E64 \\
\hline U059 & II. Noncommunicable diseases ${ }^{a}$ & $\begin{array}{l}140-242,244-259,270-279 \\
\text { (minus 279.5), 282-285 } \\
\text { (minus 285.9), 286-319, 324-380, } \\
\text { 383-459, 470-478, 490-611, } \\
617-629,680-759\end{array}$ & $\begin{array}{l}\text { C00-C97, D00-D48, D55-D64 } \\
\text { (minus D 64.9) D65-D89, E03-E07, } \\
\text { E10-E16, E20-E34, E65-E88, F01-F99, } \\
\text { G06-G98, H00-H61, H68-H93, I00-I99, } \\
\text { J30-J98, K00-K92, N00-N64, N75-N98, } \\
\text { L00-L98, M00-M99, O00-099 }\end{array}$ \\
\hline U060 & A. Malignant neoplasms & 140-208 & COO-C97 \\
\hline U061 & 1. Mouth and oropharynx cancers ${ }^{b}$ & $140-149$ & $\mathrm{C} 00-\mathrm{C} 14$ \\
\hline U062 & 2. Esophageal cancer ${ }^{b}$ & 150 & C15 \\
\hline U063 & 3. Stomach cancer ${ }^{b}$ & 151 & C16 \\
\hline U064 & 4. Colon and rectal cancers ${ }^{b}$ & $153-154$ & C18-C21 \\
\hline U065 & 5. Liver cancer & 155 & $\mathrm{C} 22$ \\
\hline U066 & 6. Pancreas cancer & 157 & $\mathrm{C} 25$ \\
\hline U067 & 7. Trachea, bronchus, and lung cancers & 162 & C33-C34 \\
\hline U068 & 8. Melanoma and other skin cancers ${ }^{b}$ & $172-173$ & C43-C44 \\
\hline U069 & 9. Breast cancer ${ }^{b}$ & $174-175$ & C50 \\
\hline U070 & 10. Cervix uteri cancer ${ }^{b}$ & 180 & C53 \\
\hline
\end{tabular}


Table 3A.2 Continued

\begin{tabular}{|c|c|c|c|}
\hline Code & GBD cause name & ICD-9 code & ICD-10 code \\
\hline U071 & 11. Corpus uteri cancer ${ }^{b}$ & 179,182 & C54-C55 \\
\hline U072 & 12. Ovarian cancer & 183 & C56 \\
\hline U073 & 13. Prostate cancer ${ }^{b}$ & 185 & C61 \\
\hline U074 & 14. Bladder cancer ${ }^{b}$ & 188 & C67 \\
\hline U075 & 15. Lymphomas and multiple myeloma ${ }^{b}$ & 200-203 & C81-C90, C96 \\
\hline U076 & 16. Leukemia ${ }^{b}$ & 204-208 & C91-C95 \\
\hline U077 & Other malignant neoplasms ${ }^{b}$ & $\begin{array}{l}152,156,158-161,163-171,181 \\
184,186-187,189-199\end{array}$ & $\begin{array}{l}\text { C17, C23, C24, C26-C32, C37-C41, } \\
\text { C45-C49, C51, C52, C57-C60, C62-C66, } \\
\text { C68-C80, C97 }\end{array}$ \\
\hline U078 & B. Other neoplasms & $210-239$ & D00-D48 \\
\hline U079 & C. Diabetes mellitus & 250 & E10-E14 \\
\hline U080 & D. Endocrine disorders & $\begin{array}{l}\text { 240-242, 244-246, 251-259, } \\
270-279 \text { (minus 274, 279.5), 282-285 } \\
\text { (minus 285.9), 286-289 }\end{array}$ & $\begin{array}{l}\text { D55-D64 (minus D64.9), D65-D89, } \\
\text { E03-E07, E15-E16, E20-E34, E65-E88 }\end{array}$ \\
\hline U081 & E. Neuropsychiatric conditions & $290-319,324-359$ & F01-F99, G06-G98 \\
\hline U082 & 1. Unipolar depressive disorders & $296.1,311$ & F32-F33 \\
\hline U083 & 2. Bipolar affective disorder & 296 (minus 296.1) & F30-F31 \\
\hline U084 & 3. Schizophrenia & 295 & F20-F29 \\
\hline U085 & 4. Epilepsy & 345 & G40-G41 \\
\hline U086 & 5. Alcohol use disorders & $291,303,305.0$ & F10 \\
\hline U087 & 6. Alzheimer's disease and other dementias & $290,330,331$ & F01, F03, G30-G31 \\
\hline U088 & 7. Parkinson's disease & 332 & G20-G21 \\
\hline U089 & 8. Multiple sclerosis & 340 & G35 \\
\hline U090 & 9. Drug use disorders & $304,305.2-305.9$ & F11-F16, F18-F19 \\
\hline U091 & 10. Post-traumatic stress disorder & 308-309 & F43.1 \\
\hline U092 & 11. Obsessive-compulsive disorder & 300.3 & $\mathrm{~F} 42$ \\
\hline U093 & 12. Panic disorder & 300.2 & F40.0, F41.0 \\
\hline U094 & 13. Insomnia (primary) & 307.4 & F51 \\
\hline U095 & 14. Migraine & 346 & G43 \\
\hline U096 & 15. Mental retardation, lead-caused & $317-319$ & F70-F79 \\
\hline U097 & Other neuropsychiatric disorders & $\begin{array}{l}\text { 292-294, 297-300.1, 300.4-302, } \\
\text { 305.1, 306-307 (minus 307.4), 310, } \\
\text { 312-316, 324-326, 333-337, } \\
\text { 341-344, 347-349, 350-359 }\end{array}$ & $\begin{array}{l}\text { F04-F09, F17, F34-F39, F401-F409, } \\
\text { F411-F419, F43 (minus F43.1), F44-F50, } \\
\text { F52-F69, F80-F99, G06-G12, G23-G25, } \\
\text { G36, G37, G44-G98 }\end{array}$ \\
\hline U098 & F. Sense organ diseases & $360-380,383-389$ & H00-H61, H68-H93 \\
\hline U099 & 1. Glaucoma & 365 & $\mathrm{H} 40$ \\
\hline U100 & 2. Cataracts & 366 & $\mathrm{H} 25-\mathrm{H} 26$ \\
\hline U101 & 3. Vision disorders, age-related & 367.4 & $\mathrm{H} 524$ \\
\hline U102 & 4. Hearing loss, adult onset & 389 & H90-H91 \\
\hline U103 & Other sense organ disorders & $\begin{array}{l}\text { 360-364, 367-380 (minus 367.4), } \\
383-388\end{array}$ & $\begin{array}{l}\mathrm{H} 00-\mathrm{H} 21, \mathrm{H} 27-\mathrm{H} 35, \mathrm{H} 43-\mathrm{H} 61 \\
\text { (minus H524), H68-H83, H92-H93 }\end{array}$ \\
\hline U104 & G. Cardiovascular diseases & $390-459$ & $100-199$ \\
\hline U105 & 1. Rheumatic heart disease & 390-398 & $101-109$ \\
\hline U106 & 2. Hypertensive heart disease & $401-405$ & $\mid 10-113$ \\
\hline U107 & 3. Ischemic heart disease $\mathrm{C}^{\mathrm{C}}$ & $410-414$ & $120-\mid 25$ \\
\hline U108 & 4. Cerebrovascular disease & 430-438 & $160-169$ \\
\hline U109 & 5. Inflammatory heart diseases & $420,421,422,425$ & $|30-| 33,|38| 40,, \mid 42$ \\
\hline
\end{tabular}


Table 3A.2 Continued

\begin{tabular}{|c|c|c|c|}
\hline Code & GBD cause name & ICD-9 code & ICD-10 code \\
\hline U110 & Other cardiovascular diseases ${ }^{c}$ & $\begin{array}{l}415-417,423-424,426-429 \\
440-448,451-459\end{array}$ & I00, I26-I28, I34-I37, |44-I51, I70-199 \\
\hline U111 & H. Respiratory diseases & $470-478,490-519$ & J30-J98 \\
\hline U112 & 1. Chronic obstructive pulmonary disease & $490-492,495-496$ & $J 40-J 44$ \\
\hline U113 & 2. Asthma & 493 & J45-J46 \\
\hline U114 & Other respiratory diseases & $470-478,494,500-508,510-519$ & J30-J39, J47-J98 \\
\hline U115 & I. Digestive diseases & $530-579$ & K20-K92 \\
\hline U116 & 1. Peptic ulcer disease & $531-533$ & K25-K27 \\
\hline U117 & 2. Cirrhosis of the liver & 571 & K70, K74 \\
\hline U118 & 3. Appendicitis & $540-543$ & K35-K37 \\
\hline U119 & Other digestive diseases & $\begin{array}{l}530,534-537,550-553,555-558 \\
560-570,572-579\end{array}$ & $\begin{array}{l}\text { K20-K22, K28-K31, K38, K40-K66, } \\
\text { K71-K73, K75-K92 }\end{array}$ \\
\hline U120 & J. Genitourinary diseases & $580-611,617-629$ & N00-N64, N75-N98 \\
\hline U121 & 1. Nephritis and nephrosis & $580-589$ & N00-N19 \\
\hline U122 & 2. Benign prostatic hypertrophy & 600 & $\mathrm{~N} 40$ \\
\hline U123 & Other genitourinary system diseases & $590-599,601-611,617-629$ & N20-N39, N41-N64, N75-N98 \\
\hline U124 & K. Skin diseases & $680-709$ & L00-L98 \\
\hline U125 & L. Musculoskeletal diseases & $710-739,274$ & M00-M99 \\
\hline U126 & 1. Rheumatoid arthritis & 714 & M05-M06 \\
\hline U127 & 2. Osteoarthritis & 715 & M15-M19 \\
\hline U128 & 3. Gout & 274 & M10 \\
\hline U129 & 4. Low back pain & 720-724 (minus 721.1, 722.0, 722.4) & M45-M48, M54 (minus M54.2) \\
\hline U130 & Other musculoskeletal disorders & $\begin{array}{l}710-713,716-719,721.1,722.0 \\
722.4,723,725-739\end{array}$ & $\begin{array}{l}\text { M00-M02, M08, M11-M13, M20-M43, } \\
\text { M50-M53, M54.2, M55-M99 }\end{array}$ \\
\hline U131 & M. Congenital anomalies & $740-759$ & 000-099 \\
\hline U132 & 1. Abdominal wall defect & 756.7 & $079.2-079.5$ \\
\hline U133 & 2. Anencephaly & 740.0 & 000 \\
\hline U134 & 3. Anorectal atresia & 751.2 & 042 \\
\hline U135 & 4. Cleft lip & 749.1 & 036 \\
\hline U136 & 5. Cleft palate & 749.0 & 035,037 \\
\hline U137 & 6. Esophageal atresia & 750.3 & 039.0-039.1 \\
\hline U138 & 7. Renal agenesis & 753.0 & 060 \\
\hline U139 & 8. Down syndrome & 758.0 & 090 \\
\hline U140 & 9. Congenital heart anomalies & $745-747$ & 020-028 \\
\hline U141 & 10. Spina bifida & 741 & 005 \\
\hline U142 & Other congenital anomalies & $\begin{array}{l}740.1,740.2,742-744,748,749.2 \\
750.0,750.1,750.2,750.4-751.1 \\
751.3-751.9,752,753.1-753.9,754 \\
755,756.0-756.6,756.8,756.9,757 \\
758.1-758.9,759\end{array}$ & $\begin{array}{l}\text { 001-004, 006-018, 030-034, 038, } \\
\text { 0392-0399, 040-041, 043-056, } \\
\text { 061-078, 0790, 0791, 0796, 0798, } \\
\text { 0799, 080-089, 091-099 }\end{array}$ \\
\hline U143 & N. Oral conditions & $520-529$ & K00-K14 \\
\hline U144 & 1. Dental caries & 521.0 & K02 \\
\hline U145 & 2. Periodontal disease & 523 & K05 \\
\hline U146 & 3. Edentulism & - & - \\
\hline U147 & Other oral diseases & $520,521.1-521.9,522,524-529$ & K00, K01, K03, K04, K06-K14 \\
\hline
\end{tabular}


Table 3A.2 Continued

\begin{tabular}{|c|c|c|c|}
\hline Code & GBD Cause Name & ICD-9 code & ICD-10 code \\
\hline U148 & III. Injuries & E800-999 & V01-Y89 \\
\hline U149 & A. Unintentional injuries ${ }^{d}$ & E800-949 & V01-X59, Y40-Y86, Y88, Y89 \\
\hline U150 & 1. Road traffic accidents & E810-819, E826-829, E929.0 & e \\
\hline U151 & 2. Poisonings & E850-869 & X40-X49 \\
\hline U152 & 3. Falls & E880-888 & W00-W19 \\
\hline U153 & 4. Fires & E890-899 & X00-X09 \\
\hline U154 & 5. Drownings & E910 & W65-W74 \\
\hline U155 & 6. Other unintentional injuries & $\begin{array}{l}\text { E800-E807, E820-E848, E870-E879, } \\
\text { E900-E909, E911-E949 }\end{array}$ & $\begin{array}{l}\text { Rest of V, W20-W64, W75-W99, } \\
\text { X10-X39, X50-X59, Y40-Y86, Y88, Y89 }\end{array}$ \\
\hline U156 & B. Intentional injuries ${ }^{d}$ & E950-978, 990-999 & X60-Y09, Y35-Y36, Y870, Y871 \\
\hline U157 & 1. Self-inflicted injuries & E950-959 & X60-X84, Y870 \\
\hline U158 & 2. Violence & E960-969 & X85-Y09, Y871 \\
\hline U159 & 3. War & E990-999 & Y36 \\
\hline U160 & Other intentional injuries & E970-E978 & Y35 \\
\hline
\end{tabular}

Source: Mathers, Lopez and others 2004.

a. Deaths coded to "Symptoms, signs and ill-defined conditions" (780-799 in ICD-9 and R00-R99 in ICD-10) are distributed proportionately to all causes within Group I and Group II.

b. Cancer deaths coded to ICD categories for malignant neoplasms of other and unspecified sites including those whose point of origin cannot be determined, and secondary and unspecified neoplasms (ICD-10 C76, C80, C97 or ICD-9 195, 199) were redistributed pro rata across the footnoted malignant neoplasm categories within each age-sex group, so that the category "Other malignant neoplasms" includes only malignant neoplasms of other specified sites.

c. Ischemic heart disease deaths may be miscoded to a number of so-called cardiovascular "garbage" codes. These include heart failure, ventricular dysrhythmias, generalized atherosclerosis, and illdefined descriptions and complications of heart disease. Proportions of deaths coded to these causes were redistributed to ischemic heart disease as described by Lozano and others (2001). Relevant ICD-9 codes are 427.1, 427.4, 427.5, 428, 429.0, 429.1, 429.2, 429.9, 440.9; relevant ICD-10 codes are 147.2, 149.0, I46, I50, I51.4, I51.5, I51.6, I51.9, I70.9.

d. Injury deaths where the intent is not determined (E980-989 of ICD-9 and Y10-Y34, Y872 in ICD-10) are distributed proportionately to all causes below the group level for injuries.

e. For countries with three-digit ICD-10 data, use: V01-V04, V06, V09-V80, V87, V89, V99. For countries with four-digit ICD-10 data, use: V01.1-V01.9, V02.1-V02.9, V03.1-V03.9, V04.1-V04.9,

V06.1-V06.9, V09.2, V09.3, V10.4-V10.9, V11.4-V11.9, V12.3-V12.9, V13.3-V13.9, V14.3-V14.9, V15.4-V15.9, V16.4-V16.9, V17.4-V17.9, V18.4-V18.9, V19.4-V19.6, V20.3-V20.9, V21.3-V21.9, V22.3-V22.9, V23.3-V23.9, V24.3-V24.9, V25.3-V25.9, V26.3-V26.9, V27.3-V27.9, V28.3-V28.9, V29.4-V29.9, V30.4.V30.9, V31.4-V31.9, V32.4-V32.9, V33.4-V33.9, V34.4-V34.9, V35.4-V35.9, V36.4-V36.9, V37.4-V37.9, V38.4-V38.9, V39.4-V39.9, V40.4-V40.9, V41.4-V41.9, V42.4-V42.9, V43.4-V43.9, V44.4-V44.9, V45.4-V45.9, V46.4-V46.9, V47.4-V47.9, V48.4-V48.9, V49.4-V49.9, V50.4-V50.9, V51.4-V51.9, V52.4-V52.9, V53.4-V53.9, V54.4-V54.9, V55.4-V55.9, V56.4-V56.9, V57.4-V57.9, V58.4-V58.9, V59.4-V59.9, V60.4-V60.9, V61.4-V61.9, V62.4-V62.9, V63.4-V63.9, V64.4-V64.9, V65.4-V65.9, V66.4-V66.9, V67.4-V67.9, V68.4-V68.9, V69.4-V69.9, V70.4-V70.9, V71.4-V71.9, V72.4-V72.9, V73.4-V73.9, V74.4-V74.9, V75.4-V75.9, V76.4-V76.9, V77.4-V77.9, V78.4-V78.9, V79.4-V79.9, V80.3-V80.5, V81.1, V82.1, V83.0-V83.3, V84.0-V84.3, V85.0-V85.3, V86.0-V86.3, V87.0-V87.8, V89.2, V89.9, V99, Y850. 
Table 3A.3 Data Sources and Methods for Estimation of Mortality by Cause, Age, and Sex

\begin{tabular}{|c|c|c|c|c|c|}
\hline Country & Vital registration data & Year used & $\begin{array}{l}\text { Other sources } \\
\text { of information }\end{array}$ & Method & $\begin{array}{l}\text { Cause of death } \\
\text { distribution } \\
\text { pattern used }\end{array}$ \\
\hline Afghanistan & & & $\mathrm{a}$ & CodMod & $\begin{array}{l}\text { Arab Rep. of Egypt 2000, } \\
\text { Islamic Rep. of Iran } 2001\end{array}$ \\
\hline Albania & 1987-9, 1992-2000 & 2000 & a & CodMod & 2000 \\
\hline Algeria & & & a & CodMod & South Africa 1996 \\
\hline Andorra & & & $\mathrm{b}$ & $\begin{array}{l}\text { Based on } 2000 \text { data from } \\
\text { Aragon, Navarra, and } \\
\text { Cataluna provinces of Spain }\end{array}$ & \\
\hline Angola & & & a & CodMod & South Africa 1996 \\
\hline $\begin{array}{l}\text { Antigua and } \\
\text { Barbuda }\end{array}$ & $\begin{array}{l}\text { 1961-4, 1966, 1969-78, } \\
1983,1985-95\end{array}$ & $1993-5$ & c & Vital registration & Vital registration \\
\hline Argentina & 1966-70, 1977-2001 & 2001 & $b$ & Vital registration & Vital registration \\
\hline Armenia & 1981-2, 1985-2001 & 2001 & $a$ & CodMod & 2001 \\
\hline Australia & 1950-2000 & 2000 & $b$ & Vital registration & Vital registration \\
\hline Austria & 1955-2001 & 2001 & $b$ & Vital registration & Vital registration \\
\hline Azerbaijan & 1981-2, 1985-2001 & 2001 & a & CodMod & 2001 \\
\hline Bahamas, The & $\begin{array}{l}\text { 1969, 1971-2, 1974-7, } \\
\text { 1979-81, 1983-5, 1987, } \\
1993-8\end{array}$ & $1996-8$ & c & Vital registration & Vital registration \\
\hline Bahrain & $1985,1987-8,1997-2001$ & $2000-1$ & b & Vital registration & Vital registration \\
\hline Bangladesh & & & a & CodMod & India, Philippines \\
\hline Barbados & 1955-95 & $1993-5$ & $\begin{array}{l}\text { Preliminary vital registration } \\
\text { data for } 2000^{c}\end{array}$ & Vital registration & Vital registration \\
\hline Belarus & 1981-2, 1985-2001 & 2001 & c & Vital registration & Vital registration \\
\hline Belgium & $1954-97$ & 1997 & $b$ & Vital registration & Vital registration \\
\hline Belize & $\begin{array}{l}1964-84,1986-7 \\
1989-91,1993-8\end{array}$ & 1997-8 & c & Vital registration & Vital registration \\
\hline Benin & & & a & CodMod & South Africa 1996 \\
\hline Bhutan & & & a & CodMod & India \\
\hline Bolivia & & & a & CodMod & Peru 2000 \\
\hline $\begin{array}{l}\text { Bosnia and } \\
\text { Herzegovina }\end{array}$ & 1985-91, 1999 & 1999 & c & Vital registration & Vital registration \\
\hline Botswana & $1995-8$ & & a & CodMod & South Africa 1996 \\
\hline Brazil & 1977-2000 & 2000 & a & CodMod & 2000 \\
\hline $\begin{array}{l}\text { Brunei } \\
\text { Darussalam }\end{array}$ & 1996-2000 & 1998-2000 & $\mathrm{b}$ & Vital registration & Vital registration \\
\hline Bulgaria & 1964-2001 & 2001 & c & Vital registration & Vital registration \\
\hline Burkina Faso & & & a & CodMod & South Africa 1996 \\
\hline Burundi & & & a & CodMod & South Africa 1996 \\
\hline Cambodia & & & a & CodMod & Philippines, Thailand \\
\hline Cameroon & & & a & CodMod & South Africa 1996 \\
\hline Canada & 1950-2000 & 2000 & $b$ & Vital registration & Vital registration \\
\hline Cape Verde & 1980 & & a & CodMod & South Africa 1996 \\
\hline $\begin{array}{l}\text { Central African } \\
\text { Republic }\end{array}$ & & & a & CodMod & South Africa 1996 \\
\hline Chad & & & a & CodMod & South Africa 1996 \\
\hline Chile & 1954-99 & 1999 & $\begin{array}{l}\text { Preliminary vital registration } \\
\text { data for } 2000^{b}\end{array}$ & Vital registration & Vital registration \\
\hline
\end{tabular}

100 I Global Burden of Disease and Risk Factors I Colin D. Mathers, Alan D. Lopez, and Christopher J. L. Murray 
Table 3A.3 Continued

\begin{tabular}{|c|c|c|c|c|c|}
\hline Country & Vital registration data & Year used & $\begin{array}{l}\text { Other sources } \\
\text { of information }\end{array}$ & Method & $\begin{array}{l}\text { Cause of death } \\
\text { distribution } \\
\text { pattern used }\end{array}$ \\
\hline China & 1987-2000 & 2000 & DSPa & Vital registration & Vital registration \\
\hline Colombia & $\begin{array}{l}\text { 1953-70, 1972, 1974-7, } \\
\text { 1979, 1981, 1984-99 }\end{array}$ & 1999 & a & CodMod & 1999 \\
\hline Comoros & & & a & CodMod & South Africa 1996 \\
\hline $\begin{array}{l}\text { Congo, Dem. } \\
\text { Rep. of }\end{array}$ & & & a & CodMod & South Africa 1996 \\
\hline Congo, Rep. of & & & a & CodMod & South Africa 1996 \\
\hline Cook Islands & 1995-2001 & 1999-2001 & a & Vital registration & Vital registration \\
\hline Costa Rica & 1956-2002 & 2002 & $b$ & Vital registration & Vital registration \\
\hline Côte d'Ivoire & & & $\begin{array}{l}\text { Abidjan, 1973-92: deaths } \\
\text { assessed by medical } \\
\text { personnel in city hospitals. } \\
\text { Source: ENSEA, Abidjana }\end{array}$ & CodMod & South Africa 1996 \\
\hline Croatia & 1985-2001 & 2001 & $\mathrm{~b}$ & Vital registration & Vital registration \\
\hline Cuba & 1959, 1964-5, 1968-2001 & 2001 & $b$ & Vital registration & Vital registration \\
\hline Cyprus & $1996-9$ & $1997-9$ & a & CodMod & $1997-9$ \\
\hline Czech Republic & 1985-2001 & 2001 & $\mathrm{~b}$ & Vital registration & Vital registration \\
\hline Denmark & $1951-99$ & 1999 & b & Vital registration & Vital registration \\
\hline Djibouti & & & a & CodMod & $\begin{array}{l}\text { Arab Rep. of Egypt } 2000 \text {, } \\
\text { Islamic Rep. of Iran } 2000\end{array}$ \\
\hline Dominica & $1961-2,1967-94$ & $1992-4$ & c & Vital registration & Vital registration \\
\hline $\begin{array}{l}\text { Dominican } \\
\text { Republic }\end{array}$ & $\begin{array}{l}\text { 1956-63, 1965-92, } \\
1994-8\end{array}$ & 1998 & a & CodMod & 1998 \\
\hline Ecuador & 1961, 1963-75, 1977-2000 & 2000 & a & CodMod & 2000 \\
\hline $\begin{array}{l}\text { Egypt, Arab } \\
\text { Rep. of }\end{array}$ & $\begin{array}{l}\text { 1954-67, 1970-80, 1987, } \\
1991-2,1996-2000\end{array}$ & 2000 & a & CodMod & 2000 \\
\hline El Salvador & $\begin{array}{l}\text { 1950-74, 1981-4, 1990-3, } \\
1995-9\end{array}$ & 1999 & a & CodMod & 1999 \\
\hline $\begin{array}{c}\text { Equatorial } \\
\text { Guinea }\end{array}$ & & & a & CodMod & South Africa 1996 \\
\hline Eritrea & $1998-9$ & & a & CodMod & South Africa 1996 \\
\hline Estonia & 1981-2, 1985-2001 & 2001 & $b$ & Vital registration & Vital registration \\
\hline Ethiopia & & & a & CodMod & South Africa 1996 \\
\hline Fiji & 1978, 1992-7, 1999-2000 & 2000 & a & Vital registration & Vital registration \\
\hline Finland & 1952-2001 & 2001 & $\mathrm{~b}$ & Vital registration & Vital registration \\
\hline France & 1950-99 & 1999 & $b$ & Vital registration & Vital registration \\
\hline Gabon & & & a & CodMod & South Africa 1996 \\
\hline Gambia, The & & & a & CodMod & South Africa 1996 \\
\hline Georgia & $\begin{array}{l}1981-2,1985-92 \\
1994-2000\end{array}$ & 2000 & a & CodMod & 2000 \\
\hline Germany & 1969-2000 & 2000 & $b$ & Vital registration & Vital registration \\
\hline Ghana & & & $\begin{array}{l}\text { Hospital mortality data for } \\
\text { Eastern Region, 1990-2000a }\end{array}$ & CodMod & South Africa 1996 \\
\hline Greece & $1956-99$ & 1999 & b & Vital registration & Vital registration \\
\hline Grenada & $\begin{array}{l}\text { 1974-8, 1984, 1988, } \\
1994-6\end{array}$ & $1994-6$ & c & Vital registration & Vital registration \\
\hline
\end{tabular}


Table 3A.3 Continued

\begin{tabular}{|c|c|c|c|c|c|}
\hline Country & Vital registration data & Year used & $\begin{array}{l}\text { Other sources } \\
\text { of information }\end{array}$ & Method & $\begin{array}{l}\text { Cause of death } \\
\text { distribution } \\
\text { pattern used }\end{array}$ \\
\hline Guatemala & 1958-71, 1974-81, 1984 & 1996 & $\begin{array}{l}\text { Preliminary vital registration } \\
\text { data for } 1996^{a}\end{array}$ & CodMod & 1996 \\
\hline Guinea & & & a & CodMod & South Africa 1996 \\
\hline Guinea-Bissau & & & a & CodMod & South Africa 1996 \\
\hline Guyana & $\begin{array}{l}\text { 1975-7, 1979, 1984, 1988, } \\
1990,1993-6\end{array}$ & $1994-6$ & c & Vital registration & Vital registration \\
\hline Haiti & 1980-1, 1983, 1997, 1999 & 1999 & a & CodMod & 1999 \\
\hline Honduras & 1966, 1968-83 & & a & CodMod & $\begin{array}{l}\text { Nicaragua, El Salvador, } \\
\text { Guatemala }\end{array}$ \\
\hline Hungary & 1955-2001 & 2001 & $b$ & Vital registration & Vital registration \\
\hline Iceland & 1951-99 & $1997-9$ & $\mathrm{~b}$ & Vital registration & Vital registration \\
\hline India & $\begin{array}{l}\text { Survey of Cause of Death } \\
\text { (Rural) }\end{array}$ & $1996-8$ & $\begin{array}{l}\text { Urban Medical Certification } \\
\text { of Cause of Death System, } \\
1995^{\mathrm{a}}\end{array}$ & $\begin{array}{l}\text { Proportionate mortality for } \\
\text { urban and rural summed up } \\
\text { to national estimate }\end{array}$ & $\begin{array}{l}\text { Cause of death informa- } \\
\text { tion from urban and rural } \\
\text { data sources }\end{array}$ \\
\hline Indonesia & & & a & CodMod & $\begin{array}{l}\text { Singapore, India, } \\
\text { Thailand, Philippines }\end{array}$ \\
\hline $\begin{array}{l}\text { Iran, Islamic } \\
\text { Rep. of }\end{array}$ & 1999-2001 & 2001 & a & CodMod & $\begin{array}{l}2001 \text { (18 provinces' } \\
\text { mortality data) }\end{array}$ \\
\hline Iraq & & & a & CodMod & $\begin{array}{l}\text { Arab Rep. of Egypt 2000, } \\
\text { Islamic Rep. of Iran } 2001\end{array}$ \\
\hline Ireland & 1950-2000 & 2000 & c & Vital registration & Vital registration \\
\hline Israel & 1975-98 & 1998 & b & Vital registration & Vital registration \\
\hline Italy & 1951-99 & 1999 & $\mathrm{~b}$ & Vital registration & Vital registration \\
\hline Jamaica & $\begin{array}{l}1960-1,1964-5 \\
1967-71,1975,1977 \\
1980-91\end{array}$ & 1991 & a & CodMod & 1991 \\
\hline Japan & 1950-2000 & 2000 & $b$ & Vital registration & Vital registration \\
\hline Jordan & $\begin{array}{l}\text { 1959-60, 1962-6, 1968, } \\
1970-5,1978-9\end{array}$ & & $\begin{array}{l}\text { Mortality and causes of } \\
\text { death in Jordan 1995-6: } \\
\text { assessment by verbal } \\
\text { autopsy. Source: S.A. Khoury, } \\
\text { D. Massad, and T. Fardous, } \\
\text { Bulletin of the World Health } \\
\text { Organization } 77(8)^{\mathrm{a}}\end{array}$ & Verbal autopsy data & Verbal autopsy data \\
\hline Kazakhstan & 1981-2, 1985-2001 & 2001 & a & CodMod & 2001 \\
\hline Kenya & & & $\begin{array}{l}\text { Ministry of Health, hospital } \\
\text { data, 1996, 1998-2000a }\end{array}$ & CodMod & South Africa 1996 \\
\hline Kiribati & 1999-2002 & $2000-2$ & $\begin{array}{l}\text { Ministry of Health, Family } \\
\text { Planning and Social Welfare, } \\
\text { Third National Health, Family } \\
\text { Planning and Social Welfare } \\
\text { Plan 1992-5a }\end{array}$ & Vital registration & $2000-2$ \\
\hline $\begin{array}{l}\text { Korea, } \\
\text { Democratic } \\
\text { People's Rep. of }\end{array}$ & & & a & CodMod & Philippines, India \\
\hline Korea, Rep. of & 1985-2001 & 2001 & $b$ & Vital registration & Vital registration \\
\hline Kuwait & 1972, 1975-87, 1993-2001 & 1999-2001 & $b$ & Vital registration & Vital registration \\
\hline Kyrgyz Republic & $1981-2,1985-2001$ & 2001 & a & CodMod & 2001 \\
\hline Lao PDR & & & a & CodMod & Philippines, Thailand \\
\hline
\end{tabular}

102 | Colin D. Mathers, Alan D. Lopez, and Christopher J. L. Murray 
Table 3A.3 Continued

\begin{tabular}{|c|c|c|c|c|c|}
\hline Country & Vital registration data & Year used & $\begin{array}{l}\text { Other sources } \\
\text { of information }\end{array}$ & Method & $\begin{array}{l}\text { Cause of death } \\
\text { distribution } \\
\text { pattern used }\end{array}$ \\
\hline Latvia & 1980-2001 & 2001 & $\mathrm{~b}$ & Vital registration & Vital registration \\
\hline Lebanon & $1997-9$ & & a & CodMod & $\begin{array}{l}\text { Arab Rep. of Egypt 2000, } \\
\text { Islamic Rep. of Iran } 2001\end{array}$ \\
\hline Lesotho & & & a & CodMod & South Africa 1996 \\
\hline Liberia & & & a & CodMod & South Africa 1996 \\
\hline Libya & & & a & CodMod & $\begin{array}{l}\text { Arab Rep. of Egypt 2000, } \\
\text { Islamic Rep. of Iran } 2001\end{array}$ \\
\hline Lithuania & 1981-2, 1985-2001 & 2001 & $\mathrm{~b}$ & Vital registration & Vital registration \\
\hline Luxembourg & $1955-62,1965-2001$ & 1999-2001 & $\mathrm{b}$ & Vital registration & Vital registration \\
\hline Macedonia, FYR & $1991-2000$ & 2000 & c & Vital registration & Vital registration \\
\hline Madagascar & & & $\begin{array}{l}\text { Antananarivo, 1976-95: } \\
\text { deaths certified by medical } \\
\text { personnel. Source: CEPED, } \\
\text { Paris }^{\text {a }}\end{array}$ & CodMod & South Africa 1996 \\
\hline Malawi & & & a & CodMod & South Africa 1996 \\
\hline Malaysia & $1986,1990-8$ & & a & CodMod & $\begin{array}{l}\text { Singapore, China, } \\
\text { Thailand }\end{array}$ \\
\hline Maldives & & & a & CodMod & India, Philippines \\
\hline Mali & & & a & CodMod & South Africa 1996 \\
\hline Malta & 1955-2001 & 1999-2001 & $\mathrm{b}$ & Vital registration & Vital registration \\
\hline Marshall Islands & & & a & & $\begin{array}{l}\text { Cook Islands, Marshall } \\
\text { Islands, Niue, Samoa, } \\
\text { Tonga, Tuvalu, Vanuatu, } \\
\text { Kiribati, Nauru, Fiji }\end{array}$ \\
\hline Mauritania & & & a & CodMod & South Africa 1996 \\
\hline Mauritius & $1957-2000$ & 1998-2000 & c & Vital registration & Vital registration \\
\hline Mexico & 1955-2001 & 2001 & $\mathrm{~b}$ & Vital registration & Vital registration \\
\hline $\begin{array}{l}\text { Micronesia, } \\
\text { Federated } \\
\text { States of }\end{array}$ & & & $\begin{array}{l}1999 \text { Federated States of } \\
\text { Micronesia Statistical }_{\text {Yearbook }^{\mathrm{a}}}\end{array}$ & & $\begin{array}{l}\text { Cook Islands, Marshall } \\
\text { Islands, Niue, Samoa, } \\
\text { Tonga, Tuvalu, Vanuatu, } \\
\text { Kiribati, Nauru, Fiji }\end{array}$ \\
\hline Moldova & $1981-2,1985-2001$ & 2001 & $\mathrm{~b}$ & Vital registration & Vital registration \\
\hline Monaco & & & $\mathrm{b}$ & & $\begin{array}{l}\text { Based on } 1998 \text { data } \\
\text { from Provence Alpes } \\
\text { Côte d'Azur, Department } \\
\text { of France }\end{array}$ \\
\hline Mongolia & 1990-2000 & 2000 & a & CodMod & 2000 \\
\hline Morocco & $1990-7$ & & a & CodMod & $\begin{array}{l}\text { Arab Rep. of Egypt 2000, } \\
\text { Islamic Rep. of Iran } 2001\end{array}$ \\
\hline Mozambique & & & a & CodMod & $\begin{array}{l}\text { Zimbabwe 1995, South } \\
\text { Africa } 1996\end{array}$ \\
\hline Myanmar & $1977-8$ & & a & CodMod & Philippines, India \\
\hline Namibia & & & a & CodMod & South Africa 1996 \\
\hline Nauru & $1994-6$ & $1994-6$ & $\begin{array}{l}\text { Mortality decline in Nauru. } \\
\text { Source: R. Taylor and K. } \\
\text { Thoma, unpublished 1998a }\end{array}$ & Vital registration & $1994-6$ \\
\hline Nepal & & & a & CodMod & Philippines, India \\
\hline Netherlands & 1950-2000 & 2000 & $\mathrm{~b}$ & Vital registration & $\begin{array}{l}\text { Vital registration } \\
\text { inues on the following }\end{array}$ \\
\hline
\end{tabular}


Table 3A.3 Continued

\begin{tabular}{|c|c|c|c|c|c|}
\hline Country & Vital registration data & Year used & $\begin{array}{l}\text { Other sources } \\
\text { of information }\end{array}$ & Method & $\begin{array}{l}\text { Cause of death } \\
\text { distribution } \\
\text { pattern used }\end{array}$ \\
\hline New Zealand & 1950-99 & 1999 & $\mathrm{~b}$ & Vital registration & Vital registration \\
\hline Nicaragua & $\begin{array}{l}\text { 1959, 1961-5, 1968-9, } \\
\text { 1973-8, 1988-94, } \\
1996-2000\end{array}$ & 2000 & a & CodMod & 2000 \\
\hline Niger & & & a & CodMod & South Africa 1996 \\
\hline Nigeria & & & a & CodMod & South Africa 1996 \\
\hline Niue & 1995-2000 & 1998-2000 & a & Vital registration & Vital registration \\
\hline Norway & $1951-2000$ & 2000 & $\mathrm{~b}$ & Vital registration & Vital registration \\
\hline Oman & 1997 & & a & CodMod & $\begin{array}{l}\text { Bahrain, Kuwait, } \\
\text { 1997-2001 }\end{array}$ \\
\hline Pakistan & & & a & CodMod & India \\
\hline Palau & & & a & & $\begin{array}{l}\text { Cook Islands, Marshall } \\
\text { Islands, Niue, Samoa, } \\
\text { Tonga, Tuvalu, Vanuatu, } \\
\text { Kiribati, Nauru, Fiji }\end{array}$ \\
\hline Panama & 1954-89, 1996-2000 & 2000 & a & CodMod & 2000 \\
\hline $\begin{array}{l}\text { Papua New } \\
\text { Guinea }\end{array}$ & 1977, 1980 & & a & CodMod & Philippines, India \\
\hline Paraguay & $\begin{array}{l}\text { 1961-3, 1965-91, 1994, } \\
1996-2000\end{array}$ & 2000 & a & CodMod & 2000 \\
\hline Peru & $\begin{array}{l}1966-73,1977-8 \\
1980-3,1986-92 \\
1994-2000\end{array}$ & 2000 & a & CodMod & 2000 \\
\hline Philippines & 1963-78, 1981, 1992-8 & 1998 & a & CodMod & 1998 \\
\hline Poland & 1959-2001 & 2001 & $\mathrm{~b}$ & Vital registration & Vital registration \\
\hline Portugal & $1955-2000$ & 2000 & $b$ & Vital registration & Vital registration \\
\hline Qatar & $1995,2000-1$ & 2001 & a & CodMod & 2001 \\
\hline Romania & 1959-2001 & 2001 & $\mathrm{~b}$ & Vital registration & Vital registration \\
\hline $\begin{array}{l}\text { Russian } \\
\text { Federation }\end{array}$ & 1980-2001 & 2001 & c & Vital registration & Vital registration \\
\hline Rwanda & & & a & CodMod & South Africa 1996 \\
\hline $\begin{array}{l}\text { St. Kitts and } \\
\text { Nevis }\end{array}$ & $\begin{array}{l}1961-3,1965-7 \\
1969-95\end{array}$ & $1993-5$ & c & Vital registration & Vital registration \\
\hline St. Lucia & 1968-81, 1983, 1986-95 & $1993-5$ & c & Vital registration & Vital registration \\
\hline $\begin{array}{l}\text { St. Vincent and } \\
\text { the Grenadines }\end{array}$ & $\begin{array}{l}1970-2,1974,1977,1979 \\
1982-7,1995-9\end{array}$ & $1997-9$ & c & Vital registration & Vital registration \\
\hline Samoa & & & $\begin{array}{l}\text { Department of Health } \\
\text { Statistics, Demographic } \\
\text { and Health Survey, } 1999 \\
\text { and } 2000^{\mathrm{a}}\end{array}$ & & $\begin{array}{l}\text { Cook Islands, Marshall } \\
\text { Islands, Niue, Samoa, } \\
\text { Tonga, Tuvalu, Vanuatu, } \\
\text { Kiribati, Nauru, Fiji }\end{array}$ \\
\hline San Marino & 1995-2000 & 1998-2000 & $\mathrm{b}$ & Vital registration & Vital registration \\
\hline $\begin{array}{l}\text { São Tomé and } \\
\text { Principe }\end{array}$ & $1984-5,1987$ & & a & CodMod & South Africa 1996 \\
\hline Saudi Arabia & & & a & CodMod & $\begin{array}{l}\text { Bahrain, Kuwait, } \\
\text { 1997-2001 }\end{array}$ \\
\hline Senegal & & & $\begin{array}{l}\text { Niakhar 1983-90: deaths } \\
\text { assessed by verbal autopsy. } \\
\text { Source: CEPED, Paris }{ }^{\mathrm{a}}\end{array}$ & CodMod & South Africa 1996 \\
\hline
\end{tabular}

104 I Global Burden of Disease and Risk Factors I Colin D. Mathers, Alan D. Lopez, and Christopher J. L. Murray 
Table 3A.3 Continued

\begin{tabular}{|c|c|c|c|c|c|}
\hline Country & Vital registration data & Year used & $\begin{array}{l}\text { Other sources } \\
\text { of information }\end{array}$ & Method & $\begin{array}{l}\text { Cause of death } \\
\text { distribution } \\
\text { pattern used }\end{array}$ \\
\hline $\begin{array}{l}\text { Serbia and } \\
\text { Montenegro }\end{array}$ & 2000 & 2000 & $b$ & Vital registration & Vital registration \\
\hline Seychelles & $\begin{array}{l}1981-2,1985-7 \\
1997-2000\end{array}$ & 1998-2000 & $b$ & Vital registration & Vital registration \\
\hline Sierra Leone & & & a & CodMod & South Africa 1996 \\
\hline Singapore & 1955-2001 & 2001 & c & Vital registration & Vital registration \\
\hline Slovak Republic & 1992-2001 & 2001 & $b$ & Vital registration & Vital registration \\
\hline Slovenia & 1985-2001 & 2001 & $b$ & Vital registration & Vital registration \\
\hline Solomon Islands & & & a & & $\begin{array}{l}\text { Cook Islands, Marshall } \\
\text { Islands, Niue, Samoa, } \\
\text { Tonga, Tuvalu, Vanuatu, } \\
\text { Kiribati, Nauru, Fiji }\end{array}$ \\
\hline Somalia & & & a & CodMod & $\begin{array}{l}\text { Arab Rep. of Egypt 2000, } \\
\text { South Africa } 1996\end{array}$ \\
\hline South Africa & $1993-6$ & 1996 & $\begin{array}{l}\text { National Injury Mortality } \\
\text { Surveillance System: } \\
\text { Summary Report 2000; } \\
\text { K. Kahn, S. M. Tollman, } \\
\text { M. Garenne, and J. S. Gear, } \\
\text { "Causes of Death in a Rural } \\
\text { Area of South Africa: An } \\
\text { International Perspective, } \\
\text { Journal of Tropical } \\
\text { Pediatrics, } 46 \text { (June)c; and } \\
\text { Violence and Injury } \\
\text { Surveillance Consortium, } \\
\text { Rapid Assessment of } \\
\text { Trauma Facilities at State } \\
\text { Hospitals in South Africa, } \\
\text { 2000a }\end{array}$ & CodMod & South Africa 1996 \\
\hline Spain & 1951-2000 & 2000 & $b$ & Vital registration & Vital registration \\
\hline Sri Lanka & $\begin{array}{l}\text { 1950-68, 1977, 1980-9, } \\
1991-2,1995-6\end{array}$ & 1996 & a & CodMod & 1996 \\
\hline Sudan & & & a & CodMod & $\begin{array}{l}\text { Arab Rep. of Egypt 2000, } \\
\text { South Africa } 1996\end{array}$ \\
\hline Suriname & $\begin{array}{l}\text { 1963-6, 1971-3, 1975-82, } \\
1984-92\end{array}$ & 1990-2 & a & Vital registration & Vital registration \\
\hline Swaziland & & & a & CodMod & South Africa 1996 \\
\hline Sweden & 1951-2000 & 2000 & $b$ & Vital registration & Vital registration \\
\hline Switzerland & 1951-99 & 1999 & $b$ & Vital registration & Vital registration \\
\hline $\begin{array}{l}\text { Syrian Arab } \\
\text { Republic }\end{array}$ & $\begin{array}{l}1973-8,1980-1,1984-5, \\
2000-1\end{array}$ & 2001 & a & CodMod & 2001 \\
\hline Tajikistan & 1981-2, 1985-95, 1999 & 1999 & a & CodMod & 1999 \\
\hline Tanzania & & & a & CodMod & $\begin{array}{l}\text { Zimbabwe 1995, South } \\
\text { Africa } 1996\end{array}$ \\
\hline Thailand & 1955-87, 1990-2000 & 2000 & $\begin{array}{l}\text { Ministry of Health, verbal } \\
\text { autopsy study }\end{array}$ & $\begin{array}{l}\text { Vital registration corrected } \\
\text { by verbal autopsy study }\end{array}$ & \\
\hline Timor-Leste & & & a & CodMod & India, Philippines \\
\hline Togo & & & a & CodMod & $\begin{array}{l}\text { South Africa } 1996 \\
\text { nues on the following page }\end{array}$ \\
\hline
\end{tabular}


Table 3A.3 Continued

\begin{tabular}{|c|c|c|c|c|c|}
\hline Country & Vital registration data & Year used & $\begin{array}{l}\text { Other sources } \\
\text { of information }\end{array}$ & Method & $\begin{array}{l}\text { Cause of death } \\
\text { distribution } \\
\text { pattern used }\end{array}$ \\
\hline Tonga & 1998 & 1998 & $\begin{array}{l}\text { Report of the Minister of } \\
\text { Health for } 1994\end{array}$ & Vital registration & Vital registration \\
\hline $\begin{array}{l}\text { Trinidad and } \\
\text { Tobago }\end{array}$ & 1951-98 & $1996-8$ & $\mathrm{~b}$ & Vital registration & Vital registration \\
\hline Tunisia & & & a & CodMod & $\begin{array}{l}\text { Arab Rep. of Egypt } 2000 \\
\text { Islamic Rep. of Iran } 2001\end{array}$ \\
\hline Turkey & 1987-98 & 1998 & $\mathrm{a}$ & CodMod & 1998 \\
\hline Turkmenistan & $1981-2,1985-98$ & 1998 & a & CodMod & 1998 \\
\hline Tuvalu & & & a & & $\begin{array}{l}\text { Cook Islands, Marshall } \\
\text { Islands, Niue, Samoa, } \\
\text { Tonga, Tuvalu, Vanuatu, } \\
\text { Kiribati, Nauru, Fiji }\end{array}$ \\
\hline Uganda & & & a & CodMod & South Africa 1996 \\
\hline Ukraine & 1981-2, 1985-2000 & 2000 & c & Vital registration & Vital registration \\
\hline $\begin{array}{l}\text { United Arab } \\
\text { Emirates }\end{array}$ & & & a & CodMod & $\begin{array}{l}\text { Bahrain and Kuwait, } \\
\text { 1997-2001 }\end{array}$ \\
\hline United Kingdom & $1950-2000$ & 2000 & $b$ & Vital registration & Vital registration \\
\hline United States & 1950-2000 & 2000 & $b$ & Vital registration & Vital registration \\
\hline Uruguay & $\begin{array}{l}\text { 1955-60, 1963-78, } \\
\text { 1980-91, 1993-2000 }\end{array}$ & 2000 & $b$ & Vital registration & Vital registration \\
\hline Uzbekistan & 1981-2, 1985-2000 & 2000 & a & CodMod & 2000 \\
\hline Vanuatu & & & $\begin{array}{l}\text { Ministry of Health, hospital } \\
\text { data, } 2001^{\mathrm{a}}\end{array}$ & & $\begin{array}{l}\text { Cook Islands, Marshall } \\
\text { Islands, Niue, Samoa, } \\
\text { Tonga, Tuvalu, Vanuatu, } \\
\text { Kiribati, Nauru and Fiji }\end{array}$ \\
\hline $\begin{array}{l}\text { Venezuela } \\
\text { (R.B. de) }\end{array}$ & $\begin{array}{l}\text { 1955-83, 1985-90, } \\
\text { 1992-2000 }\end{array}$ & 2000 & $\mathrm{~b}$ & Vital registration & Vital registration \\
\hline Vietnam & & & a & CodMod & $\begin{array}{l}\text { China, India, and } \\
\text { Thailand }\end{array}$ \\
\hline $\begin{array}{l}\text { Yemen, } \\
\text { Republic of }\end{array}$ & & & a & CodMod & $\begin{array}{l}\text { Arab Rep. of Egypt } 2000, \\
\text { Islamic Rep. of Iran } 2001\end{array}$ \\
\hline Zambia & 1999-2000 & & a & CodMod & South Africa 1996 \\
\hline Zimbabwe & $1990,1994-5$ & & a & CodMod & $\begin{array}{l}\text { Zimbabwe 1995, South } \\
\text { Africa } 1996\end{array}$ \\
\hline
\end{tabular}

Source: Mathers, Lopez, and others 2004

Note: CEPED = Centre Population et Développement; ENSEA = I'Ecole Nationale Supérieure de Statistiques et d'Economie Appliquée.

a. Epidemiological estimates obtained from studies, WHO technical programs, and UNAIDS for the following conditions: HIV/AIDS, tuberculosis, measles, pertussis, poliomyelitis, tetanus, lower

respiratory infections, Chagas' disease, maternal conditions, perinatal conditions, cancers, drug use disorders, rheumatoid arthritis, and war.

b. Epidemiological estimates obtained from studies, WHO technical programs, and UNAIDS for the following conditions: drug use disorders and war.

c. Epidemiological estimates obtained from studies, WHO technical programs, and UNAIDS for the following conditions: HIV/AIDS, drug use disorders, and war. 
Table 3A.4 GBD Regional Epidemiological Analysis Categories

\begin{tabular}{|c|c|c|c|c|}
\hline $\begin{array}{l}\text { GBD } \\
\text { region }\end{array}$ & $\begin{array}{l}\text { Mortality } \\
\text { stratum }\end{array}$ & $\begin{array}{l}\text { Region } \\
\text { code }\end{array}$ & WHO member states & $\begin{array}{l}\text { WHO } \\
\text { region }\end{array}$ \\
\hline \multirow[t]{2}{*}{ AFRO } & \multirow[t]{2}{*}{$D^{\mathrm{a}}$} & \multirow[t]{2}{*}{1} & $\begin{array}{l}\text { Algeria, Angola, Benin, Burkina Faso, Cameroon, Cape Verde, Chad, Comoros, } \\
\text { Equatorial Guinea, Gabon, The Gambia, Ghana, Guinea, Guinea-Bissau, Liberia, } \\
\text { Madagascar, Mali, Mauritania, Mauritius, Niger, Nigeria, São Tomé and Principe, } \\
\text { Senegal, Seychelles, Sierra Leone, Togo }\end{array}$ & AFRO \\
\hline & & & Djibouti, Somalia, Sudan & EMRO \\
\hline AFRO & $E$ & 2 & $\begin{array}{l}\text { Botswana, Burundi, Central African Republic, Democratic Republic of Congo, Republic } \\
\text { of Congo, Côte d'Ivoire, Eritrea, Ethiopia, Kenya, Lesotho, Malawi, Mozambique, } \\
\text { Namibia, Rwanda, South Africa, Swaziland, Tanzania, Uganda, Zambia, Zimbabwe }\end{array}$ & AFRO \\
\hline AMRO & $A^{b}$ & 3 & Canada, United States of America & AMRO \\
\hline AMRO & $\mathrm{B}^{\mathrm{C}}$ & 4 & $\begin{array}{l}\text { Antigua and Barbuda, Argentina, The Bahamas, Barbados, Belize, Brazil, Chile, } \\
\text { Colombia, Costa Rica, Cuba, Dominica, Dominican Republic, El Salvador, Grenada, } \\
\text { Guyana, Honduras, Jamaica, Mexico, Panama, Paraguay, St. Kitts and Nevis, St. Lucia, } \\
\text { St. Vincent and the Grenadines, Suriname, Trinidad and Tobago, Uruguay, República } \\
\text { Bolivariana de Venezuela }\end{array}$ & AMRO \\
\hline AMRO & D & 5 & Bolivia, Ecuador, Guatemala, Haiti, Nicaragua, Peru & AMRO \\
\hline \multirow[t]{2}{*}{ EMRO } & \multirow[t]{2}{*}{ B } & \multirow[t]{2}{*}{6} & $\begin{array}{l}\text { Bahrain, Islamic Republic of Iran, Jordan, Kuwait, Lebanon, Libya, Oman, Qatar, Saudi } \\
\text { Arabia, Syrian Arab Republic, Tunisia, United Arab Emirates }\end{array}$ & EMRO \\
\hline & & & Cyprus & EURO \\
\hline EMRO & $D^{d}$ & 7 & Arab Republic of Egypt, Iraq, Morocco, Republic of Yemen & EMRO \\
\hline EURO & $A^{e}$ & 8 & $\begin{array}{l}\text { Andorra, Austria, Belgium, Croatia, Czech Republic, Denmark, Finland, France, } \\
\text { Germany, Greece, Iceland, Ireland, Israel, Italy, Luxembourg, Malta, Monaco, } \\
\text { Netherlands, Norway, Portugal, San Marino, Slovenia, Spain, Sweden, Switzerland, } \\
\text { United Kingdom }\end{array}$ & EURO \\
\hline EURO & B1 & 9 & $\begin{array}{l}\text { Albania, Bosnia and Herzegovina, Bulgaria, Georgia, Former Yugoslav Republic of } \\
\text { Macedonia, Poland, Romania, Serbia and Montenegro, Slovak Republic, Turkey }\end{array}$ & EURO \\
\hline EURO & B2 & 10 & Armenia, Azerbaijan, Republic of Kyrgyz, Tajikistan, Turkmenistan, Uzbekistan & EURO \\
\hline EURO & C & 11 & $\begin{array}{l}\text { Belarus, Estonia, Hungary, Kazakhstan, Latvia, Lithuania, Moldova, } \\
\text { Russian Federation, Ukraine }\end{array}$ & EURO \\
\hline \multirow[t]{2}{*}{ SEARO } & \multirow[t]{2}{*}{ B } & \multirow[t]{2}{*}{12} & Indonesia, Sri Lanka, Thailand & SEARO \\
\hline & & & Brunei Darussalam, Malaysia, the Philippines, Singapore & WPRO \\
\hline \multirow[t]{2}{*}{ SEARO } & \multirow[t]{2}{*}{ D } & \multirow[t]{2}{*}{13} & Bangladesh, Bhutan, India, Maldives, Nepal, Timor-Leste & SEARO \\
\hline & & & Afghanistan, Pakistan & EMRO \\
\hline WPRO & A & 14 & Australia, Japan, New Zealand & WPRO \\
\hline \multirow[t]{2}{*}{ WPRO } & \multirow[t]{2}{*}{ B1 } & \multirow[t]{2}{*}{15} & China, Mongolia, Republic of Korea & WPRO \\
\hline & & & Democratic People's Republic of Korea & SEARO \\
\hline \multirow[t]{2}{*}{ WPRO } & \multirow[t]{2}{*}{ B2 } & \multirow[t]{2}{*}{16} & Cambodia, Lao People's Democratic Republic, Vietnam & WPRO \\
\hline & & & Myanmar & SEARO \\
\hline WPRO & $B 3^{f}$ & 17 & $\begin{array}{l}\text { Cook Islands, Fiji, Kiribati, Marshall Islands, Federated States of Micronesia, Nauru, } \\
\text { Niue, Palau, Papua New Guinea, Samoa, Solomon Islands, Tonga, Tuvalu, Vanuatu }\end{array}$ & WPRO \\
\hline
\end{tabular}

Source: Authors' compilation.

a. Réunion and St. Helena are assumed to have the same burden of disease rates as the WHO member states shown.

b. St. Pierre et Miquelon is assumed to have the same burden of disease rates as the WHO member states shown.

c. Anguilla, Aruba, Bermuda, British Virgin Islands, Caymen Islands, Falkland Islands, French Guiana, Guadeloupe, Martinique, Montserrat, Netherlands Antilles, Puerto Rico, Turks and Caicos Islands, and U.S. Virgin Islands are assumed to have the same burden of disease rates as the WHO member states shown.

d. Western Sahara is assumed to have the same burden of disease rates as the WHO member states shown. Burden of disease rates for the West Bank and Gaza were estimated using death registration data and separate estimates of war deaths.

e. Channel Islands, Faeroe Islands, Isle of Man, Gibraltar, Greenland, Holy See (Vatican City), and Liechtenstein are assumed to have the same burden of disease rates as the WHO member states shown. f. Samoa, French Polynesia, Guam, New Caledonia, Northern Mariana Islands, Pitcairn, Tokelau, and Wallis and Futuna Islands are assumed to have the same burden of disease rates as the WHO member states shown. 
Table 3A.5 GBD Cause Categories, Sequelae, and Case Definitions

\section{GBD cause/sequela \\ I. Communicable, maternal, perinatal, and nutritional conditions}

A1. Tuberculosis

HIV sero-negative cases

HIV sero-positive cases

A2a. Syphilis

Congenital syphilis

Low birthweight

Primary

Secondary

Tertiary—neurologic

A2b. Chlamydia

Cervicitis

Neonatal pneumonia

Ophthalmia neonatorum

Low birthweight

Pelvic inflammatory disease

Ectopic pregnancy

Tubo-ovarian abscess

Chronic pelvic pain

Infertility

Symptomatic urethritis

Epididymitis

A2c. Gonorrhea

Ophthalmia neonatorum

Low birthweight

Corneal scar—blindness

Corneal scar-low vision

Cervicitis

Pelvic inflammatory disease

Ectopic pregnancy

Tubo-ovarian abscess

Chronic pelvic pain

Infertility

Symptomatic urethritis

Epididymitis

Stricture
Case definition

Version $^{\mathrm{a}}$

Cases refer to individuals with clinical tuberculosis, normally pulmonary sputum culture positives and extra-pulmonary cases

HIV sero-negative cases

HIV sero-positive cases

Acute and chronic infection with Treponema pallidum

Syphilis in the newborn due to maternal-fetal transmission in utero

Birthweight of less than 2,500 g

Initial infection in adults resulting in primary chancre at site of inoculation

Disseminated disease, which appears 2-8 weeks after the primary stage and is usually marked by a rash

Late stage of disease with varied neurological manifestations

Bacterial infection transmitted vaginally, anally, or perinatally with Chlamydia trachomatis (excludes ocular trachoma)

Inflammation of the cervix uteri due to Chlamydia trachomatis

Pneumonia in infants due to infection with Chlamydia

Purulent conjunctivitis in infants less than 30 days, acquired during passage through an infected birth canal

Birthweight of less than $2,500 \mathrm{~g}$

Inflammation of the adnexa of the uterus (includes endometritis)

Pregnancy located outside the uterus

Abscess located in the fallopian tubes or ovaries

Chronic pelvic pain following reproductive tract infection with Chlamydia

Total infertility due to Chlamydia-related pelvic inflammatory disease and ectopic pregnancy in women and epididymitis in men.

Inflammation of the urethra causing symptoms including dysuria and/or hematuria Inflammation of the sperm ducts

Bacterial infection transmitted vaginally, anally, or perinatally with Neisseria gonorrhea

Purulent conjunctivitis in infants less than 30 days, acquired during passage through an infected birth canal

Birthweight of less than $2,500 \mathrm{~g}$

Permanent corneal scar resulting from corneal ulceration due to infection with Neisseria gonorrhea and leading to blindness

Permanent corneal scar resulting from corneal ulceration due to infection with Neisseria gonorrhea and leading to low vision

Inflammation of the cervix uteri due to Neisseria gonorrhea

Includes both acute and recurrent pelvic inflammatory disease due to gonorrhea

Pregnancy located outside the uterus

Abscess located in the fallopian tubes or ovaries

Chronic pelvic pain following reproductive tract infection with Neisseria gonorrhea

Total infertility due to gonorrhea-related pelvic inflammatory disease and ectopic pregnancy in women and epididymitis in men

Inflammation of the urethra causing symptoms including dysuria and/or hematuria Inflammation of the sperm ducts

Narrowing of the urethra due to urethritis 
Table 3A.5 Continued

\begin{tabular}{|c|c|c|c|}
\hline \multicolumn{2}{|c|}{ GBD cause/sequela } & \multirow[t]{2}{*}{ Case definition } & \multirow{2}{*}{$\frac{\text { Version }^{\mathbf{a}}}{2}$} \\
\hline A3. & HIV/AIDS & & \\
\hline & HIV cases & HIV sero-positive, not yet progressed to AIDS & \\
\hline & AIDS cases & HIV sero-positive and progressed to AIDS & \\
\hline A4. & Diarrheal diseases_-episodes & $\begin{array}{l}\text { Episodes of diarrhea including acute watery diarrhea, persistent diarrhea, and } \\
\text { dysentery; deaths of children with both measles and diarrhea or both lower respi- } \\
\text { ratory infection and diarrhea are not included in estimates of diarrhea mortality }\end{array}$ & 2 \\
\hline A5a. & Pertussis & $\begin{array}{l}\text { Acute bacterial infection of the respiratory tract with Bordetella pertussis or } \\
\text { parapertussis }\end{array}$ & 2 \\
\hline & Episodes & $\begin{array}{l}\text { Acute bacterial infection of the respiratory tract with Bordetella pertussis or } \\
\text { parapertussis, characterized by paroxysmal, violent coughs followed by high- } \\
\text { pitched inspiratory whoop }\end{array}$ & \\
\hline & Encephalopathy & $\begin{array}{l}\text { Degenerative disease of the brain, which in pertussis is usually a result of } \\
\text { hypoxia, leading to mental retardation }\end{array}$ & \\
\hline A5b. & Poliomyelitis-lameness & $\begin{array}{l}\text { Viral infection characterized by acute flaccid paralysis and proven by isolation of } \\
\text { polio virus from stool }\end{array}$ & 2 \\
\hline A5c. & Diphtheria & Acute disease caused by toxin-producing Corynebacterium diphtheriae & 2 \\
\hline & Episodes & $\begin{array}{l}\text { Acute bacterial disease involving primarily tonsils, pharynx, larynx, nose, and } \\
\text { other sites, characterized by grayish plaques or membranes with surrounding } \\
\text { tissue inflammation }\end{array}$ & \\
\hline & Neurological complications & $\begin{array}{l}\text { Polyneuritis involving both cranial and peripheral nerve palsies, which are largely } \\
\text { reversible }\end{array}$ & \\
\hline & Myocarditis & $\begin{array}{l}\text { Inflammation of the heart muscle leading to electrocardiographic aberrations and } \\
\text { sometimes permanent damage with congestive heart failure, which may be fatal }\end{array}$ & \\
\hline A5d. & Measles-episodes & $\begin{array}{l}\text { Acute and highly contagious infection with measles virus characterized by red, } \\
\text { blotchy rash, fever, cough, coryza, and conjunctivitis }\end{array}$ & 2 \\
\hline \multirow[t]{2}{*}{ A5e. } & Tetanus_episodes & $\begin{array}{l}\text { Neonatal: infection with Clostridium tetani in infants less than } 30 \text { days with pro- } \\
\text { gressive difficulty and inability to feed because of trismus, generalized stiffness, } \\
\text { spasms, and opisthotonus }\end{array}$ & 2 \\
\hline & & $\begin{array}{l}\text { Non-neonatal: infection with Clostridium tetani in non-neonates with initial } \\
\text { localized spasms lead to general rigidity, opisthotonus, and risus sardonicus }\end{array}$ & \\
\hline A6. & Meningitis & $\begin{array}{l}\text { Acute bacterial disease with sudden onset and fever, intense headache, nausea, } \\
\text { vomiting, neck stiffness, and-in meningococcal disease—-petechial rash with } \\
\text { pink macules; must be accompanied by laboratory evidence (in cerebrospinal fluid } \\
\text { or blood) of Neisseria meningitidis, Strep pneumoniae, or Haemophilus influenzae } \\
\text { type B }\end{array}$ & 2 \\
\hline & Streptococcus pneumoniae-episodes & $\begin{array}{l}\text { Acute bacterial disease with sudden onset and fever, intense headache, nausea, } \\
\text { vomiting, and neck stiffness; must be accompanied by laboratory evidence (in } \\
\text { cerebrospinal fluid or blood) of Strep pneumoniae }\end{array}$ & \\
\hline & Haemophilus influenzae—episodes & $\begin{array}{l}\text { Acute bacterial disease with sudden onset and fever, intense headache, nausea, } \\
\text { vomiting, and neck stiffness; must be accompanied by laboratory evidence (in } \\
\text { cerebrospinal fluid or blood) of Haemophilus influenza type B }\end{array}$ & \\
\hline & Neisseria meningitidis—episodes & $\begin{array}{l}\text { Acute bacterial disease with sudden onset and fever, intense headache, nausea, } \\
\text { vomiting, and neck stiffness; must be accompanied by laboratory evidence (in } \\
\text { cerebrospinal fluid or blood) of Neisseria meningitidis }\end{array}$ & \\
\hline & $\begin{array}{l}\text { Meningococcaemia without } \\
\text { meningitis—episodes }\end{array}$ & Invasion of the bloodstream with Neisseria meningitidis. & \\
\hline & Deafness & $\begin{array}{l}\text { At least moderate impairment, where person is able to hear and repeat words } \\
\text { using raised voice at } 1 \text { meter, resulting from meningitis }\end{array}$ & \\
\hline & Seizure disorder & $\begin{array}{l}\text { Seizures of any type that were present at least six months after hospitalization, } \\
\text { resulting from meningitis }\end{array}$ & \\
\hline
\end{tabular}


Table 3A.5 Continued

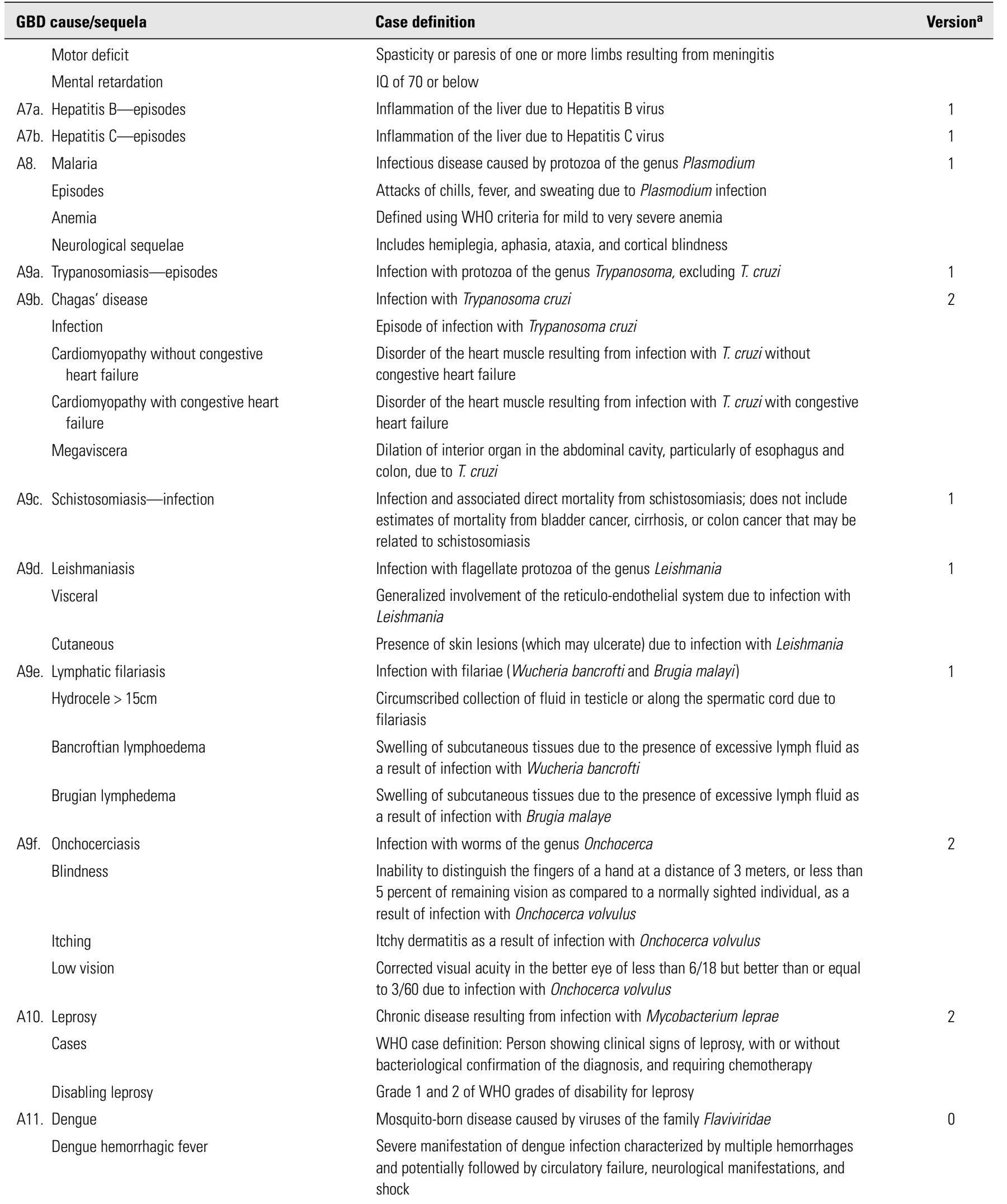

110 | Global Burden of Disease and Risk Factors I Colin D. Mathers, Alan D. Lopez, and Christopher J. L. Murray 
Table 3A.5 Continued

\section{GBD cause/sequela}

A12. Japanese encephalitis

Episodes

Cognitive impairment

Neurological sequelae

A13. Trachoma

Blindness

Low vision

A14a. Ascariasis

High-intensity infection

Contemporaneous cognitive deficit

Cognitive impairment

Intestinal obstruction

A14b. Trichuriasis

High-intensity infection

Contemporaneous cognitive deficit

Massive dysentery syndrome

Cognitive impairment

A14c. Hookworm disease

High-intensity infection

Anemia

Cognitive impairment

B1. Lower respiratory infections

Episodes

Chronic sequelae

B2. Upper respiratory infections Episodes

Pharyngitis

B3. Otitis media

Episodes

Deafness

C1. Maternal hemorrhage

Episodes

Severe anemia

\section{Case definition}

Version $^{\mathrm{a}}$

Mosquito-born encephalitis caused by Japanese encephalitis virus

0

Episode of Japanese encephalitis infection

Reduced cognitive function resulting from encephalitis due to Japanese

encephalitis virus

Neurological deficits resulting from encephalitis due to Japanese encephalitis virus

Cases of follicular or inflammatory trachoma

Corrected visual acuity in the better eye of less than 3/60

Corrected visual acuity in the better eye of less than $6 / 18$ but better than or equal to $3 / 60$

Infection with roundworms of the genus Ascaris

1

Infection resulting in at least 20-40 worms per stool load

Reduction in cognitive ability in school-age children, which occurs only while infection persists

Delayed psychomotor development and impaired performance in language skills, motor skills, and coordination equivalent to a 5- to 10-point deficit in 10

Blockage of the intestines due to worm mass

Infection with the whipworm Trichuris trichiura

1

Infection resulting in at least 250-500 worms per stool load

Reduction in cognitive ability in school-age children, which occurs only while infection persists

Rectal prolapse and/or tenesmus and/or bloody mucoid stools due to carpeting of intestinal mucosa by worms

Delayed psychomotor development and impaired performance in language skills, motor skills, and coordination equivalent to a 5- to 10-point deficit in 10

Infection with hookworms of the genus Ancylostomiasis and Necatoriasis

1

Infection resulting in at least 80-160 worms per stool load

Anemia due to hookworm infection

Delayed psychomotor development and impaired performance in language skills, motor skills, and coordination equivalent to a 5- to 10-point deficit in I0

Episode of lower respiratory infection

Includes bronchiectasis and impaired lung function as measured by a decrease in forced expiratory volume

Episode of upper respiratory infection

Inflammation of the pharynx

Inflammation of the middle ear

Episodes of acute otitis media

At least moderate impairment, where person is able to hear and repeat words using raised voice at 1 meter, resulting from otitis media

All episodes of antepartum and postpartum hemorrhage

Blood hemoglobin level $<10 \mathrm{mg} / \mathrm{dl}$ following postpartum hemorrhage

(Continues on the following page.) 
Table 3A.5 Continued

\section{GBD cause/sequela}

C2. Maternal sepsis

Episodes

Infertility

C3. Hypertensive disorders of pregnancyepisodes

C4. Obstructed labor

Episodes

Cesarean section for obstructed labor

Stress incontinence

Rectovaginal fistula

C5. Abortion

Episodes

Infertility

Reproductive tract infection

D1. Low birthweight_all sequelae

D2. Birth asphyxia and birth trauma -all sequelae

E1. Protein-energy malnutrition

Wasting

Stunting

Developmental disability

E2. Iodine deficiency

Goiter grades 1 and 2

Mild developmental disability

Cretinoidism

Cretinism

E3. Vitamin A deficiency

Xerophthalmia

Corneal scar
Case definition

Version $^{\mathbf{a}}$

Major puerperal infection, excluding infection following abortion, minor genital tract infection following delivery, and urinary tract infections following delivery

Failure to conceive again after a previous conception (secondary infertility), caused by maternal sepsis

Includes pre-eclampsia and eclampsia

Labor with no advance of the presenting part of the fetus despite strong uterine contractions

Cases of obstructed labor for which cesarean section has been performed

Cases with leaking of urine during coughing or sneezing

Cases with a communication between the vaginal wall and the bladder/rectum resulting from obstructed labor

Episodes of unsafe abortion (termination of an unwanted pregnancy either by persons lacking the necessary skills or in an environment lacking the necessary standards or both)

Failure to conceive following unsafe abortion

Cases of reproductive tract infection resulting from unsafe abortion

Birthweight below 2,500 g, including small-for-gestational-age infants and premature infants (all developmental sequelae due to low birthweight have been clustered into one outcome, which includes cerebral palsy, mental retardation, epilepsy, hearing loss, and vision loss)

All developmental sequelae due to birth asphyxia and birth trauma have been clustered into one outcome, which includes cerebral palsy, mental retardation, epilepsy, hearing loss, and vision loss

Observed weight for height at least 2 standard deviations below the mean for children ages $0-5$

Observed height for age at least 2 standard deviations below the mean for children ages $0-5$

Limited physical and mental ability to perform most activities in all of the following areas: recreation, education, procreation, or occupation

Cases of goiter grade 1 (a mass in the neck consistent with an enlarged thyroidgrade 1: palpable but not visible) and grade 2 (a mass in the neck consistent with an enlarged thyroid — grade 2: palpable and visible in neutral neck position)

Any of the following due to iodine deficiency:

Bilateral hearing loss, delay of walking ability, mild intellectual impairment

Hypothyroid cretinism: Hypothyroidism and stunting as a result of iodine deficiency

Neurological cretinism: Mental deficiency (I0 below 70), deaf-mutism, and spastic paralysis as a result of iodine deficiency

Some but not all features of full cretinism as a result of iodine deficiency

All ocular manifestations of vitamin A deficiency: night blindness, Bitot's spots, corneal xerosis, corneal ulceration, and corneal scarring

Permanent corneal scar resulting from corneal ulceration due to vitamin A deficiency and potentially leading to blindness

112 | Global Burden of Disease and Risk Factors I Colin D. Mathers, Alan D. Lopez, and Christopher J. L. Murray 
Table 3A.5 Continued

\begin{tabular}{l}
\hline GBD cause/sequela \\
\hline E4. Iron-deficiency anemia \\
Mild \\
Moderate \\
Severe \\
Cognitive impairment
\end{tabular}

\section{Noncommunicable diseases}

A. Malignant neoplasms sequelae

Diagnosis and primary therapy

Control

Preterminal (metastasis)

Terminal

Mastectomy

Infertility

Incontinence or impotence

Stoma

C. Diabetes mellitus

Cases

Diabetic foot

Neuropathy

Retinopathy_-blindness

Amputation

E1. Unipolar depressive disorders

Mild episode

Moderate episode

Severe episode

Dysthymia

E2. Bipolar affective disorder-cases

E3. Schizophrenia-cases

E4. Epilepsy-cases

E6. Alzheimer's and other dementias — cases

E7. Parkinson's disease-cases

E8. Multiple sclerosis-cases

\section{Case definition}

Version $^{\mathrm{a}}$

2

Hemoglobin of 100-109 $\mathrm{g} / \mathrm{l}$ in pregnant women, $110-119 \mathrm{~g} / \mathrm{l}$ in children and adult women, and $120-129 \mathrm{~g} / \mathrm{l}$ in adult men

Hemoglobin of 70-99 $\mathrm{g} / \mathrm{l}$ in pregnant women, 80-109 $\mathrm{g} / \mathrm{l}$ in children and adult women, and 90-119 $\mathrm{g} / \mathrm{l}$ in adult men

Hemoglobin of 40-69 $\mathrm{g} / \mathrm{l}$ in pregnant women, $50-79 \mathrm{~g} / \mathrm{l}$ in children and adult women, and $60-89 \mathrm{~g} / \mathrm{l}$ in adult men

Delayed psychomotor development and impaired performance in language skills, motor skills, and coordination equivalent to a 5- to 10-point deficit in 10

Chemotherapy, radiotherapy, surgery

Clinical observation during control/remission phase

Metastatic dissemination of the disease

Terminal stage prior to death

Mastectomy in five-year breast cancer survivor

Infertility in five-year survivor of cervical, uterine, or ovarian cancer

Incontinence or impotence in five-year survivor of prostate cancer

Stoma in five-year survivor of digestive system cancer

Venous plasma concentration of $\mu 11.1 \mathrm{mmol} / \mathrm{l} 2 \mathrm{~h}$ after a $75 \mathrm{~g}$ oral glucose challenge

Chronic or recurring diabetic foot ulcers

Loss of reflexes and vibration; damage and dysfunction of sensory, motor, or autonomic nerves attributable to diabetes

Retinopathy: Microaneurysms or worse lesions in at least one eye; progressive damage of the small blood vessels of the retina

Blindness: Unable to distinguish the fingers of a hand at the distance of 3 meters, or has less than 5 percent of remaining vision as compared to a normally sighted individual; visual acuity of less than $3 / 60$ or corresponding visual field loss in the better eye with best possible correction

Surgical elimination of the lower extremity or part of it due to gangrene

Mild major depressive episode (ICD-10 codes F 32.0 and F 33.0)

Moderate major depressive episode (ICD-10 codes F 32.1 and F 33.1)

Severe major depressive episode (ICD-10 codes F 32.2 , F 32.3, F 33.2 and F 33.3)

Dysthymia case with no concurrent major depressive episode

Cases meeting ICD-10 criteria

Cases meeting ICD-10 criteria 2

Cases meeting ILAE definition 1

Cases meeting ICD-10 criteria for alcohol dependence and harmful use (F10.1 and

F 10.2), excluding cases with comorbid depressive episode

Mild, moderate, and severe Alzheimer's disease; senility; and other dementias 2

Cases meeting clinical criteria for Parkinson's disease 1

Cases of chronic or intermittent relapsing multiple sclerosis 
Table 3A.5 Continued

\section{GBD cause/sequela}

E9. Drug use disorders

E10. Post-traumatic stress disorder-cases

E11. Obsessive-compulsive disorder—cases

E12. Panic disorder—cases

E13. Insomnia (primary)

E14. Migraine
E15. Mild mental retardation attributable
to lead exposure
F1. Glaucoma
Low vision
Blindness
F2. Cataracts
Low vision
Blindness
F3. Vision disorders, age-related

Low vision

Blindness

F4. Hearing loss, adult onset

Mild hearing loss, untreated

Moderate hearing loss, treated

Severe or profound hearing loss, untreated

Severe or profound hearing loss, treated

G1. Rheumatic heart disease

G2. Hypertensive heart disease
Case definition

Version $^{\mathrm{a}}$

Cases meeting ICD-10 criteria for opioid dependence and harmful use

( F 11.1 F 11.2) or cocaine dependence and harmful use (F 14.1 and F 14.2), excluding cases with comorbid depressive episode

Cases meeting DSM IV criteria ${ }^{b}$ for post-traumatic stress disorder, excluding cases with comorbid depressive episode or alcohol and drug use (harmful and/or dependence)

Cases meeting ICD-10 criteria (F 42), excluding cases with comorbid depressive episode

Cases meeting ICD-10 criteria for panic disorder (F 41.0) or agoraphobia with panic disorder (F 40.01), excluding cases with comorbid depressive episode.

Cases meeting DSM IV criteria for primary insomnia (307.42) where the insomnia causes problems with usual activities; excludes cases with comorbid depressive episode or alcohol and drug use (harmful and/or dependence)

Cases meeting IHS definition for migraine

10 in the range of 50-69 attributable to childhood lead exposure

Cases of primary angle closure glaucoma and primary open angle glaucoma

Corrected visual acuity in the better eye of less than $6 / 18$ but better than or equal to $3 / 60$

Corrected visual acuity in the better eye of less than $3 / 60$

Cases of senile cataract causing progressive visual impairment

Corrected visual acuity in the better eye of less than $6 / 18$ but better than or equal to $3 / 60$

Corrected visual acuity in the better eye of less than $3 / 60$

Low vision or blindness due to macular degeneration, refractive errors, or other age-related causes; excludes sight loss due to congenital causes, other diseases, or injury

Corrected visual acuity in the better eye of less than $6 / 18$ but better than or equal to $3 / 60$

Corrected visual acuity in the better eye of less than $3 / 60$

Cases of adult onset hearing loss due to aging or noise exposure; excludes hearing loss due to congenital causes, infectious diseases, other diseases, or injury

Hearing threshold level in the better ear is 41-60 dBHTL (averaged over 0.5, 1, 2, $4 \mathrm{kHz}$ ) (some difficulty understanding or actively participating in a conversation with one person, great difficulty with more than one person); person does not use a hearing aid

Hearing threshold level in the better ear is 41-60 dBHTL (averaged over 0.5, 1, $2,4 \mathrm{kHz}$ ) (some difficulty understanding or actively participating in a conversation with one person, great difficulty with more than one person); person uses a hearing aid

Hearing threshold level in the better ear is $61 \mathrm{dBHTL}$ or more laveraged over 0.5 , $1,2,4 \mathrm{kHz}$ ) (great difficulty or unable to understand or participate in a conversation with one other person); person does not use a hearing aid

Hearing threshold level in the better ear is $61 \mathrm{dBHTL}$ or more laveraged over 0.5, $1,2,4 \mathrm{kHz}$ ) (great difficulty or unable to understand or participate in a conversation with one other person); person uses a hearing aid

Symptomatic cases of congestive heart failure due to rheumatic heart disease

Symptomatic cases of congestive heart failure due to hypertensive heart disease
2

114 | Global Burden of Disease and Risk Factors I Colin D. Mathers, Alan D. Lopez, and Christopher J. L. Murray 
Table 3A.5 Continued

\section{GBD cause/sequela}

G3. Ischemic heart disease

Acute myocardial infarction

Angina pectoris

Congestive heart failure

G4. Cerebrovascular disease

First-ever stroke cases

Long-term stroke survivors

G5. Inflammatory heart diseases

Myocarditis

Pericarditis

Endocarditis

Cardiomyopathy

H1. Chronic obstructive pulmonary disease-symptomatic cases

H2. Asthma_cases

11. Peptic ulcer disease

Cases with antibiotic treatment

Cases not treated with antibiotic

12. Cirrhosis of the liver-symptomatic cases

13. Appendicitis-episodes

J1. Nephritis and nephrosis

Acute glomerulonephritis

End-stage renal disease

J2. Benign prostatic hypertrophysymptomatic cases

L1. Rheumatoid arthritis_-cases

L2. Osteoarthritis

Hip

Knee

L3. Gout

L4. Low back pain

Episode of limiting low back pain

Case definition

Version $^{\mathrm{a}}$

Definite and possible episodes of acute myocardial infarction according to MONICA study criteria

Cases of clinically diagnosed angina pectoris or definite angina pectoris according to Rose questionnaire

Mild and greater (Killip scale k2-k4)

First-ever stroke according to WHO definition; includes subarachnoid hemorrhage, but excludes transient ischemic attacks, subdural hematoma, and hemorrhage or infarction due to infection or tumor

Persons who survive more than 28 days after first-ever stroke

Symptomatic cases of congestive heart failure due to myocarditis

Symptomatic cases of congestive heart failure due to pericarditis

Symptomatic cases of congestive heart failure due to endocarditis

Symptomatic cases of congestive heart failure due to cardiomyopathy

Chronic (stable) airways obstruction with FEV $<1$ liter (corresponding to

symptomatic disability

Reported wheeze in the last 12 months plus current bronchial hyperresponsiveness, defined as a 20 percent fall in $\mathrm{FEV}_{1}$ with a provoking concentration of histamine (PC20) at $8 \mathrm{mg} / \mathrm{ml}$ or less

Individuals with peptic ulcers, most of whom have recurrent intermittent symptoms

Active gastric or peptic duodenal ulcer receiving appropriate antibiotic treatment

Other active gastric or peptic duodenal ulcer; includes untreated cases and cases receiving symptomatic treatment

Individuals with symptomatic cirrhosis

Episodes of acute appendicitis (treated or untreated)

Acute episode of glomerulonephritis

End-stage renal failure with or without dialysis, excluding diabetic nephropathy and nephropathy due to cancers, congenital conditions, and injury

Individuals with some, albeit intermittent, symptoms from benign prostatic hypertrophy

Definite or classical rheumatoid arthritis by 1958 ARA or 1987 ACR criteria

Symptomatic osteoarthritis of the hip, radiologically confirmed as KellgrenLawrence grade 2-4

Symptomatic osteoarthritis of the knee, radiologically confirmed as KellgrenLawrence grade 2-4

Cases of gout (ARA 1977 survey criteria; at least 6 of 11 symptoms)

Acute episode of low back pain resulting in moderate or greater limitations to mobility and usual activities; excludes low back pain due to intervertebral disc displacement or herniation, and low back pain that does not result in some limitations to mobility and usual activities

(Continues on the following page.) 
Table 3A.5 Continued

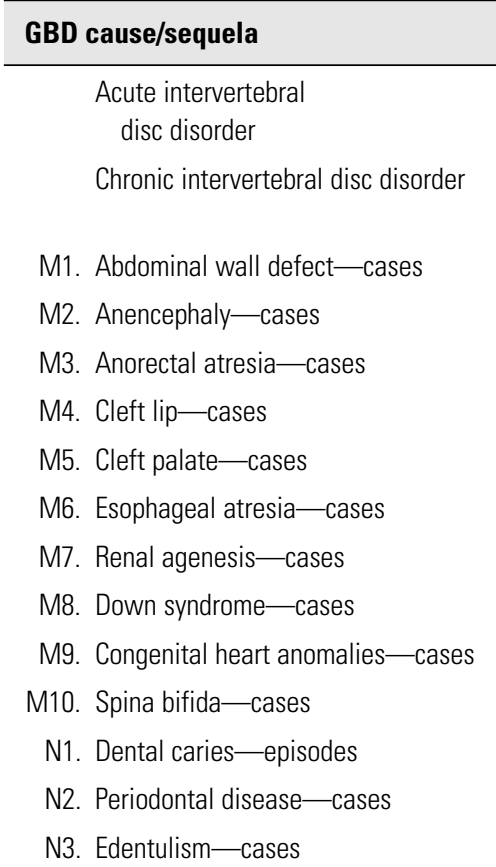

Episode of intervertebral disc displacement or herniation

Disorder of intervertebral disc resulting in pain and disability that does not resolve within six weeks following treatment (medical or surgical)

Live-born cases with exomphalos or gastroschisis 0

Live-born cases with anencephaly 0

Live-born cases with anorectal atresia 0

Live-born cases, includes individuals who have had surgical correction 0

Live-born cases, includes individuals who have had surgical correction 0

Live-born cases with esophageal atresia 0

Live-born cases with renal agenesis 0

Live-born cases with Down syndrome 0

Live-born cases with major congenital malformations of the heart 0

Live-born cases with spina bifida aperta (low, medium, or high level) 0

Episodes per person, not per tooth, quadrant, or sextant 0

Pockets greater than $6 \mathrm{~mm}$ deep 0

Cases of treated and untreated edentulism (absence of all teeth) 0

\section{Injuries}

External cause categories

Includes injury severe enough to warrant medical attention or that leads immediately to death. In other words, injuries that are severe enough that if an individual had access to a medical facility he or she would seek attention ${ }^{\mathrm{C}}$

A1. Road traffic accidents

Includes crashes and pedestrian injuries due to motor vehicles

Only one outcome is included for poisonings

Includes falls resulting from osteoporotic fractures

A3. Falls

Most of the sequelae of fires are due to burns; some individuals, however, jump

from buildings or are otherwise injured because of fires

A5. Drownings

Other than drowning and near-drowning rates, the only other major disabling sequelae from near-drowning included is quadriplegia

A6. Other unintentional injuries

This is not a residual category, but includes injuries due to environmental factors, machinery and electrical equipment, cutting and piercing implements, and various other external causes of unintentional injury

B1. Self-inflicted injuries

Suicide attempts, whether or not resulting in death

B2. Violence

Interpersonal violence, including assault and homicide

Injuries directly attributable to war or organized civil conflict in combatants and noncombatants

For each of the external cause categories, injury sequelae defined in terms of type

\section{Type of injury-sequelae} of injury were analyzed. The type of injury sequelae were defined in terms of ICD-9 and ICD-10 nature of injury codes ( $\mathrm{N}$-codes) as follows:
ICD-9 Code
ICD-10 Code 
Table 3A.5 Continued

\begin{tabular}{|c|c|c|}
\hline GBD cause/sequela & ICD-9 code & ICD-10 code \\
\hline \multicolumn{3}{|l|}{ 1. Fractures } \\
\hline Skull—short-term ${ }^{d}$ & 800 to 801 & S02.0/1/7/9, T90.2 \\
\hline Skull-long-term ${ }^{\mathrm{d}}$ & 800 to 801 & S02.0/1/7/9, T90.2 \\
\hline Face bones $^{d}$ & 802 & S02.2/6/8 \\
\hline Vertebral column & 805 & S12, S22.0/1, S32.0/7, T91.1 \\
\hline Rib or sternum ${ }^{\mathrm{e}}$ & 807 & S22.2-9 \\
\hline Pelvis ${ }^{\mathrm{e}}$ & 808 & S32.1-5/8, T91.2 \\
\hline Clavicle, scapula or humerus ${ }^{\dagger}$ & 810-812 & S42, S49.7 \\
\hline Radius or ulna ${ }^{f}$ & 813 & S52, S59.7, T10, T92.1 \\
\hline Hand bones ${ }^{f}$ & 814-817 & S62, S69.7, T92.2 \\
\hline Femur-short-term ${ }^{g}$ & $820-821$ & S72, S79.7 \\
\hline Femur-long-term ${ }^{g}$ & $820-821$ & S72, S79.7 \\
\hline Patella, tibia, or fibulag & $822-823$ & S82.0-4, S82.7/9, S89.7, T12 \\
\hline Ankleg & 824 & S82.5-6/8 \\
\hline Foot bones ${ }^{g}$ & $825-826$ & S92, S99.7 \\
\hline 2. Injured spinal cord & 806 and 952 & S14, S24, S34, T06.0/1, T08, T91.3 \\
\hline \multicolumn{3}{|l|}{ 3. Dislocations } \\
\hline Shoulder, elbow, or hip & $831,832,835$ & S43, S73 \\
\hline \multirow[t]{2}{*}{ Other dislocation } & 830, 833-834, 836-839 & $\begin{array}{l}\text { S03.0-3, S13, S23, S33, S53, S63.0/1, S83.1-3, S93.1-3, T03, T11.2, T13.2, } \\
\text { T14.3, T92.3, T93.3 }\end{array}$ \\
\hline & 840-848 & $\begin{array}{l}\text { S03.4/5, S16, S29.0, S39.0, S46, S56, S63.5-7, S66, S76, S83.4/7, S86, } \\
\text { S93.4/6, S96, T06.4, T11.5, T13.5, T14.6, T92.5, T93.5 }\end{array}$ \\
\hline \multicolumn{3}{|l|}{ 4. Sprains } \\
\hline \multicolumn{3}{|l|}{ 5. Intracranial injuries } \\
\hline Short-term & $850-854$ & S06, T90.5 \\
\hline Long-term & $850-854$ & SO6, T90.5 \\
\hline 6. Internal injuries & 860-869 & S25-S27, S35-S37, S39.6, T06.4, T91.4/5 \\
\hline 7. Open wound & $870,872-884,890-894$ & $\begin{array}{l}\text { S01, S08, S11, S15, S21, S31, S41, S45, S51, S55, S61, S65, S71, S75, } \\
\text { S81, S85, S91, S95, T01, T11.1/4, T13.5, T14.6, T90.1, T92.5, T93.5 }\end{array}$ \\
\hline \multicolumn{3}{|l|}{ 8. Injury to eyes } \\
\hline Short-term & 871,950 & S05, T90.4 \\
\hline Long-term & 871,950 & S05, T90.4 \\
\hline \multicolumn{3}{|l|}{ 9. Amputations } \\
\hline Thumb & 885 & S68.0 \\
\hline Finger & 886 & S68.1/2 \\
\hline Arm & 887 & S48, S58, S68.3-9, T05.0/2, T11.6 \\
\hline$T_{0 e^{h}}$ & 895 & $\$ 98.1 / 2$ \\
\hline Foot $^{\text {h }}$ & 896, 897.0-1 & S98.0/3/4, T05.3 \\
\hline Leg $^{\text {h }}$ & $897.2-3$ & S78, S88, T05.4/6, T13.6 \\
\hline 10. Crushing & $925-929$ & S07, S17, S28, S38, S47, S57, S67, S77, S87, S97, T04, T14.7, T92.6, T93.6 \\
\hline \multicolumn{3}{|l|}{ 11. Burns } \\
\hline Less than $20 \%$ —short-term & $940-947,948.0-1$ & T31.0/1 \\
\hline Less than $20 \%$-long-term ${ }^{i}$ & 940-947, 948.0-1 & T31.0/1 \\
\hline 20 to $60 \%$ - short-term ${ }^{i}$ & $948.2-5$ & T331.2/5 \\
\hline
\end{tabular}

(Continues on the following page.) 
Table 3A.5 Continued

\begin{tabular}{|c|c|c|}
\hline GBD cause/sequela & ICD-9 code & ICD-10 code \\
\hline 20 to $60 \%$ - long-termi & $948.2-5$ & T331.2/5 \\
\hline Greater than $60 \%$ —short-term ${ }^{i}$ & $948.6-9$ & T31.6/9 \\
\hline Greater than $60 \%$-long-termi & $948.6-9$ & T31.6/9 \\
\hline \multicolumn{3}{|l|}{ 12. Injured nerves } \\
\hline Short-term & $951,953-957$ & S04, S44, S54, S64, S74, S84, S94, T06.2, T11.3, T13.3, T14.4 \\
\hline Long-term & $951,953-957$ & S04, S44, S54, S64, S74, S84, S94, T06.2, T11.3, T13.3, T14.4 \\
\hline 13. Poisoning & 960-979, 980-989 & T36-T65, T96-T97 \\
\hline
\end{tabular}

Source: Authors' compilation.

a. Version 0 estimates for YLD are based on epidemiological reviews and disease models from the GBD 1990, adjusted for time trends and internal consistency with 2001 population estimates, and cause-specific and background mortality for 2001. Version 1 estimates for YLD are provisional revised estimates based on new epidemiological reviews and disease models for 2001. These estimates may change with further revisions. Version 2 estimates for YLD are final estimates based on new epidemiological reviews and disease models for 2001. YLL for all causes are based on complete analysis of available mortality data for years up to and including 2002.

b. DSM IV is the Diagnostic and Statistical Manual of Mental Disorders_ Fourth Edition (Washington, DC: American Psychiatric Association, C., 1994).

c. See table 3A.2 for ICD-9 and ICD-10 definitions.

d. The $\mathrm{N}$-codes 803 and 804 were assigned to fractured skull following the distribution of $\mathrm{N}$-codes 801 and 802

e. The $\mathrm{N}$-code 809 was assigned to fractured rib, sternum, and pelvis following the distribution of $\mathrm{N}$-codes 807 and 808 .

f. The $\mathrm{N}$-codes 818 and 819 were assigned to fractured clavicle, scapula, humerus, radius, ulna, and hand bones following the distribution of $\mathrm{N}$-codes $810-817$.

g. The $\mathrm{N}$-codes 827 and 828 were assigned to fractured patella, tibia, fibula, ankle, and foot bones following the distribution of N-codes $822-826$.

h. The N-codes 897.4-897.7 were assigned to amputated toe, foot, and leg following the distribution of N-codes 895, 896, and 897.0-897.3.

i. The N-code 949 was assigned to bums following the N-codes 940-948. In ICD-10, burns are classified by site (T20-T30) and/or proportion of body surface affected (T31). 
Table 3A.6 Disability Weights for Diseases and Conditions (Except Cancers and Injuries)

\begin{tabular}{|c|c|c|c|}
\hline Sequela & $\begin{array}{l}\text { Average disability } \\
\text { weight }^{\mathrm{a}}\end{array}$ & Range $^{b}$ & Source \\
\hline Tuberculosis—cases & 0.271 & $0.264-0.294$ & GBD $1990^{\circ}$, varies with age \\
\hline \multicolumn{4}{|l|}{ Syphilis } \\
\hline Congenital syphilis & 0.315 & & GBD 1990 \\
\hline Primary & 0.015 & $0.014-0.015$ & GBD $1990^{\circ}$, varies with age \\
\hline Secondary & 0.048 & $0.044-0.048$ & GBD $1990^{\circ}$, varies with age \\
\hline Tertiary-neurologic & 0.283 & & GBD 1990 \\
\hline \multicolumn{4}{|l|}{ Chlamydia } \\
\hline Cervicitis & 0.049 & & GBD 1990 \\
\hline Neonatal pneumonia & 0.280 & & GBD 1990 \\
\hline Ophthalmia neonatorum & 0.180 & & GBD 1990 \\
\hline Pelvic inflammatory disease & 0.327 & $0.194-0.382$ & GBD 1990с: untreated 0.420, treated 0.169 \\
\hline Ectopic pregnancy & 0.549 & & GBD 1990 \\
\hline Tubo-ovarian abscess & 0.548 & & GBD 1990 \\
\hline Chronic pelvic pain & 0.122 & & GBD 1990 \\
\hline Infertility & 0.180 & & GBD 1990 \\
\hline Symptomatic urethritis & 0.067 & & GBD 1990 \\
\hline Epididymitis & 0.167 & & GBD 1990 \\
\hline \multicolumn{4}{|l|}{ Gonorrhea } \\
\hline Cervicitis & 0.049 & & GBD 1990 \\
\hline Corneal scar-blindness & 0.600 & & GBD 1990 \\
\hline Ophthalmia neonatorum & 0.180 & & GBD 1990 \\
\hline Pelvic inflammatory disease & 0.169 & & GBD 1990 \\
\hline Ectopic pregnancy & 0.549 & & GBD 1990 \\
\hline Tubo-ovarian abscess & 0.548 & & GBD 1990 \\
\hline Chronic pelvic pain & 0.122 & & GBD 1990 \\
\hline Infertility & 0.180 & & GBD 1990 \\
\hline Symptomatic urethritis & 0.067 & & GBD 1990 \\
\hline Epididymitis & 0.167 & & GBD 1990 \\
\hline Corneal scar-low vision & 0.233 & $0.233-0.245$ & GBD $1990^{\circ}$, varies with age \\
\hline Stricture & 0.151 & & GBD 1990 \\
\hline \multicolumn{4}{|l|}{ HIV/AIDS } \\
\hline HIV cases & 0.135 & $0.123-0.136$ & GBD 1990c, varies with age \\
\hline AIDS cases & 0.505 & & GBD 1990 \\
\hline Diarrheal diseases_episodes & 0.105 & $0.086-0.119$ & GBD $1990^{\circ}$, varies with age \\
\hline \multicolumn{4}{|l|}{ Pertussis } \\
\hline Episodes & 0.129 & $0.016-0.160$ & GBD 1990 \\
\hline Encephalopathy & 0.450 & $0.402-0.484$ & GBD $1990^{\circ}$, varies with age and treatment \\
\hline Poliomyelitis_cases_lameness & 0.369 & & GBD 1990 \\
\hline \multicolumn{4}{|l|}{ Diphtheria } \\
\hline Episodes & 0.231 & & GBD 1990 \\
\hline Neurological complications & 0.078 & & GBD 1990 \\
\hline Myocarditis & 0.323 & & GBD 1990 \\
\hline Measles_-episodes & 0.152 & & GBD 1990 \\
\hline Tetanus-episodes & 0.633 & $0.604-0.640$ & GBD $1990^{\circ}$, varies with age \\
\hline \multicolumn{4}{|l|}{ Meningitis } \\
\hline Streptococcus pneumoniae—episodes & 0.615 & $0.613-0.616$ & GBD $1990^{\circ}$, varies with age \\
\hline Haemophilus influenzae—episodes & 0.616 & $0.613-0.616$ & GBD $1990^{\circ}$, varies with age \\
\hline Neisseria meningitidis—episodes & 0.615 & $0.613-0.616$ & GBD $1990^{\circ}$, varies with age \\
\hline Meningococcaemia without meningitis—episodes & 0.152 & & GBD 1990 \\
\hline Deafness & 0.229 & $0.213-0.233$ & GBD $1990^{\circ}$, varies with age and treatment \\
\hline Mental retardation & 0.456 & $0.402-0.484$ & GBD $1990^{\circ}$, varies with age and treatment \\
\hline Motor deficit & 0.380 & $0.339-0.460$ & GBD $1990^{\circ}$, varies with age and treatment \\
\hline Seizure disorder & 0.097 & $0.046-0.142$ & GBD $1990^{\circ}$, varies with age and treatment \\
\hline Hepatitis B-episodes & 0.211 & $0.170-0.212$ & GBD $1990^{\circ}$, varies with age \\
\hline Hepatitis C-episodes & 0.211 & $0.170-0.212$ & GBD $1990^{\circ}$, varies with age \\
\hline
\end{tabular}


Table 3A.6 Continued

\begin{tabular}{|c|c|c|c|}
\hline Sequela & $\begin{array}{l}\text { Average disability } \\
\text { weight }^{\mathrm{a}}\end{array}$ & Range $^{b}$ & Source \\
\hline \multicolumn{4}{|l|}{ Malaria } \\
\hline Episodes & 0.191 & $0.172-0.211$ & GBD $1990^{\circ}$, varies with age and treatment \\
\hline Neurological sequelae & 0.471 & $0.443-0.471$ & GBD $1990^{\circ}$, varies with age and treatment \\
\hline Anemia & 0.012 & $0.012-0.013$ & GBD $1990^{\circ}$, varies with age \\
\hline Trypanosomiasis—episodes & 0.350 & & GBD 1990 \\
\hline \multicolumn{4}{|l|}{ Chagas' disease } \\
\hline Infection & 0.000 & & GBD 1990 \\
\hline Cardiomyopathy without congestive heart failure & 0.062 & & GBD 1990 \\
\hline Cardiomyopathy with congestive heart failure & 0.270 & $0.186-0.308$ & GBD 1990c: untreated 0.323 , treated 0.171 \\
\hline Megaviscera & 0.240 & & GBD 1990 \\
\hline Schistosomiasis_infection & 0.006 & $0.005-0.006$ & GBD $1990^{\circ}$, varies with age \\
\hline \multicolumn{4}{|l|}{ Leishmaniasis } \\
\hline Visceral & 0.243 & & GBD 1990 \\
\hline Cutaneous & 0.023 & & GBD 1990 \\
\hline \multicolumn{4}{|l|}{ Lymphatic filariasis } \\
\hline Hydrocele $>15 \mathrm{~cm}$ & 0.073 & $0.066-0.075$ & GBD $1990^{c}$, varies with age \\
\hline Bancroftian lymphedema & 0.106 & $0.067-0.128$ & GBD $1990^{\circ}$, varies with age \\
\hline Brugian lymphedema & 0.116 & $0.064-0.128$ & GBD $1990^{\circ}$, varies with age \\
\hline \multicolumn{4}{|l|}{ Onchocerciasis } \\
\hline Blindness & 0.600 & & GBD 1990 \\
\hline Itching & 0.068 & & GBD 1990 \\
\hline Low vision & 0.260 & & GBD 1990d \\
\hline \multicolumn{4}{|l|}{ Leprosy } \\
\hline Cases & 0.000 & & GBD 1990 \\
\hline Disabling leprosy & 0.152 & & GBD 1990 \\
\hline Dengue — dengue hemorrhagic fever & 0.210 & $0.195-0.211$ & GBD $1990^{\circ}$, varies with age \\
\hline \multicolumn{4}{|l|}{ Japanese encephalitis } \\
\hline Episodes & 0.616 & $0.613-0.616$ & GBD $1990^{\circ}$, varies with age \\
\hline Cognitive impairment & 0.468 & $0.402-0.484$ & GBD $1990^{\circ}$, varies with age and treatment \\
\hline Neurological sequelae & 0.380 & $0.339-0.460$ & GBD $1990^{\circ}$, varies with age and treatment \\
\hline \multicolumn{4}{|l|}{ Trachoma } \\
\hline Blindness & 0.600 & & GBD 1990 \\
\hline Low vision & 0.278 & $0.227-0.282$ & GBD 1990d: untreated 0.282, treated 0.227 \\
\hline \multicolumn{4}{|l|}{ Ascariasis } \\
\hline High-intensity infection & 0.000 & & GBD 1990 \\
\hline Contemporaneous cognitive deficit & 0.006 & & GBD 1990 \\
\hline Cognitive impairment & 0.463 & & GBD 1990 \\
\hline Intestinal obstruction & 0.024 & & GBD 1990 \\
\hline \multicolumn{4}{|l|}{ Trichuriasis } \\
\hline High-intensity infection & 0.000 & & GBD 1990 \\
\hline Contemporaneous cognitive deficit & 0.006 & & GBD 1990 \\
\hline Massive dysentery syndrome & 0.116 & $0.114-0.138$ & GBD $1990^{\circ}$, varies with age \\
\hline Cognitive impairment & 0.024 & & GBD 1990 \\
\hline \multicolumn{4}{|l|}{$\begin{array}{l}\text { Hookworm disease (ancylostomiasis and } \\
\text { necatoriasis) }\end{array}$} \\
\hline High-intensity infection & 0.000 & & GBD 1990 \\
\hline Anemia & 0.024 & & GBD 1990 \\
\hline Cognitive impairment & 0.024 & & GBD 1990 \\
\hline \multicolumn{4}{|l|}{ Lower respiratory infections } \\
\hline Episodes & 0.279 & & GBD 1990 \\
\hline Chronic sequelae & 0.099 & & GBD 1990 \\
\hline \multicolumn{4}{|l|}{ Upper respiratory infections } \\
\hline Episodes & 0.000 & & GBD 1990 \\
\hline Pharyngitis & 0.070 & & GBD 1990 \\
\hline \multicolumn{4}{|l|}{ Otitis media } \\
\hline Episodes & 0.023 & & GBD 1990 \\
\hline Deafness & 0.229 & $0.213-0.233$ & GBD $1990^{\circ}$, varies with age and treatment \\
\hline
\end{tabular}

120 | Global Burden of Disease and Risk Factors I Colin D. Mathers, Alan D. Lopez, and Christopher J. L. Murray 
Table 3A.6 Continued

\begin{tabular}{|c|c|c|c|}
\hline Sequela & $\begin{array}{l}\text { Average disability } \\
\text { weight }^{\mathrm{a}}\end{array}$ & Range $^{b}$ & Source \\
\hline \multicolumn{4}{|l|}{ Maternal hemorrhage } \\
\hline Episodes & 0.000 & & GBD 1990 \\
\hline Severe anemia & 0.093 & $0.087-0.093$ & GBD $1990^{\circ}$, varies with age \\
\hline \multicolumn{4}{|l|}{ Maternal sepsis } \\
\hline Episodes & 0.000 & & GBD 1990 \\
\hline Infertility & 0.180 & & GBD 1990 \\
\hline $\begin{array}{l}\text { Hypertensive disorders of pregnancy- } \\
\text { episodes }\end{array}$ & 0.000 & & GBD 1990 \\
\hline \multicolumn{4}{|l|}{ Obstructed labor } \\
\hline Episodes & 0.000 & & GBD 1990 \\
\hline Cesarean section for obstructed labor & 0.349 & & GBD 1990 \\
\hline Stress incontinence & 0.025 & & GBD 1990 \\
\hline Rectovaginal fistula & 0.430 & & GBD 1990 \\
\hline \multicolumn{4}{|l|}{ Abortion } \\
\hline Episodes & 0.000 & & GBD 1990 \\
\hline Infertility & 0.180 & & GBD 1990 \\
\hline Reproductive tract infection & 0.067 & & GBD 1990 \\
\hline \multicolumn{4}{|l|}{ Other maternal conditions } \\
\hline Stress incontinence & 0.025 & & GBD 1990 \\
\hline Low birthweight_-all sequelae & 0.106 & & GBD 1990 \\
\hline $\begin{array}{l}\text { Birth asphyxia and birth trauma- } \\
\text { all sequelae }\end{array}$ & 0.372 & $0.343-0.379$ & GBD 1990c: untreated 0.381, treated 0.334 \\
\hline \multicolumn{4}{|l|}{ Protein-energy malnutrition } \\
\hline Wasting & 0.053 & & GBD 1990 \\
\hline Stunting & 0.002 & & GBD 1990 \\
\hline Developmental disability & 0.024 & & GBD 1990 \\
\hline \multicolumn{4}{|l|}{ lodine deficiency } \\
\hline Goiter grades 1 and 2 & 0.000 & & GBD 1990 \\
\hline Mild developmental disability & 0.006 & & GBD 1990 \\
\hline Cretinoidism & 0.255 & & GBD 1990 \\
\hline Cretinism & 0.804 & & GBD 1990 \\
\hline \multicolumn{4}{|l|}{ Vitamin A deficiency } \\
\hline Xerophthalmia & 0.000 & & GBD 1990 \\
\hline Corneal scar & 0.276 & $0.274-0.282$ & GBD $1990^{\circ}$, varies with age \\
\hline \multicolumn{4}{|l|}{ Iron-deficiency anemia } \\
\hline Mild & 0.000 & & GBD 1990 \\
\hline Moderate & 0.011 & $0.011-0.012$ & GBD 1990', varies with age \\
\hline Severe & 0.090 & $0.087-0.093$ & GBD $1990^{\circ}$, varies with age \\
\hline Cognitive impairment & 0.024 & & GBD 1990 \\
\hline \multicolumn{4}{|l|}{ Diabetes mellitus } \\
\hline Cases & 0.015 & $0.012-0.018$ & GBD 1990c: untreated 0.012, treated 0.033 \\
\hline Diabetic foot & 0.133 & $0.130-0.136$ & GBD 1990c: untreated 0.137, treated 0.129 \\
\hline Neuropathy & 0.072 & $0.066-0.076$ & GBD 1990c: untreated 0.078, treated 0.064 \\
\hline Retinopathy_-blindness & 0.550 & $0.511-0.595$ & GBD 1990c: untreated 0.600, treated 0.493 \\
\hline Amputation & 0.102 & $0.086-0.151$ & GBD 1990c: untreated 0.155 , treated 0.068 \\
\hline \multicolumn{4}{|l|}{ Unipolar depressive disorders } \\
\hline Mild episode & 0.140 & & Netherlands studye \\
\hline Moderate episode & 0.350 & & Netherlands studye \\
\hline Severe episode & 0.760 & & Netherlands studye \\
\hline Dysthymia & 0.140 & & Netherlands studye \\
\hline Bipolar affective disorder — cases & 0.367 & $0.309-0.387$ & Untreated 0.400 , treated 0.140 \\
\hline Schizophrenia—cases & 0.528 & $0.406-0.572$ & GBD $1990^{\circ}$, varies with age and treatment \\
\hline Epilepsy_cases & 0.113 & $0.052-0.142$ & GBD $1990^{\circ}$, varies with age and treatment \\
\hline Alcohol use disorders—cases & 0.155 & & d \\
\hline Alzheimer's disease and & 0.666 & $0.627-0.667$ & GBD $1990^{\circ}$, varies with age \\
\hline
\end{tabular}

(Continues on the following page.) 
Table 3A.6 Continued

\begin{tabular}{|c|c|c|c|}
\hline Sequela & $\begin{array}{l}\text { Average disability } \\
\text { weight }^{\mathrm{a}}\end{array}$ & Range $^{b}$ & Source \\
\hline Parkinson's disease — cases & 0.351 & $0.324-0.395$ & GBD $1990^{\circ}$, varies with age and treatment \\
\hline Multiple sclerosis—cases & 0.411 & $0.410-0.437$ & GBD $1990^{\circ}$, varies with age \\
\hline Drug use disorders - cases & 0.252 & & GBD 1990 \\
\hline Post-traumatic stress disorder—cases & 0.105 & & GBD 1990 \\
\hline Obsessive-compulsive disorder—cases & 0.127 & $0.122-0.129$ & GBD 1990: untreated 0.129, treated 0.080 \\
\hline Panic disorder—cases & 0.165 & $0.153-0.171$ & GBD 1990c: untreated 0.173 , treated 0.091 \\
\hline Insomnia (primary)—cases & 0.100 & & f \\
\hline Migraine_cases & 0.029 & $0.025-0.030$ & $f$ \\
\hline Mild mental retardation, lead-caused—cases & 0.361 & & Netherlands studye \\
\hline \multicolumn{4}{|l|}{ Glaucoma } \\
\hline Low vision & 0.247 & $0.227-0.282$ & GBD 1990d: untreated 0.282, treated 0.227 \\
\hline Blindness & 0.600 & & GBD 1990 \\
\hline \multicolumn{4}{|l|}{ Cataracts } \\
\hline Low vision & 0.271 & $0.234-0.280$ & GBD 1990d. untreated 0.282, treated 0.227 \\
\hline Blindness & 0.568 & $0.511-0.595$ & GBD 1990: untreated 0.600, treated 0.488 \\
\hline \multicolumn{4}{|l|}{ Vision disorders, age-related and other } \\
\hline Low vision & 0.263 & $0.227-0.282$ & GBD 1990d: untreated 0.282, treated 0.227 \\
\hline Blindness & 0.600 & & GBD 1990 \\
\hline \multicolumn{4}{|l|}{ Hearing loss, adult onset } \\
\hline Mild & 0.000 & & Assumed to have no disability for GBD \\
\hline Moderate, treated & 0.040 & & Assumed similar to mild hearing loss ${ }^{f}$ \\
\hline Moderate, untreated & 0.120 & & Netherlands studye \\
\hline Severe or profound, treated & 0.120 & & Assumed similar to moderate loss ${ }^{\dagger}$ \\
\hline Severe or profound, untreated & 0.333 & & GBD 1990 deafness weight ${ }^{c}$ \\
\hline Rheumatic heart disease—cases & 0.253 & $0.186-0.300$ & GBD 1990c: untreated 0.323, treated 0.171 \\
\hline Hypertensive heart disease-cases & 0.243 & $0.201-0.300$ & f: untreated 0.323, treated 0.171 \\
\hline \multicolumn{4}{|l|}{ Ischemic heart disease } \\
\hline Acute myocardial infarction & 0.437 & $0.405-0.477$ & GBD 1990c: untreated 0.491, treated 0.395 \\
\hline Angina pectoris & 0.137 & $0.108-0.207$ & GBD 1990c: untreated 0.227, treated 0.095 \\
\hline Congestive heart failure & 0.234 & $0.186-0.300$ & GBD 1990c: untreated 0.323, treated 0.171 \\
\hline \multicolumn{4}{|l|}{ Cerebrovascular disease } \\
\hline First-ever stroke cases & 0.920 & & $f$ \\
\hline Long-term stroke survivors & 0.270 & $0.228-0.295$ & ${ }^{d}$, varies with age and treatment \\
\hline Inflammatory heart disease_-all sequelae & 0.252 & $0.201-0.300$ & GBD 1990c: untreated 0.323 , treated 0.171 \\
\hline \multicolumn{4}{|l|}{ Chronic obstructive pulmonary disease } \\
\hline Mild and moderate symptomatic cases & 0.170 & & Netherlands studye \\
\hline Severe symptomatic cases & 0.530 & & Netherlands studye \\
\hline Asthma-cases & 0.043 & $0.036-0.050$ & f. untreated 0.054 , treated 0.043 \\
\hline Peptic ulcer disease & & & f \\
\hline Cases with antibiotic treatment & 0.003 & & GBD 1990 \\
\hline Cases not treated with antibiotic & 0.115 & & GBD 1990 \\
\hline Cirrhosis of the liver-symptomatic cases & 0.330 & & GBD 1990 \\
\hline Appendicitis —episodes & 0.463 & & GBD 1990 \\
\hline \multicolumn{4}{|l|}{ Nephritis and nephrosis } \\
\hline Acute glomerulonephritis & 0.091 & $0.082-0.104$ & GBD $1990^{\circ}$, varies with age and treatment \\
\hline End-stage renal disease & 0.098 & $0.087-0.107$ & GBD $1990^{\circ}$, varies with age and treatment \\
\hline $\begin{array}{l}\text { Benign prostatic hypertrophy—symptomatic } \\
\text { cases }\end{array}$ & 0.038 & & GBD 1990 \\
\hline Skin diseases_-cases & 0.056 & & $f$ \\
\hline Rheumatoid arthritis—cases & 0.199 & $0.185-0.221$ & GBD 1990c: untreated 0.233 , treated 0.174 \\
\hline \multicolumn{4}{|l|}{ Osteoarthritis } \\
\hline Hip & 0.126 & $0.118-0.147$ & GBD 1990c: untreated 0.156, treated 0.108 \\
\hline Knee & 0.129 & $0.118-0.147$ & GBD 1990c: untreated 0.156, treated 0.108 \\
\hline Gout-cases & 0.132 & $0.061-0.189$ & f \\
\hline
\end{tabular}

122 | Global Burden of Disease and Risk Factors I Colin D. Mathers, Alan D. Lopez, and Christopher J. L. Murray 
Table 3A.6 Continued

\begin{tabular}{|c|c|c|c|}
\hline Sequela & $\begin{array}{c}\text { Average disability } \\
\text { weight }^{\mathrm{a}}\end{array}$ & Range $^{b}$ & Source \\
\hline \multicolumn{4}{|l|}{ Low back pain } \\
\hline Episode of limiting low back pain & 0.061 & & e \\
\hline Acute intervertebral disc disorder & 0.061 & & f \\
\hline Chronic intervertebral disc disorder & 0.121 & $0.103-0.125$ & f \\
\hline Abdominal wall defect—cases & 0.850 & & GBD 1990 \\
\hline Anencephaly—cases & 0.850 & & GBD 1990 \\
\hline Anorectal atresia—cases & 0.850 & & GBD 1990 \\
\hline Cleft lip—cases & 0.049 & $0.002-0.082$ & GBD 1990': untreated 0.016, treated 0.098 \\
\hline Cleft palate_-cases & 0.101 & $0.036-0.187$ & GBD 1990c: untreated 0.015 , treated 0.231 \\
\hline Esophageal atresia—cases & 0.850 & & GBD 1990 \\
\hline Renal agenesis—cases & 0.850 & & GBD 1990 \\
\hline Down syndrome_-cases & 0.593 & & GBD 1990 \\
\hline Congenital heart anomalies_-cases & 0.323 & & GBD 1990 \\
\hline Spina bifida—cases & 0.593 & & GBD 1990 \\
\hline Dental caries_-episodes & 0.081 & & GBD 1990 \\
\hline Periodontal disease_-cases & 0.001 & & GBD 1990 \\
\hline Edentulism—cases & 0.020 & $0.007-0.052$ & GBD 1990c: untreated 0.062, treated 0.001 \\
\hline
\end{tabular}

Source: Authors' compilation.

a. Global average disability weight.

b. Minimum and maximum disability weights if there is variation across age-sex-region categories. For disability weights based on the GBD 1990 study, further details of age-sex variation, treated and untreated weights, are given in annex tables 3 and 4 of Murray and Lopez 1996a.

c. Disability weights from GBD 1990 (Murray and Lopez 1996a).

d. Disability weights based on GBD 1990 (Murray and Lopez 1996a) with some revisions.

e. Disability weights drawn from Netherlands disability weights study (Stouthard and others 1997).

f. Provisional disability weights based on GBD 1990 or Netherlands weights for comparable health states. 
Table 3A.7 Disability Weights for Malignant Neoplasms and Their Long-Term Sequelae

\begin{tabular}{|c|c|c|c|c|c|c|c|}
\hline \multirow[b]{3}{*}{ Site } & \multicolumn{3}{|c|}{ Stage } & \multirow{2}{*}{\multicolumn{4}{|c|}{ Mortality stratum (for WHO subregions) }} \\
\hline & \multirow{2}{*}{$\begin{array}{c}\text { Diagnosis/ } \\
\text { therapy }\end{array}$} & \multirow[b]{2}{*}{ Control } & \multirow[b]{2}{*}{ Long-term sequela } & & & & \\
\hline & & & & A & B & C & D/E \\
\hline Mouth and oropharynx & 0.09 & 0.09 & & & & & \\
\hline Esophagus & 0.20 & 0.20 & & & & & \\
\hline Stomach & 0.20 & 0.20 & & & & & \\
\hline Liver & 0.20 & 0.20 & & & & & \\
\hline Pancreas & 0.20 & 0.20 & & & & & \\
\hline Trachea, bronchus, and lung & 0.15 & 0.15 & & & & & \\
\hline Melanoma and other skin & 0.05 & 0.05 & & & & & \\
\hline Breast & 0.09 & 0.09 & Mastectomy & 0.03 & 0.05 & 0.06 & 0.08 \\
\hline Prostate & 0.13 & 0.13 & Impotence/incontinence & 0.06 & 0.06 & 0.06 & 0.06 \\
\hline Bladder & 0.09 & 0.09 & & & & & \\
\hline Non-Hodgkin's lymphoma & 0.06 & 0.06 & & & & & \\
\hline Hodgkin's lymphoma & 0.06 & 0.06 & & & & & \\
\hline Leukemia & 0.09 & 0.09 & & & & & \\
\hline Other & 0.09 & 0.09 & & & & & \\
\hline
\end{tabular}

Sources: Mathers, Vos, and Stevenson 1999; Stouthard and others 1997.

Note: For all cancer sites, the disability weight is 0.75 for the preterminal metastasis stage, and 0.81 in the terminal stage. For definitions of the mortality strata, see WHO $2002 \mathrm{e}$. 
Table 3A.8 Disability Weights for Injuries

\begin{tabular}{|c|c|c|c|c|}
\hline \multirow[b]{2}{*}{ Injury category } & \multicolumn{2}{|c|}{ Short-term weight } & \multicolumn{2}{|c|}{ Long-term weight } \\
\hline & Treated & Untreated & Treated & Untreated \\
\hline Fractured skull & 0.431 & 0.431 & & \\
\hline Ages 0-44 & & & 0.350 & 0.410 \\
\hline Ages 45-59 & & & 0.350 & 0.419 \\
\hline Ages $60+$ & & & 0.404 & 0.471 \\
\hline Intracranial injuries & 0.359 & 0.359 & & \\
\hline Ages 0-44 & & & 0.350 & 0.410 \\
\hline Ages 45-59 & & & 0.350 & 0.419 \\
\hline Ages 60+ & & & 0.404 & 0.471 \\
\hline \multicolumn{5}{|l|}{ Fracture } \\
\hline Face bones & 0.223 & 0.223 & & \\
\hline Vertebral column & 0.266 & 0.266 & & \\
\hline Rib or sternum & 0.199 & 0.199 & & \\
\hline Pelvis & 0.247 & 0.247 & & \\
\hline Clavicle, scapula, or humerus & 0.153 & 0.153 & & \\
\hline Ulna or radius & 0.180 & 0.180 & & \\
\hline Hand bones & 0.100 & 0.100 & & \\
\hline Femur & 0.372 & 0.372 & 0.272 & 0.272 \\
\hline Patella, tibia, or fibula & 0.271 & 0.271 & & \\
\hline Ankle & 0.196 & 0.196 & & \\
\hline Foot bones & 0.077 & 0.077 & & \\
\hline Injured spinal cord & & & 0.725 & 0.725 \\
\hline Dislocation of shoulder, elbow, or hip & 0.074 & 0.074 & & \\
\hline Other dislocation & 0.074 & 0.074 & & \\
\hline Sprains & 0.064 & 0.064 & & \\
\hline \multicolumn{5}{|l|}{ Amputation } \\
\hline Thumb & & & 0.165 & 0.165 \\
\hline Finger & & & 0.102 & 0.102 \\
\hline Arm & & & 0.257 & 0.308 \\
\hline Toe & & & 0.102 & 0.102 \\
\hline Foot & & & 0.300 & 0.300 \\
\hline Leg & & & 0.300 & 0.300 \\
\hline Internal injuries & 0.208 & 0.208 & & \\
\hline Open wound & 0.108 & 0.108 & & \\
\hline Injury to eyes & 0.108 & 0.108 & 0.300 & 0.354 \\
\hline Crushing & 0.218 & 0.218 & & \\
\hline \multicolumn{5}{|l|}{ Burns } \\
\hline$<20 \%$ & 0.158 & 0.156 & 0.001 & 0.002 \\
\hline$>20 \%$ and $<60 \%$ & 0.441 & 0.469 & 0.255 & 0.255 \\
\hline$>60 \%$ & 0.441 & 0.469 & 0.255 & 0.255 \\
\hline Injured nerves & 0.064 & 0.078 & 0.064 & 0.078 \\
\hline \multicolumn{5}{|l|}{ Poisoning } \\
\hline Ages 0-14 & 0.611 & 0.611 & & \\
\hline Ages 14+ & 0.608 & 0.608 & & \\
\hline
\end{tabular}

Source: Murray and Lopez 1996a. 
ANNEX 3B: Deaths by Cause, Sex, Age, and Region, 2001

Table 3B.1 Deaths by Cause, Sex, and Age in Low- and Middle-Income Countries, 2001 (thousands)

\begin{tabular}{|c|c|c|c|c|c|c|c|c|c|c|}
\hline \multirow[b]{2}{*}{ Cause } & \multicolumn{10}{|c|}{ Male } \\
\hline & Total & $0-4$ & 5-14 & $15-29$ & 30-44 & $45-59$ & $60-69$ & 70-79 & $80+$ & Total \\
\hline Population (millions) & 5,219 & 288 & 563 & 712 & 545 & 326 & 124 & 61 & 15 & 2,636 \\
\hline $\begin{array}{l}\text { All causes } \\
\text { I. Communicable, maternal, perinatal, } \\
\text { and nutritional conditions }\end{array}$ & $\begin{array}{l}48,351 \\
17,613\end{array}$ & $\begin{array}{l}5,407 \\
4,837\end{array}$ & $\begin{array}{l}733 \\
375\end{array}$ & $\begin{array}{r}1,835 \\
585\end{array}$ & $\begin{array}{l}2,786 \\
1,150\end{array}$ & $\begin{array}{r}3,998 \\
853\end{array}$ & $\begin{array}{r}4,069 \\
529\end{array}$ & $\begin{array}{r}4,376 \\
469\end{array}$ & $\begin{array}{r}2,349 \\
268\end{array}$ & $\begin{array}{r}25,554 \\
9,068\end{array}$ \\
\hline A. Infectious and parasitic diseases & 10,686 & 2,360 & 295 & 539 & 1,085 & 734 & 348 & 249 & 111 & 5,724 \\
\hline 1. Tuberculosis & 1,590 & 22 & 16 & 138 & 256 & 281 & 184 & 115 & 31 & 1,043 \\
\hline $\begin{array}{l}\text { 2. Sexually transmitted diseases } \\
\text { excluding HIV/AIDS }\end{array}$ & 176 & 31 & 0 & 2 & 11 & 26 & 10 & 6 & 3 & 89 \\
\hline a. Syphilis & 155 & 30 & 0 & 1 & 10 & 23 & 10 & 6 & 3 & 83 \\
\hline b. Chlamydia & 9 & - & - & - & - & - & - & - & - & - \\
\hline c. Gonorrhea & 1 & - & 0 & 0 & 0 & 0 & 0 & 0 & 0 & 0 \\
\hline d. Other sexually transmitted diseases & 12 & 1 & 0 & 0 & 1 & 4 & 0 & 0 & 0 & 6 \\
\hline 3. HIV/AIDS & 2,552 & 173 & 52 & 258 & 640 & 221 & 29 & 4 & 0 & 1,377 \\
\hline 4. Diarrheal diseases & 1,777 & 837 & 3 & 6 & 11 & 15 & 15 & 19 & 23 & 930 \\
\hline 5. Childhood-cluster diseases & 1,362 & 524 & 109 & 24 & 11 & 6 & 3 & 1 & 1 & 679 \\
\hline a. Pertussis & 301 & 150 & 0 & - & - & - & 0 & - & - & 150 \\
\hline b. Poliomyelitis ${ }^{a}$ & 0 & 0 & 0 & 0 & 0 & 0 & 0 & 0 & 0 & 0 \\
\hline c. Diphtheria & 6 & 3 & 0 & 0 & 0 & 0 & 0 & 0 & 0 & 3 \\
\hline d. Measles & 762 & 277 & 91 & 11 & 0 & 0 & 0 & - & - & 379 \\
\hline e. Tetanus & 293 & 94 & 18 & 13 & 11 & 6 & 3 & 1 & 1 & 147 \\
\hline 6. Meningitis & 169 & 29 & 14 & 11 & 9 & 10 & 5 & 6 & 3 & 87 \\
\hline 7. Hepatitis $\mathbf{B}^{\mathbf{b}}$ & 95 & 3 & 4 & 8 & 16 & 22 & 7 & 4 & 2 & 66 \\
\hline Hepatitis $\mathbf{C}^{\mathrm{b}}$ & 39 & 1 & 1 & 3 & 7 & 9 & 3 & 2 & 1 & 27 \\
\hline 8. Malaria & 1,207 & 521 & 7 & 10 & 11 & 11 & 7 & 7 & 4 & 579 \\
\hline 9. Tropical-cluster diseases & 128 & 4 & 21 & 15 & 13 & 13 & 7 & 4 & 1 & 78 \\
\hline a. Trypanosomiasis & 48 & 2 & 11 & 6 & 6 & 5 & 1 & 0 & 0 & 31 \\
\hline b. Chagas' disease & 14 & 0 & 0 & 0 & 1 & 2 & 2 & 1 & 1 & 8 \\
\hline c. Schistosomiasis & 14 & 0 & 0 & 0 & 1 & 3 & 3 & 2 & 0 & 9 \\
\hline d. Leishmaniasis & 51 & 2 & 10 & 8 & 5 & 3 & 1 & 1 & 0 & 30 \\
\hline e. Lymphatic filariasis & 0 & 0 & 0 & 0 & 0 & 0 & 0 & 0 & 0 & 0 \\
\hline f. Onchocerciasis & 0 & 0 & - & - & - & - & - & - & - & 0 \\
\hline 10. Leprosy & 6 & 0 & 0 & 0 & 1 & 1 & 1 & 1 & 0 & 4 \\
\hline 11. Dengue & 19 & 2 & 5 & 0 & 0 & 0 & 0 & 0 & 0 & 9 \\
\hline 12. Japanese encephalitis & 14 & 2 & 0 & 1 & 2 & 0 & 0 & 0 & $\mathbf{0}$ & 7 \\
\hline 13. Trachoma & 0 & 0 & 0 & 0 & - & 0 & - & $\mathbf{0}$ & - & 0 \\
\hline 14. Intestinal nematode infections & 12 & 1 & 3 & $\mathbf{0}$ & 0 & 1 & 0 & 0 & 0 & 6 \\
\hline a. Ascariasis & 3 & 0 & 1 & 0 & 0 & 0 & 0 & 0 & 0 & 1 \\
\hline b. Trichuriasis & 3 & 0 & 1 & 0 & 0 & 0 & 0 & 0 & 0 & 2 \\
\hline c. Hookworm disease & 3 & 0 & 0 & 0 & 0 & 0 & 0 & 0 & 0 & 2 \\
\hline Other intestinal infections & 2 & 1 & 0 & 0 & 0 & 0 & 0 & 0 & 0 & 1 \\
\hline Other infectious diseases & 1,540 & 210 & 59 & 62 & 97 & 117 & 76 & 80 & 42 & 744 \\
\hline B. Respiratory infections & 3,481 & 1,003 & 58 & 37 & 54 & 94 & 168 & 203 & 145 & 1,761 \\
\hline 1. Lower respiratory infections & 3,408 & 989 & 55 & 35 & 51 & 90 & 163 & 198 & 141 & 1,724 \\
\hline 2. Upper respiratory infections & 69 & 14 & 2 & 2 & 2 & 4 & 5 & 5 & 3 & 35 \\
\hline 3. Otitis media & 3 & 0 & 1 & 0 & 0 & 0 & 0 & 0 & 0 & 2 \\
\hline C. Maternal conditions & 507 & - & - & - & - & - & - & - & - & - \\
\hline 1. Maternal hemorrhage & 141 & - & - & - & - & - & - & - & - & - \\
\hline 2. Maternal sepsis & 75 & - & - & - & - & - & - & - & - & - \\
\hline 3. Hypertensive disorders of pregnancy & 71 & - & - & - & - & - & - & - & - & - \\
\hline 4. Obstructed labor & 43 & - & - & - & - & - & - & - & - & - \\
\hline 5. Abortion & 66 & - & - & - & - & - & - & - & - & - \\
\hline Other maternal conditions & 111 & - & - & - & - & - & - & - & - & - \\
\hline D. Perinatal conditions ${ }^{\mathrm{c}}$ & 2,489 & 1,381 & 0 & 0 & 0 & 0 & 0 & 0 & - & 1,381 \\
\hline 1. Low birthweight & 1,291 & 704 & 0 & - & 0 & - & 0 & - & - & 704 \\
\hline 2. Birth asphyxia and birth trauma & 728 & 426 & 0 & 0 & 0 & 0 & - & - & - & 426 \\
\hline Other perinatal conditions & 470 & 251 & 0 & 0 & 0 & 0 & - & 0 & - & 251 \\
\hline E. Nutritional deficiencies & 450 & 93 & 22 & 9 & 10 & 25 & 13 & 16 & 13 & 201 \\
\hline 1. Protein-energy malnutrition & 241 & 70 & 15 & 4 & 3 & 6 & 7 & 8 & 7 & 121 \\
\hline 2. lodine deficiency & 7 & 2 & 1 & 0 & 0 & 0 & 0 & 0 & 0 & 3 \\
\hline 3. Vitamin A deficiency & 23 & 7 & 2 & 0 & 0 & 1 & 0 & 0 & 0 & 11 \\
\hline 4. Iron-deficiency anemia & 126 & 9 & 3 & 4 & 6 & 15 & 2 & 2 & 2 & 43 \\
\hline Other nutritional disorders & 54 & 5 & 1 & 1 & 1 & 3 & 3 & 6 & 3 & 23 \\
\hline
\end{tabular}


Table 3B.1 Continued

\begin{tabular}{|c|c|c|c|c|c|c|c|c|}
\hline \multicolumn{9}{|c|}{ Female } \\
\hline $0-4$ & 5-14 & $15-29$ & 30-44 & $45-59$ & $60-69$ & $70-79$ & $80+$ & Total \\
\hline 274 & 533 & 682 & 530 & 326 & 136 & 78 & 25 & 2,583 \\
\hline $\begin{array}{l}5,123 \\
4,555\end{array}$ & $\begin{array}{l}738 \\
451\end{array}$ & $\begin{array}{r}1,576 \\
950\end{array}$ & $\begin{array}{r}1,831 \\
959\end{array}$ & $\begin{array}{r}2,521 \\
512\end{array}$ & $\begin{array}{r}2,960 \\
358\end{array}$ & $\begin{array}{r}4,319 \\
408\end{array}$ & $\begin{array}{r}3,729 \\
353\end{array}$ & $\begin{array}{r}22,797 \\
8,546\end{array}$ \\
\hline 2,406 & 347 & 628 & 666 & 400 & 205 & 191 & 118 & 4,962 \\
\hline 18 & 16 & 106 & 135 & 117 & 79 & 56 & 19 & 547 \\
\hline 37 & 0 & 13 & 10 & 14 & 7 & 4 & 1 & 87 \\
\hline 37 & 0 & 12 & 8 & 6 & 4 & 4 & 1 & 72 \\
\hline- & - & 0 & 1 & 6 & 1 & 0 & 0 & 9 \\
\hline 0 & - & 0 & 0 & 0 & 0 & 0 & 0 & 1 \\
\hline 0 & 0 & 0 & 1 & 2 & 1 & 0 & 0 & 6 \\
\hline 166 & 51 & 384 & 423 & 128 & 20 & 4 & 0 & 1,175 \\
\hline 762 & 3 & 3 & 5 & 10 & 14 & 20 & 30 & 847 \\
\hline 525 & 111 & 24 & 11 & 6 & 3 & 1 & 1 & 682 \\
\hline 151 & 0 & 0 & - & 0 & 0 & - & - & 151 \\
\hline 0 & 0 & 0 & 0 & 0 & 0 & 0 & 0 & 0 \\
\hline 2 & 1 & 0 & 0 & 0 & 0 & 0 & 0 & 3 \\
\hline 279 & 93 & 11 & - & 0 & 0 & - & - & 383 \\
\hline 93 & 18 & 13 & 11 & 6 & 3 & 1 & 1 & 146 \\
\hline 35 & 18 & 9 & 5 & 5 & 4 & 4 & 2 & 82 \\
\hline 5 & 2 & 5 & 4 & 6 & 3 & 2 & 2 & 29 \\
\hline 2 & 1 & 2 & 2 & 3 & 1 & 1 & 1 & 12 \\
\hline 566 & 8 & $1 \overline{2}$ & 12 & 13 & 8 & 7 & 4 & 629 \\
\hline 4 & 15 & 10 & 6 & 7 & 4 & 3 & 2 & 50 \\
\hline$i$ & 6 & 4 & 3 & 3 & 0 & 0 & - & 17 \\
\hline 0 & 0 & 0 & 1 & 2 & 1 & 1 & 1 & 7 \\
\hline 0 & 0 & 0 & 0 & 1 & 1 & 2 & 1 & 5 \\
\hline 3 & 8 & 5 & 1 & 1 & 1 & 0 & 0 & 21 \\
\hline 0 & 0 & 0 & 0 & 0 & 0 & 0 & 0 & 0 \\
\hline- & - & 0 & - & - & 0 & - & - & 0 \\
\hline 0 & 0 & 0 & 0 & 0 & 0 & 0 & 0 & 2 \\
\hline 2 & 6 & 1 & 0 & 0 & 0 & 0 & 0 & 10 \\
\hline 4 & 2 & 1 & 1 & 0 & 0 & 0 & 0 & 7 \\
\hline- & - & - & - & - & - & - & - & - \\
\hline 2 & 3 & 0 & 0 & 0 & 0 & 0 & 0 & 6 \\
\hline 1 & 1 & 0 & 0 & 0 & 0 & 0 & 0 & 2 \\
\hline 0 & 1 & 0 & 0 & 0 & 0 & 0 & 0 & 1 \\
\hline- & 0 & 0 & 0 & 0 & 0 & 0 & 0 & 1 \\
\hline 1 & 0 & 0 & 0 & 0 & 0 & 0 & 0 & 1 \\
\hline 277 & 113 & 59 & 53 & 90 & 62 & 87 & 55 & 796 \\
\hline 939 & 78 & 57 & 44 & 57 & 131 & 197 & 215 & 1,719 \\
\hline 924 & 73 & 56 & 44 & 57 & 128 & 194 & 209 & 1,684 \\
\hline 15 & 5 & 1 & 1 & 1 & 3 & 3 & 6 & 34 \\
\hline 0 & 0 & 0 & 0 & 0 & 0 & 0 & 0 & 1 \\
\hline 0 & 0 & 257 & 232 & 18 & 0 & 0 & 0 & 507 \\
\hline 0 & 0 & 63 & 71 & 7 & - & - & - & 141 \\
\hline- & 0 & 37 & 35 & 4 & - & - & - & 75 \\
\hline- & 0 & 41 & 28 & 2 & - & - & 0 & 71 \\
\hline- & - & 26 & 17 & 0 & - & - & - & 43 \\
\hline- & 0 & 44 & 22 & 0 & - & - & - & 66 \\
\hline- & 0 & 46 & 59 & 5 & 0 & 0 & 0 & 111 \\
\hline 1,109 & 0 & 0 & 0 & 0 & 0 & 0 & 0 & 1,109 \\
\hline 587 & 0 & - & - & - & - & - & - & 587 \\
\hline 302 & 0 & 0 & - & 0 & - & 0 & 0 & 302 \\
\hline 219 & 0 & 0 & 0 & 0 & 0 & 0 & 0 & 219 \\
\hline 101 & 25 & 8 & 16 & 37 & 22 & 20 & 20 & 249 \\
\hline 69 & 17 & 2 & 3 & 5 & 7 & 7 & 10 & 119 \\
\hline 3 & 0 & 0 & 0 & 0 & 0 & 0 & 0 & 3 \\
\hline 9 & 2 & 0 & 0 & 0 & 0 & 0 & 0 & 12 \\
\hline 11 & 5 & 5 & 13 & 30 & 12 & 4 & 4 & 83 \\
\hline \multirow[t]{2}{*}{9} & 1 & 0 & 0 & 2 & 3 & 8 & 6 & 31 \\
\hline & & & & & & ontinues & the foll & ng page. \\
\hline
\end{tabular}

The Burden of Disease and Mortality by Condition: Data, Methods, and Results for 2001 | 127 
Table 3B.1 Continued

\begin{tabular}{|c|c|c|c|c|c|c|c|c|c|c|}
\hline \multirow[b]{2}{*}{ Cause } & \multicolumn{10}{|c|}{ Male } \\
\hline & Total & $0-4$ & $5-14$ & $15-29$ & $30-44$ & $45-59$ & $60-69$ & 70-79 & $80+$ & Total \\
\hline II. Noncommunicable diseases & 26,023 & 416 & 116 & 339 & 834 & 2,575 & 3,290 & 3,743 & 2,006 & 13,317 \\
\hline A. Malignant neoplasms & 4,955 & 18 & 25 & 68 & 186 & 671 & 834 & 708 & 250 & 2,762 \\
\hline 1. Mouth and oropharynx cancers & 271 & 0 & 0 & 4 & 16 & 54 & 62 & 37 & 13 & 187 \\
\hline 2. Esophageal cancer & 380 & 0 & 0 & 1 & 13 & 59 & 84 & 62 & 17 & 235 \\
\hline 3. Stomach cancer & 696 & 0 & 0 & 4 & 25 & 110 & 131 & 117 & 42 & 429 \\
\hline 4. Colon and rectal cancers & 357 & 0 & 0 & 4 & 15 & 39 & 53 & 52 & 22 & 185 \\
\hline 5. Liver cancer & 505 & 1 & 0 & 8 & 38 & 122 & 97 & 69 & 16 & 350 \\
\hline 6. Pancreas cancer & 117 & 0 & 0 & 0 & 4 & 18 & 19 & 16 & 6 & 64 \\
\hline 7. Trachea, bronchus, and lung cancers & 771 & 0 & 0 & 2 & 24 & 138 & 205 & 160 & 39 & 569 \\
\hline 8. Melanoma and other skin cancers & 35 & 0 & 0 & 1 & 2 & 4 & 4 & 4 & 2 & 17 \\
\hline 9. Breast cancer & 317 & 0 & 0 & 0 & 0 & 0 & 0 & 0 & 0 & 1 \\
\hline 10. Cervix uteri cancer & 218 & - & - & - & - & - & - & - & - & - \\
\hline 11. Corpus uteri cancer & 44 & - & - & - & - & - & - & - & - & - \\
\hline 12. Ovarian cancer & 86 & - & - & - & - & - & - & - & - & - \\
\hline 13. Prostate cancer & 145 & 0 & 0 & 0 & 0 & 9 & 35 & 65 & 35 & 145 \\
\hline 14. Bladder cancer & 117 & 0 & 0 & 0 & 2 & 12 & 24 & 30 & 14 & 81 \\
\hline 15. Lymphomas and multiple myeloma & 216 & 2 & 7 & 11 & 15 & 22 & 23 & 19 & 8 & 107 \\
\hline 16. Leukemia & 190 & 7 & 12 & 23 & 12 & 16 & 16 & 14 & 6 & 105 \\
\hline Other malignant neoplasms & 490 & 8 & 5 & 9 & 22 & 69 & 80 & 64 & 29 & 285 \\
\hline B. Other neoplasms & 89 & 2 & 2 & 5 & 5 & 10 & 9 & 9 & 4 & 45 \\
\hline C. Diabetes mellitus & 757 & 1 & 1 & 7 & 18 & 75 & 92 & 94 & 47 & 337 \\
\hline D. Endocrine disorders & 170 & 21 & 4 & 6 & 8 & 11 & 9 & 10 & 8 & 76 \\
\hline E. Neuropsychiatric conditions & 701 & 24 & 16 & 42 & 76 & 69 & 36 & 82 & 53 & 398 \\
\hline 1. Unipolar depressive disorders & 10 & 0 & 0 & 0 & 2 & 2 & 1 & 0 & 0 & 5 \\
\hline 2. Bipolar affective disorder & 0 & 0 & 0 & 0 & 0 & 0 & 0 & 0 & 0 & 0 \\
\hline 3. Schizophrenia & 21 & 0 & 0 & 0 & 3 & 4 & 1 & 1 & 0 & 10 \\
\hline 4. Epilepsy & 116 & 12 & 7 & 14 & 14 & 9 & 4 & 3 & 2 & 65 \\
\hline 5. Alcohol use disorders & 62 & 0 & 0 & 5 & 16 & 19 & 9 & 4 & 1 & 54 \\
\hline 6. Alzheimer's and other dementias & 173 & 1 & 0 & 0 & 1 & 3 & 4 & 39 & 28 & 77 \\
\hline 7. Parkinson's disease & 51 & 0 & 0 & 0 & 1 & 1 & 3 & 12 & 8 & 25 \\
\hline 8. Multiple sclerosis & 8 & 0 & 0 & 0 & 1 & 1 & 1 & 0 & 0 & 4 \\
\hline 9. Drug use disorders & 73 & 0 & 0 & 11 & 28 & 19 & 2 & 0 & 0 & 60 \\
\hline 10. Post-traumatic stress disorder & 0 & 0 & 0 & 0 & 0 & 0 & 0 & 0 & 0 & 0 \\
\hline 11. Obsessive-compulsive disorder & - & - & - & - & - & - & - & - & - & - \\
\hline 12. Panic disorder & - & - & - & - & - & - & - & - & - & - \\
\hline 13. Insomnia (primary) & - & - & - & - & - & - & - & - & - & - \\
\hline 14. Migraine & - & - & - & - & - & - & - & - & - & - \\
\hline 15. Mental retardation, lead-caused & 5 & 1 & 1 & 1 & 0 & 0 & 0 & 0 & 0 & 3 \\
\hline Other neuropsychiatric disorders & 183 & 11 & 8 & 10 & 9 & 12 & 12 & 23 & 13 & 97 \\
\hline F. Sense organ diseases & 3 & 0 & $\mathbf{0}$ & 0 & $\mathbf{0}$ & $\mathbf{0}$ & $\mathbf{0}$ & $\mathbf{0}$ & $\mathbf{0}$ & 1 \\
\hline 1. Glaucoma & 0 & 0 & - & 0 & 0 & 0 & 0 & 0 & 0 & 0 \\
\hline 2. Cataracts & - & - & - & - & - & - & - & - & - & - \\
\hline 3. Vision disorders, age-related & - & - & - & - & - & - & - & - & - & - \\
\hline 4. Hearing loss, adult onset & - & - & - & - & - & - & - & - & - & - \\
\hline Other sense organ disorders & 3 & 0 & 0 & 0 & 0 & 0 & 0 & 0 & 0 & 1 \\
\hline G. Cardiovascular diseases & 13,354 & 38 & 24 & 103 & 320 & 1,170 & 1,670 & 2,047 & 1,168 & 6,541 \\
\hline 1. Rheumatic heart disease & 307 & 7 & 5 & 18 & 17 & 28 & 24 & 22 & 10 & 131 \\
\hline 2. Hypertensive heart disease & 760 & 1 & 1 & 4 & 17 & 65 & 97 & 113 & 64 & 361 \\
\hline 3. Ischemic heart disease & 5,699 & 3 & 3 & 26 & 144 & 620 & 818 & 928 & 467 & 3,010 \\
\hline 4. Cerebrovascular disease & 4,608 & 7 & 5 & 21 & 68 & 328 & 575 & 758 & 410 & 2,171 \\
\hline 5. Inflammatory heart diseases & 319 & 7 & 2 & 9 & 18 & 31 & 31 & 38 & 27 & 163 \\
\hline Other cardiovascular diseases & 1,661 & 12 & 7 & 26 & 56 & 98 & 126 & 189 & 191 & 704 \\
\hline H. Respiratory diseases & 3,125 & 33 & 9 & 23 & 55 & 236 & 368 & 537 & 342 & 1,604 \\
\hline 1. Chronic obstructive pulmonary disease & e 2,378 & 2 & 0 & 2 & 16 & 167 & 297 & 449 & 275 & 1,209 \\
\hline 2. Asthma & 205 & 2 & 4 & 10 & 18 & 29 & 19 & 17 & 7 & 106 \\
\hline Other respiratory diseases & 542 & 29 & 5 & 11 & 20 & 40 & 52 & 71 & 60 & 289 \\
\hline
\end{tabular}


Table 3B.1 Continued

\begin{tabular}{|c|c|c|c|c|c|c|c|c|}
\hline & & & & Fema & & & & \\
\hline $0-4$ & 5-14 & $15-29$ & $30-44$ & $45-59$ & $60-69$ & $70-79$ & $80+$ & Total \\
\hline 419 & 123 & 285 & 584 & 1,760 & 2,466 & 3,779 & 3,289 & 12,705 \\
\hline 19 & 22 & 54 & 194 & 524 & 537 & 550 & 293 & 2,193 \\
\hline 0 & 0 & 1 & 5 & 20 & 22 & 21 & 14 & 84 \\
\hline 0 & 0 & 1 & 5 & 33 & 41 & 42 & 22 & 144 \\
\hline 0 & 0 & 3 & 21 & 52 & 61 & 80 & 50 & 266 \\
\hline 0 & 0 & 2 & 13 & 32 & 42 & 50 & 34 & 172 \\
\hline 1 & 1 & 5 & 15 & 36 & 39 & 41 & 16 & 154 \\
\hline 0 & 0 & 0 & 3 & 10 & 14 & 17 & 9 & 53 \\
\hline 0 & 1 & 1 & 11 & 46 & 58 & 64 & 21 & 203 \\
\hline 0 & 0 & 0 & 2 & 3 & 4 & 5 & 3 & 18 \\
\hline 0 & 0 & 2 & 44 & 108 & 74 & 57 & 31 & 316 \\
\hline 0 & 0 & 11 & 19 & 68 & 59 & 44 & 17 & 218 \\
\hline 0 & 0 & 0 & 3 & 10 & 12 & 12 & 6 & 44 \\
\hline 0 & 1 & 3 & 9 & 24 & 22 & 19 & 8 & 86 \\
\hline- & - & - & - & - & - & - & - & - \\
\hline 1 & 0 & 0 & 3 & 5 & 8 & 10 & 8 & 35 \\
\hline 3 & 6 & 8 & 12 & 19 & 23 & 25 & 14 & 109 \\
\hline 8 & 9 & 12 & 12 & 14 & 12 & 12 & 7 & 85 \\
\hline 6 & 4 & 5 & 17 & 42 & 46 & 51 & 34 & 204 \\
\hline 2 & 2 & 3 & 5 & 11 & 8 & 8 & 5 & 44 \\
\hline 2 & 2 & 6 & 14 & 76 & 121 & 133 & 67 & 421 \\
\hline 22 & 3 & 7 & 10 & 11 & 12 & 14 & 14 & 94 \\
\hline 19 & 15 & 23 & 27 & 34 & 25 & 81 & 78 & 303 \\
\hline 0 & 0 & 0 & 1 & 3 & 1 & 0 & 0 & 5 \\
\hline 0 & 0 & 0 & 0 & 0 & 0 & 0 & 0 & 0 \\
\hline 0 & 0 & 0 & 2 & 4 & 2 & 2 & 1 & 10 \\
\hline 9 & 8 & 12 & 8 & 6 & 3 & 3 & 2 & 51 \\
\hline 0 & 0 & 0 & 2 & 3 & 2 & 1 & 0 & 8 \\
\hline 1 & 0 & 1 & 1 & 2 & 5 & 40 & 47 & 96 \\
\hline 0 & 0 & 0 & 0 & 1 & 2 & 11 & 11 & 26 \\
\hline 0 & 0 & 0 & 1 & 2 & 1 & 1 & 0 & 5 \\
\hline - & 0 & 2 & 6 & 4 & 0 & 0 & 0 & 13 \\
\hline 0 & 0 & 0 & 0 & 0 & 0 & 0 & 0 & 0 \\
\hline- & - & - & - & - & - & - & - & - \\
\hline- & - & - & - & - & - & - & - & - \\
\hline- & - & - & - & - & - & - & - & - \\
\hline- & - & - & - & - & - & - & - & - \\
\hline 0 & 1 & 1 & 0 & 0 & 0 & 0 & 0 & 2 \\
\hline 8 & 6 & 7 & 6 & 9 & 10 & 24 & 16 & 86 \\
\hline 0 & 0 & 0 & 0 & 0 & 0 & 0 & 0 & 1 \\
\hline- & - & - & 0 & 0 & 0 & 0 & 0 & 0 \\
\hline - & - & - & - & - & - & - & - & - \\
\hline- & - & - & - & - & - & - & - & - \\
\hline- & - & - & - & - & - & - & - & - \\
\hline 0 & 0 & 0 & 0 & 0 & 0 & 0 & 0 & 1 \\
\hline 45 & 26 & 91 & 192 & 723 & 1,312 & 2,274 & 2,150 & 6,814 \\
\hline 8 & 8 & 19 & 21 & 35 & 31 & 35 & 18 & 175 \\
\hline 1 & 1 & 3 & 12 & 52 & 85 & 132 & 114 & 399 \\
\hline 2 & 2 & 27 & 68 & 290 & 581 & 911 & 808 & 2,689 \\
\hline 5 & 5 & 13 & 45 & 238 & 463 & 869 & 799 & 2,437 \\
\hline 6 & 3 & 6 & 9 & 20 & 24 & 43 & 45 & 156 \\
\hline 23 & 8 & 24 & 36 & 88 & 127 & 284 & 366 & 957 \\
\hline 30 & 9 & 22 & 44 & 173 & 249 & 483 & 512 & 1,521 \\
\hline 2 & 0 & 1 & 13 & 122 & 199 & 411 & 421 & 1,169 \\
\hline 2 & 4 & 12 & 19 & 26 & 14 & 13 & 10 & 99 \\
\hline \multirow[t]{2}{*}{26} & 5 & 9 & 12 & 25 & 36 & 58 & 81 & 252 \\
\hline & & & & & & \multicolumn{3}{|c|}{ (Continues on the following page. } \\
\hline
\end{tabular}


Table 3B.1 Continued

\begin{tabular}{|c|c|c|c|c|c|c|c|c|c|c|}
\hline \multirow[b]{2}{*}{ Cause } & \multicolumn{10}{|c|}{ Male } \\
\hline & Total & $0-4$ & 5-14 & $15-29$ & $30-44$ & $45-59$ & $60-69$ & 70-79 & $80+$ & Total \\
\hline I. Digestive diseases & 1,600 & 47 & 16 & 48 & 123 & 252 & 183 & 156 & 74 & 899 \\
\hline 1. Peptic ulcer disease & 234 & 3 & 2 & 7 & 18 & 40 & 29 & 28 & 14 & 140 \\
\hline 2. Cirrhosis of the liver & 654 & 8 & 4 & 16 & 63 & 143 & 94 & 65 & 21 & 413 \\
\hline 3. Appendicitis & 19 & 0 & 1 & 1 & 1 & 3 & 2 & 2 & 1 & 11 \\
\hline Other digestive diseases & 694 & 37 & 10 & 23 & 41 & 66 & 58 & 61 & 38 & 335 \\
\hline J. Genitourinary diseases & 676 & 11 & 7 & 21 & 35 & 70 & 79 & 87 & 53 & 363 \\
\hline 1. Nephritis and nephrosis & 552 & 9 & 6 & 19 & 31 & 58 & 61 & 64 & 37 & 285 \\
\hline 2. Benign prostatic hypertrophy & 29 & - & - & 0 & 0 & 5 & 6 & 10 & 7 & 29 \\
\hline Other genitourinary system diseases & 96 & 2 & 1 & 2 & 4 & 8 & 11 & 13 & 8 & 50 \\
\hline K. Skin diseases & 53 & 1 & $\mathbf{0}$ & 1 & 3 & 4 & 3 & 5 & 4 & 21 \\
\hline L. Musculoskeletal diseases & 60 & 1 & 1 & 2 & 2 & 4 & 4 & 6 & 4 & 24 \\
\hline 1. Rheumatoid arthritis & 15 & 0 & 0 & 0 & 0 & 1 & 1 & 1 & 1 & 5 \\
\hline 2. Osteoarthritis & 2 & 0 & 0 & 0 & 0 & 0 & 0 & 0 & 0 & 1 \\
\hline 3. Gout & 1 & 0 & 0 & 0 & 0 & 0 & 0 & 0 & 0 & 1 \\
\hline 4. Low back pain & 1 & 0 & 0 & 0 & 0 & 0 & 0 & 0 & 0 & 1 \\
\hline Other musculoskeletal disorders & 41 & 1 & 1 & 2 & 1 & 3 & 3 & 4 & 3 & 16 \\
\hline M. Congenital anomalies & 477 & 217 & 10 & 12 & 3 & 2 & 1 & 1 & 0 & 246 \\
\hline 1. Abdominal wall defect & 4 & 2 & 0 & 0 & 0 & 0 & 0 & 0 & 0 & 2 \\
\hline 2. Anencephaly & 18 & 9 & 0 & 0 & 0 & 0 & 0 & 0 & 0 & 9 \\
\hline 3. Anorectal atresia & 1 & 1 & 0 & 0 & 0 & 0 & 0 & 0 & 0 & 1 \\
\hline 4. Cleft lip & 0 & 0 & 0 & 0 & 0 & 0 & 0 & 0 & 0 & 0 \\
\hline 5. Cleft palate & 1 & 1 & 0 & 0 & 0 & 0 & 0 & 0 & 0 & 1 \\
\hline 6. Esophageal atresia & 1 & 1 & 0 & 0 & 0 & 0 & 0 & 0 & 0 & 1 \\
\hline 7. Renal agenesis & 2 & 1 & 0 & 0 & 0 & 0 & 0 & 0 & 0 & 1 \\
\hline 8. Down syndrome & 22 & 8 & 1 & 2 & 0 & 0 & 0 & 0 & 0 & 11 \\
\hline 9. Congenital heart anomalies & 257 & 112 & 6 & 7 & 2 & 1 & 0 & 0 & 0 & 128 \\
\hline 10. Spina bifida & 24 & 11 & 0 & 0 & 0 & 0 & 0 & 0 & 0 & 12 \\
\hline Other congenital anomalies & 148 & 73 & 3 & 3 & 1 & 1 & 0 & 0 & 0 & 82 \\
\hline N. Oral conditions & 1 & $\mathbf{0}$ & $\mathbf{0}$ & $\mathbf{0}$ & $\mathbf{0}$ & $\mathbf{0}$ & $\mathbf{0}$ & $\mathbf{0}$ & $\mathbf{0}$ & 1 \\
\hline 1. Dental caries & 0 & - & - & - & - & - & - & 0 & 0 & 0 \\
\hline 2. Periodontal disease & 0 & - & - & 0 & 0 & 0 & 0 & 0 & 0 & 0 \\
\hline 3. Edentulism & - & - & - & - & - & - & - & - & - & - \\
\hline Other oral diseases & 1 & 0 & 0 & 0 & 0 & 0 & 0 & 0 & 0 & 1 \\
\hline III. Injuries & 4,715 & 154 & 242 & 911 & 802 & 571 & 250 & 164 & 75 & 3,169 \\
\hline A. Unintentional injuries & 3,214 & 147 & 222 & 511 & 478 & 386 & 174 & 119 & 56 & 2,095 \\
\hline 1. Road traffic accidents & 1,069 & 28 & 82 & 217 & 204 & 143 & 58 & 37 & 13 & 781 \\
\hline 2. Poisonings & 328 & 9 & 11 & 35 & 52 & 65 & 26 & 10 & 3 & 211 \\
\hline 3. Falls & 316 & 9 & 13 & 28 & 33 & 38 & 26 & 30 & 21 & 199 \\
\hline 4. Fires & 300 & 17 & 16 & 23 & 24 & 15 & 8 & 6 & 3 & 113 \\
\hline 5. Drownings & 368 & 33 & 57 & 66 & 44 & 30 & 11 & 7 & 3 & 252 \\
\hline 6. Other unintentional injuries & 832 & 50 & 43 & 141 & 121 & 95 & 45 & 29 & 14 & 539 \\
\hline B. Intentional injuries & 1,501 & 7 & 20 & 400 & 324 & 184 & 76 & 45 & 18 & 1,074 \\
\hline 1. Self-inflicted injuries & 749 & 0 & 8 & 137 & 124 & 94 & 47 & 31 & 11 & 453 \\
\hline 2. Violence & 532 & 6 & 9 & 179 & 131 & 66 & 19 & 10 & 5 & 425 \\
\hline 3. War & 207 & 0 & 2 & 80 & 66 & 24 & 10 & 3 & 2 & 187 \\
\hline Other intentional injuries & 12 & 1 & 0 & 3 & 3 & 1 & 1 & 1 & 0 & 10 \\
\hline
\end{tabular}


Table 3B.1 Continued

\begin{tabular}{|c|c|c|c|c|c|c|c|c|}
\hline \multicolumn{9}{|c|}{ Female } \\
\hline $0-4$ & 5-14 & $15-29$ & 30-44 & 45-59 & $60-69$ & $70-79$ & $80+$ & Total \\
\hline 67 & 26 & 47 & 62 & 132 & 123 & 143 & 102 & 702 \\
\hline 3 & 1 & 4 & 9 & 18 & 18 & 22 & 18 & 94 \\
\hline 17 & 8 & 15 & 24 & 61 & 50 & 46 & 20 & 240 \\
\hline 0 & 0 & 1 & 1 & 2 & 2 & 2 & 2 & 9 \\
\hline 47 & 17 & 27 & 28 & 52 & 53 & 72 & 63 & 359 \\
\hline 9 & 7 & 16 & 26 & 61 & 67 & 75 & 52 & 313 \\
\hline 7 & 6 & 14 & 22 & 54 & 58 & 64 & 43 & 267 \\
\hline- & - & - & - & - & - & - & - & - \\
\hline 1 & 1 & 3 & 4 & 8 & 9 & 12 & 9 & 46 \\
\hline 1 & 0 & 1 & 3 & 5 & 6 & 8 & 8 & 32 \\
\hline 1 & 1 & 4 & 5 & 6 & 5 & 8 & 7 & 37 \\
\hline 0 & 0 & 0 & 1 & 2 & 2 & 4 & 1 & 10 \\
\hline 0 & 0 & 0 & 0 & 0 & 0 & 0 & 0 & 1 \\
\hline 0 & 0 & 0 & 0 & 0 & 0 & 0 & 0 & 0 \\
\hline 0 & 0 & 0 & 0 & 0 & 0 & 0 & 0 & 0 \\
\hline 1 & 1 & 4 & 4 & 4 & 3 & 4 & 5 & 25 \\
\hline 204 & 10 & 10 & 3 & 2 & 1 & 1 & 0 & 231 \\
\hline 2 & 0 & 0 & 0 & 0 & 0 & 0 & 0 & 2 \\
\hline 9 & 0 & 0 & 0 & 0 & 0 & 0 & 0 & 9 \\
\hline 0 & 0 & 0 & 0 & 0 & 0 & 0 & 0 & 0 \\
\hline 0 & 0 & 0 & 0 & 0 & 0 & 0 & 0 & 0 \\
\hline 1 & 0 & 0 & 0 & 0 & 0 & 0 & 0 & 1 \\
\hline 1 & 0 & 0 & 0 & 0 & 0 & 0 & 0 & 1 \\
\hline 1 & 0 & 0 & 0 & 0 & 0 & 0 & 0 & 1 \\
\hline 7 & 1 & 2 & 0 & 0 & 0 & 0 & 0 & 11 \\
\hline 112 & 6 & 6 & 2 & 1 & 0 & 0 & 0 & 128 \\
\hline 12 & 0 & 0 & 0 & 0 & 0 & 0 & 0 & 12 \\
\hline 60 & 3 & 1 & 1 & 1 & 0 & 0 & 0 & 66 \\
\hline 0 & 0 & 0 & 0 & 0 & 0 & 0 & 0 & 1 \\
\hline- & - & - & - & 0 & - & - & 0 & 0 \\
\hline- & 0 & 0 & 0 & 0 & 0 & 0 & 0 & 0 \\
\hline- & - & - & - & - & - & - & - & - \\
\hline 0 & 0 & 0 & 0 & 0 & 0 & 0 & 0 & 1 \\
\hline 148 & 164 & 342 & 288 & 249 & 136 & 132 & 87 & 1,546 \\
\hline 142 & 148 & 212 & 179 & 166 & 99 & 101 & 73 & 1,119 \\
\hline 22 & 48 & 52 & 57 & 54 & 25 & 20 & 9 & 288 \\
\hline 7 & 10 & 16 & 21 & 26 & 22 & 10 & 5 & 117 \\
\hline 7 & 8 & 9 & 9 & 15 & 13 & 29 & 28 & 117 \\
\hline 23 & 18 & 66 & 38 & 19 & 9 & 10 & 6 & 187 \\
\hline 25 & 31 & 21 & 13 & 10 & 6 & 6 & 4 & 116 \\
\hline 59 & 34 & 48 & 40 & 41 & 23 & 26 & 22 & 293 \\
\hline 6 & 16 & 130 & 109 & 83 & 37 & 31 & 14 & 427 \\
\hline 0 & 6 & 98 & 75 & 57 & 25 & 23 & 11 & 296 \\
\hline 6 & 9 & 28 & 28 & 20 & 9 & 6 & 2 & 108 \\
\hline 0 & 1 & 4 & 5 & 5 & 3 & 1 & 1 & 20 \\
\hline 0 & 0 & 1 & 0 & 1 & 0 & 0 & 0 & 3 \\
\hline
\end{tabular}

Source: Authors' compilation.

Note: $-=$ an estimate of zero; the number zero in a cell indicates a non-zero estimate of less than 500 .

a. For East Asia and Pacific, Europe and Central Asia, and Latin America and the Caribbean regions, these figures include

late effects of polio cases with onset prior to regional certification of polio eradication in 1994, 2000, and 2002, respectively.

b. Does not include liver cancer and cirrhosis deaths resulting from chronic hepatitis virus infection.

c. This cause category includes "Causes arising in the perinatal period" as defined in the International Classification of

Diseases, principally low birthweight, prematurity, birth asphyxia, and birth trauma, and does not include all causes of deaths occurring in the perinatal period. 
Table 3B.2 Deaths by Cause, Sex, and Age in the East Asia and Pacific Region, 2001

\begin{tabular}{|c|c|c|c|c|c|c|c|c|c|c|}
\hline \multirow[b]{2}{*}{ Cause } & \multicolumn{10}{|c|}{ Male } \\
\hline & Total & $0-4$ & 5-14 & $15-29$ & $30-44$ & $45-59$ & $60-69$ & 70-79 & $80+$ & Total \\
\hline Population (millions) & 1,849 & 80 & 175 & 244 & 224 & 136 & 51 & 25 & 6 & 942 \\
\hline $\begin{array}{l}\text { All causes } \\
\text { I. Communicable, maternal, perinatal, } \\
\text { and nutritional conditions }\end{array}$ & $\begin{array}{r}13,070 \\
2,470\end{array}$ & $\begin{array}{l}701 \\
535\end{array}$ & $\begin{array}{r}121 \\
35\end{array}$ & $\begin{array}{r}416 \\
83\end{array}$ & $\begin{array}{l}624 \\
153\end{array}$ & $\begin{array}{r}1,182 \\
160\end{array}$ & $\begin{array}{r}1,388 \\
137\end{array}$ & $\begin{array}{r}1,619 \\
135\end{array}$ & $\begin{array}{r}895 \\
86\end{array}$ & $\begin{array}{l}6,945 \\
1,324\end{array}$ \\
\hline A. Infectious and parasitic diseases & 1,299 & 196 & 25 & 70 & 139 & 132 & 102 & 88 & 37 & 790 \\
\hline 1. Tuberculosis & 534 & 2 & 3 & 29 & 64 & 82 & 82 & 65 & 19 & 347 \\
\hline $\begin{array}{l}\text { 2. Sexually transmitted diseases } \\
\text { excluding HIV/AIDS }\end{array}$ & 9 & 1 & 0 & $\mathbf{0}$ & 0 & 1 & 1 & 1 & 1 & 4 \\
\hline a. Syphilis & 5 & 1 & 0 & 0 & 0 & 0 & 1 & 1 & 0 & 4 \\
\hline b. Chlamydia & 1 & - & - & - & - & - & - & - & - & - \\
\hline c. Gonorrhea & 0 & - & - & 0 & 0 & - & - & - & - & 0 \\
\hline d. Other sexually transmitted diseases & 3 & 0 & - & 0 & 0 & 0 & 0 & - & 0 & 1 \\
\hline 3. HIV/AIDS & 106 & 3 & $\mathbf{0}$ & 15 & 43 & 16 & 1 & $\mathbf{0}$ & $\mathbf{0}$ & 79 \\
\hline 4. Diarrheal diseases & 226 & 105 & 1 & 1 & 2 & 2 & 2 & 2 & 3 & 118 \\
\hline 5. Childhood-cluster diseases & 107 & 32 & 14 & 5 & 1 & 1 & $\mathbf{0}$ & $\mathbf{0}$ & 0 & 54 \\
\hline a. Pertussis & 3 & 2 & - & - & - & - & - & - & - & 2 \\
\hline b. Poliomyelitis ${ }^{a}$ & 0 & 0 & - & - & - & - & - & - & - & 0 \\
\hline c. Diphtheria & 1 & 0 & 0 & 0 & 0 & 0 & 0 & - & 0 & 0 \\
\hline d. Measles & 76 & 22 & 12 & 3 & - & - & - & - & - & 38 \\
\hline e. Tetanus & 27 & 8 & 2 & 1 & 1 & 1 & 0 & 0 & 0 & 14 \\
\hline 6. Meningitis & 33 & 7 & 1 & 3 & 2 & 2 & 1 & 1 & 1 & 16 \\
\hline 7. Hepatitis $\mathbf{B}^{\mathbf{b}}$ & 32 & 1 & $\mathbf{0}$ & 3 & 7 & 11 & 3 & 1 & 1 & 26 \\
\hline Hepatitis $\mathbf{c}^{\mathbf{b}}$ & 13 & 0 & 0 & 1 & 3 & 5 & 1 & 0 & 0 & 11 \\
\hline 8. Malaria & 30 & 18 & $\mathbf{0}$ & $\mathbf{0}$ & $\mathbf{0}$ & 0 & $\mathbf{0}$ & $\mathbf{0}$ & 0 & 20 \\
\hline 9. Tropical-cluster diseases & 5 & $\mathbf{0}$ & $\mathbf{0}$ & $\mathbf{0}$ & 0 & 1 & 1 & 0 & $\mathbf{0}$ & 4 \\
\hline a. Trypanosomiasis & - & - & - & - & - & - & - & - & - & - \\
\hline b. Chagas' disease & 0 & - & - & - & - & - & - & - & - & - \\
\hline c. Schistosomiasis & 3 & - & 0 & 0 & 0 & 1 & 1 & 0 & 0 & 3 \\
\hline d. Leishmaniasis & 2 & 0 & 0 & 0 & 0 & 0 & 0 & 0 & - & 1 \\
\hline e. Lymphatic filariasis & 0 & 0 & - & 0 & 0 & 0 & 0 & - & 0 & 0 \\
\hline f. Onchocerciasis & - & - & - & - & - & - & - & - & - & - \\
\hline 10. Leprosy & 2 & 0 & $\mathbf{0}$ & 0 & 0 & 0 & 1 & 1 & $\mathbf{0}$ & 2 \\
\hline 11. Dengue & 8 & 1 & 2 & 0 & 0 & $\mathbf{0}$ & $\mathbf{0}$ & $\mathbf{0}$ & 0 & 3 \\
\hline 12. Japanese encephalitis & 4 & 1 & $\mathbf{0}$ & $\mathbf{0}$ & 0 & 0 & $\mathbf{0}$ & 0 & $\mathbf{0}$ & 2 \\
\hline 13. Trachoma & $\mathbf{0}$ & $\mathbf{0}$ & - & $\mathbf{0}$ & - & $\mathbf{0}$ & - & $\mathbf{0}$ & - & 0 \\
\hline 14. Intestinal nematode infections & 2 & 1 & $\mathbf{0}$ & $\mathbf{0}$ & $\mathbf{0}$ & 0 & $\mathbf{0}$ & 0 & $\mathbf{0}$ & 1 \\
\hline a. Ascariasis & 1 & 0 & 0 & - & - & 0 & - & 0 & - & 0 \\
\hline b. Trichuriasis & 0 & 0 & 0 & - & - & - & - & - & - & 0 \\
\hline c. Hookworm disease & 0 & - & - & 0 & 0 & 0 & 0 & 0 & 0 & 0 \\
\hline Other intestinal infections & 1 & 0 & 0 & 0 & 0 & 0 & 0 & 0 & 0 & 0 \\
\hline Other infectious diseases & 190 & 24 & 3 & 13 & 16 & 11 & 8 & 15 & 12 & 102 \\
\hline B. Respiratory infections & 571 & 68 & 8 & 10 & 11 & 25 & 33 & 45 & 48 & 248 \\
\hline 1. Lower respiratory infections & 544 & 63 & 8 & 9 & 10 & 22 & 32 & 44 & 46 & 233 \\
\hline 2. Upper respiratory infections & 27 & 6 & 0 & 1 & 1 & 2 & 2 & 1 & 2 & 14 \\
\hline 3. Otitis media & 1 & 0 & 0 & 0 & 0 & 0 & 0 & 0 & 0 & 1 \\
\hline C. Maternal conditions & 37 & - & - & - & - & - & - & - & - & - \\
\hline 1. Maternal hemorrhage & 12 & - & - & - & - & - & - & - & - & - \\
\hline 2. Maternal sepsis & 3 & - & - & - & - & - & - & - & - & - \\
\hline 3. Hypertensive disorders of pregnancy & 5 & - & - & - & - & - & - & - & - & - \\
\hline 4. Obstructed labor & 2 & - & - & - & - & - & - & - & - & - \\
\hline 5. Abortion & 5 & - & - & - & - & - & - & - & - & - \\
\hline Other maternal conditions & 10 & - & - & - & - & - & - & - & - & - \\
\hline D. Perinatal conditions ${ }^{\mathbf{c}}$ & 502 & 262 & $\mathbf{0}$ & - & - & - & - & - & - & 262 \\
\hline 1. Low birthweight & 193 & 100 & - & - & - & - & - & - & - & 100 \\
\hline 2. Birth asphyxia and birth trauma & 158 & 83 & - & - & - & - & - & - & - & 83 \\
\hline Other perinatal conditions & 152 & 78 & 0 & - & - & - & - & - & - & 78 \\
\hline E. Nutritional deficiencies & 61 & 9 & 1 & 3 & 2 & 3 & 2 & 2 & 2 & 24 \\
\hline 1. Protein-energy malnutrition & 27 & 7 & 1 & 1 & 1 & 1 & 1 & 1 & 1 & 14 \\
\hline 2. lodine deficiency & 0 & 0 & 0 & - & 0 & - & - & - & 0 & 0 \\
\hline 3. Vitamin A deficiency & 0 & 0 & 0 & - & 0 & 0 & - & - & - & 0 \\
\hline 4. Iron-deficiency anemia & 25 & 1 & 0 & 1 & 1 & 2 & 0 & 0 & 0 & 6 \\
\hline Other nutritional disorders & 9 & 1 & 0 & 0 & 0 & 0 & 0 & 1 & 1 & 3 \\
\hline
\end{tabular}


Table 3B.2 Continued

\begin{tabular}{|c|c|c|c|c|c|c|c|c|}
\hline \multicolumn{9}{|c|}{ Female } \\
\hline $0-4$ & 5-14 & $15-29$ & $30-44$ & $45-59$ & $60-69$ & $70-79$ & $80+$ & Total \\
\hline 74 & 161 & 232 & 217 & 131 & 52 & 30 & 10 & 907 \\
\hline $\begin{array}{l}697 \\
542\end{array}$ & $\begin{array}{l}97 \\
34\end{array}$ & $\begin{array}{r}220 \\
67\end{array}$ & $\begin{array}{l}408 \\
100\end{array}$ & $\begin{array}{r}747 \\
85\end{array}$ & $\begin{array}{r}936 \\
80\end{array}$ & $\begin{array}{r}1,530 \\
106\end{array}$ & $\begin{array}{r}1,491 \\
130\end{array}$ & $\begin{array}{l}6,124 \\
1,145\end{array}$ \\
\hline 168 & 25 & 44 & 69 & 67 & 52 & 53 & 32 & 509 \\
\hline 2 & 3 & 22 & 39 & 42 & 36 & 31 & 12 & 187 \\
\hline 0 & - & 0 & 1 & 2 & 1 & 1 & 0 & 4 \\
\hline 0 & - & 0 & 0 & 0 & 0 & 0 & 0 & 1 \\
\hline- & - & - & 0 & 1 & 0 & - & - & 1 \\
\hline 0 & - & 0 & 0 & 0 & - & - & - & 0 \\
\hline 0 & - & 0 & 0 & 1 & 0 & 0 & 0 & 2 \\
\hline 2 & 0 & 5 & 9 & 8 & 2 & 1 & 0 & 27 \\
\hline 95 & 1 & 1 & 1 & 1 & 2 & 3 & 4 & 107 \\
\hline 32 & 14 & 5 & 1 & 1 & 0 & 0 & 0 & 53 \\
\hline 2 & - & - & - & - & - & - & - & 2 \\
\hline 0 & - & - & - & 0 & - & - & - & 0 \\
\hline 0 & 0 & 0 & 0 & 0 & - & - & - & 0 \\
\hline 23 & 12 & 3 & - & - & - & - & - & 38 \\
\hline 7 & 2 & 1 & 1 & 1 & 0 & 0 & 0 & 13 \\
\hline 6 & 1 & 3 & 3 & 2 & 1 & 1 & 0 & 17 \\
\hline 1 & 0 & 1 & 2 & 1 & $i$ & 0 & 1 & 6 \\
\hline 0 & 0 & 0 & 1 & 0 & 0 & 0 & 0 & 2 \\
\hline 8 & 0 & 0 & 0 & 0 & 0 & 0 & 0 & 9 \\
\hline 0 & 0 & 0 & 0 & 0 & 0 & 0 & 0 & 1 \\
\hline- & - & - & - & - & - & - & - & - \\
\hline- & - & - & 0 & - & - & - & - & 0 \\
\hline- & 0 & 0 & 0 & 0 & 0 & 0 & 0 & 1 \\
\hline 0 & 0 & 0 & 0 & 0 & 0 & 0 & - & 1 \\
\hline- & - & - & - & 0 & - & 0 & 0 & 0 \\
\hline- & - & - & - & - & - & - & - & - \\
\hline 0 & 0 & 0 & 0 & 0 & 0 & 0 & 0 & 0 \\
\hline 1 & 2 & 0 & 0 & 0 & 0 & 0 & 0 & 5 \\
\hline 1 & 0 & 0 & 0 & 0 & 0 & 0 & 0 & 2 \\
\hline- & - & - & - & - & - & - & - & - \\
\hline 1 & 0 & 0 & 0 & 0 & 0 & 0 & 0 & 1 \\
\hline 0 & 0 & 0 & 0 & - & 0 & - & - & 0 \\
\hline- & 0 & - & - & - & - & - & - & 0 \\
\hline- & - & 0 & 0 & 0 & 0 & 0 & 0 & 0 \\
\hline 0 & 0 & 0 & 0 & - & 0 & 0 & 0 & 0 \\
\hline 18 & 3 & 7 & 12 & 8 & 9 & 17 & 13 & 87 \\
\hline 127 & 8 & 5 & 6 & 10 & 24 & 49 & 93 & 323 \\
\hline 122 & 8 & 5 & 5 & 10 & 24 & 48 & 89 & 311 \\
\hline 6 & 0 & 0 & 0 & 0 & 0 & 1 & 5 & 12 \\
\hline 0 & 0 & 0 & 0 & 0 & 0 & - & 0 & 0 \\
\hline- & 0 & 16 & 20 & 0 & - & - & - & 37 \\
\hline- & - & 5 & 6 & 0 & - & - & - & 12 \\
\hline- & - & 2 & 2 & 0 & - & - & - & 3 \\
\hline- & 0 & 2 & 2 & 0 & - & - & - & 5 \\
\hline- & - & 1 & 1 & 0 & - & - & - & 2 \\
\hline- & - & 3 & 2 & 0 & - & - & - & 5 \\
\hline- & - & 3 & 6 & 0 & - & - & - & 10 \\
\hline 241 & 0 & - & - & - & - & - & - & 241 \\
\hline 92 & 0 & - & - & - & - & - & - & 92 \\
\hline 74 & - & - & - & - & - & - & - & 74 \\
\hline 74 & - & - & - & - & - & - & - & 74 \\
\hline 6 & 1 & 2 & 6 & 8 & 5 & 4 & 5 & 37 \\
\hline 5 & 1 & 0 & 1 & 1 & 1 & 1 & 2 & 12 \\
\hline 0 & 0 & - & 0 & - & 0 & - & - & 0 \\
\hline 0 & 0 & - & - & - & - & - & - & 0 \\
\hline 1 & 0 & 2 & 5 & 7 & 3 & 1 & 1 & 19 \\
\hline 0 & 0 & 0 & 0 & 0 & 1 & 2 & 2 & 6 \\
\hline & & & & & & ntinues & he fo & $g p a$ \\
\hline
\end{tabular}


Table 3B.2 Continued

\begin{tabular}{|c|c|c|c|c|c|c|c|c|c|c|}
\hline \multirow[b]{2}{*}{ Cause } & \multicolumn{10}{|c|}{ Male } \\
\hline & Total & $0-4$ & $5-14$ & $15-29$ & $30-44$ & $45-59$ & $60-69$ & $70-79$ & $80+$ & Total \\
\hline II. Noncommunicable diseases & 9,221 & 125 & 26 & 96 & 254 & 860 & 1,168 & 1,429 & 783 & 4,741 \\
\hline A. Malignant neoplasms & 2,143 & 6 & 8 & 26 & 90 & 346 & 382 & 316 & 91 & 1,264 \\
\hline 1. Mouth and oropharynx cancers & 66 & 0 & 0 & 1 & 5 & 16 & 12 & 8 & 2 & 45 \\
\hline 2. Esophageal cancer & 234 & - & - & 1 & 8 & 36 & 52 & 41 & 9 & 147 \\
\hline 3. Stomach cancer & 442 & 0 & 0 & 3 & 16 & 76 & 84 & 75 & 25 & 278 \\
\hline 4. Colon and rectal cancers & 159 & 0 & 0 & 2 & 7 & 20 & 24 & 22 & 9 & 83 \\
\hline 5. Liver cancer & 373 & 0 & 0 & 5 & 29 & 99 & 74 & 51 & 11 & 269 \\
\hline 6. Pancreas cancer & 37 & 0 & 0 & 0 & 2 & 7 & 6 & 5 & 2 & 22 \\
\hline 7. Trachea, bronchus, and lung cancers & 387 & 0 & 0 & 1 & 12 & 60 & 94 & 77 & 18 & 262 \\
\hline 8. Melanoma and other skin cancers & 5 & 0 & 0 & 0 & 0 & 1 & 1 & 1 & 0 & 2 \\
\hline 9. Breast cancer & 93 & - & - & - & 0 & 0 & 0 & 0 & 0 & 0 \\
\hline 10. Cervix uteri cancer & 47 & - & - & - & - & - & - & - & - & - \\
\hline 11. Corpus uteri cancer & 8 & - & - & - & - & - & - & - & - & - \\
\hline 12. Ovarian cancer & 25 & - & - & - & - & - & - & - & - & - \\
\hline 13. Prostate cancer & 16 & 0 & 0 & 0 & 0 & 1 & 4 & 8 & 3 & 16 \\
\hline 14. Bladder cancer & 30 & 0 & 0 & 0 & 0 & 2 & 6 & 9 & 4 & 21 \\
\hline 15. Lymphomas and multiple myeloma & 42 & 0 & 1 & 2 & 4 & 6 & 5 & 3 & 1 & 24 \\
\hline 16. Leukemia & 76 & 3 & 5 & 10 & 4 & 7 & 6 & 5 & 2 & 42 \\
\hline Other malignant neoplasms & 104 & 2 & 1 & 1 & 3 & 15 & 15 & 12 & 5 & 54 \\
\hline B. Other neoplasms & 21 & 1 & 1 & 1 & 1 & 3 & 2 & 2 & 1 & 9 \\
\hline C. Diabetes mellitus & 233 & 0 & 0 & 1 & 5 & 20 & 29 & 29 & 12 & 97 \\
\hline D. Endocrine disorders & 61 & 9 & 1 & 2 & 2 & 3 & 2 & 3 & 3 & 25 \\
\hline E. Neuropsychiatric conditions & 186 & 3 & 2 & 8 & 15 & 14 & 11 & 21 & 18 & 91 \\
\hline 1. Unipolar depressive disorders & 1 & 0 & 0 & 0 & 0 & 0 & 0 & 0 & 0 & 1 \\
\hline 2. Bipolar affective disorder & 0 & 0 & 0 & 0 & 0 & 0 & 0 & 0 & 0 & 0 \\
\hline 3. Schizophrenia & 6 & 0 & 0 & 0 & 1 & 1 & 0 & 0 & 0 & 3 \\
\hline 4. Epilepsy & 24 & 2 & 1 & 3 & 4 & 2 & 1 & 0 & 0 & 13 \\
\hline 5. Alcohol use disorders & 12 & 0 & 0 & 1 & 3 & 4 & 2 & 1 & 0 & 11 \\
\hline 6. Alzheimer's and other dementias & 58 & 0 & 0 & 0 & 1 & 1 & 2 & 8 & 9 & 21 \\
\hline 7. Parkinson's disease & 26 & 0 & 0 & 0 & 1 & 0 & 1 & 5 & 4 & 11 \\
\hline 8. Multiple sclerosis & 1 & 0 & 0 & 0 & 0 & 0 & 0 & 0 & 0 & 0 \\
\hline 9. Drug use disorders & 7 & 0 & 0 & 1 & 3 & 2 & 0 & 0 & 0 & 6 \\
\hline 10. Post-traumatic stress disorder & 0 & 0 & 0 & 0 & 0 & 0 & 0 & 0 & 0 & 0 \\
\hline 11. Obsessive-compulsive disorder & - & - & - & - & - & - & - & - & - & - \\
\hline 12. Panic disorder & - & - & - & - & - & - & - & - & - & - \\
\hline 13. Insomnia (primary) & - & - & - & - & - & - & - & - & - & - \\
\hline 14. Migraine & - & - & - & - & - & - & - & - & - & - \\
\hline 15. Mental retardation, lead-caused & 0 & 0 & 0 & 0 & 0 & 0 & 0 & 0 & 0 & 0 \\
\hline Other neuropsychiatric disorders & 50 & 1 & 1 & 2 & 2 & 4 & 5 & 7 & 4 & 26 \\
\hline F. Sense organ diseases & $\mathbf{0}$ & $\mathbf{0}$ & 0 & $\mathbf{0}$ & $\mathbf{0}$ & 0 & $\mathbf{0}$ & $\mathbf{0}$ & $\mathbf{0}$ & $\mathbf{0}$ \\
\hline 1. Glaucoma & 0 & 0 & - & 0 & 0 & 0 & 0 & - & - & 0 \\
\hline 2. Cataracts & - & - & - & - & - & - & - & - & - & - \\
\hline 3. Vision disorders, age-related & - & - & - & - & - & - & - & - & - & - \\
\hline 4. Hearing loss, adult onset & - & - & - & - & - & - & - & - & - & - \\
\hline Other sense organ disorders & 0 & 0 & 0 & 0 & 0 & 0 & 0 & 0 & 0 & 0 \\
\hline G. Cardiovascular diseases & 4,003 & 7 & 4 & 27 & 81 & 310 & 493 & 664 & 389 & 1,976 \\
\hline 1. Rheumatic heart disease & 121 & 1 & 1 & 5 & 6 & 11 & 10 & 10 & 4 & 47 \\
\hline 2. Hypertensive heart disease & 333 & 0 & 0 & 1 & 7 & 28 & 47 & 54 & 29 & 166 \\
\hline 3. Ischemic heart disease & 1,151 & 1 & 1 & 8 & 31 & 104 & 149 & 189 & 109 & 591 \\
\hline 4. Cerebrovascular disease & 1,902 & 2 & 1 & 6 & 25 & 139 & 249 & 351 & 188 & 959 \\
\hline 5. Inflammatory heart diseases & 81 & 1 & 0 & 1 & 2 & 5 & 7 & 11 & 10 & 36 \\
\hline Other cardiovascular diseases & 415 & 3 & 2 & 6 & 10 & 25 & 33 & 50 & 49 & 177 \\
\hline H. Respiratory diseases & 1,660 & 7 & 1 & 5 & 11 & 62 & 158 & 308 & 225 & 775 \\
\hline 1. Chronic obstructive pulmonary disease & 1,415 & 0 & 0 & 0 & 2 & 46 & 136 & 275 & 191 & 651 \\
\hline 2. Asthma & 56 & 1 & 1 & 3 & 5 & 8 & 5 & 5 & 2 & 28 \\
\hline Other respiratory diseases & 189 & 5 & 1 & 2 & 3 & 8 & 16 & 27 & 32 & 96 \\
\hline
\end{tabular}


Table 3B.2 Continued

\begin{tabular}{|c|c|c|c|c|c|c|c|c|}
\hline \multicolumn{9}{|c|}{ Female } \\
\hline $0-4$ & 5-14 & $15-29$ & $30-44$ & 45-59 & $60-69$ & $70-79$ & $80+$ & Total \\
\hline 115 & 25 & 67 & 195 & 571 & 808 & 1,375 & 1,324 & 4,479 \\
\hline 6 & 6 & 15 & 79 & 217 & 211 & 229 & 114 & 879 \\
\hline 0 & 0 & 0 & 1 & 6 & 5 & 6 & 3 & 22 \\
\hline 0 & 0 & 0 & 2 & 20 & 25 & 27 & 12 & 87 \\
\hline 0 & 0 & 2 & 13 & 33 & 36 & 49 & 31 & 164 \\
\hline 0 & 0 & 1 & 7 & 16 & 19 & 20 & 14 & 76 \\
\hline 1 & 0 & 2 & 10 & 25 & 28 & 27 & 10 & 104 \\
\hline 0 & 0 & 0 & 1 & 3 & 4 & 5 & 2 & 16 \\
\hline 0 & 0 & 0 & 6 & 27 & 36 & 42 & 12 & 125 \\
\hline 0 & 0 & 0 & 0 & 0 & 0 & 1 & 0 & 2 \\
\hline 0 & 0 & 1 & 16 & 38 & 19 & 13 & 7 & 93 \\
\hline 0 & 0 & 2 & 3 & 14 & 13 & 11 & 4 & 47 \\
\hline 0 & 0 & 0 & 1 & 2 & 2 & 2 & 1 & 8 \\
\hline 0 & 0 & 1 & 4 & 8 & 6 & 5 & 2 & 25 \\
\hline- & - & - & - & - & - & - & - & - \\
\hline 0 & 0 & 0 & 0 & 1 & 2 & 3 & 2 & 8 \\
\hline 0 & 1 & 1 & 3 & 4 & 3 & 4 & 2 & 18 \\
\hline 3 & 4 & 4 & 6 & 7 & 4 & 4 & 2 & 35 \\
\hline 2 & 1 & 1 & 5 & 12 & 10 & 11 & 8 & 50 \\
\hline 1 & 0 & 0 & 1 & 3 & 2 & 2 & 1 & 11 \\
\hline 0 & 0 & 1 & 5 & 23 & 39 & 46 & 22 & 136 \\
\hline 9 & 1 & 4 & 6 & 3 & 3 & 4 & 6 & 36 \\
\hline 2 & 2 & 5 & 7 & 9 & 9 & 24 & 38 & 95 \\
\hline- & - & 0 & 0 & 0 & 0 & 0 & 0 & 1 \\
\hline 0 & - & 0 & 0 & 0 & 0 & 0 & 0 & 0 \\
\hline 0 & 0 & 0 & 1 & 1 & 0 & 0 & 0 & 3 \\
\hline 1 & 1 & 3 & 3 & 1 & 0 & 0 & 0 & 11 \\
\hline- & - & 0 & 1 & 1 & 0 & 0 & 0 & 2 \\
\hline 0 & 0 & 0 & 1 & 1 & 2 & 9 & 24 & 37 \\
\hline 0 & - & 0 & 0 & 0 & 1 & 6 & 7 & 15 \\
\hline 0 & 0 & 0 & 0 & 0 & 0 & 0 & 0 & 1 \\
\hline- & - & 0 & 1 & 0 & 0 & 0 & 0 & 1 \\
\hline- & - & - & - & 0 & 0 & 0 & 0 & 0 \\
\hline- & - & - & - & - & - & - & - & - \\
\hline- & - & - & - & - & - & - & - & - \\
\hline- & - & - & - & - & - & - & - & - \\
\hline- & - & - & - & - & - & - & - & - \\
\hline 0 & 0 & 0 & 0 & 0 & 0 & 0 & 0 & 0 \\
\hline 0 & 1 & 1 & 1 & 3 & 4 & 8 & 7 & 25 \\
\hline 0 & 0 & 0 & 0 & 0 & 0 & 0 & 0 & 0 \\
\hline- & - & - & - & - & - & - & - & - \\
\hline- & - & - & - & - & - & - & - & - \\
\hline- & - & - & - & - & - & - & - & - \\
\hline- & - & - & - & - & - & - & - & - \\
\hline 0 & 0 & 0 & 0 & 0 & 0 & 0 & 0 & 0 \\
\hline 5 & 4 & 19 & 54 & 209 & 355 & 684 & 695 & 2,027 \\
\hline 1 & 1 & 5 & 8 & 16 & 14 & 18 & 10 & 74 \\
\hline 0 & 0 & 1 & 4 & 20 & 34 & 57 & 51 & 167 \\
\hline 0 & 0 & 6 & 16 & 58 & 111 & 181 & 186 & 560 \\
\hline 1 & 1 & 3 & 15 & 90 & 158 & 343 & 332 & 943 \\
\hline 1 & 0 & 1 & 1 & 4 & 6 & 14 & 20 & 46 \\
\hline 2 & 1 & 4 & 9 & 22 & 32 & 71 & 96 & 237 \\
\hline 5 & 1 & 4 & 11 & 48 & 126 & 307 & 382 & 884 \\
\hline 0 & 0 & 0 & 3 & 35 & 109 & 282 & 333 & 763 \\
\hline 1 & 1 & 3 & 6 & 8 & 5 & 3 & 2 & 28 \\
\hline 4 & 1 & 1 & 2 & 5 & 11 & 21 & 47 & 93 \\
\hline
\end{tabular}


Table 3B.2 Continued

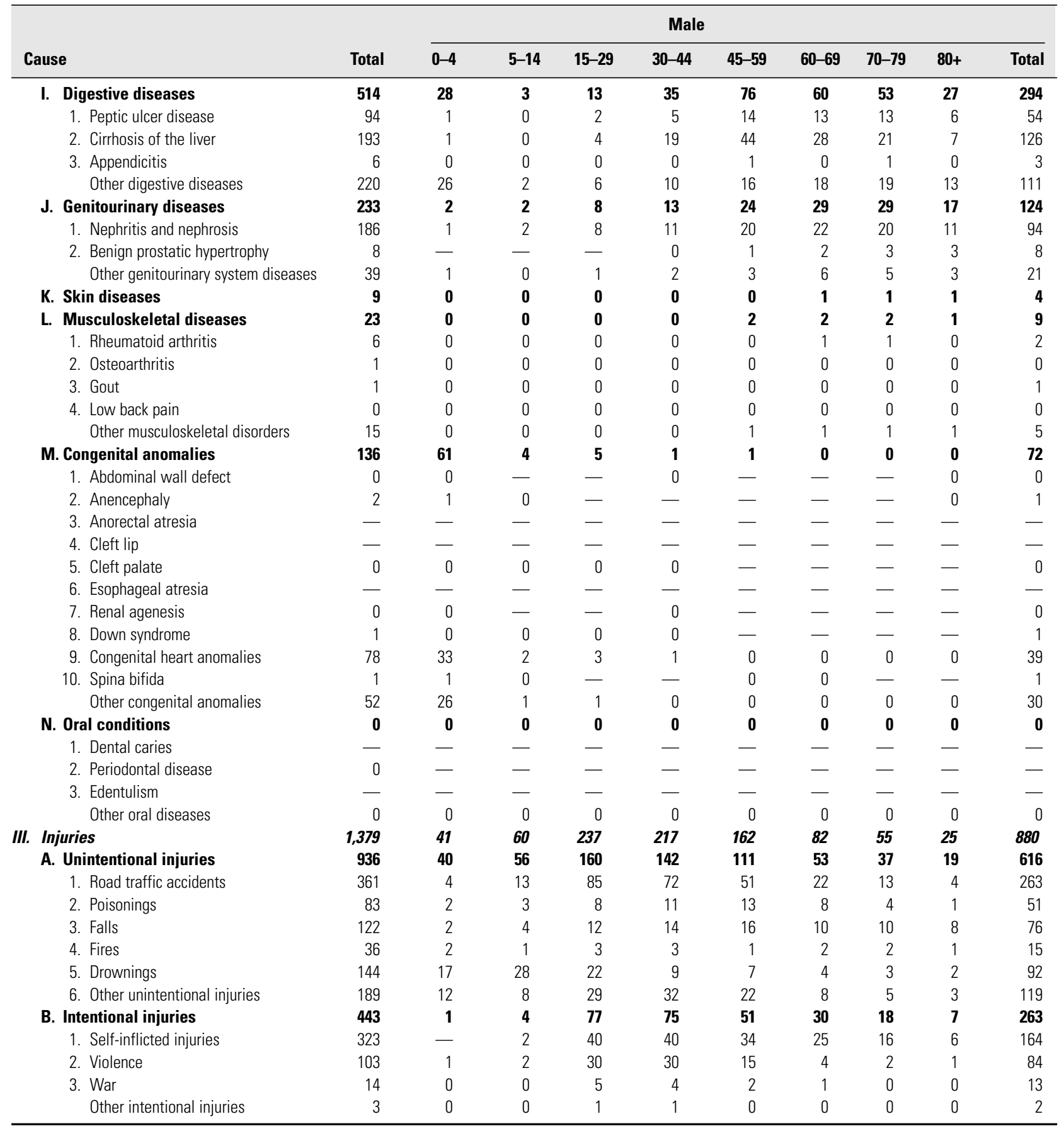


Table 3B.2 Continued

\begin{tabular}{|c|c|c|c|c|c|c|c|c|}
\hline \multicolumn{9}{|c|}{ Female } \\
\hline $0-4$ & 5-14 & $15-29$ & $30-44$ & 45-59 & $60-69$ & $70-79$ & $80+$ & Total \\
\hline 31 & 3 & 7 & 16 & 34 & 37 & 49 & 43 & 219 \\
\hline 1 & 0 & 1 & 4 & 7 & 8 & 10 & 9 & 40 \\
\hline 2 & 1 & 2 & 7 & 17 & 15 & 16 & 8 & 68 \\
\hline 0 & 0 & 0 & 0 & 1 & 0 & 1 & 1 & 3 \\
\hline 28 & 2 & 4 & 5 & 10 & 13 & 22 & 25 & 109 \\
\hline 1 & 2 & 6 & 11 & 21 & 24 & 26 & 18 & 109 \\
\hline 1 & 2 & 5 & 10 & 18 & 20 & 21 & 15 & 91 \\
\hline- & - & - & - & - & - & - & - & - \\
\hline 0 & 0 & 1 & 2 & 3 & 3 & 5 & 3 & 18 \\
\hline 0 & 0 & 0 & 0 & 1 & 1 & 1 & 1 & 5 \\
\hline 0 & 0 & 1 & 2 & 2 & 2 & 3 & 3 & 14 \\
\hline 0 & 0 & 0 & 0 & 1 & 1 & 1 & 0 & 4 \\
\hline 0 & 0 & 0 & 0 & 0 & 0 & 0 & 0 & 0 \\
\hline 0 & 0 & 0 & 0 & 0 & 0 & 0 & 0 & 0 \\
\hline 0 & 0 & 0 & 0 & 0 & 0 & 0 & 0 & 0 \\
\hline 0 & 0 & 1 & 2 & 1 & 1 & 1 & 2 & 9 \\
\hline 53 & 4 & 3 & 2 & 1 & 0 & 0 & 0 & 64 \\
\hline 0 & - & - & - & - & - & 0 & - & 0 \\
\hline 1 & 0 & 0 & 0 & 0 & - & - & - & 1 \\
\hline- & - & - & - & - & - & - & - & - \\
\hline- & - & - & - & - & - & - & - & - \\
\hline 0 & 0 & 0 & 0 & 0 & - & - & - & 0 \\
\hline- & - & - & - & - & - & - & - & - \\
\hline 0 & - & - & - & - & - & - & - & 0 \\
\hline 0 & 0 & 0 & 0 & 0 & - & - & - & 1 \\
\hline 30 & 3 & 3 & 1 & 0 & 0 & 0 & 0 & 39 \\
\hline 1 & 0 & 0 & 0 & 0 & - & 0 & 0 & 1 \\
\hline 21 & 1 & 0 & 0 & 0 & 0 & 0 & 0 & 23 \\
\hline 0 & 0 & 0 & 0 & 0 & 0 & 0 & 0 & 0 \\
\hline- & - & - & - & - & - & - & - & - \\
\hline- & 0 & 0 & 0 & 0 & - & - & - & 0 \\
\hline- & - & - & - & - & - & - & - & - \\
\hline 0 & 0 & 0 & 0 & 0 & 0 & 0 & 0 & 0 \\
\hline 40 & 38 & 86 & 113 & 91 & 47 & 48 & 36 & 499 \\
\hline 40 & 35 & 45 & 61 & 52 & 27 & 31 & 28 & 319 \\
\hline 3 & 8 & 16 & 28 & 24 & 9 & 7 & 3 & 98 \\
\hline 3 & 3 & 4 & 7 & 6 & 4 & 3 & 2 & 32 \\
\hline 2 & 1 & 3 & 4 & 6 & 5 & 10 & 14 & 46 \\
\hline 2 & 2 & 7 & 5 & 2 & 1 & 1 & 1 & 22 \\
\hline 11 & 16 & 6 & 6 & 4 & 3 & 3 & 2 & 53 \\
\hline 20 & 4 & 8 & 11 & 9 & 5 & 5 & 6 & 69 \\
\hline 1 & 3 & 40 & 52 & 39 & 20 & 17 & 8 & 180 \\
\hline- & 2 & 35 & 45 & 35 & 18 & 16 & 7 & 159 \\
\hline 1 & 1 & 5 & 6 & 4 & 2 & 1 & 0 & 19 \\
\hline 0 & 0 & 0 & 0 & 0 & 0 & 0 & 0 & 1 \\
\hline 0 & 0 & 0 & 0 & 0 & 0 & 0 & 0 & 1 \\
\hline
\end{tabular}

Source: Authors' compilation.

Note: - = an estimate of zero; the number zero in a cell indicates a non-zero estimate of less than 500.

a. These figures include late effects of polio cases with onset prior to regional certification of polio eradication in 1994.

b. Does not include liver cancer and cirrhosis deaths resulting from chronic hepatitis virus infection.

c. This cause category includes "Causes arising in the perinatal period" as defined in the International Classification of Diseases, principally low birthweight, prematurity, birth asphyxia, and birth trauma, and does not include all causes of deaths occurring in the perinatal period. 
Table 3B.3 Deaths by Cause, Sex, and Age in the Europe and Central Asia Region, 2001 (thousands)

\begin{tabular}{|c|c|c|c|c|c|c|c|c|c|c|}
\hline \multirow[b]{2}{*}{ Cause } & \multicolumn{10}{|c|}{ Male } \\
\hline & Total & $0-4$ & $5-14$ & $15-29$ & 30-44 & $45-59$ & $60-69$ & 70-79 & $80+$ & Total \\
\hline Population (millions) & 477 & 15 & 38 & 59 & 51 & 37 & 17 & 9 & 2 & 230 \\
\hline All causes & 5,669 & 97 & 21 & 148 & 323 & 619 & 707 & 720 & 348 & 2,985 \\
\hline $\begin{array}{l}\text { I. Communicable, maternal, perinatal, } \\
\text { and nutritional conditions }\end{array}$ & 326 & 70 & 4 & 15 & 40 & 42 & 21 & 13 & 7 & 211 \\
\hline A. Infectious and parasitic diseases & 152 & 17 & 2 & 13 & 36 & 29 & 10 & 4 & 1 & 112 \\
\hline 1. Tuberculosis & 66 & 0 & $\mathbf{0}$ & 5 & 19 & 20 & 7 & 2 & 0 & 55 \\
\hline $\begin{array}{l}\text { 2. Sexually transmitted diseases } \\
\text { excluding HIV/AIDS }\end{array}$ & 1 & 0 & 0 & 0 & $\mathbf{0}$ & $\mathbf{0}$ & 0 & $\mathbf{0}$ & 0 & 0 \\
\hline a. Syphilis & 0 & 0 & 0 & 0 & 0 & 0 & 0 & 0 & 0 & 0 \\
\hline b. Chlamydia & - & - & - & - & - & - & - & - & - & 一 \\
\hline c. Gonorrhea & 0 & - & - & 0 & 0 & 0 & - & 0 & - & 0 \\
\hline d. Other sexually transmitted diseases & 0 & 0 & - & 0 & 0 & 0 & 0 & 0 & - & 0 \\
\hline 3. HIV/AIDS & 28 & 0 & $\mathbf{0}$ & 5 & 13 & 5 & 1 & $\mathbf{0}$ & 0 & 24 \\
\hline 4. Diarrheal diseases & 15 & 6 & 0 & 0 & 0 & 0 & 0 & $\mathbf{0}$ & 0 & 8 \\
\hline 5. Childhood-cluster diseases & 8 & 3 & 1 & 0 & $\mathbf{0}$ & $\mathbf{0}$ & $\mathbf{0}$ & $\mathbf{0}$ & 0 & 4 \\
\hline a. Pertussis & 0 & 0 & 0 & - & - & - & - & - & - & 0 \\
\hline b. Poliomyelitis ${ }^{a}$ & 0 & 0 & 0 & 0 & 0 & 0 & 0 & 0 & 0 & 0 \\
\hline c. Diphtheria & 0 & 0 & 0 & 0 & 0 & 0 & 0 & 0 & 0 & 0 \\
\hline d. Measles & 8 & 3 & 1 & 0 & 0 & 0 & 0 & - & - & 4 \\
\hline e. Tetanus & 0 & 0 & 0 & 0 & 0 & 0 & 0 & 0 & 0 & 0 \\
\hline 6. Meningitis & 14 & 5 & 0 & 1 & 1 & 1 & 0 & $\mathbf{0}$ & 0 & 8 \\
\hline 7. Hepatitis $\mathbf{B}^{\mathbf{b}}$ & 3 & 0 & 0 & 1 & 0 & 0 & 0 & $\mathbf{0}$ & 0 & 2 \\
\hline Hepatitis $\mathbf{C}^{\mathbf{b}}$ & 1 & 0 & 0 & 0 & $\mathbf{0}$ & $\mathbf{0}$ & 0 & $\mathbf{0}$ & 0 & 1 \\
\hline 8. Malaria & 0 & 0 & 0 & 0 & 0 & $\mathbf{0}$ & $\mathbf{0}$ & $\mathbf{0}$ & 0 & 0 \\
\hline 9. Tropical-cluster diseases & 0 & 0 & - & 0 & 0 & $\mathbf{0}$ & $\mathbf{0}$ & $\mathbf{0}$ & 0 & 0 \\
\hline a. Trypanosomiasis & 0 & - & - & - & - & - & - & - & - & 一 \\
\hline b. Chagas' disease & - & - & - & - & - & - & - & - & - & - \\
\hline c. Schistosomiasis & 0 & 0 & - & 0 & - & 0 & 0 & 0 & - & 0 \\
\hline d. Leishmaniasis & 0 & 0 & - & - & 0 & 0 & 0 & 0 & 0 & 0 \\
\hline e. Lymphatic filariasis & 0 & 0 & - & 0 & 0 & - & 0 & - & - & 0 \\
\hline f. Onchocerciasis & - & - & - & - & - & - & - & - & - & - \\
\hline 10. Leprosy & $\mathbf{0}$ & - & - & - & 0 & 0 & $\mathbf{0}$ & $\mathbf{0}$ & $\mathbf{0}$ & 0 \\
\hline 11. Dengue & 0 & - & - & - & - & $\mathbf{0}$ & - & - & - & 0 \\
\hline 12. Japanese encephalitis & - & - & - & - & - & - & - & - & - & 一 \\
\hline 13. Trachoma & - & - & - & - & - & - & - & - & - & - \\
\hline 14. Intestinal nematode infections & $\mathbf{0}$ & $\mathbf{0}$ & - & 0 & - & 0 & 0 & $\mathbf{0}$ & $\mathbf{0}$ & 0 \\
\hline a. Ascariasis & 0 & - & - & - & - & - & - & - & 0 & 0 \\
\hline b. Trichuriasis & - & - & - & - & - & - & - & - & - & - \\
\hline c. Hookworm disease & 0 & - & - & - & - & 0 & 0 & 0 & 0 & 0 \\
\hline Other intestinal infections & 0 & 0 & - & 0 & - & - & - & - & 0 & 0 \\
\hline Other infectious diseases & 15 & 2 & 0 & 1 & 2 & 1 & 1 & 1 & 0 & 9 \\
\hline B. Respiratory infections & 109 & 19 & 2 & 2 & 4 & 13 & 11 & 8 & 5 & 64 \\
\hline 1. Lower respiratory infections & 104 & 18 & 1 & 1 & 3 & 13 & 11 & 8 & 5 & 61 \\
\hline 2. Upper respiratory infections & 4 & 1 & 0 & 0 & 0 & 0 & 0 & 0 & 0 & 3 \\
\hline 3. Otitis media & 0 & 0 & 0 & 0 & 0 & 0 & 0 & 0 & 0 & 0 \\
\hline C. Maternal conditions & 3 & - & - & - & - & - & - & - & - & 一 \\
\hline 1. Maternal hemorrhage & 1 & - & - & - & - & - & - & - & - & - \\
\hline 2. Maternal sepsis & 0 & - & - & - & - & - & - & - & - & - \\
\hline 3. Hypertensive disorders of pregnancy & 0 & - & - & - & - & - & - & - & - & 一 \\
\hline 4. Obstructed labor & 0 & - & - & - & - & - & - & - & - & - \\
\hline 5. Abortion & 0 & - & - & - & - & - & - & - & - & - \\
\hline Other maternal conditions & 1 & - & - & - & - & - & - & - & - & - \\
\hline D. Perinatal conditions $\mathbf{c}^{\mathrm{c}}$ & 57 & 33 & 0 & 0 & $\mathbf{0}$ & 0 & 0 & $\mathbf{0}$ & - & 33 \\
\hline 1. Low birthweight & 24 & 13 & - & - & - & - & 0 & - & - & 13 \\
\hline 2. Birth asphyxia and birth trauma & 17 & 10 & 0 & 0 & - & 0 & - & - & - & 10 \\
\hline Other perinatal conditions & 16 & 10 & 0 & 0 & 0 & 0 & - & 0 & - & 10 \\
\hline E. Nutritional deficiencies & 5 & 0 & 0 & 0 & $\mathbf{0}$ & 0 & $\mathbf{0}$ & $\mathbf{0}$ & 0 & 3 \\
\hline 1. Protein-energy malnutrition & 2 & 0 & 0 & 0 & 0 & 0 & 0 & 0 & 0 & 1 \\
\hline 2. Iodine deficiency & 0 & 0 & 0 & 0 & 0 & 0 & 0 & 0 & 0 & 0 \\
\hline 3. Vitamin A deficiency & 0 & - & - & - & - & - & - & - & 0 & 0 \\
\hline 4. Iron-deficiency anemia & 3 & 0 & 0 & 0 & 0 & 0 & 0 & 0 & 0 & 2 \\
\hline Other nutritional disorders & 0 & 0 & 0 & 0 & 0 & 0 & 0 & 0 & 0 & 0 \\
\hline
\end{tabular}


Table 3B.3 Continued

\begin{tabular}{|c|c|c|c|c|c|c|c|c|}
\hline \multicolumn{9}{|c|}{ Female } \\
\hline $0-4$ & 5-14 & $15-29$ & $30-44$ & $45-59$ & $60-69$ & $70-79$ & $80+$ & Total \\
\hline 14 & 36 & 58 & 52 & 41 & 23 & 17 & 6 & 248 \\
\hline 77 & 13 & 46 & 105 & 257 & 434 & 845 & 907 & 2,685 \\
\hline 56 & 3 & 7 & 9 & 9 & 7 & 11 & 12 & 115 \\
\hline 15 & 2 & 4 & 7 & 5 & 3 & 3 & 2 & 41 \\
\hline 0 & 0 & 1 & 4 & 3 & 1 & 1 & 1 & 11 \\
\hline 0 & 0 & 0 & 0 & 0 & 0 & 0 & 0 & 0 \\
\hline 0 & - & 0 & 0 & 0 & 0 & 0 & 0 & 0 \\
\hline- & - & - & - & - & - & - & - & - \\
\hline 0 & - & - & - & 0 & 0 & - & - & 0 \\
\hline 0 & 0 & 0 & 0 & 0 & 0 & 0 & 0 & 0 \\
\hline 0 & 0 & 1 & 1 & 1 & 0 & 0 & 0 & 4 \\
\hline 6 & 0 & 0 & 0 & 0 & 0 & 0 & 0 & 7 \\
\hline 3 & 1 & 0 & 0 & 0 & 0 & 0 & 0 & 4 \\
\hline 0 & - & - & - & 0 & 0 & - & - & 0 \\
\hline 0 & 0 & 0 & 0 & 0 & 0 & 0 & 0 & 0 \\
\hline 0 & 0 & 0 & 0 & 0 & 0 & 0 & 0 & 0 \\
\hline 2 & 1 & 0 & - & 0 & 0 & - & - & 4 \\
\hline 0 & - & 0 & 0 & 0 & 0 & 0 & 0 & 0 \\
\hline 4 & 0 & 0 & 0 & 0 & 0 & 0 & 0 & 6 \\
\hline 0 & 0 & 0 & 0 & 0 & 0 & 0 & 0 & 1 \\
\hline 0 & 0 & 0 & 0 & 0 & 0 & 0 & 0 & 0 \\
\hline 0 & 0 & 0 & 0 & 0 & 0 & 0 & 0 & 0 \\
\hline- & 0 & 0 & 0 & 0 & 0 & 0 & 0 & 0 \\
\hline- & - & - & 0 & - & 0 & - & - & 0 \\
\hline- & - & - & - & - & - & - & - & - \\
\hline- & - & - & - & 0 & 0 & - & - & 0 \\
\hline- & - & 0 & 0 & - & 0 & 0 & 0 & 0 \\
\hline- & 0 & - & - & - & - & - & - & 0 \\
\hline- & - & - & - & - & - & - & - & - \\
\hline- & - & - & - & - & 0 & - & 0 & 0 \\
\hline- & - & - & - & - & - & - & - & - \\
\hline- & - & - & - & - & - & - & - & - \\
\hline- & - & - & - & - & - & - & - & - \\
\hline 0 & - & - & 0 & 0 & - & 0 & 0 & 0 \\
\hline 0 & - & - & - & - & - & - & - & 0 \\
\hline- & - & - & - & - & - & - & - & - \\
\hline- & - & - & 0 & - & - & - & - & 0 \\
\hline 0 & - & - & - & 0 & - & 0 & 0 & 0 \\
\hline 2 & 0 & 1 & 1 & 1 & 1 & 1 & 1 & 6 \\
\hline 16 & 1 & 1 & 2 & 4 & 4 & 7 & 9 & 45 \\
\hline 15 & 1 & 1 & 2 & 4 & 4 & 7 & 9 & 43 \\
\hline 1 & 0 & 0 & 0 & 0 & 0 & 0 & 0 & 2 \\
\hline 0 & 0 & 0 & 0 & 0 & 0 & 0 & 0 & 0 \\
\hline- & 0 & 2 & 1 & 0 & 0 & - & - & 3 \\
\hline- & - & 0 & 0 & 0 & - & - & - & 1 \\
\hline- & - & 0 & 0 & 0 & - & - & - & 0 \\
\hline- & - & 0 & 0 & 0 & - & - & - & 0 \\
\hline- & - & 0 & 0 & 0 & - & - & - & 0 \\
\hline- & 0 & 0 & 0 & 0 & - & - & - & 0 \\
\hline- & - & 1 & 0 & 0 & 0 & - & - & 1 \\
\hline 24 & 0 & 0 & 0 & 0 & 0 & - & - & 24 \\
\hline 11 & - & - & - & - & - & - & - & 11 \\
\hline 7 & 0 & 0 & - & - & - & - & - & 7 \\
\hline 6 & 0 & 0 & 0 & 0 & 0 & - & - & 6 \\
\hline 0 & 0 & 0 & 0 & 0 & 0 & 1 & 0 & 3 \\
\hline 0 & 0 & 0 & 0 & 0 & 0 & 0 & 0 & 1 \\
\hline 0 & 0 & 0 & 0 & 0 & 0 & 0 & 0 & 0 \\
\hline 0 & 0 & 0 & 0 & 0 & 0 & 0 & 0 & 0 \\
\hline 0 & 0 & 0 & 0 & 0 & 0 & 0 & 0 & 2 \\
\hline \multirow[t]{2}{*}{0} & 0 & 0 & 0 & 0 & 0 & 0 & 0 & 0 \\
\hline & & & & & & inues & fol & $\operatorname{age}$ \\
\hline
\end{tabular}


Table 3B.3 Continued

\begin{tabular}{|c|c|c|c|c|c|c|c|c|c|c|}
\hline \multirow[b]{2}{*}{ Cause } & \multicolumn{10}{|c|}{ Male } \\
\hline & Total & $0-4$ & $5-14$ & $15-29$ & $30-44$ & $45-59$ & $60-69$ & $70-79$ & $80+$ & Total \\
\hline II. Noncommunicable diseases & 4,736 & 20 & 7 & 34 & 143 & 445 & 633 & 685 & 336 & 2,304 \\
\hline A. Malignant neoplasms & 825 & 1 & 2 & 7 & 24 & 113 & 158 & 129 & 32 & 465 \\
\hline 1. Mouth and oropharynx cancers & 27 & 0 & 0 & 0 & 1 & 9 & 7 & 3 & 1 & 22 \\
\hline 2. Esophageal cancer & 21 & 0 & 0 & 0 & 0 & 5 & 6 & 4 & 1 & 15 \\
\hline 3. Stomach cancer & 101 & 0 & 0 & 0 & 3 & 14 & 21 & 18 & 4 & 60 \\
\hline 4. Colon and rectal cancers & 96 & 0 & 0 & 0 & 2 & 8 & 15 & 17 & 5 & 47 \\
\hline 5. Liver cancer & 28 & 0 & 0 & 0 & 1 & 4 & 6 & 5 & 1 & 17 \\
\hline 6. Pancreas cancer & 35 & 0 & 0 & 0 & 1 & 5 & 6 & 5 & 1 & 19 \\
\hline 7. Trachea, bronchus, and lung cancers & 165 & 0 & 0 & 0 & 5 & 36 & 53 & 37 & 6 & 137 \\
\hline 8. Melanoma and other skin cancers & 11 & 0 & 0 & 0 & 1 & 1 & 1 & 1 & 1 & 5 \\
\hline 9. Breast cancer & 63 & - & 0 & 0 & 0 & 0 & 0 & 0 & 0 & 1 \\
\hline 10. Cervix uteri cancer & 19 & - & - & - & - & - & - & - & - & - \\
\hline 11. Corpus uteri cancer & 17 & - & - & - & - & - & - & - & - & - \\
\hline 12. Ovarian cancer & 21 & - & - & - & - & - & - & - & - & - \\
\hline 13. Prostate cancer & 25 & 0 & 0 & 0 & 0 & 2 & 7 & 11 & 5 & 25 \\
\hline 14. Bladder cancer & 24 & 0 & 0 & 0 & 0 & 3 & 6 & 7 & 2 & 19 \\
\hline 15. Lymphomas and multiple myeloma & 23 & 0 & 0 & 1 & 2 & 3 & 3 & 2 & 1 & 12 \\
\hline 16. Leukemia & 27 & 0 & 1 & 2 & 2 & 3 & 3 & 3 & 1 & 15 \\
\hline Other malignant neoplasms & 123 & 0 & 1 & 3 & 6 & 20 & 22 & 15 & 4 & 71 \\
\hline B. Other neoplasms & 8 & 0 & 0 & $\mathbf{0}$ & 0 & 1 & 1 & 1 & 0 & 4 \\
\hline C. Diabetes mellitus & 51 & 0 & $\mathbf{0}$ & 1 & 2 & 4 & 6 & 6 & 2 & 21 \\
\hline D. Endocrine disorders & 6 & 0 & 0 & 0 & $\mathbf{0}$ & 1 & 0 & 0 & 0 & 3 \\
\hline E. Neuropsychiatric conditions & 66 & 2 & 2 & 5 & 10 & 12 & 6 & 4 & 2 & 41 \\
\hline 1. Unipolar depressive disorders & 0 & 0 & 0 & 0 & 0 & 0 & 0 & 0 & 0 & 0 \\
\hline 2. Bipolar affective disorder & 0 & - & - & - & 0 & 0 & 0 & 0 & 0 & 0 \\
\hline 3. Schizophrenia & 1 & 0 & 0 & 0 & 0 & 0 & 0 & 0 & 0 & 1 \\
\hline 4. Epilepsy & 9 & 0 & 0 & 1 & 2 & 1 & 0 & 0 & 0 & 6 \\
\hline 5. Alcohol use disorders & 10 & 0 & 0 & 0 & 2 & 3 & 2 & 1 & 0 & 8 \\
\hline 6. Alzheimer's and other dementias & 10 & 0 & 0 & 0 & 0 & 1 & 1 & 1 & 1 & 4 \\
\hline 7. Parkinson's disease & 4 & 0 & 0 & 0 & 0 & 0 & 0 & 1 & 0 & 2 \\
\hline 8. Multiple sclerosis & 4 & 0 & 0 & 0 & 0 & 1 & 0 & 0 & 0 & 2 \\
\hline 9. Drug use disorders & 11 & 0 & 0 & 2 & 4 & 3 & 1 & 0 & 0 & 9 \\
\hline 10. Post-traumatic stress disorder & 0 & 0 & 0 & 0 & 0 & 0 & 0 & 0 & 0 & 0 \\
\hline 11. Obsessive-compulsive disorder & - & - & - & - & - & - & - & - & - & - \\
\hline 12. Panic disorder & - & - & - & - & - & - & - & - & - & - \\
\hline 13. Insomnia (primary) & - & - & - & - & - & - & - & - & - & - \\
\hline 14. Migraine & - & - & - & - & - & - & - & - & - & - \\
\hline 15. Mental retardation, lead-caused & 1 & 0 & 0 & 0 & 0 & 0 & 0 & 0 & 0 & 0 \\
\hline Other neuropsychiatric disorders & 17 & 1 & 1 & 2 & 1 & 2 & 1 & 1 & 0 & 10 \\
\hline F. Sense organ diseases & 0 & 0 & 0 & 0 & 0 & 0 & 0 & 0 & 0 & 0 \\
\hline 1. Glaucoma & 0 & - & - & - & - & 0 & 0 & 0 & 0 & 0 \\
\hline 2. Cataracts & - & - & - & - & - & - & - & - & - & - \\
\hline 3. Vision disorders, age-related & - & - & - & - & - & - & - & - & - & - \\
\hline 4. Hearing loss, adult onset & - & - & - & - & - & - & - & - & - & - \\
\hline Other sense organ disorders & 0 & 0 & 0 & 0 & 0 & 0 & 0 & 0 & 0 & 0 \\
\hline G. Cardiovascular diseases & 3,295 & 1 & 1 & 12 & 75 & 250 & 389 & 477 & 274 & 1,480 \\
\hline 1. Rheumatic heart disease & 22 & 0 & 0 & 0 & 2 & 3 & 2 & 1 & 0 & 9 \\
\hline 2. Hypertensive heart disease & 109 & 0 & 0 & 0 & 2 & 9 & 13 & 15 & 7 & 47 \\
\hline 3. Ischemic heart disease & 1,685 & 0 & 0 & 3 & 38 & 153 & 226 & 266 & 131 & 817 \\
\hline 4. Cerebrovascular disease & 1,029 & 0 & 1 & 3 & 13 & 54 & 109 & 144 & 80 & 405 \\
\hline 5. Inflammatory heart diseases & 67 & 0 & 0 & 2 & 7 & 11 & 8 & 7 & 5 & 40 \\
\hline Other cardiovascular diseases & 383 & 1 & 0 & 4 & 14 & 20 & 30 & 44 & 50 & 162 \\
\hline H. Respiratory diseases & 190 & 1 & 0 & 2 & 5 & 17 & 37 & 41 & 17 & 121 \\
\hline 1. Chronic obstructive pulmonary disease & 130 & 0 & 0 & 0 & 2 & 10 & 25 & 32 & 13 & 84 \\
\hline 2. Asthma & 27 & 0 & 0 & 0 & 1 & 3 & 6 & 5 & 2 & 17 \\
\hline Other respiratory diseases & 33 & 1 & 0 & 1 & 3 & 4 & 5 & 4 & 2 & 21 \\
\hline
\end{tabular}


Table 3B.3 Continued

\begin{tabular}{|c|c|c|c|c|c|c|c|c|}
\hline \multicolumn{9}{|c|}{ Female } \\
\hline $0-4$ & 5-14 & $15-29$ & 30-44 & $45-59$ & $60-69$ & $70-79$ & $80+$ & Total \\
\hline 17 & 5 & 19 & 68 & 214 & 407 & 818 & 884 & 2,432 \\
\hline 1 & 2 & 5 & 25 & 73 & 94 & 113 & 48 & 360 \\
\hline 0 & 0 & 0 & 0 & 1 & 1 & 1 & 1 & 5 \\
\hline 0 & - & 0 & 0 & 1 & 1 & 2 & 1 & 6 \\
\hline 0 & 0 & 0 & 2 & 6 & 11 & 15 & 6 & 41 \\
\hline 0 & 0 & 0 & 2 & 7 & 13 & 18 & 9 & 49 \\
\hline 0 & 0 & 0 & 0 & 2 & 3 & 4 & 2 & 11 \\
\hline 0 & 0 & 0 & 0 & 2 & 5 & 6 & 3 & 16 \\
\hline 0 & 0 & 0 & 1 & 6 & 8 & 10 & 3 & 29 \\
\hline 0 & 0 & 0 & 1 & 1 & 1 & 1 & 1 & 6 \\
\hline 0 & 0 & 0 & 6 & 19 & 16 & 15 & 7 & 63 \\
\hline- & 0 & 0 & 3 & 6 & 4 & 4 & 2 & 19 \\
\hline 0 & 0 & 0 & 1 & 3 & 5 & 5 & 2 & 17 \\
\hline 0 & 0 & 0 & 2 & 6 & 6 & 5 & 2 & 21 \\
\hline- & - & - & - & - & - & - & - & - \\
\hline 0 & 0 & 0 & 0 & 0 & 1 & 2 & 1 & 5 \\
\hline 0 & 0 & 1 & 1 & 2 & 3 & 3 & 1 & 10 \\
\hline 0 & 1 & 1 & 1 & 2 & 3 & 3 & 1 & 12 \\
\hline 0 & 1 & 1 & 4 & 10 & 13 & 16 & 7 & 52 \\
\hline 0 & 0 & 0 & 0 & 1 & 1 & 1 & 1 & 4 \\
\hline 0 & 0 & 1 & 1 & 4 & 9 & 12 & 4 & 31 \\
\hline 0 & 0 & 0 & 0 & 1 & 1 & 1 & 0 & 3 \\
\hline 1 & 1 & 2 & 4 & 5 & 3 & 4 & 4 & 25 \\
\hline 0 & 0 & 0 & 0 & 0 & 0 & 0 & 0 & 0 \\
\hline 0 & 0 & 0 & 0 & 0 & 0 & 0 & 0 & 0 \\
\hline 0 & 0 & 0 & 0 & 0 & 0 & 0 & 0 & 1 \\
\hline 0 & 0 & 1 & 1 & 1 & 0 & 0 & 0 & 3 \\
\hline 0 & 0 & 0 & 0 & 1 & 0 & 0 & 0 & 2 \\
\hline 0 & 0 & 0 & 0 & 0 & 1 & 2 & 2 & 6 \\
\hline 0 & 0 & 0 & 0 & 0 & 0 & 1 & 0 & 2 \\
\hline 0 & 0 & 0 & 1 & 1 & 0 & 0 & 0 & 2 \\
\hline- & 0 & 0 & 1 & 1 & 0 & 0 & 0 & 2 \\
\hline 0 & 0 & 0 & 0 & 0 & 0 & 0 & 0 & 0 \\
\hline- & - & - & - & - & - & - & - & - \\
\hline- & - & - & - & - & - & - & - & - \\
\hline- & - & - & - & - & - & - & - & - \\
\hline- & - & - & - & - & - & - & - & - \\
\hline 0 & 0 & 0 & 0 & 0 & 0 & 0 & 0 & 0 \\
\hline 1 & 1 & 1 & 1 & 1 & 1 & 1 & 1 & 7 \\
\hline 0 & 0 & 0 & 0 & 0 & 0 & 0 & 0 & 0 \\
\hline- & - & - & - & 0 & 0 & 0 & 0 & 0 \\
\hline- & - & - & - & - & - & - & - & - \\
\hline- & - & - & - & - & - & - & - & - \\
\hline- & - & - & - & - & - & - & - & - \\
\hline 0 & 0 & 0 & 0 & 0 & 0 & 0 & 0 & 0 \\
\hline 1 & 1 & 5 & 24 & 101 & 262 & 634 & 788 & 1,815 \\
\hline 0 & 0 & 0 & 1 & 3 & 4 & 3 & 1 & 13 \\
\hline 0 & 0 & 0 & 1 & 6 & 13 & 23 & 19 & 62 \\
\hline 0 & 0 & 1 & 7 & 44 & 129 & 314 & 374 & 868 \\
\hline 0 & 0 & 2 & 7 & 35 & 92 & 224 & 264 & 624 \\
\hline 0 & 0 & 1 & 2 & 3 & 4 & 8 & 9 & 27 \\
\hline 0 & 0 & 1 & 5 & 10 & 20 & 62 & 122 & 221 \\
\hline 1 & 0 & 1 & 2 & 6 & 12 & 24 & 23 & 69 \\
\hline 0 & 0 & 0 & 1 & 3 & 7 & 17 & 18 & 46 \\
\hline 0 & 0 & 0 & 1 & 1 & 3 & 3 & 3 & 11 \\
\hline 1 & 0 & 1 & 1 & 1 & 2 & 3 & 3 & 12 \\
\hline & & & & & & ntinue & he fo & $g$ page \\
\hline
\end{tabular}


Table 3B.3 Continued

\begin{tabular}{|c|c|c|c|c|c|c|c|c|c|c|}
\hline \multirow[b]{2}{*}{ Cause } & \multicolumn{10}{|c|}{ Male } \\
\hline & Total & $0-4$ & $5-14$ & $15-29$ & $30-44$ & $45-59$ & $60-69$ & $70-79$ & $80+$ & Total \\
\hline I. Digestive diseases & 205 & 1 & 1 & 4 & 21 & 40 & 30 & 20 & 6 & 122 \\
\hline 1. Peptic ulcer disease & 23 & 0 & 0 & 1 & 2 & 4 & 4 & 3 & 1 & 16 \\
\hline 2. Cirrhosis of the liver & 103 & 0 & 0 & 2 & 12 & 24 & 17 & 9 & 1 & 65 \\
\hline 3. Appendicitis & 1 & 0 & 0 & 0 & 0 & 0 & 0 & 0 & 0 & 1 \\
\hline Other digestive diseases & 77 & 1 & 0 & 2 & 7 & 10 & 9 & 8 & 3 & 41 \\
\hline J. Genitourinary diseases & 53 & $\mathbf{0}$ & 0 & 2 & 3 & 6 & 6 & 7 & 3 & 28 \\
\hline 1. Nephritis and nephrosis & 36 & 0 & 0 & 1 & 3 & 4 & 4 & 4 & 2 & 18 \\
\hline 2. Benign prostatic hypertrophy & 3 & - & - & 0 & 0 & 0 & 1 & 1 & 1 & 3 \\
\hline Other genitourinary system diseases & 14 & 0 & 0 & 0 & 1 & 1 & 2 & 2 & 1 & 7 \\
\hline K. Skin diseases & 3 & $\mathbf{0}$ & 0 & $\mathbf{0}$ & 0 & 0 & 0 & 0 & 0 & 2 \\
\hline L. Musculoskeletal diseases & 6 & 0 & 0 & 0 & 0 & 1 & 0 & $\mathbf{0}$ & $\mathbf{0}$ & 2 \\
\hline 1. Rheumatoid arthritis & 2 & 0 & 0 & 0 & 0 & 0 & 0 & 0 & 0 & 0 \\
\hline 2. Osteoarthritis & 0 & 0 & 0 & 0 & 0 & 0 & 0 & 0 & 0 & 0 \\
\hline 3. Gout & 0 & 0 & 0 & 0 & 0 & 0 & 0 & 0 & 0 & 0 \\
\hline 4. Low back pain & 0 & 0 & 0 & 0 & 0 & 0 & 0 & 0 & 0 & 0 \\
\hline Other musculoskeletal disorders & 3 & 0 & 0 & 0 & 0 & 0 & 0 & 0 & 0 & 1 \\
\hline M. Congenital anomalies & 28 & 13 & 1 & 1 & $\mathbf{0}$ & 0 & 0 & 0 & $\mathbf{0}$ & 16 \\
\hline 1. Abdominal wall defect & 0 & 0 & 0 & 0 & 0 & 0 & 0 & 0 & 0 & 0 \\
\hline 2. Anencephaly & 0 & 0 & 0 & 0 & 0 & 0 & 0 & 0 & 0 & 0 \\
\hline 3. Anorectal atresia & 0 & 0 & 0 & 0 & 0 & 0 & 0 & 0 & 0 & 0 \\
\hline 4. Cleft lip & 0 & 0 & 0 & 0 & 0 & 0 & 0 & 0 & - & 0 \\
\hline 5. Cleft palate & 0 & 0 & 0 & 0 & 0 & 0 & 0 & 0 & - & 0 \\
\hline 6. Esophageal atresia & 0 & 0 & 0 & 0 & 0 & 0 & 0 & 0 & 0 & 0 \\
\hline 7. Renal agenesis & 0 & 0 & 0 & 0 & 0 & 0 & 0 & 0 & 0 & 0 \\
\hline 8. Down syndrome & 1 & 0 & 0 & 0 & 0 & 0 & 0 & 0 & 0 & 0 \\
\hline 9. Congenital heart anomalies & 13 & 6 & 0 & 0 & 0 & 0 & 0 & 0 & 0 & 7 \\
\hline 10. Spina bifida & 1 & 1 & 0 & 0 & 0 & 0 & 0 & 0 & 0 & 1 \\
\hline Other congenital anomalies & 12 & 6 & 0 & 0 & 0 & 0 & 0 & 0 & 0 & 7 \\
\hline N. Oral conditions & $\mathbf{0}$ & $\mathbf{0}$ & - & 0 & $\mathbf{0}$ & 0 & 0 & $\mathbf{0}$ & $\mathbf{0}$ & $\mathbf{0}$ \\
\hline 1. Dental caries & 0 & - & - & - & - & - & - & - & - & - \\
\hline 2. Periodontal disease & 0 & - & - & - & 0 & - & - & - & - & 0 \\
\hline 3. Edentulism & - & - & - & - & - & - & - & - & - & - \\
\hline Other oral diseases & 0 & 0 & - & 0 & 0 & 0 & 0 & 0 & 0 & 0 \\
\hline III. Injuries & 607 & 6 & 10 & 99 & 140 & 132 & 53 & 22 & 6 & 470 \\
\hline A. Unintentional injuries & 401 & 6 & 8 & 59 & 87 & 89 & 37 & 14 & 4 & 304 \\
\hline 1. Road traffic accidents & 83 & 1 & 2 & 18 & 18 & 13 & 6 & 3 & 1 & 62 \\
\hline 2. Poisonings & 106 & 1 & 0 & 12 & 26 & 30 & 11 & 2 & 0 & 82 \\
\hline 3. Falls & 35 & 0 & 0 & 3 & 5 & 7 & 4 & 3 & 2 & 24 \\
\hline 4. Fires & 20 & 1 & 0 & 2 & 4 & 4 & 2 & 1 & 0 & 14 \\
\hline 5. Drownings & 35 & 1 & 2 & 7 & 9 & 6 & 3 & 1 & 0 & 29 \\
\hline 6. Other unintentional injuries & 121 & 3 & 3 & 18 & 25 & 28 & 12 & 4 & 1 & 93 \\
\hline B. Intentional injuries & 207 & 0 & 2 & 40 & 53 & 43 & 16 & 8 & 2 & 165 \\
\hline 1. Self-inflicted injuries & 121 & 0 & 1 & 22 & 29 & 27 & 11 & 6 & 2 & 99 \\
\hline 2. Violence & 68 & 0 & 0 & 11 & 18 & 14 & 4 & 1 & 0 & 50 \\
\hline 3. War & 17 & 0 & 0 & 7 & 6 & 2 & 1 & 0 & 0 & 16 \\
\hline Other intentional injuries & 1 & 0 & 0 & 0 & 0 & 0 & 0 & - & 0 & 0 \\
\hline
\end{tabular}


Table 3B.3 Continued

\begin{tabular}{|c|c|c|c|c|c|c|c|c|}
\hline \multicolumn{9}{|c|}{ Female } \\
\hline $0-4$ & 5-14 & $15-29$ & 30-44 & $45-59$ & 60-69 & $70-79$ & $80+$ & Total \\
\hline 1 & 0 & 2 & 8 & 18 & 19 & 22 & 13 & 83 \\
\hline 0 & 0 & 0 & 0 & 1 & 1 & 2 & 2 & 7 \\
\hline 0 & 0 & 1 & 4 & 12 & 11 & 8 & 3 & 39 \\
\hline 0 & 0 & 0 & 0 & 0 & 0 & 0 & 0 & 1 \\
\hline 1 & 0 & 1 & 3 & 5 & 7 & 11 & 8 & 36 \\
\hline 0 & 0 & 1 & 3 & 4 & 6 & 6 & 4 & 24 \\
\hline 0 & 0 & 1 & 2 & 3 & 4 & 5 & 3 & 18 \\
\hline- & - & - & - & - & - & - & - & - \\
\hline 0 & 0 & 0 & 1 & 1 & 2 & 2 & 1 & 7 \\
\hline 0 & 0 & 0 & 0 & 0 & 0 & 0 & 0 & 2 \\
\hline 0 & 0 & 0 & 0 & 1 & 1 & 1 & 0 & 4 \\
\hline 0 & 0 & 0 & 0 & 0 & 1 & 1 & 0 & 2 \\
\hline 0 & 0 & 0 & 0 & 0 & 0 & 0 & 0 & 0 \\
\hline 0 & 0 & 0 & 0 & 0 & 0 & 0 & 0 & 0 \\
\hline 0 & 0 & 0 & 0 & 0 & 0 & 0 & 0 & 0 \\
\hline 0 & 0 & 0 & 0 & 1 & 0 & 0 & 0 & 2 \\
\hline 10 & 1 & 0 & 0 & 0 & 0 & 0 & 0 & 13 \\
\hline 0 & 0 & 0 & 0 & 0 & 0 & 0 & 0 & 0 \\
\hline 0 & 0 & 0 & 0 & 0 & 0 & 0 & 0 & 0 \\
\hline 0 & 0 & 0 & 0 & 0 & 0 & 0 & 0 & 0 \\
\hline 0 & 0 & 0 & 0 & 0 & 0 & 0 & - & 0 \\
\hline 0 & 0 & 0 & 0 & 0 & 0 & 0 & 0 & 0 \\
\hline 0 & 0 & 0 & 0 & 0 & 0 & 0 & 0 & 0 \\
\hline 0 & 0 & 0 & 0 & 0 & 0 & 0 & 0 & 0 \\
\hline 0 & 0 & 0 & 0 & 0 & 0 & 0 & 0 & 0 \\
\hline 5 & 0 & 0 & 0 & 0 & 0 & 0 & 0 & 6 \\
\hline 1 & 0 & 0 & 0 & 0 & 0 & 0 & 0 & 1 \\
\hline 4 & 0 & 0 & 0 & 0 & 0 & 0 & 0 & 5 \\
\hline 0 & - & 0 & 0 & 0 & 0 & 0 & 0 & 0 \\
\hline- & - & - & - & 0 & - & - & 0 & 0 \\
\hline- & - & - & - & - & - & - & 0 & 0 \\
\hline- & - & - & - & - & - & - & - & - \\
\hline 0 & - & 0 & 0 & 0 & 0 & 0 & 0 & 0 \\
\hline 5 & 5 & 20 & 27 & 34 & 20 & 17 & 11 & 138 \\
\hline 5 & 4 & 12 & 17 & 24 & 14 & 12 & 9 & 96 \\
\hline 1 & 1 & 5 & 4 & 4 & 3 & 3 & 1 & 21 \\
\hline 0 & 0 & 3 & 6 & 9 & 4 & 2 & 1 & 24 \\
\hline 0 & 0 & 1 & 1 & 1 & 1 & 3 & 4 & 12 \\
\hline 1 & 0 & 0 & 1 & 1 & 1 & 1 & 1 & 5 \\
\hline 1 & 1 & 1 & 1 & 1 & 1 & 1 & 0 & 6 \\
\hline 2 & 1 & 3 & 5 & 7 & 4 & 3 & 2 & 27 \\
\hline 0 & 1 & 8 & 10 & 10 & 5 & 5 & 3 & 42 \\
\hline- & 1 & 4 & 4 & 5 & 3 & 3 & 2 & 22 \\
\hline 0 & 0 & 3 & 5 & 4 & 2 & 2 & 1 & 18 \\
\hline 0 & 0 & 0 & 0 & 0 & 0 & 0 & 0 & 2 \\
\hline 0 & 0 & 0 & 0 & 0 & 0 & 0 & 0 & 0 \\
\hline
\end{tabular}

Source: Authors' compilation.

Note: - = an estimate of zero; the number zero in a cell indicates a non-zero estimate of less than 500.

a. These figures include late effects of polio cases with onset prior to regional certification of polio eradication in 2000.

b. Does not include liver cancer and cirrhosis deaths resulting from chronic hepatitis virus infection.

c. This cause category includes "Causes arising in the perinatal period" as defined in the International Classification of Diseases, principally low birthweight, prematurity, birth asphyxia, and birth trauma, and does not include all causes of deaths occurring in the perinatal period. 
Table 3B.4 Deaths by Cause, Sex, and Age in the Latin America and the Caribbean Region, 2001 (thousands)

\begin{tabular}{|c|c|c|c|c|c|c|c|c|c|c|}
\hline \multirow[b]{2}{*}{ Cause } & \multicolumn{10}{|c|}{ Male } \\
\hline & Total & $0-4$ & $5-14$ & $15-29$ & 30-44 & $45-59$ & $60-69$ & 70-79 & $80+$ & Total \\
\hline Population (millions) & 526 & 28 & 56 & 74 & 52 & 31 & 11 & 6 & 2 & 260 \\
\hline All causes & 3,277 & 226 & 30 & 178 & 211 & 288 & 276 & 336 & 288 & 1,833 \\
\hline $\begin{array}{l}\text { I. Communicable, maternal, perinatal, } \\
\text { and nutritional conditions }\end{array}$ & 716 & 177 & 8 & 26 & 50 & 37 & 24 & 31 & 38 & 390 \\
\hline A. Infectious and parasitic diseases & 324 & 52 & 5 & 22 & 44 & 27 & 14 & 14 & 11 & 189 \\
\hline 1. Tuberculosis & 45 & 1 & 0 & 4 & 6 & 7 & 5 & 4 & 2 & 29 \\
\hline $\begin{array}{l}\text { 2. Sexually transmitted diseases } \\
\text { excluding HIV/AIDS }\end{array}$ & 2 & 1 & $\mathbf{0}$ & 0 & $\mathbf{0}$ & 0 & 0 & $\mathbf{0}$ & 0 & 1 \\
\hline a. Syphilis & 1 & 1 & 0 & 0 & 0 & 0 & 0 & 0 & 0 & 1 \\
\hline b. Chlamydia & 0 & - & - & - & - & - & - & - & - & - \\
\hline c. Gonorrhea & 0 & - & 0 & 0 & - & - & - & 0 & 0 & 0 \\
\hline d. Other sexually transmitted diseases & 1 & 0 & - & - & 0 & 0 & - & 0 & 0 & 0 \\
\hline 3. HIV/AIDS & 83 & 3 & 1 & 11 & 27 & 9 & 1 & $\mathbf{0}$ & $\mathbf{0}$ & 54 \\
\hline 4. Diarrheal diseases & 55 & 24 & 0 & 0 & 0 & 1 & 1 & 1 & 1 & 29 \\
\hline 5. Childhood-cluster diseases & 7 & 4 & 0 & 0 & 0 & 0 & 0 & 0 & $\mathbf{0}$ & 4 \\
\hline a. Pertussis & 6 & 3 & - & - & - & - & 0 & - & - & 3 \\
\hline b. Poliomyelitis ${ }^{a}$ & 0 & 0 & 0 & 0 & 0 & 0 & 0 & - & 0 & 0 \\
\hline c. Diphtheria & 0 & 0 & - & - & 0 & - & - & - & 0 & 0 \\
\hline d. Measles & - & - & - & - & - & - & - & - & - & - \\
\hline e. Tetanus & 1 & 0 & 0 & 0 & 0 & 0 & 0 & 0 & 0 & 0 \\
\hline 6. Meningitis & 17 & 5 & 1 & 1 & 1 & 1 & 0 & $\mathbf{0}$ & $\mathbf{0}$ & 10 \\
\hline 7. Hepatitis $\mathbf{B}^{\mathbf{b}}$ & 4 & 0 & 0 & 1 & 1 & 1 & 0 & 0 & 0 & 3 \\
\hline Hepatitis $\mathbf{C}^{\mathbf{b}}$ & 2 & 0 & $\mathbf{0}$ & 0 & 0 & 0 & 0 & 0 & 0 & 1 \\
\hline 8. Malaria & 2 & 1 & 0 & 0 & 0 & 0 & 0 & 0 & 0 & 1 \\
\hline 9. Tropical-cluster diseases & 15 & 0 & 0 & 0 & 1 & 3 & 2 & 1 & 1 & 8 \\
\hline a. Trypanosomiasis & 0 & - & - & - & - & - & 0 & - & - & 0 \\
\hline b. Chagas' disease & 14 & 0 & 0 & 0 & 1 & 2 & 2 & 1 & 1 & 8 \\
\hline c. Schistosomiasis & 1 & 0 & 0 & 0 & 0 & 0 & 0 & 0 & 0 & 0 \\
\hline d. Leishmaniasis & 0 & 0 & 0 & 0 & 0 & 0 & 0 & 0 & 0 & 0 \\
\hline e. Lymphatic filariasis & 0 & - & - & - & 0 & - & 0 & 0 & - & 0 \\
\hline f. Onchocerciasis & 0 & 0 & - & - & - & - & - & - & - & 0 \\
\hline 10. Leprosy & 1 & - & - & 0 & 0 & 0 & 0 & 0 & $\mathbf{0}$ & 0 \\
\hline 11. Dengue & 2 & $\mathbf{0}$ & 1 & 0 & 0 & 0 & 0 & 0 & 0 & 1 \\
\hline 12. Japanese encephalitis & - & - & - & - & - & - & - & - & - & - \\
\hline 13. Trachoma & $\mathbf{0}$ & 0 & $\mathbf{0}$ & - & - & - & - & - & - & 0 \\
\hline 14. Intestinal nematode infections & 2 & 0 & 0 & $\mathbf{0}$ & 0 & 0 & $\mathbf{0}$ & 0 & $\mathbf{0}$ & 1 \\
\hline a. Ascariasis & 0 & 0 & 0 & 0 & 0 & 0 & 0 & 0 & 0 & 0 \\
\hline b. Trichuriasis & 0 & - & - & - & 0 & - & - & - & - & 0 \\
\hline c. Hookworm disease & 0 & - & 0 & - & 0 & 0 & - & - & - & 0 \\
\hline Other intestinal infections & 1 & 0 & 0 & 0 & 0 & 0 & 0 & 0 & 0 & 1 \\
\hline Other infectious diseases & 87 & 13 & 2 & 4 & 6 & 6 & 5 & 6 & 6 & 48 \\
\hline B. Respiratory infections & 160 & 24 & 2 & 3 & 5 & 7 & 8 & 13 & 21 & 82 \\
\hline 1. Lower respiratory infections & 157 & 23 & 2 & 3 & 5 & 7 & 8 & 13 & 20 & 81 \\
\hline 2. Upper respiratory infections & 3 & 1 & 0 & 0 & 0 & 0 & 0 & 0 & 0 & 1 \\
\hline 3. Otitis media & 0 & 0 & 0 & 0 & 0 & 0 & 0 & 0 & 0 & 0 \\
\hline C. Maternal conditions & 16 & - & - & - & - & - & - & - & - & - \\
\hline 1. Maternal hemorrhage & 4 & - & - & - & - & - & - & - & - & - \\
\hline 2. Maternal sepsis & 1 & - & - & - & - & - & - & - & - & - \\
\hline 3. Hypertensive disorders of pregnancy & 4 & - & - & - & - & - & - & - & - & - \\
\hline 4. Obstructed labor & 0 & - & - & - & - & - & - & - & - & - \\
\hline 5. Abortion & 2 & - & - & - & - & - & - & - & - & - \\
\hline Other maternal conditions & 5 & - & - & - & - & - & - & - & - & - \\
\hline D. Perinatal conditions ${ }^{\mathbf{C}}$ & 164 & 93 & 0 & 0 & 0 & 0 & - & - & - & 93 \\
\hline 1. Low birthweight & 20 & 11 & - & - & - & - & - & - & - & 11 \\
\hline 2. Birth asphyxia and birth trauma & 89 & 51 & 0 & 0 & 0 & 0 & - & - & - & 51 \\
\hline Other perinatal conditions & 54 & 31 & 0 & 0 & - & - & - & - & - & 31 \\
\hline E. Nutritional deficiencies & 52 & 8 & 1 & 1 & 2 & 2 & 2 & 4 & 6 & 26 \\
\hline 1. Protein-energy malnutrition & 37 & 7 & 1 & 1 & 1 & 1 & 1 & 3 & 4 & 19 \\
\hline 2. lodine deficiency & 0 & 0 & - & - & - & 0 & 0 & 0 & 0 & 0 \\
\hline 3. Vitamin A deficiency & 0 & 0 & - & - & - & - & 0 & - & 0 & 0 \\
\hline 4. Iron-deficiency anemia & 13 & 1 & 0 & 1 & 1 & 1 & 1 & 1 & 1 & 6 \\
\hline Other nutritional disorders & 3 & 0 & 0 & 0 & 0 & 0 & 0 & 0 & 0 & 2 \\
\hline
\end{tabular}


Table 3B.4 Continued

\begin{tabular}{|c|c|c|c|c|c|c|c|c|}
\hline \multicolumn{9}{|c|}{ Female } \\
\hline $0-4$ & 5-14 & $15-29$ & $30-44$ & $45-59$ & $60-69$ & $70-79$ & $80+$ & Total \\
\hline 27 & 54 & 73 & 55 & 33 & 13 & 8 & 3 & 266 \\
\hline $\begin{array}{l}181 \\
140\end{array}$ & $\begin{array}{r}23 \\
8\end{array}$ & $\begin{array}{l}66 \\
26\end{array}$ & $\begin{array}{r}104 \\
31\end{array}$ & $\begin{array}{r}185 \\
21\end{array}$ & $\begin{array}{r}206 \\
18\end{array}$ & $\begin{array}{r}294 \\
28\end{array}$ & $\begin{array}{r}386 \\
52\end{array}$ & $\begin{array}{r}1,444 \\
326\end{array}$ \\
\hline $\begin{array}{r}42 \\
0 \\
0\end{array}$ & $\begin{array}{l}5 \\
0 \\
0\end{array}$ & $\begin{array}{r}15 \\
3 \\
0\end{array}$ & $\begin{array}{r}20 \\
3 \\
0\end{array}$ & $\begin{array}{r}15 \\
3 \\
0\end{array}$ & $\begin{array}{r}10 \\
2 \\
0\end{array}$ & $\begin{array}{r}12 \\
2 \\
0\end{array}$ & $\begin{array}{r}15 \\
1 \\
0\end{array}$ & $\begin{array}{r}135 \\
16 \\
1\end{array}$ \\
\hline 0 & - & 0 & 0 & 0 & 0 & 0 & 0 & 0 \\
\hline- & - & - & - & - & 0 & 0 & 0 & 0 \\
\hline 0 & - & - & - & 0 & - & - & - & 0 \\
\hline 0 & 0 & 0 & 0 & 0 & 0 & 0 & 0 & 1 \\
\hline 3 & 1 & 9 & 13 & 3 & 0 & 0 & 0 & 30 \\
\hline 22 & 0 & 0 & 0 & 0 & 1 & 1 & 2 & 26 \\
\hline 3 & 0 & 0 & 0 & 0 & 0 & 0 & 0 & 4 \\
\hline 3 & 0 & 0 & - & - & - & - & - & 3 \\
\hline 0 & 0 & 0 & 0 & 0 & 0 & - & 0 & 0 \\
\hline 0 & 0 & - & - & 0 & - & - & - & 0 \\
\hline- & - & - & - & - & - & - & - & - \\
\hline 0 & 0 & 0 & 0 & 0 & 0 & 0 & 0 & 0 \\
\hline 4 & 1 & 0 & 0 & 1 & 0 & 0 & 0 & 7 \\
\hline 0 & 0 & 0 & 0 & 0 & 0 & 0 & 0 & 2 \\
\hline 0 & 0 & 0 & 0 & 0 & 0 & 0 & 0 & 1 \\
\hline 1 & 0 & 0 & 0 & 0 & 0 & 0 & 0 & 1 \\
\hline 0 & 0 & 0 & 1 & 2 & 2 & 1 & 1 & 7 \\
\hline- & 0 & - & - & - & - & - & - & 0 \\
\hline 0 & 0 & 0 & 1 & 2 & 1 & 1 & 1 & 7 \\
\hline 0 & 0 & 0 & 0 & 0 & 0 & 0 & 0 & 0 \\
\hline 0 & 0 & 0 & 0 & 0 & 0 & 0 & 0 & 0 \\
\hline- & - & - & 0 & 0 & 0 & - & 0 & 0 \\
\hline- & - & 0 & - & - & 0 & - & - & 0 \\
\hline- & 0 & 0 & 0 & 0 & 0 & 0 & 0 & 0 \\
\hline 0 & 1 & 0 & 0 & 0 & 0 & 0 & 0 & 1 \\
\hline- & - & - & - & - & - & - & - & - \\
\hline- & - & - & - & - & - & - & - & - \\
\hline 0 & 0 & 0 & 0 & 0 & 0 & 0 & 0 & 1 \\
\hline 0 & 0 & 0 & 0 & 0 & 0 & 0 & 0 & 0 \\
\hline 0 & - & - & 0 & - & - & - & - & 0 \\
\hline- & - & - & - & - & 0 & - & - & 0 \\
\hline 0 & 0 & 0 & 0 & 0 & 0 & 0 & 0 & 1 \\
\hline 8 & 2 & 2 & 2 & 5 & 5 & 7 & 10 & 39 \\
\hline 20 & 2 & 2 & 3 & 4 & 6 & 12 & 29 & 78 \\
\hline 20 & 2 & 2 & 3 & 4 & 6 & 12 & 29 & 76 \\
\hline 1 & 0 & 0 & 0 & 0 & 0 & 0 & 0 & 1 \\
\hline 0 & 0 & 0 & 0 & 0 & 0 & 0 & 0 & 0 \\
\hline 0 & 0 & 9 & 7 & 0 & - & 0 & 0 & 16 \\
\hline 0 & 0 & 2 & 2 & 0 & - & - & - & 4 \\
\hline- & 0 & 1 & 0 & 0 & - & - & - & 1 \\
\hline- & 0 & 2 & 2 & 0 & - & - & 0 & 4 \\
\hline- & - & 0 & 0 & - & - & - & - & 0 \\
\hline- & 0 & 2 & 1 & 0 & - & - & - & 2 \\
\hline- & 0 & 2 & 2 & 0 & - & 0 & 0 & 5 \\
\hline 71 & 0 & - & 0 & - & - & - & 0 & 71 \\
\hline 9 & - & - & - & - & - & - & - & 9 \\
\hline 38 & 0 & - & - & - & - & - & 0 & 38 \\
\hline 24 & 0 & - & 0 & - & - & - & 0 & 24 \\
\hline 7 & 1 & 1 & 1 & 2 & 2 & 4 & 9 & 26 \\
\hline 6 & 1 & 0 & 0 & 1 & 1 & 3 & 7 & 18 \\
\hline 0 & 0 & 0 & 0 & 0 & 0 & 0 & 0 & 0 \\
\hline 0 & - & - & 0 & 0 & - & - & 0 & 0 \\
\hline 1 & 0 & 0 & 0 & 1 & 0 & 1 & 2 & 6 \\
\hline 0 & 0 & 0 & 0 & 0 & 0 & 0 & 1 & 1 \\
\hline & & & & & & tinu & he fo & page \\
\hline
\end{tabular}


Table 3B.4 Continued

\begin{tabular}{|c|c|c|c|c|c|c|c|c|c|c|}
\hline \multirow[b]{2}{*}{ Cause } & \multicolumn{10}{|c|}{ Male } \\
\hline & Total & $0-4$ & $5-14$ & $15-29$ & $30-44$ & $45-59$ & $60-69$ & 70-79 & $80+$ & Total \\
\hline II. Noncommunicable diseases & 2,187 & 38 & 10 & 32 & 81 & 206 & 235 & 293 & 243 & 1,138 \\
\hline A. Malignant neoplasms & 484 & 2 & 3 & 7 & 14 & 47 & 60 & 70 & 44 & 247 \\
\hline 1. Mouth and oropharynx cancers & 14 & 0 & 0 & 0 & 1 & 3 & 3 & 2 & 1 & 10 \\
\hline 2. Esophageal cancer & 16 & - & 0 & 0 & 1 & 3 & 3 & 3 & 2 & 12 \\
\hline 3. Stomach cancer & 57 & 0 & 0 & 0 & 2 & 7 & 9 & 10 & 6 & 34 \\
\hline 4. Colon and rectal cancers & 37 & 0 & 0 & 0 & 1 & 3 & 4 & 5 & 3 & 18 \\
\hline 5. Liver cancer & 21 & 0 & 0 & 0 & 1 & 2 & 3 & 3 & 2 & 11 \\
\hline 6. Pancreas cancer & 20 & 0 & 0 & 0 & 0 & 2 & 3 & 3 & 2 & 10 \\
\hline 7. Trachea, bronchus, and lung cancers & 55 & 0 & 0 & 0 & 1 & 8 & 12 & 12 & 5 & 38 \\
\hline 8. Melanoma and other skin cancers & 7 & 0 & 0 & 0 & 0 & 1 & 1 & 1 & 1 & 4 \\
\hline 9. Breast cancer & 37 & 0 & - & 0 & 0 & 0 & 0 & 0 & 0 & 0 \\
\hline 10. Cervix uteri cancer & 26 & - & - & - & - & - & - & - & - & - \\
\hline 11. Corpus uteri cancer & 12 & - & - & - & - & - & - & - & - & - \\
\hline 12. Ovarian cancer & 9 & - & - & - & - & - & - & - & - & - \\
\hline 13. Prostate cancer & 37 & 0 & 0 & 0 & 0 & 2 & 7 & 15 & 13 & 37 \\
\hline 14. Bladder cancer & 9 & 0 & 0 & 0 & 0 & 1 & 1 & 2 & 2 & 6 \\
\hline 15. Lymphomas and multiple myeloma & 24 & 0 & 1 & 1 & 2 & 3 & 3 & 3 & 1 & 13 \\
\hline 16. Leukemia & 22 & 1 & 2 & 2 & 1 & 2 & 1 & 2 & 1 & 12 \\
\hline Other malignant neoplasms & 82 & 1 & 1 & 2 & 4 & 10 & 10 & 9 & 5 & 43 \\
\hline B. Other neoplasms & 13 & 0 & 0 & 0 & 1 & 1 & 1 & 1 & 1 & 6 \\
\hline C. Diabetes mellitus & 163 & 0 & 0 & 1 & 4 & 15 & 19 & 20 & 11 & 70 \\
\hline D. Endocrine disorders & 30 & 2 & 1 & 1 & 1 & 2 & 2 & 2 & 3 & 14 \\
\hline E. Neuropsychiatric conditions & 70 & 2 & 1 & 5 & 9 & 10 & 5 & 6 & 6 & 44 \\
\hline 1. Unipolar depressive disorders & 0 & 0 & 0 & 0 & 0 & 0 & 0 & 0 & 0 & 0 \\
\hline 2. Bipolar affective disorder & 0 & 0 & 0 & 0 & 0 & 0 & 0 & 0 & 0 & 0 \\
\hline 3. Schizophrenia & 0 & 0 & 0 & 0 & 0 & 0 & 0 & 0 & 0 & 0 \\
\hline 4. Epilepsy & 8 & 0 & 0 & 1 & 1 & 1 & 0 & 0 & 0 & 5 \\
\hline 5. Alcohol use disorders & 17 & 0 & 0 & 1 & 5 & 6 & 2 & 1 & 0 & 15 \\
\hline 6. Alzheimer's and other dementias & 14 & 0 & 0 & 0 & 0 & 0 & 1 & 2 & 3 & 5 \\
\hline 7. Parkinson's disease & 5 & 0 & 0 & 0 & 0 & 0 & 0 & 1 & 1 & 3 \\
\hline 8. Multiple sclerosis & 1 & - & - & 0 & 0 & 0 & 0 & 0 & 0 & 0 \\
\hline 9. Drug use disorders & 2 & - & - & 0 & 1 & 1 & 0 & 0 & 0 & 2 \\
\hline 10. Post-traumatic stress disorder & 0 & 0 & 0 & 0 & 0 & 0 & 0 & 0 & 0 & 0 \\
\hline 11. Obsessive-compulsive disorder & - & - & - & - & - & - & - & - & - & - \\
\hline 12. Panic disorder & - & - & - & - & - & - & - & - & - & - \\
\hline 13. Insomnia (primary) & - & - & - & - & - & - & - & - & - & - \\
\hline 14. Migraine & - & - & - & - & - & - & - & - & - & - \\
\hline 15. Mental retardation, lead-caused & 0 & 0 & 0 & 0 & 0 & 0 & 0 & 0 & 0 & 0 \\
\hline Other neuropsychiatric disorders & 23 & 2 & 1 & 2 & 2 & 2 & 2 & 2 & 1 & 13 \\
\hline F. Sense organ diseases & 0 & 0 & $\mathbf{0}$ & 0 & 0 & $\mathbf{0}$ & 0 & 0 & $\mathbf{0}$ & 0 \\
\hline 1. Glaucoma & 0 & - & - & 0 & - & - & 0 & 0 & 0 & 0 \\
\hline 2. Cataracts & - & - & - & - & - & - & - & - & - & - \\
\hline 3. Vision disorders, age-related & - & - & - & - & - & - & - & - & - & - \\
\hline 4. Hearing loss, adult onset & - & - & - & - & - & - & - & - & - & - \\
\hline Other sense organ disorders & 0 & 0 & 0 & 0 & 0 & 0 & 0 & 0 & 0 & 0 \\
\hline G. Cardiovascular diseases & 910 & 2 & 1 & 7 & 25 & 79 & 97 & 130 & 120 & 462 \\
\hline 1. Rheumatic heart disease & 6 & 0 & 0 & 0 & 0 & 1 & 0 & 0 & 0 & 2 \\
\hline 2. Hypertensive heart disease & 87 & 0 & 0 & 0 & 2 & 7 & 8 & 11 & 11 & 39 \\
\hline 3. Ischemic heart disease & 358 & 0 & 0 & 2 & 9 & 39 & 48 & 59 & 44 & 202 \\
\hline 4. Cerebrovascular disease & 267 & 0 & 0 & 2 & 7 & 22 & 27 & 38 & 33 & 129 \\
\hline 5. Inflammatory heart diseases & 31 & 0 & 0 & 1 & 2 & 4 & 4 & 4 & 3 & 18 \\
\hline Other cardiovascular diseases & 160 & 1 & 0 & 2 & 5 & 8 & 9 & 17 & 28 & 72 \\
\hline H. Respiratory diseases & 195 & 5 & 1 & 3 & 5 & 11 & 19 & 32 & 32 & 107 \\
\hline 1. Chronic obstructive pulmonary disease & 99 & 0 & 0 & 0 & 1 & 5 & 11 & 20 & 20 & 58 \\
\hline 2. Asthma & 12 & 1 & 0 & 0 & 0 & 1 & 1 & 1 & 1 & 5 \\
\hline Other respiratory diseases & 84 & 4 & 1 & 2 & 4 & 6 & 7 & 10 & 11 & 44 \\
\hline
\end{tabular}


Table 3B.4 Continued

\begin{tabular}{|c|c|c|c|c|c|c|c|c|}
\hline \multicolumn{9}{|c|}{ Female } \\
\hline $0-4$ & 5-14 & 15-29 & $30-44$ & $45-59$ & $60-69$ & $70-79$ & $80+$ & Total \\
\hline 33 & 9 & 23 & 61 & 155 & 183 & 260 & 325 & 1,049 \\
\hline 2 & 2 & 6 & 23 & 55 & 51 & 56 & 42 & 237 \\
\hline 0 & 0 & 0 & 0 & 1 & 1 & 1 & 1 & 4 \\
\hline- & 0 & 0 & 0 & 1 & 1 & 1 & 1 & 4 \\
\hline 0 & 0 & 0 & 2 & 4 & 5 & 6 & 6 & 23 \\
\hline 0 & 0 & 0 & 1 & 3 & 4 & 5 & 5 & 19 \\
\hline 0 & 0 & 0 & 1 & 2 & 2 & 3 & 2 & 10 \\
\hline 0 & 0 & 0 & 0 & 2 & 2 & 3 & 2 & 10 \\
\hline 0 & 0 & 0 & 1 & 4 & 5 & 5 & 3 & 17 \\
\hline 0 & 0 & 0 & 0 & 1 & 1 & 1 & 1 & 3 \\
\hline 0 & 0 & 0 & 5 & 12 & 8 & 7 & 5 & 37 \\
\hline- & 0 & 1 & 4 & 9 & 5 & 5 & 2 & 26 \\
\hline 0 & - & 0 & 1 & 3 & 3 & 3 & 2 & 12 \\
\hline 0 & 0 & 0 & 1 & 3 & 2 & 2 & 1 & 9 \\
\hline- & - & - & - & - & - & - & - & - \\
\hline 0 & 0 & 0 & 0 & 0 & 0 & 1 & 1 & 3 \\
\hline 0 & 0 & 1 & 1 & 2 & 2 & 3 & 2 & 11 \\
\hline 1 & 1 & 1 & 1 & 2 & 1 & 2 & 1 & 10 \\
\hline 1 & 1 & 1 & 3 & 8 & 9 & 10 & 7 & 40 \\
\hline 0 & 0 & 0 & 1 & 1 & 1 & 1 & 1 & 6 \\
\hline 0 & 0 & 1 & 3 & 16 & 24 & 28 & 21 & 93 \\
\hline 1 & 1 & 1 & 1 & 2 & 2 & 3 & 4 & 16 \\
\hline 2 & 1 & 2 & 3 & 3 & 2 & 5 & 8 & 26 \\
\hline 0 & 0 & 0 & 0 & 0 & 0 & 0 & 0 & 0 \\
\hline 0 & 0 & 0 & 0 & 0 & 0 & 0 & 0 & 0 \\
\hline 0 & 0 & 0 & 0 & 0 & 0 & 0 & 0 & 0 \\
\hline 0 & 0 & 1 & 1 & 0 & 0 & 0 & 0 & 3 \\
\hline 0 & 0 & 0 & 0 & 0 & 0 & 0 & 0 & 1 \\
\hline 0 & 0 & 0 & 0 & 0 & 1 & 2 & 6 & 8 \\
\hline 0 & 0 & 0 & 0 & 0 & 0 & 1 & 1 & 2 \\
\hline- & 0 & 0 & 0 & 0 & 0 & 0 & 0 & 1 \\
\hline- & - & 0 & 0 & 0 & 0 & 0 & 0 & 0 \\
\hline 0 & 0 & 0 & 0 & 0 & 0 & 0 & 0 & 0 \\
\hline- & - & - & - & - & - & - & - & - \\
\hline- & - & - & - & - & - & - & - & - \\
\hline- & - & - & - & - & - & - & - & - \\
\hline- & - & - & - & - & - & - & - & - \\
\hline 0 & 0 & 0 & 0 & 0 & 0 & 0 & 0 & 0 \\
\hline 1 & 1 & 1 & 1 & 1 & 1 & 1 & 2 & 10 \\
\hline 0 & 0 & 0 & 0 & 0 & 0 & 0 & 0 & 0 \\
\hline- & - & - & - & - & - & - & 0 & 0 \\
\hline- & - & - & - & - & - & - & - & - \\
\hline- & - & - & - & - & - & - & - & - \\
\hline- & - & - & - & - & - & - & - & - \\
\hline 0 & 0 & 0 & 0 & 0 & 0 & 0 & 0 & 0 \\
\hline 2 & 1 & 5 & 18 & 52 & 71 & 119 & 180 & 448 \\
\hline 0 & 0 & 0 & 1 & 1 & 1 & 1 & 0 & 4 \\
\hline 0 & 0 & 0 & 2 & 6 & 8 & 13 & 18 & 48 \\
\hline 0 & 0 & 1 & 4 & 18 & 29 & 45 & 60 & 156 \\
\hline 0 & 0 & 2 & 7 & 18 & 22 & 37 & 52 & 138 \\
\hline 0 & 0 & 0 & 1 & 2 & 2 & 3 & 4 & 14 \\
\hline 1 & 1 & 2 & 4 & 7 & 9 & 20 & 46 & 88 \\
\hline 5 & 1 & 2 & 3 & 8 & 13 & 22 & 35 & 88 \\
\hline 1 & 0 & 0 & 1 & 3 & 7 & 12 & 17 & 42 \\
\hline 0 & 0 & 0 & 1 & 1 & 1 & 1 & 2 & 6 \\
\hline 3 & 1 & 1 & 2 & 4 & 5 & 9 & 16 & 40 \\
\hline
\end{tabular}


Table 3B.4 Continued

\begin{tabular}{|c|c|c|c|c|c|c|c|c|c|c|}
\hline \multirow[b]{2}{*}{ Cause } & \multicolumn{10}{|c|}{ Male } \\
\hline & Total & $\overline{0-4}$ & $5-14$ & $15-29$ & 30-44 & $45-59$ & $60-69$ & $70-79$ & $80+$ & Total \\
\hline I. Digestive diseases & 185 & 2 & 1 & 4 & 19 & 33 & 23 & 21 & 14 & 117 \\
\hline 1. Peptic ulcer disease & 14 & 0 & 0 & 0 & 1 & 2 & 2 & 2 & 2 & 8 \\
\hline 2. Cirrhosis of the liver & 74 & 0 & 0 & 2 & 12 & 21 & 12 & 7 & 3 & 57 \\
\hline 3. Appendicitis & 3 & 0 & 0 & 0 & 0 & 0 & 0 & 0 & 0 & 2 \\
\hline Other digestive diseases & 94 & 2 & 1 & 2 & 6 & 11 & 9 & 11 & 9 & 51 \\
\hline J. Genitourinary diseases & 70 & 1 & $\mathbf{0}$ & 2 & 3 & 5 & 7 & 10 & 11 & 38 \\
\hline 1. Nephritis and nephrosis & 55 & 1 & 0 & 1 & 2 & 5 & 6 & 7 & 7 & 30 \\
\hline 2. Benign prostatic hypertrophy & 2 & - & - & 0 & 0 & 0 & 0 & 1 & 1 & 2 \\
\hline Other genitourinary system diseases & 13 & 0 & 0 & 0 & 0 & 1 & 1 & 2 & 2 & 6 \\
\hline K. Skin diseases & 8 & 0 & 0 & 0 & 0 & 0 & 0 & 1 & 1 & 3 \\
\hline L. Musculoskeletal diseases & 12 & 0 & $\mathbf{0}$ & $\mathbf{0}$ & 0 & 1 & 1 & 1 & 1 & 4 \\
\hline 1. Rheumatoid arthritis & 3 & - & 0 & 0 & 0 & 0 & 0 & 0 & 0 & 1 \\
\hline 2. Osteoarthritis & 1 & 0 & - & 0 & 0 & 0 & 0 & 0 & 0 & 0 \\
\hline 3. Gout & 0 & - & - & 0 & 0 & 0 & 0 & 0 & 0 & 0 \\
\hline 4. Low back pain & 0 & - & - & 0 & 0 & 0 & 0 & 0 & 0 & 0 \\
\hline Other musculoskeletal disorders & 8 & 0 & 0 & 0 & 0 & 0 & 0 & 1 & 1 & 3 \\
\hline M. Congenital anomalies & 47 & 22 & 1 & 1 & 0 & 0 & 0 & 0 & 0 & 25 \\
\hline 1. Abdominal wall defect & 1 & 1 & 0 & 0 & 0 & - & 0 & 0 & 0 & 1 \\
\hline 2. Anencephaly & 2 & 1 & 0 & 0 & 0 & - & 0 & 0 & 0 & 1 \\
\hline 3. Anorectal atresia & 0 & 0 & 0 & 0 & 0 & - & 0 & 0 & 0 & 0 \\
\hline 4. Cleft lip & 0 & 0 & 0 & 0 & 0 & - & 0 & 0 & 0 & 0 \\
\hline 5. Cleft palate & 0 & 0 & 0 & 0 & 0 & - & 0 & 0 & 0 & 0 \\
\hline 6. Esophageal atresia & 1 & 0 & 0 & 0 & 0 & - & 0 & 0 & 0 & 0 \\
\hline 7. Renal agenesis & 0 & 0 & 0 & 0 & 0 & 0 & 0 & 0 & 0 & 0 \\
\hline 8. Down syndrome & 2 & 1 & 0 & 0 & 0 & 0 & 0 & 0 & 0 & 1 \\
\hline 9. Congenital heart anomalies & 20 & 9 & 1 & 1 & 0 & 0 & 0 & 0 & 0 & 11 \\
\hline 10. Spina bifida & 1 & 1 & 0 & 0 & 0 & 0 & 0 & 0 & 0 & 1 \\
\hline Other congenital anomalies & 19 & 9 & 0 & 0 & 0 & 0 & 0 & 0 & 0 & 10 \\
\hline N. Oral conditions & 0 & 0 & $\mathbf{0}$ & 0 & 0 & 0 & 0 & 0 & $\mathbf{0}$ & 0 \\
\hline 1. Dental caries & - & - & - & - & - & - & - & - & - & - \\
\hline 2. Periodontal disease & 0 & - & - & 0 & 0 & 0 & 0 & 0 & 0 & 0 \\
\hline 3. Edentulism & - & - & - & - & - & - & - & - & - & - \\
\hline Other oral diseases & 0 & 0 & 0 & 0 & 0 & 0 & 0 & 0 & 0 & 0 \\
\hline III. Injuries & 374 & 11 & 12 & 120 & 80 & 45 & 18 & 12 & 8 & 305 \\
\hline A. Unintentional injuries & 207 & 10 & 10 & 46 & 37 & 26 & 12 & 9 & 6 & 157 \\
\hline 1. Road traffic accidents & 88 & 2 & 4 & 23 & 19 & 12 & 5 & 3 & 1 & 69 \\
\hline 2. Poisonings & 3 & 0 & 0 & 1 & 1 & 0 & 0 & 0 & 0 & 2 \\
\hline 3. Falls & 15 & 0 & 0 & 1 & 2 & 2 & 1 & 1 & 1 & 10 \\
\hline 4. Fires & 5 & 1 & 0 & 0 & 1 & 0 & 0 & 0 & 0 & 3 \\
\hline 5. Drownings & 19 & 2 & 2 & 6 & 3 & 2 & 1 & 0 & 0 & 15 \\
\hline 6. Other unintentional injuries & 78 & 6 & 3 & 14 & 12 & 9 & 5 & 4 & 3 & 57 \\
\hline B. Intentional injuries & 167 & 1 & 2 & 74 & 43 & 19 & 6 & 3 & 1 & 148 \\
\hline 1. Self-inflicted injuries & 30 & 0 & 0 & 8 & 6 & 4 & 2 & 1 & 1 & 23 \\
\hline 2. Violence & 130 & 1 & 1 & 63 & 34 & 14 & 3 & 1 & 1 & 119 \\
\hline 3. War & 6 & 0 & 0 & 2 & 2 & 1 & 0 & 0 & 0 & 6 \\
\hline Other intentional injuries & 1 & 0 & 0 & 1 & 0 & 0 & 0 & 0 & 0 & 1 \\
\hline
\end{tabular}


Table 3B.4 Continued

\begin{tabular}{|c|c|c|c|c|c|c|c|c|}
\hline \multicolumn{9}{|c|}{ Female } \\
\hline $0-4$ & 5-14 & $15-29$ & $30-44$ & 45-59 & $60-69$ & $70-79$ & $80+$ & Total \\
\hline 1 & 1 & 2 & 5 & 12 & 12 & 16 & 18 & 68 \\
\hline 0 & 0 & 0 & 0 & 1 & 1 & 2 & 2 & 6 \\
\hline 0 & 0 & 0 & 2 & 5 & 4 & 4 & 2 & 18 \\
\hline 0 & 0 & 0 & 0 & 0 & 0 & 0 & 0 & 1 \\
\hline 1 & 0 & 1 & 3 & 6 & 7 & 11 & 13 & 43 \\
\hline 1 & 0 & 1 & 2 & 4 & 5 & 7 & 11 & 32 \\
\hline 0 & 0 & 1 & 2 & 4 & 4 & 6 & 8 & 25 \\
\hline- & - & - & - & - & - & - & - & - \\
\hline 0 & 0 & 0 & 0 & 1 & 1 & 1 & 3 & 7 \\
\hline 0 & 0 & 0 & 0 & 0 & 0 & 1 & 2 & 5 \\
\hline 0 & 0 & 1 & 1 & 1 & 1 & 1 & 2 & 8 \\
\hline- & 0 & 0 & 0 & 0 & 0 & 1 & 1 & 2 \\
\hline- & 0 & 0 & 0 & 0 & 0 & 0 & 0 & 0 \\
\hline 0 & 0 & 0 & 0 & 0 & 0 & 0 & 0 & 0 \\
\hline 0 & 0 & 0 & 0 & 0 & 0 & 0 & 0 & 0 \\
\hline 0 & 0 & 1 & 1 & 1 & 1 & 1 & 1 & 6 \\
\hline 19 & 1 & 1 & 0 & 0 & 0 & 0 & 0 & 22 \\
\hline 0 & 0 & 0 & 0 & - & - & 0 & 0 & 0 \\
\hline 1 & 0 & 0 & 0 & 0 & - & 0 & - & 1 \\
\hline 0 & 0 & 0 & 0 & 0 & 0 & 0 & - & 0 \\
\hline 0 & 0 & 0 & 0 & - & - & 0 & - & 0 \\
\hline 0 & 0 & 0 & 0 & - & - & 0 & - & 0 \\
\hline 0 & 0 & 0 & 0 & 0 & - & 0 & 0 & 0 \\
\hline 0 & 0 & 0 & 0 & 0 & 0 & 0 & 0 & 0 \\
\hline 1 & 0 & 0 & 0 & 0 & 0 & 0 & 0 & 1 \\
\hline 8 & 0 & 0 & 0 & 0 & 0 & 0 & 0 & 9 \\
\hline 1 & 0 & 0 & 0 & 0 & - & - & - & 1 \\
\hline 8 & 0 & 0 & 0 & 0 & 0 & 0 & 0 & 9 \\
\hline 0 & 0 & 0 & 0 & 0 & 0 & 0 & 0 & 0 \\
\hline- & - & - & - & - & - & - & - & - \\
\hline- & - & 0 & 0 & 0 & 0 & 0 & 0 & 0 \\
\hline- & - & - & - & - & - & - & - & - \\
\hline 0 & 0 & 0 & 0 & 0 & 0 & 0 & 0 & 0 \\
\hline 8 & 6 & 17 & 11 & 8 & 5 & 6 & 8 & 69 \\
\hline 7 & 5 & 9 & 7 & 6 & 4 & 5 & 8 & 50 \\
\hline 1 & 2 & 5 & 4 & 3 & 2 & 1 & 1 & 18 \\
\hline 0 & 0 & 0 & 0 & 0 & 0 & 0 & 0 & 1 \\
\hline 0 & 0 & 0 & 0 & 0 & 0 & 1 & 3 & 5 \\
\hline 0 & 0 & 0 & 0 & 0 & 0 & 0 & 0 & 2 \\
\hline 1 & 1 & 1 & 0 & 0 & 0 & 0 & 0 & 3 \\
\hline 5 & 2 & 3 & 2 & 2 & 2 & 2 & 4 & 21 \\
\hline 0 & 1 & 8 & 5 & 3 & 1 & 1 & 0 & 19 \\
\hline 0 & 0 & 3 & 1 & 1 & 0 & 0 & 0 & 6 \\
\hline 0 & 1 & 5 & 3 & 1 & 0 & 0 & 0 & 12 \\
\hline 0 & 0 & 0 & 0 & 0 & 0 & 0 & 0 & 1 \\
\hline 0 & - & 0 & 0 & - & 0 & 0 & 0 & 0 \\
\hline
\end{tabular}

Source: Authors' compilation.

Note: - = an estimate of zero; the number zero in a cell indicates a non-zero estimate of less than 500 .

a. These figures include late effects of polio cases with onset prior to regional certification of polio eradication in 2002.

b. Does not include liver cancer and cirrhosis deaths resulting from chronic hepatitis virus infection.

c. This cause category includes "Causes arising in the perinatal period" as defined in the International Classification of Diseases, principally low birthweight, prematurity, birth asphyxia, and birth trauma, and does not include all causes of deaths occurring in the perinatal period. 
Table 3B.5 Deaths by Cause, Sex, and Age in the Middle East and North Africa Region, 2001 (thousands)

\begin{tabular}{|c|c|c|c|c|c|c|c|c|c|c|}
\hline \multirow[b]{2}{*}{ Cause } & \multicolumn{10}{|c|}{ Male } \\
\hline & Total & $0-4$ & $5-14$ & $15-29$ & 30-44 & $45-59$ & $60-69$ & 70-79 & $80+$ & Total \\
\hline Population (millions) & 310 & 19 & 39 & 47 & 29 & 15 & 5 & 3 & 1 & 157 \\
\hline All causes & 1,914 & 231 & 33 & 78 & 85 & 158 & 173 & 206 & 103 & 1,068 \\
\hline $\begin{array}{l}\text { I. Communicable, maternal, perinatal, } \\
\text { and nutritional conditions }\end{array}$ & 464 & 178 & 7 & 5 & 8 & 12 & 11 & 14 & 8 & 243 \\
\hline A. Infectious and parasitic diseases & 216 & 69 & 5 & 4 & 6 & 10 & 8 & 8 & 4 & 113 \\
\hline 1. Tuberculosis & 23 & 1 & $\mathbf{0}$ & 1 & 2 & 3 & 2 & 2 & 1 & 13 \\
\hline $\begin{array}{l}\text { 2. Sexually transmitted diseases } \\
\text { excluding HIV/AIDS }\end{array}$ & 4 & 0 & 0 & $\mathbf{0}$ & 0 & 1 & $\mathbf{0}$ & 0 & 0 & 2 \\
\hline a. Syphilis & 3 & 0 & 0 & 0 & 0 & 1 & 0 & 0 & 0 & 1 \\
\hline b. Chlamydia & 0 & - & - & - & - & - & - & - & - & - \\
\hline c. Gonorrhea & 0 & - & - & 0 & 0 & 0 & 0 & - & - & 0 \\
\hline d. Other sexually transmitted diseases & 1 & 0 & 0 & 0 & 0 & 0 & 0 & 0 & 0 & 1 \\
\hline 3. HIV/AIDS & 4 & $\mathbf{0}$ & 0 & 1 & 1 & $\mathbf{0}$ & 0 & $\mathbf{0}$ & $\mathbf{0}$ & 2 \\
\hline 4. Diarrheal diseases & 74 & 34 & $\mathbf{0}$ & 0 & 1 & 1 & 1 & 1 & 1 & 39 \\
\hline 5. Childhood-cluster diseases & 27 & 10 & 3 & $\mathbf{0}$ & $\mathbf{0}$ & $\mathbf{0}$ & 0 & $\mathbf{0}$ & $\mathbf{0}$ & 13 \\
\hline a. Pertussis & 8 & 4 & - & - & - & - & - & - & - & 4 \\
\hline b. Poliomyelitis & 0 & 0 & - & - & - & - & - & - & - & 0 \\
\hline c. Diphtheria & 0 & 0 & - & 0 & - & - & - & 0 & - & 0 \\
\hline d. Measles & 15 & 5 & 2 & 0 & - & - & - & - & - & 8 \\
\hline e. Tetanus & 4 & 2 & 0 & 0 & 0 & 0 & 0 & 0 & 0 & 2 \\
\hline 6. Meningitis & 10 & 3 & $\mathbf{0}$ & 0 & $\mathbf{0}$ & $\mathbf{0}$ & 0 & $\mathbf{0}$ & $\mathbf{0}$ & 4 \\
\hline 7. Hepatitis $\mathbf{B}^{\mathbf{a}}$ & 6 & 0 & $\mathbf{0}$ & 0 & 0 & 1 & 1 & $\mathbf{0}$ & $\mathbf{0}$ & 3 \\
\hline Hepatitis $\mathrm{C}^{\mathrm{a}}$ & 3 & 0 & $\mathbf{0}$ & 0 & $\mathbf{0}$ & $\mathbf{0}$ & 0 & $\mathbf{0}$ & $\mathbf{0}$ & 2 \\
\hline 8. Malaria & 19 & 8 & $\mathbf{0}$ & $\mathbf{0}$ & 0 & $\mathbf{0}$ & 0 & $\mathbf{0}$ & $\mathbf{0}$ & 9 \\
\hline 9. Tropical-cluster diseases & 9 & $\mathbf{0}$ & 0 & 0 & $\mathbf{0}$ & 2 & 1 & 1 & $\mathbf{0}$ & 6 \\
\hline a. Trypanosomiasis & 1 & 0 & 0 & 0 & 0 & 0 & 0 & 0 & - & 0 \\
\hline b. Chagas' disease & - & - & - & - & - & - & - & - & - & - \\
\hline c. Schistosomiasis & 8 & 0 & 0 & 0 & 0 & 1 & 1 & 1 & 0 & 5 \\
\hline d. Leishmaniasis & 1 & 0 & 0 & 0 & 0 & 0 & 0 & 0 & 0 & 0 \\
\hline e. Lymphatic filariasis & 0 & - & - & - & 0 & - & - & - & - & 0 \\
\hline f. Onchocerciasis & - & - & - & - & - & - & - & - & - & - \\
\hline 10. Leprosy & $\mathbf{0}$ & - & - & - & - & 0 & 0 & 0 & - & 0 \\
\hline 11. Dengue & $\mathbf{0}$ & $\mathbf{0}$ & $\mathbf{0}$ & $\mathbf{0}$ & 0 & $\mathbf{0}$ & 0 & $\mathbf{0}$ & $\mathbf{0}$ & $\mathbf{0}$ \\
\hline 12. Japanese encephalitis & - & - & - & - & - & - & - & - & - & - \\
\hline 13. Trachoma & - & - & - & - & - & - & - & - & - & - \\
\hline 14. Intestinal nematode infections & $\mathbf{0}$ & $\mathbf{0}$ & 0 & $\mathbf{0}$ & $\mathbf{0}$ & $\mathbf{0}$ & $\mathbf{0}$ & $\mathbf{0}$ & $\mathbf{0}$ & 0 \\
\hline a. Ascariasis & 0 & - & 0 & 0 & 0 & - & - & - & - & 0 \\
\hline b. Trichuriasis & 0 & - & 0 & - & - & - & - & - & - & 0 \\
\hline c. Hookworm disease & 0 & 0 & - & 0 & 0 & 0 & 0 & 0 & 0 & 0 \\
\hline Other intestinal infections & 0 & - & 0 & 0 & 0 & 0 & 0 & 0 & 0 & 0 \\
\hline Other infectious diseases & 37 & 11 & 0 & 0 & 1 & 1 & 2 & 2 & 1 & 19 \\
\hline B. Respiratory infections & 110 & 40 & 2 & 1 & 1 & 2 & 3 & 5 & 4 & 58 \\
\hline 1. Lower respiratory infections & 108 & 39 & 2 & 1 & 1 & 2 & 3 & 5 & 4 & 57 \\
\hline 2. Upper respiratory infections & 2 & 1 & 0 & 0 & 0 & 0 & 0 & 0 & 0 & 1 \\
\hline 3. Otitis media & 0 & 0 & 0 & 0 & 0 & - & 0 & - & - & 0 \\
\hline C. Maternal conditions & 15 & - & - & - & - & - & - & - & - & 一 \\
\hline 1. Maternal hemorrhage & 4 & - & - & - & - & - & - & - & - & - \\
\hline 2. Maternal sepsis & 1 & - & - & - & - & - & - & - & - & - \\
\hline 3. Hypertensive disorders of pregnancy & 2 & - & - & - & - & - & - & - & - & - \\
\hline 4. Obstructed labor & 0 & - & - & - & - & - & - & - & - & - \\
\hline 5. Abortion & 1 & - & - & - & - & - & - & - & - & - \\
\hline Other maternal conditions & 6 & - & - & - & - & - & - & - & - & - \\
\hline D. Perinatal conditions ${ }^{b}$ & 106 & 64 & - & - & - & - & - & - & - & 64 \\
\hline 1. Low birthweight & 54 & 32 & - & - & - & - & - & - & - & 32 \\
\hline 2. Birth asphyxia and birth trauma & 30 & 19 & - & - & - & - & - & - & - & 19 \\
\hline Other perinatal conditions & 21 & 13 & - & - & - & - & - & - & - & 13 \\
\hline E. Nutritional deficiencies & 16 & 5 & 0 & $\mathbf{0}$ & $\mathbf{0}$ & 0 & 0 & 1 & $\mathbf{0}$ & 7 \\
\hline 1. Protein-energy malnutrition & 8 & 3 & 0 & 0 & 0 & 0 & 0 & 0 & 0 & 4 \\
\hline 2. Iodine deficiency & 0 & 0 & 0 & 0 & 0 & 0 & 0 & 0 & 0 & 0 \\
\hline 3. Vitamin A deficiency & 0 & 0 & 0 & 0 & 0 & 0 & 0 & 0 & 0 & 0 \\
\hline 4. Iron-deficiency anemia & 6 & 1 & 0 & 0 & 0 & 0 & 0 & 0 & 0 & 2 \\
\hline Other nutritional disorders & 2 & 1 & 0 & 0 & 0 & 0 & 0 & 0 & 0 & 1 \\
\hline
\end{tabular}


Table 3B.5 Continued

\begin{tabular}{|c|c|c|c|c|c|c|c|c|}
\hline \multicolumn{9}{|c|}{ Female } \\
\hline $0-4$ & 5-14 & $15-29$ & $30-44$ & $45-59$ & $60-69$ & $70-79$ & $80+$ & Total \\
\hline 18 & 37 & 45 & 28 & 15 & 6 & 3 & 1 & 153 \\
\hline $\begin{array}{l}199 \\
151\end{array}$ & $\begin{array}{r}25 \\
8\end{array}$ & $\begin{array}{l}44 \\
12\end{array}$ & $\begin{array}{l}56 \\
14\end{array}$ & $\begin{array}{r}102 \\
8\end{array}$ & $\begin{array}{r}122 \\
7\end{array}$ & $\begin{array}{r}175 \\
11\end{array}$ & $\begin{array}{r}123 \\
10\end{array}$ & $\begin{array}{l}846 \\
221\end{array}$ \\
\hline $\begin{array}{r}68 \\
1 \\
0\end{array}$ & $\begin{array}{l}5 \\
0 \\
0\end{array}$ & $\begin{array}{l}4 \\
2 \\
0\end{array}$ & $\begin{array}{l}5 \\
2 \\
0\end{array}$ & $\begin{array}{l}6 \\
2 \\
1\end{array}$ & $\begin{array}{l}5 \\
1 \\
1\end{array}$ & $\begin{array}{l}\mathbf{6} \\
1 \\
0\end{array}$ & $\begin{array}{r}5 \\
\mathbf{0} \\
-\end{array}$ & $\begin{array}{r}103 \\
10 \\
2\end{array}$ \\
\hline 0 & 0 & 0 & 0 & 0 & 0 & 0 & - & 1 \\
\hline- & - & 0 & 0 & 0 & 0 & - & - & 0 \\
\hline 0 & - & 0 & 0 & 0 & 0 & - & - & 0 \\
\hline 0 & 0 & 0 & 0 & 0 & 0 & 0 & - & 0 \\
\hline 0 & 0 & 0 & 0 & 0 & 0 & 0 & 0 & 1 \\
\hline 32 & 0 & 0 & 0 & 0 & 1 & 1 & 1 & 35 \\
\hline 10 & 2 & 0 & 0 & 0 & 0 & 0 & 0 & 13 \\
\hline 4 & - & - & - & - & - & - & - & 4 \\
\hline 0 & - & - & - & - & - & - & - & 0 \\
\hline 0 & 0 & - & - & - & - & - & 0 & 0 \\
\hline 5 & 2 & 0 & - & - & - & - & - & 8 \\
\hline 2 & 0 & 0 & 0 & 0 & 0 & 0 & 0 & 2 \\
\hline 5 & 0 & 0 & 0 & 0 & 0 & 0 & 0 & 6 \\
\hline 0 & 0 & 0 & 0 & 1 & 1 & 1 & 0 & 3 \\
\hline 0 & 0 & 0 & 0 & 0 & 0 & 0 & 0 & 1 \\
\hline 9 & 0 & 0 & 0 & 0 & 0 & 0 & 0 & 10 \\
\hline 0 & 0 & 0 & 0 & 1 & 1 & 1 & 1 & 4 \\
\hline 0 & 0 & 0 & 0 & 0 & 0 & 0 & - & 0 \\
\hline- & - & - & - & - & - & - & - & - \\
\hline 0 & 0 & 0 & 0 & 0 & 1 & 1 & 1 & 3 \\
\hline 0 & 0 & 0 & 0 & 0 & 0 & 0 & - & 0 \\
\hline- & - & - & - & 0 & - & - & 0 & 0 \\
\hline- & - & - & - & - & - & - & - & - \\
\hline 0 & - & - & - & - & 0 & 0 & 0 & 0 \\
\hline 0 & 0 & 0 & 0 & 0 & 0 & 0 & 0 & 0 \\
\hline- & - & - & - & - & - & - & - & - \\
\hline- & - & - & - & - & - & - & - & - \\
\hline- & 0 & 0 & 0 & 0 & 0 & 0 & 0 & 0 \\
\hline- & 0 & - & - & - & - & - & - & 0 \\
\hline- & 0 & - & - & - & - & - & - & 0 \\
\hline- & 0 & 0 & 0 & 0 & 0 & 0 & 0 & 0 \\
\hline- & 0 & 0 & 0 & 0 & 0 & 0 & 0 & 0 \\
\hline 10 & 1 & 1 & 1 & 1 & 1 & 2 & 2 & 18 \\
\hline 36 & 2 & 1 & 1 & 1 & 2 & 4 & 4 & 52 \\
\hline 35 & 2 & 1 & 1 & 1 & 2 & 3 & 4 & 51 \\
\hline 1 & 0 & 0 & 0 & 0 & 0 & 0 & 0 & 1 \\
\hline 0 & - & 0 & - & 0 & - & - & 0 & 0 \\
\hline- & 0 & 7 & 8 & 0 & - & - & - & 15 \\
\hline- & 0 & 2 & 2 & 0 & - & - & - & 4 \\
\hline- & 0 & 0 & 0 & 0 & - & - & - & 1 \\
\hline- & 0 & 1 & 1 & 0 & - & - & - & 2 \\
\hline- & - & 0 & 0 & 0 & - & - & - & 0 \\
\hline- & - & 1 & 1 & - & - & - & - & 1 \\
\hline- & 0 & 3 & 3 & 0 & - & - & - & 6 \\
\hline 42 & - & - & - & - & - & - & - & 42 \\
\hline 22 & - & - & - & - & - & - & - & 22 \\
\hline 11 & - & - & - & - & - & - & - & 11 \\
\hline 9 & - & - & - & - & - & - & - & 9 \\
\hline 5 & 1 & 0 & 0 & 0 & 0 & 1 & 1 & 9 \\
\hline 4 & 0 & 0 & 0 & 0 & 0 & 0 & 0 & 5 \\
\hline 0 & 0 & 0 & 0 & 0 & 0 & 0 & 0 & 0 \\
\hline 0 & 0 & 0 & 0 & 0 & 0 & 0 & 0 & 0 \\
\hline 1 & 1 & 0 & 0 & 0 & 0 & 1 & 1 & 3 \\
\hline \multirow[t]{2}{*}{1} & 0 & 0 & 0 & 0 & 0 & 0 & 0 & 1 \\
\hline & & & & & ICo & ues C & follo & page.) \\
\hline
\end{tabular}


Table 3B.5 Continued

\begin{tabular}{|c|c|c|c|c|c|c|c|c|c|c|}
\hline \multirow[b]{2}{*}{ Cause } & \multicolumn{10}{|c|}{ Male } \\
\hline & Total & $0-4$ & 5-14 & $15-29$ & 30-44 & $45-59$ & $60-69$ & 70-79 & $80+$ & Total \\
\hline II. Noncommunicable diseases & 1,235 & 38 & 11 & 26 & 49 & 123 & 149 & 183 & 91 & 671 \\
\hline A. Malignant neoplasms & 165 & 2 & 2 & 5 & 8 & 20 & 24 & 24 & 9 & 95 \\
\hline 1. Mouth and oropharynx cancers & 5 & 0 & 0 & 0 & 0 & 1 & 1 & 1 & 0 & 3 \\
\hline 2. Esophageal cancer & 5 & 0 & 0 & 0 & 0 & 1 & 1 & 1 & 0 & 3 \\
\hline 3. Stomach cancer & 18 & 0 & 0 & 0 & 1 & 2 & 3 & 4 & 1 & 11 \\
\hline 4. Colon and rectal cancers & 10 & 0 & 0 & 0 & 1 & 1 & 1 & 1 & 1 & 6 \\
\hline 5. Liver cancer & 9 & 0 & 0 & 0 & 0 & 1 & 2 & 2 & 0 & 6 \\
\hline 6. Pancreas cancer & 4 & 0 & 0 & 0 & 0 & 1 & 1 & 1 & 0 & 2 \\
\hline 7. Trachea, bronchus, and lung cancers & 20 & 0 & 0 & 0 & 1 & 4 & 5 & 4 & 1 & 15 \\
\hline 8. Melanoma and other skin cancers & 1 & 0 & 0 & 0 & 0 & 0 & 0 & 0 & 0 & 1 \\
\hline 9. Breast cancer & 14 & - & - & - & 0 & 0 & 0 & - & - & 0 \\
\hline 10. Cervix uteri cancer & 5 & - & - & - & - & - & - & - & - & - \\
\hline 11. Corpus uteri cancer & 1 & - & - & - & - & - & - & - & - & - \\
\hline 12. Ovarian cancer & 2 & - & - & - & - & - & - & - & - & - \\
\hline 13. Prostate cancer & 6 & 0 & 0 & 0 & 0 & 1 & 1 & 3 & 1 & 6 \\
\hline 14. Bladder cancer & 15 & 0 & 0 & 0 & 1 & 3 & 3 & 3 & 1 & 12 \\
\hline 15. Lymphomas and multiple myeloma & 12 & 0 & 1 & 1 & 1 & 1 & 1 & 1 & 0 & 7 \\
\hline 16. Leukemia & 14 & 1 & 1 & 2 & 1 & 1 & 1 & 1 & 0 & 8 \\
\hline Other malignant neoplasms & 26 & 1 & 1 & 1 & 2 & 3 & 4 & 3 & 1 & 15 \\
\hline B. Other neoplasms & 19 & $\mathbf{0}$ & $\mathbf{0}$ & 1 & 1 & 2 & 3 & 3 & 1 & 11 \\
\hline C. Diabetes mellitus & 31 & $\mathbf{0}$ & $\mathbf{0}$ & $\mathbf{0}$ & 1 & 3 & 4 & 5 & 2 & 14 \\
\hline D. Endocrine disorders & 20 & 1 & $\mathbf{0}$ & 1 & 1 & 2 & 2 & 2 & 1 & 9 \\
\hline E. Neuropsychiatric conditions & 51 & 2 & 1 & 6 & 10 & 7 & 3 & 3 & 2 & 34 \\
\hline 1. Unipolar depressive disorders & 0 & 0 & 0 & 0 & 0 & 0 & 0 & 0 & 0 & 0 \\
\hline 2. Bipolar affective disorder & 0 & 0 & 0 & 0 & 0 & 0 & 0 & 0 & 0 & 0 \\
\hline 3. Schizophrenia & 0 & - & 0 & 0 & 0 & 0 & 0 & 0 & 0 & 0 \\
\hline 4. Epilepsy & 5 & 0 & 0 & 1 & 1 & 0 & 0 & 0 & 0 & 3 \\
\hline 5. Alcohol use disorders & 3 & - & 0 & 1 & 1 & 1 & 0 & 0 & 0 & 3 \\
\hline 6. Alzheimer's and other dementias & 3 & 0 & 0 & 0 & 0 & 0 & 0 & 1 & 1 & 2 \\
\hline 7. Parkinson's disease & 3 & 0 & 0 & 0 & 0 & 0 & 0 & 0 & 0 & 1 \\
\hline 8. Multiple sclerosis & 1 & - & 0 & 0 & 0 & 0 & 0 & 0 & 0 & 0 \\
\hline 9. Drug use disorders & 19 & - & 0 & 3 & 7 & 5 & 0 & 0 & 0 & 16 \\
\hline 10. Post-traumatic stress disorder & 0 & 0 & 0 & 0 & 0 & 0 & 0 & 0 & 0 & 0 \\
\hline 11. Obsessive-compulsive disorder & - & - & - & - & - & - & - & - & - & - \\
\hline 12. Panic disorder & - & - & - & - & - & - & - & - & - & - \\
\hline 13. Insomnia (primary) & - & - & - & - & - & - & - & - & - & - \\
\hline 14. Migraine & - & - & - & - & - & - & - & - & - & - \\
\hline 15. Mental retardation, lead-caused & 3 & 0 & 0 & 1 & 0 & 0 & 0 & 0 & 0 & 2 \\
\hline Other neuropsychiatric disorders & 14 & 1 & 0 & 1 & 1 & 1 & 1 & 2 & 1 & 8 \\
\hline F. Sense organ diseases & 1 & $\mathbf{0}$ & 0 & - & $\mathbf{0}$ & 0 & 0 & 0 & 0 & 0 \\
\hline 1. Glaucoma & 0 & - & - & - & - & - & - & - & 0 & 0 \\
\hline 2. Cataracts & - & - & - & - & - & - & - & - & - & - \\
\hline 3. Vision disorders, age-related & - & - & - & - & - & - & - & - & - & - \\
\hline 4. Hearing loss, adult onset & - & - & - & - & - & - & - & - & - & - \\
\hline Other sense organ disorders & 1 & 0 & 0 & - & 0 & 0 & 0 & 0 & 0 & 0 \\
\hline g. Cardiovascular diseases & 671 & 5 & 3 & 8 & 20 & 65 & 84 & 111 & 60 & 356 \\
\hline 1. Rheumatic heart disease & 10 & 0 & 0 & 1 & 1 & 1 & 0 & 0 & 0 & 5 \\
\hline 2. Hypertensive heart disease & 74 & 0 & 0 & 0 & 1 & 6 & 10 & 13 & 7 & 38 \\
\hline 3. Ischemic heart disease & 323 & 0 & 0 & 2 & 11 & 41 & 49 & 59 & 26 & 188 \\
\hline 4. Cerebrovascular disease & 130 & 2 & 1 & 3 & 3 & 9 & 14 & 22 & 13 & 66 \\
\hline 5. Inflammatory heart diseases & 26 & 1 & 0 & 1 & 1 & 2 & 3 & 4 & 3 & 14 \\
\hline Other cardiovascular diseases & 108 & 2 & 1 & 2 & 3 & 6 & 8 & 13 & 11 & 46 \\
\hline H. Respiratory diseases & 80 & 4 & 1 & 1 & 3 & 6 & 10 & 14 & 8 & 46 \\
\hline 1. Chronic obstructive pulmonary disease & 41 & 0 & 0 & 0 & 1 & 3 & 6 & 9 & 5 & 23 \\
\hline 2. Asthma & 7 & 0 & 0 & 1 & 2 & 1 & 0 & 0 & 0 & 4 \\
\hline Other respiratory diseases & 33 & 3 & 0 & 1 & 1 & 2 & 4 & 5 & 3 & 18 \\
\hline
\end{tabular}


Table 3B.5 Continued

\begin{tabular}{|c|c|c|c|c|c|c|c|c|}
\hline \multicolumn{9}{|c|}{ Female } \\
\hline $0-4$ & 5-14 & $15-29$ & $30-44$ & $45-59$ & $60-69$ & $70-79$ & $80+$ & Total \\
\hline 39 & 9 & 16 & 34 & 87 & 110 & 158 & 111 & 564 \\
\hline 1 & 2 & 4 & 11 & 19 & 14 & 14 & 6 & 70 \\
\hline 0 & 0 & 0 & 0 & 0 & 0 & 0 & 0 & 1 \\
\hline 0 & 0 & 0 & 0 & 0 & 1 & 1 & 0 & 2 \\
\hline 0 & 0 & 0 & 1 & 1 & 1 & 2 & 1 & 7 \\
\hline 0 & 0 & 0 & 1 & 1 & 1 & 1 & 1 & 4 \\
\hline 0 & 0 & 0 & 0 & 1 & 1 & 1 & 0 & 3 \\
\hline 0 & 0 & 0 & 0 & 0 & 0 & 0 & 0 & 2 \\
\hline 0 & 0 & 0 & 1 & 1 & 1 & 1 & 0 & 4 \\
\hline 0 & 0 & 0 & 0 & 0 & 0 & 0 & 0 & 1 \\
\hline 0 & 0 & 0 & 3 & 5 & 2 & 2 & 1 & 14 \\
\hline 0 & 0 & 0 & 1 & 2 & 1 & 1 & 0 & 5 \\
\hline 0 & 0 & 0 & 0 & 0 & 0 & 0 & 0 & 1 \\
\hline 0 & 0 & 0 & 0 & 1 & 0 & 0 & 0 & 2 \\
\hline- & - & - & - & - & - & - & - & - \\
\hline 0 & 0 & 0 & 0 & 1 & 1 & 1 & 0 & 3 \\
\hline 0 & 0 & 1 & 1 & 1 & 1 & 1 & 0 & 5 \\
\hline 1 & 1 & 1 & 1 & 1 & 1 & 1 & 0 & 6 \\
\hline 1 & 0 & 1 & 2 & 2 & 2 & 2 & 1 & 11 \\
\hline 0 & 0 & 0 & 1 & 3 & 2 & 2 & 1 & 9 \\
\hline 0 & 0 & 0 & 1 & 3 & 5 & 6 & 3 & 17 \\
\hline 1 & 0 & 0 & 1 & 1 & 2 & 3 & 1 & 10 \\
\hline 1 & 1 & 2 & 3 & 2 & 2 & 3 & 2 & 17 \\
\hline 0 & 0 & 0 & 0 & 0 & 0 & 0 & 0 & 0 \\
\hline 0 & 0 & 0 & 0 & 0 & 0 & 0 & 0 & 0 \\
\hline- & - & 0 & 0 & 0 & 0 & 0 & 0 & 0 \\
\hline 0 & 0 & 1 & 0 & 0 & 0 & 0 & 0 & 2 \\
\hline- & - & 0 & 0 & 0 & 0 & 0 & - & 0 \\
\hline 0 & 0 & 0 & 0 & 0 & 0 & 1 & 1 & 2 \\
\hline 0 & 0 & 0 & 0 & 0 & 0 & 0 & 0 & 1 \\
\hline- & 0 & 0 & 0 & 0 & 0 & 0 & 0 & 0 \\
\hline- & - & 1 & 2 & 1 & 0 & 0 & 0 & 3 \\
\hline 0 & 0 & 0 & 0 & 0 & 0 & 0 & 0 & 0 \\
\hline- & - & - & - & - & - & - & - & - \\
\hline- & - & - & - & - & - & - & - & - \\
\hline- & - & - & - & - & - & - & - & - \\
\hline- & - & - & - & - & - & - & - & - \\
\hline 0 & 1 & 0 & 0 & 0 & 0 & 0 & 0 & 2 \\
\hline 1 & 0 & 0 & 0 & 1 & 1 & 1 & 1 & 6 \\
\hline 0 & 0 & 0 & 0 & 0 & 0 & 0 & 0 & 0 \\
\hline- & - & - & - & - & - & 0 & 0 & 0 \\
\hline- & - & - & - & - & - & - & - & - \\
\hline- & - & - & - & - & - & - & - & - \\
\hline- & - & - & - & - & - & - & - & - \\
\hline 0 & 0 & 0 & 0 & 0 & 0 & 0 & 0 & 0 \\
\hline 10 & 3 & 5 & 11 & 40 & 63 & 103 & 79 & 314 \\
\hline 0 & 0 & 1 & 1 & 1 & 0 & 1 & 0 & 5 \\
\hline 0 & 0 & 0 & 1 & 4 & 8 & 13 & 9 & 36 \\
\hline 0 & 0 & 1 & 4 & 19 & 32 & 47 & 31 & 135 \\
\hline 1 & 1 & 1 & 2 & 7 & 13 & 22 & 17 & 64 \\
\hline 0 & 0 & 0 & 1 & 1 & 2 & 4 & 3 & 12 \\
\hline 8 & 1 & 1 & 2 & 6 & 8 & 17 & 18 & 62 \\
\hline 3 & 0 & 1 & 3 & 4 & 6 & 10 & 7 & 34 \\
\hline 0 & 0 & 0 & 1 & 2 & 4 & 6 & 4 & 17 \\
\hline 0 & 0 & 0 & 1 & 1 & 0 & 0 & 0 & 3 \\
\hline 3 & 0 & 0 & 1 & 2 & 2 & 4 & 3 & 14 \\
\hline
\end{tabular}


Table 3B.5 Continued

\begin{tabular}{|c|c|c|c|c|c|c|c|c|c|c|c|}
\hline \multirow{2}{*}{ Cause } & & \multirow[b]{2}{*}{ Total } & \multicolumn{7}{|c|}{ Male } & \multirow[b]{2}{*}{$80+$} & \multirow[b]{2}{*}{ Tota } \\
\hline & & & $\overline{0-4}$ & $5-14$ & $15-29$ & $30-44$ & $45-59$ & $60-69$ & $70-79$ & & \\
\hline \multirow[t]{5}{*}{ I. } & Digestive diseases & 88 & 2 & 1 & 2 & 2 & 12 & 12 & 11 & 4 & 46 \\
\hline & 1. Peptic ulcer disease & 5 & 0 & 0 & 0 & 0 & 1 & 1 & 1 & 0 & 3 \\
\hline & 2. Cirrhosis of the liver & 37 & 0 & 0 & 1 & 1 & 6 & 6 & 5 & 2 & 21 \\
\hline & 3. Appendicitis & 0 & 0 & 0 & 0 & 0 & 0 & 0 & 0 & 0 & 0 \\
\hline & Other digestive diseases & 45 & 1 & 0 & 1 & 1 & 6 & 5 & 5 & 2 & 22 \\
\hline \multirow[t]{4}{*}{ J. } & Genitourinary diseases & 57 & 1 & 1 & 1 & 2 & 6 & 8 & 9 & 5 & 31 \\
\hline & 1. Nephritis and nephrosis & 42 & 0 & 0 & 1 & 1 & 5 & 6 & 6 & 3 & 23 \\
\hline & 2. Benign prostatic hypertrophy & 2 & - & - & - & - & 0 & 0 & 1 & 1 & 2 \\
\hline & Other genitourinary system diseases & 14 & 0 & 0 & 0 & 1 & 1 & 1 & 2 & 1 & 7 \\
\hline K. & Skin diseases & 4 & $\mathbf{0}$ & 0 & 0 & 0 & 0 & 0 & 1 & 0 & 1 \\
\hline \multirow[t]{6}{*}{ L. } & Musculoskeletal diseases & 2 & 0 & $\mathbf{0}$ & 0 & 0 & 0 & 0 & $\mathbf{0}$ & 0 & 1 \\
\hline & 1. Rheumatoid arthritis & 0 & 0 & 0 & 0 & 0 & 0 & 0 & 0 & 0 & 0 \\
\hline & 2. Osteoarthritis & 0 & 0 & - & 0 & 0 & 0 & 0 & 0 & 0 & 0 \\
\hline & 3. Gout & 0 & 0 & - & 0 & 0 & 0 & 0 & 0 & 0 & 0 \\
\hline & 4. Low back pain & 0 & 0 & - & 0 & 0 & 0 & 0 & 0 & 0 & 0 \\
\hline & Other musculoskeletal disorders & 1 & 0 & 0 & 0 & 0 & 0 & 0 & 0 & 0 & 1 \\
\hline \multicolumn{2}{|r|}{ M. Congenital anomalies } & 46 & 22 & 1 & 1 & 0 & 0 & 0 & $\mathbf{0}$ & $\mathbf{0}$ & 24 \\
\hline & 1. Abdominal wall defect & 0 & 0 & 0 & 0 & 0 & 0 & 0 & 0 & 0 & 0 \\
\hline & 2. Anencephaly & 2 & 1 & 0 & 0 & 0 & 0 & 0 & 0 & 0 & 1 \\
\hline & 3. Anorectal atresia & 0 & 0 & 0 & 0 & 0 & 0 & 0 & 0 & 0 & 0 \\
\hline & 4. Cleft lip & 0 & 0 & 0 & 0 & 0 & 0 & 0 & 0 & 0 & 0 \\
\hline & 5. Cleft palate & 0 & 0 & 0 & 0 & 0 & 0 & 0 & 0 & 0 & 0 \\
\hline & 6. Esophageal atresia & 0 & 0 & 0 & 0 & 0 & 0 & 0 & 0 & 0 & 0 \\
\hline & 7. Renal agenesis & 0 & 0 & 0 & 0 & 0 & 0 & 0 & 0 & 0 & 0 \\
\hline & 8. Down syndrome & 1 & 1 & 0 & 0 & 0 & 0 & 0 & 0 & 0 & 1 \\
\hline & 9. Congenital heart anomalies & 20 & 10 & 0 & 0 & 0 & 0 & 0 & 0 & 0 & 11 \\
\hline & 10. Spina bifida & 3 & 1 & 0 & 0 & 0 & 0 & 0 & 0 & - & 1 \\
\hline & Other congenital anomalies & 18 & 9 & 0 & 0 & 0 & 0 & 0 & 0 & 0 & 10 \\
\hline \multicolumn{2}{|r|}{ N. Oral conditions } & 0 & $\mathbf{0}$ & 0 & 0 & 0 & 0 & 0 & - & 0 & 0 \\
\hline & 1. Dental caries & - & - & - & - & - & - & - & - & - & - \\
\hline & 2. Periodontal disease & - & - & - & - & - & - & - & - & - & - \\
\hline & 3. Edentulism & - & - & - & - & - & - & - & - & - & - \\
\hline & Other oral diseases & 0 & 0 & 0 & 0 & 0 & 0 & 0 & - & 0 & 0 \\
\hline \multicolumn{2}{|c|}{ III. Injuries } & 216 & 15 & 16 & 47 & 28 & 23 & 13 & 10 & 3 & 154 \\
\hline & 181 & 14 & 16 & 36 & 21 & 20 & 11 & 9 & 3 & 129 \\
\hline \multicolumn{2}{|c|}{$\begin{array}{l}\text { A. Unintentional Injuries } \\
\text { 1. Road traffic accidents }\end{array}$} & 99 & 5 & 8 & 20 & 14 & 12 & 7 & 5 & 1 & 74 \\
\hline \multicolumn{2}{|r|}{ 2. Poisonings } & 7 & 1 & 0 & 1 & 1 & 2 & 0 & 0 & 0 & 5 \\
\hline \multicolumn{2}{|r|}{ 3. Falls } & 12 & 1 & 1 & 1 & 2 & 2 & 1 & 1 & 0 & 9 \\
\hline \multicolumn{2}{|r|}{ 4. Fires } & 13 & 1 & 1 & 2 & 1 & 1 & 0 & 0 & 0 & 6 \\
\hline \multicolumn{2}{|r|}{ 5. Drownings } & 14 & 2 & 2 & 5 & 1 & 1 & 0 & 0 & 0 & 11 \\
\hline \multicolumn{2}{|r|}{ 6. Other unintentional injuries } & 36 & 4 & 3 & 7 & 3 & 3 & 2 & 2 & 1 & 25 \\
\hline \multicolumn{2}{|c|}{ B. Intentional injuries } & 35 & 0 & 1 & 11 & 7 & 4 & 2 & 1 & 0 & 25 \\
\hline & 1. Self-inflicted injuries & 14 & 0 & 0 & 4 & 2 & 1 & 1 & 0 & 0 & 9 \\
\hline & 2. Violence & 10 & 0 & 0 & 4 & 2 & 1 & 1 & 0 & 0 & 8 \\
\hline & 3. War & 8 & 0 & 0 & 3 & 3 & 1 & 0 & 0 & 0 & 7 \\
\hline & Other intentional injuries & 2 & 0 & 0 & 1 & 0 & 0 & 0 & 0 & 0 & 1 \\
\hline
\end{tabular}


Table 3B.5 Continued

\begin{tabular}{|c|c|c|c|c|c|c|c|c|}
\hline \multicolumn{9}{|c|}{ Female } \\
\hline $0-4$ & 5-14 & $15-29$ & $30-44$ & $45-59$ & $60-69$ & $70-79$ & $80_{+}$ & Total \\
\hline 1 & 1 & 1 & 2 & 9 & 10 & 11 & 6 & 42 \\
\hline 0 & 0 & 0 & 0 & 0 & 0 & 1 & 0 & 2 \\
\hline 0 & 0 & 0 & 1 & 4 & 4 & 4 & 2 & 16 \\
\hline 0 & 0 & 0 & 0 & 0 & 0 & 0 & 0 & 0 \\
\hline 1 & 0 & 0 & 1 & 4 & 6 & 7 & 4 & 24 \\
\hline 1 & 1 & 1 & 2 & 5 & 6 & 7 & 4 & 26 \\
\hline 0 & 0 & 1 & 1 & 4 & 4 & 5 & 3 & 19 \\
\hline- & - & - & - & - & - & - & - & - \\
\hline 0 & 0 & 0 & 1 & 1 & 1 & 2 & 1 & 7 \\
\hline 0 & 0 & 0 & 0 & 0 & 1 & 1 & 0 & 2 \\
\hline 0 & 0 & 0 & 0 & 0 & 0 & 0 & 0 & 1 \\
\hline 0 & 0 & 0 & 0 & 0 & 0 & 0 & 0 & 0 \\
\hline 0 & 0 & 0 & 0 & 0 & 0 & 0 & 0 & 0 \\
\hline 0 & 0 & 0 & 0 & 0 & 0 & 0 & 0 & 0 \\
\hline 0 & 0 & 0 & 0 & 0 & 0 & 0 & 0 & 0 \\
\hline 0 & 0 & 0 & 0 & 0 & 0 & 0 & 0 & 1 \\
\hline 19 & 1 & 1 & 0 & 0 & 0 & 0 & 0 & 21 \\
\hline 0 & 0 & 0 & 0 & 0 & 0 & - & 0 & 0 \\
\hline 1 & 0 & 0 & 0 & 0 & 0 & - & 0 & 1 \\
\hline 0 & 0 & 0 & 0 & 0 & 0 & - & 0 & 0 \\
\hline 0 & 0 & 0 & 0 & 0 & 0 & - & 0 & 0 \\
\hline 0 & 0 & 0 & 0 & 0 & 0 & - & 0 & 0 \\
\hline 0 & 0 & 0 & 0 & 0 & 0 & - & 0 & 0 \\
\hline 0 & 0 & 0 & 0 & 0 & 0 & - & 0 & 0 \\
\hline 1 & 0 & 0 & 0 & 0 & 0 & - & 0 & 1 \\
\hline 8 & 0 & 0 & 0 & 0 & 0 & 0 & 0 & 9 \\
\hline 1 & 0 & 0 & 0 & - & 0 & 0 & - & 1 \\
\hline 7 & 0 & 0 & 0 & 0 & 0 & 0 & 0 & 8 \\
\hline 0 & - & - & - & - & 0 & 0 & 0 & 0 \\
\hline- & - & - & - & - & - & - & - & - \\
\hline- & - & - & - & - & - & - & - & - \\
\hline- & - & - & - & - & - & - & - & - \\
\hline 0 & - & - & - & - & 0 & 0 & 0 & 0 \\
\hline 10 & 8 & 16 & 9 & 7 & 5 & 6 & 3 & 61 \\
\hline 9 & 8 & 11 & 7 & 5 & 4 & 5 & 2 & 52 \\
\hline 3 & 4 & 5 & 4 & 3 & 2 & 2 & 1 & 25 \\
\hline 0 & 0 & 1 & 0 & 0 & 0 & 0 & 0 & 2 \\
\hline 1 & 0 & 0 & 0 & 0 & 0 & 1 & 0 & 3 \\
\hline 1 & 1 & 3 & 1 & 1 & 0 & 1 & 0 & 8 \\
\hline 1 & 1 & 1 & 0 & 0 & 0 & 0 & 0 & 3 \\
\hline 3 & 2 & 2 & 1 & 1 & 1 & 1 & 1 & 11 \\
\hline 0 & 0 & 4 & 2 & 1 & 1 & 0 & 0 & 9 \\
\hline 0 & 0 & 3 & 1 & 1 & 0 & 0 & 0 & 6 \\
\hline 0 & 0 & 1 & 1 & 0 & 0 & 0 & 0 & 2 \\
\hline 0 & 0 & 0 & 0 & 0 & 0 & 0 & 0 & 1 \\
\hline 0 & 0 & 0 & 0 & 0 & 0 & 0 & 0 & 0 \\
\hline
\end{tabular}

Source: Authors' compilation

Note: $-=$ an estimate of zero; the number zero in a cell indicates a non-zero estimate of less than 500 .

a. Does not include liver cancer and cirrhosis deaths resulting from chronic hepatitis virus infection.

b. This cause category includes "Causes arising in the perinatal period" as defined in the International Classification

of Diseases, principally low birthweight, prematurity, birth asphyxia and birth trauma, and does not include all causes

of deaths occurring in the perinatal period. 
Table 3B.6 Deaths by Cause, Sex, and Age in the South Asia Region, 2001 (thousands)

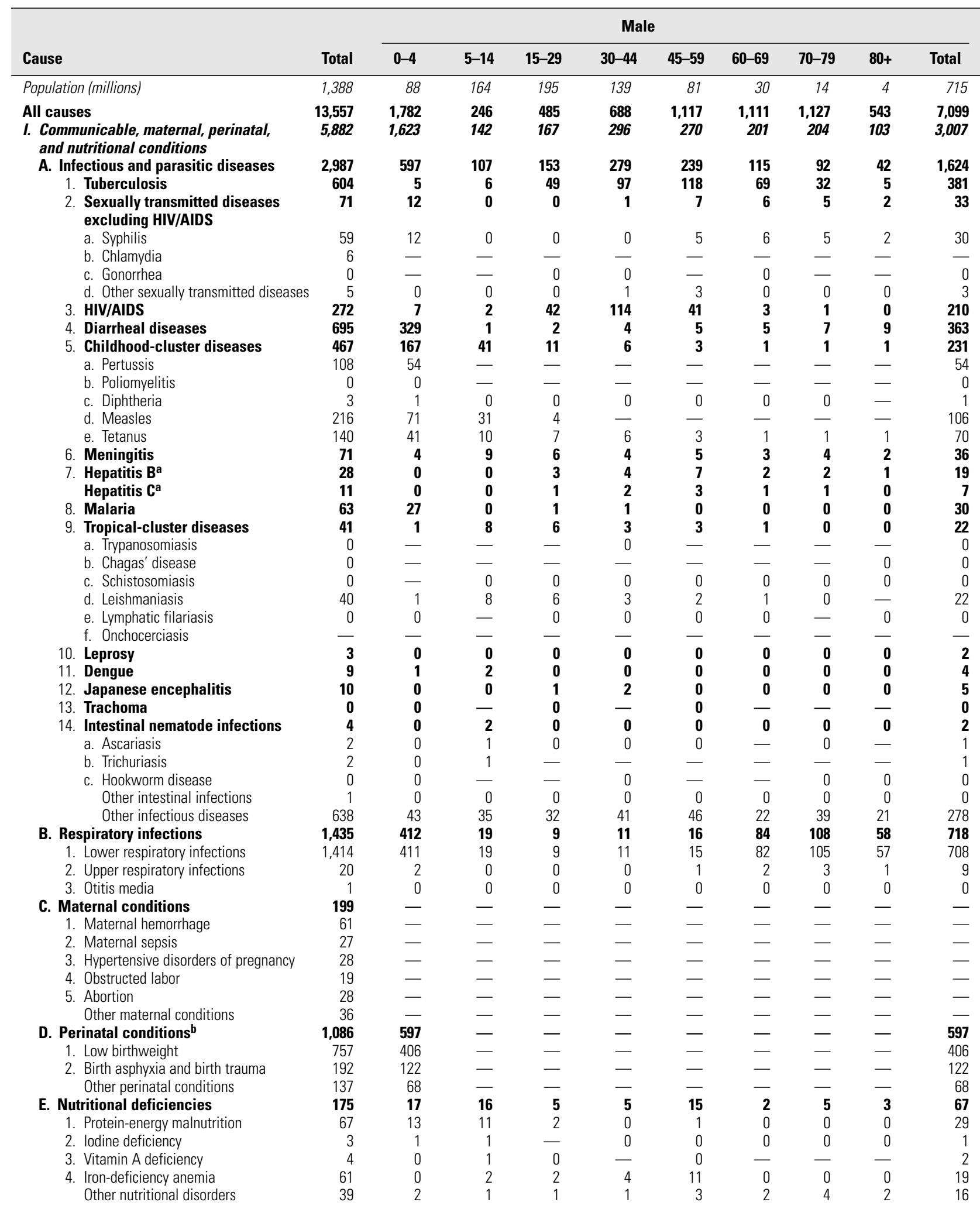


Table 3B.6 Continued

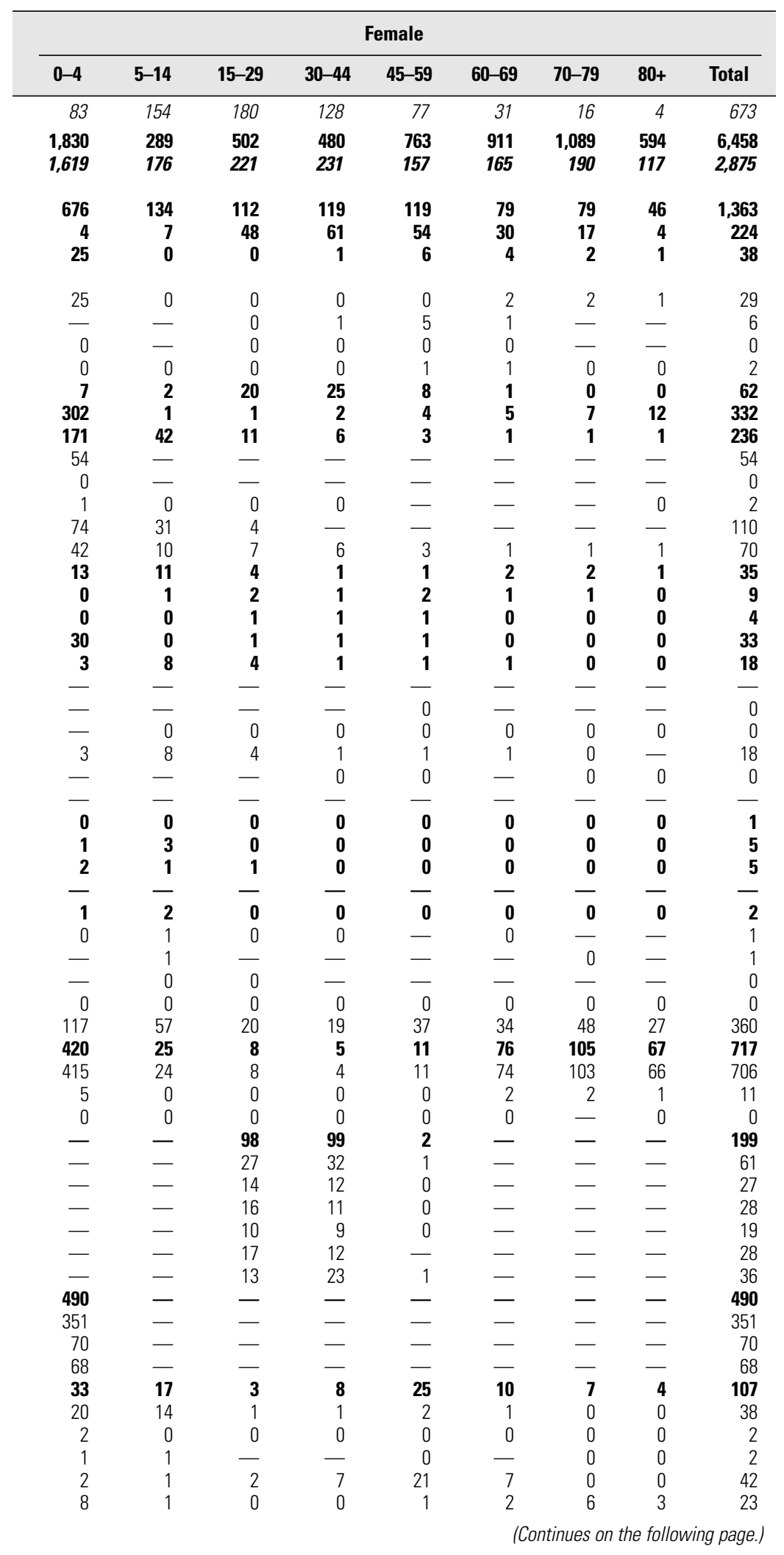

The Burden of Disease and Mortality by Condition: Data, Methods, and Results for 2001 | 157 
Table 3B.6 Continued

\begin{tabular}{|c|c|c|c|c|c|c|c|c|c|c|}
\hline \multirow[b]{2}{*}{ Cause } & \multicolumn{10}{|c|}{ Male } \\
\hline & Total & $0-4$ & $5-14$ & $15-29$ & $30-44$ & $45-59$ & $60-69$ & $70-79$ & $80+$ & Total \\
\hline II. Noncommunicable diseases & 6,346 & 129 & 44 & 107 & 203 & 714 & 854 & 872 & 412 & 3,335 \\
\hline A. Malignant neoplasms & 927 & 6 & 7 & 18 & 34 & 94 & 154 & 112 & 51 & 476 \\
\hline 1. Mouth and oropharynx cancers & 140 & 0 & 0 & 2 & 7 & 21 & 35 & 20 & 8 & 94 \\
\hline 2. Esophageal cancer & 80 & 0 & 0 & 0 & 3 & 9 & 18 & 10 & 4 & 44 \\
\hline 3. Stomach cancer & 45 & 0 & 0 & 1 & 2 & 6 & 9 & 7 & 3 & 28 \\
\hline 4. Colon and rectal cancers & 35 & 0 & 0 & 1 & 3 & 4 & 6 & 5 & 3 & 20 \\
\hline 5. Liver cancer & 27 & 0 & 0 & 1 & 2 & 4 & 5 & 3 & 1 & 17 \\
\hline 6. Pancreas cancer & 13 & 0 & 0 & 0 & 1 & 2 & 3 & 2 & 1 & 7 \\
\hline 7. Trachea, bronchus, and lung cancers & 129 & 0 & 0 & 1 & 5 & 26 & 38 & 28 & 9 & 106 \\
\hline 8. Melanoma and other skin cancers & 3 & 0 & 0 & 0 & 0 & 0 & 0 & 0 & 0 & 1 \\
\hline 9. Breast cancer & 76 & - & - & 0 & 0 & 0 & 0 & 0 & 0 & 0 \\
\hline 10. Cervix uteri cancer & 83 & - & - & - & - & - & - & - & - & - \\
\hline 11. Corpus uteri cancer & 4 & - & - & - & - & - & - & - & - & - \\
\hline 12. Ovarian cancer & 21 & - & - & - & - & - & - & - & - & 一 \\
\hline 13. Prostate cancer & 21 & 0 & 0 & 0 & 0 & 1 & 6 & 9 & 5 & 21 \\
\hline 14. Bladder cancer & 30 & 0 & 0 & 0 & 0 & 1 & 5 & 6 & 4 & 16 \\
\hline 15. Lymphomas and multiple myeloma & 82 & 1 & 2 & 3 & 4 & 4 & 7 & 5 & 3 & 29 \\
\hline 16. Leukemia & 38 & 2 & 3 & 6 & 3 & 2 & 3 & 2 & 1 & 22 \\
\hline Other malignant neoplasms & 99 & 3 & 2 & 2 & 5 & 13 & 20 & 16 & 10 & 71 \\
\hline B. Other neoplasms & 18 & 1 & 1 & 2 & 1 & 2 & 1 & 1 & 1 & 9 \\
\hline C. Diabetes mellitus & 196 & 1 & 1 & 3 & 5 & 25 & 26 & 27 & 17 & 104 \\
\hline D. Endocrine disorders & 26 & 5 & 1 & 1 & 1 & 1 & 1 & 1 & 1 & 12 \\
\hline E. Neuropsychiatric conditions & 234 & 8 & 5 & 10 & 21 & 17 & 6 & 43 & 23 & 132 \\
\hline 1. Unipolar depressive disorders & 9 & - & - & - & 2 & 2 & 1 & - & - & 4 \\
\hline 2. Bipolar affective disorder & 0 & 0 & 0 & 0 & 0 & 0 & 0 & - & - & 0 \\
\hline 3. Schizophrenia & 13 & - & 0 & 0 & 2 & 3 & 1 & 1 & 0 & 7 \\
\hline 4. Epilepsy & 32 & 7 & 3 & 2 & 1 & 1 & 0 & 1 & 0 & 15 \\
\hline 5. Alcohol use disorders & 13 & - & 0 & 2 & 3 & 4 & 2 & 1 & 0 & 12 \\
\hline 6. Alzheimer's and other dementias & 81 & 0 & 0 & 0 & 0 & 0 & 0 & 27 & 14 & 41 \\
\hline 7. Parkinson's disease & 9 & 0 & 0 & 0 & 0 & 0 & 0 & 3 & 2 & 5 \\
\hline 8. Multiple sclerosis & 1 & 0 & 0 & 0 & 0 & 0 & 0 & 0 & 0 & 1 \\
\hline 9. Drug use disorders & 29 & - & 0 & 4 & 12 & 7 & 1 & 0 & 0 & 24 \\
\hline 10. Post-traumatic stress disorder & 0 & - & - & - & 0 & 0 & - & - & 0 & 0 \\
\hline 11. Obsessive-compulsive disorder & - & - & - & - & - & - & - & - & - & - \\
\hline 12. Panic disorder & - & - & - & - & - & - & - & - & - & - \\
\hline 13. Insomnia (primary) & - & - & - & - & - & - & - & - & - & - \\
\hline 14. Migraine & - & - & - & - & - & - & - & - & - & - \\
\hline 15. Mental retardation, lead-caused & 0 & 0 & 0 & 0 & 0 & 0 & 0 & 0 & 0 & 0 \\
\hline Other neuropsychiatric disorders & 46 & 1 & 2 & 1 & 1 & 1 & 1 & 11 & 6 & 23 \\
\hline F. Sense organ diseases & 1 & 0 & 0 & 0 & 0 & 0 & 0 & 0 & 0 & $\mathbf{0}$ \\
\hline 1. Glaucoma & 0 & 0 & - & 0 & 0 & 0 & 0 & - & - & 0 \\
\hline 2. Cataracts & - & - & - & - & - & - & - & - & - & 一 \\
\hline 3. Vision disorders, age-related & - & - & - & - & - & - & - & - & - & - \\
\hline 4. Hearing loss, adult onset & - & - & - & - & - & - & - & - & - & - \\
\hline Other sense organ disorders & 1 & 0 & 0 & 0 & 0 & 0 & 0 & 0 & 0 & 0 \\
\hline G. Cardiovascular diseases & 3,421 & 18 & 11 & 36 & 84 & 374 & 495 & 527 & 250 & 1,795 \\
\hline 1. Rheumatic heart disease & 128 & 6 & 3 & 9 & 7 & 12 & 10 & 10 & 5 & 61 \\
\hline 2. Hypertensive heart disease & 90 & 0 & 0 & 1 & 3 & 10 & 12 & 13 & 6 & 47 \\
\hline 3. Ischemic heart disease & 1,838 & 2 & 3 & 10 & 49 & 244 & 293 & 297 & 135 & 1,032 \\
\hline 4. Cerebrovascular disease & 923 & 3 & 1 & 4 & 10 & 76 & 140 & 159 & 74 & 467 \\
\hline 5. Inflammatory heart diseases & 71 & 2 & 1 & 2 & 4 & 6 & 7 & 8 & 4 & 34 \\
\hline Other cardiovascular diseases & 372 & 4 & 4 & 9 & 12 & 26 & 32 & 41 & 27 & 154 \\
\hline H. Respiratory diseases & 746 & 8 & 4 & 8 & 19 & 111 & 110 & 103 & 41 & 405 \\
\hline 1. Chronic obstructive pulmonary disease & 577 & 0 & 0 & 0 & 7 & 91 & 99 & 88 & 34 & 318 \\
\hline 2. Asthma & 78 & 0 & 2 & 6 & 9 & 14 & 3 & 3 & 1 & 39 \\
\hline Other respiratory diseases & 91 & 7 & 2 & 2 & 3 & 7 & 8 & 12 & 6 & 48 \\
\hline
\end{tabular}


Table 3B.6 Continued

\begin{tabular}{|c|c|c|c|c|c|c|c|c|}
\hline \multicolumn{9}{|c|}{ Female } \\
\hline $0-4$ & 5-14 & $15-29$ & $30-44$ & 45-59 & $60-69$ & $70-79$ & $80+$ & Total \\
\hline 163 & 57 & 117 & 153 & 521 & 697 & 852 & 452 & 3,011 \\
\hline 7 & 6 & 18 & 37 & 108 & 119 & 91 & 64 & 451 \\
\hline 0 & 0 & 1 & 2 & 11 & 14 & 11 & 8 & 47 \\
\hline 0 & 0 & 0 & 2 & 8 & 11 & 8 & 6 & 36 \\
\hline 0 & 0 & 0 & 1 & 4 & 4 & 4 & 4 & 17 \\
\hline 0 & 0 & 0 & 1 & 3 & 4 & 3 & 3 & 15 \\
\hline 0 & 0 & 2 & 1 & 3 & 2 & 2 & 1 & 11 \\
\hline- & - & 0 & 0 & 1 & 1 & 1 & 1 & 5 \\
\hline 0 & 0 & 0 & 2 & 7 & 7 & 6 & 2 & 23 \\
\hline 0 & 0 & 0 & 0 & 0 & 0 & 0 & 0 & 1 \\
\hline 0 & 0 & 1 & 10 & 23 & 20 & 13 & 9 & 76 \\
\hline 0 & 0 & 6 & 5 & 26 & 26 & 14 & 7 & 83 \\
\hline 0 & 0 & 0 & 0 & 1 & 1 & 1 & 1 & 4 \\
\hline 0 & 0 & 1 & 2 & 5 & 6 & 5 & 3 & 21 \\
\hline- & - & - & - & - & - & - & - & - \\
\hline 1 & 0 & 0 & 2 & 2 & 3 & 3 & 3 & 14 \\
\hline 2 & 3 & 4 & 5 & 9 & 12 & 11 & 7 & 53 \\
\hline 3 & 2 & 3 & 2 & 2 & 2 & 1 & 1 & 16 \\
\hline 1 & 1 & 0 & 2 & 5 & 6 & 6 & 7 & 28 \\
\hline 0 & 0 & 1 & 2 & 2 & 1 & 1 & 1 & 9 \\
\hline 1 & 1 & 2 & 3 & 19 & 29 & 26 & 11 & 91 \\
\hline 5 & 1 & 1 & 1 & 1 & 2 & 2 & 1 & 13 \\
\hline 6 & 6 & 6 & 6 & 10 & 5 & 41 & 22 & 103 \\
\hline- & - & 0 & 1 & 3 & 1 & 0 & 0 & 5 \\
\hline 0 & - & 0 & 0 & 0 & 0 & 0 & 0 & 0 \\
\hline 0 & 0 & 0 & 1 & 3 & 1 & 1 & 1 & 6 \\
\hline 6 & 4 & 3 & 1 & 2 & 1 & 1 & 0 & 18 \\
\hline- & - & 0 & 0 & 0 & 0 & 0 & 0 & 1 \\
\hline 0 & 0 & 0 & 0 & 0 & 0 & 26 & 14 & 40 \\
\hline 0 & - & 0 & 0 & 0 & 0 & 3 & 1 & 4 \\
\hline 0 & 0 & 0 & 0 & 0 & 0 & 0 & 0 & 0 \\
\hline- & - & 1 & 2 & 2 & 0 & 0 & 0 & 5 \\
\hline- & - & - & - & 0 & 0 & - & - & 0 \\
\hline- & - & - & - & - & - & - & - & - \\
\hline- & - & - & - & - & - & - & - & - \\
\hline- & - & - & - & - & - & - & - & - \\
\hline- & - & - & - & - & - & - & - & - \\
\hline 0 & 0 & 0 & 0 & 0 & 0 & 0 & 0 & 0 \\
\hline 0 & 2 & 1 & 1 & 1 & 2 & 11 & 6 & 24 \\
\hline 0 & 0 & 0 & 0 & 0 & 0 & 0 & 0 & 1 \\
\hline- & - & - & 0 & - & - & - & - & 0 \\
\hline- & - & - & - & - & - & - & - & - \\
\hline- & - & - & - & - & - & - & - & - \\
\hline- & - & - & - & - & - & - & - & - \\
\hline 0 & 0 & 0 & 0 & 0 & 0 & 0 & 0 & 1 \\
\hline 21 & 13 & 42 & 58 & 229 & 427 & 553 & 281 & 1,626 \\
\hline 7 & 4 & 8 & 7 & 12 & 10 & 12 & 6 & 66 \\
\hline 0 & 1 & 1 & 2 & 9 & 12 & 12 & 7 & 43 \\
\hline 2 & 1 & 18 & 33 & 125 & 233 & 269 & 125 & 806 \\
\hline 2 & 1 & 2 & 6 & 51 & 126 & 175 & 93 & 456 \\
\hline 2 & 1 & 2 & 2 & 5 & 7 & 11 & 6 & 37 \\
\hline 9 & 4 & 10 & 9 & 27 & 40 & 75 & 44 & 217 \\
\hline 9 & 4 & 9 & 18 & 91 & 71 & 91 & 47 & 340 \\
\hline 0 & 0 & 0 & 7 & 72 & 62 & 78 & 40 & 259 \\
\hline 0 & 2 & 7 & 10 & 13 & 2 & 2 & 1 & 39 \\
\hline 9 & 2 & 2 & 2 & 5 & 7 & 10 & 6 & 42 \\
\hline
\end{tabular}


Table 3B.6 Continued

\begin{tabular}{|c|c|c|c|c|c|c|c|c|c|c|}
\hline \multirow[b]{2}{*}{ Cause } & \multicolumn{10}{|c|}{ Male } \\
\hline & Total & $0-4$ & $5-14$ & $15-29$ & 30-44 & $45-59$ & $60-69$ & $70-79$ & $80+$ & Total \\
\hline I. Digestive diseases & 444 & 11 & 9 & 18 & 27 & 65 & 40 & 35 & 18 & 222 \\
\hline 1. Peptic ulcer disease & 82 & 2 & 1 & 3 & 8 & 17 & 8 & 7 & 3 & 50 \\
\hline 2. Cirrhosis of the liver & 185 & 6 & 3 & 7 & 13 & 35 & 22 & 16 & 6 & 107 \\
\hline 3. Appendicitis & 7 & 0 & 0 & 0 & 0 & 1 & 1 & 1 & 1 & 4 \\
\hline Other digestive diseases & 169 & 4 & 5 & 7 & 6 & 12 & 10 & 10 & 8 & 62 \\
\hline J. Genitourinary diseases & 156 & 5 & 3 & 6 & 10 & 23 & 19 & 19 & 9 & 93 \\
\hline 1. Nephritis and nephrosis & 132 & 4 & 3 & 5 & 9 & 17 & 15 & 15 & 7 & 74 \\
\hline 2. Benign prostatic hypertrophy & 12 & - & - & - & 0 & 4 & 3 & 3 & 1 & 12 \\
\hline Other genitourinary system diseases & 13 & 0 & 0 & 1 & 1 & 1 & 1 & 1 & 1 & 7 \\
\hline K. Skin diseases & 10 & $\mathbf{0}$ & 0 & 0 & $\mathbf{0}$ & 1 & 1 & 1 & 1 & 5 \\
\hline L. Musculoskeletal diseases & 11 & $\mathbf{0}$ & 0 & 1 & $\mathbf{0}$ & 1 & 1 & 1 & 1 & 5 \\
\hline 1. Rheumatoid arthritis & 3 & 0 & 0 & 0 & 0 & 0 & 0 & 0 & 0 & 1 \\
\hline 2. Osteoarthritis & 0 & - & - & - & 0 & 0 & 0 & 0 & 0 & 0 \\
\hline 3. Gout & 0 & - & - & - & 0 & 0 & 0 & 0 & 0 & 0 \\
\hline 4. Low back pain & 0 & - & - & - & - & - & 0 & 0 & 0 & 0 \\
\hline Other musculoskeletal disorders & 8 & 0 & 0 & 1 & 0 & 1 & 1 & 1 & 1 & 4 \\
\hline M. Congenital anomalies & 157 & 67 & 2 & 5 & $\mathbf{0}$ & 0 & 0 & $\mathbf{0}$ & 0 & 75 \\
\hline 1. Abdominal wall defect & 1 & 0 & - & - & - & - & - & - & - & 0 \\
\hline 2. Anencephaly & 11 & 5 & - & - & - & - & - & - & - & 5 \\
\hline 3. Anorectal atresia & 0 & 0 & - & - & - & - & - & - & - & 0 \\
\hline 4. Cleft lip & 0 & 0 & - & - & - & - & - & - & - & 0 \\
\hline 5. Cleft palate & 0 & 0 & - & 0 & 0 & - & - & - & - & 0 \\
\hline 6. Esophageal atresia & - & - & - & - & - & - & - & - & - & - \\
\hline 7. Renal agenesis & 1 & 0 & 0 & - & - & - & 0 & - & - & 0 \\
\hline 8. Down syndrome & 12 & 3 & 1 & 2 & - & - & - & - & - & 5 \\
\hline 9. Congenital heart anomalies & 105 & 45 & 1 & 3 & 0 & 0 & 0 & 0 & 0 & 49 \\
\hline 10. Spina bifida & 13 & 6 & 0 & 0 & 0 & 0 & 0 & 0 & - & 6 \\
\hline Other congenital anomalies & 15 & 7 & 0 & 0 & 0 & 0 & 0 & 0 & 0 & 8 \\
\hline N. Oral conditions & 1 & 0 & 0 & 0 & $\mathbf{0}$ & 0 & 0 & 0 & 0 & $\mathbf{0}$ \\
\hline 1. Dental caries & - & - & - & - & - & - & - & - & - & - \\
\hline 2. Periodontal disease & 0 & - & - & - & - & - & - & - & - & - \\
\hline 3. Edentulism & - & - & - & - & - & - & - & - & - & - \\
\hline Other oral diseases & 0 & 0 & 0 & 0 & 0 & 0 & 0 & 0 & 0 & 0 \\
\hline III. Injuries & 1,329 & 30 & 60 & 212 & 189 & 132 & 56 & 51 & 28 & 758 \\
\hline A. Unintentional injuries & 994 & 28 & 55 & 131 & 126 & 100 & 45 & 41 & 22 & 548 \\
\hline 1. Road traffic accidents & 238 & 4 & 14 & 45 & 53 & 36 & 11 & 9 & 4 & 176 \\
\hline 2. Poisonings & 90 & 1 & 3 & 8 & 8 & 17 & 6 & 3 & 1 & 47 \\
\hline 3. Falls & 112 & 4 & 4 & 8 & 8 & 9 & 9 & 14 & 9 & 67 \\
\hline 4. Fires & 183 & 5 & 4 & 15 & 15 & 7 & 3 & 2 & 1 & 52 \\
\hline 5. Drownings & 90 & 5 & 12 & 16 & 9 & 6 & 3 & 3 & 1 & 55 \\
\hline 6. Other unintentional injuries & 280 & 10 & 18 & 38 & 33 & 24 & 13 & 11 & 5 & 152 \\
\hline B. Intentional injuries & 335 & 2 & 5 & 81 & 63 & 32 & 11 & 10 & 6 & 210 \\
\hline 1. Self-inflicted injuries & 224 & - & 3 & 53 & 39 & 20 & 6 & 6 & 3 & 130 \\
\hline 2. Violence & 79 & 1 & 2 & 17 & 15 & 9 & 3 & 3 & 3 & 52 \\
\hline 3. War & 26 & 0 & 0 & 10 & 8 & 3 & 1 & 0 & 0 & 23 \\
\hline Other intentional injuries & 6 & 0 & 0 & 1 & 1 & 1 & 1 & 0 & 0 & 5 \\
\hline
\end{tabular}


Table 3B.6 Continued

\begin{tabular}{|c|c|c|c|c|c|c|c|c|}
\hline \multicolumn{9}{|c|}{ Female } \\
\hline $0-4$ & 5-14 & $15-29$ & 30-44 & 45-59 & $60-69$ & $70-79$ & $80+$ & Total \\
\hline 31 & 19 & 29 & 23 & 45 & 29 & 30 & 16 & 221 \\
\hline 2 & 1 & 2 & 4 & 8 & 5 & 6 & 3 & 32 \\
\hline 14 & 6 & 10 & 8 & 17 & 10 & 10 & 4 & 79 \\
\hline 0 & 0 & 0 & 0 & 1 & 1 & 1 & 1 & 3 \\
\hline 15 & 12 & 16 & 11 & 19 & 13 & 13 & 8 & 107 \\
\hline 4 & 2 & 5 & 5 & 14 & 13 & 13 & 7 & 63 \\
\hline 4 & 2 & 4 & 4 & 13 & 12 & 12 & 6 & 57 \\
\hline- & - & - & - & - & - & - & - & - \\
\hline 0 & 0 & 1 & 1 & 1 & 1 & 1 & 0 & 6 \\
\hline 0 & 0 & 0 & 0 & 1 & 1 & 1 & 1 & 5 \\
\hline 1 & 0 & 0 & 0 & 1 & 1 & 1 & 1 & 6 \\
\hline 0 & 0 & 0 & 0 & 0 & 0 & 1 & 0 & 2 \\
\hline 0 & - & - & 0 & 0 & 0 & 0 & 0 & 0 \\
\hline- & - & - & - & 0 & 0 & 0 & 0 & 0 \\
\hline 0 & 0 & 0 & - & 0 & 0 & - & 0 & 0 \\
\hline 1 & 0 & 0 & 0 & 0 & 0 & 1 & 1 & 4 \\
\hline 75 & 3 & 4 & 0 & 0 & 0 & 0 & 0 & 83 \\
\hline 0 & - & - & - & - & - & - & - & 0 \\
\hline 6 & - & - & - & - & 0 & - & - & 6 \\
\hline 0 & - & - & - & - & - & - & - & 0 \\
\hline 0 & - & - & - & - & - & - & - & 0 \\
\hline 0 & - & - & - & 0 & - & - & - & 0 \\
\hline- & - & - & - & - & - & - & - & - \\
\hline 0 & 0 & - & - & - & 0 & - & - & 0 \\
\hline 4 & 1 & 2 & - & - & - & - & - & 6 \\
\hline 52 & 2 & 2 & 0 & 0 & 0 & 0 & 0 & 56 \\
\hline 7 & 0 & 0 & 0 & 0 & - & 0 & 0 & 7 \\
\hline 6 & 0 & 0 & 0 & 0 & 0 & 0 & 0 & 7 \\
\hline 0 & 0 & 0 & 0 & 0 & 0 & 0 & 0 & 0 \\
\hline- & - & - & - & - & - & - & - & - \\
\hline- & 0 & 0 & 0 & 0 & - & - & - & 0 \\
\hline- & - & - & - & - & - & - & - & - \\
\hline 0 & 0 & 0 & 0 & 0 & 0 & 0 & 0 & 0 \\
\hline 49 & 56 & 164 & 95 & 86 & 48 & 47 & 25 & 571 \\
\hline 47 & 52 & 109 & 68 & 64 & 41 & 41 & 23 & 445 \\
\hline 7 & 8 & 11 & 10 & 14 & 5 & 5 & 2 & 62 \\
\hline 1 & 4 & 5 & 6 & 9 & 12 & 4 & 2 & 43 \\
\hline 4 & 4 & 4 & 3 & 6 & 5 & 13 & 6 & 45 \\
\hline 9 & 10 & 54 & 30 & 13 & 6 & 6 & 3 & 131 \\
\hline 6 & 9 & 8 & 4 & 3 & 2 & 2 & 1 & 36 \\
\hline 20 & 16 & 27 & 16 & 18 & 11 & 12 & 8 & 128 \\
\hline 2 & 5 & 55 & 27 & 21 & 7 & 6 & 2 & 125 \\
\hline 0 & 3 & 49 & 21 & 14 & 3 & 3 & 2 & 95 \\
\hline 2 & 1 & 6 & 6 & 6 & 4 & 2 & 0 & 27 \\
\hline 0 & 0 & 0 & 1 & 1 & 0 & 0 & 0 & 3 \\
\hline 0 & 0 & 0 & 0 & 0 & 0 & 0 & 0 & 1 \\
\hline
\end{tabular}

Source: Authors' compilation.

Note: $-=$ an estimate of zero; the number zero in a cell indicates a non-zero estimate of less than 500 .

a. Does not include liver cancer and cirrhosis deaths resulting from chronic hepatitis virus infection.

b. This cause category includes "Causes arising in the perinatal period" as defined in the International Classification of

Diseases, principally low birthweight, prematurity, birth asphyxia, and birth trauma, and does not include all causes of deaths occurring in the perinatal period. 
Table 3B.7 Deaths by Cause, Sex, and Age in the Sub-Saharan Africa Region, 2001 (thousands)

\begin{tabular}{|c|c|c|c|c|c|c|c|c|c|c|}
\hline \multirow[b]{2}{*}{ Cause } & \multicolumn{10}{|c|}{ Male } \\
\hline & Total & $0-4$ & $5-14$ & $15-29$ & $30-44$ & 45-59 & $60-69$ & $70-79$ & $80+$ & Total \\
\hline Population (millions) & 668 & 57 & 92 & 93 & 49 & 26 & 9 & 4 & 1 & 331 \\
\hline All causes & 10,837 & 2,367 & 281 & 529 & 852 & 632 & 412 & 366 & 170 & 5,611 \\
\hline $\begin{array}{l}\text { I. Communicable, maternal, perinatal, } \\
\text { and nutritional conditions }\end{array}$ & 7,747 & 2,252 & 179 & 289 & 603 & 331 & 135 & 73 & 26 & 3,888 \\
\hline A. Infectious and parasitic diseases & 5,702 & 1,428 & 150 & 277 & 580 & 297 & 100 & 45 & 16 & 2,893 \\
\hline 1. Tuberculosis & 317 & 12 & 7 & 49 & 68 & 51 & 19 & 9 & 3 & 218 \\
\hline $\begin{array}{l}\text { 2. Sexually transmitted diseases } \\
\text { excluding HIV/AIDS }\end{array}$ & 90 & 17 & $\mathbf{0}$ & 1 & 10 & 17 & 3 & $\mathbf{0}$ & $\mathbf{0}$ & 48 \\
\hline a. Syphilis & 87 & 17 & 0 & 1 & 9 & 17 & 3 & 0 & 0 & 47 \\
\hline b. Chlamydia & 1 & - & - & - & - & - & - & - & - & — \\
\hline c. Gonorrhea & 1 & - & - & 0 & 0 & 0 & 0 & - & - & 0 \\
\hline d. Other sexually transmitted diseases & 2 & 0 & 0 & 0 & 0 & 0 & 0 & 0 & 0 & 1 \\
\hline 3. HIV/AIDS & 2,058 & 159 & 48 & 185 & 440 & 148 & 23 & 3 & $\mathbf{0}$ & 1,007 \\
\hline 4. Diarrheal diseases & 712 & 337 & 1 & 2 & 4 & 6 & 6 & 8 & 8 & 373 \\
\hline 5. Childhood-cluster diseases & 745 & 308 & 50 & 8 & 4 & 2 & 1 & $\mathbf{0}$ & 0 & 373 \\
\hline a. Pertussis & 176 & 88 & - & - & - & - & - & - & - & 88 \\
\hline b. Poliomyelitis & 0 & 0 & - & - & - & - & - & - & - & 0 \\
\hline c. Diphtheria & 1 & 1 & - & 0 & - & - & - & - & - & 1 \\
\hline d. Measles & 447 & 177 & 44 & 3 & - & - & - & - & - & 224 \\
\hline e. Tetanus & 121 & 43 & 6 & 5 & 4 & 2 & 1 & 0 & 0 & 60 \\
\hline 6. Meningitis & 23 & 5 & 2 & 1 & 2 & 2 & 1 & $\mathbf{0}$ & 0 & 12 \\
\hline 7. Hepatitis $\mathbf{B}^{\mathbf{a}}$ & 21 & 1 & 3 & 1 & 3 & 2 & 1 & 0 & $\mathbf{0}$ & 12 \\
\hline Hepatitis $\mathbf{C}^{\mathbf{a}}$ & 8 & 1 & 1 & 0 & 1 & 1 & 0 & $\mathbf{0}$ & 0 & 5 \\
\hline 8. Malaria & 1,093 & 467 & 7 & 9 & 10 & 10 & 7 & 6 & 3 & 518 \\
\hline 9. Tropical-cluster diseases & 58 & 3 & 12 & 8 & 7 & 5 & 1 & 1 & $\mathbf{0}$ & 38 \\
\hline a. Trypanosomiasis & 48 & 2 & 11 & 6 & 6 & 5 & 1 & 0 & 0 & 31 \\
\hline b. Chagas' disease & - & - & - & - & - & - & - & - & - & - \\
\hline c. Schistosomiasis & 2 & 0 & 0 & 0 & 0 & 0 & 1 & 0 & 0 & 1 \\
\hline d. Leishmaniasis & 8 & 1 & 2 & 2 & 1 & 0 & 0 & 0 & - & 6 \\
\hline e. Lymphatic filariasis & 0 & 0 & 0 & 0 & 0 & 0 & 0 & 0 & - & 0 \\
\hline f. Onchocerciasis & - & - & - & - & - & - & - & - & - & - \\
\hline 10. Leprosy & 1 & - & - & - & 0 & $\mathbf{0}$ & 0 & 0 & - & 0 \\
\hline 11. Dengue & 0 & $\mathbf{0}$ & $\mathbf{0}$ & $\mathbf{0}$ & 0 & 0 & 0 & 0 & $\mathbf{0}$ & 0 \\
\hline 12. Japanese encephalitis & - & - & - & - & - & - & - & - & - & - \\
\hline 13. Trachoma & - & - & - & - & - & - & - & - & - & - \\
\hline 14. Intestinal nematode infections & 4 & 0 & $\mathbf{0}$ & 0 & 0 & 0 & 0 & 0 & 0 & 2 \\
\hline a. Ascariasis & 1 & 0 & 0 & 0 & 0 & - & - & - & - & 0 \\
\hline b. Trichuriasis & 1 & - & 0 & 0 & 0 & 0 & 0 & 0 & 0 & 0 \\
\hline c. Hookworm disease & 2 & 0 & 0 & 0 & 0 & 0 & 0 & 0 & 0 & 1 \\
\hline Other intestinal infections & 0 & - & 0 & 0 & 0 & 0 & 0 & 0 & 0 & 0 \\
\hline Other infectious diseases & 572 & 117 & 18 & 12 & 31 & 52 & 38 & 17 & 1 & 287 \\
\hline B. Respiratory infections & 1,094 & 439 & 26 & 12 & 22 & 31 & 29 & 23 & 9 & 590 \\
\hline 1. Lower respiratory infections & 1,080 & 435 & 24 & 11 & 21 & 31 & 28 & 23 & 9 & 583 \\
\hline 2. Upper respiratory infections & 13 & 3 & 1 & 0 & 0 & 0 & 1 & 0 & 0 & 6 \\
\hline 3. Otitis media & 1 & 0 & 1 & 0 & 0 & - & 0 & - & - & 1 \\
\hline C. Maternal conditions & 237 & - & - & - & - & - & - & - & - & 一 \\
\hline 1. Maternal hemorrhage & 60 & - & - & - & - & - & - & - & - & - \\
\hline 2. Maternal sepsis & 44 & - & - & - & - & - & - & - & - & - \\
\hline 3. Hypertensive disorders of pregnancy & 32 & - & - & - & - & - & - & - & - & - \\
\hline 4. Obstructed labor & 22 & - & - & - & - & - & - & - & - & - \\
\hline 5. Abortion & 28 & - & - & - & - & - & - & - & - & - \\
\hline Other maternal conditions & 52 & - & - & - & - & - & - & - & - & - \\
\hline D. Perinatal conditions ${ }^{b}$ & 573 & 332 & - & - & - & - & - & - & - & 332 \\
\hline 1. Low birthweight & 243 & 141 & - & - & - & - & - & - & - & 141 \\
\hline 2. Birth asphyxia and birth trauma & 240 & 139 & - & - & - & - & - & - & - & 139 \\
\hline Other perinatal conditions & 90 & 52 & - & - & - & - & - & - & - & 52 \\
\hline E. Nutritional deficiencies & 140 & 53 & 3 & 0 & 1 & 4 & 5 & 4 & 2 & 73 \\
\hline 1. Protein-energy malnutrition & 99 & 40 & 2 & 0 & 0 & 2 & 4 & 4 & 2 & 55 \\
\hline 2. Iodine deficiency & 3 & 1 & 0 & 0 & 0 & 0 & 0 & 0 & 0 & 1 \\
\hline 3. Vitamin A deficiency & 18 & 7 & 1 & 0 & 0 & 0 & 0 & 0 & 0 & 8 \\
\hline 4. Iron-deficiency anemia & 18 & 5 & 1 & 0 & 0 & 1 & 1 & 0 & 0 & 8 \\
\hline Other nutritional disorders & 2 & 1 & 0 & 0 & 0 & 0 & 0 & 0 & 0 & 1 \\
\hline
\end{tabular}


Table 3B.7 Continued

\begin{tabular}{|c|c|c|c|c|c|c|c|c|}
\hline \multicolumn{9}{|c|}{ Female } \\
\hline $0-4$ & 5-14 & $15-29$ & $30-44$ & $45-59$ & $60-69$ & $70-79$ & $80+$ & Total \\
\hline 56 & 91 & 93 & 51 & 28 & 11 & 5 & 1 & 336 \\
\hline $\begin{array}{l}2,137 \\
2,046\end{array}$ & $\begin{array}{l}289 \\
221\end{array}$ & $\begin{array}{l}698 \\
616\end{array}$ & $\begin{array}{l}678 \\
573\end{array}$ & $\begin{array}{l}466 \\
231\end{array}$ & $\begin{array}{r}350 \\
79\end{array}$ & $\begin{array}{r}384 \\
62\end{array}$ & $\begin{array}{r}225 \\
31\end{array}$ & $\begin{array}{l}5,227 \\
3,859\end{array}$ \\
\hline 1,436 & 176 & 449 & 447 & 188 & 57 & 37 & 18 & 2,809 \\
\hline 11 & 6 & 30 & 26 & 13 & 9 & 4 & 1 & 99 \\
\hline 12 & 0 & 12 & 8 & 6 & 2 & 1 & 0 & 42 \\
\hline 11 & 0 & 12 & 8 & 6 & 2 & 1 & 0 & 40 \\
\hline- & - & 0 & 0 & 0 & 0 & - & - & 1 \\
\hline- & - & 0 & 0 & 0 & 0 & - & - & 0 \\
\hline 0 & 0 & 0 & 0 & 0 & 0 & 0 & 0 & 1 \\
\hline 154 & 47 & 349 & 374 & 108 & 17 & 4 & 0 & 1,051 \\
\hline 306 & 1 & 1 & 2 & 4 & 6 & 8 & 11 & 339 \\
\hline 305 & 52 & 8 & 4 & 2 & 1 & 0 & 0 & 372 \\
\hline 88 & - & - & - & - & - & - & - & 88 \\
\hline 0 & - & - & - & - & - & - & - & 0 \\
\hline 1 & 0 & - & - & - & - & - & - & 1 \\
\hline 175 & 45 & 3 & - & - & - & - & - & 223 \\
\hline 42 & 6 & 5 & 4 & 2 & 1 & 0 & 0 & 60 \\
\hline 4 & 4 & 2 & 0 & 1 & 0 & 0 & 0 & 11 \\
\hline 4 & 1 & 1 & 1 & 1 & 0 & 0 & 0 & 9 \\
\hline 2 & 0 & 1 & 0 & 1 & 0 & 0 & 0 & 3 \\
\hline 518 & 7 & 11 & 11 & 12 & 7 & 6 & 4 & 575 \\
\hline 1 & 7 & 5 & 3 & 3 & 0 & 0 & 0 & 20 \\
\hline 1 & 6 & 4 & 3 & 2 & 0 & 0 & - & 17 \\
\hline- & - & - & - & - & - & - & - & - \\
\hline 0 & 0 & 0 & 0 & 0 & 0 & 0 & 0 & 1 \\
\hline 0 & 1 & 1 & 0 & 0 & 0 & 0 & - & 2 \\
\hline 0 & 0 & 0 & 0 & 0 & 0 & 0 & 0 & 0 \\
\hline- & - & - & - & - & - & - & - & - \\
\hline 0 & - & - & - & 0 & 0 & 0 & 0 & 0 \\
\hline 0 & 0 & 0 & 0 & 0 & 0 & 0 & 0 & 0 \\
\hline- & - & - & - & - & - & - & - & - \\
\hline- & - & - & - & - & - & - & - & - \\
\hline 0 & 1 & 0 & 0 & 0 & 0 & 0 & 0 & 2 \\
\hline 0 & 0 & - & - & - & - & - & - & 0 \\
\hline- & 0 & 0 & 0 & 0 & 0 & 0 & 0 & 0 \\
\hline- & 0 & 0 & 0 & 0 & 0 & 0 & 0 & 1 \\
\hline- & 0 & 0 & 0 & 0 & 0 & 0 & 0 & 0 \\
\hline 121 & 51 & 29 & 18 & 38 & 14 & 12 & 2 & 285 \\
\hline 318 & 40 & 39 & 29 & 26 & 19 & 21 & 12 & 504 \\
\hline 317 & 36 & 39 & 28 & 26 & 19 & 21 & 12 & 497 \\
\hline 2 & 4 & 0 & 0 & 0 & 0 & 0 & 0 & 6 \\
\hline 0 & - & 0 & 0 & - & - & - & - & 0 \\
\hline- & 0 & 126 & 97 & 14 & - & - & - & 237 \\
\hline- & - & 26 & 28 & 5 & - & - & - & 60 \\
\hline- & - & 20 & 20 & 4 & - & - & - & 44 \\
\hline- & 0 & 19 & 11 & 1 & - & - & - & 32 \\
\hline- & - & 14 & 7 & 0 & - & - & - & 22 \\
\hline- & - & 22 & 7 & - & - & - & - & 28 \\
\hline- & - & 24 & 24 & 4 & - & - & - & 52 \\
\hline 241 & - & - & - & - & - & - & - & 241 \\
\hline 102 & - & - & - & - & - & - & - & 102 \\
\hline 101 & - & - & - & - & - & - & - & 101 \\
\hline 38 & - & - & - & - & - & - & - & 38 \\
\hline 50 & 5 & 1 & 1 & 2 & 4 & 3 & 1 & 67 \\
\hline 34 & 1 & 1 & 1 & 1 & 4 & 3 & 1 & 45 \\
\hline 1 & 0 & 0 & 0 & 0 & 0 & 0 & 0 & 1 \\
\hline 8 & 1 & 0 & 0 & 0 & 0 & 0 & 0 & 10 \\
\hline 7 & 2 & 0 & 0 & 1 & 0 & 0 & 0 & 11 \\
\hline 1 & 0 & 0 & 0 & 0 & 0 & 0 & 0 & 1 \\
\hline & & & & & & nues & the follc & page. \\
\hline
\end{tabular}


Table 3B.7 Continued

\begin{tabular}{|c|c|c|c|c|c|c|c|c|c|c|}
\hline \multirow[b]{2}{*}{ Cause } & \multicolumn{10}{|c|}{ Male } \\
\hline & Total & $0-4$ & $5-14$ & $15-29$ & $30-44$ & $45-59$ & $60-69$ & 70-79 & $80+$ & Total \\
\hline II. Noncommunicable diseases & 2,283 & 65 & 18 & 45 & 102 & 225 & 249 & 279 & 140 & 1,122 \\
\hline A. Malignant neoplasms & 409 & 2 & 3 & 6 & 16 & 51 & 57 & 57 & 23 & 214 \\
\hline 1. Mouth and oropharynx cancers & 19 & 0 & 0 & 0 & 1 & 4 & 4 & 3 & 1 & 13 \\
\hline 2. Esophageal cancer & 24 & 0 & 0 & 0 & 1 & 5 & 5 & 3 & 1 & 15 \\
\hline 3. Stomach cancer & 33 & 0 & 0 & 0 & 1 & 5 & 5 & 4 & 2 & 18 \\
\hline 4. Colon and rectal cancers & 20 & 0 & 0 & 0 & 1 & 3 & 3 & 3 & 1 & 11 \\
\hline 5. Liver cancer & 46 & 0 & 0 & 1 & 4 & 11 & 8 & 5 & 1 & 31 \\
\hline 6. Pancreas cancer & 8 & 0 & 0 & 0 & 0 & 1 & 1 & 1 & 0 & 4 \\
\hline 7. Trachea, bronchus, and lung cancers & 15 & 0 & 0 & 0 & 1 & 4 & 4 & 2 & 1 & 11 \\
\hline 8. Melanoma and other skin cancers & 8 & 0 & 0 & 0 & 0 & 1 & 1 & 1 & 0 & 4 \\
\hline 9. Breast cancer & 34 & - & - & - & 0 & 0 & 0 & 0 & 0 & 0 \\
\hline 10. Cervix uteri cancer & 38 & - & - & - & - & - & - & - & - & - \\
\hline 11. Corpus uteri cancer & 3 & - & - & - & - & - & - & - & - & - \\
\hline 12. Ovarian cancer & 9 & - & - & - & - & - & - & - & - & - \\
\hline 13. Prostate cancer & 40 & 0 & 0 & 0 & 0 & 3 & 11 & 18 & 8 & 40 \\
\hline 14. Bladder cancer & 10 & 0 & 0 & 0 & 0 & 1 & 2 & 2 & 1 & 7 \\
\hline 15. Lymphomas and multiple myeloma & 34 & 1 & 2 & 2 & 3 & 4 & 4 & 4 & 2 & 21 \\
\hline 16. Leukemia & 14 & 0 & 0 & 1 & 1 & 1 & 1 & 1 & 1 & 7 \\
\hline Other malignant neoplasms & 55 & 1 & 1 & 1 & 2 & 8 & 9 & 8 & 4 & 32 \\
\hline B. Other neoplasms & 10 & 1 & $\mathbf{0}$ & 1 & 1 & 1 & 1 & 1 & 0 & 5 \\
\hline C. Diabetes mellitus & 82 & 0 & $\mathbf{0}$ & 1 & 3 & 7 & 8 & 8 & 3 & 30 \\
\hline D. Endocrine disorders & 28 & 3 & 1 & 1 & 2 & 2 & 1 & 1 & 1 & 12 \\
\hline E. Neuropsychiatric conditions & 93 & 8 & 5 & 9 & 10 & 9 & 6 & 6 & 3 & 56 \\
\hline 1. Unipolar depressive disorders & 0 & - & - & - & 0 & 0 & - & - & - & 0 \\
\hline 2. Bipolar affective disorder & - & - & - & - & - & - & - & - & - & - \\
\hline 3. Schizophrenia & 0 & - & 0 & - & 0 & - & 0 & 0 & - & 0 \\
\hline 4. Epilepsy & 38 & 2 & 2 & 6 & 5 & 4 & 2 & 1 & 1 & 23 \\
\hline 5. Alcohol use disorders & 7 & - & - & 0 & 2 & 2 & 1 & 1 & 0 & 5 \\
\hline 6. Alzheimer's and other dementias & 7 & 0 & 0 & 0 & 0 & 0 & 0 & 1 & 1 & 4 \\
\hline 7. Parkinson's disease & 5 & 0 & 0 & - & 0 & 0 & 1 & 1 & 1 & 3 \\
\hline 8. Multiple sclerosis & 0 & - & 0 & 0 & 0 & 0 & 0 & 0 & 0 & 0 \\
\hline 9. Drug use disorders & 4 & - & - & 1 & 1 & 1 & 0 & 0 & 0 & 3 \\
\hline 10. Post-traumatic stress disorder & - & - & - & - & - & - & - & - & - & - \\
\hline 11. Obsessive-compulsive disorder & - & - & - & - & - & - & - & - & - & 一 \\
\hline 12. Panic disorder & - & - & - & - & - & - & - & - & - & - \\
\hline 13. Insomnia (primary) & - & - & - & - & - & - & - & - & - & - \\
\hline 14. Migraine & - & - & - & - & - & - & - & - & - & - \\
\hline 15. Mental retardation, lead-caused & 0 & 0 & 0 & 0 & 0 & 0 & 0 & - & - & 0 \\
\hline Other neuropsychiatric disorders & 32 & 5 & 3 & 2 & 2 & 2 & 2 & 1 & 0 & 18 \\
\hline F. Sense organ diseases & $\mathbf{0}$ & $\mathbf{0}$ & 0 & 0 & $\mathbf{0}$ & 0 & $\mathbf{0}$ & $\mathbf{0}$ & 0 & 0 \\
\hline 1. Glaucoma & 0 & - & - & - & 0 & - & - & - & - & 0 \\
\hline 2. Cataracts & - & - & - & - & - & - & - & - & - & - \\
\hline 3. Vision disorders, age-related & - & - & - & - & - & - & - & - & - & - \\
\hline 4. Hearing loss, adult onset & - & - & - & - & - & - & - & - & - & - \\
\hline Other sense organ disorders & 0 & 0 & 0 & 0 & 0 & 0 & 0 & 0 & 0 & 0 \\
\hline G. Cardiovascular diseases & 1,048 & 4 & 3 & 12 & 35 & 91 & 112 & 137 & 76 & 469 \\
\hline 1. Rheumatic heart disease & 19 & 0 & 1 & 2 & 1 & 1 & 0 & 0 & 0 & 6 \\
\hline 2. Hypertensive heart disease & 66 & 0 & 0 & 0 & 2 & 5 & 6 & 7 & 4 & 24 \\
\hline 3. Ischemic heart disease & 343 & 0 & 0 & 1 & 7 & 39 & 52 & 58 & 22 & 179 \\
\hline 4. Cerebrovascular disease & 355 & 0 & 1 & 4 & 11 & 28 & 36 & 44 & 22 & 145 \\
\hline 5. Inflammatory heart diseases & 43 & 2 & 1 & 2 & 3 & 4 & 3 & 4 & 2 & 22 \\
\hline Other cardiovascular diseases & 223 & 1 & 1 & 4 & 11 & 13 & 15 & 24 & 25 & 93 \\
\hline H. Respiratory diseases & 253 & 9 & 2 & 5 & 12 & 28 & 35 & 39 & 19 & 149 \\
\hline 1. Chronic obstructive pulmonary disease & 116 & 0 & 0 & 1 & 3 & 13 & 20 & 25 & 12 & 74 \\
\hline 2. Asthma & 26 & 1 & 0 & 1 & 2 & 3 & 3 & 3 & 1 & 13 \\
\hline Other respiratory diseases & 112 & 8 & 1 & 4 & 7 & 12 & 12 & 12 & 6 & 62 \\
\hline
\end{tabular}


Table 3B.7 Continued

\begin{tabular}{|c|c|c|c|c|c|c|c|c|}
\hline \multicolumn{9}{|c|}{ Female } \\
\hline $0-4$ & 5-14 & $15-29$ & $30-44$ & $45-59$ & 60-69 & 70-79 & $80+$ & Total \\
\hline 54 & 18 & 43 & 72 & 211 & 259 & 314 & 190 & 1,161 \\
\hline 2 & 3 & 5 & 18 & 53 & 48 & 47 & 19 & 194 \\
\hline 0 & 0 & 0 & 0 & 1 & 2 & 2 & 1 & 6 \\
\hline 0 & 0 & 0 & 1 & 2 & 2 & 2 & 1 & 9 \\
\hline 0 & 0 & 0 & 1 & 4 & 4 & 4 & 2 & 15 \\
\hline 0 & 0 & 0 & 1 & 2 & 2 & 2 & 1 & 9 \\
\hline 0 & 0 & 1 & 2 & 4 & 3 & 4 & 1 & 15 \\
\hline 0 & 0 & 0 & 0 & 1 & 1 & 1 & 0 & 4 \\
\hline 0 & 0 & 0 & 0 & 1 & 1 & 1 & 0 & 4 \\
\hline 0 & 0 & 0 & 0 & 1 & 1 & 2 & 1 & 5 \\
\hline 0 & 0 & 0 & 4 & 11 & 9 & 7 & 3 & 34 \\
\hline 0 & 0 & 1 & 2 & 12 & 10 & 9 & 2 & 38 \\
\hline 0 & 0 & 0 & 0 & 1 & 1 & 1 & 0 & 3 \\
\hline 0 & 0 & 0 & 1 & 3 & 2 & 2 & 1 & 9 \\
\hline- & - & - & - & - & - & - & - & - \\
\hline 0 & 0 & 0 & 0 & 1 & 1 & 1 & 0 & 3 \\
\hline 1 & 2 & 1 & 1 & 2 & 2 & 3 & 1 & 13 \\
\hline 0 & 0 & 1 & 1 & 2 & 1 & 1 & 1 & 6 \\
\hline 1 & 1 & 0 & 2 & 5 & 5 & 6 & 3 & 23 \\
\hline 0 & 1 & 0 & 0 & 1 & 1 & 1 & 0 & 5 \\
\hline 0 & 0 & 1 & 2 & 12 & 16 & 15 & 5 & 52 \\
\hline 4 & 0 & 1 & 1 & 3 & 3 & 2 & 1 & 16 \\
\hline 7 & 4 & 6 & 4 & 5 & 4 & 5 & 3 & 37 \\
\hline- & - & 0 & - & - & - & - & - & 0 \\
\hline- & - & - & - & - & - & - & - & - \\
\hline- & - & - & - & 0 & 0 & 0 & - & 0 \\
\hline 2 & 2 & 4 & 2 & 2 & 1 & 1 & 1 & 15 \\
\hline- & - & 0 & 1 & 1 & 0 & 0 & - & 2 \\
\hline 0 & 0 & 0 & 0 & 0 & 1 & 1 & 1 & 3 \\
\hline 0 & - & 0 & 0 & 0 & 0 & 1 & 1 & 2 \\
\hline- & - & - & 0 & 0 & 0 & 0 & 0 & 0 \\
\hline- & - & 0 & 0 & 0 & 0 & 0 & 0 & 1 \\
\hline- & - & - & - & - & - & - & - & - \\
\hline- & - & - & - & - & - & - & - & - \\
\hline- & - & - & - & - & - & - & - & - \\
\hline- & - & - & - & - & - & - & - & - \\
\hline- & - & - & - & - & - & - & - & - \\
\hline 0 & 0 & 0 & 0 & 0 & - & - & - & 0 \\
\hline 5 & 2 & 2 & 1 & 2 & 1 & 1 & 0 & 14 \\
\hline 0 & 0 & 0 & 0 & 0 & 0 & 0 & 0 & 0 \\
\hline- & - & - & - & - & - & - & - & - \\
\hline- & - & - & - & - & - & - & - & - \\
\hline- & - & - & - & - & - & - & - & - \\
\hline- & - & - & - & - & - & - & - & - \\
\hline 0 & 0 & 0 & 0 & 0 & 0 & 0 & 0 & 0 \\
\hline 5 & 4 & 14 & 26 & 91 & 133 & 180 & 125 & 579 \\
\hline 0 & 1 & 3 & 2 & 3 & 1 & 1 & 0 & 13 \\
\hline 0 & 0 & 0 & 2 & 7 & 10 & 14 & 9 & 42 \\
\hline 0 & 0 & 1 & 3 & 26 & 46 & 55 & 31 & 163 \\
\hline 0 & 1 & 3 & 8 & 37 & 53 & 67 & 41 & 209 \\
\hline 2 & 1 & 2 & 2 & 4 & 4 & 4 & 3 & 21 \\
\hline 2 & 1 & 6 & 8 & 15 & 19 & 38 & 41 & 130 \\
\hline 7 & 1 & 5 & 7 & 16 & 22 & 29 & 17 & 104 \\
\hline 0 & 0 & 0 & 1 & 6 & 10 & 15 & 9 & 42 \\
\hline 0 & 0 & 1 & 1 & 2 & 3 & 3 & 2 & 12 \\
\hline 6 & 1 & 4 & 5 & 7 & 9 & 11 & 7 & 50 \\
\hline
\end{tabular}


Table 3B.7 Continued

\begin{tabular}{|c|c|c|c|c|c|c|c|c|c|c|}
\hline \multirow[b]{2}{*}{ Cause } & \multicolumn{10}{|c|}{ Male } \\
\hline & Total & $0-4$ & $5-14$ & $15-29$ & 30-44 & $45-59$ & $60-69$ & $70-79$ & $80+$ & Total \\
\hline I. Digestive diseases & 164 & 3 & 2 & 7 & 18 & 26 & 18 & 16 & 5 & 95 \\
\hline 1. Peptic ulcer disease & 15 & 0 & 0 & 1 & 2 & 2 & 2 & 1 & 1 & 8 \\
\hline 2. Cirrhosis of the liver & 59 & 0 & 0 & 1 & 6 & 13 & 9 & 7 & 2 & 38 \\
\hline 3. Appendicitis & 2 & 0 & 0 & 0 & 0 & 0 & 0 & 0 & 0 & 1 \\
\hline Other digestive diseases & 88 & 3 & 2 & 5 & 11 & 11 & 8 & 7 & 3 & 48 \\
\hline J. Genitourinary diseases & 107 & 3 & 1 & 3 & 4 & 7 & 10 & 13 & 8 & 48 \\
\hline 1. Nephritis and nephrosis & 101 & 2 & 1 & 3 & 4 & 7 & 10 & 11 & 7 & 45 \\
\hline 2. Benign prostatic hypertrophy & 2 & - & - & - & - & 0 & 0 & 1 & 1 & 2 \\
\hline Other genitourinary system diseases & 4 & 0 & 0 & 0 & 0 & 0 & 0 & 1 & 0 & 2 \\
\hline K. Skin diseases & 20 & 0 & 0 & 0 & 1 & 1 & 1 & 1 & 1 & 6 \\
\hline L. Musculoskeletal diseases & 7 & 0 & $\mathbf{0}$ & $\mathbf{0}$ & 0 & $\mathbf{0}$ & $\mathbf{0}$ & 1 & 0 & 3 \\
\hline 1. Rheumatoid arthritis & 1 & - & - & 0 & 0 & 0 & 0 & 0 & 0 & 0 \\
\hline 2. Osteoarthritis & 0 & - & - & - & - & 0 & - & 0 & 0 & 0 \\
\hline 3. Gout & 0 & - & - & - & - & - & - & 0 & - & 0 \\
\hline 4. Low back pain & 0 & - & - & - & 0 & 0 & 0 & 0 & - & 0 \\
\hline Other musculoskeletal disorders & 5 & 0 & 0 & 0 & 0 & 0 & 0 & 1 & 0 & 2 \\
\hline M. Congenital anomalies & 63 & 32 & 2 & 1 & 0 & 0 & 0 & 0 & 0 & 35 \\
\hline 1. Abdominal wall defect & 1 & 1 & - & - & - & - & - & - & - & 1 \\
\hline 2. Anencephaly & 2 & 1 & 0 & - & - & - & - & - & - & 1 \\
\hline 3. Anorectal atresia & 0 & 0 & 0 & - & - & - & - & - & - & 0 \\
\hline 4. Cleft lip & 0 & 0 & - & - & - & - & - & - & - & 0 \\
\hline 5. Cleft palate & 0 & 0 & - & - & - & - & - & - & - & 0 \\
\hline 6. Esophageal atresia & 0 & 0 & 0 & - & - & - & - & - & - & 0 \\
\hline 7. Renal agenesis & 0 & 0 & - & - & - & - & 0 & - & - & 0 \\
\hline 8. Down syndrome & 5 & 3 & 0 & 0 & 0 & 0 & - & - & - & 3 \\
\hline 9. Congenital heart anomalies & 20 & 10 & 1 & 0 & 0 & 0 & 0 & 0 & 0 & 11 \\
\hline 10.Spina bifida & 4 & 2 & 0 & 0 & - & - & - & - & - & 2 \\
\hline Other congenital anomalies & 31 & 15 & 1 & 0 & 0 & 0 & 0 & 0 & 0 & 17 \\
\hline N. Oral conditions & 0 & 0 & 0 & 0 & 0 & 0 & 0 & 0 & - & 0 \\
\hline 1. Dental caries & - & - & - & - & - & - & - & - & - & - \\
\hline 2. Periodontal disease & - & - & - & - & - & - & - & - & - & - \\
\hline 3. Edentulism & - & - & - & - & - & - & - & - & - & - \\
\hline Other oral diseases & 0 & 0 & 0 & 0 & 0 & 0 & 0 & 0 & - & 0 \\
\hline III. Injuries & 807 & 51 & 84 & 196 & 147 & 76 & 29 & 14 & 4 & 600 \\
\hline A. Unintentional injuries & 494 & 48 & 77 & 80 & 65 & 41 & 16 & 9 & 3 & 339 \\
\hline 1. Road traffic accidents & 200 & 12 & 41 & 27 & 28 & 18 & 7 & 4 & 1 & 137 \\
\hline 2. Poisonings & 37 & 5 & 4 & 5 & 6 & 3 & 1 & - & - & 23 \\
\hline 3. Falls & 20 & 1 & 3 & 2 & 2 & 2 & 1 & 1 & 0 & 14 \\
\hline 4. Fires & 44 & 8 & 11 & 1 & 1 & 1 & 1 & 1 & 0 & 24 \\
\hline 5. Drownings & 66 & 7 & 11 & 11 & 13 & 8 & 1 & 0 & 0 & 50 \\
\hline 6. Other unintentional injuries & 127 & 15 & 8 & 35 & 16 & 9 & 5 & 3 & 1 & 92 \\
\hline B. Intentional injuries & 313 & 3 & 7 & 116 & 82 & 35 & 12 & 5 & 2 & 261 \\
\hline 1. Self-inflicted injuries & 36 & - & 2 & 9 & 7 & 6 & 2 & 1 & 0 & 28 \\
\hline 2. Violence & 141 & 3 & 4 & 53 & 32 & 13 & 4 & 2 & 0 & 111 \\
\hline 3. War & 136 & 0 & 1 & 53 & 43 & 16 & 7 & 2 & 1 & 123 \\
\hline Other intentional injuries & 0 & 0 & 0 & 0 & 0 & 0 & 0 & 0 & 0 & 0 \\
\hline
\end{tabular}


Table 3B.7 Continued

\begin{tabular}{|c|c|c|c|c|c|c|c|c|}
\hline \multicolumn{9}{|c|}{ Female } \\
\hline $0-4$ & 5-14 & $15-29$ & $30-44$ & 45-59 & $60-69$ & $70-79$ & $80+$ & Total \\
\hline 1 & 2 & 6 & 8 & 15 & 15 & 14 & 7 & 69 \\
\hline 0 & 0 & 0 & 1 & 1 & 1 & 2 & 1 & 7 \\
\hline 0 & 0 & 1 & 2 & 6 & 6 & 4 & 1 & 22 \\
\hline- & 0 & 0 & 0 & 0 & 0 & 0 & 0 & 1 \\
\hline 1 & 1 & 5 & 5 & 7 & 8 & 8 & 5 & 40 \\
\hline 2 & 1 & 2 & 3 & 12 & 14 & 16 & 9 & 59 \\
\hline 1 & 1 & 2 & 3 & 11 & 14 & 15 & 8 & 56 \\
\hline- & - & - & - & - & - & - & - & - \\
\hline 0 & 0 & 0 & 0 & 0 & 0 & 1 & 1 & 2 \\
\hline 0 & 0 & 0 & 1 & 3 & 3 & 3 & 3 & 13 \\
\hline 0 & 0 & 1 & 1 & 1 & 1 & 1 & 0 & 4 \\
\hline- & 0 & - & 0 & 0 & 0 & 0 & 0 & 1 \\
\hline- & - & - & - & 0 & 0 & 0 & 0 & 0 \\
\hline- & - & - & - & - & 0 & - & - & 0 \\
\hline 0 & 0 & 0 & - & 0 & 0 & - & 0 & 0 \\
\hline 0 & 0 & 1 & 1 & 1 & 0 & 0 & 0 & 3 \\
\hline 26 & 1 & 0 & 0 & 0 & 0 & 0 & 0 & 28 \\
\hline 1 & - & 0 & - & - & - & - & - & 1 \\
\hline 1 & - & 0 & - & - & 0 & - & - & 1 \\
\hline 0 & - & - & - & - & - & - & - & 0 \\
\hline 0 & - & - & - & - & - & - & - & 0 \\
\hline 0 & - & - & - & - & - & - & - & 0 \\
\hline- & - & - & - & - & - & - & - & - \\
\hline 0 & - & 0 & - & - & 0 & - & - & 0 \\
\hline 1 & 0 & 0 & - & 0 & - & - & - & 2 \\
\hline 8 & 0 & 0 & 0 & 0 & 0 & 0 & 0 & 9 \\
\hline 2 & 0 & 0 & 0 & - & - & - & - & 2 \\
\hline 13 & 1 & 0 & 0 & 0 & 0 & 0 & 0 & 14 \\
\hline 0 & - & - & - & - & 0 & 0 & 0 & 0 \\
\hline- & - & - & - & - & - & - & - & - \\
\hline- & - & - & - & - & - & - & - & - \\
\hline- & - & - & - & - & - & - & - & - \\
\hline 0 & - & - & - & - & 0 & 0 & 0 & 0 \\
\hline 37 & 51 & 39 & 32 & 23 & 11 & 8 & 4 & 207 \\
\hline 34 & 45 & 25 & 19 & 15 & 8 & 6 & 3 & 155 \\
\hline 7 & 25 & 11 & 8 & 7 & 4 & 2 & 1 & 63 \\
\hline 2 & 2 & 3 & 3 & 2 & 2 & 1 & 0 & 15 \\
\hline 1 & 2 & 0 & 0 & 1 & 1 & 1 & 1 & 6 \\
\hline 10 & 4 & 2 & 2 & 1 & 1 & 1 & 0 & 20 \\
\hline 4 & 3 & 4 & 1 & 2 & 1 & 0 & 0 & 15 \\
\hline 11 & 8 & 5 & 5 & 3 & 1 & 1 & 1 & 36 \\
\hline 3 & 6 & 15 & 13 & 9 & 3 & 2 & 1 & 52 \\
\hline 0 & 0 & 3 & 2 & 2 & 1 & 0 & 0 & 8 \\
\hline 3 & 5 & 9 & 7 & 4 & 1 & 1 & 0 & 30 \\
\hline 0 & 1 & 2 & 4 & 4 & 2 & 1 & 1 & 13 \\
\hline 0 & 0 & 0 & 0 & 0 & 0 & - & 0 & 0 \\
\hline
\end{tabular}

Source: Authors' compilation.

Note: $-=$ an estimate of zero; the number zero in a cell indicates a non-zero estimate of less than 500.

a. Does not include liver cancer and cirrhosis deaths resulting from chronic hepatitis virus infection.

b. This cause category includes "Causes arising in the perinatal period" as defined in the International Classification

of Diseases, principally low birthweight, prematurity, birth asphyxia, and birth trauma, and does not include all causes

of deaths occurring in the perinatal period. 
Table 3B.8 Deaths by Cause, Sex, and Age in High-Income Countries, 2001 (thousands)

\begin{tabular}{|c|c|c|c|c|c|c|c|c|c|c|}
\hline \multirow[b]{2}{*}{ Cause } & \multirow[b]{2}{*}{ Total } & \multicolumn{8}{|c|}{ Male } & \multirow[b]{2}{*}{ Total } \\
\hline & & $0-4$ & 5-14 & $15-29$ & 30-44 & $45-59$ & $60-69$ & 70-79 & $80+$ & \\
\hline Population (millions) & 929 & 28 & 60 & 96 & 107 & 88 & 40 & 27 & 10 & 457 \\
\hline All causes & 7,891 & 41 & 11 & 90 & 192 & 520 & 698 & 1,170 & 1,281 & 4,002 \\
\hline $\begin{array}{l}\text { I. Communicable, maternal, perinatal, } \\
\text { and nutritional conditions }\end{array}$ & 552 & 21 & 1 & 3 & 16 & 24 & 26 & 60 & 117 & 268 \\
\hline A. Infectious and parasitic diseases & 152 & 2 & $\mathbf{0}$ & 2 & 13 & 16 & 12 & 18 & 18 & 81 \\
\hline 1. Tuberculosis & 16 & 0 & $\mathbf{0}$ & 0 & 1 & 2 & 2 & 3 & 3 & 10 \\
\hline $\begin{array}{l}\text { 2. Sexually transmitted diseases } \\
\text { excluding HIV/AIDS }\end{array}$ & 1 & 0 & 0 & 0 & 0 & $\mathbf{0}$ & 0 & $\mathbf{0}$ & 0 & 0 \\
\hline a. Syphilis & 0 & 0 & 0 & 0 & 0 & 0 & 0 & 0 & 0 & 0 \\
\hline b. Chlamydia & 0 & - & - & - & - & - & - & - & - & - \\
\hline c. Gonorrhea & 0 & - & 0 & 0 & 0 & - & 0 & 0 & 0 & 0 \\
\hline d. Other sexually transmitted diseases & 0 & 0 & - & - & 0 & 0 & 0 & 0 & 0 & 0 \\
\hline 3. HIV/AIDS & 22 & 0 & $\mathbf{0}$ & 1 & 9 & 5 & 1 & $\mathbf{0}$ & $\mathbf{0}$ & 17 \\
\hline 4. Diarrheal diseases & 6 & 0 & 0 & 0 & 0 & 0 & 0 & 1 & 1 & 2 \\
\hline 5. Childhood-cluster diseases & 2 & 0 & $\mathbf{0}$ & $\mathbf{0}$ & 0 & $\mathbf{0}$ & 0 & $\mathbf{0}$ & $\mathbf{0}$ & $\mathbf{0}$ \\
\hline a. Pertussis & 0 & 0 & 0 & - & - & - & 0 & - & - & 0 \\
\hline b. Poliomyelitis & 1 & 0 & 0 & 0 & 0 & 0 & 0 & 0 & 0 & 0 \\
\hline c. Diphtheria & 0 & 0 & - & - & 0 & - & - & 0 & 0 & 0 \\
\hline d. Measles & 1 & 0 & 0 & 0 & 0 & 0 & - & 0 & - & 0 \\
\hline e. Tetanus & 0 & 0 & 0 & 0 & 0 & 0 & 0 & 0 & 0 & 0 \\
\hline 6. Meningitis & 4 & 0 & $\mathbf{0}$ & $\mathbf{0}$ & 0 & 0 & 0 & $\mathbf{0}$ & $\mathbf{0}$ & 2 \\
\hline 7. Hepatitis $\mathbf{B}^{\mathrm{a}}$ & 5 & 0 & 0 & 0 & 0 & 1 & 1 & 1 & 0 & 3 \\
\hline Hepatitis $\mathbf{C}^{\mathbf{a}}$ & 12 & 0 & $\mathbf{0}$ & $\mathbf{0}$ & 1 & 2 & 1 & 1 & 1 & 7 \\
\hline 8. Malaria & 0 & 0 & 0 & 0 & 0 & $\mathbf{0}$ & 0 & $\mathbf{0}$ & 0 & $\mathbf{0}$ \\
\hline 9. Tropical-cluster diseases & $\mathbf{0}$ & 0 & $\mathbf{0}$ & $\mathbf{0}$ & 0 & $\mathbf{0}$ & 0 & $\mathbf{0}$ & 0 & $\mathbf{0}$ \\
\hline a. Trypanosomiasis & 0 & - & - & - & - & - & 0 & - & - & 0 \\
\hline b. Chagas' disease & 0 & 0 & 0 & 0 & 0 & 0 & 0 & 0 & 0 & 0 \\
\hline c. Schistosomiasis & 0 & 0 & 0 & 0 & 0 & 0 & 0 & 0 & 0 & 0 \\
\hline d. Leishmaniasis & 0 & 0 & 0 & 0 & 0 & 0 & 0 & 0 & 0 & 0 \\
\hline e. Lymphatic filariasis & 0 & 0 & - & 0 & 0 & 0 & 0 & 0 & 0 & 0 \\
\hline f. Onchocerciasis & 0 & 0 & - & - & - & - & - & - & - & 0 \\
\hline 10. Leprosy & $\mathbf{0}$ & 0 & $\mathbf{0}$ & 0 & 0 & 0 & $\mathbf{0}$ & $\mathbf{0}$ & $\mathbf{0}$ & 0 \\
\hline 11. Dengue & $\mathbf{0}$ & 0 & $\mathbf{0}$ & 0 & 0 & 0 & 0 & $\mathbf{0}$ & $\mathbf{0}$ & $\mathbf{0}$ \\
\hline 12. Japanese encephalitis & 0 & 0 & 0 & 0 & 0 & 0 & 0 & 0 & 0 & 0 \\
\hline 13. Trachoma & $\mathbf{0}$ & 0 & $\mathbf{0}$ & $\mathbf{0}$ & - & 0 & - & - & - & $\mathbf{0}$ \\
\hline 14. Intestinal nematode infections & $\mathbf{0}$ & 0 & $\mathbf{0}$ & $\mathbf{0}$ & 0 & $\mathbf{0}$ & $\mathbf{0}$ & $\mathbf{0}$ & $\mathbf{0}$ & $\mathbf{0}$ \\
\hline a. Ascariasis & 0 & 0 & 0 & 0 & 0 & 0 & 0 & 0 & 0 & 0 \\
\hline b. Trichuriasis & 0 & - & 0 & - & - & - & - & - & - & 0 \\
\hline c. Hookworm disease & 0 & - & 0 & - & 0 & 0 & - & 0 & 0 & 0 \\
\hline Other intestinal infections & 0 & 0 & 0 & 0 & 0 & 0 & 0 & 0 & 0 & 0 \\
\hline Other infectious diseases & 84 & 1 & 0 & 1 & 2 & 5 & 6 & 11 & 13 & 39 \\
\hline B. Respiratory infections & 349 & 1 & $\mathbf{0}$ & 1 & 2 & 7 & 14 & 41 & 96 & 162 \\
\hline 1. Lower respiratory infections & 345 & 1 & 0 & 1 & 2 & 7 & 14 & 41 & 95 & 160 \\
\hline 2. Upper respiratory infections & 4 & 0 & 0 & 0 & 0 & 0 & 0 & 0 & 1 & 2 \\
\hline 3. Otitis media & 0 & 0 & 0 & 0 & 0 & 0 & 0 & 0 & 0 & 0 \\
\hline C. Maternal conditions & 1 & - & - & - & - & - & - & - & - & - \\
\hline 1. Maternal hemorrhage & 0 & - & - & - & - & - & - & - & - & - \\
\hline 2. Maternal sepsis & 0 & - & - & - & - & - & - & - & - & - \\
\hline 3. Hypertensive disorders of pregnancy & 0 & - & - & - & - & - & - & - & - & 一 \\
\hline 4. Obstructed labor & 0 & - & - & - & - & - & - & - & - & - \\
\hline 5. Abortion & 0 & - & - & - & - & - & - & - & - & - \\
\hline Other maternal conditions & 1 & - & - & - & - & - & - & - & - & - \\
\hline D. Perinatal conditions ${ }^{b}$ & 32 & 18 & $\mathbf{0}$ & $\mathbf{0}$ & 0 & $\mathbf{0}$ & $\mathbf{0}$ & - & - & 18 \\
\hline 1. Low birthweight & 10 & 5 & 0 & - & 0 & - & 0 & - & - & 5 \\
\hline 2. Birth asphyxia and birth trauma & 11 & 6 & 0 & 0 & 0 & 0 & - & - & - & 6 \\
\hline Other perinatal conditions & 12 & 7 & 0 & 0 & 0 & 0 & - & - & - & 7 \\
\hline E. Nutritional deficiencies & 18 & $\mathbf{0}$ & $\mathbf{0}$ & 0 & 0 & $\mathbf{0}$ & 1 & 1 & 4 & 6 \\
\hline 1. Protein-energy malnutrition & 9 & 0 & 0 & 0 & 0 & 0 & 0 & 1 & 2 & 3 \\
\hline 2. Iodine deficiency & 0 & 0 & 0 & - & 0 & 0 & 0 & 0 & 0 & 0 \\
\hline 3. Vitamin A deficiency & 0 & 0 & 0 & - & - & - & 0 & - & 0 & 0 \\
\hline 4. Iron-deficiency anemia & 7 & 0 & 0 & 0 & 0 & 0 & 0 & 0 & 1 & 2 \\
\hline Other nutritional disorders & 2 & 0 & 0 & 0 & 0 & 0 & 0 & 0 & 0 & 1 \\
\hline
\end{tabular}


Table 3B.8 Continued

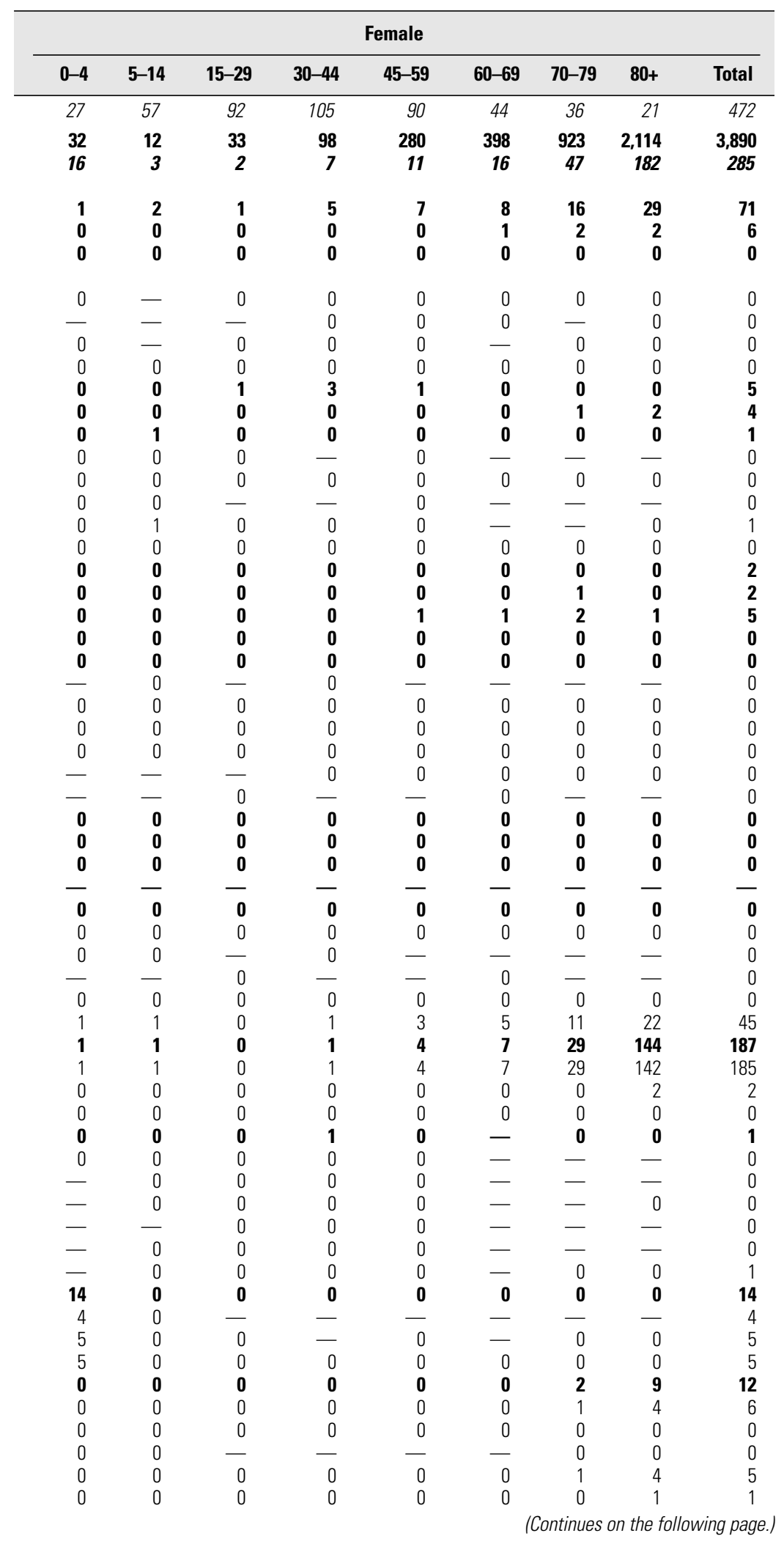


Table 3B.8 Continued

\begin{tabular}{|c|c|c|c|c|c|c|c|c|c|c|}
\hline \multirow[b]{2}{*}{ Cause } & \multicolumn{10}{|c|}{ Male } \\
\hline & Total & $0-4$ & 5-14 & $15-29$ & $30-44$ & $45-59$ & $60-69$ & $70-79$ & $80+$ & Total \\
\hline II. Noncommunicable diseases & 6,868 & 15 & 5 & 24 & 106 & 431 & 638 & 1,074 & 1,127 & 3,420 \\
\hline A. Malignant neoplasms & 2,066 & 1 & 2 & 7 & 31 & 180 & 284 & 393 & 258 & 1,155 \\
\hline 1. Mouth and oropharynx cancers & 41 & 0 & 0 & 0 & 2 & 10 & 8 & 7 & 3 & 30 \\
\hline 2. Esophageal cancer & 58 & 0 & 0 & 0 & 1 & 10 & 13 & 13 & 6 & 44 \\
\hline 3. Stomach cancer & 146 & 0 & 0 & 0 & 2 & 14 & 23 & 30 & 20 & 89 \\
\hline 4. Colon and rectal cancers & 257 & 0 & 0 & 0 & 3 & 18 & 32 & 46 & 33 & 133 \\
\hline 5. Liver cancer & 102 & 0 & 0 & 0 & 2 & 14 & 21 & 22 & 9 & 69 \\
\hline 6. Pancreas cancer & 110 & 0 & 0 & 0 & 1 & 10 & 15 & 19 & 11 & 56 \\
\hline 7. Trachea, bronchus, and lung cancers & 456 & 0 & 0 & 0 & 5 & 50 & 86 & 115 & 53 & 311 \\
\hline 8. Melanoma and other skin cancers & 30 & 0 & 0 & 0 & 1 & 4 & 4 & 5 & 4 & 18 \\
\hline 9. Breast cancer & 155 & 0 & - & 0 & 0 & 0 & 0 & 1 & 0 & 2 \\
\hline 10. Cervix uteri cancer & 17 & - & - & - & - & - & - & - & - & - \\
\hline 11. Corpus uteri cancer & 27 & - & - & - & - & - & - & - & - & - \\
\hline 12. Ovarian cancer & 46 & - & - & - & - & - & - & - & - & - \\
\hline 13. Prostate cancer & 119 & 0 & 0 & 0 & 0 & 4 & 16 & 44 & 56 & 119 \\
\hline 14. Bladder cancer & 59 & 0 & 0 & 0 & 0 & 3 & 8 & 16 & 14 & 42 \\
\hline 15. Lymphomas, and multiple myeloma & 115 & 0 & 0 & 1 & 3 & 9 & 14 & 20 & 13 & 60 \\
\hline 16. Leukemia & 73 & 0 & 1 & 2 & 2 & 5 & 8 & 13 & 9 & 41 \\
\hline Other malignant neoplasms & 257 & 0 & 1 & 3 & 7 & 28 & 36 & 43 & 25 & 143 \\
\hline B. Other neoplasms & 57 & $\mathbf{0}$ & $\mathbf{0}$ & $\mathbf{0}$ & 1 & 3 & 5 & 9 & 10 & 28 \\
\hline C. Diabetes mellitus & 202 & $\mathbf{0}$ & $\mathbf{0}$ & $\mathbf{0}$ & 3 & 12 & 19 & 30 & 26 & 91 \\
\hline D. Endocrine disorders & 70 & 1 & $\mathbf{0}$ & 1 & 2 & 5 & 5 & 7 & 9 & 31 \\
\hline E. Neuropsychiatric conditions & 378 & 1 & 1 & 6 & 13 & 19 & 17 & 38 & 65 & 160 \\
\hline 1. Unipolar depressive disorders & 3 & 0 & 0 & 0 & 0 & 0 & 0 & 0 & 1 & 1 \\
\hline 2. Bipolar affective disorder & 0 & 0 & 0 & 0 & 0 & 0 & 0 & 0 & 0 & 0 \\
\hline 3. Schizophrenia & 2 & 0 & 0 & 0 & 0 & 0 & 0 & 0 & 0 & 1 \\
\hline 4. Epilepsy & 9 & 0 & 0 & 1 & 1 & 1 & 1 & 1 & 1 & 5 \\
\hline 5. Alcohol use disorders & 23 & 0 & 0 & 0 & 4 & 8 & 4 & 2 & 1 & 18 \\
\hline 6. Alzheimer's and other dementias & 207 & 0 & 0 & 0 & 0 & 1 & 3 & 17 & 42 & 64 \\
\hline 7. Parkinson's disease & 45 & 0 & 0 & 0 & 0 & 0 & 2 & 9 & 13 & 24 \\
\hline 8. Multiple sclerosis & 8 & 0 & 0 & 0 & 0 & 1 & 1 & 0 & 0 & 3 \\
\hline 9. Drug use disorders & 13 & 0 & 0 & 3 & 5 & 2 & 0 & 0 & 0 & 10 \\
\hline 10. Post-traumatic stress disorder & 0 & 0 & 0 & 0 & 0 & 0 & 0 & 0 & 0 & 0 \\
\hline 11. Obsessive-compulsive disorder & - & - & - & - & - & - & - & - & - & - \\
\hline 12. Panic disorder & - & - & - & - & - & - & - & - & - & - \\
\hline 13. Insomnia (primary) & - & - & - & - & - & - & - & - & - & - \\
\hline 14. Migraine & - & - & - & - & - & - & - & - & - & - \\
\hline 15. Mental retardation, lead-caused & 1 & 0 & 0 & 0 & 0 & 0 & 0 & 0 & 0 & 1 \\
\hline Other neuropsychiatric disorders & 68 & 1 & 1 & 2 & 2 & 5 & 6 & 9 & 8 & 33 \\
\hline F. Sense organ diseases & $\mathbf{0}$ & 0 & $\mathbf{0}$ & $\mathbf{0}$ & 0 & 0 & 0 & 0 & $\mathbf{0}$ & 0 \\
\hline 1. Glaucoma & 0 & 0 & - & 0 & 0 & 0 & 0 & 0 & 0 & 0 \\
\hline 2. Cataracts & - & - & - & - & - & - & - & - & - & - \\
\hline 3. Vision disorders, age-related & - & - & - & - & - & - & - & - & - & - \\
\hline 4. Hearing loss, adult onset & - & - & - & - & - & - & - & - & - & - \\
\hline Other sense organ disorders & 0 & 0 & 0 & 0 & 0 & 0 & 0 & 0 & 0 & 0 \\
\hline G. Cardiovascular diseases & 3,039 & 1 & 1 & 5 & 35 & 148 & 224 & 434 & 564 & 1,412 \\
\hline 1. Rheumatic heart disease & 17 & 0 & 0 & 0 & 0 & 1 & 1 & 2 & 2 & 5 \\
\hline 2. Hypertensive heart disease & 129 & 0 & 0 & 0 & 2 & 6 & 7 & 14 & 19 & 48 \\
\hline 3. Ischemic heart disease & 1,364 & 0 & 0 & 1 & 16 & 88 & 130 & 231 & 250 & 716 \\
\hline 4. Cerebrovascular disease & 781 & 0 & 0 & 1 & 6 & 25 & 44 & 101 & 145 & 323 \\
\hline 5. Inflammatory heart diseases & 72 & 0 & 0 & 1 & 3 & 7 & 8 & 11 & 10 & 41 \\
\hline Other cardiovascular diseases & 676 & 1 & 0 & 2 & 8 & 21 & 34 & 74 & 139 & 279 \\
\hline H. Respiratory diseases & 477 & 1 & $\mathbf{0}$ & 1 & 3 & 14 & 37 & 94 & 112 & 262 \\
\hline 1. Chronic obstructive pulmonary disease & 297 & 0 & 0 & 0 & 1 & 8 & 24 & 65 & 73 & 171 \\
\hline 2. Asthma & 28 & 0 & 0 & 0 & 1 & 1 & 2 & 4 & 4 & 12 \\
\hline Other respiratory diseases & 152 & 0 & 0 & 1 & 2 & 5 & 11 & 25 & 35 & 79 \\
\hline
\end{tabular}


Table 3B.8 Continued

\begin{tabular}{|c|c|c|c|c|c|c|c|c|}
\hline \multicolumn{9}{|c|}{ Female } \\
\hline $0-4$ & 5-14 & $15-29$ & 30-44 & 45-59 & $60-69$ & $70-79$ & $80+$ & Total \\
\hline 13 & 5 & 14 & 69 & 248 & 368 & 851 & 1,880 & 3,448 \\
\hline 1 & 2 & 5 & 34 & 136 & 172 & 275 & 287 & 911 \\
\hline 0 & 0 & 0 & 0 & 2 & 2 & 3 & 4 & 11 \\
\hline 0 & 0 & 0 & 0 & 2 & 2 & 4 & 5 & 13 \\
\hline 0 & 0 & 0 & 2 & 6 & 9 & 17 & 23 & 57 \\
\hline 0 & 0 & 0 & 2 & 13 & 20 & 37 & 52 & 124 \\
\hline 0 & 0 & 0 & 1 & 3 & 7 & 12 & 10 & 33 \\
\hline 0 & 0 & 0 & 1 & 6 & 10 & 19 & 19 & 54 \\
\hline 0 & 0 & 0 & 4 & 23 & 34 & 50 & 35 & 145 \\
\hline 0 & 0 & 0 & 1 & 2 & 2 & 3 & 4 & 13 \\
\hline 0 & 0 & 0 & 11 & 37 & 30 & 37 & 38 & 154 \\
\hline 0 & 0 & 0 & 3 & 5 & 3 & 3 & 3 & 17 \\
\hline- & 0 & 0 & 1 & 4 & 6 & 8 & 8 & 27 \\
\hline 0 & 0 & 0 & 2 & 9 & 11 & 14 & 10 & 46 \\
\hline- & - & - & - & - & - & - & - & - \\
\hline 0 & 0 & 0 & 0 & 1 & 2 & 5 & 9 & 17 \\
\hline 0 & 0 & 1 & 2 & 6 & 10 & 18 & 18 & 55 \\
\hline 0 & 1 & 1 & 2 & 4 & 5 & 9 & 11 & 32 \\
\hline 0 & 1 & 1 & 4 & 14 & 21 & 35 & 37 & 114 \\
\hline 0 & 0 & 0 & 1 & 2 & 3 & 8 & 14 & 29 \\
\hline 0 & 0 & 0 & 2 & 7 & 14 & 34 & 54 & 112 \\
\hline 1 & 0 & 1 & 2 & 3 & 4 & 8 & 21 & 40 \\
\hline 1 & 1 & 2 & 5 & 10 & 11 & 40 & 148 & 218 \\
\hline 0 & 0 & 0 & 0 & 0 & 0 & 0 & 1 & 2 \\
\hline 0 & 0 & 0 & 0 & 0 & 0 & 0 & 0 & 0 \\
\hline 0 & 0 & 0 & 0 & 0 & 0 & 0 & 0 & 1 \\
\hline 0 & 0 & 0 & 1 & 1 & 0 & 1 & 1 & 4 \\
\hline 0 & 0 & 0 & 1 & 2 & 1 & 1 & 0 & 4 \\
\hline 0 & 0 & 0 & 0 & 1 & 3 & 23 & 117 & 143 \\
\hline 0 & 0 & 0 & 0 & 0 & 1 & 6 & 13 & 21 \\
\hline 0 & 0 & 0 & 1 & 2 & 1 & 1 & 0 & 5 \\
\hline 0 & 0 & 1 & 1 & 1 & 0 & 0 & 0 & 2 \\
\hline 0 & 0 & 0 & 0 & 0 & 0 & 0 & 0 & 0 \\
\hline- & - & - & - & - & - & - & - & - \\
\hline- & - & - & - & - & - & - & - & - \\
\hline- & - & - & - & - & - & - & - & - \\
\hline- & - & - & - & - & - & - & - & - \\
\hline 0 & 0 & 0 & 0 & 0 & 0 & 0 & 0 & 0 \\
\hline 1 & 1 & 1 & 2 & 4 & 5 & 8 & 14 & 34 \\
\hline 0 & 0 & 0 & 0 & 0 & 0 & 0 & 0 & 0 \\
\hline- & - & - & - & 0 & 0 & 0 & 0 & 0 \\
\hline- & - & - & - & - & - & - & - & - \\
\hline- & - & - & - & - & - & - & - & - \\
\hline- & - & - & - & - & - & - & - & - \\
\hline 0 & 0 & 0 & 0 & 0 & 0 & 0 & 0 & 0 \\
\hline 1 & 1 & 3 & 15 & 56 & 111 & 358 & 1,082 & 1,627 \\
\hline 0 & 0 & 0 & 0 & 1 & 2 & 4 & 5 & 12 \\
\hline 0 & 0 & 0 & 1 & 3 & 5 & 17 & 54 & 80 \\
\hline 0 & 0 & 0 & 4 & 23 & 52 & 158 & 412 & 648 \\
\hline 0 & 0 & 1 & 4 & 16 & 28 & 101 & 307 & 458 \\
\hline 0 & 0 & 0 & 1 & 3 & 4 & 8 & 15 & 31 \\
\hline 0 & 0 & 1 & 4 & 11 & 20 & 71 & 289 & 397 \\
\hline 0 & 0 & 1 & 2 & 10 & 23 & 62 & 118 & 215 \\
\hline 0 & 0 & 0 & 0 & 5 & 15 & 41 & 64 & 127 \\
\hline 0 & 0 & 0 & 1 & 2 & 2 & 4 & 7 & 15 \\
\hline 0 & 0 & 0 & 1 & 3 & 6 & 16 & 46 & 73 \\
\hline
\end{tabular}

(Continues on the following page.) 
Table 3B.8 Continued

\begin{tabular}{|c|c|c|c|c|c|c|c|c|c|c|}
\hline \multirow[b]{2}{*}{ Cause } & \multicolumn{10}{|c|}{ Male } \\
\hline & Total & $\overline{0-4}$ & 5-14 & $15-29$ & $30-44$ & $45-59$ & $60-69$ & $70-79$ & $80+$ & Total \\
\hline I. Digestive diseases & 335 & 1 & 0 & 1 & 14 & 42 & 37 & 42 & 40 & 177 \\
\hline 1. Peptic ulcer disease & 27 & 0 & 0 & 0 & 0 & 2 & 2 & 4 & 5 & 13 \\
\hline 2. Cirrhosis of the liver & 118 & 0 & 0 & 0 & 10 & 30 & 21 & 14 & 5 & 80 \\
\hline 3. Appendicitis & 1 & 0 & 0 & 0 & 0 & 0 & 0 & 0 & 0 & 1 \\
\hline Other digestive diseases & 189 & 1 & 0 & 1 & 4 & 11 & 13 & 24 & 30 & 83 \\
\hline J. Genitourinary diseases & 153 & 0 & $\mathbf{0}$ & 0 & 1 & 5 & 8 & 20 & 35 & 70 \\
\hline 1. Nephritis and nephrosis & 111 & 0 & 0 & 0 & 1 & 4 & 7 & 16 & 25 & 53 \\
\hline 2. Benign prostatic hypertrophy & 2 & - & - & 0 & 0 & 0 & 0 & 0 & 1 & 2 \\
\hline Other genitourinary system diseases & 40 & 0 & 0 & 0 & 0 & 1 & 1 & 4 & 9 & 15 \\
\hline K. Skin diseases & 15 & 0 & 0 & 0 & $\mathbf{0}$ & 0 & 0 & 1 & 2 & 5 \\
\hline L. Musculoskeletal diseases & 44 & 0 & $\mathbf{0}$ & $\mathbf{0}$ & 1 & 1 & 2 & 4 & 5 & 13 \\
\hline 1. Rheumatoid arthritis & 9 & 0 & 0 & 0 & 0 & 0 & 0 & 1 & 1 & 2 \\
\hline 2. Osteoarthritis & 3 & 0 & 0 & 0 & 0 & 0 & 0 & 0 & 0 & 1 \\
\hline 3. Gout & 0 & - & 0 & 0 & 0 & 0 & 0 & 0 & 0 & 0 \\
\hline 4. Low back pain & 2 & 0 & 0 & 0 & 0 & 0 & 0 & 0 & 0 & 1 \\
\hline Other musculoskeletal disorders & 30 & 0 & 0 & 0 & 0 & 1 & 1 & 3 & 4 & 9 \\
\hline M. Congenital anomalies & 30 & 10 & 1 & 1 & 1 & 1 & 1 & 1 & 1 & 16 \\
\hline 1. Abdominal wall defect & 0 & 0 & 0 & 0 & 0 & 0 & 0 & 0 & 0 & 0 \\
\hline 2. Anencephaly & 1 & 0 & 0 & 0 & 0 & 0 & 0 & 0 & 0 & 0 \\
\hline 3. Anorectal atresia & 0 & 0 & 0 & 0 & 0 & 0 & 0 & 0 & 0 & 0 \\
\hline 4. Cleft lip & 0 & 0 & 0 & 0 & 0 & 0 & 0 & 0 & 0 & 0 \\
\hline 5. Cleft palate & 0 & 0 & 0 & 0 & 0 & 0 & 0 & 0 & 0 & 0 \\
\hline 6. Esophageal atresia & 0 & 0 & 0 & 0 & 0 & 0 & 0 & 0 & 0 & 0 \\
\hline 7. Renal agenesis & 1 & 0 & 0 & 0 & 0 & 0 & 0 & 0 & 0 & 0 \\
\hline 8. Down syndrome & 2 & 0 & 0 & 0 & 0 & 0 & 0 & 0 & 0 & 1 \\
\hline 9. Congenital heart anomalies & 13 & 4 & 0 & 1 & 1 & 1 & 0 & 0 & 0 & 7 \\
\hline 10. Spina bifida & 0 & 0 & 0 & 0 & 0 & 0 & 0 & 0 & 0 & 0 \\
\hline Other congenital anomalies & 13 & 5 & 0 & 0 & 0 & 0 & 0 & 0 & 0 & 7 \\
\hline N. Oral conditions & 0 & 0 & $\mathbf{0}$ & 0 & 0 & 0 & 0 & 0 & 0 & 0 \\
\hline 1. Dental caries & 0 & - & - & - & - & 0 & - & 0 & 0 & 0 \\
\hline 2. Periodontal disease & 0 & - & - & 0 & 0 & 0 & 0 & 0 & 0 & 0 \\
\hline 3. Edentulism & - & - & - & - & - & - & - & - & - & - \\
\hline Other oral diseases & 0 & 0 & 0 & 0 & 0 & 0 & 0 & 0 & 0 & 0 \\
\hline III. Injuries & 471 & 4 & 5 & 63 & 70 & 65 & 34 & 35 & 36 & 314 \\
\hline A. Unintentional injuries & 321 & 4 & 4 & 40 & 39 & 36 & 22 & 26 & 31 & 202 \\
\hline 1. Road traffic accidents & 121 & 1 & 2 & 28 & 20 & 15 & 8 & 7 & 4 & 86 \\
\hline 2. Poisonings & 21 & 0 & 0 & 3 & 7 & 4 & 1 & 0 & 0 & 15 \\
\hline 3. Falls & 71 & 0 & 0 & 1 & 3 & 5 & 4 & 7 & 13 & 34 \\
\hline 4. Fires & 9 & 0 & 0 & 1 & 1 & 1 & 1 & 1 & 1 & 6 \\
\hline 5. Drownings & 16 & 1 & 1 & 2 & 2 & 2 & 1 & 1 & 1 & 11 \\
\hline 6. Other unintentional injuries & 82 & 1 & 1 & 5 & 7 & 9 & 7 & 9 & 11 & 49 \\
\hline B. Intentional injuries & 151 & 1 & 1 & 23 & 31 & 29 & 12 & 9 & 6 & 112 \\
\hline 1. Self-inflicted injuries & 126 & 0 & 0 & 16 & 25 & 27 & 12 & 9 & 6 & 94 \\
\hline 2. Violence & 24 & 1 & 0 & 7 & 5 & 3 & 1 & 0 & 0 & 17 \\
\hline 3. War & 0 & 0 & 0 & 0 & 0 & 0 & 0 & 0 & 0 & 0 \\
\hline Other intentional injuries & 0 & 0 & 0 & 0 & 0 & 0 & 0 & 0 & 0 & 0 \\
\hline
\end{tabular}


Table 3B.8 Continued

\begin{tabular}{|c|c|c|c|c|c|c|c|c|}
\hline \multicolumn{9}{|c|}{ Female } \\
\hline $0-4$ & 5-14 & $15-29$ & $30-44$ & 45-59 & $60-69$ & $70-79$ & $80+$ & Total \\
\hline 0 & 0 & 1 & 6 & 16 & 18 & 38 & 79 & 158 \\
\hline 0 & 0 & 0 & 0 & 1 & 1 & 3 & 9 & 14 \\
\hline 0 & 0 & 0 & 3 & 10 & 8 & 10 & 6 & 37 \\
\hline 0 & 0 & 0 & 0 & 0 & 0 & 0 & 0 & 1 \\
\hline 0 & 0 & 0 & 2 & 6 & 9 & 25 & 64 & 106 \\
\hline 0 & 0 & 0 & 1 & 3 & 7 & 19 & 53 & 83 \\
\hline 0 & 0 & 0 & 1 & 3 & 5 & 14 & 35 & 58 \\
\hline- & - & - & - & - & - & - & - & - \\
\hline 0 & 0 & 0 & 0 & 1 & 1 & 5 & 18 & 25 \\
\hline 0 & 0 & 0 & 0 & 0 & 1 & 2 & 7 & 10 \\
\hline 0 & 0 & 0 & 1 & 2 & 3 & 8 & 16 & 31 \\
\hline 0 & 0 & 0 & 0 & 0 & 1 & 3 & 3 & 7 \\
\hline 0 & 0 & 0 & 0 & 0 & 0 & 0 & 2 & 2 \\
\hline 0 & 0 & 0 & 0 & 0 & 0 & 0 & 0 & 0 \\
\hline 0 & 0 & 0 & 0 & 0 & 0 & 0 & 0 & 1 \\
\hline 0 & 0 & 0 & 1 & 2 & 2 & 4 & 11 & 21 \\
\hline 8 & 1 & 1 & 1 & 1 & 1 & 1 & 1 & 14 \\
\hline 0 & 0 & 0 & 0 & 0 & 0 & 0 & 0 & 0 \\
\hline 0 & 0 & 0 & 0 & 0 & 0 & 0 & 0 & 0 \\
\hline 0 & 0 & 0 & 0 & 0 & 0 & 0 & 0 & 0 \\
\hline 0 & 0 & 0 & 0 & 0 & 0 & 0 & 0 & 0 \\
\hline 0 & 0 & 0 & 0 & 0 & 0 & 0 & 0 & 0 \\
\hline 0 & 0 & 0 & 0 & 0 & 0 & 0 & 0 & 0 \\
\hline 0 & 0 & 0 & 0 & 0 & 0 & 0 & 0 & 0 \\
\hline 0 & 0 & 0 & 0 & 0 & 0 & 0 & 0 & 1 \\
\hline 3 & 0 & 0 & 1 & 0 & 0 & 0 & 0 & 6 \\
\hline 0 & 0 & 0 & 0 & 0 & 0 & 0 & 0 & 0 \\
\hline 4 & 0 & 0 & 0 & 0 & 0 & 0 & 0 & 6 \\
\hline 0 & 0 & 0 & 0 & 0 & 0 & 0 & 0 & 0 \\
\hline- & - & - & - & 0 & - & - & 0 & 0 \\
\hline- & 0 & 0 & 0 & 0 & 0 & 0 & 0 & 0 \\
\hline- & - & - & - & - & - & - & - & - \\
\hline 0 & 0 & 0 & 0 & 0 & 0 & 0 & 0 & 0 \\
\hline 3 & 4 & 17 & 21 & 22 & 14 & 24 & 52 & 157 \\
\hline 2 & 3 & 11 & 11 & 12 & 10 & 20 & 49 & 119 \\
\hline 1 & 2 & 8 & 6 & 6 & 4 & 5 & 3 & 35 \\
\hline 0 & 0 & 1 & 2 & 2 & 0 & 0 & 1 & 6 \\
\hline 0 & 0 & 0 & 1 & 1 & 2 & 6 & 26 & 37 \\
\hline 0 & 0 & 0 & 0 & 1 & 0 & 1 & 1 & 4 \\
\hline 0 & 0 & 0 & 0 & 1 & 1 & 1 & 1 & 5 \\
\hline 1 & 1 & 1 & 1 & 3 & 3 & 6 & 17 & 32 \\
\hline 0 & 0 & 6 & 10 & 10 & 5 & 4 & 3 & 38 \\
\hline 0 & 0 & 4 & 7 & 8 & 4 & 4 & 3 & 32 \\
\hline 0 & 0 & 2 & 2 & 1 & 0 & 0 & 0 & 7 \\
\hline 0 & 0 & 0 & 0 & 0 & 0 & 0 & 0 & 0 \\
\hline 0 & 0 & 0 & 0 & 0 & 0 & 0 & 0 & 0 \\
\hline
\end{tabular}

Source: Authors' compilation.

Note: - = an estimate of zero; the number zero in a cell indicates a non-zero estimate of less than 500 .

a. Does not include liver cancer and cirrhosis deaths resulting from chronic hepatitis virus infection.

b. This cause category includes "Causes arising in the perinatal period" as defined in the International Classification of

Diseases, principally low birthweight, prematurity, birth asphyxia, and birth trauma, and does not include all causes of deaths

occurring in the perinatal period. 
Table 3B.9 Deaths by Cause, Sex, and Age in the World, 2001 (thousands)

\begin{tabular}{|c|c|c|c|c|c|c|c|c|c|c|}
\hline \multirow[b]{2}{*}{ Cause } & \multicolumn{10}{|c|}{ Male } \\
\hline & Total & $0-4$ & $5-14$ & $15-29$ & $30-44$ & $45-59$ & $60-69$ & $70-79$ & $80+$ & Total $^{\mathbf{a}}$ \\
\hline Population (millions) & 6,148 & 317 & 623 & 808 & 653 & 415 & 164 & 88 & 25 & 3,093 \\
\hline $\begin{array}{l}\text { All causes } \\
\text { I. Communicable, maternal, perinatal, } \\
\text { and nutritional conditions }\end{array}$ & $\begin{array}{l}56,242 \\
18,166\end{array}$ & $\begin{array}{l}5,448 \\
4,858\end{array}$ & $\begin{array}{l}744 \\
376\end{array}$ & $\begin{array}{r}1,925 \\
588\end{array}$ & $\begin{array}{l}2,978 \\
1,166\end{array}$ & $\begin{array}{r}4,518 \\
877\end{array}$ & $\begin{array}{r}4,767 \\
555\end{array}$ & $\begin{array}{r}5,546 \\
529\end{array}$ & $\begin{array}{r}3,630 \\
386\end{array}$ & $\begin{array}{r}29,555 \\
9,335\end{array}$ \\
\hline A. Infectious and parasitic diseases & 10,838 & 2,362 & 296 & 541 & 1,099 & 750 & 360 & 267 & 129 & $\mathbf{5 , 8 0 5}$ \\
\hline 1. Tuberculosis & 1,606 & 22 & 16 & 138 & 257 & 282 & 186 & 118 & 34 & 1,053 \\
\hline $\begin{array}{l}\text { 2. Sexually transmitted diseases } \\
\text { excluding HIV/AIDS }\end{array}$ & 177 & 31 & 0 & 2 & 11 & 26 & 10 & 6 & 3 & 89 \\
\hline a. Syphilis & 155 & 30 & 0 & 1 & 10 & 23 & 10 & 6 & 3 & 83 \\
\hline b. Chlamydia & 9 & - & - & - & - & - & - & - & - & - \\
\hline c. Gonorrhea & 1 & - & 0 & 0 & 0 & 0 & 0 & 0 & 0 & 0 \\
\hline d. Other sexually transmitted diseases & 12 & 1 & 0 & 0 & 1 & 4 & 0 & 0 & 0 & 6 \\
\hline 3. HIV/AIDS & 2,574 & 173 & 52 & 260 & 649 & 226 & 30 & 4 & 0 & 1,394 \\
\hline 4. Diarrheal diseases & 1,783 & 837 & 3 & 6 & 12 & 15 & 15 & 20 & 24 & 932 \\
\hline 5. Childhood-cluster diseases & 1,363 & 524 & 110 & 24 & 11 & 6 & 3 & 1 & 1 & 680 \\
\hline a. Pertussis & 301 & 150 & 0 & - & - & - & 0 & - & - & 150 \\
\hline b. Poliomyelitis ${ }^{b}$ & 1 & 0 & 0 & 0 & 0 & 0 & 0 & 0 & 0 & 0 \\
\hline c. Diphtheria & 6 & 3 & 0 & 0 & 0 & 0 & 0 & 0 & 0 & 3 \\
\hline d. Measles & 763 & 277 & 91 & 11 & 0 & 0 & 0 & 0 & - & 379 \\
\hline e. Tetanus & 293 & 94 & 18 & 13 & 11 & 6 & 3 & 1 & 1 & 147 \\
\hline 6. Meningitis & 173 & 30 & 14 & 12 & 10 & 10 & 5 & 6 & 3 & 89 \\
\hline 7. Hepatitis $\mathbf{B}^{\mathbf{c}}$ & 100 & 3 & 4 & 8 & 17 & 23 & 8 & 5 & 2 & 69 \\
\hline Hepatitis Cc & 51 & 1 & 1 & 3 & 7 & 12 & 5 & 3 & $\overline{1}$ & 33 \\
\hline 8. Malaria & 1,208 & 521 & 7 & 10 & 11 & 11 & 7 & 7 & 4 & 579 \\
\hline 9. Tropical-cluster diseases & 128 & 4 & 21 & 15 & 13 & 13 & 7 & 4 & 1 & 78 \\
\hline a. Trypanosomiasis & 48 & 2 & 11 & 6 & 6 & 5 & 1 & 0 & 0 & 31 \\
\hline b. Chagas' disease & 14 & 0 & 0 & 0 & 1 & 2 & 2 & 1 & 1 & 8 \\
\hline c. Schistosomiasis & 14 & 0 & 0 & 0 & 1 & 3 & 3 & 2 & 0 & 9 \\
\hline d. Leishmaniasis & 51 & 2 & 10 & 8 & 5 & 3 & 1 & 1 & 0 & 30 \\
\hline e. Lymphatic filariasis & 0 & 0 & 0 & 0 & 0 & 0 & 0 & 0 & 0 & 0 \\
\hline f. Onchocerciasis & 0 & 0 & - & - & - & - & - & - & - & 0 \\
\hline 10. Leprosy & 6 & 0 & 0 & $\mathbf{0}$ & 1 & 1 & 1 & 1 & $\mathbf{0}$ & 4 \\
\hline 11. Dengue & 19 & 2 & 5 & $\mathbf{0}$ & 0 & 0 & 0 & 0 & 0 & 9 \\
\hline 12. Japanese encephalitis & 14 & 2 & $\mathbf{0}$ & 1 & 2 & 0 & 0 & $\mathbf{0}$ & 0 & 7 \\
\hline 13. Trachoma & $\mathbf{0}$ & 0 & 0 & $\mathbf{0}$ & - & 0 & - & $\mathbf{0}$ & - & 0 \\
\hline 14. Intestinal nematode infections & 12 & 1 & 3 & $\mathbf{0}$ & 0 & 1 & 0 & $\mathbf{0}$ & 0 & 6 \\
\hline a. Ascariasis & 3 & 0 & 1 & 0 & 0 & 0 & 0 & 0 & 0 & 1 \\
\hline b. Trichuriasis & 3 & 0 & 1 & 0 & 0 & 0 & 0 & 0 & 0 & 2 \\
\hline c. Hookworm disease & 3 & 0 & 0 & 0 & 0 & 0 & 0 & 0 & 0 & 2 \\
\hline Other intestinal infections & 2 & 1 & 0 & 0 & 0 & 0 & 0 & 0 & 0 & 1 \\
\hline Other infectious diseases & 1,624 & 211 & 59 & 63 & 99 & 122 & 82 & 91 & 54 & 783 \\
\hline B. Respiratory infections & 3,830 & 1,004 & 58 & 37 & 56 & 101 & 182 & 244 & 240 & 1,923 \\
\hline 1. Lower respiratory infections & 3,753 & 990 & 55 & 35 & 54 & 97 & 177 & 239 & 236 & 1,884 \\
\hline 2. Upper respiratory infections & 73 & 14 & 2 & 2 & 2 & 4 & 5 & 5 & 4 & 37 \\
\hline 3. Otitis media & 4 & 0 & 1 & 0 & 0 & 0 & 0 & 0 & 0 & 2 \\
\hline C. Maternal conditions & 508 & - & - & - & - & - & - & - & - & - \\
\hline 1. Maternal hemorrhage & 141 & - & - & - & - & - & - & - & - & 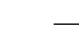 \\
\hline 2. Maternal sepsis & 75 & - & - & - & - & - & - & - & - & - \\
\hline 3. Hypertensive disorders of pregnancy & 71 & - & - & - & - & - & - & - & - & - \\
\hline 4. Obstructed labor & 43 & - & - & - & - & - & - & - & - & - \\
\hline 5. Abortion & 66 & - & - & - & - & - & - & - & - & - \\
\hline Other maternal conditions & 111 & - & - & - & - & - & - & - & - & - \\
\hline D. Perinatal conditions ${ }^{\mathrm{d}}$ & 2,522 & 1,399 & 0 & 0 & 0 & 0 & 0 & $\mathbf{0}$ & - & 1,399 \\
\hline 1. Low birthweight & 1,301 & 709 & 0 & - & 0 & - & 0 & - & - & 709 \\
\hline 2. Birth asphyxia and birth trauma & 739 & 432 & 0 & 0 & 0 & 0 & - & - & - & 432 \\
\hline Other perinatal conditions & 482 & 258 & 0 & 0 & 0 & 0 & - & 0 & - & 258 \\
\hline E. Nutritional deficiencies & 468 & 93 & 22 & 10 & 11 & 26 & 13 & 18 & 16 & 208 \\
\hline 1. Protein-energy malnutrition & 250 & 70 & 15 & 4 & 3 & 7 & 8 & 9 & 9 & 125 \\
\hline 2. lodine deficiency & 7 & 2 & 1 & 0 & 0 & 0 & 0 & 0 & 0 & 3 \\
\hline 3. Vitamin A deficiency & 23 & 7 & 2 & 0 & 0 & 1 & 0 & 0 & 0 & 11 \\
\hline 4. Iron-deficiency anemia & 133 & 9 & 3 & 4 & 6 & 15 & 2 & 3 & 3 & 46 \\
\hline Other nutritional disorders & 56 & 5 & 1 & 1 & 1 & 3 & 3 & 6 & 4 & 24 \\
\hline
\end{tabular}


Table 3B.9 Continued

\begin{tabular}{|c|c|c|c|c|c|c|c|c|}
\hline \multicolumn{9}{|c|}{ Female } \\
\hline $0-4$ & 5-14 & $15-29$ & $30-44$ & 45-59 & $60-69$ & $70-79$ & $80+$ & Total \\
\hline 301 & 589 & 774 & 635 & 415 & 180 & 115 & 47 & 3,055 \\
\hline $\begin{array}{l}5,155 \\
4,571\end{array}$ & $\begin{array}{l}750 \\
454\end{array}$ & $\begin{array}{r}1,610 \\
952\end{array}$ & $\begin{array}{r}1,928 \\
966\end{array}$ & $\begin{array}{r}2,802 \\
523\end{array}$ & $\begin{array}{r}3,358 \\
373\end{array}$ & $\begin{array}{r}5,242 \\
455\end{array}$ & $\begin{array}{r}5,843 \\
535\end{array}$ & $\begin{array}{r}26,687 \\
8,830\end{array}$ \\
\hline 2,407 & 350 & 630 & 672 & 406 & 213 & 207 & 147 & 5,033 \\
\hline 18 & 17 & 106 & 135 & 117 & 80 & 58 & 22 & 552 \\
\hline 37 & 0 & 13 & 10 & 14 & 7 & 4 & 1 & 88 \\
\hline 37 & 0 & 12 & 8 & 6 & 4 & 4 & 1 & 72 \\
\hline- & - & 0 & 1 & 6 & 1 & 0 & 0 & 9 \\
\hline 0 & - & 0 & 0 & 0 & 0 & 0 & 0 & 1 \\
\hline 0 & 0 & 0 & 1 & 2 & 1 & 1 & 0 & 6 \\
\hline 166 & 51 & 385 & 426 & 129 & 20 & 4 & 0 & 1,180 \\
\hline 763 & 3 & 3 & 5 & 10 & 14 & 21 & 33 & 851 \\
\hline 525 & 112 & 24 & 11 & 6 & 3 & 2 & 1 & 684 \\
\hline 151 & 0 & 0 & - & 0 & 0 & - & - & 151 \\
\hline 0 & 0 & 0 & 0 & 0 & 0 & 0 & 0 & 0 \\
\hline 2 & 1 & 0 & 0 & 0 & 0 & 0 & 0 & 3 \\
\hline 279 & 93 & 11 & 0 & 0 & 0 & - & 0 & 383 \\
\hline 93 & 18 & 13 & 11 & 6 & 3 & 1 & 1 & 146 \\
\hline 35 & 18 & 9 & 6 & 5 & 4 & 4 & 2 & 84 \\
\hline 5 & 2 & 5 & 4 & 6 & 3 & 3 & 2 & 31 \\
\hline 2 & 1 & 2 & 2 & 3 & 3 & 3 & 2 & 17 \\
\hline 566 & 8 & 12 & 12 & 13 & 8 & 7 & 4 & 629 \\
\hline 4 & 15 & 10 & 6 & 7 & 4 & 3 & 2 & 50 \\
\hline 1 & 6 & 4 & 3 & 3 & 0 & 0 & - & 17 \\
\hline 0 & 0 & 0 & 1 & 2 & 1 & 1 & 1 & 7 \\
\hline 0 & 0 & 0 & 0 & 1 & 1 & 2 & 1 & 5 \\
\hline 3 & 9 & 5 & 2 & 1 & 1 & 0 & 0 & 21 \\
\hline 0 & 0 & 0 & 0 & 0 & 0 & 0 & 0 & 0 \\
\hline- & - & 0 & - & - & 0 & - & - & 0 \\
\hline 0 & 0 & 0 & 0 & 0 & 0 & 0 & 0 & 2 \\
\hline 2 & 6 & 1 & 0 & 0 & 0 & 0 & 0 & 10 \\
\hline 4 & 2 & 1 & 1 & 0 & 0 & 0 & 0 & 7 \\
\hline- & - & - & - & - & - & - & - & - \\
\hline 2 & 3 & 0 & 0 & 0 & 0 & 0 & 0 & 6 \\
\hline 1 & 1 & 0 & 0 & 0 & 0 & 0 & 0 & 2 \\
\hline 0 & 1 & 0 & 0 & 0 & 0 & 0 & 0 & 1 \\
\hline- & 0 & 0 & 0 & 0 & 0 & 0 & 0 & 1 \\
\hline 1 & 0 & 0 & 0 & 0 & 0 & 0 & 0 & 1 \\
\hline 277 & 115 & 59 & 54 & 94 & 67 & 98 & 77 & 841 \\
\hline 940 & 79 & 57 & 46 & 61 & 138 & 226 & 359 & 1,906 \\
\hline 925 & 74 & 56 & 45 & 60 & 135 & 223 & 351 & 1,869 \\
\hline 15 & 5 & 1 & 1 & 1 & 3 & 3 & 8 & 36 \\
\hline 0 & 0 & 0 & 0 & 0 & 0 & 0 & 0 & 1 \\
\hline 0 & 0 & 257 & 233 & 18 & 0 & 0 & 0 & 508 \\
\hline 0 & 0 & 63 & 72 & 7 & - & - & - & 141 \\
\hline- & 0 & 37 & 35 & 4 & - & - & - & 75 \\
\hline- & 0 & 41 & 28 & 2 & - & - & 0 & 71 \\
\hline- & - & 26 & 17 & 0 & - & - & - & 43 \\
\hline- & 0 & 44 & 22 & 0 & - & - & - & 66 \\
\hline- & 0 & 47 & 60 & 5 & 0 & 0 & 0 & 111 \\
\hline 1,122 & 0 & 0 & 0 & 0 & 0 & 0 & 0 & 1,123 \\
\hline 591 & 0 & - & - & - & - & - & - & 591 \\
\hline 307 & 0 & 0 & - & 0 & - & 0 & 0 & 307 \\
\hline 225 & 0 & 0 & 0 & 0 & 0 & 0 & 0 & 225 \\
\hline 101 & 25 & 8 & 16 & 37 & 22 & 21 & 29 & 261 \\
\hline 69 & 17 & 2 & 3 & 5 & 7 & 8 & 14 & 125 \\
\hline 3 & 0 & 0 & 0 & 0 & 0 & 0 & 0 & 3 \\
\hline 9 & 2 & 0 & 0 & 0 & 0 & 0 & 0 & 12 \\
\hline 11 & 5 & 5 & 13 & 30 & 12 & 5 & 7 & 88 \\
\hline 9 & 1 & 0 & 0 & 2 & 3 & 9 & 7 & 33 \\
\hline & & & & & & nues & follo & page.) \\
\hline
\end{tabular}


Table 3B.9 Continued

\begin{tabular}{|c|c|c|c|c|c|c|c|c|c|c|}
\hline \multirow[b]{2}{*}{ Cause } & \multicolumn{10}{|c|}{ Male } \\
\hline & Total & $0-4$ & 5-14 & $15-29$ & $30-44$ & $45-59$ & $60-69$ & 70-79 & $80+$ & Total \\
\hline II. Noncommunicable diseases & 32,891 & 431 & 121 & 363 & 939 & 3,005 & 3,927 & 4,817 & 3,134 & 16,737 \\
\hline A. Malignant neoplasms & 7,021 & 19 & 27 & 75 & 217 & 851 & 1,118 & 1,102 & 508 & 3,917 \\
\hline 1. Mouth and oropharynx cancers & 312 & 0 & 0 & 4 & 17 & 64 & 71 & 44 & 16 & 217 \\
\hline 2. Esophageal cancer & 438 & 0 & 0 & 1 & 14 & 69 & 97 & 75 & 23 & 280 \\
\hline 3. Stomach cancer & 842 & 0 & 0 & 5 & 27 & 124 & 153 & 147 & 62 & 519 \\
\hline 4. Colon and rectal cancers & 614 & 0 & 0 & 4 & 18 & 57 & 85 & 98 & 55 & 318 \\
\hline 5. Liver cancer & 607 & 1 & 1 & 8 & 40 & 136 & 118 & 91 & 26 & 420 \\
\hline 6. Pancreas cancer & 227 & 0 & 0 & 0 & 5 & 28 & 34 & 35 & 16 & 119 \\
\hline 7. Trachea, bronchus, and lung cancers & 1,227 & 0 & 0 & 2 & 29 & 188 & 291 & 275 & 93 & 879 \\
\hline 8. Melanoma and other skin cancers & 65 & 0 & 0 & 1 & 3 & 8 & 8 & 9 & 6 & 35 \\
\hline 9. Breast cancer & 473 & 0 & 0 & 0 & 0 & 1 & 1 & 1 & 1 & 3 \\
\hline 10. Cervix uteri cancer & 235 & - & - & - & - & - & - & - & - & - \\
\hline 11. Corpus uteri cancer & 71 & - & - & - & - & - & - & - & - & - \\
\hline 12. Ovarian cancer & 132 & - & - & - & - & - & - & - & - & 一 \\
\hline 13. Prostate cancer & 264 & 0 & 0 & 0 & 1 & 12 & 51 & 108 & 91 & 264 \\
\hline 14. Bladder cancer & 175 & 0 & 0 & 0 & 2 & 15 & 32 & 45 & 29 & 123 \\
\hline 15. Lymphomas and multiple myeloma & 331 & 2 & 7 & 12 & 18 & 32 & 37 & 39 & 21 & 167 \\
\hline 16. Leukemia & 263 & 8 & 12 & 24 & 14 & 21 & 24 & 27 & 15 & 146 \\
\hline Other malignant neoplasms & 746 & 8 & 6 & 12 & 29 & 97 & 116 & 107 & 54 & 428 \\
\hline B. Other neoplasms & 146 & 2 & 2 & 5 & 6 & 13 & 14 & 18 & 13 & 73 \\
\hline C. Diabetes mellitus & 960 & 1 & 1 & 8 & 21 & 88 & 111 & 124 & 73 & 428 \\
\hline D. Endocrine disorders & 240 & 21 & 4 & 7 & 10 & 15 & 13 & 18 & 18 & 107 \\
\hline E. Neuropsychiatric conditions & 1,079 & 26 & 16 & 48 & 89 & 88 & 53 & 121 & 117 & 558 \\
\hline 1. Unipolar depressive disorders & 13 & 0 & 0 & 0 & 2 & 2 & 1 & 0 & 1 & 6 \\
\hline 2. Bipolar affective disorder & 1 & 0 & 0 & 0 & 0 & 0 & 0 & 0 & 0 & 0 \\
\hline 3. Schizophrenia & 23 & 0 & 0 & 0 & 4 & 4 & 1 & 1 & 1 & 11 \\
\hline 4. Epilepsy & 125 & 12 & 7 & 15 & 15 & 10 & 5 & 4 & 2 & 70 \\
\hline 5. Alcohol use disorders & 84 & 0 & 0 & 5 & 20 & 27 & 13 & 6 & 2 & 72 \\
\hline 6. Alzheimer's and other dementias & 380 & 1 & 0 & 0 & 1 & 3 & 8 & 56 & 71 & 141 \\
\hline 7. Parkinson's disease & 95 & 0 & 0 & 0 & 1 & 1 & 4 & 20 & 21 & 49 \\
\hline 8. Multiple sclerosis & 16 & 0 & 0 & 0 & 1 & 2 & 1 & 1 & 0 & 6 \\
\hline 9. Drug use disorders & 86 & 0 & 0 & 13 & 33 & 21 & 2 & 0 & 0 & 70 \\
\hline 10. Post-traumatic stress disorder & 0 & 0 & 0 & 0 & 0 & 0 & 0 & 0 & 0 & 0 \\
\hline 11. Obsessive-compulsive disorder & - & - & - & - & - & - & - & - & - & - \\
\hline 12. Panic disorder & - & - & - & - & - & - & - & - & - & 一 \\
\hline 13. Insomnia (primary) & - & - & - & - & - & - & - & - & - & - \\
\hline 14. Migraine & - & - & - & - & - & - & - & - & - & - \\
\hline 15. Mental retardation, lead-caused & 6 & 1 & 1 & 1 & 0 & 0 & 0 & 0 & 0 & 3 \\
\hline Other neuropsychiatric disorders & 251 & 12 & 8 & 12 & 11 & 17 & 18 & 32 & 20 & 130 \\
\hline F. Sense organ diseases & 3 & $\mathbf{0}$ & 0 & 0 & $\mathbf{0}$ & 0 & 0 & 0 & 0 & 2 \\
\hline 1. Glaucoma & 0 & 0 & - & 0 & 0 & 0 & 0 & 0 & 0 & 0 \\
\hline 2. Cataracts & - & - & - & - & - & - & - & - & - & 一 \\
\hline 3. Vision disorders, age-related & - & - & - & - & - & - & - & - & - & - \\
\hline 4. Hearing loss, adult onset & - & - & - & - & - & - & - & - & - & 一 \\
\hline Other sense organ disorders & 3 & 0 & 0 & 0 & 0 & 0 & 0 & 0 & 0 & 2 \\
\hline G. Cardiovascular diseases & 16,394 & 39 & 24 & 109 & 355 & 1,318 & 1,894 & 2,481 & 1,733 & 7,953 \\
\hline 1. Rheumatic heart disease & 324 & 7 & 5 & 18 & 17 & 29 & 25 & 24 & 11 & 136 \\
\hline 2. Hypertensive heart disease & 889 & 1 & 1 & 4 & 19 & 71 & 104 & 126 & 83 & 409 \\
\hline 3. Ischemic heart disease & 7,063 & 3 & 3 & 27 & 160 & 707 & 947 & 1,160 & 717 & 3,726 \\
\hline 4. Cerebrovascular disease & 5,390 & 7 & 5 & 22 & 74 & 353 & 619 & 859 & 555 & 2,494 \\
\hline 5. Inflammatory heart diseases & 391 & 7 & 2 & 10 & 21 & 39 & 39 & 49 & 37 & 204 \\
\hline Other cardiovascular diseases & 2,337 & 13 & 8 & 28 & 64 & 119 & 160 & 263 & 330 & 983 \\
\hline H. Respiratory diseases & 3,603 & 34 & 9 & 24 & 58 & 250 & 405 & 632 & 454 & 1,866 \\
\hline 1. Chronic obstructive pulmonary disease & 2,676 & 2 & 0 & 2 & 17 & 175 & 322 & 515 & 348 & 1,380 \\
\hline 2. Asthma & 233 & 2 & 4 & 11 & 19 & 30 & 21 & 20 & 11 & 119 \\
\hline Other respiratory diseases & 694 & 30 & 5 & 12 & 22 & 45 & 62 & 97 & 95 & 368 \\
\hline
\end{tabular}


Table 3B.9 Continued

\begin{tabular}{|c|c|c|c|c|c|c|c|c|}
\hline \multicolumn{9}{|c|}{ Female } \\
\hline $0-4$ & 5-14 & $15-29$ & $30-44$ & 45-59 & $60-69$ & $70-79$ & $80+$ & Total \\
\hline 432 & 128 & 299 & 653 & 2,008 & 2,834 & 4,630 & 5,169 & 16,153 \\
\hline 19 & 23 & 59 & 228 & 660 & 709 & 825 & 580 & 3,104 \\
\hline 0 & 0 & 1 & 5 & 22 & 24 & 24 & 18 & 95 \\
\hline 0 & 0 & 1 & 5 & 34 & 44 & 46 & 27 & 158 \\
\hline 0 & 0 & 4 & 23 & 58 & 70 & 96 & 72 & 323 \\
\hline 0 & 0 & 2 & 15 & 45 & 62 & 87 & 85 & 296 \\
\hline 1 & 1 & 5 & 15 & 40 & 46 & 53 & 26 & 187 \\
\hline 0 & 0 & 0 & 4 & 16 & 24 & 35 & 28 & 108 \\
\hline 0 & 1 & 1 & 15 & 69 & 92 & 114 & 55 & 347 \\
\hline 0 & 0 & 1 & 3 & 6 & 6 & 8 & 8 & 30 \\
\hline 0 & 0 & 2 & 55 & 145 & 104 & 94 & 69 & 470 \\
\hline 0 & 0 & 11 & 22 & 73 & 62 & 47 & 20 & 235 \\
\hline 0 & 0 & 0 & 4 & 14 & 17 & 21 & 15 & 71 \\
\hline 0 & 1 & 3 & 11 & 34 & 33 & 33 & 19 & 132 \\
\hline- & - & - & - & - & - & - & - & - \\
\hline 1 & 0 & 0 & 3 & 6 & 10 & 16 & 17 & 53 \\
\hline 3 & 6 & 8 & 13 & 25 & 33 & 43 & 32 & 164 \\
\hline 8 & 10 & 13 & 14 & 18 & 16 & 21 & 18 & 117 \\
\hline 6 & 5 & 6 & 22 & 56 & 66 & 85 & 71 & 318 \\
\hline 2 & 2 & 3 & 6 & 13 & 11 & 16 & 20 & 73 \\
\hline 2 & 2 & 6 & 16 & 84 & 135 & 167 & 121 & 532 \\
\hline 22 & 3 & 8 & 12 & 15 & 16 & 22 & 35 & 133 \\
\hline 20 & 16 & 26 & 32 & 44 & 36 & 121 & 226 & 520 \\
\hline 0 & 0 & 0 & 1 & 3 & 1 & 0 & 1 & 7 \\
\hline 0 & 0 & 0 & 0 & 0 & 0 & 0 & 0 & 1 \\
\hline 0 & 0 & 0 & 2 & 4 & 2 & 2 & 1 & 11 \\
\hline 9 & 8 & 13 & 8 & 7 & 3 & 3 & 3 & 55 \\
\hline 0 & 0 & 0 & 3 & 5 & 2 & 2 & 0 & 12 \\
\hline 1 & 0 & 1 & 1 & 3 & 8 & 63 & 164 & 240 \\
\hline 0 & 0 & 0 & 0 & 1 & 3 & 17 & 25 & 46 \\
\hline 0 & 0 & 0 & 2 & 3 & 2 & 2 & 1 & 9 \\
\hline 0 & 0 & 3 & 7 & 5 & 0 & 0 & 0 & 15 \\
\hline 0 & 0 & 0 & 0 & 0 & 0 & 0 & 0 & 0 \\
\hline- & - & - & - & - & - & - & - & - \\
\hline- & - & - & - & - & - & - & - & - \\
\hline- & - & - & - & - & - & - & - & - \\
\hline- & - & - & - & - & - & - & - & - \\
\hline 0 & 1 & 1 & 0 & 0 & 0 & 0 & 0 & 3 \\
\hline 9 & 7 & 8 & 7 & 13 & 15 & 32 & 30 & 120 \\
\hline 0 & 0 & 0 & 0 & 0 & 0 & 0 & 0 & 2 \\
\hline- & - & - & 0 & 0 & 0 & 0 & 0 & 0 \\
\hline- & - & - & - & - & - & - & - & - \\
\hline- & - & - & - & - & - & - & - & - \\
\hline- & - & - & - & - & - & - & - & - \\
\hline 0 & 0 & 0 & 0 & 0 & 0 & 0 & 0 & 2 \\
\hline 45 & 27 & 94 & 206 & 779 & 1,424 & 2,633 & 3,233 & 8,441 \\
\hline 8 & 8 & 19 & 21 & 36 & 33 & 39 & 24 & 188 \\
\hline 1 & 1 & 3 & 13 & 55 & 91 & 149 & 168 & 480 \\
\hline 2 & 2 & 27 & 72 & 312 & 633 & 1,069 & 1,220 & 3,338 \\
\hline 5 & 5 & 13 & 50 & 254 & 492 & 970 & 1,106 & 2,896 \\
\hline 6 & 3 & 7 & 10 & 23 & 28 & 51 & 60 & 187 \\
\hline 23 & 8 & 25 & 41 & 99 & 147 & 355 & 655 & 1,354 \\
\hline 30 & 9 & 22 & 46 & 183 & 272 & 545 & 629 & 1,736 \\
\hline 2 & 0 & 1 & 13 & 127 & 214 & 453 & 485 & 1,296 \\
\hline 2 & 4 & 12 & 20 & 28 & 16 & 17 & 17 & 115 \\
\hline 26 & 5 & 9 & 13 & 28 & 42 & 74 & 127 & 325 \\
\hline
\end{tabular}


Table 3B.9 Continued

\begin{tabular}{|c|c|c|c|c|c|c|c|c|c|c|}
\hline \multirow[b]{2}{*}{ Cause } & \multicolumn{10}{|c|}{ Male } \\
\hline & Total & $0-4$ & 5-14 & $15-29$ & 30-44 & $45-59$ & $60-69$ & $70-79$ & $80+$ & Total \\
\hline I. Digestive diseases & 1,935 & 48 & 17 & 49 & 137 & 294 & 219 & 198 & 114 & 1,075 \\
\hline 1. Peptic ulcer disease & 261 & 3 & 2 & 7 & 18 & 42 & 31 & 32 & 19 & 153 \\
\hline 2. Cirrhosis of the liver & 771 & 8 & 4 & 17 & 72 & 173 & 115 & 80 & 25 & 493 \\
\hline 3. Appendicitis & 21 & 0 & 1 & 1 & 1 & 3 & 2 & 2 & 1 & 11 \\
\hline Other digestive diseases & 883 & 37 & 11 & 24 & 45 & 77 & 71 & 85 & 68 & 418 \\
\hline J. Genitourinary diseases & 830 & 11 & 7 & 22 & 37 & 75 & 87 & 107 & 87 & 433 \\
\hline 1. Nephritis and nephrosis & 663 & 9 & 6 & 19 & 32 & 62 & 68 & 80 & 62 & 338 \\
\hline 2. Benign prostatic hypertrophy & 31 & - & - & 0 & 1 & 5 & 6 & 10 & 9 & 31 \\
\hline Other genitourinary system diseases & 136 & 2 & 1 & 2 & 4 & 9 & 13 & 17 & 17 & 65 \\
\hline K. Skin diseases & 67 & 1 & 0 & 1 & 3 & 4 & 4 & 6 & 6 & 26 \\
\hline L. Musculoskeletal diseases & 105 & 1 & 1 & 2 & 3 & 6 & 6 & 10 & 9 & 37 \\
\hline 1. Rheumatoid arthritis & 25 & 0 & 0 & 0 & 0 & 1 & 2 & 2 & 1 & 7 \\
\hline 2. Osteoarthritis & 5 & 0 & 0 & 0 & 0 & 0 & 0 & 1 & 1 & 2 \\
\hline 3. Gout & 1 & 0 & 0 & 0 & 0 & 0 & 0 & 0 & 0 & 1 \\
\hline 4. Low back pain & 3 & 0 & 0 & 0 & 0 & 0 & 0 & 0 & 0 & 2 \\
\hline Other musculoskeletal disorders & 71 & 1 & 1 & 2 & 2 & 4 & 4 & 6 & 6 & 25 \\
\hline M. Congenital anomalies & 507 & 227 & 11 & 13 & 4 & 3 & 2 & 1 & 1 & 262 \\
\hline 1. Abdominal wall defect & 4 & 2 & 0 & 0 & 0 & 0 & 0 & 0 & 0 & 2 \\
\hline 2. Anencephaly & 19 & 9 & 0 & 0 & 0 & 0 & 0 & 0 & 0 & 9 \\
\hline 3. Anorectal atresia & 1 & 1 & 0 & 0 & 0 & 0 & 0 & 0 & 0 & 1 \\
\hline 4. Cleft lip & 0 & 0 & 0 & 0 & 0 & 0 & 0 & 0 & 0 & 0 \\
\hline 5. Cleft palate & 1 & 1 & 0 & 0 & 0 & 0 & 0 & 0 & 0 & 1 \\
\hline 6. Esophageal atresia & 1 & 1 & 0 & 0 & 0 & 0 & 0 & 0 & 0 & 1 \\
\hline 7. Renal agenesis & 2 & 1 & 0 & 0 & 0 & 0 & 0 & 0 & 0 & 1 \\
\hline 8. Down syndrome & 24 & 8 & 1 & 2 & 0 & 1 & 0 & 0 & 0 & 12 \\
\hline 9. Congenital heart anomalies & 269 & 116 & 6 & 8 & 2 & 1 & 1 & 1 & 0 & 135 \\
\hline 10. Spina bifida & 24 & 11 & 0 & 0 & 0 & 0 & 0 & 0 & 0 & 12 \\
\hline Other congenital anomalies & 161 & 78 & 4 & 3 & 1 & 1 & 1 & 1 & 0 & 88 \\
\hline N. Oral conditions & 2 & 0 & 0 & 0 & 0 & 0 & 0 & 0 & 0 & 1 \\
\hline 1. Dental caries & 0 & - & - & - & - & 0 & - & 0 & 0 & 0 \\
\hline 2. Periodontal disease & 0 & - & - & 0 & 0 & 0 & 0 & 0 & 0 & 0 \\
\hline 3. Edentulism & - & - & - & - & - & - & - & - & - & - \\
\hline Other oral diseases & 2 & 0 & 0 & 0 & 0 & 0 & 0 & 0 & 0 & 1 \\
\hline III. Injuries & 5,186 & 158 & 247 & 974 & 872 & 636 & 284 & 200 & 111 & 3,483 \\
\hline A. Unintentional injuries & 3,535 & 151 & 227 & 551 & 518 & 422 & 196 & 145 & 87 & 2,296 \\
\hline 1. Road traffic accidents & 1,189 & 29 & 84 & 246 & 224 & 158 & 65 & 44 & 17 & 867 \\
\hline 2. Poisonings & 349 & 9 & 11 & 38 & 59 & 68 & 27 & 10 & 4 & 226 \\
\hline 3. Falls & 387 & 10 & 13 & 29 & 36 & 43 & 31 & 37 & 34 & 234 \\
\hline 4. Fires & 310 & 18 & 17 & 24 & 25 & 16 & 8 & 7 & 3 & 119 \\
\hline 5. Drownings & 385 & 34 & 58 & 68 & 45 & 32 & 12 & 8 & 4 & 263 \\
\hline 6. Other unintentional injuries & 914 & 51 & 44 & 146 & 128 & 104 & 52 & 39 & 25 & 588 \\
\hline B. Intentional injuries & 1,651 & 8 & 21 & 423 & 355 & 213 & 89 & 54 & 24 & 1,187 \\
\hline 1. Self-inflicted injuries & 875 & 0 & 9 & 153 & 149 & 120 & 59 & 40 & 17 & 547 \\
\hline 2. Violence & 556 & 7 & 10 & 186 & 136 & 68 & 20 & 10 & 5 & 442 \\
\hline 3. War & 208 & 0 & 2 & 80 & 66 & 24 & 10 & 3 & 2 & 187 \\
\hline Other intentional injuries & 13 & 1 & 0 & 3 & 3 & 1 & 1 & 1 & 0 & 10 \\
\hline
\end{tabular}


Table 3B.9 Continued

\begin{tabular}{|c|c|c|c|c|c|c|c|c|}
\hline \multicolumn{9}{|c|}{ Female } \\
\hline $0-4$ & 5-14 & $15-29$ & 30-44 & $45-59$ & $60-69$ & $70-79$ & $80+$ & Total \\
\hline 68 & 27 & 48 & 67 & 148 & 141 & 181 & 181 & 860 \\
\hline 3 & 1 & 4 & 9 & 19 & 19 & 25 & 27 & 108 \\
\hline 17 & 8 & 15 & 27 & 70 & 58 & 56 & 26 & 278 \\
\hline 0 & 0 & 1 & 1 & 2 & 2 & 2 & 2 & 9 \\
\hline 48 & 17 & 27 & 30 & 57 & 62 & 97 & 127 & 465 \\
\hline 9 & 7 & 17 & 27 & 65 & 73 & 94 & 105 & 396 \\
\hline 7 & 6 & 14 & 22 & 56 & 63 & 77 & 78 & 325 \\
\hline- & - & - & - & - & - & - & - & - \\
\hline 1 & 1 & 3 & 5 & 8 & 10 & 17 & 27 & 71 \\
\hline 1 & 1 & 1 & 3 & 5 & 7 & 10 & 15 & 42 \\
\hline 1 & 1 & 4 & 6 & 9 & 9 & 15 & 23 & 68 \\
\hline 0 & 0 & 0 & 1 & 3 & 3 & 6 & 4 & 17 \\
\hline 0 & 0 & 0 & 0 & 0 & 0 & 1 & 2 & 3 \\
\hline 0 & 0 & 0 & 0 & 0 & 0 & 0 & 0 & 0 \\
\hline 0 & 0 & 0 & 0 & 0 & 0 & 0 & 1 & 1 \\
\hline 1 & 1 & 4 & 5 & 6 & 5 & 8 & 16 & 46 \\
\hline 212 & 11 & 10 & 4 & 3 & 1 & 2 & 1 & 245 \\
\hline 2 & 0 & 0 & 0 & 0 & 0 & 0 & 0 & 2 \\
\hline 10 & 0 & 0 & 0 & 0 & 0 & 0 & 0 & 10 \\
\hline 0 & 0 & 0 & 0 & 0 & 0 & 0 & 0 & 0 \\
\hline 0 & 0 & 0 & 0 & 0 & 0 & 0 & 0 & 0 \\
\hline 1 & 0 & 0 & 0 & 0 & 0 & 0 & 0 & 1 \\
\hline 1 & 0 & 0 & 0 & 0 & 0 & 0 & 0 & 1 \\
\hline 1 & 0 & 0 & 0 & 0 & 0 & 0 & 0 & 1 \\
\hline 7 & 1 & 2 & 0 & 1 & 0 & 0 & 0 & 12 \\
\hline 115 & 7 & 7 & 3 & 1 & 1 & 1 & 1 & 134 \\
\hline 12 & 0 & 0 & 0 & 0 & 0 & 0 & 0 & 12 \\
\hline 64 & 3 & 2 & 1 & 1 & 1 & 1 & 1 & 73 \\
\hline 0 & 0 & 0 & 0 & 0 & 0 & 0 & 0 & 1 \\
\hline- & - & - & - & 0 & - & - & 0 & 0 \\
\hline- & 0 & 0 & 0 & 0 & 0 & 0 & 0 & 0 \\
\hline- & - & - & - & - & - & - & - & - \\
\hline 0 & 0 & 0 & 0 & 0 & 0 & 0 & 0 & 1 \\
\hline 151 & 168 & 359 & 309 & 271 & 150 & 156 & 139 & 1,703 \\
\hline 145 & 151 & 223 & 190 & 178 & 108 & 121 & 122 & 1,238 \\
\hline 23 & 50 & 61 & 63 & 60 & 28 & 25 & 12 & 323 \\
\hline 7 & 10 & 17 & 24 & 28 & 23 & 10 & 5 & 124 \\
\hline 7 & 8 & 9 & 9 & 16 & 15 & 35 & 54 & 154 \\
\hline 23 & 18 & 66 & 39 & 19 & 9 & 10 & 7 & 191 \\
\hline 25 & 31 & 21 & 13 & 11 & 7 & 7 & 5 & 122 \\
\hline 60 & 35 & 49 & 42 & 44 & 26 & 32 & 38 & 326 \\
\hline 7 & 16 & 136 & 118 & 92 & 42 & 36 & 17 & 465 \\
\hline 0 & 6 & 102 & 83 & 66 & 30 & 27 & 14 & 328 \\
\hline 6 & 9 & 30 & 30 & 21 & 9 & 7 & 2 & 114 \\
\hline 0 & 1 & 4 & 5 & 5 & 3 & 1 & 1 & 20 \\
\hline 0 & 0 & 1 & 0 & 1 & 0 & 0 & 0 & 3 \\
\hline
\end{tabular}

Source: Authors' compilation.

Note: $-=$ an estimate of zero; the number zero in a cell indicates a non-zero estimate of less than 500 .

a. World totals for males and females include residual populations not included in the World Bank regions.

b. For East Asia and Pacific, Europe and Central Asia, and Latin America and the Caribbean regions, these figures include

late effects of polio cases with onset prior to regional certification of polio eradication in 1994, 2000, and 2002, respectively.

c. Does not include liver cancer and cirrhosis deaths resulting from chronic hepatitis virus infection.

d. This cause category includes "Causes arising in the perinatal period" as defined in the International Classification of

Diseases, principally low birthweight, prematurity, birth asphyxia, and birth trauma, and does not include all causes of deaths occurring in the perinatal period. 
ANNEX 3C: DALYs(3,0) by Cause, Sex, Age, and Region, 2001

Table 3C.1 DALYs $(3,0)$ by Cause, Sex, and Age in Low- and Middle-Income Countries, 2001 (thousands)

\begin{tabular}{|c|c|c|c|c|c|c|c|c|c|c|}
\hline \multirow[b]{2}{*}{ Cause } & \multicolumn{10}{|c|}{ Male } \\
\hline & Total & $0-4$ & 5-14 & 15-29 & 30-44 & $45-59$ & $60-69$ & 70-79 & $80+$ & Total \\
\hline Population (millions) & 5,219 & 288 & 563 & 712 & 545 & 326 & 124 & 61 & 15 & 2,636 \\
\hline All causes & $1,386,709$ & 217,652 & 42,491 & 97,880 & 106,062 & 114,028 & 74,490 & 49,024 & 14,065 & 715,692 \\
\hline $\begin{array}{l}\text { I. Communicable, maternal, perinatal, } \\
\text { and nutritional conditions }\end{array}$ & 552,376 & 169,032 & 16,353 & 22,553 & 32,261 & 18,503 & 7,975 & 4,567 & 1,387 & 272,631 \\
\hline A. Infectious and parasitic diseases & 320,663 & 78,874 & 12,391 & 20,370 & 30,127 & 15,899 & 5,391 & 2,551 & 623 & 166,227 \\
\hline 1. Tuberculosis & 35,874 & 730 & 615 & 4,394 & 6,966 & 5,933 & 2,771 & 1,162 & 188 & 22,760 \\
\hline $\begin{array}{l}\text { 2. Sexually transmitted diseases } \\
\text { excluding HIV/AIDS }\end{array}$ & 9,338 & 1,502 & 19 & 703 & 537 & 505 & 145 & 55 & 16 & 3,483 \\
\hline a. Syphilis & 4,122 & 1,004 & 2 & 105 & 267 & 428 & 140 & 52 & 15 & 2,014 \\
\hline b. Chlamydia & 2,438 & 34 & 5 & 154 & 32 & 1 & 0 & - & - & 227 \\
\hline c. Gonorrhea & 2,550 & 448 & 12 & 441 & 216 & 7 & 1 & 0 & 0 & 1,125 \\
\hline $\begin{array}{l}\text { d. Other sexually transmitted } \\
\text { diseases }\end{array}$ & 228 & 15 & 0 & 3 & 22 & 69 & 4 & 2 & 1 & 117 \\
\hline 3. HIV/AIDS & 70,796 & 5,322 & 1,570 & 8,834 & 16,592 & 4,497 & 424 & 40 & 1 & 37,280 \\
\hline 4. Diarrheal diseases & 58,697 & 27,757 & 691 & 528 & 564 & 463 & 270 & 203 & 115 & 30,592 \\
\hline 5. Childhood-cluster diseases & 43,131 & 16,976 & 3,305 & 684 & 278 & 126 & 35 & 12 & 6 & 21,422 \\
\hline a. Pertussis & 11,403 & 5,623 & 49 & - & - & - & 0 & - & 一 & 5,672 \\
\hline b. Poliomyelitis ${ }^{a}$ & 136 & 15 & 8 & 25 & 17 & 4 & 0 & 0 & 0 & 69 \\
\hline c. Diphtheria & 164 & 76 & 6 & 1 & 1 & 1 & 0 & 0 & 0 & 86 \\
\hline d. Measles & 23,091 & 8,432 & 2,716 & 302 & 0 & 0 & 0 & - & - & 11,450 \\
\hline e. Tetanus & 8,336 & 2,831 & 526 & 356 & 260 & 121 & 34 & 12 & 5 & 4,145 \\
\hline 6. Meningitis & 5,475 & 1,308 & 472 & 352 & 244 & 206 & 73 & 54 & 14 & 2,723 \\
\hline 7. Hepatitis $\mathbf{B}^{\mathbf{b}}$ & 2,082 & 92 & 108 & 225 & 398 & 430 & 111 & 38 & 9 & 1,411 \\
\hline Hepatitis $\mathbf{C}^{\mathbf{b}}$ & 844 & 31 & 44 & 82 & 163 & 182 & 47 & 17 & 4 & 570 \\
\hline 8. Malaria & 39,961 & 17,344 & 497 & 455 & 384 & 276 & 125 & 70 & 20 & 19,172 \\
\hline 9. Tropical-cluster diseases & 10,094 & 358 & 1,918 & 2,175 & 1,300 & 695 & 160 & 62 & 13 & 6,680 \\
\hline a. Trypanosomiasis & 1,333 & 67 & 322 & 189 & 149 & 104 & 9 & 3 & 0 & 844 \\
\hline b. Chagas' disease & 584 & 0 & 0 & 125 & 62 & 67 & 32 & 12 & 4 & 303 \\
\hline c. Schistosomiasis & 1,525 & 88 & 279 & 208 & 143 & 113 & 58 & 25 & 5 & 920 \\
\hline d. Leishmaniasis & 1,757 & 88 & 382 & 306 & 171 & 66 & 19 & 6 & 0 & 1,038 \\
\hline e. Lymphatic filariasis & 4,455 & 106 & 914 & 1,310 & 704 & 261 & 17 & 5 & 1 & 3,319 \\
\hline f. Onchocerciasis & 439 & 7 & 20 & 37 & 71 & 84 & 25 & 10 & 3 & 257 \\
\hline 10. Leprosy & 191 & 7 & 19 & 16 & 23 & 29 & 11 & 8 & 1 & 115 \\
\hline 11. Dengue & 529 & 61 & 143 & 12 & 9 & 6 & 3 & 2 & 1 & 238 \\
\hline 12. Japanese encephalitis & 598 & 90 & 67 & 46 & 65 & 11 & 3 & 2 & 1 & 285 \\
\hline 13. Trachoma & 2,620 & 2 & 2 & 23 & 152 & 211 & 150 & 88 & 21 & 649 \\
\hline 14. Intestinal nematode infections & 2,339 & 228 & 910 & 12 & 8 & 10 & 6 & 3 & 1 & 1,178 \\
\hline a. Ascariasis & 1,153 & 112 & 462 & 1 & 0 & 0 & 0 & 0 & 0 & 574 \\
\hline b. Trichuriasis & 489 & 45 & 205 & 1 & 1 & 1 & 1 & 0 & 0 & 253 \\
\hline c. Hookworm disease & 634 & 56 & 237 & 10 & 6 & 8 & 5 & 2 & 1 & 323 \\
\hline Other intestinal infections & 63 & 16 & 6 & 1 & 1 & 2 & 1 & 0 & 0 & 27 \\
\hline Other infectious diseases & 38,095 & 7,065 & 2,010 & 1,829 & 2,442 & 2,318 & 1,058 & 735 & 213 & 17,669 \\
\hline B. Respiratory infections & 86,710 & 32,320 & 2,475 & 1,080 & 1,356 & 1,878 & 2,299 & 1,829 & 698 & 43,936 \\
\hline 1. Lower respiratory infections & 83,606 & 31,654 & 1,930 & 1,006 & 1,274 & 1,799 & 2,227 & 1,786 & 681 & 42,357 \\
\hline 2. Upper respiratory infections & 1,680 & 425 & 67 & 64 & 71 & 76 & 71 & 43 & 17 & 833 \\
\hline 3. Otitis media & 1,424 & 241 & 478 & 11 & 11 & 3 & 2 & 0 & 0 & 747 \\
\hline C. Maternal conditions & 26,383 & - & - & - & - & - & - & - & - & - \\
\hline 1. Maternal hemorrhage & 3,922 & - & - & - & - & - & - & - & - & 一 \\
\hline 2. Maternal sepsis & 5,267 & - & - & - & - & - & - & - & 一 & 一 \\
\hline $\begin{array}{l}\text { 3. Hypertensive disorders of } \\
\text { pregnancy }\end{array}$ & 1889 & - & - & - & - & - & - & - & - & - \\
\hline 4. Obstructed labor & 2,495 & - & - & - & - & - & - & - & - & - \\
\hline 5. Abortion & 3,502 & - & - & - & - & - & - & - & - & - \\
\hline Other maternal conditions & 9,308 & - & - & - & - & - & - & - & - & - \\
\hline D. Perinatal conditions ${ }^{c}$ & 89,068 & 48,595 & 0 & 0 & 0 & $\mathbf{0}$ & 0 & 0 & - & 48,596 \\
\hline 1. Low birthweight & 42,597 & 22,984 & 0 & - & 0 & - & 0 & - & - & 22,984 \\
\hline 2. Birth asphyxia and birth trauma & 31,429 & 17,646 & 0 & 0 & 0 & 0 & - & - & - & 17,646 \\
\hline Other perinatal conditions & 15,043 & 7,965 & 0 & 0 & 0 & 0 & - & 0 & - & 7,966 \\
\hline
\end{tabular}


Table 3C.1 Continued

\begin{tabular}{|c|c|c|c|c|c|c|c|c|}
\hline \multicolumn{9}{|c|}{ Female } \\
\hline $0-4$ & 5-14 & $15-29$ & $30-44$ & $45-59$ & $60-69$ & $70-79$ & $80+$ & Total \\
\hline 274 & 533 & 682 & 530 & 326 & 136 & 78 & 25 & 2,583 \\
\hline 206,246 & 41,746 & 97,168 & 85,867 & 89,704 & 67,948 & 58,445 & 23,893 & 671,017 \\
\hline 160,933 & 18,395 & 43,617 & 30,916 & 12,740 & 6,393 & 4,809 & 1,944 & 279,745 \\
\hline 80,661 & 13,212 & 23,628 & 19,916 & 9,880 & 3,908 & 2,474 & 758 & 154,436 \\
\hline 654 & 659 & 3,386 & 3,752 & 2,596 & 1,307 & 639 & 121 & 13,114 \\
\hline 1,692 & 69 & 2,742 & 887 & 304 & 108 & 47 & 5 & 5,855 \\
\hline 1,215 & 3 & 407 & 244 & 127 & 65 & 42 & 4 & 2,107 \\
\hline 33 & 49 & 1,674 & 307 & 127 & 21 & 0 & 0 & 2,211 \\
\hline 433 & 17 & 651 & 315 & 7 & 2 & 0 & 0 & 1,426 \\
\hline 10 & 0 & 10 & 22 & 43 & 19 & 5 & 1 & 111 \\
\hline 5,142 & 1,543 & 12,402 & 11,328 & 2,738 & 312 & 49 & 1 & 33,516 \\
\hline 25,568 & 654 & 424 & 398 & 378 & 281 & 244 & 159 & 28,105 \\
\hline 17,133 & 3,391 & 708 & 285 & 133 & 39 & 14 & 6 & 21,709 \\
\hline 5,682 & 49 & 0 & - & 0 & 0 & - & - & 5,731 \\
\hline 15 & 7 & 24 & 17 & 4 & 0 & 0 & 0 & 68 \\
\hline 61 & 16 & 0 & 0 & 0 & 0 & 0 & 0 & 78 \\
\hline 8,544 & 2,781 & 315 & - & 0 & 0 & - & - & 11,641 \\
\hline 2,830 & 538 & 368 & 268 & 129 & 38 & 14 & 6 & 4,191 \\
\hline 1,474 & 591 & 289 & 157 & 120 & 68 & 41 & 12 & 2,752 \\
\hline 163 & 50 & 145 & 106 & 122 & 48 & 26 & 10 & 670 \\
\hline 62 & 19 & 57 & 44 & 53 & 22 & 13 & 4 & 273 \\
\hline 18,795 & 500 & 516 & 399 & 321 & 145 & 87 & 26 & 20,789 \\
\hline 295 & 952 & 939 & 497 & 548 & 108 & 58 & 17 & 3,414 \\
\hline 37 & 187 & 121 & 79 & 57 & 6 & 2 & 0 & 490 \\
\hline 0 & 0 & 138 & 46 & 56 & 24 & 13 & 5 & 282 \\
\hline 59 & 190 & 151 & 92 & 57 & 23 & 24 & 9 & 606 \\
\hline 115 & 298 & 192 & 58 & 34 & 17 & 4 & 0 & 719 \\
\hline 77 & 256 & 305 & 171 & 295 & 22 & 8 & 1 & 1,136 \\
\hline 7 & 20 & 32 & 51 & 49 & 15 & 7 & 2 & 183 \\
\hline 9 & 18 & 13 & 15 & 10 & 7 & 4 & 1 & 76 \\
\hline 70 & 176 & 19 & 11 & 7 & 4 & 3 & 1 & 291 \\
\hline 144 & 100 & 32 & 24 & 8 & 4 & 2 & 0 & 313 \\
\hline 2 & 5 & 50 & 441 & 598 & 470 & 313 & 93 & 1,971 \\
\hline 243 & 886 & 10 & 5 & 7 & 5 & 2 & 1 & 1,161 \\
\hline 125 & 453 & 0 & 0 & 0 & 0 & 0 & 0 & 579 \\
\hline 39 & 195 & 1 & 0 & 1 & 0 & 0 & 0 & 236 \\
\hline 54 & 231 & 8 & 5 & 6 & 4 & 2 & 0 & 310 \\
\hline 25 & 7 & 1 & 0 & 1 & 1 & 0 & 0 & 35 \\
\hline 9,215 & 3,599 & 1,894 & 1,568 & 1,935 & 980 & 934 & 301 & 20,426 \\
\hline 30,485 & 3,044 & 1,669 & 1,154 & 1,268 & 2,012 & 2,064 & 1,077 & 42,774 \\
\hline 29,776 & 2,450 & 1,621 & 1,121 & 1,243 & 1,963 & 2,031 & 1,045 & 41,249 \\
\hline 478 & 160 & 40 & 32 & 24 & 48 & 33 & 32 & 847 \\
\hline 231 & 434 & 8 & 1 & 1 & 1 & 0 & 0 & 678 \\
\hline 0 & 158 & 17,028 & 8,748 & 448 & 0 & 0 & 0 & 26,383 \\
\hline 0 & 0 & 1,913 & 1,857 & 152 & - & - & - & 3,922 \\
\hline- & 0 & 3,767 & 1,417 & 83 & - & - & - & 5,267 \\
\hline - & 1 & 1,145 & 698 & 46 & - & - & 0 & 1,889 \\
\hline - & - & 1,744 & 737 & 14 & - & - & - & 2,495 \\
\hline - & 155 & 2,699 & 646 & 2 & - & - & - & 3,502 \\
\hline- & 2 & 5,760 & 3,393 & 153 & 0 & 0 & 0 & 9,308 \\
\hline 40,473 & 0 & 0 & 0 & 0 & 0 & 0 & 0 & 40,473 \\
\hline 19,613 & 0 & - & - & - & - & - & - & 19,613 \\
\hline 13,782 & 0 & 0 & - & 0 & - & 0 & 0 & 13,782 \\
\hline 7,077 & 0 & 0 & 0 & 0 & 0 & 0 & 0 & 7,077 \\
\hline & & & & & & & & $a p$ \\
\hline
\end{tabular}

The Burden of Disease and Mortality by Condition: Data, Methods, and Results for 2001 | 181 
Table 3C.1 Continued

\begin{tabular}{|c|c|c|c|c|c|c|c|c|c|c|}
\hline \multirow[b]{2}{*}{ Cause } & \multicolumn{10}{|c|}{ Male } \\
\hline & Total & $0-4$ & $5-14$ & $15-29$ & $30-44$ & $45-59$ & $60-69$ & $70-79$ & $80+$ & Total \\
\hline E. Nutritional deficiencies & 29,552 & 9,242 & 1,486 & 1,102 & 779 & 726 & 285 & 186 & 66 & 13,872 \\
\hline 1. Protein-energy malnutrition & 15,449 & 6,891 & 433 & 123 & 66 & 120 & 101 & 73 & 34 & 7,842 \\
\hline 2. Iodine deficiency & 2,873 & 1,074 & 352 & 0 & 1 & 2 & 1 & 0 & 0 & 1,430 \\
\hline 3. Vitamin A deficiency & 711 & 233 & 70 & 3 & 6 & 10 & 5 & 2 & 0 & 328 \\
\hline 4. Iron-deficiency anemia & 9,487 & 879 & 598 & 953 & 672 & 530 & 139 & 60 & 15 & 3,847 \\
\hline Other nutritional disorders & 1,032 & 165 & 33 & 23 & 33 & 65 & 38 & 51 & 16 & 424 \\
\hline II. Noncommunicable diseases & 678,483 & 40,662 & 12,508 & 39,898 & 48,592 & 82,245 & 62,479 & 42,709 & 12,241 & 341,334 \\
\hline A. Malignant neoplasms & 74,753 & 560 & 757 & 1,898 & 4,465 & 12,873 & 11,531 & 6,552 & 1,296 & 39,933 \\
\hline 1. Mouth and oropharynx cancers & 4,078 & 5 & 12 & 115 & 382 & 1,050 & 866 & 347 & 69 & 2,846 \\
\hline 2. Esophageal cancer & 5,252 & 1 & 2 & 29 & 302 & 1,108 & 1,146 & 571 & 87 & 3,245 \\
\hline 3. Stomach cancer & 9,616 & 3 & 8 & 121 & 597 & 2,095 & 1,801 & 1,073 & 214 & 5,913 \\
\hline 4. Colon and rectal cancers & 5,060 & 1 & 4 & 109 & 358 & 773 & 758 & 488 & 114 & 2,605 \\
\hline 5. Liver cancer & 7,945 & 20 & 15 & 216 & 893 & 2,337 & 1,327 & 633 & 85 & 5,525 \\
\hline 6. Pancreas cancer & 1,621 & 0 & 2 & 5 & 99 & 340 & 267 & 150 & 29 & 892 \\
\hline 7. Trachea, bronchus, and lung cancers & 10,701 & 3 & 7 & 64 & 564 & 2,600 & 2,811 & 1,481 & 208 & 7,738 \\
\hline 8. Melanoma and other skin cancers & 501 & 1 & 2 & 14 & 38 & 83 & 62 & 38 & 11 & 249 \\
\hline 9. Breast cancer & 5,527 & 0 & 0 & 0 & 2 & 5 & 4 & 3 & 1 & 15 \\
\hline 10. Cervix uteri cancer & 3,799 & - & - & - & - & - & - & - & - & - \\
\hline 11. Corpus uteri cancer & 908 & - & - & - & - & - & - & - & - & - \\
\hline 12. Ovarian cancer & 1,488 & - & - & - & - & - & - & - & - & - \\
\hline 13. Prostate cancer & 1,479 & 1 & 1 & 3 & 12 & 173 & 503 & 601 & 185 & 1,479 \\
\hline 14. Bladder cancer & 1,504 & 2 & 2 & 8 & 46 & 237 & 343 & 282 & 77 & 997 \\
\hline 15. Lymphomas and multiple myeloma & 3,770 & 66 & 196 & 313 & 369 & 432 & 321 & 171 & 41 & 1,909 \\
\hline 16. Leukemia & 3,965 & 224 & 347 & 636 & 290 & 311 & 214 & 132 & 30 & 2,184 \\
\hline Other malignant neoplasms & 7,538 & 235 & 162 & 261 & 514 & 1,330 & 1,106 & 582 & 145 & 4,335 \\
\hline B. Other neoplasms & 1,540 & 69 & 66 & 126 & 116 & 185 & 117 & 77 & 18 & 774 \\
\hline C. Diabetes mellitus & 15,804 & 42 & 70 & 446 & 1,228 & 2,315 & 1,622 & 1,011 & 269 & 7,002 \\
\hline D. Endocrine disorders & 10,943 & 3,663 & 216 & 305 & 297 & 411 & 193 & 138 & 55 & 5,278 \\
\hline E. Neuropsychiatric conditions & 137,074 & 10,291 & 5,938 & 23,898 & 13,022 & 7,037 & 2,731 & 2,323 & 949 & 66,189 \\
\hline 1. Unipolar depressive disorders & 43,427 & 0 & 2,452 & 5,692 & 4,992 & 3,076 & 906 & 180 & 33 & 17,331 \\
\hline 2. Bipolar affective disorder & 8,678 & 0 & 247 & 3,653 & 454 & 14 & 4 & 0 & 0 & 4,372 \\
\hline 3. Schizophrenia & 10,528 & 0 & 781 & 3,731 & 568 & 157 & 32 & 14 & 3 & 5,287 \\
\hline 4. Epilepsy & 5,759 & 501 & 636 & 861 & 591 & 294 & 105 & 48 & 12 & 3,049 \\
\hline 5. Alcohol use disorders & 11,007 & 2 & 101 & 4,029 & 3,427 & 1,621 & 289 & 62 & 8 & 9,540 \\
\hline 6. Alzheimer's and other dementias & 9,640 & 181 & 76 & 85 & 68 & 255 & 788 & 1,513 & 742 & 3,707 \\
\hline 7. Parkinson's disease & 1,239 & 5 & 3 & 10 & 66 & 150 & 139 & 156 & 49 & 578 \\
\hline 8. Multiple sclerosis & 916 & 1 & 43 & 165 & 126 & 40 & 11 & 4 & 1 & 391 \\
\hline 9. Drug use disorders & 4,405 & 1 & 68 & 1,765 & 1,170 & 455 & 27 & 4 & 1 & 3,491 \\
\hline 10. Post-traumatic stress disorder & 2,013 & 0 & 23 & 269 & 177 & 89 & 2 & 1 & 0 & 562 \\
\hline 11. Obsessive-compulsive disorder & 3,136 & - & 158 & 698 & 347 & 103 & 20 & 8 & 1 & 1,336 \\
\hline 12. Panic disorder & 4,015 & - & 70 & 1,209 & 16 & 56 & 7 & 4 & 0 & 1,362 \\
\hline 13. Insomnia (primary) & 2,219 & - & 29 & 278 & 297 & 188 & 95 & 32 & 6 & 925 \\
\hline 14. Migraine & 4,851 & 44 & 511 & 670 & 80 & 6 & 0 & 0 & 0 & 1,311 \\
\hline 15. Mental retardation, lead-caused & 8,599 & 4,319 & 17 & 22 & 7 & 4 & 1 & 0 & 0 & 4,370 \\
\hline Other neuropsychiatric disorders & 16,644 & 5,236 & 724 & 762 & 637 & 527 & 303 & 296 & 92 & 8,577 \\
\hline F. Sense organ diseases & 72,275 & 30 & 165 & 1,621 & 8,069 & 12,018 & 7,042 & 3,113 & 604 & 32,662 \\
\hline 1. Glaucoma & 4,112 & 7 & 30 & 128 & 346 & 649 & 381 & 191 & 45 & 1,776 \\
\hline 2. Cataracts & 28,150 & 17 & 100 & 606 & 2,450 & 4,569 & 2,674 & 1,259 & 281 & 11,955 \\
\hline 3. Vision disorders, age-related & 15,364 & 3 & 34 & 305 & 1,263 & 2,465 & 1,599 & 774 & 175 & 6,617 \\
\hline 4. Hearing loss, adult onset & 24,607 & - & - & 581 & 4,007 & 4,331 & 2,387 & 887 & 101 & 12,293 \\
\hline Other sense organ disorders & 42 & 3 & 2 & 2 & 2 & 4 & 2 & 4 & 1 & 20 \\
\hline G. Cardiovascular diseases & 178,929 & 1,417 & 870 & 3,522 & 8,988 & 24,986 & 25,652 & 20,136 & 6,079 & 91,650 \\
\hline 1. Rheumatic heart disease & 6,151 & 230 & 170 & 553 & 453 & 603 & 344 & 204 & 50 & 2,607 \\
\hline 2. Hypertensive heart disease & 9,969 & 27 & 21 & 110 & 434 & 1,294 & 1,408 & 1,141 & 405 & 4,840 \\
\hline 3. Ischemic heart disease & 71,882 & 103 & 217 & 1,033 & 3,782 & 12,275 & 11,574 & 8,509 & 2,270 & 39,761 \\
\hline 4. Cerebrovascular disease & 62,669 & 215 & 146 & 563 & 2,055 & 7,924 & 9,867 & 8,000 & 2,202 & 30,972 \\
\hline 5. Inflammatory heart diseases & 5,811 & 260 & 80 & 389 & 615 & 756 & 549 & 420 & 153 & 3,222 \\
\hline Other cardiovascular diseases & 22,446 & 582 & 237 & 875 & 1,648 & 2,134 & 1,910 & 1,862 & 998 & 10,248 \\
\hline
\end{tabular}


Table 3C.1 Continued

\begin{tabular}{|c|c|c|c|c|c|c|c|c|}
\hline & & & & Female & & & & \\
\hline $0-4$ & 5-14 & $15-29$ & $30-44$ & 45-59 & $60-69$ & $70-79$ & $80+$ & Total \\
\hline 9,314 & 1,980 & 1,292 & 1,097 & 1,143 & 474 & 271 & 108 & 15,680 \\
\hline 6,674 & 489 & 54 & 68 & 97 & 105 & 75 & 45 & 7,607 \\
\hline 1,100 & 338 & 1 & 1 & 2 & 1 & 1 & 0 & 1,443 \\
\hline 288 & 69 & 9 & 4 & 8 & 3 & 2 & 0 & 382 \\
\hline 945 & 1,039 & 1,210 & 1,006 & 989 & 316 & 106 & 30 & 5,640 \\
\hline 308 & 45 & 19 & 19 & 46 & 49 & 88 & 33 & 607 \\
\hline 38,101 & 13,010 & 38,561 & 44,802 & 70,363 & 58,972 & 51,956 & 21,385 & 337,149 \\
\hline 587 & 669 & 1,602 & 5,079 & 11,048 & 8,382 & 5,836 & 1,618 & 34,820 \\
\hline 9 & 5 & 32 & 123 & 415 & 346 & 224 & 78 & 1,232 \\
\hline 1 & 2 & 21 & 127 & 665 & 632 & 442 & 118 & 2,007 \\
\hline 1 & 4 & 97 & 512 & 1,061 & 930 & 832 & 266 & 3,704 \\
\hline 1 & 3 & 47 & 328 & 685 & 672 & 537 & 182 & 2,456 \\
\hline 32 & 19 & 145 & 363 & 740 & 602 & 426 & 91 & 2,420 \\
\hline 0 & 1 & 9 & 70 & 212 & 211 & 176 & 50 & 729 \\
\hline 6 & 29 & 29 & 280 & 935 & 883 & 682 & 120 & 2,963 \\
\hline 1 & 3 & 12 & 38 & 69 & 59 & 52 & 17 & 251 \\
\hline 1 & 2 & 62 & 1,169 & 2,297 & 1,180 & 624 & 178 & 5,512 \\
\hline 0 & 2 & 341 & 517 & 1,448 & 923 & 470 & 99 & 3,799 \\
\hline 2 & 2 & 18 & 162 & 318 & 226 & 143 & 36 & 908 \\
\hline 2 & 18 & 90 & 258 & 523 & 348 & 203 & 46 & 1,488 \\
\hline- & - & - & - & - & - & - & - & 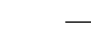 \\
\hline 21 & 7 & 13 & 78 & 109 & 124 & 112 & 44 & 507 \\
\hline 88 & 171 & 216 & 293 & 398 & 360 & 258 & 77 & 1,861 \\
\hline 238 & 272 & 333 & 303 & 298 & 178 & 121 & 37 & 1,781 \\
\hline 184 & 129 & 136 & 457 & 876 & 708 & 534 & 179 & 3,203 \\
\hline 50 & 51 & 70 & 132 & 227 & 121 & 88 & 28 & 766 \\
\hline 60 & 78 & 400 & 1,231 & 2,623 & 2,313 & 1,660 & 438 & 8,802 \\
\hline 3,354 & 213 & 433 & 454 & 526 & 333 & 241 & 111 & 5,665 \\
\hline 9,880 & 6,118 & 23,919 & 13,362 & 8,016 & 3,919 & 3,487 & 2,185 & 70,885 \\
\hline 0 & 2,313 & 7,821 & 8,164 & 5,445 & 1,815 & 432 & 105 & 26,096 \\
\hline 0 & 212 & 3,609 & 462 & 17 & 5 & 1 & 0 & 4,306 \\
\hline 1 & 191 & 3,922 & 800 & 241 & 49 & 29 & 6 & 5,241 \\
\hline 415 & 642 & 793 & 437 & 257 & 92 & 57 & 16 & 2,710 \\
\hline 0 & 41 & 661 & 460 & 244 & 44 & 15 & 2 & 1,467 \\
\hline 163 & 72 & 89 & 74 & 281 & 1,150 & 2,282 & 1,821 & 5,933 \\
\hline 6 & 4 & 7 & 56 & 159 & 165 & 189 & 74 & 661 \\
\hline 1 & 56 & 213 & 174 & 57 & 15 & 6 & 2 & 525 \\
\hline- & 42 & 453 & 295 & 114 & 7 & 2 & 1 & 913 \\
\hline 0 & 16 & 724 & 491 & 200 & 11 & 6 & 2 & 1,450 \\
\hline - & 304 & 769 & 504 & 168 & 37 & 15 & 3 & 1,800 \\
\hline- & 71 & 2,416 & 25 & 114 & 15 & 11 & 1 & 2,653 \\
\hline - & 28 & 304 & 412 & 300 & 168 & 65 & 16 & 1,293 \\
\hline 132 & 1,567 & 1,421 & 416 & 4 & 0 & 0 & 0 & 3,540 \\
\hline 4,182 & 19 & 16 & 8 & 4 & 1 & 1 & 0 & 4,229 \\
\hline 4,980 & 539 & 700 & 585 & 411 & 342 & 376 & 135 & 8,067 \\
\hline 30 & 114 & 1,268 & 8,483 & 14,213 & 9,493 & 4,835 & 1,176 & 39,613 \\
\hline 4 & 26 & 141 & 401 & 812 & 547 & 309 & 95 & 2,336 \\
\hline 14 & 62 & 560 & 2,826 & 6,114 & 4,023 & 2,055 & 542 & 16,195 \\
\hline 9 & 24 & 225 & 1,417 & 3,092 & 2,238 & 1,358 & 384 & 8,746 \\
\hline - & - & 338 & 3,838 & 4,192 & 2,684 & 1,109 & 153 & 12,314 \\
\hline 3 & 2 & 3 & 2 & 3 & 2 & 4 & 2 & 22 \\
\hline 1,614 & 931 & 3,134 & 5,748 & 16,720 & 22,339 & 25,431 & 11,362 & 87,279 \\
\hline 246 & 247 & 589 & 587 & 809 & 556 & 398 & 113 & 3,544 \\
\hline 23 & 25 & 79 & 308 & 1,100 & 1,371 & 1,502 & 720 & 5,129 \\
\hline 74 & 166 & 1,003 & 1,893 & 6,198 & 9,196 & 9,592 & 3,998 & 32,121 \\
\hline 156 & 139 & 349 & 1,480 & 6,136 & 8,728 & 10,344 & 4,366 & 31,697 \\
\hline 203 & 84 & 256 & 322 & 488 & 453 & 520 & 262 & 2,589 \\
\hline 912 & 269 & 857 & 1,157 & 1,989 & 2,035 & 3,076 & 1,903 & 12,199 \\
\hline
\end{tabular}

The Burden of Disease and Mortality by Condition: Data, Methods, and Results for 2001 | 183 
Table 3C.1 Continued

\begin{tabular}{|c|c|c|c|c|c|c|c|c|c|c|c|}
\hline \multirow[b]{2}{*}{ Cause } & & \multicolumn{10}{|c|}{ Male } \\
\hline & & Total & $0-4$ & 5-14 & $15-29$ & $30-44$ & $45-59$ & $60-69$ & 70-79 & $80+$ & Total \\
\hline \multicolumn{2}{|c|}{ H. Respiratory diseases } & 58,086 & 3,254 & 1,699 & 2,368 & 2,918 & 7,262 & 6,468 & 5,466 & 1,889 & 31,324 \\
\hline & $\begin{array}{l}\text { 1. Chronic obstructive pulmonary } \\
\text { disease }\end{array}$ & 33,453 & 48 & 15 & 120 & 1,307 & 5,359 & 5,162 & 4,466 & 1,499 & 17,977 \\
\hline \multicolumn{2}{|r|}{$\begin{array}{l}\text { disease } \\
\text { 2. Asthma }\end{array}$} & 11,514 & 1,013 & 1,348 & 1,783 & 829 & 714 & 285 & 156 & 36 & 6,165 \\
\hline & Other respiratory diseases & 13,119 & 2,193 & 336 & 464 & 781 & 1,188 & 1,021 & 845 & 354 & 7,182 \\
\hline \multirow[t]{5}{*}{ I. } & Digestive diseases & 52,402 & 7,366 & 859 & 2,588 & 4,573 & 7,021 & 3,436 & 1,998 & 571 & 28,411 \\
\hline & 1. Peptic ulcer disease & 4,801 & 91 & 67 & 394 & 667 & 967 & 454 & 269 & 74 & 2,983 \\
\hline & 2. Cirrhosis of the liver & 13,633 & 256 & 152 & 621 & 1,835 & 3,171 & 1,447 & 674 & 121 & 8,278 \\
\hline & 3. Appendicitis & 377 & 7 & 28 & 36 & 38 & 55 & 24 & 19 & 6 & 213 \\
\hline & Other digestive diseases & 33,591 & 7,012 & 612 & 1,536 & 2,033 & 2,829 & 1,510 & 1,036 & 370 & 16,938 \\
\hline \multirow[t]{4}{*}{ J. } & Genitourinary diseases & 16,381 & 975 & 345 & 725 & 1,000 & 3,616 & 1,389 & 983 & 320 & 9,352 \\
\hline & 1. Nephritis and nephrosis & 9,076 & 327 & 283 & 583 & 777 & 1,104 & 837 & 578 & 180 & 4,669 \\
\hline & 2. Benign prostatic hypertrophy & 2,613 & - & - & 0 & 12 & 2,118 & 255 & 173 & 55 & 2,613 \\
\hline & $\begin{array}{l}\text { Other genitourinary system } \\
\text { diseases }\end{array}$ & 4,691 & 648 & 62 & 142 & 211 & 395 & 296 & 233 & 84 & 2,070 \\
\hline $\mathbf{K}$. & Skin diseases & 3,696 & 498 & 225 & 302 & 287 & 256 & 130 & 95 & 36 & 1,828 \\
\hline & Musculoskeletal diseases & 25,693 & 202 & 432 & 1,293 & 3,307 & 3,561 & 1,589 & 689 & 137 & 11,210 \\
\hline & 1. Rheumatoid arthritis & 3,645 & 11 & 75 & 171 & 246 & 308 & 150 & 71 & 14 & 1,046 \\
\hline & 2. Osteoarthritis & 13,666 & 0 & 3 & 367 & 1,348 & 1,970 & 1,060 & 403 & 52 & 5,203 \\
\hline & 3. Gout & 2,785 & 0 & 0 & 123 & 1,307 & 854 & 147 & 39 & 6 & 2,476 \\
\hline & 4. Low back pain & 1,692 & 69 & 167 & 184 & 212 & 184 & 55 & 23 & 4 & 899 \\
\hline & Other musculoskeletal disorders & 3,905 & 122 & 187 & 448 & 195 & 245 & 178 & 153 & 60 & 1,587 \\
\hline \multicolumn{2}{|r|}{ M. Congenital anomalies } & 23,533 & 11,352 & 302 & 339 & 67 & 35 & 12 & 6 & 1 & 12,115 \\
\hline \multicolumn{2}{|r|}{ 1. Abdominal wall defect } & 110 & 59 & 0 & 0 & 0 & 0 & 0 & 0 & 0 & 60 \\
\hline \multicolumn{2}{|r|}{ 2. Anencephaly } & 545 & 258 & 0 & 0 & 0 & 0 & 0 & 0 & 0 & 259 \\
\hline \multicolumn{2}{|r|}{ 3. Anorectal atresia } & 31 & 20 & 0 & 0 & 0 & 0 & 0 & 0 & 0 & 20 \\
\hline \multicolumn{2}{|r|}{ 4. Cleft lip } & 117 & 61 & 0 & 0 & 0 & 0 & 0 & 0 & 0 & 61 \\
\hline \multicolumn{2}{|r|}{ 5. Cleft palate } & 131 & 67 & 0 & 0 & 0 & 0 & 0 & 0 & 0 & 67 \\
\hline \multicolumn{2}{|r|}{ 6. Esophageal atresia } & 46 & 23 & 0 & 0 & 0 & 0 & 0 & 0 & 0 & 23 \\
\hline \multicolumn{2}{|r|}{ 7. Renal agenesis } & 53 & 30 & 0 & 1 & 0 & 0 & 0 & 0 & 0 & 31 \\
\hline \multicolumn{2}{|r|}{ 8. Down syndrome } & 3,416 & 1,736 & 28 & 57 & 3 & 3 & 0 & 0 & 0 & 1,827 \\
\hline & 9. Congenital heart anomalies & 13,191 & 6,198 & 164 & 202 & 42 & 15 & 5 & 3 & 1 & 6,629 \\
\hline \multicolumn{2}{|r|}{ 10. Spina bifida } & 1,488 & 706 & 10 & 4 & 0 & 1 & 0 & 0 & 0 & 721 \\
\hline & Other congenital anomalies & 4,405 & 2,196 & 98 & 74 & 21 & 17 & 6 & 3 & 1 & 2,417 \\
\hline & Oral conditions & 7,375 & 942 & 564 & 467 & 256 & 670 & 569 & 121 & 18 & 3,607 \\
\hline & 1. Dental caries & 4,752 & 919 & 558 & 341 & 144 & 250 & 123 & 60 & 15 & 2,409 \\
\hline & 2. Periodontal disease & 207 & - & - & 26 & 47 & 20 & 7 & 4 & 1 & 103 \\
\hline & 3. Edentulism & 2,293 & - & - & 95 & 59 & 397 & 438 & 57 & 2 & 1,047 \\
\hline & Other oral diseases & 123 & 24 & 5 & 6 & 6 & 3 & 1 & 1 & 0 & 47 \\
\hline III. $\ln$ & juries & 155,850 & 7,959 & 13,630 & 35,429 & 25,209 & 13,279 & 4,036 & 1,747 & 437 & 101,727 \\
\hline & Unintentional injuries & 113,235 & 7,608 & 12,447 & 21,335 & 15,467 & 9,335 & 2,931 & 1,311 & 338 & 70,773 \\
\hline & 1. Road traffic accidents & 32,017 & 1,186 & 3,267 & 7,571 & 5,925 & 3,203 & 860 & 362 & 67 & 22,441 \\
\hline & 2. Poisonings & 7,115 & 286 & 320 & 962 & 1,267 & 1,273 & 368 & 91 & 16 & 4,583 \\
\hline & 3. Falls & 13,582 & 983 & 1,670 & 2,159 & 1,384 & 1,002 & 468 & 360 & 130 & 8,157 \\
\hline & 4. Fires & 10,080 & 923 & 865 & 884 & 738 & 375 & 112 & 55 & 14 & 3,967 \\
\hline & 5. Drownings & 9,391 & 1,010 & 1,680 & 1,835 & 1,057 & 583 & 151 & 62 & 17 & 6,395 \\
\hline & 6. Other unintentional injuries & 41,050 & 3,219 & 4,644 & 7,923 & 5,096 & 2,899 & 972 & 381 & 95 & 25,229 \\
\hline & Intentional injuries & 42,615 & 351 & 1,183 & 14,094 & 9,742 & 3,944 & 1,105 & 436 & 99 & 30,954 \\
\hline & 1. Self-inflicted injuries & 17,674 & 3 & 338 & 3,938 & 3,054 & 1,849 & 646 & 294 & 58 & 10,181 \\
\hline & 2. Violence & 18,132 & 240 & 760 & 7,424 & 4,360 & 1,506 & 291 & 102 & 28 & 14,711 \\
\hline & 3. War & 6,492 & 91 & 71 & 2,628 & 2,254 & 563 & 157 & 35 & 12 & 5,809 \\
\hline & Other intentional injuries & 317 & 17 & 15 & 104 & 74 & 26 & 11 & 5 & 2 & 253 \\
\hline
\end{tabular}


Table 3C.1 Continued

\begin{tabular}{|c|c|c|c|c|c|c|c|c|}
\hline \multicolumn{9}{|c|}{ Female } \\
\hline $0-4$ & 5-14 & $15-29$ & $30-44$ & $45-59$ & 60-69 & $70-79$ & $80+$ & Total \\
\hline 2,709 & 1,790 & 1,938 & 2,405 & 4,997 & 4,615 & 5,539 & 2,770 & 26,762 \\
\hline 63 & 21 & 129 & 1,269 & 3,543 & 3,578 & 4,618 & 2,255 & 15,476 \\
\hline 740 & 1,521 & 1,349 & 604 & 658 & 264 & 159 & 54 & 5,349 \\
\hline 1,907 & 247 & 459 & 533 & 795 & 772 & 762 & 461 & 5,937 \\
\hline 7,072 & 1,164 & 2,371 & 2,749 & 4,630 & 2,867 & 2,261 & 878 & 23,990 \\
\hline 98 & 56 & 194 & 332 & 500 & 299 & 244 & 96 & 1,819 \\
\hline 597 & 299 & 501 & 804 & 1,523 & 914 & 581 & 136 & 5,355 \\
\hline 6 & 21 & 28 & 20 & 35 & 24 & 22 & 8 & 164 \\
\hline 6,371 & 788 & 1,648 & 1,592 & 2,573 & 1,629 & 1,413 & 638 & 16,653 \\
\hline 626 & 333 & 720 & 1,015 & 1,685 & 1,280 & 999 & 371 & 7,028 \\
\hline 266 & 275 & 436 & 564 & 1,095 & 883 & 663 & 224 & 4,407 \\
\hline- & - & - & - & - & - & - & - & - \\
\hline 360 & 58 & 284 & 452 & 589 & 396 & 336 & 147 & 2,621 \\
\hline 348 & 158 & 284 & 295 & 311 & 200 & 180 & 93 & 1,868 \\
\hline 187 & 544 & 1,685 & 3,504 & 4,619 & 2,437 & 1,186 & 322 & 14,483 \\
\hline 21 & 183 & 476 & 616 & 759 & 338 & 165 & 41 & 2,599 \\
\hline 0 & 4 & 632 & 2,133 & 3,133 & 1,719 & 724 & 120 & 8,463 \\
\hline 0 & 0 & 21 & 146 & 84 & 34 & 20 & 4 & 309 \\
\hline 35 & 201 & 125 & 199 & 155 & 51 & 22 & 5 & 794 \\
\hline 131 & 156 & 430 & 410 & 488 & 295 & 256 & 151 & 2,318 \\
\hline 10,700 & 306 & 269 & 78 & 41 & 13 & 9 & 2 & 11,418 \\
\hline 50 & 0 & 0 & 0 & 0 & 0 & 0 & 0 & 50 \\
\hline 284 & 1 & 1 & 0 & 0 & 0 & 0 & 0 & 286 \\
\hline 11 & 0 & 0 & 0 & 0 & 0 & 0 & 0 & 11 \\
\hline 56 & 0 & 0 & 0 & 0 & 0 & 0 & 0 & 56 \\
\hline 63 & 0 & 0 & 0 & 0 & 0 & 0 & 0 & 64 \\
\hline 22 & 0 & 0 & 0 & 0 & 0 & 0 & 0 & 23 \\
\hline 20 & 0 & 0 & 0 & 0 & 0 & 0 & 0 & 22 \\
\hline 1,497 & 30 & 53 & 3 & 4 & 0 & 0 & 0 & 1,588 \\
\hline 6,115 & 187 & 174 & 53 & 21 & 6 & 5 & 1 & 6,562 \\
\hline 755 & 8 & 3 & 1 & 1 & 0 & 0 & 0 & 768 \\
\hline 1,826 & 79 & 37 & 21 & 15 & 6 & 3 & 1 & 1,988 \\
\hline 883 & 541 & 470 & 266 & 709 & 662 & 205 & 32 & 3,768 \\
\hline 860 & 534 & 327 & 141 & 251 & 135 & 73 & 22 & 2,343 \\
\hline- & 0 & 25 & 45 & 20 & 8 & 4 & 1 & 103 \\
\hline- & - & 97 & 63 & 434 & 517 & 126 & 8 & 1,246 \\
\hline 23 & 7 & 20 & 17 & 4 & 2 & 1 & 0 & 76 \\
\hline 7,212 & 10,342 & 14,990 & 10,149 & 6,602 & 2,583 & 1,680 & 564 & 54,123 \\
\hline 7,006 & 9,322 & 10,464 & 7,096 & 4,763 & 1,995 & 1,334 & 481 & 42,462 \\
\hline 1,088 & 2,342 & 2,178 & 1,880 & 1,374 & 426 & 237 & 52 & 9,576 \\
\hline 205 & 293 & 472 & 537 & 549 & 345 & 105 & 26 & 2,532 \\
\hline 953 & 1,422 & 997 & 569 & 503 & 330 & 446 & 205 & 5,424 \\
\hline 1,079 & 1,075 & 2,066 & 1,139 & 472 & 144 & 105 & 33 & 6,113 \\
\hline 762 & 922 & 585 & 328 & 215 & 99 & 64 & 22 & 2,995 \\
\hline 2,919 & 3,269 & 4,167 & 2,643 & 1,651 & 652 & 378 & 143 & 15,821 \\
\hline 206 & 1,019 & 4,526 & 3,053 & 1,839 & 587 & 347 & 83 & 11,661 \\
\hline 1 & 409 & 3,181 & 1,974 & 1,213 & 394 & 257 & 64 & 7,493 \\
\hline 187 & 412 & 1,205 & 920 & 464 & 146 & 73 & 14 & 3,421 \\
\hline 6 & 190 & 126 & 148 & 151 & 43 & 14 & 5 & 683 \\
\hline 12 & 7 & 15 & 11 & 12 & 4 & 2 & 1 & 64 \\
\hline
\end{tabular}

Source: Authors' compilation.

Note: $-=$ an estimate of zero; the number zero in a cell indicates a non-zero estimate of less than 500 .

a. For East Asia and Pacific, Europe and Central Asia, and Latin America and the Caribbean regions, these figures include

late effects of polio cases with onset prior to regional certification of polio eradication in 1994, 2000, and 2002, respectively.

b. Does not include liver cancer and cirrhosis $\operatorname{DALYs}(3,0)$ resulting from chronic hepatitis virus infection.

c. This cause category includes "Causes arising in the perinatal period" as defined in the International Classification of

Diseases, principally low birthweight, prematurity, birth asphyxia, and birth trauma, and does not include all causes of

$\operatorname{DALYs}(3,0)$ occurring in the perinatal period. 
Table 3C.2 DALYs(3,0) by Cause, Sex, and Age in the East Asia and Pacific Region, 2001 (thousands)

\begin{tabular}{|c|c|c|c|c|c|c|c|c|c|c|}
\hline \multirow[b]{2}{*}{ Cause } & \multicolumn{10}{|c|}{ Male } \\
\hline & Total & $0-4$ & $5-14$ & $15-29$ & $30-44$ & 45-59 & $60-69$ & 70-79 & $80+$ & Total \\
\hline Population (millions) & 1,849 & 80 & 175 & 244 & 224 & 136 & 51 & 25 & 6 & 942 \\
\hline $\begin{array}{l}\text { All causes } \\
\text { I. Communicable, maternal, perinatal, } \\
\text { and nutritional conditions }\end{array}$ & $\begin{array}{r}346,225 \\
76,710\end{array}$ & $\begin{array}{l}32,713 \\
20,685\end{array}$ & $\begin{array}{l}8,127 \\
2,069\end{array}$ & $\begin{array}{r}24,304 \\
3,489\end{array}$ & $\begin{array}{r}27,851 \\
4,693\end{array}$ & $\begin{array}{r}37,655 \\
3,712\end{array}$ & $\begin{array}{r}26,822 \\
2,155\end{array}$ & $\begin{array}{r}18,363 \\
1,345\end{array}$ & $\begin{array}{r}5,448 \\
456\end{array}$ & $\begin{array}{r}181,284 \\
38,605\end{array}$ \\
\hline A. Infectious and parasitic diseases & 36,941 & 7,035 & 1,394 & 2,822 & 4,207 & 3,032 & 1,626 & 909 & 214 & 21,238 \\
\hline 1. Tuberculosis & 10,878 & 74 & 110 & 997 & 1,812 & 1,817 & 1,256 & 660 & 117 & 6,842 \\
\hline $\begin{array}{l}\text { 2. Sexually transmitted diseases } \\
\text { excluding HIV/AIDS }\end{array}$ & 848 & 78 & 2 & 86 & 46 & 19 & 11 & 7 & 3 & 252 \\
\hline a. Syphilis & 129 & 37 & 0 & 9 & 7 & 9 & 10 & 7 & 2 & 82 \\
\hline b. Chlamydia & 409 & 4 & 1 & 27 & 6 & 0 & 0 & - & - & 38 \\
\hline c. Gonorrhea & 263 & 36 & 1 & 49 & 31 & 1 & 0 & - & - & 118 \\
\hline $\begin{array}{l}\text { d. Other sexually transmitted } \\
\text { diseases }\end{array}$ & 48 & 0 & - & 1 & 2 & 9 & 0 & - & 1 & 14 \\
\hline 3. HIV/AIDS & 3,087 & 94 & 12 & 657 & 1,204 & 337 & 21 & 2 & $\mathbf{0}$ & 2,328 \\
\hline 4. Diarrheal diseases & 8,782 & 3,661 & 174 & 224 & 228 & 156 & 73 & 44 & 19 & 4,579 \\
\hline 5. Childhood-cluster diseases & 3,707 & 1,201 & 447 & 138 & 40 & 16 & 5 & 2 & 1 & 1,849 \\
\hline a. Pertussis & 579 & 276 & 12 & - & - & - & - & - & - & 288 \\
\hline b. Poliomyelitis ${ }^{a}$ & 49 & 1 & 3 & 10 & 8 & 2 & - & - & - & 24 \\
\hline c. Diphtheria & 18 & 12 & 1 & 0 & 1 & 0 & 0 & - & 0 & 13 \\
\hline d. Measles & 2,303 & 680 & 370 & 90 & - & - & - & - & - & 1,140 \\
\hline e. Tetanus & 758 & 233 & 61 & 37 & 30 & 13 & 5 & 2 & 1 & 383 \\
\hline 6. Meningitis & 1,067 & 295 & 47 & 78 & 49 & 34 & 12 & 10 & 3 & 528 \\
\hline 7. Hepatitis $\mathbf{B}^{\mathbf{b}}$ & 673 & 17 & 2 & 70 & 179 & 218 & 52 & 10 & 3 & 551 \\
\hline Hepatitis $\mathbf{C}^{\mathbf{b}}$ & 275 & 4 & 0 & 29 & 76 & 91 & 21 & 4 & 1 & 228 \\
\hline 8. Malaria & 1,090 & 596 & 43 & 27 & 22 & 14 & 6 & 3 & 1 & 711 \\
\hline 9. Tropical-cluster diseases & 483 & 8 & 51 & 118 & 76 & 63 & 17 & 3 & 1 & 336 \\
\hline a. Trypanosomiasis & - & - & - & - & - & - & - & - & - & - \\
\hline b. Chagas' disease & 0 & - & - & - & - & - & - & - & - & - \\
\hline c. Schistosomiasis & 64 & 0 & 2 & 5 & 7 & 22 & 13 & 1 & 0 & 51 \\
\hline d. Leishmaniasis & 48 & 2 & 8 & 10 & 5 & 2 & 1 & 0 & 0 & 28 \\
\hline e. Lymphatic filariasis & 371 & 6 & 41 & 103 & 63 & 39 & 4 & 1 & 0 & 257 \\
\hline f. Onchocerciasis & - & - & - & - & - & - & - & - & - & - \\
\hline 10. Leprosy & 34 & $\mathbf{0}$ & 1 & 2 & 4 & 6 & 8 & 4 & $\mathbf{0}$ & 25 \\
\hline 11. Dengue & 217 & 28 & 48 & 7 & 3 & 2 & 1 & 1 & 0 & 90 \\
\hline 12. Japanese encephalitis & 301 & 74 & 48 & 17 & 12 & 3 & 1 & 1 & 0 & 155 \\
\hline 13. Trachoma & 500 & $\mathbf{0}$ & $\mathbf{0}$ & 1 & 22 & 38 & 29 & 20 & 5 & 115 \\
\hline 14. Intestinal nematode infections & 680 & 79 & 263 & 1 & 1 & 1 & 1 & 0 & $\mathbf{0}$ & 347 \\
\hline a. Ascariasis & 301 & 34 & 117 & - & - & 0 & - & 0 & - & 151 \\
\hline b. Trichuriasis & 197 & 24 & 79 & - & - & - & - & - & - & 103 \\
\hline c. Hookworm disease & 168 & 16 & 65 & 1 & 1 & 1 & 1 & 0 & 0 & 86 \\
\hline Other intestinal infections & 14 & 5 & 2 & 0 & 0 & 0 & 0 & 0 & 0 & 7 \\
\hline Other infectious diseases & 4,318 & 825 & 146 & 371 & 433 & 217 & 114 & 138 & 60 & 2,302 \\
\hline B. Respiratory infections & 11,800 & 2,414 & 434 & 310 & 300 & 492 & 459 & 402 & 230 & 5,044 \\
\hline 1. Lower respiratory infections & 10,786 & 2,171 & 282 & 274 & 262 & 442 & 435 & 394 & 222 & 4,482 \\
\hline 2. Upper respiratory infections & 598 & 177 & 8 & 31 & 36 & 49 & 23 & 8 & 8 & 341 \\
\hline 3. Otitis media & 416 & 67 & 143 & 5 & 3 & 1 & 1 & 0 & 0 & 221 \\
\hline C. Maternal conditions & 3,475 & - & - & - & - & - & - & - & - & - \\
\hline 1. Maternal hemorrhage & 322 & - & - & - & - & - & - & - & - & - \\
\hline 2. Maternal sepsis & 881 & - & - & - & - & - & - & - & - & 一 \\
\hline 3. Hypertensive disorders & & & & & & & & & & \\
\hline of pregnancy & 128 & - & - & - & - & - & - & - & - & - \\
\hline 4. Obstructed labor & 239 & - & - & - & - & - & - & - & - & - \\
\hline 5. Abortion & 191 & - & - & - & - & - & - & - & - & - \\
\hline Other maternal conditions & 1,714 & - & - & - & - & - & - & - & - & - \\
\hline D. Perinatal conditions ${ }^{c}$ & 18,696 & 9,697 & 0 & - & - & - & - & - & - & 9,697 \\
\hline 1. Low birthweight & 6,226 & 3,233 & - & - & - & - & - & - & - & 3,233 \\
\hline 2. Birth asphyxia and birth trauma & 7,737 & 4,044 & - & - & - & - & - & - & - & 4,044 \\
\hline Other perinatal conditions & 4,734 & 2,420 & 0 & - & - & - & - & - & - & 2,420 \\
\hline
\end{tabular}


Table 3C.2 Continued

\begin{tabular}{|c|c|c|c|c|c|c|c|c|}
\hline & & & & Female & & & & \\
\hline $0-4$ & 5-14 & $15-29$ & $30-44$ & 45-59 & $60-69$ & $70-79$ & $80+$ & Total \\
\hline 74 & 161 & 232 & 217 & 131 & 52 & 30 & 10 & 907 \\
\hline 32,207 & 7,531 & 20,001 & 22,375 & 29,536 & 22,927 & 20,849 & 9,516 & 164,941 \\
\hline 20,993 & 2,310 & 5,035 & 3,961 & 2,359 & 1,490 & 1,253 & 703 & 38,104 \\
\hline 6,177 & 1,331 & 2,122 & 2,392 & 1,773 & 1,000 & 692 & 215 & 15,702 \\
\hline 68 & 116 & 749 & 1,104 & 963 & 602 & 357 & 77 & 4,036 \\
\hline 58 & 9 & 360 & 115 & 34 & 12 & 6 & 1 & 596 \\
\hline 19 & 0 & 11 & 7 & 0 & 4 & 3 & 1 & 46 \\
\hline 4 & 7 & 278 & 59 & 20 & 3 & - & - & 371 \\
\hline 35 & 2 & 69 & 39 & 0 & 0 & - & - & 145 \\
\hline 0 & - & 2 & 9 & 13 & 6 & 3 & 1 & 34 \\
\hline 73 & 11 & 210 & 259 & 171 & 29 & 6 & 0 & 759 \\
\hline 3,356 & 159 & 199 & 198 & 138 & 73 & 52 & 28 & 4,203 \\
\hline 1,201 & 450 & 143 & 40 & 17 & 5 & 2 & 1 & 1,859 \\
\hline 280 & 12 & - & - & - & - & - & - & 292 \\
\hline 1 & 3 & 10 & 8 & 2 & - & - & - & 24 \\
\hline 4 & 1 & 0 & 0 & 0 & - & - & - & 5 \\
\hline 698 & 372 & 94 & - & - & - & - & - & 1,163 \\
\hline 219 & 62 & 39 & 32 & 14 & 5 & 2 & 1 & 375 \\
\hline 263 & 37 & 83 & 80 & 46 & 17 & 9 & 2 & 539 \\
\hline 16 & 2 & 22 & 39 & 25 & 11 & 4 & 3 & 122 \\
\hline 6 & 0 & 9 & 17 & 9 & 4 & 2 & 1 & 48 \\
\hline 287 & 36 & 20 & 17 & 11 & 5 & 3 & 1 & 379 \\
\hline 6 & 16 & 48 & 32 & 37 & 4 & 1 & 2 & 146 \\
\hline- & - & - & - & - & - & - & - & - \\
\hline- & - & - & 0 & - & - & - & - & 0 \\
\hline 0 & 1 & 2 & 3 & 3 & 2 & 0 & 2 & 12 \\
\hline 2 & 5 & 7 & 4 & 1 & 0 & 0 & 0 & 20 \\
\hline 4 & 11 & 39 & 25 & 32 & 2 & 1 & 0 & 114 \\
\hline- & - & - & - & - & - & - & - & - \\
\hline 0 & 1 & 1 & 3 & 2 & 1 & 0 & 0 & 8 \\
\hline 40 & 60 & 14 & 6 & 4 & 2 & 1 & 1 & 127 \\
\hline 67 & 47 & 14 & 13 & 3 & 1 & 0 & 0 & 146 \\
\hline 0 & 0 & 4 & 76 & 116 & 94 & 70 & 24 & 385 \\
\hline 76 & 252 & 2 & 1 & 1 & 1 & 0 & 0 & 333 \\
\hline 37 & 112 & 0 & 0 & - & 0 & - & - & 149 \\
\hline 18 & 76 & - & - & - & - & - & - & 94 \\
\hline 15 & 62 & 2 & 1 & 1 & 1 & 0 & 0 & 82 \\
\hline 6 & 1 & 0 & 0 & - & 0 & 0 & 0 & 8 \\
\hline 659 & 136 & 245 & 392 & 197 & 137 & 177 & 73 & 2,016 \\
\hline 4,412 & 450 & 172 & 159 & 235 & 373 & 498 & 458 & 6,756 \\
\hline 4,176 & 305 & 157 & 147 & 227 & 365 & 492 & 434 & 6,304 \\
\hline 175 & 12 & 14 & 12 & 7 & 8 & 6 & 24 & 258 \\
\hline 62 & 133 & 0 & 0 & 0 & 0 & - & 0 & 195 \\
\hline- & 6 & 2,342 & 1,116 & 12 & - & - & - & 3,475 \\
\hline- & - & 155 & 165 & 2 & - & - & - & 322 \\
\hline- & - & 721 & 159 & 1 & - & - & - & 881 \\
\hline- & 0 & 66 & 61 & 1 & - & - & - & 128 \\
\hline- & - & 177 & 62 & 0 & - & - & - & 239 \\
\hline- & 6 & 123 & 61 & 2 & - & - & - & 191 \\
\hline- & - & 1,100 & 608 & 6 & - & - & - & 1,714 \\
\hline 8,999 & 0 & - & - & - & - & - & - & 8,999 \\
\hline 2,993 & 0 & - & - & - & - & - & - & 2,993 \\
\hline 3,693 & - & - & - & - & - & - & - & 3,693 \\
\hline \multirow[t]{2}{*}{2,313} & - & - & - & - & - & - & - & 2,313 \\
\hline & & & & & \multicolumn{4}{|c|}{ (Continues on the following page.) } \\
\hline
\end{tabular}


Table 3C.2 Continued

\begin{tabular}{|c|c|c|c|c|c|c|c|c|c|c|}
\hline \multirow[b]{2}{*}{ Cause } & \multicolumn{10}{|c|}{ Male } \\
\hline & Total & $0-4$ & 5-14 & $15-29$ & $30-44$ & $45-59$ & $60-69$ & $70-79$ & 80+ & Total \\
\hline E. Nutritional deficiencies & 5,797 & 1,539 & 241 & 357 & 186 & 188 & 69 & 34 & 12 & 2,626 \\
\hline 1. Protein-energy malnutrition & 2,725 & 1,303 & 27 & 29 & 22 & 24 & 13 & 8 & 5 & 1,431 \\
\hline 2. Iodine deficiency & 251 & 92 & 32 & 0 & 0 & 0 & 0 & 0 & 0 & 125 \\
\hline 3. Vitamin A deficiency & 10 & 2 & 3 & - & 1 & 0 & - & - & - & 7 \\
\hline 4. Iron-deficiency anemia & 2,695 & 124 & 176 & 325 & 160 & 157 & 50 & 17 & 3 & 1,013 \\
\hline Other nutritional disorders & 116 & 18 & 2 & 3 & 3 & 7 & 6 & 9 & 3 & 51 \\
\hline II. Noncommunicable diseases & 228,073 & 10,262 & 3,138 & 12,214 & 16,524 & 30,234 & 23,387 & 16,449 & 4,848 & 117,055 \\
\hline A. Malignant neoplasms & 32,341 & 181 & 247 & 718 & 2,158 & 6,608 & 5,244 & 2,915 & 482 & 18,553 \\
\hline 1. Mouth and oropharynx cancers & 1,064 & 0 & 1 & 33 & 133 & 313 & 170 & 72 & 12 & 736 \\
\hline 2. Esophageal cancer & 3,217 & 0 & 0 & 16 & 189 & 678 & 703 & 377 & 50 & 2,013 \\
\hline 3. Stomach cancer & 6,134 & 1 & 2 & 74 & 375 & 1,437 & 1,155 & 686 & 131 & 3,861 \\
\hline 4. Colon and rectal cancers & 2,330 & 0 & 0 & 49 & 173 & 394 & 339 & 201 & 46 & 1,202 \\
\hline 5. Liver cancer & 5,923 & 7 & 6 & 145 & 689 & 1,905 & 1,009 & 472 & 57 & 4,290 \\
\hline 6. Pancreas cancer & 544 & 0 & 1 & 2 & 39 & 137 & 82 & 47 & 8 & 317 \\
\hline 7. Trachea, bronchus, and lung cancers & 5,333 & 2 & 3 & 25 & 276 & 1,124 & 1,281 & 709 & 98 & 3,518 \\
\hline 8. Melanoma and other skin cancers & 66 & 0 & 0 & 1 & 3 & 12 & 10 & 5 & 1 & 32 \\
\hline 9. Breast cancer & 1,730 & - & - & - & 0 & 0 & 0 & 0 & 0 & 1 \\
\hline 10. Cervix uteri cancer & 805 & - & - & - & - & - & - & - & - & - \\
\hline 11. Corpus uteri cancer & 175 & - & - & - & - & - & - & - & - & - \\
\hline 12. Ovarian cancer & 464 & - & - & - & - & - & - & - & - & - \\
\hline 13. Prostate cancer & 164 & 0 & 0 & 0 & 1 & 18 & 55 & 73 & 17 & 164 \\
\hline 14. Bladder cancer & 348 & 0 & 0 & 1 & 6 & 50 & 84 & 85 & 21 & 248 \\
\hline 15. Lymphomas and multiple myeloma & 753 & 15 & 44 & 69 & 92 & 121 & 70 & 32 & 7 & 451 \\
\hline 16. Leukemia & 1,652 & 98 & 156 & 269 & 109 & 136 & 77 & 45 & 9 & 900 \\
\hline Other malignant neoplasms & 1,640 & 57 & 33 & 34 & 72 & 282 & 207 & 111 & 25 & 820 \\
\hline B. Other neoplasms & 354 & 15 & 16 & 17 & 18 & 53 & 24 & 16 & 3 & 163 \\
\hline C. Diabetes mellitus & 4,918 & 5 & 14 & 116 & 391 & 702 & 514 & 313 & 72 & 2,126 \\
\hline D. Endocrine disorders & 2,560 & 585 & 57 & 71 & 75 & 93 & 42 & 33 & 18 & 975 \\
\hline E. Neuropsychiatric conditions & 42,926 & 2,395 & 1,636 & 7,643 & 4,601 & 2,490 & 1,083 & 847 & 354 & 21,050 \\
\hline 1. Unipolar depressive disorders & 14,037 & 0 & 746 & 1,902 & 1,720 & 1,221 & 351 & 68 & 11 & 6,019 \\
\hline 2. Bipolar affective disorder & 3,115 & 0 & 64 & 1,271 & 201 & 6 & 2 & 0 & 0 & 1,544 \\
\hline 3. Schizophrenia & 3,930 & 0 & 362 & 1,331 & 284 & 60 & 11 & 3 & 1 & 2,051 \\
\hline 4. Epilepsy & 1,303 & 80 & 126 & 199 & 178 & 71 & 25 & 11 & 3 & 693 \\
\hline 5. Alcohol use disorders & 4,303 & 0 & 33 & 1,475 & 1,510 & 657 & 100 & 18 & 3 & 3,796 \\
\hline 6. Alzheimer's and other dementias & 4,110 & 65 & 28 & 35 & 39 & 123 & 437 & 598 & 287 & 1,613 \\
\hline 7. Parkinson's disease & 435 & 1 & 0 & 3 & 21 & 48 & 46 & 61 & 23 & 202 \\
\hline 8. Multiple sclerosis & 317 & 0 & 14 & 58 & 46 & 11 & 2 & 1 & 0 & 131 \\
\hline 9. Drug use disorders & 425 & 0 & 17 & 172 & 109 & 40 & 2 & 0 & 0 & 340 \\
\hline 10. Post-traumatic stress disorder & 748 & 0 & 8 & 84 & 75 & 38 & 1 & 1 & 0 & 207 \\
\hline 11. Obsessive-compulsive disorder & 667 & - & 10 & 134 & 94 & 29 & 3 & 1 & 0 & 270 \\
\hline 12. Panic disorder & 1,400 & - & 26 & 411 & 6 & 25 & 3 & 2 & 0 & 472 \\
\hline 13. Insomnia (primary) & 596 & - & 12 & 46 & 51 & 39 & 18 & 11 & 2 & 179 \\
\hline 14. Migraine & 1,691 & 7 & 89 & 295 & 28 & 2 & 0 & 0 & 0 & 421 \\
\hline 15. Mental retardation, lead-caused & 2,598 & 1,335 & 1 & 0 & 0 & 0 & 0 & 0 & 0 & 1,336 \\
\hline Other neuropsychiatric disorders & 3,255 & 907 & 100 & 227 & 241 & 120 & 82 & 73 & 24 & 1,775 \\
\hline F. Sense organ diseases & 27,758 & 1 & 26 & 453 & 2,895 & 5,007 & 3,116 & 1,244 & 223 & 12,964 \\
\hline 1. Glaucoma & 1,703 & 0 & 2 & 13 & 97 & 336 & 200 & 85 & 19 & 753 \\
\hline 2. Cataracts & 9,727 & 0 & 1 & 42 & 609 & 1,766 & 1,214 & 519 & 95 & 4,246 \\
\hline 3. Vision disorders, age-related & 7,608 & 0 & 23 & 166 & 617 & 1,323 & 828 & 345 & 80 & 3,383 \\
\hline 4. Hearing loss, adult onset & 8,712 & - & - & 231 & 1,571 & 1,580 & 873 & 294 & 29 & 4,578 \\
\hline Other sense organ disorders & 8 & 0 & 0 & 1 & 1 & 1 & 0 & 0 & 0 & 4 \\
\hline G. Cardiovascular diseases & 52,872 & 249 & 182 & 951 & 2,381 & 6,987 & 7,874 & 6,652 & 2,090 & 27,365 \\
\hline 1. Rheumatic heart disease & 2,244 & 24 & 32 & 160 & 159 & 228 & 150 & 90 & 21 & 864 \\
\hline 2. Hypertensive heart disease & 4,234 & 6 & 3 & 30 & 170 & 558 & 676 & 545 & 191 & 2,180 \\
\hline 3. Ischemic heart disease & 14,242 & 26 & 65 & 328 & 807 & 2,071 & 2,097 & 1,725 & 541 & 7,661 \\
\hline 4. Cerebrovascular disease & 25,832 & 49 & 20 & 157 & 830 & 3,509 & 4,377 & 3,714 & 1,037 & 13,692 \\
\hline 5. Inflammatory heart diseases & 1,147 & 25 & 11 & 52 & 71 & 121 & 123 & 118 & 56 & 577 \\
\hline Other cardiovascular diseases & 5,173 & 119 & 50 & 224 & 345 & 500 & 449 & 461 & 244 & 2,392 \\
\hline
\end{tabular}


Table 3C.2 Continued

\begin{tabular}{|c|c|c|c|c|c|c|c|c|}
\hline & & & & Female & & & & \\
\hline $0-4$ & 5-14 & $15-29$ & $30-44$ & $45-59$ & $60-69$ & $70-79$ & $80+$ & Total \\
\hline 1,405 & 523 & 399 & 294 & 340 & 117 & 63 & 30 & 3,171 \\
\hline 1,183 & 22 & 6 & 17 & 20 & 20 & 15 & 11 & 1,294 \\
\hline 94 & 33 & 0 & 0 & 0 & 0 & 0 & 0 & 127 \\
\hline 2 & 2 & - & - & - & - & - & - & 3 \\
\hline 116 & 465 & 391 & 275 & 311 & 86 & 29 & 8 & 1,682 \\
\hline 10 & 2 & 1 & 3 & 9 & 11 & 19 & 11 & 65 \\
\hline 9,423 & 2,874 & 11,115 & 14,652 & 24,822 & 20,560 & 18,988 & 8,584 & 111,018 \\
\hline 196 & 207 & 453 & 2,065 & 4,548 & 3,266 & 2,415 & 637 & 13,788 \\
\hline 1 & 1 & 6 & 38 & 126 & 79 & 59 & 17 & 329 \\
\hline 0 & 0 & 5 & 53 & 407 & 384 & 288 & 67 & 1,204 \\
\hline 0 & 0 & 53 & 329 & 670 & 543 & 509 & 169 & 2,273 \\
\hline 0 & 0 & 19 & 172 & 345 & 303 & 211 & 77 & 1,128 \\
\hline 19 & 7 & 69 & 254 & 517 & 424 & 285 & 58 & 1,633 \\
\hline 0 & 1 & 3 & 31 & 70 & 59 & 49 & 14 & 227 \\
\hline 4 & 24 & 10 & 156 & 559 & 541 & 446 & 74 & 1,814 \\
\hline 0 & 0 & 1 & 5 & 10 & 8 & 7 & 2 & 34 \\
\hline 0 & 0 & 16 & 423 & 805 & 304 & 143 & 39 & 1,730 \\
\hline 0 & 0 & 65 & 93 & 308 & 196 & 120 & 22 & 805 \\
\hline 0 & 0 & 2 & 39 & 72 & 38 & 19 & 5 & 175 \\
\hline 1 & 5 & 28 & 104 & 175 & 91 & 50 & 11 & 464 \\
\hline - & - & - & - & - & - & - & - & - \\
\hline 0 & 0 & 1 & 7 & 17 & 29 & 34 & 12 & 100 \\
\hline 7 & 15 & 31 & 65 & 80 & 49 & 42 & 12 & 303 \\
\hline 101 & 123 & 123 & 155 & 136 & 62 & 39 & 13 & 753 \\
\hline 62 & 30 & 21 & 142 & 250 & 158 & 114 & 44 & 819 \\
\hline 17 & 6 & 13 & 29 & 67 & 30 & 22 & 7 & 191 \\
\hline 7 & 15 & 106 & 399 & 830 & 734 & 559 & 142 & 2,792 \\
\hline 884 & 52 & 162 & 207 & 112 & 75 & 56 & 36 & 1,585 \\
\hline 2,053 & 1,405 & 7,489 & 4,191 & 3,047 & 1,465 & 1,301 & 925 & 21,877 \\
\hline- & 688 & 1,767 & 2,472 & 2,214 & 671 & 163 & 43 & 8,017 \\
\hline 0 & 83 & 1,277 & 201 & 8 & 2 & 0 & 0 & 1,571 \\
\hline 0 & 88 & 1,389 & 275 & 98 & 17 & 10 & 2 & 1,879 \\
\hline 55 & 113 & 187 & 145 & 66 & 25 & 14 & 4 & 609 \\
\hline - & 17 & 217 & 190 & 75 & 7 & 2 & 0 & 507 \\
\hline 54 & 26 & 38 & 42 & 131 & 522 & 900 & 783 & 2,496 \\
\hline 0 & - & 3 & 10 & 42 & 55 & 81 & 42 & 233 \\
\hline 0 & 24 & 75 & 67 & 16 & 3 & 1 & 0 & 186 \\
\hline - & 9 & 38 & 27 & 10 & 0 & 0 & 0 & 85 \\
\hline - & 5 & 241 & 212 & 75 & 4 & 2 & 1 & 540 \\
\hline - & 4 & 200 & 144 & 42 & 4 & 1 & 0 & 396 \\
\hline- & 20 & 840 & 10 & 47 & 6 & 4 & 1 & 927 \\
\hline- & 10 & 81 & 142 & 97 & 59 & 22 & 6 & 417 \\
\hline 19 & 258 & 945 & 47 & 1 & 0 & 0 & 0 & 1,270 \\
\hline 1,261 & 0 & 0 & 0 & 0 & 0 & 0 & 0 & 1,261 \\
\hline 663 & 60 & 192 & 206 & 126 & 90 & 100 & 43 & 1,480 \\
\hline 1 & 4 & 273 & 2,661 & 5,620 & 3,964 & 1,841 & 431 & 14,794 \\
\hline- & 2 & 13 & 99 & 404 & 269 & 125 & 37 & 949 \\
\hline 0 & 1 & 18 & 529 & 2,175 & 1,756 & 817 & 185 & 5,481 \\
\hline 0 & 0 & 98 & 784 & 1,645 & 1,030 & 514 & 154 & 4,225 \\
\hline - & - & 143 & 1,248 & 1,395 & 908 & 384 & 55 & 4,134 \\
\hline 1 & 1 & 1 & 0 & 1 & 0 & 0 & 0 & 4 \\
\hline 206 & 165 & 706 & 1,647 & 5,019 & 6,227 & 7,757 & 3,780 & 25,507 \\
\hline 24 & 38 & 166 & 232 & 355 & 285 & 212 & 67 & 1,380 \\
\hline 4 & 3 & 18 & 104 & 416 & 546 & 638 & 324 & 2,054 \\
\hline 14 & 44 & 245 & 452 & 1,246 & 1,756 & 1,890 & 935 & 6,582 \\
\hline 37 & 24 & 82 & 532 & 2,430 & 3,057 & 4,121 & 1,858 & 12,140 \\
\hline 19 & 9 & 32 & 44 & 93 & 102 & 158 & 112 & 570 \\
\hline 108 & 47 & 163 & 282 & 479 & 481 & 738 & 484 & 2,781 \\
\hline
\end{tabular}


Table 3C.2 Continued

\begin{tabular}{|c|c|c|c|c|c|c|c|c|c|c|}
\hline \multirow[b]{2}{*}{ Cause } & \multicolumn{10}{|c|}{ Male } \\
\hline & Total & $0-4$ & $5-14$ & $15-29$ & $30-44$ & $45-59$ & $60-69$ & $70-79$ & $80+$ & Total \\
\hline H. Respiratory diseases & 23,551 & 660 & 391 & 613 & 729 & 2,813 & 2,854 & 3,065 & 1,227 & 12,353 \\
\hline $\begin{array}{l}\text { 1. Chronic obstructive pulmonary } \\
\text { disease }\end{array}$ & 17,181 & 7 & 2 & 13 & 308 & 2,346 & 2,476 & 2,724 & 1,049 & 8,924 \\
\hline 2. Asthma & 3,203 & 254 & 335 & 513 & 267 & 209 & 77 & 46 & 9 & 1,709 \\
\hline Other respiratory diseases & 3,167 & 399 & 54 & 88 & 154 & 258 & 302 & 296 & 169 & 1,720 \\
\hline I. Digestive diseases & 15,419 & 2,427 & 129 & 667 & 1,378 & 2,162 & 1,091 & 687 & 210 & 8,750 \\
\hline 1. Peptic ulcer disease & 1,800 & 32 & 11 & 104 & 229 & 365 & 202 & 119 & 34 & 1,096 \\
\hline 2. Cirrhosis of the liver & 3,882 & 39 & 17 & 163 & 579 & 994 & 437 & 217 & 41 & 2,487 \\
\hline 3. Appendicitis & 122 & 2 & 6 & 12 & 14 & 23 & 7 & 5 & 2 & 70 \\
\hline Other digestive diseases & 9,615 & 2,354 & 96 & 388 & 556 & 780 & 445 & 346 & 133 & 5,097 \\
\hline J. Genitourinary diseases & 5,388 & 185 & 90 & 271 & 347 & 1,328 & 509 & 326 & 99 & 3,154 \\
\hline 1. Nephritis and nephrosis & 3,041 & 52 & 69 & 230 & 287 & 384 & 294 & 186 & 53 & 1,555 \\
\hline 2. Benign prostatic hypertrophy & 997 & - & - & - & 5 & 820 & 91 & 62 & 20 & 997 \\
\hline Other genitourinary system diseases & 1,350 & 132 & 21 & 41 & 55 & 124 & 125 & 78 & 26 & 602 \\
\hline K. Skin diseases & 960 & 92 & 58 & 86 & 71 & 70 & 34 & 23 & 8 & 442 \\
\hline L. Musculoskeletal diseases & 10,389 & 45 & 119 & 382 & 1,372 & 1,666 & 774 & 287 & 57 & 4,702 \\
\hline 1. Rheumatoid arthritis & 1,225 & 1 & 11 & 29 & 86 & 141 & 70 & 31 & 6 & 375 \\
\hline 2. Osteoarthritis & 5,724 & 0 & 1 & 105 & 475 & 909 & 514 & 146 & 15 & 2,166 \\
\hline 3. Gout & 1,414 & 0 & 0 & 52 & 667 & 444 & 76 & 20 & 3 & 1,262 \\
\hline 4. Low back pain & 633 & 20 & 54 & 64 & 82 & 79 & 24 & 10 & 2 & 335 \\
\hline Other musculoskeletal disorders & 1,394 & 24 & 52 & 131 & 63 & 93 & 90 & 80 & 32 & 565 \\
\hline M. Congenital anomalies & 6,208 & 2,992 & 103 & 128 & 28 & 13 & 4 & 2 & 1 & 3,272 \\
\hline 1. Abdominal wall defect & 6 & 4 & - & - & 0 & - & - & - & 0 & 4 \\
\hline 2. Anencephaly & 49 & 28 & 0 & - & - & - & - & - & 0 & 29 \\
\hline 3. Anorectal atresia & 1 & 1 & - & - & - & - & - & - & - & 1 \\
\hline 4. Cleft lip & 56 & 29 & - & - & - & - & - & - & - & 29 \\
\hline 5. Cleft palate & 35 & 22 & 0 & 0 & 0 & - & - & - & - & 22 \\
\hline 6. Esophageal atresia & 1 & 0 & - & - & - & - & - & - & - & 0 \\
\hline 7. Renal agenesis & 2 & 1 & - & - & 0 & - & - & - & - & 1 \\
\hline 8. Down syndrome & 447 & 244 & 3 & 5 & 0 & - & - & - & - & 252 \\
\hline 9. Congenital heart anomalies & 3,848 & 1,781 & 65 & 84 & 21 & 7 & 2 & 1 & 0 & 1,960 \\
\hline 10. Spina bifida & 208 & 102 & 0 & - & - & 0 & 0 & - & - & 102 \\
\hline Other congenital anomalies & 1,555 & 779 & 36 & 39 & 7 & 7 & 2 & 1 & 0 & 872 \\
\hline N. Oral conditions & 2,429 & 430 & 69 & 97 & 80 & 243 & 223 & 40 & 4 & 1,187 \\
\hline 1. Dental caries & 1,400 & 424 & 66 & 54 & 43 & 83 & 31 & 15 & 4 & 720 \\
\hline 2. Periodontal disease & 48 & - & - & 6 & 12 & 4 & 1 & 1 & 0 & 24 \\
\hline 3. Edentulism & 935 & - & - & 36 & 22 & 155 & 190 & 24 & 1 & 428 \\
\hline Other oral diseases & 45 & 7 & 3 & 2 & 2 & 1 & 0 & 0 & 0 & 15 \\
\hline III. Injuries & 41,442 & 1,765 & 2,921 & 8,601 & 6,634 & 3,709 & 1,281 & 569 & 144 & 25,624 \\
\hline A. Unintentional injuries & 30,638 & 1,718 & 2,701 & 6,138 & 4,545 & 2,666 & 866 & 394 & 108 & 19,136 \\
\hline 1. Road traffic accidents & 10,243 & 207 & 543 & 2,812 & 2,065 & 1,129 & 322 & 122 & 20 & 7,221 \\
\hline 2. Poisonings & 1,793 & 76 & 78 & 235 & 276 & 256 & 105 & 40 & 7 & 1,072 \\
\hline 3. Falls & 4,675 & 245 & 420 & 832 & 574 & 413 & 172 & 117 & 47 & 2,821 \\
\hline 4. Fires & 1,135 & 69 & 52 & 111 & 99 & 43 & 22 & 16 & 4 & 416 \\
\hline 5. Drownings & 3,740 & 521 & 817 & 614 & 224 & 138 & 51 & 26 & 9 & 2,399 \\
\hline 6. Other unintentional injuries & 9,052 & 599 & 792 & 1,535 & 1,307 & 688 & 193 & 71 & 21 & 5,207 \\
\hline B. Intentional injuries & 10,804 & 47 & 219 & 2,462 & 2,089 & 1,043 & 415 & 175 & 36 & 6,487 \\
\hline 1. Self-inflicted injuries & 7,074 & - & 98 & 1,135 & 992 & 673 & 338 & 154 & 31 & 3,421 \\
\hline 2. Violence & 3,118 & 39 & 113 & 1,105 & 882 & 315 & 63 & 18 & 4 & 2,539 \\
\hline 3. War & 532 & 7 & 6 & 195 & 190 & 47 & 12 & 3 & 1 & 461 \\
\hline Other intentional injuries & 79 & 1 & 2 & 28 & 24 & 7 & 2 & 1 & 0 & 65 \\
\hline
\end{tabular}


Table 3C.2 Continued

\begin{tabular}{|c|c|c|c|c|c|c|c|c|}
\hline \multicolumn{9}{|c|}{ Female } \\
\hline $0-4$ & 5-14 & $15-29$ & $30-44$ & $45-59$ & $60-69$ & $70-79$ & $80+$ & Tota \\
\hline 534 & 425 & 439 & 671 & 1,355 & 2,300 & 3,452 & 2,022 & 11,199 \\
\hline 5 & 0 & 15 & 364 & 967 & 1,978 & 3,156 & 1,772 & 8,257 \\
\hline 204 & 388 & 349 & 200 & 202 & 95 & 41 & 13 & 1,494 \\
\hline 325 & 36 & 75 & 106 & 187 & 227 & 255 & 237 & 1,448 \\
\hline 2,268 & 139 & 407 & 716 & 1,243 & 805 & 751 & 340 & 6,669 \\
\hline 36 & 11 & 54 & 111 & 201 & 137 & 106 & 48 & 704 \\
\hline 56 & 27 & 82 & 249 & 442 & 278 & 204 & 56 & 1,395 \\
\hline 2 & 4 & 6 & 9 & 12 & 7 & 8 & 3 & 52 \\
\hline 2,173 & 96 & 265 & 348 & 588 & 383 & 432 & 233 & 4,518 \\
\hline 78 & 83 & 214 & 388 & 570 & 441 & 341 & 117 & 2,234 \\
\hline 46 & 71 & 149 & 248 & 372 & 305 & 219 & 76 & 1,486 \\
\hline- & - & - & - & - & - & - & - & - \\
\hline 32 & 13 & 65 & 140 & 199 & 135 & 122 & 41 & 748 \\
\hline 78 & 54 & 98 & 102 & 88 & 46 & 35 & 18 & 518 \\
\hline 51 & 135 & 559 & 1,445 & 2,053 & 949 & 378 & 117 & 5,687 \\
\hline 2 & 26 & 72 & 175 & 338 & 157 & 63 & 17 & 850 \\
\hline 0 & 1 & 303 & 962 & 1,435 & 638 & 191 & 28 & 3,558 \\
\hline 0 & 0 & 8 & 76 & 41 & 16 & 9 & 2 & 152 \\
\hline 10 & 64 & 44 & 84 & 65 & 20 & 9 & 2 & 298 \\
\hline 38 & 44 & 133 & 148 & 173 & 118 & 106 & 68 & 829 \\
\hline 2,660 & 120 & 89 & 43 & 15 & 4 & 3 & 1 & 2,936 \\
\hline 2 & - & - & - & - & - & 0 & - & 2 \\
\hline 20 & 0 & 0 & 0 & 0 & - & - & - & 20 \\
\hline 0 & - & - & - & - & - & - & - & 0 \\
\hline 27 & - & - & - & - & - & - & - & 27 \\
\hline 13 & 0 & 0 & 0 & 0 & - & - & - & 13 \\
\hline 0 & - & - & - & - & - & - & - & c \\
\hline 1 & - & - & - & - & - & - & - & 1 \\
\hline 186 & 3 & 6 & 0 & 0 & - & - & - & 195 \\
\hline 1,671 & 94 & 73 & 35 & 10 & 3 & 2 & 1 & 1,888 \\
\hline 106 & 0 & 0 & 0 & 0 & - & 0 & 0 & 106 \\
\hline 633 & 23 & 10 & 8 & 5 & 2 & 1 & 0 & 683 \\
\hline 390 & 64 & 105 & 88 & 254 & 254 & 76 & 10 & 1,242 \\
\hline 385 & 62 & 52 & 43 & 83 & 33 & 18 & 6 & 681 \\
\hline- & 0 & 6 & 12 & 4 & 1 & 1 & 0 & 24 \\
\hline - & - & 37 & 24 & 166 & 218 & 57 & 4 & 506 \\
\hline 6 & 2 & 10 & 10 & 2 & 0 & 0 & 0 & 30 \\
\hline 1,792 & 2,347 & 3,851 & 3,761 & 2,354 & 877 & 608 & 229 & 15,819 \\
\hline 1,773 & 2,108 & 2,586 & 2,366 & 1,503 & 567 & 415 & 184 & 11,502 \\
\hline 189 & 396 & 728 & 865 & 584 & 158 & 83 & 18 & 3,022 \\
\hline 82 & 94 & 128 & 176 & 136 & 61 & 35 & 9 & 722 \\
\hline 266 & 372 & 377 & 250 & 205 & 127 & 161 & 96 & 1,854 \\
\hline 88 & 125 & 250 & 149 & 62 & 19 & 17 & 8 & 718 \\
\hline 345 & 482 & 178 & 159 & 91 & 40 & 31 & 13 & 1,341 \\
\hline 802 & 639 & 925 & 766 & 424 & 161 & 88 & 40 & 3,845 \\
\hline 19 & 239 & 1,265 & 1,395 & 851 & 310 & 193 & 45 & 4,317 \\
\hline - & 153 & 1,064 & 1,188 & 743 & 281 & 180 & 43 & 3,653 \\
\hline 18 & 59 & 185 & 190 & 89 & 25 & 12 & 1 & 579 \\
\hline 0 & 25 & 11 & 12 & 17 & 3 & 1 & 0 & 71 \\
\hline 1 & 1 & 4 & 4 & 3 & 1 & 0 & 0 & 14 \\
\hline
\end{tabular}

Source: Authors' compilation.

Note: - = an estimate of zero; the number zero in a cell indicates a non-zero estimate of less than 500 .

a. These figures include late effects of polio cases with onset prior to regional certification of polio eradication in 1994 .

b. Does not include liver cancer and cirrhosis $\operatorname{DALYS}(3,0)$ resulting from chronic hepatitis virus infection.

c. This cause category includes "Causes arising in the perinatal period" as defined in the International Classification of

Diseases, principally low birthweight, prematurity, birth asphyxia, and birth trauma, and does not include all causes

of DALYS $(3,0)$ occurring in the perinatal period. 
Table 3C.3 DALYs(3,0) by Cause, Sex, and Age in the Europe and Central Asia Region, 2001 (thousands)

\begin{tabular}{|c|c|c|c|c|c|c|c|c|c|c|}
\hline \multirow[b]{2}{*}{ Cause } & \multirow[b]{2}{*}{ Total } & \multicolumn{8}{|c|}{ Male } & \multirow[b]{2}{*}{ Total } \\
\hline & & $0-4$ & $5-14$ & $15-29$ & 30-44 & $45-59$ & $60-69$ & 70-79 & $80+$ & \\
\hline Population (millions) & 477 & 15 & 38 & 59 & 51 & 37 & 17 & $g$ & 2 & 230 \\
\hline All causes & 116,502 & 5,211 & 1,902 & 8,184 & 11,371 & 15,315 & 11,968 & 8,004 & 1,950 & 63,904 \\
\hline $\begin{array}{l}\text { I. Communicable, maternal, perinatal, } \\
\text { and nutritional conditions }\end{array}$ & 10,908 & 2,829 & 284 & 631 & 1,135 & 905 & 329 & 128 & 32 & 6,274 \\
\hline A. Infectious and parasitic diseases & 4,760 & 676 & 102 & 554 & 1,021 & 624 & 153 & 45 & 8 & 3,183 \\
\hline 1. Tuberculosis & 1,536 & 4 & 4 & 174 & 498 & 431 & 110 & 27 & 3 & 1,251 \\
\hline $\begin{array}{l}\text { 2. Sexually transmitted diseases } \\
\text { excluding HIV/AIDS }\end{array}$ & 200 & 8 & 0 & 20 & 13 & 1 & 0 & 0 & 0 & 43 \\
\hline a. Syphilis & 12 & 4 & 0 & 1 & 1 & 0 & 0 & 0 & 0 & 6 \\
\hline b. Chlamydia & 122 & 1 & 0 & 6 & 3 & 0 & 0 & - & - & 10 \\
\hline c. Gonorrhea & 59 & 1 & 0 & 13 & 10 & 0 & 0 & 0 & - & 24 \\
\hline $\begin{array}{l}\text { d. Other sexually transmitted } \\
\text { diseases }\end{array}$ & 8 & 2 & - & 0 & 0 & 0 & 0 & 0 & - & 3 \\
\hline 3. HIV/AIDS & 982 & 6 & 7 & 254 & 403 & 121 & 10 & 1 & 0 & 802 \\
\hline 4. Diarrheal diseases & 657 & 278 & 13 & 15 & 15 & 10 & 5 & 3 & 1 & 341 \\
\hline 5. Childhood-cluster diseases & 323 & 121 & 36 & 5 & 0 & 1 & 0 & 0 & 0 & 164 \\
\hline a. Pertussis & 81 & 39 & 1 & - & - & - & - & - & - & 40 \\
\hline b. Poliomyelitis ${ }^{a}$ & 2 & 0 & 0 & 0 & 0 & 0 & 0 & 0 & 0 & 1 \\
\hline c. Diphtheria & 2 & 0 & 0 & 0 & 0 & 0 & 0 & 0 & 0 & 1 \\
\hline d. Measles & 236 & 82 & 34 & 4 & 0 & 0 & 0 & - & - & 121 \\
\hline e. Tetanus & 2 & 0 & 0 & 0 & 0 & 0 & 0 & 0 & 0 & 1 \\
\hline 6. Meningitis & 403 & 149 & 10 & 18 & 20 & 19 & 7 & 2 & 1 & 225 \\
\hline 7. Hepatitis $\mathbf{B}^{\mathbf{b}}$ & 79 & 7 & 2 & 18 & 12 & 5 & 2 & 1 & 0 & 48 \\
\hline Hepatitis $\mathbf{C}^{\mathbf{b}}$ & 31 & 3 & 1 & 7 & 5 & 2 & 1 & 0 & 0 & 20 \\
\hline 8. Malaria & 18 & 3 & 3 & 1 & 1 & $\mathbf{0}$ & 0 & 0 & 0 & 9 \\
\hline 9. Tropical-cluster diseases & 7 & 2 & 1 & 1 & 0 & 0 & 0 & 0 & 0 & 5 \\
\hline a. Trypanosomiasis & 0 & - & - & - & - & - & - & - & - & - \\
\hline b. Chagas' disease & - & - & - & - & - & - & - & - & - & - \\
\hline c. Schistosomiasis & 1 & 0 & - & 0 & - & 0 & 0 & 0 & - & 1 \\
\hline d. Leishmaniasis & 6 & 1 & 1 & 0 & 0 & 0 & 0 & 0 & 0 & 4 \\
\hline e. Lymphatic filariasis & 1 & 1 & - & 0 & 0 & - & 0 & - & - & 1 \\
\hline f. Onchocerciasis & - & - & - & - & - & - & - & - & - & - \\
\hline 10. Leprosy & 0 & - & - & 0 & 0 & 0 & 0 & 0 & 0 & $\mathbf{0}$ \\
\hline 11. Dengue & 0 & - & - & - & - & $\mathbf{0}$ & - & - & - & $\mathbf{0}$ \\
\hline 12. Japanese encephalitis & - & - & - & - & - & - & - & - & - & - \\
\hline 13. Trachoma & - & - & - & - & - & - & - & - & - & - \\
\hline 14. Intestinal nematode infections & 1 & $\mathbf{0}$ & 0 & 0 & - & $\mathbf{0}$ & 0 & 0 & 0 & 0 \\
\hline a. Ascariasis & 0 & 0 & 0 & - & - & - & - & - & 0 & 0 \\
\hline b. Trichuriasis & 0 & 0 & 0 & - & - & - & - & - & - & 0 \\
\hline c. Hookworm disease & 0 & - & - & - & - & 0 & 0 & 0 & 0 & 0 \\
\hline Other intestinal infections & 0 & 0 & - & 0 & - & - & - & - & 0 & 0 \\
\hline Other infectious diseases & 522 & 94 & 23 & 40 & 54 & 34 & 16 & 9 & 2 & 273 \\
\hline B. Respiratory infections & 2,305 & 626 & 78 & 48 & 87 & 260 & 152 & 76 & 23 & 1,350 \\
\hline 1. Lower respiratory infections & 2,111 & 573 & 49 & 44 & 81 & 254 & 148 & 74 & 23 & 1,245 \\
\hline 2. Upper respiratory infections & 129 & 44 & 7 & 4 & 5 & 6 & 4 & 2 & 0 & 72 \\
\hline 3. Otitis media & 65 & 9 & 22 & 0 & 0 & 0 & 0 & 0 & 0 & 33 \\
\hline C. Maternal conditions & 486 & - & - & - & - & - & - & - & - & - \\
\hline 1. Maternal hemorrhage & 17 & - & - & - & - & - & - & - & - & - \\
\hline 2. Maternal sepsis & 124 & - & - & - & - & - & - & - & - & - \\
\hline $\begin{array}{l}\text { 3. Hypertensive disorders } \\
\text { of pregnancy }\end{array}$ & 11 & - & - & - & - & - & - & - & - & - \\
\hline 4. Obstructed labor & 2 & - & - & - & - & - & - & - & - & - \\
\hline 5. Abortion & 17 & - & - & - & - & - & - & - & - & - \\
\hline Other maternal conditions & 315 & - & - & - & - & - & - & - & - & - \\
\hline D. Perinatal conditions ${ }^{\mathrm{C}}$ & 2,125 & 1,203 & 0 & 0 & $\mathbf{0}$ & 0 & $\mathbf{0}$ & 0 & - & 1,203 \\
\hline 1. Low birthweight & 822 & 448 & - & - & - & - & 0 & - & - & 448 \\
\hline 2. Birth asphyxia and birth trauma & 779 & 444 & 0 & 0 & - & 0 & - & - & - & 444 \\
\hline Other perinatal conditions & 524 & 311 & 0 & 0 & 0 & 0 & - & 0 & - & 311 \\
\hline
\end{tabular}


Table 3C.3 Continued

\begin{tabular}{|c|c|c|c|c|c|c|c|c|}
\hline \multicolumn{9}{|c|}{ Female } \\
\hline $0-4$ & 5-14 & 15-29 & 30-44 & $45-59$ & $60-69$ & 70-79 & $80+$ & Total \\
\hline 14 & 36 & 58 & 52 & 41 & 23 & 17 & 6 & 248 \\
\hline 4,372 & 1,425 & 5,019 & 6,106 & 9,168 & 9,818 & 11,359 & 5,333 & 52,598 \\
\hline 2,414 & 297 & 854 & 485 & 254 & 142 & 129 & 59 & 4,634 \\
\hline 621 & 97 & 330 & 272 & 138 & 62 & 43 & 13 & 1,577 \\
\hline 4 & 6 & 59 & 107 & 64 & 26 & 15 & 3 & 284 \\
\hline 8 & 2 & 111 & 34 & 2 & 0 & 1 & 0 & 158 \\
\hline 4 & 0 & 1 & 1 & 0 & 0 & 0 & 0 & 6 \\
\hline 1 & 2 & 89 & 19 & 1 & 0 & - & - & 112 \\
\hline 1 & 0 & 20 & 13 & 0 & 0 & - & - & 35 \\
\hline 2 & 0 & 1 & 1 & 1 & 0 & 0 & 0 & 5 \\
\hline 6 & 6 & 90 & 60 & 16 & 2 & 0 & 0 & 180 \\
\hline 254 & 13 & 13 & 12 & 10 & 6 & 6 & 2 & 315 \\
\hline 115 & 37 & 5 & 0 & 1 & 1 & 0 & 0 & 159 \\
\hline 39 & 1 & - & - & 0 & 0 & - & - & 41 \\
\hline 0 & 0 & 0 & 0 & 0 & 0 & 0 & 0 & 1 \\
\hline 0 & 0 & 0 & 0 & 0 & 0 & 0 & 0 & 1 \\
\hline 75 & 35 & 5 & - & 0 & 0 & - & - & 115 \\
\hline 0 & 0 & 0 & 0 & 0 & 0 & 0 & 0 & 1 \\
\hline 136 & 8 & 7 & 7 & 9 & 6 & 3 & 1 & 178 \\
\hline 5 & 2 & 8 & 7 & 6 & 2 & 1 & 0 & 31 \\
\hline 2 & 1 & 3 & 2 & 2 & 1 & 0 & 0 & 11 \\
\hline 3 & 3 & 1 & 1 & 0 & 0 & 0 & 0 & 9 \\
\hline 0 & 1 & 0 & 0 & 0 & 0 & 0 & 0 & 2 \\
\hline- & - & - & 0 & - & 0 & - & - & 0 \\
\hline- & - & - & - & - & - & - & - & - \\
\hline- & - & - & - & 0 & 0 & - & - & 0 \\
\hline 0 & 1 & 0 & 0 & 0 & 0 & 0 & 0 & 2 \\
\hline- & 0 & - & - & - & - & - & - & 0 \\
\hline- & - & - & - & - & - & - & - & - \\
\hline- & - & 0 & 0 & 0 & 0 & - & 0 & 0 \\
\hline- & - & - & - & - & - & - & - & - \\
\hline- & - & - & - & - & - & - & - & - \\
\hline- & - & - & - & - & - & - & - & - \\
\hline 0 & 0 & - & 0 & 0 & - & 0 & 0 & 0 \\
\hline 0 & 0 & - & - & - & - & - & - & 0 \\
\hline 0 & 0 & - & - & - & - & - & - & 0 \\
\hline- & - & - & 0 & - & - & - & - & 0 \\
\hline 0 & - & - & - & 0 & - & 0 & 0 & 0 \\
\hline 89 & 19 & 32 & 40 & 28 & 19 & 16 & 6 & 249 \\
\hline 537 & 70 & 36 & 44 & 85 & 65 & 75 & 43 & 954 \\
\hline 490 & 43 & 32 & 41 & 81 & 62 & 73 & 42 & 865 \\
\hline 38 & 5 & 3 & 3 & 3 & 2 & 2 & 1 & 57 \\
\hline 9 & 22 & 0 & 0 & 0 & 0 & 0 & 0 & 32 \\
\hline- & 1 & 357 & 127 & 1 & 0 & - & - & 486 \\
\hline- & - & 12 & 5 & 0 & - & - & - & 17 \\
\hline- & - & 107 & 16 & 0 & - & - & - & 124 \\
\hline- & - & 8 & 3 & 0 & - & - & - & 11 \\
\hline- & - & 1 & 0 & 0 & - & - & - & 2 \\
\hline- & 1 & 12 & 4 & 0 & - & - & - & 17 \\
\hline- & - & 216 & 99 & 1 & 0 & - & - & 315 \\
\hline 923 & 0 & 0 & 0 & 0 & 0 & - & - & 923 \\
\hline 374 & - & - & - & - & - & - & - & 374 \\
\hline 336 & 0 & 0 & - & - & - & - & - & 336 \\
\hline 213 & 0 & 0 & 0 & 0 & 0 & - & - & 213 \\
\hline
\end{tabular}

The Burden of Disease and Mortality by Condition: Data, Methods, and Results for 2001 | 193 


\begin{tabular}{|c|c|c|c|c|c|c|c|c|c|c|}
\hline \multirow[b]{2}{*}{ Cause } & \multicolumn{10}{|c|}{ Male } \\
\hline & Total & $0-4$ & $5-14$ & $15-29$ & $30-44$ & $45-59$ & $60-69$ & $70-79$ & $80+$ & Total \\
\hline E. Nutritional deficiencies & 1,232 & 325 & 104 & 28 & 27 & 21 & 24 & 8 & 1 & 538 \\
\hline 1. Protein-energy malnutrition & 173 & 75 & 1 & 2 & 3 & 4 & 2 & 1 & 0 & 88 \\
\hline 2. Iodine deficiency & 566 & 198 & 73 & 0 & 0 & 0 & 0 & 0 & 0 & 272 \\
\hline 3. Vitamin A deficiency & 1 & 0 & 0 & - & - & - & - & - & 0 & 0 \\
\hline 4. Iron-deficiency anemia & 421 & 34 & 25 & 24 & 22 & 16 & 21 & 7 & 1 & 150 \\
\hline Other nutritional disorders & 72 & 18 & 5 & 2 & 1 & 1 & 1 & 0 & 0 & 29 \\
\hline II. Noncommunicable diseases & 88,969 & 1,998 & 868 & 3,869 & 6,195 & 11,529 & 10,810 & 7,624 & 1,877 & 44,768 \\
\hline A. Malignant neoplasms & 12,159 & 32 & 63 & 186 & 573 & 2,195 & 2,211 & 1,217 & 162 & 6,638 \\
\hline 1. Mouth and oropharynx cancers & 426 & 0 & 1 & 4 & 34 & 179 & 101 & 33 & 4 & 357 \\
\hline 2. Esophageal cancer & 288 & 0 & 0 & 1 & 11 & 88 & 78 & 34 & 4 & 216 \\
\hline 3. Stomach cancer & 1,376 & 0 & 0 & 8 & 74 & 266 & 289 & 165 & 20 & 824 \\
\hline 4. Colon and rectal cancers & 1,290 & 0 & 0 & 8 & 47 & 170 & 224 & 159 & 26 & 633 \\
\hline 5. Liver cancer & 379 & 1 & 1 & 4 & 20 & 75 & 80 & 44 & 5 & 230 \\
\hline 6. Pancreas cancer & 481 & 0 & 0 & 1 & 28 & 100 & 87 & 46 & 6 & 270 \\
\hline 7. Trachea, bronchus, and lung cancers & 2,323 & 0 & 1 & 8 & 110 & 684 & 736 & 347 & 29 & 1,917 \\
\hline 8. Melanoma and other skin cancers & 160 & 0 & 0 & 4 & 15 & 29 & 18 & 11 & 3 & 80 \\
\hline 9. Breast cancer & 1,058 & - & 0 & 0 & 1 & 3 & 3 & 2 & 0 & 10 \\
\hline 10. Cervix uteri cancer & 356 & - & - & - & - & - & - & - & - & - \\
\hline 11. Corpus uteri cancer & 349 & - & - & - & - & - & - & - & - & - \\
\hline 12. Ovarian cancer & 350 & - & - & - & - & - & - & - & - & - \\
\hline 13. Prostate cancer & 283 & 0 & 0 & 1 & 3 & 37 & 105 & 111 & 26 & 283 \\
\hline 14. Bladder cancer & 300 & 0 & 0 & 1 & 9 & 58 & 91 & 68 & 13 & 240 \\
\hline 15. Lymphomas and multiple myeloma & 375 & 3 & 10 & 26 & 39 & 58 & 46 & 23 & 3 & 208 \\
\hline 16. Leukemia & 462 & 12 & 25 & 47 & 39 & 52 & 48 & 30 & 5 & 256 \\
\hline Other malignant neoplasms & 1,901 & 15 & 24 & 71 & 143 & 395 & 305 & 142 & 18 & 1,114 \\
\hline B. Other neoplasms & 126 & 2 & 2 & 5 & 9 & 18 & 14 & 9 & 2 & 61 \\
\hline C. Diabetes mellitus & 1,375 & 1 & 4 & 34 & 109 & 183 & 137 & 78 & 13 & 560 \\
\hline D. Endocrine disorders & 534 & 131 & 14 & 27 & 28 & 24 & 9 & 5 & 1 & 239 \\
\hline E. Neuropsychiatric conditions & 14,106 & 595 & 520 & 2,346 & 1,554 & 936 & 374 & 297 & 113 & 6,735 \\
\hline 1. Unipolar depressive disorders & 4,268 & 0 & 170 & 488 & 446 & 316 & 110 & 27 & 4 & 1,561 \\
\hline 2. Bipolar affective disorder & 668 & - & 17 & 279 & 36 & 1 & 0 & 0 & 0 & 334 \\
\hline 3. Schizophrenia & 778 & 0 & 76 & 277 & 20 & 6 & 4 & 2 & 0 & 386 \\
\hline 4. Epilepsy & 354 & 9 & 24 & 56 & 60 & 38 & 11 & 4 & 1 & 202 \\
\hline 5. Alcohol use disorders & 1,849 & 2 & 14 & 620 & 561 & 294 & 56 & 10 & 1 & 1,557 \\
\hline 6. Alzheimer's and other dementias & 1,612 & 13 & 7 & 10 & 12 & 37 & 99 & 199 & 97 & 474 \\
\hline 7. Parkinson's disease & 228 & 1 & 1 & 1 & 8 & 21 & 33 & 23 & 4 & 93 \\
\hline 8. Multiple sclerosis & 143 & 0 & 3 & 16 & 21 & 14 & 4 & 2 & 0 & 60 \\
\hline 9. Drug use disorders & 559 & 1 & 5 & 188 & 154 & 73 & 8 & 1 & 0 & 430 \\
\hline 10. Post-traumatic stress disorder & 192 & 0 & 0 & 25 & 17 & 10 & 0 & 0 & 0 & 52 \\
\hline 11. Obsessive-compulsive disorder & 419 & - & 29 & 73 & 62 & 11 & 2 & 4 & 0 & 181 \\
\hline 12. Panic disorder & 340 & - & 5 & 100 & 1 & 6 & 1 & 1 & 0 & 114 \\
\hline 13. Insomnia (primary) & 255 & - & 2 & 24 & 28 & 24 & 13 & 7 & 1 & 99 \\
\hline 14. Migraine & 414 & 4 & 62 & 50 & 10 & 1 & 0 & 0 & 0 & 128 \\
\hline 15. Mental retardation, lead-caused & 312 & 148 & 1 & 3 & 2 & 1 & 0 & 0 & 0 & 156 \\
\hline Other neuropsychiatric disorders & 1,716 & 417 & 103 & 136 & 116 & 84 & 31 & 16 & 4 & 907 \\
\hline F. Sense organ diseases & 5,091 & 1 & 2 & 73 & 304 & 596 & 659 & 390 & 62 & 2,086 \\
\hline 1. Glaucoma & 280 & 0 & 1 & 5 & 10 & 26 & 37 & 27 & 6 & 112 \\
\hline 2. Cataracts & 455 & 0 & 0 & 3 & 16 & 44 & 63 & 46 & 10 & 182 \\
\hline 3. Vision disorders, age-related & 1,787 & 0 & 1 & 14 & 27 & 181 & 257 & 168 & 33 & 680 \\
\hline 4. Hearing loss, adult onset & 2,564 & - & - & 51 & 250 & 345 & 301 & 149 & 13 & 1,109 \\
\hline Other sense organ disorders & 5 & 0 & 0 & 1 & 1 & 1 & 0 & 0 & 0 & 3 \\
\hline G. Cardiovascular diseases & 38,281 & 49 & 39 & 390 & 1,993 & 5,225 & 5,843 & 4,694 & 1,335 & 19,569 \\
\hline 1. Rheumatic heart disease & 408 & 1 & 2 & 15 & 45 & 68 & 33 & 12 & 2 & 177 \\
\hline 2. Hypertensive heart disease & 1,346 & 1 & 1 & 8 & 53 & 173 & 192 & 143 & 39 & 611 \\
\hline 3. Ischemic heart disease & 18,510 & 0 & 5 & 98 & 944 & 3,016 & 3,174 & 2,444 & 603 & 10,286 \\
\hline 4. Cerebrovascular disease & 12,616 & 13 & 15 & 80 & 371 & 1,283 & 1,852 & 1,562 & 405 & 5,581 \\
\hline 5. Inflammatory heart diseases & 1,166 & 15 & 5 & 75 & 202 & 252 & 130 & 79 & 25 & 783 \\
\hline Other cardiovascular diseases & 4,234 & 20 & 10 & 114 & 378 & 432 & 461 & 454 & 261 & 2,131 \\
\hline
\end{tabular}


Table 3C.3 Continued

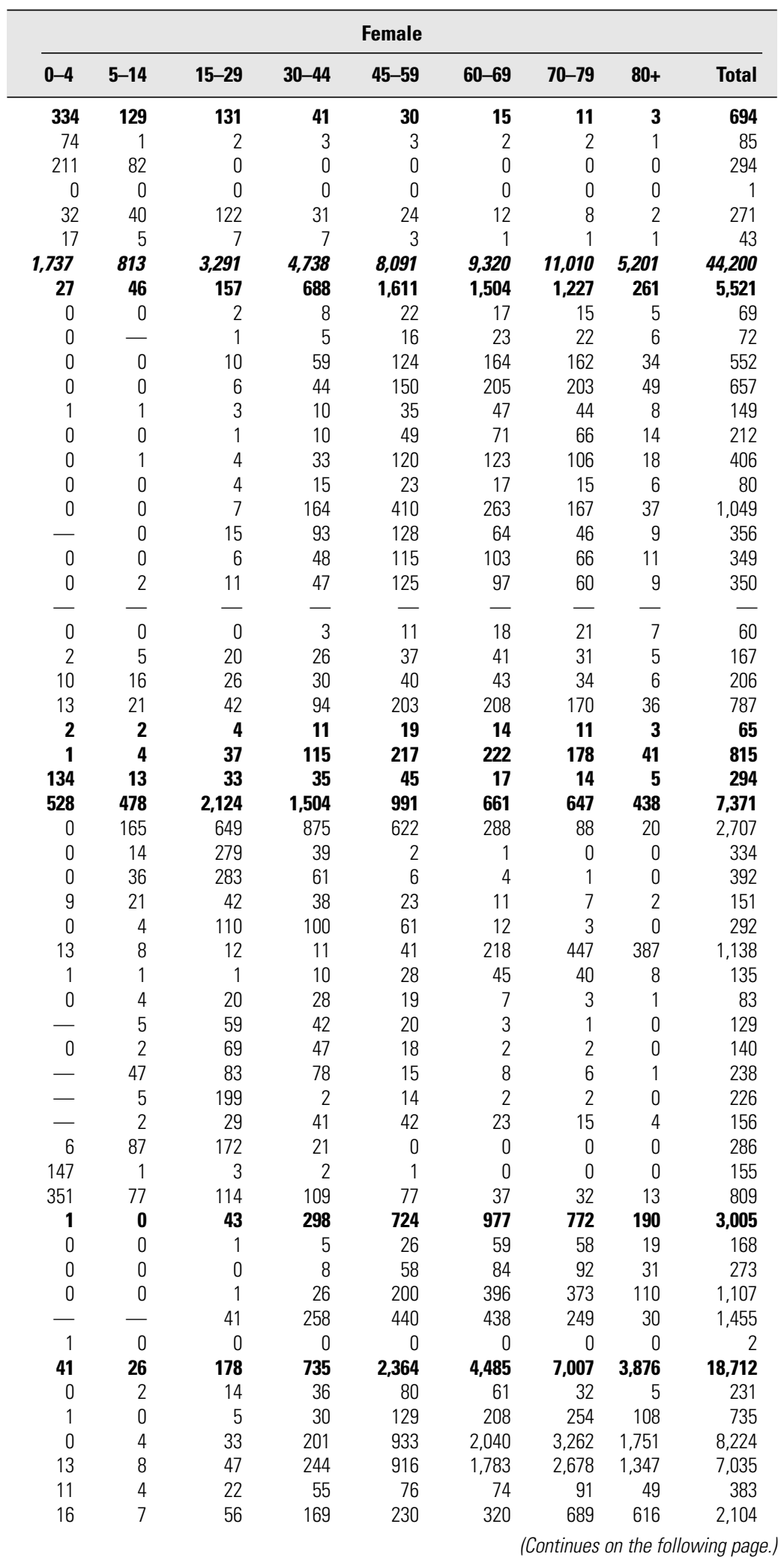


Table 3C.3 Continued

\begin{tabular}{|c|c|c|c|c|c|c|c|c|c|c|}
\hline \multirow[b]{2}{*}{ Cause } & \multicolumn{10}{|c|}{ Male } \\
\hline & Total & $0-4$ & $5-14$ & $15-29$ & $30-44$ & $45-59$ & $60-69$ & 70-79 & $80+$ & Total \\
\hline H. Respiratory diseases & 4,284 & 173 & 81 & 151 & 288 & 533 & 653 & 481 & 103 & 2,462 \\
\hline $\begin{array}{l}\text { 1. Chronic obstructive pulmonary } \\
\text { disease }\end{array}$ & 2,362 & 2 & 2 & 31 & 147 & 312 & 430 & 350 & 74 & 1,349 \\
\hline 2. Asthma & 688 & 36 & 49 & 74 & 39 & 61 & 89 & 45 & 8 & 400 \\
\hline Other respiratory diseases & 1,234 & 136 & 30 & 45 & 102 & 160 & 133 & 86 & 22 & 713 \\
\hline I. Digestive diseases & 5,675 & 267 & 34 & 301 & 749 & 1,058 & 510 & 240 & 43 & 3,202 \\
\hline 1. Peptic ulcer disease & 442 & 0 & 3 & 30 & 81 & 102 & 61 & 30 & 5 & 313 \\
\hline 2. Cirrhosis of the liver & 2,084 & 2 & 7 & 64 & 336 & 540 & 255 & 87 & 8 & 1,301 \\
\hline 3. Appendicitis & 31 & 1 & 2 & 4 & 4 & 4 & 3 & 2 & 0 & 18 \\
\hline Other digestive diseases & 3,119 & 263 & 22 & 203 & 329 & 412 & 191 & 121 & 29 & 1,570 \\
\hline J. Genitourinary diseases & 1,417 & 42 & 13 & 67 & 109 & 234 & 145 & 101 & 25 & 737 \\
\hline 1. Nephritis and nephrosis & 585 & 5 & 8 & 38 & 68 & 82 & 56 & 37 & 9 & 303 \\
\hline 2. Benign prostatic hypertrophy & 176 & - & - & 0 & 0 & 87 & 48 & 33 & 8 & 176 \\
\hline Other genitourinary system diseases & 656 & 37 & 5 & 29 & 40 & 66 & 42 & 31 & 8 & 258 \\
\hline K. Skin diseases & 237 & 8 & 6 & 20 & 28 & 29 & 13 & 6 & 1 & 112 \\
\hline L. Musculoskeletal diseases & 3,726 & 10 & 38 & 188 & 400 & 408 & 205 & 95 & 15 & 1,361 \\
\hline 1. Rheumatoid arthritis & 578 & 1 & 11 & 32 & 32 & 33 & 21 & 11 & 2 & 141 \\
\hline 2. Osteoarthritis & 2,281 & 0 & 0 & 63 & 256 & 278 & 153 & 68 & 9 & 827 \\
\hline 3. Gout & 137 & 0 & 0 & 6 & 52 & 45 & 8 & 3 & 1 & 115 \\
\hline 4. Low back pain & 143 & 3 & 9 & 14 & 24 & 17 & 6 & 3 & 0 & 76 \\
\hline Other musculoskeletal disorders & 587 & 7 & 18 & 74 & 35 & 35 & 17 & 11 & 4 & 201 \\
\hline M. Congenital anomalies & 1,285 & 638 & 19 & 19 & 11 & 8 & 2 & 1 & $\mathbf{0}$ & 699 \\
\hline 1. Abdominal wall defect & 7 & 4 & 0 & 0 & 0 & 0 & 0 & 0 & 0 & 5 \\
\hline 2. Anencephaly & 8 & 3 & 0 & 0 & 0 & 0 & 0 & 0 & 0 & 3 \\
\hline 3. Anorectal atresia & 2 & 2 & 0 & 0 & 0 & 0 & 0 & 0 & 0 & 2 \\
\hline 4. Cleft lip & 3 & 1 & 0 & 0 & 0 & 0 & 0 & 0 & - & 1 \\
\hline 5. Cleft palate & 3 & 1 & 0 & 0 & 0 & 0 & 0 & 0 & - & 1 \\
\hline 6. Esophageal atresia & 6 & 3 & 0 & 0 & 0 & 0 & 0 & 0 & 0 & 3 \\
\hline 7. Renal agenesis & 6 & 4 & 0 & 0 & 0 & 0 & 0 & 0 & 0 & 4 \\
\hline 8. Down syndrome & 180 & 99 & 1 & 1 & 1 & 1 & 0 & 0 & 0 & 102 \\
\hline 9. Congenital heart anomalies & 670 & 327 & 9 & 12 & 7 & 3 & 1 & 0 & 0 & 358 \\
\hline 10. Spina bifida & 49 & 23 & 1 & 0 & 0 & 0 & 0 & 0 & 0 & 25 \\
\hline Other congenital anomalies & 351 & 171 & 9 & 6 & 3 & 4 & 1 & 1 & 0 & 195 \\
\hline N. Oral conditions & 672 & 47 & 33 & 61 & 38 & 82 & 34 & 11 & 2 & 308 \\
\hline 1. Dental caries & 375 & 47 & 33 & 35 & 15 & 29 & 14 & 8 & 2 & 181 \\
\hline 2. Periodontal disease & 19 & - & - & 2 & 5 & 1 & 1 & 0 & 0 & 9 \\
\hline 3. Edentulism & 276 & - & - & 24 & 18 & 52 & 19 & 3 & 0 & 116 \\
\hline Other oral diseases & 3 & 0 & 0 & 0 & 0 & 0 & 0 & 0 & 0 & 1 \\
\hline III. Injuries & 16,626 & 384 & 750 & 3,684 & 4,042 & 2,881 & 829 & 252 & 41 & 12,862 \\
\hline A. Unintentional injuries & 11,366 & 369 & 665 & 2,312 & 2,512 & 1,975 & 597 & 175 & 30 & 8,635 \\
\hline 1. Road traffic accidents & 2,264 & 29 & 101 & 608 & 509 & 289 & 81 & 29 & 4 & 1,650 \\
\hline 2. Poisonings & 2,251 & 17 & 15 & 318 & 626 & 594 & 148 & 23 & 2 & 1,742 \\
\hline 3. Falls & 1,372 & 64 & 127 & 265 & 225 & 186 & 76 & 43 & 13 & 998 \\
\hline 4. Fires & 571 & 49 & 38 & 83 & 119 & 101 & 32 & 10 & 1 & 433 \\
\hline 5. Drownings & 806 & 32 & 65 & 186 & 209 & 125 & 35 & 8 & 1 & 661 \\
\hline 6. Other unintentional injuries & 4,103 & 179 & 320 & 853 & 823 & 679 & 225 & 62 & 9 & 3,151 \\
\hline B. Intentional injuries & 5,259 & 14 & 85 & 1,372 & 1,530 & 906 & 232 & 77 & 11 & 4,227 \\
\hline 1. Self-inflicted injuries & 2,625 & 0 & 49 & 631 & 719 & 543 & 156 & 58 & 8 & 2,165 \\
\hline 2. Violence & 2,030 & 9 & 29 & 504 & 591 & 308 & 62 & 16 & 2 & 1,520 \\
\hline 3. War & 585 & 5 & 6 & 233 & 214 & 53 & 14 & 3 & 1 & 530 \\
\hline Other intentional injuries & 19 & 0 & 0 & 4 & 6 & 3 & 0 & 0 & 0 & 12 \\
\hline
\end{tabular}


Table 3C.3 Continued

\begin{tabular}{|c|c|c|c|c|c|c|c|c|}
\hline \multicolumn{9}{|c|}{ Female } \\
\hline $0-4$ & 5-14 & $15-29$ & 30-44 & 45-59 & $60-69$ & 70-79 & $80+$ & Total \\
\hline 155 & 91 & 136 & 292 & 358 & 319 & 331 & 140 & 1,822 \\
\hline 1 & 13 & 41 & 214 & 244 & 194 & 214 & 93 & 1,013 \\
\hline 30 & 60 & 51 & 21 & 34 & 44 & 35 & 13 & 288 \\
\hline 124 & 18 & 44 & 57 & 80 & 81 & 82 & 35 & 521 \\
\hline 222 & 25 & 184 & 360 & 717 & 463 & 377 & 125 & 2,474 \\
\hline 0 & 1 & 11 & 21 & 33 & 26 & 28 & 9 & 129 \\
\hline 2 & 5 & 33 & 149 & 293 & 184 & 99 & 18 & 783 \\
\hline 0 & 1 & 2 & 2 & 2 & 2 & 2 & 0 & 13 \\
\hline 220 & 17 & 138 & 188 & 389 & 251 & 248 & 98 & 1,549 \\
\hline 28 & 16 & 81 & 120 & 153 & 130 & 111 & 40 & 680 \\
\hline 5 & 10 & 27 & 51 & 66 & 61 & 48 & 14 & 282 \\
\hline- & - & - & - & - & - & - & - & - \\
\hline 23 & 7 & 54 & 69 & 87 & 69 & 63 & 26 & 398 \\
\hline 9 & 6 & 20 & 24 & 28 & 19 & 12 & 8 & 126 \\
\hline 9 & 57 & 218 & 505 & 754 & 456 & 297 & 67 & 2,365 \\
\hline 2 & 30 & 107 & 108 & 97 & 51 & 33 & 9 & 437 \\
\hline 0 & 0 & 30 & 304 & 538 & 341 & 211 & 29 & 1,453 \\
\hline 0 & 0 & 1 & 6 & 6 & 4 & 3 & 1 & 22 \\
\hline 1 & 10 & 9 & 17 & 17 & 8 & 4 & 1 & 67 \\
\hline 6 & 16 & 72 & 71 & 96 & 51 & 46 & 28 & 386 \\
\hline 536 & 15 & 13 & 9 & 9 & 3 & 1 & 0 & 586 \\
\hline 3 & 0 & 0 & 0 & 0 & 0 & 0 & 0 & 3 \\
\hline 5 & 0 & 0 & 0 & 0 & 0 & 0 & 0 & 6 \\
\hline 1 & 0 & 0 & 0 & 0 & 0 & 0 & 0 & 1 \\
\hline 1 & 0 & 0 & 0 & 0 & 0 & 0 & - & 1 \\
\hline 1 & 0 & 0 & 0 & 0 & 0 & 0 & 0 & 1 \\
\hline 3 & 0 & 0 & 0 & 0 & 0 & 0 & 0 & 3 \\
\hline 2 & 0 & 0 & 0 & 0 & 0 & 0 & 0 & 2 \\
\hline 74 & 0 & 1 & 1 & 1 & 0 & 0 & 0 & 78 \\
\hline 286 & 7 & 8 & 5 & 4 & 1 & 0 & 0 & 311 \\
\hline 23 & 1 & 0 & 0 & 0 & 0 & 0 & 0 & 24 \\
\hline 136 & 7 & 4 & 3 & 4 & 1 & 1 & 0 & 156 \\
\hline 45 & 32 & 63 & 42 & 102 & 51 & 25 & 6 & 365 \\
\hline 45 & 32 & 34 & 15 & 32 & 19 & 14 & 5 & 194 \\
\hline- & - & 2 & 5 & 1 & 1 & 1 & 0 & 10 \\
\hline- & - & 26 & 21 & 69 & 31 & 11 & 1 & 160 \\
\hline 0 & 0 & 0 & 0 & 0 & 0 & 0 & 0 & 1 \\
\hline 221 & 316 & 873 & 884 & 823 & 355 & 220 & 73 & 3,764 \\
\hline 211 & 262 & 592 & 584 & 593 & 266 & 164 & 58 & 2,731 \\
\hline 27 & 66 & 195 & 137 & 103 & 48 & 32 & 6 & 614 \\
\hline 15 & 11 & 73 & 137 & 185 & 66 & 19 & 3 & 508 \\
\hline 35 & 43 & 75 & 55 & 49 & 36 & 50 & 31 & 374 \\
\hline 25 & 12 & 20 & 26 & 29 & 13 & 10 & 3 & 138 \\
\hline 19 & 24 & 32 & 29 & 23 & 10 & 6 & 1 & 145 \\
\hline 90 & 105 & 198 & 200 & 204 & 93 & 47 & 13 & 952 \\
\hline 10 & 54 & 281 & 299 & 229 & 89 & 56 & 15 & 1,032 \\
\hline- & 23 & 123 & 117 & 105 & 49 & 34 & 9 & 460 \\
\hline 8 & 19 & 147 & 166 & 107 & 36 & 21 & 5 & 510 \\
\hline 0 & 11 & 10 & 14 & 14 & 4 & 1 & 0 & 55 \\
\hline 1 & 0 & 0 & 2 & 3 & 0 & 0 & 0 & 7 \\
\hline
\end{tabular}

Source: Authors' compilation.

Note: $-=$ an estimate of zero: the number zero in a cell indicates a non-zero estimate of less than 500

a. These figures include late effects of polio cases with onset prior to regional certification of polio eradication in 2000

b. Does not include liver cancer and cirrhosis DALYS $(3,0)$ resulting from chronic hepatitis virus infection.

c. This cause category includes "Causes arising in the perinatal period" as defined in the International Classification of

Diseases, principally low birthweight, prematurity, birth asphyxia, and birth trauma, and does not include all causes

of $\operatorname{DALYS}(3,0)$ occurring in the perinatal period. 
Table 3C.4 DALYs $(3,0)$ by Cause, Sex, and Age in the Latin America and the Caribbean Region, 2001 (thousands)

\begin{tabular}{|c|c|c|c|c|c|c|c|c|c|c|}
\hline \multirow[b]{2}{*}{ Cause } & \multicolumn{10}{|c|}{ Male } \\
\hline & Total & $0-4$ & $5-14$ & $15-29$ & 30-44 & $45-59$ & $60-69$ & 70-79 & $80+$ & Total \\
\hline Population (millions) & 526 & 28 & 56 & 74 & 52 & 31 & 11 & 6 & 2 & 260 \\
\hline All causes & 104,287 & 12,782 & 3,168 & 11,429 & 9,087 & 8,940 & 5,505 & 4,078 & 1,730 & 56,718 \\
\hline $\begin{array}{l}\text { I. Communicable, maternal, perinatal, } \\
\text { and nutritional conditions }\end{array}$ & 22,741 & 6,922 & 548 & 1,126 & 1,436 & 806 & 365 & 287 & 176 & 11,666 \\
\hline A. Infectious and parasitic diseases & 10,288 & 2,057 & 371 & 965 & 1,268 & 614 & 223 & 136 & 57 & 5,694 \\
\hline 1. Tuberculosis & 966 & 24 & 20 & 115 & 169 & 150 & 70 & 39 & 11 & 599 \\
\hline $\begin{array}{l}\text { 2. Sexually transmitted diseases } \\
\text { excluding HIV/AIDS }\end{array}$ & 430 & 44 & 1 & 49 & 28 & 1 & $\mathbf{0}$ & $\mathbf{0}$ & $\mathbf{0}$ & 123 \\
\hline a. Syphilis & 77 & 30 & 0 & 9 & 6 & 0 & 0 & 0 & 0 & 46 \\
\hline b. Chlamydia & 183 & 2 & 0 & 10 & 4 & 0 & 0 & - & - & 16 \\
\hline c. Gonorrhea & 157 & 11 & 1 & 30 & 18 & 0 & 0 & 0 & 0 & 60 \\
\hline d. Other sexually transmitted diseases & 12 & 0 & - & - & 0 & 0 & - & 0 & 0 & 0 \\
\hline 3. HIV/AIDS & 2,354 & 91 & 41 & 430 & 735 & 194 & 16 & 2 & $\mathbf{0}$ & 1,510 \\
\hline 4. Diarrheal diseases & 2,362 & 983 & 113 & 39 & 34 & 25 & 13 & 10 & 6 & 1,224 \\
\hline 5. Childhood-cluster diseases & 397 & 191 & 5 & 2 & 1 & 1 & $\mathbf{0}$ & $\mathbf{0}$ & $\mathbf{0}$ & 200 \\
\hline a. Pertussis & 366 & 179 & 3 & - & - & - & 0 & - & - & 182 \\
\hline b. Poliomyelitis ${ }^{a}$ & 6 & 0 & 1 & 1 & 1 & 0 & 0 & 0 & 0 & 3 \\
\hline c. Diphtheria & 8 & 6 & 0 & - & 0 & - & - & - & 0 & 6 \\
\hline d. Measles & 0 & 0 & 0 & - & - & - & - & - & - & 0 \\
\hline e. Tetanus & 17 & 6 & 1 & 0 & 0 & 1 & 0 & 0 & 0 & 9 \\
\hline 6. Meningitis & 591 & 214 & 31 & 35 & 27 & 17 & 6 & 4 & 1 & 335 \\
\hline 7. Hepatitis $\mathbf{B}^{\mathbf{b}}$ & 95 & 10 & 3 & 17 & 14 & 11 & 4 & 2 & 0 & 61 \\
\hline Hepatitis $\mathbf{C}^{\mathbf{b}}$ & 37 & 0 & 0 & 1 & 6 & 9 & 3 & 1 & 0 & 20 \\
\hline 8. Malaria & 111 & 34 & 10 & 6 & 5 & 3 & 1 & 1 & 0 & 60 \\
\hline 9. Tropical-cluster diseases & 696 & 7 & 12 & 145 & 80 & 76 & 34 & 14 & 4 & 372 \\
\hline a. Trypanosomiasis & 0 & - & - & - & - & - & 0 & - & - & 0 \\
\hline b. Chagas' disease & 583 & 0 & 0 & 125 & 62 & 67 & 32 & 12 & 4 & 302 \\
\hline c. Schistosomiasis & 66 & 2 & 6 & 9 & 9 & 6 & 2 & 1 & 0 & 34 \\
\hline d. Leishmaniasis & 37 & 5 & 4 & 8 & 7 & 2 & 0 & 0 & 0 & 28 \\
\hline e. Lymphatic filariasis & 9 & 0 & 2 & 2 & 1 & 1 & 0 & 0 & 0 & 7 \\
\hline f. Onchocerciasis & 2 & 0 & 0 & 0 & 0 & 0 & 0 & 0 & 0 & 1 \\
\hline 10. Leprosy & 18 & $\mathbf{0}$ & 2 & 2 & 2 & 2 & 1 & 1 & 0 & 10 \\
\hline 11. Dengue & 59 & 7 & 19 & 1 & 1 & 1 & 0 & 0 & $\mathbf{0}$ & 29 \\
\hline 12. Japanese encephalitis & - & - & - & - & - & - & - & - & - & 一 \\
\hline 13. Trachoma & 191 & $\mathbf{0}$ & $\mathbf{0}$ & 1 & 11 & 16 & 11 & 6 & 2 & 47 \\
\hline 14. Intestinal nematode infections & 139 & 22 & 43 & $\mathbf{0}$ & 1 & 1 & 1 & 0 & 0 & 69 \\
\hline a. Ascariasis & 46 & 6 & 16 & 0 & 0 & 0 & 0 & 0 & 0 & 23 \\
\hline b. Trichuriasis & 50 & 5 & 20 & 0 & 0 & 0 & 0 & 0 & 0 & 25 \\
\hline c. Hookworm disease & 12 & 1 & 5 & 0 & 0 & 0 & 0 & 0 & 0 & 6 \\
\hline Other intestinal infections & 31 & 9 & 2 & 0 & 1 & 0 & 1 & 0 & 0 & 14 \\
\hline Other infectious diseases & 1,842 & 429 & 71 & 122 & 154 & 109 & 63 & 56 & 31 & 1,035 \\
\hline B. Respiratory infections & 3,271 & 933 & 125 & 93 & 119 & 147 & 107 & 117 & 93 & 1,733 \\
\hline 1. Lower respiratory infections & 3,043 & 886 & 75 & 88 & 116 & 144 & 105 & 115 & 92 & 1,621 \\
\hline 2. Upper respiratory infections & 80 & 22 & 3 & 4 & 3 & 2 & 1 & 1 & 1 & 38 \\
\hline 3. Otitis media & 147 & 26 & 46 & 1 & 1 & 1 & 0 & 0 & 0 & 74 \\
\hline C. Maternal conditions & 1,329 & - & - & - & - & - & - & - & - & 一 \\
\hline 1. Maternal hemorrhage & 98 & - & - & - & - & - & - & - & - & - \\
\hline 2. Maternal sepsis & 337 & - & - & - & - & - & - & - & - & - \\
\hline 3. Hypertensive disorders of pregnancy & 112 & - & - & - & - & - & - & - & - & - \\
\hline 4. Obstructed labor & 52 & - & - & - & - & - & - & - & - & - \\
\hline 5. Abortion & 117 & - & - & - & - & - & - & - & - & - \\
\hline Other maternal conditions & 613 & - & - & - & - & - & - & - & - & - \\
\hline D. Perinatal conditions ${ }^{\mathrm{C}}$ & 6,296 & 3,473 & 0 & 0 & 0 & $\mathbf{0}$ & - & - & - & 3,473 \\
\hline 1. Low birthweight & 795 & 432 & - & - & - & - & - & - & - & 432 \\
\hline 2. Birth asphyxia and birth trauma & 3,765 & 2,072 & 0 & 0 & 0 & 0 & - & - & - & 2,072 \\
\hline Other perinatal conditions & 1,736 & 969 & 0 & 0 & - & - & - & - & - & 969 \\
\hline E. Nutritional deficiencies & 1,558 & 459 & 51 & 68 & 48 & 44 & 35 & 34 & 26 & 766 \\
\hline 1. Protein-energy malnutrition & 916 & 345 & 15 & 18 & 20 & 23 & 20 & 23 & 19 & 482 \\
\hline 2. Iodine deficiency & 110 & 41 & 13 & 0 & 0 & 0 & 0 & 0 & 0 & 54 \\
\hline 3. Vitamin A deficiency & 1 & 0 & - & - & - & - & 0 & - & 0 & 0 \\
\hline 4. Iron-deficiency anemia & 477 & 62 & 22 & 48 & 25 & 18 & 12 & 9 & 5 & 201 \\
\hline Other nutritional disorders & 55 & 11 & 1 & 2 & 4 & 3 & 3 & 3 & 1 & 29 \\
\hline
\end{tabular}


Table 3C.4 Continued

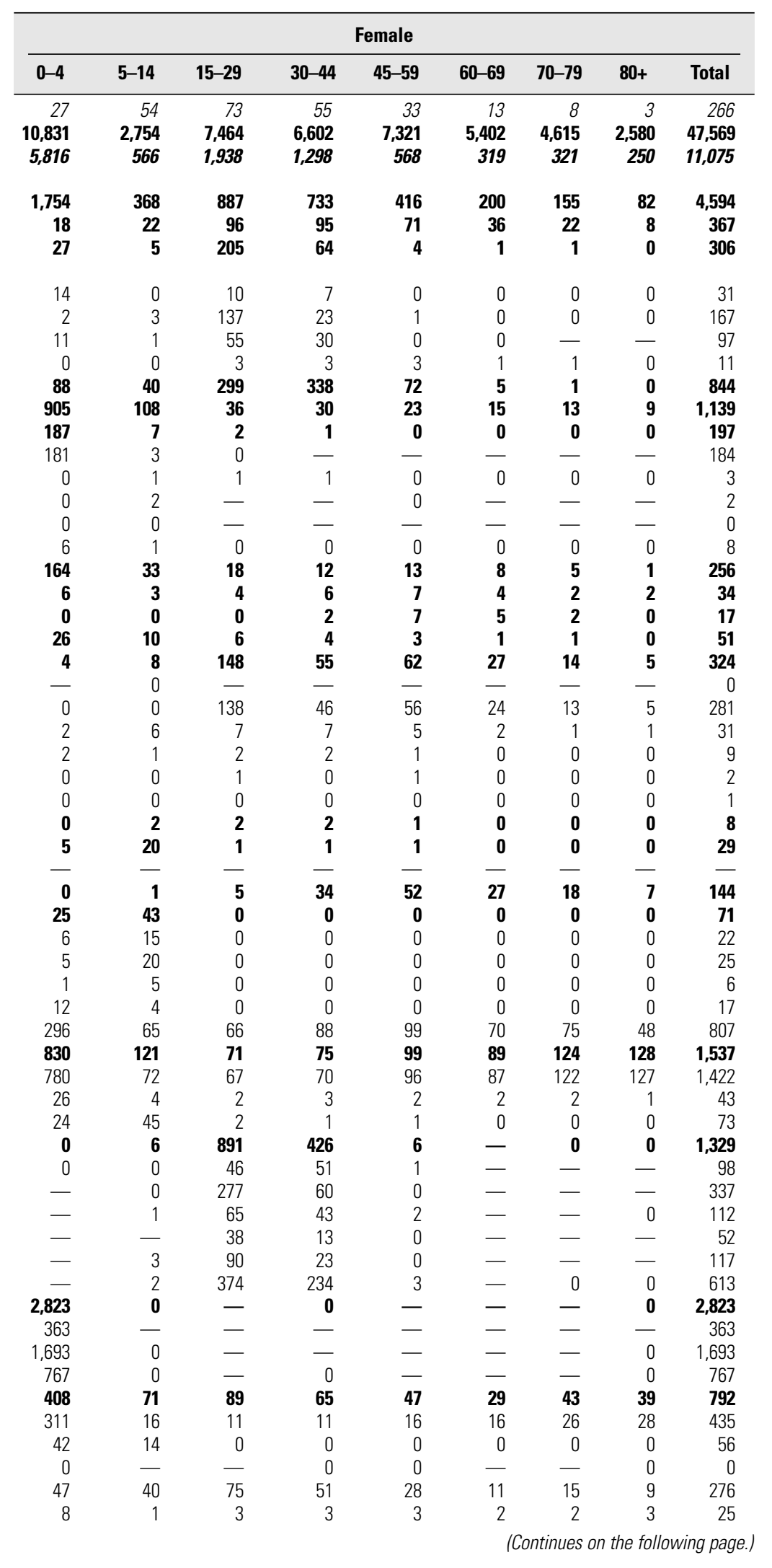

The Burden of Disease and Mortality by Condition: Data, Methods, and Results for 2001 | 199 
Table 3C.4 Continued

\begin{tabular}{|c|c|c|c|c|c|c|c|c|c|c|}
\hline \multirow[b]{2}{*}{ Cause } & \multicolumn{10}{|c|}{ Male } \\
\hline & Total & $0-4$ & $5-14$ & $15-29$ & 30-44 & $45-59$ & $60-69$ & $70-79$ & $80+$ & Total \\
\hline II. Noncommunicable diseases & 67,815 & 5,265 & 1,686 & 5,194 & 4,811 & 6,982 & 4,831 & 3,653 & 1,508 & 33,930 \\
\hline A. Malignant neoplasms & 7,060 & 56 & 94 & 205 & 338 & 905 & 835 & 639 & 218 & 3,289 \\
\hline 1. Mouth and oropharynx cancers & 204 & 0 & 0 & 3 & 18 & 67 & 40 & 19 & 5 & 153 \\
\hline 2. Esophageal cancer & 215 & - & 0 & 1 & 13 & 64 & 48 & 28 & 8 & 160 \\
\hline 3. Stomach cancer & 735 & 0 & 1 & 10 & 45 & 133 & 121 & 88 & 29 & 427 \\
\hline 4. Colon and rectal cancers & 485 & 0 & 0 & 9 & 28 & 66 & 60 & 48 & 17 & 229 \\
\hline 5. Liver cancer & 277 & 2 & 1 & 4 & 15 & 43 & 39 & 27 & 8 & 139 \\
\hline 6. Pancreas cancer & 248 & 0 & 0 & 1 & 10 & 37 & 37 & 26 & 8 & 120 \\
\hline 7. Trachea, bronchus, and lung cancers & 728 & 0 & 1 & 5 & 31 & 157 & 160 & 107 & 25 & 486 \\
\hline 8. Melanoma and other skin cancers & 97 & 0 & 0 & 3 & 10 & 16 & 12 & 9 & 4 & 53 \\
\hline 9. Breast cancer & 642 & 0 & - & 0 & 1 & 2 & 1 & 1 & 0 & 4 \\
\hline 10. Cervix uteri cancer & 494 & - & - & - & - & - & - & - & - & - \\
\hline 11. Corpus uteri cancer & 254 & - & - & - & - & - & - & - & - & - \\
\hline 12. Ovarian cancer & 152 & - & - & - & - & - & - & - & - & - \\
\hline 13. Prostate cancer & 340 & 0 & 0 & 1 & 2 & 34 & 98 & 137 & 67 & 340 \\
\hline 14. Bladder cancer & 100 & 0 & 0 & 1 & 3 & 15 & 20 & 21 & 9 & 68 \\
\hline 15. Lymphomas and multiple myeloma & 383 & 5 & 16 & 33 & 37 & 53 & 40 & 26 & 7 & 217 \\
\hline 16. Leukemia & 444 & 24 & 45 & 66 & 36 & 30 & 20 & 16 & 6 & 242 \\
\hline Other malignant neoplasms & 1,263 & 23 & 29 & 67 & 90 & 190 & 141 & 86 & 25 & 651 \\
\hline B. Other neoplasms & 196 & 9 & 7 & 12 & 13 & 22 & 17 & 13 & 5 & 97 \\
\hline C. Diabetes mellitus & 2,775 & 2 & 3 & 35 & 152 & 386 & 305 & 203 & 61 & 1,149 \\
\hline D. Endocrine disorders & 3,150 & 1,221 & 64 & 81 & 71 & 92 & 47 & 37 & 19 & 1,632 \\
\hline E. Neuropsychiatric conditions & 18,781 & 1,522 & 758 & 3,667 & 1,717 & 951 & 355 & 248 & 144 & 9,362 \\
\hline 1. Unipolar depressive disorders & 5,219 & 0 & 266 & 621 & 591 & 320 & 94 & 20 & 5 & 1,917 \\
\hline 2. Bipolar affective disorder & 883 & 0 & 23 & 362 & 41 & 1 & 0 & 0 & 0 & 428 \\
\hline 3. Schizophrenia & 1,078 & 0 & 77 & 383 & 43 & 9 & 2 & 1 & 0 & 516 \\
\hline 4. Epilepsy & 737 & 33 & 85 & 121 & 85 & 43 & 13 & 7 & 2 & 388 \\
\hline 5. Alcohol use disorders & 2,883 & 1 & 30 & 1,271 & 590 & 371 & 72 & 18 & 2 & 2,355 \\
\hline 6. Alzheimer's and other dementias & 1,215 & 20 & 9 & 10 & 7 & 28 & 104 & 165 & 119 & 463 \\
\hline 7. Parkinson's disease & 90 & 0 & 0 & 0 & 4 & 12 & 11 & 12 & 6 & 46 \\
\hline 8. Multiple sclerosis & 97 & - & 4 & 18 & 12 & 4 & 1 & 1 & 0 & 41 \\
\hline 9. Drug use disorders & 746 & - & 7 & 370 & 152 & 28 & 1 & 0 & 0 & 559 \\
\hline 10. Post-traumatic stress disorder & 177 & 0 & 2 & 29 & 17 & 8 & 0 & 0 & 0 & 58 \\
\hline 11. Obsessive-compulsive disorder & 480 & - & 4 & 121 & 59 & 30 & 8 & 2 & 0 & 225 \\
\hline 12. Panic disorder & 409 & - & 3 & 123 & 1 & 5 & 1 & 0 & 0 & 134 \\
\hline 13. Insomnia (primary) & 312 & - & 4 & 39 & 45 & 29 & 15 & 3 & 1 & 136 \\
\hline 14. Migraine & 736 & 6 & 68 & 114 & 5 & 0 & 0 & 0 & 0 & 193 \\
\hline 15. Mental retardation, lead-caused & 1,500 & 753 & 0 & 1 & 0 & 0 & 0 & 0 & 0 & 755 \\
\hline Other neuropsychiatric disorders & 2,217 & 710 & 177 & 83 & 64 & 61 & 29 & 19 & 8 & 1,150 \\
\hline F. Sense organ diseases & 5,465 & 6 & 18 & 82 & 484 & 801 & 589 & 384 & 91 & 2,453 \\
\hline 1. Glaucoma & 304 & 1 & 2 & 5 & 24 & 37 & 27 & 22 & 7 & 124 \\
\hline 2. Cataracts & 1,813 & 3 & 11 & 42 & 122 & 248 & 166 & 103 & 35 & 731 \\
\hline 3. Vision disorders, age-related & 1,639 & 1 & 5 & 34 & 140 & 222 & 143 & 107 & 25 & 677 \\
\hline 4. Hearing loss, adult onset & 1,706 & - & - & 1 & 198 & 293 & 253 & 152 & 23 & 920 \\
\hline Other sense organ disorders & 3 & 0 & 0 & 0 & 0 & 0 & 0 & 0 & 0 & 2 \\
\hline G. Cardiovascular diseases & 11,827 & 84 & 45 & 243 & 710 & 1,733 & 1,531 & 1,285 & 606 & 6,237 \\
\hline 1. Rheumatic heart disease & 133 & 0 & 3 & 12 & 10 & 12 & 6 & 3 & 1 & 49 \\
\hline 2. Hypertensive heart disease & 1,052 & 1 & 1 & 12 & 46 & 128 & 122 & 112 & 62 & 484 \\
\hline 3. Ischemic heart disease & 4,328 & 1 & 10 & 70 & 245 & 781 & 696 & 541 & 206 & 2,550 \\
\hline 4. Cerebrovascular disease & 3,936 & 11 & 10 & 47 & 214 & 561 & 501 & 419 & 176 & 1,939 \\
\hline 5. Inflammatory heart diseases & 557 & 15 & 5 & 33 & 62 & 87 & 63 & 44 & 17 & 326 \\
\hline Other cardiovascular diseases & 1,821 & 56 & 15 & 69 & 133 & 164 & 142 & 164 & 144 & 889 \\
\hline H. Respiratory diseases & 5,198 & 427 & 252 & 352 & 312 & 495 & 398 & 347 & 172 & 2,754 \\
\hline 1. Chronic obstructive pulmonary disease & 2,037 & 14 & 2 & 18 & 126 & 304 & 262 & 224 & 107 & 1,057 \\
\hline 2. Asthma & 1,547 & 189 & 216 & 262 & 73 & 39 & 16 & 12 & 6 & 812 \\
\hline Other respiratory diseases & 1,614 & 224 & 33 & 73 & 113 & 152 & 121 & 111 & 59 & 885 \\
\hline
\end{tabular}


Table 3C.4 Continued

\begin{tabular}{|c|c|c|c|c|c|c|c|c|}
\hline & & & & male & & & & \\
\hline $0-4$ & 5-14 & $15-29$ & $30-44$ & $45-59$ & $60-69$ & $70-79$ & $80+$ & Total \\
\hline 4,644 & 1,770 & 4,680 & 4,819 & 6,496 & 4,981 & 4,215 & 2,280 & 33,885 \\
\hline 49 & 74 & 178 & 641 & 1,194 & 812 & 602 & 222 & 3,771 \\
\hline 0 & 0 & 2 & 6 & 14 & 13 & 12 & 5 & 52 \\
\hline - & 0 & 0 & 4 & 15 & 16 & 13 & 5 & 54 \\
\hline 0 & 1 & 9 & 46 & 82 & 72 & 67 & 29 & 307 \\
\hline 0 & 0 & 6 & 34 & 70 & 62 & 57 & 27 & 256 \\
\hline 2 & 1 & 4 & 13 & 40 & 38 & 31 & 11 & 139 \\
\hline 0 & 0 & 1 & 9 & 35 & 38 & 33 & 13 & 128 \\
\hline 0 & 0 & 3 & 27 & 77 & 71 & 49 & 15 & 242 \\
\hline 0 & 0 & 2 & 8 & 12 & 8 & 8 & 5 & 44 \\
\hline 0 & 0 & 8 & 144 & 262 & 123 & 73 & 27 & 638 \\
\hline - & 0 & 33 & 124 & 191 & 84 & 49 & 13 & 494 \\
\hline 0 & 0 & 7 & 58 & 95 & 52 & 32 & 10 & 254 \\
\hline 0 & 1 & 10 & 26 & 55 & 33 & 21 & 5 & 152 \\
\hline - & - & - & - & - & - & - & - & - \\
\hline 0 & 0 & 0 & 2 & 8 & 8 & 9 & 5 & 32 \\
\hline 3 & 9 & 15 & 23 & 43 & 36 & 29 & 9 & 166 \\
\hline 22 & 35 & 38 & 34 & 31 & 19 & 16 & 6 & 201 \\
\hline 21 & 26 & 37 & 84 & 163 & 139 & 103 & 38 & 611 \\
\hline 6 & 6 & 10 & 16 & 23 & 16 & 14 & 6 & 98 \\
\hline 3 & 5 & 37 & 160 & 492 & 450 & 349 & 130 & 1,625 \\
\hline 980 & 60 & 99 & 96 & 127 & 64 & 56 & 36 & 1,518 \\
\hline 1,420 & 813 & 3,207 & 1,936 & 951 & 419 & 355 & 317 & 9,418 \\
\hline 0 & 256 & 1,110 & 1,091 & 602 & 186 & 44 & 14 & 3,303 \\
\hline 0 & 23 & 383 & 47 & 2 & 1 & 0 & 0 & 455 \\
\hline 0 & 10 & 430 & 108 & 10 & 2 & 2 & 0 & 562 \\
\hline 30 & 79 & 107 & 72 & 38 & 14 & 7 & 2 & 350 \\
\hline 0 & 8 & 296 & 129 & 77 & 15 & 3 & 1 & 528 \\
\hline 20 & 9 & 11 & 8 & 33 & 136 & 256 & 281 & 753 \\
\hline 0 & 0 & 0 & 4 & 12 & 10 & 11 & 6 & 44 \\
\hline- & 5 & 25 & 18 & 6 & 1 & 1 & 0 & 56 \\
\hline - & 7 & 122 & 50 & 9 & 1 & 0 & 0 & 188 \\
\hline 0 & 1 & 54 & 47 & 15 & 1 & 1 & 0 & 119 \\
\hline - & 2 & 146 & 54 & 42 & 6 & 4 & 1 & 255 \\
\hline - & 8 & 251 & 2 & 11 & 1 & 1 & 0 & 275 \\
\hline - & 3 & 51 & 52 & 38 & 24 & 6 & 2 & 176 \\
\hline 20 & 241 & 115 & 166 & 0 & 0 & 0 & 0 & 543 \\
\hline 743 & 0 & 1 & 1 & 0 & 0 & 0 & 0 & 745 \\
\hline 607 & 163 & 107 & 88 & 54 & 21 & 18 & 9 & 1,067 \\
\hline 2 & 8 & 48 & 298 & 978 & 899 & 606 & 172 & 3,012 \\
\hline 0 & 1 & 4 & 25 & 42 & 48 & 45 & 16 & 180 \\
\hline 1 & 5 & 18 & 83 & 384 & 314 & 200 & 78 & 1,082 \\
\hline 0 & 1 & 25 & 56 & 283 & 297 & 239 & 60 & 962 \\
\hline - & - & 1 & 134 & 269 & 240 & 122 & 19 & 785 \\
\hline 0 & 0 & 0 & 0 & 0 & 0 & 0 & 0 & 2 \\
\hline 67 & 48 & 180 & 546 & 1,251 & 1,244 & 1,337 & 916 & 5,590 \\
\hline 0 & 4 & 13 & 20 & 24 & 12 & 8 & 3 & 84 \\
\hline 1 & 1 & 12 & 45 & 125 & 130 & 146 & 108 & 568 \\
\hline 1 & 12 & 38 & 114 & 392 & 469 & 471 & 282 & 1,778 \\
\hline 8 & 9 & 44 & 238 & 510 & 451 & 457 & 280 & 1,997 \\
\hline 13 & 5 & 21 & 31 & 51 & 45 & 41 & 23 & 231 \\
\hline 43 & 17 & 52 & 98 & 149 & 136 & 214 & 222 & 933 \\
\hline 384 & 300 & 290 & 361 & 366 & 284 & 277 & 181 & 2,444 \\
\hline 36 & 5 & 50 & 254 & 223 & 165 & 155 & 91 & 980 \\
\hline 158 & 270 & 180 & 33 & 40 & 26 & 19 & 8 & 735 \\
\hline 190 & 25 & 59 & 74 & 103 & 93 & 103 & 82 & 729 \\
\hline
\end{tabular}


Table 3C.4 Continued

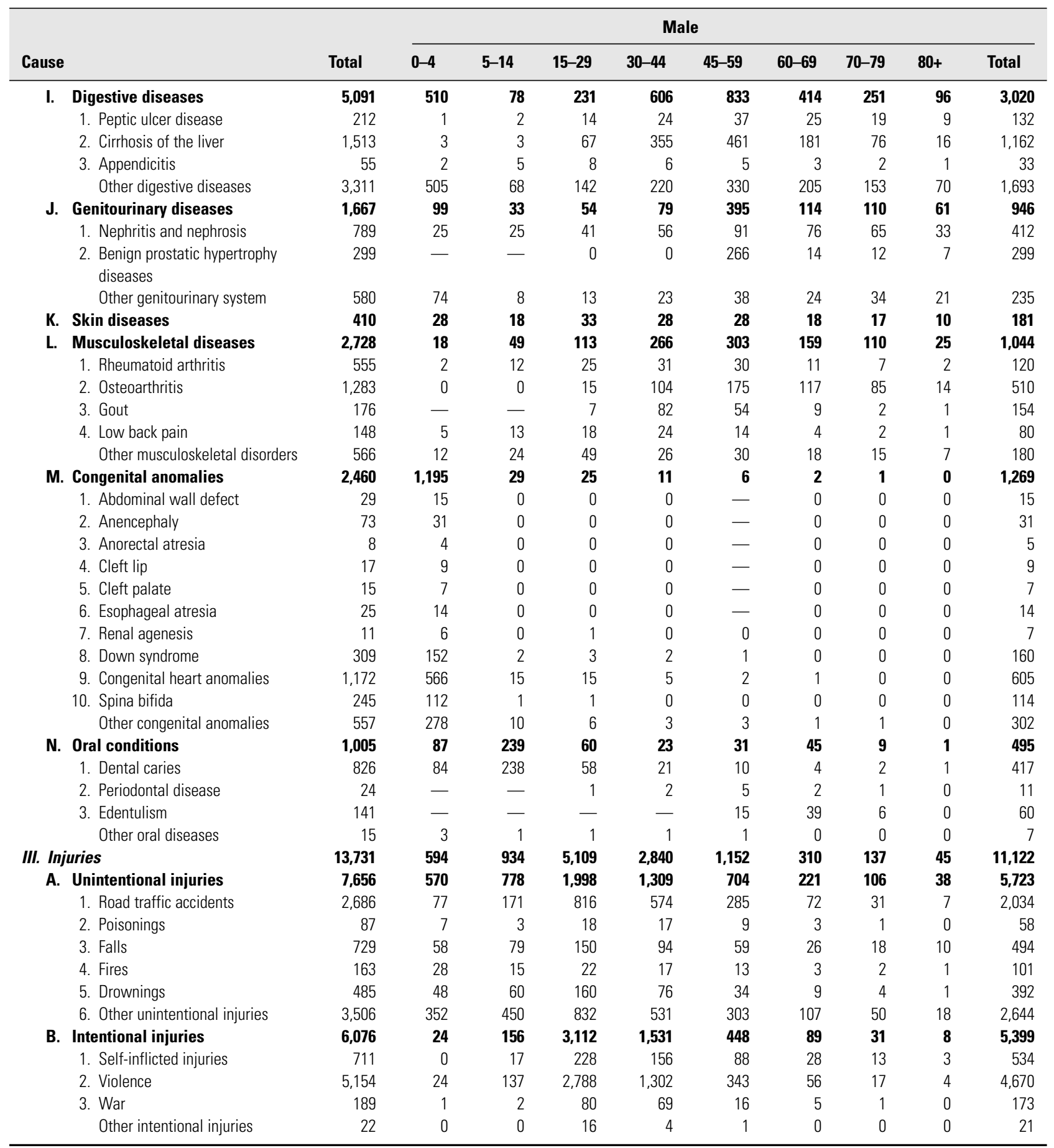


Table 3C.4 Continued

\begin{tabular}{|c|c|c|c|c|c|c|c|c|}
\hline \multicolumn{9}{|c|}{ Female } \\
\hline $0-4$ & 5-14 & $15-29$ & $30-44$ & $45-59$ & $60-69$ & $70-79$ & $80+$ & Total \\
\hline 401 & 59 & 188 & 270 & 459 & 301 & 258 & 136 & 2,071 \\
\hline 1 & 1 & 8 & 12 & 18 & 15 & 17 & 11 & 81 \\
\hline 3 & 2 & 15 & 70 & 121 & 77 & 48 & 13 & 351 \\
\hline 2 & 4 & 5 & 4 & 4 & 2 & 2 & 1 & 22 \\
\hline 396 & 52 & 160 & 184 & 317 & 207 & 192 & 111 & 1,618 \\
\hline 82 & 43 & 89 & 96 & 140 & 103 & 98 & 71 & 722 \\
\hline 24 & 30 & 38 & 47 & 78 & 65 & 58 & 36 & 377 \\
\hline- & - & - & - & - & - & - & - & - \\
\hline 58 & 13 & 51 & 49 & 62 & 38 & 39 & 34 & 345 \\
\hline 35 & 17 & 38 & 38 & 36 & 22 & 23 & 20 & 230 \\
\hline 13 & 79 & 235 & 323 & 438 & 308 & 219 & 69 & 1,685 \\
\hline 3 & 43 & 132 & 114 & 85 & 33 & 19 & 7 & 435 \\
\hline- & 0 & 5 & 94 & 245 & 228 & 163 & 38 & 774 \\
\hline 0 & 0 & 1 & 11 & 6 & 2 & 2 & 0 & 22 \\
\hline 2 & 15 & 12 & 18 & 14 & 4 & 2 & 1 & 67 \\
\hline 8 & 22 & 85 & 87 & 87 & 41 & 33 & 23 & 387 \\
\hline 1,117 & 27 & 22 & 12 & 8 & 3 & 2 & 0 & 1,191 \\
\hline 14 & 0 & 0 & 0 & - & - & 0 & 0 & 14 \\
\hline 41 & 0 & 0 & 0 & 0 & - & 0 & - & 41 \\
\hline 3 & 0 & 0 & 0 & 0 & 0 & 0 & - & 3 \\
\hline 8 & 0 & 0 & 0 & - & - & 0 & - & 8 \\
\hline 7 & 0 & 0 & 0 & - & - & 0 & - & 7 \\
\hline 10 & 0 & 0 & 0 & 0 & - & 0 & 0 & 10 \\
\hline 3 & 0 & 0 & 0 & 0 & 0 & 0 & 0 & 4 \\
\hline 142 & 2 & 2 & 2 & 2 & 0 & 0 & 0 & 149 \\
\hline 527 & 15 & 13 & 6 & 4 & 1 & 1 & 0 & 566 \\
\hline 129 & 1 & 1 & 0 & 0 & - & - & - & 132 \\
\hline 232 & 8 & 6 & 4 & 3 & 1 & 1 & 0 & 255 \\
\hline 84 & 231 & 60 & 24 & 34 & 56 & 18 & 3 & 510 \\
\hline 81 & 230 & 58 & 22 & 11 & 4 & 3 & 1 & 409 \\
\hline- & - & 1 & 2 & 6 & 2 & 1 & 1 & 13 \\
\hline- & - & - & - & 17 & 49 & 14 & 1 & 81 \\
\hline 3 & 1 & 1 & 1 & 0 & 0 & 0 & 0 & 8 \\
\hline 371 & 418 & 846 & 485 & 258 & 103 & 79 & 50 & 2,609 \\
\hline 355 & 358 & 514 & 308 & 192 & 87 & 71 & 48 & 1,932 \\
\hline 64 & 111 & 217 & 134 & 77 & 28 & 17 & 5 & 651 \\
\hline 6 & 4 & 8 & 6 & 3 & 1 & 1 & 0 & 29 \\
\hline 47 & 50 & 43 & 21 & 17 & 15 & 22 & 20 & 235 \\
\hline 22 & 11 & 11 & 7 & 6 & 2 & 2 & 1 & 63 \\
\hline 31 & 27 & 20 & 8 & 4 & 2 & 1 & 0 & 93 \\
\hline 185 & 156 & 214 & 132 & 85 & 39 & 29 & 21 & 862 \\
\hline 16 & 59 & 332 & 177 & 66 & 16 & 8 & 2 & 677 \\
\hline 0 & 16 & 90 & 38 & 22 & 6 & 3 & 1 & 177 \\
\hline 16 & 42 & 239 & 134 & 40 & 8 & 4 & 1 & 484 \\
\hline 0 & 2 & 3 & 4 & 4 & 1 & 0 & 0 & 16 \\
\hline 0 & - & 0 & 0 & 0 & 0 & 0 & 0 & 1 \\
\hline
\end{tabular}

Source: Authors' compilation.

Note: $-=$ an estimate of zero; the number zero in a cell indicates a non-zero estimate of less than 500 .

a. These figures include late effects of polio cases with onset prior to regional certification of polio eradication in 2002.

b. Does not include liver cancer and cirrhosis $\operatorname{DALYS}(3,0)$ resulting from chronic hepatitis virus infection.

c. This cause category includes "Causes arising in the perinatal period" as defined in the International Classification of

Diseases, principally low birthweight, prematurity, birth asphyxia, and birth trauma, and does not include all causes of

$\operatorname{DALY}(3,0)$ occurring in the perinatal period. 
Table 3C.5 DALYs(3,0) by Cause, Sex, and Age in the Middle East and North Africa Region, 2001 (thousands)

\begin{tabular}{|c|c|c|c|c|c|c|c|c|c|c|}
\hline \multirow[b]{2}{*}{ Cause } & \multicolumn{10}{|c|}{ Male } \\
\hline & Total & $0-4$ & $5-14$ & $15-29$ & 30-44 & $45-59$ & $60-69$ & $70-79$ & $80+$ & Total \\
\hline Population (millions) & 310 & 19 & 39 & 47 & 29 & 15 & 5 & 3 & 1 & 157 \\
\hline All causes & 65,570 & 11,155 & 2,606 & 5,127 & 4,351 & 5,113 & 3,267 & 2,310 & 630 & 34,559 \\
\hline $\begin{array}{l}\text { I. Communicable, maternal, perinatal, } \\
\text { and nutritional conditions }\end{array}$ & 17,739 & 6,784 & 503 & 309 & 298 & 286 & 187 & 139 & 45 & 8,552 \\
\hline A. Infectious and parasitic diseases & 7,320 & 2,424 & 275 & 219 & 235 & 228 & 131 & 84 & 23 & 3,619 \\
\hline 1. Tuberculosis & 522 & 37 & 12 & 42 & 67 & 57 & 35 & 21 & 5 & 275 \\
\hline $\begin{array}{l}\text { 2. Sexually transmitted diseases } \\
\text { excluding HIV/AIDS }\end{array}$ & 342 & 16 & 1 & 33 & 19 & 17 & 7 & 3 & 1 & 97 \\
\hline a. Syphilis & 64 & 3 & 0 & 4 & 6 & 12 & 4 & 1 & 0 & 30 \\
\hline b. Chlamydia & 166 & 2 & 0 & 11 & 3 & 0 & 0 & - & - & 16 \\
\hline c. Gonorrhea & 97 & 10 & 0 & 18 & 9 & 0 & 0 & - & - & 39 \\
\hline d. Other sexually transmitted diseases & 15 & 0 & 0 & 0 & 1 & 5 & 3 & 2 & 0 & 11 \\
\hline 3. HIV/AIDS & 105 & 9 & 1 & 22 & 25 & 7 & $\mathbf{0}$ & 0 & 0 & 64 \\
\hline 4. Diarrheal diseases & 2,571 & 1,207 & 47 & 23 & 22 & 18 & 10 & 8 & 5 & 1,339 \\
\hline 5. Childhood-cluster diseases & 915 & 357 & 81 & 15 & 2 & 1 & $\mathbf{0}$ & 0 & $\mathbf{0}$ & 457 \\
\hline a. Pertussis & 326 & 161 & 2 & - & - & - & - & - & - & 164 \\
\hline b. Poliomyelitis & 8 & 0 & 1 & 2 & 1 & 0 & 0 & 0 & 0 & 4 \\
\hline c. Diphtheria & 1 & 0 & 0 & 0 & - & - & - & 0 & - & 0 \\
\hline d. Measles & 470 & 148 & 74 & 12 & - & - & - & - & - & 234 \\
\hline e. Tetanus & 110 & 48 & 4 & 1 & 1 & 1 & 0 & 0 & 0 & 55 \\
\hline 6. Meningitis & 328 & 113 & 13 & 10 & 4 & 3 & 2 & 1 & $\mathbf{0}$ & 146 \\
\hline 7. Hepatitis $\mathbf{B}^{\mathbf{a}}$ & 111 & 14 & 3 & 4 & 9 & 18 & 9 & 5 & 0 & 63 \\
\hline Hepatitis Ca & 55 & 6 & 1 & 2 & 4 & 9 & 5 & 2 & 0 & 30 \\
\hline 8. Malaria & 668 & 265 & 21 & 14 & 10 & 7 & 2 & 1 & 1 & 322 \\
\hline 9. Tropical-cluster diseases & 281 & 13 & 33 & 29 & 27 & 37 & 22 & 13 & 2 & 176 \\
\hline a. Trypanosomiasis & 22 & 1 & 4 & 4 & 2 & 2 & 0 & 0 & 0 & 13 \\
\hline b. Chagas' disease & - & - & - & - & - & - & - & - & - & - \\
\hline c. Schistosomiasis & 207 & 4 & 18 & 18 & 20 & 34 & 21 & 12 & 2 & 129 \\
\hline d. Leishmaniasis & 48 & 8 & 11 & 6 & 4 & 2 & 0 & 0 & 0 & 31 \\
\hline e. Lymphatic filariasis & 4 & 0 & 0 & 1 & 1 & 0 & 0 & 0 & 0 & 2 \\
\hline f. Onchocerciasis & 0 & 0 & 0 & 0 & 0 & 0 & 0 & 0 & 0 & 0 \\
\hline 10. Leprosy & 2 & $\mathbf{0}$ & $\mathbf{0}$ & $\mathbf{0}$ & $\mathbf{0}$ & $\mathbf{0}$ & $\mathbf{0}$ & 0 & - & 1 \\
\hline 11. Dengue & 8 & 1 & 3 & $\mathbf{0}$ & $\mathbf{0}$ & $\mathbf{0}$ & $\mathbf{0}$ & 0 & $\mathbf{0}$ & 5 \\
\hline 12. Japanese encephalitis & - & - & - & - & - & - & - & - & - & - \\
\hline 13. Trachoma & 273 & $\mathbf{0}$ & $\mathbf{0}$ & 3 & 22 & 23 & 15 & 9 & 2 & 74 \\
\hline 14. Intestinal nematode infections & 64 & 5 & 27 & $\mathbf{0}$ & 0 & $\mathbf{0}$ & $\mathbf{0}$ & 0 & $\mathbf{0}$ & 33 \\
\hline a. Ascariasis & 47 & 4 & 20 & 0 & 0 & - & - & - & - & 24 \\
\hline b. Trichuriasis & 0 & - & 0 & - & - & - & - & - & - & 0 \\
\hline c. Hookworm disease & 17 & 1 & 7 & 0 & 0 & 0 & 0 & 0 & 0 & 9 \\
\hline Other intestinal infections & 0 & - & 0 & 0 & 0 & 0 & 0 & 0 & 0 & 0 \\
\hline Other infectious diseases & 1,073 & 381 & 31 & 22 & 24 & 30 & 24 & 21 & 7 & 539 \\
\hline B. Respiratory infections & 3,141 & 1,323 & 92 & 33 & 31 & 38 & 44 & 47 & 20 & 1,629 \\
\hline 1. Lower respiratory infections & 2,974 & 1,283 & 57 & 31 & 29 & 37 & 43 & 46 & 19 & 1,545 \\
\hline 2. Upper respiratory infections & 72 & 24 & 3 & 2 & 1 & 1 & 2 & 2 & 0 & 36 \\
\hline 3. Otitis media & 95 & 16 & 32 & 0 & 0 & - & 0 & - & - & 48 \\
\hline C. Maternal conditions & 1,266 & - & - & - & - & - & 一 & - & 一 & 一 \\
\hline 1. Maternal hemorrhage & 121 & - & - & - & - & - & - & - & - & 一 \\
\hline 2. Maternal sepsis & 222 & - & - & - & - & - & - & - & - & 一 \\
\hline 3. Hypertensive disorders of pregnancy & 54 & - & - & - & - & - & - & - & - & - \\
\hline 4. Obstructed labor & 96 & - & - & - & - & - & - & - & - & 一 \\
\hline 5. Abortion & 152 & - & - & - & - & - & - & - & - & - \\
\hline Other maternal conditions & 620 & - & - & - & - & - & - & - & - & - \\
\hline D. Perinatal conditions ${ }^{b}$ & 4,155 & 2,415 & - & - & - & - & - & - & - & 2,415 \\
\hline 1. Low birthweight & 1,839 & 1,072 & - & - & - & - & - & - & - & 1,072 \\
\hline 2. Birth asphyxia and birth trauma & 1,595 & 928 & - & - & - & - & - & - & - & 928 \\
\hline Other perinatal conditions & 722 & 414 & - & - & - & - & - & - & - & 414 \\
\hline E. Nutritional deficiencies & 1,857 & 622 & 136 & 57 & 32 & 19 & 11 & 8 & 2 & 889 \\
\hline 1. Protein-energy malnutrition & 712 & 351 & 3 & 0 & 0 & 1 & 1 & 1 & 1 & 358 \\
\hline 2. Iodine deficiency & 506 & 192 & 62 & 0 & 0 & 0 & 0 & 0 & 0 & 254 \\
\hline 3. Vitamin A deficiency & 5 & 1 & 0 & 0 & 0 & 0 & 0 & 0 & 0 & 3 \\
\hline 4. Iron-deficiency anemia & 587 & 56 & 70 & 57 & 32 & 17 & 10 & 7 & 2 & 249 \\
\hline Other nutritional disorders & 47 & 23 & 1 & 0 & 0 & 0 & 0 & 0 & 0 & 25 \\
\hline
\end{tabular}


Table 3C.5 Continued

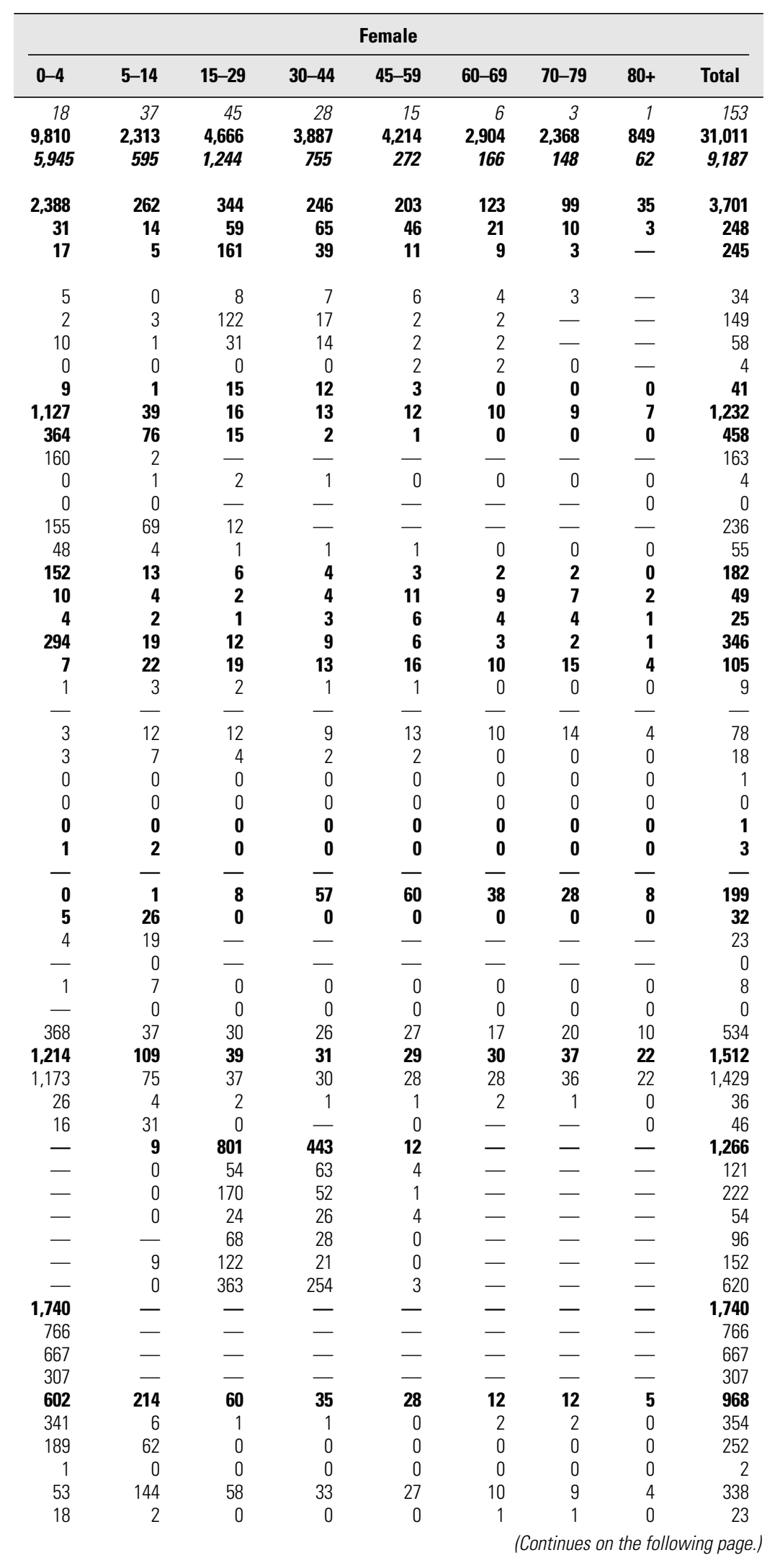




\begin{tabular}{|c|c|c|c|c|c|c|c|c|c|c|}
\hline \multirow[b]{2}{*}{ Cause } & \multicolumn{10}{|c|}{ Male } \\
\hline & Total & $0-4$ & $5-14$ & $15-29$ & 30-44 & $45-59$ & $60-69$ & $70-79$ & $80+$ & Total \\
\hline II. Noncommunicable diseases & 38,860 & 3,650 & 1,005 & 2,643 & 2,949 & 4,212 & 2,871 & 2,069 & 565 & 19,964 \\
\hline A. Malignant neoplasms & 2,747 & 54 & 71 & 128 & 206 & 390 & 334 & 226 & 48 & 1,456 \\
\hline 1. Mouth and oropharynx cancers & 78 & 1 & 1 & 4 & 12 & 17 & 12 & 6 & 1 & 54 \\
\hline 2. Esophageal cancer & 72 & 0 & 0 & 1 & 5 & 14 & 12 & 8 & 1 & 41 \\
\hline 3. Stomach cancer & 252 & 1 & 2 & 5 & 18 & 42 & 43 & 32 & 6 & 149 \\
\hline 4. Colon and rectal cancers & 164 & 0 & 1 & 8 & 20 & 27 & 19 & 12 & 3 & 90 \\
\hline 5. Liver cancer & 138 & 1 & 1 & 3 & 11 & 26 & 23 & 14 & 2 & 82 \\
\hline 6. Pancreas cancer & 55 & 0 & 0 & 0 & 4 & 11 & 9 & 6 & 1 & 31 \\
\hline 7. Trachea, bronchus, and lung cancers & 283 & 1 & 1 & 5 & 23 & 68 & 66 & 41 & 7 & 211 \\
\hline 8. Melanoma and other skin cancers & 19 & 0 & 0 & 1 & 1 & 2 & 2 & 2 & 1 & 9 \\
\hline 9. Breast cancer & 273 & - & - & - & 0 & 0 & 0 & - & - & 0 \\
\hline 10. Cervix uteri cancer & 93 & - & - & - & - & - & - & - & - & - \\
\hline 11. Corpus uteri cancer & 22 & - & - & - & - & - & - & - & - & - \\
\hline 12. Ovarian cancer & 42 & - & - & - & - & - & - & - & - & - \\
\hline 13. Prostate cancer & 64 & 0 & 0 & 1 & 2 & 12 & 19 & 24 & 6 & 64 \\
\hline 14. Bladder cancer & 214 & 0 & 0 & 3 & 18 & 61 & 50 & 33 & 8 & 174 \\
\hline 15. Lymphomas and multiple myeloma & 232 & 7 & 20 & 27 & 30 & 27 & 16 & 9 & 2 & 138 \\
\hline 16. Leukemia & 307 & 21 & 30 & 51 & 24 & 21 & 14 & 9 & 2 & 173 \\
\hline Other malignant neoplasms & 440 & 22 & 15 & 19 & 37 & 65 & 48 & 28 & 7 & 241 \\
\hline B. Other neoplasms & 324 & 6 & 10 & 20 & 34 & 41 & 34 & 23 & 4 & 172 \\
\hline C. Diabetes mellitus & 843 & 3 & 4 & 31 & 83 & 111 & 78 & 52 & 11 & 372 \\
\hline D. Endocrine disorders & 1,152 & 392 & 27 & 30 & 28 & 50 & 31 & 23 & $\mathbf{5}$ & 586 \\
\hline E. Neuropsychiatric conditions & 8,310 & 1,096 & 426 & 1,408 & 727 & 351 & 116 & 87 & 32 & 4,244 \\
\hline 1. Unipolar depressive disorders & 2,027 & 0 & 130 & 256 & 267 & 131 & 37 & 8 & 1 & 831 \\
\hline 2. Bipolar affective disorder & 567 & 0 & 17 & 246 & 24 & 1 & 0 & 0 & 0 & 288 \\
\hline 3. Schizophrenia & 696 & - & 30 & 292 & 29 & 1 & 1 & 0 & 0 & 353 \\
\hline 4. Epilepsy & 248 & 16 & 28 & 46 & 25 & 12 & 4 & 2 & 1 & 134 \\
\hline 5. Alcohol use disorders & 79 & - & 0 & 23 & 34 & 13 & 2 & 1 & 0 & 73 \\
\hline 6. Alzheimer's and other dementias & 292 & 11 & 5 & 5 & 3 & 10 & 26 & 48 & 22 & 130 \\
\hline 7. Parkinson's disease & 81 & 3 & 2 & 5 & 9 & 12 & 8 & 5 & 1 & 44 \\
\hline 8. Multiple sclerosis & 55 & - & 3 & 12 & 7 & 1 & 1 & 0 & 0 & 24 \\
\hline 9. Drug use disorders & 786 & - & 6 & 278 & 241 & 117 & 6 & 1 & 0 & 649 \\
\hline 10. Post-traumatic stress disorder & 124 & 0 & 1 & 20 & 9 & 4 & 0 & 0 & 0 & 34 \\
\hline 11. Obsessive-compulsive disorder & 300 & - & 45 & 56 & 26 & 4 & 1 & 0 & 0 & 132 \\
\hline 12. Panic disorder & 264 & - & 3 & 84 & 1 & 2 & 0 & 0 & 0 & 90 \\
\hline 13. Insomnia (primary) & 74 & - & 1 & 6 & 9 & 5 & 4 & 1 & 0 & 26 \\
\hline 14. Migraine & 227 & 6 & 54 & 7 & 2 & 0 & 0 & 0 & 0 & 69 \\
\hline 15. Mental retardation, lead-caused & 725 & 336 & 13 & 15 & 3 & 1 & 0 & 0 & 0 & 369 \\
\hline Other neuropsychiatric disorders & 1,764 & 725 & 88 & 57 & 40 & 36 & 26 & 20 & 5 & 997 \\
\hline F. Sense organ diseases & 5,380 & 3 & 37 & 239 & 681 & 886 & 433 & 171 & 35 & 2,485 \\
\hline 1. Glaucoma & 681 & 1 & 10 & 48 & 78 & 82 & 42 & 20 & 4 & 286 \\
\hline 2. Cataracts & 1,491 & 2 & 26 & 120 & 179 & 189 & 92 & 49 & 11 & 667 \\
\hline 3. Vision disorders, age-related & 1,801 & 0 & 1 & 58 & 233 & 296 & 159 & 58 & 14 & 819 \\
\hline 4. Hearing loss, adult onset & 1,398 & - & - & 13 & 191 & 319 & 138 & 43 & 4 & 708 \\
\hline Other sense organ disorders & 8 & 0 & 0 & - & 0 & 0 & 1 & 2 & 1 & 4 \\
\hline G. Cardiovascular diseases & 9,528 & 169 & 105 & 267 & 550 & 1,353 & 1,246 & 1,063 & 318 & 5,071 \\
\hline 1. Rheumatic heart disease & 250 & 6 & 17 & 35 & 32 & 18 & 5 & 4 & 1 & 118 \\
\hline 2. Hypertensive heart disease & 933 & 6 & 3 & 10 & 36 & 120 & 134 & 126 & 41 & 474 \\
\hline 3. Ischemic heart disease & 4,315 & 7 & 10 & 90 & 281 & 810 & 696 & 535 & 132 & 2,561 \\
\hline 4. Cerebrovascular disease & 1,948 & 49 & 43 & 69 & 83 & 215 & 247 & 233 & 69 & 1,008 \\
\hline 5. Inflammatory heart diseases & 401 & 24 & 9 & 17 & 26 & 48 & 42 & 42 & 14 & 222 \\
\hline Other cardiovascular diseases & 1,680 & 77 & 23 & 46 & 91 & 142 & 122 & 123 & 62 & 687 \\
\hline H. Respiratory diseases & 2,285 & 260 & 107 & 156 & 252 & 218 & 168 & 143 & 42 & 1,346 \\
\hline 1. Chronic obstructive pulmonary disease & 816 & 9 & 2 & 26 & 163 & 127 & 96 & 85 & 24 & 532 \\
\hline 2. Asthma & 553 & 47 & 79 & 102 & 56 & 20 & 4 & 2 & 1 & 311 \\
\hline Other respiratory diseases & 916 & 204 & 26 & 28 & 33 & 71 & 68 & 56 & 17 & 502 \\
\hline
\end{tabular}


Table 3C.5 Continued

\begin{tabular}{|c|c|c|c|c|c|c|c|c|}
\hline & & & & emale & & & & \\
\hline $0-4$ & 5-14 & $15-29$ & $30-44$ & $45-59$ & $60-69$ & $70-79$ & $80+$ & Total \\
\hline 3,355 & 1,009 & 2,544 & 2,720 & 3,713 & 2,640 & 2,147 & 768 & 18,897 \\
\hline 41 & 56 & 116 & 284 & 394 & 218 & 147 & 33 & 1,290 \\
\hline 0 & 0 & 2 & 5 & 8 & 5 & 3 & 1 & 24 \\
\hline 0 & 0 & 1 & 4 & 10 & 8 & 7 & 1 & 31 \\
\hline 0 & 1 & 7 & 20 & 30 & 23 & 18 & 4 & 103 \\
\hline 0 & 1 & 5 & 18 & 21 & 15 & 11 & 3 & 74 \\
\hline 1 & 1 & 4 & 8 & 20 & 12 & 8 & 2 & 56 \\
\hline 0 & 0 & 1 & 3 & 8 & 6 & 5 & 1 & 23 \\
\hline 0 & 1 & 3 & 13 & 24 & 16 & 11 & 2 & 72 \\
\hline 0 & 0 & 1 & 2 & 3 & 2 & 1 & 0 & 9 \\
\hline 0 & 0 & 6 & 85 & 117 & 40 & 20 & 4 & 272 \\
\hline 0 & 0 & 12 & 14 & 38 & 19 & 9 & 1 & 93 \\
\hline 0 & 0 & 2 & 5 & 8 & 4 & 3 & 1 & 22 \\
\hline 0 & 1 & 5 & 11 & 14 & 6 & 4 & 1 & 42 \\
\hline - & - & - & - & - & - & - & - & - \\
\hline 0 & 0 & 1 & 7 & 12 & 9 & 8 & 2 & 40 \\
\hline 4 & 11 & 16 & 19 & 19 & 13 & 9 & 2 & 94 \\
\hline 16 & 22 & 37 & 24 & 20 & 9 & 6 & 1 & 135 \\
\hline 19 & 15 & 15 & 45 & 43 & 31 & 24 & 7 & 199 \\
\hline 5 & 7 & 13 & 26 & 54 & 26 & 17 & 4 & 152 \\
\hline 2 & 5 & 37 & 99 & 136 & 99 & 75 & 18 & 471 \\
\hline 360 & 25 & 29 & 28 & 46 & 39 & 31 & 9 & 566 \\
\hline 903 & 426 & 1,411 & 682 & 351 & 144 & 99 & 50 & 4,066 \\
\hline 0 & 124 & 388 & 402 & 202 & 62 & 14 & 3 & 1,196 \\
\hline 0 & 15 & 238 & 25 & 1 & 0 & 0 & 0 & 279 \\
\hline - & 16 & 273 & 37 & 15 & 2 & 0 & 0 & 343 \\
\hline 13 & 27 & 36 & 22 & 11 & 3 & 2 & 1 & 114 \\
\hline- & 0 & 1 & 2 & 2 & 0 & 0 & 0 & 5 \\
\hline 10 & 4 & 5 & 2 & 11 & 38 & 55 & 36 & 162 \\
\hline 4 & 3 & 3 & 6 & 9 & 6 & 4 & 2 & 37 \\
\hline- & 3 & 16 & 8 & 2 & 1 & 1 & 0 & 31 \\
\hline - & 5 & 55 & 50 & 25 & 1 & 0 & 0 & 137 \\
\hline 0 & 1 & 53 & 27 & 8 & 0 & 0 & 0 & 90 \\
\hline- & 47 & 75 & 34 & 8 & 4 & 0 & 0 & 168 \\
\hline - & 6 & 161 & 1 & 5 & 1 & 0 & 0 & 174 \\
\hline- & 2 & 6 & 12 & 21 & 6 & 2 & 0 & 48 \\
\hline 8 & 107 & 36 & 7 & 0 & 0 & 0 & 0 & 158 \\
\hline 323 & 15 & 10 & 5 & 2 & 0 & 0 & 0 & 355 \\
\hline 544 & 51 & 56 & 40 & 29 & 21 & 19 & 7 & 767 \\
\hline 20 & 78 & 277 & 752 & 1,002 & 501 & 213 & 51 & 2,895 \\
\hline 3 & 20 & 70 & 104 & 111 & 57 & 24 & 7 & 395 \\
\hline 9 & 39 & 137 & 193 & 236 & 125 & 67 & 19 & 824 \\
\hline 8 & 20 & 54 & 268 & 365 & 172 & 76 & 20 & 982 \\
\hline - & - & 16 & 188 & 290 & 145 & 45 & 6 & 690 \\
\hline 0 & 0 & 0 & 0 & 0 & 1 & 2 & 1 & 4 \\
\hline 366 & 93 & 178 & 315 & 889 & 1,038 & 1,129 & 449 & 4,456 \\
\hline 7 & 18 & 37 & 33 & 21 & 8 & 6 & 2 & 132 \\
\hline 4 & 3 & 9 & 22 & 93 & 123 & 144 & 59 & 458 \\
\hline 8 & 8 & 54 & 110 & 411 & 500 & 493 & 171 & 1,754 \\
\hline 31 & 25 & 29 & 66 & 191 & 238 & 259 & 100 & 940 \\
\hline 16 & 7 & 12 & 20 & 30 & 33 & 42 & 19 & 179 \\
\hline 300 & 33 & 37 & 64 & 142 & 136 & 185 & 98 & 993 \\
\hline 208 & 105 & 105 & 122 & 130 & 112 & 114 & 42 & 939 \\
\hline 6 & 2 & 6 & 55 & 65 & 60 & 65 & 24 & 284 \\
\hline 33 & 86 & 70 & 31 & 16 & 5 & 2 & 1 & 242 \\
\hline 169 & 17 & 29 & 36 & 50 & 47 & 46 & 18 & 413 \\
\hline
\end{tabular}


Table 3C.5 Continued

\begin{tabular}{|c|c|c|c|c|c|c|c|c|c|c|}
\hline \multirow[b]{2}{*}{ Cause } & \multirow[b]{2}{*}{ Total } & \multicolumn{8}{|c|}{ Male } & \multirow[b]{2}{*}{ Total } \\
\hline & & $0-4$ & $5-14$ & $15-29$ & $30-44$ & $45-59$ & $60-69$ & $70-79$ & $80+$ & \\
\hline I. Digestive diseases & 2,948 & 425 & 65 & 132 & 119 & 342 & 226 & 149 & 34 & 1,492 \\
\hline 1. Peptic ulcer disease & 100 & 1 & 1 & 9 & 11 & 17 & 10 & 9 & 3 & 61 \\
\hline 2. Cirrhosis of the liver & 686 & 17 & 10 & 24 & 26 & 134 & 95 & 52 & 10 & 368 \\
\hline 3. Appendicitis & 11 & 0 & 1 & 2 & 1 & 1 & 0 & 1 & 0 & 6 \\
\hline Other digestive diseases & 2,152 & 406 & 52 & 97 & 81 & 190 & 121 & 87 & 22 & 1,057 \\
\hline J. Genitourinary diseases & 1,283 & 91 & 24 & 46 & 55 & 258 & 119 & 94 & 29 & 717 \\
\hline 1. Nephritis and nephrosis & 634 & 15 & 17 & 31 & 33 & 86 & 82 & 57 & 15 & 335 \\
\hline 2. Benign prostatic hypertrophy & 156 & - & - & - & - & 134 & 10 & 8 & 4 & 156 \\
\hline Other genitourinary system diseases & 493 & 76 & 7 & 16 & 22 & 39 & 28 & 28 & 11 & 226 \\
\hline K. Skin diseases & 234 & 36 & 15 & 18 & 13 & 10 & 7 & 7 & 2 & 109 \\
\hline L. Musculoskeletal diseases & 1,080 & 16 & 29 & 76 & 164 & 129 & 45 & 21 & 4 & 484 \\
\hline 1. Rheumatoid arthritis & 185 & 1 & 6 & 14 & 12 & 13 & 6 & 3 & 1 & 55 \\
\hline 2. Osteoarthritis & 517 & 0 & 0 & 16 & 73 & 64 & 26 & 12 & 2 & 193 \\
\hline 3. Gout & 122 & 0 & 0 & 7 & 60 & 34 & 5 & 2 & 0 & 108 \\
\hline 4. Low back pain & 101 & 5 & 12 & 12 & 11 & 9 & 3 & 1 & 0 & 53 \\
\hline Other musculoskeletal disorders & 155 & 10 & 11 & 26 & 9 & 9 & 5 & 4 & 1 & 75 \\
\hline M. Congenital anomalies & 2,026 & 1,006 & 32 & 19 & 6 & 3 & 2 & 1 & $\mathbf{0}$ & 1,068 \\
\hline 1. Abdominal wall defect & 14 & 7 & 0 & 0 & 0 & 0 & 0 & 0 & 0 & 7 \\
\hline 2. Anencephaly & 47 & 20 & 0 & 0 & 0 & 0 & 0 & 0 & 0 & 21 \\
\hline 3. Anorectal atresia & 5 & 3 & 0 & 0 & 0 & 0 & 0 & 0 & 0 & 3 \\
\hline 4. Cleft lip & 5 & 3 & 0 & 0 & 0 & 0 & 0 & 0 & 0 & 3 \\
\hline 5. Cleft palate & 9 & 5 & 0 & 0 & 0 & 0 & 0 & 0 & 0 & 5 \\
\hline 6. Esophageal atresia & 6 & 1 & 0 & 0 & 0 & 0 & 0 & 0 & 0 & 1 \\
\hline 7. Renal agenesis & 13 & 8 & 0 & 0 & 0 & 0 & 0 & 0 & 0 & 8 \\
\hline 8. Down syndrome & 306 & 157 & 1 & 1 & 0 & 0 & 0 & 0 & 0 & 160 \\
\hline 9. Congenital heart anomalies & 964 & 477 & 14 & 9 & 3 & 1 & 1 & 1 & 0 & 506 \\
\hline 10. Spina bifida & 104 & 45 & 4 & 2 & 0 & 0 & 0 & 0 & - & 51 \\
\hline Other congenital anomalies & 551 & 281 & 12 & 6 & 2 & 1 & 1 & 0 & 0 & 302 \\
\hline N. Oral conditions & 721 & 91 & 54 & 73 & 32 & 69 & 31 & 9 & 1 & 361 \\
\hline 1. Dental caries & 463 & 90 & 54 & 38 & 11 & 23 & 11 & 6 & 1 & 235 \\
\hline 2. Periodontal disease & 9 & - & - & 2 & 2 & 0 & 0 & 0 & 0 & 4 \\
\hline 3. Edentulism & 241 & - & - & 34 & 18 & 45 & 20 & 3 & 0 & 119 \\
\hline Other oral diseases & 7 & 1 & 0 & 0 & 0 & 0 & 0 & 0 & 0 & 3 \\
\hline III. Injuries & 8,971 & 721 & 1,098 & 2,175 & 1,104 & 615 & 209 & 102 & 20 & 6,044 \\
\hline A. Unintentional injuries & 7,854 & 704 & 1,062 & 1,752 & 864 & 531 & 185 & 95 & 18 & 5,211 \\
\hline 1. Road traffic accidents & 3,002 & 183 & 348 & 744 & 455 & 277 & 97 & 51 & 8 & 2,162 \\
\hline 2. Poisonings & 184 & 20 & 5 & 42 & 20 & 32 & 5 & 2 & 0 & 125 \\
\hline 3. Falls & 915 & 86 & 140 & 156 & 68 & 44 & 16 & 12 & 3 & 525 \\
\hline 4. Fires & 564 & 64 & 43 & 71 & 33 & 19 & 5 & 3 & 1 & 238 \\
\hline 5. Drownings & 378 & 55 & 67 & 126 & 27 & 15 & 4 & 2 & 0 & 296 \\
\hline 6. Other unintentional injuries & 2,810 & 296 & 460 & 613 & 262 & 144 & 59 & 26 & 6 & 1,865 \\
\hline B. Intentional injuries & 1,117 & 17 & 36 & 423 & 240 & 84 & 25 & 7 & 2 & 833 \\
\hline 1. Self-inflicted injuries & 364 & 3 & 8 & 107 & 57 & 26 & 9 & 3 & 1 & 213 \\
\hline 2. Violence & 440 & 8 & 23 & 194 & 86 & 31 & 8 & 2 & 1 & 353 \\
\hline 3. War & 272 & 5 & 4 & 106 & 87 & 23 & 6 & 1 & 1 & 233 \\
\hline Other intentional injuries & 41 & 2 & 2 & 15 & 11 & 4 & 1 & 0 & 0 & 35 \\
\hline
\end{tabular}


Table 3C.5 Continued

\begin{tabular}{|c|c|c|c|c|c|c|c|c|}
\hline \multicolumn{9}{|c|}{ Female } \\
\hline $0-4$ & 5-14 & $15-29$ & 30-44 & 45-59 & $60-69$ & $70-79$ & $80+$ & Total \\
\hline 364 & 53 & 129 & 131 & 324 & 229 & 174 & 54 & 1,456 \\
\hline 0 & 1 & 5 & 7 & 9 & 7 & 7 & 3 & 39 \\
\hline 15 & 8 & 16 & 26 & 112 & 76 & 51 & 13 & 317 \\
\hline 0 & 1 & 1 & 1 & 0 & 0 & 0 & 0 & 5 \\
\hline 348 & 43 & 106 & 98 & 202 & 145 & 115 & 37 & 1,095 \\
\hline 58 & 25 & 51 & 64 & 140 & 106 & 89 & 34 & 566 \\
\hline 11 & 16 & 23 & 30 & 85 & 66 & 52 & 16 & 299 \\
\hline- & - & - & - & - & - & - & - & - \\
\hline 47 & 9 & 28 & 34 & 55 & 40 & 37 & 17 & 267 \\
\hline 24 & 10 & 18 & 15 & 16 & 13 & 13 & 16 & 125 \\
\hline 19 & 41 & 94 & 163 & 159 & 81 & 34 & 6 & 596 \\
\hline 7 & 16 & 27 & 31 & 30 & 11 & 5 & 1 & 130 \\
\hline 0 & 1 & 38 & 100 & 104 & 58 & 21 & 3 & 325 \\
\hline 0 & 0 & 1 & 7 & 3 & 1 & 1 & 0 & 13 \\
\hline 3 & 15 & 9 & 11 & 8 & 2 & 1 & 0 & 48 \\
\hline 9 & 9 & 18 & 14 & 14 & 8 & 6 & 2 & 80 \\
\hline 898 & 33 & 15 & 7 & 2 & 1 & 1 & 0 & 958 \\
\hline 6 & 0 & 0 & 0 & 0 & 0 & - & 0 & 7 \\
\hline 24 & 1 & 1 & 0 & 0 & 0 & - & 0 & 26 \\
\hline 2 & 0 & 0 & 0 & 0 & 0 & - & 0 & 2 \\
\hline 2 & 0 & 0 & 0 & 0 & 0 & - & 0 & 3 \\
\hline 4 & 0 & 0 & 0 & 0 & 0 & - & 0 & 4 \\
\hline 5 & 0 & 0 & 0 & 0 & 0 & - & 0 & 5 \\
\hline 5 & 0 & 0 & 0 & 0 & 0 & - & 0 & 5 \\
\hline 144 & 2 & 0 & 0 & 0 & 0 & - & 0 & 146 \\
\hline 429 & 14 & 8 & 4 & 1 & 1 & 1 & 0 & 458 \\
\hline 48 & 3 & 2 & 0 & - & 0 & 0 & - & 53 \\
\hline 228 & 12 & 5 & 2 & 1 & 1 & 0 & 0 & 249 \\
\hline 87 & 52 & 72 & 31 & 70 & 34 & 11 & 2 & 359 \\
\hline 86 & 52 & 36 & 11 & 23 & 12 & 6 & 2 & 228 \\
\hline- & - & 2 & 2 & 0 & 0 & 0 & 0 & 4 \\
\hline- & - & 33 & 17 & 46 & 22 & 4 & 0 & 122 \\
\hline 1 & 0 & 1 & 1 & 0 & 0 & 0 & 0 & 5 \\
\hline 510 & 709 & 878 & 412 & 229 & 98 & 72 & 19 & 2,928 \\
\hline 503 & 673 & 733 & 356 & 203 & 89 & 68 & 18 & 2,643 \\
\hline 140 & 223 & 196 & 129 & 82 & 37 & 27 & 5 & 840 \\
\hline 11 & 4 & 21 & 13 & 5 & 3 & 2 & 1 & 59 \\
\hline 96 & 130 & 89 & 29 & 19 & 10 & 13 & 5 & 390 \\
\hline 57 & 56 & 128 & 43 & 27 & 6 & 7 & 2 & 326 \\
\hline 35 & 19 & 18 & 6 & 3 & 2 & 1 & 0 & 83 \\
\hline 165 & 241 & 282 & 136 & 68 & 31 & 19 & 5 & 946 \\
\hline 7 & 35 & 145 & 57 & 26 & 9 & 4 & 1 & 284 \\
\hline 0 & 8 & 96 & 30 & 11 & 4 & 2 & 0 & 151 \\
\hline 5 & 8 & 40 & 20 & 8 & 3 & 1 & 1 & 88 \\
\hline 0 & 19 & 6 & 6 & 7 & 2 & 1 & 0 & 40 \\
\hline 1 & 1 & 2 & 1 & 0 & 0 & 0 & 0 & 5 \\
\hline
\end{tabular}

Source: Authors' compilation.

Note: $-=$ an estimate of zero; the number zero in a cell indicates a non-zero estimate of less than 500 .

a. Does not include liver cancer and cirrhosis $\operatorname{DALYS}(3,0)$ resulting from chronic hepatitis virus infection.

b. This cause category includes "Causes arising in the perinatal period" as defined in the International Classification

of Diseases, principally low birthweight, prematurity, birth asphyxia, and birth trauma, and does not include all causes of

$\operatorname{DALYs}(3,0)$ occurring in the perinatal period. 
Table 3C.6 DALYs $(3,0)$ by Cause, Sex, and Age in the South Asia Region, 2001 (thousands)

\begin{tabular}{|c|c|c|c|c|c|c|c|c|c|c|}
\hline \multirow[b]{2}{*}{ Cause } & \multicolumn{10}{|c|}{ Male } \\
\hline & Total & $0-4$ & 5-14 & $15-29$ & 30-44 & $45-59$ & $60-69$ & $70-79$ & $80+$ & Total \\
\hline Population (millions) & 1,388 & 88 & 164 & 195 & 139 & 81 & 30 & 14 & 4 & 715 \\
\hline All causes & 408,655 & 71,133 & 13,630 & 25,697 & 27,281 & 30,913 & 19,697 & 12,168 & 3,233 & 203,753 \\
\hline $\begin{array}{l}\text { I. Communicable, maternal, perinatal, } \\
\text { and nutritional conditions }\end{array}$ & 181,180 & 56,837 & 5,771 & 6,612 & 8,602 & 5,776 & 2,890 & 1,913 & 523 & 88,924 \\
\hline A. Infectious and parasitic diseases & 87,705 & 20,242 & 4,416 & 5,937 & 7,938 & 5,060 & 1,692 & 882 & 221 & 46,388 \\
\hline 1. Tuberculosis & 13,875 & 194 & 228 & 1,575 & 2,626 & 2,439 & 1,014 & 324 & 34 & $\mathbf{8 , 4 3 5}$ \\
\hline $\begin{array}{l}\text { 2. Sexually transmitted diseases } \\
\text { excluding HIV/AIDS }\end{array}$ & 3,670 & 581 & 8 & 276 & 146 & 131 & 89 & 43 & 12 & 1,286 \\
\hline a. Syphilis & 1,490 & 369 & 0 & 20 & 12 & 79 & 89 & 43 & 12 & 625 \\
\hline b. Chlamydia & 997 & 12 & 2 & 67 & 12 & 0 & 0 & - & - & 94 \\
\hline c. Gonorrhea & 1,079 & 199 & 5 & 190 & 108 & 2 & 0 & - & - & 504 \\
\hline $\begin{array}{l}\text { d. Other sexually transmitted } \\
\text { diseases }\end{array}$ & 105 & 0 & 0 & 0 & 14 & 49 & 0 & 0 & 0 & 64 \\
\hline 3. HIV/AIDS & 7,413 & 226 & 56 & 1,422 & 2,953 & 840 & 50 & 6 & 0 & 5,553 \\
\hline 4. Diarrheal diseases & 22,257 & 10,821 & 184 & 134 & 139 & 124 & 80 & 67 & 44 & 11,592 \\
\hline 5. Childhood-cluster diseases & 14,566 & 5,389 & 1,233 & 311 & 149 & 67 & 18 & 6 & 3 & 7,175 \\
\hline a. Pertussis & 3,930 & 1,934 & 15 & - & - & - & - & - & - & 1,949 \\
\hline b. Poliomyelitis & 55 & 6 & 3 & 11 & 7 & 1 & - & - & - & 28 \\
\hline c. Diphtheria & 90 & 32 & 6 & 1 & 0 & 1 & 0 & 0 & - & 40 \\
\hline d. Measles & 6,527 & 2,163 & 924 & 110 & - & - & - & - & - & 3,197 \\
\hline e. Tetanus & 3,965 & 1,254 & 286 & 189 & 142 & 65 & 18 & 6 & 3 & 1,961 \\
\hline 6. Meningitis & 2,142 & 274 & 285 & 177 & 101 & 100 & 39 & 34 & 9 & 1,019 \\
\hline 7. Hepatitis $\mathbf{B}^{\mathrm{a}}$ & 585 & 2 & 2 & 90 & 107 & 137 & 27 & 18 & 5 & 387 \\
\hline Hepatitis Ca & 228 & 1 & 1 & 34 & 41 & 54 & 11 & 7 & 2 & 149 \\
\hline 8. Malaria & 2,603 & 950 & 132 & 68 & 52 & 32 & 13 & 7 & 3 & 1,258 \\
\hline 9. Tropical-cluster diseases & 3,721 & 112 & 801 & 877 & 521 & 167 & 22 & 7 & 1 & 2,509 \\
\hline a. Trypanosomiasis & 0 & - & - & - & 0 & - & - & - & - & 0 \\
\hline b. Chagas' disease & 0 & - & - & - & - & - & - & - & 0 & 0 \\
\hline c. Schistosomiasis & 3 & - & 0 & 1 & 1 & 0 & 0 & 0 & 0 & 2 \\
\hline d. Leishmaniasis & 1,306 & 43 & 273 & 211 & 123 & 54 & 15 & 4 & 0 & 724 \\
\hline e. Lymphatic filariasis & 2,412 & 69 & 528 & 666 & 397 & 113 & 7 & 2 & 0 & 1,782 \\
\hline f. Onchocerciasis & - & - & - & - & - & - & - & - & - & - \\
\hline 10. Leprosy & 113 & 6 & 14 & 10 & 16 & 13 & 3 & 2 & 1 & 64 \\
\hline 11. Dengue & 240 & 25 & 73 & 4 & 4 & 3 & 1 & 1 & 0 & 111 \\
\hline 12. Japanese encephalitis & 298 & 16 & 19 & 30 & 53 & 8 & 2 & 2 & 0 & 130 \\
\hline 13. Trachoma & 197 & 1 & $\mathbf{0}$ & 3 & 13 & 18 & 13 & 6 & 2 & 55 \\
\hline 14. Intestinal nematode infections & 548 & 41 & 233 & 1 & 0 & 1 & 0 & $\mathbf{0}$ & 0 & 277 \\
\hline a. Ascariasis & 283 & 22 & 118 & 0 & 0 & 0 & - & 0 & - & 140 \\
\hline b. Trichuriasis & 122 & 6 & 60 & - & - & - & - & - & - & 66 \\
\hline c. Hookworm disease & 127 & 12 & 53 & - & 0 & - & - & 0 & 0 & 65 \\
\hline Other intestinal infections & 16 & 2 & 2 & 0 & 0 & 1 & 0 & 0 & 0 & 6 \\
\hline Other infectious diseases & 15,249 & 1,601 & 1,147 & 926 & 1,020 & 927 & 310 & 352 & 105 & 6,388 \\
\hline B. Respiratory infections & 35,044 & 13,238 & 774 & 274 & 285 & 348 & 1,136 & 975 & 285 & 17,316 \\
\hline 1. Lower respiratory infections & 34,196 & 13,112 & 621 & 259 & 270 & 334 & 1,107 & 949 & 278 & 16,929 \\
\hline 2. Upper respiratory infections & 428 & 53 & 16 & 14 & 14 & 14 & 29 & 26 & 7 & 173 \\
\hline 3. Otitis media & 421 & 73 & 137 & 2 & 1 & 1 & 0 & 0 & 0 & 214 \\
\hline C. Maternal conditions & 10,069 & - & - & - & - & - & - & - & - & - \\
\hline 1. Maternal hemorrhage & 1,718 & - & - & - & - & - & - & - & - & - \\
\hline 2. Maternal sepsis & 1,857 & - & - & - & - & - & - & - & - & - \\
\hline 3. Hypertensive disorders of pregnancy & 742 & - & - & - & - & - & - & - & - & - \\
\hline 4. Obstructed labor & 1,185 & - & - & - & - & - & - & - & - & - \\
\hline 5. Abortion & 1,467 & - & - & - & - & - & - & - & - & - \\
\hline Other maternal conditions & 3,100 & - & - & - & - & - & - & - & - & - \\
\hline D. Perinatal conditions ${ }^{b}$ & 37,721 & 20,442 & - & - & - & - & - & - & - & 20,442 \\
\hline 1. Low birthweight & 25,015 & 13,292 & - & - & - & - & - & - & - & 13,292 \\
\hline 2. Birth asphyxia and birth trauma & 8,283 & 4,957 & - & - & - & - & - & - & - & 4,957 \\
\hline Other perinatal conditions & 4,423 & 2,193 & - & - & - & - & - & - & - & 2,193 \\
\hline E. Nutritional deficiencies & 10,640 & 2,915 & 580 & 400 & 380 & 368 & 62 & 56 & 16 & 4,777 \\
\hline 1. Protein-energy malnutrition & 5,695 & 2,319 & 334 & 66 & 10 & 22 & 3 & 3 & 1 & 2,759 \\
\hline 2. Iodine deficiency & 490 & 168 & 71 & 0 & 0 & 0 & 0 & 0 & 0 & 239 \\
\hline 3. Vitamin A deficiency & 146 & 23 & 46 & 0 & - & 0 & - & - & - & 70 \\
\hline 4. Iron-deficiency anemia & 3,616 & 330 & 106 & 318 & 346 & 294 & 31 & 15 & 4 & 1,443 \\
\hline Other nutritional disorders & 693 & 75 & 22 & 16 & 24 & 52 & 28 & 38 & 11 & 267 \\
\hline
\end{tabular}


Table 3C.6 Continued

\begin{tabular}{|c|c|c|c|c|c|c|c|c|}
\hline & & & & Female & & & & \\
\hline $0-4$ & 5-14 & $15-29$ & $30-44$ & $45-59$ & $60-69$ & $70-79$ & $80+$ & Total \\
\hline 83 & 154 & 180 & 128 & 77 & 31 & 16 & 4 & 673 \\
\hline 72,151 & 15,087 & 30,361 & 24,094 & 25,792 & 19,349 & 14,059 & 4,009 & 204,902 \\
\hline 56,809 & 6,543 & 11,886 & 7,825 & 3,777 & 2,688 & 2,088 & 638 & 92,256 \\
\hline 22,702 & 4,846 & 4,800 & 3,721 & 2,812 & 1,301 & 878 & 257 & 41,317 \\
\hline 175 & 268 & 1,479 & 1,664 & 1,161 & 480 & 191 & 23 & 5,440 \\
\hline 979 & 26 & 904 & 275 & 124 & 53 & 19 & 4 & 2,384 \\
\hline 778 & 1 & 22 & 14 & 0 & 27 & 19 & 3 & 865 \\
\hline 12 & 19 & 631 & 127 & 98 & 15 & - & - & 903 \\
\hline 189 & 7 & 249 & 128 & 2 & 0 & - & - & 575 \\
\hline 0 & 0 & 1 & 6 & 23 & 10 & 0 & 0 & 41 \\
\hline 217 & 54 & 719 & 686 & 174 & 10 & 1 & 0 & 1,860 \\
\hline 10,007 & 174 & 98 & 77 & 93 & 85 & 75 & 56 & 10,665 \\
\hline 5,557 & 1,259 & 323 & 153 & 70 & 20 & 7 & 3 & 7,391 \\
\hline 1,966 & 15 & - & - & - & - & - & - & 1,981 \\
\hline 6 & 3 & 10 & 7 & 1 & - & - & - & 27 \\
\hline 37 & 13 & 0 & 0 & - & - & - & 0 & 50 \\
\hline 2,276 & 938 & 116 & - & - & - & - & - & 3,330 \\
\hline 1,272 & 290 & 196 & 146 & 69 & 20 & 7 & 3 & 2,004 \\
\hline 527 & 348 & 122 & 38 & 32 & 31 & 19 & 6 & 1,123 \\
\hline 4 & 16 & 67 & 36 & 47 & 16 & 10 & 3 & 198 \\
\hline 2 & 7 & 27 & 14 & 19 & 6 & 4 & 1 & 79 \\
\hline 1,042 & 127 & 66 & 50 & 34 & 15 & 8 & 3 & 1,345 \\
\hline 127 & 416 & 302 & 129 & 203 & 27 & 8 & 1 & 1,212 \\
\hline - & - & - & - & - & - & - & - & - \\
\hline- & - & - & - & 0 & - & - & - & 0 \\
\hline- & 0 & 0 & 0 & 0 & 0 & 0 & 0 & 1 \\
\hline 97 & 249 & 151 & 38 & 28 & 16 & 3 & 0 & 581 \\
\hline 30 & 167 & 151 & 92 & 174 & 11 & 4 & 1 & 630 \\
\hline- & - & - & - & - & - & - & - & - \\
\hline 9 & 13 & 9 & 8 & 7 & 2 & 2 & 0 & 49 \\
\hline 23 & 92 & 4 & 4 & 3 & 1 & 1 & 0 & 129 \\
\hline 77 & 52 & 19 & 11 & 5 & 3 & 1 & 0 & 168 \\
\hline 0 & 1 & 7 & 37 & 40 & 32 & 20 & 5 & 142 \\
\hline 57 & 212 & 1 & 0 & 0 & 0 & 0 & 0 & 271 \\
\hline 34 & 109 & 0 & 0 & - & 0 & - & - & 143 \\
\hline 6 & 50 & - & - & - & - & 0 & - & 56 \\
\hline 11 & 50 & 0 & - & - & - & - & - & 62 \\
\hline 7 & 2 & 0 & 0 & 0 & 0 & 0 & 0 & 10 \\
\hline 3,899 & 1,782 & 655 & 539 & 801 & 522 & 513 & 150 & 8,861 \\
\hline 13,535 & 937 & 241 & 124 & 270 & 1,160 & 1,106 & 356 & 17,728 \\
\hline 13,307 & 787 & 231 & 116 & 260 & 1,130 & 1,086 & 350 & 17,267 \\
\hline 153 & 19 & 10 & 8 & 10 & 29 & 20 & 6 & 255 \\
\hline 75 & 131 & 0 & 0 & 0 & 1 & - & 0 & 206 \\
\hline- & 67 & 6,461 & 3,474 & 67 & - & - & - & 10,069 \\
\hline- & - & 847 & 839 & 32 & - & - & - & 1,718 \\
\hline - & - & 1,360 & 497 & 1 & - & - & - & 1,857 \\
\hline- & - & 451 & 284 & 7 & - & - & - & 742 \\
\hline- & - & 803 & 381 & 2 & - & - & - & 1,185 \\
\hline - & 67 & 1,070 & 330 & 0 & - & - & - & 1,467 \\
\hline - & - & 1,931 & 1,144 & 26 & - & - & - & 3,100 \\
\hline 17,279 & - & - & - & - & - & - & - & 17,279 \\
\hline 11,723 & - & - & - & - & - & - & - & 11,723 \\
\hline 3,326 & - & - & - & - & - & - & - & 3,326 \\
\hline 2,230 & - & - & - & - & - & - & - & 2,230 \\
\hline 3,293 & 693 & 384 & 507 & 628 & 228 & 104 & 25 & 5,863 \\
\hline 2,441 & 403 & 15 & 23 & 40 & 12 & 3 & 1 & 2,937 \\
\hline 198 & 52 & 0 & 0 & 0 & 0 & 0 & 0 & 251 \\
\hline 46 & 30 & - & - & 0 & - & 0 & 0 & 77 \\
\hline 374 & 175 & 363 & 479 & 558 & 182 & 36 & 6 & 2,172 \\
\hline \multirow[t]{2}{*}{234} & 32 & 6 & 6 & 31 & 34 & 64 & 19 & 426 \\
\hline & & & & & & \multicolumn{3}{|c|}{ (Continues on the following page } \\
\hline
\end{tabular}


Table 3C.6 Continued

\begin{tabular}{|c|c|c|c|c|c|c|c|c|c|c|}
\hline \multirow[b]{2}{*}{ Cause } & \multirow[b]{2}{*}{ Total } & \multicolumn{8}{|c|}{ Male } & \multirow[b]{2}{*}{ Total } \\
\hline & & $0-4$ & $5-14$ & $15-29$ & $30-44$ & $45-59$ & $60-69$ & $70-79$ & $80+$ & \\
\hline II. Noncommunicable diseases & 181,339 & 12,192 & 3,875 & 10,614 & 12,752 & 22,030 & 15,881 & 9,726 & 2,553 & 89,623 \\
\hline A. Malignant neoplasms & 14,127 & 185 & 196 & 487 & 811 & 1,797 & 2,123 & 1,028 & 261 & 6,887 \\
\hline 1. Mouth and oropharynx cancers & 2,020 & 2 & 7 & 63 & 161 & 400 & 489 & 189 & 42 & 1,353 \\
\hline 2. Esophageal cancer & 1,116 & 1 & 1 & 9 & 63 & 174 & 242 & 93 & 19 & 602 \\
\hline 3. Stomach cancer & 629 & 0 & 2 & 16 & 53 & 114 & 131 & 60 & 17 & 393 \\
\hline 4. Colon and rectal cancers & 499 & 0 & 1 & 26 & 61 & 69 & 81 & 41 & 14 & 292 \\
\hline 5. Liver cancer & 464 & 5 & 3 & 30 & 52 & 83 & 65 & 28 & 6 & 272 \\
\hline 6. Pancreas cancer & 176 & 0 & 0 & 1 & 12 & 32 & 36 & 16 & 4 & 102 \\
\hline 7. Trachea, bronchus, and lung cancers & 1,807 & 0 & 1 & 19 & 110 & 494 & 515 & 256 & 45 & 1,441 \\
\hline 8. Melanoma and other skin cancers & 41 & 1 & 1 & 3 & 3 & 5 & 5 & 3 & 1 & 21 \\
\hline 9. Breast cancer & 1,246 & - & - & 0 & 0 & 0 & 0 & 0 & 0 & 0 \\
\hline 10. Cervix uteri cancer & 1,423 & - & - & - & - & - & - & - & - & - \\
\hline 11. Corpus uteri cancer & 66 & - & - & - & - & - & - & - & - & - \\
\hline 12. Ovarian cancer & 327 & - & - & - & - & - & - & - & - & - \\
\hline 13. Prostate cancer & 210 & 0 & 0 & 0 & 2 & 20 & 79 & 85 & 24 & 210 \\
\hline 14. Bladder cancer & 408 & 0 & 0 & 1 & 6 & 28 & 72 & 52 & 19 & 178 \\
\hline 15. Lymphomas and multiple myeloma & 1,401 & 19 & 55 & 96 & 101 & 87 & 91 & 46 & 13 & 507 \\
\hline 16. Leukemia & 851 & 62 & 80 & 171 & 66 & 44 & 38 & 18 & 5 & 484 \\
\hline Other malignant neoplasms & 1,444 & 94 & 46 & 52 & 123 & 248 & 280 & 141 & 50 & 1,033 \\
\hline B. Other neoplasms & 350 & 19 & 18 & 56 & 29 & 33 & 16 & 10 & 4 & 185 \\
\hline C. Diabetes mellitus & 4,433 & 26 & 37 & 190 & 392 & 750 & 464 & 290 & 94 & 2,243 \\
\hline D. Endocrine disorders & 828 & 257 & 21 & 35 & 31 & 34 & 20 & 13 & 4 & 415 \\
\hline E. Neuropsychiatric conditions & 37,734 & 2,909 & 1,794 & 5,854 & 3,294 & 1,746 & 603 & 713 & 260 & 17,173 \\
\hline 1. Unipolar depressive disorders & 14,582 & - & 834 & 1,997 & 1,633 & 918 & 266 & 49 & 10 & 5,706 \\
\hline 2. Bipolar affective disorder & 2,237 & 0 & 78 & 970 & 110 & 3 & 1 & - & - & 1,163 \\
\hline 3. Schizophrenia & 2,896 & - & 208 & 954 & 158 & 80 & 14 & 7 & 2 & 1,423 \\
\hline 4. Epilepsy & 1,741 & 257 & 229 & 211 & 88 & 40 & 16 & 11 & 3 & 854 \\
\hline 5. Alcohol use disorders & 1,202 & - & 15 & 363 & 533 & 189 & 43 & 9 & 2 & 1,153 \\
\hline 6. Alzheimer's and other dementias & 1,955 & 43 & 17 & 17 & 2 & 41 & 85 & 437 & 185 & 828 \\
\hline 7. Parkinson's disease & 303 & 0 & 0 & 0 & 19 & 43 & 26 & 39 & 10 & 138 \\
\hline 8. Multiple sclerosis & 227 & 0 & 14 & 48 & 30 & 8 & 3 & 1 & 0 & 105 \\
\hline 9. Drug use disorders & 957 & - & 23 & 285 & 347 & 148 & 7 & 1 & 0 & 812 \\
\hline 10. Post-traumatic stress disorder & 548 & - & 7 & 76 & 45 & 21 & 0 & 0 & 0 & 149 \\
\hline 11. Obsessive-compulsive disorder & 649 & - & 11 & 168 & 75 & 15 & 3 & 1 & 0 & 273 \\
\hline 12. Panic disorder & 1,081 & - & 21 & 336 & 4 & 13 & 2 & 1 & 0 & 377 \\
\hline 13. Insomnia (primary) & 747 & - & 8 & 105 & 125 & 68 & 35 & 7 & 2 & 349 \\
\hline 14. Migraine & 1,452 & 3 & 196 & 183 & 25 & 2 & 0 & 0 & 0 & 408 \\
\hline 15. Mental retardation, lead-caused & 1,955 & 992 & 1 & 2 & 1 & 0 & 0 & 0 & 0 & 997 \\
\hline Other neuropsychiatric disorders & 5,202 & 1,614 & 133 & 137 & 100 & 157 & 101 & 149 & 46 & 2,437 \\
\hline F. Sense organ diseases & 19,602 & 4 & 11 & 278 & 2,430 & 3,489 & 1,673 & 655 & 135 & 8,675 \\
\hline 1. Glaucoma & 203 & 3 & 5 & 7 & 12 & 27 & 19 & 8 & 2 & 84 \\
\hline 2. Cataracts & 9,478 & 0 & 4 & 143 & 900 & 1,574 & 810 & 375 & 91 & 3,898 \\
\hline 3. Vision disorders, age-related & 1,600 & 0 & 0 & 14 & 128 & 281 & 151 & 67 & 16 & 657 \\
\hline 4. Hearing loss, adult onset & 8,305 & - & - & 113 & 1,388 & 1,606 & 693 & 203 & 26 & 4,030 \\
\hline Other sense organ disorders & 16 & 1 & 1 & 1 & 1 & 1 & 1 & 1 & 0 & 7 \\
\hline G. Cardiovascular diseases & 51,264 & 704 & 392 & 1,288 & 2,434 & 7,821 & 7,517 & 5,149 & 1,325 & 26,629 \\
\hline 1. Rheumatic heart disease & 2,635 & 179 & 79 & 276 & 182 & 259 & 144 & 91 & 23 & 1,233 \\
\hline 2. Hypertensive heart disease & 1,460 & 11 & 10 & 41 & 87 & 210 & 195 & 147 & 47 & 749 \\
\hline 3. Ischemic heart disease & 25,877 & 66 & 114 & 405 & 1,314 & 4,819 & 4,172 & 2,723 & 674 & 14,287 \\
\hline 4. Cerebrovascular disease & 13,184 & 80 & 35 & 110 & 284 & 1,764 & 2,346 & 1,648 & 397 & 6,663 \\
\hline 5. Inflammatory heart diseases & 1,591 & 102 & 31 & 142 & 150 & 149 & 129 & 94 & 25 & 822 \\
\hline Other cardiovascular diseases & 6,517 & 266 & 122 & 315 & 417 & 620 & 531 & 446 & 158 & 2,875 \\
\hline H. Respiratory diseases & 16,590 & 968 & 517 & 687 & 943 & 2,568 & 1,858 & 1,054 & 241 & $\mathbf{8 , 8 3 6}$ \\
\hline $\begin{array}{l}\text { 1. Chronic obstructive pulmonary } \\
\text { disease }\end{array}$ & 9,416 & 2 & 1 & 2 & 449 & 1,972 & 1,589 & 848 & 182 & 5,045 \\
\hline 2. Asthma & 3,593 & 200 & 383 & 569 & 314 & 304 & 52 & 27 & 6 & 1,855 \\
\hline Other respiratory diseases & 3,581 & 766 & 133 & 116 & 180 & 291 & 217 & 179 & 54 & 1,936 \\
\hline
\end{tabular}


Table 3C.6 Continued

\begin{tabular}{|c|c|c|c|c|c|c|c|c|}
\hline \multicolumn{9}{|c|}{ Female } \\
\hline $0-4$ & 5-14 & $15-29$ & 30-44 & 45-59 & $60-69$ & $70-79$ & $80+$ & Total \\
\hline 12,653 & 4,468 & 11,756 & 12,784 & 19,735 & 15,734 & 11,378 & 3,207 & 91,715 \\
\hline 218 & 193 & 540 & 947 & 2,214 & 1,837 & 938 & 353 & 7,239 \\
\hline 6 & 3 & 18 & 56 & 214 & 209 & 115 & 46 & 667 \\
\hline 1 & 1 & 12 & 49 & 169 & 164 & 85 & 33 & 515 \\
\hline 0 & 0 & 11 & 28 & 71 & 69 & 38 & 19 & 236 \\
\hline 0 & 1 & 8 & 36 & 54 & 57 & 33 & 18 & 207 \\
\hline 7 & 7 & 43 & 28 & 51 & 32 & 19 & 6 & 192 \\
\hline 0 & 0 & 2 & 10 & 24 & 20 & 13 & 6 & 74 \\
\hline 0 & 1 & 8 & 44 & 131 & 113 & 59 & 9 & 366 \\
\hline 1 & 1 & 1 & 2 & 5 & 6 & 3 & 1 & 20 \\
\hline 0 & 0 & 18 & 247 & 472 & 315 & 139 & 54 & 1,246 \\
\hline 0 & 1 & 175 & 129 & 534 & 397 & 148 & 40 & 1,423 \\
\hline 2 & 2 & 1 & 7 & 16 & 19 & 13 & 6 & 66 \\
\hline 1 & 6 & 26 & 44 & 99 & 87 & 49 & 16 & 327 \\
\hline - & - & - & - & - & - & - & - & - \\
\hline 20 & 6 & 10 & 54 & 49 & 46 & 30 & 15 & 230 \\
\hline 55 & 82 & 105 & 128 & 179 & 187 & 116 & 41 & 893 \\
\hline 83 & 63 & 92 & 46 & 36 & 25 & 14 & 7 & 367 \\
\hline 43 & 18 & 10 & 38 & 109 & 92 & 64 & 37 & 410 \\
\hline 15 & 14 & 22 & 38 & 40 & 19 & 13 & 6 & 165 \\
\hline 44 & 40 & 141 & 358 & 657 & 545 & 331 & 73 & 2,190 \\
\hline 248 & 22 & 25 & 23 & 39 & 30 & 20 & 6 & 414 \\
\hline 3,267 & 2,059 & 6,882 & 3,947 & 2,113 & 991 & 925 & 376 & 20,561 \\
\hline- & 778 & 3,194 & 2,753 & 1,502 & 521 & 106 & 23 & 8,876 \\
\hline 0 & 64 & 902 & 104 & 3 & 1 & 0 & 0 & 1,075 \\
\hline 1 & 34 & 1,076 & 215 & 107 & 23 & 14 & 4 & 1,473 \\
\hline 210 & 255 & 240 & 83 & 61 & 21 & 14 & 3 & 887 \\
\hline - & 6 & 13 & 12 & 10 & 6 & 2 & 1 & 49 \\
\hline 40 & 17 & 17 & 6 & 48 & 185 & 538 & 276 & 1,127 \\
\hline 0 & - & 0 & 22 & 54 & 37 & 42 & 10 & 165 \\
\hline 1 & 13 & 59 & 37 & 9 & 2 & 1 & 0 & 121 \\
\hline - & 7 & 37 & 65 & 33 & 2 & 1 & 0 & 145 \\
\hline - & 5 & 209 & 119 & 61 & 2 & 1 & 0 & 398 \\
\hline - & 7 & 171 & 137 & 51 & 8 & 2 & 0 & 376 \\
\hline - & 19 & 647 & 6 & 26 & 3 & 2 & 0 & 703 \\
\hline - & 7 & 115 & 128 & 82 & 45 & 18 & 2 & 398 \\
\hline 70 & 752 & 57 & 163 & 3 & 0 & 0 & 0 & 1,044 \\
\hline 953 & 2 & 2 & 0 & 0 & 0 & 0 & 0 & 957 \\
\hline 1,993 & 93 & 145 & 96 & 62 & 136 & 183 & 57 & 2,765 \\
\hline 2 & 2 & 200 & 2,950 & 4,221 & 2,321 & 1,002 & 230 & 10,926 \\
\hline - & 0 & 5 & 23 & 42 & 30 & 15 & 4 & 119 \\
\hline 0 & 1 & 157 & 1,208 & 2,242 & 1,207 & 606 & 159 & 5,580 \\
\hline 0 & 0 & 18 & 153 & 379 & 253 & 112 & 28 & 943 \\
\hline - & - & 18 & 1,565 & 1,558 & 829 & 267 & 39 & 4,275 \\
\hline 1 & 1 & 2 & 1 & 1 & 1 & 2 & 0 & 9 \\
\hline 769 & 447 & 1,433 & 1,762 & 5,206 & 7,157 & 6,238 & 1,623 & 24,635 \\
\hline 199 & 139 & 261 & 198 & 274 & 169 & 127 & 35 & 1,402 \\
\hline 12 & 16 & 26 & 54 & 188 & 197 & 159 & 59 & 712 \\
\hline 49 & 77 & 610 & 922 & 2,670 & 3,690 & 2,887 & 684 & 11,590 \\
\hline 59 & 44 & 65 & 178 & 1,261 & 2,301 & 2,072 & 540 & 6,521 \\
\hline 90 & 36 & 107 & 97 & 138 & 126 & 135 & 40 & 769 \\
\hline 359 & 136 & 364 & 312 & 676 & 674 & 857 & 265 & 3,642 \\
\hline 890 & 528 & 603 & 749 & 2,414 & 1,239 & 1,052 & 279 & 7,755 \\
\hline 3 & 0 & 2 & 348 & 1,904 & 1,022 & 870 & 223 & 4,372 \\
\hline 163 & 427 & 480 & 278 & 306 & 48 & 28 & 8 & 1,737 \\
\hline 725 & 101 & 121 & 124 & 204 & 169 & 154 & 49 & 1,646 \\
\hline
\end{tabular}


Table 3C.6 Continued

\begin{tabular}{|c|c|c|c|c|c|c|c|c|c|c|c|}
\hline \multirow[b]{2}{*}{ Cause } & & \multicolumn{10}{|c|}{ Male } \\
\hline & & Total & $0-4$ & 5-14 & $15-29$ & $30-44$ & $45-59$ & $60-69$ & $70-79$ & $80+$ & Total \\
\hline \multirow[t]{5}{*}{ I. } & Digestive diseases & 16,010 & 2,597 & 389 & 838 & 1,126 & 1,809 & 778 & 426 & 133 & 8,096 \\
\hline & 1. Peptic ulcer disease & 1,901 & 54 & 48 & 196 & 270 & 389 & 129 & 76 & 19 & 1,182 \\
\hline & 2. Cirrhosis of the liver & 4,249 & 192 & 110 & 239 & 364 & 761 & 339 & 165 & 35 & 2,205 \\
\hline & 3. Appendicitis & 113 & 1 & 4 & 8 & 7 & 19 & 10 & 8 & 3 & 60 \\
\hline & Other digestive diseases & 9,747 & 2,349 & 227 & 396 & 484 & 640 & 300 & 176 & 76 & 4,649 \\
\hline \multirow[t]{4}{*}{ J. } & Genitourinary diseases & 3,991 & 328 & 114 & 186 & 285 & 946 & 326 & 210 & 56 & 2,452 \\
\hline & 1. Nephritis and nephrosis & 2,387 & 133 & 106 & 165 & 236 & 331 & 199 & 132 & 34 & 1,334 \\
\hline & 2. Benign prostatic hypertrophy & 692 & - & - & - & 7 & 546 & 80 & 47 & 12 & 692 \\
\hline & $\begin{array}{l}\text { Other genitourinary system } \\
\text { diseases }\end{array}$ & 913 & 195 & 9 & 21 & 43 & 69 & 47 & 32 & 11 & 426 \\
\hline K. & Skin diseases & 896 & 199 & 78 & 73 & 53 & 55 & 24 & 15 & 5 & 502 \\
\hline \multirow[t]{6}{*}{ L. } & Musculoskeletal diseases & 5,586 & 77 & 129 & 373 & 850 & 778 & 289 & 117 & 26 & 2,639 \\
\hline & 1. Rheumatoid arthritis & 848 & 5 & 27 & 56 & 54 & 69 & 31 & 15 & 3 & 261 \\
\hline & 2. Osteoarthritis & 2,577 & - & 0 & 111 & 297 & 349 & 164 & 52 & 8 & 981 \\
\hline & 3. Gout & 841 & - & - & 42 & 405 & 254 & 40 & 10 & 1 & 752 \\
\hline & 4. Low back pain & 453 & 22 & 51 & 51 & 51 & 46 & 14 & 5 & 1 & 241 \\
\hline & Other musculoskeletal disorders & 868 & 49 & 51 & 112 & 44 & 60 & 40 & 34 & 13 & 403 \\
\hline \multicolumn{2}{|r|}{ M. Congenital anomalies } & 8,101 & 3,771 & 73 & 128 & 7 & 3 & 1 & 1 & $\mathbf{0}$ & 3,984 \\
\hline & 1. Abdominal wall defect & 18 & 8 & - & - & - & - & - & - & - & 8 \\
\hline & 2. Anencephaly & 322 & 153 & - & - & - & - & - & - & - & 153 \\
\hline & 3. Anorectal atresia & 2 & 1 & - & - & - & - & - & - & - & 1 \\
\hline & 4. Cleft lip & 24 & 13 & - & - & - & - & - & - & - & 13 \\
\hline & 5. Cleft palate & 41 & 20 & - & 0 & 0 & - & - & - & - & 21 \\
\hline & 6. Esophageal atresia & 7 & 3 & - & - & - & - & - & - & - & 3 \\
\hline & 7. Renal agenesis & 18 & 9 & 0 & - & - & - & 0 & - & - & 9 \\
\hline & 8. Down syndrome & 1,753 & 838 & 21 & 46 & - & - & - & - & - & 905 \\
\hline & 9. Congenital heart anomalies & 4,881 & 2,223 & 43 & 76 & 4 & 1 & 1 & 1 & 0 & 2,348 \\
\hline & 10. Spina bifida & 588 & 281 & 1 & 0 & 0 & 0 & 0 & 0 & - & 283 \\
\hline & Other congenital anomalies & 449 & 222 & 8 & 6 & 3 & 2 & 1 & 1 & 0 & 241 \\
\hline & Oral conditions & 1,824 & 149 & 107 & 141 & 68 & 200 & 187 & 46 & 8 & 907 \\
\hline & 1. Dental caries & 1,189 & 144 & 107 & 126 & 46 & 93 & 58 & 27 & 7 & 607 \\
\hline & 2. Periodontal disease & 84 & - & - & 11 & 20 & 8 & 3 & 1 & 0 & 43 \\
\hline & 3. Edentulism & 518 & - & - & 1 & 1 & 99 & 126 & 16 & 1 & 244 \\
\hline & Other oral diseases & 32 & 6 & 1 & 3 & 2 & 1 & 0 & 1 & 0 & 12 \\
\hline \multicolumn{2}{|c|}{ III. Injuries } & 46,136 & 2,103 & 3,984 & 8,472 & 5,926 & 3,107 & 926 & 529 & 157 & 25,205 \\
\hline \multicolumn{2}{|c|}{ A. Unintentional injuries } & 36,774 & 2,003 & 3,717 & 5,865 & 4,192 & 2,432 & 772 & 438 & 127 & 19,545 \\
\hline & 1. Road traffic accidents & 7,424 & 266 & 691 & 1,624 & 1,511 & 796 & 174 & 91 & 23 & 5,176 \\
\hline & 2. Poisonings & 1,844 & 24 & 100 & 224 & 182 & 331 & 92 & 25 & 6 & 985 \\
\hline & 3. Falls & 4,907 & 421 & 680 & 644 & 358 & 238 & 152 & 152 & 54 & 2,697 \\
\hline & 4. Fires & 5,905 & 272 & 264 & 528 & 433 & 162 & 38 & 19 & 5 & 1,722 \\
\hline & 5. Drownings & 2,256 & 149 & 349 & 456 & 215 & 117 & 36 & 23 & 6 & 1,351 \\
\hline & 6. Other unintentional injuries & 14,439 & 872 & 1,633 & 2,389 & 1,492 & 788 & 280 & 127 & 33 & 7,613 \\
\hline B. & Intentional injuries & 9,362 & 100 & 267 & 2,607 & 1,734 & 674 & 154 & 92 & 31 & 5,660 \\
\hline & 1. Self-inflicted injuries & 6,015 & - & 109 & 1,564 & 956 & 404 & 86 & 53 & 13 & 3,183 \\
\hline & 2. Violence & 2,376 & 44 & 140 & 665 & 474 & 195 & 43 & 31 & 15 & 1,607 \\
\hline & 3. War & 819 & 43 & 8 & 337 & 275 & 65 & 19 & 4 & 1 & 754 \\
\hline & Other intentional injuries & 152 & 13 & 10 & 41 & 29 & 11 & 7 & 4 & 1 & 116 \\
\hline
\end{tabular}


Table 3C.6 Continued

\begin{tabular}{|c|c|c|c|c|c|c|c|c|}
\hline \multicolumn{9}{|c|}{ Female } \\
\hline $0-4$ & 5-14 & $15-29$ & $30-44$ & 45-59 & $60-69$ & $70-79$ & $80+$ & Total \\
\hline 2,717 & 682 & 1,081 & 923 & 1,305 & 638 & 424 & 143 & 7,914 \\
\hline 60 & 36 & 93 & 148 & 205 & 90 & 68 & 18 & 719 \\
\hline 513 & 247 & 319 & 241 & 397 & 179 & 120 & 27 & 2,043 \\
\hline 1 & 4 & 5 & 4 & 14 & 12 & 10 & 3 & 53 \\
\hline 2,143 & 395 & 664 & 529 & 690 & 357 & 226 & 94 & 5,098 \\
\hline 224 & 94 & 151 & 217 & 372 & 256 & 175 & 51 & 1,539 \\
\hline 118 & 88 & 131 & 110 & 263 & 181 & 128 & 34 & 1,053 \\
\hline- & - & - & - & - & - & - & - & - \\
\hline 106 & 7 & 19 & 107 & 109 & 74 & 47 & 17 & 486 \\
\hline 132 & 47 & 58 & 55 & 48 & 28 & 20 & 7 & 395 \\
\hline 68 & 163 & 371 & 747 & 907 & 467 & 175 & 48 & 2,946 \\
\hline 3 & 57 & 116 & 145 & 165 & 66 & 31 & 5 & 587 \\
\hline 0 & 1 & 138 & 446 & 597 & 318 & 82 & 13 & 1,595 \\
\hline- & - & 6 & 45 & 24 & 9 & 5 & 1 & 89 \\
\hline 11 & 61 & 34 & 50 & 38 & 12 & 4 & 1 & 212 \\
\hline 54 & 45 & 77 & 62 & 83 & 62 & 53 & 28 & 464 \\
\hline 3,917 & 75 & 116 & 4 & 4 & 1 & 1 & 0 & 4,118 \\
\hline 9 & - & - & - & - & - & - & - & 9 \\
\hline 169 & - & - & - & - & 0 & - & - & 169 \\
\hline 1 & - & - & - & - & - & - & - & 1 \\
\hline 11 & - & - & - & - & - & - & - & 11 \\
\hline 20 & - & - & - & 0 & - & - & - & 20 \\
\hline 3 & - & - & - & - & - & - & - & 3 \\
\hline 9 & 0 & - & - & - & 0 & - & - & 9 \\
\hline 784 & 21 & 43 & - & - & - & - & - & 848 \\
\hline 2,417 & 45 & 67 & 2 & 1 & 1 & 1 & 0 & 2,533 \\
\hline 303 & 1 & 0 & 0 & 0 & - & 0 & 0 & 305 \\
\hline 190 & 8 & 6 & 2 & 2 & 0 & 0 & 0 & 208 \\
\hline 141 & 101 & 134 & 65 & 195 & 205 & 65 & 11 & 917 \\
\hline 136 & 100 & 117 & 42 & 89 & 61 & 30 & 8 & 582 \\
\hline- & 0 & 10 & 18 & 8 & 3 & 2 & 0 & 41 \\
\hline- & - & 1 & 1 & 98 & 140 & 33 & 2 & 274 \\
\hline 5 & 2 & 6 & 4 & 1 & 1 & 0 & 0 & 20 \\
\hline 2,689 & 4,076 & 6,718 & 3,484 & 2,280 & 928 & 592 & 163 & 20,931 \\
\hline 2,624 & 3,770 & 4,782 & 2,733 & 1,824 & 819 & 527 & 150 & 17,229 \\
\hline 331 & 586 & 456 & 353 & 358 & 94 & 55 & 15 & 2,248 \\
\hline 30 & 111 & 152 & 139 & 189 & 189 & 39 & 11 & 858 \\
\hline 427 & 677 & 374 & 190 & 191 & 124 & 181 & 46 & 2,209 \\
\hline 538 & 693 & 1,595 & 868 & 316 & 95 & 60 & 17 & 4,182 \\
\hline 194 & 266 & 224 & 98 & 59 & 33 & 24 & 7 & 905 \\
\hline 1,104 & 1,438 & 1,982 & 1,084 & 711 & 285 & 168 & 55 & 6,826 \\
\hline 65 & 306 & 1,937 & 751 & 455 & 109 & 65 & 13 & 3,702 \\
\hline 0 & 196 & 1,697 & 549 & 300 & 45 & 35 & 10 & 2,832 \\
\hline 55 & 96 & 218 & 181 & 133 & 56 & 28 & 2 & 769 \\
\hline 1 & 8 & 14 & 18 & 17 & 5 & 2 & 1 & 65 \\
\hline 9 & 6 & 7 & 4 & 5 & 3 & 1 & 0 & 36 \\
\hline
\end{tabular}

Source: Authors' compilation.

Note: $-=$ an estimate of zero; the number zero in a cell indicates a non-zero estimate of less than 500 .

a. Does not include liver cancer and cirrhosis $\operatorname{DALY}(3,0)$ resulting from chronic hepatitis virus infection.

b. This cause category includes "Causes arising in the perinatal period" as defined in the International Classification of

Diseases, principally low birthweight, prematurity, birth asphyxia, and birth trauma, and does not include all causes of

$\operatorname{DALYS}(3,0)$ occurring in the perinatal period. 
Table 3C.7 DALYs $(3,0)$ by Cause, Sex, and Age in the Sub-Saharan Africa Region, 2001 (thousands)

\begin{tabular}{|c|c|c|c|c|c|c|c|c|c|c|}
\hline \multirow[b]{2}{*}{ Cause } & \multicolumn{10}{|c|}{ Male } \\
\hline & Total & $0-4$ & 5-14 & $15-29$ & $30-44$ & $45-59$ & $60-69$ & $70-79$ & 80+ & Total \\
\hline Population (millions) & 668 & 57 & 92 & 93 & 49 & 26 & 9 & 4 & 1 & 331 \\
\hline All causes & 344,754 & 84,575 & 13,040 & 23,098 & 26,058 & 16,033 & 7,197 & 4,076 & 1,064 & 175,141 \\
\hline $\begin{array}{l}\text { I. Communicable, maternal, perinatal, } \\
\text { and nutritional conditions }\end{array}$ & 242,837 & 74,911 & 7,172 & 10,377 & 16,077 & 7,005 & 2,046 & 752 & 154 & 118,494 \\
\hline A. Infectious and parasitic diseases & 173,484 & 46,407 & 5,827 & 9,866 & 15,439 & 6,331 & 1,563 & 495 & 99 & 86,027 \\
\hline 1. Tuberculosis & 8,084 & 397 & 241 & 1,489 & 1,792 & 1,038 & 285 & 91 & 18 & 5,350 \\
\hline $\begin{array}{l}\text { 2. Sexually transmitted diseases } \\
\text { excluding HIV/AIDS }\end{array}$ & 3,842 & 775 & 6 & 238 & 285 & 336 & 38 & 0 & $\mathbf{0}$ & 1,679 \\
\hline a. Syphilis & 2,347 & 560 & 1 & 63 & 235 & 327 & 37 & 0 & 0 & 1,223 \\
\hline b. Chlamydia & 559 & 12 & 1 & 34 & 5 & 0 & 0 & - & - & 52 \\
\hline c. Gonorrhea & 894 & 191 & 4 & 141 & 40 & 3 & 0 & - & - & 379 \\
\hline $\begin{array}{l}\text { d. Other sexually transmitted } \\
\text { diseases }\end{array}$ & 41 & 12 & 0 & 1 & 5 & 6 & 1 & 0 & 0 & 24 \\
\hline 3. HIV/AIDS & 56,820 & 4,894 & 1,452 & 6,047 & 11,262 & 2,995 & 325 & 29 & 0 & 27,005 \\
\hline 4. Diarrheal diseases & 22,046 & 10,797 & 160 & 93 & 127 & 130 & 88 & 71 & 39 & 11,506 \\
\hline 5. Childhood-cluster diseases & 23,198 & 9,709 & 1,502 & 213 & 86 & 41 & 11 & 4 & 1 & 11,568 \\
\hline a. Pertussis & 6,116 & 3,031 & 15 & - & - & - & - & - & - & 3,047 \\
\hline b. Poliomyelitis & 17 & 8 & - & - & - & - & - & - & - & 8 \\
\hline c. Diphtheria & 45 & 26 & 0 & 0 & - & - & - & - & - & 26 \\
\hline d. Measles & 13,539 & 5,354 & 1,312 & 85 & - & - & - & - & - & 6,752 \\
\hline e. Tetanus & 3,481 & 1,289 & 174 & 128 & 86 & 41 & 11 & 4 & 1 & 1,735 \\
\hline 6. Meningitis & 941 & 261 & 85 & 35 & 43 & 32 & 8 & 3 & 0 & 469 \\
\hline 7. Hepatitis $\mathbf{B}^{\mathbf{a}}$ & 536 & 43 & 95 & 25 & 77 & 41 & 16 & 3 & 0 & 301 \\
\hline Hepatitis $\mathbf{C}^{\mathbf{a}}$ & 217 & 17 & 40 & 10 & 32 & 17 & 7 & 1 & 0 & 124 \\
\hline 8. Malaria & 35,447 & 15,487 & 287 & 338 & 294 & 220 & 102 & 58 & 15 & 16,801 \\
\hline 9. Tropical-cluster diseases & 4,897 & 215 & 1,019 & 1,003 & 594 & 350 & 64 & 26 & 5 & 3,277 \\
\hline a. Trypanosomiasis & 1,310 & 66 & 318 & 185 & 146 & 102 & 9 & 3 & 0 & 830 \\
\hline b. Chagas' disease & - & - & - & - & - & - & - & - & - & - \\
\hline c. Schistosomiasis & 1,184 & 82 & 253 & 175 & 106 & 51 & 22 & 10 & 2 & 701 \\
\hline d. Leishmaniasis & 312 & 29 & 85 & 70 & 31 & 5 & 2 & 1 & 0 & 223 \\
\hline e. Lymphatic filariasis & 1,656 & 31 & 343 & 537 & 241 & 108 & 6 & 2 & 0 & 1,269 \\
\hline f. Onchocerciasis & 436 & 7 & 20 & 36 & 70 & 83 & 25 & 10 & 3 & 255 \\
\hline 10. Leprosy & 24 & 0 & 2 & 1 & 1 & 9 & 0 & 1 & - & 14 \\
\hline 11. Dengue & 4 & $\mathbf{0}$ & 1 & 0 & 0 & 0 & $\mathbf{0}$ & 0 & $\mathbf{0}$ & 2 \\
\hline 12. Japanese encephalitis & - & - & - & - & - & - & - & - & - & - \\
\hline 13. Trachoma & 1,455 & 0 & 1 & 15 & 84 & 116 & 83 & 48 & 11 & 357 \\
\hline 14. Intestinal nematode infections & 905 & 80 & 343 & 10 & 5 & 7 & 4 & 2 & 0 & 452 \\
\hline a. Ascariasis & 476 & 45 & 191 & 0 & 0 & 0 & 0 & 0 & 0 & 235 \\
\hline b. Trichuriasis & 119 & 10 & 45 & 1 & 1 & 1 & 1 & 0 & 0 & 58 \\
\hline c. Hookworm disease & 309 & 26 & 107 & 8 & 5 & 6 & 4 & 1 & 0 & 158 \\
\hline Other intestinal infections & 1 & - & 0 & 0 & 0 & 0 & 0 & 0 & 0 & 1 \\
\hline Other infectious diseases & 15,068 & 3,731 & 592 & 348 & 756 & 1,000 & 530 & 158 & 7 & 7,123 \\
\hline B. Respiratory infections & 31,107 & 13,774 & 971 & 320 & 533 & 590 & 399 & 212 & 46 & 16,846 \\
\hline 1. Lower respiratory infections & 30,455 & 13,619 & 845 & 310 & 514 & 586 & 389 & 208 & 46 & 16,517 \\
\hline 2. Upper respiratory infections & 371 & 105 & 31 & 8 & 11 & 4 & 11 & 4 & 0 & 173 \\
\hline 3. Otitis media & 281 & 50 & 96 & 3 & 7 & - & 0 & - & - & 156 \\
\hline C. Maternal conditions & 9,743 & - & - & - & - & - & - & - & - & - \\
\hline 1. Maternal hemorrhage & 1,643 & - & - & - & - & - & - & - & - & - \\
\hline 2. Maternal sepsis & 1,843 & - & - & - & - & - & - & - & - & - \\
\hline 3. Hypertensive disorders of pregnancy & 842 & - & - & - & - & - & - & - & - & - \\
\hline 4. Obstructed labor & 919 & - & - & - & - & - & - & - & - & - \\
\hline 5. Abortion & 1,557 & - & - & - & - & - & - & - & - & - \\
\hline Other maternal conditions & 2,940 & - & - & - & - & - & - & - & - & - \\
\hline D. Perinatal conditions $\mathbf{s}^{\mathrm{b}}$ & 20,047 & 11,351 & - & - & - & - & - & - & - & 11,351 \\
\hline 1. Low birthweight & 7,891 & 4,501 & - & - & - & - & - & - & - & 4,501 \\
\hline 2. Birth asphyxia and birth trauma & 9,256 & 5,195 & - & - & - & - & - & - & - & 5,195 \\
\hline Other perinatal conditions & 2,899 & 1,655 & - & - & - & - & - & - & - & 1,655 \\
\hline E. Nutritional deficiencies & 8,455 & 3,378 & 373 & 191 & 105 & 84 & 84 & 46 & 9 & 4,271 \\
\hline 1. Protein-energy malnutrition & 5,220 & 2,496 & 53 & 8 & 11 & 46 & 62 & 38 & 8 & 2,722 \\
\hline 2. lodine deficiency & 951 & 383 & 100 & 0 & 1 & 1 & 1 & 0 & 0 & 487 \\
\hline 3. Vitamin A deficiency & 548 & 206 & 20 & 2 & 5 & 9 & 5 & 1 & 0 & 249 \\
\hline 4. Iron-deficiency anemia & 1,688 & 273 & 199 & 180 & 88 & 27 & 16 & 6 & 1 & 789 \\
\hline Other nutritional disorders & 49 & 20 & 1 & 0 & 0 & 1 & 1 & 0 & 0 & 24 \\
\hline
\end{tabular}


Table 3C.7 Continued

\begin{tabular}{|c|c|c|c|c|c|c|c|c|}
\hline & & & & Female & & & & \\
\hline $0-4$ & 5-14 & $15-29$ & 30-44 & $45-59$ & $60-69$ & $70-79$ & $80+$ & Total \\
\hline 56 & 91 & 93 & 51 & 28 & 11 & 5 & 1 & 336 \\
\hline 76,795 & 12,562 & 29,616 & 22,752 & 13,624 & 7,514 & 5,165 & 1,585 & 169,613 \\
\hline 68,898 & 8,056 & 22,639 & 16,570 & 5,500 & 1,586 & 866 & 228 & 124,343 \\
\hline 46,986 & 6,290 & 15,133 & 12,539 & 4,531 & 1,219 & 605 & 154 & 87,458 \\
\hline 358 & 233 & 945 & 715 & 290 & 142 & 45 & 7 & 2,734 \\
\hline 602 & 21 & 1,001 & 359 & 130 & 32 & 17 & 0 & 2,163 \\
\hline 394 & 1 & 355 & 207 & 121 & 30 & 16 & 0 & 1,124 \\
\hline 12 & 14 & 415 & 60 & 5 & 1 & - & - & 507 \\
\hline 188 & 7 & 227 & 90 & 2 & 0 & - & - & 515 \\
\hline 8 & 0 & 3 & 2 & 2 & 1 & 1 & 0 & 17 \\
\hline 4,748 & 1,432 & 11,064 & 9,966 & 2,299 & 266 & 41 & 0 & 29,815 \\
\hline 9,910 & 160 & 63 & 69 & 100 & 93 & 89 & 56 & 10,540 \\
\hline 9,702 & 1,558 & 220 & 88 & 44 & 13 & 5 & 2 & 11,630 \\
\hline 3,054 & 15 & - & - & - & - & - & - & 3,069 \\
\hline 9 & - & - & - & - & - & - & - & 9 \\
\hline 19 & 0 & - & - & - & - & - & - & 19 \\
\hline 5,336 & 1,363 & 89 & - & - & - & - & - & 6,787 \\
\hline 1,284 & 180 & 131 & 88 & 44 & 13 & 5 & 2 & 1,746 \\
\hline 231 & 150 & 53 & 15 & 17 & 4 & 2 & 0 & 473 \\
\hline 123 & 22 & 42 & 14 & 26 & 6 & 2 & 0 & 235 \\
\hline 48 & 9 & 16 & 6 & 10 & 3 & 1 & 0 & 93 \\
\hline 17,133 & 304 & 409 & 317 & 267 & 121 & 73 & 21 & 18,646 \\
\hline 151 & 487 & 422 & 267 & 230 & 39 & 20 & 5 & 1,620 \\
\hline 37 & 184 & 119 & 77 & 55 & 6 & 2 & 0 & 480 \\
\hline- & - & - & - & - & - & - & - & - \\
\hline 55 & 170 & 129 & 74 & 36 & 10 & 8 & 2 & 483 \\
\hline 10 & 36 & 28 & 12 & 2 & 1 & 0 & 0 & 89 \\
\hline 42 & 78 & 114 & 54 & 88 & 8 & 2 & 0 & 387 \\
\hline 7 & 20 & 32 & 50 & 49 & 15 & 7 & 2 & 181 \\
\hline 0 & 2 & 1 & 1 & 1 & 3 & 1 & 0 & 9 \\
\hline 0 & 2 & 0 & 0 & 0 & 0 & 0 & 0 & 3 \\
\hline- & - & - & - & - & - & - & - & - \\
\hline 1 & 2 & 27 & 236 & 330 & 278 & 175 & 48 & 1,097 \\
\hline 79 & 352 & 7 & 4 & 5 & 4 & 1 & 0 & 453 \\
\hline 44 & 196 & 0 & 0 & 0 & 0 & 0 & 0 & 241 \\
\hline 10 & 49 & 1 & 0 & 1 & 0 & 0 & 0 & 61 \\
\hline 25 & 107 & 6 & 3 & 5 & 3 & 1 & 0 & 151 \\
\hline- & 0 & 0 & 0 & 0 & 0 & 0 & 0 & 1 \\
\hline 3,900 & 1,556 & 864 & 481 & 781 & 215 & 133 & 13 & 7,945 \\
\hline 9,948 & 1,349 & 1,108 & 720 & 550 & 294 & 222 & 69 & 14,261 \\
\hline 9,841 & 1,159 & 1,094 & 716 & 549 & 289 & 221 & 69 & 13,938 \\
\hline 60 & 117 & 9 & 4 & 1 & 5 & 2 & 0 & 198 \\
\hline 46 & 74 & 5 & 0 & - & - & - & - & 125 \\
\hline- & 69 & 6,169 & 3,156 & 350 & - & - & - & 9,743 \\
\hline- & - & 797 & 734 & 112 & - & - & - & 1,643 \\
\hline- & - & 1,131 & 632 & 80 & - & - & - & 1,843 \\
\hline - & 0 & 530 & 280 & 32 & - & - & - & 842 \\
\hline- & - & 655 & 253 & 11 & - & - & - & 919 \\
\hline- & 69 & 1,281 & 207 & 0 & - & - & - & 1,557 \\
\hline- & 0 & 1,774 & 1,051 & 114 & - & - & - & 2,940 \\
\hline 8,696 & - & - & - & - & - & - & - & $\mathbf{8 , 6 9 6}$ \\
\hline 3,391 & - & - & - & - & - & - & - & 3,391 \\
\hline 4,062 & - & - & - & - & - & - & - & 4,062 \\
\hline 1,244 & - & - & - & - & - & - & - & 1,244 \\
\hline 3,269 & 347 & 229 & 154 & 70 & 72 & 38 & 5 & 4,184 \\
\hline 2,322 & 40 & 18 & 13 & 19 & 54 & 28 & 4 & 2,498 \\
\hline 365 & 95 & 1 & 0 & 1 & 0 & 0 & 0 & 464 \\
\hline 238 & 37 & 8 & 4 & 8 & 3 & 1 & 0 & 299 \\
\hline 323 & 173 & 200 & 137 & 41 & 15 & 8 & 1 & 899 \\
\hline \multirow[t]{2}{*}{20} & 2 & 1 & 0 & 1 & 0 & 0 & 0 & 25 \\
\hline & & & & & & \multicolumn{3}{|c|}{ (Continues on the following page } \\
\hline
\end{tabular}

The Burden of Disease and Mortality by Condition: Data, Methods, and Results for 2001 | 217 
Table 3C.7 Continued

\begin{tabular}{|c|c|c|c|c|c|c|c|c|c|c|}
\hline \multirow[b]{2}{*}{ Cause } & \multicolumn{10}{|c|}{ Male } \\
\hline & Total & $0-4$ & 5-14 & $15-29$ & 30-44 & $45-59$ & $60-69$ & 70-79 & $80+$ & Total \\
\hline II. Noncommunicable diseases & 73,069 & 7,276 & 1,930 & 5,350 & 5,335 & 7,218 & 4,673 & 3,166 & 880 & 35,829 \\
\hline A. Malignant neoplasms & 6,281 & 53 & 87 & 174 & 377 & 972 & 780 & 524 & 125 & 3,092 \\
\hline 1. Mouth and oropharynx cancers & 284 & 0 & 2 & 8 & 24 & 73 & 54 & 27 & 5 & 193 \\
\hline 2. Esophageal cancer & 343 & 0 & 0 & 2 & 21 & 90 & 62 & 31 & 6 & 212 \\
\hline 3. Stomach cancer & 487 & 1 & 2 & 8 & 32 & 102 & 63 & 41 & 10 & 258 \\
\hline 4. Colon and rectal cancers & 291 & 0 & 1 & 9 & 29 & 48 & 35 & 27 & 8 & 158 \\
\hline 5. Liver cancer & 762 & 3 & 3 & 29 & 107 & 204 & 112 & 47 & 7 & 512 \\
\hline 6. Pancreas cancer & 117 & 0 & 0 & 0 & 5 & 23 & 15 & 7 & 2 & 52 \\
\hline 7. Trachea, bronchus, and lung cancers & 225 & 0 & 0 & 1 & 13 & 72 & 52 & 19 & 3 & 162 \\
\hline 8. Melanoma and other skin cancers & 118 & 0 & 0 & 3 & 6 & 19 & 15 & 7 & 2 & 53 \\
\hline 9. Breast cancer & 574 & - & - & - & 0 & 0 & 0 & 0 & 0 & 1 \\
\hline 10. Cervix uteri cancer & 627 & - & - & - & - & - & - & - & - & - \\
\hline 11. Corpus uteri cancer & 41 & - & - & - & - & - & - & - & - & - \\
\hline 12. Ovarian cancer & 152 & - & - & - & - & - & - & - & - & - \\
\hline 13. Prostate cancer & 416 & 0 & 0 & 1 & 2 & 53 & 146 & 170 & 44 & 416 \\
\hline 14. Bladder cancer & 133 & 0 & 0 & 1 & 5 & 25 & 26 & 23 & 7 & 88 \\
\hline 15. Lymphomas and multiple myeloma & 622 & 17 & 51 & 61 & 69 & 85 & 58 & 36 & 9 & 386 \\
\hline 16. Leukemia & 245 & 7 & 12 & 32 & 16 & 28 & 16 & 14 & 3 & 128 \\
\hline Other malignant neoplasms & 844 & 24 & 15 & 19 & 48 & 149 & 124 & 73 & 19 & 472 \\
\hline B. Other neoplasms & 188 & 16 & 13 & 16 & 12 & 19 & 12 & 6 & 1 & 95 \\
\hline C. Diabetes mellitus & 1,448 & 4 & 8 & 39 & 100 & 181 & 122 & 75 & 18 & 547 \\
\hline D. Endocrine disorders & 2,706 & 1,074 & 34 & 61 & 64 & 117 & 42 & 26 & 8 & 1,425 \\
\hline E. Neuropsychiatric conditions & 15,151 & 1,768 & 802 & 2,970 & 1,122 & 558 & 199 & 129 & 45 & 7,593 \\
\hline 1. Unipolar depressive disorders & 3,275 & - & 306 & 426 & 334 & 169 & 46 & 8 & 1 & 1,291 \\
\hline 2. Bipolar affective disorder & 1,204 & - & 48 & 524 & 41 & 1 & 0 & - & - & 615 \\
\hline 3. Schizophrenia & 1,146 & - & 28 & 492 & 34 & 2 & 0 & 0 & 0 & 556 \\
\hline 4. Epilepsy & 1,373 & 107 & 145 & 228 & 155 & 89 & 36 & 12 & 4 & 777 \\
\hline 5. Alcohol use disorders & 685 & - & 10 & 274 & 197 & 97 & 15 & 6 & 1 & 600 \\
\hline 6. Alzheimer's and other dementias & 450 & 29 & 9 & 6 & 5 & 16 & 36 & 65 & 30 & 197 \\
\hline 7. Parkinson's disease & 100 & 0 & 0 & - & 5 & 15 & 15 & 15 & 4 & 54 \\
\hline 8. Multiple sclerosis & 77 & - & 4 & 13 & 9 & 1 & 0 & 0 & 0 & 29 \\
\hline 9. Drug use disorders & 929 & - & 11 & 472 & 165 & 49 & 2 & 0 & 0 & 699 \\
\hline 10. Post-traumatic stress disorder & 224 & - & 4 & 35 & 14 & 8 & 0 & 0 & 0 & 61 \\
\hline 11. Obsessive-compulsive disorder & 619 & - & 59 & 145 & 32 & 14 & 3 & 0 & 0 & 254 \\
\hline 12. Panic disorder & 519 & - & 11 & 155 & 2 & 5 & 1 & 0 & 0 & 174 \\
\hline 13. Insomnia (primary) & 234 & - & 1 & 58 & 41 & 23 & 10 & 3 & 0 & 136 \\
\hline 14. Migraine & 329 & 18 & 42 & 21 & 10 & 0 & 0 & 0 & 0 & 92 \\
\hline 15. Mental retardation, lead-caused & 1,505 & 753 & 0 & 0 & 0 & 0 & 0 & - & - & 753 \\
\hline Other neuropsychiatric disorders & 2,481 & 861 & 122 & 120 & 77 & 68 & 34 & 19 & 4 & 1,306 \\
\hline F. Sense organ diseases & 8,939 & 15 & 71 & 495 & 1,270 & 1,233 & 569 & 268 & 58 & 3,980 \\
\hline 1. Glaucoma & 937 & 3 & 10 & 49 & 124 & 140 & 55 & 28 & 7 & 416 \\
\hline 2. Cataracts & 5,169 & 11 & 57 & 254 & 623 & 746 & 327 & 167 & 39 & 2,224 \\
\hline 3. Vision disorders, age-related & 920 & 1 & 4 & 20 & 116 & 161 & 60 & 28 & 7 & 397 \\
\hline 4. Hearing loss, adult onset & 1,912 & - & - & 171 & 407 & 187 & 126 & 46 & 5 & 942 \\
\hline Other sense organ disorders & 2 & 0 & 0 & 0 & 0 & 0 & 0 & 0 & 0 & 1 \\
\hline G. Cardiovascular diseases & 15,069 & 160 & 108 & 382 & 916 & 1,856 & 1,630 & 1,286 & 401 & 6,738 \\
\hline 1. Rheumatic heart disease & 479 & 20 & 37 & 54 & 24 & 18 & 4 & 5 & 2 & 165 \\
\hline 2. Hypertensive heart disease & 937 & 1 & 2 & 9 & 42 & 103 & 88 & 68 & 24 & 338 \\
\hline 3. Ischemic heart disease & 4,579 & 2 & 13 & 42 & 188 & 772 & 733 & 537 & 112 & 2,399 \\
\hline 4. Cerebrovascular disease & 5,125 & 14 & 22 & 100 & 272 & 590 & 541 & 422 & 118 & 2,077 \\
\hline 5. Inflammatory heart diseases & 945 & 79 & 18 & 71 & 105 & 100 & 59 & 43 & 15 & 490 \\
\hline Other cardiovascular diseases & 3,004 & 43 & 16 & 107 & 284 & 273 & 204 & 212 & 129 & 1,268 \\
\hline H. Respiratory diseases & 6,150 & 764 & 351 & 407 & 393 & 633 & 535 & 374 & 102 & 3,559 \\
\hline $\begin{array}{l}\text { 1. Chronic obstructive pulmonary } \\
\text { disease }\end{array}$ & 1,631 & 14 & 6 & 30 & 113 & 296 & 308 & 234 & 62 & 1,065 \\
\hline 2. Asthma & 1,925 & 286 & 285 & 263 & 81 & 80 & 47 & 25 & 6 & 1,074 \\
\hline Other respiratory diseases & 2,595 & 464 & 59 & 114 & 199 & 256 & 180 & 115 & 33 & 1,420 \\
\hline
\end{tabular}


Table 3C.7 Continued

\begin{tabular}{|c|c|c|c|c|c|c|c|c|}
\hline \multicolumn{9}{|c|}{ Female } \\
\hline $0-4$ & 5-14 & $15-29$ & $30-44$ & $45-59$ & $60-69$ & $70-79$ & $80+$ & Total \\
\hline 6,272 & 2,049 & 5,159 & 5,064 & 7,469 & 5,708 & 4,191 & 1,327 & 37,240 \\
\hline 55 & 90 & 158 & 450 & 1,081 & 740 & 504 & 110 & 3,189 \\
\hline 1 & 1 & 2 & 10 & 30 & 23 & 20 & 4 & 91 \\
\hline 0 & 0 & 2 & 12 & 48 & 38 & 26 & 6 & 131 \\
\hline 0 & 2 & 7 & 29 & 82 & 59 & 39 & 11 & 229 \\
\hline 0 & 1 & 4 & 24 & 45 & 30 & 22 & 7 & 133 \\
\hline 3 & 2 & 22 & 51 & 77 & 49 & 39 & 7 & 250 \\
\hline 0 & 0 & 1 & 8 & 26 & 17 & 10 & 2 & 64 \\
\hline 0 & 1 & 1 & 7 & 22 & 18 & 11 & 2 & 63 \\
\hline 0 & 0 & 2 & 7 & 15 & 19 & 18 & 3 & 64 \\
\hline 0 & 1 & 7 & 104 & 229 & 133 & 81 & 18 & 574 \\
\hline 0 & 0 & 41 & 64 & 247 & 162 & 98 & 14 & 627 \\
\hline 0 & 0 & 1 & 6 & 12 & 10 & 10 & 2 & 41 \\
\hline 0 & 3 & 10 & 26 & 55 & 34 & 20 & 4 & 152 \\
\hline- & - & - & - & - & - & - & - & - \\
\hline 0 & 0 & 1 & 5 & 13 & 15 & 9 & 3 & 45 \\
\hline 17 & 48 & 29 & 31 & 39 & 34 & 30 & 7 & 236 \\
\hline 7 & 12 & 17 & 13 & 35 & 19 & 12 & 3 & 118 \\
\hline 26 & 18 & 11 & 54 & 107 & 79 & 59 & 18 & 372 \\
\hline 5 & 17 & 7 & 12 & 24 & 16 & 10 & 2 & 93 \\
\hline 2 & 7 & 41 & 100 & 290 & 261 & 168 & 33 & 901 \\
\hline 745 & 38 & 84 & 66 & 156 & 107 & 64 & 20 & 1,281 \\
\hline 1,704 & 932 & 2,797 & 1,095 & 560 & 236 & 158 & 75 & 7,558 \\
\hline- & 301 & 711 & 567 & 300 & 87 & 16 & 3 & 1,985 \\
\hline- & 14 & 529 & 45 & 2 & 0 & - & - & 590 \\
\hline- & 8 & 469 & 103 & 5 & 2 & 1 & 0 & 590 \\
\hline 98 & 147 & 180 & 77 & 58 & 19 & 13 & 4 & 596 \\
\hline- & 6 & 25 & 26 & 19 & 4 & 5 & 0 & 85 \\
\hline 26 & 8 & 7 & 5 & 18 & 51 & 84 & 55 & 253 \\
\hline 0 & - & 0 & 4 & 14 & 12 & 10 & 6 & 46 \\
\hline- & 7 & 20 & 15 & 5 & 1 & 0 & 0 & 48 \\
\hline- & 9 & 143 & 62 & 16 & 1 & 0 & 0 & 230 \\
\hline- & 3 & 97 & 40 & 22 & 1 & 0 & 0 & 162 \\
\hline- & 197 & 94 & 56 & 10 & 7 & 2 & 0 & 365 \\
\hline- & 13 & 317 & 4 & 10 & 1 & 1 & 0 & 346 \\
\hline- & 5 & 22 & 37 & 20 & 11 & 2 & 1 & 98 \\
\hline 9 & 121 & 97 & 10 & 0 & 0 & 0 & 0 & 237 \\
\hline 752 & 0 & 0 & 0 & 0 & - & - & - & 752 \\
\hline 820 & 94 & 87 & 46 & 61 & 37 & 23 & 5 & 1,174 \\
\hline 5 & 22 & 428 & 1,519 & 1,661 & 828 & 398 & 100 & 4,959 \\
\hline 1 & 3 & 49 & 144 & 187 & 82 & 42 & 12 & 522 \\
\hline 3 & 17 & 230 & 804 & 1,015 & 534 & 271 & 69 & 2,944 \\
\hline 0 & 2 & 29 & 128 & 219 & 89 & 43 & 13 & 522 \\
\hline- & - & 119 & 443 & 239 & 122 & 41 & 5 & 970 \\
\hline 0 & 0 & 0 & 0 & 0 & 0 & 0 & 0 & 1 \\
\hline 165 & 145 & 458 & 739 & 1,983 & 2,180 & 1,953 & 710 & 8,331 \\
\hline 15 & 45 & 96 & 68 & 54 & 22 & 12 & 2 & 314 \\
\hline 1 & 1 & 9 & 52 & 148 & 166 & 160 & 62 & 599 \\
\hline 2 & 21 & 23 & 93 & 545 & 738 & 586 & 173 & 2,180 \\
\hline 8 & 27 & 82 & 221 & 825 & 894 & 752 & 239 & 3,048 \\
\hline 53 & 22 & 62 & 74 & 100 & 72 & 53 & 19 & 455 \\
\hline 86 & 28 & 186 & 231 & 312 & 288 & 391 & 216 & 1,736 \\
\hline 537 & 338 & 365 & 208 & 372 & 358 & 311 & 103 & 2,592 \\
\hline 12 & 0 & 15 & 32 & 141 & 157 & 157 & 52 & 566 \\
\hline 152 & 289 & 218 & 41 & 61 & 47 & 33 & 10 & 851 \\
\hline 374 & 48 & 132 & 135 & 170 & 154 & 121 & 41 & 1,175 \\
\hline
\end{tabular}

The Burden of Disease and Mortality by Condition: Data, Methods, and Results for 2001 । 219 
Table 3C.7 Continued

\begin{tabular}{|c|c|c|c|c|c|c|c|c|c|c|c|}
\hline \multirow{2}{*}{ Cause } & & \multirow[b]{2}{*}{ Total } & \multicolumn{8}{|c|}{ Male } & \multirow[b]{2}{*}{ Total } \\
\hline & & & $0-4$ & 5-14 & $15-29$ & $30-44$ & $45-59$ & $60-69$ & $70-79$ & $80+$ & \\
\hline \multirow[t]{5}{*}{ I. } & Digestive diseases & 7,226 & 1,139 & 163 & 419 & 592 & 812 & 413 & 244 & 54 & 3,836 \\
\hline & 1. Peptic ulcer disease & 345 & 2 & 2 & 42 & 51 & 57 & 26 & 14 & 5 & 199 \\
\hline & 2. Cirrhosis of the liver & 1,212 & 3 & 4 & 64 & 173 & 278 & 139 & 78 & 12 & 750 \\
\hline & 3. Appendicitis & 44 & 0 & 10 & 4 & 5 & 3 & 2 & 1 & 0 & 25 \\
\hline & Other digestive diseases & 5,626 & 1,134 & 147 & 309 & 363 & 474 & 246 & 151 & 38 & 2,863 \\
\hline \multirow[t]{4}{*}{ J. } & Genitourinary diseases & 2,623 & 230 & 70 & 100 & 124 & 452 & 174 & 142 & 50 & 1,341 \\
\hline & 1. Nephritis and nephrosis & 1,633 & 96 & 59 & 79 & 96 & 129 & 131 & 101 & 35 & 727 \\
\hline & 2. Benign prostatic hypertrophy & 292 & - & - & - & - & 263 & 13 & 11 & 5 & 292 \\
\hline & $\begin{array}{l}\text { Other genitourinary system } \\
\text { diseases }\end{array}$ & 697 & 134 & 11 & 21 & 28 & 59 & 30 & 30 & 9 & 322 \\
\hline K. & Skin diseases & 956 & 135 & 51 & 71 & 93 & 63 & 34 & 26 & 9 & 482 \\
\hline \multirow[t]{6}{*}{ L. } & Musculoskeletal diseases & 2,171 & 36 & 67 & 160 & 253 & 275 & 116 & 59 & 9 & 975 \\
\hline & 1. Rheumatoid arthritis & 252 & 2 & 7 & 15 & 31 & 23 & 11 & 4 & 1 & 94 \\
\hline & 2. Osteoarthritis & 1,278 & - & 1 & 57 & 143 & 194 & 84 & 40 & 5 & 523 \\
\hline & 3. Gout & 94 & - & 0 & 8 & 41 & 23 & 7 & 3 & 1 & 83 \\
\hline & 4. Low back pain & 214 & 14 & 28 & 25 & 20 & 18 & 4 & 2 & 0 & 113 \\
\hline & Other musculoskeletal disorders & 333 & 20 & 31 & 55 & 18 & 18 & 8 & 9 & 3 & 162 \\
\hline \multicolumn{2}{|r|}{ M. Congenital anomalies } & 3,441 & 1,746 & 46 & 21 & 5 & 2 & 0 & $\mathbf{0}$ & 0 & 1,819 \\
\hline & 1. Abdominal wall defect & 36 & 20 & - & - & - & - & - & - & - & 20 \\
\hline & 2. Anencephaly & 47 & 23 & 0 & - & - & - & - & - & - & 23 \\
\hline & 3. Anorectal atresia & 14 & 9 & 0 & - & - & - & - & - & - & 9 \\
\hline & 4. Cleft lip & 12 & 6 & - & - & - & - & - & - & - & 6 \\
\hline & 5. Cleft palate & 28 & 11 & - & - & - & - & - & - & - & 11 \\
\hline & 6. Esophageal atresia & 2 & 1 & 0 & - & - & - & - & - & - & 1 \\
\hline & 7. Renal agenesis & 2 & 2 & - & - & - & - & 0 & - & - & 2 \\
\hline & 8. Down syndrome & 419 & 244 & 1 & 2 & 0 & 0 & - & - & - & 248 \\
\hline & 9. Congenital heart anomalies & 1,651 & 821 & 18 & 7 & 2 & 1 & 0 & 0 & 0 & 849 \\
\hline & 10. Spina bifida & 293 & 142 & 3 & 1 & - & - & - & - & - & 146 \\
\hline & Other congenital anomalies & 938 & 465 & 23 & 11 & 3 & 1 & 0 & 0 & 0 & 504 \\
\hline & Oral conditions & 720 & 137 & 60 & 35 & 14 & 45 & 48 & 7 & 1 & 347 \\
\hline & 1. Dental caries & 496 & 130 & 60 & 30 & 8 & 13 & 5 & 2 & 0 & 248 \\
\hline & 2. Periodontal disease & 23 & - & - & 4 & 5 & 1 & 0 & 0 & 0 & 11 \\
\hline & 3. Edentulism & 181 & - & - & - & - & 31 & 43 & 5 & 0 & 80 \\
\hline & Other oral diseases & 21 & 6 & 1 & 0 & 0 & 0 & 0 & 0 & 0 & 8 \\
\hline \multicolumn{2}{|c|}{ III. Injuries } & 28,848 & 2,388 & 3,937 & 7,371 & 4,647 & 1,809 & 479 & 157 & 29 & 20,819 \\
\hline & Unintentional injuries & 18,876 & 2,241 & 3,518 & 3,262 & 2,036 & 1,023 & 290 & 104 & 18 & 12,491 \\
\hline & 1. Road traffic accidents & 6,374 & 424 & 1,412 & 964 & 807 & 425 & 113 & 37 & 5 & 4,186 \\
\hline & 2. Poisonings & 954 & 143 & 120 & 126 & 145 & 51 & 15 & 0 & 0 & 599 \\
\hline & 3. Falls & 976 & 109 & 224 & 113 & 65 & 60 & 27 & 17 & 4 & 619 \\
\hline & 4. Fires & 1,739 & 441 & 453 & 69 & 37 & 38 & 12 & 5 & 1 & 1,057 \\
\hline & 5. Drownings & 1,720 & 204 & 322 & 293 & 305 & 153 & 15 & 0 & 0 & 1,294 \\
\hline & 6. Other unintentional injuries & 7,112 & 919 & 988 & 1,698 & 677 & 296 & 108 & 44 & 7 & 4,736 \\
\hline & Intentional injuries & 9,972 & 148 & 419 & 4,109 & 2,611 & 787 & 189 & 54 & 12 & 8,328 \\
\hline & 1. Self-inflicted injuries & 882 & - & 57 & 272 & 174 & 115 & 29 & 13 & 2 & 663 \\
\hline & 2. Violence & 4,996 & 116 & 318 & 2,161 & 1,019 & 314 & 59 & 18 & 2 & 4,007 \\
\hline & 3. War & 4,090 & 31 & 44 & 1,675 & 1,418 & 357 & 101 & 22 & 7 & 3,655 \\
\hline & Other intentional injuries & 3 & 1 & 0 & 1 & 0 & 0 & 0 & 0 & 0 & 2 \\
\hline
\end{tabular}


Table 3C.7 Continued

\begin{tabular}{|c|c|c|c|c|c|c|c|c|}
\hline \multicolumn{9}{|c|}{ Female } \\
\hline $0-4$ & 5-14 & $15-29$ & $30-44$ & $45-59$ & $60-69$ & $70-79$ & $80+$ & Total \\
\hline 1,097 & 204 & 381 & 347 & 578 & 428 & 275 & 79 & 3,390 \\
\hline 0 & 5 & 24 & 34 & 35 & 24 & 18 & 6 & 146 \\
\hline 7 & 9 & 35 & 69 & 157 & 119 & 58 & 9 & 462 \\
\hline 0 & 7 & 8 & 0 & 2 & 1 & 1 & 0 & 19 \\
\hline 1,090 & 184 & 314 & 244 & 384 & 285 & 198 & 64 & 2,763 \\
\hline 155 & 69 & 133 & 130 & 308 & 243 & 184 & 58 & 1,281 \\
\hline 62 & 60 & 68 & 78 & 230 & 204 & 158 & 47 & 907 \\
\hline- & - & - & - & - & - & - & - & - \\
\hline 93 & 10 & 66 & 53 & 78 & 39 & 26 & 10 & 375 \\
\hline 71 & 25 & 51 & 61 & 94 & 73 & 75 & 24 & 473 \\
\hline 27 & 68 & 206 & 319 & 306 & 175 & 81 & 14 & 1,196 \\
\hline 3 & 11 & 21 & 45 & 43 & 20 & 13 & 2 & 158 \\
\hline- & 1 & 118 & 225 & 213 & 134 & 55 & 9 & 755 \\
\hline- & 0 & 3 & 1 & 3 & 2 & 1 & 0 & 11 \\
\hline 8 & 36 & 18 & 20 & 14 & 4 & 1 & 0 & 101 \\
\hline 16 & 20 & 46 & 29 & 33 & 14 & 11 & 3 & 172 \\
\hline 1,569 & 33 & 13 & 2 & 2 & 1 & 1 & 0 & 1,622 \\
\hline 16 & - & 0 & - & - & - & - & - & 16 \\
\hline 24 & - & 0 & - & - & 0 & - & - & 24 \\
\hline 4 & - & - & - & - & - & - & - & 4 \\
\hline 6 & - & - & - & - & - & - & - & 6 \\
\hline 17 & - & - & - & - & - & - & - & 17 \\
\hline 1 & - & - & - & - & - & - & - & 1 \\
\hline 0 & - & 0 & - & - & 0 & - & - & 0 \\
\hline 166 & 3 & 2 & - & 1 & - & - & - & 171 \\
\hline 782 & 11 & 5 & 2 & 1 & 0 & 1 & 0 & 802 \\
\hline 146 & 0 & 1 & 0 & - & - & - & - & 147 \\
\hline 407 & 19 & 6 & 1 & 1 & 1 & 0 & 0 & 434 \\
\hline 135 & 61 & 37 & 15 & 53 & 62 & 10 & 1 & 373 \\
\hline 128 & 59 & 30 & 8 & 14 & 5 & 3 & 1 & 248 \\
\hline- & - & 4 & 6 & 1 & 0 & 0 & 0 & 12 \\
\hline- & - & - & - & 37 & 56 & 7 & 0 & 101 \\
\hline 7 & 2 & 2 & 1 & 0 & 0 & 0 & 0 & 13 \\
\hline 1,625 & 2,457 & 1,817 & 1,117 & 655 & 221 & 108 & 29 & 8,030 \\
\hline 1,536 & 2,132 & 1,252 & 745 & 445 & 167 & 87 & 22 & 6,385 \\
\hline 336 & 950 & 384 & 261 & 169 & 62 & 22 & 4 & 2,188 \\
\hline 60 & 70 & 90 & 65 & 31 & 25 & 10 & 2 & 355 \\
\hline 82 & 148 & 39 & 23 & 23 & 17 & 19 & 6 & 357 \\
\hline 348 & 177 & 62 & 46 & 30 & 8 & 9 & 2 & 682 \\
\hline 137 & 103 & 113 & 28 & 34 & 12 & 0 & 0 & 426 \\
\hline 572 & 685 & 564 & 322 & 158 & 43 & 26 & 8 & 2,377 \\
\hline 89 & 325 & 565 & 373 & 210 & 54 & 21 & 7 & 1,645 \\
\hline 0 & 13 & 110 & 52 & 32 & 8 & 4 & 1 & 220 \\
\hline 85 & 187 & 375 & 227 & 86 & 18 & 8 & 3 & 989 \\
\hline 4 & 125 & 81 & 93 & 92 & 27 & 9 & 3 & 435 \\
\hline 0 & 0 & 0 & 0 & 0 & 0 & - & 0 & 1 \\
\hline
\end{tabular}

Source: Authors' compilation.

Note: $-=$ an estimate of zero; the number zero in a cell indicates a non-zero estimate of less than 500 .

a. Does not include liver cancer and cirrhosis $\operatorname{DALY}(3,0)$ resulting from chronic hepatitis virus infection.

b. This cause category includes "Causes arising in the perinatal period" as defined in the International Classification

of Diseases, principally low birthweight prematurity, birth asphyxia, and birth trauma, and does not include all causes of

DALYS $(3,0)$ occurring in the perinatal period. 
Table 3C.8 DALYs $(3,0)$ by Cause, Sex, and Age in High-Income Countries, 2001 (thousands)

\begin{tabular}{|c|c|c|c|c|c|c|c|c|c|c|}
\hline \multirow[b]{2}{*}{ Cause } & \multicolumn{10}{|c|}{ Male } \\
\hline & Total & $0-4$ & 5-14 & $15-29$ & 30-44 & $45-59$ & 60-69 & 70-79 & $80+$ & Total \\
\hline Population (millions) & 929 & 28 & 60 & 96 & 107 & 88 & 40 & 27 & 10 & 457 \\
\hline All causes & 149,161 & 3,641 & 1,706 & 8,093 & 10,396 & 16,700 & 14,284 & 14,522 & 7,444 & 76,786 \\
\hline $\begin{array}{l}\text { I. Communicable, maternal, perinatal, } \\
\text { and nutritional conditions }\end{array}$ & 8,561 & 1,170 & 126 & 365 & 601 & 561 & 411 & 573 & 503 & 4,310 \\
\hline A. Infectious and parasitic diseases & 3,375 & 288 & 68 & 228 & 506 & 375 & 182 & 176 & 86 & 1,909 \\
\hline 1. Tuberculosis & 219 & 1 & 1 & 8 & 23 & 36 & 28 & 33 & 15 & 146 \\
\hline $\begin{array}{l}\text { 2. Sexually transmitted diseases } \\
\text { excluding HIV/AIDS }\end{array}$ & 145 & 7 & 0 & 9 & 9 & 1 & $\mathbf{0}$ & $\mathbf{0}$ & 0 & 26 \\
\hline a. Syphilis & 13 & 5 & 0 & 0 & 0 & 0 & 0 & 0 & 0 & 7 \\
\hline b. Chlamydia & 100 & 1 & 0 & 4 & 4 & 0 & 0 & - & - & 9 \\
\hline c. Gonorrhea & 27 & 0 & 0 & 5 & 5 & 0 & 0 & 0 & 0 & 10 \\
\hline d. Other sexually transmitted diseases & 4 & 0 & - & - & 0 & 0 & 0 & 0 & 0 & 0 \\
\hline 3. HIV/AIDS & 665 & 2 & 1 & 84 & 288 & 119 & 14 & 3 & $\mathbf{0}$ & 510 \\
\hline 4. Diarrheal diseases & 444 & 121 & 13 & 20 & 22 & 19 & 11 & 9 & 6 & 222 \\
\hline 5. Childhood-cluster diseases & 175 & 70 & 2 & 1 & 1 & 1 & 1 & 1 & 0 & 76 \\
\hline a. Pertussis & 139 & 68 & $\overline{1}$ & - & - & - & 0 & - & - & 69 \\
\hline b. Poliomyelitis & 8 & 0 & 0 & 0 & 0 & 1 & 1 & 1 & 0 & 4 \\
\hline c. Diphtheria & 0 & 0 & 0 & - & 0 & - & - & 0 & 0 & 0 \\
\hline d. Measles & 23 & 1 & 1 & 0 & 0 & 0 & - & 0 & - & 3 \\
\hline e. Tetanus & 5 & 0 & 0 & 0 & 0 & 0 & 0 & 0 & 0 & 1 \\
\hline 6. Meningitis & 131 & 27 & 5 & 7 & 7 & 9 & 5 & 4 & 1 & 65 \\
\hline 7. Hepatitis $\mathbf{B}^{\mathbf{a}}$ & 86 & 0 & $\mathbf{0}$ & 2 & 12 & 23 & 10 & 6 & 2 & 55 \\
\hline Hepatitis $\mathbf{C}^{\mathrm{a}}$ & 185 & 0 & 0 & 1 & 19 & 53 & 22 & 15 & 3 & 113 \\
\hline 8. Malaria & 9 & 1 & $\mathbf{0}$ & $\mathbf{0}$ & 1 & 2 & $\mathbf{0}$ & $\mathbf{0}$ & 0 & 6 \\
\hline 9. Tropical-cluster diseases & 219 & 4 & 22 & 75 & 65 & 11 & 2 & 1 & $\mathbf{0}$ & 180 \\
\hline a. Trypanosomiasis & 0 & - & - & - & - & - & 0 & - & - & 0 \\
\hline b. Chagas' disease & 1 & 0 & 0 & 0 & 0 & 0 & 0 & 0 & 0 & 0 \\
\hline c. Schistosomiasis & 1 & 0 & 0 & 0 & 0 & 0 & 0 & 0 & 0 & 1 \\
\hline d. Leishmaniasis & 5 & 0 & 0 & 0 & 0 & 0 & 0 & 0 & 0 & 1 \\
\hline e. Lymphatic filariasis & 212 & 4 & 21 & 75 & 64 & 11 & 2 & 1 & 0 & 178 \\
\hline f. Onchocerciasis & 0 & 0 & 0 & 0 & 0 & 0 & 0 & 0 & 0 & 0 \\
\hline 10. Leprosy & 1 & $\mathbf{0}$ & $\mathbf{0}$ & $\mathbf{0}$ & 0 & $\mathbf{0}$ & $\mathbf{0}$ & $\mathbf{0}$ & $\mathbf{0}$ & $\mathbf{0}$ \\
\hline 11. Dengue & 0 & $\mathbf{0}$ & $\mathbf{0}$ & $\mathbf{0}$ & 0 & $\mathbf{0}$ & $\mathbf{0}$ & 0 & 0 & $\mathbf{0}$ \\
\hline 12. Japanese encephalitis & 6 & 1 & 1 & $\mathbf{0}$ & $\mathbf{0}$ & $\mathbf{0}$ & $\mathbf{0}$ & $\mathbf{0}$ & $\mathbf{0}$ & 3 \\
\hline 13. Trachoma & 10 & $\mathbf{0}$ & $\mathbf{0}$ & $\mathbf{0}$ & 3 & 2 & $\mathbf{0}$ & $\mathbf{0}$ & $\mathbf{0}$ & 5 \\
\hline 14. Intestinal nematode infections & 11 & 1 & 4 & $\mathbf{0}$ & 0 & $\mathbf{0}$ & $\mathbf{0}$ & $\mathbf{0}$ & $\mathbf{0}$ & 5 \\
\hline a. Ascariasis & 5 & 0 & 2 & 0 & 0 & 0 & 0 & 0 & 0 & 2 \\
\hline b. Trichuriasis & 3 & 0 & 1 & - & - & - & - & - & - & 1 \\
\hline c. Hookworm disease & 2 & 0 & 1 & - & 0 & 0 & - & 0 & 0 & 1 \\
\hline Other intestinal infections & 1 & 0 & 0 & 0 & 0 & 0 & 0 & 0 & 0 & 0 \\
\hline Other infectious diseases & 1,070 & 53 & 18 & 21 & 56 & 101 & 87 & 104 & 58 & 497 \\
\hline B. Respiratory infections & 2,474 & 52 & 46 & 24 & 61 & 141 & 188 & 356 & 396 & 1,264 \\
\hline 1. Lower respiratory infections & 2,314 & 33 & 10 & 21 & 57 & 137 & 184 & 351 & 391 & 1,183 \\
\hline 2. Upper respiratory infections & 60 & 3 & 2 & 3 & 4 & 4 & 4 & 5 & 4 & 30 \\
\hline 3. Otitis media & 100 & 17 & 34 & 0 & 0 & 0 & 0 & 0 & 0 & 51 \\
\hline C. Maternal conditions & 391 & - & - & - & - & - & - & - & - & - \\
\hline 1. Maternal hemorrhage & 4 & - & - & - & - & - & - & - & - & - \\
\hline 2. Maternal sepsis & 78 & - & - & - & - & - & - & - & - & - \\
\hline 3. Hypertensive disorders of pregnancy & 4 & - & - & - & - & - & - & - & - & - \\
\hline 4. Obstructed labor & 9 & - & - & - & - & - & - & - & - & - \\
\hline 5. Abortion & 4 & - & - & - & - & - & - & - & - & - \\
\hline Other maternal conditions & 292 & - & - & - & - & - & - & - & - & - \\
\hline D. Perinatal conditions ${ }^{\mathrm{b}}$ & 1,408 & 773 & 1 & 1 & 0 & $\mathbf{0}$ & $\mathbf{0}$ & - & - & 775 \\
\hline 1. Low birthweight & 467 & 252 & 0 & - & 0 & - & 0 & - & - & 252 \\
\hline 2. Birth asphyxia and birth trauma & 530 & 291 & 1 & 0 & 0 & 0 & - & - & - & 292 \\
\hline Other perinatal conditions & 412 & 229 & 0 & 1 & 0 & 0 & - & - & - & 230 \\
\hline E. Nutritional deficiencies & 912 & 57 & 12 & 112 & 33 & 44 & 41 & 41 & 21 & 362 \\
\hline 1. Protein-energy malnutrition & 130 & 35 & 0 & 1 & 2 & 6 & 5 & 6 & 7 & 61 \\
\hline 2. lodine deficiency & 2 & 1 & 0 & 0 & 0 & 0 & 0 & 0 & 0 & 1 \\
\hline 3. Vitamin A deficiency & 1 & 0 & 0 & - & - & - & 0 & - & 0 & 0 \\
\hline 4. Iron-deficiency anemia & 758 & 20 & 11 & 111 & 31 & 37 & 35 & 33 & 13 & 291 \\
\hline Other nutritional disorders & 21 & 1 & 0 & 0 & 1 & 2 & 1 & 1 & 2 & 9 \\
\hline
\end{tabular}


Table 3C.8 Continued

\begin{tabular}{|c|c|c|c|c|c|c|c|c|}
\hline & & & & Female & & & & \\
\hline $0-4$ & 5-14 & $15-29$ & $30-44$ & 45-59 & $60-69$ & $70-79$ & $80+$ & Total \\
\hline 27 & 57 & 92 & 105 & 90 & 44 & 36 & 21 & 472 \\
\hline 3,163 & 1,636 & 6,661 & 8,187 & 12,971 & 11,408 & 15,017 & 13,333 & 72,375 \\
\hline 1,007 & 206 & 626 & 532 & 350 & 290 & 511 & 729 & 4,251 \\
\hline 275 & 113 & 192 & 252 & 174 & 140 & 184 & 136 & 1,466 \\
\hline 1 & 4 & 7 & 9 & 10 & 11 & 20 & 12 & 73 \\
\hline 6 & 2 & 79 & 27 & 2 & 1 & 1 & 1 & 119 \\
\hline 5 & 0 & 0 & 0 & 0 & 0 & 0 & 0 & 6 \\
\hline 1 & 2 & 69 & 18 & 1 & 0 & - & 0 & 91 \\
\hline 0 & 0 & 9 & 8 & 0 & 0 & 0 & 0 & 18 \\
\hline 0 & 0 & 0 & 1 & 1 & 1 & 1 & 1 & 4 \\
\hline 2 & 2 & 32 & 88 & 28 & 3 & 1 & 0 & 155 \\
\hline 114 & 13 & 19 & 22 & 18 & 11 & 13 & 13 & 222 \\
\hline 71 & 22 & 1 & 1 & 1 & 1 & 1 & 1 & 99 \\
\hline 70 & 1 & 0 & - & 0 & - & - & - & 70 \\
\hline 0 & 0 & 0 & 0 & 1 & 1 & 1 & 0 & 4 \\
\hline 0 & 0 & - & - & 0 & - & - & - & 0 \\
\hline 2 & 18 & 0 & 0 & 0 & - & - & 0 & 20 \\
\hline 0 & 3 & 0 & 0 & 0 & 0 & 0 & 0 & 4 \\
\hline 23 & 12 & 7 & 6 & 7 & 5 & 5 & 2 & 66 \\
\hline 0 & 0 & 1 & 4 & 8 & 8 & 7 & 2 & 30 \\
\hline 0 & 0 & 1 & 7 & 18 & 20 & 20 & 6 & 72 \\
\hline 1 & 1 & 0 & 0 & 0 & 0 & 0 & 0 & 4 \\
\hline 1 & 7 & 15 & 13 & 2 & 0 & 0 & 0 & 38 \\
\hline- & 0 & - & 0 & - & - & - & - & 0 \\
\hline 0 & 0 & 0 & 0 & 0 & 0 & 0 & 0 & 0 \\
\hline 0 & 0 & 0 & 0 & 0 & 0 & 0 & 0 & 0 \\
\hline 0 & 4 & 0 & 0 & 0 & 0 & 0 & 0 & 4 \\
\hline 1 & 3 & 14 & 13 & 1 & 0 & 0 & 0 & 34 \\
\hline- & 0 & 0 & 0 & 0 & 0 & 0 & 0 & 0 \\
\hline 0 & 0 & 0 & 0 & 0 & 0 & 0 & 0 & 0 \\
\hline 0 & 0 & 0 & 0 & 0 & 0 & 0 & 0 & 0 \\
\hline 1 & 2 & 0 & 0 & 0 & 0 & 0 & 0 & 3 \\
\hline 0 & 0 & 0 & 2 & 1 & 0 & 0 & 0 & 5 \\
\hline 1 & 4 & 0 & 0 & 0 & 0 & 0 & 0 & 5 \\
\hline 0 & 2 & 0 & 0 & 0 & 0 & 0 & 0 & 2 \\
\hline 0 & 1 & - & 0 & - & - & - & - & 2 \\
\hline 0 & 1 & 0 & - & - & 0 & - & - & 1 \\
\hline 0 & 0 & 0 & 0 & 0 & 0 & 0 & 0 & 0 \\
\hline 53 & 44 & 31 & 74 & 79 & 78 & 115 & 99 & 573 \\
\hline 46 & 59 & 19 & 41 & 86 & 113 & 291 & 555 & 1,210 \\
\hline 28 & 25 & 15 & 37 & 82 & 110 & 286 & 548 & 1,131 \\
\hline 2 & 2 & 4 & 4 & 3 & 3 & 6 & 7 & 31 \\
\hline 16 & 32 & 0 & 0 & 0 & 0 & 0 & 0 & 49 \\
\hline 0 & 0 & 241 & 150 & 1 & - & 0 & 0 & 391 \\
\hline 0 & 0 & 1 & 2 & 0 & - & - & - & 4 \\
\hline- & 0 & 56 & 22 & 0 & - & - & - & 78 \\
\hline- & 0 & 2 & 2 & 0 & - & - & 0 & 4 \\
\hline- & - & 6 & 3 & 0 & - & - & - & 9 \\
\hline- & 0 & 3 & 1 & 0 & - & - & - & 4 \\
\hline- & 0 & 172 & 119 & 0 & - & 0 & 0 & 292 \\
\hline 632 & 1 & 0 & 0 & 0 & 0 & 0 & 0 & 634 \\
\hline 215 & 0 & - & - & - & - & - & - & 215 \\
\hline 237 & 0 & 0 & - & 0 & - & 0 & 0 & 237 \\
\hline 181 & 0 & 0 & 0 & 0 & 0 & 0 & 0 & 182 \\
\hline 54 & 33 & 174 & 89 & 89 & 37 & 35 & 38 & 550 \\
\hline 33 & 3 & 1 & 2 & 3 & 4 & 8 & 16 & 69 \\
\hline 1 & 0 & 0 & 0 & 0 & 0 & 0 & 0 & 1 \\
\hline 0 & 0 & - & - & - & - & 0 & 0 & 1 \\
\hline 20 & 28 & 173 & 85 & 85 & 33 & 25 & 18 & 467 \\
\hline 1 & 1 & 1 & 2 & 1 & 1 & 2 & 4 & 12 \\
\hline
\end{tabular}

The Burden of Disease and Mortality by Condition: Data, Methods, and Results for 2001 | 223 
Table 3C.8 Continued

\begin{tabular}{|c|c|c|c|c|c|c|c|c|c|c|}
\hline \multirow[b]{2}{*}{ Cause } & \multicolumn{10}{|c|}{ Male } \\
\hline & Total & $0-4$ & $5-14$ & $15-29$ & $30-44$ & $45-59$ & $60-69$ & $70-79$ & $80+$ & Total \\
\hline II. Noncommunicable diseases & 129,356 & 2,238 & 1,189 & 5,421 & 7,675 & 14,623 & 13,311 & 13,573 & 6,756 & 64,784 \\
\hline A. Malignant neoplasms & 25,888 & 30 & 55 & 189 & 764 & 3,587 & 4,150 & 3,840 & 1,334 & 13,949 \\
\hline 1. Mouth and oropharynx cancers & 576 & 0 & 0 & 4 & 38 & 193 & 120 & 65 & 17 & 437 \\
\hline 2. Esophageal cancer & 702 & 0 & 0 & 1 & 25 & 195 & 184 & 124 & 32 & 561 \\
\hline 3. Stomach cancer & 1,628 & 0 & 0 & 6 & 59 & 258 & 313 & 282 & 99 & 1,017 \\
\hline 4. Colon and rectal cancers & 3,175 & 0 & 0 & 9 & 79 & 422 & 519 & 484 & 179 & 1,692 \\
\hline 5. Liver cancer & 1,223 & 1 & 1 & 6 & 49 & 265 & 291 & 204 & 46 & 863 \\
\hline 6. Pancreas cancer & 1,232 & 0 & 0 & 1 & 30 & 191 & 207 & 172 & 53 & 654 \\
\hline 7. Trachea, bronchus, and lung cancers & 5,397 & 0 & 0 & 4 & 125 & 945 & 1,193 & 1,066 & 271 & 3,606 \\
\hline 8. Melanoma and other skin cancers & 409 & 0 & 0 & 7 & 36 & 80 & 55 & 46 & 19 & 244 \\
\hline 9. Breast cancer & 2,509 & 0 & - & 0 & 2 & 5 & 4 & 4 & 2 & 17 \\
\hline 10. Cervix uteri cancer & 319 & - & - & - & - & - & - & - & - & - \\
\hline 11. Corpus uteri cancer & 586 & - & - & - & - & - & - & - & - & - \\
\hline 12. Ovarian cancer & 651 & - & - & - & - & - & - & - & - & - \\
\hline 13. Prostate cancer & 1,212 & 0 & 0 & 0 & 4 & 104 & 309 & 500 & 295 & 1,212 \\
\hline 14. Bladder cancer & 670 & 0 & 0 & 2 & 12 & 94 & 137 & 172 & 80 & 497 \\
\hline 15. Lymphomas and multiple myeloma & 1,362 & 1 & 4 & 27 & 68 & 184 & 192 & 189 & 66 & 730 \\
\hline 16. Leukemia & 919 & 11 & 20 & 47 & 57 & 105 & 113 & 118 & 47 & 518 \\
\hline Other malignant neoplasms & 3,316 & 16 & 29 & 74 & 182 & 546 & 512 & 414 & 128 & 1,901 \\
\hline B. Other neoplasms & 556 & 6 & 5 & 11 & 23 & 55 & 65 & 78 & 43 & 286 \\
\hline C. Diabetes mellitus & 4,192 & 1 & 3 & 43 & 300 & 676 & 465 & 384 & 159 & 2,032 \\
\hline D. Endocrine disorders & 2,442 & 355 & 46 & 73 & 127 & 190 & 114 & 117 & 69 & 1,090 \\
\hline E. Neuropsychiatric conditions & 31,230 & 520 & 689 & 4,235 & 3,162 & 1,975 & 1,202 & 1,445 & 1,020 & 14,248 \\
\hline 1. Unipolar depressive disorders & 8,408 & 0 & 268 & 873 & 963 & 662 & 259 & 75 & 24 & 3,126 \\
\hline 2. Bipolar affective disorder & 1,056 & 0 & 20 & 436 & 69 & 3 & 1 & 0 & 0 & 531 \\
\hline 3. Schizophrenia & 1,115 & 0 & 120 & 401 & 43 & 11 & 4 & 2 & 1 & 582 \\
\hline 4. Epilepsy & 464 & 13 & 31 & 55 & 66 & 47 & 21 & 14 & 5 & 252 \\
\hline 5. Alcohol use disorders & 4,171 & 0 & 25 & 1,289 & 1,269 & 592 & 107 & 38 & 7 & 3,328 \\
\hline 6. Alzheimer's and other dementias & 7,468 & 22 & 11 & 15 & 15 & 92 & 455 & 957 & 817 & 2,384 \\
\hline 7. Parkinson's disease & 1,086 & 0 & 0 & 0 & 8 & 91 & 159 & 196 & 91 & 546 \\
\hline 8. Multiple sclerosis & 293 & 0 & 6 & 29 & 40 & 34 & 10 & 5 & 1 & 124 \\
\hline 9. Drug use disorders & 1,242 & 0 & 10 & 490 & 336 & 104 & 4 & 0 & 0 & 944 \\
\hline 10. Post-traumatic stress disorder & 369 & 0 & 2 & 39 & 36 & 17 & 1 & 1 & 0 & 96 \\
\hline 11. Obsessive-compulsive disorder & 399 & - & 2 & 91 & 56 & 10 & 3 & 2 & 0 & 164 \\
\hline 12. Panic disorder & 532 & - & 8 & 149 & 3 & 14 & 2 & 2 & 0 & 178 \\
\hline 13. Insomnia (primary) & 691 & - & 4 & 57 & 77 & 77 & 46 & 26 & 6 & 292 \\
\hline 14. Migraine & 1,129 & 8 & 101 & 162 & 14 & 0 & 0 & 0 & 0 & 285 \\
\hline 15. Mental retardation, lead-caused & 187 & 87 & 1 & 2 & 2 & 2 & 1 & 1 & 0 & 96 \\
\hline Other neuropsychiatric disorders & 2,619 & 390 & 78 & 146 & 165 & 218 & 128 & 126 & 67 & 1,319 \\
\hline F. Sense organ diseases & 7,676 & 0 & 1 & 38 & 577 & 1,100 & 1,024 & 718 & 130 & 3,589 \\
\hline 1. Glaucoma & 268 & 0 & 0 & 1 & 9 & 41 & 33 & 17 & 6 & 107 \\
\hline 2. Cataracts & 493 & 0 & 0 & 4 & 30 & 67 & 61 & 30 & 9 & 201 \\
\hline 3. Vision disorders, age-related & 1,525 & 0 & 1 & 9 & 59 & 212 & 191 & 103 & 34 & 611 \\
\hline 4. Hearing loss, adult onset & 5,387 & - & - & 24 & 479 & 779 & 739 & 567 & 81 & 2,669 \\
\hline Other sense organ disorders & 3 & 0 & 0 & 0 & 0 & 0 & 0 & 0 & 0 & 1 \\
\hline G. Cardiovascular diseases & 29,859 & 43 & 22 & 189 & 1,089 & 3,491 & 3,771 & 4,524 & 2,787 & 15,916 \\
\hline 1. Rheumatic heart disease & 199 & 1 & 0 & 2 & 5 & 15 & 18 & 17 & 8 & 65 \\
\hline 2. Hypertensive heart disease & 1,209 & 0 & 0 & 4 & 39 & 119 & 108 & 135 & 119 & 526 \\
\hline 3. Ischemic heart disease & 12,390 & 1 & 3 & 37 & 425 & 1,797 & 1,879 & 2,132 & 1,126 & 7,400 \\
\hline 4. Cerebrovascular disease & 9,354 & 6 & 4 & 27 & 279 & 937 & 1,105 & 1,360 & 792 & 4,510 \\
\hline 5. Inflammatory heart diseases & 982 & 9 & 5 & 34 & 91 & 160 & 135 & 122 & 53 & 610 \\
\hline Other cardiovascular diseases & 5,724 & 26 & 9 & 85 & 251 & 463 & 526 & 758 & 689 & 2,806 \\
\hline H. Respiratory diseases & 9,801 & 312 & 236 & 370 & 492 & 1,141 & 950 & 1,151 & 609 & 5,261 \\
\hline 1. Chronic obstructive pulmonary disease & 5,282 & 1 & 4 & 48 & 249 & 785 & 614 & 750 & 367 & 2,818 \\
\hline 2. Asthma & 1,660 & 121 & 198 & 272 & 101 & 79 & 38 & 38 & 18 & 865 \\
\hline Other respiratory diseases & 2,859 & 190 & 34 & 50 & 143 & 277 & 298 & 363 & 224 & 1,579 \\
\hline
\end{tabular}


Table 3C.8 Continued

\begin{tabular}{|c|c|c|c|c|c|c|c|c|}
\hline & & & & Female & & & & \\
\hline $0-4$ & 5-14 & $15-29$ & $30-44$ & $45-59$ & $60-69$ & $70-79$ & $80+$ & Total \\
\hline 1,992 & 1,160 & 5,264 & 6,885 & 11,998 & 10,807 & 14,159 & 12,308 & 64,572 \\
\hline 24 & 48 & 159 & 1,068 & 3,204 & 2,886 & 3,090 & 1,458 & 11,939 \\
\hline 0 & 0 & 2 & 12 & 43 & 33 & 30 & 18 & 139 \\
\hline 0 & 0 & 0 & 4 & 31 & 38 & 44 & 24 & 142 \\
\hline 0 & 0 & 7 & 53 & 131 & 139 & 174 & 107 & 611 \\
\hline 0 & 0 & 8 & 74 & 324 & 357 & 451 & 270 & 1,483 \\
\hline 1 & 1 & 3 & 15 & 65 & 103 & 124 & 49 & 360 \\
\hline 0 & 0 & 1 & 17 & 115 & 156 & 196 & 92 & 578 \\
\hline 0 & 0 & 3 & 87 & 466 & 521 & 534 & 181 & 1,792 \\
\hline 0 & 0 & 6 & 27 & 48 & 32 & 32 & 20 & 165 \\
\hline 0 & 0 & 12 & 371 & 916 & 534 & 456 & 202 & 2,492 \\
\hline 0 & 0 & 11 & 90 & 117 & 50 & 37 & 15 & 319 \\
\hline 0 & 0 & 4 & 55 & 204 & 152 & 125 & 47 & 586 \\
\hline 0 & 1 & 10 & 56 & 214 & 170 & 147 & 54 & 651 \\
\hline- & - & - & - & - & - & - & - & - \\
\hline 0 & 0 & 0 & 6 & 23 & 36 & 62 & 47 & 174 \\
\hline 1 & 3 & 18 & 40 & 123 & 155 & 198 & 94 & 632 \\
\hline 9 & 16 & 29 & 41 & 74 & 77 & 100 & 55 & 401 \\
\hline 13 & 27 & 47 & 119 & 311 & 334 & 381 & 184 & 1,416 \\
\hline 6 & 6 & 9 & 20 & 43 & 48 & 78 & 62 & 271 \\
\hline 1 & 2 & 28 & 227 & 570 & 467 & 535 & 330 & 2,161 \\
\hline 365 & 37 & 101 & 152 & 260 & 122 & 162 & 152 & 1,351 \\
\hline 456 & 621 & 4,183 & 2,837 & 2,139 & 1,419 & 2,303 & 3,024 & 16,982 \\
\hline 0 & 253 & 1,479 & 1,654 & 1,228 & 411 & 172 & 86 & 5,283 \\
\hline 0 & 16 & 427 & 74 & 4 & 2 & 1 & 1 & 526 \\
\hline 0 & 61 & 407 & 41 & 12 & 4 & 4 & 2 & 533 \\
\hline 11 & 30 & 45 & 46 & 35 & 19 & 17 & 9 & 212 \\
\hline 0 & 8 & 341 & 312 & 143 & 26 & 12 & 2 & 843 \\
\hline 22 & 11 & 15 & 15 & 117 & 596 & 1,679 & 2,631 & 5,084 \\
\hline 0 & 0 & 0 & 7 & 68 & 140 & 197 & 129 & 540 \\
\hline 0 & 7 & 32 & 52 & 48 & 18 & 10 & 3 & 169 \\
\hline 0 & 6 & 151 & 103 & 35 & 2 & 0 & 0 & 297 \\
\hline 0 & 2 & 108 & 87 & 69 & 3 & 4 & 1 & 273 \\
\hline- & 4 & 120 & 89 & 19 & 3 & 1 & 0 & 235 \\
\hline- & 7 & 301 & 5 & 31 & 4 & 4 & 1 & 354 \\
\hline- & 3 & 63 & 100 & 104 & 69 & 50 & 10 & 398 \\
\hline 11 & 153 & 585 & 94 & 0 & 0 & 0 & 0 & 844 \\
\hline 83 & 1 & 1 & 2 & 2 & 1 & 1 & 0 & 91 \\
\hline 329 & 60 & 110 & 157 & 225 & 121 & 152 & 147 & 1,301 \\
\hline 1 & 3 & 41 & 556 & 1,179 & 1,217 & 851 & 239 & 4,087 \\
\hline 0 & 1 & 2 & 15 & 48 & 51 & 31 & 14 & 161 \\
\hline 0 & 1 & 4 & 32 & 91 & 87 & 54 & 22 & 292 \\
\hline 1 & 1 & 10 & 95 & 300 & 246 & 176 & 85 & 915 \\
\hline- & - & 24 & 414 & 740 & 832 & 589 & 118 & 2,718 \\
\hline 0 & 0 & 0 & 0 & 0 & 0 & 0 & 0 & 1 \\
\hline 35 & 24 & 117 & 566 & 1,602 & 2,212 & 4,337 & 5,048 & 13,943 \\
\hline 0 & 2 & 2 & 6 & 21 & 33 & 43 & 27 & 134 \\
\hline 0 & 0 & 3 & 20 & 65 & 86 & 186 & 323 & 684 \\
\hline 1 & 3 & 19 & 121 & 537 & 881 & 1,677 & 1,751 & 4,991 \\
\hline 5 & 6 & 20 & 215 & 648 & 813 & 1,548 & 1,591 & 4,844 \\
\hline 10 & 4 & 17 & 40 & 66 & 69 & 93 & 74 & 372 \\
\hline 20 & 8 & 57 & 164 & 266 & 329 & 791 & 1,283 & 2,918 \\
\hline 257 & 262 & 322 & 576 & 904 & 699 & 861 & 660 & 4,540 \\
\hline 1 & 1 & 57 & 435 & 656 & 452 & 522 & 341 & 2,465 \\
\hline 90 & 238 & 212 & 42 & 66 & 55 & 55 & 37 & 796 \\
\hline 166 & 23 & 53 & 99 & 182 & 192 & 283 & 282 & 1,280 \\
\hline
\end{tabular}


Table 3C.8 Continued

\begin{tabular}{|c|c|c|c|c|c|c|c|c|c|c|}
\hline \multirow[b]{2}{*}{ Cause } & \multicolumn{10}{|c|}{ Male } \\
\hline & Total & $0-4$ & $5-14$ & $15-29$ & $30-44$ & $45-59$ & $60-69$ & $70-79$ & $80+$ & Total \\
\hline I. Digestive diseases & 6,536 & 270 & 21 & 98 & 521 & 1,182 & 682 & 532 & 259 & 3,566 \\
\hline 1. Peptic ulcer disease & 295 & 0 & 1 & 7 & 18 & 43 & 33 & 37 & 22 & 160 \\
\hline 2. Cirrhosis of the liver & 2,146 & 3 & 2 & 13 & 297 & 657 & 328 & 144 & 27 & 1,471 \\
\hline 3. Appendicitis & 35 & 1 & 2 & 4 & 5 & 4 & 2 & 2 & 1 & 20 \\
\hline Other digestive diseases & 4,060 & 267 & 16 & 75 & 202 & 478 & 319 & 350 & 209 & 1,916 \\
\hline J. Genitourinary diseases & 2,074 & 23 & 2 & 19 & 53 & 292 & 237 & 276 & 198 & 1,101 \\
\hline 1. Nephritis and nephrosis & 929 & 6 & 2 & 10 & 31 & 78 & 95 & 139 & 105 & 467 \\
\hline 2. Benign prostatic hypertrophy & 342 & - & - & 0 & 0 & 159 & 90 & 64 & 29 & 342 \\
\hline Other genitourinary system diseases & 803 & 17 & 1 & 9 & 22 & 55 & 51 & 73 & 64 & 292 \\
\hline K. Skin diseases & 288 & 2 & 2 & 5 & 11 & 18 & 17 & 28 & 27 & 110 \\
\hline L. Musculoskeletal diseases & 6,437 & 7 & 18 & 76 & 478 & 776 & 531 & 447 & 113 & 2,447 \\
\hline 1. Rheumatoid arthritis & 1,051 & 1 & 5 & 10 & 45 & 103 & 66 & 51 & 14 & 295 \\
\hline 2. Osteoarthritis & 3,786 & 0 & 0 & 20 & 188 & 444 & 370 & 320 & 54 & 1,397 \\
\hline 3. Gout & 480 & - & 0 & 12 & 181 & 145 & 42 & 20 & 8 & 408 \\
\hline 4. Low back pain & 246 & 4 & 9 & 18 & 39 & 36 & 14 & 9 & 3 & 132 \\
\hline Other musculoskeletal disorders & 875 & 2 & 4 & 16 & 25 & 49 & 39 & 47 & 33 & 215 \\
\hline M. Congenital anomalies & 1,420 & 623 & 17 & 32 & 28 & 24 & 9 & 5 & 2 & 740 \\
\hline 1. Abdominal wall defect & 6 & 3 & 0 & 0 & 0 & 0 & 0 & 0 & 0 & 3 \\
\hline 2. Anencephaly & 18 & 8 & 0 & 0 & 0 & 0 & 0 & 0 & 0 & 8 \\
\hline 3. Anorectal atresia & 2 & 1 & 0 & 0 & 0 & 0 & 0 & 0 & 0 & 1 \\
\hline 4. Cleft lip & 6 & 3 & 0 & 0 & 0 & 0 & 0 & 0 & 0 & 3 \\
\hline 5. Cleft palate & 7 & 3 & 0 & 0 & 0 & 0 & 0 & 0 & 0 & 3 \\
\hline 6. Esophageal atresia & 3 & 1 & 0 & 0 & 0 & 0 & 0 & 0 & 0 & 1 \\
\hline 7. Renal agenesis & 15 & 10 & 0 & 0 & 0 & 0 & 0 & 0 & 0 & 10 \\
\hline 8. Down syndrome & 196 & 88 & 1 & 3 & 3 & 8 & 2 & 0 & 0 & 104 \\
\hline 9. Congenital heart anomalies & 758 & 335 & 8 & 18 & 16 & 10 & 4 & 3 & 1 & 394 \\
\hline 10. Spina bifida & 63 & 26 & 1 & 1 & 1 & 0 & 0 & 0 & 0 & 29 \\
\hline Other congenital anomalies & 347 & 144 & 8 & 10 & 8 & 6 & 3 & 2 & 1 & 183 \\
\hline N. Oral conditions & 957 & 45 & 70 & 44 & 49 & 115 & 94 & 26 & 5 & 448 \\
\hline 1. Dental caries & 462 & 44 & 70 & 39 & 23 & 23 & 17 & 11 & 4 & 230 \\
\hline 2. Periodontal disease & 28 & - & - & 3 & 8 & 2 & 1 & 0 & 0 & 14 \\
\hline 3. Edentulism & 454 & - & - & 1 & 18 & 89 & 76 & 14 & 1 & 198 \\
\hline Other oral diseases & 12 & 2 & 0 & 0 & 1 & 1 & 0 & 0 & 0 & 5 \\
\hline III. Injuries & 11,244 & 234 & 391 & 2,308 & 2,120 & 1,517 & 562 & 376 & 185 & 7,692 \\
\hline A. Unintentional injuries & 7,876 & 215 & 334 & 1,579 & 1,302 & 928 & 385 & 289 & 158 & 5,189 \\
\hline 1. Road traffic accidents & 3,045 & 38 & 90 & 921 & 571 & 336 & 113 & 67 & 20 & 2,157 \\
\hline 2. Poisonings & 494 & 3 & 3 & 88 & 162 & 75 & 10 & 5 & 2 & 347 \\
\hline 3. Falls & 1,459 & 41 & 57 & 176 & 163 & 159 & 91 & 96 & 72 & 856 \\
\hline 4. Fires & 215 & 15 & 13 & 21 & 31 & 33 & 10 & 8 & 3 & 136 \\
\hline 5. Drownings & 304 & 23 & 20 & 58 & 45 & 40 & 18 & 13 & 5 & 222 \\
\hline 6. Other unintentional injuries & 2,360 & 94 & 151 & 315 & 330 & 285 & 142 & 100 & 55 & 1,471 \\
\hline B. Intentional injuries & 3,368 & 19 & 57 & 729 & 818 & 588 & 176 & 87 & 27 & 2,503 \\
\hline 1. Self-inflicted injuries & 2,581 & 0 & 38 & 441 & 635 & 523 & 165 & 83 & 26 & 1,911 \\
\hline 2. Violence & 765 & 19 & 19 & 278 & 175 & 63 & 11 & 4 & 1 & 571 \\
\hline 3. War & 10 & 0 & 0 & 4 & 4 & 1 & 0 & 0 & 0 & 9 \\
\hline Other intentional injuries & 12 & 0 & 0 & 5 & 5 & 2 & 0 & 0 & 0 & 12 \\
\hline
\end{tabular}


Table 3C.8 Continued

\begin{tabular}{|c|c|c|c|c|c|c|c|c|}
\hline \multicolumn{9}{|c|}{ Female } \\
\hline $0-4$ & 5-14 & $15-29$ & 30-44 & $45-59$ & $60-69$ & $70-79$ & $80+$ & Total \\
\hline 204 & 25 & 94 & 298 & 681 & 488 & 637 & 542 & 2,969 \\
\hline 0 & 1 & 4 & 10 & 30 & 19 & 34 & 37 & 135 \\
\hline 1 & 2 & 8 & 117 & 237 & 151 & 123 & 37 & 675 \\
\hline 0 & 2 & 2 & 3 & 2 & 1 & 2 & 2 & 15 \\
\hline 203 & 20 & 80 & 168 & 411 & 318 & 478 & 466 & 2,145 \\
\hline 22 & 6 & 22 & 57 & 153 & 157 & 262 & 294 & 973 \\
\hline 5 & 4 & 7 & 20 & 56 & 83 & 142 & 146 & 462 \\
\hline- & - & - & - & - & - & - & - & - \\
\hline 17 & 2 & 15 & 37 & 97 & 74 & 121 & 148 & 511 \\
\hline 3 & 3 & 6 & 12 & 20 & 22 & 44 & 70 & 178 \\
\hline 7 & 37 & 118 & 443 & 1,092 & 945 & 938 & 411 & 3,990 \\
\hline 3 & 21 & 34 & 121 & 285 & 140 & 102 & 51 & 755 \\
\hline 0 & 0 & 15 & 187 & 605 & 676 & 688 & 217 & 2,389 \\
\hline 0 & 0 & 4 & 13 & 21 & 15 & 15 & 4 & 72 \\
\hline 2 & 11 & 12 & 28 & 32 & 14 & 10 & 5 & 114 \\
\hline 2 & 5 & 52 & 94 & 149 & 100 & 123 & 134 & 660 \\
\hline 568 & 19 & 23 & 23 & 24 & 10 & 8 & 4 & 679 \\
\hline 2 & 0 & 0 & 0 & 0 & 0 & 0 & 0 & 2 \\
\hline 9 & 0 & 0 & 0 & 0 & 0 & 0 & 0 & 9 \\
\hline 1 & 0 & 0 & 0 & 0 & 0 & 0 & 0 & 1 \\
\hline 3 & 0 & 0 & 0 & 0 & 0 & 0 & 0 & 3 \\
\hline 4 & 0 & 0 & 0 & 0 & 0 & 0 & 0 & 4 \\
\hline 1 & 0 & 0 & 0 & 0 & 0 & 0 & 0 & 2 \\
\hline 5 & 0 & 0 & 0 & 0 & 0 & 0 & 0 & 6 \\
\hline 74 & 1 & 2 & 3 & 8 & 3 & 0 & 0 & 92 \\
\hline 311 & 8 & 12 & 13 & 10 & 4 & 4 & 2 & 364 \\
\hline 30 & 1 & 1 & 1 & 0 & 0 & 0 & 0 & 34 \\
\hline 129 & 9 & 7 & 6 & 5 & 3 & 3 & 2 & 164 \\
\hline 43 & 67 & 42 & 48 & 126 & 115 & 54 & 15 & 509 \\
\hline 41 & 66 & 37 & 22 & 23 & 18 & 15 & 9 & 232 \\
\hline- & 0 & 3 & 8 & 2 & 1 & 1 & 0 & 14 \\
\hline- & - & 1 & 18 & 100 & 96 & 37 & 4 & 256 \\
\hline 2 & 0 & 0 & 1 & 1 & 0 & 1 & 1 & 7 \\
\hline 164 & 269 & 771 & 770 & 623 & 311 & 347 & 296 & 3,552 \\
\hline 149 & 237 & 578 & 501 & 413 & 233 & 298 & 279 & 2,687 \\
\hline 30 & 69 & 308 & 195 & 145 & 66 & 57 & 18 & 888 \\
\hline 2 & 3 & 26 & 63 & 37 & 8 & 5 & 3 & 147 \\
\hline 29 & 33 & 64 & 56 & 64 & 61 & 129 & 167 & 603 \\
\hline 10 & 13 & 11 & 14 & 13 & 6 & 7 & 4 & 79 \\
\hline 13 & 13 & 8 & 9 & 11 & 8 & 13 & 6 & 82 \\
\hline 65 & 106 & 162 & 163 & 142 & 84 & 87 & 80 & 889 \\
\hline 15 & 32 & 193 & 270 & 211 & 79 & 49 & 17 & 865 \\
\hline 0 & 20 & 130 & 203 & 185 & 73 & 45 & 15 & 670 \\
\hline 15 & 12 & 63 & 66 & 26 & 6 & 4 & 1 & 194 \\
\hline 0 & 0 & 0 & 0 & 0 & 0 & 0 & 0 & 1 \\
\hline 0 & 0 & 0 & 0 & 0 & 0 & 0 & 0 & 0 \\
\hline
\end{tabular}

Source: Authors' compilation.

Note: $-=$ an estimate of zero; the number zero in a cell indicates a non-zero estimate of less than 500 .

a. Does not include liver cancer and cirrhosis $\operatorname{DALY}(3,0)$ resulting from chronic hepatitis virus infection.

b. This cause category includes "Causes arising in the perinatal period" as defined in the International Classification

of Diseases, principally low birthweight, prematurity, birth asphyxia, and birth trauma, and does not include all causes of

DALYs $(3,0)$ occurring in the perinatal period. 
Table 3C.9 DALYs(3,0) by Cause, Sex, and Age in the World, 2001 (thousands)

\begin{tabular}{|c|c|c|c|c|c|c|c|c|c|c|}
\hline \multirow[b]{2}{*}{ Cause } & \multirow[b]{2}{*}{ Total } & \multicolumn{8}{|c|}{ Male } & \multirow[b]{2}{*}{ Total $^{\mathbf{a}}$} \\
\hline & & $0-4$ & $5-14$ & $15-29$ & $30-44$ & $45-59$ & $60-69$ & 70-79 & $80+$ & \\
\hline Population (millions) & 6,148 & 317 & 623 & 808 & 653 & 415 & 164 & 88 & 25 & 3,093 \\
\hline All causes & $1,535,871$ & 221,294 & 44,197 & 105,973 & 116,457 & 130,728 & 88,774 & 63,545 & 21,509 & 792,478 \\
\hline $\begin{array}{l}\text { I. Communicable, maternal, perinatal, } \\
\text { and nutritional conditions }\end{array}$ & 560,937 & 170,202 & 16,479 & 22,918 & 32,862 & 19,064 & 8,386 & 5,140 & 1,890 & 276,941 \\
\hline A. Infectious and parasitic diseases & 324,038 & 79,163 & 12,459 & 20,598 & 30,632 & 16,275 & 5,573 & 2,727 & 709 & 168,136 \\
\hline 1. Tuberculosis & 36,093 & 731 & 616 & 4,401 & 6,990 & 5,969 & 2,799 & 1,195 & 204 & 22,906 \\
\hline $\begin{array}{l}\text { 2. Sexually transmitted diseases } \\
\text { excluding HIV/AIDS }\end{array}$ & 9,483 & 1,509 & 19 & 712 & 546 & 506 & 146 & 55 & 16 & 3,509 \\
\hline a. Syphilis & 4,134 & 1,009 & 2 & 106 & 268 & 429 & 140 & 53 & 15 & 2,021 \\
\hline b. Chlamydia & 2,538 & 36 & 5 & 158 & 36 & 1 & 0 & - & - & 236 \\
\hline c. Gonorrhea & 2,578 & 449 & 12 & 445 & 220 & 7 & 1 & 0 & 0 & 1,135 \\
\hline $\begin{array}{l}\text { d. Other sexually transmitted } \\
\text { diseases }\end{array}$ & 233 & 15 & 0 & 3 & 22 & 69 & 4 & 2 & 1 & 117 \\
\hline 3. HIV/AIDS & 71,461 & 5,324 & 1,571 & 8,918 & 16,879 & 4,616 & 438 & 43 & 1 & 37,790 \\
\hline 4. Diarrheal diseases & 59,141 & 27,878 & 705 & 548 & 587 & 482 & 280 & 213 & 121 & 30,814 \\
\hline 5. Childhood-cluster diseases & 43,305 & 17,046 & 3,307 & 685 & 279 & 127 & 36 & 13 & 6 & 21,498 \\
\hline a. Pertussis & 11,543 & 5,691 & 50 & - & - & - & 0 & - & - & 5,741 \\
\hline b. Poliomyelitis ${ }^{b}$ & 144 & 15 & 8 & 25 & 17 & 5 & 1 & 1 & 0 & 72 \\
\hline c. Diphtheria & 164 & 76 & 6 & 1 & 1 & 1 & 0 & 0 & 0 & 86 \\
\hline d. Measles & 23,113 & 8,433 & 2,717 & 302 & 0 & 0 & 0 & 0 & - & 11,452 \\
\hline e. Tetanus & 8,341 & 2,831 & 526 & 356 & 260 & 121 & 34 & 12 & 6 & 4,146 \\
\hline 6. Meningitis & 5,607 & 1,335 & 477 & 360 & 252 & 214 & 78 & 58 & 15 & 2,788 \\
\hline 7. Hepatitis $\mathbf{B}^{\mathbf{c}}$ & 2,167 & 93 & 108 & 226 & 410 & 453 & 121 & 45 & 11 & 1,467 \\
\hline Hepatitis Cc & 1,029 & 31 & 44 & 83 & 182 & 235 & 70 & 32 & 7 & 684 \\
\hline 8. Malaria & 39,970 & 17,345 & 498 & 455 & 385 & 278 & 125 & 71 & 20 & 19,178 \\
\hline 9. Tropical-cluster diseases & 10,312 & 362 & 1,939 & 2,250 & 1,365 & 706 & 162 & 63 & 13 & 6,860 \\
\hline a. Trypanosomiasis & 1,333 & 67 & 322 & 189 & 149 & 104 & 9 & 3 & 0 & 844 \\
\hline b. Chagas' disease & 585 & 0 & 0 & 126 & 62 & 67 & 32 & 12 & 4 & 303 \\
\hline c. Schistosomiasis & 1,526 & 88 & 279 & 208 & 144 & 113 & 58 & 25 & 5 & 920 \\
\hline d. Leishmaniasis & 1,762 & 89 & 383 & 306 & 171 & 66 & 19 & 6 & 0 & 1,039 \\
\hline e. Lymphatic filariasis & 4,667 & 111 & 935 & 1,385 & 769 & 272 & 19 & 6 & 1 & 3,497 \\
\hline f. Onchocerciasis & 439 & 7 & 20 & 37 & 71 & 84 & 25 & 10 & 3 & 257 \\
\hline 10. Leprosy & 192 & 7 & 19 & 16 & 23 & 29 & 11 & 8 & 1 & 115 \\
\hline 11. Dengue & 529 & 61 & 143 & 12 & 9 & 6 & 3 & 2 & 1 & 238 \\
\hline 12. Japanese encephalitis & 604 & 91 & 69 & 47 & 65 & 11 & 3 & 2 & 1 & 288 \\
\hline 13. Trachoma & 2,630 & 2 & 2 & 23 & 155 & 213 & 150 & 88 & 21 & 654 \\
\hline 14. Intestinal nematode infections & 2,349 & 229 & 914 & 12 & 8 & 10 & 6 & 3 & 1 & 1,183 \\
\hline a. Ascariasis & 1,158 & 112 & 463 & 1 & 0 & 0 & 0 & 0 & 0 & 577 \\
\hline b. Trichuriasis & 492 & 45 & 206 & 1 & 1 & 1 & 1 & 0 & 0 & 254 \\
\hline c. Hookworm disease & 636 & 56 & 238 & 10 & 6 & 8 & 5 & 2 & 1 & 325 \\
\hline Other intestinal infections & 63 & 16 & 6 & 1 & 1 & 2 & 1 & 0 & 0 & 28 \\
\hline Other infectious diseases & 39,165 & 7,118 & 2,028 & 1,850 & 2,498 & 2,419 & 1,145 & 839 & 270 & 18,166 \\
\hline B. Respiratory infections & 89,184 & 32,372 & 2,521 & 1,104 & 1,417 & 2,019 & 2,487 & 2,185 & 1,094 & 45,200 \\
\hline 1. Lower respiratory infections & 85,920 & 31,687 & 1,940 & 1,026 & 1,330 & 1,936 & 2,411 & 2,137 & 1,072 & 43,540 \\
\hline 2. Upper respiratory infections & 1,740 & 428 & 69 & 67 & 75 & 80 & 74 & 48 & 21 & 862 \\
\hline 3. Otitis media & 1,525 & 258 & 511 & 11 & 12 & 3 & 2 & 1 & 0 & 798 \\
\hline C. Maternal conditions & 26,774 & - & - & - & - & - & - & - & - & - \\
\hline 1. Maternal hemorrhage & 3,926 & - & - & - & - & - & - & - & - & - \\
\hline 2. Maternal sepsis & 5,345 & - & - & - & - & - & - & - & - & - \\
\hline $\begin{array}{l}\text { 3. Hypertensive disorders } \\
\text { of pregnancy }\end{array}$ & 1,894 & - & - & - & - & - & - & - & - & - \\
\hline 4. Obstructed labor & 2,504 & - & - & - & - & - & - & - & - & - \\
\hline 5. Abortion & 3,506 & - & - & - & - & - & - & - & - & - \\
\hline Other maternal conditions & 9,599 & - & - & - & - & - & - & - & - & - \\
\hline D. Perinatal conditions ${ }^{d}$ & 90,477 & 49,368 & 1 & 1 & 0 & 0 & 0 & 0 & - & 49,370 \\
\hline 1. Low birthweight & 43,064 & 23,236 & 0 & - & 0 & - & 0 & - & - & 23,236 \\
\hline 2. Birth asphyxia and birth trauma & 31,958 & 17,938 & 1 & 0 & 0 & 0 & - & - & - & 17,939 \\
\hline Other perinatal conditions & 15,455 & 8,195 & 0 & 1 & 0 & 0 & - & 0 & - & 8,196 \\
\hline
\end{tabular}


Table 3C.9 Continued

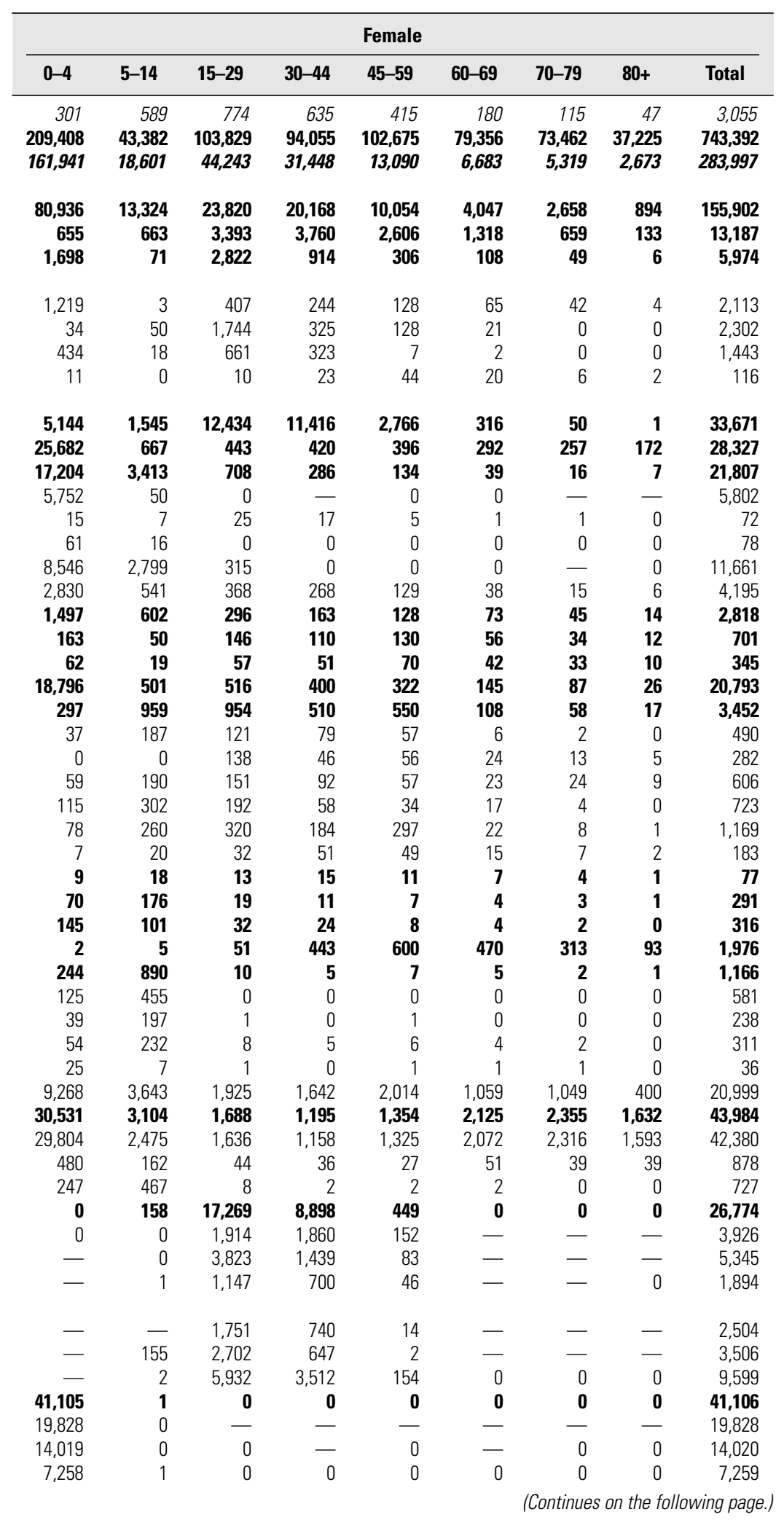


Table 3C.9 Continued

\begin{tabular}{|c|c|c|c|c|c|c|c|c|c|c|}
\hline \multirow[b]{2}{*}{ Cause } & \multicolumn{10}{|c|}{ Male } \\
\hline & Total & $0-4$ & $5-14$ & $15-29$ & $30-44$ & $45-59$ & $60-69$ & 70-79 & $80+$ & Total $^{\mathrm{a}}$ \\
\hline E. Nutritional deficiencies & 30,463 & 9,299 & 1,498 & 1,215 & 812 & 770 & 326 & 227 & 87 & 14,234 \\
\hline 1. Protein-energy malnutrition & 15,578 & 6,926 & 433 & 124 & 68 & 125 & 106 & 80 & 41 & 7,903 \\
\hline 2. Iodine deficiency & 2,876 & 1,074 & 352 & 0 & 1 & 2 & 1 & 0 & 0 & 1,431 \\
\hline 3. Vitamin A deficiency & 711 & 233 & 70 & 3 & 6 & 10 & 5 & 2 & 0 & 329 \\
\hline 4. Iron-deficiency anemia & 10,245 & 899 & 609 & 1,064 & 704 & 567 & 174 & 93 & 28 & 4,138 \\
\hline Other nutritional disorders & 1,053 & 166 & 34 & 23 & 34 & 67 & 40 & 52 & 18 & 433 \\
\hline II. Noncommunicable diseases & 807,839 & 42,899 & 13,697 & 45,319 & 56,267 & 96,868 & 75,790 & 56,282 & 18,997 & 406,118 \\
\hline A. Malignant neoplasms & 100,641 & 590 & 812 & 2,086 & 5,229 & 16,460 & 15,681 & 10,393 & 2,630 & 53,882 \\
\hline 1. Mouth and oropharynx cancers & 4,654 & 5 & 12 & 119 & 420 & 1,243 & 986 & 412 & 86 & 3,284 \\
\hline 2. Esophageal cancer & 5,955 & 1 & 2 & 30 & 326 & 1,303 & 1,330 & 695 & 119 & 3,806 \\
\hline 3. Stomach cancer & 11,244 & 3 & 8 & 127 & 656 & 2,353 & 2,114 & 1,355 & 313 & 6,930 \\
\hline 4. Colon and rectal cancers & 8,236 & 1 & 4 & 118 & 437 & 1,195 & 1,277 & 972 & 293 & 4,297 \\
\hline 5. Liver cancer & 9,169 & 21 & 16 & 221 & 942 & 2,602 & 1,619 & 837 & 131 & 6,388 \\
\hline 6. Pancreas cancer & 2,853 & 0 & 2 & 7 & 129 & 530 & 474 & 322 & 82 & 1,547 \\
\hline 7. Trachea, bronchus, and lung cancers & 16,099 & 3 & 7 & 68 & 689 & 3,545 & 4,005 & 2,547 & 479 & 11,344 \\
\hline 8. Melanoma and other skin cancers & 909 & 1 & 2 & 22 & 74 & 163 & 116 & 84 & 30 & 493 \\
\hline 9. Breast cancer & 8,036 & 0 & 0 & 0 & 3 & 11 & 9 & 7 & 2 & 33 \\
\hline 10. Cervix uteri cancer & 4,119 & - & - & - & - & - & - & - & - & - \\
\hline 11. Corpus uteri cancer & 1,494 & - & - & - & - & - & - & - & - & - \\
\hline 12. Ovarian cancer & 2,139 & - & - & - & - & - & - & - & - & - \\
\hline 13. Prostate cancer & 2,691 & 1 & 1 & 4 & 16 & 276 & 812 & 1,101 & 480 & 2,691 \\
\hline 14. Bladder cancer & 2,174 & 2 & 2 & 10 & 58 & 331 & 480 & 454 & 157 & 1,494 \\
\hline 15. Lymphomas and multiple myeloma & 5,131 & 67 & 200 & 340 & 436 & 616 & 513 & 360 & 107 & 2,639 \\
\hline 16. Leukemia & 4,883 & 235 & 368 & 683 & 346 & 415 & 327 & 250 & 77 & 2,702 \\
\hline Other malignant neoplasms & 10,854 & 251 & 190 & 336 & 696 & 1,876 & 1,618 & 996 & 273 & 6,235 \\
\hline B. Other neoplasms & 2,096 & 74 & 71 & 137 & 140 & 240 & 182 & 155 & 61 & 1,060 \\
\hline C. Diabetes mellitus & 19,997 & 43 & 73 & 489 & 1,528 & 2,990 & 2,087 & 1,395 & 428 & 9,033 \\
\hline D. Endocrine disorders & 13,385 & 4,018 & 262 & 378 & 423 & 601 & 306 & 255 & 124 & 6,368 \\
\hline E. Neuropsychiatric conditions & 168,305 & 10,811 & 6,627 & 28,133 & 16,184 & 9,011 & 3,933 & 3,768 & 1,969 & 80,437 \\
\hline 1. Unipolar depressive disorders & 51,835 & 0 & 2,720 & 6,565 & 5,955 & 3,739 & 1,165 & 255 & 57 & 20,457 \\
\hline 2. Bipolar affective disorder & 9,734 & 0 & 267 & 4,090 & 523 & 17 & 5 & 0 & 0 & 4,903 \\
\hline 3. Schizophrenia & 11,642 & 0 & 901 & 4,132 & 611 & 168 & 36 & 16 & 4 & 5,869 \\
\hline 4. Epilepsy & 6,223 & 514 & 668 & 916 & 657 & 341 & 127 & 62 & 17 & 3,301 \\
\hline 5. Alcohol use disorders & 15,178 & 2 & 126 & 5,319 & 4,696 & 2,214 & 396 & 100 & 15 & 12,868 \\
\hline 6. Alzheimer's and other dementias & 17,108 & 203 & 87 & 100 & 84 & 346 & 1,243 & 2,470 & 1,558 & 6,092 \\
\hline 7. Parkinson's disease & 2,325 & 5 & 3 & 10 & 74 & 241 & 299 & 351 & 140 & 1,124 \\
\hline 8. Multiple sclerosis & 1,209 & 1 & 49 & 194 & 165 & 74 & 21 & 9 & 2 & 515 \\
\hline 9. Drug use disorders & 5,647 & 1 & 79 & 2,255 & 1,506 & 559 & 31 & 4 & 1 & 4,436 \\
\hline 10. Post-traumatic stress disorder & 2,382 & 0 & 25 & 308 & 212 & 106 & 3 & 2 & 1 & 658 \\
\hline 11. Obsessive-compulsive disorder & 3,535 & - & 160 & 789 & 403 & 114 & 23 & 10 & 1 & 1,500 \\
\hline 12. Panic disorder & 4,547 & - & 78 & 1,358 & 19 & 71 & 9 & 6 & 1 & 1,540 \\
\hline 13. Insomnia (primary) & 2,909 & - & 32 & 335 & 375 & 265 & 142 & 58 & 12 & 1,218 \\
\hline 14. Migraine & 5,980 & 52 & 613 & 832 & 94 & 6 & 0 & 0 & 0 & 1,596 \\
\hline 15. Mental retardation, lead-caused & 8,786 & 4,406 & 17 & 24 & 9 & 6 & 2 & 1 & 0 & 4,466 \\
\hline Other neuropsychiatric disorders & 19,263 & 5,627 & 802 & 908 & 802 & 745 & 431 & 422 & 160 & 9,895 \\
\hline F. Sense organ diseases & 79,951 & 30 & 167 & 1,659 & 8,646 & 13,118 & 8,066 & 3,831 & 734 & 36,251 \\
\hline 1. Glaucoma & 4,380 & 7 & 30 & 129 & 356 & 690 & 414 & 207 & 51 & 1,883 \\
\hline 2. Cataracts & 28,643 & 17 & 101 & 610 & 2,480 & 4,636 & 2,734 & 1,289 & 290 & 12,157 \\
\hline 3. Vision disorders, age-related & 16,889 & 3 & 35 & 314 & 1,322 & 2,678 & 1,790 & 877 & 210 & 7,228 \\
\hline 4. Hearing loss, adult onset & 29,994 & - & - & 605 & 4,486 & 5,110 & 3,125 & 1,454 & 182 & 14,962 \\
\hline Other sense organ disorders & 45 & 3 & 2 & 3 & 2 & 4 & 3 & 4 & 2 & 22 \\
\hline G. Cardiovascular diseases & 208,787 & 1,460 & 892 & 3,711 & 10,077 & 28,477 & 29,423 & 24,660 & 8,866 & 107,566 \\
\hline 1. Rheumatic heart disease & 6,350 & 231 & 171 & 554 & 458 & 618 & 361 & 221 & 58 & 2,672 \\
\hline 2. Hypertensive heart disease & 11,178 & 27 & 21 & 114 & 473 & 1,413 & 1,516 & 1,276 & 524 & 5,365 \\
\hline 3. Ischemic heart disease & 84,273 & 104 & 219 & 1,070 & 4,207 & 14,072 & 13,452 & 10,641 & 3,396 & 47,161 \\
\hline 4. Cerebrovascular disease & 72,024 & 221 & 150 & 590 & 2,334 & 8,861 & 10,972 & 9,360 & 2,995 & 35,482 \\
\hline 5. Inflammatory heart diseases & 6,793 & 269 & 85 & 423 & 706 & 917 & 684 & 542 & 206 & 3,832 \\
\hline Other cardiovascular diseases & 28,170 & 608 & 246 & 959 & 1,899 & 2,597 & 2,436 & 2,620 & 1,687 & 13,054 \\
\hline
\end{tabular}


Table 3C.9 Continued

\begin{tabular}{|c|c|c|c|c|c|c|c|c|}
\hline & & & & Female & & & & \\
\hline $0-4$ & 5-14 & $15-29$ & $30-44$ & 45-59 & $60-69$ & $70-79$ & $80+$ & Total \\
\hline 9,368 & 2,013 & 1,467 & 1,186 & 1,232 & 511 & 306 & 146 & 16,230 \\
\hline 6,707 & 492 & 54 & 69 & 100 & 109 & 83 & 61 & 7,676 \\
\hline 1,101 & 338 & 1 & 1 & 2 & 1 & 1 & 0 & 1,445 \\
\hline 288 & 70 & 9 & 4 & 8 & 3 & 2 & 0 & 383 \\
\hline 965 & 1,067 & 1,383 & 1,091 & 1,075 & 349 & 131 & 48 & 6,107 \\
\hline 309 & 46 & 20 & 21 & 48 & 50 & 90 & 37 & 620 \\
\hline 40,092 & 14,171 & 43,825 & 51,687 & 82,360 & 69,779 & 66,115 & 33,693 & 401,721 \\
\hline 611 & 717 & 1,761 & 6,147 & 14,252 & 11,268 & 8,926 & 3,076 & 46,759 \\
\hline 9 & 6 & 34 & 135 & 458 & 379 & 254 & 95 & 1,371 \\
\hline 1 & 2 & 21 & 131 & 696 & 669 & 485 & 142 & 2,148 \\
\hline 1 & 4 & 104 & 564 & 1,192 & 1,069 & 1,007 & 374 & 4,315 \\
\hline 1 & 3 & 55 & 402 & 1,009 & 1,029 & 988 & 452 & 3,939 \\
\hline 33 & 20 & 148 & 378 & 805 & 705 & 551 & 141 & 2,780 \\
\hline 0 & 1 & 10 & 88 & 327 & 367 & 371 & 142 & 1,306 \\
\hline 6 & 29 & 32 & 368 & 1,401 & 1,404 & 1,216 & 301 & 4,755 \\
\hline 1 & 3 & 17 & 65 & 116 & 91 & 85 & 38 & 416 \\
\hline 1 & 2 & 74 & 1,540 & 3,213 & 1,714 & 1,080 & 380 & 8,004 \\
\hline 0 & 2 & 352 & 608 & 1,564 & 972 & 507 & 113 & 4,119 \\
\hline 2 & 3 & 22 & 217 & 522 & 378 & 268 & 82 & 1,494 \\
\hline 2 & 19 & 100 & 313 & 737 & 518 & 350 & 100 & 2,139 \\
\hline- & - & - & - & - & - & - & - & - \\
\hline 21 & 7 & 14 & 83 & 132 & 160 & 174 & 91 & 681 \\
\hline 89 & 175 & 234 & 333 & 520 & 514 & 456 & 171 & 2,492 \\
\hline 247 & 288 & 362 & 345 & 372 & 255 & 221 & 91 & 2,181 \\
\hline 196 & 155 & 183 & 577 & 1,187 & 1,043 & 915 & 363 & 4,618 \\
\hline 55 & 57 & 79 & 152 & 270 & 168 & 166 & 89 & 1,037 \\
\hline 60 & 80 & 428 & 1,458 & 3,193 & 2,781 & 2,195 & 767 & 10,963 \\
\hline 3,720 & 250 & 534 & 606 & 786 & 454 & 403 & 263 & 7,017 \\
\hline 10,335 & 6,740 & 28,102 & 16,199 & 10,155 & 5,338 & 5,790 & 5,208 & 87,868 \\
\hline 0 & 2,565 & 9,300 & 9,818 & 6,672 & 2,226 & 604 & 191 & 31,378 \\
\hline 0 & 229 & 4,036 & 536 & 21 & 7 & 1 & 1 & 4,831 \\
\hline 1 & 252 & 4,329 & 842 & 253 & 54 & 33 & 9 & 5,773 \\
\hline 426 & 672 & 837 & 483 & 293 & 111 & 74 & 26 & 2,922 \\
\hline 0 & 49 & 1,003 & 771 & 386 & 70 & 27 & 4 & 2,310 \\
\hline 184 & 82 & 104 & 89 & 398 & 1,746 & 3,961 & 4,452 & 11,016 \\
\hline 6 & 4 & 7 & 63 & 228 & 305 & 386 & 203 & 1,202 \\
\hline 1 & 63 & 245 & 225 & 105 & 34 & 17 & 5 & 695 \\
\hline 0 & 48 & 604 & 398 & 148 & 10 & 2 & 1 & 1,211 \\
\hline 0 & 18 & 832 & 578 & 269 & 14 & 10 & 3 & 1,724 \\
\hline- & 308 & 889 & 593 & 187 & 40 & 16 & 4 & 2,036 \\
\hline- & 78 & 2,717 & 30 & 145 & 19 & 15 & 3 & 3,007 \\
\hline- & 31 & 367 & 512 & 403 & 237 & 115 & 26 & 1,692 \\
\hline 143 & 1,720 & 2,006 & 510 & 4 & 0 & 0 & 0 & 4,384 \\
\hline 4,265 & 19 & 17 & 10 & 6 & 1 & 1 & 0 & 4,320 \\
\hline 5,309 & 599 & 809 & 742 & 635 & 463 & 529 & 283 & 9,368 \\
\hline 31 & 117 & 1,309 & 9,039 & 15,393 & 10,710 & 5,686 & 1,415 & 43,700 \\
\hline 5 & 26 & 143 & 415 & 861 & 598 & 340 & 109 & 2,496 \\
\hline 14 & 63 & 564 & 2,858 & 6,205 & 4,110 & 2,109 & 563 & 16,487 \\
\hline 9 & 25 & 236 & 1,512 & 3,392 & 2,484 & 1,534 & 469 & 9,661 \\
\hline- & - & 363 & 4,252 & 4,932 & 3,516 & 1,698 & 271 & 15,032 \\
\hline 3 & 2 & 4 & 2 & 3 & 3 & 4 & 2 & 23 \\
\hline 1,650 & 955 & 3,251 & 6,314 & 18,322 & 24,551 & 29,769 & 16,410 & 101,222 \\
\hline 246 & 249 & 591 & 593 & 829 & 590 & 441 & 140 & 3,679 \\
\hline 24 & 25 & 81 & 328 & 1,165 & 1,458 & 1,688 & 1,043 & 5,812 \\
\hline 75 & 170 & 1,022 & 2,014 & 6,735 & 10,077 & 11,270 & 5,749 & 37,112 \\
\hline 161 & 145 & 369 & 1,695 & 6,784 & 9,541 & 11,892 & 5,957 & 36,542 \\
\hline 213 & 88 & 274 & 362 & 554 & 522 & 612 & 335 & 2,961 \\
\hline 932 & 277 & 914 & 1,321 & 2,255 & 2,365 & 3,866 & 3,186 & 15,116 \\
\hline
\end{tabular}


Table 3C.9 Continued

\begin{tabular}{|c|c|c|c|c|c|c|c|c|c|c|c|}
\hline \multirow{2}{*}{ Cause } & & \multirow[b]{2}{*}{ Total } & \multicolumn{8}{|c|}{ Male } & \multirow[b]{2}{*}{ Total $^{\mathrm{a}}$} \\
\hline & & & $0-4$ & 5-14 & $15-29$ & $30-44$ & $45-59$ & $60-69$ & 70-79 & $80+$ & \\
\hline \multirow[t]{4}{*}{ H. } & Respiratory diseases & 67,887 & 3,566 & 1,935 & 2,738 & 3,410 & 8,403 & 7,418 & 6,618 & 2,497 & 36,585 \\
\hline & $\begin{array}{l}\text { 1. Chronic obstructive pulmonary } \\
\text { disease }\end{array}$ & 38,736 & 49 & 19 & 168 & 1,556 & 6,144 & 5,776 & 5,216 & 1,866 & 20,795 \\
\hline & 2. Asthma & 13,174 & 1,134 & 1,546 & 2,056 & 930 & 793 & 323 & 194 & 54 & 7,030 \\
\hline & Other respiratory diseases & 15,977 & 2,383 & 370 & 514 & 924 & 1,465 & 1,319 & 1,208 & 578 & 8,761 \\
\hline \multirow[t]{5}{*}{ I. } & Digestive diseases & 58,937 & 7,636 & 879 & 2,686 & 5,094 & 8,203 & 4,118 & 2,531 & 830 & 31,977 \\
\hline & 1. Peptic ulcer disease & 5,096 & 91 & 67 & 401 & 685 & 1,009 & 487 & 306 & 96 & 3,142 \\
\hline & 2. Cirrhosis of the liver & 15,778 & 259 & 154 & 634 & 2,131 & 3,828 & 1,776 & 818 & 148 & 9,749 \\
\hline & 3. Appendicitis & 412 & 7 & 30 & 40 & 42 & 58 & 26 & 21 & 7 & 232 \\
\hline & Other digestive diseases & 37,651 & 7,279 & 628 & 1,611 & 2,236 & 3,307 & 1,829 & 1,386 & 579 & 18,854 \\
\hline \multirow[t]{4}{*}{ J. } & Genitourinary diseases & 18,455 & 999 & 347 & 744 & 1,053 & 3,908 & 1,626 & 1,259 & 518 & 10,453 \\
\hline & 1. Nephritis and nephrosis & 10,005 & 333 & 285 & 593 & 808 & 1,182 & 933 & 717 & 285 & 5,136 \\
\hline & 2. Benign prostatic hypertrophy & 2,955 & - & - & 0 & 12 & 2,277 & 346 & 236 & 84 & 2,955 \\
\hline & $\begin{array}{l}\text { Other genitourinary system } \\
\text { diseases }\end{array}$ & 5,495 & 665 & 63 & 150 & 233 & 449 & 347 & 306 & 148 & 2,362 \\
\hline K. & Skin diseases & 3,985 & 501 & 227 & 306 & 298 & 274 & 147 & 123 & 63 & 1,939 \\
\hline \multirow[t]{6}{*}{ L. } & Musculoskeletal diseases & 32,130 & 209 & 450 & 1,369 & 3,786 & 4,338 & 2,120 & 1,136 & 249 & 13,657 \\
\hline & 1. Rheumatoid arthritis & 4,695 & 12 & 80 & 181 & 291 & 411 & 215 & 121 & 28 & 1,341 \\
\hline & 2. Osteoarthritis & 17,452 & 0 & 3 & 387 & 1,536 & 2,414 & 1,430 & 723 & 106 & 6,600 \\
\hline & 3. Gout & 3,265 & 0 & 0 & 135 & 1,488 & 999 & 188 & 59 & 14 & 2,884 \\
\hline & 4. Low back pain & 1,938 & 72 & 176 & 202 & 251 & 220 & 70 & 32 & 8 & 1,030 \\
\hline & Other musculoskeletal disorders & 4,780 & 124 & 190 & 464 & 220 & 294 & 217 & 200 & 92 & 1,802 \\
\hline \multicolumn{2}{|r|}{ M. Congenital anomalies } & 24,952 & 11,975 & 319 & 371 & 95 & 59 & 21 & 12 & 4 & 12,855 \\
\hline & 1. Abdominal wall defect & 116 & 63 & 0 & 0 & 0 & 0 & 0 & 0 & 0 & 63 \\
\hline & 2. Anencephaly & 563 & 266 & 0 & 0 & 0 & 0 & 0 & 0 & 0 & 267 \\
\hline & 3. Anorectal atresia & 33 & 21 & 0 & 0 & 0 & 0 & 0 & 0 & 0 & 21 \\
\hline & 4. Cleft lip & 122 & 64 & 0 & 0 & 0 & 0 & 0 & 0 & 0 & 64 \\
\hline & 5. Cleft palate & 138 & 70 & 0 & 0 & 0 & 0 & 0 & 0 & 0 & 70 \\
\hline & 6. Esophageal atresia & 49 & 24 & 0 & 0 & 0 & 0 & 0 & 0 & 0 & 24 \\
\hline & 7. Renal agenesis & 68 & 39 & 0 & 1 & 0 & 0 & 0 & 0 & 0 & 41 \\
\hline & 8. Down syndrome & 3,612 & 1,824 & 29 & 60 & 6 & 10 & 2 & 0 & 0 & 1,932 \\
\hline & 9. Congenital heart anomalies & 13,949 & 6,533 & 172 & 219 & 58 & 25 & 9 & 5 & 2 & 7,023 \\
\hline & 10. Spina bifida & 1,551 & 732 & 10 & 5 & 1 & 1 & 0 & 0 & 0 & 750 \\
\hline & Other congenital anomalies & 4,751 & 2,340 & 106 & 85 & 29 & 23 & 9 & 6 & 2 & 2,599 \\
\hline \multicolumn{2}{|r|}{ N. Oral conditions } & 8,331 & 988 & 633 & 511 & 305 & 786 & 662 & 147 & 23 & 4,054 \\
\hline & 1. Dental caries & 5,214 & 962 & 628 & 380 & 167 & 274 & 139 & 71 & 19 & 2,640 \\
\hline & 2. Periodontal disease & 235 & - & - & 29 & 55 & 21 & 8 & 4 & 1 & 118 \\
\hline & 3. Edentulism & 2,747 & - & - & 96 & 77 & 486 & 514 & 71 & 2 & 1,245 \\
\hline & Other oral diseases & 135 & 25 & 5 & 7 & 6 & 5 & 2 & 1 & 1 & 52 \\
\hline \multicolumn{2}{|c|}{ III. Injuries } & 167,094 & 8,193 & 14,022 & 37,737 & 27,329 & 14,796 & 4,598 & 2,124 & 623 & 109,420 \\
\hline \multicolumn{2}{|c|}{ A. Unintentional injuries } & 121,111 & 7,822 & 12,781 & 22,914 & 16,768 & 10,264 & 3,317 & 1,600 & 496 & 75,962 \\
\hline & 1. Road traffic accidents & 35,063 & 1,224 & 3,358 & 8,492 & 6,496 & 3,539 & 973 & 429 & 88 & 24,598 \\
\hline & 2. Poisonings & 7,608 & 289 & 322 & 1,051 & 1,429 & 1,348 & 378 & 95 & 17 & 4,930 \\
\hline & 3. Falls & 15,041 & 1,024 & 1,727 & 2,336 & 1,547 & 1,161 & 560 & 456 & 202 & 9,013 \\
\hline & 4. Fires & 10,295 & 938 & 878 & 905 & 769 & 409 & 123 & 63 & 17 & 4,103 \\
\hline & 5. Drownings & 9,695 & 1,034 & 1,700 & 1,893 & 1,103 & 622 & 169 & 75 & 22 & 6,618 \\
\hline & 6. Other unintentional injuries & 43,410 & 3,313 & 4,795 & 8,238 & 5,426 & 3,184 & 1,114 & 481 & 150 & 26,700 \\
\hline & Intentional injuries & 45,983 & 370 & 1,240 & 14,822 & 10,560 & 4,533 & 1,281 & 523 & 127 & 33,457 \\
\hline & 1. Self-inflicted injuries & 20,255 & 3 & 376 & 4,379 & 3,689 & 2,372 & 811 & 377 & 84 & 12,092 \\
\hline & 2. Violence & 18,897 & 259 & 779 & 7,703 & 4,535 & 1,569 & 302 & 106 & 29 & 15,282 \\
\hline & 3. War & 6,502 & 91 & 71 & 2,632 & 2,258 & 563 & 157 & 35 & 12 & 5,818 \\
\hline & Other intentional injuries & 329 & 17 & 15 & 109 & 79 & 28 & 11 & 5 & 2 & 265 \\
\hline
\end{tabular}


Table 3C.9 Continued

\begin{tabular}{|c|c|c|c|c|c|c|c|c|}
\hline \multicolumn{9}{|c|}{ Female } \\
\hline $0-4$ & 5-14 & $15-29$ & $30-44$ & $45-59$ & $60-69$ & $70-79$ & $80+$ & Total \\
\hline 2,966 & 2,051 & 2,259 & 2,982 & 5,901 & 5,313 & 6,400 & 3,430 & 31,302 \\
\hline 64 & 22 & 186 & 1,704 & 4,199 & 4,030 & 5,140 & 2,596 & 17,941 \\
\hline 830 & 1,759 & 1,561 & 646 & 724 & 319 & 214 & 91 & 6,145 \\
\hline 2,072 & 270 & 512 & 632 & 977 & 964 & 1,045 & 744 & 7,217 \\
\hline 7,276 & 1,189 & 2,465 & 3,047 & 5,311 & 3,355 & 2,898 & 1,420 & 26,960 \\
\hline 98 & 57 & 198 & 343 & 530 & 318 & 278 & 132 & 1,954 \\
\hline 597 & 301 & 509 & 921 & 1,760 & 1,065 & 704 & 173 & 6,030 \\
\hline 6 & 23 & 30 & 23 & 37 & 25 & 24 & 10 & 179 \\
\hline 6,575 & 808 & 1,728 & 1,760 & 2,984 & 1,947 & 1,891 & 1,104 & 18,797 \\
\hline 648 & 339 & 742 & 1,072 & 1,838 & 1,436 & 1,261 & 665 & 8,002 \\
\hline 271 & 279 & 443 & 584 & 1,152 & 966 & 805 & 370 & 4,869 \\
\hline- & - & - & - & - & - & - & - & - \\
\hline 377 & 60 & 299 & 489 & 686 & 470 & 457 & 295 & 3,132 \\
\hline 351 & 161 & 290 & 307 & 330 & 221 & 223 & 163 & 2,046 \\
\hline 194 & 582 & 1,803 & 3,946 & 5,710 & 3,382 & 2,124 & 733 & 18,473 \\
\hline 24 & 203 & 510 & 738 & 1,043 & 478 & 266 & 92 & 3,354 \\
\hline 0 & 4 & 647 & 2,319 & 3,738 & 2,395 & 1,412 & 337 & 10,852 \\
\hline 0 & 0 & 25 & 158 & 105 & 49 & 35 & 9 & 381 \\
\hline 37 & 213 & 138 & 227 & 187 & 65 & 32 & 10 & 908 \\
\hline 133 & 161 & 482 & 504 & 637 & 395 & 379 & 286 & 2,978 \\
\hline 11,269 & 325 & 292 & 101 & 64 & 23 & 16 & 6 & 12,097 \\
\hline 52 & 0 & 0 & 0 & 0 & 0 & 0 & 0 & 53 \\
\hline 293 & 1 & 1 & 0 & 0 & 0 & 0 & 0 & 295 \\
\hline 12 & 0 & 0 & 0 & 0 & 0 & 0 & 0 & 12 \\
\hline 59 & 0 & 0 & 0 & 0 & 0 & 0 & 0 & 59 \\
\hline 67 & 0 & 0 & 0 & 0 & 0 & 0 & 0 & 67 \\
\hline 24 & 0 & 0 & 0 & 0 & 0 & 0 & 0 & 24 \\
\hline 25 & 0 & 0 & 0 & 0 & 0 & 0 & 0 & 27 \\
\hline 1,571 & 31 & 56 & 6 & 12 & 3 & 0 & 0 & 1,680 \\
\hline 6,426 & 195 & 186 & 65 & 30 & 10 & 9 & 4 & 6,926 \\
\hline 785 & 8 & 5 & 2 & 1 & 0 & 0 & 0 & 801 \\
\hline 1,955 & 88 & 44 & 27 & 20 & 9 & 6 & 2 & 2,152 \\
\hline 926 & 608 & 511 & 315 & 835 & 777 & 259 & 47 & 4,277 \\
\hline 901 & 600 & 364 & 163 & 274 & 153 & 88 & 31 & 2,574 \\
\hline - & 0 & 28 & 53 & 21 & 8 & 5 & 2 & 117 \\
\hline- & - & 98 & 81 & 534 & 613 & 163 & 13 & 1,502 \\
\hline 25 & 8 & 21 & 18 & 5 & 3 & 2 & 1 & 83 \\
\hline 7,376 & 10,611 & 15,762 & 10,920 & 7,225 & 2,894 & 2,027 & 860 & 57,675 \\
\hline 7,155 & 9,559 & 11,043 & 7,597 & 5,176 & 2,228 & 1,631 & 759 & 45,149 \\
\hline 1,118 & 2,411 & 2,486 & 2,076 & 1,519 & 492 & 293 & 70 & 10,465 \\
\hline 207 & 296 & 498 & 600 & 586 & 353 & 111 & 29 & 2,679 \\
\hline 983 & 1,455 & 1,061 & 624 & 567 & 391 & 574 & 372 & 6,027 \\
\hline 1,089 & 1,088 & 2,077 & 1,154 & 485 & 150 & 112 & 37 & 6,192 \\
\hline 775 & 935 & 593 & 337 & 226 & 107 & 77 & 28 & 3,077 \\
\hline 2,984 & 3,374 & 4,329 & 2,806 & 1,793 & 736 & 464 & 223 & 16,710 \\
\hline 221 & 1,051 & 4,719 & 3,323 & 2,050 & 666 & 396 & 100 & 12,526 \\
\hline 1 & 429 & 3,311 & 2,177 & 1,398 & 467 & 302 & 79 & 8,163 \\
\hline 202 & 425 & 1,267 & 986 & 489 & 152 & 77 & 16 & 3,615 \\
\hline 6 & 191 & 126 & 148 & 151 & 43 & 14 & 5 & 683 \\
\hline 12 & 7 & 15 & 11 & 12 & 4 & 2 & 1 & 64 \\
\hline
\end{tabular}

Source: Authors' compilation.

Note: $-=$ an estimate of zero; the number zero in a cell indicates a non-zero estimate of less than 500 .

a. World totals for males and females include residual populations not included in the World Bank regions.

b. For East Asia and Pacific, Europe and Central Asia, and Latin America and the Caribbean regions, these figures include

late effects of polio cases with onset prior to regional certification of polio eradication in 1994, 2000, and 2002, respectively.

c. Does not include liver cancer and cirrhosis DALYs $(3,0)$ resulting from chronic hepatitis virus infection

d. This cause category includes "Causes arising in the perinatal period" as defined in the International Classification

of Diseases, principally low birthweight, prematurity, birth asphyxia, and birth trauma, and does not include all causes of $\operatorname{DALYS}(3,0)$ occurring in the perinatal period. 


\section{ACKNOWLEDGMENTS}

In writing this chapter we have drawn on documentation prepared by the many people involved in the GBD studies and acknowledged this as far as possible by means of citations. We would especially like to acknowledge the assistance of Doris Ma Fat and Mie Inoue, who compiled comprehensive summaries of mortality data sources for GBD 2001 (Mathers, Lopez, and others 2004), which we have drawn on here, and the assistance of Chalapati Rao, who carried out analyses of the latest mortality data for China, India, Iran, Turkey, and various other countries.

Many people, both inside and outside WHO, contributed to the GBD analyses reported here. Apart from the authors, current and former staff of the former WHO Global Program on Evidence for Health Policy who worked directly on the GBD 2001 include Omar Ahmad, José Ayuso, Prerna Banati, Stephen Begg, Christina Bernard, Cynthia Boschi-Pinto, Marisol Concha, Carmen Dolea, Majid Ezzati, Doris Ma Fat, Brodie Ferguson, Kim Moesgaard Iburg, Mie Inoue, Jeremy Lauer, Matilde Leonardi, Steve Lim, Rafael Lozano, Sue Piccolo, Chalapati Rao, Tanuja Rastogi, Eduardo Sabaté, Joshua Salomon, Toshi Satoh, Kenji Shibuya, Claudia Stein, Lana Tomaskovic, Niels Tomijima, Thomas Truelsen, Bedirhan Ustün, Marie-Claude von Rulach, Sarah Wild, and Hongyi Xu. We also gratefully acknowledge the support of David Evans, director of the Global Program on Evidence for Health Policy.

We also wish to acknowledge the contributions of staff in various WHO programs and of expert groups outside WHO who provided advice and collaborated in the reviews of epidemiological data and in the estimation of the burden of disease. While it is not possible to name all those who contributed to this effort, we would like to note the considerable assistance and inputs provided by Carla AbouZahr, Elisabeth Aahman, Jan Barendregt, Maureen Birmingham, Jennifer Bryce, Mercedes de Onis, Chris Dye, Jacques Ferlay, Anthony Gerbase, Ken Hill, Yvan Hutin, Gareth Jones, Hilary King, Eline Korenromp, Daniel Lavanchy, Silvio Mariotti, Mike McKenna, Catherine Michaud, Chris Nelson, Tomoko Ono, Donatella Pascolini, Margie Peden, Bruce Pfleger, Paola Pisani, Annette Pruss-Ustun, Juergen Rehm, Serge Resnikoff, Sue Robertson, Gojke Roglic, Kate Strong, Deborah Symmonds, Theo Vos, Neff Walker, Catherine Watt, and Lara Wolfson.

We would also like to thank the Child Health Epidemiology Reference Group, chaired by Robert Black, and established to provide technical advice to WHO on the estimation of child mortality and the burden of disease; the
Bone and Joint Decade's Bone and Joint Monitor Project coordinated by Anthony Woolf; and the Joint United Nations Programme on HIVAIDS and WHO Working Group on Global HIV/AIDS and STI Surveillance. The authors gratefully acknowledge financial support for this project from the National Institute on Aging (research grant number PO1 AG17625). The authors alone are responsible for the views expressed in this chapter, which do not necessarily reflect the opinions of the WHO or of its member states.

\section{REFERENCES}

Abdallah, M. B., and S. Zehani. 2000. Registre des Cancers Nord-Tunisie 1994. Tunis, Tunisia:Ministry of Public Health, Salah Azaiz Institute, and National Institute of Public Health.

Ahman, E., C. Dolea, and I. Shah. 2003. "Global Burden of Unsafe Abortion in the Year 2000." GBD 2000 Working Paper, World Health Organization, Geneva. http://www.who.int/evidence/bod.

Alley, W. S., G. J. van Oortmarssen, B. A. Boatin, N. J. Nagelkerke, A. P. Plaisier, J. H. Remme, J. Lazdins, G. J. Borsboom, and J. D. Habb. 2001. "Macrofilaricides and Onchocerciasis Control, Mathematical Modeling of the Prospects for Elimination." BMC Public Health 1 (1): 12.

American Psychiatric Association. 1994. Diagnostic and Statistical Manual on Mental Disorders, 4th ed. Washington, DC: American Psychiatric Press.

Anand, S., and K. Hanson. 1997. "Disability-Adjusted Life Years: A Critical Review.” Journal of Health Economics 16 (6): 685-702.

1998. "DALYs: Efficiency Versus Equity." World Development 26 (2): 307-10.

Ayuso-Mateos, J. L. 2002a. "Global Burden of Bipolar Disorder in the Year 2000.” GBD 2000 Working Paper, World Health Organization, Geneva. http://www.who.int/evidence/bod.

2002b. "Global Burden of Obsessive Compulsive Disorders in the Year 2000.” GBD 2000 Working Paper, World Health Organization, Geneva. http://www.who.int/evidence/bod.

2002c. "Global Burden of Post-Traumatic Stress Disorder in the Year 2000: Version 1 Estimates." GBD 2000 Working Paper, World Health Organization, Geneva. http://www.who.int/evidence/bod.

.2002d. "Global Burden of Schizophrenia in the Year 2000: Version 1 Estimates." GBD 2000 Working Paper, World Health Organization, Geneva. http://www.who.int/evidence/bod.

Ayuso-Mateos, J. L, J. L. Vazquez-Barquero, C. Dowrick, V. Lehtinen, O. S. Dalgard, P. Casey, C. Wilkinson, L. Lasa, H. Page, G. Dunn, G. Wilkinson, and the ODIN Group. 2001. "Depressive Disorders in Europe: Prevalence Figures from the ODIN Study." British Journal of Psychiatry 179 (4): 308-16.

Bannister, J., and K. Hill. 2004. "Mortality in China, 1964-2000." Population Studies 58 (1): 55-75.

Barendregt, J., G. J. van Oortmarssen, T. Vos, and C. J. L. Murray. 2003. “A Generic Model for the Assessment of Disease Epidemiology: The Computational Basis of DisMod II.” Population Health Metrics 1: 4.

Baskent University. 2005. Burden of Disease Final Report. Ankara: Turkish Ministry of Health and Baskent University, Refik Saydam Hygiene Presidency, School of Public Health.

Begg, S., N. Tomijima, T. Vos, and C. D. Mathers. 2002. "Global Burden of Injury in the Year 2000: An Overview of Methods.” GBD 2000 Working 
Paper, World Health Organization, Geneva. http://www.who.int/ evidence/bod.

Bern, C. 2004. "Diarrhoeal Diseases." In The Global Epidemiology of Infectious Diseases, ed. C. J. L. Murray, A. D. Lopez, and C. D. Mathers, 1-27. Vol. 4 of Global Burden of Disease and Injury Series. Geneva: World Health Organization. http://whqlibdoc.who.int/publications/ 2004/9241592303.pdf.

Boschi-Pinto, C., L. Tomaskovic, E. Gouws, and K Shibuya. Forthcoming. "Estimates of the Distribution of Child Deaths Due to Diarrhea in Developing Regions of the World."

Bradshaw, D., P. Groenewald, R. Laubscher, N. Nannan, B. Nojilana, R. Norman, D. Pieterse, M. Schneider, D. E. Bourne, I. M. Timaeus, R. Dorrington, and L. Johnson. 2003. Initial Burden of Disease Estimates for South Africa, 2000. Cape Town: South African Medical Research Council.

Brooker, S., M. Rowlands, L. Haller, L. Savioli, and D. A. P. Bundy. 2000. "Towards an Atlas of Human Helminth Infection in Sub-Saharan Africa: The Use of Geographical Information systems (GIS)." Parasitology Today 16 (7): 303-7.

Brooker, S., M. Beasley, M. Ndinaromtan, E. M. Madjiouroum, M. Baboguel, E. Djenguinabe, S. I. Hay, and D. A. Bundy. 2002. "Use of Remote Sensing and a Geographical Information System in a National Helminth Control Programme in Chad." Bulletin of the World Health Organization 80 (10): 783-9.

Bundhamcharoen, K., Y. Teerawatananon, T. Vos, and S. Begg. 2002. Burden of Disease and Injuries in Thailand: Priority Setting for Policy. Bangkok: Ministry of Public Health.

Bundy, D. A. P., M. S. Chan, G. F. Medley, D. Jamison, and L. Savioli. 2004. "Intestinal Nematode Infections." In The Global Epidemiology of Infectious Diseases, ed. C. J. L. Murray, A. D. Lopez, and C. D. Mathers, 243-300. Vol. 4 of Global Burden of Disease and Injury Series. Geneva: World Health Organization. http://whqlibdoc.who.int/publications/ 2004/9241592303.pdf.

Center for Research on the Epidemiology of Disasters. 2001. "EM-DAT: The OFDA/CRED International Disaster Database." Brussels, Belgium: Université Catholique de Louvain. http://www.em-dat.net.

Chan, M. S. 1997. "The Global Burden of Intestinal Nematode Infections: Fifty Years On." Parasitology Today 13: 438-43.

Chenet, L., M. McKee, D. Leon, V. Shkolnikov, and S. Vassin. 1998. "Alcohol and Cardiovascular Mortality in Moscow: New Evidence of a Causal Association." Journal of Epidemiology and Community Health 52 (12): $772-4$.

Chinn, S., P. Burney, D. Jarvis, and C. Luczynska. 1997. "Variation in Bronchial Responsiveness in the European Community Respiratory Health Survey (ECRHS)." European Respiratory Journal 10 (11): 2495-2501.

Cooper, R. S., B. Osotimehin, J. S. Kaufman, and T. Forrester. 1998. "Disease Burden in Sub-Saharan Africa: What Should We Conclude in the Absence of Data?" Lancet 351 (9097): 208-10.

Corbett, E. L, C. J. Watt, N. Walker, D. Maher, B. G. Williams, M. C. Raviglione, and C. Dye. 2003. "The Growing Burden of Tuberculosis: Global Trends and Interactions with the HIV Epidemic." Archives of Internal Medicine 163: 1009-21.

Crowcroft, N. S., C. Stein, P. Duclos, and M. Birmingham. 2003. "How Best to Estimate the Global Burden of Pertussis?" Lancet Infectious Diseases 3 (7): 413-8.

Degenhardt, L., W. Hall, M. Warner-Smith, and M. Lynskey. 2003. "Illicit Drugs." In Comparative Quantification of Health Risks: Global and Regional Burden of Disease Attributable to Selected Major Risk Factors, ed. M. Ezzati, A. D. Lopez, A. Rodgers, and C. J. L. Murray, 1109-75. Geneva: World Health Organization.

Dempsey, M. 1947. "Decline in Tuberculosis: The Death Rate Fails to Tell the Entire Story." American Review of Tuberculosis 56: 143-51. de Onis, M., and M. Blossner. 2003. "The World Health Organization Global Database on Child Growth and Malnutrition: Methodology and Applications." International Journal of Epidemiology 32 (4): 518-26.

de Onis, M., E. A. Frongillo, and M. Blossner. 2000. "Is Malnutrition Declining? An Analysis of Changes in Levels of Child Malnutrition Since 1980." Bulletin of the World Health Organization 78 (10): 1222-33.

de Onis, M., M. Blossner, E. Borghi, R. Morris, and E. A. Frongillo. 2004. "Methodology for Estimating Regional and Global Trends of Child Malnutrition." International Journal of Epidemiology 33 (6): 1260-70.

de Silva, N. R., S. Brooker, P. J. Hotez, A. Montresor, D. Engels, and L. Savioli. 2003. "Soil-Transmitted Helminth Infections: Updating the Global Picture." Trends in Parasitology 19 (12): 547-51.

Dolea, C., and C. AbouZahr. 2003a. "Global Burden of Hypertensive Disorders in Pregnancy in the Year 2000." GBD 2000 Working Paper, World Health Organization, Geneva. http://www.who.int/ evidence/ bod.

2003b. "Global Burden of Obstructed Labor in the Year 2000." GBD 2000 Working Paper, World Health Organization, Geneva. http:// www.who.int/evidence/bod.

Dolea, C., and C. Stein. 2003. "Global Burden of Maternal Sepsis in the Year 2000." GBD 2000 Working Paper, World Health Organization, Geneva. http://www.who.int/evidence/bod.

Dolea, C., C. AbouZahr, and C. Stein. 2003. "Global Burden of Maternal Hemorrhage in the Year 2000." GBD 2000 Working Paper, World Health Organization, Geneva. http://www.who.int/evidence/bod.

Dolin, P. J., H. Faal, G. J. Johnson, D. Minassian, S. Sowa, S. Day, J. Ajewale, A. A. Mohamed, and A. Foster. 1997. "Reduction of Trachoma in a SubSaharan Village in Absence of a Disease Control Programme." Lancet 349 (9064): 1511-2.

Doumenge, J. P., K. E. Mott, C. Cheung, D. Villenave, O. Chapuis, M. F. Perrin, and G. Reaud-Thomas. 1987. CEGET/WHO Atlas de la Répartition Mondiale des Schistosomiases (Atlas of the Global Distribution of Schistosomiasis). Bordeaux, France: Presses Universitaires de Bordeaux.

Dye, C., S. Scheele, P. Dolin, V. Pathania, and M. C. Raviglione. 1999. "Global Burden of Tuberculosis: Estimated Incidence, Prevalence, and Mortality by Country." Journal of the American Medical Association 282 (7): 677-86.

European Monitoring Centre for Drugs and Drug Addiction. 2002. Annual Report on the State of the Drug Problem in the European Union and Norway. Lisbon: European Monitoring Centre for Drugs and Drug Addiction.

Ezzati, M., A. D. Lopez, S. Vander Hoorn, A. Rodgers, C. J. L Murray, and Comparative Risk Assessment Collaborative Group. 2002. "Selected Major Risk Factors and Global and Regional Burden of Disease." Lancet 360 (9343): 1347-60.

Ezzati, M., S. Vander Hoorn, A. Rodgers, A. D. Lopez, C. D. Mathers, C. J. L. Murray, and Comparative Risk Factors Collaborating Group. 2003. "Estimates of Global and Regional Potential Health Gains from Reducing Multiple Major Risk Factors.” Lancet 362 (9380): 271-80.

Ferlay, J., F. Bray, P. Pisani, and D. M. Parkin. 2001. “Globocan 2000: Cancer Incidence, Mortality, and Prevalence Worldwide." Version 1.0. International Agency for Research on Cancer CancerBase No. 5. Lyon, France: International Agency for Research on Cancer Press.

Fewtrell, L. J., A. Pruss-Ustun, P. Landrigan, and J. L Ayuso-Mateos. 2004. "Estimating the Global Burden of Disease of Mild Mental Retardation and Cardiovascular Diseases from Environmental Lead Exposure." Environmental Research 94 (2): 120-33.

Field, M. J., and G. M. Gold, eds. 1998. Summarizing Population Health: Directions for the Development and Application of Population Metrics. Washington, DC: National Academy Press. 
Frick, K., E. Basilion, C. Hanson, and A. Colchero. 2003. "Estimating the Burden and Economic Impact of Trachomatous Visual Loss." Ophthalmic Epidemiology 10 (2): 121-32.

Gajalakshmi, V., R. Peto, S. Kanaka, and S. Balasubramian. 2002. "Verbal Autopsy of 48,000 Adult Deaths Attributable to Medical Causes in Chennai (formerly Madras).” BioMed Central 16, no. 2(1): 7.

Galazka, A. M., and S. E. Robertson. 2004. "Pertussis." In The Global Epidemiology of Infectious Diseases, ed. C. J. L. Murray, A. D. Lopez, and C. D. Mathers, 29-54. Vol. 4 of Global Burden of Disease and Injury Series. Geneva: World Health Organization. http://whqlibdoc. who.int/publications/2004/9241592303.pdf.

Gavrilova, N. S., V. G. Semyonova, G. N. Evdokushkina, and L. A. Gavrilov. 2000. "The Response of Violent Mortality to Economic Crisis in Russia." Population Research and Policy Review 19 (5): 397-419.

Gerbase, A. C., J. T. Rowley, D. H. L. Heymann, S. F. B. Berkley, and P. Piot. 1998. "Global Prevalence and Incidence Estimates of Selected Curable STDs." Sexually Transmitted Diseases 74 (Suppl 1): S12-S16.

Ghana Health Assessment Project Team. 1981. "A Quantitative Method of Assessing the Health Impact of Different Diseases in Less Developed Countries." International Journal of Epidemiology 10 (1): 72-80.

Gleditsch, N. P., P. Wallensteen, M. Eriksson, M. Sollenberg, and H. Strand. 2002. "Armed Conflict 1946-2001: A New Dataset." Journal of Peace Research 39 (5): 615-37.

Global Burden of Hepatitis C Working Group. 2004. "Global Burden of Disease (GBD) for Hepatitis C." Journal of Clinical Pharmacology 44 (1): 20-9.

Handicap International. 2001. Landmine Victim Assistance: World Report 2001. Lyon, France: Handicap International.

Hill, K., C. AbouZahr, and T. Wardlaw. 2001. "Estimates of Maternal Mortality for 1995." Bulletin of the World Health Organization 79 (3): 182-93.

Horton, R. 2005. “The Ellison Institute: Monitoring Health, Challenging WHO." Lancet 366 (9481): 179-81.

Human Rights Watch. 2001. Landmine Monitor Report: 2001-Toward a Mine-Free World. New York: Human Rights Watch.

Hyder, A. A., G. Rotllanat, and R. H. Morrow. 1998. "Measuring the Burden of Disease: Healthy Life-Years." American Journal of Public Health 88 (2): 196-202.

ISAAC Steering Committee. 1998a. "Worldwide Variation in the Prevalence of Symptoms of Asthma, Allergic Rhinoconjunctivitis, and Atopic Eczema: The International Study of Asthma and Allergies in Childhood (ISAAC).” Lancet 351 (9111): 1225-32.

1998b. "Worldwide Variations in the Prevalence of Asthma Symptoms: The International Study of Asthma and Allergies in Childhood (ISAAC)." European Respiratory Journal 12 (2): 315-35.

Jamison, D. T. 1996. "Foreword to the Global Burden of Disease and Injury Series." In The Global Burden of Disease: A Comprehensive Assessment of Mortality and Disability from Diseases, Injuries, and Risk Factors in 1990 and Projected to 2020, ed. C. J. L. Murray and A. D. Lopez, xvi-xxiii. Vol. 1 of Global Burden of Disease and Injury. Cambridge, MA: Harvard School of Public Health.

Jamison, D. T., W. H. Mosley, A. R. Measham, and J. L. Bobadilla. 1993. Disease Control Priorities in Developing Countries. New York: Oxford University Press.

Jamison, D. T., J.G. Breman, A. R. Measham, G. Alleyne, M. Claeson, D. B. Evans, P. Jha, and P. Mills and P. Mubgrove. 2006. Disease Control Priorities in Developing Countries, 2nd ed. New York: Oxford University Press.

Karvonen, M., M. Viik-Kajander, E. Moltchanova, I. Libman, R. LaPorte, and J. Tuomilehto. 2000. "Incidence of Childhood Type 1 Diabetes Worldwide. Diabetes Mondiale (DiaMond) Project Group." Diabetes Care 23 (10): 1516-26.
Katz, J., and G. King. 1999. "A Statistical Model for Multiparty Electoral Data." American Political Science Review 93 (1): 15-32.

Kauhanen, J., G. A. Kaplan, D. E. Goldberg, and J. T. Salonen. 1997. "Beer Binging and Mortality: Results from the Kuopio Ischaemic Heart Disease Risk Factor Study, A Prospective Population-Based Study." British Medical Journal 315 (7112): 846-51.

Korenromp, E. L. 2005. "Malaria Incidence Estimates at Country Level for the Year 2004." Proposed estimates and draft report, World Health Organization, Roll Back Malaria Monitoring and Evaluation Reference Group and Malaria Epidemiology Reference Group Task Force on Malaria Morbidity, Geneva.

Korenromp, E., B. G. Williams, E. Gouws, C. Dye, and R. W. Snow. 2003. "Measurement of Trends in Childhood Malaria Mortality in Africa: An Assessment of Progress Toward Targets Based on Verbal Autopsy." Lancet Infectious Diseases 3 (6): 349-58.

Kruijshaar, M., N. Hoeymans, J. Skijker, M. E. A. Stouthard, and M. EssinkBot. 2005. "Has the Burden of Depression Been Overestimated?" Bulletin of the World Health Organization 83 (8): 443-8.

Lavanchy, D. 2004. "Hepatitis B Virus Epidemiology, Disease Burden, Treatment, and Current and Emerging Prevention and Control Measures." Journal of Viral Hepatitis 11 (2): 97-107.

Lawn, J. E., S. Cousens, and J. Zupan. 2005. "Four Million Neonatal Deaths: When? Where? Why?" Lancet 365 (9462): 891-900.

LeDuc, J. W., K. Esteves, and N. G. Gratz. 2004. "Dengue and Dengue Haemorrhagic Fever." In The Global Epidemiology of Infectious Diseases, ed. C. J. L. Murray, A. D. Lopez, and C. D. Mathers, 219-42. Vol. 4 of Global Burden of Disease and Injury Series. Geneva: World Health Organization. http://whqlibdoc.who.int/publications/2004/ 9241592303.pdf.

Leonardi, M., and C. D. Mathers. 2003. "Global Burden of Migraine in the Year 2000: Summary of Methods and Data Sources." GBD 2000 Working Paper, World Health Organization, Geneva. http://www. who.int/evidence/bod.

Lopez, A. D., K. Shibuya, C. Rao, and C. D. Mathers. Forthcoming. "Estimating the Burden of Chronic Obstructive Pulmonary Disease: Methods and Results from the Global Burden of Disease Study." European Respiratory Journal.

Lopez, A. D., O. Ahmad, M. Guillot, B. Ferguson, J. Salomon, C. J. L. Murray, and K. H. Hill. 2002. World Mortality in 2000: Life Tables for 191 Countries. Geneva: World Health Organization.

Lozano, R., C. J. L. Murray, J. Frenk, and J. Bobadilla. 1995. "Burden of Disease Assessment and Health System Reform: Results of a Study in Mexico." Journal of International Development 7 (3): 555-64.

Lozano, R., C. J. L. Murray, A. D. Lopez, and T. Satoh. 2001. "Miscoding and Misclassification of Ischaemic Heart Disease Mortality." Global Program on Evidence for Health Policy Discussion Paper 12, World Health Organization, Geneva. http://www.who.int/evidence.

Mahapatra, P. 2002. Estimating National Burden of Disease: The Burden of Disease in Andhra Pradesh, 1990's. Hyderabad, India: Institute of Health Systems.

Mari Bhat, P. N. 2002. "Completeness of India's Sample Registration System: An Assessment Using the General Growth Balance Method." Population Studies 56 (2): 119-34.

Marshall, M. G., and T. R. Gurr. 2003. Peace and Conflict 2003: A Global Survey of Armed Conflicts, Self-Determination Movements, and Democracy. College Park, MD: Center for International Development and Conflict Management.

Mathers, C. D., and J. L. Ayuso-Mateos. 2003. "Global Burden of Alcohol Use Disorders in the Year 2000: Summary of Methods and Data Sources.” GBD 2000 Working Paper, World Health Organization, Geneva. http://www.who.int/evidence/bod.

Mathers, C. D., and A. de Francisco. 2004. “The Global Burden of Disease.” In Monitoring Financial Flows for Health Research, vol. 2, ed. Global 
Forum for Health Research, 55-67. Geneva: Global Forum for Health Research.

Mathers, C. D., and M. Leonardi. 2003. "Global Burden of Dementia in the Year 2000: Summary of Methods and Data Sources." GBD 2000 Working Paper, World Health Organization, Geneva. http://www. who.int/evidence/bod.

Mathers, C. D., C. J. L. Murray, and J. A. Salomon. 2003. "Methods for Measuring Healthy Life Expectancy." In Health Systems Performance Assessment: Debates, Methods, and Empiricism, ed. C. J. L. Murray and D. Evans, 437-70. Geneva: World Health Organization.

Mathers, C. D., A. Smith, and M. Concha. 2003. "Global Burden of Hearing Loss in the Year 2000." GBD 2000 Working Paper, World Health Organization, Geneva. http://www.who.int/evidence/bod.

Mathers, C. D., T. Vos, and C. Stevenson. 1999. The Burden of Disease and Injury in Australia. Canberra: Australian Institute of Health and Welfare.

Mathers, C. D., J. A. Salomon, C. J. L. Murray, and A. D. Lopez. 2003. "Alternative Summary Measures of Average Population Health." In Health Systems Performance Assessment: Debates, Methods, and Empiricism, ed. C. J. L. Murray and D. Evans, 319-34. Geneva: World Health Organization.

Mathers, C. D., T. Truelsen, S. Begg, and T. Satoh. 2004. “Global Burden of Ischaemic Heart Disease in the Year 2000.” GBD 2000 Working Paper, World Health Organization, Geneva. http://www.who.int/evidence/ bod.

Mathers, C. D., M. Ezzati, A. D. Lopez, C. J. L Murray, and A. Rogers. 2002. "Causal Decomposition of Summary Measures of Population Health." In Summary Measures of Population Health: Concepts, Ethics, Measurement, and Applications, ed. C. J. L. Murray, J. A. Salomon, C. D. Mathers, and A. D. Lopez, 273-90. Geneva: World Health Organization. http://www.who.int/pub/smph/en/index.html.

Mathers, C. D., D. Ma Fat, M. Inoue, C. Rao, and A. D. Lopez. 2005. "Counting the Dead and What They Died from: An Assessment of the Global Status of Cause of Death Data." Bulletin of the World Health Organization 83 (3): 171-7.

Mathers, C. D., C. J. L. Murray, A. D. Lopez, J. A. Salomon, and R. Sadana. 2003. "Global Patterns of Health Expectancy in the Year 2000." In Determining Health Expectancies, ed. J. M. Robine, C. Jagger, C. D. Mathers, E. M. Crimmins, and R. M. Suzman, 335-58. Chichester, U.K.: John Wiley \& Sons.

Mathers, C. D., K. Shibuya, C. Boschi-Pinto, A. D. Lopez, and C. J. Murray. 2002. "Global and Regional Estimates of Cancer Mortality and Incidence by Site: I. Application of Regional Cancer Survival Model to Estimate Cancer Mortality Distribution by Site." BMC Cancer 2 (1): 36.

Mathers, C. D., A. D. Lopez, C. Stein, D. Ma Fat, C. Rao, M. Inoue, K. Shibuya, N. Tomijima, C. Bernard and H. Xu. 2004. "Deaths and Disease Burden by Cause: Global Burden of Disease Estimates for 2001 by World Bank Country Groups." Working Papers Series 18, Second Project on Disease Control Priorities in Developing Countries, World Health Organization; World Bank; and Fogarty International Center, U.S. National Institutes of Health, Washington, DC.

McDowell, I., and C. Newell. 1996. Measuring Health. A Guide to Rating Scales and Questionnaires, 2nd ed. Oxford, U.K.: Oxford University Press.

McKenna, M. T., C. M. Michaud, C. J. L. Murray, and J. S. Marks. 2005. "Assessing the Burden of Disease in the United States Using DisabilityAdjusted Life Years." American Journal of Preventive Medicine 28 (5): $415-23$.

Men, T., P. Brennan, P. Boffetta, and D. Zaridze. 2003. "Russian Mortality Trends for 1991-2001: Analysis by Cause and Region." British Medical Journal 327 (7421): 964.

Ministry of Public Health. 2002. Burden of Disease and Injuries in Thailand: Priority Setting for Policy. Bangkok: Government of Thailand.
Moncayo, A. 2003. “Chagas' Disease: Current Epidemiological Trends after the Interruption of Vectorial and Transfusional Transmission in the Southern Cone Countries." Memórias do Instituto Oswaldo Cruz 98 (5): 577-91.

Moncayo, A., F. Guhl, and C. Stein. 2002. "The Global Burden of Chagas' Disease in the Year 2000." GBD 2000 Working Paper, World Health Organization, Geneva. http://www.who.int/evidence/bod.

Murray, C. J. L. 1996. "Rethinking DALYs.” In The Global Burden of Disease, ed. C. J. L. Murray and A. D. Lopez, 1-98. Vol. 1 of Global Burden of Disease and Injury Series. Cambridge, MA: Harvard University Press.

Murray, C. J. L., and A. K. Acharya. 1997. "Understanding DALYs." Journal of Health Economics 16 (6): 703-30.

Murray, C. J. L., and A. D. Lopez. 1996a. "Estimating Causes of Death: New Methods and Global and Regional Applications for 1990." In The Global Burden of Disease, ed. C. J. L. Murray and A. D. Lopez, 117-200. Vol. 1 of Global Burden of Disease and Injury Series. Cambridge, MA: Harvard University Press.

1996b. "Evidence-Based Health Policy: Lessons from the Global Burden of Disease Study." Science 274 (5288):740-3.

eds. 1996c. The Global Burden of Disease: A Comprehensive Assessment of Mortality and Disability from Diseases, Injuries, and Risk Factors in 1990 and Projected to 2020. Vol. 1 of Global Burden of Disease and Injury Series. Cambridge, MA: Harvard University Press.

1996d. Global Health Statistics. Vol. 2 of Global Burden of Disease and Injury Series. Cambridge, MA: Harvard University Press.

1997a. "Global Mortality, Disability, and the Contribution of Risk Factors: Global Burden of Disease Study." Lancet 349 (9063): 1436-42.

1997b. "Mortality by Cause for Eight Regions of the World:

Global Burden of Disease Study." Lancet 349 (9061): 1269-76.

eds. 1998. Health Dimensions of Sex and Reproduction. Vol. 3 of Global Burden of Disease and Injury Series. Cambridge, MA: Harvard University Press.

2000. "Progress and Directions in Refining the Global Burden of Disease Approach: A Response to Williams." Health Economics 9 (1): 69-82.

Murray, C. J. L., A. D. Lopez, and D. T. Jamison. 1994. “The Global Burden of Disease in 1990: Summary Results, Sensitivity Analysis, and Future Directions." Bulletin of the World Health Organization 72 (3): 495-509.

Murray, C. J., A. D. Lopez, and S. Wibulpolprasert. 2004. "Monitoring Global Health: Time for New Solutions." British Medical Journal 329 (7333): 1096-100.

Murray, C. J. L, C. D. Mathers, and J. A. Salomon. 2003. "Towards Evidence-based Public Health." In Health Systems Performance Assessment: Debates, Methods, and Empiricism, ed. C. J. L. Murray and D. Evans, 715-26. Geneva: World Health Organization.

Murray, C. J. L., J. A. Salomon, and C. D. Mathers. 2000. "A Critical Examination of Summary Measures of Population Health." Bulletin of the World Health Organization 78 (8): 981-94.

Murray, C. J. L, J. A. Salomon, C. D. Mathers, and A. D. Lopez, eds. 2002a Summary Measures of Population Health: Concepts, Ethics, Measurement, and Applications. Geneva: World Health Organization.

2002b. "Summary Measures of Population Health: Conclusions and Recommendations." In Summary Measures of Population Health: Concepts, Ethics, Measurement, and Applications, ed. C. J. L. Murray, J. A. Salomon, C. D. Mathers, and A. D. Lopez, 731-56. Geneva: World Health Organization.

Murray, C. J. L., A. Tandon, J. A. Salomon, and C. D. Mathers. 2002. "New Approaches to Enhance Cross-Population Comparability of Survey Results." In Summary Measures of Population Health: Concepts, Ethics, Measurement, and Applications, ed. C. J. L. Murray, J. A. Salomon, C. D. Mathers, and A. D. Lopez, 421-31. Geneva: World Health Organization. 
Murray, C. J., G. King, A. D. Lopez, N. Tomijima, and E. G. Krug. 2002. "Armed Conflict as a Public Health Problem." British Medical Journal 324 (7333): 346-9.

Murray, C. J. L., B. D. Ferguson, A. D. Lopez, M. Guillot, J. A. Salomon, and O. Ahmad. 2003. "Modified Logit Life Table System: Principles, Empirical Validation, and Application." Population Studies 57 (2): 1-18.

Pearce, N., J. Sunyer, S. Cheng, S. Chinn, B. Bjorksten, M. Burr, U. Keil, H. R. Anderson, and P. Burney. 2000. "Comparison of Asthma Prevalence in the ISAAC and the ECRHS. ISAAC Steering Committee and the European Community Respiratory Health Survey. International Study of Asthma and Allergies in Childhood." European Respiratory Journal 16 (3): 420-6.

Peto, R., A. D. Lopez, J. Boreham, M. Thun, and C. Heath. 1992. "Mortality from Tobacco in Developed Countries: Indirect Estimation from National Vital Statistics." Lancet 339 (8804): 1268-78.

Preston, S. H. 1976. Mortality Patterns in National Populations. New York: Academic Press.

Project Ploughshares. 2001. Armed Conflicts Report 2001. Waterloo, Canada: Project Ploughshares.

2002. Armed Conflicts Report 2002. Waterloo, Canada: Project Ploughshares.

Pruss-Ustun, A., L. J. Fewtrell, P. Landrigan, and J. L Ayuso-Mateos. 2004. "Lead Exposure." In Comparative Quantification of Health Risks: Global and Regional Burden of Disease Attributable to Selected Major Risk Factors, ed. M. Ezzati, A. D. Lopez, A. Rodgers, and C. J. L Murray, 1495-592. Geneva: World Health Organization.

Ranson, M. K., and T. G. Evans. 1995. "The Global Burden of Trachomatous Visual Impairment: I. Assessing Prevalence." International Ophthalmology 19 (5): 261-70.

Rao, C., D. Bradshaw, and C. D. Mathers. 2004. "Improving Death Registration and Statistics in Developing Countries: Lessons from SubSaharan Africa." Southern African Journal of Demography 9 (2): 79-97.

Rastogi, T., and C. D. Mathers. 2002a. "Global Burden of Iodine Deficiency Disorders in the Year 2000." GBD 2000 Working Paper, World Health Organization, Geneva. http://www.who.int/evidence/bod.

2002b. "Global Burden of Iron Deficiency Anaemia in the Year 2000." GBD 2000 Working Paper, World Health Organization, Geneva. http://www.who.int/evidence/bod.

.2002c. "Global Burden of Vitamin A Deficiency in the Year 2000." GBD 2000 Working Paper, World Health Organization, Geneva. http://www.who.int/evidence/bod.

Rehm, J., R. Room, M. Monteiro, G. Gmel, K. Graham, N. Rehn, C. Sempos, U. Frick, and D. Jernigan. 2004. "Alcohol Use." In Comparative Quantification of Health Risks: Global and Regional Burden of Disease Attributable to Selected Major Risk Factors, ed. M. Ezzati, A. D. Lopez, A. Rodgers, and C. J. L Murray, 959-1108. Geneva: World Health Organization.

Resnikoff, S., D. Pascolini, D. Etya’ale, I. Kocur, R. Pararajasegaram, G. P. Pokharel, and S. P. Mariotti. 2004. "Global Data on Visual Impairment in the Year 2002." Bulletin of the World Health Organization 82 (11): 844-51.

Richards, F. O., B. Boatin, M. Sauerbrey, and A. Seketeli. 2001. "Control of Onchocerciasis Today: Status and Challenges." Trends in Parasitology 17 (12): 558-63.

Ries, L. A. G., M. P. Eisner, C. L. Kosary, B. F. Hankey, B. A. Biller, L. Clegg, B. K. Edwards, eds. 2002. SEER Cancer Statistics Review, 1973-1999. Bethesda, MD: National Cancer Institute.

Robine, J. M., C. Jagger, C. D. Mathers, E. M. Crimmins, and R. M. Suzman. 2003. Determining Health Expectancies. Chichester, U.K.: John Wiley \& Sons.

Roglic, G., N. Unwin, P. H. Bennett, C. D. Mathers, J. Tuomilheto, and H. King. 2005. "The Realistic Burden of Mortality Attributable to Diabetes: Estimates for the Year 2000." Diabetes Care 28 (9): 2130-5.
Rowe, A. K., R. W. Steketee, S. Y. Rowe, R. W. Snow, E. Korenromp, J. A. Schellenberg, C. Stein, B. Nahler, J. Bryce, and R. Black. 2004. "Estimates of the Burden of Mortality Directly Attributable to Malaria for Children under Five Years of Age in Africa for the Year 2000." Final report for the Child Health Epidemiology Reference Group. http://www.who.int/child-adolescent-health/New_Publications/ CHILD_HEALTH/EPI/CHERG_Malaria_Mortality.pdf.

Rudan, I., L. Tomaskovic, C. Boschi-Pinto, and H. Campbell. 2004. "Global Estimate of the Incidence of Clinical Pneumonia among Children under Five Years of Age." Bulletin of the World Health Organization 82 (12): 895-903.

Sadana, R. 2002. "Development of Standardized Health State Descriptions." In Summary Measures of Population Health: Concepts, Ethics, Measurement, and Applications, ed. C. J. L. Murray, J. A. Salomon, C. D. Mathers, and A. D. Lopez, 315-28. Geneva: World Health Organization.

Sadana, R., C. D. Mathers, A. D. Lopez, C. J. L Murray, and K. MoesgaardIburg. 2002. "Comparative Analyses of More Than 50 Household Surveys on Health Status." In Summary Measures of Population Health: Concepts, Ethics, Measurement, and Applications, ed. C. J. L. Murray, J. A. Salomon, C. D. Mathers, and A. D. Lopez, 369-86. Geneva: World Health Organization.

Salomon, J. A., and C. J. L. Murray. 2001a. "Compositional Models for Mortality by Age, Sex, and Cause." Global Program on Evidence for Health Policy Discussion Paper 11, World Health Organization, Geneva. http://www.who.int/evidence.

. 2001b. "Modelling HIV/AIDS Epidemics in Sub-Saharan Africa Using Seroprevalence Data from Antenatal Clinics." Bulletin of the World Health Organization 79 (7): 596-607.

2002a. "The Epidemiologic Transition Revisited: Compositional Models for Causes of Death by Age and Sex." Population and Development Review 28 (2): 205-28.

2002b. "Estimating Health State Valuations Using a MultipleMethod Protocol." In Summary Measures of Population Health: Concepts, Ethics, Measurement, and Applications, ed. C. J. L. Murray, J. A. Salomon, C. D. Mathers, and A. D. Lopez, 487-99. Geneva: World Health Organization.

2004. "A Multimethod Approach to Estimating Health State Valuations." Health Economics 13 (3): 281-90

Salomon, J. A., C. J. L. Murray, T. B. Ustun, and S. Chatterji. 2003. "Health State Valuations in Summary Measures of Population Health.” In Health Systems Performance Assessment: Debate, Methods, and Empiricism, ed. C. J. L. Murray and D. Evans, 409-36. Geneva: World Health Organization.

Salomon, J. A., C. D. Mathers, S. Chatterji, R. Sadana, T. B. Ustun, and C. J. L. Murray. 2003. "Quantifying Individual Levels of Health: Definitions, Concepts, and Measurement Issues." In Health Systems Performance Assessment: Debate, Methods, and Empiricism, ed. C. J. L. Murray, and D. Evans, 301-18. Geneva: World Health Organization.

Schwartlander, B., K. A. Stanecki, T. Brown, P. O. Way, R. Monasch, J. Chin, D. Tarantola, and N. Walker. 1999. "Country-Specific Estimates and Models of HIV and AIDS: Methods and Limitations." AIDS 13 (17): 2445-58.

Semenova, V. G., N. S. Gavrilova, Y. A. Varavikova, L. A. Gavrilov, and G. N. Yevdokushkina. 2000. "Rise in Violent Death Rates in Russia as a Consequence of the Economic Crisis." Diseases Prevention and Health Promotion 4: 3-10.

Setel, P. W., O. Sankoh, C. Rao, V. A. Velkoff, C. D. Mathers, Y. Gonghuan, I. Hemed, P. Jha, and A. D. Lopez. 2005. "Sample Registration of Vital Events with Verbal Autopsy: A Renewed Commitment to Measuring and Monitoring Vital Statistics." Bulletin of the World Health Organization 83 (8): 611-17.

Shibuya, K., and M. Ezzati. 2003. "Global Burden of Onchocerciasis in the Year 2000: Summary of Methods and Data Sources." GBD 2000 
Working Paper, World Health Organization, Geneva. http://www .who.int/evidence/bod.

Shibuya, K., and C. D. Mathers. 2003. "Global Burden of Trachoma in the Year 2000: Summary of Methods and Data Sources." GBD 2000 Working Paper, World Health Organization, Geneva. http://www .who.int/evidence/bod.

Shibuya, K., C. D. Mathers, C. Boschi-Pinto, A. D. Lopez, and C. J. L. Murray. 2002. "Global and Regional Estimates of Cancer Mortality and Incidence by Site: II. Results for the Global Burden of Disease Study 2000." BMC Cancer 2: 37.

Shkolnikov, V., M. McKee, and D. A. Leon. 2001. "Changes in Life Expectancy in Russia in the Mid-1990s." Lancet 357 (9260): 917-21.

Single, E., L. Robson, X. Xie, and J. Rehm. 2002. The Costs of Substance Abuse in Canada. Ottawa: Canadian Centre on Substance Abuse.

Snow, R. W., M. Craig, U. Deichmann, and K. Marsh. 1999. "Estimating Mortality, Morbidity, and Disability Due to Malaria among Africa's Non-pregnant Population." Bulletin of the World Health Organization 77 (8): 624-40.

Stein, C. 2002a. "Global Burden of Leprosy in the Year 2000." GBD 2000 Working Paper, World Health Organization, Geneva. http://www .who.int/evidence/bod.

2002b. "Global Burden of Poliomyelitis in the Year 2000." GBD 2000 Working Paper, World Health Organization, Geneva. http://www. who.int/evidence/bod.

2002c. "Global Burden of Protein-Energy Malnutrition in the Year 2000.” GBD 2000 Working Paper, World Health Organization, Geneva. http://www.who.int/evidence/bod.

Stein, C., and M. Robertson. 2002. "Global Burden of Diphtheria in the Year 2000.” GBD 2000 Working Paper, World Health Organization, Geneva. http://www.who.int/evidence/bod.

Stouthard, M., M. Essink-Bot, G. Bonsel, J. Barendregt, and P. Kramers. 1997. Disability Weights for Diseases in the Netherlands. Rotterdam, Netherlands: Erasmus University, Department of Public Health.

Stover, J., N. Walker, G. P. Garnett, J. A. Salomon, K. A. Stanecki, P. D. Ghys, N. C. Grassly, R. M. Anderson, and B. Schwartlander. 2002. "Can We Reverse the HIV/AIDS Pandemic with an Expanded Response?" Lancet 360 (9326): 73-77.

Symmons, D. P., C. D. Mathers, and B. Pfleger. 2002a. "The Global Burden of Osteoarthritis in the Year 2000.” GBD 2000 Working Paper, World Health Organization, Geneva. http://www.who.int/evidence/bod.

. 2002b. "The Global Burden of Rheumatoid Arthritis in the Year 2000." GBD 2000 Working Paper, World Health Organization, Geneva. http://www.who.int/evidence/bod.

Tan-Torres Edejer, T. R. Baltussen, T. Adam, R. Hutubessy, A Acharya, D. B. Evans, and C. J. L. Murray. 2003. WHO Guide to Cost-Effectiveness Analysis. Geneva: World Health Organization.

Thylefors, B., A. D. Negrel, R. Pararajasegaram, and K. Y. Dadzie. 1995. "Global Data on Blindness." Bulletin of the World Health Organization 73 (1): 115-21.

Truelsen, T., S. Begg, C. D. Mathers, and T. Satoh. 2002. "Global Burden of Cerebrovascular Disease in the Year 2000." GBD 2000 Working Paper, World Health Organization, Geneva. http://www.who.int/ evidence/bod.

UNAIDS (Joint United Nations Programme on HIV/AIDS) Reference Group on Estimates Model and Projections. 2002. "Improved Methods and Assumptions for Estimation and Projection of HIV/AIDS Epidemics." AIDS 16: 1-16.

UNICEF (United Nations Children's Fund) and WHO (World Health Organization). 2005. Low Birthweight: Country, Regional, and Global Estimates. New York: UNICEF.

United Nations Population Division. 2003. World Population Prospects: The 2002 Revision. New York: United Nations.
Ustun, T. B., and D. Chisholm. 2001. "Global Burden of Disease Study for Psychiatric Disorders.” Psychiatrisch Prax 28 (Suppl 1): S7-S11.

Ustun, T. B., J. L. Ayuso-Mateos, S. Chatterji, C. D. Mathers, and C. J. L. Murray. 2005. "Global Burden of Depressive Disorders in the Year 2000." British Journal of Psychiatry 184: 386-92.

Ustun, T. B., S. Chatterji, A. Mechbal, C. J. L. Murray, and World Health Survey Collaborating Groups. 2003. "The World Health Surveys." In Health Systems Performance Assessment: Debates, Methods, and Empiricism, ed. C. J. L. Murray and D. Evans, 797-808. Geneva: World Health Organization.

Ustun, T. B., S. Chatterji, M. Villanueva, L. Bendib, C. Celik, R. Sadana, N. Valentine, C. Mathers, J. P. Ortiz, A. Tandon, J. Salomon, Y. Cao, X. W. Jun, and C. J. L. Murray. 2003. "The WHO Multicountry Household Survey Study on Health and Responsiveness 2000-2001." In Health Systems Performance Assessment: Debates, Methods, and Empiricism, ed. C. J. L. Murray and D. Evans, 761-96. Geneva: World Health Organization.

van der Werf, M. J., and S. J. de Vlas. 2001. Morbidity and Infection with Schistosomes or Soil-Transmitted Helminths. Report prepared for the World Health Organization Parasitic Diseases and Vector Control. Rotterdam, Netherlands: Erasmus University.

Vos, T., M. Tobias, H. Gareeboo, F. Roussety, S. Huttley, and C. J. L. Murray. 1995. Mauritius HealthSectorReform, National Burden ofDisease Study, Final Report of Consultancy. Port Louis, Mauritius: Ministry of Health and Ministry of Economic Planning and Development.

Walker, N., K. A. Stanecki, T. Brown, J. Stover, S. Lazzari, J. M. Garcia-Calleja, B. Schwartlander, and P. D. Ghys. 2003. "Methods and Procedures for Estimating HIV/AIDS and Its Impact: The UNAIDS/WHO Estimates for the End of 2001." AIDS 17 (15): 2215-25.

Ware, J. E., and C. D. Sherbourne. 1992. "The MOS 36-Item Short Form Health Survey (SF-36): I. Conceptual Framework and Item Selection." Medical Care 30 (6): 473-83.

Warren, S., and K. G. Warren. 2001. Multiple Sclerosis. Geneva: World Health Organization.

WHO (World Health Organization). 1977. Manual of the International Statistical Classification of Diseases, Injuries, and Causes of Death, 9th rev, vol. 1. Geneva: WHO.

1992. International Classification of Diseases and Related Health Problems, 10th rev. Geneva: WHO.

1995. "Onchocerciasis and Its Control: Report of a WHO Expert Committee on Onchocerciasis Control." WHO Technical Report Series 852, World Health Organization, Geneva.

1996. Investing in Health Research and Development. Report of the Ad Hoc Committee on Health Research Relating to Future Intervention Options. Geneva: WHO.

2000. World Health Report 2000. Health Systems: Improving Performance. Geneva: WHO.

2001a. Assessment of Iodine Deficiency Disorders and Monitoring their Elimination. Document WHO/NHD/01.1. Geneva: WHO.

2001b. "Epidemics of Meningococcal Disease, African Meningitis Belt, 2001.” Weekly Epidemiological Record 76 (37): 281-8.

2001c. Global Prevalence and Incidence of Selected Curable Sexually Transmitted Infections: Overview and Estimates. WHO/CDS/CSR/ EDC/2001.10. Geneva: WHO.

2001d. World Health Report 2001: Mental Health-New Understanding, New Hope. Geneva: WHO.

2002a. "Global Distribution of Hepatitis A, B, and C, 2001." Weekly Epidemiological Record 77 (6): 41-8.

2002b. Hepatitis B. WHO/CDS/CSR/LYO/2002.2:Hepatitis B. Geneva: WHO, Department of Communicable Diseases Surveillance and Response. 
.2002c. "Leprosy: Global Situation." Weekly Epidemiological Record 77 (1): $1-8$.

2002d. World Health Report 2002. Reducing Risks, Promoting Healthy Life. Geneva: WHO.

2003a. Global Tuberculosis Control: Surveillance, Planning, and Financing. Geneva: WHO. WHO.

2003b. World Health Report 2003: Shaping the Future. Geneva:

. 2004a. Unsafe Abortion. Geneva: WHO.

. 2004b. World Health Report 2004: Changing History. Geneva: WHO.

WHO Nutrition Program. 2002a. "Database on Anaemia." Geneva: WHO. http://www.who.int/nut/db_mdis.htm.

2002b. "Database on Vitamin A Deficiency." Geneva: WHO. http://www.who.int/nut/db_mdis.htm.

2005. "Database on Iodine Deficiency Disorders." Geneva: WHO. http://www3.who.int/whosis/micronutrient/.

WHO, UNICEF, and UNFPA (World Health Organization, United Nations Children's Fund, and United Nations Population Fund). 2003. Maternal Mortality in 2000: Estimates Developed by WHO, UNICEF and UNFPA. Geneva: WHO.

Wild, S., G. Roglic, A. Green, R. Sicree, and H. King. 2004. "Global Prevalence of Diabetes: Estimates for the Year 2000 and Projections for 2030." Diabetes Care 27 (5): 1047-53.
Williams, A. 1997. "Intergenerational Equity: An Exploration of the 'Fair Innings' Argument." Health Economics 6 (2): 117-32.

1999. Calculating the Global Burden of Disease: Time for a Strategic Reappraisal. Health Economics 8 (1): 1-8.

Williams, B. G., E. Gouws, C. Boschi-Pinto, J. Bryce, and C. Dye. 2002. "Estimates of World-wide Distribution of Child Deaths from Acute Respiratory Infections." Lancet 2 (1): 25-32.

Wolfson, M. C. 1999. "Measuring Health: Visions and Practicalities." Statistical Journal of the United Nations Economic Commission for Europe 16 (1): 1-17.

World Bank. 1993. World Development Report 1993. Investing in Health. New York: Oxford University Press.

2003. World Development Report 2003: Sustainable Development in a Dynamic World: Transforming Institutions, Growth, and Quality of Life. New York: Oxford University Press.

Yang, G. H., J. Hu, K. Q. Rao, J. Ma, C. Rao, and A. D. Lopez. 2005. "Mortality Registration and Surveillance in China: History, Current Situation, and Challenges." Population Health Metrics 3: 3.

Zatonski, W. 1998. "Alcohol and Health: What Is Good for the French May Not Be for the Russians." Journal of Epidemiology and Community Health 52 (12): 766-7.

Zeckhauser, R., and D. Shepard. 1976. "Where Now for Saving Lives?” Law and Contemporary Problems 40 (4): 5-45. 
Chapter

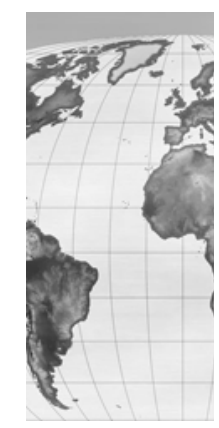

Detailed descriptions of the level and distribution of diseases and injuries and their causes are important inputs into strategies for improving population health. A substantial body of work has focused on quantifying causes of mortality and, more recently, the burden of disease (Murray and Lopez 1997; Preston, 1976; see also chapter 3 in this volume). Data on disease or injury outcomes alone, such as death or hospitalization, tend to focus on the need for curative or palliative services. Reliable and comparable analyses of risks to health are critical for preventing disease and injury. Investigators have frequently analyzed morbidity and mortality due to risk factors in the context of methodological traditions of individual risk factors and for selected populations (Kunzli and others 2000; Leigh and others 1999; McGinnis and Foege 1993; Peto and others 1992; Single and others 1999; Smith 2000; Smith, Corvalan, and Kjellstrom 1999; Willet 2002). As a result, most estimates have been affected by the following shortcomings, which limit comparability:

- The causal attribution of morbidity and mortality due to risk factors has been estimated relative to arbitrary exposure levels without standardizing baseline exposure

\section{Comparative Quantification of Mortality and Burden of Disease Attributable to Selected Risk Factors}

Majid Ezzati, Stephen Vander Hoorn, Alan D. Lopez, Goodarz Danaei, Anthony Rodgers, Colin D. Mathers, and Christopher J. L. Murray

across risk factors. For example, the implicit baseline for the burden of injuries attributable to occupational factors has been "no work," because estimates have been based on occupational registries intended to register all injuries, regardless of whether they are avoidable (Leigh and others 1999).

- The intermediate stages and interactions in the causal process have not been considered when calculating the disease burden attributable to risk factors. As a result, attributable burden could only be calculated for those risk factor and disease combinations for which epidemiological studies had been conducted.

- The outcomes of analyses have been morbidity or mortality from specific diseases without conversion to a standard unit, making comparisons among different diseases and/or risk factors difficult.

To permit the assessment of risk factors in a unified framework while acknowledging characteristics specific to individual risk factors, the Comparative Risk Assessment (CRA) project initiated a systematic evaluation of the changes in population health that would result from 
modifying the population distribution of exposure to a risk factor or to a group of risk factors (Murray and others 2003; Murray and Lopez 1999; Ezzati and others 2004). In particular, the CRA framework

- compares the burden of disease due to the observed distribution of exposure in a population with the burden from an alternative distribution consistently defined across risk factors;

- considers multiple stages in the causal network of multiple risk factors and disease outcomes to allow inferences about combinations of risk factors for which epidemiological studies have not been conducted, including the joint effects of changes in multiple risk factors;

- converts the burden of disease and injury into a summary measure of population health that permits comparing fatal and nonfatal outcomes while also taking severity and duration into account (the summary measure used in this chapter is the disability-adjusted life year [DALY], whose definition and calculation are described in chapter 3).

Therefore, even though CRA is similar to other risk assessment exercises in the sense that it applies knowledge about the hazardous effects of risk factors from epidemiological research to data on exposure in the broader population, it creates conceptual and methodological consistency in measuring the impacts of various risk factors on population health. Furthermore, we have attempted to use consistent and comparable criteria for evaluating the scientific evidence on prevalence, causality, and magnitude of hazardous effects across risk factors. As a result, the unified framework for describing population exposure to risk factors and their consequences for population health is an important step in linking the growing interest in the causal determinants of health across a variety of disciplines from natural, physical, and medical sciences to the social sciences and humanities.

We note that risk assessment as defined here is distinct from intervention analysis, whose purpose is to estimate the benefits of a given intervention or group of interventions in a specific population at a particular time. Rather, risk assessment aims at mapping alternative population health scenarios that arise from changes in the distribution of exposure to risk factors, irrespective of whether exposure change is achievable using existing interventions. The alternative visions of population health in turn contribute to identifying those risk factors for which effective or cost-effective interventions should be implemented or new interventions should be developed.

\section{BURDEN OF DISEASE ATTRIBUTABLE TO RISK FACTORS}

Mathers and others (2002) describe two traditions for the causal attribution of health outcomes or states: categorical attribution and counterfactual analysis. In categorical attribution, an event such as death is attributed to a single cause, such as a disease or a risk factor, or to a group of causes, according to a defined set of rules such as the International Classification of Diseases (ICD) system (WHO 1992). In counterfactual analysis, the effects of one or a group of diseases or risk factors is estimated by comparing the current or future disease burden with the levels that would be expected under some alternative hypothetical scenario, referred to as the counterfactual, including the absence of or reduction in the diseases or risk factors of interest (see Maldonado and Greenland 2002 for a discussion of the conceptual and methodological issues involved in the use of counterfactuals). In theory, causal attribution of the burden of disease to risk factors can be done using both categorical and counterfactual approaches. For example, researchers have used categorical attribution for attributing diseases and injuries to occupational risk factors in occupational health registries (Leigh and others 1999) and motor vehicle accidents to alcohol use. However, categorical attribution to risk factors overlooks that many diseases have multiple causes (Rothman 1976).

The CRA estimates of the burden of disease and injuries due to risk factors are based on a counterfactual exposure distribution that would result in the lowest population risk, irrespective of whether currently attainable in practice, referred to as the theoretical-minimum-risk exposure distribution (Murray and Lopez 1999). Using the theoretical-minimumrisk exposure distribution as the counterfactual has the advantage of providing an indication of potential gains in population health from reducing the risk from all levels of suboptimal exposure in a consistent way across risk factors.

\section{RISK FACTOR SELECTION}

The CRA project included a selected group of risk factors, presented in table 4.1. The criteria for selecting risk factors included the following:

- they were likely to be among the leading causes of the disease burden globally or regionally;

- they were not too specific, for example, every one of the hundreds of air pollutants or fruits and vegetables, or too 


\begin{tabular}{|c|c|}
\hline Risk factor & Exposure variable \\
\hline \multicolumn{2}{|c|}{ Childhood and maternal undernutrition } \\
\hline Childhood underweight & $\begin{array}{l}\text { Children }<-1 \text { SD weight-for-age } \\
\text { compared to the international refer- } \\
\text { ence group in } 1 \text { SD increments }\end{array}$ \\
\hline Iron-deficiency anemia & $\begin{array}{l}\text { Hemoglobin concentration distribution, } \\
\text { estimated from prevalence of anemia }\end{array}$ \\
\hline Vitamin A deficiency & $\begin{array}{l}\text { Prevalence of vitamin A deficiency, } \\
\text { estimated as low serum retinol } \\
\text { concentrations }(<0.70 \mu \mathrm{mol} / \mathrm{L} \text { ) among } \\
\text { children aged } 0-4 \text { years and among } \\
\text { pregnant women (aged } 15-44 \text { years) }\end{array}$ \\
\hline
\end{tabular}

Zinc deficiency

Less than the U.S. recommended dietary allowances for zinc

Other nutrition-related risk factors and physical activity

High blood pressure Usual level of systolic blood pressure

High cholesterol Usual level of total blood cholesterol

Overweight and

obesity (high BMI)

BMI (height/weight squared)

Low fruit and vegetable intake

Physical inactivity

Addictive substances Smoking

Alcohol use

Theoretical-minimum-risk exposure distribution
$3.8 \mathrm{SD} 0.6 \mathrm{mmol} / \mathrm{L}$

$21 \mathrm{SD} 1 \mathrm{~kg} / \mathrm{m}^{2}$

600 SD $50 \mathrm{~g}$ intake per day for adults

Same proportion of children below -1 $\mathrm{SD}$ weight-for-age as the international reference group

Hemoglobin distributions that are estimated to occur if all iron deficiency were eliminated ${ }^{b}$

No vitamin A deficiency

The entire population consuming sufficient dietary zinc to meet physiological needs, taking into account routine and illness-related losses and bioavailability

$115 \mathrm{SD} 6 \mathrm{mmHg}$
$3.8 \mathrm{SD} 0.6 \mathrm{mmol} / \mathrm{L}$
$21 \mathrm{SD} 1 \mathrm{~kg} / \mathrm{m}^{2}$

All having at least 2.5 hours per week of moderate-intensity activity or equivalent (4,000 KJ/week) (indirect indicator of accumulated

Disease outcomes ${ }^{\mathrm{a}}$

Mortality and acute morbidity from diarrhea, malaria, measles, pneumonia, and selected other Group I (communicable, maternal, perinatal, and nutritional) diseases; long-term risks of undernutrition

Anemia and its sequelae (including cognitive impairment), maternal and perinatal mortality

Mortality due to diarrhea, measles, malaria, and miscellaneous infectious causes of disease (children under five), morbidity due to malaria (children under five), maternal mortality (pregnant women), vitamin A deficiency and its sequelae (all age groups); maternal morbidity, low birthweight, and other perinatal conditions

Diarrhea, pneumonia, malaria; adult and pregnancy outcomes

IHD, stroke, hypertensive disease, and selected other cardiovascular diseases; renal failure

IHD, stroke; other cardiovascular diseases

IHD, stroke, hypertensive disease, diabetes, osteoarthritis, endometrial and colon cancers, postmenopausal breast cancer; gallbladder cancer, kidney cancer, breathlessness, back pain, dermatitis, menstrual disorders and infertility, gallstones, psychological effects

IHD, stroke, colorectal cancer, gastric cancer, lung cancer, esophageal cancer

IHD, breast cancer, colorectal cancer, diabetes; falls and osteoporosis, osteoarthritis, lower back pain, prostate cancer ciently active $(<2.5$ hours per week of moderate-intensity activity, or less than 4,000 KJ/week), and sufficiently active. Activity in discretionary-time, work, and transport considered

Current levels of smoking impact ratio smoking risk based on excess lung cancer mortality)

No smoking

Lung cancer, upper aerodigestive cancer, stomach cancer, liver cancer, pancreas cancer, cervix uteri cancer, bladder cancer, leukemia, COPD, other respiratory diseases, IHD, stroke, selected other cardiovascular diseases except hypertensive heart disease, and selected other medical causes in adults over 30 years of age; fire injuries, maternal outcomes, and perinatal conditions ${ }^{c}$

IHD, stroke, hypertensive disease, diabetes, liver cancer, mouth and oropharynx cancer, breast cancer, esophageal cancer, selected other cancers, cirrhosis of the liver, epilepsy, alcohol use disorders, depression, intentional and unintentional injuries; selected other cardiovascular diseases and cancers, social consequences 
Table 4.1 Continued

\begin{tabular}{|c|c|c|c|}
\hline Risk factor & Exposure variable & $\begin{array}{l}\text { Theoretical-minimum-risk } \\
\text { exposure distribution }\end{array}$ & Disease outcomes $^{a}$ \\
\hline Illicit drug use & $\begin{array}{l}\text { Use of amphetamine, cocaine, heroin, } \\
\text { or other opioids, and intravenous } \\
\text { drug use }\end{array}$ & No illicit drug use & $\begin{array}{l}\text { HIV/AIDS, overdose, drug use disorder, suicide, and } \\
\text { trauma; other neuropsychological diseases, social } \\
\text { consequences, hepatitis B and hepatitis C }\end{array}$ \\
\hline \multicolumn{4}{|c|}{ Sexual and reproductive health } \\
\hline Unsafe sex & $\begin{array}{l}\text { Sex with an infected partner without } \\
\text { any measures to prevent infection }\end{array}$ & No unsafe sex & $\begin{array}{l}\text { HIV/AIDS, sexually transmitted infections, and } \\
\text { cervical cancer }\end{array}$ \\
\hline $\begin{array}{l}\text { Non-use and use of } \\
\text { ineffective methods } \\
\text { of contraception }\end{array}$ & $\begin{array}{l}\text { Prevalence of traditional methods or } \\
\text { non-use of contraception }\end{array}$ & $\begin{array}{l}\text { Use of modern contraceptives for all } \\
\text { women who want to space or limit } \\
\text { future pregnancies }\end{array}$ & $\begin{array}{l}\text { Maternal mortality and morbidity; increased perinatal } \\
\text { and child mortality with lower birth intervals }\end{array}$ \\
\hline \multicolumn{4}{|l|}{ Environmental risks } \\
\hline $\begin{array}{l}\text { Unsafe water, sanita- } \\
\text { tion, and hygiene }\end{array}$ & $\begin{array}{l}\text { Six scenarios, ranging from regulated } \\
\text { water and sanitation with hygiene to } \\
\text { no improved water supply and no } \\
\text { improved sanitation }\end{array}$ & $\begin{array}{l}\text { Absence of transmission of diarrheal } \\
\text { disease through water, sanitation, and } \\
\text { hygiene }\end{array}$ & Diarrheal diseases \\
\hline Urban air pollution & $\begin{array}{l}\text { Estimated annual average particulate } \\
\text { matter concentration for particles with } \\
\text { aerodynamic diameters less than } \\
2.5 \text { or } 10 \text { microns }\left(\mathrm{PM}_{2.5} \text { or } \mathrm{PM}_{10}\right)\end{array}$ & $\begin{array}{l}7.5 \mu \mathrm{g} / \mathrm{m}^{3} \text { for } \mathrm{PM}_{2.5} \\
15 \mu \mathrm{g} / \mathrm{m}^{3} \text { for } \mathrm{PM}_{10}\end{array}$ & $\begin{array}{l}\text { Mortality from combined respiratory and selected } \\
\text { cardiovascular causes in adults over } 30 \text {, lung cancer, } \\
\text { acute respiratory infection mortality in children under } \\
\text { five; cardiovascular and respiratory morbidity }\end{array}$ \\
\hline $\begin{array}{l}\text { Indoor smoke from } \\
\text { household use } \\
\text { of solid fuels }\end{array}$ & $\begin{array}{l}\text { Household use of solid fuels and } \\
\text { ventilation }\end{array}$ & No household solid fuel use & $\begin{array}{l}\text { Acute lower respiratory infections in children under five, } \\
\text { COPD, lung cancer (coal):e low birthweight, cataracts, } \\
\text { tuberculosis, asthma, lung cancer from biomass }\end{array}$ \\
\hline \multicolumn{4}{|l|}{ Other selected risks } \\
\hline $\begin{array}{l}\text { Contaminated } \\
\text { injections in health } \\
\text { care settings }\end{array}$ & $\begin{array}{l}\text { Exposure to at least one contaminated } \\
\text { injection }\end{array}$ & No contaminated injections & $\begin{array}{l}\text { Acute infection with hepatitis B virus (HBV), hepatitis C } \\
\text { virus (HCV), and HIV, cirrhosis and liver cancer }\end{array}$ \\
\hline Child sexual abuse & $\begin{array}{l}\text { Prevalence of noncontact abuse, } \\
\text { contact abuse, and intercourse }\end{array}$ & No abuse & $\begin{array}{l}\text { Depression, panic disorder, alcohol abuse/dependence, } \\
\text { drug abuse/dependence, post-traumatic stress disorder } \\
\text { and suicide in adulthood; non-mental health outcomes, } \\
\text { such as sexually transmitted diseases, unwanted } \\
\text { pregnancies, and injuries }\end{array}$ \\
\hline
\end{tabular}

Sources: Table 1 in Ezzati and others 2002, and individual risk factor chapters in Ezzati and others 2004 for data sources.

Note: $\mathrm{BMI}=$ body mass index, $\mathrm{COPD}=$ chronic obstructive pulmonary disease, $\mathrm{IHD}=$ ischemic heart disease, $\mathrm{KJ}=$ kilo joules, $\mathrm{SD}=$ standard deviation. New disease outcomes are used here when more recent epidemiological analyses enabled improvements over those of the CRA project (for example, multiple cancer sites assessed separately, versus grouped together, for smoking). Several risk factors (lead exposure, global climate change, and selected occupational risks) were included in the CRA project but not in the current analysis because of conceptual and empirical difficulties in converting estimates of exposure and/or hazards from the GBD analysis subregions to the World Bank regions.

a. Outcomes in italic are those that are likely to be causal but not quantified due to lack of sufficient evidence on the magnitude of hazardous effect.

b. The resulting hemoglobin levels vary across regions and age-sex groups because the other risks for anemia (for example, malaria) vary.

c. In estimating years of life lost because of disability (YLD) due to smoking in the CRA project (Ezzati and Lopez 2004), population attributable fractions (PAFs) for disease incidence were assumed to be the same as PAFs for mortality for cancers and COPD, and one-half of PAFs for mortality for all other diseases. In the current analysis, PAFs for disease incidence were assumed to be the same as those for mortality for cancers, COPD, and cardiovascular diseases- for which smoking is expected to increase mortality through increasing incidence-and zero for all other diseases. The estimated total disease burden from smoking is robust to assumptions about the effects of smoking on incidence of diseases other than major chronic diseases (cancers, COPD, and cardiovascular diseases). d. The theoretical-minimum-risk exposure level for alcohol is zero, the global theoretical minimum. Specific population subgroups or diseases may have a non-zero theoretical-minimum-risk exposure (see figure 4.2) (Rehm and others 2004).

e. In the CRA project, PAFs for indoor smoke from household use of solid fuels were applied to COPD and lung cancer mortality and disease burden after subtracting the smoking-attributable burden (Smith, Mehta, and Maeusezahl-Feuz 2004). This overlooks multi-causality of COPD and lung cancer, which is also illustrated empirically in the analysis of the hazards of smoking in China (Liu and others 1998). Therefore, we applied PAFs to total mortality from COPD and lung cancer.

broad, such as the environment or diet taken as a single exposure;

- the likelihood of causality was high based on collective scientific knowledge;
- reasonably complete data on exposure and risk levels were available or sufficient data were available to extrapolate information when necessary;

- they were potentially modifiable. 
The risks to health examined in the CRA project cover many of the important hazards to health addressed in various fields of scientific inquiry. Arguably, hundreds of risk exposures are harmful to health. We selected only a relatively small number of exposures for quantification, largely determined by the availability of data and scientific research about their level and health effects in different parts of the world.

We also had to make choices about the definition of each risk factor. Given the close interrelationships among diet, exercise, and physiological risks on the one hand, or among water, sanitation, and personal hygiene on the other, the exact definition of what a risk factor is requires careful attention. The absence of a particular risk factor like dietary fat intake from table 4.1 does not imply that it is of limited relevance. Similarly, the assessment of unsafe sex separately from that of non-use and use of ineffective methods of contraception does not override their close linkages. Rather, we focused the analysis on risk factors for which we were likely to be able to satisfactorily quantify their population exposure distributions and health effects using existing scientific evidence and available data and for which intervention strategies are available or might be envisioned.

\section{Estimating Population Attributable Fractions}

The contribution of a risk factor to disease or mortality is expressed as the fraction of disease or death attributable to the risk factor in a population and is referred to as the population attributable fraction (PAF), and is given by the generalized potential impact fraction in equation 4.1 (Eide and Heuch 2001; Walter 1980).

$$
P A F=\frac{\int_{x=0}^{m} R R(x) P(x) d x-\int_{x=0}^{m} R R(x) P^{\prime}(x) d x}{\int_{x=0}^{m} R R(x) P(x) d x},
$$

where $R R(x)$ is the relative risk at exposure level $x, P(x)$ is the population distribution of exposure, $P^{\prime}(x)$ is the counterfactual distribution of exposure, and $m$ is the maximum exposure level.

The corresponding relationship when exposure is described as a discrete variable with $n$ levels is given by

$$
P A F=\frac{\sum_{i=1}^{n} P_{i} R R_{i}-\sum_{i=1}^{n} P_{i}^{\prime} R R_{i}}{\sum_{i=1}^{n} P_{i} R R_{i}} .
$$

PAFs obtained in this way estimate the proportional reduction in disease or death that would occur if exposure to the risk factor were reduced to the counterfactual distribution. The alternative (counterfactual) scenario used is the exposure distribution that would result in the lowest population risk, referred to as the theoretical-minimumrisk exposure distribution (Ezzati and others 2002; Murray and Lopez 1999). For risk factors for which the assumption of constant relative risk was not appropriate, we estimated PAFs by accounting for the determinants of hazard heterogeneity. For example, the PAFs for injuries as a result of alcohol use accounted for alcohol drinking patterns (moderate versus binge).

Because most diseases are caused by multiple risk factors, PAFs for individual risk factors for the same disease overlap and can add to more than 100 percent (Murray and Lopez 1999; Rothman 1976). For example, some deaths from childhood pneumonia may have been avoided by preventing exposure to indoor smoke from household use of solid fuels, childhood underweight, and zinc deficiency (which itself affects weight-for-age); and some cardiovascular disease events may be due to a combination of smoking, physical inactivity, and low fruit and vegetable intake. Such cases would be attributed to all these risk factors.

\section{Attributable Mortality and Burden of Disease}

For each risk factor and disease pair, we calculated PAFs for each age and sex group, and in each region, using the relationships in equations $4.1 \mathrm{a}$ and $4.1 \mathrm{~b}$, separately for mortality $\left(P A F_{M}\right)$ and incidence $\left(P A F_{I}\right)$ when the relative risks for mortality and incidence were different. For each of these age, sex, and region groups, we obtained estimates of mortality $\left(A M_{i j}\right)$ and the burden of disease $\left(A B_{i j}\right)$ from disease $j$ attributable to risk factor $i$ as follows:

$$
\begin{aligned}
& A M_{i j}=P A F_{M-i j} \times M_{j}, \\
& A-Y L L_{i j}=P A F_{M-i j} \times Y L L_{j}, \\
& A-Y L D_{i j}=P A F_{I-i j} \times Y L D_{j}, \\
& A B_{i j}=A-Y L L_{i j}+A-Y L D_{i j},
\end{aligned}
$$

where $Y L L$ denotes years of life lost because of premature mortality and YLD denotes years of healthy life lost as a result of disability.

\section{Data on Exposure and Hazard}

Between 1999 and 2002, for each risk factor, an expert working group conducted a comprehensive review of the published 
literature and other sources (government reports, international databases, and so on) to obtain data on the prevalence of risk factor exposure and hazard size (relative risk or absolute hazard size when appropriate, such as the effects of lead on blood pressure) (Ezzati and others 2004). The work included collecting primary data and undertaking a number of reanalyses of original data, systematic reviews, and meta-analyses. To increase comparability while acknowledging the fundamental differences in exposure and hazard quantification across risk factors, the criteria for using the scientific evidence included consistency of exposure variables used in exposure data sources with those used in epidemiological studies on hazard, population representativeness of exposure data, and study design for estimating the magnitude of hazardous effects (including minimizing the effects of confounders).

Data were initially presented separately for males and females and broken down into eight age groups $(0-4,5-14$, $15-29,30-44,45-59,60-69,70-79$, and 80 years old and older) and the 14 epidemiological subregions of the Global Burden of Disease (GBD) study (see chapter 3), which are based on a combination of World Health Organization regions and child and adult mortality levels, as described in the annexes of the annual World Health Report 2002 (WHO 2002). Data sources, models, and assumptions used to extrapolate exposure or relative risk across countries or regions are described in detail in chapters devoted to individual risk factors elsewhere (Ezzati and others 2004). External reviewers anonymously peer reviewed each risk factor chapter, including conducting re-reviews as appropriate.

In this reanalysis, estimates of mortality and disease burden attributable to risk factors were needed in World Bank regions (see map 1 inside the front cover). For six risk factors (childhood underweight, high blood pressure, high cholesterol, overweight and obesity, smoking, and indoor smoke from household use of solid fuels), country-level data were available and allowed reestimating exposure directly for World Bank regions. In such cases, we used newly available data sources on exposure to update CRA project estimations. For seven risk factors (unsafe water, sanitation, and hygiene; zinc deficiency; vitamin A deficiency; iron deficiency anemia; physical inactivity; low fruit and vegetable intake; and child sexual abuse), we estimated exposure in World Bank regions from the 14 GBD subregions using population-weighted averages. For another five risk factors (unsafe sex, urban air pollution, illicit drug use, non-use and use of ineffective methods of contraception, and contaminated injections in health care settings), where both exposure and hazards change across populations, we converted PAFs from GBD subregions to World Bank regions, with PAFs weighted by age-, sex-, and diseasespecific mortality rates. The prevalence of alcohol use was converted from GBD subregions to World Bank regions and was used to estimate exposure and PAFs in World Bank regions for most disease outcomes, because relative risks did not vary across populations. For all injury outcomes, ischemic heart disease, depression, stroke, and diabetes, whose hazards varied across regions, PAFs were converted from GBD subregions to World Bank regions using mortality weighting.

\section{Theoretical-Minimum-Risk Exposure Distributions}

The theoretical-minimum-risk exposure distribution was zero for risk factors for which zero exposure could be defined and reflected minimum risk, such as no smoking. For some risk factors, zero exposure was an inappropriate choice, either because it is physiologically impossible, as in the case of body mass index (BMI) or high cholesterol, or because physical lower limits to exposure reduction exist, as for concentrations of ambient particulate matter. For the latter risk factors, we used the lowest levels observed in specific populations and epidemiological studies to choose the theoretical-minimum-risk exposure distribution. For example, counterfactual exposure distributions of $115 \mathrm{mmHg}$ for systolic blood pressure and $3.8 \mathrm{mmol} / \mathrm{L}$ for total cholesterol, each with a small standard deviation, are the lowest levels at which meta-analyses of cohort studies have characterized dose-response relationships (Chen and others 1991; Eastern Stroke and Coronary Heart Disease Collaborative Research Group 1998; Law, Wald, and Thompson 1994).

Alcohol has benefits as well as causing harm for different diseases depending on the disease and on patterns of alcohol consumption (Corrao and others 2000; Puddey and others 1999). Rehm and others (2004) chose a counterfactual of zero for alcohol use. This was because despite its benefits for cardiovascular diseases in some populations, the global and regional burden of disease due to alcohol use was dominated by its impacts on neuropsychiatric diseases and injuries that are considerably larger than these benefits.

Finally, for factors with protective effects, namely, fruit and vegetable intake and physical activity, we chose a counterfactual exposure distribution based on a combination of levels observed in high-intake populations and the level to which the benefits may continue given current scientific 
evidence. Table 4.1 reports the theoretical-minimum-risk exposure distributions for the risk factors.

\section{BURDEN OF DISEASE ATTRIBUTABLE TO INDIVIDUAL RISK FACTORS}

Detailed results by risk factor, disease outcome, age, sex, and region are provided in annex 4A. Figure 4.1 shows the contributions of the leading global risk factors to all-cause mortality and burden of disease. The different ordering of risk factors in their contributions to mortality and to the disease burden expressed in DALYs reflects the age profile of mortality, such as the higher contribution to the disease burden from mortality among children as a result of underweight, and of nonfatal outcomes, such as neuropsychiatric diseases caused by alcohol use.

The leading causes of mortality and the disease burden include risk factors for communicable, maternal, perinatal, and nutritional conditions (Group I as defined in chapter 3), such as undernutrition; indoor smoke from household use of solid fuels; unsafe water, sanitation, and hygiene, whose burden is primarily concentrated in low-income regions of South Asia and Sub-Saharan Africa; and unsafe sex. They also include risk factors for noncommunicable diseases and injuries (Groups II and III as defined in chapter 3), such as high blood pressure and cholesterol, smoking, alcohol use, and overweight and obesity, which affect most regions.

Undernutrition is the single leading global cause of health loss, as it was in 1990 (the 2001 results disaggregate undernutrition into underweight and micronutrient deficiencies). Even though the prevalence of underweight has decreased in most regions in the past decade, it has increased in Sub-Saharan Africa (de Onis, Frongilla, and Blossner 2000; de Onis and others 2004), where its effects are disproportionately large because of simultaneous exposure to other risk factors for childhood disease. Three-quarters of the burden of disease attributable to unsafe sex is also in Sub-Saharan Africa, primarily as a result of HIV/AIDS, followed by South Asia (13 percent). The burden of disease attributable to unsafe water, sanitation, and hygiene has declined since 1990, mostly because of a worldwide decline in mortality from diarrheal disease, which is partly a result of improved case management interventions, particularly oral rehydration therapy. The increase in the global burden of disease attributable to smoking since 1990 mostly reflects the increased accumulated hazards of this risk, which is most noticeable in developing countries, but the increase is also partially due to methodological changes based on new evidence on the magnitude of the hazard after correction for confounding (Ezzati, Henley, Lopez, and others 2005; Ezzati, Henley, Thun, and others 2005; Ezzati and Lopez 2003; Thun, Apicella, and Henley 2000). The large increase in the burden of disease due to high blood pressure is likely to be an outcome of major methodological improvements, that is, relative risks that account for regression dilution bias and choice of theoretical-minimum-risk exposure distribution based on epidemiological evidence versus clinical definitions.

Table 4.2 shows the distributions of mortality and the disease burden attributable to the risk factors by age and sex. The disease burden attributable to underweight and micronutrient deficiencies in children was equally distributed among males and females, but the total all-age disease burden from iron and vitamin A deficiencies was slightly greater among females because of the effects on maternal mortality and morbidity conditions. Other diet-related risks, physical inactivity, environmental risks, and unsafe sex contributed almost equally to the disease burden in males and females. Approximately 77 to 86 percent of the disease burden from addictive substances occured among men, reflecting the social and economic forces that have so far made addictive substances more widely used by men, especially in developing countries. Women suffered an estimated twothirds of the disease burden from child sexual abuse and all of the burden caused by non-use and use of ineffective methods of contraception.

The estimated disease burdens from childhood undernutrition and unsafe water, sanitation, and hygiene were almost exclusively among children under five years of age. For these risks, more than 90 percent of the total attributable burden occurred in this age group, with the exception of iron deficiency, where adults bore more than 40 percent of burden, especially women of childbearing age. The disease burdens attributable to overweight and obesity and smoking were almost equally distributed among adults below and above the age of 60 years. The disease burdens attributable to other diet-related risks and physical inactivity were higher among those older than 60 (see also chapter 5).

More than 90 percent of the disease burden attributable to non-use and use of ineffective methods of contraception, illicit drug use, and child sexual abuse and more than 75 percent of the disease burden attributable to alcohol use and unsafe sex occurred in adults younger than 60. Most of the risks whose burden is concentrated among younger adults are those with outcomes that include HIV/AIDS, 


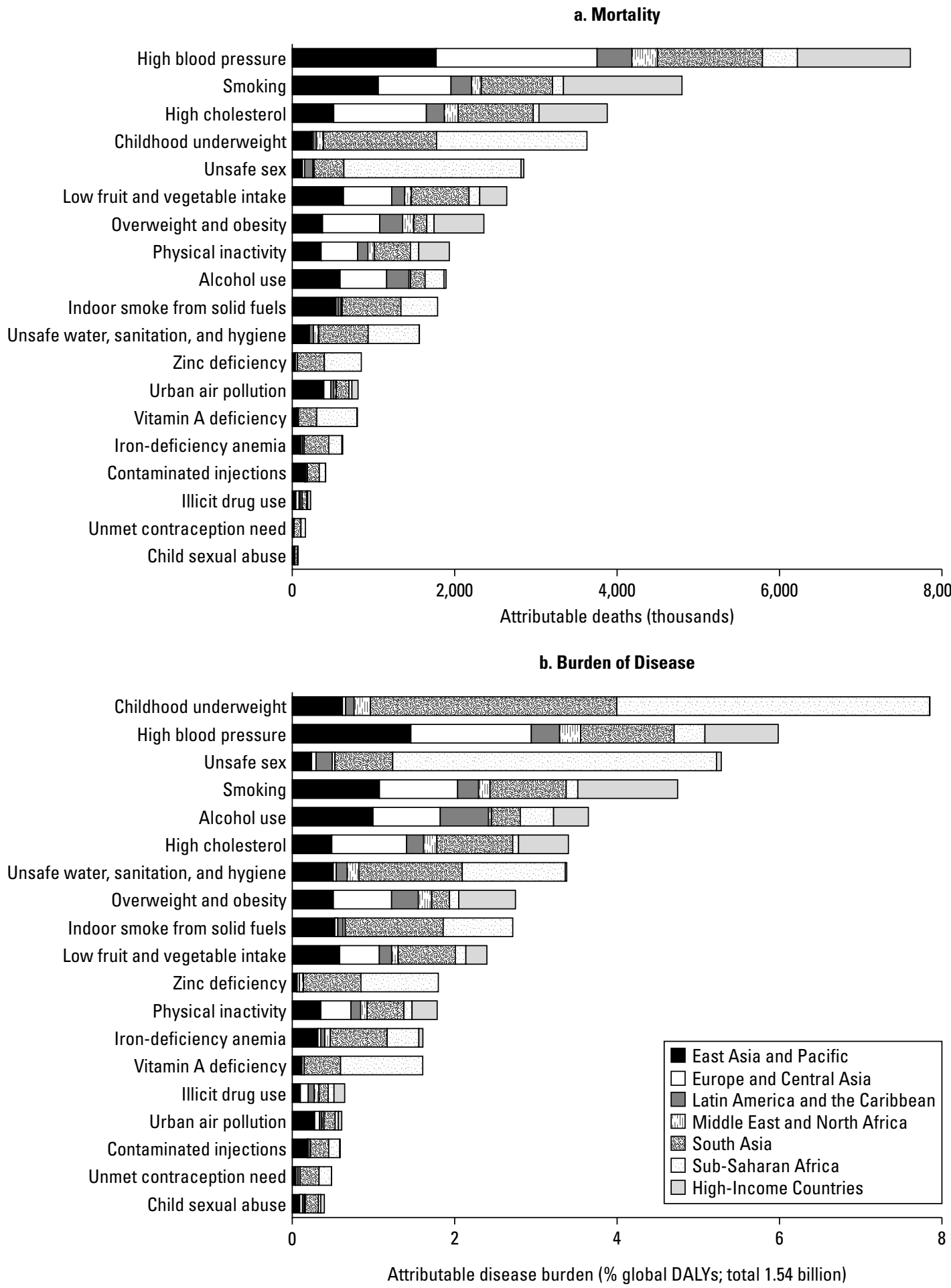

Source: Authors' calculations.

Note: The figure shows estimated mortality and disease burden attributable to each risk factor considered individually, relative to its own theoretical minimum risk exposure distribution. These risks act in part through, or jointly with, other risks. Consequently, the burden due to groups of risk factors will usually be less than the sum of individual risks. "Unmet contraception need" refers to "Non-use and use of ineffective methods of contraception" in table 4.1.

Figure 4.1 Mortality and the Burden of Disease Attributable to Leading Global Risk Factors, by World Bank Region 
Table 4.2 Distribution of Risk Factor-Attributable Mortality and Burden of Disease, by Age and Sex.

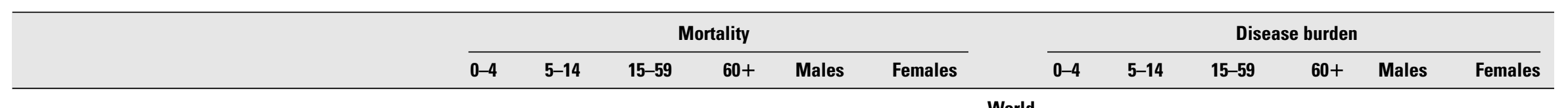

Childhood and maternal undernutrition

Childhood underweight

Iron-deficiency anemia

Vitamin A deficiency

Zinc deficiency

$\begin{array}{rl}100 & 0 \\ 68 & 1 \\ 93 & 0 \\ 100 & 0\end{array}$

0
25
7
0

World

Other nutrition-related risk factors and physical inactivity High blood pressure

High cholesterol

Overweight and obesity (high BMI)

Low fruit and vegetable consumption

Physical inactivity

Addictive substances

Smoking

Alcohol use

Illicit drugs use

Sexual and reproductive health

Unsafe sex

Non-use and use of ineffective methods of contraception

Environmental risk factors

Unsafe water, sanitation, and hygiene

Urban air pollution

Indoor smoke from household use of solid fuels

Other selected risk factors

Contaminated injections in health care settings

Child sexual abuse

Childhood and maternal undernutrition

Childhood underweight

Iron-deficiency anemia

Vitamin A deficiency

Zinc deficiency

Other nutrition-related risk factors and physical inactivity

High blood pressure

High cholesterol

Overweight and obesity (high BMI)

Low fruit and vegetable consumption

Physical inactivity

Addictive substances

Smoking

Alcohol use

Illicit drugs use

\begin{tabular}{|c|c|c|c|c|c|c|c|c|c|c|c|}
\hline 0 & 0 & 13 & 87 & 47 & 53 & 0 & 0 & 25 & 75 & 50 & 50 \\
\hline 0 & 0 & 23 & 77 & 48 & 52 & 0 & 0 & 40 & 60 & 52 & 48 \\
\hline 0 & 0 & 27 & 73 & 45 & 55 & 0 & 0 & 47 & 53 & 45 & 55 \\
\hline 0 & 0 & 23 & 77 & 53 & 47 & 0 & 0 & 38 & 62 & 55 & 45 \\
\hline 0 & 0 & 21 & 79 & 50 & 50 & 0 & 0 & 38 & 62 & 52 & 48 \\
\hline 0 & 0 & 31 & 69 & 76 & 24 & 0 & 0 & 50 & 50 & 77 & 23 \\
\hline 1 & 1 & 65 & 33 & 90 & 10 & 1 & 2 & 82 & 15 & 86 & 14 \\
\hline 0 & 0 & 99 & 1 & 79 & 21 & 0 & 1 & 98 & 0 & 77 & 23 \\
\hline 14 & 4 & 75 & 8 & 48 & 52 & 17 & 4 & 76 & 3 & 47 & 53 \\
\hline 0 & 0 & 100 & 0 & 0 & 100 & 0 & 0 & 100 & 0 & 0 & 100 \\
\hline 90 & 0 & 3 & 7 & 52 & 48 & 91 & 2 & 5 & 2 & 52 & 48 \\
\hline 3 & 0 & 16 & 81 & 52 & 48 & 7 & 0 & 28 & 65 & 53 & 47 \\
\hline 52 & 0 & 8 & 40 & 45 & 55 & 71 & 0 & 10 & 18 & 48 & 52 \\
\hline 12 & 3 & 52 & 32 & 63 & 37 & 17 & 5 & 60 & 18 & 60 & 40 \\
\hline 0 & 0 & 80 & 20 & 50 & 50 & 0 & 0 & 93 & 7 & 36 & 64 \\
\hline
\end{tabular}

Low- and middle-income countries

$\begin{array}{rlrl}100 & 0 & 0 & 0 \\ 69 & 1 & 26 & 4 \\ 93 & 0 & 7 & 0 \\ 100 & 0 & 0 & 0\end{array}$

$\begin{array}{lrl}50 & 100 & 0 \\ 57 & 59 & 7 \\ 53 & 94 & 0 \\ 49 & 100 & 0\end{array}$

$\begin{array}{rlll}0 & 0 & 50 & 50 \\ 32 & 3 & 44 & 56 \\ 6 & 0 & 47 & 53 \\ 0 & 0 & 51 & 49\end{array}$

$\begin{array}{lll}0 & 0 & 14 \\ 0 & 0 & 26 \\ 0 & 0 & 30 \\ 0 & 0 & 24 \\ 0 & 0 & 23 \\ & & \\ 0 & 0 & 38 \\ 1 & 1 & 59 \\ 0 & 0 & 98\end{array}$

53
52
56
47
50

18
16
19

$\begin{array}{lll}0 & 0 & 26 \\ 0 & 0 & 42 \\ 0 & 0 & 49 \\ 0 & 0 & 39 \\ 0 & 0 & 39 \\ & & \\ 0 & 0 & 55 \\ 1 & 2 & 79 \\ 0 & 1 & 98\end{array}$

$\begin{array}{rll}74 & 49 & 51 \\ 58 & 52 & 48 \\ 51 & 43 & 57 \\ 61 & 54 & 46 \\ 61 & 51 & 49 \\ & & \\ 45 & 82 & 18 \\ 17 & 84 & 16 \\ 1 & 79 & 21\end{array}$




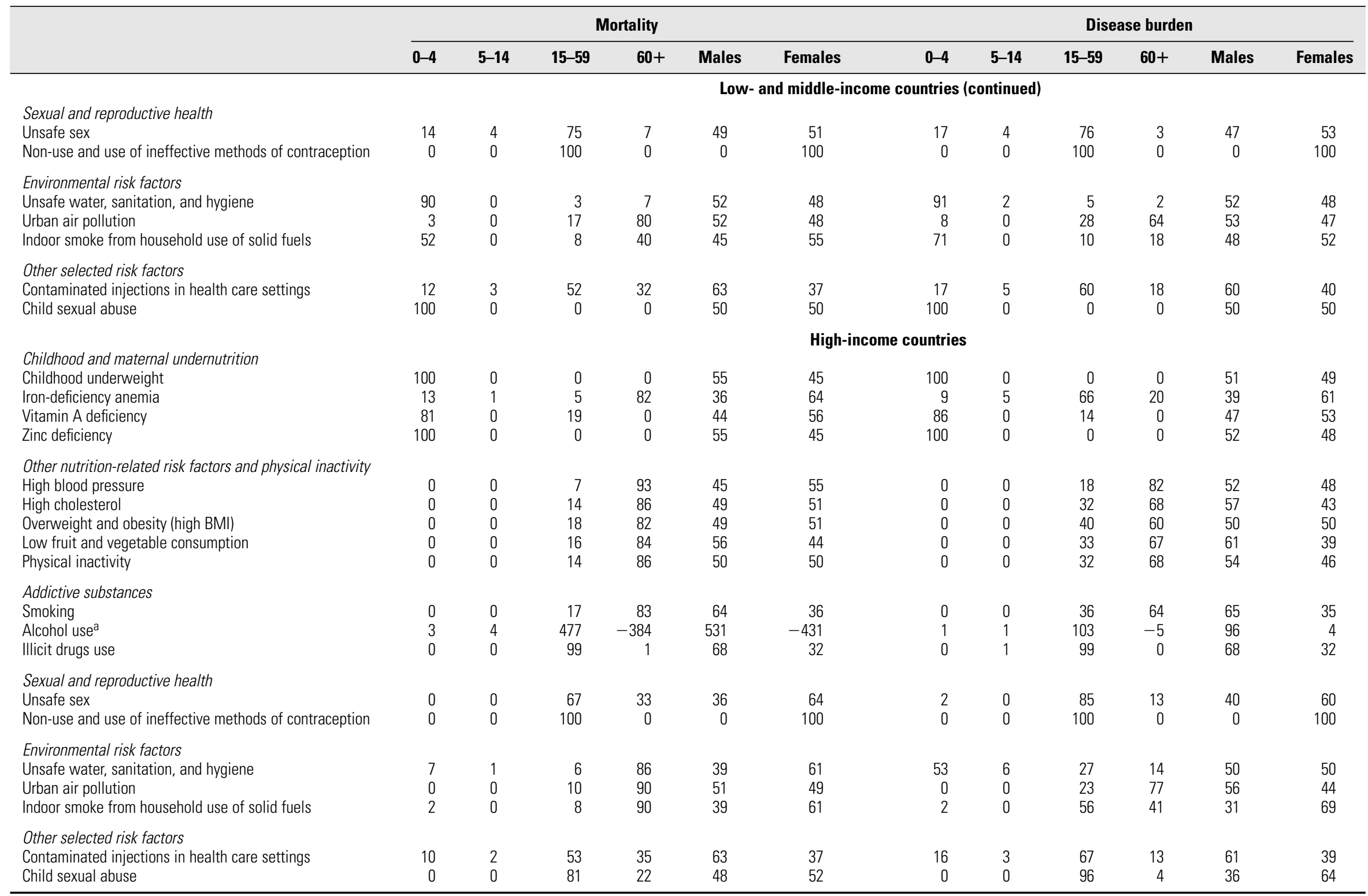

\section{Source: Authors' calculations.}

Note: $\mathrm{BMI}=$ body mass index. Numbers show percentage of total death or disease burden in each age group or for each sex

a. The figures are the ratio of deaths in each age group or for each sex to the total alcohol-attributable deaths and disease burden. Because the beneficial effects of alcohol are age dependent (more benefits for cardiovascular diseases in older ages) and because

the benefit-harm ratio is larger for women than for men (smaller alcohol-attributable burden from injuries), the ratios in younger ages or for males are larger than 100 percent and those in older ages or for females are negative. 
maternal conditions, neuropsychiatric diseases, and injuries. This illustrates the large, and at times neglected, disease burden from risks that affect young adults, especially in lowand-middle-income countries, with important consequences for economic development.

Only a small fraction of the disease burden from the risk factors considered was among those aged 5 to 14 years. This was because some of the leading conditions that affect this age group, such as motor vehicle accidents and other injuries and depression, have complex and heterogeneous causes that could not easily be included in the risk-based framework used. For other leading diseases of this group, such as diarrhea and lower respiratory infections, most epidemiological studies have focused on children younger than five and do not provide estimates of hazardous effects for older children.

Figure 4.2 presents the burden of disease due to the 10 leading risk factors for low- and middle-income countries and for high-income countries by disease or disease group. Leading causes of the burden of disease in low- and middleincome countries include the risk factors affecting the poor and associated with communicable, maternal, perinatal, and nutritional conditions (Group I) — such as childhood
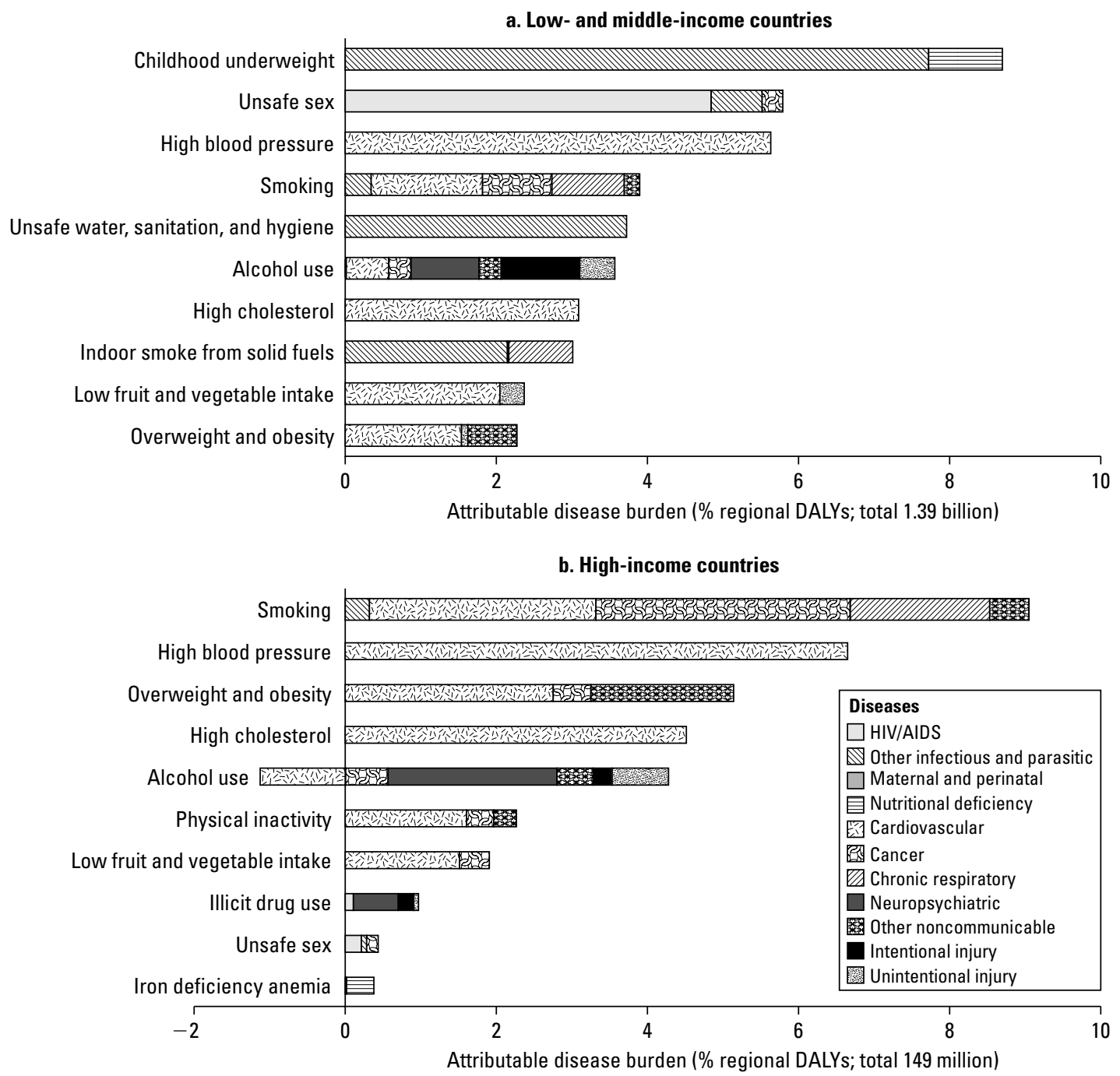

Source: Authors' calculations.

Figure 4.2 Burden of Disease Attributable to 10 Leading Regional Risk Factors, by Disease Type 
underweight (8.7 percent); unsafe water, sanitation, and hygiene (3.7 percent); indoor smoke from household use of solid fuels (3.0 percent); and unsafe sex (5.8 percent) along with risk factors for noncommunicable diseases (Group II), including addictive substances, nutrition related risks, and physical inactivity.

The relative contribution of unsafe sex was disproportionately larger in Sub-Saharan Africa (17.8 percent) than in all other regions, because HIV/AIDS prevalence and mortality are higher in Sub-Saharan Africa than anywhere else. This makes unsafe sex a leading cause of the burden of disease in this region together with childhood underweight (17.1 percent). The outcomes of these two risk factors were mostly communicable, maternal, perinatal, and nutritional conditions, which dominate the disease burden in highmortality developing regions.

In addition to their relative magnitude, the absolute loss of healthy life years attributed to risk factors in low- and middle-income regions is enormous. In these regions, which account for 85 percent of the global population, childhood underweight and unsafe sex alone contributed more to the loss of healthy life (200 million DALYs[3,0]) than all diseases and injuries in high-income countries (149 million DALYs $[3,0])$. In high-income countries, smoking (12.9 percent), high blood pressure (9.3 percent), overweight and obesity ( 7.2 percent), high cholesterol (6.3 percent), and alcohol use (4.4 percent) were the leading causes of loss of healthy life, contributing mainly to noncommunicable diseases and injuries (groups II and III).

\section{JOINT EFFECTS OF MULTIPLE RISK FACTORS}

Many users of risk assessment, who may be familiar with categorical attribution systems such as the ICD, desire information characterized by additive decomposition. In other words, they would like to know what fraction of the disease burden is related to a particular risk factor or group of risk factors independent of changes in other risk factors. As Mathers and others (2002) discuss, additive decomposition is not generally a property of counterfactual attribution, because many diseases are caused by the interaction of multiple determinants acting simultaneously (Rothman 1976; Rothman and Greenland 1998; Walter 1980; Yerushalmy and Palmer 1959). Indeed, the sum of PAFs for a single disease due to multiple risk factors is theoretically unbounded.

Although epidemiologically unavoidable and conceptually acceptable, the lack of additivity adds to policy complexity and implies the need for great care when interpreting and communicating estimates of PAF and attributable burden. With multiple attribution, a reduction in one risk factor would seem to make other, equally important, risk factors potentially irrelevant from a perspective with limited scope in relation to interpreting quantitative results. It also necessitates the development of methods to quantify the effects of joint counterfactual distributions for multiple risk factors. Estimating the joint effects of multiple distal and proximal risks is particularly important, because many factors act through other intermediate factors (Murray and Lopez 1999; Yerushalmy and Palmer 1959) or in combination with other factors. For example, education, occupation, and income may affect smoking, physical activity, and diet, which are risk factors for cardiovascular diseases, both directly and through further layers of such intermediate factors as BMI, blood pressure, and high cholesterol. Multicausality also means that a range of interventions can be used for disease prevention, with the specific choices determined by factors such as cost, technology availability, infrastructure, and preferences.

In equations $4.1 \mathrm{a}$ and $4.1 \mathrm{~b}, R R, P$, and $P^{\prime}$ may represent joint relative risks and exposure distributions for multiple risk factors, that is, $x$ may be a vector of risk factors, with $R R$ for each risk factor estimated at the appropriate level of the remaining ones (Eide and Heuch 2001). While such data have been gathered for a small number of risk factor combinations, for example, alcohol and smoking for oral cancer (Rothman and Keller 1972) and some cardiovascular risks (Neaton and Wentworth 1992; Yusuf and others 2004), they are generally rare in epidemiological studies. Alternatively, for $n$ biologically independent and uncorrelated risk factors, the joint PAF is given by equation 4.3 (Miettinen 1974; Walter 1976):

$$
P A F=1-\prod_{i=1}^{n}\left(1-P A F_{i}\right)
$$

where $\mathrm{PAF}_{i}$ shows the PAFs of individual risk factors.

If risk factors are independent and uncorrelated, the proportion of the remaining disease that is attributed to the ith additional risk factor equals $\mathrm{PAF}_{i}$, and hence $\left(1-\mathrm{PAF}_{i}\right)$ is not attributable to this factor. Therefore, the second term in the right-hand-side of equation 4.3 , that is, the product of all $\left(1-\mathrm{PAF}_{i}\right)$ terms, is the fraction of disease not attributable to any of the $n$ risk factors. One minus this term is the fraction attributable to the combined effects of the $n$ risk factors.

Estimating the joint effects of multiple risk factors is, in practice, complex and does not follow the simple, independent, and uncorrelated relationship of equation 4.3 for 


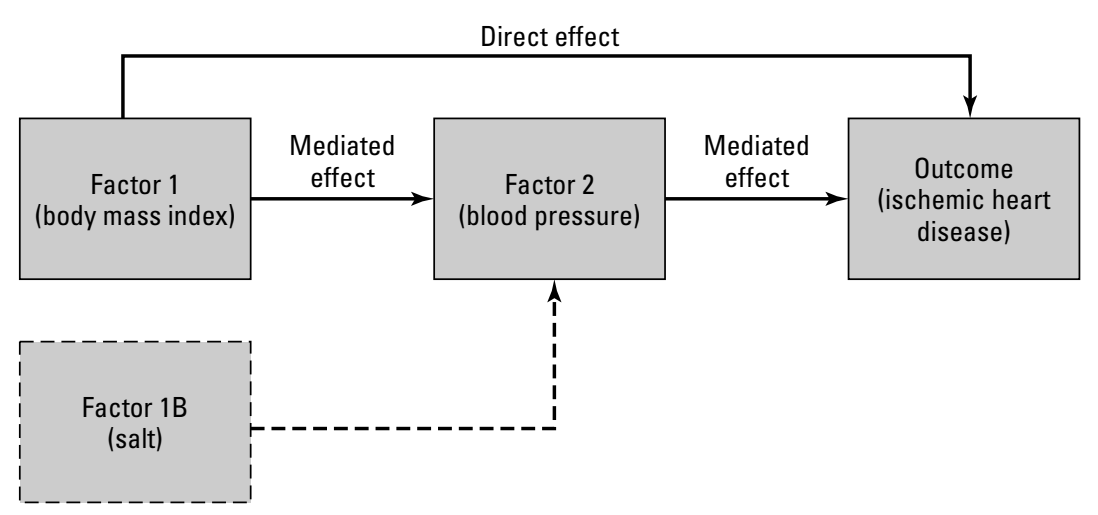

Note: Some of the effects of a risk factor (for example, body mass index-BMI) may be mediated through other factors (for example, blood pressure). When estimating the total effects of individual distal factors on disease, both mediated and direct effects should be considered, because, in the presence of mediated effects, controlling for the intermediated factor would attenuate the effects of the more distal one (Greenland 1987). When estimating the joint effects of the more distal factor (for example, BMI) and the intermediate one (for example, blood pressure), the direct and mediated effects must be separated, especially if the intermediate factor is affected by other distal factors (for example, dietary salt).

Figure 4.3 Mediated and Direct Effects of a Risk Factor

several reasons. First, some of the effects of the more distal factors, such as physical inactivity, are mediated through intermediate factors. For instance, a proportion of the hazards of physical inactivity is mediated through overweight and obesity, which is itself mediated through elevated blood pressure (figure 4.3). Estimating the joint effects of distal and intermediate factors requires knowledge of independent hazards of the distal ones (versus individual risk factor effects, which are based on total hazard). Second, the hazard due to a risk factor may depend on the presence of other risk factors (Koopman 1981; Rothman and Greenland 1998) (effect modification). ${ }^{1}$ Third, correlation may exist between exposures to multiple risk factors because they are affected by the same distal factors and policies. For example, undernutrition, unsafe water and sanitation, and use of solid fuels are more common among poor rural households in developing countries and smokers generally have higher and more harmful patterns of alcohol consumption and worse diets than nonsmokers.

The epidemiological literature refers to the first and second issues as biological interaction and the third issue as statistical interaction (Miettinen 1974; Rothman and Greenland 1998; Rothman, Greenland, and Walker 1980). This distinction is, however, somewhat arbitrary, and the three scenarios may occur simultaneously. For example, zinc deficiency affects mortality from diarrhea directly as well as by reducing growth (first issue) (Brown and others 2002; Zinc Investigators' Collaborative Group 1999), and may also be correlated with underweight, other micronutrient deficiencies, and unsafe water and sanitation (third issue). Similarly, alcohol and smoking may not only be correlated (third issue), but also affect each other's hazard for some diseases (second issue) (Rothman and Keller 1972).

\section{Data Sources for Mediated Effects and Effect Modification}

Despite the emphasis on removing or minimizing the effects of confounding in epidemiological research, mediated and stratified hazards have received disproportionately little empirical attention. We therefore reviewed the literature and reanalyzed cohort data to strengthen the empirical basis for considering interactions. The sensitivity of estimates to these assumptions were negligible as described in detail elsewhere (Ezzati, Vander Hoorn, and others 2004; Ezzati and others 2003).

Joint Hazards of Cardiovascular Disease Risk Factors. Epidemiological studies of the effects of overweight and obesity, physical inactivity, and low fruit and vegetable intake on cardiovascular diseases have illustrated some attenuation of the effects after adjustment for intermediate factors such as blood pressure or cholesterol (Berlin and Colditz 1990; Blair, Cheng, and Holder 2001; Eaton 1992; Gaziano and others 1995; Jarrett, Shipley, and Rose 1982; Jousilahti and others 1999; Khaw and Barrett-Connor 1987; Liu and others 2000, 2001; Manson and others 1990, 2002; 
Rosengren, Wedel, and Wilhelmsen 1999; Tate, Manfreda, and Cuddy 1998). This attenuation confirms that some of the hazard of the more distal factors is mediated through the intermediate ones (figure 4.3). The extent of attenuation has varied from study to study, but has consistently been less than half of the excess risk of the distal factors. We used an estimate of 50 percent as the proportion of the excess risk from these risk factors mediated through intermediate factors that are themselves among the selected risks. To include effect modification, we used deviations from the multiplicative model of 10 percent for ischemic heart disease and 30 percent for ischemic stroke based on existing studies, both submultiplicative (Eastern Stroke and Coronary Heart Disease Collaborative Research Group 1998; Neaton and Wentworth 1992).

Joint Hazards of Smoking and Other Risk Factors. Liu and others (1998, figures 4 and 6) find that in China, the relative risks of mortality from lung and other cancers, respiratory diseases, and vascular diseases are approximately constant in different cities where mortality rates for these diseases among nonsmokers varied by a factor of 4 to 10 . Studies that stratified hazards of smoking on serum cholesterol have confirmed this finding (Jee and others 1999).

Joint Hazards of Childhood Undernutrition for Infectious Diseases. Zinc affects growth in children (Brown and others 2002), and some of its effects on infectious diseases may be mediated through reducing growth. Because no published source for such mediated effects existed, data from some of the available zinc trials were reanalyzed (Zinc Investigators' Collaborative Group 1999). We used an upper bound of 50 percent on the proportion of zinc deficiency risk mediated through underweight.

Investigators have found that vitamin A deficiency, which affects some of the same diseases as underweight and zinc deficiency, does not change the hazard size for the other two risk factors based on stratified results from clinical trials and recent reviews of the literature on micronutrient deficiencies (Christian and West 1998; Ramakrishnan, Latham, and Abel 1995; Ramakrishnan and Martorell 1998; West and others 1991).

Joint Hazards of Undernutrition and Environmental Risk Factors in Childhood Diseases. Anthropometric (growth) indicators of childhood nutrition, such as weight-for-age, are aggregate measures of multiple factors that include nutrition and previous infection (Pelletier, Frongillo, and Habicht 1993; Scrimshaw, Taylor, and Gordon 1968; UNICEF 1990). Therefore, some of the risks from indoor smoke from household use of solid fuels and unsafe water, sanitation, and hygiene, which result in lower respiratory infections and diarrhea respectively, may be mediated through underweight. In a review of the literature, Briend (1990) concludes that attempts to disentangle direct and mediated contributions, especially over the long periods needed to affect population-level anthropometry, have not established diarrhea as a significant cause of underweight. Other works, however, have found evidence that infection, especially diarrhea, could reduce growth and increase the prevalence of underweight (Black 1991; Guerrant and others 1992; Lutter and others 1989, 1992; Martorell, Habicht and others 1975; Martorell, Yarbrough, and others 1975; Stephensen 1999). To account for potential mediated effects, we considered an upper bound of 50 percent on the proportion of the excess risks from indoor smoke from household use of solid fuels and unsafe water, sanitation, and hygiene mediated through underweight in regions where underweight was present.

\section{Risk Factor Correlation}

To estimate the joint effects of risk factors with a continuous exposure variable, for instance, blood pressure and cholesterol, each integral in the $P A F$ relationship may be replaced with $\int_{x_{1}=0}^{m_{1}} \int_{x_{2}=0}^{m_{2}} R R_{1}\left(x_{1}\right) R R_{2}\left(x_{2}\right) P\left(x_{1}, x_{2}\right) d x_{1} d x_{2}$, where subscripts 1 and 2 denote the two risk factors and $P$ is the joint distribution of the two exposures. If joint $R R$ were a linear function of exposure levels $\left(x_{1}\right.$ and $\left.x_{2}\right)$, then correlation between the two risk factors would not affect total hazard. Because individual RRs are nonlinear functions of exposure, for example, in a Cox proportional hazard model, and joint $R R s$ are the product of such nonlinear terms, positive correlation between risk factors would, in general, imply a larger PAF than zero correlation, ${ }^{2}$ which in turn would be larger than negative correlation. Similarly, for categorical risk factors, positive correlation would in general result in a larger PAF (see also Greenland 1984). For the range of exposures and relative risks in the CRA, this secondary effect of risk factor correlation would be considerably smaller than the joint attributable fraction, as described in detail elsewhere (Ezzati and others 2003). 
Table 4.3 Joint Contributions (PAFs) of the Leading Risk Factors to Mortality and Burden of Disease, by Region

\begin{tabular}{|c|c|c|c|c|c|c|}
\hline \multirow[b]{2}{*}{ Region } & \multicolumn{3}{|c|}{ Mortality } & \multicolumn{3}{|c|}{ Burden of disease $[\mathrm{DALYs}(3,0)]$} \\
\hline & Male & Female & Both & Male & Female & Both \\
\hline Low- and middle-income countries & $46 \%(25.5)$ & $45 \%(22.8)$ & $46 \%(48.3)$ & $37 \%(715)$ & $35 \%(671)$ & $36 \%(1,386)$ \\
\hline East Asia and Pacific & $39 \%(6.9)$ & $35 \%(6.1)$ & $37 \%(13.1)$ & $29 \%(181)$ & $24 \%(165)$ & $27 \%(346)$ \\
\hline Europe and Central Asia & $63 \%(3.0)$ & $61 \%(2.7)$ & $62 \%(5.7)$ & $50 \%(64)$ & $41 \%(53)$ & $46 \%(116)$ \\
\hline Latin America and the Caribbean & $42 \%(1.8)$ & $40 \%(1.4)$ & $41 \%(3.3)$ & $32 \%(57)$ & $25 \%(48)$ & $29 \%(104)$ \\
\hline Middle East and North Africa & $40 \%(1.1)$ & $40 \%(0.8)$ & $40 \%(1.9)$ & $26 \%(35)$ & $25 \%(31)$ & $25 \%(66)$ \\
\hline South Asia & $44 \%(7.1)$ & $40 \%(6.5)$ & $42 \%(13.6)$ & $35 \%(204)$ & $33 \%(205)$ & $34 \%(409)$ \\
\hline Sub-Saharan Africa & $52 \%(5.6)$ & $56 \%(5.2)$ & $54 \%(10.8)$ & $48 \%(175)$ & $50 \%(170)$ & $49 \%(345)$ \\
\hline High-income countries & $48 \%(4.0)$ & $40 \%(3.9)$ & $44 \%(7.9)$ & $40 \%(77)$ & $28 \%(72)$ & $34 \%(149)$ \\
\hline World & $46 \%(29.5)$ & $44 \%(26.7)$ & $45 \%(56.2)$ & $38 \%(792)$ & $34 \%(743)$ & $36 \%(1,535)$ \\
\hline
\end{tabular}

Source: Authors' calculations

Note: The risk factors are those listed in table 4.1. Numbers in parentheses show total number of deaths and DALYs $(3,0)$, in millions.

\section{BURDEN OF DISEASE ATTRIBUTABLE TO MULTIPLE RISK FACTORS}

This section presents the disease burden attributable to the joint hazards of the risk factors in table 4.1.

\section{All Selected Risk Factors}

Table 4.3 shows the joint contributions of all the risk factors shown in table 4.1 to the total mortality and disease burden in different regions. Globally, an estimated 45 percent of mortality and 36 percent of the disease burden were attributable to the joint effects of the 19 selected risk factors. SubSaharan Africa (49 percent of the disease burden) and Europe and Central Asia (46 percent of the disease burden) had the largest regional PAFs, and the Middle East and North Africa (25 percent of the disease burden) and East Asia and the Pacific (27 percent of the disease burden) had the smallest. The regions with large joint PAFs are those where a relatively small number of diseases and their risk factors are responsible for large losses of life, for example, HIV/AIDS and childhood disease risk factors in SubSaharan Africa and cardiovascular risks, smoking, and alcohol consumption in Europe and Central Asia. Those with smaller joint PAFs are regions where the causes of health loss are distributed among a larger number of diseases and their risk factors.

Table 4.4 shows the individual and joint contributions of the selected risk factors to the 10 leading diseases in the world and in low- and middle-income and high-income countries. As the table shows, for most diseases the joint effects of these risk factors were substantially less than the crude sum of their individual effects. For example, globally four separate risk factors were each responsible for 88,50 , 20 , and 11 percent of the diarrheal disease burden, but with a joint PAF of 92 percent; or seven separate risk factors were each responsible for 45, 46, 18, 28, 21, 17, and 17 percent of ischemic heart disease, but with a joint PAF of 80 percent. This confirms that the joint actions of more than one of these risk factors acting simultaneously or through other factors cause a large proportion of disease.

Globally, large fractions of the burden of HIV/AIDS (96 percent), diarrhea ( 92 percent), ischemic heart disease (80 percent), lung cancer (74 percent), stroke (65 percent), chronic obstructive pulmonary disease (64 percent), and lower respiratory infections (53 percent) were attributable to the joint effects of the 19 risk factors considered here. The joint PAFs for a number of other important diseases and injuries, such as perinatal and maternal conditions, certain other cancers, and intentional and unintentional injuries, which have more diverse risk factors, were smaller but nonnegligible. Even though the fraction of the total malaria burden attributable to childhood undernutrition was relatively large (59 percent), this was because of the contribution of mortality at younger ages to the malaria burden. No adult malaria was attributed to the risk factors in table 4.1, because the epidemiological literature has focused on quantifying increased risk of malaria as a result of childhood undernutrition only. Finally, with the exception of alcohol and drug dependence, which were fully attributable to their namesake risk factors, small or zero fractions of neuropsychiatric conditions, tuberculosis, congenital anomalies, and a number of other diseases were attributed to the risk factors considered here. 
Table 4.4 Individual and Joint Contributions of Risk Factors to 10 Leading Diseases and Total Burden of Disease

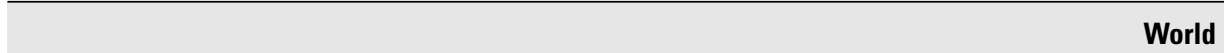

World

\begin{tabular}{|c|c|c|c|c|c|}
\hline Disease & $\begin{array}{l}\text { Proportion of } \\
\text { global disease } \\
\text { burden }(\%) \\
\text { [total: } 1.54 \text { billion } \\
\text { DALYs(3,0)] }\end{array}$ & $\begin{array}{l}\text { Proportion of global } \\
\text { mortality (\%) (total: } \\
56.2 \text { million deaths) }\end{array}$ & PAFs for individual risk factors (for disease burden) & $\begin{array}{l}\text { Joint PAF - } \\
\text { disease burden }(\%)\end{array}$ & $\begin{array}{l}\text { Joint PAF - } \\
\text { mortality (\%) }\end{array}$ \\
\hline Lower respiratory infections & 5.6 & 6.7 & $\begin{array}{l}\text { Childhood underweight ( } 37 \%) \text {; zinc deficiency }(15 \%) \text {; indoor smoke from } \\
\text { solid fuels (35\%); smoking }(4 \%)^{\text {a }} \text {; urban air pollution }(1 \%)^{b}\end{array}$ & 53 & 42 \\
\hline Ischemic heart disease & 5.5 & 12.6 & $\begin{array}{l}\text { High blood pressure ( } 45 \%) \text {; high cholesterol ( } 48 \%) \text {; overweight and } \\
\text { obesity (18\%); low fruit and vegetable intake (28\%); physical inactivity } \\
(21 \%) \text {; smoking (17\%); alcohol use (2\%); urban air pollution }(2 \%)^{\text {b }}\end{array}$ & 80 & 79 \\
\hline Stroke & 4.7 & 9.6 & $\begin{array}{l}\text { High blood pressure (54\%); high cholesterol (16\%); overweight and } \\
\text { obesity ( } 12 \%) \text {; low fruit and vegetable intake }(11 \%) \text {; physical } \\
\text { inactivity }(7 \%) \text {; smoking }(13 \%) \text {; alcohol use }(3 \%) \text {; urban air } \\
\text { pollution }(3 \%)^{\text {b }}\end{array}$ & 65 & 60 \\
\hline HIV/AIDS & 4.7 & 4.6 & $\begin{array}{l}\text { Unsafe sex (95\%); contaminated injections in health care settings }(5 \%) \text {; } \\
\text { illicit drug use }(3 \%)\end{array}$ & 96 & 96 \\
\hline Diarrheal diseases & 3.9 & 3.2 & $\begin{array}{l}\text { Childhood underweight (50\%); vitamin A deficiency }(22 \%) \text {; zinc deficiency } \\
(12 \%) \text {; unsafe water, sanitation, and hygiene ( } 88 \%)\end{array}$ & 92 & 93 \\
\hline Unipolar depressive disorders & 3.4 & 0.0 & Alcohol use (2\%); childhood sexual abuse (5\%) & 6 & $N A^{c}$ \\
\hline Low birthweight & 2.8 & 2.3 & Iron-deficiency anemia (14\%); alcohol use (0.5\%) & 16 & 17 \\
\hline Malaria & 2.6 & 2.1 & $\begin{array}{l}\text { Childhood underweight (51\%); vitamin A deficiency (19\%); } \\
\text { zinc deficiency (22\%) }\end{array}$ & 59 & 61 \\
\hline $\begin{array}{l}\text { Chronic obstructive } \\
\text { pulmonary disease }\end{array}$ & 2.5 & 4.8 & $\begin{array}{l}\text { Indoor smoke from solid fuels }(30 \%) \text {; urban air pollution }(2 \%)^{\text {b) }} \text {; } \\
\text { smoking }(44 \%)\end{array}$ & 64 & 62 \\
\hline Tuberculosis & 2.4 & 2.9 & Smoking $(4 \%)^{\mathrm{a}}$ & 9 & 10 \\
\hline $\begin{array}{l}\text { Communicable, maternal, perinatal, } \\
\text { and nutritional conditions }\end{array}$ & 36.5 & 32.3 & Multiple risks (see annex 4A) & 46 & 47 \\
\hline Noncommunicable diseases & 52.6 & 58.5 & Multiple risks (see annex 4A) & 33 & 49 \\
\hline Injuries & 10.9 & 9.2 & Multiple risks (see annex 4A) & 15 & 18 \\
\hline All causes & 100 & 100 & All 19 selected risks (see annex 4A) & 36 & 46 \\
\hline
\end{tabular}




\begin{tabular}{|c|c|c|c|c|c|}
\hline \multicolumn{6}{|c|}{ Low- and middle-income countries } \\
\hline Disease & $\begin{array}{l}\text { Proportion of } \\
\text { regional disease } \\
\text { burden }(\%) \text { [total: } \\
1.39 \text { billion } \\
\text { DALYs }(3,0)]\end{array}$ & $\begin{array}{l}\text { Proportion of regional } \\
\text { mortality (\%) (total: } \\
48.3 \text { million deaths) }\end{array}$ & PAFs for individual risk factors (for disease burden) & $\begin{array}{l}\text { Joint PAF - } \\
\text { disease burden (\%) }\end{array}$ & $\begin{array}{l}\text { Joint PAF - } \\
\text { mortality (\%) }\end{array}$ \\
\hline Lower respiratory infections & 6.0 & 7.0 & $\begin{array}{l}\text { Childhood underweight (38\%); zinc deficiency }(16 \%) \text {; indoor smoke from } \\
\text { solid fuels }(36 \%) \text {; smoking }(4 \%)^{a} \text {; urban air pollution }(4 \%)^{b}\end{array}$ & 54 & 44 \\
\hline Ischemic heart disease & 5.2 & 11.8 & $\begin{array}{l}\text { High blood pressure (44\%); high cholesterol ( } 46 \%) \text {; overweight and } \\
\text { obesity (16\%); low fruit and vegetable intake }(30 \%) \text {; physical inactivity } \\
(21 \%) \text {; smoking (15\%); alcohol use (4\%); urban air pollution }(2 \%)^{b}\end{array}$ & 80 & 78 \\
\hline HIV/AIDS & 5.1 & 5.3 & $\begin{array}{l}\text { Unsafe sex (95\%); contaminated injections in health care settings }(5 \%) \text {; } \\
\text { illicit drug use }(3 \%)\end{array}$ & 97 & 97 \\
\hline Stroke & 4.5 & 9.5 & $\begin{array}{l}\text { High blood pressure ( } 54 \%) \text {; high cholesterol }(15 \%) \text {; overweight and } \\
\text { obesity }(10 \%) \text {; low fruit and vegetable intake }(11 \%) \text {; physical } \\
\text { inactivity }(6 \%) \text {; smoking }(12 \%) \text {; alcohol use }(5 \%) \text {; urban air pollution }(4 \%)^{b}\end{array}$ & 64 & 61 \\
\hline Diarrheal diseases & 4.2 & 3.7 & $\begin{array}{l}\text { Childhood underweight ( } 56 \%) \text {; vitamin A deficiency }(22 \%) \text {; zinc } \\
\text { deficiency }(12 \%) \text {; unsafe water, sanitation, and hygiene }(88 \%)\end{array}$ & 93 & 94 \\
\hline Unipolar major depression & 3.1 & 0.0 & Alcohol use (1\%); childhood sexual abuse (5\%) & 6 & $N A^{c}$ \\
\hline Low birthweight & 3.1 & 2.7 & Iron-deficiency anemia (14\%); alcohol use (0.5\%) & 16 & 17 \\
\hline Malaria & 2.9 & 2.5 & Childhood underweight (51\%); vitamin A deficiency (19\%); zinc deficiency (22\%) & 59 & 61 \\
\hline Tuberculosis & 2.6 & 3.3 & Smoking $(4 \%)^{c}$ & 9 & 10 \\
\hline $\begin{array}{l}\text { Chronic obstructive } \\
\text { pulmonary disease }\end{array}$ & 2.4 & 4.9 & Indoor smoke from solid fuels (35\%); urban air pollution $(2 \%)^{b}$; smoking $(40 \%)$ & 63 & 60 \\
\hline $\begin{array}{l}\text { Communicable, maternal, } \\
\text { perinatal, and nutritional } \\
\text { conditions }\end{array}$ & 39.8 & 36.4 & Multiple risks (see annex $4 A$ ) & 47 & 48 \\
\hline Noncommunicable diseases & 48.9 & 53.8 & Multiple risks (see annex 4A) & 33 & 50 \\
\hline Injuries & 11.2 & 9.8 & Multiple risks (see annex 4A) & 15 & 18 \\
\hline All causes & 100 & 100 & All 19 selected risks (see annex 4A) & 36 & 46 \\
\hline
\end{tabular}


Table 4.4 Continued

High-income countries

\begin{tabular}{|c|c|c|c|c|c|}
\hline Disease & $\begin{array}{l}\text { Proportion of } \\
\text { regional disease } \\
\text { burden }(\%) \text { [total: } \\
149.2 \text { million } \\
\text { DALYs }(3,0)]\end{array}$ & $\begin{array}{l}\text { Proportion of regional } \\
\text { mortality (\%) (total: } \\
7.9 \text { million deaths) }\end{array}$ & PAFs for individual risk factors (for disease burden) & $\begin{array}{l}\text { Joint PAF - } \\
\text { disease burden (\%) }\end{array}$ & $\begin{array}{l}\text { Joint PAF - } \\
\text { mortality (\%) }\end{array}$ \\
\hline Ischemic heart disease & 8.3 & 17.3 & $\begin{array}{l}\text { High blood pressure (48\%); high cholesterol (57\%); overweight and } \\
\text { obesity ( } 27 \%) \text {; low fruit and vegetable intake (19\%); physical } \\
\text { inactivity ( } 21 \%) \text {; smoking }(23 \%) \text {; alcohol use }(-13 \%) \text {; urban air } \\
\text { pollution }(1 \%)^{\text {b }}\end{array}$ & 84 & 80 \\
\hline Stroke & 6.3 & 9.9 & $\begin{array}{l}\text { High blood pressure (56\%); high cholesterol }(25 \%) \text {; overweight and } \\
\text { obesity }(20 \%) \text {; low fruit and vegetable intake }(9 \%) \text {; physical } \\
\text { inactivity }(8 \%) \text {; smoking }(21 \%) \text {; alcohol use }(-11 \%) \text {; urban air } \\
\text { pollution }(1 \%)^{b}\end{array}$ & 68 & 54 \\
\hline Unipolar depressive disorders & 5.6 & 0.0 & Alcohol use (4\%); childhood sexual abuse (4\%) & 7 & $N A^{c}$ \\
\hline $\begin{array}{l}\text { Dementia and other } \\
\text { degenerative and } \\
\text { hereditary central nervous } \\
\text { system disorders }\end{array}$ & 5.0 & 2.6 & None of the selected risks & NA & NA \\
\hline $\begin{array}{l}\text { Trachea, bronchus, and } \\
\text { lung cancers }\end{array}$ & 3.6 & 5.8 & $\begin{array}{l}\text { Indoor smoke from solid fuels (coal only) (0\%); smoking }(84 \%) \text {; } \\
\text { low fruit and vegetable intake }(9 \%) \text {; urban air pollution }(3 \%)^{b}\end{array}$ & 86 & 87 \\
\hline Hearing loss & 3.6 & 0.0 & None of the selected risks & NA & NA \\
\hline $\begin{array}{l}\text { Chronic obstructive } \\
\text { pulmonary disease }\end{array}$ & 3.5 & 3.8 & $\begin{array}{l}\text { Indoor smoke from solid fuels }(0 \%) \text {; urban air pollution }(1 \%)^{b} \text {; } \\
\text { smoking }(73 \%)\end{array}$ & 73 & 76 \\
\hline Diabetes mellitus & 2.8 & 2.6 & $\begin{array}{l}\text { Overweight and obesity }(76 \%) \text {; physical inactivity }(15 \%) \text {; } \\
\text { smoking }(4 \%)^{\text {a }} \text {; alcohol use }(-5 \%)\end{array}$ & 78 & 74 \\
\hline Alcohol use disorders & 2.8 & 0.3 & Alcohol use (100\%); childhood sexual abuse $(6 \%)$ & 100 & 100 \\
\hline Osteoarthritis & 2.7 & 2.4 & Overweight and obesity (20\%); smoking $(4 \%)^{a}$ & 20 & $N A^{c}$ \\
\hline $\begin{array}{l}\text { Communicable, maternal, } \\
\text { perinatal, and nutritional } \\
\text { conditions }\end{array}$ & 5.7 & 7.0 & Multiple risks (see annex 4A) & 18 & 18 \\
\hline Noncommunicable diseases & 86.7 & 87.0 & Multiple risks (see annex 4A) & 37 & 48 \\
\hline Injuries & 7.5 & 6.0 & Multiple risks (see annex 4A) & 21 & 22 \\
\hline All causes & 100 & 100 & All 19 selected risks (see annex 4A) & 34 & 44 \\
\hline
\end{tabular}

Source: Authors' calculations.

Note: $\mathrm{NA}=$ not applicable. Risk factors are those listed in table 4.1. These factors also contribute to other diseases that are not among the leading 10.

a. Affected by smoking in the category "other respiratory diseases" or "selected other medical causes" (Ezzati and Lopez 2003, 2004; Peto and others 1992). The PAF has large uncertainty.

. Affected by urban air pollution in the category "cardiopulmonary diseases." The PAF for individual causes has large uncertainty.

c. The number of deaths coded to "hearing loss," "unipolar depressive disorders," "osteoarthritis," and "dementia and other degenerative and hereditary central nervous system disorders" is zero or very small in the GBD database, making the mortality PAF for

these diseases undefined or unstable. 
An important finding of this analysis is the key role of nutrition in health worldwide. Approximately 11 percent of the global disease burden was attributable to the joint effects of underweight or micronutrient deficiencies. In addition, almost 16 percent of the burden ( 28 percent for those aged 30 years and older) can be attributed to risk factors that have substantial dietary determinants (high blood pressure, high cholesterol, overweight and obesity, and low fruit and vegetable intake) and to physical inactivity. These patterns are not uniform within regions, however, and the transition has been healthier in some countries than in others (Lee, Popkin, and Kim 2000; Popkin 2002a, 2002b; Popkin and others 2001). Furthermore, the major nutritional and related risk factors show interregional heterogeneity, for instance, the relative contributions of blood pressure, cholesterol, and BMI differed across regions.

At the same time, the joint contributions of these risk factors left an important part of the global disease burden unexplained, because only a small fraction of some important diseases was attributable to the risk factors considered here. These include diseases whose determinants (a) are diffuse among environmental and behavioral factors, for example, some cancers, perinatal conditions, and neuropsychiatric diseases; (b) have more complex, multifactor etiology and often heterogeneous determinants in different populations, and are therefore difficult to quantify without data on a small scale, such as tuberculosis and injuries; (c) involve long delays between risk factor exposure and disease outcome; or (d) have limited quantitative research at the population level, for instance, neuropsychiatric diseases, often as a result of the previous three factors as well as difficulties in measuring exposure or outcome (Evans 1976, 1978). The mitigation of many such conditions, including malaria, tuberculosis, and injuries, may be better guided by analyses of the effects of interventions tailored to individual settings than by risk factor analysis.

\section{Risk Factor Clusters}

In addition to estimating the joint contributions of all the risk factors in table 4.1 to the all-cause mortality and disease burden, we also examined the role of selected clusters of risks that may be of particular interest to disease prevention policies and programs. The risk factor clusters were those affecting cancers (alcohol use, smoking, low fruit and vegetable intake, indoor smoke from household use of solid fuels, urban air pollution, overweight and obesity, physical inactivity, contaminated injections in health care settings, and unsafe sex), cardiovascular diseases (high blood pressure, high cholesterol, smoking, overweight and obesity, alcohol use, physical inactivity, low fruit and vegetable intake, and urban air pollution), and child mortality (childhood underweight; vitamin A deficiency; zinc deficiency; iron deficiency anemia; unsafe water, sanitation, and hygiene; and indoor smoke from household use of solid fuels). Tables 4.5 through 4.7 show the individual and joint contributions of these risk factors to mortality and to the burden of disease for specific diseases within each cluster.

Globally, the cancers with the largest mortality fraction attributable to the risk factors in table 4.1 were cervix uteri cancer (100 percent); trachea, bronchus, and lung cancers (74 percent); and esophagus cancer (62 percent), and those with the smallest joint PAFs were colon and rectum cancers (13 percent) and leukemia ( 9 percent) (table 4.5 ). The largest number of deaths attributable to the joint effects of the risk factors was from trachea, bronchus, and lung cancer (930,000 deaths) and liver cancer (283,000 deaths), which reflects both the relatively large joint PAF and the total number of deaths from these cancers. Except for cervix uteri cancer, which was by definition fully attributable to the risk factor unsafe sex, joint PAFs were larger in high-income countries than in low- and middle-income countries for all cancer sites, mostly because of the higher contribution of smoking and alcohol use. The joint PAFs for all cancers combined, however, were similar for the two groups of countries (34 percent versus 37 percent for the disease burden), because of the distributions of total mortality from various site-specific cancers.

Almost two-thirds of all cardiovascular deaths were attributable to eight of the selected risk factors that affect these outcomes (table 4.6). The joint effects of these risk factors were much lower than the crude sum of individual effects (64 percent versus 126 percent for the disease burden), pointing to the extensive overlap in their hazards for cardiovascular diseases compared with cancers. The overlap is partly because the hazardous effects of some risks are mediated through others and partly because multiple risk factors act in combination. The joint PAF differed little between low- and middle-income and high-income countries, reflecting the high levels of multiple cardiovascular risk factors in many middle-income nations (Ezzati and others 2005). Coupled with substantially more cardiovascular deaths and a larger disease burden in low- and middle-income countries, these risk factors result in a much larger loss of healthy life in these nations.

Worldwide, approximately half of the mortality among children under five years of age (about 5 million deaths) was attributable to six major risk factors, with childhood underweight alone accounting for more than a quarter of all child 
Table 4.5 Individual and Joint Contributions of Risk Factors to Mortality and Burden of Disease from Site-Specific Cancers

\begin{tabular}{|c|c|c|c|c|c|}
\hline \multicolumn{6}{|c|}{ World } \\
\hline Cancer type & Deaths & $\begin{array}{l}\text { Proportion of } \\
\text { global disease } \\
\text { burden }(\%) \text { [total: } \\
1.54 \text { billion } \\
\text { DALYs }(3,0)]\end{array}$ & $\begin{array}{l}\text { PAFs for individual risk factors for mortality (first number) and } \\
\text { disease burden (second number) }\end{array}$ & $\begin{array}{l}\text { Joint PAF - } \\
\text { mortality (\%) }\end{array}$ & $\begin{array}{l}\text { Joint PAF - } \\
\text { disease burden (\%) }\end{array}$ \\
\hline Mouth and oropharynx & 311,633 & 0.30 & Alcohol use (16\%; $18 \%)$, smoking (42\%; 42\%) & 52 & 54 \\
\hline Esophagus & 437,511 & 0.39 & $\begin{array}{l}\text { Alcohol use (26\%; } 27 \%) \text {, smoking (42\%; } 42 \%) \text {, low fruit and vegetable } \\
\text { intake }(18 \% ; 19 \%)\end{array}$ & 62 & 63 \\
\hline Colon and rectal & 613,740 & 0.53 & $\begin{array}{l}\text { Overweight and obesity }(11 \% ; 12 \%) \text {, physical inactivity }(15 \% ; 16 \%) \text {, } \\
\text { low fruit and vegetable intake }(2 \% ; 2 \%)\end{array}$ & 13 & 14 \\
\hline Liver & 606,441 & 0.59 & $\begin{array}{l}\text { Smoking }(14 \% ; 14 \%) \text {, alcohol use }(25 \% ; 25 \%) \text {, contaminated injections } \\
\text { in health care setting }(18 \% ; 19 \%)\end{array}$ & 47 & 47 \\
\hline Pancreas & 226,981 & 0.19 & Smoking (22\%; 22\%) & 22 & 22 \\
\hline $\begin{array}{l}\text { Trachea, bronchus, } \\
\text { and lung }\end{array}$ & $1,226,574$ & 1.04 & $\begin{array}{l}\text { Smoking }(70 \% ; 67 \%) \text {, low fruit and vegetable intake }(11 \% ; 12 \%) \text {, } \\
\text { indoor smoke from household use of solid fuels }(1 \% ; 1 \%) \text {, } \\
\text { urban air pollution (5\%; } 5 \%)\end{array}$ & 76 & 74 \\
\hline Corpus uteri & 70,881 & 0.10 & Overweight and obesity $(40 \% ; 46 \%)$ & 40 & 42 \\
\hline Bladder & 175,318 & 0.14 & Smoking $(28 \% ; 27 \%)$ & 28 & 27 \\
\hline Leukemia & 263,169 & 0.32 & Smoking (9\%; 6\%) & 9 & 6 \\
\hline Selected other neoplasms ${ }^{b}$ & 145,802 & 0.14 & Alcohol use $(6 \% ; 5 \%)$ & 6 & 5 \\
\hline All other neoplasms & $1,391,507$ & 1.27 & None of the selected risk factors & 0 & 0 \\
\hline All cancers & $7,018,402$ & 6.53 & $\begin{array}{l}\text { Alcohol use }(5 \% ; 5 \%) \text {, smoking }(21 \% ; 21 \%) \text {, low fruit and vegetable } \\
\text { intake }(5 \% ; 5 \%) \text {, indoor smoke from household use of solid fuels } \\
(<0.5 \% ;<0.5 \%) \text {, urban air pollution }(1 \% ; 1 \%) \text {, overweight and obesity } \\
(2 \% ; 2 \%) \text {, physical inactivity }(2 \% ; 2 \%) \text {, contaminated injections in } \\
\text { health care setting }(2 \% ; 2 \%) \text {, unsafe sex }(3 \% ; 4 \%)\end{array}$ & 35 & 34 \\
\hline
\end{tabular}


Low- and middle-income countries

\begin{tabular}{|c|c|c|c|c|c|}
\hline \multicolumn{6}{|c|}{ Low- and middle-income countries } \\
\hline Cancer type & Deaths & $\begin{array}{l}\text { Proportion of } \\
\text { regional disease } \\
\text { burden }(\%) \text { [total: } \\
1.39 \text { billion } \\
\text { DALYs }(3,0)]\end{array}$ & $\begin{array}{l}\text { PAFs for individual risk factors for mortality (first number) and } \\
\text { disease burden (second number) }\end{array}$ & $\begin{array}{l}\text { Joint PAF- } \\
\text { mortality (\%) }\end{array}$ & $\begin{array}{l}\text { Joint PAF- } \\
\text { disease burden (\%) }\end{array}$ \\
\hline Mouth and oropharynx & 271,074 & 0.29 & Alcohol use $(14 \% ; 15 \%)$, smoking $(37 \% ; 39 \%)$ & 48 & 50 \\
\hline Esophagus & 379,760 & 0.38 & $\begin{array}{l}\text { Alcohol use }(24 \% ; 25 \%) \text {, smoking }(37 \% ; 39 \%) \text {, low fruit and vegetable } \\
\text { intake }(19 \% ; 20 \%)\end{array}$ & 58 & 60 \\
\hline Stomach & 695,426 & 0.69 & Smoking $(11 \% ; 11 \%)$, low fruit and vegetable intake $(19 \% ; 20 \%)$ & 27 & 29 \\
\hline Colon and rectal & 356,949 & 0.36 & $\begin{array}{l}\text { Overweight and obesity }(9 \% ; 9 \%) \text {, physical inactivity }(15 \% ; 16 \%) \text {, } \\
\text { low fruit and vegetable intake }(2 \% ; 3 \%)\end{array}$ & 11 & 12 \\
\hline Liver & 504,407 & 0.57 & $\begin{array}{l}\text { Smoking }(11 \% ; 12 \%) \text {, alcohol use }(23 \% ; 24 \%) \text {, contaminated injections } \\
\text { in health care setting }(21 \% ; 22 \%)\end{array}$ & 45 & 46 \\
\hline Pancreas & 116,827 & 0.12 & Smoking (15\%; 16\%) & 15 & 16 \\
\hline Trachea, bronchus, and lung & 770,938 & 0.77 & $\begin{array}{l}\text { Smoking ( } 60 \% \text {; } 59 \%) \text {, low fruit and vegetable intake }(13 \% ; 13 \%) \text {, } \\
\text { indoor smoke from household use of solid fuels }(2 \% ; 2 \%) \text {, } \\
\text { urban air pollution }(7 \% ; 7 \%)\end{array}$ & 69 & 68 \\
\hline Breast & 317,195 & 0.40 & $\begin{array}{l}\text { Alcohol use }(4 \% ; 4 \%) \text {, overweight and obesity }(7 \% ; 7 \%) \text {, } \\
\text { physical inactivity }(10 \% ; 10 \%)\end{array}$ & 18 & 17 \\
\hline Cervix uteri & 218,064 & 0.27 & Smoking $(2 \% ; 2 \%)$, unsafe sex $(100 \% ; 100 \%)^{a}$ & 100 & 100 \\
\hline Corpus uteri & 43,926 & 0.07 & Overweight and obesity (37\%; 39\%) & 37 & 39 \\
\hline Bladder & 116,682 & 0.11 & Smoking (21\%; $21 \%)$ & 21 & 21 \\
\hline Leukemia & 190,059 & 0.28 & Smoking $(6 \% ; 4 \%)$ & 6 & 4 \\
\hline Selected other neoplasms ${ }^{b}$ & 88,706 & 0.11 & Alcohol use $(4 \% ; 4 \%)$ & 4 & 4 \\
\hline All other neoplasms & 882,001 & 0.95 & None of the selected risk factors & 0 & 0 \\
\hline All cancers & $4,952,014$ & 5.37 & $\begin{array}{l}\text { Alcohol use }(5 \% ; 6 \%) \text {, smoking }(18 \% ; 19 \%) \text {, low fruit and vegetable } \\
\text { intake }(6 \% ; 6 \%) \text {, indoor smoke from household use of solid fuels } \\
(<0.5 \% ;<0.5 \%) \text {, urban air pollution }(1 \% ; 1 \%) \text {, overweight and obesity } \\
(1 \% ; 2 \%) \text {, physical inactivity }(2 \% ; 2 \%) \text {, contaminated injections in } \\
\text { health care setting }(2 \% ; 2 \%) \text {, unsafe sex }(4 \% ; 5 \%)\end{array}$ & 34 & (Continues on the followina page) \\
\hline
\end{tabular}




\begin{tabular}{|c|c|c|c|c|c|}
\hline \multicolumn{6}{|c|}{ High-income countries } \\
\hline Cancer type & Deaths & $\begin{array}{l}\text { Proportion of } \\
\text { regional disease } \\
\text { burden }(\%) \text { [total: } \\
149.2 \text { million } \\
\text { DALYs }(3,0)]\end{array}$ & $\begin{array}{l}\text { PAFs for individual risk factors for mortality (first number) and } \\
\text { disease burden (second number) }\end{array}$ & $\begin{array}{l}\text { Joint PAF - } \\
\text { mortality (\%) }\end{array}$ & $\begin{array}{l}\text { Joint PAF - } \\
\text { disease burden (\%) }\end{array}$ \\
\hline Mouth and oropharynx & 40,559 & 0.39 & Alcohol use (33\%; 35\%), smoking (71\%; 71\%) & 80 & 80 \\
\hline Esophagus & 57,752 & 0.47 & $\begin{array}{l}\text { Alcohol use }(41 \% ; 43 \%) \text {, smoking }(71 \% ; 71 \%) \text {, low fruit and vegetable } \\
\text { intake }(12 \% ; 13 \%)\end{array}$ & 85 & 86 \\
\hline Stomach & 146,267 & 1.09 & Smoking (25\%; $25 \%)$, low fruit and vegetable intake (12\%; 13\%) & 34 & 35 \\
\hline Colon and rectal & 256,791 & 2.13 & $\begin{array}{l}\text { Overweight and obesity }(14 \% ; 15 \%) \text {, physical inactivity }(14 \% ; 16 \%) \text {, } \\
\text { low fruit and vegetable intake }(1 \% ; 2 \%)\end{array}$ & 15 & 17 \\
\hline Liver & 102,033 & 0.82 & $\begin{array}{l}\text { Smoking }(29 \% ; 29 \%) \text {, alcohol use }(32 \% ; 33 \%) \text {, contaminated injections } \\
\text { in health care setting }(3 \% ; 4 \%)\end{array}$ & 52 & 54 \\
\hline Pancreas & 110,154 & 0.83 & Smoking $(30 \% ; 30 \%)$ & 30 & 30 \\
\hline Trachea, bronchus, and lung & 455,636 & 3.62 & $\begin{array}{l}\text { Smoking }(86 \% ; 84 \%) \text {, low fruit and vegetable intake }(8 \% ; 9 \%) \text {, } \\
\text { indoor smoke from household use of solid fuels }(0 \% ; 0 \%) \text {, } \\
\text { urban air pollution }(3 \% ; 3 \%)\end{array}$ & 87 & 86 \\
\hline Breast & 155,230 & 1.68 & $\begin{array}{l}\text { Alcohol use (9\%; } 9 \%) \text {, overweight and obesity (13\%; 12\%), } \\
\text { physical inactivity (9\%; } 10 \%)\end{array}$ & 27 & 26 \\
\hline Cervix uteri & 16,663 & 0.21 & Smoking (11\%; $10 \%)$, unsafe sex $(100 \% ; 100 \%)^{a}$ & 100 & 100 \\
\hline Corpus uteri & 26,955 & 0.39 & Overweight and obesity (43\%; 46\%) & 43 & 46 \\
\hline Bladder & 58,636 & 0.45 & Smoking (41\%; $41 \%)$ & 41 & 41 \\
\hline Leukemia & 73,110 & 0.62 & Smoking (17\%; 15\%) & 17 & 15 \\
\hline Selected other neoplasms ${ }^{b}$ & 57,095 & 0.37 & Alcohol use (8\%; 9\%) & 8 & 9 \\
\hline All other neoplasms & 509,507 & 4.28 & None of the selected risk factors & 0 & 0 \\
\hline All cancers & $2,066,388$ & 17.35 & $\begin{array}{l}\text { Alcohol use }(4 \% ; 5 \%) \text {, smoking }(29 \% \text {; } 29 \%) \text {, low fruit and vegetable } \\
\text { intake }(3 \% ; 3 \%) \text {, indoor smoke from household use of solid fuels } \\
(0 \% ; 0 \%) \text {, urban air pollution }(1 \% ; 1 \%) \text {, overweight and obesity } \\
(3 \% ; 4 \%) \text {, physical inactivity }(2 \% ; 3 \%) \text {, contaminated injections in } \\
\text { health care setting }(<0.5 \% ;<0.5 \%) \text {, unsafe sex }(1 \% ; 1 \%)\end{array}$ & 37 & 37 \\
\hline
\end{tabular}

Source: Authors' calculations.

Note: Risk factors are those listed in table 4.1

a. Currently, a proportion of human papilloma virus (HPV) infections that lead to cervix uteri cancer are transmitted through routes other than sexual contact. The PAF for unsafe sex, as defined in the CRA project (Slaymaker and others 2004), measures the current population-level cervix cancer mortality that would be reduced, had there never been any sexual transmission of infection (that is, the consequences of past and current exposure, as we do for accumulated hazards of smoking). By considering the health consequences of past and current exposure, nearly all sexually transmitted diseases are attributable to unsafe sex. This is because, in the absence of sexual transmission in the past, current infections transmitted through other forms of contact would not occur if the infected hosts acquired their infection sexually (and so on in the sequence of past infected hosts).

b. This category includes neoplasms under the ICD-9 three-digit codes 210-239. 
Table 4.6 Individual and Joint Contributions of Risk Factors to Mortality and Burden of Disease from Cardiovascular Diseases

\begin{tabular}{|c|c|c|c|c|c|}
\hline \multicolumn{6}{|c|}{ World } \\
\hline Disease & Deaths & $\begin{array}{l}\text { Proportion of } \\
\text { global disease } \\
\text { burden }(\%) \text { [total: } \\
1.54 \text { billion } \\
\text { DALYs }(3,0) \text { ] }\end{array}$ & $\begin{array}{l}\text { PAFs for individual risk factors for mortality (first number) and } \\
\text { disease burden (second number) }\end{array}$ & $\begin{array}{l}\text { Joint PAF - } \\
\text { mortality (\%) }\end{array}$ & $\begin{array}{l}\text { Joint PAF - } \\
\text { disease burden (\%) }\end{array}$ \\
\hline Ischemic heart disease & $7,060,851$ & 5.5 & $\begin{array}{l}\text { High blood pressure }(47 \% \text {; } 45 \%) \text {, high cholesterol }(45 \% ; 48 \%) \text {, } \\
\text { smoking }(12 \% ; 17 \%) \text {, overweight and obesity }(15 \% ; 18 \%) \text {, } \\
\text { alcohol use (1\%; } 2 \%) \text {, physical inactivity }(19 \% ; 21 \%) \text {, low fruit and } \\
\text { vegetable intake (25\%; } 28 \%) \text {, urban air pollution }(3 \% ; 2 \%)^{a}\end{array}$ & 79 & 80 \\
\hline Stroke & $5,387,500$ & 4.7 & $\begin{array}{l}\text { High blood pressure (54\%; } 54 \%) \text {, high cholesterol }(13 \% ; 16 \%) \text {, } \\
\text { smoking }(8 \% ; 13 \%) \text {, overweight and obesity }(8 \% ; 12 \%) \text {, alcohol } \\
\text { use }(2 \% ; 3 \%) \text {, physical inactivity }(6 \% ; 7 \%) \text {, low fruit and vegetable } \\
\text { intake }(9 \% ; 11 \%) \text {, urban air pollution }(3 \% ; 2 \%)^{a}\end{array}$ & 60 & 65 \\
\hline Hypertensive disease & 888,144 & 0.7 & $\begin{array}{l}\text { High blood pressure }(79 \% ; 75 \%) \text {, overweight and obesity } \\
(29 \% ; 33 \%) \text {, alcohol use }(15 \% ; 15 \%) \text {, urban air pollution }(3 \% ; 2 \%)^{\text {a }}\end{array}$ & 85 & 83 \\
\hline $\begin{array}{l}\text { Selected other cardiovascular } \\
\text { diseases }^{b}\end{array}$ & $2,335,514$ & 1.8 & High blood pressure $(28 \% ; 24 \%)$, smoking $(14 \% ; 12 \%)$ & 37 & 33 \\
\hline All cardiovascular diseases & $16,386,531$ & 13.5 & $\begin{array}{l}\text { High blood pressure (46\%; } 44 \%) \text {, high cholesterol }(24 \% ; 25 \%) \text {, } \\
\text { smoking }(10 \% ; 13 \%) \text {, overweight and obesity }(11 \% ; 13 \%) \text {, } \\
\text { alcohol use }(2 \% ; 3 \%) \text {, physical inactivity }(10 \% ; 11 \%) \text {, low fruit } \\
\text { and vegetable intake }(14 \% ; 15 \%) \text {, urban air pollution }(2 \% ; 2 \%)^{\text {a }}\end{array}$ & 63 & 64 \\
\hline
\end{tabular}

\begin{tabular}{|c|c|c|c|c|c|}
\hline \multicolumn{6}{|c|}{ Low- and middle-income countries } \\
\hline Disease & Deaths & $\begin{array}{l}\text { Proportion of } \\
\text { regional disease } \\
\text { burden }(\%) \text { [total: } \\
1.39 \text { billion } \\
\text { DALYs(3,0)] }\end{array}$ & $\begin{array}{l}\text { PAFs for individual risk factors for mortality (first number) and } \\
\text { disease burden (second number) }\end{array}$ & $\begin{array}{l}\text { Joint PAF - } \\
\text { mortality (\%) }\end{array}$ & $\begin{array}{l}\text { Joint PAF - } \\
\text { disease burden (\%) }\end{array}$ \\
\hline Ischemic heart disease & $5,696,844$ & 5.2 & $\begin{array}{l}\text { High blood pressure (47\%; } 44 \%) \text {, high cholesterol }(43 \% ; 46 \%) \text {, } \\
\text { smoking }(11 \% ; 15 \%) \text {, overweight and obesity }(14 \% ; 16 \%) \text {, } \\
\text { alcohol use (4\%; } 4 \%) \text {, physical inactivity }(20 \% ; 21 \%) \text {, low fruit and } \\
\text { vegetable intake (27\%; 30\%), urban air pollution }(4 \% ; 2 \%)^{\mathrm{a}}\end{array}$ & 78 & 80 \\
\hline Stroke & $4,606,426$ & 4.5 & $\begin{array}{l}\text { High blood pressure }(54 \% ; 54 \%) \text {, high cholesterol }(12 \% ; 15 \%) \text {, } \\
\text { smoking }(8 \% ; 12 \%) \text {, overweight and obesity }(7 \% ; 10 \%) \text {, alcohol } \\
\text { use }(5 \% ; 5 \%) \text {, physical inactivity }(6 \% ; 6 \%) \text {, low fruit and vegetable } \\
\text { intake }(10 \% ; 11 \%) \text {, urban air pollution }(4 \% ; 2 \%)^{a}\end{array}$ & 61 & 64 \\
\hline Hypertensive disease & 759,487 & 0.7 & $\begin{array}{l}\text { High blood pressure }(79 \% ; 75 \%) \text {, overweight and obesity } \\
(28 \% ; 31 \%) \text {, alcohol use }(14 \% ; 14 \%) \text {, urban air pollution }(4 \% ; 2 \%)^{a}\end{array}$ & 84 & 82 \\
\hline
\end{tabular}




\begin{tabular}{|c|c|c|c|c|c|}
\hline \multicolumn{6}{|c|}{ Low- and middle-income countries } \\
\hline Disease & Deaths & $\begin{array}{l}\text { Proportion of } \\
\text { regional disease } \\
\text { burden }(\%) \text { [total: } \\
1.39 \text { billion } \\
\text { DALYs }(3,0) \text { ] }\end{array}$ & $\begin{array}{l}\text { PAFs for individual risk factors for mortality (first number) and } \\
\text { disease burden (second number) }\end{array}$ & $\begin{array}{l}\text { Joint PAF - } \\
\text { mortality (\%) }\end{array}$ & $\begin{array}{l}\text { Joint PAF - } \\
\text { disease burden (\%) }\end{array}$ \\
\hline $\begin{array}{l}\text { Selected other cardiovascular } \\
\text { diseases }^{b}\end{array}$ & $1,659,570$ & 1.6 & High blood pressure (27\%; 23\%), smoking (9\%; 9\%) & 33 & 29 \\
\hline All cardiovascular diseases & $13,347,471$ & 12.9 & $\begin{array}{l}\text { High blood pressure (47\%; } 44 \%) \text {, high cholesterol }(23 \% ; 24 \%) \text {, } \\
\text { smoking }(9 \% ; 11 \%) \text {, overweight and obesity }(10 \% ; 12 \%) \text {, } \\
\text { alcohol use }(4 \% ; 4 \%) \text {, physical inactivity }(10 \% ; 11 \%) \text {, low fruit } \\
\text { and vegetable intake }(15 \% ; 16 \%) \text {, urban air pollution }(3 \% ; 2 \%)^{a}\end{array}$ & 64 & 63 \\
\hline \multicolumn{6}{|c|}{ High-income countries } \\
\hline Disease & Deaths & $\begin{array}{l}\text { Proportion of } \\
\text { regional disease } \\
\text { burden }(\%) \text { [total; } \\
142.9 \text { million } \\
\text { DALYs }(3,0) \text { ] }\end{array}$ & $\begin{array}{l}\text { PAFs for individual risk factors for mortality (first number) and } \\
\text { disease burden (second number) }\end{array}$ & $\begin{array}{l}\text { Joint PAF - } \\
\text { mortality (\%) }\end{array}$ & $\begin{array}{l}\text { Joint PAF - } \\
\text { disease burden (\%) }\end{array}$ \\
\hline Ischemic heart disease & $1,364,007$ & 8.3 & $\begin{array}{l}\text { High blood pressure (49\%; } 48 \%) \text {, high cholesterol }(52 \% ; 57 \%) \text {, smoking } \\
(15 \% ; 23 \%) \text {, overweight and obesity }(19 \% ; 27 \%) \text {, alcohol use } \\
(-12 \% ;-13 \%) \text {, physical inactivity }(19 \% ; 21 \%) \text {, low fruit and vegetable } \\
\text { intake }(16 \% ; 19 \%) \text {, urban air pollution }(2 \% ; 1 \%)^{\text {a }}\end{array}$ & 80 & 84 \\
\hline Stroke & 781,074 & 6.3 & $\begin{array}{l}\text { High blood pressure (54\%; } 56 \%) \text {, high cholesterol }(17 \% ; 25 \%) \text {, } \\
\text { smoking }(10 \% ; 21 \%) \text {, overweight and obesity }(12 \% ; 20 \%) \text {, } \\
\text { alcohol use }(-13 \% ;-11 \%) \text {, physical inactivity }(6 \% ; 8 \%) \text {, low fruit } \\
\text { and vegetable intake }(6 \% ; 9 \%) \text {, urban air pollution }(2 \% ; 1 \%)^{a}\end{array}$ & 54 & 68 \\
\hline Hypertensive disease & 128,657 & 0.8 & $\begin{array}{l}\text { High blood pressure }(84 \% ; 82 \%) \text {, overweight and obesity }(38 \% ; 45 \%) \text {, } \\
\text { alcohol use }(23 \% ; 24 \%) \text {, urban air pollution }(2 \% ; 1 \%)^{a}\end{array}$ & 91 & 91 \\
\hline $\begin{array}{l}\text { Selected other cardiovascular } \\
\text { diseases }^{b}\end{array}$ & 675,944 & 3.8 & High blood pressure (29\%; 29\%), smoking (26\%; 25\%) & 48 & 46 \\
\hline All cardiovascular diseases & $3,039,060$ & 20.0 & $\begin{array}{l}\text { High blood pressure }(46 \% ; 46 \%) \text {, high cholesterol }(28 \% ; 32 \%) \text {, } \\
\text { smoking }(15 \% ; 21 \%) \text {, overweight and obesity }(13 \% ; 19 \%) \text {, alcohol } \\
\text { use }(-8 \% ;-8 \%) \text {, physical inactivity }(10 \% ; 11 \%) \text {, low fruit and } \\
\text { vegetable intake }(9 \% ; 11 \%) \text {, urban air pollution }(1 \% ; 1 \%)^{\text {a }}\end{array}$ & 64 & 69 \\
\hline
\end{tabular}

Source: Authors' calculations.

Note: Risk factors are those listed in table 4.1.

a. Affected by urban air pollution in the category "cardiopulmonary diseases." The PAFs for individual causes have large uncertainty.

b. This category includes ICD-9 three-digit codes 415-417, 423-424, 426-429, 440-448, 451-459. 
Table 4.7 Individual and Joint Contributions of Risk Factors to Mortality and Burden of Disease from Major Diseases of Children

\begin{tabular}{|c|c|c|c|c|c|}
\hline \multicolumn{6}{|c|}{ World } \\
\hline Disease $^{a}$ & Deaths & $\begin{array}{l}\text { Proportion of } \\
\text { global disease } \\
\text { burden }(\%) \text { [total: } \\
1.54 \text { billion } \\
\text { DALYs }(3,0)]\end{array}$ & $\begin{array}{l}\text { PAFs for individual risk factors for mortality (first number) and } \\
\text { disease burden (second number) }\end{array}$ & $\begin{array}{l}\text { Joint PAF - } \\
\text { mortality (\%) }\end{array}$ & $\begin{array}{l}\text { Joint PAF - } \\
\text { disease burden (\%) }\end{array}$ \\
\hline Measles & 556,384 & 1.1 & Childhood underweight (44\%; 44\%), vitamin A deficiency (20\%; 20\%) & 55 & 55 \\
\hline Diarrheal diseases & $1,598,754$ & 3.5 & $\begin{array}{l}\text { Childhood underweight ( } 61 \% ; 55 \%) \text {, vitamin A deficiency }(24 \% ; 22 \%) \text {, } \\
\text { zinc deficiency (13\%; } 12 \%) \text {, unsafe water, sanitation, and } \\
\text { hygiene ( } 88 \% ; 88 \%)\end{array}$ & 94 & 93 \\
\hline Lower respiratory infections & $1,914,189$ & 4.0 & $\begin{array}{l}\text { Childhood underweight (54\%; } 52 \%) \text {, zinc deficiency }(21 \% ; 21 \%) \text {, } \\
\text { indoor air pollution (49\%; } 48 \%)\end{array}$ & 72 & 70 \\
\hline Malaria & $1,086,298$ & 2.3 & $\begin{array}{l}\text { Childhood underweight (55\%; } 51 \%) \text {, vitamin A deficiency (19\%; 19\%), } \\
\text { zinc deficiency (22\%; 22\%) }\end{array}$ & 68 & 65 \\
\hline $\begin{array}{l}\text { Conditions arising during } \\
\text { the perinatal period }\end{array}$ & $2,520,986$ & 5.9 & Iron-deficiency anemia (16\%; 14\%) & 16 & 14 \\
\hline All other childhood diseases & $2,921,834$ & 11.2 & $\begin{array}{l}\text { Partially attributable to childhood underweight and vitamin A deficiency } \\
\text { (see annex } 4 \mathrm{~A} \text { for details). }\end{array}$ & Not estimated & Not estimated \\
\hline All causes & $10,598,444$ & 28.0 & $\begin{array}{l}\text { Childhood underweight (27\%; } 20 \%) \text {, vitamin A deficiency }(7 \% ; 5 \%) \text {, } \\
\text { zinc deficiency }(8 \% ; 6 \%) \text {, unsafe water, sanitation, and hygiene } \\
(13 \% ; 11 \%) \text {, indoor air pollution }(9 \% ; 7 \%) \text {, iron-deficiency } \\
\text { anemia }(4 \% ; 3 \%)\end{array}$ & 49 & 40 \\
\hline \multicolumn{6}{|c|}{ Low- and middle-income countries } \\
\hline Disease $^{a}$ & Deaths & $\begin{array}{l}\text { Proportion of } \\
\text { regional disease } \\
\text { burden }(\%) \text { [total: } \\
1.39 \text { billion } \\
\text { DALYs }(3,0) \text { ] }\end{array}$ & $\begin{array}{l}\text { PAFs for individual risk factors for mortality (first number) and } \\
\text { disease burden (second number) }\end{array}$ & $\begin{array}{l}\text { Joint PAF - } \\
\text { mortality (\%) }\end{array}$ & $\begin{array}{l}\text { Joint PAF - } \\
\text { disease burden (\%) }\end{array}$ \\
\hline Measles & 556,312 & 1.2 & Childhood underweight (44\%; 44\%), vitamin A deficiency (20\%; 20\%) & 55 & 55 \\
\hline Diarrheal diseases & $1,598,336$ & 3.8 & $\begin{array}{l}\text { Childhood underweight ( } 61 \% ; 56 \%) \text {, vitamin A deficiency }(24 \% ; 22 \%) \text {, } \\
\text { zinc deficiency (13\%; } 12 \%) \text {, unsafe water, sanitation, and } \\
\text { hygiene (88\%; } 88 \%)\end{array}$ & 94 & 93 \\
\hline Lower respiratory infections & $1,912,599$ & 4.4 & $\begin{array}{l}\text { Childhood underweight (54\%; } 52 \%) \text {, zinc deficiency }(21 \% ; 21 \%) \text {, } \\
\text { indoor air pollution (49\%; } 48 \%)\end{array}$ & 72 & 70 \\
\hline Malaria & $1,086,237$ & 2.6 & $\begin{array}{l}\text { Childhood underweight (55\%; } 51 \%) \text {, vitamin A deficiency (19\%; 19\%), } \\
\text { zinc deficiency (22\%; 22\%) }\end{array}$ & 68 & 65 \\
\hline $\begin{array}{l}\text { Conditions arising during } \\
\text { the perinatal period }\end{array}$ & $2,488,718$ & 6.4 & Iron-deficiency anemia (16\%; 14\%) & 16 & 14 \\
\hline
\end{tabular}




\begin{tabular}{|c|c|c|c|c|c|}
\hline \multicolumn{6}{|c|}{ Low- and middle-income countries } \\
\hline Disease $^{a}$ & Deaths & $\begin{array}{l}\text { Proportion of } \\
\text { regional disease } \\
\text { burden }(\%) \text { [total: } \\
1.39 \text { billion } \\
\text { DALYs(3,0)] }\end{array}$ & $\begin{array}{l}\text { PAFs for individual risk factors for mortality (first number) and } \\
\text { disease burden (second number) }\end{array}$ & $\begin{array}{l}\text { Joint PAF - } \\
\text { mortality (\%) }\end{array}$ & $\begin{array}{l}\text { Joint PAF - } \\
\text { disease burden (\%) }\end{array}$ \\
\hline All other childhood diseases & $2,883,496$ & 12.0 & $\begin{array}{l}\text { Partially attributable to childhood underweight and vitamin A } \\
\text { deficiency (see annex } 4 \text { A for details). }\end{array}$ & Not estimated & Not estimated \\
\hline All causes & $10,525,699$ & 30.5 & $\begin{array}{l}\text { Childhood underweight (27\%; } 21 \%) \text {, vitamin A deficiency }(7 \% ; 5 \%) \text {, } \\
\text { zinc deficiency (8\%; } 7 \%) \text {, unsafe water, sanitation, and hygiene (13\%; } 11 \%) \text {, } \\
\text { indoor air pollution }(9 \% ; 7 \%) \text {, iron-deficiency anemia }(4 \% ; 3 \%)\end{array}$ & 49 & 41 \\
\hline \multicolumn{6}{|c|}{ High-income countries } \\
\hline Disease $^{a}$ & Deaths & $\begin{array}{l}\text { Proportion of } \\
\text { regional disease } \\
\text { burden }(\%) \text { [total: } \\
149.2 \text { million } \\
\text { DALYs }(3,0)]\end{array}$ & $\begin{array}{l}\text { PAFs for individual risk factors for mortality (first number) and } \\
\text { disease burden (second number) }\end{array}$ & $\begin{array}{l}\text { Joint PAF - } \\
\text { mortality (\%) }\end{array}$ & $\begin{array}{l}\text { Joint PAF - } \\
\text { disease burden (\%) }\end{array}$ \\
\hline Measles & 73 & 0.0 & Childhood underweight (0\%; $0 \%)$, vitamin A deficiency (1\%; $0 \%)$ & 1 & 0 \\
\hline Diarrheal diseases & 418 & 0.2 & $\begin{array}{l}\text { Childhood underweight }(0 \% ; 0 \%) \text {, vitamin A deficiency }(1 \% ; 0 \%) \text {, } \\
\text { zinc deficiency }(1 \% ; 1 \%) \text {, unsafe water, sanitation, and hygiene } \\
(65 \% ; 65 \%)\end{array}$ & 66 & 66 \\
\hline Lower respiratory infections & 1,590 & 0.0 & $\begin{array}{l}\text { Childhood underweight }(0 \% ; 0 \%) \text {, zinc deficiency }(3 \% ; 3 \%) \text {, } \\
\text { indoor air pollution }(0 \% ; 0 \%)\end{array}$ & 1 & 1 \\
\hline Malaria & 61 & 0.0 & $\begin{array}{l}\text { Childhood underweight (0\%; } 0 \%) \text {, vitamin A deficiency }(1 \% ; 1 \%) \text {, } \\
\text { zinc deficiency }(3 \% ; 3 \%)\end{array}$ & 2 & 2 \\
\hline $\begin{array}{l}\text { Conditions arising during } \\
\text { the perinatal period }\end{array}$ & 32,268 & 0.9 & Iron-deficiency anemia (3\%; 2\%) & 3 & 2 \\
\hline All other childhood diseases & 38,337 & 3.4 & $\begin{array}{l}\text { Partially attributable to childhood underweight and vitamin A } \\
\text { deficiency (see annex } 4 \text { A for details). }\end{array}$ & Not estimated & Not estimated \\
\hline All causes & 72,746 & 4.6 & $\begin{array}{l}\text { Childhood underweight }(0 \% ; 0 \%) \text {, vitamin A deficiency }(0 \% ; 0 \%) \text {, } \\
\text { zinc deficiency }(0 \% ; 0 \%) \text {, unsafe water, sanitation, and hygiene } \\
(0 \% ; 2 \%) \text {, indoor air pollution }(0 \% ; 0 \%) \text {, iron-deficiency anemia } \\
(1 \% ; 0 \%)\end{array}$ & 2 & 4 \\
\hline
\end{tabular}

Source: Authors' calculations.

Note: Risk factors are those listed in table 4.1.

a. By definition, the diseases protein-energy malnutrition (ICD-9 three-digit codes 260-263), iron-deficiency anemia (ICD-9 three-digit codes 280-285), and vitamin A deficiency (ICD-9 three-digit code 264) are 100 percent attributable to their corresponding namesake risk factors. Thus, the PAFs for these diseases are not shown. PAFs, attributable mortality, and attributable disease burden are reported in annex 4A. 
deaths. Practically all the mortality and disease burden from childhood diseases attributable to major risk factors occurred in low- and middle-income countries (table 4.7). The reasons for this large disparity in the disease burden attributable to risk factors are higher risk factor exposure coupled with lower access to case management, which affects child mortality together with risk factor exposure.

\section{DIRECTIONS FOR FUTURE RESEARCH}

Health research has at times focused on topics that, while scientifically intriguing, have not always taken population health consequences into account when shaping specific research questions (Editorial 2001; Gross, Anserson, and Powe 1999; Horton 2003). The collation of evidence on exposure and hazard for different risks and the existing data gaps revealed the areas where data and monitoring need to be improved for better quantification of important risks and for more effective intervention. This includes the need for more detailed and higher quality data on exposure to most risks using exposure variables that capture the full distribution of hazards in the population. Important examples include detailed data on alcohol consumption volumes and patterns, dietary and biological markers for micronutrients, physical activity, and indoor smoke from household use of solid fuels, all of which were quantified using indirect measures with limited resolution. Furthermore, assumptions and extrapolations were needed in quantifying the relationships between risk factors and disease because of research gaps for some important global risk factors. Examples include limited quantitative assessments of the hazards of specific sexual behaviors for HIV/AIDS and other sexually transmitted diseases (UNAIDS 2001), of alcohol drinking patterns (Puddey and others 1999), or of exposure to indoor smoke from household use of solid fuels (Ezzati and Kammen 2002). Equally important are detailed exposure data for risks that have traditionally been studied in developed countries, but have global importance and require more detailed data and hazard quantification in developing regions, including smoking, body mass index, blood pressure, and cholesterol (Yusuf and others 2004).

The limited evidence on the effects of multiple risk factors and risk factor interactions also points to important gaps in research on multirisk and stratified hazards. Including multiple layers of causality in epidemiological research and risk assessment would allow investigators to estimate the benefits of reducing combinations of distal and proximal exposures using multiple interventions. Examples of such integrated strategies include using education and economic tools to promote physical activity and a healthier diet coupled with screening and lowering cholesterol, and addressing the overall childhood nutrition and physical environment instead of focusing on individual components. In such research, risk factor groups should be selected based on both biological relationships and socioeconomic factors that affect multiple diseases. Examples include those risk factors that are affected by the same policies and distal socioeconomic factors, such as malnutrition; unsafe water, sanitation, and hygiene; indoor smoke from household use of solid fuels; and rural development policies, or affect the same group of diseases, for instance, the previous example for childhood infectious diseases and smoking, diet, physical activity, and blood pressure for vascular diseases. Once risk factors are selected, the emphasis on reducing confounding should be matched by equally important inquiry into independent and mediated hazard sizes that are stratified based on the levels of other risks.

Finally, to inform interventions and policies, similar analyses should take place at smaller scales than global or regional levels, for example, rural and urban areas or different geographical regions of individual countries, and should include micro-level data and possibly a more comprehensive list of both distal and proximal risk factors, such as adverse life events and stress, risk factors for injuries, salt and fat intake, and blood glucose.

\section{DISCUSSION}

Despite inherent uncertainties in population health risk assessment, described in chapter 5 and in chapters devoted to individual risk factors elsewhere (Ezzati and others 2004), the quantification of the burden of disease attributable to the individual and joint hazards of selected risk factors illustrates that those risk factors that affect the poorest regions and populations, such as undernutrition; unsafe water, sanitation, and hygiene; and indoor smoke from household use of solid fuels, continue to dominate the loss of health worldwide. These are coupled with hazards such as alcohol use, smoking, high blood pressure, high cholesterol, and overweight and obesity that are globally widespread and have large health effects.

The large remaining burden due to childhood mortality risks such as undernutrition; unsafe water, sanitation, and hygiene; and indoor smoke from household use of solid 
fuels indicates the persistent need for developing and delivering effective interventions, including lowering the costs of pertinent technological interventions. At the same time, four of the five leading causes of lost healthy life affect adults: high blood pressure, unsafe sex, smoking, and alcohol use (figure 4.1). Risk factors for both adult communicable and noncommunicable diseases already make substantial contributions to the disease burden even in regions with low income and high infant mortality. Therefore, the public health community should continually reassess the need for interventions addressing both childhood disease risk factors and those that affect adult health. Dynamic and systematic policy responses can, to a large extent, mitigate the spread of such risk factors and their more distal causes throughout the development process, for example, through cleaner environmental or healthier nutritional transitions (Arrow and others 1995; Lee, Popkin, and Kim 2000). In addition, as illustrated by the persistence of diseases such as malaria or the large increase in the disease burden due to HIV/AIDS and its risk factors since 1990, as well as the potential for generalized HIV/AIDS epidemics in some Eastern European countries (MacLehose, McKee, and Weinberg 2002) and China (Kaufman and Jing 2002), risk factors for important communicable diseases also require dynamic monitoring and policy responses.

Risk factors that were not among the leading global causes of the disease burden should not be neglected for a number of reasons. First, the analysis could be expanded with other risk factors that are both prevalent and hazardous. Second, although smaller than other risk factors, many make non-negligible contributions to the burden of disease in various populations. For example, in the low- and middle-income countries of East Asia and the Pacific, which is dominated by China in terms of population, urban air pollution from transportation and industrial and household energy use based on coal has health effects comparable to those of micronutrient deficiencies. Similarly, non-use and use of ineffective methods of contraception was associated with a larger disease burden than most chronic disease risk factors among females in South Asia and Sub-Saharan Africa. Third, for other risk factors, such as child sexual abuse, ethical considerations may outweigh direct contribu- tions to the disease burden in policy debate. Finally, while the burden of disease due to a risk factor may be comparatively small, effective or cost-effective interventions may be known. Examples include reducing the number of unnecessary injections at health facilities coupled with the use of sterile syringes and the reduction in exposure to urban air pollution in industrial countries in the second half of the 20th century, which often also led to benefits such as energy savings.

A small number of risks account for large contributions to the global loss of healthy life. Furthermore, several are relatively prominent in regions at all stages of development. While reducing all the risks discussed to their theoretical minimums may not be possible using current interventions, the results illustrate that preventing disease by addressing known distal and proximal risk factors can provide substantial and underutilized public health gains. Treating established disease will always have a role in public health, especially in the case of diseases such as tuberculosis, where treatment contributes to prevention. At the same time, the current devotion of a disproportionately small share of resources to prevention by reducing major known risk factors through personal and nonpersonal interventions should be reconsidered in a more systematic way in light of the evidence presented here.

The estimates of the joint contributions of 19 selected global risk factors showed that these risks together contributed to a considerable loss of healthy life in different regions of the world. In particular, for some of the leading global diseases, such as lower respiratory infections, diarrhea, HIV/AIDS, lung cancer, ischemic heart disease, and stroke, substantial proportions were attributable to these selected risk factors. This concentration of the disease burden further emphasizes the contribution of leading risks such as undernutrition, unsafe sex, high blood pressure, and smoking and alcohol use to the loss of healthy life globally. The results further emphasize that for more effective and affordable implementation of a prevention paradigm, policies, programs, and scientific research should acknowledge and take advantage of the interactive and correlated role of major risks to health, across and within causality layers. 
ANNEX 4A: Population Attributable Fractions, Attributable Deaths, Years of Life Lost Because of Premature Mortality (YLL), and Disability-Adjusted Life Years (DALYs) by Risk Factor, Disease Outcome, Age, Sex, and Region

Table 4A.1

Risk factor:

Childhood underweight

Disease:

Diarrheal diseases

\begin{tabular}{|c|c|c|c|c|c|c|c|c|c|c|c|c|c|c|c|c|c|c|c|}
\hline \multirow[b]{2}{*}{ Region } & \multicolumn{2}{|c|}{$0-4$ years } & \multicolumn{2}{|c|}{ 5-14 years } & \multicolumn{2}{|c|}{$15-29$ years } & \multicolumn{2}{|c|}{$30-44$ years } & \multicolumn{2}{|c|}{ 45-59 years } & \multicolumn{2}{|c|}{$60-69$ years } & \multicolumn{2}{|c|}{$70-79$ years } & \multicolumn{2}{|c|}{$80+$ years } & \multicolumn{3}{|c|}{ Total } \\
\hline & Male & Female & Male & Female & Male & Female & Male & Female & Male & Female & Male & Female & Male & Female & Male & Female & Male & Female & All \\
\hline \multicolumn{20}{|l|}{ PAF of Mortality (\%) } \\
\hline East Asia and Pacific & 46 & 46 & NA & NA & NA & NA & NA & NA & NA & NA & NA & NA & NA & NA & NA & NA & 41 & 41 & 41 \\
\hline Europe and Central Asia & 27 & 27 & NA & NA & NA & NA & NA & NA & NA & NA & NA & NA & NA & NA & NA & NA & 22 & 21 & 22 \\
\hline Latin America and the Caribbean & 24 & 24 & NA & NA & NA & NA & NA & NA & NA & NA & NA & NA & NA & NA & NA & NA & 21 & 21 & 21 \\
\hline Middle East and North Africa & 42 & 42 & NA & NA & NA & NA & NA & NA & NA & NA & NA & NA & NA & NA & NA & NA & 37 & 38 & 38 \\
\hline South Asia & 71 & 71 & NA & NA & NA & NA & NA & NA & NA & NA & NA & NA & NA & NA & NA & NA & 64 & 64 & 64 \\
\hline Sub-Saharan Africa & 60 & 60 & NA & NA & NA & NA & NA & NA & NA & NA & NA & NA & NA & NA & NA & NA & 54 & 54 & 54 \\
\hline Low- and middle-income countries & 61 & 61 & NA & NA & NA & NA & NA & NA & NA & NA & NA & NA & NA & NA & NA & NA & 54 & 54 & 54 \\
\hline High-income countries & 0 & 0 & NA & NA & NA & NA & NA & NA & NA & NA & NA & NA & NA & NA & NA & NA & 0 & 0 & 0 \\
\hline WORLD & 60 & 61 & NA & NA & NA & NA & NA & NA & NA & NA & NA & NA & NA & NA & NA & NA & 54 & 54 & 54 \\
\hline
\end{tabular}

PAF of YLL (\%)

East Asia and Pacific

Europe and Central Asia

Latin America and the Caribbean

Middle East and North Africa

South Asia

Sub-Saharan Africa

$\begin{array}{lllllll}46 & 46 & \text { NA } & \text { NA } & \text { NA } & \text { NA } & \text { NA } \\ 27 & 27 & \text { NA } & \text { NA } & \text { NA } & \text { NA } & \text { NA } \\ 24 & 24 & \text { NA } & \text { NA } & \text { NA } & \text { NA } & \text { NA }\end{array}$

4242 NA NA NA NA NA NA

Low- and middle-income countries

High-income countries

$71 \quad 71$ NA NA NA NA NA NA NA 11 NA NA NA NA

WORLD

PAF of DALYs (\%)

East Asia and Pacific

Europe and Central Asia

Latin America and the Caribbea

Middle East and North Africa

South Asia

Sub-Saharan Africa

$61 \quad 61 \quad N A$

NA NA NA NA NA

Low- and middle-income countries

High-income countries

WORLD

Attributable Mortality (thousands

East Asia and Pacilic

Europe and Central Asia

Latin America and the Caribbean

Middle East and North Africa

South Asia

Sub-Saharan Africa

$0 \quad 0 \quad N A$

NA NA NA

NA NA NA NA

$\begin{array}{lllll}\text { NA } & \text { NA } & \text { NA } & \text { NA } & \text { NA } \\ \text { NA } & \text { NA } & \text { NA } & \text { NA } & \text { NA } \\ \text { NA } & \text { NA } & \text { NA } & \text { NA } & \text { NA } \\ \text { NA } & \text { NA } & \text { NA } & \text { NA } & \text { NA } \\ \text { NA } & \text { NA } & \text { NA } & \text { NA } & \text { NA } \\ \text { NA } & \text { NA } & \text { NA } & \text { NA } & \text { NA } \\ \text { NA } & \text { NA } & \text { NA } & \text { NA } & \text { NA }\end{array}$

$\begin{array}{lllllll}\text { NA } & \text { NA } & \text { NA } & \text { NA } & 43 & 44 & 44 \\ \text { NA } & \text { NA } & \text { NA } & \text { NA } & 24 & 24 & 24 \\ \text { NA } & \text { NA } & \text { NA } & \text { NA } & 23 & 23 & 23 \\ \text { NA } & \text { NA } & \text { NA } & \text { NA } & 40 & 40 & 40 \\ \text { NA } & \text { NA } & \text { NA } & \text { NA } & 68 & 68 & 68 \\ \text { NA } & \text { NA } & \text { NA } & \text { NA } & 57 & 58 & 57 \\ \text { NA } & \text { NA } & \text { NA } & \text { NA } & 58 & 58 & 58\end{array}$

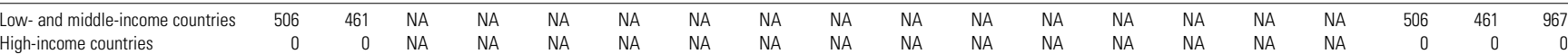

\begin{tabular}{llllllllllllllllllllllll}
\hline WORLD & 506 & 461 & NA & NA & NA & NA & NA & NA & NA & NA & NA & NA & NA & NA & NA & NA & 506 & 461 & 967
\end{tabular}

Attributable YLL (thousands)

East Asia and Pacific

Europe and Central Asia

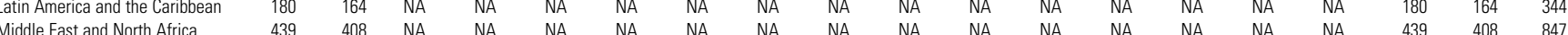

$\begin{array}{lllllllllllllllllllllll}\text { South Asia } & 7,050 & 6,510 & \text { NA } & \text { NA } & \text { NA } & \text { NA } & \text { NA } & \text { NA } & \text { NA } & \text { NA } & \text { NA } & \text { NA } & \text { NA } & \text { NA } & \text { NA } & \text { NA } & 7,050 & 6,510 & 13,560\end{array}$

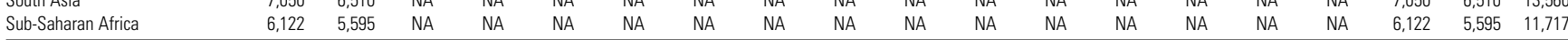

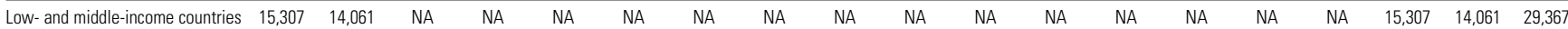

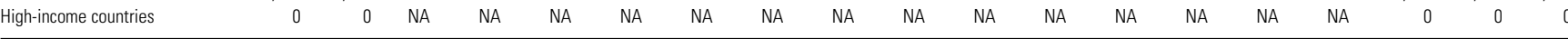

\begin{tabular}{llllllllllllllllllllll}
\hline WORLD & 15,307 & 14,061 & NA & NA & NA & NA & NA & NA & NA & NA & NA & NA & NA & NA & NA & NA & 15,307 & 14,061 & 29,367 \\
\hline
\end{tabular}

Attributable DALYs (thousands)

\begin{tabular}{|c|c|c|c|c|c|c|c|c|c|c|c|c|c|c|c|c|c|c|c|}
\hline East Asia and Pacific & 1,480 & 1,354 & NA & NA & NA & NA & NA & NA & NA & NA & NA & NA & NA & NA & NA & NA & 1,480 & 1,354 & 2,834 \\
\hline Europe and Central Asia & 52 & 47 & NA & NA & NA & NA & NA & NA & NA & NA & NA & NA & NA & NA & NA & NA & 52 & 47 & 99 \\
\hline Latin America and the Caribbean & 183 & 166 & NA & NA & NA & NA & NA & NA & NA & NA & NA & NA & NA & NA & NA & NA & 183 & 166 & 349 \\
\hline Middle East and North Africa & 444 & 413 & NA & NA & NA & NA & NA & NA & NA & NA & NA & NA & NA & NA & NA & NA & 444 & 413 & 856 \\
\hline South Asia & 7,127 & 6,582 & NA & NA & NA & NA & NA & NA & NA & NA & NA & NA & NA & NA & NA & NA & 7,127 & 6,582 & 13,709 \\
\hline Sub-Saharan Africa & 6,158 & 5,630 & NA & NA & NA & NA & NA & NA & NA & NA & NA & NA & NA & NA & NA & NA & 6,158 & 5,630 & 11,788 \\
\hline Low- and middle-income countries & 15,444 & 14,192 & NA & NA & NA & NA & NA & NA & NA & NA & NA & NA & NA & NA & NA & NA & 15,444 & 14,192 & 29,636 \\
\hline High-income countries & 0 & 0 & NA & NA & NA & NA & NA & NA & NA & NA & NA & NA & NA & NA & NA & NA & 0 & 0 & 0 \\
\hline WORLD & 15,444 & 14,192 & NA & NA & NA & NA & NA & NA & NA & NA & NA & NA & NA & NA & NA & NA & 15,444 & 14,192 & 29,636 \\
\hline
\end{tabular}

Source: Authors' calculations.

Note: $\mathrm{NA}=$ not applicable 
Table 4A.2

Risk factor:

Childhood underweight

Disease:

Measles

0-4 years $\quad 5-14$ years

15-29 years 30-44 years

45-59 years

60-69 years

70-79 years

$80+$ years

Total

Region

Male Female Male Female Male Female Male Female Male Female Male Female Male Female Male Female Male Female All

\section{PAF of Mortality (\%)}

East Asia and Pacific

Latin America and the Caribbean

Middle East and North Africa

South Asia

Sub-Saharan Africa

Low- and middle-income countries

$31 \quad 31 \quad N A \quad N A \quad N A$

\section{PAF of YLL (\%)}

East Asia and Pacific

Europe and Central Asia

Latin America and the Caribbean

Middle East and North Africa

South Asia

Sub-Saharan Africa

Low- and middle-income countries

High-income countries

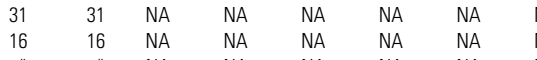

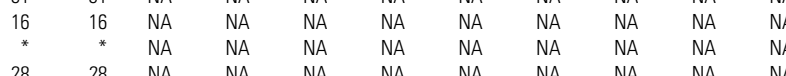

53 53 NA NA NA NA NA NA

4343 NA NA NA NA NA NA NA NA

PAF of DALYs (\%)

East Asia and Pacific

Europe and

Latin America and the Caribbe

Middle East and North Africa

South Asia

Sub-Saharan Africa

Low- and middle-income countries

High-income co

WORLD

Attributable Mortality (thousands

East Asia and Pacific

Europe and Central Asia

Latin America and the Caribbean

Middle East and North Africa

South Asia

Sub-Saharan Africa

$\begin{array}{lll}0 & 0 & N A\end{array}$

NA NA NA

NA NA NA

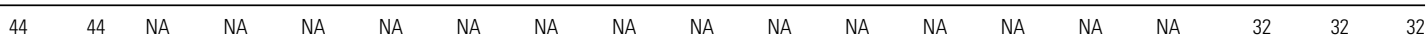

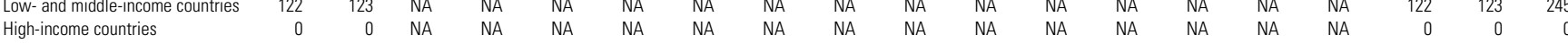

\begin{tabular}{lllllllllllllllllllll}
\hline WORLD & 122 & 123 & NA & NA & NA & NA & NA & NA & NA & NA & NA & NA & NA & NA & NA & NA & 122 & 123 & 245
\end{tabular}

Attributable YLL (thousands)

East Asia and Pacific

Europe and Central Asia

atin America and the Caribbean

Middle East and North Africa

South Asia

Sub-Saharan Africa

$\begin{array}{rrr}31 & 31 & N A \\ 16 & 16 & N A \\ * & * & N A \\ 28 & 28 & N A \\ 53 & 53 & N A \\ 43 & 43 & N A\end{array}$

NA NA

$\begin{array}{lllll}\text { NA } & \text { NA } & \text { NA } & \text { NA } & \text { NA } \\ \text { NA } & \text { NA } & \text { NA } & \text { NA } & \text { NA } \\ \text { NA } & \text { NA } & \text { NA } & \text { NA } & \text { NA } \\ \text { NA } & \text { NA } & \text { NA } & \text { NA } & \text { NA } \\ \text { NA } & \text { NA } & \text { NA } & \text { NA } & \text { NA } \\ \text { NA } & \text { NA } & \text { NA } & \text { NA } & \text { NA } \\ \text { NA } & \text { NA } & \text { NA } & \text { NA } & \text { NA }\end{array}$

\begin{tabular}{|c|c|c|c|c|c|c|c|c|}
\hline NA & NA & NA & NA & NA & NA & 18 & 18 & 18 \\
\hline NA & NA & NA & NA & NA & NA & 11 & 11 & 11 \\
\hline NA & NA & NA & NA & NA & NA & * & * & * \\
\hline NA & NA & NA & NA & NA & NA & 17 & 18 & 18 \\
\hline NA & NA & NA & NA & NA & NA & 36 & 37 & 36 \\
\hline NA & NA & NA & NA & NA & NA & 34 & 34 & 34 \\
\hline NA & NA & NA & NA & NA & NA & 32 & 32 & 32 \\
\hline NA & NA & NA & NA & NA & NA & 0 & 0 & 0 \\
\hline NA & NA & NA & NA & NA & NA & 32 & 32 & 32 \\
\hline
\end{tabular}

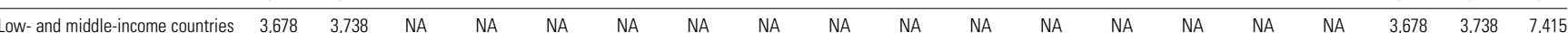

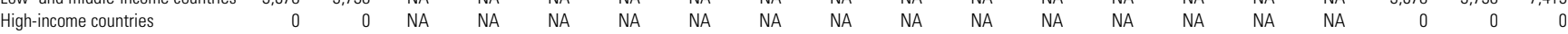

\begin{tabular}{lllllllllllllllllllll}
\hline WORLD & 3,678 & 3,738 & NA & NA & NA & NA & NA & NA & NA & NA & NA & NA & NA & NA & NA & NA & 3,678 & 3,738 & 7,415
\end{tabular}

\section{Attributable DALYs (thousands)}

East Asia

Europe and Central Asia

Latin America and the Caribbean

Middle East and North Africa

South Asia

Sub-Saharan Africa

\section{$205 \quad 212 \quad N A$}

\section{NA $\quad$ NA}

NA NA NA NA

$\begin{array}{lllllllrrr}\text { NA } & \text { NA } & \text { NA } & \text { NA } & \text { NA } & \text { NA } & \text { NA } & 205 & 212 & 416 \\ \text { NA } & \text { NA } & \text { NA } & \text { NA } & \text { NA } & \text { NA } & \text { NA } & 13 & 12 & 25 \\ \text { NA } & \text { NA } & \text { NA } & \text { NA } & \text { NA } & \text { NA } & \text { NA } & 0 & 0 & 0 \\ \text { NA } & \text { NA } & \text { NA } & \text { NA } & \text { NA } & \text { NA } & \text { NA } & 40 & 42 & 82 \\ \text { NA } & \text { NA } & \text { NA } & \text { NA } & \text { NA } & \text { NA } & \text { NA } & 1,145 & 1,205 & 2,350 \\ \text { NA } & \text { NA } & \text { NA } & \text { NA } & \text { NA } & \text { NA } & \text { NA } & 2,275 & 2,267 & 4,542 \\ \text { NA } & \text { NA } & \text { NA } & \text { NA } & \text { NA } & \text { NA } & \text { NA } & 3,678 & 3,738 & 7,415 \\ \text { NA } & \text { NA } & \text { NA } & \text { NA } & \text { NA } & \text { NA } & \text { NA } & 0 & 0 & 0 \\ \text { NA } & \text { NA } & \text { NA } & \text { NA } & \text { NA } & \text { NA } & \text { NA } & 3,678 & 3,738 & 7,415\end{array}$

High-income countries

WORLD

Source: Authors' calculations.

Note: $\mathrm{NA}=$ not applicable.

*The number of deaths (and hence YLL) directly coded to a number of diseases, especially neuropsychiatric and musculoskeletal diseases, is zero or very small. For other diseases, mortality or disease burden may be zero in some

region-age-sex groups. In such cases, the population attributable fractions would be undefined or unstable and have not been calculated. 
Table 4A.3

Risk factor: Childhood underweight

Disease:

Malaria

\begin{tabular}{|c|c|c|c|c|c|c|c|c|c|c|c|c|c|c|c|c|c|c|c|}
\hline \multirow[b]{2}{*}{ Region } & \multicolumn{2}{|c|}{$0-4$ years } & \multicolumn{2}{|c|}{ 5-14 years } & \multicolumn{2}{|c|}{$15-29$ years } & \multicolumn{2}{|c|}{ 30-44 years } & \multicolumn{2}{|c|}{$45-59$ years } & \multicolumn{2}{|c|}{ 60-69 years } & \multicolumn{2}{|c|}{ 70-79 years } & \multicolumn{2}{|c|}{$80+$ years } & \multicolumn{3}{|c|}{ Total } \\
\hline & Male & Female & Male & Female & Male & Female & Male & Female & Male & Female & Male & Female & Male & Female & Male & Female & Male & Female & All \\
\hline \multicolumn{20}{|l|}{ PAF of Mortality (\%) } \\
\hline East Asia and Pacific & 41 & 41 & NA & NA & NA & NA & NA & NA & NA & NA & NA & NA & NA & NA & NA & NA & 37 & 37 & 37 \\
\hline Europe and Central Asia & 23 & 23 & NA & NA & NA & NA & NA & NA & NA & NA & NA & NA & NA & NA & NA & NA & 15 & 16 & 16 \\
\hline Latin America and the Caribbean & 21 & 21 & NA & NA & NA & NA & NA & NA & NA & NA & NA & NA & NA & NA & NA & NA & 18 & 19 & 19 \\
\hline Middle East and North Africa & 38 & 38 & NA & NA & NA & NA & NA & NA & NA & NA & NA & NA & NA & NA & NA & NA & 33 & 34 & 33 \\
\hline South Asia & 66 & 66 & NA & NA & NA & NA & NA & NA & NA & NA & NA & NA & NA & NA & NA & NA & 60 & 60 & 60 \\
\hline Sub-Saharan Africa & 55 & 55 & NA & NA & NA & NA & NA & NA & NA & NA & NA & NA & NA & NA & NA & NA & 50 & 50 & 50 \\
\hline Low- and middle-income countries & 55 & 55 & NA & NA & NA & NA & NA & NA & NA & NA & NA & NA & NA & NA & NA & NA & 49 & 50 & 50 \\
\hline High-income countries & 0 & 0 & NA & NA & NA & NA & NA & NA & NA & NA & NA & NA & NA & NA & NA & NA & 0 & 0 & 0 \\
\hline WORLD & 55 & 55 & NA & NA & NA & NA & NA & NA & NA & NA & NA & NA & NA & NA & NA & NA & 49 & 50 & 50 \\
\hline
\end{tabular}

PAF of YLL (\%)

\begin{tabular}{|c|c|c|c|c|c|c|c|c|c|c|c|c|c|c|c|c|c|c|c|}
\hline East Asia and Pacific & 41 & 41 & NA & NA & NA & NA & NA & NA & NA & NA & NA & NA & NA & NA & NA & NA & 39 & 39 & 39 \\
\hline Europe and Central Asia & 23 & 23 & NA & NA & NA & NA & NA & NA & NA & NA & NA & NA & NA & NA & NA & NA & 17 & 19 & 18 \\
\hline Latin America and the Caribbean & 21 & 21 & NA & NA & NA & NA & NA & NA & NA & NA & NA & NA & NA & NA & NA & NA & 19 & 19 & 19 \\
\hline Middle East and North Africa & 38 & 38 & NA & NA & NA & NA & NA & NA & NA & NA & NA & NA & NA & NA & NA & NA & 34 & 35 & 35 \\
\hline South Asia & 66 & 66 & NA & NA & NA & NA & NA & NA & NA & NA & NA & NA & NA & NA & NA & NA & 62 & 62 & 52 \\
\hline Sub-Saharan Africa & 55 & 55 & NA & NA & NA & NA & NA & NA & NA & NA & NA & NA & NA & NA & NA & NA & 51 & 51 & 51 \\
\hline Low- and middle-income countries & 55 & 55 & NA & NA & NA & NA & NA & NA & NA & NA & NA & NA & NA & NA & NA & NA & 51 & 51 & 51 \\
\hline High-income countries & 0 & 0 & NA & NA & NA & NA & NA & NA & NA & NA & NA & NA & NA & NA & NA & NA & 0 & 0 & 0 \\
\hline WORLD & 55 & 55 & NA & NA & NA & NA & NA & NA & NA & NA & NA & NA & NA & NA & NA & NA & 51 & 51 & 1 \\
\hline
\end{tabular}

PAF of DALYs (\%)

\begin{tabular}{|c|c|c|c|c|c|c|c|c|c|c|c|c|c|c|c|c|c|c|}
\hline East Asia and Pacific & 39 & 37 & NA & NA & NA & NA & NA & NA & NA & NA & NA & NA & NA & NA & NA & NA & 33 & 28 \\
\hline Europe and Central Asia & 6 & 7 & NA & NA & NA & NA & NA & NA & NA & NA & NA & NA & NA & NA & NA & NA & 2 & 2 \\
\hline Latin America and the Caribbean & 16 & 14 & NA & NA & NA & NA & NA & NA & NA & NA & NA & NA & NA & NA & NA & NA & 9 & 7 \\
\hline Middle East and North Africa & 35 & 35 & NA & NA & NA & NA & NA & NA & NA & NA & NA & NA & NA & NA & NA & NA & 28 & 30 \\
\hline South Asia & 59 & 60 & NA & NA & NA & NA & NA & NA & NA & NA & NA & NA & NA & NA & NA & NA & 44 & 46 \\
\hline Sub-Saharan Africa & 51 & 51 & NA & NA & NA & NA & NA & NA & NA & NA & NA & NA & NA & NA & NA & NA & 47 & 47 \\
\hline Low- and middle-income countries & 51 & 51 & NA & NA & NA & NA & NA & NA & NA & NA & NA & NA & NA & NA & NA & NA & 46 & 46 \\
\hline High-income countries & 0 & 0 & NA & NA & NA & NA & NA & NA & NA & NA & NA & NA & NA & NA & NA & NA & 0 & 0 \\
\hline RLD & 51 & 51 & NA & NA & NA & NA & NA & NA & NA & NA & NA & NA & NA & NA & NA & NA & 46 & 46 \\
\hline
\end{tabular}

Attributable Mortality (thousands)

East Asia an

Europe and Central Asia

Latin America and the Caribbean

Middle East and North Africa

South Asia

Sub-Saharan Africa

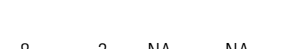

\begin{tabular}{|c|c|c|c|c|c|c|c|c|c|c|c|c|c|c|c|c|c|c|c|}
\hline \multicolumn{20}{|l|}{ Attributable Mortality (thousands) } \\
\hline East Asia and Pacific & 8 & 3 & NA & NA & NA & NA & NA & NA & NA & NA & NA & NA & NA & NA & NA & NA & 8 & 3 & 11 \\
\hline Europe and Central Asia & 0 & 0 & NA & NA & NA & NA & NA & NA & NA & NA & NA & NA & NA & NA & NA & NA & 0 & 0 & 0 \\
\hline Latin America and the Caribbean & 0 & 0 & NA & NA & NA & NA & NA & NA & NA & NA & NA & NA & NA & NA & NA & NA & 0 & 0 & 0 \\
\hline Middle East and North Africa & 3 & 3 & NA & NA & NA & NA & NA & NA & NA & NA & NA & NA & NA & NA & NA & NA & 3 & 3 & 6 \\
\hline South Asia & 18 & 20 & NA & NA & NA & NA & NA & NA & NA & NA & NA & NA & NA & NA & NA & NA & 18 & 20 & 38 \\
\hline Sub-Saharan Africa & 257 & 285 & NA & NA & NA & NA & NA & NA & NA & NA & NA & NA & NA & NA & NA & NA & 257 & 285 & 542 \\
\hline Low- and middle-income countries & 286 & 312 & NA & NA & NA & NA & NA & NA & NA & NA & NA & NA & NA & NA & NA & NA & 286 & 312 & 598 \\
\hline High-income countries & 0 & 0 & NA & NA & NA & NA & NA & NA & NA & NA & NA & NA & NA & NA & NA & NA & 0 & 0 & 0 \\
\hline WORLD & 286 & 312 & NA & NA & NA & NA & NA & NA & NA & NA & NA & NA & NA & NA & NA & NA & 286 & 312 & 598 \\
\hline
\end{tabular}

Attributable YLL (thousands)

\begin{tabular}{|c|c|c|c|c|c|c|c|c|c|c|c|c|c|c|c|c|c|c|c|}
\hline East Asia and Pacific & 231 & 104 & NA & NA & NA & NA & NA & NA & NA & NA & NA & NA & NA & NA & NA & NA & 231 & 104 & 335 \\
\hline Europe and Central Asia & 0 & 0 & NA & NA & NA & NA & NA & NA & NA & NA & NA & NA & NA & NA & NA & NA & 0 & 0 & 0 \\
\hline Latin America and the Caribbean & 5 & 4 & NA & NA & NA & NA & NA & NA & NA & NA & NA & NA & NA & NA & NA & NA & 5 & 4 & 9 \\
\hline Middle East and North Africa & 91 & 102 & NA & NA & NA & NA & NA & NA & NA & NA & NA & NA & NA & NA & NA & NA & 91 & 102 & 193 \\
\hline South Asia & 542 & 606 & NA & NA & NA & NA & NA & NA & NA & NA & NA & NA & NA & NA & NA & NA & 542 & 606 & 1,148 \\
\hline Sub-Saharan Africa & 7,770 & 8,683 & NA & NA & NA & NA & NA & NA & NA & NA & NA & NA & NA & NA & NA & NA & 7,770 & 8,683 & 16,452 \\
\hline Low- and middle-income countries & 8,638 & 9,499 & NA & NA & NA & NA & NA & NA & NA & NA & NA & NA & NA & NA & NA & NA & 8,638 & 9,499 & 18,137 \\
\hline High-income countries & 0 & 0 & NA & NA & NA & NA & NA & NA & NA & NA & NA & NA & NA & NA & NA & NA & 0 & 0 & 0 \\
\hline WORLD & 8,638 & 9,499 & NA & NA & NA & NA & NA & NA & NA & NA & NA & NA & NA & NA & NA & NA & 8,638 & 9,499 & 18,137 \\
\hline \multicolumn{20}{|l|}{ Attributable DALYs (thousands) } \\
\hline East Asia and Pacific & 232 & 106 & NA & NA & NA & NA & NA & NA & NA & NA & NA & NA & NA & NA & NA & NA & 232 & 106 & 338 \\
\hline Europe and Central Asia & 0 & 0 & NA & NA & NA & NA & NA & NA & NA & NA & NA & NA & NA & NA & NA & NA & 0 & 0 & 0 \\
\hline Latin America and the Caribbean & 5 & 4 & NA & NA & NA & NA & NA & NA & NA & NA & NA & NA & NA & NA & NA & NA & 5 & 4 & 9 \\
\hline Middle East and North Africa & 92 & 103 & NA & NA & NA & NA & NA & NA & NA & NA & NA & NA & NA & NA & NA & NA & 92 & 103 & 195 \\
\hline South Asia & 557 & 620 & NA & NA & NA & NA & NA & NA & NA & NA & NA & NA & NA & NA & NA & NA & 557 & 620 & 1,177 \\
\hline Sub-Saharan Africa & 7,876 & 8,789 & NA & NA & NA & NA & NA & NA & NA & NA & NA & NA & NA & NA & NA & NA & 7,876 & 8,789 & 16,665 \\
\hline Low- and middle-income countries & 8,763 & 9,622 & NA & NA & NA & NA & NA & NA & NA & NA & NA & NA & NA & NA & NA & NA & 8,763 & 9,622 & 18,385 \\
\hline High-income countries & 0 & 0 & NA & NA & NA & NA & NA & NA & NA & NA & NA & NA & NA & NA & NA & NA & 0 & 0 & 0 \\
\hline$\overline{\text { WORLD }}$ & 8,763 & 9,622 & NA & NA & NA & NA & NA & NA & NA & NA & NA & NA & NA & NA & NA & NA & 8,763 & 9,622 & 18,385 \\
\hline
\end{tabular}

Source: Authors' calculations.

Note: $\mathrm{NA}=$ not applicable. 
Table 4A.4

Risk factor: Childhood underweight

Disease:

Lower respiratory infections

\begin{tabular}{|c|c|c|c|c|c|c|c|c|c|c|c|c|c|c|c|c|c|c|c|}
\hline \multirow[b]{2}{*}{ Region } & \multicolumn{2}{|c|}{$0-4$ years } & \multicolumn{2}{|c|}{ 5-14 years } & \multicolumn{2}{|c|}{$15-29$ years } & \multicolumn{2}{|c|}{ 30-44 years } & \multicolumn{2}{|c|}{$45-59$ years } & \multicolumn{2}{|c|}{$60-69$ years } & \multicolumn{2}{|c|}{ 70-79 years } & \multicolumn{2}{|c|}{$80+$ years } & \multicolumn{3}{|c|}{ Total } \\
\hline & Male & Female & Male & Female & Male & Female & Male & Female & Male & Female & Male & Female & Male & Female & Male & Female & Male & Female & All \\
\hline \multicolumn{20}{|l|}{ PAF of Mortality (\%) } \\
\hline East Asia and Pacific & 39 & 39 & NA & NA & NA & NA & NA & NA & NA & NA & NA & NA & NA & NA & NA & NA & 10 & 15 & 13 \\
\hline Europe and Central Asia & 21 & 21 & NA & NA & NA & NA & NA & NA & NA & NA & NA & NA & NA & NA & NA & NA & 6 & 8 & 7 \\
\hline Latin America and the Caribbean & 20 & 20 & NA & NA & NA & NA & NA & NA & NA & NA & NA & NA & NA & NA & NA & NA & 6 & 5 & 5 \\
\hline Middle East and North Africa & 35 & 35 & NA & NA & NA & NA & NA & NA & NA & NA & NA & NA & NA & NA & NA & NA & 24 & 24 & 24 \\
\hline South Asia & 63 & 63 & NA & NA & NA & NA & NA & NA & NA & NA & NA & NA & NA & NA & NA & NA & 37 & 37 & 37 \\
\hline Sub-Saharan Africa & 52 & 52 & NA & NA & NA & NA & NA & NA & NA & NA & NA & NA & NA & NA & NA & NA & 39 & 33 & 36 \\
\hline Low- and middle-income countries & 54 & 53 & NA & NA & NA & NA & NA & NA & NA & NA & NA & NA & NA & NA & NA & NA & 31 & 29 & 30 \\
\hline High-income countries & 0 & 0 & NA & NA & NA & NA & NA & NA & NA & NA & NA & NA & NA & NA & NA & NA & 0 & 0 & 0 \\
\hline WORLD & 54 & 53 & NA & NA & NA & NA & NA & NA & NA & NA & NA & NA & NA & NA & NA & NA & 28 & 26 & 27 \\
\hline
\end{tabular}

PAF of YLL (\%)

\begin{tabular}{|c|c|c|c|c|c|c|c|c|c|c|c|c|c|c|c|c|c|c|c|}
\hline East Asia and Pacific & 39 & 39 & NA & NA & NA & NA & NA & NA & NA & NA & NA & NA & NA & NA & NA & NA & 18 & 25 & 22 \\
\hline Europe and Central Asia & 21 & 21 & NA & NA & NA & NA & NA & NA & NA & NA & NA & NA & NA & NA & NA & NA & 10 & 12 & 11 \\
\hline Latin America and the Caribbean & 20 & 20 & NA & NA & NA & NA & NA & NA & NA & NA & NA & NA & NA & NA & NA & NA & 10 & 10 & 10 \\
\hline Middle East and North Africa & 35 & 35 & NA & NA & NA & NA & NA & NA & NA & NA & NA & NA & NA & NA & NA & NA & 29 & 29 & 29 \\
\hline South Asia & 63 & 63 & NA & NA & NA & NA & NA & NA & NA & NA & NA & NA & NA & NA & NA & NA & 49 & 48 & 49 \\
\hline Sub-Saharan Africa & 52 & 52 & NA & NA & NA & NA & NA & NA & NA & NA & NA & NA & NA & NA & NA & NA & 43 & 37 & 40 \\
\hline Low- and middle-income countries & 54 & 53 & NA & NA & NA & NA & NA & NA & NA & NA & NA & NA & NA & NA & NA & NA & 40 & 38 & 39 \\
\hline High-income countries & 0 & 0 & NA & NA & NA & NA & NA & NA & NA & NA & NA & NA & NA & NA & NA & NA & 0 & 0 & 0 \\
\hline WORLD & 54 & 53 & NA & NA & NA & NA & NA & NA & NA & NA & NA & NA & NA & NA & NA & NA & 39 & 37 & 38 \\
\hline
\end{tabular}

PAF of DALYs $(\%)$

\begin{tabular}{|c|c|c|c|c|c|c|c|c|c|c|c|c|c|c|c|c|c|c|}
\hline East Asia and Pacific & 35 & 35 & NA & NA & NA & NA & NA & NA & NA & NA & NA & NA & NA & NA & NA & NA & 17 & 23 \\
\hline Europe and Central Asia & 20 & 20 & NA & NA & NA & NA & NA & NA & NA & NA & NA & NA & NA & NA & NA & NA & 9 & 12 \\
\hline Latin America and the Caribbean & 16 & 16 & NA & NA & NA & NA & NA & NA & NA & NA & NA & NA & NA & NA & NA & NA & 9 & 9 \\
\hline Middle East and North Africa & 33 & 33 & NA & NA & NA & NA & NA & NA & NA & NA & NA & NA & NA & NA & NA & NA & 27 & 27 \\
\hline South Asia & 61 & 61 & NA & NA & NA & NA & NA & NA & NA & NA & NA & NA & NA & NA & NA & NA & 47 & 47 \\
\hline Sub-Saharan Africa & 51 & 51 & NA & NA & NA & NA & NA & NA & NA & NA & NA & NA & NA & NA & NA & NA & 42 & 36 \\
\hline Low- and middle-income countries & 52 & 51 & NA & NA & NA & NA & NA & NA & NA & NA & NA & NA & NA & NA & NA & NA & 39 & 37 \\
\hline High-income countries & 0 & 0 & NA & NA & NA & NA & NA & NA & NA & NA & NA & NA & NA & NA & NA & NA & 0 & 0 \\
\hline RLD & 52 & 51 & NA & NA & NA & NA & NA & NA & NA & NA & NA & NA & NA & NA & NA & NA & 38 & 36 \\
\hline
\end{tabular}

Attributable Mortality (thousands)

$\begin{array}{lllllllllllllllllllllll}\text { East Asia and Pacific } & 24 & 47 & \text { NA } & \text { NA } & \text { NA } & \text { NA } & \text { NA } & \text { NA } & \text { NA } & \text { NA } & \text { NA } & \text { NA } & \text { NA } & \text { NA } & \text { NA } & \text { NA } & 24 & 47 & 71\end{array}$

Europe and Central Asia

$\begin{array}{lllllllllllllllllllllll}\text { Latin America and the Caribbean } & 5 & 4 & \text { NA } & \text { NA } & \text { NA } & \text { NA } & \text { NA } & \text { NA } & \text { NA } & \text { NA } & \text { NA } & \text { NA } & \text { NA } & \text { NA } & \text { NA } & \text { NA } & 5 & 4\end{array}$

$\begin{array}{lllllllllllllllllllllll}\text { Middle East and North Africa } & 14 & 12 & \text { NA } & \text { NA } & \text { NA } & \text { NA } & \text { NA } & \text { NA } & \text { NA } & \text { NA } & \text { NA } & \text { NA } & \text { NA } & \text { NA } & \text { NA } & \text { NA } & 14 & 12 & 26\end{array}$

$\begin{array}{lllllllllllllllllllllll}\text { South Asia } & 259 & 262 & \text { NA } & \text { NA } & \text { NA } & \text { NA } & \text { NA } & \text { NA } & \text { NA } & \text { NA } & \text { NA } & \text { NA } & \text { NA } & \text { NA } & \text { NA } & \text { NA } & 259 & 262 & 521\end{array}$

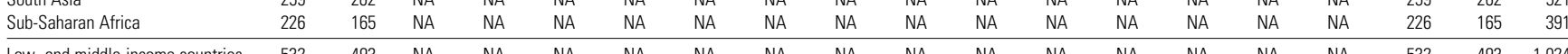

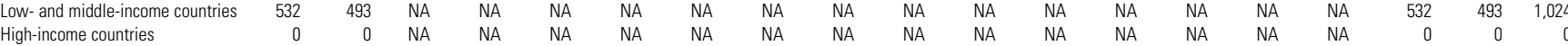

\begin{tabular}{llllllllllllllllllll}
\hline WORLD & 532 & 493 & NA & NA & NA & NA & NA & NA & NA & NA & NA & NA & NA & NA & NA & NA & 532 & 493 & 1,024
\end{tabular}

Attributable YLL (thousands)

\begin{tabular}{|c|c|c|c|c|c|c|c|c|c|c|c|c|c|c|c|c|c|c|c|}
\hline East Asia and Pacific & 730 & 1,430 & NA & NA & NA & NA & NA & NA & NA & NA & NA & NA & NA & NA & NA & NA & 730 & 1,430 & 2,160 \\
\hline Europe and Central Asia & 116 & 98 & NA & NA & NA & NA & NA & NA & NA & NA & NA & NA & NA & NA & NA & NA & 116 & 98 & 214 \\
\hline Latin America and the Caribbean & 137 & 117 & NA & NA & NA & NA & NA & NA & NA & NA & NA & NA & NA & NA & NA & NA & 137 & 117 & 254 \\
\hline Middle East and North Africa & 414 & 379 & NA & NA & NA & NA & NA & NA & NA & NA & NA & NA & NA & NA & NA & NA & 414 & 379 & 793 \\
\hline South Asia & 7,838 & 7,974 & NA & NA & NA & NA & NA & NA & NA & NA & NA & NA & NA & NA & NA & NA & 7,838 & 7,974 & 15,812 \\
\hline Sub-Saharan Africa & 6,851 & 5,016 & NA & NA & NA & NA & NA & NA & NA & NA & NA & NA & NA & NA & NA & NA & 6,851 & 5,016 & 11,867 \\
\hline Low- and middle-income countries & 16,086 & 15,014 & NA & NA & NA & NA & NA & NA & NA & NA & NA & NA & NA & NA & NA & NA & 16,086 & 15,014 & 31,100 \\
\hline High-income countries & 0 & 0 & NA & NA & NA & NA & NA & NA & NA & NA & NA & NA & NA & NA & NA & NA & 0 & 0 & 0 \\
\hline WORLD & 16,086 & 15,014 & NA & NA & NA & NA & NA & NA & NA & NA & NA & NA & NA & NA & NA & NA & 16,086 & 15,014 & 31,100 \\
\hline \multicolumn{20}{|l|}{ Attributable DALYs (thousands) } \\
\hline East Asia and Pacific & 762 & 1,486 & NA & NA & NA & NA & NA & NA & NA & NA & NA & NA & NA & NA & NA & NA & 762 & 1,486 & 2,248 \\
\hline Europe and Central Asia & 117 & 100 & NA & NA & NA & NA & NA & NA & NA & NA & NA & NA & NA & NA & NA & NA & 117 & 100 & 217 \\
\hline Latin America and the Caribbean & 144 & 124 & NA & NA & NA & NA & NA & NA & NA & NA & NA & NA & NA & NA & NA & NA & 144 & 124 & 269 \\
\hline Middle East and North Africa & 424 & 388 & NA & NA & NA & NA & NA & NA & NA & NA & NA & NA & NA & NA & NA & NA & 424 & 388 & 812 \\
\hline South Asia & 8,016 & 8,147 & NA & NA & NA & NA & NA & NA & NA & NA & NA & NA & NA & NA & NA & NA & 8,016 & 8,147 & 16,162 \\
\hline Sub-Saharan Africa & 6,935 & 5,053 & NA & NA & NA & NA & NA & NA & NA & NA & NA & NA & NA & NA & NA & NA & 6,935 & 5,053 & 11,988 \\
\hline Low- and middle-income countries & 16,398 & 15,298 & NA & NA & NA & NA & NA & NA & NA & NA & NA & NA & NA & NA & NA & NA & 16,398 & 15,298 & 31,696 \\
\hline High-income countries & 0 & 0 & NA & NA & NA & NA & NA & NA & NA & NA & NA & NA & NA & NA & NA & NA & 0 & 0 & 0 \\
\hline WORLD & 16,398 & 15,298 & NA & NA & NA & NA & NA & NA & NA & NA & NA & NA & NA & NA & NA & NA & 16,398 & 15,298 & 31,696 \\
\hline
\end{tabular}

Source: Authors' calculations.

Note: $\mathrm{NA}=$ not applicable.

272 | Global Burden of Disease and Risk Factors I Majid Ezzati, Stephen Vander Hoorn, Alan D. Lopez, and others 
Table 4A.5

Risk factor: Childhood underweight

Disease:

Protein-energy malnutrition

\begin{tabular}{|c|c|c|c|c|c|c|c|c|c|c|c|c|c|c|c|c|c|c|c|}
\hline \multirow[b]{2}{*}{ Region } & \multicolumn{2}{|c|}{$0-4$ years } & \multicolumn{2}{|c|}{ 5-14 years } & \multicolumn{2}{|c|}{$15-29$ years } & \multicolumn{2}{|c|}{ 30-44 years } & \multicolumn{2}{|c|}{$45-59$ years } & \multicolumn{2}{|c|}{$60-69$ years } & \multicolumn{2}{|c|}{$70-79$ years } & \multicolumn{2}{|c|}{$80+$ years } & \multicolumn{3}{|c|}{ Total } \\
\hline & Male & Female & Male & Female & Male & Female & Male & Female & Male & Female & Male & Female & Male & Female & Male & Female & Male & Female & All \\
\hline \multicolumn{20}{|l|}{ PAF of Mortality (\%) } \\
\hline East Asia and Pacific & 100 & 100 & NA & NA & NA & NA & NA & NA & NA & NA & NA & NA & NA & NA & NA & NA & 50 & 40 & 45 \\
\hline Europe and Central Asia & 100 & 100 & NA & NA & NA & NA & NA & NA & NA & NA & NA & NA & NA & NA & NA & NA & 14 & 14 & 14 \\
\hline Latin America and the Caribbean & 100 & 100 & NA & NA & NA & NA & NA & NA & NA & NA & NA & NA & NA & NA & NA & NA & 37 & 32 & 35 \\
\hline Middle East and North Africa & 100 & 100 & NA & NA & NA & NA & NA & NA & NA & NA & NA & NA & NA & NA & NA & NA & 88 & 85 & 87 \\
\hline South Asia & 100 & 100 & NA & NA & NA & NA & NA & NA & NA & NA & NA & NA & NA & NA & NA & NA & 45 & 52 & 49 \\
\hline Sub-Saharan Africa & 100 & 100 & NA & NA & NA & NA & NA & NA & NA & NA & NA & NA & NA & NA & NA & NA & 73 & 77 & 74 \\
\hline Low- and middle-income countries & 100 & 100 & NA & NA & NA & NA & NA & NA & NA & NA & NA & NA & NA & NA & NA & NA & 58 & 58 & 58 \\
\hline High-income countries & 100 & 100 & NA & NA & NA & NA & NA & NA & NA & NA & NA & NA & NA & NA & NA & NA & 2 & 1 & 1 \\
\hline WORLD & 100 & 100 & NA & NA & NA & NA & NA & NA & NA & NA & NA & NA & NA & NA & NA & NA & 56 & 55 & 56 \\
\hline
\end{tabular}

PAF of YLL (\%)

\begin{tabular}{|c|c|c|c|c|c|c|c|c|c|c|c|c|c|c|c|c|c|c|c|}
\hline East Asia and Pacific & 100 & 100 & NA & NA & NA & NA & NA & NA & NA & NA & NA & NA & NA & NA & NA & NA & 62 & 57 & 60 \\
\hline Europe and Central Asia & 100 & 100 & NA & NA & NA & NA & NA & NA & NA & NA & NA & NA & NA & NA & NA & NA & 22 & 24 & 23 \\
\hline Latin America and the Caribbean & 100 & 100 & NA & NA & NA & NA & NA & NA & NA & NA & NA & NA & NA & NA & NA & NA & 61 & 59 & 60 \\
\hline Middle East and North Africa & 100 & 100 & NA & NA & NA & NA & NA & NA & NA & NA & NA & NA & NA & NA & NA & NA & 93 & 90 & 92 \\
\hline South Asia & 100 & 100 & NA & NA & NA & NA & NA & NA & NA & NA & NA & NA & NA & NA & NA & NA & 47 & 55 & 52 \\
\hline Sub-Saharan Africa & 100 & 100 & NA & NA & NA & NA & NA & NA & NA & NA & NA & NA & NA & NA & NA & NA & 84 & 86 & 85 \\
\hline Low- and middle-income countries & 100 & 100 & NA & NA & NA & NA & NA & NA & NA & NA & NA & NA & NA & NA & NA & NA & 69 & 69 & 9 \\
\hline High-income countries & 100 & 100 & NA & NA & NA & NA & NA & NA & NA & NA & NA & NA & NA & NA & NA & NA & 6 & 4 & 4 \\
\hline WORLD & 100 & 100 & NA & NA & NA & NA & NA & NA & NA & NA & NA & NA & NA & NA & NA & NA & 69 & 68 & 9 \\
\hline
\end{tabular}

PAF of DALYs (\%)

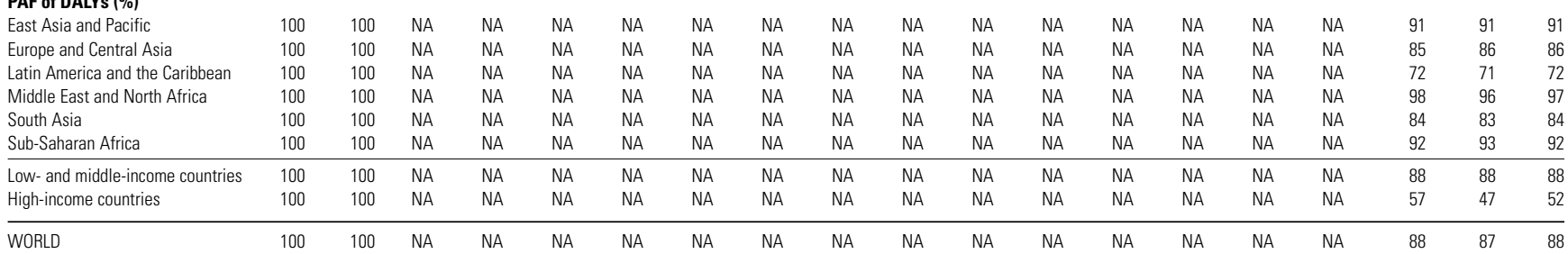

Attributable Mortality (thousands)

East Asia and Pacific
Europe and Central Asi

Latin America and the Caribbe

Middle East and North Africa

South Asia

Sub-Saharan Africa

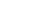

\begin{tabular}{lllllllllllllllllllll}
\hline Low- and middle-income countries & 70 & 69 & NA & NA & NA & NA & NA & NA & NA & NA & NA & NA & NA & NA & NA & NA & 70 & 69 & 139
\end{tabular}

$\begin{array}{lllllllllllllllllllllllll}\text { High-income countries } & 0 & 0 & \text { NA } & \text { NA } & \text { NA } & \text { NA } & \text { NA } & \text { NA } & \text { NA } & \text { NA } & \text { NA } & \text { NA } & \text { NA } & \text { NA } & \text { NA } & \text { NA } & 0 & 0 & 0\end{array}$

\begin{tabular}{llllllllllllllllllllll}
\hline WORLD & 70 & 69 & NA & NA & NA & NA & NA & NA & NA & NA & NA & NA & NA & NA & NA & NA & 70 & 69 & 140 \\
\hline
\end{tabular}

Attributable YLL (thousands)

\begin{tabular}{|c|c|c|c|c|c|c|c|c|c|c|c|c|c|c|c|c|c|c|c|}
\hline \multicolumn{20}{|l|}{ East Asia and Pacific } \\
\hline East Asia and Pacific & 212 & 150 & NA & NA & NA & NA & NA & NA & NA & NA & NA & NA & NA & NA & NA & NA & 212 & 150 & 363 \\
\hline Europe and Central Asia & 4 & 4 & NA & NA & NA & NA & NA & NA & NA & NA & NA & NA & NA & NA & NA & NA & 4 & 4 & 7 \\
\hline Latin America and the Caribbean & 212 & 180 & NA & NA & NA & NA & NA & NA & NA & NA & NA & NA & NA & NA & NA & NA & 212 & 180 & 392 \\
\hline Middle East and North Africa & 103 & 119 & NA & NA & NA & NA & NA & NA & NA & NA & NA & NA & NA & NA & NA & NA & 103 & 119 & 221 \\
\hline South Asia & 392 & 608 & NA & NA & NA & NA & NA & NA & NA & NA & NA & NA & NA & NA & NA & NA & 392 & 608 & 1,000 \\
\hline Sub-Saharan Africa & 1,202 & 1,042 & NA & NA & NA & NA & NA & NA & NA & NA & NA & NA & NA & NA & NA & NA & 1,202 & 1,042 & 2,244 \\
\hline Low- and middle-income countries & 2,124 & 2,102 & NA & NA & NA & NA & NA & NA & NA & NA & NA & NA & NA & NA & NA & NA & 2,124 & 2,102 & 4,226 \\
\hline High-income countries & 2 & 1 & NA & NA & NA & NA & NA & NA & NA & NA & NA & NA & NA & NA & NA & NA & 2 & 1 & 3 \\
\hline WORLD & 2,126 & 2,104 & NA & NA & NA & NA & NA & NA & NA & NA & NA & NA & NA & NA & NA & NA & 2,126 & 2,104 & 4,229 \\
\hline \multicolumn{20}{|l|}{ Attributable DALYs (thousands) } \\
\hline East Asia and Pacific & 1,303 & 1,184 & NA & NA & NA & NA & NA & NA & NA & NA & NA & NA & NA & NA & NA & NA & 1,303 & 1,184 & 2,487 \\
\hline Europe and Central Asia & 75 & 74 & NA & NA & NA & NA & NA & NA & NA & NA & NA & NA & NA & NA & NA & NA & 75 & 74 & 149 \\
\hline Latin America and the Caribbean & 345 & 311 & NA & NA & NA & NA & NA & NA & NA & NA & NA & NA & NA & NA & NA & NA & 345 & 311 & 655 \\
\hline Middle East and North Africa & 351 & 341 & NA & NA & NA & NA & NA & NA & NA & NA & NA & NA & NA & NA & NA & NA & 351 & 341 & 692 \\
\hline South Asia & 2,319 & 2,441 & NA & NA & NA & NA & NA & NA & NA & NA & NA & NA & NA & NA & NA & NA & 2,319 & 2,441 & 4,759 \\
\hline Sub-Saharan Africa & 2,497 & 2,322 & NA & NA & NA & NA & NA & NA & NA & NA & NA & NA & NA & NA & NA & NA & 2,497 & 2,322 & 4,819 \\
\hline Low- and middle-income countries & 6,889 & 6,672 & NA & NA & NA & NA & NA & NA & NA & NA & NA & NA & NA & NA & NA & NA & 6,889 & 6,672 & 13,561 \\
\hline High-income countries & 35 & 33 & NA & NA & NA & NA & NA & NA & NA & NA & NA & NA & NA & NA & NA & NA & 35 & 33 & 67 \\
\hline WORLD & 6,924 & 6,705 & NA & NA & NA & NA & NA & NA & NA & NA & NA & NA & NA & NA & NA & NA & 6,924 & 6,705 & 13,628 \\
\hline
\end{tabular}

Source: Authors' calculations.

Note: $\mathrm{NA}=$ not applicable. 
Table 4A.6

Risk factor: Childhood underweight

Disease:

Selected other Group I diseases

\begin{tabular}{|c|c|c|c|c|c|c|c|c|c|c|c|c|c|c|c|c|c|c|c|}
\hline \multirow[b]{2}{*}{ Region } & \multicolumn{2}{|c|}{$0-4$ years } & \multicolumn{2}{|c|}{ 5-14 years } & \multicolumn{2}{|c|}{$15-29$ years } & \multicolumn{2}{|c|}{ 30-44 years } & \multicolumn{2}{|c|}{$45-59$ years } & \multicolumn{2}{|c|}{$60-69$ years } & \multicolumn{2}{|c|}{ 70-79 years } & \multicolumn{2}{|c|}{$80+$ years } & \multicolumn{3}{|c|}{ Total } \\
\hline & Male & Female & Male & Female & Male & Female & Male & Female & Male & Female & Male & Female & Male & Female & Male & Female & Male & Female & All \\
\hline \multicolumn{20}{|l|}{ PAF of Mortality (\%) } \\
\hline East Asia and Pacific & 40 & 40 & NA & NA & NA & NA & NA & NA & NA & NA & NA & NA & NA & NA & NA & NA & 4 & 5 & 4 \\
\hline Europe and Central Asia & 22 & 22 & NA & NA & NA & NA & NA & NA & NA & NA & NA & NA & NA & NA & NA & NA & 3 & 6 & 3 \\
\hline Latin America and the Caribbean & 20 & 20 & NA & NA & NA & NA & NA & NA & NA & NA & NA & NA & NA & NA & NA & NA & 5 & 4 & 4 \\
\hline Middle East and North Africa & 36 & 36 & NA & NA & NA & NA & NA & NA & NA & NA & NA & NA & NA & NA & NA & NA & 14 & 13 & 13 \\
\hline South Asia & 64 & 64 & NA & NA & NA & NA & NA & NA & NA & NA & NA & NA & NA & NA & NA & NA & 11 & 17 & 14 \\
\hline Sub-Saharan Africa & 53 & 53 & NA & NA & NA & NA & NA & NA & NA & NA & NA & NA & NA & NA & NA & NA & 20 & 18 & 19 \\
\hline Low- and middle-income countries & 53 & 55 & NA & NA & NA & NA & NA & NA & NA & NA & NA & NA & NA & NA & NA & NA & 12 & 14 & 13 \\
\hline High-income countries & 0 & 0 & NA & NA & NA & NA & NA & NA & NA & NA & NA & NA & NA & NA & NA & NA & 0 & 0 & 0 \\
\hline WORLD & 53 & 55 & NA & NA & NA & NA & NA & NA & NA & NA & NA & NA & NA & NA & NA & NA & 12 & 14 & 13 \\
\hline
\end{tabular}

PAF of YLL (\%)

\begin{tabular}{|c|c|c|c|c|c|c|c|c|c|c|c|c|c|c|c|c|c|c|c|}
\hline East Asia and Pacific & 40 & 40 & NA & NA & NA & NA & NA & NA & NA & NA & NA & NA & NA & NA & NA & NA & 6 & 7 & 7 \\
\hline Europe and Central Asia & 22 & 22 & NA & NA & NA & NA & NA & NA & NA & NA & NA & NA & NA & NA & NA & NA & 4 & 8 & 5 \\
\hline Latin America and the Caribbean & 20 & 20 & NA & NA & NA & NA & NA & NA & NA & NA & NA & NA & NA & NA & NA & NA & 7 & 5 & 6 \\
\hline Middle East and North Africa & 36 & 36 & NA & NA & NA & NA & NA & NA & NA & NA & NA & NA & NA & NA & NA & NA & 20 & 16 & 18 \\
\hline South Asia & 64 & 64 & NA & NA & NA & NA & NA & NA & NA & NA & NA & NA & NA & NA & NA & NA & 16 & 21 & 19 \\
\hline Sub-Saharan Africa & 53 & 53 & NA & NA & NA & NA & NA & NA & NA & NA & NA & NA & NA & NA & NA & NA & 24 & 20 & 22 \\
\hline Low- and middle-income countries & 53 & 55 & NA & NA & NA & NA & NA & NA & NA & NA & NA & NA & NA & NA & NA & NA & 17 & 18 & 17 \\
\hline High-income countries & 0 & 0 & NA & NA & NA & NA & NA & NA & NA & NA & NA & NA & NA & NA & NA & NA & 0 & 0 & 0 \\
\hline WORLD & 53 & 55 & NA & NA & NA & NA & NA & NA & NA & NA & NA & NA & NA & NA & NA & NA & 16 & 18 & 17 \\
\hline
\end{tabular}

PAF of DALYs $(\%)$

\begin{tabular}{|c|c|c|c|c|c|c|c|c|c|c|c|c|c|c|c|c|c|c|}
\hline East Asia and Pacific & 29 & 27 & NA & NA & NA & NA & NA & NA & NA & NA & NA & NA & NA & NA & NA & NA & 5 & 4 \\
\hline Europe and Central Asia & 17 & 16 & NA & NA & NA & NA & NA & NA & NA & NA & NA & NA & NA & NA & NA & NA & 3 & 3 \\
\hline Latin America and the Caribbean & 15 & 14 & NA & NA & NA & NA & NA & NA & NA & NA & NA & NA & NA & NA & NA & NA & 5 & 3 \\
\hline Middle East and North Africa & 30 & 29 & NA & NA & NA & NA & NA & NA & NA & NA & NA & NA & NA & NA & NA & NA & 14 & 8 \\
\hline South Asia & 52 & 56 & NA & NA & NA & NA & NA & NA & NA & NA & NA & NA & NA & NA & NA & NA & 13 & 15 \\
\hline Sub-Saharan Africa & 47 & 47 & NA & NA & NA & NA & NA & NA & NA & NA & NA & NA & NA & NA & NA & NA & 19 & 15 \\
\hline Low- and middle-income countries & 44 & 47 & NA & NA & NA & NA & NA & NA & NA & NA & NA & NA & NA & NA & NA & NA & 13 & 12 \\
\hline High-income countries & 0 & 0 & NA & NA & NA & NA & NA & NA & NA & NA & NA & NA & NA & NA & NA & NA & 0 & 0 \\
\hline RLD & 43 & 46 & NA & NA & NA & NA & NA & NA & NA & NA & NA & NA & NA & NA & NA & NA & 13 & 12 \\
\hline
\end{tabular}

Attributable Mortality (thousands)

Atribusio Asia

Europe and Central Asia

Latin America and the Caribbean

Middle East and North Africa

South Asia

Sub-Saharan Africa

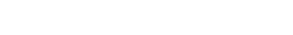

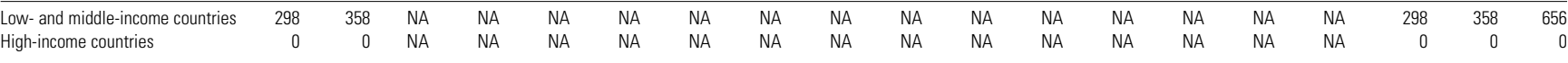

\begin{tabular}{lllllllllllllllllllllll}
\hline WORLD & 298 & 358 & NA & NA & NA & NA & NA & NA & NA & NA & NA & NA & NA & NA & NA & NA & 298 & 358 & 656 \\
\hline
\end{tabular}

Attributable YLL (thousands)

\begin{tabular}{|c|c|c|c|c|c|c|c|c|c|c|c|c|c|c|c|c|c|c|c|}
\hline East Asia and Pacific & 646 & 546 & NA & NA & NA & NA & NA & NA & NA & NA & NA & NA & NA & NA & NA & NA & 646 & 546 & 1,193 \\
\hline Europe and Central Asia & 61 & 53 & NA & NA & NA & NA & NA & NA & NA & NA & NA & NA & NA & NA & NA & NA & 61 & 53 & 114 \\
\hline Latin America and the Caribbean & 154 & 109 & NA & NA & NA & NA & NA & NA & NA & NA & NA & NA & NA & NA & NA & NA & 154 & 109 & 263 \\
\hline Middle East and North Africa & 247 & 249 & NA & NA & NA & NA & NA & NA & NA & NA & NA & NA & NA & NA & NA & NA & 247 & 249 & 497 \\
\hline South Asia & 3,197 & 5,265 & NA & NA & NA & NA & NA & NA & NA & NA & NA & NA & NA & NA & NA & NA & 3,197 & 5,265 & 8,462 \\
\hline Sub-Saharan Africa & 4,700 & 4,658 & NA & NA & NA & NA & NA & NA & NA & NA & NA & NA & NA & NA & NA & NA & 4,700 & 4,658 & 9,358 \\
\hline Low- and middle-income countries & 9,005 & 10,881 & NA & NA & NA & NA & NA & NA & NA & NA & NA & NA & NA & NA & NA & NA & 9,005 & 10,881 & 19,887 \\
\hline High-income countries & 0 & 0 & NA & NA & NA & NA & NA & NA & NA & NA & NA & NA & NA & NA & NA & NA & 0 & 0 & 0 \\
\hline WORLD & 9,005 & 10,881 & NA & NA & NA & NA & NA & NA & NA & NA & NA & NA & NA & NA & NA & NA & 9,005 & 10,881 & 19,887 \\
\hline \multicolumn{20}{|l|}{ Attributable DALYs (thousands) } \\
\hline East Asia and Pacific & 646 & 546 & NA & NA & NA & NA & NA & NA & NA & NA & NA & NA & NA & NA & NA & NA & 646 & 546 & 1,193 \\
\hline Europe and Central Asia & 61 & 53 & NA & NA & NA & NA & NA & NA & NA & NA & NA & NA & NA & NA & NA & NA & 61 & 53 & 114 \\
\hline Latin America and the Caribbean & 154 & 109 & NA & NA & NA & NA & NA & NA & NA & NA & NA & NA & NA & NA & NA & NA & 154 & 109 & 263 \\
\hline Middle East and North Africa & 247 & 249 & NA & NA & NA & NA & NA & NA & NA & NA & NA & NA & NA & NA & NA & NA & 247 & 249 & 497 \\
\hline South Asia & 3,197 & 5,265 & NA & NA & NA & NA & NA & NA & NA & NA & NA & NA & NA & NA & NA & NA & 3,197 & 5,265 & 8,462 \\
\hline Sub-Saharan Africa & 4,700 & 4,658 & NA & NA & NA & NA & NA & NA & NA & NA & NA & NA & NA & NA & NA & NA & 4,700 & 4,658 & 9,358 \\
\hline Low- and middle-income countries & 9,005 & 10,881 & NA & NA & NA & NA & NA & NA & NA & NA & NA & NA & NA & NA & NA & NA & 9,005 & 10,881 & 19,887 \\
\hline High-income countries & 0 & 0 & NA & NA & NA & NA & NA & NA & NA & NA & NA & NA & NA & NA & NA & NA & 0 & 0 & 0 \\
\hline WORLD & 9,005 & 10,881 & NA & NA & NA & NA & NA & NA & NA & NA & NA & NA & NA & NA & NA & NA & 9,005 & 10,881 & 19,887 \\
\hline
\end{tabular}

Source: Authors' calculations.

Note: $\mathrm{NA}=$ not applicable 
Table 4A.7

Risk factor: Childhood underweight

Disease:

All causes

\begin{tabular}{|c|c|c|c|c|c|c|c|c|c|c|c|c|c|c|c|c|c|c|c|}
\hline \multirow[b]{2}{*}{ Region } & \multicolumn{2}{|c|}{$0-4$ years } & \multicolumn{2}{|c|}{ 5-14 years } & \multicolumn{2}{|c|}{$15-29$ years } & \multicolumn{2}{|c|}{ 30-44 years } & \multicolumn{2}{|c|}{ 45-59 years } & \multicolumn{2}{|c|}{ 60-69 years } & \multicolumn{2}{|c|}{ 70-79 years } & \multicolumn{2}{|c|}{$80+$ years } & \multicolumn{3}{|c|}{ Total } \\
\hline & Male & Female & Male & Female & Male & Female & Male & Female & Male & Female & Male & Female & Male & Female & Male & Female & Male & Female & All \\
\hline \multicolumn{20}{|l|}{ PAF of Mortality (\%) } \\
\hline East Asia and Pacific & 16 & 18 & NA & NA & NA & NA & NA & NA & NA & NA & NA & NA & NA & NA & NA & NA & 2 & 2 & 2 \\
\hline Europe and Central Asia & 8 & 9 & NA & NA & NA & NA & NA & NA & NA & NA & NA & NA & NA & NA & NA & NA & 0 & 0 & 0 \\
\hline Latin America and the Caribbean & 10 & 10 & NA & NA & NA & NA & NA & NA & NA & NA & NA & NA & NA & NA & NA & NA & 1 & 1 & 1 \\
\hline Middle East and North Africa & 19 & 21 & NA & NA & NA & NA & NA & NA & NA & NA & NA & NA & NA & NA & NA & NA & 4 & 5 & 5 \\
\hline South Asia & 37 & 40 & NA & NA & NA & NA & NA & NA & NA & NA & NA & NA & NA & NA & NA & NA & 9 & 11 & 10 \\
\hline Sub-Saharan Africa & 40 & 42 & NA & NA & NA & NA & NA & NA & NA & NA & NA & NA & NA & NA & NA & NA & 17 & 17 & 17 \\
\hline Low- and middle-income countries & 34 & 35 & NA & NA & NA & NA & NA & NA & NA & NA & NA & NA & NA & NA & NA & NA & 7 & 8 & 8 \\
\hline High-income countries & 0 & 0 & NA & NA & NA & NA & NA & NA & NA & NA & NA & NA & NA & NA & NA & NA & 0 & 0 & 0 \\
\hline WORLD & 33 & 35 & NA & NA & NA & NA & NA & NA & NA & NA & NA & NA & NA & NA & NA & NA & 6 & 7 & 6 \\
\hline
\end{tabular}

PAF of YLL (\%)

\begin{tabular}{|c|c|c|c|c|c|c|c|c|c|c|c|c|c|c|c|c|c|c|c|}
\hline East Asia and Pacific & 16 & 18 & NA & NA & NA & NA & NA & NA & NA & NA & NA & NA & NA & NA & NA & NA & 3 & 4 & 4 \\
\hline Europe and Central Asia & 8 & 9 & NA & NA & NA & NA & NA & NA & NA & NA & NA & NA & NA & NA & NA & NA & 1 & 1 & 1 \\
\hline Latin America and the Caribbean & 10 & 10 & NA & NA & NA & NA & NA & NA & NA & NA & NA & NA & NA & NA & NA & NA & 2 & 3 & 2 \\
\hline Middle East and North Africa & 19 & 21 & NA & NA & NA & NA & NA & NA & NA & NA & NA & NA & NA & NA & NA & NA & 7 & 8 & 7 \\
\hline South Asia & 37 & 40 & NA & NA & NA & NA & NA & NA & NA & NA & NA & NA & NA & NA & NA & NA & 14 & 17 & 15 \\
\hline Sub-Saharan Africa & 40 & 42 & NA & NA & NA & NA & NA & NA & NA & NA & NA & NA & NA & NA & NA & NA & 21 & 21 & 21 \\
\hline Low- and middle-income countries & 34 & 35 & NA & NA & NA & NA & NA & NA & NA & NA & NA & NA & NA & NA & NA & NA & 11 & 13 & 12 \\
\hline High-income countries & 0 & 0 & NA & NA & NA & NA & NA & NA & NA & NA & NA & NA & NA & NA & NA & NA & 0 & 0 & 0 \\
\hline WORLD & 33 & 35 & NA & NA & NA & NA & NA & NA & NA & NA & NA & NA & NA & NA & NA & NA & 10 & 12 & 11 \\
\hline
\end{tabular}

PAF of DALYs $(\%)$

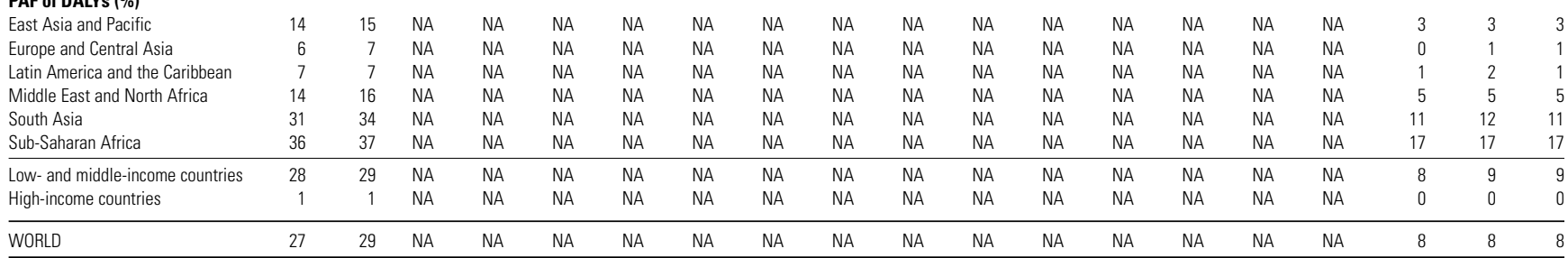

Attributable Mortality (thousands)

\begin{tabular}{|c|c|c|c|c|c|c|c|c|c|c|c|c|c|c|c|c|c|c|c|}
\hline East Asia and Pacific & 115 & 124 & NA & NA & NA & NA & NA & NA & NA & NA & NA & NA & NA & NA & NA & NA & 115 & 124 & 239 \\
\hline Europe and Central Asia & 8 & 7 & NA & NA & NA & NA & NA & NA & NA & NA & NA & NA & NA & NA & NA & NA & 8 & 7 & 15 \\
\hline Latin America and the Caribbean & 23 & 19 & NA & NA & NA & NA & NA & NA & NA & NA & NA & NA & NA & NA & NA & NA & 23 & 19 & 42 \\
\hline Middle East and North Africa & 44 & 43 & NA & NA & NA & NA & NA & NA & NA & NA & NA & NA & NA & NA & NA & NA & 44 & 43 & 87 \\
\hline South Asia & 666 & 728 & NA & NA & NA & NA & NA & NA & NA & NA & NA & NA & NA & NA & NA & NA & 666 & 728 & 1,394 \\
\hline Sub-Saharan Africa & 957 & 896 & NA & NA & NA & NA & NA & NA & NA & NA & NA & NA & NA & NA & NA & NA & 957 & 896 & 1,853 \\
\hline Low- and middle-income countries & 1,814 & 1,816 & NA & NA & NA & NA & NA & NA & NA & NA & NA & NA & NA & NA & NA & NA & 1,814 & 1,816 & 3,630 \\
\hline High-income countries & 0 & 0 & NA & NA & NA & NA & NA & NA & NA & NA & NA & NA & NA & NA & NA & NA & 0 & 0 & 0 \\
\hline WORLD & 1,814 & 1,816 & NA & NA & NA & NA & NA & NA & NA & NA & NA & NA & NA & NA & NA & NA & 1,814 & 1,816 & 0 \\
\hline
\end{tabular}

Attributable YLL (thousands)

\begin{tabular}{|c|c|c|c|c|c|c|c|c|c|c|c|c|c|c|c|c|c|c|c|}
\hline East Asia and Pacific & 3,488 & 3,781 & NA & NA & NA & NA & NA & NA & NA & NA & NA & NA & NA & NA & NA & NA & 3,488 & 3,781 & 7,269 \\
\hline Europe and Central Asia & 245 & 213 & NA & NA & NA & NA & NA & NA & NA & NA & NA & NA & NA & NA & NA & NA & 245 & 213 & 458 \\
\hline Latin America and the Caribbean & 687 & 573 & NA & NA & NA & NA & NA & NA & NA & NA & NA & NA & NA & NA & NA & NA & 687 & 573 & 1,261 \\
\hline Middle East and North Africa & 1,334 & 1,299 & NA & NA & NA & NA & NA & NA & NA & NA & NA & NA & NA & NA & NA & NA & 1,334 & 1,299 & 2,633 \\
\hline South Asia & 20,164 & 22,167 & NA & NA & NA & NA & NA & NA & NA & NA & NA & NA & NA & NA & NA & NA & 20,164 & 22,167 & 42,331 \\
\hline Sub-Saharan Africa & 28,919 & 27,261 & NA & NA & NA & NA & NA & NA & NA & NA & NA & NA & NA & NA & NA & NA & 28,919 & 27,261 & 56,181 \\
\hline Low- and middle-income countries & 54,838 & 55,295 & NA & NA & NA & NA & NA & NA & NA & NA & NA & NA & NA & NA & NA & NA & 54,838 & 55,295 & 110,132 \\
\hline High-income countries & 2 & 1 & NA & NA & NA & NA & NA & NA & NA & NA & NA & NA & NA & NA & NA & NA & 2 & 1 & 3 \\
\hline WORLD & 54,839 & 55,296 & NA & NA & NA & NA & NA & NA & NA & NA & NA & NA & NA & NA & NA & NA & 54,839 & 55,296 & 110,135 \\
\hline \multicolumn{20}{|l|}{ Attributable DALYs (thousands) } \\
\hline East Asia and Pacific & 4,628 & 4,887 & NA & NA & NA & NA & NA & NA & NA & NA & NA & NA & NA & NA & NA & NA & 4,628 & 4,887 & 9,516 \\
\hline Europe and Central Asia & 319 & 285 & NA & NA & NA & NA & NA & NA & NA & NA & NA & NA & NA & NA & NA & NA & 319 & 285 & 604 \\
\hline Latin America and the Caribbean & 831 & 714 & NA & NA & NA & NA & NA & NA & NA & NA & NA & NA & NA & NA & NA & NA & 831 & 714 & 1,546 \\
\hline Middle East and North Africa & 1,598 & 1,537 & NA & NA & NA & NA & NA & NA & NA & NA & NA & NA & NA & NA & NA & NA & 1,598 & 1,537 & 3,134 \\
\hline South Asia & 22,361 & 24,259 & NA & NA & NA & NA & NA & NA & NA & NA & NA & NA & NA & NA & NA & NA & 22,361 & 24,259 & 46,620 \\
\hline Sub-Saharan Africa & 30,440 & 28,720 & NA & NA & NA & NA & NA & NA & NA & NA & NA & NA & NA & NA & NA & NA & 30,440 & 28,720 & 59,160 \\
\hline Low- and middle-income countries & 60,177 & 60,403 & NA & NA & NA & NA & NA & NA & NA & NA & NA & NA & NA & NA & NA & NA & 60,177 & 60,403 & 120,579 \\
\hline High-income countries & 35 & 33 & NA & NA & NA & NA & NA & NA & NA & NA & NA & NA & NA & NA & NA & NA & 35 & 33 & 67 \\
\hline$\overline{\text { WORLD }}$ & 60,211 & 60,436 & NA & NA & NA & NA & NA & NA & NA & NA & NA & NA & NA & NA & NA & NA & 60,211 & 60,436 & 120,647 \\
\hline
\end{tabular}


Table 4A.8

Risk factor: Iron-deficiency anemia

Disease:

Maternal conditions

\begin{tabular}{|c|c|c|c|c|c|c|c|c|c|c|c|c|c|c|c|c|c|c|c|}
\hline \multirow[b]{2}{*}{ Region } & \multicolumn{2}{|c|}{$0-4$ years } & \multicolumn{2}{|c|}{ 5-14 years } & \multicolumn{2}{|c|}{$15-29$ years } & \multicolumn{2}{|c|}{ 30-44 years } & \multicolumn{2}{|c|}{$45-59$ years } & \multicolumn{2}{|c|}{$60-69$ years } & \multicolumn{2}{|c|}{ 70-79 years } & \multicolumn{2}{|c|}{$80+$ years } & \multicolumn{3}{|c|}{ Total } \\
\hline & Male & Female & Male & Female & Male & Female & Male & Female & Male & Female & Male & Female & Male & Female & Male & Female & Male & Female & All \\
\hline \multicolumn{20}{|l|}{ PAF of Mortality (\%) } \\
\hline East Asia and Pacific & NA & NA & NA & NA & NA & 17 & NA & 17 & NA & NA & NA & NA & NA & NA & NA & NA & NA & 16 & 16 \\
\hline Europe and Central Asia & NA & NA & NA & NA & NA & 8 & NA & 8 & NA & NA & NA & NA & NA & NA & NA & NA & NA & 8 & 8 \\
\hline Latin America and the Caribbean & NA & NA & NA & NA & NA & 7 & NA & 7 & NA & NA & NA & NA & NA & NA & NA & NA & NA & 7 & 7 \\
\hline Middle East and North Africa & NA & NA & NA & NA & NA & 14 & NA & 15 & NA & NA & NA & NA & NA & NA & NA & NA & NA & 14 & 14 \\
\hline South Asia & NA & NA & NA & NA & NA & 22 & NA & 23 & NA & NA & NA & NA & NA & NA & NA & NA & NA & 22 & 22 \\
\hline Sub-Saharan Africa & NA & NA & NA & NA & NA & 14 & NA & 14 & NA & NA & NA & NA & NA & NA & NA & NA & NA & 13 & 13 \\
\hline Low- and middle-income countries & NA & NA & NA & NA & NA & 17 & NA & 18 & NA & NA & NA & NA & NA & NA & NA & NA & NA & 17 & 17 \\
\hline High-income countries & NA & NA & NA & NA & NA & 4 & NA & 4 & NA & NA & NA & NA & NA & NA & NA & NA & NA & 4 & 4 \\
\hline WORLD & NA & NA & NA & NA & NA & 17 & NA & 18 & NA & NA & NA & NA & NA & NA & NA & NA & NA & 17 & 17 \\
\hline
\end{tabular}

PAF of YLL (\%)

\begin{tabular}{|c|c|c|c|c|c|c|c|c|c|c|c|c|c|c|c|c|c|c|c|}
\hline East Asia and Pacific & NA & NA & NA & NA & NA & 17 & NA & 17 & NA & NA & NA & NA & NA & NA & NA & NA & NA & 16 & 16 \\
\hline Europe and Central Asia & NA & NA & NA & NA & NA & 8 & NA & 8 & NA & NA & NA & NA & NA & NA & NA & NA & NA & 8 & 8 \\
\hline Latin America and the Caribbean & NA & NA & NA & NA & NA & 7 & NA & 7 & NA & NA & NA & NA & NA & NA & NA & NA & NA & 7 & 7 \\
\hline Middle East and North Africa & NA & NA & NA & NA & NA & 14 & NA & 15 & NA & NA & NA & NA & NA & NA & NA & NA & NA & 14 & 14 \\
\hline South Asia & NA & NA & NA & NA & NA & 22 & NA & 23 & NA & NA & NA & NA & NA & NA & NA & NA & NA & 22 & 22 \\
\hline Sub-Saharan Africa & NA & NA & NA & NA & NA & 14 & NA & 14 & NA & NA & NA & NA & NA & NA & NA & NA & NA & 13 & 13 \\
\hline Low- and middle-income countries & NA & NA & NA & NA & NA & 17 & NA & 18 & NA & NA & NA & NA & NA & NA & NA & NA & NA & 17 & 17 \\
\hline High-income countries & NA & NA & NA & NA & NA & 4 & NA & 4 & NA & NA & NA & NA & NA & NA & NA & NA & NA & 4 & 4 \\
\hline WORLD & NA & NA & NA & NA & NA & 17 & NA & 18 & NA & NA & NA & NA & NA & NA & NA & NA & NA & 17 & 17 \\
\hline
\end{tabular}

PAF of DALYs $(\%)$

\begin{tabular}{|c|c|c|c|c|c|c|c|c|c|c|c|c|c|c|c|c|c|c|}
\hline East Asia and Pacific & NA & NA & NA & NA & NA & 3 & NA & 8 & NA & NA & NA & NA & NA & NA & NA & NA & NA & 5 \\
\hline Europe and Central Asia & NA & NA & NA & NA & NA & 1 & NA & 1 & NA & NA & NA & NA & NA & NA & NA & NA & NA & 1 \\
\hline Latin America and the Caribbean & NA & NA & NA & NA & NA & 2 & NA & 3 & NA & NA & NA & NA & NA & NA & NA & NA & NA & 2 \\
\hline Middle East and North Africa & NA & NA & NA & NA & NA & 3 & NA & 6 & NA & NA & NA & NA & NA & NA & NA & NA & NA & 4 \\
\hline South Asia & NA & NA & NA & NA & NA & 9 & NA & 16 & NA & NA & NA & NA & NA & NA & NA & NA & NA & 12 \\
\hline Sub-Saharan Africa & NA & NA & NA & NA & NA & 8 & NA & 11 & NA & NA & NA & NA & NA & NA & NA & NA & NA & 8 \\
\hline Low- and middle-income countries & NA & NA & NA & NA & NA & 7 & NA & 12 & NA & NA & NA & NA & NA & NA & NA & NA & NA & 9 \\
\hline High-income countries & NA & NA & NA & NA & NA & 0 & NA & 0 & NA & NA & NA & NA & NA & NA & NA & NA & NA & 0 \\
\hline RLD & NA & NA & NA & NA & NA & 7 & NA & 12 & NA & NA & NA & NA & NA & NA & NA & NA & NA & 8 \\
\hline
\end{tabular}

Attributable Mortality (thousands

\begin{tabular}{|c|c|c|c|c|c|c|c|c|c|c|c|c|c|c|c|c|c|c|}
\hline East Asia and Pacific & NA & NA & NA & NA & NA & 3 & NA & 3 & NA & NA & NA & NA & NA & NA & NA & NA & NA & 6 \\
\hline Europe and Central Asia & NA & NA & NA & NA & NA & 0 & NA & 0 & NA & NA & NA & NA & NA & NA & NA & NA & NA & 0 \\
\hline Latin America and the Caribbean & NA & NA & NA & NA & NA & 1 & NA & 0 & NA & NA & NA & NA & NA & NA & NA & NA & NA & 1 \\
\hline Middle East and North Africa & NA & NA & NA & NA & NA & 1 & NA & 1 & NA & NA & NA & NA & NA & NA & NA & NA & NA & 2 \\
\hline South Asia & NA & NA & NA & NA & NA & 22 & NA & 22 & NA & NA & NA & NA & NA & NA & NA & NA & NA & 44 \\
\hline Sub-Saharan Africa & NA & NA & NA & NA & NA & 17 & NA & 13 & NA & NA & NA & NA & NA & NA & NA & NA & NA & 31 \\
\hline Low- and middle-income countries & NA & NA & NA & NA & NA & 44 & NA & 41 & NA & NA & NA & NA & NA & NA & NA & NA & NA & 85 \\
\hline High-income countries & NA & NA & NA & NA & NA & 0 & NA & 0 & NA & NA & NA & NA & NA & NA & NA & NA & NA & 0 \\
\hline & NA & NA & NA & NA & NA & 44 & NA & 41 & NA & NA & NA & NA & NA & NA & NA & NA & NA & 85 \\
\hline
\end{tabular}

Attributable YLL (thousands)

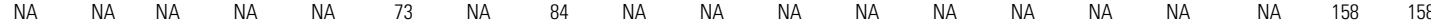

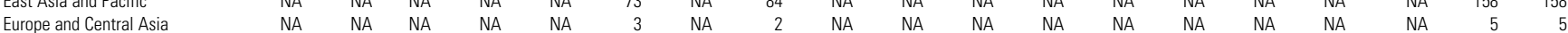
$\begin{array}{lllllllllllllllllllllllll}\text { Latin America and the Caribbean } & \text { NA } & \text { NA } & \text { NA } & \text { NA } & \text { NA } & 16 & \text { NA } & 12 & \text { NA } & \text { NA } & \text { NA } & \text { NA } & \text { NA } & \text { NA } & \text { NA } & \text { NA } & \text { NA } & 29 & 29\end{array}$

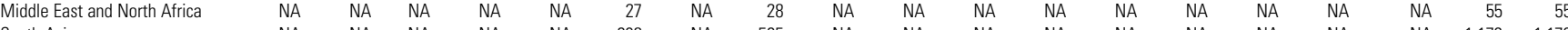

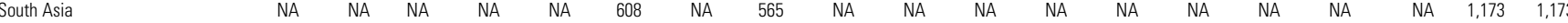

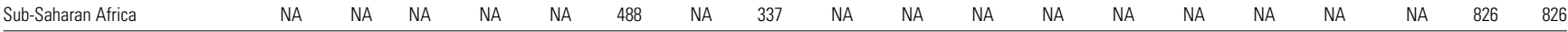

\begin{tabular}{llllllllllllllllllll}
\hline Low- and middle-income countries & NA & NA & NA & NA & NA & 1,217 & NA & 1,029 & NA & NA & NA & NA & NA & NA & NA & NA & NA & 2,246 & 2,246
\end{tabular}

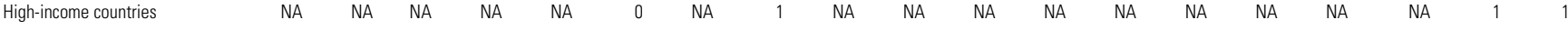

\begin{tabular}{llllllllllllllllllll}
\hline WORLD & NA & NA & NA & NA & NA & 1,217 & NA & 1,030 & NA & NA & NA & NA & NA & NA & NA & NA & NA & 2,247 & 2,247
\end{tabular}

Attributable DALYs (thousands)

\begin{tabular}{|c|c|c|c|c|c|c|c|c|c|c|c|c|c|c|c|c|c|c|c|}
\hline East Asia and Pacific & NA & NA & NA & NA & NA & 73 & NA & 84 & NA & NA & NA & NA & NA & NA & NA & NA & NA & 158 & 158 \\
\hline Europe and Central Asia & NA & NA & NA & NA & NA & 3 & NA & 2 & NA & NA & NA & NA & NA & NA & NA & NA & NA & 5 & 5 \\
\hline Latin America and the Caribbean & NA & NA & NA & NA & NA & 16 & NA & 12 & NA & NA & NA & NA & NA & NA & NA & NA & NA & 29 & 29 \\
\hline Middle East and North Africa & NA & NA & NA & NA & NA & 27 & NA & 28 & NA & NA & NA & NA & NA & NA & NA & NA & NA & 55 & 55 \\
\hline South Asia & NA & NA & NA & NA & NA & 608 & NA & 565 & NA & NA & NA & NA & NA & NA & NA & NA & NA & 1,173 & 1,173 \\
\hline Sub-Saharan Africa & NA & NA & NA & NA & NA & 488 & NA & 337 & NA & NA & NA & NA & NA & NA & NA & NA & NA & 826 & 826 \\
\hline Low- and middle-income countries & NA & NA & NA & NA & NA & 1,217 & NA & 1,029 & NA & NA & NA & NA & NA & NA & NA & NA & NA & 2,246 & 2,246 \\
\hline High-income countries & NA & NA & NA & NA & NA & 0 & NA & 1 & NA & NA & NA & NA & NA & NA & NA & NA & NA & 1 & 1 \\
\hline WORLD & NA & NA & NA & NA & NA & 1,217 & NA & 1,030 & NA & NA & NA & NA & NA & NA & NA & NA & NA & 2,247 & 2,247 \\
\hline
\end{tabular}

Source: Authors' calculations.

Note: $\mathrm{NA}=$ not applicable 
Table 4A.9

Risk factor: Iron-deficiency anemia

Disease:

Perinatal conditions

\begin{tabular}{|c|c|c|c|c|c|c|c|c|c|c|c|c|c|c|c|c|c|c|c|}
\hline \multirow[b]{2}{*}{ Region } & \multicolumn{2}{|c|}{$0-4$ years } & \multicolumn{2}{|c|}{ 5-14 years } & \multicolumn{2}{|c|}{$15-29$ years } & \multicolumn{2}{|c|}{ 30-44 years } & \multicolumn{2}{|c|}{$45-59$ years } & \multicolumn{2}{|c|}{$60-69$ years } & \multicolumn{2}{|c|}{$70-79$ years } & \multicolumn{2}{|c|}{$80+$ years } & \multicolumn{3}{|c|}{ Total } \\
\hline & Male & Female & Male & Female & Male & Female & Male & Female & Male & Female & Male & Female & Male & Female & Male & Female & Male & Female & All \\
\hline \multicolumn{20}{|l|}{ PAF of Mortality (\%) } \\
\hline East Asia and Pacific & 13 & 13 & NA & NA & NA & NA & NA & NA & NA & NA & NA & NA & NA & NA & NA & NA & 13 & 13 & 13 \\
\hline Europe and Central Asia & 6 & 6 & NA & NA & NA & NA & NA & NA & NA & NA & NA & NA & NA & NA & NA & NA & 6 & 6 & 6 \\
\hline Latin America and the Caribbean & 5 & 5 & NA & NA & NA & NA & NA & NA & NA & NA & NA & NA & NA & NA & NA & NA & 5 & 5 & 5 \\
\hline Middle East and North Africa & 12 & 12 & NA & NA & NA & NA & NA & NA & NA & NA & NA & NA & NA & NA & NA & NA & 12 & 12 & 12 \\
\hline South Asia & 18 & 18 & NA & NA & NA & NA & NA & NA & NA & NA & NA & NA & NA & NA & NA & NA & 18 & 18 & 18 \\
\hline Sub-Saharan Africa & 20 & 20 & NA & NA & NA & NA & NA & NA & NA & NA & NA & NA & NA & NA & NA & NA & 20 & 20 & 20 \\
\hline Low- and middle-income countries & 16 & 16 & NA & NA & NA & NA & NA & NA & NA & NA & NA & NA & NA & NA & NA & NA & 16 & 16 & 16 \\
\hline High-income countries & 3 & 3 & NA & NA & NA & NA & NA & NA & NA & NA & NA & NA & NA & NA & NA & NA & 3 & 3 & 3 \\
\hline WORLD & 16 & 16 & NA & NA & NA & NA & NA & NA & NA & NA & NA & NA & NA & NA & NA & NA & 16 & 16 & 16 \\
\hline
\end{tabular}

PAF of YLL (\%)

\begin{tabular}{|c|c|c|c|c|c|c|c|c|c|c|c|c|c|c|c|c|c|c|c|}
\hline East Asia and Pacific & 13 & 13 & NA & NA & NA & NA & NA & NA & NA & NA & NA & NA & NA & NA & NA & NA & 13 & 13 & 13 \\
\hline Europe and Central Asia & 6 & 6 & NA & NA & NA & NA & NA & NA & NA & NA & NA & NA & NA & NA & NA & NA & 6 & 6 & 6 \\
\hline Latin America and the Caribbean & 5 & 5 & NA & NA & NA & NA & NA & NA & NA & NA & NA & NA & NA & NA & NA & NA & 5 & 5 & 5 \\
\hline Middle East and North Africa & 12 & 12 & NA & NA & NA & NA & NA & NA & NA & NA & NA & NA & NA & NA & NA & NA & 12 & 12 & 12 \\
\hline South Asia & 18 & 18 & NA & NA & NA & NA & NA & NA & NA & NA & NA & NA & NA & NA & NA & NA & 18 & 18 & 18 \\
\hline Sub-Saharan Africa & 20 & 20 & NA & NA & NA & NA & NA & NA & NA & NA & NA & NA & NA & NA & NA & NA & 20 & 20 & 20 \\
\hline Low- and middle-income countries & 16 & 16 & NA & NA & NA & NA & NA & NA & NA & NA & NA & NA & NA & NA & NA & NA & 16 & 16 & 16 \\
\hline High-income countries & 3 & 3 & NA & NA & NA & NA & NA & NA & NA & NA & NA & NA & NA & NA & NA & NA & 3 & 3 & 3 \\
\hline WORLD & 16 & 16 & NA & NA & NA & NA & NA & NA & NA & NA & NA & NA & NA & NA & NA & NA & 16 & 16 & 16 \\
\hline
\end{tabular}

PAF of DALYs (\%)

\begin{tabular}{|c|c|c|c|c|c|c|c|c|c|c|c|c|c|c|c|c|c|c|c|}
\hline & & & & & & & & & & & & & & & & & & & \\
\hline East Asia and Pacific & 11 & 11 & NA & NA & NA & NA & NA & NA & NA & NA & NA & NA & NA & NA & NA & NA & 11 & 11 & 11 \\
\hline Europe and Central Asia & 5 & 5 & NA & NA & NA & NA & NA & NA & NA & NA & NA & NA & NA & NA & NA & NA & 5 & 5 & 5 \\
\hline Latin America and the Caribbean & 4 & 4 & NA & NA & NA & NA & NA & NA & NA & NA & NA & NA & NA & NA & NA & NA & 4 & 4 & 4 \\
\hline Middle East and North Africa & 9 & 8 & NA & NA & NA & NA & NA & NA & NA & NA & NA & NA & NA & NA & NA & NA & 9 & 8 & 9 \\
\hline South Asia & 16 & 16 & NA & NA & NA & NA & NA & NA & NA & NA & NA & NA & NA & NA & NA & NA & 16 & 16 & 16 \\
\hline Sub-Saharan Africa & 18 & 17 & NA & NA & NA & NA & NA & NA & NA & NA & NA & NA & NA & NA & NA & NA & 18 & 17 & 17 \\
\hline Low- and middle-income countries & 14 & 13 & NA & NA & NA & NA & NA & NA & NA & NA & NA & NA & NA & NA & NA & NA & 14 & 13 & 14 \\
\hline High-income countries & 2 & 2 & NA & NA & NA & NA & NA & NA & NA & NA & NA & NA & NA & NA & NA & NA & 2 & 2 & 2 \\
\hline WORLD & 14 & 13 & NA & NA & NA & NA & NA & NA & NA & NA & NA & NA & NA & NA & NA & NA & 14 & 13 & 14 \\
\hline
\end{tabular}

Attributable Mortality (thousands)

\begin{tabular}{|c|c|c|c|c|c|c|c|c|c|c|c|c|c|c|c|c|c|c|c|}
\hline East Asia and Pacific & 35 & 32 & NA & NA & NA & NA & NA & NA & NA & NA & NA & NA & NA & NA & NA & NA & 35 & 32 & 66 \\
\hline Europe and Central Asia & 2 & 1 & NA & NA & NA & NA & NA & NA & NA & NA & NA & NA & NA & NA & NA & NA & 2 & 1 & 3 \\
\hline Latin America and the Caribbean & 5 & 4 & NA & NA & NA & NA & NA & NA & NA & NA & NA & NA & NA & NA & NA & NA & 5 & 4 & 9 \\
\hline Middle East and North Africa & 7 & 5 & NA & NA & NA & NA & NA & NA & NA & NA & NA & NA & NA & NA & NA & NA & 7 & 5 & 12 \\
\hline South Asia & 108 & 89 & NA & NA & NA & NA & NA & NA & NA & NA & NA & NA & NA & NA & NA & NA & 108 & 89 & 197 \\
\hline Sub-Saharan Africa & 66 & 48 & NA & NA & NA & NA & NA & NA & NA & NA & NA & NA & NA & NA & NA & NA & 66 & 48 & 114 \\
\hline Low- and middle-income countries & 223 & 179 & NA & NA & NA & NA & NA & NA & NA & NA & NA & NA & NA & NA & NA & NA & 223 & 179 & 402 \\
\hline High-income countries & 1 & 0 & NA & NA & NA & NA & NA & NA & NA & NA & NA & NA & NA & NA & NA & NA & 1 & 0 & 1 \\
\hline WORLD & 224 & 179 & NA & NA & NA & NA & NA & NA & NA & NA & NA & NA & NA & NA & NA & NA & 224 & 179 & 403 \\
\hline
\end{tabular}

Attributable YLL (thousands)

\begin{tabular}{|c|c|c|c|c|c|c|c|c|c|c|c|c|c|c|c|c|c|c|c|}
\hline \multicolumn{20}{|l|}{ Attributable YLL (the } \\
\hline East Asia and Pacific & 1,047 & 970 & NA & NA & NA & NA & NA & NA & NA & NA & NA & NA & NA & NA & NA & NA & 1,047 & 970 & 2,016 \\
\hline Europe and Central Asia & 61 & 45 & NA & NA & NA & NA & NA & NA & NA & NA & NA & NA & NA & NA & NA & NA & 61 & 45 & 106 \\
\hline Latin America and the Caribbean & 151 & 117 & NA & NA & NA & NA & NA & NA & NA & NA & NA & NA & NA & NA & NA & NA & 151 & 117 & 268 \\
\hline Middle East and North Africa & 225 & 147 & NA & NA & NA & NA & NA & NA & NA & NA & NA & NA & NA & NA & NA & NA & 225 & 147 & 372 \\
\hline South Asia & 3,272 & 2,704 & NA & NA & NA & NA & NA & NA & NA & NA & NA & NA & NA & NA & NA & NA & 3,272 & 2,704 & 5,976 \\
\hline Sub-Saharan Africa & 2,001 & 1,466 & NA & NA & NA & NA & NA & NA & NA & NA & NA & NA & NA & NA & NA & NA & 2,001 & 1,466 & 3,466 \\
\hline Low- and middle-income countries & 6,757 & 5,448 & NA & NA & NA & NA & NA & NA & NA & NA & NA & NA & NA & NA & NA & NA & 6,757 & 5,448 & 12,205 \\
\hline High-income countries & 17 & 13 & NA & NA & NA & NA & NA & NA & NA & NA & NA & NA & NA & NA & NA & NA & 17 & 13 & 30 \\
\hline WORLD & 6,774 & 5,461 & NA & NA & NA & NA & NA & NA & NA & NA & NA & NA & NA & NA & NA & NA & 6,774 & 5,461 & 12,235 \\
\hline \multicolumn{20}{|l|}{ Attributable DALYs (thousands) } \\
\hline East Asia and Pacific & 1,047 & 970 & NA & NA & NA & NA & NA & NA & NA & NA & NA & NA & NA & NA & NA & NA & 1,047 & 970 & 2,016 \\
\hline Europe and Central Asia & 61 & 45 & NA & NA & NA & NA & NA & NA & NA & NA & NA & NA & NA & NA & NA & NA & 61 & 45 & 106 \\
\hline Latin America and the Caribbean & 151 & 117 & NA & NA & NA & NA & NA & NA & NA & NA & NA & NA & NA & NA & NA & NA & 151 & 117 & 268 \\
\hline Middle East and North Africa & 225 & 147 & NA & NA & NA & NA & NA & NA & NA & NA & NA & NA & NA & NA & NA & NA & 225 & 147 & 372 \\
\hline South Asia & 3,272 & 2,704 & NA & NA & NA & NA & NA & NA & NA & NA & NA & NA & NA & NA & NA & NA & 3,272 & 2,704 & 5,976 \\
\hline Sub-Saharan Africa & 2,001 & 1,466 & NA & NA & NA & NA & NA & NA & NA & NA & NA & NA & NA & NA & NA & NA & 2,001 & 1,466 & 3,466 \\
\hline Low- and middle-income countries & 6,757 & 5,448 & NA & NA & NA & NA & NA & NA & NA & NA & NA & NA & NA & NA & NA & NA & 6,757 & 5,448 & 12,205 \\
\hline High-income countries & 17 & 13 & NA & NA & NA & NA & NA & NA & NA & NA & NA & NA & NA & NA & NA & NA & 17 & 13 & 30 \\
\hline WORLD & 6,774 & 5,461 & NA & NA & NA & NA & NA & NA & NA & NA & NA & NA & NA & NA & NA & NA & 6,774 & 5,461 & 12,235 \\
\hline
\end{tabular}

Source: Authors' calculations.

Note: $\mathrm{NA}=$ not applicable 
Table 4A.10

Risk factor: Iron-deficiency anemia

Disease: Iron-deficiency anemia

\begin{tabular}{|c|c|c|c|c|c|c|c|c|c|c|c|c|c|c|c|c|c|c|c|}
\hline \multirow[b]{2}{*}{ Region } & \multicolumn{2}{|c|}{$0-4$ years } & \multicolumn{2}{|c|}{ 5-14 years } & \multicolumn{2}{|c|}{$15-29$ years } & \multicolumn{2}{|c|}{ 30-44 years } & \multicolumn{2}{|c|}{$45-59$ years } & \multicolumn{2}{|c|}{ 60-69 years } & \multicolumn{2}{|c|}{$70-79$ years } & \multicolumn{2}{|c|}{$80+$ years } & \multicolumn{3}{|c|}{ Total } \\
\hline & Male & Female & Male & Female & Male & Female & Male & Female & Male & Female & Male & Female & Male & Female & Male & Female & Male & Female & All \\
\hline \multicolumn{20}{|l|}{ PAF of Mortality (\%) } \\
\hline East Asia and Pacific & 100 & 100 & 100 & 100 & 100 & 100 & 100 & 100 & 100 & 100 & 100 & 100 & 100 & 100 & 100 & 100 & 100 & 100 & 100 \\
\hline Europe and Central Asia & 100 & 100 & 100 & 100 & 100 & 100 & 100 & 100 & 100 & 100 & 100 & 100 & 100 & 100 & 100 & 100 & 100 & 100 & 100 \\
\hline Latin America and the Caribbean & 100 & 100 & 100 & 100 & 100 & 100 & 100 & 100 & 100 & 100 & 100 & 100 & 100 & 100 & 100 & 100 & 100 & 100 & 100 \\
\hline Middle East and North Africa & 100 & 100 & 100 & 100 & 100 & 100 & 100 & 100 & 100 & 100 & 100 & 100 & 100 & 100 & 100 & 100 & 100 & 100 & 100 \\
\hline South Asia & 100 & 100 & 100 & 100 & 100 & 100 & 100 & 100 & 100 & 100 & 100 & 100 & 100 & 100 & 100 & 100 & 100 & 100 & 100 \\
\hline Sub-Saharan Africa & 100 & 100 & 100 & 100 & 100 & 100 & 100 & 100 & 100 & 100 & 100 & 100 & 100 & 100 & 100 & 100 & 100 & 100 & 100 \\
\hline Low- and middle-income countries & 100 & 100 & 100 & 100 & 100 & 100 & 100 & 100 & 100 & 100 & 100 & 100 & 100 & 100 & 100 & 100 & 100 & 100 & 100 \\
\hline High-income countries & 100 & 100 & 100 & 100 & 100 & 100 & 100 & 100 & 100 & 100 & 100 & 100 & 100 & 100 & 100 & 100 & 100 & 100 & 100 \\
\hline WORLD & 100 & 100 & 100 & 100 & 100 & 100 & 100 & 100 & 100 & 100 & 100 & 100 & 100 & 100 & 100 & 100 & 100 & 100 & 100 \\
\hline
\end{tabular}

PAF of YLL (\%)

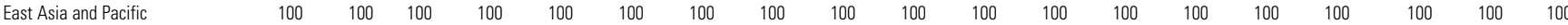

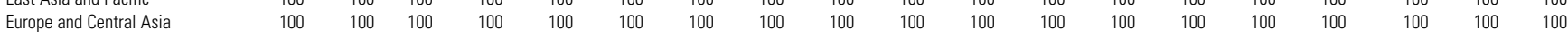

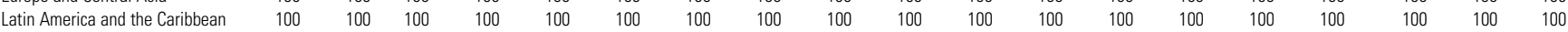

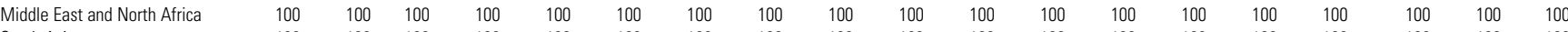

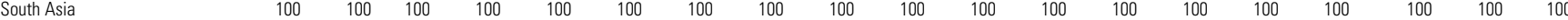

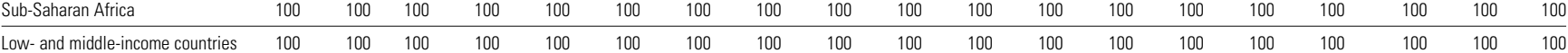

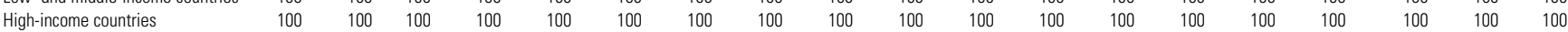

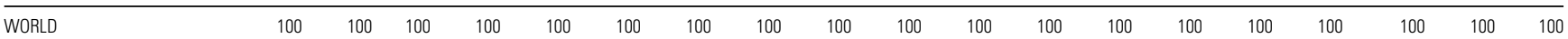

PAF of DALYs $(\%)$

\begin{tabular}{|c|c|c|c|c|c|c|c|c|c|c|c|c|c|c|c|c|c|c|c|}
\hline East Asia and Pacific & 100 & 100 & 100 & 100 & 100 & 100 & 100 & 100 & 100 & 100 & 100 & 100 & 100 & 100 & 100 & 100 & 100 & 100 & 100 \\
\hline Europe and Central Asia & 100 & 100 & 100 & 100 & 100 & 100 & 100 & 100 & 100 & 100 & 100 & 100 & 100 & 100 & 100 & 100 & 100 & 100 & 100 \\
\hline Latin America and the Caribbean & 100 & 100 & 100 & 100 & 100 & 100 & 100 & 100 & 100 & 100 & 100 & 100 & 100 & 100 & 100 & 100 & 100 & 100 & 100 \\
\hline Middle East and North Africa & 100 & 100 & 100 & 100 & 100 & 100 & 100 & 100 & 100 & 100 & 100 & 100 & 100 & 100 & 100 & 100 & 100 & 100 & 100 \\
\hline South Asia & 100 & 100 & 100 & 100 & 100 & 100 & 100 & 100 & 100 & 100 & 100 & 100 & 100 & 100 & 100 & 100 & 100 & 100 & 100 \\
\hline Sub-Saharan Africa & 100 & 100 & 100 & 100 & 100 & 100 & 100 & 100 & 100 & 100 & 100 & 100 & 100 & 100 & 100 & 100 & 100 & 100 & 100 \\
\hline Low- and middle-income countries & 100 & 100 & 100 & 100 & 100 & 100 & 100 & 100 & 100 & 100 & 100 & 100 & 100 & 100 & 100 & 100 & 100 & 100 & 100 \\
\hline High-income countries & 100 & 100 & 100 & 100 & 100 & 100 & 100 & 100 & 100 & 100 & 100 & 100 & 100 & 100 & 100 & 100 & 100 & 100 & 100 \\
\hline WORLD & 100 & 100 & 100 & 100 & 100 & 100 & 100 & 100 & 100 & 100 & 100 & 100 & 100 & 100 & 100 & 100 & 100 & 100 & JU \\
\hline
\end{tabular}

Attributable Mortality (thousands)

Europe and Central Asia

Latin America and the Caribbean

Middle East and North Africa

South Asia

Sub-Saharan Africa

Low- and middle-income countries

High-income countries

WORLD

\begin{tabular}{rrrrrrrrrrrrrrrrrrrrrr}
1 & 1 & 0 & 0 & 1 & 2 & 1 & 5 & 2 & 7 & 0 & 3 & 0 & 1 & 0 & 1 & 6 & 19 & 25 \\
0 & 0 & 0 & 0 & 0 & 0 & 0 & 0 & 0 & 0 & 0 & 0 & 0 & 0 & 0 & 0 & 2 & 2 & 3 \\
1 & 1 & 0 & 0 & 1 & 0 & 1 & 0 & 1 & 1 & 1 & 0 & 1 & 1 & 1 & 2 & 6 & 6 & 13 \\
1 & 1 & 0 & 1 & 0 & 0 & 0 & 0 & 0 & 0 & 0 & 0 & 0 & 1 & 0 & 1 & 2 & 3 & 6 \\
0 & 2 & 2 & 1 & 2 & 2 & 4 & 7 & 11 & 21 & 0 & 7 & 0 & 0 & 0 & 0 & 19 & 42 & 61 \\
5 & 7 & 1 & 2 & 0 & 0 & 0 & 0 & 1 & 1 & 1 & 0 & 0 & 0 & 0 & 0 & 8 & 11 & 18 \\
\hline 9 & 11 & 3 & 5 & 4 & 5 & 6 & 13 & 15 & 30 & 2 & 12 & 2 & 4 & 2 & 4 & 43 & 83 & 126 \\
0 & 0 & 0 & 0 & 0 & 0 & 0 & 0 & 0 & 0 & 0 & 0 & 0 & 1 & 1 & 4 & 2 & 5 & 7 \\
9 & 11 & 3 & 5 & 4 & 5 & 6 & 13 & 15 & 30 & 2 & 12 & 3 & 5 & 3 & 7 & 46 & 88 & 133 \\
\hline
\end{tabular}

Attributable YLL (thousands)

East Asia and Pacific

Europe and Central Asia

Latin America and the Caribbean

Middle East and North Africa

South Asia

Sub-Saharan Africa

High-income countries

\begin{tabular}{|c|c|c|c|c|c|c|c|c|c|c|c|c|c|c|c|c|c|c|}
\hline 28 & 17 & 10 & 8 & 40 & 43 & 21 & 118 & 35 & 134 & 6 & 45 & 3 & 12 & 1 & 4 & 145 & 381 & 526 \\
\hline 8 & 6 & 3 & 2 & 6 & 3 & 5 & 4 & 4 & 4 & 3 & 4 & 2 & 4 & 1 & 1 & 31 & 29 & 60 \\
\hline 30 & 18 & 10 & 12 & 19 & 14 & 20 & 11 & 15 & 21 & 8 & 7 & 7 & 12 & 5 & 8 & 114 & 104 & 218 \\
\hline 26 & 21 & 6 & 16 & 3 & 5 & 2 & 4 & 2 & 4 & 3 & 4 & 3 & 7 & 1 & 4 & 47 & 64 & 111 \\
\hline 12 & 64 & 47 & 43 & 44 & 70 & 88 & 171 & 215 & 434 & 2 & 122 & 2 & 3 & 0 & 1 & 411 & 908 & 1,319 \\
\hline 159 & 208 & 22 & 60 & 3 & 13 & 7 & 4 & 12 & 12 & 7 & 4 & 2 & 2 & 0 & 0 & 212 & 303 & 515 \\
\hline 265 & 334 & 98 & 139 & 114 & 148 & 142 & 312 & 284 & 610 & 29 & 186 & 20 & 40 & 8 & 19 & 960 & 1,789 & 2,748 \\
\hline 0 & 0 & 0 & 2 & 0 & 0 & 1 & 1 & 2 & 2 & 2 & 3 & 4 & 7 & 6 & 14 & 17 & 29 & 45 \\
\hline 265 & 334 & 98 & 141 & 114 & 148 & 143 & 314 & 287 & 612 & 31 & 189 & 24 & 47 & 14 & 33 & 976 & 1,817 & 2,794 \\
\hline
\end{tabular}

Attributable DALYs (thousands

East Asia and Pacific

Europe and Central Asia

Latin America and the Caribbea

Middle East and North Africa

South Asia

Sub-Saharan Africa

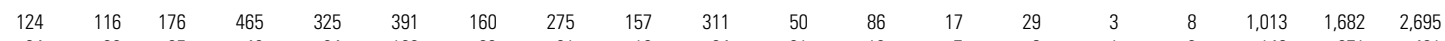

High-income countrie

WORLD

Source: Authors' calculations. 
Table 4A.11

Risk factor:

Iron-deficiency anemia

Disease:

All causes

\begin{tabular}{|c|c|c|c|c|c|c|c|c|c|c|c|c|c|c|c|c|c|c|c|}
\hline \multirow[b]{2}{*}{ Region } & \multicolumn{2}{|c|}{$0-4$ years } & \multicolumn{2}{|c|}{ 5-14 years } & \multicolumn{2}{|c|}{$15-29$ years } & \multicolumn{2}{|c|}{ 30-44 years } & \multicolumn{2}{|c|}{ 45-59 years } & \multicolumn{2}{|c|}{ 60-69 years } & \multicolumn{2}{|c|}{ 70-79 years } & \multicolumn{2}{|c|}{$80+$ years } & \multicolumn{3}{|c|}{ Total } \\
\hline & Male & Female & Male & Female & Male & Female & Male & Female & Male & Female & Male & Female & Male & Female & Male & Female & Male & Female & All \\
\hline \multicolumn{20}{|l|}{ PAF of Mortality (\%) } \\
\hline East Asia and Pacific & 5 & 5 & 0 & 0 & 0 & 2 & 0 & 2 & 0 & 1 & 0 & 0 & 0 & 0 & 0 & 0 & 1 & 1 & 1 \\
\hline Europe and Central Asia & 2 & 2 & 0 & 1 & 0 & 1 & 0 & 0 & 0 & 0 & 0 & 0 & 0 & 0 & 0 & 0 & 0 & 0 & 0 \\
\hline Latin America and the Caribbean & 3 & 2 & 1 & 2 & 0 & 2 & 0 & 1 & 0 & 1 & 0 & 0 & 0 & 0 & 0 & 0 & 1 & 1 & 1 \\
\hline Middle East and North Africa & 4 & 3 & 1 & 2 & 0 & 3 & 0 & 2 & 0 & 0 & 0 & 0 & 0 & 0 & 0 & 1 & 1 & 1 & 1 \\
\hline South Asia & 6 & 5 & 1 & 0 & 0 & 5 & 1 & 6 & 1 & 3 & 0 & 1 & 0 & 0 & 0 & 0 & 2 & 3 & 2 \\
\hline Sub-Saharan Africa & 3 & 3 & 0 & 1 & 0 & 3 & 0 & 2 & 0 & 0 & 0 & 0 & 0 & 0 & 0 & 0 & 1 & 2 & 2 \\
\hline Low- and middle-income countries & 4 & 4 & 0 & 1 & 0 & 3 & 0 & 3 & 0 & 1 & 0 & 0 & 0 & 0 & 0 & 0 & 1 & 2 & $\overline{1}$ \\
\hline High-income countries & 1 & 1 & 0 & 1 & 0 & 0 & 0 & 0 & 0 & 0 & 0 & 0 & 0 & 0 & 0 & 0 & 0 & 0 & 0 \\
\hline WORLD & 4 & 4 & 0 & 1 & 0 & 3 & 0 & 3 & 0 & 1 & 0 & 0 & 0 & 0 & 0 & 0 & 1 & 1 & 1 \\
\hline
\end{tabular}

PAF of YLL (\%)

East Asia and Pacific

Europe and Central Asia

Latin America and the Caribbean

Middle East and North Africa

South Asia

Sub-Saharan Africa

Low- and middle-income countries

High-income countries

\begin{tabular}{llllllllllllllllllll} 
East Asia and Pacific & 5 & 5 & 0 & 0 & 0 & 2 & 0 & 2 & 0 & 1 & 0 & 0 & 0 & 0 & 0 & 0 & 1 & 2 & 1 \\
Europe and Central Asia & 2 & 2 & 0 & 1 & 0 & 1 & 0 & 0 & 0 & 0 & 0 & 0 & 0 & 0 & 0 & 0 & 0 & 0 & 0 \\
Latin America and the Caribbean & 3 & 2 & 1 & 2 & 0 & 2 & 0 & 1 & 0 & 1 & 0 & 0 & 0 & 0 & 0 & 0 & 1 & 1 & 1 \\
Middle East and North Africa & 4 & 3 & 1 & 2 & 0 & 3 & 0 & 2 & 0 & 0 & 0 & 0 & 0 & 0 & 0 & 1 & 1 & 2 & 2 \\
South Asia & 6 & 5 & 1 & 0 & 0 & 5 & 1 & 6 & 1 & 3 & 0 & 1 & 0 & 0 & 0 & 0 & 3 & 4 & 3 \\
Sub-Saharan Africa & 3 & 3 & 0 & 1 & 0 & 3 & 0 & 2 & 0 & 0 & 0 & 0 & 0 & 0 & 0 & 0 & 2 & 2 & 2 \\
\hline Low- and middle-income countries & 4 & 4 & 0 & 1 & 0 & 3 & 0 & 3 & 0 & 1 & 0 & 0 & 0 & 0 & 0 & 0 & 2 & 2 & 2 \\
High-income countries & 1 & 1 & 0 & 1 & 0 & 0 & 0 & 0 & 0 & 0 & 0 & 0 & 0 \\
\hline WORLD & 4 & 4 & 0 & 1 & 0 & 3 & 0 & 3 & 0 & 1 & 0 & 0 & 0 & 0 & 0 & 0 \\
\hline
\end{tabular}

\section{PAF of DALYs (\%)}

East Asia and Pacific

Europe and Central Asia

Latin America and the Caribbean

Middle East and North Africa

South Asia

Sub-Saharan Africa

Low- and middle-income countries

High-income countries

WORLD

Attributable Mortality (thousands

East Asia and Pacific

Europe and Central Asia

Latin America and the Caribbean

Middle East and North Africa

South Asia

Sub-Saharan Africa

Low- and middle-income countries

High-income countries

WORLD

$\begin{array}{lll}4 & 3 & 2 \\ 2 & 2 & 1 \\ 2 & 2 & 1 \\ 3 & 2 & 3 \\ 5 & 4 & 1 \\ 3 & 2 & 2 \\ 4 & 3 & 1 \\ 1 & 1 & 1\end{array}$

\begin{tabular}{lll}
6 & 1 & 2 \\
3 & 0 & 2 \\
1 & 0 & 1 \\
6 & 1 & 2 \\
1 & 1 & 3 \\
1 & 1 & 2 \\
\hline
\end{tabular}

$\begin{array}{llllll}1 & 2 & 0 & 1 & 0 & 0 \\ 0 & 1 & 0 & 0 & 0 & 0 \\ 0 & 1 & 0 & 0 & 0 & 0 \\ 1 & 2 & 0 & 1 & 0 & 0 \\ 1 & 4 & 1 & 2 & 0 & 1 \\ 0 & 2 & 0 & 0 & 0 & 0 \\ 1 & 2 & 0 & 1 & 0 & 0 \\ 0 & 1 & 0 & 1 & 0 & 0\end{array}$

\begin{tabular}{lllllll}
0 & 0 & 0 & 0 & 1 & 2 & 1 \\
0 & 0 & 0 & 0 & 0 & 1 & 0 \\
0 & 0 & 0 & 0 & 1 & 1 & 1 \\
0 & 0 & 0 & 0 & 1 & 2 & 2 \\
0 & 0 & 0 & 0 & 2 & 3 & 3 \\
0 & 0 & 0 & 0 & 2 & 2 & 2 \\
0 & 0 & 0 & 0 & 1 & 2 & 2 \\
0 & 0 & 0 & 0 & 0 & 1 & 1 \\
0 & 0 & 0 & 0 & 1 & 2 & 2 \\
\hline
\end{tabular}

Attributable YLL (thousands

\begin{tabular}{|c|c|c|c|c|c|c|c|c|c|c|c|c|c|c|c|c|c|c|c|}
\hline East Asia and Pacific & 1,075 & 987 & 10 & 8 & 40 & 117 & 21 & 202 & 35 & 134 & 6 & 45 & 3 & 12 & 1 & 4 & 1,192 & 1,509 & 2,700 \\
\hline Europe and Central Asia & 70 & 51 & 3 & 2 & 6 & 7 & 5 & 6 & 4 & 4 & 3 & 4 & 2 & 4 & 1 & 1 & 92 & 79 & 171 \\
\hline Latin America and the Caribbean & 182 & 135 & 10 & 12 & 19 & 30 & 20 & 24 & 15 & 21 & 8 & 7 & 7 & 12 & 5 & 8 & 265 & 249 & 515 \\
\hline Middle East and North Africa & 251 & 168 & 6 & 16 & 3 & 32 & 2 & 32 & 2 & 4 & 3 & 4 & 3 & 7 & 1 & 4 & 272 & 267 & 538 \\
\hline South Asia & 3,284 & 2,768 & 47 & 43 & 44 & 678 & 88 & 736 & 215 & 434 & 2 & 122 & 2 & 3 & 0 & 1 & 3,683 & 4,785 & 8,468 \\
\hline Sub-Saharan Africa & 2,160 & 1,673 & 22 & 60 & 3 & 501 & 7 & 342 & 12 & 12 & 7 & 4 & 2 & 2 & 0 & 0 & 2,213 & 2,594 & 4,807 \\
\hline Low- and middle-income countries & 7,022 & 5,782 & 98 & 139 & 114 & 1,364 & 142 & 1,341 & 284 & 610 & 29 & 186 & 20 & 40 & 8 & 19 & 7,717 & 9,482 & 17,199 \\
\hline High-income countries & 18 & 13 & 0 & 2 & 0 & 1 & 1 & 2 & 2 & 2 & 2 & 3 & 4 & 7 & 6 & 14 & 34 & 43 & 77 \\
\hline WORLD & 7,039 & 5,795 & 98 & 141 & 114 & 1,365 & 143 & 1,343 & 287 & 612 & 31 & 189 & 24 & 47 & 14 & 33 & 7,750 & 9,525 & 17,275 \\
\hline
\end{tabular}

Attributable DALYs (thousands

\begin{tabular}{|c|c|c|c|c|c|c|c|c|c|c|c|c|c|c|c|c|c|c|c|}
\hline East Asia and Pacific & 1,171 & 1,086 & 176 & 465 & 325 & 464 & 160 & 359 & 157 & 311 & 50 & 86 & 17 & 29 & 3 & 8 & 2,060 & 2,809 & 4,869 \\
\hline Europe and Central Asia & 95 & 76 & 25 & 40 & 24 & 125 & 22 & 33 & 16 & 24 & 21 & 12 & 7 & 8 & 1 & 2 & 211 & 321 & 532 \\
\hline Latin America and the Caribbean & 214 & 164 & 22 & 40 & 48 & 91 & 25 & 63 & 18 & 28 & 12 & 11 & 9 & 15 & 5 & 9 & 352 & 421 & 774 \\
\hline Middle East and North Africa & 281 & 200 & 70 & 144 & 57 & 85 & 32 & 61 & 17 & 27 & 10 & 10 & 7 & 9 & 2 & 4 & 475 & 540 & 1,015 \\
\hline South Asia & 3,602 & 3,078 & 106 & 175 & 318 & 971 & 346 & 1,044 & 294 & 558 & 31 & 182 & 15 & 36 & 4 & 6 & 4,715 & 6,049 & 10,764 \\
\hline Sub-Saharan Africa & 2,273 & 1,788 & 199 & 173 & 180 & 689 & 88 & 474 & 27 & 41 & 16 & 15 & 6 & 8 & 1 & 1 & 2,790 & 3,190 & 5,980 \\
\hline Low- and middle-income countries & 7,636 & 6,393 & 598 & 1,037 & 953 & 2,426 & 672 & 2,034 & 530 & 989 & 139 & 316 & 60 & 106 & 15 & 30 & 10,602 & 13,331 & 23,933 \\
\hline High-income countries & 37 & 33 & 11 & 28 & 111 & 173 & 31 & 86 & 37 & 85 & 35 & 33 & 33 & 25 & 13 & 18 & 308 & 481 & 789 \\
\hline WORLD & 7,673 & 6,425 & 609 & 1,066 & 1,064 & 2,599 & 703 & 2,120 & 567 & 1,074 & 174 & 348 & 93 & 131 & 28 & 48 & 10,911 & 13,812 & 24,722 \\
\hline
\end{tabular}

Source: Authors' calculations. 
Table 4A.12

Risk factor:

Vitamin A deficiency

Disease:

Diarrheal diseases

\begin{tabular}{|c|c|c|c|c|c|c|c|c|c|c|c|c|c|c|c|c|c|c|c|}
\hline \multirow[b]{2}{*}{ Region } & \multicolumn{2}{|c|}{$0-4$ years } & \multicolumn{2}{|c|}{ 5-14 years } & \multicolumn{2}{|c|}{$15-29$ years } & \multicolumn{2}{|c|}{ 30-44 years } & \multicolumn{2}{|c|}{$45-59$ years } & \multicolumn{2}{|c|}{$60-69$ years } & \multicolumn{2}{|c|}{ 70-79 years } & \multicolumn{2}{|c|}{$80+$ years } & \multicolumn{3}{|c|}{ Total } \\
\hline & Male & Female & Male & Female & Male & Female & Male & Female & Male & Female & Male & Female & Male & Female & Male & Female & Male & Female & All \\
\hline \multicolumn{20}{|l|}{ PAF of Mortality (\%) } \\
\hline East Asia and Pacific & 19 & 19 & NA & NA & NA & NA & NA & NA & NA & NA & NA & NA & NA & NA & NA & NA & 17 & 17 & 17 \\
\hline Europe and Central Asia & 0 & 0 & NA & NA & NA & NA & NA & NA & NA & NA & NA & NA & NA & NA & NA & NA & 0 & 0 & 0 \\
\hline Latin America and the Caribbean & 15 & 15 & NA & NA & NA & NA & NA & NA & NA & NA & NA & NA & NA & NA & NA & NA & 13 & 12 & 12 \\
\hline Middle East and North Africa & 15 & 15 & NA & NA & NA & NA & NA & NA & NA & NA & NA & NA & NA & NA & NA & NA & 13 & 13 & 13 \\
\hline South Asia & 25 & 25 & NA & NA & NA & NA & NA & NA & NA & NA & NA & NA & NA & NA & NA & NA & 23 & 23 & 23 \\
\hline Sub-Saharan Africa & 27 & 27 & NA & NA & NA & NA & NA & NA & NA & NA & NA & NA & NA & NA & NA & NA & 24 & 24 & 24 \\
\hline Low- and middle-income countries & 24 & 24 & NA & NA & NA & NA & NA & NA & NA & NA & NA & NA & NA & NA & NA & NA & 22 & 22 & 22 \\
\hline High-income countries & 1 & 1 & NA & NA & NA & NA & NA & NA & NA & NA & NA & NA & NA & NA & NA & NA & 0 & 0 & 0 \\
\hline WORLD & 24 & 24 & NA & NA & NA & NA & NA & NA & NA & NA & NA & NA & NA & NA & NA & NA & 22 & 22 & 22 \\
\hline
\end{tabular}

PAF of YLL (\%)

\begin{tabular}{|c|c|c|c|c|c|c|c|c|c|c|c|c|c|c|c|c|c|c|c|}
\hline East Asia and Pacific & 19 & 19 & NA & NA & NA & NA & NA & NA & NA & NA & NA & NA & NA & NA & NA & NA & 18 & 18 & 18 \\
\hline Europe and Central Asia & 0 & 0 & NA & NA & NA & NA & NA & NA & NA & NA & NA & NA & NA & NA & NA & NA & 0 & 0 & 0 \\
\hline Latin America and the Caribbean & 15 & 15 & NA & NA & NA & NA & NA & NA & NA & NA & NA & NA & NA & NA & NA & NA & 14 & 14 & 14 \\
\hline Middle East and North Africa & 15 & 15 & NA & NA & NA & NA & NA & NA & NA & NA & NA & NA & NA & NA & NA & NA & 14 & 14 & 14 \\
\hline South Asia & 25 & 25 & NA & NA & NA & NA & NA & NA & NA & NA & NA & NA & NA & NA & NA & NA & 24 & 24 & 24 \\
\hline Sub-Saharan Africa & 27 & 27 & NA & NA & NA & NA & NA & NA & NA & NA & NA & NA & NA & NA & NA & NA & 25 & 26 & 25 \\
\hline Low- and middle-income countries & 24 & 24 & NA & NA & NA & NA & NA & NA & NA & NA & NA & NA & NA & NA & NA & NA & 23 & 23 & 23 \\
\hline High-income countries & 1 & 1 & NA & NA & NA & NA & NA & NA & NA & NA & NA & NA & NA & NA & NA & NA & 0 & 0 & 0 \\
\hline WORLD & 24 & 24 & NA & NA & NA & NA & NA & NA & NA & NA & NA & NA & NA & NA & NA & NA & 23 & 23 & 23 \\
\hline
\end{tabular}

PAF of DALYs $(\%)$

\begin{tabular}{|c|c|c|c|c|c|c|c|c|c|c|c|c|c|c|c|c|c|c|c|}
\hline 18 & & & & & & & & & & & & & & & & & & & \\
\hline East Asia and Pacific & 16 & 16 & NA & NA & NA & NA & NA & NA & NA & NA & NA & NA & NA & NA & NA & NA & 13 & 13 & 13 \\
\hline Europe and Central Asia & 0 & 0 & NA & NA & NA & NA & NA & NA & NA & NA & NA & NA & NA & NA & NA & NA & 0 & 0 & 0 \\
\hline Latin America and the Caribbean & 11 & 11 & NA & NA & NA & NA & NA & NA & NA & NA & NA & NA & NA & NA & NA & NA & 9 & 9 & 9 \\
\hline Middle East and North Africa & 13 & 13 & NA & NA & NA & NA & NA & NA & NA & NA & NA & NA & NA & NA & NA & NA & 12 & 12 & 12 \\
\hline South Asia & 23 & 23 & NA & NA & NA & NA & NA & NA & NA & NA & NA & NA & NA & NA & NA & NA & 22 & 22 & 22 \\
\hline Sub-Saharan Africa & 25 & 25 & NA & NA & NA & NA & NA & NA & NA & NA & NA & NA & NA & NA & NA & NA & 24 & 24 & 24 \\
\hline Low- and middle-income countries & 22 & 22 & NA & NA & NA & NA & NA & NA & NA & NA & NA & NA & NA & NA & NA & NA & 20 & 20 & 20 \\
\hline High-income countries & 0 & 0 & NA & NA & NA & NA & NA & NA & NA & NA & NA & NA & NA & NA & NA & NA & 0 & 0 & 0 \\
\hline WORLD & 22 & 22 & NA & NA & NA & NA & NA & NA & NA & NA & NA & NA & NA & NA & NA & NA & 20 & 20 & $=0$ \\
\hline
\end{tabular}

Attributable Mortality (thousands)

East Asia and Pacific
Europe and Central Asi

Latin America and the Caribbe

Middle East and North Africa

$\begin{array}{rrrrr}20 & 18 & \text { NA } & \text { NA } & \text { NA } \\ 0 & 0 & \text { NA } & \text { NA } & \text { NA }\end{array}$

South Asia

Sub-Saharan Africa

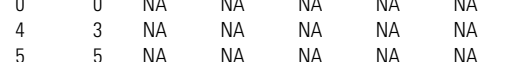

\begin{tabular}{|c|c|c|c|c|c|c|c|c|c|c|c|c|c|c|c|c|c|c|c|}
\hline Low- and middle-income countries & 201 & 184 & NA & NA & NA & NA & NA & NA & NA & NA & NA & NA & NA & NA & NA & NA & 201 & 184 & 385 \\
\hline High-income countries & 0 & 0 & NA & NA & NA & NA & NA & NA & NA & NA & NA & NA & NA & NA & NA & NA & 0 & 0 & 0 \\
\hline WORLD & 201 & 184 & NA & NA & NA & NA & NA & NA & NA & NA & NA & NA & NA & NA & NA & NA & 201 & 184 & 35 \\
\hline
\end{tabular}

Attributable YLL (thousands)

\begin{tabular}{|c|c|c|c|c|c|c|c|c|c|c|c|c|c|c|c|c|c|c|c|}
\hline East Asia and Pacific & 599 & 555 & NA & NA & NA & NA & NA & NA & NA & NA & NA & NA & NA & NA & NA & NA & 599 & 555 & 1,154 \\
\hline Europe and Central Asia & 0 & 0 & NA & NA & NA & NA & NA & NA & NA & NA & NA & NA & NA & NA & NA & NA & 0 & 0 & 1 \\
\hline Latin America and the Caribbean & 108 & 98 & NA & NA & NA & NA & NA & NA & NA & NA & NA & NA & NA & NA & NA & NA & 108 & 98 & 206 \\
\hline Middle East and North Africa & 155 & 144 & NA & NA & NA & NA & NA & NA & NA & NA & NA & NA & NA & NA & NA & NA & 155 & 144 & 299 \\
\hline South Asia & 2,500 & 2,308 & NA & NA & NA & NA & NA & NA & NA & NA & NA & NA & NA & NA & NA & NA & 2,500 & 2,308 & 4,808 \\
\hline Sub-Saharan Africa & 2,719 & 2,486 & NA & NA & NA & NA & NA & NA & NA & NA & NA & NA & NA & NA & NA & NA & 2,719 & 2,486 & 5,205 \\
\hline Low- and middle-income countries & 6,081 & 5,592 & NA & NA & NA & NA & NA & NA & NA & NA & NA & NA & NA & NA & NA & NA & 6,081 & 5,592 & 11,673 \\
\hline High-income countries & 0 & 0 & NA & NA & NA & NA & NA & NA & NA & NA & NA & NA & NA & NA & NA & NA & 0 & 0 & 0 \\
\hline WORLD & 6,081 & 5,592 & NA & NA & NA & NA & NA & NA & NA & NA & NA & NA & NA & NA & NA & NA & 6,081 & 5,592 & 11,673 \\
\hline \multicolumn{20}{|l|}{ Attributable DALYs (thousands) } \\
\hline East Asia and Pacific & 599 & 555 & NA & NA & NA & NA & NA & NA & NA & NA & NA & NA & NA & NA & NA & NA & 599 & 555 & 1,154 \\
\hline Europe and Central Asia & 0 & 0 & NA & NA & NA & NA & NA & NA & NA & NA & NA & NA & NA & NA & NA & NA & 0 & 0 & 1 \\
\hline Latin America and the Caribbean & 108 & 98 & NA & NA & NA & NA & NA & NA & NA & NA & NA & NA & NA & NA & NA & NA & 108 & 98 & 206 \\
\hline Middle East and North Africa & 155 & 144 & NA & NA & NA & NA & NA & NA & NA & NA & NA & NA & NA & NA & NA & NA & 155 & 144 & 299 \\
\hline South Asia & 2,500 & 2,308 & NA & NA & NA & NA & NA & NA & NA & NA & NA & NA & NA & NA & NA & NA & 2,500 & 2,308 & 4,808 \\
\hline Sub-Saharan Africa & 2,719 & 2,486 & NA & NA & NA & NA & NA & NA & NA & NA & NA & NA & NA & NA & NA & NA & 2,719 & 2,486 & 5,205 \\
\hline Low- and middle-income countries & 6,081 & 5,592 & NA & NA & NA & NA & NA & NA & NA & NA & NA & NA & NA & NA & NA & NA & 6,081 & 5,592 & 11,673 \\
\hline High-income countries & 0 & 0 & NA & NA & NA & NA & NA & NA & NA & NA & NA & NA & NA & NA & NA & NA & 0 & 0 & 0 \\
\hline WORLD & 6,081 & 5,592 & NA & NA & NA & NA & NA & NA & NA & NA & NA & NA & NA & NA & NA & NA & 6,081 & 5,592 & $\overline{11,673}$ \\
\hline
\end{tabular}

Source: Authors' calculations.

Note: $\mathrm{NA}=$ not applicable 
Table 4A.13

Risk factor:

Disease:

Vitamin A deficiency

Measles

\begin{tabular}{|c|c|c|c|c|c|c|c|c|c|c|c|c|c|c|c|c|c|c|c|}
\hline \multirow[b]{2}{*}{ Region } & \multicolumn{2}{|c|}{$0-4$ years } & \multicolumn{2}{|c|}{ 5-14 years } & \multicolumn{2}{|c|}{$15-29$ years } & \multicolumn{2}{|c|}{$30-44$ years } & \multicolumn{2}{|c|}{ 45-59 years } & \multicolumn{2}{|c|}{$60-69$ years } & \multicolumn{2}{|c|}{ 70-79 years } & \multicolumn{2}{|c|}{$80+$ years } & \multicolumn{3}{|c|}{ Total } \\
\hline & Male & Female & Male & Female & Male & Female & Male & Female & Male & Female & Male & Female & Male & Female & Male & Female & Male & Female & All \\
\hline \multicolumn{20}{|l|}{ PAF of Mortality (\%) } \\
\hline East Asia and Pacific & 15 & 15 & NA & NA & NA & NA & NA & NA & NA & NA & NA & NA & NA & NA & NA & NA & 9 & 9 & 9 \\
\hline Europe and Central Asia & 0 & 0 & NA & NA & NA & NA & NA & NA & NA & NA & NA & NA & NA & NA & NA & NA & 0 & 0 & 0 \\
\hline Latin America and the Caribbean & * & * & NA & NA & NA & NA & NA & NA & NA & NA & NA & NA & NA & NA & NA & NA & * & * & * \\
\hline Middle East and North Africa & 12 & 12 & NA & NA & NA & NA & NA & NA & NA & NA & NA & NA & NA & NA & NA & NA & 7 & 8 & 7 \\
\hline South Asia & 20 & 20 & NA & NA & NA & NA & NA & NA & NA & NA & NA & NA & NA & NA & NA & NA & 13 & 14 & 13 \\
\hline Sub-Saharan Africa & 21 & 21 & NA & NA & NA & NA & NA & NA & NA & NA & NA & NA & NA & NA & NA & NA & 17 & 17 & 17 \\
\hline Low- and middle-income countries & 20 & 20 & NA & NA & NA & NA & NA & NA & NA & NA & NA & NA & NA & NA & NA & NA & 15 & 15 & 15 \\
\hline High-income countries & 1 & 1 & NA & NA & NA & NA & NA & NA & NA & NA & NA & NA & NA & NA & NA & NA & 0 & 0 & 0 \\
\hline WORLD & 20 & 20 & NA & NA & NA & NA & NA & NA & NA & NA & NA & NA & NA & NA & NA & NA & 15 & 15 & 15 \\
\hline
\end{tabular}

PAF of YLL (\%)

\begin{tabular}{|c|c|c|c|c|c|c|c|c|c|c|c|c|c|c|c|c|c|c|c|}
\hline East Asia and Pacific & 15 & 15 & NA & NA & NA & NA & NA & NA & NA & NA & NA & NA & NA & NA & NA & NA & 9 & 9 & 9 \\
\hline Europe and Central Asia & 0 & 0 & NA & NA & NA & NA & NA & NA & NA & NA & NA & NA & NA & NA & NA & NA & 0 & 0 & 0 \\
\hline Latin America and the Caribbean & * & * & NA & NA & NA & NA & NA & NA & NA & NA & NA & NA & NA & NA & NA & NA & * & * & * \\
\hline Middle East and North Africa & 12 & 12 & NA & NA & NA & NA & NA & NA & NA & NA & NA & NA & NA & NA & NA & NA & 7 & 8 & 7 \\
\hline South Asia & 20 & 20 & NA & NA & NA & NA & NA & NA & NA & NA & NA & NA & NA & NA & NA & NA & 14 & 14 & 14 \\
\hline Sub-Saharan Africa & 21 & 21 & NA & NA & NA & NA & NA & NA & NA & NA & NA & NA & NA & NA & NA & NA & 17 & 17 & 17 \\
\hline Low- and middle-income countries & 20 & 20 & NA & NA & NA & NA & NA & NA & NA & NA & NA & NA & NA & NA & NA & NA & 15 & 15 & 15 \\
\hline High-income countries & 1 & 1 & NA & NA & NA & NA & NA & NA & NA & NA & NA & NA & NA & NA & NA & NA & 0 & 0 & 0 \\
\hline WORLD & 20 & 20 & NA & NA & NA & NA & NA & NA & NA & NA & NA & NA & NA & NA & NA & NA & 15 & 15 & 15 \\
\hline
\end{tabular}

PAF of DALYs (\%)

\begin{tabular}{|c|c|c|c|c|c|c|c|c|c|c|c|c|c|c|c|c|c|c|}
\hline East Asia and Pacific & 15 & 15 & NA & NA & NA & NA & NA & NA & NA & NA & NA & NA & NA & NA & NA & NA & 9 & 9 \\
\hline Europe and Central Asia & 0 & 0 & NA & NA & NA & NA & NA & NA & NA & NA & NA & NA & NA & NA & NA & NA & 0 & 0 \\
\hline Latin America and the Caribbean & * & * & NA & NA & NA & NA & NA & NA & NA & NA & NA & NA & NA & NA & NA & NA & 0 & 0 \\
\hline Middle East and North Africa & 11 & 11 & NA & NA & NA & NA & NA & NA & NA & NA & NA & NA & NA & NA & NA & NA & 7 & 7 \\
\hline South Asia & 20 & 20 & NA & NA & NA & NA & NA & NA & NA & NA & NA & NA & NA & NA & NA & NA & 13 & 14 \\
\hline Sub-Saharan Africa & 21 & 21 & NA & NA & NA & NA & NA & NA & NA & NA & NA & NA & NA & NA & NA & NA & 17 & 17 \\
\hline Low- and middle-income countries & 20 & 20 & NA & NA & NA & NA & NA & NA & NA & NA & NA & NA & NA & NA & NA & NA & 15 & 15 \\
\hline High-income countries & 0 & 0 & NA & NA & NA & NA & NA & NA & NA & NA & NA & NA & NA & NA & NA & NA & 0 & 0 \\
\hline RLD & 20 & 20 & NA & NA & NA & NA & NA & NA & NA & NA & NA & NA & NA & NA & NA & NA & 15 & 15 \\
\hline
\end{tabular}

Attributable Mortality (thousands)

East Asia an

Europe and Central Asia

Latin America and the Caribbean

Middle East and North Africa

South Asia

Sub-Saharan Africa

Low- and middle-income countries

High-income countries

\begin{tabular}{|c|c|c|c|c|c|c|c|c|c|c|c|c|c|c|c|c|c|c|c|}
\hline \multicolumn{20}{|l|}{ Attributable Mortality (thousands) } \\
\hline East Asia and Pacific & 3 & 3 & NA & NA & NA & NA & NA & NA & NA & NA & NA & NA & NA & NA & NA & NA & 3 & 3 & 7 \\
\hline Europe and Central Asia & 0 & 0 & NA & NA & NA & NA & NA & NA & NA & NA & NA & NA & NA & NA & NA & NA & 0 & 0 & 0 \\
\hline Latin America and the Caribbean & 0 & 0 & NA & NA & NA & NA & NA & NA & NA & NA & NA & NA & NA & NA & NA & NA & 0 & 0 & 0 \\
\hline Middle East and North Africa & 1 & 1 & NA & NA & NA & NA & NA & NA & NA & NA & NA & NA & NA & NA & NA & NA & 1 & 1 & 1 \\
\hline South Asia & 14 & 15 & NA & NA & NA & NA & NA & NA & NA & NA & NA & NA & NA & NA & NA & NA & 14 & 15 & 29 \\
\hline Sub-Saharan Africa & 38 & 37 & NA & NA & NA & NA & NA & NA & NA & NA & NA & NA & NA & NA & NA & NA & 38 & 37 & 75 \\
\hline Low- and middle-income countries & 56 & 56 & NA & NA & NA & NA & NA & NA & NA & NA & NA & NA & NA & NA & NA & NA & 56 & 56 & 112 \\
\hline High-income countries & 0 & 0 & NA & NA & NA & NA & NA & NA & NA & NA & NA & NA & NA & NA & NA & NA & 0 & 0 & 0 \\
\hline WORLD & 56 & 56 & NA & NA & NA & NA & NA & NA & NA & NA & NA & NA & NA & NA & NA & NA & 56 & 56 & 112 \\
\hline
\end{tabular}

Attributable YLL (thousands)

\begin{tabular}{|c|c|c|c|c|c|c|c|c|c|c|c|c|c|c|c|c|c|c|c|}
\hline East Asia and Pacific & 99 & 104 & NA & NA & NA & NA & NA & NA & NA & NA & NA & NA & NA & NA & NA & NA & 99 & 104 & 203 \\
\hline Europe and Central Asia & 0 & 0 & NA & NA & NA & NA & NA & NA & NA & NA & NA & NA & NA & NA & NA & NA & 0 & 0 & 0 \\
\hline Latin America and the Caribbean & 0 & 0 & NA & NA & NA & NA & NA & NA & NA & NA & NA & NA & NA & NA & NA & NA & 0 & 0 & 0 \\
\hline Middle East and North Africa & 17 & 18 & NA & NA & NA & NA & NA & NA & NA & NA & NA & NA & NA & NA & NA & NA & 17 & 18 & 34 \\
\hline South Asia & 428 & 451 & NA & NA & NA & NA & NA & NA & NA & NA & NA & NA & NA & NA & NA & NA & 428 & 451 & 879 \\
\hline Sub-Saharan Africa & 1,137 & 1,134 & NA & NA & NA & NA & NA & NA & NA & NA & NA & NA & NA & NA & NA & NA & 1,137 & 1,134 & 2,271 \\
\hline Low- and middle-income countries & 1,681 & 1,706 & NA & NA & NA & NA & NA & NA & NA & NA & NA & NA & NA & NA & NA & NA & 1,681 & 1,706 & 3,388 \\
\hline High-income countries & 0 & 0 & NA & NA & NA & NA & NA & NA & NA & NA & NA & NA & NA & NA & NA & NA & 0 & 0 & 0 \\
\hline WORLD & 1,681 & 1,706 & NA & NA & NA & NA & NA & NA & NA & NA & NA & NA & NA & NA & NA & NA & 1,681 & 1,706 & 3,388 \\
\hline \multicolumn{20}{|l|}{ Attributable DALYs (thousands) } \\
\hline East Asia and Pacific & 99 & 104 & NA & NA & NA & NA & NA & NA & NA & NA & NA & NA & NA & NA & NA & NA & 99 & 104 & 203 \\
\hline Europe and Central Asia & 0 & 0 & NA & NA & NA & NA & NA & NA & NA & NA & NA & NA & NA & NA & NA & NA & 0 & 0 & 0 \\
\hline Latin America and the Caribbean & 0 & 0 & NA & NA & NA & NA & NA & NA & NA & NA & NA & NA & NA & NA & NA & NA & 0 & 0 & 0 \\
\hline Middle East and North Africa & 17 & 18 & NA & NA & NA & NA & NA & NA & NA & NA & NA & NA & NA & NA & NA & NA & 17 & 18 & 34 \\
\hline South Asia & 428 & 451 & NA & NA & NA & NA & NA & NA & NA & NA & NA & NA & NA & NA & NA & NA & 428 & 451 & 879 \\
\hline Sub-Saharan Africa & 1,137 & 1,134 & NA & NA & NA & NA & NA & NA & NA & NA & NA & NA & NA & NA & NA & NA & 1,137 & 1,134 & 2,271 \\
\hline Low- and middle-income countries & 1,681 & 1,706 & NA & NA & NA & NA & NA & NA & NA & NA & NA & NA & NA & NA & NA & NA & 1,681 & 1,706 & 3,388 \\
\hline High-income countries & 0 & 0 & NA & NA & NA & NA & NA & NA & NA & NA & NA & NA & NA & NA & NA & NA & 0 & 0 & 0 \\
\hline WORLD & 1,681 & 1,706 & NA & NA & NA & NA & NA & NA & NA & NA & NA & NA & NA & NA & NA & NA & 1,681 & 1,706 & 3,388 \\
\hline
\end{tabular}

Source: Authors' calculations.

Note: NA = not applicable

*The number of deaths (and hence YLL) directly coded to a number of diseases, especially neuropsychiatric and musculoskeletal diseases, is zero or very small. For other diseases, mortality or disease burden may be zero in some

region-age-sex groups. In such cases, the population attributable fractions would be undefined or unstable and have not been calculated. 
Table 4A.14

Risk factor:

Disease:

Vitamin A deficiency

Malaria

\begin{tabular}{|c|c|c|c|c|c|c|c|c|c|c|c|c|c|c|c|c|c|c|c|}
\hline \multirow[b]{2}{*}{ Region } & \multicolumn{2}{|c|}{$0-4$ years } & \multicolumn{2}{|c|}{ 5-14 years } & \multicolumn{2}{|c|}{$15-29$ years } & \multicolumn{2}{|c|}{$30-44$ years } & \multicolumn{2}{|c|}{ 45-59 years } & \multicolumn{2}{|c|}{$60-69$ years } & \multicolumn{2}{|c|}{ 70-79 years } & \multicolumn{2}{|c|}{$80+$ years } & \multicolumn{3}{|c|}{ Total } \\
\hline & Male & Female & Male & Female & Male & Female & Male & Female & Male & Female & Male & Female & Male & Female & Male & Female & Male & Female & All \\
\hline \multicolumn{20}{|l|}{ PAF of Mortality (\%) } \\
\hline East Asia and Pacific & 14 & 14 & NA & NA & NA & NA & NA & NA & NA & NA & NA & NA & NA & NA & NA & NA & 12 & 13 & 12 \\
\hline Europe and Central Asia & 0 & 0 & NA & NA & NA & NA & NA & NA & NA & NA & NA & NA & NA & NA & NA & NA & 0 & 0 & 0 \\
\hline Latin America and the Caribbean & 10 & 10 & NA & NA & NA & NA & NA & NA & NA & NA & NA & NA & NA & NA & NA & NA & 9 & 9 & 9 \\
\hline Middle East and North Africa & 11 & 11 & NA & NA & NA & NA & NA & NA & NA & NA & NA & NA & NA & NA & NA & NA & 9 & 10 & 9 \\
\hline South Asia & 19 & 19 & NA & NA & NA & NA & NA & NA & NA & NA & NA & NA & NA & NA & NA & NA & 17 & 17 & 17 \\
\hline Sub-Saharan Africa & 20 & 20 & NA & NA & NA & NA & NA & NA & NA & NA & NA & NA & NA & NA & NA & NA & 18 & 18 & 18 \\
\hline Low- and middle-income countries & 19 & 20 & NA & NA & NA & NA & NA & NA & NA & NA & NA & NA & NA & NA & NA & NA & 17 & 18 & 18 \\
\hline High-income countries & 1 & 1 & NA & NA & NA & NA & NA & NA & NA & NA & NA & NA & NA & NA & NA & NA & 0 & 0 & 0 \\
\hline WORLD & 19 & 20 & NA & NA & NA & NA & NA & NA & NA & NA & NA & NA & NA & NA & NA & NA & 17 & 18 & 18 \\
\hline
\end{tabular}

PAF of YLL (\%)

\begin{tabular}{|c|c|c|c|c|c|c|c|c|c|c|c|c|c|c|c|c|c|c|c|}
\hline East Asia and Pacific & 14 & 14 & NA & NA & NA & NA & NA & NA & NA & NA & NA & NA & NA & NA & NA & NA & 13 & 13 & 13 \\
\hline Europe and Central Asia & 0 & 0 & NA & NA & NA & NA & NA & NA & NA & NA & NA & NA & NA & NA & NA & NA & 0 & 0 & 0 \\
\hline Latin America and the Caribbean & 10 & 10 & NA & NA & NA & NA & NA & NA & NA & NA & NA & NA & NA & NA & NA & NA & 9 & 10 & 9 \\
\hline Middle East and North Africa & 11 & 11 & NA & NA & NA & NA & NA & NA & NA & NA & NA & NA & NA & NA & NA & NA & 10 & 10 & 10 \\
\hline South Asia & 19 & 19 & NA & NA & NA & NA & NA & NA & NA & NA & NA & NA & NA & NA & NA & NA & 17 & 17 & 17 \\
\hline Sub-Saharan Africa & 20 & 20 & NA & NA & NA & NA & NA & NA & NA & NA & NA & NA & NA & NA & NA & NA & 18 & 18 & 18 \\
\hline Low- and middle-income countries & 19 & 20 & NA & NA & NA & NA & NA & NA & NA & NA & NA & NA & NA & NA & NA & NA & 18 & 18 & 18 \\
\hline High-income countries & 1 & 1 & NA & NA & NA & NA & NA & NA & NA & NA & NA & NA & NA & NA & NA & NA & 0 & 0 & 0 \\
\hline WORLD & 19 & 20 & NA & NA & NA & NA & NA & NA & NA & NA & NA & NA & NA & NA & NA & NA & 18 & 18 & 18 \\
\hline
\end{tabular}

\section{PAF of DALYs (\%)}

\begin{tabular}{|c|c|c|c|c|c|c|c|c|c|c|c|c|c|c|c|c|c|c|c|}
\hline East Asia and Pacific & 14 & 14 & NA & NA & NA & NA & NA & NA & NA & NA & NA & NA & NA & NA & NA & NA & 11 & 10 & 11 \\
\hline Europe and Central Asia & 0 & 0 & NA & NA & NA & NA & NA & NA & NA & NA & NA & NA & NA & NA & NA & NA & 0 & 0 & 0 \\
\hline Latin America and the Caribbean & 10 & 10 & NA & NA & NA & NA & NA & NA & NA & NA & NA & NA & NA & NA & NA & NA & 6 & 5 & 6 \\
\hline Middle East and North Africa & 11 & 11 & NA & NA & NA & NA & NA & NA & NA & NA & NA & NA & NA & NA & NA & NA & 9 & 9 & 9 \\
\hline South Asia & 19 & 19 & NA & NA & NA & NA & NA & NA & NA & NA & NA & NA & NA & NA & NA & NA & 14 & 14 & 14 \\
\hline Sub-Saharan Africa & 20 & 20 & NA & NA & NA & NA & NA & NA & NA & NA & NA & NA & NA & NA & NA & NA & 18 & 18 & 18 \\
\hline Low- and middle-income countries & 19 & 20 & NA & NA & NA & NA & NA & NA & NA & NA & NA & NA & NA & NA & NA & NA & 18 & 18 & 18 \\
\hline High-income countries & 1 & 1 & NA & NA & NA & NA & NA & NA & NA & NA & NA & NA & NA & NA & NA & NA & 0 & 0 & 0 \\
\hline WORLD & 19 & 20 & NA & NA & NA & NA & NA & NA & NA & NA & NA & NA & NA & NA & NA & NA & 18 & 18 & 18 \\
\hline
\end{tabular}

Attributable Mortality (thousands)

\begin{tabular}{|c|c|c|c|c|c|c|c|c|c|c|c|c|c|c|c|c|c|c|c|}
\hline East Asia and Pacific & 3 & 1 & NA & NA & NA & NA & NA & NA & NA & NA & NA & NA & NA & NA & NA & NA & 3 & 1 & 4 \\
\hline Europe and Central Asia & 0 & 0 & NA & NA & NA & NA & NA & NA & NA & NA & NA & NA & NA & NA & NA & NA & 0 & 0 & 0 \\
\hline Latin America and the Caribbean & 0 & 0 & NA & NA & NA & NA & NA & NA & NA & NA & NA & NA & NA & NA & NA & NA & 0 & 0 & 0 \\
\hline Middle East and North Africa & 1 & 1 & NA & NA & NA & NA & NA & NA & NA & NA & NA & NA & NA & NA & NA & NA & 1 & 1 & 2 \\
\hline South Asia & 5 & 6 & NA & NA & NA & NA & NA & NA & NA & NA & NA & NA & NA & NA & NA & NA & 5 & 6 & 11 \\
\hline Sub-Saharan Africa & 92 & 103 & NA & NA & NA & NA & NA & NA & NA & NA & NA & NA & NA & NA & NA & NA & 92 & 103 & 195 \\
\hline Low- and middle-income countries & 101 & 110 & NA & NA & NA & NA & NA & NA & NA & NA & NA & NA & NA & NA & NA & NA & 101 & 110 & 211 \\
\hline High-income countries & 0 & 0 & NA & NA & NA & NA & NA & NA & NA & NA & NA & NA & NA & NA & NA & NA & 0 & 0 & 0 \\
\hline WORLD & 101 & 110 & NA & NA & NA & NA & NA & NA & NA & NA & NA & NA & NA & NA & NA & NA & 101 & 110 & 211 \\
\hline
\end{tabular}

Attributable YLL (thousands)

\begin{tabular}{|c|c|c|c|c|c|c|c|c|c|c|c|c|c|c|c|c|c|c|c|}
\hline East Asia and Pacific & 76 & 35 & NA & NA & NA & NA & NA & NA & NA & NA & NA & NA & NA & NA & NA & NA & 76 & 35 & 111 \\
\hline Europe and Central Asia & 0 & 0 & NA & NA & NA & NA & NA & NA & NA & NA & NA & NA & NA & NA & NA & NA & 0 & 0 & 0 \\
\hline Latin America and the Caribbean & 3 & 2 & NA & NA & NA & NA & NA & NA & NA & NA & NA & NA & NA & NA & NA & NA & 3 & 2 & 4 \\
\hline Middle East and North Africa & 26 & 29 & NA & NA & NA & NA & NA & NA & NA & NA & NA & NA & NA & NA & NA & NA & 26 & 29 & 54 \\
\hline South Asia & 152 & 170 & NA & NA & NA & NA & NA & NA & NA & NA & NA & NA & NA & NA & NA & NA & 152 & 170 & 322 \\
\hline Sub-Saharan Africa & 2,794 & 3,125 & NA & NA & NA & NA & NA & NA & NA & NA & NA & NA & NA & NA & NA & NA & 2,794 & 3,125 & 5,919 \\
\hline Low- and middle-income countries & 3,050 & 3,360 & NA & NA & NA & NA & NA & NA & NA & NA & NA & NA & NA & NA & NA & NA & 3,050 & 3,360 & 6,410 \\
\hline High-income countries & 0 & 0 & NA & NA & NA & NA & NA & NA & NA & NA & NA & NA & NA & NA & NA & NA & 0 & 0 & 0 \\
\hline WORLD & 3,050 & 3,360 & NA & NA & NA & NA & NA & NA & NA & NA & NA & NA & NA & NA & NA & NA & 3,050 & 3,360 & 6,410 \\
\hline \multicolumn{20}{|l|}{ Attributable DALYs (thousands) } \\
\hline East Asia and Pacific & 81 & 40 & NA & NA & NA & NA & NA & NA & NA & NA & NA & NA & NA & NA & NA & NA & 81 & 40 & 121 \\
\hline Europe and Central Asia & 0 & 0 & NA & NA & NA & NA & NA & NA & NA & NA & NA & NA & NA & NA & NA & NA & 0 & 0 & 0 \\
\hline Latin America and the Caribbean & 4 & 3 & NA & NA & NA & NA & NA & NA & NA & NA & NA & NA & NA & NA & NA & NA & 4 & 3 & 6 \\
\hline Middle East and North Africa & 28 & 31 & NA & NA & NA & NA & NA & NA & NA & NA & NA & NA & NA & NA & NA & NA & 28 & 31 & 59 \\
\hline South Asia & 176 & 193 & NA & NA & NA & NA & NA & NA & NA & NA & NA & NA & NA & NA & NA & NA & 176 & 193 & 369 \\
\hline Sub-Saharan Africa & 3,069 & 3,397 & NA & NA & NA & NA & NA & NA & NA & NA & NA & NA & NA & NA & NA & NA & 3,069 & 3,397 & 6,466 \\
\hline Low- and middle-income countries & 3,358 & 3,663 & NA & NA & NA & NA & NA & NA & NA & NA & NA & NA & NA & NA & NA & NA & 3,358 & 3,663 & 7,021 \\
\hline High-income countries & 0 & 0 & NA & NA & NA & NA & NA & NA & NA & NA & NA & NA & NA & NA & NA & NA & 0 & 0 & 0 \\
\hline WORLD & 3,358 & 3,663 & NA & NA & NA & NA & NA & NA & NA & NA & NA & NA & NA & NA & NA & NA & 3,358 & 3,663 & 7,021 \\
\hline
\end{tabular}

Source: Authors' calculations.

Note: $\mathrm{NA}=$ not applicable. 
Table 4A.15

Risk factor:

Vitamin A deficiency

Disease:

Other infectious diseases

\begin{tabular}{|c|c|c|c|c|c|c|c|c|c|c|c|c|c|c|c|c|c|c|c|}
\hline \multirow[b]{2}{*}{ Region } & \multicolumn{2}{|c|}{$0-4$ years } & \multicolumn{2}{|c|}{ 5-14 years } & \multicolumn{2}{|c|}{$15-29$ years } & \multicolumn{2}{|c|}{ 30-44 years } & \multicolumn{2}{|c|}{$45-59$ years } & \multicolumn{2}{|c|}{ 60-69 years } & \multicolumn{2}{|c|}{ 70-79 years } & \multicolumn{2}{|c|}{$80+$ years } & \multicolumn{3}{|c|}{ Total } \\
\hline & Male & Female & Male & Female & Male & Female & Male & Female & Male & Female & Male & Female & Male & Female & Male & Female & Male & Female & All \\
\hline \multicolumn{20}{|l|}{ PAF of Mortality (\%) } \\
\hline East Asia and Pacific & 3 & 3 & NA & NA & NA & NA & NA & NA & NA & NA & NA & NA & NA & NA & NA & NA & 1 & 1 & 1 \\
\hline Europe and Central Asia & 0 & 0 & NA & NA & NA & NA & NA & NA & NA & NA & NA & NA & NA & NA & NA & NA & 0 & 0 & 0 \\
\hline Latin America and the Caribbean & 2 & 2 & NA & NA & NA & NA & NA & NA & NA & NA & NA & NA & NA & NA & NA & NA & 1 & 0 & 0 \\
\hline Middle East and North Africa & 2 & 2 & NA & NA & NA & NA & NA & NA & NA & NA & NA & NA & NA & NA & NA & NA & 1 & 1 & 1 \\
\hline South Asia & 4 & 4 & NA & NA & NA & NA & NA & NA & NA & NA & NA & NA & NA & NA & NA & NA & 1 & 1 & 1 \\
\hline Sub-Saharan Africa & 4 & 4 & NA & NA & NA & NA & NA & NA & NA & NA & NA & NA & NA & NA & NA & NA & 2 & 2 & 2 \\
\hline Low- and middle-income countries & 3 & 4 & NA & NA & NA & NA & NA & NA & NA & NA & NA & NA & NA & NA & NA & NA & 1 & 1 & $\overline{1}$ \\
\hline High-income countries & 0 & 0 & NA & NA & NA & NA & NA & NA & NA & NA & NA & NA & NA & NA & NA & NA & 0 & 0 & 0 \\
\hline WORLD & 3 & 4 & NA & NA & NA & NA & NA & NA & NA & NA & NA & NA & NA & NA & NA & NA & 1 & 1 & 1 \\
\hline
\end{tabular}

PAF of YLL (\%)

\begin{tabular}{|c|c|c|c|c|c|c|c|c|c|c|c|c|c|c|c|c|c|c|}
\hline East Asia and Pacific & 3 & 3 & NA & NA & NA & NA & NA & NA & NA & NA & NA & NA & NA & NA & NA & NA & 1 & 1 \\
\hline Europe and Central Asia & 0 & 0 & NA & NA & NA & NA & NA & NA & NA & NA & NA & NA & NA & NA & NA & NA & 0 & 0 \\
\hline Latin America and the Caribbean & 2 & 2 & NA & NA & NA & NA & NA & NA & NA & NA & NA & NA & NA & NA & NA & NA & 1 & 1 \\
\hline Middle East and North Africa & 2 & 2 & NA & NA & NA & NA & NA & NA & NA & NA & NA & NA & NA & NA & NA & NA & 1 & 1 \\
\hline South Asia & 4 & 4 & NA & NA & NA & NA & NA & NA & NA & NA & NA & NA & NA & NA & NA & NA & 1 & 2 \\
\hline Sub-Saharan Africa & 4 & 4 & NA & NA & NA & NA & NA & NA & NA & NA & NA & NA & NA & NA & NA & NA & 2 & 2 \\
\hline Low- and middle-income countries & 3 & 4 & NA & NA & NA & NA & NA & NA & NA & NA & NA & NA & NA & NA & NA & NA & 1 & 2 \\
\hline High-income countries & 0 & 0 & NA & NA & NA & NA & NA & NA & NA & NA & NA & NA & NA & NA & NA & NA & 0 & 0 \\
\hline WORLD & 3 & 4 & NA & NA & NA & NA & NA & NA & NA & NA & NA & NA & NA & NA & NA & NA & 1 & 2 \\
\hline
\end{tabular}

PAF of DALYs (\%)

Latin America and the Caribbean

Middle East and North Africa

South Asia

Sub-Saharan Africa

Low- and middle-income countries

$\begin{array}{llllllllllll}2 & 2 & \text { NA } & \text { NA } & \text { NA } & \text { NA } & \text { NA } & \text { NA } & \text { NA } & \text { NA } & \text { NA } & \text { NA } \\ 0 & 0 & \text { NA } & \text { NA } & \text { NA } & \text { NA } & \text { NA } & \text { NA } & \text { NA } & \text { NA } & \text { NA } & \text { NA } \\ 2 & 2 & \text { NA } & \text { NA } & \text { NA } & \text { NA } & \text { NA } & \text { NA } & \text { NA } & \text { NA } & \text { NA } & \text { NA } \\ 2 & 2 & \text { NA } & \text { NA } & \text { NA } & \text { NA } & \text { NA } & \text { NA } & \text { NA } & \text { NA } & \text { NA } & \text { NA } \\ 3 & 3 & \text { NA } & \text { NA } & \text { NA } & \text { NA } & \text { NA } & \text { NA } & \text { NA } & \text { NA } & \text { NA } & \text { NA } \\ 4 & 4 & \text { NA } & \text { NA } & \text { NA } & \text { NA } & \text { NA } & \text { NA } & \text { NA } & \text { NA } & \text { NA } & \text { NA } \\ 3 & 3 & \text { NA } & \text { NA } & \text { NA } & \text { NA } & \text { NA } & \text { NA } & \text { NA } & \text { NA } & \text { NA } & \text { NA }\end{array}$

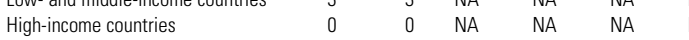

\begin{tabular}{lllllllllllllllllllll}
\hline WORLD & 3 & 3 & NA & NA & NA & NA & NA & NA & NA & NA & NA & NA & NA & NA & NA & NA & 1 & 1 & 1 \\
\hline
\end{tabular}

Attributable Mortality (thousands)

East Asia and Pacific
Europe and Central Asi

Latin America and the Caribbean

Middle East and North Africa

South Asia

Sub-Saharan Africa

Low- and middle-income countries

High-income countries

\begin{tabular}{|c|c|c|c|c|c|c|c|c|c|c|c|c|c|c|c|c|c|c|}
\hline 1 & 0 & NA & NA & NA & NA & NA & NA & NA & NA & NA & NA & NA & NA & NA & NA & 1 & 0 & 1 \\
\hline 0 & 0 & NA & NA & NA & NA & NA & NA & NA & NA & NA & NA & NA & NA & NA & NA & 0 & 0 & 0 \\
\hline 0 & 0 & NA & NA & NA & NA & NA & NA & NA & NA & NA & NA & NA & NA & NA & NA & 0 & 0 & 0 \\
\hline 0 & 0 & NA & NA & NA & NA & NA & NA & NA & NA & NA & NA & NA & NA & NA & NA & 0 & 0 & 0 \\
\hline 2 & 4 & NA & NA & NA & NA & NA & NA & NA & NA & NA & NA & NA & NA & NA & NA & 2 & 4 & 6 \\
\hline 5 & 5 & NA & NA & NA & NA & NA & NA & NA & NA & NA & NA & NA & NA & NA & NA & 5 & 5 & 9 \\
\hline 7 & 10 & NA & NA & NA & NA & NA & NA & NA & NA & NA & NA & NA & NA & NA & NA & 7 & 10 & 17 \\
\hline 0 & 0 & NA & NA & NA & NA & NA & NA & NA & NA & NA & NA & NA & NA & NA & NA & 0 & 0 & 0 \\
\hline 7 & 10 & NA & NA & NA & NA & NA & NA & NA & NA & NA & NA & NA & NA & NA & NA & 7 & 10 & 17 \\
\hline
\end{tabular}

Attributable YLL (thousands)

East Asia and Pacific

Europe and Central Asia

Latin America and the Caribbe

Middle East and North Afric
South Asia

$\begin{array}{lrrrrr}\text { South Asia } & 47 & 129 & \text { NA } & \text { NA } & \text { NA } \\ \text { Sub-Saharan Africa } & 138 & 144 & \text { NA } & \text { NA } & \text { NA }\end{array}$

$\begin{array}{rrrrr}19 & 14 & \text { NA } & \text { NA } & \text { NA } \\ 0 & 0 & \text { NA } & \text { NA } & \text { NA }\end{array}$

$\begin{array}{llllllllllllllllllllll}\text { Low- and middle-income countries } & 217 & 297 & \text { NA } & \text { NA } & \text { NA } & \text { NA } & \text { NA } & \text { NA } & \text { NA } & \text { NA } & \text { NA } & \text { NA } & \text { NA } & \text { NA } & \text { NA } & \text { NA } & 217 & 297 & 515\end{array}$

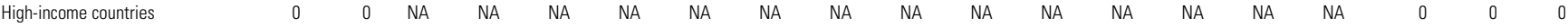

\begin{tabular}{lllllllllllllllllllll}
\hline WORLD & 217 & 298 & NA & NA & NA & NA & NA & NA & NA & NA & NA & NA & NA & NA & NA & NA & 217 & 298 & 515 \\
\hline
\end{tabular}

Attributable DALYs (thousands)

East Asia and Pacific

Europe and Central Asia

Latin America and the Caribbean

Middle East and North Africa

South Asia

Sub-Saharan Africa

$\begin{array}{llllll}19 & 14 & N A & N A & N A & N A\end{array}$

NA NA NA NA

NA NA NA NA NA NA NA $\quad 19 \quad 14 \quad 33$

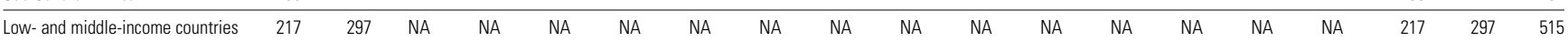

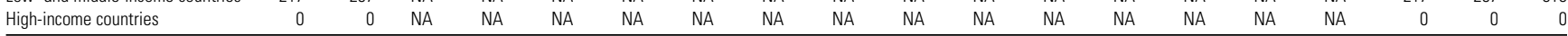

\begin{tabular}{llllllllllllllllllll}
\hline WORLD & 217 & 298 & NA & NA & NA & NA & NA & NA & NA & NA & NA & NA & NA & NA & NA & NA & 217 & 298 & 515
\end{tabular}

Source: Authors' calculations.

Note: $\mathrm{NA}=$ not applicable. 
Table 4A.16

Risk factor: Vitamin A deficiency

Disease: Selected maternal conditions

\begin{tabular}{|c|c|c|c|c|c|c|c|c|c|c|c|c|c|c|c|c|c|c|c|}
\hline \multirow[b]{2}{*}{ Region } & \multicolumn{2}{|c|}{$0-4$ years } & \multicolumn{2}{|c|}{ 5-14 years } & \multicolumn{2}{|c|}{$15-29$ years } & \multicolumn{2}{|c|}{ 30-44 years } & \multicolumn{2}{|c|}{$45-59$ years } & \multicolumn{2}{|c|}{$60-69$ years } & \multicolumn{2}{|c|}{$70-79$ years } & \multicolumn{2}{|c|}{$80+$ years } & \multicolumn{3}{|c|}{ Total } \\
\hline & Male & Female & Male & Female & Male & Female & Male & Female & Male & Female & Male & Female & Male & Female & Male & Female & Male & Female & All \\
\hline \multicolumn{20}{|l|}{ PAF of Mortality (\%) } \\
\hline East Asia and Pacific & NA & NA & NA & NA & NA & 16 & NA & 16 & NA & NA & NA & NA & NA & NA & NA & NA & NA & 16 & 16 \\
\hline Europe and Central Asia & NA & NA & NA & NA & NA & 0 & NA & 0 & NA & NA & NA & NA & NA & NA & NA & NA & NA & 0 & 0 \\
\hline Latin America and the Caribbean & NA & NA & NA & NA & NA & 10 & NA & 10 & NA & NA & NA & NA & NA & NA & NA & NA & NA & 10 & 10 \\
\hline Middle East and North Africa & NA & NA & NA & NA & NA & 13 & NA & 14 & NA & NA & NA & NA & NA & NA & NA & NA & NA & 13 & 13 \\
\hline South Asia & NA & NA & NA & NA & NA & 17 & NA & 17 & NA & NA & NA & NA & NA & NA & NA & NA & NA & 17 & 17 \\
\hline Sub-Saharan Africa & NA & NA & NA & NA & NA & 26 & NA & 26 & NA & NA & NA & NA & NA & NA & NA & NA & NA & 24 & 24 \\
\hline Low- and middle-income countries & NA & NA & NA & NA & NA & 21 & NA & 21 & NA & NA & NA & NA & NA & NA & NA & NA & NA & 20 & 20 \\
\hline High-income countries & NA & NA & NA & NA & NA & 1 & NA & 1 & NA & NA & NA & NA & NA & NA & NA & NA & NA & 1 & 1 \\
\hline WORLD & NA & NA & NA & NA & NA & 21 & NA & 21 & NA & NA & NA & NA & NA & NA & NA & NA & NA & 20 & 20 \\
\hline
\end{tabular}

PAF of YLL (\%)

\begin{tabular}{|c|c|c|c|c|c|c|c|c|c|c|c|c|c|c|c|c|c|c|c|}
\hline East Asia and Pacific & NA & NA & NA & NA & NA & 16 & NA & 16 & NA & NA & NA & NA & NA & NA & NA & NA & NA & 16 & 16 \\
\hline Europe and Central Asia & NA & NA & NA & NA & NA & 0 & NA & 0 & NA & NA & NA & NA & NA & NA & NA & NA & NA & 0 & 0 \\
\hline Latin America and the Caribbean & NA & NA & NA & NA & NA & 10 & NA & 10 & NA & NA & NA & NA & NA & NA & NA & NA & NA & 10 & 10 \\
\hline Middle East and North Africa & NA & NA & NA & NA & NA & 13 & NA & 14 & NA & NA & NA & NA & NA & NA & NA & NA & NA & 13 & 13 \\
\hline South Asia & NA & NA & NA & NA & NA & 17 & NA & 17 & NA & NA & NA & NA & NA & NA & NA & NA & NA & 17 & 17 \\
\hline Sub-Saharan Africa & NA & NA & NA & NA & NA & 26 & NA & 26 & NA & NA & NA & NA & NA & NA & NA & NA & NA & 24 & 24 \\
\hline Low- and middle-income countries & NA & NA & NA & NA & NA & 21 & NA & 21 & NA & NA & NA & NA & NA & NA & NA & NA & NA & 20 & 20 \\
\hline High-income countries & NA & NA & NA & NA & NA & 1 & NA & 1 & NA & NA & NA & NA & NA & NA & NA & NA & NA & 1 & 1 \\
\hline WORLD & NA & NA & NA & NA & NA & 21 & NA & 21 & NA & NA & NA & NA & NA & NA & NA & NA & NA & 20 & 20 \\
\hline
\end{tabular}

PAF of DALYs $(\%)$

\begin{tabular}{|c|c|c|c|c|c|c|c|c|c|c|c|c|c|c|c|c|c|c|c|}
\hline East Asia and Pacific & NA & NA & NA & NA & NA & 3 & NA & 10 & NA & NA & NA & NA & NA & NA & NA & NA & NA & 5 & 5 \\
\hline Europe and Central Asia & NA & NA & NA & NA & NA & 0 & NA & 0 & NA & NA & NA & NA & NA & NA & NA & NA & NA & 0 & 0 \\
\hline Latin America and the Caribbean & NA & NA & NA & NA & NA & 2 & NA & 5 & NA & NA & NA & NA & NA & NA & NA & NA & NA & 2 & 2 \\
\hline Middle East and North Africa & NA & NA & NA & NA & NA & 3 & NA & 7 & NA & NA & NA & NA & NA & NA & NA & NA & NA & 4 & 4 \\
\hline South Asia & NA & NA & NA & NA & NA & 8 & NA & 14 & NA & NA & NA & NA & NA & NA & NA & NA & NA & 10 & 10 \\
\hline Sub-Saharan Africa & NA & NA & NA & NA & NA & 17 & NA & 22 & NA & NA & NA & NA & NA & NA & NA & NA & NA & 18 & 18 \\
\hline Low- and middle-income countries & NA & NA & NA & NA & NA & 10 & NA & 16 & NA & NA & NA & NA & NA & NA & NA & NA & NA & 12 & 12 \\
\hline High-income countries & NA & NA & NA & NA & NA & 0 & NA & 0 & NA & NA & NA & NA & NA & NA & NA & NA & NA & 0 & 0 \\
\hline VORLD & NA & NA & NA & NA & NA & 10 & NA & 16 & NA & NA & NA & NA & NA & NA & NA & NA & NA & 12 & 12 \\
\hline
\end{tabular}

Attributable Mortality (thousands)

\begin{tabular}{|c|c|c|c|c|c|c|c|c|c|c|c|c|c|c|c|c|c|c|c|}
\hline East Asia and Pacific & NA & NA & NA & NA & NA & 1 & NA & 1 & NA & NA & NA & NA & NA & NA & NA & NA & NA & 3 & 3 \\
\hline Europe and Central Asia & NA & NA & NA & NA & NA & 0 & NA & 0 & NA & NA & NA & NA & NA & NA & NA & NA & NA & 0 & 0 \\
\hline Latin America and the Caribbean & NA & NA & NA & NA & NA & 0 & NA & 0 & NA & NA & NA & NA & NA & NA & NA & NA & NA & 0 & 0 \\
\hline Middle East and North Africa & NA & NA & NA & NA & NA & 0 & NA & 0 & NA & NA & NA & NA & NA & NA & NA & NA & NA & 1 & 1 \\
\hline South Asia & NA & NA & NA & NA & NA & 9 & NA & 9 & NA & NA & NA & NA & NA & NA & NA & NA & NA & 18 & 18 \\
\hline Sub-Saharan Africa & NA & NA & NA & NA & NA & 16 & NA & 14 & NA & NA & NA & NA & NA & NA & NA & NA & NA & 30 & 30 \\
\hline Low- and middle-income countries & NA & NA & NA & NA & NA & 27 & NA & 26 & NA & NA & NA & NA & NA & NA & NA & NA & NA & 52 & 52 \\
\hline High-income countries & NA & NA & NA & NA & NA & 0 & NA & 0 & NA & NA & NA & NA & NA & NA & NA & NA & NA & 0 & 0 \\
\hline WORLD & NA & NA & NA & NA & NA & 27 & NA & 26 & NA & NA & NA & NA & NA & NA & NA & NA & NA & 52 & 52 \\
\hline
\end{tabular}

Attributable YLL (thousands)

\begin{tabular}{|c|c|c|c|c|c|c|c|c|c|c|c|c|c|c|c|c|c|c|c|}
\hline East Asia and Pacific & NA & NA & NA & NA & NA & 35 & NA & 37 & NA & NA & NA & NA & NA & NA & NA & NA & NA & 72 & 72 \\
\hline Europe and Central Asia & NA & NA & NA & NA & NA & 0 & NA & 0 & NA & NA & NA & NA & NA & NA & NA & NA & NA & 0 & 0 \\
\hline Latin America and the Caribbean & NA & NA & NA & NA & NA & 6 & NA & 6 & NA & NA & NA & NA & NA & NA & NA & NA & NA & 12 & 12 \\
\hline Middle East and North Africa & NA & NA & NA & NA & NA & 9 & NA & 10 & NA & NA & NA & NA & NA & NA & NA & NA & NA & 19 & 19 \\
\hline South Asia & NA & NA & NA & NA & NA & 249 & NA & 234 & NA & NA & NA & NA & NA & NA & NA & NA & NA & 482 & 482 \\
\hline Sub-Saharan Africa & NA & NA & NA & NA & NA & 438 & NA & 355 & NA & NA & NA & NA & NA & NA & NA & NA & NA & 794 & 794 \\
\hline Low- and middle-income countries & NA & NA & NA & NA & NA & 737 & NA & 642 & NA & NA & NA & NA & NA & NA & NA & NA & NA & 1,379 & 1,379 \\
\hline High-income countries & NA & NA & NA & NA & NA & 0 & NA & 0 & NA & NA & NA & NA & NA & NA & NA & NA & NA & 0 & 0 \\
\hline WORLD & NA & NA & NA & NA & NA & 737 & NA & 642 & NA & NA & NA & NA & NA & NA & NA & NA & NA & 1,379 & 1,379 \\
\hline \multicolumn{20}{|l|}{ Attributable DALYs (thousands) } \\
\hline East Asia and Pacific & NA & NA & NA & NA & NA & 35 & NA & 37 & NA & NA & NA & NA & NA & NA & NA & NA & NA & 72 & 72 \\
\hline Europe and Central Asia & NA & NA & NA & NA & NA & 0 & NA & 0 & NA & NA & NA & NA & NA & NA & NA & NA & NA & 0 & 0 \\
\hline Latin America and the Caribbean & NA & NA & NA & NA & NA & 6 & NA & 6 & NA & NA & NA & NA & NA & NA & NA & NA & NA & 12 & 12 \\
\hline Middle East and North Africa & NA & NA & NA & NA & NA & 9 & NA & 10 & NA & NA & NA & NA & NA & NA & NA & NA & NA & 19 & 19 \\
\hline South Asia & NA & NA & NA & NA & NA & 249 & NA & 234 & NA & NA & NA & NA & NA & NA & NA & NA & NA & 482 & 482 \\
\hline Sub-Saharan Africa & NA & NA & NA & NA & NA & 438 & NA & 355 & NA & NA & NA & NA & NA & NA & NA & NA & NA & 794 & 794 \\
\hline Low- and middle-income countries & NA & NA & NA & NA & NA & 737 & NA & 642 & NA & NA & NA & NA & NA & NA & NA & NA & NA & 1,379 & 1,379 \\
\hline High-income countries & NA & NA & NA & NA & NA & 0 & NA & 0 & NA & NA & NA & NA & NA & NA & NA & NA & NA & 0 & 0 \\
\hline WORLD & NA & NA & NA & NA & NA & 737 & NA & 642 & NA & NA & NA & NA & NA & NA & NA & NA & NA & 1,379 & 1,379 \\
\hline
\end{tabular}

Source: Authors' calculations.

Note: $\mathrm{NA}=$ not applicable 
Table 4A.18

Risk factor:

Vitamin A deficiency

Disease:

All causes

\begin{tabular}{|c|c|c|c|c|c|c|c|c|c|c|c|c|c|c|c|c|c|c|c|}
\hline \multirow[b]{2}{*}{ Region } & \multicolumn{2}{|c|}{$0-4$ years } & \multicolumn{2}{|c|}{ 5-14 years } & \multicolumn{2}{|c|}{$15-29$ years } & \multicolumn{2}{|c|}{ 30-44 years } & \multicolumn{2}{|c|}{ 45-59 years } & \multicolumn{2}{|c|}{$60-69$ years } & \multicolumn{2}{|c|}{ 70-79 years } & \multicolumn{2}{|c|}{$80+$ years } & \multicolumn{3}{|c|}{ Total } \\
\hline & Male & Female & Male & Female & Male & Female & Male & Female & Male & Female & Male & Female & Male & Female & Male & Female & Male & Female & All \\
\hline \multicolumn{20}{|l|}{ PAF of Mortality (\%) } \\
\hline East Asia and Pacific & 4 & 3 & 0 & 0 & 0 & 1 & 0 & 0 & 0 & 0 & 0 & 0 & 0 & 0 & 0 & 0 & 0 & 0 & 0 \\
\hline Europe and Central Asia & 0 & 0 & 0 & 0 & 0 & 0 & 0 & 0 & 0 & 0 & 0 & 0 & 0 & 0 & 0 & 0 & 0 & 0 & 0 \\
\hline Latin America and the Caribbean & 2 & 2 & 0 & 0 & 0 & 0 & 0 & 0 & 0 & 0 & 0 & 0 & 0 & 0 & 0 & 0 & 0 & 0 & 0 \\
\hline Middle East and North Africa & 3 & 3 & 0 & 0 & 0 & 1 & 0 & 1 & 0 & 0 & 0 & 0 & 0 & 0 & 0 & 0 & 1 & 1 & 1 \\
\hline South Asia & 6 & 6 & 1 & 0 & 0 & 2 & 0 & 2 & 0 & 0 & 0 & 0 & 0 & 0 & 0 & 0 & 1 & 2 & 2 \\
\hline Sub-Saharan Africa & 10 & 11 & 0 & 0 & 0 & 2 & 0 & 2 & 0 & 0 & 0 & 0 & 0 & 0 & 0 & 0 & 4 & 5 & 5 \\
\hline Low- and middle-income countries & 7 & 7 & 0 & 0 & 0 & 2 & 0 & 1 & 0 & 0 & 0 & 0 & 0 & 0 & 0 & 0 & 1 & 2 & 2 \\
\hline High-income countries & 0 & 0 & 0 & 0 & 0 & 0 & 0 & 0 & 0 & 0 & 0 & 0 & 0 & 0 & 0 & 0 & 0 & 0 & 0 \\
\hline WORLD & 7 & 7 & 0 & 0 & 0 & 2 & 0 & 1 & 0 & 0 & 0 & 0 & 0 & 0 & 0 & 0 & 1 & 2 & 1 \\
\hline
\end{tabular}

PAF of YLL (\%)

East Asia and Pacific

Europe and Central Asia

Latin America and the Caribbean

Middle East and North Africa

South Asia

Sub-Saharan Africa

Low- and middle-income countries

High-income countries

\begin{tabular}{|c|c|c|c|c|c|c|c|c|c|c|c|c|c|c|c|c|c|}
\hline East Asia and Pacific & 4 & 3 & 0 & 0 & 0 & 1 & 0 & 0 & 0 & 0 & 0 & 0 & 0 & 0 & 0 & 0 & \\
\hline Europe and Central Asia & 0 & 0 & 0 & 0 & 0 & 0 & 0 & 0 & 0 & 0 & 0 & 0 & 0 & 0 & 0 & 0 & \\
\hline Latin America and the Caribbean & 2 & 2 & 0 & 0 & 0 & 0 & 0 & 0 & 0 & 0 & 0 & 0 & 0 & 0 & 0 & 0 & \\
\hline Middle East and North Africa & 3 & 3 & 0 & 0 & 0 & 1 & 0 & 1 & 0 & 0 & 0 & 0 & 0 & 0 & 0 & 0 & \\
\hline South Asia & 6 & 6 & 0 & 0 & 0 & 2 & 0 & 2 & 0 & 0 & 0 & 0 & 0 & 0 & 0 & 0 & \\
\hline Sub-Saharan Africa & 10 & 11 & 0 & 0 & 0 & 2 & 0 & 2 & 0 & 0 & 0 & 0 & 0 & 0 & 0 & 0 & \\
\hline Low- and middle-income countries & 7 & 7 & 0 & 0 & 0 & 2 & 0 & 1 & 0 & 0 & 0 & 0 & 0 & 0 & 0 & 0 & \\
\hline High-income countries & 0 & 0 & 0 & 0 & 0 & 0 & 0 & 0 & 0 & 0 & 0 & 0 & 0 & 0 & 0 & 0 & \\
\hline$\overline{\text { WORLD }}$ & 7 & 7 & 0 & 0 & 0 & 2 & 0 & 1 & 0 & 0 & 0 & 0 & 0 & 0 & 0 & 0 & \\
\hline
\end{tabular}

\section{PAF of DALYs (\%)}

East Asia and Pacific

Europe and Central Asia

Latin America and the Caribbean

Middle East and North Africa

South Asia

Sub-Saharan Africa

Low- and middle-income countries

High-income countries

WORLD

Attributable Mortality (thousands)

East Asia and Pacific

Europe and Central Asia

Latin America and the Caribbean

Middle East and North Africa

South Asia

Sub-Saharan Africa

Low- and middle-income countries

High-income countries

$\begin{array}{lrlllllll}2 & 2 & 0 & 0 & 0 & 0 & 0 & 0 & 0 \\ 0 & 0 & 0 & 0 & 0 & 0 & 0 & 0 & 0 \\ 1 & 1 & 0 & 0 & 0 & 0 & 0 & 0 & 0 \\ 2 & 2 & 0 & 0 & 0 & 0 & 0 & 0 & 0 \\ 4 & 4 & 0 & 0 & 0 & 1 & 0 & 1 & 0 \\ 9 & 10 & 0 & 0 & 0 & 2 & 0 & 2 & 0 \\ 5 & 6 & 0 & 0 & 0 & 1 & 0 & 1 & 0 \\ 0 & 0 & 0 & 0 & 0 & 0 & 0 & 0 & 0\end{array}$

$\begin{array}{lll}0 & 0 & 0 \\ 0 & 0 & 0 \\ 0 & 0 & 0 \\ 0 & 0 & 0 \\ 0 & 0 & 0 \\ 0 & 0 & 0 \\ 0 & 0 & 0 \\ 0 & 0 & 0\end{array}$

$\begin{array}{lllllll}0 & 0 & 0 & 0 & 0 & 0 & 0 \\ 0 & 0 & 0 & 0 & 0 & 0 & 0 \\ 0 & 0 & 0 & 0 & 0 & 0 & 0 \\ 0 & 0 & 0 & 0 & 1 & 1 & 1 \\ 0 & 0 & 0 & 0 & 2 & 2 & 2 \\ 0 & 0 & 0 & 0 & 4 & 5 & 5 \\ 0 & 0 & 0 & 0 & 2 & 2 & 2 \\ 0 & 0 & 0 & 0 & 0 & 0 & 0 \\ 0 & 0 & 0 & 0 & 1 & 2 & 2\end{array}$

\begin{tabular}{lllllllllllllllllllll}
\hline WORLD & 372 & 369 & 2 & 2 & 0 & 27 & 0 & 26 & 1 & 0 & 0 & 0 & 0 & 0 & 0 & 0 & 376 & 424 & 800
\end{tabular}

Attributable YLL (thousands)

\begin{tabular}{|c|c|c|c|c|c|c|c|c|c|c|c|c|c|c|c|c|c|c|c|}
\hline East Asia and Pacific & 794 & 708 & 2 & 1 & 0 & 35 & 1 & 37 & 0 & 0 & 0 & 0 & 0 & 0 & 0 & 0 & 798 & 781 & 1,578 \\
\hline Europe and Central Asia & 0 & 0 & 0 & 0 & 0 & 0 & 0 & 0 & 0 & 0 & 0 & 0 & 0 & 0 & 0 & 0 & 0 & 0 & 1 \\
\hline Latin America and the Caribbean & 118 & 105 & 0 & 0 & 0 & 6 & 0 & 6 & 0 & 0 & 0 & 0 & 0 & 0 & 0 & 0 & 118 & 117 & 235 \\
\hline Middle East and North Africa & 204 & 197 & 0 & 0 & 0 & 9 & 0 & 10 & 0 & 0 & 0 & 0 & 0 & 0 & 0 & 0 & 206 & 218 & 423 \\
\hline South Asia & 3,140 & 3,095 & 43 & 27 & 0 & 249 & 0 & 234 & 0 & 0 & 0 & 0 & 0 & 0 & 0 & 0 & 3,184 & 3,605 & 6,788 \\
\hline$\underline{\text { Sub-Saharan Africa }}$ & 6,985 & 7,117 & 16 & 33 & 2 & 447 & 5 & 359 & 9 & 8 & 5 & 3 & 1 & 1 & 0 & 0 & 7,023 & 7,968 & 14,991 \\
\hline Low- and middle-income countries & 11,241 & 11,222 & 61 & 61 & 3 & 746 & 6 & 646 & 10 & 8 & 5 & 3 & 2 & 2 & 0 & 0 & 11,329 & 12,688 & 24,017 \\
\hline High-income countries & 0 & 0 & 0 & 0 & 0 & 0 & 0 & 0 & 0 & 0 & 0 & 0 & 0 & 0 & 0 & 0 & 0 & 1 & 1 \\
\hline WORLD & 11,241 & 11,222 & 61 & 61 & 3 & 746 & 6 & 646 & 10 & 8 & 5 & 3 & 2 & 2 & 0 & 0 & 11,329 & 12,689 & 24,017 \\
\hline
\end{tabular}

Attributable DALYs (thousands)

East Asia and Pacific

Europe and Central Asia

Latin America and the Caribbea

Middle East and North Africa

South Asia

Sub-Saharan Africa

$\begin{array}{rrrr}800 & 714 & 3 & 2 \\ 1 & 1 & 0 & 0 \\ 119 & 106 & 0 & 0 \\ 207 & 200 & 0 & 0 \\ 3,174 & 3,127 & 46 & 30 \\ 7,269 & 7,399 & 20 & 37\end{array}$

Low- and middle-income countries $\quad 11,570 \quad 11,546$

High-income countries

WORLD

$\begin{array}{lll}11,570 & 11,546 & 70\end{array}$

Source: Authors' calculations. 
Table 4A.19

Risk factor:

Zinc deficiency

Disease:

Diarrheal diseases

\begin{tabular}{|c|c|c|c|c|c|c|c|c|c|c|c|c|c|c|c|c|c|c|c|}
\hline \multirow[b]{2}{*}{ Region } & \multicolumn{2}{|c|}{$0-4$ years } & \multicolumn{2}{|c|}{ 5-14 years } & \multicolumn{2}{|c|}{ 15-29 years } & \multicolumn{2}{|c|}{ 30-44 years } & \multicolumn{2}{|c|}{ 45-59 years } & \multicolumn{2}{|c|}{ 60-69 years } & \multicolumn{2}{|c|}{ 70-79 years } & \multicolumn{2}{|c|}{$80+$ years } & \multicolumn{3}{|c|}{ Total } \\
\hline & Male & Female & Male & Female & Male & Female & Male & Female & Male & Female & Male & Female & Male & Female & Male & Female & Male & Female & All \\
\hline \multicolumn{20}{|l|}{ PAF of Mortality (\%) } \\
\hline East Asia and Pacific & 4 & 4 & NA & NA & NA & NA & NA & NA & NA & NA & NA & NA & NA & NA & NA & NA & 4 & 4 & 4 \\
\hline Europe and Central Asia & 3 & 3 & NA & NA & NA & NA & NA & NA & NA & NA & NA & NA & NA & NA & NA & NA & 2 & 2 & 2 \\
\hline Latin America and the Caribbean & 8 & 8 & NA & NA & NA & NA & NA & NA & NA & NA & NA & NA & NA & NA & NA & NA & 7 & 7 & 7 \\
\hline Middle East and North Africa & 10 & 10 & NA & NA & NA & NA & NA & NA & NA & NA & NA & NA & NA & NA & NA & NA & 9 & 9 & 9 \\
\hline South Asia & 16 & 16 & NA & NA & NA & NA & NA & NA & NA & NA & NA & NA & NA & NA & NA & NA & 15 & 15 & 15 \\
\hline Sub-Saharan Africa & 12 & 12 & NA & NA & NA & NA & NA & NA & NA & NA & NA & NA & NA & NA & NA & NA & 11 & 11 & 11 \\
\hline Low- and middle-income countries & 13 & 13 & NA & NA & NA & NA & NA & NA & NA & NA & NA & NA & NA & NA & NA & NA & 11 & 11 & 11 \\
\hline High-income countries & 1 & 1 & NA & NA & NA & NA & NA & NA & NA & NA & NA & NA & NA & NA & NA & NA & 0 & 0 & 0 \\
\hline WORLD & 13 & 13 & NA & NA & NA & NA & NA & NA & NA & NA & NA & NA & NA & NA & NA & NA & 11 & 11 & 11 \\
\hline
\end{tabular}

PAF of YLL (\%)

East Asia and Pacific

Europe and Central Asia

Latin America and the Caribbean

Middle East and North Africa

South Asia

Sub-Saharan Africa

\begin{tabular}{|c|c|c|c|c|}
\hline 4 & 4 & NA & NA & NA \\
\hline 3 & 3 & NA & NA & NA \\
\hline 8 & 8 & NA & NA & NA \\
\hline 10 & 10 & NA & NA & NA \\
\hline 16 & 16 & NA & NA & NA \\
\hline 12 & 12 & NA & NA & NA \\
\hline
\end{tabular}

Low- and middle-income countrie

1313 NA NA NA NA

High-income countries

\begin{tabular}{|c|c|c|c|c|c|c|c|c|c|c|c|c|c|c|c|c|c|c|c|}
\hline WORLD & 13 & 13 & NA & NA & NA & NA & NA & NA & NA & NA & NA & NA & NA & NA & NA & NA & 12 & 12 & $\overline{12}$ \\
\hline \multicolumn{20}{|l|}{ PAF of DALYs (\%) } \\
\hline East Asia and Pacific & 4 & 4 & NA & NA & NA & NA & NA & NA & NA & NA & NA & NA & NA & NA & NA & NA & 3 & 3 & 3 \\
\hline Europe and Central Asia & 3 & 3 & NA & NA & NA & NA & NA & NA & NA & NA & NA & NA & NA & NA & NA & NA & 2 & 2 & 2 \\
\hline Latin America and the Caribbean & 8 & 8 & NA & NA & NA & NA & NA & NA & NA & NA & NA & NA & NA & NA & NA & NA & 7 & 7 & 7 \\
\hline Middle East and North Africa & 10 & 10 & NA & NA & NA & NA & NA & NA & NA & NA & NA & NA & NA & NA & NA & NA & 9 & 9 & 9 \\
\hline South Asia & 16 & 16 & NA & NA & NA & NA & NA & NA & NA & NA & NA & NA & NA & NA & NA & NA & 15 & 15 & 15 \\
\hline Sub-Saharan Africa & 12 & 12 & NA & NA & NA & NA & NA & NA & NA & NA & NA & NA & NA & NA & NA & NA & 12 & 12 & 12 \\
\hline Low- and middle-income countries & 12 & 12 & NA & NA & NA & NA & NA & NA & NA & NA & NA & NA & NA & NA & NA & NA & 11 & 11 & 11 \\
\hline High-income countries & 1 & 1 & NA & NA & NA & NA & NA & NA & NA & NA & NA & NA & NA & NA & NA & NA & 1 & 1 & 1 \\
\hline WORLD & 12 & 12 & NA & NA & NA & NA & NA & NA & NA & NA & NA & NA & NA & NA & NA & NA & 11 & 11 & 11 \\
\hline
\end{tabular}

Attributable Mortality (thousands)

East Asia and Pacific

Europe and Central Asia

Latin America and the Caribbean

Middle East and North Africa

South Asia

Sub-Saharan Africa

Low- and middle-income countries 105

WORLD

\begin{tabular}{|c|c|c|c|c|c|c|c|c|c|c|c|c|c|c|c|c|c|c|}
\hline 4 & 4 & NA & NA & NA & NA & NA & NA & NA & NA & NA & NA & NA & NA & NA & NA & 4 & 4 & 9 \\
\hline 0 & 0 & NA & NA & NA & NA & NA & NA & NA & NA & NA & NA & NA & NA & NA & NA & 0 & 0 & 0 \\
\hline 2 & 2 & NA & NA & NA & NA & NA & NA & NA & NA & NA & NA & NA & NA & NA & NA & 2 & 2 & 4 \\
\hline 3 & 3 & NA & NA & NA & NA & NA & NA & NA & NA & NA & NA & NA & NA & NA & NA & 3 & 3 & 7 \\
\hline 53 & 49 & NA & NA & NA & NA & NA & NA & NA & NA & NA & NA & NA & NA & NA & NA & 53 & 49 & 102 \\
\hline 42 & 38 & NA & NA & NA & NA & NA & NA & NA & NA & NA & NA & NA & NA & NA & NA & 42 & 38 & 80 \\
\hline 105 & 96 & NA & NA & NA & NA & NA & NA & NA & NA & NA & NA & NA & NA & NA & NA & 105 & 96 & 201 \\
\hline 0 & 0 & NA & NA & NA & NA & NA & NA & NA & NA & NA & NA & NA & NA & NA & NA & 0 & 0 & 0 \\
\hline 105 & 96 & NA & NA & NA & NA & NA & NA & NA & NA & NA & NA & NA & NA & NA & NA & 105 & 96 & 201 \\
\hline
\end{tabular}

Attributable YLL (thousands)

Europe and Central Asia

Latin America and the Caribbea

Middle East and North Africa

South Asia

Sub-Saharan Africa

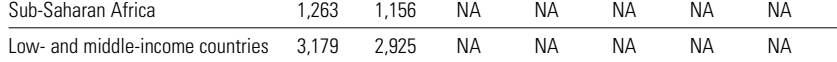

\begin{tabular}{llllllllllllllllllll}
\hline WORLD & 3,179 & 2,925 & NA & NA & NA & NA & NA & NA & NA & NA & NA & NA & NA & NA & NA & NA & 3,179 & 2,925 & 6,104
\end{tabular}

Attributable DALYs (thousands)

East Asia and Pacific

Europe and Central Asia

tatin America and the Caiblean

Middle East and North Africa

South Asia

Sub-Saharan Africa

NA NA NA

NA NA NA

NA NA NA NA NA NA NA $\quad 156 \quad 146 \quad 301$

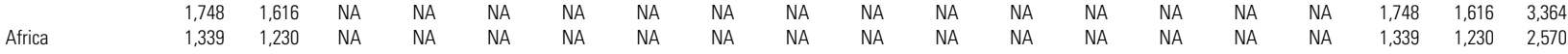

\begin{tabular}{lllllllllllllllllllll}
\hline Low- and middle-income countries & 3,453 & 3,188 & NA & NA & NA & NA & NA & NA & NA & NA & NA & NA & NA & NA & NA & NA & 3,453 & 3,188 & 6,641
\end{tabular}

$\begin{array}{llllllllllllllllllll}\text { High-income countries } & 2 & 2 & \text { NA } & \text { NA } & \text { NA } & \text { NA } & \text { NA } & \text { NA } & \text { NA } & \text { NA } & \text { NA } & \text { NA } & \text { NA } & \text { NA } & \text { NA } & \text { NA } & 2 & 2 & 3\end{array}$

\begin{tabular}{lllllllllllllllllllll}
\hline WORLD & 3,455 & 3,190 & NA & NA & NA & NA & NA & NA & NA & NA & NA & NA & NA & NA & NA & NA & 3,455 & 3,190 & 6,645
\end{tabular}

Source: Authors' calculations.

Note: NA = not applicable 
Table 4A.20

Risk factor:

Disease:

Zinc deficiency

Malaria

\begin{tabular}{|c|c|c|c|c|c|c|c|c|c|c|c|c|c|c|c|c|c|c|c|}
\hline \multirow[b]{2}{*}{ Region } & \multicolumn{2}{|c|}{$0-4$ years } & \multicolumn{2}{|c|}{ 5-14 years } & \multicolumn{2}{|c|}{$15-29$ years } & \multicolumn{2}{|c|}{ 30-44 years } & \multicolumn{2}{|c|}{$45-59$ years } & \multicolumn{2}{|c|}{$60-69$ years } & \multicolumn{2}{|c|}{$70-79$ years } & \multicolumn{2}{|c|}{$80+$ years } & \multicolumn{3}{|c|}{ Total } \\
\hline & Male & Female & Male & Female & Male & Female & Male & Female & Male & Female & Male & Female & Male & Female & Male & Female & Male & Female & All \\
\hline \multicolumn{20}{|l|}{ PAF of Mortality (\%) } \\
\hline East Asia and Pacific & 8 & 8 & NA & NA & NA & NA & NA & NA & NA & NA & NA & NA & NA & NA & NA & NA & 7 & 7 & 7 \\
\hline Europe and Central Asia & 5 & 5 & NA & NA & NA & NA & NA & NA & NA & NA & NA & NA & NA & NA & NA & NA & 3 & 4 & 4 \\
\hline Latin America and the Caribbean & 16 & 16 & NA & NA & NA & NA & NA & NA & NA & NA & NA & NA & NA & NA & NA & NA & 14 & 14 & 14 \\
\hline Middle East and North Africa & 18 & 18 & NA & NA & NA & NA & NA & NA & NA & NA & NA & NA & NA & NA & NA & NA & 16 & 16 & 16 \\
\hline South Asia & 28 & 28 & NA & NA & NA & NA & NA & NA & NA & NA & NA & NA & NA & NA & NA & NA & 25 & 25 & 25 \\
\hline Sub-Saharan Africa & 22 & 22 & NA & NA & NA & NA & NA & NA & NA & NA & NA & NA & NA & NA & NA & NA & 20 & 20 & 20 \\
\hline Low- and middle-income countries & 22 & 22 & NA & NA & NA & NA & NA & NA & NA & NA & NA & NA & NA & NA & NA & NA & 20 & 20 & 20 \\
\hline High-income countries & 3 & 3 & NA & NA & NA & NA & NA & NA & NA & NA & NA & NA & NA & NA & NA & NA & 1 & 1 & 1 \\
\hline WORLD & 22 & 22 & NA & NA & NA & NA & NA & NA & NA & NA & NA & NA & NA & NA & NA & NA & 20 & 20 & 20 \\
\hline
\end{tabular}

PAF of YLL (\%)

\begin{tabular}{|c|c|c|c|c|c|c|c|c|c|c|c|c|c|c|c|c|c|c|c|}
\hline East Asia and Pacific & 8 & 8 & NA & NA & NA & NA & NA & NA & NA & NA & NA & NA & NA & NA & NA & NA & 8 & 8 & 8 \\
\hline Europe and Central Asia & 5 & 5 & NA & NA & NA & NA & NA & NA & NA & NA & NA & NA & NA & NA & NA & NA & 4 & 4 & 4 \\
\hline Latin America and the Caribbean & 16 & 16 & NA & NA & NA & NA & NA & NA & NA & NA & NA & NA & NA & NA & NA & NA & 14 & 14 & 14 \\
\hline Middle East and North Africa & 18 & 18 & NA & NA & NA & NA & NA & NA & NA & NA & NA & NA & NA & NA & NA & NA & 17 & 17 & 17 \\
\hline South Asia & 28 & 28 & NA & NA & NA & NA & NA & NA & NA & NA & NA & NA & NA & NA & NA & NA & 26 & 26 & 26 \\
\hline Sub-Saharan Africa & 22 & 22 & NA & NA & NA & NA & NA & NA & NA & NA & NA & NA & NA & NA & NA & NA & 21 & 20 & 21 \\
\hline Low- and middle-income countries & 22 & 22 & NA & NA & NA & NA & NA & NA & NA & NA & NA & NA & NA & NA & NA & NA & 20 & 21 & 20 \\
\hline High-income countries & 3 & 3 & NA & NA & NA & NA & NA & NA & NA & NA & NA & NA & NA & NA & NA & NA & 1 & 1 & 1 \\
\hline WORLD & 22 & 22 & NA & NA & NA & NA & NA & NA & NA & NA & NA & NA & NA & NA & NA & NA & 20 & 21 & 20 \\
\hline
\end{tabular}

PAF of DALYs (\%)

Latin America and the Caribbean

Middle East and North Africa

South Asia

Sub-Saharan Africa

Low- and middle-income countries

\section{$\begin{array}{llllll}8 & 8 & N A & N A & N A & N A\end{array}$}

NA NA NA NA

NA NA NA NA NA NA NA

7

\begin{tabular}{|c|c|c|c|c|c|c|c|c|c|c|c|c|c|c|c|c|c|c|c|}
\hline $\begin{array}{l}\text { Low-and midale-Income countries } \\
\text { High-income countries }\end{array}$ & $\begin{array}{r}2 L \\
3\end{array}$ & $\begin{array}{r}2 L \\
3\end{array}$ & NA & NA & NA & NA & $\begin{array}{l}\text { NA } \\
\text { NA }\end{array}$ & NA & NA & NA & NA & NA & NA & NA & NA & NA & $\begin{array}{r}20 \\
1\end{array}$ & $\begin{array}{r}20 \\
1\end{array}$ & 1 \\
\hline WORLD & 22 & 22 & NA & NA & NA & NA & NA & NA & NA & NA & NA & NA & NA & NA & NA & NA & 20 & 20 & 20 \\
\hline
\end{tabular}

Attributable Mortality (thousands)

\begin{tabular}{|c|c|c|c|c|c|c|c|c|c|c|c|c|c|c|c|c|c|c|c|}
\hline $\begin{array}{l}\text { Attributable Mortality (thousa } \\
\text { East Asia and Pacific }\end{array}$ & 1 & 1 & NA & NA & NA & NA & NA & NA & NA & NA & NA & NA & NA & NA & NA & NA & 1 & 1 & 2 \\
\hline Europe and Central Asia & 0 & 0 & NA & NA & NA & NA & NA & NA & NA & NA & NA & NA & NA & NA & NA & NA & 0 & 0 & 0 \\
\hline Latin America and the Caribbean & 0 & 0 & NA & NA & NA & NA & NA & NA & NA & NA & NA & NA & NA & NA & NA & NA & 0 & 0 & 0 \\
\hline Middle East and North Africa & 1 & 2 & NA & NA & NA & NA & NA & NA & NA & NA & NA & NA & NA & NA & NA & NA & 1 & 2 & 3 \\
\hline South Asia & 8 & 8 & NA & NA & NA & NA & NA & NA & NA & NA & NA & NA & NA & NA & NA & NA & 8 & 8 & 16 \\
\hline Sub-Saharan Africa & 103 & 114 & NA & NA & NA & NA & NA & NA & NA & NA & NA & NA & NA & NA & NA & NA & 103 & 114 & 217 \\
\hline Low- and middle-income countries & 114 & 125 & NA & NA & NA & NA & NA & NA & NA & NA & NA & NA & NA & NA & NA & NA & 114 & 125 & 239 \\
\hline High-income countries & 0 & 0 & NA & NA & NA & NA & NA & NA & NA & NA & NA & NA & NA & NA & NA & NA & 0 & 0 & 0 \\
\hline WORLD & 114 & 125 & NA & NA & NA & NA & NA & NA & NA & NA & NA & NA & NA & NA & NA & NA & 114 & 125 & 239 \\
\hline
\end{tabular}

Attributable YLL (thousands)

\begin{tabular}{|c|c|c|c|c|c|c|c|c|c|c|c|c|c|c|c|c|c|c|c|}
\hline East Asia and Pacific & 45 & 21 & NA & NA & NA & NA & NA & NA & NA & NA & NA & NA & NA & NA & NA & NA & 45 & 21 & 66 \\
\hline Europe and Central Asia & 0 & 0 & NA & NA & NA & NA & NA & NA & NA & NA & NA & NA & NA & NA & NA & NA & 0 & 0 & 0 \\
\hline Latin America and the Caribbean & 4 & 3 & NA & NA & NA & NA & NA & NA & NA & NA & NA & NA & NA & NA & NA & NA & 4 & 3 & 6 \\
\hline Middle East and North Africa & 43 & 49 & NA & NA & NA & NA & NA & NA & NA & NA & NA & NA & NA & NA & NA & NA & 43 & 49 & 93 \\
\hline South Asia & 228 & 255 & NA & NA & NA & NA & NA & NA & NA & NA & NA & NA & NA & NA & NA & NA & 228 & 255 & 484 \\
\hline Sub-Saharan Africa & 3,112 & 3,480 & NA & NA & NA & NA & NA & NA & NA & NA & NA & NA & NA & NA & NA & NA & 3,112 & 3,480 & 6,592 \\
\hline Low- and middle-income countries & 3,432 & 3,808 & NA & NA & NA & NA & NA & NA & NA & NA & NA & NA & NA & NA & NA & NA & 3,432 & 3,808 & 7,240 \\
\hline High-income countries & 0 & 0 & NA & NA & NA & NA & NA & NA & NA & NA & NA & NA & NA & NA & NA & NA & 0 & 0 & 0 \\
\hline WORLD & 3,433 & 3,808 & NA & NA & NA & NA & NA & NA & NA & NA & NA & NA & NA & NA & NA & NA & 3,433 & 3,808 & 7,241 \\
\hline
\end{tabular}

Attributable DALYs (thousands)

\begin{tabular}{|c|c|c|c|c|c|c|c|c|c|c|c|c|c|c|c|c|c|c|c|}
\hline East Asia and Pacific & 48 & 24 & NA & NA & NA & NA & NA & NA & NA & NA & NA & NA & NA & NA & NA & NA & 48 & 24 & 72 \\
\hline Europe and Central Asia & 0 & 0 & NA & NA & NA & NA & NA & NA & NA & NA & NA & NA & NA & NA & NA & NA & 0 & 0 & 0 \\
\hline Latin America and the Caribbean & 5 & 4 & NA & NA & NA & NA & NA & NA & NA & NA & NA & NA & NA & NA & NA & NA & 5 & 4 & 9 \\
\hline Middle East and North Africa & 48 & 53 & NA & NA & NA & NA & NA & NA & NA & NA & NA & NA & NA & NA & NA & NA & 48 & 53 & 101 \\
\hline South Asia & 264 & 290 & NA & NA & NA & NA & NA & NA & NA & NA & NA & NA & NA & NA & NA & NA & 264 & 290 & 554 \\
\hline Sub-Saharan Africa & 3,417 & 3,783 & NA & NA & NA & NA & NA & NA & NA & NA & NA & NA & NA & NA & NA & NA & 3,417 & 3,783 & 7,201 \\
\hline Low- and middle-income countries & 3,783 & 4,154 & NA & NA & NA & NA & NA & NA & NA & NA & NA & NA & NA & NA & NA & NA & 3,783 & 4,154 & 7,938 \\
\hline High-income countries & 0 & 0 & NA & NA & NA & NA & NA & NA & NA & NA & NA & NA & NA & NA & NA & NA & 0 & 0 & 0 \\
\hline WORLD & 3,783 & 4,154 & NA & NA & NA & NA & NA & NA & NA & NA & NA & NA & NA & NA & NA & NA & 3,783 & 4,154 & 7,938 \\
\hline
\end{tabular}

Source: Authors' calculations.

Note: $\mathrm{NA}=$ not applicable. 
Table 4A.21

Risk factor:

Disease:

Zinc deficiency

Lower respiratory infections

\begin{tabular}{|c|c|c|c|c|c|c|c|c|c|c|c|c|c|c|c|c|c|c|c|}
\hline \multirow[b]{2}{*}{ Region } & \multicolumn{2}{|c|}{$0-4$ years } & \multicolumn{2}{|c|}{ 5-14 years } & \multicolumn{2}{|c|}{$15-29$ years } & \multicolumn{2}{|c|}{ 30-44 years } & \multicolumn{2}{|c|}{$45-59$ years } & \multicolumn{2}{|c|}{$60-69$ years } & \multicolumn{2}{|c|}{$70-79$ years } & \multicolumn{2}{|c|}{$80+$ years } & \multicolumn{3}{|c|}{ Total } \\
\hline & Male & Female & Male & Female & Male & Female & Male & Female & Male & Female & Male & Female & Male & Female & Male & Female & Male & Female & All \\
\hline \multicolumn{20}{|l|}{ PAF of Mortality (\%) } \\
\hline East Asia and Pacific & 8 & 8 & NA & NA & NA & NA & NA & NA & NA & NA & NA & NA & NA & NA & NA & NA & 2 & 3 & 3 \\
\hline Europe and Central Asia & 5 & 5 & NA & NA & NA & NA & NA & NA & NA & NA & NA & NA & NA & NA & NA & NA & 1 & 2 & 2 \\
\hline Latin America and the Caribbean & 15 & 15 & NA & NA & NA & NA & NA & NA & NA & NA & NA & NA & NA & NA & NA & NA & 4 & 4 & 4 \\
\hline Middle East and North Africa & 17 & 17 & NA & NA & NA & NA & NA & NA & NA & NA & NA & NA & NA & NA & NA & NA & 12 & 12 & 12 \\
\hline South Asia & 26 & 26 & NA & NA & NA & NA & NA & NA & NA & NA & NA & NA & NA & NA & NA & NA & 15 & 16 & 15 \\
\hline Sub-Saharan Africa & 21 & 21 & NA & NA & NA & NA & NA & NA & NA & NA & NA & NA & NA & NA & NA & NA & 16 & 13 & 15 \\
\hline Low- and middle-income countries & 22 & 21 & NA & NA & NA & NA & NA & NA & NA & NA & NA & NA & NA & NA & NA & NA & 12 & 12 & 12 \\
\hline High-income countries & 3 & 3 & NA & NA & NA & NA & NA & NA & NA & NA & NA & NA & NA & NA & NA & NA & 0 & 0 & 0 \\
\hline WORLD & 22 & 21 & NA & NA & NA & NA & NA & NA & NA & NA & NA & NA & NA & NA & NA & NA & 11 & 10 & 11 \\
\hline
\end{tabular}

PAF of YLL (\%)

\begin{tabular}{|c|c|c|c|c|c|c|c|c|c|c|c|c|c|c|c|c|c|c|c|}
\hline East Asia and Pacific & 8 & 8 & NA & NA & NA & NA & NA & NA & NA & NA & NA & NA & NA & NA & NA & NA & 4 & 5 & 4 \\
\hline Europe and Central Asia & 5 & 5 & NA & NA & NA & NA & NA & NA & NA & NA & NA & NA & NA & NA & NA & NA & 2 & 3 & 2 \\
\hline Latin America and the Caribbean & 15 & 15 & NA & NA & NA & NA & NA & NA & NA & NA & NA & NA & NA & NA & NA & NA & 7 & 7 & 7 \\
\hline Middle East and North Africa & 17 & 17 & NA & NA & NA & NA & NA & NA & NA & NA & NA & NA & NA & NA & NA & NA & 14 & 14 & 14 \\
\hline South Asia & 26 & 26 & NA & NA & NA & NA & NA & NA & NA & NA & NA & NA & NA & NA & NA & NA & 20 & 20 & 20 \\
\hline Sub-Saharan Africa & 21 & 21 & NA & NA & NA & NA & NA & NA & NA & NA & NA & NA & NA & NA & NA & NA & 17 & 15 & 16 \\
\hline Low- and middle-income countries & 22 & 21 & NA & NA & NA & NA & NA & NA & NA & NA & NA & NA & NA & NA & NA & NA & 16 & 15 & 16 \\
\hline High-income countries & 3 & 3 & NA & NA & NA & NA & NA & NA & NA & NA & NA & NA & NA & NA & NA & NA & 0 & 0 & 0 \\
\hline WORLD & 22 & 21 & NA & NA & NA & NA & NA & NA & NA & NA & NA & NA & NA & NA & NA & NA & 16 & 15 & 15 \\
\hline
\end{tabular}

PAF of DALYs (\%)

\begin{tabular}{|c|c|c|c|c|c|c|c|c|c|c|c|c|c|c|c|c|c|c|}
\hline East Asia and Pacific & 8 & 8 & NA & NA & NA & NA & NA & NA & NA & NA & NA & NA & NA & NA & NA & NA & 4 & 5 \\
\hline Europe and Central Asia & 5 & 5 & NA & NA & NA & NA & NA & NA & NA & NA & NA & NA & NA & NA & NA & NA & 2 & 3 \\
\hline Latin America and the Caribbean & 15 & 15 & NA & NA & NA & NA & NA & NA & NA & NA & NA & NA & NA & NA & NA & NA & 8 & 8 \\
\hline Middle East and North Africa & 17 & 17 & NA & NA & NA & NA & NA & NA & NA & NA & NA & NA & NA & NA & NA & NA & 14 & 14 \\
\hline South Asia & 26 & 26 & NA & NA & NA & NA & NA & NA & NA & NA & NA & NA & NA & NA & NA & NA & 20 & 20 \\
\hline Sub-Saharan Africa & 21 & 21 & NA & NA & NA & NA & NA & NA & NA & NA & NA & NA & NA & NA & NA & NA & 17 & 15 \\
\hline Low- and middle-income countries & 22 & 21 & NA & NA & NA & NA & NA & NA & NA & NA & NA & NA & NA & NA & NA & NA & 16 & 15 \\
\hline High-income countries & 3 & 3 & NA & NA & NA & NA & NA & NA & NA & NA & NA & NA & NA & NA & NA & NA & 0 & 0 \\
\hline WORLD & 22 & 21 & NA & NA & NA & NA & NA & NA & NA & NA & NA & NA & NA & NA & NA & NA & 16 & 15 \\
\hline
\end{tabular}

Attributable Mortality (thousands)

East Asia an

Europe and Central Asia

Latin America and the Caribbean

Middle East and North Africa

South Asia

Sub-Saharan Africa

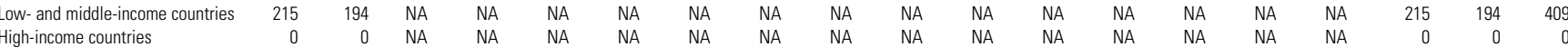

\begin{tabular}{|c|c|c|c|c|c|c|c|c|c|c|c|c|c|c|c|c|c|c|}
\hline High-income countries & 0 & 0 & NA & NA & NA & NA & NA & NA & NA & NA & NA & NA & NA & NA & NA & NA & 0 & 0 \\
\hline & 215 & 194 & NA & NA & NA & NA & NA & NA & NA & NA & NA & NA & NA & NA & NA & NA & 215 & 194 \\
\hline
\end{tabular}

Attributable YLL (thousands)

East Asia and Pacific

Europe and Central Asia

Latin America and the Caribbea

Middle East and North Africa

South Asia

Sub-Saharan Africa

$\begin{array}{rrrrrrrr}144 & 287 & \text { NA } & \text { NA } & \text { NA } & \text { NA } & \text { NA } & \text { NA } \\ 26 & 22 & \text { NA } & \text { NA } & \text { NA } & \text { NA } & \text { NA } & \text { NA } \\ 102 & 88 & \text { NA } & \text { NA } & \text { NA } & \text { NA } & \text { NA } & \text { NA }\end{array}$

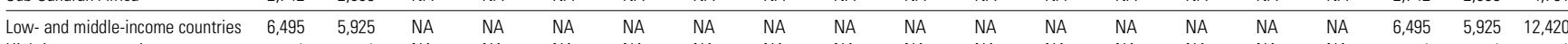

$\begin{array}{llllllllllllllllllll}\text { High-income countries } & 1 & 1 & \text { NA } & \text { NA } & \text { NA } & \text { NA } & \text { NA } & \text { NA } & \text { NA } & \text { NA } & \text { NA } & \text { NA } & \text { NA } & \text { NA } & \text { NA } & \text { NA } & 1 & 1 & 1\end{array}$

\begin{tabular}{llllllllllllllllllllll}
\hline WORLD & 6,495 & 5,926 & NA & NA & NA & NA & NA & NA & NA & NA & NA & NA & NA & NA & NA & NA & 6,495 & 5,926 & 12,421
\end{tabular}

Attributable DALYs (thousands)

East Asia and Pacific

Europe and Central Asia

atin America and the Caibben

Middle East and North Africa

South Asia

Sub-Saharan Africa

$\begin{array}{llllll}165 & 325 & \text { NA } & \text { NA } & \text { NA } & \text { NA } \\ 28 & 24 & \text { NA } & \text { NA } & \text { NA } & \text { NA }\end{array}$

NA NA NA NA

NA NA NA NA NA NA NA $\quad 165 \quad 325 \quad 490$

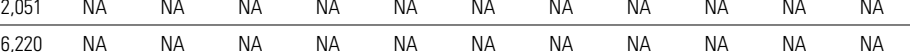

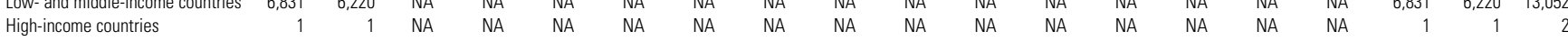

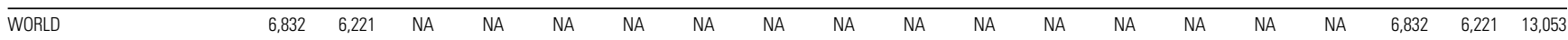

Source: Authors' calculations.

Note: $\mathrm{NA}=$ not applicable 
Table 4A.22

Risk factor:

Disease:

Zinc deficiency

All causes

\begin{tabular}{|c|c|c|c|c|c|c|c|c|c|c|c|c|c|c|c|c|c|c|c|}
\hline \multirow[b]{2}{*}{ Region } & \multicolumn{2}{|c|}{$0-4$ years } & \multicolumn{2}{|c|}{ 5-14 years } & \multicolumn{2}{|c|}{$15-29$ years } & \multicolumn{2}{|c|}{ 30-44 years } & \multicolumn{2}{|c|}{$45-59$ years } & \multicolumn{2}{|c|}{$60-69$ years } & \multicolumn{2}{|c|}{ 70-79 years } & \multicolumn{2}{|c|}{$80+$ years } & \multicolumn{3}{|c|}{ Total } \\
\hline & Male & Female & Male & Female & Male & Female & Male & Female & Male & Female & Male & Female & Male & Female & Male & Female & Male & Female & All \\
\hline \multicolumn{20}{|l|}{ PAF of Mortality $(\%)$} \\
\hline East Asia and Pacific & 2 & 2 & NA & NA & NA & NA & NA & NA & NA & NA & NA & NA & NA & NA & NA & NA & 0 & 0 & 0 \\
\hline Europe and Central Asia & 1 & 1 & NA & NA & NA & NA & NA & NA & NA & NA & NA & NA & NA & NA & NA & NA & 0 & 0 & 0 \\
\hline Latin America and the Caribbean & 2 & 3 & NA & NA & NA & NA & NA & NA & NA & NA & NA & NA & NA & NA & NA & NA & 0 & 0 & 0 \\
\hline Middle East and North Africa & 5 & 5 & NA & NA & NA & NA & NA & NA & NA & NA & NA & NA & NA & NA & NA & NA & 1 & 1 & 1 \\
\hline South Asia & 9 & 9 & NA & NA & NA & NA & NA & NA & NA & NA & NA & NA & NA & NA & NA & NA & 2 & 3 & 2 \\
\hline Sub-Saharan Africa & 10 & 10 & NA & NA & NA & NA & NA & NA & NA & NA & NA & NA & NA & NA & NA & NA & 4 & 4 & 4 \\
\hline Low- and middle-income countries & 8 & 8 & NA & NA & NA & NA & NA & NA & NA & NA & NA & NA & NA & NA & NA & NA & 2 & 2 & 2 \\
\hline High-income countries & 0 & 0 & NA & NA & NA & NA & NA & NA & NA & NA & NA & NA & NA & NA & NA & NA & 0 & 0 & 0 \\
\hline WORLD & 8 & 8 & NA & NA & NA & NA & NA & NA & NA & NA & NA & NA & NA & NA & NA & NA & 1 & 2 & 2 \\
\hline
\end{tabular}

PAF of YLL (\%)

\begin{tabular}{|c|c|c|c|c|c|c|c|c|c|c|c|c|c|c|c|c|c|c|c|}
\hline East Asia and Pacific & 2 & 2 & NA & NA & NA & NA & NA & NA & NA & NA & NA & NA & NA & NA & NA & NA & 0 & 0 & 0 \\
\hline Europe and Central Asia & 1 & 1 & NA & NA & NA & NA & NA & NA & NA & NA & NA & NA & NA & NA & NA & NA & 0 & 0 & 0 \\
\hline Latin America and the Caribbean & 2 & 3 & NA & NA & NA & NA & NA & NA & NA & NA & NA & NA & NA & NA & NA & NA & 1 & 1 & 1 \\
\hline Middle East and North Africa & 5 & 5 & NA & NA & NA & NA & NA & NA & NA & NA & NA & NA & NA & NA & NA & NA & 2 & 2 & 2 \\
\hline South Asia & 9 & 9 & NA & NA & NA & NA & NA & NA & NA & NA & NA & NA & NA & NA & NA & NA & 4 & 4 & 4 \\
\hline Sub-Saharan Africa & 10 & 10 & NA & NA & NA & NA & NA & NA & NA & NA & NA & NA & NA & NA & NA & NA & 5 & 5 & 5 \\
\hline Low- and middle-income countries & 8 & 8 & NA & NA & NA & NA & NA & NA & NA & NA & NA & NA & NA & NA & NA & NA & 3 & 3 & 3 \\
\hline High-income countries & 0 & 0 & NA & NA & NA & NA & NA & NA & NA & NA & NA & NA & NA & NA & NA & NA & 0 & 0 & 0 \\
\hline WORLD & 8 & 8 & NA & NA & NA & NA & NA & NA & NA & NA & NA & NA & NA & NA & NA & NA & 2 & 3 & 3 \\
\hline
\end{tabular}

PAF of DALYs (\%)

Latin America and the Caribbean

Middle East and North Africa

South Asia

Sub-Saharan Africa

Low- and middle-income countries

\begin{tabular}{lllllllllllllllllllll}
\hline WORLD & 6 & 6 & NA & NA & NA & NA & NA & NA & NA & NA & NA & NA & NA & NA & NA & NA & 2 & 2 & 2
\end{tabular}

Attributable Mortality (thousands)

\begin{tabular}{|c|c|c|c|c|c|c|c|c|c|c|c|c|c|c|c|c|c|c|c|}
\hline East Asia and Pacific & 11 & 14 & NA & NA & NA & NA & NA & NA & NA & NA & NA & NA & NA & NA & NA & NA & 11 & 14 & 25 \\
\hline Europe and Central Asia & 1 & 1 & NA & NA & NA & NA & NA & NA & NA & NA & NA & NA & NA & NA & NA & NA & 1 & 1 & 2 \\
\hline Latin America and the Caribbean & 6 & 5 & NA & NA & NA & NA & NA & NA & NA & NA & NA & NA & NA & NA & NA & NA & 6 & 5 & 10 \\
\hline Middle East and North Africa & 11 & 11 & NA & NA & NA & NA & NA & NA & NA & NA & NA & NA & NA & NA & NA & NA & 11 & 11 & 22 \\
\hline South Asia & 169 & 167 & NA & NA & NA & NA & NA & NA & NA & NA & NA & NA & NA & NA & NA & NA & 169 & 167 & 336 \\
\hline Sub-Saharan Africa & 235 & 218 & NA & NA & NA & NA & NA & NA & NA & NA & NA & NA & NA & NA & NA & NA & 235 & 218 & 454 \\
\hline Low- and middle-income countries & 433 & 416 & NA & NA & NA & NA & NA & NA & NA & NA & NA & NA & NA & NA & NA & NA & 433 & 416 & 849 \\
\hline High-income countries & 0 & 0 & NA & NA & NA & NA & NA & NA & NA & NA & NA & NA & NA & NA & NA & NA & 0 & 0 & 0 \\
\hline WORLD & 433 & 416 & NA & NA & NA & NA & NA & NA & NA & NA & NA & NA & NA & NA & NA & NA & 433 & 416 & 349 \\
\hline
\end{tabular}

Attributable YLL (thousands)

\begin{tabular}{|c|c|c|c|c|c|c|c|c|c|c|c|c|c|c|c|c|c|c|c|}
\hline East Asia and Pacific & 323 & 433 & NA & NA & NA & NA & NA & NA & NA & NA & NA & NA & NA & NA & NA & NA & 323 & 433 & 756 \\
\hline Europe and Central Asia & 32 & 27 & NA & NA & NA & NA & NA & NA & NA & NA & NA & NA & NA & NA & NA & NA & 32 & 27 & 59 \\
\hline Latin America and the Caribbean & 169 & 147 & NA & NA & NA & NA & NA & NA & NA & NA & NA & NA & NA & NA & NA & NA & 169 & 147 & 316 \\
\hline Middle East and North Africa & 348 & 329 & NA & NA & NA & NA & NA & NA & NA & NA & NA & NA & NA & NA & NA & NA & 348 & 329 & 677 \\
\hline South Asia & 5,118 & 5,077 & NA & NA & NA & NA & NA & NA & NA & NA & NA & NA & NA & NA & NA & NA & 5,118 & 5,077 & 10,195 \\
\hline$\underline{\text { Sub-Saharan Africa }}$ & 7,117 & 6,645 & NA & NA & NA & NA & NA & NA & NA & NA & NA & NA & NA & NA & NA & NA & 7,117 & 6,645 & 13,762 \\
\hline Low- and middle-income countries & 13,106 & 12,658 & NA & NA & NA & NA & NA & NA & NA & NA & NA & NA & NA & NA & NA & NA & 13,106 & 12,658 & 25,765 \\
\hline High-income countries & 1 & 1 & NA & NA & NA & NA & NA & NA & NA & NA & NA & NA & NA & NA & NA & NA & 1 & 1 & 2 \\
\hline WORLD & 13,107 & 12,659 & NA & NA & NA & NA & NA & NA & NA & NA & NA & NA & NA & NA & NA & NA & 13,107 & 12,659 & 25,766 \\
\hline
\end{tabular}

Attributable DALYs (thousands)

\begin{tabular}{|c|c|c|c|c|c|c|c|c|c|c|c|c|c|c|c|c|c|c|c|}
\hline East Asia and Pacific & 369 & 494 & NA & NA & NA & NA & NA & NA & NA & NA & NA & NA & NA & NA & NA & NA & 369 & 494 & 863 \\
\hline Europe and Central Asia & 35 & 31 & NA & NA & NA & NA & NA & NA & NA & NA & NA & NA & NA & NA & NA & NA & 35 & 31 & 66 \\
\hline Latin America and the Caribbean & 218 & 195 & NA & NA & NA & NA & NA & NA & NA & NA & NA & NA & NA & NA & NA & NA & 218 & 195 & 413 \\
\hline Middle East and North Africa & 387 & 366 & NA & NA & NA & NA & NA & NA & NA & NA & NA & NA & NA & NA & NA & NA & 387 & 366 & 752 \\
\hline South Asia & 5,467 & 5,412 & NA & NA & NA & NA & NA & NA & NA & NA & NA & NA & NA & NA & NA & NA & 5,467 & 5,412 & 10,878 \\
\hline Sub-Saharan Africa & 7,592 & 7,065 & NA & NA & NA & NA & NA & NA & NA & NA & NA & NA & NA & NA & NA & NA & 7,592 & 7,065 & 14,657 \\
\hline Low- and middle-income countries & 14,068 & 13,563 & NA & NA & NA & NA & NA & NA & NA & NA & NA & NA & NA & NA & NA & NA & 14,068 & 13,563 & 27,631 \\
\hline High-income countries & 3 & 2 & NA & NA & NA & NA & NA & NA & NA & NA & NA & NA & NA & NA & NA & NA & 3 & 2 & 5 \\
\hline WORLD & 14,071 & 13,565 & NA & NA & NA & NA & NA & NA & NA & NA & NA & NA & NA & NA & NA & NA & 14,071 & 13,565 & 27,636 \\
\hline
\end{tabular}

Source: Authors' calculations.

Note: NA = not applicable 
Table 4A.23

Risk factor: High blood pressure

Disease:

Hypertensive heart disease

\begin{tabular}{|c|c|c|c|c|c|c|c|c|c|c|c|c|c|c|c|c|c|c|c|}
\hline \multirow[b]{2}{*}{ Region } & \multicolumn{2}{|c|}{$0-4$ years } & \multicolumn{2}{|c|}{ 5-14 years } & \multicolumn{2}{|c|}{$15-29$ years } & \multicolumn{2}{|c|}{ 30-44 years } & \multicolumn{2}{|c|}{$45-59$ years } & \multicolumn{2}{|c|}{$60-69$ years } & \multicolumn{2}{|c|}{$70-79$ years } & \multicolumn{2}{|c|}{$80+$ years } & \multicolumn{3}{|c|}{ Total } \\
\hline & Male & Female & Male & Female & Male & Female & Male & Female & Male & Female & Male & Female & Male & Female & Male & Female & Male & Female & All \\
\hline \multicolumn{20}{|l|}{ PAF of Mortality (\%) } \\
\hline East Asia and Pacific & NA & NA & NA & NA & NA & NA & 24 & 17 & 64 & 69 & 79 & 85 & 80 & 86 & 73 & 81 & 73 & 80 & 76 \\
\hline Europe and Central Asia & NA & NA & NA & NA & NA & NA & 63 & 54 & 86 & 90 & 92 & 96 & 92 & 97 & 87 & 94 & 88 & 94 & 91 \\
\hline Latin America and the Caribbean & NA & NA & NA & NA & NA & NA & 49 & 32 & 76 & 75 & 85 & 88 & 86 & 89 & 79 & 84 & 79 & 82 & 81 \\
\hline Middle East and North Africa & NA & NA & NA & NA & NA & NA & 38 & 31 & 72 & 79 & 84 & 91 & 85 & 92 & 79 & 88 & 78 & 86 & 82 \\
\hline South Asia & NA & NA & NA & NA & NA & NA & 29 & 24 & 68 & 62 & 81 & 76 & 82 & 78 & 76 & 71 & 70 & 67 & 69 \\
\hline Sub-Saharan Africa & NA & NA & NA & NA & NA & NA & 56 & 37 & 73 & 70 & 80 & 82 & 81 & 83 & 75 & 77 & 75 & 76 & 76 \\
\hline Low- and middle-income countries & NA & NA & NA & NA & NA & NA & 37 & 28 & 70 & 72 & 82 & 86 & 83 & 88 & 77 & 83 & 76 & 81 & 79 \\
\hline High-income countries & NA & NA & NA & NA & NA & NA & 55 & 21 & 75 & 70 & 85 & 87 & 86 & 90 & 82 & 87 & 82 & 86 & 84 \\
\hline WORLD & NA & NA & NA & NA & NA & NA & 38 & 28 & 71 & 72 & 82 & 86 & 83 & 88 & 78 & 84 & 76 & 82 & 79 \\
\hline
\end{tabular}

PAF of YLL $(\%)$

\begin{tabular}{|c|c|c|c|c|c|c|c|c|c|c|c|c|c|c|c|c|c|c|c|}
\hline East Asia and Pacific & NA & NA & NA & NA & NA & NA & 24 & 17 & 64 & 69 & 79 & 85 & 80 & 86 & 73 & 81 & 69 & 77 & 73 \\
\hline Europe and Central Asia & NA & NA & NA & NA & NA & NA & 63 & 54 & 86 & 90 & 92 & 96 & 92 & 97 & 87 & 94 & 86 & 92 & 89 \\
\hline Latin America and the Caribbean & NA & NA & NA & NA & NA & NA & 49 & 32 & 76 & 75 & 85 & 88 & 86 & 89 & 79 & 84 & 76 & 78 & 77 \\
\hline Middle East and North Africa & NA & NA & NA & NA & NA & NA & 38 & 31 & 72 & 79 & 84 & 91 & 85 & 92 & 79 & 88 & 74 & 82 & 78 \\
\hline South Asia & NA & NA & NA & NA & NA & NA & 29 & 24 & 68 & 62 & 81 & 76 & 82 & 78 & 76 & 71 & 63 & 61 & 62 \\
\hline Sub-Saharan Africa & NA & NA & NA & NA & NA & NA & 56 & 37 & 73 & 70 & 80 & 82 & 81 & 83 & 75 & 77 & 72 & 73 & 72 \\
\hline Low- and middle-income countries & NA & NA & NA & NA & NA & NA & 37 & 28 & 70 & 72 & 82 & 86 & 83 & 88 & 77 & 83 & 71 & 77 & 74 \\
\hline High-income countries & NA & NA & NA & NA & NA & NA & 55 & 21 & 75 & 70 & 85 & 87 & 86 & 90 & 82 & 87 & 79 & 83 & 81 \\
\hline WORLD & NA & NA & NA & NA & NA & NA & 38 & 28 & 71 & 72 & 82 & 86 & 83 & 88 & 78 & 84 & 72 & 78 & 75 \\
\hline
\end{tabular}

PAF of DALYs (\%)

\begin{tabular}{|c|c|c|c|c|c|c|c|c|c|c|c|c|c|c|c|c|c|c|}
\hline East Asia and Pacific & NA & NA & NA & NA & NA & NA & 24 & 17 & 64 & 69 & 79 & 85 & 80 & 86 & 73 & 81 & 69 & 77 \\
\hline Europe and Central Asia & NA & NA & NA & NA & NA & NA & 63 & 54 & 86 & 90 & 92 & 96 & 92 & 97 & 87 & 94 & 86 & 92 \\
\hline Latin America and the Caribbean & NA & NA & NA & NA & NA & NA & 49 & 32 & 76 & 75 & 85 & 88 & 86 & 89 & 79 & 84 & 76 & 78 \\
\hline Middle East and North Africa & NA & NA & NA & NA & NA & NA & 38 & 31 & 72 & 79 & 84 & 91 & 85 & 92 & 79 & 88 & 74 & 82 \\
\hline South Asia & NA & NA & NA & NA & NA & NA & 29 & 24 & 68 & 62 & 81 & 76 & 82 & 78 & 76 & 71 & 64 & 62 \\
\hline Sub-Saharan Africa & NA & NA & NA & NA & NA & NA & 56 & 37 & 73 & 70 & 80 & 82 & 81 & 83 & 75 & 77 & 72 & 73 \\
\hline Low- and middle-income countries & NA & NA & NA & NA & NA & NA & 37 & 28 & 70 & 72 & 82 & 86 & 83 & 88 & 76 & 83 & 72 & 77 \\
\hline High-income countries & NA & NA & NA & NA & NA & NA & 55 & 21 & 75 & 70 & 85 & 87 & 86 & 90 & 82 & 87 & 79 & 84 \\
\hline NORLD & NA & NA & NA & NA & NA & NA & 38 & 28 & 71 & 72 & 82 & 86 & 83 & 88 & 78 & 84 & 73 & 78 \\
\hline
\end{tabular}

Attributable Mortality (thousands

\begin{tabular}{|c|c|c|c|c|c|c|c|c|c|c|c|c|c|c|c|c|c|c|c|}
\hline East Asia and Pacific & NA & NA & NA & NA & NA & NA & 2 & 1 & 18 & 14 & 37 & 29 & 43 & 49 & 21 & 41 & 120 & 134 & 254 \\
\hline Europe and Central Asia & NA & NA & NA & NA & NA & NA & 1 & 1 & 8 & 6 & 12 & 13 & 13 & 22 & 6 & 18 & 41 & 59 & 100 \\
\hline Latin America and the Caribbean & NA & NA & NA & NA & NA & NA & 1 & 1 & 5 & 4 & 7 & 7 & 10 & 12 & 8 & 16 & 31 & 39 & 71 \\
\hline Middle East and North Africa & NA & NA & NA & NA & NA & NA & 1 & 0 & 4 & 4 & 8 & 7 & 11 & 12 & 6 & 8 & 30 & 31 & 61 \\
\hline South Asia & NA & NA & NA & NA & NA & NA & 1 & 0 & 7 & 5 & 10 & 9 & 11 & 9 & 4 & 5 & 33 & 29 & 62 \\
\hline Sub-Saharan Africa & NA & NA & NA & NA & NA & NA & 1 & 1 & 4 & 5 & 5 & 8 & 5 & 12 & 3 & 7 & 18 & 32 & 50 \\
\hline Low- and middle-income countries & NA & NA & NA & NA & NA & NA & 6 & 3 & 46 & 37 & 79 & 73 & 93 & 116 & 49 & 94 & 273 & 324 & 598 \\
\hline High-income countries & NA & NA & NA & NA & NA & NA & 1 & 0 & 5 & 2 & 6 & 5 & 12 & 15 & 16 & 47 & 39 & 69 & 109 \\
\hline WORLD & NA & NA & NA & NA & NA & NA & 7 & 4 & 50 & 40 & 85 & 78 & 105 & 131 & 65 & 141 & 313 & 393 & 706 \\
\hline
\end{tabular}

Attributable YLL (thousands)

\begin{tabular}{|c|c|c|c|c|c|c|c|c|c|c|c|c|c|c|c|c|c|c|c|}
\hline East Asia and Pacific & NA & NA & NA & NA & NA & NA & 38 & 17 & 334 & 270 & 494 & 438 & 381 & 503 & 107 & 215 & 1,354 & 1,443 & 2,797 \\
\hline Europe and Central Asia & NA & NA & NA & NA & NA & NA & 33 & 15 & 143 & 111 & 168 & 190 & 121 & 228 & 29 & 84 & 493 & 629 & 1,121 \\
\hline Latin America and the Caribbean & NA & NA & NA & NA & NA & NA & 22 & 14 & 94 & 90 & 98 & 109 & 86 & 120 & 39 & 73 & 338 & 406 & 744 \\
\hline Middle East and North Africa & NA & NA & NA & NA & NA & NA & 13 & 7 & 83 & 70 & 107 & 107 & 99 & 124 & 28 & 44 & 331 & 351 & 683 \\
\hline South Asia & NA & NA & NA & NA & NA & NA & 23 & 12 & 131 & 108 & 137 & 133 & 94 & 98 & 22 & 26 & 407 & 377 & 785 \\
\hline Sub-Saharan Africa & NA & NA & NA & NA & NA & NA & 23 & 18 & 72 & 97 & 66 & 126 & 48 & 119 & 14 & 37 & 222 & 397 & 619 \\
\hline Low- and middle-income countries & NA & NA & NA & NA & NA & NA & 151 & 83 & 857 & 747 & 1,069 & 1,102 & 830 & 1,191 & 239 & 479 & 3,146 & 3,603 & 6,749 \\
\hline High-income countries & NA & NA & NA & NA & NA & NA & 21 & 4 & 86 & 44 & 86 & 71 & 102 & 148 & 67 & 188 & 361 & 456 & 816 \\
\hline WORLD & NA & NA & NA & NA & NA & NA & 172 & 87 & 943 & 792 & 1,155 & 1,173 & 932 & 1,340 & 306 & 668 & 3,507 & 4,059 & 7,566 \\
\hline \multicolumn{20}{|l|}{ Attributable DALYs (thousands) } \\
\hline East Asia and Pacific & NA & NA & NA & NA & NA & NA & 40 & 18 & 355 & 286 & 532 & 465 & 434 & 552 & 139 & 261 & 1,501 & 1,581 & 3,083 \\
\hline Europe and Central Asia & NA & NA & NA & NA & NA & NA & 34 & 16 & 148 & 115 & 176 & 199 & 131 & 246 & 35 & 101 & 524 & 677 & 1201 \\
\hline Latin America and the Caribbean & NA & NA & NA & NA & NA & NA & 22 & 14 & 98 & 94 & 104 & 115 & 96 & 131 & 49 & 90 & 369 & 444 & 813 \\
\hline Middle East and North Africa & NA & NA & NA & NA & NA & NA & 13 & 7 & 86 & 74 & 112 & 112 & 107 & 132 & 32 & 52 & 350 & 377 & 728 \\
\hline South Asia & NA & NA & NA & NA & NA & NA & 25 & 13 & 142 & 116 & 159 & 151 & 121 & 123 & 36 & 42 & 482 & 444 & 926 \\
\hline Sub-Saharan Africa & NA & NA & NA & NA & NA & NA & 24 & 19 & 76 & 103 & 71 & 135 & 54 & 133 & 18 & 47 & 243 & 438 & 681 \\
\hline Low- and middle-income countries & NA & NA & NA & NA & NA & NA & 159 & 87 & 905 & 787 & 1,153 & 1,177 & 943 & 1,317 & 309 & 594 & 3,470 & 3,962 & 7,432 \\
\hline High-income countries & NA & NA & NA & NA & NA & NA & 21 & 4 & 90 & 46 & 92 & 75 & 116 & 166 & 98 & 280 & 418 & 572 & 990 \\
\hline WORLD & NA & NA & NA & NA & NA & NA & 180 & 91 & 995 & 833 & 1,245 & 1,252 & 1,060 & 1,483 & 407 & 874 & 3,888 & 4,534 & 8,421 \\
\hline
\end{tabular}

Source: Authors' calculations.

Note: $\mathrm{NA}=$ not applicable 
Risk factor:

High blood pressure

Disease:

Ischemic heart disease

\begin{tabular}{|c|c|c|c|c|c|c|c|c|c|c|c|c|c|c|c|c|c|c|c|}
\hline \multirow[b]{2}{*}{ Region } & \multicolumn{2}{|c|}{$0-4$ years } & \multicolumn{2}{|c|}{ 5-14 years } & \multicolumn{2}{|c|}{$15-29$ years } & \multicolumn{2}{|c|}{ 30-44 years } & \multicolumn{2}{|c|}{$45-59$ years } & \multicolumn{2}{|c|}{ 60-69 years } & \multicolumn{2}{|c|}{ 70-79 years } & \multicolumn{2}{|c|}{$80+$ years } & \multicolumn{3}{|c|}{ Total } \\
\hline & Male & Female & Male & Female & Male & Female & Male & Female & Male & Female & Male & Female & Male & Female & Male & Female & Male & Female & All \\
\hline \multicolumn{20}{|l|}{ PAF of Mortality (\%) } \\
\hline East Asia and Pacific & NA & NA & NA & NA & NA & NA & 11 & 7 & 34 & 37 & 45 & 51 & 43 & 50 & 35 & 41 & 38 & 44 & 41 \\
\hline Europe and Central Asia & NA & NA & NA & NA & NA & NA & 34 & 27 & 55 & 60 & 62 & 72 & 58 & 70 & 50 & 61 & 56 & 65 & 61 \\
\hline Latin America and the Caribbean & NA & NA & NA & NA & NA & NA & 24 & 15 & 45 & 44 & 52 & 56 & 50 & 55 & 41 & 45 & 46 & 49 & 47 \\
\hline Middle East and North Africa & NA & NA & NA & NA & NA & NA & 18 & 15 & 41 & 48 & 51 & 61 & 49 & 59 & 41 & 50 & 44 & 54 & 48 \\
\hline South Asia & NA & NA & NA & NA & NA & NA & 13 & 10 & 36 & 32 & 47 & 43 & 45 & 41 & 37 & 34 & 40 & 37 & 39 \\
\hline Sub-Saharan Africa & NA & NA & NA & NA & NA & NA & 29 & 17 & 42 & 39 & 47 & 48 & 44 & 47 & 37 & 39 & 43 & 44 & 43 \\
\hline Low- and middle-income countries & NA & NA & NA & NA & NA & NA & 20 & 12 & 42 & 40 & 51 & 53 & 49 & 55 & 41 & 50 & 45 & 49 & 47 \\
\hline High-income countries & NA & NA & NA & NA & NA & NA & 28 & 9 & 44 & 40 & 53 & 55 & 51 & 56 & 44 & 49 & 47 & 51 & 49 \\
\hline WORLD & NA & NA & NA & NA & NA & NA & 21 & 12 & 42 & 40 & 52 & 53 & 49 & 55 & 42 & 49 & 45 & 50 & 47 \\
\hline
\end{tabular}

PAF of YLL $(\%)$

\begin{tabular}{|c|c|c|c|c|c|c|c|c|c|c|c|c|c|c|c|c|c|c|}
\hline \multicolumn{19}{|l|}{ East Asia and Pacific } \\
\hline Europe and Central Asia & NA & NA & NA & NA & NA & NA & 34 & 27 & 55 & 60 & 62 & 72 & 58 & 70 & 50 & 61 & 55 & 66 \\
\hline Latin America and the Caribbean & NA & NA & NA & NA & NA & NA & 24 & 15 & 45 & 44 & 52 & 56 & 50 & 55 & 41 & 45 & 45 & 48 \\
\hline Middle East and North Africa & NA & NA & NA & NA & NA & NA & 18 & 15 & 41 & 48 & 51 & 61 & 49 & 59 & 41 & 50 & 42 & 52 \\
\hline South Asia & NA & NA & NA & NA & NA & NA & 13 & 10 & 36 & 32 & 47 & 43 & 45 & 41 & 37 & 34 & 38 & 35 \\
\hline Sub-Saharan Africa & NA & NA & NA & NA & NA & NA & 29 & 17 & 42 & 39 & 47 & 48 & 44 & 47 & 37 & 39 & 43 & 43 \\
\hline Low- and middle-income countries & NA & NA & NA & NA & NA & NA & 20 & 12 & 42 & 40 & 51 & 53 & 49 & 54 & 40 & 49 & 43 & 47 \\
\hline High-income countries & NA & NA & NA & NA & NA & NA & 28 & 9 & 44 & 40 & 53 & 55 & 51 & 56 & 44 & 49 & 47 & 51 \\
\hline WORLD & NA & NA & NA & NA & NA & NA & 21 & 12 & 42 & 40 & 52 & 53 & 49 & 55 & 42 & 49 & 44 & 47 \\
\hline
\end{tabular}

\section{PAF of DALYs $(\%)$}

\begin{tabular}{|c|c|c|c|c|c|c|c|c|c|c|c|c|c|c|c|c|c|c|}
\hline East Asia and Pacific & NA & NA & NA & NA & NA & NA & 11 & 7 & 34 & 37 & 45 & 51 & 43 & 50 & 35 & 41 & 34 & 41 \\
\hline Europe and Central Asia & NA & NA & NA & NA & NA & NA & 34 & 27 & 55 & 60 & 62 & 72 & 58 & 70 & 50 & 61 & 55 & 66 \\
\hline Latin America and the Caribbean & NA & NA & NA & NA & NA & NA & 24 & 15 & 45 & 44 & 52 & 56 & 50 & 55 & 41 & 45 & 44 & 47 \\
\hline Middle East and North Africa & NA & NA & NA & NA & NA & NA & 18 & 15 & 41 & 48 & 51 & 61 & 49 & 59 & 41 & 50 & 41 & 51 \\
\hline South Asia & NA & NA & NA & NA & NA & NA & 13 & 10 & 36 & 32 & 47 & 43 & 45 & 41 & 37 & 34 & 37 & 34 \\
\hline Sub-Saharan Africa & NA & NA & NA & NA & NA & NA & 29 & 17 & 42 & 39 & 47 & 48 & 44 & 47 & 37 & 39 & 42 & 42 \\
\hline Low- and middle-income countries & NA & NA & NA & NA & NA & NA & 20 & 12 & 42 & 40 & 51 & 53 & 49 & 54 & 40 & 49 & 42 & 46 \\
\hline High-income countries & NA & NA & NA & NA & NA & NA & 28 & 9 & 44 & 40 & 53 & 55 & 51 & 56 & 44 & 49 & 47 & 50 \\
\hline WORLD & NA & NA & NA & NA & NA & NA & 20 & 12 & 42 & 40 & 51 & 53 & 49 & 55 & 42 & 49 & 43 & 46 \\
\hline
\end{tabular}

Attributable Mortality (thousands)

\begin{tabular}{|c|c|c|c|c|c|c|c|c|c|c|c|c|c|c|c|c|c|c|c|}
\hline East Asia and Pacific & NA & NA & NA & NA & NA & NA & 3 & 1 & 35 & 21 & 66 & 57 & 81 & 91 & 38 & 77 & 223 & 247 & 471 \\
\hline Europe and Central Asia & NA & NA & NA & NA & NA & NA & 13 & 2 & 84 & 26 & 141 & 92 & 155 & 218 & 65 & 227 & 459 & 565 & 1,024 \\
\hline Latin America and the Caribbean & NA & NA & NA & NA & NA & NA & 2 & 1 & 18 & 8 & 25 & 16 & 29 & 24 & 18 & 27 & 93 & 76 & 169 \\
\hline Middle East and North Africa & NA & NA & NA & NA & NA & NA & 2 & 1 & 17 & 9 & 25 & 19 & 29 & 28 & 11 & 16 & 83 & 72 & 155 \\
\hline South Asia & NA & NA & NA & NA & NA & NA & 6 & 3 & 89 & 40 & 138 & 99 & 133 & 111 & 50 & 42 & 415 & 296 & 711 \\
\hline Sub-Saharan Africa & NA & NA & NA & NA & NA & NA & 2 & 1 & 17 & 10 & 24 & 22 & 26 & 26 & 8 & 12 & 77 & 71 & 148 \\
\hline Low- and middle-income countries & NA & NA & NA & NA & NA & NA & 29 & 8 & 258 & 115 & 419 & 307 & 454 & 497 & 190 & 401 & 1,350 & 1,328 & 2,678 \\
\hline High-income countries & NA & NA & NA & NA & NA & NA & 5 & 0 & 39 & 9 & 69 & 29 & 118 & 88 & 110 & 202 & 340 & 328 & 668 \\
\hline WORLD & NA & NA & NA & NA & NA & NA & 33 & 9 & 297 & 124 & 488 & 336 & 571 & 585 & 300 & 603 & 1,690 & 1,657 & 3,346 \\
\hline
\end{tabular}

Attributable YLL (thousands)

\begin{tabular}{|c|c|c|c|c|c|c|c|c|c|c|c|c|c|c|c|c|c|c|c|}
\hline East Asia and Pacific & NA & NA & NA & NA & NA & NA & 77 & 29 & 652 & 427 & 890 & 859 & 721 & 927 & 186 & 382 & 2,527 & 2,624 & 5,151 \\
\hline Europe and Central Asia & NA & NA & NA & NA & NA & NA & 299 & 47 & 1,591 & 519 & 1,911 & 1,388 & 1,405 & 2,227 & 296 & 1,049 & 5,502 & 5,229 & 10,731 \\
\hline Latin America and the Caribbean & NA & NA & NA & NA & NA & NA & 52 & 14 & 328 & 156 & 342 & 248 & 262 & 250 & 83 & 125 & 1,067 & 794 & 1,861 \\
\hline Middle East and North Africa & NA & NA & NA & NA & NA & NA & 45 & 14 & 312 & 183 & 337 & 291 & 256 & 286 & 53 & 84 & 1,003 & 858 & 1,861 \\
\hline South Asia & NA & NA & NA & NA & NA & NA & 149 & 83 & 1,652 & 797 & 1,862 & 1,497 & 1,193 & 1,160 & 244 & 226 & 5,100 & 3,764 & 8,864 \\
\hline Sub-Saharan Africa & NA & NA & NA & NA & NA & NA & 49 & 13 & 308 & 197 & 332 & 339 & 233 & 269 & 40 & 66 & 962 & 885 & 1,846 \\
\hline Low- and middle-income countries & NA & NA & NA & NA & NA & NA & 671 & 200 & 4,842 & 2,280 & 5,673 & 4,622 & 4,071 & 5,120 & 903 & 1,933 & 16,160 & 14,154 & 30,315 \\
\hline High-income countries & NA & NA & NA & NA & NA & NA & 106 & 8 & 723 & 177 & 921 & 432 & 1,030 & 876 & 480 & 830 & 3,261 & 2,324 & 5,585 \\
\hline WORLD & NA & NA & NA & NA & NA & NA & 777 & 209 & 5,566 & 2,457 & 6,594 & 5,054 & 5,101 & 5,996 & 1,383 & 2,763 & 19,421 & 16,478 & 35,899 \\
\hline \multicolumn{20}{|l|}{ Attributable DALYs (thousands) } \\
\hline East Asia and Pacific & NA & NA & NA & NA & NA & NA & 85 & 32 & 696 & 459 & 934 & 901 & 737 & 945 & 189 & 387 & 2,642 & 2,723 & 5,365 \\
\hline Europe and Central Asia & NA & NA & NA & NA & NA & NA & 321 & 53 & 1,652 & 557 & 1,972 & 1,453 & 1,429 & 2,269 & 300 & 1,062 & 5,675 & 5,395 & 11,070 \\
\hline Latin America and the Caribbean & NA & NA & NA & NA & NA & NA & 59 & 17 & 350 & 170 & 364 & 263 & 270 & 257 & 85 & 128 & 1,127 & 835 & 1,962 \\
\hline Middle East and North Africa & NA & NA & NA & NA & NA & NA & 50 & 16 & 329 & 196 & 353 & 304 & 261 & 290 & 54 & 85 & 1,048 & 891 & 1,939 \\
\hline South Asia & NA & NA & NA & NA & NA & NA & 170 & 95 & 1,753 & 860 & 1,960 & 1,577 & 1,223 & 1,186 & 248 & 230 & 5,354 & 3,948 & 9,302 \\
\hline Sub-Saharan Africa & NA & NA & NA & NA & NA & NA & 54 & 16 & 327 & 210 & 348 & 357 & 239 & 275 & 41 & 67 & 1,010 & 924 & 1,934 \\
\hline Low- and middle-income countries & NA & NA & NA & NA & NA & NA & 740 & 229 & 5,108 & 2,451 & 5,932 & 4,854 & 4,159 & 5,221 & 917 & 1,960 & 16,856 & 14,716 & 31,572 \\
\hline High-income countries & NA & NA & NA & NA & NA & NA & 121 & 11 & 797 & 213 & 991 & 485 & 1,084 & 934 & 497 & 861 & 3,490 & 2,504 & 5,994 \\
\hline ORLD & NA & NA & $\mathrm{N}$ & NA & NA & NA & 861 & 240 & 5,905 & 2,664 & 6,923 & 5,339 & 5,243 & 6,156 & 1,414 & 2,821 & 20,346 & 17,220 & 37,566 \\
\hline
\end{tabular}

Source: Authors' calculations.

Note: $\mathrm{NA}=$ not applicable 
Risk factor: High blood pressure

Disease:

Cerebrovascular disease

\begin{tabular}{|c|c|c|c|c|c|c|c|c|c|c|c|c|c|c|c|c|c|c|c|}
\hline \multirow[b]{2}{*}{ Region } & \multicolumn{2}{|c|}{$0-4$ years } & \multicolumn{2}{|c|}{ 5-14 years } & \multicolumn{2}{|c|}{$15-29$ years } & \multicolumn{2}{|c|}{ 30-44 years } & \multicolumn{2}{|c|}{$45-59$ years } & \multicolumn{2}{|c|}{$60-69$ years } & \multicolumn{2}{|c|}{$70-79$ years } & \multicolumn{2}{|c|}{$80+$ years } & \multicolumn{3}{|c|}{ Total } \\
\hline & Male & Female & Male & Female & Male & Female & Male & Female & Male & Female & Male & Female & Male & Female & Male & Female & Male & Female & All \\
\hline \multicolumn{20}{|l|}{ PAF of Mortality (\%) } \\
\hline East Asia and Pacific & NA & NA & NA & NA & NA & NA & 15 & 10 & 45 & 49 & 56 & 63 & 53 & 61 & 35 & 41 & 48 & 52 & 50 \\
\hline Europe and Central Asia & NA & NA & NA & NA & NA & NA & 45 & 36 & 68 & 73 & 74 & 83 & 70 & 80 & 50 & 61 & 65 & 71 & 69 \\
\hline Latin America and the Caribbean & NA & NA & NA & NA & NA & NA & 33 & 21 & 57 & 56 & 64 & 68 & 61 & 66 & 41 & 45 & 53 & 54 & 54 \\
\hline Middle East and North Africa & NA & NA & NA & NA & NA & NA & 25 & 20 & 52 & 60 & 62 & 73 & 60 & 70 & 41 & 50 & 49 & 59 & 54 \\
\hline South Asia & NA & NA & NA & NA & NA & NA & 18 & 15 & 48 & 43 & 59 & 54 & 56 & 52 & 37 & 34 & 51 & 46 & 49 \\
\hline Sub-Saharan Africa & NA & NA & NA & NA & NA & NA & 39 & 24 & 54 & 50 & 59 & 60 & 55 & 58 & 37 & 39 & 50 & 51 & 51 \\
\hline Low- and middle-income countries & NA & NA & NA & NA & NA & NA & 27 & 19 & 51 & 52 & 61 & 65 & 58 & 65 & 39 & 47 & 52 & 56 & 54 \\
\hline High-income countries & NA & NA & NA & NA & NA & NA & 38 & 13 & 57 & 51 & 64 & 67 & 62 & 67 & 44 & 49 & 53 & 54 & 54 \\
\hline WORLD & NA & NA & NA & NA & NA & NA & 28 & 19 & 51 & 52 & 61 & 65 & 58 & 65 & 40 & 48 & 52 & 56 & 54 \\
\hline
\end{tabular}

PAF of YLL (\%)

\begin{tabular}{|c|c|c|c|c|c|c|c|c|c|c|c|c|c|c|c|c|c|c|c|}
\hline East Asia and Pacific & NA & NA & NA & NA & NA & NA & 15 & 10 & 45 & 49 & 56 & 63 & 53 & 61 & 35 & 41 & 47 & 53 & 50 \\
\hline Europe and Central Asia & NA & NA & NA & NA & NA & NA & 45 & 36 & 68 & 73 & 74 & 83 & 70 & 80 & 50 & 61 & 66 & 74 & 70 \\
\hline Latin America and the Caribbean & NA & NA & NA & NA & NA & NA & 33 & 21 & 57 & 56 & 64 & 68 & 61 & 66 & 41 & 45 & 53 & 53 & 53 \\
\hline Middle East and North Africa & NA & NA & NA & NA & NA & NA & 25 & 20 & 52 & 60 & 62 & 73 & 60 & 70 & 41 & 50 & 44 & 56 & 50 \\
\hline South Asia & NA & NA & NA & NA & NA & NA & 18 & 15 & 48 & 43 & 59 & 54 & 56 & 52 & 37 & 34 & 50 & 47 & 48 \\
\hline Sub-Saharan Africa & NA & NA & NA & NA & NA & NA & 39 & 24 & 54 & 50 & 59 & 60 & 55 & 58 & 37 & 39 & 49 & 50 & 49 \\
\hline Low- and middle-income countries & NA & NA & NA & NA & NA & NA & 27 & 19 & 51 & 52 & 61 & 65 & 58 & 64 & 39 & 47 & 52 & 56 & 54 \\
\hline High-income countries & NA & NA & NA & NA & NA & NA & 38 & 13 & 57 & 51 & 64 & 67 & 62 & 67 & 44 & 49 & 55 & 56 & 56 \\
\hline WORLD & NA & NA & NA & NA & NA & NA & 28 & 19 & 52 & 52 & 61 & 65 & 58 & 65 & 40 & 47 & 52 & 56 & 54 \\
\hline
\end{tabular}

\section{PAF of DALYs $(\%)$}

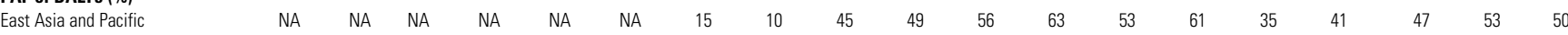

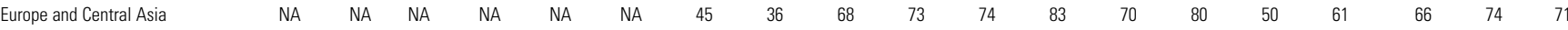

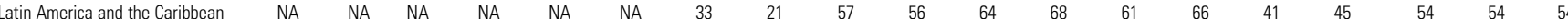

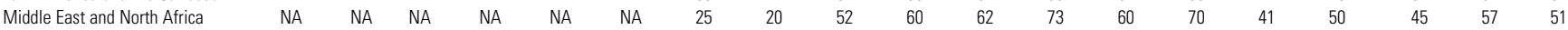

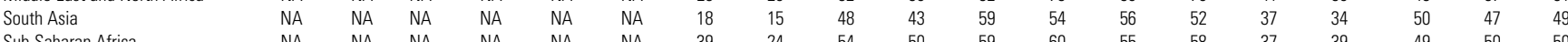

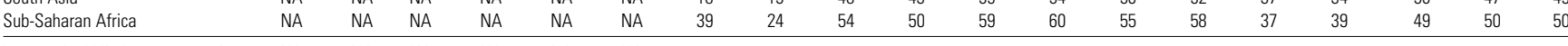

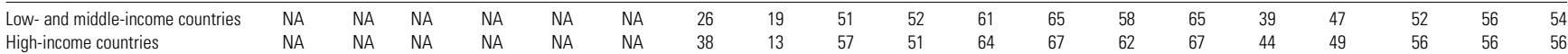

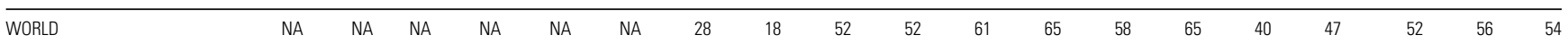

Attributable Mortality (thousands)

\begin{tabular}{|c|c|c|c|c|c|c|c|c|c|c|c|c|c|c|c|c|c|c|c|}
\hline East Asia and Pacific & NA & NA & NA & NA & NA & NA & 4 & 2 & 62 & 44 & 139 & 100 & 187 & 211 & 66 & 137 & 458 & 493 & 951 \\
\hline Europe and Central Asia & NA & NA & NA & NA & NA & NA & 6 & 3 & 37 & 26 & 81 & 76 & 101 & 180 & 40 & 160 & 264 & 444 & 709 \\
\hline Latin America and the Caribbean & NA & NA & NA & NA & NA & NA & 2 & 1 & 12 & 10 & 17 & 15 & 23 & 25 & 14 & 24 & 69 & 75 & 144 \\
\hline Middle East and North Africa & NA & NA & NA & NA & NA & NA & 1 & 0 & 5 & 4 & 9 & 9 & 13 & 16 & 5 & 9 & 32 & 38 & 71 \\
\hline South Asia & NA & NA & NA & NA & NA & NA & 2 & 1 & 36 & 22 & 82 & 68 & 89 & 90 & 27 & 31 & 237 & 212 & 449 \\
\hline Sub-Saharan Africa & NA & NA & NA & NA & NA & NA & 4 & 2 & 15 & 18 & 21 & 31 & 24 & 39 & 8 & 16 & 73 & 107 & 179 \\
\hline Low- and middle-income countries & NA & NA & NA & NA & NA & NA & 18 & 9 & 167 & 124 & 350 & 300 & 438 & 560 & 160 & 377 & 1,133 & 1,369 & 2,502 \\
\hline High-income countries & NA & NA & NA & NA & NA & NA & 2 & 1 & 14 & 8 & 28 & 19 & 63 & 68 & 64 & 151 & 172 & 247 & 418 \\
\hline WORLD & NA & NA & NA & NA & NA & NA & 21 & 9 & 182 & 132 & 378 & 319 & 501 & 628 & 224 & 528 & 1,305 & 1,616 & 2,921 \\
\hline
\end{tabular}

\section{Attributable YLL (thousands)}

\begin{tabular}{|c|c|c|c|c|c|c|c|c|c|c|c|c|c|c|c|c|c|c|c|}
\hline East Asia and Pacific & NA & NA & NA & NA & NA & NA & 89 & 39 & 1,149 & 871 & 1,868 & 1,502 & 1,665 & 2,152 & 330 & 701 & 5,100 & 5,264 & 10,364 \\
\hline Europe and Central Asia & NA & NA & NA & NA & NA & NA & 136 & 65 & 698 & 511 & 1,088 & 1,139 & 911 & 1,843 & 182 & 749 & 3,015 & 4,306 & 7,321 \\
\hline Latin America and the Caribbean & NA & NA & NA & NA & NA & NA & 52 & 33 & 231 & 206 & 233 & 228 & 205 & 250 & 64 & 113 & 785 & 830 & 1,615 \\
\hline Middle East and North Africa & NA & NA & NA & NA & NA & NA & 15 & 10 & 85 & 89 & 120 & 140 & 118 & 160 & 25 & 46 & 362 & 445 & 808 \\
\hline South Asia & NA & NA & NA & NA & NA & NA & 42 & 21 & 677 & 436 & 1,113 & 1,022 & 798 & 942 & 134 & 167 & 2,762 & 2,588 & 5,350 \\
\hline Sub-Saharan Africa & NA & NA & NA & NA & NA & NA & 100 & 48 & 288 & 366 & 282 & 475 & 214 & 402 & 41 & 88 & 926 & 1,379 & 2,305 \\
\hline Low- and middle-income countries & NA & NA & NA & NA & NA & NA & 433 & 216 & 3,128 & 2,479 & 4,704 & 4,506 & 3,910 & 5,749 & 776 & 1,863 & 12,951 & 14,812 & 27,763 \\
\hline High-income countries & NA & NA & NA & NA & NA & NA & 56 & 14 & 269 & 161 & 379 & 285 & 544 & 675 & 275 & 621 & 1,523 & 1,756 & 3,279 \\
\hline WORLD & NA & NA & NA & NA & NA & NA & 489 & 230 & 3,397 & 2,640 & 5,083 & 4,791 & 4,454 & 6,424 & 1,051 & 2,484 & 14,474 & 16,568 & 31,042 \\
\hline \multicolumn{20}{|l|}{ Attributable DALYs (thousands) } \\
\hline East Asia and Pacific & NA & NA & NA & NA & NA & NA & 124 & 55 & 1,559 & 1,178 & 2,451 & 1,938 & 1,987 & 2,532 & 363 & 769 & 6,483 & 6,471 & 12,955 \\
\hline Europe and Central Asia & NA & NA & NA & NA & NA & NA & 168 & 89 & 870 & 668 & 1,369 & 1,466 & 1,093 & 2,153 & 202 & 817 & 3,702 & 5,193 & 8,895 \\
\hline Latin America and the Caribbean & NA & NA & NA & NA & NA & NA & 71 & 49 & 321 & 285 & 321 & 308 & 255 & 302 & 72 & 127 & 1,041 & 1,071 & 2,112 \\
\hline Middle East and North Africa & NA & NA & NA & NA & NA & NA & 21 & 13 & 113 & 115 & 154 & 173 & 140 & 183 & 28 & 50 & 456 & 534 & 990 \\
\hline South Asia & NA & NA & NA & NA & NA & NA & 52 & 26 & 844 & 538 & 1,379 & 1,239 & 922 & 1,068 & 146 & 182 & 3,343 & 3,054 & 6,397 \\
\hline Sub-Saharan Africa & NA & NA & NA & NA & NA & NA & 106 & 52 & 321 & 414 & 318 & 536 & 233 & 435 & 43 & 93 & 1,021 & 1,530 & 2,551 \\
\hline Low- and middle-income countries & NA & NA & NA & NA & NA & NA & 542 & 285 & 4,027 & 3,199 & 5,993 & 5,660 & 4,630 & 6,673 & 854 & 2,038 & 16,046 & 17,854 & 33,899 \\
\hline High-income countries & NA & NA & NA & NA & NA & NA & 107 & 28 & 530 & 332 & 712 & 544 & 843 & 1,038 & 350 & 782 & 2,542 & 2,724 & 5,265 \\
\hline ORLD & NA & NA & NA & NA & NA & NA & 649 & 312 & 4,557 & 3,531 & 6,705 & 6,204 & 5,473 & 7,711 & 1,204 & 2,820 & 18,587 & 20,577 & 39,164 \\
\hline
\end{tabular}

Source: Authors' calculations.

Note: NA = not applicable 


\begin{tabular}{|c|c|c|c|c|c|c|c|c|c|c|c|c|c|c|c|c|c|c|c|}
\hline \multirow[b]{2}{*}{ Region } & \multicolumn{2}{|c|}{$0-4$ years } & \multicolumn{2}{|c|}{ 5-14 years } & \multicolumn{2}{|c|}{$15-29$ years } & \multicolumn{2}{|c|}{ 30-44 years } & \multicolumn{2}{|c|}{$45-59$ years } & \multicolumn{2}{|c|}{$60-69$ years } & \multicolumn{2}{|c|}{ 70-79 years } & \multicolumn{2}{|c|}{$80+$ years } & \multicolumn{3}{|c|}{ Total } \\
\hline & Male & Female & Male & Female & Male & Female & Male & Female & Male & Female & Male & Female & Male & Female & Male & Female & Male & Female & All \\
\hline \multicolumn{20}{|l|}{ PAF of Mortality (\%) } \\
\hline East Asia and Pacific & NA & NA & NA & NA & NA & NA & 7 & 4 & 22 & 24 & 30 & 36 & 24 & 29 & 19 & 23 & 21 & 25 & 23 \\
\hline Europe and Central Asia & NA & NA & NA & NA & NA & NA & 24 & 18 & 40 & 45 & 46 & 57 & 37 & 48 & 30 & 39 & 35 & 43 & 39 \\
\hline Latin America and the Caribbean & NA & NA & NA & NA & NA & NA & 16 & 9 & 31 & 30 & 36 & 40 & 29 & 33 & 23 & 26 & 25 & 28 & 27 \\
\hline Middle East and North Africa & NA & NA & NA & NA & NA & NA & 12 & 9 & 27 & 33 & 35 & 45 & 28 & 37 & 23 & 30 & 24 & 28 & 27 \\
\hline South Asia & NA & NA & NA & NA & NA & NA & 8 & 6 & 24 & 20 & 32 & 28 & 25 & 23 & 20 & 18 & 21 & 19 & 20 \\
\hline Sub-Saharan Africa & NA & NA & NA & NA & NA & NA & 20 & 11 & 29 & 25 & 32 & 33 & 25 & 27 & 20 & 22 & 23 & 23 & 23 \\
\hline Low- and middle-income countries & NA & NA & NA & NA & NA & NA & 15 & 9 & 28 & 27 & 35 & 37 & 28 & 32 & 23 & 28 & 25 & 28 & 27 \\
\hline High-income countries & NA & NA & NA & NA & NA & NA & 20 & 6 & 30 & 26 & 37 & 39 & 30 & 34 & 25 & 29 & 28 & 30 & 29 \\
\hline WORLD & NA & NA & NA & NA & NA & NA & 16 & 9 & 28 & 27 & 36 & 37 & 29 & 32 & 24 & 29 & 26 & 29 & 28 \\
\hline
\end{tabular}

\section{PAF of YLL $(\%)$}

\begin{tabular}{|c|c|c|c|c|c|c|c|c|c|c|c|c|c|c|c|c|c|c|}
\hline \multirow{2}{*}{$\begin{array}{l}\text { East Asia and Pacific } \\
\text { Europe and Central Asia }\end{array}$} & NA & NA & NA & NA & NA & NA & 7 & 4 & 22 & 24 & 30 & 36 & 24 & 29 & 19 & 23 & 19 & 24 \\
\hline & NA & NA & NA & NA & NA & NA & 24 & 18 & 40 & 45 & 46 & 57 & 37 & 48 & 30 & 39 & 34 & 43 \\
\hline Latin America and the Caribbean & NA & NA & NA & NA & NA & NA & 16 & 9 & 31 & 30 & 36 & 40 & 29 & 33 & 23 & 26 & 23 & 26 \\
\hline Middle East and North Africa & NA & NA & NA & NA & NA & NA & 12 & 9 & 27 & 33 & 35 & 45 & 28 & 37 & 23 & 30 & 21 & 22 \\
\hline South Asia & NA & NA & NA & NA & NA & NA & 8 & 6 & 24 & 20 & 32 & 28 & 25 & 23 & 20 & 18 & 18 & 17 \\
\hline Sub-Saharan Africa & NA & NA & NA & NA & NA & NA & 20 & 11 & 29 & 25 & 32 & 33 & 25 & 27 & 20 & 22 & 22 & 21 \\
\hline Low- and middle-income countries & NA & NA & NA & NA & NA & NA & 15 & 9 & 28 & 27 & 35 & 37 & 28 & 32 & 23 & 28 & 23 & 25 \\
\hline High-income countries & NA & NA & NA & NA & NA & NA & 20 & 6 & 30 & 26 & 37 & 39 & 30 & 34 & 25 & 29 & 28 & 30 \\
\hline WORLD & NA & NA & NA & NA & NA & NA & 16 & 9 & 28 & 27 & 36 & 37 & 29 & 32 & 24 & 28 & 24 & 26 \\
\hline
\end{tabular}

\section{PAF of DALYs $(\%)$}

East Asia and Pacific

Europe and Central Asia

Latin America and the Caribbe

Middle East and North Africa

South Asia

Sub-Saharan Africa

Low- and middle-income countries

High-income countries

$\begin{array}{llllll}\text { NA } & \text { NA } & \text { NA } & \text { NA } & \text { NA } & \text { NA } \\ \text { NA } & \text { NA } & \text { NA } & \text { NA } & \text { NA } & \text { NA } \\ \text { NA } & \text { NA } & \text { NA } & \text { NA } & \text { NA } & \text { NA } \\ \text { NA } & \text { NA } & \text { NA } & \text { NA } & \text { NA } & \text { NA } \\ \text { NA } & \text { NA } & \text { NA } & \text { NA } & \text { NA } & \text { NA } \\ \text { NA } & \text { NA } & \text { NA } & \text { NA } & \text { NA } & \text { NA } \\ \text { NA } & \text { NA } & \text { NA } & \text { NA } & \text { NA } & \text { NA } \\ \text { NA } & \text { NA } & \text { NA } & \text { NA } & \text { NA } & \text { NA }\end{array}$

$\begin{array}{lr}\text { NA } & 7 \\ \text { NA } & 24\end{array}$

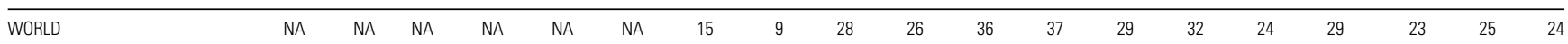

Attributable Mortality (thousands)

East Asia and Pacific

NA NA NA NA NA NA

NA NA NA NA NA NA

Middle East and North Africa

South Asia

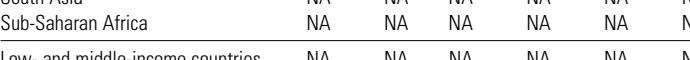

NA NA NA NA NA NA

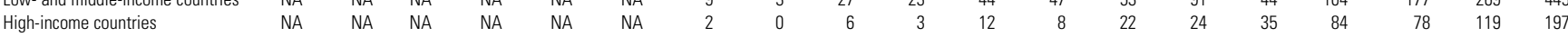

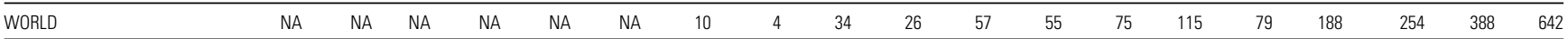

Attributable YLL (thousands)

East Asia and Pacific

Europe and Central Asia

Latin America and the Caribbea

Middle East and North Africa

South Asia

Sub-Saharan Africa

Low- and middle-income countries

High-income countr

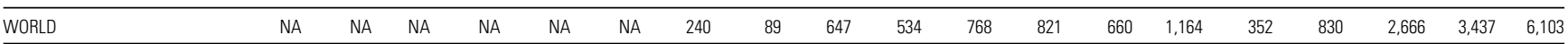

Attributable DALYs (thousands)

East Asia and Pacific

Europe and Central Asia

Latin America and the Caribbean

Middle East and North Africa

South Asia

Sub-Saharan Africa

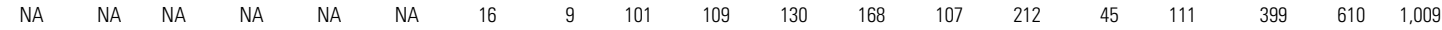

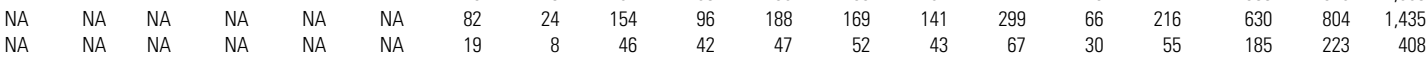

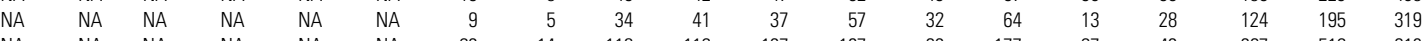

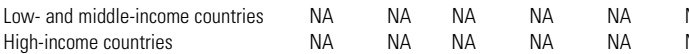

WORLD

NA NA NA NA

$\begin{array}{lllllll}\text { NA } & \text { NA } & 287 & 113 & 734 & 597 & 868\end{array}$

Source: Authors' calculations.

Note: $\mathrm{NA}=$ not applicable

\begin{tabular}{|c|c|c|c|c|c|c|c|c|c|c|c|c|c|c|c|c|c|c|}
\hline NA & NA & NA & NA & NA & NA & 23 & 12 & 108 & 115 & 133 & 171 & 110 & 216 & 46 & 113 & 419 & 626 & 1,045 \\
\hline NA & NA & NA & NA & NA & NA & 91 & 31 & 174 & 104 & 214 & 182 & 166 & 328 & 78 & 242 & 722 & 887 & 1,609 \\
\hline NA & NA & NA & NA & NA & NA & 22 & 9 & 50 & 44 & 52 & 55 & 48 & 71 & 33 & 58 & 205 & 237 & 442 \\
\hline NA & NA & NA & NA & NA & NA & 11 & 6 & 39 & 47 & 43 & 61 & 35 & 68 & 14 & 29 & 141 & 211 & 352 \\
\hline NA & NA & NA & NA & NA & NA & 34 & 20 & 147 & 138 & 168 & 188 & 113 & 194 & 32 & 48 & 494 & 588 & 1,082 \\
\hline NA & NA & NA & NA & NA & NA & 57 & 25 & 78 & 79 & 65 & 94 & 53 & 105 & 26 & 46 & 279 & 350 & 630 \\
\hline NA & NA & NA & NA & NA & NA & 238 & 104 & 595 & 527 & 674 & 751 & 525 & 982 & 229 & 536 & 2,260 & 2,901 & 5,161 \\
\hline NA & NA & NA & NA & NA & NA & 49 & 9 & 140 & 70 & 194 & 129 & 227 & 269 & 175 & 375 & 785 & 852 & 1,638 \\
\hline NA & NA & NA & NA & NA & NA & 287 & 113 & 734 & 597 & 868 & 880 & 752 & 1,251 & 404 & 912 & 3,045 & 3,753 & 6,798 \\
\hline
\end{tabular}


Table 4A.27

Risk factor:

High blood pressure

Disease:

All causes

\begin{tabular}{|c|c|c|c|c|c|c|c|c|c|c|c|c|c|c|c|c|c|c|c|}
\hline \multirow[b]{2}{*}{ Region } & \multicolumn{2}{|c|}{$0-4$ years } & \multicolumn{2}{|c|}{ 5-14 years } & \multicolumn{2}{|c|}{$15-29$ years } & \multicolumn{2}{|c|}{ 30-44 years } & \multicolumn{2}{|c|}{ 45-59 years } & \multicolumn{2}{|c|}{ 60-69 years } & \multicolumn{2}{|c|}{ 70-79 years } & \multicolumn{2}{|c|}{$80+$ years } & \multicolumn{3}{|c|}{ Total } \\
\hline & Male & Female & Male & Female & Male & Female & Male & Female & Male & Female & Male & Female & Male & Female & Male & Female & Male & Female & All \\
\hline \multicolumn{20}{|l|}{ PAF of Mortality (\%) } \\
\hline East Asia and Pacific & NA & NA & NA & NA & NA & NA & 1 & 1 & 10 & 11 & 18 & 21 & 20 & 24 & 15 & 19 & 12 & 15 & 14 \\
\hline Europe and Central Asia & NA & NA & NA & NA & NA & NA & 7 & 6 & 22 & 24 & 35 & 44 & 40 & 53 & 36 & 50 & 27 & 43 & 35 \\
\hline Latin America and the Caribbean & NA & NA & NA & NA & NA & NA & 3 & 3 & 13 & 13 & 19 & 20 & 20 & 23 & 16 & 20 & 12 & 15 & 13 \\
\hline Middle East and North Africa & NA & NA & NA & NA & NA & NA & 4 & 3 & 17 & 19 & 26 & 32 & 27 & 35 & 23 & 31 & 15 & 19 & 16 \\
\hline South Asia & NA & NA & NA & NA & NA & NA & 1 & 1 & 12 & 10 & 22 & 21 & 22 & 21 & 16 & 15 & 10 & 9 & 10 \\
\hline Sub-Saharan Africa & NA & NA & NA & NA & NA & NA & 1 & 1 & 6 & 8 & 13 & 20 & 17 & 23 & 14 & 19 & 3 & 5 & 4 \\
\hline Low- and middle-income countries & NA & NA & NA & NA & NA & NA & 2 & 1 & 12 & 12 & 22 & 25 & 24 & 29 & 19 & 26 & 11 & 14 & 13 \\
\hline High-income countries & NA & NA & NA & NA & NA & NA & 5 & 1 & 12 & 8 & 17 & 15 & 18 & 21 & 18 & 23 & 16 & 20 & 18 \\
\hline WORLD & NA & NA & NA & NA & NA & NA & 2 & 1 & 12 & 11 & 21 & 23 & 23 & 28 & 18 & 25 & 12 & 15 & 14 \\
\hline
\end{tabular}

\section{PAF of YLL (\%)}

East Asia and Pacific

Europe and Central Asia

Latin America and the Caribbea

Middle East and North Africa

South Asia

Sub-Saharan Africa

$\begin{array}{llllll}\text { NA } & \text { NA } & \text { NA } & \text { NA } & \text { NA } & \text { NA } \\ \text { NA } & \text { NA } & \text { NA } & \text { NA } & \text { NA } & \text { NA }\end{array}$

NA

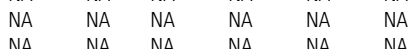

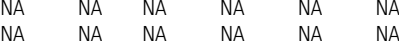

\begin{tabular}{lllllll}
\hline Low- and middle-income countries & NA & NA & NA & NA & NA & NA \\
& NA & NA & NA & NA & NA &
\end{tabular}

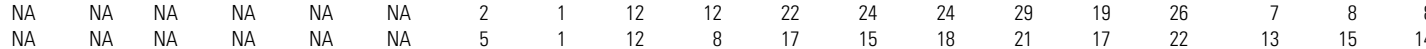

\begin{tabular}{llllllllllllllllllll}
\hline WORLD & NA & NA & NA & NA & NA & NA & 2 & 1 & 12 & 11 & 21 & 23 & 23 & 28 & 18 & 25 & 8 & 9 & 8
\end{tabular}

\section{PAF of DALYs $(\%)$}

\begin{tabular}{|c|c|c|c|c|c|c|c|c|c|c|c|c|c|c|c|c|c|c|c|}
\hline East Asia and Pacific & NA & NA & NA & NA & NA & NA & 1 & 1 & 7 & 7 & 15 & 15 & 18 & 20 & 14 & 16 & 6 & 7 & 6 \\
\hline Europe and Central Asia & NA & NA & NA & NA & NA & NA & 5 & 3 & 19 & 16 & 31 & 34 & 35 & 44 & 31 & 42 & 17 & 23 & 20 \\
\hline Latin America and the Caribbean & NA & NA & NA & NA & NA & NA & 2 & 1 & 9 & 8 & 15 & 14 & 16 & 16 & 14 & 16 & 5 & 5 & 5 \\
\hline Middle East and North Africa & NA & NA & NA & NA & NA & NA & 2 & 1 & 11 & 10 & 20 & 22 & 23 & 28 & 20 & 25 & 6 & 6 & 6 \\
\hline South Asia & NA & NA & NA & NA & NA & NA & 1 & 1 & 9 & 6 & 19 & 16 & 20 & 18 & 14 & 13 & 5 & 4 & 4 \\
\hline Sub-Saharan Africa & NA & NA & NA & NA & NA & NA & 1 & 0 & 5 & 6 & 11 & 15 & 14 & 18 & 12 & 16 & 1 & 2 & 2 \\
\hline Low- and middle-income countries & NA & NA & NA & NA & NA & NA & 2 & 1 & 9 & 8 & 18 & 18 & 21 & 24 & 16 & 21 & 5 & 6 & 6 \\
\hline High-income countries & NA & NA & NA & NA & NA & NA & 3 & 1 & 9 & 5 & 14 & 11 & 16 & 16 & 15 & 17 & 9 & 9 & 9 \\
\hline WORLD & NA & NA & NA & NA & NA & NA & 2 & 1 & 9 & 7 & 18 & 17 & 20 & 23 & 16 & 20 & 6 & 6 & 6 \\
\hline \multicolumn{20}{|l|}{ Attributable Mortality (thousands) } \\
\hline East Asia and Pacific & NA & NA & NA & NA & NA & NA & 9 & 4 & 120 & 84 & 252 & 198 & 323 & 371 & 134 & 278 & 838 & 935 & 1,773 \\
\hline Europe and Central Asia & NA & NA & NA & NA & NA & NA & 23 & 6 & 137 & 62 & 248 & 192 & 286 & 451 & 126 & 452 & 820 & 1,163 & 1,983 \\
\hline Latin America and the Caribbean & NA & NA & NA & NA & NA & NA & 6 & 3 & 37 & 25 & 53 & 42 & 67 & 67 & 47 & 78 & 211 & 215 & 426 \\
\hline Middle East and North Africa & NA & NA & NA & NA & NA & NA & 3 & 1 & 27 & 19 & 45 & 39 & 56 & 61 & 24 & 38 & 156 & 159 & 315 \\
\hline South Asia & NA & NA & NA & NA & NA & NA & 10 & 5 & 138 & 73 & 240 & 187 & 243 & 227 & 87 & 86 & 718 & 579 & 1,297 \\
\hline Sub-Saharan Africa & NA & NA & NA & NA & NA & NA & 9 & 4 & 40 & 37 & 55 & 68 & 61 & 87 & 24 & 43 & 189 & 240 & 429 \\
\hline Low- and middle-income countries & NA & NA & NA & NA & NA & NA & 62 & 24 & 499 & 300 & 893 & 727 & 1,037 & 1,264 & 442 & 976 & 2,933 & 3,290 & 6,223 \\
\hline High-income countries & NA & NA & NA & NA & NA & NA & 9 & 1 & 64 & 22 & 116 & 60 & 214 & 195 & 225 & 485 & 629 & 763 & 1,392 \\
\hline WORLD & NA & NA & NA & NA & NA & NA & 71 & 25 & 563 & 322 & 1,008 & 787 & 1,252 & 1,459 & 667 & 1,461 & 3,561 & 4,054 & 7,615 \\
\hline
\end{tabular}

Attributable YLL (thousands)

East Asia and Pacific

Europe and Central Asia

Latin America and the Caribbean

Middle East and North Africa

South Asia

Sub-Saharan Africa

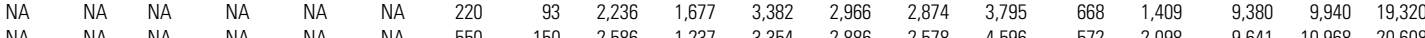

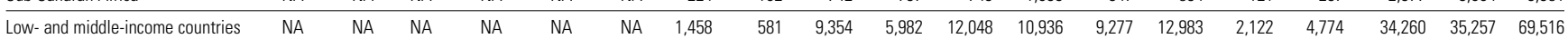

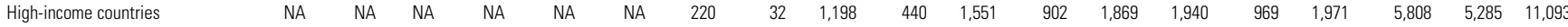

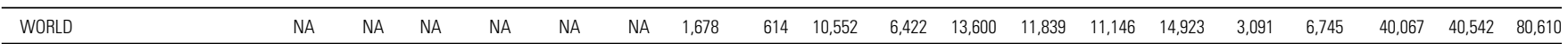

Attributable DALYs (thousands)

\begin{tabular}{|c|c|c|c|c|c|c|c|c|c|c|c|c|c|c|c|c|c|c|c|}
\hline East Asia and Pacific & NA & NA & NA & NA & NA & NA & 272 & 117 & 2,718 & 2,038 & 4,049 & 3,474 & 3,268 & 4,244 & 738 & 1,530 & 11,045 & 11,403 & 22,447 \\
\hline Europe and Central Asia & NA & NA & NA & NA & NA & NA & 614 & 189 & 2,844 & 1,444 & 3,732 & 3,301 & 2,819 & 4,995 & 614 & 2,222 & 10,623 & 12,152 & 22,774 \\
\hline Latin America and the Caribbean & NA & NA & NA & NA & NA & NA & 174 & 89 & 819 & 594 & 841 & 741 & 669 & 761 & 239 & 403 & 2,742 & 2,587 & 5,330 \\
\hline Middle East and North Africa & NA & NA & NA & NA & NA & NA & 95 & 42 & 566 & 432 & 663 & 650 & 542 & 674 & 128 & 216 & 1,995 & 2,014 & 4,009 \\
\hline South Asia & NA & NA & NA & NA & NA & NA & 281 & 154 & 2,885 & 1,652 & 3,665 & 3,155 & 2,380 & 2,572 & 462 & 501 & 9,673 & 8,034 & 17,707 \\
\hline Sub-Saharan Africa & NA & NA & NA & NA & NA & NA & 241 & 113 & 802 & 806 & 802 & 1,122 & 579 & 947 & 129 & 254 & 2,554 & 3,241 & 5,795 \\
\hline Low- a & NA & NA & NA & NA & NA & NA & 1,678 & 705 & 10,635 & 6,964 & 13,752 & 12,442 & 10,257 & 14,192 & 2,309 & 5,128 & 38,632 & 39,431 & 78,063 \\
\hline High-income countries & NA & NA & NA & NA & NA & NA & 298 & 52 & 1,556 & 660 & 1,989 & 1,233 & 2,271 & 2,408 & 1,120 & 2,299 & 7,234 & 6,652 & 13,887 \\
\hline ORLD & NA & NA & NA & NA & NA & NA & 1,976 & 756 & 12,191 & 7,625 & 15,741 & 13,675 & 12,528 & 16,600 & 3,429 & 7,427 & 45,866 & 46,084 & 91,950 \\
\hline
\end{tabular}

Source: Authors' calculations.

Note: $\mathrm{NA}=$ not applicable 
Risk factor:

High cholesterol

Disease:

Ischemic heart disease

\begin{tabular}{|c|c|c|c|c|c|c|c|c|c|c|c|c|c|c|c|c|c|c|c|}
\hline \multirow[b]{2}{*}{ Region } & \multicolumn{2}{|c|}{$0-4$ years } & \multicolumn{2}{|c|}{ 5-14 years } & \multicolumn{2}{|c|}{$15-29$ years } & \multicolumn{2}{|c|}{ 30-44 years } & \multicolumn{2}{|c|}{ 45-59 years } & \multicolumn{2}{|c|}{ 60-69 years } & \multicolumn{2}{|c|}{ 70-79 years } & \multicolumn{2}{|c|}{$80+$ years } & \multicolumn{3}{|c|}{ Total } \\
\hline & Male & Female & Male & Female & Male & Female & Male & Female & Male & Female & Male & Female & Male & Female & Male & Female & Male & Female & All \\
\hline \multicolumn{20}{|l|}{ PAF of Mortality (\%) } \\
\hline East Asia and Pacific & NA & NA & NA & NA & NA & NA & 49 & 41 & 44 & 51 & 29 & 41 & 21 & 35 & 15 & 32 & 27 & 37 & 32 \\
\hline Europe and Central Asia & NA & NA & NA & NA & NA & NA & 86 & 83 & 74 & 77 & 54 & 64 & 44 & 55 & 40 & 54 & 54 & 57 & 55 \\
\hline Latin America and the Caribbean & NA & NA & NA & NA & NA & NA & 84 & 76 & 70 & 69 & 51 & 56 & 41 & 48 & 34 & 42 & 49 & 50 & 49 \\
\hline Middle East and North Africa & NA & NA & NA & NA & NA & NA & 75 & 70 & 62 & 67 & 45 & 54 & 36 & 46 & 32 & 43 & 45 & 51 & 47 \\
\hline South Asia & NA & NA & NA & NA & NA & NA & 71 & 76 & 59 & 70 & 41 & 45 & 32 & 38 & 26 & 38 & 42 & 46 & 43 \\
\hline Sub-Saharan Africa & NA & NA & NA & NA & NA & NA & 10 & 18 & 14 & 36 & 8 & 28 & 2 & 20 & 0 & 18 & 7 & 24 & 15 \\
\hline Low- and middle-income countries & NA & NA & NA & NA & NA & NA & 69 & 65 & 58 & 64 & 41 & 48 & 32 & 43 & 27 & 43 & 41 & 47 & 43 \\
\hline High-income countries & NA & NA & NA & NA & NA & NA & 89 & 84 & 76 & 76 & 56 & 63 & 45 & 53 & 42 & 52 & 51 & 54 & 52 \\
\hline WORLD & NA & NA & NA & NA & NA & NA & 71 & 66 & 61 & 65 & 43 & 49 & 34 & 45 & 32 & 46 & 43 & 48 & 45 \\
\hline
\end{tabular}

PAF of YLL $(\%)$

\begin{tabular}{|c|c|c|c|c|c|c|c|c|c|c|c|c|c|c|c|c|c|c|c|}
\hline East Asia and Pacific & NA & NA & NA & NA & NA & NA & 49 & 41 & 44 & 51 & 29 & 41 & 21 & 35 & 15 & 32 & 31 & 39 & 35 \\
\hline Europe and Central Asia & NA & NA & NA & NA & NA & NA & 86 & 83 & 74 & 77 & 54 & 64 & 44 & 55 & 40 & 54 & 59 & 60 & 60 \\
\hline Latin America and the Caribbean & NA & NA & NA & NA & NA & NA & 84 & 76 & 70 & 69 & 51 & 56 & 41 & 48 & 34 & 42 & 55 & 55 & 55 \\
\hline Middle East and North Africa & NA & NA & NA & NA & NA & NA & 75 & 70 & 62 & 67 & 45 & 54 & 36 & 46 & 32 & 43 & 49 & 53 & 51 \\
\hline South Asia & NA & NA & NA & NA & NA & NA & 71 & 76 & 59 & 70 & 41 & 45 & 32 & 38 & 26 & 38 & 46 & 48 & 47 \\
\hline Sub-Saharan Africa & NA & NA & NA & NA & NA & NA & 10 & 18 & 14 & 36 & 8 & 28 & 2 & 20 & 0 & 18 & 8 & 26 & 17 \\
\hline Low- and middle-income countries & NA & NA & NA & NA & NA & NA & 68 & 65 & 59 & 64 & 41 & 48 & 32 & 43 & 27 & 43 & 45 & 48 & 47 \\
\hline High-income countries & NA & NA & NA & NA & NA & NA & 89 & 84 & 76 & 76 & 56 & 63 & 45 & 53 & 42 & 52 & 57 & 57 & 57 \\
\hline WORLD & NA & NA & NA & NA & NA & NA & 70 & 66 & 61 & 65 & 43 & 49 & 34 & 45 & 32 & 46 & 47 & 50 & 48 \\
\hline
\end{tabular}

\section{PAF of DALYs (\%)}

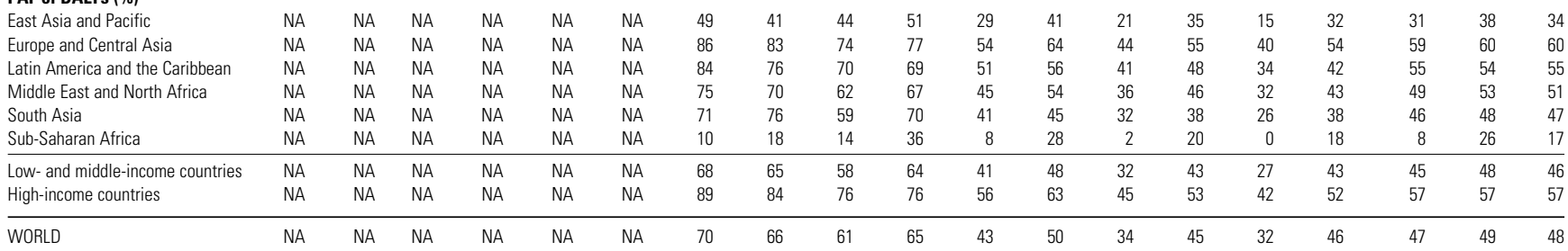

Attributable Mortality (thousands)

\begin{tabular}{|c|c|c|c|c|c|c|c|c|c|c|c|c|c|c|c|c|c|c|c|}
\hline East Asia and Pacific & NA & NA & NA & NA & NA & NA & 15 & 7 & 46 & 30 & 43 & 46 & 39 & 63 & 16 & 59 & 159 & 205 & 364 \\
\hline Europe and Central Asia & NA & NA & NA & NA & NA & NA & 32 & 6 & 113 & 34 & 123 & 83 & 116 & 174 & 53 & 200 & 438 & 497 & 935 \\
\hline Latin America and the Caribbean & NA & NA & NA & NA & NA & NA & 8 & 3 & 28 & 12 & 25 & 16 & 24 & 21 & 15 & 25 & 99 & 78 & 177 \\
\hline Middle East and North Africa & NA & NA & NA & NA & NA & NA & 8 & 3 & 25 & 13 & 22 & 17 & 21 & 22 & 8 & 14 & 85 & 68 & 153 \\
\hline South Asia & NA & NA & NA & NA & NA & NA & 35 & 25 & 144 & 87 & 122 & 105 & 94 & 102 & 35 & 48 & 430 & 367 & 797 \\
\hline Sub-Saharan Africa & NA & NA & NA & NA & NA & NA & 1 & 1 & 6 & 9 & 4 & 13 & 1 & 11 & 0 & 6 & 12 & 40 & 51 \\
\hline Low- and middle-income countries & NA & NA & NA & NA & NA & NA & 99 & 44 & 362 & 186 & 339 & 280 & 296 & 393 & 128 & 351 & 1,223 & 1,254 & 2,477 \\
\hline High-income countries & NA & NA & NA & NA & NA & NA & 14 & 3 & 66 & 17 & 73 & 33 & 104 & 84 & 104 & 212 & 361 & 350 & 711 \\
\hline WORLD & NA & NA & NA & NA & NA & NA & 113 & 47 & 428 & 203 & 412 & 313 & 400 & 477 & 232 & 564 & 1,585 & 1,604 & 3,189 \\
\hline
\end{tabular}

Attributable YLL (thousands)

\begin{tabular}{|c|c|c|c|c|c|c|c|c|c|c|c|c|c|c|c|c|c|c|c|}
\hline East Asia and Pacific & NA & NA & NA & NA & NA & NA & 360 & 166 & 858 & 593 & 581 & 690 & 347 & 645 & 79 & 293 & 2,225 & 2,387 & 4,612 \\
\hline Europe and Central Asia & NA & NA & NA & NA & NA & NA & 758 & 145 & 2,143 & 669 & 1,671 & 1,245 & 1,052 & 1,774 & 241 & 927 & 5,865 & 4,760 & 10,624 \\
\hline Latin America and the Caribbean & NA & NA & NA & NA & NA & NA & 182 & 74 & 515 & 248 & 334 & 247 & 214 & 218 & 69 & 116 & 1,313 & 903 & 2,216 \\
\hline Middle East and North Africa & NA & NA & NA & NA & NA & NA & 191 & 68 & 474 & 259 & 299 & 261 & 187 & 224 & 41 & 73 & 1,192 & 885 & 2,077 \\
\hline South Asia & NA & NA & NA & NA & NA & NA & 823 & 610 & 2,692 & 1,736 & 1,644 & 1,580 & 842 & 1,072 & 174 & 256 & 6,175 & 5,253 & 11,428 \\
\hline Sub-Saharan Africa & NA & NA & NA & NA & NA & NA & 17 & 15 & 105 & 184 & 53 & 194 & 13 & 116 & 0 & 31 & 187 & 539 & 726 \\
\hline Low- and middle-income countries & NA & NA & NA & NA & NA & NA & 2,330 & 1,077 & 6,787 & 3,688 & 4,581 & 4,217 & 2,655 & 4,048 & 603 & 1,695 & 16,957 & 14,726 & 31,683 \\
\hline High-income countries & NA & NA & NA & NA & NA & NA & 330 & 76 & 1,234 & 341 & 979 & 493 & 909 & 842 & 455 & 870 & 3,908 & 2,623 & 6,531 \\
\hline WORLD & NA & NA & NA & NA & NA & NA & 2,661 & 1,154 & 8,021 & 4,029 & 5,561 & 4,709 & 3,565 & 4,890 & 1,058 & 2,566 & 20,865 & 17,348 & 38,214 \\
\hline \multicolumn{20}{|l|}{ Attributable DALYs (thousands) } \\
\hline East Asia and Pacific & NA & NA & NA & NA & NA & NA & 399 & 185 & 916 & 637 & 609 & 724 & 355 & 657 & 80 & 297 & 2,359 & 2,499 & 4,858 \\
\hline Europe and Central Asia & NA & NA & NA & NA & NA & NA & 814 & 167 & 2,226 & 717 & 1,725 & 1,304 & 1,070 & 1,807 & 244 & 940 & 6,077 & 4,934 & 11,011 \\
\hline Latin America and the Caribbean & NA & NA & NA & NA & NA & NA & 207 & 86 & 550 & 271 & 356 & 262 & 220 & 223 & 70 & 118 & 1,402 & 960 & 2,362 \\
\hline Middle East and North Africa & NA & NA & NA & NA & NA & NA & 212 & 77 & 501 & 276 & 314 & 273 & 191 & 228 & 42 & 74 & 1,259 & 927 & 2,186 \\
\hline South Asia & NA & NA & NA & NA & NA & NA & 936 & 698 & 2,856 & 1,871 & 1,731 & 1,664 & 864 & 1,096 & 177 & 260 & 6,563 & 5,589 & 12,152 \\
\hline Sub-Saharan Africa & NA & NA & NA & NA & NA & NA & 18 & 17 & 111 & 196 & 55 & 205 & 13 & 118 & 0 & 31 & 199 & 567 & 766 \\
\hline Low- and middle-income countries & NA & NA & NA & NA & NA & NA & 2,585 & 1,229 & 7,159 & 3,969 & 4,789 & 4,430 & 2,713 & 4,129 & 613 & 1,719 & 17,860 & 15,476 & 33,336 \\
\hline High-income countries & NA & NA & NA & NA & NA & NA & 377 & 102 & 1,360 & 409 & 1,054 & 553 & 956 & 898 & 471 & 903 & 4,218 & 2,866 & 7,084 \\
\hline ORLD & NA & NA & NA & NA & NA & NA & 2,963 & 1,331 & 8,519 & 4,378 & 5,843 & 4,983 & 3,669 & 5,027 & 1,083 & 2,622 & 22,078 & 18,342 & 40,420 \\
\hline
\end{tabular}

Source: Authors' calculations.

Note: $\mathrm{NA}=$ not applicable. 
Risk factor:

High cholesterol

Disease:

Cerebrovascular disease

\begin{tabular}{|c|c|c|c|c|c|c|c|c|c|c|c|c|c|c|c|c|c|c|c|}
\hline \multirow[b]{2}{*}{ Region } & \multicolumn{2}{|c|}{$0-4$ years } & \multicolumn{2}{|c|}{ 5-14 years } & \multicolumn{2}{|c|}{$15-29$ years } & \multicolumn{2}{|c|}{ 30-44 years } & \multicolumn{2}{|c|}{$45-59$ years } & \multicolumn{2}{|c|}{$60-69$ years } & \multicolumn{2}{|c|}{ 70-79 years } & \multicolumn{2}{|c|}{$80+$ years } & \multicolumn{3}{|c|}{ Total } \\
\hline & Male & Female & Male & Female & Male & Female & Male & Female & Male & Female & Male & Female & Male & Female & Male & Female & Male & Female & All \\
\hline \multicolumn{20}{|l|}{ PAF of Mortality (\%) } \\
\hline East Asia and Pacific & NA & NA & NA & NA & NA & NA & 6 & 5 & 9 & 11 & 8 & 12 & 6 & 11 & 3 & 6 & 6 & 9 & 8 \\
\hline Europe and Central Asia & NA & NA & NA & NA & NA & NA & 25 & 23 & 27 & 29 & 23 & 28 & 18 & 24 & 10 & 14 & 19 & 21 & 20 \\
\hline Latin America and the Caribbean & NA & NA & NA & NA & NA & NA & 22 & 18 & 23 & 23 & 20 & 22 & 16 & 19 & 8 & 10 & 16 & 16 & 16 \\
\hline Middle East and North Africa & NA & NA & NA & NA & NA & NA & 18 & 16 & 19 & 22 & 17 & 22 & 14 & 18 & 7 & 10 & 13 & 16 & 15 \\
\hline South Asia & NA & NA & NA & NA & NA & NA & 16 & 18 & 18 & 23 & 16 & 17 & 12 & 14 & 6 & 9 & 13 & 15 & 14 \\
\hline Sub-Saharan Africa & NA & NA & NA & NA & NA & NA & 2 & 3 & 4 & 10 & 3 & 10 & 1 & 7 & 0 & 4 & 2 & 8 & 5 \\
\hline Low- and middle-income countries & NA & NA & NA & NA & NA & NA & 12 & 12 & 15 & 17 & 13 & 17 & 10 & 15 & 5 & 9 & 11 & 14 & 12 \\
\hline High-income countries & NA & NA & NA & NA & NA & NA & 27 & 24 & 28 & 28 & 24 & 27 & 19 & 23 & 10 & 13 & 16 & 17 & 17 \\
\hline WORLD & NA & NA & NA & NA & NA & NA & 14 & 13 & 16 & 18 & 14 & 18 & 11 & 16 & 6 & 10 & 11 & 14 & 13 \\
\hline
\end{tabular}

PAF of YLL (\%)

\begin{tabular}{|c|c|c|c|c|c|c|c|c|c|c|c|c|c|c|c|c|c|c|c|}
\hline East Asia and Pacific & NA & NA & NA & NA & NA & NA & 6 & 5 & 9 & 11 & 8 & 12 & 6 & 11 & 3 & 6 & 7 & 10 & 8 \\
\hline Europe and Central Asia & NA & NA & NA & NA & NA & NA & 25 & 23 & 27 & 29 & 23 & 28 & 18 & 24 & 10 & 14 & 21 & 23 & 22 \\
\hline Latin America and the Caribbean & NA & NA & NA & NA & NA & NA & 22 & 18 & 23 & 23 & 20 & 22 & 16 & 19 & 8 & 10 & 18 & 18 & 18 \\
\hline Middle East and North Africa & NA & NA & NA & NA & NA & NA & 18 & 16 & 19 & 22 & 17 & 22 & 14 & 18 & 7 & 10 & 13 & 17 & 15 \\
\hline South Asia & NA & NA & NA & NA & NA & NA & 16 & 18 & 18 & 23 & 16 & 17 & 12 & 14 & 6 & 9 & 14 & 16 & 15 \\
\hline Sub-Saharan Africa & NA & NA & NA & NA & NA & NA & 2 & 3 & 4 & 10 & 3 & 10 & 1 & 7 & 0 & 4 & 2 & 8 & 6 \\
\hline Low- and middle-income countries & NA & NA & NA & NA & NA & NA & 12 & 12 & 15 & 17 & 13 & 17 & 10 & 15 & 5 & 9 & 12 & 15 & 13 \\
\hline High-income countries & NA & NA & NA & NA & NA & NA & 27 & 24 & 28 & 28 & 24 & 27 & 19 & 23 & 10 & 13 & 20 & 20 & 20 \\
\hline WORLD & NA & NA & NA & NA & NA & NA & 14 & 13 & 16 & 18 & 14 & 18 & 11 & 16 & 6 & 10 & 12 & 15 & 14 \\
\hline
\end{tabular}

\section{PAF of DALYs (\%)}

\begin{tabular}{|c|c|c|c|c|c|c|c|c|c|c|c|c|c|c|c|c|c|c|}
\hline East Asia and Pacific & NA & NA & NA & NA & NA & NA & 9 & 7 & 12 & 14 & 10 & 14 & 7 & 12 & 3 & 6 & 9 & 12 \\
\hline Europe and Central Asia & NA & NA & NA & NA & NA & NA & 29 & 28 & 30 & 33 & 26 & 32 & 20 & 26 & 10 & 15 & 24 & 26 \\
\hline Latin America and the Caribbean & NA & NA & NA & NA & NA & NA & 27 & 23 & 28 & 27 & 23 & 26 & 17 & 20 & 8 & 10 & 21 & 22 \\
\hline Middle East and North Africa & NA & NA & NA & NA & NA & NA & 22 & 19 & 23 & 25 & 20 & 24 & 15 & 19 & 7 & 10 & 15 & 19 \\
\hline South Asia & NA & NA & NA & NA & NA & NA & 19 & 21 & 21 & 26 & 17 & 19 & 13 & 15 & 6 & 9 & 16 & 18 \\
\hline Sub-Saharan Africa & NA & NA & NA & NA & NA & NA & 2 & 3 & 4 & 11 & 3 & 11 & 1 & 8 & 0 & 4 & 2 & 9 \\
\hline Low- and middle-income countries & NA & NA & NA & NA & NA & NA & 15 & 15 & 18 & 20 & 15 & 20 & 11 & 16 & 5 & 9 & 14 & 17 \\
\hline High-income countries & NA & NA & NA & NA & NA & NA & 36 & 32 & 36 & 36 & 29 & 34 & 21 & 26 & 11 & 14 & 25 & 25 \\
\hline WORLD & NA & NA & NA & NA & NA & NA & 18 & 17 & 20 & 22 & 17 & 21 & 12 & 18 & 7 & 11 & 15 & 18 \\
\hline
\end{tabular}

Attributable Mortality (thousands)

\begin{tabular}{|c|c|c|c|c|c|c|c|c|c|c|c|c|c|c|c|c|c|c|c|}
\hline East Asia and Pacific & NA & NA & NA & NA & NA & NA & 2 & 1 & 13 & 10 & 20 & 19 & 21 & 37 & 5 & 19 & 61 & 85 & 146 \\
\hline Europe and Central Asia & NA & NA & NA & NA & NA & NA & 3 & 2 & 15 & 10 & 25 & 26 & 26 & 54 & 8 & 38 & 78 & 130 & 207 \\
\hline Latin America and the Caribbean & NA & NA & NA & NA & NA & NA & 1 & 1 & 5 & 4 & 5 & 5 & 6 & 7 & 3 & 5 & 20 & 22 & 43 \\
\hline Middle East and North Africa & NA & NA & NA & NA & NA & NA & 0 & 0 & 2 & 2 & 2 & 3 & 3 & 4 & 1 & 2 & 8 & 10 & 19 \\
\hline South Asia & NA & NA & NA & NA & NA & NA & 2 & 1 & 14 & 12 & 22 & 22 & 19 & 25 & 4 & 8 & 60 & 67 & 128 \\
\hline Sub-Saharan Africa & NA & NA & NA & NA & NA & NA & 0 & 0 & 1 & 4 & 1 & 5 & 0 & 5 & 0 & 2 & 3 & 16 & 18 \\
\hline Low- and middle-income countries & NA & NA & NA & NA & NA & NA & 8 & 5 & 49 & 41 & 76 & 79 & 76 & 132 & 20 & 73 & 230 & 331 & 561 \\
\hline High-income countries & NA & NA & NA & NA & NA & NA & 2 & 1 & 7 & 4 & 10 & 8 & 19 & 23 & 15 & 41 & 53 & 78 & 131 \\
\hline WORLD & NA & NA & NA & NA & NA & NA & 10 & 6 & 56 & 45 & 87 & 87 & 95 & 155 & 35 & 115 & 283 & 409 & 692 \\
\hline
\end{tabular}

Attributable YLL (thousands)

\begin{tabular}{|c|c|c|c|c|c|c|c|c|c|c|c|c|c|c|c|c|c|c|c|}
\hline East Asia and Pacific & NA & NA & NA & NA & NA & NA & 38 & 19 & 236 & 195 & 269 & 280 & 190 & 375 & 24 & 99 & 757 & 968 & 1,725 \\
\hline Europe and Central Asia & NA & NA & NA & NA & NA & NA & 76 & 41 & 278 & 202 & 342 & 392 & 238 & 552 & 36 & 176 & 971 & 1,364 & 2,335 \\
\hline Latin America and the Caribbean & NA & NA & NA & NA & NA & NA & 34 & 29 & 93 & 83 & 73 & 74 & 53 & 71 & 12 & 24 & 265 & 282 & 548 \\
\hline Middle East and North Africa & NA & NA & NA & NA & NA & NA & 10 & 7 & 31 & 32 & 33 & 41 & 27 & 42 & 4 & 9 & 106 & 132 & 238 \\
\hline South Asia & NA & NA & NA & NA & NA & NA & 36 & 25 & 256 & 231 & 295 & 325 & 169 & 264 & 20 & 43 & 777 & 887 & 1,664 \\
\hline Sub-Saharan Africa & NA & NA & NA & NA & NA & NA & 4 & 6 & 20 & 74 & 13 & 81 & 3 & 51 & 0 & 9 & 40 & 221 & 261 \\
\hline Low- and middle-income countries & NA & NA & NA & NA & NA & NA & 199 & 129 & 915 & 817 & 1,026 & 1,193 & 680 & 1,356 & 97 & 360 & 2,917 & 3,855 & 6,772 \\
\hline High-income countries & NA & NA & NA & NA & NA & NA & 39 & 26 & 131 & 88 & 140 & 116 & 165 & 233 & 64 & 169 & 539 & 632 & 1,171 \\
\hline WORLD & NA & NA & NA & NA & NA & NA & 238 & 155 & 1,046 & 905 & 1,166 & 1,309 & 845 & 1,588 & 161 & 529 & 3,456 & 4,487 & 7,942 \\
\hline \multicolumn{20}{|l|}{ Attributable DALYs (thousands) } \\
\hline East Asia and Pacific & NA & NA & NA & NA & NA & NA & 76 & 39 & 420 & 344 & 434 & 439 & 253 & 490 & 28 & 115 & 1,210 & 1,426 & 2,637 \\
\hline Europe and Central Asia & NA & NA & NA & NA & NA & NA & 107 & 69 & 388 & 302 & 475 & 562 & 305 & 684 & 42 & 197 & 1,317 & 1,814 & 3,131 \\
\hline Latin America and the Caribbean & NA & NA & NA & NA & NA & NA & 58 & 55 & 155 & 138 & 117 & 116 & 73 & 93 & 14 & 29 & 417 & 430 & 847 \\
\hline Middle East and North Africa & NA & NA & NA & NA & NA & NA & 18 & 13 & 49 & 48 & 49 & 57 & 34 & 50 & 5 & 11 & 155 & 179 & 334 \\
\hline South Asia & NA & NA & NA & NA & NA & NA & 53 & 37 & 364 & 324 & 409 & 436 & 209 & 317 & 23 & 48 & 1,059 & 1,162 & 2,221 \\
\hline Sub-Saharan Africa & NA & NA & NA & NA & NA & NA & 5 & 7 & 24 & 91 & 15 & 97 & 4 & 58 & 0 & 9 & 48 & 262 & 310 \\
\hline Low- and middle-income countries & NA & NA & NA & NA & NA & NA & 317 & 219 & 1,400 & 1,247 & 1,500 & 1,707 & 878 & 1,692 & 112 & 408 & 4,206 & 5,273 & 9,479 \\
\hline High-income countries & NA & NA & NA & NA & NA & NA & 100 & 70 & 333 & 235 & 324 & 274 & 291 & 406 & 87 & 228 & 1,134 & 1,212 & 2,347 \\
\hline ORLD & NA & NA & NA & NA & NA & NA & 417 & 289 & 1,733 & 1,482 & 1,823 & 1,981 & 1,169 & 2,098 & 199 & 636 & 5,340 & 6,486 & 11,826 \\
\hline
\end{tabular}

Source: Authors' calculations.

Note: $\mathrm{NA}=$ not applicable. 
Table 4A.30

Risk factor: High cholesterol

Disease: All causes

\begin{tabular}{|c|c|c|c|c|c|c|c|c|c|c|c|c|c|c|c|c|c|c|c|}
\hline \multirow[b]{2}{*}{ Region } & \multicolumn{2}{|c|}{$0-4$ years } & \multicolumn{2}{|c|}{ 5-14 years } & \multicolumn{2}{|c|}{$15-29$ years } & \multicolumn{2}{|c|}{ 30-44 years } & \multicolumn{2}{|c|}{ 45-59 years } & \multicolumn{2}{|c|}{$60-69$ years } & \multicolumn{2}{|c|}{$70-79$ years } & \multicolumn{2}{|c|}{$80+$ years } & \multicolumn{3}{|c|}{ Total } \\
\hline & Male & Female & Male & Female & Male & Female & Male & Female & Male & Female & Male & Female & Male & Female & Male & Female & Male & Female & All \\
\hline \multicolumn{20}{|l|}{ PAF of Mortality (\%) } \\
\hline East Asia and Pacific & NA & NA & NA & NA & NA & NA & 3 & 2 & 5 & 5 & 5 & 7 & 4 & 7 & 2 & 5 & 3 & 5 & 4 \\
\hline Europe and Central Asia & NA & NA & NA & NA & NA & NA & 11 & 7 & 21 & 17 & 21 & 25 & 20 & 27 & 18 & 26 & 17 & 23 & 20 \\
\hline Latin America and the Caribbean & NA & NA & NA & NA & NA & NA & 4 & 4 & 11 & 9 & 11 & 10 & 9 & 10 & 6 & 8 & 7 & 7 & 7 \\
\hline Middle East and North Africa & NA & NA & NA & NA & NA & NA & 10 & 6 & 17 & 14 & 14 & 16 & 12 & 15 & 9 & 12 & 9 & 9 & 9 \\
\hline South Asia & NA & NA & NA & NA & NA & NA & 5 & 5 & 14 & 13 & 13 & 14 & 10 & 12 & 7 & 9 & 7 & 7 & 7 \\
\hline Sub-Saharan Africa & NA & NA & NA & NA & NA & NA & 0 & 0 & 1 & 3 & 1 & 5 & 0 & 4 & 0 & 3 & 0 & 1 & 1 \\
\hline Low- and middle-income countries & NA & NA & NA & NA & NA & NA & 4 & 3 & 10 & 9 & 10 & 12 & 9 & 12 & 6 & 11 & 6 & 7 & 6 \\
\hline High-income countries & NA & NA & NA & NA & NA & NA & 8 & 4 & 14 & 8 & 12 & 10 & 11 & 12 & 9 & 12 & 10 & 11 & 11 \\
\hline WORLD & NA & NA & NA & NA & NA & NA & 4 & 3 & 11 & 9 & 10 & 12 & 9 & 12 & 7 & 12 & 6 & 8 & 7 \\
\hline
\end{tabular}

PAF of YLL (\%)

\begin{tabular}{|c|c|c|c|c|c|c|c|c|c|c|c|c|c|c|c|c|c|c|c|}
\hline East Asia and Pacific & NA & NA & NA & NA & NA & NA & 3 & 2 & 5 & 5 & 5 & 7 & 4 & 7 & 2 & 5 & 3 & 4 & 3 \\
\hline Europe and Central Asia & NA & NA & NA & NA & NA & NA & 11 & 7 & 20 & 17 & 21 & 25 & 20 & 27 & 17 & 26 & 15 & 20 & 17 \\
\hline Latin America and the Caribbean & NA & NA & NA & NA & NA & NA & 4 & 4 & 11 & 9 & 11 & 10 & 9 & 10 & 6 & 8 & 5 & 5 & 5 \\
\hline Middle East and North Africa & NA & NA & NA & NA & NA & NA & 10 & 5 & 17 & 14 & 14 & 16 & 12 & 15 & 9 & 12 & 7 & 6 & 6 \\
\hline South Asia & NA & NA & NA & NA & NA & NA & 5 & 5 & 14 & 13 & 13 & 14 & 10 & 12 & 7 & 9 & 5 & 5 & 5 \\
\hline$\underline{\text { Sub-Saharan Africa }}$ & NA & NA & NA & NA & NA & NA & 0 & 0 & 1 & 3 & 1 & 5 & 0 & 4 & 0 & 3 & 0 & 1 & 0 \\
\hline Low- and middle-income countries & NA & NA & NA & NA & NA & NA & 4 & 3 & 10 & 9 & 10 & 12 & 9 & 12 & 6 & 11 & 4 & 4 & 4 \\
\hline High-income countries & NA & NA & NA & NA & NA & NA & 8 & 4 & 14 & 8 & 12 & 10 & 10 & 12 & 9 & 12 & 10 & 9 & 10 \\
\hline WORLD & NA & NA & NA & NA & NA & NA & 4 & 3 & 11 & 9 & 10 & 12 & 9 & 12 & 7 & 11 & 5 & 5 & 5 \\
\hline
\end{tabular}

PAF of DALYs (\%)

East Asia and Pacific

Latin America and the Caribbea

Middle East and North Africa

South Asia

Sub-Saharan Africa

Low- and middle-income countries

High-income countries

$\begin{array}{lllllll}\text { NA } & \text { NA } & \text { NA } & \text { NA } & \text { NA } & \text { NA } & 2 \\ \text { NA } & \text { NA } & \text { NA } & \text { NA } & \text { NA } & \text { NA } & 8 \\ \text { NA } & \text { NA } & \text { NA } & \text { NA } & \text { NA } & \text { NA } & 3 \\ \text { NA } & \text { NA } & \text { NA } & \text { NA } & \text { NA } & \text { NA } & 5 \\ \text { NA } & \text { NA } & \text { NA } & \text { NA } & \text { NA } & \text { NA } & 4 \\ \text { NA } & \text { NA } & \text { NA } & \text { NA } & \text { NA } & \text { NA } & 0 \\ \text { NA } & \text { NA } & \text { NA } & \text { NA } & \text { NA } & \text { NA } & 3\end{array}$

$\begin{array}{rr}1 & 4 \\ 4 & 17 \\ 2 & 8 \\ 2 & 11 \\ 3 & 10 \\ 0 & 1 \\ 2 & 8\end{array}$

\begin{tabular}{llllllllll}
\hline WORLD & NA & NA & NA & NA & NA & NA & 3 & 2
\end{tabular}

Attributable Mortality (thousands)

\begin{tabular}{|c|c|c|c|c|c|c|c|c|c|c|c|c|c|c|c|c|c|c|c|}
\hline East Asia and Pacific & NA & NA & NA & NA & NA & NA & 17 & 8 & 59 & 39 & 63 & 65 & 60 & 100 & 21 & 79 & 220 & 290 & 510 \\
\hline Europe and Central Asia & NA & NA & NA & NA & NA & NA & 36 & 8 & 128 & 44 & 149 & 109 & 143 & 228 & 61 & 238 & 516 & 626 & 1,142 \\
\hline Latin America and the Caribbean & NA & NA & NA & NA & NA & NA & 9 & 4 & 33 & 17 & 30 & 21 & 30 & 28 & 18 & 30 & 119 & 100 & 220 \\
\hline Middle East and North Africa & NA & NA & NA & NA & NA & NA & 8 & 3 & 27 & 15 & 25 & 20 & 24 & 26 & 9 & 15 & 93 & 79 & 172 \\
\hline South Asia & NA & NA & NA & NA & NA & NA & 37 & 26 & 158 & 99 & 143 & 126 & 113 & 127 & 39 & 56 & 491 & 434 & 925 \\
\hline Sub-Saharan Africa & NA & NA & NA & NA & NA & NA & 1 & 1 & 7 & 13 & 5 & 18 & 2 & 16 & 0 & 7 & 14 & 56 & 70 \\
\hline Low- and middle-income countries & NA & NA & NA & NA & NA & NA & 107 & 49 & 411 & 227 & 415 & 360 & 372 & 525 & 148 & 425 & 1,453 & 1,585 & 3,038 \\
\hline High-income countries & NA & NA & NA & NA & NA & NA & 16 & 4 & 73 & 22 & 83 & 41 & 123 & 108 & 119 & 253 & 415 & 428 & 842 \\
\hline WORLD & NA & NA & NA & NA & NA & NA & 123 & 53 & 484 & 248 & 498 & 400 & 495 & 633 & 267 & 678 & 1,868 & 2,013 & 3,880 \\
\hline
\end{tabular}

Attributable YLL (thousands)

\begin{tabular}{|c|c|c|c|c|c|c|c|c|c|c|c|c|c|c|c|c|c|c|c|}
\hline East Asia and Pacific & NA & NA & NA & NA & NA & NA & 398 & 185 & 1,094 & 787 & 850 & 970 & 537 & 1,020 & 102 & 392 & 2,982 & 3,354 & 6,337 \\
\hline Europe and Central Asia & NA & NA & NA & NA & NA & NA & 834 & 186 & 2,422 & 871 & 2,013 & 1,637 & 1,290 & 2,326 & 277 & 1,103 & 6,836 & 6,124 & 12,959 \\
\hline Latin America and the Caribbean & NA & NA & NA & NA & NA & NA & 216 & 103 & 608 & 331 & 407 & 321 & 267 & 289 & 81 & 140 & 1,579 & 1,185 & 2,764 \\
\hline Middle East and North Africa & NA & NA & NA & NA & NA & NA & 201 & 75 & 506 & 291 & 332 & 302 & 214 & 266 & 46 & 82 & 1,298 & 1,017 & 2,315 \\
\hline South Asia & NA & NA & NA & NA & NA & NA & 859 & 635 & 2,948 & 1,967 & 1,940 & 1,905 & 1,011 & 1,335 & 194 & 299 & 6,952 & 6,140 & 13,092 \\
\hline Sub-Saharan Africa & NA & NA & NA & NA & NA & NA & 21 & 21 & 125 & 258 & 66 & 275 & 16 & 167 & 0 & 40 & 227 & 760 & 988 \\
\hline Low- and middle-income countries & NA & NA & NA & NA & NA & NA & 2,529 & 1,206 & 7,702 & 4,505 & 5,607 & 5,410 & 3,335 & 5,404 & 700 & 2,055 & 19,874 & 18,581 & 38,455 \\
\hline High-income countries & NA & NA & NA & NA & NA & NA & 369 & 102 & 1,365 & 429 & 1,119 & 609 & 1,074 & 1,075 & 519 & 1,040 & 4,447 & 3,254 & 7,701 \\
\hline WORLD & NA & NA & NA & NA & NA & NA & 2,898 & 1,308 & 9,067 & 4,934 & 6,727 & 6,019 & 4,410 & 6,479 & 1,219 & 3,095 & 24,321 & 21,835 & 46,156 \\
\hline \multicolumn{20}{|l|}{ Attributable DALYs (thousands) } \\
\hline East Asia and Pacific & NA & NA & NA & NA & NA & NA & 474 & 224 & 1,336 & 981 & 1,044 & 1,163 & 608 & 1,147 & 108 & 411 & 3,570 & 3,925 & 7,495 \\
\hline Europe and Central Asia & NA & NA & NA & NA & NA & NA & 921 & 235 & 2,614 & 1,019 & 2,200 & 1,865 & 1,375 & 2,491 & 286 & 1,137 & 7,395 & 6,748 & 14,142 \\
\hline Latin America and the Caribbean & NA & NA & NA & NA & NA & NA & 265 & 141 & 705 & 408 & 473 & 378 & 293 & 317 & 84 & 146 & 1,819 & 1,390 & 3,209 \\
\hline Middle East and North Africa & NA & NA & NA & NA & NA & NA & 230 & 89 & 550 & 325 & 362 & 330 & 225 & 278 & 47 & 84 & 1,414 & 1,106 & 2,520 \\
\hline South Asia & NA & NA & NA & NA & NA & NA & 989 & 735 & 3,220 & 2,196 & 2,140 & 2,100 & 1,073 & 1,413 & 200 & 309 & 7,622 & 6,751 & 14,373 \\
\hline Sub-Saharan Africa & NA & NA & NA & NA & NA & NA & 23 & 24 & 135 & 287 & 71 & 302 & 17 & 175 & 0 & 41 & 246 & 829 & 1,075 \\
\hline Low- and middle-income countries & NA & NA & NA & NA & NA & NA & 2,902 & 1,448 & 8,559 & 5,216 & 6,289 & 6,137 & 3,591 & 5,821 & 725 & 2,128 & 22,066 & 20,750 & 42,815 \\
\hline High-income countries & NA & NA & NA & NA & NA & NA & 477 & 172 & 1,693 & 644 & 1,378 & 827 & 1,247 & 1,304 & 558 & 1,131 & 5,353 & 4,078 & 9,431 \\
\hline /ORLD & NA & NA & NA & NA & NA & NA & 3,379 & 1,620 & 10,252 & 5,860 & 7,667 & 6,964 & 4,838 & 7,125 & 1,282 & 3,259 & 27,419 & 24,828 & 52,246 \\
\hline
\end{tabular}

Source: Authors' calculations.

Note: NA = not applicable 
Table 4A.31

Risk factor: Overweight and obesity

Disease:

Colon and rectal cancers

\begin{tabular}{|c|c|c|c|c|c|c|c|c|c|c|c|c|c|c|c|c|c|c|c|}
\hline \multirow[b]{2}{*}{ Region } & \multicolumn{2}{|c|}{$0-4$ years } & \multicolumn{2}{|c|}{ 5-14 years } & \multicolumn{2}{|c|}{$15-29$ years } & \multicolumn{2}{|c|}{ 30-44 years } & \multicolumn{2}{|c|}{$45-59$ years } & \multicolumn{2}{|c|}{$60-69$ years } & \multicolumn{2}{|c|}{ 70-79 years } & \multicolumn{2}{|c|}{$80+$ years } & \multicolumn{3}{|c|}{ Total } \\
\hline & Male & Female & Male & Female & Male & Female & Male & Female & Male & Female & Male & Female & Male & Female & Male & Female & Male & Female & All \\
\hline \multicolumn{20}{|l|}{ PAF of Mortality (\%) } \\
\hline East Asia and Pacific & NA & NA & NA & NA & NA & NA & 6 & 7 & 6 & 8 & 6 & 9 & 5 & 6 & 0 & 1 & 5 & 6 & 6 \\
\hline Europe and Central Asia & NA & NA & NA & NA & NA & NA & 12 & 15 & 15 & 20 & 15 & 21 & 13 & 18 & 12 & 14 & 14 & 18 & 16 \\
\hline Latin America and the Caribbean & NA & NA & NA & NA & NA & NA & 12 & 15 & 13 & 17 & 13 & 17 & 12 & 16 & 9 & 12 & 12 & 15 & 13 \\
\hline Middle East and North Africa & NA & NA & NA & NA & NA & NA & 12 & 17 & 13 & 17 & 10 & 14 & 9 & 11 & 7 & 9 & 10 & 13 & 11 \\
\hline South Asia & NA & NA & NA & NA & NA & NA & 2 & 4 & 2 & 5 & 2 & 2 & 1 & 4 & 1 & 0 & 1 & 3 & 2 \\
\hline Sub-Saharan Africa & NA & NA & NA & NA & NA & NA & 4 & 7 & 5 & 6 & 3 & 5 & 2 & 4 & 1 & 1 & 3 & 5 & 4 \\
\hline Low- and middle-income countries & NA & NA & NA & NA & NA & NA & 7 & 9 & 8 & 11 & 9 & 13 & 8 & 11 & 5 & 6 & 8 & 10 & 9 \\
\hline High-income countries & NA & NA & NA & NA & NA & NA & 14 & 14 & 16 & 17 & 16 & 18 & 14 & 16 & 11 & 12 & 14 & 15 & 14 \\
\hline WORLD & NA & NA & NA & NA & NA & NA & 8 & 10 & 11 & 13 & 11 & 14 & 11 & 13 & 9 & 10 & 10 & 12 & 11 \\
\hline
\end{tabular}

PAF of YLL (\%)

\begin{tabular}{|c|c|c|c|c|c|c|c|c|c|c|c|c|c|c|c|c|c|c|c|}
\hline East Asia and Pacific & NA & NA & NA & NA & NA & NA & 6 & 7 & 6 & 8 & 6 & 9 & 5 & 6 & 0 & 1 & 5 & 7 & 6 \\
\hline Europe and Central Asia & NA & NA & NA & NA & NA & NA & 12 & 15 & 15 & 20 & 15 & 21 & 13 & 18 & 12 & 14 & 14 & 19 & 16 \\
\hline Latin America and the Caribbean & NA & NA & NA & NA & NA & NA & 12 & 15 & 13 & 17 & 13 & 17 & 12 & 16 & 9 & 12 & 12 & 16 & 14 \\
\hline Middle East and North Africa & NA & NA & NA & NA & NA & NA & 12 & 17 & 13 & 17 & 10 & 14 & 9 & 11 & 7 & 9 & 10 & 14 & 12 \\
\hline South Asia & NA & NA & NA & NA & NA & NA & 2 & 4 & 2 & 5 & 2 & 2 & 1 & 4 & 1 & 0 & 2 & 3 & 2 \\
\hline Sub-Saharan Africa & NA & NA & NA & NA & NA & NA & 4 & 7 & 5 & 6 & 3 & 5 & 2 & 4 & 1 & 1 & 4 & 5 & 4 \\
\hline Low- and middle-income countries & NA & NA & NA & NA & NA & NA & 7 & 9 & 8 & 11 & 9 & 13 & 8 & 11 & 5 & 6 & 8 & 11 & 9 \\
\hline High-income countries & NA & NA & NA & NA & NA & NA & 14 & 14 & 16 & 17 & 16 & 18 & 14 & 16 & 11 & 12 & 15 & 16 & 15 \\
\hline WORLD & NA & NA & NA & NA & NA & NA & 8 & 10 & 11 & 13 & 11 & 14 & 11 & 13 & 9 & 9 & 10 & 12 & 11 \\
\hline
\end{tabular}

PAF of DALYs $(\%)$

\begin{tabular}{|c|c|c|c|c|c|c|c|c|c|c|c|c|c|c|c|c|c|c|c|}
\hline East Asia and Pacific & NA & NA & NA & NA & NA & NA & 6 & 7 & 6 & 8 & 6 & 9 & 5 & 6 & 0 & 1 & 5 & 7 & 6 \\
\hline Europe and Central Asia & NA & NA & NA & NA & NA & NA & 12 & 15 & 15 & 20 & 15 & 21 & 13 & 18 & 12 & 14 & 14 & 19 & 16 \\
\hline Latin America and the Caribbean & NA & NA & NA & NA & NA & NA & 12 & 15 & 13 & 17 & 13 & 17 & 12 & 16 & 9 & 12 & 12 & 16 & 14 \\
\hline Middle East and North Africa & NA & NA & NA & NA & NA & NA & 12 & 17 & 13 & 17 & 10 & 14 & 9 & 11 & 7 & 9 & 10 & 14 & 12 \\
\hline South Asia & NA & NA & NA & NA & NA & NA & 2 & 4 & 2 & 5 & 2 & 2 & 1 & 4 & 1 & 0 & 2 & 3 & 2 \\
\hline Sub-Saharan Africa & NA & NA & NA & NA & NA & NA & 4 & 7 & 5 & 6 & 3 & 5 & 2 & 4 & 1 & 1 & 4 & 5 & 4 \\
\hline Low- and middle-income countries & NA & NA & NA & NA & NA & NA & 7 & 9 & 8 & 11 & 9 & 13 & 8 & 12 & 5 & 6 & 8 & 11 & 9 \\
\hline High-income countries & NA & NA & NA & NA & NA & NA & 14 & 14 & 16 & 17 & 16 & 18 & 14 & 16 & 11 & 12 & 15 & 16 & 15 \\
\hline WORLD & NA & NA & NA & NA & NA & NA & 8 & 10 & 11 & 13 & 12 & 15 & 11 & 14 & 9 & 10 & 10 & 13 & 12 \\
\hline
\end{tabular}

Attributable Mortality (thousands)

\begin{tabular}{llllllllllllllrrrrrrr} 
East Asia and Pacific & NA & NA & NA & NA & NA & NA & 0 & 0 & 1 & 1 & 1 & 2 & 1 & 1 & 0 & 0 & 4 & 5 & 9 \\
Europe and Central Asia & NA & NA & NA & NA & NA & NA & 0 & 0 & 1 & 1 & 2 & 3 & 2 & 3 & 1 & 1 & 6 & 9 & 15 \\
Latin America and the Caribbean & NA & NA & NA & NA & NA & NA & 0 & 0 & 0 & 1 & 1 & 1 & 1 & 1 & 0 & 1 & 2 & 3 & 5 \\
Middle East and North Africa & NA & NA & NA & NA & NA & NA & 0 & 0 & 0 & 0 & 0 & 0 & 0 & 0 & 0 & 0 & 1 & 1 & 1 \\
South Asia & NA & NA & NA & NA & NA & NA & 0 & 0 & 0 & 0 & 0 & 0 & 0 & 0 & 0 & 0 & 0 & 0 & 1 \\
Sub-Saharan Africa & NA & NA & NA & NA & NA & NA & 0 & 0 & 0 & 0 & 0 & 0 & 0 & 0 & 0 & 0 & 0 & 0 & 1 \\
\hline Low- and middle-income countries & NA & NA & NA & NA & NA & NA & 1 & 1 & 3 & 4 & 5 & 5 & 4 & 6 & 1 & 2 & 14 & 18 & 32 \\
High-income countries & NA & NA & NA & NA & NA & NA & 0 & 0 & 3 & 2 & 5 & 3 & 7 & 6 & 4 & 6 \\
\hline WORLD & NA & NA & NA & NA & NA & NA & 1 & 1 & 6 & 6 & 10 & 9 & 11 & 12 & 5 & 8 \\
\hline
\end{tabular}

Attributable YLL (thousands)

\begin{tabular}{|c|c|c|c|c|c|c|c|c|c|c|c|c|c|c|c|c|c|c|c|}
\hline East Asia and Pacific & NA & NA & NA & NA & NA & NA & 10 & 11 & 23 & 25 & 20 & 27 & 9 & 12 & 0 & 1 & 63 & 75 & 138 \\
\hline Europe and Central Asia & NA & NA & NA & NA & NA & NA & 5 & 6 & 23 & 28 & 30 & 40 & 20 & 35 & 3 & 6 & 81 & 116 & 197 \\
\hline Latin America and the Caribbean & NA & NA & NA & NA & NA & NA & 3 & 5 & 8 & 11 & 7 & 10 & 6 & 9 & 1 & 3 & 26 & 38 & 63 \\
\hline Middle East and North Africa & NA & NA & NA & NA & NA & NA & 2 & 3 & 3 & 3 & 2 & 2 & 1 & 1 & 0 & 0 & 9 & 10 & 18 \\
\hline South Asia & NA & NA & NA & NA & NA & NA & 1 & 1 & 1 & 3 & 1 & 1 & 0 & 1 & 0 & 0 & 4 & 6 & 11 \\
\hline Sub-Saharan Africa & NA & NA & NA & NA & NA & NA & 1 & 2 & 3 & 3 & 1 & 2 & 0 & 1 & 0 & 0 & 5 & 7 & 12 \\
\hline Low- and middle-income countries & NA & NA & NA & NA & NA & NA & 23 & 27 & 61 & 73 & 62 & 81 & 36 & 59 & 5 & 11 & 188 & 251 & 439 \\
\hline High-income countries & NA & NA & NA & NA & NA & NA & 10 & 8 & 55 & 43 & 67 & 53 & 59 & 61 & 17 & 28 & 208 & 191 & 399 \\
\hline WORLD & NA & NA & NA & NA & NA & NA & 33 & 35 & 117 & 115 & 129 & 134 & 95 & 120 & 22 & 38 & 396 & 442 & 839 \\
\hline
\end{tabular}

Attributable DALYs (thousands)

\begin{tabular}{|c|c|c|c|c|c|c|c|c|c|c|c|c|c|c|c|c|c|c|c|}
\hline East Asia and Pacific & NA & NA & NA & NA & NA & NA & 10 & 11 & 25 & 27 & 21 & 28 & 10 & 12 & 0 & 1 & 66 & 79 & 145 \\
\hline Europe and Central Asia & NA & NA & NA & NA & NA & NA & 6 & 7 & 25 & 30 & 33 & 43 & 21 & 37 & 3 & 7 & 87 & 124 & 211 \\
\hline Latin America and the Caribbean & NA & NA & NA & NA & NA & NA & 3 & 5 & 9 & 12 & 8 & 10 & 6 & 9 & 1 & 3 & 27 & 40 & 67 \\
\hline Middle East and North Africa & NA & NA & NA & NA & NA & NA & 2 & 3 & 3 & 4 & 2 & 2 & 1 & 1 & 0 & 0 & 9 & 10 & 19 \\
\hline South Asia & NA & NA & NA & NA & NA & NA & 1 & 1 & 1 & 3 & 1 & 1 & 0 & 1 & 0 & 0 & 5 & 6 & 11 \\
\hline Sub-Saharan Africa & NA & NA & NA & NA & NA & NA & 1 & 2 & 3 & 3 & 1 & 2 & 0 & 1 & 0 & 0 & 6 & 7 & 13 \\
\hline Low- and middle-income countries & NA & NA & NA & NA & NA & NA & 24 & 29 & 65 & 78 & 66 & 86 & 38 & 62 & 5 & 11 & 199 & 266 & 465 \\
\hline High-income countries & NA & NA & NA & NA & NA & NA & 11 & 10 & 68 & 54 & 81 & 64 & 69 & 73 & 20 & 32 & 251 & 233 & 483 \\
\hline WORLD & NA & NA & NA & NA & NA & NA & 35 & 39 & 134 & 132 & 147 & 150 & 108 & 135 & 26 & 43 & 449 & 499 & 48 \\
\hline
\end{tabular}

Source: Authors' calculations.

Note: $\mathrm{NA}=$ not applicable. 
Table 4A.32

Risk factor: Overweight and obesity

Disease:

Breast cancer

\begin{tabular}{|c|c|c|c|c|c|c|c|c|c|c|c|c|c|c|c|c|c|c|c|}
\hline \multirow[b]{2}{*}{ Region } & \multicolumn{2}{|c|}{$0-4$ years } & \multicolumn{2}{|c|}{ 5-14 years } & \multicolumn{2}{|c|}{$15-29$ years } & \multicolumn{2}{|c|}{ 30-44 years } & \multicolumn{2}{|c|}{ 45-59 years } & \multicolumn{2}{|c|}{ 60-69 years } & \multicolumn{2}{|c|}{ 70-79 years } & \multicolumn{2}{|c|}{ 80+ years } & \multicolumn{3}{|c|}{ Total } \\
\hline & Male & Female & Male & Female & Male & Female & Male & Female & Male & Female & Male & Female & Male & Female & Male & Female & Male & Female & All \\
\hline \multicolumn{20}{|l|}{ PAF of Mortality (\%) } \\
\hline East Asia and Pacific & NA & NA & NA & NA & NA & NA & NA & NA & NA & 5 & NA & 9 & NA & 6 & NA & 1 & NA & 5 & 5 \\
\hline Europe and Central Asia & NA & NA & NA & NA & NA & NA & NA & NA & NA & 13 & NA & 21 & NA & 18 & NA & 14 & NA & 15 & 15 \\
\hline Latin America and the Caribbean & NA & NA & NA & NA & NA & NA & NA & NA & NA & 12 & NA & 17 & NA & 16 & NA & 12 & NA & 12 & 12 \\
\hline Middle East and North Africa & NA & NA & NA & NA & NA & NA & NA & NA & NA & 11 & NA & 14 & NA & 11 & NA & 9 & NA & 9 & 9 \\
\hline South Asia & NA & NA & NA & NA & NA & NA & NA & NA & NA & 3 & NA & 2 & NA & 4 & NA & 0 & NA & 2 & 2 \\
\hline Sub-Saharan Africa & NA & NA & NA & NA & NA & NA & NA & NA & NA & 4 & NA & 5 & NA & 4 & NA & 1 & NA & 4 & 4 \\
\hline Low- and middle-income countries & NA & NA & NA & NA & NA & NA & NA & NA & NA & 7 & NA & 10 & NA & 10 & NA & 5 & NA & 7 & 7 \\
\hline High-income countries & NA & NA & NA & NA & NA & NA & NA & NA & NA & 11 & NA & 18 & NA & 16 & NA & 12 & NA & 13 & 13 \\
\hline WORLD & NA & NA & NA & NA & NA & NA & NA & NA & NA & 8 & NA & 12 & NA & 12 & NA & 9 & NA & 9 & $\overline{9}$ \\
\hline
\end{tabular}

PAF of YLL (\%)

\begin{tabular}{|c|c|c|c|c|c|c|c|c|c|c|c|c|c|c|c|c|c|c|c|}
\hline East Asia and Pacific & NA & NA & NA & NA & NA & NA & NA & NA & NA & 5 & NA & 9 & NA & 6 & NA & 1 & NA & 5 & 5 \\
\hline Europe and Central Asia & NA & NA & NA & NA & NA & NA & NA & NA & NA & 13 & NA & 21 & NA & 18 & NA & 14 & NA & 14 & 14 \\
\hline Latin America and the Caribbean & NA & NA & NA & NA & NA & NA & NA & NA & NA & 12 & NA & 17 & NA & 16 & NA & 12 & NA & 10 & 10 \\
\hline Middle East and North Africa & NA & NA & NA & NA & NA & NA & NA & NA & NA & 11 & NA & 14 & NA & 11 & NA & 9 & NA & 8 & 8 \\
\hline South Asia & NA & NA & NA & NA & NA & NA & NA & NA & NA & 3 & NA & 2 & NA & 4 & NA & 0 & NA & 2 & 2 \\
\hline Sub-Saharan Africa & NA & NA & NA & NA & NA & NA & NA & NA & NA & 4 & NA & 5 & NA & 4 & NA & 1 & NA & 3 & 3 \\
\hline Low- and middle-income countries & NA & NA & NA & NA & NA & NA & NA & NA & NA & 7 & NA & 10 & NA & 10 & NA & 5 & NA & 7 & 7 \\
\hline High-income countries & NA & NA & NA & NA & NA & NA & NA & NA & NA & 11 & NA & 18 & NA & 16 & NA & 12 & NA & 12 & 12 \\
\hline WORLD & NA & NA & NA & NA & NA & NA & NA & NA & NA & 8 & NA & 12 & NA & 12 & NA & 9 & NA & 8 & 8 \\
\hline
\end{tabular}

PAF of DALYs (\%)

\begin{tabular}{|c|c|c|c|c|c|c|c|c|c|c|c|c|c|c|c|c|c|c|c|}
\hline East Asia and Pacific & NA & NA & NA & NA & NA & NA & NA & NA & NA & 5 & NA & 9 & NA & 6 & NA & 1 & NA & 5 & 5 \\
\hline Europe and Central Asia & NA & NA & NA & NA & NA & NA & NA & NA & NA & 13 & NA & 21 & NA & 18 & NA & 14 & NA & 14 & 14 \\
\hline Latin America and the Caribbean & NA & NA & NA & NA & NA & NA & NA & NA & NA & 12 & NA & 17 & NA & 16 & NA & 12 & NA & 10 & 10 \\
\hline Middle East and North Africa & NA & NA & NA & NA & NA & NA & NA & NA & NA & 11 & NA & 14 & NA & 11 & NA & 9 & NA & 8 & 8 \\
\hline South Asia & NA & NA & NA & NA & NA & NA & NA & NA & NA & 3 & NA & 2 & NA & 4 & NA & 0 & NA & 2 & 2 \\
\hline Sub-Saharan Africa & NA & NA & NA & NA & NA & NA & NA & NA & NA & 4 & NA & 5 & NA & 4 & NA & 1 & NA & 3 & 3 \\
\hline Low- and middle-income countries & NA & NA & NA & NA & NA & NA & NA & NA & NA & 7 & NA & 10 & NA & 10 & NA & 5 & NA & 7 & 7 \\
\hline High-income countries & NA & NA & NA & NA & NA & NA & NA & NA & NA & 11 & NA & 18 & NA & 16 & NA & 12 & NA & 12 & 12 \\
\hline WORLL & NA & NA & NA & NA & NA & NA & NA & NA & NA & 8 & NA & 13 & NA & 13 & NA & 9 & NA & 8 & 8 \\
\hline
\end{tabular}

Attributable Mortality (thousands

\begin{tabular}{|c|c|c|c|c|c|c|c|c|c|c|c|c|c|c|c|c|c|c|c|}
\hline East Asia and Pacific & NA & NA & NA & NA & NA & NA & NA & NA & NA & 2 & NA & 2 & NA & 1 & NA & 0 & NA & 5 & 5 \\
\hline Europe and Central Asia & NA & NA & NA & NA & NA & NA & NA & NA & NA & 3 & NA & 3 & NA & 3 & NA & 1 & NA & 10 & 10 \\
\hline Latin America and the Caribbean & NA & NA & NA & NA & NA & NA & NA & NA & NA & 1 & NA & 1 & NA & 1 & NA & 1 & NA & 4 & 4 \\
\hline Middle East and North Africa & NA & NA & NA & NA & NA & NA & NA & NA & NA & 1 & NA & 0 & NA & 0 & NA & 0 & NA & 1 & 1 \\
\hline South Asia & NA & NA & NA & NA & NA & NA & NA & NA & NA & 1 & NA & 0 & NA & 1 & NA & 0 & NA & 2 & 2 \\
\hline Sub-Saharan Africa & NA & NA & NA & NA & NA & NA & NA & NA & NA & 0 & NA & 0 & NA & 0 & NA & 0 & NA & 1 & 1 \\
\hline Low- and middle-income countries & NA & NA & NA & NA & NA & NA & NA & NA & NA & 8 & NA & 8 & NA & 6 & NA & 2 & NA & 23 & 23 \\
\hline High-income countries & NA & NA & NA & NA & NA & NA & NA & NA & NA & 4 & NA & 5 & NA & 6 & NA & 5 & NA & 20 & 20 \\
\hline WORLD & NA & NA & NA & NA & NA & NA & NA & NA & NA & 12 & NA & 13 & NA & 12 & NA & 6 & NA & 43 & 43 \\
\hline
\end{tabular}

Attributable YLL (thousands)

\begin{tabular}{|c|c|c|c|c|c|c|c|c|c|c|c|c|c|c|c|c|c|c|c|}
\hline East Asia and Pacific & NA & NA & NA & NA & NA & NA & NA & NA & NA & 40 & NA & 27 & NA & 8 & NA & 0 & NA & 75 & 75 \\
\hline Europe and Central Asia & NA & NA & NA & NA & NA & NA & NA & NA & NA & 51 & NA & 52 & NA & 29 & NA & 5 & NA & 137 & 137 \\
\hline Latin America and the Caribbean & NA & NA & NA & NA & NA & NA & NA & NA & NA & 29 & NA & 20 & NA & 11 & NA & 3 & NA & 62 & 62 \\
\hline Middle East and North Africa & NA & NA & NA & NA & NA & NA & NA & NA & NA & 12 & NA & 5 & NA & 2 & NA & 0 & NA & 20 & 20 \\
\hline South Asia & NA & NA & NA & NA & NA & NA & NA & NA & NA & 16 & NA & 5 & NA & 6 & NA & 0 & NA & 26 & 26 \\
\hline Sub-Saharan Africa & NA & NA & NA & NA & NA & NA & NA & NA & NA & 9 & NA & 7 & NA & 3 & NA & 0 & NA & 19 & 19 \\
\hline Low- and middle-income countries & NA & NA & NA & NA & NA & NA & NA & NA & NA & 157 & NA & 116 & NA & 58 & NA & 9 & NA & 339 & 339 \\
\hline High-income countries & NA & NA & NA & NA & NA & NA & NA & NA & NA & 83 & NA & 82 & NA & 61 & NA & 21 & NA & 247 & 247 \\
\hline WORLD & NA & NA & NA & NA & NA & NA & NA & NA & NA & 239 & NA & 198 & NA & 120 & NA & 29 & NA & 586 & 586 \\
\hline
\end{tabular}

Attributable DALYs (thousands)

\begin{tabular}{|c|c|c|c|c|c|c|c|c|c|c|c|c|c|c|c|c|c|c|c|}
\hline East Asia and Pacific & NA & NA & NA & NA & NA & NA & NA & NA & NA & 42 & NA & 28 & NA & 8 & NA & 0 & NA & 79 & 79 \\
\hline Europe and Central Asia & NA & NA & NA & NA & NA & NA & NA & NA & NA & 55 & NA & 55 & NA & 31 & NA & 5 & NA & 146 & 146 \\
\hline Latin America and the Caribbean & NA & NA & NA & NA & NA & NA & NA & NA & NA & 31 & NA & 21 & NA & 12 & NA & 3 & NA & 66 & 66 \\
\hline Middle East and North Africa & NA & NA & NA & NA & NA & NA & NA & NA & NA & 13 & NA & 5 & NA & 2 & NA & 0 & NA & 21 & 21 \\
\hline South Asia & NA & NA & NA & NA & NA & NA & NA & NA & NA & 16 & NA & 5 & NA & 6 & NA & 0 & NA & 27 & 27 \\
\hline$\underline{\text { Sub-Saharan Africa }}$ & NA & NA & NA & NA & NA & NA & NA & NA & NA & 9 & NA & 7 & NA & 3 & NA & 0 & NA & 20 & 20 \\
\hline Low- and middle-income countries & NA & NA & NA & NA & NA & NA & NA & NA & NA & 166 & NA & 122 & NA & 62 & NA & 9 & NA & 359 & 359 \\
\hline High-income countries & NA & NA & NA & NA & NA & NA & NA & NA & NA & 102 & NA & 95 & NA & 73 & NA & 24 & NA & 294 & 294 \\
\hline WORLD & NA & NA & NA & NA & NA & NA & NA & NA & NA & 267 & NA & 217 & NA & 135 & NA & 33 & NA & 653 & 653 \\
\hline
\end{tabular}

Source: Authors' calculations.

Note: $\mathrm{NA}=$ not applicable. 
Table 4A.33

Risk factor: Overweight and obesity

Disease: Corpus uteri cancer

\begin{tabular}{|c|c|c|c|c|c|c|c|c|c|c|c|c|c|c|c|c|c|c|c|}
\hline \multirow[b]{2}{*}{ Region } & \multicolumn{2}{|c|}{$0-4$ years } & \multicolumn{2}{|c|}{ 5-14 years } & \multicolumn{2}{|c|}{$15-29$ years } & \multicolumn{2}{|c|}{ 30-44 years } & \multicolumn{2}{|c|}{ 45-59 years } & \multicolumn{2}{|c|}{ 60-69 years } & \multicolumn{2}{|c|}{$70-79$ years } & \multicolumn{2}{|c|}{$80+$ years } & \multicolumn{3}{|c|}{ Total } \\
\hline & Male & Female & Male & Female & Male & Female & Male & Female & Male & Female & Male & Female & Male & Female & Male & Female & Male & Female & All \\
\hline \multicolumn{20}{|l|}{ PAF of Mortality (\%) } \\
\hline East Asia and Pacific & NA & NA & NA & NA & NA & NA & NA & 21 & NA & 24 & NA & 29 & NA & 19 & NA & 3 & NA & 21 & 21 \\
\hline Europe and Central Asia & NA & NA & NA & NA & NA & NA & NA & 44 & NA & 54 & NA & 56 & NA & 51 & NA & 41 & NA & 51 & 51 \\
\hline Latin America and the Caribbean & NA & NA & NA & NA & NA & NA & NA & 44 & NA & 49 & NA & 48 & NA & 46 & NA & 35 & NA & 44 & 44 \\
\hline Middle East and North Africa & NA & NA & NA & NA & NA & NA & NA & 49 & NA & 48 & NA & 42 & NA & 34 & NA & 29 & NA & 38 & 38 \\
\hline South Asia & NA & NA & NA & NA & NA & NA & NA & 13 & NA & 18 & NA & 6 & NA & 16 & NA & 0 & NA & 10 & 10 \\
\hline Sub-Saharan Africa & NA & NA & NA & NA & NA & NA & NA & 23 & NA & 21 & NA & 18 & NA & 15 & NA & 5 & NA & 16 & 16 \\
\hline Low- and middle-income countries & NA & NA & NA & NA & NA & NA & NA & 35 & NA & 41 & NA & 42 & NA & 39 & NA & 25 & NA & 37 & 37 \\
\hline High-income countries & NA & NA & NA & NA & NA & NA & NA & 41 & NA & 47 & NA & 49 & NA & 46 & NA & 36 & NA & 43 & 43 \\
\hline WORLD & NA & NA & NA & NA & NA & NA & NA & 36 & NA & 43 & NA & 45 & NA & 41 & NA & 31 & NA & 40 & 40 \\
\hline
\end{tabular}

PAF of YLL (\%)

\begin{tabular}{|c|c|c|c|c|c|c|c|c|c|c|c|c|c|c|c|c|c|c|c|}
\hline East Asia and Pacific & NA & NA & NA & NA & NA & NA & NA & 21 & NA & 24 & NA & 29 & NA & 19 & NA & 3 & NA & 23 & 23 \\
\hline Europe and Central Asia & NA & NA & NA & NA & NA & NA & NA & 44 & NA & 54 & NA & 56 & NA & 51 & NA & 41 & NA & 52 & 52 \\
\hline Latin America and the Caribbean & NA & NA & NA & NA & NA & NA & NA & 44 & NA & 49 & NA & 48 & NA & 46 & NA & 35 & NA & 45 & 45 \\
\hline Middle East and North Africa & NA & NA & NA & NA & NA & NA & NA & 49 & NA & 48 & NA & 42 & NA & 34 & NA & 29 & NA & 39 & 39 \\
\hline South Asia & NA & NA & NA & NA & NA & NA & NA & 13 & NA & 18 & NA & 6 & NA & 16 & NA & 0 & NA & 11 & 11 \\
\hline Sub-Saharan Africa & NA & NA & NA & NA & NA & NA & NA & 23 & NA & 21 & NA & 18 & NA & 15 & NA & 5 & NA & 17 & 17 \\
\hline Low- and middle-income countries & NA & NA & NA & NA & NA & NA & NA & 35 & NA & 41 & NA & 42 & NA & 39 & NA & 24 & NA & 39 & 39 \\
\hline High-income countries & NA & NA & NA & NA & NA & NA & NA & 41 & NA & 47 & NA & 49 & NA & 46 & NA & 36 & NA & 45 & 45 \\
\hline WORLD & NA & NA & NA & NA & NA & NA & NA & 36 & NA & 43 & NA & 45 & NA & 41 & NA & 30 & NA & 41 & 41 \\
\hline
\end{tabular}

PAF of DALYs $(\%)$

\begin{tabular}{|c|c|c|c|c|c|c|c|c|c|c|c|c|c|c|c|c|c|c|}
\hline East Asia and Pacific & NA & NA & NA & NA & NA & NA & NA & 21 & NA & 24 & NA & 29 & NA & 19 & NA & 3 & NA & 23 \\
\hline Europe and Central Asia & NA & NA & NA & NA & NA & NA & NA & 44 & NA & 54 & NA & 56 & NA & 51 & NA & 41 & NA & 51 \\
\hline Latin America and the Caribbean & NA & NA & NA & NA & NA & NA & NA & 44 & NA & 49 & NA & 48 & NA & 46 & NA & 35 & NA & 45 \\
\hline Middle East and North Africa & NA & NA & NA & NA & NA & NA & NA & 49 & NA & 48 & NA & 42 & NA & 34 & NA & 29 & NA & 40 \\
\hline South Asia & NA & NA & NA & NA & NA & NA & NA & 13 & NA & 18 & NA & 6 & NA & 16 & NA & 0 & NA & 11 \\
\hline Sub-Saharan Africa & NA & NA & NA & NA & NA & NA & NA & 23 & NA & 21 & NA & 18 & NA & 15 & NA & 5 & NA & 17 \\
\hline Low- and middle-income countries & NA & NA & NA & NA & NA & NA & NA & 36 & NA & 43 & NA & 43 & NA & 39 & NA & 24 & NA & 39 \\
\hline High-income countries & NA & NA & NA & NA & NA & NA & NA & 41 & NA & 47 & NA & 49 & NA & 46 & NA & 36 & NA & 46 \\
\hline RLD & NA & NA & NA & NA & NA & NA & NA & 38 & NA & 44 & NA & 46 & NA & 42 & NA & 31 & NA & 42 \\
\hline
\end{tabular}

Attributable Mortality (thousands)

\begin{tabular}{|c|c|c|c|c|c|c|c|c|c|c|c|c|c|c|c|c|c|c|c|}
\hline East Asia and Pacific & NA & NA & NA & NA & NA & NA & NA & 0 & NA & 1 & NA & 1 & NA & 0 & NA & 0 & NA & 2 & 2 \\
\hline Europe and Central Asia & NA & NA & NA & NA & NA & NA & NA & 0 & NA & 2 & NA & 3 & NA & 3 & NA & 1 & NA & 8 & 8 \\
\hline Latin America and the Caribbean & NA & NA & NA & NA & NA & NA & NA & 1 & NA & 1 & NA & 1 & NA & 1 & NA & 1 & NA & 5 & 5 \\
\hline Middle East and North Africa & NA & NA & NA & NA & NA & NA & NA & 0 & NA & 0 & NA & 0 & NA & 0 & NA & 0 & NA & 0 & 0 \\
\hline South Asia & NA & NA & NA & NA & NA & NA & NA & 0 & NA & 0 & NA & 0 & NA & 0 & NA & 0 & NA & 0 & 0 \\
\hline Sub-Saharan Africa & NA & NA & NA & NA & NA & NA & NA & 0 & NA & 0 & NA & 0 & NA & 0 & NA & 0 & NA & 0 & 0 \\
\hline Low- and middle-income countries & NA & NA & NA & NA & NA & NA & NA & 1 & NA & 4 & NA & 5 & NA & 5 & NA & 2 & NA & 16 & 16 \\
\hline High-income countries & NA & NA & NA & NA & NA & NA & NA & 0 & NA & 2 & NA & 3 & NA & 4 & NA & 3 & NA & 12 & 12 \\
\hline WORLD & NA & NA & NA & NA & NA & NA & NA & 2 & NA & 6 & NA & 8 & NA & 9 & NA & 5 & NA & 28 & 28 \\
\hline
\end{tabular}

Attributable YLL (thousands)

\begin{tabular}{|c|c|c|c|c|c|c|c|c|c|c|c|c|c|c|c|c|c|c|c|}
\hline East Asia and Pacific & NA & NA & NA & NA & NA & NA & NA & 4 & NA & 11 & NA & 9 & NA & 3 & NA & 0 & NA & 28 & 28 \\
\hline Europe and Central Asia & NA & NA & NA & NA & NA & NA & NA & 10 & NA & 35 & NA & 42 & NA & 29 & NA & 4 & NA & 120 & 120 \\
\hline Latin America and the Caribbean & NA & NA & NA & NA & NA & NA & NA & 13 & NA & 28 & NA & 20 & NA & 13 & NA & 3 & NA & 78 & 78 \\
\hline Middle East and North Africa & NA & NA & NA & NA & NA & NA & NA & 1 & NA & 2 & NA & 1 & NA & 1 & NA & 0 & NA & 6 & 6 \\
\hline South Asia & NA & NA & NA & NA & NA & NA & NA & 1 & NA & 2 & NA & 1 & NA & 2 & NA & 0 & NA & 6 & 6 \\
\hline Sub-Saharan Africa & NA & NA & NA & NA & NA & NA & NA & 1 & NA & 2 & NA & 2 & NA & 1 & NA & 0 & NA & 6 & 6 \\
\hline Low- and middle-income countries & NA & NA & NA & NA & NA & NA & NA & 30 & NA & 81 & NA & 75 & NA & 49 & NA & 8 & NA & 243 & 243 \\
\hline High-income countries & NA & NA & NA & NA & NA & NA & NA & 7 & NA & 35 & NA & 42 & NA & 40 & NA & 14 & NA & 137 & 137 \\
\hline WORLD & NA & NA & NA & NA & NA & NA & NA & 37 & NA & 116 & NA & 116 & NA & 89 & NA & 22 & NA & 380 & 380 \\
\hline
\end{tabular}

Attributable DALYs (thousands)

\begin{tabular}{|c|c|c|c|c|c|c|c|c|c|c|c|c|c|c|c|c|c|c|c|}
\hline East Asia and Pacific & NA & NA & NA & NA & NA & NA & NA & 8 & NA & 17 & NA & 11 & NA & 3 & NA & 0 & NA & 40 & 40 \\
\hline Europe and Central Asia & NA & NA & NA & NA & NA & NA & NA & 21 & NA & 62 & NA & 57 & NA & 33 & NA & 5 & NA & 178 & 178 \\
\hline Latin America and the Caribbean & NA & NA & NA & NA & NA & NA & NA & 25 & NA & 46 & NA & 25 & NA & 14 & NA & 4 & NA & 115 & 115 \\
\hline Middle East and North Africa & NA & NA & NA & NA & NA & NA & NA & 2 & NA & 4 & NA & 2 & NA & 1 & NA & 0 & NA & 9 & 9 \\
\hline South Asia & NA & NA & NA & NA & NA & NA & NA & 1 & NA & 3 & NA & 1 & NA & 2 & NA & 0 & NA & 7 & 7 \\
\hline Sub-Saharan Africa & NA & NA & NA & NA & NA & NA & NA & 1 & NA & 2 & NA & 2 & NA & 1 & NA & 0 & NA & 7 & 7 \\
\hline Low- and middle-income countries & NA & NA & NA & NA & NA & NA & NA & 59 & NA & 135 & NA & 98 & NA & 56 & NA & 9 & NA & 356 & 356 \\
\hline High-income countries & NA & NA & NA & NA & NA & NA & NA & 23 & NA & 96 & NA & 75 & NA & 57 & NA & 17 & NA & 267 & 267 \\
\hline WORLD & NA & NA & NA & NA & NA & NA & NA & 82 & NA & 231 & NA & 173 & NA & 113 & NA & 25 & NA & 623 & 623 \\
\hline
\end{tabular}


Table 4A.34

Risk factor: Overweight and obesity

Disease: Diabetes mellitus

\begin{tabular}{|c|c|c|c|c|c|c|c|c|c|c|c|c|c|c|c|c|c|c|c|}
\hline \multirow[b]{2}{*}{ Region } & \multicolumn{2}{|c|}{$0-4$ years } & \multicolumn{2}{|c|}{ 5-14 years } & \multicolumn{2}{|c|}{$15-29$ years } & \multicolumn{2}{|c|}{ 30-44 years } & \multicolumn{2}{|c|}{ 45-59 years } & \multicolumn{2}{|c|}{$60-69$ years } & \multicolumn{2}{|c|}{$70-79$ years } & \multicolumn{2}{|c|}{$80+$ years } & \multicolumn{3}{|c|}{ Total } \\
\hline & Male & Female & Male & Female & Male & Female & Male & Female & Male & Female & Male & Female & Male & Female & Male & Female & Male & Female & All \\
\hline \multicolumn{20}{|l|}{ PAF of Mortality (\%) } \\
\hline East Asia and Pacific & NA & NA & NA & NA & NA & NA & 54 & 73 & 42 & 63 & 33 & 55 & 38 & 35 & 4 & 7 & 33 & 42 & 38 \\
\hline Europe and Central Asia & NA & NA & NA & NA & NA & NA & 80 & 96 & 73 & 95 & 62 & 83 & 73 & 77 & 72 & 67 & 68 & 78 & 74 \\
\hline Latin America and the Caribbean & NA & NA & NA & NA & NA & NA & 81 & 96 & 71 & 92 & 58 & 76 & 71 & 72 & 60 & 59 & 65 & 74 & 70 \\
\hline Middle East and North Africa & NA & NA & NA & NA & NA & NA & 86 & 99 & 70 & 93 & 52 & 72 & 65 & 60 & 55 & 55 & 61 & 69 & 65 \\
\hline South Asia & NA & NA & NA & NA & NA & NA & 30 & 61 & 13 & 56 & 11 & 14 & 8 & 34 & 14 & 0 & 12 & 27 & 19 \\
\hline Sub-Saharan Africa & NA & NA & NA & NA & NA & NA & 53 & 80 & 43 & 64 & 19 & 36 & 18 & 30 & 10 & 10 & 26 & 39 & 34 \\
\hline Low- and middle-income countries & NA & NA & NA & NA & NA & NA & 57 & 80 & 41 & 70 & 34 & 50 & 38 & 47 & 26 & 29 & 36 & 49 & 43 \\
\hline High-income countries & NA & NA & NA & NA & NA & NA & 87 & 96 & 77 & 91 & 64 & 78 & 76 & 72 & 69 & 60 & 72 & 68 & 70 \\
\hline WORLD & NA & NA & NA & NA & NA & NA & 61 & 82 & 46 & 72 & 39 & 53 & 47 & 52 & 41 & 43 & 43 & 53 & 49 \\
\hline
\end{tabular}

PAF of YLL (\%)

\begin{tabular}{|c|c|c|c|c|c|c|c|c|c|c|c|c|c|c|c|c|c|c|c|}
\hline East Asia and Pacific & NA & NA & NA & NA & NA & NA & 54 & 73 & 42 & 63 & 33 & 55 & 38 & 35 & 4 & 7 & 36 & 48 & 43 \\
\hline Europe and Central Asia & NA & NA & NA & NA & NA & NA & 80 & 96 & 73 & 95 & 62 & 83 & 73 & 77 & 72 & 67 & 66 & 79 & 73 \\
\hline Latin America and the Caribbean & NA & NA & NA & NA & NA & NA & 81 & 96 & 71 & 92 & 58 & 76 & 71 & 72 & 60 & 59 & 65 & 77 & 72 \\
\hline Middle East and North Africa & NA & NA & NA & NA & NA & NA & 86 & 99 & 70 & 93 & 52 & 72 & 65 & 60 & 55 & 55 & 61 & 72 & 67 \\
\hline South Asia & NA & NA & NA & NA & NA & NA & 30 & 61 & 13 & 56 & 11 & 14 & 8 & 34 & 14 & 0 & 12 & 30 & 21 \\
\hline Sub-Saharan Africa & NA & NA & NA & NA & NA & NA & 53 & 80 & 43 & 64 & 19 & 36 & 18 & 30 & 10 & 10 & 30 & 44 & 39 \\
\hline Low- and middle-income countries & NA & NA & NA & NA & NA & NA & 57 & 80 & 41 & 70 & 34 & 50 & 38 & 47 & 25 & 28 & 37 & 53 & 46 \\
\hline High-income countries & NA & NA & NA & NA & NA & NA & 87 & 96 & 77 & 91 & 64 & 78 & 76 & 72 & 69 & 60 & 72 & 74 & 73 \\
\hline WORLD & NA & NA & NA & NA & NA & NA & 61 & 82 & 46 & 72 & 39 & 53 & 47 & 52 & 40 & 41 & 43 & 56 & 50 \\
\hline
\end{tabular}

PAF of DALYs $(\%)$

\begin{tabular}{|c|c|c|c|c|c|c|c|c|c|c|c|c|c|c|c|c|c|c|c|}
\hline East Asia and Pacific & NA & NA & NA & NA & NA & NA & 54 & 73 & 42 & 63 & 33 & 55 & 38 & 35 & 4 & 7 & 38 & 51 & 45 \\
\hline Europe and Central Asia & NA & NA & NA & NA & NA & NA & 80 & 96 & 73 & 95 & 62 & 83 & 73 & 77 & 72 & 67 & 67 & 82 & 76 \\
\hline Latin America and the Caribbean & NA & NA & NA & NA & NA & NA & 81 & 96 & 71 & 92 & 58 & 76 & 71 & 72 & 60 & 59 & 66 & 79 & 73 \\
\hline Middle East and North Africa & NA & NA & NA & NA & NA & NA & 86 & 99 & 70 & 93 & 52 & 72 & 65 & 60 & 55 & 55 & 61 & 74 & 69 \\
\hline South Asia & NA & NA & NA & NA & NA & NA & 30 & 61 & 13 & 56 & 11 & 14 & 8 & 34 & 14 & 0 & 14 & 35 & 24 \\
\hline Sub-Saharan Africa & NA & NA & NA & NA & NA & NA & 53 & 80 & 43 & 64 & 19 & 36 & 18 & 30 & 10 & 10 & 31 & 46 & 40 \\
\hline Low- and middle-income countries & NA & NA & NA & NA & NA & NA & 54 & 77 & 41 & 71 & 34 & 51 & 39 & 48 & 26 & 29 & 38 & 56 & 48 \\
\hline High-income countries & NA & NA & NA & NA & NA & NA & 87 & 96 & 77 & 91 & 64 & 78 & 76 & 72 & 69 & 60 & 73 & 78 & 76 \\
\hline RLD & NA & NA & NA & NA & NA & NA & 61 & 80 & 49 & 75 & 41 & 55 & 49 & 54 & 42 & 43 & 46 & 60 & 54 \\
\hline
\end{tabular}

Attributable Mortality (thousands)

\begin{tabular}{|c|c|c|c|c|c|c|c|c|c|c|c|c|c|c|c|c|c|c|c|}
\hline East Asia and Pacific & NA & NA & NA & NA & NA & NA & 3 & 3 & 8 & 14 & 10 & 21 & 11 & 16 & 1 & 1 & 32 & 57 & 89 \\
\hline Europe and Central Asia & NA & NA & NA & NA & NA & NA & 1 & 1 & 3 & 3 & 4 & 8 & 4 & 9 & 1 & 3 & 14 & 24 & 38 \\
\hline Latin America and the Caribbean & NA & NA & NA & NA & NA & NA & 3 & 3 & 11 & 15 & 11 & 18 & 14 & 20 & 7 & 13 & 46 & 69 & 114 \\
\hline Middle East and North Africa & NA & NA & NA & NA & NA & NA & 1 & 1 & 2 & 3 & 2 & 3 & 3 & 3 & 1 & 1 & 9 & 12 & 20 \\
\hline South Asia & NA & NA & NA & NA & NA & NA & 2 & 2 & 3 & 11 & 3 & 4 & 2 & 9 & 2 & 0 & 12 & 25 & 37 \\
\hline Sub-Saharan Africa & NA & NA & NA & NA & NA & NA & 1 & 2 & 3 & 8 & 1 & 6 & 1 & 5 & 0 & 1 & 8 & 21 & 28 \\
\hline Low- and middle-income countries & NA & NA & NA & NA & NA & NA & 10 & 11 & 31 & 54 & 31 & 60 & 36 & 63 & 12 & 19 & 121 & 207 & 327 \\
\hline High-income countries & NA & NA & NA & NA & NA & NA & 3 & 2 & 9 & 7 & 12 & 11 & 23 & 25 & 18 & 32 & 65 & 76 & 142 \\
\hline WORLD & NA & NA & NA & NA & NA & NA & 13 & 13 & 40 & 60 & 43 & 71 & 59 & 87 & 30 & 52 & 186 & 283 & 469 \\
\hline
\end{tabular}

Attributable YLL (thousands)

\begin{tabular}{|c|c|c|c|c|c|c|c|c|c|c|c|c|c|c|c|c|c|c|c|}
\hline East Asia and Pacific & NA & NA & NA & NA & NA & NA & 60 & 82 & 159 & 284 & 131 & 321 & 101 & 170 & 3 & 8 & 454 & 865 & 1,319 \\
\hline Europe and Central Asia & NA & NA & NA & NA & NA & NA & 33 & 28 & 61 & 69 & 53 & 115 & 38 & 93 & 6 & 16 & 192 & 321 & 512 \\
\hline Latin America and the Caribbean & NA & NA & NA & NA & NA & NA & 71 & 70 & 201 & 288 & 149 & 271 & 128 & 211 & 32 & 65 & 580 & 906 & 1,486 \\
\hline Middle East and North Africa & NA & NA & NA & NA & NA & NA & 14 & 15 & 36 & 55 & 29 & 52 & 27 & 36 & 5 & 8 & 111 & 166 & 278 \\
\hline South Asia & NA & NA & NA & NA & NA & NA & 36 & 38 & 62 & 212 & 39 & 60 & 18 & 94 & 11 & 0 & 166 & 404 & 570 \\
\hline Sub-Saharan Africa & NA & NA & NA & NA & NA & NA & 32 & 46 & 60 & 155 & 20 & 88 & 12 & 47 & 2 & 3 & 126 & 339 & 465 \\
\hline Low- and middle-income countries & NA & NA & NA & NA & NA & NA & 248 & 280 & 579 & 1,062 & 421 & 906 & 324 & 652 & 58 & 100 & 1,630 & 3,001 & 4,631 \\
\hline High-income countries & NA & NA & NA & NA & NA & NA & 63 & 39 & 176 & 133 & 165 & 167 & 201 & 248 & 81 & 144 & 686 & 730 & 1,416 \\
\hline WORLD & NA & NA & NA & NA & NA & NA & 310 & 319 & 755 & 1,195 & 586 & 1,073 & 525 & 900 & 139 & 244 & 2,316 & 3,731 & 6,047 \\
\hline
\end{tabular}

Attributable DALYs (thousands)

\begin{tabular}{|c|c|c|c|c|c|c|c|c|c|c|c|c|c|c|c|c|c|c|c|}
\hline East Asia and Pacific & NA & NA & NA & NA & NA & NA & 212 & 290 & 293 & 522 & 172 & 402 & 120 & 197 & 3 & 9 & 800 & 1,422 & 2,221 \\
\hline Europe and Central Asia & NA & NA & NA & NA & NA & NA & 87 & 110 & 134 & 205 & 85 & 185 & 57 & 137 & 9 & 28 & 373 & 665 & 1,038 \\
\hline Latin America and the Caribbean & NA & NA & NA & NA & NA & NA & 124 & 153 & 273 & 454 & 176 & 343 & 145 & 253 & 36 & 77 & 754 & 1,281 & 2,034 \\
\hline Middle East and North Africa & NA & NA & NA & NA & NA & NA & 71 & 98 & 78 & 126 & 40 & 71 & 33 & 45 & 6 & 10 & 228 & 350 & 578 \\
\hline South Asia & NA & NA & NA & NA & NA & NA & 119 & 218 & 98 & 371 & 52 & 75 & 22 & 113 & 13 & 0 & 304 & 777 & 1,081 \\
\hline Sub-Saharan Africa & NA & NA & NA & NA & NA & NA & 53 & 80 & 78 & 186 & 23 & 95 & 13 & 50 & 2 & 3 & 170 & 414 & 584 \\
\hline Low- and middle-income countries & NA & NA & NA & NA & NA & NA & 666 & 950 & 954 & 1,864 & 548 & 1,172 & 391 & 794 & 70 & 128 & 2,628 & 4,908 & 7,536 \\
\hline High-income countries & NA & NA & NA & NA & NA & NA & 261 & 218 & 521 & 520 & 300 & 364 & 294 & 386 & 109 & 198 & 1,484 & 1,686 & 3,171 \\
\hline WORLD & NA & NA & NA & NA & NA & NA & 927 & 1,168 & 1,474 & 2,384 & 848 & 1,536 & 684 & 1,181 & 179 & 326 & 4,112 & 6,595 & 10,707 \\
\hline
\end{tabular}

Source: Authors' calculations.

Note: $\mathrm{NA}=$ not applicable. 
Table 4A.35

Risk factor: Overweight and obesity

Disease: Hypertensive heart disease

\begin{tabular}{|c|c|c|c|c|c|c|c|c|c|c|c|c|c|c|c|c|c|c|c|}
\hline \multirow[b]{2}{*}{ Region } & \multicolumn{2}{|c|}{$0-4$ years } & \multicolumn{2}{|c|}{ 5-14 years } & \multicolumn{2}{|c|}{$15-29$ years } & \multicolumn{2}{|c|}{ 30-44 years } & \multicolumn{2}{|c|}{ 45-59 years } & \multicolumn{2}{|c|}{$60-69$ years } & \multicolumn{2}{|c|}{$70-79$ years } & \multicolumn{2}{|c|}{$80+$ years } & \multicolumn{3}{|c|}{ Total } \\
\hline & Male & Female & Male & Female & Male & Female & Male & Female & Male & Female & Male & Female & Male & Female & Male & Female & Male & Female & All \\
\hline \multicolumn{20}{|l|}{ PAF of Mortality (\%) } \\
\hline East Asia and Pacific & NA & NA & NA & NA & NA & NA & 37 & 42 & 32 & 39 & 26 & 39 & 17 & 20 & 1 & 3 & 20 & 21 & 21 \\
\hline Europe and Central Asia & NA & NA & NA & NA & NA & NA & 62 & 74 & 61 & 75 & 52 & 68 & 41 & 54 & 28 & 32 & 46 & 53 & 50 \\
\hline Latin America and the Caribbean & NA & NA & NA & NA & NA & NA & 62 & 74 & 58 & 70 & 48 & 59 & 38 & 49 & 21 & 27 & 40 & 45 & 43 \\
\hline Middle East and North Africa & NA & NA & NA & NA & NA & NA & 66 & 81 & 57 & 70 & 42 & 54 & 31 & 37 & 17 & 22 & 36 & 41 & 39 \\
\hline South Asia & NA & NA & NA & NA & NA & NA & 18 & 30 & 9 & 30 & 9 & 9 & 3 & 17 & 4 & 0 & 7 & 15 & 11 \\
\hline Sub-Saharan Africa & NA & NA & NA & NA & NA & NA & 33 & 47 & 32 & 36 & 14 & 24 & 7 & 16 & 3 & 4 & 15 & 20 & 18 \\
\hline Low- and middle-income countries & NA & NA & NA & NA & NA & NA & 41 & 51 & 37 & 47 & 30 & 41 & 22 & 30 & 10 & 13 & 25 & 30 & 28 \\
\hline High-income countries & NA & NA & NA & NA & NA & NA & 70 & 72 & 65 & 68 & 54 & 61 & 44 & 49 & 26 & 28 & 42 & 36 & 38 \\
\hline WORLD & NA & NA & NA & NA & NA & NA & 44 & 53 & 40 & 49 & 32 & 42 & 24 & 32 & 14 & 18 & 27 & 31 & 29 \\
\hline
\end{tabular}

PAF of YLL (\%)

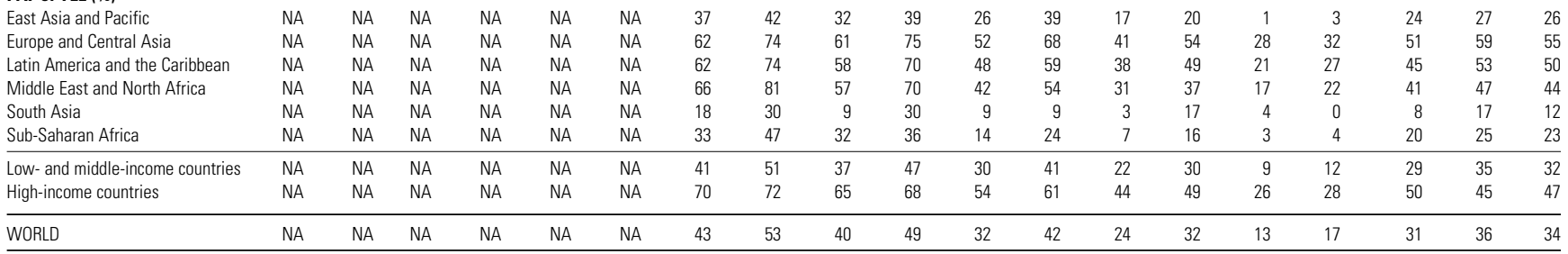

\section{PAF of DALYs (\%)}

East Asia and Pacific

Europe and Central Asia

Latin America and the Caribbean

Middle East and North Africa

South Asia

Sub-Saharan Africa

Low- and middle-income countries

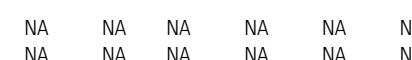

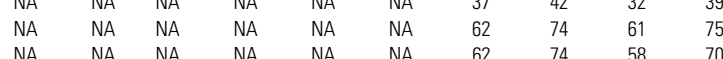

\begin{tabular}{rr}
26 \\
5 & 52 \\
0 & 48 \\
0 & 42 \\
30 & 9 \\
6 & 14 \\
\hline 7 & 30
\end{tabular}

$\begin{array}{lll}39 & 17 & 20 \\ 68 & 41 & 54\end{array}$

$\begin{array}{lllll}1 & 3 & 24 & 27 & 25\end{array}$

\begin{tabular}{lllllllllllllllllllll} 
& NA & NA & NA & NA & NA & NA & 70 & 72 & 65 & 68 & 54 & 61 & 44 & 49 & 26 & 28 & 48 & 43 & 45 \\
\hline WORLD & NA & NA & NA & NA & NA & NA & 43 & 52 & 39 & 48 & 32 & 42 & 24 & 32 & 13 & 17 & 30 & 35 & 33 \\
\hline
\end{tabular}

Attributable Mortality (thousands)

\begin{tabular}{|c|c|c|c|c|c|c|c|c|c|c|c|c|c|c|c|c|c|c|c|}
\hline East Asia and Pacific & NA & NA & NA & NA & NA & NA & 2 & 2 & 9 & 8 & 12 & 13 & 9 & 11 & 0 & 1 & 33 & 35 & 68 \\
\hline Europe and Central Asia & NA & NA & NA & NA & NA & NA & 1 & 1 & 5 & 5 & 7 & 9 & 6 & 12 & 2 & 6 & 22 & 33 & 55 \\
\hline Latin America and the Caribbean & NA & NA & NA & NA & NA & NA & 1 & 1 & 4 & 4 & 4 & 5 & 4 & 6 & 2 & 5 & 16 & 22 & 37 \\
\hline Middle East and North Africa & NA & NA & NA & NA & NA & NA & 1 & 1 & 4 & 3 & 4 & 4 & 4 & 5 & 1 & 2 & 14 & 15 & 29 \\
\hline South Asia & NA & NA & NA & NA & NA & NA & 1 & 1 & 1 & 3 & 1 & 1 & 0 & 2 & 0 & 0 & 3 & 6 & 10 \\
\hline Sub-Saharan Africa & NA & NA & NA & NA & NA & NA & 1 & 1 & 2 & 2 & 1 & 2 & 0 & 2 & 0 & 0 & 4 & 8 & 12 \\
\hline Low- and middle-income countries & NA & NA & NA & NA & NA & NA & 7 & 6 & 24 & 25 & 29 & 35 & 24 & 39 & 6 & 15 & 91 & 120 & 211 \\
\hline High-income countries & NA & NA & NA & NA & NA & NA & 1 & 1 & 4 & 2 & 4 & 3 & 6 & 8 & 5 & 15 & 20 & 29 & 49 \\
\hline WORLD & NA & NA & NA & NA & NA & NA & 8 & 7 & 28 & 27 & 33 & 38 & 30 & 47 & 11 & 30 & 111 & 149 & 260 \\
\hline
\end{tabular}

Attributable YLL (thousands)

\begin{tabular}{|c|c|c|c|c|c|c|c|c|c|c|c|c|c|c|c|c|c|c|c|}
\hline East Asia and Pacific & NA & NA & NA & NA & NA & NA & 59 & 42 & 168 & 152 & 165 & 199 & 80 & 117 & 2 & 7 & 474 & 516 & 990 \\
\hline Europe and Central Asia & NA & NA & NA & NA & NA & NA & 32 & 21 & 101 & 93 & 95 & 134 & 54 & 127 & 9 & 29 & 291 & 404 & 695 \\
\hline Latin America and the Caribbean & NA & NA & NA & NA & NA & NA & 27 & 32 & 71 & 84 & 55 & 73 & 39 & 65 & 10 & 24 & 202 & 278 & 480 \\
\hline Middle East and North Africa & NA & NA & NA & NA & NA & NA & 23 & 17 & 66 & 62 & 53 & 63 & 37 & 49 & 6 & 11 & 185 & 202 & 388 \\
\hline South Asia & NA & NA & NA & NA & NA & NA & 15 & 15 & 18 & 53 & 14 & 15 & 3 & 22 & 1 & 0 & 52 & 105 & 157 \\
\hline Sub-Saharan Africa & NA & NA & NA & NA & NA & NA & 13 & 23 & 31 & 50 & 12 & 37 & 4 & 23 & 1 & 2 & 61 & 135 & 195 \\
\hline Low- and middle-income countries & NA & NA & NA & NA & NA & NA & 169 & 150 & 456 & 495 & 394 & 522 & 217 & 403 & 29 & 72 & 1,265 & 1,641 & 2,906 \\
\hline High-income countries & NA & NA & NA & NA & NA & NA & 26 & 14 & 74 & 43 & 55 & 50 & 52 & 81 & 21 & 60 & 228 & 248 & 476 \\
\hline WORLD & NA & NA & NA & NA & NA & NA & 195 & 164 & 530 & 537 & 449 & 572 & 268 & 484 & 50 & 132 & 1,493 & 1,889 & 3,382 \\
\hline \multicolumn{20}{|l|}{ Attributable DALYs (thousands) } \\
\hline East Asia and Pacific & NA & NA & NA & NA & NA & NA & 62 & 44 & 179 & 161 & 178 & 211 & 91 & 128 & 2 & 8 & 513 & 552 & 1,065 \\
\hline Europe and Central Asia & NA & NA & NA & NA & NA & NA & 33 & 22 & 105 & 97 & 100 & 141 & 58 & 137 & 11 & 35 & 307 & 431 & 738 \\
\hline Latin America and the Caribbean & NA & NA & NA & NA & NA & NA & 28 & 33 & 74 & 88 & 58 & 77 & 43 & 71 & 13 & 29 & 217 & 299 & 515 \\
\hline Middle East and North Africa & NA & NA & NA & NA & NA & NA & 23 & 18 & 68 & 65 & 56 & 66 & 39 & 53 & 7 & 13 & 194 & 215 & 409 \\
\hline South Asia & NA & NA & NA & NA & NA & NA & 16 & 16 & 20 & 57 & 17 & 17 & 4 & 28 & 2 & 0 & 58 & 118 & 176 \\
\hline Sub-Saharan Africa & NA & NA & NA & NA & NA & NA & 14 & 24 & 33 & 53 & 13 & 40 & 5 & 26 & 1 & 2 & 65 & 145 & 209 \\
\hline Low- and middle-income countries & NA & NA & NA & NA & NA & NA & 177 & 157 & 479 & 520 & 420 & 552 & 241 & 442 & 36 & 87 & 1,353 & 1,759 & 3,112 \\
\hline High-income countries & NA & NA & NA & NA & NA & NA & 27 & 15 & 78 & 44 & 59 & 53 & 59 & 90 & 32 & 90 & 254 & 292 & 546 \\
\hline WORLD & NA & NA & NA & NA & NA & NA & 204 & 172 & 557 & 564 & 479 & 605 & 300 & 532 & 67 & 177 & 1,607 & 2,051 & 3,658 \\
\hline
\end{tabular}

Source: Authors' calculations.

Note: $\mathrm{NA}=$ not applicable. 
Table 4A.36

Risk factor: Overweight and obesity

Disease:

Ischemic heart disease

\begin{tabular}{|c|c|c|c|c|c|c|c|c|c|c|c|c|c|c|c|c|c|c|c|}
\hline \multirow[b]{2}{*}{ Region } & \multicolumn{2}{|c|}{$0-4$ years } & \multicolumn{2}{|c|}{ 5-14 years } & \multicolumn{2}{|c|}{$15-29$ years } & \multicolumn{2}{|c|}{ 30-44 years } & \multicolumn{2}{|c|}{$45-59$ years } & \multicolumn{2}{|c|}{$60-69$ years } & \multicolumn{2}{|c|}{ 70-79 years } & \multicolumn{2}{|c|}{$80+$ years } & \multicolumn{3}{|c|}{ Total } \\
\hline & Male & Female & Male & Female & Male & Female & Male & Female & Male & Female & Male & Female & Male & Female & Male & Female & Male & Female & All \\
\hline \multicolumn{20}{|l|}{ PAF of Mortality (\%) } \\
\hline East Asia and Pacific & NA & NA & NA & NA & NA & NA & 23 & 26 & 19 & 23 & 14 & 20 & 7 & 9 & 0 & 1 & 10 & 10 & 10 \\
\hline Europe and Central Asia & NA & NA & NA & NA & NA & NA & 42 & 52 & 39 & 51 & 30 & 42 & 20 & 27 & 9 & 10 & 25 & 23 & 24 \\
\hline Latin America and the Caribbean & NA & NA & NA & NA & NA & NA & 42 & 52 & 36 & 46 & 26 & 34 & 18 & 24 & 6 & 8 & 22 & 23 & 23 \\
\hline Middle East and North Africa & NA & NA & NA & NA & NA & NA & 43 & 58 & 35 & 45 & 22 & 29 & 14 & 16 & 5 & 6 & 21 & 22 & 22 \\
\hline South Asia & NA & NA & NA & NA & NA & NA & 10 & 17 & 5 & 16 & 4 & 4 & 1 & 7 & 1 & 0 & 3 & 7 & 5 \\
\hline Sub-Saharan Africa & NA & NA & NA & NA & NA & NA & 19 & 28 & 17 & 19 & 7 & 12 & 3 & 7 & 1 & 1 & 7 & 9 & 8 \\
\hline Low- and middle-income countries & NA & NA & NA & NA & NA & NA & 26 & 28 & 20 & 27 & 16 & 19 & 10 & 16 & 4 & 6 & 13 & 15 & 14 \\
\hline High-income countries & NA & NA & NA & NA & NA & NA & 49 & 49 & 43 & 44 & 32 & 36 & 21 & 24 & 8 & 9 & 22 & 16 & 19 \\
\hline WORLD & NA & NA & NA & NA & NA & NA & 28 & 29 & 23 & 28 & 18 & 20 & 12 & 17 & 5 & 7 & 15 & 15 & $\overline{15}$ \\
\hline
\end{tabular}

PAF of YLL (\%)

\begin{tabular}{|c|c|c|c|c|c|c|c|c|c|c|c|c|c|c|c|c|c|c|c|}
\hline East Asia and Pacific & NA & NA & NA & NA & NA & NA & 23 & 26 & 19 & 23 & 14 & 20 & 7 & 9 & 0 & 1 & 13 & 14 & 13 \\
\hline Europe and Central Asia & NA & NA & NA & NA & NA & NA & 42 & 52 & 39 & 51 & 30 & 42 & 20 & 27 & 9 & 10 & 30 & 30 & 30 \\
\hline Latin America and the Caribbean & NA & NA & NA & NA & NA & NA & 42 & 52 & 36 & 46 & 26 & 34 & 18 & 24 & 6 & 8 & 27 & 30 & 28 \\
\hline Middle East and North Africa & NA & NA & NA & NA & NA & NA & 43 & 58 & 35 & 45 & 22 & 29 & 14 & 16 & 5 & 6 & 25 & 28 & 26 \\
\hline South Asia & NA & NA & NA & NA & NA & NA & 10 & 17 & 5 & 16 & 4 & 4 & 1 & 7 & 1 & 0 & 4 & 8 & 6 \\
\hline Sub-Saharan Africa & NA & NA & NA & NA & NA & NA & 19 & 28 & 17 & 19 & 7 & 12 & 3 & 7 & 1 & 1 & 10 & 12 & 11 \\
\hline Low- and middle-income countries & NA & NA & NA & NA & NA & NA & 26 & 28 & 21 & 27 & 16 & 19 & 10 & 15 & 4 & 6 & 16 & 18 & 17 \\
\hline High-income countries & NA & NA & NA & NA & NA & NA & 49 & 49 & 43 & 44 & 32 & 36 & 21 & 24 & 8 & 9 & 28 & 23 & 26 \\
\hline WORLD & NA & NA & NA & NA & NA & NA & 28 & 29 & 23 & 28 & 18 & 20 & 12 & 17 & 5 & 6 & 18 & 18 & 18 \\
\hline
\end{tabular}

PAF of DALYs $(\%)$

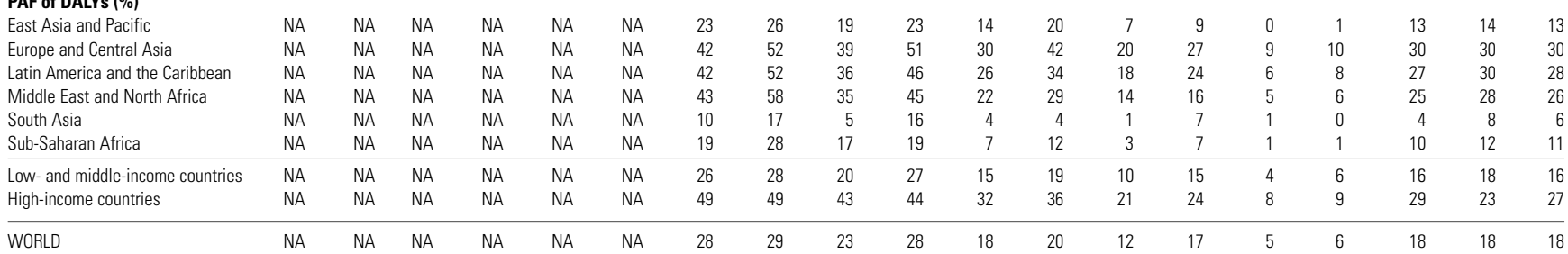

Attributable Mortality (thousands)

\begin{tabular}{|c|c|c|c|c|c|c|c|c|c|c|c|c|c|c|c|c|c|c|c|}
\hline East Asia and Pacific & NA & NA & NA & NA & NA & NA & 7 & 4 & 19 & 13 & 20 & 22 & 14 & 16 & 0 & 1 & 60 & 57 & 118 \\
\hline Europe and Central Asia & NA & NA & NA & NA & NA & NA & 16 & 4 & 60 & 22 & 68 & 54 & 52 & 86 & 11 & 38 & 207 & 203 & 410 \\
\hline Latin America and the Caribbean & NA & NA & NA & NA & NA & NA & 4 & 2 & 14 & 8 & 13 & 10 & 11 & 11 & 3 & 5 & 44 & 36 & 81 \\
\hline Middle East and North Africa & NA & NA & NA & NA & NA & NA & 5 & 2 & 14 & 9 & 11 & 9 & 8 & 8 & 1 & 2 & 39 & 30 & 69 \\
\hline South Asia & NA & NA & NA & NA & NA & NA & 5 & 6 & 12 & 20 & 12 & 9 & 4 & 18 & 1 & 0 & 34 & 53 & 88 \\
\hline Sub-Saharan Africa & NA & NA & NA & NA & NA & NA & 1 & 1 & 7 & 5 & 4 & 5 & 2 & 4 & 0 & 0 & 13 & 15 & 29 \\
\hline Low- and middle-income countries & NA & NA & NA & NA & NA & NA & 37 & 19 & 127 & 78 & 127 & 110 & 91 & 142 & 17 & 47 & 399 & 395 & 794 \\
\hline High-income countries & NA & NA & NA & NA & NA & NA & 8 & 2 & 37 & 10 & 41 & 19 & 50 & 38 & 21 & 36 & 156 & 104 & 261 \\
\hline WORLD & NA & NA & NA & NA & NA & NA & 45 & 21 & 164 & 88 & 168 & 129 & 140 & 180 & 38 & 82 & 555 & 499 & 1,055 \\
\hline
\end{tabular}

Attributable YLL (thousands)

\begin{tabular}{|c|c|c|c|c|c|c|c|c|c|c|c|c|c|c|c|c|c|c|c|}
\hline East Asia and Pacific & NA & NA & NA & NA & NA & NA & 165 & 105 & 359 & 262 & 270 & 336 & 124 & 163 & 2 & 7 & 920 & 874 & 1,793 \\
\hline Europe and Central Asia & NA & NA & NA & NA & NA & NA & 367 & 91 & 1,135 & 444 & 917 & 806 & 472 & 873 & 52 & 175 & 2,943 & 2,388 & 5,332 \\
\hline Latin America and the Caribbean & NA & NA & NA & NA & NA & NA & 90 & 51 & 265 & 166 & 173 & 152 & 97 & 109 & 13 & 23 & 637 & 502 & 1,139 \\
\hline Middle East and North Africa & NA & NA & NA & NA & NA & NA & 110 & 56 & 271 & 174 & 146 & 140 & 74 & 80 & 7 & 10 & 608 & 460 & 1,068 \\
\hline South Asia & NA & NA & NA & NA & NA & NA & 119 & 135 & 228 & 403 & 163 & 139 & 32 & 193 & 6 & 0 & 548 & 870 & 1,419 \\
\hline Sub-Saharan Africa & NA & NA & NA & NA & NA & NA & 32 & 23 & 124 & 99 & 48 & 82 & 15 & 38 & 1 & 2 & 219 & 243 & 462 \\
\hline Low- and middle-income countries & NA & NA & NA & NA & NA & NA & 883 & 461 & 2,382 & 1,547 & 1,717 & 1,655 & 814 & 1,456 & 80 & 218 & 5,875 & 5,337 & 11,213 \\
\hline High-income countries & NA & NA & NA & NA & NA & NA & 182 & 45 & 695 & 199 & 552 & 283 & 434 & 377 & 90 & 146 & 1,953 & 1,050 & 3,003 \\
\hline WORLD & NA & NA & NA & NA & NA & NA & 1,065 & 506 & 3,077 & 1,747 & 2,268 & 1,938 & 1,248 & 1,833 & 170 & 364 & 7,828 & 6,387 & 14,216 \\
\hline
\end{tabular}

Attributable DALYs (thousands)

\begin{tabular}{|c|c|c|c|c|c|c|c|c|c|c|c|c|c|c|c|c|c|c|c|}
\hline East Asia and Pacific & NA & NA & NA & NA & NA & NA & 183 & 117 & 383 & 282 & 283 & 353 & 126 & 166 & 2 & 7 & 978 & 925 & 1,902 \\
\hline Europe and Central Asia & NA & NA & NA & NA & NA & NA & 394 & 104 & 1,179 & 476 & 946 & 844 & 480 & 889 & 52 & 178 & 3,052 & 2,491 & 5,543 \\
\hline Latin America and the Caribbean & NA & NA & NA & NA & NA & NA & 102 & 59 & 283 & 181 & 184 & 161 & 99 & 112 & 13 & 24 & 681 & 538 & 1,219 \\
\hline Middle East and North Africa & NA & NA & NA & NA & NA & NA & 122 & 64 & 286 & 185 & 153 & 146 & 76 & 81 & 7 & 11 & 644 & 487 & 1,131 \\
\hline South Asia & NA & NA & NA & NA & NA & NA & 136 & 155 & 241 & 434 & 172 & 146 & 33 & 198 & 7 & 0 & 588 & 933 & 1,521 \\
\hline Sub-Saharan Africa & NA & NA & NA & NA & NA & NA & 36 & 26 & 132 & 105 & 50 & 86 & 15 & 39 & 1 & 2 & 234 & 258 & 492 \\
\hline Low- and middle-income countries & NA & NA & NA & NA & NA & NA & 972 & 525 & 2,505 & 1,664 & 1,789 & 1,737 & 830 & 1,484 & 81 & 221 & 6,177 & 5,631 & 11,808 \\
\hline High-income countries & NA & NA & NA & NA & NA & NA & 208 & 60 & 766 & 239 & 594 & 317 & 456 & 402 & 93 & 152 & 2,117 & 1,170 & 3,287 \\
\hline ORLD & NA & NA & NA & NA & NA & NA & 1,180 & 585 & 3,270 & 1,903 & 2,383 & 2,055 & 1,286 & 1,887 & 174 & 372 & 8,294 & 6,802 & 15,096 \\
\hline
\end{tabular}

Source: Authors' calculations.

Note: $\mathrm{NA}=$ not applicable. 
Table 4A.37

Risk factor: Overweight and obesity

Disease: Cerebrovascular disease

\begin{tabular}{|c|c|c|c|c|c|c|c|c|c|c|c|c|c|c|c|c|c|c|c|}
\hline \multirow[b]{2}{*}{ Region } & \multicolumn{2}{|c|}{$0-4$ years } & \multicolumn{2}{|c|}{ 5-14 years } & \multicolumn{2}{|c|}{$15-29$ years } & \multicolumn{2}{|c|}{ 30-44 years } & \multicolumn{2}{|c|}{ 45-59 years } & \multicolumn{2}{|c|}{$60-69$ years } & \multicolumn{2}{|c|}{$70-79$ years } & \multicolumn{2}{|c|}{$80+$ years } & \multicolumn{3}{|c|}{ Total } \\
\hline & Male & Female & Male & Female & Male & Female & Male & Female & Male & Female & Male & Female & Male & Female & Male & Female & Male & Female & All \\
\hline \multicolumn{20}{|l|}{ PAF of Mortality (\%) } \\
\hline East Asia and Pacific & NA & NA & NA & NA & NA & NA & 7 & 8 & 7 & 9 & 6 & 9 & 4 & 5 & 0 & 0 & 4 & 4 & 4 \\
\hline Europe and Central Asia & NA & NA & NA & NA & NA & NA & 21 & 27 & 23 & 30 & 19 & 27 & 14 & 20 & 7 & 8 & 16 & 17 & 16 \\
\hline Latin America and the Caribbean & NA & NA & NA & NA & NA & NA & 19 & 24 & 20 & 25 & 16 & 21 & 13 & 16 & 5 & 7 & 13 & 15 & 14 \\
\hline Middle East and North Africa & NA & NA & NA & NA & NA & NA & 20 & 27 & 19 & 24 & 13 & 18 & 10 & 11 & 4 & 5 & 10 & 12 & 11 \\
\hline South Asia & NA & NA & NA & NA & NA & NA & 5 & 8 & 3 & 9 & 2 & 2 & 1 & 5 & 1 & 0 & 2 & 4 & 3 \\
\hline Sub-Saharan Africa & NA & NA & NA & NA & NA & NA & 9 & 13 & 9 & 11 & 4 & 7 & 2 & 5 & 1 & 1 & 4 & 6 & 5 \\
\hline Low- and middle-income countries & NA & NA & NA & NA & NA & NA & 11 & 15 & 10 & 14 & 8 & 11 & 6 & 9 & 2 & 4 & 7 & 8 & 7 \\
\hline High-income countries & NA & NA & NA & NA & NA & NA & 25 & 25 & 25 & 26 & 20 & 23 & 16 & 17 & 7 & 7 & 13 & 11 & 12 \\
\hline WORLD & NA & NA & NA & NA & NA & NA & 13 & 16 & 11 & 15 & 9 & 12 & 7 & 10 & 3 & 5 & 7 & 9 & 8 \\
\hline
\end{tabular}

PAF of YLL (\%)

\begin{tabular}{|c|c|c|c|c|c|c|c|c|c|c|c|c|c|c|c|c|c|c|c|}
\hline East Asia and Pacific & NA & NA & NA & NA & NA & NA & 7 & 8 & 7 & 9 & 6 & 9 & 4 & 5 & 0 & 0 & 5 & 6 & 6 \\
\hline Europe and Central Asia & NA & NA & NA & NA & NA & NA & 21 & 27 & 23 & 30 & 19 & 27 & 14 & 20 & 7 & 8 & 17 & 20 & 19 \\
\hline Latin America and the Caribbean & NA & NA & NA & NA & NA & NA & 19 & 24 & 20 & 25 & 16 & 21 & 13 & 16 & 5 & 7 & 15 & 18 & 16 \\
\hline Middle East and North Africa & NA & NA & NA & NA & NA & NA & 20 & 27 & 19 & 24 & 13 & 18 & 10 & 11 & 4 & 5 & 11 & 14 & 13 \\
\hline South Asia & NA & NA & NA & NA & NA & NA & 5 & 8 & 3 & 9 & 2 & 2 & 1 & 5 & 1 & 0 & 2 & 4 & 3 \\
\hline Sub-Saharan Africa & NA & NA & NA & NA & NA & NA & 9 & 13 & 9 & 11 & 4 & 7 & 2 & 5 & 1 & 1 & 5 & 7 & 6 \\
\hline Low- and middle-income countries & NA & NA & NA & NA & NA & NA & 11 & 15 & 10 & 14 & 8 & 11 & 6 & 9 & 2 & 3 & 7 & 10 & 9 \\
\hline High-income countries & NA & NA & NA & NA & NA & NA & 25 & 25 & 25 & 26 & 20 & 23 & 16 & 17 & 7 & 7 & 16 & 15 & 16 \\
\hline WORLD & NA & NA & NA & NA & NA & NA & 13 & 16 & 11 & 15 & 9 & 12 & 7 & 10 & 3 & 4 & 8 & 10 & 9 \\
\hline
\end{tabular}

PAF of DALYs $(\%)$

\begin{tabular}{|c|c|c|c|c|c|c|c|c|c|c|c|c|c|c|c|c|c|c|c|}
\hline East Asia and Pacific & NA & NA & NA & NA & NA & NA & 10 & 11 & 9 & 11 & 8 & 11 & 5 & 5 & 0 & 1 & 7 & 8 & 7 \\
\hline Europe and Central Asia & NA & NA & NA & NA & NA & NA & 25 & 32 & 26 & 34 & 21 & 30 & 15 & 21 & 7 & 9 & 19 & 23 & 21 \\
\hline Latin America and the Caribbean & NA & NA & NA & NA & NA & NA & 24 & 31 & 23 & 30 & 19 & 24 & 14 & 18 & 5 & 7 & 18 & 22 & 20 \\
\hline Middle East and North Africa & NA & NA & NA & NA & NA & NA & 25 & 33 & 22 & 28 & 15 & 20 & 11 & 12 & 4 & 5 & 13 & 17 & 15 \\
\hline South Asia & NA & NA & NA & NA & NA & NA & 6 & 9 & 3 & 10 & 3 & 3 & 1 & 5 & 1 & 0 & 2 & 5 & 4 \\
\hline Sub-Saharan Africa & NA & NA & NA & NA & NA & NA & 9 & 14 & 10 & 12 & 4 & 8 & 2 & 5 & 1 & 1 & 6 & 8 & 7 \\
\hline Low- and middle-income countries & NA & NA & NA & NA & NA & NA & 14 & 19 & 12 & 17 & 10 & 13 & 6 & 10 & 2 & 3 & 9 & 12 & 10 \\
\hline High-income countries & NA & NA & NA & NA & NA & NA & 33 & 34 & 32 & 33 & 25 & 29 & 18 & 20 & 7 & 8 & 21 & 20 & 20 \\
\hline WORLD & NA & NA & NA & NA & NA & NA & 16 & 21 & 14 & 18 & 11 & 15 & 8 & 11 & 4 & 5 & 10 & 13 & 12 \\
\hline
\end{tabular}

Attributable Mortality (thousands)

\begin{tabular}{|c|c|c|c|c|c|c|c|c|c|c|c|c|c|c|c|c|c|c|c|}
\hline East Asia and Pacific & NA & NA & NA & NA & NA & NA & 2 & 1 & 10 & 8 & 15 & 15 & 14 & 17 & 0 & 2 & 42 & 42 & 84 \\
\hline Europe and Central Asia & NA & NA & NA & NA & NA & NA & 3 & 2 & 13 & 10 & 21 & 25 & 21 & 44 & 6 & 22 & 63 & 104 & 166 \\
\hline Latin America and the Caribbean & NA & NA & NA & NA & NA & NA & 1 & 2 & 4 & 5 & 4 & 5 & 5 & 6 & 2 & 3 & 16 & 20 & 37 \\
\hline Middle East and North Africa & NA & NA & NA & NA & NA & NA & 1 & 1 & 2 & 2 & 2 & 2 & 2 & 3 & 1 & 1 & 7 & 8 & 15 \\
\hline South Asia & NA & NA & NA & NA & NA & NA & 0 & 0 & 2 & 5 & 3 & 3 & 1 & 8 & 1 & 0 & 8 & 16 & 24 \\
\hline Sub-Saharan Africa & NA & NA & NA & NA & NA & NA & 1 & 1 & 3 & 4 & 2 & 4 & 1 & 3 & 0 & 0 & 6 & 12 & 18 \\
\hline Low- and middle-income countries & NA & NA & NA & NA & NA & NA & 8 & 7 & 33 & 33 & 48 & 53 & 44 & 81 & 9 & 28 & 142 & 202 & 344 \\
\hline High-income countries & NA & NA & NA & NA & NA & NA & 2 & 1 & 6 & 4 & 9 & 7 & 16 & 18 & 10 & 22 & 42 & 51 & 94 \\
\hline WORLD & NA & NA & NA & NA & NA & NA & 9 & 8 & 39 & 37 & 57 & 59 & 60 & 99 & 19 & 50 & 184 & 254 & 438 \\
\hline
\end{tabular}

Attributable YLL (thousands)

\begin{tabular}{|c|c|c|c|c|c|c|c|c|c|c|c|c|c|c|c|c|c|c|c|}
\hline East Asia and Pacific & NA & NA & NA & NA & NA & NA & 42 & 30 & 185 & 157 & 207 & 218 & 127 & 171 & 2 & 8 & 563 & 585 & 1,148 \\
\hline Europe and Central Asia & NA & NA & NA & NA & NA & NA & 65 & 47 & 237 & 210 & 286 & 371 & 186 & 452 & 26 & 104 & 799 & 1,183 & 1,983 \\
\hline Latin America and the Caribbean & NA & NA & NA & NA & NA & NA & 30 & 39 & 79 & 92 & 58 & 70 & 42 & 62 & 8 & 17 & 218 & 278 & 496 \\
\hline Middle East and North Africa & NA & NA & NA & NA & NA & NA & 12 & 13 & 31 & 36 & 26 & 34 & 19 & 26 & 3 & 5 & 90 & 113 & 203 \\
\hline South Asia & NA & NA & NA & NA & NA & NA & 11 & 11 & 38 & 90 & 47 & 46 & 12 & 86 & 3 & 0 & 111 & 234 & 345 \\
\hline Sub-Saharan Africa & NA & NA & NA & NA & NA & NA & 23 & 27 & 50 & 78 & 20 & 57 & 8 & 32 & 1 & 2 & 101 & 195 & 296 \\
\hline Low- and middle-income countries & NA & NA & NA & NA & NA & NA & 182 & 167 & 621 & 663 & 644 & 795 & 394 & 829 & 42 & 135 & 1,882 & 2,589 & 4,471 \\
\hline High-income countries & NA & NA & NA & NA & NA & NA & 36 & 27 & 117 & 81 & 119 & 98 & 136 & 175 & 43 & 90 & 451 & 472 & 923 \\
\hline WORLD & NA & NA & NA & NA & NA & NA & 218 & 194 & 738 & 743 & 763 & 894 & 530 & 1,004 & 85 & 225 & 2,333 & 3,060 & 5,394 \\
\hline \multicolumn{20}{|l|}{ Attributable DALYs (thousands) } \\
\hline East Asia and Pacific & NA & NA & NA & NA & NA & NA & 82 & 61 & 329 & 278 & 333 & 342 & 170 & 223 & 2 & 10 & 917 & 914 & 1,831 \\
\hline Europe and Central Asia & NA & NA & NA & NA & NA & NA & 91 & 78 & 330 & 313 & 397 & 531 & 239 & 560 & 30 & 116 & 1,086 & 1,599 & 2,685 \\
\hline Latin America and the Caribbean & NA & NA & NA & NA & NA & NA & 51 & 73 & 132 & 152 & 93 & 108 & 58 & 81 & 9 & 20 & 343 & 433 & 776 \\
\hline Middle East and North Africa & NA & NA & NA & NA & NA & NA & 21 & 22 & 48 & 54 & 37 & 47 & 25 & 31 & 3 & 5 & 134 & 159 & 293 \\
\hline South Asia & NA & NA & NA & NA & NA & NA & 16 & 16 & 55 & 127 & 65 & 62 & 15 & 104 & 3 & 0 & 154 & 308 & 462 \\
\hline Sub-Saharan Africa & NA & NA & NA & NA & NA & NA & 26 & 31 & 59 & 95 & 24 & 68 & 9 & 36 & 1 & 2 & 119 & 233 & 351 \\
\hline Low- and middle-income countries & NA & NA & NA & NA & NA & NA & 287 & 281 & 954 & 1,018 & 950 & 1,159 & 513 & 1,035 & 49 & 153 & 2,754 & 3,646 & 6,399 \\
\hline High-income countries & NA & NA & NA & NA & NA & NA & 93 & 73 & 297 & 217 & 275 & 232 & 240 & 306 & 58 & 121 & 963 & 949 & 1,912 \\
\hline WORLD & NA & NA & NA & NA & NA & NA & 380 & 355 & 1,251 & 1,235 & 1,226 & 1,391 & 753 & 1,340 & 107 & 274 & 3,717 & 4,595 & 8,311 \\
\hline
\end{tabular}

Source: Authors' calculations.

Note: NA = not applicable. 
Table 4A.38

Risk factor: Overweight and obesity

Disease: Osteoarthritis

\begin{tabular}{|c|c|c|c|c|c|c|c|c|c|c|c|c|c|c|c|c|c|c|c|}
\hline \multirow[b]{2}{*}{ Region } & \multicolumn{2}{|c|}{$0-4$ years } & \multicolumn{2}{|c|}{ 5-14 years } & \multicolumn{2}{|c|}{$15-29$ years } & \multicolumn{2}{|c|}{ 30-44 years } & \multicolumn{2}{|c|}{ 45-59 years } & \multicolumn{2}{|c|}{ 60-69 years } & \multicolumn{2}{|c|}{ 70-79 years } & \multicolumn{2}{|c|}{$80+$ years } & \multicolumn{3}{|c|}{ Total } \\
\hline & Male & Female & Male & Female & Male & Female & Male & Female & Male & Female & Male & Female & Male & Female & Male & Female & Male & Female & All \\
\hline \multicolumn{20}{|l|}{ PAF of Mortality (\%) } \\
\hline East Asia and Pacific & NA & NA & NA & NA & NA & NA & 8 & 9 & 8 & 10 & 8 & 12 & 6 & 8 & 1 & 1 & 5 & 6 & 6 \\
\hline Europe and Central Asia & NA & NA & NA & NA & NA & NA & 16 & 20 & 19 & 26 & 19 & 27 & 17 & 24 & 16 & 18 & 17 & 23 & 21 \\
\hline Latin America and the Caribbean & NA & NA & NA & NA & NA & NA & 15 & 20 & 17 & 23 & 17 & 22 & 16 & 21 & 12 & 15 & 14 & 17 & 16 \\
\hline Middle East and North Africa & NA & NA & NA & NA & NA & NA & 15 & 22 & 17 & 22 & 14 & 18 & 12 & 14 & 9 & 12 & 10 & 16 & 13 \\
\hline South Asia & NA & NA & NA & NA & NA & NA & 3 & 5 & 2 & 7 & 2 & 2 & 1 & 6 & 2 & 0 & 2 & 3 & 3 \\
\hline Sub-Saharan Africa & NA & NA & NA & NA & NA & NA & ${ }^{*}$ & * & 7 & 8 & * & 7 & 2 & 6 & 2 & 2 & 3 & 5 & 4 \\
\hline Low- and middle-income countries & NA & NA & NA & NA & NA & NA & 12 & 14 & 12 & 16 & 12 & 20 & 10 & 15 & 7 & 12 & 9 & 14 & 12 \\
\hline High-income countries & NA & NA & NA & NA & NA & NA & 19 & 18 & 21 & 22 & 20 & 23 & 19 & 21 & 15 & 16 & 17 & 17 & 17 \\
\hline WORLD & NA & NA & NA & NA & NA & NA & 15 & 14 & 14 & 18 & 15 & 21 & 14 & 19 & 12 & 15 & 13 & 16 & 15 \\
\hline
\end{tabular}

PAF of YLL (\%)

\begin{tabular}{|c|c|c|c|c|c|c|c|c|c|c|c|c|c|c|c|c|c|c|c|}
\hline East Asia and Pacific & NA & NA & NA & NA & NA & NA & 8 & 9 & 8 & 10 & 8 & 12 & 6 & 8 & 1 & 1 & 7 & 7 & 7 \\
\hline Europe and Central Asia & NA & NA & NA & NA & NA & NA & 16 & 20 & 19 & 26 & 19 & 27 & 17 & 24 & 16 & 18 & 16 & 24 & 21 \\
\hline Latin America and the Caribbean & NA & NA & NA & NA & NA & NA & 15 & 20 & 17 & 23 & 17 & 22 & 16 & 21 & 12 & 15 & 14 & 19 & 17 \\
\hline Middle East and North Africa & NA & NA & NA & NA & NA & NA & 15 & 22 & 17 & 22 & 14 & 18 & 12 & 14 & 9 & 12 & 9 & 18 & 13 \\
\hline South Asia & NA & NA & NA & NA & NA & NA & 3 & 5 & 2 & 7 & 2 & 2 & 1 & 6 & 2 & 0 & 2 & 4 & 3 \\
\hline Sub-Saharan Africa & NA & NA & NA & NA & NA & NA & * & $*$ & 7 & 8 & * & 7 & 2 & 6 & 2 & 2 & 4 & 6 & 6 \\
\hline Low- and middle-income countries & NA & NA & NA & NA & NA & NA & 12 & 14 & 12 & 16 & 12 & 20 & 10 & 15 & 7 & 11 & 10 & 15 & 12 \\
\hline High-income countries & NA & NA & NA & NA & NA & NA & 19 & 18 & 21 & 22 & 20 & 23 & 19 & 21 & 15 & 16 & 18 & 18 & 18 \\
\hline WORLD & NA & NA & NA & NA & NA & NA & 14 & 14 & 14 & 18 & 15 & 21 & 14 & 18 & 12 & 15 & 13 & 17 & 15 \\
\hline
\end{tabular}

PAF of DALYs $(\%)$

\begin{tabular}{|c|c|c|c|c|c|c|c|c|c|c|c|c|c|c|c|c|c|c|c|}
\hline East Asia and Pacific & NA & NA & NA & NA & NA & NA & 8 & 9 & 8 & 10 & 8 & 12 & 6 & 8 & 1 & 1 & 8 & 9 & 9 \\
\hline Europe and Central Asia & NA & NA & NA & NA & NA & NA & 16 & 20 & 19 & 26 & 19 & 27 & 17 & 24 & 16 & 18 & 16 & 24 & 21 \\
\hline Latin America and the Caribbean & NA & NA & NA & NA & NA & NA & 15 & 20 & 17 & 23 & 17 & 22 & 16 & 21 & 12 & 15 & 16 & 21 & 19 \\
\hline Middle East and North Africa & NA & NA & NA & NA & NA & NA & 15 & 22 & 17 & 22 & 14 & 18 & 12 & 14 & 9 & 12 & 14 & 18 & 16 \\
\hline South Asia & NA & NA & NA & NA & NA & NA & 3 & 5 & 2 & 7 & 2 & 2 & 1 & 6 & 2 & 0 & 2 & 5 & 4 \\
\hline Sub-Saharan Africa & NA & NA & NA & NA & NA & NA & 6 & 9 & 7 & 8 & 4 & 7 & 2 & 6 & 2 & 2 & 5 & 7 & 6 \\
\hline Low- and middle-income countries & NA & NA & NA & NA & NA & NA & 9 & 11 & 10 & 14 & 10 & 14 & 9 & 15 & 7 & 10 & 9 & 12 & 11 \\
\hline High-income countries & NA & NA & NA & NA & NA & NA & 19 & 18 & 21 & 22 & 20 & 23 & 19 & 21 & 15 & 16 & 19 & 21 & 20 \\
\hline JRLD & NA & NA & NA & NA & NA & NA & 10 & 11 & 12 & 15 & 12 & 17 & 13 & 18 & 11 & 14 & 11 & 14 & 10 \\
\hline
\end{tabular}

Attributable Mortality (thousands)

\begin{tabular}{llllllllllllllllllll} 
East Asia and Pacific & NA & NA & NA & NA & NA & NA & 0 & 0 & 0 & 0 & 0 & 0 & 0 & 0 & 0 & 0 & 0 & 0 & 0 \\
Europe and Central Asia & NA & NA & NA & NA & NA & NA & 0 & 0 & 0 & 0 & 0 & 0 & 0 & 0 & 0 & 0 & 0 & 0 & 0 \\
Latin America and the Caribbean & NA & NA & NA & NA & NA & NA & 0 & 0 & 0 & 0 & 0 & 0 & 0 & 0 & 0 & 0 & 0 & 0 & 0 \\
Middle East and North Africa & NA & NA & NA & NA & NA & NA & 0 & 0 & 0 & 0 & 0 & 0 & 0 & 0 & 0 & 0 & 0 & 0 & 0 \\
South Asia & NA & NA & NA & NA & NA & NA & 0 & 0 & 0 & 0 & 0 & 0 & 0 & 0 & 0 & 0 & 0 & 0 & 0 \\
Sub-Saharan Africa & NA & NA & NA & NA & NA & NA & 0 & 0 & 0 & 0 & 0 & 0 & 0 & 0 & 0 & 0 & 0 & 0 & 0 \\
\hline Low- and middle-income countries & NA & NA & NA & NA & NA & NA & 0 & 0 & 0 & 0 & 0 & 0 & 0 & 0 & 0 & 0 & 0 & 0 & 0 \\
High-income countries & NA & NA & NA & NA & NA & NA & 0 & 0 & 0 & 0 & 0 & 0 & 0 \\
\hline WORLD & NA & NA & NA & NA & NA & NA & 0 & 0 & 0 & 0 & 0 & 0 & 0 & 0 & 0 & 0 \\
\hline
\end{tabular}

Attributable YLL (thousands)

\begin{tabular}{lllllllllllllllllllll} 
East Asia and Pacific & NA & NA & NA & NA & NA & NA & 0 & 0 & 0 & 0 & 0 & 0 & 0 & 0 & 0 & 0 & 0 & 0 & 0 \\
Europe and Central Asia & NA & NA & NA & NA & NA & NA & 0 & 0 & 0 & 0 & 0 & 0 & 0 & 0 & 0 & 0 & 0 & 0 & 0 \\
Latin America and the Caribbean & NA & NA & NA & NA & NA & NA & 0 & 0 & 0 & 0 & 0 & 0 & 0 & 0 & 0 & 0 & 0 & 1 & 1 \\
Middle East and North Africa & NA & NA & NA & NA & NA & NA & 0 & 0 & 0 & 0 & 0 & 0 & 0 & 0 & 0 & 0 & 0 & 0 & 0 \\
South Asia & NA & NA & NA & NA & NA & NA & 0 & 0 & 0 & 0 & 0 & 0 & 0 & 0 & 0 & 0 & 0 & 0 & 0 \\
Sub-Saharan Africa & NA & NA & NA & NA & NA & NA & 0 & 0 & 0 & 0 & 0 & 0 & 0 & 0 & 0 & 0 & 0 & 0 & 0 \\
\hline Low- and middle-income countries & NA & NA & NA & NA & NA & NA & 0 & 0 & 0 & 0 & 0 & 0 & 0 & 0 & 0 & 0 & 1 & 1 & 2 \\
High-income countries & NA & NA & NA & NA & NA & NA & 0 & 0 & 0 & 0 & 0 & 0 & 0 & 1 \\
\hline WORLD & NA & NA & NA & NA & NA & NA & 0 & 0 & 0 & 0 & 0 & 1 & 1 & 1 & 0 & 1 \\
\hline
\end{tabular}

Attributable DALYs (thousands)

\begin{tabular}{|c|c|c|c|c|c|c|c|c|c|c|c|c|c|c|c|c|c|c|c|}
\hline East Asia and Pacific & NA & NA & NA & NA & NA & NA & 37 & 84 & 76 & 148 & 42 & 78 & 9 & 14 & 0 & 0 & 164 & 325 & 489 \\
\hline Europe and Central Asia & NA & NA & NA & NA & NA & NA & 40 & 60 & 53 & 139 & 29 & 93 & 12 & 50 & 1 & 5 & 135 & 348 & 483 \\
\hline Latin America and the Caribbean & NA & NA & NA & NA & NA & NA & 16 & 19 & 30 & 56 & 20 & 50 & 14 & 34 & 2 & 6 & 81 & 164 & 245 \\
\hline Middle East and North Africa & NA & NA & NA & NA & NA & NA & 11 & 22 & 11 & 23 & 4 & 11 & 1 & 3 & 0 & 0 & 27 & 58 & 85 \\
\hline South Asia & NA & NA & NA & NA & NA & NA & 10 & 23 & 7 & 41 & 4 & 7 & 1 & 5 & 0 & 0 & 22 & 76 & 98 \\
\hline Sub-Saharan Africa & NA & NA & NA & NA & NA & NA & 8 & 21 & 14 & 18 & 3 & 9 & 1 & 3 & 0 & 0 & 27 & 51 & 78 \\
\hline Low- and middle-income countries & NA & NA & NA & NA & NA & NA & 121 & 228 & 191 & 424 & 102 & 248 & 37 & 110 & 3 & 12 & 455 & 1,023 & 1,478 \\
\hline High-income countries & NA & NA & NA & NA & NA & NA & 36 & 34 & 93 & 131 & 75 & 156 & 60 & 144 & 8 & 34 & 272 & 500 & 772 \\
\hline WORLD & NA & NA & NA & NA & NA & NA & 157 & 262 & 285 & 556 & 177 & 405 & 97 & 254 & 12 & 46 & 727 & 1,523 & 2,250 \\
\hline
\end{tabular}

Source: Authors' calculations.

Note: NA = not applicable

*The number of deaths (and hence YLL) directly coded to a number of diseases, especially neuropsychiatric and musculoskeletal diseases, is zero or very small. For other diseases, mortality or disease burden may be zero in some region-age-sex groups. In such cases, the population attributable fractions would be undefined or unstable and have not been calculated. 
Table 4A.39

Risk factor: Overweight and obesity

Disease:

All causes

\begin{tabular}{|c|c|c|c|c|c|c|c|c|c|c|c|c|c|c|c|c|c|c|c|}
\hline \multirow[b]{2}{*}{ Region } & \multicolumn{2}{|c|}{$0-4$ years } & \multicolumn{2}{|c|}{ 5-14 years } & \multicolumn{2}{|c|}{$15-29$ years } & \multicolumn{2}{|c|}{ 30-44 years } & \multicolumn{2}{|c|}{$45-59$ years } & \multicolumn{2}{|c|}{$60-69$ years } & \multicolumn{2}{|c|}{$70-79$ years } & \multicolumn{2}{|c|}{$80+$ years } & \multicolumn{3}{|c|}{ Total } \\
\hline & Male & Female & Male & Female & Male & Female & Male & Female & Male & Female & Male & Female & Male & Female & Male & Female & Male & Female & All \\
\hline \multicolumn{20}{|l|}{ PAF of Mortality (\%) } \\
\hline East Asia and Pacific & NA & NA & NA & NA & NA & NA & 2 & 3 & 4 & 6 & 4 & 8 & 3 & 4 & 0 & 0 & 2 & 3 & 3 \\
\hline Europe and Central Asia & NA & NA & NA & NA & NA & NA & 7 & 8 & 13 & 18 & 14 & 24 & 12 & 19 & 6 & 8 & 10 & 15 & 12 \\
\hline Latin America and the Caribbean & NA & NA & NA & NA & NA & NA & 4 & 8 & 12 & 19 & 12 & 20 & 10 & 16 & 5 & 7 & 7 & 11 & 9 \\
\hline Middle East and North Africa & NA & NA & NA & NA & NA & NA & 8 & 8 & 14 & 17 & 11 & 16 & 9 & 11 & 4 & 5 & 6 & 8 & 7 \\
\hline South Asia & NA & NA & NA & NA & NA & NA & 1 & 2 & 2 & 5 & 2 & 2 & 1 & 4 & 1 & 0 & 1 & 2 & 1 \\
\hline Sub-Saharan Africa & NA & NA & NA & NA & NA & NA & 1 & 1 & 2 & 4 & 2 & 5 & 1 & 4 & 0 & 1 & 1 & 1 & 1 \\
\hline Low- and middle-income countries & NA & NA & NA & NA & NA & NA & 2 & 2 & 5 & 8 & 6 & 9 & 5 & 8 & 2 & 3 & 3 & 4 & 4 \\
\hline High-income countries & NA & NA & NA & NA & NA & NA & 7 & 6 & 12 & 11 & 10 & 13 & 9 & 11 & 4 & 6 & 8 & 8 & 8 \\
\hline WORLD & NA & NA & NA & NA & NA & NA & 3 & 3 & 6 & 8 & 7 & 10 & 5 & 8 & 3 & 4 & 4 & 5 & 4 \\
\hline
\end{tabular}

PAF of YLL (\%)

\begin{tabular}{lllllllllrrrrrrrrrrr} 
East Asia and Pacific & NA & NA & NA & NA & NA & NA & 2 & 3 & 4 & 6 & 4 & 8 & 3 & 4 & 0 & 0 & 2 & 3 & 3 \\
Europe and Central Asia & NA & NA & NA & NA & NA & NA & 7 & 8 & 13 & 18 & 14 & 24 & 12 & 19 & 6 & 8 & 10 & 15 & 12 \\
Latin America and the Caribbean & NA & NA & NA & NA & NA & NA & 4 & 8 & 11 & 19 & 12 & 20 & 10 & 16 & 5 & 8 & 5 & 10 & 7 \\
Middle East and North Africa & NA & NA & NA & NA & NA & NA & 8 & 8 & 14 & 17 & 11 & 16 & 9 & 11 & 4 & 5 & 5 & 6 & 6 \\
South Asia & NA & NA & NA & NA & NA & NA & 1 & 2 & 2 & 5 & 2 & 2 & 1 & 4 & 1 & 0 & 1 & 1 & 1 \\
Sub-Saharan Africa & NA & NA & NA & NA & NA & NA & 0 & 1 & 2 & 4 & 2 & 5 & 1 & 4 & 0 & 1 & 0 & 1 & 1 \\
Low- and middle-income countries & NA & NA & NA & NA & NA & NA & 2 & 2 & 5 & 8 & 6 & 9 & 5 & 8 & 2 & 3 & 2 & 3 & 3 \\
High-income countries & NA & NA & NA & NA & NA & NA & 7 & 6 & 11 & 11 & 10 & 13 & 9 & 11 & 5 & 6 & 8 & 9 & 8 \\
\hline WORLD & NA & NA & NA & NA & NA & NA & 3 & 3 & 6 & 8 & 7 & 10 & 5 & 8 & 3 & 4 & 3 & 4 & 3 \\
\hline
\end{tabular}

PAF of DALYs (\%)

Latin America and the Caribbea

Middle East and North Afric

South Asia

Sub-Saharan Africa

Low- and middle-income countries

High-income countries

$\begin{array}{llllll}\text { NA } & \text { NA } & \text { NA } & \text { NA } & \text { NA } & \text { NA } \\ \text { NA } & \text { NA } & \text { NA } & \text { NA } & \text { NA } & \text { NA } \\ \text { NA } & \text { NA } & \text { NA } & \text { NA } & \text { NA } & \text { NA } \\ \text { NA } & \text { NA } & \text { NA } & \text { NA } & \text { NA } & \text { NA } \\ \text { NA } & \text { NA } & \text { NA } & \text { NA } & \text { NA } & \text { NA } \\ \text { NA } & \text { NA } & \text { NA } & \text { NA } & \text { NA } & \text { NA } \\ \text { NA } & \text { NA } & \text { NA } & \text { NA } & \text { NA } & \text { NA }\end{array}$

Attributable Mortality (thousands

\begin{tabular}{|c|c|c|c|c|c|c|c|c|c|c|c|c|c|c|c|c|c|c|c|}
\hline East Asia and Pacific & NA & NA & NA & NA & NA & NA & 14 & 11 & 48 & 47 & 59 & 76 & 50 & 63 & 2 & 6 & 172 & 202 & 374 \\
\hline Europe and Central Asia & NA & NA & NA & NA & NA & NA & 21 & 8 & 82 & 47 & 102 & 104 & 85 & 160 & 21 & 72 & 312 & 391 & 702 \\
\hline Latin America and the Caribbean & NA & NA & NA & NA & NA & NA & 9 & 9 & 33 & 35 & 33 & 41 & 35 & 47 & 14 & 28 & 124 & 159 & 283 \\
\hline Middle East and North Africa & NA & NA & NA & NA & NA & NA & 7 & 4 & 22 & 17 & 19 & 20 & 18 & 19 & 4 & 6 & 69 & 67 & 136 \\
\hline South Asia & NA & NA & NA & NA & NA & NA & 8 & 8 & 19 & 39 & 20 & 18 & 7 & 39 & 4 & 0 & 58 & 104 & 161 \\
\hline Sub-Saharan Africa & NA & NA & NA & NA & NA & NA & 4 & 5 & 14 & 20 & 7 & 18 & 4 & 14 & 1 & 2 & 31 & 59 & 90 \\
\hline Low- and middle-income countries & NA & NA & NA & NA & NA & NA & 64 & 46 & 218 & 205 & 240 & 275 & 199 & 341 & 46 & 114 & 766 & 981 & 1,747 \\
\hline High-income countries & NA & NA & NA & NA & NA & NA & 13 & 6 & 60 & 31 & 71 & 52 & 101 & 104 & 57 & 119 & 303 & 311 & 614 \\
\hline WORLD & NA & NA & NA & NA & NA & NA & 77 & 51 & 278 & 236 & 311 & 327 & 300 & 445 & 103 & 233 & 1,069 & 1,292 & 2,361 \\
\hline
\end{tabular}

Attributable YLL (thousands)

\begin{tabular}{|c|c|c|c|c|c|c|c|c|c|c|c|c|c|c|c|c|c|c|c|}
\hline East Asia and Pacific & NA & NA & NA & NA & NA & NA & 336 & 274 & 895 & 932 & 793 & 1,137 & 441 & 644 & 9 & 31 & 2,473 & 3,018 & 5,491 \\
\hline Europe and Central Asia & NA & NA & NA & NA & NA & NA & 502 & 203 & 1,558 & 929 & 1,381 & 1,561 & 770 & 1,637 & 96 & 339 & 4,306 & 4,670 & 8,976 \\
\hline Latin America and the Caribbean & NA & NA & NA & NA & NA & NA & 221 & 209 & 624 & 699 & 442 & 615 & 311 & 480 & 64 & 138 & 1,663 & 2,142 & 3,805 \\
\hline Middle East and North Africa & NA & NA & NA & NA & NA & NA & 161 & 106 & 408 & 345 & 256 & 298 & 158 & 195 & 21 & 35 & 1,003 & 978 & 1,982 \\
\hline South Asia & NA & NA & NA & NA & NA & NA & 182 & 201 & 347 & 778 & 265 & 267 & 66 & 405 & 22 & 0 & 882 & 1,651 & 2,533 \\
\hline Sub-Saharan Africa & NA & NA & NA & NA & NA & NA & 102 & 121 & 267 & 395 & 101 & 273 & 39 & 146 & 4 & 9 & 513 & 944 & 1,457 \\
\hline Low- and middle-income countries & NA & NA & NA & NA & NA & NA & 1,505 & 1,114 & 4,099 & 4,077 & 3,238 & 4,151 & 1,786 & 3,508 & 215 & 553 & 10,841 & 13,403 & 24,244 \\
\hline High-income countries & NA & NA & NA & NA & NA & NA & 317 & 140 & 1,118 & 616 & 958 & 774 & 882 & 1,044 & 252 & 503 & 3,526 & 3,077 & 6,603 \\
\hline WORLD & NA & NA & NA & NA & NA & NA & 1,822 & 1,254 & 5,217 & 4,693 & 4,195 & 4,925 & 2,667 & 4,551 & 467 & 1,056 & 14,368 & 16,480 & 30,848 \\
\hline \multicolumn{20}{|l|}{ Attributable DALYs (thousands) } \\
\hline East Asia and Pacific & NA & NA & NA & NA & NA & NA & 586 & 616 & 1,285 & 1,477 & 1,029 & 1,454 & 526 & 753 & 10 & 36 & 3,437 & 4,336 & 7,773 \\
\hline Europe and Central Asia & NA & NA & NA & NA & NA & NA & 651 & 402 & 1,826 & 1,377 & 1,590 & 1,950 & 867 & 1,875 & 107 & 378 & 5,040 & 5,981 & 11,022 \\
\hline Latin America and the Caribbean & NA & NA & NA & NA & NA & NA & 324 & 368 & 801 & 1,020 & 539 & 796 & 364 & 586 & 75 & 166 & 2,102 & 2,935 & 5,038 \\
\hline Middle East and North Africa & NA & NA & NA & NA & NA & NA & 251 & 228 & 495 & 473 & 292 & 351 & 176 & 217 & 23 & 40 & 1,236 & 1,308 & 2,545 \\
\hline South Asia & NA & NA & NA & NA & NA & NA & 298 & 430 & 422 & 1,052 & 311 & 315 & 74 & 456 & 25 & 0 & 1,130 & 2,253 & 3,384 \\
\hline Sub-Saharan Africa & NA & NA & NA & NA & NA & NA & 139 & 186 & 319 & 471 & 114 & 308 & 43 & 159 & 4 & 10 & 620 & 1,134 & 1,754 \\
\hline Low- and middle-income countries & NA & NA & NA & NA & NA & NA & 2,248 & 2,230 & 5,148 & 5,869 & 3,875 & 5,174 & 2,051 & 4,045 & 244 & 630 & 13,566 & 17,948 & 31,515 \\
\hline High-income countries & NA & NA & NA & NA & NA & NA & 636 & 433 & 1,823 & 1,403 & 1,384 & 1,356 & 1,178 & 1,531 & 321 & 668 & 5,341 & 5,392 & 10,733 \\
\hline WORLD & NA & NA & NA & NA & NA & NA & 2,884 & 2,663 & 6,971 & 7,272 & 5,259 & 6,530 & 3,229 & 5,577 & 564 & 1,298 & 18,907 & 23,340 & 42,248 \\
\hline
\end{tabular}

Source: Authors' calculations.

Note: NA = not applicable. 
Risk factor: Low fruit and vegetable intake

\begin{tabular}{|c|c|c|c|c|c|c|c|c|c|c|c|c|c|c|c|c|c|c|c|}
\hline \multirow[b]{2}{*}{ Region } & \multicolumn{2}{|c|}{$0-4$ years } & \multicolumn{2}{|c|}{ 5-14 years } & \multicolumn{2}{|c|}{$15-29$ years } & \multicolumn{2}{|c|}{ 30-44 years } & \multicolumn{2}{|c|}{ 45-59 years } & \multicolumn{2}{|c|}{$60-69$ years } & \multicolumn{2}{|c|}{$70-79$ years } & \multicolumn{2}{|c|}{ 80+ years } & \multicolumn{3}{|c|}{ Total } \\
\hline & Male & Female & Male & Female & Male & Female & Male & Female & Male & Female & Male & Female & Male & Female & Male & Female & Male & Female & All \\
\hline \multicolumn{20}{|l|}{ PAF of Mortality (\%) } \\
\hline East Asia and Pacific & NA & NA & NA & NA & 20 & 22 & 20 & 21 & 19 & 20 & 20 & 22 & 17 & 18 & 13 & 13 & 18 & 19 & 19 \\
\hline Europe and Central Asia & NA & NA & NA & NA & 20 & 22 & 21 & 23 & 20 & 22 & 21 & 23 & 16 & 18 & 12 & 13 & 19 & 19 & 19 \\
\hline Latin America and the Caribbean & NA & NA & NA & NA & 26 & 27 & 27 & 23 & 25 & 26 & 24 & 25 & 18 & 19 & 13 & 13 & 22 & 20 & 21 \\
\hline Middle East and North Africa & NA & NA & NA & NA & 22 & 20 & 17 & 18 & 16 & 20 & 15 & 17 & 12 & 14 & 8 & 9 & 14 & 16 & 15 \\
\hline South Asia & NA & NA & NA & NA & 23 & 25 & 23 & 25 & 23 & 25 & 23 & 25 & 18 & 19 & 13 & 14 & 21 & 22 & 21 \\
\hline Sub-Saharan Africa & NA & NA & NA & NA & 25 & 24 & 19 & 22 & 19 & 24 & 17 & 21 & 12 & 17 & 7 & 11 & 16 & 20 & 17 \\
\hline Low- and middle-income countries & NA & NA & NA & NA & 22 & 24 & 21 & 23 & 20 & 22 & 21 & 23 & 17 & 18 & 12 & 13 & 19 & 20 & $\overline{19}$ \\
\hline High-income countries & NA & NA & NA & NA & 19 & 20 & 18 & 18 & 16 & 16 & 14 & 14 & 11 & 11 & 8 & 8 & 13 & 11 & 12 \\
\hline WORLD & NA & NA & NA & NA & 22 & 24 & 21 & 23 & 19 & 22 & 20 & 22 & 16 & 18 & 11 & 12 & 18 & 19 & 18 \\
\hline \multicolumn{20}{|l|}{ PAF of YLL (\%) } \\
\hline East Asia and Pacific & NA & NA & NA & NA & 20 & 22 & 20 & 21 & 19 & 20 & 20 & 22 & 17 & 18 & 13 & 13 & 19 & 20 & 19 \\
\hline Europe and Central Asia & NA & NA & NA & NA & 20 & 22 & 21 & 23 & 20 & 22 & 21 & 23 & 16 & 18 & 12 & 13 & 20 & 21 & 20 \\
\hline Latin America and the Caribbean & NA & NA & NA & NA & 26 & 27 & 27 & 23 & 25 & 26 & 24 & 25 & 18 & 19 & 13 & 13 & 23 & 22 & 23 \\
\hline Middle East and North Africa & NA & NA & NA & NA & 22 & 20 & 17 & 18 & 16 & 20 & 15 & 17 & 12 & 14 & 8 & 9 & 15 & 17 & 16 \\
\hline South Asia & NA & NA & NA & NA & 23 & 25 & 23 & 25 & 23 & 25 & 23 & 25 & 18 & 19 & 13 & 14 & 22 & 23 & 22 \\
\hline Sub-Saharan Africa & NA & NA & NA & NA & 25 & 24 & 19 & 22 & 19 & 24 & 17 & 21 & 12 & 17 & 7 & 11 & 17 & 21 & 19 \\
\hline Low- and middle-income countries & NA & NA & NA & NA & 22 & 24 & 21 & 23 & 20 & 22 & 21 & 23 & 17 & 18 & 12 & 13 & 20 & 21 & 20 \\
\hline High-income countries & NA & NA & NA & NA & 19 & 20 & 18 & 18 & 16 & 16 & 14 & 14 & 11 & 11 & 8 & 8 & 14 & 13 & 14 \\
\hline WORLD & NA & NA & NA & NA & 22 & 24 & 21 & 23 & 19 & 22 & 20 & 22 & 16 & 18 & 11 & 13 & 19 & 20 & 19 \\
\hline
\end{tabular}

\section{PAF of DALYs (\%)}

\begin{tabular}{|c|c|c|c|c|c|c|c|c|c|c|c|c|c|c|c|c|c|c|c|}
\hline 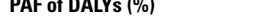 & & & & & & & & & & & & & & & & & & & \\
\hline East Asia and Pacific & NA & NA & NA & NA & 20 & 22 & 20 & 21 & 19 & 20 & 20 & 22 & 17 & 18 & 13 & 13 & 19 & 20 & 19 \\
\hline Europe and Central Asia & NA & NA & NA & NA & 20 & 22 & 21 & 23 & 20 & 22 & 21 & 23 & 16 & 18 & 12 & 13 & 20 & 21 & 20 \\
\hline Latin America and the Caribbean & NA & NA & NA & NA & 26 & 27 & 27 & 23 & 25 & 26 & 24 & 25 & 18 & 19 & 13 & 13 & 23 & 22 & 23 \\
\hline Middle East and North Africa & NA & NA & NA & NA & 22 & 20 & 17 & 18 & 16 & 20 & 15 & 17 & 12 & 14 & 8 & 9 & 15 & 17 & 16 \\
\hline South Asia & NA & NA & NA & NA & 23 & 25 & 23 & 25 & 23 & 25 & 23 & 25 & 18 & 19 & 13 & 14 & 22 & 23 & 22 \\
\hline Sub-Saharan Africa & NA & NA & NA & NA & 25 & 24 & 19 & 22 & 19 & 24 & 17 & 21 & 12 & 17 & 7 & 11 & 17 & 21 & 19 \\
\hline Low- and middle-income countries & NA & NA & NA & NA & 22 & 24 & 21 & 23 & 20 & 22 & 21 & 23 & 17 & 18 & 12 & 13 & 20 & 21 & 20 \\
\hline High-income countries & NA & NA & NA & NA & 19 & 20 & 18 & 18 & 16 & 16 & 14 & 14 & 11 & 11 & 8 & 8 & 14 & 13 & 13 \\
\hline WORLD & NA & NA & NA & NA & 22 & 24 & 21 & 23 & 19 & 22 & 20 & 22 & 16 & 18 & 11 & 13 & 19 & 20 & 9 \\
\hline
\end{tabular}

Attributable Mortality (thousands)

\begin{tabular}{lllllllllrrrrrrrrrrrrr} 
East Asia and Pacific & NA & NA & NA & NA & 0 & 0 & 2 & 0 & 7 & 4 & 10 & 6 & 7 & 5 & 1 & 2 & 27 & 17 & 44 \\
Europe and Central Asia & NA & NA & NA & NA & 0 & 0 & 0 & 0 & 1 & 0 & 1 & 0 & 1 & 0 & 0 & 0 & 3 & 1 & 4 \\
Latin America and the Caribbean & NA & NA & NA & NA & 0 & 0 & 0 & 0 & 1 & 0 & 1 & 0 & 1 & 0 & 0 & 0 & 3 & 1 & 3 \\
Middle East and North Africa & NA & NA & NA & NA & 0 & 0 & 0 & 0 & 0 & 0 & 0 & 0 & 0 & 0 & 0 & 0 & 0 & 0 & 1 \\
South Asia & NA & NA & NA & NA & 0 & 0 & 1 & 1 & 2 & 2 & 4 & 3 & 2 & 2 & 0 & 1 & 9 & 8 & 17 \\
Sub-Saharan Africa & NA & NA & NA & NA & 0 & 0 & 0 & 0 & 1 & 1 & 1 & 1 & 0 & 0 & 0 & 0 & 2 & 2 & 4 \\
\hline Low- and middle-income countries & NA & NA & NA & NA & 0 & 0 & 3 & 1 & 12 & 7 & 17 & 10 & 10 & 8 & 2 & 3 & 44 & 29 & 73 \\
High-income countries & NA & NA & NA & NA & 0 & 0 & 0 & 0 & 2 & 0 & 2 & 0 & 1 & 0 & 1 & 0 \\
\hline WORLD & NA & NA & NA & NA & 0 & 0 & 3 & 1 & 13 & 7 & 19 & 10 & 12 & 8 & 3 & 3 \\
\hline
\end{tabular}

Attributable YLL (thousands)

\begin{tabular}{|c|c|c|c|c|c|c|c|c|c|c|c|c|c|c|c|c|c|c|c|}
\hline East Asia and Pacific & NA & NA & NA & NA & 3 & 1 & 37 & 11 & 126 & 81 & 141 & 85 & 63 & 52 & 6 & 9 & 377 & 240 & 616 \\
\hline Europe and Central Asia & NA & NA & NA & NA & 0 & 0 & 2 & 1 & 17 & 3 & 16 & 5 & 5 & 4 & 0 & 1 & 42 & 15 & 57 \\
\hline Latin America and the Caribbean & NA & NA & NA & NA & 0 & 0 & 3 & 1 & 16 & 4 & 11 & 4 & 5 & 2 & 1 & 1 & 36 & 12 & 48 \\
\hline Middle East and North Africa & NA & NA & NA & NA & 0 & 0 & 1 & 1 & 2 & 2 & 2 & 1 & 1 & 1 & 0 & 0 & 6 & 5 & 11 \\
\hline South Asia & NA & NA & NA & NA & 2 & 3 & 14 & 12 & 40 & 43 & 55 & 41 & 16 & 16 & 2 & 5 & 130 & 119 & 249 \\
\hline Sub-Saharan Africa & NA & NA & NA & NA & 1 & 0 & 4 & 3 & 17 & 11 & 10 & 8 & 4 & 4 & 0 & 1 & 36 & 27 & 63 \\
\hline Low- and middle-income countries & NA & NA & NA & NA & 6 & 5 & 62 & 29 & 218 & 145 & 236 & 144 & 94 & 81 & 11 & 16 & 627 & 419 & 1,045 \\
\hline High-income countries & NA & NA & NA & NA & 0 & 0 & 4 & 1 & 30 & 5 & 25 & 5 & 13 & 5 & 2 & 2 & 75 & 18 & 92 \\
\hline WORLD & NA & NA & NA & NA & 7 & 5 & 66 & 29 & 248 & 149 & 260 & 149 & 107 & 85 & 13 & 18 & 702 & 436 & 1,138 \\
\hline
\end{tabular}

Attributable DALYs (thousands)

\begin{tabular}{|c|c|c|c|c|c|c|c|c|c|c|c|c|c|c|c|c|c|c|c|}
\hline East Asia and Pacific & NA & NA & NA & NA & 3 & 1 & 37 & 11 & 128 & 82 & 142 & 86 & 63 & 53 & 6 & 9 & 380 & 242 & 622 \\
\hline Europe and Central Asia & NA & NA & NA & NA & 0 & 0 & 2 & 1 & 18 & 3 & 16 & 5 & 6 & 4 & 0 & 1 & 43 & 15 & 57 \\
\hline Latin America and the Caribbean & NA & NA & NA & NA & 0 & 0 & 3 & 1 & 16 & 4 & 12 & 4 & 5 & 3 & 1 & 1 & 37 & 12 & 49 \\
\hline Middle East and North Africa & NA & NA & NA & NA & 0 & 0 & 1 & 1 & 2 & 2 & 2 & 1 & 1 & 1 & 0 & 0 & 6 & 5 & 11 \\
\hline South Asia & NA & NA & NA & NA & 2 & 3 & 14 & 12 & 40 & 43 & 55 & 41 & 17 & 17 & 2 & 5 & 131 & 120 & 250 \\
\hline Sub-Saharan Africa & NA & NA & NA & NA & 1 & 0 & 4 & 3 & 17 & 12 & 10 & 8 & 4 & 4 & 0 & 1 & 36 & 28 & 64 \\
\hline Low- and middle-income countries & NA & NA & NA & NA & 6 & 5 & 62 & 29 & 220 & 145 & 238 & 145 & 95 & 81 & 11 & 16 & 632 & 421 & 1,054 \\
\hline High-income countries & NA & NA & NA & NA & 0 & 0 & 4 & 1 & 31 & 5 & 25 & 5 & 13 & 5 & 3 & 2 & 77 & 18 & 95 \\
\hline WORLD & NA & NA & NA & NA & 7 & 5 & 67 & 29 & 251 & 150 & 263 & 150 & 108 & 86 & 13 & 18 & 709 & 439 & 1,148 \\
\hline
\end{tabular}

Source: Authors' calculations.

Note: $\mathrm{NA}=$ not applicable. 
Table 4A.41

Risk factor: Low fruit and vegetable intake

Disease:

Stomach cancer

\begin{tabular}{|c|c|c|c|c|c|c|c|c|c|c|c|c|c|c|c|c|c|c|c|}
\hline \multirow[b]{2}{*}{ Region } & \multicolumn{2}{|c|}{$0-4$ years } & \multicolumn{2}{|c|}{ 5-14 years } & \multicolumn{2}{|c|}{$15-29$ years } & \multicolumn{2}{|c|}{ 30-44 years } & \multicolumn{2}{|c|}{$45-59$ years } & \multicolumn{2}{|c|}{ 60-69 years } & \multicolumn{2}{|c|}{ 70-79 years } & \multicolumn{2}{|c|}{ 80+ years } & \multicolumn{3}{|c|}{ Total } \\
\hline & Male & Female & Male & Female & Male & Female & Male & Female & Male & Female & Male & Female & Male & Female & Male & Female & Male & Female & All \\
\hline \multicolumn{20}{|l|}{ PAF of Mortality (\%) } \\
\hline East Asia and Pacific & NA & NA & NA & NA & 20 & 22 & 20 & 21 & 19 & 20 & 20 & 22 & 17 & 18 & 13 & 13 & 18 & 19 & 18 \\
\hline Europe and Central Asia & NA & NA & NA & NA & 20 & 22 & 21 & 23 & 20 & 22 & 21 & 23 & 16 & 18 & 12 & 13 & 19 & 20 & 19 \\
\hline Latin America and the Caribbean & NA & NA & NA & NA & 26 & 27 & 27 & 23 & 25 & 26 & 24 & 25 & 18 & 19 & 13 & 13 & 21 & 20 & 21 \\
\hline Middle East and North Africa & NA & NA & NA & NA & 22 & 20 & 17 & 18 & 16 & 20 & 15 & 17 & 12 & 14 & 8 & 9 & 14 & 16 & 15 \\
\hline South Asia & NA & NA & NA & NA & 23 & 25 & 23 & 25 & 23 & 25 & 23 & 25 & 18 & 19 & 13 & 14 & 20 & 21 & 21 \\
\hline Sub-Saharan Africa & NA & NA & NA & NA & 25 & 24 & 19 & 22 & 19 & 24 & 17 & 21 & 12 & 17 & 7 & 11 & 15 & 20 & 17 \\
\hline Low- and middle-income countries & NA & NA & NA & NA & 21 & 23 & 21 & 22 & 20 & 21 & 21 & 23 & 17 & 18 & 13 & 13 & 18 & 19 & 19 \\
\hline High-income countries & NA & NA & NA & NA & 19 & 20 & 18 & 18 & 16 & 16 & 14 & 14 & 11 & 11 & 8 & 8 & 12 & 11 & 12 \\
\hline WORLD & NA & NA & NA & NA & 21 & 23 & 20 & 21 & 19 & 21 & 20 & 22 & 15 & 17 & 11 & 12 & 17 & 18 & 18 \\
\hline \multicolumn{20}{|l|}{ PAF of YLL (\%) } \\
\hline East Asia and Pacific & NA & NA & NA & NA & 20 & 22 & 20 & 21 & 19 & 20 & 20 & 22 & 17 & 18 & 13 & 13 & 19 & 20 & 19 \\
\hline Europe and Central Asia & NA & NA & NA & NA & 20 & 22 & 21 & 23 & 20 & 22 & 21 & 23 & 16 & 18 & 12 & 13 & 20 & 21 & 20 \\
\hline Latin America and the Caribbean & NA & NA & NA & NA & 26 & 27 & 27 & 23 & 25 & 26 & 24 & 25 & 18 & 19 & 13 & 13 & 23 & 22 & 22 \\
\hline Middle East and North Africa & NA & NA & NA & NA & 22 & 20 & 17 & 18 & 16 & 20 & 15 & 17 & 12 & 14 & 8 & 9 & 15 & 17 & 16 \\
\hline South Asia & NA & NA & NA & NA & 23 & 25 & 23 & 25 & 23 & 25 & 23 & 25 & 18 & 19 & 13 & 14 & 22 & 23 & 22 \\
\hline Sub-Saharan Africa & NA & NA & NA & NA & 25 & 24 & 19 & 22 & 19 & 24 & 17 & 21 & 12 & 17 & 7 & 11 & 17 & 21 & 19 \\
\hline Low- and middle-income countries & NA & NA & NA & NA & 21 & 23 & 21 & 22 & 20 & 21 & 21 & 23 & 17 & 18 & 13 & 13 & 19 & 20 & $\overline{20}$ \\
\hline High-income countries & NA & NA & NA & NA & 19 & 20 & 18 & 18 & 16 & 16 & 14 & 14 & 11 & 11 & 8 & 8 & 13 & 13 & 13 \\
\hline WORLD & NA & NA & NA & NA & 21 & 23 & 20 & 21 & 19 & 21 & 20 & 22 & 15 & 17 & 11 & 12 & 18 & 19 & 19 \\
\hline
\end{tabular}

PAF of DALYs (\%)

\begin{tabular}{|c|c|c|c|c|c|c|c|c|c|c|c|c|c|c|c|c|c|c|c|}
\hline 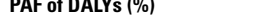 & & & & & & & & & & & & & & & & & & & \\
\hline East Asia and Pacific & NA & NA & NA & NA & 20 & 22 & 20 & 21 & 19 & 20 & 20 & 22 & 17 & 18 & 13 & 13 & 19 & 20 & 19 \\
\hline Europe and Central Asia & NA & NA & NA & NA & 20 & 22 & 21 & 23 & 20 & 22 & 21 & 23 & 16 & 18 & 12 & 13 & 20 & 21 & 20 \\
\hline Latin America and the Caribbean & NA & NA & NA & NA & 26 & 27 & 27 & 23 & 25 & 26 & 24 & 25 & 18 & 19 & 13 & 13 & 23 & 22 & 22 \\
\hline Middle East and North Africa & NA & NA & NA & NA & 22 & 20 & 17 & 18 & 16 & 20 & 15 & 17 & 12 & 14 & 8 & 9 & 15 & 17 & 16 \\
\hline South Asia & NA & NA & NA & NA & 23 & 25 & 23 & 25 & 23 & 25 & 23 & 25 & 18 & 19 & 13 & 14 & 22 & 23 & 22 \\
\hline Sub-Saharan Africa & NA & NA & NA & NA & 25 & 24 & 19 & 22 & 19 & 24 & 17 & 21 & 12 & 17 & 7 & 11 & 17 & 21 & 19 \\
\hline Low- and middle-income countries & NA & NA & NA & NA & 21 & 23 & 21 & 22 & 20 & 21 & 21 & 23 & 17 & 18 & 13 & 13 & 19 & 20 & 20 \\
\hline High-income countries & NA & NA & NA & NA & 19 & 20 & 18 & 18 & 16 & 16 & 14 & 14 & 11 & 11 & 8 & 8 & 13 & 13 & 13 \\
\hline WORLD & NA & NA & NA & NA & 21 & 23 & 20 & 21 & 19 & 21 & 20 & 22 & 15 & 17 & 11 & 12 & 18 & 19 & 19 \\
\hline
\end{tabular}

Attributable Mortality (thousands)

\begin{tabular}{lllllllllrrrrrrrrrrrr} 
East Asia and Pacific & NA & NA & NA & NA & 1 & 0 & 3 & 3 & 14 & 7 & 17 & 8 & 13 & 9 & 3 & 4 & 51 & 31 & 82 \\
Europe and Central Asia & NA & NA & NA & NA & 0 & 0 & 1 & 1 & 3 & 1 & 4 & 2 & 3 & 3 & 0 & 1 & 11 & 8 & 19 \\
Latin America and the Caribbean & NA & NA & NA & NA & 0 & 0 & 1 & 0 & 2 & 1 & 2 & 1 & 2 & 1 & 1 & 1 & 7 & 5 & 12 \\
Middle East and North Africa & NA & NA & NA & NA & 0 & 0 & 0 & 0 & 0 & 0 & 0 & 0 & 0 & 0 & 0 & 0 & 2 & 1 & 3 \\
South Asia & NA & NA & NA & NA & 0 & 0 & 1 & 0 & 1 & 1 & 2 & 1 & 1 & 1 & 0 & 1 & 6 & 4 & 9 \\
Sub-Saharan Africa & NA & NA & NA & NA & 0 & 0 & 0 & 0 & 1 & 1 & 1 & 1 & 1 & 1 & 0 & 0 & 3 & 3 & 6 \\
\hline Low- and middle-income countries & NA & NA & NA & NA & 1 & 1 & 5 & 4 & 22 & 11 & 27 & 14 & 19 & 15 & 5 & 7 & 79 & 51 & 130 \\
High-income countries & NA & NA & NA & NA & 0 & 0 & 0 & 0 & 2 & 1 & 3 & 1 & 3 & 2 & 2 & 2 & 11 & 6 & 17 \\
\hline WORLD & NA & NA & NA & NA & 1 & 1 & 6 & 5 & 24 & 12 & 30 & 15 & 23 & 16 & 7 & 8 & 90 & 58 & 147 \\
\hline
\end{tabular}

Attributable YLL (thousands)

\begin{tabular}{|c|c|c|c|c|c|c|c|c|c|c|c|c|c|c|c|c|c|c|c|}
\hline East Asia and Pacific & NA & NA & NA & NA & 15 & 11 & 73 & 68 & 267 & 134 & 231 & 120 & 113 & 92 & 17 & 22 & 716 & 447 & 1,163 \\
\hline Europe and Central Asia & NA & NA & NA & NA & 2 & 2 & 16 & 14 & 53 & 27 & 59 & 38 & 27 & 28 & 2 & 4 & 158 & 113 & 271 \\
\hline Latin America and the Caribbean & NA & NA & NA & NA & 3 & 2 & 12 & 10 & 32 & 21 & 29 & 18 & 16 & 13 & 4 & 4 & 95 & 68 & 163 \\
\hline Middle East and North Africa & NA & NA & NA & NA & 1 & 1 & 3 & 4 & 7 & 6 & 6 & 4 & 4 & 3 & 0 & 0 & 21 & 18 & 39 \\
\hline South Asia & NA & NA & NA & NA & 4 & 3 & 12 & 7 & 26 & 18 & 30 & 17 & 10 & 7 & 2 & 3 & 84 & 55 & 139 \\
\hline Sub-Saharan Africa & NA & NA & NA & NA & 2 & 2 & 6 & 6 & 19 & 19 & 10 & 12 & 5 & 6 & 1 & 1 & 43 & 48 & 91 \\
\hline Low- and middle-income countries & NA & NA & NA & NA & 26 & 22 & 122 & 109 & 404 & 225 & 364 & 208 & 175 & 149 & 26 & 35 & 1,117 & 748 & 1,865 \\
\hline High-income countries & NA & NA & NA & NA & 1 & 1 & 10 & 9 & 40 & 20 & 41 & 19 & 29 & 19 & 7 & 8 & 129 & 77 & 206 \\
\hline WORLD & NA & NA & NA & NA & 27 & 23 & 133 & 118 & 443 & 245 & 406 & 228 & 204 & 169 & 34 & 43 & 1,246 & 825 & 2,072 \\
\hline
\end{tabular}

Attributable DALYs (thousands)

\begin{tabular}{|c|c|c|c|c|c|c|c|c|c|c|c|c|c|c|c|c|c|c|c|}
\hline East Asia and Pacific & NA & NA & NA & NA & 15 & 12 & 74 & 69 & 271 & 135 & 234 & 121 & 115 & 93 & 17 & 23 & 726 & 453 & 1,178 \\
\hline Europe and Central Asia & NA & NA & NA & NA & 2 & 2 & 16 & 14 & 54 & 27 & 60 & 38 & 27 & 29 & 2 & 4 & 161 & 115 & 275 \\
\hline Latin America and the Caribbean & NA & NA & NA & NA & 3 & 2 & 12 & 11 & 33 & 21 & 29 & 18 & 16 & 13 & 4 & 4 & 96 & 69 & 165 \\
\hline Middle East and North Africa & NA & NA & NA & NA & 1 & 1 & 3 & 4 & 7 & 6 & 6 & 4 & 4 & 3 & 0 & 0 & 22 & 18 & 39 \\
\hline South Asia & NA & NA & NA & NA & 4 & 3 & 12 & 7 & 26 & 18 & 30 & 17 & 11 & 7 & 2 & 3 & 85 & 55 & 140 \\
\hline Sub-Saharan Africa & NA & NA & NA & NA & 2 & 2 & 6 & 6 & 19 & 20 & 11 & 13 & 5 & 7 & 1 & 1 & 44 & 48 & 92 \\
\hline Low- and middle-income countries & NA & NA & NA & NA & 26 & 22 & 123 & 110 & 409 & 228 & 370 & 211 & 178 & 151 & 27 & 35 & 1,132 & 757 & 1,889 \\
\hline High-income countries & NA & NA & NA & NA & 1 & 1 & 10 & 10 & 41 & 20 & 43 & 20 & 30 & 20 & 8 & 9 & 134 & 80 & 214 \\
\hline WORLD & NA & NA & NA & NA & 27 & 23 & 134 & 120 & 450 & 248 & 412 & 231 & 208 & 171 & 35 & 44 & 1,266 & 837 & 2,103 \\
\hline
\end{tabular}


Risk factor: Low fruit and vegetable intake

Disease:

Colon and rectal cancers

\begin{tabular}{|c|c|c|c|c|c|c|c|c|c|c|c|c|c|c|c|c|c|c|c|}
\hline \multirow[b]{2}{*}{ Region } & \multicolumn{2}{|c|}{$0-4$ years } & \multicolumn{2}{|c|}{ 5-14 years } & \multicolumn{2}{|c|}{$15-29$ years } & \multicolumn{2}{|c|}{ 30-44 years } & \multicolumn{2}{|c|}{ 45-59 years } & \multicolumn{2}{|c|}{$60-69$ years } & \multicolumn{2}{|c|}{ 70-79 years } & \multicolumn{2}{|c|}{$80+$ years } & \multicolumn{3}{|c|}{ Total } \\
\hline & Male & Female & Male & Female & Male & Female & Male & Female & Male & Female & Male & Female & Male & Female & Male & Female & Male & Female & All \\
\hline \multicolumn{20}{|l|}{ PAF of Mortality (\%) } \\
\hline East Asia and Pacific & NA & NA & NA & NA & 3 & 3 & 3 & 3 & 3 & 3 & 3 & 3 & 3 & 3 & 0 & 0 & 2 & 2 & 2 \\
\hline Europe and Central Asia & NA & NA & NA & NA & 3 & 3 & 3 & 3 & 3 & 3 & 3 & 3 & 3 & 3 & 0 & 0 & 2 & 3 & 3 \\
\hline Latin America and the Caribbean & NA & NA & NA & NA & 3 & 4 & 4 & 1 & 3 & 4 & 3 & 3 & 3 & 3 & 0 & 0 & 3 & 2 & 2 \\
\hline Middle East and North Africa & NA & NA & NA & NA & 3 & 3 & 2 & 2 & 2 & 3 & 2 & 2 & 2 & 2 & 0 & 0 & 2 & 2 & 2 \\
\hline South Asia & NA & NA & NA & NA & 3 & 3 & 3 & 3 & 3 & 3 & 3 & 3 & 3 & 3 & 0 & 0 & 3 & 3 & 3 \\
\hline Sub-Saharan Africa & NA & NA & NA & NA & 3 & 3 & 3 & 3 & 2 & 3 & 2 & 3 & 2 & 3 & 0 & 0 & 2 & 3 & 2 \\
\hline Low- and middle-income countries & NA & NA & NA & NA & 3 & 3 & 3 & 3 & 3 & 3 & 3 & 3 & 3 & 3 & 0 & 0 & 2 & 2 & 2 \\
\hline High-income countries & NA & NA & NA & NA & 3 & 3 & 2 & 2 & 2 & 2 & 2 & 2 & 2 & 2 & 0 & 0 & 1 & 1 & 1 \\
\hline WORLD & NA & NA & NA & NA & 3 & 3 & 3 & 3 & 2 & 3 & 2 & 3 & 2 & 3 & 0 & 0 & 2 & 2 & 2 \\
\hline
\end{tabular}

PAF of YLL (\%)

\begin{tabular}{llllllllllllllllllll} 
East Asia and Pacific & NA & NA & NA & NA & 3 & 3 & 3 & 3 & 3 & 3 & 3 & 3 & 3 & 3 & 0 & 0 & 3 & 3 & 3 \\
Europe and Central Asia & NA & NA & NA & NA & 3 & 3 & 3 & 3 & 3 & 3 & 3 & 3 & 3 & 3 & 0 & 0 & 3 & 3 & 3 \\
Latin America and the Caribbean & NA & NA & NA & NA & 3 & 4 & 4 & 1 & 3 & 4 & 3 & 3 & 3 & 3 & 0 & 0 & 3 & 3 & 3 \\
Middle East and North Africa & NA & NA & NA & NA & 3 & 3 & 2 & 2 & 2 & 3 & 2 & 2 & 2 & 2 & 0 & 0 & 2 & 2 & 2 \\
South Asia & NA & NA & NA & NA & 3 & 3 & 3 & 3 & 3 & 3 & 3 & 3 & 3 & 3 & 0 & 0 & 3 & 3 & 3 \\
Sub-Saharan Africa & NA & NA & NA & NA & 3 & 3 & 3 & 3 & 2 & 3 & 2 & 3 & 2 & 3 & 0 & 0 & 2 & 3 & 3 \\
\hline Low- and middle-income countries & NA & NA & NA & NA & 3 & 3 & 3 & 3 & 3 & 3 & 3 & 3 & 3 & 3 & 0 & 0 & 3 & 3 & 3 \\
High-income countries & NA & NA & NA & NA & 3 & 3 & 2 & 2 & 2 & 2 & 2 & 2 & 2 & 2 & 0 & 0 \\
\hline WORLD & NA & NA & NA & NA & 3 & 3 & 3 & 3 & 2 & 3 & 2 & 3 & 2 & 3 & 0 & 0 \\
\hline
\end{tabular}

\section{PAF of DALYs (\%)}

East Asia and Pacific

Europe and Central Asia

Latin America and the Caribbean

Middle East and North Africa

South Asia

Sub-Saharan Africa

Low- and middle-income countries

High-income countries

$\begin{array}{llll}\text { NA } & \text { NA } & \text { NA } & \text { NA } \\ \text { NA } & \text { NA } & \text { NA } & \text { NA } \\ \text { NA } & \text { NA } & \text { NA } & \text { NA } \\ \text { NA } & \text { NA } & \text { NA } & \text { NA } \\ \text { NA } & \text { NA } & \text { NA } & \text { NA } \\ \text { NA } & \text { NA } & \text { NA } & \text { NA } \\ \text { NA } & \text { NA } & \text { NA } & \text { NA }\end{array}$

\begin{tabular}{ll}
3 & 3 \\
3 & 3 \\
3 & 4 \\
3 & 3 \\
3 & 3 \\
3 & 3 \\
\hline 3 & 3
\end{tabular}

\begin{tabular}{llllllllllllllllll}
\hline WORLD & NA & NA & NA & NA & 3 & 3 & 3 & 3 & 2 & 3 & 2 & 3 & 2 & 3 & 0 & 0 & 2 \\
\hline
\end{tabular}

Attributable Mortality (thousands)

\begin{tabular}{llllllllllllllllllllll} 
East Asia and Pacific & NA & NA & NA & NA & 0 & 0 & 0 & 0 & 0 & 0 & 1 & 1 & 1 & 1 & 0 & 0 & 2 & 2 & 4 \\
Europe and Central Asia & NA & NA & NA & NA & 0 & 0 & 0 & 0 & 0 & 0 & 0 & 0 & 0 & 1 & 0 & 0 & 1 & 1 & 2 \\
Latin America and the Caribbean & NA & NA & NA & NA & 0 & 0 & 0 & 0 & 0 & 0 & 0 & 0 & 0 & 0 & 0 & 0 & 0 & 0 & 1 \\
Middle East and North Africa & NA & NA & NA & NA & 0 & 0 & 0 & 0 & 0 & 0 & 0 & 0 & 0 & 0 & 0 & 0 & 0 & 0 & 0 \\
South Asia & NA & NA & NA & NA & 0 & 0 & 0 & 0 & 0 & 0 & 0 & 0 & 0 & 0 & 0 & 0 & 1 & 0 & 1 \\
Sub-Saharan Africa & NA & NA & NA & NA & 0 & 0 & 0 & 0 & 0 & 0 & 0 & 0 & 0 & 0 & 0 & 0 & 0 & 0 & 0 \\
\hline Low- and middle-income countries & NA & NA & NA & NA & 0 & 0 & 0 & 0 & 1 & 1 & 1 & 1 & 2 & 2 & 0 & 0 & 5 & 4 & 9 \\
High-income countries & NA & NA & NA & NA & 0 & 0 & 0 & 0 & 0 & 0 & 1 & 0 & 1 & 1 & 0 & 0 \\
\hline WORLD & NA & NA & NA & NA & 0 & 0 & 0 & 0 & 1 & 1 & 2 & 2 & 2 & 2 & 0 & 0 \\
\hline
\end{tabular}

Attributable YLL (thousands)

\begin{tabular}{llllllllrrrrrrrrrrrrr} 
East Asia and Pacific & NA & NA & NA & NA & 1 & 1 & 4 & 5 & 9 & 9 & 9 & 9 & 6 & 7 & 0 & 0 & 30 & 29 & 59 \\
Europe and Central Asia & NA & NA & NA & NA & 0 & 0 & 1 & 1 & 4 & 4 & 6 & 6 & 4 & 6 & 0 & 0 & 16 & 18 & 33 \\
Latin America and the Caribbean & NA & NA & NA & NA & 0 & 0 & 1 & 0 & 2 & 2 & 2 & 2 & 1 & 2 & 0 & 0 & 7 & 7 & 13 \\
Middle East and North Africa & NA & NA & NA & NA & 0 & 0 & 0 & 0 & 1 & 1 & 0 & 0 & 0 & 0 & 0 & 0 & 2 & 2 & 3 \\
South Asia & NA & NA & NA & NA & 1 & 0 & 2 & 1 & 2 & 2 & 2 & 2 & 1 & 1 & 0 & 0 & 8 & 6 & 15 \\
Sub-Saharan Africa & NA & NA & NA & NA & 0 & 0 & 1 & 1 & 1 & 1 & 1 & 1 & 1 & 1 & 0 & 0 & 4 & 4 & 7 \\
\hline Low- and middle-income countries & NA & NA & NA & NA & 3 & 1 & 10 & 8 & 19 & 19 & 20 & 20 & 14 & 17 & 0 & 0 & 66 & 65 & 131 \\
High-income countries & NA & NA & NA & NA & 0 & 0 & 2 & 1 & 7 & 5 & 7 & 5 & 7 & 7 \\
\hline WORLD & NA & NA & NA & NA & 3 & 2 & 11 & 10 & 26 & 24 & 27 & 25 & 21 & 24 & 0 & 0 & 89 & 84 & 173 \\
\hline
\end{tabular}

Attributable DALYs (thousands)

\begin{tabular}{lrllllllrrrrrrrrrrrr} 
East Asia and Pacific & NA & NA & NA & NA & 1 & 1 & 5 & 5 & 10 & 9 & 9 & 9 & 6 & 7 & 0 & 0 & 31 & 31 & 62 \\
Europe and Central Asia & NA & NA & NA & NA & 0 & 0 & 1 & 1 & 5 & 4 & 6 & 7 & 5 & 6 & 0 & 0 & 17 & 19 & 36 \\
Latin America and the Caribbean & NA & NA & NA & NA & 0 & 0 & 1 & 0 & 2 & 2 & 2 & 2 & 2 & 2 & 0 & 0 & 7 & 7 & 14 \\
Middle East and North Africa & NA & NA & NA & NA & 0 & 0 & 0 & 0 & 1 & 1 & 0 & 0 & 0 & 0 & 0 & 0 & 2 & 2 & 4 \\
South Asia & NA & NA & NA & NA & 1 & 0 & 2 & 1 & 2 & 2 & 2 & 2 & 1 & 1 & 0 & 0 & 9 & 6 & 15 \\
Sub-Saharan Africa & NA & NA & NA & NA & 0 & 0 & 1 & 1 & 1 & 1 & 1 & 1 & 1 & 1 & 0 & 0 & 4 & 4 & 7 \\
\hline Low- and middle-income countries & NA & NA & NA & NA & 3 & 1 & 10 & 9 & 20 & 20 & 21 & 21 & 14 & 17 & 0 & 0 & 69 & 69 & 138 \\
High-income countries & NA & NA & NA & NA & 0 & 0 & 2 & 2 & 8 & 6 & 9 & 6 & 8 & 9 & 0 & 0 & 28 & 23 & 51 \\
\hline WORLD & NA & NA & NA & NA & 3 & 2 & 12 & 11 & 29 & 27 & 30 & 27 & 23 & 26 & 0 & 0 & 96 & 92 & 189
\end{tabular}

Source: Authors' calculations.

Note: $\mathrm{NA}=$ not applicable. 
Risk factor: Low fruit and vegetable intake

Disease:

Trachea, bronchus, and lung cancers

\begin{tabular}{|c|c|c|c|c|c|c|c|c|c|c|c|c|c|c|c|c|c|c|c|}
\hline \multirow[b]{2}{*}{ Region } & \multicolumn{2}{|c|}{$0-4$ years } & \multicolumn{2}{|c|}{ 5-14 years } & \multicolumn{2}{|c|}{$15-29$ years } & \multicolumn{2}{|c|}{ 30-44 years } & \multicolumn{2}{|c|}{ 45-59 years } & \multicolumn{2}{|c|}{$60-69$ years } & \multicolumn{2}{|c|}{$70-79$ years } & \multicolumn{2}{|c|}{$80+$ years } & \multicolumn{3}{|c|}{ Total } \\
\hline & Male & Female & Male & Female & Male & Female & Male & Female & Male & Female & Male & Female & Male & Female & Male & Female & Male & Female & All \\
\hline \multicolumn{20}{|l|}{ PAF of Mortality (\%) } \\
\hline East Asia and Pacific & NA & NA & NA & NA & 13 & 14 & 13 & 14 & 12 & 13 & 13 & 15 & 11 & 13 & 10 & 10 & 12 & 13 & 12 \\
\hline Europe and Central Asia & NA & NA & NA & NA & 13 & 15 & 14 & 15 & 13 & 14 & 13 & 15 & 11 & 12 & 9 & 10 & 13 & 13 & 13 \\
\hline Latin America and the Caribbean & NA & NA & NA & NA & 17 & 18 & 17 & 14 & 16 & 17 & 16 & 16 & 12 & 13 & 10 & 10 & 14 & 14 & 14 \\
\hline Middle East and North Africa & NA & NA & NA & NA & 14 & 13 & 11 & 12 & 10 & 13 & 9 & 11 & 8 & 10 & 6 & 7 & 9 & 11 & 9 \\
\hline South Asia & NA & NA & NA & NA & 15 & 16 & 15 & 16 & 15 & 17 & 15 & 16 & 12 & 13 & 10 & 11 & 14 & 15 & 14 \\
\hline Sub-Saharan Africa & NA & NA & NA & NA & 16 & 16 & 12 & 14 & 12 & 16 & 11 & 14 & 8 & 11 & 5 & 8 & 11 & 13 & 11 \\
\hline Low- and middle-income countries & NA & NA & NA & NA & 14 & 15 & 14 & 14 & 13 & 14 & 13 & 15 & 11 & 13 & 10 & 10 & 13 & 13 & 13 \\
\hline High-income countries & NA & NA & NA & NA & 12 & 13 & 11 & 12 & 10 & 10 & 9 & 9 & 7 & 8 & 6 & 6 & 8 & 8 & 8 \\
\hline WORLD & NA & NA & NA & NA & 14 & 15 & 13 & 14 & 12 & 13 & 12 & 13 & 10 & 10 & 8 & 8 & 11 & 11 & 11 \\
\hline
\end{tabular}

PAF of YLL (\%)

\begin{tabular}{|c|c|c|c|c|c|c|c|c|c|c|c|c|c|c|c|c|c|c|c|}
\hline East Asia and Pacific & NA & NA & NA & NA & 13 & 14 & 13 & 14 & 12 & 13 & 13 & 15 & 11 & 13 & 10 & 10 & 12 & 13 & 13 \\
\hline Europe and Central Asia & NA & NA & NA & NA & 13 & 15 & 14 & 15 & 13 & 14 & 13 & 15 & 11 & 12 & 9 & 10 & 13 & 14 & 13 \\
\hline Latin America and the Caribbean & NA & NA & NA & NA & 17 & 18 & 17 & 14 & 16 & 17 & 16 & 16 & 12 & 13 & 10 & 10 & 15 & 15 & 15 \\
\hline Middle East and North Africa & NA & NA & NA & NA & 14 & 13 & 11 & 12 & 10 & 13 & 9 & 11 & 8 & 10 & 6 & 7 & 9 & 11 & 10 \\
\hline South Asia & NA & NA & NA & NA & 15 & 16 & 15 & 16 & 15 & 17 & 15 & 16 & 12 & 13 & 10 & 11 & 14 & 16 & 15 \\
\hline Sub-Saharan Africa & NA & NA & NA & NA & 16 & 16 & 12 & 14 & 12 & 16 & 11 & 14 & 8 & 11 & 5 & 8 & 11 & 14 & 12 \\
\hline Low- and middle-income countries & NA & NA & NA & NA & 14 & 15 & 14 & 14 & 13 & 14 & 13 & 15 & 11 & 13 & 10 & 10 & 13 & 14 & 13 \\
\hline High-income countries & NA & NA & NA & NA & 12 & 13 & 11 & 12 & 10 & 10 & 9 & 9 & 7 & 8 & 6 & 6 & 8 & 9 & 9 \\
\hline WORLD & NA & NA & NA & NA & 14 & 15 & 13 & 14 & 12 & 13 & 12 & 13 & 10 & 10 & 8 & 8 & 11 & 12 & 12 \\
\hline
\end{tabular}

PAF of DALYs (\%)

\begin{tabular}{|c|c|c|c|c|c|c|c|c|c|c|c|c|c|c|c|c|c|c|c|}
\hline East Asia and Pacific & NA & NA & NA & NA & 13 & 14 & 13 & 14 & 12 & 13 & 13 & 15 & 11 & 13 & 10 & 10 & 12 & 13 & 13 \\
\hline Europe and Central Asia & NA & NA & NA & NA & 13 & 15 & 14 & 15 & 13 & 14 & 13 & 15 & 11 & 12 & 9 & 10 & 13 & 14 & 13 \\
\hline Latin America and the Caribbean & NA & NA & NA & NA & 17 & 18 & 17 & 14 & 16 & 17 & 16 & 16 & 12 & 13 & 10 & 10 & 15 & 15 & 15 \\
\hline Middle East and North Africa & NA & NA & NA & NA & 14 & 13 & 11 & 12 & 10 & 13 & 9 & 11 & 8 & 10 & 6 & 7 & 9 & 11 & 10 \\
\hline South Asia & NA & NA & NA & NA & 15 & 16 & 15 & 16 & 15 & 17 & 15 & 16 & 12 & 13 & 10 & 11 & 14 & 16 & 15 \\
\hline Sub-Saharan Africa & NA & NA & NA & NA & 16 & 16 & 12 & 14 & 12 & 16 & 11 & 14 & 8 & 11 & 5 & 8 & 11 & 14 & 12 \\
\hline Low- and middle-income countries & NA & NA & NA & NA & 14 & 15 & 14 & 14 & 13 & 14 & 13 & 15 & 11 & 13 & 10 & 10 & 13 & 14 & 13 \\
\hline High-income countries & NA & NA & NA & NA & 12 & 13 & 11 & 12 & 10 & 10 & 9 & 9 & 7 & 8 & 6 & 6 & 8 & 9 & 9 \\
\hline WORLD & NA & NA & NA & NA & 14 & 15 & 13 & 14 & 12 & 13 & 12 & 13 & 10 & 10 & 8 & 8 & 11 & 12 & 12 \\
\hline
\end{tabular}

Attributable Mortality (thousands)

\begin{tabular}{lrlllllllrrrrrrrrrrr} 
East Asia and Pacific & NA & NA & NA & NA & 0 & 0 & 1 & 1 & 7 & 4 & 12 & 5 & 9 & 5 & 2 & 1 & 32 & 16 & 48 \\
Europe and Central Asia & NA & NA & NA & NA & 0 & 0 & 1 & 0 & 5 & 1 & 7 & 1 & 4 & 1 & 1 & 0 & 17 & 4 & 21 \\
Latin America and the Caribbean & NA & NA & NA & NA & 0 & 0 & 0 & 0 & 1 & 1 & 2 & 1 & 1 & 1 & 0 & 0 & 5 & 2 & 8 \\
Middle East and North Africa & NA & NA & NA & NA & 0 & 0 & 0 & 0 & 0 & 0 & 0 & 0 & 0 & 0 & 0 & 0 & 1 & 0 & 2 \\
South Asia & NA & NA & NA & NA & 0 & 0 & 1 & 0 & 4 & 1 & 6 & 1 & 3 & 1 & 1 & 0 & 15 & 4 & 18 \\
Sub-Saharan Africa & NA & NA & NA & NA & 0 & 0 & 0 & 0 & 0 & 0 & 0 & 0 & 0 & 0 & 0 & 0 & 1 & 1 & 2 \\
\hline Low- and middle-income countries & NA & NA & NA & NA & 0 & 0 & 3 & 2 & 18 & 6 & 28 & 9 & 18 & 8 & 4 & 2 & 71 & 27 & 98 \\
High-income countries & NA & NA & NA & NA & 0 & 0 & 1 & 0 & 5 & 2 & 7 & 3 & 8 & 4 & 3 & 2 & 25 & 12 & 36 \\
\hline WORLD & NA & NA & NA & NA & 0 & 0 & 4 & 2 & 23 & 9 & 35 & 12 & 27 & 12 & 7 & 4 & 96 & 39 & 135 \\
\hline
\end{tabular}

Attributable YLL (thousands)

\begin{tabular}{|c|c|c|c|c|c|c|c|c|c|c|c|c|c|c|c|c|c|c|c|}
\hline East Asia and Pacific & NA & NA & NA & NA & 3 & 1 & 35 & 21 & 135 & 72 & 166 & 78 & 80 & 55 & 9 & 7 & 428 & 235 & 664 \\
\hline Europe and Central Asia & NA & NA & NA & NA & 1 & 1 & 15 & 5 & 87 & 17 & 97 & 19 & 38 & 13 & 3 & 2 & 241 & 56 & 297 \\
\hline Latin America and the Caribbean & NA & NA & NA & NA & 1 & 1 & 5 & 4 & 25 & 13 & 24 & 11 & 13 & 6 & 2 & 1 & 71 & 36 & 107 \\
\hline Middle East and North Africa & NA & NA & NA & NA & 1 & 0 & 3 & 2 & 7 & 3 & 6 & 2 & 3 & 1 & 0 & 0 & 20 & 8 & 28 \\
\hline South Asia & NA & NA & NA & NA & 3 & 1 & 16 & 7 & 73 & 22 & 76 & 18 & 31 & 8 & 4 & 1 & 203 & 57 & 260 \\
\hline Sub-Saharan Africa & NA & NA & NA & NA & 0 & 0 & 2 & 1 & 9 & 3 & 6 & 2 & 2 & 1 & 0 & 0 & 18 & 8 & 26 \\
\hline Low- and middle-income countries & NA & NA & NA & NA & 9 & 4 & 76 & 39 & 335 & 130 & 375 & 130 & 167 & 84 & 19 & 12 & 980 & 400 & 1,381 \\
\hline High-income countries & NA & NA & NA & NA & 1 & 0 & 14 & 10 & 93 & 45 & 100 & 46 & 74 & 40 & 15 & 11 & 296 & 151 & 447 \\
\hline WORLD & NA & NA & NA & NA & 9 & 5 & 90 & 49 & 427 & 175 & 474 & 176 & 240 & 124 & 35 & 23 & 1,276 & 552 & 1,828 \\
\hline
\end{tabular}

Attributable DALYs (thousands)

\begin{tabular}{|c|c|c|c|c|c|c|c|c|c|c|c|c|c|c|c|c|c|c|c|}
\hline East Asia and Pacific & NA & NA & NA & NA & 3 & 1 & 35 & 21 & 136 & 73 & 168 & 79 & 81 & 56 & 10 & 8 & 433 & 238 & 671 \\
\hline Europe and Central Asia & NA & NA & NA & NA & 1 & 1 & 15 & 5 & 88 & 17 & 98 & 19 & 39 & 13 & 3 & 2 & 245 & 56 & 301 \\
\hline Latin America and the Caribbean & NA & NA & NA & NA & 1 & 1 & 5 & 4 & 25 & 13 & 25 & 11 & 13 & 6 & 3 & 1 & 72 & 36 & 108 \\
\hline Middle East and North Africa & NA & NA & NA & NA & 1 & 0 & 3 & 2 & 7 & 3 & 6 & 2 & 3 & 1 & 0 & 0 & 20 & 8 & 28 \\
\hline South Asia & NA & NA & NA & NA & 3 & 1 & 16 & 7 & 73 & 22 & 77 & 18 & 31 & 8 & 4 & 1 & 205 & 57 & 262 \\
\hline Sub-Saharan Africa & NA & NA & NA & NA & 0 & 0 & 2 & 1 & 9 & 4 & 6 & 2 & 2 & 1 & 0 & 0 & 18 & 9 & 27 \\
\hline Low- and middle-income countries & NA & NA & NA & NA & 9 & 4 & 76 & 40 & 338 & 131 & 379 & 131 & 170 & 86 & 20 & 12 & 992 & 405 & 1,397 \\
\hline High-income countries & NA & NA & NA & NA & 1 & 0 & 14 & 10 & 95 & 46 & 102 & 47 & 76 & 41 & 16 & 11 & 304 & 155 & 460 \\
\hline WORLD & NA & NA & NA & NA & 10 & 5 & 90 & 50 & 433 & 177 & 482 & 179 & 246 & 126 & 36 & 23 & 1,297 & 560 & 1,856 \\
\hline
\end{tabular}


Risk factor: Low fruit and vegetable intake

Disease: Ischemic heart disease

\begin{tabular}{|c|c|c|c|c|c|c|c|c|c|c|c|c|c|c|c|c|c|c|c|}
\hline \multirow[b]{2}{*}{ Region } & \multicolumn{2}{|c|}{$0-4$ years } & \multicolumn{2}{|c|}{ 5-14 years } & \multicolumn{2}{|c|}{$15-29$ years } & \multicolumn{2}{|c|}{ 30-44 years } & \multicolumn{2}{|c|}{ 45-59 years } & \multicolumn{2}{|c|}{$60-69$ years } & \multicolumn{2}{|c|}{$70-79$ years } & \multicolumn{2}{|c|}{$80+$ years } & \multicolumn{3}{|c|}{ Total } \\
\hline & Male & Female & Male & Female & Male & Female & Male & Female & Male & Female & Male & Female & Male & Female & Male & Female & Male & Female & All \\
\hline \multicolumn{20}{|l|}{ PAF of Mortality (\%) } \\
\hline East Asia and Pacific & NA & NA & NA & NA & 31 & 33 & 30 & 32 & 29 & 31 & 31 & 34 & 24 & 26 & 19 & 19 & 26 & 26 & 26 \\
\hline Europe and Central Asia & NA & NA & NA & NA & 31 & 34 & 33 & 36 & 31 & 34 & 32 & 35 & 24 & 26 & 17 & 19 & 27 & 25 & 26 \\
\hline Latin America and the Caribbean & NA & NA & NA & NA & 40 & 40 & 40 & 36 & 38 & 39 & 37 & 38 & 27 & 27 & 20 & 19 & 30 & 28 & 29 \\
\hline Middle East and North Africa & NA & NA & NA & NA & 33 & 31 & 27 & 29 & 26 & 31 & 23 & 26 & 18 & 21 & 12 & 14 & 21 & 22 & 21 \\
\hline South Asia & NA & NA & NA & NA & 36 & 38 & 35 & 38 & 35 & 38 & 35 & 37 & 25 & 28 & 19 & 20 & 30 & 31 & 31 \\
\hline Sub-Saharan Africa & NA & NA & NA & NA & 38 & 37 & 30 & 34 & 29 & 36 & 26 & 33 & 18 & 24 & 11 & 16 & 23 & 27 & 25 \\
\hline Low- and middle-income countries & NA & NA & NA & NA & 34 & 36 & 33 & 35 & 32 & 35 & 32 & 35 & 24 & 26 & 18 & 19 & 27 & 27 & 27 \\
\hline High-income countries & NA & NA & NA & NA & 30 & 31 & 28 & 28 & 25 & 25 & 22 & 23 & 16 & 17 & 12 & 12 & 17 & 15 & 16 \\
\hline WORLD & NA & NA & NA & NA & 34 & 36 & 32 & 35 & 31 & 35 & 31 & 34 & 22 & 25 & 16 & 17 & 25 & 25 & 25 \\
\hline
\end{tabular}

PAF of YLL (\%)

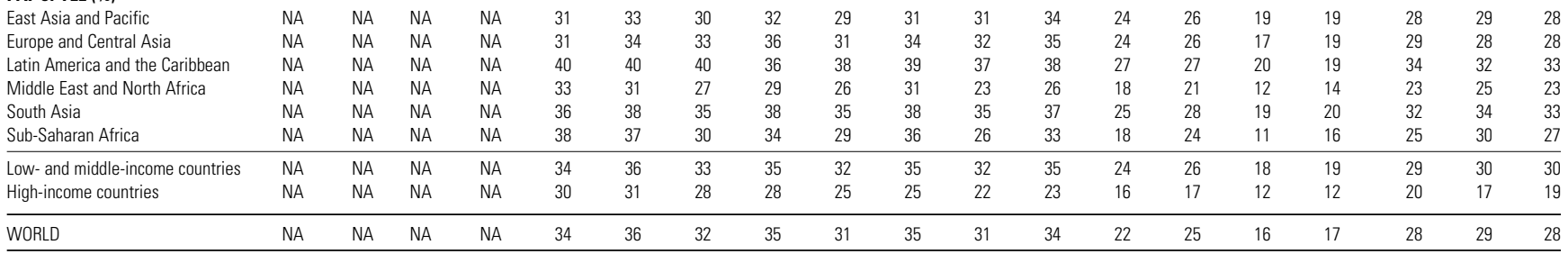

\section{PAF of DALYs $(\%)$}

East Asia and Pacific

Europe and Central Asia

Latin America and the Caribbean

Middle East and North Africa

South Asia

Sub-Saharan Africa

Low- and middle-income countries

NA NA NA NA

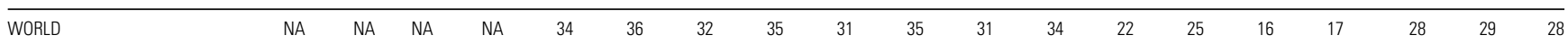

Attributable Mortality (thousands)

\begin{tabular}{|c|c|c|c|c|c|c|c|c|c|c|c|c|c|c|c|c|c|c|c|}
\hline East Asia and Pacific & NA & NA & NA & NA & 3 & 2 & 9 & 5 & 30 & 18 & 46 & 38 & 46 & 48 & 20 & 36 & 155 & 147 & 301 \\
\hline Europe and Central Asia & NA & NA & NA & NA & 1 & 0 & 12 & 3 & 48 & 15 & 72 & 46 & 63 & 81 & 23 & 73 & 220 & 216 & 436 \\
\hline Latin America and the Caribbean & NA & NA & NA & NA & 1 & 0 & 4 & 1 & 15 & 7 & 18 & 11 & 16 & 12 & 9 & 11 & 61 & 43 & 105 \\
\hline Middle East and North Africa & NA & NA & NA & NA & 1 & 0 & 3 & 1 & 10 & 6 & 11 & 8 & 10 & 10 & 3 & 4 & 39 & 30 & 69 \\
\hline South Asia & NA & NA & NA & NA & 4 & 7 & 17 & 12 & 85 & 47 & 102 & 87 & 76 & 75 & 25 & 25 & 309 & 254 & 563 \\
\hline Sub-Saharan Africa & NA & NA & NA & NA & 0 & 0 & 2 & 1 & 11 & 9 & 14 & 15 & 11 & 13 & 2 & 5 & 41 & 44 & 85 \\
\hline Low- and middle-income countries & NA & NA & NA & NA & 9 & 10 & 48 & 24 & 200 & 103 & 263 & 205 & 221 & 238 & 83 & 155 & 824 & 735 & 1,559 \\
\hline High-income countries & NA & NA & NA & NA & 0 & 0 & 4 & 1 & 22 & 6 & 29 & 12 & 37 & 27 & 30 & 51 & 123 & 96 & 219 \\
\hline WORLD & NA & NA & NA & NA & 9 & 10 & 52 & 25 & 222 & 108 & 292 & 217 & 259 & 265 & 113 & 206 & 947 & 831 & 1,777 \\
\hline
\end{tabular}

Attributable YLL (thousands)

\begin{tabular}{|c|c|c|c|c|c|c|c|c|c|c|c|c|c|c|c|c|c|c|c|}
\hline East Asia and Pacific & NA & NA & NA & NA & 70 & 52 & 221 & 129 & 565 & 358 & 622 & 568 & 408 & 488 & 100 & 179 & 1,987 & 1,774 & 3,761 \\
\hline Europe and Central Asia & NA & NA & NA & NA & 25 & 7 & 290 & 62 & 905 & 292 & 980 & 688 & 572 & 821 & 104 & 336 & 2,877 & 2,207 & 5,083 \\
\hline Latin America and the Caribbean & NA & NA & NA & NA & 16 & 7 & 86 & 36 & 275 & 140 & 241 & 167 & 140 & 125 & 40 & 53 & 798 & 527 & 1,325 \\
\hline Middle East and North Africa & NA & NA & NA & NA & 22 & 11 & 67 & 28 & 196 & 120 & 153 & 125 & 93 & 101 & 16 & 23 & 548 & 407 & 955 \\
\hline South Asia & NA & NA & NA & NA & 98 & 193 & 406 & 303 & 1,587 & 940 & 1,380 & 1,311 & 677 & 783 & 124 & 137 & 4,273 & 3,667 & 7,940 \\
\hline$\underline{\text { Sub-Saharan Africa }}$ & NA & NA & NA & NA & 9 & 6 & 51 & 27 & 211 & 185 & 184 & 228 & 96 & 140 & 12 & 27 & 564 & 614 & 1,178 \\
\hline Low- and middle-income & NA & NA & NA & NA & 242 & 275 & 1,122 & 586 & 3,739 & 2,035 & 3,560 & 3,087 & 1,986 & 2,458 & 396 & 755 & 11,046 & 9,196 & 20,242 \\
\hline High-income countries & NA & NA & NA & NA & 8 & 2 & 104 & 26 & 415 & 110 & 385 & 180 & 325 & 267 & 130 & 208 & 1,367 & 793 & 2,160 \\
\hline WORLD & NA & NA & NA & NA & 250 & 277 & 1,227 & 611 & 4,154 & 2,145 & 3,945 & 3,267 & 2,311 & 2,726 & 527 & 963 & 12,413 & 9,989 & 22,402 \\
\hline
\end{tabular}

Attributable DALYs (thousands)

\begin{tabular}{|c|c|c|c|c|c|c|c|c|c|c|c|c|c|c|c|c|c|c|c|}
\hline East Asia and Pacific & NA & NA & NA & NA & 101 & 82 & 245 & 144 & 603 & 385 & 652 & 595 & 417 & 498 & 102 & 181 & 2,121 & 1,885 & 4,006 \\
\hline Europe and Central Asia & NA & NA & NA & NA & 31 & 11 & 311 & 72 & 939 & 313 & 1,012 & 721 & 582 & 837 & 105 & 340 & 2,981 & 2,294 & 5,275 \\
\hline Latin America and the Caribbean & NA & NA & NA & NA & 28 & 15 & 98 & 41 & 293 & 152 & 257 & 177 & 144 & 128 & 41 & 54 & 861 & 568 & 1,429 \\
\hline Middle East and North Africa & NA & NA & NA & NA & 30 & 17 & 75 & 32 & 207 & 127 & 160 & 131 & 95 & 103 & 16 & 23 & 584 & 433 & 1,016 \\
\hline South Asia & NA & NA & NA & NA & 145 & 230 & 462 & 347 & 1,684 & 1,013 & 1,453 & 1,381 & 694 & 800 & 127 & 139 & 4,565 & 3,911 & 8,476 \\
\hline Sub-Saharan Africa & NA & NA & NA & NA & 16 & 8 & 57 & 32 & 225 & 198 & 193 & 241 & 98 & 142 & 12 & 28 & 601 & 649 & 1,250 \\
\hline Low- and middle-income countries & NA & NA & NA & NA & 351 & 364 & 1,248 & 667 & 3,952 & 2,190 & 3,728 & 3,245 & 2,030 & 2,508 & 403 & 765 & 11,712 & 9,739 & 21,452 \\
\hline High-income countries & NA & NA & NA & NA & 11 & 6 & 119 & 34 & 457 & 132 & 414 & 202 & 342 & 285 & 135 & 216 & 1,478 & 875 & 2,353 \\
\hline WORLD & NA & NA & NA & NA & 363 & 370 & 1,367 & 701 & 4,409 & 2,322 & 4,142 & 3,447 & 2,373 & 2,793 & 538 & 981 & 13,191 & 10,614 & 23,805 \\
\hline
\end{tabular}

Source: Authors' calculations.

Note: $\mathrm{NA}=$ not applicable. 
Risk factor: Low fruit and vegetable intake

Disease:

Cerebrovascular disease

\begin{tabular}{|c|c|c|c|c|c|c|c|c|c|c|c|c|c|c|c|c|c|c|c|}
\hline \multirow[b]{2}{*}{ Region } & \multicolumn{2}{|c|}{$0-4$ years } & \multicolumn{2}{|c|}{ 5-14 years } & \multicolumn{2}{|c|}{$15-29$ years } & \multicolumn{2}{|c|}{ 30-44 years } & \multicolumn{2}{|c|}{$45-59$ years } & \multicolumn{2}{|c|}{$60-69$ years } & \multicolumn{2}{|c|}{ 70-79 years } & \multicolumn{2}{|c|}{$80+$ years } & \multicolumn{3}{|c|}{ Total } \\
\hline & Male & Female & Male & Female & Male & Female & Male & Female & Male & Female & Male & Female & Male & Female & Male & Female & Male & Female & All \\
\hline \multicolumn{20}{|l|}{ PAF of Mortality (\%) } \\
\hline East Asia and Pacific & NA & NA & NA & NA & 6 & 6 & 6 & 6 & 7 & 7 & 8 & 9 & 8 & 9 & 7 & 7 & 8 & 8 & 8 \\
\hline Europe and Central Asia & NA & NA & NA & NA & 10 & 11 & 10 & 11 & 11 & 12 & 12 & 14 & 11 & 12 & 8 & 9 & 11 & 11 & 11 \\
\hline Latin America and the Caribbean & NA & NA & NA & NA & 11 & 12 & 11 & 10 & 12 & 13 & 13 & 14 & 11 & 12 & 9 & 9 & 11 & 11 & 11 \\
\hline Middle East and North Africa & NA & NA & NA & NA & 9 & 9 & 7 & 8 & 8 & 10 & 8 & 9 & 7 & 9 & 6 & 6 & 7 & 8 & 7 \\
\hline South Asia & NA & NA & NA & NA & 10 & 11 & 10 & 11 & 11 & 12 & 12 & 13 & 11 & 12 & 8 & 9 & 11 & 12 & 11 \\
\hline Sub-Saharan Africa & NA & NA & NA & NA & 11 & 10 & 8 & 10 & 9 & 12 & 9 & 12 & 8 & 10 & 5 & 7 & 8 & 10 & 9 \\
\hline Low- and middle-income countries & NA & NA & NA & NA & 9 & 10 & 8 & 9 & 9 & 10 & 10 & 12 & 9 & 10 & 8 & 8 & 9 & 10 & 10 \\
\hline High-income countries & NA & NA & NA & NA & 9 & 9 & 8 & 9 & 9 & 8 & 8 & 8 & 7 & 7 & 6 & 6 & 7 & 6 & 6 \\
\hline WORLD & NA & NA & NA & NA & 9 & 10 & 8 & 9 & 9 & 10 & 10 & 12 & 9 & 10 & 7 & 8 & 9 & 9 & 9 \\
\hline
\end{tabular}

PAF of YLL (\%)

\begin{tabular}{lllllrrrrrrrrrrrrrrr} 
East Asia and Pacific & NA & NA & NA & NA & 6 & 6 & 6 & 6 & 7 & 7 & 8 & 9 & 8 & 9 & 7 & 7 & 8 & 8 & 8 \\
Europe and Central Asia & NA & NA & NA & NA & 10 & 11 & 10 & 11 & 11 & 12 & 12 & 14 & 11 & 12 & 8 & 9 & 11 & 12 & 11 \\
Latin America and the Caribbean & NA & NA & NA & NA & 11 & 12 & 11 & 10 & 12 & 13 & 13 & 14 & 11 & 12 & 9 & 9 & 12 & 12 & 12 \\
Middle East and North Africa & NA & NA & NA & NA & 9 & 9 & 7 & 8 & 8 & 10 & 8 & 9 & 7 & 9 & 6 & 6 & 7 & 8 & 7 \\
South Asia & NA & NA & NA & NA & 10 & 11 & 10 & 11 & 11 & 12 & 12 & 13 & 11 & 12 & 8 & 9 & 11 & 12 & 11 \\
Sub-Saharan Africa & NA & NA & NA & NA & 11 & 10 & 8 & 10 & 9 & 12 & 9 & 12 & 8 & 10 & 5 & 7 & 8 & 11 & 10 \\
\hline Low- and middle-income countries & NA & NA & NA & NA & 9 & 10 & 8 & 9 & 9 & 10 & 10 & 12 & 9 & 10 & 7 & 8 & 9 & 10 & 10 \\
High-income countries & NA & NA & NA & NA & 9 & 9 & 8 & 9 & 9 & 8 & 8 & 8 & 7 & 7 & 6 & 6 & 7 & 7 & 7 \\
\hline WORLD & NA & NA & NA & NA & 9 & 10 & 8 & 9 & 9 & 10 & 10 & 12 & 9 & 10 & 7 & 8 & 9 & 10 & 9 \\
\hline
\end{tabular}

\section{PAF of DALYs (\%)}

Latin America and the Caribbean

Middle East and North Afric

South Asia

Sub-Saharan Africa

Low- and middle-income countries

High-income countries

$\begin{array}{llll}\text { NA } & \text { NA } & \text { NA } & \text { NA } \\ \text { NA } & \text { NA } & \text { NA } & \text { NA } \\ \text { NA } & \text { NA } & \text { NA } & \text { NA } \\ \text { NA } & \text { NA } & \text { NA } & \text { NA } \\ \text { NA } & \text { NA } & \text { NA } & \text { NA } \\ \text { NA } & \text { NA } & \text { NA } & \text { NA } \\ \text { NA } & \text { NA } & \text { NA } & \text { NA }\end{array}$

$\begin{array}{rrrr}6 & 6 & 8 & 8 \\ 10 & 11 & 12 & 14 \\ 11 & 12 & 14 & 13 \\ 9 & 9 & 9 & 10 \\ 10 & 11 & 12 & 12 \\ 11 & 10 & 9 & 10 \\ 9 & 10 & 10 & 11 \\ 9 & 9 & 11 & 12\end{array}$

9
12
15
10
13
10
11
11

$\begin{array}{rr}9 & 10 \\ 14 & 14 \\ 15 & 15 \\ 12 & 9 \\ 14 & 14 \\ 13 & 10 \\ 12 & 12 \\ 11 & 10\end{array}$

\begin{tabular}{rrrrrrrr}
11 & 9 & 10 & 7 & 8 & 9 & 10 & 9 \\
15 & 11 & 12 & 9 & 10 & 12 & 13 & 12 \\
16 & 12 & 12 & 9 & 9 & 14 & 13 & 13 \\
10 & 8 & 9 & 6 & 6 & 8 & 9 & 8 \\
15 & 11 & 12 & 9 & 9 & 12 & 13 & 13 \\
12 & 8 & 11 & 5 & 7 & 9 & 11 & 10 \\
13 & 10 & 11 & 8 & 9 & 11 & 11 & 11 \\
10 & 8 & 8 & 6 & 6 & 9 & 8 & 9 \\
13 & 10 & 11 & 7 & 8 & 10 & 11 & 11 \\
\hline
\end{tabular}

Attributable Mortality (thousands)

\begin{tabular}{|c|c|c|c|c|c|c|c|c|c|c|c|c|c|c|c|c|c|c|c|}
\hline East Asia and Pacific & NA & NA & NA & NA & 0 & 0 & 1 & 1 & 9 & 6 & 21 & 15 & 29 & 31 & 13 & 24 & 74 & 77 & 151 \\
\hline Europe and Central Asia & NA & NA & NA & NA & 0 & 0 & 1 & 1 & 6 & 4 & 13 & 13 & 15 & 26 & 7 & 24 & 43 & 68 & 112 \\
\hline Latin America and the Caribbean & NA & NA & NA & NA & 0 & 0 & 1 & 1 & 3 & 2 & 4 & 3 & 4 & 4 & 3 & 5 & 14 & 15 & 29 \\
\hline Middle East and North Africa & NA & NA & NA & NA & 0 & 0 & 0 & 0 & 1 & 1 & 1 & 1 & 2 & 2 & 1 & 1 & 5 & 5 & 10 \\
\hline South Asia & NA & NA & NA & NA & 0 & 0 & 1 & 1 & 9 & 6 & 17 & 17 & 17 & 20 & 6 & 9 & 50 & 53 & 104 \\
\hline Sub-Saharan Africa & NA & NA & NA & NA & 0 & 0 & 1 & 1 & 3 & 4 & 3 & 6 & 3 & 7 & 1 & 3 & 12 & 21 & 33 \\
\hline Low- and middle-income countries & NA & NA & NA & NA & 2 & 1 & 6 & 4 & 30 & 25 & 60 & 55 & 71 & 91 & 31 & 66 & 198 & 241 & 439 \\
\hline High-income countries & NA & NA & NA & NA & 0 & 0 & 1 & 0 & 2 & 1 & 4 & 2 & 7 & 8 & 8 & 18 & 21 & 29 & 51 \\
\hline WORLD & NA & NA & NA & NA & 2 & 1 & 6 & 4 & 32 & 26 & 63 & 57 & 78 & 98 & 39 & 83 & 220 & 270 & 489 \\
\hline
\end{tabular}

Attributable YLL (thousands)

\begin{tabular}{|c|c|c|c|c|c|c|c|c|c|c|c|c|c|c|c|c|c|c|c|}
\hline East Asia and Pacific & NA & NA & NA & NA & 9 & 5 & 33 & 22 & 173 & 128 & 280 & 219 & 258 & 317 & 66 & 123 & 819 & 815 & 1,634 \\
\hline Europe and Central Asia & NA & NA & NA & NA & 8 & 5 & 31 & 20 & 113 & 84 & 181 & 192 & 139 & 265 & 30 & 115 & 503 & 681 & 1,184 \\
\hline Latin America and the Caribbean & NA & NA & NA & NA & 5 & 5 & 18 & 16 & 50 & 48 & 48 & 46 & 38 & 44 & 14 & 21 & 172 & 179 & 352 \\
\hline Middle East and North Africa & NA & NA & NA & NA & 6 & 3 & 4 & 4 & 13 & 15 & 15 & 18 & 14 & 20 & 3 & 6 & 57 & 64 & 121 \\
\hline South Asia & NA & NA & NA & NA & 11 & 7 & 22 & 15 & 159 & 127 & 235 & 256 & 152 & 213 & 31 & 45 & 610 & 664 & 1,274 \\
\hline Sub-Saharan Africa & NA & NA & NA & NA & 11 & 9 & 21 & 19 & 50 & 87 & 44 & 93 & 29 & 71 & 5 & 16 & 161 & 296 & 457 \\
\hline Low- and middle-income countries & NA & NA & NA & NA & 50 & 33 & 130 & 97 & 557 & 489 & 805 & 824 & 630 & 931 & 150 & 326 & 2,321 & 2,700 & 5,021 \\
\hline High-income countries & NA & NA & NA & NA & 2 & 2 & 12 & 9 & 41 & 26 & 47 & 36 & 61 & 75 & 34 & 72 & 198 & 220 & 418 \\
\hline WORLD & NA & NA & NA & NA & 52 & 35 & 142 & 106 & 598 & 515 & 852 & 859 & 691 & 1,006 & 184 & 399 & 2,519 & 2,920 & 5,439 \\
\hline \multicolumn{20}{|l|}{ Attributable DALYs (thousands) } \\
\hline East Asia and Pacific & NA & NA & NA & NA & 9 & 5 & 66 & 45 & 307 & 227 & 451 & 344 & 343 & 414 & 77 & 142 & 1,254 & 1,178 & 2,431 \\
\hline Europe and Central Asia & NA & NA & NA & NA & 8 & 5 & 44 & 33 & 157 & 126 & 252 & 275 & 178 & 329 & 35 & 129 & 674 & 896 & 1,570 \\
\hline Latin America and the Caribbean & NA & NA & NA & NA & 5 & 5 & 31 & 30 & 82 & 79 & 77 & 71 & 51 & 57 & 16 & 25 & 263 & 267 & 531 \\
\hline Middle East and North Africa & NA & NA & NA & NA & 6 & 3 & 8 & 6 & 20 & 22 & 22 & 24 & 19 & 24 & 4 & 6 & 79 & 86 & 165 \\
\hline South Asia & NA & NA & NA & NA & 11 & 7 & 33 & 22 & 227 & 178 & 326 & 344 & 188 & 256 & 35 & 51 & 818 & 858 & 1,677 \\
\hline Sub-Saharan Africa & NA & NA & NA & NA & 11 & 9 & 24 & 23 & 59 & 107 & 53 & 112 & 33 & 80 & 6 & 18 & 186 & 348 & 534 \\
\hline Low- and middle-income countries & NA & NA & NA & NA & 50 & 33 & 204 & 160 & 853 & 738 & 1,182 & 1,170 & 812 & 1,160 & 172 & 371 & 3,275 & 3,633 & 6,907 \\
\hline High-income countries & NA & NA & NA & NA & 2 & 2 & 31 & 25 & 103 & 70 & 109 & 84 & 108 & 130 & 47 & 97 & 401 & 409 & 810 \\
\hline WORLD & NA & NA & NA & NA & 53 & 35 & 236 & 185 & 956 & 808 & 1,291 & 1,255 & 920 & 1,290 & 219 & 468 & 3,676 & 4,042 & 7,718 \\
\hline
\end{tabular}


Risk factor: Low fruit and vegetable intake

\begin{tabular}{|c|c|c|c|c|c|c|c|c|c|c|c|c|c|c|c|c|c|c|c|}
\hline \multirow[b]{2}{*}{ Region } & \multicolumn{2}{|c|}{$0-4$ years } & \multicolumn{2}{|c|}{ 5-14 years } & \multicolumn{2}{|c|}{$15-29$ years } & \multicolumn{2}{|c|}{ 30-44 years } & \multicolumn{2}{|c|}{$45-59$ years } & \multicolumn{2}{|c|}{$60-69$ years } & \multicolumn{2}{|c|}{$70-79$ years } & \multicolumn{2}{|c|}{$80+$ years } & \multicolumn{3}{|c|}{ Total } \\
\hline & Male & Female & Male & Female & Male & Female & Male & Female & Male & Female & Male & Female & Male & Female & Male & Female & Male & Female & All \\
\hline \multicolumn{20}{|l|}{ PAF of Mortality (\%) } \\
\hline East Asia and Pacific & NA & NA & NA & NA & 1 & 1 & 3 & 3 & 6 & 5 & 8 & 8 & 6 & 6 & 4 & 5 & 5 & 5 & 5 \\
\hline Europe and Central Asia & NA & NA & NA & NA & 1 & 1 & 5 & 4 & 10 & 8 & 14 & 15 & 12 & 13 & 9 & 11 & 10 & 11 & 10 \\
\hline Latin America and the Caribbean & NA & NA & NA & NA & 1 & 1 & 3 & 3 & 7 & 6 & 10 & 8 & 7 & 6 & 5 & 4 & 5 & 5 & 5 \\
\hline Middle East and North Africa & NA & NA & NA & NA & 1 & 1 & 4 & 3 & 8 & 7 & 8 & 8 & 6 & 7 & 4 & 4 & 4 & 4 & 4 \\
\hline South Asia & NA & NA & NA & NA & 1 & 1 & 3 & 3 & 9 & 8 & 12 & 12 & 9 & 9 & 6 & 6 & 5 & 5 & 5 \\
\hline Sub-Saharan Africa & NA & NA & NA & NA & 0 & 0 & 0 & 0 & 3 & 3 & 5 & 7 & 4 & 6 & 2 & 4 & 1 & 1 & 1 \\
\hline Low- and middle-income countries & NA & NA & NA & NA & 1 & 1 & 2 & 2 & 7 & 6 & 10 & 10 & 8 & 8 & 5 & 6 & 5 & 5 & 5 \\
\hline High-income countries & NA & NA & NA & NA & 1 & 1 & 3 & 2 & 6 & 4 & 6 & 5 & 5 & 4 & 3 & 3 & 5 & 4 & 4 \\
\hline WORLD & NA & NA & NA & NA & 1 & 1 & 2 & 2 & 7 & 6 & 9 & 9 & 7 & 8 & 5 & 5 & 5 & 5 & 5 \\
\hline
\end{tabular}

PAF of YLL (\%)

\begin{tabular}{lllllllllrrrrrrrrrrr} 
East Asia and Pacific & NA & NA & NA & NA & 1 & 1 & 3 & 3 & 6 & 5 & 8 & 8 & 6 & 6 & 4 & 4 & 4 & 4 & 4 \\
Europe and Central Asia & NA & NA & NA & NA & 1 & 1 & 5 & 4 & 10 & 8 & 14 & 14 & 12 & 13 & 9 & 11 & 9 & 10 & 9 \\
Latin America and the Caribbean & NA & NA & NA & NA & 1 & 1 & 2 & 3 & 7 & 6 & 10 & 8 & 7 & 6 & 5 & 4 & 4 & 4 & 4 \\
Middle East and North Africa & NA & NA & NA & NA & 1 & 1 & 4 & 3 & 8 & 7 & 8 & 8 & 6 & 7 & 4 & 4 & 3 & 3 & 3 \\
South Asia & NA & NA & NA & NA & 1 & 1 & 3 & 3 & 9 & 8 & 12 & 12 & 9 & 9 & 6 & 6 & 4 & 3 & 4 \\
Sub-Saharan Africa & NA & NA & NA & NA & 0 & 0 & 0 & 0 & 3 & 3 & 5 & 6 & 4 & 6 & 2 & 4 & 1 & 1 & 1 \\
\hline Low- and middle-income countries & NA & NA & NA & NA & 1 & 1 & 2 & 2 & 7 & 6 & 10 & 10 & 8 & 8 & 5 & 6 & 3 & 3 & 3 \\
High-income countries & NA & NA & NA & NA & 0 & 1 & 3 & 2 & 6 & 4 & 6 & 5 & 5 & 4 & 3 & 3 & 5 & 4 & 4 \\
\hline WORLD & NA & NA & NA & NA & 1 & 1 & 2 & 2 & 7 & 6 & 9 & 9 & 7 & 5 \\
\hline
\end{tabular}

\section{PAF of DALYs (\%)}

East Asia and Pacific

Europe and Central Asia

NA NA NA NA

South Asia

Sub-Saharan Africa

Low- and middle-income countries

High-income countries

$\begin{array}{ll}\text { NA } & 1 \\ \text { NA } & 1 \\ \text { NA } & 0 \\ \text { NA } & 1 \\ \text { NA } & 1 \\ \text { NA } & 0 \\ \text { NA } & 0 \\ \text { NA } & 0\end{array}$

$\begin{array}{lll}1 & 2 & 1 \\ 0 & 3 & 2 \\ 0 & 2 & 1 \\ 0 & 2 & 1 \\ 1 & 2 & 2 \\ 0 & 0 & 0 \\ 0 & 2 & 1 \\ 0 & 2 & 1\end{array}$

\begin{tabular}{l}
4 \\
8 \\
5 \\
5 \\
7 \\
2 \\
\hline 5 \\
4
\end{tabular}

\begin{tabular}{rr}
3 & 6 \\
5 & 12 \\
4 & 7 \\
4 & 6 \\
5 & 10 \\
3 & 4 \\
\hline 4 & 8 \\
2 & 5
\end{tabular}

WORLD

\section{Attributable Mortality (thousands)}

\begin{tabular}{|c|c|c|c|c|c|c|c|c|c|c|c|c|c|c|c|c|c|c|c|}
\hline East Asia and Pacific & NA & NA & NA & NA & 4 & 3 & 17 & 10 & 68 & 39 & 108 & 72 & 104 & 99 & 40 & 67 & 340 & 290 & 630 \\
\hline Europe and Central Asia & NA & NA & NA & NA & 1 & 1 & 15 & 4 & 62 & 21 & 99 & 63 & 87 & 111 & 31 & 98 & 295 & 299 & 594 \\
\hline Latin America and the Caribbean & NA & NA & NA & NA & 1 & 1 & 5 & 3 & 21 & 11 & 26 & 16 & 24 & 19 & 13 & 17 & 91 & 67 & 158 \\
\hline Middle East and North Africa & NA & NA & NA & NA & 1 & 1 & 3 & 2 & 12 & 7 & 14 & 10 & 13 & 12 & 4 & 5 & 47 & 37 & 84 \\
\hline South Asia & NA & NA & NA & NA & 4 & 7 & 20 & 14 & 101 & 58 & 131 & 109 & 99 & 98 & 33 & 36 & 389 & 322 & 712 \\
\hline Sub-Saharan Africa & NA & NA & NA & NA & 1 & 1 & 4 & 2 & 16 & 16 & 19 & 23 & 15 & 22 & 4 & 8 & 59 & 71 & 130 \\
\hline Low- and middle-income countries & NA & NA & NA & NA & 12 & 12 & 65 & 35 & 282 & 153 & 397 & 293 & 342 & 361 & 125 & 232 & 1,222 & 1,086 & 2,308 \\
\hline High-income countries & NA & NA & NA & NA & 0 & 0 & 6 & 2 & 34 & 11 & 45 & 19 & 58 & 41 & 43 & 73 & 187 & 146 & 333 \\
\hline WORLD & NA & NA & NA & NA & 13 & 13 & 71 & 38 & 315 & 163 & 442 & 313 & 399 & 402 & 168 & 305 & 1,408 & 1,233 & 2,641 \\
\hline
\end{tabular}

Attributable YLL (thousands)

\begin{tabular}{|c|c|c|c|c|c|c|c|c|c|c|c|c|c|c|c|c|c|c|c|}
\hline East Asia and Pacific & NA & NA & NA & NA & 102 & 71 & 405 & 256 & 1,276 & 783 & 1,448 & 1,078 & 927 & 1,013 & 199 & 340 & 4,355 & 3,542 & 7,897 \\
\hline Europe and Central Asia & NA & NA & NA & NA & 36 & 15 & 356 & 103 & 1,179 & 428 & 1,339 & 948 & 786 & 1,138 & 140 & 457 & 3,836 & 3,089 & 6,925 \\
\hline Latin America and the Caribbean & NA & NA & NA & NA & 26 & 15 & 125 & 67 & 399 & 227 & 356 & 248 & 213 & 191 & 61 & 80 & 1,179 & 829 & 2,008 \\
\hline Middle East and North Africa & NA & NA & NA & NA & 31 & 15 & 78 & 38 & 226 & 146 & 182 & 149 & 116 & 126 & 21 & 29 & 654 & 504 & 1,158 \\
\hline South Asia & NA & NA & NA & NA & 119 & 207 & 474 & 346 & 1,886 & 1,151 & 1,778 & 1,645 & 887 & 1,029 & 164 & 191 & 5,308 & 4,568 & 9,876 \\
\hline Sub-Saharan Africa & NA & NA & NA & NA & 23 & 17 & 85 & 58 & 306 & 309 & 256 & 345 & 137 & 224 & 18 & 46 & 825 & 998 & 1,822 \\
\hline Low- and middle-income countries & NA & NA & NA & NA & 336 & 341 & 1,523 & 868 & 5,272 & 3,043 & 5,359 & 4,414 & 3,065 & 3,720 & 602 & 1,143 & 16,157 & 13,529 & 29,686 \\
\hline High-income countries & NA & NA & NA & NA & 12 & 6 & 146 & 56 & 625 & 211 & 605 & 291 & 509 & 413 & 190 & 301 & 2,088 & 1,278 & 3,366 \\
\hline WORLD & NA & NA & NA & NA & 348 & 347 & 1,669 & 924 & 5,897 & 3,254 & 5,965 & 4,705 & 3,574 & 4,133 & 792 & 1,444 & 18,245 & 14,806 & 33,051 \\
\hline \multicolumn{20}{|l|}{ Attributable DALYs (thousands) } \\
\hline East Asia and Pacific & NA & NA & NA & NA & 133 & 102 & 462 & 295 & 1,455 & 911 & 1,657 & 1,234 & 1,026 & 1,121 & 212 & 363 & 4,945 & 4,026 & 8,970 \\
\hline Europe and Central Asia & NA & NA & NA & NA & 42 & 20 & 390 & 126 & 1,261 & 491 & 1,444 & 1,065 & 836 & 1,217 & 146 & 476 & 4,119 & 3,395 & 7,514 \\
\hline Latin America and the Caribbean & NA & NA & NA & NA & 37 & 24 & 150 & 86 & 451 & 272 & 402 & 284 & 231 & 208 & 65 & 85 & 1,336 & 959 & 2,295 \\
\hline Middle East and North Africa & NA & NA & NA & NA & 39 & 21 & 89 & 45 & 244 & 161 & 197 & 162 & 122 & 132 & 21 & 30 & 713 & 551 & 1,264 \\
\hline South Asia & NA & NA & NA & NA & 166 & 245 & 540 & 397 & 2,052 & 1,276 & 1,943 & 1,803 & 941 & 1,090 & 171 & 199 & 5,812 & 5,008 & 10,820 \\
\hline Sub-Saharan Africa & NA & NA & NA & NA & 29 & 19 & 93 & 65 & 330 & 341 & 274 & 376 & 143 & 235 & 19 & 47 & 888 & 1,085 & 1,973 \\
\hline Low- and middle-income countries & NA & NA & NA & NA & 446 & 430 & 1,725 & 1,014 & 5,792 & 3,453 & 5,918 & 4,924 & 3,299 & 4,004 & 633 & 1,200 & 17,812 & 15,024 & 32,836 \\
\hline High-income countries & NA & NA & NA & NA & 16 & 10 & 181 & 81 & 735 & 280 & 703 & 365 & 578 & 490 & 208 & 335 & 2,422 & 1,561 & 3,982 \\
\hline WORLD & NA & NA & NA & NA & 462 & 440 & 1,906 & 1,095 & 6,527 & 3,732 & 6,620 & 5,289 & 3,878 & 4,493 & 841 & 1,535 & 20,234 & 16,584 & 36,819 \\
\hline
\end{tabular}

Source: Authors' calculations.

Note: $\mathrm{NA}=$ not applicable. 
Table 4A.47

Risk factor: Physical inactivity

Disease:

Colon and rectal cancers

\begin{tabular}{|c|c|c|c|c|c|c|c|c|c|c|c|c|c|c|c|c|c|c|c|}
\hline \multirow[b]{2}{*}{ Region } & \multicolumn{2}{|c|}{$0-4$ years } & \multicolumn{2}{|c|}{ 5-14 years } & \multicolumn{2}{|c|}{$15-29$ years } & \multicolumn{2}{|c|}{ 30-44 years } & \multicolumn{2}{|c|}{ 45-59 years } & \multicolumn{2}{|c|}{$60-69$ years } & \multicolumn{2}{|c|}{$70-79$ years } & \multicolumn{2}{|c|}{ 80+ years } & \multicolumn{3}{|c|}{ Total } \\
\hline & Male & Female & Male & Female & Male & Female & Male & Female & Male & Female & Male & Female & Male & Female & Male & Female & Male & Female & All \\
\hline \multicolumn{20}{|l|}{ PAF of Mortality (\%) } \\
\hline East Asia and Pacific & NA & NA & NA & NA & 14 & 15 & 15 & 16 & 15 & 16 & 16 & 17 & 12 & 13 & 8 & 8 & 14 & 14 & 14 \\
\hline Europe and Central Asia & NA & NA & NA & NA & 15 & 16 & 16 & 18 & 17 & 18 & 19 & 22 & 16 & 17 & 11 & 12 & 17 & 17 & 17 \\
\hline Latin America and the Caribbean & NA & NA & NA & NA & 15 & 17 & 16 & 19 & 16 & 19 & 17 & 23 & 14 & 19 & 10 & 13 & 15 & 18 & 17 \\
\hline Middle East and North Africa & NA & NA & NA & NA & 14 & 16 & 16 & 16 & 16 & 16 & 17 & 18 & 13 & 15 & 9 & 10 & 15 & 15 & 15 \\
\hline South Asia & NA & NA & NA & NA & 14 & 15 & 15 & 16 & 15 & 16 & 17 & 17 & 13 & 14 & 8 & 10 & 14 & 14 & 14 \\
\hline Sub-Saharan Africa & NA & NA & NA & NA & 13 & 14 & 14 & 15 & 15 & 15 & 16 & 16 & 12 & 12 & 8 & 8 & 13 & 13 & 13 \\
\hline Low- and middle-income countries & NA & NA & NA & NA & 14 & 15 & 15 & 16 & 16 & 17 & 17 & 19 & 14 & 15 & 9 & 10 & 15 & 15 & $\overline{15}$ \\
\hline High-income countries & NA & NA & NA & NA & 16 & 17 & 17 & 18 & 17 & 18 & 18 & 19 & 13 & 15 & 10 & 11 & 14 & 14 & 14 \\
\hline WORLD & NA & NA & NA & NA & 14 & 15 & 16 & 16 & 16 & 17 & 17 & 19 & 14 & 15 & 10 & 10 & 14 & 15 & 15 \\
\hline \multicolumn{20}{|l|}{ PAF of YLL (\%) } \\
\hline East Asia and Pacific & NA & NA & NA & NA & 14 & 15 & 15 & 16 & 15 & 16 & 16 & 17 & 12 & 13 & 8 & 8 & 15 & 15 & 15 \\
\hline Europe and Central Asia & NA & NA & NA & NA & 15 & 16 & 16 & 18 & 17 & 18 & 19 & 22 & 16 & 17 & 11 & 12 & 17 & 18 & 18 \\
\hline Latin America and the Caribbean & NA & NA & NA & NA & 15 & 17 & 16 & 19 & 16 & 19 & 17 & 23 & 14 & 19 & 10 & 13 & 16 & 19 & 18 \\
\hline Middle East and North Africa & NA & NA & NA & NA & 14 & 16 & 16 & 16 & 16 & 16 & 17 & 18 & 13 & 15 & 9 & 10 & 15 & 16 & 16 \\
\hline South Asia & NA & NA & NA & NA & 14 & 15 & 15 & 16 & 15 & 16 & 17 & 17 & 13 & 14 & 8 & 10 & 15 & 15 & 15 \\
\hline Sub-Saharan Africa & NA & NA & NA & NA & 13 & 14 & 14 & 15 & 15 & 15 & 16 & 16 & 12 & 12 & 8 & 8 & 14 & 14 & 14 \\
\hline Low- and middle-income countries & NA & NA & NA & NA & 14 & 15 & 15 & 16 & 16 & 17 & 17 & 19 & 14 & 15 & 9 & 10 & 15 & 16 & $\overline{16}$ \\
\hline High-income countries & NA & NA & NA & NA & 16 & 17 & 17 & 18 & 17 & 18 & 18 & 19 & 13 & 15 & 10 & 11 & 15 & 16 & 16 \\
\hline WORLD & NA & NA & NA & NA & 14 & 15 & 16 & 16 & 16 & 17 & 17 & 19 & 14 & 15 & 10 & 10 & 15 & 16 & 16 \\
\hline
\end{tabular}

PAF of DALYs (\%)

\begin{tabular}{|c|c|c|c|c|c|c|c|c|c|c|c|c|c|c|c|c|c|c|c|}
\hline East Asia and Pacific & NA & NA & NA & NA & 14 & 15 & 15 & 16 & 15 & 16 & 16 & 17 & 12 & 13 & 8 & 8 & 15 & 15 & 15 \\
\hline Europe and Central Asia & NA & NA & NA & NA & 15 & 16 & 16 & 18 & 17 & 18 & 19 & 22 & 16 & 17 & 11 & 12 & 17 & 18 & 18 \\
\hline Latin America and the Caribbean & NA & NA & NA & NA & 15 & 17 & 16 & 19 & 16 & 19 & 17 & 23 & 14 & 19 & 10 & 13 & 16 & 19 & 18 \\
\hline Middle East and North Africa & NA & NA & NA & NA & 14 & 16 & 16 & 16 & 16 & 16 & 17 & 18 & 13 & 15 & 9 & 10 & 15 & 16 & 16 \\
\hline South Asia & NA & NA & NA & NA & 14 & 15 & 15 & 16 & 15 & 16 & 17 & 17 & 13 & 14 & 8 & 10 & 15 & 15 & 15 \\
\hline Sub-Saharan Africa & NA & NA & NA & NA & 13 & 14 & 14 & 15 & 15 & 15 & 16 & 16 & 12 & 12 & 8 & 8 & 14 & 14 & 14 \\
\hline Low- and middle-income countries & NA & NA & NA & NA & 14 & 15 & 15 & 16 & 16 & 17 & 17 & 19 & 14 & 15 & 9 & 10 & 15 & 16 & 16 \\
\hline High-income countries & NA & NA & NA & NA & 16 & 17 & 17 & 18 & 17 & 18 & 18 & 19 & 13 & 15 & 10 & 11 & 15 & 16 & 16 \\
\hline WORLD & NA & NA & NA & NA & 14 & 15 & 16 & 17 & 16 & 17 & 17 & 19 & 14 & 15 & 10 & 10 & 15 & 16 & 16 \\
\hline
\end{tabular}

Attributable Mortality (thousands)

\begin{tabular}{|c|c|c|c|c|c|c|c|c|c|c|c|c|c|c|c|c|c|c|c|}
\hline & & & & & & & & & & & & & & & & & & & \\
\hline East Asia and Pacific & NA & NA & NA & NA & 0 & 0 & 1 & 1 & 3 & 3 & 4 & 3 & 3 & 3 & 1 & 1 & 12 & 11 & 22 \\
\hline Europe and Central Asia & NA & NA & NA & NA & 0 & 0 & 0 & 0 & 1 & 1 & 3 & 3 & 3 & 3 & 1 & 1 & 8 & 8 & 16 \\
\hline Latin America and the Caribbean & NA & NA & NA & NA & 0 & 0 & 0 & 0 & 1 & 1 & 1 & 1 & 1 & 1 & 0 & 1 & 3 & 3 & 6 \\
\hline Middle East and North Africa & NA & NA & NA & NA & 0 & 0 & 0 & 0 & 0 & 0 & 0 & 0 & 0 & 0 & 0 & 0 & 1 & 1 & 2 \\
\hline South Asia & NA & NA & NA & NA & 0 & 0 & 0 & 0 & 1 & 0 & 1 & 1 & 1 & 0 & 0 & 0 & 3 & 2 & 5 \\
\hline Sub-Saharan Africa & NA & NA & NA & NA & 0 & 0 & 0 & 0 & 0 & 0 & 0 & 0 & 0 & 0 & 0 & 0 & 1 & 1 & 3 \\
\hline Low- and middle-income countries & NA & NA & NA & NA & 1 & 0 & 2 & 2 & 6 & 5 & 9 & 8 & 7 & 8 & 2 & 3 & 27 & 26 & 54 \\
\hline High-income countries & NA & NA & NA & NA & 0 & 0 & 0 & 0 & 3 & 2 & 6 & 4 & 6 & 5 & 3 & 6 & 19 & 17 & 36 \\
\hline WORLD & NA & NA & NA & NA & 1 & 0 & 3 & 2 & 9 & 8 & 15 & 12 & 13 & 13 & 5 & 9 & 46 & 44 & 90 \\
\hline
\end{tabular}

Attributable YLL (thousands)

\begin{tabular}{|c|c|c|c|c|c|c|c|c|c|c|c|c|c|c|c|c|c|c|c|}
\hline East Asia and Pacific & NA & NA & NA & NA & 7 & 3 & 25 & 25 & 56 & 50 & 52 & 48 & 24 & 26 & 4 & 6 & 168 & 159 & 326 \\
\hline Europe and Central Asia & NA & NA & NA & NA & 1 & 1 & 7 & 7 & 26 & 25 & 40 & 41 & 24 & 33 & 3 & 5 & 101 & 112 & 213 \\
\hline Latin America and the Caribbean & NA & NA & NA & NA & 1 & 1 & 4 & 6 & 10 & 13 & 10 & 14 & 7 & 10 & 2 & 3 & 34 & 47 & 81 \\
\hline Middle East and North Africa & NA & NA & NA & NA & 1 & 1 & 3 & 3 & 4 & 3 & 3 & 3 & 2 & 2 & 0 & 0 & 13 & 11 & 24 \\
\hline South Asia & NA & NA & NA & NA & 4 & 1 & 9 & 6 & 10 & 8 & 13 & 9 & 5 & 5 & 1 & 2 & 43 & 31 & 73 \\
\hline Sub-Saharan Africa & NA & NA & NA & NA & 1 & 1 & 4 & 3 & 7 & 6 & 5 & 5 & 3 & 3 & 1 & 1 & 22 & 18 & 40 \\
\hline Low- and middle-income countries & NA & NA & NA & NA & 15 & 7 & 54 & 50 & 113 & 106 & 123 & 120 & 64 & 78 & 10 & 18 & 380 & 378 & 758 \\
\hline High-income countries & NA & NA & NA & NA & 1 & 1 & 12 & 10 & 58 & 45 & 75 & 56 & 55 & 55 & 15 & 25 & 216 & 192 & 408 \\
\hline WORLD & NA & NA & NA & NA & 16 & 8 & 65 & 60 & 172 & 151 & 199 & 176 & 119 & 133 & 25 & 42 & 596 & 570 & 1,166 \\
\hline
\end{tabular}

Attributable DALYs (thousands)

\begin{tabular}{lrlllrrrrrrrrrrrrrrr} 
East Asia and Pacific & NA & NA & NA & NA & 7 & 3 & 26 & 27 & 59 & 54 & 54 & 51 & 25 & 27 & 4 & 6 & 175 & 168 & 343 \\
Europe and Central Asia & NA & NA & NA & NA & 1 & 1 & 7 & 8 & 28 & 27 & 43 & 44 & 25 & 34 & 3 & 6 & 108 & 120 & 228 \\
Latin America and the Caribbean & NA & NA & NA & NA & 1 & 1 & 5 & 6 & 11 & 14 & 11 & 14 & 7 & 11 & 2 & 4 & 36 & 50 & 85 \\
Middle East and North Africa & NA & NA & NA & NA & 1 & 1 & 3 & 3 & 4 & 3 & 3 & 3 & 2 & 2 & 0 & 0 & 14 & 12 & 25 \\
South Asia & NA & NA & NA & NA & 4 & 1 & 9 & 6 & 11 & 9 & 13 & 10 & 5 & 5 & 1 & 2 & 43 & 32 & 75 \\
Sub-Saharan Africa & NA & NA & NA & NA & 1 & 1 & 4 & 3 & 7 & 6 & 6 & 5 & 3 & 3 & 1 & 1 & 22 & 19 & 41 \\
\hline Low- and middle-income countries & NA & NA & NA & NA & 16 & 7 & 55 & 53 & 120 & 113 & 130 & 126 & 67 & 81 & 11 & 18 & 397 & 400 & 797 \\
High-income countries & NA & NA & NA & NA & 1 & 1 & 13 & 13 & 72 & 57 & 91 & 67 & 65 & 66 & 17 & 29 & 261 & 234 & 494 \\
\hline WORLD & NA & NA & NA & NA & 17 & 9 & 68 & 66 & 192 & 170 & 221 & 194 & 132 & 148 & 28 & 47 & 658 & 633 & 1,291
\end{tabular}


Risk factor: Physical inactivity

Disease: Breast cancer

\begin{tabular}{|c|c|c|c|c|c|c|c|c|c|c|c|c|c|c|c|c|c|c|c|}
\hline \multirow[b]{2}{*}{ Region } & \multicolumn{2}{|c|}{$0-4$ years } & \multicolumn{2}{|c|}{ 5-14 years } & \multicolumn{2}{|c|}{$15-29$ years } & \multicolumn{2}{|c|}{ 30-44 years } & \multicolumn{2}{|c|}{$45-59$ years } & \multicolumn{2}{|c|}{$60-69$ years } & \multicolumn{2}{|c|}{$70-79$ years } & \multicolumn{2}{|c|}{$80+$ years } & \multicolumn{3}{|c|}{ Total } \\
\hline & Male & Female & Male & Female & Male & Female & Male & Female & Male & Female & Male & Female & Male & Female & Male & Female & Male & Female & All \\
\hline \multicolumn{20}{|l|}{ PAF of Mortality (\%) } \\
\hline East Asia and Pacific & NA & NA & NA & NA & NA & 8 & NA & 8 & NA & 10 & NA & 10 & NA & 8 & NA & 5 & NA & 9 & 9 \\
\hline Europe and Central Asia & NA & NA & NA & NA & NA & 8 & NA & 9 & NA & 11 & NA & 13 & NA & 10 & NA & 7 & NA & 11 & 11 \\
\hline Latin America and the Caribbean & NA & NA & NA & NA & NA & 9 & NA & 9 & NA & 12 & NA & 14 & NA & 11 & NA & 8 & NA & 11 & 11 \\
\hline Middle East and North Africa & NA & NA & NA & NA & NA & 8 & NA & 9 & NA & 10 & NA & 11 & NA & 9 & NA & 6 & NA & 9 & 9 \\
\hline South Asia & NA & NA & NA & NA & NA & 8 & NA & 8 & NA & 10 & NA & 10 & NA & 8 & NA & 6 & NA & 9 & 9 \\
\hline Sub-Saharan Africa & NA & NA & NA & NA & NA & 8 & NA & 9 & NA & 9 & NA & 10 & NA & 8 & NA & 5 & NA & 9 & 9 \\
\hline Low- and middle-income countries & NA & NA & NA & NA & NA & 8 & NA & 9 & NA & 10 & NA & 11 & NA & 9 & NA & 6 & NA & 10 & $\overline{10}$ \\
\hline High-income countries & NA & NA & NA & NA & NA & 9 & NA & 10 & NA & 11 & NA & 12 & NA & 9 & NA & 7 & NA & 10 & 9 \\
\hline$\overline{\text { WORLD }}$ & NA & NA & NA & NA & NA & 8 & NA & 9 & NA & 10 & NA & 11 & NA & 9 & NA & 6 & NA & 10 & $\overline{10}$ \\
\hline
\end{tabular}

PAF of YLL (\%)

\begin{tabular}{|c|c|c|c|c|c|c|c|c|c|c|c|c|c|c|c|c|c|c|c|}
\hline East Asia and Pacific & NA & NA & NA & NA & NA & 8 & NA & 8 & NA & 10 & NA & 10 & NA & 8 & NA & 5 & NA & 9 & 9 \\
\hline Europe and Central Asia & NA & NA & NA & NA & NA & 8 & NA & 9 & NA & 11 & NA & 13 & NA & 10 & NA & 7 & NA & 11 & 11 \\
\hline Latin America and the Caribbean & NA & NA & NA & NA & NA & 9 & NA & 9 & NA & 12 & NA & 14 & NA & 11 & NA & 8 & NA & 11 & 11 \\
\hline Middle East and North Africa & NA & NA & NA & NA & NA & 8 & NA & 9 & NA & 10 & NA & 11 & NA & 9 & NA & 6 & NA & 10 & 10 \\
\hline South Asia & NA & NA & NA & NA & NA & 8 & NA & 8 & NA & 10 & NA & 10 & NA & 8 & NA & 6 & NA & 9 & 9 \\
\hline Sub-Saharan Africa & NA & NA & NA & NA & NA & 8 & NA & 9 & NA & 9 & NA & 10 & NA & 8 & NA & 5 & NA & 9 & 9 \\
\hline Low- and middle-income countries & NA & NA & NA & NA & NA & 8 & NA & 9 & NA & 10 & NA & 11 & NA & 9 & NA & 6 & NA & 10 & 10 \\
\hline High-income countries & NA & NA & NA & NA & NA & 9 & NA & 10 & NA & 11 & NA & 12 & NA & 9 & NA & 7 & NA & 10 & 10 \\
\hline WORLD & NA & NA & NA & NA & NA & 8 & NA & 9 & NA & 10 & NA & 11 & NA & 9 & NA & 6 & NA & 10 & 10 \\
\hline
\end{tabular}

PAF of DALYs $(\%)$

\begin{tabular}{|c|c|c|c|c|c|c|c|c|c|c|c|c|c|c|c|c|c|c|c|}
\hline East Asia and Pacific & NA & NA & NA & NA & NA & 8 & NA & 8 & NA & 10 & NA & 10 & NA & 8 & NA & 5 & NA & 9 & 9 \\
\hline Europe and Central Asia & NA & NA & NA & NA & NA & 8 & NA & 9 & NA & 11 & NA & 13 & NA & 10 & NA & 7 & NA & 11 & 11 \\
\hline Latin America and the Caribbean & NA & NA & NA & NA & NA & 9 & NA & 9 & NA & 12 & NA & 14 & NA & 11 & NA & 8 & NA & 11 & 11 \\
\hline Middle East and North Africa & NA & NA & NA & NA & NA & 8 & NA & 9 & NA & 10 & NA & 11 & NA & 9 & NA & 6 & NA & 9 & 9 \\
\hline South Asia & NA & NA & NA & NA & NA & 8 & NA & 8 & NA & 10 & NA & 10 & NA & 8 & NA & 6 & NA & 9 & 9 \\
\hline Sub-Saharan Africa & NA & NA & NA & NA & NA & 8 & NA & 9 & NA & 9 & NA & 10 & NA & 8 & NA & 5 & NA & 9 & 9 \\
\hline Low- and middle-income countries & NA & NA & NA & NA & NA & 8 & NA & 9 & NA & 10 & NA & 11 & NA & 9 & NA & 6 & NA & 10 & 10 \\
\hline High-income countries & NA & NA & NA & NA & NA & 9 & NA & 10 & NA & 11 & NA & 12 & NA & 9 & NA & 7 & NA & 10 & 10 \\
\hline WORLD & NA & NA & NA & NA & NA & 8 & NA & 9 & NA & 10 & NA & 11 & NA & 9 & NA & 6 & NA & 10 & 10 \\
\hline
\end{tabular}

Attributable Mortality (thousands)

\begin{tabular}{|c|c|c|c|c|c|c|c|c|c|c|c|c|c|c|c|c|c|c|c|}
\hline East Asia and Pacific & NA & NA & NA & NA & NA & 0 & NA & 1 & NA & 4 & NA & 2 & NA & 1 & NA & 0 & NA & 8 & 8 \\
\hline Europe and Central Asia & NA & NA & NA & NA & NA & 0 & NA & 1 & NA & 2 & NA & 2 & NA & 2 & NA & 0 & NA & 7 & 7 \\
\hline Latin America and the Caribbean & NA & NA & NA & NA & NA & 0 & NA & 0 & NA & 1 & NA & 1 & NA & 1 & NA & 0 & NA & 4 & 4 \\
\hline Middle East and North Africa & NA & NA & NA & NA & NA & 0 & NA & 0 & NA & 1 & NA & 0 & NA & 0 & NA & 0 & NA & 1 & 1 \\
\hline South Asia & NA & NA & NA & NA & NA & 0 & NA & 1 & NA & 2 & NA & 2 & NA & 1 & NA & 1 & NA & 7 & 7 \\
\hline Sub-Saharan Africa & NA & NA & NA & NA & NA & 0 & NA & 0 & NA & 1 & NA & 1 & NA & 1 & NA & 0 & NA & 3 & 3 \\
\hline Low- and middle-income countries & NA & NA & NA & NA & NA & 0 & NA & 4 & NA & 11 & NA & 8 & NA & 5 & NA & 2 & NA & 30 & 30 \\
\hline High-income countries & NA & NA & NA & NA & NA & 0 & NA & 1 & NA & 4 & NA & 4 & NA & 3 & NA & 3 & NA & 15 & 15 \\
\hline WORLD & NA & NA & NA & NA & NA & 0 & NA & 5 & NA & 15 & NA & 12 & NA & 9 & NA & 4 & NA & 45 & 45 \\
\hline
\end{tabular}

Attributable YLL (thousands)

\begin{tabular}{|c|c|c|c|c|c|c|c|c|c|c|c|c|c|c|c|c|c|c|c|}
\hline East Asia and Pacific & NA & NA & NA & NA & NA & 1 & NA & 33 & NA & 74 & NA & 30 & NA & 11 & NA & 2 & NA & 151 & 151 \\
\hline Europe and Central Asia & NA & NA & NA & NA & NA & 1 & NA & 14 & NA & 42 & NA & 32 & NA & 16 & NA & 2 & NA & 107 & 107 \\
\hline Latin America and the Caribbean & NA & NA & NA & NA & NA & 1 & NA & 12 & NA & 28 & NA & 16 & NA & 8 & NA & 2 & NA & 67 & 67 \\
\hline Middle East and North Africa & NA & NA & NA & NA & NA & 0 & NA & 7 & NA & 11 & NA & 4 & NA & 2 & NA & 0 & NA & 24 & 24 \\
\hline South Asia & NA & NA & NA & NA & NA & 1 & NA & 20 & NA & 44 & NA & 31 & NA & 11 & NA & 3 & NA & 111 & 111 \\
\hline Sub-Saharan Africa & NA & NA & NA & NA & NA & 1 & NA & 9 & NA & 21 & NA & 13 & NA & 6 & NA & 1 & NA & 50 & 50 \\
\hline Low- and middle-income countries & NA & NA & NA & NA & NA & 5 & NA & 93 & NA & 221 & NA & 127 & NA & 54 & NA & 10 & NA & 510 & 510 \\
\hline High-income countries & NA & NA & NA & NA & NA & 1 & NA & 26 & NA & 82 & NA & 54 & NA & 35 & NA & 11 & NA & 209 & 209 \\
\hline WORLD & NA & NA & NA & NA & NA & 5 & NA & 119 & NA & 303 & NA & 181 & NA & 88 & NA & 22 & NA & 719 & 719 \\
\hline
\end{tabular}

\section{Attributable DALYs (thousands)}

\begin{tabular}{|c|c|c|c|c|c|c|c|c|c|c|c|c|c|c|c|c|c|c|c|}
\hline East Asia and Pacific & NA & NA & NA & NA & NA & 1 & NA & 36 & NA & 78 & NA & 32 & NA & 11 & NA & 2 & NA & 160 & 160 \\
\hline Europe and Central Asia & NA & NA & NA & NA & NA & 1 & NA & 15 & NA & 45 & NA & 34 & NA & 17 & NA & 3 & NA & 115 & 115 \\
\hline Latin America and the Caribbean & NA & NA & NA & NA & NA & 1 & NA & 13 & NA & 30 & NA & 17 & NA & 8 & NA & 2 & NA & 72 & 72 \\
\hline Middle East and North Africa & NA & NA & NA & NA & NA & 0 & NA & 7 & NA & 12 & NA & 4 & NA & 2 & NA & 0 & NA & 26 & 26 \\
\hline South Asia & NA & NA & NA & NA & NA & 1 & NA & 21 & NA & 46 & NA & 33 & NA & 12 & NA & 3 & NA & 115 & 115 \\
\hline Sub-Saharan Africa & NA & NA & NA & NA & NA & 1 & NA & 9 & NA & 22 & NA & 13 & NA & 6 & NA & 1 & NA & 52 & 52 \\
\hline Low- and middle-income countries & NA & NA & NA & NA & NA & 5 & NA & 101 & NA & 233 & NA & 133 & NA & 57 & NA & 11 & NA & 540 & 540 \\
\hline High-income countries & NA & NA & NA & NA & NA & 1 & NA & 36 & NA & 101 & NA & 63 & NA & 42 & NA & 13 & NA & 256 & 256 \\
\hline WORLD & NA & NA & NA & NA & NA & 6 & NA & 138 & NA & 334 & NA & 196 & NA & 98 & NA & 24 & NA & 796 & 796 \\
\hline
\end{tabular}

Source: Authors' calculations.

Note: $\mathrm{NA}=$ not applicable. 
Table 4A.49

Risk factor: Physical inactivity

Disease: Diabetes mellitus

\begin{tabular}{|c|c|c|c|c|c|c|c|c|c|c|c|c|c|c|c|c|c|c|c|}
\hline \multirow[b]{2}{*}{ Region } & \multicolumn{2}{|c|}{$0-4$ years } & \multicolumn{2}{|c|}{ 5-14 years } & \multicolumn{2}{|c|}{$15-29$ years } & \multicolumn{2}{|c|}{ 30-44 years } & \multicolumn{2}{|c|}{ 45-59 years } & \multicolumn{2}{|c|}{ 60-69 years } & \multicolumn{2}{|c|}{$70-79$ years } & \multicolumn{2}{|c|}{$80+$ years } & \multicolumn{3}{|c|}{ Total } \\
\hline & Male & Female & Male & Female & Male & Female & Male & Female & Male & Female & Male & Female & Male & Female & Male & Female & Male & Female & All \\
\hline \multicolumn{20}{|l|}{ PAF of Mortality (\%) } \\
\hline East Asia and Pacific & NA & NA & NA & NA & 14 & 14 & 14 & 15 & 14 & 15 & 15 & 15 & 12 & 12 & 8 & 8 & 13 & 13 & 13 \\
\hline Europe and Central Asia & NA & NA & NA & NA & 14 & 15 & 15 & 16 & 15 & 16 & 16 & 18 & 13 & 14 & 9 & 10 & 14 & 15 & 15 \\
\hline Latin America and the Caribbean & NA & NA & NA & NA & 14 & 15 & 15 & 16 & 15 & 16 & 15 & 19 & 12 & 15 & 9 & 11 & 13 & 15 & 14 \\
\hline Middle East and North Africa & NA & NA & NA & NA & 14 & 14 & 15 & 15 & 15 & 15 & 15 & 15 & 12 & 13 & 8 & 9 & 13 & 13 & 13 \\
\hline South Asia & NA & NA & NA & NA & 14 & 14 & 14 & 14 & 14 & 14 & 15 & 15 & 11 & 12 & 7 & 8 & 12 & 13 & 13 \\
\hline Sub-Saharan Africa & NA & NA & NA & NA & 14 & 14 & 15 & 15 & 15 & 15 & 15 & 16 & 12 & 12 & 8 & 8 & 13 & 14 & 13 \\
\hline Low- and middle-income countries & NA & NA & NA & NA & 14 & 14 & 15 & 15 & 14 & 15 & 15 & 16 & 12 & 13 & 8 & 9 & 13 & 14 & 13 \\
\hline High-income countries & NA & NA & NA & NA & 15 & 16 & 17 & 17 & 16 & 16 & 17 & 17 & 13 & 13 & 9 & 10 & 13 & 12 & 13 \\
\hline WORLD & NA & NA & NA & NA & 14 & 14 & 15 & 15 & 15 & 15 & 15 & 16 & 12 & 13 & 8 & 9 & 13 & 13 & 13 \\
\hline
\end{tabular}

PAF of YLL (\%)

\begin{tabular}{|c|c|c|c|c|c|c|c|c|c|c|c|c|c|c|c|c|c|c|c|}
\hline East Asia and Pacific & NA & NA & NA & NA & 14 & 14 & 14 & 15 & 14 & 15 & 15 & 15 & 12 & 12 & 8 & 8 & 14 & 14 & 14 \\
\hline Europe and Central Asia & NA & NA & NA & NA & 14 & 15 & 15 & 16 & 15 & 16 & 16 & 18 & 13 & 14 & 9 & 10 & 15 & 15 & 15 \\
\hline Latin America and the Caribbean & NA & NA & NA & NA & 14 & 15 & 15 & 16 & 15 & 16 & 15 & 19 & 12 & 15 & 9 & 11 & 14 & 16 & 15 \\
\hline Middle East and North Africa & NA & NA & NA & NA & 14 & 14 & 15 & 15 & 15 & 15 & 15 & 15 & 12 & 13 & 8 & 9 & 14 & 14 & 14 \\
\hline South Asia & NA & NA & NA & NA & 14 & 14 & 14 & 14 & 14 & 14 & 15 & 15 & 11 & 12 & 7 & 8 & 13 & 13 & 13 \\
\hline Sub-Saharan Africa & NA & NA & NA & NA & 14 & 14 & 15 & 15 & 15 & 15 & 15 & 16 & 12 & 12 & 8 & 8 & 14 & 14 & 14 \\
\hline Low- and middle-income countries & NA & NA & NA & NA & 14 & 14 & 15 & 15 & 14 & 15 & 15 & 16 & 12 & 13 & 8 & 9 & 14 & 14 & 14 \\
\hline High-income countries & NA & NA & NA & NA & 15 & 16 & 17 & 17 & 16 & 16 & 17 & 17 & 13 & 13 & 9 & 10 & 15 & 14 & 14 \\
\hline WORLD & NA & NA & NA & NA & 14 & 14 & 15 & 15 & 15 & 15 & 15 & 16 & 12 & 13 & 8 & 9 & 14 & 14 & 14 \\
\hline
\end{tabular}

PAF of DALYs $(\%)$

\begin{tabular}{|c|c|c|c|c|c|c|c|c|c|c|c|c|c|c|c|c|c|c|c|}
\hline & & & & & & & & & & & & & & & & & & & \\
\hline East Asia and Pacific & NA & NA & NA & NA & 14 & 14 & 14 & 15 & 14 & 15 & 15 & 15 & 12 & 12 & 8 & 8 & 14 & 14 & 14 \\
\hline Europe and Central Asia & NA & NA & NA & NA & 14 & 15 & 15 & 16 & 15 & 16 & 16 & 18 & 13 & 14 & 9 & 10 & 15 & 16 & 15 \\
\hline Latin America and the Caribbean & NA & NA & NA & NA & 14 & 15 & 15 & 16 & 15 & 16 & 15 & 19 & 12 & 15 & 9 & 11 & 14 & 16 & 15 \\
\hline Middle East and North Africa & NA & NA & NA & NA & 14 & 14 & 15 & 15 & 15 & 15 & 15 & 15 & 12 & 13 & 8 & 9 & 14 & 14 & 14 \\
\hline South Asia & NA & NA & NA & NA & 14 & 14 & 14 & 14 & 14 & 14 & 15 & 15 & 11 & 12 & 7 & 8 & 13 & 13 & 13 \\
\hline Sub-Saharan Africa & NA & NA & NA & NA & 14 & 14 & 15 & 15 & 15 & 15 & 15 & 16 & 12 & 12 & 8 & 8 & 14 & 14 & 14 \\
\hline Low- and middle-income countries & NA & NA & NA & NA & 14 & 14 & 14 & 15 & 14 & 15 & 15 & 16 & 12 & 13 & 8 & 9 & 14 & 14 & 14 \\
\hline High-income countries & NA & NA & NA & NA & 15 & 16 & 17 & 17 & 16 & 16 & 17 & 17 & 13 & 13 & 9 & 10 & 15 & 15 & 15 \\
\hline WORLD & NA & NA & NA & NA & 14 & 14 & 15 & 15 & 15 & 15 & 16 & 16 & 12 & 13 & 8 & 9 & 14 & 14 & 14 \\
\hline
\end{tabular}

Attributable Mortality (thousands)

\begin{tabular}{lrlllllllrrrrrrrrrrr} 
East Asia and Pacific & NA & NA & NA & NA & 0 & 0 & 1 & 1 & 3 & 3 & 4 & 6 & 4 & 6 & 1 & 2 & 13 & 18 & 30 \\
Europe and Central Asia & NA & NA & NA & NA & 0 & 0 & 0 & 0 & 1 & 1 & 1 & 2 & 1 & 2 & 0 & 0 & 3 & 5 & 8 \\
Latin America and the Caribbean & NA & NA & NA & NA & 0 & 0 & 1 & 0 & 2 & 3 & 3 & 4 & 2 & 4 & 1 & 2 & 9 & 14 & 24 \\
Middle East and North Africa & NA & NA & NA & NA & 0 & 0 & 0 & 0 & 0 & 0 & 1 & 1 & 1 & 1 & 0 & 0 & 2 & 2 & 4 \\
South Asia & NA & NA & NA & NA & 0 & 0 & 1 & 0 & 4 & 3 & 4 & 4 & 3 & 3 & 1 & 1 & 13 & 12 & 24 \\
Sub-Saharan Africa & NA & NA & NA & NA & 0 & 0 & 0 & 0 & 1 & 2 & 1 & 2 & 1 & 2 & 0 & 0 & 4 & 7 & 11 \\
\hline Low- and middle-income countries & NA & NA & NA & NA & 1 & 1 & 3 & 2 & 11 & 11 & 14 & 20 & 11 & 17 & 4 & 6 & 44 & 57 & 11 \\
High-income countries & NA & NA & NA & NA & 0 & 0 & 1 & 0 & 2 & 1 & 3 & 2 & 4 & 5 & 2 & 5 & 12 & 14 & 26 \\
\hline WORLD & NA & NA & NA & NA & 1 & 1 & 3 & 2 & 13 & 13 & 17 & 22 & 15 & 22 & 6 & 11 & 55 & 71 & 126 \\
\hline
\end{tabular}

Attributable YLL (thousands)

\begin{tabular}{|c|c|c|c|c|c|c|c|c|c|c|c|c|c|c|c|c|c|c|c|}
\hline East Asia and Pacific & NA & NA & NA & NA & 5 & 5 & 16 & 16 & 54 & 66 & 60 & 91 & 31 & 58 & 5 & 10 & 172 & 245 & 417 \\
\hline Europe and Central Asia & NA & NA & NA & NA & 3 & 3 & 6 & 5 & 13 & 12 & 14 & 25 & 7 & 17 & 1 & 2 & 43 & 63 & 106 \\
\hline Latin America and the Caribbean & NA & NA & NA & NA & 3 & 3 & 13 & 11 & 42 & 50 & 40 & 67 & 22 & 45 & 5 & 12 & 125 & 189 & 314 \\
\hline Middle East and North Africa & NA & NA & NA & NA & 1 & 1 & 2 & 2 & 8 & 9 & 9 & 11 & 5 & 8 & 1 & 1 & 25 & 32 & 57 \\
\hline South Asia & NA & NA & NA & NA & 12 & 8 & 17 & 9 & 67 & 54 & 52 & 64 & 27 & 33 & 6 & 5 & 181 & 173 & 353 \\
\hline Sub-Saharan Africa & NA & NA & NA & NA & 3 & 4 & 9 & 9 & 20 & 36 & 16 & 37 & 8 & 19 & 1 & 3 & 58 & 107 & 165 \\
\hline Low- and middle-income countries & NA & NA & NA & NA & 27 & 23 & 63 & 52 & 203 & 226 & 190 & 296 & 100 & 180 & 19 & 32 & 603 & 810 & 1,413 \\
\hline High-income countries & NA & NA & NA & NA & 2 & 1 & 12 & 7 & 38 & 24 & 42 & 37 & 34 & 46 & 11 & 23 & 138 & 138 & 276 \\
\hline WORLD & NA & NA & NA & NA & 29 & 25 & 75 & 59 & 241 & 250 & 233 & 332 & 134 & 226 & 29 & 55 & 741 & 947 & 1,689 \\
\hline
\end{tabular}

Attributable DALYs (thousands)

\begin{tabular}{|c|c|c|c|c|c|c|c|c|c|c|c|c|c|c|c|c|c|c|c|}
\hline East Asia and Pacific & NA & NA & NA & NA & 16 & 15 & 56 & 58 & 101 & 121 & 78 & 114 & 37 & 68 & 6 & 11 & 294 & 386 & 680 \\
\hline Europe and Central Asia & NA & NA & NA & NA & 5 & 5 & 16 & 18 & 27 & 34 & 23 & 40 & 10 & 25 & 1 & 4 & 82 & 127 & 209 \\
\hline Latin America and the Caribbean & NA & NA & NA & NA & 5 & 5 & 23 & 25 & 57 & 79 & 47 & 85 & 25 & 54 & 5 & 14 & 162 & 263 & 425 \\
\hline Middle East and North Africa & NA & NA & NA & NA & 4 & 5 & 12 & 15 & 16 & 20 & 12 & 15 & 6 & 10 & 1 & 2 & 52 & 66 & 118 \\
\hline South Asia & NA & NA & NA & NA & 26 & 20 & 56 & 52 & 106 & 94 & 69 & 81 & 32 & 39 & 7 & 6 & 296 & 292 & 589 \\
\hline Sub-Saharan Africa & NA & NA & NA & NA & 5 & 6 & 15 & 15 & 26 & 43 & 18 & 40 & 9 & 20 & 1 & 3 & 75 & 127 & 202 \\
\hline Low- and middle-income countries & NA & NA & NA & NA & 62 & 56 & 178 & 182 & 334 & 392 & 247 & 376 & 120 & 215 & 22 & 39 & 962 & 1,261 & 2,223 \\
\hline High-income countries & NA & NA & NA & NA & 7 & 4 & 50 & 38 & 111 & 94 & 77 & 80 & 49 & 72 & 14 & 31 & 308 & 319 & 628 \\
\hline WORLD & NA & NA & NA & NA & 68 & 61 & 227 & 220 & 445 & 486 & 324 & 456 & 169 & 287 & 36 & 71 & 1,270 & 1,581 & 2,851 \\
\hline
\end{tabular}


Table 4A.50

Risk factor: Physical inactivity

Disease:

Ischemic heart disease

\begin{tabular}{|c|c|c|c|c|c|c|c|c|c|c|c|c|c|c|c|c|c|c|c|}
\hline \multirow[b]{2}{*}{ Region } & \multicolumn{2}{|c|}{$0-4$ years } & \multicolumn{2}{|c|}{ 5-14 years } & \multicolumn{2}{|c|}{$15-29$ years } & \multicolumn{2}{|c|}{ 30-44 years } & \multicolumn{2}{|c|}{ 45-59 years } & \multicolumn{2}{|c|}{$60-69$ years } & \multicolumn{2}{|c|}{$70-79$ years } & \multicolumn{2}{|c|}{$80+$ years } & \multicolumn{3}{|c|}{ Total } \\
\hline & Male & Female & Male & Female & Male & Female & Male & Female & Male & Female & Male & Female & Male & Female & Male & Female & Male & Female & All \\
\hline \multicolumn{20}{|l|}{ PAF of Mortality (\%) } \\
\hline East Asia and Pacific & NA & NA & NA & NA & 22 & 22 & 22 & 23 & 22 & 23 & 24 & 24 & 19 & 19 & 13 & 12 & 20 & 18 & 19 \\
\hline Europe and Central Asia & NA & NA & NA & NA & 22 & 22 & 23 & 24 & 23 & 24 & 25 & 27 & 20 & 21 & 14 & 14 & 21 & 19 & 20 \\
\hline Latin America and the Caribbean & NA & NA & NA & NA & 22 & 22 & 23 & 24 & 23 & 24 & 24 & 28 & 19 & 23 & 13 & 16 & 20 & 21 & 20 \\
\hline Middle East and North Africa & NA & NA & NA & NA & 22 & 22 & 23 & 23 & 23 & 23 & 23 & 23 & 18 & 19 & 12 & 13 & 20 & 20 & 20 \\
\hline South Asia & NA & NA & NA & NA & 22 & 22 & 22 & 22 & 22 & 22 & 23 & 23 & 17 & 18 & 12 & 13 & 19 & 19 & 19 \\
\hline Sub-Saharan Africa & NA & NA & NA & NA & 22 & 22 & 23 & 24 & 23 & 24 & 24 & 24 & 18 & 19 & 12 & 13 & 20 & 20 & 20 \\
\hline Low- and middle-income countries & NA & NA & NA & NA & 22 & 22 & 22 & 23 & 22 & 23 & 24 & 24 & 19 & 20 & 13 & 14 & 20 & 19 & 20 \\
\hline High-income countries & NA & NA & NA & NA & 24 & 24 & 26 & 26 & 25 & 25 & 25 & 26 & 20 & 20 & 14 & 15 & 20 & 17 & 19 \\
\hline WORLD & NA & NA & NA & NA & 22 & 22 & 23 & 23 & 23 & 23 & 24 & 24 & 19 & 20 & 13 & 14 & 20 & 19 & 19 \\
\hline
\end{tabular}

PAF of YLL (\%)

\begin{tabular}{|c|c|c|c|c|c|c|c|c|c|c|c|c|c|c|c|c|c|c|c|}
\hline East Asia and Pacific & NA & NA & NA & NA & 22 & 22 & 22 & 23 & 22 & 23 & 24 & 24 & 19 & 19 & 13 & 12 & 21 & 20 & 21 \\
\hline Europe and Central Asia & NA & NA & NA & NA & 22 & 22 & 23 & 24 & 23 & 24 & 25 & 27 & 20 & 21 & 14 & 14 & 22 & 21 & 22 \\
\hline Latin America and the Caribbean & NA & NA & NA & NA & 22 & 22 & 23 & 24 & 23 & 24 & 24 & 28 & 19 & 23 & 13 & 16 & 21 & 23 & 22 \\
\hline Middle East and North Africa & NA & NA & NA & NA & 22 & 22 & 23 & 23 & 23 & 23 & 23 & 23 & 18 & 19 & 12 & 13 & 21 & 21 & 21 \\
\hline South Asia & NA & NA & NA & NA & 22 & 22 & 22 & 22 & 22 & 22 & 23 & 23 & 17 & 18 & 12 & 13 & 21 & 21 & 21 \\
\hline Sub-Saharan Africa & NA & NA & NA & NA & 22 & 22 & 23 & 24 & 23 & 24 & 24 & 24 & 18 & 19 & 12 & 13 & 21 & 21 & 21 \\
\hline Low- and middle-income countries & NA & NA & NA & NA & 22 & 22 & 22 & 23 & 22 & 23 & 24 & 24 & 19 & 20 & 13 & 14 & 21 & 21 & 21 \\
\hline High-income countries & NA & NA & NA & NA & 24 & 24 & 26 & 26 & 25 & 25 & 25 & 26 & 20 & 20 & 14 & 15 & 22 & 20 & 21 \\
\hline WORLD & NA & NA & NA & NA & 22 & 22 & 23 & 23 & 23 & 23 & 24 & 24 & 19 & 20 & 13 & 14 & 21 & 21 & 21 \\
\hline
\end{tabular}

PAF of DALYs (\%)

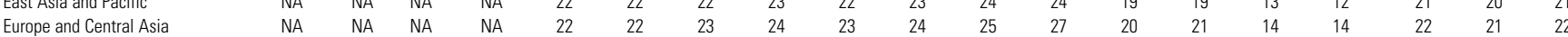

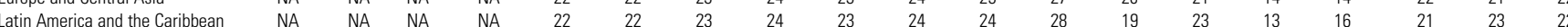

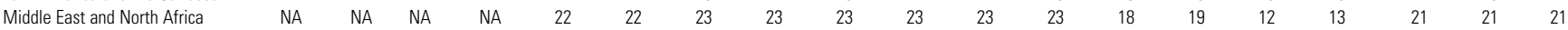
South Asia

Sub-Saharan Africa

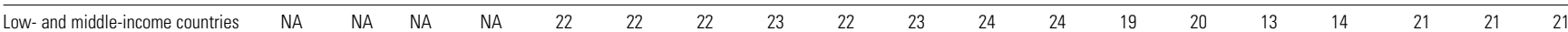

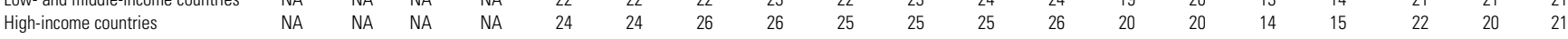

\begin{tabular}{|c|c|c|c|c|c|c|c|c|c|c|c|c|c|c|c|c|c|c|c|}
\hline WORLD & NA & NA & NA & NA & 22 & 22 & 23 & 23 & 23 & 23 & 24 & 24 & 19 & 20 & 13 & 14 & 21 & 21 & 21 \\
\hline
\end{tabular}

Attributable Mortality (thousands)

\begin{tabular}{|c|c|c|c|c|c|c|c|c|c|c|c|c|c|c|c|c|c|c|c|}
\hline East Asia and Pacific & NA & NA & NA & NA & 2 & 1 & 7 & 4 & 23 & 13 & 35 & 27 & 35 & 34 & 14 & 23 & 116 & 102 & 218 \\
\hline Europe and Central Asia & NA & NA & NA & NA & 1 & 0 & 8 & 2 & 35 & 10 & 56 & 34 & 53 & 66 & 18 & 54 & 172 & 166 & 338 \\
\hline Latin America and the Caribbean & NA & NA & NA & NA & 0 & 0 & 2 & 1 & 9 & 4 & 11 & 8 & 11 & 10 & 6 & 9 & 40 & 33 & 73 \\
\hline Middle East and North Africa & NA & NA & NA & NA & 1 & 0 & 2 & 1 & 9 & 4 & 12 & 7 & 10 & 9 & 3 & 4 & 37 & 26 & 64 \\
\hline South Asia & NA & NA & NA & NA & 2 & 4 & 11 & 7 & 54 & 28 & 66 & 53 & 51 & 49 & 16 & 16 & 200 & 157 & 356 \\
\hline Sub-Saharan Africa & NA & NA & NA & NA & 0 & 0 & 2 & 1 & 9 & 6 & 12 & 11 & 11 & 10 & 3 & 4 & 36 & 33 & 69 \\
\hline Low- and middle-income countries & NA & NA & NA & NA & 6 & 6 & 32 & 15 & 139 & 66 & 193 & 141 & 172 & 178 & 59 & 111 & 601 & 517 & 1,118 \\
\hline High-income countries & NA & NA & NA & NA & 0 & 0 & 4 & 1 & 22 & 6 & 33 & 14 & 46 & 32 & 35 & 60 & 140 & 113 & 253 \\
\hline WORLD & NA & NA & NA & NA & 6 & 6 & 36 & 16 & 161 & 72 & 226 & 154 & 218 & 211 & 94 & 171 & 741 & 630 & 1,371 \\
\hline
\end{tabular}

Attributable YLL (thousands)

\begin{tabular}{|c|c|c|c|c|c|c|c|c|c|c|c|c|c|c|c|c|c|c|c|}
\hline East Asia and Pacific & NA & NA & NA & NA & 49 & 34 & 163 & 92 & 433 & 261 & 471 & 399 & 315 & 349 & 68 & 115 & 1,499 & 1,249 & 2,749 \\
\hline Europe and Central Asia & NA & NA & NA & NA & 18 & 4 & 199 & 42 & 664 & 208 & 762 & 516 & 481 & 671 & 83 & 250 & 2,206 & 1,691 & 3,897 \\
\hline Latin America and the Caribbean & NA & NA & NA & NA & 9 & 4 & 50 & 23 & 166 & 87 & 154 & 123 & 99 & 103 & 27 & 43 & 505 & 383 & 889 \\
\hline Middle East and North Africa & NA & NA & NA & NA & 15 & 8 & 57 & 22 & 174 & 87 & 155 & 112 & 93 & 94 & 16 & 23 & 511 & 346 & 857 \\
\hline South Asia & NA & NA & NA & NA & 59 & 111 & 256 & 179 & 999 & 549 & 899 & 799 & 458 & 512 & 76 & 85 & 2,746 & 2,235 & 4,981 \\
\hline Sub-Saharan Africa & NA & NA & NA & NA & 5 & 3 & 39 & 19 & 166 & 120 & 165 & 169 & 95 & 108 & 14 & 22 & 484 & 441 & 925 \\
\hline Low- and middle-income countries & NA & NA & NA & NA & 155 & 164 & 764 & 377 & 2,601 & 1,312 & 2,607 & 2,117 & 1,541 & 1,837 & 283 & 538 & 7,952 & 6,346 & 14,298 \\
\hline High-income countries & NA & NA & NA & NA & 6 & 2 & 95 & 23 & 414 & 113 & 444 & 204 & 400 & 323 & 151 & 246 & 1,511 & 911 & 2,422 \\
\hline ORLD & NA & NA & NA & NA & 161 & 166 & 859 & 400 & 3,016 & 1,425 & 3,051 & 2,321 & 1,942 & 2,160 & 435 & 784 & 9,464 & 7,256 & 16,720 \\
\hline
\end{tabular}

Attributable DALYs (thousands)

\begin{tabular}{|c|c|c|c|c|c|c|c|c|c|c|c|c|c|c|c|c|c|c|c|}
\hline East Asia and Pacific & NA & NA & NA & NA & 71 & 54 & 181 & 102 & 463 & 281 & 494 & 419 & 322 & 355 & 69 & 116 & 1,599 & 1,326 & 2,926 \\
\hline Europe and Central Asia & NA & NA & NA & NA & 22 & 7 & 213 & 48 & 689 & 223 & 787 & 540 & 489 & 684 & 84 & 253 & 2,284 & 1,755 & 4,040 \\
\hline Latin America and the Caribbean & NA & NA & NA & NA & 16 & 8 & 57 & 27 & 177 & 95 & 164 & 130 & 102 & 106 & 27 & 44 & 543 & 411 & 954 \\
\hline Middle East and North Africa & NA & NA & NA & NA & 20 & 12 & 64 & 25 & 184 & 93 & 163 & 117 & 95 & 96 & 16 & 23 & 542 & 366 & 908 \\
\hline South Asia & NA & NA & NA & NA & 87 & 132 & 291 & 205 & 1,059 & 592 & 946 & 841 & 470 & 524 & 78 & 86 & 2,931 & 2,380 & 5,312 \\
\hline Sub-Saharan Africa & NA & NA & NA & NA & 9 & 5 & 43 & 22 & 177 & 128 & 173 & 178 & 98 & 110 & 14 & 22 & 514 & 465 & 979 \\
\hline Low- and middle-income countries & NA & NA & NA & NA & 225 & 219 & 849 & 429 & 2,749 & 1,412 & 2,728 & 2,225 & 1,575 & 1,874 & 288 & 545 & 8,413 & 6,704 & 15,117 \\
\hline High-income countries & NA & NA & NA & NA & 9 & 5 & 109 & 31 & 457 & 136 & 478 & 229 & 421 & 344 & 157 & 256 & 1,630 & 999 & 2,629 \\
\hline WORLD & NA & NA & NA & NA & 234 & 223 & 958 & 460 & 3,205 & 1,547 & 3,206 & 2,454 & 1,996 & 2,218 & 444 & 801 & 10,043 & 7,704 & 17,747 \\
\hline
\end{tabular}

Source: Authors' calculations.

Note: $\mathrm{NA}=$ not applicable. 
Table 4A.51

Risk factor Physical inactivity

Disease:

Cerebrovascular disease

\begin{tabular}{|c|c|c|c|c|c|c|c|c|c|c|c|c|c|c|c|c|c|c|c|}
\hline \multirow[b]{2}{*}{ Region } & \multicolumn{2}{|c|}{$0-4$ years } & \multicolumn{2}{|c|}{ 5-14 years } & \multicolumn{2}{|c|}{$15-29$ years } & \multicolumn{2}{|c|}{ 30-44 years } & \multicolumn{2}{|c|}{$45-59$ years } & \multicolumn{2}{|c|}{ 60-69 years } & \multicolumn{2}{|c|}{ 70-79 years } & \multicolumn{2}{|c|}{$80+$ years } & \multicolumn{3}{|c|}{ Total } \\
\hline & Male & Female & Male & Female & Male & Female & Male & Female & Male & Female & Male & Female & Male & Female & Male & Female & Male & Female & All \\
\hline \multicolumn{20}{|l|}{ PAF of Mortality (\%) } \\
\hline East Asia and Pacific & NA & NA & NA & NA & 3 & 3 & 3 & 3 & 4 & 4 & 5 & 5 & 4 & 5 & 3 & 3 & 4 & 4 & 4 \\
\hline Europe and Central Asia & NA & NA & NA & NA & 5 & 6 & 6 & 7 & 7 & 8 & 9 & 10 & 8 & 9 & 6 & 6 & 7 & 8 & 8 \\
\hline Latin America and the Caribbean & NA & NA & NA & NA & 5 & 5 & 5 & 6 & 6 & 8 & 7 & 10 & 7 & 9 & 5 & 7 & 6 & 8 & 7 \\
\hline Middle East and North Africa & NA & NA & NA & NA & 4 & 5 & 5 & 5 & 6 & 6 & 7 & 7 & 6 & 7 & 5 & 5 & 6 & 6 & 6 \\
\hline South Asia & NA & NA & NA & NA & 4 & 5 & 5 & 5 & 6 & 6 & 7 & 7 & 6 & 6 & 4 & 5 & 6 & 6 & 6 \\
\hline Sub-Saharan Africa & NA & NA & NA & NA & 4 & 4 & 4 & 4 & 5 & 5 & 6 & 6 & 5 & 5 & 4 & 4 & 5 & 5 & 5 \\
\hline Low- and middle-income countries & NA & NA & NA & NA & 4 & 4 & 4 & 5 & 5 & 5 & 6 & 7 & 6 & 6 & 4 & 5 & 5 & 6 & $\overline{6}$ \\
\hline High-income countries & NA & NA & NA & NA & 5 & 6 & 6 & 6 & 7 & 7 & 8 & 8 & 6 & 7 & 5 & 6 & 6 & 6 & 6 \\
\hline WORLD & NA & NA & NA & NA & 4 & 5 & 4 & 5 & 5 & 6 & 6 & 7 & 6 & 6 & 4 & 5 & 5 & 6 & 6 \\
\hline
\end{tabular}

PAF of YLL $(\%)$

\begin{tabular}{|c|c|c|c|c|c|c|c|c|c|c|c|c|c|c|c|c|c|c|c|}
\hline East Asia and Pacific & NA & NA & NA & NA & 3 & 3 & 3 & 3 & 4 & 4 & 5 & 5 & 4 & 5 & 3 & 3 & 4 & 4 & 4 \\
\hline Europe and Central Asia & NA & NA & NA & NA & 5 & 6 & 6 & 7 & 7 & 8 & 9 & 10 & 8 & 9 & 6 & 6 & 8 & 8 & 8 \\
\hline Latin America and the Caribbean & NA & NA & NA & NA & 5 & 5 & 5 & 6 & 6 & 8 & 7 & 10 & 7 & 9 & 5 & 7 & 6 & 8 & 7 \\
\hline Middle East and North Africa & NA & NA & NA & NA & 4 & 5 & 5 & 5 & 6 & 6 & 7 & 7 & 6 & 7 & 5 & 5 & 5 & 6 & 6 \\
\hline South Asia & NA & NA & NA & NA & 4 & 5 & 5 & 5 & 6 & 6 & 7 & 7 & 6 & 6 & 4 & 5 & 6 & 6 & 6 \\
\hline Sub-Saharan Africa & NA & NA & NA & NA & 4 & 4 & 4 & 4 & 5 & 5 & 6 & 6 & 5 & 5 & 4 & 4 & 5 & 5 & 5 \\
\hline Low- and middle-income countries & NA & NA & NA & NA & 4 & 4 & 4 & 5 & 5 & 5 & 6 & 7 & 6 & 6 & 4 & 5 & 5 & 6 & $\overline{6}$ \\
\hline High-Income Countries & NA & NA & NA & NA & 5 & 6 & 6 & 6 & 7 & 7 & 8 & 8 & 6 & 7 & 5 & 6 & 6 & 7 & 6 \\
\hline WORLD & NA & NA & NA & NA & 4 & 5 & 4 & 5 & 5 & 6 & 6 & 7 & 6 & 6 & 4 & 5 & 5 & 6 & 6 \\
\hline
\end{tabular}

PAF of DALYs $(\%)$

Latin America and the Caribbea

Middle East and North Afric

South Asia

Sub-Saharan Africa

Low- and middle-income countries

High-income countries

$\begin{array}{llll}\text { NA } & \text { NA } & \text { NA } & \text { NA } \\ \text { NA } & \text { NA } & \text { NA } & \text { NA } \\ \text { NA } & \text { NA } & \text { NA } & \text { NA } \\ \text { NA } & \text { NA } & \text { NA } & \text { NA } \\ \text { NA } & \text { NA } & \text { NA } & \text { NA } \\ \text { NA } & \text { NA } & \text { NA } & \text { NA } \\ \text { NA } & \text { NA } & \text { NA } & \text { NA }\end{array}$

\begin{tabular}{lll}
3 & 3 & 4 \\
5 & 6 & 6 \\
5 & 5 & 6 \\
4 & 5 & 6 \\
4 & 5 & 6 \\
4 & 4 & 5 \\
\hline 4 & 4 & 5 \\
5 & 6 & 8
\end{tabular}

$\begin{array}{ll}5 & 5 \\ 8 & 8 \\ 8 & 7 \\ 6 & 7 \\ 6 \\ 5 \\ 6 \\ 8\end{array}$

$\begin{array}{rrrr}5 & 5 & 6 & 6 \\ 8 & 9 & 10 & 11 \\ 7 & 9 & 8 & 12 \\ 7 & 7 & 8 & 8 \\ 6 & 7 & 7 & 8 \\ 5 & 5 & 7 & 7 \\ 6 & 6 & 7 & 8 \\ 9 & 9 & 9 & 10\end{array}$

$\begin{array}{rrrrrrr}5 & 5 & 3 & 3 & 5 & 5 & 5 \\ 8 & 9 & 6 & 6 & 8 & 9 & 9 \\ 7 & 10 & 5 & 7 & 7 & 9 & 8 \\ 7 & 7 & 5 & 5 & 6 & 7 & 6 \\ 6 & 7 & 4 & 5 & 6 & 7 & 7 \\ 6 & 6 & 4 & 4 & 5 & 6 & 6 \\ 6 & 7 & 4 & 5 & 6 & 7 & 6 \\ 7 & 8 & 5 & 6 & 8 & 8 & 8 \\ 6 & 7 & 5 & 5 & 6 & 7 & 7\end{array}$

Attributable Mortality (thousands

\begin{tabular}{|c|c|c|c|c|c|c|c|c|c|c|c|c|c|c|c|c|c|c|c|}
\hline East Asia and Pacific & NA & NA & NA & NA & 0 & 0 & 1 & 0 & 5 & 4 & 12 & 8 & 15 & 16 & 6 & 11 & 40 & 39 & 79 \\
\hline Europe and Central Asia & NA & NA & NA & NA & 0 & 0 & 1 & 0 & 4 & 3 & 9 & 9 & 11 & 19 & 5 & 17 & 30 & 48 & 79 \\
\hline Latin America and the Caribbean & NA & NA & NA & NA & 0 & 0 & 0 & 0 & 1 & 1 & 2 & 2 & 3 & 3 & 2 & 4 & 8 & 11 & 19 \\
\hline Middle East and North Africa & NA & NA & NA & NA & 0 & 0 & 0 & 0 & 1 & 0 & 1 & 1 & 1 & 2 & 1 & 1 & 4 & 4 & 8 \\
\hline South Asia & NA & NA & NA & NA & 0 & 0 & 0 & 0 & 4 & 3 & 9 & 9 & 9 & 11 & 3 & 4 & 26 & 28 & 54 \\
\hline Sub-Saharan Africa & NA & NA & NA & NA & 0 & 0 & 0 & 0 & 1 & 2 & 2 & 3 & 2 & 4 & 1 & 2 & 7 & 11 & 18 \\
\hline Low- and middle-income countries & NA & NA & NA & NA & 1 & 1 & 3 & 2 & 17 & 13 & 36 & 32 & 42 & 55 & 17 & 38 & 116 & 141 & 256 \\
\hline High-income countries & NA & NA & NA & NA & 0 & 0 & 0 & 0 & 2 & 1 & 3 & 2 & 6 & 7 & 7 & 17 & 19 & 28 & 47 \\
\hline WORLD & NA & NA & NA & NA & 1 & 1 & 3 & 2 & 18 & 14 & 39 & 35 & 49 & 62 & 24 & 55 & 135 & 169 & 303 \\
\hline
\end{tabular}

Attributable YLL (thousands)

\begin{tabular}{|c|c|c|c|c|c|c|c|c|c|c|c|c|c|c|c|c|c|c|c|}
\hline East Asia and Pacific & NA & NA & NA & NA & 4 & 2 & 19 & 12 & 100 & 73 & 161 & 121 & 136 & 160 & 31 & 55 & 451 & 424 & 875 \\
\hline Europe and Central Asia & NA & NA & NA & NA & 4 & 3 & 17 & 12 & 70 & 53 & 128 & 138 & 103 & 198 & 22 & 78 & 344 & 480 & 824 \\
\hline Latin America and the Caribbean & NA & NA & NA & NA & 2 & 2 & 8 & 10 & 24 & 28 & 26 & 34 & 23 & 35 & 8 & 17 & 91 & 126 & 217 \\
\hline Middle East and North Africa & NA & NA & NA & NA & 3 & 1 & 3 & 2 & 9 & 9 & 13 & 14 & 12 & 16 & 3 & 5 & 44 & 48 & 92 \\
\hline South Asia & NA & NA & NA & NA & 5 & 3 & 11 & 7 & 79 & 59 & 127 & 132 & 81 & 115 & 15 & 24 & 318 & 341 & 658 \\
\hline Sub-Saharan Africa & NA & NA & NA & NA & 4 & 3 & 11 & 9 & 27 & 37 & 29 & 49 & 21 & 38 & 4 & 9 & 97 & 145 & 242 \\
\hline Low- and middle-income countries & NA & NA & NA & NA & 23 & 15 & 68 & 52 & 309 & 258 & 485 & 488 & 376 & 562 & 83 & 188 & 1,344 & 1,563 & 2,908 \\
\hline High-income countries & NA & NA & NA & NA & 1 & 1 & 8 & 7 & 32 & 22 & 44 & 35 & 56 & 71 & 31 & 70 & 172 & 206 & 378 \\
\hline WORLD & NA & NA & NA & NA & 24 & 17 & 77 & 59 & 341 & 279 & 529 & 523 & 432 & 633 & 113 & 258 & 1,517 & 1,769 & 3,286 \\
\hline \multicolumn{20}{|l|}{ Attributable DALYs (thousands) } \\
\hline East Asia and Pacific & NA & NA & NA & NA & 4 & 2 & 37 & 25 & 177 & 128 & 260 & 190 & 182 & 209 & 36 & 64 & 696 & 619 & 1,315 \\
\hline Europe and Central Asia & NA & NA & NA & NA & 4 & 3 & 24 & 19 & 97 & 78 & 178 & 197 & 133 & 245 & 25 & 87 & 460 & 630 & 1,090 \\
\hline Latin America and the Caribbean & NA & NA & NA & NA & 2 & 2 & 14 & 19 & 40 & 46 & 42 & 53 & 31 & 46 & 9 & 20 & 138 & 186 & 324 \\
\hline Middle East and North Africa & NA & NA & NA & NA & 3 & 1 & 5 & 4 & 15 & 13 & 20 & 19 & 15 & 19 & 3 & 5 & 61 & 63 & 124 \\
\hline South Asia & NA & NA & NA & NA & 5 & 3 & 16 & 10 & 113 & 83 & 176 & 178 & 101 & 139 & 17 & 27 & 426 & 439 & 866 \\
\hline Sub-Saharan Africa & NA & NA & NA & NA & 4 & 3 & 13 & 10 & 32 & 45 & 35 & 59 & 23 & 43 & 5 & 10 & 112 & 170 & 282 \\
\hline Low- and middle-income countries & NA & NA & NA & NA & 23 & 15 & 108 & 88 & 474 & 394 & 711 & 696 & 484 & 700 & 95 & 214 & 1,895 & 2,107 & 4,001 \\
\hline High-income countries & NA & NA & NA & NA & 1 & 1 & 22 & 18 & 80 & 58 & 102 & 83 & 98 & 125 & 42 & 94 & 346 & 379 & 725 \\
\hline WORLD & NA & NA & NA & NA & 24 & 17 & 130 & 106 & 555 & 452 & 813 & 779 & 583 & 824 & 137 & 308 & 2,241 & 2,485 & 4,726 \\
\hline
\end{tabular}

Source: Authors' calculations.

Note: NA = not applicable. 
Table 4A.52

Risk factor: Physical inactivity

Disease:

All causes

\begin{tabular}{|c|c|c|c|c|c|c|c|c|c|c|c|c|c|c|c|c|c|c|c|}
\hline \multirow[b]{2}{*}{ Region } & \multicolumn{2}{|c|}{$0-4$ years } & \multicolumn{2}{|c|}{ 5-14 years } & \multicolumn{2}{|c|}{$15-29$ years } & \multicolumn{2}{|c|}{ 30-44 years } & \multicolumn{2}{|c|}{ 45-59 years } & \multicolumn{2}{|c|}{$60-69$ years } & \multicolumn{2}{|c|}{ 70-79 years } & \multicolumn{2}{|c|}{$80+$ years } & \multicolumn{3}{|c|}{ Total } \\
\hline & Male & Female & Male & Female & Male & Female & Male & Female & Male & Female & Male & Female & Male & Female & Male & Female & Male & Female & All \\
\hline \multicolumn{20}{|l|}{ PAF of Mortality (\%) } \\
\hline East Asia and Pacific & NA & NA & NA & NA & 1 & 1 & 2 & 2 & 3 & 4 & 4 & 5 & 4 & 4 & 2 & 3 & 3 & 3 & 3 \\
\hline Europe and Central Asia & NA & NA & NA & NA & 1 & 1 & 3 & 3 & 7 & 7 & 10 & 12 & 9 & 11 & 7 & 8 & 7 & 9 & 8 \\
\hline Latin America and the Caribbean & NA & NA & NA & NA & 0 & 1 & 2 & 2 & 4 & 6 & 6 & 8 & 5 & 7 & 3 & 4 & 3 & 5 & 4 \\
\hline Middle East and North Africa & NA & NA & NA & NA & 1 & 1 & 3 & 3 & 7 & 6 & 8 & 8 & 6 & 7 & 4 & 4 & 4 & 4 & 4 \\
\hline South Asia & NA & NA & NA & NA & 1 & 1 & 2 & 2 & 6 & 5 & 7 & 8 & 6 & 6 & 4 & 4 & 3 & 3 & 3 \\
\hline Sub-Saharan Africa & NA & NA & NA & NA & 0 & 0 & 0 & 0 & 2 & 2 & 4 & 5 & 4 & 4 & 2 & 3 & 1 & 1 & 1 \\
\hline Low- and middle-income countries & NA & NA & NA & NA & 0 & 0 & 1 & 1 & 4 & 4 & 6 & 7 & 5 & 6 & 4 & 4 & 3 & 3 & 3 \\
\hline High-income countries & NA & NA & NA & NA & 0 & 1 & 3 & 3 & 6 & 5 & 6 & 6 & 5 & 6 & 4 & 4 & 5 & 5 & 5 \\
\hline WORLD & NA & NA & NA & NA & 0 & 0 & 2 & 1 & 4 & 4 & 6 & 7 & 5 & 6 & 4 & 4 & 3 & 4 & 3 \\
\hline
\end{tabular}

PAF of YLL (\%)

\begin{tabular}{lllllllllllrrrrrrrrrr} 
East Asia and Pacific & NA & NA & NA & NA & 1 & 1 & 1 & 2 & 3 & 3 & 4 & 5 & 4 & 4 & 2 & 2 & 2 & 2 & 2 \\
Europe and Central Asia & NA & NA & NA & NA & 1 & 1 & 3 & 3 & 7 & 7 & 10 & 11 & 9 & 11 & 7 & 8 & 6 & 8 & 7 \\
Latin America and the Caribbean & NA & NA & NA & NA & 0 & 1 & 1 & 2 & 4 & 6 & 6 & 8 & 5 & 7 & 3 & 4 & 2 & 4 & 3 \\
Middle East and North Africa & NA & NA & NA & NA & 1 & 1 & 3 & 3 & 6 & 6 & 8 & 8 & 6 & 7 & 4 & 4 & 3 & 3 & 3 \\
South Asia & NA & NA & NA & NA & 1 & 1 & 2 & 2 & 5 & 5 & 7 & 8 & 6 & 6 & 4 & 4 & 2 & 2 & 2 \\
Sub-Saharan Africa & NA & NA & NA & NA & 0 & 0 & 0 & 0 & 2 & 2 & 4 & 5 & 4 & 4 & 2 & 3 & 0 & 1 & 1 \\
\hline Low- and middle-income countries & NA & NA & NA & NA & 0 & 0 & 1 & 1 & 4 & 4 & 6 & 7 & 5 & 6 & 3 & 4 & 2 & 2 & 2 \\
High-income countries & NA & NA & NA & NA & 0 & 1 & 3 & 3 & 6 & 5 & 6 & 6 & 5 & 6 \\
\hline WORLD & NA & NA & NA & NA & 0 & 0 & 2 & 1 & 4 & 4 & 6 & 7 & 5 & 6 & 4 & 4 & 2 & 2 & 2 \\
\hline
\end{tabular}

PAF of DALYs $(\%)$

Latin America and the Caribbean

Middle East and North Africa

South Asia

Sub-Saharan Africa

Low- and middle-income countries

High-income countries

$\begin{array}{llll}\text { NA } & \text { NA } & \text { NA } & \text { NA } \\ \text { NA } & \text { NA } & \text { NA } & \text { NA } \\ \text { NA } & \text { NA } & \text { NA } & \text { NA } \\ \text { NA } & \text { NA } & \text { NA } & \text { NA } \\ \text { NA } & \text { NA } & \text { NA } & \text { NA } \\ \text { NA } & \text { NA } & \text { NA } & \text { NA } \\ \text { NA } & \text { NA } & \text { NA } & \text { NA }\end{array}$

Attributable Mortality (thousands)

\begin{tabular}{|c|c|c|c|c|c|c|c|c|c|c|c|c|c|c|c|c|c|c|c|}
\hline \multicolumn{20}{|c|}{ Attributable Mortality (thousands) } \\
\hline East Asia and Pacific & NA & NA & NA & NA & 2 & 2 & 9 & 7 & 34 & 26 & 55 & 46 & 57 & 59 & 22 & 37 & 180 & 177 & 357 \\
\hline Europe and Central Asia & NA & NA & NA & NA & 1 & 0 & 10 & 3 & 41 & 17 & 70 & 50 & 68 & 91 & 24 & 73 & 213 & 235 & 448 \\
\hline Latin America and the Caribbean & NA & NA & NA & NA & 1 & 0 & 3 & 3 & 13 & 10 & 17 & 17 & 17 & 20 & 9 & 16 & 60 & 66 & 125 \\
\hline Middle East and North Africa & NA & NA & NA & NA & 1 & 0 & 3 & 1 & 10 & 6 & 13 & 10 & 12 & 12 & 4 & 5 & 44 & 35 & 78 \\
\hline South Asia & NA & NA & NA & NA & 3 & 4 & 12 & 9 & 62 & 36 & 81 & 69 & 64 & 65 & 20 & 22 & 242 & 205 & 446 \\
\hline Sub-Saharan Africa & NA & NA & NA & NA & 0 & 0 & 3 & 2 & 12 & 11 & 16 & 18 & 14 & 17 & 4 & 6 & 49 & 55 & 104 \\
\hline Low- and middle-income countries & NA & NA & NA & NA & 8 & 8 & 40 & 26 & 172 & 107 & 252 & 209 & 232 & 263 & 82 & 160 & 787 & 772 & 1,559 \\
\hline High-income countries & NA & NA & NA & NA & 0 & 0 & 5 & 3 & 29 & 14 & 45 & 26 & 62 & 53 & 47 & 90 & 190 & 186 & 376 \\
\hline WORLD & NA & NA & NA & NA & 8 & 8 & 46 & 28 & 201 & 121 & 297 & 235 & 294 & 316 & 130 & 250 & 977 & 958 & 1,935 \\
\hline
\end{tabular}

Attributable YLL (thousands)

\begin{tabular}{|c|c|c|c|c|c|c|c|c|c|c|c|c|c|c|c|c|c|c|c|}
\hline East Asia and Pacific & NA & NA & NA & NA & 65 & 45 & 223 & 178 & 643 & 524 & 744 & 690 & 506 & 604 & 108 & 188 & 2,290 & 2,228 & 4,518 \\
\hline Europe and Central Asia & NA & NA & NA & NA & 26 & 11 & 229 & 79 & 772 & 339 & 944 & 751 & 615 & 934 & 108 & 338 & 2,694 & 2,453 & 5,147 \\
\hline Latin America and the Caribbean & NA & NA & NA & NA & 16 & 11 & 76 & 63 & 241 & 206 & 231 & 254 & 151 & 201 & 41 & 77 & 755 & 812 & 1,568 \\
\hline Middle East and North Africa & NA & NA & NA & NA & 20 & 11 & 66 & 37 & 195 & 120 & 180 & 144 & 111 & 121 & 20 & 30 & 592 & 462 & 1,054 \\
\hline South Asia & NA & NA & NA & NA & 79 & 124 & 293 & 220 & 1,155 & 715 & 1,091 & 1,036 & 572 & 676 & 98 & 118 & 3,287 & 2,890 & 6,177 \\
\hline Sub-Saharan Africa & NA & NA & NA & NA & 14 & 12 & 63 & 48 & 220 & 220 & 216 & 273 & 127 & 174 & 20 & 35 & 660 & 762 & 1,423 \\
\hline He-income countries & NA & NA & NA & NA & 220 & 214 & 950 & 624 & 3,227 & 2,124 & 3,406 & 3,148 & 2,082 & 2,711 & 394 & 786 & 10,279 & 9,607 & 19,887 \\
\hline High-income countries & NA & NA & NA & NA & 11 & 6 & 127 & 73 & 542 & 286 & 606 & 385 & 545 & 530 & 207 & 375 & 2,038 & 1,655 & 3,693 \\
\hline WORLD & NA & NA & NA & NA & 231 & 220 & 1,077 & 697 & 3,769 & 2,409 & 4,012 & 3,534 & 2,627 & 3,241 & 602 & 1,161 & 12,318 & 11,262 & 23,580 \\
\hline
\end{tabular}

Attributable DALYs (thousands)

\begin{tabular}{|c|c|c|c|c|c|c|c|c|c|c|c|c|c|c|c|c|c|c|c|}
\hline East Asia and Pacific & NA & NA & NA & NA & 98 & 75 & 300 & 247 & 799 & 662 & 887 & 805 & 566 & 670 & 115 & 200 & 2,765 & 2,659 & 5,424 \\
\hline Europe and Central Asia & NA & NA & NA & NA & 32 & 17 & 260 & 109 & 842 & 408 & 1,029 & 855 & 657 & 1,005 & 113 & 353 & 2,935 & 2,747 & 5,681 \\
\hline Latin America and the Caribbean & NA & NA & NA & NA & 24 & 18 & 98 & 91 & 284 & 264 & 264 & 300 & 165 & 225 & 44 & 84 & 879 & 980 & 1,860 \\
\hline Middle East and North Africa & NA & NA & NA & NA & 28 & 20 & 84 & 54 & 219 & 142 & 198 & 159 & 118 & 128 & 21 & 31 & 668 & 534 & 1,202 \\
\hline South Asia & NA & NA & NA & NA & 122 & 158 & 372 & 293 & 1,289 & 824 & 1,204 & 1,142 & 608 & 718 & 103 & 124 & 3,697 & 3,259 & 6,956 \\
\hline Sub-Saharan Africa & NA & NA & NA & NA & 20 & 15 & 75 & 59 & 243 & 245 & 233 & 296 & 133 & 182 & 21 & 36 & 723 & 833 & 1,556 \\
\hline Low- and middle-income countries & NA & NA & NA & NA & 325 & 303 & 1,189 & 853 & 3,676 & 2,544 & 3,815 & 3,556 & 2,247 & 2,928 & 415 & 828 & 11,667 & 11,012 & 22,679 \\
\hline High-income countries & NA & NA & NA & NA & 18 & 12 & 193 & 137 & 721 & 445 & 748 & 521 & 634 & 648 & 230 & 424 & 2,545 & 2,187 & 4,732 \\
\hline WORLD & NA & NA & NA & NA & 343 & 315 & 1,383 & 990 & 4,397 & 2,989 & 4,564 & 4,078 & 2,881 & 3,576 & 645 & 1,251 & 14,212 & 13,199 & 1 \\
\hline
\end{tabular}

Source: Authors' calculations.

Note: $\mathrm{NA}=$ not applicable. 
Risk factor:

Unsafe sex

Sexually transmitted diseases excluding HIV/AIDS

\begin{tabular}{|c|c|c|c|c|c|c|c|c|c|c|c|c|c|c|c|c|c|c|c|}
\hline \multirow[b]{2}{*}{ Region } & \multicolumn{2}{|c|}{$0-4$ years } & \multicolumn{2}{|c|}{ 5-14 years } & \multicolumn{2}{|c|}{$15-29$ years } & \multicolumn{2}{|c|}{ 30-44 years } & \multicolumn{2}{|c|}{$45-59$ years } & \multicolumn{2}{|c|}{$60-69$ years } & \multicolumn{2}{|c|}{ 70-79 years } & \multicolumn{2}{|c|}{$80+$ years } & \multicolumn{3}{|c|}{ Total } \\
\hline & Male & Female & Male & Female & Male & Female & Male & Female & Male & Female & Male & Female & Male & Female & Male & Female & Male & Female & All \\
\hline \multicolumn{20}{|l|}{ PAF of Mortality (\%) } \\
\hline East Asia and Pacific & 100 & 100 & 100 & 100 & 100 & 100 & 100 & 100 & 100 & 100 & 100 & 100 & 100 & 100 & 100 & 100 & 100 & 100 & 100 \\
\hline Europe and Central Asia & 100 & 100 & 100 & 100 & 100 & 100 & 100 & 100 & 100 & 100 & 100 & 100 & 100 & 100 & 100 & 100 & 100 & 100 & 100 \\
\hline Latin America and the Caribbean & 100 & 100 & 100 & 100 & 100 & 100 & 100 & 100 & 100 & 100 & 100 & 100 & 100 & 100 & 100 & 100 & 100 & 100 & 100 \\
\hline Middle East and North Africa & 100 & 100 & 100 & 100 & 100 & 100 & 100 & 100 & 100 & 100 & 100 & 100 & 100 & 100 & 100 & 100 & 100 & 100 & 100 \\
\hline South Asia & 100 & 100 & 100 & 100 & 100 & 100 & 100 & 100 & 100 & 100 & 100 & 100 & 100 & 100 & 100 & 100 & 100 & 100 & 100 \\
\hline Sub-Saharan Africa & 100 & 100 & 100 & 100 & 100 & 100 & 100 & 100 & 100 & 100 & 100 & 100 & 100 & 100 & 100 & 100 & 100 & 100 & 100 \\
\hline Low- and middle-income countries & 100 & 100 & 100 & 100 & 100 & 100 & 100 & 100 & 100 & 100 & 100 & 100 & 100 & 100 & 100 & 100 & 100 & 100 & 100 \\
\hline High-income countries & 100 & 100 & 100 & 100 & 100 & 100 & 100 & 100 & 100 & 100 & 100 & 100 & 100 & 100 & 100 & 100 & 100 & 100 & 100 \\
\hline WORLD & 100 & 100 & 100 & 100 & 100 & 100 & 100 & 100 & 100 & 100 & 100 & 100 & 100 & 100 & 100 & 100 & 100 & 100 & 100 \\
\hline \multicolumn{20}{|l|}{ PAF of YLL (\%) } \\
\hline East Asia and Pacific & 100 & 100 & 100 & 100 & 100 & 100 & 100 & 100 & 100 & 100 & 100 & 100 & 100 & 100 & 100 & 100 & 100 & 100 & 100 \\
\hline Europe and Central Asia & 100 & 100 & 100 & 100 & 100 & 100 & 100 & 100 & 100 & 100 & 100 & 100 & 100 & 100 & 100 & 100 & 100 & 100 & 100 \\
\hline Latin America and the Caribbean & 100 & 100 & 100 & 100 & 100 & 100 & 100 & 100 & 100 & 100 & 100 & 100 & 100 & 100 & 100 & 100 & 100 & 100 & 100 \\
\hline Middle East and North Africa & 100 & 100 & 100 & 100 & 100 & 100 & 100 & 100 & 100 & 100 & 100 & 100 & 100 & 100 & 100 & 100 & 100 & 100 & 100 \\
\hline South Asia & 100 & 100 & 100 & 100 & 100 & 100 & 100 & 100 & 100 & 100 & 100 & 100 & 100 & 100 & 100 & 100 & 100 & 100 & 100 \\
\hline Sub-Saharan Africa & 100 & 100 & 100 & 100 & 100 & 100 & 100 & 100 & 100 & 100 & 100 & 100 & 100 & 100 & 100 & 100 & 100 & 100 & 100 \\
\hline Low- and middle-income countries & 100 & 100 & 100 & 100 & 100 & 100 & 100 & 100 & 100 & 100 & 100 & 100 & 100 & 100 & 100 & 100 & 100 & 100 & 100 \\
\hline High-income countries & 100 & 100 & 100 & 100 & 100 & 100 & 100 & 100 & 100 & 100 & 100 & 100 & 100 & 100 & 100 & 100 & 100 & 100 & 100 \\
\hline WORLD & 100 & 100 & 100 & 100 & 100 & 100 & 100 & 100 & 100 & 100 & 100 & 100 & 100 & 100 & 100 & 100 & 100 & 100 & 100 \\
\hline \multicolumn{20}{|l|}{ PAF of DALYs (\%) } \\
\hline East Asia and Pacific & 100 & 100 & 100 & 100 & 100 & 100 & 100 & 100 & 100 & 100 & 100 & 100 & 100 & 100 & 100 & 100 & 100 & 100 & 100 \\
\hline Europe and Central Asia & 100 & 100 & 100 & 100 & 100 & 100 & 100 & 100 & 100 & 100 & 100 & 100 & 100 & 100 & 100 & 100 & 100 & 100 & 100 \\
\hline Latin America and the Caribbean & 100 & 100 & 100 & 100 & 100 & 100 & 100 & 100 & 100 & 100 & 100 & 100 & 100 & 100 & 100 & 100 & 100 & 100 & 100 \\
\hline Middle East and North Africa & 100 & 100 & 100 & 100 & 100 & 100 & 100 & 100 & 100 & 100 & 100 & 100 & 100 & 100 & 100 & 100 & 100 & 100 & 100 \\
\hline South Asia & 100 & 100 & 100 & 100 & 100 & 100 & 100 & 100 & 100 & 100 & 100 & 100 & 100 & 100 & 100 & 100 & 100 & 100 & 100 \\
\hline Sub-Saharan Africa & 100 & 100 & 100 & 100 & 100 & 100 & 100 & 100 & 100 & 100 & 100 & 100 & 100 & 100 & 100 & 100 & 100 & 100 & 100 \\
\hline Low- and middle-income countries & 100 & 100 & 100 & 100 & 100 & 100 & 100 & 100 & 100 & 100 & 100 & 100 & 100 & 100 & 100 & 100 & 100 & 100 & 100 \\
\hline High-income countries & 100 & 100 & 100 & 100 & 100 & 100 & 100 & 100 & 100 & 100 & 100 & 100 & 100 & 100 & 100 & 100 & 100 & 100 & 100 \\
\hline WORLD & 100 & 100 & 100 & 100 & 100 & 100 & 100 & 100 & 100 & 100 & 100 & 100 & 100 & 100 & 100 & 100 & 100 & 100 & 100 \\
\hline
\end{tabular}

Attributable Mortality (thousands)

East Asia and Pacific

Europe and Central Asia

Latin America and the Caribbean

Middle East and North Africa

South Asia

Sub-Saharan Africa

\begin{tabular}{lrrrrrrrrrrrrrrrrrrrrrrrr} 
East Asia and Pacific & 1 & 0 & 0 & 0 & 0 & 0 & 0 & 1 & 1 & 2 & 1 & 1 & 1 & 1 & 1 & 0 & 4 & 4 & 9 \\
Europe and Central Asia & 0 & 0 & 0 & 0 & 0 & 0 & 0 & 0 & 0 & 0 & 0 & 0 & 0 & 0 & 0 & 0 & 0 & 0 & 1 \\
Latin America and the Caribbean & 1 & 0 & 0 & 0 & 0 & 0 & 0 & 0 & 0 & 0 & 0 & 0 & 0 & 0 & 0 & 0 & 1 & 1 & 2 \\
Middle East and North Africa & 0 & 0 & 0 & 0 & 0 & 0 & 0 & 0 & 1 & 1 & 0 & 1 & 0 & 0 & 0 & 0 & 2 & 2 & 4 \\
South Asia & 12 & 25 & 0 & 0 & 0 & 0 & 1 & 1 & 7 & 6 & 6 & 4 & 5 & 2 & 2 & 1 & 33 & 38 & 71 \\
Sub-Saharan Africa & 17 & 12 & 0 & 0 & 1 & 12 & 10 & 8 & 17 & 6 & 3 & 2 & 0 & 1 & 0 & 0 & 48 & 42 & 90 \\
\hline Low- and middle-income countries & 31 & 37 & 0 & 0 & 2 & 13 & 11 & 10 & 26 & 14 & 10 & 7 & 6 & 4 & 3 & 1 & 89 & 87 & 176 \\
High-income countries & 0 & 0 & 0 & 0 & 0 & 0 & 0 & 0 & 0 & 0 & 0 & 0 & 0 & 0 & 0 & 0 \\
\hline WORLD & 31 & 37 & 0 & 0 & 2 & 13 & 11 & 10 & 26 & 14 & 10 & 7 & 6 & 4 & 3 & 1 \\
\hline
\end{tabular}

Attributable YLL (thousands)

East Asia and Pacific
Europe and Central Asia

Europe and Central Asia
Latin America and the Caribbea

Middle East and North Africa

South Asia

Sub-Saharan Africa

29
6

Low- and middle-income

High-income countries

\begin{tabular}{|c|c|c|}
\hline WORLD & 929 & 11 \\
\hline
\end{tabular}

Attributable DALYs (thousands)

East Asia and Pacific

Europe and Central Asia

Latin America and the Caribbean

Middle East and North Africa

South Asia

Sub-Saharan Africa

\begin{tabular}{|c|c|c|c|c|c|c|c|c|c|c|c|c|c|c|c|c|c|c|c|}
\hline East Asia and Pacific & 78 & 58 & 2 & 9 & 86 & 360 & 46 & 115 & 19 & 34 & 11 & 12 & 7 & 6 & 3 & 1 & 252 & 596 & 848 \\
\hline Europe and Central Asia & 8 & 8 & 0 & 2 & 20 & 111 & 13 & 34 & 1 & 2 & 0 & 0 & 0 & 1 & 0 & 0 & 43 & 158 & 200 \\
\hline Latin America and the Caribbean & 44 & 27 & 1 & 5 & 49 & 205 & 28 & 64 & 1 & 4 & 0 & 1 & 0 & 1 & 0 & 0 & 123 & 306 & 430 \\
\hline Middle East and North Africa & 16 & 17 & 1 & 5 & 33 & 161 & 19 & 39 & 17 & 11 & 7 & 9 & 3 & 3 & 1 & 0 & 97 & 245 & 342 \\
\hline South Asia & 581 & 978 & 8 & 26 & 276 & 904 & 146 & 276 & 131 & 124 & 89 & 53 & 43 & 19 & 12 & 4 & 1,286 & 2,383 & 3,669 \\
\hline Sub-Saharan Africa & 775 & 602 & 6 & 21 & 238 & 1,001 & 285 & 359 & 336 & 130 & 38 & 32 & 0 & 17 & 0 & 0 & 1,680 & 2,163 & 3,842 \\
\hline Low- and middle-income countries & 1,502 & 1,691 & 19 & 69 & 702 & 2,741 & 537 & 886 & 504 & 304 & 145 & 107 & 55 & 47 & 16 & 5 & 3,481 & 5,851 & 9,332 \\
\hline$\underline{\text { High-income countries }}$ & 7 & 6 & 0 & 2 & 9 & 79 & 9 & 27 & 1 & 2 & 0 & 1 & 0 & 1 & 0 & 1 & 26 & 119 & 145 \\
\hline WORLD & 1,509 & 1,697 & 19 & 71 & 711 & 2,820 & 546 & 913 & 505 & 306 & 146 & 108 & 55 & 49 & 16 & 6 & 3,507 & 5,970 & 9,477 \\
\hline
\end{tabular}

Source: Authors' calculations. 
Table 4A.54

Risk factor:

Disease:

Unsafe sex

HIV/AIDS

\begin{tabular}{|c|c|c|c|c|c|c|c|c|c|c|c|c|c|c|c|c|c|c|c|}
\hline \multirow[b]{2}{*}{ Region } & \multicolumn{2}{|c|}{$0-4$ years } & \multicolumn{2}{|c|}{ 5-14 years } & \multicolumn{2}{|c|}{$15-29$ years } & \multicolumn{2}{|c|}{ 30-44 years } & \multicolumn{2}{|c|}{$45-59$ years } & \multicolumn{2}{|c|}{$60-69$ years } & \multicolumn{2}{|c|}{ 70-79 years } & \multicolumn{2}{|c|}{$80+$ years } & \multicolumn{3}{|c|}{ Total } \\
\hline & Male & Female & Male & Female & Male & Female & Male & Female & Male & Female & Male & Female & Male & Female & Male & Female & Male & Female & All \\
\hline \multicolumn{20}{|l|}{ PAF of Mortality (\%) } \\
\hline East Asia and Pacific & 66 & 66 & 65 & 66 & 66 & 67 & 66 & 66 & 67 & 66 & 67 & 0 & 66 & 0 & 52 & 0 & 66 & 60 & 65 \\
\hline Europe and Central Asia & 27 & 30 & 54 & 59 & 28 & 29 & 28 & 29 & 27 & 28 & 28 & 28 & 27 & 27 & 29 & 59 & 28 & 30 & 28 \\
\hline Latin America and the Caribbean & 90 & 90 & 90 & 90 & 89 & 90 & 89 & 89 & 89 & 89 & 89 & 89 & 89 & 89 & 88 & 88 & 89 & 89 & 89 \\
\hline Middle East and North Africa & 94 & 94 & 95 & 90 & 94 & 95 & 95 & 95 & 94 & 95 & 94 & 96 & 95 & 96 & 97 & 97 & 94 & 95 & 94 \\
\hline South Asia & 80 & 80 & 80 & 79 & 79 & 79 & 79 & 79 & 79 & 79 & 79 & 85 & 79 & 85 & 85 & 85 & 79 & 79 & 79 \\
\hline Sub-Saharan Africa & 100 & 100 & 100 & 100 & 100 & 100 & 100 & 100 & 100 & 100 & 100 & 100 & 100 & 100 & 100 & 100 & 100 & 100 & 100 \\
\hline Low- and middle-income countries & 98 & 98 & 99 & 99 & 93 & 98 & 92 & 97 & 91 & 96 & 94 & 90 & 94 & 87 & 95 & 37 & 93 & 97 & 95 \\
\hline High-income countries & 62 & 60 & 70 & 66 & 63 & 65 & 67 & 68 & 69 & 70 & 67 & 68 & 68 & 67 & 69 & 68 & 67 & 68 & 67 \\
\hline WORLD & 98 & 98 & 99 & 99 & 93 & 98 & 92 & 97 & 91 & 96 & 93 & 89 & 92 & 87 & 90 & 43 & 93 & 97 & 95 \\
\hline
\end{tabular}

PAF of YLL (\%)

\begin{tabular}{|c|c|c|c|c|c|c|c|c|c|c|c|c|c|c|c|c|c|c|c|}
\hline East Asia and Pacific & 66 & 66 & 65 & 66 & 66 & 67 & 66 & 66 & 67 & 66 & 67 & 0 & 66 & 0 & 52 & 0 & 66 & 63 & 65 \\
\hline Europe and Central Asia & 27 & 30 & 54 & 59 & 28 & 29 & 28 & 29 & 27 & 28 & 28 & 28 & 27 & 27 & 29 & 59 & 28 & 30 & 28 \\
\hline Latin America and the Caribbean & 90 & 90 & 90 & 90 & 89 & 90 & 89 & 89 & 89 & 89 & 89 & 89 & 89 & 89 & 88 & 88 & 89 & 89 & 89 \\
\hline Middle East and North Africa & 94 & 94 & 95 & 90 & 94 & 95 & 95 & 95 & 94 & 95 & 94 & 96 & 95 & 96 & 97 & 97 & 94 & 95 & 94 \\
\hline South Asia & 80 & 80 & 80 & 79 & 79 & 79 & 79 & 79 & 79 & 79 & 79 & 85 & 79 & 85 & 85 & 85 & 79 & 79 & 79 \\
\hline Sub-Saharan Africa & 100 & 100 & 100 & 100 & 100 & 100 & 100 & 100 & 100 & 100 & 100 & 100 & 100 & 100 & 100 & 100 & 100 & 100 & 100 \\
\hline Low- and middle-income countries & 98 & 98 & 99 & 99 & 93 & 98 & 92 & 97 & 91 & 96 & 94 & 90 & 94 & 88 & 94 & 36 & 93 & 98 & 95 \\
\hline High-income countries & 62 & 60 & 70 & 66 & 63 & 65 & 67 & 68 & 69 & 70 & 67 & 68 & 68 & 67 & 69 & 68 & 67 & 68 & 67 \\
\hline WORLD & 98 & 98 & 99 & 99 & 93 & 98 & 92 & 97 & 91 & 96 & 93 & 89 & 92 & 87 & 89 & 41 & 93 & 97 & 95 \\
\hline
\end{tabular}

\section{PAF of DALYs (\%)}

East Asia and Pacific

Europe and Central Asia

Latin America and the Caribbean

Middle East and North Africa

South Asia

Sub-Saharan Africa

Low- and middle-income countries

Attributable Mortality (thousands)

East Asia and Pacific

Europe and Central Asia

Latin America and the Caribbean

Middle East and North Africa

South Asia

Sub-Saharan Africa

$\begin{array}{rrr}66 & 66 & 65 \\ 27 & 30 & 54 \\ 90 & 90 & 90 \\ 94 & 94 & 95 \\ 80 & 80 & 80 \\ 100 & 100 & 100 \\ 98 & 98 & 98 \\ 62 & 60 & 70\end{array}$

$\begin{array}{llll}66 & 66 & 67 & 66 \\ 59 & 28 & 29 & 28\end{array}$

$\begin{array}{ll}66 & 67 \\ 29 & 27\end{array}$

\section{(2)}

\begin{tabular}{|c|c|c|c|c|c|c|c|c|c|c|c|c|c|c|c|c|c|c|c|}
\hline Sub-Saharan Africa & 159 & 153 & 48 & 47 & 185 & 349 & 440 & 374 & 148 & 108 & 23 & 17 & 3 & 4 & 0 & 0 & 1,006 & 1,051 & 2,057 \\
\hline Low- and middle-income countries & 170 & 163 & 51 & 50 & 240 & 376 & 587 & 411 & 201 & 123 & 27 & 17 & 4 & 4 & 0 & 0 & 1,281 & 1,145 & 2,425 \\
\hline High-income countries & 0 & 0 & 0 & 0 & 1 & 0 & 6 & 2 & 4 & 1 & 1 & 0 & 0 & 0 & 0 & 0 & 11 & 3 & 15 \\
\hline WORLD & 170 & 163 & 51 & 50 & 240 & 377 & 594 & 413 & 205 & 123 & 28 & 18 & 4 & 4 & 0 & 0 & 1,292 & 1,148 & 2,440 \\
\hline
\end{tabular}

Attributable YLL (thousands)

\begin{tabular}{|c|c|c|c|c|c|c|c|c|c|c|c|c|c|c|c|c|c|c|c|}
\hline $\begin{array}{l}\text { Attributable YLL (thousands) } \\
\text { East Asia and Pacific }\end{array}$ & 60 & 47 & 7 & 6 & 269 & 86 & 689 & 148 & 213 & 110 & 12 & 0 & 1 & 0 & 0 & 0 & 1,251 & 396 & 1,647 \\
\hline Europe and Central Asia & 2 & 2 & 3 & 3 & 35 & 10 & 89 & 11 & 30 & 4 & 2 & 0 & 0 & 0 & 0 & 0 & 161 & 30 & 190 \\
\hline Latin America and the Caribbean & 80 & 78 & 36 & 35 & 276 & 223 & 592 & 282 & 163 & 62 & 13 & 4 & 2 & 1 & 0 & 0 & 1,162 & 685 & 1,847 \\
\hline Middle East and North Africa & 8 & 8 & 1 & 1 & 14 & 12 & 21 & 11 & 6 & 3 & 0 & 0 & 0 & 0 & 0 & 0 & 50 & 35 & 85 \\
\hline South Asia & 177 & 169 & 42 & 40 & 882 & 425 & 2,181 & 493 & 649 & 133 & 37 & 8 & 4 & 1 & 0 & 0 & 3,972 & 1,267 & 5,239 \\
\hline Sub-Saharan Africa & 4,816 & 4,672 & 1,420 & 1,395 & 4,989 & 9,577 & 10,702 & 9,414 & 2,903 & 2,237 & 317 & 260 & 29 & 41 & 0 & 0 & 25,176 & 27,596 & 52,771 \\
\hline Low- and middle-income countries & 5,144 & 4,976 & 1,510 & 1,480 & 6,464 & 10,332 & 14,272 & 10,358 & 3,963 & 2,548 & 383 & 272 & 36 & 42 & 1 & 0 & 31,772 & 30,008 & 61,780 \\
\hline High-income countries & 1 & 1 & 1 & 1 & 19 & 10 & 147 & 50 & 73 & 18 & 8 & 2 & 2 & 1 & 0 & 0 & 250 & 82 & 332 \\
\hline WORLD & 5,144 & 4,976 & 1,510 & 1,481 & 6,483 & 10,343 & 14,419 & 10,407 & 4,036 & 2,566 & 391 & 274 & 37 & 43 & 1 & 0 & 32,022 & 30,090 & 62,112 \\
\hline
\end{tabular}

Attributable DALYs (thousands)

\begin{tabular}{|c|c|c|c|c|c|c|c|c|c|c|c|c|c|c|c|c|c|c|c|}
\hline East Asia and Pacific & 62 & 48 & 8 & 7 & 437 & 141 & 799 & 171 & 225 & 113 & 14 & 0 & 1 & 0 & 0 & 0 & 1,546 & 480 & 2,026 \\
\hline Europe and Central Asia & 2 & 2 & 4 & 3 & 70 & 26 & 112 & 17 & 33 & 5 & 3 & 0 & 0 & 0 & 0 & 0 & 223 & 53 & 277 \\
\hline Latin America and the Caribbean & 82 & 80 & 37 & 36 & 385 & 268 & 653 & 300 & 172 & 64 & 14 & 5 & 2 & 1 & 0 & 0 & 1,345 & 753 & 2,099 \\
\hline Middle East and North Africa & 9 & 8 & 1 & 1 & 20 & 15 & 23 & 12 & 7 & 3 & 0 & 0 & 0 & 0 & 0 & 0 & 60 & 39 & 99 \\
\hline South Asia & 181 & 173 & 45 & 43 & 1,117 & 568 & 2,322 & 544 & 663 & 138 & 39 & 8 & 5 & 1 & 0 & 0 & 4,371 & 1,475 & 5,846 \\
\hline Sub-Saharan Africa & 4,894 & 4,748 & 1,451 & 1,431 & 6,041 & 11,057 & 11,257 & 9,961 & 2,994 & 2,298 & 325 & 266 & 29 & 41 & 0 & 0 & 26,992 & 29,803 & 56,795 \\
\hline Low- and middle-income countries & 5,229 & 5,059 & 1,546 & 1,521 & 8,070 & 12,075 & 15,165 & 11,006 & 4,093 & 2,621 & 396 & 280 & 37 & 43 & 1 & 0 & 34,537 & 32,604 & 67,141 \\
\hline High-income countries & 1 & 1 & 1 & 1 & 53 & 21 & 192 & 59 & 82 & 19 & 9 & 2 & 2 & 1 & 0 & 0 & 341 & 104 & 445 \\
\hline WORLD & 5,230 & 5,060 & 1,547 & 1,522 & 8,123 & 12,095 & 15,357 & 11,065 & 4,176 & 2,641 & 406 & 282 & 39 & 43 & 1 & 0 & 34,878 & 32,708 & 67,586 \\
\hline
\end{tabular}

Source: Authors' calculations. 
Table 4A.55

Risk factor: Unsafe sex

Disease:

Cervix uteri cancer

\begin{tabular}{|c|c|c|c|c|c|c|c|c|c|c|c|c|c|c|c|c|c|c|c|}
\hline \multirow[b]{2}{*}{ Region } & \multicolumn{2}{|c|}{$0-4$ years } & \multicolumn{2}{|c|}{ 5-14 years } & \multicolumn{2}{|c|}{$15-29$ years } & \multicolumn{2}{|c|}{ 30-44 years } & \multicolumn{2}{|c|}{$45-59$ years } & \multicolumn{2}{|c|}{$60-69$ years } & \multicolumn{2}{|c|}{$70-79$ years } & \multicolumn{2}{|c|}{$80+$ years } & \multicolumn{3}{|c|}{ Total } \\
\hline & Male & Female & Male & Female & Male & Female & Male & Female & Male & Female & Male & Female & Male & Female & Male & Female & Male & Female & All \\
\hline \multicolumn{20}{|l|}{ PAF of Mortality (\%) } \\
\hline East Asia and Pacific & NA & 100 & NA & 100 & NA & 100 & NA & 100 & NA & 100 & NA & 100 & NA & 100 & NA & 100 & NA & 100 & 100 \\
\hline Europe and Central Asia & NA & 100 & NA & 100 & NA & 100 & NA & 100 & NA & 100 & NA & 100 & NA & 100 & NA & 100 & NA & 100 & 100 \\
\hline Latin America and the Caribbean & NA & 100 & NA & 100 & NA & 100 & NA & 100 & NA & 100 & NA & 100 & NA & 100 & NA & 100 & NA & 100 & 100 \\
\hline Middle East and North Africa & NA & 100 & NA & 100 & NA & 100 & NA & 100 & NA & 100 & NA & 100 & NA & 100 & NA & 100 & NA & 100 & 100 \\
\hline South Asia & NA & 100 & NA & 100 & NA & 100 & NA & 100 & NA & 100 & NA & 100 & NA & 100 & NA & 100 & NA & 100 & 100 \\
\hline Sub-Saharan Africa & NA & 100 & NA & 100 & NA & 100 & NA & 100 & NA & 100 & NA & 100 & NA & 100 & NA & 100 & NA & 100 & 100 \\
\hline Low- and middle-income countries & NA & 100 & NA & 100 & NA & 100 & NA & 100 & NA & 100 & NA & 100 & NA & 100 & NA & 100 & NA & 100 & 100 \\
\hline High-income countries & NA & 100 & NA & 100 & NA & 100 & NA & 100 & NA & 100 & NA & 100 & NA & 100 & NA & 100 & NA & 100 & 100 \\
\hline WORLD & NA & 100 & NA & 100 & NA & 100 & NA & 100 & NA & 100 & NA & 100 & NA & 100 & NA & 100 & NA & 100 & 100 \\
\hline
\end{tabular}

PAF of YLL (\%)

\begin{tabular}{|c|c|c|c|c|c|c|c|c|c|c|c|c|c|c|c|c|c|c|c|}
\hline East Asia and Pacific & NA & 100 & NA & 100 & NA & 100 & NA & 100 & NA & 100 & NA & 100 & NA & 100 & NA & 100 & NA & 100 & 100 \\
\hline Europe and Central Asia & NA & 100 & NA & 100 & NA & 100 & NA & 100 & NA & 100 & NA & 100 & NA & 100 & NA & 100 & NA & 100 & 100 \\
\hline Latin America and the Caribbean & NA & 100 & NA & 100 & NA & 100 & NA & 100 & NA & 100 & NA & 100 & NA & 100 & NA & 100 & NA & 100 & 100 \\
\hline Middle East and North Africa & NA & 100 & NA & 100 & NA & 100 & NA & 100 & NA & 100 & NA & 100 & NA & 100 & NA & 100 & NA & 100 & 100 \\
\hline South Asia & NA & 100 & NA & 100 & NA & 100 & NA & 100 & NA & 100 & NA & 100 & NA & 100 & NA & 100 & NA & 100 & 100 \\
\hline$\underline{\text { Sub-Saharan Africa }}$ & NA & 100 & NA & 100 & NA & 100 & NA & 100 & NA & 100 & NA & 100 & NA & 100 & NA & 100 & NA & 100 & 100 \\
\hline Low- and middle-income countries & NA & 100 & NA & 100 & NA & 100 & NA & 100 & NA & 100 & NA & 100 & NA & 100 & NA & 100 & NA & 100 & 100 \\
\hline High-income countries & NA & 100 & NA & 100 & NA & 100 & NA & 100 & NA & 100 & NA & 100 & NA & 100 & NA & 100 & NA & 100 & 100 \\
\hline WORLD & NA & 100 & NA & 100 & NA & 100 & NA & 100 & NA & 100 & NA & 100 & NA & 100 & NA & 100 & NA & 100 & 100 \\
\hline \multicolumn{20}{|l|}{ PAF of DALYs (\%) } \\
\hline East Asia and Pacific & NA & 100 & NA & 100 & NA & 100 & NA & 100 & NA & 100 & NA & 100 & NA & 100 & NA & 100 & NA & 100 & 100 \\
\hline Europe and Central Asia & NA & 100 & NA & 100 & NA & 100 & NA & 100 & NA & 100 & NA & 100 & NA & 100 & NA & 100 & NA & 100 & 100 \\
\hline Latin America and the Caribbean & NA & 100 & NA & 100 & NA & 100 & NA & 100 & NA & 100 & NA & 100 & NA & 100 & NA & 100 & NA & 100 & 100 \\
\hline Middle East and North Africa & NA & 100 & NA & 100 & NA & 100 & NA & 100 & NA & 100 & NA & 100 & NA & 100 & NA & 100 & NA & 100 & 100 \\
\hline South Asia & NA & 100 & NA & 100 & NA & 100 & NA & 100 & NA & 100 & NA & 100 & NA & 100 & NA & 100 & NA & 100 & 100 \\
\hline$\underline{\text { Sub-Saharan Africa }}$ & NA & 100 & NA & 100 & NA & 100 & NA & 100 & NA & 100 & NA & 100 & NA & 100 & NA & 100 & NA & 100 & 100 \\
\hline Low- and middle-income countries & NA & 100 & NA & 100 & NA & 100 & NA & 100 & NA & 100 & NA & 100 & NA & 100 & NA & 100 & NA & 100 & 100 \\
\hline High-income countries & NA & 100 & NA & 100 & NA & 100 & NA & 100 & NA & 100 & NA & 100 & NA & 100 & NA & 100 & NA & 100 & 100 \\
\hline WORLD & NA & 100 & NA & 100 & NA & 100 & NA & 100 & NA & 100 & NA & 100 & NA & 100 & NA & 100 & NA & 100 & 100 \\
\hline
\end{tabular}

Attributable Mortality (thousands)

\begin{tabular}{|c|c|c|c|c|c|c|c|c|c|c|c|c|c|c|c|c|c|c|c|}
\hline East Asia and Pacific & NA & 0 & NA & 0 & NA & 2 & NA & 3 & NA & 14 & NA & 13 & NA & 11 & NA & 4 & NA & 47 & 47 \\
\hline Europe and Central Asia & NA & 0 & NA & 0 & NA & 0 & NA & 3 & NA & 6 & NA & 4 & NA & 4 & NA & 2 & NA & 19 & 19 \\
\hline Latin America and the Caribbean & NA & 0 & NA & 0 & NA & 1 & NA & 4 & NA & 9 & NA & 5 & NA & 5 & NA & 2 & NA & 26 & 26 \\
\hline Middle East and North Africa & NA & 0 & NA & 0 & NA & 0 & NA & 1 & NA & 2 & NA & 1 & NA & 1 & NA & 0 & NA & 5 & 5 \\
\hline South Asia & NA & 0 & NA & 0 & NA & 6 & NA & 5 & NA & 26 & NA & 26 & NA & 14 & NA & 7 & NA & 83 & 83 \\
\hline Sub-Saharan Africa & NA & 0 & NA & 0 & NA & 1 & NA & 2 & NA & 12 & NA & 10 & NA & 9 & NA & 2 & NA & 38 & 38 \\
\hline Low- and middle-income countries & NA & 0 & NA & 0 & NA & 11 & NA & 19 & NA & 68 & NA & 59 & NA & 44 & NA & 17 & NA & 218 & 218 \\
\hline High-income countries & NA & 0 & NA & 0 & NA & 0 & NA & 3 & NA & 5 & NA & 3 & NA & 3 & NA & 3 & NA & 17 & 17 \\
\hline WORLD & NA & 0 & NA & 0 & NA & 11 & NA & 22 & NA & 73 & NA & 62 & NA & 47 & NA & 20 & NA & 235 & 235 \\
\hline
\end{tabular}

Attributable YLL (thousands)

\begin{tabular}{|c|c|c|c|c|c|c|c|c|c|c|c|c|c|c|c|c|c|c|c|}
\hline East Asia and Pacific & NA & 0 & NA & 0 & NA & 53 & NA & 82 & NA & 287 & NA & 189 & NA & 117 & NA & 22 & NA & 750 & 750 \\
\hline Europe and Central Asia & NA & 0 & NA & 0 & NA & 11 & NA & 79 & NA & 117 & NA & 61 & NA & 45 & NA & 8 & NA & 321 & 321 \\
\hline Latin America and the Caribbean & NA & 0 & NA & 0 & NA & 27 & NA & 109 & NA & 177 & NA & 80 & NA & 48 & NA & 12 & NA & 454 & 454 \\
\hline Middle East and North Africa & NA & 0 & NA & 0 & NA & 10 & NA & 12 & NA & 36 & NA & 18 & NA & 8 & NA & 1 & NA & 86 & 86 \\
\hline South Asia & NA & 0 & NA & 0 & NA & 159 & NA & 123 & NA & 519 & NA & 389 & NA & 145 & NA & 39 & NA & 1,375 & 1,375 \\
\hline Sub-Saharan Africa & NA & 0 & NA & 0 & NA & 37 & NA & 62 & NA & 239 & NA & 158 & NA & 96 & NA & 14 & NA & 606 & 606 \\
\hline Low- and middle-income countries & NA & 0 & NA & 1 & NA & 298 & NA & 468 & NA & 1,373 & NA & 896 & NA & 459 & NA & 96 & NA & 3,592 & 3,592 \\
\hline High-income countries & NA & 0 & NA & 0 & NA & 6 & NA & 66 & NA & 96 & NA & 44 & NA & 34 & NA & 13 & NA & 260 & 260 \\
\hline WORLD & NA & 0 & NA & 1 & NA & 304 & NA & 534 & NA & 1,469 & NA & 941 & NA & 494 & NA & 109 & NA & 3,852 & 3,852 \\
\hline \multicolumn{20}{|l|}{ Attributable DALYs (thousands) } \\
\hline East Asia and Pacific & NA & 0 & NA & 0 & NA & 65 & NA & 93 & NA & 308 & NA & 196 & NA & 120 & NA & 22 & NA & 805 & 805 \\
\hline Europe and Central Asia & NA & 0 & NA & 0 & NA & 15 & NA & 93 & NA & 128 & NA & 64 & NA & 46 & NA & 9 & NA & 356 & 356 \\
\hline Latin America and the Caribbean & NA & 0 & NA & 0 & NA & 33 & NA & 124 & NA & 191 & NA & 84 & NA & 49 & NA & 13 & NA & 494 & 494 \\
\hline Middle East and North Africa & NA & 0 & NA & 0 & NA & 12 & NA & 14 & NA & 38 & NA & 19 & NA & 9 & NA & 1 & NA & 93 & 93 \\
\hline South Asia & NA & 0 & NA & 1 & NA & 175 & NA & 129 & NA & 534 & NA & 397 & NA & 148 & NA & 40 & NA & 1,423 & 1,423 \\
\hline Sub-Saharan Africa & NA & 0 & NA & 0 & NA & 41 & NA & 64 & NA & 247 & NA & 162 & NA & 98 & NA & 14 & NA & 627 & 627 \\
\hline Low- and middle-income countries & NA & 0 & NA & 2 & NA & 341 & NA & 517 & NA & 1,446 & NA & 922 & NA & 469 & NA & 99 & NA & 3,797 & 3,797 \\
\hline High-income countries & NA & 0 & NA & 0 & NA & 11 & NA & 90 & NA & 117 & NA & 50 & NA & 37 & NA & 14 & NA & 319 & 319 \\
\hline WORLD & NA & 0 & NA & 2 & NA & 352 & NA & 607 & NA & 1,563 & NA & 972 & NA & 506 & NA & 113 & NA & 4,116 & 4,116 \\
\hline
\end{tabular}

Source: Authors' calculations.

Note: $\mathrm{NA}=$ not applicable. 
Table 4A.56

Risk factor: Unsafe sex

Disease: All causes

\begin{tabular}{|c|c|c|c|c|c|c|c|c|c|c|c|c|c|c|c|c|c|c|c|}
\hline \multirow[b]{2}{*}{ Region } & \multicolumn{2}{|c|}{$0-4$ years } & \multicolumn{2}{|c|}{ 5-14 years } & \multicolumn{2}{|c|}{$15-29$ years } & \multicolumn{2}{|c|}{ 30-44 years } & \multicolumn{2}{|c|}{ 45-59 years } & \multicolumn{2}{|c|}{$60-69$ years } & \multicolumn{2}{|c|}{$70-79$ years } & \multicolumn{2}{|c|}{$80+$ years } & \multicolumn{3}{|c|}{ Total } \\
\hline & Male & Female & Male & Female & Male & Female & Male & Female & Male & Female & Male & Female & Male & Female & Male & Female & Male & Female & All \\
\hline \multicolumn{20}{|l|}{ PAF of Mortality (\%) } \\
\hline East Asia and Pacific & 0 & 0 & 0 & 0 & 2 & 2 & 5 & 2 & 1 & 3 & 0 & 1 & 0 & 1 & 0 & 0 & 1 & 1 & 1 \\
\hline Europe and Central Asia & 0 & 0 & 1 & 1 & 1 & 2 & 1 & 4 & 0 & 2 & 0 & 1 & 0 & 1 & 0 & 0 & 0 & 1 & 0 \\
\hline Latin America and the Caribbean & 1 & 2 & 4 & 5 & 6 & 14 & 12 & 15 & 3 & 6 & 0 & 3 & 0 & 2 & 0 & 1 & 3 & 4 & 3 \\
\hline Middle East and North Africa & 0 & 0 & 0 & 0 & 1 & 2 & 1 & 2 & 1 & 2 & 0 & 1 & 0 & 1 & 0 & 0 & 0 & 1 & 1 \\
\hline South Asia & 1 & 2 & 1 & 0 & 7 & 4 & 13 & 5 & 4 & 5 & 1 & 3 & 0 & 1 & 0 & 1 & 3 & 3 & 3 \\
\hline Sub-Saharan Africa & 7 & 8 & 17 & 16 & 35 & 52 & 53 & 57 & 26 & 27 & 6 & 8 & 1 & 4 & 0 & 1 & 19 & 22 & 20 \\
\hline Low- and middle-income countries & 4 & 4 & 7 & 7 & 13 & 25 & 21 & 24 & 6 & 8 & 1 & 3 & 0 & 1 & 0 & 0 & 5 & 6 & 6 \\
\hline High-income countries & 0 & 0 & 0 & 0 & 1 & 2 & 3 & 5 & 1 & 2 & 0 & 1 & 0 & 0 & 0 & 0 & 0 & 1 & 0 \\
\hline WORLD & 4 & 4 & 7 & 7 & 13 & 25 & 20 & 23 & 5 & 8 & 1 & 3 & 0 & 1 & 0 & 0 & 5 & 6 & 5 \\
\hline
\end{tabular}

PAF of YLL (\%)

\begin{tabular}{|c|c|c|c|c|c|c|c|c|c|c|c|c|c|c|c|c|c|c|c|}
\hline East Asia and Pacific & 0 & 0 & 0 & 0 & 2 & 2 & 5 & 2 & 1 & 3 & 0 & 1 & 0 & 1 & 0 & 0 & 1 & 1 & 1 \\
\hline Europe and Central Asia & 0 & 0 & 1 & 1 & 1 & 2 & 1 & 4 & 0 & 2 & 0 & 1 & 0 & 1 & 0 & 0 & 0 & 1 & 1 \\
\hline Latin America and the Caribbean & 1 & 2 & 4 & 5 & 6 & 14 & 12 & 15 & 3 & 6 & 0 & 3 & 0 & 2 & 0 & 1 & 4 & 5 & 4 \\
\hline Middle East and North Africa & 0 & 0 & 0 & 0 & 1 & 2 & 1 & 2 & 1 & 2 & 0 & 1 & 0 & 1 & 0 & 0 & 0 & 1 & 1 \\
\hline South Asia & 1 & 2 & 1 & 0 & 7 & 4 & 13 & 5 & 4 & 5 & 1 & 3 & 0 & 1 & 0 & 1 & 3 & 3 & 3 \\
\hline Sub-Saharan Africa & 7 & 8 & 17 & 16 & 35 & 52 & 53 & 57 & 27 & 27 & 6 & 8 & 1 & 4 & 0 & 1 & 19 & 23 & 21 \\
\hline Low- and middle-income countries & 4 & 4 & 7 & 7 & 13 & 25 & 22 & 24 & 6 & 8 & 1 & 3 & 0 & 1 & 0 & 1 & 7 & 8 & 8 \\
\hline High-income countries & 0 & 0 & 0 & 0 & 1 & 2 & 3 & 5 & 1 & 2 & 0 & 1 & 0 & 0 & 0 & 0 & 1 & 1 & 1 \\
\hline WORLD & 4 & 4 & 7 & 7 & 12 & 25 & 21 & 23 & 5 & 8 & 1 & 3 & 0 & 1 & 0 & 0 & 6 & 8 & 7 \\
\hline
\end{tabular}

PAF of DALYs $(\%)$

East Asia and Pacific

Europe and Central Asia

Latin America and the Caribbean

Middle East and North Africa

South Asia

Sub-Saharan Africa

Low- and middle-income countries

High-income countries

\begin{tabular}{rrrrrrrrrrrrrrrrrrrr}
0 & 0 & 0 & 0 & 2 & 3 & 3 & 2 & 1 & 2 & 0 & 1 & 0 & 1 & 0 & 0 & 1 & 1 & 1 \\
0 & 0 & 0 & 0 & 1 & 3 & 1 & 2 & 0 & 1 & 0 & 1 & 0 & 0 & 0 & 0 & 0 & 1 & 1 \\
1 & 1 & 1 & 1 & 4 & 7 & 7 & 7 & 2 & 4 & 0 & 2 & 0 & 1 & 0 & 1 & 3 & 3 & 3 \\
0 & 0 & 0 & 0 & 1 & 4 & 1 & 2 & 0 & 1 & 0 & 1 & 0 & 0 & 0 & 0 & 0 & 1 & 1 \\
1 & 2 & 0 & 0 & 5 & 5 & 9 & 4 & 3 & 3 & 1 & 2 & 0 & 1 & 0 & 1 & 3 & 3 & 3 \\
7 & 7 & 11 & 12 & 27 & 41 & 44 & 46 & 21 & 20 & 5 & 6 & 1 & 3 & 0 & 1 & 16 & 19 & 18 \\
\hline 3 & 3 & 4 & 4 & 9 & 16 & 15 & 14 & 4 & 5 & 1 & 2 & 0 & 1 & 0 & 0 & 5 & 6 & 6 \\
0 & 0 & 0 & 0 & 1 & 2 & 2 & 2 & 0 & 1 & 0 & 0 & 0 & 0 & 0 & 0 & 0 & 1 & 1 \\
\hline 3 & 3 & 4 & 4 & 8 & 15 & 14 & 13 & 4 & 4 & 1 & 2 & 0 & 1 & 0 & 0 & 5 & 6 & 5 \\
\hline
\end{tabular}

Attributable Mortality (thousands)

\begin{tabular}{|c|c|c|c|c|c|c|c|c|c|c|c|c|c|c|c|c|c|c|c|}
\hline \multicolumn{20}{|l|}{ Attributable Mortality (thousands) } \\
\hline East Asia and Pacific & 3 & 2 & 0 & 0 & 10 & 5 & 29 & 10 & 12 & 21 & 2 & 13 & 1 & 12 & 1 & 4 & 57 & 67 & 124 \\
\hline Europe and Central Asia & 0 & 0 & 0 & 0 & 1 & 1 & 4 & 4 & 2 & 6 & 0 & 4 & 0 & 4 & 0 & 2 & 7 & 21 & 28 \\
\hline Latin America and the Caribbean & 3 & 3 & 1 & 1 & 10 & 9 & 24 & 16 & 8 & 12 & 1 & 6 & 0 & 5 & 0 & 2 & 49 & 53 & 102 \\
\hline Middle East and North Africa & 0 & 0 & 0 & 0 & 1 & 1 & 1 & 1 & 1 & 2 & 1 & 2 & 0 & 1 & 0 & 0 & 4 & 8 & 12 \\
\hline South Asia & 17 & 30 & 1 & 1 & 33 & 21 & 91 & 26 & 40 & 38 & 9 & 30 & 5 & 16 & 2 & 8 & 199 & 170 & 369 \\
\hline Sub-Saharan Africa & 177 & 165 & 48 & 47 & 186 & 362 & 450 & 384 & 165 & 126 & 25 & 29 & 3 & 14 & 0 & 2 & 1,054 & 1,130 & 2,185 \\
\hline Low- and middle-income countries & 201 & 201 & 51 & 50 & 241 & 400 & 598 & 441 & 227 & 205 & 37 & 84 & 10 & 52 & 3 & 18 & 1,370 & 1,450 & 2,819 \\
\hline High-income countries & 0 & 0 & 0 & 0 & 1 & 1 & 6 & 5 & 4 & 6 & 1 & 3 & 0 & 4 & 0 & 3 & 11 & 21 & 32 \\
\hline WORLD & 201 & 201 & 51 & 50 & 242 & 400 & 604 & 445 & 231 & 210 & 38 & 87 & 10 & 56 & 3 & 21 & 1,381 & 1,470 & 2,851 \\
\hline
\end{tabular}

Attributable YLL (thousands)

\begin{tabular}{|c|c|c|c|c|c|c|c|c|c|c|c|c|c|c|c|c|c|c|c|}
\hline \multicolumn{20}{|l|}{ Attributable YLL (the } \\
\hline East Asia and Pacific & 89 & 58 & 7 & 6 & 270 & 140 & 692 & 246 & 231 & 429 & 23 & 201 & 9 & 123 & 3 & 23 & 1,324 & 1,227 & 2,551 \\
\hline Europe and Central Asia & 7 & 7 & 3 & 3 & 35 & 22 & 89 & 91 & 31 & 121 & 3 & 62 & 0 & 45 & 0 & 9 & 169 & 360 & 529 \\
\hline Latin America and the Caribbean & 101 & 83 & 36 & 35 & 278 & 253 & 592 & 395 & 163 & 242 & 13 & 86 & 2 & 50 & 0 & 13 & 1,186 & 1,156 & 2,342 \\
\hline Middle East and North Africa & 9 & 10 & 1 & 2 & 15 & 28 & 26 & 30 & 23 & 49 & 7 & 27 & 3 & 12 & 1 & 1 & 86 & 159 & 244 \\
\hline South Asia & 525 & 928 & 43 & 41 & 883 & 587 & 2,195 & 640 & 777 & 770 & 126 & 449 & 48 & 165 & 12 & 42 & 4,609 & 3,623 & 8,231 \\
\hline Sub-Saharan Africa & 5,341 & 5,027 & 1,420 & 1,396 & 5,027 & 9,955 & 10,931 & 9,678 & 3,238 & 2,603 & 356 & 450 & 29 & 154 & 1 & 14 & 26,341 & 29,278 & 55,619 \\
\hline Low- and middle-income countries & 6,072 & 6,114 & 1,510 & 1,482 & 6,508 & 10,986 & 14,526 & 11,081 & 4,463 & 4,215 & 527 & 1,274 & 90 & 549 & 17 & 101 & 33,714 & 35,802 & 69,516 \\
\hline High-income countries & 1 & 1 & 1 & 1 & 19 & 17 & 147 & 116 & 73 & 115 & 8 & 47 & 2 & 36 & 0 & 14 & 252 & 347 & 599 \\
\hline WORLD & 6,073 & 6,115 & 1,511 & 1,483 & 6,528 & 11,002 & 14,673 & 11,197 & 4,536 & 4,330 & 536 & 1,322 & 92 & 585 & 17 & 116 & 33,965 & 36,149 & 70,115 \\
\hline
\end{tabular}

Attributable DALYs (thousands)

\begin{tabular}{lrrrrrrrrrrrrrrrrrrr} 
East Asia and Pacific & 139 & 107 & 10 & 16 & 523 & 566 & 845 & 379 & 243 & 455 & 25 & 209 & 9 & 126 & 3 & 24 & 1,797 & 1,881 & 3,678 \\
Europe and Central Asia & 10 & 10 & 4 & 6 & 90 & 151 & 125 & 144 & 34 & 135 & 3 & 65 & 0 & 47 & 0 & 9 & 266 & 567 & 833 \\
Latin America and the Caribbean & 126 & 107 & 38 & 41 & 434 & 505 & 681 & 488 & 173 & 259 & 15 & 89 & 2 & 51 & 0 & 13 & 1,468 & 1,554 & 3,022 \\
Middle East and North Africa & 25 & 26 & 2 & 6 & 53 & 187 & 43 & 64 & 24 & 52 & 7 & 28 & 3 & 12 & 1 & 1 & 157 & 376 & 534 \\
South Asia & 761 & 1,151 & 53 & 70 & 1,394 & 1,647 & 2,468 & 948 & 793 & 795 & 128 & 458 & 48 & 168 & 12 & 43 & 5,657 & 5,281 & 10,938 \\
Sub-Saharan Africa & 5,670 & 5,350 & 1,458 & 1,452 & 6,280 & 12,100 & 11,541 & 10,385 & 3,330 & 2,675 & 363 & 460 & 29 & 156 & 1 & 14 & 28,672 & 32,593 & 61,265 \\
\hline Low- and middle-income countries & 6,731 & 6,750 & 1,565 & 1,591 & 8,773 & 15,157 & 15,702 & 12,409 & 4,598 & 4,372 & 542 & 1,309 & 92 & 559 & 17 & 104 & 38,018 & 42,252 & 80,270 \\
High-income countries & 8 & 7 & 1 & 3 & 62 & 111 & 201 & 176 & 83 & 138 & 10 & 53 & 2 & 39 & 0 & 15 & 367 & 542 & 909 \\
\hline WORLD & 6,739 & 6,757 & 1,566 & 1,594 & 8,834 & 15,268 & 15,903 & 12,586 & 4,681 & 4,510 & 551 & 1,362 & 94 & 598 & 17 & 120 & 38,385 & 42,794 & 81,179 \\
\hline
\end{tabular}

Source: Authors' calculations. 
Table 4A.57

Risk factor:

Alcohol use

Disease:

Low birthweight

\begin{tabular}{|c|c|c|c|c|c|c|c|c|c|c|c|c|c|c|c|c|c|c|c|}
\hline \multirow[b]{2}{*}{ Region } & \multicolumn{2}{|c|}{$0-4$ years } & \multicolumn{2}{|c|}{ 5-14 years } & \multicolumn{2}{|c|}{$15-29$ years } & \multicolumn{2}{|c|}{ 30-44 years } & \multicolumn{2}{|c|}{$45-59$ years } & \multicolumn{2}{|c|}{$60-69$ years } & \multicolumn{2}{|c|}{$70-79$ years } & \multicolumn{2}{|c|}{$80+$ years } & \multicolumn{3}{|c|}{ Total } \\
\hline & Male & Female & Male & Female & Male & Female & Male & Female & Male & Female & Male & Female & Male & Female & Male & Female & Male & Female & All \\
\hline \multicolumn{20}{|l|}{ PAF of Mortality (\%) } \\
\hline East Asia and Pacific & 0 & 0 & NA & NA & NA & NA & NA & NA & NA & NA & NA & NA & NA & NA & NA & NA & 0 & 0 & 0 \\
\hline Europe and Central Asia & 4 & 4 & NA & NA & NA & NA & NA & NA & NA & NA & NA & NA & NA & NA & NA & NA & 4 & 4 & 4 \\
\hline Latin America and the Caribbean & 3 & 3 & NA & NA & NA & NA & NA & NA & NA & NA & NA & NA & NA & NA & NA & NA & 3 & 3 & 3 \\
\hline Middle East and North Africa & 3 & 3 & NA & NA & NA & NA & NA & NA & NA & NA & NA & NA & NA & NA & NA & NA & 3 & 3 & 3 \\
\hline South Asia & 0 & 0 & NA & NA & NA & NA & NA & NA & NA & NA & NA & NA & NA & NA & NA & NA & 0 & 0 & 0 \\
\hline Sub-Saharan Africa & 0 & 0 & NA & NA & NA & NA & NA & NA & NA & NA & NA & NA & NA & NA & NA & NA & 0 & 0 & 0 \\
\hline Low- and middle-income countries & 0 & 0 & NA & NA & NA & NA & NA & NA & NA & NA & NA & NA & NA & NA & NA & NA & 0 & 0 & 0 \\
\hline High-income countries & 2 & 2 & NA & NA & NA & NA & NA & NA & NA & NA & NA & NA & NA & NA & NA & NA & 2 & 2 & 2 \\
\hline WORLD & 0 & 0 & NA & NA & NA & NA & NA & NA & NA & NA & NA & NA & NA & NA & NA & NA & 0 & 0 & 0 \\
\hline
\end{tabular}

PAF of YLL (\%)

\begin{tabular}{|c|c|c|c|c|c|c|c|c|c|c|c|c|c|c|c|c|c|c|c|}
\hline East Asia and Pacific & 0 & 0 & NA & NA & NA & NA & NA & NA & NA & NA & NA & NA & NA & NA & NA & NA & 0 & 0 & 0 \\
\hline Europe and Central Asia & 4 & 4 & NA & NA & NA & NA & NA & NA & NA & NA & NA & NA & NA & NA & NA & NA & 4 & 4 & 4 \\
\hline Latin America and the Caribbean & 3 & 3 & NA & NA & NA & NA & NA & NA & NA & NA & NA & NA & NA & NA & NA & NA & 3 & 3 & 3 \\
\hline Middle East and North Africa & 3 & 3 & NA & NA & NA & NA & NA & NA & NA & NA & NA & NA & NA & NA & NA & NA & 3 & 3 & 3 \\
\hline South Asia & 0 & 0 & NA & NA & NA & NA & NA & NA & NA & NA & NA & NA & NA & NA & NA & NA & 0 & 0 & 0 \\
\hline Sub-Saharan Africa & 0 & 0 & NA & NA & NA & NA & NA & NA & NA & NA & NA & NA & NA & NA & NA & NA & 0 & 0 & 0 \\
\hline Low- and middle-income countries & 0 & 0 & NA & NA & NA & NA & NA & NA & NA & NA & NA & NA & NA & NA & NA & NA & 0 & 0 & 0 \\
\hline High-income countries & 2 & 2 & NA & NA & NA & NA & NA & NA & NA & NA & NA & NA & NA & NA & NA & NA & 2 & 2 & 2 \\
\hline WORLD & 1 & 0 & NA & NA & NA & NA & NA & NA & NA & NA & NA & NA & NA & NA & NA & NA & 1 & 0 & 0 \\
\hline
\end{tabular}

PAF of DALYs (\%)

Europe and Central Asia

Latin America and the Cribbean

Middle East and North Africa

South Asia

Sub-Saharan Africa

Low- and middle-income countries

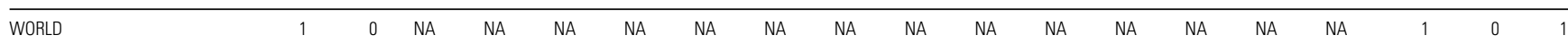

Attributable Mortality (thousands)

\begin{tabular}{|c|c|c|c|c|c|c|c|c|c|c|c|c|c|c|c|c|c|c|}
\hline East Asia and Pacific & 0 & 0 & NA & NA & NA & NA & NA & NA & NA & NA & NA & NA & NA & NA & NA & NA & 0 & 0 \\
\hline Europe and Central Asia & 1 & 0 & NA & NA & NA & NA & NA & NA & NA & NA & NA & NA & NA & NA & NA & NA & 1 & 0 \\
\hline Latin America and the Caribbean & 0 & 0 & NA & NA & NA & NA & NA & NA & NA & NA & NA & NA & NA & NA & NA & NA & 0 & 0 \\
\hline Middle East and North Africa & 1 & 1 & NA & NA & NA & NA & NA & NA & NA & NA & NA & NA & NA & NA & NA & NA & 1 & 1 \\
\hline South Asia & 1 & 1 & NA & NA & NA & NA & NA & NA & NA & NA & NA & NA & NA & NA & NA & NA & 1 & 1 \\
\hline Sub-Saharan Africa & 0 & 0 & NA & NA & NA & NA & NA & NA & NA & NA & NA & NA & NA & NA & NA & NA & 0 & 0 \\
\hline Low- and middle-income countries & 3 & 3 & NA & NA & NA & NA & NA & NA & NA & NA & NA & NA & NA & NA & NA & NA & 3 & 3 \\
\hline High-income countries & 0 & 0 & NA & NA & NA & NA & NA & NA & NA & NA & NA & NA & NA & NA & NA & NA & 0 & 0 \\
\hline WORLD & 4 & 3 & NA & NA & NA & NA & NA & NA & NA & NA & NA & NA & NA & NA & NA & NA & 4 & 3 \\
\hline
\end{tabular}

Attributable YLL (thousands)

\begin{tabular}{|c|c|c|c|c|c|c|c|c|c|c|c|c|c|c|c|c|c|c|c|}
\hline East Asia and Pacific & 3 & 2 & NA & NA & NA & NA & NA & NA & NA & NA & NA & NA & NA & NA & NA & NA & 3 & 2 & 5 \\
\hline Europe and Central Asia & 17 & 14 & NA & NA & NA & NA & NA & NA & NA & NA & NA & NA & NA & NA & NA & NA & 17 & 14 & 30 \\
\hline Latin America and the Caribbean & 12 & 9 & NA & NA & NA & NA & NA & NA & NA & NA & NA & NA & NA & NA & NA & NA & 12 & 9 & 21 \\
\hline Middle East and North Africa & 27 & 18 & NA & NA & NA & NA & NA & NA & NA & NA & NA & NA & NA & NA & NA & NA & 27 & 18 & 46 \\
\hline South Asia & 35 & 30 & NA & NA & NA & NA & NA & NA & NA & NA & NA & NA & NA & NA & NA & NA & 35 & 30 & 65 \\
\hline$\underline{\text { Sub-Saharan Africa }}$ & 12 & 9 & NA & NA & NA & NA & NA & NA & NA & NA & NA & NA & NA & NA & NA & NA & 12 & 9 & 21 \\
\hline Low- and middle-income countries & 105 & 83 & NA & NA & NA & NA & NA & NA & NA & NA & NA & NA & NA & NA & NA & NA & 105 & 83 & 188 \\
\hline High-income countries & 2 & 2 & NA & NA & NA & NA & NA & NA & NA & NA & NA & NA & NA & NA & NA & NA & 2 & 2 & 4 \\
\hline WORLD & 107 & 85 & NA & NA & NA & NA & NA & NA & NA & NA & NA & NA & NA & NA & NA & NA & 107 & 85 & 192 \\
\hline
\end{tabular}

Attributable DALYs (thousands)

\begin{tabular}{|c|c|c|c|c|c|c|c|c|c|c|c|c|c|c|c|c|c|c|c|}
\hline East Asia and Pacific & 3 & 3 & NA & NA & NA & NA & NA & NA & NA & NA & NA & NA & NA & NA & NA & NA & 3 & 3 & 5 \\
\hline Europe and Central Asia & 19 & 16 & NA & NA & NA & NA & NA & NA & NA & NA & NA & NA & NA & NA & NA & NA & 19 & 16 & 34 \\
\hline Latin America and the Caribbean & 15 & 13 & NA & NA & NA & NA & NA & NA & NA & NA & NA & NA & NA & NA & NA & NA & 15 & 13 & 28 \\
\hline Middle East and North Africa & 30 & 21 & NA & NA & NA & NA & NA & NA & NA & NA & NA & NA & NA & NA & NA & NA & 30 & 21 & 51 \\
\hline South Asia & 37 & 33 & NA & NA & NA & NA & NA & NA & NA & NA & NA & NA & NA & NA & NA & NA & 37 & 33 & 71 \\
\hline Sub-Saharan Africa & 13 & 10 & NA & NA & NA & NA & NA & NA & NA & NA & NA & NA & NA & NA & NA & NA & 13 & 10 & 22 \\
\hline Low- and middle-income countries & 116 & 95 & NA & NA & NA & NA & NA & NA & NA & NA & NA & NA & NA & NA & NA & NA & 116 & 95 & 211 \\
\hline High-income countries & 4 & 3 & NA & NA & NA & NA & NA & NA & NA & NA & NA & NA & NA & NA & NA & NA & 4 & 3 & 7 \\
\hline WORLD & 120 & 98 & NA & NA & NA & NA & NA & NA & NA & NA & NA & NA & NA & NA & NA & NA & 120 & 98 & 218 \\
\hline
\end{tabular}

Source: Authors' calculations.

Note: $\mathrm{NA}=$ not applicable. 
Risk factor:

Disease:

Alcohol use

Mouth and oropharynx cancers

\begin{tabular}{|c|c|c|c|c|c|c|c|c|c|c|c|c|c|c|c|c|c|c|c|}
\hline \multirow[b]{2}{*}{ Region } & \multicolumn{2}{|c|}{$0-4$ years } & \multicolumn{2}{|c|}{ 5-14 years } & \multicolumn{2}{|c|}{$15-29$ years } & \multicolumn{2}{|c|}{ 30-44 years } & \multicolumn{2}{|c|}{$45-59$ years } & \multicolumn{2}{|c|}{$60-69$ years } & \multicolumn{2}{|c|}{ 70-79 years } & \multicolumn{2}{|c|}{$80+$ years } & \multicolumn{3}{|c|}{ Total } \\
\hline & Male & Female & Male & Female & Male & Female & Male & Female & Male & Female & Male & Female & Male & Female & Male & Female & Male & Female & All \\
\hline \multicolumn{20}{|l|}{ PAF of Mortality (\%) } \\
\hline East Asia and Pacific & NA & NA & NA & NA & 30 & 11 & 32 & 11 & 32 & 11 & 29 & 8 & 27 & 4 & 27 & 4 & 30 & 8 & 23 \\
\hline Europe and Central Asia & NA & NA & NA & NA & 44 & 31 & 42 & 31 & 43 & 31 & 38 & 26 & 33 & 24 & 32 & 24 & 39 & 26 & 37 \\
\hline Latin America and the Caribbean & NA & NA & NA & NA & 38 & 29 & 39 & 31 & 39 & 29 & 35 & 26 & 25 & 20 & 25 & 20 & 34 & 24 & 31 \\
\hline Middle East and North Africa & NA & NA & NA & NA & 10 & 4 & 9 & 3 & 9 & 3 & 6 & 2 & 4 & 1 & 4 & 2 & 7 & 2 & 5 \\
\hline South Asia & NA & NA & NA & NA & 11 & 4 & 15 & 2 & 8 & 2 & 2 & 0 & 0 & 0 & 0 & 0 & 4 & 1 & 3 \\
\hline Sub-Saharan Africa & NA & NA & NA & NA & 23 & 12 & 28 & 16 & 27 & 17 & 25 & 14 & 22 & 11 & 22 & 11 & 25 & 13 & 21 \\
\hline Low- and middle-income countries & NA & NA & NA & NA & 19 & 10 & 25 & 9 & 25 & 8 & 15 & 5 & 12 & 5 & 10 & 4 & 18 & 6 & 14 \\
\hline High-income countries & NA & NA & NA & NA & 41 & 32 & 41 & 31 & 41 & 31 & 35 & 26 & 32 & 23 & 32 & 23 & 36 & 26 & 33 \\
\hline WORLD & NA & NA & NA & NA & 20 & 11 & 27 & 11 & 27 & 10 & 17 & 7 & 15 & 7 & 14 & 8 & 20 & 8 & 16 \\
\hline
\end{tabular}

PAF of YLL (\%)

\begin{tabular}{|c|c|c|c|c|c|c|c|c|c|c|c|c|c|c|c|c|c|c|c|}
\hline East Asia and Pacific & NA & NA & NA & NA & 30 & 11 & 32 & 11 & 32 & 11 & 29 & 8 & 27 & 4 & 27 & 4 & 31 & 9 & 24 \\
\hline Europe and Central Asia & NA & NA & NA & NA & 44 & 31 & 42 & 31 & 43 & 31 & 38 & 26 & 33 & 24 & 32 & 24 & 40 & 27 & 38 \\
\hline Latin America and the Caribbean & NA & NA & NA & NA & 38 & 29 & 39 & 31 & 39 & 29 & 35 & 26 & 25 & 20 & 25 & 20 & 36 & 26 & 33 \\
\hline Middle East and North Africa & NA & NA & NA & NA & 10 & 4 & 9 & 3 & 9 & 3 & 6 & 2 & 4 & 1 & 4 & 2 & 7 & 2 & 6 \\
\hline South Asia & NA & NA & NA & NA & 11 & 4 & 15 & 2 & 8 & 2 & 2 & 0 & 0 & 0 & 0 & 0 & 6 & 1 & 4 \\
\hline Sub-Saharan Africa & NA & NA & NA & NA & 23 & 12 & 28 & 16 & 27 & 17 & 25 & 14 & 22 & 11 & 22 & 11 & 25 & 14 & 22 \\
\hline Low- and middle-income countries & NA & NA & NA & NA & 19 & 10 & 25 & 9 & 25 & 8 & 15 & 5 & 12 & 5 & 10 & 4 & 19 & 6 & 15 \\
\hline High-income countries & NA & NA & NA & NA & 41 & 32 & 41 & 31 & 41 & 31 & 35 & 26 & 32 & 23 & 32 & 23 & 38 & 27 & 35 \\
\hline WORLD & NA & NA & NA & NA & 20 & 11 & 27 & 11 & 27 & 10 & 17 & 7 & 15 & 7 & 14 & 8 & 22 & 8 & 18 \\
\hline
\end{tabular}

\section{PAF of DALYs $(\%)$}

\begin{tabular}{|c|c|c|c|c|c|c|c|c|c|c|c|c|c|c|c|c|c|c|c|}
\hline & & & & & & & & & & & & & & & & & & & \\
\hline East Asia and Pacific & NA & NA & NA & NA & 30 & 11 & 32 & 11 & 32 & 11 & 29 & 8 & 27 & 4 & 27 & 4 & 31 & 9 & 24 \\
\hline Europe and Central Asia & NA & NA & NA & NA & 44 & 31 & 42 & 31 & 43 & 31 & 38 & 26 & 33 & 24 & 32 & 24 & 40 & 27 & 38 \\
\hline Latin America and the Caribbean & NA & NA & NA & NA & 38 & 29 & 39 & 31 & 39 & 29 & 35 & 26 & 25 & 20 & 25 & 20 & 36 & 25 & 33 \\
\hline Middle East and North Africa & NA & NA & NA & NA & 10 & 4 & 9 & 3 & 9 & 3 & 6 & 2 & 4 & 1 & 4 & 2 & 7 & 2 & 6 \\
\hline South Asia & NA & NA & NA & NA & 11 & 4 & 15 & 2 & 8 & 2 & 2 & 0 & 0 & 0 & 0 & 0 & 6 & 1 & 4 \\
\hline Sub-Saharan Africa & NA & NA & NA & NA & 23 & 12 & 28 & 16 & 27 & 17 & 25 & 14 & 22 & 11 & 22 & 11 & 25 & 14 & 22 \\
\hline Low- and middle-income countries & NA & NA & NA & NA & 19 & 10 & 25 & 9 & 25 & 8 & 15 & 5 & 12 & 5 & 10 & 4 & 19 & 6 & $\overline{15}$ \\
\hline High-income countries & NA & NA & NA & NA & 41 & 32 & 41 & 31 & 41 & 31 & 35 & 26 & 32 & 23 & 32 & 23 & 38 & 27 & 35 \\
\hline WORLD & NA & NA & NA & NA & 20 & 11 & 27 & 11 & 27 & 10 & 17 & 7 & 15 & 7 & 14 & 8 & 22 & 9 & 10 \\
\hline
\end{tabular}

Attributable Mortality (thousands)

\begin{tabular}{llllllllllllllllrrrrrrrrr} 
East Asia and Pacific & NA & NA & NA & NA & 0 & 0 & 2 & 0 & 5 & 1 & 4 & 0 & 2 & 0 & 1 & 0 & 13 & 2 & 15 \\
Europe and Central Asia & NA & NA & NA & NA & 0 & 0 & 1 & 0 & 4 & 0 & 3 & 0 & 1 & 0 & 0 & 0 & 9 & 1 & 10 \\
Latin America and the Caribbean & NA & NA & NA & NA & 0 & 0 & 0 & 0 & 1 & 0 & 1 & 0 & 1 & 0 & 0 & 0 & 3 & 1 & 4 \\
Middle East and North Africa & NA & NA & NA & NA & 0 & 0 & 0 & 0 & 0 & 0 & 0 & 0 & 0 & 0 & 0 & 0 & 0 & 0 & 0 \\
South Asia & NA & NA & NA & NA & 0 & 0 & 1 & 0 & 2 & 0 & 1 & 0 & 0 & 0 & 0 & 0 & 4 & 0 & 4 \\
Sub-Saharan Africa & NA & NA & NA & NA & 0 & 0 & 0 & 0 & 1 & 0 & 1 & 0 & 1 & 0 & 0 & 0 & 3 & 1 & 4 \\
\hline Low- and middle-income countries & NA & NA & NA & NA & 1 & 0 & 4 & 0 & 13 & 2 & 9 & 1 & 4 & 1 & 1 & 1 & 33 & 5 & 38 \\
High-income countries & NA & NA & NA & NA & 0 & 0 & 1 & 0 & 4 & 1 & 3 & 1 & 2 & 1 & 1 & 1 & 11 & 3 & 14 \\
\hline WORLD & NA & NA & NA & NA & 1 & 0 & 5 & 1 & 17 & 2 & 12 & 2 & 7 & 2 & 2 & 1 & 44 & 8 & 51 \\
\hline
\end{tabular}

Attributable YLL (thousands)

\begin{tabular}{lrrrrrrrrrrrrrrrrrrr} 
East Asia and Pacific & NA & NA & NA & NA & 9 & 1 & 42 & 4 & 97 & 14 & 48 & 6 & 19 & 2 & 3 & 1 & 219 & 28 & 247 \\
Europe and Central Asia & NA & NA & NA & NA & 2 & 1 & 14 & 2 & 75 & 7 & 37 & 4 & 10 & 3 & 1 & 1 & 140 & 18 & 158 \\
Latin America and the Caribbean & NA & NA & NA & NA & 1 & 1 & 7 & 2 & 25 & 4 & 14 & 3 & 5 & 2 & 1 & 1 & 53 & 13 & 65 \\
Middle East and North Africa & NA & NA & NA & NA & 0 & 0 & 1 & 0 & 1 & 0 & 1 & 0 & 0 & 0 & 0 & 0 & 4 & 1 & 4 \\
South Asia & NA & NA & NA & NA & 7 & 1 & 24 & 1 & 33 & 3 & 10 & 0 & 0 & 0 & 0 & 0 & 74 & 5 & 79 \\
Sub-Saharan Africa & NA & NA & NA & NA & 2 & 0 & 7 & 1 & 19 & 5 & 13 & 3 & 6 & 2 & 1 & 0 & 48 & 12 & 60 \\
\hline Low- and middle-income countries & NA & NA & NA & NA & 22 & 3 & 95 & 11 & 252 & 33 & 124 & 17 & 40 & 10 & 6 & 3 & 538 & 76 & 614 \\
High-income countries & NA & NA & NA & NA & 2 & 1 & 15 & 3 & 75 & 13 & 40 & 8 & 19 & 7 & 5 & 4 & 155 & 35 & 190 \\
\hline WORLD & NA & NA & NA & NA & 23 & 3 & 110 & 14 & 327 & 45 & 163 & 25 & 59 & 17 & 11 & 7 & 693 & 111 & 804 \\
\hline
\end{tabular}

Attributable DALYs (thousands)

\begin{tabular}{|c|c|c|c|c|c|c|c|c|c|c|c|c|c|c|c|c|c|c|c|}
\hline East Asia and Pacific & NA & NA & NA & NA & 10 & 1 & 43 & 4 & 100 & 14 & 50 & 6 & 19 & 2 & 3 & 1 & 225 & 29 & 253 \\
\hline Europe and Central Asia & NA & NA & NA & NA & 2 & 1 & 14 & 2 & 77 & 7 & 38 & 4 & 11 & 3 & 1 & 1 & 144 & 19 & 163 \\
\hline Latin America and the Caribbean & NA & NA & NA & NA & 1 & 1 & 7 & 2 & 26 & 4 & 14 & 3 & 5 & 2 & 1 & 1 & 54 & 13 & 67 \\
\hline Middle East and North Africa & NA & NA & NA & NA & 0 & 0 & 1 & 0 & 1 & 0 & 1 & 0 & 0 & 0 & 0 & 0 & 4 & 1 & 5 \\
\hline South Asia & NA & NA & NA & NA & 7 & 1 & 24 & 1 & 34 & 3 & 11 & 0 & 0 & 0 & 0 & 0 & 76 & 5 & 81 \\
\hline Sub-Saharan Africa & NA & NA & NA & NA & 2 & 0 & 7 & 2 & 20 & 5 & 13 & 3 & 6 & 2 & 1 & 0 & 49 & 13 & 61 \\
\hline Low- and middle-income countries & NA & NA & NA & NA & 22 & 3 & 96 & 11 & 258 & 34 & 127 & 17 & 41 & 10 & 7 & 3 & 552 & 79 & 631 \\
\hline High-income countries & NA & NA & NA & NA & 2 & 1 & 16 & 4 & 79 & 13 & 42 & 9 & 21 & 7 & 5 & 4 & 164 & 38 & 202 \\
\hline WORLD & NA & NA & NA & NA & 24 & 4 & 112 & 15 & 337 & 47 & 169 & 26 & 62 & 18 & 12 & 7 & 716 & 117 & 833 \\
\hline
\end{tabular}

Source: Authors' calculations.

Note: $\mathrm{NA}=$ not applicable. 
Table 4A.59

Risk factor:

Alcohol use

Disease:

Esophageal cancer

\begin{tabular}{|c|c|c|c|c|c|c|c|c|c|c|c|c|c|c|c|c|c|c|c|}
\hline \multirow[b]{2}{*}{ Region } & \multicolumn{2}{|c|}{$0-4$ years } & \multicolumn{2}{|c|}{ 5-14 years } & \multicolumn{2}{|c|}{$15-29$ years } & \multicolumn{2}{|c|}{ 30-44 years } & \multicolumn{2}{|c|}{ 45-59 years } & \multicolumn{2}{|c|}{$60-69$ years } & \multicolumn{2}{|c|}{$70-79$ years } & \multicolumn{2}{|c|}{$80+$ years } & \multicolumn{3}{|c|}{ Total } \\
\hline & Male & Female & Male & Female & Male & Female & Male & Female & Male & Female & Male & Female & Male & Female & Male & Female & Male & Female & All \\
\hline \multicolumn{20}{|l|}{ PAF of Mortality (\%) } \\
\hline East Asia and Pacific & NA & NA & NA & NA & 40 & 18 & 42 & 18 & 41 & 19 & 39 & 13 & 36 & 7 & 36 & 7 & 39 & 12 & 29 \\
\hline Europe and Central Asia & NA & NA & NA & NA & 49 & 41 & 48 & 40 & 49 & 40 & 45 & 36 & 41 & 34 & 41 & 34 & 45 & 35 & 42 \\
\hline Latin America and the Caribbean & NA & NA & NA & NA & 44 & 36 & 45 & 37 & 46 & 36 & 43 & 33 & 35 & 29 & 35 & 29 & 41 & 31 & 38 \\
\hline Middle East and North Africa & NA & NA & NA & NA & 15 & 6 & 14 & 5 & 13 & 4 & 10 & 3 & 5 & 2 & 6 & 3 & 9 & 3 & 7 \\
\hline South Asia & NA & NA & NA & NA & 18 & 6 & 23 & 3 & 14 & 3 & 4 & 0 & 0 & 0 & 0 & 0 & 6 & 1 & 4 \\
\hline Sub-Saharan Africa & NA & NA & NA & NA & 33 & 19 & 37 & 22 & 34 & 23 & 32 & 19 & 29 & 16 & 29 & 16 & 32 & 19 & 27 \\
\hline Low- and middle-income countries & NA & NA & NA & NA & 32 & 12 & 37 & 14 & 37 & 16 & 31 & 11 & 30 & 8 & 27 & 8 & 32 & 11 & 24 \\
\hline High-income countries & NA & NA & NA & NA & 48 & 41 & 48 & 41 & 47 & 40 & 43 & 36 & 41 & 33 & 41 & 33 & 43 & 34 & 41 \\
\hline WORLD & NA & NA & NA & NA & 33 & 12 & 38 & 14 & 38 & 17 & 33 & 13 & 32 & 10 & 31 & 12 & 34 & 13 & 26 \\
\hline \multicolumn{20}{|l|}{ PAF of YLL (\%) } \\
\hline East Asia and Pacific & NA & NA & NA & NA & 40 & 18 & 42 & 18 & 41 & 19 & 39 & 13 & 36 & 7 & 36 & 7 & 39 & 13 & 30 \\
\hline Europe and Central Asia & NA & NA & NA & NA & 49 & 41 & 48 & 40 & 49 & 40 & 45 & 36 & 41 & 34 & 41 & 34 & 46 & 36 & 44 \\
\hline Latin America and the Caribbean & NA & NA & NA & NA & 44 & 36 & 45 & 37 & 46 & 36 & 43 & 33 & 35 & 29 & 35 & 29 & 43 & 33 & 40 \\
\hline Middle East and North Africa & NA & NA & NA & NA & 15 & 6 & 14 & 5 & 13 & 4 & 10 & 3 & 5 & 2 & 6 & 3 & 10 & 3 & 7 \\
\hline South Asia & NA & NA & NA & NA & 18 & 6 & 23 & 3 & 14 & 3 & 4 & 0 & 0 & 0 & 0 & 0 & 8 & 1 & 5 \\
\hline Sub-Saharan Africa & NA & NA & NA & NA & 33 & 19 & 37 & 22 & 34 & 23 & 32 & 19 & 29 & 16 & 29 & 16 & 33 & 20 & 28 \\
\hline Low- and middle-income countries & NA & NA & NA & NA & 32 & 12 & 37 & 14 & 37 & 16 & 31 & 11 & 30 & 8 & 28 & 8 & 33 & 12 & 25 \\
\hline High-income countries & NA & NA & NA & NA & 48 & 41 & 48 & 41 & 47 & 40 & 43 & 36 & 41 & 33 & 41 & 33 & 44 & 36 & 43 \\
\hline WORLD & NA & NA & NA & NA & 33 & 12 & 38 & 14 & 38 & 17 & 33 & 13 & 32 & 10 & 31 & 12 & 35 & 13 & 27 \\
\hline
\end{tabular}

PAF of DALYs (\%)

\begin{tabular}{|c|c|c|c|c|c|c|c|c|c|c|c|c|c|c|c|c|c|c|c|}
\hline East Asia and Pacific & NA & NA & NA & NA & 40 & 18 & 42 & 18 & 41 & 19 & 39 & 13 & 36 & 7 & 36 & 7 & 39 & 13 & 30 \\
\hline Europe and Central Asia & NA & NA & NA & NA & 49 & 41 & 48 & 40 & 49 & 40 & 45 & 36 & 41 & 34 & 41 & 34 & 46 & 36 & 44 \\
\hline Latin America and the Caribbean & NA & NA & NA & NA & 44 & 36 & 45 & 37 & 46 & 36 & 43 & 33 & 35 & 29 & 35 & 29 & 43 & 33 & 40 \\
\hline Middle East and North Africa & NA & NA & NA & NA & 15 & 6 & 14 & 5 & 13 & 4 & 10 & 3 & 5 & 2 & 6 & 3 & 10 & 3 & 7 \\
\hline South Asia & NA & NA & NA & NA & 18 & 6 & 23 & 3 & 14 & 3 & 4 & 0 & 0 & 0 & 0 & 0 & 8 & 1 & 5 \\
\hline Sub-Saharan Africa & NA & NA & NA & NA & 33 & 19 & 37 & 22 & 34 & 23 & 32 & 19 & 29 & 16 & 29 & 16 & 33 & 20 & 28 \\
\hline Low- and middle-income countries & NA & NA & NA & NA & 32 & 12 & 37 & 14 & 37 & 16 & 31 & 11 & 30 & 8 & 28 & 8 & 33 & 12 & 25 \\
\hline High-income countries & NA & NA & NA & NA & 48 & 41 & 48 & 41 & 47 & 40 & 43 & 36 & 41 & 33 & 41 & 33 & 44 & 36 & 43 \\
\hline WORLD & NA & NA & NA & NA & 33 & 12 & 38 & 14 & 38 & 17 & 33 & 13 & 32 & 10 & 31 & 12 & 35 & 13 & \\
\hline
\end{tabular}

Attributable Mortality (thousands

East Asia an

Europe and Central As

Latin America and the Caribbean

Middle East and North Africa

South Asia

Sub-Saharan Africa

Low- and middle-income countries

High-income countries

NA NA NA NA

$\begin{array}{llll}\text { NA } & \text { NA } & \text { NA } & \text { NA } \\ \text { NA } & \text { NA } & \text { NA } & \text { NA } \\ \text { NA } & \text { NA } & \text { NA } & \text { NA }\end{array}$

NA NA NA NA

NA NA NA NA

NA NA NA NA

WORLD

NA NA NA NA

Attributable YLL (thousands)

East Asia and Pacific

Europe and Central Asia

Latin America and the Caribbea

Middle East and North Africa

South Asia

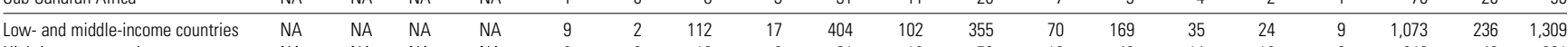

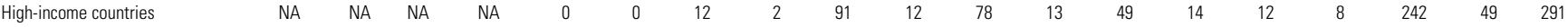

\begin{tabular}{|c|c|c|c|c|c|c|c|c|c|c|c|c|c|c|c|c|c|}
\hline WORLD & NA & NA & NA & NA & 10 & o & 124 & 19 & 495 & 115 & 433 & 83 & 218 & 49 & 36 & 17 & 1,315 \\
\hline
\end{tabular}

Attributable DALYs (thousands)

East Asia and Pacific

Europe and Central Asia

Latin America and the Caribbean

Middle East and North Africa

South Asia

Sub-Saharan Africa

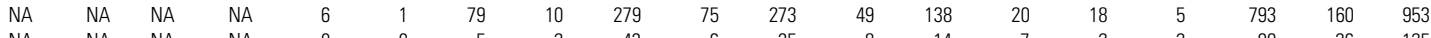

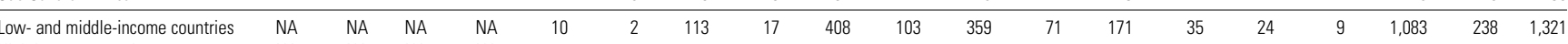

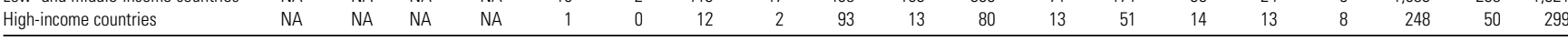

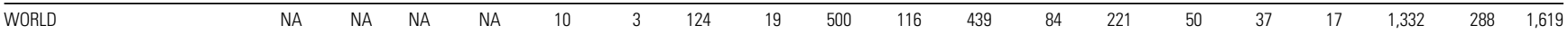

Source: Authors' calculations.

Note: $\mathrm{NA}=$ not applicable. 
Table 4A.60

Risk factor:

Alcohol use

Disease:

Liver cancer

\begin{tabular}{|c|c|c|c|c|c|c|c|c|c|c|c|c|c|c|c|c|c|c|c|}
\hline \multirow[b]{2}{*}{ Region } & \multicolumn{2}{|c|}{$0-4$ years } & \multicolumn{2}{|c|}{ 5-14 years } & \multicolumn{2}{|c|}{$15-29$ years } & \multicolumn{2}{|c|}{ 30-44 years } & \multicolumn{2}{|c|}{$45-59$ years } & \multicolumn{2}{|c|}{$60-69$ years } & \multicolumn{2}{|c|}{ 70-79 years } & \multicolumn{2}{|c|}{$80+$ years } & \multicolumn{3}{|c|}{ Total } \\
\hline & Male & Female & Male & Female & Male & Female & Male & Female & Male & Female & Male & Female & Male & Female & Male & Female & Male & Female & All \\
\hline \multicolumn{20}{|l|}{ PAF of Mortality (\%) } \\
\hline East Asia and Pacific & NA & NA & NA & NA & 31 & 12 & 33 & 11 & 32 & 12 & 30 & 8 & 27 & 4 & 27 & 4 & 30 & 8 & 24 \\
\hline Europe and Central Asia & NA & NA & NA & NA & 43 & 34 & 41 & 33 & 43 & 34 & 38 & 29 & 32 & 26 & 32 & 26 & 37 & 28 & 34 \\
\hline Latin America and the Caribbean & NA & NA & NA & NA & 35 & 27 & 36 & 28 & 36 & 27 & 33 & 25 & 25 & 21 & 26 & 21 & 31 & 23 & 27 \\
\hline Middle East and North Africa & NA & NA & NA & NA & 10 & 4 & 10 & 3 & 9 & 3 & 7 & 2 & 4 & 1 & 4 & 2 & 7 & 2 & 5 \\
\hline South Asia & NA & NA & NA & NA & 12 & 4 & 16 & 2 & 8 & 2 & 2 & 0 & 0 & 0 & 0 & 0 & 6 & 1 & 4 \\
\hline Sub-Saharan Africa & NA & NA & NA & NA & 28 & 14 & 33 & 18 & 31 & 18 & 28 & 15 & 24 & 12 & 24 & 12 & 29 & 15 & 24 \\
\hline Low- and middle-income countries & NA & NA & NA & NA & 28 & 10 & 32 & 13 & 31 & 13 & 29 & 11 & 26 & 8 & 25 & 8 & 29 & 10 & 23 \\
\hline High-income countries & NA & NA & NA & NA & 41 & 34 & 41 & 32 & 40 & 32 & 35 & 28 & 32 & 25 & 32 & 25 & 35 & 26 & 32 \\
\hline WORLD & NA & NA & NA & NA & 28 & 11 & 32 & 13 & 32 & 15 & 30 & 13 & 27 & 12 & 27 & 15 & 30 & 13 & 25 \\
\hline
\end{tabular}

PAF of YLL $(\%)$

\begin{tabular}{|c|c|c|c|c|c|c|c|c|c|c|c|c|c|c|c|c|c|c|c|}
\hline East Asia and Pacific & NA & NA & NA & NA & 31 & 12 & 33 & 11 & 32 & 12 & 30 & 8 & 27 & 4 & 27 & 4 & 31 & 9 & 25 \\
\hline Europe and Central Asia & NA & NA & NA & NA & 43 & 34 & 41 & 33 & 43 & 34 & 38 & 29 & 32 & 26 & 32 & 26 & 39 & 29 & 35 \\
\hline Latin America and the Caribbean & NA & NA & NA & NA & 35 & 27 & 36 & 28 & 36 & 27 & 33 & 25 & 25 & 21 & 26 & 21 & 32 & 24 & 28 \\
\hline Middle East and North Africa & NA & NA & NA & NA & 10 & 4 & 10 & 3 & 9 & 3 & 7 & 2 & 4 & 1 & 4 & 2 & 7 & 3 & 5 \\
\hline South Asia & NA & NA & NA & NA & 12 & 4 & 16 & 2 & 8 & 2 & 2 & 0 & 0 & 0 & 0 & 0 & 7 & 2 & 5 \\
\hline Sub-Saharan Africa & NA & NA & NA & NA & 28 & 14 & 33 & 18 & 31 & 18 & 28 & 15 & 24 & 12 & 24 & 12 & 29 & 15 & 25 \\
\hline Low- and middle-income countries & NA & NA & NA & NA & 28 & 10 & 32 & 13 & 31 & 13 & 29 & 11 & 26 & 8 & 25 & 8 & 30 & 11 & 24 \\
\hline High-income countries & NA & NA & NA & NA & 41 & 34 & 41 & 32 & 40 & 32 & 35 & 28 & 32 & 25 & 32 & 25 & 36 & 27 & 33 \\
\hline WORLD & NA & NA & NA & NA & 28 & 11 & 32 & 13 & 32 & 15 & 30 & 13 & 27 & 12 & 27 & 14 & 30 & 13 & 25 \\
\hline
\end{tabular}

\section{PAF of DALYs (\%)}

East Asia and Pacific

Europe and Central Asia

Latin America and the Caribbe

Middle East and North Africa

South Asia

Sub-Saharan Africa

Low- and middle-income countries

High-income countries

$\begin{array}{llll}\text { NA } & \text { NA } & \text { NA } & \text { NA } \\ \text { NA } & \text { NA } & \text { NA } & \text { NA } \\ \text { NA } & \text { NA } & \text { NA } & \text { NA } \\ \text { NA } & \text { NA } & \text { NA } & \text { NA } \\ \text { NA } & \text { NA } & \text { NA } & \text { NA } \\ \text { NA } & \text { NA } & \text { NA } & \text { NA } \\ \text { NA } & \text { NA } & \text { NA } & \text { NA } \\ \text { NA } & \text { NA } & \text { NA } & \text { NA }\end{array}$

$\begin{array}{ll}31 & 12 \\ 43 & 34 \\ 35 & 27 \\ 10 & \\ 12 & 4 \\ 28 & 14 \\ 28 & 10 \\ 41 & 34\end{array}$

$\begin{array}{rrrrrrr}12 & 33 & 11 & 32 & 12 & 30 & 8 \\ 34 & 41 & 33 & 43 & 34 & 38 & 29 \\ 27 & 36 & 28 & 36 & 27 & 33 & 25 \\ 4 & 10 & 3 & 9 & 3 & 7 & 2 \\ 4 & 16 & 2 & 8 & 2 & 2 & 0 \\ 14 & 33 & 18 & 31 & 18 & 28 & 15 \\ 10 & 32 & 13 & 31 & 13 & 29 & 11 \\ 34 & 41 & 32 & 40 & 32 & 35 & 28\end{array}$

\begin{tabular}{rrrrrrr}
27 & 4 & 27 & 4 & 31 & 9 & 25 \\
32 & 26 & 32 & 26 & 38 & 29 & 35 \\
25 & 21 & 26 & 21 & 32 & 24 & 28 \\
4 & 1 & 4 & 2 & 7 & 3 & 5 \\
0 & 0 & 0 & 0 & 7 & 2 & 5 \\
24 & 12 & 24 & 12 & 29 & 15 & 25 \\
26 & 8 & 25 & 8 & 30 & 11 & 24 \\
32 & 25 & 32 & 25 & 36 & 27 & 33 \\
27 & 12 & 27 & 14 & 30 & 13 & 25 \\
\hline
\end{tabular}

Attributable Mortality (thousands

\begin{tabular}{|c|c|c|c|c|c|c|c|c|c|c|c|c|c|c|c|c|c|c|c|}
\hline \multicolumn{20}{|l|}{ Attributable Mortality (thousands) } \\
\hline East Asia and Pacific & NA & NA & NA & NA & 2 & 0 & 9 & 1 & 32 & 3 & 22 & 2 & 14 & 1 & 3 & 0 & 82 & 8 & 90 \\
\hline Europe and Central Asia & NA & NA & NA & NA & 0 & 0 & 0 & 0 & 2 & 1 & 2 & 1 & 2 & 1 & 0 & 0 & 6 & 3 & 9 \\
\hline Latin America and the Caribbean & NA & NA & NA & NA & 0 & 0 & 0 & 0 & 1 & 1 & 1 & 1 & 1 & 1 & 0 & 0 & 3 & 2 & 6 \\
\hline Middle East and North Africa & NA & NA & NA & NA & 0 & 0 & 0 & 0 & 0 & 0 & 0 & 0 & 0 & 0 & 0 & 0 & 0 & 0 & 0 \\
\hline South Asia & NA & NA & NA & NA & 0 & 0 & 0 & 0 & 0 & 0 & 0 & 0 & 0 & 0 & 0 & 0 & 1 & 0 & 1 \\
\hline Sub-Saharan Africa & NA & NA & NA & NA & 0 & 0 & 1 & 0 & 3 & 1 & 2 & 0 & 1 & 0 & 0 & 0 & 9 & 2 & 11 \\
\hline Low- and middle-income countries & NA & NA & NA & NA & 2 & 1 & 12 & 2 & 38 & 5 & 28 & 4 & 18 & 3 & 4 & 1 & 102 & 16 & 117 \\
\hline High-income countries & NA & NA & NA & NA & 0 & 0 & 1 & 0 & 6 & 1 & 8 & 2 & 7 & 3 & 3 & 3 & 24 & 9 & 33 \\
\hline WORLD & NA & NA & NA & NA & 2 & 1 & 13 & 2 & 44 & 6 & 35 & 6 & 25 & 6 & 7 & 4 & 126 & 25 & 150 \\
\hline
\end{tabular}

Attributable YLL (thousands)

\begin{tabular}{|c|c|c|c|c|c|c|c|c|c|c|c|c|c|c|c|c|c|c|c|}
\hline East Asia and Pacific & NA & NA & NA & NA & 44 & 8 & 223 & 29 & 607 & 59 & 299 & 33 & 128 & 11 & 15 & 2 & 1,317 & 142 & 1,460 \\
\hline Europe and Central Asia & NA & NA & NA & NA & 2 & 1 & 8 & 3 & 32 & 12 & 30 & 14 & 14 & 11 & 2 & 2 & 88 & 43 & 131 \\
\hline Latin America and the Caribbean & NA & NA & NA & NA & 2 & 1 & 5 & 4 & 16 & 11 & 13 & 9 & 7 & 6 & 2 & 2 & 44 & 33 & 77 \\
\hline Middle East and North Africa & NA & NA & NA & NA & 0 & 0 & 1 & 0 & 2 & 1 & 2 & 0 & 1 & 0 & 0 & 0 & 6 & 1 & 7 \\
\hline South Asia & NA & NA & NA & NA & 3 & 2 & 8 & 1 & 7 & 1 & 1 & 0 & 0 & 0 & 0 & 0 & 20 & 3 & 24 \\
\hline Sub-Saharan Africa & NA & NA & NA & NA & 8 & 3 & 35 & 9 & 62 & 14 & 31 & 7 & 11 & 4 & 2 & 1 & 150 & 38 & 188 \\
\hline Low- and middle-income countries & NA & NA & NA & NA & 59 & 15 & 281 & 45 & 726 & 97 & 376 & 63 & 161 & 34 & 21 & 7 & 1,625 & 262 & 1,887 \\
\hline High-income countries & NA & NA & NA & NA & 2 & 1 & 20 & 5 & 104 & 21 & 101 & 28 & 63 & 31 & 14 & 12 & 305 & 97 & 402 \\
\hline WORLD & NA & NA & NA & NA & 62 & 16 & 301 & 50 & 831 & 118 & 477 & 91 & 225 & 64 & 35 & 19 & 1,930 & 359 & 2,289 \\
\hline
\end{tabular}

Attributable DALYs (thousands)

\begin{tabular}{|c|c|c|c|c|c|c|c|c|c|c|c|c|c|c|c|c|c|c|c|}
\hline East Asia and Pacific & NA & NA & NA & NA & 45 & 8 & 224 & 29 & 610 & 60 & 301 & 33 & 130 & 11 & 16 & 2 & 1,326 & 143 & 1,469 \\
\hline Europe and Central Asia & NA & NA & NA & NA & 2 & 1 & 8 & 3 & 32 & 12 & 30 & 14 & 14 & 11 & 2 & 2 & 88 & 43 & 132 \\
\hline Latin America and the Caribbean & NA & NA & NA & NA & 2 & 1 & 5 & 4 & 16 & 11 & 13 & 9 & 7 & 6 & 2 & 2 & 44 & 33 & 78 \\
\hline Middle East and North Africa & NA & NA & NA & NA & 0 & 0 & 1 & 0 & 2 & 1 & 2 & 0 & 1 & 0 & 0 & 0 & 6 & 1 & 7 \\
\hline South Asia & NA & NA & NA & NA & 3 & 2 & 8 & 1 & 7 & 1 & 1 & 0 & 0 & 0 & 0 & 0 & 20 & 3 & 24 \\
\hline Sub-Saharan Africa & NA & NA & NA & NA & 8 & 3 & 35 & 9 & 62 & 14 & 32 & 7 & 12 & 5 & 2 & 1 & 150 & 38 & 189 \\
\hline Low- and middle-income countries & NA & NA & NA & NA & 60 & 15 & 282 & 46 & 730 & 98 & 379 & 63 & 163 & 34 & 21 & 8 & 1,635 & 264 & 1,898 \\
\hline High-income countries & NA & NA & NA & NA & 2 & 1 & 20 & 5 & 106 & 21 & 102 & 29 & 65 & 31 & 15 & 12 & 310 & 99 & 409 \\
\hline WORLD & NA & NA & NA & NA & 62 & 16 & 302 & 51 & 836 & 119 & 482 & 92 & 227 & 65 & 36 & 20 & 1,945 & 362 & 2,307 \\
\hline
\end{tabular}

Source: Authors' calculations.

Note: $\mathrm{NA}=$ not applicable. 
Table 4A.61

Risk factor:

Alcohol use

Disease:

Breast cancer

\begin{tabular}{|c|c|c|c|c|c|c|c|c|c|c|c|c|c|c|c|c|c|c|c|}
\hline \multirow[b]{2}{*}{ Region } & \multicolumn{2}{|c|}{ 0-4 years } & \multicolumn{2}{|c|}{ 5-14 years } & \multicolumn{2}{|c|}{ 15-29 years } & \multicolumn{2}{|c|}{ 30-44 years } & \multicolumn{2}{|c|}{$45-59$ years } & \multicolumn{2}{|c|}{$60-69$ years } & \multicolumn{2}{|c|}{ 70-79 years } & \multicolumn{2}{|c|}{$80+$ years } & \multicolumn{3}{|c|}{ Total } \\
\hline & Male & Female & Male & Female & Male & Female & Male & Female & Male & Female & Male & Female & Male & Female & Male & Female & Male & Female & All \\
\hline \multicolumn{20}{|l|}{ PAF of Mortality (\%) } \\
\hline East Asia and Pacific & NA & NA & NA & NA & NA & 0 & NA & 0 & NA & 4 & NA & 3 & NA & 1 & NA & 1 & NA & 3 & 3 \\
\hline Europe and Central Asia & NA & NA & NA & NA & NA & 0 & NA & 0 & NA & 12 & NA & 10 & NA & 9 & NA & 9 & NA & 9 & 9 \\
\hline Latin America and the Caribbean & NA & NA & NA & NA & NA & 0 & NA & 0 & NA & 9 & NA & 8 & NA & 7 & NA & 7 & NA & 7 & 7 \\
\hline Middle East and North Africa & NA & NA & NA & NA & NA & 0 & NA & 0 & NA & 1 & NA & 0 & NA & 0 & NA & 1 & NA & 1 & 1 \\
\hline South Asia & NA & NA & NA & NA & NA & 0 & NA & 0 & NA & 1 & NA & 0 & NA & 0 & NA & 0 & NA & 0 & 0 \\
\hline Sub-Saharan Africa & NA & NA & NA & NA & NA & 0 & NA & 0 & NA & 6 & NA & 4 & NA & 3 & NA & 3 & NA & 4 & 4 \\
\hline Low- and middle-income countries & NA & NA & NA & NA & NA & 0 & NA & 0 & NA & 5 & NA & 4 & NA & 4 & NA & 4 & NA & 4 & 4 \\
\hline High-income countries & NA & NA & NA & NA & NA & 0 & NA & 0 & NA & 12 & NA & 10 & NA & 8 & NA & 8 & NA & 9 & 9 \\
\hline WORLD & NA & NA & NA & NA & NA & 0 & NA & 0 & NA & 7 & NA & 6 & NA & 6 & NA & 6 & NA & 5 & 5 \\
\hline
\end{tabular}

PAF of YLL $(\%)$

\begin{tabular}{|c|c|c|c|c|c|c|c|c|c|c|c|c|c|c|c|c|c|c|c|}
\hline East Asia and Pacific & NA & NA & NA & NA & NA & 0 & NA & 0 & NA & 4 & NA & 3 & NA & 1 & NA & 1 & NA & 3 & 3 \\
\hline Europe and Central Asia & NA & NA & NA & NA & NA & 0 & NA & 0 & NA & 12 & NA & 10 & NA & 9 & NA & 9 & NA & 9 & 9 \\
\hline Latin America and the Caribbean & NA & NA & NA & NA & NA & 0 & NA & 0 & NA & 9 & NA & 8 & NA & 7 & NA & 7 & NA & 7 & 7 \\
\hline Middle East and North Africa & NA & NA & NA & NA & NA & 0 & NA & 0 & NA & 1 & NA & 0 & NA & 0 & NA & 1 & NA & 1 & 1 \\
\hline South Asia & NA & NA & NA & NA & NA & 0 & NA & 0 & NA & 1 & NA & 0 & NA & 0 & NA & 0 & NA & 0 & 0 \\
\hline Sub-Saharan Africa & NA & NA & NA & NA & NA & 0 & NA & 0 & NA & 6 & NA & 4 & NA & 3 & NA & 3 & NA & 4 & 4 \\
\hline Low- and middle-income countries & NA & NA & NA & NA & NA & 0 & NA & 0 & NA & 5 & NA & 4 & NA & 4 & NA & 3 & NA & 4 & 4 \\
\hline High-income countries & NA & NA & NA & NA & NA & 0 & NA & 0 & NA & 12 & NA & 10 & NA & 8 & NA & 8 & NA & 9 & 9 \\
\hline WORLD & NA & NA & NA & NA & NA & 0 & NA & 0 & NA & 7 & NA & 6 & NA & 6 & NA & 6 & NA & 5 & 5 \\
\hline
\end{tabular}

PAF of DALYs $(\%)$

\begin{tabular}{|c|c|c|c|c|c|c|c|c|c|c|c|c|c|c|c|c|c|c|c|}
\hline East Asia and Pacific & NA & NA & NA & NA & NA & 0 & NA & 0 & NA & 4 & NA & 3 & NA & 1 & NA & 1 & NA & 3 & 3 \\
\hline Europe and Central Asia & NA & NA & NA & NA & NA & 0 & NA & 0 & NA & 12 & NA & 10 & NA & 9 & NA & 9 & NA & 9 & 9 \\
\hline Latin America and the Caribbean & NA & NA & NA & NA & NA & 0 & NA & 0 & NA & 9 & NA & 8 & NA & 7 & NA & 7 & NA & 6 & 6 \\
\hline Middle East and North Africa & NA & NA & NA & NA & NA & 0 & NA & 0 & NA & 1 & NA & 0 & NA & 0 & NA & 1 & NA & 0 & 0 \\
\hline South Asia & NA & NA & NA & NA & NA & 0 & NA & 0 & NA & 1 & NA & 0 & NA & 0 & NA & 0 & NA & 0 & 0 \\
\hline Sub-Saharan Africa & NA & NA & NA & NA & NA & 0 & NA & 0 & NA & 6 & NA & 4 & NA & 3 & NA & 3 & NA & 4 & 4 \\
\hline Low- and middle-income countries & NA & NA & NA & NA & NA & 0 & NA & 0 & NA & 5 & NA & 4 & NA & 4 & NA & 3 & NA & 4 & 4 \\
\hline High-income countries & NA & NA & NA & NA & NA & 0 & NA & 0 & NA & 12 & NA & 10 & NA & 8 & NA & 8 & NA & 9 & 9 \\
\hline WORLD & NA & NA & NA & NA & NA & 0 & NA & 0 & NA & 7 & NA & 6 & NA & 6 & NA & 6 & NA & 5 & 5 \\
\hline \multicolumn{20}{|l|}{ Attributable Mortality (thousands) } \\
\hline East Asia and Pacific & NA & NA & NA & NA & NA & 0 & NA & 0 & NA & 2 & NA & 1 & NA & 0 & NA & 0 & NA & 2 & 2 \\
\hline Europe and Central Asia & NA & NA & NA & NA & NA & 0 & NA & 0 & NA & 2 & NA & 2 & NA & 1 & NA & 1 & NA & 6 & 6 \\
\hline Latin America and the Caribbean & NA & NA & NA & NA & NA & 0 & NA & 0 & NA & 1 & NA & 1 & NA & 0 & NA & 0 & NA & 3 & 3 \\
\hline Middle East and North Africa & NA & NA & NA & NA & NA & 0 & NA & 0 & NA & 0 & NA & 0 & NA & 0 & NA & 0 & NA & 0 & 0 \\
\hline South Asia & NA & NA & NA & NA & NA & 0 & NA & 0 & NA & 0 & NA & 0 & NA & 0 & NA & 0 & NA & 0 & 0 \\
\hline Sub-Saharan Africa & NA & NA & NA & NA & NA & 0 & NA & 0 & NA & 1 & NA & 0 & NA & 0 & NA & 0 & NA & 1 & 1 \\
\hline Low- and middle-income countries & NA & NA & NA & NA & NA & 0 & NA & 0 & NA & 6 & NA & 3 & NA & 2 & NA & 1 & NA & 12 & 12 \\
\hline High-income countries & NA & NA & NA & NA & NA & 0 & NA & 0 & NA & 4 & NA & 3 & NA & 3 & NA & 3 & NA & 14 & 14 \\
\hline WORLD & NA & NA & NA & NA & NA & 0 & NA & 0 & NA & 10 & NA & 6 & NA & 5 & NA & 4 & NA & 26 & 26 \\
\hline
\end{tabular}

Attributable YLL (thousands)

\begin{tabular}{|c|c|c|c|c|c|c|c|c|c|c|c|c|c|c|c|c|c|c|c|}
\hline East Asia and Pacific & NA & NA & NA & NA & NA & 0 & NA & 0 & NA & 31 & NA & 8 & NA & 2 & NA & 0 & NA & 42 & 42 \\
\hline Europe and Central Asia & NA & NA & NA & NA & NA & 0 & NA & 0 & NA & 46 & NA & 25 & NA & 14 & NA & 3 & NA & 87 & 87 \\
\hline Latin America and the Caribbean & NA & NA & NA & NA & NA & 0 & NA & 0 & NA & 23 & NA & 10 & NA & 5 & NA & 2 & NA & 39 & 39 \\
\hline Middle East and North Africa & NA & NA & NA & NA & NA & 0 & NA & 0 & NA & 1 & NA & 0 & NA & 0 & NA & 0 & NA & 1 & 1 \\
\hline South Asia & NA & NA & NA & NA & NA & 0 & NA & 0 & NA & 3 & NA & 0 & NA & 0 & NA & 0 & NA & 3 & 3 \\
\hline Sub-Saharan Africa & NA & NA & NA & NA & NA & 0 & NA & 0 & NA & 12 & NA & 6 & NA & 3 & NA & 1 & NA & 21 & 21 \\
\hline Low- and middle-income countries & NA & NA & NA & NA & NA & 0 & NA & 0 & NA & 116 & NA & 49 & NA & 23 & NA & 6 & NA & 193 & 193 \\
\hline High-income countries & NA & NA & NA & NA & NA & 0 & NA & 0 & NA & 87 & NA & 46 & NA & 32 & NA & 14 & NA & 179 & 179 \\
\hline WORLD & NA & NA & NA & NA & NA & 0 & NA & 0 & NA & 203 & NA & 95 & NA & 55 & NA & 20 & NA & 373 & 373 \\
\hline
\end{tabular}

Attributable DALYs (thousands)

\begin{tabular}{|c|c|c|c|c|c|c|c|c|c|c|c|c|c|c|c|c|c|c|c|}
\hline East Asia and Pacific & NA & NA & NA & NA & NA & 0 & NA & 0 & NA & 33 & NA & 8 & NA & 2 & NA & 1 & NA & 44 & 44 \\
\hline Europe and Central Asia & NA & NA & NA & NA & NA & 0 & NA & 0 & NA & 49 & NA & 27 & NA & 15 & NA & 3 & NA & 93 & 93 \\
\hline Latin America and the Caribbean & NA & NA & NA & NA & NA & 0 & NA & 0 & NA & 24 & NA & 10 & NA & 5 & NA & 2 & NA & 41 & 41 \\
\hline Middle East and North Africa & NA & NA & NA & NA & NA & 0 & NA & 0 & NA & 1 & NA & 0 & NA & 0 & NA & 0 & NA & 1 & 1 \\
\hline South Asia & NA & NA & NA & NA & NA & 0 & NA & 0 & NA & 3 & NA & 0 & NA & 0 & NA & 0 & NA & 3 & 3 \\
\hline Sub-Saharan Africa & NA & NA & NA & NA & NA & 0 & NA & 0 & NA & 13 & NA & 6 & NA & 3 & NA & 1 & NA & 22 & 22 \\
\hline Low- and middle-income countries & NA & NA & NA & NA & NA & 0 & NA & 0 & NA & 123 & NA & 52 & NA & 24 & NA & 6 & NA & 205 & 205 \\
\hline High-income countries & NA & NA & NA & NA & NA & 0 & NA & 0 & NA & 107 & NA & 53 & NA & 38 & NA & 17 & NA & 215 & 215 \\
\hline WORLD & NA & NA & NA & NA & NA & 0 & NA & 0 & NA & 230 & NA & 105 & NA & 62 & NA & 23 & NA & 420 & 420 \\
\hline
\end{tabular}


Risk factor:

Alcohol use

Selected other neoplasms

\begin{tabular}{|c|c|c|c|c|c|c|c|c|c|c|c|c|c|c|c|c|c|c|c|}
\hline \multirow[b]{2}{*}{ Region } & \multicolumn{2}{|c|}{$0-4$ years } & \multicolumn{2}{|c|}{ 5-14 years } & \multicolumn{2}{|c|}{$15-29$ years } & \multicolumn{2}{|c|}{ 30-44 years } & \multicolumn{2}{|c|}{$45-59$ years } & \multicolumn{2}{|c|}{$60-69$ years } & \multicolumn{2}{|c|}{ 70-79 years } & \multicolumn{2}{|c|}{$80+$ years } & \multicolumn{3}{|c|}{ Total } \\
\hline & Male & Female & Male & Female & Male & Female & Male & Female & Male & Female & Male & Female & Male & Female & Male & Female & Male & Female & All \\
\hline \multicolumn{20}{|l|}{ PAF of Mortality (\%) } \\
\hline East Asia and Pacific & NA & NA & NA & NA & 9 & 3 & 9 & 3 & 9 & 3 & 8 & 2 & 7 & 1 & 7 & 1 & 8 & 2 & 4 \\
\hline Europe and Central Asia & NA & NA & NA & NA & 14 & 9 & 13 & 9 & 14 & 9 & 12 & 8 & 9 & 7 & 9 & 7 & 11 & 8 & 9 \\
\hline Latin America and the Caribbean & NA & NA & NA & NA & 11 & 8 & 11 & 8 & 11 & 8 & 10 & 7 & 7 & 5 & 7 & 5 & 8 & 6 & 7 \\
\hline Middle East and North Africa & NA & NA & NA & NA & 2 & 1 & 2 & 1 & 2 & 1 & 2 & 0 & 1 & 0 & 1 & 0 & 1 & 0 & 1 \\
\hline South Asia & NA & NA & NA & NA & 3 & 1 & 4 & 0 & 2 & 0 & 0 & 0 & 0 & 0 & 0 & 0 & 2 & 0 & 1 \\
\hline Sub-Saharan Africa & NA & NA & NA & NA & 7 & 3 & 9 & 4 & 8 & 4 & 7 & 3 & 6 & 3 & 6 & 3 & 6 & 3 & 4 \\
\hline Low- and middle-income countries & NA & NA & NA & NA & 5 & 3 & 6 & 3 & 7 & 3 & 6 & 3 & 4 & 2 & 4 & 2 & 5 & 3 & 4 \\
\hline High-income countries & NA & NA & NA & NA & 13 & 9 & 13 & 9 & 13 & 9 & 10 & 7 & 9 & 7 & 9 & 6 & 10 & 7 & 8 \\
\hline WORLD & NA & NA & NA & NA & 6 & 4 & 7 & 4 & 8 & 4 & 7 & 4 & 7 & 4 & 8 & 5 & 7 & 4 & 6 \\
\hline
\end{tabular}

PAF of YLL (\%)

\begin{tabular}{lllllrrrrrrrrrrrrrrr} 
East Asia and Pacific & NA & NA & NA & NA & 9 & 3 & 9 & 3 & 9 & 3 & 8 & 2 & 7 & 1 & 7 & 1 & 7 & 2 & 4 \\
Europe and Central Asia & NA & NA & NA & NA & 14 & 9 & 13 & 9 & 14 & 9 & 12 & 8 & 9 & 7 & 9 & 7 & 11 & 8 & 10 \\
Latin America and the Caribbean & NA & NA & NA & NA & 11 & 8 & 11 & 8 & 11 & 8 & 10 & 7 & 7 & 5 & 7 & 5 & 8 & 6 & 7 \\
Middle East and North Africa & NA & NA & NA & NA & 2 & 1 & 2 & 1 & 2 & 1 & 2 & 0 & 1 & 0 & 1 & 0 & 2 & 1 & 1 \\
South Asia & NA & NA & NA & NA & 3 & 1 & 4 & 0 & 2 & 0 & 0 & 0 & 0 & 0 & 0 & 0 & 2 & 0 & 1 \\
Sub-Saharan Africa & NA & NA & NA & NA & 7 & 3 & 9 & 4 & 8 & 4 & 7 & 3 & 6 & 3 & 6 & 3 & 5 & 3 & 4 \\
\hline Low- and middle-income countries & NA & NA & NA & NA & 5 & 3 & 6 & 3 & 7 & 3 & 6 & 3 & 4 & 2 & 4 & 2 & 5 & 2 & 4 \\
High-income countries & NA & NA & NA & NA & 13 & 9 & 13 & 9 & 13 & 9 & 10 & 7 & 9 & 7 & 9 & 6 & 10 & 7 & 9 \\
\hline WORLD & NA & NA & NA & NA & 6 & 4 & 7 & 4 & 8 & 4 & 7 & 4 & 7 & 4 & 8 & 5 & 6 & 4 & 5 \\
\hline
\end{tabular}

\section{PAF of DALYs (\%)}

East Asia and Pacific

Europe and Central Asia

Latin America and the Caribbea

Middle East and North Afric

South Asia

Sub-Saharan Africa

Low- and middle-income countries

High-income countries

$\begin{array}{rrrrrrrrr}\text { NA } & \text { NA } & \text { NA } & \text { NA } & 9 & 3 & 9 & 3 & 9 \\ \text { NA } & \text { NA } & \text { NA } & \text { NA } & 14 & 9 & 13 & 9 & 14 \\ \text { NA } & \text { NA } & \text { NA } & \text { NA } & 11 & 8 & 11 & 8 & 11 \\ \text { NA } & \text { NA } & \text { NA } & \text { NA } & 2 & 1 & 2 & 1 & 2 \\ \text { NA } & \text { NA } & \text { NA } & \text { NA } & 3 & 1 & 4 & 0 & 2 \\ \text { NA } & \text { NA } & \text { NA } & \text { NA } & 7 & 3 & 9 & 4 & 8 \\ \text { NA } & \text { NA } & \text { NA } & \text { NA } & 5 & 3 & 6 & 3 & 7\end{array}$

WORLD

NA NA

$\begin{array}{rrr}3 & 8 & 2 \\ 9 & 12 & 8 \\ 8 & 10 & 7 \\ 1 & 2 & 0 \\ 0 & 0 & 0 \\ 4 & 7 & 3 \\ 3 & 6 & 3 \\ 9 & 10 & 7\end{array}$

\begin{tabular}{rrrrrrr}
7 & 1 & 7 & 1 & 7 & 2 & 4 \\
9 & 7 & 9 & 7 & 11 & 8 & 10 \\
7 & 5 & 7 & 5 & 8 & 6 & 7 \\
1 & 0 & 1 & 0 & 2 & 1 & 1 \\
0 & 0 & 0 & 0 & 2 & 0 & 1 \\
6 & 3 & 6 & 3 & 5 & 3 & 4 \\
4 & 2 & 4 & 2 & 5 & 2 & 4 \\
9 & 7 & 9 & 6 & 10 & 7 & 9 \\
\hline
\end{tabular}

Attributable Mortality (thousands

\begin{tabular}{lllllllllllllllllllll} 
East Asia and Pacific & NA & NA & NA & NA & 0 & 0 & 0 & 0 & 0 & 0 & 0 & 0 & 0 & 0 & 0 & 0 & 1 & 0 & 1 \\
Europe and Central Asia & NA & NA & NA & NA & 0 & 0 & 0 & 0 & 0 & 0 & 0 & 0 & 0 & 0 & 0 & 0 & 0 & 0 & 1 \\
Latin America and the Caribbean & NA & NA & NA & NA & 0 & 0 & 0 & 0 & 0 & 0 & 0 & 0 & 0 & 0 & 0 & 0 & 1 & 0 & 1 \\
Middle East and North Africa & NA & NA & NA & NA & 0 & 0 & 0 & 0 & 0 & 0 & 0 & 0 & 0 & 0 & 0 & 0 & 0 & 0 & 0 \\
South Asia & NA & NA & NA & NA & 0 & 0 & 0 & 0 & 0 & 0 & 0 & 0 & 0 & 0 & 0 & 0 & 0 & 0 & 0 \\
Sub-Saharan Africa & NA & NA & NA & NA & 0 & 0 & 0 & 0 & 0 & 0 & 0 & 0 & 0 & 0 & 0 & 0 & 0 & 0 & 0 \\
\hline Low- and middle-income countries & NA & NA & NA & NA & 0 & 0 & 0 & 0 & 1 & 0 & 1 & 0 & 0 & 0 & 0 & 0 & 2 & 1 & 3 \\
High-income countries & NA & NA & NA & NA & 0 & 0 & 0 & 0 & 0 & 0 & 0 & 0 & 1 & 1 & 1 & 1 \\
\hline WORLD & NA & NA & NA & NA & 0 & 0 & 0 & 0 & 1 & 1 & 1 & 0 & 1 & 1 & 1 & 1 \\
\hline
\end{tabular}

Attributable YLL (thousands)

East Asia and Pacific

Europe and Central Asia

Latin America and the Caribbean

Middle East and North Africa

South Asia

Sub-Saharan

Low- and middle-income countries

High-income

\section{NA NA NA NA}

NA

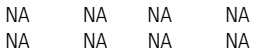

$\begin{array}{llll}N A & N A & N A & N A \\ N A & N A & N A & N A\end{array}$

NA NA NA NA

NA NA NA NA

\begin{tabular}{|c|c|c|c|c|c|c|c|c|c|c|c|c|c|c|c|c|c|c|c|}
\hline WORLD & NA & NA & NA & NA & 8 & 3 & 10 & 6 & 20 & 11 & 13 & 7 & 11 & 7 & 5 & 5 & 67 & 38 & 105 \\
\hline
\end{tabular}

Attributable DALYs (thousands)

East Asia and Pacific

Europe and Central Asia

Latin America and the Caribbean

Middle East and North Africa

South Asia

Sub-Saharan Africa

\section{NA NA NA NA}

NA NA NA NA

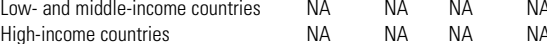

WORLD NA NA NA NA

Source: Authors' calculations.

Note: $\mathrm{NA}=$ not applicable. 
Risk factor:

Disease:

Alcohol use

Diabetes mellitus

\begin{tabular}{|c|c|c|c|c|c|c|c|c|c|c|c|c|c|c|c|c|c|c|c|}
\hline \multirow[b]{2}{*}{ Region } & \multicolumn{2}{|c|}{$0-4$ years } & \multicolumn{2}{|c|}{ 5-14 years } & \multicolumn{2}{|c|}{$15-29$ years } & \multicolumn{2}{|c|}{$30-44$ years } & \multicolumn{2}{|c|}{ 45-59 years } & \multicolumn{2}{|c|}{$60-69$ years } & \multicolumn{2}{|c|}{ 70-79 years } & \multicolumn{2}{|c|}{$80+$ years } & \multicolumn{3}{|c|}{ Total } \\
\hline & Male & Female & Male & Female & Male & Female & Male & Female & Male & Female & Male & Female & Male & Female & Male & Female & Male & Female & All \\
\hline \multicolumn{20}{|l|}{ PAF of Mortality (\%) } \\
\hline East Asia and Pacific & NA & NA & NA & NA & 0 & 0 & 0 & 0 & 0 & 0 & 0 & 0 & 0 & 0 & 0 & 0 & 0 & 0 & 0 \\
\hline Europe and Central Asia & NA & NA & NA & NA & 0 & 0 & 0 & 0 & 0 & 0 & 0 & 0 & 0 & 0 & -1 & -1 & 0 & 0 & 0 \\
\hline Latin America and the Caribbean & NA & NA & NA & NA & 0 & 0 & 0 & 1 & 0 & 0 & 0 & 0 & 0 & 0 & 0 & 0 & 0 & 0 & 0 \\
\hline Middle East and North Africa & NA & NA & NA & NA & 0 & 0 & 0 & 0 & 0 & 0 & 0 & 0 & 0 & 0 & 0 & 0 & 0 & 0 & 0 \\
\hline South Asia & NA & NA & NA & NA & 0 & 0 & 0 & 0 & 0 & 0 & 0 & 0 & 0 & 0 & 0 & 0 & 0 & 0 & 0 \\
\hline Sub-Saharan Africa & NA & NA & NA & NA & 0 & 0 & 0 & 0 & 0 & 0 & 0 & 0 & 0 & 0 & 0 & 0 & 0 & 0 & 0 \\
\hline Low- and middle-income countries & NA & NA & NA & NA & 0 & 0 & 0 & 0 & 0 & 0 & 0 & 0 & 0 & 0 & 0 & 0 & 0 & 0 & 0 \\
\hline High-income countries & NA & NA & NA & NA & -7 & -5 & -7 & -6 & -6 & -5 & -4 & -4 & -3 & -4 & -3 & -5 & -4 & -5 & -4 \\
\hline WORLD & NA & NA & NA & NA & 0 & 0 & -1 & 0 & -1 & 0 & -1 & 0 & -1 & -1 & -1 & -2 & -1 & -1 & -1 \\
\hline
\end{tabular}

PAF of YLL (\%)

\begin{tabular}{llllllllllllrrrrrrrr} 
East Asia and Pacific & NA & NA & NA & NA & 0 & 0 & 0 & 0 & 0 & 0 & 0 & 0 & 0 & 0 & 0 & 0 & 0 & 0 & 0 \\
Europe and Central Asia & NA & NA & NA & NA & 0 & 0 & 0 & 0 & 0 & 0 & 0 & 0 & 0 & 0 & -1 & -1 & 0 & 0 & 0 \\
Latin America and the Caribbean & NA & NA & NA & NA & 0 & 0 & 0 & 1 & 0 & 0 & 0 & 0 & 0 & 0 & 0 & 0 & 0 & 0 & 0 \\
Middle East and North Africa & NA & NA & NA & NA & 0 & 0 & 0 & 0 & 0 & 0 & 0 & 0 & 0 & 0 & 0 & 0 & 0 & 0 & 0 \\
South Asia & NA & NA & NA & NA & 0 & 0 & 0 & 0 & 0 & 0 & 0 & 0 & 0 & 0 & 0 & 0 & 0 & 0 & 0 \\
Sub-Saharan Africa & NA & NA & NA & NA & 0 & 0 & 0 & 0 & 0 & 0 & 0 & 0 & 0 & 0 & 0 & 0 & 0 & 0 & 0 \\
\hline Low- and middle-income countries & NA & NA & NA & NA & 0 & 0 & 0 & 0 & 0 & 0 & 0 & 0 & 0 & 0 & 0 & 0 & 0 & 0 & 0 \\
High-income countries & NA & NA & NA & NA & -7 & -5 & -7 & -6 & -6 & -5 & -4 & -4 & -3 & -4 & -3 & -5 & -4 & -4 & -4 \\
\hline WORLD & NA & NA & NA & NA & 0 & 0 & -1 & 0 & -1 & 0 & -1 & 0 & -1 & -1 & -1 & -2 & -1 & -1 & -1 \\
\hline
\end{tabular}

\section{PAF of DALYs (\%)}

\begin{tabular}{lllllllllllrrrrrrrrrrr} 
East Asia and Pacific & NA & NA & NA & NA & 0 & 0 & 0 & 0 & 0 & 0 & 0 & 0 & 0 & 0 & 0 & 0 & 0 & 0 & 0 \\
Europe and Central Asia & NA & NA & NA & NA & 0 & 0 & 0 & 0 & 0 & 0 & 0 & 0 & 0 & 0 & -1 & -1 & 0 & 0 & 0 \\
Latin America and the Caribbean & NA & NA & NA & NA & 0 & 0 & 0 & 1 & 0 & 0 & 0 & 0 & 0 & 0 & 0 & 0 & 0 & 0 & 0 \\
Middle East and North Africa & NA & NA & NA & NA & 0 & 0 & 0 & 0 & 0 & 0 & 0 & 0 & 0 & 0 & 0 & 0 & 0 & 0 & 0 \\
South Asia & NA & NA & NA & NA & 0 & 0 & 0 & 0 & 0 & 0 & 0 & 0 & 0 & 0 & 0 & 0 & 0 & 0 & 0 \\
Sub-Saharan Africa & NA & NA & NA & NA & 0 & 0 & 0 & 0 & 0 & 0 & 0 & 0 & 0 & 0 & 0 & 0 & 0 & 0 & 0 \\
\hline Low- and middle-income countries & NA & NA & NA & NA & 0 & 0 & 0 & 0 & 0 & 0 & 0 & 0 & 0 & 0 & 0 & 0 & 0 & 0 & 0 \\
High-income countries & NA & NA & NA & NA & -7 & -5 & -7 & -6 & -6 & -5 & -4 & -4 & -3 & -4 & -3 & -5 & -5 & -5 & -5 \\
\hline WORLD & NA & NA & NA & NA & -1 & 0 & -1 & -1 & -1 & -1 & -1 & -1 & -1 & -1 & -1 & -2 & -1 & -1 & -1
\end{tabular}

Attributable Mortality (thousands

\begin{tabular}{|c|c|c|c|c|c|c|c|c|c|c|c|c|c|c|c|c|c|c|c|}
\hline \multicolumn{20}{|l|}{ Attributable Mortality (thousands) } \\
\hline East Asia and Pacific & NA & NA & NA & NA & 0 & 0 & 0 & 0 & 0 & 0 & 0 & 0 & 0 & 0 & 0 & 0 & 0 & 0 & 0 \\
\hline Europe and Central Asia & NA & NA & NA & NA & 0 & 0 & 0 & 0 & 0 & 0 & 0 & 0 & 0 & 0 & 0 & 0 & 0 & 0 & 0 \\
\hline Latin America and the Caribbean & NA & NA & NA & NA & 0 & 0 & 0 & 0 & 0 & 0 & 0 & 0 & 0 & 0 & 0 & 0 & 0 & 0 & 0 \\
\hline Middle East and North Africa & NA & NA & NA & NA & 0 & 0 & 0 & 0 & 0 & 0 & 0 & 0 & 0 & 0 & 0 & 0 & 0 & 0 & 0 \\
\hline South Asia & NA & NA & NA & NA & 0 & 0 & 0 & 0 & 0 & 0 & 0 & 0 & 0 & 0 & 0 & 0 & 0 & 0 & 0 \\
\hline Sub-Saharan Africa & NA & NA & NA & NA & 0 & 0 & 0 & 0 & 0 & 0 & 0 & 0 & 0 & 0 & 0 & 0 & 0 & 0 & 0 \\
\hline Low- and middle-income countries & NA & NA & NA & NA & 0 & 0 & 0 & 0 & 0 & 0 & 0 & 0 & 0 & 0 & 0 & 0 & 0 & 0 & 0 \\
\hline High-income countries & NA & NA & NA & NA & 0 & 0 & 0 & 0 & -1 & 0 & -1 & -1 & -1 & -1 & -1 & -3 & -3 & -5 & -9 \\
\hline WORLD & NA & NA & NA & NA & 0 & 0 & 0 & 0 & -1 & 0 & -1 & -1 & -1 & -1 & -1 & -3 & -3 & -5 & -9 \\
\hline
\end{tabular}

Attributable YLL (thousands)

\begin{tabular}{|c|c|c|c|c|c|c|c|c|c|c|c|c|c|c|c|c|c|c|c|}
\hline East Asia and Pacific & NA & NA & NA & NA & 0 & 0 & 0 & 0 & 0 & 0 & 0 & 0 & 0 & 0 & 0 & 0 & 0 & 0 & 0 \\
\hline Europe and Central Asia & NA & NA & NA & NA & 0 & 0 & 0 & 0 & 0 & 0 & 0 & 0 & 0 & 0 & 0 & 0 & 0 & 0 & 0 \\
\hline Latin America and the Caribbean & NA & NA & NA & NA & 0 & 0 & 0 & 1 & 0 & 0 & 0 & 0 & 0 & 0 & 0 & 0 & 0 & 1 & 1 \\
\hline Middle East and North Africa & NA & NA & NA & NA & 0 & 0 & 0 & 0 & 0 & 0 & 0 & 0 & 0 & 0 & 0 & 0 & 0 & 0 & 0 \\
\hline South Asia & NA & NA & NA & NA & 0 & 0 & 0 & 0 & 0 & 0 & 0 & 0 & 0 & 0 & 0 & 0 & 0 & 0 & 0 \\
\hline Sub-Saharan Africa & NA & NA & NA & NA & 0 & 0 & 0 & 0 & 0 & 0 & 0 & 0 & 0 & 0 & 0 & 0 & 0 & 0 & 0 \\
\hline Low- and middle-income countries & NA & NA & NA & NA & 0 & 0 & 0 & 1 & 0 & 0 & 0 & 0 & 0 & 0 & 0 & 0 & 0 & 0 & 0 \\
\hline High-income countries & NA & NA & NA & NA & -1 & 0 & -5 & -2 & -14 & -7 & -10 & -9 & -8 & -14 & -4 & -12 & -41 & -44 & -86 \\
\hline WORLD & NA & NA & NA & NA & -1 & 0 & -5 & -2 & -14 & -7 & -10 & -9 & -8 & -14 & -4 & -12 & -41 & -44 & -85 \\
\hline
\end{tabular}

Attributable DALYs (thousands)

\begin{tabular}{lrrrrrrrrrrrrrrrrrrrr} 
East Asia and Pacific & NA & NA & NA & NA & 0 & 0 & 0 & 0 & 0 & 0 & 0 & 0 & 0 & 0 & 0 & 0 & 0 & 0 & 0 \\
Europe and Central Asia & NA & NA & NA & NA & 0 & 0 & 0 & 0 & 0 & 0 & 0 & 0 & 0 & 0 & 0 & 0 & 0 & 0 & -1 \\
Latin America and the Caribbean & NA & NA & NA & NA & 0 & 0 & 0 & 2 & 0 & 0 & 0 & 0 & 0 & 0 & 0 & 0 & 0 & 2 & 2 \\
Middle East and North Africa & NA & NA & NA & NA & 0 & 0 & 0 & 0 & 0 & 0 & 0 & 0 & 0 & 0 & 0 & 0 & 0 & 0 & 0 \\
South Asia & NA & NA & NA & NA & 0 & 0 & 0 & 0 & 0 & 0 & 0 & 0 & 0 & 0 & 0 & 0 & 0 & 0 & 0 \\
Sub-Saharan Africa & NA & NA & NA & NA & 0 & 0 & 0 & 0 & 0 & 0 & 0 & 0 & 0 & 0 & 0 & 0 & 0 & 0 & 0 \\
\hline Low- and middle-income countries & NA & NA & NA & NA & 0 & 0 & 0 & 2 & 0 & 0 & 0 & 0 & 0 & 0 & 0 & 0 & 0 & 1 & 1 \\
High-income countries & NA & NA & NA & NA & -3 & -1 & -21 & -14 & -41 & -29 & -19 & -19 & -12 & -21 & -5 & -16 & -99 & -100 & -200 \\
\hline WORLD & NA & NA & NA & NA & -3 & -1 & -21 & -12 & -41 & -29 & -19 & -19 & -12 & -21 & -5 & -17 & -100 & -99 & -199
\end{tabular}


Risk factor:

Disease:

Alcohol use

Unipolar depressive disorders

\begin{tabular}{|c|c|c|c|c|c|c|c|c|c|c|c|c|c|c|c|c|c|c|c|}
\hline \multirow[b]{2}{*}{ Region } & \multicolumn{2}{|c|}{$0-4$ years } & \multicolumn{2}{|c|}{ 5-14 years } & \multicolumn{2}{|c|}{ 15-29 years } & \multicolumn{2}{|c|}{ 30-44 years } & \multicolumn{2}{|c|}{$45-59$ years } & \multicolumn{2}{|c|}{$60-69$ years } & \multicolumn{2}{|c|}{$70-79$ years } & \multicolumn{2}{|c|}{$80+$ years } & \multicolumn{3}{|c|}{ Total } \\
\hline & Male & Female & Male & Female & Male & Female & Male & Female & Male & Female & Male & Female & Male & Female & Male & Female & Male & Female & All \\
\hline \multicolumn{20}{|l|}{ PAF of Mortality (\%) } \\
\hline East Asia and Pacific & NA & NA & NA & NA & 2 & 0 & 2 & 0 & 2 & 0 & 2 & 0 & 2 & 0 & 2 & 0 & 2 & 0 & 1 \\
\hline Europe and Central Asia & NA & NA & NA & NA & 6 & 1 & 6 & 1 & 6 & 1 & 6 & 1 & 6 & 1 & 6 & 1 & 6 & 1 & 3 \\
\hline Latin America and the Caribbean & NA & NA & NA & NA & 7 & 1 & 7 & 1 & 7 & 1 & 7 & 1 & 7 & 1 & 7 & 1 & 7 & 1 & 3 \\
\hline Middle East and North Africa & NA & NA & NA & NA & 0 & 0 & 0 & 0 & 0 & 0 & 0 & 0 & 0 & 0 & 0 & 0 & 0 & 0 & 0 \\
\hline South Asia & NA & NA & NA & NA & * & 0 & 2 & 0 & 2 & 0 & 2 & 0 & * & 0 & ${ }^{*}$ & 0 & 2 & 0 & 1 \\
\hline Sub-Saharan Africa & NA & NA & NA & NA & ${ }^{*}$ & 0 & 2 & ${ }^{*}$ & 2 & * & ${ }^{*}$ & ${ }^{*}$ & ${ }^{*}$ & ${ }^{*}$ & ${ }^{*}$ & ${ }^{*}$ & 2 & 0 & 1 \\
\hline Low- and middle-income countries & NA & NA & NA & NA & 4 & 0 & 2 & 0 & 2 & 0 & 2 & 0 & 6 & 1 & 6 & 1 & 2 & 0 & 1 \\
\hline High-income countries & NA & NA & NA & NA & 8 & 2 & 8 & 2 & 7 & 2 & 8 & 2 & 8 & 2 & 8 & 2 & 8 & 2 & 4 \\
\hline WORLD & NA & NA & NA & NA & 6 & 1 & 2 & 0 & 2 & 0 & 3 & 0 & 8 & 2 & 8 & 2 & 3 & 1 & 2 \\
\hline
\end{tabular}

PAF of YLL (\%)

\begin{tabular}{|c|c|c|c|c|c|c|c|c|c|c|c|c|c|c|c|c|c|c|c|}
\hline East Asia and Pacific & NA & NA & NA & NA & 2 & 0 & 2 & 0 & 2 & 0 & 2 & 0 & 2 & 0 & 2 & 0 & 2 & 0 & 1 \\
\hline Europe and Central Asia & NA & NA & NA & NA & 6 & 1 & 6 & 1 & 6 & 1 & 6 & 1 & 6 & 1 & 6 & 1 & 6 & 1 & 3 \\
\hline Latin America and the Caribbean & NA & NA & NA & NA & 7 & 1 & 7 & 1 & 7 & 1 & 7 & 1 & 7 & 1 & 7 & 1 & 7 & 1 & 3 \\
\hline Middle East and North Africa & NA & NA & NA & NA & 0 & 0 & 0 & 0 & 0 & 0 & 0 & 0 & 0 & 0 & 0 & 0 & 0 & 0 & 0 \\
\hline South Asia & NA & NA & NA & NA & * & 0 & 2 & 0 & 2 & 0 & 2 & 0 & * & 0 & * & 0 & 2 & 0 & 1 \\
\hline Sub-Saharan Africa & NA & NA & NA & NA & * & 0 & 2 & * & 2 & ${ }^{*}$ & ${ }^{*}$ & ${ }^{*}$ & * & ${ }^{*}$ & * & ${ }^{*}$ & 2 & 0 & 1 \\
\hline Low- and middle-income countries & NA & NA & NA & NA & 4 & 0 & 2 & 0 & 2 & 0 & 2 & 0 & 6 & 1 & 6 & 1 & 2 & 0 & 1 \\
\hline High-income countries & NA & NA & NA & NA & 8 & 2 & 8 & 2 & 7 & 2 & 8 & 2 & 8 & 2 & 8 & 2 & 8 & 2 & 4 \\
\hline WORLD & NA & NA & NA & NA & 6 & 1 & 2 & 0 & 2 & 0 & 3 & 0 & 8 & 2 & 8 & 2 & 2 & 0 & 1 \\
\hline
\end{tabular}

\section{PAF of DALYs (\%)}

Latin America and the Caribbean

Middle East and North Africa

South Asia

Sub-Saharan Africa

Low- and middle-income countries

High-income countries

$\begin{array}{llll}\text { NA } & \text { NA } & \text { NA } & \text { NA } \\ \text { NA } & \text { NA } & \text { NA } & \text { NA } \\ \text { NA } & \text { NA } & \text { NA } & \text { NA } \\ \text { NA } & \text { NA } & \text { NA } & \text { NA } \\ \text { NA } & \text { NA } & \text { NA } & \text { NA } \\ \text { NA } & \text { NA } & \text { NA } & \text { NA } \\ \text { NA } & \text { NA } & \text { NA } & \text { NA }\end{array}$

\begin{tabular}{lllll} 
& & NA & NA & NA \\
\hline WORLD & NA & NA & NA & NA
\end{tabular}

$\begin{array}{ll}2 & 0 \\ 6 & 1 \\ 7 & 1 \\ 0 & 0 \\ 2 & 0 \\ 2 & 0 \\ 3 & 0 \\ 8 & 2\end{array}$

2
6
7
0
2
2
3
8

\begin{tabular}{ll}
0 & 2 \\
1 & 6 \\
1 & 7 \\
0 & 0 \\
0 & 2 \\
0 & 2 \\
0 & 3 \\
\hline
\end{tabular}

\begin{tabular}{ll}
0 & 2 \\
1 & 6 \\
1 & 7 \\
0 & 0 \\
0 & 2 \\
0 & 2 \\
0 & 3 \\
\hline
\end{tabular}

\begin{tabular}{|c|c|c|c|c|c|c|c|c|c|c|c|c|c|c|c|c|c|c|c|}
\hline WORLD & NA & NA & NA & NA & 3 & 1 & 4 & 1 & 4 & 1 & 4 & 1 & 5 & 1 & 5 & 1 & 3 & 0 & 2 \\
\hline \multicolumn{20}{|l|}{ Attributable Mortality (thousands) } \\
\hline East Asia and Pacific & NA & NA & NA & NA & 0 & 0 & 0 & 0 & 0 & 0 & 0 & 0 & 0 & 0 & 0 & 0 & 0 & 0 & 0 \\
\hline Europe and Central Asia & NA & NA & NA & NA & 0 & 0 & 0 & 0 & 0 & 0 & 0 & 0 & 0 & 0 & 0 & 0 & 0 & 0 & 0 \\
\hline Latin America and the Caribbean & NA & NA & NA & NA & 0 & 0 & 0 & 0 & 0 & 0 & 0 & 0 & 0 & 0 & 0 & 0 & 0 & 0 & 0 \\
\hline Middle East and North Africa & NA & NA & NA & NA & 0 & 0 & 0 & 0 & 0 & 0 & 0 & 0 & 0 & 0 & 0 & 0 & 0 & 0 & 0 \\
\hline South Asia & NA & NA & NA & NA & 0 & 0 & 0 & 0 & 0 & 0 & 0 & 0 & 0 & 0 & 0 & 0 & 0 & 0 & 0 \\
\hline Sub-Saharan Africa & NA & NA & NA & NA & 0 & 0 & 0 & 0 & 0 & 0 & 0 & 0 & 0 & 0 & 0 & 0 & 0 & 0 & 0 \\
\hline Low- and middle-income countries & NA & NA & NA & NA & 0 & 0 & 0 & 0 & 0 & 0 & 0 & 0 & 0 & 0 & 0 & 0 & 0 & 0 & 0 \\
\hline High-income countries & NA & NA & NA & NA & 0 & 0 & 0 & 0 & 0 & 0 & 0 & 0 & 0 & 0 & 0 & 0 & 0 & 0 & 0 \\
\hline WORLD & NA & NA & NA & NA & 0 & 0 & 0 & 0 & 0 & 0 & 0 & 0 & 0 & 0 & 0 & 0 & 0 & 0 & 0 \\
\hline
\end{tabular}

\section{Attributable YLL (thousands)}

\begin{tabular}{llllllllllllllllllll} 
East Asia and Pacific & NA & NA & NA & NA & 0 & 0 & 0 & 0 & 0 & 0 & 0 & 0 & 0 & 0 & 0 & 0 & 0 & 0 & 0 \\
Europe and Central Asia & NA & NA & NA & NA & 0 & 0 & 0 & 0 & 0 & 0 & 0 & 0 & 0 & 0 & 0 & 0 & 0 & 0 & 0 \\
Latin America and the Caribbean & NA & NA & NA & NA & 0 & 0 & 0 & 0 & 0 & 0 & 0 & 0 & 0 & 0 & 0 & 0 & 0 & 0 & 0 \\
Middle East and North Africa & NA & NA & NA & NA & 0 & 0 & 0 & 0 & 0 & 0 & 0 & 0 & 0 & 0 & 0 & 0 & 0 & 0 & 0 \\
South Asia & NA & NA & NA & NA & 0 & 0 & 1 & 0 & 1 & 0 & 0 & 0 & 0 & 0 & 0 & 0 & 2 & 0 & 2 \\
Sub-Saharan Africa & NA & NA & NA & NA & 0 & 0 & 0 & 0 & 0 & 0 & 0 & 0 & 0 & 0 & 0 & 0 & 0 & 0 & 0 \\
\hline Low- and middle-income countries & NA & NA & NA & NA & 0 & 0 & 1 & 0 & 1 & 0 & 0 & 0 & 0 & 0 & 0 & 0 & 2 & 0 & 2 \\
High-income countries & NA & NA & NA & NA & 0 & 0 & 0 & 0 & 0 & 0 & 0 & 0 & 0 & 0 \\
\hline WORLD & NA & NA & NA & NA & 0 & 0 & 1 & 0 & 1 & 0 & 0 & 0 & 0 & 0 & 0 & 0 \\
\hline
\end{tabular}

Attributable DALYs (thousands)

\begin{tabular}{|c|c|c|c|c|c|c|c|c|c|c|c|c|c|c|c|c|c|c|c|}
\hline East Asia and Pacific & NA & NA & NA & NA & 38 & 0 & 34 & 0 & 24 & 0 & 7 & 0 & 1 & 0 & 0 & 0 & 105 & 0 & 105 \\
\hline Europe and Central Asia & NA & NA & NA & NA & 29 & 6 & 27 & 9 & 19 & 6 & 7 & 3 & 2 & 1 & 0 & 0 & 83 & 25 & 109 \\
\hline Latin America and the Caribbean & NA & NA & NA & NA & 43 & 11 & 41 & 11 & 22 & 6 & 7 & 2 & 1 & 0 & 0 & 0 & 116 & 30 & 146 \\
\hline Middle East and North Africa & NA & NA & NA & NA & 0 & 0 & 0 & 0 & 0 & 0 & 0 & 0 & 0 & 0 & 0 & 0 & 0 & 0 & 0 \\
\hline South Asia & NA & NA & NA & NA & 40 & 0 & 33 & 0 & 18 & 0 & 5 & 0 & 1 & 0 & 0 & 0 & 97 & 0 & 97 \\
\hline Sub-Saharan Africa & NA & NA & NA & NA & 9 & 0 & 7 & 0 & 3 & 0 & 1 & 0 & 0 & 0 & 0 & 0 & 20 & 0 & 20 \\
\hline Low- and middle-income countries & NA & NA & NA & NA & 159 & 18 & 142 & 20 & 88 & 12 & 27 & 5 & 6 & 1 & 1 & 0 & 422 & 56 & 478 \\
\hline High-income countries & NA & NA & NA & NA & 70 & 30 & 77 & 33 & 46 & 25 & 21 & 8 & 6 & 3 & 2 & 2 & 222 & 101 & 323 \\
\hline WORLD & NA & NA & NA & NA & 229 & 47 & 219 & 53 & 134 & 37 & 47 & 13 & 12 & 5 & 3 & 2 & 644 & 156 & 800 \\
\hline
\end{tabular}

Source: Authors' calculations.

Note: NA = not applicable.

${ }^{*}$ The number of deaths (and hence YLL) directly coded to a number of diseases, especially neuropsychiatric and musculoskeletal diseases, is zero or very small. For other diseases, mortality or disease burden may be zero in some region-age-sex groups. In such cases, the population attributable fractions would be undefined or unstable and have not been calculated. 
Table 4A.65

Risk factor: Alcohol use

Disease:

Epilepsy

\begin{tabular}{|c|c|c|c|c|c|c|c|c|c|c|c|c|c|c|c|c|c|c|c|}
\hline \multirow[b]{2}{*}{ Region } & \multicolumn{2}{|c|}{$0-4$ years } & \multicolumn{2}{|c|}{ 5-14 years } & \multicolumn{2}{|c|}{$15-29$ years } & \multicolumn{2}{|c|}{ 30-44 years } & \multicolumn{2}{|c|}{$45-59$ years } & \multicolumn{2}{|c|}{ 60-69 years } & \multicolumn{2}{|c|}{$70-79$ years } & \multicolumn{2}{|c|}{$80+$ years } & \multicolumn{3}{|c|}{ Total } \\
\hline & Male & Female & Male & Female & Male & Female & Male & Female & Male & Female & Male & Female & Male & Female & Male & Female & Male & Female & All \\
\hline \multicolumn{20}{|l|}{ PAF of Mortality (\%) } \\
\hline East Asia and Pacific & NA & NA & NA & NA & 36 & 8 & 39 & 7 & 41 & 10 & 38 & 6 & 34 & 3 & 34 & 3 & 30 & 6 & 19 \\
\hline Europe and Central Asia & NA & NA & NA & NA & 59 & 46 & 57 & 45 & 61 & 48 & 54 & 41 & 42 & 35 & 42 & 35 & 52 & 39 & 48 \\
\hline Latin America and the Caribbean & NA & NA & NA & NA & 43 & 34 & 46 & 36 & 48 & 37 & 43 & 34 & 30 & 25 & 30 & 25 & 38 & 29 & 34 \\
\hline Middle East and North Africa & NA & NA & NA & NA & 13 & 6 & 12 & 5 & 13 & 5 & 10 & 2 & 6 & 2 & 6 & 3 & 9 & 3 & 7 \\
\hline South Asia & NA & NA & NA & NA & 9 & 7 & 18 & 3 & 8 & 3 & 2 & 0 & 0 & 0 & 0 & 0 & 3 & 2 & 2 \\
\hline Sub-Saharan Africa & NA & NA & NA & NA & 43 & 20 & 50 & 26 & 48 & 29 & 45 & 22 & 38 & 17 & 38 & 17 & 38 & 17 & 30 \\
\hline Low- and middle-income countries & NA & NA & NA & NA & 36 & 15 & 44 & 18 & 45 & 20 & 40 & 16 & 27 & 11 & 27 & 11 & 28 & 11 & 21 \\
\hline High-income countries & NA & NA & NA & NA & 55 & 44 & 55 & 40 & 55 & 44 & 47 & 37 & 41 & 33 & 41 & 33 & 49 & 35 & 43 \\
\hline WORLD & NA & NA & NA & NA & 37 & 16 & 45 & 20 & 46 & 22 & 41 & 19 & 29 & 15 & 30 & 18 & 30 & 13 & 22 \\
\hline \multicolumn{20}{|l|}{ PAF of YLL (\%) } \\
\hline East Asia and Pacific & NA & NA & NA & NA & 36 & 8 & 39 & 7 & 41 & 10 & 38 & 6 & 34 & 3 & 34 & 3 & 28 & 6 & 18 \\
\hline Europe and Central Asia & NA & NA & NA & NA & 59 & 46 & 57 & 45 & 61 & 48 & 54 & 41 & 42 & 35 & 42 & 35 & 51 & 38 & 46 \\
\hline Latin America and the Caribbean & NA & NA & NA & NA & 43 & 34 & 46 & 36 & 48 & 37 & 43 & 34 & 30 & 25 & 30 & 25 & 37 & 28 & 33 \\
\hline Middle East and North Africa & NA & NA & NA & NA & 13 & 6 & 12 & 5 & 13 & 5 & 10 & 2 & 6 & 2 & 6 & 3 & 9 & 3 & 7 \\
\hline South Asia & NA & NA & NA & NA & 9 & 7 & 18 & 3 & 8 & 3 & 2 & 0 & 0 & 0 & 0 & 0 & 2 & 2 & 2 \\
\hline Sub-Saharan Africa & NA & NA & NA & NA & 43 & 20 & 50 & 26 & 48 & 29 & 45 & 22 & 38 & 17 & 38 & 17 & 36 & 15 & 27 \\
\hline Low- and middle-income countries & NA & NA & NA & NA & 36 & 15 & 44 & 18 & 45 & 20 & 40 & 16 & 27 & 11 & 27 & 11 & 26 & 10 & 19 \\
\hline High-income countries & NA & NA & NA & NA & 55 & 44 & 55 & 40 & 55 & 44 & 47 & 37 & 41 & 33 & 41 & 33 & 49 & 35 & 44 \\
\hline WORLD & NA & NA & NA & NA & 37 & 16 & 45 & 20 & 46 & 22 & 41 & 19 & 29 & 15 & 30 & 17 & 27 & 11 & 20 \\
\hline
\end{tabular}

PAF of DALYs (\%)

\begin{tabular}{|c|c|c|c|c|c|c|c|c|c|c|c|c|c|c|c|c|c|c|c|}
\hline East Asia and Pacific & NA & NA & NA & NA & 36 & 8 & 39 & 7 & 41 & 10 & 38 & 6 & 34 & 3 & 34 & 3 & 27 & 5 & 17 \\
\hline Europe and Central Asia & NA & NA & NA & NA & 59 & 46 & 57 & 45 & 61 & 48 & 54 & 41 & 42 & 35 & 42 & 35 & 48 & 36 & 43 \\
\hline Latin America and the Caribbean & NA & NA & NA & NA & 43 & 34 & 46 & 36 & 48 & 37 & 43 & 34 & 30 & 25 & 30 & 25 & 31 & 24 & 28 \\
\hline Middle East and North Africa & NA & NA & NA & NA & 13 & 6 & 12 & 5 & 13 & 5 & 10 & 2 & 6 & 2 & 6 & 3 & 8 & 3 & 6 \\
\hline South Asia & NA & NA & NA & NA & 9 & 7 & 18 & 3 & 8 & 3 & 2 & 0 & 0 & 0 & 0 & 0 & 5 & 2 & 3 \\
\hline Sub-Saharan Africa & NA & NA & NA & NA & 43 & 20 & 50 & 26 & 48 & 29 & 45 & 22 & 38 & 17 & 38 & 17 & 31 & 13 & 23 \\
\hline Low- and middle-income countries & NA & NA & NA & NA & 32 & 16 & 40 & 18 & 41 & 20 & 36 & 16 & 26 & 12 & 26 & 12 & 23 & 10 & 17 \\
\hline High-income countries & NA & NA & NA & NA & 55 & 44 & 55 & 40 & 55 & 44 & 47 & 37 & 41 & 33 & 41 & 33 & 44 & 33 & 39 \\
\hline WORLD & NA & NA & NA & NA & 34 & 17 & 42 & 20 & 43 & 23 & 38 & 20 & 29 & 17 & 30 & 20 & 24 & 12 & \\
\hline
\end{tabular}

Attributable Mortality (thousands)

\begin{tabular}{|c|c|c|c|c|c|c|c|c|c|c|c|c|c|c|c|c|c|c|c|}
\hline East Asia and Pacific & NA & NA & NA & NA & 1 & 0 & 2 & 0 & 1 & 0 & 0 & 0 & 0 & 0 & 0 & 0 & 4 & 1 & 5 \\
\hline Europe and Central Asia & NA & NA & NA & NA & 1 & 0 & 1 & 0 & 1 & 0 & 0 & 0 & 0 & 0 & 0 & 0 & 3 & 1 & 4 \\
\hline Latin America and the Caribbean & NA & NA & NA & NA & 1 & 0 & 1 & 0 & 0 & 0 & 0 & 0 & 0 & 0 & 0 & 0 & 2 & 1 & 3 \\
\hline Middle East and North Africa & NA & NA & NA & NA & 0 & 0 & 0 & 0 & 0 & 0 & 0 & 0 & 0 & 0 & 0 & 0 & 0 & 0 & 0 \\
\hline South Asia & NA & NA & NA & NA & 0 & 0 & 0 & 0 & 0 & 0 & 0 & 0 & 0 & 0 & 0 & 0 & 0 & 0 & 1 \\
\hline Sub-Saharan Africa & NA & NA & NA & NA & 2 & 1 & 3 & 1 & 2 & 1 & 1 & 0 & 0 & 0 & 0 & 0 & 9 & 2 & 11 \\
\hline Low- and middle-income countries & NA & NA & NA & NA & 5 & 2 & 6 & 1 & 4 & 1 & 2 & 0 & 1 & 0 & 0 & 0 & 18 & 6 & 24 \\
\hline High-income countries & NA & NA & NA & NA & 0 & 0 & 1 & 0 & 1 & 0 & 0 & 0 & 0 & 0 & 0 & 0 & 3 & 1 & 4 \\
\hline WORLD & NA & NA & NA & NA & 6 & 2 & 7 & 2 & 5 & 2 & 2 & 1 & 1 & 1 & 1 & 0 & 21 & 7 & 28 \\
\hline
\end{tabular}

Attributable YLL (thousands)

\begin{tabular}{|c|c|c|c|c|c|c|c|c|c|c|c|c|c|c|c|c|c|c|c|}
\hline East Asia and Pacific & NA & NA & NA & NA & 34 & 7 & 40 & 5 & 12 & 3 & 3 & 0 & 1 & 0 & 0 & 0 & 90 & 15 & 106 \\
\hline Europe and Central Asia & NA & NA & NA & NA & 21 & 11 & 25 & 10 & 17 & 6 & 3 & 1 & 1 & 1 & 0 & 0 & 67 & 28 & 95 \\
\hline Latin America and the Caribbean & NA & NA & NA & NA & 15 & 7 & 15 & 7 & 7 & 3 & 2 & 1 & 1 & 0 & 0 & 0 & 40 & 19 & 58 \\
\hline Middle East and North Africa & NA & NA & NA & NA & 3 & 1 & 1 & 0 & 1 & 0 & 0 & 0 & 0 & 0 & 0 & 0 & 6 & 2 & 7 \\
\hline South Asia & NA & NA & NA & NA & 5 & 7 & 4 & 1 & 1 & 1 & 0 & 0 & 0 & 0 & 0 & 0 & 10 & 8 & 18 \\
\hline Sub-Saharan Africa & NA & NA & NA & NA & 65 & 20 & 63 & 13 & 39 & 14 & 15 & 3 & 4 & 2 & 1 & 1 & 188 & 53 & 241 \\
\hline Low- and middle-income countries & NA & NA & NA & NA & 143 & 53 & 148 & 35 & 77 & 26 & 23 & 7 & 7 & 3 & 2 & 1 & 401 & 125 & 526 \\
\hline High-income countries & NA & NA & NA & NA & 9 & 5 & 18 & 6 & 13 & 6 & 4 & 2 & 2 & 2 & 1 & 1 & 48 & 22 & 71 \\
\hline WORLD & NA & NA & NA & NA & 152 & 58 & 167 & 41 & 90 & 32 & 27 & 9 & 10 & 5 & 3 & 2 & 449 & 147 & 596 \\
\hline
\end{tabular}

Attributable DALYs (thousands)

\begin{tabular}{|c|c|c|c|c|c|c|c|c|c|c|c|c|c|c|c|c|c|c|c|}
\hline East Asia and Pacific & NA & NA & NA & NA & 71 & 14 & 69 & 10 & 29 & 6 & 9 & 2 & 4 & 0 & 1 & 0 & 184 & 33 & 217 \\
\hline Europe and Central Asia & NA & NA & NA & NA & 33 & 20 & 34 & 17 & 23 & 11 & 6 & 4 & 2 & 2 & 0 & 1 & 98 & 55 & 153 \\
\hline Latin America and the Caribbean & NA & NA & NA & NA & 52 & 37 & 39 & 26 & 21 & 14 & 6 & 5 & 2 & 2 & 0 & 1 & 120 & 84 & 204 \\
\hline Middle East and North Africa & NA & NA & NA & NA & 6 & 2 & 3 & 1 & 2 & 1 & 0 & 0 & 0 & 0 & 0 & 0 & 11 & 4 & 15 \\
\hline South Asia & NA & NA & NA & NA & 20 & 17 & 16 & 2 & 3 & 2 & 0 & 0 & 0 & 0 & 0 & 0 & 39 & 22 & 60 \\
\hline Sub-Saharan Africa & NA & NA & NA & NA & 98 & 36 & 77 & 20 & 43 & 17 & 16 & 4 & 5 & 2 & 1 & 1 & 240 & 79 & 319 \\
\hline Low- and middle-income countries & NA & NA & NA & NA & 279 & 126 & 238 & 77 & 120 & 51 & 38 & 15 & 12 & 7 & 3 & 2 & 692 & 277 & 968 \\
\hline High-income countries & NA & NA & NA & NA & 30 & 20 & 36 & 18 & 26 & 16 & 10 & 7 & 6 & 6 & 2 & 3 & 110 & 69 & 179 \\
\hline WORLD & NA & NA & NA & NA & 310 & 145 & 274 & 95 & 146 & 66 & 48 & 22 & 18 & 13 & 5 & 5 & 802 & 346 & 1,147 \\
\hline
\end{tabular}

Source: Authors' calculations.

Note: $\mathrm{NA}=$ not applicable. 
Risk factor:

Alcohol use

Alcohol use disorders

\begin{tabular}{|c|c|c|c|c|c|c|c|c|c|c|c|c|c|c|c|c|c|c|c|}
\hline \multirow[b]{2}{*}{ Region } & \multicolumn{2}{|c|}{$0-4$ years } & \multicolumn{2}{|c|}{ 5-14 years } & \multicolumn{2}{|c|}{$15-29$ years } & \multicolumn{2}{|c|}{ 30-44 years } & \multicolumn{2}{|c|}{$45-59$ years } & \multicolumn{2}{|c|}{ 60-69 years } & \multicolumn{2}{|c|}{ 70-79 years } & \multicolumn{2}{|c|}{$80+$ years } & \multicolumn{3}{|c|}{ Total } \\
\hline & Male & Female & Male & Female & Male & Female & Male & Female & Male & Female & Male & Female & Male & Female & Male & Female & Male & Female & All \\
\hline \multicolumn{20}{|l|}{ PAF of Mortality (\%) } \\
\hline East Asia and Pacific & 100 & 100 & 100 & 100 & 100 & 100 & 100 & 100 & 100 & 100 & 100 & 100 & 100 & 100 & 100 & 100 & 100 & 100 & 100 \\
\hline Europe and Central Asia & 100 & 100 & 100 & 100 & 100 & 100 & 100 & 100 & 100 & 100 & 100 & 100 & 100 & 100 & 100 & 100 & 100 & 100 & 100 \\
\hline Latin America and the Caribbean & 100 & 100 & 100 & 100 & 100 & 100 & 100 & 100 & 100 & 100 & 100 & 100 & 100 & 100 & 100 & 100 & 100 & 100 & 100 \\
\hline Middle East and North Africa & 100 & 100 & 100 & 100 & 100 & 100 & 100 & 100 & 100 & 100 & 100 & 100 & 100 & 100 & 100 & 100 & 100 & 100 & 100 \\
\hline South Asia & 100 & 100 & 100 & 100 & 100 & 100 & 100 & 100 & 100 & 100 & 100 & 100 & 100 & 100 & 100 & 100 & 100 & 100 & 100 \\
\hline Sub-Saharan Africa & 100 & 100 & 100 & 100 & 100 & 100 & 100 & 100 & 100 & 100 & 100 & 100 & 100 & 100 & 100 & 100 & 100 & 100 & 100 \\
\hline Low- and middle-income countries & 100 & 100 & 100 & 100 & 100 & 100 & 100 & 100 & 100 & 100 & 100 & 100 & 100 & 100 & 100 & 100 & 100 & 100 & 100 \\
\hline High-income countries & 100 & 100 & 100 & 100 & 100 & 100 & 100 & 100 & 100 & 100 & 100 & 100 & 100 & 100 & 100 & 100 & 100 & 100 & 100 \\
\hline WORLD & 100 & 100 & 100 & 100 & 100 & 100 & 100 & 100 & 100 & 100 & 100 & 100 & 100 & 100 & 100 & 100 & 100 & 100 & 100 \\
\hline \multicolumn{20}{|l|}{ PAF of YLL (\%) } \\
\hline East Asia and Pacific & 100 & 100 & 100 & 100 & 100 & 100 & 100 & 100 & 100 & 100 & 100 & 100 & 100 & 100 & 100 & 100 & 100 & 100 & 100 \\
\hline Europe and Central Asia & 100 & 100 & 100 & 100 & 100 & 100 & 100 & 100 & 100 & 100 & 100 & 100 & 100 & 100 & 100 & 100 & 100 & 100 & 100 \\
\hline Latin America and the Caribbean & 100 & 100 & 100 & 100 & 100 & 100 & 100 & 100 & 100 & 100 & 100 & 100 & 100 & 100 & 100 & 100 & 100 & 100 & 100 \\
\hline Middle East and North Africa & 100 & 100 & 100 & 100 & 100 & 100 & 100 & 100 & 100 & 100 & 100 & 100 & 100 & 100 & 100 & 100 & 100 & 100 & 100 \\
\hline South Asia & 100 & 100 & 100 & 100 & 100 & 100 & 100 & 100 & 100 & 100 & 100 & 100 & 100 & 100 & 100 & 100 & 100 & 100 & 100 \\
\hline Sub-Saharan Africa & 100 & 100 & 100 & 100 & 100 & 100 & 100 & 100 & 100 & 100 & 100 & 100 & 100 & 100 & 100 & 100 & 100 & 100 & 100 \\
\hline Low- and middle-income countries & 100 & 100 & 100 & 100 & 100 & 100 & 100 & 100 & 100 & 100 & 100 & 100 & 100 & 100 & 100 & 100 & 100 & 100 & 100 \\
\hline High-income countries & 100 & 100 & 100 & 100 & 100 & 100 & 100 & 100 & 100 & 100 & 100 & 100 & 100 & 100 & 100 & 100 & 100 & 100 & 100 \\
\hline WORLD & 100 & 100 & 100 & 100 & 100 & 100 & 100 & 100 & 100 & 100 & 100 & 100 & 100 & 100 & 100 & 100 & 100 & 100 & 100 \\
\hline \multicolumn{20}{|l|}{ PAF of DALYs (\%) } \\
\hline East Asia and Pacific & 100 & 100 & 100 & 100 & 100 & 100 & 100 & 100 & 100 & 100 & 100 & 100 & 100 & 100 & 100 & 100 & 100 & 100 & 100 \\
\hline Europe and Central Asia & 100 & 100 & 100 & 100 & 100 & 100 & 100 & 100 & 100 & 100 & 100 & 100 & 100 & 100 & 100 & 100 & 100 & 100 & 100 \\
\hline Latin America and the Caribbean & 100 & 100 & 100 & 100 & 100 & 100 & 100 & 100 & 100 & 100 & 100 & 100 & 100 & 100 & 100 & 100 & 100 & 100 & 100 \\
\hline Middle East and North Africa & 100 & 100 & 100 & 100 & 100 & 100 & 100 & 100 & 100 & 100 & 100 & 100 & 100 & 100 & 100 & 100 & 100 & 100 & 100 \\
\hline South Asia & 100 & 100 & 100 & 100 & 100 & 100 & 100 & 100 & 100 & 100 & 100 & 100 & 100 & 100 & 100 & 100 & 100 & 100 & 100 \\
\hline Sub-Saharan Africa & 100 & 100 & 100 & 100 & 100 & 100 & 100 & 100 & 100 & 100 & 100 & 100 & 100 & 100 & 100 & 100 & 100 & 100 & 100 \\
\hline Low- and middle-income countries & 100 & 100 & 100 & 100 & 100 & 100 & 100 & 100 & 100 & 100 & 100 & 100 & 100 & 100 & 100 & 100 & 100 & 100 & 100 \\
\hline High-income countries & 100 & 100 & 100 & 100 & 100 & 100 & 100 & 100 & 100 & 100 & 100 & 100 & 100 & 100 & 100 & 100 & 100 & 100 & 100 \\
\hline WORLD & 100 & 100 & 100 & 100 & 100 & 100 & 100 & 100 & 100 & 100 & 100 & 100 & 100 & 100 & 100 & 100 & 100 & 100 & 100 \\
\hline
\end{tabular}

Attributable Mortality (thousands)

\begin{tabular}{lrlllllrrrrrrrrrrrrrrr} 
East Asia and Pacific & 0 & 0 & 0 & 0 & 1 & 0 & 3 & 1 & 4 & 1 & 2 & 0 & 1 & 0 & 0 & 0 & 11 & 2 & 12 \\
Europe and Central Asia & 0 & 0 & 0 & 0 & 0 & 0 & 2 & 0 & 3 & 1 & 2 & 0 & 1 & 0 & 0 & 0 & 8 & 2 & 10 \\
Latin America and the Caribbean & 0 & 0 & 0 & 0 & 1 & 0 & 5 & 0 & 6 & 0 & 2 & 0 & 1 & 0 & 0 & 0 & 15 & 1 & 17 \\
Middle East and North Africa & 0 & 0 & 0 & 0 & 1 & 0 & 1 & 0 & 1 & 0 & 0 & 0 & 0 & 0 & 0 & 0 & 3 & 0 & 3 \\
South Asia & 0 & 0 & 0 & 0 & 2 & 0 & 3 & 0 & 4 & 0 & 2 & 0 & 1 & 0 & 0 & 0 & 12 & 1 & 13 \\
Sub-Saharan Africa & 0 & 0 & 0 & 0 & 0 & 0 & 2 & 1 & 2 & 1 & 1 & 0 & 1 & 0 & 0 & 0 & 5 & 2 & 7 \\
\hline Low- and middle-income countries & 0 & 0 & 0 & 0 & 5 & 0 & 16 & 2 & 19 & 3 & 9 & 2 & 4 & 1 & 1 & 0 & 54 & 8 & 62 \\
High-income countries & 0 & 0 & 0 & 0 & 0 & 0 & 4 & 1 & 8 & 2 & 4 & 1 & 2 & 1 & 1 & 0 \\
\hline WORLD & 0 & 0 & 0 & 0 & 5 & 0 & 20 & 3 & 27 & 5 & 13 & 2 & 6 & 2 & 2 & 0 \\
\hline
\end{tabular}

Attributable YLL (thousands)

\begin{tabular}{lrlllrrrrrrrrrrrrrrr} 
East Asia and Pacific & 0 & 0 & 0 & 0 & 17 & 4 & 73 & 13 & 80 & 14 & 25 & 3 & 7 & 1 & 1 & 0 & 205 & 35 & 239 \\
Europe and Central Asia & 1 & 0 & 1 & 0 & 5 & 1 & 44 & 7 & 66 & 15 & 26 & 7 & 5 & 2 & 0 & 0 & 148 & 32 & 180 \\
Latin America and the Caribbean & 1 & 0 & 0 & 0 & 27 & 2 & 114 & 10 & 111 & 9 & 31 & 4 & 12 & 1 & 2 & 0 & 297 & 27 & 324 \\
Middle East and North Africa & 0 & 0 & 0 & 0 & 22 & 1 & 33 & 2 & 12 & 2 & 2 & 0 & 1 & 0 & 0 & 0 & 70 & 5 & 75 \\
South Asia & 0 & 0 & 0 & 0 & 52 & 0 & 79 & 0 & 68 & 8 & 29 & 6 & 5 & 2 & 1 & 1 & 236 & 16 & 252 \\
Sub-Saharan Africa & 0 & 0 & 0 & 0 & 6 & 2 & 41 & 13 & 31 & 13 & 9 & 4 & 5 & 5 & 1 & 0 & 92 & 37 & 128 \\
\hline Low- and middle-income countries & 2 & 0 & 1 & 0 & 129 & 10 & 385 & 45 & 369 & 61 & 122 & 23 & 35 & 11 & 5 & 1 & 1,048 & 152 & 1,200 \\
High-income countries & 0 & 0 & 0 & 0 & 9 & 2 & 90 & 21 & 146 & 38 & 55 & 13 & 18 & 6 & 3 & 1 \\
\hline WORLD & 2 & 0 & 1 & 0 & 138 & 12 & 475 & 67 & 514 & 98 & 177 & 36 & 53 & 17 & 8 & 2 & 1,368 & 233 & 1,601 \\
\hline
\end{tabular}

Attributable DALYs (thousands)

\begin{tabular}{lrrrrrrrrrrrrrrrrrrr} 
East Asia and Pacific & 0 & 0 & 33 & 17 & 1,475 & 217 & 1,510 & 190 & 657 & 75 & 100 & 7 & 18 & 2 & 3 & 0 & 3,796 & 507 & 4,303 \\
Europe and Central Asia & 1 & 0 & 14 & 4 & 620 & 110 & 561 & 100 & 294 & 61 & 56 & 13 & 10 & 3 & 1 & 0 & 1,557 & 292 & 1,849 \\
Latin America and the Caribbean & 1 & 0 & 30 & 8 & 1,271 & 296 & 590 & 129 & 371 & 77 & 72 & 15 & 18 & 3 & 2 & 1 & 2,355 & 528 & 2,883 \\
Middle East and North Africa & 0 & 0 & 0 & 0 & 23 & 1 & 34 & 2 & 13 & 2 & 2 & 0 & 1 & 0 & 0 & 0 & 73 & 5 & 79 \\
South Asia & 0 & 0 & 15 & 6 & 363 & 13 & 533 & 12 & 189 & 10 & 43 & 6 & 9 & 2 & 2 & 1 & 1,153 & 49 & 1,202 \\
Sub-Saharan Africa & 0 & 0 & 10 & 6 & 274 & 25 & 197 & 26 & 97 & 19 & 15 & 4 & 6 & 5 & 1 & 0 & 600 & 85 & 685 \\
\hline Low- and middle-income countries & 2 & 0 & 101 & 41 & 4,027 & 661 & 3,425 & 460 & 1,620 & 243 & 289 & 44 & 62 & 15 & 8 & 2 & 9,534 & 1,467 & 11,001 \\
High-income countries & 0 & 0 & 25 & 8 & 1,289 & 341 & 1,269 & 312 & 592 & 143 & 107 & 26 & 38 & 12 & 7 & 2 & 3,328 & 843 & 4,171 \\
\hline WORLD & 2 & 0 & 126 & 49 & 5,316 & 1,003 & 4,694 & 771 & 2,213 & 386 & 396 & 70 & 100 & 27 & 15 & 4 & 12,862 & 2,310 & 15,172
\end{tabular}

Source: Authors' calculations. 
Table 4A.67

Risk factor:

Alcohol use

Disease:

Hypertensive heart disease

\begin{tabular}{|c|c|c|c|c|c|c|c|c|c|c|c|c|c|c|c|c|c|c|c|}
\hline \multirow[b]{2}{*}{ Region } & \multicolumn{2}{|c|}{$0-4$ years } & \multicolumn{2}{|c|}{ 5-14 years } & \multicolumn{2}{|c|}{$15-29$ years } & \multicolumn{2}{|c|}{ 30-44 years } & \multicolumn{2}{|c|}{ 45-59 years } & \multicolumn{2}{|c|}{$60-69$ years } & \multicolumn{2}{|c|}{$70-79$ years } & \multicolumn{2}{|c|}{$80+$ years } & \multicolumn{3}{|c|}{ Total } \\
\hline & Male & Female & Male & Female & Male & Female & Male & Female & Male & Female & Male & Female & Male & Female & Male & Female & Male & Female & All \\
\hline \multicolumn{20}{|l|}{ PAF of Mortality (\%) } \\
\hline East Asia and Pacific & NA & NA & NA & NA & 27 & 10 & 29 & 10 & 26 & 10 & 24 & 7 & 22 & 4 & 22 & 4 & 24 & 5 & 14 \\
\hline Europe and Central Asia & NA & NA & NA & NA & 39 & 29 & 38 & 28 & 31 & 26 & 28 & 23 & 25 & 21 & 25 & 21 & 28 & 22 & 24 \\
\hline Latin America and the Caribbean & NA & NA & NA & NA & 33 & 25 & 35 & 27 & 28 & 21 & 26 & 19 & 21 & 17 & 21 & 17 & 24 & 18 & 21 \\
\hline Middle East and North Africa & NA & NA & NA & NA & 9 & 3 & 8 & 3 & 7 & 2 & 5 & 1 & 3 & 1 & 3 & 2 & 4 & 1 & 3 \\
\hline South Asia & NA & NA & NA & NA & 10 & 4 & 14 & 1 & 7 & 1 & 2 & 0 & 0 & 0 & 0 & 0 & 3 & 0 & 2 \\
\hline Sub-Saharan Africa & NA & NA & NA & NA & 22 & 11 & 26 & 14 & 22 & 13 & 20 & 11 & 17 & 9 & 17 & 9 & 20 & 10 & 14 \\
\hline Low- and middle-income countries & NA & NA & NA & NA & 20 & 11 & 26 & 13 & 22 & 11 & 20 & 10 & 17 & 8 & 18 & 9 & 19 & 9 & $\overline{14}$ \\
\hline High-income countries & NA & NA & NA & NA & 37 & 29 & 37 & 28 & 30 & 25 & 27 & 22 & 25 & 20 & 25 & 20 & 26 & 20 & 23 \\
\hline WORLD & NA & NA & NA & NA & 21 & 12 & 27 & 14 & 22 & 12 & 20 & 10 & 18 & 9 & 20 & 12 & 20 & 11 & 15 \\
\hline
\end{tabular}

PAF of YLL (\%)

\begin{tabular}{|c|c|c|c|c|c|c|c|c|c|c|c|c|c|c|c|c|c|c|c|}
\hline East Asia and Pacific & NA & NA & NA & NA & 27 & 10 & 29 & 10 & 26 & 10 & 24 & 7 & 22 & 4 & 22 & 4 & 24 & 6 & 16 \\
\hline Europe and Central Asia & NA & NA & NA & NA & 39 & 29 & 38 & 28 & 31 & 26 & 28 & 23 & 25 & 21 & 25 & 21 & 29 & 23 & 26 \\
\hline Latin America and the Caribbean & NA & NA & NA & NA & 33 & 25 & 35 & 27 & 28 & 21 & 26 & 19 & 21 & 17 & 21 & 17 & 26 & 19 & 22 \\
\hline Middle East and North Africa & NA & NA & NA & NA & 9 & 3 & 8 & 3 & 7 & 2 & 5 & 1 & 3 & 1 & 3 & 2 & 5 & 2 & 3 \\
\hline South Asia & NA & NA & NA & NA & 10 & 4 & 14 & 1 & 7 & 1 & 2 & 0 & 0 & 0 & 0 & 0 & 5 & 1 & 3 \\
\hline Sub-Saharan Africa & NA & NA & NA & NA & 22 & 11 & 26 & 14 & 22 & 13 & 20 & 11 & 17 & 9 & 17 & 9 & 20 & 11 & 14 \\
\hline Low- and middle-income countries & NA & NA & NA & NA & 20 & 11 & 26 & 13 & 22 & 11 & 20 & 10 & 17 & 8 & 18 & 8 & 20 & 9 & 15 \\
\hline High-income countries & NA & NA & NA & NA & 37 & 29 & 37 & 28 & 30 & 25 & 27 & 22 & 25 & 20 & 25 & 20 & 28 & 21 & 24 \\
\hline WORLD & NA & NA & NA & NA & 21 & 12 & 27 & 14 & 22 & 12 & 20 & 10 & 18 & 9 & 19 & 11 & 21 & 11 & 16 \\
\hline
\end{tabular}

\section{PAF of DALYs (\%)}

East Asia and Pacific

Europe and Central Asia

Latin America and the Caribbean

Middle East and North Africa

South Asia

Sub-Saharan Africa

Low- and middle-income countries

WORLD

NA NA NA NA

NA 27

\begin{tabular}{ll}
10 & 29 \\
\hline & 38
\end{tabular}

\begin{tabular}{|c|c|c|c|c|c|c|c|c|c|c|c|c|c|c|c|c|c|c|}
\hline NA & NA & NA & NA & 27 & 10 & 29 & 10 & 26 & 10 & 24 & 7 & 22 & 4 & 22 & 4 & 24 & 6 & 15 \\
\hline NA & NA & NA & NA & 39 & 29 & 38 & 28 & 31 & 26 & 28 & 23 & 25 & 21 & 25 & 21 & 29 & 22 & 25 \\
\hline NA & NA & NA & NA & 9 & 3 & 8 & 3 & 7 & 2 & 5 & 1 & 3 & 1 & 3 & 2 & 5 & 2 & 3 \\
\hline NA & NA & NA & NA & 22 & 11 & 26 & 14 & 22 & 13 & 20 & 11 & 17 & 9 & 17 & 9 & 20 & 11 & 14 \\
\hline NA & NA & NA & NA & 20 & 11 & 26 & 13 & 22 & 11 & 20 & 9 & 17 & 8 & 18 & 8 & 20 & 9 & 14 \\
\hline NA & NA & NA & NA & 37 & 29 & 37 & 28 & 30 & 25 & 27 & 22 & 25 & 20 & 25 & 20 & 28 & 21 & 24 \\
\hline
\end{tabular}

Attributable Mortality (thousands)

\begin{tabular}{lrllllllrrrrrrrrrrrr} 
East Asia and Pacific & NA & NA & NA & NA & 0 & 0 & 2 & 0 & 7 & 2 & 11 & 2 & 12 & 2 & 6 & 2 & 39 & 9 & 48 \\
Europe and Central Asia & NA & NA & NA & NA & 0 & 0 & 1 & 0 & 3 & 2 & 4 & 3 & 4 & 5 & 2 & 4 & 13 & 14 & 27 \\
Latin America and the Caribbean & NA & NA & NA & NA & 0 & 0 & 1 & 0 & 2 & 1 & 2 & 2 & 2 & 2 & 2 & 3 & 10 & 9 & 18 \\
Middle East and North Africa & NA & NA & NA & NA & 0 & 0 & 0 & 0 & 0 & 0 & 0 & 0 & 0 & 0 & 0 & 0 & 2 & 1 & 2 \\
South Asia & NA & NA & NA & NA & 0 & 0 & 0 & 0 & 1 & 0 & 0 & 0 & 0 & 0 & 0 & 0 & 2 & 0 & 2 \\
Sub-Saharan Africa & NA & NA & NA & NA & 0 & 0 & 0 & 0 & 1 & 1 & 1 & 1 & 1 & 1 & 1 & 1 & 5 & 4 & 9 \\
\hline Low- and middle-income countries & NA & NA & NA & NA & 1 & 0 & 4 & 2 & 14 & 6 & 19 & 8 & 20 & 10 & 11 & 10 & 70 & 36 & 106 \\
High-income countries & NA & NA & NA & NA & 0 & 0 & 1 & 0 & 2 & 1 & 2 & 1 & 3 & 3 & 5 & 11 & 13 & 16 & 29 \\
\hline WORLD & NA & NA & NA & NA & 1 & 0 & 5 & 2 & 16 & 7 & 21 & 9 & 23 & 14 & 16 & 21 & 82 & 52 & 13 \\
\hline
\end{tabular}

Attributable YLL (thousands)

\begin{tabular}{|c|c|c|c|c|c|c|c|c|c|c|c|c|c|c|c|c|c|c|c|}
\hline East Asia and Pacific & NA & NA & NA & NA & 8 & 2 & 47 & 10 & 135 & 40 & 151 & 36 & 106 & 21 & 33 & 10 & 479 & 118 & 597 \\
\hline Europe and Central Asia & NA & NA & NA & NA & 3 & 1 & 19 & 8 & 52 & 32 & 52 & 45 & 33 & 49 & 8 & 19 & 168 & 154 & 321 \\
\hline Latin America and the Caribbean & NA & NA & NA & NA & 4 & 3 & 15 & 12 & 34 & 25 & 30 & 23 & 22 & 22 & 10 & 14 & 115 & 99 & 215 \\
\hline Middle East and North Africa & NA & NA & NA & NA & 1 & 0 & 3 & 1 & 8 & 2 & 7 & 2 & 3 & 2 & 1 & 1 & 23 & 7 & 30 \\
\hline South Asia & NA & NA & NA & NA & 4 & 1 & 11 & 1 & 14 & 3 & 3 & 0 & 0 & 0 & 0 & 0 & 33 & 4 & 37 \\
\hline Sub-Saharan Africa & NA & NA & NA & NA & 2 & 1 & 11 & 7 & 21 & 18 & 16 & 17 & 10 & 13 & 3 & 4 & 63 & 60 & 123 \\
\hline Low- and middle-income countries & NA & NA & NA & NA & 21 & 8 & 106 & 38 & 265 & 120 & 259 & 122 & 175 & 106 & 56 & 48 & 881 & 442 & 1,323 \\
\hline High-income countries & NA & NA & NA & NA & 2 & 1 & 14 & 6 & 34 & 16 & 28 & 18 & 30 & 33 & 20 & 43 & 127 & 117 & 244 \\
\hline WORLD & NA & NA & NA & NA & 23 & 9 & 120 & 44 & 299 & 136 & 286 & 140 & 204 & 139 & 76 & 91 & 1,009 & 558 & 1,567 \\
\hline
\end{tabular}

Attributable DALYs (thousands)

\begin{tabular}{lrlllllrrrrrrrrrrrrr} 
East Asia and Pacific & NA & NA & NA & NA & 8 & 2 & 50 & 11 & 144 & 43 & 162 & 38 & 121 & 23 & 42 & 12 & 527 & 127 & 655 \\
Europe and Central Asia & NA & NA & NA & NA & 3 & 2 & 20 & 8 & 54 & 33 & 55 & 47 & 36 & 52 & 10 & 22 & 178 & 165 & 342 \\
Latin America and the Caribbean & NA & NA & NA & NA & 4 & 3 & 16 & 12 & 36 & 26 & 32 & 25 & 24 & 24 & 13 & 18 & 125 & 108 & 232 \\
Middle East and North Africa & NA & NA & NA & NA & 1 & 0 & 3 & 1 & 9 & 2 & 7 & 2 & 4 & 2 & 1 & 1 & 24 & 7 & 31 \\
South Asia & NA & NA & NA & NA & 4 & 1 & 12 & 1 & 15 & 3 & 4 & 0 & 0 & 0 & 0 & 0 & 35 & 5 & 40 \\
Sub-Saharan Africa & NA & NA & NA & NA & 2 & 1 & 11 & 7 & 22 & 19 & 17 & 18 & 12 & 14 & 4 & 5 & 69 & 65 & 134 \\
\hline Low- and middle-income countries & NA & NA & NA & NA & 22 & 9 & 111 & 40 & 280 & 126 & 277 & 129 & 196 & 115 & 71 & 58 & 958 & 477 & 1,434 \\
High-income countries & NA & NA & NA & NA & 2 & 1 & 14 & 6 & 36 & 17 & 30 & 19 & 34 & 37 & 30 & 64 & 145 & 144 & 289 \\
\hline WORLD & NA & NA & NA & NA & 24 & 9 & 126 & 46 & 316 & 142 & 307 & 148 & 230 & 152 & 101 & 122 & 1,103 & 620 & 1,723
\end{tabular}


Risk factor:

Alcohol use

Disease:

Ischemic heart disease

\begin{tabular}{|c|c|c|c|c|c|c|c|c|c|c|c|c|c|c|c|c|c|c|c|}
\hline \multirow[b]{2}{*}{ Region } & \multicolumn{2}{|c|}{$0-4$ years } & \multicolumn{2}{|c|}{ 5-14 years } & \multicolumn{2}{|c|}{$15-29$ years } & \multicolumn{2}{|c|}{ 30-44 years } & \multicolumn{2}{|c|}{$45-59$ years } & \multicolumn{2}{|c|}{$60-69$ years } & \multicolumn{2}{|c|}{$70-79$ years } & \multicolumn{2}{|c|}{$80+$ years } & \multicolumn{3}{|c|}{ Total } \\
\hline & Male & Female & Male & Female & Male & Female & Male & Female & Male & Female & Male & Female & Male & Female & Male & Female & Male & Female & All \\
\hline \multicolumn{20}{|l|}{ PAF of Mortality (\%) } \\
\hline East Asia and Pacific & NA & NA & NA & NA & 2 & 0 & 3 & 0 & 4 & 0 & 2 & 0 & 1 & 0 & 1 & 0 & 2 & 0 & 1 \\
\hline Europe and Central Asia & NA & NA & NA & NA & 13 & 2 & 14 & 2 & 13 & 2 & 13 & 2 & 13 & 2 & 13 & 2 & 13 & 2 & 7 \\
\hline Latin America and the Caribbean & NA & NA & NA & NA & 14 & 2 & 14 & 2 & 15 & 2 & 15 & 2 & 13 & 1 & 12 & 1 & 14 & 1 & 8 \\
\hline Middle East and North Africa & NA & NA & NA & NA & 1 & 0 & 1 & 0 & 1 & 0 & 1 & 0 & 0 & 0 & 0 & 0 & 1 & 0 & 0 \\
\hline South Asia & NA & NA & NA & NA & 5 & 0 & 11 & 0 & 13 & 0 & 3 & 0 & 0 & 0 & 0 & 0 & 4 & 0 & 3 \\
\hline Sub-Saharan Africa & NA & NA & NA & NA & 5 & 0 & 5 & 0 & 5 & 0 & 5 & 0 & 4 & 0 & 4 & 0 & 5 & 0 & 2 \\
\hline Low- and middle-income countries & NA & NA & NA & NA & 5 & 0 & 9 & 0 & 10 & 0 & 6 & 1 & 5 & 1 & 5 & 1 & 7 & 1 & 4 \\
\hline High-income & NA & NA & NA & NA & -13 & -11 & -15 & -13 & -15 & -12 & -14 & -11 & -14 & -10 & -14 & -10 & -14 & -10 & -12 \\
\hline WORLD & NA & NA & NA & NA & 4 & 0 & 7 & 0 & 7 & 0 & 4 & 0 & 1 & -1 & -1 & -3 & 3 & -1 & 1 \\
\hline
\end{tabular}

PAF of YLL (\%)

\begin{tabular}{|c|c|c|c|c|c|c|c|c|c|c|c|c|c|c|c|c|c|c|c|}
\hline East Asia and Pacific & NA & NA & NA & NA & 2 & 0 & 3 & 0 & 4 & 0 & 2 & 0 & 1 & 0 & 1 & 0 & 2 & 0 & 1 \\
\hline Europe and Central Asia & NA & NA & NA & NA & 13 & 2 & 14 & 2 & 13 & 2 & 13 & 2 & 13 & 2 & 13 & 2 & 13 & 2 & 8 \\
\hline Latin America and the Caribbean & NA & NA & NA & NA & 14 & 2 & 14 & 2 & 15 & 2 & 15 & 2 & 13 & 1 & 12 & 1 & 14 & 2 & 9 \\
\hline Middle East and North Africa & NA & NA & NA & NA & 1 & 0 & 1 & 0 & 1 & 0 & 1 & 0 & 0 & 0 & 0 & 0 & 1 & 0 & 0 \\
\hline South Asia & NA & NA & NA & NA & 5 & 0 & 11 & 0 & 13 & 0 & 3 & 0 & 0 & 0 & 0 & 0 & 6 & 0 & 4 \\
\hline Sub-Saharan Africa & NA & NA & NA & NA & 5 & 0 & 5 & 0 & 5 & 0 & 5 & 0 & 4 & 0 & 4 & 0 & 5 & 0 & 2 \\
\hline Low- and middle-income countries & NA & NA & NA & NA & 5 & 0 & 9 & 0 & 10 & 0 & 6 & 1 & 5 & 1 & 5 & 1 & 7 & 1 & 4 \\
\hline High-income countries & NA & NA & NA & NA & -13 & -11 & -15 & -13 & -15 & -12 & -14 & -11 & -14 & -10 & -14 & -10 & -14 & -10 & -13 \\
\hline WORLD & NA & NA & NA & NA & 4 & 0 & 7 & 0 & 7 & 0 & 4 & 0 & 1 & -1 & -1 & -2 & 4 & -1 & 2 \\
\hline
\end{tabular}

\section{PAF of DALYs (\%)}

East Asia and Pacific

Europe and Central Asia

Latin America and the Caribbea

Middle East and North Africa

South Asia

Sub-Saharan Africa

Low- and middle-income countries

$\begin{array}{llll}\text { NA } & \text { NA } & \text { NA } & \text { NA } \\ \text { NA } & \text { NA } & \text { NA } & \text { NA } \\ \text { NA } & \text { NA } & \text { NA } & \text { NA } \\ \text { NA } & \text { NA } & \text { NA } & \text { NA } \\ \text { NA } & \text { NA } & \text { NA } & \text { NA } \\ \text { NA } & \text { NA } & \text { NA } & \text { NA } \\ \text { NA } & \text { NA } & \text { NA } & \text { NA } \\ \text { NA } & \text { NA } & \text { NA } & \text { NA }\end{array}$

$\begin{array}{rrrrr}2 & 0 & 3 & 0 & 4 \\ 13 & 2 & 14 & 2 & 13 \\ 14 & 2 & 14 & 2 & 15 \\ 1 & 0 & 1 & 0 & 1 \\ 5 & 0 & 11 & 0 & 13 \\ 5 & 0 & 5 & 0 & 5 \\ 5 & 0 & 9 & 0 & 10\end{array}$

\begin{tabular}{rrrrrrrrrr}
0 & 2 & 0 & 1 & 0 & 1 & 0 & 2 & 0 & 1 \\
2 & 13 & 2 & 13 & 2 & 13 & 2 & 13 & 2 & 8 \\
2 & 15 & 2 & 13 & 1 & 12 & 1 & 14 & 2 & 9 \\
0 & 1 & 0 & 0 & 0 & 0 & 0 & 1 & 0 & 0 \\
0 & 3 & 0 & 0 & 0 & 0 & 0 & 6 & 0 & 4 \\
0 & 5 & 0 & 4 & 0 & 4 & 0 & 5 & 0 & 2 \\
\hline 0 & 6 & 1 & 5 & 1 & 5 & 1 & 7 & 1 & 4 \\
-12 & -14 & -11 & -14 & -10 & -14 & -10 & -14 & -10 & -13 \\
-1 & 3 & 0 & 1 & -1 & -1 & -2 & 4 & -1 & 2
\end{tabular}

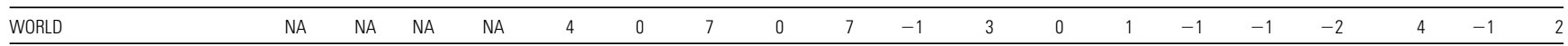

Attributable Mortality (thousands)

\begin{tabular}{lrlllllllrrrrrrrrrrr} 
East Asia and Pacific & NA & NA & NA & NA & 0 & 0 & 1 & 0 & 4 & 0 & 3 & 0 & 2 & 0 & 1 & 0 & 11 & 0 & 11 \\
Europe and Central Asia & NA & NA & NA & NA & 0 & 0 & 5 & 0 & 20 & 1 & 29 & 3 & 35 & 6 & 17 & 7 & 107 & 17 & 124 \\
Latin America and the Caribbean & NA & NA & NA & NA & 0 & 0 & 1 & 0 & 6 & 0 & 7 & 1 & 8 & 0 & 5 & 1 & 28 & 2 & 30 \\
Middle East and North Africa & NA & NA & NA & NA & 0 & 0 & 0 & 0 & 0 & 0 & 0 & 0 & 0 & 0 & 0 & 0 & 1 & 0 & 1 \\
South Asia & NA & NA & NA & NA & 1 & 0 & 5 & 0 & 32 & 0 & 9 & 0 & 0 & 0 & 0 & 0 & 46 & 0 & 46 \\
Sub-Saharan Africa & NA & NA & NA & NA & 0 & 0 & 0 & 0 & 2 & 0 & 3 & 0 & 2 & 0 & 1 & 0 & 8 & 0 & 8 \\
\hline Low- and middle-income countries & NA & NA & NA & NA & 1 & 0 & 13 & 0 & 64 & 1 & 52 & 3 & 46 & 7 & 24 & 8 & 201 & 19 & 220 \\
High-income countries & NA & NA & NA & NA & 0 & 0 & -2 & 0 & -13 & -3 & -18 & -6 & -32 & -16 & -35 & -41 & -101 & -66 & -167 \\
\hline WORLD & NA & NA & NA & NA & 1 & 0 & 11 & 0 & 51 & -1 & 33 & -3 & 14 & -9 & -11 & -33 & 100 & -46 & 53 \\
\hline
\end{tabular}

Attributable YLL (thousands)

\begin{tabular}{|c|c|c|c|c|c|c|c|c|c|c|c|c|c|c|c|c|c|c|c|}
\hline East Asia and Pacific & NA & NA & NA & NA & 5 & 0 & 22 & 0 & 78 & 0 & 40 & 0 & 17 & 0 & 5 & 0 & 166 & 0 & 166 \\
\hline Europe and Central Asia & NA & NA & NA & NA & 11 & 0 & 123 & 4 & 376 & 17 & 399 & 39 & 312 & 64 & 77 & 35 & 1,299 & 159 & 1,458 \\
\hline Latin America and the Caribbean & NA & NA & NA & NA & 6 & 0 & 30 & 2 & 110 & 7 & 98 & 9 & 68 & 5 & 24 & 3 & 336 & 26 & 362 \\
\hline Middle East and North Africa & NA & NA & NA & NA & 1 & 0 & 3 & 0 & 8 & 0 & 7 & 0 & 0 & 0 & 0 & 0 & 17 & 0 & 17 \\
\hline South Asia & NA & NA & NA & NA & 14 & 0 & 127 & 0 & 590 & 0 & 119 & 0 & 0 & 0 & 0 & 0 & 850 & 0 & 850 \\
\hline Sub-Saharan Africa & NA & NA & NA & NA & 1 & 0 & 8 & 0 & 36 & 0 & 35 & 0 & 21 & 0 & 4 & 0 & 106 & 0 & 106 \\
\hline Low- and middle-income countries & NA & NA & NA & NA & 36 & 1 & 314 & 5 & 1,198 & 24 & 698 & 48 & 419 & 69 & 111 & 37 & 2,775 & 184 & 2,960 \\
\hline High-income countries & NA & NA & NA & NA & -3 & -1 & -56 & -12 & -245 & -54 & -244 & -86 & -284 & -157 & -152 & -169 & -984 & -479 & $-1,463$ \\
\hline WORLD & NA & NA & NA & NA & 33 & 0 & 258 & -6 & 953 & -29 & 453 & -39 & 135 & -89 & -41 & -131 & 1,791 & -295 & 1,497 \\
\hline
\end{tabular}

Attributable DALYs (thousands)

\begin{tabular}{lllllrlrrrrrrrrrrrrr} 
East Asia and Pacific & NA & NA & NA & NA & 7 & 0 & 24 & 0 & 83 & 0 & 42 & 0 & 17 & 0 & 5 & 0 & 178 & 0 & 178 \\
Europe and Central Asia & NA & NA & NA & NA & 13 & 1 & 132 & 4 & 391 & 19 & 412 & 41 & 318 & 65 & 78 & 35 & 1,344 & 164 & 1,508 \\
Latin America and the Caribbean & NA & NA & NA & NA & 10 & 1 & 34 & 2 & 117 & 8 & 104 & 9 & 70 & 5 & 25 & 3 & 361 & 28 & 388 \\
Middle East and North Africa & NA & NA & NA & NA & 1 & 0 & 3 & 0 & 8 & 0 & 7 & 0 & 0 & 0 & 0 & 0 & 19 & 0 & 19 \\
South Asia & NA & NA & NA & NA & 20 & 0 & 145 & 0 & 626 & 0 & 125 & 0 & 0 & 0 & 0 & 0 & 916 & 0 & 916 \\
Sub-Saharan Africa & NA & NA & NA & NA & 2 & 0 & 9 & 0 & 39 & 0 & 37 & 0 & 21 & 0 & 4 & 0 & 113 & 0 & 113 \\
\hline Low- and middle-income countries & NA & NA & NA & NA & 52 & 1 & 348 & 6 & 1,264 & 26 & 727 & 50 & 427 & 70 & 113 & 38 & 2,931 & 192 & 3,123 \\
High-income countries & NA & NA & NA & NA & -5 & -2 & -64 & -16 & -270 & -64 & -263 & -97 & -299 & -168 & -158 & -175 & $-1,057$ & -522 & $-1,579$ \\
\hline WORLD & NA & NA & NA & NA & 48 & -1 & 284 & -9 & 994 & -38 & 464 & -47 & 128 & -98 & -45 & -137 & 1,873 & -330 & 1,543
\end{tabular}

Source: Authors' calculations.

Note: $\mathrm{NA}=$ not applicable. 
Table 4A.69

Risk factor:

Alcohol use

Disease:

Cerebrovascular disease

\begin{tabular}{|c|c|c|c|c|c|c|c|c|c|c|c|c|c|c|c|c|c|c|c|}
\hline \multirow[b]{2}{*}{ Region } & \multicolumn{2}{|c|}{$0-4$ years } & \multicolumn{2}{|c|}{ 5-14 years } & \multicolumn{2}{|c|}{ 15-29 years } & \multicolumn{2}{|c|}{ 30-44 years } & \multicolumn{2}{|c|}{$45-59$ years } & \multicolumn{2}{|c|}{$60-69$ years } & \multicolumn{2}{|c|}{ 70-79 years } & \multicolumn{2}{|c|}{$80+$ years } & \multicolumn{3}{|c|}{ Total } \\
\hline & Male & Female & Male & Female & Male & Female & Male & Female & Male & Female & Male & Female & Male & Female & Male & Female & Male & Female & All \\
\hline \multicolumn{20}{|l|}{ PAF of Mortality (\%) } \\
\hline East Asia and Pacific & NA & NA & NA & NA & 14 & 1 & 17 & 0 & 15 & 0 & 13 & 0 & 11 & 0 & 10 & 0 & 12 & 0 & 6 \\
\hline Europe and Central Asia & NA & NA & NA & NA & 19 & 6 & 19 & 7 & 19 & 6 & 15 & 3 & 10 & 2 & 9 & 1 & 13 & 2 & 7 \\
\hline Latin America and the Caribbean & NA & NA & NA & NA & 15 & 11 & 17 & 14 & 15 & 10 & 13 & 7 & 7 & 3 & 6 & 2 & 10 & 5 & 7 \\
\hline Middle East and North Africa & NA & NA & NA & NA & 3 & 1 & 5 & 1 & 3 & 0 & 2 & 0 & 1 & 0 & 1 & 0 & 2 & 0 & 1 \\
\hline South Asia & NA & NA & NA & NA & 3 & 2 & 6 & 0 & 3 & 0 & 0 & 0 & 0 & 0 & 0 & 0 & 1 & 0 & 0 \\
\hline Sub-Saharan Africa & NA & NA & NA & NA & 13 & 2 & 16 & 3 & 13 & 5 & 10 & 3 & 8 & 1 & 7 & 1 & 10 & 2 & 5 \\
\hline Low- and middle-income countries & NA & NA & NA & NA & 11 & 3 & 15 & 4 & 12 & 2 & 10 & 1 & 8 & 1 & 7 & 1 & 9 & 1 & 5 \\
\hline High-income countries & NA & NA & NA & NA & 16 & -28 & 16 & -32 & 14 & -27 & 11 & -26 & 7 & -25 & 6 & -28 & 8 & -27 & -13 \\
\hline WORLD & NA & NA & NA & NA & 11 & 1 & 15 & 1 & 12 & 1 & 10 & 0 & 8 & -2 & 7 & -7 & 9 & -3 & 2 \\
\hline
\end{tabular}

PAF of YLL $(\%)$

\begin{tabular}{lrrrrrrrrrrrrrrrrrrr} 
East Asia and Pacific & NA & NA & NA & NA & 14 & 1 & 17 & 0 & 15 & 0 & 13 & 0 & 11 & 0 & 10 & 0 & 13 & 0 & 7 \\
Europe and Central Asia & NA & NA & NA & NA & 19 & 6 & 19 & 7 & 19 & 6 & 15 & 3 & 10 & 2 & 9 & 1 & 14 & 3 & 8 \\
Latin America and the Caribbean & NA & NA & NA & NA & 15 & 11 & 17 & 14 & 15 & 10 & 13 & 7 & 7 & 3 & 6 & 2 & 12 & 7 & 9 \\
Middle East and North Africa & NA & NA & NA & NA & 3 & 1 & 5 & 1 & 3 & 0 & 2 & 0 & 1 & 0 & 1 & 0 & 2 & 0 & 1 \\
South Asia & NA & NA & NA & NA & 3 & 2 & 6 & 0 & 3 & 0 & 0 & 0 & 0 & 0 & 0 & 0 & 1 & 0 & 1 \\
Sub-Saharan Africa & NA & NA & NA & NA & 13 & 2 & 16 & 3 & 13 & 5 & 10 & 3 & 8 & 1 & 7 & 1 & 11 & 3 & 6 \\
\hline Low- and middle-income countries & NA & NA & NA & NA & 11 & 3 & 15 & 4 & 12 & 2 & 10 & 1 & 8 & 1 & 7 & 1 & 10 & 1 & 6 \\
High-income countries & NA & NA & NA & NA & 16 & -28 & 16 & -32 & 14 & -27 & 11 & -26 & 7 & -25 & 6 & -28 & 9 & -27 & -10 \\
\hline WORLD & NA & NA & NA & NA & 11 & 1 & 15 & 1 & 12 & 1 & 10 & 0 & 8 & -2 & 7 & -6 & 10 & -2 & 10 \\
\hline
\end{tabular}

\section{PAF of DALYs (\%)}

East Asia and Pacific

Europe and Central Asia

Latin America and the Caribbean

Middle East and North Africa

South Asia

Sub-Saharan Africa

Low- and middle-income counties

$\begin{array}{llllrrrrrr}\text { NA } & \text { NA } & \text { NA } & \text { NA } & 14 & 1 & 14 & 0 & 13 & \\ \text { NA } & \text { NA } & \text { NA } & \text { NA } & 19 & 6 & 17 & 5 & 18 & \\ \text { NA } & \text { NA } & \text { NA } & \text { NA } & 15 & 11 & 15 & 11 & 13 & \\ \text { NA } & \text { NA } & \text { NA } & \text { NA } & 3 & 1 & 4 & 0 & 3 & 0 \\ \text { NA } & \text { NA } & \text { NA } & \text { NA } & 3 & 2 & 6 & 0 & 2 & 0 \\ \text { NA } & \text { NA } & \text { NA } & \text { NA } & 13 & 2 & 15 & 3 & 12 & \\ \text { NA } & \text { NA } & \text { NA } & \text { NA } & 11 & 3 & 13 & 3 & 11 & \\ \text { NA } & \text { NA } & \text { NA } & \text { NA } & 16 & -28 & 12 & -37 & 10 & -32\end{array}$

$\begin{array}{rr}0 & 11 \\ 5 & 14 \\ 8 & 11 \\ 0 & 2 \\ 0 & 0 \\ 4 & 10 \\ 2 & 9 \\ -32 & 7\end{array}$

\begin{tabular}{|c|c|c|c|c|c|c|c|c|c|c|c|c|c|c|c|c|c|c|c|}
\hline WORLD & NA & NA & NA & NA & 11 & 1 & 13 & -2 & 11 & -1 & 9 & -1 & 7 & -3 & 6 & -7 & 9 & -3 & 3 \\
\hline
\end{tabular}

Attributable Mortality (thousands)

\begin{tabular}{lrllllllrrrrrrrrrrrr} 
East Asia and Pacific & NA & NA & NA & NA & 1 & 0 & 4 & 0 & 21 & 0 & 32 & 0 & 37 & 0 & 18 & 0 & 114 & 0 & 114 \\
Europe and Central Asia & NA & NA & NA & NA & 1 & 0 & 2 & 0 & 10 & 2 & 16 & 3 & 15 & 5 & 7 & 4 & 52 & 15 & 67 \\
Latin America and the Caribbean & NA & NA & NA & NA & 0 & 0 & 1 & 1 & 3 & 2 & 3 & 2 & 3 & 1 & 2 & 1 & 13 & 7 & 20 \\
Middle East and North Africa & NA & NA & NA & NA & 0 & 0 & 0 & 0 & 0 & 0 & 0 & 0 & 0 & 0 & 0 & 0 & 1 & 0 & 1 \\
South Asia & NA & NA & NA & NA & 0 & 0 & 1 & 0 & 2 & 0 & 1 & 0 & 0 & 0 & 0 & 0 & 3 & 0 & 3 \\
Sub-Saharan Africa & NA & NA & NA & NA & 0 & 0 & 2 & 0 & 4 & 2 & 4 & 1 & 3 & 1 & 2 & 0 & 14 & 5 & 19 \\
\hline Low- and middle-income countries & NA & NA & NA & NA & 2 & 0 & 10 & 2 & 40 & 6 & 57 & 6 & 58 & 7 & 30 & 5 & 197 & 27 & 224 \\
High-income countries & NA & NA & NA & NA & 0 & 0 & 1 & -1 & 3 & -4 & 5 & -7 & 7 & -25 & 9 & -87 & 26 & -126 & -100 \\
\hline WORLD & NA & NA & NA & NA & 2 & 0 & 11 & 0 & 44 & 2 & 61 & -1 & 66 & -18 & 39 & -82 & 223 & -99 & 124 \\
\hline
\end{tabular}

Attributable YLL (thousands)

\begin{tabular}{|c|c|c|c|c|c|c|c|c|c|c|c|c|c|c|c|c|c|c|c|}
\hline East Asia and Pacific & NA & NA & NA & NA & 22 & 1 & 99 & 0 & 394 & 0 & 432 & 0 & 330 & 0 & 92 & 0 & 1,369 & 1 & 1,370 \\
\hline Europe and Central Asia & NA & NA & NA & NA & 15 & 3 & 57 & 12 & 195 & 44 & 222 & 45 & 134 & 56 & 34 & 18 & 656 & 178 & 834 \\
\hline Latin America and the Caribbean & NA & NA & NA & NA & 7 & 5 & 26 & 23 & 60 & 39 & 46 & 24 & 24 & 12 & 10 & 6 & 174 & 108 & 282 \\
\hline Middle East and North Africa & NA & NA & NA & NA & 2 & 0 & 3 & 0 & 6 & 1 & 3 & 1 & 2 & 0 & 0 & 0 & 16 & 2 & 18 \\
\hline South Asia & NA & NA & NA & NA & 4 & 1 & 14 & 0 & 36 & 0 & 9 & 0 & 0 & 0 & 0 & 0 & 62 & 1 & 64 \\
\hline Sub-Saharan Africa & NA & NA & NA & NA & 12 & 1 & 40 & 7 & 66 & 33 & 49 & 21 & 30 & 8 & 8 & 2 & 206 & 73 & 279 \\
\hline Low- and middle-income countries & NA & NA & NA & NA & 63 & 11 & 239 & 42 & 757 & 117 & 761 & 91 & 520 & 76 & 144 & 26 & 2,484 & 363 & 2,847 \\
\hline High-income countries & NA & NA & NA & NA & 4 & -5 & 23 & -34 & 65 & -85 & 62 & -109 & 64 & -252 & 40 & -359 & 259 & -845 & -586 \\
\hline WORLD & NA & NA & NA & NA & 67 & 5 & 262 & 8 & 822 & 32 & 823 & -18 & 584 & -177 & 184 & -333 & 2,743 & -482 & 2,261 \\
\hline \multicolumn{20}{|l|}{ Attributable DALYs (thousands) } \\
\hline East Asia and Pacific & NA & NA & NA & NA & 22 & 1 & 119 & 0 & 462 & 0 & 497 & 0 & 357 & 0 & 96 & 0 & 1,553 & 1 & 1,553 \\
\hline Europe and Central Asia & NA & NA & NA & NA & 15 & 3 & 65 & 13 & 225 & 48 & 256 & 48 & 148 & 58 & 36 & 17 & 745 & 188 & 932 \\
\hline Latin America and the Caribbean & NA & NA & NA & NA & 7 & 5 & 32 & 26 & 73 & 43 & 54 & 26 & 26 & 12 & 10 & 6 & 203 & 119 & 322 \\
\hline Middle East and North Africa & NA & NA & NA & NA & 2 & 0 & 3 & 0 & 7 & 1 & 4 & 1 & 2 & 0 & 0 & 0 & 18 & 2 & 20 \\
\hline South Asia & NA & NA & NA & NA & 4 & 1 & 16 & 0 & 39 & 0 & 9 & 0 & 0 & 0 & 0 & 0 & 67 & 1 & 68 \\
\hline Sub-Saharan Africa & NA & NA & NA & NA & 12 & 1 & 42 & 7 & 71 & 34 & 53 & 22 & 31 & 8 & 8 & 2 & 217 & 75 & 292 \\
\hline Low- and middle-income countries & NA & NA & NA & NA & 63 & 11 & 276 & 47 & 875 & 126 & 873 & 97 & 565 & 79 & 150 & 26 & 2,802 & 386 & 3,188 \\
\hline High-income countries & NA & NA & NA & NA & 4 & -5 & 33 & -79 & 95 & -204 & 82 & -228 & 75 & -411 & 43 & -466 & 331 & $-1,394$ & $-1,063$ \\
\hline WORLD & NA & NA & NA & NA & 67 & 5 & 309 & -32 & 970 & -78 & 955 & -131 & 639 & -332 & 194 & -440 & 3,134 & $-1,008$ & 2,126 \\
\hline
\end{tabular}


Table 4A.70

Risk factor:

Alcohol use

Disease:

Cirrhosis of the liver

\begin{tabular}{|c|c|c|c|c|c|c|c|c|c|c|c|c|c|c|c|c|c|c|c|}
\hline \multirow[b]{2}{*}{ Region } & \multicolumn{2}{|c|}{$0-4$ years } & \multicolumn{2}{|c|}{ 5-14 years } & \multicolumn{2}{|c|}{$15-29$ years } & \multicolumn{2}{|c|}{ 30-44 years } & \multicolumn{2}{|c|}{$45-59$ years } & \multicolumn{2}{|c|}{$60-69$ years } & \multicolumn{2}{|c|}{ 70-79 years } & \multicolumn{2}{|c|}{$80+$ years } & \multicolumn{3}{|c|}{ Total } \\
\hline & Male & Female & Male & Female & Male & Female & Male & Female & Male & Female & Male & Female & Male & Female & Male & Female & Male & Female & All \\
\hline \multicolumn{20}{|l|}{ PAF of Mortality (\%) } \\
\hline East Asia and Pacific & NA & NA & NA & NA & 45 & 10 & 49 & 9 & 48 & 9 & 45 & 6 & 40 & 3 & 40 & 3 & 45 & 6 & 31 \\
\hline Europe and Central Asia & NA & NA & NA & NA & 69 & 55 & 67 & 54 & 70 & 55 & 62 & 47 & 50 & 40 & 50 & 41 & 64 & 48 & 58 \\
\hline Latin America and the Caribbean & NA & NA & NA & NA & 56 & 46 & 59 & 49 & 57 & 45 & 51 & 42 & 34 & 30 & 34 & 30 & 52 & 39 & 49 \\
\hline Middle East and North Africa & NA & NA & NA & NA & 17 & 8 & 16 & 7 & 16 & 7 & 12 & 3 & 8 & 2 & 8 & 3 & 12 & 4 & 8 \\
\hline South Asia & NA & NA & NA & NA & 12 & 11 & 23 & 4 & 8 & 4 & 2 & 0 & 0 & 0 & 0 & 0 & 7 & 3 & 5 \\
\hline Sub-Saharan Africa & NA & NA & NA & NA & 50 & 25 & 58 & 33 & 56 & 35 & 53 & 27 & 46 & 20 & 46 & 20 & 53 & 28 & 44 \\
\hline Low- and middle-income countries & NA & NA & NA & NA & 34 & 15 & 49 & 21 & 43 & 22 & 37 & 19 & 29 & 13 & 26 & 11 & 38 & 16 & 30 \\
\hline High-income countries & NA & NA & NA & NA & 66 & 53 & 65 & 49 & 64 & 51 & 56 & 43 & 49 & 39 & 49 & 39 & 58 & 44 & 54 \\
\hline WORLD & NA & NA & NA & NA & 34 & 15 & 52 & 24 & 46 & 26 & 41 & 22 & 33 & 17 & 31 & 18 & 41 & 20 & 34 \\
\hline
\end{tabular}

PAF of YLL (\%)

\begin{tabular}{|c|c|c|c|c|c|c|c|c|c|c|c|c|c|c|c|c|c|c|c|}
\hline East Asia and Pacific & NA & NA & NA & NA & 45 & 10 & 49 & 9 & 48 & 9 & 45 & 6 & 40 & 3 & 40 & 3 & 45 & 7 & 32 \\
\hline Europe and Central Asia & NA & NA & NA & NA & 69 & 55 & 67 & 54 & 70 & 55 & 62 & 47 & 50 & 40 & 50 & 41 & 66 & 50 & 60 \\
\hline Latin America and the Caribbean & NA & NA & NA & NA & 56 & 46 & 59 & 49 & 57 & 45 & 51 & 42 & 34 & 30 & 34 & 30 & 54 & 42 & 52 \\
\hline Middle East and North Africa & NA & NA & NA & NA & 17 & 8 & 16 & 7 & 16 & 7 & 12 & 3 & 8 & 2 & 8 & 3 & 12 & 4 & 9 \\
\hline South Asia & NA & NA & NA & NA & 12 & 11 & 23 & 4 & 8 & 4 & 2 & 0 & 0 & 0 & 0 & 0 & 8 & 3 & 6 \\
\hline Sub-Saharan Africa & NA & NA & NA & NA & 50 & 25 & 58 & 33 & 56 & 35 & 53 & 27 & 46 & 20 & 46 & 20 & 54 & 29 & 45 \\
\hline Low- and middle-income countries & NA & NA & NA & NA & 34 & 15 & 49 & 21 & 43 & 22 & 37 & 19 & 29 & 13 & 26 & 11 & 39 & 16 & 30 \\
\hline High-income countries & NA & NA & NA & NA & 66 & 53 & 65 & 49 & 64 & 51 & 56 & 43 & 49 & 39 & 49 & 39 & 60 & 46 & 56 \\
\hline WORLD & NA & NA & NA & NA & 34 & 15 & 52 & 24 & 46 & 26 & 41 & 22 & 33 & 17 & 30 & 17 & 42 & 19 & 34 \\
\hline
\end{tabular}

\section{PAF of DALYs $(\%)$}

\begin{tabular}{|c|c|c|c|c|c|c|c|c|c|c|c|c|c|c|c|c|c|c|}
\hline East Asia and Pacific & NA & NA & NA & NA & 45 & 10 & 49 & 9 & 48 & 9 & 45 & 6 & 40 & 3 & 40 & 3 & 45 & 7 \\
\hline Europe and Central Asia & NA & NA & NA & NA & 69 & 55 & 67 & 54 & 70 & 55 & 62 & 47 & 50 & 40 & 50 & 41 & 66 & 50 \\
\hline Latin America and the Caribbean & NA & NA & NA & NA & 56 & 46 & 59 & 49 & 57 & 45 & 51 & 42 & 34 & 30 & 34 & 30 & 55 & 42 \\
\hline Middle East and North Africa & NA & NA & NA & NA & 17 & 8 & 16 & 7 & 16 & 7 & 12 & 3 & 8 & 2 & 8 & 3 & 12 & 4 \\
\hline South Asia & NA & NA & NA & NA & 12 & 11 & 23 & 4 & 8 & 4 & 2 & 0 & 0 & 0 & 0 & 0 & 8 & 3 \\
\hline Sub-Saharan Africa & NA & NA & NA & NA & 50 & 25 & 58 & 33 & 56 & 35 & 53 & 27 & 46 & 20 & 46 & 20 & 54 & 29 \\
\hline Low- and middle-income countries & NA & NA & NA & NA & 36 & 15 & 49 & 21 & 43 & 22 & 37 & 19 & 29 & 13 & 27 & 11 & 39 & 16 \\
\hline High-income countries & NA & NA & NA & NA & 66 & 53 & 65 & 49 & 64 & 51 & 56 & 43 & 49 & 39 & 49 & 39 & 60 & 46 \\
\hline WORLD & NA & NA & NA & NA & 36 & 16 & 52 & 25 & 46 & 26 & 41 & 22 & 33 & 17 & 31 & 17 & 42 & 19 \\
\hline
\end{tabular}

Attributable Mortality (thousands)

\begin{tabular}{lrrrrrrrrrrrrrrrrrrr} 
East Asia and Pacific & NA & NA & NA & NA & 2 & 0 & 9 & 1 & 21 & 1 & 13 & 1 & 8 & 0 & 3 & 0 & 56 & 4 & 60 \\
Europe and Central Asia & NA & NA & NA & NA & 1 & 0 & 8 & 2 & 17 & 7 & 11 & 5 & 4 & 3 & 1 & 1 & 42 & 19 & 60 \\
Latin America and the Caribbean & NA & NA & NA & NA & 1 & 0 & 7 & 1 & 12 & 2 & 6 & 2 & 3 & 1 & 1 & 1 & 29 & 7 & 36 \\
Middle East and North Africa & NA & NA & NA & NA & 0 & 0 & 0 & 0 & 1 & 0 & 1 & 0 & 0 & 0 & 0 & 0 & 2 & 1 & 3 \\
South Asia & NA & NA & NA & NA & 1 & 1 & 3 & 0 & 3 & 1 & 0 & 0 & 0 & 0 & 0 & 0 & 7 & 2 & 9 \\
Sub-Saharan Africa & NA & NA & NA & NA & 1 & 0 & 3 & 1 & 7 & 2 & 5 & 2 & 3 & 1 & 1 & 0 & 20 & 6 & 26 \\
\hline Low- and middle-income countries & NA & NA & NA & NA & 6 & 2 & 31 & 5 & 61 & 13 & 35 & 9 & 19 & 6 & 5 & 2 & 157 & 38 & 195 \\
High-income countries & NA & NA & NA & NA & 0 & 0 & 6 & 2 & 19 & 5 & 12 & 4 & 7 & 4 & 2 & 2 & 47 & 16 & 63 \\
\hline WORLD & NA & NA & NA & NA & 6 & 2 & 37 & 7 & 80 & 18 & 47 & 13 & 26 & 10 & 8 & 5 & 204 & 55 & 258 \\
\hline
\end{tabular}

Attributable YLL (thousands)

\begin{tabular}{|c|c|c|c|c|c|c|c|c|c|c|c|c|c|c|c|c|c|c|c|}
\hline East Asia and Pacific & NA & NA & NA & NA & 50 & 6 & 224 & 15 & 406 & 30 & 173 & 13 & 76 & 5 & 14 & 1 & 943 & 71 & 1,014 \\
\hline Europe and Central Asia & NA & NA & NA & NA & 32 & 13 & 184 & 58 & 328 & 133 & 145 & 76 & 39 & 35 & 4 & 6 & 732 & 320 & 1,052 \\
\hline Latin America and the Caribbean & NA & NA & NA & NA & 23 & 5 & 168 & 24 & 230 & 44 & 83 & 27 & 23 & 12 & 5 & 3 & 530 & 116 & 646 \\
\hline Middle East and North Africa & NA & NA & NA & NA & 3 & 1 & 3 & 1 & 18 & 6 & 10 & 2 & 3 & 1 & 1 & 0 & 39 & 11 & 50 \\
\hline South Asia & NA & NA & NA & NA & 24 & 30 & 69 & 7 & 51 & 13 & 6 & 0 & 0 & 0 & 0 & 0 & 151 & 51 & 202 \\
\hline Sub-Saharan Africa & NA & NA & NA & NA & 19 & 6 & 81 & 16 & 136 & 46 & 62 & 25 & 31 & 10 & 4 & 1 & 334 & 104 & 438 \\
\hline Low- and middle-income countries & NA & NA & NA & NA & 151 & 60 & 730 & 122 & 1,168 & 271 & 480 & 143 & 172 & 62 & 27 & 12 & 2,729 & 672 & 3,401 \\
\hline High-income countries & NA & NA & NA & NA & 5 & 2 & 151 & 40 & 362 & 99 & 163 & 55 & 62 & 40 & 11 & 11 & 755 & 247 & 1,002 \\
\hline WORLD & NA & NA & NA & NA & 156 & 62 & 881 & 163 & 1,531 & 370 & 642 & 198 & 235 & 102 & 38 & 24 & 3,483 & 919 & 4,403 \\
\hline \multicolumn{20}{|l|}{ Attributable DALYs (thousands) } \\
\hline East Asia and Pacific & NA & NA & NA & NA & 73 & 8 & 282 & 22 & 476 & 39 & 196 & 16 & 87 & 6 & 16 & 2 & 1,131 & 93 & 1,224 \\
\hline Europe and Central Asia & NA & NA & NA & NA & 45 & 18 & 226 & 80 & 375 & 162 & 160 & 87 & 44 & 40 & 4 & 7 & 853 & 394 & 1,247 \\
\hline Latin America and the Caribbean & NA & NA & NA & NA & 37 & 7 & 208 & 34 & 265 & 55 & 92 & 32 & 26 & 14 & 6 & 4 & 634 & 147 & 781 \\
\hline Middle East and North Africa & NA & NA & NA & NA & 4 & 1 & 4 & 2 & 21 & 7 & 11 & 2 & 4 & 1 & 1 & 0 & 46 & 14 & 60 \\
\hline South Asia & NA & NA & NA & NA & 30 & 34 & 84 & 9 & 59 & 15 & 7 & 0 & 0 & 0 & 0 & 0 & 180 & 59 & 240 \\
\hline Sub-Saharan Africa & NA & NA & NA & NA & 32 & 9 & 101 & 23 & 156 & 56 & 74 & 32 & 36 & 12 & 5 & 2 & 404 & 133 & 537 \\
\hline Low- and middle-income countries & NA & NA & NA & NA & 221 & 78 & 905 & 170 & 1,352 & 335 & 541 & 170 & 197 & 73 & 32 & 15 & 3,248 & 841 & 4,089 \\
\hline High-income countries & NA & NA & NA & NA & 9 & 4 & 194 & 57 & 421 & 121 & 182 & 65 & 70 & 48 & 13 & 14 & 889 & 310 & 1,199 \\
\hline WORLD & NA & NA & NA & NA & 230 & 82 & 1,099 & 227 & 1,773 & 456 & 723 & 235 & 267 & 121 & 45 & 29 & 4,137 & 1,150 & 5,287 \\
\hline
\end{tabular}

Source: Authors' calculations.

Note: $\mathrm{NA}=$ not applicable. 
Table 4A.71

Risk factor:

Disease:

Alcohol use

Road traffic accidents

\begin{tabular}{|c|c|c|c|c|c|c|c|c|c|c|c|c|c|c|c|c|c|c|c|}
\hline \multirow[b]{2}{*}{ Region } & \multicolumn{2}{|c|}{$0-4$ years } & \multicolumn{2}{|c|}{ 5-14 years } & \multicolumn{2}{|c|}{$15-29$ years } & \multicolumn{2}{|c|}{$30-44$ years } & \multicolumn{2}{|c|}{$45-59$ years } & \multicolumn{2}{|c|}{ 60-69 years } & \multicolumn{2}{|c|}{ 70-79 years } & \multicolumn{2}{|c|}{$80+$ years } & \multicolumn{3}{|c|}{ Total } \\
\hline & Male & Female & Male & Female & Male & Female & Male & Female & Male & Female & Male & Female & Male & Female & Male & Female & Male & Female & All \\
\hline \multicolumn{20}{|l|}{ PAF of Mortality (\%) } \\
\hline East Asia and Pacific & 9 & 5 & 9 & 6 & 27 & 7 & 30 & 10 & 13 & 8 & 11 & 6 & 11 & 6 & 11 & 6 & 22 & 8 & 18 \\
\hline Europe and Central Asia & 23 & 15 & 26 & 18 & 66 & 23 & 70 & 32 & 47 & 26 & 40 & 19 & 39 & 19 & 36 & 19 & 57 & 23 & 48 \\
\hline Latin America and the Caribbean & 16 & 11 & 17 & 11 & 54 & 13 & 58 & 19 & 34 & 15 & 28 & 11 & 28 & 11 & 28 & 11 & 45 & 14 & 39 \\
\hline Middle East and North Africa & 3 & 2 & 3 & 2 & 8 & 3 & 10 & 4 & 4 & 3 & 3 & 2 & 3 & 2 & 3 & 1 & 6 & 3 & 5 \\
\hline South Asia & 4 & 2 & 4 & 3 & 20 & 3 & 23 & 5 & 10 & 4 & 8 & 3 & 8 & 3 & 8 & 3 & 16 & 3 & 12 \\
\hline Sub-Saharan Africa & 12 & 8 & 13 & 8 & 38 & 10 & 42 & 14 & 22 & 11 & 18 & 8 & 17 & 8 & 17 & 7 & 25 & 9 & 20 \\
\hline Low- and middle-income countries & 9 & 5 & 10 & 7 & 31 & 8 & 35 & 11 & 18 & 9 & 15 & 7 & 13 & 7 & 13 & 7 & 24 & 8 & 20 \\
\hline High-income countries & 19 & 12 & 19 & 12 & 40 & 15 & 43 & 20 & 22 & 16 & 18 & 12 & 19 & 13 & 19 & 13 & 32 & 15 & 27 \\
\hline WORLD & 10 & 5 & 11 & 7 & 32 & 9 & 35 & 12 & 18 & 9 & 15 & 8 & 14 & 8 & 14 & 8 & 25 & 9 & 21 \\
\hline
\end{tabular}

PAF of YLL (\%)

\begin{tabular}{|c|c|c|c|c|c|c|c|c|c|c|c|c|c|c|c|c|c|c|c|}
\hline East Asia and Pacific & 9 & 5 & 9 & 6 & 27 & 7 & 30 & 10 & 13 & 8 & 11 & 6 & 11 & 6 & 11 & 6 & 23 & 8 & 19 \\
\hline Europe and Central Asia & 23 & 15 & 26 & 18 & 66 & 23 & 70 & 32 & 47 & 26 & 40 & 19 & 39 & 19 & 36 & 19 & 59 & 24 & 50 \\
\hline Latin America and the Caribbean & 16 & 11 & 17 & 11 & 54 & 13 & 58 & 19 & 34 & 15 & 28 & 11 & 28 & 11 & 28 & 11 & 47 & 14 & 40 \\
\hline Middle East and North Africa & 3 & 2 & 3 & 2 & 8 & 3 & 10 & 4 & 4 & 3 & 3 & 2 & 3 & 2 & 3 & 1 & 6 & 3 & 5 \\
\hline South Asia & 4 & 2 & 4 & 3 & 20 & 3 & 23 & 5 & 10 & 4 & 8 & 3 & 8 & 3 & 8 & 3 & 16 & 3 & 13 \\
\hline Sub-Saharan Africa & 12 & 8 & 13 & 8 & 38 & 10 & 42 & 14 & 22 & 11 & 18 & 8 & 17 & 8 & 17 & 7 & 25 & 9 & 20 \\
\hline Low- and middle-income countries & 9 & 5 & 10 & 7 & 31 & 8 & 35 & 11 & 18 & 9 & 15 & 7 & 13 & 7 & 13 & 7 & 25 & 8 & 21 \\
\hline High-income countries & 19 & 12 & 19 & 12 & 40 & 15 & 43 & 20 & 22 & 16 & 18 & 12 & 19 & 13 & 19 & 13 & 35 & 16 & 29 \\
\hline WORLD & 10 & 5 & 11 & 7 & 32 & 9 & 35 & 12 & 18 & 9 & 15 & 8 & 14 & 8 & 14 & 8 & 26 & 9 & 21 \\
\hline
\end{tabular}

\section{PAF of DALYs (\%)}

East Asia and Pacific

Europe and Central Asia

Latin America and the Caribbean

Middle East and North Africa

South Asia

Sub-Saharan Africa

Low- and middle-income countries

High-income countries

\begin{tabular}{rrrrrrrrr}
8 & 4 & 8 & 5 & 25 & 6 & 28 & 9 & 13 \\
21 & 14 & 23 & 15 & 62 & 21 & 66 & 29 & 45 \\
14 & 9 & 15 & 9 & 50 & 12 & 54 & 17 & 32 \\
3 & 2 & 3 & 2 & 7 & 3 & 9 & 4 & 4 \\
3 & 2 & 4 & 2 & 18 & 3 & 22 & 4 & 10 \\
11 & 7 & 12 & 8 & 35 & 9 & 40 & 13 & 21 \\
\hline 8 & 5 & 9 & 6 & 29 & 7 & 33 & 11 & 17
\end{tabular}

\begin{tabular}{rrrrrrrrrr}
8 & 11 & 6 & 11 & 6 & 11 & 6 & 22 & 7 & 17 \\
24 & 39 & 18 & 38 & 18 & 35 & 19 & 56 & 22 & 47 \\
14 & 27 & 10 & 27 & 10 & 27 & 11 & 43 & 12 & 36 \\
3 & 3 & 2 & 3 & 2 & 3 & 1 & 6 & 2 & 5 \\
4 & 8 & 3 & 8 & 3 & 8 & 3 & 15 & 3 & 11 \\
10 & 17 & 8 & 16 & 8 & 16 & 7 & 24 & 9 & 19 \\
\hline 8 & 14 & 7 & 13 & 7 & 13 & 6 & 23 & 7 & 19 \\
15 & 18 & 12 & 18 & 12 & 19 & 13 & 33 & 14 & 28 \\
9 & 15 & 7 & 14 & 8 & 14 & 8 & 24 & 8 & 19
\end{tabular}

Atributable Mortality (thousands)

\begin{tabular}{|c|c|c|c|c|c|c|c|c|c|c|c|c|c|c|c|c|c|c|c|}
\hline \multicolumn{20}{|l|}{ Attributable Mortality (thousands) } \\
\hline East Asia and Pacific & 0 & 0 & 1 & 0 & 23 & 1 & 21 & 3 & 7 & 2 & 2 & 1 & 1 & 0 & 0 & 0 & 57 & 8 & 64 \\
\hline Europe and Central Asia & 0 & 0 & 1 & 0 & 12 & 1 & 13 & 1 & 6 & 1 & 2 & 1 & 1 & 1 & 0 & 0 & 35 & 5 & 40 \\
\hline Latin America and the Caribbean & 0 & 0 & 1 & 0 & 13 & 1 & 11 & 1 & 4 & 0 & 1 & 0 & 1 & 0 & 0 & 0 & 31 & 3 & 34 \\
\hline Middle East and North Africa & 0 & 0 & 0 & 0 & 2 & 0 & 1 & 0 & 0 & 0 & 0 & 0 & 0 & 0 & 0 & 0 & 4 & 1 & 5 \\
\hline South Asia & 0 & 0 & 1 & 0 & 9 & 0 & 12 & 0 & 4 & 1 & 1 & 0 & 1 & 0 & 0 & 0 & 27 & 2 & 30 \\
\hline Sub-Saharan Africa & 1 & 1 & 5 & 2 & 10 & 1 & 12 & 1 & 4 & 1 & 1 & 0 & 1 & 0 & 0 & 0 & 35 & 6 & 40 \\
\hline Low- and middle-income countries & 3 & 1 & 9 & 3 & 68 & 4 & 70 & 7 & 25 & 5 & 8 & 2 & 5 & 1 & 2 & 1 & 190 & 24 & 213 \\
\hline High-income countries & 0 & 0 & 0 & 0 & 11 & 1 & 9 & 1 & 3 & 1 & 1 & 0 & 1 & 1 & 1 & 0 & 28 & 5 & 33 \\
\hline WORLD & 3 & 1 & 9 & 3 & 79 & 6 & 79 & 8 & 28 & 6 & 10 & 2 & 6 & 2 & 2 & 1 & 217 & 29 & 246 \\
\hline
\end{tabular}

Attributable YLL (thousands)

\begin{tabular}{|c|c|c|c|c|c|c|c|c|c|c|c|c|c|c|c|c|c|c|c|}
\hline & & & & & & & & & & & & & & & & & & & \\
\hline East Asia and Pacific & 12 & 5 & 34 & 13 & 628 & 32 & 521 & 68 & 129 & 39 & 33 & 8 & 13 & 4 & 2 & 1 & 1,372 & 172 & 1,544 \\
\hline Europe and Central Asia & 5 & 3 & 19 & 7 & 329 & 31 & 303 & 32 & 121 & 21 & 31 & 8 & 11 & 5 & 1 & 1 & 820 & 109 & 929 \\
\hline Latin America and the Caribbean & 8 & 4 & 19 & 7 & 342 & 18 & 267 & 18 & 81 & 9 & 18 & 3 & 8 & 2 & 2 & 0 & 745 & 60 & 805 \\
\hline Middle East and North Africa & 5 & 2 & 7 & 2 & 43 & 4 & 35 & 4 & 9 & 2 & 3 & 1 & 1 & 1 & 0 & 0 & 104 & 16 & 119 \\
\hline South Asia & 5 & 4 & 16 & 8 & 242 & 9 & 296 & 12 & 69 & 12 & 12 & 2 & 7 & 1 & 2 & 0 & 650 & 49 & 698 \\
\hline Sub-Saharan Africa & 42 & 17 & 156 & 58 & 278 & 30 & 281 & 29 & 76 & 15 & 18 & 4 & 6 & 2 & 1 & 0 & 859 & 155 & 1,013 \\
\hline Low- and middle-income countries & 76 & 34 & 251 & 95 & 1,863 & 124 & 1,703 & 163 & 487 & 98 & 116 & 27 & 45 & 15 & 8 & 3 & 4,549 & 559 & 5,108 \\
\hline High-income countries & 6 & 3 & 13 & 6 & 312 & 36 & 210 & 31 & 65 & 19 & 19 & 7 & 12 & 7 & 4 & 2 & 639 & 109 & 748 \\
\hline WORLD & 82 & 37 & 264 & 101 & 2,175 & 159 & 1,913 & 193 & 552 & 116 & 135 & 34 & 57 & 22 & 12 & 5 & 5,188 & 668 & 5,856 \\
\hline
\end{tabular}

Attributable DALYs (thousands)

\begin{tabular}{|c|c|c|c|c|c|c|c|c|c|c|c|c|c|c|c|c|c|c|c|}
\hline East Asia and Pacific & 16 & 8 & 44 & 20 & 715 & 43 & 587 & 81 & 141 & 44 & 35 & 9 & 13 & 5 & 2 & 1 & 1,554 & 211 & 1,765 \\
\hline Europe and Central Asia & 6 & 4 & 24 & 10 & 377 & 40 & 338 & 40 & 131 & 25 & 32 & 9 & 11 & 6 & 1 & 1 & 920 & 134 & 1,055 \\
\hline Latin America and the Caribbean & 11 & 6 & 26 & 10 & 408 & 25 & 310 & 23 & 91 & 11 & 20 & 3 & 8 & 2 & 2 & 0 & 876 & 80 & 956 \\
\hline Middle East and North Africa & 5 & 2 & 9 & 3 & 54 & 5 & 42 & 5 & 11 & 2 & 3 & 1 & 2 & 1 & 0 & 0 & 126 & 20 & 145 \\
\hline South Asia & 8 & 7 & 25 & 14 & 296 & 12 & 330 & 15 & 77 & 14 & 13 & 3 & 7 & 2 & 2 & 0 & 757 & 67 & 824 \\
\hline Sub-Saharan Africa & 48 & 23 & 175 & 72 & 336 & 35 & 320 & 34 & 88 & 17 & 20 & 5 & 6 & 2 & 1 & 0 & 993 & 187 & 1,181 \\
\hline Low- and middle-income countries & 94 & 49 & 302 & 130 & 2,186 & 160 & 1,927 & 198 & 539 & 113 & 122 & 29 & 47 & 16 & 8 & 3 & 5,226 & 699 & 5,924 \\
\hline High-income countries & 7 & 3 & 16 & 7 & 350 & 43 & 233 & 36 & 71 & 22 & 20 & 8 & 12 & 7 & 4 & 2 & 712 & 128 & 840 \\
\hline WORLD & 101 & 53 & 318 & 138 & 2,536 & 203 & 2,160 & 234 & 610 & 134 & 142 & 37 & 60 & 23 & 12 & 6 & 5,938 & 827 & 6,765 \\
\hline
\end{tabular}

Source: Authors' calculations. 
Table 4A.72

Risk factor:

Disease:

Alcohol use

Poisonings

\begin{tabular}{|c|c|c|c|c|c|c|c|c|c|c|c|c|c|c|c|c|c|c|c|}
\hline \multirow[b]{2}{*}{ Region } & \multicolumn{2}{|c|}{$0-4$ years } & \multicolumn{2}{|c|}{ 5-14 years } & \multicolumn{2}{|c|}{$15-29$ years } & \multicolumn{2}{|c|}{ 30-44 years } & \multicolumn{2}{|c|}{$45-59$ years } & \multicolumn{2}{|c|}{$60-69$ years } & \multicolumn{2}{|c|}{ 70-79 years } & \multicolumn{2}{|c|}{$80+$ years } & \multicolumn{3}{|c|}{ Total } \\
\hline & Male & Female & Male & Female & Male & Female & Male & Female & Male & Female & Male & Female & Male & Female & Male & Female & Male & Female & All \\
\hline \multicolumn{20}{|l|}{ PAF of Mortality (\%) } \\
\hline East Asia and Pacific & NA & NA & NA & NA & 20 & 14 & 10 & 9 & 10 & 9 & 10 & 8 & 5 & 4 & 5 & 4 & 10 & 7 & 9 \\
\hline Europe and Central Asia & NA & NA & NA & NA & 63 & 40 & 44 & 29 & 44 & 29 & 44 & 29 & 26 & 15 & 25 & 14 & 46 & 28 & 41 \\
\hline Latin America and the Caribbean & NA & NA & NA & NA & 44 & 23 & 25 & 15 & 24 & 16 & 25 & 15 & 15 & 7 & 15 & 7 & 26 & 11 & 22 \\
\hline Middle East and North Africa & NA & NA & NA & NA & 5 & 4 & 3 & 3 & 2 & 2 & 3 & 2 & 1 & 1 & 1 & 1 & 3 & 2 & 3 \\
\hline South Asia & NA & NA & NA & NA & 16 & 7 & 8 & 4 & 8 & 4 & 8 & 4 & 4 & 2 & 4 & 2 & 8 & 4 & 6 \\
\hline Sub-Saharan Africa & NA & NA & NA & NA & 27 & 17 & 17 & 11 & 16 & 11 & 16 & 10 & * & 4 & * & 0 & 12 & 8 & 11 \\
\hline Low- and middle-income countries & NA & NA & NA & NA & 34 & 16 & 28 & 13 & 25 & 14 & 24 & 10 & 10 & 5 & 7 & 4 & 24 & 10 & 19 \\
\hline High-income countries & NA & NA & NA & NA & 32 & 25 & 18 & 16 & 17 & 16 & 17 & 16 & 9 & 8 & 10 & 9 & 20 & 16 & 19 \\
\hline WORLD & NA & NA & NA & NA & 34 & 17 & 27 & 13 & 25 & 14 & 23 & 10 & 10 & 5 & 7 & 5 & 24 & 11 & 19 \\
\hline
\end{tabular}

PAF of YLL $(\%)$

\begin{tabular}{|c|c|c|c|c|c|c|c|c|c|c|c|c|c|c|c|c|c|c|c|}
\hline East Asia and Pacific & NA & NA & NA & NA & 20 & 14 & 10 & 9 & 10 & 9 & 10 & 8 & 5 & 4 & 5 & 4 & 11 & 7 & 9 \\
\hline Europe and Central Asia & NA & NA & NA & NA & 63 & 40 & 44 & 29 & 44 & 29 & 44 & 29 & 26 & 15 & 25 & 14 & 46 & 29 & 42 \\
\hline Latin America and the Caribbean & NA & NA & NA & NA & 44 & 23 & 25 & 15 & 24 & 16 & 25 & 15 & 15 & 7 & 15 & 7 & 27 & 11 & 22 \\
\hline Middle East and North Africa & NA & NA & NA & NA & 5 & 4 & 3 & 3 & 2 & 2 & 3 & 2 & 1 & 1 & 1 & 1 & 3 & 2 & 3 \\
\hline South Asia & NA & NA & NA & NA & 16 & 7 & 8 & 4 & 8 & 4 & 8 & 4 & 4 & 2 & 4 & 2 & 9 & 4 & 6 \\
\hline Sub-Saharan Africa & NA & NA & NA & NA & 27 & 17 & 17 & 11 & 16 & 11 & 16 & 10 & * & 4 & * & 0 & 12 & 8 & 10 \\
\hline Low- and middle-income countries & NA & NA & NA & NA & 34 & 16 & 28 & 13 & 25 & 14 & 24 & 10 & 10 & 5 & 7 & 4 & 24 & 10 & 19 \\
\hline High-income countries & NA & NA & NA & NA & 32 & 25 & 18 & 16 & 17 & 16 & 17 & 16 & 9 & 8 & 10 & 9 & 21 & 17 & 20 \\
\hline WORLD & NA & NA & NA & NA & 34 & 17 & 26 & 13 & 25 & 14 & 23 & 10 & 10 & 5 & 7 & 5 & 24 & 11 & 19 \\
\hline
\end{tabular}

\section{PAF of DALYs $(\%)$}

East Asia and Pacific

Europe and Central Asia

Latin America and the Caribbean

Middle East and North Afric

South Asia

Sub-Saharan Africa

Low- and middle-income countries

\begin{tabular}{|c|c|c|c|c|c|c|c|c|c|c|c|c|c|c|c|c|c|c|c|}
\hline East Asia and Pacific & NA & NA & NA & NA & 20 & 14 & 10 & 9 & 10 & 9 & 10 & 8 & 5 & 4 & 5 & 4 & 10 & 7 & 9 \\
\hline Europe and Central Asia & NA & NA & NA & NA & 63 & 40 & 44 & 29 & 44 & 28 & 44 & 29 & 26 & 15 & 25 & 14 & 46 & 28 & 42 \\
\hline Latin America and the Caribbean & NA & NA & NA & NA & 41 & 19 & 24 & 12 & 23 & 13 & 24 & 13 & 14 & 7 & 14 & 7 & 25 & 10 & 20 \\
\hline Middle East and North Africa & NA & NA & NA & NA & 5 & 4 & 3 & 3 & 2 & 2 & 3 & 2 & 1 & 1 & 1 & 1 & 3 & 2 & 3 \\
\hline South Asia & NA & NA & NA & NA & 16 & 7 & 8 & 4 & 8 & 4 & 8 & 4 & 4 & 2 & 4 & 2 & 9 & 4 & 6 \\
\hline Sub-Saharan Africa & NA & NA & NA & NA & 27 & 17 & 17 & 11 & 16 & 11 & 16 & 10 & 2 & 4 & 2 & 0 & 11 & 8 & 10 \\
\hline Low- and middle-income countries & NA & NA & NA & NA & 34 & 16 & 27 & 13 & 25 & 14 & 23 & 10 & 10 & 5 & 7 & 4 & 24 & 10 & 19 \\
\hline High-income countries & NA & NA & NA & NA & 31 & 23 & 18 & 15 & 16 & 15 & 16 & 14 & 9 & 8 & 10 & 9 & 21 & 16 & 19 \\
\hline WORLD & NA & NA & NA & NA & 34 & 16 & 26 & 13 & 25 & 14 & 23 & 10 & 10 & 5 & 7 & 5 & 24 & 11 & 19 \\
\hline
\end{tabular}

Attributable Mortality (thousands)

\begin{tabular}{lrrrrrrrrrrrrrrrrrrr} 
East Asia and Pacific & NA & NA & NA & NA & 2 & 1 & 1 & 1 & 1 & 1 & 1 & 0 & 0 & 0 & 0 & 0 & 5 & 2 & 7 \\
Europe and Central Asia & NA & NA & NA & NA & 7 & 1 & 12 & 2 & 13 & 3 & 5 & 1 & 1 & 0 & 0 & 0 & 37 & 7 & 44 \\
Latin America and the Caribbean & NA & NA & NA & NA & 0 & 0 & 0 & 0 & 0 & 0 & 0 & 0 & 0 & 0 & 0 & 0 & 1 & 0 & 1 \\
Middle East and North Africa & NA & NA & NA & NA & 0 & 0 & 0 & 0 & 0 & 0 & 0 & 0 & 0 & 0 & 0 & 0 & 0 & 0 & 0 \\
South Asia & NA & NA & NA & NA & 1 & 0 & 1 & 0 & 1 & 0 & 1 & 0 & 0 & 0 & 0 & 0 & 4 & 2 & 6 \\
Sub-Saharan Africa & NA & NA & NA & NA & 1 & 1 & 1 & 0 & 0 & 0 & 0 & 0 & 0 & 0 & 0 & 0 & 3 & 1 & 4 \\
\hline Low- and middle-income countries & NA & NA & NA & NA & 12 & 3 & 14 & 3 & 16 & 4 & 6 & 2 & 1 & 1 & 0 & 0 & 50 & 12 & 62 \\
High-income countries & NA & NA & NA & NA & 1 & 0 & 1 & 0 & 1 & 0 & 0 & 0 & 0 & 0 & 0 & 0 \\
\hline WORLD & NA & NA & NA & NA & 13 & 3 & 16 & 3 & 17 & 4 & 6 & 2 & 1 & 1 & 0 & 0 \\
\hline
\end{tabular}

Attributable YLL (thousands)

\begin{tabular}{|c|c|c|c|c|c|c|c|c|c|c|c|c|c|c|c|c|c|c|c|}
\hline East Asia and Pacific & NA & NA & NA & NA & 46 & 17 & 27 & 15 & 25 & 12 & 10 & 5 & 2 & 1 & 0 & 0 & 111 & 51 & 162 \\
\hline Europe and Central Asia & NA & NA & NA & NA & 200 & 28 & 275 & 39 & 258 & 52 & 65 & 19 & 6 & 3 & 0 & 0 & 804 & 142 & 946 \\
\hline Latin America and the Caribbean & NA & NA & NA & NA & 7 & 1 & 4 & 1 & 2 & 0 & 1 & 0 & 0 & 0 & 0 & 0 & 14 & 2 & 16 \\
\hline Middle East and North Africa & NA & NA & NA & NA & 2 & 1 & 1 & 0 & 1 & 0 & 0 & 0 & 0 & 0 & 0 & 0 & 3 & 1 & 5 \\
\hline South Asia & NA & NA & NA & NA & 35 & 10 & 14 & 5 & 26 & 7 & 7 & 8 & 1 & 1 & 0 & 0 & 85 & 32 & 116 \\
\hline$\underline{\text { Sub-Saharan Africa }}$ & NA & NA & NA & NA & 33 & 15 & 24 & 7 & 8 & 3 & 2 & 3 & 0 & 0 & 0 & 0 & 68 & 28 & 97 \\
\hline Low- and middle-income countries & NA & NA & NA & NA & 323 & 73 & 346 & 68 & 319 & 75 & 86 & 34 & 9 & 5 & 1 & 1 & 1,085 & 257 & 1,342 \\
\hline High-income countries & NA & NA & NA & NA & 27 & 6 & 29 & 9 & 12 & 5 & 2 & 1 & 0 & 0 & 0 & 0 & 70 & 22 & 92 \\
\hline WORLD & NA & NA & NA & NA & 351 & 79 & 374 & 78 & 331 & 80 & 88 & 35 & 9 & 6 & 1 & 1 & 1,155 & 279 & 1,434 \\
\hline
\end{tabular}

Attributable DALYs (thousands)

\begin{tabular}{|c|c|c|c|c|c|c|c|c|c|c|c|c|c|c|c|c|c|c|c|}
\hline East Asia and Pacific & NA & NA & NA & NA & 46 & 17 & 27 & 16 & 25 & 12 & 10 & 5 & 2 & 1 & 0 & 0 & 112 & 52 & 164 \\
\hline Europe and Central Asia & NA & NA & NA & NA & 200 & 29 & 275 & 40 & 259 & 53 & 65 & 19 & 6 & 3 & 0 & 0 & 807 & 143 & 950 \\
\hline Latin America and the Caribbean & NA & NA & NA & NA & 8 & 2 & 4 & 1 & 2 & 0 & 1 & 0 & 0 & 0 & 0 & 0 & 14 & 3 & 17 \\
\hline Middle East and North Africa & NA & NA & NA & NA & 2 & 1 & 1 & 0 & 1 & 0 & 0 & 0 & 0 & 0 & 0 & 0 & 3 & 1 & 5 \\
\hline South Asia & NA & NA & NA & NA & 36 & 10 & 15 & 6 & 26 & 8 & 7 & 8 & 1 & 1 & 0 & 0 & 85 & 32 & 117 \\
\hline Sub-Saharan Africa & NA & NA & NA & NA & 34 & 15 & 25 & 7 & 8 & 3 & 2 & 3 & 0 & 0 & 0 & 0 & 69 & 29 & 97 \\
\hline Low- and middle-income countries & NA & NA & NA & NA & 325 & 74 & 347 & 69 & 322 & 76 & 87 & 34 & 9 & 5 & 1 & 1 & 1,090 & 260 & 1,350 \\
\hline High-income countries & NA & NA & NA & NA & 28 & 6 & 29 & 10 & 12 & 6 & 2 & 1 & 0 & 0 & 0 & 0 & 71 & 23 & 94 \\
\hline WORLD & NA & NA & NA & NA & 353 & 80 & 375 & 79 & 334 & 82 & 88 & 35 & 9 & 6 & 1 & 1 & 1,161 & 283 & 1,444 \\
\hline
\end{tabular}

Source: Authors' calculations.

Note: NA = not applicable.

*The number of deaths (and hence YLL) directly coded to a number of diseases, especially neuropsychiatric and musculoskeletal diseases, is zero or very small. For other diseases, mortality or disease burden may be zero in some

region-age-sex groups. In such cases, the population attributable fractions would be undefined or unstable and have not been calculated. 
Table 4A.73

Risk factor:

Alcohol use

Disease:

Falls

\begin{tabular}{|c|c|c|c|c|c|c|c|c|c|c|c|c|c|c|c|c|c|c|c|}
\hline \multirow[b]{2}{*}{ Region } & \multicolumn{2}{|c|}{$0-4$ years } & \multicolumn{2}{|c|}{ 5-14 years } & \multicolumn{2}{|c|}{$15-29$ years } & \multicolumn{2}{|c|}{ 30-44 years } & \multicolumn{2}{|c|}{ 45-59 years } & \multicolumn{2}{|c|}{$60-69$ years } & \multicolumn{2}{|c|}{$70-79$ years } & \multicolumn{2}{|c|}{$80+$ years } & \multicolumn{3}{|c|}{ Total } \\
\hline & Male & Female & Male & Female & Male & Female & Male & Female & Male & Female & Male & Female & Male & Female & Male & Female & Male & Female & All \\
\hline \multicolumn{20}{|l|}{ PAF of Mortality (\%) } \\
\hline East Asia and Pacific & NA & NA & NA & NA & 15 & 8 & 15 & 8 & 15 & 8 & 11 & 5 & 8 & 2 & 8 & 2 & 12 & 4 & 9 \\
\hline Europe and Central Asia & NA & NA & NA & NA & 51 & 26 & 52 & 25 & 51 & 25 & 42 & 17 & 31 & 7 & 28 & 7 & 44 & 12 & 34 \\
\hline Latin America and the Caribbean & NA & NA & NA & NA & 36 & 14 & 37 & 15 & 37 & 15 & 30 & 10 & 22 & 4 & 22 & 4 & 29 & 6 & 22 \\
\hline Middle East and North Africa & NA & NA & NA & NA & 3 & 2 & 4 & 3 & 4 & 2 & 3 & 1 & 2 & 1 & 2 & 1 & 3 & 1 & 2 \\
\hline South Asia & NA & NA & NA & NA & 12 & 4 & 12 & 4 & 12 & 4 & 9 & 2 & 6 & 1 & 6 & 1 & 8 & 2 & 5 \\
\hline Sub-Saharan Africa & NA & NA & NA & NA & 21 & 8 & 23 & 10 & 23 & 10 & 18 & 6 & 13 & 3 & 12 & 3 & 13 & 4 & 10 \\
\hline Low- and middle-income countries & NA & NA & NA & NA & 18 & 7 & 21 & 9 & 22 & 8 & 16 & 5 & 10 & 2 & 10 & 3 & 15 & 4 & 11 \\
\hline High-income countries & NA & NA & NA & NA & 23 & 15 & 25 & 16 & 25 & 17 & 20 & 11 & 15 & 5 & 15 & 5 & 18 & 6 & 12 \\
\hline WORLD & NA & NA & NA & NA & 18 & 8 & 22 & 9 & 22 & 9 & 16 & 6 & 11 & 3 & 12 & 4 & 15 & 4 & 11 \\
\hline
\end{tabular}

PAF of YLL $(\%)$

\begin{tabular}{|c|c|c|c|c|c|c|c|c|c|c|c|c|c|c|c|c|c|c|c|}
\hline East Asia and Pacific & NA & NA & NA & NA & 15 & 8 & 15 & 8 & 15 & 8 & 11 & 5 & 8 & 2 & 8 & 2 & 12 & 5 & 10 \\
\hline Europe and Central Asia & NA & NA & NA & NA & 51 & 26 & 52 & 25 & 51 & 25 & 42 & 17 & 31 & 7 & 28 & 7 & 46 & 16 & 39 \\
\hline Latin America and the Caribbean & NA & NA & NA & NA & 36 & 14 & 37 & 15 & 37 & 15 & 30 & 10 & 22 & 4 & 22 & 4 & 30 & 7 & 25 \\
\hline Middle East and North Africa & NA & NA & NA & NA & 3 & 2 & 4 & 3 & 4 & 2 & 3 & 1 & 2 & 1 & 2 & 1 & 2 & 1 & 2 \\
\hline South Asia & NA & NA & NA & NA & 12 & 4 & 12 & 4 & 12 & 4 & 9 & 2 & 6 & 1 & 6 & 1 & 8 & 2 & 6 \\
\hline Sub-Saharan Africa & NA & NA & NA & NA & 21 & 8 & 23 & 10 & 23 & 10 & 18 & 6 & 13 & 3 & 12 & 3 & 11 & 3 & 9 \\
\hline Low- and middle-income countries & NA & NA & NA & NA & 18 & 7 & 21 & 9 & 22 & 8 & 16 & 5 & 10 & 2 & 9 & 3 & 15 & 4 & 12 \\
\hline High-income countries & NA & NA & NA & NA & 23 & 15 & 25 & 16 & 25 & 17 & 20 & 11 & 15 & 5 & 15 & 5 & 20 & 8 & 15 \\
\hline WORLD & NA & NA & NA & NA & 18 & 8 & 22 & 9 & 23 & 9 & 17 & 6 & 11 & 3 & 11 & 4 & 16 & 5 & 12 \\
\hline
\end{tabular}

\section{PAF of DALYs (\%)}

East Asia and Pacific

Europe and Central Asia

Latin America and the Caribbean

Middle East and North Afric

South Asia

Sub-Saharan Africa

Low- and middle-income countries

High-income countries

$\begin{array}{llllrrr}\text { NA } & \text { NA } & \text { NA } & \text { NA } & 10 & 4 & 12 \\ \text { NA } & \text { NA } & \text { NA } & \text { NA } & 30 & 14 & 39 \\ \text { NA } & \text { NA } & \text { NA } & \text { NA } & 21 & 7 & 26 \\ \text { NA } & \text { NA } & \text { NA } & \text { NA } & 2 & 1 & 3 \\ \text { NA } & \text { NA } & \text { NA } & \text { NA } & 7 & 3 & 9 \\ \text { NA } & \text { NA } & \text { NA } & \text { NA } & 14 & 5 & 18 \\ \text { NA } & \text { NA } & \text { NA } & \text { NA } & 12 & 4 & 16\end{array}$

$\begin{array}{rr}6 & 13 \\ 17 & 43 \\ 8 & 30 \\ 1 & 3 \\ 3 & 10 \\ 6 & 20 \\ 6 & 19\end{array}$

\begin{tabular}{rrrrrrrrrr}
6 & 10 & 4 & 7 & 2 & 7 & 2 & 8 & 3 & 6 \\
19 & 35 & 12 & 23 & 5 & 22 & 5 & 29 & 10 & 24 \\
10 & 24 & 6 & 17 & 3 & 18 & 3 & 17 & 4 & 13 \\
1 & 2 & 1 & 2 & 1 & 2 & 0 & 1 & 0 & 1 \\
3 & 8 & 2 & 5 & 1 & 6 & 1 & 5 & 1 & 3 \\
7 & 15 & 5 & 11 & 2 & 9 & 2 & 7 & 2 & 5 \\
6 & 14 & 4 & 9 & 2 & 9 & 2 & 10 & 2 & 7 \\
11 & 16 & 8 & 12 & 3 & 13 & 4 & 14 & 5 & 10 \\
\hline 7 & 14 & 5 & 9 & 2 & 10 & 3 & 10 & 3 & 7 \\
\hline
\end{tabular}

Attributable Mortality (thousands)

\begin{tabular}{|c|c|c|c|c|c|c|c|c|c|c|c|c|c|c|c|c|c|c|c|}
\hline \multicolumn{20}{|l|}{ Attributable Mortality (thousands) } \\
\hline East Asia and Pacific & NA & NA & NA & NA & 2 & 0 & 2 & 0 & 2 & 0 & 1 & 0 & 1 & 0 & 1 & 0 & 9 & 2 & 11 \\
\hline Europe and Central Asia & NA & NA & NA & NA & 1 & 0 & 3 & 0 & 4 & 0 & 2 & 0 & 1 & 0 & 0 & 0 & 11 & 1 & 12 \\
\hline Latin America and the Caribbean & NA & NA & NA & NA & 1 & 0 & 1 & 0 & 1 & 0 & 0 & 0 & 0 & 0 & 0 & 0 & 3 & 0 & 3 \\
\hline Middle East and North Africa & NA & NA & NA & NA & 0 & 0 & 0 & 0 & 0 & 0 & 0 & 0 & 0 & 0 & 0 & 0 & 0 & 0 & 0 \\
\hline South Asia & NA & NA & NA & NA & 1 & 0 & 1 & 0 & 1 & 0 & 1 & 0 & 1 & 0 & 1 & 0 & 5 & 1 & 6 \\
\hline Sub-Saharan Africa & NA & NA & NA & NA & 0 & 0 & 0 & 0 & 1 & 0 & 0 & 0 & 0 & 0 & 0 & 0 & 2 & 0 & 2 \\
\hline Low- and middle-income countries & NA & NA & NA & NA & 5 & 1 & 7 & 1 & 8 & 1 & 4 & 1 & 3 & 1 & 2 & 1 & 30 & 5 & 34 \\
\hline High-income countries & NA & NA & NA & NA & 0 & 0 & 1 & 0 & 1 & 0 & 1 & 0 & 1 & 0 & 2 & 1 & 6 & 2 & 8 \\
\hline WORLD & NA & NA & NA & NA & 5 & 1 & 8 & 1 & 10 & 1 & 5 & 1 & 4 & 1 & 4 & 2 & 36 & 7 & 43 \\
\hline
\end{tabular}

Attributable YLL (thousands)

\begin{tabular}{|c|c|c|c|c|c|c|c|c|c|c|c|c|c|c|c|c|c|c|c|}
\hline East Asia and Pacific & NA & NA & NA & NA & 51 & 7 & 51 & 8 & 47 & 10 & 15 & 4 & 7 & 2 & 3 & 1 & 174 & 32 & 206 \\
\hline Europe and Central Asia & NA & NA & NA & NA & 36 & 4 & 66 & 6 & 69 & 7 & 22 & 3 & 7 & 2 & 2 & 1 & 203 & 24 & 226 \\
\hline Latin America and the Caribbean & NA & NA & NA & NA & 14 & 1 & 17 & 1 & 14 & 1 & 5 & 1 & 2 & 0 & 1 & 0 & 55 & 4 & 58 \\
\hline Middle East and North Africa & NA & NA & NA & NA & 1 & 0 & 1 & 0 & 1 & 0 & 0 & 0 & 0 & 0 & 0 & 0 & 4 & 1 & 5 \\
\hline South Asia & NA & NA & NA & NA & 27 & 4 & 24 & 3 & 20 & 5 & 11 & 2 & 8 & 1 & 3 & 0 & 93 & 16 & 109 \\
\hline Sub-Saharan Africa & NA & NA & NA & NA & 9 & 1 & 9 & 1 & 11 & 1 & 3 & 1 & 2 & 0 & 0 & 0 & 34 & 4 & 38 \\
\hline Low- and middle-income countries & NA & NA & NA & NA & 139 & 18 & 168 & 18 & 163 & 24 & 57 & 10 & 26 & 6 & 10 & 4 & 563 & 80 & 643 \\
\hline High-income countries & NA & NA & NA & NA & 9 & 1 & 18 & 2 & 24 & 5 & 12 & 3 & 9 & 3 & 8 & 5 & 80 & 19 & 99 \\
\hline WORLD & NA & NA & NA & NA & 148 & 19 & 186 & 21 & 188 & 29 & 68 & 13 & 36 & 9 & 18 & 9 & 643 & 99 & 742 \\
\hline
\end{tabular}

Attributable DALYs (thousands)

\begin{tabular}{lrrrrrrrrrrrrrrrrrrr} 
East Asia and Pacific & NA & NA & NA & NA & 85 & 16 & 67 & 14 & 53 & 13 & 17 & 5 & 8 & 3 & 3 & 2 & 234 & 52 & 286 \\
Europe and Central Asia & NA & NA & NA & NA & 79 & 11 & 88 & 9 & 81 & 9 & 27 & 4 & 10 & 3 & 3 & 2 & 287 & 38 & 325 \\
Latin America and the Caribbean & NA & NA & NA & NA & 32 & 3 & 25 & 2 & 18 & 2 & 6 & 1 & 3 & 1 & 2 & 1 & 86 & 8 & 94 \\
Middle East and North Africa & NA & NA & NA & NA & 4 & 1 & 2 & 0 & 1 & 0 & 0 & 0 & 0 & 0 & 0 & 0 & 8 & 2 & 10 \\
South Asia & NA & NA & NA & NA & 48 & 10 & 32 & 5 & 24 & 6 & 12 & 2 & 8 & 1 & 3 & 0 & 127 & 25 & 152 \\
Sub-Saharan Africa & NA & NA & NA & NA & 15 & 2 & 12 & 1 & 12 & 2 & 4 & 1 & 2 & 0 & 0 & 0 & 45 & 6 & 52 \\
\hline Low- and middle-income countries & NA & NA & NA & NA & 263 & 42 & 226 & 32 & 189 & 32 & 66 & 13 & 31 & 8 & 11 & 4 & 787 & 132 & 919 \\
High-income countries & NA & NA & NA & NA & 23 & 5 & 28 & 5 & 31 & 7 & 15 & 5 & 12 & 5 & 9 & 6 & 117 & 33 & 150 \\
\hline WORLD & NA & NA & NA & NA & 286 & 47 & 254 & 37 & 220 & 39 & 81 & 18 & 43 & 12 & 21 & 11 & 904 & 165 & 1,069
\end{tabular}

Source: Authors' calculations.

Note: $\mathrm{NA}=$ not applicable. 
Table 4A.74

Risk factor:

Alcohol use

Disease:

Drownings

\begin{tabular}{|c|c|c|c|c|c|c|c|c|c|c|c|c|c|c|c|c|c|c|c|}
\hline \multirow[b]{2}{*}{ Region } & \multicolumn{2}{|c|}{$0-4$ years } & \multicolumn{2}{|c|}{ 5-14 years } & \multicolumn{2}{|c|}{$15-29$ years } & \multicolumn{2}{|c|}{ 30-44 years } & \multicolumn{2}{|c|}{ 45-59 years } & \multicolumn{2}{|c|}{ 60-69 years } & \multicolumn{2}{|c|}{$70-79$ years } & \multicolumn{2}{|c|}{$80+$ years } & \multicolumn{3}{|c|}{ Total } \\
\hline & Male & Female & Male & Female & Male & Female & Male & Female & Male & Female & Male & Female & Male & Female & Male & Female & Male & Female & All \\
\hline \multicolumn{20}{|l|}{ PAF of Mortality (\%) } \\
\hline East Asia and Pacific & NA & NA & NA & NA & 18 & 15 & 22 & 19 & 21 & 19 & 17 & 15 & 17 & 15 & 17 & 15 & 10 & 8 & 9 \\
\hline Europe and Central Asia & NA & NA & NA & NA & 57 & 40 & 64 & 48 & 64 & 47 & 56 & 40 & 54 & 39 & 53 & 40 & 54 & 33 & 50 \\
\hline Latin America and the Caribbean & NA & NA & NA & NA & 42 & 25 & 48 & 30 & 48 & 30 & 40 & 23 & 39 & 23 & 39 & 24 & 34 & 11 & 30 \\
\hline Middle East and North Africa & NA & NA & NA & NA & 5 & 5 & 8 & 9 & 9 & 8 & 8 & 7 & 7 & 4 & 5 & 2 & 4 & 2 & 4 \\
\hline South Asia & NA & NA & NA & NA & 13 & 7 & 17 & 9 & 17 & 9 & 13 & 7 & 14 & 7 & 14 & 7 & 10 & 4 & 8 \\
\hline Sub-Saharan Africa & NA & NA & NA & NA & 27 & 18 & 33 & 23 & 33 & 23 & 27 & 18 & 27 & 16 & 24 & 3 & 20 & 10 & 18 \\
\hline Low- and middle-income countries & NA & NA & NA & NA & 23 & 14 & 34 & 19 & 34 & 20 & 27 & 15 & 22 & 14 & 18 & 14 & 18 & 8 & 15 \\
\hline High-income countries & NA & NA & NA & NA & 26 & 23 & 33 & 29 & 33 & 31 & 27 & 25 & 27 & 25 & 26 & 24 & 25 & 21 & 24 \\
\hline WORLD & NA & NA & NA & NA & 23 & 14 & 34 & 19 & 34 & 20 & 27 & 16 & 23 & 16 & 20 & 17 & 18 & 9 & 15 \\
\hline
\end{tabular}

PAF of YLL $(\%)$

\begin{tabular}{|c|c|c|c|c|c|c|c|c|c|c|c|c|c|c|c|c|c|c|c|}
\hline East Asia and Pacific & NA & NA & NA & NA & 18 & 15 & 22 & 19 & 21 & 19 & 17 & 15 & 17 & 15 & 17 & 15 & 8 & 6 & 8 \\
\hline Europe and Central Asia & NA & NA & NA & NA & 57 & 40 & 64 & 48 & 64 & 47 & 56 & 40 & 54 & 39 & 53 & 40 & 52 & 31 & 48 \\
\hline Latin America and the Caribbean & NA & NA & NA & NA & 42 & 25 & 48 & 30 & 48 & 30 & 40 & 23 & 39 & 23 & 39 & 24 & 32 & 10 & 28 \\
\hline Middle East and North Africa & NA & NA & NA & NA & 5 & 5 & 8 & 9 & 9 & 8 & 8 & 7 & 7 & 4 & 5 & 2 & 3 & 2 & 3 \\
\hline South Asia & NA & NA & NA & NA & 13 & 7 & 17 & 9 & 17 & 9 & 13 & 7 & 14 & 7 & 14 & 7 & 9 & 4 & 7 \\
\hline Sub-Saharan Africa & NA & NA & NA & NA & 27 & 18 & 33 & 23 & 33 & 23 & 27 & 18 & 27 & 16 & 24 & 3 & 18 & 9 & 16 \\
\hline Low- and middle-income countries & NA & NA & NA & NA & 23 & 14 & 34 & 19 & 34 & 20 & 27 & 15 & 22 & 14 & 18 & 14 & 16 & 7 & 13 \\
\hline High-income countries & NA & NA & NA & NA & 26 & 23 & 33 & 29 & 33 & 31 & 27 & 25 & 27 & 25 & 26 & 24 & 24 & 18 & 22 \\
\hline WORLD & NA & NA & NA & NA & 23 & 14 & 34 & 19 & 34 & 20 & 27 & 16 & 23 & 16 & 20 & 16 & 17 & 7 & 14 \\
\hline
\end{tabular}

\section{PAF of DALYs $(\%)$}

\begin{tabular}{|c|c|c|c|c|c|c|c|c|c|c|c|c|c|c|c|c|c|c|c|}
\hline East Asia and Pacific & NA & NA & NA & NA & 18 & 15 & 22 & 19 & 21 & 19 & 17 & 15 & 17 & 15 & 17 & 15 & 8 & 6 & 8 \\
\hline Europe and Central Asia & NA & NA & NA & NA & 57 & 40 & 64 & 48 & 64 & 47 & 56 & 40 & 54 & 39 & 53 & 40 & 52 & 30 & 48 \\
\hline Latin America and the Caribbean & NA & NA & NA & NA & 42 & 24 & 48 & 30 & 48 & 30 & 40 & 22 & 39 & 22 & 39 & 24 & 32 & 10 & 28 \\
\hline Middle East and North Africa & NA & NA & NA & NA & 5 & 5 & 8 & 9 & 9 & 8 & 8 & 7 & 7 & 4 & 5 & 2 & 3 & 2 & 3 \\
\hline South Asia & NA & NA & NA & NA & 13 & 7 & 17 & 9 & 17 & 9 & 13 & 7 & 14 & 7 & 14 & 7 & 9 & 4 & 7 \\
\hline Sub-Saharan Africa & NA & NA & NA & NA & 27 & 18 & 33 & 23 & 33 & 23 & 27 & 18 & 25 & 15 & 24 & 3 & 18 & 9 & 16 \\
\hline Low- and middle-income countries & NA & NA & NA & NA & 23 & 14 & 34 & 19 & 34 & 20 & 27 & 15 & 22 & 14 & 18 & 14 & 16 & 7 & 13 \\
\hline High-income countries & NA & NA & NA & NA & 26 & 22 & 33 & 29 & 33 & 31 & 27 & 24 & 27 & 25 & 26 & 24 & 24 & 18 & 22 \\
\hline WORLD & NA & NA & NA & NA & 23 & 14 & 34 & 19 & 34 & 20 & 27 & 16 & 23 & 16 & 20 & 16 & 17 & 7 & 14 \\
\hline
\end{tabular}

Attributable Mortality (thousands)

\begin{tabular}{lrrrrrrrrrrrrrrrrrrrr} 
East Asia and Pacific & NA & NA & NA & NA & 4 & 1 & 2 & 1 & 1 & 1 & 1 & 0 & 0 & 0 & 0 & 0 & 9 & 4 & 13 \\
Europe and Central Asia & NA & NA & NA & NA & 4 & 0 & 6 & 1 & 4 & 1 & 1 & 0 & 0 & 0 & 0 & 0 & 15 & 2 & 18 \\
Latin America and the Caribbean & NA & NA & NA & NA & 2 & 0 & 1 & 0 & 1 & 0 & 0 & 0 & 0 & 0 & 0 & 0 & 5 & 0 & 6 \\
Middle East and North Africa & NA & NA & NA & NA & 0 & 0 & 0 & 0 & 0 & 0 & 0 & 0 & 0 & 0 & 0 & 0 & 0 & 0 & 0 \\
South Asia & NA & NA & NA & NA & 2 & 1 & 2 & 0 & 1 & 0 & 0 & 0 & 0 & 0 & 0 & 0 & 6 & 2 & 7 \\
Sub-Saharan Africa & NA & NA & NA & NA & 3 & 1 & 4 & 0 & 3 & 0 & 0 & 0 & 0 & 0 & 0 & 0 & 10 & 1 & 12 \\
\hline Low- and middle-income countries & NA & NA & NA & NA & 15 & 3 & 15 & 2 & 10 & 2 & 3 & 1 & 2 & 1 & 1 & 1 & 46 & 10 & 55 \\
High-income countries & NA & NA & NA & NA & 1 & 0 & 1 & 0 & 1 & 0 & 0 & 0 & 0 & 0 & 0 & 0 \\
\hline WORLD & NA & NA & NA & NA & 16 & 3 & 15 & 3 & 11 & 2 & 3 & 1 & 2 & 1 & 1 & 1 \\
\hline
\end{tabular}

Attributable YLL (thousands)

\begin{tabular}{lrrrrrrrrrrrrrrrrrrr} 
East Asia and Pacific & NA & NA & NA & NA & 110 & 26 & 49 & 30 & 29 & 17 & 9 & 6 & 4 & 5 & 1 & 2 & 202 & 86 & 288 \\
Europe and Central Asia & NA & NA & NA & NA & 105 & 13 & 134 & 14 & 80 & 11 & 20 & 4 & 5 & 2 & 0 & 1 & 344 & 44 & 388 \\
Latin America and the Caribbean & NA & NA & NA & NA & 67 & 5 & 36 & 2 & 16 & 1 & 4 & 0 & 1 & 0 & 0 & 0 & 125 & 9 & 134 \\
Middle East and North Africa & NA & NA & NA & NA & 6 & 1 & 2 & 1 & 1 & 0 & 0 & 0 & 0 & 0 & 0 & 0 & 10 & 2 & 12 \\
South Asia & NA & NA & NA & NA & 59 & 16 & 37 & 9 & 20 & 5 & 5 & 2 & 3 & 2 & 1 & 0 & 124 & 34 & 158 \\
Sub-Saharan Africa & NA & NA & NA & NA & 79 & 20 & 101 & 6 & 51 & 8 & 4 & 2 & 0 & 0 & 0 & 0 & 234 & 36 & 271 \\
\hline Low- and middle-income countries & NA & NA & NA & NA & 426 & 80 & 358 & 62 & 197 & 43 & 41 & 15 & 14 & 9 & 3 & 3 & 1,040 & 211 & 1,251 \\
High-income countries & NA & NA & NA & NA & 15 & 2 & 15 & 3 & 13 & 3 & 5 & 2 & 3 & 3 & 1 & 2 \\
\hline WORLD & NA & NA & NA & NA & 441 & 82 & 373 & 64 & 210 & 46 & 46 & 17 & 17 & 12 & 4 & 5 & 1,092 & 226 & 1,317 \\
\hline
\end{tabular}

Attributable DALYs (thousands)

\begin{tabular}{|c|c|c|c|c|c|c|c|c|c|c|c|c|c|c|c|c|c|c|c|}
\hline East Asia and Pacific & NA & NA & NA & NA & 110 & 26 & 49 & 30 & 29 & 17 & 9 & 6 & 4 & 5 & 1 & 2 & 203 & 86 & 289 \\
\hline Europe and Central Asia & NA & NA & NA & NA & 106 & 13 & 134 & 14 & 80 & 11 & 20 & 4 & 5 & 2 & 0 & 1 & 344 & 44 & 389 \\
\hline Latin America and the Caribbean & NA & NA & NA & NA & 67 & 5 & 36 & 2 & 16 & 1 & 4 & 0 & 1 & 0 & 0 & 0 & 125 & 9 & 134 \\
\hline Middle East and North Africa & NA & NA & NA & NA & 6 & 1 & 2 & 1 & 1 & 0 & 0 & 0 & 0 & 0 & 0 & 0 & 10 & 2 & 12 \\
\hline South Asia & NA & NA & NA & NA & 59 & 16 & 37 & 9 & 20 & 5 & 5 & 2 & 3 & 2 & 1 & 0 & 124 & 34 & 158 \\
\hline Sub-Saharan Africa & NA & NA & NA & NA & 79 & 20 & 101 & 6 & 51 & 8 & 4 & 2 & 0 & 0 & 0 & 0 & 235 & 37 & 271 \\
\hline Low- and middle-income countries & NA & NA & NA & NA & 427 & 81 & 359 & 62 & 197 & 43 & 41 & 15 & 14 & 9 & 3 & 3 & 1,041 & 212 & 1,253 \\
\hline High-income countries & NA & NA & NA & NA & 15 & 2 & 15 & 3 & 13 & 3 & 5 & 2 & 3 & 3 & 1 & 2 & 52 & 15 & 67 \\
\hline WORLD & NA & NA & NA & NA & 442 & 82 & 374 & 65 & 210 & 46 & 46 & 17 & 17 & 12 & 4 & 5 & 1,093 & 227 & 1,320 \\
\hline
\end{tabular}

Source: Authors' calculations.

Note: NA = not applicable. 
Table 4A.75

Risk factor:

Alcohol use

Disease:

Other unintentional injuries

\begin{tabular}{|c|c|c|c|c|c|c|c|c|c|c|c|c|c|c|c|c|c|c|c|}
\hline \multirow[b]{2}{*}{ Region } & \multicolumn{2}{|c|}{$0-4$ years } & \multicolumn{2}{|c|}{ 5-14 years } & \multicolumn{2}{|c|}{$15-29$ years } & \multicolumn{2}{|c|}{ 30-44 years } & \multicolumn{2}{|c|}{$45-59$ years } & \multicolumn{2}{|c|}{$60-69$ years } & \multicolumn{2}{|c|}{ 70-79 years } & \multicolumn{2}{|c|}{$80+$ years } & \multicolumn{3}{|c|}{ Total } \\
\hline & Male & Female & Male & Female & Male & Female & Male & Female & Male & Female & Male & Female & Male & Female & Male & Female & Male & Female & All \\
\hline \multicolumn{20}{|l|}{ PAF of Mortality (\%) } \\
\hline East Asia and Pacific & 10 & 3 & 10 & 3 & 20 & 13 & 20 & 13 & 17 & 10 & 16 & 11 & 16 & 11 & 16 & 11 & 17 & 9 & 14 \\
\hline Europe and Central Asia & 22 & 7 & 23 & 7 & 57 & 34 & 58 & 36 & 53 & 31 & 53 & 32 & 50 & 31 & 47 & 29 & 53 & 30 & 48 \\
\hline Latin America and the Caribbean & 15 & 5 & 16 & 5 & 45 & 22 & 45 & 23 & 38 & 19 & 38 & 19 & 38 & 19 & 38 & 19 & 37 & 16 & 32 \\
\hline Middle East and North Africa & 3 & 1 & 3 & 1 & 6 & 4 & 6 & 5 & 5 & 3 & 5 & 4 & 5 & 3 & 4 & 3 & 5 & 3 & 4 \\
\hline South Asia & 4 & 1 & 4 & 1 & 16 & 7 & 16 & 7 & 13 & 5 & 13 & 5 & 13 & 5 & 13 & 5 & 13 & 5 & 9 \\
\hline Sub-Saharan Africa & 13 & 4 & 13 & 4 & 30 & 17 & 32 & 18 & 27 & 14 & 25 & 14 & 25 & 12 & 23 & 11 & 25 & 10 & 21 \\
\hline Low- and middle-income countries & 10 & 3 & 9 & 2 & 28 & 11 & 31 & 14 & 29 & 12 & 28 & 13 & 23 & 11 & 22 & 12 & 25 & 9 & 20 \\
\hline High-income countries & 17 & 5 & 17 & 5 & 31 & 25 & 31 & 25 & 27 & 22 & 27 & 22 & 28 & 22 & 27 & 22 & 28 & 21 & 25 \\
\hline WORLD & 11 & 3 & 9 & 2 & 28 & 12 & 31 & 15 & 29 & 13 & 28 & 14 & 25 & 13 & 24 & 16 & 25 & 10 & 20 \\
\hline
\end{tabular}

PAF of YLL (\%)

\begin{tabular}{|c|c|c|c|c|c|c|c|c|c|c|c|c|c|c|c|c|c|c|c|}
\hline East Asia and Pacific & 10 & 3 & 10 & 3 & 20 & 13 & 20 & 13 & 17 & 10 & 16 & 11 & 16 & 11 & 16 & 11 & 17 & 8 & 14 \\
\hline Europe and Central Asia & 22 & 7 & 23 & 7 & 57 & 34 & 58 & 36 & 53 & 31 & 53 & 32 & 50 & 31 & 47 & 29 & 53 & 29 & 48 \\
\hline Latin America and the Caribbean & 15 & 5 & 16 & 5 & 45 & 22 & 45 & 23 & 38 & 19 & 38 & 19 & 38 & 19 & 38 & 19 & 37 & 14 & 31 \\
\hline Middle East and North Africa & 3 & 1 & 3 & 1 & 6 & 4 & 6 & 5 & 5 & 3 & 5 & 4 & 5 & 3 & 4 & 3 & 5 & 2 & 4 \\
\hline South Asia & 4 & 1 & 4 & 1 & 16 & 7 & 16 & 7 & 13 & 5 & 13 & 5 & 13 & 5 & 13 & 5 & 12 & 4 & 9 \\
\hline Sub-Saharan Africa & 13 & 4 & 13 & 4 & 30 & 17 & 32 & 18 & 27 & 14 & 25 & 14 & 25 & 12 & 23 & 11 & 25 & 9 & 20 \\
\hline Low- and middle-income countries & 10 & 3 & 9 & 2 & 28 & 11 & 30 & 14 & 29 & 12 & 28 & 13 & 24 & 11 & 21 & 11 & 24 & 8 & 19 \\
\hline High-income countries & 17 & 5 & 17 & 5 & 31 & 25 & 31 & 25 & 27 & 22 & 27 & 22 & 28 & 22 & 27 & 22 & 28 & 20 & 25 \\
\hline WORLD & 11 & 3 & 9 & 2 & 28 & 12 & 30 & 15 & 29 & 13 & 28 & 14 & 25 & 13 & 24 & 15 & 25 & 9 & 19 \\
\hline
\end{tabular}

PAF of DALYs (\%)

East Asia and Pacific

Europe and Central Asia

Latin America and the Caribbean

Middle East and North Africa

South Asia

Sub-Saharan Africa

Low- and middle-income countries

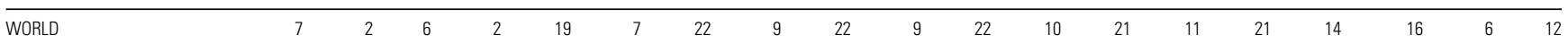

Attributable Mortality (thousands)

East Asia and Pacific

Europe and Central Asia

Latin America and the Caribbean

Middle East and North Africa

South Asia

Sub-Saharan Africa

Low- and middle-income countries

High-income countries

\begin{tabular}{rrrrrrrrrrrrrrrrrrrrr} 
s) & 1 & 1 & 0 & 6 & 1 & 6 & 1 & 4 & 1 & 1 & 1 & 1 & 1 & 0 & 1 & 21 & 6 & 27 \\
1 & 0 & 1 & 0 & 10 & 1 & 14 & 2 & 15 & 2 & 6 & 1 & 2 & 1 & 0 & 1 & 50 & 8 & 58 \\
1 & 0 & 1 & 0 & 6 & 1 & 5 & 0 & 4 & 0 & 2 & 0 & 2 & 0 & 1 & 1 & 21 & 3 & 25 \\
0 & 0 & 0 & 0 & 0 & 0 & 0 & 0 & 0 & 0 & 0 & 0 & 0 & 0 & 0 & 0 & 1 & 0 & 1 \\
0 & 0 & 1 & 0 & 6 & 2 & 5 & 1 & 3 & 1 & 2 & 1 & 1 & 1 & 1 & 0 & 19 & 6 & 25 \\
2 & 0 & 1 & 0 & 11 & 1 & 5 & 1 & 2 & 0 & 1 & 0 & 1 & 0 & 0 & 0 & 23 & 3 & 27 \\
\hline 5 & 2 & 4 & 1 & 39 & 5 & 37 & 6 & 28 & 5 & 13 & 3 & 7 & 3 & 3 & 3 & 135 & 27 & 162 \\
0 & 0 & 0 & 0 & 1 & 0 & 2 & 0 & 2 & 1 & 2 & 1 & 3 & 1 & 3 & 4 & 14 & 7 & 21 \\
5 & 2 & 4 & 1 & 41 & 6 & 39 & 6 & 30 & 6 & 14 & 3 & 9 & 4 & 6 & 6 & 149 & 34 & 183 \\
\hline
\end{tabular}

WORLD

Attributable YLL (thousands)

\begin{tabular}{|c|c|c|c|c|c|c|c|c|c|c|c|c|c|c|c|c|c|c|c|}
\hline East Asia and Pacific & 37 & 18 & 23 & 4 & 160 & 31 & 158 & 36 & 73 & 19 & 17 & 8 & 7 & 6 & 2 & 3 & 477 & 125 & 602 \\
\hline Europe and Central Asia & 18 & 4 & 17 & 2 & 276 & 27 & 346 & 42 & 288 & 47 & 90 & 21 & 21 & 11 & 2 & 3 & 1,058 & 156 & 1,214 \\
\hline Latin America and the Caribbean & 27 & 7 & 16 & 3 & 174 & 16 & 131 & 11 & 70 & 8 & 26 & 5 & 14 & 4 & 5 & 4 & 462 & 58 & 520 \\
\hline Middle East and North Africa & 4 & 1 & 3 & 1 & 11 & 2 & 4 & 1 & 3 & 1 & 2 & 1 & 1 & 0 & 0 & 0 & 27 & 7 & 34 \\
\hline South Asia & 12 & 6 & 22 & 5 & 167 & 53 & 127 & 28 & 61 & 19 & 23 & 8 & 12 & 6 & 3 & 2 & 427 & 127 & 554 \\
\hline$\underline{\text { Sub-Saharan Africa }}$ & 59 & 13 & 29 & 10 & 290 & 23 & 126 & 25 & 46 & 10 & 16 & 2 & 8 & 2 & 1 & 1 & 574 & 84 & 658 \\
\hline Low- and middle-income countries & 157 & 49 & 109 & 24 & 1,077 & 152 & 891 & 142 & 541 & 103 & 173 & 44 & 63 & 30 & 14 & 13 & 3,025 & 557 & 3,582 \\
\hline High-income countries & 7 & 1 & 5 & 1 & 38 & 6 & 51 & 9 & 46 & 11 & 25 & 9 & 22 & 14 & 13 & 15 & 206 & 66 & 272 \\
\hline WORLD & 164 & 50 & 114 & 25 & 1,116 & 158 & 942 & 151 & 586 & 114 & 199 & 53 & 85 & 44 & 27 & 27 & 3,232 & 623 & 3,854 \\
\hline
\end{tabular}

Attributable DALYs (thousands)

East Asia and Pacific

Europe and Central Asia

Latin America and the Caribbean

Middle East and North Africa

South Asia

Sub-Saharan Africa

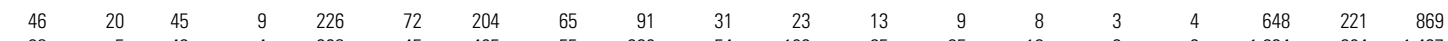

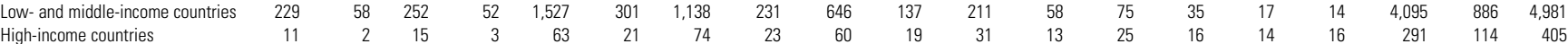

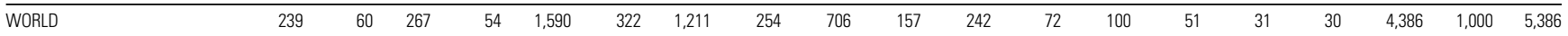

Source: Authors' calculations. 
Table 4A.76

Risk factor:

Alcohol use

Disease:

Self-inflicted injuries

\begin{tabular}{|c|c|c|c|c|c|c|c|c|c|c|c|c|c|c|c|c|c|c|c|}
\hline \multirow[b]{2}{*}{ Region } & \multicolumn{2}{|c|}{$0-4$ years } & \multicolumn{2}{|c|}{ 5-14 years } & \multicolumn{2}{|c|}{$15-29$ years } & \multicolumn{2}{|c|}{ 30-44 years } & \multicolumn{2}{|c|}{$45-59$ years } & \multicolumn{2}{|c|}{$60-69$ years } & \multicolumn{2}{|c|}{ 70-79 years } & \multicolumn{2}{|c|}{$80+$ years } & \multicolumn{3}{|c|}{ Total } \\
\hline & Male & Female & Male & Female & Male & Female & Male & Female & Male & Female & Male & Female & Male & Female & Male & Female & Male & Female & All \\
\hline \multicolumn{20}{|l|}{ PAF of Mortality (\%) } \\
\hline East Asia and Pacific & NA & NA & NA & NA & 10 & 6 & 10 & 6 & 7 & 5 & 7 & 5 & 3 & 3 & 3 & 3 & 8 & 5 & 7 \\
\hline Europe and Central Asia & NA & NA & NA & NA & 40 & 17 & 40 & 18 & 32 & 15 & 33 & 16 & 17 & 10 & 16 & 11 & 35 & 15 & 31 \\
\hline Latin America and the Caribbean & NA & NA & NA & NA & 27 & 10 & 27 & 11 & 21 & 9 & 21 & 9 & 10 & 6 & 10 & 5 & 23 & 9 & 20 \\
\hline Middle East and North Africa & NA & NA & NA & NA & 2 & 1 & 2 & 1 & 2 & 1 & 2 & 1 & 1 & 1 & 1 & 1 & 2 & 1 & 2 \\
\hline South Asia & NA & NA & NA & NA & 8 & 3 & 8 & 3 & 6 & 2 & 5 & 2 & 2 & 1 & 2 & 1 & 7 & 3 & 5 \\
\hline Sub-Saharan Africa & NA & NA & NA & NA & 17 & 6 & 18 & 7 & 13 & 5 & 13 & 6 & 6 & 3 & 6 & 3 & 14 & 6 & 12 \\
\hline Low- and middle-income countries & NA & NA & NA & NA & 15 & 5 & 18 & 6 & 15 & 5 & 14 & 6 & 6 & 4 & 5 & 4 & 15 & 5 & 11 \\
\hline High-income countries & NA & NA & NA & NA & 17 & 11 & 18 & 11 & 13 & 9 & 13 & 9 & 6 & 6 & 6 & 6 & 14 & 9 & 13 \\
\hline WORLD & NA & NA & NA & NA & 16 & 5 & 18 & 6 & 15 & 6 & 14 & 6 & 6 & 4 & 5 & 4 & 14 & 5 & 11 \\
\hline
\end{tabular}

PAF of YLL (\%)

\begin{tabular}{|c|c|c|c|c|c|c|c|c|c|c|c|c|c|c|c|c|c|c|c|}
\hline East Asia and Pacific & NA & NA & NA & NA & 10 & 6 & 10 & 6 & 7 & 5 & 7 & 5 & 3 & 3 & 3 & 3 & 9 & 5 & 7 \\
\hline Europe and Central Asia & NA & NA & NA & NA & 40 & 17 & 40 & 18 & 32 & 15 & 33 & 16 & 17 & 10 & 16 & 11 & 36 & 15 & 33 \\
\hline Latin America and the Caribbean & NA & NA & NA & NA & 27 & 10 & 27 & 11 & 21 & 9 & 21 & 9 & 10 & 6 & 10 & 5 & 25 & 9 & 21 \\
\hline Middle East and North Africa & NA & NA & NA & NA & 2 & 1 & 2 & 1 & 2 & 1 & 2 & 1 & 1 & 1 & 1 & 1 & 2 & 1 & 2 \\
\hline South Asia & NA & NA & NA & NA & 8 & 3 & 8 & 3 & 6 & 2 & 5 & 2 & 2 & 1 & 2 & 1 & 7 & 3 & 5 \\
\hline Sub-Saharan Africa & NA & NA & NA & NA & 17 & 6 & 18 & 7 & 13 & 5 & 13 & 6 & 6 & 3 & 6 & 3 & 15 & 6 & 13 \\
\hline Low- and middle-income countries & NA & NA & NA & NA & 15 & 5 & 18 & 6 & 15 & 5 & 14 & 6 & 6 & 4 & 5 & 4 & 15 & 5 & 11 \\
\hline High-income countries & NA & NA & NA & NA & 17 & 11 & 18 & 11 & 13 & 9 & 13 & 9 & 6 & 6 & 6 & 6 & 15 & 10 & 14 \\
\hline WORLD & NA & NA & NA & NA & 16 & 5 & 18 & 6 & 15 & 6 & 14 & 7 & 6 & 4 & 5 & 4 & 15 & 5 & 11 \\
\hline
\end{tabular}

\section{PAF of DALYs (\%)}

East Asia and Pacific

Europe and Central Asia

Latin America and the Caribbean

Middle East and North Africa

South Asia

Sub-Saharan Africa

Low- and middle-income countries

High-income countries

$\begin{array}{llllrrrrr}\text { NA } & \text { NA } & \text { NA } & \text { NA } & 10 & 6 & 10 & 6 & 7 \\ \text { NA } & \text { NA } & \text { NA } & \text { NA } & 39 & 16 & 39 & 17 & 32 \\ \text { NA } & \text { NA } & \text { NA } & \text { NA } & 27 & 9 & 27 & 11 & 21 \\ \text { NA } & \text { NA } & \text { NA } & \text { NA } & 2 & 1 & 2 & 1 & 2 \\ \text { NA } & \text { NA } & \text { NA } & \text { NA } & 8 & 3 & 8 & 3 & 6 \\ \text { NA } & \text { NA } & \text { NA } & \text { NA } & 16 & 5 & 18 & 7 & 13 \\ \text { NA } & \text { NA } & \text { NA } & \text { NA } & 15 & 4 & 17 & 6 & 15\end{array}$

\begin{tabular}{rrrrrrrrrr}
5 & 7 & 5 & 3 & 3 & 3 & 3 & 8 & 5 & 7 \\
15 & 33 & 16 & 17 & 10 & 16 & 11 & 35 & 15 & 32 \\
9 & 21 & 9 & 10 & 6 & 10 & 5 & 24 & 8 & 20 \\
1 & 2 & 1 & 1 & 1 & 1 & 1 & 2 & 1 & 1 \\
2 & 5 & 2 & 2 & 1 & 2 & 1 & 7 & 2 & 5 \\
5 & 13 & 6 & 6 & 3 & 6 & 3 & 14 & 5 & 12 \\
\hline 5 & 14 & 6 & 6 & 4 & 5 & 4 & 15 & 5 & 10 \\
9 & 13 & 9 & 6 & 6 & 6 & 6 & 15 & 9 & 13 \\
\hline 6 & 14 & 6 & 6 & 4 & 5 & 4 & 15 & 5 & 11 \\
\hline
\end{tabular}

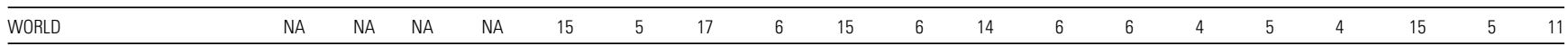

Attributable Mortality (thousands)

\begin{tabular}{lrrrrrrrrrrrrrrrrrrrr} 
East Asia and Pacific & NA & NA & NA & NA & 4 & 2 & 4 & 3 & 2 & 2 & 2 & 1 & 0 & 0 & 0 & 0 & 13 & 8 & 21 \\
Europe and Central Asia & NA & NA & NA & NA & 9 & 1 & 12 & 1 & 9 & 1 & 4 & 0 & 1 & 0 & 0 & 0 & 34 & 3 & 38 \\
Latin America and the Caribbean & NA & NA & NA & NA & 2 & 0 & 2 & 0 & 1 & 0 & 0 & 0 & 0 & 0 & 0 & 0 & 5 & 1 & 6 \\
Middle East and North Africa & NA & NA & NA & NA & 0 & 0 & 0 & 0 & 0 & 0 & 0 & 0 & 0 & 0 & 0 & 0 & 0 & 0 & 0 \\
South Asia & NA & NA & NA & NA & 4 & 1 & 3 & 1 & 1 & 0 & 0 & 0 & 0 & 0 & 0 & 0 & 9 & 2 & 12 \\
Sub-Saharan Africa & NA & NA & NA & NA & 2 & 0 & 1 & 0 & 1 & 0 & 0 & 0 & 0 & 0 & 0 & 0 & 4 & 0 & 4 \\
\hline Low- and middle-income countries & NA & NA & NA & NA & 21 & 5 & 22 & 4 & 14 & 3 & 6 & 2 & 2 & 1 & 1 & 0 & 66 & 15 & 81 \\
High-income countries & NA & NA & NA & NA & 3 & 0 & 5 & 1 & 3 & 1 & 2 & 0 & 1 & 0 & 0 & 0 \\
\hline WORLD & NA & NA & NA & NA & 24 & 5 & 26 & 5 & 18 & 4 & 8 & 2 & 2 & 1 & 1 \\
\hline
\end{tabular}

Attributable YLL (thousands)

\begin{tabular}{|c|c|c|c|c|c|c|c|c|c|c|c|c|c|c|c|c|c|c|c|}
\hline East Asia and Pacific & NA & NA & NA & NA & 110 & 59 & 97 & 68 & 46 & 36 & 23 & 14 & 4 & 5 & 1 & 1 & 283 & 182 & 465 \\
\hline Europe and Central Asia & NA & NA & NA & NA & 243 & 20 & 281 & 20 & 172 & 15 & 51 & 8 & 10 & 3 & 1 & 1 & 758 & 66 & 824 \\
\hline Latin America and the Caribbean & NA & NA & NA & NA & 61 & 8 & 41 & 4 & 18 & 2 & 6 & 1 & 1 & 0 & 0 & 0 & 128 & 14 & 142 \\
\hline Middle East and North Africa & NA & NA & NA & NA & 2 & 1 & 1 & 0 & 1 & 0 & 0 & 0 & 0 & 0 & 0 & 0 & 4 & 1 & 5 \\
\hline South Asia & NA & NA & NA & NA & 116 & 41 & 75 & 16 & 24 & 6 & 4 & 1 & 1 & 0 & 0 & 0 & 220 & 64 & 284 \\
\hline Sub-Saharan Africa & NA & NA & NA & NA & 44 & 5 & 31 & 3 & 15 & 2 & 4 & 0 & 1 & 0 & 0 & 0 & 94 & 11 & 105 \\
\hline Low- and middle-income countries & NA & NA & NA & NA & 576 & 133 & 526 & 111 & 275 & 61 & 88 & 23 & 17 & 9 & 3 & 2 & 1,486 & 339 & 1,825 \\
\hline High-income countries & NA & NA & NA & NA & 73 & 13 & 110 & 20 & 66 & 16 & 21 & 6 & 5 & 2 & 2 & 1 & 276 & 58 & 335 \\
\hline WORLD & NA & NA & NA & NA & 649 & 146 & 636 & 131 & 342 & 76 & 109 & 29 & 22 & 11 & 4 & 3 & 1,762 & 398 & 2,160 \\
\hline
\end{tabular}

Attributable DALYs (thousands

\begin{tabular}{|c|c|c|c|c|c|c|c|c|c|c|c|c|c|c|c|c|c|c|c|}
\hline East Asia and Pacific & NA & NA & NA & NA & 111 & 60 & 98 & 70 & 47 & 37 & 23 & 14 & 5 & 5 & 1 & 1 & 285 & 187 & 472 \\
\hline Europe and Central Asia & NA & NA & NA & NA & 247 & 20 & 284 & 20 & 173 & 15 & 51 & 8 & 10 & 3 & 1 & 1 & 766 & 68 & 834 \\
\hline Latin America and the Caribbean & NA & NA & NA & NA & 61 & 8 & 42 & 4 & 18 & 2 & 6 & 1 & 1 & 0 & 0 & 0 & 129 & 15 & 144 \\
\hline Middle East and North Africa & NA & NA & NA & NA & 2 & 1 & 1 & 0 & 1 & 0 & 0 & 0 & 0 & 0 & 0 & 0 & 4 & 1 & 5 \\
\hline South Asia & NA & NA & NA & NA & 119 & 44 & 76 & 16 & 24 & 6 & 4 & 1 & 1 & 0 & 0 & 0 & 225 & 68 & 292 \\
\hline Sub-Saharan Africa & NA & NA & NA & NA & 45 & 6 & 31 & 4 & 15 & 2 & 4 & 0 & 1 & 0 & 0 & 0 & 95 & 12 & 107 \\
\hline Low- and middle-income countries & NA & NA & NA & NA & 586 & 140 & 531 & 114 & 277 & 62 & 89 & 24 & 17 & 9 & 3 & 2 & 1,504 & 350 & 1,854 \\
\hline High-income countries & NA & NA & NA & NA & 74 & 14 & 112 & 21 & 67 & 16 & 21 & 6 & 5 & 3 & 2 & 1 & 280 & 61 & 341 \\
\hline WORLD & NA & NA & NA & NA & 660 & 154 & 643 & 135 & 344 & 78 & 110 & 30 & 22 & 12 & 4 & 3 & 1,784 & 411 & 2,195 \\
\hline
\end{tabular}

Source: Authors' calculations.

Note: $\mathrm{NA}=$ not applicable. 
Table 4A.77

Risk factor:

Alcohol use

Disease:

Violence

\begin{tabular}{|c|c|c|c|c|c|c|c|c|c|c|c|c|c|c|c|c|c|c|c|}
\hline \multirow[b]{2}{*}{ Region } & \multicolumn{2}{|c|}{$0-4$ years } & \multicolumn{2}{|c|}{ 5-14 years } & \multicolumn{2}{|c|}{$15-29$ years } & \multicolumn{2}{|c|}{ 30-44 years } & \multicolumn{2}{|c|}{$45-59$ years } & \multicolumn{2}{|c|}{ 60-69 years } & \multicolumn{2}{|c|}{ 70-79 years } & \multicolumn{2}{|c|}{$80+$ years } & \multicolumn{3}{|c|}{ Total } \\
\hline & Male & Female & Male & Female & Male & Female & Male & Female & Male & Female & Male & Female & Male & Female & Male & Female & Male & Female & All \\
\hline \multicolumn{20}{|l|}{ PAF of Mortality (\%) } \\
\hline East Asia and Pacific & 7 & 7 & 7 & 7 & 18 & 16 & 19 & 16 & 19 & 16 & 19 & 16 & 18 & 16 & 18 & 16 & 18 & 15 & 18 \\
\hline Europe and Central Asia & 26 & 25 & 24 & 25 & 59 & 45 & 60 & 46 & 60 & 45 & 60 & 46 & 59 & 46 & 58 & 46 & 59 & 45 & 56 \\
\hline Latin America and the Caribbean & 14 & 14 & 14 & 14 & 44 & 28 & 44 & 29 & 44 & 28 & 44 & 28 & 43 & 28 & 43 & 29 & 43 & 27 & 42 \\
\hline Middle East and North Africa & 3 & 3 & 3 & 4 & 8 & 10 & 8 & 9 & 7 & 7 & 8 & 4 & 9 & 6 & 5 & 8 & 8 & 8 & 8 \\
\hline South Asia & 3 & 3 & 3 & 4 & 14 & 8 & 14 & 8 & 14 & 8 & 14 & 8 & 15 & 8 & 15 & 8 & 13 & 7 & 11 \\
\hline Sub-Saharan Africa & 11 & 11 & 11 & 11 & 31 & 22 & 32 & 22 & 32 & 21 & 31 & 21 & 30 & 22 & 30 & 23 & 30 & 19 & 28 \\
\hline Low- and middle-income countries & 9 & 9 & 10 & 10 & 33 & 21 & 34 & 22 & 35 & 22 & 34 & 21 & 29 & 23 & 22 & 29 & 32 & 20 & 30 \\
\hline High-income countries & 15 & 15 & 15 & 15 & 28 & 29 & 29 & 29 & 29 & 30 & 29 & 30 & 29 & 30 & 30 & 31 & 28 & 28 & 28 \\
\hline WORLD & 10 & 9 & 10 & 10 & 33 & 22 & 34 & 23 & 34 & 22 & 34 & 21 & 29 & 23 & 22 & 29 & 32 & 21 & 30 \\
\hline
\end{tabular}

PAF of YLL (\%)

East Asia and Pacific

Europe and Central Asia

Latin America and the Caribbea

Middle East and North Afric

South Asia

Sub-Saharan Africa

\begin{tabular}{|c|c|c|c|c|c|c|c|c|c|c|c|c|c|c|c|c|c|c|}
\hline 7 & 7 & 7 & 7 & 18 & 16 & 19 & 16 & 19 & 16 & 19 & 16 & 18 & 16 & 18 & 16 & 18 & 15 & 18 \\
\hline 14 & 14 & 14 & 14 & 44 & 28 & 44 & 29 & 44 & 28 & 44 & 28 & 43 & 28 & 43 & 29 & 43 & 27 & 42 \\
\hline 3 & 3 & 3 & 4 & 8 & 10 & 8 & 9 & 7 & 7 & 8 & 4 & 9 & 6 & 5 & 8 & 8 & 8 & 8 \\
\hline 3 & 3 & 3 & 4 & 14 & 8 & 14 & 8 & 14 & 8 & 14 & 8 & 15 & 8 & 15 & 8 & 13 & 7 & 11 \\
\hline 9 & 9 & 10 & 10 & 33 & 21 & 34 & 22 & 35 & 22 & 34 & 21 & 29 & 22 & 22 & 28 & 32 & 20 & 30 \\
\hline 15 & 15 & 15 & 15 & 28 & 29 & 29 & 29 & 29 & 30 & 29 & 30 & 29 & 30 & 30 & 31 & 28 & 27 & 27 \\
\hline
\end{tabular}

Low- and middle-income countries

High-income counties

WORLD

PAF of DALYs (\%)

East Asia and Pacific

Europe and Central Asia

Latin America and the Caribbean

Middle East and North Africa

South Asia

Sub-Saharan Africa

Low- and middle-income co

High-income countries

\begin{tabular}{|c|c|c|c|c|c|c|c|c|c|c|c|c|c|c|c|c|c|c|}
\hline 7 & 7 & 5 & 5 & 16 & 13 & 17 & 14 & 18 & 15 & 18 & 16 & 17 & 16 & 17 & 14 & 16 & 13 & 15 \\
\hline 24 & 24 & 16 & 18 & 46 & 35 & 51 & 38 & 56 & 42 & 57 & 44 & 55 & 43 & 49 & 41 & 50 & 38 & 47 \\
\hline 12 & 13 & 8 & 10 & 35 & 21 & 35 & 23 & 39 & 25 & 40 & 26 & 38 & 25 & 35 & 24 & 34 & 21 & 33 \\
\hline 3 & 3 & 2 & 3 & 5 & 7 & 6 & 7 & 6 & 6 & 7 & 4 & 8 & 6 & 5 & 7 & 5 & 6 & 6 \\
\hline 3 & 3 & 2 & 3 & 12 & 7 & 12 & 7 & 13 & 8 & 13 & 8 & 14 & 8 & 15 & 7 & 11 & 6 & 9 \\
\hline 9 & 11 & 7 & 10 & 26 & 18 & 28 & 20 & 29 & 20 & 29 & 20 & 26 & 21 & 23 & 22 & 24 & 17 & 23 \\
\hline 8 & 8 & 6 & 8 & 27 & 18 & 29 & 20 & 32 & 21 & 32 & 21 & 27 & 22 & 21 & 25 & 27 & 17 & 25 \\
\hline 15 & 15 & 11 & 12 & 24 & 25 & 25 & 26 & 26 & 28 & 27 & 29 & 26 & 28 & 25 & 26 & 24 & 24 & 24 \\
\hline
\end{tabular}

Attributable Mortality (thousands)

\begin{tabular}{lllllrrrrrrrrrrrrrrrr} 
Attributable Mortality (thousands) & & 0 & 0 & 0 & 0 & 5 & 1 & 6 & 1 & 3 & 1 & 1 & 0 & 0 & 0 & 0 & 0 & 15 & 3 & 18 \\
East Asia and Pacific & 0 & 0 & 0 & 0 & 7 & 1 & 11 & 2 & 8 & 2 & 2 & 1 & 1 & 1 & 0 & 0 & 30 & 8 & 38 \\
Europe and Central Asia & 0 & 0 & 0 & 0 & 28 & 1 & 15 & 1 & 6 & 0 & 2 & 0 & 1 & 0 & 0 & 0 & 52 & 3 & 55 \\
Latin America and the Caribbean & 0 & 0 & 0 & 0 & 0 & 0 & 0 & 0 & 0 & 0 & 0 & 0 & 0 & 0 & 0 & 0 & 1 & 0 & 1 \\
Middle East and North Africa & 0 & 0 & 0 & 0 & 2 & 0 & 2 & 0 & 1 & 0 & 0 & 0 & 0 & 0 & 0 & 0 & 7 & 2 & 9 \\
South Asia & 0 & 0 & 0 & 1 & 17 & 2 & 10 & 2 & 4 & 1 & 1 & 0 & 0 & 0 & 0 & 0 & 33 & 6 & 39 \\
Sub-Saharan Africa & 1 & 0 & 1 & 1 & 59 & 6 & 44 & 6 & 23 & 4 & 6 & 2 & 3 & 1 & 1 & 1 & 138 & 22 & 160 \\
\hline Low- and middle-income countries & 0 & 0 & 0 & 0 & 2 & 0 & 2 & 1 & 1 & 0 & 0 & 0 & 0 & 0 & 0 & 0 \\
High-income countries & 1 & 1 & 1 & 1 & 61 & 7 & 46 & 7 & 23 & 5 & 7 & 2 & 3 & 2 & 1 & 1 \\
\hline WORLD & 143 & 24 & 166 \\
\hline
\end{tabular}

Attributable YLL (thousands)

East Asia and Pacific

Europe and Central Asia

Latin America and the Caribbean

Middle East and North Africa

South Asia

Sub-Saharan A

Low- and middle-income countries

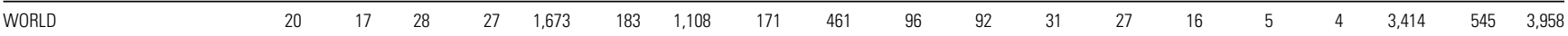

Attributable DALYs (thousands)

East Asia and Pacific

Europe and Central Asia

Latin America and the Caribbean

Middle East and North Africa

South Asia

Sub-Saharan Africa

Low- and middle-income countries

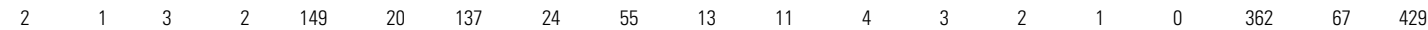

\begin{tabular}{|c|c|c|c|c|c|c|c|c|c|c|c|c|c|c|c|c|c|c|}
\hline 3 & 1 & 5 & 3 & 171 & 24 & 150 & 27 & 57 & 14 & 12 & 4 & 3 & 2 & 1 & 0 & 402 & 75 & 477 \\
\hline 2 & 2 & 5 & 3 & 233 & 51 & 304 & 64 & 173 & 45 & 36 & 16 & 9 & 9 & 1 & 2 & 762 & 192 & 955 \\
\hline 3 & 2 & 12 & 4 & 972 & 50 & 456 & 31 & 132 & 10 & 23 & 2 & 7 & 1 & 1 & 0 & 1,606 & 100 & 1,706 \\
\hline 0 & 0 & 0 & 0 & 11 & 3 & 5 & 2 & 2 & 1 & 1 & 0 & 0 & 0 & 0 & 0 & 19 & 6 & 24 \\
\hline 1 & 2 & 3 & 3 & 77 & 15 & 57 & 13 & 25 & 10 & 6 & 4 & 4 & 2 & 2 & 0 & 176 & 49 & 225 \\
\hline 11 & 9 & 23 & 18 & 551 & 69 & 282 & 45 & 91 & 17 & 17 & 4 & 5 & 2 & 1 & 1 & 980 & 163 & 1,143 \\
\hline 20 & 16 & 48 & 32 & 2,015 & 213 & 1,254 & 181 & 481 & 96 & 93 & 30 & 28 & 16 & 6 & 4 & 3,944 & 586 & 4,530 \\
\hline 3 & 2 & 2 & 2 & 66 & 15 & 43 & 17 & 17 & 7 & 3 & 2 & 1 & 1 & 0 & 0 & 135 & 47 & 181 \\
\hline 22 & 18 & 50 & 33 & 2,081 & 228 & 1,297 & 198 & 497 & 103 & 96 & 32 & 29 & 17 & 6 & 4 & 4,079 & 633 & 4,711 \\
\hline
\end{tabular}

Source: Authors' calculations. 
Table 4A.78

Risk factor:

Alcohol use

Disease:

Other intentional injuries

\begin{tabular}{|c|c|c|c|c|c|c|c|c|c|c|c|c|c|c|c|c|c|c|c|}
\hline \multirow[b]{2}{*}{ Region } & \multicolumn{2}{|c|}{$0-4$ years } & \multicolumn{2}{|c|}{ 5-14 years } & \multicolumn{2}{|c|}{$15-29$ years } & \multicolumn{2}{|c|}{ 30-44 years } & \multicolumn{2}{|c|}{$45-59$ years } & \multicolumn{2}{|c|}{$60-69$ years } & \multicolumn{2}{|c|}{ 70-79 years } & \multicolumn{2}{|c|}{$80+$ years } & \multicolumn{3}{|c|}{ Total } \\
\hline & Male & Female & Male & Female & Male & Female & Male & Female & Male & Female & Male & Female & Male & Female & Male & Female & Male & Female & All \\
\hline \multicolumn{20}{|l|}{ PAF of Mortality (\%) } \\
\hline East Asia and Pacific & NA & NA & NA & NA & 13 & 11 & 13 & 10 & 12 & 9 & 12 & 9 & 5 & 4 & 6 & 4 & 12 & 9 & 11 \\
\hline Europe and Central Asia & NA & NA & NA & NA & 46 & 31 & 45 & 33 & 44 & 26 & 41 & 35 & * & 19 & 33 & 16 & 45 & 28 & 39 \\
\hline Latin America and the Caribbean & NA & NA & NA & NA & 36 & 22 & 35 & 21 & 35 & * & 32 & 22 & 20 & 11 & 18 & 7 & 35 & 16 & 34 \\
\hline Middle East and North Africa & NA & NA & NA & NA & 2 & 2 & 3 & 2 & 2 & 2 & 2 & 2 & 1 & 1 & 1 & 1 & 2 & 1 & 2 \\
\hline South Asia & NA & NA & NA & NA & 10 & 5 & 10 & 6 & 10 & 5 & 10 & 6 & 5 & 3 & 5 & 3 & 8 & 4 & 7 \\
\hline Sub-Saharan Africa & NA & NA & NA & NA & 2 & 2 & 3 & 4 & 3 & 2 & 2 & 5 & 3 & * & 1 & 1 & 2 & 2 & 2 \\
\hline Low- and middle-income countries & NA & NA & NA & NA & 15 & 7 & 14 & 12 & 13 & 11 & 10 & 7 & 5 & 3 & 6 & 3 & 12 & 7 & 11 \\
\hline High-income countries & NA & NA & NA & NA & 21 & 12 & 20 & 20 & 21 & 18 & 21 & 11 & 9 & 6 & 5 & 5 & 20 & 13 & 20 \\
\hline WORLD & NA & NA & NA & NA & 15 & 7 & 14 & 12 & 14 & 11 & 10 & 7 & 5 & 3 & 6 & 3 & 12 & 7 & 11 \\
\hline
\end{tabular}

PAF of YLL $(\%)$

\begin{tabular}{|c|c|c|c|c|c|c|c|c|c|c|c|c|c|c|c|c|c|c|c|}
\hline East Asia and Pacific & NA & NA & NA & NA & 13 & 11 & 13 & 10 & 12 & 9 & 12 & 9 & 5 & 4 & 6 & 4 & 12 & 9 & 12 \\
\hline Europe and Central Asia & NA & NA & NA & NA & 46 & 31 & 45 & 33 & 44 & 26 & 41 & 35 & * & 19 & 33 & 16 & 45 & 29 & 39 \\
\hline Latin America and the Caribbean & NA & NA & NA & NA & 36 & 22 & 35 & 21 & 35 & * & 32 & 22 & 20 & 11 & 18 & 7 & 35 & 18 & 35 \\
\hline Middle East and North Africa & NA & NA & NA & NA & 2 & 2 & 3 & 2 & 2 & 2 & 2 & 2 & 1 & 1 & 1 & 1 & 2 & 1 & 2 \\
\hline South Asia & NA & NA & NA & NA & 10 & 5 & 10 & 6 & 10 & 5 & 10 & 6 & 5 & 3 & 5 & 3 & 8 & 3 & 7 \\
\hline Sub-Saharan Africa & NA & NA & NA & NA & 2 & 2 & 3 & 4 & 3 & 2 & 2 & 5 & 3 & ${ }^{*}$ & 1 & 1 & 1 & 1 & 1 \\
\hline Low- and middle-income countries & NA & NA & NA & NA & 15 & 7 & 14 & 12 & 13 & 11 & 10 & 7 & 5 & 3 & 6 & 3 & 12 & 7 & 11 \\
\hline High-income countries & NA & NA & NA & NA & 21 & 12 & 20 & 20 & 21 & 18 & 21 & 11 & 9 & 6 & 5 & 5 & 20 & 13 & 20 \\
\hline WORLD & NA & NA & NA & NA & 15 & 7 & 14 & 12 & 14 & 11 & 10 & 7 & 5 & 3 & 6 & 3 & 12 & 7 & 11 \\
\hline
\end{tabular}

\section{PAF of DALYs $(\%)$}

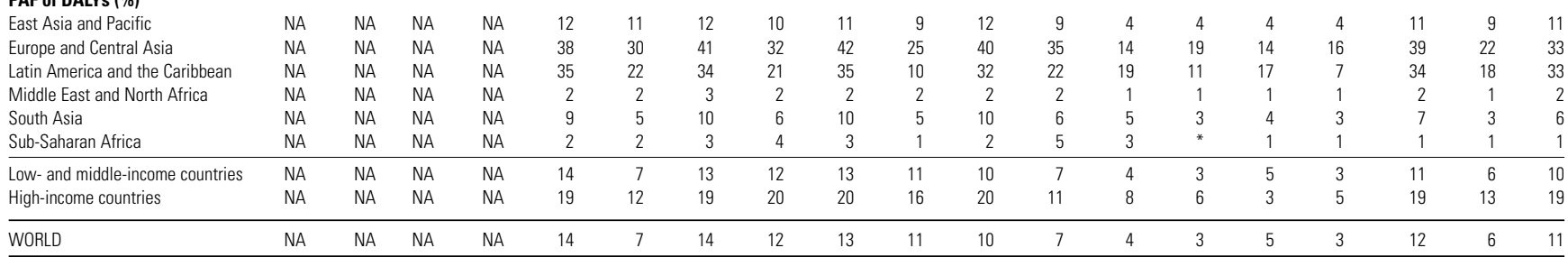

Attributable Mortality (thousands)

\begin{tabular}{llllllllllllllllllllll} 
East Asia and Pacific & NA & NA & NA & NA & 0 & 0 & 0 & 0 & 0 & 0 & 0 & 0 & 0 & 0 & 0 & 0 & 0 & 0 & 0 \\
Europe and Central Asia & NA & NA & NA & NA & 0 & 0 & 0 & 0 & 0 & 0 & 0 & 0 & 0 & 0 & 0 & 0 & 0 & 0 & 0 \\
Latin America and the Caribbean & NA & NA & NA & NA & 0 & 0 & 0 & 0 & 0 & 0 & 0 & 0 & 0 & 0 & 0 & 0 & 0 & 0 & 0 \\
Middle East and North Africa & NA & NA & NA & NA & 0 & 0 & 0 & 0 & 0 & 0 & 0 & 0 & 0 & 0 & 0 & 0 & 0 & 0 & 0 \\
South Asia & NA & NA & NA & NA & 0 & 0 & 0 & 0 & 0 & 0 & 0 & 0 & 0 & 0 & 0 & 0 & 0 & 0 & 0 \\
Sub-Saharan Africa & NA & NA & NA & NA & 0 & 0 & 0 & 0 & 0 & 0 & 0 & 0 & 0 & 0 & 0 & 0 & 0 & 0 & 0 \\
\hline Low- and middle-income countries & NA & NA & NA & NA & 0 & 0 & 0 & 0 & 0 & 0 & 0 & 0 & 0 & 0 & 0 & 0 & 1 & 0 & 1 \\
High-income countries & NA & NA & NA & NA & 0 & 0 & 0 & 0 & 0 & 0 & 0 & 0 & 0 & 0 \\
\hline WORLD & NA & NA & NA & NA & 1 & 0 & 0 & 0 & 0 & 0 & 0 & 0 & 0 & 0 & 0 & 0 \\
\hline
\end{tabular}

Attributable YLL (thousands)

\begin{tabular}{|c|c|c|c|c|c|c|c|c|c|c|c|c|c|c|c|c|c|c|c|}
\hline East Asia and Pacific & NA & NA & NA & NA & 3 & 0 & 3 & 0 & 1 & 0 & 0 & 0 & 0 & 0 & 0 & 0 & 7 & 1 & 8 \\
\hline Europe and Central Asia & NA & NA & NA & NA & 1 & 0 & 2 & 1 & 1 & 1 & 0 & 0 & 0 & 0 & 0 & 0 & 4 & 2 & 6 \\
\hline Latin America and the Caribbean & NA & NA & NA & NA & 5 & 0 & 1 & 0 & 0 & 0 & 0 & 0 & 0 & 0 & 0 & 0 & 7 & 0 & 7 \\
\hline Middle East and North Africa & NA & NA & NA & NA & 0 & 0 & 0 & 0 & 0 & 0 & 0 & 0 & 0 & 0 & 0 & 0 & 1 & ( & 1 \\
\hline South Asia & NA & NA & NA & NA & 3 & 0 & 3 & 0 & 1 & 0 & 1 & 0 & 0 & 0 & 0 & 0 & 8 & (1) & 9 \\
\hline Sub-Saharan Africa & NA & NA & NA & NA & 0 & 0 & 0 & 0 & 0 & 0 & 0 & 0 & 0 & 0 & 0 & 0 & 0 & ( & 0 \\
\hline Low- and middle-income countries & NA & NA & NA & NA & 13 & 1 & 9 & 1 & 3 & 1 & 1 & 0 & 0 & 0 & 0 & 0 & 27 & the & 31 \\
\hline High-income countries & NA & NA & NA & NA & 1 & 0 & 1 & 0 & 0 & 0 & 0 & 0 & 0 & 0 & 0 & 0 & 2 & ( & 2 \\
\hline WORLD & NA & NA & NA & NA & 14 & 1 & 10 & 1 & 4 & 1 & 1 & 0 & 0 & 0 & 0 & 0 & 29 & ra & 33 \\
\hline
\end{tabular}

Attributable DALYs (thousands)

\begin{tabular}{lrrrrrrrrrrrrrrrrrrr} 
East Asia and Pacific & NA & NA & NA & NA & 3 & 0 & 3 & 0 & 1 & 0 & 0 & 0 & 0 & 0 & 0 & 0 & 7 & 1 & 8 \\
Europe and Central Asia & NA & NA & NA & NA & 1 & 0 & 2 & 1 & 1 & 1 & 0 & 0 & 0 & 0 & 0 & 0 & 5 & 2 & 6 \\
Latin America and the Caribbean & NA & NA & NA & NA & 6 & 0 & 1 & 0 & 0 & 0 & 0 & 0 & 0 & 0 & 0 & 0 & 7 & 0 & 7 \\
Middle East and North Africa & NA & NA & NA & NA & 0 & 0 & 0 & 0 & 0 & 0 & 0 & 0 & 0 & 0 & 0 & 0 & 1 & 0 & 1 \\
South Asia & NA & NA & NA & NA & 4 & 0 & 3 & 0 & 1 & 0 & 1 & 0 & 0 & 0 & 0 & 0 & 8 & 1 & 10 \\
Sub-Saharan Africa & NA & NA & NA & NA & 0 & 0 & 0 & 0 & 0 & 0 & 0 & 0 & 0 & 0 & 0 & 0 & 0 & 0 & 0 \\
\hline Low- and middle-income countries & NA & NA & NA & NA & 14 & 1 & 10 & 1 & 3 & 1 & 1 & 0 & 0 & 0 & 0 & 0 & 29 & 4 & 33 \\
High-income countries & NA & NA & NA & NA & 1 & 0 & 1 & 0 & 0 & 0 & 0 & 0 & 0 & 0 & 0 & 0 \\
\hline WORLD & NA & NA & NA & NA & 15 & 1 & 11 & 1 & 4 & 1 & 1 & 0 & 0 & 0 & 0 & 0 & 31 & 4 & 35
\end{tabular}

Source: Authors' calculations.

Note: $\mathrm{NA}=$ not applicable.

*The number of deaths (and hence YLL) directly coded to a number of diseases, especially neuropsychiatric and musculoskeletal diseases, is zero or very small. For other diseases, mortality or disease burden may be zero in some region-age-sex groups. In such cases, the population attributable fractions would be undefined or unstable and have not been calculated. 
Table 4A.79

Risk factor:

Alcohol use

Disease:

All causes

\begin{tabular}{|c|c|c|c|c|c|c|c|c|c|c|c|c|c|c|c|c|c|c|c|}
\hline \multirow[b]{2}{*}{ Region } & \multicolumn{2}{|c|}{$0-4$ years } & \multicolumn{2}{|c|}{ 5-14 years } & \multicolumn{2}{|c|}{$15-29$ years } & \multicolumn{2}{|c|}{ 30-44 years } & \multicolumn{2}{|c|}{ 45-59 years } & \multicolumn{2}{|c|}{ 60-69 years } & \multicolumn{2}{|c|}{ 70-79 years } & \multicolumn{2}{|c|}{$80+$ years } & \multicolumn{3}{|c|}{ Total } \\
\hline & Male & Female & Male & Female & Male & Female & Male & Female & Male & Female & Male & Female & Male & Female & Male & Female & Male & Female & All \\
\hline \multicolumn{20}{|l|}{ PAF of Mortality (\%) } \\
\hline East Asia and Pacific & 0 & 0 & 2 & 1 & 13 & 4 & 13 & 3 & 11 & 3 & 8 & 1 & 6 & 1 & 4 & 0 & 7 & 1 & 4 \\
\hline Europe and Central Asia & 1 & 1 & 6 & 3 & 36 & 15 & 28 & 12 & 20 & 10 & 13 & 5 & 10 & 3 & 9 & 2 & 15 & 4 & 10 \\
\hline Latin America and the Caribbean & 1 & 0 & 5 & 2 & 31 & 6 & 25 & 6 & 17 & 5 & 11 & 4 & 7 & 3 & 5 & 2 & 12 & 3 & 8 \\
\hline Middle East and North Africa & 1 & 0 & 1 & 0 & 5 & 1 & 5 & 1 & 3 & 1 & 2 & 0 & 1 & 0 & 1 & 0 & 2 & 0 & 1 \\
\hline South Asia & 0 & 0 & 1 & 0 & 6 & 1 & 6 & 1 & 5 & 1 & 2 & 0 & 0 & 0 & 0 & 0 & 2 & 0 & 1 \\
\hline Sub-Saharan Africa & 0 & 0 & 2 & 1 & 9 & 1 & 5 & 1 & 6 & 2 & 6 & 2 & 4 & 1 & 3 & 1 & 3 & 1 & 2 \\
\hline Low- and middle-income countries & 0 & 0 & 2 & 1 & 13 & 2 & 11 & 2 & 10 & 3 & 7 & 2 & 5 & 1 & 4 & 1 & 6 & 1 & 4 \\
\hline High-income countries & 1 & 1 & 6 & 2 & 23 & 9 & 16 & 5 & 9 & 4 & 4 & 0 & 1 & -2 & 0 & -5 & 3 & -3 & 0 \\
\hline WORLD & 0 & 0 & 2 & 1 & 14 & 2 & 12 & 3 & 10 & 3 & 7 & 2 & 4 & 1 & 2 & -1 & 6 & 1 & 3 \\
\hline
\end{tabular}

PAF of YLL (\%)

East Asia and Pacific

Europe and Central Asia

Latin America and the Caribbean

Middle East and North Africa

South Asia

Sub-Saharan Africa

Low- and middle-income countries

High-income countries

\section{WORLD \\ PAF of DALYs (\%)}

East Asia and Pacific

Europe and Central Asia

Latin America and the Caribbean

Middle East and North Africa

South Asia

Sub-Saharan Africa

Low- and middle-income countries

High-income countries

\begin{tabular}{rrrrrrrrrrrrrrr}
13 & 4 & 13 & 3 & 11 & 3 & 8 & 1 & 6 & 1 & 4 & 0 & 8 & 1 & 5 \\
36 & 15 & 28 & 12 & 20 & 10 & 13 & 5 & 10 & 3 & 9 & 2 & 18 & 6 & 13 \\
31 & 6 & 25 & 6 & 17 & 5 & 11 & 4 & 7 & 3 & 5 & 2 & 14 & 3 & 10 \\
5 & 1 & 5 & 1 & 3 & 1 & 2 & 0 & 1 & 0 & 1 & 0 & 2 & 1 & 1 \\
6 & 1 & 6 & 1 & 5 & 1 & 2 & 0 & 0 & 0 & 0 & 0 & 2 & 0 & 1 \\
9 & 1 & 5 & 1 & 6 & 2 & 6 & 2 & 5 & 1 & 3 & 1 & 3 & 1 & 2 \\
13 & 2 & 11 & 2 & 10 & 3 & 7 & 2 & 5 & 1 & 4 & 1 & 6 & 1 & 4 \\
23 & 9 & 17 & 5 & 9 & 4 & 4 & 0 & 1 & -2 & 0 & -5 & 6 & -1 & 3 \\
\hline 14 & 2 & 12 & 3 & 10 & 3 & 7 & 2 & 4 & 1 & 2 & -1 & 6 & 1 & 4 \\
\hline
\end{tabular}

WORLD

Attributable Mortality (thousands

East Asia and Pacific

Europe and Central Asia

Latin America and the Caribbean

Middle East and North Africa

South Asia

Sub-Saharan Africa

Low- and middle-income countries

High-income countries

$0 \quad 0$

$\begin{array}{ll}2 & 1 \\ 6 & 3 \\ 5 & 2 \\ 1 & 0 \\ 1 & 0 \\ 2 & 1 \\ 2 & 1 \\ 6 & 2\end{array}$


Risk factor: Illicit drug use

\begin{tabular}{|c|c|c|c|c|c|c|c|c|c|c|c|c|c|c|c|c|c|c|c|}
\hline \multirow[b]{2}{*}{ Region } & \multicolumn{2}{|c|}{$0-4$ years } & \multicolumn{2}{|c|}{ 5-14 years } & \multicolumn{2}{|c|}{$15-29$ years } & \multicolumn{2}{|c|}{ 30-44 years } & \multicolumn{2}{|c|}{ 45-59 years } & \multicolumn{2}{|c|}{ 60-69 years } & \multicolumn{2}{|c|}{$70-79$ years } & \multicolumn{2}{|c|}{$80+$ years } & \multicolumn{3}{|c|}{ Total } \\
\hline & Male & Female & Male & Female & Male & Female & Male & Female & Male & Female & Male & Female & Male & Female & Male & Female & Male & Female & All \\
\hline \multicolumn{20}{|l|}{ PAF of Mortality (\%) } \\
\hline East Asia and Pacific & NA & NA & NA & NA & 27 & 26 & 27 & 27 & 27 & 27 & NA & NA & NA & NA & NA & NA & 25 & 22 & 24 \\
\hline Europe and Central Asia & NA & NA & NA & NA & 74 & 74 & 74 & 74 & 74 & 75 & NA & NA & NA & NA & NA & NA & 71 & 66 & 70 \\
\hline Latin America and the Caribbean & NA & NA & NA & NA & 9 & 9 & 10 & 10 & 11 & 10 & NA & NA & NA & NA & NA & NA & 9 & 8 & 9 \\
\hline Middle East and North Africa & NA & NA & NA & NA & 1 & 0 & 1 & 0 & 1 & 1 & NA & NA & NA & NA & NA & NA & 1 & 0 & 1 \\
\hline South Asia & NA & NA & NA & NA & 2 & 2 & 2 & 2 & 2 & 2 & NA & NA & NA & NA & NA & NA & 2 & 2 & 2 \\
\hline Sub-Saharan Africa & NA & NA & NA & NA & 0 & 0 & 0 & 0 & 0 & 0 & NA & NA & NA & NA & NA & NA & 0 & 0 & 0 \\
\hline Low- and middle-income countries & NA & NA & NA & NA & 4 & 1 & 4 & 1 & 5 & 2 & NA & NA & NA & NA & NA & NA & 3 & 1 & 2 \\
\hline High-income countries & NA & NA & NA & NA & 38 & 36 & 35 & 34 & 32 & 32 & NA & NA & NA & NA & NA & NA & 32 & 31 & 32 \\
\hline WORLD & NA & NA & NA & NA & 4 & 1 & 5 & 1 & 5 & 3 & NA & NA & NA & NA & NA & NA & 4 & 1 & 3 \\
\hline
\end{tabular}

PAF of YLL (\%)

\begin{tabular}{|c|c|c|c|c|c|c|c|c|c|c|c|c|c|c|c|c|c|c|c|}
\hline East Asia and Pacific & NA & NA & NA & NA & 27 & 26 & 27 & 27 & 27 & 27 & NA & NA & NA & NA & NA & NA & 25 & 22 & 24 \\
\hline Europe and Central Asia & NA & NA & NA & NA & 74 & 74 & 74 & 74 & 74 & 75 & NA & NA & NA & NA & NA & NA & 71 & 65 & 70 \\
\hline Latin America and the Caribbean & NA & NA & NA & NA & 9 & 9 & 10 & 10 & 11 & 10 & NA & NA & NA & NA & NA & NA & 9 & 8 & 9 \\
\hline Middle East and North Africa & NA & NA & NA & NA & 1 & 0 & 1 & 0 & 1 & 1 & NA & NA & NA & NA & NA & NA & 1 & 0 & 1 \\
\hline South Asia & NA & NA & NA & NA & 2 & 2 & 2 & 2 & 2 & 2 & NA & NA & NA & NA & NA & NA & 2 & 2 & 2 \\
\hline Sub-Saharan Africa & NA & NA & NA & NA & 0 & 0 & 0 & 0 & 0 & 0 & NA & NA & NA & NA & NA & NA & 0 & 0 & 0 \\
\hline Low- and middle-income countries & NA & NA & NA & NA & 4 & 1 & 4 & 1 & 5 & 2 & NA & NA & NA & NA & NA & NA & 3 & 1 & 2 \\
\hline High-income countries & NA & NA & NA & NA & 38 & 36 & 35 & 34 & 32 & 32 & NA & NA & NA & NA & NA & NA & 33 & 32 & 33 \\
\hline WORLD & NA & NA & NA & NA & 4 & 1 & 5 & 1 & 5 & 3 & NA & NA & NA & NA & NA & NA & 4 & 1 & 2 \\
\hline
\end{tabular}

\section{PAF of DALYs (\%)}

\begin{tabular}{|c|c|c|c|c|c|c|c|c|c|c|c|c|c|c|c|c|c|c|}
\hline East Asia and Pacific & NA & NA & NA & NA & 27 & 26 & 27 & 27 & 27 & 27 & NA & NA & NA & NA & NA & NA & 26 & 22 \\
\hline Europe and Central Asia & NA & NA & NA & NA & 74 & 74 & 74 & 74 & 74 & 75 & NA & NA & NA & NA & NA & NA & 72 & 68 \\
\hline Latin America and the Caribbean & NA & NA & NA & NA & 9 & 9 & 10 & 10 & 11 & 10 & NA & NA & NA & NA & NA & NA & 9 & 8 \\
\hline Middle East and North Africa & NA & NA & NA & NA & 1 & 0 & 1 & 0 & 1 & 1 & NA & NA & NA & NA & NA & NA & 1 & 0 \\
\hline South Asia & NA & NA & NA & NA & 2 & 2 & 2 & 2 & 2 & 2 & NA & NA & NA & NA & NA & NA & 2 & 2 \\
\hline Sub-Saharan Africa & NA & NA & NA & NA & 0 & 0 & 0 & 0 & 0 & 0 & NA & NA & NA & NA & NA & NA & 0 & 0 \\
\hline Low- and middle-income countries & NA & NA & NA & NA & 5 & 1 & 5 & 1 & 5 & 3 & NA & NA & NA & NA & NA & NA & 4 & 1 \\
\hline High-income countries & NA & NA & NA & NA & 38 & 36 & 35 & 34 & 32 & 32 & NA & NA & NA & NA & NA & NA & 34 & 32 \\
\hline WORLD & NA & NA & NA & NA & 5 & 1 & 5 & 2 & 6 & 3 & NA & NA & NA & NA & NA & NA & 4 & 1 \\
\hline
\end{tabular}

Attributable Mortality (thousands)

\begin{tabular}{|c|c|c|c|c|c|c|c|c|c|c|c|c|c|c|c|c|c|c|c|}
\hline East Asia and Pacific & NA & NA & NA & NA & 4 & 1 & 12 & 2 & 4 & 2 & NA & NA & NA & NA & NA & NA & 20 & 6 & 26 \\
\hline Europe and Central Asia & NA & NA & NA & NA & 3 & 1 & 10 & 1 & 4 & 0 & NA & NA & NA & NA & NA & NA & 17 & 3 & 20 \\
\hline Latin America and the Caribbean & NA & NA & NA & NA & 1 & 1 & 3 & 1 & 1 & 0 & NA & NA & NA & NA & NA & NA & 5 & 2 & 7 \\
\hline Middle East and North Africa & NA & NA & NA & NA & 0 & 0 & 0 & 0 & 0 & 0 & NA & NA & NA & NA & NA & NA & 0 & 0 & 0 \\
\hline South Asia & NA & NA & NA & NA & 1 & 0 & 3 & 1 & 1 & 0 & NA & NA & NA & NA & NA & NA & 4 & 1 & 5 \\
\hline Sub-Saharan Africa & NA & NA & NA & NA & 0 & 0 & 0 & 0 & 0 & 0 & NA & NA & NA & NA & NA & NA & 0 & 0 & 0 \\
\hline Low- and middle-income countries & NA & NA & NA & NA & 10 & 3 & 27 & 5 & 10 & 3 & NA & NA & NA & NA & NA & NA & 47 & 12 & 59 \\
\hline High-income countries & NA & NA & NA & NA & 0 & 0 & 3 & 1 & 2 & 0 & NA & NA & NA & NA & NA & NA & 5 & 2 & 7 \\
\hline WORLD & NA & NA & NA & NA & 10 & 4 & 30 & 6 & 12 & 4 & NA & NA & NA & NA & NA & NA & 52 & 14 & 65 \\
\hline
\end{tabular}

Attributable YLL (thousands)

\begin{tabular}{|c|c|c|c|c|c|c|c|c|c|c|c|c|c|c|c|c|c|c|c|}
\hline East Asia and Pacific & NA & NA & NA & NA & 111 & 33 & 282 & 60 & 85 & 45 & NA & NA & NA & NA & NA & NA & 478 & 138 & 616 \\
\hline Europe and Central Asia & NA & NA & NA & NA & 93 & 27 & 236 & 27 & 81 & 10 & NA & NA & NA & NA & NA & NA & 410 & 64 & 474 \\
\hline Latin America and the Caribbean & NA & NA & NA & NA & 29 & 23 & 68 & 32 & 20 & 7 & NA & NA & NA & NA & NA & NA & 117 & 63 & 180 \\
\hline Middle East and North Africa & NA & NA & NA & NA & 0 & 0 & 0 & 0 & 0 & 0 & NA & NA & NA & NA & NA & NA & 0 & 0 & 0 \\
\hline South Asia & NA & NA & NA & NA & 25 & 11 & 62 & 13 & 18 & 4 & NA & NA & NA & NA & NA & NA & 105 & 28 & 133 \\
\hline Sub-Saharan Africa & NA & NA & NA & NA & 0 & 0 & 0 & 0 & 0 & 0 & NA & NA & NA & NA & NA & NA & 1 & 1 & 2 \\
\hline Low- and middle-income countries & NA & NA & NA & NA & 258 & 95 & 649 & 133 & 204 & 65 & NA & NA & NA & NA & NA & NA & 1,111 & 293 & 1,404 \\
\hline High-income countries & NA & NA & NA & NA & 12 & 6 & 77 & 25 & 34 & 8 & NA & NA & NA & NA & NA & NA & 123 & 39 & 161 \\
\hline WORLD & NA & NA & NA & NA & 270 & 101 & 726 & 158 & 238 & 73 & NA & NA & NA & NA & NA & NA & 1,234 & 332 & 1,566 \\
\hline
\end{tabular}

Attributable DALYs (thousands)

\begin{tabular}{|c|c|c|c|c|c|c|c|c|c|c|c|c|c|c|c|c|c|c|c|}
\hline East Asia and Pacific & NA & NA & NA & NA & 180 & 54 & 327 & 70 & 90 & 46 & NA & NA & NA & NA & NA & NA & 597 & 170 & 767 \\
\hline Europe and Central Asia & NA & NA & NA & NA & 188 & 67 & 297 & 44 & 90 & 12 & NA & NA & NA & NA & NA & NA & 574 & 123 & 697 \\
\hline Latin America and the Caribbean & NA & NA & NA & NA & 41 & 28 & 76 & 34 & 21 & 8 & NA & NA & NA & NA & NA & NA & 137 & 70 & 207 \\
\hline Middle East and North Africa & NA & NA & NA & NA & 0 & 0 & 0 & 0 & 0 & 0 & NA & NA & NA & NA & NA & NA & 0 & 0 & 1 \\
\hline South Asia & NA & NA & NA & NA & 32 & 15 & 66 & 14 & 18 & 4 & NA & NA & NA & NA & NA & NA & 116 & 33 & 149 \\
\hline Sub-Saharan Africa & NA & NA & NA & NA & 0 & 0 & 0 & 0 & 0 & 0 & NA & NA & NA & NA & NA & NA & 1 & 1 & 2 \\
\hline Low- and middle-income countries & NA & NA & NA & NA & 440 & 164 & 766 & 163 & 219 & 69 & NA & NA & NA & NA & NA & NA & 1,425 & 397 & 1,822 \\
\hline High-income countries & NA & NA & NA & NA & 32 & 12 & 101 & 30 & 39 & 9 & NA & NA & NA & NA & NA & NA & 171 & 50 & 221 \\
\hline WORLD & NA & NA & NA & NA & 472 & 176 & 867 & 193 & 257 & 78 & NA & NA & NA & NA & NA & NA & 1,596 & 447 & 2,043 \\
\hline
\end{tabular}

Source: Authors' calculations.

Note: $\mathrm{NA}=$ not applicable. 
Table 4A.81

Risk factor: Illicit drug use

Disease:

Drug use disorders

\begin{tabular}{|c|c|c|c|c|c|c|c|c|c|c|c|c|c|c|c|c|c|c|c|}
\hline \multirow[b]{2}{*}{ Region } & \multicolumn{2}{|c|}{$0-4$ years } & \multicolumn{2}{|c|}{ 5-14 years } & \multicolumn{2}{|c|}{$15-29$ years } & \multicolumn{2}{|c|}{ 30-44 years } & \multicolumn{2}{|c|}{$45-59$ years } & \multicolumn{2}{|c|}{$60-69$ years } & \multicolumn{2}{|c|}{ 70-79 years } & \multicolumn{2}{|c|}{$80+$ years } & \multicolumn{3}{|c|}{ Total } \\
\hline & Male & Female & Male & Female & Male & Female & Male & Female & Male & Female & Male & Female & Male & Female & Male & Female & Male & Female & All \\
\hline \multicolumn{20}{|l|}{ PAF of Mortality (\%) } \\
\hline East Asia and Pacific & 100 & 100 & 100 & 100 & 100 & 100 & 100 & 100 & 100 & 100 & 100 & 100 & 100 & 100 & 100 & 100 & 100 & 100 & 100 \\
\hline Europe and Central Asia & 100 & 100 & 100 & 100 & 100 & 100 & 100 & 100 & 100 & 100 & 100 & 100 & 100 & 100 & 100 & 100 & 100 & 100 & 100 \\
\hline Latin America and the Caribbean & 100 & 100 & 100 & 100 & 100 & 100 & 100 & 100 & 100 & 100 & 100 & 100 & 100 & 100 & 100 & 100 & 100 & 100 & 100 \\
\hline Middle East and North Africa & 100 & 100 & 100 & 100 & 100 & 100 & 100 & 100 & 100 & 100 & 100 & 100 & 100 & 100 & 100 & 100 & 100 & 100 & 100 \\
\hline South Asia & 100 & 100 & 100 & 100 & 100 & 100 & 100 & 100 & 100 & 100 & 100 & 100 & 100 & 100 & 100 & 100 & 100 & 100 & 100 \\
\hline Sub-Saharan Africa & 100 & 100 & 100 & 100 & 100 & 100 & 100 & 100 & 100 & 100 & 100 & 100 & 100 & 100 & 100 & 100 & 100 & 100 & 100 \\
\hline Low- and middle-income countries & 100 & 100 & 100 & 100 & 100 & 100 & 100 & 100 & 100 & 100 & 100 & 100 & 100 & 100 & 100 & 100 & 100 & 100 & 100 \\
\hline High-income countries & 100 & 100 & 100 & 100 & 100 & 100 & 100 & 100 & 100 & 100 & 100 & 100 & 100 & 100 & 100 & 100 & 100 & 100 & 100 \\
\hline WORLD & 100 & 100 & 100 & 100 & 100 & 100 & 100 & 100 & 100 & 100 & 100 & 100 & 100 & 100 & 100 & 100 & 100 & 100 & 100 \\
\hline \multicolumn{20}{|l|}{ PAF of YLL (\%) } \\
\hline East Asia and Pacific & 100 & 100 & 100 & 100 & 100 & 100 & 100 & 100 & 100 & 100 & 100 & 100 & 100 & 100 & 100 & 100 & 100 & 100 & 100 \\
\hline Europe and Central Asia & 100 & 100 & 100 & 100 & 100 & 100 & 100 & 100 & 100 & 100 & 100 & 100 & 100 & 100 & 100 & 100 & 100 & 100 & 100 \\
\hline Latin America and the Caribbean & 100 & 100 & 100 & 100 & 100 & 100 & 100 & 100 & 100 & 100 & 100 & 100 & 100 & 100 & 100 & 100 & 100 & 100 & 100 \\
\hline Middle East and North Africa & 100 & 100 & 100 & 100 & 100 & 100 & 100 & 100 & 100 & 100 & 100 & 100 & 100 & 100 & 100 & 100 & 100 & 100 & 100 \\
\hline South Asia & 100 & 100 & 100 & 100 & 100 & 100 & 100 & 100 & 100 & 100 & 100 & 100 & 100 & 100 & 100 & 100 & 100 & 100 & 100 \\
\hline Sub-Saharan Africa & 100 & 100 & 100 & 100 & 100 & 100 & 100 & 100 & 100 & 100 & 100 & 100 & 100 & 100 & 100 & 100 & 100 & 100 & 100 \\
\hline Low- and middle-income countries & 100 & 100 & 100 & 100 & 100 & 100 & 100 & 100 & 100 & 100 & 100 & 100 & 100 & 100 & 100 & 100 & 100 & 100 & 100 \\
\hline High-income countries & 100 & 100 & 100 & 100 & 100 & 100 & 100 & 100 & 100 & 100 & 100 & 100 & 100 & 100 & 100 & 100 & 100 & 100 & 100 \\
\hline WORLD & 100 & 100 & 100 & 100 & 100 & 100 & 100 & 100 & 100 & 100 & 100 & 100 & 100 & 100 & 100 & 100 & 100 & 100 & 100 \\
\hline \multicolumn{20}{|l|}{ PAF of DALYs (\%) } \\
\hline East Asia and Pacific & 100 & 100 & 100 & 100 & 100 & 100 & 100 & 100 & 100 & 100 & 100 & 100 & 100 & 100 & 100 & 100 & 100 & 100 & 100 \\
\hline Europe and Central Asia & 100 & 100 & 100 & 100 & 100 & 100 & 100 & 100 & 100 & 100 & 100 & 100 & 100 & 100 & 100 & 100 & 100 & 100 & 100 \\
\hline Latin America and the Caribbean & 100 & 100 & 100 & 100 & 100 & 100 & 100 & 100 & 100 & 100 & 100 & 100 & 100 & 100 & 100 & 100 & 100 & 100 & 100 \\
\hline Middle East and North Africa & 100 & 100 & 100 & 100 & 100 & 100 & 100 & 100 & 100 & 100 & 100 & 100 & 100 & 100 & 100 & 100 & 100 & 100 & 100 \\
\hline South Asia & 100 & 100 & 100 & 100 & 100 & 100 & 100 & 100 & 100 & 100 & 100 & 100 & 100 & 100 & 100 & 100 & 100 & 100 & 100 \\
\hline Sub-Saharan Africa & 100 & 100 & 100 & 100 & 100 & 100 & 100 & 100 & 100 & 100 & 100 & 100 & 100 & 100 & 100 & 100 & 100 & 100 & 100 \\
\hline Low- and middle-income countries & 100 & 100 & 100 & 100 & 100 & 100 & 100 & 100 & 100 & 100 & 100 & 100 & 100 & 100 & 100 & 100 & 100 & 100 & 100 \\
\hline High-income countries & 100 & 100 & 100 & 100 & 100 & 100 & 100 & 100 & 100 & 100 & 100 & 100 & 100 & 100 & 100 & 100 & 100 & 100 & 100 \\
\hline WORLD & 100 & 100 & 100 & 100 & 100 & 100 & 100 & 100 & 100 & 100 & 100 & 100 & 100 & 100 & 100 & 100 & 100 & 100 & 100 \\
\hline
\end{tabular}

Attributable Mortality (thousands)

\begin{tabular}{lrrrrrrrrrrrrrrrrrrrrrr} 
East Asia and Pacific & 0 & 0 & 0 & 0 & 1 & 0 & 3 & 1 & 2 & 0 & 0 & 0 & 0 & 0 & 0 & 0 & 6 & 1 & 7 \\
Europe and Central Asia & 0 & 0 & 0 & 0 & 2 & 0 & 4 & 1 & 3 & 1 & 1 & 0 & 0 & 0 & 0 & 0 & 9 & 2 & 11 \\
Latin America and the Caribbean & 0 & 0 & 0 & 0 & 0 & 0 & 1 & 0 & 1 & 0 & 0 & 0 & 0 & 0 & 0 & 0 & 2 & 0 & 2 \\
Middle East and North Africa & 0 & 0 & 0 & 0 & 3 & 1 & 7 & 2 & 5 & 1 & 0 & 0 & 0 & 0 & 0 & 0 & 16 & 3 & 19 \\
South Asia & 0 & 0 & 0 & 0 & 4 & 1 & 12 & 2 & 7 & 2 & 1 & 0 & 0 & 0 & 0 & 0 & 24 & 5 & 29 \\
Sub-Saharan Africa & 0 & 0 & 0 & 0 & 1 & 0 & 1 & 0 & 1 & 0 & 0 & 0 & 0 & 0 & 0 & 0 & 3 & 1 & 4 \\
\hline Low- and middle-income countries & 0 & 0 & 0 & 0 & 11 & 2 & 28 & 6 & 19 & 4 & 2 & 0 & 0 & 0 & 0 & 0 & 60 & 13 & 73 \\
High-income countries & 0 & 0 & 0 & 0 & 3 & 1 & 5 & 1 & 2 & 1 & 0 & 0 & 0 & 0 & 0 & 0 & 10 & 2 & 13 \\
\hline WORLD & 0 & 0 & 0 & 0 & 13 & 3 & 33 & 7 & 21 & 5 & 2 & 0 & 0 & 0 & 0 & 0 & 70 & 15 & 85 \\
\hline
\end{tabular}

Attributable YLL (thousands)

\begin{tabular}{|c|c|c|c|c|c|c|c|c|c|c|c|c|c|c|c|c|c|c|c|}
\hline East Asia and Pacific & 0 & 0 & 0 & 0 & 30 & 5 & 67 & 17 & 37 & 10 & 2 & 0 & 0 & 0 & 0 & 0 & 136 & 33 & 169 \\
\hline Europe and Central Asia & 1 & 0 & 0 & 0 & 41 & 8 & 96 & 22 & 59 & 15 & 8 & 2 & 1 & 1 & 0 & 0 & 206 & 47 & 253 \\
\hline Latin America and the Caribbean & 0 & 0 & 0 & 0 & 11 & 1 & 23 & 5 & 12 & 3 & 1 & 0 & 0 & 0 & 0 & 0 & 46 & 10 & 56 \\
\hline Middle East and North Africa & 0 & 0 & 0 & 0 & 75 & 16 & 176 & 37 & 104 & 23 & 5 & 1 & 1 & 0 & 0 & 0 & 362 & 78 & 440 \\
\hline South Asia & 0 & 0 & 0 & 0 & 118 & 21 & 276 & 58 & 146 & 33 & 7 & 2 & 1 & 1 & 0 & 0 & 550 & 115 & 665 \\
\hline Sub-Saharan Africa & 0 & 0 & 0 & 0 & 15 & 3 & 34 & 8 & 20 & 5 & 1 & 0 & 0 & 0 & 0 & 0 & 70 & 16 & 86 \\
\hline Low- and middle-income countries & 1 & 0 & 0 & 0 & 290 & 54 & 672 & 148 & 378 & 89 & 23 & 6 & 4 & 2 & 1 & 1 & 1,369 & 299 & 1,668 \\
\hline High-income countries & 0 & 0 & 0 & 0 & 72 & 16 & 117 & 29 & 50 & 14 & 1 & 1 & 0 & 0 & 0 & 0 & 240 & 59 & 299 \\
\hline WORLD & 1 & 0 & 1 & 0 & 362 & 70 & 789 & 177 & 427 & 102 & 24 & 7 & 4 & 2 & 1 & 1 & 1,609 & 358 & 1,968 \\
\hline \multicolumn{20}{|l|}{ Attributable DALYs (thousands) } \\
\hline East Asia and Pacific & 0 & 0 & 17 & 9 & 172 & 38 & 109 & 27 & 40 & 10 & 2 & 0 & 0 & 0 & 0 & 0 & 340 & 85 & 425 \\
\hline Europe and Central Asia & 1 & 0 & 5 & 5 & 188 & 59 & 154 & 42 & 73 & 20 & 9 & 3 & 1 & 1 & 0 & 0 & 431 & 129 & 559 \\
\hline Latin America and the Caribbean & 0 & 0 & 7 & 7 & 370 & 122 & 152 & 50 & 28 & 9 & 1 & 1 & 0 & 0 & 0 & 0 & 559 & 188 & 746 \\
\hline Middle East and North Africa & 0 & 0 & 6 & 5 & 278 & 55 & 241 & 50 & 117 & 26 & 6 & 1 & 1 & 0 & 0 & 0 & 649 & 137 & 786 \\
\hline South Asia & 0 & 0 & 23 & 7 & 285 & 37 & 347 & 65 & 148 & 33 & 7 & 2 & 1 & 1 & 0 & 0 & 812 & 145 & 957 \\
\hline Sub-Saharan Africa & 0 & 0 & 11 & 9 & 472 & 143 & 165 & 62 & 49 & 16 & 2 & 1 & 0 & 0 & 0 & 0 & 699 & 230 & 929 \\
\hline Low- and middle-income countries & 1 & 0 & 68 & 42 & 1,764 & 453 & 1,169 & 295 & 455 & 114 & 27 & 8 & 4 & 2 & 1 & 1 & 3,489 & 913 & 4,402 \\
\hline High-income countries & 0 & 0 & 10 & 6 & 490 & 151 & 336 & 103 & 104 & 35 & 4 & 2 & 0 & 0 & 0 & 0 & 944 & 297 & 1,242 \\
\hline WORLD & 1 & 0 & 79 & 48 & 2,254 & 604 & 1,505 & 398 & 559 & 148 & 31 & 10 & 4 & 2 & 1 & 1 & 4,434 & 1,210 & $\overline{5,644}$ \\
\hline
\end{tabular}

Source: Authors' calculations. 
Risk factor: Illicit drug use

Disease:

Unintentional injuries

\begin{tabular}{|c|c|c|c|c|c|c|c|c|c|c|c|c|c|c|c|c|c|c|c|}
\hline \multirow[b]{2}{*}{ Region } & \multicolumn{2}{|c|}{$0-4$ years } & \multicolumn{2}{|c|}{ 5-14 years } & \multicolumn{2}{|c|}{$15-29$ years } & \multicolumn{2}{|c|}{$30-44$ years } & \multicolumn{2}{|c|}{$45-59$ years } & \multicolumn{2}{|c|}{$60-69$ years } & \multicolumn{2}{|c|}{ 70-79 years } & \multicolumn{2}{|c|}{$80+$ years } & \multicolumn{3}{|c|}{ Total } \\
\hline & Male & Female & Male & Female & Male & Female & Male & Female & Male & Female & Male & Female & Male & Female & Male & Female & Male & Female & All \\
\hline \multicolumn{20}{|l|}{ PAF of Mortality (\%) } \\
\hline East Asia and Pacific & NA & NA & NA & NA & 1 & 0 & 3 & 1 & 3 & 0 & NA & NA & NA & NA & NA & NA & 2 & 0 & 1 \\
\hline Europe and Central Asia & NA & NA & NA & NA & 0 & 1 & 1 & 4 & 1 & 2 & NA & NA & NA & NA & NA & NA & 0 & 1 & 1 \\
\hline Latin America and the Caribbean & NA & NA & NA & NA & 1 & 3 & 3 & 11 & 3 & 9 & NA & NA & NA & NA & NA & NA & 1 & 3 & 2 \\
\hline Middle East and North Africa & NA & NA & NA & NA & 1 & 1 & 4 & 3 & 3 & 2 & NA & NA & NA & NA & NA & NA & 1 & 1 & 1 \\
\hline South Asia & NA & NA & NA & NA & 1 & 0 & 3 & 1 & 2 & 1 & NA & NA & NA & NA & NA & NA & 1 & 0 & 1 \\
\hline Sub-Saharan Africa & NA & NA & NA & NA & 1 & 0 & 3 & 1 & 3 & 1 & NA & NA & NA & NA & NA & NA & 1 & 0 & 1 \\
\hline Low- and middle-income countries & NA & NA & NA & NA & 1 & 0 & 3 & 2 & 2 & 1 & NA & NA & NA & NA & NA & NA & 1 & 0 & $\overline{1}$ \\
\hline High-income countries & NA & NA & NA & NA & 2 & 7 & 7 & 26 & 7 & 18 & NA & NA & NA & NA & NA & NA & 3 & 5 & 4 \\
\hline WORLD & NA & NA & NA & NA & 1 & 1 & 3 & 3 & 3 & 2 & NA & NA & NA & NA & NA & NA & 1 & 1 & 1 \\
\hline \multicolumn{20}{|l|}{ PAF of YLL (\%) } \\
\hline East Asia and Pacific & NA & NA & NA & NA & 1 & 0 & 3 & 1 & 3 & 0 & NA & NA & NA & NA & NA & NA & 2 & 0 & 1 \\
\hline Europe and Central Asia & NA & NA & NA & NA & 0 & 1 & 1 & 4 & 1 & 2 & NA & NA & NA & NA & NA & NA & 0 & 2 & 1 \\
\hline Latin America and the Caribbean & NA & NA & NA & NA & 1 & 3 & 3 & 11 & 3 & 9 & NA & NA & NA & NA & NA & NA & 1 & 3 & 2 \\
\hline Middle East and North Africa & NA & NA & NA & NA & 1 & 1 & 4 & 3 & 3 & 2 & NA & NA & NA & NA & NA & NA & 1 & 1 & 1 \\
\hline South Asia & NA & NA & NA & NA & 1 & 0 & 3 & 1 & 2 & 1 & NA & NA & NA & NA & NA & NA & 1 & 0 & 1 \\
\hline Sub-Saharan Africa & NA & NA & NA & NA & 1 & 0 & 3 & 1 & 3 & 1 & NA & NA & NA & NA & NA & NA & 1 & 0 & 1 \\
\hline Low- and middle-income countries & NA & NA & NA & NA & 1 & 0 & 3 & 2 & 2 & 1 & NA & NA & NA & NA & NA & NA & 1 & 1 & $\overline{1}$ \\
\hline High-income countries & NA & NA & NA & NA & 2 & 7 & 7 & 26 & 7 & 18 & NA & NA & NA & NA & NA & NA & 4 & 9 & 5 \\
\hline WORLD & NA & NA & NA & NA & 1 & 1 & 3 & 3 & 3 & 2 & NA & NA & NA & NA & NA & NA & 1 & 1 & 1 \\
\hline
\end{tabular}

\section{PAF of DALYs (\%)}

East Asia and Pacific

Europe and Central Asia

Latin America and the Caribbea

Middle East and North Africa

South Asia

Sub-Saharan Africa

Low- and middle-income countries

High-income co

\begin{tabular}{|c|c|c|c|c|c|c|c|c|c|c|c|c|c|c|c|c|c|c|}
\hline WORLD & NA & NA & NA & NA & 1 & 1 & 3 & 3 & 3 & 3 & NA & NA & NA & NA & NA & NA & 1 & 1 \\
\hline
\end{tabular}

Attributable Mortality (thousands)

\begin{tabular}{|c|c|c|c|c|c|c|c|c|c|c|c|c|c|c|c|c|c|c|c|}
\hline East Asia and Pacific & NA & NA & NA & NA & 1 & 0 & 5 & 0 & 3 & 0 & NA & NA & NA & NA & NA & NA & 9 & 1 & 10 \\
\hline Europe and Central Asia & NA & NA & NA & NA & 0 & 0 & 1 & 1 & 1 & 1 & NA & NA & NA & NA & NA & NA & 1 & 1 & 3 \\
\hline Latin America and the Caribbean & NA & NA & NA & NA & 0 & 0 & 1 & 1 & 1 & 1 & NA & NA & NA & NA & NA & NA & 2 & 1 & 4 \\
\hline Middle East and North Africa & NA & NA & NA & NA & 0 & 0 & 1 & 0 & 1 & 0 & NA & NA & NA & NA & NA & NA & 2 & 0 & 2 \\
\hline South Asia & NA & NA & NA & NA & 1 & 0 & 3 & 1 & 2 & 0 & NA & NA & NA & NA & NA & NA & 6 & 1 & 7 \\
\hline Sub-Saharan Africa & NA & NA & NA & NA & 1 & 0 & 2 & 0 & 1 & 0 & NA & NA & NA & NA & NA & NA & 4 & 0 & 4 \\
\hline Low- and middle-income countries & NA & NA & NA & NA & 4 & 1 & 12 & 3 & 9 & 2 & NA & NA & NA & NA & NA & NA & 25 & 5 & 30 \\
\hline High-income countries & NA & NA & NA & NA & 1 & 1 & 3 & 3 & 2 & 2 & NA & NA & NA & NA & NA & NA & 6 & 6 & 12 \\
\hline WORLD & NA & NA & NA & NA & 5 & 2 & 15 & 6 & 11 & 4 & NA & NA & NA & NA & NA & NA & 31 & 11 & 42 \\
\hline
\end{tabular}

Attributable YLL (thousands)

\begin{tabular}{|c|c|c|c|c|c|c|c|c|c|c|c|c|c|c|c|c|c|c|c|}
\hline 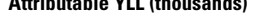 & & & & & & & & & & & & & & & & & & & \\
\hline East Asia and Pacific & NA & NA & NA & NA & 41 & 3 & 113 & 8 & 65 & 4 & NA & NA & NA & NA & NA & NA & 218 & 15 & 233 \\
\hline Europe and Central Asia & NA & NA & NA & NA & 5 & 5 & 14 & 17 & 10 & 11 & NA & NA & NA & NA & NA & NA & 29 & 33 & 62 \\
\hline Latin America and the Caribbean & NA & NA & NA & NA & 9 & 6 & 23 & 18 & 17 & 10 & NA & NA & NA & NA & NA & NA & 49 & 34 & 83 \\
\hline Middle East and North Africa & NA & NA & NA & NA & 10 & 2 & 22 & 4 & 13 & 2 & NA & NA & NA & NA & NA & NA & 45 & 8 & 53 \\
\hline South Asia & NA & NA & NA & NA & 24 & 6 & 77 & 14 & 42 & 8 & NA & NA & NA & NA & NA & NA & 142 & 27 & 170 \\
\hline Sub-Saharan Africa & NA & NA & NA & NA & 20 & 3 & 47 & 6 & 21 & 3 & NA & NA & NA & NA & NA & NA & 87 & 12 & 99 \\
\hline Low- and middle-income countries & NA & NA & NA & NA & 109 & 24 & 295 & 68 & 167 & 39 & NA & NA & NA & NA & NA & NA & 570 & 130 & 700 \\
\hline High-income countries & NA & NA & NA & NA & 23 & 21 & 66 & 73 & 48 & 46 & NA & NA & NA & NA & NA & NA & 137 & 139 & 276 \\
\hline WORLD & NA & NA & NA & NA & 132 & 44 & 360 & 141 & 215 & 84 & NA & NA & NA & NA & NA & NA & 707 & 270 & $9 / 6$ \\
\hline
\end{tabular}

Attributable DALYs (thousands)

\begin{tabular}{|c|c|c|c|c|c|c|c|c|c|c|c|c|c|c|c|c|c|c|c|}
\hline East Asia and Pacific & NA & NA & NA & NA & 57 & 6 & 149 & 12 & 80 & 6 & NA & NA & NA & NA & NA & NA & 286 & 24 & 310 \\
\hline Europe and Central Asia & NA & NA & NA & NA & 8 & 8 & 17 & 24 & 11 & 13 & NA & NA & NA & NA & NA & NA & 36 & 46 & 82 \\
\hline Latin America and the Caribbean & NA & NA & NA & NA & 14 & 13 & 33 & 34 & 24 & 17 & NA & NA & NA & NA & NA & NA & 71 & 64 & 134 \\
\hline Middle East and North Africa & NA & NA & NA & NA & 18 & 4 & 37 & 9 & 18 & 4 & NA & NA & NA & NA & NA & NA & 73 & 17 & 90 \\
\hline South Asia & NA & NA & NA & NA & 39 & 9 & 106 & 23 & 53 & 11 & NA & NA & NA & NA & NA & NA & 198 & 42 & 240 \\
\hline Sub-Saharan Africa & NA & NA & NA & NA & 29 & 5 & 60 & 9 & 27 & 5 & NA & NA & NA & NA & NA & NA & 117 & 19 & 135 \\
\hline Low- and middle-income countries & NA & NA & NA & NA & 166 & 44 & 402 & 112 & 212 & 56 & NA & NA & NA & NA & NA & NA & 779 & 212 & 991 \\
\hline High-income countries & NA & NA & NA & NA & 33 & 39 & 90 & 130 & 64 & 75 & NA & NA & NA & NA & NA & NA & 188 & 244 & 432 \\
\hline WORLD & NA & NA & NA & NA & 199 & 83 & 492 & 242 & 276 & 131 & NA & NA & NA & NA & NA & NA & 967 & 456 & 1,423 \\
\hline
\end{tabular}

Source: Authors' calculations.

Note: NA = not applicable. 
Risk factor: Illicit drug use

Disease:

Self-inflicted injuries

\begin{tabular}{|c|c|c|c|c|c|c|c|c|c|c|c|c|c|c|c|c|c|c|c|}
\hline \multirow[b]{2}{*}{ Region } & \multicolumn{2}{|c|}{$0-4$ years } & \multicolumn{2}{|c|}{ 5-14 years } & \multicolumn{2}{|c|}{$15-29$ years } & \multicolumn{2}{|c|}{ 30-44 years } & \multicolumn{2}{|c|}{$45-59$ years } & \multicolumn{2}{|c|}{$60-69$ years } & \multicolumn{2}{|c|}{$70-79$ years } & \multicolumn{2}{|c|}{$80+$ years } & \multicolumn{3}{|c|}{ Total } \\
\hline & Male & Female & Male & Female & Male & Female & Male & Female & Male & Female & Male & Female & Male & Female & Male & Female & Male & Female & All \\
\hline \multicolumn{20}{|l|}{ PAF of Mortality (\%) } \\
\hline East Asia and Pacific & NA & NA & NA & NA & 1 & 0 & 2 & 0 & 2 & 0 & NA & NA & NA & NA & NA & NA & 1 & 0 & 1 \\
\hline Europe and Central Asia & NA & NA & NA & NA & 3 & 6 & 7 & 16 & 5 & 10 & NA & NA & NA & NA & NA & NA & 4 & 7 & 5 \\
\hline Latin America and the Caribbean & NA & NA & NA & NA & 1 & 2 & 5 & 15 & 5 & 14 & NA & NA & NA & NA & NA & NA & 3 & 7 & 4 \\
\hline Middle East and North Africa & NA & NA & NA & NA & 5 & 1 & 20 & 7 & 17 & 7 & NA & NA & NA & NA & NA & NA & 10 & 3 & 7 \\
\hline South Asia & NA & NA & NA & NA & 5 & 1 & 18 & 7 & 21 & 6 & NA & NA & NA & NA & NA & NA & 11 & 3 & 7 \\
\hline Sub-Saharan Africa & NA & NA & NA & NA & 3 & 1 & 10 & 3 & 8 & 4 & NA & NA & NA & NA & NA & NA & 5 & 2 & 4 \\
\hline Low- and middle-income countries & NA & NA & NA & NA & 3 & 1 & 9 & 3 & 8 & 3 & NA & NA & NA & NA & NA & NA & 5 & 2 & 4 \\
\hline High-income countries & NA & NA & NA & NA & 3 & 7 & 7 & 12 & 4 & 8 & NA & NA & NA & NA & NA & NA & 4 & 6 & 4 \\
\hline WORLD & NA & NA & NA & NA & 3 & 1 & 9 & 4 & 7 & 4 & NA & NA & NA & NA & NA & NA & 5 & 2 & 4 \\
\hline
\end{tabular}

PAF of YLL (\%)

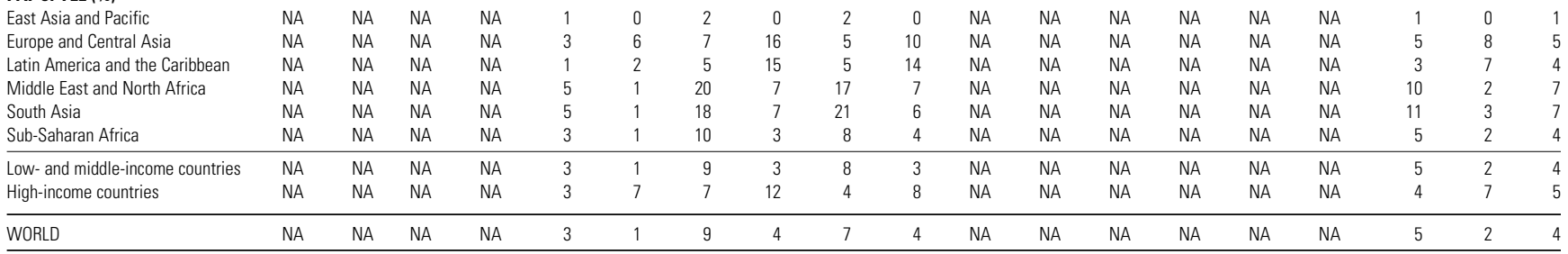

PAF of DALYs (\%)

\begin{tabular}{|c|c|c|c|c|c|c|c|c|c|c|c|c|c|c|c|c|c|c|c|}
\hline East Asia and Pacific & NA & NA & NA & NA & 1 & 0 & 2 & 0 & 2 & 0 & NA & NA & NA & NA & NA & NA & 1 & 0 & 1 \\
\hline Europe and Central Asia & NA & NA & NA & NA & 3 & 6 & 7 & 16 & 5 & 10 & NA & NA & NA & NA & NA & NA & 5 & 8 & 5 \\
\hline Latin America and the Caribbean & NA & NA & NA & NA & 1 & 2 & 5 & 15 & 5 & 14 & NA & NA & NA & NA & NA & NA & 3 & 6 & 4 \\
\hline Middle East and North Africa & NA & NA & NA & NA & 5 & 1 & 20 & 7 & 17 & 7 & NA & NA & NA & NA & NA & NA & 10 & 2 & 7 \\
\hline South Asia & NA & NA & NA & NA & 5 & 1 & 18 & 7 & 21 & 6 & NA & NA & NA & NA & NA & NA & 10 & 3 & 7 \\
\hline Sub-Saharan Africa & NA & NA & NA & NA & 3 & 1 & 10 & 3 & 8 & 4 & NA & NA & NA & NA & NA & NA & 5 & 2 & 4 \\
\hline Low- and middle-income countries & NA & NA & NA & NA & 3 & 1 & 9 & 3 & 8 & 3 & NA & NA & NA & NA & NA & NA & 5 & 2 & 4 \\
\hline High-income countries & NA & NA & NA & NA & 3 & 7 & 7 & 12 & 4 & 8 & NA & NA & NA & NA & NA & NA & 4 & 7 & 5 \\
\hline WORLD & NA & NA & NA & NA & 3 & 1 & 9 & 4 & 7 & 4 & NA & NA & NA & NA & NA & NA & 5 & 2 & 4 \\
\hline
\end{tabular}

Attributable Mortality (thousands)

\begin{tabular}{|c|c|c|c|c|c|c|c|c|c|c|c|c|c|c|c|c|c|c|}
\hline East Asia and Pacific & NA & NA & NA & NA & 0 & 0 & 1 & 0 & 1 & 0 & NA & NA & NA & NA & NA & NA & 2 & 0 \\
\hline Europe and Central Asia & NA & NA & NA & NA & 1 & 0 & 2 & 1 & 1 & 0 & NA & NA & NA & NA & NA & NA & 4 & 1 \\
\hline Latin America and the Caribbean & NA & NA & NA & NA & 0 & 0 & 0 & 0 & 0 & 0 & NA & NA & NA & NA & NA & NA & 1 & 0 \\
\hline Middle East and North Africa & NA & NA & NA & NA & 0 & 0 & 0 & 0 & 0 & 0 & NA & NA & NA & NA & NA & NA & 1 & 0 \\
\hline South Asia & NA & NA & NA & NA & 3 & 0 & 7 & 1 & 4 & 1 & NA & NA & NA & NA & NA & NA & 14 & 3 \\
\hline Sub-Saharan Africa & NA & NA & NA & NA & 0 & 0 & 1 & 0 & 0 & 0 & NA & NA & NA & NA & NA & NA & 1 & 0 \\
\hline Low- and middle-income countries & NA & NA & NA & NA & 4 & 1 & 11 & 3 & 7 & 2 & NA & NA & NA & NA & NA & NA & 23 & 5 \\
\hline High-income countries & NA & NA & NA & NA & 1 & 0 & 2 & 1 & 1 & 1 & NA & NA & NA & NA & NA & NA & 3 & 2 \\
\hline WORLD & NA & NA & NA & NA & 5 & 1 & 13 & 3 & 8 & 2 & NA & NA & NA & NA & NA & NA & 26 & 7 \\
\hline
\end{tabular}

Attributable YLL (thousands)

\begin{tabular}{|c|c|c|c|c|c|c|c|c|c|c|c|c|c|c|c|c|c|c|c|}
\hline East Asia and Pacific & NA & NA & NA & NA & 8 & 1 & 20 & 3 & 11 & 2 & NA & NA & NA & NA & NA & NA & 39 & 5 & 44 \\
\hline Europe and Central Asia & NA & NA & NA & NA & 20 & 7 & 49 & 17 & 28 & 10 & NA & NA & NA & NA & NA & NA & 98 & 34 & 132 \\
\hline Latin America and the Caribbean & NA & NA & NA & NA & 3 & 2 & 8 & 5 & 5 & 3 & NA & NA & NA & NA & NA & NA & 16 & 10 & 26 \\
\hline Middle East and North Africa & NA & NA & NA & NA & 5 & 1 & 11 & 2 & 4 & 1 & NA & NA & NA & NA & NA & NA & 21 & 4 & 24 \\
\hline South Asia & NA & NA & NA & NA & 71 & 13 & 167 & 34 & 84 & 18 & NA & NA & NA & NA & NA & NA & 323 & 66 & 389 \\
\hline Sub-Saharan Africa & NA & NA & NA & NA & 8 & 1 & 16 & 2 & 9 & 1 & NA & NA & NA & NA & NA & NA & 33 & 4 & 37 \\
\hline Low- and middle-income countries & NA & NA & NA & NA & 115 & 25 & 272 & 63 & 142 & 35 & NA & NA & NA & NA & NA & NA & 528 & 123 & 651 \\
\hline High-income countries & NA & NA & NA & NA & 15 & 8 & 41 & 23 & 23 & 13 & NA & NA & NA & NA & NA & NA & 79 & 44 & 123 \\
\hline WORLD & NA & NA & NA & NA & 130 & 34 & 313 & 86 & 164 & 48 & NA & NA & NA & NA & NA & NA & 607 & 167 & 774 \\
\hline
\end{tabular}

Attributable DALYs (thousands)

\begin{tabular}{|c|c|c|c|c|c|c|c|c|c|c|c|c|c|c|c|c|c|c|c|}
\hline East Asia and Pacific & NA & NA & NA & NA & 8 & 1 & 21 & 3 & 11 & 2 & NA & NA & NA & NA & NA & NA & 40 & 5 & 45 \\
\hline Europe and Central Asia & NA & NA & NA & NA & 21 & 8 & 50 & 18 & 29 & 10 & NA & NA & NA & NA & NA & NA & 100 & 36 & 136 \\
\hline Latin America and the Caribbean & NA & NA & NA & NA & 3 & 2 & 8 & 6 & 5 & 3 & NA & NA & NA & NA & NA & NA & 16 & 11 & 27 \\
\hline Middle East and North Africa & NA & NA & NA & NA & 5 & 1 & 11 & 2 & 4 & 1 & NA & NA & NA & NA & NA & NA & 21 & 4 & 25 \\
\hline South Asia & NA & NA & NA & NA & 77 & 16 & 170 & 36 & 86 & 19 & NA & NA & NA & NA & NA & NA & 333 & 71 & 404 \\
\hline Sub-Saharan Africa & NA & NA & NA & NA & 8 & 1 & 17 & 2 & 9 & 1 & NA & NA & NA & NA & NA & NA & 34 & 5 & 38 \\
\hline Low- and middle-income countries & NA & NA & NA & NA & 122 & 29 & 277 & 67 & 144 & 36 & NA & NA & NA & NA & NA & NA & 543 & 132 & 675 \\
\hline High-income countries & NA & NA & NA & NA & 15 & 9 & 43 & 25 & 23 & 14 & NA & NA & NA & NA & NA & NA & 81 & 48 & 130 \\
\hline WORLD & NA & NA & NA & NA & 137 & 39 & 320 & 92 & 167 & 50 & NA & NA & NA & NA & NA & NA & 624 & 180 & 804 \\
\hline
\end{tabular}


Risk factor: Illicit drug use

Disease: All causes

\begin{tabular}{|c|c|c|c|c|c|c|c|c|c|c|c|c|c|c|c|c|c|c|c|}
\hline \multirow[b]{2}{*}{ Region } & \multicolumn{2}{|c|}{$0-4$ years } & \multicolumn{2}{|c|}{ 5-14 years } & \multicolumn{2}{|c|}{$15-29$ years } & \multicolumn{2}{|c|}{ 30-44 years } & \multicolumn{2}{|c|}{$45-59$ years } & \multicolumn{2}{|c|}{ 60-69 years } & \multicolumn{2}{|c|}{$70-79$ years } & \multicolumn{2}{|c|}{$80+$ years } & \multicolumn{3}{|c|}{ Total } \\
\hline & Male & Female & Male & Female & Male & Female & Male & Female & Male & Female & Male & Female & Male & Female & Male & Female & Male & Female & All \\
\hline \multicolumn{20}{|l|}{ PAF of Mortality (\%) } \\
\hline East Asia and Pacific & 0 & 0 & 0 & 0 & 2 & 1 & 3 & 1 & 1 & 0 & 0 & 0 & 0 & 0 & 0 & 0 & 1 & 0 & 0 \\
\hline Europe and Central Asia & 0 & 0 & 0 & 0 & 4 & 4 & 5 & 3 & 1 & 1 & 0 & 0 & 0 & 0 & 0 & 0 & 1 & 0 & 1 \\
\hline Latin America and the Caribbean & 0 & 0 & 0 & 0 & 1 & 2 & 2 & 2 & 1 & 1 & 0 & 0 & 0 & 0 & 0 & 0 & 1 & 0 & 0 \\
\hline Middle East and North Africa & 0 & 0 & 0 & 0 & 4 & 2 & 10 & 3 & 4 & 1 & 0 & 0 & 0 & 0 & 0 & 0 & 2 & 0 & 1 \\
\hline South Asia & 0 & 0 & 0 & 0 & 2 & 0 & 4 & 1 & 1 & 0 & 0 & 0 & 0 & 0 & 0 & 0 & 1 & 0 & 0 \\
\hline Sub-Saharan Africa & 0 & 0 & 0 & 0 & 0 & 0 & 0 & 0 & 0 & 0 & 0 & 0 & 0 & 0 & 0 & 0 & 0 & 0 & 0 \\
\hline Low- and middle-income countries & 0 & 0 & 0 & 0 & 2 & 0 & 3 & 1 & 1 & 0 & 0 & 0 & 0 & 0 & 0 & 0 & 1 & 0 & 0 \\
\hline High-income countries & 0 & 0 & 0 & 0 & 5 & 5 & 7 & 6 & 2 & 1 & 0 & 0 & 0 & 0 & 0 & 0 & 1 & 0 & 0 \\
\hline WORLD & 0 & 0 & 0 & 0 & 2 & 1 & 3 & 1 & 1 & 1 & 0 & 0 & 0 & 0 & 0 & 0 & 1 & 0 & 0 \\
\hline
\end{tabular}

PAF of YLL (\%)

East Asia and Pacific

Europe and Central Asia

Latin America and the Caribbean

Middle East and North Africa

South Asia

Sub-Saharan Africa

Low- and middle-income countries

High-income countries

\begin{tabular}{llllllllllllllllllll} 
East Asia and Pacific & 0 & 0 & 0 & 0 & 2 & 1 & 3 & 1 & 1 & 0 & 0 & 0 & 0 & 0 & 0 & 0 & 1 & 0 & 1 \\
Europe and Central Asia & 0 & 0 & 0 & 0 & 4 & 4 & 5 & 3 & 2 & 1 & 0 & 0 & 0 & 0 & 0 & 0 & 2 & 1 & 1 \\
Latin America and the Caribbean & 0 & 0 & 0 & 0 & 1 & 2 & 2 & 2 & 1 & 1 & 0 & 0 & 0 & 0 & 0 & 0 & 1 & 1 & 1 \\
Middle East and North Africa & 0 & 0 & 0 & 0 & 4 & 2 & 10 & 3 & 4 & 1 & 0 & 0 & 0 & 0 & 0 & 0 & 2 & 1 & 1 \\
South Asia & 0 & 0 & 0 & 0 & 2 & 0 & 4 & 1 & 1 & 0 & 0 & 0 & 0 & 0 & 0 & 0 & 1 & 0 & 0 \\
Sub-Saharan Africa & 0 & 0 & 0 & 0 & 0 & 0 & 0 & 0 & 0 & 0 & 0 & 0 & 0 & 0 & 0 & 0 & 0 & 0 & 0 \\
\hline Low- and middle-income countries & 0 & 0 & 0 & 0 & 2 & 0 & 3 & 1 & 1 & 0 & 0 & 0 & 0 & 0 & 0 & 0 & 1 & 0 & 0 \\
High-income countries & 0 & 0 & 0 & 0 & 5 & 5 & 7 & 6 & 2 & 1 & 0 & 0 & 0 & 0 & 0 & 0 \\
\hline WORLD & 0 & 0 & 0 & 0 & 2 & 1 & 3 & 1 & 1 & 1 & 0 & 0 & 0 & 0 & 0 & 0 & 1 & 0 & 1 \\
\hline
\end{tabular}

PAF of DALYs (\%)

East Asia and Pacific

Europe and Central Asia

Latin America and the Caribbean

Middle East and North Africa

South Asia

Sub-Saharan Africa

Low- and middle-income countries

High-income countries

WORLD

Attributable Mortality (thousands

\begin{tabular}{lrrrrrrrrrrrrrrrrrrrr} 
East Asia and Pacific & 0 & 0 & 0 & 0 & 7 & 2 & 20 & 4 & 10 & 3 & 0 & 0 & 0 & 0 & 0 & 0 & 37 & 8 & 45 \\
Europe and Central Asia & 0 & 0 & 0 & 0 & 6 & 2 & 16 & 3 & 9 & 2 & 1 & 0 & 0 & 0 & 0 & 0 & 32 & 7 & 39 \\
Latin America and the Caribbean & 0 & 0 & 0 & 0 & 2 & 1 & 5 & 2 & 3 & 1 & 0 & 0 & 0 & 0 & 0 & 0 & 10 & 5 & 14 \\
Middle East and North Africa & 0 & 0 & 0 & 0 & 3 & 1 & 9 & 2 & 6 & 1 & 0 & 0 & 0 & 0 & 0 & 0 & 19 & 4 & 22 \\
South Asia & 0 & 0 & 0 & 0 & 9 & 2 & 24 & 5 & 15 & 3 & 1 & 0 & 0 & 0 & 0 & 0 & 48 & 10 & 58 \\
Sub-Saharan Africa & 0 & 0 & 0 & 0 & 2 & 0 & 4 & 1 & 3 & 0 & 0 & 0 & 0 & 0 & 0 & 0 & 8 & 1 & 10 \\
\hline Low- and middle-income countries & 0 & 0 & 0 & 0 & 28 & 7 & 78 & 17 & 45 & 11 & 2 & 0 & 0 & 0 & 0 & 0 & 154 & 35 & 189 \\
High-income countries & 0 & 0 & 0 & 0 & 4 & 2 & 13 & 6 & 8 & 4 & 0 & 0 & 0 & 0 & 0 & 0 \\
\hline WORLD & 0 & 0 & 0 & 0 & 33 & 9 & 91 & 23 & 53 & 15 & 2 & 0 & 0 & 0 & 0 & 0 \\
\hline
\end{tabular}

Attributable YLL (thousands)

\begin{tabular}{|c|c|c|c|c|c|c|c|c|c|c|c|c|c|c|c|c|c|c|c|}
\hline \multicolumn{20}{|l|}{ Attributable YLL (the } \\
\hline East Asia and Pacific & 0 & 0 & 0 & 0 & 190 & 42 & 482 & 88 & 197 & 60 & 2 & 0 & 0 & 0 & 0 & 0 & 871 & 191 & 1,061 \\
\hline Europe and Central Asia & 1 & 0 & 0 & 0 & 159 & 46 & 395 & 83 & 179 & 45 & 8 & 2 & 1 & 1 & 0 & 0 & 743 & 178 & 921 \\
\hline Latin America and the Caribbean & 0 & 0 & 0 & 0 & 52 & 33 & 122 & 61 & 53 & 23 & 1 & 0 & 0 & 0 & 0 & 0 & 227 & 117 & 345 \\
\hline Middle East and North Africa & 0 & 0 & 0 & 0 & 90 & 19 & 210 & 44 & 121 & 26 & 5 & 1 & 1 & 0 & 0 & 0 & 428 & 90 & 517 \\
\hline South Asia & 0 & 0 & 0 & 0 & 238 & 51 & 582 & 120 & 290 & 63 & 7 & 2 & 1 & 1 & 0 & 0 & 1,120 & 236 & 1,356 \\
\hline Sub-Saharan Africa & 0 & 0 & 0 & 0 & 43 & 7 & 98 & 16 & 49 & 10 & 1 & 0 & 0 & 0 & 0 & 0 & 191 & 33 & 223 \\
\hline Low- and middle-income countries & 1 & 0 & 0 & 0 & 772 & 198 & 1,888 & 411 & 890 & 227 & 23 & 6 & 4 & 2 & 1 & 1 & 3,579 & 845 & 4,424 \\
\hline High-income countries & 0 & 0 & 0 & 0 & 121 & 51 & 301 & 150 & 155 & 80 & 1 & 1 & 0 & 0 & 0 & 0 & 578 & 282 & 860 \\
\hline WORLD & 1 & 0 & 1 & 0 & 893 & 248 & 2,189 & 561 & 1,044 & 308 & 24 & 7 & 4 & 2 & 1 & 1 & 4,157 & 1,127 & 5,284 \\
\hline \multicolumn{20}{|l|}{ Attributable DALYs (thousands) } \\
\hline East Asia and Pacific & 0 & 0 & 17 & 9 & 417 & 98 & 606 & 112 & 220 & 64 & 2 & 0 & 0 & 0 & 0 & 0 & 1,262 & 284 & 1,547 \\
\hline Europe and Central Asia & 1 & 0 & 5 & 5 & 404 & 141 & 518 & 128 & 203 & 56 & 9 & 3 & 1 & 1 & 0 & 0 & 1,140 & 334 & 1,474 \\
\hline Latin America and the Caribbean & 0 & 0 & 7 & 7 & 427 & 165 & 269 & 123 & 77 & 36 & 1 & 1 & 0 & 0 & 0 & 0 & 782 & 332 & 1,114 \\
\hline Middle East and North Africa & 0 & 0 & 6 & 5 & 302 & 60 & 290 & 61 & 139 & 30 & 6 & 1 & 1 & 0 & 0 & 0 & 743 & 158 & 901 \\
\hline South Asia & 0 & 0 & 23 & 7 & 432 & 77 & 689 & 139 & 305 & 66 & 7 & 2 & 1 & 1 & 0 & 0 & 1,458 & 292 & 1,750 \\
\hline Sub-Saharan Africa & 0 & 0 & 11 & 9 & 509 & 149 & 242 & 73 & 86 & 22 & 2 & 1 & 0 & 0 & 0 & 0 & 851 & 254 & 1,104 \\
\hline Low- and middle-income countries & 1 & 0 & 68 & 42 & 2,492 & 690 & 2,614 & 636 & 1,030 & 275 & 27 & 8 & 4 & 2 & 1 & 1 & 6,237 & 1,653 & 7,890 \\
\hline High-income countries & 0 & 0 & 10 & 6 & 571 & 211 & 570 & 287 & 230 & 132 & 4 & 2 & 0 & 0 & 0 & 0 & 1,385 & 640 & 2,024 \\
\hline WORLD & 1 & 0 & 79 & 48 & 3,062 & 901 & 3,184 & 924 & 1,260 & 407 & 31 & 10 & 4 & 2 & 1 & 1 & 7,621 & 2,293 & 9,914 \\
\hline
\end{tabular}

Source: Authors' calculations. 
Risk factor: Unsafe water, sanitation, and hygiene

\begin{tabular}{|c|c|c|c|c|c|c|c|c|c|c|c|c|c|c|c|c|c|c|c|}
\hline \multirow[b]{2}{*}{ Region } & \multicolumn{2}{|c|}{$0-4$ years } & \multicolumn{2}{|c|}{ 5-14 years } & \multicolumn{2}{|c|}{$15-29$ years } & \multicolumn{2}{|c|}{ 30-44 years } & \multicolumn{2}{|c|}{ 45-59 years } & \multicolumn{2}{|c|}{ 60-69 years } & \multicolumn{2}{|c|}{ 70-79 years } & \multicolumn{2}{|c|}{$80+$ years } & \multicolumn{3}{|c|}{ Total } \\
\hline & Male & Female & Male & Female & Male & Female & Male & Female & Male & Female & Male & Female & Male & Female & Male & Female & Male & Female & All \\
\hline \multicolumn{20}{|l|}{ PAF of Mortality (\%) } \\
\hline East Asia and Pacific & 88 & 88 & 88 & 88 & 88 & 88 & 88 & 88 & 88 & 88 & 88 & 88 & 88 & 88 & 88 & 88 & 88 & 88 & 88 \\
\hline Europe and Central Asia & 86 & 86 & 86 & 86 & 86 & 86 & 86 & 86 & 86 & 86 & 86 & 86 & 86 & 86 & 86 & 85 & 86 & 86 & 86 \\
\hline Latin America and the Caribbean & 87 & 87 & 87 & 87 & 87 & 87 & 87 & 87 & 87 & 87 & 87 & 87 & 87 & 87 & 86 & 87 & 87 & 87 & 87 \\
\hline Middle East and North Africa & 87 & 87 & 87 & 87 & 87 & 87 & 87 & 87 & 87 & 87 & 87 & 87 & 87 & 87 & 87 & 87 & 87 & 87 & 87 \\
\hline South Asia & 88 & 88 & 88 & 88 & 88 & 88 & 88 & 88 & 88 & 88 & 88 & 88 & 88 & 88 & 88 & 88 & 88 & 88 & 88 \\
\hline Sub-Saharan Africa & 88 & 88 & 88 & 88 & 88 & 88 & 88 & 88 & 88 & 88 & 88 & 88 & 88 & 88 & 88 & 88 & 88 & 88 & 88 \\
\hline Low- and middle-income countries & 88 & 88 & 88 & 88 & 88 & 88 & 88 & 88 & 88 & 88 & 88 & 88 & 88 & 88 & 88 & 88 & 88 & 88 & 88 \\
\hline High-income countries & 66 & 65 & 66 & 65 & 66 & 66 & 66 & 65 & 64 & 64 & 64 & 64 & 62 & 63 & 61 & 62 & 63 & 62 & 62 \\
\hline WORLD & 88 & 88 & 88 & 88 & 88 & 88 & 88 & 88 & 88 & 88 & 88 & 88 & 87 & 87 & 87 & 86 & 88 & 88 & 88 \\
\hline \multicolumn{20}{|l|}{ PAF of YLL (\%) } \\
\hline East Asia and Pacific & 88 & 88 & 88 & 88 & 88 & 88 & 88 & 88 & 88 & 88 & 88 & 88 & 88 & 88 & 88 & 88 & 88 & 88 & 88 \\
\hline Europe and Central Asia & 86 & 86 & 86 & 86 & 86 & 86 & 86 & 86 & 86 & 86 & 86 & 86 & 86 & 86 & 86 & 85 & 86 & 86 & 86 \\
\hline Latin America and the Caribbean & 87 & 87 & 87 & 87 & 87 & 87 & 87 & 87 & 87 & 87 & 87 & 87 & 87 & 87 & 86 & 87 & 87 & 87 & 87 \\
\hline Middle East and North Africa & 87 & 87 & 87 & 87 & 87 & 87 & 87 & 87 & 87 & 87 & 87 & 87 & 87 & 87 & 87 & 87 & 87 & 87 & 87 \\
\hline South Asia & 88 & 88 & 88 & 88 & 88 & 88 & 88 & 88 & 88 & 88 & 88 & 88 & 88 & 88 & 88 & 88 & 88 & 88 & 88 \\
\hline Sub-Saharan Africa & 88 & 88 & 88 & 88 & 88 & 88 & 88 & 88 & 88 & 88 & 88 & 88 & 88 & 88 & 88 & 88 & 88 & 88 & 88 \\
\hline Low- and middle-income countries & 88 & 88 & 88 & 88 & 88 & 88 & 88 & 88 & 88 & 88 & 88 & 88 & 88 & 88 & 88 & 88 & 88 & 88 & 88 \\
\hline High-income countries & 66 & 65 & 66 & 65 & 66 & 66 & 66 & 65 & 64 & 64 & 64 & 64 & 62 & 63 & 61 & 62 & 64 & 63 & 63 \\
\hline WORLD & 88 & 88 & 88 & 88 & 88 & 88 & 88 & 88 & 88 & 88 & 88 & 88 & 87 & 87 & 87 & 86 & 88 & 88 & 88 \\
\hline
\end{tabular}

\section{PAF of DALYs (\%)}

East Asia and Pacific

Europe and Central Asia

Latin America and the Caribbean

Middle East and North Africa

South Asia

Sub-Saharan Africa

Low- and middle-income countries

High-income countries

\begin{tabular}{llllllllllllllllllll}
\hline WORLD & 88 & 88 & 87 & 87 & 87 & 87 & 87 & 87 & 87 & 87 & 87 & 87 & 87 & 87 & 87 & 86 & 88 & 88 & 88 \\
\hline
\end{tabular}

Attributable Mortality (thousands)

\begin{tabular}{lrrrrrrrrrrrrrrrrrrr} 
East Asia and Pacific & 92 & 84 & 1 & 1 & 1 & 1 & 1 & 1 & 2 & 1 & 2 & 2 & 2 & 2 & 3 & 4 & 104 & 94 & 199 \\
Europe and Central Asia & 5 & 5 & 0 & 0 & 0 & 0 & 0 & 0 & 0 & 0 & 0 & 0 & 0 & 0 & 0 & 0 & 7 & 6 & 13 \\
Latin America and the Caribbean & 21 & 19 & 0 & 0 & 0 & 0 & 0 & 0 & 1 & 0 & 1 & 1 & 1 & 1 & 1 & 1 & 25 & 23 & 47 \\
Middle East and North Africa & 30 & 28 & 0 & 0 & 0 & 0 & 0 & 0 & 1 & 0 & 1 & 0 & 1 & 1 & 1 & 1 & 34 & 31 & 64 \\
South Asia & 289 & 265 & 1 & 1 & 2 & 1 & 4 & 1 & 5 & 3 & 5 & 4 & 6 & 6 & 8 & 10 & 319 & 292 & 611 \\
Sub-Saharan Africa & 297 & 270 & 1 & 1 & 2 & 1 & 4 & 2 & 6 & 4 & 6 & 5 & 7 & 7 & 7 & 10 & 329 & 299 & 629 \\
\hline Low- and middle-income countries & 736 & 670 & 3 & 3 & 5 & 2 & 10 & 4 & 13 & 9 & 13 & 12 & 17 & 17 & 20 & 27 & 818 & 745 & 1,563 \\
High-income countries & 0 & 0 & 0 & 0 & 0 & 0 & 0 & 0 & 0 & 0 & 0 & 0 & 0 & 0 \\
\hline WORLD & 736 & 671 & 3 & 3 & 5 & 2 & 10 & 4 & 14 & 9 & 13 & 12 & 17 & 13 & 21 & 28 & 819 & 747 & 1,567 \\
\hline
\end{tabular}

Attributable YLL (thousands)

\begin{tabular}{lrrrrrrrrrrrrrrrrrrr} 
East Asia and Pacific & 2,796 & 2,556 & 22 & 19 & 29 & 16 & 35 & 16 & 35 & 25 & 24 & 25 & 19 & 23 & 12 & 17 & 2,973 & 2,697 & 5,670 \\
Europe and Central Asia & 166 & 148 & 3 & 3 & 5 & 3 & 6 & 3 & 4 & 3 & 2 & 2 & 1 & 2 & 0 & 1 & 187 & 166 & 353 \\
Latin America and the Caribbean & 641 & 581 & 4 & 4 & 6 & 3 & 9 & 5 & 10 & 8 & 7 & 8 & 7 & 8 & 5 & 7 & 689 & 623 & 1,312 \\
Middle East and North Africa & 903 & 840 & 12 & 6 & 7 & 2 & 12 & 4 & 11 & 7 & 7 & 7 & 6 & 7 & 4 & 5 & 962 & 878 & 1,840 \\
South Asia & 8,757 & 8,086 & 24 & 24 & 44 & 18 & 87 & 35 & 88 & 62 & 62 & 66 & 55 & 62 & 38 & 48 & 9,155 & 8,402 & 17,557 \\
Sub-Saharan Africa & 8,990 & 8,217 & 25 & 26 & 47 & 20 & 93 & 42 & 104 & 77 & 74 & 78 & 61 & 77 & 34 & 49 & 9,429 & 8,585 & 18,014 \\
\hline Low- and middle-income countries & 22,254 & 20,428 & 89 & 82 & 137 & 61 & 242 & 105 & 253 & 182 & 177 & 185 & 150 & 178 & 94 & 128 & 23,396 & 21,350 & 44,746 \\
High-income countries & 5 & 4 & 0 & 0 & 0 & 0 & 1 & 0 & 2 & 1 & 2 & 2 & 3 & 4 & 3 & 6 & 15 & 18 & 33 \\
\hline WORLD & 22,259 & 20,432 & 89 & 83 & 137 & 61 & 243 & 105 & 255 & 183 & 180 & 187 & 153 & 183 & 97 & 133 & 23,412 & 21,368 & 44,780 \\
\hline
\end{tabular}

Attributable DALYs (thousands)

\begin{tabular}{lrrrrrrrrrrrrrrrrrrr} 
East Asia and Pacific & 3,237 & 2,967 & 153 & 140 & 197 & 175 & 201 & 174 & 138 & 122 & 64 & 64 & 38 & 46 & 17 & 25 & 4,046 & 3,713 & 7,758 \\
Europe and Central Asia & 238 & 217 & 11 & 11 & 13 & 11 & 13 & 11 & 9 & 9 & 5 & 5 & 3 & 5 & 1 & 2 & 292 & 270 & 561 \\
Latin America and the Caribbean & 855 & 787 & 98 & 94 & 34 & 31 & 29 & 26 & 22 & 20 & 12 & 13 & 9 & 11 & 5 & 8 & 1,063 & 990 & 2,053 \\
Middle East and North Africa & 1,051 & 982 & 41 & 34 & 20 & 14 & 19 & 11 & 15 & 11 & 8 & 9 & 7 & 8 & 4 & 6 & 1,166 & 1,073 & 2,238 \\
South Asia & 9,505 & 8,791 & 162 & 153 & 118 & 86 & 122 & 67 & 109 & 82 & 70 & 74 & 59 & 66 & 39 & 50 & 10,183 & 9,370 & 19,552 \\
Sub-Saharan Africa & 9,530 & 8,748 & 141 & 141 & 82 & 55 & 112 & 61 & 114 & 88 & 78 & 82 & 63 & 79 & 35 & 49 & 10,156 & 9,303 & 19,459 \\
\hline Low- and middle-income countries & 24,416 & 22,491 & 606 & 573 & 464 & 372 & 496 & 350 & 407 & 332 & 237 & 247 & 179 & 214 & 101 & 139 & 26,905 & 24,717 & 51,622 \\
High-income countries & 79 & 74 & 9 & 8 & 13 & 12 & 15 & 14 & 12 & 12 & 7 & 7 & 6 & 8 & 4 & 8 & 145 & 144 & 289 \\
\hline WORLD & 24,495 & 22,565 & 615 & 582 & 477 & 384 & 511 & 364 & 419 & 343 & 244 & 254 & 185 & 222 & 105 & 147 & 27,050 & 24,862 & 51,911
\end{tabular}


Risk factor: Unsafe water, sanitation, and hygiene

Disease:

All causes

\begin{tabular}{|c|c|c|c|c|c|c|c|c|c|c|c|c|c|c|c|c|c|c|c|}
\hline \multirow[b]{2}{*}{ Region } & \multicolumn{2}{|c|}{$0-4$ years } & \multicolumn{2}{|c|}{ 5-14 years } & \multicolumn{2}{|c|}{$15-29$ years } & \multicolumn{2}{|c|}{$30-44$ years } & \multicolumn{2}{|c|}{$45-59$ years } & \multicolumn{2}{|c|}{ 60-69 years } & \multicolumn{2}{|c|}{$70-79$ years } & \multicolumn{2}{|c|}{$80+$ years } & \multicolumn{3}{|c|}{ Total } \\
\hline & Male & Female & Male & Female & Male & Female & Male & Female & Male & Female & Male & Female & Male & Female & Male & Female & Male & Female & All \\
\hline \multicolumn{20}{|l|}{ PAF of Mortality (\%) } \\
\hline East Asia and Pacific & 13 & 12 & 1 & 1 & 0 & 0 & 0 & 0 & 0 & 0 & 0 & 0 & 0 & 0 & 0 & 0 & 2 & 2 & 2 \\
\hline Europe and Central Asia & 6 & 6 & 0 & 1 & 0 & 0 & 0 & 0 & 0 & 0 & 0 & 0 & 0 & 0 & 0 & 0 & 0 & 0 & 0 \\
\hline Latin America and the Caribbean & 9 & 11 & 0 & 1 & 0 & 0 & 0 & 0 & 0 & 0 & 0 & 0 & 0 & 0 & 0 & 0 & 1 & 2 & 1 \\
\hline Middle East and North Africa & 13 & 14 & 1 & 1 & 0 & 0 & 1 & 0 & 0 & 0 & 0 & 0 & 0 & 0 & 1 & 1 & 3 & 4 & 3 \\
\hline South Asia & 16 & 14 & 0 & 0 & 0 & 0 & 1 & 0 & 0 & 0 & 0 & 0 & 1 & 1 & 2 & 2 & 4 & 5 & 5 \\
\hline Sub-Saharan Africa & 13 & 13 & 0 & 0 & 0 & 0 & 0 & 0 & 1 & 1 & 1 & 1 & 2 & 2 & 4 & 4 & 6 & 6 & 6 \\
\hline Low- and middle-income countries & 14 & 13 & 0 & 0 & 0 & 0 & 0 & 0 & 0 & 0 & 0 & 0 & 0 & 0 & 1 & 1 & 3 & 3 & 3 \\
\hline High-income countries & 0 & 0 & 0 & 0 & 0 & 0 & 0 & 0 & 0 & 0 & 0 & 0 & 0 & 0 & 0 & 0 & 0 & 0 & 0 \\
\hline WORLD & 14 & 13 & 0 & 0 & 0 & 0 & 0 & 0 & 0 & 0 & 0 & 0 & 0 & 0 & 1 & 0 & 3 & 3 & 3 \\
\hline
\end{tabular}

PAF of YLL (\%)

\begin{tabular}{lrrrrrrrrrrrrrrrrrrrrr} 
East Asia and Pacific & 13 & 12 & 1 & 1 & 0 & 0 & 0 & 0 & 0 & 0 & 0 & 0 & 0 & 0 & 0 & 0 & 3 & 3 & 3 \\
Europe and Central Asia & 6 & 6 & 0 & 1 & 0 & 0 & 0 & 0 & 0 & 0 & 0 & 0 & 0 & 0 & 0 & 0 & 0 & 1 & 0 \\
Latin America and the Caribbean & 9 & 11 & 0 & 1 & 0 & 0 & 0 & 0 & 0 & 0 & 0 & 0 & 0 & 0 & 0 & 0 & 2 & 3 & 2 \\
Middle East and North Africa & 13 & 14 & 1 & 1 & 0 & 0 & 1 & 0 & 0 & 0 & 0 & 0 & 0 & 0 & 1 & 1 & 5 & 6 & 5 \\
South Asia & 16 & 15 & 0 & 0 & 0 & 0 & 1 & 0 & 0 & 0 & 0 & 0 & 1 & 1 & 1 & 2 & 7 & 6 & 6 \\
Sub-Saharan Africa & 13 & 13 & 0 & 0 & 0 & 0 & 0 & 0 & 1 & 1 & 1 & 1 & 2 & 2 & 4 & 4 & 7 & 7 & 7 \\
\hline Low- and middle-income countries & 14 & 13 & 0 & 0 & 0 & 0 & 0 & 0 & 0 & 0 & 0 & 0 & 0 & 0 & 1 & 1 & 5 & 5 & 5 \\
High-income countries & 0 & 0 & 0 & 0 & 0 & 0 & 0 & 0 & 0 & 0 & 0 & 0 & 0 & 0 & 0 & 0 \\
\hline WORLD & 14 & 13 & 0 & 0 & 0 & 0 & 0 & 0 & 0 & 0 & 0 & 0 & 0 & 0 & 1 & 0 \\
\hline
\end{tabular}

\section{PAF of DALYs (\%)}

East Asia and Pacific

Europe and Central Asia

Latin America and the Caribbean

Middle East and North Africa

South Asia

Sub-Saharan Africa

Low- and middle-income countries

High-income countries

$10 \quad 9 \quad 2$

\begin{tabular}{lllllllllllllllllll}
\hline WORLD & 11 & 11 & 1 & 1 & 0 & 0 & 0 & 0 & 0 & 0 & 0 & 0 & 0 & 0 & 0 & 0 & 3 & 3 \\
\hline
\end{tabular}

Attributable Mortality (thousands)

\begin{tabular}{lrrrrrrrrrrrrrrrrrrr} 
East Asia and Pacific & 92 & 84 & 1 & 1 & 1 & 1 & 1 & 1 & 2 & 1 & 2 & 2 & 2 & 2 & 3 & 4 & 104 & 94 & 199 \\
Europe and Central Asia & 5 & 5 & 0 & 0 & 0 & 0 & 0 & 0 & 0 & 0 & 0 & 0 & 0 & 0 & 0 & 0 & 7 & 6 & 13 \\
Latin America and the Caribbean & 21 & 19 & 0 & 0 & 0 & 0 & 0 & 0 & 1 & 0 & 1 & 1 & 1 & 1 & 1 & 1 & 25 & 23 & 47 \\
Middle East and North Africa & 30 & 28 & 0 & 0 & 0 & 0 & 0 & 0 & 1 & 0 & 1 & 0 & 1 & 1 & 1 & 1 & 34 & 31 & 64 \\
South Asia & 289 & 265 & 1 & 1 & 2 & 1 & 4 & 1 & 5 & 3 & 5 & 4 & 6 & 6 & 8 & 10 & 319 & 292 & 611 \\
Sub-Saharan Africa & 297 & 270 & 1 & 1 & 2 & 1 & 4 & 2 & 6 & 4 & 6 & 5 & 7 & 7 & 7 & 10 & 329 & 299 & 629 \\
\hline Low- and middle-income countries & 736 & 670 & 3 & 3 & 5 & 2 & 10 & 4 & 13 & 9 & 13 & 12 & 17 & 17 & 20 & 27 & 818 & 745 & 1,563 \\
High-income countries & 0 & 0 & 0 & 0 & 0 & 0 & 0 & 0 & 0 & 0 & 0 & 0 & 0 & 0 & 1 \\
\hline WORLD & 736 & 671 & 3 & 3 & 5 & 2 & 10 & 4 & 14 & 9 & 13 & 12 & 17 & 18 & 21 & 28 & 819 & 747 & 1,567 \\
\hline
\end{tabular}

Attributable YLL (thousands)

\begin{tabular}{lrrrrrrrrrrrrrrrrrrr} 
East Asia and Pacific & 2,796 & 2,556 & 22 & 19 & 29 & 16 & 35 & 16 & 35 & 25 & 24 & 25 & 19 & 23 & 12 & 17 & 2,973 & 2,697 & 5,670 \\
Europe and Central Asia & 166 & 148 & 3 & 3 & 5 & 3 & 6 & 3 & 4 & 3 & 2 & 2 & 1 & 2 & 0 & 1 & 187 & 166 & 353 \\
Latin America and the Caribbean & 641 & 581 & 4 & 4 & 6 & 3 & 9 & 5 & 10 & 8 & 7 & 8 & 7 & 8 & 5 & 7 & 689 & 623 & 1,312 \\
Middle East and North Africa & 903 & 840 & 12 & 6 & 7 & 2 & 12 & 4 & 11 & 7 & 7 & 7 & 6 & 7 & 4 & 5 & 962 & 878 & 1,840 \\
South Asia & 8,757 & 8,086 & 24 & 24 & 44 & 18 & 87 & 35 & 88 & 62 & 62 & 66 & 55 & 62 & 38 & 48 & 9,155 & 8,402 & 17,557 \\
Sub-Saharan Africa & 8,990 & 8,217 & 25 & 26 & 47 & 20 & 93 & 42 & 104 & 77 & 74 & 78 & 61 & 77 & 34 & 49 & 9,429 & 8,585 & 18,014 \\
\hline Low- and middle-income countries & 22,254 & 20,428 & 89 & 82 & 137 & 61 & 242 & 105 & 253 & 182 & 177 & 185 & 150 & 178 & 94 & 128 & 23,396 & 21,350 & 44,746 \\
High-income countries & 5 & 4 & 0 & 0 & 0 & 0 & 1 & 0 & 2 & 1 & 2 & 2 & 3 & 4 & 3 & 6 & 15 & 18 & 33 \\
\hline WORLD & 22,259 & 20,432 & 89 & 83 & 137 & 61 & 243 & 105 & 255 & 183 & 180 & 187 & 153 & 183 & 97 & 133 & 23,412 & 21,368 & 44,780 \\
\hline
\end{tabular}

Attributable DALYs (thousands)

\begin{tabular}{lrrrrrrrrrrrrrrrrrrr} 
East Asia and Pacific & 3,237 & 2,967 & 153 & 140 & 197 & 175 & 201 & 174 & 138 & 122 & 64 & 64 & 38 & 46 & 17 & 25 & 4,046 & 3,713 & 7,758 \\
Europe and Central Asia & 238 & 217 & 11 & 11 & 13 & 11 & 13 & 11 & 9 & 9 & 5 & 5 & 3 & 5 & 1 & 2 & 292 & 270 & 561 \\
Latin America and the Caribbean & 855 & 787 & 98 & 94 & 34 & 31 & 29 & 26 & 22 & 20 & 12 & 13 & 9 & 11 & 5 & 8 & 1,063 & 990 & 2,053 \\
Middle East and North Africa & 1,051 & 982 & 41 & 34 & 20 & 14 & 19 & 11 & 15 & 11 & 8 & 9 & 7 & 8 & 4 & 6 & 1,166 & 1,073 & 2,238 \\
South Asia & 9,505 & 8,791 & 162 & 153 & 118 & 86 & 122 & 67 & 109 & 82 & 70 & 74 & 59 & 66 & 39 & 50 & 10,183 & 9,370 & 19,552 \\
Sub-Saharan Africa & 9,530 & 8,748 & 141 & 141 & 82 & 55 & 112 & 61 & 114 & 88 & 78 & 82 & 63 & 79 & 35 & 49 & 10,156 & 9,303 & 19,459 \\
\hline Low- and middle-income countries & 24,416 & 22,491 & 606 & 573 & 464 & 372 & 496 & 350 & 407 & 332 & 237 & 247 & 179 & 214 & 101 & 139 & 26,905 & 24,717 & 51,622 \\
High-income countries & 79 & 74 & 9 & 8 & 13 & 12 & 15 & 14 & 12 & 12 & 7 & 7 & 6 & 8 & 4 & 8 & 145 & 144 & 289 \\
\hline WORLD & 24,495 & 22,565 & 615 & 582 & 477 & 384 & 511 & 364 & 419 & 343 & 244 & 254 & 185 & 222 & 105 & 147 & 27,050 & 24,862 & 51,911
\end{tabular}

Source: Authors' calculations. 
Risk factor:

Child sexual abuse

Disease:

Unipolar depressive disorders

\begin{tabular}{|c|c|c|c|c|c|c|c|c|c|c|c|c|c|c|c|c|c|c|c|}
\hline \multirow[b]{2}{*}{ Region } & \multicolumn{2}{|c|}{$0-4$ years } & \multicolumn{2}{|c|}{ 5-14 years } & \multicolumn{2}{|c|}{$15-29$ years } & \multicolumn{2}{|c|}{ 30-44 years } & \multicolumn{2}{|c|}{ 45-59 years } & \multicolumn{2}{|c|}{$60-69$ years } & \multicolumn{2}{|c|}{$70-79$ years } & \multicolumn{2}{|c|}{ 80+ years } & \multicolumn{3}{|c|}{ Total } \\
\hline & Male & Female & Male & Female & Male & Female & Male & Female & Male & Female & Male & Female & Male & Female & Male & Female & Male & Female & All \\
\hline \multicolumn{20}{|l|}{ PAF of Mortality (\%) } \\
\hline East Asia and Pacific & NA & NA & NA & NA & 3 & 4 & 4 & 5 & 4 & 5 & 4 & 5 & 4 & 5 & 4 & 5 & 4 & 5 & 4 \\
\hline Europe and Central Asia & NA & NA & NA & NA & 2 & 5 & 3 & 7 & 3 & 7 & 3 & 7 & 3 & 7 & 3 & 7 & 2 & 6 & 5 \\
\hline Latin America and the Caribbean & NA & NA & NA & NA & 2 & 2 & 4 & 3 & 4 & 3 & 4 & 3 & 4 & 3 & 4 & 3 & 3 & 3 & 3 \\
\hline Middle East and North Africa & NA & NA & NA & NA & 2 & 5 & 3 & 7 & 3 & 7 & 3 & 7 & 3 & 7 & 3 & 7 & 2 & 5 & 4 \\
\hline South Asia & NA & NA & NA & NA & $*$ & 9 & 6 & 13 & 6 & 13 & 6 & 13 & * & 13 & * & 13 & 6 & 13 & 10 \\
\hline Sub-Saharan Africa & NA & NA & NA & NA & * & 6 & 4 & $*$ & 4 & $*$ & * & * & * & * & * & $*$ & 4 & 6 & 5 \\
\hline Low- and middle-income countries & NA & NA & NA & NA & 2 & 5 & 6 & 11 & 6 & 11 & 6 & 11 & 3 & 5 & 3 & 4 & 6 & 11 & $\overline{9}$ \\
\hline High-income countries & NA & NA & NA & NA & 1 & 4 & 1 & 6 & 2 & 6 & 1 & 6 & 1 & 6 & 1 & 6 & 1 & 6 & 4 \\
\hline WORLD & NA & NA & NA & NA & 2 & 5 & 6 & 11 & 6 & 11 & 6 & 11 & 2 & 6 & 2 & 6 & 5 & 10 & $\overline{8}$ \\
\hline
\end{tabular}

PAF of YLL (\%)

\begin{tabular}{llllllllrrrrrrrrrrrrr} 
East Asia and Pacific & NA & NA & NA & NA & 3 & 4 & 4 & 5 & 4 & 5 & 4 & 5 & 4 & 5 & 4 & 5 & 4 & 5 & 4 \\
Europe and Central Asia & NA & NA & NA & NA & 2 & 5 & 3 & 7 & 3 & 7 & 3 & 7 & 3 & 7 & 3 & 7 & 2 & 6 & 5 \\
Latin America and the Caribbean & NA & NA & NA & NA & 2 & 2 & 4 & 3 & 4 & 3 & 4 & 3 & 4 & 3 & 4 & 3 & 3 & 3 & 3 \\
Middle East and North Africa & NA & NA & NA & NA & 2 & 5 & 3 & 7 & 3 & 7 & 3 & 7 & 3 & 7 & 3 & 7 & 2 & 4 & 3 \\
South Asia & NA & NA & NA & NA & ${ }^{*}$ & 9 & 6 & 13 & 6 & 13 & 6 & 13 & $*$ & 13 & $*$ & 13 & 6 & 13 & 10 \\
Sub-Saharan Africa & NA & NA & NA & NA & ${ }^{*}$ & 6 & 4 & ${ }^{*}$ & 4 & ${ }^{*}$ & ${ }^{*}$ & ${ }^{*}$ & ${ }^{*}$ & ${ }^{*}$ & ${ }^{*}$ & ${ }^{*}$ & 4 & 6 & 5 \\
\hline Low- and middle-income countries & NA & NA & NA & NA & 2 & 5 & 6 & 11 & 6 & 11 & 6 & 11 & 3 & 5 & 3 & 4 & 6 & 11 & 9 \\
High-income countries & NA & NA & NA & NA & 1 & 4 & 1 & 6 & 2 & 6 & 1 & 6 & 1 & 6 & 1 & 6 \\
\hline WORLD & NA & NA & NA & NA & 2 & 5 & 6 & 11 & 6 & 11 & 6 & 11 & 2 & 6 & 2 & 6 & 6 & 11 & 8 \\
\hline
\end{tabular}

\section{PAF of DALYs (\%)}

Latin America and the Caribbean

Middle East and North Africa

South Asia

Sub-Saharan Africa

Low- and middle-income countries

High-income countries

$\begin{array}{llll}\text { NA } & \text { NA } & \text { NA } & \text { NA } \\ \text { NA } & \text { NA } & \text { NA } & \text { NA } \\ \text { NA } & \text { NA } & \text { NA } & \text { NA } \\ \text { NA } & \text { NA } & \text { NA } & \text { NA } \\ \text { NA } & \text { NA } & \text { NA } & \text { NA } \\ \text { NA } & \text { NA } & \text { NA } & \text { NA } \\ \text { NA } & \text { NA } & \text { NA } & \text { NA }\end{array}$

\begin{tabular}{rrrrrrrrrrrrrrr}
3 & 4 & 4 & 5 & 4 & 5 & 4 & 5 & 4 & 5 & 4 & 5 & 3 & 4 & 4 \\
2 & 5 & 3 & 7 & 3 & 7 & 3 & 7 & 3 & 7 & 3 & 7 & 2 & 6 & 4 \\
2 & 2 & 4 & 3 & 4 & 3 & 4 & 3 & 4 & 3 & 4 & 3 & 3 & 2 & 2 \\
2 & 5 & 3 & 7 & 3 & 7 & 3 & 7 & 3 & 7 & 3 & 7 & 2 & 5 & 4 \\
5 & 9 & 6 & 13 & 6 & 13 & 6 & 13 & 6 & 13 & 6 & 13 & 5 & 10 & 8 \\
3 & 6 & 4 & 8 & 4 & 8 & 4 & 8 & 4 & 8 & 4 & 8 & 3 & 6 & 5 \\
3 & 6 & 4 & 8 & 4 & 7 & 4 & 7 & 4 & 7 & 4 & 7 & 3 & 6 & 5 \\
1 & 4 & 1 & 6 & 2 & 6 & 1 & 6 & 1 & 6 & 1 & 6 & 1 & 5 & 4 \\
3 & 6 & 4 & 7 & 4 & 7 & 4 & 7 & 3 & 7 & 3 & 6 & 3 & 6 & 5 \\
\hline
\end{tabular}

Attributable Mortality (thousands)

\begin{tabular}{llllllllllllllllllllll} 
East Asia and Pacific & NA & NA & NA & NA & 0 & 0 & 0 & 0 & 0 & 0 & 0 & 0 & 0 & 0 & 0 & 0 & 0 & 0 & 0 \\
Europe and Central Asia & NA & NA & NA & NA & 0 & 0 & 0 & 0 & 0 & 0 & 0 & 0 & 0 & 0 & 0 & 0 & 0 & 0 & 0 \\
Latin America and the Caribbean & NA & NA & NA & NA & 0 & 0 & 0 & 0 & 0 & 0 & 0 & 0 & 0 & 0 & 0 & 0 & 0 & 0 & 0 \\
Middle East and North Africa & NA & NA & NA & NA & 0 & 0 & 0 & 0 & 0 & 0 & 0 & 0 & 0 & 0 & 0 & 0 & 0 & 0 & 0 \\
South Asia & NA & NA & NA & NA & 0 & 0 & 0 & 0 & 0 & 0 & 0 & 0 & 0 & 0 & 0 & 0 & 0 & 1 & 1 \\
Sub-Saharan Africa & NA & NA & NA & NA & 0 & 0 & 0 & 0 & 0 & 0 & 0 & 0 & 0 & 0 & 0 & 0 & 0 & 0 & 0 \\
\hline Low- and middle-income countries & NA & NA & NA & NA & 0 & 0 & 0 & 0 & 0 & 0 & 0 & 0 & 0 & 0 & 0 & 0 & 0 & 1 & 1 \\
High-income countries & NA & NA & NA & NA & 0 & 0 & 0 & 0 & 0 & 0 & 0 & 0 & 0 & 0 & 0 & 0 \\
\hline WORLD & NA & NA & NA & NA & 0 & 0 & 0 & 0 & 0 & 0 & 0 & 0 & 0 & 0 & 0 & 0 \\
\hline
\end{tabular}

Attributable YLL (thousands)

\begin{tabular}{|c|c|c|c|c|c|c|c|c|c|c|c|c|c|c|c|c|c|c|c|}
\hline Attributable YLL (thousands) & & & & & & & & & & & & & & & & & & & \\
\hline East Asia and Pacific & NA & NA & NA & NA & 0 & 0 & 0 & 0 & 0 & 0 & 0 & 0 & 0 & 0 & 0 & 0 & 0 & 1 & 1 \\
\hline Europe and Central Asia & NA & NA & NA & NA & 0 & 0 & 0 & 0 & 0 & 0 & 0 & 0 & 0 & 0 & 0 & 0 & 0 & 0 & 0 \\
\hline Latin America and the Caribbean & NA & NA & NA & NA & 0 & 0 & 0 & 0 & 0 & 0 & 0 & 0 & 0 & 0 & 0 & 0 & 0 & 0 & 0 \\
\hline Middle East and North Africa & NA & NA & NA & NA & 0 & 0 & 0 & 0 & 0 & 0 & 0 & 0 & 0 & 0 & 0 & 0 & 0 & 0 & 0 \\
\hline South Asia & NA & NA & NA & NA & 0 & 0 & 3 & 3 & 2 & 6 & 1 & 2 & 0 & 0 & 0 & 0 & 5 & 11 & 17 \\
\hline Sub-Saharan Africa & NA & NA & NA & NA & 0 & 0 & 0 & 0 & 0 & 0 & 0 & 0 & 0 & 0 & 0 & 0 & 0 & 0 & 0 \\
\hline Low- and middle-income countries & NA & NA & NA & NA & 0 & 0 & 3 & 3 & 2 & 7 & 1 & 2 & 0 & 0 & 0 & 0 & 6 & 12 & 18 \\
\hline High-income countries & NA & NA & NA & NA & 0 & 0 & 0 & 0 & 0 & 0 & 0 & 0 & 0 & 0 & 0 & 0 & 0 & 1 & 1 \\
\hline WORLD & NA & NA & NA & NA & 0 & 0 & 3 & 3 & 2 & 7 & 1 & 2 & 0 & 0 & 0 & 0 & 6 & 13 & 19 \\
\hline
\end{tabular}

Attributable DALYs (thousands)

\begin{tabular}{|c|c|c|c|c|c|c|c|c|c|c|c|c|c|c|c|c|c|c|c|}
\hline East Asia and Pacific & NA & NA & NA & NA & 50 & 64 & 61 & 120 & 43 & 108 & 12 & 33 & 2 & 8 & 0 & 2 & 169 & 335 & 504 \\
\hline Europe and Central Asia & NA & NA & NA & NA & 9 & 32 & 11 & 58 & 8 & 41 & 3 & 19 & 1 & 6 & 0 & 1 & 32 & 158 & 190 \\
\hline Latin America and the Caribbean & NA & NA & NA & NA & 15 & 23 & 21 & 31 & 11 & 17 & 3 & 5 & 1 & 1 & 0 & 0 & 51 & 77 & 129 \\
\hline Middle East and North Africa & NA & NA & NA & NA & 5 & 19 & 8 & 27 & 4 & 14 & 1 & 4 & 0 & 1 & 0 & 0 & 18 & 65 & 83 \\
\hline South Asia & NA & NA & NA & NA & 92 & 292 & 105 & 348 & 58 & 189 & 17 & 66 & 3 & 13 & 1 & 3 & 275 & 911 & 1,186 \\
\hline Sub-Saharan Africa & NA & NA & NA & NA & 13 & 40 & 14 & 44 & 7 & 23 & 2 & 7 & 0 & 1 & 0 & 0 & 37 & 116 & 153 \\
\hline Low- and middle-income countries & NA & NA & NA & NA & 184 & 470 & 220 & 629 & 132 & 392 & 38 & 133 & 7 & 31 & 1 & 7 & 583 & 1,662 & 2,245 \\
\hline High-income countries & NA & NA & NA & NA & 9 & 63 & 14 & 95 & 10 & 71 & 4 & 24 & 1 & 10 & 0 & 5 & 39 & 267 & 305 \\
\hline WORLD & NA & NA & NA & NA & 193 & 532 & 234 & 724 & 142 & 463 & 42 & 157 & 9 & 40 & 2 & 12 & 622 & 1,928 & 2,550 \\
\hline
\end{tabular}

Source: Authors' calculations.

Note: NA = not applicable.

* The number of deaths (and hence YLL) directly coded to a number of diseases, especially neuropsychiatric and musculoskeletal diseases, is zero or very small. For other diseases, mortality or disease burden may be zero in some

region-age-sex groups. In such cases, the population attributable fractions would be undefined or unstable and have not been calculated. 
Risk factor:

Disease:

Child sexual abuse

Alcohol use disorders

\begin{tabular}{|c|c|c|c|c|c|c|c|c|c|c|c|c|c|c|c|c|c|c|c|}
\hline \multirow[b]{2}{*}{ Region } & \multicolumn{2}{|c|}{$0-4$ years } & \multicolumn{2}{|c|}{ 5-14 years } & \multicolumn{2}{|c|}{$15-29$ years } & \multicolumn{2}{|c|}{ 30-44 years } & \multicolumn{2}{|c|}{ 45-59 years } & \multicolumn{2}{|c|}{$60-69$ years } & \multicolumn{2}{|c|}{ 70-79 years } & \multicolumn{2}{|c|}{$80+$ years } & \multicolumn{3}{|c|}{ Total } \\
\hline & Male & Female & Male & Female & Male & Female & Male & Female & Male & Female & Male & Female & Male & Female & Male & Female & Male & Female & All \\
\hline \multicolumn{20}{|l|}{ PAF of Mortality (\%) } \\
\hline East Asia and Pacific & NA & NA & NA & NA & 4 & 5 & 6 & 7 & 6 & 7 & 6 & 7 & 6 & 7 & 6 & 7 & 6 & 7 & 6 \\
\hline Europe and Central Asia & NA & NA & NA & NA & 3 & 7 & 4 & 10 & 4 & 10 & 4 & 10 & 4 & 10 & 4 & 10 & 4 & 10 & 5 \\
\hline Latin America and the Caribbean & NA & NA & NA & NA & 4 & 3 & 5 & 4 & 5 & 4 & 5 & 4 & 5 & 4 & 5 & 4 & 5 & 4 & 5 \\
\hline Middle East and North Africa & NA & NA & NA & NA & 3 & 7 & 4 & 10 & 4 & 10 & 4 & 10 & 4 & 10 & 4 & * & 4 & 9 & 4 \\
\hline South Asia & NA & NA & NA & NA & 7 & 13 & 9 & 17 & 9 & 17 & 9 & 17 & 9 & 17 & 9 & 17 & 9 & 17 & 10 \\
\hline Sub-Saharan Africa & NA & NA & NA & NA & 5 & 8 & 7 & 11 & 7 & 11 & 7 & 11 & 7 & 11 & 7 & * & 7 & 11 & 8 \\
\hline Low- and middle-income countries & NA & NA & NA & NA & 5 & 6 & 6 & 8 & 6 & 10 & 6 & 11 & 6 & 11 & 6 & 11 & 6 & 9 & 6 \\
\hline High-income countries & NA & NA & NA & NA & 2 & 6 & 2 & 8 & 2 & 8 & 2 & 8 & 2 & 8 & 2 & 8 & 2 & 8 & 3 \\
\hline WORLD & NA & NA & NA & NA & 5 & 6 & 5 & 8 & 5 & 9 & 5 & 10 & 5 & 10 & 5 & 10 & 5 & 9 & $\overline{6}$ \\
\hline
\end{tabular}

PAF of YLL (\%)

\begin{tabular}{|c|c|c|c|c|c|c|c|c|c|c|c|c|c|c|c|c|c|c|}
\hline \multirow{2}{*}{$\begin{array}{l}\text { East Asia and Pacific } \\
\text { Europe and Central Asia }\end{array}$} & NA & NA & NA & NA & 4 & 5 & 6 & 7 & 6 & 7 & 6 & 7 & 6 & 7 & 6 & 7 & 5 & 7 \\
\hline & NA & NA & NA & NA & 3 & 7 & 4 & 10 & 4 & 10 & 4 & 10 & 4 & 10 & 4 & 10 & 4 & 9 \\
\hline Latin America and the Caribbean & NA & NA & NA & NA & 4 & 3 & 5 & 4 & 5 & 4 & 5 & 4 & 5 & 4 & 5 & 4 & 5 & 4 \\
\hline Middle East and North Africa & NA & NA & NA & NA & 3 & 7 & 4 & 10 & 4 & 10 & 4 & 10 & 4 & 10 & 4 & * & 4 & 9 \\
\hline South Asia & NA & NA & NA & NA & 7 & 13 & 9 & 17 & 9 & 17 & 9 & 17 & 9 & 17 & 9 & 17 & 9 & 17 \\
\hline Sub-Saharan Africa & NA & NA & NA & NA & 5 & 8 & 7 & 11 & 7 & 11 & 7 & 11 & 7 & 11 & 7 & * & 7 & 11 \\
\hline Low- and middle-income countries & NA & NA & NA & NA & 5 & 6 & 6 & 8 & 6 & 10 & 6 & 11 & 6 & 11 & 6 & 11 & 6 & 9 \\
\hline High-income countries & NA & NA & NA & NA & 2 & 6 & 2 & 8 & 2 & 8 & 2 & 8 & 2 & 8 & 2 & 8 & 2 & 8 \\
\hline WORLD & NA & NA & NA & NA & 5 & 6 & 5 & 8 & 5 & 9 & 5 & 10 & 5 & 10 & 5 & 10 & 5 & 9 \\
\hline
\end{tabular}

\section{PAF of DALYs (\%)}

East Asia and Pacific

Europe and Central Asia

Latin America and the Caribbean

Middle East and North Afric

South Asia

Sub-Saharan Africa

Low- and middle-income countries

High-income countries

$\begin{array}{llll}\text { NA } & \text { NA } & \text { NA } & \text { NA } \\ \text { NA } & \text { NA } & \text { NA } & \text { NA } \\ \text { NA } & \text { NA } & \text { NA } & \text { NA } \\ \text { NA } & \text { NA } & \text { NA } & \text { NA } \\ \text { NA } & \text { NA } & \text { NA } & \text { NA } \\ \text { NA } & \text { NA } & \text { NA } & \text { NA } \\ \text { NA } & \text { NA } & \text { NA } & \text { NA }\end{array}$

Attributable Mortality (thousands)

\begin{tabular}{lllllllllllllllllllll} 
East Asia and Pacific & NA & NA & NA & NA & 0 & 0 & 0 & 0 & 0 & 0 & 0 & 0 & 0 & 0 & 0 & 0 & 1 & 0 & 1 \\
Europe and Central Asia & NA & NA & NA & NA & 0 & 0 & 0 & 0 & 0 & 0 & 0 & 0 & 0 & 0 & 0 & 0 & 0 & 0 & 0 \\
Latin America and the Caribbean & NA & NA & NA & NA & 0 & 0 & 0 & 0 & 0 & 0 & 0 & 0 & 0 & 0 & 0 & 0 & 1 & 0 & 1 \\
Middle East and North Africa & NA & NA & NA & NA & 0 & 0 & 0 & 0 & 0 & 0 & 0 & 0 & 0 & 0 & 0 & 0 & 0 & 0 & 0 \\
South Asia & NA & NA & NA & NA & 0 & 0 & 0 & 0 & 0 & 0 & 0 & 0 & 0 & 0 & 0 & 0 & 1 & 0 & 1 \\
Sub-Saharan Africa & NA & NA & NA & NA & 0 & 0 & 0 & 0 & 0 & 0 & 0 & 0 & 0 & 0 & 0 & 0 & 0 & 0 & 1 \\
\hline Low- and middle-income countries & NA & NA & NA & NA & 0 & 0 & 1 & 0 & 1 & 0 & 1 & 0 & 0 & 0 & 0 & 0 & 3 & 1 & 4 \\
High-income countries & NA & NA & NA & NA & 0 & 0 & 0 & 0 & 0 & 0 & 0 & 0 & 0 & 0 & 0 & 0 \\
\hline WORLD & NA & NA & NA & NA & 0 & 0 & 1 & 0 & 1 & 0 & 1 & 0 & 0 & 0 & 0 & 0 \\
\hline
\end{tabular}

Attributable YLL (thousands)

\begin{tabular}{lrrrrrrrrrrrrrrrrrrr} 
East Asia and Pacific & NA & NA & NA & NA & 1 & 0 & 4 & 1 & 5 & 1 & 1 & 0 & 0 & 0 & 0 & 0 & 11 & 2 & 14 \\
Europe and Central Asia & NA & NA & NA & NA & 0 & 0 & 2 & 1 & 3 & 1 & 1 & 1 & 0 & 0 & 0 & 0 & 6 & 3 & 9 \\
Latin America and the Caribbean & NA & NA & NA & NA & 1 & 0 & 6 & 0 & 6 & 0 & 2 & 0 & 1 & 0 & 0 & 0 & 16 & 1 & 17 \\
Middle East and North Africa & NA & NA & NA & NA & 1 & 0 & 1 & 0 & 1 & 0 & 0 & 0 & 0 & 0 & 0 & 0 & 3 & 0 & 3 \\
South Asia & NA & NA & NA & NA & 4 & 0 & 8 & 0 & 6 & 1 & 3 & 1 & 1 & 0 & 0 & 0 & 21 & 3 & 24 \\
Sub-Saharan Africa & NA & NA & NA & NA & 0 & 0 & 3 & 1 & 2 & 1 & 1 & 0 & 0 & 1 & 0 & 0 & 6 & 4 & 10 \\
\hline Low- and middle-income countries & NA & NA & NA & NA & 6 & 1 & 24 & 4 & 22 & 6 & 7 & 2 & 2 & 1 & 0 & 0 & 62 & 14 & 76 \\
High-income countries & NA & NA & NA & NA & 0 & 0 & 2 & 2 & 3 & 3 & 1 & 1 & 0 & 1 & 0 & 0 \\
\hline WORLD & NA & NA & NA & NA & 7 & 1 & 26 & 5 & 25 & 9 & 9 & 4 & 3 & 2 & 0 & 0 & 69 & 21 & 90
\end{tabular}

Attributable DALYs (thousands

\begin{tabular}{lrrrrrrrrrrrrrrrrrrr} 
East Asia and Pacific & NA & NA & NA & NA & 61 & 11 & 84 & 13 & 37 & 5 & 6 & 0 & 1 & 0 & 0 & 0 & 189 & 31 & 220 \\
Europe and Central Asia & NA & NA & NA & NA & 18 & 8 & 22 & 10 & 11 & 6 & 2 & 1 & 0 & 0 & 0 & 0 & 53 & 25 & 78 \\
Latin America and the Caribbean & NA & NA & NA & NA & 48 & 9 & 32 & 5 & 20 & 3 & 4 & 1 & 1 & 0 & 0 & 0 & 105 & 18 & 123 \\
Middle East and North Africa & NA & NA & NA & NA & 1 & 0 & 1 & 0 & 1 & 0 & 0 & 0 & 0 & 0 & 0 & 0 & 3 & 0 & 3 \\
South Asia & NA & NA & NA & NA & 25 & 2 & 51 & 2 & 18 & 2 & 4 & 1 & 1 & 0 & 0 & 0 & 98 & 7 & 105 \\
Sub-Saharan Africa & NA & NA & NA & NA & 13 & 2 & 13 & 3 & 7 & 2 & 1 & 0 & 0 & 1 & 0 & 0 & 35 & 8 & 43 \\
\hline Low- and middle-income countries & NA & NA & NA & NA & 166 & 32 & 204 & 34 & 93 & 18 & 17 & 4 & 4 & 1 & 1 & 0 & 484 & 89 & 573 \\
High-income countries & NA & NA & NA & NA & 20 & 21 & 29 & 26 & 14 & 12 & 2 & 2 & 1 & 1 & 0 & 0 & 66 & 63 & 129 \\
\hline WORLD & NA & NA & NA & NA & 186 & 53 & 232 & 60 & 107 & 30 & 19 & 6 & 5 & 2 & 1 & 0 & 550 & 152 & 702
\end{tabular}

Source: Authors' calculations.

Note: $\mathrm{NA}=$ not applicable.

* The number of deaths (and hence YLL) directly coded to a number of diseases, especially neuropsychiatric and musculoskeletal diseases, is zero or very small. For other diseases, mortality or disease burden may be zero in some

region-age-sex groups. In such cases, the population attributable fractions would be undefined or unstable and have not been calculated. 
Table 4A.89

Risk factor: Child sexual abuse

Disease: Drug use disorders

\begin{tabular}{|c|c|c|c|c|c|c|c|c|c|c|c|c|c|c|c|c|c|c|c|}
\hline \multirow[b]{2}{*}{ Region } & \multicolumn{2}{|c|}{$0-4$ years } & \multicolumn{2}{|c|}{ 5-14 years } & \multicolumn{2}{|c|}{$15-29$ years } & \multicolumn{2}{|c|}{ 30-44 years } & \multicolumn{2}{|c|}{ 45-59 years } & \multicolumn{2}{|c|}{$60-69$ years } & \multicolumn{2}{|c|}{$70-79$ years } & \multicolumn{2}{|c|}{$80+$ years } & \multicolumn{3}{|c|}{ Total } \\
\hline & Male & Female & Male & Female & Male & Female & Male & Female & Male & Female & Male & Female & Male & Female & Male & Female & Male & Female & All \\
\hline \multicolumn{20}{|l|}{ PAF of Mortality (\%) } \\
\hline East Asia and Pacific & NA & NA & NA & NA & 4 & 6 & 5 & 8 & 5 & 8 & 5 & 8 & 5 & 8 & 5 & 8 & 5 & 8 & 6 \\
\hline Europe and Central Asia & NA & NA & NA & NA & 3 & 8 & 4 & 11 & 4 & 11 & 4 & 11 & 4 & 11 & 4 & 11 & 4 & 10 & 5 \\
\hline Latin America and the Caribbean & NA & NA & NA & NA & 4 & 3 & 6 & 5 & 6 & 5 & 6 & 5 & 6 & 5 & 6 & 5 & 6 & 5 & 5 \\
\hline Middle East and North Africa & NA & NA & NA & NA & 4 & 8 & 5 & 11 & 5 & 11 & 5 & 11 & 5 & 11 & 5 & 11 & 5 & 10 & 6 \\
\hline South Asia & NA & NA & NA & NA & 8 & 15 & 11 & 20 & 11 & 20 & 11 & 20 & 10 & 20 & 10 & 20 & 10 & 19 & 12 \\
\hline Sub-Saharan Africa & NA & NA & NA & NA & 5 & 9 & 7 & 12 & 7 & 12 & 7 & 12 & 7 & 12 & 7 & 12 & 7 & 12 & 8 \\
\hline Low- and middle-income countries & NA & NA & NA & NA & 5 & 10 & 7 & 14 & 7 & 14 & 7 & 13 & 7 & 13 & 7 & 14 & 7 & 13 & 8 \\
\hline High-income countries & NA & NA & NA & NA & 2 & 7 & 3 & 9 & 3 & 9 & 3 & 9 & 3 & 9 & 3 & 9 & 2 & 9 & 4 \\
\hline WORLD & NA & NA & NA & NA & 5 & 10 & 7 & 13 & 7 & 13 & 6 & 13 & 7 & 13 & 7 & 13 & 6 & 13 & 7 \\
\hline
\end{tabular}

PAF of YLL (\%)

\begin{tabular}{llllllrrrrrrrrrrrrrr} 
East Asia and Pacific & NA & NA & NA & NA & 4 & 6 & 5 & 8 & 5 & 8 & 5 & 8 & 5 & 8 & 5 & 8 & 5 & 8 & 6 \\
Europe and Central Asia & NA & NA & NA & NA & 3 & 8 & 4 & 11 & 4 & 11 & 4 & 11 & 4 & 11 & 4 & 11 & 4 & 10 & 5 \\
Latin America and the Caribbean & NA & NA & NA & NA & 4 & 3 & 6 & 5 & 6 & 5 & 6 & 5 & 6 & 5 & 6 & 5 & 5 & 5 & 5 \\
Middle East and North Africa & NA & NA & NA & NA & 4 & 8 & 5 & 11 & 5 & 11 & 5 & 11 & 5 & 11 & 5 & 11 & 5 & 10 & 6 \\
South Asia & NA & NA & NA & NA & 8 & 15 & 11 & 20 & 11 & 20 & 11 & 20 & 10 & 20 & 10 & 20 & 10 & 19 & 12 \\
Sub-Saharan Africa & NA & NA & NA & NA & 5 & 9 & 7 & 12 & 7 & 12 & 7 & 12 & 7 & 12 & 7 & 12 & 7 & 12 & 8 \\
\hline Low- and middle-income countries & NA & NA & NA & NA & 5 & 10 & 7 & 14 & 7 & 14 & 7 & 13 & 7 & 13 & 7 & 14 & 7 & 13 & 8 \\
High-income countries & NA & NA & NA & NA & 2 & 7 & 3 & 9 & 3 & 9 & 3 & 9 & 3 & 9 & 3 & 9 & 2 & 9 & 4 \\
\hline WORLD & NA & NA & NA & NA & 5 & 10 & 7 & 13 & 7 & 13 & 6 & 13 & 7 & 13 & 7 & 13 & 6 & 12 & 7 \\
\hline
\end{tabular}

PAF of DALYs $(\%)$

East Asia and Pacific

NA $\quad$ NA NA NA

Latin America and the Caribbean NA NA NA NA

Middle East and North Africa NA NA NA NA

South Asia

Sub-Saharan Africa

Low- and middle-income countries NA NA NA NA

$\begin{array}{lll}\text { High-income countries } & \text { NA NA NA NA }\end{array}$

\begin{tabular}{llllllllllllllllllll}
\hline WORLD & NA & NA & NA & NA & 4 & 7 & 6 & 11 & 6 & 12 & 6 & 12 & 7 & 13 & 7 & 13 & 5 & 9 & 6
\end{tabular}

Attributable Mortality (thousands)

\begin{tabular}{lllllllllllllllllllll} 
East Asia and Pacific & NA & NA & NA & NA & 0 & 0 & 0 & 0 & 0 & 0 & 0 & 0 & 0 & 0 & 0 & 0 & 0 & 0 & 0 \\
Europe and Central Asia & NA & NA & NA & NA & 0 & 0 & 0 & 0 & 0 & 0 & 0 & 0 & 0 & 0 & 0 & 0 & 0 & 0 & 1 \\
Latin America and the Caribbean & NA & NA & NA & NA & 0 & 0 & 0 & 0 & 0 & 0 & 0 & 0 & 0 & 0 & 0 & 0 & 0 & 0 & 0 \\
Middle East and North Africa & NA & NA & NA & NA & 0 & 0 & 0 & 0 & 0 & 0 & 0 & 0 & 0 & 0 & 0 & 0 & 1 & 0 & 1 \\
South Asia & NA & NA & NA & NA & 0 & 0 & 1 & 0 & 1 & 0 & 0 & 0 & 0 & 0 & 0 & 0 & 2 & 1 & 3 \\
Sub-Saharan Africa & NA & NA & NA & NA & 0 & 0 & 0 & 0 & 0 & 0 & 0 & 0 & 0 & 0 & 0 & 0 & 0 & 0 & 0 \\
\hline Low- and middle-income countries & NA & NA & NA & NA & 1 & 0 & 2 & 1 & 1 & 1 & 0 & 0 & 0 & 0 & 0 & 0 & 4 & 2 & 6 \\
High-income countries & NA & NA & NA & NA & 0 & 0 & 0 & 0 & 0 & 0 & 0 & 0 & 0 & 0 & 0 & 0 \\
\hline WORLD & NA & NA & NA & NA & 1 & 0 & 2 & 1 & 1 & 1 & 0 & 0 & 0 & 0 & 0 & 0 \\
\hline
\end{tabular}

Attributable YLL (thousands)

\begin{tabular}{lrrrrrrrrrrrrrrrrrrrrr} 
East Asia and Pacific & NA & NA & NA & NA & 1 & 0 & 4 & 1 & 2 & 1 & 0 & 0 & 0 & 0 & 0 & 0 & 7 & 3 & 9 \\
Europe and Central Asia & NA & NA & NA & NA & 1 & 1 & 4 & 2 & 3 & 2 & 0 & 0 & 0 & 0 & 0 & 0 & 9 & 5 & 13 \\
Latin America and the Caribbean & NA & NA & NA & NA & 0 & 0 & 1 & 0 & 1 & 0 & 0 & 0 & 0 & 0 & 0 & 0 & 3 & 0 & 3 \\
Middle East and North Africa & NA & NA & NA & NA & 3 & 1 & 9 & 4 & 5 & 2 & 0 & 0 & 0 & 0 & 0 & 0 & 17 & 8 & 25 \\
South Asia & NA & NA & NA & NA & 9 & 3 & 30 & 12 & 15 & 7 & 1 & 0 & 0 & 0 & 0 & 0 & 55 & 22 & 77 \\
Sub-Saharan Africa & NA & NA & NA & NA & 1 & 0 & 2 & 1 & 1 & 1 & 0 & 0 & 0 & 0 & 0 & 0 & 5 & 2 & 7 \\
\hline Low- and middle-income countries & NA & NA & NA & NA & 16 & 6 & 50 & 21 & 27 & 12 & 2 & 1 & 0 & 0 & 0 & 0 & 95 & 39 & 134 \\
High-income countries & NA & NA & NA & NA & 1 & 1 & 3 & 3 & 1 & 1 & 0 & 0 & 0 & 0 & 0 & 0 \\
\hline WORLD & NA & NA & NA & NA & 17 & 7 & 53 & 23 & 29 & 13 & 2 & 1 & 0 & 0 & 0 & 0 & 11 \\
\hline
\end{tabular}

Attributable DALYs (thousands)

\begin{tabular}{|c|c|c|c|c|c|c|c|c|c|c|c|c|c|c|c|c|c|c|c|}
\hline East Asia and Pacific & NA & NA & NA & NA & 7 & 2 & 6 & 2 & 2 & 1 & 0 & 0 & 0 & 0 & 0 & 0 & 15 & 5 & 20 \\
\hline Europe and Central Asia & NA & NA & NA & NA & 6 & 5 & 7 & 4 & 3 & 2 & 0 & 0 & 0 & 0 & 0 & 0 & 17 & 12 & 28 \\
\hline Latin America and the Caribbean & NA & NA & NA & NA & 15 & 4 & 9 & 2 & 2 & 0 & 0 & 0 & 0 & 0 & 0 & 0 & 26 & 7 & 33 \\
\hline Middle East and North Africa & NA & NA & NA & NA & 10 & 4 & 12 & 5 & 6 & 3 & 0 & 0 & 0 & 0 & 0 & 0 & 28 & 13 & 41 \\
\hline South Asia & NA & NA & NA & NA & 22 & 5 & 37 & 13 & 16 & 7 & 1 & 0 & 0 & 0 & 0 & 0 & 76 & 25 & 101 \\
\hline Sub-Saharan Africa & NA & NA & NA & NA & 24 & 13 & 12 & 8 & 4 & 2 & 0 & 0 & 0 & 0 & 0 & 0 & 40 & 22 & 62 \\
\hline Low- and middle-income countries & NA & NA & NA & NA & 85 & 34 & 83 & 35 & 32 & 15 & 2 & 1 & 0 & 0 & 0 & 0 & 201 & 84 & 286 \\
\hline High-income countries & NA & NA & NA & NA & 9 & 10 & 9 & 9 & 3 & 3 & 0 & 0 & 0 & 0 & 0 & 0 & 20 & 23 & 43 \\
\hline WORLD & NA & NA & NA & NA & 93 & 44 & 91 & 44 & 35 & 18 & 2 & 1 & 0 & 0 & 0 & 0 & 221 & 108 & 29 \\
\hline
\end{tabular}

Source: Authors' calculations.

Note: $\mathrm{NA}=$ not applicable. 
Table 4A.90

Risk factor:

Child sexual abuse

Disease:

Post-traumatic stress disorder

\begin{tabular}{|c|c|c|c|c|c|c|c|c|c|c|c|c|c|c|c|c|c|c|c|}
\hline \multirow[b]{2}{*}{ Region } & \multicolumn{2}{|c|}{$0-4$ years } & \multicolumn{2}{|c|}{ 5-14 years } & \multicolumn{2}{|c|}{$15-29$ years } & \multicolumn{2}{|c|}{ 30-44 years } & \multicolumn{2}{|c|}{ 45-59 years } & \multicolumn{2}{|c|}{$60-69$ years } & \multicolumn{2}{|c|}{ 70-79 years } & \multicolumn{2}{|c|}{$80+$ years } & \multicolumn{3}{|c|}{ Total } \\
\hline & Male & Female & Male & Female & Male & Female & Male & Female & Male & Female & Male & Female & Male & Female & Male & Female & Male & Female & All \\
\hline \multicolumn{20}{|l|}{ PAF of Mortality (\%) } \\
\hline East Asia and Pacific & NA & NA & NA & NA & 18 & * & 23 & * & 23 & 28 & 23 & 28 & 23 & 28 & 24 & 28 & 24 & 28 & 26 \\
\hline Europe and Central Asia & NA & NA & NA & NA & 12 & 28 & 16 & 35 & 16 & 35 & 16 & 35 & 16 & 35 & 16 & 35 & 15 & 32 & 28 \\
\hline Latin America and the Caribbean & NA & NA & NA & NA & 16 & 13 & 22 & 18 & 22 & 18 & 22 & 18 & 22 & 18 & 22 & 18 & 22 & 1 & 20 \\
\hline Middle East and North Africa & NA & NA & NA & NA & 14 & 28 & 18 & 35 & 18 & 35 & 18 & 35 & 18 & 35 & 18 & 35 & 14 & 23 & 19 \\
\hline South Asia & NA & NA & NA & NA & * & * & 34 & * & 33 & 52 & * & 52 & * & * & 33 & * & 33 & 52 & 40 \\
\hline Sub-Saharan Africa & NA & NA & NA & NA & * & * & $*$ & * & * & * & * & * & * & * & $*$ & $*$ & * & * & * \\
\hline Low- and middle-income countries & NA & NA & NA & NA & 13 & 28 & 27 & 35 & 24 & 32 & 19 & 31 & 18 & 34 & 24 & 35 & 23 & 30 & 26 \\
\hline High-income countries & NA & NA & NA & NA & 7 & 25 & 10 & 32 & 10 & 32 & 10 & 32 & 10 & 32 & 10 & 32 & 10 & 32 & 22 \\
\hline WORLD & NA & NA & NA & NA & 9 & 28 & 27 & 35 & 19 & 32 & 16 & 31 & 10 & 32 & 24 & 34 & 18 & 31 & 25 \\
\hline
\end{tabular}

PAF of YLL (\%)

East Asia and Pacific

Europe and Central Asia

Latin America and the Caribbean

Middle East and North Africa

South Asia

Sub-Saharan Africa

Low- and middle-income countries

$\begin{array}{llll}N A & N A & N A & N A \\ N A & N A & N A & N A\end{array}$

$\begin{array}{ll}\text { NA } & 18 \\ \text { NA } & 12\end{array}$

\begin{tabular}{rr}
18 & $*$ \\
12 & 28 \\
16 & 13 \\
14 & 28 \\
$*$ & $*$ \\
$*$ & $*$ \\
\hline
\end{tabular}

\begin{tabular}{|c|c|c|c|c|c|c|c|c|c|c|c|c|c|c|c|c|c|c|}
\hline WORLD & NA & NA & NA & NA & 9 & 28 & 27 & 35 & 18 & 32 & 16 & 31 & 10 & 32 & 24 & 34 & 18 & 29 \\
\hline
\end{tabular}

PAF of DALYs $(\%)$

\begin{tabular}{|c|c|c|c|c|c|c|c|c|c|c|c|c|c|c|c|c|c|c|c|}
\hline East Asia and Pacific & NA & NA & NA & NA & 18 & 22 & 23 & 28 & 23 & 28 & 23 & 28 & 23 & 28 & 24 & 28 & 20 & 25 & 24 \\
\hline Europe and Central Asia & NA & NA & NA & NA & 12 & 28 & 16 & 35 & 16 & 35 & 16 & 35 & 16 & 35 & 16 & 35 & 14 & 31 & 26 \\
\hline Latin America and the Caribbean & NA & NA & NA & NA & 16 & 13 & 22 & 18 & 22 & 18 & 22 & 18 & 22 & 18 & 22 & 18 & 18 & 16 & 16 \\
\hline Middle East and North Africa & NA & NA & NA & NA & 14 & 28 & 18 & 35 & 18 & 35 & 18 & 35 & 18 & 35 & 18 & 35 & 15 & 30 & 26 \\
\hline South Asia & NA & NA & NA & NA & 26 & 43 & 34 & 52 & 33 & 52 & 33 & 52 & 33 & 51 & 33 & 52 & 28 & 46 & 41 \\
\hline Sub-Saharan Africa & NA & NA & NA & NA & 19 & 31 & 26 & 39 & 26 & 39 & 26 & 39 & 26 & 39 & 26 & 40 & 20 & 34 & 30 \\
\hline Low- and middle-income countries & NA & NA & NA & NA & 19 & 30 & 25 & 35 & 25 & 36 & 24 & 33 & 23 & 34 & 25 & 33 & 21 & 32 & 29 \\
\hline High-income countries & NA & NA & NA & NA & 7 & 25 & 10 & 32 & 10 & 32 & 10 & 32 & 10 & 32 & 10 & 32 & 9 & 29 & 24 \\
\hline WORLD & NA & NA & NA & NA & 18 & 29 & 23 & 34 & 22 & 35 & 19 & 33 & 18 & 33 & 19 & 32 & 19 & 31 & 28 \\
\hline
\end{tabular}

Attributable Mortality (thousands)

\begin{tabular}{lllllllllllllllllllll} 
Attributable Mortality (thousands) & NA & NA & NA & NA & 0 & 0 & 0 & 0 & 0 & 0 & 0 & 0 & 0 & 0 & 0 & 0 & 0 & 0 & 0 \\
Europe and Central Asia & NA & NA & NA & NA & 0 & 0 & 0 & 0 & 0 & 0 & 0 & 0 & 0 & 0 & 0 & 0 & 0 & 0 & 0 \\
Latin America and the Caribbean & NA & NA & NA & NA & 0 & 0 & 0 & 0 & 0 & 0 & 0 & 0 & 0 & 0 & 0 & 0 & 0 & 0 & 0 \\
Middle East and North Africa & NA & NA & NA & NA & 0 & 0 & 0 & 0 & 0 & 0 & 0 & 0 & 0 & 0 & 0 & 0 & 0 & 0 & 0 \\
South Asia & NA & NA & NA & NA & 0 & 0 & 0 & 0 & 0 & 0 & 0 & 0 & 0 & 0 & 0 & 0 & 0 & 0 & 0 \\
Sub-Saharan Africa & NA & NA & NA & NA & 0 & 0 & 0 & 0 & 0 & 0 & 0 & 0 & 0 & 0 & 0 & 0 & 0 & 0 & 0 \\
\hline Low- and middle-income countries & NA & NA & NA & NA & 0 & 0 & 0 & 0 & 0 & 0 & 0 & 0 & 0 & 0 & 0 & 0 & 0 & 0 & 0 \\
High-income countries & NA & NA & NA & NA & 0 & 0 & 0 & 0 & 0 & 0 & 0 & 0 & 0 & 0 & 0 & 0 \\
\hline WORLD & NA & NA & NA & NA & 0 & 0 & 0 & 0 & 0 & 0 & 0 & 0 & 0 & 0 & 0 & 0 \\
\hline
\end{tabular}

Attributable YLL (thousands)

East Asia and Pacific

Latin America and the Caribbean

Middle East and North Africa

South Asia

Sub-Saharan Africa

Low- and middle-income countries

High-income countries

NA NA NA NA

\begin{tabular}{rrrrrrrrrrrrr}
23 & $*$ & 23 & 28 & 23 & 28 & 23 & 28 & 24 & 28 & 23 & 28 & 26 \\
16 & 35 & 16 & 35 & 16 & 35 & 16 & 35 & 16 & 35 & 14 & 31 & 27 \\
22 & 18 & 22 & 18 & 22 & 18 & 22 & 18 & 22 & 18 & 22 & 1 & 18 \\
18 & 35 & 18 & 35 & 18 & 35 & 18 & 35 & 18 & 35 & 12 & 18 & 15 \\
34 & ${ }^{*}$ & 33 & 52 & $*$ & 52 & $*$ & $*$ & 33 & $*$ & 33 & 52 & 39 \\
${ }^{*}$ & ${ }^{*}$ & ${ }^{*}$ & ${ }^{*}$ & ${ }^{*}$ & ${ }^{*}$ & ${ }^{*}$ & ${ }^{*}$ & ${ }^{*}$ & ${ }^{*}$ & ${ }^{*}$ & ${ }^{*}$ & $*$ \\
27 & 35 & 24 & 32 & 18 & 31 & 18 & 34 & 24 & 35 & 22 & 28 & 25 \\
10 & 32 & 10 & 32 & 10 & 32 & 10 & 32 & 10 & 32 & 9 & 32 & 20 \\
27 & 35 & 18 & 32 & 16 & 31 & 10 & 32 & 24 & 34 & 18 & 29 & 24 \\
\hline
\end{tabular}

WORLD

$\begin{array}{llllllllllllllllllll}\text { NA } & \text { NA } & \text { NA } & \text { NA } & 0 & 0 & 0 & 0 & 0 & 0 & 0 & 0 & 0 & 0 & 0 & 0 & 0 & 0 & 0 \\ \text { NA } & \text { NA } & \text { NA } & \text { NA } & 0 & 0 & 0 & 0 & 0 & 0 & 0 & 0 & 0 & 0 & 0 & 0 & 0 & 0 & 0 \\ \text { NA } & \text { NA } & \text { NA } & \text { NA } & 0 & 0 & 0 & 0 & 0 & 0 & 0 & 0 & 0 & 0 & 0 & 0 & 0 & 0 & 0 \\ \text { NA } & \text { NA } & \text { NA } & \text { NA } & 0 & 0 & 0 & 0 & 0 & 0 & 0 & 0 & 0 & 0 & 0 & 0 & 0 & 0 & 0 \\ \text { NA } & \text { NA } & \text { NA } & \text { NA } & 0 & 0 & 0 & 0 & 0 & 0 & 0 & 0 & 0 & 0 & 0 & 0 & 0 & 0 & 0 \\ \text { NA } & \text { NA } & \text { NA } & \text { NA } & 0 & 0 & 0 & 0 & 0 & 0 & 0 & 0 & 0 & 0 & 0 & 0 & 0 & 0 & 0 \\ \text { NA } & \text { NA } & \text { NA } & \text { NA } & 0 & 0 & 0 & 0 & 0 & 0 & 0 & 0 & 0 & 0 & 0 & 0 & 0 & 0 & 0 \\ \text { NA } & \text { NA } & \text { NA } & \text { NA } & 0 & 0 & 0 & 0 & 0 & 0 & 0 & 0 & 0 & 0 & 0 & 0 & 0 & 0 \\ \text { NA } & \text { NA } & \text { NA } & \text { NA } & 0 & 0 & 0 & 0 & 0 & 0 & 0 & 0 & 0 & 0 & 0 & 0 & 0 & 0 & 0\end{array}$

Attributable DALYs (thousands

East Asia and Pacific

Europe and Central Asia

Latin America and the Caribbean

Middle East and North Africa

South Asia

Sub-Saharan Africa

Low- and middle-income countries

High-income countries

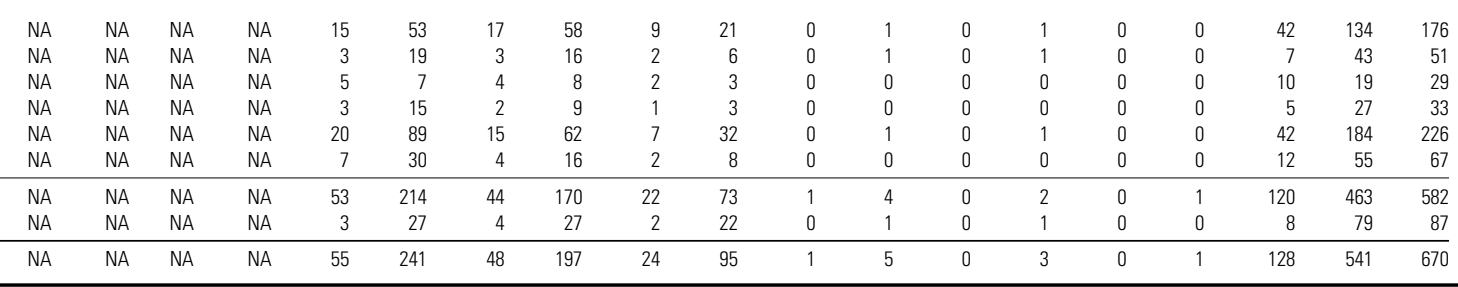

Source: Authors' calculations.

Note: NA = not applicable.

* The number of deaths (and hence YLL) directly coded to a number of diseases, especially neuropsychiatric and musculoskeletal diseases, is zero or very small. For other diseases, mortality or disease burden may be zero in some region-age-sex groups. In such cases, the population attributable fractions would be undefined or unstable and have not been calculated. 
Table 4A.91

Risk factor: Child sexual abuse

Disease: Panic disorder

\begin{tabular}{|c|c|c|c|c|c|c|c|c|c|c|c|c|c|c|c|c|c|c|c|}
\hline \multirow[b]{2}{*}{ Region } & \multicolumn{2}{|c|}{$0-4$ years } & \multicolumn{2}{|c|}{ 5-14 years } & \multicolumn{2}{|c|}{$15-29$ years } & \multicolumn{2}{|c|}{ 30-44 years } & \multicolumn{2}{|c|}{ 45-59 years } & \multicolumn{2}{|c|}{$60-69$ years } & \multicolumn{2}{|c|}{$70-79$ years } & \multicolumn{2}{|c|}{$80+$ years } & \multicolumn{3}{|c|}{ Total } \\
\hline & Male & Female & Male & Female & Male & Female & Male & Female & Male & Female & Male & Female & Male & Female & Male & Female & Male & Female & All \\
\hline \multicolumn{20}{|l|}{ PAF of Mortality (\%) } \\
\hline East Asia and Pacific & NA & NA & NA & NA & * & * & * & * & * & * & * & * & * & * & * & * & * & * & * \\
\hline Europe and Central Asia & NA & NA & NA & NA & * & * & * & * & * & * & * & * & * & * & * & * & * & * & * \\
\hline Latin America and the Caribbean & NA & NA & NA & NA & * & * & * & * & * & * & * & * & * & * & * & * & * & * & * \\
\hline Middle East and North Africa & NA & NA & NA & NA & * & * & * & * & * & * & * & * & * & * & * & * & * & * & * \\
\hline South Asia & NA & NA & NA & NA & * & * & * & * & * & * & * & * & * & * & * & * & * & * & * \\
\hline Sub-Saharan Africa & NA & NA & NA & NA & * & * & * & * & * & * & $*$ & * & * & * & * & * & * & * & * \\
\hline Low- and middle-income countries & NA & NA & NA & NA & * & * & * & * & * & * & * & * & * & * & * & * & * & * & * \\
\hline High-income countries & NA & NA & NA & NA & * & * & * & * & * & * & * & * & * & * & * & * & * & * & * \\
\hline WORLD & NA & NA & NA & NA & * & * & * & * & * & * & * & * & * & * & * & * & * & * & * \\
\hline
\end{tabular}

PAF of YLL $(\%)$

\begin{tabular}{|c|c|c|c|c|c|c|c|c|c|c|c|c|c|c|c|c|c|c|c|}
\hline East Asia and Pacific & NA & NA & NA & NA & * & * & * & * & * & * & * & * & * & * & * & * & * & * & * \\
\hline Europe and Central Asia & NA & NA & NA & NA & * & * & * & * & * & * & * & * & * & * & * & * & * & * & * \\
\hline Latin America and the Caribbean & NA & NA & NA & NA & * & * & * & * & * & * & * & * & * & * & * & $*$ & * & * & * \\
\hline Middle East and North Africa & NA & NA & NA & NA & * & * & * & * & * & * & * & * & * & * & * & * & * & the & * \\
\hline South Asia & NA & NA & NA & NA & * & * & * & * & * & * & * & * & * & * & * & * & * & , & * \\
\hline Sub-Saharan Africa & NA & NA & NA & NA & * & * & * & * & * & * & * & * & * & * & * & * & * & * & * \\
\hline Low- and middle-income countries & NA & NA & NA & NA & * & * & * & * & * & * & * & * & * & * & * & * & * & * & * \\
\hline High-income countries & NA & NA & NA & NA & * & * & * & * & * & * & * & * & * & * & * & * & * & * & * \\
\hline WORLD & NA & NA & NA & NA & * & * & * & * & * & * & * & * & * & * & * & * & * & the & * \\
\hline
\end{tabular}

\section{PAF of DALYs (\%)}

\begin{tabular}{|c|c|c|c|c|c|c|c|c|c|c|c|c|c|c|c|c|c|c|c|}
\hline East Asia and Pacific & NA & NA & NA & NA & 6 & 9 & 8 & 11 & 8 & 11 & 8 & 11 & 8 & 11 & 8 & 11 & 6 & 9 & 8 \\
\hline Europe and Central Asia & NA & NA & NA & NA & 4 & 11 & 6 & 15 & 6 & 15 & 6 & 15 & 6 & 15 & 6 & 15 & 4 & 11 & 9 \\
\hline Latin America and the Caribbean & NA & NA & NA & NA & 6 & 5 & 8 & 7 & 8 & 7 & 8 & 7 & 8 & 7 & 8 & 7 & 6 & 5 & 5 \\
\hline Middle East and North Africa & NA & NA & NA & NA & 5 & 11 & 7 & 15 & 7 & 15 & 7 & 15 & 7 & 15 & 7 & 15 & 5 & 11 & 9 \\
\hline South Asia & NA & NA & NA & NA & 10 & 20 & 14 & 27 & 14 & 27 & 14 & 27 & 14 & 27 & 13 & 27 & 10 & 20 & 16 \\
\hline Sub-Saharan Africa & NA & NA & NA & NA & 7 & 13 & 9 & 18 & 9 & 18 & 9 & 18 & 9 & 18 & 9 & 18 & 6 & 13 & 11 \\
\hline Low- and middle-income countries & NA & NA & NA & NA & 7 & 12 & 9 & 16 & 9 & 16 & 9 & 16 & 9 & 15 & 9 & 15 & 7 & 12 & 10 \\
\hline High-income countries & NA & NA & NA & NA & 2 & 10 & 4 & 13 & 4 & 13 & 4 & 13 & 3 & 13 & 3 & 13 & 2 & 10 & 8 \\
\hline WORLD & NA & NA & NA & NA & 6 & 12 & 8 & 15 & 8 & 15 & 8 & 15 & 7 & 15 & 7 & 14 & 6 & 12 & 10 \\
\hline
\end{tabular}

Attributable Mortality (thousands

\begin{tabular}{|c|c|c|c|c|c|c|c|c|c|c|c|c|c|c|c|c|c|c|c|}
\hline \multicolumn{20}{|l|}{ Attributable Mortality (thousands) } \\
\hline East Asia and Pacific & NA & NA & NA & NA & 0 & 0 & 0 & 0 & 0 & 0 & 0 & 0 & 0 & 0 & 0 & 0 & 0 & 0 & 0 \\
\hline Europe and Central Asia & NA & NA & NA & NA & 0 & 0 & 0 & 0 & 0 & 0 & 0 & 0 & 0 & 0 & 0 & 0 & 0 & 0 & 0 \\
\hline Latin America and the Caribbean & NA & NA & NA & NA & 0 & 0 & 0 & 0 & 0 & 0 & 0 & 0 & 0 & 0 & 0 & 0 & 0 & 0 & 0 \\
\hline Middle East and North Africa & NA & NA & NA & NA & 0 & 0 & 0 & 0 & 0 & 0 & 0 & 0 & 0 & 0 & 0 & 0 & 0 & 0 & 0 \\
\hline South Asia & NA & NA & NA & NA & 0 & 0 & 0 & 0 & 0 & 0 & 0 & 0 & 0 & 0 & 0 & 0 & 0 & 0 & 0 \\
\hline Sub-Saharan Africa & NA & NA & NA & NA & 0 & 0 & 0 & 0 & 0 & 0 & 0 & 0 & 0 & 0 & 0 & 0 & 0 & 0 & 0 \\
\hline Low- and middle-income countries & NA & NA & NA & NA & 0 & 0 & 0 & 0 & 0 & 0 & 0 & 0 & 0 & 0 & 0 & 0 & 0 & 0 & 0 \\
\hline High-income countries & NA & NA & NA & NA & 0 & 0 & 0 & 0 & 0 & 0 & 0 & 0 & 0 & 0 & 0 & 0 & 0 & 0 & 0 \\
\hline WORLD & NA & NA & NA & NA & 0 & 0 & 0 & 0 & 0 & 0 & 0 & 0 & 0 & 0 & 0 & 0 & 0 & 0 & 0 \\
\hline
\end{tabular}

Attributable YLL (thousands)

\begin{tabular}{|c|c|c|c|c|c|c|c|c|c|c|c|c|c|c|c|c|c|c|c|}
\hline East Asia and Pacific & NA & NA & NA & NA & 0 & 0 & 0 & 0 & 0 & 0 & 0 & 0 & 0 & 0 & 0 & 0 & 0 & 0 & 0 \\
\hline Europe and Central Asia & NA & NA & NA & NA & 0 & 0 & 0 & 0 & 0 & 0 & 0 & 0 & 0 & 0 & 0 & 0 & 0 & 0 & 0 \\
\hline Latin America and the Caribbean & NA & NA & NA & NA & 0 & 0 & 0 & 0 & 0 & 0 & 0 & 0 & 0 & 0 & 0 & 0 & 0 & 0 & 0 \\
\hline Middle East and North Africa & NA & NA & NA & NA & 0 & 0 & 0 & 0 & 0 & 0 & 0 & 0 & 0 & 0 & 0 & 0 & 0 & 0 & 0 \\
\hline South Asia & NA & NA & NA & NA & 0 & 0 & 0 & 0 & 0 & 0 & 0 & 0 & 0 & 0 & 0 & 0 & 0 & 0 & 0 \\
\hline Sub-Saharan Africa & NA & NA & NA & NA & 0 & 0 & 0 & 0 & 0 & 0 & 0 & 0 & 0 & 0 & 0 & 0 & 0 & 0 & 0 \\
\hline Low- and middle-income countries & NA & NA & NA & NA & 0 & 0 & 0 & 0 & 0 & 0 & 0 & 0 & 0 & 0 & 0 & 0 & 0 & 0 & 0 \\
\hline High-income countries & NA & NA & NA & NA & 0 & 0 & 0 & 0 & 0 & 0 & 0 & 0 & 0 & 0 & 0 & 0 & 0 & 0 & 0 \\
\hline WORLD & NA & NA & NA & NA & 0 & 0 & 0 & 0 & 0 & 0 & 0 & 0 & 0 & 0 & 0 & 0 & 0 & 0 & 0 \\
\hline
\end{tabular}

Attributable DALYs (thousands)

\begin{tabular}{lrrrrrrrrrrrrrrrrrrr} 
East Asia and Pacific & NA & NA & NA & NA & 24 & 72 & 0 & 1 & 2 & 5 & 0 & 1 & 0 & 0 & 0 & 0 & 27 & 79 & 106 \\
Europe and Central Asia & NA & NA & NA & NA & 4 & 22 & 0 & 0 & 0 & 2 & 0 & 0 & 0 & 0 & 0 & 0 & 5 & 26 & 31 \\
Latin America and the Caribbean & NA & NA & NA & NA & 7 & 12 & 0 & 0 & 0 & 1 & 0 & 0 & 0 & 0 & 0 & 0 & 7 & 13 & 21 \\
Middle East and North Africa & NA & NA & NA & NA & 4 & 18 & 0 & 0 & 0 & 1 & 0 & 0 & 0 & 0 & 0 & 0 & 4 & 19 & 24 \\
South Asia & NA & NA & NA & NA & 35 & 131 & 1 & 2 & 2 & 7 & 0 & 1 & 0 & 1 & 0 & 0 & 37 & 141 & 178 \\
Sub-Saharan Africa & NA & NA & NA & NA & 10 & 42 & 0 & 1 & 0 & 2 & 0 & 0 & 0 & 0 & 0 & 0 & 11 & 45 & 55 \\
\hline Low- and middle-income countries & NA & NA & NA & NA & 84 & 297 & 1 & 4 & 5 & 18 & 1 & 2 & 0 & 2 & 0 & 0 & 92 & 323 & 415 \\
High-income countries & NA & NA & NA & NA & 4 & 30 & 0 & 1 & 1 & 4 & 0 & 1 & 0 & 1 & 0 & 0 \\
\hline WORLD & NA & NA & NA & NA & 88 & 327 & 2 & 5 & 6 & 22 & 1 & 3 & 0 & 2 & 0 & 0 & 96 & 359 & 455
\end{tabular}

Source: Authors' calculations.

Note: $\mathrm{NA}=$ not applicable.

* The number of deaths (and hence YLL) directly coded to a number of diseases, especially neuropsychiatric and musculoskeletal diseases, is zero or very small. For other diseases, mortality or disease burden may be zero in some region-age-sex groups. In such cases, the population attributable fractions would be undefined or unstable and have not been calculated. 
Risk factor:

Disease:

Child sexual abuse

Self-inflicted injuries

\begin{tabular}{|c|c|c|c|c|c|c|c|c|c|c|c|c|c|c|c|c|c|c|c|}
\hline \multirow[b]{2}{*}{ Region } & \multicolumn{2}{|c|}{$0-4$ years } & \multicolumn{2}{|c|}{ 5-14 years } & \multicolumn{2}{|c|}{$15-29$ years } & \multicolumn{2}{|c|}{ 30-44 years } & \multicolumn{2}{|c|}{$45-59$ years } & \multicolumn{2}{|c|}{$60-69$ years } & \multicolumn{2}{|c|}{ 70-79 years } & \multicolumn{2}{|c|}{$80+$ years } & \multicolumn{3}{|c|}{ Total } \\
\hline & Male & Female & Male & Female & Male & Female & Male & Female & Male & Female & Male & Female & Male & Female & Male & Female & Male & Female & All \\
\hline \multicolumn{20}{|l|}{ PAF of Mortality (\%) } \\
\hline East Asia and Pacific & NA & NA & NA & NA & 4 & 6 & 5 & 7 & 5 & 7 & 5 & 7 & 5 & 7 & 5 & 8 & 5 & 7 & 6 \\
\hline Europe and Central Asia & NA & NA & NA & NA & 3 & 7 & 4 & 10 & 4 & 10 & 4 & 10 & 4 & 10 & 4 & 10 & 4 & 9 & 5 \\
\hline Latin America and the Caribbean & NA & NA & NA & NA & 4 & 3 & 5 & 4 & 5 & 4 & 5 & 4 & 5 & 4 & 5 & 4 & 5 & 4 & 4 \\
\hline Middle East and North Africa & NA & NA & NA & NA & 3 & 8 & 4 & 10 & 4 & 10 & 4 & 10 & 4 & 10 & 4 & 10 & 4 & 8 & 6 \\
\hline South Asia & NA & NA & NA & NA & 7 & 14 & 10 & 19 & 10 & 18 & 10 & 18 & 10 & 18 & 9 & 18 & 8 & 15 & 11 \\
\hline Sub-Saharan Africa & NA & NA & NA & NA & 5 & 8 & 6 & 12 & 6 & 12 & 6 & 12 & 6 & 12 & 7 & 12 & 5 & 10 & 6 \\
\hline Low- and middle-income countries & NA & NA & NA & NA & 5 & 10 & 6 & 11 & 6 & 11 & 5 & 9 & 6 & 9 & 6 & 10 & 6 & 10 & 7 \\
\hline High-income countries & NA & NA & NA & NA & 2 & 6 & 2 & 9 & 2 & 9 & 2 & 9 & 2 & 9 & 2 & 9 & 2 & 8 & 4 \\
\hline WORLD & NA & NA & NA & NA & 5 & 10 & 6 & 11 & 5 & 10 & 5 & 9 & 5 & 9 & 5 & 9 & 5 & 10 & 7 \\
\hline
\end{tabular}

PAF of YLL (\%)

\begin{tabular}{|c|c|c|c|c|c|c|c|c|c|c|c|c|c|c|c|c|c|c|c|}
\hline East Asia and Pacific & NA & NA & NA & NA & 4 & 6 & 5 & 7 & 5 & 7 & 5 & 7 & 5 & 7 & 5 & 8 & 5 & 7 & 6 \\
\hline Europe and Central Asia & NA & NA & NA & NA & 3 & 7 & 4 & 10 & 4 & 10 & 4 & 10 & 4 & 10 & 4 & 10 & 4 & 9 & 5 \\
\hline Latin America and the Caribbean & NA & NA & NA & NA & 4 & 3 & 5 & 4 & 5 & 4 & 5 & 4 & 5 & 4 & 5 & 4 & 5 & 3 & 4 \\
\hline Middle East and North Africa & NA & NA & NA & NA & 3 & 8 & 4 & 10 & 4 & 10 & 4 & 10 & 4 & 10 & 4 & 10 & 4 & 8 & 5 \\
\hline South Asia & NA & NA & NA & NA & 7 & 14 & 10 & 19 & 10 & 18 & 10 & 18 & 10 & 18 & 9 & 18 & 8 & 15 & 11 \\
\hline Sub-Saharan Africa & NA & NA & NA & NA & 5 & 8 & 6 & 12 & 6 & 12 & 6 & 12 & 6 & 12 & 7 & 12 & 5 & 9 & 6 \\
\hline Low- and middle-income countries & NA & NA & NA & NA & 5 & 10 & 6 & 11 & 6 & 10 & 5 & 9 & 6 & 9 & 6 & 10 & 5 & 10 & 7 \\
\hline High-income countries & NA & NA & NA & NA & 2 & 6 & 2 & 9 & 2 & 9 & 2 & 9 & 2 & 9 & 2 & 9 & 2 & 8 & 4 \\
\hline WORLD & NA & NA & NA & NA & 5 & 10 & 6 & 11 & 5 & 10 & 5 & 9 & 5 & 9 & 5 & 9 & 5 & 10 & 7 \\
\hline
\end{tabular}

\section{PAF of DALYs (\%)}

East Asia and Pacific

Europe and Central Asia

Latin America and the Caribbean

Middle East and North Afric

South Asia

Sub-Saharan Africa

Low- and middle-income countries

High-income countries

$\begin{array}{llll}\text { NA } & \text { NA } & \text { NA } & \text { NA } \\ \text { NA } & \text { NA } & \text { NA } & \text { NA } \\ \text { NA } & \text { NA } & \text { NA } & \text { NA } \\ \text { NA } & \text { NA } & \text { NA } & \text { NA } \\ \text { NA } & \text { NA } & \text { NA } & \text { NA } \\ \text { NA } & \text { NA } & \text { NA } & \text { NA } \\ \text { NA } & \text { NA } & \text { NA } & \text { NA }\end{array}$

Attributable Mortality (thousands)

\begin{tabular}{lrrrrrrrrrrrrrrrrrrrr} 
East Asia and Pacific & NA & NA & NA & NA & 2 & 2 & 2 & 3 & 2 & 3 & 1 & 1 & 1 & 1 & 0 & 1 & 8 & 11 & 19 \\
Europe and Central Asia & NA & NA & NA & NA & 1 & 0 & 1 & 0 & 1 & 0 & 0 & 0 & 0 & 0 & 0 & 0 & 4 & 2 & 6 \\
Latin America and the Caribbean & NA & NA & NA & NA & 0 & 0 & 0 & 0 & 0 & 0 & 0 & 0 & 0 & 0 & 0 & 0 & 1 & 0 & 1 \\
Middle East and North Africa & NA & NA & NA & NA & 0 & 0 & 0 & 0 & 0 & 0 & 0 & 0 & 0 & 0 & 0 & 0 & 0 & 0 & 1 \\
South Asia & NA & NA & NA & NA & 4 & 7 & 4 & 4 & 2 & 3 & 1 & 1 & 1 & 1 & 0 & 0 & 11 & 15 & 26 \\
Sub-Saharan Africa & NA & NA & NA & NA & 0 & 0 & 0 & 0 & 0 & 0 & 0 & 0 & 0 & 0 & 0 & 0 & 2 & 1 & 2 \\
\hline Low- and middle-income countries & NA & NA & NA & NA & 7 & 10 & 8 & 8 & 5 & 6 & 3 & 2 & 2 & 2 & 1 & 1 & 25 & 29 & 54 \\
High-income countries & NA & NA & NA & NA & 0 & 0 & 1 & 1 & 1 & 1 & 0 & 0 & 0 & 0 & 0 & 0 \\
\hline WORLD & NA & NA & NA & NA & 7 & 10 & 8 & 9 & 6 & 7 & 3 & 3 & 2 & 3 & 1 & 1 \\
\hline
\end{tabular}

Attributable YLL (thousands)

\begin{tabular}{|c|c|c|c|c|c|c|c|c|c|c|c|c|c|c|c|c|c|c|c|}
\hline East Asia and Pacific & NA & NA & NA & NA & 41 & 54 & 49 & 84 & 34 & 54 & 17 & 20 & 8 & 13 & 2 & 3 & 150 & 228 & 377 \\
\hline Europe and Central Asia & NA & NA & NA & NA & 18 & 9 & 28 & 11 & 22 & 10 & 6 & 5 & 2 & 3 & 0 & 1 & 77 & 39 & 115 \\
\hline Latin America and the Caribbean & NA & NA & NA & NA & 8 & 2 & 8 & 2 & 5 & 1 & 1 & 0 & 1 & 0 & 0 & 0 & 24 & 5 & 29 \\
\hline Middle East and North Africa & NA & NA & NA & NA & 3 & 7 & 2 & 3 & 1 & 1 & 0 & 0 & 0 & 0 & 0 & 0 & 8 & 12 & 19 \\
\hline South Asia & NA & NA & NA & NA & 103 & 188 & 92 & 96 & 38 & 54 & 8 & 8 & 5 & 6 & 1 & 2 & 247 & 355 & 602 \\
\hline$\underline{\text { Sub-Saharan Africa }}$ & NA & NA & NA & NA & 12 & 8 & 11 & 6 & 7 & 4 & 2 & 1 & 1 & 0 & 0 & 0 & 33 & 18 & 51 \\
\hline Low- and middle-income countries & NA & NA & NA & NA & 185 & 268 & 191 & 202 & 106 & 124 & 35 & 35 & 16 & 23 & 3 & 6 & 538 & 657 & 1,194 \\
\hline High-income countries & NA & NA & NA & NA & 7 & 8 & 14 & 16 & 12 & 15 & 4 & 6 & 2 & 3 & 1 & 1 & 39 & 49 & 88 \\
\hline WORLD & NA & NA & NA & NA & 192 & 276 & 205 & 217 & 118 & 139 & 39 & 40 & 18 & 26 & 4 & 7 & 577 & 706 & 1,283 \\
\hline
\end{tabular}

Attributable DALYs (thousands)

\begin{tabular}{|c|c|c|c|c|c|c|c|c|c|c|c|c|c|c|c|c|c|c|c|}
\hline East Asia and Pacific & NA & NA & NA & NA & 42 & 59 & 50 & 88 & 34 & 55 & 17 & 21 & 8 & 13 & 2 & 3 & 153 & 240 & 393 \\
\hline Europe and Central Asia & NA & NA & NA & NA & 19 & 9 & 29 & 12 & 22 & 11 & 6 & 5 & 2 & 3 & 0 & 1 & 78 & 41 & 119 \\
\hline Latin America and the Caribbean & NA & NA & NA & NA & 9 & 3 & 8 & 2 & 5 & 1 & 2 & 0 & 1 & 0 & 0 & 0 & 24 & 6 & 30 \\
\hline Middle East and North Africa & NA & NA & NA & NA & 4 & 7 & 3 & 3 & 1 & 1 & 0 & 0 & 0 & 0 & 0 & 0 & 8 & 12 & 20 \\
\hline South Asia & NA & NA & NA & NA & 111 & 233 & 94 & 102 & 39 & 55 & 8 & 8 & 5 & 6 & 1 & 2 & 258 & 407 & 664 \\
\hline Sub-Saharan Africa & NA & NA & NA & NA & 12 & 9 & 11 & 6 & 7 & 4 & 2 & 1 & 1 & 0 & 0 & 0 & 34 & 20 & 54 \\
\hline Low- and middle-income countries & NA & NA & NA & NA & 196 & 320 & 195 & 212 & 108 & 127 & 35 & 36 & 17 & 24 & 3 & 6 & 555 & 726 & 1,280 \\
\hline High-income countries & NA & NA & NA & NA & 7 & 8 & 15 & 17 & 12 & 16 & 4 & 6 & 2 & 4 & 1 & 1 & 41 & 53 & 94 \\
\hline WORLD & NA & NA & NA & NA & 203 & 329 & 209 & 230 & 120 & 143 & 39 & 42 & 19 & 28 & 4 & 7 & 595 & 779 & 1,374 \\
\hline
\end{tabular}

Source: Authors' calculations.

Note: NA = not applicable. 
Table 4A.93

Risk factor: Child sexual abuse

Disease: All causes

\begin{tabular}{|c|c|c|c|c|c|c|c|c|c|c|c|c|c|c|c|c|c|c|c|}
\hline \multirow[b]{2}{*}{ Region } & \multicolumn{2}{|c|}{$0-4$ years } & \multicolumn{2}{|c|}{ 5-14 years } & \multicolumn{2}{|c|}{$15-29$ years } & \multicolumn{2}{|c|}{$30-44$ years } & \multicolumn{2}{|c|}{$45-59$ years } & \multicolumn{2}{|c|}{$60-69$ years } & \multicolumn{2}{|c|}{ 70-79 years } & \multicolumn{2}{|c|}{$80+$ years } & \multicolumn{3}{|c|}{ Total } \\
\hline & Male & Female & Male & Female & Male & Female & Male & Female & Male & Female & Male & Female & Male & Female & Male & Female & Male & Female & All \\
\hline \multicolumn{20}{|l|}{ PAF of Mortality (\%) } \\
\hline East Asia and Pacific & NA & NA & NA & NA & 0 & 1 & 0 & 1 & 0 & 0 & 0 & 0 & 0 & 0 & 0 & 0 & 0 & 0 & 0 \\
\hline Europe and Central Asia & NA & NA & NA & NA & 0 & 1 & 0 & 1 & 0 & 0 & 0 & 0 & 0 & 0 & 0 & 0 & 0 & 0 & 0 \\
\hline Latin America and the Caribbean & NA & NA & NA & NA & 0 & 0 & 0 & 0 & 0 & 0 & 0 & 0 & 0 & 0 & 0 & 0 & 0 & 0 & 0 \\
\hline Middle East and North Africa & NA & NA & NA & NA & 0 & 1 & 1 & 1 & 0 & 0 & 0 & 0 & 0 & 0 & 0 & 0 & 0 & 0 & 0 \\
\hline South Asia & NA & NA & NA & NA & 1 & 1 & 1 & 1 & 0 & 0 & 0 & 0 & 0 & 0 & 0 & 0 & 0 & 0 & 0 \\
\hline Sub-Saharan Africa & NA & NA & NA & NA & 0 & 0 & 0 & 0 & 0 & 0 & 0 & 0 & 0 & 0 & 0 & 0 & 0 & 0 & 0 \\
\hline Low- and middle-income countries & NA & NA & NA & NA & 0 & 1 & 0 & 1 & 0 & 0 & 0 & 0 & 0 & 0 & 0 & 0 & 0 & 0 & $\overline{0}$ \\
\hline High-income countries & NA & NA & NA & NA & 0 & 1 & 0 & 1 & 0 & 0 & 0 & 0 & 0 & 0 & 0 & 0 & 0 & 0 & 0 \\
\hline WORLD & NA & NA & NA & NA & 0 & 1 & 0 & 1 & 0 & 0 & 0 & 0 & 0 & 0 & 0 & 0 & 0 & 0 & $\overline{0}$ \\
\hline
\end{tabular}

PAF of YLL (\%)

\begin{tabular}{|c|c|c|c|c|c|c|c|c|c|c|c|c|c|c|c|c|c|c|c|}
\hline East Asia and Pacific & NA & NA & NA & NA & 0 & 1 & 0 & 1 & 0 & 0 & 0 & 0 & 0 & 0 & 0 & 0 & 0 & 0 & 0 \\
\hline Europe and Central Asia & NA & NA & NA & NA & 0 & 1 & 0 & 1 & 0 & 0 & 0 & 0 & 0 & 0 & 0 & 0 & 0 & ( & 0 \\
\hline Latin America and the Caribbean & NA & NA & NA & NA & 0 & 0 & 0 & 0 & 0 & 0 & 0 & 0 & 0 & 0 & 0 & 0 & 0 & ( & 0 \\
\hline Middle East and North Africa & NA & NA & NA & NA & 0 & 1 & 1 & 1 & 0 & 0 & 0 & 0 & 0 & 0 & 0 & 0 & 0 & ( & 0 \\
\hline South Asia & NA & NA & NA & NA & 1 & 1 & 1 & 1 & 0 & 0 & 0 & 0 & 0 & 0 & 0 & 0 & 0 & ( & 0 \\
\hline Sub-Saharan Africa & NA & NA & NA & NA & 0 & 0 & 0 & 0 & 0 & 0 & 0 & 0 & 0 & 0 & 0 & 0 & 0 & ( & 0 \\
\hline Low- and middle-income countries & NA & NA & NA & NA & 0 & 1 & 0 & 1 & 0 & 0 & 0 & 0 & 0 & 0 & 0 & 0 & 0 & ( & 0 \\
\hline High-income countries & NA & NA & NA & NA & 0 & 1 & 0 & 1 & 0 & 0 & 0 & 0 & 0 & 0 & 0 & 0 & 0 & 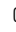 & 0 \\
\hline WORLD & NA & NA & NA & NA & 0 & 1 & 0 & 1 & 0 & 0 & 0 & 0 & 0 & 0 & 0 & 0 & 0 & 0 & 0 \\
\hline
\end{tabular}

\section{PAF of DALYs (\%)}

East Asia and Pacific

Europe and Central Asia

NA NA NA NA

South Asia

Sub-Saharan Africa

Low- and middle-income countries

High-income countries

$\begin{array}{llll}\text { NA } & \text { NA } & \text { NA } & \text { NA } \\ \text { NA } & \text { NA } & \text { NA } & \text { NA } \\ \text { NA } & \text { NA } & \text { NA } & \text { NA } \\ \text { NA } & \text { NA } & \text { NA } & \text { NA } \\ \text { NA } & \text { NA } & \text { NA } & \text { NA } \\ \text { NA } & \text { NA } & \text { NA } & \text { NA } \\ \text { NA } & \text { NA } & \text { NA } & \text { NA }\end{array}$

Attributable Mortality (thousands)

\begin{tabular}{llllllrrrrrrrrrrrrrrrr} 
East Asia and Pacific & NA & NA & NA & NA & 2 & 2 & 2 & 3 & 2 & 3 & 1 & 1 & 1 & 1 & 0 & 1 & 9 & 11 & 20 \\
Europe and Central Asia & NA & NA & NA & NA & 1 & 0 & 1 & 1 & 1 & 1 & 1 & 0 & 0 & 0 & 0 & 0 & 4 & 2 & 7 \\
Latin America and the Caribbean & NA & NA & NA & NA & 0 & 0 & 1 & 0 & 1 & 0 & 0 & 0 & 0 & 0 & 0 & 0 & 2 & 0 & 2 \\
Middle East and North Africa & NA & NA & NA & NA & 0 & 0 & 1 & 0 & 0 & 0 & 0 & 0 & 0 & 0 & 0 & 0 & 1 & 1 & 2 \\
South Asia & NA & NA & NA & NA & 4 & 7 & 5 & 4 & 3 & 3 & 1 & 1 & 1 & 1 & 0 & 0 & 15 & 16 & 31 \\
Sub-Saharan Africa & NA & NA & NA & NA & 0 & 0 & 1 & 0 & 1 & 0 & 0 & 0 & 0 & 0 & 0 & 0 & 2 & 1 & 3 \\
\hline Low- and middle-income countries & NA & NA & NA & NA & 8 & 10 & 11 & 9 & 8 & 7 & 3 & 3 & 2 & 2 & 1 & 1 & 33 & 32 & 65 \\
High-income countries & NA & NA & NA & NA & 0 & 0 & 1 & 1 & 1 & 1 & 0 & 0 & 0 & 0 & 0 & 0 \\
\hline WORLD & NA & NA & NA & NA & 8 & 10 & 12 & 10 & 9 & 8 & 4 & 3 & 2 & 3 & 1 & 1 \\
\hline
\end{tabular}

Attributable YLL (thousands)

\begin{tabular}{|c|c|c|c|c|c|c|c|c|c|c|c|c|c|c|c|c|c|c|c|}
\hline East Asia and Pacific & NA & NA & NA & NA & 43 & 55 & 57 & 86 & 40 & 56 & 18 & 21 & 8 & 13 & 2 & 3 & 168 & 234 & 402 \\
\hline Europe and Central Asia & NA & NA & NA & NA & 19 & 9 & 34 & 14 & 27 & 13 & 8 & 6 & 3 & 3 & 0 & 1 & 91 & 47 & 137 \\
\hline Latin America and the Caribbean & NA & NA & NA & NA & 10 & 2 & 16 & 2 & 11 & 1 & 3 & 0 & 1 & 0 & 0 & 0 & 42 & 7 & 49 \\
\hline Middle East and North Africa & NA & NA & NA & NA & 7 & 9 & 13 & 7 & 7 & 4 & 1 & 1 & 0 & 0 & 0 & 0 & 27 & 20 & 48 \\
\hline South Asia & NA & NA & NA & NA & 116 & 191 & 132 & 111 & 62 & 69 & 12 & 11 & 6 & 7 & 1 & 2 & 329 & 391 & 720 \\
\hline Sub-Saharan Africa & NA & NA & NA & NA & 13 & 8 & 16 & 8 & 11 & 6 & 2 & 1 & 1 & 1 & 0 & 0 & 44 & 24 & 68 \\
\hline Low- and middle-income countries & NA & NA & NA & NA & 208 & 274 & 267 & 229 & 158 & 149 & 45 & 40 & 19 & 24 & 4 & 6 & 700 & 722 & 1,423 \\
\hline High-income countries & NA & NA & NA & NA & 8 & 9 & 19 & 20 & 17 & 19 & 5 & 7 & 2 & 4 & 1 & 2 & 52 & 61 & 114 \\
\hline WORLD & NA & NA & NA & NA & 216 & 283 & 287 & 249 & 175 & 168 & 50 & 47 & 21 & 29 & 5 & 8 & 753 & 784 & 1,536 \\
\hline
\end{tabular}

Attributable DALYs (thousands)

\begin{tabular}{|c|c|c|c|c|c|c|c|c|c|c|c|c|c|c|c|c|c|c|c|}
\hline East Asia and Pacific & NA & NA & NA & NA & 200 & 261 & 219 & 284 & 127 & 195 & 36 & 56 & 12 & 23 & 2 & 6 & 596 & 824 & 1,420 \\
\hline Europe and Central Asia & NA & NA & NA & NA & 59 & 95 & 72 & 101 & 47 & 68 & 12 & 27 & 4 & 10 & 0 & 2 & 193 & 303 & 497 \\
\hline Latin America and the Caribbean & NA & NA & NA & NA & 99 & 58 & 74 & 49 & 40 & 25 & 9 & 7 & 2 & 2 & 1 & 0 & 224 & 141 & 365 \\
\hline Middle East and North Africa & NA & NA & NA & NA & 27 & 64 & 25 & 45 & 12 & 21 & 2 & 5 & 0 & 1 & 0 & 0 & 66 & 137 & 203 \\
\hline South Asia & NA & NA & NA & NA & 305 & 752 & 302 & 528 & 138 & 291 & 30 & 77 & 9 & 21 & 2 & 5 & 786 & 1,675 & 2,461 \\
\hline Sub-Saharan Africa & NA & NA & NA & NA & 79 & 136 & 55 & 77 & 27 & 41 & 5 & 9 & 2 & 3 & 0 & 0 & 168 & 266 & 434 \\
\hline Low- and middle-income countries & NA & NA & NA & NA & 768 & 1,366 & 746 & 1,083 & 392 & 643 & 94 & 180 & 29 & 60 & 6 & 14 & 2,034 & 3,346 & 5,381 \\
\hline High-income countries & NA & NA & NA & NA & 52 & 160 & 70 & 176 & 41 & 127 & 10 & 34 & 4 & 16 & 1 & 7 & 178 & 520 & 699 \\
\hline$\overline{\text { WORLD }}$ & NA & NA & NA & NA & 819 & 1,526 & 817 & 1,259 & 433 & 770 & 104 & 213 & 33 & 76 & 7 & 21 & 2,212 & 3,867 & 6,079 \\
\hline
\end{tabular}

Source: Authors' calculations.

Note: NA = not applicable. 
Risk factor: Indoor smoke from household use of solid fuels

\begin{tabular}{|c|c|c|c|c|c|c|c|c|c|c|c|c|c|c|c|c|c|c|c|}
\hline \multirow[b]{2}{*}{ Region } & \multicolumn{2}{|c|}{$0-4$ years } & \multicolumn{2}{|c|}{ 5-14 years } & \multicolumn{2}{|c|}{$15-29$ years } & \multicolumn{2}{|c|}{ 30-44 years } & \multicolumn{2}{|c|}{$45-59$ years } & \multicolumn{2}{|c|}{$60-69$ years } & \multicolumn{2}{|c|}{ 70-79 years } & \multicolumn{2}{|c|}{$80+$ years } & \multicolumn{3}{|c|}{ Total } \\
\hline & Male & Female & Male & Female & Male & Female & Male & Female & Male & Female & Male & Female & Male & Female & Male & Female & Male & Female & All \\
\hline \multicolumn{20}{|l|}{ PAF of Mortality (\%) } \\
\hline East Asia and Pacific & 41 & 41 & NA & NA & NA & NA & NA & NA & NA & NA & NA & NA & NA & NA & NA & NA & 11 & 16 & 14 \\
\hline Europe and Central Asia & 20 & 20 & NA & NA & NA & NA & NA & NA & NA & NA & NA & NA & NA & NA & NA & NA & 6 & 7 & 6 \\
\hline Latin America and the Caribbean & 28 & 28 & NA & NA & NA & NA & NA & NA & NA & NA & NA & NA & NA & NA & NA & NA & 8 & 7 & 8 \\
\hline Middle East and North Africa & 14 & 14 & NA & NA & NA & NA & NA & NA & NA & NA & NA & NA & NA & NA & NA & NA & 10 & 10 & 10 \\
\hline South Asia & 52 & 52 & NA & NA & NA & NA & NA & NA & NA & NA & NA & NA & NA & NA & NA & NA & 30 & 31 & 30 \\
\hline Sub-Saharan Africa & 53 & 53 & NA & NA & NA & NA & NA & NA & NA & NA & NA & NA & NA & NA & NA & NA & 39 & 34 & 37 \\
\hline Low- and middle-income countries & 49 & 48 & NA & NA & NA & NA & NA & NA & NA & NA & NA & NA & NA & NA & NA & NA & 28 & 27 & 27 \\
\hline High-income countries & 0 & 0 & NA & NA & NA & NA & NA & NA & NA & NA & NA & NA & NA & NA & NA & NA & 0 & 0 & 0 \\
\hline WORLD & 49 & 48 & NA & NA & NA & NA & NA & NA & NA & NA & NA & NA & NA & NA & NA & NA & 26 & 24 & 25 \\
\hline
\end{tabular}

PAF of YLL (\%)

\begin{tabular}{|c|c|c|c|c|c|c|c|c|c|c|c|c|c|c|c|c|c|c|c|}
\hline East Asia and Pacific & 41 & 41 & NA & NA & NA & NA & NA & NA & NA & NA & NA & NA & NA & NA & NA & NA & 19 & 27 & 24 \\
\hline Europe and Central Asia & 20 & 20 & NA & NA & NA & NA & NA & NA & NA & NA & NA & NA & NA & NA & NA & NA & 9 & 11 & 10 \\
\hline Latin America and the Caribbean & 28 & 28 & NA & NA & NA & NA & NA & NA & NA & NA & NA & NA & NA & NA & NA & NA & 14 & 14 & 14 \\
\hline Middle East and North Africa & 14 & 14 & NA & NA & NA & NA & NA & NA & NA & NA & NA & NA & NA & NA & NA & NA & 11 & 11 & 11 \\
\hline South Asia & 52 & 52 & NA & NA & NA & NA & NA & NA & NA & NA & NA & NA & NA & NA & NA & NA & 40 & 40 & 40 \\
\hline Sub-Saharan Africa & 53 & 53 & NA & NA & NA & NA & NA & NA & NA & NA & NA & NA & NA & NA & NA & NA & 44 & 37 & 41 \\
\hline Low- and middle-income countries & 49 & 48 & NA & NA & NA & NA & NA & NA & NA & NA & NA & NA & NA & NA & NA & NA & 37 & 35 & 36 \\
\hline High-income countries & 0 & 0 & NA & NA & NA & NA & NA & NA & NA & NA & NA & NA & NA & NA & NA & NA & 0 & 0 & 0 \\
\hline WORLD & 49 & 48 & NA & NA & NA & NA & NA & NA & NA & NA & NA & NA & NA & NA & NA & NA & 36 & 34 & 35 \\
\hline
\end{tabular}

\section{PAF of DALYs (\%)}

\begin{tabular}{|c|c|c|c|c|c|c|c|c|c|c|c|c|c|c|c|c|c|c|c|}
\hline East Asia and Pacific & 41 & 41 & NA & NA & NA & NA & NA & NA & NA & NA & NA & NA & NA & NA & NA & NA & 20 & 27 & 24 \\
\hline Europe and Central Asia & 20 & 20 & NA & NA & NA & NA & NA & NA & NA & NA & NA & NA & NA & NA & NA & NA & 9 & 11 & 10 \\
\hline Latin America and the Caribbean & 28 & 28 & NA & NA & NA & NA & NA & NA & NA & NA & NA & NA & NA & NA & NA & NA & 16 & 16 & 16 \\
\hline Middle East and North Africa & 14 & 14 & NA & NA & NA & NA & NA & NA & NA & NA & NA & NA & NA & NA & NA & NA & 12 & 11 & 11 \\
\hline South Asia & 52 & 52 & NA & NA & NA & NA & NA & NA & NA & NA & NA & NA & NA & NA & NA & NA & 40 & 40 & 40 \\
\hline Sub-Saharan Africa & 53 & 53 & NA & NA & NA & NA & NA & NA & NA & NA & NA & NA & NA & NA & NA & NA & 44 & 37 & 41 \\
\hline Low- and middle-income countries & 49 & 48 & NA & NA & NA & NA & NA & NA & NA & NA & NA & NA & NA & NA & NA & NA & 36 & 35 & 36 \\
\hline High-income countries & 0 & 0 & NA & NA & NA & NA & NA & NA & NA & NA & NA & NA & NA & NA & NA & NA & 0 & 0 & 0 \\
\hline RLLD & 49 & 48 & NA & NA & NA & NA & NA & NA & NA & NA & NA & NA & NA & NA & NA & NA & 35 & 34 & 35 \\
\hline
\end{tabular}

Attributable Mortality (thousands)

\begin{tabular}{|c|c|c|c|c|c|c|c|c|c|c|c|c|c|c|c|c|c|c|c|}
\hline East Asia and Pacific & 26 & 50 & NA & NA & NA & NA & NA & NA & NA & NA & NA & NA & NA & NA & NA & NA & 26 & 50 & 76 \\
\hline Europe and Central Asia & 4 & 3 & NA & NA & NA & NA & NA & NA & NA & NA & NA & NA & NA & NA & NA & NA & 4 & 3 & 7 \\
\hline Latin America and the Caribbean & 7 & 6 & NA & NA & NA & NA & NA & NA & NA & NA & NA & NA & NA & NA & NA & NA & 7 & 6 & 12 \\
\hline Middle East and North Africa & 5 & 5 & NA & NA & NA & NA & NA & NA & NA & NA & NA & NA & NA & NA & NA & NA & 5 & 5 & 10 \\
\hline South Asia & 213 & 215 & NA & NA & NA & NA & NA & NA & NA & NA & NA & NA & NA & NA & NA & NA & 213 & 215 & 429 \\
\hline Sub-Saharan Africa & 230 & 167 & NA & NA & NA & NA & NA & NA & NA & NA & NA & NA & NA & NA & NA & NA & 230 & 167 & 397 \\
\hline Low- and middle-income countries & 485 & 447 & NA & NA & NA & NA & NA & NA & NA & NA & NA & NA & NA & NA & NA & NA & 485 & 447 & 931 \\
\hline High-income countries & 0 & 0 & NA & NA & NA & NA & NA & NA & NA & NA & NA & NA & NA & NA & NA & NA & 0 & 0 & 0 \\
\hline WORLD & 485 & 447 & NA & NA & NA & NA & NA & NA & NA & NA & NA & NA & NA & NA & NA & NA & 485 & 447 & 931 \\
\hline
\end{tabular}

Attributable YLL (thousands)

\begin{tabular}{|c|c|c|c|c|c|c|c|c|c|c|c|c|c|c|c|c|c|c|c|}
\hline \multicolumn{20}{|l|}{ Attributable YLL (the } \\
\hline East Asia and Pacific & 776 & 1,530 & NA & NA & NA & NA & NA & NA & NA & NA & NA & NA & NA & NA & NA & NA & 776 & 1,530 & 2,306 \\
\hline Europe and Central Asia & 110 & 93 & NA & NA & NA & NA & NA & NA & NA & NA & NA & NA & NA & NA & NA & NA & 110 & 93 & 203 \\
\hline Latin America and the Caribbean & 199 & 170 & NA & NA & NA & NA & NA & NA & NA & NA & NA & NA & NA & NA & NA & NA & 199 & 170 & 370 \\
\hline Middle East and North Africa & 164 & 150 & NA & NA & NA & NA & NA & NA & NA & NA & NA & NA & NA & NA & NA & NA & 164 & 150 & 314 \\
\hline South Asia & 6,454 & 6,566 & NA & NA & NA & NA & NA & NA & NA & NA & NA & NA & NA & NA & NA & NA & 6,454 & 6,566 & 13,020 \\
\hline Sub-Saharan Africa & 6,961 & 5,098 & NA & NA & NA & NA & NA & NA & NA & NA & NA & NA & NA & NA & NA & NA & 6,961 & 5,098 & 12,059 \\
\hline Low- and middle-income countries & 14,663 & 13,608 & NA & NA & NA & NA & NA & NA & NA & NA & NA & NA & NA & NA & NA & NA & 14,663 & 13,608 & 28,271 \\
\hline High-income countries & & 0 & NA & NA & NA & NA & NA & NA & NA & NA & NA & NA & NA & NA & NA & NA & 0 & 0 & c \\
\hline WORLD & 14,663 & 13,608 & NA & NA & NA & NA & NA & NA & NA & NA & NA & NA & NA & NA & NA & NA & 14,663 & 13,608 & 28,271 \\
\hline \multicolumn{20}{|l|}{ Attributable DALYs (thousands) } \\
\hline East Asia and Pacific & 892 & 1,734 & NA & NA & NA & NA & NA & NA & NA & NA & NA & NA & NA & NA & NA & NA & 892 & 1,734 & 2,626 \\
\hline Europe and Central Asia & 115 & 99 & NA & NA & NA & NA & NA & NA & NA & NA & NA & NA & NA & NA & NA & NA & 115 & 99 & 214 \\
\hline Latin America and the Caribbean & 252 & 222 & NA & NA & NA & NA & NA & NA & NA & NA & NA & NA & NA & NA & NA & NA & 252 & 222 & 475 \\
\hline Middle East and North Africa & 178 & 163 & NA & NA & NA & NA & NA & NA & NA & NA & NA & NA & NA & NA & NA & NA & 178 & 163 & 341 \\
\hline South Asia & 6,799 & 6,902 & NA & NA & NA & NA & NA & NA & NA & NA & NA & NA & NA & NA & NA & NA & 6,799 & 6,902 & 13,700 \\
\hline Sub-Saharan Africa & 7,199 & 5,204 & NA & NA & NA & NA & NA & NA & NA & NA & NA & NA & NA & NA & NA & NA & 7,199 & 5,204 & 12,403 \\
\hline Low- and middle-income countries & 15,435 & 14,325 & NA & NA & NA & NA & NA & NA & NA & NA & NA & NA & NA & NA & NA & NA & 15,435 & 14,325 & 29,761 \\
\hline High-income countries & 0 & 0 & NA & NA & NA & NA & NA & NA & NA & NA & NA & NA & NA & NA & NA & NA & 0 & 0 & c \\
\hline WORLD & 15,436 & 14,325 & NA & NA & NA & NA & NA & NA & NA & $\mathrm{NA}$ & NA & NA & NA & NA & NA & NA & 15,436 & 14,325 & 29,761 \\
\hline
\end{tabular}

Source: Authors' calculations.

Note: NA = not applicable. 
Risk factor: Indoor smoke from household use of solid fuels

Disease:

Trachea, bronchus, and lung cancers

\begin{tabular}{|c|c|c|c|c|c|c|c|c|c|c|c|c|c|c|c|c|c|c|c|}
\hline \multirow[b]{2}{*}{ Region } & \multicolumn{2}{|c|}{$0-4$ years } & \multicolumn{2}{|c|}{ 5-14 years } & \multicolumn{2}{|c|}{$15-29$ years } & \multicolumn{2}{|c|}{$30-44$ years } & \multicolumn{2}{|c|}{$45-59$ years } & \multicolumn{2}{|c|}{$60-69$ years } & \multicolumn{2}{|c|}{ 70-79 years } & \multicolumn{2}{|c|}{$80+$ years } & \multicolumn{3}{|c|}{ Total } \\
\hline & Male & Female & Male & Female & Male & Female & Male & Female & Male & Female & Male & Female & Male & Female & Male & Female & Male & Female & All \\
\hline \multicolumn{20}{|l|}{ PAF of Mortality (\%) } \\
\hline East Asia and Pacific & NA & NA & NA & NA & NA & NA & 3 & 5 & 3 & 5 & 3 & 5 & 3 & 5 & 3 & 5 & 3 & 5 & 4 \\
\hline Europe and Central Asia & NA & NA & NA & NA & NA & NA & 0 & 0 & 0 & 0 & 0 & 0 & 0 & 0 & 0 & 0 & 0 & 0 & 0 \\
\hline Latin America and the Caribbean & NA & NA & NA & NA & NA & NA & 0 & 0 & 0 & 0 & 0 & 0 & 0 & 0 & 0 & 0 & 0 & 0 & 0 \\
\hline Middle East and North Africa & NA & NA & NA & NA & NA & NA & 0 & 0 & 0 & 0 & 0 & 0 & 0 & 0 & 0 & 0 & 0 & 0 & 0 \\
\hline South Asia & NA & NA & NA & NA & NA & NA & 1 & 2 & 1 & 2 & 1 & 2 & 1 & 2 & 1 & 3 & 1 & 2 & 2 \\
\hline Sub-Saharan Africa & NA & NA & NA & NA & NA & NA & 0 & 0 & 0 & 0 & 0 & 0 & 0 & 0 & 0 & 0 & 0 & 0 & 0 \\
\hline Low- and middle-income countries & NA & NA & NA & NA & NA & NA & 2 & 3 & 2 & 3 & 2 & 3 & 2 & 4 & 2 & 3 & 2 & 3 & 2 \\
\hline High-income countries & NA & NA & NA & NA & NA & NA & 0 & 0 & 0 & 0 & 0 & 0 & 0 & 0 & 0 & 0 & 0 & 0 & 0 \\
\hline WORLD & NA & NA & NA & NA & NA & NA & 1 & 2 & 1 & 2 & 1 & 2 & 1 & 2 & 1 & 1 & 1 & 2 & 1 \\
\hline
\end{tabular}

PAF of YLL (\%)

\begin{tabular}{|c|c|c|c|c|c|c|c|c|c|c|c|c|c|c|c|c|c|c|c|}
\hline East Asia and Pacific & NA & NA & NA & NA & NA & NA & 3 & 5 & 3 & 5 & 3 & 5 & 3 & 5 & 3 & 5 & 3 & 5 & 4 \\
\hline Europe and Central Asia & NA & NA & NA & NA & NA & NA & 0 & 0 & 0 & 0 & 0 & 0 & 0 & 0 & 0 & 0 & 0 & ( & 0 \\
\hline Latin America and the Caribbean & NA & NA & NA & NA & NA & NA & 0 & 0 & 0 & 0 & 0 & 0 & 0 & 0 & 0 & 0 & 0 & ( & 0 \\
\hline Middle East and North Africa & NA & NA & NA & NA & NA & NA & 0 & 0 & 0 & 0 & 0 & 0 & 0 & 0 & 0 & 0 & 0 & ( & 0 \\
\hline South Asia & NA & NA & NA & NA & NA & NA & 1 & 2 & 1 & 2 & 1 & 2 & 1 & 2 & 1 & 3 & 1 & 2 & 2 \\
\hline Sub-Saharan Africa & NA & NA & NA & NA & NA & NA & 0 & 0 & 0 & 0 & 0 & 0 & 0 & 0 & 0 & 0 & 0 & ( & 0 \\
\hline Low- and middle-income countries & NA & NA & NA & NA & NA & NA & 2 & 3 & 2 & 3 & 2 & 3 & 2 & 4 & 2 & 3 & 2 & 3 & 2 \\
\hline High-income countries & NA & NA & NA & NA & NA & NA & 0 & 0 & 0 & 0 & 0 & 0 & 0 & 0 & 0 & 0 & 0 & 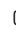 & 0 \\
\hline WORLD & NA & NA & NA & NA & NA & NA & 1 & 2 & 1 & 2 & 1 & 2 & 1 & 2 & 1 & 1 & 1 & 2 & 1 \\
\hline
\end{tabular}

\section{PAF of DALYs (\%)}

Latin America and the Caribbean

Middle East and North Afric

South Asia

Sub-Saharan Africa

Low- and middle-income countries

High-income countries

$\begin{array}{lllllllll}\text { NA } & \text { NA } & \text { NA } & \text { NA } & \text { NA } & \text { NA } & 3 & 5 & 3 \\ \text { NA } & \text { NA } & \text { NA } & \text { NA } & \text { NA } & \text { NA } & 0 & 0 & 0 \\ \text { NA } & \text { NA } & \text { NA } & \text { NA } & \text { NA } & \text { NA } & 0 & 0 & 0 \\ \text { NA } & \text { NA } & \text { NA } & \text { NA } & \text { NA } & \text { NA } & 0 & 0 & 0 \\ \text { NA } & \text { NA } & \text { NA } & \text { NA } & \text { NA } & \text { NA } & 1 & 2 & 1 \\ \text { NA } & \text { NA } & \text { NA } & \text { NA } & \text { NA } & \text { NA } & 0 & 0 & 0 \\ \text { NA } & \text { NA } & \text { NA } & \text { NA } & \text { NA } & \text { NA } & 2 & 3 & 2\end{array}$

\begin{tabular}{ll}
5 & 3 \\
0 & 0 \\
0 & 0 \\
0 & 0 \\
2 & 1 \\
0 & 0 \\
\hline 3 & 2
\end{tabular}

WORLD

NA NA

NA NA

Attributable Mortality (thousands)

\begin{tabular}{|c|c|c|c|c|c|c|c|c|c|c|c|c|c|c|c|c|c|c|c|}
\hline East Asia and Pacific & NA & NA & NA & NA & NA & NA & 0 & 0 & 2 & 1 & 3 & 2 & 2 & 2 & 1 & 1 & 8 & 6 & 14 \\
\hline Europe and Central Asia & NA & NA & NA & NA & NA & NA & 0 & 0 & 0 & 0 & 0 & 0 & 0 & 0 & 0 & 0 & 0 & 0 & 0 \\
\hline Latin America and the Caribbean & NA & NA & NA & NA & NA & NA & 0 & 0 & 0 & 0 & 0 & 0 & 0 & 0 & 0 & 0 & 0 & 0 & 0 \\
\hline Middle East and North Africa & NA & NA & NA & NA & NA & NA & 0 & 0 & 0 & 0 & 0 & 0 & 0 & 0 & 0 & 0 & 0 & 0 & 0 \\
\hline South Asia & NA & NA & NA & NA & NA & NA & 0 & 0 & 0 & 0 & 1 & 0 & 0 & 0 & 0 & 0 & 1 & 1 & 2 \\
\hline Sub-Saharan Africa & NA & NA & NA & NA & NA & NA & 0 & 0 & 0 & 0 & 0 & 0 & 0 & 0 & 0 & 0 & 0 & 0 & 0 \\
\hline Low- and middle-income countries & NA & NA & NA & NA & NA & NA & 0 & 0 & 2 & 2 & 3 & 2 & 3 & 2 & 1 & 1 & 9 & 7 & 16 \\
\hline High-income countries & NA & NA & NA & NA & NA & NA & 0 & 0 & 0 & 0 & 0 & 0 & 0 & 0 & 0 & 0 & 0 & 0 & 0 \\
\hline WORLD & NA & NA & NA & NA & NA & NA & 0 & 0 & 2 & 2 & 3 & 2 & 3 & 2 & 1 & 1 & 9 & 7 & 16 \\
\hline
\end{tabular}

Attributable YLL (thousands)

East Asia and Pacific

Europe and Central Asia

Latin America and the Caribbean

Middle East and North Africa

South Asia

NA NA NA NA NA NA

Sub-Saharan Africa NA NA NA NA

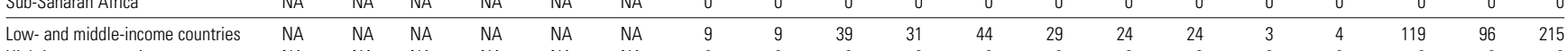

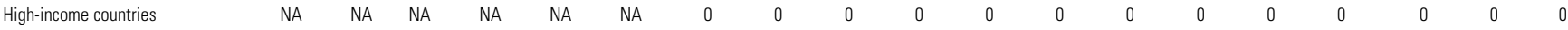

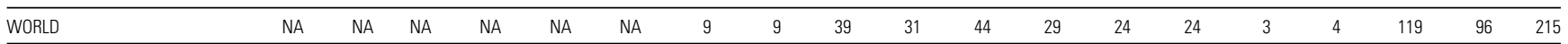

Attributable DALYs (thousands)

East Asia and Pacific

Europe and Central Asia

Latin America and the Caribbean

Middle East and North Africa

South Asia

Sub-Saharan Africa

NA NA NA NA NA NA

NA NA NA NA NA NA

NA NA NA NA NA NA NA

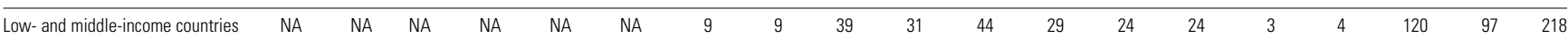

\begin{tabular}{llllllllrrrrrrrrrr} 
High-income countries & NA & NA & NA & NA & NA & NA & 0 & 0 & 0 & 0 & 0 & 0 & 0 & 0 & 0 & 0 & 0 \\
\hline
\end{tabular}


Risk factor: Indoor smoke from household use of solid fuels

Disease: Chronic obstructive pulmonary disease

\begin{tabular}{|c|c|c|c|c|c|c|c|c|c|c|c|c|c|c|c|c|c|c|c|}
\hline \multirow[b]{2}{*}{ Region } & \multicolumn{2}{|c|}{$0-4$ years } & \multicolumn{2}{|c|}{ 5-14 years } & \multicolumn{2}{|c|}{$15-29$ years } & \multicolumn{2}{|c|}{ 30-44 years } & \multicolumn{2}{|c|}{ 45-59 years } & \multicolumn{2}{|c|}{$60-69$ years } & \multicolumn{2}{|c|}{ 70-79 years } & \multicolumn{2}{|c|}{$80+$ years } & \multicolumn{3}{|c|}{ Total } \\
\hline & Male & Female & Male & Female & Male & Female & Male & Female & Male & Female & Male & Female & Male & Female & Male & Female & Male & Female & All \\
\hline \multicolumn{20}{|l|}{ PAF of Mortality (\%) } \\
\hline East Asia and Pacific & NA & NA & NA & NA & NA & NA & 21 & 43 & 21 & 42 & 21 & 43 & 20 & 42 & 21 & 41 & 21 & 41 & 32 \\
\hline Europe and Central Asia & NA & NA & NA & NA & NA & NA & 10 & 24 & 8 & 20 & 9 & 19 & 8 & 18 & 8 & 18 & 8 & 18 & 12 \\
\hline Latin America and the Caribbean & NA & NA & NA & NA & NA & NA & 18 & 38 & 18 & 38 & 18 & 37 & 17 & 36 & 17 & 35 & 17 & 35 & 25 \\
\hline Middle East and North Africa & NA & NA & NA & NA & NA & NA & 7 & 18 & 6 & 17 & 7 & 17 & 7 & 17 & 7 & 17 & 7 & 17 & 11 \\
\hline South Asia & NA & NA & NA & NA & NA & NA & 40 & 65 & 40 & 64 & 40 & 64 & 40 & 64 & 40 & 64 & 40 & 64 & 51 \\
\hline Sub-Saharan Africa & NA & NA & NA & NA & NA & NA & 40 & 65 & 40 & 65 & 40 & 65 & 40 & 65 & 40 & 64 & 40 & 63 & 48 \\
\hline Low- and middle-income countries & NA & NA & NA & NA & NA & NA & 31 & 54 & 31 & 55 & 27 & 49 & 24 & 45 & 23 & 42 & 26 & 46 & 35 \\
\hline High-income countries & NA & NA & NA & NA & NA & NA & 0 & 0 & 0 & 0 & 0 & 0 & 0 & 0 & 0 & 0 & 0 & 0 & 0 \\
\hline WORLD & NA & NA & NA & NA & NA & NA & 30 & 52 & 30 & 53 & 25 & 46 & 21 & 41 & 18 & 36 & 22 & 41 & 32 \\
\hline
\end{tabular}

PAF of YLL (\%)

\begin{tabular}{|c|c|c|c|c|c|c|c|c|c|c|c|c|c|c|c|c|c|c|c|}
\hline East Asia and Pacific & NA & NA & NA & NA & NA & NA & 21 & 43 & 21 & 42 & 21 & 43 & 20 & 42 & 21 & 41 & 21 & 42 & 32 \\
\hline Europe and Central Asia & NA & NA & NA & NA & NA & NA & 10 & 24 & 8 & 20 & 9 & 19 & 8 & 18 & 8 & 18 & 8 & 19 & 12 \\
\hline Latin America and the Caribbean & NA & NA & NA & NA & NA & NA & 18 & 38 & 18 & 38 & 18 & 37 & 17 & 36 & 17 & 35 & 17 & 32 & 24 \\
\hline Middle East and North Africa & NA & NA & NA & NA & NA & NA & 7 & 18 & 6 & 17 & 7 & 17 & 7 & 17 & 7 & 17 & 6 & 16 & 11 \\
\hline South Asia & NA & NA & NA & NA & NA & NA & 40 & 65 & 40 & 64 & 40 & 64 & 40 & 64 & 40 & 64 & 40 & 64 & 51 \\
\hline Sub-Saharan Africa & NA & NA & NA & NA & NA & NA & 40 & 65 & 40 & 65 & 40 & 65 & 40 & 65 & 40 & 64 & 39 & 62 & 47 \\
\hline Low- and middle-income countries & NA & NA & NA & NA & NA & NA & 31 & 54 & 31 & 55 & 27 & 49 & 24 & 45 & 23 & 42 & 27 & 48 & 37 \\
\hline High-income countries & NA & NA & NA & NA & NA & NA & 0 & 0 & 0 & 0 & 0 & 0 & 0 & 0 & 0 & 0 & 0 & 0 & 0 \\
\hline WORLD & NA & NA & NA & NA & NA & NA & 30 & 52 & 30 & 53 & 25 & 46 & 21 & 41 & 19 & 37 & 24 & 44 & 34 \\
\hline
\end{tabular}

\section{PAF of DALYs (\%)}

\begin{tabular}{|c|c|c|c|c|c|c|c|c|c|c|c|c|c|c|c|c|c|c|c|}
\hline East Asia and Pacific & NA & NA & NA & NA & NA & NA & 21 & 43 & 21 & 42 & 21 & 43 & 20 & 42 & 21 & 41 & 21 & 42 & 31 \\
\hline Europe and Central Asia & NA & NA & NA & NA & NA & NA & 10 & 24 & 8 & 20 & 9 & 19 & 8 & 18 & 8 & 18 & 8 & 19 & 13 \\
\hline Latin America and the Caribbean & NA & NA & NA & NA & NA & NA & 18 & 38 & 18 & 38 & 18 & 37 & 17 & 36 & 17 & 35 & 17 & 34 & 25 \\
\hline Middle East and North Africa & NA & NA & NA & NA & NA & NA & 7 & 18 & 6 & 17 & 7 & 17 & 7 & 17 & 7 & 17 & 6 & 16 & 10 \\
\hline South Asia & NA & NA & NA & NA & NA & NA & 40 & 65 & 40 & 64 & 40 & 64 & 40 & 64 & 40 & 64 & 40 & 64 & 51 \\
\hline Sub-Saharan Africa & NA & NA & NA & NA & NA & NA & 40 & 65 & 40 & 65 & 40 & 65 & 40 & 65 & 40 & 64 & 38 & 62 & 46 \\
\hline Low- and middle-income countries & NA & NA & NA & NA & NA & NA & 26 & 44 & 28 & 53 & 26 & 48 & 24 & 45 & 23 & 42 & 25 & 46 & 35 \\
\hline High-income countries & NA & NA & NA & NA & NA & NA & 0 & 0 & 0 & 0 & 0 & 0 & 0 & 0 & 0 & 0 & 0 & 0 & 0 \\
\hline NORLD & NA & NA & NA & NA & NA & NA & 22 & 33 & 24 & 45 & 23 & 43 & 20 & 41 & 19 & 37 & 22 & 40 & 30 \\
\hline
\end{tabular}

Attributable Mortality (thousands)

\begin{tabular}{|c|c|c|c|c|c|c|c|c|c|c|c|c|c|c|c|c|c|c|c|}
\hline East Asia and Pacific & NA & NA & NA & NA & NA & NA & 0 & 1 & 10 & 15 & 28 & 47 & 56 & 118 & 40 & 136 & 135 & 316 & 451 \\
\hline Europe and Central Asia & NA & NA & NA & NA & NA & NA & 0 & 0 & 1 & 1 & 2 & 1 & 3 & 3 & 1 & 3 & 7 & 9 & 16 \\
\hline Latin America and the Caribbean & NA & NA & NA & NA & NA & NA & 0 & 0 & 1 & 1 & 2 & 3 & 3 & 4 & 4 & 6 & 10 & 14 & 24 \\
\hline Middle East and North Africa & NA & NA & NA & NA & NA & NA & 0 & 0 & 0 & 0 & 0 & 1 & 1 & 1 & 0 & 1 & 2 & 3 & 4 \\
\hline South Asia & NA & NA & NA & NA & NA & NA & 3 & 4 & 36 & 46 & 39 & 40 & 35 & 51 & 14 & 25 & 126 & 167 & 293 \\
\hline Sub-Saharan Africa & NA & NA & NA & NA & NA & NA & 1 & 1 & 5 & 4 & 8 & 6 & 10 & 10 & 5 & 6 & 29 & 26 & 56 \\
\hline Low- and middle-income countries & NA & NA & NA & NA & NA & NA & 5 & 7 & 53 & 68 & 80 & 97 & 108 & 186 & 64 & 177 & 309 & 535 & 844 \\
\hline High-income countries & NA & NA & NA & NA & NA & NA & 0 & 0 & 0 & 0 & 0 & 0 & 0 & 0 & 0 & 0 & 0 & 0 & 0 \\
\hline WORLD & NA & NA & NA & NA & NA & NA & 5 & 7 & 53 & 68 & 80 & 97 & 108 & 186 & 64 & 177 & 309 & 535 & 844 \\
\hline
\end{tabular}

Attributable YLL (thousands)

\begin{tabular}{|c|c|c|c|c|c|c|c|c|c|c|c|c|c|c|c|c|c|c|c|}
\hline East Asia and Pacific & NA & NA & NA & NA & NA & NA & 11 & 33 & 173 & 290 & 374 & 695 & 493 & 1,189 & 201 & 686 & 1,253 & 2,892 & 4,145 \\
\hline Europe and Central Asia & NA & NA & NA & NA & NA & NA & 5 & 4 & 16 & 12 & 29 & 21 & 24 & 33 & 5 & 15 & 79 & 85 & 164 \\
\hline Latin America and the Caribbean & NA & NA & NA & NA & NA & NA & 4 & 7 & 15 & 24 & 26 & 38 & 31 & 44 & 16 & 28 & 92 & 142 & 234 \\
\hline Middle East and North Africa & NA & NA & NA & NA & NA & NA & 1 & 2 & 3 & 7 & 5 & 9 & 5 & 11 & 2 & 4 & 17 & 34 & 50 \\
\hline South Asia & NA & NA & NA & NA & NA & NA & 64 & 104 & 653 & 909 & 535 & 600 & 314 & 531 & 68 & 136 & 1,633 & 2,281 & 3,914 \\
\hline Sub-Saharan Africa & NA & NA & NA & NA & NA & NA & 32 & 17 & 95 & 79 & 107 & 95 & 89 & 98 & 24 & 32 & 347 & 322 & 669 \\
\hline Low- and middle-income countries & NA & NA & NA & NA & NA & NA & 118 & 167 & 955 & 1,322 & 1,077 & 1,459 & 956 & 1,907 & 315 & 902 & 3,421 & 5,756 & 9,177 \\
\hline High-income countries & NA & NA & NA & NA & NA & NA & 0 & 0 & 0 & 0 & 0 & 0 & 0 & 0 & 0 & 0 & 0 & 1 & 1 \\
\hline NORLD & NA & NA & NA & NA & NA & NA & 118 & 167 & 955 & 1,322 & 1,077 & 1,459 & 956 & 1,907 & 316 & 902 & 3,421 & 5,756 & 9,178 \\
\hline
\end{tabular}

Attributable DALYs (thousands)

\begin{tabular}{|c|c|c|c|c|c|c|c|c|c|c|c|c|c|c|c|c|c|c|c|}
\hline East Asia and Pacific & NA & NA & NA & NA & NA & NA & 66 & 158 & 483 & 410 & 509 & 845 & 555 & 1,315 & 222 & 721 & 1,836 & 3,448 & 5,284 \\
\hline Europe and Central Asia & NA & NA & NA & NA & NA & NA & 15 & 51 & 26 & 49 & 37 & 37 & 29 & 39 & 6 & 17 & 113 & 193 & 306 \\
\hline Latin America and the Caribbean & NA & NA & NA & NA & NA & NA & 23 & 98 & 54 & 84 & 46 & 61 & 39 & 56 & 19 & 32 & 181 & 330 & 512 \\
\hline Middle East and North Africa & NA & NA & NA & NA & NA & NA & 12 & 10 & 7 & 11 & 6 & 10 & 6 & 11 & 2 & 4 & 33 & 47 & 80 \\
\hline South Asia & NA & NA & NA & NA & NA & NA & 179 & 225 & 784 & 1,227 & 631 & 659 & 337 & 560 & 72 & 143 & 2,003 & 2,814 & 4,817 \\
\hline Sub-Saharan Africa & NA & NA & NA & NA & NA & NA & 45 & 21 & 118 & 91 & 124 & 102 & 94 & 102 & 25 & 33 & 407 & 349 & 755 \\
\hline Low- and middle-income countries & NA & NA & NA & NA & NA & NA & 340 & 562 & 1,474 & 1,872 & 1,353 & 1,714 & 1,059 & 2,083 & 346 & 950 & 4,572 & 7,181 & 11,753 \\
\hline High-income countries & NA & NA & NA & NA & NA & NA & 0 & 0 & 0 & 1 & 0 & 0 & 0 & 0 & 0 & 0 & 1 & 2 & 2 \\
\hline WORLD & NA & NA & NA & NA & NA & NA & 340 & 562 & 1,474 & 1,872 & 1,354 & 1,715 & 1,060 & 2,083 & 346 & 950 & 4,573 & 7,182 & 11,755 \\
\hline
\end{tabular}

Source: Authors' calculations.

Note: $\mathrm{NA}=$ not applicable. 
Risk factor:

Disease:

Indoor smoke from household use of solid fuels

All causes

\begin{tabular}{|c|c|c|c|c|c|c|c|c|c|c|c|c|c|c|c|c|c|c|c|}
\hline \multirow[b]{2}{*}{ Region } & \multicolumn{2}{|c|}{$0-4$ years } & \multicolumn{2}{|c|}{ 5-14 years } & \multicolumn{2}{|c|}{$15-29$ years } & \multicolumn{2}{|c|}{ 30-44 years } & \multicolumn{2}{|c|}{$45-59$ years } & \multicolumn{2}{|c|}{$60-69$ years } & \multicolumn{2}{|c|}{ 70-79 years } & \multicolumn{2}{|c|}{$80+$ years } & \multicolumn{3}{|c|}{ Total } \\
\hline & Male & Female & Male & Female & Male & Female & Male & Female & Male & Female & Male & Female & Male & Female & Male & Female & Male & Female & All \\
\hline \multicolumn{20}{|l|}{ PAF of Mortality (\%) } \\
\hline East Asia and Pacific & 4 & 7 & NA & NA & NA & NA & 0 & 0 & 1 & 2 & 2 & 5 & 4 & 8 & 5 & 9 & 2 & 6 & 4 \\
\hline Europe and Central Asia & 4 & 4 & NA & NA & NA & NA & 0 & 0 & 0 & 0 & 0 & 0 & 0 & 0 & 0 & 0 & 0 & 0 & 0 \\
\hline Latin America and the Caribbean & 3 & 3 & NA & NA & NA & NA & 0 & 0 & 0 & 1 & 1 & 1 & 1 & 1 & 1 & 2 & 1 & 1 & 1 \\
\hline Middle East and North Africa & 2 & 2 & NA & NA & NA & NA & 0 & 0 & 0 & 0 & 0 & 1 & 0 & 1 & 0 & 1 & 1 & 1 & 1 \\
\hline South Asia & 12 & 12 & NA & NA & NA & NA & 0 & 1 & 3 & 6 & 4 & 4 & 3 & 5 & 3 & 4 & 5 & 6 & 5 \\
\hline Sub-Saharan Africa & 10 & 8 & NA & NA & NA & NA & 0 & 0 & 1 & 1 & 2 & 2 & 3 & 3 & 3 & 3 & 5 & 4 & 4 \\
\hline Low- and middle-income countries & 9 & 9 & NA & NA & NA & NA & 0 & 0 & 1 & 3 & 2 & 3 & 3 & 4 & 3 & 5 & 3 & 4 & 4 \\
\hline High-income countries & 0 & 0 & NA & NA & NA & NA & 0 & 0 & 0 & 0 & 0 & 0 & 0 & 0 & 0 & 0 & 0 & 0 & 0 \\
\hline WORLD & 9 & 9 & NA & NA & NA & NA & 0 & 0 & 1 & 2 & 2 & 3 & 2 & 4 & 2 & 3 & 3 & 4 & 3 \\
\hline
\end{tabular}

PAF of YLL (\%)

\begin{tabular}{|c|c|c|c|c|c|c|c|c|c|c|c|c|c|c|c|c|c|c|}
\hline \multirow{2}{*}{$\begin{array}{l}\text { East Asia and Pacific } \\
\text { Europe and Central Asia }\end{array}$} & 4 & 7 & NA & NA & NA & NA & 0 & 0 & 1 & 2 & 2 & 5 & 4 & 8 & 5 & 9 & 2 & 5 \\
\hline & 4 & 4 & NA & NA & NA & NA & 0 & 0 & 0 & 0 & 0 & 0 & 0 & 0 & 0 & 0 & 0 & 1 \\
\hline Latin America and the Caribbean & 3 & 3 & NA & NA & NA & NA & 0 & 0 & 0 & 1 & 1 & 1 & 1 & 1 & 1 & 2 & 1 & 1 \\
\hline Middle East and North Africa & 2 & 2 & NA & NA & NA & NA & 0 & 0 & 0 & 0 & 0 & 1 & 0 & 1 & 0 & 1 & 1 & 1 \\
\hline South Asia & 12 & 12 & NA & NA & NA & NA & 0 & 1 & 3 & 6 & 4 & 4 & 3 & 5 & 3 & 4 & 6 & 7 \\
\hline Sub-Saharan Africa & 10 & 8 & NA & NA & NA & NA & 0 & 0 & 1 & 1 & 2 & 2 & 3 & 2 & 3 & 3 & 5 & 4 \\
\hline Low- and middle-income countries & 9 & 9 & NA & NA & NA & NA & 0 & 0 & 1 & 3 & 2 & 3 & 3 & 4 & 3 & 5 & 4 & 5 \\
\hline High-income countries & 0 & 0 & NA & NA & NA & NA & 0 & 0 & 0 & 0 & 0 & 0 & 0 & 0 & 0 & 0 & 0 & 0 \\
\hline WORLD & 9 & 9 & NA & NA & NA & NA & 0 & 0 & 1 & 2 & 2 & 3 & 2 & 4 & 2 & 3 & 3 & 4 \\
\hline
\end{tabular}

PAF of DALYs $(\%)$

Latin America and the Caribbean

Middle East and North Africa

South Asia

Sub-Saharan Africa

Low- and middle-income countries

High-income countries

$\begin{array}{lll}N A & N A & N A \\ N A & N A & N A\end{array}$

NA
NA
NA

0

$\begin{array}{llllllllllll}1 & 1 & 1 & 2 & 4 & 3 & 6 & 4 & 8 & 2 & 3 & 2 \\ 1 & 0 & 1 & 0 & 0 & 0 & 0 & 0 & 0 & 0 & 1 & 0 \\ 1 & 1 & 1 & 1 & 1 & 1 & 1 & 1 & 1 & 1 & 1 & 1 \\ 0 & 0 & 0 & 0 & 0 & 0 & 0 & 0 & 0 & 1 & 1 & 1 \\ 1 & 3 & 5 & 3 & 3 & 3 & 4 & 2 & 4 & 4 & 5 & 5 \\ 0 & 1 & 1 & 2 & 1 & 2 & 2 & 2 & 2 & 4 & 3 & 4 \\ 1 & 1 & 2 & 2 & 3 & 2 & 4 & 2 & 4 & 3 & 3 & 3 \\ 0 & 0 & 0 & 0 & 0 & 0 & 0 & 0 & 0 & 0 & 0 & 0\end{array}$

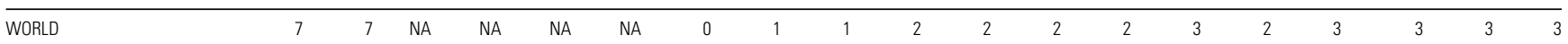

Attributable Mortality (thousands)

\begin{tabular}{|c|c|c|c|c|c|c|c|c|c|c|c|c|c|c|c|c|c|c|c|}
\hline East Asia and Pacific & 26 & 50 & NA & NA & NA & NA & 1 & 2 & 11 & 16 & 31 & 48 & 58 & 120 & 41 & 136 & 168 & 373 & 540 \\
\hline Europe and Central Asia & 4 & 3 & NA & NA & NA & NA & 0 & 0 & 1 & 1 & 2 & 1 & 3 & 3 & 1 & 3 & 11 & 12 & 22 \\
\hline Latin America and the Caribbean & 7 & 6 & NA & NA & NA & NA & 0 & 0 & 1 & 1 & 2 & 3 & 3 & 4 & 4 & 6 & 17 & 20 & 37 \\
\hline Middle East and North Africa & 5 & 5 & NA & NA & NA & NA & 0 & 0 & 0 & 0 & 0 & 1 & 1 & 1 & 0 & 1 & 7 & 8 & 15 \\
\hline South Asia & 213 & 215 & NA & NA & NA & NA & 3 & 4 & 36 & 47 & 40 & 40 & 35 & 51 & 14 & 26 & 341 & 383 & 724 \\
\hline Sub-Saharan Africa & 230 & 167 & NA & NA & NA & NA & 1 & 1 & 5 & 4 & 8 & 6 & 10 & 10 & 5 & 6 & 259 & 194 & 453 \\
\hline Low- and middle-income countries & 485 & 447 & NA & NA & NA & NA & 5 & 7 & 55 & 69 & 83 & 99 & 110 & 189 & 64 & 177 & 802 & 988 & 1,791 \\
\hline High-income countries & 0 & 0 & NA & NA & NA & NA & 0 & 0 & 0 & 0 & 0 & 0 & 0 & 0 & 0 & 0 & 0 & 0 & 0 \\
\hline WORLD & 485 & 447 & NA & NA & NA & NA & 5 & 7 & 55 & 69 & 83 & 99 & 110 & 189 & 64 & 177 & 802 & 989 & 1,791 \\
\hline
\end{tabular}

Attributable YLL (thousands)

\begin{tabular}{|c|c|c|c|c|c|c|c|c|c|c|c|c|c|c|c|c|c|c|c|}
\hline East Asia and Pacific & 776 & 1,530 & NA & NA & NA & NA & 19 & 40 & 205 & 317 & 411 & 721 & 514 & 1,212 & 204 & 690 & 2,128 & 4,510 & 6,638 \\
\hline Europe and Central Asia & 110 & 93 & NA & NA & NA & NA & 5 & 4 & 16 & 12 & 29 & 21 & 24 & 33 & 5 & 15 & 189 & 178 & 367 \\
\hline Latin America and the Caribbean & 199 & 170 & NA & NA & NA & NA & 4 & 7 & 15 & 24 & 26 & 38 & 31 & 44 & 16 & 28 & 291 & 313 & 604 \\
\hline Middle East and North Africa & 164 & 150 & NA & NA & NA & NA & 1 & 2 & 3 & 7 & 5 & 9 & 5 & 11 & 2 & 4 & 180 & 184 & 364 \\
\hline South Asia & 6,454 & 6,566 & NA & NA & NA & NA & 66 & 105 & 659 & 912 & 542 & 603 & 317 & 533 & 68 & 137 & 8,106 & 8,855 & 16,961 \\
\hline Sub-Saharan Africa & 6,961 & 5,098 & NA & NA & NA & NA & 32 & 17 & 95 & 79 & 107 & 95 & 89 & 98 & 24 & 32 & 7,308 & 5,420 & 12,729 \\
\hline Low- and middle-income countries & 14,663 & 13,608 & NA & NA & NA & NA & 127 & 175 & 994 & 1,352 & 1,120 & 1,488 & 980 & 1,931 & 319 & 906 & 18,203 & 19,460 & 37,663 \\
\hline High-income countries & 0 & 0 & NA & NA & NA & NA & 0 & 0 & 0 & 0 & 0 & 0 & 0 & 0 & 0 & 0 & 0 & 1 & 1 \\
\hline IORLD & 14,663 & 13,608 & NA & NA & NA & NA & 127 & 175 & 994 & 1,352 & 1,120 & 1,488 & 980 & 1,931 & 319 & 906 & 18,203 & 19,461 & 37,664 \\
\hline
\end{tabular}

Attributable DALYs (thousands)

\begin{tabular}{|c|c|c|c|c|c|c|c|c|c|c|c|c|c|c|c|c|c|c|c|}
\hline East Asia and Pacific & 892 & 1,734 & NA & NA & NA & NA & 74 & 165 & 516 & 437 & 546 & 871 & 576 & 1,338 & 225 & 725 & 2,828 & 5,271 & 8,100 \\
\hline Europe and Central Asia & 115 & 99 & NA & NA & NA & NA & 15 & 51 & 26 & 49 & 37 & 37 & 29 & 39 & 6 & 17 & 228 & 292 & 520 \\
\hline Latin America and the Caribbean & 252 & 222 & NA & NA & NA & NA & 23 & 98 & 54 & 84 & 46 & 61 & 39 & 56 & 19 & 32 & 434 & 553 & 986 \\
\hline Middle East and North Africa & 178 & 163 & NA & NA & NA & NA & 12 & 10 & 7 & 11 & 6 & 10 & 6 & 11 & 2 & 4 & 211 & 210 & 421 \\
\hline South Asia & 6,799 & 6,902 & NA & NA & NA & NA & 180 & 226 & 790 & 1,230 & 638 & 662 & 340 & 562 & 73 & 144 & 8,821 & 9,724 & 18,545 \\
\hline Sub-Saharan Africa & 7,199 & 5,204 & NA & NA & NA & NA & 45 & 21 & 118 & 91 & 124 & 102 & 94 & 102 & 25 & 33 & 7,606 & 5,553 & 13,159 \\
\hline Low- and middle-income countries & 15,435 & 14,325 & NA & NA & NA & NA & 349 & 570 & 1,513 & 1,903 & 1,398 & 1,744 & 1,084 & 2,107 & 349 & 954 & 20,128 & 21,603 & 41,731 \\
\hline High-income countries & 0 & 0 & NA & NA & NA & NA & 0 & 0 & 0 & 1 & 0 & 0 & 0 & 0 & 0 & 0 & 1 & 2 & 2 \\
\hline WORLD & 15,436 & 14,325 & NA & NA & NA & NA & 349 & 571 & 1,513 & 1,903 & 1,398 & 1,744 & 1,084 & 2,107 & 349 & 954 & 20,129 & 21,605 & 41,734 \\
\hline
\end{tabular}

Source: Authors' calculations.

Note: $\mathrm{NA}=$ not applicable. 
Risk factor: Contaminated injections in health care setting

Disease:

HIV/AIDS

\begin{tabular}{|c|c|c|c|c|c|c|c|c|c|c|c|c|c|c|c|c|c|c|c|}
\hline \multirow[b]{2}{*}{ Region } & \multicolumn{2}{|c|}{$0-4$ years } & \multicolumn{2}{|c|}{ 5-14 years } & \multicolumn{2}{|c|}{$15-29$ years } & \multicolumn{2}{|c|}{$30-44$ years } & \multicolumn{2}{|c|}{$45-59$ years } & \multicolumn{2}{|c|}{$60-69$ years } & \multicolumn{2}{|c|}{$70-79$ years } & \multicolumn{2}{|c|}{$80+$ years } & \multicolumn{3}{|c|}{ Total } \\
\hline & Male & Female & Male & Female & Male & Female & Male & Female & Male & Female & Male & Female & Male & Female & Male & Female & Male & Female & All \\
\hline \multicolumn{20}{|l|}{ PAF of Mortality (\%) } \\
\hline East Asia and Pacific & 29 & 30 & 20 & 20 & 5 & 10 & 5 & 9 & 5 & 10 & 5 & 0 & 5 & 0 & 2 & 0 & 6 & 10 & 7 \\
\hline Europe and Central Asia & 0 & 0 & 0 & 0 & 0 & 1 & 0 & 2 & 0 & 2 & 0 & 2 & 0 & 2 & 0 & 0 & 0 & 2 & 0 \\
\hline Latin America and the Caribbean & 4 & 4 & 3 & 4 & 0 & 1 & 0 & 1 & 0 & 1 & 0 & 1 & 0 & 1 & 0 & 1 & 1 & 1 & 1 \\
\hline Middle East and North Africa & 5 & 5 & 4 & 4 & 4 & 4 & 3 & 4 & 3 & 4 & 3 & 4 & 3 & 4 & 3 & 3 & 4 & 4 & 4 \\
\hline South Asia & 55 & 56 & 50 & 58 & 14 & 24 & 14 & 23 & 13 & 23 & 14 & 11 & 14 & 11 & 5 & 11 & 15 & 28 & 18 \\
\hline Sub-Saharan Africa & 9 & 9 & 6 & 8 & 2 & 1 & 2 & 1 & 2 & 1 & 2 & 1 & 2 & 1 & 2 & 1 & 3 & 3 & 3 \\
\hline Low- and middle-income countries & 11 & 11 & 8 & 9 & 4 & 3 & 4 & 3 & 4 & 3 & 3 & 1 & 4 & 1 & 2 & 0 & 5 & 4 & 5 \\
\hline High-income countries & 0 & 0 & 0 & 0 & 0 & 1 & 0 & 0 & 0 & 0 & 0 & 0 & 0 & 0 & 0 & 0 & 0 & 0 & 0 \\
\hline WORLD & 11 & 11 & 8 & 9 & 4 & 3 & 4 & 3 & 4 & 3 & 3 & 1 & 3 & 1 & 1 & 0 & 5 & 4 & 5 \\
\hline
\end{tabular}

PAF of YLL (\%)

\begin{tabular}{|c|c|c|c|c|c|c|c|c|c|c|c|c|c|c|c|c|c|c|c|}
\hline East Asia and Pacific & 29 & 30 & 20 & 20 & 5 & 10 & 5 & 9 & 5 & 10 & 5 & 0 & 5 & 0 & 2 & 0 & 6 & 11 & 8 \\
\hline Europe and Central Asia & 0 & 0 & 0 & 0 & 0 & 1 & 0 & 2 & 0 & 2 & 0 & 2 & 0 & 2 & 0 & 0 & 0 & 2 & 0 \\
\hline Latin America and the Caribbean & 4 & 4 & 3 & 4 & 0 & 1 & 0 & 1 & 0 & 1 & 0 & 1 & 0 & 1 & 0 & 1 & 1 & 1 & 1 \\
\hline Middle East and North Africa & 5 & 5 & 4 & 4 & 4 & 4 & 3 & 4 & 3 & 4 & 3 & 4 & 3 & 4 & 3 & 3 & 4 & 4 & 4 \\
\hline South Asia & 55 & 56 & 50 & 58 & 14 & 24 & 14 & 23 & 13 & 23 & 14 & 11 & 14 & 11 & 5 & 11 & 16 & 29 & 19 \\
\hline Sub-Saharan Africa & 9 & 9 & 6 & 8 & 2 & 1 & 2 & 1 & 2 & 1 & 2 & 1 & 2 & 1 & 2 & 1 & 3 & 3 & 3 \\
\hline Low- and middle-income countries & 11 & 11 & 8 & 9 & 4 & 3 & 4 & 3 & 4 & 3 & 3 & 1 & 4 & 1 & 2 & 0 & 5 & 4 & 5 \\
\hline High-income countries & 0 & 0 & 0 & 0 & 0 & 1 & 0 & 0 & 0 & 0 & 0 & 0 & 0 & 0 & 0 & 0 & 0 & 0 & 0 \\
\hline WORLD & 11 & 11 & 8 & 9 & 4 & 3 & 4 & 3 & 4 & 3 & 3 & 1 & 3 & 1 & 1 & 0 & 5 & 4 & 5 \\
\hline
\end{tabular}

\section{PAF of DALYs $(\%)$}

East Asia and Pacific

Europe and Central Asia

Latin America and the Caribbean

Middle East and North Africa

South Asia

Sub-Saharan Africa

Low- and middle-income countries

High-income countries

\begin{tabular}{rrrrrrrrrrrrrrrrrrr}
29 & 30 & 20 & 20 & 5 & 10 & 5 & 9 & 5 & 10 & 5 & 0 & 5 & 0 & 2 & 0 & 6 & 11 & 7 \\
0 & 0 & 0 & 0 & 0 & 1 & 0 & 2 & 0 & 2 & 0 & 2 & 0 & 2 & 0 & 0 & 0 & 2 & 1 \\
4 & 4 & 3 & 4 & 0 & 1 & 0 & 1 & 0 & 1 & 0 & 1 & 0 & 1 & 0 & 1 & 1 & 1 & 1 \\
5 & 5 & 4 & 4 & 4 & 4 & 3 & 4 & 3 & 4 & 3 & 4 & 3 & 4 & 3 & 3 & 4 & 4 & 4 \\
55 & 56 & 50 & 58 & 14 & 24 & 14 & 23 & 13 & 23 & 14 & 11 & 14 & 11 & 5 & 11 & 16 & 28 & 19 \\
9 & 9 & 6 & 8 & 2 & 1 & 2 & 1 & 2 & 1 & 2 & 1 & 2 & 1 & 2 & 1 & 3 & 3 & 3 \\
\hline 11 & 11 & 8 & 9 & 4 & 3 & 4 & 3 & 4 & 3 & 3 & 1 & 4 & 1 & 2 & 0 & 5 & 4 & 5 \\
0 & 0 & 0 & 0 & 0 & 1 & 0 & 0 & 0 & 0 & 0 & 0 & 0 & 0 & 0 & 0 & 0 & 0 & 0 \\
11 & 11 & 8 & 9 & 4 & 3 & 4 & 3 & 4 & 3 & 3 & 1 & 3 & 1 & 1 & 0 & 5 & 4 & 5
\end{tabular}

Attributable Mortality (thousands)

\begin{tabular}{|c|c|c|c|c|c|c|c|c|c|c|c|c|c|c|c|c|c|c|c|}
\hline \multicolumn{20}{|l|}{ Attributable Mortality (thousands) } \\
\hline East Asia and Pacific & 1 & 1 & 0 & 0 & 1 & 0 & 2 & 1 & 1 & 1 & 0 & 0 & 0 & 0 & 0 & 0 & 5 & 3 & 8 \\
\hline Europe and Central Asia & 0 & 0 & 0 & 0 & 0 & 0 & 0 & 0 & 0 & 0 & 0 & 0 & 0 & 0 & 0 & 0 & 0 & 0 & 0 \\
\hline Latin America and the Caribbean & 0 & 0 & 0 & 0 & 0 & 0 & 0 & 0 & 0 & 0 & 0 & 0 & 0 & 0 & 0 & 0 & 0 & 0 & 1 \\
\hline Middle East and North Africa & 0 & 0 & 0 & 0 & 0 & 0 & 0 & 0 & 0 & 0 & 0 & 0 & 0 & 0 & 0 & 0 & 0 & 0 & 0 \\
\hline South Asia & 4 & 4 & 1 & 1 & 6 & 5 & 16 & 6 & 6 & 2 & 0 & 0 & 0 & 0 & 0 & 0 & 32 & 17 & 49 \\
\hline Sub-Saharan Africa & 14 & 14 & 3 & 4 & 4 & 5 & 8 & 5 & 3 & 1 & 0 & 0 & 0 & 0 & 0 & 0 & 33 & 28 & 61 \\
\hline Low- and middle-income countries & 19 & 18 & 4 & 5 & 10 & 10 & 26 & 12 & 9 & 4 & 1 & 0 & 0 & 0 & 0 & 0 & 70 & 49 & 119 \\
\hline High-income countries & 0 & 0 & 0 & 0 & 0 & 0 & 0 & 0 & 0 & 0 & 0 & 0 & 0 & 0 & 0 & 0 & 0 & 0 & 0 \\
\hline WORLD & 19 & 18 & 4 & 5 & 10 & 10 & 26 & 12 & 9 & 4 & 1 & 0 & 0 & 0 & 0 & 0 & 70 & 49 & 119 \\
\hline
\end{tabular}

Attributable YLL (thousands)

\begin{tabular}{|c|c|c|c|c|c|c|c|c|c|c|c|c|c|c|c|c|c|c|c|}
\hline East Asia and Pacific & 27 & 21 & 2 & 2 & 20 & 12 & 51 & 21 & 17 & 16 & 1 & 0 & 0 & 0 & 0 & 0 & 118 & 72 & 190 \\
\hline Europe and Central Asia & 0 & 0 & 0 & 0 & 0 & 1 & 1 & 1 & 0 & 0 & 0 & 0 & 0 & 0 & 0 & 0 & 2 & 2 & 3 \\
\hline Latin America and the Caribbean & 4 & 4 & 1 & 1 & 1 & 2 & 2 & 2 & 1 & 1 & 0 & 0 & 0 & 0 & 0 & 0 & 10 & 10 & 20 \\
\hline Middle East and North Africa & 0 & 0 & 0 & 0 & 1 & 1 & 1 & 0 & 0 & 0 & 0 & 0 & 0 & 0 & 0 & 0 & 2 & 2 & 4 \\
\hline South Asia & 122 & 118 & 27 & 29 & 154 & 128 & 378 & 143 & 110 & 39 & 6 & 1 & 1 & 0 & 0 & 0 & 797 & 458 & 1,255 \\
\hline Sub-Saharan Africa & 428 & 415 & 90 & 109 & 96 & 125 & 205 & 123 & 56 & 29 & 6 & 3 & 1 & 1 & 0 & 0 & 881 & 806 & 1,687 \\
\hline Low- and middle-income countries & 582 & 558 & 121 & 141 & 271 & 269 & 638 & 291 & 183 & 85 & 14 & 4 & 1 & 1 & 0 & 0 & 1,809 & 1,349 & 3,159 \\
\hline High-income countries & 0 & 0 & 0 & 0 & 0 & 0 & 0 & 0 & 0 & 0 & 0 & 0 & 0 & 0 & 0 & 0 & 0 & 0 & 1 \\
\hline WORLD & 582 & 558 & 121 & 141 & 272 & 269 & 638 & 291 & 183 & 85 & 14 & 4 & 1 & 1 & 0 & 0 & 1,810 & 1,350 & 3,159 \\
\hline
\end{tabular}

Attributable DALYs (thousands)

East Asia and Pacific

Europe and Central Asia

Latin America and the Caribbean

Middle East and North Africa

South Asia

Sub-Saharan Africa

$\begin{array}{lllllllllllllllllll}28 & 22 & 2 & 2 & 32 & 20 & 59 & 24 & 18 & 16 & 1 & 0 & 0 & 0 & 0 & 0 & 140 & 85 & 225\end{array}$

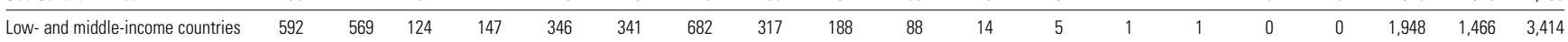

High-income countries

WORLD

Source: Authors' calculations. 
Table 4A.99

Risk factor: Contaminated injections in health care setting

Disease:

Hepatitis B

\begin{tabular}{|c|c|c|c|c|c|c|c|c|c|c|c|c|c|c|c|c|c|c|c|}
\hline \multirow[b]{2}{*}{ Region } & \multicolumn{2}{|c|}{$0-4$ years } & \multicolumn{2}{|c|}{ 5-14 years } & \multicolumn{2}{|c|}{$15-29$ years } & \multicolumn{2}{|c|}{ 30-44 years } & \multicolumn{2}{|c|}{$45-59$ years } & \multicolumn{2}{|c|}{$60-69$ years } & \multicolumn{2}{|c|}{$70-79$ years } & \multicolumn{2}{|c|}{$80+$ years } & \multicolumn{3}{|c|}{ Total } \\
\hline & Male & Female & Male & Female & Male & Female & Male & Female & Male & Female & Male & Female & Male & Female & Male & Female & Male & Female & All \\
\hline \multicolumn{20}{|l|}{ PAF of Mortality (\%) } \\
\hline East Asia and Pacific & 42 & 42 & 25 & 32 & 27 & 34 & 27 & 29 & 29 & 24 & 28 & 24 & 26 & 24 & 29 & 29 & 28 & 29 & 29 \\
\hline Europe and Central Asia & 2 & 2 & 2 & 1 & 4 & 3 & 4 & 4 & 4 & 4 & 3 & 3 & 2 & 3 & 2 & 3 & 4 & 3 & 3 \\
\hline Latin America and the Caribbean & 5 & 4 & 6 & 4 & 6 & 6 & 4 & 4 & 3 & 5 & 5 & 5 & 4 & 5 & 5 & 3 & 5 & 4 & 5 \\
\hline Middle East and North Africa & 15 & 39 & 30 & 39 & 43 & 32 & 37 & 33 & 23 & 19 & 21 & 24 & 35 & 48 & 45 & 54 & 27 & 35 & 31 \\
\hline South Asia & 52 & 53 & 52 & 52 & 52 & 52 & 51 & 52 & 52 & 51 & 51 & 50 & 51 & 52 & 52 & 52 & 51 & 52 & 52 \\
\hline Sub-Saharan Africa & 13 & 12 & 9 & 11 & 12 & 11 & 15 & 14 & 14 & 11 & 10 & 15 & 12 & 38 & 44 & 44 & 12 & 13 & 12 \\
\hline Low- and middle-income countries & 18 & 17 & 10 & 27 & 32 & 33 & 30 & 32 & 34 & 29 & 29 & 29 & 36 & 39 & 41 & 36 & 30 & 29 & 30 \\
\hline High-income countries & 23 & 37 & 8 & 6 & 11 & 5 & 8 & 4 & 6 & 2 & 6 & 2 & 1 & 0 & 1 & 2 & 5 & 2 & 4 \\
\hline WORLD & 18 & 17 & 10 & 27 & 32 & 33 & 30 & 31 & 32 & 27 & 28 & 25 & 32 & 31 & 36 & 29 & 29 & 27 & 29 \\
\hline
\end{tabular}

PAF of YLL $(\%)$

\begin{tabular}{lrrrrrrrrrrrrrrrrrrr} 
East Asia and Pacific & 42 & 42 & 25 & 32 & 27 & 34 & 27 & 29 & 29 & 24 & 28 & 24 & 26 & 24 & 29 & 29 & 28 & 30 & 29 \\
Europe and Central Asia & 2 & 2 & 2 & 1 & 4 & 3 & 4 & 4 & 4 & 4 & 3 & 3 & 2 & 3 & 2 & 3 & 4 & 3 & 3 \\
Latin America and the Caribbean & 5 & 4 & 6 & 4 & 6 & 6 & 4 & 4 & 3 & 5 & 5 & 5 & 4 & 5 & 5 & 3 & 5 & 5 & 5 \\
Middle East and North Africa & 15 & 39 & 30 & 39 & 43 & 32 & 37 & 33 & 23 & 19 & 21 & 24 & 35 & 48 & 45 & 54 & 26 & 33 & 29 \\
South Asia & 52 & 53 & 52 & 52 & 52 & 52 & 51 & 52 & 52 & 51 & 51 & 50 & 51 & 52 & 52 & 52 & 52 & 52 & 52 \\
Sub-Saharan Africa & 13 & 12 & 9 & 11 & 12 & 11 & 15 & 14 & 14 & 11 & 10 & 15 & 12 & 38 & 44 & 44 & 12 & 12 & 12 \\
\hline Low- and middle-income countries & 18 & 17 & 10 & 27 & 32 & 33 & 30 & 32 & 34 & 29 & 29 & 29 & 36 & 39 & 41 & 36 & 29 & 28 & 29 \\
High-income countries & 23 & 37 & 8 & 6 & 11 & 5 & 8 & 4 & 6 & 2 & 6 & 2 & 1 & 0 & 1 & 2 & 6 & 2 & 5 \\
\hline WORLD & 18 & 17 & 10 & 27 & 32 & 33 & 29 & 31 & 32 & 28 & 28 & 25 & 32 & 31 & 36 & 30 & 28 & 27 & 28 \\
\hline
\end{tabular}

\section{PAF of DALYs $(\%)$}

East Asia and Pacific

Europe and Central Asia

Latin America and the Caribbean

Middle East and North Africa

South Asia

Sub-Saharan Africa

Low- and middle-income countries

$\begin{array}{rrrrrrrr}42 & 42 & 25 & 32 & 27 & 34 & 27 & 29 \\ 2 & 2 & 2 & 1 & 4 & 3 & 4 & 4 \\ 5 & 4 & 6 & 4 & 6 & 6 & 4 & \\ 15 & 39 & 30 & 39 & 43 & 32 & 37 & 33 \\ 52 & 53 & 52 & 52 & 52 & 52 & 51 & 52 \\ 13 & 12 & 9 & 11 & 12 & 11 & 15 & 14 \\ 18 & 17 & 10 & 27 & 32 & 33 & 30 & 32\end{array}$

\begin{tabular}{rrrrrrrrrrrr}
29 & 29 & 24 & 28 & 24 & 26 & 24 & 29 & 29 & 28 & 30 & 29 \\
4 & 4 & 4 & 3 & 3 & 2 & 3 & 2 & 3 & 4 & 3 & 4 \\
4 & 3 & 5 & 5 & 5 & 4 & 5 & 5 & 3 & 5 & 5 & 5 \\
33 & 23 & 19 & 21 & 24 & 35 & 48 & 45 & 54 & 26 & 33 & 29 \\
52 & 52 & 51 & 51 & 50 & 51 & 52 & 52 & 52 & 52 & 52 & 52 \\
14 & 14 & 11 & 10 & 15 & 12 & 38 & 44 & 44 & 12 & 12 & 12 \\
32 & 34 & 29 & 29 & 29 & 36 & 39 & 41 & 36 & 29 & 28 & 29 \\
4 & 6 & 2 & 6 & 2 & 1 & 0 & 1 & 2 & 6 & 2 & 5 \\
31 & 32 & 28 & 27 & 25 & 31 & 31 & 35 & 30 & 28 & 27 & 28 \\
\hline
\end{tabular}

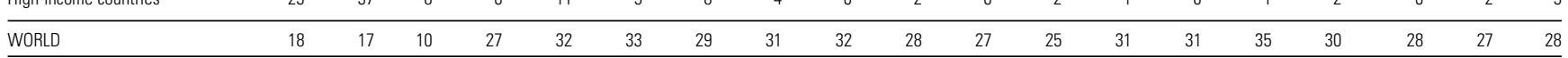

Attributable Mortality (thousands)

\begin{tabular}{|c|c|c|c|c|c|c|c|c|c|c|c|c|c|c|c|c|c|c|c|}
\hline East Asia and Pacific & 0 & 0 & 0 & 0 & 1 & 0 & 2 & 0 & 3 & 0 & 1 & 0 & 0 & 0 & 0 & 0 & 8 & 2 & 9 \\
\hline Europe and Central Asia & 0 & 0 & 0 & 0 & 0 & 0 & 0 & 0 & 0 & 0 & 0 & 0 & 0 & 0 & 0 & 0 & 0 & 0 & 0 \\
\hline Latin America and the Caribbean & 0 & 0 & 0 & 0 & 0 & 0 & 0 & 0 & 0 & 0 & 0 & 0 & 0 & 0 & 0 & 0 & 0 & 0 & 0 \\
\hline Middle East and North Africa & 0 & 0 & 0 & 0 & 0 & 0 & 0 & 0 & 0 & 0 & 0 & 0 & 0 & 0 & 0 & 0 & 1 & 1 & 2 \\
\hline South Asia & 0 & 0 & 0 & 0 & 2 & 1 & 2 & 1 & 4 & 1 & 1 & 0 & 1 & 0 & 1 & 0 & 10 & 5 & 15 \\
\hline Sub-Saharan Africa & 0 & 0 & 0 & 0 & 0 & 0 & 0 & 0 & 0 & 0 & 0 & 0 & 0 & 0 & 0 & 0 & 1 & 1 & 3 \\
\hline Low- and middle-income countries & 1 & 1 & 0 & 0 & 3 & 2 & 5 & 1 & 7 & 2 & 2 & 1 & 1 & 1 & 1 & 1 & 20 & 8 & 28 \\
\hline High-income countries & 0 & 0 & 0 & 0 & 0 & 0 & 0 & 0 & 0 & 0 & 0 & 0 & 0 & 0 & 0 & 0 & 0 & 0 & 0 \\
\hline WORLD & 1 & 1 & 0 & 0 & 3 & 2 & 5 & 1 & 7 & 2 & 2 & 1 & 1 & 1 & 1 & 1 & 20 & 9 & 29 \\
\hline
\end{tabular}

Attributable YLL (thousands)

East Asia and Pacific

Europe and Central Asia

Latin America and the Caribbean

Middle East and North Africa

South Asia

Sub-Saharan Afric

Low- and middle-income countries

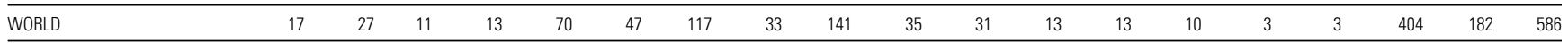

Attributable DALYs (thousands)

East Asia and Pacific

Europe and Central Asia

Latin America and the Caribbean

Middle East and North Africa

South Asia

Sub-Saharan Africa

Low- and middle-income countries

High-income countries

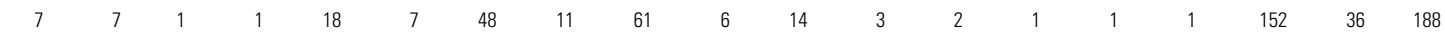

WORLD

Source: Authors' calculations. 
Table 4A.100

Risk factor:

Contaminated injections in health care setting

Disease:

Hepatitis C

\begin{tabular}{|c|c|c|c|c|c|c|c|c|c|c|c|c|c|c|c|c|c|c|c|}
\hline \multirow[b]{2}{*}{ Region } & \multicolumn{2}{|c|}{$0-4$ years } & \multicolumn{2}{|c|}{ 5-14 years } & \multicolumn{2}{|c|}{$15-29$ years } & \multicolumn{2}{|c|}{ 30-44 years } & \multicolumn{2}{|c|}{ 45-59 years } & \multicolumn{2}{|c|}{$60-69$ years } & \multicolumn{2}{|c|}{$70-79$ years } & \multicolumn{2}{|c|}{$80+$ years } & \multicolumn{3}{|c|}{ Total } \\
\hline & Male & Female & Male & Female & Male & Female & Male & Female & Male & Female & Male & Female & Male & Female & Male & Female & Male & Female & All \\
\hline \multicolumn{20}{|l|}{ PAF of Mortality (\%) } \\
\hline East Asia and Pacific & 55 & 55 & 32 & 39 & 34 & 41 & 34 & 36 & 35 & 32 & 35 & 32 & 33 & 33 & 35 & 36 & 35 & 37 & 35 \\
\hline Europe and Central Asia & 5 & 5 & 6 & 0 & 11 & 8 & 10 & 13 & 11 & 13 & 7 & 8 & 6 & 6 & 6 & 8 & 9 & 9 & 9 \\
\hline Latin America and the Caribbean & 7 & 7 & 7 & 6 & 1 & 1 & 2 & 5 & 2 & 3 & 2 & 2 & 1 & 1 & 1 & 1 & 2 & 2 & 2 \\
\hline Middle East and North Africa & 70 & 57 & 45 & 56 & 62 & 43 & 64 & 39 & 40 & 27 & 30 & 36 & 54 & 67 & 52 & 77 & 48 & 50 & 49 \\
\hline South Asia & 72 & 73 & 66 & 62 & 60 & 59 & 59 & 61 & 60 & 61 & 61 & 61 & 59 & 63 & 61 & 62 & 60 & 61 & 60 \\
\hline Sub-Saharan Africa & 20 & 18 & 13 & 17 & 18 & 16 & 22 & 19 & 21 & 17 & 15 & 22 & 18 & 54 & 62 & 63 & 18 & 19 & 18 \\
\hline Low- and middle-income countries & 33 & 25 & 15 & 37 & 41 & 40 & 37 & 40 & 40 & 34 & 35 & 32 & 43 & 47 & 47 & 50 & 37 & 36 & 37 \\
\hline High-income countries & 35 & 49 & 8 & 21 & 6 & 3 & 2 & 1 & 2 & 0 & 1 & 0 & 0 & 0 & 0 & 0 & 1 & 0 & 1 \\
\hline WORLD & 33 & 25 & 15 & 37 & 40 & 40 & 33 & 35 & 32 & 25 & 25 & 18 & 24 & 19 & 27 & 20 & 30 & 26 & 29 \\
\hline
\end{tabular}

PAF of YLL $(\%)$

\begin{tabular}{lrrrrrrrrrrrrrrrrrrr} 
East Asia and Pacific & 55 & 55 & 32 & 39 & 34 & 41 & 34 & 36 & 35 & 32 & 35 & 32 & 33 & 33 & 35 & 36 & 35 & 38 & 36 \\
Europe and Central Asia & 5 & 5 & 6 & 0 & 11 & 8 & 10 & 13 & 11 & 13 & 7 & 8 & 6 & 6 & 6 & 8 & 9 & 9 & 9 \\
Latin America and the Caribbean & 7 & 7 & 7 & 6 & 1 & 1 & 2 & 5 & 2 & 3 & 2 & 2 & 1 & 1 & 1 & 1 & 2 & 3 & 2 \\
Middle East and North Africa & 70 & 57 & 45 & 56 & 62 & 43 & 64 & 39 & 40 & 27 & 30 & 36 & 54 & 67 & 52 & 77 & 50 & 46 & 48 \\
South Asia & 72 & 73 & 66 & 62 & 60 & 59 & 59 & 61 & 60 & 61 & 61 & 61 & 59 & 63 & 61 & 62 & 60 & 61 & 60 \\
Sub-Saharan Africa & 20 & 18 & 13 & 17 & 18 & 16 & 22 & 19 & 21 & 17 & 15 & 22 & 18 & 54 & 62 & 63 & 18 & 18 & 18 \\
\hline Low- and middle-income countries & 33 & 25 & 15 & 37 & 41 & 40 & 37 & 40 & 40 & 34 & 35 & 32 & 43 & 46 & 47 & 51 & 36 & 35 & 36 \\
High-income countries & 35 & 49 & 8 & 21 & 6 & 3 & 2 & 1 & 2 & 0 & 1 & 0 & 0 & 0 & 0 & 0 & 2 & 0 & 1 \\
\hline WORLD & 33 & 25 & 15 & 37 & 41 & 40 & 33 & 35 & 32 & 26 & 25 & 18 & 24 & 19 & 27 & 22 & 31 & 28 & 30 \\
\hline
\end{tabular}

\section{PAF of DALYs $(\%)$}

East Asia and Pacific

Europe and Central Asia

Latin America and the Caribbean

Middle East and North Africa

South Asia

Sub-Saharan Africa

Low- and middle-income countries

$\begin{array}{rrrrrrrr}55 & 55 & 32 & 39 & 34 & 41 & 34 & 36 \\ 5 & 5 & 6 & 0 & 11 & 8 & 10 & 13 \\ 7 & 7 & 7 & 6 & 1 & 1 & 2 & \\ 70 & 57 & 45 & 56 & 62 & 43 & 64 & 39 \\ 72 & 73 & 66 & 62 & 60 & 59 & 59 & 61 \\ 20 & 18 & 13 & 17 & 18 & 16 & 22 & \\ 33 & 25 & 15 & 37 & 41 & 40 & 37 & 40\end{array}$

$\begin{array}{rrrrr}36 & 35 & 32 & 35 & 32 \\ 13 & 11 & 13 & 7 & 8 \\ 5 & 2 & 3 & 2 & 2 \\ 39 & 40 & 27 & 30 & 36 \\ 61 & 60 & 61 & 61 & 61 \\ 19 & 21 & 17 & 15 & 22 \\ 40 & 40 & 34 & 35 & 32\end{array}$

\begin{tabular}{|c|c|c|c|c|c|c|c|c|c|c|c|c|c|c|c|c|c|c|c|}
\hline WORLD & 33 & 25 & 15 & 37 & 40 & 40 & 33 & 35 & 31 & 26 & 24 & 17 & 23 & 18 & 25 & 20 & 31 & 28 & 30 \\
\hline
\end{tabular}

Attributable Mortality (thousands)

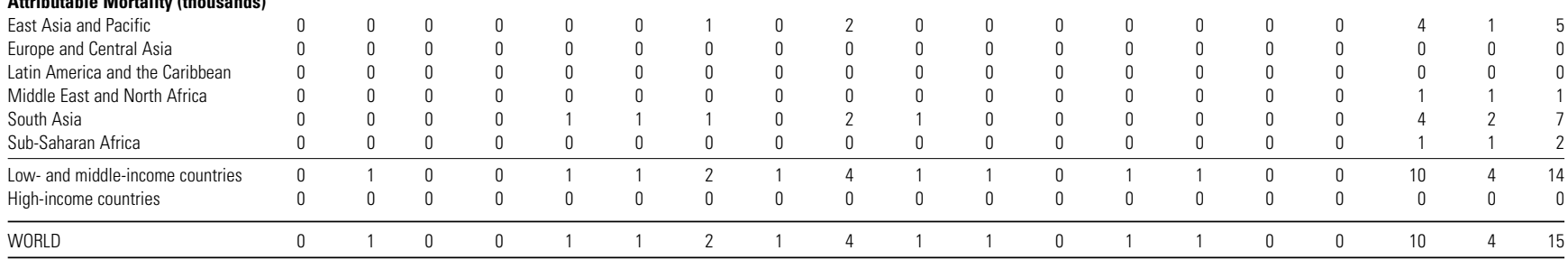

Attributable YLL (thousands)

East Asia and Pacific

Europe and Central Asia

Latin America and the Caribbean

Middle East and North Africa

South Asia

Sub-Saharan Africa

Low- and middle-income countries

\begin{tabular}{llllllllllllllllllll}
\hline WORLD & 10 & 16 & 7 & 7 & 33 & 22 & 59 & 17 & 71 & 18 & 16 & 7 & 7 & 6 & 2 & 2 & 203 & 94 & 298
\end{tabular}

Attributable DALYs (thousands)

East Asia and Pacific

Europe and Central Asia

Latin America and the Caribbean

Middle East and North Africa

South Asia

Sub-Saharan Africa

Low- and middle-income countries

23

0

$\begin{array}{rr}0 & 10 \\ 0 & 1\end{array}$

$4 \quad 25$

\begin{tabular}{rr}
6 & 31 \\
0 & 0 \\
0 & 0 \\
1 & 4 \\
8 & 31 \\
1 & 3 \\
\hline 17 & 70 \\
\hline
\end{tabular}

3
0
0
1
11
2
18

countries

WORLD

Source: Authors' calculations. 
Table 4A.101

Risk factor:

Contaminated injections in health care setting

Disease:

Liver cancer

\begin{tabular}{|c|c|c|c|c|c|c|c|c|c|c|c|c|c|c|c|c|c|c|c|}
\hline \multirow[b]{2}{*}{ Region } & \multicolumn{2}{|c|}{$0-4$ years } & \multicolumn{2}{|c|}{ 5-14 years } & \multicolumn{2}{|c|}{$15-29$ years } & \multicolumn{2}{|c|}{ 30-44 years } & \multicolumn{2}{|c|}{$45-59$ years } & \multicolumn{2}{|c|}{$60-69$ years } & \multicolumn{2}{|c|}{ 70-79 years } & $80+$ & years & & Total & \\
\hline & Male & Female & Male & Female & Male & Female & Male & Female & Male & Female & Male & Female & Male & Female & Male & Female & Male & Female & All \\
\hline PAF of Mortality (\%) & & & & & & & & & & & & & & & & & & & \\
\hline East Asia and Pacific & 37 & 38 & 24 & 25 & 24 & 25 & 24 & 24 & 24 & 24 & 24 & 24 & 24 & 24 & 24 & 24 & 24 & 24 & 24 \\
\hline Europe and Central Asia & 6 & 5 & 4 & 2 & 4 & 4 & 5 & 6 & 5 & 6 & 5 & 6 & 5 & 6 & 4 & 6 & 5 & 6 & 5 \\
\hline Latin America and the Caribbean & 2 & 3 & 3 & 3 & 3 & 3 & 2 & 2 & 2 & 2 & 2 & 2 & 2 & 2 & 2 & 2 & 2 & 2 & 2 \\
\hline Middle East and North Africa & 29 & 14 & 26 & 13 & 21 & 22 & 22 & 21 & 22 & 28 & 22 & 23 & 20 & 21 & 19 & 20 & 21 & 23 & 22 \\
\hline South Asia & 42 & 38 & 38 & 35 & 35 & 35 & 36 & 35 & 35 & 36 & 36 & 36 & 36 & 36 & 36 & 35 & 36 & 36 & 36 \\
\hline Sub-Saharan Africa & 10 & 9 & 9 & 9 & 6 & 8 & 6 & 8 & 7 & 8 & 7 & 8 & 7 & 8 & 7 & 8 & 7 & 8 & 7 \\
\hline Low- and middle-income countries & 29 & 32 & 22 & 24 & 23 & 24 & 22 & 21 & 22 & 21 & 21 & 21 & 21 & 20 & 20 & 19 & 22 & 21 & 21 \\
\hline High-income countries & 4 & 15 & 3 & 7 & 12 & 11 & 10 & 11 & 5 & 6 & 3 & 4 & 1 & 2 & 1 & 1 & 3 & 3 & 3 \\
\hline WORLD & 27 & 32 & 21 & 24 & 22 & 24 & 22 & 21 & 20 & 20 & 18 & 18 & 16 & 16 & 13 & 12 & 19 & 18 & 18 \\
\hline PAF of YLL (\%) & & & & & & & & & & & & & & & & & & & \\
\hline East Asia and Pacific & 37 & 38 & 24 & 25 & 24 & 25 & 24 & 24 & 24 & 24 & 24 & 24 & 24 & 24 & 24 & 24 & 24 & 24 & 24 \\
\hline Europe and Central Asia & 6 & 5 & 4 & 2 & 4 & 4 & 5 & 6 & 5 & 6 & 5 & 6 & 5 & 6 & 4 & 6 & 5 & 6 & 5 \\
\hline Latin America and the Caribbean & 2 & 3 & 3 & 3 & 3 & 3 & 2 & 2 & 2 & 2 & 2 & 2 & 2 & 2 & 2 & 2 & 2 & 2 & 2 \\
\hline Middle East and North Africa & 29 & 14 & 26 & 13 & 21 & 22 & 22 & 21 & 22 & 28 & 22 & 23 & 20 & 21 & 19 & 20 & 22 & 24 & 22 \\
\hline South Asia & 42 & 38 & 38 & 35 & 35 & 35 & 36 & 35 & 35 & 36 & 36 & 36 & 36 & 36 & 36 & 35 & 36 & 36 & 36 \\
\hline Sub-Saharan Africa & 10 & 9 & 9 & 9 & 6 & 8 & 6 & 8 & 7 & 8 & 7 & 8 & 7 & 8 & 7 & 8 & 7 & 8 & 7 \\
\hline Low- and middle-income countries & 29 & 32 & 22 & 24 & 23 & 24 & 22 & 22 & 22 & 21 & 21 & 21 & 21 & 20 & 20 & 19 & 22 & 21 & 22 \\
\hline High-income countries & 4 & 15 & 3 & 7 & 12 & 11 & 10 & 11 & 5 & 6 & 3 & 4 & 1 & 2 & 1 & 1 & 4 & 3 & 4 \\
\hline WORLD & 27 & 32 & 21 & 24 & 22 & 24 & 22 & 21 & 20 & 20 & 18 & 18 & 16 & 16 & 14 & 13 & 19 & 19 & 19 \\
\hline PAF of DALYs (\%) & & & & & & & & & & & & & & & & & & & \\
\hline East Asia and Pacific & 37 & 38 & 24 & 25 & 24 & 25 & 24 & 24 & 24 & 24 & 24 & 24 & 24 & 24 & 24 & 24 & 24 & 24 & 24 \\
\hline Europe and Central Asia & 6 & 5 & 4 & 2 & 4 & 4 & 5 & 6 & 5 & 6 & 5 & 6 & 5 & 6 & 4 & 6 & 5 & 6 & 5 \\
\hline Latin America and the Caribbean & 2 & 3 & 3 & 3 & 3 & 3 & 2 & 2 & 2 & 2 & 2 & 2 & 2 & 2 & 2 & 2 & 2 & 2 & 2 \\
\hline Middle East and North Africa & 29 & 14 & 26 & 13 & 21 & 22 & 22 & 21 & 22 & 28 & 22 & 23 & 20 & 21 & 19 & 20 & 22 & 24 & 22 \\
\hline South Asia & 42 & 38 & 38 & 35 & 35 & 35 & 36 & 35 & 35 & 36 & 36 & 36 & 36 & 36 & 36 & 35 & 36 & 36 & 36 \\
\hline$\underline{\text { Sub-Saharan Africa }}$ & 10 & 9 & 9 & 9 & 6 & 8 & 6 & 8 & 7 & 8 & 7 & 8 & 7 & 8 & 7 & 8 & 7 & 8 & 7 \\
\hline Low- and middle-income countries & 29 & 32 & 22 & 24 & 23 & 24 & 22 & 22 & 22 & 21 & 21 & 21 & 21 & 20 & 20 & 19 & 22 & 21 & 22 \\
\hline High-income countries & 4 & 15 & 3 & 7 & 12 & 11 & 10 & 11 & 5 & 6 & 3 & 4 & 1 & 2 & 1 & 1 & 4 & 3 & 4 \\
\hline WORLD & 27 & 32 & 21 & 24 & 22 & 24 & 22 & 21 & 20 & 20 & 18 & 18 & 16 & 16 & 13 & 13 & 19 & 19 & 19 \\
\hline Attributable Mortality (thousand & & & & & & & & & & & & & & & & & & & \\
\hline East Asia and Pacific & 0 & 0 & 0 & 0 & 1 & 1 & 7 & 2 & 24 & 6 & 18 & 7 & 12 & 7 & 3 & 2 & 65 & 25 & 91 \\
\hline Europe and Central Asia & 0 & 0 & 0 & 0 & 0 & 0 & 0 & 0 & 0 & 0 & 0 & 0 & 0 & 0 & 0 & 0 & 1 & 1 & 1 \\
\hline Latin America and the Caribbean & 0 & 0 & 0 & 0 & 0 & 0 & 0 & 0 & 0 & 0 & 0 & 0 & 0 & 0 & 0 & 0 & 0 & 0 & 0 \\
\hline Middle East and North Africa & 0 & 0 & 0 & 0 & 0 & 0 & 0 & 0 & 0 & 0 & 0 & 0 & 0 & 0 & 0 & 0 & 1 & 1 & 2 \\
\hline South Asia & 0 & 0 & 0 & 0 & 0 & 1 & 1 & 0 & 2 & 1 & 2 & 1 & 1 & 1 & 0 & 0 & 6 & 4 & 10 \\
\hline Sub-Saharan Africa & 0 & 0 & 0 & 0 & 0 & 0 & 0 & 0 & 1 & 0 & 1 & 0 & 0 & 0 & 0 & 0 & 2 & 1 & 3 \\
\hline Low- and middle-income countries & 0 & 0 & 0 & 0 & 2 & 1 & 8 & 3 & 27 & 8 & 21 & 8 & 15 & 8 & 3 & 3 & 76 & 32 & 108 \\
\hline High-income countries & 0 & 0 & 0 & 0 & 0 & 0 & 0 & 0 & 1 & 0 & 1 & 0 & 0 & 0 & 0 & 0 & 2 & 1 & 3 \\
\hline WORLD & 0 & 0 & 0 & 0 & 2 & 1 & 9 & 3 & 28 & 8 & 21 & 8 & 15 & 8 & 3 & 3 & 78 & 33 & 111 \\
\hline Attributable YLL (thousands) & & & & & & & & & & & & & & & & & & & \\
\hline East Asia and Pacific & 3 & 7 & 1 & 2 & 35 & 17 & 168 & 61 & 459 & 125 & 242 & 102 & 114 & 69 & 14 & 14 & 1,035 & 397 & 1,432 \\
\hline Europe and Central Asia & 0 & 0 & 0 & 0 & 0 & 0 & 1 & 1 & 4 & 2 & 4 & 3 & 2 & 3 & 0 & 0 & 11 & 9 & 20 \\
\hline Latin America and the Caribbean & 0 & 0 & 0 & 0 & 0 & 0 & 0 & 0 & 1 & 1 & 1 & 1 & 0 & 1 & 0 & 0 & 3 & 3 & 5 \\
\hline Middle East and North Africa & 0 & 0 & 0 & 0 & 1 & 1 & 2 & 2 & 6 & 5 & 5 & 3 & 3 & 2 & 0 & 0 & 18 & 13 & 31 \\
\hline South Asia & 2 & 3 & 1 & 2 & 10 & 15 & 18 & 10 & 29 & 18 & 23 & 11 & 10 & 7 & 2 & 2 & 96 & 68 & 164 \\
\hline Sub-Saharan Africa & 0 & 0 & 0 & 0 & 2 & 2 & 7 & 4 & 14 & 6 & 8 & 4 & 3 & 3 & 1 & 1 & 36 & 20 & 56 \\
\hline Low- and middle-income countries & 6 & 10 & 3 & 5 & 48 & 35 & 196 & 77 & 513 & 158 & 283 & 124 & 132 & 84 & 17 & 17 & 1,198 & 510 & 1,708 \\
\hline High-income countries & 0 & 0 & 0 & 0 & 1 & 0 & 5 & 2 & 14 & 4 & 8 & 4 & 3 & 2 & 0 & 0 & 31 & 12 & 43 \\
\hline WORLD & 6 & 10 & 3 & 5 & 49 & 35 & 201 & 79 & 527 & 161 & 290 & 128 & 135 & 86 & 17 & 18 & 1,229 & 523 & 1,751 \\
\hline Attributable DALYs (thousands) & & & & & & & & & & & & & & & & & & & \\
\hline East Asia and Pacific & 3 & 7 & 1 & 2 & 35 & 17 & 168 & 62 & 462 & 125 & 244 & 103 & 115 & 70 & 14 & 14 & 1,041 & 400 & 1,441 \\
\hline Europe and Central Asia & 0 & 0 & 0 & 0 & 0 & 0 & 1 & 1 & 4 & 2 & 4 & 3 & 2 & 3 & 0 & 1 & 11 & 9 & 20 \\
\hline Latin America and the Caribbean & 0 & 0 & 0 & 0 & 0 & 0 & 0 & 0 & 1 & 1 & 1 & 1 & 0 & 1 & 0 & 0 & 3 & 3 & 5 \\
\hline Middle East and North Africa & 0 & 0 & 0 & 0 & 1 & 1 & 2 & 2 & 6 & 6 & 5 & 3 & 3 & 2 & 0 & 0 & 18 & 13 & 31 \\
\hline South Asia & 2 & 3 & 1 & 2 & 10 & 15 & 18 & 10 & 29 & 18 & 23 & 11 & 10 & 7 & 2 & 2 & 97 & 68 & 165 \\
\hline Sub-Saharan Africa & 0 & 0 & 0 & 0 & 2 & 2 & 7 & 4 & 15 & 6 & 8 & 4 & 3 & 3 & 1 & 1 & 36 & 20 & 56 \\
\hline Low- and middle-income countries & 6 & 10 & 3 & 5 & 49 & 35 & 197 & 78 & 515 & 159 & 285 & 125 & 134 & 84 & 17 & 18 & 1,206 & 513 & 1,719 \\
\hline High-income countries & 0 & 0 & 0 & 0 & 1 & 0 & 5 & 2 & 14 & 4 & 8 & 4 & 3 & 3 & 0 & 0 & 31 & 12 & 44 \\
\hline WORLD & 6 & 11 & 3 & 5 & 49 & 35 & 202 & 80 & 530 & 162 & 292 & 128 & 136 & 87 & 18 & 18 & 1,237 & 526 & 1,762 \\
\hline
\end{tabular}

Source: Authors' calculations. 
Table 4A.102

Risk factor:

Disease:

Contaminated injections in health care setting

Cirrhosis of the liver

\begin{tabular}{|c|c|c|c|c|c|c|c|c|c|c|c|c|c|c|c|c|c|c|c|}
\hline \multirow[b]{2}{*}{ Region } & \multicolumn{2}{|c|}{$0-4$ years } & \multicolumn{2}{|c|}{ 5-14 years } & \multicolumn{2}{|c|}{$15-29$ years } & \multicolumn{2}{|c|}{ 30-44 years } & \multicolumn{2}{|c|}{$45-59$ years } & \multicolumn{2}{|c|}{$60-69$ years } & \multicolumn{2}{|c|}{$70-79$ years } & \multicolumn{2}{|c|}{$80+$ years } & \multicolumn{3}{|c|}{ Total } \\
\hline & Male & Female & Male & Female & Male & Female & Male & Female & Male & Female & Male & Female & Male & Female & Male & Female & Male & Female & All \\
\hline \multicolumn{20}{|l|}{ PAF of Mortality (\%) } \\
\hline East Asia and Pacific & 32 & 34 & 24 & 28 & 22 & 25 & 21 & 22 & 21 & 21 & 21 & 21 & 22 & 22 & 22 & 22 & 22 & 22 & 22 \\
\hline Europe and Central Asia & 7 & 7 & 3 & 1 & 6 & 5 & 7 & 9 & 7 & 9 & 7 & 8 & 6 & 7 & 5 & 6 & 7 & 8 & 7 \\
\hline Latin America and the Caribbean & 3 & 3 & 3 & 1 & 1 & 2 & 1 & 1 & 1 & 1 & 1 & 2 & 2 & 2 & 2 & 2 & 1 & 2 & 1 \\
\hline Middle East and North Africa & 38 & 55 & 36 & 48 & 39 & 41 & 33 & 36 & 39 & 41 & 37 & 39 & 34 & 38 & 32 & 34 & 36 & 39 & 37 \\
\hline South Asia & 39 & 38 & 35 & 35 & 35 & 34 & 34 & 35 & 35 & 36 & 36 & 38 & 37 & 38 & 37 & 37 & 36 & 36 & 36 \\
\hline Sub-Saharan Africa & 41 & 45 & 25 & 51 & 9 & 13 & 7 & 10 & 10 & 13 & 10 & 10 & 9 & 10 & 9 & 12 & 9 & 12 & 10 \\
\hline Low- and middle-income countries & 37 & 38 & 32 & 34 & 24 & 29 & 16 & 21 & 19 & 22 & 20 & 20 & 21 & 21 & 22 & 21 & 20 & 23 & 21 \\
\hline High-income countries & 13 & 17 & 7 & 13 & 7 & 5 & 2 & 1 & 1 & 1 & 1 & 1 & 1 & 1 & 1 & 1 & 1 & 1 & 1 \\
\hline WORLD & 37 & 38 & 32 & 34 & 23 & 29 & 14 & 19 & 16 & 19 & 16 & 18 & 17 & 18 & 18 & 17 & 17 & 20 & 18 \\
\hline \multicolumn{20}{|l|}{ PAF of YLL (\%) } \\
\hline East Asia and Pacific & 32 & 34 & 24 & 28 & 22 & 25 & 21 & 22 & 21 & 21 & 21 & 21 & 22 & 22 & 22 & 22 & 22 & 22 & 22 \\
\hline Europe and Central Asia & 7 & 7 & 3 & 1 & 6 & 5 & 7 & 9 & 7 & 9 & 7 & 8 & 6 & 7 & 5 & 6 & 7 & 8 & 7 \\
\hline Latin America and the Caribbean & 3 & 3 & 3 & 1 & 1 & 2 & 1 & 1 & 1 & 1 & 1 & 2 & 2 & 2 & 2 & 2 & 1 & 2 & 1 \\
\hline Middle East and North Africa & 38 & 55 & 36 & 48 & 39 & 41 & 33 & 36 & 39 & 41 & 37 & 39 & 34 & 38 & 32 & 34 & 37 & 40 & 38 \\
\hline South Asia & 39 & 38 & 35 & 35 & 35 & 34 & 34 & 35 & 35 & 36 & 36 & 38 & 37 & 38 & 37 & 37 & 36 & 36 & 36 \\
\hline Sub-Saharan Africa & 41 & 45 & 25 & 51 & 9 & 13 & 7 & 10 & 10 & 13 & 10 & 10 & 9 & 10 & 9 & 12 & 9 & 13 & 11 \\
\hline Low- and middle-income countries & 37 & 38 & 32 & 34 & 24 & 29 & 16 & 21 & 19 & 22 & 20 & 20 & 20 & 21 & 22 & 22 & 20 & 25 & $\overline{22}$ \\
\hline High-income countries & 13 & 17 & 7 & 13 & 7 & 5 & 2 & 1 & 1 & 1 & 1 & 1 & 1 & 1 & 1 & 1 & 1 & 1 & 1 \\
\hline WORLD & 37 & 38 & 32 & 34 & 23 & 29 & 14 & 19 & 16 & 19 & 16 & 18 & 17 & 18 & 18 & 17 & 17 & 22 & 19 \\
\hline
\end{tabular}

\section{PAF of DALYs (\%)}

East Asia and Pacific

Europe and Central Asia

Latin America and the Caribbean

Middle East and North Africa

South Asia

Sub-Saharan Africa

Low- and middle-income countries

High-income countries

$32 \quad 34 \quad 24$

$\begin{array}{lll}37 & 38 \\ & 13 & 17\end{array}$

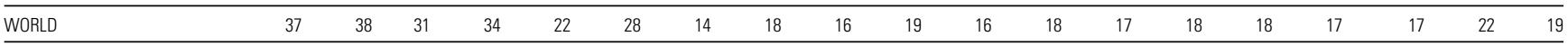

Attributable Mortality (thousands)

East Asia and Pacific

Europe and Central Asia

Latin America and the Caribbean

Middle East and North Africa

South Asia

Sub-Saharan Africa

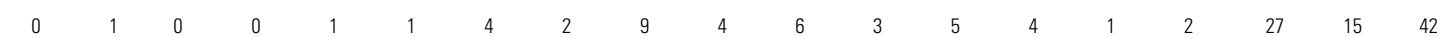

Low- and middle-income countries

High-income countries

\begin{tabular}{llllllllllllllllllll}
\hline WORLD & 3 & 6 & 1 & 3 & 4 & 4 & 10 & 5 & 28 & 13 & 19 & 10 & 14 & 10 & 5 & 4 & 82 & 56 & 139
\end{tabular}

Attributable YLL (thousands)

East Asia and Pacific

Europe and Central Asia

Latin America and the Caribbean

Middle East and North Africa

South Asia

Sub-Saharan Africa

Low- and middle-income countries

High-income co

\begin{tabular}{|c|c|c|c|c|c|c|c|c|c|c|c|c|c|c|c|c|c|c|c|}
\hline East Asia and Pacific & 12 & 18 & 3 & 6 & 24 & 15 & 97 & 38 & 180 & 72 & 83 & 49 & 41 & 37 & 8 & 10 & 447 & 244 & 692 \\
\hline Europe and Central Asia & 0 & 0 & 0 & 0 & 3 & 1 & 20 & 10 & 34 & 22 & 15 & 13 & 5 & 6 & 0 & 1 & 77 & 53 & 130 \\
\hline Latin America and the Caribbean & 0 & 0 & 0 & 0 & 1 & 0 & 3 & 1 & 5 & 1 & 2 & 1 & 1 & 1 & 0 & 0 & 13 & 4 & 17 \\
\hline Middle East and North Africa & 5 & 7 & 2 & 3 & 8 & 5 & 7 & 7 & 44 & 35 & 31 & 24 & 15 & 16 & 3 & 4 & 115 & 101 & 216 \\
\hline South Asia & 66 & 167 & 28 & 65 & 68 & 96 & 101 & 66 & 233 & 121 & 110 & 57 & 53 & 38 & 11 & 8 & 669 & 617 & 1,286 \\
\hline Sub-Saharan Africa & 1 & 3 & 0 & 4 & 4 & 3 & 10 & 5 & 24 & 17 & 12 & 10 & 6 & 5 & 1 & 1 & 57 & 47 & 104 \\
\hline Low- and middle-income countries & 84 & 195 & 34 & 77 & 107 & 121 & 239 & 125 & 519 & 269 & 252 & 154 & 121 & 103 & 23 & 23 & 1,378 & 1,067 & 2,445 \\
\hline High-income countries & 0 & 0 & 0 & 0 & 1 & 0 & 3 & 1 & 6 & 2 & 3 & 2 & 1 & 1 & 0 & 0 & 14 & 7 & 21 \\
\hline WORLD & 84 & 195 & 34 & 78 & 107 & 121 & 242 & 126 & 524 & 271 & 255 & 156 & 122 & 104 & 23 & 24 & 1,392 & 1,074 & 2,466 \\
\hline
\end{tabular}

Attributable DALYs (thousands)

East Asia and Pacific

Europe and Central Asia

Latin America and the Caribbean

Middle East and North Africa

South Asia

Sub-Saharan Africa

Low- and middle-income countries

High-income countries

WORLD

\begin{tabular}{|c|c|c|c|c|c|c|c|c|c|c|c|c|c|c|c|c|c|c|}
\hline 13 & 19 & 4 & 8 & 36 & 20 & 122 & 54 & 211 & 94 & 94 & 59 & 47 & 45 & 9 & 12 & 536 & 312 & 847 \\
\hline 0 & 0 & 0 & 0 & 4 & 2 & 25 & 13 & 38 & 27 & 17 & 15 & 5 & 7 & 0 & 1 & 89 & 65 & 155 \\
\hline 0 & 0 & 0 & 0 & 1 & 0 & 4 & 1 & 6 & 2 & 3 & 1 & 1 & 1 & 0 & 0 & 15 & 5 & 21 \\
\hline 7 & 8 & 4 & 4 & 10 & 7 & 9 & 9 & 52 & 46 & 35 & 30 & 17 & 19 & 3 & 5 & 135 & 127 & 262 \\
\hline 74 & 194 & 39 & 85 & 83 & 110 & 124 & 83 & 268 & 144 & 123 & 68 & 60 & 45 & 13 & 10 & 785 & 740 & 1,524 \\
\hline 1 & 3 & 1 & 4 & 6 & 4 & 13 & 7 & 27 & 21 & 14 & 12 & 7 & 6 & 1 & 1 & 70 & 59 & 129 \\
\hline 95 & 225 & 48 & 101 & 139 & 143 & 296 & 168 & 603 & 333 & 285 & 185 & 138 & 123 & 27 & 29 & 1,630 & 1,308 & 2,938 \\
\hline 0 & 0 & 0 & 0 & 1 & 0 & 4 & 2 & 7 & 3 & 3 & 2 & 1 & 1 & 0 & 0 & 17 & 9 & 26 \\
\hline 95 & 225 & 48 & 102 & 140 & 143 & 300 & 169 & 610 & 336 & 288 & 187 & 140 & 125 & 27 & 30 & 1,647 & 1,317 & 2,964 \\
\hline
\end{tabular}

Source: Authors' calculations. 
Table 4A.103

Risk factor:

Contaminated injections in health care setting

Disease:

All causes

\begin{tabular}{|c|c|c|c|c|c|c|c|c|c|c|c|c|c|c|c|c|c|c|c|}
\hline \multirow[b]{2}{*}{ Region } & \multicolumn{2}{|c|}{$0-4$ years } & \multicolumn{2}{|c|}{ 5-14 years } & \multicolumn{2}{|c|}{$15-29$ years } & \multicolumn{2}{|c|}{$30-44$ years } & \multicolumn{2}{|c|}{$45-59$ years } & \multicolumn{2}{|c|}{$60-69$ years } & \multicolumn{2}{|c|}{ 70-79 years } & $80+$ & years & & Total & \\
\hline & Male & Female & Male & Female & Male & Female & Male & Female & Male & Female & Male & Female & Male & Female & Male & Female & Male & Female & All \\
\hline PAF of Mortality (\%) & & & & & & & & & & & & & & & & & & & \\
\hline East Asia and Pacific & 0 & 0 & 0 & 0 & 1 & 1 & 3 & 1 & 3 & 1 & 2 & 1 & 1 & 1 & 0 & 0 & 2 & 1 & 1 \\
\hline Europe and Central Asia & 0 & 0 & 0 & 0 & 0 & 0 & 0 & 0 & 0 & 0 & 0 & 0 & 0 & 0 & 0 & 0 & 0 & 0 & 0 \\
\hline Latin America and the Caribbean & 0 & 0 & 0 & 0 & 0 & 0 & 0 & 0 & 0 & 0 & 0 & 0 & 0 & 0 & 0 & 0 & 0 & 0 & 0 \\
\hline Middle East and North Africa & 0 & 0 & 0 & 1 & 1 & 1 & 1 & 1 & 2 & 2 & 2 & 2 & 1 & 1 & 1 & 1 & 1 & 1 & 1 \\
\hline South Asia & 0 & 1 & 1 & 1 & 2 & 2 & 3 & 2 & 2 & 1 & 1 & 1 & 1 & 0 & 1 & 0 & 1 & 1 & 1 \\
\hline Sub-Saharan Africa & 1 & 1 & 1 & 1 & 1 & 1 & 1 & 1 & 1 & 1 & 1 & 0 & 0 & 0 & 0 & 0 & 1 & 1 & 1 \\
\hline Low- and middle-income countries & 0 & 1 & 1 & 1 & 1 & 1 & 2 & 1 & 2 & 1 & 1 & 1 & 1 & 0 & 0 & 0 & 1 & 1 & 1 \\
\hline High-income countries & 0 & 0 & 0 & 0 & 0 & 0 & 0 & 0 & 0 & 0 & 0 & 0 & 0 & 0 & 0 & 0 & 0 & 0 & 0 \\
\hline WORLD & 0 & 1 & 1 & 1 & 1 & 1 & 2 & 1 & 2 & 1 & 1 & 1 & 1 & 0 & 0 & 0 & 1 & 1 & 1 \\
\hline PAF of YLL (\%) & & & & & & & & & & & & & & & & & & & \\
\hline East Asia and Pacific & 0 & 0 & 0 & 0 & 1 & 1 & 3 & 1 & 3 & 1 & 2 & 1 & 1 & 1 & 1 & 0 & 2 & 1 & 1 \\
\hline Europe and Central Asia & 0 & 0 & 0 & 0 & 0 & 0 & 0 & 0 & 0 & 0 & 0 & 0 & 0 & 0 & 0 & 0 & 0 & 0 & 0 \\
\hline Latin America and the Caribbean & 0 & 0 & 0 & 0 & 0 & 0 & 0 & 0 & 0 & 0 & 0 & 0 & 0 & 0 & 0 & 0 & 0 & 0 & 0 \\
\hline Middle East and North Africa & 0 & 0 & 0 & 1 & 1 & 1 & 1 & 1 & 2 & 2 & 2 & 2 & 1 & 1 & 1 & 1 & 1 & 1 & 1 \\
\hline South Asia & 0 & 1 & 1 & 1 & 2 & 2 & 3 & 2 & 2 & 1 & 1 & 1 & 1 & 0 & 1 & 0 & 1 & 1 & 1 \\
\hline$\underline{\text { Sub-Saharan Africa }}$ & 1 & 1 & 1 & 1 & 1 & 1 & 1 & 1 & 1 & 1 & 1 & 0 & 0 & 0 & 0 & 0 & 1 & 1 & 1 \\
\hline Low- and middle-income countries & 0 & 1 & 1 & 1 & 1 & 1 & 2 & 1 & 2 & 1 & 1 & 1 & 1 & 0 & 0 & 0 & 1 & 1 & 1 \\
\hline High-income countries & 0 & 0 & 0 & 0 & 0 & 0 & 0 & 0 & 0 & 0 & 0 & 0 & 0 & 0 & 0 & 0 & 0 & 0 & 0 \\
\hline WORLD & 0 & 1 & 1 & 1 & 1 & 1 & 2 & 1 & 2 & 1 & 1 & 1 & 1 & 0 & 0 & 0 & 1 & 1 & 1 \\
\hline PAF of DALYs (\%) & & & & & & & & & & & & & & & & & & & \\
\hline East Asia and Pacific & 0 & 0 & 0 & 0 & 1 & 0 & 2 & 1 & 2 & 1 & 1 & 1 & 1 & 1 & 0 & 0 & 1 & 1 & 1 \\
\hline Europe and Central Asia & 0 & 0 & 0 & 0 & 0 & 0 & 0 & 0 & 0 & 0 & 0 & 0 & 0 & 0 & 0 & 0 & 0 & 0 & 0 \\
\hline Latin America and the Caribbean & 0 & 0 & 0 & 0 & 0 & 0 & 0 & 0 & 0 & 0 & 0 & 0 & 0 & 0 & 0 & 0 & 0 & 0 & 0 \\
\hline Middle East and North Africa & 0 & 0 & 0 & 0 & 0 & 0 & 0 & 0 & 1 & 1 & 1 & 1 & 1 & 1 & 1 & 1 & 1 & 1 & 1 \\
\hline South Asia & 0 & 0 & 1 & 1 & 1 & 1 & 2 & 1 & 2 & 1 & 1 & 0 & 1 & 0 & 1 & 0 & 1 & 1 & 1 \\
\hline Sub-Saharan Africa & 1 & 1 & 1 & 1 & 1 & 1 & 1 & 1 & 1 & 0 & 0 & 0 & 0 & 0 & 0 & 0 & 1 & 1 & 1 \\
\hline Low- and middle-income countries & 0 & 0 & 0 & 1 & 1 & 1 & 1 & 1 & 1 & 1 & 1 & 0 & 1 & 0 & 0 & 0 & 1 & 1 & 1 \\
\hline High-income countries & 0 & 0 & 0 & 0 & 0 & 0 & 0 & 0 & 0 & 0 & 0 & 0 & 0 & 0 & 0 & 0 & 0 & 0 & 0 \\
\hline WORLD & 0 & 0 & 0 & 1 & 1 & 1 & 1 & 1 & 1 & 1 & 1 & 0 & 0 & 0 & 0 & 0 & 1 & 0 & 1 \\
\hline Attributable Mortality (thousand & & & & & & & & & & & & & & & & & & & \\
\hline East Asia and Pacific & 2 & 2 & 0 & 0 & 4 & 2 & 16 & 6 & 39 & 11 & 25 & 10 & 18 & 10 & 4 & 4 & 109 & 46 & 154 \\
\hline Europe and Central Asia & 0 & 0 & 0 & 0 & 0 & 0 & 1 & 0 & 2 & 1 & 1 & 1 & 1 & 1 & 0 & 0 & 5 & 4 & 9 \\
\hline Latin America and the Caribbean & 0 & 0 & 0 & 0 & 0 & 0 & 0 & 0 & 0 & 0 & 0 & 0 & 0 & 0 & 0 & 0 & 1 & 1 & 2 \\
\hline Middle East and North Africa & 0 & 0 & 0 & 0 & 0 & 0 & 1 & 0 & 3 & 2 & 3 & 2 & 2 & 2 & 1 & 1 & 10 & 9 & 19 \\
\hline South Asia & 6 & 10 & 2 & 4 & 11 & 10 & 24 & 10 & 24 & 10 & 12 & 5 & 8 & 5 & 3 & 2 & 91 & 56 & 147 \\
\hline Sub-Saharan Africa & 15 & 15 & 4 & 4 & 4 & 5 & 10 & 5 & 5 & 3 & 2 & 1 & 1 & 1 & 0 & 0 & 41 & 34 & 75 \\
\hline Low- and middle-income countries & 23 & 27 & 6 & 8 & 19 & 18 & 52 & 22 & 74 & 28 & 44 & 20 & 30 & 19 & 9 & 8 & 257 & 150 & 407 \\
\hline High-income countries & 0 & 0 & 0 & 0 & 0 & 0 & 0 & 0 & 1 & 0 & 1 & 0 & 0 & 0 & 0 & 0 & 3 & 1 & 4 \\
\hline WORLD & 23 & 27 & 6 & 8 & 20 & 18 & 53 & 22 & 75 & 28 & 44 & 20 & 31 & 20 & 9 & 8 & 260 & 151 & 412 \\
\hline Attributable YLL (thousands) & & & & & & & & & & & & & & & & & & & \\
\hline East Asia and Pacific & 51 & 56 & 7 & 11 & 107 & 56 & 388 & 137 & 748 & 221 & 346 & 155 & 159 & 108 & 22 & 25 & 1,829 & 767 & 2,596 \\
\hline Europe and Central Asia & 1 & 0 & 0 & 0 & 4 & 2 & 23 & 11 & 38 & 25 & 19 & 16 & 7 & 9 & 1 & 1 & 93 & 66 & 159 \\
\hline Latin America and the Caribbean & 4 & 4 & 2 & 2 & 3 & 3 & 7 & 4 & 7 & 3 & 3 & 2 & 2 & 1 & 0 & 0 & 28 & 19 & 48 \\
\hline Middle East and North Africa & 12 & 14 & 4 & 6 & 11 & 7 & 16 & 11 & 57 & 44 & 39 & 31 & 21 & 24 & 3 & 6 & 164 & 143 & 307 \\
\hline South Asia & 192 & 290 & 58 & 109 & 297 & 289 & 575 & 246 & 471 & 213 & 158 & 80 & 76 & 51 & 17 & 12 & 1,843 & 1,290 & 3,133 \\
\hline Sub-Saharan Africa & 438 & 442 & 104 & 117 & 106 & 137 & 239 & 135 & 103 & 57 & 29 & 18 & 11 & 9 & 1 & 2 & 1,031 & 917 & 1,949 \\
\hline Low- and middle-income countries & 698 & 807 & 175 & 243 & 529 & 493 & 1,248 & 544 & 1,424 & 564 & 595 & 302 & 274 & 203 & 45 & 46 & 4,988 & 3,203 & 8,191 \\
\hline High-income countries & 0 & 0 & 0 & 0 & 2 & 1 & 10 & 3 & 22 & 6 & 11 & 5 & 4 & 4 & 1 & 1 & 49 & 20 & 70 \\
\hline WORLD & 699 & 807 & 175 & 244 & 530 & 494 & 1,258 & 547 & 1,446 & 570 & 605 & 308 & 278 & 206 & 45 & 47 & 5,038 & 3,223 & 8,261 \\
\hline Attributable DALYs (thousands) & & & & & & & & & & & & & & & & & & & \\
\hline East Asia and Pacific & 53 & 58 & 8 & 12 & 132 & 69 & 424 & 158 & 786 & 244 & 360 & 166 & 166 & 116 & 24 & 28 & 1,954 & 851 & 2,805 \\
\hline Europe and Central Asia & 1 & 0 & 0 & 0 & 6 & 4 & 28 & 16 & 43 & 30 & 21 & 18 & 7 & 10 & 1 & 2 & 107 & 79 & 186 \\
\hline Latin America and the Caribbean & 5 & 4 & 2 & 2 & 4 & 3 & 8 & 4 & 8 & 4 & 4 & 2 & 2 & 2 & 0 & 0 & 32 & 21 & 53 \\
\hline Middle East and North Africa & 14 & 15 & 6 & 7 & 14 & 9 & 18 & 14 & 66 & 55 & 43 & 36 & 23 & 27 & 4 & 7 & 186 & 170 & 356 \\
\hline South Asia & 202 & 321 & 70 & 132 & 355 & 346 & 623 & 279 & 512 & 239 & 174 & 91 & 84 & 59 & 19 & 14 & 2,040 & 1,481 & 3,521 \\
\hline Sub-Saharan Africa & 445 & 449 & 107 & 120 & 128 & 158 & 253 & 144 & 108 & 62 & 31 & 21 & 12 & 11 & 2 & 2 & 1,087 & 967 & 2,054 \\
\hline Low- and middle-income countries & 719 & 847 & 193 & 273 & 639 & 589 & 1,353 & 614 & 1,523 & 634 & 633 & 335 & 294 & 225 & 49 & 53 & 5,404 & 3,570 & 8,974 \\
\hline High-income countries & 1 & 0 & 0 & 0 & 2 & 1 & 11 & 4 & 24 & 7 & 11 & 6 & 4 & 4 & 1 & 1 & 54 & 23 & 76 \\
\hline WORLD & 720 & 848 & 194 & 273 & 641 & 590 & 1,364 & 618 & 1,547 & 640 & 644 & 341 & 299 & 229 & 50 & 53 & 5,458 & 3,592 & 9,050 \\
\hline
\end{tabular}

Source: Authors' calculations. 
Table 4A.104

Risk factor:

Urban air pollution

Disease:

Respiratory infections

\begin{tabular}{|c|c|c|c|c|c|c|c|c|c|c|c|c|c|c|c|c|c|c|c|}
\hline \multirow[b]{2}{*}{ Region } & \multicolumn{2}{|c|}{$0-4$ years } & \multicolumn{2}{|c|}{ 5-14 years } & \multicolumn{2}{|c|}{$15-29$ years } & \multicolumn{2}{|c|}{ 30-44 years } & \multicolumn{2}{|c|}{ 45-59 years } & \multicolumn{2}{|c|}{$60-69$ years } & \multicolumn{2}{|c|}{ 70-79 years } & $80+$ & years & & Total & \\
\hline & Male & Female & Male & Female & Male & Female & Male & Female & Male & Female & Male & Female & Male & Female & Male & Female & Male & Female & All \\
\hline PAF of Mortality (\%) & & & & & & & & & & & & & & & & & & & \\
\hline East Asia and Pacific & 2 & 2 & NA & NA & NA & NA & NA & NA & NA & NA & NA & NA & NA & NA & NA & NA & 1 & 1 & 1 \\
\hline Europe and Central Asia & 1 & 1 & NA & NA & NA & NA & NA & NA & NA & NA & NA & NA & NA & NA & NA & NA & 0 & 0 & 0 \\
\hline Latin America and the Caribbean & 1 & 1 & NA & NA & NA & NA & NA & NA & NA & NA & NA & NA & NA & NA & NA & NA & 0 & 0 & 0 \\
\hline Middle East and North Africa & 2 & 2 & NA & NA & NA & NA & NA & NA & NA & NA & NA & NA & NA & NA & NA & NA & 1 & 1 & 1 \\
\hline South Asia & 1 & 1 & NA & NA & NA & NA & NA & NA & NA & NA & NA & NA & NA & NA & NA & NA & 1 & 1 & 1 \\
\hline Sub-Saharan Africa & 1 & 1 & NA & NA & NA & NA & NA & NA & NA & NA & NA & NA & NA & NA & NA & NA & 1 & 1 & 1 \\
\hline Low- and middle-income countries & 1 & 1 & NA & NA & NA & NA & NA & NA & NA & NA & NA & NA & NA & NA & NA & NA & 1 & 1 & 1 \\
\hline High-income countries & 2 & 2 & NA & NA & NA & NA & NA & NA & NA & NA & NA & NA & NA & NA & NA & NA & 0 & 0 & 0 \\
\hline WORLD & 1 & 1 & NA & NA & NA & NA & NA & NA & NA & NA & NA & NA & NA & NA & NA & NA & 1 & 1 & 1 \\
\hline PAF of YLL (\%) & & & & & & & & & & & & & & & & & & & \\
\hline East Asia and Pacific & 2 & 2 & NA & NA & NA & NA & NA & NA & NA & NA & NA & NA & NA & NA & NA & NA & 1 & 1 & 1 \\
\hline Europe and Central Asia & 1 & 1 & NA & NA & NA & NA & NA & NA & NA & NA & NA & NA & NA & NA & NA & NA & 1 & 1 & 1 \\
\hline Latin America and the Caribbean & 1 & 1 & NA & NA & NA & NA & NA & NA & NA & NA & NA & NA & NA & NA & NA & NA & 1 & 1 & 1 \\
\hline Middle East and North Africa & 2 & 2 & NA & NA & NA & NA & NA & NA & NA & NA & NA & NA & NA & NA & NA & NA & 1 & 1 & 1 \\
\hline South Asia & 1 & 1 & NA & NA & NA & NA & NA & NA & NA & NA & NA & NA & NA & NA & NA & NA & 1 & 1 & 1 \\
\hline Sub-Saharan Africa & 1 & 1 & NA & NA & NA & NA & NA & NA & NA & NA & NA & NA & NA & NA & NA & NA & 1 & 1 & 1 \\
\hline Low- and middle-income countries & 1 & 1 & NA & NA & NA & NA & NA & NA & NA & NA & NA & NA & NA & NA & NA & NA & 1 & 1 & 1 \\
\hline High-income countries & 2 & 2 & NA & NA & NA & NA & NA & NA & NA & NA & NA & NA & NA & NA & NA & NA & 0 & 0 & 0 \\
\hline WORLD & 1 & 1 & NA & NA & NA & NA & NA & NA & NA & NA & NA & NA & NA & NA & NA & NA & 1 & 1 & 1 \\
\hline PAF of DALYs (\%) & & & & & & & & & & & & & & & & & & & \\
\hline East Asia and Pacific & 2 & 2 & NA & NA & NA & NA & NA & NA & NA & NA & NA & NA & NA & NA & NA & NA & 1 & 1 & 1 \\
\hline Europe and Central Asia & 1 & 1 & NA & NA & NA & NA & NA & NA & NA & NA & NA & NA & NA & NA & NA & NA & 1 & 1 & 1 \\
\hline Latin America and the Caribbean & 1 & 1 & NA & NA & NA & NA & NA & NA & NA & NA & NA & NA & NA & NA & NA & NA & 1 & 1 & 1 \\
\hline Middle East and North Africa & 2 & 2 & NA & NA & NA & NA & NA & NA & NA & NA & NA & NA & NA & NA & NA & NA & 1 & 1 & 1 \\
\hline South Asia & 1 & 1 & NA & NA & NA & NA & NA & NA & NA & NA & NA & NA & NA & NA & NA & NA & 1 & 1 & 1 \\
\hline Sub-Saharan Africa & 1 & 1 & NA & NA & NA & NA & NA & NA & NA & NA & NA & NA & NA & NA & NA & NA & 1 & 1 & 1 \\
\hline Low- and middle-income countries & 1 & 1 & NA & NA & NA & NA & NA & NA & NA & NA & NA & NA & NA & NA & NA & NA & 1 & 1 & 1 \\
\hline High-income countries & 1 & 1 & NA & NA & NA & NA & NA & NA & NA & NA & NA & NA & NA & NA & NA & NA & 0 & 0 & 0 \\
\hline WORLD & 1 & 1 & NA & NA & NA & NA & NA & NA & NA & NA & NA & NA & NA & NA & NA & NA & 1 & 1 & 1 \\
\hline Attributable Mortality (thousand & & & & & & & & & & & & & & & & & & & \\
\hline East Asia and Pacific & 1 & 3 & NA & NA & NA & NA & NA & NA & NA & NA & NA & NA & NA & NA & NA & NA & 1 & 3 & 4 \\
\hline Europe and Central Asia & 0 & 0 & NA & NA & NA & NA & NA & NA & NA & NA & NA & NA & NA & NA & NA & NA & 0 & 0 & 0 \\
\hline Latin America and the Caribbean & 0 & 0 & NA & NA & NA & NA & NA & NA & NA & NA & NA & NA & NA & NA & NA & NA & 0 & 0 & 1 \\
\hline Middle East and North Africa & 1 & 1 & NA & NA & NA & NA & NA & NA & NA & NA & NA & NA & NA & NA & NA & NA & 1 & 1 & 1 \\
\hline South Asia & 5 & 5 & NA & NA & NA & NA & NA & NA & NA & NA & NA & NA & NA & NA & NA & NA & 5 & 5 & 10 \\
\hline Sub-Saharan Africa & 4 & 3 & NA & NA & NA & NA & NA & NA & NA & NA & NA & NA & NA & NA & NA & NA & 4 & 3 & 6 \\
\hline Low- and middle-income countries & 11 & 11 & NA & NA & NA & NA & NA & NA & NA & NA & NA & NA & NA & NA & NA & NA & 11 & 11 & 23 \\
\hline High-income countries & 0 & 0 & NA & NA & NA & NA & NA & NA & NA & NA & NA & NA & NA & NA & NA & NA & 0 & 0 & 0 \\
\hline WORLD & 11 & 11 & NA & NA & NA & NA & NA & NA & NA & NA & NA & NA & NA & NA & NA & NA & 11 & 11 & 23 \\
\hline Attributable YLL (thousands) & & & & & & & & & & & & & & & & & & & \\
\hline East Asia and Pacific & 44 & 83 & NA & NA & NA & NA & NA & NA & NA & NA & NA & NA & NA & NA & NA & NA & 44 & 83 & 127 \\
\hline Europe and Central Asia & 7 & 6 & NA & NA & NA & NA & NA & NA & NA & NA & NA & NA & NA & NA & NA & NA & 7 & 6 & 13 \\
\hline Latin America and the Caribbean & 9 & 8 & NA & NA & NA & NA & NA & NA & NA & NA & NA & NA & NA & NA & NA & NA & 9 & 8 & 17 \\
\hline Middle East and North Africa & 20 & 19 & NA & NA & NA & NA & NA & NA & NA & NA & NA & NA & NA & NA & NA & NA & 20 & 19 & 39 \\
\hline South Asia & 152 & 154 & NA & NA & NA & NA & NA & NA & NA & NA & NA & NA & NA & NA & NA & NA & 152 & 154 & 306 \\
\hline Sub-Saharan Africa & 107 & 79 & NA & NA & NA & NA & NA & NA & NA & NA & NA & NA & NA & NA & NA & NA & 107 & 79 & 186 \\
\hline Low- and middle-income countries & 340 & 349 & NA & NA & NA & NA & NA & NA & NA & NA & NA & NA & NA & NA & NA & NA & 340 & 349 & 688 \\
\hline High-income countries & 1 & 0 & NA & NA & NA & NA & NA & NA & NA & NA & NA & NA & NA & NA & NA & NA & 1 & 0 & 1 \\
\hline WORLD & 340 & 349 & NA & NA & NA & NA & NA & NA & NA & NA & NA & NA & NA & NA & NA & NA & 340 & 349 & 689 \\
\hline Attributable DALYs (thousands) & & & & & & & & & & & & & & & & & & & \\
\hline East Asia and Pacific & 44 & 83 & NA & NA & NA & NA & NA & NA & NA & NA & NA & NA & NA & NA & NA & NA & 44 & 83 & 127 \\
\hline Europe and Central Asia & 7 & 6 & NA & NA & NA & NA & NA & NA & NA & NA & NA & NA & NA & NA & NA & NA & 7 & 6 & 13 \\
\hline Latin America and the Caribbean & 9 & 8 & NA & NA & NA & NA & NA & NA & NA & NA & NA & NA & NA & NA & NA & NA & 9 & 8 & 17 \\
\hline Middle East and North Africa & 20 & 19 & NA & NA & NA & NA & NA & NA & NA & NA & NA & NA & NA & NA & NA & NA & 20 & 19 & 39 \\
\hline South Asia & 152 & 154 & NA & NA & NA & NA & NA & NA & NA & NA & NA & NA & NA & NA & NA & NA & 152 & 154 & 306 \\
\hline Sub-Saharan Africa & 107 & 79 & NA & NA & NA & NA & NA & NA & NA & NA & NA & NA & NA & NA & NA & NA & 107 & 79 & 186 \\
\hline Low- and middle-income countries & 340 & 349 & NA & NA & NA & NA & NA & NA & NA & NA & NA & NA & NA & NA & NA & NA & 340 & 349 & 688 \\
\hline High-income countries & 1 & 0 & NA & NA & NA & NA & NA & NA & NA & NA & NA & NA & NA & NA & NA & NA & 1 & 0 & 1 \\
\hline$\overline{\text { WORLD }}$ & 340 & 349 & NA & NA & NA & NA & NA & NA & NA & NA & NA & NA & NA & NA & NA & NA & 340 & 349 & 689 \\
\hline
\end{tabular}

Source: Authors' calculations.

Note: $\mathrm{NA}=$ not applicable

372 I Global Burden of Disease and Risk Factors I Majid Ezzati, Stephen Vander Hoorn, Alan D. Lopez, and others 
Table 4A.105

Risk factor:

Urban air pollution

Disease:

Trachea, bronchus, and lung cancers

\begin{tabular}{|c|c|c|c|c|c|c|c|c|c|c|c|c|c|c|c|c|c|c|c|}
\hline \multirow[b]{2}{*}{ Region } & \multicolumn{2}{|c|}{$0-4$ years } & \multicolumn{2}{|c|}{ 5-14 years } & \multicolumn{2}{|c|}{$15-29$ years } & \multicolumn{2}{|c|}{ 30-44 years } & \multicolumn{2}{|c|}{ 45-59 years } & \multicolumn{2}{|c|}{ 60-69 years } & \multicolumn{2}{|c|}{ 70-79 years } & $80+$ & years & & Total & \\
\hline & Male & Female & Male & Female & Male & Female & Male & Female & Male & Female & Male & Female & Male & Female & Male & Female & Male & Female & All \\
\hline PAF of Mortality (\%) & & & & & & & & & & & & & & & & & & & \\
\hline East Asia and Pacific & NA & NA & NA & NA & NA & NA & 9 & 9 & 9 & 9 & 9 & 9 & 9 & 10 & 9 & 10 & 9 & 9 & 9 \\
\hline Europe and Central Asia & NA & NA & NA & NA & NA & NA & 4 & 4 & 4 & 4 & 4 & 4 & 4 & 3 & 4 & 3 & 4 & 4 & 4 \\
\hline Latin America and the Caribbean & NA & NA & NA & NA & NA & NA & 4 & 4 & 4 & 4 & 4 & 4 & 4 & 4 & 4 & 4 & 4 & 4 & 4 \\
\hline Middle East and North Africa & NA & NA & NA & NA & NA & NA & 6 & 6 & 6 & 6 & 6 & 6 & 6 & 6 & 6 & 6 & 6 & 6 & 6 \\
\hline South Asia & NA & NA & NA & NA & NA & NA & 5 & 5 & 5 & 5 & 5 & 5 & 5 & 5 & 5 & 5 & 5 & 5 & 5 \\
\hline Sub-Saharan Africa & NA & NA & NA & NA & NA & NA & 4 & 3 & 3 & 3 & 3 & 3 & 3 & 3 & 4 & 3 & 3 & 3 & 3 \\
\hline Low- and middle-income countries & NA & NA & NA & NA & NA & NA & 7 & 7 & 6 & 7 & 6 & 7 & 7 & 8 & 7 & 7 & 6 & 7 & 7 \\
\hline High-income countries & NA & NA & NA & NA & NA & NA & 3 & 3 & 3 & 3 & 3 & 3 & 3 & 3 & 3 & 3 & 3 & 3 & 3 \\
\hline WORLD & NA & NA & NA & NA & NA & NA & 6 & 6 & 5 & 6 & 5 & 6 & 5 & 5 & 4 & 5 & 5 & 5 & 5 \\
\hline PAF of YLL (\%) & & & & & & & & & & & & & & & & & & & \\
\hline East Asia and Pacific & NA & NA & NA & NA & NA & NA & 9 & 9 & 9 & 9 & 9 & 9 & 9 & 10 & 9 & 10 & 9 & 9 & 9 \\
\hline Europe and Central Asia & NA & NA & NA & NA & NA & NA & 4 & 4 & 4 & 4 & 4 & 4 & 4 & 3 & 4 & 3 & 4 & 4 & 4 \\
\hline Latin America and the Caribbean & NA & NA & NA & NA & NA & NA & 4 & 4 & 4 & 4 & 4 & 4 & 4 & 4 & 4 & 4 & 4 & 4 & 4 \\
\hline Middle East and North Africa & NA & NA & NA & NA & NA & NA & 6 & 6 & 6 & 6 & 6 & 6 & 6 & 6 & 6 & 6 & 6 & 5 & 6 \\
\hline South Asia & NA & NA & NA & NA & NA & NA & 5 & 5 & 5 & 5 & 5 & 5 & 5 & 5 & 5 & 5 & 5 & 5 & 5 \\
\hline Sub-Saharan Africa & NA & NA & NA & NA & NA & NA & 4 & 3 & 3 & 3 & 3 & 3 & 3 & 3 & 4 & 3 & 3 & 3 & 3 \\
\hline Low- and middle-income countries & NA & NA & NA & NA & NA & NA & 7 & 7 & 6 & 7 & 6 & 7 & 7 & 8 & 7 & 7 & 6 & 7 & 7 \\
\hline High-income countries & NA & NA & NA & NA & NA & NA & 3 & 3 & 3 & 3 & 3 & 3 & 3 & 3 & 3 & 3 & 3 & 3 & 3 \\
\hline WORLD & NA & NA & NA & NA & NA & NA & 6 & 6 & 5 & 6 & 5 & 6 & 5 & 6 & 4 & 5 & 5 & 6 & 5 \\
\hline PAF of DALYs (\%) & & & & & & & & & & & & & & & & & & & \\
\hline East Asia and Pacific & NA & NA & NA & NA & NA & NA & 9 & 9 & 9 & 9 & 9 & 9 & 9 & 9 & 9 & 9 & 9 & 9 & 9 \\
\hline Europe and Central Asia & NA & NA & NA & NA & NA & NA & 4 & 4 & 4 & 4 & 3 & 4 & 3 & 3 & 3 & 3 & 3 & 4 & 3 \\
\hline Latin America and the Caribbean & NA & NA & NA & NA & NA & NA & 4 & 4 & 4 & 4 & 4 & 4 & 4 & 4 & 4 & 4 & 4 & 4 & 4 \\
\hline Middle East and North Africa & NA & NA & NA & NA & NA & NA & 6 & 6 & 6 & 6 & 6 & 6 & 6 & 6 & 5 & 6 & 6 & 5 & 6 \\
\hline South Asia & NA & NA & NA & NA & NA & NA & 5 & 5 & 5 & 5 & 5 & 5 & 5 & 5 & 5 & 5 & 5 & 5 & 5 \\
\hline Sub-Saharan Africa & NA & NA & NA & NA & NA & NA & 4 & 3 & 3 & 3 & 3 & 3 & 3 & 3 & 3 & 3 & 3 & 3 & 3 \\
\hline Low- and middle-income countries & NA & NA & NA & NA & NA & NA & 7 & 7 & 6 & 7 & 6 & 7 & 6 & 8 & 6 & 7 & 6 & 7 & 7 \\
\hline High-income countries & NA & NA & NA & NA & NA & NA & 3 & 3 & 3 & 3 & 3 & 3 & 3 & 3 & 3 & 3 & 3 & 3 & 3 \\
\hline WORLD & NA & NA & NA & NA & NA & NA & 6 & 6 & 5 & 6 & 5 & 6 & 5 & 5 & 4 & 5 & 5 & 6 & 5 \\
\hline Attributable Mortality (thousand & & & & & & & & & & & & & & & & & & & \\
\hline East Asia and Pacific & NA & NA & NA & NA & NA & NA & 1 & 1 & 5 & 3 & 9 & 3 & 7 & 4 & 2 & 1 & 24 & 12 & 36 \\
\hline Europe and Central Asia & NA & NA & NA & NA & NA & NA & 0 & 0 & 1 & 0 & 2 & 0 & 1 & 0 & 0 & 0 & 5 & 1 & 6 \\
\hline Latin America and the Caribbean & NA & NA & NA & NA & NA & NA & 0 & 0 & 0 & 0 & 1 & 0 & 1 & 0 & 0 & 0 & 2 & 1 & 2 \\
\hline Middle East and North Africa & NA & NA & NA & NA & NA & NA & 0 & 0 & 0 & 0 & 0 & 0 & 0 & 0 & 0 & 0 & 1 & 0 & 1 \\
\hline South Asia & NA & NA & NA & NA & NA & NA & 0 & 0 & 1 & 0 & 2 & 0 & 1 & 0 & 0 & 0 & 5 & 1 & 6 \\
\hline Sub-Saharan Africa & NA & NA & NA & NA & NA & NA & 0 & 0 & 0 & 0 & 0 & 0 & 0 & 0 & 0 & 0 & 0 & 0 & 0 \\
\hline Low- and middle-income countries & NA & NA & NA & NA & NA & NA & 2 & 1 & 9 & 3 & 13 & 4 & 11 & 5 & 3 & 2 & 37 & 15 & 52 \\
\hline High-income countries & NA & NA & NA & NA & NA & NA & 0 & 0 & 1 & 1 & 2 & 1 & 3 & 1 & 1 & 1 & 8 & 4 & 12 \\
\hline$\overline{\text { WORLD }}$ & NA & NA & NA & NA & NA & NA & 2 & 1 & 10 & 4 & 16 & 5 & 14 & 6 & 4 & 2 & 45 & 19 & 64 \\
\hline Attributable YLL (thousands) & & & & & & & & & & & & & & & & & & & \\
\hline East Asia and Pacific & NA & NA & NA & NA & NA & NA & 25 & 14 & 101 & 52 & 117 & 51 & 65 & 42 & 9 & 7 & 316 & 166 & 482 \\
\hline Europe and Central Asia & NA & NA & NA & NA & NA & NA & 4 & 1 & 24 & 4 & 26 & 4 & 12 & 4 & 1 & 1 & 67 & 14 & 81 \\
\hline Latin America and the Caribbean & NA & NA & NA & NA & NA & NA & 1 & 1 & 7 & 3 & 7 & 3 & 5 & 2 & 1 & 1 & 21 & 10 & 31 \\
\hline Middle East and North Africa & NA & NA & NA & NA & NA & NA & 2 & 1 & 4 & 1 & 4 & 1 & 2 & 1 & 0 & 0 & 12 & 4 & 16 \\
\hline South Asia & NA & NA & NA & NA & NA & NA & 5 & 2 & 23 & 6 & 24 & 5 & 12 & 3 & 2 & 0 & 67 & 17 & 84 \\
\hline Sub-Saharan Africa & NA & NA & NA & NA & NA & NA & 0 & 0 & 2 & 1 & 2 & 1 & 1 & 0 & 0 & 0 & 5 & 2 & 7 \\
\hline Low- and middle-income countries & NA & NA & NA & NA & NA & NA & 38 & 20 & 162 & 68 & 179 & 65 & 96 & 51 & 13 & 9 & 488 & 213 & 701 \\
\hline High-income countries & NA & NA & NA & NA & NA & NA & 3 & 3 & 24 & 13 & 31 & 14 & 27 & 14 & 7 & 5 & 92 & 49 & 141 \\
\hline WORLD & NA & NA & NA & NA & NA & NA & 41 & 23 & 186 & 81 & 209 & 79 & 123 & 65 & 20 & 14 & 580 & 262 & 842 \\
\hline Attributable DALYs (thousands) & & & & & & & & & & & & & & & & & & & \\
\hline East Asia and Pacific & NA & NA & NA & NA & NA & NA & 25 & 14 & 101 & 52 & 117 & 51 & 65 & 42 & 9 & 7 & 316 & 166 & 482 \\
\hline Europe and Central Asia & NA & NA & NA & NA & NA & NA & 4 & 1 & 24 & 4 & 26 & 4 & 12 & 4 & 1 & 1 & 67 & 14 & 81 \\
\hline Latin America and the Caribbean & NA & NA & NA & NA & NA & NA & 1 & 1 & 7 & 3 & 7 & 3 & 5 & 2 & 1 & 1 & 21 & 10 & 31 \\
\hline Middle East and North Africa & NA & NA & NA & NA & NA & NA & 2 & 1 & 4 & 1 & 4 & 1 & 2 & 1 & 0 & 0 & 12 & 4 & 16 \\
\hline South Asia & NA & NA & NA & NA & NA & NA & 5 & 2 & 23 & 6 & 24 & 5 & 12 & 3 & 2 & 0 & 67 & 17 & 84 \\
\hline Sub-Saharan Africa & NA & NA & NA & NA & NA & NA & 0 & 0 & 2 & 1 & 2 & 1 & 1 & 0 & 0 & 0 & 5 & 2 & 7 \\
\hline Low- and middle-income countries & NA & NA & NA & NA & NA & NA & 38 & 20 & 162 & 68 & 179 & 65 & 96 & 51 & 13 & 9 & 488 & 213 & 701 \\
\hline High-income countries & NA & NA & NA & NA & NA & NA & 3 & 3 & 24 & 13 & 31 & 14 & 27 & 14 & 7 & 5 & 92 & 49 & 141 \\
\hline$\overline{\text { WORLD }}$ & NA & NA & NA & NA & NA & NA & 41 & 23 & 186 & 81 & 209 & 79 & 123 & 65 & 20 & 14 & 580 & 262 & $\overline{842}$ \\
\hline
\end{tabular}

Source: Authors' calculations.

Note: NA = not applicable. 
Table 4A.106

Risk factor:

Urban air pollution

Disease:

Selected cardiopulmonary causes

\begin{tabular}{|c|c|c|c|c|c|c|c|c|c|c|c|c|c|c|c|c|c|c|c|}
\hline \multirow[b]{2}{*}{ Region } & \multicolumn{2}{|c|}{$0-4$ years } & \multicolumn{2}{|c|}{ 5-14 years } & \multicolumn{2}{|c|}{$15-29$ years } & \multicolumn{2}{|c|}{ 30-44 years } & \multicolumn{2}{|c|}{$45-59$ years } & \multicolumn{2}{|c|}{ 60-69 years } & \multicolumn{2}{|c|}{$70-79$ years } & $80+$ & years & & Total & \\
\hline & Male & Female & Male & Female & Male & Female & Male & Female & Male & Female & Male & Female & Male & Female & Male & Female & Male & Female & All \\
\hline PAF of Mortality (\%) & & & & & & & & & & & & & & & & & & & \\
\hline East Asia and Pacific & NA & NA & NA & NA & NA & NA & 6 & 6 & 6 & 6 & 6 & 6 & 6 & 6 & 7 & 7 & 6 & 6 & 6 \\
\hline Europe and Central Asia & NA & NA & NA & NA & NA & NA & 2 & 3 & 2 & 3 & 3 & 3 & 3 & 2 & 3 & 2 & 2 & 2 & 2 \\
\hline Latin America and the Caribbean & NA & NA & NA & NA & NA & NA & 3 & 3 & 3 & 3 & 3 & 3 & 3 & 3 & 3 & 3 & 3 & 3 & 3 \\
\hline Middle East and North Africa & NA & NA & NA & NA & NA & NA & 4 & 4 & 4 & 5 & 4 & 5 & 4 & 5 & 4 & 4 & 4 & 4 & 4 \\
\hline South Asia & NA & NA & NA & NA & NA & NA & 4 & 3 & 3 & 3 & 3 & 3 & 3 & 3 & 4 & 3 & 3 & 3 & 3 \\
\hline Sub-Saharan Africa & NA & NA & NA & NA & NA & NA & 2 & 2 & 2 & 2 & 2 & 2 & 3 & 2 & 3 & 2 & 1 & 1 & 1 \\
\hline Low- and middle-income countries & NA & NA & NA & NA & NA & NA & 4 & 4 & 4 & 4 & 4 & 4 & 4 & 4 & 5 & 4 & 4 & 4 & $\overline{4}$ \\
\hline High-income countries & NA & NA & NA & NA & NA & NA & 2 & 2 & 2 & 2 & 2 & 2 & 2 & 2 & 2 & 2 & 2 & 2 & 2 \\
\hline WORLD & NA & NA & NA & NA & NA & NA & 4 & 4 & 4 & 4 & 4 & 4 & 4 & 4 & 4 & 4 & 3 & 3 & 3 \\
\hline PAF of YLL (\%) & & & & & & & & & & & & & & & & & & & \\
\hline East Asia and Pacific & NA & NA & NA & NA & NA & NA & 6 & 6 & 6 & 6 & 6 & 6 & 6 & 6 & 7 & 7 & 6 & 5 & 6 \\
\hline Europe and Central Asia & NA & NA & NA & NA & NA & NA & 2 & 3 & 2 & 3 & 3 & 3 & 3 & 2 & 3 & 2 & 2 & 2 & 2 \\
\hline Latin America and the Caribbean & NA & NA & NA & NA & NA & NA & 3 & 3 & 3 & 3 & 3 & 3 & 3 & 3 & 3 & 3 & 3 & 3 & 3 \\
\hline Middle East and North Africa & NA & NA & NA & NA & NA & NA & 4 & 4 & 4 & 5 & 4 & 5 & 4 & 5 & 4 & 4 & 3 & 3 & 3 \\
\hline South Asia & NA & NA & NA & NA & NA & NA & 4 & 3 & 3 & 3 & 3 & 3 & 3 & 3 & 4 & 3 & 2 & 2 & 2 \\
\hline Sub-Saharan Africa & NA & NA & NA & NA & NA & NA & 2 & 2 & 2 & 2 & 2 & 2 & 3 & 2 & 3 & 2 & 1 & 1 & 1 \\
\hline Low- and middle-income countries & NA & NA & NA & NA & NA & NA & 4 & 4 & 4 & 4 & 4 & 4 & 4 & 4 & 5 & 5 & 3 & 3 & 3 \\
\hline High-income countries & NA & NA & NA & NA & NA & NA & 2 & 2 & 2 & 2 & 2 & 2 & 2 & 2 & 2 & 2 & 2 & 2 & 2 \\
\hline WORLD & NA & NA & NA & NA & NA & NA & 4 & 4 & 4 & 4 & 4 & 4 & 4 & 4 & 4 & 4 & 3 & 3 & 3 \\
\hline PAF of DALYs (\%) & & & & & & & & & & & & & & & & & & & \\
\hline East Asia and Pacific & NA & NA & NA & NA & NA & NA & 4 & 4 & 4 & 5 & 5 & 5 & 6 & 6 & 6 & 6 & 4 & 5 & 4 \\
\hline Europe and Central Asia & NA & NA & NA & NA & NA & NA & 2 & 2 & 2 & 2 & 2 & 2 & 2 & 2 & 2 & 2 & 2 & 2 & 2 \\
\hline Latin America and the Caribbean & NA & NA & NA & NA & NA & NA & 2 & 2 & 3 & 2 & 3 & 3 & 3 & 3 & 3 & 3 & 2 & 2 & 2 \\
\hline Middle East and North Africa & NA & NA & NA & NA & NA & NA & 3 & 3 & 4 & 4 & 4 & 4 & 4 & 4 & 4 & 4 & 3 & 3 & 3 \\
\hline South Asia & NA & NA & NA & NA & NA & NA & 3 & 3 & 3 & 3 & 3 & 3 & 3 & 3 & 3 & 3 & 2 & 2 & 2 \\
\hline Sub-Saharan Africa & NA & NA & NA & NA & NA & NA & 2 & 2 & 2 & 2 & 2 & 2 & 2 & 2 & 2 & 2 & 1 & 1 & 1 \\
\hline Low- and middle-income countries & NA & NA & NA & NA & NA & NA & 3 & 3 & 3 & 3 & 4 & 4 & 4 & 4 & 4 & 4 & 2 & 3 & 2 \\
\hline High-income countries & NA & NA & NA & NA & NA & NA & 1 & 1 & 1 & 1 & 2 & 2 & 2 & 2 & 2 & 2 & 1 & 1 & 1 \\
\hline WORLD & NA & NA & NA & NA & NA & NA & 3 & 3 & 3 & 3 & 3 & 3 & 4 & 4 & 4 & 4 & 2 & 2 & 2 \\
\hline Attributable Mortality (thousand & & & & & & & & & & & & & & & & & & & \\
\hline East Asia and Pacific & NA & NA & NA & NA & NA & NA & 5 & 3 & 23 & 14 & 41 & 29 & 62 & 62 & 40 & 71 & 171 & 179 & 349 \\
\hline Europe and Central Asia & NA & NA & NA & NA & NA & NA & 2 & 1 & 6 & 3 & 10 & 6 & 12 & 15 & 6 & 17 & 37 & 41 & 78 \\
\hline Latin America and the Caribbean & NA & NA & NA & NA & NA & NA & 1 & 1 & 3 & 2 & 4 & 3 & 5 & 4 & 5 & 6 & 18 & 16 & 34 \\
\hline Middle East and North Africa & NA & NA & NA & NA & NA & NA & 1 & 0 & 3 & 2 & 4 & 3 & 5 & 5 & 3 & 3 & 15 & 13 & 28 \\
\hline South Asia & NA & NA & NA & NA & NA & NA & 3 & 2 & 16 & 10 & 22 & 18 & 24 & 23 & 11 & 12 & 76 & 65 & 141 \\
\hline Sub-Saharan Africa & NA & NA & NA & NA & NA & NA & 1 & 1 & 3 & 3 & 4 & 4 & 4 & 5 & 2 & 3 & 15 & 15 & 30 \\
\hline Low- and middle-income countries & NA & NA & NA & NA & NA & NA & 13 & 8 & 54 & 33 & 85 & 62 & 113 & 113 & 67 & 112 & 332 & 329 & 660 \\
\hline High-income countries & NA & NA & NA & NA & NA & NA & 1 & 0 & 3 & 1 & 5 & 3 & 10 & 8 & 12 & 20 & 31 & 33 & 64 \\
\hline WORLD & NA & NA & NA & NA & NA & NA & 14 & 9 & 57 & 34 & 90 & 65 & 123 & 121 & 79 & 132 & 363 & 362 & 724 \\
\hline Attributable YLL (thousands) & & & & & & & & & & & & & & & & & & & \\
\hline East Asia and Pacific & NA & NA & NA & NA & NA & NA & 125 & 79 & 421 & 282 & 547 & 431 & 550 & 628 & 198 & 356 & 1,842 & 1,776 & 3,618 \\
\hline Europe and Central Asia & NA & NA & NA & NA & NA & NA & 40 & 14 & 121 & 50 & 137 & 97 & 111 & 152 & 29 & 78 & 438 & 391 & 829 \\
\hline Latin America and the Caribbean & NA & NA & NA & NA & NA & NA & 23 & 16 & 55 & 38 & 51 & 40 & 46 & 45 & 22 & 30 & 197 & 169 & 365 \\
\hline Middle East and North Africa & NA & NA & NA & NA & NA & NA & 21 & 12 & 53 & 35 & 53 & 44 & 46 & 47 & 13 & 17 & 186 & 155 & 341 \\
\hline South Asia & NA & NA & NA & NA & NA & NA & 80 & 56 & 294 & 199 & 299 & 268 & 211 & 237 & 55 & 63 & 939 & 824 & 1,763 \\
\hline Sub-Saharan Africa & NA & NA & NA & NA & NA & NA & 31 & 30 & 60 & 55 & 54 & 58 & 39 & 49 & 10 & 15 & 194 & 206 & 401 \\
\hline Low- and middle-income countries & NA & NA & NA & NA & NA & NA & 319 & 207 & 1,006 & 658 & 1,141 & 939 & 1,005 & 1,157 & 326 & 560 & 3,797 & 3,520 & 7,317 \\
\hline High-income countries & NA & NA & NA & NA & NA & NA & 16 & 8 & 59 & 27 & 69 & 43 & 85 & 80 & 53 & 83 & 282 & 240 & 522 \\
\hline WORLD & NA & NA & NA & NA & NA & NA & 336 & 214 & 1,065 & 685 & 1,209 & 981 & 1,090 & 1,237 & 379 & 644 & 4,079 & 3,761 & 7,839 \\
\hline Attributable DALYs (thousands) & & & & & & & & & & & & & & & & & & & \\
\hline East Asia and Pacific & NA & NA & NA & NA & NA & NA & 125 & 79 & 421 & 282 & 547 & 431 & 550 & 628 & 198 & 356 & 1,842 & 1,776 & 3,618 \\
\hline Europe and Central Asia & NA & NA & NA & NA & NA & NA & 40 & 14 & 121 & 50 & 137 & 97 & 111 & 152 & 29 & 78 & 438 & 391 & 829 \\
\hline Latin America and the Caribbean & NA & NA & NA & NA & NA & NA & 23 & 16 & 55 & 38 & 51 & 40 & 46 & 45 & 22 & 30 & 197 & 169 & 365 \\
\hline Middle East and North Africa & NA & NA & NA & NA & NA & NA & 21 & 12 & 53 & 35 & 53 & 44 & 46 & 47 & 13 & 17 & 186 & 155 & 341 \\
\hline South Asia & NA & NA & NA & NA & NA & NA & 80 & 56 & 294 & 199 & 299 & 268 & 211 & 237 & 55 & 63 & 939 & 824 & 1,763 \\
\hline Sub-Saharan Africa & NA & NA & NA & NA & NA & NA & 31 & 30 & 60 & 55 & 54 & 58 & 39 & 49 & 10 & 15 & 194 & 206 & 401 \\
\hline Low- and middle-income countries & NA & NA & NA & NA & NA & NA & 319 & 207 & 1,006 & 658 & 1,141 & 939 & 1,005 & 1,157 & 326 & 560 & 3,797 & 3,520 & 7,317 \\
\hline High-income countries & NA & NA & NA & NA & NA & NA & 16 & 8 & 59 & 27 & 69 & 43 & 85 & 80 & 53 & 83 & 282 & 240 & 522 \\
\hline$\overline{\text { WORLD }}$ & NA & NA & NA & NA & NA & NA & 336 & 214 & 1,065 & 685 & 1,209 & 981 & 1,090 & 1,237 & 379 & 644 & 4,079 & 3,761 & 7,839 \\
\hline
\end{tabular}

Source: Authors' calculations.

Note: $\mathrm{NA}=$ not applicable.

374 | Global Burden of Disease and Risk Factors I Majid Ezzati, Stephen Vander Hoorn, Alan D. Lopez, and others 
Table 4A.107

Risk factor:

Urban air pollution

Disease:

All causes

\begin{tabular}{|c|c|c|c|c|c|c|c|c|c|c|c|c|c|c|c|c|c|c|c|}
\hline \multirow[b]{2}{*}{ Region } & \multicolumn{2}{|c|}{$0-4$ years } & \multicolumn{2}{|c|}{ 5-14 years } & \multicolumn{2}{|c|}{$15-29$ years } & \multicolumn{2}{|c|}{ 30-44 years } & \multicolumn{2}{|c|}{$45-59$ years } & \multicolumn{2}{|c|}{ 60-69 years } & \multicolumn{2}{|c|}{$70-79$ years } & $80+$ & years & & Total & \\
\hline & Male & Female & Male & Female & Male & Female & Male & Female & Male & Female & Male & Female & Male & Female & Male & Female & Male & Female & All \\
\hline PAF of Mortality (\%) & & & & & & & & & & & & & & & & & & & \\
\hline East Asia and Pacific & 0 & 0 & NA & NA & NA & NA & 1 & 1 & 2 & 2 & 4 & 3 & 4 & 4 & 5 & 5 & 3 & 3 & 3 \\
\hline Europe and Central Asia & 0 & 0 & NA & NA & NA & NA & 1 & 1 & 1 & 1 & 2 & 2 & 2 & 2 & 2 & 2 & 1 & 2 & 1 \\
\hline Latin America and the Caribbean & 0 & 0 & NA & NA & NA & NA & 0 & 1 & 1 & 1 & 2 & 1 & 2 & 2 & 2 & 2 & 1 & 1 & 1 \\
\hline Middle East and North Africa & 0 & 0 & NA & NA & NA & NA & 1 & 1 & 2 & 2 & 2 & 2 & 3 & 3 & 3 & 3 & 2 & 2 & 2 \\
\hline South Asia & 0 & 0 & NA & NA & NA & NA & 1 & 0 & 2 & 1 & 2 & 2 & 2 & 2 & 2 & 2 & 1 & 1 & 1 \\
\hline Sub-Saharan Africa & 0 & 0 & NA & NA & NA & NA & 0 & 0 & 1 & 1 & 1 & 1 & 1 & 1 & 1 & 1 & 0 & 0 & 0 \\
\hline Low- and middle-income countries & 0 & 0 & NA & NA & NA & NA & 1 & 1 & 2 & 1 & 2 & 2 & 3 & 3 & 3 & 3 & 1 & 2 & 2 \\
\hline High-income countries & 0 & 0 & NA & NA & NA & NA & 0 & 0 & 1 & 1 & 1 & 1 & 1 & 1 & 1 & 1 & 1 & 1 & 1 \\
\hline WORLD & 0 & 0 & NA & NA & NA & NA & 1 & 1 & 1 & 1 & 2 & 2 & 2 & 2 & 2 & 2 & 1 & 1 & 1 \\
\hline PAF of YLL (\%) & & & & & & & & & & & & & & & & & & & \\
\hline East Asia and Pacific & 0 & 0 & NA & NA & NA & NA & 1 & 1 & 2 & 2 & 4 & 3 & 4 & 4 & 5 & 5 & 2 & 2 & 2 \\
\hline Europe and Central Asia & 0 & 0 & NA & NA & NA & NA & 1 & 1 & 1 & 1 & 2 & 2 & 2 & 2 & 2 & 2 & 1 & 1 & 1 \\
\hline Latin America and the Caribbean & 0 & 0 & NA & NA & NA & NA & 0 & 1 & 1 & 1 & 2 & 1 & 2 & 2 & 2 & 2 & 1 & 1 & 1 \\
\hline Middle East and North Africa & 0 & 0 & NA & NA & NA & NA & 1 & 1 & 2 & 2 & 2 & 2 & 3 & 3 & 3 & 3 & 1 & 1 & 1 \\
\hline South Asia & 0 & 0 & NA & NA & NA & NA & 1 & 0 & 2 & 1 & 2 & 2 & 2 & 2 & 2 & 2 & 1 & 1 & 1 \\
\hline Sub-Saharan Africa & 0 & 0 & NA & NA & NA & NA & 0 & 0 & 1 & 1 & 1 & 1 & 1 & 1 & 1 & 1 & 0 & 0 & 0 \\
\hline Low- and middle-income countries & 0 & 0 & NA & NA & NA & NA & 1 & 0 & 2 & 1 & 2 & 2 & 3 & 3 & 3 & 3 & 1 & 1 & $\overline{1}$ \\
\hline High-income countries & 0 & 0 & NA & NA & NA & NA & 0 & 0 & 1 & 1 & 1 & 1 & 1 & 1 & 1 & 1 & 1 & 1 & 1 \\
\hline WORLD & 0 & 0 & NA & NA & NA & NA & 1 & 0 & 1 & 1 & 2 & 2 & 2 & 2 & 2 & 2 & 1 & 1 & 1 \\
\hline PAF of DALYs (\%) & & & & & & & & & & & & & & & & & & & \\
\hline East Asia and Pacific & 0 & 0 & NA & NA & NA & NA & 1 & 0 & 1 & 1 & 2 & 2 & 3 & 3 & 4 & 4 & 1 & 1 & 1 \\
\hline Europe and Central Asia & 0 & 0 & NA & NA & NA & NA & 0 & 0 & 1 & 1 & 1 & 1 & 2 & 1 & 2 & 1 & 1 & 1 & 1 \\
\hline Latin America and the Caribbean & 0 & 0 & NA & NA & NA & NA & 0 & 0 & 1 & 1 & 1 & 1 & 1 & 1 & 1 & 1 & 0 & 0 & 0 \\
\hline Middle East and North Africa & 0 & 0 & NA & NA & NA & NA & 1 & 0 & 1 & 1 & 2 & 2 & 2 & 2 & 2 & 2 & 1 & 1 & 1 \\
\hline South Asia & 0 & 0 & NA & NA & NA & NA & 0 & 0 & 1 & 1 & 2 & 1 & 2 & 2 & 2 & 2 & 1 & 0 & 1 \\
\hline Sub-Saharan Africa & 0 & 0 & NA & NA & NA & NA & 0 & 0 & 0 & 0 & 1 & 1 & 1 & 1 & 1 & 1 & 0 & 0 & 0 \\
\hline Low- and middle-income countries & 0 & 0 & NA & NA & NA & NA & 0 & 0 & 1 & 1 & 2 & 1 & 2 & 2 & 2 & 2 & 1 & 1 & $\overline{1}$ \\
\hline High-income countries & 0 & 0 & NA & NA & NA & NA & 0 & 0 & 0 & 0 & 1 & 0 & 1 & 1 & 1 & 1 & 0 & 0 & 0 \\
\hline WORLD & 0 & 0 & NA & NA & NA & NA & 0 & 0 & 1 & 1 & 2 & 1 & 2 & 2 & 2 & 2 & 1 & 1 & 1 \\
\hline Attributable Mortality (thousand & & & & & & & & & & & & & & & & & & & \\
\hline East Asia and Pacific & 1 & 3 & NA & NA & NA & NA & 6 & 4 & 28 & 17 & 49 & 32 & 69 & 66 & 42 & 72 & 196 & 193 & 389 \\
\hline Europe and Central Asia & 0 & 0 & NA & NA & NA & NA & 2 & 1 & 8 & 3 & 12 & 7 & 14 & 15 & 7 & 17 & 42 & 42 & 84 \\
\hline Latin America and the Caribbean & 0 & 0 & NA & NA & NA & NA & 1 & 1 & 3 & 2 & 4 & 3 & 6 & 5 & 5 & 7 & 20 & 17 & 37 \\
\hline Middle East and North Africa & 1 & 1 & NA & NA & NA & NA & 1 & 1 & 3 & 2 & 4 & 3 & 5 & 5 & 3 & 3 & 17 & 14 & 31 \\
\hline South Asia & 5 & 5 & NA & NA & NA & NA & 4 & 2 & 17 & 10 & 24 & 18 & 25 & 23 & 12 & 12 & 86 & 71 & 157 \\
\hline Sub-Saharan Africa & 4 & 3 & NA & NA & NA & NA & 1 & 1 & 3 & 3 & 4 & 4 & 4 & 5 & 2 & 3 & 19 & 18 & 37 \\
\hline Low- and middle-income countries & 11 & 11 & NA & NA & NA & NA & 15 & 9 & 63 & 36 & 98 & 67 & 123 & 118 & 69 & 113 & 380 & 355 & 735 \\
\hline High-income countries & 0 & 0 & NA & NA & NA & NA & 1 & 0 & 4 & 2 & 7 & 4 & 13 & 9 & 14 & 21 & 39 & 37 & 76 \\
\hline WORLD & 11 & 11 & NA & NA & NA & NA & 16 & 10 & 67 & 38 & 105 & 71 & 136 & 127 & 83 & 135 & 419 & 392 & 811 \\
\hline Attributable YLL (thousands) & & & & & & & & & & & & & & & & & & & \\
\hline East Asia and Pacific & 44 & 83 & NA & NA & NA & NA & 150 & 94 & 522 & 334 & 664 & 482 & 615 & 670 & 207 & 363 & 2,202 & 2,025 & 4,227 \\
\hline Europe and Central Asia & 7 & 6 & NA & NA & NA & NA & 44 & 15 & 146 & 54 & 163 & 102 & 123 & 155 & 30 & 79 & 512 & 411 & 923 \\
\hline Latin America and the Caribbean & 9 & 8 & NA & NA & NA & NA & 24 & 17 & 62 & 41 & 58 & 43 & 51 & 47 & 23 & 31 & 227 & 187 & 414 \\
\hline Middle East and North Africa & 20 & 19 & NA & NA & NA & NA & 22 & 13 & 57 & 36 & 57 & 45 & 49 & 48 & 13 & 17 & 218 & 177 & 395 \\
\hline South Asia & 152 & 154 & NA & NA & NA & NA & 86 & 58 & 318 & 205 & 323 & 274 & 223 & 240 & 57 & 64 & 1,159 & 995 & 2,154 \\
\hline Sub-Saharan Africa & 107 & 79 & NA & NA & NA & NA & 31 & 30 & 63 & 56 & 55 & 58 & 40 & 49 & 10 & 15 & 307 & 287 & 594 \\
\hline Low- and middle-income countries & 340 & 349 & NA & NA & NA & NA & 357 & 227 & 1,167 & 726 & 1,320 & 1,003 & 1,101 & 1,209 & 340 & 569 & 4,625 & 4,082 & 8,707 \\
\hline High-income countries & 1 & 0 & NA & NA & NA & NA & 20 & 10 & 83 & 40 & 99 & 57 & 112 & 94 & 60 & 88 & 374 & 290 & 664 \\
\hline WORLD & 340 & 349 & NA & NA & NA & NA & 377 & 237 & 1,251 & 766 & 1,419 & 1,060 & 1,213 & 1,302 & 400 & 657 & 4,999 & 4,372 & 9,371 \\
\hline Attributable DALYs (thousands) & & & & & & & & & & & & & & & & & & & \\
\hline East Asia and Pacific & 44 & 83 & NA & NA & NA & NA & 150 & 94 & 522 & 334 & 664 & 482 & 615 & 670 & 207 & 363 & 2,202 & 2,025 & 4,227 \\
\hline Europe and Central Asia & 7 & 6 & NA & NA & NA & NA & 44 & 15 & 146 & 54 & 163 & 102 & 123 & 155 & 30 & 79 & 512 & 411 & 923 \\
\hline Latin America and the Caribbean & 9 & 8 & NA & NA & NA & NA & 24 & 17 & 62 & 41 & 58 & 43 & 51 & 47 & 23 & 31 & 227 & 187 & 414 \\
\hline Middle East and North Africa & 20 & 19 & NA & NA & NA & NA & 22 & 13 & 57 & 36 & 57 & 45 & 49 & 48 & 13 & 17 & 218 & 177 & 395 \\
\hline South Asia & 152 & 154 & NA & NA & NA & NA & 86 & 58 & 318 & 205 & 323 & 274 & 223 & 240 & 57 & 64 & 1,159 & 995 & 2,154 \\
\hline Sub-Saharan Africa & 107 & 79 & NA & NA & NA & NA & 31 & 30 & 63 & 56 & 55 & 58 & 40 & 49 & 10 & 15 & 307 & 287 & 594 \\
\hline Low- and middle-income countries & 340 & 349 & NA & NA & NA & NA & 357 & 227 & 1,167 & 726 & 1,320 & 1,003 & 1,101 & 1,209 & 340 & 569 & 4,625 & 4,082 & 8,707 \\
\hline High-income countries & 1 & 0 & NA & NA & NA & NA & 20 & 10 & 83 & 40 & 99 & 57 & 112 & 94 & 60 & 88 & 374 & 290 & 664 \\
\hline$\overline{\text { WORLD }}$ & 340 & 349 & NA & NA & NA & NA & 377 & 237 & 1,251 & 766 & 1,419 & 1,060 & 1,213 & 1,302 & 400 & 657 & 4,999 & 4,372 & 9,371 \\
\hline
\end{tabular}

Source: Authors' calculations.

Note: $\mathrm{NA}=$ not applicable 
Table 4A.108

Risk factor:

Smoking

Disease:

Chronic obstructive pulmonary disease

\begin{tabular}{|c|c|c|c|c|c|c|c|c|c|c|c|c|c|c|c|c|c|c|c|}
\hline \multirow[b]{2}{*}{ Region } & \multicolumn{2}{|c|}{$0-4$ years } & \multicolumn{2}{|c|}{ 5-14 years } & \multicolumn{2}{|c|}{$15-29$ years } & \multicolumn{2}{|c|}{ 30-44 years } & \multicolumn{2}{|c|}{$45-59$ years } & \multicolumn{2}{|c|}{ 60-69 years } & \multicolumn{2}{|c|}{$70-79$ years } & $80+$ & years & & Total & \\
\hline & Male & Female & Male & Female & Male & Female & Male & Female & Male & Female & Male & Female & Male & Female & Male & Female & Male & Female & All \\
\hline PAF of Mortality (\%) & & & & & & & & & & & & & & & & & & & \\
\hline East Asia and Pacific & NA & NA & NA & NA & NA & NA & 70 & 42 & 54 & 21 & 48 & 21 & 41 & 17 & 44 & 13 & 44 & 16 & 29 \\
\hline Europe and Central Asia & NA & NA & NA & NA & NA & NA & 87 & 44 & 89 & 51 & 86 & 43 & 78 & 45 & 64 & 27 & 79 & 38 & 64 \\
\hline Latin America and the Caribbean & NA & NA & NA & NA & NA & NA & 52 & 23 & 68 & 45 & 64 & 44 & 59 & 42 & 60 & 47 & 60 & 43 & 53 \\
\hline Middle East and North Africa & NA & NA & NA & NA & NA & NA & 69 & 63 & 67 & 38 & 64 & 28 & 59 & 26 & 60 & 11 & 60 & 25 & 45 \\
\hline South Asia & NA & NA & NA & NA & NA & NA & 52 & 0 & 70 & 25 & 66 & 25 & 59 & 9 & 58 & 0 & 64 & 16 & 42 \\
\hline Sub-Saharan Africa & NA & NA & NA & NA & NA & NA & 23 & 15 & 46 & 22 & 42 & 18 & 35 & 13 & 33 & 13 & 38 & 15 & 29 \\
\hline Low- and middle-income countries & NA & NA & NA & NA & NA & NA & 53 & 18 & 65 & 25 & 58 & 24 & 48 & 17 & 47 & 14 & 53 & 18 & $\overline{36}$ \\
\hline High-income countries & NA & NA & NA & NA & NA & NA & 71 & 62 & 81 & 69 & 80 & 70 & 79 & 73 & 79 & 74 & 79 & 73 & 76 \\
\hline WORLD & NA & NA & NA & NA & NA & NA & 54 & 19 & 65 & 27 & 59 & 27 & 52 & 22 & 54 & 22 & 56 & 23 & 40 \\
\hline PAF of YLL (\%) & & & & & & & & & & & & & & & & & & & \\
\hline East Asia and Pacific & NA & NA & NA & NA & NA & NA & 70 & 42 & 54 & 21 & 48 & 21 & 41 & 17 & 44 & 13 & 46 & 18 & 31 \\
\hline Europe and Central Asia & NA & NA & NA & NA & NA & NA & 87 & 44 & 89 & 51 & 86 & 43 & 78 & 45 & 64 & 27 & 81 & 41 & 68 \\
\hline Latin America and the Caribbean & NA & NA & NA & NA & NA & NA & 52 & 23 & 68 & 45 & 64 & 44 & 59 & 42 & 60 & 47 & 59 & 38 & 50 \\
\hline Middle East and North Africa & NA & NA & NA & NA & NA & NA & 69 & 63 & 67 & 38 & 64 & 28 & 59 & 26 & 60 & 11 & 59 & 28 & 46 \\
\hline South Asia & NA & NA & NA & NA & NA & NA & 52 & 0 & 70 & 25 & 66 & 25 & 59 & 9 & 58 & 0 & 65 & 19 & 44 \\
\hline Sub-Saharan Africa & NA & NA & NA & NA & NA & NA & 23 & 15 & 46 & 22 & 42 & 18 & 35 & 13 & 33 & 13 & 38 & 16 & 30 \\
\hline Low- and middle-income countries & NA & NA & NA & NA & NA & NA & 53 & 18 & 65 & 25 & 58 & 24 & 48 & 17 & 47 & 14 & 55 & 20 & $\overline{38}$ \\
\hline High-income countries & NA & NA & NA & NA & NA & NA & 71 & 62 & 81 & 69 & 80 & 70 & 79 & 73 & 79 & 74 & 79 & 72 & 76 \\
\hline WORLD & NA & NA & NA & NA & NA & NA & 54 & 19 & 65 & 27 & 60 & 27 & 52 & 22 & 53 & 21 & 57 & 24 & 41 \\
\hline PAF of DALYs (\%) & & & & & & & & & & & & & & & & & & & \\
\hline East Asia and Pacific & NA & NA & NA & NA & NA & NA & 70 & 42 & 54 & 21 & 48 & 21 & 41 & 17 & 44 & 13 & 48 & 19 & 34 \\
\hline Europe and Central Asia & NA & NA & NA & NA & NA & NA & 87 & 44 & 89 & 51 & 86 & 43 & 78 & 45 & 64 & 27 & 81 & 42 & 64 \\
\hline Latin America and the Caribbean & NA & NA & NA & NA & NA & NA & 52 & 23 & 68 & 45 & 64 & 44 & 59 & 42 & 60 & 47 & 60 & 35 & 48 \\
\hline Middle East and North Africa & NA & NA & NA & NA & NA & NA & 69 & 63 & 67 & 38 & 64 & 28 & 59 & 26 & 60 & 11 & 61 & 34 & 52 \\
\hline South Asia & NA & NA & NA & NA & NA & NA & 52 & 0 & 70 & 25 & 66 & 25 & 59 & 9 & 58 & 0 & 65 & 18 & 43 \\
\hline Sub-Saharan Africa & NA & NA & NA & NA & NA & NA & 23 & 15 & 46 & 22 & 42 & 18 & 35 & 13 & 33 & 13 & 37 & 16 & 30 \\
\hline Low- and middle-income countries & NA & NA & NA & NA & NA & NA & 60 & 27 & 62 & 27 & 58 & 25 & 48 & 18 & 47 & 14 & 55 & 21 & $\overline{40}$ \\
\hline High-income countries & NA & NA & NA & NA & NA & NA & 71 & 62 & 81 & 69 & 80 & 70 & 79 & 73 & 79 & 74 & 77 & 68 & 73 \\
\hline WORLD & NA & NA & NA & NA & NA & NA & 62 & 36 & 65 & 34 & 60 & 30 & 53 & 23 & 54 & 22 & 58 & 28 & 44 \\
\hline Attributable Mortality (thousand & & & & & & & & & & & & & & & & & & & \\
\hline East Asia and Pacific & NA & NA & NA & NA & NA & NA & 2 & 1 & 25 & 7 & 66 & 23 & 113 & 48 & 83 & 44 & 289 & 123 & 412 \\
\hline Europe and Central Asia & NA & NA & NA & NA & NA & NA & 2 & 0 & 9 & 2 & 22 & 3 & 25 & 8 & 9 & 5 & 66 & 18 & 84 \\
\hline Latin America and the Caribbean & NA & NA & NA & NA & NA & NA & 0 & 0 & 3 & 1 & 7 & 3 & 12 & 5 & 12 & 8 & 35 & 18 & 53 \\
\hline Middle East and North Africa & NA & NA & NA & NA & NA & NA & 1 & 0 & 2 & 1 & 4 & 1 & 5 & 2 & 3 & 0 & 14 & 4 & 18 \\
\hline South Asia & NA & NA & NA & NA & NA & NA & 3 & 0 & 63 & 18 & 65 & 15 & 52 & 7 & 20 & 0 & 204 & 40 & 244 \\
\hline Sub-Saharan Africa & NA & NA & NA & NA & NA & NA & 1 & 0 & 6 & 1 & 8 & 2 & 9 & 2 & 4 & 1 & 28 & 6 & 34 \\
\hline Low- and middle-income countries & NA & NA & NA & NA & NA & NA & 9 & 2 & 108 & 30 & 172 & 48 & 216 & 71 & 131 & 58 & 635 & 210 & 845 \\
\hline High-income countries & NA & NA & NA & NA & NA & NA & 0 & 0 & 6 & 4 & 20 & 11 & 51 & 30 & 58 & 48 & 135 & 93 & 227 \\
\hline WORLD & NA & NA & NA & NA & NA & NA & 9 & 3 & 114 & 34 & 191 & 58 & 267 & 102 & 188 & 106 & 770 & 302 & 1,072 \\
\hline Attributable YLL (thousands) & & & & & & & & & & & & & & & & & & & \\
\hline East Asia and Pacific & NA & NA & NA & NA & NA & NA & 37 & 31 & 453 & 144 & 874 & 347 & 998 & 483 & 414 & 221 & 2,776 & 1,226 & 4,002 \\
\hline Europe and Central Asia & NA & NA & NA & NA & NA & NA & 40 & 7 & 168 & 30 & 294 & 47 & 227 & 80 & 40 & 23 & 769 & 188 & 957 \\
\hline Latin America and the Caribbean & NA & NA & NA & NA & NA & NA & 11 & 4 & 58 & 29 & 95 & 46 & 105 & 52 & 55 & 38 & 325 & 168 & 493 \\
\hline Middle East and North Africa & NA & NA & NA & NA & NA & NA & 12 & 8 & 35 & 16 & 51 & 15 & 47 & 17 & 14 & 3 & 158 & 58 & 216 \\
\hline South Asia & NA & NA & NA & NA & NA & NA & 83 & 0 & 1,144 & 350 & 890 & 231 & 465 & 75 & 100 & 0 & 2,683 & 657 & 3,339 \\
\hline Sub-Saharan Africa & NA & NA & NA & NA & NA & NA & 18 & 4 & 110 & 27 & 112 & 26 & 78 & 19 & 20 & 7 & 338 & 83 & 420 \\
\hline Low- and middle-income countries & NA & NA & NA & NA & NA & NA & 202 & 55 & 1,968 & 596 & 2,317 & 712 & 1,920 & 726 & 643 & 290 & 7,048 & 2,380 & 9,428 \\
\hline High-income countries & NA & NA & NA & NA & NA & NA & 11 & 6 & 111 & 71 & 259 & 159 & 446 & 307 & 256 & 213 & 1,083 & 756 & 1,838 \\
\hline WORLD & NA & NA & NA & NA & NA & NA & 212 & 61 & 2,079 & 667 & 2,576 & 871 & 2,365 & 1,033 & 899 & 503 & 8,131 & 3,136 & 11,267 \\
\hline Attributable DALYs (thousands) & & & & & & & & & & & & & & & & & & & \\
\hline East Asia and Pacific & NA & NA & NA & NA & NA & NA & 215 & 152 & 1,264 & 204 & 1,190 & 421 & 1,123 & 534 & 457 & 232 & 4,250 & 1,543 & 5,793 \\
\hline Europe and Central Asia & NA & NA & NA & NA & NA & NA & 127 & 94 & 277 & 123 & 369 & 83 & 272 & 96 & 47 & 25 & 1,093 & 422 & 1,514 \\
\hline Latin America and the Caribbean & NA & NA & NA & NA & NA & NA & 65 & 59 & 206 & 99 & 168 & 73 & 133 & 65 & 64 & 43 & 636 & 339 & 976 \\
\hline Middle East and North Africa & NA & NA & NA & NA & NA & NA & 113 & 35 & 85 & 24 & 61 & 17 & 50 & 17 & 14 & 3 & 325 & 96 & 420 \\
\hline South Asia & NA & NA & NA & NA & NA & NA & 232 & 0 & 1,375 & 473 & 1,050 & 253 & 499 & 79 & 106 & 0 & 3,262 & 806 & 4,068 \\
\hline Sub-Saharan Africa & NA & NA & NA & NA & NA & NA & 26 & 5 & 137 & 31 & 129 & 28 & 83 & 20 & 21 & 7 & 395 & 90 & 485 \\
\hline Low- and middle-income countries & NA & NA & NA & NA & NA & NA & 779 & 345 & 3,344 & 954 & 2,968 & 876 & 2,160 & 811 & 710 & 310 & 9,961 & 3,296 & 13,257 \\
\hline High-income countries & NA & NA & NA & NA & NA & NA & 178 & 268 & 632 & 453 & 491 & 316 & 590 & 383 & 289 & 253 & 2,181 & 1,673 & 3,853 \\
\hline$\overline{\text { WORLD }}$ & NA & NA & NA & NA & NA & NA & 957 & 612 & 3,976 & 1,407 & 3,459 & 1,192 & 2,750 & 1,194 & 1,000 & 563 & 12,142 & 4,968 & 17,110 \\
\hline
\end{tabular}

Source: Authors' calculations.

Note: $\mathrm{NA}=$ not applicable

376 | Global Burden of Disease and Risk Factors I Majid Ezzati, Stephen Vander Hoorn, Alan D. Lopez, and others 
Table 4A.109

Risk factor:

Smoking

Disease:

Trachea, bronchus, and lung cancers

\begin{tabular}{|c|c|c|c|c|c|c|c|c|c|c|c|c|c|c|c|c|c|c|c|}
\hline \multirow[b]{2}{*}{ Region } & \multicolumn{2}{|c|}{$0-4$ years } & \multicolumn{2}{|c|}{ 5-14 years } & \multicolumn{2}{|c|}{$15-29$ years } & \multicolumn{2}{|c|}{$30-44$ years } & \multicolumn{2}{|c|}{$45-59$ years } & \multicolumn{2}{|c|}{$60-69$ years } & \multicolumn{2}{|c|}{ 70-79 years } & $80+$ & years & & Total & \\
\hline & Male & Female & Male & Female & Male & Female & Male & Female & Male & Female & Male & Female & Male & Female & Male & Female & Male & Female & All \\
\hline PAF of Mortality (\%) & & & & & & & & & & & & & & & & & & & \\
\hline East Asia and Pacific & NA & NA & NA & NA & NA & NA & 51 & 19 & 52 & 20 & 60 & 25 & 59 & 19 & 60 & 12 & 57 & 20 & 45 \\
\hline Europe and Central Asia & NA & NA & NA & NA & NA & NA & 76 & 21 & 93 & 55 & 95 & 54 & 93 & 51 & 83 & 26 & 92 & 48 & 85 \\
\hline Latin America and the Caribbean & NA & NA & NA & NA & NA & NA & 36 & 10 & 78 & 49 & 85 & 55 & 84 & 49 & 81 & 45 & 80 & 47 & 70 \\
\hline Middle East and North Africa & NA & NA & NA & NA & NA & NA & 52 & 36 & 77 & 42 & 85 & 38 & 84 & 33 & 80 & 13 & 79 & 34 & 69 \\
\hline South Asia & NA & NA & NA & NA & NA & NA & 33 & 0 & 79 & 29 & 86 & 34 & 84 & 12 & 80 & 0 & 80 & 22 & 70 \\
\hline Sub-Saharan Africa & NA & NA & NA & NA & NA & NA & 18 & 10 & 58 & 27 & 69 & 27 & 66 & 20 & 58 & 18 & 61 & 23 & 51 \\
\hline Low- and middle-income countries & NA & NA & NA & NA & NA & NA & 51 & 16 & 71 & 29 & 76 & 33 & 74 & 26 & 71 & 18 & 72 & 27 & 60 \\
\hline High-income countries & NA & NA & NA & NA & NA & NA & 55 & 33 & 88 & 73 & 92 & 78 & 93 & 78 & 91 & 72 & 91 & 74 & 86 \\
\hline$\overline{\text { WORLD }}$ & NA & NA & NA & NA & NA & NA & 51 & 20 & 75 & 44 & 81 & 50 & 82 & 49 & 83 & 51 & 79 & 47 & 70 \\
\hline PAF of YLL (\%) & & & & & & & & & & & & & & & & & & & \\
\hline East Asia and Pacific & NA & NA & NA & NA & NA & NA & 51 & 19 & 52 & 20 & 60 & 25 & 59 & 19 & 60 & 12 & 56 & 21 & 44 \\
\hline Europe and Central Asia & NA & NA & NA & NA & NA & NA & 76 & 21 & 93 & 55 & 95 & 54 & 93 & 51 & 83 & 26 & 92 & 49 & 85 \\
\hline Latin America and the Caribbean & NA & NA & NA & NA & NA & NA & 36 & 10 & 78 & 49 & 85 & 55 & 84 & 49 & 81 & 45 & 78 & 45 & 67 \\
\hline Middle East and North Africa & NA & NA & NA & NA & NA & NA & 52 & 36 & 77 & 42 & 85 & 38 & 84 & 33 & 80 & 13 & 76 & 35 & 65 \\
\hline South Asia & NA & NA & NA & NA & NA & NA & 33 & 0 & 79 & 29 & 86 & 34 & 84 & 12 & 80 & 0 & 78 & 23 & 67 \\
\hline Sub-Saharan Africa & NA & NA & NA & NA & NA & NA & 18 & 10 & 58 & 27 & 69 & 27 & 66 & 20 & 58 & 18 & 59 & 23 & 49 \\
\hline Low- and middle-income countries & NA & NA & NA & NA & NA & NA & 51 & 16 & 71 & 29 & 76 & 33 & 74 & 26 & 71 & 17 & 71 & 27 & 59 \\
\hline High-income countries & NA & NA & NA & NA & NA & NA & 55 & 33 & 88 & 73 & 92 & 78 & 93 & 78 & 91 & 72 & 90 & 74 & 84 \\
\hline WORLD & NA & NA & NA & NA & NA & NA & 51 & 20 & 75 & 43 & 81 & 50 & 82 & 48 & 82 & 50 & 77 & 45 & 67 \\
\hline PAF of DALYs (\%) & & & & & & & & & & & & & & & & & & & \\
\hline East Asia and Pacific & NA & NA & NA & NA & NA & NA & 51 & 19 & 52 & 20 & 60 & 25 & 59 & 19 & 60 & 12 & 56 & 21 & 44 \\
\hline Europe and Central Asia & NA & NA & NA & NA & NA & NA & 76 & 21 & 93 & 55 & 95 & 54 & 93 & 51 & 83 & 26 & 92 & 49 & 85 \\
\hline Latin America and the Caribbean & NA & NA & NA & NA & NA & NA & 36 & 10 & 78 & 49 & 85 & 55 & 84 & 49 & 81 & 45 & 78 & 45 & 67 \\
\hline Middle East and North Africa & NA & NA & NA & NA & NA & NA & 52 & 36 & 77 & 42 & 85 & 38 & 84 & 33 & 80 & 13 & 76 & 35 & 65 \\
\hline South Asia & NA & NA & NA & NA & NA & NA & 33 & 0 & 79 & 29 & 86 & 34 & 84 & 12 & 80 & 0 & 78 & 23 & 67 \\
\hline Sub-Saharan Africa & NA & NA & NA & NA & NA & NA & 18 & 10 & 58 & 27 & 69 & 27 & 66 & 20 & 58 & 18 & 59 & 23 & 49 \\
\hline Low- and middle-income countries & NA & NA & NA & NA & NA & NA & 51 & 16 & 71 & 29 & 76 & 33 & 74 & 26 & 71 & 17 & 71 & 27 & 59 \\
\hline High-income countries & NA & NA & NA & NA & NA & NA & 55 & 33 & 88 & 73 & 92 & 78 & 93 & 78 & 91 & 72 & 90 & 74 & 84 \\
\hline WORLD & NA & NA & NA & NA & NA & NA & 51 & 20 & 75 & 44 & 81 & 50 & 82 & 49 & 82 & 50 & 77 & 45 & 67 \\
\hline Attributable Mortality (thousand & & & & & & & & & & & & & & & & & & & \\
\hline East Asia and Pacific & NA & NA & NA & NA & NA & NA & 6 & 1 & 31 & 6 & 57 & 9 & 45 & 8 & 11 & 2 & 150 & 25 & 176 \\
\hline Europe and Central Asia & NA & NA & NA & NA & NA & NA & 4 & 0 & 34 & 3 & 50 & 4 & 34 & 5 & 5 & 1 & 126 & 14 & 140 \\
\hline Latin America and the Caribbean & NA & NA & NA & NA & NA & NA & 0 & 0 & 6 & 2 & 10 & 3 & 10 & 2 & 4 & 1 & 31 & 8 & 39 \\
\hline Middle East and North Africa & NA & NA & NA & NA & NA & NA & 1 & 0 & 3 & 0 & 4 & 0 & 4 & 0 & 1 & 0 & 12 & 1 & 14 \\
\hline South Asia & NA & NA & NA & NA & NA & NA & 2 & 0 & 21 & 2 & 32 & 2 & 23 & 1 & 7 & 0 & 85 & 5 & 90 \\
\hline Sub-Saharan Africa & NA & NA & NA & NA & NA & NA & 0 & 0 & 2 & 0 & 3 & 0 & 1 & 0 & 0 & 0 & 7 & 1 & 8 \\
\hline Low- and middle-income countries & NA & NA & NA & NA & NA & NA & 12 & 2 & 97 & 13 & 156 & 19 & 117 & 17 & 28 & 4 & 411 & 55 & 466 \\
\hline High-income countries & NA & NA & NA & NA & NA & NA & 3 & 1 & 44 & 17 & 80 & 26 & 107 & 39 & 49 & 25 & 283 & 108 & 391 \\
\hline$\overline{\text { WORLD }}$ & NA & NA & NA & NA & NA & NA & 15 & 3 & 141 & 30 & 236 & 45 & 225 & 56 & 77 & 29 & 693 & 163 & 856 \\
\hline Attributable YLL (thousands) & & & & & & & & & & & & & & & & & & & \\
\hline East Asia and Pacific & NA & NA & NA & NA & NA & NA & 139 & 29 & 583 & 112 & 765 & 136 & 410 & 85 & 58 & 9 & 1,953 & 371 & 2,324 \\
\hline Europe and Central Asia & NA & NA & NA & NA & NA & NA & 83 & 7 & 629 & 66 & 688 & 66 & 316 & 53 & 23 & 5 & 1,740 & 196 & 1,936 \\
\hline Latin America and the Caribbean & NA & NA & NA & NA & NA & NA & 11 & 3 & 120 & 38 & 133 & 39 & 89 & 23 & 19 & 6 & 373 & 109 & 482 \\
\hline Middle East and North Africa & NA & NA & NA & NA & NA & NA & 12 & 5 & 51 & 10 & 55 & 6 & 34 & 4 & 5 & 0 & 158 & 25 & 183 \\
\hline South Asia & NA & NA & NA & NA & NA & NA & 36 & 0 & 389 & 37 & 437 & 38 & 212 & 7 & 35 & 0 & 1,109 & 82 & 1,191 \\
\hline Sub-Saharan Africa & NA & NA & NA & NA & NA & NA & 2 & 1 & 42 & 6 & 35 & 5 & 13 & 2 & 2 & 0 & 94 & 14 & 108 \\
\hline Low- and middle-income countries & NA & NA & NA & NA & NA & NA & 284 & 45 & 1,814 & 269 & 2,114 & 290 & 1,073 & 174 & 143 & 20 & 5,427 & 797 & 6,224 \\
\hline High-income countries & NA & NA & NA & NA & NA & NA & 67 & 28 & 810 & 331 & 1,074 & 396 & 957 & 402 & 233 & 123 & 3,141 & 1,281 & 4,422 \\
\hline WORLD & NA & NA & NA & NA & NA & NA & 351 & 73 & 2,624 & 599 & 3,187 & 686 & 2,030 & 576 & 376 & 144 & 8,568 & 2,078 & $\overline{10,646}$ \\
\hline Attributable DALYs (thousands) & & & & & & & & & & & & & & & & & & & \\
\hline East Asia and Pacific & NA & NA & NA & NA & NA & NA & 140 & 30 & 588 & 113 & 774 & 138 & 416 & 86 & 59 & 9 & 1,978 & 375 & 2,353 \\
\hline Europe and Central Asia & NA & NA & NA & NA & NA & NA & 84 & 7 & 636 & 66 & 698 & 67 & 322 & 54 & 24 & 5 & 1,764 & 199 & 1,963 \\
\hline Latin America and the Caribbean & NA & NA & NA & NA & NA & NA & 11 & 3 & 122 & 38 & 135 & 39 & 90 & 24 & 20 & 7 & 379 & 110 & 488 \\
\hline Middle East and North Africa & NA & NA & NA & NA & NA & NA & 12 & 5 & 52 & 10 & 56 & 6 & 35 & 4 & 6 & 0 & 160 & 25 & 185 \\
\hline South Asia & NA & NA & NA & NA & NA & NA & 36 & 0 & 392 & 37 & 442 & 38 & 215 & 7 & 36 & 0 & 1,121 & 83 & 1,203 \\
\hline Sub-Saharan Africa & NA & NA & NA & NA & NA & NA & 2 & 1 & 42 & 6 & 36 & 5 & 13 & 2 & 2 & 0 & 95 & 14 & 109 \\
\hline Low- and middle-income countries & NA & NA & NA & NA & NA & NA & 285 & 45 & 1,833 & 271 & 2,141 & 292 & 1,090 & 176 & 147 & 21 & 5,496 & 805 & 6,302 \\
\hline High-income countries & NA & NA & NA & NA & NA & NA & 68 & 29 & 828 & 338 & 1,103 & 407 & 991 & 416 & 247 & 129 & 3,237 & 1,319 & 4,556 \\
\hline$\overline{\text { WORLD }}$ & NA & NA & NA & NA & NA & NA & 354 & 74 & 2,661 & 609 & 3,244 & 699 & 2,081 & 592 & 394 & 150 & 8,734 & 2,124 & $\overline{10,858}$ \\
\hline
\end{tabular}

Source: Authors' calculations.

Note: $\mathrm{NA}=$ not applicable. 
Table 4A.110

Risk factor:

Smoking

Disease:

Liver cancer

\begin{tabular}{|c|c|c|c|c|c|c|c|c|c|c|c|c|c|c|c|c|c|c|c|}
\hline \multirow[b]{2}{*}{ Region } & \multicolumn{2}{|c|}{$0-4$ years } & \multicolumn{2}{|c|}{ 5-14 years } & \multicolumn{2}{|c|}{$15-29$ years } & \multicolumn{2}{|c|}{ 30-44 years } & \multicolumn{2}{|c|}{ 45-59 years } & \multicolumn{2}{|c|}{ 60-69 years } & \multicolumn{2}{|c|}{$70-79$ years } & $80+$ & years & & Total & \\
\hline & Male & Female & Male & Female & Male & Female & Male & Female & Male & Female & Male & Female & Male & Female & Male & Female & Male & Female & All \\
\hline PAF of Mortality (\%) & & & & & & & & & & & & & & & & & & & \\
\hline East Asia and Pacific & NA & NA & NA & NA & NA & NA & 26 & 4 & 15 & 1 & 12 & 1 & 9 & 1 & 10 & 1 & 14 & 1 & 10 \\
\hline Europe and Central Asia & NA & NA & NA & NA & NA & NA & 49 & 4 & 55 & 5 & 47 & 4 & 34 & 4 & 21 & 2 & 43 & 4 & 27 \\
\hline Latin America and the Caribbean & NA & NA & NA & NA & NA & NA & 14 & 2 & 24 & 4 & 21 & 4 & 18 & 4 & 18 & 5 & 19 & 4 & 12 \\
\hline Middle East and North Africa & NA & NA & NA & NA & NA & NA & 25 & 8 & 23 & 3 & 21 & 2 & 18 & 2 & 18 & 1 & 20 & 3 & 13 \\
\hline South Asia & NA & NA & NA & NA & NA & NA & 14 & 0 & 25 & 2 & 22 & 2 & 18 & 1 & 17 & 0 & 19 & 1 & 12 \\
\hline Sub-Saharan Africa & NA & NA & NA & NA & NA & NA & 4 & 1 & 11 & 1 & 10 & 1 & 8 & 1 & 7 & 1 & 9 & 1 & 6 \\
\hline Low- and middle-income countries & NA & NA & NA & NA & NA & NA & 23 & 3 & 16 & 2 & 15 & 2 & 12 & 2 & 12 & 1 & 15 & 2 & $\overline{11}$ \\
\hline High-income countries & NA & NA & NA & NA & NA & NA & 27 & 8 & 38 & 11 & 37 & 11 & 35 & 13 & 36 & 14 & 36 & 12 & 29 \\
\hline WORLD & NA & NA & NA & NA & NA & NA & 23 & 3 & 19 & 3 & 19 & 3 & 18 & 4 & 21 & 6 & 19 & 4 & 14 \\
\hline PAF of YLL (\%) & & & & & & & & & & & & & & & & & & & \\
\hline East Asia and Pacific & NA & NA & NA & NA & NA & NA & 26 & 4 & 15 & 1 & 12 & 1 & 9 & 1 & 10 & 1 & 15 & 2 & 11 \\
\hline Europe and Central Asia & NA & NA & NA & NA & NA & NA & 49 & 4 & 55 & 5 & 47 & 4 & 34 & 4 & 21 & 2 & 46 & 4 & 29 \\
\hline Latin America and the Caribbean & NA & NA & NA & NA & NA & NA & 14 & 2 & 24 & 4 & 21 & 4 & 18 & 4 & 18 & 5 & 19 & 4 & 11 \\
\hline Middle East and North Africa & NA & NA & NA & NA & NA & NA & 25 & 8 & 23 & 3 & 21 & 2 & 18 & 2 & 18 & 1 & 20 & 3 & 13 \\
\hline South Asia & NA & NA & NA & NA & NA & NA & 14 & 0 & 25 & 2 & 22 & 2 & 18 & 1 & 17 & 0 & 18 & 1 & 11 \\
\hline Sub-Saharan Africa & NA & NA & NA & NA & NA & NA & 4 & 1 & 11 & 1 & 10 & 1 & 8 & 1 & 7 & 1 & 8 & 1 & 6 \\
\hline Low- and middle-income countries & NA & NA & NA & NA & NA & NA & 23 & 3 & 16 & 2 & 15 & 2 & 12 & 2 & 12 & 1 & 16 & 2 & $\overline{12}$ \\
\hline High-income countries & NA & NA & NA & NA & NA & NA & 27 & 8 & 38 & 11 & 37 & 11 & 35 & 13 & 36 & 14 & 36 & 12 & 29 \\
\hline WORLD & NA & NA & NA & NA & NA & NA & 23 & 3 & 19 & 3 & 19 & 3 & 18 & 4 & 20 & 6 & 18 & 3 & 14 \\
\hline PAF of DALYs (\%) & & & & & & & & & & & & & & & & & & & \\
\hline East Asia and Pacific & NA & NA & NA & NA & NA & NA & 26 & 4 & 15 & 1 & 12 & 1 & 9 & 1 & 10 & 1 & 15 & 2 & 11 \\
\hline Europe and Central Asia & NA & NA & NA & NA & NA & NA & 49 & 4 & 55 & 5 & 47 & 4 & 34 & 4 & 21 & 2 & 45 & 4 & 29 \\
\hline Latin America and the Caribbean & NA & NA & NA & NA & NA & NA & 14 & 2 & 24 & 4 & 21 & 4 & 18 & 4 & 18 & 5 & 19 & 4 & 11 \\
\hline Middle East and North Africa & NA & NA & NA & NA & NA & NA & 25 & 8 & 23 & 3 & 21 & 2 & 18 & 2 & 18 & 1 & 20 & 3 & 13 \\
\hline South Asia & NA & NA & NA & NA & NA & NA & 14 & 0 & 25 & 2 & 22 & 2 & 18 & 1 & 17 & 0 & 18 & 1 & 11 \\
\hline Sub-Saharan Africa & NA & NA & NA & NA & NA & NA & 4 & 1 & 11 & 1 & 10 & 1 & 8 & 1 & 7 & 1 & 8 & 1 & 6 \\
\hline Low- and middle-income countries & NA & NA & NA & NA & NA & NA & 23 & 3 & 16 & 2 & 15 & 2 & 12 & 2 & 12 & 1 & 16 & 2 & $\overline{12}$ \\
\hline High-income countries & NA & NA & NA & NA & NA & NA & 27 & 8 & 38 & 11 & 37 & 11 & 35 & 13 & 36 & 14 & 36 & 12 & 29 \\
\hline WORLD & NA & NA & NA & NA & NA & NA & 23 & 3 & 19 & 3 & 19 & 3 & 18 & 4 & 20 & 6 & 18 & 3 & 14 \\
\hline Attributable Mortality (thousand & & & & & & & & & & & & & & & & & & & \\
\hline East Asia and Pacific & NA & NA & NA & NA & NA & NA & 7 & 0 & 15 & 0 & 9 & 0 & 5 & 0 & 1 & 0 & 37 & 2 & 38 \\
\hline Europe and Central Asia & NA & NA & NA & NA & NA & NA & 0 & 0 & 2 & 0 & 3 & 0 & 2 & 0 & 0 & 0 & 7 & 0 & 8 \\
\hline Latin America and the Caribbean & NA & NA & NA & NA & NA & NA & 0 & 0 & 1 & 0 & 1 & 0 & 1 & 0 & 0 & 0 & 2 & 0 & 2 \\
\hline Middle East and North Africa & NA & NA & NA & NA & NA & NA & 0 & 0 & 0 & 0 & 0 & 0 & 0 & 0 & 0 & 0 & 1 & 0 & 1 \\
\hline South Asia & NA & NA & NA & NA & NA & NA & 0 & 0 & 1 & 0 & 1 & 0 & 1 & 0 & 0 & 0 & 3 & 0 & 3 \\
\hline Sub-Saharan Africa & NA & NA & NA & NA & NA & NA & 0 & 0 & 1 & 0 & 1 & 0 & 0 & 0 & 0 & 0 & 3 & 0 & 3 \\
\hline Low- and middle-income countries & NA & NA & NA & NA & NA & NA & 9 & 0 & 20 & 1 & 14 & 1 & 8 & 1 & 2 & 0 & 53 & 3 & $\overline{56}$ \\
\hline High-income countries & NA & NA & NA & NA & NA & NA & 1 & 0 & 5 & 0 & 8 & 1 & 8 & 2 & 3 & 1 & 25 & 4 & 29 \\
\hline$\overline{\text { WORLD }}$ & NA & NA & NA & NA & NA & NA & 9 & 1 & 25 & 1 & 22 & 1 & 16 & 2 & 5 & 2 & 78 & 7 & 85 \\
\hline Attributable YLL (thousands) & & & & & & & & & & & & & & & & & & & \\
\hline East Asia and Pacific & NA & NA & NA & NA & NA & NA & 176 & 9 & 280 & 7 & 121 & 6 & 44 & 3 & 6 & 0 & 627 & 26 & 654 \\
\hline Europe and Central Asia & NA & NA & NA & NA & NA & NA & 10 & 0 & 41 & 2 & 37 & 2 & 15 & 2 & 1 & 0 & 104 & 6 & 110 \\
\hline Latin America and the Caribbean & NA & NA & NA & NA & NA & NA & 2 & 0 & 10 & 2 & 8 & 2 & 5 & 1 & 1 & 0 & 26 & 5 & 32 \\
\hline Middle East and North Africa & NA & NA & NA & NA & NA & NA & 3 & 1 & 6 & 1 & 5 & 0 & 3 & 0 & 0 & 0 & 16 & 2 & 18 \\
\hline South Asia & NA & NA & NA & NA & NA & NA & 7 & 0 & 21 & 1 & 14 & 1 & 5 & 0 & 1 & 0 & 48 & 2 & 50 \\
\hline Sub-Saharan Africa & NA & NA & NA & NA & NA & NA & 4 & 0 & 23 & 1 & 11 & 1 & 4 & 0 & 1 & 0 & 42 & 3 & 45 \\
\hline Low- and middle-income countries & NA & NA & NA & NA & NA & NA & 202 & 11 & 381 & 14 & 197 & 11 & 75 & 7 & 10 & 1 & 864 & 44 & $\overline{908}$ \\
\hline High-income countries & NA & NA & NA & NA & NA & NA & 13 & 1 & 100 & 7 & 107 & 11 & 70 & 16 & 16 & 7 & 306 & 42 & 348 \\
\hline WORLD & NA & NA & NA & NA & NA & NA & 215 & 12 & 481 & 20 & 303 & 22 & 145 & 23 & 26 & 8 & 1,170 & 85 & 1,256 \\
\hline Attributable DALYs (thousands) & & & & & & & & & & & & & & & & & & & \\
\hline East Asia and Pacific & NA & NA & NA & NA & NA & NA & 177 & 9 & 282 & 7 & 122 & 6 & 44 & 3 & 6 & 0 & 631 & 27 & 657 \\
\hline Europe and Central Asia & NA & NA & NA & NA & NA & NA & 10 & 0 & 41 & 2 & 38 & 2 & 15 & 2 & 1 & 0 & 104 & 6 & 111 \\
\hline Latin America and the Caribbean & NA & NA & NA & NA & NA & NA & 2 & 0 & 10 & 2 & 8 & 2 & 5 & 1 & 1 & 0 & 27 & 5 & 32 \\
\hline Middle East and North Africa & NA & NA & NA & NA & NA & NA & 3 & 1 & 6 & 1 & 5 & 0 & 3 & 0 & 0 & 0 & 16 & 2 & 18 \\
\hline South Asia & NA & NA & NA & NA & NA & NA & 7 & 0 & 21 & 1 & 15 & 1 & 5 & 0 & 1 & 0 & 49 & 2 & 50 \\
\hline Sub-Saharan Africa & NA & NA & NA & NA & NA & NA & 4 & 0 & 23 & 1 & 11 & 1 & 4 & 0 & 1 & 0 & 42 & 3 & 45 \\
\hline Low- and middle-income countries & NA & NA & NA & NA & NA & NA & 202 & 11 & 383 & 14 & 198 & 11 & 75 & 7 & 10 & 1 & 869 & 44 & $\overline{913}$ \\
\hline High-income countries & NA & NA & NA & NA & NA & NA & 13 & 1 & 101 & 7 & 108 & 12 & 72 & 16 & 16 & 7 & 311 & 43 & 354 \\
\hline$\overline{\text { WORLD }}$ & NA & NA & NA & NA & NA & NA & 216 & 12 & 484 & 21 & 306 & 22 & 147 & 23 & 27 & 8 & 1,180 & 86 & 1,267 \\
\hline
\end{tabular}

Source: Authors' calculations.

Note: $\mathrm{NA}=$ not applicable. 
Table 4A.111

Risk factor:

Smoking

Disease:

Cervix uteri cancer

\begin{tabular}{|c|c|c|c|c|c|c|c|c|c|c|c|c|c|c|c|c|c|c|c|}
\hline \multirow[b]{2}{*}{ Region } & \multicolumn{2}{|c|}{$0-4$ years } & \multicolumn{2}{|c|}{ 5-14 years } & \multicolumn{2}{|c|}{$15-29$ years } & \multicolumn{2}{|c|}{ 30-44 years } & \multicolumn{2}{|c|}{ 45-59 years } & \multicolumn{2}{|c|}{$60-69$ years } & \multicolumn{2}{|c|}{ 70-79 years } & \multicolumn{2}{|c|}{$80+$ years } & \multicolumn{3}{|c|}{ Total } \\
\hline & Male & Female & Male & Female & Male & Female & Male & Female & Male & Female & Male & Female & Male & Female & Male & Female & Male & Female & All \\
\hline \multicolumn{20}{|l|}{ PAF of Mortality (\%) } \\
\hline East Asia and Pacific & NA & NA & NA & NA & NA & NA & NA & 4 & NA & 1 & NA & 1 & NA & 1 & NA & 1 & NA & 1 & 1 \\
\hline Europe and Central Asia & NA & NA & NA & NA & NA & NA & NA & 4 & NA & 5 & NA & 4 & NA & 4 & NA & 2 & NA & 4 & 4 \\
\hline Latin America and the Caribbean & NA & NA & NA & NA & NA & NA & NA & 2 & NA & 4 & NA & 4 & NA & 4 & NA & 5 & NA & 4 & 4 \\
\hline Middle East and North Africa & NA & NA & NA & NA & NA & NA & NA & 8 & NA & 3 & NA & 2 & NA & 2 & NA & 1 & NA & 3 & 3 \\
\hline South Asia & NA & NA & NA & NA & NA & NA & NA & 0 & NA & 2 & NA & 2 & NA & 1 & NA & 0 & NA & 1 & 1 \\
\hline Sub-Saharan Africa & NA & NA & NA & NA & NA & NA & NA & 1 & NA & 1 & NA & 1 & NA & 1 & NA & 1 & NA & 1 & 1 \\
\hline Low- and middle-income countries & NA & NA & NA & NA & NA & NA & NA & 2 & NA & 2 & NA & 2 & NA & 1 & NA & 1 & NA & 2 & 2 \\
\hline High-income countries & NA & NA & NA & NA & NA & NA & NA & 8 & NA & 11 & NA & 11 & NA & 13 & NA & 14 & NA & 11 & 11 \\
\hline WORLD & NA & NA & NA & NA & NA & NA & NA & 3 & NA & 3 & NA & 2 & NA & 2 & NA & 3 & NA & 2 & 2 \\
\hline \multicolumn{20}{|l|}{ PAF of YLL (\%) } \\
\hline East Asia and Pacific & NA & NA & NA & NA & NA & NA & NA & 4 & NA & 1 & NA & 1 & NA & 1 & NA & 1 & NA & 2 & 2 \\
\hline Europe and Central Asia & NA & NA & NA & NA & NA & NA & NA & 4 & NA & 5 & NA & 4 & NA & 4 & NA & 2 & NA & 4 & 4 \\
\hline Latin America and the Caribbean & NA & NA & NA & NA & NA & NA & NA & 2 & NA & 4 & NA & 4 & NA & 4 & NA & 5 & NA & 3 & 3 \\
\hline Middle East and North Africa & NA & NA & NA & NA & NA & NA & NA & 8 & NA & 3 & NA & 2 & NA & 2 & NA & 1 & NA & 3 & 3 \\
\hline South Asia & NA & NA & NA & NA & NA & NA & NA & 0 & NA & 2 & NA & 2 & NA & 1 & NA & 0 & NA & 1 & 1 \\
\hline Sub-Saharan Africa & NA & NA & NA & NA & NA & NA & NA & 1 & NA & 1 & NA & 1 & NA & 1 & NA & 1 & NA & 1 & 1 \\
\hline Low- and middle-income countries & NA & NA & NA & NA & NA & NA & NA & 2 & NA & 2 & NA & 2 & NA & 1 & NA & 1 & NA & 2 & 2 \\
\hline High-income countries & NA & NA & NA & NA & NA & NA & NA & 8 & NA & 11 & NA & 11 & NA & 13 & NA & 14 & NA & 10 & 10 \\
\hline WORLD & NA & NA & NA & NA & NA & NA & NA & 3 & NA & 3 & NA & 2 & NA & 2 & NA & 3 & NA & 2 & 2 \\
\hline \multicolumn{20}{|l|}{ PAF of DALYs (\%) } \\
\hline East Asia and Pacific & NA & NA & NA & NA & NA & NA & NA & 4 & NA & 1 & NA & 1 & NA & 1 & NA & 1 & NA & 2 & 2 \\
\hline Europe and Central Asia & NA & NA & NA & NA & NA & NA & NA & 4 & NA & 5 & NA & 4 & NA & 4 & NA & 2 & NA & 4 & 4 \\
\hline Latin America and the Caribbean & NA & NA & NA & NA & NA & NA & NA & 2 & NA & 4 & NA & 4 & NA & 4 & NA & 5 & NA & 3 & 3 \\
\hline Middle East and North Africa & NA & NA & NA & NA & NA & NA & NA & 8 & NA & 3 & NA & 2 & NA & 2 & NA & 1 & NA & 3 & 3 \\
\hline South Asia & NA & NA & NA & NA & NA & NA & NA & 0 & NA & 2 & NA & 2 & NA & 1 & NA & 0 & NA & 1 & 1 \\
\hline Sub-Saharan Africa & NA & NA & NA & NA & NA & NA & NA & 1 & NA & 1 & NA & 1 & NA & 1 & NA & 1 & NA & 1 & 1 \\
\hline Low- and middle-income countries & NA & NA & NA & NA & NA & NA & NA & 2 & NA & 2 & NA & 2 & NA & 1 & NA & 1 & NA & 2 & 2 \\
\hline High-income countries & NA & NA & NA & NA & NA & NA & NA & 8 & NA & 11 & NA & 11 & NA & 13 & NA & 14 & NA & 10 & 10 \\
\hline WORLD & NA & NA & NA & NA & NA & NA & NA & 3 & NA & 3 & NA & 2 & NA & 2 & NA & 3 & NA & 2 & 2 \\
\hline
\end{tabular}

Attributable Mortality (thousands)

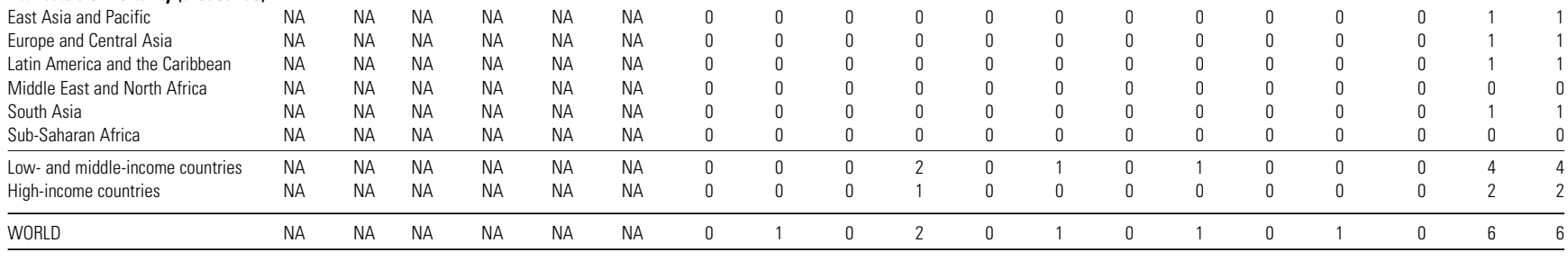

Attributable YLL (thousands)

Europe and Central Asia

Latin America and the Caribbea

Middle East and North Africa

South Asia

Sub-Saharan Africa

Low- and middle-income countries

High-incomed

NA NA NA NA NA NA

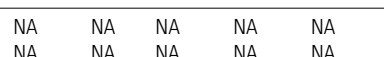

\begin{tabular}{|c|c|c|c|c|c|c|c|c|c|c|}
\hline WORLL & \multicolumn{2}{|l|}{ NA } & 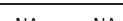 & NA & NA & \multicolumn{2}{|c|}{15} & 0 & 42 & 2 \\
\hline
\end{tabular}

Attributable DALYs (thousands)

East Asia and Pacific

Europe and Central Asia

Latin America and the Caribbean

Middle East and North Africa

South Asia

Sub-Saharan Africa

Low- and middle-income countrie

High-income countries

$\begin{array}{llllll}\text { NA } & \text { NA } & \text { NA } & \text { NA } & \text { NA } & \text { NA } \\ \text { NA } & \text { NA } & \text { NA } & \text { NA } & \text { NA } & \text { NA } \\ \text { NA } & \text { NA } & \text { NA } & \text { NA } & \text { NA } & \text { NA } \\ \text { NA } & \text { NA } & \text { NA } & \text { NA } & \text { NA } & \text { NA } \\ \text { NA } & \text { NA } & \text { NA } & \text { NA } & \text { NA } & \text { NA } \\ \text { NA } & \text { NA } & \text { NA } & \text { NA } & \text { NA } & \text { NA } \\ \text { NA } & \text { NA } & \text { NA } & \text { NA } & \text { NA } & \text { NA } \\ \text { NA } & \text { NA } & \text { NA } & \text { NA } & \text { NA } & \text { NA } \\ \text { NA } & \text { NA } & \text { NA } & \text { NA } & \text { NA } & \text { NA }\end{array}$

Source: Authors' calculations.

Note: $\mathrm{NA}=$ not applicable 
Table 4A.112

Risk factor:

Smoking

Disease:

Bladder cancer

\begin{tabular}{|c|c|c|c|c|c|c|c|c|c|c|c|c|c|c|c|c|c|c|c|}
\hline \multirow[b]{2}{*}{ Region } & \multicolumn{2}{|c|}{$0-4$ years } & \multicolumn{2}{|c|}{ 5-14 years } & \multicolumn{2}{|c|}{$15-29$ years } & \multicolumn{2}{|c|}{$30-44$ years } & \multicolumn{2}{|c|}{ 45-59 years } & \multicolumn{2}{|c|}{ 60-69 years } & \multicolumn{2}{|c|}{ 70-79 years } & \multicolumn{2}{|c|}{ 80+ years } & \multicolumn{3}{|c|}{ Total } \\
\hline & Male & Female & Male & Female & Male & Female & Male & Female & Male & Female & Male & Female & Male & Female & Male & Female & Male & Female & All \\
\hline \multicolumn{20}{|l|}{ PAF of Mortality (\%) } \\
\hline East Asia and Pacific & NA & NA & NA & NA & NA & NA & 34 & 10 & 21 & 4 & 17 & 4 & 14 & 3 & 15 & 2 & 16 & 3 & 12 \\
\hline Europe and Central Asia & NA & NA & NA & NA & NA & NA & 59 & 11 & 64 & 13 & 57 & 10 & 44 & 11 & 28 & 5 & 49 & 10 & 41 \\
\hline Latin America and the Caribbean & NA & NA & NA & NA & NA & NA & 19 & 4 & 32 & 11 & 29 & 11 & 25 & 10 & 25 & 12 & 26 & 11 & 21 \\
\hline Middle East and North Africa & NA & NA & NA & NA & NA & NA & 33 & 20 & 31 & 8 & 28 & 6 & 24 & 5 & 25 & 2 & 28 & 7 & 24 \\
\hline South Asia & NA & NA & NA & NA & NA & NA & 19 & 0 & 34 & 5 & 30 & 5 & 24 & 1 & 24 & 0 & 27 & 2 & 15 \\
\hline Sub-Saharan Africa & NA & NA & NA & NA & NA & NA & 6 & 3 & 16 & 4 & 14 & 3 & 11 & 2 & 10 & 2 & 12 & 3 & 9 \\
\hline Low- and middle-income countries & NA & NA & NA & NA & NA & NA & 33 & 3 & 35 & 6 & 32 & 6 & 25 & 5 & 21 & 3 & 28 & 4 & 21 \\
\hline High-income countries & NA & NA & NA & NA & NA & NA & 36 & 19 & 48 & 25 & 47 & 26 & 45 & 29 & 45 & 30 & 46 & 29 & 41 \\
\hline WORLD & NA & NA & NA & NA & NA & NA & 33 & 4 & 38 & 9 & 36 & 10 & 32 & 13 & 33 & 18 & 34 & 13 & 28 \\
\hline \multicolumn{20}{|l|}{ PAF of YLL (\%) } \\
\hline East Asia and Pacific & NA & NA & NA & NA & NA & NA & 34 & 10 & 21 & 4 & 17 & 4 & 14 & 3 & 15 & 2 & 17 & 4 & 13 \\
\hline Europe and Central Asia & NA & NA & NA & NA & NA & NA & 59 & 11 & 64 & 13 & 57 & 10 & 44 & 11 & 28 & 5 & 53 & 10 & 45 \\
\hline Latin America and the Caribbean & NA & NA & NA & NA & NA & NA & 19 & 4 & 32 & 11 & 29 & 11 & 25 & 10 & 25 & 12 & 27 & 10 & 21 \\
\hline Middle East and North Africa & NA & NA & NA & NA & NA & NA & 33 & 20 & 31 & 8 & 28 & 6 & 24 & 5 & 25 & 2 & 28 & 9 & 25 \\
\hline South Asia & NA & NA & NA & NA & NA & NA & 19 & 0 & 34 & 5 & 30 & 5 & 24 & 1 & 24 & 0 & 28 & 2 & 13 \\
\hline Sub-Saharan Africa & NA & NA & NA & NA & NA & NA & 6 & 3 & 16 & 4 & 14 & 3 & 11 & 2 & 10 & 2 & 13 & 3 & 9 \\
\hline Low- and middle-income countries & NA & NA & NA & NA & NA & NA & 33 & 3 & 35 & 6 & 32 & 6 & 25 & 5 & 21 & 3 & 30 & 5 & 21 \\
\hline High-income countries & NA & NA & NA & NA & NA & NA & 36 & 19 & 48 & 25 & 47 & 26 & 45 & 29 & 45 & 30 & 46 & 28 & 41 \\
\hline WORLD & NA & NA & NA & NA & NA & NA & 33 & 4 & 38 & 9 & 36 & 10 & 32 & 13 & 33 & 17 & 34 & 10 & 26 \\
\hline \multicolumn{20}{|l|}{ PAF of DALYs (\%) } \\
\hline East Asia and Pacific & NA & NA & NA & NA & NA & NA & 34 & 10 & 21 & 4 & 17 & 4 & 14 & 3 & 15 & 2 & 17 & 4 & 13 \\
\hline Europe and Central Asia & NA & NA & NA & NA & NA & NA & 59 & 11 & 64 & 13 & 57 & 10 & 44 & 11 & 28 & 5 & 53 & 10 & 45 \\
\hline Latin America and the Caribbean & NA & NA & NA & NA & NA & NA & 19 & 4 & 32 & 11 & 29 & 11 & 25 & 10 & 25 & 12 & 27 & 10 & 21 \\
\hline Middle East and North Africa & NA & NA & NA & NA & NA & NA & 33 & 20 & 31 & 8 & 28 & 6 & 24 & 5 & 25 & 2 & 28 & 9 & 25 \\
\hline South Asia & NA & NA & NA & NA & NA & NA & 19 & 0 & 34 & 5 & 30 & 5 & 24 & 1 & 24 & 0 & 28 & 2 & 13 \\
\hline Sub-Saharan Africa & NA & NA & NA & NA & NA & NA & 6 & 3 & 16 & 4 & 14 & 3 & 11 & 2 & 10 & 2 & 13 & 3 & 9 \\
\hline Low- and middle-income countries & NA & NA & NA & NA & NA & NA & 33 & 3 & 36 & 6 & 33 & 6 & 25 & 5 & 21 & 3 & 30 & 5 & 21 \\
\hline High-income countries & NA & NA & NA & NA & NA & NA & 36 & 19 & 48 & 25 & 47 & 26 & 45 & 29 & 45 & 30 & 46 & 28 & 41 \\
\hline WORLD & NA & NA & NA & NA & NA & NA & 34 & 5 & 39 & 9 & 37 & 10 & 32 & 13 & 34 & 17 & 35 & 11 & 27 \\
\hline
\end{tabular}

Attributable Mortality (thousands

\begin{tabular}{|c|c|c|c|c|c|c|c|c|c|c|c|c|c|c|c|c|c|c|c|}
\hline East Asia and Pacific & NA & NA & NA & NA & NA & NA & 0 & 0 & 1 & 0 & 1 & 0 & 1 & 0 & 1 & 0 & 3 & 0 & 4 \\
\hline Europe and Central Asia & NA & NA & NA & NA & NA & NA & 0 & 0 & 2 & 0 & 4 & 0 & 3 & 0 & 1 & 0 & 9 & 0 & 10 \\
\hline Latin America and the Caribbean & NA & NA & NA & NA & NA & NA & 0 & 0 & 0 & 0 & 0 & 0 & 1 & 0 & 0 & 0 & 2 & 0 & 2 \\
\hline Middle East and North Africa & NA & NA & NA & NA & NA & NA & 0 & 0 & 1 & 0 & 1 & 0 & 1 & 0 & 0 & 0 & 3 & 0 & 4 \\
\hline South Asia & NA & NA & NA & NA & NA & NA & 0 & 0 & 0 & 0 & 2 & 0 & 1 & 0 & 1 & 0 & 4 & 0 & 5 \\
\hline$\underline{\text { Sub-Saharan Africa }}$ & NA & NA & NA & NA & NA & NA & 0 & 0 & 0 & 0 & 0 & 0 & 0 & 0 & 0 & 0 & 1 & 0 & 1 \\
\hline Low- and middle-income countries & NA & NA & NA & NA & NA & NA & 1 & 0 & 4 & 0 & 8 & 0 & 7 & 0 & 3 & 0 & 23 & 2 & 24 \\
\hline High-income countries & NA & NA & NA & NA & NA & NA & 0 & 0 & 2 & 0 & 4 & 1 & 7 & 1 & 7 & 3 & 19 & 5 & 24 \\
\hline WORLD & NA & NA & NA & NA & NA & NA & 1 & 0 & 6 & 1 & 11 & 1 & 14 & 2 & 10 & 3 & 42 & 7 & 48 \\
\hline
\end{tabular}

Attributable YLL (thousands)

East Asia and Pacific

Europe and Central Asia

Latin America and the Caribbean

Middle East and North Africa

South Asia

Sub-Saharan Africa

Low- and middle-income countries

-

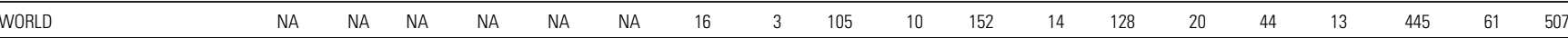

Attributable DALYs (thousands)

East Asia and Pacific

Europe and Central Asia

Latin America and the Caribbean

Middle East and North Africa

South Asia

Sub-Saharan Africa

NA NA NA NA NA NA

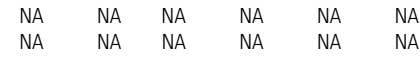

NA NA NA NA NA NA

$\begin{array}{lllllllllllll}5 & 1 & 18 & 1 & 14 & 1 & 8 & 0 & 2 & 0 & 46 & 3 & 49\end{array}$

\begin{tabular}{rrrrrrrrrrrrrrrrr} 
& NA & NA & NA & NA & NA & NA & 13 & 2 & 77 & 6 & 104 & 7 & 65 & 5 & 15 & 1 \\
\hline
\end{tabular}

Low- and middle-income countries

High-income countries

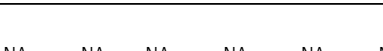

\begin{tabular}{rrrrrrrrr}
49 & 8 & 62 & 15 & 29 & 12 & 171 & 40 & 211 \\
152 & 14 & 128 & 20 & 44 & 13 & 445 & 61 & 507 \\
\hline
\end{tabular}

WORLD

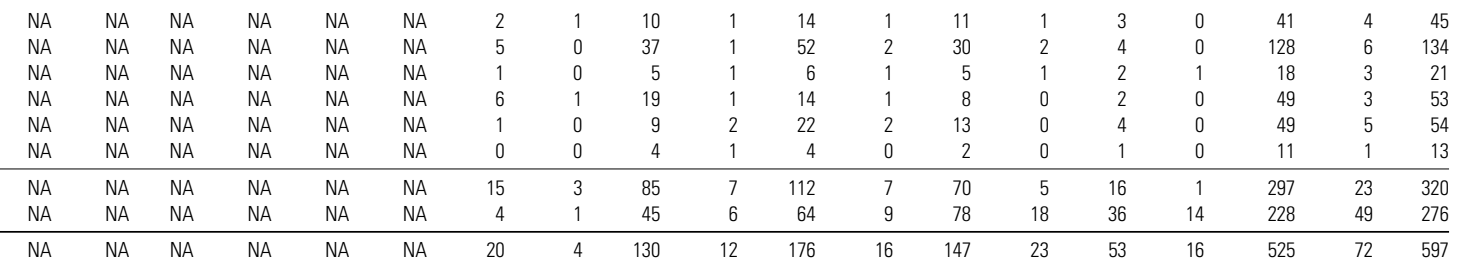

Source: Authors' calculations.

Note: $\mathrm{NA}=$ not applicable 
Table 4A.113

Risk factor:

Smoking

Disease:

Pancreas cancer

\begin{tabular}{|c|c|c|c|c|c|c|c|c|c|c|c|c|c|c|c|c|c|c|c|}
\hline \multirow[b]{2}{*}{ Region } & \multicolumn{2}{|c|}{$0-4$ years } & \multicolumn{2}{|c|}{ 5-14 years } & \multicolumn{2}{|c|}{$15-29$ years } & \multicolumn{2}{|c|}{ 30-44 years } & \multicolumn{2}{|c|}{ 45-59 years } & \multicolumn{2}{|c|}{$60-69$ years } & \multicolumn{2}{|c|}{$70-79$ years } & \multicolumn{2}{|c|}{$80+$ years } & \multicolumn{3}{|c|}{ Total } \\
\hline & Male & Female & Male & Female & Male & Female & Male & Female & Male & Female & Male & Female & Male & Female & Male & Female & Male & Female & All \\
\hline \multicolumn{20}{|l|}{ PAF of Mortality (\%) } \\
\hline East Asia and Pacific & NA & NA & NA & NA & NA & NA & 24 & 8 & 14 & 3 & 11 & 3 & 9 & 3 & 9 & 2 & 12 & 3 & 8 \\
\hline Europe and Central Asia & NA & NA & NA & NA & NA & NA & 46 & 9 & 52 & 12 & 45 & 9 & 32 & 10 & 19 & 5 & 42 & 9 & 26 \\
\hline Latin America and the Caribbean & NA & NA & NA & NA & NA & NA & 13 & 4 & 22 & 9 & 19 & 9 & 16 & 9 & 17 & 10 & 18 & 9 & 14 \\
\hline Middle East and North Africa & NA & NA & NA & NA & NA & NA & 23 & 18 & 21 & 7 & 19 & 5 & 16 & 4 & 16 & 2 & 19 & 6 & 14 \\
\hline South Asia & NA & NA & NA & NA & NA & NA & 13 & 0 & 24 & 4 & 21 & 4 & 16 & 1 & 16 & 0 & 19 & 2 & 12 \\
\hline Sub-Saharan Africa & NA & NA & NA & NA & NA & NA & 4 & 2 & 10 & 3 & 9 & 3 & 7 & 2 & 6 & 2 & 8 & 3 & 5 \\
\hline Low- and middle-income countries & NA & NA & NA & NA & NA & NA & 27 & 7 & 27 & 7 & 25 & 6 & 18 & 6 & 14 & 5 & 23 & 6 & 15 \\
\hline High-income countries & NA & NA & NA & NA & NA & NA & 25 & 17 & 36 & 22 & 35 & 23 & 33 & 26 & 33 & 27 & 34 & 26 & 30 \\
\hline WORLD & NA & NA & NA & NA & NA & NA & 26 & 9 & 30 & 12 & 29 & 13 & 26 & 17 & 27 & 20 & 28 & 16 & 22 \\
\hline \multicolumn{20}{|l|}{ PAF of YLL (\%) } \\
\hline East Asia and Pacific & NA & NA & NA & NA & NA & NA & 24 & 8 & 14 & 3 & 11 & 3 & 9 & 3 & 9 & 2 & 13 & 4 & 9 \\
\hline Europe and Central Asia & NA & NA & NA & NA & NA & NA & 46 & 9 & 52 & 12 & 45 & 9 & 32 & 10 & 19 & 5 & 45 & 9 & 29 \\
\hline Latin America and the Caribbean & NA & NA & NA & NA & NA & NA & 13 & 4 & 22 & 9 & 19 & 9 & 16 & 9 & 17 & 10 & 19 & 9 & 14 \\
\hline Middle East and North Africa & NA & NA & NA & NA & NA & NA & 23 & 18 & 21 & 7 & 19 & 5 & 16 & 4 & 16 & 2 & 20 & 7 & 14 \\
\hline South Asia & NA & NA & NA & NA & NA & NA & 13 & 0 & 24 & 4 & 21 & 4 & 16 & 1 & 16 & 0 & 20 & 3 & 12 \\
\hline Sub-Saharan Africa & NA & NA & NA & NA & NA & NA & 4 & 2 & 10 & 3 & 9 & 3 & 7 & 2 & 6 & 2 & 9 & 3 & 5 \\
\hline Low- and middle-income countries & NA & NA & NA & NA & NA & NA & 27 & 7 & 27 & 7 & 25 & 6 & 18 & 6 & 14 & 5 & 24 & 6 & 16 \\
\hline High-income countries & NA & NA & NA & NA & NA & NA & 25 & 17 & 36 & 22 & 35 & 23 & 33 & 26 & 33 & 27 & 34 & 24 & 30 \\
\hline WORLD & NA & NA & NA & NA & NA & NA & 26 & 9 & 30 & 12 & 29 & 13 & 26 & 17 & 26 & 19 & 28 & 14 & 22 \\
\hline \multicolumn{20}{|l|}{ PAF of DALYs (\%) } \\
\hline East Asia and Pacific & NA & NA & NA & NA & NA & NA & 24 & 8 & 14 & 3 & 11 & 3 & 9 & 3 & 9 & 2 & 13 & 4 & 9 \\
\hline Europe and Central Asia & NA & NA & NA & NA & NA & NA & 46 & 9 & 52 & 12 & 45 & 9 & 32 & 10 & 19 & 5 & 45 & 9 & 29 \\
\hline Latin America and the Caribbean & NA & NA & NA & NA & NA & NA & 13 & 4 & 22 & 9 & 19 & 9 & 16 & 9 & 17 & 10 & 19 & 9 & 14 \\
\hline Middle East and North Africa & NA & NA & NA & NA & NA & NA & 23 & 18 & 21 & 7 & 19 & 5 & 16 & 4 & 16 & 2 & 20 & 7 & 14 \\
\hline South Asia & NA & NA & NA & NA & NA & NA & 13 & 0 & 24 & 4 & 21 & 4 & 16 & 1 & 16 & 0 & 20 & 3 & 12 \\
\hline Sub-Saharan Africa & NA & NA & NA & NA & NA & NA & 4 & 2 & 10 & 3 & 9 & 3 & 7 & 2 & 6 & 2 & 9 & 3 & 5 \\
\hline Low- and middle-income countries & NA & NA & NA & NA & NA & NA & 27 & 7 & 27 & 7 & 25 & 6 & 18 & 6 & 14 & 5 & 24 & 6 & 16 \\
\hline High-income countries & NA & NA & NA & NA & NA & NA & 25 & 17 & 36 & 22 & 35 & 23 & 33 & 26 & 33 & 27 & 34 & 25 & 30 \\
\hline WORLD & NA & NA & NA & NA & NA & NA & 26 & 9 & 30 & 12 & 29 & 14 & 26 & 17 & 27 & 19 & 28 & 14 & 22 \\
\hline
\end{tabular}

Attributable Mortality (thousands

\begin{tabular}{llllllllllllllllllll} 
East Asia and Pacific & NA & NA & NA & NA & NA & NA & 0 & 0 & 1 & 0 & 1 & 0 & 0 & 0 & 0 & 0 & 3 & 1 & 3 \\
Europe and Central Asia & NA & NA & NA & NA & NA & NA & 1 & 0 & 3 & 0 & 3 & 0 & 2 & 1 & 0 & 0 & 8 & 1 & 9 \\
Latin America and the Caribbean & NA & NA & NA & NA & NA & NA & 0 & 0 & 0 & 0 & 1 & 0 & 0 & 0 & 0 & 0 & 2 & 1 & 3 \\
Middle East and North Africa & NA & NA & NA & NA & NA & NA & 0 & 0 & 0 & 0 & 0 & 0 & 0 & 0 & 0 & 0 & 0 & 0 & 1 \\
South Asia & NA & NA & NA & NA & NA & NA & 0 & 0 & 0 & 0 & 1 & 0 & 0 & 0 & 0 & 0 & 1 & 0 & 2 \\
Sub-Saharan Africa & NA & NA & NA & NA & NA & NA & 0 & 0 & 0 & 0 & 0 & 0 & 0 & 0 & 0 & 0 & 0 & 0 & 0 \\
\hline Low- and middle-income countries & NA & NA & NA & NA & NA & NA & 1 & 0 & 5 & 1 & 5 & 1 & 3 & 1 & 1 & 0 & 14 & 3 & 18 \\
High-income countries & NA & NA & NA & NA & NA & NA & 0 & 0 & 4 & 1 & 5 & 2 & 6 & 5 & 4 & 5 \\
\hline WORLD & NA & NA & NA & NA & NA & NA & 1 & 0 & 8 & 2 & 10 & 3 & 9 & 6 & 4 & 6 & 33 & 17 & 50 \\
\hline
\end{tabular}

Attributable YLL (thousands)

\begin{tabular}{|c|c|c|c|c|c|c|c|c|c|c|c|c|c|c|c|c|c|c|c|}
\hline \multicolumn{20}{|l|}{ Attributable YLL (thousands) } \\
\hline East Asia and Pacific & NA & NA & NA & NA & NA & NA & 9 & 3 & 18 & 2 & 9 & 2 & 4 & 1 & 1 & 0 & 41 & 8 & 50 \\
\hline Europe and Central Asia & NA & NA & NA & NA & NA & NA & 13 & 1 & 51 & 6 & 39 & 6 & 14 & 6 & 1 & 1 & 118 & 20 & 138 \\
\hline Latin America and the Caribbean & NA & NA & NA & NA & NA & NA & 1 & 0 & 8 & 3 & 7 & 4 & 4 & 3 & 1 & 1 & 22 & 11 & 33 \\
\hline Middle East and North Africa & NA & NA & NA & NA & NA & NA & 1 & 1 & 2 & 1 & 2 & 0 & 1 & 0 & 0 & 0 & 6 & 2 & 8 \\
\hline South Asia & NA & NA & NA & NA & NA & NA & 2 & 0 & 7 & 1 & 7 & 1 & 3 & 0 & 1 & 0 & 20 & 2 & 22 \\
\hline Sub-Saharan Africa & NA & NA & NA & NA & NA & NA & 0 & 0 & 2 & 1 & 1 & 0 & 1 & 0 & 0 & 0 & 4 & 2 & 6 \\
\hline Low- and middle-income countries & NA & NA & NA & NA & NA & NA & 26 & 5 & 90 & 14 & 65 & 13 & 27 & 11 & 4 & 2 & 212 & 45 & 256 \\
\hline High-income countries & NA & NA & NA & NA & NA & NA & 7 & 3 & 67 & 25 & 70 & 35 & 55 & 50 & 17 & 24 & 216 & 138 & 354 \\
\hline WORLD & NA & NA & NA & NA & NA & NA & 34 & 7 & 157 & 39 & 135 & 49 & 82 & 61 & 21 & 26 & 428 & 183 & 611 \\
\hline \multicolumn{20}{|l|}{ Attributable DALYs (thousands) } \\
\hline East Asia and Pacific & NA & NA & NA & NA & NA & NA & 9 & 3 & 19 & 2 & 9 & 2 & 4 & 1 & 1 & 0 & 42 & 9 & 50 \\
\hline Europe and Central Asia & NA & NA & NA & NA & NA & NA & 13 & 1 & 52 & 6 & 39 & 6 & 15 & 6 & 1 & 1 & 120 & 20 & 140 \\
\hline Latin America and the Caribbean & NA & NA & NA & NA & NA & NA & 1 & 0 & 8 & 3 & 7 & 4 & 4 & 3 & 1 & 1 & 22 & 11 & 34 \\
\hline Middle East and North Africa & NA & NA & NA & NA & NA & NA & 1 & 1 & 2 & 1 & 2 & 0 & 1 & 0 & 0 & 0 & 6 & 2 & 8 \\
\hline South Asia & NA & NA & NA & NA & NA & NA & 2 & 0 & 8 & 1 & 7 & 1 & 3 & 0 & 1 & 0 & 20 & 2 & 22 \\
\hline Sub-Saharan Africa & NA & NA & NA & NA & NA & NA & 0 & 0 & 2 & 1 & 1 & 0 & 1 & 0 & 0 & 0 & 4 & 2 & 6 \\
\hline Low- and middle-income countries & NA & NA & NA & NA & NA & NA & 26 & 5 & 91 & 14 & 66 & 13 & 27 & 11 & 4 & 2 & 214 & 45 & 259 \\
\hline High-income countries & NA & NA & NA & NA & NA & NA & 8 & 3 & 68 & 26 & 72 & 36 & 57 & 51 & 18 & 25 & 222 & 142 & 364 \\
\hline WORLD & NA & NA & NA & NA & NA & NA & 34 & 8 & 159 & 40 & 138 & 50 & 84 & 62 & 22 & 28 & 436 & 187 & 623 \\
\hline
\end{tabular}

Source: Authors' calculations.

Note: $\mathrm{NA}=$ not applicable 
Table 4A.114

Risk factor:

Smoking

Disease:

Stomach cancer

\begin{tabular}{|c|c|c|c|c|c|c|c|c|c|c|c|c|c|c|c|c|c|c|c|}
\hline \multirow[b]{2}{*}{ Region } & \multicolumn{2}{|c|}{$0-4$ years } & \multicolumn{2}{|c|}{ 5-14 years } & \multicolumn{2}{|c|}{$15-29$ years } & \multicolumn{2}{|c|}{ 30-44 years } & \multicolumn{2}{|c|}{ 45-59 years } & \multicolumn{2}{|c|}{$60-69$ years } & \multicolumn{2}{|c|}{$70-79$ years } & \multicolumn{2}{|c|}{$80+$ years } & \multicolumn{3}{|c|}{ Total } \\
\hline & Male & Female & Male & Female & Male & Female & Male & Female & Male & Female & Male & Female & Male & Female & Male & Female & Male & Female & All \\
\hline \multicolumn{20}{|l|}{ PAF of Mortality (\%) } \\
\hline East Asia and Pacific & NA & NA & NA & NA & NA & NA & 23 & 4 & 13 & 1 & 11 & 1 & 8 & 1 & 9 & 1 & 11 & 1 & 8 \\
\hline Europe and Central Asia & NA & NA & NA & NA & NA & NA & 45 & 4 & 51 & 5 & 44 & 4 & 31 & 4 & 19 & 2 & 40 & 4 & 25 \\
\hline Latin America and the Caribbean & NA & NA & NA & NA & NA & NA & 12 & 2 & 21 & 4 & 19 & 4 & 16 & 4 & 16 & 5 & 17 & 4 & 12 \\
\hline Middle East and North Africa & NA & NA & NA & NA & NA & NA & 23 & 8 & 21 & 3 & 19 & 2 & 16 & 2 & 16 & 1 & 18 & 3 & 12 \\
\hline South Asia & NA & NA & NA & NA & NA & NA & 12 & 0 & 23 & 2 & 20 & 2 & 16 & 1 & 15 & 0 & 18 & 1 & 12 \\
\hline Sub-Saharan Africa & NA & NA & NA & NA & NA & NA & 4 & 1 & 10 & 1 & 9 & 1 & 7 & 1 & 6 & 1 & 8 & 1 & 5 \\
\hline Low- and middle-income countries & NA & NA & NA & NA & NA & NA & 23 & 3 & 19 & 2 & 17 & 2 & 13 & 2 & 12 & 1 & 16 & 2 & 11 \\
\hline High-income countries & NA & NA & NA & NA & NA & NA & 24 & 8 & 35 & 11 & 34 & 11 & 32 & 13 & 33 & 13 & 33 & 12 & 25 \\
\hline WORLD & NA & NA & NA & NA & NA & NA & 23 & 4 & 21 & 3 & 20 & 3 & 17 & 4 & 18 & 5 & 19 & 4 & 13 \\
\hline \multicolumn{20}{|l|}{ PAF of YLL (\%) } \\
\hline East Asia and Pacific & NA & NA & NA & NA & NA & NA & 23 & 4 & 13 & 1 & 11 & 1 & 8 & 1 & 9 & 1 & 12 & 2 & 8 \\
\hline Europe and Central Asia & NA & NA & NA & NA & NA & NA & 45 & 4 & 51 & 5 & 44 & 4 & 31 & 4 & 19 & 2 & 43 & 4 & 27 \\
\hline Latin America and the Caribbean & NA & NA & NA & NA & NA & NA & 12 & 2 & 21 & 4 & 19 & 4 & 16 & 4 & 16 & 5 & 18 & 4 & 12 \\
\hline Middle East and North Africa & NA & NA & NA & NA & NA & NA & 23 & 8 & 21 & 3 & 19 & 2 & 16 & 2 & 16 & 1 & 18 & 3 & 12 \\
\hline South Asia & NA & NA & NA & NA & NA & NA & 12 & 0 & 23 & 2 & 20 & 2 & 16 & 1 & 15 & 0 & 18 & 1 & 12 \\
\hline Sub-Saharan Africa & NA & NA & NA & NA & NA & NA & 4 & 1 & 10 & 1 & 9 & 1 & 7 & 1 & 6 & 1 & 8 & 1 & 5 \\
\hline Low- and middle-income countries & NA & NA & NA & NA & NA & NA & 23 & 3 & 19 & 2 & 17 & 2 & 13 & 2 & 12 & 1 & 17 & 2 & 11 \\
\hline High-income countries & NA & NA & NA & NA & NA & NA & 24 & 8 & 35 & 11 & 34 & 11 & 32 & 13 & 33 & 13 & 33 & 11 & 25 \\
\hline WORLD & NA & NA & NA & NA & NA & NA & 23 & 4 & 21 & 3 & 20 & 3 & 17 & 4 & 18 & 5 & 19 & 3 & 13 \\
\hline \multicolumn{20}{|l|}{ PAF of DALYs (\%) } \\
\hline East Asia and Pacific & NA & NA & NA & NA & NA & NA & 23 & 4 & 13 & 1 & 11 & 1 & 8 & 1 & 9 & 1 & 12 & 2 & 8 \\
\hline Europe and Central Asia & NA & NA & NA & NA & NA & NA & 45 & 4 & 51 & 5 & 44 & 4 & 31 & 4 & 19 & 2 & 43 & 4 & 27 \\
\hline Latin America and the Caribbean & NA & NA & NA & NA & NA & NA & 12 & 2 & 21 & 4 & 19 & 4 & 16 & 4 & 16 & 5 & 18 & 4 & 12 \\
\hline Middle East and North Africa & NA & NA & NA & NA & NA & NA & 23 & 8 & 21 & 3 & 19 & 2 & 16 & 2 & 16 & 1 & 18 & 3 & 12 \\
\hline South Asia & NA & NA & NA & NA & NA & NA & 12 & 0 & 23 & 2 & 20 & 2 & 16 & 1 & 15 & 0 & 18 & 1 & 12 \\
\hline Sub-Saharan Africa & NA & NA & NA & NA & NA & NA & 4 & 1 & 10 & 1 & 9 & 1 & 7 & 1 & 6 & 1 & 8 & 1 & 5 \\
\hline Low- and middle-income countries & NA & NA & NA & NA & NA & NA & 23 & 3 & 19 & 2 & 17 & 2 & 13 & 2 & 12 & 1 & 17 & 2 & $\overline{11}$ \\
\hline High-income countries & NA & NA & NA & NA & NA & NA & 24 & 8 & 35 & 11 & 34 & 11 & 32 & 13 & 33 & 13 & 33 & 11 & 25 \\
\hline WORLD & NA & NA & NA & NA & NA & NA & 23 & 4 & 21 & 3 & 20 & 3 & 17 & 4 & 18 & 5 & 19 & 3 & 13 \\
\hline
\end{tabular}

Attributable Mortality (thousands)

\begin{tabular}{|c|c|c|c|c|c|c|c|c|c|c|c|c|c|c|c|c|c|c|c|}
\hline East Asia and Pacific & NA & NA & NA & NA & NA & NA & 4 & 0 & 10 & 0 & 9 & 1 & 6 & 1 & 2 & 0 & 31 & 2 & 33 \\
\hline Europe and Central Asia & NA & NA & NA & NA & NA & NA & 1 & 0 & 7 & 0 & 9 & 0 & 5 & 1 & 1 & 0 & 24 & 2 & 25 \\
\hline Latin America and the Caribbean & NA & NA & NA & NA & NA & NA & 0 & 0 & 1 & 0 & 2 & 0 & 2 & 0 & 1 & 0 & 6 & 1 & 7 \\
\hline Middle East and North Africa & NA & NA & NA & NA & NA & NA & 0 & 0 & 0 & 0 & 1 & 0 & 1 & 0 & 0 & 0 & 2 & 0 & 2 \\
\hline South Asia & NA & NA & NA & NA & NA & NA & 0 & 0 & 1 & 0 & 2 & 0 & 1 & 0 & 1 & 0 & 5 & 0 & 5 \\
\hline Sub-Saharan Africa & NA & NA & NA & NA & NA & NA & 0 & 0 & 1 & 0 & 0 & 0 & 0 & 0 & 0 & 0 & 1 & 0 & 2 \\
\hline Low- and middle-income countries & NA & NA & NA & NA & NA & NA & 6 & 1 & 21 & 1 & 23 & 1 & 15 & 1 & 5 & 1 & 69 & 5 & 74 \\
\hline High-income countries & NA & NA & NA & NA & NA & NA & 1 & 0 & 5 & 1 & 8 & 1 & 10 & 2 & 7 & 3 & 29 & 7 & 36 \\
\hline WORLD & NA & NA & NA & NA & NA & NA & 6 & 1 & 26 & 2 & 30 & 2 & 25 & 4 & 11 & 4 & 99 & 12 & 111 \\
\hline
\end{tabular}

\section{Attributable YLL (thousands)}

East Asia and Pacific

Europe and Central Asia

Latin America and the Caribbean

Middle East and North Africa

South Asia

Sub-Saharan Africa

Low- and middle-income countries

High-income co

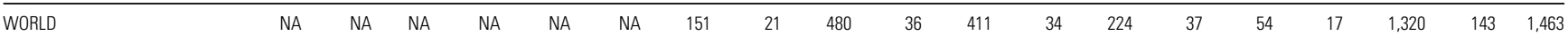

Attributable DALYs (thousands)

East Asia and Pacific

Europe and Central Asia

Latin America and the Caribbean

Middle East and North Africa

South Asia

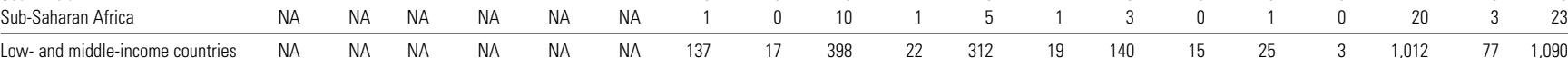

$\begin{array}{lllll}\text { NA } & \text { NA } & \text { NA } & \text { NA } & \text { NA } \\ \text { NA } & \text { NA } & \text { NA } & \text { NA } & \text { NA }\end{array}$

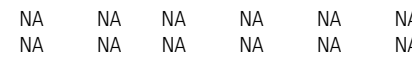

NA NA NA NA NA NA

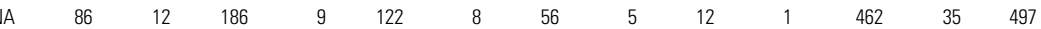

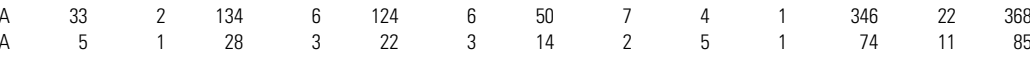

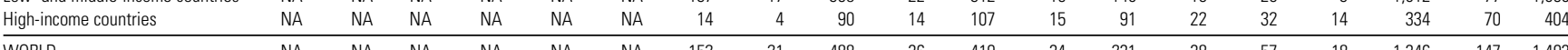

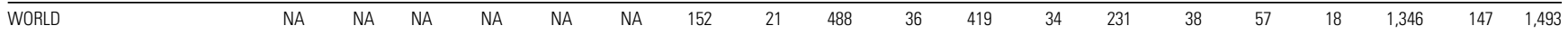

Source: Authors' calculations.

Note: $\mathrm{NA}=$ not applicable 
Table 4A.115

Risk factor:

Smoking

Disease:

Upper aerodigestive cancer

\begin{tabular}{|c|c|c|c|c|c|c|c|c|c|c|c|c|c|c|c|c|c|c|c|}
\hline \multirow[b]{2}{*}{ Region } & \multicolumn{2}{|c|}{$0-4$ years } & \multicolumn{2}{|c|}{ 5-14 years } & \multicolumn{2}{|c|}{$15-29$ years } & \multicolumn{2}{|c|}{$30-44$ years } & \multicolumn{2}{|c|}{$45-59$ years } & \multicolumn{2}{|c|}{ 60-69 years } & \multicolumn{2}{|c|}{ 70-79 years } & \multicolumn{2}{|c|}{$80+$ years } & \multicolumn{3}{|c|}{ Total } \\
\hline & Male & Female & Male & Female & Male & Female & Male & Female & Male & Female & Male & Female & Male & Female & Male & Female & Male & Female & All \\
\hline \multicolumn{20}{|l|}{ PAF of Mortality (\%) } \\
\hline East Asia and Pacific & NA & NA & NA & NA & NA & NA & 65 & 28 & 48 & 13 & 42 & 13 & 36 & 10 & 38 & 8 & 43 & 12 & 32 \\
\hline Europe and Central Asia & NA & NA & NA & NA & NA & NA & 84 & 30 & 87 & 36 & 83 & 29 & 73 & 30 & 58 & 17 & 81 & 28 & 69 \\
\hline Latin America and the Caribbean & NA & NA & NA & NA & NA & NA & 46 & 14 & 62 & 30 & 59 & 30 & 54 & 28 & 54 & 32 & 57 & 29 & 49 \\
\hline Middle East and North Africa & NA & NA & NA & NA & NA & NA & 64 & 48 & 62 & 25 & 59 & 18 & 53 & 16 & 54 & 6 & 56 & 21 & 43 \\
\hline South Asia & NA & NA & NA & NA & NA & NA & 46 & 0 & 65 & 15 & 61 & 15 & 53 & 5 & 53 & 0 & 57 & 9 & 39 \\
\hline Sub-Saharan Africa & NA & NA & NA & NA & NA & NA & 19 & 9 & 40 & 13 & 36 & 10 & 30 & 7 & 28 & 8 & 34 & 10 & 25 \\
\hline Low- and middle-income countries & NA & NA & NA & NA & NA & NA & 56 & 15 & 58 & 15 & 53 & 15 & 45 & 10 & 46 & 6 & 51 & 12 & 37 \\
\hline High-income countries & NA & NA & NA & NA & NA & NA & 66 & 46 & 77 & 55 & 76 & 56 & 74 & 60 & 75 & 61 & 75 & 58 & 71 \\
\hline WORLD & NA & NA & NA & NA & NA & NA & 57 & 17 & 60 & 18 & 56 & 17 & 50 & 15 & 53 & 17 & 55 & 16 & 42 \\
\hline \multicolumn{20}{|l|}{ PAF of YLL (\%) } \\
\hline East Asia and Pacific & NA & NA & NA & NA & NA & NA & 65 & 28 & 48 & 13 & 42 & 13 & 36 & 10 & 38 & 8 & 45 & 13 & 33 \\
\hline Europe and Central Asia & NA & NA & NA & NA & NA & NA & 84 & 30 & 87 & 36 & 83 & 29 & 73 & 30 & 58 & 17 & 82 & 30 & 72 \\
\hline Latin America and the Caribbean & NA & NA & NA & NA & NA & NA & 46 & 14 & 62 & 30 & 59 & 30 & 54 & 28 & 54 & 32 & 57 & 28 & 50 \\
\hline Middle East and North Africa & NA & NA & NA & NA & NA & NA & 64 & 48 & 62 & 25 & 59 & 18 & 53 & 16 & 54 & 6 & 55 & 23 & 44 \\
\hline South Asia & NA & NA & NA & NA & NA & NA & 46 & 0 & 65 & 15 & 61 & 15 & 53 & 5 & 53 & 0 & 56 & 11 & 39 \\
\hline Sub-Saharan Africa & NA & NA & NA & NA & NA & NA & 19 & 9 & 40 & 13 & 36 & 10 & 30 & 7 & 28 & 8 & 34 & 10 & 25 \\
\hline Low- and middle-income countries & NA & NA & NA & NA & NA & NA & 56 & 15 & 58 & 15 & 53 & 15 & 45 & 10 & 46 & 6 & 52 & 13 & 39 \\
\hline High-income countries & NA & NA & NA & NA & NA & NA & 66 & 46 & 77 & 55 & 76 & 56 & 74 & 60 & 75 & 61 & 75 & 56 & 71 \\
\hline WORLD & NA & NA & NA & NA & NA & NA & 57 & 17 & 61 & 18 & 56 & 17 & 50 & 15 & 52 & 16 & 55 & 16 & 42 \\
\hline \multicolumn{20}{|l|}{ PAF of DALYs (\%) } \\
\hline East Asia and Pacific & NA & NA & NA & NA & NA & NA & 65 & 28 & 48 & 13 & 42 & 13 & 36 & 10 & 38 & 8 & 45 & 13 & 33 \\
\hline Europe and Central Asia & NA & NA & NA & NA & NA & NA & 84 & 30 & 87 & 36 & 83 & 29 & 73 & 30 & 58 & 17 & 82 & 30 & 72 \\
\hline Latin America and the Caribbean & NA & NA & NA & NA & NA & NA & 46 & 14 & 62 & 30 & 59 & 30 & 54 & 28 & 54 & 32 & 57 & 28 & 50 \\
\hline Middle East and North Africa & NA & NA & NA & NA & NA & NA & 64 & 48 & 62 & 25 & 59 & 18 & 53 & 16 & 54 & 6 & 55 & 23 & 44 \\
\hline South Asia & NA & NA & NA & NA & NA & NA & 46 & 0 & 65 & 15 & 61 & 15 & 53 & 5 & 53 & 0 & 56 & 11 & 39 \\
\hline Sub-Saharan Africa & NA & NA & NA & NA & NA & NA & 19 & 9 & 40 & 13 & 36 & 10 & 30 & 7 & 28 & 8 & 34 & 10 & 25 \\
\hline Low- and middle-income countries & NA & NA & NA & NA & NA & NA & 56 & 15 & 58 & 15 & 53 & 15 & 45 & 10 & 46 & 6 & 52 & 13 & 39 \\
\hline High-income countries & NA & NA & NA & NA & NA & NA & 66 & 46 & 77 & 55 & 76 & 56 & 74 & 60 & 75 & 61 & 75 & 56 & 71 \\
\hline WORLD & NA & NA & NA & NA & NA & NA & 57 & 17 & 61 & 18 & 56 & 17 & 50 & 15 & 53 & 16 & 55 & 17 & 42 \\
\hline
\end{tabular}

Attributable Mortality (thousands

\begin{tabular}{|c|c|c|c|c|c|c|c|c|c|c|c|c|c|c|c|c|c|c|c|}
\hline East Asia and Pacific & NA & NA & NA & NA & NA & NA & 9 & 1 & 25 & 3 & 27 & 4 & 17 & 3 & 4 & 1 & 83 & 13 & 95 \\
\hline Europe and Central Asia & NA & NA & NA & NA & NA & NA & 2 & 0 & 12 & 1 & 11 & 1 & 5 & 1 & 1 & 0 & 30 & 3 & 33 \\
\hline Latin America and the Caribbean & NA & NA & NA & NA & NA & NA & 1 & 0 & 4 & 0 & 4 & 1 & 3 & 1 & 1 & 1 & 13 & 2 & 15 \\
\hline Middle East and North Africa & NA & NA & NA & NA & NA & NA & 0 & 0 & 1 & 0 & 1 & 0 & 1 & 0 & 0 & 0 & 4 & 1 & 4 \\
\hline South Asia & NA & NA & NA & NA & NA & NA & 4 & 0 & 19 & 3 & 32 & 4 & 16 & 1 & 6 & 0 & 78 & 8 & 86 \\
\hline Sub-Saharan Africa & NA & NA & NA & NA & NA & NA & 0 & 0 & 3 & 1 & 3 & 0 & 2 & 0 & 1 & 0 & 9 & 1 & 11 \\
\hline Low- and middle-income countries & NA & NA & NA & NA & NA & NA & 16 & 1 & 65 & 8 & 78 & 9 & 44 & 6 & 14 & 2 & 216 & 28 & 244 \\
\hline High-income countries & NA & NA & NA & NA & NA & NA & 2 & 0 & 15 & 2 & 16 & 2 & 15 & 4 & 7 & 5 & 56 & 14 & 70 \\
\hline WORLD & NA & NA & NA & NA & NA & NA & 18 & 2 & 80 & 10 & 94 & 12 & 59 & 11 & 21 & 8 & 272 & 42 & 314 \\
\hline
\end{tabular}

\section{Attributable YLL (thousands)}

East Asia and Pacific

Europe and Central Asia

Latin America and the Caribbean

Middle East and North Africa

South Asia

Sub-Saharan Africa

Low- and middle-income countries

High-income

\begin{tabular}{|c|c|c|c|c|c|c|c|c|c|c|c|c|c|c|c|c|c|c|}
\hline NA & NA & NA & NA & NA & NA & 206 & 25 & 470 & 67 & 364 & 58 & 158 & 34 & 23 & 6 & 1,222 & 190 & 1,412 \\
\hline NA & NA & NA & NA & NA & NA & 14 & 1 & 80 & 9 & 50 & 9 & 24 & 7 & 7 & 3 & 175 & 29 & 204 \\
\hline NA & NA & NA & NA & NA & NA & 11 & 5 & 18 & 4 & 14 & 2 & 7 & 2 & 1 & 0 & 52 & 13 & 64 \\
\hline NA & NA & NA & NA & NA & NA & 102 & 0 & 365 & 57 & 436 & 56 & 147 & 10 & 31 & 0 & 1,080 & 123 & 1,203 \\
\hline NA & NA & NA & NA & NA & NA & 378 & 36 & 1,224 & 160 & 1,051 & 142 & 401 & 67 & 69 & 12 & 3,124 & 417 & 3,541 \\
\hline NA & NA & NA & NA & NA & NA & 41 & 7 & 287 & 39 & 222 & 38 & 134 & 42 & 34 & 24 & 717 & 150 & 867 \\
\hline
\end{tabular}

WORLD

East Asia and Pacific

Europe and Central Asia

Middle East and North Africa

South Asia

Sub-Saharan Africa

NA NA NA NA

NA NA

\begin{tabular}{|c|c|c|c|c|c|c|c|c|c|c|c|c|c|c|c|c|c|}
\hline \multirow{2}{*}{$\begin{array}{l}\text { NA } \\
\text { NA }\end{array}$} & NA & NA & NA & NA & NA & 38 & 4 & 231 & 13 & 148 & 12 & 49 & 11 & 4 & 2 & 471 & 42 \\
\hline & NA & NA & NA & NA & NA & 14 & 1 & 82 & 9 & 51 & 9 & 25 & 7 & 7 & 3 & 179 & 29 \\
\hline NA & NA & NA & NA & NA & NA & 11 & 5 & 19 & 4 & 14 & 2 & 8 & 2 & 1 & 0 & 52 & 13 \\
\hline NA & NA & NA & NA & NA & NA & 102 & 0 & 371 & 58 & 444 & 56 & 150 & 10 & 32 & 0 & 1,099 & 125 \\
\hline NA & NA & NA & NA & NA & NA & 8 & 2 & 66 & 10 & 42 & 6 & 18 & 3 & 3 & 1 & 137 & 23 \\
\hline NA & NA & NA & NA & NA & NA & 382 & 37 & 1,244 & 162 & 1,069 & 144 & 409 & 68 & 71 & 12 & 3,176 & 424 \\
\hline NA & NA & NA & NA & NA & NA & 42 & 8 & 298 & 41 & 231 & 39 & 141 & 44 & 36 & 26 & 747 & 157 \\
\hline NA & NA & NA & NA & NA & NA & 424 & 45 & 1,541 & 203 & 1,300 & 183 & 550 & 112 & 108 & 38 & 3,923 & 581 \\
\hline
\end{tabular}

High-income countries

NA NA NA NA

Source: Authors' calculations.

Note: $\mathrm{NA}=$ not applicable. 
Table 4A.116

Risk factor:

Smoking

Disease:

Leukemia

\begin{tabular}{|c|c|c|c|c|c|c|c|c|c|c|c|c|c|c|c|c|c|c|c|}
\hline \multirow[b]{2}{*}{ Region } & \multicolumn{2}{|c|}{$0-4$ years } & \multicolumn{2}{|c|}{ 5-14 years } & \multicolumn{2}{|c|}{$15-29$ years } & \multicolumn{2}{|c|}{ 30-44 years } & \multicolumn{2}{|c|}{$45-59$ years } & \multicolumn{2}{|c|}{ 60-69 years } & \multicolumn{2}{|c|}{ 70-79 years } & $80+$ & years & & Total & \\
\hline & Male & Female & Male & Female & Male & Female & Male & Female & Male & Female & Male & Female & Male & Female & Male & Female & Male & Female & All \\
\hline PAF of Mortality (\%) & & & & & & & & & & & & & & & & & & & \\
\hline East Asia and Pacific & NA & NA & NA & NA & NA & NA & 19 & 2 & 10 & 1 & 8 & 1 & 7 & 1 & 7 & 0 & 6 & 1 & 4 \\
\hline Europe and Central Asia & NA & NA & NA & NA & NA & NA & 39 & 2 & 45 & 3 & 38 & 2 & 26 & 2 & 15 & 1 & 28 & 2 & 16 \\
\hline Latin America and the Caribbean & NA & NA & NA & NA & NA & NA & 10 & 1 & 17 & 2 & 15 & 2 & 13 & 2 & 13 & 2 & 8 & 1 & 5 \\
\hline Middle East and North Africa & NA & NA & NA & NA & NA & NA & 18 & 4 & 17 & 2 & 15 & 1 & 13 & 1 & 13 & 0 & 9 & 1 & 6 \\
\hline South Asia & NA & NA & NA & NA & NA & NA & 10 & 0 & 19 & 1 & 16 & 1 & 12 & 0 & 12 & 0 & 7 & 0 & 4 \\
\hline Sub-Saharan Africa & NA & NA & NA & NA & NA & NA & 3 & 0 & 8 & 1 & 7 & 1 & 5 & 0 & 5 & 0 & 4 & 0 & 3 \\
\hline Low- and middle-income countries & NA & NA & NA & NA & NA & NA & 17 & 2 & 18 & 1 & 17 & 1 & 13 & 1 & 11 & 1 & 10 & 1 & $\overline{6}$ \\
\hline High-income countries & NA & NA & NA & NA & NA & NA & 20 & 4 & 29 & 5 & 28 & 6 & 27 & 7 & 27 & 7 & 25 & 6 & 17 \\
\hline WORLD & NA & NA & NA & NA & NA & NA & 18 & 2 & 21 & 2 & 21 & 2 & 19 & 3 & 21 & 5 & 14 & 2 & 9 \\
\hline PAF of YLL (\%) & & & & & & & & & & & & & & & & & & & \\
\hline East Asia and Pacific & NA & NA & NA & NA & NA & NA & 19 & 2 & 10 & 1 & 8 & 1 & 7 & 1 & 7 & 0 & 5 & 1 & 3 \\
\hline Europe and Central Asia & NA & NA & NA & NA & NA & NA & 39 & 2 & 45 & 3 & 38 & 2 & 26 & 2 & 15 & 1 & 25 & 2 & 15 \\
\hline Latin America and the Caribbean & NA & NA & NA & NA & NA & NA & 10 & 1 & 17 & 2 & 15 & 2 & 13 & 2 & 13 & 2 & 6 & 1 & 4 \\
\hline Middle East and North Africa & NA & NA & NA & NA & NA & NA & 18 & 4 & 17 & 2 & 15 & 1 & 13 & 1 & 13 & 0 & 7 & 1 & 4 \\
\hline South Asia & NA & NA & NA & NA & NA & NA & 10 & 0 & 19 & 1 & 16 & 1 & 12 & 0 & 12 & 0 & 5 & 0 & 3 \\
\hline Sub-Saharan Africa & NA & NA & NA & NA & NA & NA & 3 & 0 & 8 & 1 & 7 & 1 & 5 & 0 & 5 & 0 & 4 & 0 & 2 \\
\hline Low- and middle-income countries & NA & NA & NA & NA & NA & NA & 17 & 2 & 18 & 1 & 17 & 1 & 13 & 1 & 10 & 1 & 7 & 1 & 4 \\
\hline High-income countries & NA & NA & NA & NA & NA & NA & 20 & 4 & 29 & 5 & 28 & 6 & 27 & 7 & 27 & 7 & 23 & 5 & 15 \\
\hline WORLD & NA & NA & NA & NA & NA & NA & 18 & 2 & 21 & 2 & 21 & 2 & 19 & 3 & 20 & 4 & 10 & 1 & 6 \\
\hline PAF of DALYs (\%) & & & & & & & & & & & & & & & & & & & \\
\hline East Asia and Pacific & NA & NA & NA & NA & NA & NA & 19 & 2 & 10 & 1 & 8 & 1 & 7 & 1 & 7 & 0 & 5 & 1 & 3 \\
\hline Europe and Central Asia & NA & NA & NA & NA & NA & NA & 39 & 2 & 45 & 3 & 38 & 2 & 26 & 2 & 15 & 1 & 25 & 2 & 15 \\
\hline Latin America and the Caribbean & NA & NA & NA & NA & NA & NA & 10 & 1 & 17 & 2 & 15 & 2 & 13 & 2 & 13 & 2 & 6 & 1 & 4 \\
\hline Middle East and North Africa & NA & NA & NA & NA & NA & NA & 18 & 4 & 17 & 2 & 15 & 1 & 13 & 1 & 13 & 0 & 7 & 1 & 4 \\
\hline South Asia & NA & NA & NA & NA & NA & NA & 10 & 0 & 19 & 1 & 16 & 1 & 12 & 0 & 12 & 0 & 5 & 0 & 3 \\
\hline Sub-Saharan Africa & NA & NA & NA & NA & NA & NA & 3 & 0 & 8 & 1 & 7 & 1 & 5 & 0 & 5 & 0 & 4 & 0 & 2 \\
\hline Low- and middle-income countries & NA & NA & NA & NA & NA & NA & 17 & 2 & 18 & 1 & 17 & 1 & 13 & 1 & 10 & 1 & 7 & 1 & 4 \\
\hline High-income countries & NA & NA & NA & NA & NA & NA & 20 & 4 & 29 & 5 & 28 & 6 & 27 & 7 & 27 & 7 & 23 & 5 & 15 \\
\hline WORLD & NA & NA & NA & NA & NA & NA & 18 & 2 & 21 & 2 & 21 & 2 & 19 & 4 & 21 & 4 & 10 & 1 & 6 \\
\hline Attributable Mortality (thousand & & & & & & & & & & & & & & & & & & & \\
\hline East Asia and Pacific & NA & NA & NA & NA & NA & NA & 1 & 0 & 1 & 0 & 0 & 0 & 0 & 0 & 0 & 0 & 2 & 0 & 3 \\
\hline Europe and Central Asia & NA & NA & NA & NA & NA & NA & 1 & 0 & 1 & 0 & 1 & 0 & 1 & 0 & 0 & 0 & 4 & 0 & 4 \\
\hline Latin America and the Caribbean & NA & NA & NA & NA & NA & NA & 0 & 0 & 0 & 0 & 0 & 0 & 0 & 0 & 0 & 0 & 1 & 0 & 1 \\
\hline Middle East and North Africa & NA & NA & NA & NA & NA & NA & 0 & 0 & 0 & 0 & 0 & 0 & 0 & 0 & 0 & 0 & 1 & 0 & 1 \\
\hline South Asia & NA & NA & NA & NA & NA & NA & 0 & 0 & 0 & 0 & 0 & 0 & 0 & 0 & 0 & 0 & 2 & 0 & 2 \\
\hline Sub-Saharan Africa & NA & NA & NA & NA & NA & NA & 0 & 0 & 0 & 0 & 0 & 0 & 0 & 0 & 0 & 0 & 0 & 0 & 0 \\
\hline Low- and middle-income countries & NA & NA & NA & NA & NA & NA & 2 & 0 & 3 & 0 & 3 & 0 & 2 & 0 & 1 & 0 & 10 & 1 & 11 \\
\hline High-income countries & NA & NA & NA & NA & NA & NA & 0 & 0 & 2 & 0 & 2 & 0 & 3 & 1 & 3 & 1 & 10 & 2 & 12 \\
\hline WORLD & NA & NA & NA & NA & NA & NA & 3 & 0 & 4 & 0 & 5 & 0 & 5 & 1 & 3 & 1 & 20 & 3 & 23 \\
\hline Attributable YLL (thousands) & & & & & & & & & & & & & & & & & & & \\
\hline East Asia and Pacific & NA & NA & NA & NA & NA & NA & 20 & 3 & 14 & 1 & 6 & 0 & 3 & 0 & 1 & 0 & 44 & 4 & 49 \\
\hline Europe and Central Asia & NA & NA & NA & NA & NA & NA & 15 & 1 & 23 & 1 & 18 & 1 & 8 & 1 & 1 & 0 & 63 & 3 & 66 \\
\hline Latin America and the Caribbean & NA & NA & NA & NA & NA & NA & 3 & 0 & 5 & 1 & 3 & 0 & 2 & 0 & 1 & 0 & 14 & 2 & 16 \\
\hline Middle East and North Africa & NA & NA & NA & NA & NA & NA & 4 & 1 & 3 & 0 & 2 & 0 & 1 & 0 & 0 & 0 & 11 & 1 & 13 \\
\hline South Asia & NA & NA & NA & NA & NA & NA & 6 & 0 & 8 & 0 & 6 & 0 & 2 & 0 & 1 & 0 & 23 & 1 & 24 \\
\hline Sub-Saharan Africa & NA & NA & NA & NA & NA & NA & 0 & 0 & 2 & 0 & 1 & 0 & 1 & 0 & 0 & 0 & 5 & 0 & 5 \\
\hline Low- and middle-income countries & NA & NA & NA & NA & NA & NA & 50 & 5 & 55 & 3 & 36 & 2 & 16 & 1 & 3 & 0 & 161 & 11 & 172 \\
\hline High-income countries & NA & NA & NA & NA & NA & NA & 11 & 2 & 29 & 4 & 31 & 4 & 30 & 6 & 12 & 4 & 113 & 19 & 132 \\
\hline WORLD & NA & NA & NA & NA & NA & NA & 61 & 6 & 85 & 7 & 67 & 6 & 46 & 7 & 15 & 4 & 274 & 31 & 304 \\
\hline Attributable DALYs (thousands) & & & & & & & & & & & & & & & & & & & \\
\hline East Asia and Pacific & NA & NA & NA & NA & NA & NA & 21 & 3 & 14 & 1 & 7 & 0 & 3 & 0 & 1 & 0 & 45 & 4 & 49 \\
\hline Europe and Central Asia & NA & NA & NA & NA & NA & NA & 15 & 1 & 23 & 1 & 18 & 1 & 8 & 1 & 1 & 0 & 64 & 3 & 68 \\
\hline Latin America and the Caribbean & NA & NA & NA & NA & NA & NA & 3 & 0 & 5 & 1 & 3 & 0 & 2 & 0 & 1 & 0 & 14 & 2 & 16 \\
\hline Middle East and North Africa & NA & NA & NA & NA & NA & NA & 4 & 1 & 3 & 0 & 2 & 0 & 1 & 0 & 0 & 0 & 12 & 1 & 13 \\
\hline South Asia & NA & NA & NA & NA & NA & NA & 6 & 0 & 8 & 0 & 6 & 0 & 2 & 0 & 1 & 0 & 24 & 1 & 24 \\
\hline Sub-Saharan Africa & NA & NA & NA & NA & NA & NA & 0 & 0 & 2 & 0 & 1 & 0 & 1 & 0 & 0 & 0 & 5 & 0 & 5 \\
\hline Low- and middle-income countries & NA & NA & NA & NA & NA & NA & 50 & 5 & 56 & 3 & 37 & 2 & 17 & 1 & 3 & 0 & 163 & 12 & 175 \\
\hline High-income countries & NA & NA & NA & NA & NA & NA & 11 & 2 & 30 & 4 & 32 & 4 & 32 & 6 & 13 & 4 & 118 & 20 & 139 \\
\hline$\overline{\text { WORLD }}$ & NA & NA & NA & NA & NA & NA & 62 & 6 & 87 & 7 & 69 & 6 & 48 & 8 & 16 & 4 & 282 & 32 & $\overline{313}$ \\
\hline
\end{tabular}

Source: Authors' calculations.

Note: $\mathrm{NA}=$ not applicable 
Risk factor:

Smoking

Disease:

Ischemic heart disease

\begin{tabular}{|c|c|c|c|c|c|c|c|c|c|c|c|c|c|c|c|c|c|c|c|}
\hline \multirow[b]{2}{*}{ Region } & \multicolumn{2}{|c|}{$0-4$ years } & \multicolumn{2}{|c|}{ 5-14 years } & \multicolumn{2}{|c|}{$15-29$ years } & \multicolumn{2}{|c|}{ 30-44 years } & \multicolumn{2}{|c|}{$45-59$ years } & \multicolumn{2}{|c|}{$60-69$ years } & \multicolumn{2}{|c|}{ 70-79 years } & $80+$ & years & & Total & \\
\hline & Male & Female & Male & Female & Male & Female & Male & Female & Male & Female & Male & Female & Male & Female & Male & Female & Male & Female & All \\
\hline PAF of Mortality (\%) & & & & & & & & & & & & & & & & & & & \\
\hline East Asia and Pacific & NA & NA & NA & NA & NA & NA & 54 & 9 & 21 & 7 & 8 & 4 & 3 & 1 & 0 & 1 & 10 & 2 & 6 \\
\hline Europe and Central Asia & NA & NA & NA & NA & NA & NA & 76 & 10 & 65 & 23 & 37 & 11 & 13 & 5 & 0 & 1 & 30 & 5 & 17 \\
\hline Latin America and the Caribbean & NA & NA & NA & NA & NA & NA & 35 & 4 & 32 & 19 & 15 & 11 & 6 & 4 & 0 & 3 & 13 & 7 & 10 \\
\hline Middle East and North Africa & NA & NA & NA & NA & NA & NA & 53 & 19 & 32 & 15 & 15 & 6 & 6 & 2 & 0 & 0 & 16 & 5 & 11 \\
\hline South Asia & NA & NA & NA & NA & NA & NA & 35 & 0 & 34 & 9 & 16 & 5 & 6 & 1 & 0 & 0 & 16 & 3 & 10 \\
\hline Sub-Saharan Africa & NA & NA & NA & NA & NA & NA & 13 & 2 & 16 & 8 & 7 & 3 & 2 & 1 & 0 & 1 & 7 & 3 & 5 \\
\hline Low- and middle-income countries & NA & NA & NA & NA & NA & NA & 50 & 5 & 38 & 12 & 20 & 6 & 7 & 2 & 0 & 1 & 18 & 4 & 11 \\
\hline High-income countries & NA & NA & NA & NA & NA & NA & 56 & 18 & 49 & 40 & 28 & 27 & 14 & 15 & 0 & 10 & 17 & 13 & 15 \\
\hline$\overline{\text { WORLD }}$ & NA & NA & NA & NA & NA & NA & 50 & 5 & 40 & 14 & 21 & 8 & 9 & 4 & 0 & 4 & 18 & 6 & $\overline{12}$ \\
\hline PAF of YLL (\%) & & & & & & & & & & & & & & & & & & & \\
\hline East Asia and Pacific & NA & NA & NA & NA & NA & NA & 54 & 9 & 21 & 7 & 8 & 4 & 3 & 1 & 0 & 1 & 14 & 4 & 9 \\
\hline Europe and Central Asia & NA & NA & NA & NA & NA & NA & 76 & 10 & 65 & 23 & 37 & 11 & 13 & 5 & 0 & 1 & 40 & 8 & 26 \\
\hline Latin America and the Caribbean & NA & NA & NA & NA & NA & NA & 35 & 4 & 32 & 19 & 15 & 11 & 6 & 4 & 0 & 3 & 19 & 9 & 15 \\
\hline Middle East and North Africa & NA & NA & NA & NA & NA & NA & 53 & 19 & 32 & 15 & 15 & 6 & 6 & 2 & 0 & 0 & 21 & 7 & 15 \\
\hline South Asia & NA & NA & NA & NA & NA & NA & 35 & 0 & 34 & 9 & 16 & 5 & 6 & 1 & 0 & 0 & 21 & 4 & 13 \\
\hline Sub-Saharan Africa & NA & NA & NA & NA & NA & NA & 13 & 2 & 16 & 8 & 7 & 3 & 2 & 1 & 0 & 1 & 9 & 3 & 6 \\
\hline Low- and middle-income countries & NA & NA & NA & NA & NA & NA & 50 & 5 & 38 & 12 & 20 & 6 & 7 & 2 & 0 & 1 & 24 & 5 & $\overline{15}$ \\
\hline High-income countries & NA & NA & NA & NA & NA & NA & 56 & 18 & 49 & 40 & 28 & 27 & 14 & 15 & 0 & 10 & 26 & 17 & 22 \\
\hline WORLD & NA & NA & NA & NA & NA & NA & 50 & 5 & 40 & 14 & 21 & 8 & 9 & 4 & 0 & 4 & 24 & 7 & 17 \\
\hline PAF of DALYs (\%) & & & & & & & & & & & & & & & & & & & \\
\hline East Asia and Pacific & NA & NA & NA & NA & NA & NA & 54 & 9 & 21 & 7 & 8 & 4 & 3 & 1 & 0 & 1 & 14 & 4 & 9 \\
\hline Europe and Central Asia & NA & NA & NA & NA & NA & NA & 76 & 10 & 65 & 23 & 37 & 11 & 13 & 5 & 0 & 1 & 41 & 8 & 26 \\
\hline Latin America and the Caribbean & NA & NA & NA & NA & NA & NA & 35 & 4 & 32 & 19 & 15 & 11 & 6 & 4 & 0 & 3 & 19 & 9 & 15 \\
\hline Middle East and North Africa & NA & NA & NA & NA & NA & NA & 53 & 19 & 32 & 15 & 15 & 6 & 6 & 2 & 0 & 0 & 21 & 7 & 15 \\
\hline South Asia & NA & NA & NA & NA & NA & NA & 35 & 0 & 34 & 9 & 16 & 5 & 6 & 1 & 0 & 0 & 21 & 4 & 13 \\
\hline Sub-Saharan Africa & NA & NA & NA & NA & NA & NA & 13 & 2 & 16 & 8 & 7 & 3 & 2 & 1 & 0 & 1 & 9 & 3 & 6 \\
\hline Low- and middle-income countries & NA & NA & NA & NA & NA & NA & 50 & 5 & 38 & 12 & 20 & 6 & 7 & 2 & 0 & 1 & 24 & 5 & $\overline{15}$ \\
\hline High-income countries & NA & NA & NA & NA & NA & NA & 56 & 18 & 49 & 40 & 28 & 27 & 14 & 15 & 0 & 10 & 26 & 18 & 23 \\
\hline WORLD & NA & NA & NA & NA & NA & NA & 50 & 5 & 40 & 14 & 21 & 8 & 9 & 4 & 0 & 4 & 24 & 7 & 17 \\
\hline Attributable Mortality (thousand & & & & & & & & & & & & & & & & & & & \\
\hline East Asia and Pacific & NA & NA & NA & NA & NA & NA & 16 & 1 & 22 & 4 & 12 & 5 & 6 & 2 & 0 & 1 & 56 & 14 & 70 \\
\hline Europe and Central Asia & NA & NA & NA & NA & NA & NA & 29 & 1 & 99 & 10 & 84 & 14 & 36 & 15 & 0 & 5 & 248 & 45 & 293 \\
\hline Latin America and the Caribbean & NA & NA & NA & NA & NA & NA & 3 & 0 & 13 & 3 & 7 & 3 & 4 & 2 & 0 & 2 & 27 & 11 & 38 \\
\hline Middle East and North Africa & NA & NA & NA & NA & NA & NA & 6 & 1 & 13 & 3 & 7 & 2 & 4 & 1 & 0 & 0 & 30 & 7 & 36 \\
\hline South Asia & NA & NA & NA & NA & NA & NA & 17 & 0 & 84 & 11 & 47 & 12 & 18 & 2 & 0 & 0 & 166 & 24 & 190 \\
\hline Sub-Saharan Africa & NA & NA & NA & NA & NA & NA & 1 & 0 & 6 & 2 & 3 & 2 & 1 & 0 & 0 & 0 & 12 & 4 & 16 \\
\hline Low- and middle-income countries & NA & NA & NA & NA & NA & NA & 72 & 3 & 237 & 34 & 161 & 37 & 68 & 22 & 1 & 8 & 539 & 105 & 644 \\
\hline High-income countries & NA & NA & NA & NA & NA & NA & 9 & 1 & 43 & 9 & 36 & 14 & 33 & 23 & 1 & 40 & 122 & 86 & 208 \\
\hline$\overline{\text { WORLD }}$ & NA & NA & NA & NA & NA & NA & 81 & 4 & 280 & 43 & 197 & 51 & 100 & 45 & 2 & 48 & 661 & 191 & 852 \\
\hline Attributable YLL (thousands) & & & & & & & & & & & & & & & & & & & \\
\hline East Asia and Pacific & NA & NA & NA & NA & NA & NA & 392 & 36 & 409 & 86 & 165 & 69 & 51 & 23 & 1 & 5 & 1,018 & 220 & 1,238 \\
\hline Europe and Central Asia & NA & NA & NA & NA & NA & NA & 672 & 17 & 1,881 & 202 & 1,138 & 209 & 323 & 153 & 1 & 24 & 4,015 & 605 & 4,620 \\
\hline Latin America and the Caribbean & NA & NA & NA & NA & NA & NA & 75 & 4 & 237 & 69 & 97 & 50 & 32 & 20 & 0 & 9 & 442 & 151 & 594 \\
\hline Middle East and North Africa & NA & NA & NA & NA & NA & NA & 134 & 19 & 244 & 59 & 98 & 28 & 32 & 10 & 0 & 1 & 508 & 117 & 625 \\
\hline South Asia & NA & NA & NA & NA & NA & NA & 403 & 0 & 1,566 & 223 & 632 & 175 & 159 & 17 & 1 & 0 & 2,761 & 415 & 3,176 \\
\hline Sub-Saharan Africa & NA & NA & NA & NA & NA & NA & 22 & 2 & 119 & 39 & 46 & 23 & 13 & 5 & 0 & 1 & 199 & 70 & 269 \\
\hline Low- and middle-income countries & NA & NA & NA & NA & NA & NA & 1,698 & 78 & 4,455 & 679 & 2,177 & 553 & 610 & 229 & 4 & 39 & 8,944 & 1,578 & $\overline{10,522}$ \\
\hline High-income countries & NA & NA & NA & NA & NA & NA & 207 & 16 & 793 & 179 & 489 & 211 & 286 & 229 & 5 & 162 & 1,780 & 798 & 2,577 \\
\hline WORLD & NA & NA & NA & NA & NA & NA & 1,905 & 94 & 5,247 & 858 & 2,666 & 764 & 896 & 458 & 9 & 201 & 10,724 & 2,376 & 13,099 \\
\hline Attributable DALYs (thousands) & & & & & & & & & & & & & & & & & & & \\
\hline East Asia and Pacific & NA & NA & NA & NA & NA & NA & 434 & 41 & 437 & 92 & 174 & 72 & 52 & 24 & 1 & 5 & 1,097 & 234 & 1,331 \\
\hline Europe and Central Asia & NA & NA & NA & NA & NA & NA & 722 & 20 & 1,953 & 217 & 1,175 & 219 & 328 & 156 & 1 & 24 & 4,179 & 635 & 4,815 \\
\hline Latin America and the Caribbean & NA & NA & NA & NA & NA & NA & 86 & 5 & 253 & 76 & 104 & 53 & 33 & 20 & 0 & 9 & 476 & 162 & 638 \\
\hline Middle East and North Africa & NA & NA & NA & NA & NA & NA & 149 & 21 & 257 & 63 & 103 & 29 & 33 & 11 & 0 & 1 & 542 & 124 & 667 \\
\hline South Asia & NA & NA & NA & NA & NA & NA & 458 & 0 & 1,661 & 240 & 666 & 184 & 163 & 18 & 1 & 0 & 2,949 & 442 & 3,391 \\
\hline Sub-Saharan Africa & NA & NA & NA & NA & NA & NA & 24 & 2 & 126 & 42 & 48 & 24 & 13 & 5 & 0 & 1 & 212 & 74 & 286 \\
\hline Low- and middle-income countries & NA & NA & NA & NA & NA & NA & 1,872 & 88 & 4,687 & 730 & 2,269 & 581 & 622 & 233 & 4 & 40 & 9,455 & 1,672 & 11,127 \\
\hline High-income countries & NA & NA & NA & NA & NA & NA & 237 & 22 & 873 & 215 & 526 & 237 & 300 & 244 & 6 & 168 & 1,942 & 886 & 2,829 \\
\hline WORLD & NA & NA & NA & NA & NA & NA & 2,109 & 110 & 5,561 & 945 & 2,795 & 818 & 923 & 478 & 10 & 208 & 11,397 & 2,559 & $\overline{13,956}$ \\
\hline
\end{tabular}

Source: Authors' calculations.

Note: $\mathrm{NA}=$ not applicable. 
Risk factor:

Smoking

Selected other cardiovascular diseases

\begin{tabular}{|c|c|c|c|c|c|c|c|c|c|c|c|c|c|c|c|c|c|c|c|}
\hline \multirow[b]{2}{*}{ Region } & \multicolumn{2}{|c|}{$0-4$ years } & \multicolumn{2}{|c|}{ 5-14 years } & \multicolumn{2}{|c|}{$15-29$ years } & \multicolumn{2}{|c|}{ 30-44 years } & \multicolumn{2}{|c|}{$45-59$ years } & \multicolumn{2}{|c|}{ 60-69 years } & \multicolumn{2}{|c|}{$70-79$ years } & $80+$ & years & & Total & \\
\hline & Male & Female & Male & Female & Male & Female & Male & Female & Male & Female & Male & Female & Male & Female & Male & Female & Male & Female & All \\
\hline PAF of Mortality (\%) & & & & & & & & & & & & & & & & & & & \\
\hline East Asia and Pacific & NA & NA & NA & NA & NA & NA & 22 & 7 & 13 & 3 & 10 & 3 & 8 & 2 & 9 & 2 & 10 & 2 & 5 \\
\hline Europe and Central Asia & NA & NA & NA & NA & NA & NA & 44 & 7 & 50 & 9 & 43 & 7 & 30 & 8 & 18 & 4 & 32 & 5 & 16 \\
\hline Latin America and the Caribbean & NA & NA & NA & NA & NA & NA & 12 & 3 & 21 & 8 & 18 & 8 & 15 & 7 & 16 & 8 & 15 & 7 & 11 \\
\hline Middle East and North Africa & NA & NA & NA & NA & NA & NA & 22 & 15 & 20 & 6 & 18 & 4 & 15 & 4 & 15 & 1 & 15 & 3 & 8 \\
\hline South Asia & NA & NA & NA & NA & NA & NA & 12 & 0 & 22 & 3 & 19 & 3 & 15 & 1 & 15 & 0 & 15 & 1 & 7 \\
\hline Sub-Saharan Africa & NA & NA & NA & NA & NA & NA & 4 & 2 & 10 & 3 & 8 & 2 & 6 & 1 & 6 & 2 & 6 & 2 & 4 \\
\hline Low- and middle-income countries & NA & NA & NA & NA & NA & NA & 21 & 4 & 23 & 4 & 21 & 4 & 16 & 3 & 13 & 3 & 16 & 3 & 9 \\
\hline High-income countries & NA & NA & NA & NA & NA & NA & 24 & 14 & 34 & 19 & 33 & 19 & 31 & 22 & 32 & 23 & 31 & 22 & 26 \\
\hline WORLD & NA & NA & NA & NA & NA & NA & 21 & 5 & 25 & 6 & 24 & 6 & 20 & 7 & 21 & 12 & 21 & 9 & 14 \\
\hline PAF of YLL (\%) & & & & & & & & & & & & & & & & & & & \\
\hline East Asia and Pacific & NA & NA & NA & NA & NA & NA & 22 & 7 & 13 & 3 & 10 & 3 & 8 & 2 & 9 & 2 & 10 & 2 & 6 \\
\hline Europe and Central Asia & NA & NA & NA & NA & NA & NA & 44 & 7 & 50 & 9 & 43 & 7 & 30 & 8 & 18 & 4 & 36 & 6 & 21 \\
\hline Latin America and the Caribbean & NA & NA & NA & NA & NA & NA & 12 & 3 & 21 & 8 & 18 & 8 & 15 & 7 & 16 & 8 & 14 & 6 & 10 \\
\hline Middle East and North Africa & NA & NA & NA & NA & NA & NA & 22 & 15 & 20 & 6 & 18 & 4 & 15 & 4 & 15 & 1 & 14 & 3 & 8 \\
\hline South Asia & NA & NA & NA & NA & NA & NA & 12 & 0 & 22 & 3 & 19 & 3 & 15 & 1 & 15 & 0 & 14 & 1 & 7 \\
\hline Sub-Saharan Africa & NA & NA & NA & NA & NA & NA & 4 & 2 & 10 & 3 & 8 & 2 & 6 & 1 & 6 & 2 & 6 & 2 & 3 \\
\hline Low- and middle-income countries & NA & NA & NA & NA & NA & NA & 21 & 4 & 23 & 4 & 21 & 4 & 16 & 3 & 13 & 3 & 17 & 3 & 9 \\
\hline High-income countries & NA & NA & NA & NA & NA & NA & 24 & 14 & 34 & 19 & 33 & 19 & 31 & 22 & 32 & 23 & 30 & 21 & 25 \\
\hline WORLD & NA & NA & NA & NA & NA & NA & 21 & 5 & 25 & 6 & 24 & 6 & 20 & 7 & 20 & 11 & 20 & 6 & 12 \\
\hline PAF of DALYs (\%) & & & & & & & & & & & & & & & & & & & \\
\hline East Asia and Pacific & NA & NA & NA & NA & NA & NA & 22 & 7 & 13 & 3 & 10 & 3 & 8 & 2 & 9 & 2 & 10 & 2 & 6 \\
\hline Europe and Central Asia & NA & NA & NA & NA & NA & NA & 44 & 7 & 50 & 9 & 43 & 7 & 30 & 8 & 18 & 4 & 36 & 6 & 21 \\
\hline Latin America and the Caribbean & NA & NA & NA & NA & NA & NA & 12 & 3 & 21 & 8 & 18 & 8 & 15 & 7 & 16 & 8 & 14 & 6 & 10 \\
\hline Middle East and North Africa & NA & NA & NA & NA & NA & NA & 22 & 15 & 20 & 6 & 18 & 4 & 15 & 4 & 15 & 1 & 14 & 3 & 8 \\
\hline South Asia & NA & NA & NA & NA & NA & NA & 12 & 0 & 22 & 3 & 19 & 3 & 15 & 1 & 15 & 0 & 13 & 1 & 7 \\
\hline Sub-Saharan Africa & NA & NA & NA & NA & NA & NA & 4 & 2 & 10 & 3 & 8 & 2 & 6 & 1 & 6 & 2 & 6 & 2 & 3 \\
\hline Low- and middle-income countries & NA & NA & NA & NA & NA & NA & 21 & 4 & 24 & 4 & 22 & 4 & 16 & 3 & 13 & 3 & 16 & 3 & $\overline{9}$ \\
\hline High-income countries & NA & NA & NA & NA & NA & NA & 24 & 14 & 34 & 19 & 33 & 19 & 31 & 22 & 32 & 23 & 30 & 21 & 25 \\
\hline WORLD & NA & NA & NA & NA & NA & NA & 21 & 5 & 26 & 6 & 24 & 6 & 20 & 7 & 21 & 11 & 19 & 6 & 12 \\
\hline Attributable Mortality (thousand & & & & & & & & & & & & & & & & & & & \\
\hline East Asia and Pacific & NA & NA & NA & NA & NA & NA & 2 & 1 & 3 & 1 & 3 & 1 & 4 & 1 & 4 & 1 & 17 & 5 & 22 \\
\hline Europe and Central Asia & NA & NA & NA & NA & NA & NA & 6 & 0 & 10 & 1 & 13 & 1 & 13 & 5 & 9 & 5 & 51 & 12 & 63 \\
\hline Latin America and the Caribbean & NA & NA & NA & NA & NA & NA & 1 & 0 & 2 & 1 & 2 & 1 & 3 & 1 & 4 & 4 & 11 & 6 & 17 \\
\hline Middle East and North Africa & NA & NA & NA & NA & NA & NA & 1 & 0 & 1 & 0 & 1 & 0 & 2 & 1 & 2 & 0 & 7 & 2 & 9 \\
\hline South Asia & NA & NA & NA & NA & NA & NA & 1 & 0 & 6 & 1 & 6 & 1 & 6 & 1 & 4 & 0 & 23 & 3 & 26 \\
\hline Sub-Saharan Africa & NA & NA & NA & NA & NA & NA & 0 & 0 & 1 & 0 & 1 & 0 & 1 & 1 & 1 & 1 & 6 & 2 & 8 \\
\hline Low- and middle-income countries & NA & NA & NA & NA & NA & NA & 12 & 2 & 23 & 4 & 27 & 5 & 29 & 10 & 25 & 11 & 115 & 30 & $\overline{146}$ \\
\hline High-income countries & NA & NA & NA & NA & NA & NA & 2 & 1 & 7 & 2 & 11 & 4 & 23 & 16 & 44 & 66 & 87 & 88 & 175 \\
\hline WORLD & NA & NA & NA & NA & NA & NA & 14 & 2 & 30 & 6 & 38 & 9 & 53 & 25 & 69 & 77 & 203 & 119 & 321 \\
\hline Attributable YLL (thousands) & & & & & & & & & & & & & & & & & & & \\
\hline East Asia and Pacific & NA & NA & NA & NA & NA & NA & 55 & 14 & 59 & 12 & 45 & 13 & 36 & 15 & 21 & 7 & 217 & 61 & 278 \\
\hline Europe and Central Asia & NA & NA & NA & NA & NA & NA & 151 & 10 & 192 & 20 & 173 & 21 & 116 & 48 & 40 & 21 & 672 & 120 & 792 \\
\hline Latin America and the Caribbean & NA & NA & NA & NA & NA & NA & 14 & 3 & 31 & 11 & 23 & 10 & 23 & 14 & 20 & 17 & 111 & 54 & 165 \\
\hline Middle East and North Africa & NA & NA & NA & NA & NA & NA & 17 & 8 & 25 & 7 & 19 & 5 & 17 & 6 & 9 & 1 & 87 & 27 & 114 \\
\hline South Asia & NA & NA & NA & NA & NA & NA & 33 & 0 & 110 & 18 & 84 & 19 & 55 & 8 & 20 & 0 & 302 & 45 & 347 \\
\hline Sub-Saharan Africa & NA & NA & NA & NA & NA & NA & 9 & 4 & 25 & 8 & 16 & 6 & 13 & 6 & 7 & 3 & 71 & 27 & 98 \\
\hline Low- and middle-income countries & NA & NA & NA & NA & NA & NA & 279 & 38 & 443 & 76 & 361 & 74 & 259 & 97 & 116 & 50 & 1,458 & 335 & 1,794 \\
\hline High-income countries & NA & NA & NA & NA & NA & NA & 45 & 15 & 134 & 41 & 149 & 56 & 201 & 155 & 184 & 260 & 713 & 527 & 1,240 \\
\hline WORLD & NA & NA & NA & NA & NA & NA & 324 & 54 & 577 & 117 & 510 & 131 & 461 & 252 & 300 & 309 & 2,171 & 863 & 3,034 \\
\hline Attributable DALYs (thousands) & & & & & & & & & & & & & & & & & & & \\
\hline East Asia and Pacific & NA & NA & NA & NA & NA & NA & 77 & 19 & 63 & 13 & 46 & 13 & 37 & 15 & 21 & 7 & 244 & 67 & 312 \\
\hline Europe and Central Asia & NA & NA & NA & NA & NA & NA & 168 & 13 & 217 & 22 & 197 & 23 & 137 & 53 & 47 & 23 & 765 & 133 & 899 \\
\hline Latin America and the Caribbean & NA & NA & NA & NA & NA & NA & 16 & 3 & 34 & 11 & 26 & 10 & 25 & 15 & 22 & 19 & 123 & 58 & 181 \\
\hline Middle East and North Africa & NA & NA & NA & NA & NA & NA & 20 & 9 & 29 & 8 & 22 & 5 & 19 & 7 & 9 & 1 & 99 & 31 & 130 \\
\hline South Asia & NA & NA & NA & NA & NA & NA & 49 & 0 & 137 & 22 & 103 & 22 & 67 & 9 & 23 & 0 & 380 & 53 & 432 \\
\hline Sub-Saharan Africa & NA & NA & NA & NA & NA & NA & 10 & 4 & 26 & 9 & 17 & 6 & 13 & 6 & 8 & 3 & 74 & 28 & 102 \\
\hline Low- and middle-income countries & NA & NA & NA & NA & NA & NA & 339 & 48 & 506 & 85 & 411 & 80 & 298 & 104 & 131 & 53 & 1,685 & 370 & 2,055 \\
\hline High-income countries & NA & NA & NA & NA & NA & NA & 59 & 23 & 157 & 49 & 174 & 63 & 237 & 174 & 218 & 294 & 845 & 604 & 1,448 \\
\hline$\overline{\text { WORLD }}$ & NA & NA & NA & NA & NA & NA & 398 & 72 & 663 & 134 & 585 & 143 & 535 & 278 & 349 & 347 & 2,530 & 974 & $\overline{3,503}$ \\
\hline
\end{tabular}

Source: Authors' calculations.

Note: $\mathrm{NA}=$ not applicable 
Table 4A.119

Risk factor:

Smoking

Disease:

Cerebrovascular disease

\begin{tabular}{|c|c|c|c|c|c|c|c|c|c|c|c|c|c|c|c|c|c|c|c|}
\hline \multirow[b]{2}{*}{ Region } & \multicolumn{2}{|c|}{$0-4$ years } & \multicolumn{2}{|c|}{ 5-14 years } & \multicolumn{2}{|c|}{$15-29$ years } & \multicolumn{2}{|c|}{ 30-44 years } & \multicolumn{2}{|c|}{$45-59$ years } & \multicolumn{2}{|c|}{$60-69$ years } & \multicolumn{2}{|c|}{ 70-79 years } & $80+$ & years & & Total & \\
\hline & Male & Female & Male & Female & Male & Female & Male & Female & Male & Female & Male & Female & Male & Female & Male & Female & Male & Female & All \\
\hline PAF of Mortality (\%) & & & & & & & & & & & & & & & & & & & \\
\hline East Asia and Pacific & NA & NA & NA & NA & NA & NA & 35 & 22 & 22 & 9 & 8 & 5 & 3 & 2 & 0 & 0 & 7 & 3 & 5 \\
\hline Europe and Central Asia & NA & NA & NA & NA & NA & NA & 60 & 23 & 66 & 28 & 37 & 12 & 12 & 7 & 0 & 0 & 25 & 6 & 14 \\
\hline Latin America and the Caribbean & NA & NA & NA & NA & NA & NA & 20 & 10 & 33 & 24 & 15 & 13 & 5 & 6 & 0 & 0 & 11 & 7 & 9 \\
\hline Middle East and North Africa & NA & NA & NA & NA & NA & NA & 35 & 39 & 32 & 19 & 15 & 7 & 5 & 3 & 0 & 0 & 11 & 6 & 8 \\
\hline South Asia & NA & NA & NA & NA & NA & NA & 20 & 0 & 35 & 11 & 16 & 6 & 5 & 1 & 0 & 0 & 13 & 3 & 8 \\
\hline Sub-Saharan Africa & NA & NA & NA & NA & NA & NA & 6 & 6 & 17 & 10 & 6 & 4 & 2 & 1 & 0 & 0 & 6 & 3 & 4 \\
\hline Low- and middle-income countries & NA & NA & NA & NA & NA & NA & 32 & 15 & 33 & 14 & 16 & 7 & 5 & 3 & 0 & 0 & 12 & 4 & $\overline{8}$ \\
\hline High-income countries & NA & NA & NA & NA & NA & NA & 37 & 38 & 49 & 46 & 28 & 30 & 13 & 20 & 0 & 0 & 12 & 8 & 10 \\
\hline WORLD & NA & NA & NA & NA & NA & NA & 32 & 17 & 34 & 16 & 17 & 8 & 6 & 5 & 0 & 0 & 12 & 5 & 8 \\
\hline PAF of YLL (\%) & & & & & & & & & & & & & & & & & & & \\
\hline East Asia and Pacific & NA & NA & NA & NA & NA & NA & 35 & 22 & 22 & 9 & 8 & 5 & 3 & 2 & 0 & 0 & 10 & 4 & 7 \\
\hline Europe and Central Asia & NA & NA & NA & NA & NA & NA & 60 & 23 & 66 & 28 & 37 & 12 & 12 & 7 & 0 & 0 & 34 & 10 & 20 \\
\hline Latin America and the Caribbean & NA & NA & NA & NA & NA & NA & 20 & 10 & 33 & 24 & 15 & 13 & 5 & 6 & 0 & 0 & 16 & 11 & 13 \\
\hline Middle East and North Africa & NA & NA & NA & NA & NA & NA & 35 & 39 & 32 & 19 & 15 & 7 & 5 & 3 & 0 & 0 & 13 & 8 & 11 \\
\hline South Asia & NA & NA & NA & NA & NA & NA & 20 & 0 & 35 & 11 & 16 & 6 & 5 & 1 & 0 & 0 & 16 & 4 & 10 \\
\hline Sub-Saharan Africa & NA & NA & NA & NA & NA & NA & 6 & 6 & 17 & 10 & 6 & 4 & 2 & 1 & 0 & 0 & 8 & 4 & 6 \\
\hline Low- and middle-income countries & NA & NA & NA & NA & NA & NA & 32 & 15 & 33 & 14 & 16 & 7 & 5 & 3 & 0 & 0 & 16 & 6 & $\overline{11}$ \\
\hline High-income countries & NA & NA & NA & NA & NA & NA & 37 & 38 & 49 & 46 & 28 & 30 & 13 & 20 & 0 & 0 & 20 & 16 & 18 \\
\hline WORLD & NA & NA & NA & NA & NA & NA & 32 & 17 & 34 & 16 & 17 & 8 & 6 & 5 & 0 & 0 & 17 & 7 & 12 \\
\hline PAF of DALYs (\%) & & & & & & & & & & & & & & & & & & & \\
\hline East Asia and Pacific & NA & NA & NA & NA & NA & NA & 35 & 22 & 22 & 9 & 8 & 5 & 3 & 2 & 0 & 0 & 11 & 5 & 8 \\
\hline Europe and Central Asia & NA & NA & NA & NA & NA & NA & 60 & 23 & 66 & 28 & 37 & 12 & 12 & 7 & 0 & 0 & 35 & 10 & 21 \\
\hline Latin America and the Caribbean & NA & NA & NA & NA & NA & NA & 20 & 10 & 33 & 24 & 15 & 13 & 5 & 6 & 0 & 0 & 17 & 12 & 14 \\
\hline Middle East and North Africa & NA & NA & NA & NA & NA & NA & 35 & 39 & 32 & 19 & 15 & 7 & 5 & 3 & 0 & 0 & 15 & 9 & 12 \\
\hline South Asia & NA & NA & NA & NA & NA & NA & 20 & 0 & 35 & 11 & 16 & 6 & 5 & 1 & 0 & 0 & 17 & 5 & 11 \\
\hline Sub-Saharan Africa & NA & NA & NA & NA & NA & NA & 6 & 6 & 17 & 10 & 6 & 4 & 2 & 1 & 0 & 0 & 8 & 5 & 6 \\
\hline Low- and middle-income countries & NA & NA & NA & NA & NA & NA & 32 & 16 & 32 & 14 & 16 & 7 & 5 & 3 & 0 & 0 & 17 & 6 & 12 \\
\hline High-income countries & NA & NA & NA & NA & NA & NA & 37 & 38 & 49 & 46 & 28 & 30 & 13 & 20 & 0 & 0 & 23 & 19 & 21 \\
\hline WORLD & NA & NA & NA & NA & NA & NA & 33 & 19 & 34 & 17 & 17 & 9 & 6 & 5 & 0 & 0 & 18 & 8 & 13 \\
\hline Attributable Mortality (thousand & & & & & & & & & & & & & & & & & & & \\
\hline East Asia and Pacific & NA & NA & NA & NA & NA & NA & 9 & 3 & 30 & 8 & 20 & 8 & 9 & 6 & 0 & 0 & 68 & 26 & 94 \\
\hline Europe and Central Asia & NA & NA & NA & NA & NA & NA & 8 & 2 & 36 & 10 & 40 & 11 & 17 & 16 & 0 & 0 & 101 & 39 & 139 \\
\hline Latin America and the Caribbean & NA & NA & NA & NA & NA & NA & 1 & 1 & 7 & 4 & 4 & 3 & 2 & 2 & 0 & 0 & 14 & 10 & 25 \\
\hline Middle East and North Africa & NA & NA & NA & NA & NA & NA & 1 & 1 & 3 & 1 & 2 & 1 & 1 & 1 & 0 & 0 & 7 & 4 & 11 \\
\hline South Asia & NA & NA & NA & NA & NA & NA & 2 & 0 & 27 & 6 & 22 & 7 & 8 & 2 & 0 & 0 & 59 & 15 & 74 \\
\hline Sub-Saharan Africa & NA & NA & NA & NA & NA & NA & 1 & 1 & 5 & 4 & 2 & 2 & 1 & 1 & 0 & 0 & 9 & 7 & 16 \\
\hline Low- and middle-income countries & NA & NA & NA & NA & NA & NA & 21 & 7 & 107 & 33 & 90 & 32 & 39 & 27 & 1 & 0 & 258 & 100 & 358 \\
\hline High-income countries & NA & NA & NA & NA & NA & NA & 2 & 2 & 13 & 7 & 12 & 9 & 13 & 20 & 1 & 0 & 40 & 38 & 78 \\
\hline WORLD & NA & NA & NA & NA & NA & NA & 24 & 9 & 120 & 40 & 102 & 41 & 52 & 48 & 1 & 0 & 298 & 138 & 436 \\
\hline Attributable YLL (thousands) & & & & & & & & & & & & & & & & & & & \\
\hline East Asia and Pacific & NA & NA & NA & NA & NA & NA & 210 & 81 & 557 & 167 & 270 & 115 & 83 & 64 & 1 & 0 & 1,120 & 427 & 1,548 \\
\hline Europe and Central Asia & NA & NA & NA & NA & NA & NA & 182 & 41 & 673 & 197 & 537 & 172 & 155 & 159 & 1 & 0 & 1,547 & 569 & 2,116 \\
\hline Latin America and the Caribbean & NA & NA & NA & NA & NA & NA & 31 & 17 & 133 & 87 & 53 & 44 & 18 & 24 & 0 & 0 & 236 & 171 & 407 \\
\hline Middle East and North Africa & NA & NA & NA & NA & NA & NA & 21 & 19 & 52 & 28 & 28 & 13 & 10 & 7 & 0 & 0 & 111 & 67 & 179 \\
\hline South Asia & NA & NA & NA & NA & NA & NA & 46 & 0 & 496 & 115 & 296 & 111 & 75 & 17 & 1 & 0 & 914 & 242 & 1,157 \\
\hline Sub-Saharan Africa & NA & NA & NA & NA & NA & NA & 17 & 13 & 89 & 70 & 31 & 31 & 8 & 9 & 0 & 0 & 144 & 123 & 267 \\
\hline Low- and middle-income countries & NA & NA & NA & NA & NA & NA & 506 & 171 & 2,001 & 664 & 1,215 & 486 & 350 & 280 & 3 & 0 & 4,073 & 1,600 & 5,674 \\
\hline High-income countries & NA & NA & NA & NA & NA & NA & 54 & 41 & 235 & 145 & 162 & 129 & 110 & 204 & 3 & 0 & 563 & 519 & 1,082 \\
\hline WORLD & NA & NA & NA & NA & NA & NA & 560 & 212 & 2,235 & 809 & 1,377 & 615 & 460 & 484 & 5 & 0 & 4,636 & 2,119 & 6,755 \\
\hline Attributable DALYs (thousands) & & & & & & & & & & & & & & & & & & & \\
\hline East Asia and Pacific & NA & NA & NA & NA & NA & NA & 293 & 114 & 756 & 227 & 354 & 148 & 99 & 76 & 1 & 0 & 1,503 & 565 & 2,067 \\
\hline Europe and Central Asia & NA & NA & NA & NA & NA & NA & 224 & 57 & 838 & 257 & 675 & 222 & 186 & 186 & 1 & 0 & 1,924 & 722 & 2,646 \\
\hline Latin America and the Caribbean & NA & NA & NA & NA & NA & NA & 43 & 25 & 185 & 120 & 73 & 59 & 23 & 28 & 0 & 0 & 324 & 232 & 556 \\
\hline Middle East and North Africa & NA & NA & NA & NA & NA & NA & 29 & 26 & 69 & 36 & 36 & 16 & 12 & 8 & 0 & 0 & 147 & 86 & 233 \\
\hline South Asia & NA & NA & NA & NA & NA & NA & 57 & 0 & 619 & 142 & 367 & 134 & 87 & 19 & 1 & 0 & 1,131 & 295 & 1,426 \\
\hline Sub-Saharan Africa & NA & NA & NA & NA & NA & NA & 18 & 14 & 99 & 79 & 35 & 35 & 9 & 10 & 0 & 0 & 160 & 138 & 298 \\
\hline Low- and middle-income countries & NA & NA & NA & NA & NA & NA & 663 & 236 & 2,566 & 861 & 1,541 & 615 & 416 & 327 & 3 & 0 & 5,188 & 2,038 & 7,226 \\
\hline High-income countries & NA & NA & NA & NA & NA & NA & 103 & 82 & 462 & 298 & 305 & 247 & 170 & 313 & 3 & 0 & 1,043 & 940 & 1,983 \\
\hline WORLD & NA & NA & NA & NA & NA & NA & 766 & 317 & 3,028 & 1,159 & 1,845 & 861 & 586 & 640 & 6 & 0 & 6,231 & 2,978 & 9,209 \\
\hline
\end{tabular}

Source: Authors' calculations.

Note: $\mathrm{NA}=$ not applicable. 
Table 4A.120

Risk factor:

Disease:

Smoking

Selected respiratory diseases

\begin{tabular}{|c|c|c|c|c|c|c|c|c|c|c|c|c|c|c|c|c|c|c|c|}
\hline \multirow[b]{2}{*}{ Region } & \multicolumn{2}{|c|}{$0-4$ years } & \multicolumn{2}{|c|}{ 5-14 years } & \multicolumn{2}{|c|}{$15-29$ years } & \multicolumn{2}{|c|}{ 30-44 years } & \multicolumn{2}{|c|}{$45-59$ years } & \multicolumn{2}{|c|}{$60-69$ years } & \multicolumn{2}{|c|}{$70-79$ years } & $80+$ & years & & Total & \\
\hline & Male & Female & Male & Female & Male & Female & Male & Female & Male & Female & Male & Female & Male & Female & Male & Female & Male & Female & All \\
\hline PAF of Mortality (\%) & & & & & & & & & & & & & & & & & & & \\
\hline East Asia and Pacific & NA & NA & NA & NA & NA & NA & 27 & 8 & 16 & 3 & 8 & 3 & 5 & 2 & 3 & 0 & 9 & 2 & 5 \\
\hline Europe and Central Asia & NA & NA & NA & NA & NA & NA & 51 & 9 & 56 & 11 & 36 & 8 & 21 & 6 & 7 & 1 & 33 & 4 & 24 \\
\hline Latin America and the Caribbean & NA & NA & NA & NA & NA & NA & 15 & 4 & 25 & 9 & 14 & 9 & 10 & 5 & 6 & 3 & 10 & 4 & 7 \\
\hline Middle East and North Africa & NA & NA & NA & NA & NA & NA & 26 & 18 & 24 & 7 & 14 & 5 & 10 & 3 & 6 & 0 & 7 & 2 & 5 \\
\hline South Asia & NA & NA & NA & NA & NA & NA & 15 & 0 & 27 & 4 & 15 & 4 & 10 & 1 & 6 & 0 & 9 & 1 & 5 \\
\hline Sub-Saharan Africa & NA & NA & NA & NA & NA & NA & 4 & 2 & 12 & 3 & 6 & 3 & 4 & 1 & 2 & 1 & 2 & 1 & 2 \\
\hline Low- and middle-income countries & NA & NA & NA & NA & NA & NA & 17 & 4 & 23 & 4 & 13 & 4 & 8 & 2 & 4 & 1 & 8 & 1 & 5 \\
\hline High-income countries & NA & NA & NA & NA & NA & NA & 29 & 17 & 40 & 22 & 27 & 22 & 22 & 17 & 14 & 9 & 19 & 11 & 15 \\
\hline WORLD & NA & NA & NA & NA & NA & NA & 18 & 4 & 24 & 5 & 14 & 5 & 10 & 4 & 8 & 4 & 9 & 2 & 6 \\
\hline PAF of YLL (\%) & & & & & & & & & & & & & & & & & & & \\
\hline East Asia and Pacific & NA & NA & NA & NA & NA & NA & 27 & 8 & 16 & 3 & 8 & 3 & 5 & 2 & 3 & 0 & 9 & 2 & 6 \\
\hline Europe and Central Asia & NA & NA & NA & NA & NA & NA & 51 & 9 & 56 & 11 & 36 & 8 & 21 & 6 & 7 & 1 & 32 & 4 & 23 \\
\hline Latin America and the Caribbean & NA & NA & NA & NA & NA & NA & 15 & 4 & 25 & 9 & 14 & 9 & 10 & 5 & 6 & 3 & 9 & 3 & 6 \\
\hline Middle East and North Africa & NA & NA & NA & NA & NA & NA & 26 & 18 & 24 & 7 & 14 & 5 & 10 & 3 & 6 & 0 & 5 & 2 & 3 \\
\hline South Asia & NA & NA & NA & NA & NA & NA & 15 & 0 & 27 & 4 & 15 & 4 & 10 & 1 & 6 & 0 & 7 & 1 & 4 \\
\hline Sub-Saharan Africa & NA & NA & NA & NA & NA & NA & 4 & 2 & 12 & 3 & 6 & 3 & 4 & 1 & 2 & 1 & 2 & 0 & 1 \\
\hline Low- and middle-income countries & NA & NA & NA & NA & NA & NA & 17 & 4 & 23 & 4 & 13 & 4 & 8 & 2 & 4 & 1 & 6 & 1 & $\overline{4}$ \\
\hline High-income countries & NA & NA & NA & NA & NA & NA & 29 & 17 & 40 & 22 & 27 & 22 & 22 & 17 & 14 & 9 & 22 & 14 & 18 \\
\hline WORLD & NA & NA & NA & NA & NA & NA & 18 & 4 & 24 & 5 & 14 & 5 & 10 & 4 & 7 & 3 & 7 & 1 & 4 \\
\hline PAF of DALYs (\%) & & & & & & & & & & & & & & & & & & & \\
\hline East Asia and Pacific & NA & NA & NA & NA & NA & NA & 22 & 7 & 13 & 3 & 7 & 3 & 5 & 1 & 3 & 0 & 8 & 2 & 5 \\
\hline Europe and Central Asia & NA & NA & NA & NA & NA & NA & 44 & 7 & 49 & 8 & 29 & 5 & 15 & 4 & 5 & 1 & 26 & 3 & 18 \\
\hline Latin America and the Caribbean & NA & NA & NA & NA & NA & NA & 13 & 3 & 22 & 8 & 13 & 8 & 9 & 5 & 6 & 3 & 7 & 2 & 5 \\
\hline Middle East and North Africa & NA & NA & NA & NA & NA & NA & 20 & 15 & 19 & 6 & 12 & 4 & 9 & 2 & 5 & 0 & 4 & 2 & 3 \\
\hline South Asia & NA & NA & NA & NA & NA & NA & 13 & 0 & 23 & 4 & 14 & 4 & 9 & 1 & 5 & 0 & 6 & 1 & 3 \\
\hline Sub-Saharan Africa & NA & NA & NA & NA & NA & NA & 4 & 2 & 11 & 3 & 6 & 2 & 4 & 1 & 2 & 0 & 2 & 0 & 1 \\
\hline Low- and middle-income countries & NA & NA & NA & NA & NA & NA & 15 & 3 & 20 & 4 & 11 & 4 & 7 & 1 & 4 & 1 & 6 & 1 & 4 \\
\hline High-income countries & NA & NA & NA & NA & NA & NA & 14 & 7 & 22 & 11 & 18 & 14 & 18 & 14 & 12 & 8 & 15 & 9 & 12 \\
\hline WORLD & NA & NA & NA & NA & NA & NA & 15 & 3 & 20 & 4 & 12 & 4 & 9 & 3 & 7 & 3 & 6 & 1 & 4 \\
\hline Attributable Mortality (thousand & & & & & & & & & & & & & & & & & & & \\
\hline East Asia and Pacific & NA & NA & NA & NA & NA & NA & 21 & 4 & 18 & 2 & 11 & 2 & 7 & 2 & 3 & 1 & 60 & 10 & 70 \\
\hline Europe and Central Asia & NA & NA & NA & NA & NA & NA & 13 & 1 & 21 & 1 & 8 & 1 & 3 & 1 & 1 & 0 & 46 & 3 & 49 \\
\hline Latin America and the Caribbean & NA & NA & NA & NA & NA & NA & 2 & 0 & 5 & 1 & 3 & 1 & 3 & 1 & 2 & 1 & 15 & 5 & 20 \\
\hline Middle East and North Africa & NA & NA & NA & NA & NA & NA & 1 & 1 & 2 & 0 & 1 & 0 & 1 & 0 & 0 & 0 & 6 & 2 & 7 \\
\hline South Asia & NA & NA & NA & NA & NA & NA & 16 & 0 & 38 & 3 & 25 & 4 & 15 & 1 & 4 & 0 & 98 & 8 & 106 \\
\hline Sub-Saharan Africa & NA & NA & NA & NA & NA & NA & 4 & 1 & 11 & 2 & 4 & 1 & 2 & 0 & 0 & 0 & 21 & 4 & 26 \\
\hline Low- and middle-income countries & NA & NA & NA & NA & NA & NA & 58 & 7 & 95 & 9 & 52 & 9 & 32 & 5 & 10 & 2 & 246 & 32 & 279 \\
\hline High-income countries & NA & NA & NA & NA & NA & NA & 1 & 0 & 5 & 2 & 7 & 3 & 16 & 8 & 18 & 17 & 48 & 30 & 78 \\
\hline WORLD & NA & NA & NA & NA & NA & NA & 59 & 7 & 101 & 10 & 59 & 12 & 47 & 13 & 29 & 19 & 294 & 62 & 356 \\
\hline Attributable YLL (thousands) & & & & & & & & & & & & & & & & & & & \\
\hline East Asia and Pacific & NA & NA & NA & NA & NA & NA & 510 & 96 & 341 & 39 & 143 & 34 & 64 & 16 & 15 & 4 & 1,073 & 188 & 1,261 \\
\hline Europe and Central Asia & NA & NA & NA & NA & NA & NA & 301 & 14 & 414 & 19 & 115 & 9 & 29 & 7 & 2 & 1 & 861 & 49 & 911 \\
\hline Latin America and the Caribbean & NA & NA & NA & NA & NA & NA & 51 & 7 & 97 & 21 & 38 & 17 & 25 & 12 & 9 & 6 & 220 & 64 & 284 \\
\hline Middle East and North Africa & NA & NA & NA & NA & NA & NA & 26 & 19 & 32 & 7 & 18 & 4 & 11 & 2 & 2 & 0 & 90 & 33 & 123 \\
\hline South Asia & NA & NA & NA & NA & NA & NA & 389 & 0 & 716 & 57 & 334 & 65 & 138 & 10 & 19 & 0 & 1,596 & 133 & 1,729 \\
\hline Sub-Saharan Africa & NA & NA & NA & NA & NA & NA & 105 & 33 & 214 & 32 & 52 & 14 & 16 & 4 & 2 & 1 & 389 & 84 & 473 \\
\hline Low- and middle-income countries & NA & NA & NA & NA & NA & NA & 1,382 & 170 & 1,815 & 175 & 700 & 144 & 283 & 51 & 50 & 11 & 4,229 & 551 & 4,780 \\
\hline High-income countries & NA & NA & NA & NA & NA & NA & 32 & 11 & 100 & 31 & 94 & 44 & 134 & 81 & 76 & 66 & 437 & 233 & 670 \\
\hline WORLD & NA & NA & NA & NA & NA & NA & 1,414 & 181 & 1,915 & 207 & 794 & 188 & 417 & 132 & 126 & 77 & 4,666 & 784 & $\overline{5,450}$ \\
\hline Attributable DALYs (thousands) & & & & & & & & & & & & & & & & & & & \\
\hline East Asia and Pacific & NA & NA & NA & NA & NA & NA & 510 & 96 & 341 & 39 & 143 & 34 & 64 & 16 & 15 & 4 & 1,073 & 188 & 1,261 \\
\hline Europe and Central Asia & NA & NA & NA & NA & NA & NA & 301 & 14 & 414 & 19 & 115 & 9 & 29 & 7 & 2 & 1 & 861 & 49 & 911 \\
\hline Latin America and the Caribbean & NA & NA & NA & NA & NA & NA & 51 & 7 & 97 & 21 & 38 & 17 & 25 & 12 & 9 & 6 & 220 & 64 & 284 \\
\hline Middle East and North Africa & NA & NA & NA & NA & NA & NA & 26 & 19 & 32 & 7 & 18 & 4 & 11 & 2 & 2 & 0 & 90 & 33 & 123 \\
\hline South Asia & NA & NA & NA & NA & NA & NA & 389 & 0 & 716 & 57 & 334 & 65 & 138 & 10 & 19 & 0 & 1,596 & 133 & 1,729 \\
\hline Sub-Saharan Africa & NA & NA & NA & NA & NA & NA & 105 & 33 & 214 & 32 & 52 & 14 & 16 & 4 & 2 & 1 & 389 & 84 & 473 \\
\hline Low- and middle-income countries & NA & NA & NA & NA & NA & NA & 1,382 & 170 & 1,815 & 175 & 700 & 144 & 283 & 51 & 50 & 11 & 4,229 & 551 & 4,780 \\
\hline High-income countries & NA & NA & NA & NA & NA & NA & $\begin{array}{r}1,002 \\
32\end{array}$ & 11 & 100 & 31 & 94 & 44 & 134 & 81 & 76 & 66 & 437 & 233 & 670 \\
\hline$\overline{\text { WORLD }}$ & NA & NA & NA & NA & NA & NA & 1,414 & 181 & 1,915 & 207 & 794 & 188 & 417 & 132 & 126 & 77 & 4,666 & 784 & 5,450 \\
\hline
\end{tabular}

Source: Authors' calculations.

Note: $\mathrm{NA}=$ not applicable. 
Table 4A.121

Risk factor:

Smoking

Disease:

Selected medical conditions

\begin{tabular}{|c|c|c|c|c|c|c|c|c|c|c|c|c|c|c|c|c|c|c|c|}
\hline \multirow[b]{2}{*}{ Region } & \multicolumn{2}{|c|}{$0-4$ years } & \multicolumn{2}{|c|}{ 5-14 years } & \multicolumn{2}{|c|}{$15-29$ years } & \multicolumn{2}{|c|}{ 30-44 years } & \multicolumn{2}{|c|}{$45-59$ years } & \multicolumn{2}{|c|}{ 60-69 years } & \multicolumn{2}{|c|}{$70-79$ years } & $80+$ & years & & Total & \\
\hline & Male & Female & Male & Female & Male & Female & Male & Female & Male & Female & Male & Female & Male & Female & Male & Female & Male & Female & All \\
\hline PAF of Mortality (\%) & & & & & & & & & & & & & & & & & & & \\
\hline East Asia and Pacific & NA & NA & NA & NA & NA & NA & 21 & 6 & 12 & 2 & 6 & 2 & 4 & 1 & 2 & 0 & 6 & 2 & 4 \\
\hline Europe and Central Asia & NA & NA & NA & NA & NA & NA & 42 & 7 & 48 & 8 & 29 & 6 & 16 & 4 & 5 & 1 & 28 & 5 & 17 \\
\hline Latin America and the Caribbean & NA & NA & NA & NA & NA & NA & 11 & 3 & 19 & 7 & 11 & 6 & 8 & 4 & 4 & 2 & 9 & 4 & 6 \\
\hline Middle East and North Africa & NA & NA & NA & NA & NA & NA & 20 & 13 & 19 & 5 & 11 & 3 & 7 & 2 & 4 & 0 & 10 & 3 & 6 \\
\hline South Asia & NA & NA & NA & NA & NA & NA & 11 & 0 & 21 & 3 & 12 & 3 & 7 & 1 & 4 & 0 & 10 & 1 & 5 \\
\hline Sub-Saharan Africa & NA & NA & NA & NA & NA & NA & 3 & 2 & 9 & 2 & 5 & 2 & 3 & 1 & 1 & 0 & 3 & 1 & 2 \\
\hline Low- and middle-income countries & NA & NA & NA & NA & NA & NA & 17 & 4 & 19 & 4 & 10 & 3 & 6 & 2 & 3 & 1 & 9 & 2 & 5 \\
\hline High-income countries & NA & NA & NA & NA & NA & NA & 22 & 13 & 32 & 17 & 21 & 17 & 17 & 13 & 10 & 6 & 17 & 10 & 13 \\
\hline WORLD & NA & NA & NA & NA & NA & NA & 17 & 4 & 20 & 5 & 12 & 5 & 9 & 4 & 6 & 4 & 10 & 4 & 7 \\
\hline PAF of YLL (\%) & & & & & & & & & & & & & & & & & & & \\
\hline East Asia and Pacific & NA & NA & NA & NA & NA & NA & 21 & 6 & 12 & 2 & 6 & 2 & 4 & 1 & 2 & 0 & 7 & 2 & 4 \\
\hline Europe and Central Asia & NA & NA & NA & NA & NA & NA & 42 & 7 & 48 & 8 & 29 & 6 & 16 & 4 & 5 & 1 & 31 & 5 & 19 \\
\hline Latin America and the Caribbean & NA & NA & NA & NA & NA & NA & 11 & 3 & 19 & 7 & 11 & 6 & 8 & 4 & 4 & 2 & 9 & 4 & 7 \\
\hline Middle East and North Africa & NA & NA & NA & NA & NA & NA & 20 & 13 & 19 & 5 & 11 & 3 & 7 & 2 & 4 & 0 & 10 & 3 & 6 \\
\hline South Asia & NA & NA & NA & NA & NA & NA & 11 & 0 & 21 & 3 & 12 & 3 & 7 & 1 & 4 & 0 & 9 & 1 & 5 \\
\hline Sub-Saharan Africa & NA & NA & NA & NA & NA & NA & 3 & 2 & 9 & 2 & 5 & 2 & 3 & 1 & 1 & 0 & 2 & 1 & 2 \\
\hline Low- and middle-income countries & NA & NA & NA & NA & NA & NA & 17 & 4 & 19 & 4 & 10 & 3 & 6 & 2 & 3 & 1 & 9 & 2 & 5 \\
\hline High-income countries & NA & NA & NA & NA & NA & NA & 22 & 13 & 32 & 17 & 21 & 17 & 17 & 13 & 10 & 6 & 20 & 12 & 16 \\
\hline WORLD & NA & NA & NA & NA & NA & NA & 17 & 4 & 21 & 5 & 12 & 5 & 9 & 4 & 6 & 3 & 10 & 3 & 6 \\
\hline PAF of DALYs (\%) & & & & & & & & & & & & & & & & & & & \\
\hline East Asia and Pacific & NA & NA & NA & NA & NA & NA & 3 & 1 & 2 & 0 & 1 & 0 & 1 & 0 & 1 & 0 & 1 & 0 & 1 \\
\hline Europe and Central Asia & NA & NA & NA & NA & NA & NA & 12 & 1 & 12 & 1 & 7 & 1 & 5 & 1 & 2 & 0 & 7 & 1 & 4 \\
\hline Latin America and the Caribbean & NA & NA & NA & NA & NA & NA & 3 & 1 & 6 & 2 & 4 & 2 & 3 & 1 & 2 & 1 & 3 & 1 & 2 \\
\hline Middle East and North Africa & NA & NA & NA & NA & NA & NA & 3 & 1 & 4 & 1 & 4 & 1 & 3 & 1 & 2 & 0 & 2 & 1 & 1 \\
\hline South Asia & NA & NA & NA & NA & NA & NA & 2 & 0 & 5 & 1 & 3 & 1 & 3 & 0 & 2 & 0 & 2 & 0 & 1 \\
\hline Sub-Saharan Africa & NA & NA & NA & NA & NA & NA & 1 & 0 & 2 & 1 & 2 & 1 & 1 & 0 & 1 & 0 & 1 & 0 & 0 \\
\hline Low- and middle-income countries & NA & NA & NA & NA & NA & NA & 3 & 1 & 4 & 1 & 3 & 1 & 2 & 1 & 2 & 0 & 2 & 0 & $\overline{1}$ \\
\hline High-income countries & NA & NA & NA & NA & NA & NA & 4 & 2 & 6 & 2 & 5 & 3 & 6 & 4 & 6 & 3 & 5 & 3 & 4 \\
\hline WORLD & NA & NA & NA & NA & NA & NA & 3 & 1 & 4 & 1 & 3 & 1 & 3 & 1 & 3 & 1 & 2 & 1 & 2 \\
\hline Attributable Mortality (thousand & & & & & & & & & & & & & & & & & & & \\
\hline East Asia and Pacific & NA & NA & NA & NA & NA & NA & 8 & 2 & 10 & 2 & 6 & 2 & 4 & 1 & 1 & 0 & 29 & 8 & 37 \\
\hline Europe and Central Asia & NA & NA & NA & NA & NA & NA & 7 & 1 & 14 & 1 & 8 & 2 & 4 & 1 & 1 & 0 & 34 & 5 & 39 \\
\hline Latin America and the Caribbean & NA & NA & NA & NA & NA & NA & 2 & 0 & 8 & 2 & 5 & 3 & 4 & 2 & 2 & 1 & 20 & 9 & 29 \\
\hline Middle East and North Africa & NA & NA & NA & NA & NA & NA & 1 & 1 & 4 & 1 & 2 & 1 & 2 & 0 & 0 & 0 & 10 & 3 & 13 \\
\hline South Asia & NA & NA & NA & NA & NA & NA & 4 & 0 & 20 & 3 & 8 & 2 & 5 & 0 & 2 & 0 & 40 & 5 & 45 \\
\hline Sub-Saharan Africa & NA & NA & NA & NA & NA & NA & 1 & 0 & 3 & 1 & 2 & 1 & 1 & 0 & 0 & 0 & 7 & 3 & 10 \\
\hline Low- and middle-income countries & NA & NA & NA & NA & NA & NA & 23 & 4 & 59 & 10 & 31 & 10 & 21 & 6 & 6 & 2 & 139 & 33 & $\overline{173}$ \\
\hline High-income countries & NA & NA & NA & NA & NA & NA & 3 & 1 & 13 & 4 & 12 & 7 & 17 & 14 & 13 & 16 & 57 & 42 & 99 \\
\hline WORLD & NA & NA & NA & NA & NA & NA & 26 & 5 & 71 & 15 & 42 & 17 & 38 & 20 & 19 & 18 & 196 & 76 & 272 \\
\hline Attributable YLL (thousands) & & & & & & & & & & & & & & & & & & & \\
\hline East Asia and Pacific & NA & NA & NA & NA & NA & NA & 198 & 60 & 192 & 37 & 77 & 33 & 34 & 13 & 6 & 2 & 507 & 146 & 653 \\
\hline Europe and Central Asia & NA & NA & NA & NA & NA & NA & 169 & 14 & 261 & 30 & 111 & 25 & 39 & 15 & 2 & 1 & 583 & 86 & 668 \\
\hline Latin America and the Caribbean & NA & NA & NA & NA & NA & NA & 46 & 9 & 143 & 46 & 63 & 42 & 35 & 22 & 9 & 7 & 296 & 125 & 421 \\
\hline Middle East and North Africa & NA & NA & NA & NA & NA & NA & 31 & 19 & 67 & 19 & 33 & 11 & 17 & 5 & 2 & 0 & 151 & 54 & 205 \\
\hline South Asia & NA & NA & NA & NA & NA & NA & 98 & 0 & 384 & 54 & 109 & 32 & 49 & 4 & 9 & 0 & 648 & 90 & 738 \\
\hline Sub-Saharan Africa & NA & NA & NA & NA & NA & NA & 18 & 6 & 61 & 20 & 23 & 14 & 10 & 4 & 1 & 1 & 114 & 45 & 160 \\
\hline Low- and middle-income countries & NA & NA & NA & NA & NA & NA & 561 & 108 & 1,109 & 206 & 415 & 157 & 184 & 65 & 30 & 10 & 2,299 & 545 & 2,845 \\
\hline High-income countries & NA & NA & NA & NA & NA & NA & 67 & 27 & 236 & 86 & 155 & 105 & 150 & 140 & 55 & 67 & 664 & 424 & 1,089 \\
\hline WORLD & NA & NA & NA & NA & NA & NA & 628 & 135 & 1,345 & 292 & 571 & 261 & 334 & 205 & 85 & 76 & 2,963 & 970 & 3,933 \\
\hline Attributable DALYs (thousands) & & & & & & & & & & & & & & & & & & & \\
\hline East Asia and Pacific & NA & NA & NA & NA & NA & NA & 198 & 60 & 192 & 37 & 77 & 33 & 34 & 13 & 6 & 2 & 507 & 146 & 653 \\
\hline Europe and Central Asia & NA & NA & NA & NA & NA & NA & 169 & 14 & 261 & 30 & 111 & 25 & 39 & 15 & 2 & 1 & 583 & 86 & 668 \\
\hline Latin America and the Caribbean & NA & NA & NA & NA & NA & NA & 46 & 9 & 143 & 46 & 63 & 42 & 35 & 22 & 9 & 7 & 296 & 125 & 421 \\
\hline Middle East and North Africa & NA & NA & NA & NA & NA & NA & 31 & 19 & 67 & 19 & 33 & 11 & 17 & 5 & 2 & 0 & 151 & 54 & 205 \\
\hline South Asia & NA & NA & NA & NA & NA & NA & 98 & 0 & 384 & 54 & 109 & 32 & 49 & 4 & 9 & 0 & 648 & 90 & 738 \\
\hline Sub-Saharan Africa & NA & NA & NA & NA & NA & NA & 18 & 6 & 61 & 20 & 23 & 14 & 10 & 4 & 1 & 1 & 114 & 45 & 160 \\
\hline Low- and middle-income countries & NA & NA & NA & NA & NA & NA & 561 & 108 & 1,109 & 206 & 415 & 157 & 184 & 65 & 30 & 10 & 2,299 & 545 & 2,845 \\
\hline High-income countries & NA & NA & NA & NA & NA & NA & 67 & 27 & 236 & 86 & 155 & 105 & 150 & 140 & 55 & 67 & 664 & 424 & 1,089 \\
\hline$\overline{\text { WORLD }}$ & NA & NA & NA & NA & NA & NA & 628 & 135 & 1,345 & 292 & 571 & 261 & 334 & 205 & 85 & 76 & 2,963 & 970 & $\overline{3,933}$ \\
\hline
\end{tabular}

Source: Authors' calculations.

Note: $\mathrm{NA}=$ not applicable. 
Table 4A.122

Risk factor:

Smoking

Disease:

All causes

\begin{tabular}{|c|c|c|c|c|c|c|c|c|c|c|c|c|c|c|c|c|c|c|c|}
\hline \multirow[b]{2}{*}{ Region } & \multicolumn{2}{|c|}{$0-4$ years } & \multicolumn{2}{|c|}{ 5-14 years } & \multicolumn{2}{|c|}{$15-29$ years } & \multicolumn{2}{|c|}{ 30-44 years } & \multicolumn{2}{|c|}{$45-59$ years } & \multicolumn{2}{|c|}{ 60-69 years } & \multicolumn{2}{|c|}{$70-79$ years } & $80+$ & years & & Total & \\
\hline & Male & Female & Male & Female & Male & Female & Male & Female & Male & Female & Male & Female & Male & Female & Male & Female & Male & Female & All \\
\hline PAF of Mortality (\%) & & & & & & & & & & & & & & & & & & & \\
\hline East Asia and Pacific & NA & NA & NA & NA & NA & NA & 14 & 4 & 16 & 5 & 16 & 6 & 14 & 5 & 12 & 3 & 12 & 4 & 8 \\
\hline Europe and Central Asia & NA & NA & NA & NA & NA & NA & 22 & 5 & 40 & 12 & 36 & 9 & 21 & 6 & 8 & 2 & 25 & 5 & 16 \\
\hline Latin America and the Caribbean & NA & NA & NA & NA & NA & NA & 5 & 2 & 18 & 9 & 16 & 9 & 13 & 6 & 10 & 5 & 10 & 5 & 8 \\
\hline Middle East and North Africa & NA & NA & NA & NA & NA & NA & 14 & 8 & 19 & 8 & 15 & 5 & 10 & 3 & 7 & 1 & 9 & 3 & 6 \\
\hline South Asia & NA & NA & NA & NA & NA & NA & 7 & 0 & 25 & 6 & 22 & 5 & 13 & 1 & 8 & 0 & 11 & 2 & 6 \\
\hline Sub-Saharan Africa & NA & NA & NA & NA & NA & NA & 1 & 0 & 6 & 2 & 7 & 2 & 5 & 1 & 4 & 1 & 2 & 1 & 1 \\
\hline Low- and middle-income countries & NA & NA & NA & NA & NA & NA & 9 & 2 & 21 & 6 & 20 & 6 & 14 & 4 & 10 & 2 & 11 & 3 & 7 \\
\hline High-income countries & NA & NA & NA & NA & NA & NA & 13 & 7 & 31 & 18 & 32 & 20 & 26 & 18 & 17 & 11 & 23 & 14 & 19 \\
\hline WORLD & NA & NA & NA & NA & NA & NA & 9 & 2 & 22 & 7 & 22 & 8 & 16 & 6 & 12 & 5 & 12 & 4 & 9 \\
\hline PAF of YLL (\%) & & & & & & & & & & & & & & & & & & & \\
\hline East Asia and Pacific & NA & NA & NA & NA & NA & NA & 14 & 4 & 16 & 5 & 16 & 6 & 14 & 5 & 13 & 3 & 10 & 3 & 7 \\
\hline Europe and Central Asia & NA & NA & NA & NA & NA & NA & 22 & 5 & 40 & 12 & 36 & 9 & 21 & 6 & 8 & 2 & 25 & 6 & 17 \\
\hline Latin America and the Caribbean & NA & NA & NA & NA & NA & NA & 5 & 2 & 17 & 9 & 16 & 9 & 13 & 6 & 10 & 5 & 7 & 4 & 6 \\
\hline Middle East and North Africa & NA & NA & NA & NA & NA & NA & 14 & 8 & 19 & 8 & 15 & 5 & 10 & 3 & 7 & 1 & 7 & 3 & 5 \\
\hline South Asia & NA & NA & NA & NA & NA & NA & 7 & 0 & 25 & 6 & 22 & 5 & 13 & 1 & 8 & 0 & 8 & 1 & 5 \\
\hline Sub-Saharan Africa & NA & NA & NA & NA & NA & NA & 1 & 0 & 6 & 2 & 7 & 2 & 5 & 1 & 4 & 1 & 1 & 0 & 1 \\
\hline Low- and middle-income countries & NA & NA & NA & NA & NA & NA & 9 & 2 & 21 & 6 & 20 & 6 & 14 & 4 & 10 & 2 & 8 & 2 & 5 \\
\hline High-income countries & NA & NA & NA & NA & NA & NA & 13 & 7 & 31 & 18 & 32 & 20 & 27 & 18 & 17 & 11 & 23 & 15 & 20 \\
\hline WORLD & NA & NA & NA & NA & NA & NA & 9 & 2 & 22 & 7 & 22 & 8 & 16 & 6 & 12 & 5 & 9 & 3 & 6 \\
\hline PAF of DALYs (\%) & & & & & & & & & & & & & & & & & & & \\
\hline East Asia and Pacific & NA & NA & NA & NA & NA & NA & 9 & 3 & 12 & 3 & 13 & 4 & 11 & 4 & 11 & 3 & 7 & 2 & 5 \\
\hline Europe and Central Asia & NA & NA & NA & NA & NA & NA & 17 & 4 & 33 & 8 & 31 & 7 & 19 & 5 & 7 & 2 & 19 & 4 & 13 \\
\hline Latin America and the Caribbean & NA & NA & NA & NA & NA & NA & 4 & 2 & 13 & 6 & 13 & 6 & 10 & 4 & 8 & 4 & 5 & 2 & 4 \\
\hline Middle East and North Africa & NA & NA & NA & NA & NA & NA & 9 & 4 & 13 & 4 & 11 & 3 & 9 & 2 & 6 & 1 & 5 & 2 & 3 \\
\hline South Asia & NA & NA & NA & NA & NA & NA & 5 & 0 & 19 & 4 & 18 & 4 & 12 & 1 & 7 & 0 & 6 & 1 & 4 \\
\hline Sub-Saharan Africa & NA & NA & NA & NA & NA & NA & 1 & 0 & 5 & 2 & 6 & 2 & 5 & 1 & 4 & 1 & 1 & 0 & 1 \\
\hline Low- and middle-income countries & NA & NA & NA & NA & NA & NA & 6 & 1 & 16 & 4 & 16 & 4 & 12 & 3 & 9 & 2 & 6 & 1 & $\overline{4}$ \\
\hline High-income countries & NA & NA & NA & NA & NA & NA & 8 & 6 & 23 & 12 & 24 & 13 & 21 & 13 & 14 & 8 & 16 & 9 & 13 \\
\hline WORLD & NA & NA & NA & NA & NA & NA & 6 & 2 & 17 & 5 & 18 & 6 & 14 & 5 & 10 & 4 & 7 & 2 & 5 \\
\hline Attributable Mortality (thousand & & & & & & & & & & & & & & & & & & & \\
\hline East Asia and Pacific & NA & NA & NA & NA & NA & NA & 86 & 16 & 191 & 35 & 221 & 55 & 219 & 73 & 112 & 50 & 829 & 230 & 1,059 \\
\hline Europe and Central Asia & NA & NA & NA & NA & NA & NA & 73 & 5 & 249 & 30 & 255 & 39 & 150 & 53 & 27 & 16 & 754 & 143 & 897 \\
\hline Latin America and the Caribbean & NA & NA & NA & NA & NA & NA & 11 & 2 & 51 & 16 & 44 & 18 & 43 & 18 & 28 & 19 & 177 & 73 & 250 \\
\hline Middle East and North Africa & NA & NA & NA & NA & NA & NA & 12 & 4 & 30 & 8 & 26 & 6 & 22 & 5 & 8 & 1 & 97 & 24 & 121 \\
\hline South Asia & NA & NA & NA & NA & NA & NA & 51 & 0 & 282 & 47 & 243 & 49 & 148 & 14 & 45 & 0 & 768 & 110 & 879 \\
\hline Sub-Saharan Africa & NA & NA & NA & NA & NA & NA & 9 & 3 & 41 & 11 & 28 & 9 & 20 & 5 & 7 & 2 & 105 & 30 & 135 \\
\hline Low- and middle-income countries & NA & NA & NA & NA & NA & NA & 241 & 31 & 844 & 146 & 817 & 175 & 601 & 169 & 226 & 89 & 2,730 & 610 & 3,340 \\
\hline High-income countries & NA & NA & NA & NA & NA & NA & 24 & 7 & 162 & 50 & 221 & 81 & 309 & 166 & 213 & 230 & 929 & 533 & 1,462 \\
\hline WORLD & NA & NA & NA & NA & NA & NA & 265 & 37 & 1,006 & 196 & 1,038 & 256 & 910 & 335 & 439 & 319 & 3,659 & 1,143 & 4,802 \\
\hline Attributable YLL (thousands) & & & & & & & & & & & & & & & & & & & \\
\hline East Asia and Pacific & NA & NA & NA & NA & NA & NA & 2,041 & 403 & 3,573 & 689 & 2,975 & 825 & 1,950 & 745 & 560 & 256 & 11,099 & 2,919 & 14,018 \\
\hline Europe and Central Asia & NA & NA & NA & NA & NA & NA & 1,711 & 122 & 4,725 & 598 & 3,467 & 581 & 1,367 & 546 & 124 & 77 & 11,394 & 1,925 & 13,319 \\
\hline Latin America and the Caribbean & NA & NA & NA & NA & NA & NA & 265 & 51 & 955 & 327 & 600 & 268 & 381 & 183 & 130 & 90 & 2,331 & 918 & 3,249 \\
\hline Middle East and North Africa & NA & NA & NA & NA & NA & NA & 282 & 107 & 563 & 155 & 346 & 86 & 193 & 54 & 38 & 5 & 1,421 & 408 & 1,829 \\
\hline South Asia & NA & NA & NA & NA & NA & NA & 1,211 & 0 & 5,243 & 926 & 3,294 & 738 & 1,330 & 150 & 224 & 0 & 11,302 & 1,815 & 13,117 \\
\hline Sub-Saharan Africa & NA & NA & NA & NA & NA & NA & 207 & 66 & 765 & 220 & 379 & 129 & 178 & 55 & 37 & 13 & 1,566 & 484 & 2,050 \\
\hline Low- and middle-income countries & NA & NA & NA & NA & NA & NA & 5,716 & 750 & 15,824 & 2,915 & 11,059 & 2,627 & 5,400 & 1,734 & 1,113 & 442 & 39,113 & 8,468 & 47,581 \\
\hline High-income countries & NA & NA & NA & NA & NA & NA & 572 & 168 & 3,018 & 987 & 2,963 & 1,216 & 2,723 & 1,674 & 949 & 976 & 10,225 & 5,021 & 15,246 \\
\hline WORLD & NA & NA & NA & NA & NA & NA & 6,289 & 919 & 18,842 & 3,902 & 14,023 & 3,843 & 8,123 & 3,407 & 2,062 & 1,418 & 49,338 & 13,489 & 62,827 \\
\hline Attributable DALYs (thousands) & & & & & & & & & & & & & & & & & & & \\
\hline East Asia and Pacific & NA & NA & NA & NA & NA & NA & 2,370 & 567 & 4,632 & 817 & 3,403 & 939 & 2,104 & 810 & 606 & 268 & 13,116 & 3,402 & 16,518 \\
\hline Europe and Central Asia & NA & NA & NA & NA & NA & NA & 1,909 & 231 & 5,116 & 770 & 3,762 & 679 & 1,481 & 597 & 140 & 82 & 12,408 & 2,361 & 14,769 \\
\hline Latin America and the Caribbean & NA & NA & NA & NA & NA & NA & 344 & 114 & 1,178 & 439 & 705 & 315 & 419 & 203 & 143 & 97 & 2,789 & 1,168 & 3,957 \\
\hline Middle East and North Africa & NA & NA & NA & NA & NA & NA & 410 & 146 & 650 & 176 & 374 & 93 & 203 & 56 & 40 & 5 & 1,676 & 476 & 2,153 \\
\hline South Asia & NA & NA & NA & NA & NA & NA & 1,443 & 0 & 5,727 & 1,099 & 3,591 & 797 & 1,400 & 158 & 237 & 0 & 12,397 & 2,055 & 14,452 \\
\hline Sub-Saharan Africa & NA & NA & NA & NA & NA & NA & 219 & 69 & 813 & 236 & 404 & 136 & 185 & 57 & 39 & 14 & 1,659 & 512 & 2,171 \\
\hline Low- and middle-income countries & NA & NA & NA & NA & NA & NA & 6,695 & 1,128 & 18,117 & 3,538 & 12,238 & 2,960 & 5,791 & 1,882 & 1,204 & 466 & 44,046 & 9,973 & 54,019 \\
\hline High-income countries & NA & NA & NA & NA & NA & NA & 837 & 487 & 3,920 & 1,580 & 3,463 & 1,539 & 3,043 & 1,915 & 1,047 & 1,070 & 12,309 & 6,590 & 18,900 \\
\hline$\overline{\text { WORLD }}$ & NA & NA & NA & NA & NA & NA & 7,532 & 1,615 & 22,037 & 5,118 & 15,701 & 4,499 & 8,834 & 3,796 & 2,251 & 1,536 & 56,355 & 16,564 & 72,919 \\
\hline
\end{tabular}

Source: Authors' calculations.

Note: $\mathrm{NA}=$ not applicable 
Table 4A.123

Risk factor:

Non-use and use of ineffective methods of contraception

Disease:

Abortion

\begin{tabular}{|c|c|c|c|c|c|c|c|c|c|c|c|c|c|c|c|c|c|c|c|}
\hline \multirow[b]{2}{*}{ Region } & \multicolumn{2}{|c|}{$0-4$ years } & \multicolumn{2}{|c|}{ 5-14 years } & \multicolumn{2}{|c|}{$15-29$ years } & \multicolumn{2}{|c|}{$30-44$ years } & \multicolumn{2}{|c|}{ 45-59 years } & \multicolumn{2}{|c|}{$60-69$ years } & \multicolumn{2}{|c|}{$70-79$ years } & $80+$ & years & & Total & \\
\hline & Male & Female & Male & Female & Male & Female & Male & Female & Male & Female & Male & Female & Male & Female & Male & Female & Male & Female & All \\
\hline PAF of Mortality (\%) & & & & & & & & & & & & & & & & & & & \\
\hline East Asia and Pacific & NA & NA & NA & NA & NA & 84 & NA & 87 & NA & NA & NA & NA & NA & NA & NA & NA & NA & 84 & 84 \\
\hline Europe and Central Asia & NA & NA & NA & NA & NA & 86 & NA & 86 & NA & NA & NA & NA & NA & NA & NA & NA & NA & 85 & 85 \\
\hline Latin America and the Caribbean & NA & NA & NA & NA & NA & 85 & NA & 88 & NA & NA & NA & NA & NA & NA & NA & NA & NA & 86 & 86 \\
\hline Middle East and North Africa & NA & NA & NA & NA & NA & 89 & NA & 89 & NA & NA & NA & NA & NA & NA & NA & NA & NA & 89 & 89 \\
\hline South Asia & NA & NA & NA & NA & NA & 92 & NA & 94 & NA & NA & NA & NA & NA & NA & NA & NA & NA & 93 & 93 \\
\hline Sub-Saharan Africa & NA & NA & NA & NA & NA & 88 & NA & 89 & NA & NA & NA & NA & NA & NA & NA & NA & NA & 88 & 88 \\
\hline Low- and middle-income countries & NA & NA & NA & NA & NA & 89 & NA & 91 & NA & NA & NA & NA & NA & NA & NA & NA & NA & 90 & 90 \\
\hline High-income countries & NA & NA & NA & NA & NA & 71 & NA & 74 & NA & NA & NA & NA & NA & NA & NA & NA & NA & 71 & 71 \\
\hline WORLD & NA & NA & NA & NA & NA & 89 & NA & 91 & NA & NA & NA & NA & NA & NA & NA & NA & NA & 90 & 90 \\
\hline PAF of YLL (\%) & & & & & & & & & & & & & & & & & & & \\
\hline East Asia and Pacific & NA & NA & NA & NA & NA & 84 & NA & 87 & NA & NA & NA & NA & NA & NA & NA & NA & NA & 84 & 84 \\
\hline Europe and Central Asia & NA & NA & NA & NA & NA & 86 & NA & 86 & NA & NA & NA & NA & NA & NA & NA & NA & NA & 85 & 85 \\
\hline Latin America and the Caribbean & NA & NA & NA & NA & NA & 85 & NA & 88 & NA & NA & NA & NA & NA & NA & NA & NA & NA & 86 & 86 \\
\hline Middle East and North Africa & NA & NA & NA & NA & NA & 89 & NA & 89 & NA & NA & NA & NA & NA & NA & NA & NA & NA & 89 & 89 \\
\hline South Asia & NA & NA & NA & NA & NA & 92 & NA & 94 & NA & NA & NA & NA & NA & NA & NA & NA & NA & 93 & 93 \\
\hline Sub-Saharan Africa & NA & NA & NA & NA & NA & 88 & NA & 89 & NA & NA & NA & NA & NA & NA & NA & NA & NA & 88 & 88 \\
\hline Low- and middle-income countries & NA & NA & NA & NA & NA & 89 & NA & 91 & NA & NA & NA & NA & NA & NA & NA & NA & NA & 90 & 90 \\
\hline High-income countries & NA & NA & NA & NA & NA & 71 & NA & 74 & NA & NA & NA & NA & NA & NA & NA & NA & NA & 71 & 71 \\
\hline WORLD & NA & NA & NA & NA & NA & 89 & NA & 91 & NA & NA & NA & NA & NA & NA & NA & NA & NA & 90 & 90 \\
\hline PAF of DALYs (\%) & & & & & & & & & & & & & & & & & & & \\
\hline East Asia and Pacific & NA & NA & NA & NA & NA & 84 & NA & 87 & NA & NA & NA & NA & NA & NA & NA & NA & NA & 82 & 82 \\
\hline Europe and Central Asia & NA & NA & NA & NA & NA & 86 & NA & 86 & NA & NA & NA & NA & NA & NA & NA & NA & NA & 82 & 82 \\
\hline Latin America and the Caribbean & NA & NA & NA & NA & NA & 85 & NA & 88 & NA & NA & NA & NA & NA & NA & NA & NA & NA & 83 & 83 \\
\hline Middle East and North Africa & NA & NA & NA & NA & NA & 89 & NA & 89 & NA & NA & NA & NA & NA & NA & NA & NA & NA & 84 & 84 \\
\hline South Asia & NA & NA & NA & NA & NA & 92 & NA & 94 & NA & NA & NA & NA & NA & NA & NA & NA & NA & 88 & 88 \\
\hline Sub-Saharan Africa & NA & NA & NA & NA & NA & 88 & NA & 89 & NA & NA & NA & NA & NA & NA & NA & NA & NA & 84 & 84 \\
\hline Low- and middle-income countries & NA & NA & NA & NA & NA & 89 & NA & 91 & NA & NA & NA & NA & NA & NA & NA & NA & NA & 86 & 86 \\
\hline High-income countries & NA & NA & NA & NA & NA & 71 & NA & 74 & NA & NA & NA & NA & NA & NA & NA & NA & NA & 71 & 71 \\
\hline WORLD & NA & NA & NA & NA & NA & 89 & NA & 91 & NA & NA & NA & NA & NA & NA & NA & NA & NA & 86 & 86 \\
\hline Attributable Mortality (thousand & & & & & & & & & & & & & & & & & & & \\
\hline East Asia and Pacific & NA & NA & NA & NA & NA & 2 & NA & 2 & NA & NA & NA & NA & NA & NA & NA & NA & NA & 4 & 4 \\
\hline Europe and Central Asia & NA & NA & NA & NA & NA & 0 & NA & 0 & NA & NA & NA & NA & NA & NA & NA & NA & NA & 0 & 0 \\
\hline Latin America and the Caribbean & NA & NA & NA & NA & NA & 1 & NA & 1 & NA & NA & NA & NA & NA & NA & NA & NA & NA & 2 & 2 \\
\hline Middle East and North Africa & NA & NA & NA & NA & NA & 1 & NA & 1 & NA & NA & NA & NA & NA & NA & NA & NA & NA & 1 & 1 \\
\hline South Asia & NA & NA & NA & NA & NA & 15 & NA & 11 & NA & NA & NA & NA & NA & NA & NA & NA & NA & 26 & 26 \\
\hline Sub-Saharan Africa & NA & NA & NA & NA & NA & 19 & NA & 6 & NA & NA & NA & NA & NA & NA & NA & NA & NA & 25 & 25 \\
\hline Low- and middle-income countries & NA & NA & NA & NA & NA & 39 & NA & 20 & NA & NA & NA & NA & NA & NA & NA & NA & NA & 59 & 59 \\
\hline High-income countries & NA & NA & NA & NA & NA & 0 & NA & 0 & NA & NA & NA & NA & NA & NA & NA & NA & NA & 0 & 0 \\
\hline WORLD & NA & NA & NA & NA & NA & 39 & NA & 20 & NA & NA & NA & NA & NA & NA & NA & NA & NA & 59 & 59 \\
\hline Attributable YLL (thousands) & & & & & & & & & & & & & & & & & & & \\
\hline East Asia and Pacific & NA & NA & NA & NA & NA & 61 & NA & 50 & NA & NA & NA & NA & NA & NA & NA & NA & NA & 111 & 111 \\
\hline Europe and Central Asia & NA & NA & NA & NA & NA & 3 & NA & 3 & NA & NA & NA & NA & NA & NA & NA & NA & NA & 6 & 6 \\
\hline Latin America and the Caribbean & NA & NA & NA & NA & NA & 38 & NA & 17 & NA & NA & NA & NA & NA & NA & NA & NA & NA & 55 & 55 \\
\hline Middle East and North Africa & NA & NA & NA & NA & NA & 21 & NA & 13 & NA & NA & NA & NA & NA & NA & NA & NA & NA & 35 & 35 \\
\hline South Asia & NA & NA & NA & NA & NA & 424 & NA & 276 & NA & NA & NA & NA & NA & NA & NA & NA & NA & 701 & 701 \\
\hline Sub-Saharan Africa & NA & NA & NA & NA & NA & 532 & NA & 148 & NA & NA & NA & NA & NA & NA & NA & NA & NA & 680 & 680 \\
\hline Low- and middle-income countries & NA & NA & NA & NA & NA & 1,080 & NA & 508 & NA & NA & NA & NA & NA & NA & NA & NA & NA & 1,588 & 1,588 \\
\hline High-income countries & NA & NA & NA & NA & NA & 1 & NA & 1 & NA & NA & NA & NA & NA & NA & NA & NA & NA & 2 & 2 \\
\hline WORLD & NA & NA & NA & NA & NA & 1,080 & NA & 509 & NA & NA & NA & NA & NA & NA & NA & NA & NA & 1,590 & 1,590 \\
\hline Attributable DALYs (thousands) & & & & & & & & & & & & & & & & & & & \\
\hline East Asia and Pacific & NA & NA & NA & NA & NA & 104 & NA & 53 & NA & NA & NA & NA & NA & NA & NA & NA & NA & 156 & 156 \\
\hline Europe and Central Asia & NA & NA & NA & NA & NA & 10 & NA & 3 & NA & NA & NA & NA & NA & NA & NA & NA & NA & 14 & 14 \\
\hline Latin America and the Caribbean & NA & NA & NA & NA & NA & 77 & NA & 20 & NA & NA & NA & NA & NA & NA & NA & NA & NA & 97 & 97 \\
\hline Middle East and North Africa & NA & NA & NA & NA & NA & 108 & NA & 19 & NA & NA & NA & NA & NA & NA & NA & NA & NA & 127 & 127 \\
\hline South Asia & NA & NA & NA & NA & NA & 984 & NA & 310 & NA & NA & NA & NA & NA & NA & NA & NA & NA & 1,294 & 1,294 \\
\hline Sub-Saharan Africa & NA & NA & NA & NA & NA & 1,127 & NA & 184 & NA & NA & NA & NA & NA & NA & NA & NA & NA & 1,311 & 1,311 \\
\hline Low- and middle-income countries & NA & NA & NA & NA & NA & 2,410 & NA & 589 & NA & NA & NA & NA & NA & NA & NA & NA & NA & 3,000 & 3,000 \\
\hline High-income countries & NA & NA & NA & NA & NA & 2 & NA & 1 & NA & NA & NA & NA & NA & NA & NA & NA & NA & 3 & 3 \\
\hline$\overline{\text { WORLD }}$ & NA & NA & NA & NA & NA & 2,412 & NA & 590 & NA & NA & NA & NA & NA & NA & NA & NA & NA & 3,002 & 3,002 \\
\hline
\end{tabular}

Source: Authors' calculations.

Note: $\mathrm{NA}=$ not applicable. 
Table 4A.124

Risk factor:

Non-use and use of ineffective methods of contraception

Disease:

Maternal causes other than abortion

\begin{tabular}{|c|c|c|c|c|c|c|c|c|c|c|c|c|c|c|c|c|c|c|c|}
\hline \multirow[b]{2}{*}{ Region } & \multicolumn{2}{|c|}{$0-4$ years } & \multicolumn{2}{|c|}{ 5-14 years } & \multicolumn{2}{|c|}{$15-29$ years } & \multicolumn{2}{|c|}{ 30-44 years } & \multicolumn{2}{|c|}{$45-59$ years } & \multicolumn{2}{|c|}{$60-69$ years } & \multicolumn{2}{|c|}{$70-79$ years } & $80+$ & years & & Total & \\
\hline & Male & Female & Male & Female & Male & Female & Male & Female & Male & Female & Male & Female & Male & Female & Male & Female & Male & Female & All \\
\hline PAF of Mortality (\%) & & & & & & & & & & & & & & & & & & & \\
\hline East Asia and Pacific & NA & NA & NA & NA & NA & 4 & NA & 30 & NA & NA & NA & NA & NA & NA & NA & NA & NA & 19 & 19 \\
\hline Europe and Central Asia & NA & NA & NA & NA & NA & 7 & NA & 44 & NA & NA & NA & NA & NA & NA & NA & NA & NA & 19 & 19 \\
\hline Latin America and the Caribbean & NA & NA & NA & NA & NA & 20 & NA & 51 & NA & NA & NA & NA & NA & NA & NA & NA & NA & 34 & 34 \\
\hline Middle East and North Africa & NA & NA & NA & NA & NA & 8 & NA & 40 & NA & NA & NA & NA & NA & NA & NA & NA & NA & 24 & 24 \\
\hline South Asia & NA & NA & NA & NA & NA & 8 & NA & 60 & NA & NA & NA & NA & NA & NA & NA & NA & NA & 34 & 34 \\
\hline Sub-Saharan Africa & NA & NA & NA & NA & NA & 4 & NA & 28 & NA & NA & NA & NA & NA & NA & NA & NA & NA & 14 & 14 \\
\hline Low- and middle-income countries & NA & NA & NA & NA & NA & 6 & NA & 43 & NA & NA & NA & NA & NA & NA & NA & NA & NA & 23 & 23 \\
\hline High-income countries & NA & NA & NA & NA & NA & 3 & NA & 9 & NA & NA & NA & NA & NA & NA & NA & NA & NA & 6 & 6 \\
\hline WORLD & NA & NA & NA & NA & NA & 6 & NA & 43 & NA & NA & NA & NA & NA & NA & NA & NA & NA & 23 & 23 \\
\hline PAF of YLL (\%) & & & & & & & & & & & & & & & & & & & \\
\hline East Asia and Pacific & NA & NA & NA & NA & NA & 4 & NA & 30 & NA & NA & NA & NA & NA & NA & NA & NA & NA & 18 & 18 \\
\hline Europe and Central Asia & NA & NA & NA & NA & NA & 7 & NA & 44 & NA & NA & NA & NA & NA & NA & NA & NA & NA & 19 & 19 \\
\hline Latin America and the Caribbean & NA & NA & NA & NA & NA & 20 & NA & 51 & NA & NA & NA & NA & NA & NA & NA & NA & NA & 33 & 33 \\
\hline Middle East and North Africa & NA & NA & NA & NA & NA & 8 & NA & 40 & NA & NA & NA & NA & NA & NA & NA & NA & NA & 24 & 24 \\
\hline South Asia & NA & NA & NA & NA & NA & 8 & NA & 60 & NA & NA & NA & NA & NA & NA & NA & NA & NA & 33 & 33 \\
\hline Sub-Saharan Africa & NA & NA & NA & NA & NA & 4 & NA & 28 & NA & NA & NA & NA & NA & NA & NA & NA & NA & 14 & 14 \\
\hline Low- and middle-income countries & NA & NA & NA & NA & NA & 6 & NA & 43 & NA & NA & NA & NA & NA & NA & NA & NA & NA & 23 & 23 \\
\hline High-income countries & NA & NA & NA & NA & NA & 3 & NA & 9 & NA & NA & NA & NA & NA & NA & NA & NA & NA & 6 & 6 \\
\hline WORLD & NA & NA & NA & NA & NA & 6 & NA & 43 & NA & NA & NA & NA & NA & NA & NA & NA & NA & 23 & 23 \\
\hline PAF of DALYs (\%) & & & & & & & & & & & & & & & & & & & \\
\hline East Asia and Pacific & NA & NA & NA & NA & NA & 4 & NA & 30 & NA & NA & NA & NA & NA & NA & NA & NA & NA & 12 & 12 \\
\hline Europe and Central Asia & NA & NA & NA & NA & NA & 7 & NA & 44 & NA & NA & NA & NA & NA & NA & NA & NA & NA & 17 & 17 \\
\hline Latin America and the Caribbean & NA & NA & NA & NA & NA & 20 & NA & 51 & NA & NA & NA & NA & NA & NA & NA & NA & NA & 30 & 30 \\
\hline Middle East and North Africa & NA & NA & NA & NA & NA & 8 & NA & 40 & NA & NA & NA & NA & NA & NA & NA & NA & NA & 20 & 20 \\
\hline South Asia & NA & NA & NA & NA & NA & 8 & NA & 60 & NA & NA & NA & NA & NA & NA & NA & NA & NA & 27 & 27 \\
\hline Sub-Saharan Africa & NA & NA & NA & NA & NA & 4 & NA & 28 & NA & NA & NA & NA & NA & NA & NA & NA & NA & 12 & 12 \\
\hline Low- and middle-income countries & NA & NA & NA & NA & NA & 7 & NA & 43 & NA & NA & NA & NA & NA & NA & NA & NA & NA & 19 & 19 \\
\hline High-income countries & NA & NA & NA & NA & NA & 3 & NA & 9 & NA & NA & NA & NA & NA & NA & NA & NA & NA & 5 & 5 \\
\hline WORLD & NA & NA & NA & NA & NA & 7 & NA & 42 & NA & NA & NA & NA & NA & NA & NA & NA & NA & 19 & 19 \\
\hline Attributable Mortality (thousand & & & & & & & & & & & & & & & & & & & \\
\hline East Asia and Pacific & NA & NA & NA & NA & NA & 1 & NA & 5 & NA & NA & NA & NA & NA & NA & NA & NA & NA & 6 & 6 \\
\hline Europe and Central Asia & NA & NA & NA & NA & NA & 0 & NA & 0 & NA & NA & NA & NA & NA & NA & NA & NA & NA & 0 & 0 \\
\hline Latin America and the Caribbean & NA & NA & NA & NA & NA & 1 & NA & 3 & NA & NA & NA & NA & NA & NA & NA & NA & NA & 5 & 5 \\
\hline Middle East and North Africa & NA & NA & NA & NA & NA & 0 & NA & 3 & NA & NA & NA & NA & NA & NA & NA & NA & NA & 3 & 3 \\
\hline South Asia & NA & NA & NA & NA & NA & 6 & NA & 52 & NA & NA & NA & NA & NA & NA & NA & NA & NA & 59 & 59 \\
\hline Sub-Saharan Africa & NA & NA & NA & NA & NA & 4 & NA & 25 & NA & NA & NA & NA & NA & NA & NA & NA & NA & 29 & 29 \\
\hline Low- and middle-income countries & NA & NA & NA & NA & NA & 13 & NA & 90 & NA & NA & NA & NA & NA & NA & NA & NA & NA & 103 & 103 \\
\hline High-income countries & NA & NA & NA & NA & NA & 0 & NA & 0 & NA & NA & NA & NA & NA & NA & NA & NA & NA & 0 & 0 \\
\hline WORLD & NA & NA & NA & NA & NA & 13 & NA & 90 & NA & NA & NA & NA & NA & NA & NA & NA & NA & 103 & 103 \\
\hline Attributable YLL (thousands) & & & & & & & & & & & & & & & & & & & \\
\hline East Asia and Pacific & NA & NA & NA & NA & NA & 15 & NA & 136 & NA & NA & NA & NA & NA & NA & NA & NA & NA & 150 & 150 \\
\hline Europe and Central Asia & NA & NA & NA & NA & NA & 3 & NA & 9 & NA & NA & NA & NA & NA & NA & NA & NA & NA & 12 & 12 \\
\hline Latin America and the Caribbean & NA & NA & NA & NA & NA & 39 & NA & 83 & NA & NA & NA & NA & NA & NA & NA & NA & NA & 122 & 122 \\
\hline Middle East and North Africa & NA & NA & NA & NA & NA & 13 & NA & 71 & NA & NA & NA & NA & NA & NA & NA & NA & NA & 84 & 84 \\
\hline South Asia & NA & NA & NA & NA & NA & 179 & NA & 1,320 & NA & NA & NA & NA & NA & NA & NA & NA & NA & 1,499 & 1,499 \\
\hline Sub-Saharan Africa & NA & NA & NA & NA & NA & 116 & NA & 633 & NA & NA & NA & NA & NA & NA & NA & NA & NA & 749 & 749 \\
\hline Low- and middle-income countries & NA & NA & NA & NA & NA & 365 & NA & 2,251 & NA & NA & NA & NA & NA & NA & NA & NA & NA & 2,616 & 2,616 \\
\hline High-income countries & NA & NA & NA & NA & NA & 0 & NA & 1 & NA & NA & NA & NA & NA & NA & NA & NA & NA & 2 & 2 \\
\hline WORLD & NA & NA & NA & NA & NA & 365 & NA & 2,252 & NA & NA & NA & NA & NA & NA & NA & NA & NA & 2,617 & 2,617 \\
\hline Attributable DALYs (thousands) & & & & & & & & & & & & & & & & & & & \\
\hline East Asia and Pacific & NA & NA & NA & NA & NA & 89 & NA & 317 & NA & NA & NA & NA & NA & NA & NA & NA & NA & 405 & 405 \\
\hline Europe and Central Asia & NA & NA & NA & NA & NA & 24 & NA & 54 & NA & NA & NA & NA & NA & NA & NA & NA & NA & 78 & 78 \\
\hline Latin America and the Caribbean & NA & NA & NA & NA & NA & 160 & NA & 205 & NA & NA & NA & NA & NA & NA & NA & NA & NA & 365 & 365 \\
\hline Middle East and North Africa & NA & NA & NA & NA & NA & 54 & NA & 169 & NA & NA & NA & NA & NA & NA & NA & NA & NA & 223 & 223 \\
\hline South Asia & NA & NA & NA & NA & NA & 431 & NA & 1,886 & NA & NA & NA & NA & NA & NA & NA & NA & NA & 2,318 & 2,318 \\
\hline Sub-Saharan Africa & NA & NA & NA & NA & NA & 196 & NA & 826 & NA & NA & NA & NA & NA & NA & NA & NA & NA & 1,021 & 1,021 \\
\hline Low- and middle-income countries & NA & NA & NA & NA & NA & 954 & NA & 3,457 & NA & NA & NA & NA & NA & NA & NA & NA & NA & 4,411 & 4,411 \\
\hline High-income countries & NA & NA & NA & NA & NA & 7 & NA & 13 & NA & NA & NA & NA & NA & NA & NA & NA & NA & 21 & 21 \\
\hline WORLD & NA & NA & NA & NA & NA & 961 & NA & 3,471 & NA & NA & NA & NA & NA & NA & NA & NA & NA & 4,432 & 4,432 \\
\hline
\end{tabular}

Source: Authors' calculations.

Note: $\mathrm{NA}=$ not applicable.

392 I Global Burden of Disease and Risk Factors I Majid Ezzati, Stephen Vander Hoorn, Alan D. Lopez, and others 
Table 4A.125

Risk factor:

Non-use and use of ineffective methods of contraception

Disease:

All causes

\begin{tabular}{|c|c|c|c|c|c|c|c|c|c|c|c|c|c|c|c|c|c|c|c|}
\hline \multirow[b]{2}{*}{ Region } & \multicolumn{2}{|c|}{$0-4$ years } & \multicolumn{2}{|c|}{ 5-14 years } & \multicolumn{2}{|c|}{$15-29$ years } & \multicolumn{2}{|c|}{$30-44$ years } & \multicolumn{2}{|c|}{$45-59$ years } & \multicolumn{2}{|c|}{$60-69$ years } & \multicolumn{2}{|c|}{$70-79$ years } & $80+$ & years & & Total & \\
\hline & Male & Female & Male & Female & Male & Female & Male & Female & Male & Female & Male & Female & Male & Female & Male & Female & Male & Female & All \\
\hline PAF of Mortality (\%) & & & & & & & & & & & & & & & & & & & \\
\hline East Asia and Pacific & NA & NA & NA & NA & NA & 1 & NA & 2 & NA & NA & NA & NA & NA & NA & NA & NA & NA & 0 & 0 \\
\hline Europe and Central Asia & NA & NA & NA & NA & NA & 0 & NA & 0 & NA & NA & NA & NA & NA & NA & NA & NA & NA & 0 & 0 \\
\hline Latin America and the Caribbean & NA & NA & NA & NA & NA & 4 & NA & 4 & NA & NA & NA & NA & NA & NA & NA & NA & NA & 0 & 0 \\
\hline Middle East and North Africa & NA & NA & NA & NA & NA & 3 & NA & 6 & NA & NA & NA & NA & NA & NA & NA & NA & NA & 1 & 0 \\
\hline South Asia & NA & NA & NA & NA & NA & 4 & NA & 13 & NA & NA & NA & NA & NA & NA & NA & NA & NA & 1 & 1 \\
\hline Sub-Saharan Africa & NA & NA & NA & NA & NA & 3 & NA & 5 & NA & NA & NA & NA & NA & NA & NA & NA & NA & 1 & 1 \\
\hline Low- and middle-income countries & NA & NA & NA & NA & NA & 3 & NA & 6 & NA & NA & NA & NA & NA & NA & NA & NA & NA & 1 & 0 \\
\hline High-income countries & NA & NA & NA & NA & NA & 0 & NA & 0 & NA & NA & NA & NA & NA & NA & NA & NA & NA & 0 & 0 \\
\hline WORLD & NA & NA & NA & NA & NA & 3 & NA & 6 & NA & NA & NA & NA & NA & NA & NA & NA & NA & 1 & 0 \\
\hline PAF of YLL (\%) & & & & & & & & & & & & & & & & & & & \\
\hline East Asia and Pacific & NA & NA & NA & NA & NA & 1 & NA & 2 & NA & NA & NA & NA & NA & NA & NA & NA & NA & 0 & 0 \\
\hline Europe and Central Asia & NA & NA & NA & NA & NA & 0 & NA & 0 & NA & NA & NA & NA & NA & NA & NA & NA & NA & 0 & 0 \\
\hline Latin America and the Caribbean & NA & NA & NA & NA & NA & 4 & NA & 4 & NA & NA & NA & NA & NA & NA & NA & NA & NA & 1 & 0 \\
\hline Middle East and North Africa & NA & NA & NA & NA & NA & 3 & NA & 6 & NA & NA & NA & NA & NA & NA & NA & NA & NA & 1 & 0 \\
\hline South Asia & NA & NA & NA & NA & NA & 4 & NA & 13 & NA & NA & NA & NA & NA & NA & NA & NA & NA & 2 & 1 \\
\hline Sub-Saharan Africa & NA & NA & NA & NA & NA & 3 & NA & 5 & NA & NA & NA & NA & NA & NA & NA & NA & NA & 1 & 1 \\
\hline Low- and middle-income countries & NA & NA & NA & NA & NA & 3 & NA & 6 & NA & NA & NA & NA & NA & NA & NA & NA & NA & 1 & 0 \\
\hline High-income countries & NA & NA & NA & NA & NA & 0 & NA & 0 & NA & NA & NA & NA & NA & NA & NA & NA & NA & 0 & 0 \\
\hline WORLD & NA & NA & NA & NA & NA & 3 & NA & 6 & NA & NA & NA & NA & NA & NA & NA & NA & NA & 1 & 0 \\
\hline PAF of DALYs (\%) & & & & & & & & & & & & & & & & & & & \\
\hline East Asia and Pacific & NA & NA & NA & NA & NA & 1 & NA & 2 & NA & NA & NA & NA & NA & NA & NA & NA & NA & 0 & 0 \\
\hline Europe and Central Asia & NA & NA & NA & NA & NA & 1 & NA & 1 & NA & NA & NA & NA & NA & NA & NA & NA & NA & 0 & 0 \\
\hline Latin America and the Caribbean & NA & NA & NA & NA & NA & 3 & NA & 3 & NA & NA & NA & NA & NA & NA & NA & NA & NA & 1 & 0 \\
\hline Middle East and North Africa & NA & NA & NA & NA & NA & 3 & NA & 5 & NA & NA & NA & NA & NA & NA & NA & NA & NA & 1 & 1 \\
\hline South Asia & NA & NA & NA & NA & NA & 5 & NA & 9 & NA & NA & NA & NA & NA & NA & NA & NA & NA & 2 & 1 \\
\hline Sub-Saharan Africa & NA & NA & NA & NA & NA & 4 & NA & 4 & NA & NA & NA & NA & NA & NA & NA & NA & NA & 1 & 1 \\
\hline Low- and middle-income countries & NA & NA & NA & NA & NA & 3 & NA & 5 & NA & NA & NA & NA & NA & NA & NA & NA & NA & 1 & 1 \\
\hline High-income countries & NA & NA & NA & NA & NA & 0 & NA & 0 & NA & NA & NA & NA & NA & NA & NA & NA & NA & 0 & 0 \\
\hline WORLD & NA & NA & NA & NA & NA & 3 & NA & 4 & NA & NA & NA & NA & NA & NA & NA & NA & NA & 1 & 0 \\
\hline Attributable Mortality (thousand & & & & & & & & & & & & & & & & & & & \\
\hline East Asia and Pacific & NA & NA & NA & NA & NA & 3 & NA & 7 & NA & NA & NA & NA & NA & NA & NA & NA & NA & 10 & 10 \\
\hline Europe and Central Asia & NA & NA & NA & NA & NA & 0 & NA & 0 & NA & NA & NA & NA & NA & NA & NA & NA & NA & 1 & 1 \\
\hline Latin America and the Caribbean & NA & NA & NA & NA & NA & 3 & NA & 4 & NA & NA & NA & NA & NA & NA & NA & NA & NA & 7 & 7 \\
\hline Middle East and North Africa & NA & NA & NA & NA & NA & 1 & NA & 3 & NA & NA & NA & NA & NA & NA & NA & NA & NA & 5 & 5 \\
\hline South Asia & NA & NA & NA & NA & NA & 22 & NA & 63 & NA & NA & NA & NA & NA & NA & NA & NA & NA & 85 & 85 \\
\hline$\underline{\text { Sub-Saharan Africa }}$ & NA & NA & NA & NA & NA & 23 & NA & 31 & NA & NA & NA & NA & NA & NA & NA & NA & NA & 54 & 54 \\
\hline Low- and middle-income countries & NA & NA & NA & NA & NA & 52 & NA & 110 & NA & NA & NA & NA & NA & NA & NA & NA & NA & 162 & 162 \\
\hline High-income countries & NA & NA & NA & NA & NA & 0 & NA & 0 & NA & NA & NA & NA & NA & NA & NA & NA & NA & 0 & 0 \\
\hline WORLD & NA & NA & NA & NA & NA & 52 & NA & 110 & NA & NA & NA & NA & NA & NA & NA & NA & NA & 162 & 162 \\
\hline Attributable YLL (thousands) & & & & & & & & & & & & & & & & & & & \\
\hline East Asia and Pacific & NA & NA & NA & NA & NA & 76 & NA & 186 & NA & NA & NA & NA & NA & NA & NA & NA & NA & 262 & 262 \\
\hline Europe and Central Asia & NA & NA & NA & NA & NA & 6 & NA & 12 & NA & NA & NA & NA & NA & NA & NA & NA & NA & 18 & 18 \\
\hline Latin America and the Caribbean & NA & NA & NA & NA & NA & 77 & NA & 100 & NA & NA & NA & NA & NA & NA & NA & NA & NA & 177 & 177 \\
\hline Middle East and North Africa & NA & NA & NA & NA & NA & 34 & NA & 84 & NA & NA & NA & NA & NA & NA & NA & NA & NA & 118 & 118 \\
\hline South Asia & NA & NA & NA & NA & NA & 604 & NA & 1,596 & NA & NA & NA & NA & NA & NA & NA & NA & NA & 2,200 & 2,200 \\
\hline Sub-Saharan Africa & NA & NA & NA & NA & NA & 648 & NA & 781 & NA & NA & NA & NA & NA & NA & NA & NA & NA & 1,429 & 1,429 \\
\hline Low- and middle-income countries & NA & NA & NA & NA & NA & 1,445 & NA & 2,759 & NA & NA & NA & NA & NA & NA & NA & NA & NA & 4,203 & 4,203 \\
\hline High-income countries & NA & NA & NA & NA & NA & 1 & NA & 2 & NA & NA & NA & NA & NA & NA & NA & NA & NA & 3 & 3 \\
\hline WORLD & NA & NA & NA & NA & NA & 1,446 & NA & 2,761 & NA & NA & NA & NA & NA & NA & NA & NA & NA & 4,207 & 4,207 \\
\hline Attributable DALYs (thousands) & & & & & & & & & & & & & & & & & & & \\
\hline East Asia and Pacific & NA & NA & NA & NA & NA & 192 & NA & 370 & NA & NA & NA & NA & NA & NA & NA & NA & NA & 562 & 562 \\
\hline Europe and Central Asia & NA & NA & NA & NA & NA & 35 & NA & 58 & NA & NA & NA & NA & NA & NA & NA & NA & NA & 92 & 92 \\
\hline Latin America and the Caribbean & NA & NA & NA & NA & NA & 237 & NA & 226 & NA & NA & NA & NA & NA & NA & NA & NA & NA & 462 & 462 \\
\hline Middle East and North Africa & NA & NA & NA & NA & NA & 163 & NA & 188 & NA & NA & NA & NA & NA & NA & NA & NA & NA & 350 & 350 \\
\hline South Asia & NA & NA & NA & NA & NA & 1,415 & NA & 2,196 & NA & NA & NA & NA & NA & NA & NA & NA & NA & 3,612 & 3,612 \\
\hline Sub-Saharan Africa & NA & NA & NA & NA & NA & 1,323 & NA & 1,010 & NA & NA & NA & NA & NA & NA & NA & NA & NA & 2,332 & 2,332 \\
\hline Low- and middle-income countries & NA & NA & NA & NA & NA & 3,364 & NA & 4,046 & NA & NA & NA & NA & NA & NA & NA & NA & NA & 7,411 & 7,411 \\
\hline High-income countries & NA & NA & NA & NA & NA & 9 & NA & 14 & NA & NA & NA & NA & NA & NA & NA & NA & NA & 23 & 23 \\
\hline WORLD & NA & NA & NA & NA & NA & 3,373 & NA & 4,061 & NA & NA & NA & NA & NA & NA & NA & NA & NA & 7,434 & 7,434 \\
\hline
\end{tabular}

Source: Authors' calculations.

Note: $\mathrm{NA}=$ not applicable. 


\section{NOTES}

1. Some special cases of effect modification can be identified through the terminology of "sufficient" and "component" causes (Rothman 1976; Rothman and Greenland 1998) with implications for the assessment of joint interventions as follows:

- If two risk factors are sufficient causes for a disease and a fraction of the population is affected by both sufficient causes, then the burden avoidable by reductions in both risk factors is larger than the sum of the burdens avoidable by reduction of each individual risk factor. This is because for those affected by the two risks, removal of both risks is needed to avoid disease (and hence the hazard as measured by the avoidable fraction of disease depends on the presence of the other risk). Consider, for example, the role of clean water and sanitary latrines as risk factors for diarrheal diseases. Improving water quality alone may not have much effect on the prevalence of disease without the introduction of sanitation or hygienic behavior, because fecal-oral transmission may take place through routes other than drinking water (Curtis, Cairncross, and Yonli 2000; Esrey 1996). However, the introduction of both clean water sources and sanitary latrines may reduce disease levels substantially. In the extreme, where every exposed person is affected by both sufficient causes, a change in exposure to a risk factor may result in no change in disease outcome under some circumstances. This phenomenon is known as saturation.

- If two risk factors are component causes of the same sufficient cause, then the burden avoidable by reductions in both risk factors is smaller than the sum of the burdens attributable to each individual risk factor. This is a case of synergy or positive interaction between risk factors, in which the existence of both risk factors has an effect larger than the sum of the effects from the existence of each (Rothman 1976). Synergistic interactions may be complete or partial depending on whether the risk factors are components of a single or multiple sufficient causes. Rothman (1976) uses the inheritance of the phenylketonuria gene and phenylalanine in the diet as an example of synergy.

2. Submultiplicative effect modification could result in a slightly smaller PAF even with positive correlation for some $R R$ values.

\section{REFERENCES}

Arrow, K., B. Bolin, R. Costanza, P. Dasgupta, C. Folke, C. S. Holling, B.-O. Jansson, S. Levin, K.-G. Maler, C. Perrings, and D. Pimente. 1995. "Economic Growth, Carrying Capacity, and the Environment." Science 168 (2): 520-21.

Berlin, J. A., and G. A. Colditz. 1990. "A Meta-analysis of Physical Activity in the Prevention of Coronary Heart Disease." American Journal of Epidemiology 132 (4): 612-28.

Black, R. E. 1991. "Would Control of Childhood Infectious Diseases Reduce Malnutrition? Acta Paediatrica Scandandinavica Supplement 374: $133-40$.

Blair, S. N., Y. Cheng, and J. S. Holder. 2001. "Is Physical Activity or Physical Fitness More Important in Defining Health Benefits?" Medicine and Science in Sports and Exercise 33 (6 Suppl): S379-S399.

Briend, A. 1990. "Is Diarrhoea a Major Cause of Malnutrition among the Under-Fives in Developing Countries? A Review of Available Evidence." European Journal of Clinical Nutrition 44 (9): 611-28.

Brown, K. H., J. M. Peerson, J. Rivera, and L. H. Allen. 2002. "Effect of Supplemental Zinc on the Growth and Serum Zinc Concentrations of Prepubertal Children: A Meta-analysis of Randomized Controlled Trials." American Journal of Clinical Nutrition 75 (6): 1062-71.
Chen, Z., R. Peto, R. Collins, S. MacMahon, J. Lu, and W. Li. 1991. "Serum Cholesterol Concentration and Coronary Heart Disease in Population with Low Cholesterol Concentrations." British Medical Journal 303 (6797): 276-82.

Christian, P., and K. P. West, Jr. 1998. "Interactions between Zinc and Vitamin A: An Update." American Journal of Clinical Nutrition 68 (2 Suppl): 435S-441S.

Corrao, G., L. Rubbiati, V. Bagnardi, A. Zambon, and K. Poikolainen. 2000. "Alcohol and Coronary Heart Disease: A Meta-analysis." Addiction 95 (10): 1505-23.

Curtis, V., S. Cairncross, and R. Yonli. 2000. "Domestic Hygiene and Diarrhoea: Pinpointing the Problem." Tropical Medicine and International Health 5 (1): 22-32.

de Onis, M., M. Blossner, E. Borghi, E. A. Frongillo, and R. Morris. 2004. "Estimates of Global Prevalence of Childhood Underweight in 1990 and 2015." Journal of the American Medical Association 291 (21): 2600-6.

de Onis, M., E. Frongillo, and M. Blossner. 2000. "Is Malnutrition Declining? An Analysis of Changes in Levels of Child Malnutrition since 1980." Bulletin of the World Health Organization 78 (10): 1222-33.

Eastern Stroke and Coronary Heart Disease Collaborative Research Group. 1998. "Blood Pressure, Cholesterol, and Stroke in Eastern Asia." Lancet 352 (9143): 1801-7.

Eaton, C.B. 1992. "Relation of Physical Activity and Cardiovascular Fitness to Coronary Heart Disease, Part I: A Meta-analysis of the Independent Relation of Physical Activity and Coronary Heart Disease." Journal of the American Board of Family Practice 5 (1): 31-42.

Editorial. 2001. "The Human Genome, in Proportion." Lancet 357 (9255): 489.

Eide, G. E., and I. Heuch. 2001. "Attributable Fractions: Fundamental Concepts and Their Visualization." Statistical Methods in Medical Research 10 (3): 159-93.

Esrey, S. A. 1996. "Water, Waste, and Well-Being: A Multicountry Study." American Journal of Epidemiology 143 (6): 608-23.

Evans, A. S. 1976. "Causation and Disease: The Henle-Koch Postulates Revisited." Yale Journal of Biology and Medicine 49 (2): 175-95.

1978. "Causation and Disease: A Chronological Journey." American Journal of Epidemiology 108 (4): 249-58.

Ezzati, M., and D. M. Kammen. 2002. "The Health Impacts of Exposure to Indoor Air Pollution from Solid Fuels in Developing Countries: Knowledge, Gaps, and Data Needs." Environmental Health Perspectives 110 (11): 1057-68.

Ezzati, M., and A. D. Lopez. 2003. "Estimates of Global Mortality Attributable to Smoking in 2000." Lancet 362 (9387): 847-52.

2004. "Smoking and Oral Tobacco Use." In Comparative Quantification of Health Risks: Global and Regional Burden of Disease Attributable to Selected Major Risk Factors, ed. M. Ezzati, A. D. Lopez, A. Rodgers, and C. J. L. Murray, 883-956. Geneva: World Health Organization.

Ezzati, M., S. J. Henley, A. D. Lopez, and M. J. Thun. 2005. "The Role of Smoking in Global and Regional Cancer Epidemiology: Current Patterns and Research Needs." International Journal of Cancer 116 (6): 963-71.

Ezzati, M., S. J. Henley, M. J. Thun, and A. D. Lopez. 2005. "The Role of Smoking in Global and Regional Cardiovascular Mortality." Circulation 112 (4): 489-97.

Ezzati, M., A. D. Lopez, A. Rodgers, and C. J. L. Murray. 2004. Comparative Quantification of Health Risks: Global and Regional Burden of Disease Attributable to Selected Major Risk Factors. Geneva: World Health Organization.

Ezzati, M., A. D. Lopez, A. Rodgers, S. Vander Hoorn, C. J. L. Murray, and the Comparative Risk Assessment Collaborative Group. 2002. 
"Selected Major Risk Factors and Global and Regional Burden of Disease." Lancet 360 (9343): 1347-60.

Ezzati, M., S. Vander Hoorn, A. Rodgers, A. D. Lopez, C. D. Mathers, and C. J. L. Murray. 2004. "Potential Health Gains from Reducing Multiple Risk Factors." In Comparative Quantification of Health Risks: Global and Regional Burden of Disease Attributable to Selected Major Risk Factors, ed. M. Ezzati, A. D. Lopez, A. Rodgers, and C. J. L. Murray, 2167-90. Geneva: World Health Organization.

Ezzati, M., S. Vander Hoorn, A. Rodgers, A. D. Lopez, C. D. Mathers, C. J. L. Murray, and the Comparative Risk Assessment Collaborative Group. 2003. "Estimates of Global and Regional Potential Health Gains from Reducing Multiple Major Risk Factors." Lancet 362 (9380): 271-80.

Ezzati, M., S. Vander Hoorn, C. M. M. Lawes, R. Leach, W. P. T. James, A. D. Lopez, A. Rodgers, and C. J. L. Murray. 2005. "Rethinking the 'Diseases of Affluence' Paradigm: Global Patterns of Nutritional Risks in Relation to Economic Development." PLoS Medicine 2 (5): e133.

Gaziano, J. M., J. E. Manson, L. G. Branch, G. A. Colditz, W. C. Willett, and J. E. Buring. 1995. "A Prospective Study of Consumption of Carotenoids in Fruits and Vegetables and Decreased Cardiovascular Mortality in the Elderly." Annals of Epidemiology 5 (4): 255-60.

Greenland, S. 1984. "Bias in Methods for Deriving Standardized Morbidity Ratio and Attributable Fraction Estimates." Statistics in Medicine 3: 131-41.

1987. "Quantitative Methods in the Review of Epidemiologic Literature." Epidemiologic Reviews 9: 1-30.

Gross, C. P., G. F. Anderson, and N. R. Powe. 1999. “The Relation between Funding by the National Institutes of Health and the Burden of Disease." New England Journal of Medicine 340 (24): 1881-7.

Guerrant, R. L., J. B. Schorling, J. F. McAuliffe, and M. A. de Souza. 1992. "Diarrhea as a Cause and an Effect of Malnutrition: Diarrhea Prevents Catch-Up Growth and Malnutrition Increases Diarrhea Frequency and Duration." American Journal of Tropical Medicine and Hygiene 47 (1 pt 2): 28-35.

Horton, R. 2003. "Medical Journals: Evidence of Bias against the Diseases of Poverty." Lancet 361 (9359): 712-3.

Jarrett, R. J., M. J. Shipley, and G. Rose. 1982. "Weight and Mortality in the Whitehall Study." British Medical Journal 285 (6341): 535-7.

Jee, S. H., I. Suh, I. S. Kim, and L. J. Appel. 1999. "Smoking and Atherosclerotic Cardiovascular Disease in Men with Low Levels of Serum Cholesterol: The Korea Medical Insurance Corporation Study." Journal of the American Medical Association 282 (22): 2149-55.

Jousilahti, P., E. Vartiainen, J. Tuomilehto, and P. Puska. 1999. “Sex, Age, Cardiovascular Risk Factors, and Coronary Heart Disease: A Prospective Follow-Up Study of 14,786 Middle-Aged Men and Women in Finland." Circulation 99 (9): 1165-72.

Kaufman, J., and J. Jing. 2002. "China and AIDS: The Time to Act Is Now." Science 296 (5577): 2339-40.

Khaw, K. T., and E. Barrett-Connor. 1987. "Dietary Fiber and Reduced Ischemic Heart Disease Mortality Rates in Men and Women: A 12-Year Prospective Study." American Journal of Epidemiology 126 (6): 1093-102.

Koopman, J. S. 1981. "Interaction between Discrete Causes." American Journal of Epidemiology 113 (6): 716-24.

Kunzli, N., R. Kaiser, S. Medina, M. Studnicka, O. Chanel, P. Filliger, M. Herry, F. Horak, V. Puybonnieux-Texier, P. Quenel, J. Schneider, R. Seethaler, J. C. Vergnaud, and H. Sommer. 2000. "Public-Health Impact of Outdoor and Traffic-Related Air Pollution: A European Assessment." Lancet 356 (9232): 795-801.

Law, M. R., N. J. Wald, and S. G. Thompson. 1994. "By How Much and How Quickly Does Reduction in Serum Cholesterol Concentration Lower Risk of Ischaemic Heart Disease?" British Medical Journal 308 (6925): 367-73.
Lee, M.-J., B. M. Popkin, and S. Kim. 2000. “The Unique Aspects of the Nutrition Transition in South Korea: The Retention of Healthful Elements in Their Traditional Diet." Public Health Nutrition 5 (14): 197-203.

Leigh, J., P. Macaskill, E. Kuosma, and J. Mandryk. 1999. “Global Burden of Disease and Injury Due to Occupational Factors." Epidemiology 10 (5): 626-31.

Liu, B. Q., R. Peto, Z. M. Chen, J. Boreham, Y. P. Wu, J.Y. Li, T. C. Campbell, and J. S. Chen. 1998. "Emerging Tobacco Hazards in China: 1. Retrospective Proportional Mortality Study of One Million Deaths." British Medical Journal 317 (7170): 1411-22.

Liu, S., I. M. Lee, U. Ajani, S. R. Cole, J. E. Buring, and J. E. Manson. 2001. "Intake of Vegetables Rich in Carotenoids and Risk of Coronary Heart Disease in Men: The Physicians' Health Study." International Journal of Epidemiology 30 (1): 130-5.

Liu, S., J. E. Manson, I. M. Lee, S. R. Cole, C. H. Hennekens, W. C. Willett, and J. E. Buring. 2000. "Fruit and Vegetable Intake and Risk of Cardiovascular Disease: The Women's Health Study.” American Journal of Clinical Nutrition 72 (4): 922-8.

Lutter, C. K., J. P. Habicht, J. A. Rivera, R. Martorell. 1992. "The Relationship between Energy Intake and Diarrheal Disease in Their Effects on Child Growth: Biological Model, Evidence, and Implications for Public Health Policy." Food and Nutrition Bulletin 14: 36-42.

Lutter, C. K., J. O. Mora, J. P. Habicht, K. M. Rasmussen, D. S. Robson, S. G. Sellers, M. G. Perri, D. S. Sheps, M. B. Pettinger, D. S. Siscovick. 1989. "Nutritional Supplementation: Effects on Child Stunting because of Diarrhea." American Journal of Clinical Nutrition 50 (1): 1-8.

MacLehose, L., M. McKee, and J. Weinberg. 2002. "Responding to the Challenge of Communicable Disease in Europe.” Science 295 (5562): 2047-50.

Maldonado, G., and S. Greenland. 2002. "Estimating Causal Effects." International Journal of Epidemiology 31 (2): 422-9.

Manson, J. E., G. A. Colditz, M. J. Stampfer, W. C. Willett, B. Rosner, R. R. Monson, F. E. Speizer, and C. H. Hennekens. 1990. "A Prospective Study of Obesity and Risk of Coronary Heart Disease in Women." New England Journal of Medicine 322 (13): 882-9.

Manson, J. E., P. Greenland, A. Z. LaCroix, M. L. Stefanick, C. P. Mouton, A. Oberman, M. G. Perri, D. S. Sheps, M. B. Pettinger, D. S. Siscovick. 2002. "Walking Compared with Vigorous Exercise for the Prevention of Cardiovascular Events in Women." New England Journal of Medicine 347 (10): 755-56.

Martorell, R., J. P. Habicht, C. Yarbrough, A. Lechtig, R. E. Klein, and K. A. Western. 1975. "Acute Morbidity and Physical Growth in Rural Guatemalan Children." American Journal of Diseases of Children 129 (11): 1296-301.

Martorell, R., C. Yarbrough, A. Lechtig, J. P. Habicht, and R. E. Klein. 1975. "Diarrheal Diseases and Growth Retardation in Preschool Guatemalan Children." American Journal of Physical Anthropology 43 (3): 341-6.

Mathers, C. D., M. Ezzati, A. D. Lopez, C. J. L. Murray, and A. Rodgers. 2002. "Causal Decomposition of Summary Measures of Population Health." In Summary Measures of Population Health: Concepts, Ethics, Measurement, and Applications, ed. C. J. L. Murray, J. Salomon, C. D. Mathers, and A. D. Lopez. 273-290. Geneva: World Health Organization.

McGinnis, J. M., and W.H. Foege. 1993. "Actual Causes of Death in the United States." Journal of American Medical Association 270 (18): 2207-12.

Miettinen, O. S. 1974. "Proportion of Disease Caused or Prevented by a Given Exposure, Trait, or Intervention.” American Journal of Epidemiology 99 (5): 325-32.

Murray, C. J.L., M.Ezzati,A.D.Lopez,A. Rodgers, and S.Vander Hoorn. 2003. "Comparative Quantification of Health Risks: Conceptual Framework and Methodological Issues." Population Health Metrics 1 (1): 1. 
Murray, C. J. L., and A. D. Lopez. 1997. "Global Mortality, Disability, and the Contribution of Risk Factors: Global Burden of Disease Study." Lancet 349 (9063): 1436-42.

1999. "On the Comparable Quantification of Health Risks: Lessons from the Global Burden of Disease." Epidemiology 10 (5): 594-605.

Neaton, J. D., and D. Wentworth. 1992. "Serum Cholesterol, Blood Pressure, Cigarette Smoking, and Death from Coronary Heart Disease. Overall Findings and Differences by Age for 316,099 White Men. Multiple Risk Factor Intervention Trial Research Group." Archives of Internal Medicine 152 (1): 56-64.

Pelletier, D. L., E. A. Frongillo, Jr., and J. P. Habicht. 1993. “Epidemiologic Evidence for a Potentiating Effect of Malnutrition on Child Mortality." American Journal of Public Health 83 (8): 1130-3.

Peto, R., A. D. Lopez, J. Boreham, M. Thun, and C. Heath, Jr. 1992. "Mortality from Tobacco in Developed Countries." Lancet 339 (8804): 1268-78.

Popkin, B. M. 2002a. "An Overview on the Nutrition Transition and Its Health Implications: The Bellagio Meeting." Public Health Nutrition 5 (1A): 93-103.

2002b. "The Shift in Stages of the Nutrition Transition in the Developing World Differs from Past Experiences." Public Health Nutrition 5 (1A): 205-14.

Popkin, B. M., S. Horton, S. Kim, A. Mahal, and J. Shuigao. 2001. “Trends in Diet, Nutritional Status and Diet-Related Noncommunicable Diseases in China and India: The Economic Costs of the Nutrition Transition.” Nutrition Reviews 59 (12): 379-90.

Preston, S. H. 1976. Mortality Patterns in National Populations: With Special Reference to Recorded Causes of Death. New York: Academic Press.

Puddey, I. B., V. Rakic, S. B. Dimmitt, and L. J. Beilin. 1999. "Influence of Pattern of Drinking on Cardiovascular Disease and Cardiovascular Risk Factors: A Review." Addiction 94 (5): 649-63.

Ramakrishnan, U., and R. Martorell. 1998. "The Role of Vitamin A in Reducing Child Mortality and Morbidity and Improving Growth." Salud Publica de Mexico 40 (2): 189-198.

Ramakrishnan, U., M. C. Latham, and R. Abel. 1995. "Vitamin A Supplementation Does Not Improve Growth of Preschool Children: A Randomized, Double-Blind Field Trial in South India." Journal of Nutrition 125 (2): 202-11.

Rehm, J., R. Room, M. Monteiro, G. Gmel, K. Graham, N. Rehn, C. T. Sempas, V. Frick, and D. Jerrigan. 2004. "Alcohol Use." In Comparative Quantification of Health Risks: Global and Regional Burden of Disease Attributable to Selected Major Risk Factors, ed. M. Ezzati, A. D. Lopez, C. J. Murray, and A. Rogers, 959-1108. Geneva: World Health Organization.

Rosengren, A., H. Wedel, and L. Wilhelmsen. 1999. "Body Weight and Weight Gain during Adult Life in Men in Relation to Coronary Heart Disease and Mortality: A Prospective Population.” European Health Journal 20 (4): 269-77.

Rothman, K. J. 1976. “Causes.” American Journal of Epidemiology 104 (6): 587-92.

Rothman, K. J., and S. Greenland. 1998. Modern Epidemiology. Philadelphia: Lippincott-Raven.

Rothman, K. J., and A. Keller. 1972. "The Effect of Joint Exposure to Alcohol and Tobacco on the Risk of Cancer of the Mouth and Pharynx." Journal of Chronic Disease 25 (12): 711-6.

Rothman, K. J., S. Greenland, and A. M. Walker. 1980. "Concepts of Interaction." American Journal of Epidemiology 112 (4): 467-70.

Scrimshaw, N. S., C. E. Taylor, and J. E. Gordon. 1968. Interactions of Nutrition and Infection. World Health Organization Monograph Series 57. Geneva: World Health Organization.
Single, E., L. Robson, J. Rehm, and X. Xie. 1999. "Morbidity and Mortality Attributable to Alcohol, Tobacco, and Illicit Drug Use in Canada." American Journal of Public Health 89 (3): 385-90.

Slaymaker, E., N. Walker, B. Zaba, and M. Collumbien. 2004. "Unsafe Sex." In Comparative Quantification of Health Risks: Global and Regional Burden of Disease Attributable to Selected Major Risk Factors, ed. M. Ezzati, A. D. Lopez, A. Rodgers, and C. J. L. Murray, 1177-254. Geneva: World Health Organization.

Smith, K. R. 2000. "The National Burden of Disease from Indoor Air Pollution in India." Proceedings of the National Academy of Sciences 97 (24): 13286-93.

Smith, K. R., C. F. Corvalan, and T. Kjellstrom. 1999. "How Much Global Ill Health Is Attributable to Environmental Factors." Epidemiology 10 (5): 573-84.

Smith, K. R., S. Mehta, and M. Maeusezahl-Feuz. 2004. "Indoor Air Pollution from Household Use of Solid Fuels." In Comparative Quantification of Health Risks: Global and Regional Burden of Disease Attributable to Selected Major Risk Factors, ed. M. Ezzati, A. D. Lopez, C. J. Murray, and A. Rogers, 1435-94. Geneva: World Health Organization.

Stephensen, C. B. 1999. “Burden of Infection on Growth Failure.” Journal of Nutrition 129 (25 Suppl): 534S-538S.

Tate, R. B., J. Manfreda, and T. E. Cuddy. 1998. “The Effect of Age on Risk Factors for Ischemic Heart Disease: The Manitoba Follow-up Study, 1948-1993." Annals of Epidemiology 8 (7): 415-21.

Thun, M. J., L. F. Apicella, and S. J. Henley. 2000. “Smoking vs. Other Risk Factors as the Cause of Smoking-Attributable Mortality: Confounding in the Courtroom." Journal of the American Medical Association 284 (6): 706-12.

UNAIDS (Joint United Nations Programme on HIV/AIDS). 2001. Together We Can: Leadership in a World of AIDS. Geneva: UNAIDS. http://www.unaids.org/UNGASS/leadership/English/leader_en.pdf.

UNICEF (United Nations Children's Fund). 1990. Strategy to Improve Nutrition of Children and Women in Developing Countries: A UNICEF Policy Review. New York: UNICEF.

Walter, S. D. 1976. “The Estimation and Interpretation of Attributable Risk in Health Research." Biometrics 32 (4): 829-49.

1980. "Prevention of Multifactorial Disease." American Journal of Epidemiology 112 (3): 409-16.

West, K. P., Jr, R. P. Pokhrel, J. Katz, S. C. LeClerq, S. K. Khatry, S. R. Shrestha, E. K. Pradhan, J. M. Tielsch, M. R. Pandey, and A. Sommer. 1991. "Efficacy of Vitamin A in Reducing Preschool Child Mortality in Nepal." Lancet 338 (8759): 67-71.

WHO (World Health Organization). 1992. International Statistical Classification of Disease and Related Health Problems, 10th ed. Geneva: WHO.

WHO (World Health Organization). 2002. World Health Report 2002: Reducing Risks, Promoting Healthy Life. Geneva: WHO.

Willet, W. C. 2002. "Balancing Life-Style and Genomics Research for Disease Prevention." Science 296 (5568): 695-8.

Yerushalmy, J., and C. E. Palmer. 1959. "On the Methodology of Investigations of Etiologic Factors in Chronic Diseases." Journal of Chronic Disease 108 (1): 27-40.

Yusuf, S., S. Hawken, S. Ounpuu, T. Dans, A. Avezum, F. Lanas, M. McQueen, A. Budaj, P. Pais, J. Varigos, L. Lisheng, and the INTERHEART Study Investigators. 2004. "Effect of Potentially Modifiable Risk Factors Associated with Myocardial Infarction in 52 Countries (the INTERHEART Study): Case-Control Study." Lancet 364 (9438): 937-52.

Zinc Investigators' Collaborative Group. 1999. "Prevention of Diarrhea and Pneumonia by Zinc Supplementation in Children in Developing Countries: Pooled Analysis of Randomized Controlled Trials." Journal of Pediatrics 135 (6): 689-97. 
Part II

Sensitivity Analyses 

Chapter 5

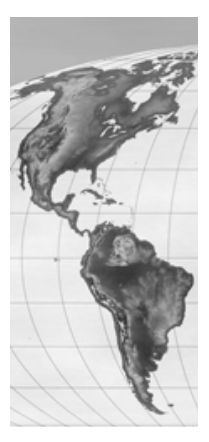

Modern epidemiological studies generally report confidence or uncertainty intervals around their estimates, often based on the variation observed in sample data. Estimates of the burden of disease and of risk factors, which extrapolate from specific data sources and epidemiological studies to population-level measures, are subject to a broader range of uncertainty because of the combination of multiple data sources and value choices. Hence, the reported uncertainty intervals should ideally include all sources of uncertainty, including those arising from measurement error, systematic biases, and modeling and extrapolation to compensate for incomplete data. In contrast to uncertainty analysis, which attempts to formally quantify the limitations of available data, sensitivity analysis examines how key analytic outputs vary when input quantities are systematically varied. Following Murray and Lopez (1996b), this chapter uses sensitivity analysis to examine the specific effects of social values that have been incorporated in the design of the disability-adjusted life year (DALY).

Taking account of uncertainty in such value parameters as the rate of time preference used to discount future outcomes is not common. Even if there is empirical evidence on

\section{Sensitivity and Uncertainty Analyses for Burden of Disease and Risk Factor Estimates}

Colin D. Mathers, Joshua A. Salomon, Majid Ezzati, Stephen Begg, Stephen Vander Hoorn, and Alan D. Lopez

population preferences for discount rates and uncertainty in these estimates, investigators have argued that the choice of discount rate for use in analysis is essentially a social value judgment and should not include uncertainty (Morgan and Henrion 1990). Although there is uncertainty about the social value judgment and about its effects on decisions based on the analysis, varying the value deterministically in the analysis and performing a sensitivity analysis to examine the impact on the outcomes of interest is usually preferable to uncertainty analysis. Thus, the 1990 Global Burden of Disease (GBD) study (Murray and Lopez 1996b) examined the sensitivity of the ranking of causes of the burden of disease globally when discount rates and age weights were varied across a range of possible values.

Health state valuations, which link mortality information with information on nonfatal health outcomes in summary measures of population health, fit somewhat more ambiguously within the framework of uncertainty analysis. If we conceptualize a health state in terms of levels in multiple domains of health, health state valuation involves the weighting of these domains to arrive at an overall assessment of the health level associated with the state. These valuations, unlike 
values such as time preference, do not have any clear normative basis; that is, while we might rely on philosophical arguments about intergenerational equity in choosing a discount rate, no obvious arguments pertain to the relative importance of mobility versus cognition in overall assessments of health levels. The choice of measurement strategies for eliciting health state valuations does sometimes introduce normative questions, but these pertain to additional considerations, such as concern for fair distribution, which are orthogonal to the assessment of the health state itself.

\section{DISCOUNTING AND AGE WEIGHTING IN THE DALY MEASURE}

This section briefly reviews the rationale and implementation of discounting and age weights in the standard DALY. To denote different choices for the discount rate and age weights, we use the notation $\operatorname{DALYs}(r, K)$, where $r$ is the discount rate in percent (not a fraction as in the GBD 1990 study) and $K$ is the age-weighting modulation factor, a parameter that allows uniform $(K=0)$ or the GBD nonuniform $(K=1)$ age weighting to be used. With this notation, DALYs $(3,0)$ denotes the DALY with a 3 percent discount rate and uniform age weights as used in the Disease Control Priorities Project (DCPP) and DALYs $(3,1)$ denotes the 3 percent discount rate and varying age weights as used in the GBD study. Similarly, we may refer to the DALY components of years of life lost due to premature mortality (YLL) and years of healthy life lost due to disability (YLD) as $\mathrm{YLL}(r, K)$ or $\mathrm{YLD}(r, K)$ using the same convention.

\section{Discounting}

Discounting future benefits is standard practice in economic analysis. Murray (1996) and Murray and Acharya (1997) review the theoretical and empirical arguments for and against discounting with a specific emphasis on health, including the possibility of negative discount rates. In addition to individual discounting and discount rates, policies dealing with risk must address the issue of benefits for different populations across time. As a result, these policies must address ethical and analytical dilemmas related to the valuation of current and future health and welfare in the form of social discount rates (Kneese 1999).

Some have argued that discounting should not be applied to future health gains or losses because health is not commensurable with money and cannot be reinvested elsewhere, but most criticisms of discounting in relation to the DALY have focused on the functional form and the level chosen (Fox-Rushby 2002). Epidemiologists and demographers, who tend to focus on measuring or estimating years of life or health without "valuing" either, rarely use discounting. Murray and Acharya (1997) conclude that the strongest argument for discounting is the disease eradication and health research paradox. According to this argument, not discounting future health would lead to the conclusion that all of society's health resources should be invested in research programs or programs for disease eradication, which produce an infinite stream of benefits, rather than any programs that improve the health of the current generation. Such an excessive intergenerational "sacrifice" is a particularly powerful argument for discounting future health (Parfit 1984). Note that this argument does not claim that future welfare or health is less valuable than current welfare or health, but rather uses discounting as a tool to avoid excessive sacrifice by the current generation to the point of investing all resources in future health.

Murray and Acharya argue that the social discount rate should be smaller than the return on capital investment, but note that the choice of a discount rate for health benefits, even if technically desirable, may result in morally unacceptable allocations between generations (see also Dasgupta, Mäler, and Barrett 1999). Because of the complexities in the choice of discount rate, the 1990 GBD study published discounted and undiscounted estimates of the global burden of disease (Murray and Lopez 1996a).

The U.S. Panel on Cost-Effectiveness in Health and Medicine has recommended that health economic analyses use a 3 percent real discount rate to adjust both costs and health outcomes (Gold and others 1996), but that analysts should examine the sensitivity of the results to the discount rate. The 1990 GBD study, the updated estimates published in recent World Health Organization (WHO) world health reports, and the DCPP have all used 3 percent discounting for DALYs.

\section{Age Weighting}

The 1990 GBD study weighted a year of healthy life lived at young ages and older ages lower than years lived at other ages. This choice was based on a number of studies that indicated a broad social preference to value a year lived by a young adult more highly than a year lived by a young child or an older adult (Murray 1996). Not all such studies agree that the youngest and oldest ages should be given less weight; nor do they agree on the relative magnitude of the differences. 
Age weights are perhaps the most controversial value choice built into the DALY. Criticisms of age weights have fallen into five categories:

- Age weighting is unacceptable on equity grounds and every year of life is of equal value (Anand and Hanson 1997).

- Age weights are not empirically based and have not been validated for large populations.

- Age weights do not reflect social values; for example, the DALY values the life of a newborn about equally to that of a 20-year-old, whereas the empirical data suggest a fourfold difference (Bobadilla 1996; see also chapter 6 in this book).

- Age weights result in more YLL for deaths at all ages from birth to 39 compared with discounted YLL not weighted by age (Barendregt, Bonneux, and van der Maas 1996).

- Age weights add an extra level of complexity to burden of disease analysis that obscures the method and makes little overall difference to the rankings of diseases and injuries.

Murray and Acharya (1997) argue that age weights are not in themselves inequitable, because everyone potentially lives through every age, and that they do reflect legitimate societal priorities. As discussed in chapter 3, the DCPP uses uniform age weights and thus values a year of healthy life equally at all ages. Chapter 6 presents an analysis in which a more extreme form of age weighting is applied to the deaths of young children.

\section{Discounting, Age Weights, and the YLL Loss Function}

DALYs are calculated as the sum of YLL from a cause and the YLD for incident cases of the health condition (see chapter 3 for more details). Murray (1996) provides general formulas for YLL and YLD that allow the annual discount rate $r$ and the age-weighting parameters $(K, C, \beta)$ to be varied. When $K$ is set equal to 1 , then the DALY includes an ageweighting function of the form $C x e^{-\beta x}$, where $x$ is the age in years and $\beta$ and $C$ are constants. For the 1990 GBD study, Murray and Lopez chose $\beta=0.04$. The value of $\beta=0.04$ was chosen to give an age pattern similar to that seen in available empirical data. $C$ is a parameter chosen to ensure that the total global DALYs are the same with and without age weighting, estimated at $C=0.1658$ for the 1990 GBD study. Figure 5.1 illustrates the form of the age-weighting function for $\beta=0.02,0.04$, and 0.06 . For the other two

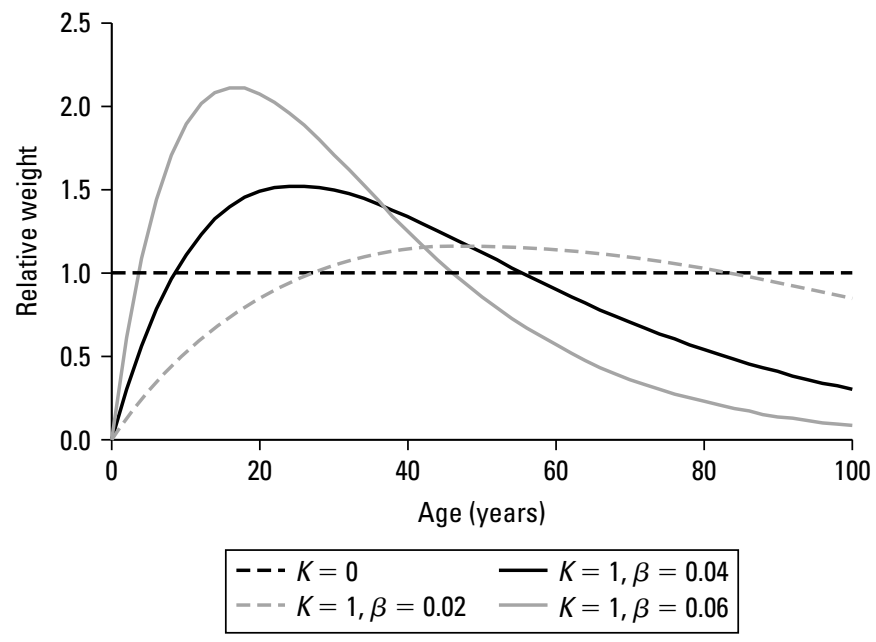

Source: Authors' calculations.

Figure 5.1 Age-Weighting Function Incorporated into the DALY

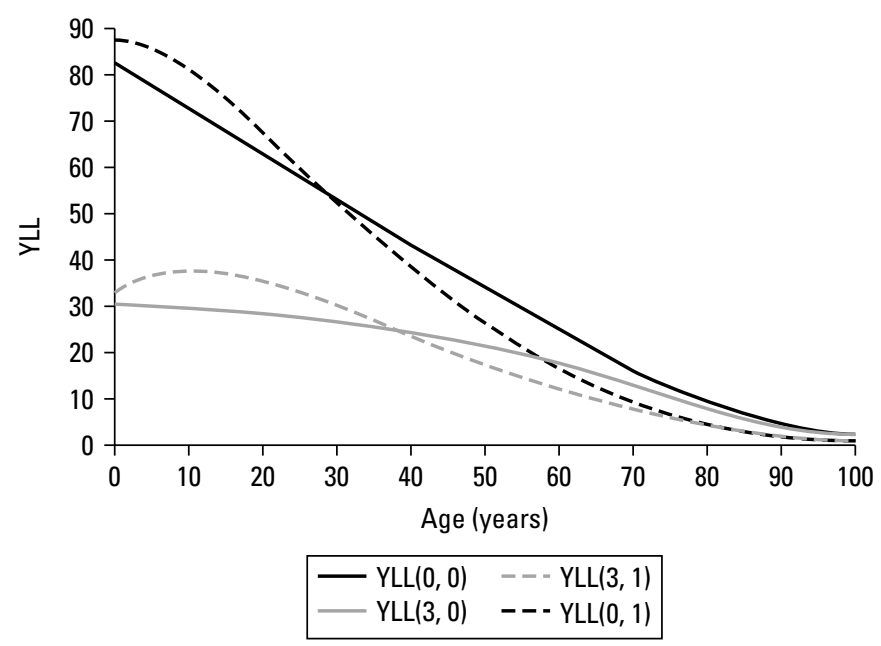

Source: Authors' calculations.

Note: $\mathrm{YLL}(r, K)$ denotes YLL calculated with discount rate $r$ (percent) and standard age weighting $(K=1)$ or uniform age weighting $(K=0)$.

Figure 5.2 Effect of Age Weighting and Discounting on the YLL per Death at Various Ages for Females

choices of $\beta$ ( 0.02 or 0.06 ), the value of $C$ was varied to ensure the same area under the curve from age 0 to 100 years.

The age-weighting function specifies the relative value of a year of life lived at different ages either for YLD or YLL estimates. To estimate the total years of life lost due to death at age $x$, the age-weighting function is integrated over all ages above $x$. Table 5.1 shows the resulting loss function for selected exact ages, also plotted in figure 5.2 for females. The male-female gap in $\operatorname{YLL}(0,0), 2.5$ years at birth, is reduced to 0.1 years for $Y L L(3,1)$ (figure 5.3). Figure 5.4 shows the effect on YLL of varying the parameter $\beta$ in the age-weighting 
Table 5.1 Standard Life Expectancies at Selected Exact Ages and Discounted YLL Due to a Death at Selected Ages

\begin{tabular}{|c|c|c|c|c|c|c|}
\hline \multirow[b]{2}{*}{ Age } & \multicolumn{2}{|c|}{$\begin{array}{c}\text { YLL }(0,0) \text { per death- } \\
\text { standard life expectancy } \\
\text { (years) }\end{array}$} & \multicolumn{2}{|c|}{$\begin{array}{c}\text { YLL }(3,0) \text { per death- } \\
3 \% \text { discounting, } \\
\text { uniform age weights (years) }\end{array}$} & \multicolumn{2}{|c|}{$\begin{array}{l}\text { YLL }(3,1) \text { per death- } \\
3 \% \text { discounting, standard } \\
\text { age weights (years) }\end{array}$} \\
\hline & Males & Females & Males & Females & Males & Females \\
\hline 0 & 80.00 & 82.50 & 30.31 & 30.53 & 33.01 & 33.13 \\
\hline 5 & 75.38 & 77.95 & 29.86 & 30.12 & 36.46 & 36.59 \\
\hline 15 & 65.41 & 68.02 & 28.65 & 29.00 & 36.80 & 36.99 \\
\hline 30 & 50.51 & 53.27 & 26.01 & 26.59 & 29.62 & 29.92 \\
\hline 45 & 35.77 & 38.72 & 21.93 & 22.90 & 20.17 & 20.66 \\
\hline 60 & 21.81 & 24.83 & 16.01 & 17.51 & 11.48 & 12.22 \\
\hline 70 & 13.58 & 16.20 & 11.15 & 12.83 & 6.69 & 7.48 \\
\hline 80 & 7.45 & 8.90 & 6.67 & 7.81 & 3.27 & 3.76 \\
\hline 90 & 3.54 & 4.25 & 3.36 & 3.99 & 1.30 & 1.53 \\
\hline 100 & 1.46 & 2.00 & 1.43 & 1.94 & 0.42 & 0.57 \\
\hline
\end{tabular}

Source: Authors' calculations.

function. Values of $\beta$ higher than 0.04 give relatively greater weight to younger ages and less to older ages; values of $\beta$ lower than 0.04 give relatively lower weight to younger ages and more to older ages.

Table 5.2 further examines the effects of varying the parameter $\beta$ in the age-weighting function on the weights applicable at different ages. For the standard DALY, $\beta=0.04$ implies a maximum age weight of 1.52 at age 25 , and the age weight is greater than 1 over the range 8.4 to 54.2 years. Compare this with $\beta=0.03$, which gives a maximum age weight of 1.29 at age 33.3 years with a prime age range (weight greater than 1) of 14.9 to 63.0 years. Note that the choice of $\beta=0.03$ gives a prime age range that matches fairly typical ages for formal entry and exit from work in

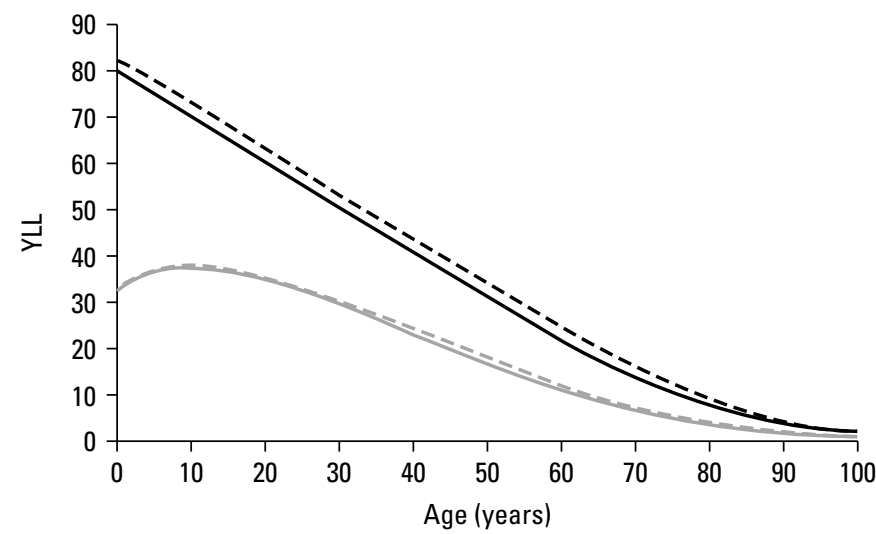

$\begin{aligned}-\operatorname{YLL}(0,0)-\text { males } & ---\operatorname{YLL}(0,0) \text {-females } \\ -\mathrm{YLL}(3,1)-\text { males } & ---\operatorname{YLL}(3,1) \text {-females }\end{aligned}$

Source: Authors' calculations.

Figure 5.3 Effect of Age Weighting and Discounting on the MaleFemale Gap in YLL per Death many societies (Mahapatra 2001). We do not consider variations in $\beta$ further here. Sensitivity analyses for GBD 2001 that follow compare standard age weights $(\beta=0.4)$ with uniform age weights.

\section{SENSITIVITY OF BURDEN OF DISEASE AND INJURY RESULTS TO VARIATIONS IN KEY PARAMETER VALUES}

This section examines the sensitivity of the DALY estimates for the global burden of disease in 2001 to alternative assumptions about the discount rate and age weighting. As discussed in chapter 3, the DALY measures the future stream

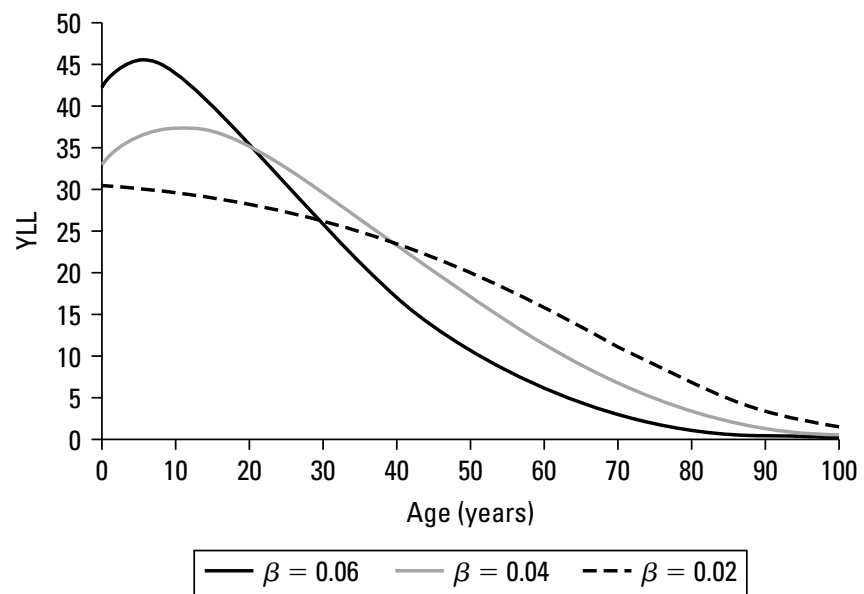

Source: Authors' calculations.

Note: The discount rate is held constant at 3 percent for the examples shown.

Figure 5.4 Effect on YLL per Death of Varying the Parameter $\beta$ in the DALY Age-Weighting Function 
Table 5.2 Implications of Variation in Choice of Age-Weight Parameter $\beta$ on the Age-Weighting Function

\begin{tabular}{ccccc}
\hline $\begin{array}{c}\text { Age-weight } \\
\text { parameter } \boldsymbol{\beta}\end{array}$ & $\begin{array}{c}\text { Age-weight } \\
\text { constant } \boldsymbol{C}\end{array}$ & $\begin{array}{c}\text { Maximum age } \\
\text { weight }\end{array}$ & $\begin{array}{c}\text { Age of maximum } \\
\text { age weight }\end{array}$ & $\begin{array}{c}\text { Age range for which } \\
\text { age weight is }>\mathbf{1}\end{array}$ \\
\hline 0.02 & 0.0634 & 1.17 & 50.0 & $27.2-83.1$ \\
0.03 & 0.1051 & 1.29 & 33.3 & $14.9-63.0$ \\
0.04 & 0.1658 & 1.52 & 25.0 & $8.4-54.2$ \\
0.05 & 0.2487 & 1.83 & 20.0 & $5.2-50.7$ \\
0.06 & 0.3560 & 2.18 & 16.7 & $3.5-46.9$ \\
\hline
\end{tabular}

Source: Authors' calculations.

Note: This form of presentation was suggested by Mahapatra 2001.

a. For values of $\beta$ other than 0.04 , the age-weight constant $C$ was chosen so that total global DALYs $(3,1)$ for 2001 were the same as for $\beta=0.04$.

of healthy years of life lost due to each incident case of disease or injury. It is thus an incidence-based measure rather than a prevalence-based measure. The GBD study applied a 3 percent time discount rate to years of life lost in the future to estimate the net present value of years of life lost. With this discount rate, a year of healthy life gained in 10 years' time is valued at 24 percent less than one gained now (note that the standard DALY uses an instantaneous 3 percent discount rate, which results in an annual discount factor that is slightly higher).

Table 5.3 summarizes the effects of varying the discount rate and age weights. Changes in the discount rate and age weights have little effect on the proportion of the burden in males and females. However, changes in the discount rate have an important effect on the proportion of the burden due to nonfatal outcomes (YLD), on the age distribution of the burden, and on the distribution of the burden by broad cause group. When the discount rate is set to zero, the proportion of burden due to YLD is just over a quarter of the total burden. When the discount rate is set to 3 percent, then 36 to 38 percent of the burden is due to YLD, depending on whether age weights are also applied.

Similarly, a nonzero discount rate significantly reduces the importance of the burden of disease or injury in children. This effect is more pronounced in low- and middle-income countries, where children bear a disproportionately large share of the total burden (figure 5.5). Because of the differences in the cause structure of the disease burden by age, these effects also influence the overall distribution of DALYs by broad cause group for low- and middle-income countries. In contrast, for high-income countries, while some changes in the age distribution of the burden are apparent, the choice

Table 5.3 Comparison of the Effects of Changing the Discount Rate $(r)$ and the Age-Weighting Factor $(K)$ on the Composition of $\operatorname{DALYs}(r, K), 2001$

\begin{tabular}{|c|c|c|c|c|c|c|}
\hline & \multicolumn{3}{|c|}{ World } & \multicolumn{3}{|c|}{ Low- and middle-income countries } \\
\hline & $\operatorname{DALY} s(0,0)$ & DALYs(3,0) & DALYs(3,1) & $\operatorname{DALYs}(0,0)$ & DALYs(3,0) & DALYs(3,1) \\
\hline Total DALYs (millions) & 2,645 & 1,536 & 1,476 & 2,447 & 1,387 & 1,357 \\
\hline \multicolumn{7}{|l|}{ By outcome $(\%)$} \\
\hline Total YLD & 27 & 36 & 38 & 26 & 34 & 36 \\
\hline Total YLL & 73 & 64 & 62 & 74 & 66 & 64 \\
\hline \multicolumn{7}{|l|}{ By cause $(\%)$} \\
\hline Group I & 47 & 37 & 41 & 50 & 40 & 44 \\
\hline Group II & 42 & 53 & 47 & 38 & 49 & 43 \\
\hline Group III & 12 & 11 & 12 & 12 & 11 & 13 \\
\hline \multicolumn{7}{|l|}{ By sex $(\%)$} \\
\hline Male & 51 & 52 & 52 & 51 & 52 & 52 \\
\hline Female & 49 & 48 & 48 & 49 & 48 & 48 \\
\hline \multicolumn{7}{|l|}{ By age group (\%) } \\
\hline $0-4$ & 39 & 28 & 30 & 41 & 31 & 32 \\
\hline $5-14$ & 6 & 6 & 8 & 7 & 6 & 8 \\
\hline $15-44$ & 26 & 27 & 35 & 26 & 28 & 35 \\
\hline $45-59$ & 12 & 15 & 14 & 12 & 15 & 13 \\
\hline $60+$ & 16 & 24 & 15 & 14 & 21 & 13 \\
\hline
\end{tabular}

Source: Authors' calculations. 

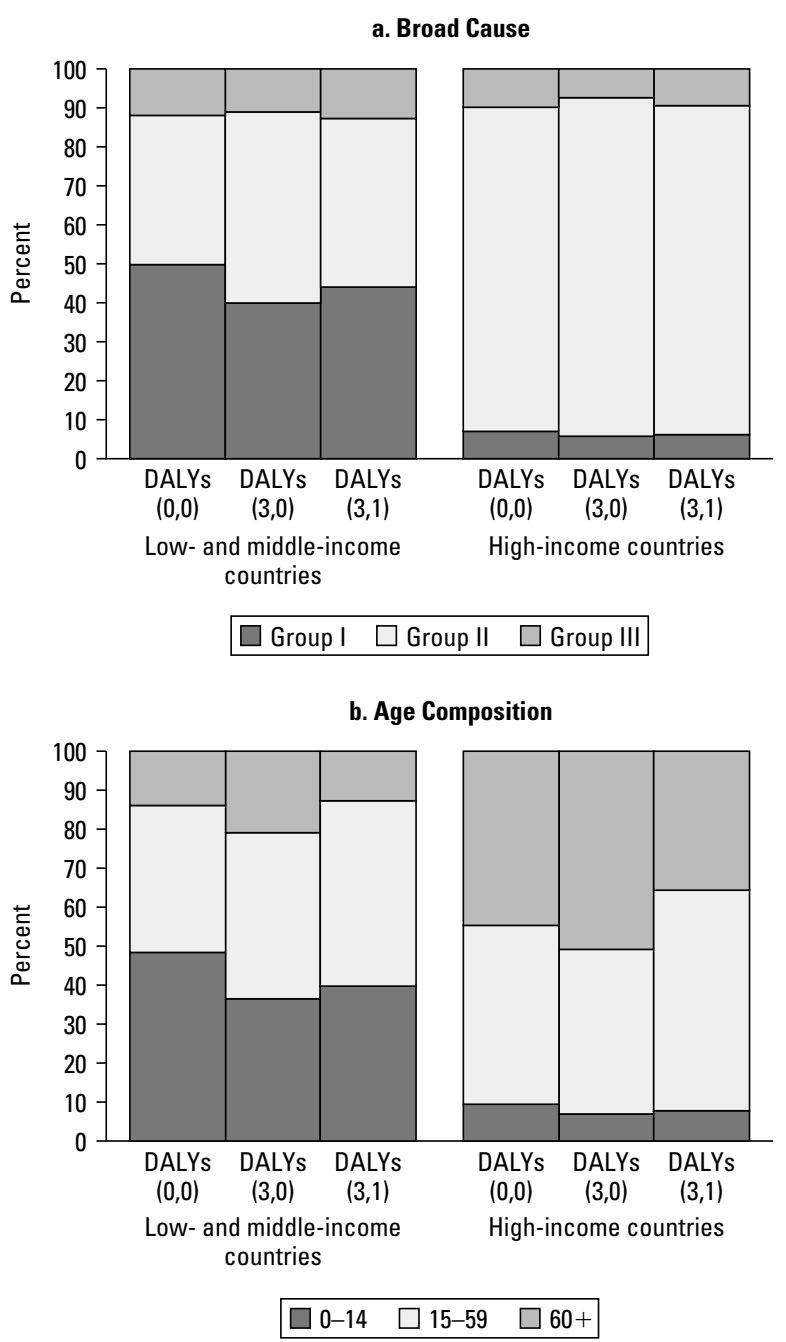

Source: Authors' calculations.

Note: The notation $\operatorname{DALY}(r, K)$ denotes DALYs calculated with discount rate $r$ (percent) or standard or uniform age weighting ( $K=1$ or 0 , respectively).

Figure 5.5 Effects of Changing the Discount Rate and Age Weighting on DALYs' Broad Cause and Age Composition, 2001

of discounting (and age weights) has relatively little influence on the broad cause group breakdown of the total burden of disease (figure 5.5).

The effects of introducing nonuniform age weights are generally much smaller than the effects of introducing nonzero discounting. A comparison of the discounted DALYs with and without age weighting in table 5.3 shows that the main effect is on the age distribution of the disease burden. For both high-income and low- and middle-income countries, age weights reduce the importance of the share of the burden borne by older people. In low- and middle-income countries, people aged 60 years and older suffer 21 percent of

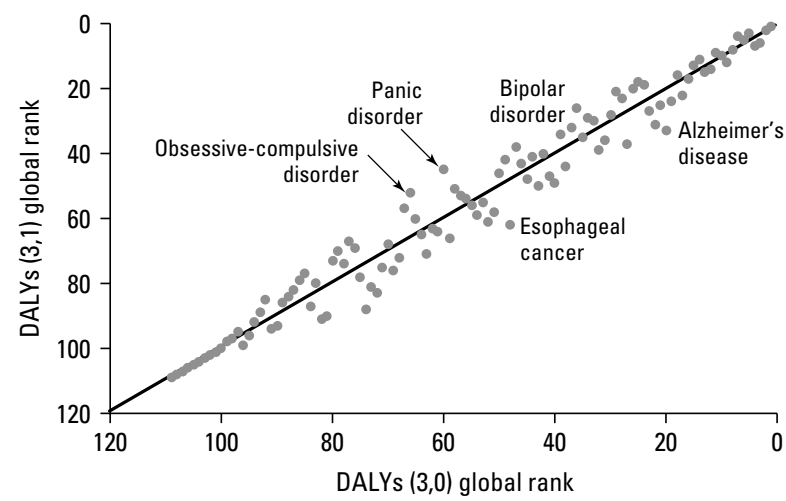

Source: Authors' calculations.

Note: Rank 1 is the largest cause.

Figure 5.6 Relationship between the Rank Order of Causes of the Global Burden Using DALYs(3,1) and DALYs(3,0) in 2001

the total burden of disease and injury. This declines to 13 percent when nonuniform age weights are used. As shown in the second part of figure 5.5, the effects of discounting and age weighting on the age structure of the burden of disease largely offset each other for older ages, so that for $\operatorname{DALYs}(0,0)$ and DALYs $(3,1)$ the share of the burden for those aged 60 years and older is quite similar. Overall, the importance of Group I conditions (communicable diseases, maternal and perinatal conditions, and nutritional deficiencies) is also slightly enhanced by age weighting and that of Group II conditions (noncommunicable diseases) is reduced. The effects on Group III (injuries) are relatively minor.

Figure 5.6 compares the rank order of causes contributing to the global burden of disease measured using $\operatorname{DALYs}(3,1)$ and DALYs $(3,0)$. The introduction of nonuniform age weights has the most impact on neuropsychiatric disorders, such as bipolar disorder, panic disorder, and obsessive-compulsive disorder, whose prevalence is greatest in younger and middle-aged people. Age-weighted DALYs give less importance to causes whose burden falls predominantly on older ages.

Figure 5.7 compares ranks for causes measured using undiscounted DALYs $(0,0)$ and discounted DALYs $(3,0)$, both with uniform age weights $(K=0)$. A zero discount rate gives greater importance to causes with a larger burden at younger ages, such as whooping cough (pertussis) and meningitis, and lower importance to causes predominantly affecting older ages. However, the different choices of discount rates and age weights do not cause any large changes in the rank ordering of diseases and injuries, which is to a large degree anchored in absolute differences in the burden arising from large differences in prevalence and mortality levels across causes. 


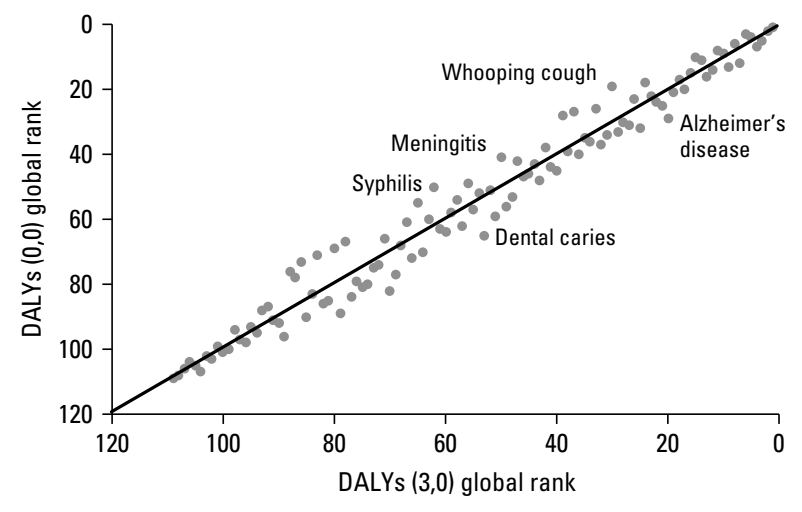

Source: Authors' calculations.

Note: Rank 1 is the largest cause.

Figure 5.7 Relationship between the Rank Order of Causes of the Global Burden of Disease in 2001, Using Uniform Age Weights and 3 Percent Discounting and No Discounting
Table 5.4 compares DALYs $(3,0)$ with $\operatorname{DALYs}(3,1)$ and $\operatorname{DALYs}(0,0)$ in more detail according to the second level of cause disaggregation within a group. These more detailed results confirm the major conclusions outlined earlier on the impacts of discounting and age weighting. $\operatorname{DALYs}(0,0)$ give greater weight to perinatal conditions (the International Classification of Diseases [ICD] category of conditions arising in the perinatal period) and respiratory infections, which primarily affect young children, than either of the discounted forms of DALYs. In contrast, the age-weighted DALYs $(3,1)$ give more weight than $\operatorname{DALYs}(3,0)$ to causes that predominantly affect younger adult ages, such as neuropsychiatric conditions and injuries. DALYs $(3,0)$ give greater weight than either $\operatorname{DALYs}(3,1)$ or DALYs $(0,0)$ to causes that predominantly affect older people, such as cardiovascular diseases and cancers.

Figure 5.8 summarizes the effects of changing the discount rate and age weighting on the global rankings for the

Table 5.4 Effects of Changing the Discount Rate $(r)$ and the Age-Weighting Factor $(K)$ on the Second-Level Cause Group Composition of DALYs $(r, K), 2001$

(percentages of total DALYs)

\begin{tabular}{|c|c|c|c|c|c|c|}
\hline \multirow[b]{2}{*}{ Group/cause } & \multicolumn{3}{|c|}{ Low- and middle-income countries } & \multicolumn{3}{|c|}{ High-income countries } \\
\hline & DALYs $(0,0)$ & DALYs(3,0) & DALYs(3,1) & $\operatorname{DALY} s(0,0)$ & DALYs(3,0) & $\operatorname{DALYs}(\mathbf{3}, 1)$ \\
\hline All causes & 100.0 & 100.0 & 100.0 & 100.0 & 100.0 & 100.0 \\
\hline $\begin{array}{l}\text { I. Communicable, maternal, perinatal, } \\
\text { and nutritional conditions }\end{array}$ & 49.8 & 39.8 & 43.9 & 6.9 & 5.7 & 6.1 \\
\hline A. Infectious and parasitic diseases & 28.0 & 23.1 & 25.5 & 2.5 & 2.3 & 2.3 \\
\hline B. Respiratory infections & 8.2 & 6.3 & 6.6 & 1.6 & 1.7 & 1.3 \\
\hline C. Maternal conditions & 1.8 & 1.9 & 2.2 & 0.3 & 0.3 & 0.4 \\
\hline D. Perinatal conditions & 9.4 & 6.4 & 7.2 & 1.9 & 0.9 & 1.3 \\
\hline E. Nutritional deficiencies & 2.2 & 2.1 & 2.3 & 0.6 & 0.6 & 0.8 \\
\hline II. Noncommunicable diseases & 38.4 & 48.9 & 43.4 & 83.1 & 86.6 & 84.7 \\
\hline A. Malignant neoplasms & 4.5 & 5.4 & 4.2 & 17.4 & 17.3 & 14.8 \\
\hline B. Other neoplasms & 0.1 & 0.1 & 0.1 & 0.4 & 0.4 & 0.3 \\
\hline C. Diabetes mellitus & 0.9 & 1.1 & 0.9 & 2.7 & 2.8 & 2.6 \\
\hline D. Endocrine disorders & 0.6 & 0.8 & 0.5 & 1.5 & 1.6 & 1.4 \\
\hline E. Neuropsychiatric conditions & 7.1 & 9.9 & 11.7 & 18.8 & 20.9 & 27.0 \\
\hline F. Sense organ diseases & 3.9 & 5.2 & 4.6 & 5.3 & 5.1 & 4.8 \\
\hline G. Cardiovascular diseases & 10.0 & 12.9 & 9.4 & 18.8 & 20.0 & 15.6 \\
\hline H. Respiratory diseases & 3.2 & 4.2 & 3.4 & 6.3 & 6.6 & 6.5 \\
\hline I. Digestive diseases & 3.0 & 3.8 & 3.0 & 4.1 & 4.4 & 4.1 \\
\hline J. Genitourinary diseases & 1.0 & 1.2 & 1.0 & 1.2 & 1.4 & 1.2 \\
\hline K. Skin diseases & 0.2 & 0.3 & 0.3 & 0.2 & 0.2 & 0.2 \\
\hline L. Musculoskeletal diseases & 1.4 & 1.9 & 1.8 & 4.2 & 4.3 & 4.1 \\
\hline M. Congenital anomalies & 2.2 & 1.7 & 2.0 & 1.7 & 1.0 & 1.3 \\
\hline N. Oral conditions & 0.3 & 0.5 & 0.5 & 0.6 & 0.6 & 0.7 \\
\hline III. Injuries & 11.9 & 11.2 & 12.7 & 9.9 & 7.5 & 9.3 \\
\hline A. Unintentional injuries & 8.7 & 8.2 & 9.3 & 6.9 & 5.3 & 6.5 \\
\hline B. Intentional injuries & 3.2 & 3.1 & 3.5 & 3.0 & 2.3 & 2.8 \\
\hline
\end{tabular}

Source: Authors' calculations. 


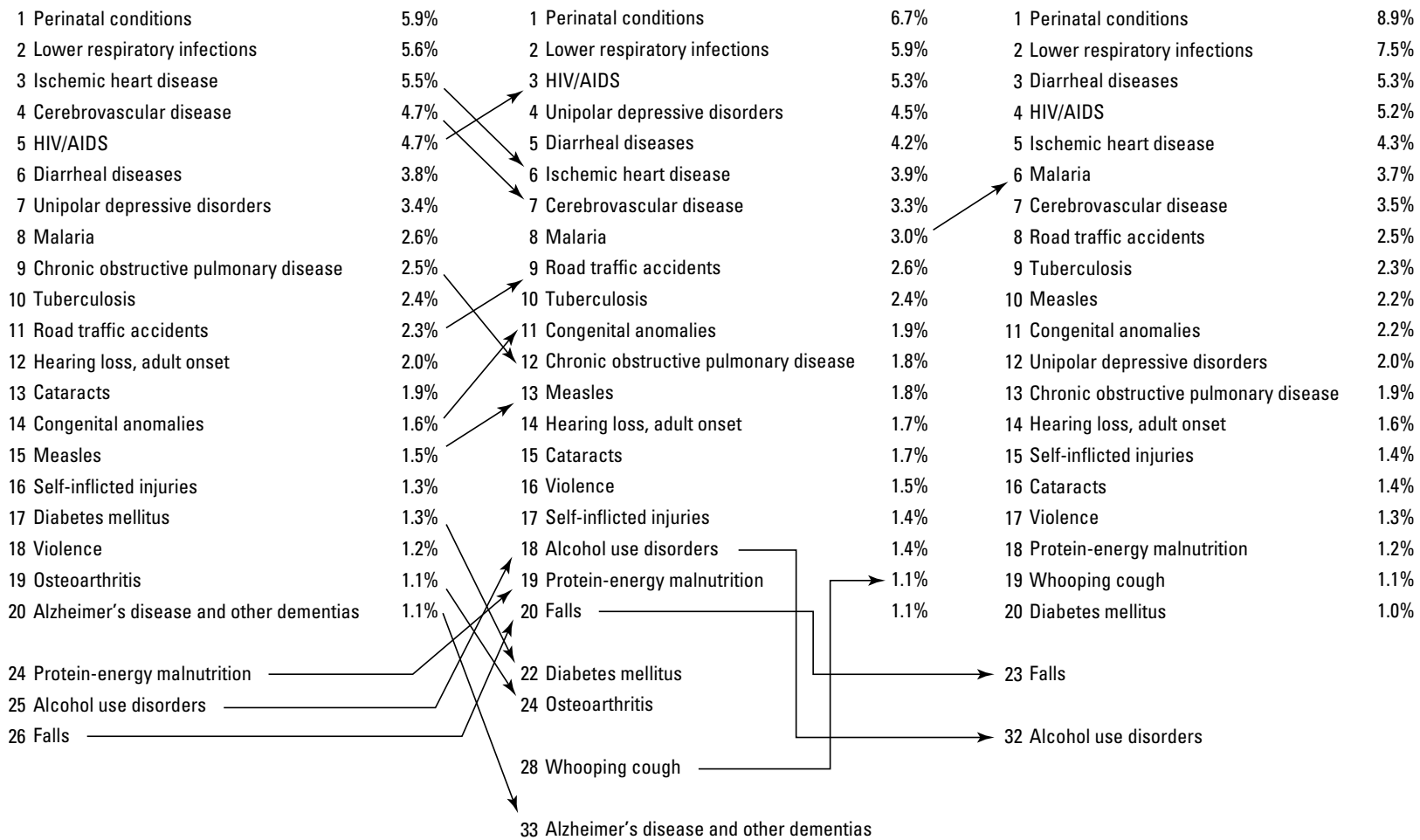

Source: Authors' calculations.

Figure 5.8 Effects of Changing the Discount Rate and Age Weighting on Global Rankings for the Top 20 Causes of the Burden of Disease, 2001

top 20 causes of the burden of disease in 2001. The left-hand column shows the rankings for causes measured using $\operatorname{DALYs}(3,0)$ as used for the DCPP. The middle column is for DALYs $(3,1)$, as used by WHO to present the GBD analysis. The principal difference is that the use of $\operatorname{DALYs}(3,0)$ results in relatively greater importance being placed on chronic diseases of middle and older ages, such as ischemic heart disease and stroke, and somewhat lesser on HIV/AIDS, road traffic accidents, congenital anomalies, and other disorders affecting children and younger adults. Undiscounted DALYs, shown in the right-hand column, give proportionately greater importance to conditions affecting children, such as malaria and measles.

\section{SENSITIVITY OF RISK FACTOR ESTIMATES TO VARIATIONS IN KEY PARAMETER VALUES}

Figures 5.9 to 5.11 examine the sensitivity of the burden of disease attributable to each of the 19 risk factors discussed in chapter 4 to key DALY discounting and age-weighting parameters for the world, for low-and-middle-income countries, and for high-income countries. The figures plot the attributable disease burden estimated by altering one key parameter against the baseline of $\operatorname{DALYs}(3,0)$ used in chapter 4 . To allow comparability, all burdens attributable to risk factors are shown as a proportion of the total global or regional disease burden, which is itself estimated with the corresponding parameters.

Including age weighting, DALYs $(3,1)$, increases the relative health consequences of risks that affect people in young and middle ages (alcohol use, illicit drug use, and unsafe sex) and lowers the relative contribution of those risks that result in death in older ages (high blood pressure, high cholesterol, low fruit and vegetable intake, overweight and obesity, physical inactivity, and smoking). In addition, the burden of disease attributable to childhood and maternal underweight increases as a proportion of the total global or regional burden of disease. This increase probably reflects a relative reduction in the total burden of those diseases that affect older adults, and hence a relative increase in the total burden of those diseases that affect young children. Because childhood and maternal underweight is a risk factor for this 
World
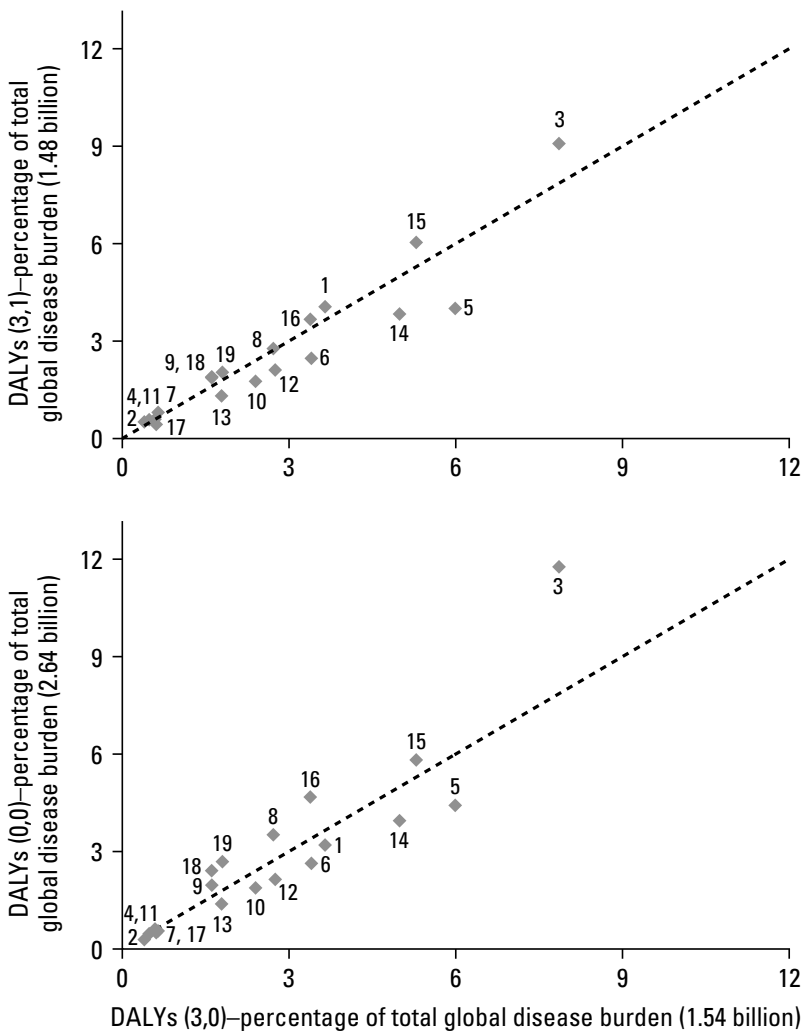

Source: Authors' calculations.

Note: Each point corresponds to the proportion of total GBD attributable to one risk factor. (1) alcohol use; (2) child sexual abuse; (3) childhood underweight; (4) contaminated injections in health care setting; (5) high blood pressure; (6) high cholesterol; (7) illicit drug use; (8) indoor smoke from household use of solid fuels: (9) iron deficiency anemia; (10) low fruit and vegetable intake; (11) non-use and use of ineffective methods of contraception; (12) overweight and obesity (high body mass index); (13) physical inactivity; (14) smoking; (15) unsafe sex; (16) unsafe water, sanitation, and hygiene; (17) urban air pollution; (18) vitamin A deficiency; (19) zinc deficiency.

Figure 5.9 Effects of Changes in Key DALY Parameters on Proportion of the Global Disease Burden Attributable to Risk Factors.

latter group of diseases, its attributable burden as a share of the total global or regional disease burden increases.

Removing discounting, $\operatorname{DALYs}(0,0)$, results in a large relative increase in the disease burden attributable to risk factors that affect young children, including childhood underweight; indoor smoke from household use of solid fuels; unsafe water, sanitation, and hygiene; vitamin A deficiency; and zinc deficiency. This is mirrored by a decrease in the disease burden attributable to the risk factors for diseases that affect adults, because the total burden of the chronic diseases affected by these risks is reduced. This effect is more noticeable in the low- and middle-income countries than in the high-income countries, where childhood mortality is low and the overall share of the disease burden is less sensitive to discounting.
Low- and middle-income countries
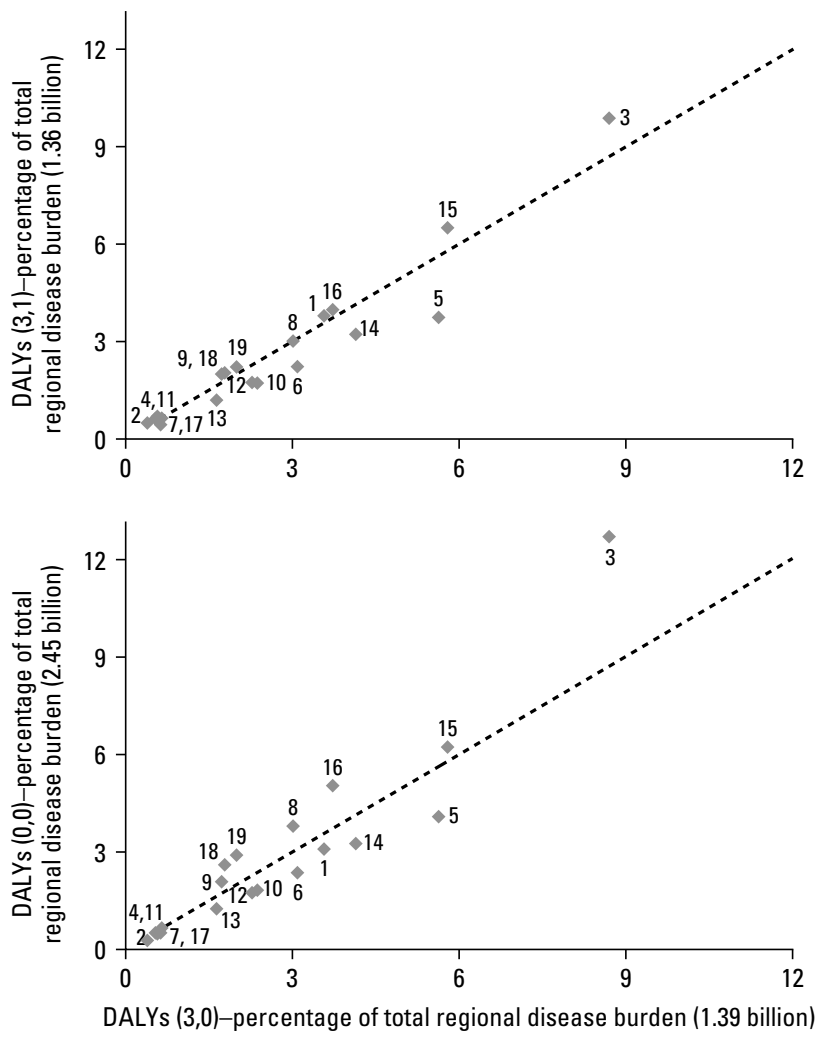

Source: Authors' calculations.

Note: Each point corresponds to the proportion of total GBD attributable to one risk factor. (1) alcohol use; (2) child sexual abuse; (3) childhood underweight; (4) contaminated injections in health care setting; (5) high blood pressure; (6) high cholesterol; (7) illicit drug use; (8) indoor smoke from household use of solid fuels; (9) iron deficiency anemia; (10) low fruit and vegetable intake; (11) non-use and use of ineffective methods of contraception; (12) overweight and obesity (high body mass index); (13) physical inactivity; (14) smoking; (15) unsafe sex; (16) unsafe water, sanitation, and hygiene; (17) urban air pollution; (18) vitamin A deficiency; (19) zinc deficiency.

Figure 5.10 Effects of Changes in Key DALY Parameters on Proportion of the Regional Disease Burden Attributable to Risk Factors for Low- and Middle-Income Countries

Sensitivity to key DALY parameters differed in the lowand middle-income countries and the high-income countries. The burden of disease attributable to risk factors for chronic diseases in adults (high blood pressure, high cholesterol, low fruit and vegetable intake, overweight and obesity, physical inactivity, and smoking) was more sensitive to these parameters in low- and middle-income countries than in high-income countries because deaths attributable to these risks occurred at younger ages in the former. By contrast, the burden of disease attributable to alcohol was much more sensitive to age-weighting in the high-income countries because many of the hazards of this risk, especially those related to injuries and neuropsychiatric conditions, occur among younger adults in this group of countries. 

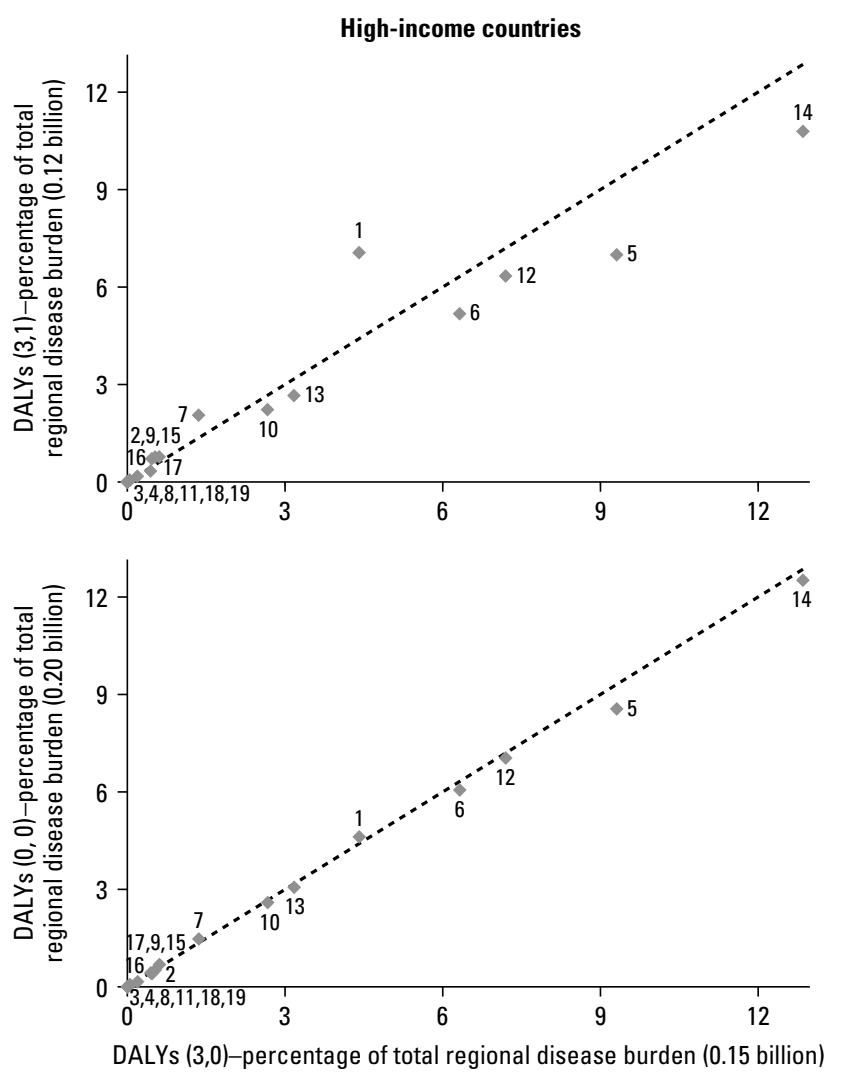

Source: Authors' calculations.

Note: Each point corresponds to the proportion of total GBD attributable to one risk factor. (1) alcohol use; (2) child sexual abuse; (3) childhood underweight; (4) contaminated injections in health care setting; (5) high blood pressure; (6) high cholesterol; (7) illicit drug use; (8) indoor smoke from household use of solid fuels; (9) iron deficiency anemia; (10) low fruit and vegetable intake; (11) non-use and use of ineffective methods of contraception; (12) overweight and obesity (high body mass index); (13) physical inactivity; (14) smoking; (15) unsafe sex; (16) unsafe water, sanitation, and hygiene; (17) urban air pollution; (18) vitamin A deficiency; (19) zinc deficiency.

Figure 5.11 Effects of Changes in Key DALY Parameters on Proportion of the Regional Disease Burden Attributable to Risk Factors for High-Income Countries

\section{UNCERTAINTY ANALYSIS OF THE GLOBAL BURDEN OF DISEASE ESTIMATES}

The 2001 GBD study estimated mortality and the burden of disease for a comprehensive set of disease and injury causes and for all regions of the world, including regions with limited, incomplete, and uncertain data. To allow users of the information to assess whether the information uncertainty range is compatible with the purpose at hand, providing some analysis and guidance on levels of uncertainty is important (Murray, Mathers, and Salomon 2003). This is difficult to do, because apart from the large number and disparate nature of the data sources used (see chapter 3 ), information or knowledge about the quality of and potential biases in the data is often limited. This and the following sections provide an overview of initial efforts to quantify the uncertainty associated with the estimation of deaths by cause, with disability weights, and with epidemiological estimates of incidence and prevalence for GBD 2001.

\section{Sources of Uncertainty}

Uncertainty in estimated disease burden may arise from the following sources:

- incomplete information, for example, when estimates for a population are based on observations from a sample;

- potential biases in information, for instance, issues concerning the representativeness for a whole population of estimates from a study of a subgroup or the validity of a survey instrument in addressing the quantity of interest;

- heterogeneity or from disagreements among information sources, as when several studies give different estimates for the same quantity of interest;

- model uncertainty, for example, the variables or functional form specified in a regression model;

- the data generation process itself; for instance, investigators may only infer risks from event counts in a population, which means that they can never know the risks themselves with certainty.

The most familiar and most commonly quantified kind of uncertainty arises from random error in the direct measurement of a quantity. An estimate of an epidemiological quantity for a population will have uncertainty arising from the finite sample used in the study as well as from random measurement error. The standard error of the mean or the confidence interval for such a quantity specifies the distribution of uncertainty in knowledge of the true mean value in the population (assuming no systematic error).

Most measurement involves not only random (stochastic) error, but also systematic error arising from biases in the measurement instrument, for instance, unrepresentativeness of a sampling frame for a survey, or from inaccuracies in the assumptions used to infer the actual quantity from the available data, for example, estimating the prevalence of a disease for a country from studies of representative subpopulations. Examinations of historical measurements reveal a consistent tendency to underestimate systematic error, perhaps because systematic error usually relates to sources of error that are unknown or about which little is known. Ignoring systematic error when estimating uncertainty is common, but this often results in substantial 
underestimation of the true uncertainty (Morgan and Henrion 1990).

Putting upper and lower bounds on the systematic error component is often possible, for example, where a disease process has biological limits or where evidence from a range of populations provides likely upper and lower limits to an epidemiological parameter such as prevalence or case fatality. In addition, consistency analysis across the various inputs for the DALY calculation (incidence, prevalence, case fatality rates or relative risk of mortality, and remission rates) often helps identify sources of systematic error and provides some basis for quantifying them (Kruijshaar, Barendregt, and Hoeymans 2002; Mathers, Murray, and Lopez 2002). This is discussed further in chapter 3.

Much of the uncertainty in estimates of deaths or DALYs for the 2001 GBD study is associated with the assessment of systematic errors in primary data. Chapter 3 examined primary data sources and their reliability in some detail and provided summary tabulations of the numbers of data sources available across regions and causes. This review clearly indicated that even though most countries have some information about prevalence, incidence, and mortality from some diseases and injuries and about population exposures to risk factors, it is generally fragmented, partial, incomparable, and diagnostically uncertain. One of the explicit aims of the GBD approach is to provide a coherent framework for integrating, validating, analyzing, and disseminating fragmentary information on the health of populations so that it is truly useful for health policy and planning. An important aspect of this framework is to assess the reliability and validity of data, particularly in relation to systematic error, and hence to provide some guide to the uncertainty in the resulting estimates.

\section{Describing and Quantifying Uncertainty}

We follow Morgan and Henrion's (1990) approach toward interpreting and using probability to describe and quantify uncertainty. The classical or frequentist view of probability defines the probability of an event occurring in a particular trial or experiment as the frequency with which it would occur during a long sequence of similar experiments. For many quantities of real interest, it is difficult to imagine how to operationalize a long sequence of relevant, similar experiments. An example of such a quantity would be the probability, estimated in late 2005, that avian influenza will cause a major global epidemic with deaths exceeding, say, 1 million in 2006. One approach has been to distinguish events whose probabilities are knowable through a series of experiments from those whose probabilities are unknowable or uncertain because no unique and operationalizable set of similar experiments exists, but this essentially limits the use of probabilities to games of chance.

Alternatively, a Bayesian view of probability defines it as the degree to which a person believes that an event will occur, or that a parameter has a certain value, given all the relevant information currently known to that person. Because different people have different information, they may legitimately assign different probabilities to the same event. These subjective probabilities must obey all the same axioms and rules as frequentist probabilities. These conceptual distinctions do not usually affect the practice of statistical inference, and essentially the same formal inference models of probability may be applied (King, Tomz, and Wittenberg 2000; Morgan and Henrion 1990). Moreover, when an empirical series of data from trials becomes available, the Bayesian assessment of probability should converge to the frequentist assessment, assuming the Bayesian approach uses the data rationally to update the assessments.

Our general approach to describing and estimating uncertainty in quantities of interest is to express them as probability distributions using a Bayesian interpretation of probability as expressing uncertainty of an observed or hypothetical event given a set of assumptions about the world. Probability distributions can therefore be used to express uncertainty about epidemiological quantities, such as the prevalence of depression in a particular population, the population values reflected in health state valuations, or the underlying risk of mortality due to a specific cause in a specific population.

Advances in computer technology have facilitated analytical methods for dealing with uncertainty enormously. One general approach to combining the uncertainties of multiple inputs into estimates relies on numerical simulation methods. The simulation approach uses multiple samples from probability distributions around uncertain inputs to allow estimates of the probability distributions around quantities of interest that may be complicated functions of these inputs, without the need to solve difficult, or in many cases insoluble, mathematical equations (King, Tomz, and Wittenberg 2000; Vose 2000).

\section{UNCERTAINTY ESTIMATES FOR ALL-CAUSE MORTALITY AND LIFE EXPECTANCIES}

Chapters 2 and 3 describe methods for estimating life tables for each of 192 WHO member states. For those countries with vital registration data projected using time series regression 
models on the parameters of the logit life table system, we accounted for uncertainty around the regression coefficients by taking 1,000 draws of the parameters using the regression estimates and variance-covariance matrix of the estimators. For each of the draws, we calculated a new life table. In cases where additional sources of information provided information on the limits of uncertainty ranges around ${ }_{5} \mathrm{q}_{0}$ (the mortality risk for children under five years of age) and ${ }_{45} \mathrm{q}_{15}$ (the mortality risk for adults between the ages of 15 and 60), the 1,000 draws were constrained so that each life table produced estimates within these specified ranges. The range of 1,000 life tables produced by these multiple draws reflects some of the uncertainty around the projected trends in mortality, notably, the imprecise quantification of systematic changes in the logit parameters over the time period captured in available vital registration data.

For countries that did not have time series data on mortality by age and sex, the following steps were undertaken. First, point estimates and ranges around ${ }_{5} \mathrm{q}_{0}$ and ${ }_{45} \mathrm{q}_{15}$ for males and females were developed on a country-by-country basis as described in chapter 2 and elsewhere (Lopez and others 2002). For countries where the ${ }_{5} \mathrm{q}_{0}$ estimate for 2001 was based on an analysis of available data sources for earlier years, such as surveys and censuses, the uncertainty range for ${ }_{5} \mathrm{q}_{0}$ was typically dominated by the uncertainty resulting from the scatter of survey-based direct and indirect estimates of child mortality for earlier years and the uncertainty in extrapolation of the trend to 2001, rather than the sampling error associated with individual estimates. For countries without usable information on levels of adult mortality, ${ }_{45} \mathrm{q}_{15}$ was estimated, along with uncertainty ranges, based on regression models of ${ }_{45} \mathrm{q}_{15}$ versus ${ }_{5} \mathrm{q}_{0}$ as observed in a set of almost 2,000 life tables judged to be of good quality. Estimated levels of child and adult mortality were then applied to a modified logit life table model, using a global standard, to estimate the full life table in 2001; HIV/AIDS deaths and war deaths were added to total mortality rates where necessary. Uncertainty ranges for HIV/AIDS were estimated as described elsewhere (Grassly and others 2004). In countries with substantial numbers of war deaths, estimates of their uncertainty range were also incorporated into the life table uncertainty analysis.

Figure 5.12 plots the final estimated uncertainty ranges for ${ }_{5} \mathrm{q}_{0}$ and ${ }_{45} \mathrm{q}_{15}$ for 192 WHO member states for males and females. Using Monte Carlo simulation methods, 1,000 random life tables were generated by drawing samples from normal distributions around these inputs with variances defined in reference to the defined ranges of uncertainty for ${ }_{5} \mathrm{q}_{0}$ and ${ }_{45} \mathrm{q}_{15}$. In countries where uncertainty around ${ }_{5} \mathrm{q}_{0}$ and
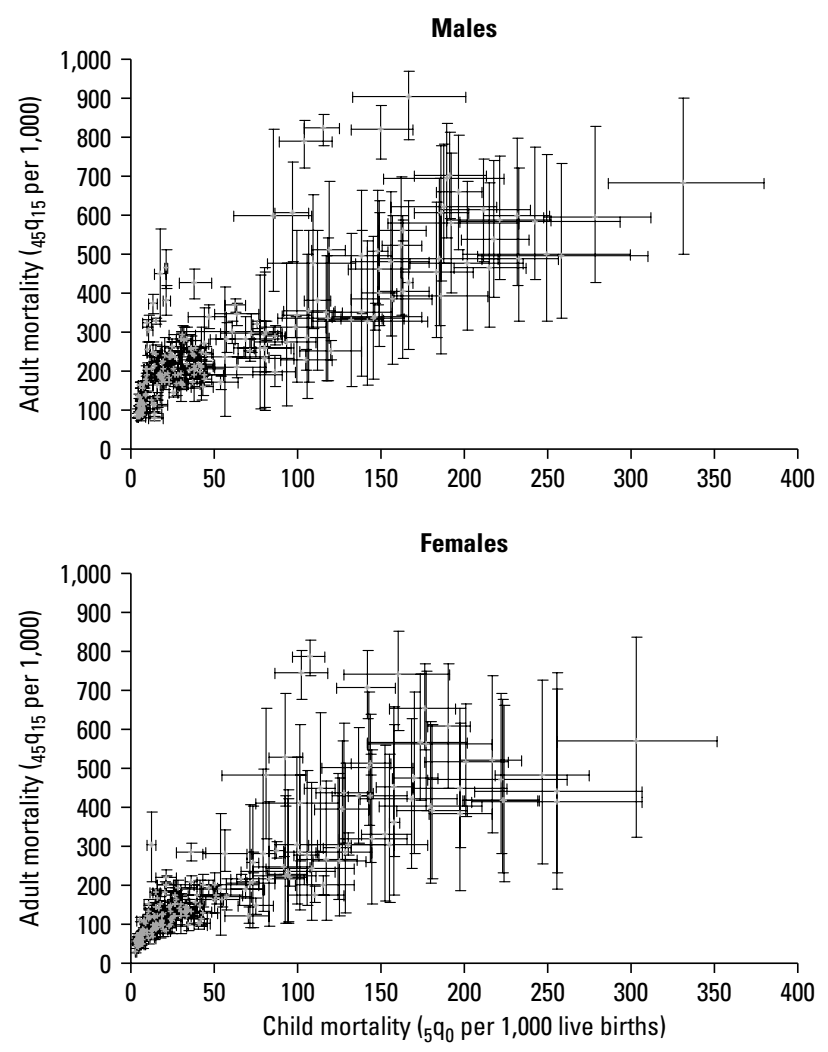

Source: Authors' calculations.

Figure 5.12 Uncertainty Ranges for Child and Adult Mortality for WHO Member States, 2001

${ }_{45} \mathrm{q}_{15}$ was considerable because of a paucity of survey or surveillance information, the samples were drawn from wide distributions, but then constrained within prior specified maximum and minimum possible values for ${ }_{5} \mathrm{q}_{0}$ and ${ }_{45} \mathrm{q}_{15}$. For each country, the results of this analysis were 1,000 different simulated life tables that were then used to describe ranges around key indicators, such as life expectancy at birth and age- and sex-specific mortality rates.

Figure 5.13 illustrates the resulting uncertainty ranges for life expectancy at birth for the World Bank regions (see map 1 inside the book's front cover). For high-income countries, where relatively complete death registration data are available, the uncertainty ranges for life expectancy at birth are around \pm 0.07 years for females and \pm 0.16 years for males. For regions such as Latin America and the Caribbean, where death registration data are available for most countries but are often incomplete, the uncertainty ranges are larger, typically around \pm 0.5 years. For regions with partial data on child mortality only, where adult mortality is predicted from child mortality, the uncertainty ranges are much larger, and 


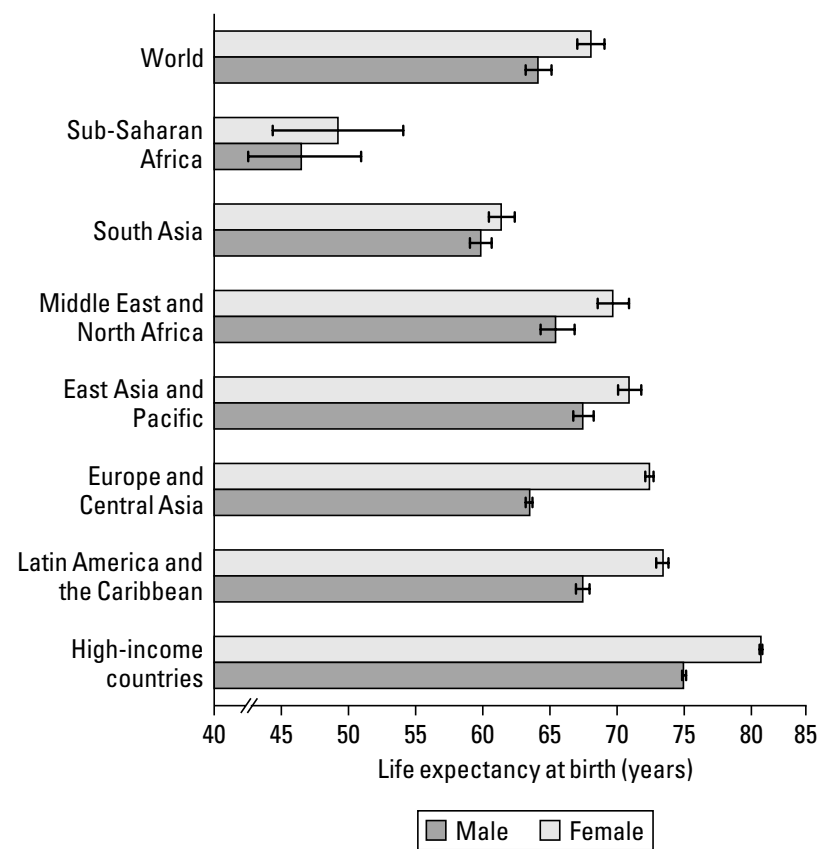

Source:Authors' calculations.

Figure 5.13 Uncertainty in Average Life Expectancy at Birth, by Sex and DCPP Region, 2001

for Sub-Saharan Africa are typically around \pm 5.0 years. Across the regions, this translates to considerable heterogeneity in uncertainty ranges for life expectancies at birth and for estimates of all-cause mortality levels.

\section{UNCERTAINTY ESTIMATES FOR REGIONAL MORTALITY BY CAUSE}

We use a simulation approach to estimate uncertainty ranges for deaths by cause for GBD 2001. These uncertainty ranges take into account uncertainty in the expected number of total deaths (life table uncertainty); uncertainty in the estimated proportions of broad cause Groups I, II, and III (where relevant for countries without vital registration data or with incomplete coverage); uncertainty in the diagnosis of underlying cause; uncertainty arising from the miscoding of underlying cause; and fundamental Poisson uncertainty in the estimated death rate arising from the observation of a finite number of deaths in a fixed time interval. This analysis was carried out by country.

As described in the previous section, a total of 1,000 life tables were developed for each of the 192 WHO member states to quantify the uncertainty distribution of key life table parameters. We then used the age-specific mortality rates from the 1,000 life tables to estimate the uncertainty distribution for the expected number of total deaths for 2001. Uncertainty in the underlying cause attribution was estimated in terms of the relative uncertainty of the proportion of deaths due to each specific cause. The estimates of cause-specific relative uncertainty were based on advice from nosologists and experts in the area of cross-country mortality analysis on the general levels of uncertainty in the attribution of specific causes within Groups I, II and III, together with detailed advice on particular causes with known higher levels of attribution uncertainty according to the ICD. Information on the latter causes derives from comparative analyses across countries, across time periods, and across ICD revisions, together with information from a variety of country-specific coding quality studies involving recoding or dual coding of deaths and comparisons with the original attributed causes.

Based on this advice, for cause distributions derived from vital registration data coded using ICD-10 (the 10th edition of the ICD), we generally assumed that diagnostic uncertainty and coding uncertainty together resulted in approximate relative 95 percent uncertainty ranges of \pm 3 percent for Group I causes, \pm 7 percent for Group II causes, and \pm 2 percent for Group III causes. Larger uncertainty ranges were assumed for specific causes known to have greater levels of diagnostic or coding error; for WHO member states that have been using ICD-10 coding for less than three years; for member states still using ICD-9 coding (with particular attention to causes where coding rates between ICD-9 and ICD-10 are known to differ); and for member states using other cause coding systems or verbal autopsy methods, or where cause of death models were used to estimate death distributions across Groups I, II, and III. In the latter case, an additional relative uncertainty for the estimation of Group I, II, and III proportions was estimated from the prediction uncertainty ranges associated with the CodMod regression model (see chapter 3).

Uncertainty estimates also took into account the redistribution of general, cancer, cardiovascular, and injury ill-defined cause codes and incomplete coverage of vital registration data. The relative uncertainty range for each cause was then combined with the estimated uncertainty distribution for allcause mortality to provide estimates of the uncertainty distributions of cause-specific mortality estimates for all ages and both sexes at the country level.

The analysis of uncertainty in cause of death estimates at the country level thus combines quantitative, countryspecific information on uncertainty in all-cause mortality 
and, in some cases, also in major cause group distributions, together with quantified average relative uncertainty ranges for specific cause attributions based on expert advice and adjusted for specific causes and for country-specific information on data sources, type of cause information available, and indicators of data quality. Here we summarize these uncertainty estimates at the regional level to provide some indication of the range of uncertainty for cause-specific mortality estimates across the World Bank regions as reported in chapter 3 . This requires some additional assumptions about the cross-country correlations in uncertainty distributions.

At one extreme, if all country-level estimates have uncorrelated uncertainty because they are derived from completely independent data sets, then even with high levels of uncertainty at the country level, there will be considerably less uncertainty at the regional or global level. At the other extreme, if the uncertainty in country-level estimates for a cause derives predominantly from a single source or assumption, for example, about the case fatality rate of malaria, that is applied in deriving each country estimate, then the uncertainty distributions will be highly correlated and the regional uncertainty will be of a similar relative magnitude as each of the country uncertainty ranges.

With respect to cross-country correlations for life table and cause of death estimates based on death registration data, we assumed that even though life table uncertainties would be uncorrelated, relative uncertainties in cause of death attribution for specific causes were likely to be correlated because of systematic errors in ICD coding practices across countries for specific causes. We arbitrarily set this correlation at 25 percent. For life table estimates not based on death registration data, we assumed some correlation in uncertainty because even though estimates of childhood mortality came from independent sources, the method for determining adult mortality was similar across countries. We therefore set this correlation at 50 percent.

We assumed that cross-country correlation for relative cause of death uncertainties in the absence of vital registration data would vary depending on the method of causal attribution. Attributions based on some data and countrylevel predictions or assumptions were assumed to have less correlation than those based simply on regional patterns. In the case of the latter, we set the correlation at 75 percent; in the former, we set it at 50 percent or 25 percent depending on the degree of independence of the underlying inputs. We assumed greater independence for cancers and maternal conditions and less independence for tuberculosis, HIV/
AIDS, sexually transmitted infections, diarrheal diseases, childhood-cluster diseases, meningitis, tropical-cluster diseases, lower respiratory infections, and perinatal conditions. We set cross-country correlations for war and drug use disorders at 25 percent for all countries, including those with vital registration data, to reflect the different methods used to obtain estimates for these causes.

We derived 95 percent uncertainty intervals by cause for World Bank regions in 2001 from the foregoing assumptions using simulation methods. We constructed 1,000 draws with the required correlation structure between countries separately for each cause, and the 2.5 th percentile and the 97.5th percentile of expected deaths were taken to be the lower and upper bounds of the corresponding uncertainty interval. Note that these ranges provide guidance on uncertainty in the underlying cause-specific death rates, as expressed in terms of expected deaths in the population in 2001. Uncertainty in population estimates is not included, and the uncertainty ranges relate to underlying death rates, not to the numbers of deaths that actually occurred in 2001.

Table 5.5 summarizes regional uncertainty ranges for total estimated deaths for selected causes for 2001. Uncertainty ranges for estimated all-cause deaths increase from around \pm 1 percent for high-income countries to $(-15$ percent, +21 percent) for Sub-Saharan Africa. For specific causes, regional uncertainty ranges are generally higher, except for those causes for which cause-specific mortality estimates were available based on country-specific data from causespecific surveillance systems (see chapter 3). For example, the uncertainty range for HIV/AIDS deaths in Sub-Saharan Africa is somewhat narrower than the all-cause mortality range, reflecting the substantial database for these estimates from antenatal clinic surveillance data and seroprevalence surveys, albeit still with considerable uncertainty arising from issues around the representativeness of the available data and the assumptions relating to survival and case fatality rates (Grassly and others 2004).

For most other causes, uncertainty ranges are greater than for the all-cause mortality estimates, because additional uncertainty is associated with cause attribution, as described earlier. For example, the relative uncertainty ranges for ischemic heart disease range from around \pm 12 percent for high-income countries to $(-24$ percent, +34 percent) for Sub-Saharan Africa (table 5.5). While the uncertainty range for high-income countries may seem surprisingly large, it reflects not only uncertainty in overall mortality levels, but also uncertainty in the attribution of 
underlying cause and in the attribution of causes coded to cancer, cardiovascular, and injury ill-defined cause codes or to the ICD chapter for symptoms, signs, and ill-defined conditions. The proportion of deaths coded to these two groups of causes is surprisingly large for some high-income countries (Mathers and others 2005).

Figure 5.14 illustrates the relative insensitivity of the regional uncertainty ranges to the assumptions about cross-country correlation of uncertainty. The broad patterns of the uncertainty ranges for causes across regions provide useful additional guidance to policy makers in interpreting regional differences, particularly in judging which policy questions these estimates can help address and for which the uncertainty levels are too great to allow useful inferences.

\section{UNCERTAINTY IN DISABILITY WEIGHTS}

Although health state valuations are often treated as value parameters without uncertainty, we argue that unlike social choices such as the discount rate, no clear normative basis is available on which to assign relative values to the different dimensions of health that collectively define the universe of health states. Ideally, these values should be derived from empirical data among representative populations (Salomon and others 2003). Numerous challenges are associated with population-based data collection for the purpose of health state valuations, particularly given the broad scope of valuations required for a comprehensive assessment of disease burden. As a result, the current empirical base for disability weights remains well short of this ideal. Given the
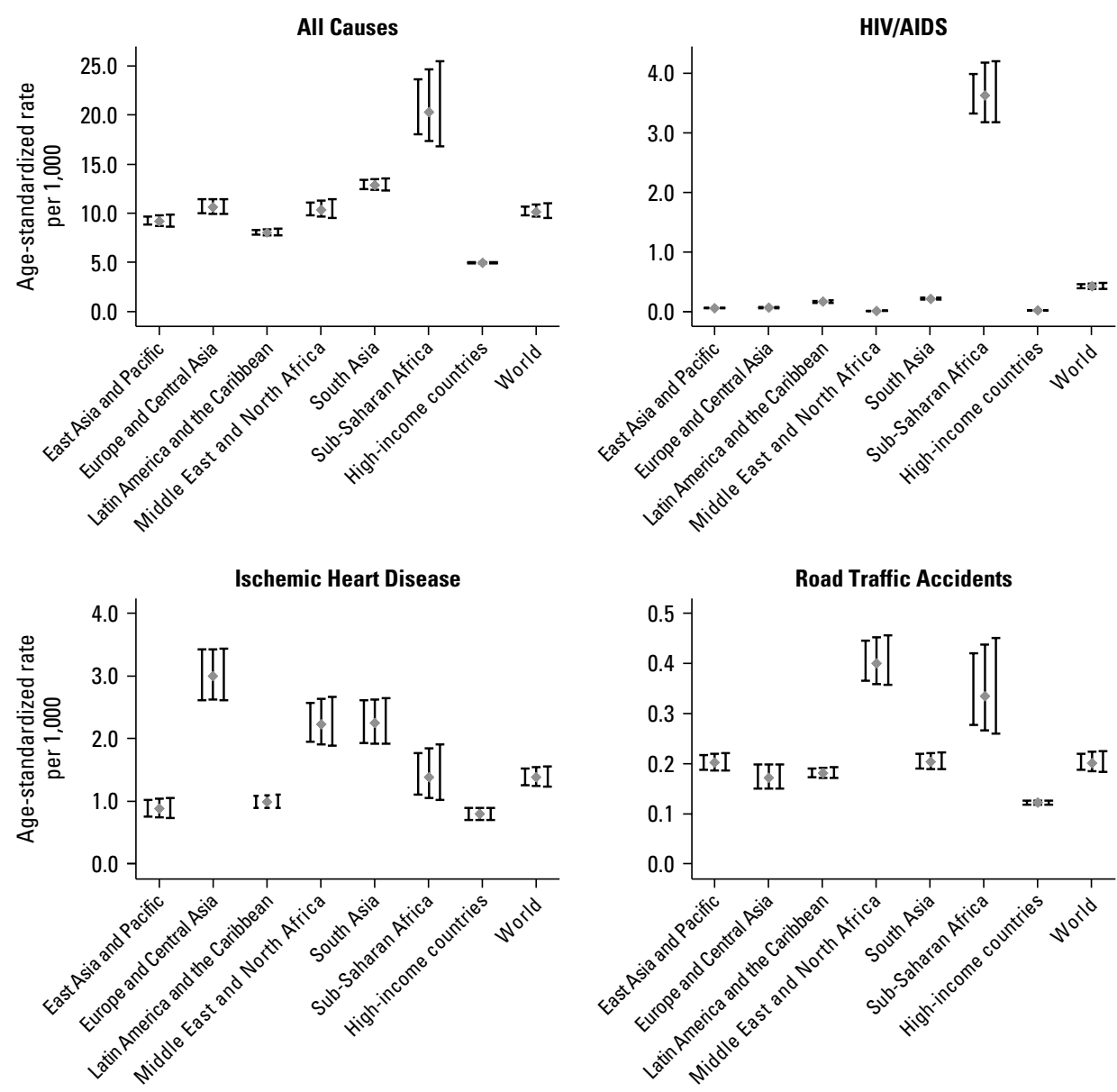

Source: Authors' calculations.

Note: Cross-country correlations in uncertainty distributions for countries without vital registration data were varied from 0 percent (left-hand bar) to 25 percent (middle bar) and 50 percent (right-hand bar) for each region.

Figure 5.14 Sensitivity of Uncertainty Ranges to Changes in Between-Country Correlation Assumptions 
Table 5.5 Estimated Total Deaths and 95 Percent Uncertainty Ranges for Selected Causes, by Region, 2001 (thousands)

\begin{tabular}{|c|c|c|c|c|c|c|c|c|}
\hline \multirow[b]{2}{*}{ Cause } & \multicolumn{2}{|c|}{ East Asia and Pacific } & \multicolumn{2}{|c|}{ Europe and Central Asia } & \multicolumn{2}{|c|}{$\begin{array}{l}\text { Latin America and } \\
\text { the Caribbean }\end{array}$} & \multicolumn{2}{|c|}{$\begin{array}{l}\text { Middle East and } \\
\text { North Africa }\end{array}$} \\
\hline & Deaths & Uncertainty & Deaths & Uncertainty & Deaths & Uncertainty & Deaths & Uncertainty \\
\hline All causes & 13,070 & $12,379-13,866$ & 5,669 & $5,334-6,122$ & 3,277 & $3,166-3,411$ & 1,914 & $1,790-2,088$ \\
\hline Tuberculosis & 534 & $497-578$ & 66 & $58-76$ & 45 & $41-50$ & 23 & $21-26$ \\
\hline HIV/AIDS & 106 & $97-116$ & 28 & $24-35$ & 83 & $74-94$ & 4 & $3-4$ \\
\hline Diarrheal diseases & 226 & 199-252 & 15 & $14-16$ & 55 & $49-61$ & 74 & $65-84$ \\
\hline Pertussis & 3 & $3-4$ & 0 & $0-0$ & 6 & $5-8$ & 8 & $6-9$ \\
\hline Diphtheria & 1 & $1-1$ & 0 & $0-0$ & 0 & $0-0$ & 0 & $0-0$ \\
\hline Measles & 76 & $66-85$ & 8 & $7-8$ & 0 & $0-0$ & 15 & $13-18$ \\
\hline Tetanus & 27 & $25-30$ & 0 & $0-0$ & 1 & $1-1$ & 4 & $3-5$ \\
\hline Meningitis & 33 & $29-39$ & 14 & $13-15$ & 17 & $15-20$ & 10 & $9-11$ \\
\hline Hepatitis B & 32 & $29-36$ & 3 & $3-4$ & 4 & $4-5$ & 6 & $5-7$ \\
\hline Hepatitis C & 13 & $12-15$ & 1 & $1-1$ & 2 & $2-2$ & 3 & $3-3$ \\
\hline Malaria & 30 & $25-36$ & 0 & $0-0$ & 2 & $1-2$ & 19 & $17-22$ \\
\hline Schistosomiasis & 3 & $3-4$ & 0 & $0-0$ & 1 & $1-1$ & 8 & $8-9$ \\
\hline Lower respiratory infections & 544 & $449-655$ & 104 & $94-116$ & 157 & 140-177 & 108 & $90-130$ \\
\hline Upper respiratory infections & 27 & $25-30$ & 4 & $4-5$ & 3 & $2-3$ & 2 & $2-3$ \\
\hline Maternal conditions & 37 & $23-56$ & 3 & $2-4$ & 16 & $12-21$ & 15 & $10-22$ \\
\hline Perinatal conditions & 502 & $447-567$ & 57 & $53-62$ & 164 & $153-177$ & 106 & $95-122$ \\
\hline Stomach cancer & 442 & $386-504$ & 101 & $89-114$ & 57 & $53-61$ & 18 & $16-20$ \\
\hline Colon and rectal cancers & 159 & $142-179$ & 96 & $87-106$ & 37 & $34-39$ & 10 & $9-11$ \\
\hline Trachea, bronchus, and lung cancers & 387 & $341-438$ & 165 & $148-187$ & 55 & $51-59$ & 20 & $18-22$ \\
\hline Breast cancer & 93 & 83-103 & 63 & $59-68$ & 37 & $34-40$ & 14 & $13-15$ \\
\hline Cervix uteri cancer & 47 & $42-52$ & 19 & $18-21$ & 26 & $24-29$ & 5 & $4-5$ \\
\hline Corpus uteri cancer & 8 & $7-9$ & 17 & $15-18$ & 12 & $11-12$ & 1 & $1-1$ \\
\hline Prostate cancer & 16 & $14-17$ & 25 & $23-29$ & 37 & $34-39$ & 6 & $5-7$ \\
\hline Lymphomas, multiple myeloma & 42 & $37-46$ & 23 & $21-24$ & 24 & $22-26$ & 12 & $11-13$ \\
\hline Leukemia & 76 & $68-86$ & 27 & $25-29$ & 22 & $21-24$ & 14 & $13-16$ \\
\hline Diabetes mellitus & 233 & $152-326$ & 51 & $45-59$ & 163 & $135-197$ & 31 & $21-44$ \\
\hline Alzheimer's and other dementias & 58 & $37-82$ & 10 & $8-11$ & 14 & $12-16$ & 3 & $2-5$ \\
\hline Parkinson's disease & 26 & $22-30$ & 4 & $3-4$ & 5 & $4-5$ & 3 & $2-3$ \\
\hline Drug use disorders & 7 & $5-11$ & 11 & $8-15$ & 2 & $2-3$ & 19 & $13-26$ \\
\hline Ischemic heart disease & 1,151 & $967-1,371$ & 1,685 & $1,473-1,928$ & 358 & $322-398$ & 323 & $276-382$ \\
\hline Cerebrovascular disease & 1,902 & $1,606-2,236$ & 1,029 & 888-1,189 & 267 & $240-298$ & 130 & $111-153$ \\
\hline $\begin{array}{l}\text { Chronic obstructive pulmonary } \\
\text { disease }\end{array}$ & 1,415 & $1,218-1,634$ & 130 & 119-143 & 99 & $92-109$ & 41 & $35-47$ \\
\hline Asthma & 56 & $41-74$ & 27 & $21-34$ & 12 & $10-14$ & 7 & $5-8$ \\
\hline Cirrhosis of the liver & 193 & $166-225$ & 103 & $94-115$ & 74 & $69-81$ & 37 & $33-43$ \\
\hline Nephritis and nephrosis & 186 & $160-217$ & 36 & $33-40$ & 55 & $50-61$ & 42 & $37-48$ \\
\hline Road traffic accidents & 361 & $334-394$ & 83 & $73-96$ & 88 & $83-93$ & 99 & $88-112$ \\
\hline Poisonings & 83 & $78-90$ & 106 & $90-127$ & 3 & $3-4$ & 7 & $7-8$ \\
\hline Falls & 122 & $114-132$ & 35 & $32-40$ & 15 & $14-16$ & 12 & $10-13$ \\
\hline Fires & 36 & $33-41$ & 20 & $16-24$ & 5 & $4-5$ & 13 & $12-15$ \\
\hline Drownings & 144 & 135-156 & 35 & $30-41$ & 19 & $18-20$ & 14 & $12-16$ \\
\hline Other unintentional injuries & 189 & $176-204$ & 121 & $106-140$ & 78 & $74-83$ & 36 & $33-41$ \\
\hline Self-inflicted injuries & 323 & $294-356$ & 121 & 105-141 & 30 & $28-32$ & 14 & $13-17$ \\
\hline Violence & 103 & 93-117 & 68 & $57-81$ & 130 & $123-138$ & 10 & $9-12$ \\
\hline War & 14 & $9-20$ & 17 & $13-23$ & 6 & $6-7$ & 8 & $5-12$ \\
\hline
\end{tabular}

Source: Authors' calculations. 
Table 5.5 Continued (thousands)

\begin{tabular}{|c|c|c|c|c|c|c|c|}
\hline \multicolumn{2}{|c|}{ South Asia } & \multicolumn{2}{|c|}{ Sub-Saharan Africa } & \multicolumn{2}{|c|}{ High-income countries } & \multicolumn{2}{|c|}{ World } \\
\hline Deaths & Uncertainty & Deaths & Uncertainty & Deaths & Uncertainty & Deaths & Uncertainty \\
\hline 13,557 & $13,053-14,240$ & 10,837 & $9,267-13,164$ & 7,891 & $7,830-7,963$ & 56,216 & $53,387-60,173$ \\
\hline 604 & $567-652$ & 317 & $258-400$ & 16 & $15-17$ & 1,605 & $1,476-1,771$ \\
\hline 272 & 255-292 & 2,058 & $1,802-2,367$ & 22 & $21-23$ & 2,573 & $2,325-2,872$ \\
\hline 695 & $628-757$ & 712 & 571-908 & 6 & $6-6$ & 1,782 & $1,557-2,065$ \\
\hline 108 & 90-130 & 176 & $134-233$ & 0 & $0-0$ & 301 & $243-382$ \\
\hline 3 & $3-3$ & 1 & $1-2$ & 0 & $0-0$ & 6 & $5-7$ \\
\hline 216 & 190-241 & 447 & $355-577$ & 1 & $1-2$ & 762 & $637-925$ \\
\hline 140 & 131-152 & 121 & $97-156$ & 0 & $0-0$ & 293 & 259-343 \\
\hline 71 & $66-79$ & 23 & $19-29$ & 4 & $4-5$ & 173 & 157-195 \\
\hline 28 & $26-31$ & 21 & $16-28$ & 5 & $5-6$ & 100 & 90-114 \\
\hline 11 & $10-12$ & 8 & $6-11$ & 12 & $11-12$ & 51 & $46-56$ \\
\hline 63 & $57-71$ & 1,093 & $841-1,465$ & 0 & $0-0$ & 1,207 & $941-1,596$ \\
\hline 0 & $0-0$ & 2 & 2-3 & 0 & $0-0$ & 14 & 13-16 \\
\hline 1,414 & $1,173-1,698$ & 1,080 & $833-1,419$ & 345 & 310-371 & 3,751 & $3,181-4,456$ \\
\hline 20 & 19-22 & 13 & 10-17 & 4 & 4-4 & 73 & 66-83 \\
\hline 199 & 158-252 & 237 & $158-341$ & 1 & $1-1$ & 508 & $381-676$ \\
\hline 1,086 & $985-1,215$ & 573 & $462-732$ & 32 & $31-34$ & 2,521 & $2,250-2,876$ \\
\hline 45 & 40-52 & 33 & $28-40$ & 146 & 135-157 & 842 & $773-917$ \\
\hline 35 & $31-40$ & 20 & $17-24$ & 257 & 238-276 & 614 & 579-648 \\
\hline 129 & $113-146$ & 15 & $13-17$ & 456 & $421-491$ & 1,227 & $1,152-1,302$ \\
\hline 76 & 67-85 & 34 & $28-43$ & 155 & 144-167 & 472 & $444-502$ \\
\hline 83 & $73-95$ & 38 & $32-46$ & 17 & 15-18 & 235 & 215-258 \\
\hline 4 & $4-5$ & 3 & $2-3$ & 27 & $25-29$ & 71 & $67-75$ \\
\hline 21 & $18-24$ & 40 & 33-48 & 119 & 110-128 & 264 & 248-282 \\
\hline 82 & 72-93 & 34 & $28-42$ & 115 & $106-124$ & 330 & 309-354 \\
\hline 38 & $33-43$ & 14 & $11-16$ & 73 & $67-79$ & 263 & 247-281 \\
\hline 196 & $127-273$ & 82 & 54-118 & 202 & $172-235$ & 959 & $744-1,207$ \\
\hline 81 & $52-113$ & 7 & $4-10$ & 207 & $175-241$ & 380 & $314-447$ \\
\hline 9 & $8-10$ & 5 & $4-6$ & 45 & $42-48$ & 95 & 88-104 \\
\hline 29 & $19-41$ & 4 & $2-6$ & 13 & $11-15$ & 85 & 64-109 \\
\hline 1,838 & $1,567-2,148$ & 343 & 260-458 & 1,364 & $1,203-1,533$ & 7,061 & $6,328-7,844$ \\
\hline 923 & $788-1,078$ & 355 & 269-474 & 781 & 689-874 & 5,388 & $4,790-6,067$ \\
\hline 577 & $502-662$ & 116 & 89-153 & 297 & $280-317$ & 2,675 & $2,370-3,030$ \\
\hline 78 & 57-101 & 26 & $19-35$ & 28 & $24-32$ & 233 & 186-287 \\
\hline 185 & $161-214$ & 59 & $45-79$ & 118 & 110-126 & 771 & 696-863 \\
\hline 132 & 114-152 & 101 & $77-135$ & 111 & $104-119$ & 662 & 586-758 \\
\hline 238 & $221-258$ & 200 & $159-261$ & 121 & 117-125 & 1,189 & $1,090-1,317$ \\
\hline 90 & $86-96$ & 37 & $29-50$ & 21 & $20-22$ & 349 & 324-381 \\
\hline 112 & $106-119$ & 20 & $16-26$ & 71 & $69-74$ & 387 & $368-412$ \\
\hline 183 & 173-194 & 44 & $35-58$ & 9 & $9-10$ & 310 & $287-339$ \\
\hline 90 & $85-96$ & 66 & $52-86$ & 16 & $16-17$ & 384 & $355-424$ \\
\hline 280 & 265-298 & 127 & 99-168 & 82 & $79-85$ & 913 & $847-1,003$ \\
\hline 224 & $206-245$ & 36 & $29-47$ & 126 & $121-131$ & 874 & $816-943$ \\
\hline 79 & $74-85$ & 141 & 114-181 & 24 & $23-25$ & 556 & $504-624$ \\
\hline 26 & $15-39$ & 136 & $54-221$ & 0 & $0-0$ & 207 & $114-308$ \\
\hline
\end{tabular}


limitations in currently available information, an examination of the contribution of uncertainty around health state valuations to overall uncertainty in burden of disease estimates measured using YLD or DALYs is useful.

Conceptually, the basis for assigning disability weights to specific sequelae requires an understanding of (a) the distribution of health states among those living with the particular sequelae, where a health state is defined by the levels on the various dimensions that constitute health; and (b) a valuation function that provides a systematic way to aggregate across multiple dimensions of health in order to arrive at a single index value that captures the overall level of health associated with a given health state (Salomon and others 2003). While disability weights may vary across regions because of variation in either component, we have proposed elsewhere that for purposes of standardization and global comparisons, computing disability weights based on an average global valuation function is the most appropriate approach (Murray and others 2002). The need to understand variation in the distribution of health states among people living with given sequelae highlights the critical link between the epidemiological inputs of burden and the estimated disability weights.

In this section, we undertake a first analysis of the contribution of uncertainty in disability weights to uncertainty in the GBD DALY estimates. Given that the current set of disability weights reflects the accumulation of a wide array of different empirical inputs rather than the result of the comprehensive and standardized approach defined earlier as the ideal, we operationalize our analysis of uncertainty in terms of error around the disability weights by sequelae rather than in terms of the uncertainty arising from the constituent components, that is, the health state distributions and the valuation function. Based on this approach, the results offer guidance on the sensitivity of burden estimates to a certain degree of uncertainty around disability weights, but do not necessarily capture all sources of uncertainty and their covariance. As noted earlier, certain specific measurement methods for eliciting health state valuations, for example, the person trade-off or standard gamble, may have important normative implications that are orthogonal to the assessment of health levels. However, undertaking a sensitivity analysis that focuses on a specific measurement approach is not appropriate here, because the weights currently used in the GBD estimates have been derived from the synthesis of multiple data sources rather than from a single measurement method.

Because of the natural constraints on the range of values that disability weights may assume, we have incorporated normal distributions with constant variance in the space of the logit of disability weights. The logit transformation is given by $\operatorname{logit}(x)=\ln [x /(1-x)]$. By allowing for normally distributed error in logit space, ranges in the natural space of valuations are constrained to fall between 0 and 1 . We chose a value of 0.6 for the standard deviation of the logits, based on the standard deviations observed across the mean valuations by country for an array of conditions included in the WHO multicountry survey study from 2000-1 (with valuation modules implemented in 14 countries) (Salomon and others 2003; Ustun and others 2003). Although the variability in country means may reflect a range of different factors, including the possibility of real valuation heterogeneity, we use this value to approximate the average level of uncertainty around the set of disability weights used in the GBD study. A constant value in logit space yields absolute ranges that widen at the midpoint of the interval and narrow as the disability weight approaches 0 or 1 (figure 5.15). In relative terms, the uncertainty is greatest for the smallest disability weights and narrows as more severe weights are attained (figure 5.16).

To trace the implications of this uncertainty through to the calculation of DALYs $(3,0)$ used in the DCPP, we took 100 draws from each of 622 independent normal distributions with a mean of 0 and a standard deviation of 0.6 (for the 622 sequelae included in the calculations). For each of the sequelae we applied a given sampled value as a perturbation of all age, sex, and region estimates of logit-transformed

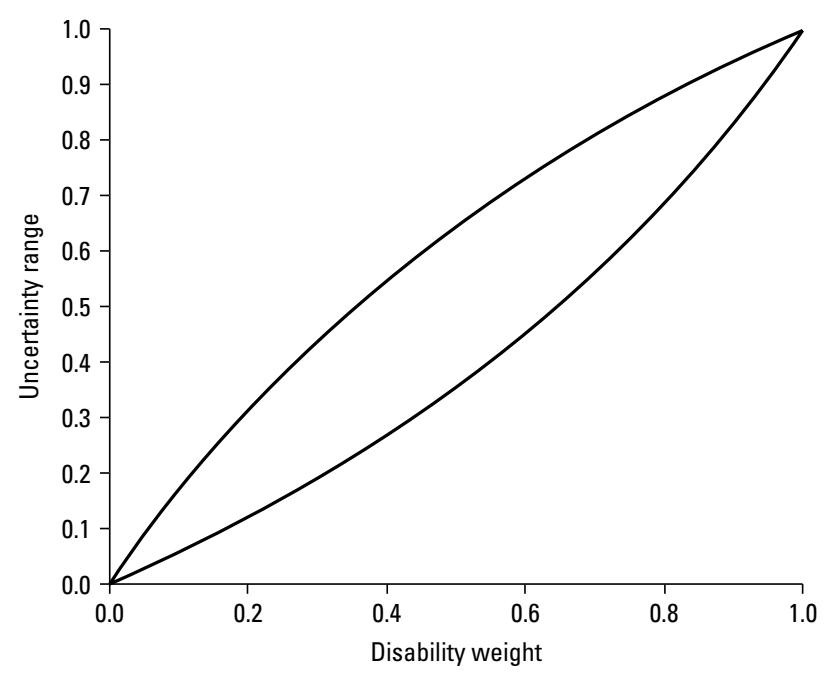

Source: Authors' calculations.

Figure 5.15 Assumed 95 Percent Uncertainty Ranges for Disability Weights Based on Constant Variance Distribution for Logit of Disability Weight 


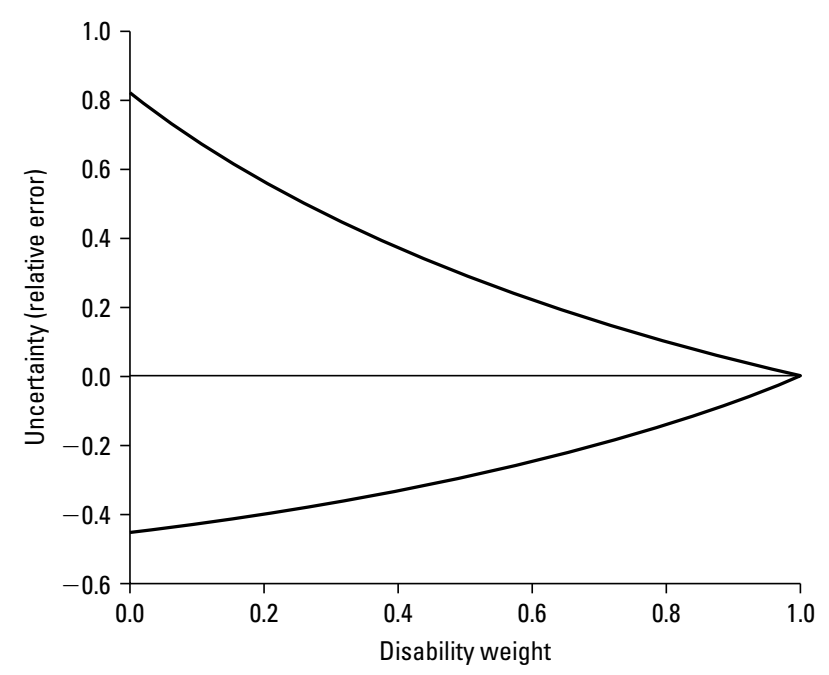

Source: Authors' calculations.

Figure 5.16 Relative 95 Percent Uncertainty Ranges for Disability Weights Based on the Assumption of a Constant Variance Distribution for Logit of Disability Weight across All Disability Weights

disability weights pertaining to that sequela, and recomputed $\operatorname{YLD}(3,0)$ based on the disability weight plus the random perturbation (after reversing the transformation for the sum). We estimated uncertainty ranges by taking the 2.5 th and 97.5th percentiles across the 100 values of the various quantities of interest based on the random draws of error around the disability weights. This method implies the simplifying assumption that errors are uncorrelated between sequelae but perfectly correlated for all estimates within a sequela. In addition to $\operatorname{YLD}(3,0)$ numbers, we recomputed YLD ranks resulting from each set of sampled values, and also calculated DALY numbers and ranks by adding each $\operatorname{YLD}(3,0)$ draw to constant $\operatorname{YLL}(3,0)$ estimates by sequela. Our intent is only to provide an indication of the sensitivity of the YLD and DALY results to disability weight uncertainty. We did not attempt either to carry out a full empirically based analysis of this issue or to combine this source of uncertainty with mortality uncertainty and uncertainty in epidemiological estimates to give a comprehensive uncertainty analysis for the DALY estimates.

Table 5.6 presents the resulting uncertainty ranges for YLD and DALYs by cause for low- and middle-income countries. As would be expected, DALY uncertainty ranges due to disability weight uncertainty are generally largest for those causes dominated by YLD and smallest for those causes dominated by YLL. Uncertainty ranges are also large for those YLD-dominated causes with high prevalence and low disability weight (with high relative uncertainty), such as hearing loss and anemia. Figure 5.17 summarizes in graphical form the uncertainty in total DALYs for low- and middle income countries for the 20 highest-ranked causes.

Table 5.7 presents the resulting 95 percent uncertainty ranges for the 40 leading causes of the burden of disease in low- and middle-income countries. Taking into account uncertainty in disability weights does not result in significant uncertainty in the ranking of the top four causes, with only the third (ischemic heart disease) and fourth (HIV/AIDS) possibly changing places. The total estimated DALYs for these two causes differ by less than 2 percent, so this is not surprising. Among the other top 10 causes, the disability weight uncertainty could change the rankings of individual causes by up to two ranks, with the exception of depressive disorders, which could change by up to four ranks. This reflects both the high relative uncertainty in the disability weight for mild depression and the fact that YLD are responsible for almost all depression DALYs. Among conditions ranked 20th to 30th in table 5.7, uncertainty ranges for most ranks are relatively narrow with the exception of nonfatal, high-prevalence conditions such as hearing loss and osteoarthritis, where the uncertainty in rank may be as much as \pm 15 places.

This analysis confirms the importance of efforts to improve the measurement of disability weights for health states close to full health, that is, with disability weights close to zero, particularly for health states with high prevalence in many populations, such as mild to moderate sense organ impairment or mild to moderate anemia. Unfortunately, most of the available choice-based or trade-off methods involving comparison in some form with death or survival present greater cognitive challenges to respondents when applied to health states close to full health.

\section{UNCERTAINTY ARISING FROM EPIDEMIOLOGICAL ESTIMATES}

Uncertainty in YLD estimates is mainly determined by the uncertainty in (a) epidemiological estimates for the prevalence and/or incidence of disability associated with specific causes or cause groups; and (b) disability weights arising from uncertainty in health state valuations and, in some cases, also in the disability severity distribution associated with a condition.

For a subset of the GBD causes, analysts carrying out reviews and analyses for the estimation of YLD also estimated 
Table 5.6 Estimated 95 Percent Uncertainty Ranges for YLD and DALYs Arising from Uncertainty in Disability Weights for Selected Causes for Low- and Middle-Income Countries, 2001 (thousands)

\begin{tabular}{|c|c|c|c|c|}
\hline Cause & $\operatorname{YLD}(3,0)$ & Uncertainty range & $\operatorname{DALYs}(3,0)$ & Uncertainty range \\
\hline \multicolumn{5}{|l|}{ Group I } \\
\hline Tuberculosis & 4,134 & $2,706-6,219$ & 35,882 & $34,400-37,900$ \\
\hline Syphilis & 407 & $310-574$ & 4,122 & $4,021-4,286$ \\
\hline Chlamydia & 2,255 & $1,766-3,073$ & 2,438 & $1,949-3,256$ \\
\hline Gonorrhea & 2,530 & $2,038-3,369$ & 2,550 & $2,058-3,390$ \\
\hline HIV/AIDS & 5,973 & $4,142-8,195$ & 70,857 & $68,900-73,000$ \\
\hline Diarrheal diseases & 7,836 & $4,236-12,900$ & 58,685 & $55,100-63,800$ \\
\hline Pertussis & 2,291 & $1,763-2,986$ & 11,408 & $10,900-12,100$ \\
\hline Poliomyelitis & 126 & 84-170 & 136 & 94-180 \\
\hline Diphtheria & 0 & $0-1$ & 164 & 164-164 \\
\hline Measles & 193 & $113-319$ & 23,097 & $23,000-23,200$ \\
\hline Tetanus & 14 & $10-16$ & 8,337 & $8,329-8,335$ \\
\hline Meningitis & 1,131 & $915-1,416$ & 5,477 & $5,255-5,756$ \\
\hline Hepatitis B & 52 & 28-96 & 2,082 & $2,056-2,124$ \\
\hline Hepatitis C & 21 & $11-39$ & 844 & $832-860$ \\
\hline Malaria & 4,501 & $3,521-5,672$ & 39,944 & $39,000-41,100$ \\
\hline Trypanosomiasis & 72 & 49-101 & 1,333 & $1,310-1,361$ \\
\hline Chagas' disease & 358 & $275-501$ & 584 & $500-727$ \\
\hline Schistosomiasis & 1,313 & $727-2,563$ & 1,525 & $938-2,774$ \\
\hline Leishmaniasis & 411 & $291-610$ & 1,757 & $1,636-1,955$ \\
\hline Lymphatic filariasis & 4,446 & $3,365-6,947$ & 4,455 & $3,374-6,956$ \\
\hline Onchocerciasis & 439 & $361-541$ & 439 & $361-541$ \\
\hline Leprosy & 93 & $56-142$ & 191 & $154-239$ \\
\hline Dengue & 5 & $3-10$ & 529 & $526-533$ \\
\hline Japanese encephalitis & 231 & $187-276$ & 598 & $554-644$ \\
\hline Trachoma & 2,618 & $2,023-3,192$ & 2,621 & $2,025-3,195$ \\
\hline Ascariasis & 1,311 & $707-2,190$ & 1,413 & $808-2,291$ \\
\hline Trichuriasis & 713 & $518-1,000$ & 800 & $604-1,087$ \\
\hline Hookworm disease & 7 & 4-13 & 63 & $60-69$ \\
\hline Lower respiratory infections & 4,430 & $3,128-6,525$ & 83,579 & $82,300-85,700$ \\
\hline Upper respiratory infections & 181 & 108-318 & 1,680 & $1,609-1,819$ \\
\hline Otitis media & 1,336 & $811-2,136$ & 1,424 & $899-2,224$ \\
\hline Maternal hemorrhage & 232 & $61-162$ & 3,923 & $3,750-3,851$ \\
\hline Maternal sepsis & 3,290 & $827-2,048$ & 5,269 & $2,804-4,025$ \\
\hline Hypertensive disorders & - & $0-0$ & 1,890 & $1,888-1,888$ \\
\hline Obstructed labor & 1,349 & $842-1,477$ & 2,495 & $1,988-2,622$ \\
\hline Abortion & 1,732 & $1,034-2,344$ & 3,503 & $2,803-4,112$ \\
\hline Perinatal causes & 13,525 & $10,300-18,100$ & 89,121 & $85,900-93,800$ \\
\hline Protein-energy malnutrition & 9,337 & $6,616-14,300$ & 15,450 & $12,700-20,400$ \\
\hline lodine deficiency & 2,685 & $1,617-2,206$ & 2,875 & $1,807-2,396$ \\
\hline Vitamin A deficiency & 58 & $34-88$ & 711 & $685-740$ \\
\hline Iron-deficiency anemia & 6,736 & $4,782-10,300$ & 9,488 & $7,530-13,000$ \\
\hline \multicolumn{5}{|l|}{ Group II } \\
\hline Mouth and oropharynx cancers & 107 & $80-127$ & 4,079 & $4,049-4,097$ \\
\hline Esophageal cancer & 42 & $29-56$ & 5,251 & $5,235-5,262$ \\
\hline Stomach cancer & 124 & $95-160$ & 9,613 & $9,577-9,643$ \\
\hline Colon and rectal cancers & 241 & 179-315 & 5,058 & $4,993-5,128$ \\
\hline Liver cancer & 49 & $37-63$ & 7,943 & $7,926-7,952$ \\
\hline Pancreas cancer & 18 & $16-19$ & 1,621 & $1,617-1,620$ \\
\hline Trachea, bronchus, and lung cancers & 137 & $117-155$ & 10,697 & $10,700-10,700$ \\
\hline Melanoma and other skin cancers & 10 & 6-15 & 501 & 497-505 \\
\hline Breast cancer & 308 & 226-386 & 5,527 & $5,440-5,600$ \\
\hline Cervix uteri cancer & 205 & 140-282 & 3,800 & $3,732-3,875$ \\
\hline Corpus uteri cancer & 276 & $200-416$ & 908 & $831-1,046$ \\
\hline Ovarian cancer & 98 & $71-138$ & 1,488 & $1,460-1,527$ \\
\hline
\end{tabular}


Table 5.6 Continued

(thousands)

\begin{tabular}{|c|c|c|c|c|}
\hline Cause & YLD(3,0) & Uncertainty range & DALYs(3,0) & Uncertainty range \\
\hline Prostate cancer & 91 & 63-109 & 1,479 & $1,448-1,494$ \\
\hline Bladder cancer & 104 & $76-134$ & 1,504 & $1,474-1,532$ \\
\hline Lymphomas, multiple myeloma & 69 & 49-98 & 3,770 & $3,746-3,795$ \\
\hline Leukemia & 58 & $33-86$ & 3,964 & $3,936-3,989$ \\
\hline Diabetes mellitus & 5,662 & $4,229-7,736$ & 15,806 & $14,400-17,900$ \\
\hline Endocrine disorders & 7,581 & $4,447-12,700$ & 10,947 & $7,814-16,100$ \\
\hline Unipolar depressive disorders & 43,223 & $30,400-53,600$ & 43,429 & $30,600-53,800$ \\
\hline Bipolar affective disorder & 8,676 & $5,636-12,100$ & 8,681 & $5,642-12,100$ \\
\hline Schizophrenia & 10,156 & $7,419-12,800$ & 10,530 & $7,793-13,200$ \\
\hline Epilepsy & 2,942 & $1,541-5,758$ & 5,759 & $4,356-8,573$ \\
\hline Alcohol use disorders & 9,808 & $6,086-15,700$ & 11,009 & $7,286-16,900$ \\
\hline Alzheimer's and other dementias & 8,172 & $6,690-9,790$ & 9,641 & $8,158-11,300$ \\
\hline Parkinson's disease & 767 & $513-1,085$ & 1,239 & $984-1,557$ \\
\hline Multiple sclerosis & 770 & $501-1,039$ & 916 & $647-1,185$ \\
\hline Drug use disorders & 2,736 & $1,693-3,825$ & 4,406 & $3,361-5,493$ \\
\hline Post-traumatic stress disorder & 2,013 & $1,217-3,918$ & 2,013 & $1,218-3,919$ \\
\hline Obsessive-compulsive disorder & 3,136 & $1,726-5,532$ & 3,136 & $1,726-5,532$ \\
\hline Panic disorder & 4,017 & $2,530-6,052$ & 4,017 & $2,530-6,052$ \\
\hline Insomnia (primary) & 2,219 & $1,314-3,883$ & 2,219 & $1,314-3,883$ \\
\hline Migraine & 4,851 & $2,720-7,503$ & 4,851 & $2,720-7,503$ \\
\hline Mental retardation, lead-caused & 8,474 & $5,358-12,100$ & 8,601 & $5,484-12,300$ \\
\hline Glaucoma & 4,110 & $2,986-5,393$ & 4,111 & $2,987-5,395$ \\
\hline Cataracts & 28,155 & $21,500-37,100$ & 28,155 & $21,500-37,100$ \\
\hline Vision disorders, age-related & 15,360 & $10,900-19,400$ & 15,360 & $10,900-19,400$ \\
\hline Hearing loss, adult onset & 24,610 & $14,000-43,800$ & 24,610 & $14,000-43,800$ \\
\hline Rheumatic heart disease & 607 & 404-863 & 6,152 & $5,945-6,404$ \\
\hline Hypertensive heart disease & 888 & $542-1,358$ & 9,969 & $9,612-10,400$ \\
\hline Ischemic heart disease & 3,921 & $2,525-5,369$ & 71,874 & $70,400-73,300$ \\
\hline Cerebrovascular disease & 11,096 & $7,209-17,100$ & 62,652 & $58,700-68,600$ \\
\hline Inflammatory heart diseases & 1,309 & $765-1,908$ & 5,812 & $5,263-6,406$ \\
\hline Chronic obstructive pulmonary disease & 8,473 & $5,670-12,400$ & 33,457 & $30,600-37,300$ \\
\hline Asthma & 7,713 & $4,479-13,600$ & 11,513 & $8,277-17,400$ \\
\hline Peptic ulcer disease & 1,154 & $556-1,737$ & 4,802 & $4,203-5,383$ \\
\hline Cirrhosis of the liver & 2,329 & $1,391-3,289$ & 13,635 & $12,700-14,600$ \\
\hline Appendicitis & 60 & $42-81$ & 377 & 358-397 \\
\hline Nephritis and nephrosis & 546 & 288-869 & 9,078 & $8,811-9,392$ \\
\hline Benign prostatic hypertrophy & 2,304 & $1,229-3,999$ & 2,613 & $1,538-4,308$ \\
\hline Skin diseases & 2,924 & $1,764-4,425$ & 3,697 & $2,535-5,197$ \\
\hline Rheumatoid arthritis & 3,433 & $2,132-5,436$ & 3,645 & $2,344-5,648$ \\
\hline Osteoarthritis & 13,651 & $8,636-22,400$ & 13,667 & $8,652-22,400$ \\
\hline Gout & 2,768 & $1,697-4,053$ & 2,785 & $1,714-4,070$ \\
\hline Low back pain & 1,676 & $1,093-2,670$ & 1,692 & $1,109-2,685$ \\
\hline Congenital anomalies & 9,295 & $7,047-11,700$ & 23,538 & $21,300-26,000$ \\
\hline Dental caries & 4,752 & $2,771-8,429$ & 4,752 & $2,771-8,429$ \\
\hline Periodontal disease & 206 & 124-368 & 207 & $125-369$ \\
\hline Edentulism & 2,293 & $1,349-3,476$ & 2,293 & $1,349-3,476$ \\
\hline \multicolumn{5}{|l|}{ Group III } \\
\hline Road traffic accidents & 7,195 & $6,489-8,063$ & 32,022 & $31,300-32,900$ \\
\hline Poisonings & 135 & 107-170 & 7,119 & $7,088-7,151$ \\
\hline Falls & 8,055 & $7,035-9,203$ & 13,582 & $12,600-14,700$ \\
\hline Fires & 2,719 & $2,199-3,286$ & 10,081 & $9,557-10,600$ \\
\hline Drownings & 37 & 33-41 & 9,389 & $9,379-9,387$ \\
\hline Self-inflicted injuries & 1,236 & $1,040-1,489$ & 17,677 & $17,500-17,900$ \\
\hline Violence & 5,405 & $4,734-6,420$ & 18,135 & $17,500-19,100$ \\
\hline War & 1,569 & $1,321-1,887$ & 6,496 & $6,240-6,806$ \\
\hline
\end{tabular}

Source: Authors' calculations. 


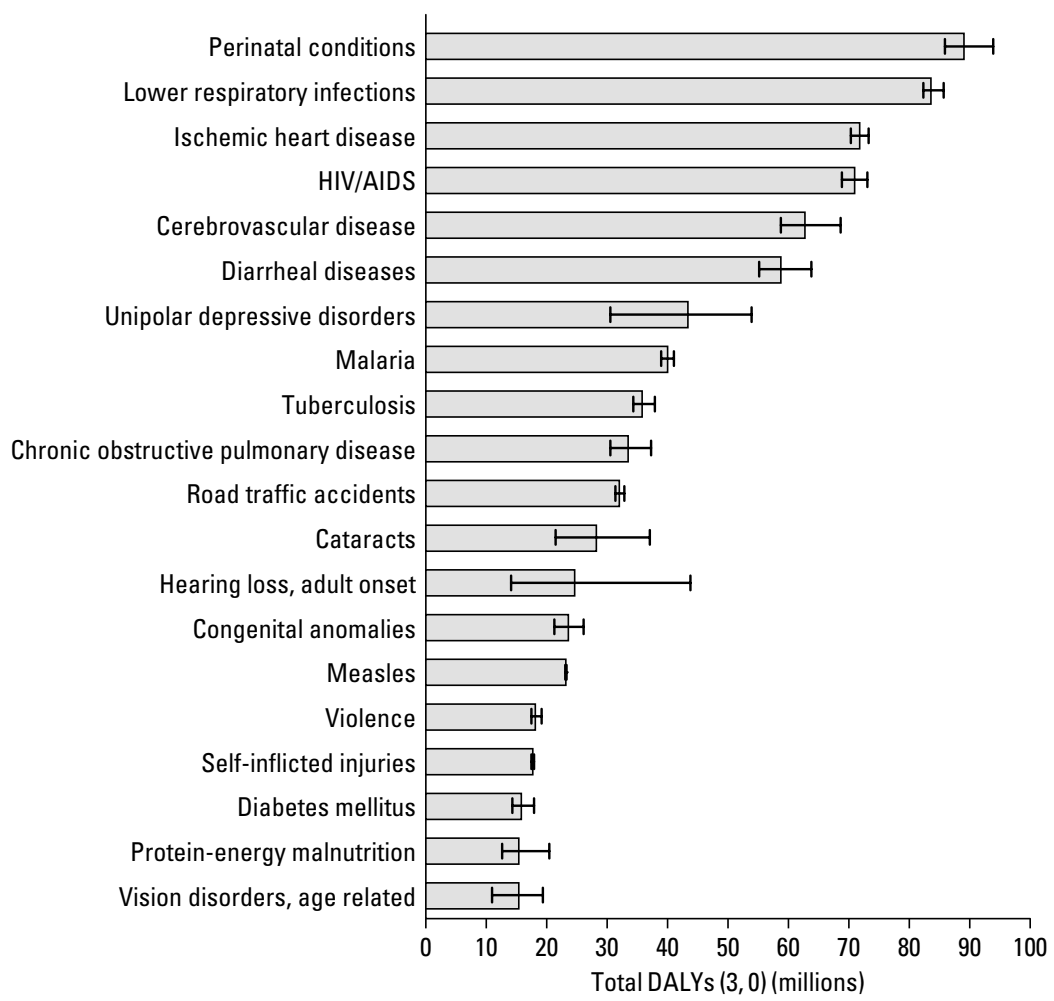

Source: Authors' calculations.

Figure 5.17 Estimated 95 Percent Uncertainty in Total DALYs $(3,0)$ Due to Uncertainty in Estimation of Disability Weights, Top 20 Causes, Low- and Middle-Income Countries

levels of uncertainty in regional prevalences. These assessments took into account not only typical levels of measurement error in the input data sets, but also expert judgment on the degree of uncertainty arising from the lack of representativeness of the available data for each region. The resulting uncertainty ranges vary considerably across causes, ranging from relatively certain estimates for some causes such as polio, for which intensive surveillance systems are in place, to highly uncertain estimates for other causes such as osteoarthritis, where for some regions not a single usable dataset was found, and where for others the latest available data were decades old. The summary tables provided in chapter 3 for numbers of data sources used for YLD estimates by cause and region provide one indication of the relative uncertainty associated with YLD estimates for different causes.

For some causes, such as stroke and ischemic heart disease, YLD estimates were essentially derived from estimates of cause-specific mortality by means of models of regional variations in case fatality rates. In such cases, YLD uncertainty will be significantly higher than the uncertainty associated with cause-specific mortality estimates given the considerable uncertainty in case fatality rates for most lowand middle-income countries and in models used to infer the burden of nonfatal disease from mortality. YLD uncertainty will generally be greater than YLL uncertainty, and will also vary across causes according to both the typical uncertainty associated with the measurement of incidence or prevalence according to GBD case definitions and with the number and representativeness of available studies. For a subset of 16 major causes of YLD for which analysts estimated indicative uncertainty ranges, the typical uncertainty for regional prevalence estimates ranged from \pm 10 percent to \pm 90 percent, with a median value of \pm 41 percent. Uncertainty ranges were generally higher for low- and middle-income countries than for high-income countries.

\section{UNCERTAINTY IN THE DISEASE BURDEN ATTRIBUTABLE TO RISK FACTORS}

The assessments of the disease burden attributable to selected risk factors reported in chapter 4 are affected by 
Table 5.7 Estimated 95 Percent Uncertainty Ranges Arising from Uncertainty in Disability Weights for the Top 40 Causes of the Burden of Disease in Low- and Middle-Income Countries, 2001

\begin{tabular}{|c|c|c|c|c|}
\hline Rank & Uncertainty range & Cause & $\begin{array}{c}\text { DALYs } \\
\text { (thousands) }\end{array}$ & Uncertainty range \\
\hline 1 & $1-1$ & Perinatal conditions & 89,121 & $85,900-93,800$ \\
\hline 2 & $2-2$ & Lower respiratory infections & 83,579 & $82,300-85,700$ \\
\hline 3 & $3-4$ & Ischemic heart disease & 71,874 & $70,400-73,300$ \\
\hline 4 & $3-4$ & HIV/AIDS & 70,857 & $68,900-73,000$ \\
\hline 5 & $5-6$ & Cerebrovascular disease & 62,652 & $58,700-68,600$ \\
\hline 6 & $5-6$ & Diarrheal diseases & 58,685 & $55,100-63,800$ \\
\hline 7 & $7-11$ & Unipolar depressive disorders & 43,429 & $30,600-53,800$ \\
\hline 8 & $7-9$ & Malaria & 39,944 & $39,000-41,100$ \\
\hline 9 & $8-10$ & Tuberculosis & 35,882 & $34,400-37,900$ \\
\hline 10 & $9-12$ & Chronic obstructive pulmonary disease & 33,457 & $30,600-37,300$ \\
\hline 11 & $10-13$ & Road traffic accidents & 32,022 & $31,300-32,900$ \\
\hline 12 & $9-14$ & Cataracts & 28,155 & $21,500-37,100$ \\
\hline 13 & $8-21$ & Hearing loss, adult onset & 24,610 & $14,000-43,800$ \\
\hline 14 & $12-15$ & Congenital anomalies & 23,538 & $21,300-26,000$ \\
\hline 15 & $13-15$ & Measles & 23,097 & $23,000-23,200$ \\
\hline 16 & $15-18$ & Violence & 18,135 & $17,500-19,100$ \\
\hline 17 & $16-19$ & Self-inflicted injuries & 17,677 & $17,500-17,900$ \\
\hline 18 & $17-22$ & Diabetes mellitus & 15,806 & $14,400-17,900$ \\
\hline 19 & $15-25$ & Protein-energy malnutrition & 15,450 & $12,700-20,400$ \\
\hline 20 & $16-27$ & Vision disorders, age-related & 15,360 & $10,900-19,400$ \\
\hline 21 & $16-36$ & Osteoarthritis & 13,667 & $8,652-22,400$ \\
\hline 22 & $20-25$ & Cirrhosis of the liver & 13,635 & $12,700-14,600$ \\
\hline 23 & $20-24$ & Falls & 13,582 & $12,600-14,700$ \\
\hline 24 & $18-38$ & Asthma & 11,513 & $8,277-17,400$ \\
\hline 25 & $23-29$ & Pertussis & 11,408 & $10,900-12,100$ \\
\hline 26 & $19-40$ & Alcohol use disorders & 11,009 & $7,286-16,900$ \\
\hline 27 & $18-40$ & Endocrine disorders & 10,947 & $7,814-16,100$ \\
\hline 28 & $25-31$ & Trachea, bronchus, and lung cancers & 10,697 & $10,700-10,700$ \\
\hline 29 & $22-39$ & Schizophrenia & 10,530 & 7,793-13,200 \\
\hline 30 & $27-34$ & Fires & 10,081 & $9,557-10,600$ \\
\hline 31 & $27-34$ & Hypertensive heart disease & 9,969 & $9,612-10,400$ \\
\hline 32 & $26-38$ & Alzheimer's and other dementias & 9,641 & $8,158-11,300$ \\
\hline 33 & $29-36$ & Stomach cancer & 9,613 & $9,577-9,643$ \\
\hline 34 & $25-40$ & Iron-deficiency anemia & 9,488 & $7,530-13,000$ \\
\hline 35 & $31-37$ & Drownings & 9,389 & $9,379-9,387$ \\
\hline 36 & $33-38$ & Nephritis and nephrosis & 9,078 & $8,811-9,392$ \\
\hline 37 & $27-46$ & Bipolar affective disorder & 8,681 & $5,642-12,100$ \\
\hline 38 & $24-47$ & Mental retardation, lead-caused & 8,601 & $5,484-12,300$ \\
\hline 39 & $36-39$ & Tetanus & 8,337 & $8,329-8,335$ \\
\hline 40 & $37-41$ & Liver cancer & 7,943 & $7,926-7,952$ \\
\hline
\end{tabular}

Source: Authors' calculations.

additional sources of uncertainty, beyond the uncertainty in DALY estimates for specific disease and injury outcomes discussed earlier. A full uncertainty analysis of such burden estimates has not yet been carried out, but would involve assessment of the following additional types of uncertainty:

- uncertainty in the estimated distributions of population risk exposure;

- uncertainty in estimates of relative risks for cause-specific mortality and incidence associated with specific expo- sures, for which a significant source of uncertainty is the extrapolation of relative risks measured at other ages to older age groups;

- uncertainty associated with estimating joint effects of risk factors.

Uncertainty in exposure and in both the existence and magnitude of hazardous effect always affects quantitative risk assessment. In one taxonomy, risk assessment uncertainty can be divided into parameter uncertainty and model uncertainty 
(National Research Council 1994). Parameter uncertainty is often quantifiable using random variable methods, for example, uncertainty due to sample size or measurement error. Model uncertainty is due to gaps in scientific theory, measurement technology, and data. It includes uncertainty in causal relationships or the form of the exposure-response relationship (for instance, threshold versus continuous or linear versus nonlinear), the level of bias in measurement, and so on. Defined broadly, model uncertainty also includes extrapolation of exposure or hazard from one population to another. Model uncertainty dominates uncertainty in risk assessment, a result of difficulty in carrying out direct studies on exposure, hazard, and background disease burden. This has motivated innovative assumptions and extrapolations even in the case of the most widely studied risk factors like smoking (Peto and others 1992).

Uncertainty around disease causation (Evans 1978; Hill 1965) was, in practice, secondary to uncertainty around hazard size, for example, relative risk, because when causality was uncertain, the estimates of relative risk needed for risk assessment were also unknown or uncertain. For example, whether the relationships between physical inactivity and lower back pain or between alcohol and violence are causal has equivalent questions on the magnitude of hazard of each risk for the disease outcome. Collective scientific knowledge from disciplines such as social and behavioral sciences, physiology and neuroscience, and epidemiology would confirm the possibility of a causal relationship in the foregoing cases, but would shift the uncertainty to hazard size. As a result, for some risk factors, we could only quantify the contribution to a subset of disease outcomes because epidemiological studies did not provide enough information for all risk factor and disease pairs, even when the causal relationship was believed or suspected.

Estimates of hazard in individual epidemiological studies were adjusted for confounding as much as possible. Extrapolation of hazard from a limited number of studies to other populations has received less attention. While the robustness of proportional measures of risk has been confirmed for more proximal factors in studies across populations (Eastern Stroke and Coronary Heart Disease Collaborative Research Group 1998; Horton 2000; Law, Wald, and Thompson 1994), their extrapolation is an important source of uncertainty for more distal risks (such as childhood sexual abuse) or those whose effects are heterogeneous (for example, alcohol and injuries versus alcohol and cancer).

Direct exposure data for many risk factors are limited both because of measurement difficulties and because of underinvestment in risk factor surveillance. To allow maximum use of available data, such risk factors were represented using indirect or aggregate indicators, for instance, smoking impact ratio for accumulated hazards of smoking, weight-for-age for childhood undernutrition, and use of solid fuels for indoor air pollution. Furthermore, for some risks multiple data sources allowed limiting the range of exposure estimates. For example, in the absence of alcohol surveys, information on total alcohol production, trade, and unrecorded consumption provided upper bounds on the fraction of the population that would be in the highest consumption category. Finally, some of the risk factors examined in chapter 4 were represented using continuous exposure variables such as high blood pressure. Others used categorical variables, for example, indoor smoke from household use of solid fuels, childhood underweight, and physical inactivity, even though the health effects occur along a continuum. This choice reflected the availability of exposure data and hazard estimates in categories. In such cases, the contribution to disease within the baseline category would not have been captured.

In addition to uncertainty in exposure and hazard, the uncertainty of estimated population attributable fractions (PAFs) is determined by the analytical properties of the PAF relationship. In particular, the PAF relationship is an increasing concave function of relative risk and exposure level, approaching 100 percent asymptotically, that is, the rate of increase declines with increasing relative risk or prevalence (figure 5.18). Therefore, if a risk factor or group of risk factors individually or jointly account for large

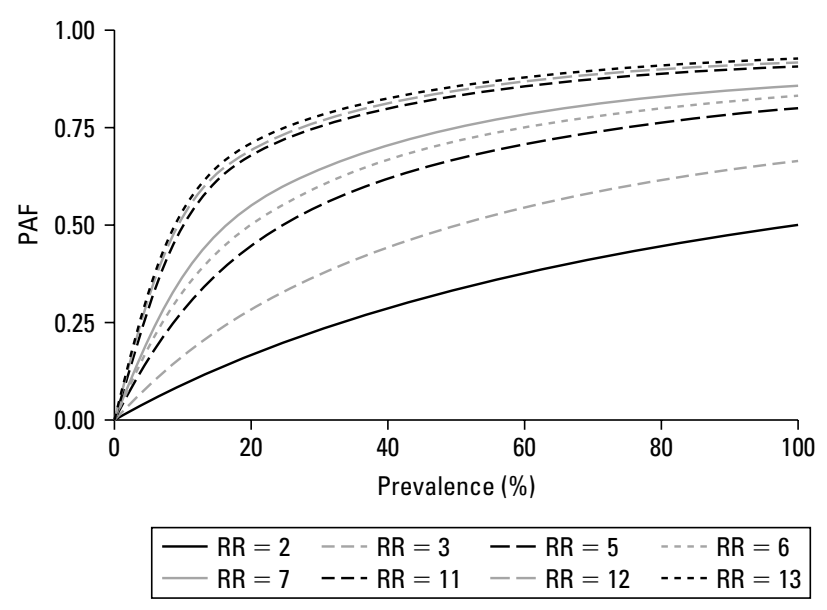

Source: Authors' calculations.

Note: The population attributable fraction (PAF) relationship is an increasing concave function of both prevalence (seen in the shape of each curve) and relative risk, $R R$ (seen in the declining distance between each adjacent pair of curves). This limits the sensitivity of individual or joint PAFs to uncertainty in input parameters, when PAFs are relatively large.

Figure 5.18 PAF Sensitivity to Exposure and Relative Risks 
fractions of specific diseases, the PAFs are more robust to uncertainty in inputs. Finally, there is uncertainty in mortality and disease burden estimates to which the estimated PAF are applied (see the previous section).

The findings in chapter 4 should therefore be considered within the context of limited available data and viewed as subject to uncertainty, which varies across risk factors and geographical regions. For further discussion of sources and quantification of uncertainty for specific risk factors see Ezzati and others (2004).

\section{DISCUSSION}

As described in chapter 3, the data requirements for adequate measurements of the global burden of disease are substantial and include information about age at death, cause of death, age-specific incidence of diseases and injuries, typical duration of life lived with the sequelae of diseases and injuries, and some quantification of the severity of disability assessed according to a common framework. While the ethical, philosophical, and conceptual issues involved in quantifying states of health other than perfect health are still very much a matter of debate, a substantial body of empirical evidence on the variations across individuals and populations in health state valuations is now available.

We have shown in this chapter that the distribution of the global burden of disease and the overall rankings of various conditions in terms of their contribution to it are largely insensitive to alternative assumptions about the discount rate and age weighting. The major effect of discounting and age weighting is to enhance the importance of neuropsychiatric conditions and sexually transmitted infections. While disease rankings are relatively unaffected, the share of the burden due to disability, the age distribution of the burden, and the distribution of the burden by broad cause group are sensitive to the discount rate but less affected by age weighting.

When compared with the discounted and age-weighted DALY used in the 1990 GBD study and the WHO updates for 2000-2, the DCPP's use of discounted but not age-weighted DALYs results in somewhat more weight being given to the chronic diseases of older ages and somewhat less weight being given to mental disorders and injuries, which affect younger adults disproportionately. Of the value choices incorporated into the standard $\operatorname{DALYs}(3,1)$, the nonuniform age weights have been the most controversial. Apart from the DCPP, a number of national burden of disease studies, including those in Australia and Canada (Mathers, Vos, and Stevenson 1999; Public Health Agency of Canada 2005), have chosen not to apply the nonuniform age weights, presumably on equity grounds. In contrast, some investigators concerned with the inequitable health burden of the low- and middleincome countries have argued for ignoring all deaths over a certain age on the grounds that they are not premature-an extreme form of age weighting (Williams 1997). Chapter 6 presents some empirical evidence in making the case for a stronger form of age weighting for infants and younger children, that is, age weights that depart further from unity than the standard age weighting used in the DALY.

Although the choices for discounting and age weighting do affect the cause and age distributions of the burden of disease to some extent, and the results of specific costeffectiveness studies may be even more sensitive to these choices, we conclude that the uncertainty of the underlying epidemiological choices is vastly more consequential than these social preferences when interpreting the results of burden of disease analysis. The validity and reliability, and hence the utility, of burden of disease studies for public policy depend much more strongly on the quality and availability of the underlying epidemiological data.

The GBD study has been criticized for making estimates of mortality and burden of disease for regions with limited, incomplete, and uncertain data (Cooper and others 1998; Gupta, Sankaranarayanan, and Ferlay 1994). Murray and Lopez describe the GBD approach as a “'meta-synthesis,' or in other words, the construction of a comprehensive and comparable view of health problems using all available sources of information" (Murray and Lopez 1996b, p. 289). The incorporation of many types of information about a comprehensive set of causes of death and disability results in estimates that are much less likely to be biased than those that emerge from an examination of specific health conditions in isolation. It also avoids the tendency to assume that if no data are available or the data are highly uncertain, then there is no disease burden.

We argue that including uncertain results (with quantified uncertainty to the extent possible) is far preferable than leaving blank cells in tables intended to provide policy makers with an overall assessment of the burden of disease in populations. We maintain that providing large volumes of unsynthesized, biased, and incomplete data relating to population health does not generally allow policy makers to make the best use of such information. Unless they have considerable analytic resources of their own, the unsynthesized products of the research enterprise are of little help to decision makers, who will often then resort to decisions on the basis of ideology, of their own beliefs about what is important, or of political imperatives. 
The quantities of interest for the GBD study are the underlying rates of incidence, remission, and mortality for defined causes for whole populations for a specified time period, and the assessment of these often requires synthesizing data from multiple studies or making adjustments for biases in relation to population, age groups, or time periods. A major source of uncertainty for the GBD estimates is the uncertainty associated with extrapolating from one or more subgroups to a regional population. For example, how representative of the incidence and prevalence patterns of dementia in Sub-Saharan Africa are two or three population-representative studies of rural or urban populations in specific regions of specific countries? The uncertainty associated with extrapolating from a set of studies in subpopulations to the regional population is related to potential systematic (selection) biases and is much more difficult to quantify than the uncertainty associated with stochastic variation due to sample size or measurement error.

Estimates of deaths from specific causes undergo continual revision as new data and syntheses become available, yet drawing a time cutoff is a necessary (if somewhat arbitrary) condition for preparing any volume such as this which reports comprehensive and consistent global and regional estimates of deaths and burden of disease (see also annex 6C). During 2001 WHO established the Child Health Epidemiology Reference Group (CHERG) to review and synthesise data on cause of deaths under age 5. While early CHERG results contributed substantially to the GBD analyses in this volume, much of their work became available well after the cutoff date for this publication. While CHERG has published revised estimates of the distribution of child deaths by cause (Bryce and others 2005), based on recent comprehensive reviews of epidemiological data, these analyses used cause categories not consistent with the GBD (including use of incompatible cause categories for neonatal and other child deaths), fewer cause categories than the GBD, and left study deaths assigned to ill-defined categories in the 'Other' category. Additionally, at the date of writing, the CHERG evidence has not been brought into the GBD analytic and consistency framework, involving consistent mapping to causal categories and checking of internal consistency between incidence, prevalence and mortality estimates for specific causes.

To the extent that they can be compared with the GBD 2001 estimates, the WHO/CHERG estimates at the global level are differ substantially for tetanus (46\% higher), lower respiratory infections (56\% higher), and are somewhat lower for measles, malaria, low birthweight and noncommunicable diseases. It is not possible at this stage, to con- clude whether or how much the WHO/CHERG analyses would modify the GBD 2001 results reported in this volume, when they are properly brought into the GBD analytic framework. However, they do give some indications that new evidence is becoming available for child deaths, and that uncertainty ranges for GBD estimates of child deaths may be greater for some causes than indicated by the analyses presented in this chapter.

The 1990 GBD study and GBD 2001 were both metasyntheses of the available data, using the best models and tools available at the time, whose primary aims were to provide a comprehensive assessment of the current burden of disease. The assessment of trends between 1990 and 2001 is a much more difficult task, as discussed in chapter 2. The comparability of best point in time estimates is difficult to assess given changes in both the availability of data and in the methods used to synthesize those data for many of the causes. Murray, Mathers, and Salomon (2003) discuss this issue in more detail and conclude that to assess change or evaluate programs, extrapolating current levels of burden of disease from past measurements is inadequate, and that the assessment must include measurements carried out at both points in time or explicit measurement of the relevant trends or rates of change.

\section{CONCLUSIONS}

The 2001 GBD study uses a summary measure of population health, the DALY, that explicitly incorporates several important social values. This has the advantage that the effects of changing preferences can be readily explored through sensitivity analysis, as illustrated in this chapter. Another advantage of the burden of disease approach is that it entails a data audit, whereby the completeness, reliability, and consistency of routinely collected data are assessed and critical gaps in health data collection are identified. One implication is that periodic quality assessments of, say, routine cause of death data are needed to ensure their continued relevance and reliability for public policy (Mathers and others 2005). Another is the need for a more rational assessment of priority data for the health care sector that places greater emphasis on data collection and data linkage to facilitate burden of disease studies rather than on routine collection of statistics of limited relevance to public health. The burden of disease framework, based on the estimated distribution and duration of health states resulting from incident cases, would benefit greatly from wider availability of linked data sets on health outcomes and further longitudinal 
research into health state transition probabilities following on from specific disease or injury causes (Kelman and Bass 2002).

A major advance with GBD 2001 has been the systematic, though as yet incomplete, attempt to quantify uncertainty in both national and global assessments of the disease burden. This uncertainty must be taken into account when making cross-national comparisons, and needs to be carefully communicated and interpreted by epidemiologists and policy makers alike. Estimates of mortality in countries without functioning vital registration systems for causes of death will always be substantially more uncertain than those derived from systems where all deaths are registered and medically certified. The same may be said for the quantification of disability due to various conditions, where the gaps in data availability across countries are likely to be even more extreme than for mortality.

Despite the progress of the past decade, the incremental gains in advancing knowledge and understanding of global descriptive epidemiology have been modest. A globally coordinated research and development effort is urgently needed to devise and implement cost-effective approaches to data collection and analysis in poor countries that are targeted to their health development needs, and that can routinely yield comparable information of sufficient quality to establish how the disease and risk factor burden is changing in populations (Murray, Lopez, and Wibulpolprasert 2004).

Much can be learned about the health of populations from relatively modest investments in sample registration systems, provided these are designed to reliably measure the causes of death in sample areas and have sufficient resources to do so. China's Disease Surveillance Points system is a good example of what can be done to improve knowledge about disease and injury control priorities in low-income countries at a modest cost (Lopez 1998; Yang and others 2005). Greater investments in getting the descriptive epidemiology of diseases and injuries correct in poor countries will do vastly more to reduce uncertainty in disease burden assessments than philosophical debate about the appropriateness of social value choices. Just as the production of global and regional estimates should not create the impression that the descriptive epidemiology of disease and injury is reliably known, so the uncertainties around these estimates must not create the impression that not enough is known reliably enough to usefully inform health priorities and programs. Health intelligence is an essential ingredient of the health development process. Those engaged in collecting, analyzing, and disseminating population health information have a responsibility to develop this evidence base using novel methods that communicate what we do know, as well, if not more convincingly, than what we do not know.

Information for policy purposes will never be perfect, but good policy makers will want to benefit from all available information to guide priority setting and action. We might well take solace in the comments of a prominent medical statistician who once cautioned that "Making the best the enemy of the good is a sure way to hinder any statistical progress. The scientific purist who will wait for medical statistics until they are nosologically exact is no wiser than Horace's rustic waiting for the river to flow away" (Greenwood 1948, p. 28).

\section{ACKNOWLEDGMENTS}

We wish to acknowledge stimulating discussions with and advice from Christopher J. L. Murray in the development of our approach and analytic tools relating to the analysis and estimation of uncertainty in mortality and GBD estimates. We also acknowledge the assistance of staff of the former Global Program on Evidence for Health Policy at WHO, who helped substantially in the analysis of uncertainty, namely: Doris Ma Fat, Brodie Ferguson, Mie Inoue, and Niels Tomijima. Finally, we thank two referees for extremely useful comments and suggestions that have substantially improved this chapter.

\section{REFERENCES}

Anand, S., and K. Hanson. 1997. "Disability-Adjusted Life Years: A Critical Review.” Journal of Health Economics 16 (6): 685-702.

Barendregt, J. J., L. Bonneux, and P. J. van der Maas. 1996. "DALYs: The Age-Weights on Balance." Bulletin of the World Health Organization 74 (4): 439-43.

Bobadilla, J. L. 1996. "Priority Setting and Cost Effectiveness." In Health Policy and Systems Development: An Agenda for Research, ed. K Janovsky, 43-60. Geneva: World Health Organization.

Bryce, J., C. Boschi-Pinto, K. Shibuya, R. E. Black and the WHO Child Health Epidemiology Reference Group. 2005. "WHO Estimates of the causes of death in children." Lancet 365 (9465): 1147-1152.

Cooper, R. S., B. Osotimehin, J. S. Kaufman, and T. Forrester. 1998. "Disease Burden in Sub-Saharan Africa: What Should We Conclude in the Absence of Data?" Lancet 351 (9097): 208-10.

Dasgupta, P., K.-G. Mäler, and S. Barrett. 1999. "Intergenerational Equity, Social Discount Rates, and Global Warming." In Discounting and Intergenerational Equity, ed. P. R. Portney and J. P. Weyant, 51-77. Washington, DC: Resources for the Future.

Eastern Stroke and Coronary Heart Disease Collaborative Research Group. 1998. "Blood Pressure, Cholesterol, and Stroke in Eastern Asia." Lancet 352 (9143): 1801-7.

Evans, A. S. 1978. "Causation and Disease: A Chronological Journey." American Journal of Epidemiology 108 (4): 248-55. 
Ezzati, M., A. D. Lopez, A. Rodgers, and C. J. L Murray. 2004. Comparative Quantification of Health Risks: Global and Regional Burden of Disease Attributable to Several Major Risk Factors. Geneva: World Health Organization.

Fox-Rushby, J. A. 2002. Disability Adjusted Life Years (DALYS) for DecisionMaking? An Overview of the Literature. London: Office of Health Economics.

Gold, M. R., J. E. Siegel, M. C. Weinstein, and L. B. Russell. 1996. CostEffectiveness in Health and Medicine. New York: Oxford University Press.

Grassly, N. C., M. Morgan, N. Walker, G. P. Garnett, K. A. Stanecki, J. Stover, T. Brown, and P. D. Ghys. 2004. "Uncertainty in Estimates of HIV/AIDS: The Estimation and Application of Plausibility Bounds." Sexually Transmitted Infections 80 (Suppl 1): S31-S38.

Greenwood, M. 1948. "Medical Statistics from Graunt to Farr: The Fitzpatrick Lectures for the Years 1941 and 1943." Cambridge, U.K.: Cambridge University Press.

Gupta, P., R. Sankaranarayanan, and J. Ferlay. 1994. "Cancer Deaths in India: Is the Model-Based Approach Valid?" Bulletin of the World Health Organization 72 (6): 943-4.

Hill, A. B. 1965. "The Environment and Disease: Association or Causation?" Proceedings of the Royal Society of Medicine 58: 295-300.

Horton, R. 2000. "Common Sense and Figures: The Rhetoric of Validity in Medicine (Bradford Hill Memorial Lecture 1999).” Statistics in Medicine 19 (23): 3149-64.

Kelman, C. W., and A. J. Bass. 2002. "Research Use of Linked Health Data: A Best Practice Protocol." Australian and New Zealand Journal of Public Health 26 (3): 251-5.

King, G., M. Tomz, and J. Wittenberg. 2000. "Making the Most of Statistical Analyses: Improving Interpretation and Presentation." American Journal of Political Science 44 (2): 341-55.

Kneese, A. V. 1999. "The Faustian Bargain." In The RFF Reader in Environment and Resource Management, ed. W. D. Oates, 55-60. Washington, DC: Resources for the Future.

Kruijshaar, M., J. Barendregt, and N. Hoeymans. 2002. "The Use of Models in the Estimation of Disease Epidemiology." Bulletin of the World Health Organization 80 (8): 622-8.

Law, M. R., N. J. Wald, and S. G. Thompson. 1994. "By How Much and How Quickly Does Reduction in Serum Cholesterol Concentration Lower Risk of Ischaemic Heart Disease?" British Medical Journal 308 (6925): 367-73.

Lopez, A. D. 1998. “Counting the Dead in China.” British Medical Journal 317 (7170): 1399-400.

Lopez, A. D., O. Ahmad, M. Guillot, B. Ferguson, J. Salomon, C. J. L. Murray, and K. H. Hill. 2002. World Mortality in 2000: Life Tables for 191 Countries. Geneva: World Health Organization.

Mahapatra, P. 2001. "Local Age Preference, Age Weight, and Discounting Parameters for Computation of DALYs." In Estimating National Burden of Disease: The Burden of Disease in Andhra Pradesh 1990s, ed. P. Mahapatra. Hyderabad, India: Institute of Health Systems.

Mathers, C. D., D. Ma Fat, M. Inoue, C. Rao, and A. D. Lopez. 2005 "Counting the Dead and What They Died from: An Assessment of the Global Status of Cause of Death Data." Bulletin of the World Health Organization 83 (3): 171-7.

Mathers, C. D., C. J. L Murray, and A. D. Lopez. 2002. "Epidemiological Evidence: Improving Validity through Consistency Analysis." Bulletin of the World Health Organization 80 (8): 611.

Mathers, C. D., T. Vos, and C. Stevenson. 1999. The Burden of Disease and Injury in Australia. Canberra: Australian Institute of Health and Welfare.
Morgan, M. G., and M. Henrion. 1990. Uncertainty: A Guide to Dealing with Uncertainty in Quantitative Risk and Policy Analysis. Cambridge, U.K.: Cambridge University Press.

Murray, C. J. L. 1996. “Rethinking DALYs.” In The Global Burden of Disease, ed. C. J. L. Murray and A. D. Lopez, 1-98. Vol. 1 of Global Burden of Disease and Injury Series. Cambridge, MA: Harvard University Press.

Murray, C. J. L., and A. K. Acharya. 1997. "Understanding DALYs." Journal of Health Economics 16 (6): 703-30.

Murray, C. J. L, and A. D. Lopez, eds. 1996a. The Global Burden of Disease: A Comprehensive Assessment of Mortality and Disability from Diseases, Injuries, and Risk Factors in 1990 and Projected to 2020. Vol. 1 of Global Burden of Disease and Injury Series. Cambridge, MA: Harvard University Press.

1996b. "The Global Burden of Disease in 1990: Final Results and Their Sensitivity to Alternative Epidemiological Perspectives, Discount Rates, Age-Weights, and Disability Weights." In The Global Burden of Disease, ed. C. J. L. Murray and A. D. Lopez, 247-93. Vol. 1 of Global Burden of Disease and Injury Series. Cambridge, MA: Harvard University Press.

Murray, C. J., A. D. Lopez, and S. Wibulpolprasert. 2004. "Monitoring Global Health: Time for New Solutions.” British Medical Journal 329 (7333): 1096-100.

Murray, C. J. L, C. D. Mathers, and J. A. Salomon. 2003. "Towards Evidence-Based Public Health.” In Health Systems Performance Assessment: Debates, Methods, and Empiricism, ed. C. J. L. Murray and D. Evans, 715-26. Geneva: World Health Organization.

Murray, C. J. L, J. A. Salomon, C. D. Mathers, and A. D. Lopez. 2002. "Summary Measures of Population Health: Conclusions and Recommendations." In Summary Measures of Population Health: Concepts, Ethics, Measurement, and Applications, ed. C. J. L. Murray, J. A. Salomon, C. D. Mathers, and A. D. Lopez, p. 731-56. Geneva: World Health Organization.

National Research Council. 1994. Science and Judgment in Risk Assessment. Washington, DC: National Academy Press.

Parfit, D. 1984. Reasons and Persons. Oxford, U.K.: Clarendon Press.

Peto, R., A. D. Lopez, J. Boreham, M. Thun, and C. Heath. 1992. "Mortality from Tobacco in Developed Countries: Indirect Estimation from National Vital Statistics." Lancet 339 (8804): 1268-78.

Public Health Agency of Canada. 2005. "Population Health Impact of Disease in Canada." http://www.phac-aspc.gc.ca/phi-isp/summary_ measures.html\#3.

Salomon, J. A., C. J. L. Murray, T. B. Ustun, and S. Chatterji. 2003. "Health State Valuations in Summary Measures of Population Health." In Health Systems Performance Assessment: Debate, Methods, and Empiricism, ed. C. J. L. Murray and D. Evans, 409-36. Geneva: World Health Organization.

Ustun, T. B., S. Chatterji, M. Villanueva, L. Bendib, C. Celik, R. Sadana, N. Valentine, C. Mathers, J. P. Ortiz, A. Tandon, J. Salomon, Y. Cao, X. W. and C. J. L. Murray. 2003. "The WHO Multicountry Household Survey Study on Health and Responsiveness 2000-2001." In Health Systems Performance Assessment: Debates, Methods, and Empiricism, ed. C. J. L. Murray and D. Evans, 761-96. Geneva: World Health Organization.

Vose, D. 2000. Risk Analysis: A Quantitative Guide. New York: Wiley.

Williams, A. 1997. "Intergenerational Equity: An Exploration of the 'Fair Innings' Argument." Health Economics 6 (2): 117-32.

Yang, G. H., J. Hu, K. Q. Rao, J. Ma, C. Rao, and A. D. Lopez. 2005 "Mortality Registration and Surveillance in China: History, Current Situation, and Challenges." Population Health Metrics 3:3. 
Chapter 6

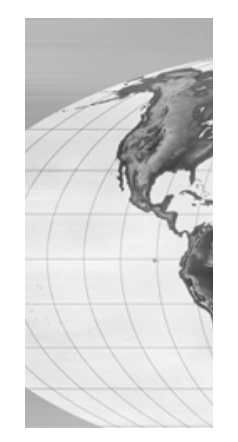

Many countries, including all high-income ones, maintain vital registration systems that provide data on the number of deaths by cause, sex, and age. Some countries also report years of life lost because of premature mortality (YLL) due to each cause, a number that depends on the age of death and on the choice of an algorithm for how YLL should depend on the age of death. The tracking of stillbirths, however, is often incomplete and variable.

As of the early 1990s, no estimates of YLL were available for many developing countries or for regional groupings of such countries. The World Bank (1993), as part of the preparation for its World Development Report 1993: Investing in Health, initiated an effort to provide estimates of deaths by age and cause, and hence YLL, for around 100 conditions for eight regional groupings, including all low- and middle-income countries. By adding years of healthy life lost as a result of disability (YLD) to YLL, the World Bank was able to generate estimates of the global burden of disease measured both in deaths by cause and in disability-adjusted life-years (DALYs) (Murray, Lopez, and Jamison 1994; World Bank 1993, appendix B). Murray and Lopez (1997) provide updated and

\section{Incorporating Deaths Near the Time of Birth Into Estimates of the Global Burden of Disease}

Dean T. Jamison, Sonbol A. Shahid-Salles, Julian Jamison, Joy E. Lawn, and Jelka Zupan

extended results and a complete description of methods. Global burden of disease estimates have subsequently been used to help guide resource allocation in the health sector and to inform debates about national and international disease control priorities (see chapter 1 in this volume); however, the global burden of disease literature currently provides little insight into the importance of deaths near the time of birth.

The purpose of this chapter is to explore the sensitivity of results within the Global Burden of Disease (GBD) framework to alternative approaches to encompassing the large number of deaths that occur near the time of birth, namely almost 4 million neonatal deaths and 3.3 million stillbirths. The sensitivity analyses in this chapter thus complement those of chapter 5, which explore the effect of variations in discount rates, age weights, and disability weights. Chapter 3 in this volume describes the GBD framework and provides estimates of deaths and DALYs by cause for 2001 using the World Bank regional grouping of countries. (Map 1, inside the front cover of this volume, shows the World Bank regional groupings used throughout this book.) This 
chapter uses the same framework and numbers to the extent possible, but with the following exceptions:

- We divide the newborn through age 4 category into neonatal (newborn through 27 days), postneonatal (28 days to less than 1 year), and child (1 through 4 years).

- We aggregate the 136 causes noted in chapter 3 into 35 causes.

- We allocate the substantial number of neonatal deaths attributed to pneumonia or sepsis to the chapter 3 category of respiratory infections.

- We explore the sensitivity of the results in chapter 3 to adding stillbirths as a new age category.

- We explore the sensitivity of the results to alternative ways of assigning YLL to deaths under the age of five.

The first section of this chapter deals with mortality: all-cause and cause specific. It uses the results presented in chapter 3, but adds to them estimates of the level of stillbirths and of the level and causes of neonatal mortality. The second section deals with estimation of the burden of disease in DALYs. The inclusion of stillbirths in the analysis highlights the more general issue of how to deal appropriately with deaths at different ages in constructing a measure of YLL.

As emphasized throughout this volume, data on causes of death and disability are fragmentary and are often inconsistent for many regions of the world. This is particularly true for the neonatal period and for stillbirths. One clear implication is the desirability of more and better data. Another implication is that any effort to construct an overall picture of population health must aggregate data of variable, often low, quality and completeness. In some instances this is done essentially as a political process, with various disease advocacy groups advancing their claims to policy makers and in the press. Alternatively, summary measures can be constructed systematically in a way that eliminates internal inconsistencies, describes methods carefully, and imposes the discipline of demographically derived totals into which cause-specific estimates must fit. This is the nature of our work on the global burden of disease.

\section{STILLBIRTHS AND NEONATAL MORTALITY IN THE CONTEXT OF THE GLOBAL BURDEN OF DISEASE}

This section first introduces the nomenclature used throughout the chapter. It then provides estimates of deaths and death rates that highlight stillbirths and neonatal deaths and discusses deaths by cause at different ages.

\section{Nomenclature}

This chapter follows standard usage where possible, but extends or tightens it as needed. Stillbirth refers to the birth of a dead fetus weighing more than 1,000 grams up to 0.25 years ( 13 weeks) prior to the expected time of birth (corresponding to 27 weeks of gestational age). Total births are the sum of the number of live births and of stillbirths. Stillbirths are conventionally divided into two categories, antepartum stillbirths, when a fetus dies before the onset of labor, and intrapartum stillbirths, when fetal death occurs during labor. The term fresh stillbirths denotes fetuses born dead but with intact skin, which are assumed to have died less than 12 hours before birth and serve as an observable surrogate measure for intrapartum stillbirths. Individuals younger than 28 days are in the neonatal period and younger than 1 year are infants. The neonatal period is divided into the early neonatal period, which refers to birth to less than 7 days old, and the remaining late neonatal period. The postneonatal period extends from 28 days to under 1 year. Child in this chapter refers to an individual from age one to under age five. (In some other usage, however, child refers to all individuals under age five).

We use standard demographic terminology to indicate death rates at different ages, that is, ${ }_{x} q_{y}$ refers to the probability that an individual aged $y$ will die before reaching age $y+x$ and is usually estimated using cross-sectional observations of age-specific mortality rates for individual ages in the age range $\mathrm{y}$ to $\mathrm{y}+\mathrm{x}$. Using this terminology, the mortality rate for those under one year old (or the infant mortality rate) is ${ }_{1} \mathrm{q}_{0}$. We extend this terminology to define the complete under one mortality rate as ${ }_{1.25} q_{-.25}$, the child mortality rate as ${ }_{4} \mathrm{q}_{1}$, the under five mortality rate as ${ }_{5} \mathrm{q}_{0}$, the stillbirth rate as ${ }_{25} \mathrm{q}_{-.25}$, the neonatal mortality rate as ${ }_{.077} \mathrm{q}_{0}$, and the complete under five mortality rate as ${ }_{5.25} \mathrm{q}_{-.25}$. This chapter uses age-specific mortality rates for 2001.

\section{Numbers of Deaths and Death Rates}

In 2001, approximately 10.6 million children born alive died before their fifth birthday ( 8.2 percent of births). Of these deaths, 3.9 million occurred during the neonatal period, that is, under the age of 28 days. Another 3.3 million stillborn children remained outside the vital registration systems of most countries (WHO 2005a). When stillbirths are included among deaths, about half of all deaths of children under five occur under the age of 28 days.

Table 6.1 provides estimates of the numbers of stillbirths in 2001, with numbers broken down by World Bank income categories. The stillbirth numbers in the table come from rates 
Table 6.1 Population Totals and Numbers of Births, 2001 thousands

\begin{tabular}{|c|c|c|c|c|}
\hline Region & Population (mid-2001) & Live births & Stillbirths & Total births \\
\hline Low- and middle-income countries & $5,221,572$ & 118,505 & 3,228 & 121,733 \\
\hline High-income countries & 928,660 & 11,371 & 45 & 11,416 \\
\hline World & $6,150,233$ & 129,876 & 3,274 & 133,150 \\
\hline
\end{tabular}

Sources: Population is calculated from United Nations Population Division 2003, table 1. Live births are calculated from population totals and crude birth rates in World Bank 2003. Stillbirths are calculated from live births, using rates from WHO 2005a.

Table 6.2 Age Distribution of Deaths under Age 5, 2001 thousands

\begin{tabular}{|c|c|c|c|c|c|c|c|c|c|c|c|}
\hline & \multirow{2}{*}{\multicolumn{3}{|c|}{ Stillbirths }} & \multirow{2}{*}{\multicolumn{3}{|c|}{ Neonatal deaths }} & \multirow{3}{*}{$\begin{array}{c}\text { Deaths ages } \\
28 \text { days to } \\
<1 \text { year }\end{array}$} & \multirow{3}{*}{$\begin{array}{l}\text { Infant deaths } \\
\text { ( } 0 \leq \text { age } \\
<1 \text { year })\end{array}$} & \multirow{3}{*}{$\begin{array}{c}\text { Child deaths } \\
\text { (1 } \leq \text { age } \\
<5 \text { years })\end{array}$} & \multicolumn{2}{|c|}{ Deaths under age 5} \\
\hline & & & & & & & & & & \multirow{2}{*}{$\begin{array}{l}\text { After live birth } \\
\text { ( } 0 \leq \text { age } \\
<5 \text { years })\end{array}$} & \multirow{2}{*}{$\begin{array}{l}\text { Including } \\
\text { stillbirth }\end{array}$} \\
\hline & Antepartum & Intrapartum & Total & Early ${ }^{a}$ & Late $^{a}$ & Total & & & & & \\
\hline Region & a & b & $\begin{array}{c}c \\
(a+b)\end{array}$ & d & e & $\begin{array}{c}f \\
(d+e)\end{array}$ & g & $\begin{array}{c}h \\
(f+g)\end{array}$ & i & $\begin{array}{c}\mathrm{j} \\
(\mathrm{h}+\mathrm{i})\end{array}$ & $\begin{array}{c}k \\
(j+c)\end{array}$ \\
\hline $\begin{array}{l}\text { Low- and middle- } \\
\text { income countries }\end{array}$ & 2,152 & 1,077 & 3,228 & 2,889 & 965 & 3,854 & 3,745 & 7,599 & 2,935 & 10,530 & 13,758 \\
\hline $\begin{array}{l}\text { High-income } \\
\text { countries }\end{array}$ & 40 & 5 & 45 & 32 & 9 & 41 & 18 & 59 & 13 & 73 & 119 \\
\hline World & 2,192 & 1,082 & 3,274 & 2,921 & 974 & 3,896 & 3,762 & 7,658 & 2,948 & 10,602 & 13,876 \\
\hline
\end{tabular}

Sources: Columns a, b, c, d, e, and f are calculated from rates provided by WHO 2005a, using live birth totals from table 6.1 of this chapter. Column $\mathrm{j}$ is from chapter 3 of this volume. Column $\mathrm{h}=$ (infant mortality rate/under-five mortality rate) $\times$ total number of deaths from column $\mathrm{j}$. Column $\mathrm{i}=$ [(under five mortality rate - infant mortality rate)/under five mortality]; under five mortality rates are from the World Bank (2003, table 2.20). The World Bank under five mortality rates are very close to, but not identical with, those reported in this volume (chapter 2, table 2.3). The World Bank numbers are used because they are accompanied by a consistently generated set of infant mortality rates.

a. The early neonatal period extends from birth to under 7 days of age; the late neonatal period extends from 7 days to under 28 days.

estimated by the World Health Organization (WHO) (WHO 2005a) applied to the birth numbers reported in the table. The table shows that in 2001, the high-income countries (those with a gross national income per capita of more than US $\$ 9,076$ in 2002) had 11.37 million live births and the lowand middle-income countries had 118.51 million live births.

Table 6.2 provides an age breakdown of deaths among children under five, again with a breakdown by World Bank income category. Early neonatal deaths account for 75 percent of all neonatal deaths. The eight-day period encompassing intrapartum stillbirths and early neonatal deaths accounts for almost 30 percent of the 13.9 million deaths occurring under the age of five. Thus, as shown in figure 6.1 for the low- and middle-income countries, roughly a quarter of the deaths under age five occur in each of the following categories: stillbirths, neonatal deaths, postneonatal infant deaths, and child deaths.

Three recent studies provide extensive literature reviews and model-based estimates of the number of stillbirths and neonatal deaths that extend the WHO estimates used here (WHO 2005a). Lawn, Shibuya, and Stein (2005, tables A-J)

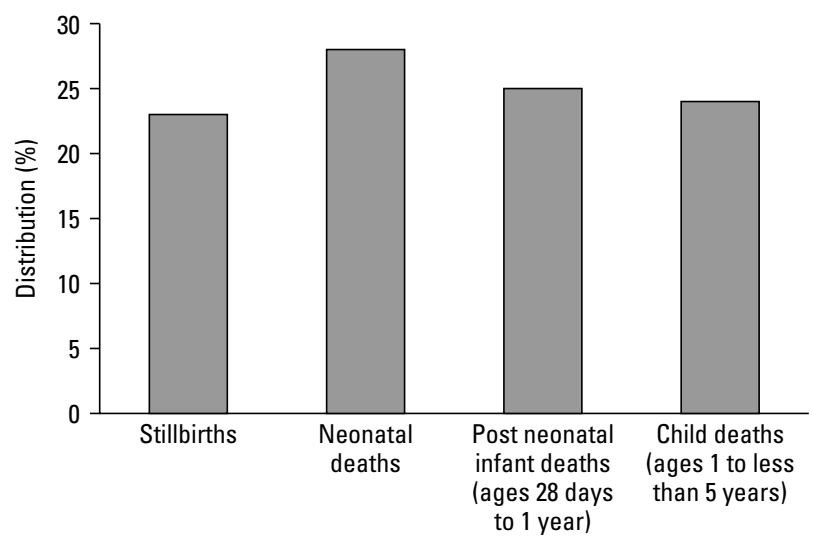

Source: Table 6.2.

Figure 6.1 Age Distribution of Deaths of Children under Five in Lowand Middle-Income Countries, 2001

focus on intrapartum stillbirths and intrapartum-related neonatal deaths. Stanton and others (forthcoming) provide estimates of the number of stillbirths for 190 countries and Hill (forthcoming) provides estimates for neonatal deaths. 
Table 6.3 Estimated Death Rates under Age 5, by Country Income Level, 2001

Probability of dying in the $x$ years following age $y\left({ }_{x} q_{y}\right)$, expressed per thousand live births

\begin{tabular}{|c|c|c|c|c|c|c|c|}
\hline & $\begin{array}{c}\text { Stillbirth rate } \\
\left({ }_{0.25} q_{-0.25}\right)\end{array}$ & $\begin{array}{c}\text { Neonatal } \\
\text { mortality rate } \\
\left({ }_{.077} q_{0}\right)\end{array}$ & $\begin{array}{c}\text { Under } 1 \\
\text { mortality rate } \\
\left({ }_{1} q_{0}\right)\end{array}$ & $\begin{array}{c}\text { Complete } \\
\text { under } 1 \\
\text { mortality rate } \\
\left(1.25 q_{-.25}\right)\end{array}$ & $\begin{array}{c}\text { Child } \\
\text { mortality rate } \\
\left({ }_{4} q_{1}\right)\end{array}$ & $\begin{array}{c}\text { Under } 5 \\
\text { mortality rate } \\
\left({ }_{5} q_{0}\right)\end{array}$ & $\begin{array}{c}\text { Complete } \\
\text { under } 5 \\
\text { mortality rate } \\
\left(5.259_{-.25}\right)\end{array}$ \\
\hline Region & a & b & c & d & e & $f$ & g \\
\hline $\begin{array}{l}\text { Low- and middle- } \\
\text { income countries }\end{array}$ & 27 & 33 & 64 & 89 & 25 & 89 & 113 \\
\hline High-income countries & 4 & 4 & 5 & 9 & 1 & 6 & 10 \\
\hline World & 25 & 30 & 58 & 82 & 23 & 82 & 104 \\
\hline
\end{tabular}

Sources: Columns $\mathrm{c}$ and $\mathrm{f}$ are based on data from the World Bank (2003, table 2.20). Data for columns a, b, d, e, and g are provided by WHO (2005a).

Note: Column $a=$ (total stillbirths)/(total births). Column $b=$ (neonatal deaths)/(live births). Column $c=$ (infant deaths)/(live births). Column $d=$ (infant deaths + stillbirths)/(total births).

Column $\mathrm{e}=$ (total deaths from ages one to four years)/(live births). Column $\mathrm{f}=$ (total deaths under age five)/(live births). Column $\mathrm{g}=$ (total deaths under age five including stillbirths)/(total births).

The midpoints of their fairly wide confidence intervals accord with the numbers we use.

Table 6.3 shows death rates, expressed per 1,000 live births, that correspond to the death totals in table 6.2. Column (c), for example, shows an under one or infant mortality rate $\left({ }_{1} q_{0}\right)$ for low- and middle-income countries of 64 per 1,000. Column (d) shows the effect of including stillbirths to give the complete under one mortality rate $\left(1.25 \mathrm{q}_{-.25}\right)$, which is markedly higher at 89 per 1,000 live births. By including stillbirths and providing relatively fine-grained age breakdowns, table 6.3 provides a more comprehensive set of estimates of mortality rates under age five than has hitherto been available. The wide confidence interval that needs to be attached to the estimates (Stanton and others forthcoming) indicates both the need for caution when using these numbers and the importance of further research. Nevertheless, the estimates in table 6.3 are reasonable given currently available information.

\section{Deaths by Cause}

Estimates of the total number of deaths in different age groups provide a starting point for breaking those totals down into deaths by cause. This task inevitably involves some degree of arbitrariness because of problems with classifying multiple causes of death or underlying versus proximal causes. That said, available data from vital registration, sentinel surveillance, and verbal autopsy can provide reasonable approximations for most causes. Chapter 3 provides background on how this was done and generates the death by cause estimates used throughout this book.

We use the estimates from chapter 3 for deaths by cause in the newborn through age four age group and aggregate chapter 3 data on age groups over age five into a single category of deaths for those age five and older. In their preparatory work for chapter 3, its authors estimated cause-specific breakdowns of deaths under age five both for infant deaths and for deaths from age one through age four, that is, deaths occurring at one year of age or older but under age five, and we have used their data in this chapter. Table 6.4 presents this information on deaths by cause aggregated, as previously indicated, into 35 groups of conditions rather than the 136 used in chapter 3 .

The aggregate numbers for neonatal deaths and for stillbirths come from WHO (2005a) as reported in table 6.2 (see also WHO 2005b, pp. 170-71). Table 6.4 breaks down neonatal deaths into six causes: diarrheal diseases, tetanus, respiratory infections, low birthweight (essentially preterm birth), birth asphyxia and birth trauma, and congenital anomalies. ${ }^{2}$ The estimates by cause were generated for WHO's Child Health Epidemiology Reference Group (CHERG) (see Bryce and others 2005 for a comprehensive presentation of data sources and methods of estimation). WHO (2005b, annex table 4) provides a summary of the numbers.

For the most part, the neonatal death categories used by CHERG align with the categories used by the GBD assessment in chapter 3; however, note the following exceptions:

- CHERG includes a pneumonia and sepsis category, which accounts for 26 percent of neonatal deaths globally and 27 percent in low- and middle-income countries. The GBD categories include respiratory infections (category I.B in our tables), which account for 1.945 million deaths worldwide in the age group 0-4. We allocate all the CHERGestimated deaths from the combined category sepsis and pneumonia to the neonatal age group's respiratory infections category in order to remain as consistent as possible with the GBD framework in chapter 3. A number of 
studies have estimated the percentage of the broad category sepsis and pneumonia that is pneumonia with a wide range of findings (see, for example, Bhutta and others 2004 and Bhutta, Ali, and Wajid 2004). Even with blood cultures and chest $\mathrm{x}$-rays, one cannot say for sure if a newborn has sepsis or pneumonia or both, and in any case, the treatment is the same, so one programmatic category is currently appropriate (Lawn, Cousens, and Wilczynska forthcoming).

- CHERG's percentage of neonatal deaths due to tetanus (7 percent) exceeds the GBD estimate for all infant deaths from tetanus but is very close to WHO and GAVI estimates for the year 2000 of 220,000. In keeping with this chapter's spirit of staying as close as possible to GBD estimates from chapter 3, we remain within the GBD envelope for the under-five age group and, as a first approximation, allocate all under one tetanus deaths to the neonatal period. However, while remaining within the under five GBD envelope for tetanus, we have modified, in this case only, the (unpublished) GBD age breakdown between ages $0-1$ and 1-4 to allocate 90 percent of under five tetanus deaths to under age one (see table 6.4, note a). The difference between the CHERG and WHO with the GBD estimates for tetanus deaths is substantial and is clearly a priority area for further work.

- The GBD work uses the category low birthweight, which is an outcome of either preterm birth or intrauterine growth retardation. Preterm birth is a major cause of neonatal death. Again in the spirit of remaining within the GBD framework, we allocate preterm births to the low birthweight GBD category. This should not cause confusion as long as it is understood that, for neonatal deaths, low birthweight refers almost entirely to preterm birth. The quantitative importance of preterm birth suggests that this is another category that could be presented separately in the next GBD effort.

We are not aware of any effort to aggregate data on causes of stillbirths that parallels the CHERG effort for neonatal deaths, hence the GBD calculations in this chapter do not attempt to allocate stillbirths by cause. However, even though this chapter does not attempt a review of the CHERG type of the causes of stillbirth, we can advance a few tentative hypotheses. First, an important cause of stillbirth is intrapartum complications. A recent systematic analysis of intrapartum stillbirths gives estimates for 192 countries based on 73 study populations ( 52 countries, $\mathrm{n}=46,779$ [73 populations]) suggesting that 1.02 million intrapartum stillbirths (uncertainty 0.66-1.48 million) occur annually, accounting for 26 percent of global stillbirths. Second, congenital anomalies constitute an important cause of antepartum stillbirth. Third, sexually transmitted diseases and other infections cause antepartum stillbirth, but systematic global estimates are currently limited.

Our categorization of neonatal deaths within the GBD framework has been deliberately conservative in that where interpretation was in any way uncertain, we assigned deaths to the not allocated category. We expect future efforts to be able to substantially reduce the not allocated component for both stillbirths and neonatal deaths, but doing so will require both improved empirical information and modification of the current GBD framework to include classifications important for deaths near the time of birth. Until such improvements are possible, table 6.4 provides a plausible extension of the GBD cause of death framework to include causes of infant and neonatal deaths.

\section{THE BURDEN OF DISEASE RESULTING FROM EVENTS NEAR THE TIME OF BIRTH}

This section explains the use of DALYs as a measure of the disease burden and identifies a number of problems associated with the traditional DALY formulation when dealing with events around the time of birth. It proposes a generalized formulation (which annex 6A describes more fully). The chapter then calculates the disease burden using two approaches to explore the sensitivity of GBD estimates to alternative formulations as follows:

- the current DALY formulation extended so as to value the DALY loss from a stillbirth the same as the DALY loss from a death at age 0 ,

- a generalized DALY formulation allowing the acquisition of life potential (ALP) to be gradual rather than instantaneous.

\section{Defining and Redefining DALYs}

The DALY family of indicators measures the disease burden from the age of onset of a condition by summing an indicator of YLL due to the condition and an indicator of disabilityadjusted YLD resulting from the condition. While, in principle, the disability weights used in this adjustment could arise from any of the procedures typically used to construct qualityadjusted life years, obtaining disability weights for a large number of causes using any procedure other than the judgments of selected reference groups is currently impractical. Chapter 3 describes the methods currently used. 
Table 6.4 Deaths by Age and Cause, 2001 (thousands)

\begin{tabular}{|c|c|c|c|c|c|c|}
\hline & \multicolumn{6}{|c|}{ Low- and middle-income countries } \\
\hline & \multicolumn{6}{|c|}{ Deaths under age 5} \\
\hline & \multicolumn{3}{|c|}{ Stillbirth } & \multirow[b]{2}{*}{ Neonatal } & \multirow{2}{*}{$\begin{array}{c}\text { Deaths aged } \\
28 \text { days to } \leq 1 \text { year }\end{array}$} & \multirow{2}{*}{$\begin{array}{c}\text { Infant deaths } \\
(0 \leq \text { age }< \\
1 \text { year })\end{array}$} \\
\hline & Antepartum & Intrapartum & Total & & & \\
\hline & \multicolumn{5}{|c|}{$(a+b)$} & \multirow[b]{2}{*}{$\mathbf{f}$} \\
\hline & a & b & c & d & e & \\
\hline \multirow{6}{*}{$\begin{array}{l}\text { Total deaths } \\
\text { I. Communicable, maternal, } \\
\text { perinatal, and nutritional conditions } \\
\text { A. Infectious and parasitic diseases } \\
\text { 1. Tuberculosis } \\
\text { 2. Sexually transmitted diseases } \\
\text { excluding HIV/AIDS } \\
\text { 3 HV/AIDS }\end{array}$} & 2,152 & 1,077 & 3,228 & 3,854 & 3,745 & 7,599 \\
\hline & & & & $\mathbf{3 , 0 8 8}$ & & 6,875 \\
\hline & & & & 284 & & 2,884 \\
\hline & & & & & & 16 \\
\hline & & & & & & 55 \\
\hline & & & & & & 202 \\
\hline 4. Diarrheal diseases & & & & 116 & 1,105 & 1,221 \\
\hline 5. Childhood-cluster diseases & & & & 170 & , 100 & 381 \\
\hline a. Pertussis & & & & & & 96 \\
\hline b. Poliomyelitis & & & & & & \\
\hline c. Diphtheria & & & & & & 2 \\
\hline d. Measles ${ }^{\mathrm{a}}$ & & & & & & 115 \\
\hline e. Tetanus ${ }^{b}$ & & & & 168 & & 168 \\
\hline 6. Meningitis & & & & & & 47 \\
\hline 8. Malaria ${ }^{\mathrm{C}}$ & & & & & & 726 \\
\hline Other ${ }^{d}$ & & & & & & 218 \\
\hline B. Respiratory infections ${ }^{\mathrm{e}}$ & & & & 1,002 & 533 & 1,535 \\
\hline $\begin{array}{l}\text { C. Maternal conditions } \\
\text { D. Perinatal conditions }\end{array}$ & & & & & & \\
\hline D. Perinatal conditions & & & & 1,802 & & 2,384 \\
\hline 1. Low birthweight ${ }^{f}$ & & & & 1,079 & 136 & 1,215 \\
\hline 2. Birth asphyxia and birth traumag & & & & 723 & & 723 \\
\hline 3. Other perinatal conditions & & & & & & 446 \\
\hline E. Nutritional deficiencies & & & & & & 96 \\
\hline II. Noncommunicable diseases & & & & 308 & & 599 \\
\hline A. Malignant neoplasms & & & & & & 11 \\
\hline C. Diabetes mellitus & & & & & & 2 \\
\hline E. Neuropsychiatric disorders & & & & & & 21 \\
\hline 1. Unipolar depressive disorders & & & & & & \\
\hline 2. Bipolar affective disorder & & & & & & \\
\hline $\begin{array}{l}\text { 3. Schizophrenia } \\
\text { Other }^{\text {h }}\end{array}$ & & & & & & \\
\hline $\begin{array}{l}\text { Other" } \\
\text { G. Cardiovascular diseases }\end{array}$ & & & & & & $\begin{array}{l}14 \\
56\end{array}$ \\
\hline 3. Ischemic heart disease & & & & & & 4 \\
\hline 4. Cerebrovascular disease & & & & & & 8 \\
\hline Otheri & & & & & & 42 \\
\hline H. Respiratory diseases & & & & & & 43 \\
\hline I. Digestive diseases & & & & & & 73 \\
\hline M. Congenital anomalies & & & & 308 & 44 & 352 \\
\hline Other ${ }^{j}$ & & & & & & 36 \\
\hline III. Injuries & & & & & & 124 \\
\hline A. Unintentional & & & & & & 121 \\
\hline 1. Road traffic accidents & & & & & & 12 \\
\hline Otherk & & & & & & 109 \\
\hline B. Intentional & & & & & & 5 \\
\hline 1. Self-inflicted & & & & & & \\
\hline Other' & & & & & & 3 \\
\hline IV. Not allocated & 2,152 & 1,077 & 3,228 & 458 & & \\
\hline
\end{tabular}

Sources: WHO 2005a for columns a-d, unreported estimates undertaken as part of the GBD study, reported in chapter 3 for columns $\mathrm{f}-\mathrm{g}$, and chapter 3 of this volume.

Note: The absence of an entry in columns a-d denotes either a value of less than 1,000 deaths or that no estimate was allocated to that entry. For columns $f-k$, a blank cell indicates that fewer than 1,000 deaths are attributable to the specific cause. Infant and child deaths in columns $f$ and $g$ are based on unreported estimates undertaken as part of the GBD study, reported in chapter 3 , of the percentage of under-five deaths that were under age one. Because the sources used for neonatal deaths left a large number unallocated, it is not appropriate to calculate values of column e by subtracting column $\mathrm{d}$ from column f except where explicitly noted.

a. WHO 2005b and Bryce and others (2005) estimate that 395,000 deaths occur due to measles. Chapter 3 provides an estimate for measles deaths age zero to four of 763,000 .

b. Lawn, Wilczynska, and Cousens (forthcoming) for the CHERG estimate (2005) that 7 percent $(260,000)$ of the 3.854 million global neonatal deaths occur due to tetanus, similar to the WHO and GAVI estimates of 220,000 for the year 2000. Chapter 3 provides an estimate for tetanus deaths ages zero to four of only 187,000. The (unpublished) GBD files used here to allocate deaths under age five to over and under age one allocated 52 percent of tetanus deaths to under age one. The CHERG review (Lawn, Wilczynska, and Cousens forthcoming) suggests this to be a major underestimate, and the 52 percent figure has thus here been revised upward to 90 percent. Consistent with the objectives of this chapter, GBD numbers have been used wherever possible, and the CHERG and WHO estimates are accordingly revised downward by allocating all infant tetanus deaths to the neonatal period.

c. WHO 2005b and Bryce and others (2005) estimate that 853,000 deaths occur due to malaria. Chapter 3 provides an estimate for malaria deaths age zero to four of 1,208,000

d. Hepatitis, tropical-cluster diseases, leprosy, dengue, Japanese encephalitis, trachoma, intestinal nematode infections, and other infectious diseases.

e. Deaths for respiratory infections in the neonatal age group are those estimated by Lawn, Cousens, and Wilczynska (forthcoming) for their category sepsis or pneumonia. This number was then subtracted from the GBD number of respiratory infections between age zero and one to derive the total in column e.

f. Low-birthweight deaths are those resulting from intrauterine growth retardation or preterm birth. Almost all low-birthweight deaths in the neonatal period result from preterm birth. 
Table 6.4 Continued

\begin{tabular}{|c|c|c|c|c|}
\hline \multicolumn{5}{|c|}{ Low- and middle-income countries } \\
\hline \multicolumn{3}{|c|}{ Deaths under age 5} & \multirow[b]{2}{*}{$\begin{array}{c}\text { Deaths age } \\
5+\end{array}$} & \multirow[b]{2}{*}{ Total } \\
\hline $\begin{array}{c}\text { Child deaths } \\
(1 \leq \text { age } \\
<5 \text { years })\end{array}$ & $\begin{array}{c}\text { After live } \\
\text { birth }\end{array}$ & $\begin{array}{l}\text { Including } \\
\text { stillbirth }\end{array}$ & & \\
\hline$(h-f)$ & $(f+g)$ & $(h+c)$ & & $(i+j)$ \\
\hline g & h & i & j & k \\
\hline 29,345 & 10,533 & 13,761 & 37,843 & 51,605 \\
\hline 2,521 & 9,396 & 9,396 & 8,226 & 17,622 \\
\hline 1,884 & 4,768 & 4,768 & 5,923 & 10,692 \\
\hline 25 & 40 & 40 & 1,550 & 1,590 \\
\hline 13 & 68 & 68 & 108 & 176 \\
\hline 138 & 340 & 340 & 2,214 & 2,554 \\
\hline 378 & 1,599 & 1,599 & 179 & 1,778 \\
\hline 667 & 1,048 & 1,048 & 313 & 1,363 \\
\hline 205 & 301 & 301 & & 301 \\
\hline 2 & 5 & 5 & 1 & 6 \\
\hline 442 & 557 & 557 & 206 & 763 \\
\hline 19 & 187 & 187 & 106 & 293 \\
\hline 18 & 64 & 64 & 105 & 169 \\
\hline 361 & 1,087 & 1,087 & 58 & 1,208 \\
\hline 299 & 518 & 518 & 1,334 & 1,854 \\
\hline 408 & 1,943 & 1,943 & 1,539 & 3,483 \\
\hline & & & 507 & 507 \\
\hline 106 & 2,490 & 2,490 & & 2,490 \\
\hline 76 & 1,291 & 1,291 & & 1,291 \\
\hline 5 & 728 & 728 & & 728 \\
\hline 25 & 471 & 471 & & 471 \\
\hline 99 & 194 & 194 & 257 & 451 \\
\hline 236 & 835 & 835 & 25,202 & 26,037 \\
\hline 26 & 37 & 37 & 4,921 & 4,957 \\
\hline 1 & 3 & 3 & 755 & 758 \\
\hline 23 & 43 & 43 & 605 & 701 \\
\hline & & & 10 & 10 \\
\hline & & & 21 & 21 \\
\hline 30 & 43 & 43 & 627 & 670 \\
\hline 26 & 82 & 82 & 13,279 & 13,362 \\
\hline 2 & 6 & 6 & 5,696 & 5,702 \\
\hline 4 & 12 & 12 & 4,598 & 4,611 \\
\hline 22 & 64 & 64 & 2,985 & 3,049 \\
\hline 21 & 63 & 63 & 3,063 & 3,127 \\
\hline 42 & 115 & 115 & 1,487 & 1,602 \\
\hline 69 & 421 & 421 & 56 & 477 \\
\hline 34 & 71 & 71 & 983 & 1,053 \\
\hline 178 & 302 & 302 & 4,415 & 4,717 \\
\hline 170 & 289 & 289 & 2,926 & 3,216 \\
\hline 38 & 49 & 49 & 1,020 & 1,070 \\
\hline 146 & 240 & 240 & 1,903 & 2,146 \\
\hline 8 & 13 & 13 & 1,488 & 1,501 \\
\hline & & & 749 & 749 \\
\hline 10 & 13 & 13 & 740 & 753 \\
\hline & & 3,228 & & 3,228 \\
\hline
\end{tabular}

g. Lawn, Wilczynska, and Cousens (forthcoming) for the CHERG estimate that 23 percent $(887,000)$ of the 4 million global neonatal deaths occur due to birth asphyxia. Chapter 3 of this volume provides an estimate for birth asphyxia and birth trauma deaths ages zero to four of only 739,000 globally, of which 734,000 were estimated to occur under age one.

Consistent with the objectives of this chapter, GBD numbers have been used wherever possible, and the CHERG and WHO estimates are accordingly revised downward by allocating all infant birth asphyxia deaths to the neonatal period. Better data in the future will allow for improved estimates.

h. Epilepsy, alcohol use disorders, Alzheimer's disease and other dementias, Parkinson's disease, multiple sclerosis, drug use disorders, post-traumatic stress disorder, obsessive-compulsive disorder, panic disorder, insomnia (primary), migraine, mental retardation attributable to lead exposure, and other neuropsychiatric disorders.

i. Rheumatic heart disease, hypertensive heart disease, inflammatory heart diseases, and other cardiovascular diseases. j. Other neoplasms, endocrine disorders, sense organ diseases, genitourinary diseases, skin diseases, musculoskeletal diseases, and oral conditions.

k. Poisonings, falls, fires, drownings, and other unintentional injuries.

I. Violence, war, and other intentional injuries. 
Table 6.4 Continued

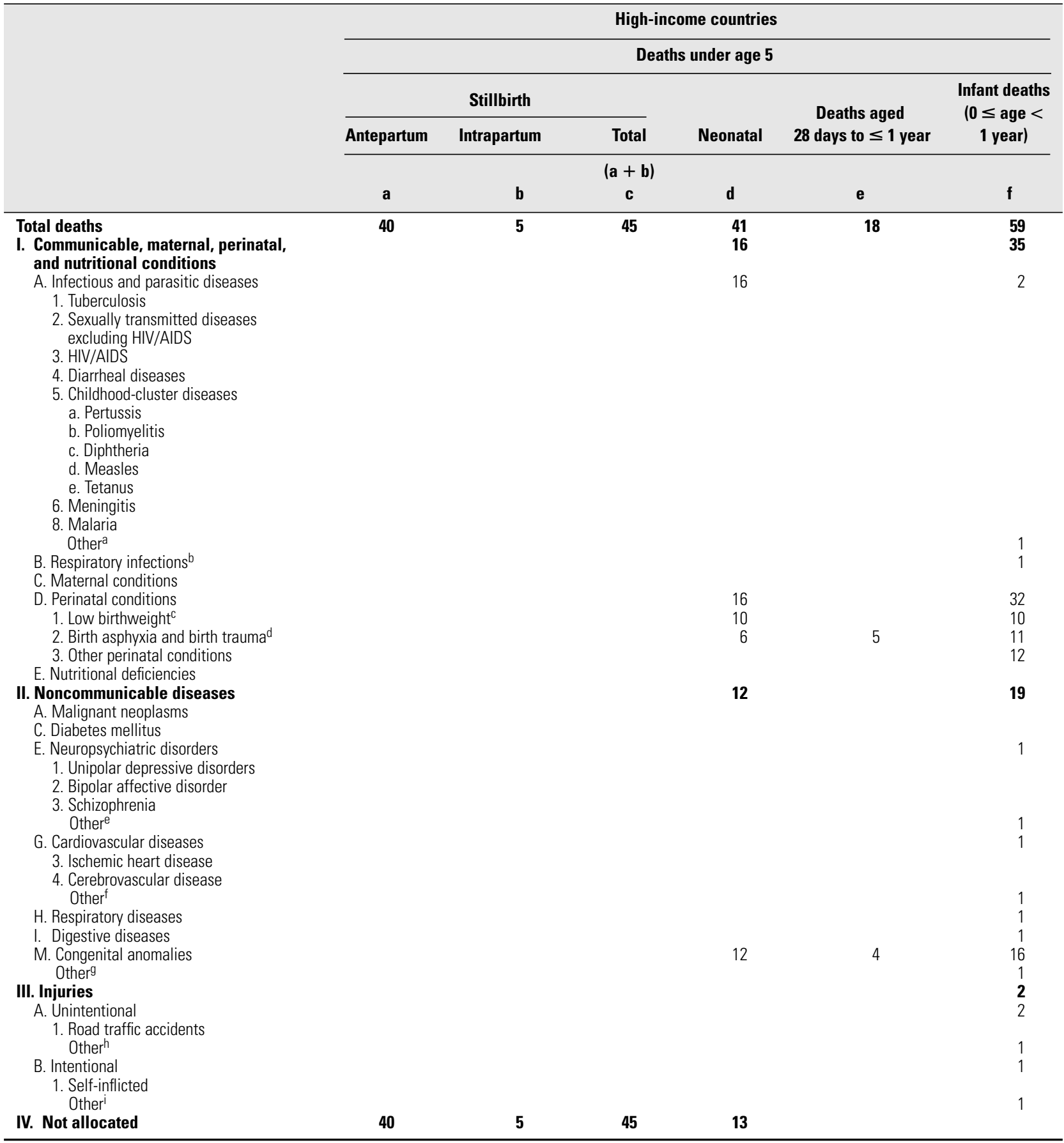

Sources: WHO 2005a for columns a-d, unreported estimates undertaken as part of the GBD study, reported in chapter 3 for columns $\mathrm{f}-\mathrm{g}$, and chapter 3 of this volume.

Note: The absence of an entry in columns a- $d$ denotes either a value of less than 1,000 deaths or that no estimate was allocated to that entry. For columns $f-k$, a blank cell indicates that fewer than 1,000 deaths are attributable to the specific cause. Infant and child deaths in columns $f$ and $g$ are based on unreported estimates undertaken as part of the GBD study, reported in chapter 3 , of the percentage of under five deaths that were under age one. Because the sources used for neonatal deaths left a large number unallocated, it is not appropriate to calculate values of column e by subtracting column d from column f except where explicitly noted.

a. Hepatitis, tropical-cluster diseases, leprosy, dengue, Japanese encephalitis, trachoma, intestinal nematode infections, and other infectious diseases.

b. This table does not attempt to partition by age the very small number of deaths from respiratory infections under age 5 .

c. Low-birthweight deaths are those resulting from intrauterine growth retardation or preterm birth. Almost all low-birthweight deaths in the neonatal period result from preterm birth.

d. The World Health Report 2005 cites that 45 percent $(19,000)$ of the 4 million global neonatal deaths occur due to pre-term birth. Chapter 3 of this volume provides an estimate for low birthweight deaths ages zero to four of only 10,000, of which 10,000 were estimated to occur under age one. Consistent with the objectives of this chapter, GBD numbers have been used wherever possible, and the World Health Report 2005 estimates are accordingly revised downward by allocating all low-birthweight deaths to the neonatal period. Better data in the future will allow for improved estimates. 
Table 6.4 Continued

\begin{tabular}{|c|c|c|c|c|}
\hline \multicolumn{5}{|c|}{ High-income countries } \\
\hline \multicolumn{3}{|c|}{ Deaths under age 5} & \multirow[b]{2}{*}{$\begin{array}{c}\text { Deaths age } \\
5+\end{array}$} & \multirow[b]{2}{*}{ Total } \\
\hline $\begin{array}{c}\text { Child deaths } \\
(1 \leq \text { age }<5 \\
\text { years })\end{array}$ & $\begin{array}{c}\text { After live } \\
\text { birth }\end{array}$ & $\begin{array}{l}\text { Including } \\
\text { stillbirth }\end{array}$ & & \\
\hline$(h-f)$ & $(f+g)$ & $(h+c)$ & & $(i+j)$ \\
\hline $\mathbf{g}$ & h & $\mathbf{i}$ & j & k \\
\hline $\begin{array}{r}13 \\
2\end{array}$ & $\begin{array}{l}73 \\
37\end{array}$ & $\begin{array}{r}118 \\
37\end{array}$ & $\begin{array}{r}7,864 \\
515\end{array}$ & $\begin{array}{r}7,982 \\
552\end{array}$ \\
\hline \multirow[t]{5}{*}{1} & 3 & 3 & $\begin{array}{r}149 \\
16 \\
1\end{array}$ & $\begin{array}{r}152 \\
16 \\
1\end{array}$ \\
\hline & & & $\begin{array}{r}22 \\
5 \\
1\end{array}$ & $\begin{array}{r}22 \\
6 \\
2\end{array}$ \\
\hline & & & 1 & 1 \\
\hline & & & 1 & 1 \\
\hline & 1 & 1 & 3 & 4 \\
\hline 1 & 2 & 2 & 100 & 101 \\
\hline \multirow[t]{6}{*}{1} & 2 & 2 & 347 & 349 \\
\hline & 32 & 32 & 1 & $\begin{array}{r}1 \\
32\end{array}$ \\
\hline & 10 & 39 & & 10 \\
\hline & 11 & 17 & & 11 \\
\hline & 12 & 12 & & 12 \\
\hline & & & 18 & 18 \\
\hline 9 & 28 & 28 & 6,840 & 6,868 \\
\hline 1 & 2 & 2 & $\begin{array}{r}2,065 \\
202\end{array}$ & $\begin{array}{r}2,066 \\
202\end{array}$ \\
\hline \multirow[t]{2}{*}{1} & 2 & 2 & $\begin{array}{r}376 \\
3\end{array}$ & $\begin{array}{r}378 \\
3\end{array}$ \\
\hline & & & 2 & 2 \\
\hline 2 & 2 & 2 & 371 & 373 \\
\hline \multirow[t]{2}{*}{1} & 2 & 2 & $\begin{array}{l}3,037 \\
1,364\end{array}$ & $\begin{array}{l}3,039 \\
1,364\end{array}$ \\
\hline & & & 781 & 781 \\
\hline \multirow[t]{3}{*}{1} & 2 & 2 & 892 & 894 \\
\hline & 1 & 1 & 476 & 477 \\
\hline & 1 & 1 & 334 & 335 \\
\hline 2 & 18 & 18 & 12 & 30 \\
\hline 1 & 2 & 2 & 338 & 340 \\
\hline 5 & 7 & 7 & 464 & 471 \\
\hline 4 & 6 & 6 & 315 & 321 \\
\hline 1 & 2 & 2 & 119 & 121 \\
\hline 3 & 4 & 4 & 196 & 200 \\
\hline \multirow[t]{2}{*}{1} & 1 & 1 & 149 & 151 \\
\hline & & & 126 & 126 \\
\hline 1 & 1 & $\begin{array}{r}1 \\
45\end{array}$ & 24 & 25 \\
\hline
\end{tabular}

e. Epilepsy, alcohol use disorders, Alzheimer's disease and other dementias, Parkinson's disease, multiple sclerosis, drug use disorders, post-traumatic stress disorder, obsessive-compulsive disorder, panic disorder, insomnia (primary), migraine, mental retardation attributable to lead exposure, and other neuropsychiatric disorders.

f. Rheumatic heart disease, hypertensive heart disease, inflammatory heart diseases, and other cardiovascular diseases. g. Other neoplasms, endocrine disorders, sense organ diseases, genitourinary diseases, skin diseases, musculoskeletal diseases, and oral conditions.

h. Poisonings, falls, fires, drownings, and other unintentional injuries.

i. Violence, war, and other intentional injuries. 
Table 6.4 Continued

\begin{tabular}{|c|c|c|c|c|c|c|}
\hline & & & & orld & & \\
\hline & & & & under age 5 & & \\
\hline & & Stillbirth & & & Deathe aued & Infant deaths \\
\hline & Antepartum & Intrapartum & Total & Neonatal & 28 days to $\leq 1$ year & 1 year) \\
\hline & & & $(\mathbf{a}+\mathbf{b})$ & & & \\
\hline & a & b & c & d & e & $f$ \\
\hline Total deaths & 2,192 & 1,082 & 3,274 & 3,896 & 3,762 & 7,658 \\
\hline $\begin{array}{l}\text { I. Communicable, maternal, perinatal, } \\
\text { and nutritional conditions }\end{array}$ & & & & 3,129 & & 6,910 \\
\hline A. Infectious and parasitic diseases & & & & 300 & & 2,886 \\
\hline 1. Tuberculosis & & & & & & 16 \\
\hline $\begin{array}{l}\text { 2. Sexually transmitted diseases } \\
\text { excluding HIV/AIDS }\end{array}$ & & & & & & 55 \\
\hline 3. HIV/AIDS & & & & & & 202 \\
\hline 4. Diarrheal diseases & & & & 116 & 1,105 & 1,222 \\
\hline 5. Childhood-cluster diseases & & & & & & 381 \\
\hline a. Pertussis & & & & & & 96 \\
\hline $\begin{array}{l}\text { b. Poliomyelitis } \\
\text { c. Diphtheria }\end{array}$ & & & & & & \\
\hline $\begin{array}{l}\text { c. Diphtheria } \\
\text { d. Measles }\end{array}$ & & & & & & $\begin{array}{r}2 \\
115\end{array}$ \\
\hline $\begin{array}{l}\text { d. Measles }{ }^{\mathrm{d}} \\
\text { e. Tetanus }\end{array}$ & & & & 168 & & $\begin{array}{l}115 \\
168\end{array}$ \\
\hline 6. Meningitis & & & & & & 47 \\
\hline 8. Malariac ${ }^{c}$ & & & & & & 726 \\
\hline Other ${ }^{d}$ & & & & & & 219 \\
\hline B. Respiratory infections ${ }^{\mathrm{e}}$ & & & & 1,013 & 523 & 1,536 \\
\hline C. Maternal conditions & & & & & & \\
\hline D. Perinatal conditions & & & & 1,832 & & 2,416 \\
\hline 1. Low birthweight ${ }^{\dagger}$ & & & & 1,098 & 136 & 1,225 \\
\hline 2. Birth asphyxia and birth trauma ${ }^{9}$ & & & & 734 & & 734 \\
\hline 3. Other perinatal conditions & & & & & & 457 \\
\hline E. Nutritional deficiencies & & & & & & 96 \\
\hline II. Noncommunicable diseases & & & & 321 & & 618 \\
\hline A. Malignant neoplasms & & & & & & 11 \\
\hline C. Diabetes mellitus & & & & & & 2 \\
\hline E. Neuropsychiatric disorders & & & & & & 22 \\
\hline 1. Unipolar depressive disorders & & & & & & \\
\hline 2. Bipolar affective disorder & & & & & & \\
\hline $\begin{array}{l}\text { 3. Schizophrenia } \\
\text { Otherh }\end{array}$ & & & & & & \\
\hline Other ${ }^{\mathrm{h}}$ & & & & & & 14 \\
\hline G. Cardiovascular diseases & & & & & & 58 \\
\hline 3. Ischemic heart disease & & & & & & 4 \\
\hline 4. Cerebrovascular disease & & & & & & 8 \\
\hline Otheri & & & & & & 43 \\
\hline H. Respiratory diseases & & & & & & 43 \\
\hline I. Digestive diseases & & & & & & 73 \\
\hline M. Congenital anomalies & & & & 321 & 48 & 368 \\
\hline Other ${ }^{i}$ & & & & & & 38 \\
\hline III. Injuries & & & & & & 126 \\
\hline A. Unintentional & & & & & & 121 \\
\hline 1. Road traffic accidents & & & & & & 12 \\
\hline Otherk ${ }^{k}$ & & & & & & 109 \\
\hline B. Intentional & & & & & & 6 \\
\hline $\begin{array}{l}\text { 1. Self-inflicted } \\
\text { Othorl }\end{array}$ & & & & & & \\
\hline Other & & & & & & 4 \\
\hline IV. Not allocated & 2,192 & 1,082 & 3,274 & 446 & & \\
\hline
\end{tabular}

Sources: WHO 2005a for columns a-d, unreported estimates undertaken as part of the GBD study, reported in chapter 3 for columns $f-g$, and chapter 3 of this volume. Note: The absence of an entry in columns a- $d$ denotes either a value of less than 1,000 deaths or that no estimate was allocated to that entry. For columns $f-k$, a blank cell indicates that fewer than 1,000 deaths are attributable to the specific cause. Infant and child deaths in columns $f$ and $g$ are based on unreported estimates undertaken as part of the GBD study, reported in chapter 3 , of the percentage of under five deaths that were under age one. Because the sources used for neonatal deaths left a large number unallocated, it is not appropriate to calculate values of column e by subtracting column d from column $\mathrm{f}$ except where explicitly noted.

a. WHO 2005b and Bryce and others (2005) estimate that 395,000 deaths occur due to measles. Chapter 3 provides an estimate for measles deaths age zero to four of 763,000

b. Lawn, Wilczynska, and Cousens (forthcoming) for the CHERG estimate $(2005)$ that 7 percent $(260,000)$ of the 3.854 million global neonatal deaths occur due to tetanus, similar to the WHO and GAVI estimates of 220,000 for the year 2000. Chapter 3 provides an estimate for tetanus deaths ages zero to four of only 187,000. The (unpublished) GBD files used here to allocate deaths under age five to over and under age one allocated 52 percent of tetanus deaths to under age one. The CHERG review (Lawn, Wilczynska, and Cousens forthcoming) suggests this to be a major underestimate, and the 52 percent figure has thus here been revised upward to 90 percent. Consistent with the objectives of this chapter, GBD numbers have been used wherever possible, and the CHERG and WHO estimates are accordingly revised downward by allocating all infant tetanus deaths to the neonatal period.

c. WHO 2005b and Bryce and others (2005) estimate that 853,000 deaths occur due to malaria. Chapter 3 provides an estimate for malaria deaths age zero to four of 1,208,000.

d. Hepatitis, tropical-cluster diseases, leprosy, dengue, Japanese encephalitis, trachoma, intestinal nematode infections, and other infectious diseases.

e. Deaths for respiratory infections in the neonatal age group are those estimated by Lawn, Cousens, and Wilczynska (forthcoming) for their category sepsis or pneumonia. This number was then subtracted from the GBD number of respiratory infections between age zero and one to derive the total in column e. 
Table 6.4 Continued

\begin{tabular}{|c|c|c|c|c|}
\hline \multicolumn{5}{|c|}{ World } \\
\hline \multicolumn{3}{|c|}{ Deaths under age 5} & \multirow[b]{2}{*}{$\begin{array}{c}\text { Deaths age } \\
5+\end{array}$} & \multirow[b]{2}{*}{ Total } \\
\hline $\begin{array}{l}\text { Child deaths } \\
\text { (1 } \leq \text { age } \\
<5 \text { years })\end{array}$ & $\begin{array}{l}\text { After live } \\
\text { birth }\end{array}$ & $\begin{array}{l}\text { Including } \\
\text { stillbirth }\end{array}$ & & \\
\hline$(h-f)$ & $(f+g)$ & $(h+c)$ & & $(i+j)$ \\
\hline $\mathbf{g}$ & h & i & j & k \\
\hline 2,948 & 10,606 & 13,880 & $\begin{array}{l}45,662 \\
\end{array}$ & 59,542 \\
\hline 2,523 & 9,433 & 9,433 & 8,741 & 18,174 \\
\hline 1,886 & 4,771 & 4,771 & 6,072 & 10,843 \\
\hline 25 & 40 & 40 & 1,566 & 1,606 \\
\hline 13 & 68 & 68 & 109 & 177 \\
\hline 138 & 340 & 340 & 2,236 & 2,576 \\
\hline 378 & 1,600 & 1,600 & 184 & 1,784 \\
\hline 668 & 1,049 & 1,049 & 315 & 1,364 \\
\hline \multirow[t]{2}{*}{205} & 301 & 301 & & 301 \\
\hline & & & 1 & 1 \\
\hline 2 & 5 & 5 & 1 & 6 \\
\hline 442 & 557 & 557 & 206 & 763 \\
\hline 19 & 187 & 187 & 107 & 293 \\
\hline 18 & 65 & 65 & 108 & 173 \\
\hline 361 & 1,087 & 1,087 & 121 & 1,208 \\
\hline 303 & 522 & 522 & 1,434 & 1,955 \\
\hline \multirow[t]{2}{*}{409} & 1,945 & 1,945 & 1,886 & 3,831 \\
\hline & & & 508 & 508 \\
\hline 107 & 2,522 & 2,522 & & 2,523 \\
\hline 76 & 1,301 & 1,301 & & 1,301 \\
\hline 5 & 739 & 739 & & 739 \\
\hline 25 & 482 & 482 & & 482 \\
\hline 99 & 194 & 194 & 274 & 469 \\
\hline 245 & 864 & 864 & 32,042 & 32,905 \\
\hline 27 & 38 & 38 & 6,986 & 7,024 \\
\hline 1 & 3 & 3 & 958 & 961 \\
\hline \multirow{4}{*}{24} & 46 & 46 & 1,034 & 1,079 \\
\hline & & & 13 & 13 \\
\hline & & & 1 & 1 \\
\hline & & & 23 & 23 \\
\hline 31 & 45 & 45 & 997 & 1,043 \\
\hline 27 & 84 & 84 & 16,316 & 16,401 \\
\hline 2 & 6 & 6 & 7,060 & 7,066 \\
\hline 4 & 13 & 13 & 5,379 & 5,392 \\
\hline 23 & 66 & 66 & 3,877 & 3,943 \\
\hline 21 & 64 & 64 & 3,540 & 3,604 \\
\hline 42 & 116 & 116 & 1,821 & 1,936 \\
\hline 71 & 439 & 439 & 68 & 507 \\
\hline 35 & 73 & 73 & 1,320 & 1,393 \\
\hline 183 & 310 & 310 & 4,879 & 5,188 \\
\hline 175 & 295 & 295 & 3,241 & 3,536 \\
\hline 39 & 51 & 51 & 1,139 & 1,190 \\
\hline 150 & 244 & 244 & 2,102 & 2,346 \\
\hline \multirow[t]{2}{*}{8} & 14 & 14 & 1,638 & 1,652 \\
\hline & & & 875 & 875 \\
\hline \multirow{2}{*}{10} & 14 & 14 & 763 & 777 \\
\hline & & 3,274 & & 3,274 \\
\hline
\end{tabular}

f. Low-birthweight deaths are those resulting from intrauterine growth retardation or preterm birth. Almost all lowbirthweight deaths in the neonatal period result from preterm birth.

g. Lawn, Wilczynska, and Cousens (forthcoming) for the CHERG estimate that 23 percent $(887,000)$ of the 4 million global neonatal deaths occur due to birth asphyxia. Chapter 3 of this volume provides an estimate for birth asphyxia and birth trauma deaths ages zero to four of only 739,000 globally, of which 734,000 were estimated to occur under age one. Consistent with the objectives of this chapter, GBD numbers have been used wherever possible, and the CHERG and WHO estimates are accordingly revised downward by allocating all infant birth asphyxia deaths to the neonatal period. Better data in the future will allow for improved estimates.

h. Epilepsy, alcohol use disorders, Alzheimer's disease and other dementias, Parkinson's disease, multiple sclerosis, drug use disorders, post-traumatic stress disorder, obsessive-compulsive disorder, panic disorder, insomnia (primary), migraine, mental retardation attributable to lead exposure, and other neuropsychiatric disorders.

i. Rheumatic heart disease, hypertensive heart disease, inflammatory heart diseases, and other cardiovascular diseases.

j. Other neoplasms, endocrine disorders, sense organ diseases, genitourinary diseases, skin diseases, musculoskeletal

diseases, and oral conditions.

k. Poisonings, falls, fires, drownings, and other unintentional injuries.

I. Violence, war, and other intentional injuries. 
DALYs generate a measure of the disease burden resulting from premature mortality by integrating a discounted, ageweighted, disability-adjusted stream of life years from the age of death (see equation 6A.2 in annex 6A). The formulation within the family of DALYs previously used to empirically assess the global burden of disease specifies a constant discount rate of 3 percent per year and an age-weighting function that gives low weight to early childhood and older ages and greater weight to middle ages. This volume reports global burden of disease estimates generated using uniform age weights. Chapter 5 provides an extensive exploration of the uncertainty and sensitivity inherent in disease burden assessment, including the results of differing assumptions about age weighting and discount rates.

To be clear about the particular form of DALY being used, the following terminology is employed throughout this volume. DALYs $(r, K)$ are DALYs constructed using a discount rate of $r$ percent per year and an amount of age weighting indexed by a parameter $K$. Two versions of the DALY are discussed at some length in chapter 5, both using a discount rate of 3 percent per year. DALYs $(3,1)$ are DALYs generated with a discount rate of 3 percent per year and with full age weighting, that is, $K=1$. DALYs $(3,0)$ are DALYs generated with a discount rate of 3 percent per year and with no age weighting, that is, $K=0$. This volume's results concerning the burden of disease (chapter 3 ) and of risk (chapter 4) are based on DALYs $(3,0)$. Annex 6B contains tables summarizing alternative calculations of the global burden of disease, and table 6B.4 presents the chapter 3 GBD results based on $\operatorname{DALYs}(3,0)$, using this chapter's aggregation of causes, for age groups under five and over five as an aggregate.

This chapter extends the DALY family by modeling a concept of ALP. The intuition behind the ALP concept is that an infant (or fetus) only gradually acquires the full life potential reflected in a stream of life years beginning at birth, that is, ALP can be gradual. The ethical understanding of the concept is based on two judgments: (a) an individual life acquires value only as it acquires self-awareness, and (b) an individual life acquires additional value as it develops bonds with others. (See the discussion in Steinbock 1992, who argues that what we label as life potential is probably acquired some time in the second trimester of pregnancy. Her position is, implicitly, that whenever it occurs, ALP is instantaneous.) To some extent, the age-weighting function of the current DALY formulation attempts to capture these judgments, and in this chapter, gradual acquisition of ALP is modeled as an alternative to age weighting. ${ }^{3}$ Mathematically, however, ALP

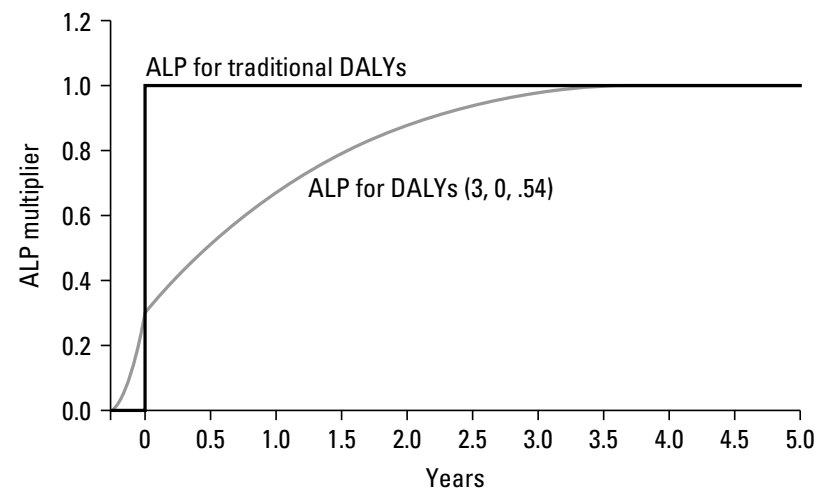

Source: Authors' calculations.

Figure 6.2 ALP, Traditional DALYs, and DALYs $(3,0, .54)$

and age weighting are independent and can be introduced simultaneously.

Our objective in this chapter is not to provide a detailed philosophical, economic, or medical rationale for gradual ALP, but to generate and apply a straightforward mechanism that allows for it. Annex 6A describes this mechanism, which essentially consists of multiplying the DALYs conventionally generated by a factor that is less than one for younger ages. This factor is zero for an age of -13 weeks (or -0.25 years), rises to a factor value of $\mathrm{f}^{0}$ at birth, then rises to 1 at time T. Figure 6.2 graphs both the ALP function used later in this chapter and the special case of ALP that jumps from 0 to 1 at age 0 (instantaneous ALP). The ALP implicit in traditional DALYs is instantaneous.

Annex 6A introduces a parameter, $A$, that indicates the speed of ALP (see equations 6A.3 through 6A.5 for a precise definition of $A$ ). $A$ is constructed so that for the fastest possible speed of ALP, namely, instantaneous ALP, $A=1 . A$ is bounded below by 0 . This chapter extends the notation $\operatorname{DALYs}(r, K)$ used elsewhere in the book in two ways. First, it explicitly indicates the level of $A$ by extending the DALY nomenclature to DALYs $(r, K, A)$. Thus using this nomenclature, DALYs $(3,0)$ become DALYs $(3,0,1)$, because the standard DALY is the special case with instantaneous ALP. Second, when stillbirths are included in the range of events to be measured in the global burden of disease, this is explicitly noted in the DALY nomenclature as $\operatorname{DALYs}_{\mathrm{SB}}(r, K, A)$. Notation around YLL is similarly extended.

Explicit modeling of ALP allows not only the reflection of the ethical judgments just indicated, but also permits three 
instrumentally useful improvements to the current family of DALYs:

- The DALY loss from a death seconds before birth is, in the current formulation, 0 ; it jumps to more than 30 years at birth. The ALP formulation allows, but does not require, this discontinuity to be avoided. See column (a) of table 6.5 for values at different ages of the ALP function associated with traditional DALYs and columns (c), (d), and (e) for values of three ALP functions defined in annex $6 \mathrm{~A}$.

- The ALP formulation allows, but does not require, a positive DALY loss associated with stillbirths.

- The ratio of the DALY loss from a death at age 20, say, to that at birth is close to 1 for any reasonable set of parameter values in the current DALY formulation. Many people's

Table 6.5 Values of Selected ALP Functions

\begin{tabular}{cccccc}
\hline $\begin{array}{c}\text { (a) } \\
\mathbf{t}(\mathbf{a g e})\end{array}$ & $\begin{array}{c}(\mathbf{b}) \\
\mathbf{f}_{\mathbf{D}}(\mathbf{t})^{\mathbf{a}}\end{array}$ & $\begin{array}{c}(\mathbf{c}) \\
\mathbf{f}_{\mathbf{D S B}}(\mathbf{t})^{\mathbf{b}}\end{array}$ & $\begin{array}{c}(\mathbf{d}) \\
\mathbf{f}_{\mathbf{1}}(\mathbf{t})\end{array}$ & $\begin{array}{c}(\mathbf{e}) \\
\mathbf{f}_{\mathbf{2}}(\mathbf{t})^{\mathbf{c}}\end{array}$ & $\begin{array}{c}(\mathbf{f}) \\
\mathbf{f}_{\mathbf{3}}(\mathbf{t})\end{array}$ \\
\hline-0.25 & 0.00 & 1.00 & 0.00 & 0.00 & 0.00 \\
-0.08 & 0.00 & 1.00 & 0.12 & 0.16 & 0.30 \\
0.00 & 1.00 & 1.00 & 0.25 & 0.30 & 0.50 \\
0.02 & 1.00 & 1.00 & 0.25 & 0.31 & 0.52 \\
0.08 & 1.00 & 1.00 & 0.26 & 0.34 & 0.59 \\
0.25 & 1.00 & 1.00 & 0.29 & 0.41 & 0.74 \\
0.30 & 1.00 & 1.00 & 0.30 & 0.43 & 0.78 \\
0.50 & 1.00 & 1.00 & 0.34 & 0.51 & 0.88 \\
1.00 & 1.00 & 1.00 & 0.41 & 0.67 & 0.98 \\
2.00 & 1.00 & 1.00 & 0.55 & 0.87 & 1.00 \\
3.00 & 1.00 & 1.00 & 0.66 & 0.97 & 1.00 \\
5.00 & 1.00 & 1.00 & 0.83 & 1.00 & 1.00 \\
\hline
\end{tabular}

Source: Authors' calculations.

a. $f_{D}(t)$ is the traditional DALY formulation that is, stillbirths are not incorporated, and ALP is instantaneous

b. $\mathrm{f}_{D S B}(t)$ is the traditional DALY formulation extended to give equal weight to stillbirths as to deaths at age 0 , that is, it leads to $\operatorname{DALY}_{S B}(3,0,1)$.

c. $f_{2}(t)$ is the ALP function used to generate the $\operatorname{DALY}_{S B}(3,0,54) \mathrm{GBD}$ estimates reported in table 6B.8. These are DALYS that incorporate stillbirth and gradual ALP.

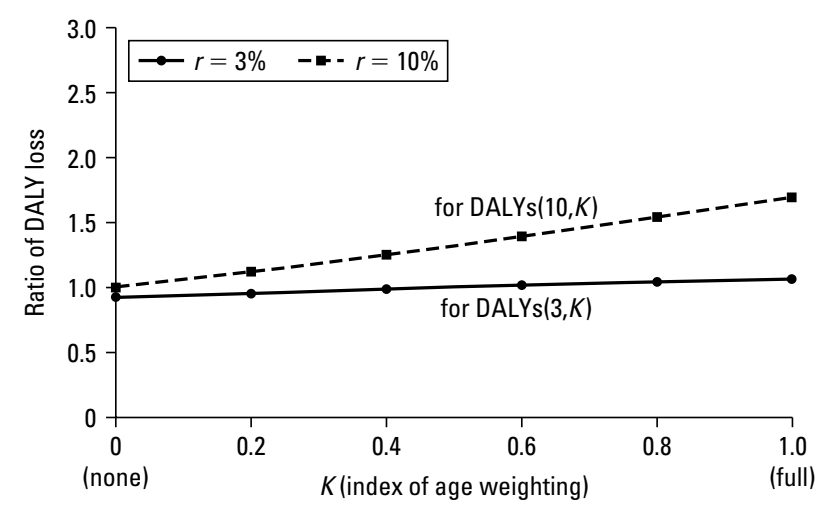

Source: Authors' calculations.

Figure 6.3 Ratio of DALYs Lost at Age 20 to Age 0 as a Function of Age Weighting ethical judgments would give this ratio a value substantially greater than 1 . The ALP formulation allows, but does not require, these judgments. Figure 6.3 shows how this ratio varies as a function of the age-weighting parameter $(K)$ for values of $r$ equal to 3 percent and 10 percent. The ratio rises only to 1.7 with full age weighting and an implausibly high discount rate of 10 percent.

\section{Alternative Calculations of the Burden of Disease}

As previously indicated, annex table 6B.4 (based on annex tables $6 \mathrm{~B} .1$ to $6 \mathrm{~B} .3$ ) presents the chapter $3 \mathrm{GBD}$ estimates in terms of DALYs $(3,0)$ - or DALYs $(3,0,1)$ - for the under and over five age groups. The $\operatorname{DALY}(3,0)$ is the sum of the YLL $(3,0,1)$ and YLD. Annex tables 6B.1, 6B.2, and 6B.3 report deaths by cause, $\operatorname{YLL}(3,0,1)$ by cause, and YLD by cause from chapter 3 . The numbers in table 6B.4 are the sum of the corresponding numbers in tables 6B.2 and 6B.3.

We generate two alternative assessments of the global burden of disease. Both incorporate stillbirths and the second permits gradual ALP. The YLD numbers that we use come from annex table 6B.3. The YLL differ from $\operatorname{YLL}(3,0,1)$ for ages under age five, but are the same for over age five.

Our first alternative is probably the simplest way to incorporate stillbirths. It does so by having an instantaneous ALP function, as with traditional DALYs, but by having that function jump from 0 to 1 at age -13 weeks $(-0.25$ years) instead of at age 0 . Stillbirths are then given the same DALY loss as a death at birth in generating YLL. Column (b) of table 6.5 shows values for this ALP function, which is uniformly 1 . We label the YLL generated from this ALP function and a 3 percent discount rate the $\mathrm{YLL}_{\mathrm{SB}}(3,0,1)$. We label the DALYs based on this YLL as DALYs $\mathrm{sB}_{\mathrm{SB}}(3,0,1)$. Table 6.6 shows values of $\mathrm{YLL}_{\mathrm{SB}}(3,0,1)$ compared with $\operatorname{YLL}(3,1)$ and YLL $(3,0)$ for different ages. Annex table 6B.5 shows values for $\mathrm{YLL}_{\mathrm{SB}}(3,0,1)$ and annex table $6 \mathrm{~B} .6$ shows the resulting burden of disease estimates in terms of DALYs $s_{\mathrm{SB}}(3,0,1)$.

Our second alternative burden of disease assessment is based on gradual ALP. Equation 6A.1 in annex 6A provides our general ALP function and the text describes the meaning of its four parameters. One of the parameters, $\mathrm{f}^{0}$, is the value of the function at age 0 . The intuitive interpretation of $\mathrm{f}^{0}$ is that it is approximately the ratio of the YLL loss associated with a death at age 0 to that from a death at age 20 . Another parameter is T, the age at which the function becomes 1. Annex 6A characterizes three alternative gradual ALPs: $\mathrm{f}_{1}, \mathrm{f}_{2}$, and $\mathrm{f}_{3}$. Figure 6.4 shows YLL at different ages for these functions and for $\operatorname{YLL}(3,0)$ and $\operatorname{YLL}(3,1)$. Table 6.5 shows values for the functions at different ages in 
Table 6.6 Discounted YLL at Different Ages of Death for Several DALY Formulations

\begin{tabular}{lccccc}
\hline Age group & $\begin{array}{c}\text { Representative } \\
\text { age of death } \\
\text { (years) }\end{array}$ & $\mathbf{Y L L}(\mathbf{3}, \mathbf{1})$ & $\mathbf{Y L L}(\mathbf{3}, \mathbf{0})$ & $\mathbf{Y L L}_{\mathbf{s B}_{\mathbf{B}} \mathbf{( 3 , 0 , \mathbf { 1 } )}}$ & $\mathbf{Y L L}_{\mathbf{s B}} \mathbf{( 3 , 0 , \mathbf { 0 }}$ \\
\hline Antepartum & -0.080 & 0 & 0 & 30.42 & 4.95 \\
Intrapartum & -0.001 & 0 & 0 & 30.42 & 9.13 \\
Neonatal & 0.020 & 33.09 & 30.42 & 30.42 & 9.40 \\
Infant & 0.300 & 33.36 & 30.40 & 30.40 & 12.95 \\
Postneonatal & 0.500 & 33.56 & 30.39 & 30.39 & 15.42 \\
Child & 2.000 & 34.81 & 30.28 & 30.28 & 26.40 \\
\hline
\end{tabular}

Source: Authors' calculations.

Note: $Y L L(3,1), Y L L(3,0)$, and $Y L L_{S B}(3,0,1)$ assume instantaneous $\operatorname{ALP}(A=1)$. $Y L L(3,1)$ assumes full age weighting $(K=1)$; the other three formulations assume uniform age weights $(K=0)$. $Y L_{S B}(3,0,54)$ assumes gradual acquisition of life potential $(A=.54)$; table $6 \mathrm{~B} .7$ reports these $Y L L$ and $6 \mathrm{~B} .8$ reports the GBD based on their use.

a. Stillbirth to age 2

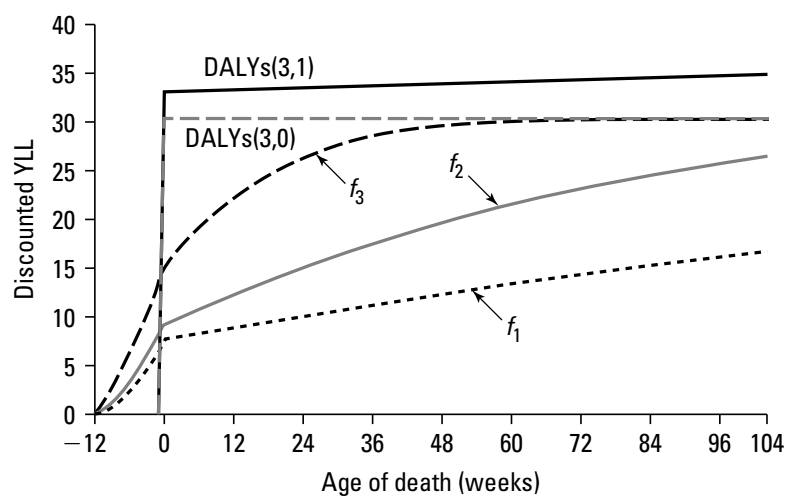

Source: Authors' calculations.

Figure 6.4 YLL for Deaths at Different Ages

columns (c), (d), and (e). We use $\mathrm{f}_{2}$ (with $A=.54$ ) to construct the disease burden estimates reported in this chapter and label the resulting YLL and DALYs as $\mathrm{YLL}_{\mathrm{SB}}(3,0, .54)$ and $\operatorname{DALYs}_{\mathrm{SB}}(3,0, .54)$. Table 6.6 shows $\mathrm{YLL}_{\mathrm{SB}}(3,0, .54)$, which are, as intended, markedly lower than $\mathrm{YLL}_{\mathrm{SB}}(3,0,1)$ for very young ages. That is, $\mathrm{YLL}_{\mathrm{SB}}(3,0, .54)$ gives less weight to deaths near the time of birth or to deaths immediately after birth than $\mathrm{YLL}_{\mathrm{SB}}(3,0,1)$.

Only a limited number of empirical studies have attempted to assess directly the views of individuals concerning deaths at different ages. In an important early study, Crawford, Salter, and Jang (1989) relate grief from a death to the concept of reproductive potential in population biology. They conclude that for several diverse human groups the relationship shows grief to be closely related to prehistoric reproductive value. Cropper, Aydede, and Portney (1994) and Johannesson and Johansson (1997) survey members of populations of high-income countries for trade-offs between deaths in middle and older ages. All three of these studies find that people judge deaths at older middle age as much less important than deaths at younger middle age, but provide no information concerning the trade-off for deaths near the time of birth.

An Institute of Medicine (1985) review of vaccine development priorities uses infant mortality equivalence in cost-effectiveness calculations. The committee members preparing the report collectively judged that the loss from a death at age 20 should be about two times that from an infant death, well above the numbers shown in figure 6.3 for any standard DALY. However, some preliminary trade-off studies by one of the authors of this chapter suggest a value closer to three or four times. What is clear is that no defensible estimate (or even range) is currently available, and hence the numbers we report should be viewed only as 
perhaps reasonable but only suggestive and as indicating the sensitivity of global burden of disease results from younger ages to better estimates of this parameter.

Annex tables 6B.7 and 6B.8 show $\mathrm{YLL}_{\mathrm{SB}}(3,0, .54)$ and DALYs $_{\mathrm{SB}}(3,0, .54)$. While table $6 \mathrm{~B} .7$ only shows the total of DALYs for ages under five, the calculations underlying those totals reflect the age distribution of deaths under age five shown in table 6.4 and the $\mathrm{YLL}_{\mathrm{SB}}(3,0, .54)$ for deaths at different ages as shown in table 6.6.

Annex tables 6B.1, 6B.6, and 6B.8 provide three alternative assessments of the global burden of disease based on deaths by cause, on DALYs(3,0), DALYs $\mathrm{DB}_{\mathrm{SB}}(3,0,1)$, and DALYs $_{\mathrm{SB}}(3,0, .54)$. Table 6.4 provides estimates of deaths by cause that include stillbirths (table 6.4, column $[\mathrm{k}]$ ). We thus have five alternative indicators of the importance of disease at different ages and from different causes. Table 6.7 provides a summary for low- and middle-income countries of the distribution of the disease burden at different ages as assessed by these different measures. DALYs $\mathrm{SB}_{\mathrm{SB}}(3,0,1)$ and $\operatorname{DALYs}_{\mathrm{SB}}(3,0, .54)$ both point to the significance of stillbirths relative to DALYs $(3,0)$, which exclude them altogether, but the gradual ALP approach of DALYs $\mathrm{SB}_{\mathrm{SB}}(3,0, .54)$ gives much less importance to stillbirths than $\operatorname{DALYs}_{\mathrm{SB}}(3,0,1)$ and substantially less importance to the under five burden than $\operatorname{DALYs}(3,0)$.

Table 6.8 provides a similar summary of how the assessed burden across groups varies with the measure used. DALYs $_{\mathrm{SB}}(3,0, .54)$ give more weight to Group II (noncommunicable diseases) and Group III (injuries) causes than do $\operatorname{DALYs}(3,0)$, while DALYs $s_{S B}(3,0,1)$ give less weight to these groups than DALYs $(3,0)$. For example, $\operatorname{DALYs}_{\mathrm{SB}}(3,0, .54)$ give about a 10 percent greater weight to cardiovascular disease than does the DALY $(3,0)$, that is, 14.2 percent versus 12.9 percent.

Table 6.7 Disease Burden at Different Ages Using Different Measures, Low- and Middle-Income Countries, 2001

\begin{tabular}{|c|c|c|c|c|c|}
\hline \multirow[b]{2}{*}{ Age group } & \multicolumn{2}{|c|}{ Percentage of deaths } & \multicolumn{3}{|c|}{ Percentage of disease burden } \\
\hline & Stillbirths excluded & Stillbirths included & $\operatorname{DALYs}(\mathbf{3}, \mathbf{0})$ & $\operatorname{DALYs}_{\mathrm{SB}}(3,0,1)$ & $\operatorname{DALYs}_{\mathrm{SB}}(3,0, .54)$ \\
\hline $\begin{array}{l}\text { Total deaths or DALYs } \\
\text { (millions) }\end{array}$ & 48.4 & 51.6 & $1,387.4$ & $1,485.6$ & $1,260.6$ \\
\hline Stillbirths & $0 \%$ & $6.3 \%$ & $0 \%$ & $6.6 \%$ & $1.6 \%$ \\
\hline Under age one & 15.7 & 21.0 & & & \\
\hline Under age five & 21.8 & 26.7 & 30.6 & 35.2 & 23.6 \\
\hline Over age five & 78.2 & 73.3 & 69.4 & 64.8 & 76.4 \\
\hline
\end{tabular}

Source: Authors' calculations.

Note: All three percentage of disease burden formulations assume a $3 \%$ discount rate and uniform age weights (that is, $K=0)$. DALYS $(3,0)$ and DALYS $(3,0,1)$ assume instantaneous $A L P(A=1)$. For $\operatorname{DALYs}(3,0,54), A=.54$.

Table 6.8 Disease Burden from Selected Groups of Causes Using Different Measures, Low- and Middle-Income Countries, 2001

\begin{tabular}{|c|c|c|c|c|c|}
\hline \multirow[b]{2}{*}{ Cause } & \multicolumn{2}{|c|}{ Percentage of deaths } & \multicolumn{3}{|c|}{ Percentage of disease burden } \\
\hline & Stillbirths excluded & Stillbirths included & DALYs(3,0) & $\operatorname{DALYs}_{\mathrm{SB}}(3,0,1)$ & $\operatorname{DALYs}_{S B}(3,0, .54)$ \\
\hline $\begin{array}{l}\text { Total deaths or DALYs } \\
\text { (millions) }\end{array}$ & 48.4 & 51.6 & $1,387.4$ & $1,485.6$ & $1,260.6$ \\
\hline Group $\mathrm{I}^{\mathrm{a}}$ & $36.4 \%$ & $40.4 \%$ & $39.8 \%$ & $43.8 \%$ & $35.5 \%$ \\
\hline Group II & 53.8 & 50.5 & 48.9 & 45.7 & 52.4 \\
\hline $\begin{array}{c}\text { (Of which cardiovascular } \\
\text { diseases) }\end{array}$ & (27.6) & (25.9) & (12.9) & (12.0) & $(14.2)$ \\
\hline Group III & 9.8 & 9.1 & 11.2 & 10.5 & 12.1 \\
\hline
\end{tabular}

Source: Authors' calculations.

Note: All three percentage of disease burden formulations assume a $3 \%$ discount rate and uniform age weights (that is, $K=0)$. DALYS $(3,0)$ and DALYS $(3,0,1)$ assume instantaneous $\mathrm{ALP}(A=1)$. For $\operatorname{DALYS}(3,0,54), A=.54$

a. The "not allocated" category (from Table 6.4) consists principally of Group I causes and is included with Group I here. 


\section{CONCLUSIONS}

Previous assessments of the global burden of disease have not included stillbirths or sufficiently emphasized the important causes of neonatal death. This was understandable given the intended focus of these studies. In addition, the inclusion of stillbirths would have highlighted issues about how to weight deaths at different ages that would have been difficult to incorporate into the DALY metrics being used to assess the global burden of disease.

Data on the numbers of stillbirths and neonatal deaths have improved, and a recent major effort by CHERG now provides a much better picture than before of the causes of neonatal death. (Annex C describes the CHERG effort and compares its results with estimates that result from fitting the CHERG estimates into the overall death envelope of chapter 3.) This chapter proposes an approach that incorporates modeling ALP, which allows flexibility in assessing how to weight stillbirths and other early deaths in constructing aggregate measures of the disease burden. This chapter combines new information and new methods into a reassessment of the global burden of disease that is based closely on, but goes beyond, what is reported in chapter 3 .

We draw the following conclusions from this exercise:

- The numbers of stillbirths and of neonatal deaths are large. This underscores the importance of implementing tools and policies for addressing them. A number of recent publications point to directions for policy (for example, Darmstadt and others 2005; Institute of Medicine 2003; Lawn and others 2006; Martines and others 2005; Stoll and Measham 2001; Tinker and others 2005; WHO 2005b; Zupan 2005).

- The inclusion of stillbirths within the standard GBD framework is now feasible, and future assessments of the global burden of disease could consider doing so.

- The GBD cause structure would need relatively minor modifications to incorporate deaths at early ages. Birth asphyxia and preterm births could be separate subcategories and sepsis and pneumonia could also be included as a separate category. Rather than reporting a single burden estimate for the under five age group, the more finegrained age breakdown of table 6.4 could be used.

- The databases on numbers and causes of stillbirths and neonatal deaths require major investments so they can be improved. Undertaking a CHERG type of review of the existing literature to gain a better understanding of the causes of stillbirths is also a priority.
- The selection of a generally appropriate ALP function requires more data on preferences or trade-offs concerning deaths at different ages.

\section{ANNEX 6A: FLEXIBLE FUNCTIONAL FORMS FOR THE ACQUISITION OF LIFE POTENTIAL}

This annex provides a technical discussion of issues raised by incorporating late fetal deaths (stillbirths) into the global burden of disease, as measured within the disabilityadjusted life year (DALY) framework. One approach is simply to take the DALY loss at birth and discount back to the time of the stillbirth, indicating that there are no life years to lose before birth, but that there are still all the postpartum life years. Essentially this is the standard DALY, but with an age-weighting function equal to 0 before birth. This is feasible, but has several potential drawbacks, in particular, any reasonable discount rate (for example, 3 percent) would thence count all late fetal losses almost the same as a loss at birth. This approach yields the DALYs $\mathrm{S}_{\mathrm{SB}}(3,0,1)$ measure as described in the main text, and table 6B.6 presents global burden of disease estimates using DALYs $\mathrm{SB}_{\mathrm{SB}}(3,0,1)$ because these are the simplest extension of $\operatorname{DALYs}(3,0)$.

However, as with traditional DALYs, DALYs $\mathrm{SB}_{\mathrm{SB}}(3,0,1)$ assume instantaneous acquisition of life potential (ALP), as illustrated in figure 6.2 and discussed in the main text. Whether or not one wishes to include stillbirths in the global burden of disease, this discontinuity (at some given age) is troublesome. The purpose of this annex is to provide a flexible, yet tractable, explicit function that allows for gradual ALP.

One natural approach is to weight the YLL from outside the integral instead of from the inside (as with age weighting), that is, to create a multiplier function (the ALP function), which takes on values between 0 and 1 as a function of age, and use it to ratchet down the YLL function, potentially starting before birth. For convenience and with some regard to the known physiological underpinnings, we take this starting point in time to be the beginning of the third trimester of pregnancy. Roughly speaking, the rate of natural fetal loss becomes noticeable after the beginning of some level of consciousness during the second half of the second trimester. One could force this function to equal 1 at birth, recovering the standard DALYs from that point onward, and this will be a special case of our formulation. However, we have no definitive reason to think that ALP is necessarily complete at birth. Indeed, quite a bit of evidence suggests that in many (if not all) societies worldwide, infants are not given full status, for instance, they are not always named immediately. Thus we 
wish to allow for continued gradual acquisition after birth and up to some time $\mathrm{T}$ that signifies full standing or full ALP. Likewise, starting the acquisition only at birth but proceeding gradually afterward is perfectly possible.

Turning to the specifics, denote the ALP multiplier function by $f(t)$, where $t$ is measured in years and ranges from -0.25 (that is, 13 weeks before birth, the beginning of the third trimester) to $\mathrm{T}$. The function is meaningfully defined for any finite value of $\mathrm{T}$, though it is natural to assume that full life potential is achieved by puberty at the latest. Thus $\mathrm{f}(-0.25)=0$ and $\mathrm{f}(\mathrm{T})=1$. We let $\mathrm{f}^{0}=\mathrm{f}(0)$ be the value at 0 . Of course, starting times other than -0.25 are perfectly legitimate as well, but -0.25 is the natural choice given the standard definitions of stillbirth and the gathering and reporting of data using that definition.

We need a functional form that smoothly begins at 0 and rises to $\mathrm{f}^{0}$, which is at least weakly convex (following the intuition that life potential is acquired increasingly rapidly as birth is approached), and whose curvature is parametrizable. The natural choice is $\mathrm{x}^{\gamma}$ with $\gamma \geq 1$. This has canonical endpoints of 0 and 1 , where $\mathrm{x}^{\gamma}$ takes on the values 0 and 1 , respectively, for any $\gamma$, so that as we change the curvature (or skewness), the endpoints remain fixed. Fitting this to our specific domain, we get $\mathrm{x}=4 \mathrm{t}+1$ for $-0.25 \leq \mathrm{t}<0$. Finally, if we wish the skewness parameter to lie between 0 and 1 as well (for clarity), we can define $g$ so that $\mathrm{g}=1 /(1-\mathrm{g})$ for $0 \leq \mathrm{g}<1$. This yields $\mathrm{f}_{-}(\mathrm{t})=$ $\mathrm{f}^{0}(4 \mathrm{t}+1)^{1 /(1-\mathrm{g})}$ for $-0.25 \leq \mathrm{t}<0$. Thus $\mathrm{g}=0$ produces a straight line (zero curvature), while $g=1$ (defined by fiat) is infinitely skewed: 0 until birth and then jumping to $\mathrm{f}^{0}$.

For $\mathrm{t} \geq 0$, we consider the symmetric version of the same polynomial, that is, $1-(1-\mathrm{x})^{\beta}$. Again we fit this to our domain, namely, from $\mathrm{t}=0$ to $\mathrm{t}=\mathrm{T}$, and define $\mathrm{b}$ so that $\mathrm{b}=1 /(1-\mathrm{b})$ for the skewness. This yields $\mathrm{f}_{+}(\mathrm{t})=$ $1-\left(1-\mathrm{f}^{0}\right)[(\mathrm{T}-\mathrm{t}) / \mathrm{T}]^{1 /(1-\mathrm{b})}$ for $0 \leq \mathrm{t} \leq \mathrm{T}$. We check that indeed $\mathrm{f}_{+}(0)=\mathrm{f}^{0}$ and $\mathrm{f}_{+}(\mathrm{T})=1$ according to this formula for any $0 \leq \beta \leq 1$. If $\mathrm{T}=1$, the formula simplifies to $\mathrm{f}_{+}(\mathrm{t})=1-\left(1-\mathrm{f}^{0}\right)(1-\mathrm{t})^{1 /(1-b)}$. This leaves four parameters: $\mathrm{f}^{0}, \mathrm{~T}, \mathrm{~g}$, and $\mathrm{b}$. We can additionally impose $\mathrm{g}=\mathrm{b}$ if we wish, but this is unnecessary.

Summarizing, the function we use for ALP is

$\mathrm{f}(\mathrm{t})=\left\{\begin{array}{l}\mathrm{f}_{-}(\mathrm{t})=\mathrm{f}^{0}(4 \mathrm{t}+1)^{1 /(1-\mathrm{g})} \\ \quad(\text { for } \quad-0.25 \leq \mathrm{t}<0) \\ \\ \mathrm{f}_{+}(\mathrm{t})=1-\left(1-\mathrm{f}^{0}\right)[(\mathrm{T}-\mathrm{t}) / \mathrm{T}]^{1 /(1-\mathrm{b})} \\ \quad(\text { for } 0 \leq \mathrm{t} \leq \mathrm{T}) .\end{array}\right.$

If $f_{D}(t)$ is the standard DALY formulation (whether or not age weighting or discounting is used), then $\mathrm{g}=\mathrm{b}=1$ (that is, discontinuous jumps around birth from 0 to 1 ) and $\mathrm{f}_{\mathrm{D}}^{0}=1$, so that technically at age 0 the value is already 1 (so the discontinuity is on the left side of age 0 only). Given these parameters, $\mathrm{T}$ is immaterial, because the function achieves its maximum immediately. However, the fact that we can replicate the standard DALY means that the gradual acquisition function does indeed generalize it.

Combining these equations with the standard definition of DALYs, the total loss $\mathrm{L}$ (a) for a death at age $\mathrm{a} \geq-0.25$ is

$$
\mathrm{L}(\mathrm{a})=\mathrm{f}(\mathrm{a}) \int_{\mathrm{a}}^{\infty} C x e^{-\beta \mathrm{x}} \mathrm{e}^{-\mathrm{r}(\mathrm{x}-\mathrm{a})} \mathrm{s}_{\mathrm{a}}(\mathrm{x}) \mathrm{dx},
$$

where $\beta$ is the age-weighting parameter (typically 0.04 ) if age weighting is used, $r$ is the discount rate (typically 0.03 ), $\mathrm{s}_{\mathrm{a}}(\mathrm{x})$ is the survival probability for reaching age $\mathrm{x} \geq \mathrm{a}$ conditional on having reached age a, and $C$ is the normalization parameter for the age weights $(C=0.16243$, see the discussion in chapter 5).

The normalization parameter $C$ in equation (6A.2) was chosen so that the total global burden of disease would be the same with and without age weighting. The index of age weighting referred to in the main text, $K$, is generated by having a weighted average-with weights of $K$ and $(1-K)$, where $0 \leq K \leq 1$ - of loss functions $\mathrm{L}$ (a) that result from equation (6A.2) with the indicated values of $\beta$ and $C$ and a loss function assuming uniform age weights. That this is at least approximately the case is apparent from figure $6.4 \mathrm{~b}$, where the two functions cross at about age 40 . Clearly this will not be true when any of the acquisition functions are used, because they reduce the YLL burden at younger ages with no corresponding increase elsewhere, leading to a reduced total burden as measured by absolute DALY levels.

Note, however, that the total burden is no longer the same even for DALYs(3,0) and DALYs(3,1), because the specific value of $C$ was calibrated to 1990 morbidity and mortality statistics. One can readily imagine more neutral (and invariant) normalizations, such as requiring a constant integral over age of death for each of these YLL functions, or perhaps weighting this integral using an idealized survival table. Any variant along these lines would raise the total level of DALYs $(3,0, .54)$ relative to both $\operatorname{DALYs}(3,0)$ and $\operatorname{DALYs}(3,1)$. Of course, we are for the most part interested only in the relative burden across ages or disease categories, so the absolute totals are of secondary importance.

Finally, to somewhat simplify the number of parameters in the ALP function, we introduce a notion of speed of acquisition, $A$. Recall that $\mathrm{f}^{0}$ can be anywhere between 0 and 1 , regardless of whether the function $f(t)$ takes on positive values before birth. If $\mathrm{f}^{0}=1$ (as in the original DALY), 
then $\mathrm{f}=1$ thereafter and the speed $A$ is in some sense as large as possible. To generalize this idea, we look at the total area between the ALP function $f(t)$ and the constant function 1.

Formally, this area is given by the integral of $1-\mathrm{f}(\mathrm{t})$, evaluated from $t^{0}$ to $T$, where $t^{0}$ is the first $t$ such that $f(t)>0$. It is thus typically either -0.25 or 0 , depending on whether we are including stillbirths. Call this integral $I$ :

$$
I=\int_{\mathrm{t}}^{\mathrm{T}}[1-\mathrm{f}(\mathrm{t})] \mathrm{dt}
$$

Substituting the second part of equation (6A.1), we can evaluate this integral as

$$
I=\mathrm{T}\left(1-\mathrm{f}^{0}\right)\left(1-\mathrm{t}^{0} / \mathrm{T}\right)(1-\mathrm{b}) /(2-\mathrm{b}) .
$$

Normalizing so that the speed $A$ lies between 0 and 1 (and higher values denote faster acquisition), we define

$$
A=1 /(1+\mathrm{I}) \text {. }
$$

For example, for $\mathrm{b}=0.7$ (a typical value) and $\mathrm{t}^{0}=0$, we obtain a simple formula for the speed parameter $A$, encapsulating the acquisition function in a single number: $A=$ $1 /\left[1+0.23 \mathrm{~T}\left(1-\mathrm{f}^{0}\right)\right]$. There is still a trade-off between $\mathrm{T}$ and $\mathrm{f}^{0}$, that is, the relationship between the underlying parameters and $A$ is not one-to-one. A single value for $A$ could have arisen from multiple combinations parameter values, but it still serves as a useful summary statistic. Figure 6A.1 graphs (as a function of $\mathrm{T}$, fixing $\mathrm{b}=0.7$ and $\mathrm{t}^{0}=0$ ) the value of $\mathrm{f}^{0}$ that yields various specified acquisition speeds $A$. The analogous figure 6.3 shows less variability in this ratio.

We evaluate three specifications (parameter choices) for the acquisition function. These are, in order of value at birth: $\mathrm{f}_{1}$, given by $\left(\mathrm{f}_{1}^{0}=0.25, \mathrm{~T}_{1}=14, \mathrm{~g}_{1}=0.5, \mathrm{~b}_{1}=0.7\right) ; \mathrm{f}_{2}$, given by $\left(\mathrm{f}_{2}^{0}=0.3, \mathrm{~T}_{2}=5, \mathrm{~g}_{2}=0.4, \mathrm{~b}_{2}=0.7\right)$; and $\mathrm{f}_{3}$, given by $\left(\mathrm{f}_{3}^{0}=0.5, \mathrm{~T}_{3}=2, \mathrm{~g}_{3}=0.3, \mathrm{~b}_{3}=0.8\right)$. The respective values for $A$ (using $\mathrm{t}^{0}=-0.25$ ) are $0.29,0.54$, and 0.84 . These three acquisition functions were graphed in figure 6.4. Representative values for specific ages were listed in table 6.5, along with the corresponding values for $f_{D}(t)$, the traditional formulation for DALYs. Figure 6A.2 shows how the ratio of years of life lost at age 20 to age 0 for these three functions varies with $A$. We view $\mathrm{f}_{2}$ (with $\mathrm{T}=5$ ) as a reasonable

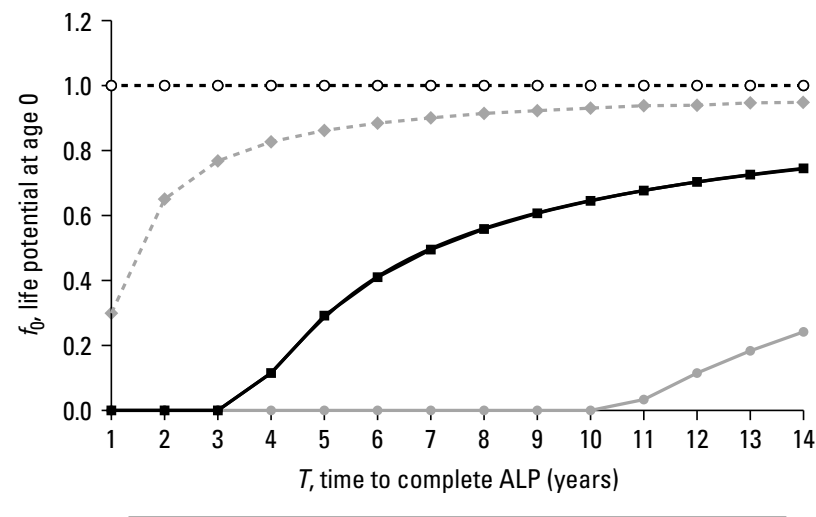

$$
\because A=0.29 \rightarrow-A=0.55 \rightarrow-A=0.86 \quad-\circ-A=1.00
$$

Source: Authors' calculations.

Note: $A$ is rate of ALP.

Figure 6A.1 Relationship between Time to Complete ALP and Life Potential at Age 0 for Several Values of $A$

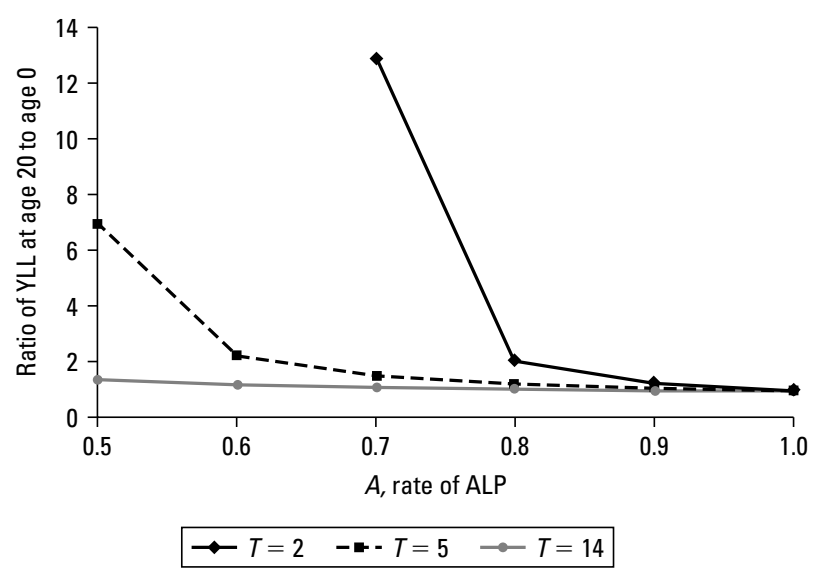

Source: Authors' calculations

Note: $A$ is rate of ALP. T is the time to complete acquisition of life potential.

Figure 6A.2 Ratio of DALYs Lost at Age 20 to Age 0 as a Function of $A$

intermediate choice and, with a 3 percent discount rate, have used $\mathrm{f}_{2}$ to generate what we define as $\operatorname{DALYs}(3,0, .54)$. Complete burden of disease calculations are reported using DALYs $(3,0, .54)$ in table 6B.8. 


\section{ANNEX B: SUPPLEMENTARY TABLES}

Table 6B.1 Deaths (Excluding Stillbirths) from Selected Causes, by Age, 2001 (thousands)

\begin{tabular}{|c|c|c|c|c|c|c|c|c|c|}
\hline \multirow[b]{3}{*}{ Cause } & \multicolumn{3}{|c|}{ Low- and middle-income countries } & \multirow{2}{*}{\multicolumn{3}{|c|}{$\begin{array}{c}\text { High-income countries } \\
\text { Deaths }\end{array}$}} & \multirow{2}{*}{\multicolumn{3}{|c|}{$\begin{array}{c}\text { World } \\
\text { Deaths }\end{array}$}} \\
\hline & \multicolumn{3}{|c|}{ Deaths } & & & & & & \\
\hline & $0-4$ & $5+$ & Total & $\overline{0-4}$ & $5+$ & $\overline{\text { Total }}$ & $0-4$ & $5+$ & Total \\
\hline Total deaths & 10,533 & 37,843 & 48,377 & 73 & 7,819 & 7,891 & 10,606 & 45,662 & 56,268 \\
\hline $\begin{array}{l}\text { I. Communicable, maternal, perinatal, } \\
\text { and nutritional conditions }\end{array}$ & 9,396 & 8,226 & 17,622 & 37 & 515 & 552 & 9,433 & 8,741 & 18,174 \\
\hline A. Infectious and parasitic diseases & 4,768 & 5,923 & 10,692 & 3 & 149 & 152 & 4,771 & 6,072 & 10,843 \\
\hline 1. Tuberculosis & 40 & 1,550 & 1,590 & & 16 & 16 & 40 & 1,566 & 1,606 \\
\hline $\begin{array}{l}\text { 2. Sexually transmitted diseases } \\
\text { excluding HIV/AIDS }\end{array}$ & 68 & 108 & 176 & & 1 & 1 & 68 & 109 & 177 \\
\hline 3. HIV/AIDS & 340 & 2,214 & 2,554 & & 22 & 22 & 340 & 2,236 & 2,576 \\
\hline 4. Diarrheal diseases & 1,599 & 179 & 1,778 & & 5 & 6 & 1,600 & 184 & 1,784 \\
\hline 5. Childhood-cluster diseases & 1,048 & 313 & 1,363 & & 1 & 2 & 1,049 & 315 & 1,364 \\
\hline a. Pertussis & 301 & & 301 & & & & 301 & & 301 \\
\hline b. Poliomyelitis & & & & & 1 & 1 & & 1 & 1 \\
\hline c. Diphtheria & 5 & 1 & 6 & & & & 5 & 1 & 6 \\
\hline d. Measles & 557 & 206 & 763 & & 1 & 1 & 557 & 206 & 763 \\
\hline e. Tetanus & 187 & 106 & 293 & & & & 187 & 107 & 293 \\
\hline 6. Meningitis & 64 & 105 & 169 & 1 & 3 & 4 & 65 & 108 & 173 \\
\hline 8. Malaria & 1,087 & 58 & 1,208 & & & & 1,087 & 121 & 1,208 \\
\hline Other I.A. $(7,9-15)^{\mathrm{a}}$ & 518 & & 1,854 & 2 & 100 & 101 & 522 & 1,434 & 1,955 \\
\hline B. Respiratory infections & 1,943 & 1,539 & 3,483 & 2 & 347 & 349 & 1,945 & 1,886 & 3,831 \\
\hline C. Maternal conditions & & 507 & 507 & & 1 & 1 & & 508 & 508 \\
\hline D. Perinatal conditions & 2,490 & & 2,490 & 32 & & 32 & 2,522 & & 2,523 \\
\hline 1. Low birthweight & 1,291 & & 1,291 & 10 & & 10 & 1,301 & & 1,301 \\
\hline 2. Birth asphyxia and birth trauma & 728 & & 728 & 11 & & 11 & 739 & & 739 \\
\hline 3. Other perinatal conditions & 471 & & 471 & 12 & & 12 & 482 & & 482 \\
\hline E. Nutritional deficiencies & 194 & 257 & 451 & & 18 & 18 & 194 & 274 & 469 \\
\hline II. Noncommunicable diseases & 835 & 25,202 & 26,037 & 28 & 6,840 & 6,868 & 864 & 32,042 & 32,905 \\
\hline A. Malignant neoplasms & 37 & 4,921 & 4,957 & 2 & 2,065 & 2,066 & 38 & 6,986 & 7,024 \\
\hline C. Diabetes mellitus & 3 & 755 & 758 & & 202 & 202 & 3 & 958 & 961 \\
\hline E. Neuropsychiatric disorders & 43 & 605 & 701 & 2 & 376 & 378 & 46 & 1,034 & 1,079 \\
\hline 1. Unipolar depressive disorders & & 10 & 10 & & 3 & 3 & & 13 & 13 \\
\hline 2. Bipolar affective disorder & & & & & & & & 1 & 1 \\
\hline 3. Schizophrenia & & 21 & 21 & & 2 & 2 & & 23 & 23 \\
\hline Other II.E. $(4-16)^{b}$ & & & 670 & 2 & 371 & 373 & 45 & 997 & 1,043 \\
\hline G. Cardiovascular diseases & 82 & 13,279 & 13,362 & 2 & 3,037 & 3,039 & 84 & 16,316 & 16,401 \\
\hline 3. Ischemic heart disease & 6 & 5,696 & 5,702 & & 1,364 & 1,364 & 6 & 7,060 & 7,066 \\
\hline 4. Cerebrovascular disease & 12 & 4,598 & 4,611 & & 781 & 781 & 13 & 5,379 & 5,392 \\
\hline Other II.G. $(1,2,5,6)^{c}$ & 64 & & 3,049 & 2 & 892 & 894 & 66 & 3,877 & 3,943 \\
\hline H. Respiratory diseases & 63 & 3,063 & 3,127 & 1 & 476 & 477 & 64 & 3,540 & 3,604 \\
\hline I. Digestive diseases & 115 & 1,487 & 1,602 & 1 & 334 & 335 & 116 & 1,821 & 1,936 \\
\hline M. Congenital anomalies & 421 & $\begin{array}{r}1,401 \\
56\end{array}$ & $\begin{array}{r}1,002 \\
477\end{array}$ & 18 & $\begin{array}{r}12 \\
12\end{array}$ & 30 & 439 & $\begin{array}{r}1,021 \\
68\end{array}$ & 507 \\
\hline Other II. $(B, D, F, J, K, L, N)^{d}$ & $\begin{array}{r}421 \\
71\end{array}$ & & 1,053 & $\begin{array}{r}10 \\
2\end{array}$ & 338 & 340 & $\begin{array}{r}439 \\
73\end{array}$ & 1,320 & 1,393 \\
\hline III. Injuries & 302 & 4,415 & 4,717 & 7 & 464 & 471 & 310 & 4,879 & 5,188 \\
\hline A. Unintentional & 289 & 2,926 & 3,216 & 6 & 315 & 321 & 295 & 3,241 & 3,536 \\
\hline 1. Road traffic accidents & 49 & 1,020 & 1,070 & 2 & 119 & 121 & 51 & 1,139 & 1,190 \\
\hline Other III.A. $(2-6)^{\mathrm{e}}$ & 240 & & 2,146 & 4 & 196 & 200 & 244 & 2,102 & 2,346 \\
\hline B. Intentional & 13 & 1,488 & 1,501 & 1 & 149 & 151 & 14 & 1,638 & 1,652 \\
\hline 1. Self-inflicted & & 749 & 749 & & 126 & 126 & & 875 & 875 \\
\hline Other III.B. $(2-4)^{f}$ & 13 & & 753 & 1 & 24 & 25 & 14 & 763 & 777 \\
\hline
\end{tabular}

Source: Chapter 3 of this volume.

Note: A blank cell indicates that fewer than 1,000 deaths are attributable to the specific cause.

a. Hepatitis, tropical-cluster diseases, leprosy, dengue, Japanese encephalitis, trachoma, intestinal nematode infections, and other infectious diseases.

b. Epilepsy, alcohol use disorders, Alzheimer's and other dementias, Parkinson's disease, multiple sclerosis, drug use disorders, post-traumatic stress disorder, obsessive-compulsive disorder, panic disorder, insomnia (primary), migraine, mental retardation attributable to lead exposure, and other neuropsychiatric disorders.

c. Rheumatic heart disease, hypertensive heart disease, inflammatory heart diseases, and other cardiovascular diseases.

d. Other neoplasms, endocrine disorders, sense organ diseases, genitourinary diseases, skin diseases, musculoskeletal diseases, and oral conditions.

e. Poisonings, falls, fires, drownings, and other unintentional injuries.

$\mathrm{f}$. Violence, war, and other intentional injuries. 
Table 6B.2 YLL(3,0) from Selected Causes, by Age, 2001 (thousands)

\begin{tabular}{|c|c|c|c|c|c|c|c|c|c|}
\hline \multirow[b]{3}{*}{ Cause } & \multicolumn{3}{|c|}{ Low- and middle-income countries } & \multicolumn{3}{|c|}{ High-income countries } & \multicolumn{3}{|c|}{ World } \\
\hline & \multicolumn{3}{|c|}{ YLL } & \multicolumn{3}{|c|}{ YLL } & \multicolumn{3}{|c|}{ YLL } \\
\hline & $0-4$ & $5+$ & Total & $0-4$ & $5+$ & $\overline{\text { Total }}$ & $0-4$ & $5+$ & Total \\
\hline Total YLL & 319,558 & 590,267 & 909,825 & 2,209 & 75,650 & 77,859 & 321,767 & 665,917 & 987,684 \\
\hline $\begin{array}{l}\text { I. Communicable, maternal, perinatal, } \\
\text { and nutritional conditions }\end{array}$ & 285,058 & 169,531 & 454,589 & 1,133 & 4,258 & 5,391 & 286,191 & 173,789 & 459,980 \\
\hline A. Infectious and parasitic diseases & 144,555 & 129,584 & 274,138 & 96 & 1,878 & 1,975 & 144,651 & 131,462 & 276,113 \\
\hline 1. Tuberculosis & 1,215 & 30,528 & 31,743 & 1 & 171 & 172 & 1,216 & 30,699 & 31,915 \\
\hline $\begin{array}{l}\text { 2. Sexually transmitted diseases } \\
\text { excluding HIV/AIDS }\end{array}$ & 2,067 & 2,079 & 4,146 & 1 & 6 & 7 & 2,068 & 2,085 & 4,153 \\
\hline 3. HIV/AIDS & 10,299 & 54,537 & 64,836 & 2 & 491 & 493 & 10,301 & 55,027 & 65,328 \\
\hline 4. Diarrheal diseases & 48,534 & 2,350 & 50,884 & 13 & 40 & 53 & 48,547 & 2,390 & 50,937 \\
\hline 5. Childhood-cluster diseases & 31,751 & 8,756 & 40,507 & 4 & 30 & 34 & 31,755 & 8,786 & 40,540 \\
\hline a. Pertussis & 9,113 & & 9,113 & 1 & & 1 & 9,114 & & 9,114 \\
\hline b. Poliomyelitis & 9 & 1 & 10 & & 6 & 6 & 9 & 7 & 16 \\
\hline c. Diphtheria & 137 & 27 & 164 & & & & 137 & 27 & 164 \\
\hline d. Measles & 16,840 & 6,057 & 22,897 & 2 & 19 & 21 & 16,843 & 6,076 & 22,918 \\
\hline e. Tetanus & 5,652 & 2,671 & 8,323 & 1 & 5 & 5 & 5,653 & 2,675 & 8,328 \\
\hline 6. Meningitis & 1,952 & 2,391 & 4,343 & 23 & 59 & 82 & 1,975 & 2,450 & 4,425 \\
\hline 8. Malaria & 32,981 & 2,481 & 35,462 & 2 & 2 & 4 & 32,982 & 2,483 & 35,466 \\
\hline Other I.A. $(7,9-15)^{\mathrm{a}}$ & 15,705 & 26,514 & 42,219 & 51 & 1,079 & 1,130 & 15,808 & 36,570 & 52,378 \\
\hline B. Respiratory infections & 58,979 & 21,810 & 80,789 & 52 & 2,227 & 2,279 & 59,031 & 24,037 & 83,068 \\
\hline C. Maternal conditions & & 13,363 & 13,363 & & 27 & 27 & & 13,390 & 13,390 \\
\hline D. Perinatal conditions & 75,642 & & 75,643 & 981 & 4 & 984 & 76,623 & 4 & 76,627 \\
\hline 1. Low birthweight & 39,228 & & 39,228 & 291 & & 291 & 39,520 & & 39,520 \\
\hline 2. Birth asphyxia and birth trauma & 22,118 & & 22,118 & 336 & 1 & 338 & 22,454 & 2 & 22,455 \\
\hline 3. Other perinatal conditions & 14,296 & & 14,297 & 353 & 2 & 355 & 14,650 & 2 & 14,652 \\
\hline E. Nutritional deficiencies & 5,882 & 4,773 & 10,656 & 4 & 122 & 126 & 5,887 & 4,895 & 10,782 \\
\hline II. Noncommunicable diseases & 25,345 & 322,376 & 347,721 & 857 & 63,397 & 64,255 & 26,203 & 385,773 & 411,976 \\
\hline A. Malignant neoplasms & 1,110 & 71,503 & 72,613 & 50 & 23,265 & 23,315 & 1,160 & 94,768 & 95,928 \\
\hline C. Diabetes mellitus & 87 & 10,054 & 10,141 & 1 & 1,942 & 1,943 & 87 & 11,997 & 12,084 \\
\hline E. Neuropsychiatric disorders & 1,317 & 10,310 & 11,626 & 63 & 3,259 & 3,322 & 1,380 & 13,569 & 14,949 \\
\hline 1. Unipolar depressive disorders & & 205 & 205 & & 21 & 21 & 1 & 226 & 227 \\
\hline 2. Bipolar affective disorder & & 5 & 5 & & 4 & 4 & & 9 & 9 \\
\hline 3. Schizophrenia & 1 & 373 & 374 & & 24 & 24 & 1 & 397 & 398 \\
\hline Other II.E. $(4-16)^{b}$ & 1,314 & 9,727 & 11,041 & 63 & 3,210 & 3,273 & 1,377 & 12,937 & 14,314 \\
\hline G. Cardiovascular diseases & 2,493 & 155,750 & 158,243 & 63 & 24,166 & 24,229 & 2,557 & 179,915 & 182,472 \\
\hline 3. Ischemic heart disease & 177 & 67,751 & 67,928 & 2 & 11,483 & 11,485 & 179 & 79,234 & 79,412 \\
\hline 4. Cerebrovascular disease & 371 & 51,170 & 51,541 & 11 & 5,886 & 5,896 & 382 & 57,056 & 57,438 \\
\hline Other II.G. $(1,2,5,6)^{c}$ & 1,946 & 36,828 & 38,774 & 50 & 6,797 & 6,848 & 1,996 & 43,626 & 45,622 \\
\hline H. Respiratory diseases & 1,925 & 34,570 & 36,495 & 30 & 3,914 & 3,945 & 1,955 & 38,484 & 40,439 \\
\hline I. Digestive diseases & 3,482 & 23,888 & 27,370 & 35 & 3,680 & 3,715 & 3,516 & 27,568 & 31,084 \\
\hline M. Congenital anomalies & 12,785 & 1,480 & 14,265 & 543 & 229 & 771 & 13,328 & 1,709 & 15,037 \\
\hline Other II. $(B, D, F, J, K, L, N)^{d}$ & 2,147 & 14,821 & 16,967 & 72 & 2,943 & 3,015 & 2,219 & 17,764 & 19,983 \\
\hline III. Injuries & 9,155 & 98,361 & 107,516 & 218 & 7,995 & 8,213 & 9,373 & 106,356 & 115,729 \\
\hline A. Unintentional & 8,757 & 64,384 & 73,141 & 186 & 5,003 & 5,189 & 8,943 & 69,387 & 78,330 \\
\hline 1. Road traffic accidents & 1,491 & 23,331 & 24,822 & 52 & 2,496 & 2,548 & 1,543 & 25,827 & 27,370 \\
\hline Other III.A. $(2-6)^{e}$ & 7,266 & 41,053 & 48,320 & 134 & 2,507 & 2,640 & 7,400 & 43,560 & 50,960 \\
\hline B. Intentional & 398 & 33,977 & 34,374 & 33 & 2,992 & 3,024 & 430 & 36,969 & 37,399 \\
\hline 1. Self-inflicted & 4 & 16,435 & 16,439 & & 2,432 & 2,433 & 4 & 18,868 & 18,871 \\
\hline Other III.B. $(2-4)^{f}$ & 394 & 17,542 & 17,936 & 32 & 559 & 592 & 426 & 18,101 & 18,527 \\
\hline
\end{tabular}

Source: Chapter 3 of this volume.

Note: A blank cell indicates that fewer than 1,000 deaths are attributable to the specific cause

a. Hepatitis, tropical-cluster diseases, leprosy, dengue, Japanese encephalitis, trachoma, intestinal nematode infections, and other infectious diseases.

b. Epilepsy, alcohol use disorders, Alzheimer's and other dementias, Parkinson's disease, multiple sclerosis, drug use disorders, post-traumatic stress disorder, obsessive-compulsive disorder, panic disorder, insomnia (primary), migraine, mental retardation attributable to lead exposure, and other neuropsychiatric disorders.

c. Rheumatic heart disease, hypertensive heart disease, inflammatory heart diseases, and other cardiovascular diseases.

d. Other neoplasms, endocrine disorders, sense organ diseases, genitourinary diseases, skin diseases, musculoskeletal diseases, and oral conditions.

e. Poisonings, falls, fires, drownings, and other unintentional injuries.

f. Violence, war, and other intentional injuries. 
Table 6B.3 YLD from Selected Causes, by Age, 2001 (thousands)

\begin{tabular}{|c|c|c|c|c|c|c|c|c|c|}
\hline \multirow[b]{3}{*}{ Cause } & \multirow{2}{*}{\multicolumn{3}{|c|}{$\frac{\text { Low- and middle-income countries }}{\text { YLD }}$}} & \multirow{2}{*}{\multicolumn{3}{|c|}{$\begin{array}{c}\text { High-income countries } \\
\text { YLD } \\
\end{array}$}} & \multirow{2}{*}{\multicolumn{3}{|c|}{$\begin{array}{c}\text { World } \\
\text { YLD } \\
\end{array}$}} \\
\hline & & & & & & & & & \\
\hline & $0-4$ & $5+$ & Total & $0-4$ & $5+$ & Total & $0-4$ & $5+$ & Total \\
\hline Total YLD & 104,557 & 372,465 & 477,022 & 4,592 & 66,717 & 71,309 & 109,148 & 439,182 & 548,330 \\
\hline $\begin{array}{l}\text { I. Communicable, maternal, perinatal, } \\
\text { and nutritional conditions }\end{array}$ & 45,068 & 52,890 & 97,958 & 1,041 & 2,127 & 3,169 & 46,109 & 55,018 & 101,127 \\
\hline A. Infectious and parasitic diseases & 15,016 & 31,552 & 46,568 & 467 & 934 & 1,401 & 15,483 & 32,486 & 47,969 \\
\hline 1. Tuberculosis & 170 & 3,964 & 4,134 & 1 & 46 & 47 & 170 & 47,799 & 47,969 \\
\hline $\begin{array}{l}\text { 2. Sexually transmitted } \\
\text { diseases excluding HIV/AIDS }\end{array}$ & 1,127 & 4,065 & 5,192 & 12 & 126 & 138 & 1,139 & 4,190 & 5,329 \\
\hline 3. HIV/AIDS & 173 & 5,802 & 5,975 & 1 & 171 & 173 & 175 & 5,974 & 6,148 \\
\hline 4. Diarrheal diseases & 4,814 & 3,022 & 7,836 & 222 & 170 & 392 & 5,036 & 3,192 & 8,228 \\
\hline 5. Childhood-cluster diseases & 2,359 & 266 & 2,625 & 138 & 4 & 141 & 2,496 & 269 & 2,766 \\
\hline a. Pertussis & 2,192 & 98 & 2,290 & 137 & 1 & 138 & 2,328 & 100 & 2,428 \\
\hline $\begin{array}{l}\text { b. Poliomyelitis } \\
\text { c. Diphtheria }\end{array}$ & 21 & 105 & 126 & & 2 & 2 & 21 & 107 & 128 \\
\hline d. Measles & 136 & 58 & 194 & 1 & 1 & 2 & 137 & 58 & 195 \\
\hline e. Tetanus & 9 & 4 & 14 & & & & 9 & 4 & 14 \\
\hline 6. Meningitis & 829 & 302 & 1,131 & 27 & 22 & 49 & 856 & 324 & 1,180 \\
\hline 8. Malaria & 3,158 & 1,341 & 4,499 & & 5 & 5 & 3,158 & 1,346 & 4,504 \\
\hline Other I.A. $(7,9-15)^{\mathrm{a}}$ & 2,386 & 12,791 & 15,177 & 66 & 391 & 457 & 2,452 & 13,182 & 15,634 \\
\hline B. Respiratory infections & 3,855 & 2,095 & 5,949 & 46 & 150 & 197 & 3,901 & 2,245 & 6,146 \\
\hline C. Maternal conditions & & & & & 364 & 364 & & 13,385 & 13,385 \\
\hline D. Perinatal conditions & 13,523 & & 13,523 & 422 & & 422 & 13,945 & & 13,945 \\
\hline 1. Low birthweight & 3,377 & & 3,377 & 175 & & 175 & 3,552 & & 3,552 \\
\hline 2. Birth asphyxia and birth trauma & 9,352 & & 9,352 & 191 & & 191 & 9,543 & & 9,543 \\
\hline 3. Other perinatal conditions & 794 & & 794 & 56 & & 56 & 850 & & 850 \\
\hline E. Nutritional deficiencies & 12,674 & 6,223 & 18,897 & 107 & 679 & 785 & 12,781 & 6,902 & 19,683 \\
\hline II. Noncommunicable diseases & 53,465 & 277,249 & 330,714 & 3,371 & 61,737 & 65,108 & 56,836 & 338,987 & 395,823 \\
\hline A. Malignant neoplasms & 37 & 2,072 & 2,109 & 4 & 2,566 & 2,570 & 41 & 4,639 & 4,680 \\
\hline C. Diabetes mellitus & 15 & 5,647 & 5,662 & 1 & 2,249 & 2,249 & 16 & 7,896 & 7,912 \\
\hline E. Neuropsychiatric disorders & 18,854 & 106,595 & 125,449 & 913 & 26,996 & 27,909 & 19,767 & 133,592 & 153,358 \\
\hline 1. Unipolar depressive disorders & & 43,222 & 43,222 & & 8,387 & 8,387 & & 51,608 & 51,608 \\
\hline 2. Bipolar affective disorder & & 8,673 & 8,673 & & 1,052 & 1,052 & & 9,725 & 9,725 \\
\hline 3. Schizophrenia & & 10,153 & 10,153 & & 1,091 & 1,091 & & 11,244 & 11,244 \\
\hline Other II.E. $(4-16)^{b}$ & 18,854 & 44,548 & 63,402 & 913 & 16,466 & 17,379 & 19,767 & 61,015 & 80,781 \\
\hline G. Cardiovascular diseases & 540 & 20,091 & 20,631 & 15 & 5,623 & 5,638 & 554 & 25,714 & 26,268 \\
\hline 3. Ischemic heart disease & 1 & 3,923 & 3,923 & & 908 & 908 & 1 & 4,831 & 4,831 \\
\hline 4. Cerebrovascular disease & & 11,102 & 11,102 & & 3,460 & 3,460 & & 14,562 & 14,562 \\
\hline Other II.G. $(1,2,5,6)^{c}$ & 539 & 5,066 & 5,605 & 15 & 1,255 & 1,270 & 554 & 6,321 & 6,875 \\
\hline H. Respiratory diseases & 4,040 & 17,546 & 21,586 & 539 & 5,319 & 5,857 & 4,578 & 22,865 & 27,443 \\
\hline I. Digestive diseases & 10,972 & 14,074 & 25,045 & 440 & 2,382 & 2,821 & 11,412 & 16,455 & 27,867 \\
\hline M. Congenital anomalies & 9,293 & & 9,293 & 647 & & 647 & 9,940 & & 9,940 \\
\hline Other II. (B, D, F, J, K, L, N) ${ }^{d}$ & 9,375 & 111,564 & 120,939 & 813 & 16,603 & 17,416 & 10,528 & 127,826 & 138,354 \\
\hline III. Injuries & 6,024 & 42,326 & 48,349 & 180 & 2,852 & 3,032 & 6,203 & 45,178 & 51,381 \\
\hline A. Unintentional & 5,864 & 34,242 & 40,106 & 178 & 2,510 & 2,688 & 6,042 & 36,752 & 42,794 \\
\hline 1. Road traffic accidents & 783 & 6,413 & 7,196 & 16 & 481 & 497 & 798 & 6,894 & 7,693 \\
\hline Other III.A. $(2-6)^{e}$ & 5,082 & 27,829 & 32,911 & 162 & 2,029 & 2,191 & 5,244 & 29,857 & 35,101 \\
\hline B. Intentional & 159 & 8,084 & 8,243 & 2 & 342 & 344 & 161 & 8,426 & 8,587 \\
\hline 1. Self-inflicted & & 1,237 & 1,237 & & 148 & 148 & & 1,385 & 1,385 \\
\hline Other III.B. $(2-4)^{f}$ & 159 & 6,847 & 7,007 & 2 & 194 & 195 & 161 & 7,041 & 7,202 \\
\hline
\end{tabular}

Source: Chapter 3 of this volume.

Note: A blank cell indicates that fewer than 1,000 deaths are attributable to the specific cause.

a. Hepatitis, tropical-cluster diseases, leprosy, dengue, Japanese encephalitis, trachoma, intestinal nematode infections, and other infectious diseases.

b. Epilepsy, alcohol use disorders, Alzheimer's and other dementias, Parkinson's disease, multiple sclerosis, drug use disorders, post-traumatic stress disorder, obsessive-compulsive disorder, panic disorder, insomnia (primary), migraine, mental retardation attributable to lead exposure, and other neuropsychiatric disorders.

c. Rheumatic heart disease, hypertensive heart disease, inflammatory heart diseases, and other cardiovascular diseases.

d. Other neoplasms, endocrine disorders, sense organ diseases, genitourinary diseases, skin diseases, musculoskeletal diseases, and oral conditions.

e. Poisonings, falls, fires, drownings, and other unintentional injuries.

f. Violence, war, and other intentional injuries. 
Table 6B.4 The Burden of Disease-DALYs(3,0) from Selected Causes, by Age, 2001 (Excluding Stillbirths) (thousands)

\begin{tabular}{|c|c|c|c|c|c|c|c|c|c|}
\hline \multirow[b]{3}{*}{ Cause } & \multicolumn{3}{|c|}{ Low- and middle-income countries } & \multirow{2}{*}{\multicolumn{3}{|c|}{$\begin{array}{c}\text { High-income countries } \\
\text { DALYs }\end{array}$}} & \multirow{2}{*}{\multicolumn{3}{|c|}{$\begin{array}{c}\text { World } \\
\text { DALYs }\end{array}$}} \\
\hline & \multicolumn{3}{|c|}{ DALYs } & & & & & & \\
\hline & $0-4$ & $5+$ & Total & $0-4$ & $5+$ & $\overline{\text { Total }}$ & $0-4$ & $5+$ & Total \\
\hline Total DALYs $(3,0)$ & 424,062 & 963,364 & $1,387,426$ & 6,804 & 142,358 & 149,161 & 430,866 & $1,105,721$ & $1,536,587$ \\
\hline $\begin{array}{l}\text { I. Communicable, maternal, perinatal, } \\
\text { and nutritional conditions }\end{array}$ & 330,086 & 222,553 & 552,639 & 2,177 & 6,384 & 8,561 & 332,263 & 228,937 & 561,200 \\
\hline A. Infectious and parasitic diseases & 159,602 & 161,226 & 320,828 & 563 & 2,812 & 3,375 & 160,165 & 164,039 & 324,203 \\
\hline 1. Tuberculosis & 1,385 & 34,502 & 35,887 & 2 & 217 & 219 & 1,387 & 34,719 & 36,106 \\
\hline $\begin{array}{l}\text { 2. Sexually transmitted diseases } \\
\text { excluding HIV/AIDS }\end{array}$ & 3,194 & 6,149 & 9,343 & 13 & 132 & 145 & 3,207 & 6,280 & 9,488 \\
\hline 3. HIV/AIDS & 10,467 & 60,362 & 70,830 & 3 & 662 & 665 & 10,471 & 61,024 & 71,495 \\
\hline 4. Diarrheal diseases & 53,343 & 5,376 & 58,719 & 235 & 210 & 444 & 53,578 & 5,586 & 59,164 \\
\hline 5. Childhood-cluster diseases & 34,124 & 9,031 & 43,155 & 141 & 33 & 175 & 34,266 & 9,064 & 43,330 \\
\hline a. Pertussis & 11,310 & 99 & 11,408 & 138 & 2 & 139 & 11,448 & 100 & 11,548 \\
\hline b. Poliomyelitis & 30 & 106 & 136 & & 8 & 8 & 30 & 114 & 144 \\
\hline c. Diphtheria & 137 & 28 & 164 & & & & 137 & 28 & 164 \\
\hline d. Measles & 16,984 & 6,121 & 23,106 & 3 & 20 & 23 & 16,988 & 6,141 & 23,129 \\
\hline e. Tetanus & 5,663 & 2,677 & 8,340 & 1 & 5 & 5 & 5,664 & 2,681 & 8,345 \\
\hline 6. Meningitis & 2,784 & 2,695 & 5,479 & 50 & 81 & 131 & 2,834 & 2,776 & 5,610 \\
\hline 8. Malaria & 36,159 & 3,827 & 39,986 & 2 & 7 & 9 & 36,161 & 3,834 & 39,995 \\
\hline Other I.A. $(7,9-15)^{\mathrm{a}}$ & 18,144 & 39,285 & 57,429 & 117 & 1,470 & 1,587 & 18,261 & 40,755 & 59,016 \\
\hline B. Respiratory infections & 62,826 & 23,926 & 86,752 & 98 & 2,376 & 2,474 & 62,924 & 26,302 & 89,227 \\
\hline C. Maternal conditions & & 26,398 & 26,398 & & 391 & 391 & & 26,789 & 26,789 \\
\hline D. Perinatal conditions & 89,096 & & 89,096 & 1,405 & 4 & 1,408 & 90,501 & 4 & 90,505 \\
\hline 1. Low birthweight & 42,606 & & 42,606 & 467 & & 467 & 43,072 & & 43,073 \\
\hline 2. Birth asphyxia and birth trauma & 31,442 & & 31,443 & 528 & 1 & 530 & 31,971 & 2 & 31,972 \\
\hline 3. Other perinatal conditions & 15,048 & & 15,048 & 410 & 2 & 412 & 15,458 & 2 & 15,460 \\
\hline E. Nutritional deficiencies & 18,562 & 11,002 & 29,564 & 111 & 801 & 912 & 18,673 & 11,803 & 30,475 \\
\hline II. Noncommunicable diseases & 78,798 & 600,044 & 678,842 & 4,229 & 125,127 & 129,356 & 83,027 & 725,171 & 808,198 \\
\hline A. Malignant neoplasms & 1,148 & 73,644 & 74,792 & 54 & 25,834 & 25,888 & 1,202 & 99,478 & 100,680 \\
\hline C. Diabetes mellitus & 102 & 15,715 & 15,817 & 1 & 4,191 & 4,192 & 103 & 19,906 & 20,009 \\
\hline E. Neuropsychiatric disorders & 20,180 & 116,960 & 137,140 & 976 & 30,254 & 31,230 & 21,156 & 147,214 & 168,371 \\
\hline 1. Unipolar depressive disorders & & 43,444 & 43,445 & & 8,408 & 8,408 & 1 & 51,852 & 51,853 \\
\hline 2. Bipolar affective disorder & & 8,681 & 8,681 & & 1,056 & 1,056 & & 9,737 & 9,737 \\
\hline 3. Schizophrenia & 1 & 10,530 & 10,531 & & 1,115 & 1,115 & 1 & 11,645 & 11,646 \\
\hline Other II.E. $(4-16)^{b}$ & 20,178 & 54,305 & 74,483 & 976 & 19,675 & 20,651 & 21,154 & 73,981 & 95,134 \\
\hline G. Cardiovascular diseases & 3,033 & 175,983 & 179,016 & 78 & 29,780 & 29,859 & 3,111 & 205,764 & 208,875 \\
\hline 3. Ischemic heart disease & 177 & 71,735 & 71,913 & 2 & 12,388 & 12,390 & 180 & 84,124 & 84,303 \\
\hline 4. Cerebrovascular disease & 371 & 62,326 & 62,697 & 11 & 9,344 & 9,354 & 382 & 71,669 & 72,051 \\
\hline Other II.G. $(1,2,5,6)^{c}$ & 2,484 & 41,922 & 44,406 & 65 & 8,049 & 8,114 & 2,550 & 49,970 & 52,520 \\
\hline H. Respiratory diseases & 5,966 & 52,146 & 58,112 & 569 & 9,233 & 9,801 & 6,535 & 61,379 & 67,914 \\
\hline I. Digestive diseases & 14,442 & 37,990 & 52,433 & 475 & 6,061 & 6,536 & 14,917 & 44,051 & 58,968 \\
\hline M. Congenital anomalies & 22,061 & 1,483 & 23,544 & 1,191 & 228 & 1,420 & 23,252 & 1,712 & 24,964 \\
\hline Other II. $(B, D, F, J, K, L, N)^{d}$ & 11,866 & 126,121 & 137,987 & 885 & 19,546 & 20,431 & 12,751 & 145,667 & 158,418 \\
\hline III. Injuries & 15,178 & 140,767 & 155,945 & 398 & 10,846 & 11,244 & 15,576 & 151,613 & 167,189 \\
\hline A. Unintentional & 14,621 & 98,684 & $1,13,306$ & 364 & 7,513 & 7,876 & 14,985 & 106,197 & 121,182 \\
\hline 1. Road traffic accidents & 2,275 & 29,766 & 32,041 & 68 & 2,978 & 3,045 & 2,343 & 32,744 & 35,087 \\
\hline Other III.A. $(2-6)^{e}$ & 12,346 & 68,918 & 81,264 & 296 & 4,535 & 4,831 & 12,642 & 73,453 & 86,095 \\
\hline B. Intentional & 557 & 42,083 & 42,640 & 34 & 3,334 & 3,368 & 591 & 45,416 & 46,007 \\
\hline 1. Self-inflicted & 4 & 17,674 & 17,678 & & 2,581 & 2,581 & 4 & 20,255 & 20,259 \\
\hline Other III.B. $(2-4)^{f}$ & 553 & 24,409 & 24,962 & 34 & 753 & 787 & 587 & 25,161 & 25,749 \\
\hline
\end{tabular}

Source: Chapter 3 of this volume.

Note: A blank cell indicates that fewer than 1,000 deaths are attributable to the specific cause.

a. Hepatitis, tropical-cluster diseases, leprosy, dengue, Japanese encephalitis, trachoma, intestinal nematode infections, and other infectious diseases.

b. Epilepsy, alcohol use disorders, Alzheimer's and other dementias, Parkinson's disease, multiple sclerosis, drug use disorders, post-traumatic stress disorder, obsessive-compulsive disorder, panic disorder, insomnia (primary), migraine, mental retardation attributable to lead exposure, and other neuropsychiatric disorders.

c. Rheumatic heart disease, hypertensive heart disease, inflammatory heart diseases, and other cardiovascular diseases.

d. Other neoplasms, endocrine disorders, sense organ diseases, genitourinary diseases, skin diseases, musculoskeletal diseases, and oral conditions.

e. Poisonings, falls, fires, drownings, and other unintentional injuries.

f. Violence, war, and other intentional injuries. 
Table 6B.5 $\mathrm{YLL}_{\mathrm{SB}}(3,0,1)$ Calculated to Include Stillbirths (Valued the Same as Newborn Deaths) (thousands)

\begin{tabular}{|c|c|c|c|c|c|c|c|}
\hline \multirow[b]{4}{*}{ Cause } & \multicolumn{7}{|c|}{ Low- and middle-income countries } \\
\hline & \multicolumn{7}{|c|}{ YLL } \\
\hline & \multicolumn{3}{|c|}{ Stillbirth } & \multicolumn{2}{|c|}{ Under age 5} & \multirow[b]{2}{*}{ age $5+$} & \multirow[b]{2}{*}{ Total } \\
\hline & Antepartum & Intrapartum & Total & $\begin{array}{l}\text { After live } \\
\text { birth }\end{array}$ & $\begin{array}{l}\text { Including } \\
\text { stillbirth }\end{array}$ & & \\
\hline $\begin{array}{l}\text { Total YLL } \\
\text { I. Communicable, maternal, perinatal, and } \\
\text { nutritional conditions }\end{array}$ & 65,463 & 32,755 & 98,198 & $\begin{array}{l}319,558 \\
285,058\end{array}$ & $\begin{array}{l}417,756 \\
285,058\end{array}$ & $\begin{array}{l}590,267 \\
169,531\end{array}$ & $\begin{array}{r}1,008,023 \\
454,589\end{array}$ \\
\hline A. Infectious and parasitic diseases & & & & 144,555 & 144,555 & 129,584 & 274,138 \\
\hline 1. Tuberculosis & & & & 1,215 & 1,215 & 30,528 & 31,743 \\
\hline $\begin{array}{l}\text { 2. Sexually transmitted diseases } \\
\text { excluding HIV/AIDS }\end{array}$ & & & & 2,067 & 2,067 & 2,079 & 4,146 \\
\hline 3. HIV/AIDS & & & & 10,299 & 10,299 & 54,537 & 64,836 \\
\hline 4. Diarrheal diseases & & & & 48,534 & 48,534 & 2,350 & 50,884 \\
\hline 5. Childhood-cluster diseases & & & & 31,751 & 31,751 & 8,756 & 40,507 \\
\hline a. Pertussis & & & & 9,113 & 9,113 & & 9,113 \\
\hline b. Poliomyelitis & & & & 9 & 9 & 1 & 10 \\
\hline c. Diphtheria & & & & 137 & 137 & 27 & 164 \\
\hline d. Measles & & & & 16,840 & 16,840 & 6,057 & 22,897 \\
\hline e. Tetanus & & & & 5,652 & 5,652 & 2,671 & 8,323 \\
\hline 6. Meningitis & & & & 1,952 & 1,952 & 2,391 & 4,343 \\
\hline 8. Malaria & & & & 32,981 & 32,981 & 2,481 & 35,462 \\
\hline Other I.A. $(7,9-15)^{a}$ & & & & 15,705 & 15,705 & 26,514 & 42,219 \\
\hline B. Respiratory infections & & & & 58,979 & 58,979 & 21,810 & 80,789 \\
\hline C. Maternal conditions & & & & & & 13,363 & 13,363 \\
\hline D. Perinatal conditions & & & & 75,642 & 75,642 & & 75,643 \\
\hline 1. Low birthweight ${ }^{b}$ & & & & 39,228 & 39,228 & & 39,228 \\
\hline 2. Birth asphyxia and birth trauma & & & & 22,118 & 22,118 & & 22,118 \\
\hline 3. Other perinatal conditions & & & & 14,296 & 14,296 & & 14,297 \\
\hline E. Nutritional deficiencies & & & & 5,882 & 5,882 & 4,773 & 10,656 \\
\hline II. Noncommunicable diseases & & & & 25,345 & 25,345 & 322,376 & 347,721 \\
\hline A. Malignant neoplasms & & & & 1,110 & 1,110 & 71,503 & 72,613 \\
\hline C. Diabetes mellitus & & & & 87 & 87 & 10,054 & 10,141 \\
\hline E. Neuropsychiatric disorders & & & & 1,317 & 1,317 & 10,310 & 11,626 \\
\hline 1. Unipolar depressive disorders & & & & & & 205 & 205 \\
\hline 2. Bipolar affective disorder & & & & & & 5 & 5 \\
\hline 3. Schizophrenia & & & & 1 & 1 & 373 & 374 \\
\hline Other II.E. $(4-16)^{c}$ & & & & 1,314 & 1,314 & 9,727 & 11,041 \\
\hline G. Cardiovascular diseases & & & & 2,493 & 2,493 & 155,750 & 158,243 \\
\hline 3. Ischemic heart disease & & & & 177 & 177 & 67,751 & 67,928 \\
\hline 4. Cerebrovascular disease & & & & 371 & 371 & 51,170 & 51,541 \\
\hline Other II.G. $(1,2,5,6)^{d}$ & & & & 1,946 & 1,946 & 36,828 & 38,774 \\
\hline H. Respiratory diseases & & & & 1,925 & 1,925 & 34,570 & 36,495 \\
\hline I. Digestive diseases & & & & 3,482 & 3,482 & 23,888 & 27,370 \\
\hline M. Congenital anomalies & & & & 12,785 & 12,785 & 1,480 & 14,265 \\
\hline Other II. (B, D, F, J, K, L, N) & & & & 2,147 & 2,147 & 14,821 & 16,967 \\
\hline III. Injuries & & & & 9,155 & 9,155 & 98,361 & 107,516 \\
\hline A. Unintentional & & & & 8,757 & 8,757 & 64,384 & 73,141 \\
\hline 1. Road traffic accidents & & & & 1,491 & 1,491 & 23,331 & 24,822 \\
\hline Other III.A. $(2-6)^{f}$ & & & & 7,266 & 7,266 & 41,053 & 48,320 \\
\hline B. Intentional & & & & 398 & 398 & 33,977 & 34,374 \\
\hline 1. Self-inflicted & & & & 4 & 4 & 16,435 & 16,439 \\
\hline Other III.B. $(2-4)^{9}$ & & & & 394 & 394 & 17,542 & 17,936 \\
\hline IV. Not allocated & 65,463 & 32,755 & 98,198 & & 98,198 & & 98,198 \\
\hline
\end{tabular}

Sources: Stillbirth data are from WHO 2005a. Neonatal and perinatal mortality are country, regional, and global estimates. Age $5+$ and total data are from table 6.4 for low- and middle-income countries. All other data are from chapter 3 of this volume.

Note: A blank cell indicates that fewer than 1,000 deaths are attributable to the specific cause.

a. Hepatitis, tropical-cluster diseases, leprosy, dengue, Japanese encephalitis, trachoma, intestinal nematode infections, and other infectious diseases.

b. Low birthweight deaths are those resulting from intrauterine growth retardation or preterm birth. Almost all low birthweight deaths in the neonatal period result from preterm birth.

c. Epilepsy, alcohol use disorders, Alzheimer's and other dementias, Parkinson's disease, multiple sclerosis, drug use disorders,

post-traumatic stress disorder, obsessive-compulsive disorder, panic disorder, insomnia (primary), migraine, mental retardation attributable to lead exposure, and other neuropsychiatric disorders.

d. Rheumatic heart disease, hypertensive heart disease, inflammatory heart diseases, and other cardiovascular diseases.

e. Other neoplasms, endocrine disorders, sense organ diseases, genitourinary diseases, skin diseases, musculoskeletal diseases, and oral conditions.

f. Poisonings, falls, fires, drownings, and other unintentional injuries.

g. Violence, war, and other intentional injuries. 
Table 6B.5 Continued

\begin{tabular}{|c|c|c|c|c|c|c|c|}
\hline \multirow[b]{4}{*}{ Cause } & \multicolumn{7}{|c|}{ High-income countries } \\
\hline & \multicolumn{7}{|c|}{ YLL } \\
\hline & \multicolumn{3}{|c|}{ Stillbirth } & \multicolumn{2}{|c|}{ Under age 5} & \multirow[b]{2}{*}{ age $5+$} & \multirow[b]{2}{*}{ Total } \\
\hline & Antepartum & Intrapartum & Total & $\begin{array}{l}\text { After live } \\
\text { birth }\end{array}$ & $\begin{array}{l}\text { Including } \\
\text { stillbirth }\end{array}$ & & \\
\hline Total YLL & 1,222 & 153 & 1,375 & 2,209 & 3,583 & 75,650 & 79,233 \\
\hline $\begin{array}{l}\text { I. Communicable, maternal, perinatal, and } \\
\text { nutritional conditions }\end{array}$ & & & & 1,133 & 1,133 & 4,258 & 5,391 \\
\hline A. Infectious and parasitic diseases & & & & 96 & 96 & 1,878 & 1,975 \\
\hline 1. Tuberculosis & & & & 1 & 1 & 171 & 172 \\
\hline $\begin{array}{l}\text { 2. Sexually transmitted diseases } \\
\text { excluding HIV/AIDS }\end{array}$ & & & & 1 & 1 & 6 & 7 \\
\hline 3. HIV/AIDS & & & & 2 & 2 & 491 & 493 \\
\hline 4. Diarrheal diseases & & & & 13 & 13 & 40 & 53 \\
\hline 5. Childhood-cluster diseases & & & & 4 & 4 & 30 & 34 \\
\hline a. Pertussis & & & & 1 & 1 & & 1 \\
\hline b. Poliomyelitis & & & & & & 6 & 6 \\
\hline c. Diphtheria & & & & & & & \\
\hline d. Measles & & & & 2 & 2 & 19 & 21 \\
\hline e. Tetanus & & & & 1 & 1 & 5 & 5 \\
\hline 6. Meningitis & & & & 23 & 23 & 59 & 82 \\
\hline 8. Malaria & & & & 2 & 2 & 2 & 4 \\
\hline Other I.A. $(7,9-15)^{a}$ & & & & 51 & 51 & 1,079 & 1,130 \\
\hline B. Respiratory infections & & & & 52 & 52 & 2,227 & 2,279 \\
\hline C. Maternal conditions & & & & & & 27 & 27 \\
\hline D. Perinatal conditions & & & & 981 & 981 & 4 & 984 \\
\hline 1. Low birthweight $t^{b}$ & & & & 291 & 291 & & 291 \\
\hline 2. Birth asphyxia and birth trauma & & & & 336 & 336 & 1 & 338 \\
\hline 3. Other perinatal conditions & & & & 353 & 353 & 2 & 355 \\
\hline E. Nutritional deficiencies & & & & 4 & 4 & 122 & 126 \\
\hline II. Noncommunicable diseases & & & & 857 & 857 & 63,397 & 64,255 \\
\hline A. Malignant neoplasms & & & & 50 & 50 & 23,265 & 23,315 \\
\hline C. Diabetes mellitus & & & & 1 & 1 & 1,942 & 1,943 \\
\hline E. Neuropsychiatric disorders & & & & 63 & 63 & 3,259 & 3,322 \\
\hline 1. Unipolar depressive disorders & & & & & & 21 & 21 \\
\hline 2. Bipolar affective disorder & & & & & & 4 & 4 \\
\hline 3. Schizophrenia & & & & & & 24 & 24 \\
\hline Other II.E. $(4-16)^{c}$ & & & & 63 & 63 & 3,210 & 3,273 \\
\hline G. Cardiovascular diseases & & & & 63 & 63 & 24,166 & 24,229 \\
\hline 3. Ischemic heart disease & & & & 2 & 2 & 11,483 & 11,485 \\
\hline 4. Cerebrovascular disease & & & & 11 & 11 & 5,886 & 5,896 \\
\hline Other II.G. $(1,2,5,6)^{d}$ & & & & 50 & 50 & 6,797 & 6,848 \\
\hline H. Respiratory diseases & & & & 30 & 30 & 3,914 & 3,945 \\
\hline I. Digestive diseases & & & & 35 & 35 & 3,680 & 3,715 \\
\hline M. Congenital anomalies & & & & 543 & 543 & 229 & 771 \\
\hline Other II. (B, D, F, J, K, L, N) $)^{\mathrm{e}}$ & & & & 72 & 72 & 2,943 & 3,015 \\
\hline III. Injuries & & & & 218 & 218 & 7,995 & 8,213 \\
\hline A. Unintentional & & & & 186 & 186 & 5,003 & 5,189 \\
\hline 1. Road traffic accidents & & & & 52 & 52 & 2,496 & 2,548 \\
\hline Other III.A. $(2-6)^{f}$ & & & & 134 & 134 & 2,507 & 2,640 \\
\hline B. Intentional & & & & 33 & 33 & 2,992 & 3,024 \\
\hline 1. Self-inflicted & & & & & & 2,432 & 2,433 \\
\hline Other III.B. $(2-4)^{g}$ & & & & 32 & 32 & 559 & 592 \\
\hline IV. Not allocated & 1,222 & 153 & 1,375 & & 1,375 & & 1,375 \\
\hline
\end{tabular}

Sources: Stillbirth data are from WHO 2005a. Neonatal and perinatal mortality are country, regional, and global estimates. Age $5+$ and total data are from table 6.4 for low- and middle-income countries. All other data are from chapter 3 of this volume.

Note: A blank cell indicates that fewer than 1,000 deaths are attributable to the specific cause.

a. Hepatitis, tropical-cluster diseases, leprosy, dengue, Japanese encephalitis, trachoma, intestinal nematode infections, and other infectious diseases.

b. Low birthweight deaths are those resulting from intrauterine growth retardation or preterm birth. Almost all low birthweight deaths in the neonatal period result from preterm birth.

c. Epilepsy, alcohol use disorders, Alzheimer's and other dementias, Parkinson's disease, multiple sclerosis, drug use disorders,

post-traumatic stress disorder, obsessive-compulsive disorder, panic disorder, insomnia (primary). migraine, mental retardation attributable to lead exposure, and other neuropsychiatric disorders.

d. Rheumatic heart disease, hypertensive heart disease, inflammatory heart diseases, and other cardiovascular diseases.

e. Other neoplasms, endocrine disorders, sense organ diseases, genitourinary diseases, skin diseases, musculoskeletal diseases, and oral conditions.

f. Poisonings, falls, fires, drownings, and other unintentional injuries.

g. Violence, war, and other intentional injuries. 


\begin{tabular}{|c|c|c|c|c|c|c|c|}
\hline \multirow[b]{4}{*}{ Cause } & \multicolumn{7}{|c|}{ World } \\
\hline & \multicolumn{7}{|c|}{ YLL } \\
\hline & \multicolumn{3}{|c|}{ Stillbirth } & \multicolumn{2}{|c|}{ Under age 5} & \multirow[b]{2}{*}{ age $5+$} & \multirow[b]{2}{*}{ Total } \\
\hline & Antepartum & Intrapartum & Total & $\begin{array}{l}\text { After live } \\
\text { birth }\end{array}$ & $\begin{array}{l}\text { Including } \\
\text { stillbirth }\end{array}$ & & \\
\hline $\begin{array}{l}\text { Total YLL } \\
\text { I. Communicable, maternal, perinatal, and } \\
\text { nutritional conditions }\end{array}$ & 66,685 & 32,907 & 99,592 & $\begin{array}{l}321,767 \\
286,191\end{array}$ & $\begin{array}{l}421,360 \\
286,191\end{array}$ & $\begin{array}{l}566,325 \\
173,789\end{array}$ & $\begin{array}{l}987,684 \\
459,980\end{array}$ \\
\hline A. Infectious and parasitic diseases & & & & 144,651 & 144,651 & 131,462 & 276,113 \\
\hline 1. Tuberculosis & & & & 1,216 & 1,216 & 30,699 & 31,915 \\
\hline $\begin{array}{l}\text { 2. Sexually transmitted diseases } \\
\text { excluding HIV/AIDS }\end{array}$ & & & & 2,068 & 2,068 & 2,085 & 4,153 \\
\hline 3. HIV/AIDS & & & & 10,301 & 10,301 & 55,027 & 65,328 \\
\hline 4. Diarrheal diseases & & & & 48,547 & 48,547 & 2,390 & 50,937 \\
\hline 5. Childhood-cluster diseases & & & & 31,755 & 31,755 & 8,786 & 40,540 \\
\hline a. Pertussis & & & & 9,114 & 9,114 & & 9,114 \\
\hline b. Poliomyelitis & & & & 9 & 9 & 7 & 16 \\
\hline c. Diphtheria & & & & 137 & 137 & 27 & 164 \\
\hline d. Measles & & & & 16,843 & 16,843 & 6,076 & 22,918 \\
\hline e. Tetanus & & & & 5,653 & 5,653 & 2,675 & 8,328 \\
\hline 6. Meningitis & & & & 1,975 & 1,975 & 2,450 & 4,425 \\
\hline 8. Malaria & & & & 32,982 & 32,982 & 2,483 & 35,466 \\
\hline Other I.A. $(7,9-15)^{a}$ & & & & 15,808 & 15,808 & 36,570 & 52,378 \\
\hline B. Respiratory infections & & & & 59,031 & 59,031 & 24,037 & 83,068 \\
\hline C. Maternal conditions & & & & & & 13,390 & 13,390 \\
\hline D. Perinatal conditions & & & & 76,623 & 76,623 & 4 & 76,627 \\
\hline 1. Low birthweight ${ }^{b}$ & & & & 39,520 & 39,520 & & 39,520 \\
\hline 2. Birth asphyxia and birth trauma & & & & 22,454 & 22,454 & 2 & 22,455 \\
\hline 3. Other perinatal conditions & & & & 14,650 & 14,650 & 2 & 14,652 \\
\hline E. Nutritional deficiencies & & & & 5,887 & 5,887 & 4,895 & 10,782 \\
\hline II. Noncommunicable diseases & & & & 26,203 & 26,203 & 385,773 & 411,976 \\
\hline A. Malignant neoplasms & & & & 1,160 & 1,160 & 94,768 & 95,928 \\
\hline C. Diabetes mellitus & & & & 87 & 87 & 11,997 & 12,084 \\
\hline E. Neuropsychiatric disorders & & & & 1,380 & 1,380 & 13,569 & 14,949 \\
\hline 1. Unipolar depressive disorders & & & & 1 & 1 & 226 & 227 \\
\hline 2. Bipolar affective disorder & & & & & & 9 & 9 \\
\hline 3. Schizophrenia & & & & 1 & 1 & 397 & 398 \\
\hline Other II.E. $(4-16)^{c}$ & & & & 1,377 & 1,377 & 12,937 & 14,314 \\
\hline G. Cardiovascular diseases & & & & 2,557 & 2,557 & 179,915 & 182,472 \\
\hline 3. Ischemic heart disease & & & & 179 & 179 & 79,234 & 79,412 \\
\hline 4. Cerebrovascular disease & & & & 382 & 382 & 57,056 & 57,438 \\
\hline Other II.G. $(1,2,5,6)^{d}$ & & & & 1,996 & 1,996 & 43,626 & 45,622 \\
\hline H. Respiratory diseases & & & & 1,955 & 1,955 & 38,484 & 40,439 \\
\hline I. Digestive diseases & & & & 3,516 & 3,516 & 27,568 & 31,084 \\
\hline M. Congenital anomalies & & & & 13,328 & 13,328 & 1,709 & 15,037 \\
\hline Other II. (B, D, F, J, K, L, N)e & & & & 2,219 & 2,219 & 17,764 & 19,983 \\
\hline III. Injuries & & & & 9,373 & 9,373 & 106,356 & 115,729 \\
\hline A. Unintentional & & & & 8,943 & 8,943 & 69,387 & 78,330 \\
\hline 1. Road traffic accidents & & & & 1,543 & 1,543 & 25,827 & 27,370 \\
\hline Other III.A. $(2-6)^{f}$ & & & & 7,400 & 7,400 & 43,560 & 50,960 \\
\hline B. Intentional & & & & 430 & 430 & 36,969 & 37,399 \\
\hline 1. Self-inflicted & & & & 4 & 4 & 18,868 & 18,871 \\
\hline Other III.B. $(2-4)^{g}$ & & & & 426 & 426 & 18,101 & 18,527 \\
\hline IV. Not allocated & 66,685 & 32,907 & 99,592 & & 99,592 & & 99,592 \\
\hline
\end{tabular}

Sources: Stillbirth data are from WHO 2005a. Neonatal and perinatal mortality are country, regional, and global estimates. Age $5+$ and total data are from table 6.4 for low- and middle-income countries. All other data are from chapter 3 of this volume.

Note: A blank cell indicates that fewer than 1,000 deaths are attributable to the specific cause.

a. Hepatitis, tropical-cluster diseases, leprosy, dengue, Japanese encephalitis, trachoma, intestinal nematode infections, and other infectious diseases.

b. Low birthweight deaths are those resulting from intrauterine growth retardation or preterm birth. Almost all low birthweight deaths in the neonatal period result from preterm birth.

c. Epilepsy, alcohol use disorders, Alzheimer's and other dementias, Parkinson's disease, multiple sclerosis, drug use disorders,

post-traumatic stress disorder, obsessive-compulsive disorder, panic disorder, insomnia (primary), migraine, mental retardation attributable to lead exposure, and other neuropsychiatric disorders.

d. Rheumatic heart disease, hypertensive heart disease, inflammatory heart diseases, and other cardiovascular diseases.

e. Other neoplasms, endocrine disorders, sense organ diseases, genitourinary diseases, skin diseases, musculoskeletal diseases, and oral conditions.

f. Poisonings, falls, fires, drownings, and other unintentional injuries.

g. Violence, war, and other intentional injuries. 
Table 6B.6 The Burden of Disease-DALYs ${ }_{S B}(3,0,1)$. Calculated to Include Stillbirths (Valued the Same as Newborn Deaths) (thousands)

\begin{tabular}{|c|c|c|c|c|c|c|c|}
\hline \multirow[b]{4}{*}{ Cause } & \multicolumn{7}{|c|}{ Low- and middle-income countries } \\
\hline & \multicolumn{7}{|c|}{ DALYs } \\
\hline & \multicolumn{3}{|c|}{ Stillbirth } & \multicolumn{2}{|c|}{ Under age 5} & \multirow[b]{2}{*}{ age $5+$} & \multirow[b]{2}{*}{ Total } \\
\hline & Antepartum & Intrapartum & Total & $\begin{array}{l}\text { After live } \\
\text { birth }\end{array}$ & $\begin{array}{l}\text { Including } \\
\text { stillbirth }\end{array}$ & & \\
\hline $\begin{array}{l}\text { Total DALYs } \\
\text { I. Communicable, maternal, perinatal, and } \\
\text { nutritional conditions }\end{array}$ & 65,463 & 32,755 & 98,198 & $\begin{array}{l}424,062 \\
330,086\end{array}$ & $\begin{array}{l}522,260 \\
330,086\end{array}$ & $\begin{array}{l}963,364 \\
222,553\end{array}$ & $\begin{array}{r}1,485,623 \\
552,639\end{array}$ \\
\hline A. Infectious and parasitic diseases & & & & 159,602 & 159,602 & 161,226 & 320,828 \\
\hline 1. Tuberculosis & & & & 1,385 & 1,385 & 34,502 & 35,887 \\
\hline $\begin{array}{l}\text { 2. Sexually transmitted diseases } \\
\text { excluding HIV/AIDS }\end{array}$ & & & & 3,194 & 3,194 & 6,149 & 9,343 \\
\hline 3. HIV/AIDS & & & & 10,467 & 10,467 & 60,362 & 70,830 \\
\hline 4. Diarrheal diseases & & & & 53,343 & 53,343 & 5,376 & 58,719 \\
\hline 5. Childhood-cluster diseases & & & & 34,124 & 34,124 & 9,031 & 43,155 \\
\hline a. Pertussis & & & & 11,310 & 11,310 & 99 & 11,408 \\
\hline b. Poliomyelitis & & & & 30 & 30 & 106 & 136 \\
\hline c. Diphtheria & & & & 137 & 137 & 28 & 164 \\
\hline d. Measles & & & & 16,984 & 16,984 & 6,121 & 23,106 \\
\hline e. Tetanus & & & & 5,663 & 5,663 & 2,677 & 8,340 \\
\hline 6. Meningitis & & & & 2,784 & 2,784 & 2,695 & 5,479 \\
\hline 8. Malaria & & & & 36,159 & 36,159 & 3,827 & 39,986 \\
\hline Other I.A. $(7,9-15)^{a}$ & & & & 18,144 & 18,144 & 8 & 18,152 \\
\hline B. Respiratory infections & & & & 62,826 & 62,826 & 23,926 & 86,752 \\
\hline C. Maternal conditions & & & & & & 26,398 & 26,398 \\
\hline D. Perinatal conditions & & & & 89,096 & 89,096 & & 89,096 \\
\hline 1. Low birthweight ${ }^{b}$ & & & & 42,606 & 42,606 & & 42,606 \\
\hline 2. Birth asphyxia and birth trauma & & & & 31,442 & 31,442 & & 31,443 \\
\hline 3. Other perinatal conditions & & & & 15,048 & 15,048 & & 15,048 \\
\hline E. Nutritional deficiencies & & & & 18,562 & 18,562 & 11,002 & 29,564 \\
\hline II. Noncommunicable diseases & & & & 78,798 & 78,798 & 600,044 & 678,842 \\
\hline A. Malignant neoplasms & & & & 1,148 & 1,148 & 73,644 & 74,792 \\
\hline C. Diabetes mellitus & & & & 102 & 102 & 15,715 & 15,817 \\
\hline E. Neuropsychiatric disorders & & & & 20,180 & 20,180 & 116,960 & 137,140 \\
\hline 1. Unipolar depressive disorders & & & & & & 43,444 & 43,445 \\
\hline 2. Bipolar affective disorder & & & & & & 8,681 & 8,681 \\
\hline 3. Schizophrenia & & & & 1 & 1 & 10,530 & 10,531 \\
\hline Other II.E. $(4-16)^{c}$ & & & & 7 & 7 & 20 & 27 \\
\hline G. Cardiovascular diseases & & & & 3,033 & 3,033 & 175,983 & 179,016 \\
\hline 3. Ischemic heart disease & & & & 177 & 177 & 71735 & 71.913 \\
\hline 4. Cerebrovascular disease & & & & 371 & 371 & 62,326 & 62,697 \\
\hline Other II.G. $(1,2,5,6)^{d}$ & & & & 2,484 & 2.484 & & 2,484 \\
\hline H. Respiratory diseases & & & & 5,966 & 5,966 & 52,146 & 58,112 \\
\hline I. Digestive diseases & & & & 14,442 & 14,442 & 37,990 & 52,433 \\
\hline M. Congenital anomalies & & & & 22,061 & 22,061 & 1,483 & 23,544 \\
\hline Other II. (B, D, F, J, K, L, N) & & & & 11,866 & 11,866 & 1 & 11,867 \\
\hline III. Injuries & & & & 15,178 & 15,178 & 140,767 & 155,945 \\
\hline A. Unintentional & & & & 14,621 & 14,621 & 98,684 & 113,306 \\
\hline 1. Road traffic accidents & & & & 2,275 & 2,275 & 29,766 & 32,041 \\
\hline Other III.A. $(2-6)^{f}$ & & & & 12,346 & 12,346 & 12 & 12,358 \\
\hline B. Intentional & & & & 557 & 557 & 42,083 & 42,640 \\
\hline 1. Self-inflicted & & & & 4 & 4 & 17,674 & 17,678 \\
\hline Other III.B. $(2-4)^{g}$ & & & & 553 & 553 & 2 & 555 \\
\hline IV. Not allocated & 65.463 & 32755 & 98.198 & & 98.198 & & 98.198 \\
\hline
\end{tabular}

Sources: Stillbirth data are from WHO 2005a. Neonatal and perinatal mortality are country, regional, and global estimates. Age 5+ and total data are from table 6.4 for low- and middle-income countries. All other data are from chapter 3 of this volume.

Note: A blank cell indicates that fewer than 1,000 deaths are attributable to the specific cause. DALYs used here: DALYs(3,0,1).

a. Hepatitis, tropical-cluster diseases, leprosy, dengue, Japanese encephalitis, trachoma, intestinal nematode infections, and other infectious diseases.

b. Low birthweight deaths are those resulting from intrauterine growth retardation or preterm birth. Almost all low birthweight deaths in the neonatal period result from preterm birth

c. Epilepsy, alcohol use disorders, Alzheimer's and other dementias, Parkinson's disease, multiple sclerosis, drug use disorders, post-traumatic stress disorder, obsessive-compulsive disorder, panic disorder, insomnia (primary), migraine, mental retardation attributable to lead exposure, and other neuropsychiatric disorders.

d. Rheumatic heart disease, hypertensive heart disease, inflammatory heart diseases, and other cardiovascular diseases.

e. Other neoplasms, endocrine disorders, sense organ diseases, genitourinary diseases, skin diseases, musculoskeletal diseases, and oral conditions.

f. Poisonings, falls, fires, drownings, and other unintentional injuries.

g. Violence, war, and other intentional injuries. 


\begin{tabular}{|c|c|c|c|c|c|c|c|}
\hline \multirow[b]{4}{*}{ Cause } & \multicolumn{7}{|c|}{ High-income countries } \\
\hline & \multicolumn{7}{|c|}{ DALYs } \\
\hline & \multicolumn{3}{|c|}{ Stillbirth } & \multicolumn{2}{|c|}{ Under age 5} & \multirow[b]{2}{*}{ age $5+$} & \multirow[b]{2}{*}{ Total } \\
\hline & Antepartum & Intrapartum & Total & $\begin{array}{l}\text { After live } \\
\text { birth }\end{array}$ & $\begin{array}{l}\text { Including } \\
\text { stillbirth }\end{array}$ & & \\
\hline $\begin{array}{l}\text { Total DALYs } \\
\text { I. Communicable, maternal, perinatal, and } \\
\text { nutritional conditions }\end{array}$ & 1,222 & 153 & 1,375 & $\begin{array}{l}6,804 \\
2,177\end{array}$ & $\begin{array}{l}8,178 \\
2,177\end{array}$ & $\begin{array}{r}142,358 \\
6,384\end{array}$ & $\begin{array}{r}150,536 \\
8,561\end{array}$ \\
\hline A. Infectious and parasitic diseases & & & & 563 & 563 & 2,812 & 3,375 \\
\hline 1. Tuberculosis & & & & 2 & 2 & 217 & 219 \\
\hline $\begin{array}{l}\text { 2. Sexually transmitted diseases } \\
\text { excluding HIV/AIDS }\end{array}$ & & & & 13 & 13 & 132 & 145 \\
\hline 3. HIV/AIDS & & & & 3 & 3 & 662 & 665 \\
\hline 4. Diarrheal diseases & & & & 235 & 235 & 210 & 444 \\
\hline 5. Childhood-cluster diseases & & & & 141 & 141 & 33 & 175 \\
\hline a. Pertussis & & & & 138 & 138 & 2 & 139 \\
\hline $\begin{array}{l}\text { b. Poliomyelitis } \\
\text { c. Diphtheria }\end{array}$ & & & & & & 8 & 8 \\
\hline d. Measles & & & & 3 & 3 & 20 & 23 \\
\hline e. Tetanus & & & & 1 & 1 & 5 & 5 \\
\hline 6. Meningitis & & & & 50 & 50 & 81 & 131 \\
\hline 8. Malaria & & & & 2 & 2 & 7 & 9 \\
\hline Other I.A. $(7,9-15)^{a}$ & & & & 117 & 117 & 1,470 & 1,587 \\
\hline B. Respiratory infections & & & & 98 & 98 & 2,376 & 2,474 \\
\hline C. Maternal conditions & & & & & & 391 & 391 \\
\hline D. Perinatal conditions & & & & 1,405 & 1,405 & 4 & 1,408 \\
\hline 1. Low birthweight ${ }^{b}$ & & & & 467 & 467 & & 467 \\
\hline 2. Birth asphyxia and birth trauma & & & & 528 & 528 & 1 & 530 \\
\hline 3. Other perinatal conditions & & & & 410 & 410 & 2 & 412 \\
\hline E. Nutritional deficiencies & & & & 111 & 111 & 801 & 912 \\
\hline II. Noncommunicable diseases & & & & 4,229 & 4,229 & 125,127 & 129,356 \\
\hline A. Malignant neoplasms & & & & 54 & 54 & 25,834 & 25,888 \\
\hline C. Diabetes mellitus & & & & 1 & 1 & 4,191 & 4,192 \\
\hline E. Neuropsychiatric disorders & & & & 976 & 976 & 30,254 & 31,230 \\
\hline 1. Unipolar depressive disorders & & & & & & 8,408 & 8,408 \\
\hline 2. Bipolar affective disorder & & & & & & 1,056 & 1,056 \\
\hline 3. Schizophrenia & & & & & & 1,115 & 1,115 \\
\hline Other II.E. $(4-16)^{c}$ & & & & 976 & 976 & 19,675 & 20,651 \\
\hline G. Cardiovascular diseases & & & & 78 & 78 & 29,780 & 29,859 \\
\hline 3. Ischemic heart disease & & & & 2 & 2 & 12,388 & 12,390 \\
\hline 4. Cerebrovascular disease & & & & 11 & 11 & 9,344 & 9,354 \\
\hline Other II.G. $(1,2,5,6)^{d}$ & & & & 65 & 65 & 8,049 & 8,114 \\
\hline H. Respiratory diseases & & & & 569 & 569 & 9,233 & 9,801 \\
\hline I. Digestive diseases & & & & 475 & 475 & 6,061 & 6,536 \\
\hline M. Congenital anomalies & & & & 1,191 & 1,191 & 228 & 1,420 \\
\hline Other II. $(B, D, F, J, K, L, N)^{e}$ & & & & 885 & 885 & 19,546 & 20,431 \\
\hline III. Injuries & & & & 398 & 398 & 10,846 & 11,244 \\
\hline A. Unintentional & & & & 364 & 364 & 7,513 & 7,876 \\
\hline 1. Road traffic accidents & & & & 68 & 68 & 2,978 & 3,045 \\
\hline Other III.A. $(2-6)^{f}$ & & & & 296 & 296 & 4,535 & 4,831 \\
\hline B. Intentional & & & & 34 & 34 & 3,334 & 3,368 \\
\hline 1. Self-inflicted & & & & & & 2,581 & 2,581 \\
\hline Other III.B. $(2-4)^{g}$ & & & & 34 & 34 & 753 & 787 \\
\hline IV. Not allocated & 1,222 & 153 & 1,375 & & 1,375 & & 1,375 \\
\hline
\end{tabular}

Sources: Stillbirth data are from WHO 2005a. Neonatal and perinatal mortality are country, regional, and global estimates. Age 5+ and total data are from table 6.4 for low- and middle-income countries. All other data are from chapter 3 of this volume.

Note: A blank cell indicates that fewer than 1,000 deaths are attributable to the specific cause. DALYs used here: DALYs $(3,0,1)$.

a. Hepatitis, tropical-cluster diseases, leprosy, dengue, Japanese encephalitis, trachoma, intestinal nematode infections, and other infectious diseases.

b. Low birthweight deaths are those resulting from intrauterine growth retardation or preterm birth. Almost all low birthweight deaths in the neonatal period result from preterm birth

c. Epilepsy, alcohol use disorders, Alzheimer's and other dementias, Parkinson's disease, multiple sclerosis, drug use disorders, post-traumatic stress disorder, obsessive-compulsive disorder, panic

disorder, insomnia (primary), migraine, mental retardation attributable to lead exposure, and other neuropsychiatric disorders.

d. Rheumatic heart disease, hypertensive heart disease, inflammatory heart diseases, and other cardiovascular diseases.

e. Other neoplasms, endocrine disorders, sense organ diseases, genitourinary diseases, skin diseases, musculoskeletal diseases, and oral conditions.

f. Poisonings, falls, fires, drownings, and other unintentional injuries.

g. Violence, war, and other intentional injuries. 
Table 6B.6 Continued

\begin{tabular}{|c|c|c|c|c|c|c|c|}
\hline \multirow[b]{4}{*}{ Cause } & \multicolumn{7}{|c|}{ World } \\
\hline & \multicolumn{7}{|c|}{ DALYs } \\
\hline & \multicolumn{3}{|c|}{ Stillbirth } & \multicolumn{2}{|c|}{ Under age 5} & \multirow[b]{2}{*}{ age $5+$} & \multirow[b]{2}{*}{ Total } \\
\hline & Antepartum & Intrapartum & Total & $\begin{array}{l}\text { After live } \\
\text { birth }\end{array}$ & $\begin{array}{l}\text { Including } \\
\text { stillbirth }\end{array}$ & & \\
\hline Total DALYs & 66,685 & 32,907 & 99,592 & 430,866 & 530,458 & $1,105,721$ & $1,636,179$ \\
\hline $\begin{array}{l}\text { I. Communicable, maternal, perinatal, and } \\
\text { nutritional conditions }\end{array}$ & & & & 332,263 & 332,263 & 228,937 & 561,200 \\
\hline A. Infectious and parasitic diseases & & & & 160,165 & 160,165 & 164,039 & 324,203 \\
\hline 1. Tuberculosis & & & & 1,387 & 1,387 & 34,719 & 36,106 \\
\hline $\begin{array}{l}\text { 2. Sexually transmitted diseases } \\
\text { excluding HIV/AIDS }\end{array}$ & & & & 3,207 & 3,207 & 6,280 & 9,488 \\
\hline 3. HIV/AIDS & & & & 10,471 & 10,471 & 61,024 & 71,495 \\
\hline 4. Diarrheal diseases & & & & 53,578 & 53,578 & 5,586 & 59,164 \\
\hline 5. Childhood-cluster diseases & & & & 34,266 & 34,266 & 9,064 & 43,330 \\
\hline a. Pertussis & & & & 11,448 & 11,448 & 100 & 11,548 \\
\hline b. Poliomyelitis & & & & 30 & 30 & 114 & 144 \\
\hline c. Diphtheria & & & & 137 & 137 & 28 & 164 \\
\hline d. Measles & & & & 16,988 & 16,988 & 6,141 & 23,129 \\
\hline e. Tetanus & & & & 5,664 & 5,664 & 2,681 & 8,345 \\
\hline 6. Meningitis & & & & 2,834 & 2,834 & 2,776 & 5,610 \\
\hline 8. Malaria & & & & 36,161 & 36,161 & 3,834 & 39,995 \\
\hline Other I.A. $(7,9-15)^{\mathrm{a}}$ & & & & 18,261 & 18,261 & 40,755 & 59,016 \\
\hline B. Respiratory infections & & & & 62,924 & 62,924 & 26,302 & 89,227 \\
\hline C. Maternal conditions & & & & & & 26,789 & 26,789 \\
\hline D. Perinatal conditions & & & & 90,501 & 90,501 & 4 & 90,505 \\
\hline 1. Low birthweight ${ }^{b}$ & & & & 43,072 & 43,072 & & 43,073 \\
\hline 2. Birth asphyxia and birth trauma & & & & 31,971 & 31,971 & 2 & 31,972 \\
\hline 3. Other perinatal conditions & & & & 15,458 & 15,458 & 2 & 15,460 \\
\hline E. Nutritional deficiencies & & & & 18,673 & 18,673 & 11,803 & 30,475 \\
\hline II. Noncommunicable diseases & & & & 83,027 & 83,027 & 725,171 & 808,198 \\
\hline A. Malignant neoplasms & & & & 1,202 & 1,202 & 99,478 & 100,680 \\
\hline C. Diabetes mellitus & & & & 103 & 103 & 19,906 & 20,009 \\
\hline E. Neuropsychiatric disorders & & & & 21,156 & 21,156 & 147,214 & 168,371 \\
\hline 1. Unipolar depressive disorders & & & & 1 & 1 & 51,852 & 51,853 \\
\hline 2. Bipolar affective disorder & & & & & & 9,737 & 9,737 \\
\hline 3. Schizophrenia & & & & 1 & 1 & 11,645 & 11,646 \\
\hline Other II.E. $(4-16)^{c}$ & & & & 21,154 & 21,154 & 73,981 & 95,134 \\
\hline G. Cardiovascular diseases & & & & 3,111 & 3,111 & 205,764 & 208,875 \\
\hline 3. Ischemic heart disease & & & & 180 & 180 & 84,124 & 84,303 \\
\hline 4. Cerebrovascular disease & & & & 382 & 382 & 71,669 & 72,051 \\
\hline Other II.G. $(1,2,5,6)^{d}$ & & & & 2,550 & 2,550 & 49,970 & 52,520 \\
\hline H. Respiratory diseases & & & & 6,535 & 6,535 & 61,379 & 67,914 \\
\hline I. Digestive diseases & & & & 14,917 & 14,917 & 44,051 & 58,968 \\
\hline M. Congenital anomalies & & & & 23,252 & 23,252 & 1,712 & 24,964 \\
\hline Other II. (B, D, F, J, K, L, N)e & & & & 12,751 & 12,751 & 145,667 & 158,418 \\
\hline III. Injuries & & & & 15,576 & 15,576 & 151,613 & 167,189 \\
\hline A. Unintentional & & & & 14,985 & 14,985 & 106,197 & 121,182 \\
\hline 1. Road traffic accidents & & & & 2,343 & 2,343 & 32,744 & 35,087 \\
\hline Other III.A. $(2-6)^{f}$ & & & & 12,642 & 12,642 & 73,453 & 86,095 \\
\hline B. Intentional & & & & 591 & 591 & 45,416 & 46,007 \\
\hline 1. Self-inflicted & & & & 4 & 4 & 20,255 & 20,259 \\
\hline Other III.B. $(2-4)^{g}$ & & & & 587 & 587 & 25,161 & 25,749 \\
\hline IV. Not allocated & 66,685 & 32,907 & 99,592 & & 99,592 & & 99,592 \\
\hline
\end{tabular}

Sources: Stillbirth data are from WHO 2005a. Neonatal and perinatal mortality are country, regional, and global estimates. Age $5+$ and total data are from table 6.4 for low- and middle-income countries. All other data are from chapter 3 of this volume.

Note: A blank cell indicates that fewer than 1,000 deaths are attributable to the specific cause. DALYs used here: DALYs $(3,0,1)$.

a. Hepatitis, tropical-cluster diseases, leprosy, dengue, Japanese encephalitis, trachoma, intestinal nematode infections, and other infectious diseases.

b. Low birthweight deaths are those resulting from intrauterine growth retardation or preterm birth. Almost all low birthweight deaths in the neonatal period result from preterm birth.

c. Epilepsy, alcohol use disorders, Alzheimer's and other dementias, Parkinson's disease, multiple sclerosis, drug use disorders, post-traumatic stress disorder, obsessive-compulsive disorder, panic

disorder, insomnia (primary), migraine, mental retardation attributable to lead exposure, and other neuropsychiatric disorders.

d. Rheumatic heart disease, hypertensive heart disease, inflammatory heart diseases, and other cardiovascular diseases.

e. Other neoplasms, endocrine disorders, sense organ diseases, genitourinary diseases, skin diseases, musculoskeletal diseases, and oral conditions.

f. Poisonings, falls, fires, drownings, and other unintentional injuries.

g. Violence, war, and other intentional injuries. 
Table 6B.7 YLL $\mathrm{SB}_{\mathrm{B}}(3,0,54)$ Calculated to Include Stillbirths and Gradual ALP (in thousands)

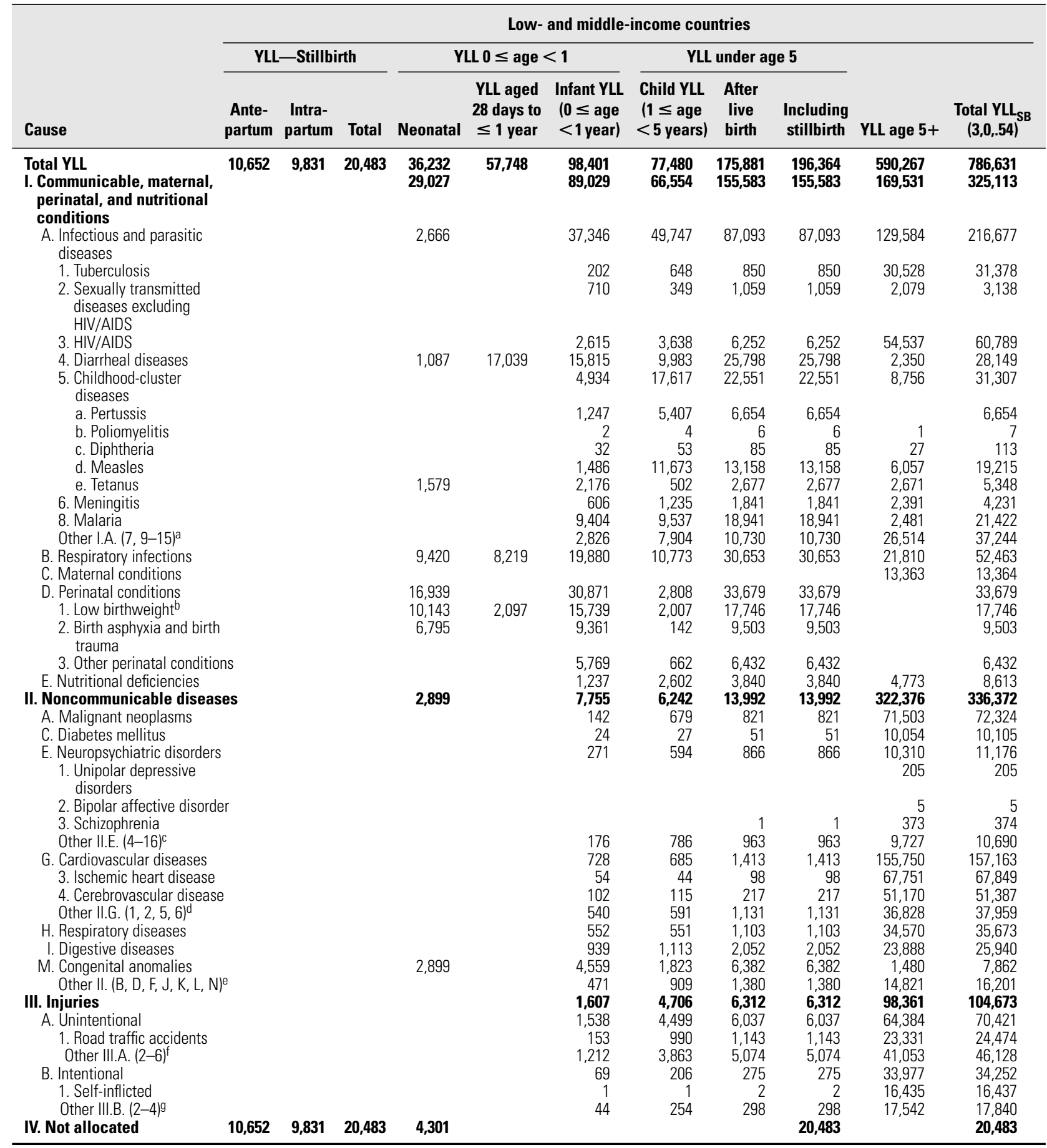

Sources: Stillbirth data are from WHO 2005a. Neonatal and perinatal mortality are country, regional, and global estimates. Age 5+ and total data are from table 6.4 for low- and middle-income countries. All other data are from chapter 3 of this volume.

Note: A blank cell indicates that fewer than 1,000 deaths are attributable to the specific cause. YLL used here: YLL(3,0,54).

a. Hepatitis, tropical-cluster diseases, leprosy, dengue, Japanese encephalitis, trachoma, intestinal nematode infections, and other infectious diseases.

b. Low birthweight deaths are those resulting from intrauterine growth retardation or preterm birth. Almost all low birthweight deaths in the neonatal period result from preterm birth.

c. Epilepsy, alcohol use disorders, Alzheimer's and other dementias, Parkinson's disease, multiple sclerosis, drug use disorders, post-traumatic stress disorder, obsessive-compulsive disorder, panic

disorder, insomnia (primary), migraine, mental retardation attributable to lead exposure, and other neuropsychiatric disorders.

d. Rheumatic heart disease, hypertensive heart disease, inflammatory heart diseases, and other cardiovascular diseases.

e. Other neoplasms, endocrine disorders, sense organ diseases, genitourinary diseases, skin diseases, musculoskeletal diseases, and oral conditions.

f. Poisonings, falls, fires, drownings, and other unintentional injuries.

g. Violence, war, and other intentional injuries. 
Table 6B.7 Continued

\begin{tabular}{|c|c|c|c|c|c|c|c|c|c|c|c|}
\hline \multirow[b]{3}{*}{ Cause } & \multicolumn{11}{|c|}{ High-income countries } \\
\hline & \multicolumn{3}{|c|}{ YLL_Stillbirth } & \multicolumn{3}{|c|}{ YLL $0 \leq$ age $<1$} & \multicolumn{3}{|c|}{ YLL under age 5} & \multirow[b]{2}{*}{ YLL age 5+ } & \multirow[b]{2}{*}{$\begin{array}{c}\text { Total YLL } \\
(3,0, .54)\end{array}$} \\
\hline & $\begin{array}{c}\text { Ante- } \\
\text { partum }\end{array}$ & $\begin{array}{l}\text { Intra- } \\
\text { partum }\end{array}$ & Total & Neonatal & $\begin{array}{l}\text { YLL aged } \\
28 \text { days to } \\
\leq 1 \text { year }\end{array}$ & $\begin{array}{c}\text { Infant } Y L L \\
\text { ( } 0 \leq \text { age } \\
<1 \text { year })\end{array}$ & $\begin{array}{l}\text { Child YLL } \\
\text { (1 } \leq \text { age } \\
<5 \text { years) }\end{array}$ & $\begin{array}{l}\text { After } \\
\text { live } \\
\text { birth }\end{array}$ & $\begin{array}{l}\text { Including } \\
\text { stillbirth }\end{array}$ & & \\
\hline Total YLL & 199 & 46 & 245 & 388 & 278 & $\begin{array}{l}769 \\
457\end{array}$ & $\begin{array}{r}353 \\
53\end{array}$ & $\begin{array}{r}1,122 \\
510\end{array}$ & $\begin{array}{r}1,366 \\
510\end{array}$ & $\begin{array}{r}75,650 \\
4,258\end{array}$ & $\begin{array}{r}77,016 \\
4,768\end{array}$ \\
\hline \multicolumn{4}{|l|}{ diseases } & 150 & & 26 & 30 & 57 & 57 & 1,878 & 1,935 \\
\hline 1. Tuberculosis & & & & & & & 1 & 1 & 1 & 171 & 172 \\
\hline $\begin{array}{l}\text { 2. Sexually transmitted } \\
\text { diseases excluding } \\
\text { HIV/AIDS }\end{array}$ & & & & & & & & & & 6 & 7 \\
\hline 5. Childhood-cluster disease & & & & & & & 2 & 2 & 3 & 30 & 32 \\
\hline a. Pertussis & & & & & & & & & 1 & & 1 \\
\hline b. Poliomyelitis & & & & & & & & & & 6 & 6 \\
\hline c. Diphtheria & & & & & & & & & & & \\
\hline d. Measles & & & & & & & 1 & 1 & 1 & 19 & 21 \\
\hline e. Tetanus & & & & & & & & & & 5 & 5 \\
\hline 6. Meningitis & & & & & & & 9 & 9 & 9 & 59 & 73 \\
\hline 8. Malaria & & & & & & & & & & 2 & 3 \\
\hline Other I.A. $(7,9-15)^{a}$ & & & & & & 10 & 24 & 34 & 34 & 1,079 & 1,113 \\
\hline B. Respiratory infections & & & & & & 14 & 16 & 30 & 30 & 2,227 & 2,258 \\
\hline C. Maternal conditions & & & & & & & & & & 27 & 27 \\
\hline D. Perinatal conditions & & & & 150 & & 415 & 5 & 420 & 420 & 4 & 424 \\
\hline 1. Low birthweight ${ }^{b}$ & & & & 90 & & 124 & & 124 & 124 & & 124 \\
\hline 2. Birth asphyxia and birth $t$ & trauma & & & 58 & 77 & 142 & 289 & 431 & 431 & 1 & 146 \\
\hline 3. Other perinatal condition & & & & & & 150 & 305 & 455 & 455 & 2 & 153 \\
\hline E. Nutritional deficiencies & & & & & & 1 & 2 & 3 & 3 & 122 & 125 \\
\hline II. Noncommunicable disease & & & & 116 & & 251 & 511 & 761 & 761 & 63,397 & 63,883 \\
\hline E. Neuropsychiatric disorders & & & & & & 14 & 26 & 40 & 40 & 3,259 & 3,299 \\
\hline 1. Unipolar depressive disor & orders & & & & & & & & & 21 & 21 \\
\hline 2. Bipolar affective disorder & & & & & & & & & & 4 & 4 \\
\hline 3. Schizophrenia & & & & & & & & & & 24 & 24 \\
\hline Other II.E. $(4-16)^{c}$ & & & & & & 7 & 19 & 26 & 26 & 3,210 & 3,236 \\
\hline G. Cardiovascular diseases & & & & & & 18 & 18 & 36 & 36 & 24,166 & 24,202 \\
\hline 3. Ischemic heart disease & & & & & & 1 & 1 & 1 & 1 & 11,483 & 11,484 \\
\hline 4. Cerebrovascular disease & & & & & & 3 & 19 & 22 & 22 & 5,886 & 5,908 \\
\hline Other II.G. $(1,2,5,6)^{d}$ & & & & & & 12 & 19 & 31 & 31 & 6,797 & 6,829 \\
\hline H. Respiratory diseases & & & & & & 8 & 10 & 18 & 18 & 3,914 & 3,932 \\
\hline I. Digestive diseases & & & & & & 11 & 7 & 19 & 19 & 3,680 & 3,698 \\
\hline M. Congenital anomalies & & & & 116 & 62 & 208 & 48 & 256 & 256 & 229 & 484 \\
\hline Other II. $(B, D, F, J, K, L, N)^{e}$ & & & & & & 17 & 28 & 45 & 45 & 2,943 & 2,988 \\
\hline III. Injuries & & & & & & 31 & 127 & 158 & 158 & 7,995 & 8,153 \\
\hline A. Unintentional & & & & & & 24 & 112 & 137 & 137 & 5,003 & 5,140 \\
\hline 1. Road traffic accidents & & & & & & 4 & 37 & 41 & 41 & 2,496 & 2,537 \\
\hline Other III.A. $(2-6)^{f}$ & & & & & & 14 & 87 & 100 & 100 & 2,507 & 2,607 \\
\hline B. Intentional & & & & & & 7 & 15 & 21 & 21 & 2,992 & 3,013 \\
\hline 1. Self-inflicted & & & & & & & & & & 2,432 & 2,433 \\
\hline Other III.B. $(2-4)^{g}$ & & & & & & 7 & 15 & 21 & 21 & 559 & 581 \\
\hline IV. Not allocated & 199 & 46 & 245 & 124 & & & & & 245 & & 245 \\
\hline
\end{tabular}

Sources: Stillbirth data are from WHO 2005a. Neonatal and perinatal mortality are country, regional, and global estimates. Age 5+ and total data are from table 6.4 for low- and middle-income countries. All other data are from chapter 3 of this volume.

Note: A blank cell indicates that fewer than 1,000 deaths are attributable to the specific cause. YLL used here: YLL(3,0,54).

a. Hepatitis, tropical-cluster diseases, leprosy, dengue, Japanese encephalitis, trachoma, intestinal nematode infections, and other infectious diseases.

b. Low birthweight deaths are those resulting from intrauterine growth retardation or preterm birth. Almost all low birthweight deaths in the neonatal period result from preterm birth.

c. Epilepsy, alcohol use disorders, Alzheimer's and other dementias, Parkinson's disease, multiple sclerosis, drug use disorders, post-traumatic stress disorder, obsessive-compulsive disorder, panic

disorder, insomnia (primary), migraine, mental retardation attributable to lead exposure, and other neuropsychiatric disorders.

d. Rheumatic heart disease, hypertensive heart disease, inflammatory heart diseases, and other cardiovascular diseases.

e. Other neoplasms, endocrine disorders, sense organ diseases, genitourinary diseases, skin diseases, musculoskeletal diseases, and oral conditions

f. Poisonings, falls, fires, drownings, and other unintentional injuries.

g. Violence, war, and other intentional injuries. 


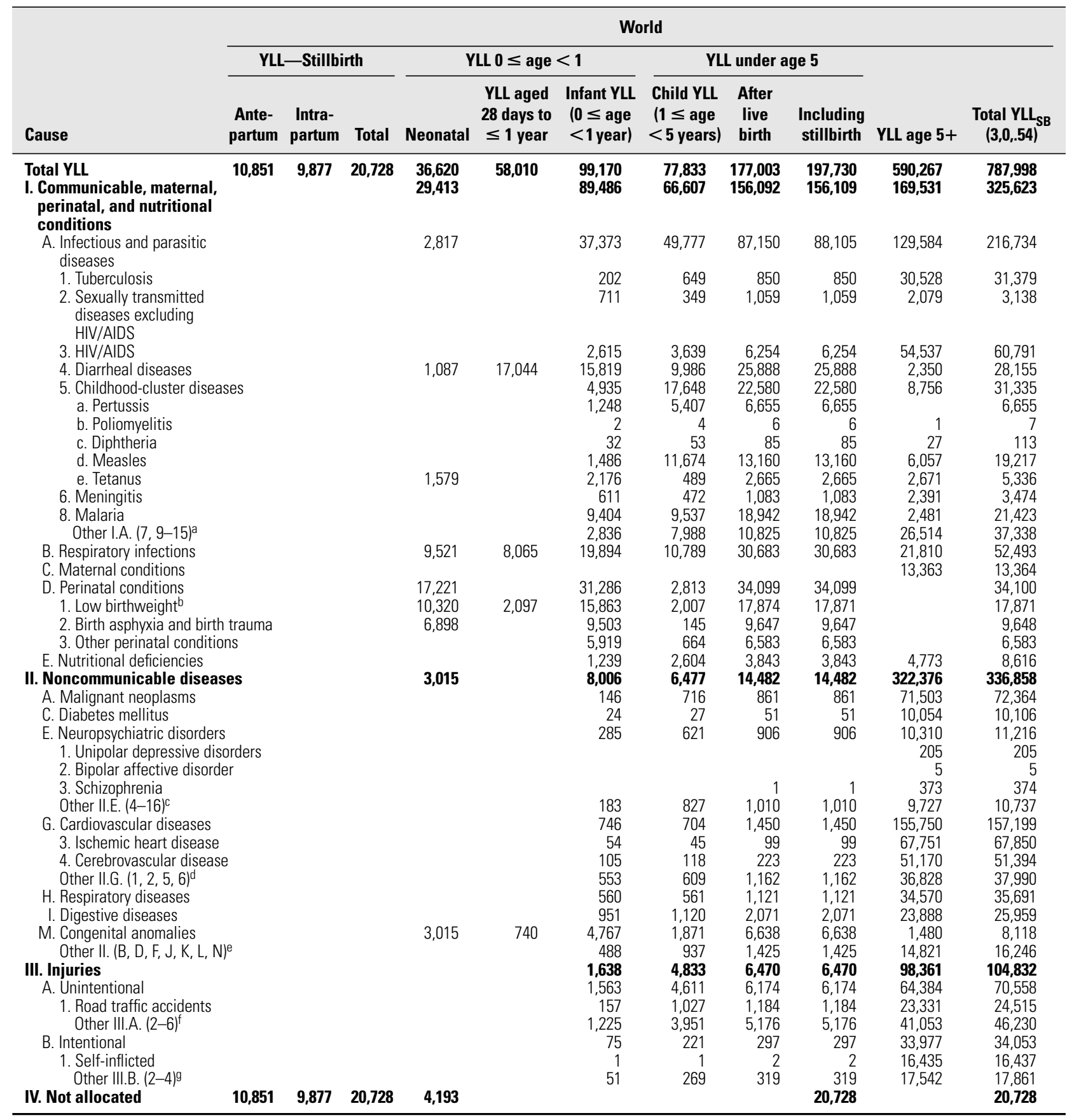

Sources: Stillbirth data are from WHO 2005a. Neonatal and perinatal mortality are country, regional, and global estimates. Age $5+$ and total data are from table 6.4 for low- and middle-income countries. All other data are from chapter 3 of this volume.

Note: A blank cell indicates that fewer than 1,000 deaths are attributable to the specific cause. YLL used here: YLL(3,0,54).

a. Hepatitis, tropical-cluster diseases, leprosy, dengue, Japanese encephalitis, trachoma, intestinal nematode infections, and other infectious diseases.

b. Low birthweight deaths are those resulting from intrauterine growth retardation or preterm birth. Almost all low birthweight deaths in the neonatal period result from preterm birth.

c. Epilepsy, alcohol use disorders, Alzheimer's and other dementias, Parkinson's disease, multiple sclerosis, drug use disorders, post-traumatic stress disorder, obsessive-compulsive disorder, panic

disorder, insomnia (primary), migraine, mental retardation attributable to lead exposure, and other neuropsychiatric disorders.

d. Rheumatic heart disease, hypertensive heart disease, inflammatory heart diseases, and other cardiovascular diseases.

e. Other neoplasms, endocrine disorders, sense organ diseases, genitourinary diseases, skin diseases, musculoskeletal diseases, and oral conditions.

f. Poisonings, falls, fires, drownings, and other unintentional injuries.

g. Violence, war, and other intentional injuries. 
Table 6B.8 The Burden of Disease-DALYs ${ }_{\mathrm{SB}}(3,0, .54)$. Calculated to Include Stillbirths and Gradual $\mathrm{ALP}(A=.54)$ (thousands)

\begin{tabular}{|c|c|c|c|c|c|c|c|}
\hline \multirow[b]{4}{*}{ Cause } & \multicolumn{7}{|c|}{ Low- and middle-income countries } \\
\hline & \multicolumn{7}{|c|}{ DALYs } \\
\hline & \multicolumn{3}{|c|}{ Stillbirth } & \multicolumn{2}{|c|}{ Under age 5} & \multirow[b]{2}{*}{ age $5+$} & \multirow{2}{*}{$\begin{array}{c}\text { Total } \\
\text { DALYs } \\
(3,0, .54)\end{array}$} \\
\hline & Antepartum & Intrapartum & Total & $\begin{array}{l}\text { After live } \\
\text { birth }\end{array}$ & $\begin{array}{l}\text { Including } \\
\text { stillbirth }\end{array}$ & & \\
\hline Total DALYs & 10,652 & 9,831 & 20,483 & 276,796 & 297,279 & 963,364 & $1,260,643$ \\
\hline $\begin{array}{l}\text { I. Communicable, maternal, perinatal, and } \\
\text { nutritional conditions }\end{array}$ & & & & 201,606 & 201,606 & 222,553 & 424,158 \\
\hline A. Infectious and parasitic diseases & & & & 103,064 & 103,064 & 161,226 & 264,291 \\
\hline 1. Tuberculosis & & & & 1,020 & 1,020 & 34,502 & 35,521 \\
\hline $\begin{array}{l}\text { 2. Sexually transmitted diseases } \\
\text { excluding HIV/AIDS }\end{array}$ & & & & 2,186 & 2,186 & 6,149 & 8,334 \\
\hline 3. HIV/AIDS & & & & 6,929 & 6,929 & 60,362 & 67,292 \\
\hline 4. Diarrheal diseases & & & & 27,489 & 27,489 & 5,376 & 32,865 \\
\hline 5. Childhood-cluster diseases & & & & 21,653 & 21,653 & 9,031 & 30,684 \\
\hline a. Pertussis & & & & 8,846 & 8,846 & 99 & 8,945 \\
\hline b. Poliomyelitis & & & & 27 & 27 & 106 & 133 \\
\hline c. Diphtheria & & & & 86 & 86 & 28 & 113 \\
\hline d. Measles & & & & 13,294 & 13,294 & 6,121 & 19,416 \\
\hline e. Tetanus & & & & 3,626 & 3,626 & 2,677 & 6,302 \\
\hline 6. Meningitis & & & & 1,898 & 1,898 & 2,695 & 4,593 \\
\hline 8. Malaria & & & & 22,099 & 22,099 & 3,827 & 25,926 \\
\hline Other I.A. $(7,9-15)^{a}$ & & & & 13,116 & 13,116 & 39,285 & 52,401 \\
\hline B. Respiratory infections & & & & 34,508 & 34,508 & 23,926 & 58,434 \\
\hline C. Maternal conditions & & & & & & 26,398 & 26,398 \\
\hline D. Perinatal conditions & & & & 47,202 & 47,202 & & 47,202 \\
\hline 1. Low birthweight ${ }^{b}$ & & & & 17,624 & 17,624 & & 17,624 \\
\hline 2. Birth asphyxia and birth trauma & & & & 18,854 & 18,854 & & 18,854 \\
\hline 3. Other perinatal conditions & & & & 7,226 & 7,226 & & 7,226 \\
\hline E. Nutritional deficiencies & & & & 16,514 & 16,514 & 11,002 & 27,516 \\
\hline II. Noncommunicable diseases & & & & 60,277 & 60,277 & 600,044 & 660,320 \\
\hline A. Malignant neoplasms & & & & 858 & 858 & 73,644 & 74,502 \\
\hline C. Diabetes mellitus & & & & 65 & 65 & 15,715 & 15,781 \\
\hline E. Neuropsychiatric disorders & & & & 19,720 & 19,720 & 116,960 & 136,680 \\
\hline 1. Unipolar depressive disorders & & & & & & 43,444 & 43,444 \\
\hline 2. Bipolar affective disorder & & & & & & 8,681 & 8,681 \\
\hline 3. Schizophrenia & & & & 1 & 1 & 10,530 & 10,531 \\
\hline Other II.E. $(4-16)^{c}$ & & & & 19,816 & 19,816 & 54,305 & 74,122 \\
\hline G. Cardiovascular diseases & & & & 2,709 & 2,709 & 175,983 & 178,692 \\
\hline 3. Ischemic heart disease & & & & 99 & 99 & 71,735 & 71,834 \\
\hline 4. Cerebrovascular disease & & & & 217 & 217 & 62,326 & 62,543 \\
\hline Other II.G. $(1,2,5,6)^{d}$ & & & & 1,670 & 1,670 & 41,922 & 43,591 \\
\hline H. Respiratory diseases & & & & 5,142 & 5,142 & 52,146 & 57,289 \\
\hline I. Digestive diseases & & & & 13,024 & 13,024 & 37,990 & 51,015 \\
\hline M. Congenital anomalies & & & & 14,689 & 14,689 & 1,483 & 16,172 \\
\hline Other II. $(B, D, F, J, K, L, N)^{e}$ & & & & 10,755 & 10,755 & 126,122 & 136,876 \\
\hline III. Injuries & & & & 12,177 & 12,177 & 140,767 & 152,944 \\
\hline A. Unintentional & & & & 11,901 & 11,901 & 98,684 & 110,586 \\
\hline 1. Road traffic accidents & & & & 1,926 & 1,926 & 29,766 & 31,692 \\
\hline Other III.A. $(2-6)^{f}$ & & & & 10,156 & 10,156 & 68,918 & 79,074 \\
\hline B. Intentional & & & & 435 & 435 & 42,083 & 42,517 \\
\hline 1. Self-inflicted & & & & 2 & 2 & 17,674 & 17,676 \\
\hline Other III.B. $(2-4)^{9}$ & & & & 457 & 457 & 24,409 & 24,866 \\
\hline IV. Not allocated & 10,652 & $\mathbf{9 , 8 3 1}$ & 20,483 & 2,599 & 23,082 & & 23,082 \\
\hline
\end{tabular}

Sources: Stillbirth data are from WHO 2005a. Neonatal and perinatal mortality are country, regional, and global estimates. Age $5+$ and total data are from table 6.4 for low- and middle-income countries. All other data are from chapter 3 of this volume.

Note: A blank cell indicates that fewer than 1,000 deaths are attributable to the specific cause. DALYs used here: DALYs(3,0,54).

a. Hepatitis, tropical-cluster diseases, leprosy, dengue, Japanese encephalitis, trachoma, intestinal nematode infections, and other infectious diseases.

b. Low birthweight deaths are those resulting from intrauterine growth retardation or preterm birth. Almost all low birthweight deaths in the neonatal period result from preterm birth

c. Epilepsy, alcohol use disorders, Alzheimer's and other dementias, Parkinson's disease, multiple sclerosis, drug use disorders, post-traumatic stress disorder, obsessive-compulsive disorder, panic

disorder, insomnia (primary), migraine, mental retardation attributable to lead exposure, and other neuropsychiatric disorders.

d. Rheumatic heart disease, hypertensive heart disease, inflammatory heart diseases, and other cardiovascular diseases.

e. Other neoplasms, endocrine disorders, sense organ diseases, genitourinary diseases, skin diseases, musculoskeletal diseases, and oral conditions.

f. Poisonings, falls, fires, drownings, and other unintentional injuries.

g. Violence, war, and other intentional injuries. 


\begin{tabular}{|c|c|c|c|c|c|c|c|}
\hline \multirow[b]{4}{*}{ Cause } & \multicolumn{7}{|c|}{ High-income countries } \\
\hline & \multicolumn{7}{|c|}{ DALYs } \\
\hline & \multicolumn{3}{|c|}{ Stillbirth } & \multicolumn{2}{|c|}{ Under age 5} & \multirow[b]{2}{*}{ age $5+$} & \multirow{2}{*}{$\begin{array}{c}\text { Total } \\
\text { DALYs } \\
(3,0, .54)\end{array}$} \\
\hline & Antepartum & Intrapartum & Total & $\begin{array}{l}\text { After live } \\
\text { birth }\end{array}$ & $\begin{array}{l}\text { Including } \\
\text { stillbirth }\end{array}$ & & \\
\hline Total DALYs & 199 & 46 & 245 & 5,713 & 5,958 & 142,358 & 148,316 \\
\hline $\begin{array}{l}\text { I. Communicable, maternal, perinatal, and } \\
\text { nutritional conditions }\end{array}$ & & & & 1,551 & 1,551 & 6,384 & 7,935 \\
\hline A. Infectious and parasitic diseases & & & & 523 & 523 & 2,812 & 3,335 \\
\hline 1. Tuberculosis & & & & 1 & 1 & 217 & 219 \\
\hline $\begin{array}{l}\text { 2. Sexually transmitted diseases } \\
\text { excluding HIV/AIDS }\end{array}$ & & & & 12 & 12 & 132 & 144 \\
\hline 3. HIV/AIDS & & & & 2 & 2 & 662 & 665 \\
\hline 4. Diarrheal diseases & & & & 224 & 224 & 210 & 438 \\
\hline 5. Childhood-cluster diseases & & & & 139 & 139 & 33 & 174 \\
\hline a. Pertussis & & & & 137 & 137 & 2 & 139 \\
\hline $\begin{array}{l}\text { b. Poliomyelitis } \\
\text { c. Diphtheria }\end{array}$ & & & & & & 8 & 8 \\
\hline d. Measles & & & & 2 & 2 & 20 & 22 \\
\hline e. Tetanus & & & & & & 5 & 5 \\
\hline 6. Meningitis & & & & 36 & 36 & 81 & 122 \\
\hline 8. Malaria & & & & 1 & 1 & 7 & 8 \\
\hline Other I.A. $(7,9-15)^{\mathrm{a}}$ & & & & 100 & 100 & 1,470 & 1,570 \\
\hline B. Respiratory infections & & & & 76 & 76 & 2,376 & 2,453 \\
\hline C. Maternal conditions & & & & & & 391 & 391 \\
\hline D. Perinatal conditions & & & & 842 & 842 & 4 & 846 \\
\hline 1. Low birthweight ${ }^{b}$ & & & & 299 & 299 & & 300 \\
\hline 2. Birth asphyxia and birth trauma & & & & 622 & 622 & 1 & 623 \\
\hline 3. Other perinatal conditions & & & & 510 & 510 & 2 & 513 \\
\hline E. Nutritional deficiencies & & & & 110 & 110 & 801 & 911 \\
\hline II. Noncommunicable diseases & & & & 4,132 & 4,132 & 125,127 & 129,860 \\
\hline A. Malignant neoplasms & & & & 14 & 14 & 25,834 & 25,848 \\
\hline C. Diabetes mellitus & & & & 1 & 1 & 4,191 & 4,192 \\
\hline E. Neuropsychiatric disorders & & & & 953 & 953 & 30,254 & 31,208 \\
\hline 1. Unipolar depressive disorders & & & & & & 8,408 & 8,408 \\
\hline 2. Bipolar affective disorder & & & & & & 1,056 & 1,056 \\
\hline 3. Schizophrenia & & & & & & 1,115 & 1,115 \\
\hline Other II.E. $(4-16)^{c}$ & & & & 939 & 939 & & 20,614 \\
\hline G. Cardiovascular diseases & & & & 51 & 51 & 29,780 & 29,832 \\
\hline 3. Ischemic heart disease & & & & 1 & 1 & 12,388 & 12,390 \\
\hline 4. Cerebrovascular disease & & & & 22 & 22 & 9,344 & 9,366 \\
\hline Other II.G. $(1,2,5,6)^{\mathrm{d}}$ & & & & 46 & 46 & 8,049 & 8,095 \\
\hline H. Respiratory diseases & & & & 557 & 557 & 9,233 & 9,789 \\
\hline I. Digestive diseases & & & & 458 & 458 & 6,061 & 6,519 \\
\hline M. Congenital anomalies & & & & 903 & 903 & 228 & 1,132 \\
\hline Other II. $(B, D, F, J, K, L, N)^{e}$ & & & & 858 & 858 & 19,546 & 20,403 \\
\hline III. Injuries & & & & 338 & 338 & 10,846 & 11,184 \\
\hline A. Unintentional & & & & 315 & 315 & 7,513 & 7,827 \\
\hline 1. Road traffic accidents & & & & 57 & 57 & 2,978 & 3,034 \\
\hline Other III.A. $(2-6)^{f}$ & & & & 263 & 263 & 4,535 & 4,798 \\
\hline B. Intentional & & & & 23 & 23 & 3,334 & 3,357 \\
\hline 1. Self-inflicted & & & & & & 2,581 & 2,581 \\
\hline Other III.B. $(2-4)^{9}$ & & & & 23 & 23 & 753 & 776 \\
\hline IV. Not allocated & 10,851 & 9,877 & 20,728 & & 20,728 & & 20,728 \\
\hline
\end{tabular}

Sources: Stillbirth data are from WHO 2005a. Neonatal and perinatal mortality are country, regional, and global estimates. Age 5+ and total data are from table 6.4 for low- and middle-income countries. All other data are from chapter 3 of this volume.

Note: A blank cell indicates that fewer than 1,000 deaths are attributable to the specific cause. DALYs used here: DALYs(3,0,.54).

a. Hepatitis, tropical-cluster diseases, leprosy, dengue, Japanese encephalitis, trachoma, intestinal nematode infections, and other infectious diseases.

b. Low birthweight deaths are those resulting from intrauterine growth retardation or preterm birth. Almost all low birthweight deaths in the neonatal period result from preterm birth.

c. Epilepsy, alcohol use disorders, Alzheimer's and other dementias, Parkinson's disease, multiple sclerosis, drug use disorders, post-traumatic stress disorder, obsessive-compulsive disorder, panic

disorder, insomnia (primary), migraine, mental retardation attributable to lead exposure, and other neuropsychiatric disorders.

d. Rheumatic heart disease, hypertensive heart disease, inflammatory heart diseases, and other cardiovascular diseases.

e. Other neoplasms, endocrine disorders, sense organ diseases, genitourinary diseases, skin diseases, musculoskeletal diseases, and oral conditions.

f. Poisonings, falls, fires, drownings, and other unintentional injuries.

g. Violence, war, and other intentional injuries.

(Continues on the following page.) 
Table 6B.8 Continued

\begin{tabular}{|c|c|c|c|c|c|c|c|}
\hline \multirow[b]{4}{*}{ Cause } & \multicolumn{7}{|c|}{ World } \\
\hline & \multicolumn{7}{|c|}{ DALYs } \\
\hline & \multicolumn{3}{|c|}{ Stillbirth } & \multicolumn{2}{|c|}{ Under age 5} & \multirow[b]{2}{*}{ age $5+$} & \multirow{2}{*}{$\begin{array}{c}\text { Total } \\
\text { DALYs } \\
(3,0,54)\end{array}$} \\
\hline & Antepartum & Intrapartum & Total & $\begin{array}{c}\text { After live } \\
\text { birth }\end{array}$ & $\begin{array}{l}\text { Including } \\
\text { stillbirth }\end{array}$ & & \\
\hline Total DALYs & 10,851 & 9,877 & 20,728 & 286,151 & 306,879 & $1,105,721$ & $1,412,600$ \\
\hline $\begin{array}{l}\text { I. Communicable, maternal, perinatal, and } \\
\text { nutritional conditions }\end{array}$ & & & & 202,202 & 202,202 & 228,937 & 431,139 \\
\hline A. Infectious and parasitic diseases & & & & 102,633 & 102,633 & 164,039 & 266,671 \\
\hline 1. Tuberculosis & & & & 1,021 & 1,021 & 34,719 & 35,740 \\
\hline $\begin{array}{l}\text { 2. Sexually transmitted diseases } \\
\text { excluding HIV/AIDS }\end{array}$ & & & & 2,198 & 2,198 & 6,280 & 8,478 \\
\hline 3. HIV/AIDS & & & & 6,428 & 6,428 & 61,024 & 67,453 \\
\hline 4. Diarrheal diseases & & & & 30,841 & 30,841 & 5,586 & 36,427 \\
\hline 5. Childhood-cluster diseases & & & & 25,076 & 25,076 & 9,064 & 34,140 \\
\hline a. Pertussis & & & & 8,983 & 8,983 & 100 & 9,083 \\
\hline b. Poliomyelitis & & & & 27 & 27 & 114 & 141 \\
\hline c. Diphtheria & & & & 86 & 86 & 28 & 113 \\
\hline d. Measles & & & & 13,297 & 13,297 & 6,141 & 19,438 \\
\hline e. Tetanus & & & & 2,674 & 2,674 & 2,681 & 5,356 \\
\hline 6. Meningitis & & & & 1,939 & 1,939 & 2,776 & 4,715 \\
\hline 8. Malaria & & & & 22,100 & 22,100 & 3,834 & 25,934 \\
\hline Other I.A. $(7,9-15)^{a}$ & & & & 13,276 & 13,276 & 40,755 & 54,032 \\
\hline B. Respiratory infections & & & & 34,584 & 34,584 & 26,302 & 60,886 \\
\hline C. Maternal conditions & & & & & & 26,789 & 26,790 \\
\hline D. Perinatal conditions & & & & 48,044 & 48,044 & 4 & 48,048 \\
\hline 1. Low birthweight $\mathrm{t}^{\mathrm{b}}$ & & & & 21,422 & 21,422 & & 21,423 \\
\hline 2. Birth asphyxia and birth trauma & & & & 19,190 & 19,190 & 2 & 19,192 \\
\hline 3. Other perinatal conditions & & & & 7,433 & 7,433 & 2 & 7,435 \\
\hline E. Nutritional deficiencies & & & & 16,623 & 16,623 & 11,803 & 28,426 \\
\hline II. Noncommunicable diseases & & & & 71,318 & 71,318 & 725,171 & 796,489 \\
\hline A. Malignant neoplasms & & & & 902 & 902 & 99,478 & 100,380 \\
\hline C. Diabetes mellitus & & & & 67 & 67 & 19,906 & 19,973 \\
\hline E. Neuropsychiatric disorders & & & & 20,673 & 20,673 & 147,214 & 167,887 \\
\hline 1. Unipolar depressive disorders & & & & & & 51,852 & 51,852 \\
\hline 2. Bipolar affective disorder & & & & & & 9,737 & 9,737 \\
\hline 3. Schizophrenia & & & & 1 & 1 & 11,645 & 11,646 \\
\hline Other II.E. $(4-16)^{c}$ & & & & 20,777 & 20,777 & 73,981 & 97,757 \\
\hline G. Cardiovascular diseases & & & & 2,044 & 2,044 & 205,764 & 207,768 \\
\hline 3. Ischemic heart disease & & & & 100 & 100 & 84,124 & 84,224 \\
\hline 4. Cerebrovascular disease & & & & 223 & 223 & 71,669 & 71,893 \\
\hline Other II.G. $(1,2,5,6)^{d}$ & & & & 1,716 & 1,716 & 49,970 & 51,686 \\
\hline H. Respiratory diseases & & & & 5,699 & 5,699 & 61,379 & 67,078 \\
\hline I. Digestive diseases & & & & 13,483 & 13,483 & 44,051 & 57,534 \\
\hline M. Congenital anomalies & & & & 16,578 & 16,578 & 1,712 & 18,290 \\
\hline Other II. $(B, D, F, J, K, L, N)^{e}$ & & & & 11,953 & 11,953 & 145,667 & 157,620 \\
\hline III. Injuries & & & & 12,674 & 12,674 & 151,613 & 164,287 \\
\hline A. Unintentional & & & & 12,216 & 12,216 & 106,197 & 118,413 \\
\hline 1. Road traffic accidents & & & & 1,983 & 1,983 & 32,744 & 34,726 \\
\hline Other III.A. $(2-6)^{f}$ & & & & 10,420 & 10,420 & 73,453 & 83,874 \\
\hline B. Intentional & & & & 237 & 237 & 45,416 & 45,654 \\
\hline 1. Self-inflicted & & & & 2 & 2 & 20,255 & 20,257 \\
\hline Other III.B. $(2-4)^{9}$ & & & & 480 & 480 & 25,161 & 25,642 \\
\hline IV. Not allocated & 10,851 & 9,877 & 20,728 & & 20,728 & & 20,728 \\
\hline
\end{tabular}

Sources: Stillbirth data are from WHO 2005a. Neonatal and perinatal mortality are country, regional, and global estimates. Age 5+ and total data are from table 6.4 for low- and middle-income countries. All other data are from chapter 3 of this volume.

Note: A blank cell indicates that fewer than 1,000 deaths are attributable to the specific cause. DALYs used here: DALYs(3,0,.54).

a. Hepatitis, tropical-cluster diseases, leprosy, dengue, Japanese encephalitis, trachoma, intestinal nematode infections, and other infectious diseases.

b. Low birthweight deaths are those resulting from intrauterine growth retardation or preterm birth. Almost all low birthweight deaths in the neonatal period result from preterm birth.

c. Epilepsy, alcohol use disorders, Alzheimer's and other dementias, Parkinson's disease, multiple sclerosis, drug use disorders, post-traumatic stress disorder, obsessive-compulsive disorder, panic

disorder, insomnia (primary), migraine, mental retardation attributable to lead exposure, and other neuropsychiatric disorders.

d. Rheumatic heart disease, hypertensive heart disease, inflammatory heart diseases, and other cardiovascular diseases.

e. Other neoplasms, endocrine disorders, sense organ diseases, genitourinary diseases, skin diseases, musculoskeletal diseases, and oral conditions.

f. Poisonings, falls, fires, drownings, and other unintentional injuries.

g. Violence, war, and other intentional injuries. 


\section{ANNEX C: CAUSES OF NEONATAL MORTALITY: COMPARISON OF NUMBERS FROM THE GLOBAL BURDEN OF DISEASE WITH THOSE FROM THE CHILD HEALTH EPIDEMIOLOGY REFERENCE GROUP}

This chapter has examined the consequences of incorporating stillbirths and neonatal deaths (deaths in the 28 days following live birth) into the Global Burden of Disease (GBD) framework. Methods and results of the GBD are presented elsewhere in this book and, in particular, chapter 3 discusses the estimates of deaths by age and cause for 2001 that form the basis for results throughout this book and in this chapter. Estimates of deaths from specific causes undergo continual revision as new data and syntheses become available, yet establishing a time cutoff is a necessary (if somewhat arbitrary) condition for preparing a volume with consistent estimates across chapters. For this volume, the cutoff date for the estimates of deaths by cause in 2001 was late 2003. That date was itself established in response to the need for a separate book—Jamison and others (2006) to have a consistent set of demographic and epidemiological numbers feeding into its highly diverse chapters.

During 2001, the World Health Organization (WHO) established the Child Health Epidemiology Reference Group (CHERG) to undertake a new synthesis of data on causes of death among children under five. While some early CHERG results influenced the GBD numbers in this volume, for the most part, CHERG's work became available well after the cutoff date for this iteration of the GBD. For this reason, the 2005 WHO estimates (Bryce and others 2005; WHO 2005b) of causes of death among children under five based on CHERG (CHERG/WHO) differ to some extent from the GBD ones used in this volume. Chapter 5 further discusses the two sets of estimates for under-five deaths, and the importance of envelope and epidemiological consistency constraints in generating the GBD numbers. In terms of data sources, the GBD uses epidemiological data together with vital registration data (where available), models extrapolating from vital registration data, and epidemiological consistency checks. CHERG relies relatively more on verbal autopsy based epidemiological data for causes of child death.

The work of CHERG, however, provides a critical input to this chapter not available from the GBD work, that is, a breakdown of the causes of death specifically for the neonatal period. One of the motivations of this chapter is that neonatal deaths account for fully 37 percent of the worldwide total of deaths among children under age five. In preparing this chapter, therefore, we needed to draw fully on the CHERG analyses of neonatal deaths while-to ensure consistency and comparability with numbers elsewhere in this volume-we use the GBD estimates of total deaths among children under five. This allows estimates of the neonatal burden to be inserted into the larger context of the GBD with its inclusion of 136 causes as well as all age groups older than age five. The specific assumptions we made to reconcile GBD and CHERG numbers are made clear in the text with table 6.4 and in the notes to table 6.4.

The CHERG/WHO results appear as percentages of deaths by one set of causes for neonates and by a mostly different set of causes for children ages 28 days to 5 years. This makes direct comparison with the GBD numbers difficult in the formats in which the two sets of numbers are presented. The difficulty is compounded by occasional differences in the labels (and content) of cause categories and by the fact that the GBD deals with far more causes than CHERG/ WHO. Even the truncated GBD cause list used in this chapter uses 35 instead of 136 causes, in contrast to the 10 used by CHERG/WHO. To facilitate comparison of the two sets of findings, annex table 6C.1 uses the 6 of the 10 CHERG/ WHO cause categories that are relevant to neonates to compare this chapter's and CHERG's findings for neonatal deaths. To construct table 6C.1 we took proportional allocations of deaths from CHERG/WHO presented in figure 2 of Bryce and others (2005) and applied those proportions to the estimated number of neonatal deaths (3.896 million) used in this chapter. The table is for the world as a whole.

Table 6C.1 Causes of Neonatal Mortality, Worldwide in 2001 (thousands)

\begin{tabular}{lrc}
\hline & \multicolumn{2}{c}{ Neonatal deaths } \\
\cline { 2 - 3 } Cause & GBD & CHERG/WHO \\
\hline Diarrheal disease & 116 & 117 \\
Tetanus $^{a}$ & 187 & 273 \\
Respiratory infection $^{b}$ & 1,013 & 1,013 \\
Low birthweightc $^{c}$ & 1,098 & 1,091 \\
Birth asphyxia and birth trauma $^{\mathrm{d}}$ & 739 & 896 \\
Congenital anomalies & 321 & 312 \\
Other & 446 & 194 \\
TOTAL & $\mathbf{3 , 8 9 6}$ & $\mathbf{3 , 8 9 6}$ \\
\hline
\end{tabular}

Sources: See text.

a. CHERG/WHO conclude that 7 percent $(273,000)$ of global neonatal deaths occur due to tetanus. Chapter 3 of this volume provides an estimate for tetanus deaths for ages zero to four of only 187,000. Consistent with the objectives of this chapter, the GBD numbers are used here, and the CHERG/WHO estimates accordingly revised downward.

b. Deaths for respiratory infections in the neonatal age group are those reported by CHERG/WHO for their category sepsis or pneumonia.

c. Low birthweight deaths are those resulting from intrauterine growth retardation or preterm birth. Almost all low birthwieght deaths in the neonatal period result from preterm birth.

d. Chapter 3 provides an estimate for birth asphyxia and birth trauma deaths for ages zero to four of only 739,000 globally. Consistent with the objectives of this chapter, the GBD numbers are used here, and the CHERG/WHO estimates accordingly revised downward. 


\section{ACKNOWLEDGMENTS}

The authors are indebted to many individuals for valuable inputs, comments, and encouragement. The late José Luis Bobadilla encouraged us concerning the importance of this work and provided guidance to the literature on causes of death among the very young. Kenji Shibuya of WHO provided helpful inputs to an early draft. At an earlier stage of this work, Nancy Hancock and Jia Wang provided valuable inputs for which we are very grateful. Elisabeth Aahman, also of WHO, provided invaluable inputs to the estimates of stillbirth and neonatal mortality rates that this chapter draws on; we owe her particular thanks. Participants at seminars at the Harvard Center for Population and Development and at the Centers for Disease Control and Prevention provided valuable comments, and in particular we would like to thank Sevgi Aral and Lincoln Chen. The editors of this volume and two peer reviewers, Arnab Acharya and Linda Martin, provided detailed and valuable critical reaction. Robert Black provided additional important critical reaction.

\section{NOTES}

1. The term child mortality rate is sometimes used to denote what we call the under five mortality rate. We try to avoid confusion by being explicit about the age range covered.

2. Murray and Lopez (1998) and Shibuya and Murray (1998a, 1998b, 1998c) provide an earlier overall assessment of the burden from some of the major causes of neonatal mortality. Low birthweight as a risk factor is further discussed in Fishman and others (2004) and in chapter 4 of this volume.

3. Allowing the use of negative age weights could achieve some of the same effects as gradual ALP.

\section{REFERENCES}

Bhutta, Z. A., N. Ali, and A. Wajid. 2004. "Perinatal and Newborn Care in Pakistan: Seeing the Unseen!" In Maternal and Child Health in Pakistan: Challenges and Opportunities, ed. Zulfiqar Bhutta, 19-46. Oxford, U.K.: Oxford University Press.

Bhutta, Z. A., F. Raza, A. Hyder, Z. Memon, S. Zaidi, and S. Rasool. 2004. Final Report: Evaluation of Community-Based Neonatal Mortality in Rural Sindh, Pakistan, Using a Verbal Autopsy Tool. Geneva: Global Forum for Health Research, Child Health and Nutrition Research Initiative.

Black, R. E., S. S. Morris, and J. Bryce. 2003. "Where and Why Are 10 Million Children Dying Every Year?” Lancet 361 (9376): 2226-34.

Bryce, J., C. Boschi-Pinto, K. Shibuya, R. E. Black, and the World Health Organization Child Health Epidemiology Reference Group. 2005. "WHO Estimates of the Causes of Death in Children" Lancet 365 (9465): 1147-52.

Crawford, C. B., B. E. Salter, and K. L. Jang. 1989. "Human Grief: Is Its Intensity Related to the Reproductive Value of the Deceased?" Ethology and Sociobiology 10 (4): 297-307.
Cropper, M. L., S. L. Aydede, and P. R. Portney. 1994. "Preferences for Life Saving Programs: How the Public Discounts Time and Age." Journal of Risk and Uncertainty 8: 243-65.

Darmstadt, G. L., Z. A. Bhutta, S. N. Cousens, T. Adam, N. Walker, and L. de Bernis. 2005. "Evidence-Based, Cost-Effective Interventions: How Many Newborns Can We Save?” Lancet 365 (9463): 977-88.

Fishman, S. M., L. E. Caulfied, M. de Onis, M. Blossner, A. A. Hyder, L. Mullany, and R. E. Black. 2004. "Childhood and Maternal Underweight." In Comparative Quantification of Health Risks: Global and Regional Burden of Disease Attributable to Selected Major Risk Factors, vol. I, ed. Majid Ezzati, Alan D. Lopez, Anthony Rodgers, and Christopher J. L. Murray, 39-161. Geneva: World Health Organization.

Kill, K. Forthcoming. "Estimates of the Numbers of Neonatal Deaths."

Jamison, D. T., J. G. Breman, A. R. Meashan, G. Alleyne, M. Claeson, D. B. Evans, P. Jha, A. Mills, and P. Musgrove. 2006. Disease Control Priorities in Developing Countries, 2nd ed. New York: Oxford University Press.

Johannesson, M., and P. O. Johansson. 1997. "Is the Valuation of a QALY Gained Independent of Age? Some Empirical Evidence." Journal of Health Economics 16 (6): 589-99.

Institute of Medicine. 1985. New Vaccine Development: Establishing Priorities. Volume 1 of Diseases of Importance in the United States. Washington, DC: National Academies Press.

2003. Improving Birth Outcomes: Meeting the Challenge in the Developing World. Washington, DC: National Academies Press.

Lawn, J. E., K. Wilczynska, and S. N. Cousens. Forthcoming. Estimating the Cause of Death for 4 Million Neonates in the Year 2000.

Lawn, J. E., S. N. Cousens, and J. Zupan. 2005. "Four Million Neonatal Deaths: When? Where? Why?" (Lancet Neonatal Series Paper 1.) Lancet 365 (9462): 891-900.

Lawn, J. E., K. Shibuya, and C. Stein. 2005. "No Cry at Birth: Global Estimates of Intrapartum Stillbirths and Intrapartum-Related Neonatal Deaths." Bulletin of the World Health Organization 83 (6): 409-16.

Lawn, J. E., J. Zupan, G. Begkoyian, and R. Knippenberg. 2006. "Newborn Survival." In Disease Control Priorities in Developing Countries, 2nd ed., ed. Dean T. Jamison, Joel G. Breman, A. R. Measham, Mariam Claeson, David B. Evans, Prabhat Jha, A. Mills, and P. Musgrove. New York: Oxford University Press.

Martines, J, V. K. Paul, Z. A. Bhutta, M. Koblinsky, A. Soucat, N. Walker, and R. Bahl. H. Foastad, A. Costello, and the Lancet Neonatal Survival Steering Team. 2005. “Neonatal Survival: A Call for Action." Lancet 365 (9465): 1189-97.

Murray, C. J. L., and A. D. Lopez. 1997. "Global Mortality, Disability, and the Contribution of Risk Factors: Global Burden of Disease Study." Lancet 349 (9063):1436-42.

1998. "Quantifying the Health Risks of Sex and Reproduction: Implications of Alternative Definitions." In Health Dimensions of Sex and Reproduction, ed. C. J. L. Murray and A. D. Lopez, 1-17. Volume 3 of Global Burden of Disease and Injury Series. Cambridge, MA: Harvard University Press.

Murray, C. J. L., A. D. Lopez, and D. T. Jamison. 1994. “The Global Burden of Disease in 1990: Summary Results, Sensitivity Analysis, and Future Directions." Bulletin of the World Health Organization 72 (3): 495-509.

Shibuya, K., and C. J. L. Murray. 1998a. "Birth Asphyxia." In Health Dimensions of Sex and Reproduction, ed. C. J. L. Murray and A. D. Lopez, 429-53. Volume 3 of Global Burden of Disease and Injury Series. Cambridge, MA: Harvard University Press.

- 1998b. "Congenital Anomalies." In Health Dimensions of Sex and Reproduction, ed. C. J. L. Murray and A. D. Lopez, 455-512. Volume 3 of Global Burden of Disease and Injury Series. Cambridge, MA: Harvard University Press.

-. 1998c. "Low Birth Weight." In Health Dimensions of Sex and Reproduction, ed. C. J. L. Murray and A. D. Lopez, 1-17. Volume 3 of 
Global Burden of Disease and Injury Series. Cambridge, MA: Harvard University Press.

Stanton, C., J. E. Lawn, H. Rahman, K. Wilczynska-Ketende, and K. Hill. Forthcoming. "Born Dead: Delivering Stillbirth Rate Estimates for 190 Countries."

Steinbock, B. 1992. Life before Birth, the Moral and Legal Status of Embryos and Fetuses. New York: Oxford University Press.

Stoll, B. J., and A. R. Measham. 2001. "Children Can't Wait: Improving the Future for the World's Poorest Infants." Journal of Pediatrics 139 (5): 729-33.

Tinker, A., P. ten Hoope-Bender, S. Azfar, F. Bustreo, and R. Bell. 2005. "A Continuum of Care to Save Newborn Lives." Lancet 365 (9462): $822-25$.
United Nations Population Division. 2003. World Population Prospects: The 2002 Revision. New York: United Nations.

WHO (World Health Organization). 2005a. "Neonatal and Perinatal Mortality. Country, Regional, and Global Estimates." Draft, WHO, Geneva.

. 2005b. World Health Report: Make Every Mother and Child Count. Geneva: WHO.

World Bank. 1993. World Development Report 1993: Investing in Health. New York: Oxford University Press.

- 2003. World Development Indicators. New York: Oxford University Press.

Zupan, J. 2005. "Perinatal Mortality in Developing Countries." New England Journal of Medicine 352 (20): 2047-48. 



\section{Glossary}

Age-standardized rate An age-standardized rate is a weighted average of the age-specific rates, where the weights are the proportions of a standard population in the corresponding age groups (q.v.). The potential confounding effect of age is removed when comparing age-standardized rates computed using the same standard population.

Age weights Factor specifying the relative value of a year of healthy life lived at different ages. The DALY can incorporate non-uniform age weights which give less weight to years of life lived in early childhood and at older ages (see Chapter 5).

AIDS: Acquired Immunodeficiency Syndrome Disease due to infection with the human immunodeficiency virus (HIV).

BMI: Body mass index A measure of underweight and overweight calculated as weight $(\mathrm{kg})$ divided by height squared $\left(\mathrm{m}^{2}\right)$.

Case Fatality Rate The proportion of cases of a disease or injury that die as a result of their disease or injury over a specified time period.

CHD: Coronary heart disease Synonymous with ischemic heart disease (q.v.).

Childhood-cluster diseases GBD (q.v.) cause group including the following vaccine-preventable diseases of childhood: pertussis, poliomyelitis, diphtheria, measles and tetanus.

CODMOD: Cause of death model A statistical model for the prediction of the broad distribution of causes of death based on observed historical data on the relationships between cause distributions, and overall levels of mortality and per-capita income (see Chapter 3).

Comorbidity Presence of more than one disease or health condition in an individual at a given time.

COPD: Chronic obstructive pulmonary disease Lung diseases that persistently obstruct bronchial airflow. COPD mainly involves two related diseases-chronic bronchitis and emphysema. COPD is also called chronic obstructive lung disease. Asthma is not included in COPD, as the obstruction to bronchial airflow is usually reversible and between asthma episodes the flow of air through the airways is usually good.

CVD: Cardiovascular disease Cardiovascular disease covers a wide array of disorders, including diseases of the cardiac muscle and of the vascular system supplying the heart, brain, and other vital organs. The most common manifestations of CVD are ischemic heart disease, congestive heart failure, and stroke. CVD is used here as an abbreviation for cardiovascular disease, not cerebrovascular disease (q.v.)

DALY: Disability Adjusted Life Year A measure of the gap in healthy years of life lived by a population as compared with a normative standard. More formally, DALYs are a time based measure which adds together years of life lost due to premature mortality with the equivalent number of years of life lived with disability or illness.

DFLE: Disability-free life expectancy A form of HE (q.v.) which gives a weight of 1 to states of health with no disability above an explicit or implicit threshold and a weight of 0 to states of health with any level of disability above that threshold.

\section{DBP: Diastolic blood pressure}

Demography The study of population size, growth and age structure, and of the forces (fertility, mortality, migration) that lead to population change.

Disability Restriction or lack of ability (resulting from an impairment or health condition) to perform an activity in the manner or within the range considered normal. Although the word "disability" is widely used, the ICF (q.v.) uses this term only as a broad umbrella term for capacity and performance in activity/participation domains. The GBD (q.v.) used the term disability, as in the DALY (q.v.), as a synonym for health states (q.v.) less than full health (q.v.). Disability is also commonly used to refer only to long-standing limitations in carrying out activities of daily living.

Disability weight Measure of the relative valuations of a health state on an interval scale. In the GBD (q.v.), health state valuations lie between 0 (full health q.v.) and 1 (states 
equivalent to death). The disability weight quantifies judgments about overall levels of health associated with different health states (q.v.), not judgments on the relative values of lives lived, persons, or of overall well-being, quality of life or utility. The GBD disability weights are intended to reflect average global valuations.

Discounting Process applied to costs, benefits, and outcomes based on the concept that there is preference for money or health in the present relative to the future.

DisMod An epidemiological disease model linking populations exposed to risk of disease with incident cases, prevalent cases, case fatality and the duration of time lived with a disease or injury, including its sequelae.

DSP Disease Surveillance Points System run by the Chinese Centre for Disease Control and Prevention for the surveillance of mortality and morbidity.

Epidemiological transition The process whereby major communicable diseases and conditions of poverty (e.g. malnutrition) are progressively replaced by non-communicable diseases such as cancers and CVD.

Epidemiology The study of the occurrence and causes of disease and injury in populations.

Full health Health state (q.v.) characterized by optimal levels of functioning or capacity in all the important domains of health, and freedom from any type of illness or disease. The "optimal" levels of functioning are defined as those levels above which further gains would not (in general) be regarded as improvements in health. States of exceptional functioning above these levels are thus considered to be talents or exceptional abilities, not higher states of health.

Garbage codes ICD codes (q.v.) for ill-defined or residual categories of major disease groups (e.g. cardiovascular diseases) that do not provide meaningful information on underlying disease or injury causes of death. Examples include ill-defined primary site of cancer and atherosclerosis.

GBD: Global burden of disease A comprehensive demographic and epidemiological framework to estimate health gaps (q.v.) for an extensive set of disease and injury causes, and for major risk factors, using all available mortality and health data and methods to ensure internal consistency and comparability of estimates. In the first global burden of disease study, Murray and Lopez estimated health gaps using DALYs (q.v.) for eight regions of the world in 1990. This book presents updated estimates for the year 2001 for the world and for World Bank regions.

Group I causes Major disease and injury cause group used in GBD (q.v.). Includes communicable, maternal, perinatal and nutritional conditions. These are causes which are characteristically common in populations who have not yet completed the epidemiological transition (q.v.).
Group II causes Major disease and injury cause group used in GBD (q.v.). Comprises non-communicable diseases, including malignant neoplasms, cardiovascular diseases, chronic respiratory diseases, digestive, musculoskeletal and genitourinary conditions, as well as mental disorders and neurological conditions.

Group III causes Major disease and injury cause group used in GBD (q.v.). Includes unintentional and intentional injuries.

HALE: Health-adjusted life expectancy Any of a number of summary measures which use explicit weights to combine health expectancies for a set of discrete health states into a single indicator of the expectation of equivalent years of good health. Also referred to as 'Healthy life expectancy'.

HE: Health expectancy Generic term for summary measures of population health which estimate the expectation of years of life lived in various health states.

Healthy life expectancy Synonym for HALE (q.v.) or Healthadjusted life expectancy.

Health state Health state refers to an individual's levels of functioning within a set of health domains such as mobility, cognition, pain, emotional functioning, self-care, etc. More specifically, in terms of ICF (q.v.) concepts, health state is defined as the capacities of an individual in all important domains of health, where such domains may include domains of body structure and function, and domains of activities/participation. Health states do not include risk factors, diseases, prognosis or the impact of health states on overall quality of life, well-being or satisfaction.

Health status A general term referring to all aspects of the health of individuals or populations. Usually understood to include mortality risks, diseases, health states (q.v.), impairments and disability. May also include some risk factors or prognosis information.

High income Category in the World Bank income grouping of countries used for countries with Gross National Income (GNI) per capita of US $\$ 9,206$ or more (exchange rate adjusted currencies) in 2001. See Table 3A-3 for list of countries included.

HIV Acronym for the Human Immunodeficiency Virus, the cause of AIDS (acquired immunodeficiency syndrome).

Ideal health Synonymous with full health (q.v.).

Incidence New cases of disease or injury occurring in a specified population in a given time period.

Incidence rate New cases of disease or injury occurring per unit of population, per unit time.

ICD: International Statistical Classification of Diseases and Related Health Problems A classification of diseases and other causes of mortality prepared by the World Health 
Organization since 1948, periodically revised as necessary. The current tenth revision was issued in 1992 to come into effect on 1 January 1993. The ICD is a member of the WHO family of international classifications.

ICF: International Classification of Functioning, Disability and Health A classification of body structures and functions (impairments) and activities/participation domains (performance and capacity). The ICF was endorsed by the WHO World Health Assembly in 2001 as a successor to the 1980 International Classification of Impairment, Disability and Handicap (ICIDH). The ICF is a member of the WHO family of international classifications.

IHD: Ischemic heart disease Any of a number of heart conditions in which heart muscle is damaged or works inefficiently because of an absence or relative deficiency of its blood supply; most often caused by atherosclerosis, it includes angina pectoris, acute myocardial infarction (heart attack), chronic ischemic heart disease and sudden death. The term coronary heart disease is synonymous with IHD.

Life expectancy The average number of years of life expected to be lived by individuals who survive to a specific age. See also: Period life expectancy.

Logit transformation A mathematical function that transforms a variable such as probability of death into another functional form, characterized by asymptotic values.

Low- and middle-income Category in the World Bank income grouping of countries used for countries with Gross National Income (GNI) per capita of less than US\$9,206 in 2001 (exchange rate adjusted currencies). See Table 3A-3 for list of countries included.

MONICA Study The MONICA (MONItoring CArdiovascular disease) Study was an international research project coordinated by the World Health Organization from the mid-1980s to the mid-1990s in which teams from 38 populations in 21 countries studied heart disease, stroke and risk factors in their populations.

Neonatal period Persons under the age of 28 days are in the neonatal period. The neonatal period is itself divided into the early neonatal period, age less than 7 days, and the remaining late neonatal period.

PAF: population attributable fraction Proportional reduction in disease or injury that would occur if population exposure to a risk factor or group of risk factors were reduced to an alternative distribution.

Perinatal deaths Includes stillbirths and neonatal deaths from any cause, including tetanus and congenital malformations. The perinatal period includes the period from 27 weeks of gestation to 28 days of life.
Perinatal causes or conditions The cause category Perinatal causes refers to the ICD cause group "Conditions arising in the perinatal period". Deaths from these causes (primarily low birth weight and birth trauma/ asphyxia) may occur at any age, but are largely confined to the perinatal period.

Period life expectancy A summary measure of a population's mortality that measures the expectation of years of life lived by a fictitious birth cohort assuming that at each age the cohort experiences the age-specific mortality rates observed in the real population during a specified time period (such as a given calendar year). See also: life expectancy.

Postneonatal period Persons between the age of 28 days and 1 year are in the postneonatal period.

Prevalence Actual number of cases of disease or injury present in a population at any particular moment in time.

Probability of death The chance that an individual, alive at age $\mathrm{x}$, will be dead before his or her $(x+n)^{\text {th }}$ birthday, usually written as ${ }_{n} \mathrm{q}_{\mathrm{x}} \cdot{ }_{5} \mathrm{q}_{0}$ denotes the probability that a newborn infant will die before his or her fifth birthday.

PTO: person trade-off A method for valuation of health states that asks respondents to choose between hypothetical interventions that offer health benefits to groups of individuals in different health states.

QALY: Quality-adjusted life year A measure of years of life lived (or gained through an intervention) adjusted for quality of life using health state preferences ranging between 0 (states equivalent to death) through to 1 (full health). QALYs were developed for the assessment of the cost-effectiveness of interventions in health economics. QALYs gained and DALYs averted through an intervention are calculated in very similar ways, and the main differences relate to the interpretation of the weights. Whereas the disability weights in the DALY quantify loss of health, the corresponding QALY weights are often interpreted in terms of well-being, quality of life, or utility.

Risk Factor A risk factor is an attribute or exposure which is causally associated with an increased probability of a disease or injury.

RR: Relative risk Relative risk is a measure of the strength of an association. It is calculated as a ratio of the risk of occurrence of a disease or death among two population groups, such as those exposed to a risk factor and those not exposed.

SD: Standard deviation A measure of the dispersion or spread of values of a variable (e.g. body weight) around a population mean value.

Sensitivity analysis Systematic investigation of the effects on estimates or outcomes of changes in data or parameter inputs or assumptions. 
Sequelae The medical conditions that can occur among people who contract a disease or suffer an injury. The GBD (q.v.) focuses on disabling sequelae of diseases and injuries; these may remain present long after the initiating disease episode or injury event.

Standard gamble (SG) A method for valuation of health states based on the axioms of expected utility theory. The standard gamble asks respondents to make choices that weigh improvements in health against mortality risks.

Standard Population A population structure that is used to provide a constant age or covariate distribution, so that the age- and sex-specific rates within different populations can be applied to it and can be compared without confounding by the different age or covariate distributions of the populations.

STD: Sexually transmitted disease See: STI.

STI: Sexually transmitted infection An infection that can be transferred from one person to another through sexual contact. Among the sexually transmitted infections (STIs) are HIV/AIDS, chlamydia, genital herpes, gonorrhea and syphilis. The term "sexually transmitted infection (STI)" corresponds to the older term "sexually transmitted disease (STD)".

SMPH: Summary measures of population health Indicators that summarize the health of a population into a single number. SMPH combine information about mortality and population health states. They may summarize either the average health level or health inequality for a population. The two main classes of summary measures are health expectancies (q.v.) and health gap measures, of which the DALY (q.v.) is the best-known example.

Stillbirth Stillbirth refers to the birth of a dead fetus weighing more than 1,000 grams up to 0.25 years ( 13 weeks) prior to the expected time of birth (corresponding to 27 weeks of gestational age).

Stroke Stroke is defined as a condition that results in a disruption of blood flow to a region of the brain causing irreversible "death" of brain tissue. There are two main types of stroke: hemorrhagic and ischemic. Stroke is the main cause of mortality and burden for cerebrovascular disease (q.v.).
Sullivan's method A method of calculating health expectancies using data on the current prevalence of health states in a population together with a period life table for the population.

Theoretical-minimum-risk exposure distribution The population distribution of exposure to a risk factor that would result in the lowest population disease burden.

TTO: time trade-off A method for valuation of health states that asks respondents to make hypothetical choices that weigh improvements in health against reduced longevity.

Uncertainty analysis Estimation of range or distribution of uncertainty in estimates based on an assessment of the uncertainty or confidence intervals for all data and parameter inputs. Uncertainty intervals should ideally include all sources of uncertainty, including those arising from systematic biases and measurement error. In contrast, generally reported confidence intervals are based solely on the variation observed in sample data.

Visual analogue scale A method for valuation of health states in which respondents are asked to directly assess health levels associated with different health states. Individuals place these on a 0 to 1 scale representing a continuum from health states considered equivalent to death through to full health (q.v.)

Verbal autopsy A method of inquiry to ascertain the likely cause of death in populations where vital registration of deaths is incomplete and unreliable. Relatives of the deceased are interviewed about symptoms and signs experienced by the deceased prior to death, from which a diagnosis of the probable cause of death is made.

Vital registration A system for the registration of vital events in a population, including births and deaths, with medical certification of the cause of death according to the rules and procedures of the ICD.

YLD: Years Lived With Disability The component of the DALY (q.v.) that measures lost years of healthy life through living in health states of less than full health (q.v.).

YLL: Years of Life Lost The component of the DALY (q.v.) that measures years of life lost due to premature mortality. 


\section{Index}

The index does not include the data provided in the annexes to the chapters.

Boxes, figures, and tables are indicated by $b, f$, and $t$ after the page numbers.

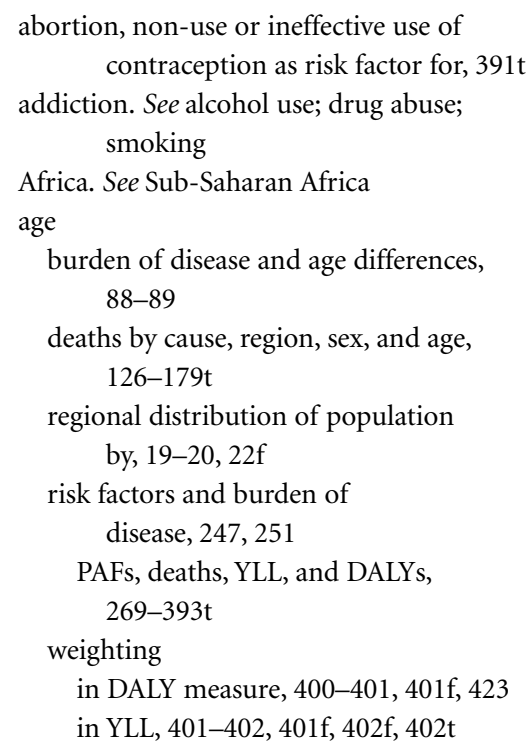

arthritis

incidence and prevalence, data sources on, $84-85$

obesity as risk factor, $306 \mathrm{t}$

Asia. See specific countries and regions asthma, data sources on incidence and prevalence, 84

avian influenza threat, 162

biases in data and uncertainty, 408

bladder cancer, smoking as risk factor, $380 \mathrm{t}$

blindness. See vision loss and blindness

blood pressure. See cardiovascular disease (CVD)

breast cancer

alcohol use as risk factor, 329t

as leading cause of cancer death for women, 90

obesity as risk factor, $300 \mathrm{t}$

physical inactivity as risk factor, $316 \mathrm{t}$

burden of disease

See also DALYs (disability-adjusted life years); Global Burden of Disease (GBD) framework; mortality rates; years of healthy life lost as result of disability (YLD); years of life lost (YLL); specific diseases and conditions analyses as information tools for health policy, $4-5$

by cause, $7-10,8 \mathrm{t}, 88,88 \mathrm{t}, 108-118 \mathrm{t}$

causes and codes, 56-59, 57f, 58t, 95-99t

history of, 2-4

income levels and, 89f, 89t

leading causes of, 85-86, 86t, 91t

discounting and age weighting. See age; discounting

risk factors, 247, 251-252, 251f, 255t

risk factors for, 5, 242, 247-252, 248f, 249-250t

sensitivity and uncertainty analyses, 399-426
See also sensitivity analysis; uncertainty analysis

cancer

air pollution as risk factor, $373 \mathrm{t}$ alcohol use as risk factor, 326-330t as cause of burden of disease, 89 contaminated injections in health care setting as risk factor, $369 t$

data sources on

deaths due to, 64 incidence and prevalence, 81 disability weights for, $124 \mathrm{t}$

ill-defined codes for death caused by, $58-59$

indoor smoke as risk factor, 363t

low fruit and vegetable intake as risk factor, 308-311t

obesity as risk factor, 299-301t

physical inactivity as risk factor, 315-316t risk factor clusters and, 259, 260-262t, 267 smoking as risk factor, $377-384 \mathrm{t}$ unsafe sex as risk factor, $323 \mathrm{t}$

cardiovascular disease (CVD) alcohol use as risk factor, 335-336t as cause of death, 69 ill-defined codes for, 56-58, 57f, 58t by region, 73

cholesterol as risk factor, 296t

disease burden of, 9,89

high blood pressure as risk factor, 291-295t

incidence and prevalence, data sources on, 83-84

joint hazards of risk factors for, 253-254 low fruit and vegetable intake as risk factor, $312 \mathrm{t}$

obesity as risk factor, 303-304t

physical inactivity as risk factor, $318 \mathrm{t}$ risk factor clusters and, 259, 263-264t smoking as risk factor, 385-386t 
Caribbean. See Latin America and Caribbean

categorical attribution, 242

cause of death models, 51

for countries with poor data, 66-68

Central Asia. See Europe and Central Asia cerebrovascular disease

alcohol use as risk factor, $337 \mathrm{t}$

as cause of death and disease burden, 89,90

cholesterol as risk factor, $297 \mathrm{t}$

high blood pressure as risk factor, 293t

incidence and prevalence, data sources on, 84

as leading cause of death, 72

low fruit and vegetable intake as risk factor, $313 \mathrm{t}$

obesity as risk factor, $305 t$

physical inactivity as risk factor, $319 \mathrm{t}$

smoking as risk factor, $387 \mathrm{t}$

cervical cancer

smoking as risk factor, $379 \mathrm{t}$

unsafe sex as risk factor, $323 \mathrm{t}$

Chagas disease, data on deaths due to, 64

CHERG. See Child Health Epidemiology Reference Group

childbirth conditions

acquisition of life potential (ALP), 431, $438-439,439 t$

burden of disease resulting from, 431-441

death rates due to, 54

low birthweight

alcohol use as risk factor, 325t

preterm birth and, 431

maternal death, 64

neonatal death and stillbirth, 427-463

See also child mortality rates

Child Health Epidemiology Reference Group (CHERG; WHO), 34, 424, $430-431,461$

childhood health

See also child mortality rates; specific diseases

environmental risk factors and child undernutrition, 254

hearing loss, 85

malnutrition, 33, 247

underweight as risk factor, 269-275t

child mortality rates

acute respiratory infections as cause, 63

communicable diseases and, 68

data available on, 21-22, 24, 32, 35, 53, 64

diarrheal disease and, 31

HIV/AIDS and, 26, 31, 33, 71

injuries as cause of death, 32, 34 leading causes of child deaths, 69-71

under age five, 28, 29-30t, 31f

trends in, 28-32, 35

lowering of, 26

malaria and, 31

neonatal mortality and stillbirths, 7, 427-463

cause of deaths, 430-431, 445-460t

cause of stillbirths, 431

childbirth conditions and, 431-441

comparison of GBD and CHERG

numbers, 461

in context of global burden of disease, 428-431, 441t, 448t

DALYs and, 431, 438-441, 438f, 439f, 441t, 442-444, 452-454t

deaths by age and cause, $432-437 \mathrm{t}$

number of deaths and death rates,

428-430, 429f, 429t, 430t

preterm birth and, 431

terminology, 428

YLL and, 440f, 440t

perinatal causes, 32, 33-34, 64, 69, 71

risk factor clusters and, 259, 265-266t, 267

vaccine-preventable diseases as cause, 31,63

child sexual abuse as risk factor, 247, 355-361t

China

death registration and reporting in, $51,53,59-60,59 f$

neonatal deaths in, 33, 34

YLL and YLD rates in, 87

cholesterol as risk factor, 296-298t

cirrhosis of liver

alcohol use as risk factor, $338 \mathrm{t}$

contaminated injections in health care setting as risk factor, $370 \mathrm{t}$

clean drinking water. See water, unsafe

CodMod software, 51, 66-68

colon and rectal cancer

low fruit and vegetable intake as risk factor, $310 \mathrm{t}$

obesity as risk factor, $299 \mathrm{t}$

physical inactivity as risk factor, $315 \mathrm{t}$

communicable diseases

See also specific diseases

as cause of burden of disease, $8 \mathrm{t}, 90$

childhood underweight as risk factor, $274 \mathrm{t}$

data sources and methods for incidence and prevalence, $79-81$

death rates due to, 53-54

risk factors for, 268

vitamin A deficiency as risk factor, $283 \mathrm{t}$
Comparative Risk Assessment (CRA) project, 241-247

See also risk factors

counterfactual analysis used in, 242

exposure and hazard, data on, 245-246

framework, 242

mortality estimates and burden of disease, 245

population attributable fractions (PAFs) and

estimating, 245

by region, risk factor, disease outcome, age, and sex, 269-393t

risk correlation, 254

risk factor selection, 242-247

congestive heart failure. See cardiovascular disease (CVD)

contaminated injections in health care setting as risk factor, 366-371t

contraception, non-use or ineffective use as risk factor, 247, 391-393t

counterfactual analysis, 242

CVD. See cardiovascular disease

DALYs (disability-adjusted life years), 3b, 47-49

age weighting in, 400-401, 401f, 423

variations in parameter values, 402-406, 402f, 403t, 404f, 405f, $405 \mathrm{t}, 406 \mathrm{f}$

by cause, $88,88 \mathrm{t}$

by cause, region, sex, and age, $180-233 \mathrm{t}$

childbirth conditions and, 431, 438-439, 438f, $439 \mathrm{f}$

comparison of high-income to lowand middle-income countries, $87 f, 88 f, 89 f$

defined, 3b, 45

discounting in, 400, 423

variations in parameter values, 402-406, 403t, 404f, 405f, 405t, 406f

by region, $87-90,87 f, 88 f$, $91 \mathrm{t}$

risk factors and burden of disease, 269-393t

variations in parameter values, $408 \mathrm{f}$

social value choices and, 49-50

stillbirths and, 442-444, 458-460t

uncertainty ranges for disability weights, 413, 416-417, 416f, 417f, 418-419t, $420 \mathrm{f}, 421 \mathrm{t}$

use of, 3b, 45, 47-49

data sources. See sources of data

death. See mortality rates

debate of appropriate values and priorities for burden of disease, 4

dehydration. See diarrheal diseases 
Demographic and Health Survey (DHS) program, 51

demographic trends of major regions (1990-2001), 18-21, 35, 36-42t

See also mortality rates

distribution by age and location, 19-20, 22f

fertility, 20-21, 20-21t

percentage of regional population covered by censuses, $18 \mathrm{t}$

population size and growth, 19, 20t

sex ratios, 20, 23f

sources of data and methodology, 18-19

urbanization, 20

dengue, data sources on incidence and prevalence, 80

depression

alcohol use as risk factor, $332 \mathrm{t}$

child sexual abuse as risk factor, $355 t$

data sources for incidence and prevalence, 82

as leading cause of disability, 7, 85

diabetes

alcohol use as risk factor, 331t

as cause of death, 69

incidence and prevalence, data sources on, $81-82$

increasing burden of, 9,90

obesity as risk factor, $302 \mathrm{t}$

physical inactivity as risk factor, $317 \mathrm{t}$

diarrheal diseases

childhood underweight as risk factor, $269 \mathrm{t}$

child deaths due to, 31,33

data sources on

deaths due to, 33, 63

incidence and prevalence, 79

deaths due to, 71, 247

unsafe water, sanitation, and hygiene as risk factors, $353 \mathrm{t}$

vitamin A deficiency as risk factor, $280 \mathrm{t}$

zinc deficiency as risk factor, $287 \mathrm{t}$

diphtheria, data on deaths due to, 63 disability

See also DALYs (disability-adjusted life years); years of healthy life lost as result of disability (YLD)

defined, 51

discounting

in DALY measure, 400, 423

variations in parameter values, 402-406, 403t, 404f, 405f, $405 t, 406 f$

in YLL measure, 401-402, 401f, 402t

Disease Control Priorities Project, regional reporting categories for, $94 \mathrm{t}$
DisMod, use to ensure consistency in YLD calculations, $74-75,75 \mathrm{f}$

drowning, alcohol use as risk factor, $342 \mathrm{t}$

drug abuse

child sexual abuse as risk factor, $357 \mathrm{t}$

data sources on

deaths due to, 64-65

incidence and prevalence, 82

as risk factor, 348-352t

drug resistance, 32

East Asia and Pacific

causes of burden of disease in, 90

DALYs by cause, sex, and age, 186-191t

deaths by cause, sex, and age, 132-137t

mortality rates in, 28

pulmonary disease in, 73

YLL and YLD rates in, 87

economic growth and health, 26-27

edentulism, data sources on incidence and prevalence, 85

Egypt, death registration and reporting in, 60

Ellison Institute for Global Health, 93

environmental risk factors and child undernutrition, 254

epidemiological assessments of cause of death, 51

epidemiological studies as data source analysis categories by region, $107 \mathrm{t}$

lack of, 92

for YLD calculations, 76

epilepsy

alcohol use as risk factor, 333t

incidence and prevalence, data sources on, $82-83$

esophageal cancer

alcohol use as risk factor, $327 \mathrm{t}$

low fruit and vegetable intake as risk factor, $308 \mathrm{t}$

estimates. See mortality rates; sensitivity analysis; uncertainty analysis

Europe and Central Asia

See also specific countries

CVD in, 73

DALYs by cause, sex, and age, 192-197t

deaths by cause, sex, and age, 138-143t

demographic structure by age, 19

disease burden in, 9, 91

injuries as cause of burden of disease in, 91

mortality rates, trends in, 26, 27

sex ratio in, 20, 23f

YLL and YLD rates in, 87 fall-related injuries. See injuries fertility trends, $20-21,20-21$ t

gender differences

age weighting and discounting for YLL, $402 \mathrm{f}$

birth ratio of boys to girls, 20

burden of disease and, 88-89

causes of death, 69, 70t, 126-179t

injuries as cause of health burden, 90, 91-92

mortality rates, $26-27 \mathrm{t}$

uncertainty estimates and, 410, 410f

neuropsychiatric disorders, 85

risk factors and burden of disease for PAFs, deaths, YLL, and DALYs, 269-393t

traffic accidents, 90, 91

Global Burden of Disease and Risk Factors (GBD). See World Bank

Global Burden of Disease (GBD) framework, 1-2, 2f, 46-47

Handicap International's annual report on landmine victims, 65

health expectancies and health gaps, $47-48,47 \mathrm{f}$

health facility data as data source for YLD calculations, 76,79

health status

how to describe, 50-51

population change and, 17

hearing loss

incidence and prevalence, data sources on, 83

as leading cause of YLD, 85, 92

heart disease. See cardiovascular disease (CVD)

helminth infections, data sources on incidence and prevalence, 80

hepatitis B

contaminated injections in health care setting as risk factor, $367 \mathrm{t}$

incidence and prevalence, data sources on, 79

hepatitis $\mathrm{C}$

contaminated injections in health care setting as risk factor, $368 \mathrm{t}$

incidence and prevalence, data sources on, 79

high-income countries' burden of disease comparison to low- and middle-income countries. See DALYs (disabilityadjusted life years); years of healthy life lost as result of disability (YLD)

DALYs by cause, sex, and age, 222-227t 
high-income countries' burden of disease (Continued)

deaths by broad cause group, 68f, $69 \mathrm{f}$ deaths by cause, sex, and age, 168-173t leading causes of, 89f, 89t

risk factors by disease type, 251, 251f, 252 HIV/AIDS

See also specific countries and regions

as cause of burden of disease, 90

child deaths due to, 26, 31, 33, 71

contaminated injections in health care setting as risk factor, $366 \mathrm{t}$

deaths due to

data on, 63

in low- and middle-income countries, $7,8,68-69,71$

drug abuse as risk factor, $348 \mathrm{t}$

incidence and prevalence, data sources for, 79

unsafe sex as risk factor, 247, 252, 322t

India

death registration and reporting in, 51, 60

neonatal deaths in, 33

indoor smoke as risk factor, 362-365t

infant mortality. See child mortality rates

infectious diseases. See communicable diseases

information and surveillance

for knowledge to implement health policy, 4-5

for priority setting, 35

injections in health care setting as risk factor, 366-371t

injuries

alcohol use as risk factor, 341t, 343-344t, 346t

as cause of health burden, 3, 7-9, $8 \mathrm{t}, 89,90$

child deaths due to, 32,34

child sexual abuse as risk factor, $360 \mathrm{t}$

data sources for incidence and prevalence, 85

disability weights for, $125 \mathrm{t}$

drug abuse as risk factor, 350-351t

intent and codes assigned to death due to, 59

insomnia, data sources on incidence and prevalence, 82

Institute of Medicine (U.S.)

on quality of medical care, 166

on vaccine development priorities, 440

International Campaign to Ban Landmines, 65

interventions, national priorities of, 4 intestinal nematode infections, data sources on incidence and prevalence, 80 iodine deficiency, data sources on incidence and prevalence, 81

Iran, death registration including cause of death, 5, 61, 61f

iron deficiency, 247

See also anemia

knowledge to implement health policy, 4-5

Landmine Monitor Report on deaths due to landmines by country, 65

Latin America and Caribbean

See also specific countries

DALYs by cause, sex, and age, 198-203t

deaths by cause, sex, and age, 144-149t

diabetes and endocrine disorders in, 9

HIV/AIDS in, 72

injuries as cause of burden of

disease in, 91

mortality rates in, 28

violence in, 9, 73

leprosy, data sources on incidence and prevalence, 80

leukemia, smoking as risk factor, $384 \mathrm{t}$

life expectancy

See also years of life lost (YLL)

age weighted and discounted YLL and, $402 \mathrm{t}$

uncertainty estimates and, 409-411, 411f

YLD and, 86-87, 87f

liver cancer

alcohol use as risk factor, $328 \mathrm{t}$

contaminated injections in health care setting as risk factor, $369 t$

as leading cause of cancer deaths, 89-90

smoking as risk factor, $378 \mathrm{t}$

low- and middle-income countries' burden of disease

See also specific regions

comparison to high-income countries. See DALYs (disability-adjusted life years); years of healthy life lost as result of disability (YLD)

DALYs by cause, sex, and age, 180-185t

deaths by broad cause group, 68f, $69 \mathrm{f}$

deaths by cause, sex, and age, 126-131t

epidemiological transition and reduction in, 90

leading causes of, 72t, 89f, $89 \mathrm{t}$

neonatal mortality and stillbirths, $441 \mathrm{t}$

noncommunicable diseases as cause of death, 7

risk factors by disease type, 251-252, 251f

lung cancer

air pollution as risk factor, $373 \mathrm{t}$

indoor smoke as risk factor, $363 \mathrm{t}$

as leading cause of cancer deaths, 69,89 low fruit and vegetable intake as risk factor, $311 \mathrm{t}$

smoking as risk factor, $377 \mathrm{t}$

lymphatic filariasis, data sources on incidence and prevalence, 80

malaria

as cause of burden of disease, 90

childhood underweight as risk factor, 271t

deaths due to

child deaths, 31

data on, 32-33, 64

in low- and middle-income countries, 8,71

incidence and prevalence, data sources for, 79

vitamin A deficiency as risk factor, $282 \mathrm{t}$

zinc deficiency as risk factor, $288 \mathrm{t}$

males. See gender differences

malignant neoplasms. See cancer

malnutrition

childhood underweight as risk factor, 273t

decline in, 33

incidence and prevalence, data sources on, 81

joint hazards of child undernutrition for infectious diseases, 254

as leading risk for burden of disease, 247

as risk factor when low fruit and vegetable intake, 308-314t

malpractice, 167

Mapping Malaria Risks in Africa International Collaboration, 64

maternal conditions

See also childbirth conditions

anemia as risk factor, $276 \mathrm{t}$

contraceptives, non-use or ineffective use as risk factor, $392 \mathrm{t}$

incidence and prevalence, data sources on, 81

vitamin A deficiency as risk factor, 284t maternal mortality, data on death rate, 64 measles

childhood underweight as risk factor, $270 \mathrm{t}$

child deaths due to, 31,33

deaths due to, 71

data on, 33, 63

malnutrition and, 33

vitamin A deficiency as risk factor, $281 \mathrm{t}$

Medical Outcomes Study (MOS) Short Form 36, 50

meningitis, data sources on incidence and prevalence, 79

mental retardation, data sources on incidence and prevalence, 83 
Middle East and North Africa

DALYs by cause, sex, and age, 204-209t

deaths by cause, sex, and age, 150-155t

fertility trends, 20

injuries as cause of burden of

disease in, 91

mortality rates in, 28

traffic injuries in, 73, 90, 91

migraine, data sources on incidence and prevalence, 83

modeling to estimate cause of death for countries with poor data, 66-68

Monte Carlo simulation techniques, use in cause of death modeling, 67

mortality rates

See also years of life lost (YLL); specific disease or condition as cause

cause of death models, 51

for countries with poor data, 66-68

causes of death, 7, 69-72, 70t

for adults, 71-72, 71t

for children, 28-32, 69, 71

in low- and middle-income countries, 126-131t

premature death, $73,73 \mathrm{f}$

by region, $72-73,72 \mathrm{t}$

changes in (1990-2001), 21-28, 32-34

characteristics by sex and region, $26,26 \mathrm{t}$

children. See child mortality rates

death registration information, 5, 22, 51, $52,53,55 \mathrm{t}, 92$

distribution by major cause group, 68-69, 68f, $69 \mathrm{f}$

epidemiological assessments of cause of, 51

estimates of, 19, 21-26, 424

differences of authors with UN data, 23-26, 24-25f

estimating by cause of death, 51-68

all-cause mortality data, 51-53, 52t

availability of data from countries, 21 , $54-56,55 \mathrm{t}$

classification of causes of disease and injury, 53-54

countries with poor data, modeling for, 66-68

data sources and methods for specific countries, 59-61, 92, 100-106t

epidemiological estimates for specific causes, 61-66, 62t

ill-defined causes and "garbage codes," $56-59,57 f, 57 t, 58 \mathrm{t}$

risk factors and attributable mortality, $245,248 f, 249-250 t$

traffic accidents as cause of, $69,71,73$

trends in, 26-28, 35

true cause not reported, 54 uncertainty estimates and

for all-cause mortality, 409-411, 410f, $411 \mathrm{f}$

for regions by cause, 411-413, 413f, 414-415t

MOS (Medical Outcomes Study) Short Form 36, 50

mouth cancer, alcohol use as risk factor, 326t

Multiple Indicator Cluster Survey program, 51

multiple sclerosis, data sources on incidence and prevalence, $82-83$

National Cancer Institute's Surveillance, Epidemiology, and End Results program, 64

neonatal conditions and deaths. See childbirth conditions; child mortality rates

neuropsychiatric disorders

as cause of health burden, 3-4, 7, 85, 90

data sources for incidence and prevalence, 83

newborn deaths. See child mortality rates

Nigeria, neonatal deaths in, 33

noncommunicable diseases

See also specific diseases

as cause of health burden, $3-4,8,8 \mathrm{t}, 9$, 89-90, 91

incidence and prevalence, data sources on, $81-85$

risk factors for, 268

obesity as risk factor, $247,299-307 t$

onchocerciasis, data sources on incidence and prevalence, 80

osteoarthritis

incidence and prevalence, data sources on, 85

obesity as risk factor, $306 t$

Pacific region. See East Asia and Pacific

PAFs. See population attributable fractions

Pakistan, neonatal deaths in, 33

pancreas cancer, smoking as risk factor, $381 \mathrm{t}$

panic disorder, child sexual abuse as risk factor, $359 t$

Parkinson's disease, data sources on incidence and prevalence, 83

performance assessment and burden of disease as indicator, 4

perinatal conditions

anemia as risk factor, $277 \mathrm{t}$

incidence and prevalence, data sources on, 81

pertussis, data on deaths due to, 63

physical inactivity as risk factor, 315-320t pneumonia, $72,430-431$

poisonings, alcohol use as risk factor, $340 \mathrm{t}$ polio, data on deaths due to, 63

population attributable fractions (PAFs), $245,269-393 t$

uncertainty and, 422-423, 422f

population surveys as data source for YLD calculations, 76

population trends. See demographic trends of major regions (1990-2001)

post-traumatic stress disorder, child sexual abuse as risk factor, $358 \mathrm{t}$

premature death, $73,73 \mathrm{f}$

pulmonary disease, 73

air pollution as risk factor, $374 \mathrm{t}$

incidence and prevalence, data sources on, 84

indoor smoke as risk factor, $364 \mathrm{t}$

smoking as risk factor, $376 \mathrm{t}$

quality-adjusted life year, defined, 47

rectal cancer. See colon and rectal cancer registration of deaths. See mortality rates resource allocation to generate greatest reduction in health loss, 5

respiratory infections

air pollution as risk factor, $372 \mathrm{t}$

childhood underweight as

risk factor, $272 \mathrm{t}$

child deaths due to, 31,33

data sources on

deaths, 63

incidence and prevalence, 80

indoor smoke as risk factor, $362 \mathrm{t}$

smoking as risk factor, $388 \mathrm{t}$

zinc deficiency as risk factor, $289 \mathrm{t}$

rheumatoid arthritis, data sources on incidence and prevalence, $84-85$

risk factors, 241-396

See also Comparative Risk Assessment (CRA) project; specific factors (e.g., alcohol use, malnutrition)

adult vs. childhood diseases, 268

air pollution as, $372-375 t$

alcohol use as, 325-347t

anemia as, 276-279t

as cause of burden of disease, 4, 5, 9-10, 10t, 242, 247-252, 248f, 249-250t

expanding analysis to include factors not among leading causes, 268

joint effects of multiple factors, 255-259, 256-258t

ordering of, 247, 251-252, 251f, 255t

as cause of death, 248f, 249-250t

childhood underweight as, 269-275t

child sexual abuse as, 355-361t 
risk factors (Continued)

cholesterol as, 296-298t

clusters, 259-267

contraceptives, non-use or ineffective use as, 391-393t

correlation, 254

data sources for mediated effects and effect modification, 253-254

drug abuse as, 348-352t

future research needs, 267

GBD framework assessing, 6

high blood pressure as, 291-295t

indoor smoke as, 362-365t

injections in health care setting as, 366-371t

joint effects of multiple factors, 252-267, 253f

low fruit and vegetable intake as, $308-314 \mathrm{t}$

obesity as, 247, 299-307t

physical inactivity as, 315-320t

risk assessment, defined, 242

sanitation and hygiene as, 353-354t

sensitivity and uncertainty analyses, 406-407, 407f

smoking as, 376-390t

theoretical-minimum-risk exposure distributions, 242, 243-244t, 246-247

uncertainty and, 420-423

unsafe water as, $247,353-354 t$

vitamin A deficiency as, 280-286t

zinc deficiency as, 287-290t

road accidents. See traffic injuries

Russian mortality rate, $26,27,87$

sanitation and hygiene as risk factor, 353-354t

schistosomiasis, data sources on incidence and prevalence, $79-80$

schizophrenia, data sources on incidence and prevalence, 82

sensitivity analysis, 399-407

age weighting

in DALY measure, 400-401, 401f, 423

variations in parameter values, 402-406, 402f, 403t, 404f, 405f, 405t, $406 \mathrm{f}$

in YLL measure, 401-402, 401f, 402f

discounting

in DALY measure, 400, 423

variations in parameter values, 402-406, 403t, 404f, 405f, 405t, 406f

in YLL measure, 401-402, 401f

risk factors, 406-407, $407 \mathrm{f}$

variations in parameter values, $408 \mathrm{f}$ sepsis and neonatal deaths, 430-431

sexual abuse. See child sexual abuse as risk factor

sexually transmitted infections

See also HIV/AIDS

incidence and prevalence, data sources on, 79

unsafe sex as risk factor, 247, 252, $321-324 t$

smoking

burden of disease attributable to, 247

joint hazards with other risk factors, 254

as risk factor, 376-390t

sources of data

criticism of GBD study based on, 423

death by cause, $51-68$

demographic trends of major regions (1990-2001), 18-19

lack of, 92-93

uncertainty and, 408-409

years of healthy life lost as result of disability (YLD), 75-79

South Asia

See also specific countries

communicable diseases in, 90

DALYs by cause, sex, and age, 210-215t

deaths by cause, sex, and age, 156-161t

HIV/AIDS in, 72

noncommunicable diseases in, 90

sex ratio in, 20, $23 \mathrm{f}$

suicide in, 73

YLL and YLD rates in, 87

statistical methods and data, use in cause of death modeling, 66-67

stillbirths. See child mortality rates stomach cancer

as leading cause of cancer deaths, 89

low fruit and vegetable intake as risk factor, 309t

smoking as risk factor, $382 \mathrm{t}$

strategic health planning, role of, 1

stroke. See cerebrovascular disease

Sub-Saharan Africa

See also specific countries

child mortality rates in, 26,32

communicable diseases in, 72

DALYs by cause, sex, and age, 216-221t

data sources on, 92

deaths by cause, sex, and age, $162-167 \mathrm{t}$

fertility trends in, 20

HIV/AIDS in, 8, 26, 33, 71, 72, 90

malaria in, 8, 31, 72, 90

malnutrition in, 247

mortality rates in, 24-25, 26, 27

traffic accidents in, 90 unsafe sex in, 247, 252

YLL and YLD rates in, 87

suicide, 73, 90, 91

surveillance. See information and surveillance

TB. See tuberculosis

tetanus and neonatal deaths, 431

Thailand

death registration and reporting in, 61

verbal autopsy study in, 5

theoretical-minimum-risk exposure distributions. See risk factors

tobacco use. See smoking

trachoma, data sources on incidence and prevalence, 80

traffic injuries

alcohol use as risk factor, $339 \mathrm{t}$

as cause of death, 9, 69, 71, 73

as cause of health burden, 90, 91

tuberculosis (TB), data sources on

deaths, 62-63

incidence and prevalence, 79

Turkey, death registration and reporting in, 60-61

uncertainty analysis, 399, 408-409

criticism of GBD study based on, 423

cross-national comparisons and, 7, 54

describing and quantifying uncertainty, 409

disability weights and, 413, 416-417, 416f, 417f, 418-419t, 420f, 421t

estimates

for all-cause mortality and life expectancies, 409-411, 410f, 411f

from epidemiological estimates, 417, 420

for regions by cause, 411-413, 413f, 414-415t

PAFs and, 422-423, 422f

risk factors and, $420-423$

sources of uncertainty, 408-409

undernutrition. See malnutrition

United Nations Population Division, 18-19, 23-26, 24-25f

United Nations Programme on HIV/AIDS, 63

unsafe sex. See sexually transmitted infections

unsafe water as risk factor, 247, 353-354t

urbanization trends, 20

uterine cancer, obesity as risk factor, $301 \mathrm{t}$

vaccine-preventable diseases

See also specific disease 
data on deaths due to, 63

incidence and prevalence, data sources on, 79

verbal autopsies, 5, 28, 32, 92

violence

alcohol use as risk factor, $345 \mathrm{t}$

as cause of death, 71,73

as cause of health burden, 9, 90, 91

vision loss and blindness

incidence and prevalence, data sources on, 83

as leading cause of YLD, 85

vitamin A deficiency

incidence and prevalence, data sources on, 81

as risk factor, $280-286 \mathrm{t}$

war, deaths due to, 56, 65-66

water, unsafe, as risk factor, 247, 353-354t women

See also childbirth conditions; gender differences; maternal conditions

breast cancer, 90

death in childbirth, 64

depression and, 7

injuries as cause of death of, 89, 91-92

world

DALYs by cause, sex, and age, 228-233t

deaths by cause, sex, and age, 174-179t
World Bank

GBD study (1990), 2-4, 35, 45, 50, 93, 399

GBD study (2001), 5-11, 35, 45-46

World Development Report 1993: Investing in Health (World Bank), 6, 427

World Development Report 2003 (World Bank), 46

World Health Organization (WHO)

on assessment of global burden of disease, 45

CHERG. See Child Health Epidemiology Reference Group

on HIV/AIDS mortality, 63

Multicountry Survey Study, 93

World Health Survey, 93

World Health Report 2001, 45

World Health Report 2002, 65, 246

World Health Report 2003, 45

years of healthy life lost as result of disability (YLD)

burden of, 85-87

comparison of high-income to low- and middle-income countries, $87 \mathrm{f}, 92$

data sources for, 75-79, 77-78t

disability weights for diseases and conditions, 119-125t

uncertainty in, 417,420 ensuring consistency by using

DisMod, 74-75, 75f

estimation of, 48, 73-74

by region (2001), 75

incidence and prevalence, calculation of, 73-85

leading causes of, 85-86, 86t

neonatal mortality and stillbirths, 439-441, 447t

regional variation in life expectancy and, $86-87,87 \mathrm{f}$

years of life lost (YLL)

age weighting in, 401-402, 401f, 402f, $402 \mathrm{t}, 405 \mathrm{f}$

defined, 48

discounting in, 401-402, 401f, 402t

estimation of, 48

neonatal mortality and stillbirths, 439-441, 440f, 440t, 446t, 449-451t, $455-457 \mathrm{t}$

premature death and, 73, 73f

by region, $87-90,87 \mathrm{f}$

risk factors and burden of disease, 269-393t

zinc deficiency as risk factor, 287-290t 



\section{Rate of Decline in Under-Five Mortality, 1990-2001}

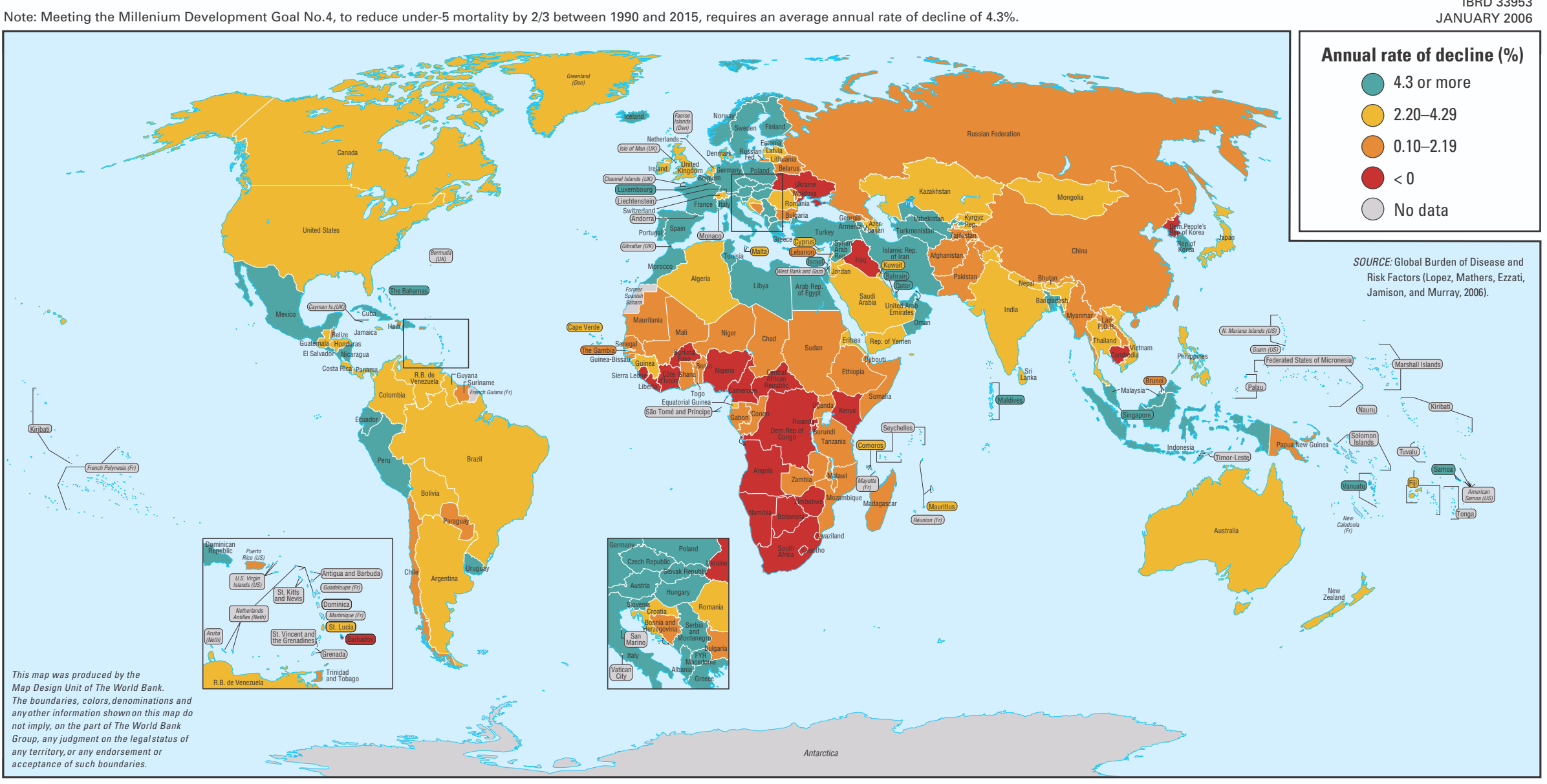


trategic health planning, the cornerstone of initiatives to facilitate the attainment of health goals in populations around the world, requires an understanding of the comparative burden of diseases and injuries, their corresponding risk factors, and the likely effects of proposed interventions. Critical to an effective assessment of risks and outcomes is a framework to integrate, validate, analyze, compare and disseminate available information to policy makers.

The Global Burden of Disease (GBD) framework, the principal instrument to do so, has been widely adopted since its publication in 1990 as the preferred method for health accounting and as the standard to guide the setting of health research priorities. Features of this framework include the development of methods for assessing the reliability of data and estimating missing data for ensuring epidemiological consistency among the various estimates available for a disease, and the use of a common metric to summarize the disease burden from diagnostic categories of the International Classification of Diseases and the major risk factors that cause those health outcomes.

The GBD framework has been refined in response to new research results. Over the last six years, the World Health Organization has undertaken a new assessment of the GBD for 2001, including substantial efforts to improve the methodological and empirical basis for the assessments. The $2001 \mathrm{GBD}$ study provides a comprehensive update of the comparative importance of diseases, injuries, and risk factors for global health, and incorporates a range of new data sources to develop consistent estimates of incidence, prevalence, severity and duration, and mortality for 136 major diseases and injuries, and estimates of exposure and hazardous effects for selected major risk factors, by sex and eight age groups. Drawing from more than 8,500 data sources that include epidemiological studies, disease registers, notifications systems, the $2001 \mathrm{GBD}$ incorporates information from more than 10,000 datasets relating to population health and mortality, representing one of the largest syntheses of global information on population health to date.

The Global Burden of Disease and Risk Factors is a culmination of the 1990 initiative and represents the end of the beginning of global disease burden assessments. It provides a single up-to-date source with a comprehensive statement of methods and a complete presentation of results. It also presents an examination of the uncertainty in the GBD estimates, and their sensitivity to variations in certain value parameters, and the inclusion of stillbirths. The growing and widespread use of disease burden concepts by national and international entities, and the heightened interest in further refining the basic descriptive epidemiology of diseases, injuries, and risk factors have laid the foundations for future population health assessments. This book provides the baseline against which future health progress will be measured.

The Global Burden of Disease and Risk Factors volume is produced by the Disease Control Priorities Project (DCPP), a partnership of the Fogarty International Center (FIC) of the U.S. National Institutes of Health, the World Bank, the World Health Organization, and the Population Reference Bureau. The project is largely funded by the Bill \& Melinda Gates Foundation.

OXFORD

UNIVERSITY PRESS

4

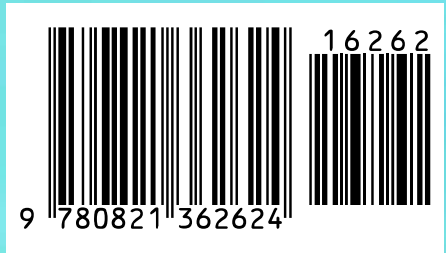

ISBN 0-8213-6262-3 